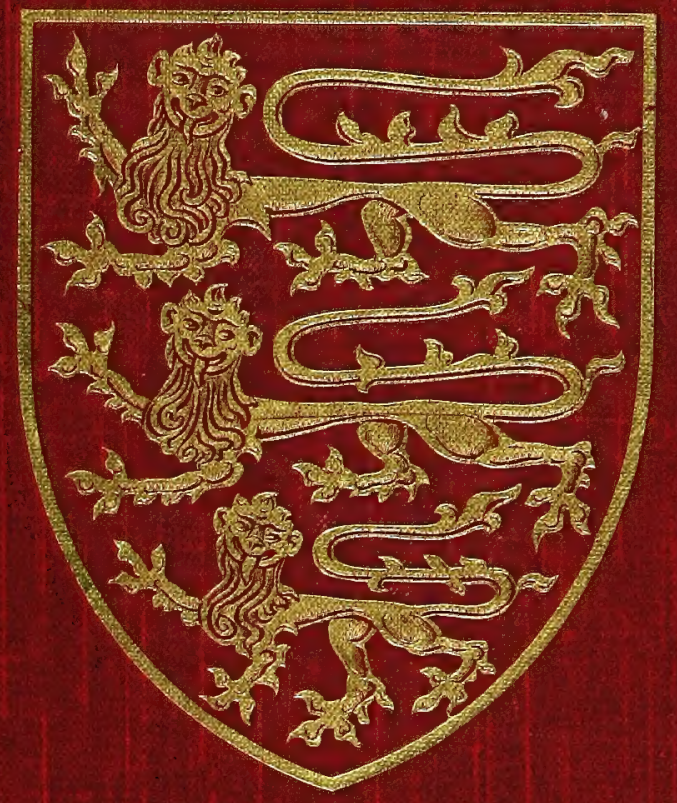




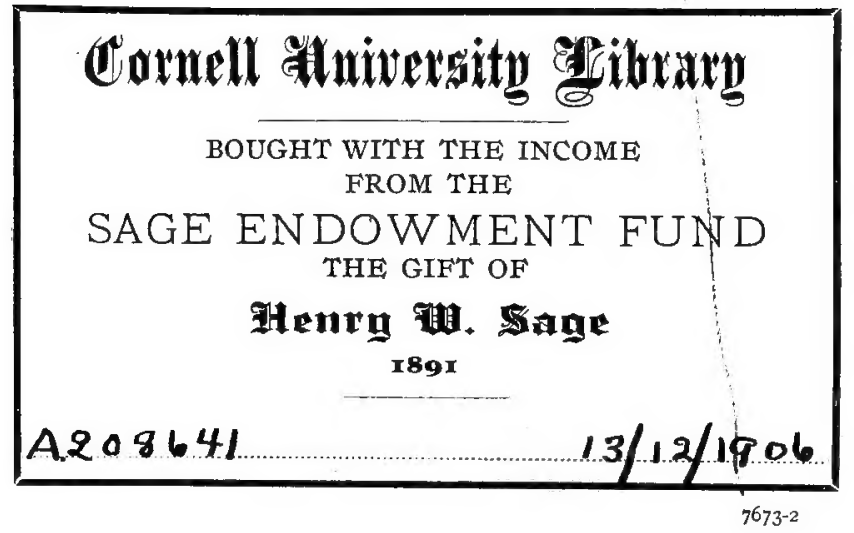




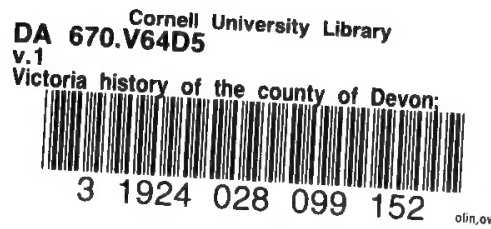




\section{Cornell University Library}

The original of this book is in the Cornell University Library.

There are no known copyright restrictions in the United States on the use of the text.

http://archive.org/details/cu31924028099152 
The Victoria Ibistorn of the Countíes of England

EDITED BY WILLIAM PAGE, F.S.A.

\section{A HISTORY OF \\ D E V O N S H R E \\ IN FIVE VOLUMES \\ VOLUME I}





\section{THE}

VICTORIA HISTORY

OF THE COUNTIES

OF ENGLAND

DEVONSHIRE

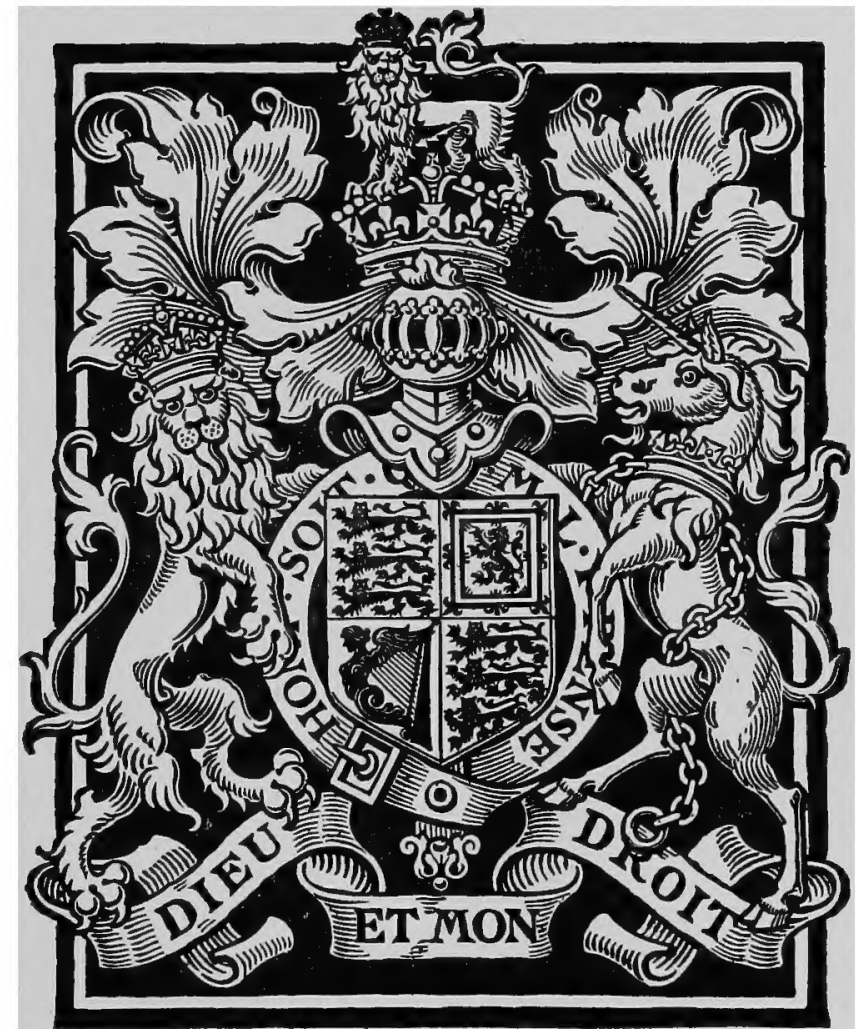

\section{LONDON}

ARCHIBALD CONSTABLE

AND COMPANY LIMITED 
A. 208641

This History is issued to Subscribers only By Arcbibald Constable \& Company Limited and printed by Eyre \& Spottiswoode H.M. Printers of London 


\author{
I N S C R I B E D \\ TO THE MEMORY OF \\ HER LATE MAJESTY \\ QUEEN VICTORIA \\ WHO GRACIOUSLY GAVE \\ THE TITLE TO AND \\ A C C EPTED THE \\ DEDICATION OF \\ THIS HISTORY
}





\section{THE ADVISORY COUNCIL OF THE VICTORIA HISTORY}

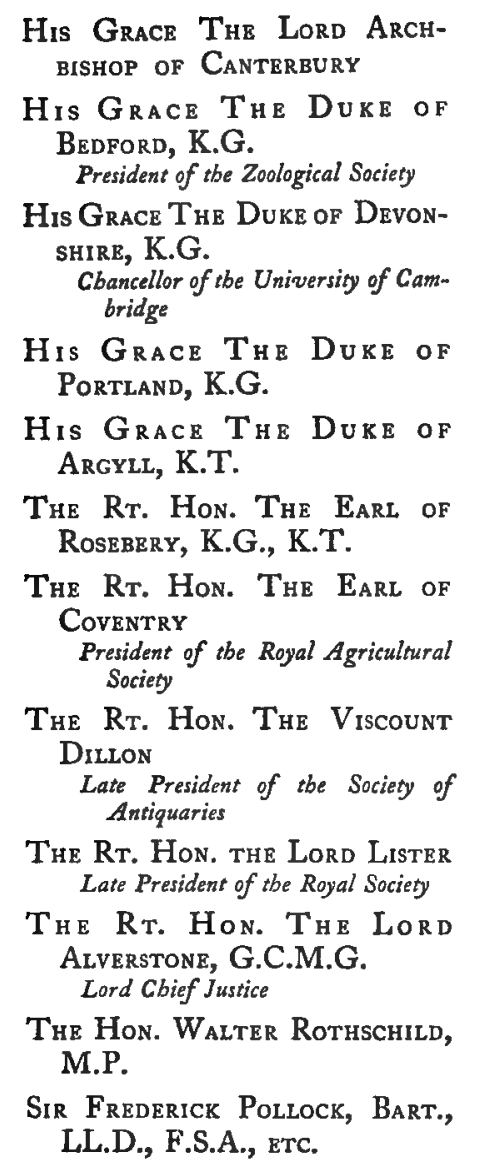

Sir John Evans, K.C.B., D.C.L., LL.D., F.R.S., ETC.

Sir Edward Maunde Thompson, K.C.B., D.C.L., LL.D., F.S.A., ETC.

Director of the Britisb Museum

Sir Clements R. Markham, K.C.B., F.R.S., F.S.A.

President of the Royal Geograpbical Society

Sir Henry C. Maxwell-Lyte, K.C.B., M.A., F.S.A., ETC. Keeper of the Public Records

Sir Jos. Hooker, G.C.S.I., M.D., D.C.L., F.R.S., ETC.

Sir Archibald Geikie, LL.D., F.R.S., ETC.

Rev. J. Charles Cox, Ll.D., F.S.A., ETC.

Lionel Cust, M.V.O., M.A., F.S.A., ETC.

Director of the National Portrait Gallery

Charles H. Firth, M.A., LL.D. Regius Professor of Modern History, Oxford

Albert C. L. G. Günther, M.A., M.D., F.R.S., PH.D.

Late President of the Linnean Society

F. Haverfield, M.A., LL.D., F.S.A.
Col. Duncan A. Johnston, C.B. R.E.

Late Director General of the Ordnance Survey

Prof. E. Ray Lankester, M.A., F.R.S., ETC.

Director of the Natural History Museum, Soutb Kensington

Reginald L. Poole, M.A.

University Lecturer in Diplomatic, Oxford

J. Horace Round, M.A., LL.D.

Walter Rye

W. H. ST. John Hope, M.A.

Assistant Secretary of the Society of Antiquaries

Among the original members of the Council were

The late Duke of Rutrand, K.G.

The late Mareurss of Salisbury

The late Dr. Mandell

CREIGuton, Bishop of London

The late Dr. Stubes, Bishop of Oxford

The late Lord Acton

The Late Sir William Flower

The late Professor F. York Powell and

The late Col. Sir J. Fareuharson, K.C.B.

General Editor-William PAGE, F.S.A.

\section{GENERAL ADVERTISEMENT}

The Victoria History of the Counties of England is a National Historic Survey which, under the direction of a large staff comprising the foremost students in science, history, and archæology, is designed to record the history of every county of England in detail. This work was, by gracious permission, dedicated to Her late Majesty Queen Victoria, who gave it her own name. It is the endeavour of all who are associated with the undertaking to make it a worthy and permanent monument to her memory.

Rich as every county of England is in materials for local history, there has hitherto been no attempt made to bring all these materials together into a coherent form.

Although from the seventeenth century down to quite recent times numerous county histories have been issued, they are very unequal in merit; the best of them are very rare and costly; most of them are imperfect and many are now out of date. Moreover, they were the work of one or two isolated scholars, who, however scholarly, could not possibly deal adequately with all the varied subjects which go to the making of a county history. 
In the Victoria History each county is not the labour of one or two men, but of many, for the work is treated scientifically, and in order to embody in it all that modern scholarship can contribute, a system of co-operation between experts and local students is applied, whereby the history acquires a completeness and definite authority hitherto lacking in similar undertakings.

The names of the distinguished men who have joined the Advisory Council are a guarantee that the work represents the results of the latest discoveries in every department of research, for the trend of modern thought insists upon the intelligent study of the past and of the social, institutional, and political developments of national life. As these histories are the first in which this object has been kept in view, and modern principles applied, it is hoped that they will form a work of reference no less indispensable to the student than welcome to the man of culture.

\title{
THE SCOPE OF THE WORK
}

The history of each county is complete in itself, and in each case its story is told from the earliest times, commencing with the natural features and the flora and fauna. Thereafter follow the antiquities, pre-Roman, Roman, and post-Roman; ancient earthworks; a new translation and critical study of the Domesday Survey ; articles on political, ecclesiastical, social, and economic history; architecture, arts, industries, sport, etc.; and topography. The greater part of each history is devoted to a detailed description and history of each parish, containing an account of the land and its owners from the Conquest to the present day. These manorial histories are compiled from original documents in the national collections and from private papers. A special feature is the wealth of illustrations afforded, for not only are buildings of interest pictured, but the coats of arms of past and present landowners are given

\section{HISTORICAL RESEARCH}

It has always been, and still is, a reproach that England, with a collection of public records greatly exceeding in extent and interest those of any other country in Europe, is yet far behind her neighbours in the study of the genesis and growth of her national and local institutions. Few Englishmen are probably aware that the national and local archives contain for a period of 800 years in an almost unbroken chain of evidence, not only the political, ecclesiastical, and constitutional history of the kingdom, but every detail of its financial and social progress and the history of the land and its successive owners from generation to generation. The neglect of our public and local records is no doubt largely due to the fact that their interest and value is known to but a small number of people, and this again is directly attributable to the absence in this country of any endowment for historical research. The government of this country has too often left to private enterprise work which our continental neighbours entrust to a government department. It is not surprising, therefore, to find that although an immense amount of work has been done by individual effort, the entire absence of organization among the workers and the lack of intelligent direction has hitherto robbed the results of much of their value.

In the VICTORIA History, for the first time, a serious attempt is made to utilize our national and local muniments to the best advantage by carefully organizing and supervising the researches required. Under the direction of the Records Committee a large staff of experts has been engaged at the Public Record Office in calendaring those classes of records which are fruitful in material for local history, and by a system of interchange of communication among workers under the direct supervision of the general editor and sub-editors a mass of information is sorted and assigned to its correct place, which would otherwise be impossible.

\section{THE RECORDS COMMITTEE}

Str Edward Maunde Thompson, K.C.B.

Sir Henry Maxwell-Lyte, K.C.B.

W. J. HaRdY, F.S.A.

F. MADAN, M.A.

F. Maitland, M.A., F.S.A.

\author{
C. T. Martin, B.A., F.S.A. \\ J. Horace Round, M.A., LL.D. \\ S. R. Scargili-Bird, F.S.A. \\ W. H. Stevenson, M.A. \\ G. F. W ARNER, M.A., F.S.A.
}




\section{FAMILY HISTORY}

Family History is, both in the Histories and in the supplementary genealogical volumes of chart Pedigrees, dealt with by genealogical experts and in the modern spirit. Every effort is made to secure accuracy of statement, and to avoid the insertion of those legendary pedigrees which have in the past brought discredit on the subject. It has been pointed out by the late Bishop of Oxford, a great master of historical research, that "the expansion and extension of genealogical study is a very remarkable feature of our own times,' that 'it is an increasing pursuit both in America and in England,' and that it can render the historian most useful service.

\section{CARTOGRAPHY}

In addition to a general map in several sections, each History contains Geological, Orographical, Botanical, Archæological, and Domesday maps; also maps illustrating the articles on Ecclesiastical and Political Histories, and the sections dealing with Topography. The Series contains many hundreds of maps in all.

\section{ARCHITECTURE}

A special feature in connexion with the Architecture is a series of ground plans, many of them coloured, showing the architectural history of castles, cathedrals, abbeys, and other monastic foundations.

In order to secure the greatest possible accuracy, the descriptions of the Architecture, ecclesiastical, military, and domestic, are under the supervision of Mr. C. R. PEERs, M.A., F.S.A., and a committee has been formed of the following students of architectural history who are referred to as may be required concerning this department of the work :-

\section{ARCHITECTURAL COMMITTEE}

J. BrLson, F.S.A., F.R.I.B.A.

R. BLOMFIEld, M.A., F.S.A., A.R.A.

HaRold BraKsPEAR, F.S.A., A.R.I.B.A.

Prof. Baldwin Brown, M.A.

Arthur S. Flower, F.S.A., A.R.I.B.A.

Grorge E. Fox, M.A., F.S.A.

J. A. Gorch, F.S.A., F.R.I.B.A.
W. H. St. John Hope, M.A.

W. H. KNowles, F.S.A., F.R.I.B.A.

J. T. Micklethwaite, F.S.A.

Roland Paul, F.S.A.

J. Horace Round, M.A., LL.D.

Percy G. Stone, F.S.A., F.R.I.B.A.

Thackeray Turner.

\section{GENEALOGICAL VOLUMES}

The genealogical volumes contain the family history and detailed genealogies of such houses as had at the end of the nineteenth century seats and landed estates, having enjoyed the like in the male line since I 760 , the first year of George III., together with an introductory section dealing with other principal families in each county. 
The general plan of Contents and the names among others of those who are contributing articles and giving assistance are as follows :

\section{Natural History}

Geology. Clement Reid, F.R.S., Horace B. Woodward, F.R.S., and others

Palæontology. R. LyDEERER, F.R.S., etc.

Contributions by G. A. Boulengrr, F.R.S., H. N. Dixon, F.L.S., G. C. Druce, M.A.,

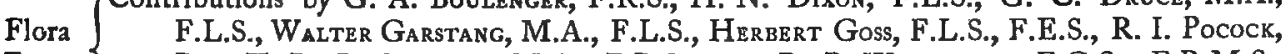

Fauna $\{$ Rev. T. R. R. Strbing, M.A., F.R.S., etc., B. B. Woodward, F.G.S., F.R.M.S., etc., and other Specialists

Prehistoric Remains. Sir John Evans, K.C.B., D.C.L., LL.D., W. Boyd Dawkins, D.Sc., LL.D., F.R.S., F.S.A., Gro. C.inch, F.G.S., John Garstang, M.A., B. Litt., F.S.A., and others

Roman Remains. F. HaVerfirLd, M.A., LL.D., F.S.A.

Anglo-Saxon Remains. C. Herculgs Rzad, F.S.A., Rgginald A. Smith, B.A., F.S.A., and others Domesday Book and other kindred Records. J. Horacr Round, M.A., LL.D., and other Specialists Architecture. C. R. Perrs, M.A., F.S.A., W. H. St. John Hope, M.A., and Harold Brakspear, F.S.A., A.R.I.B.A.

Ecclesiastical History. R. L. Poole, M.A., and others

Political History. Prof. C. H. Firth, M.A., LL.D., W. H. Struenson, M.A., J. Horace Round, M.A., LL.D., Prop. T. F. Tout, M.A., Prof. James Tait, M.A., and A. F. Pollard

History of Schools. A. F. LeAch, M.A., F.S.A.

Maritime History of Coast Counties. Prof. J. K. Laughron, M.A., M. Oppenherm, and others Topographical Accounts of Parishes and Manors. By Various Authorities

Agriculture. Sir ERnest Clarke, M.A., Sec. to the Royal Agricultural Society, and others

Forestry. John NisBet, D.CEc., and others

Industries, Arts and Manufactures
Social and Economic History By Various Authorities

Ancient and Modern Sport. E. D. Cuming and others

Hunting

$\left.\begin{array}{l}\text { Shooting } \\ \text { Fishing, etc. }\end{array}\right\}$ By Various Authorities

Cricket. Home Gordon

Football. C. W. ALcock 



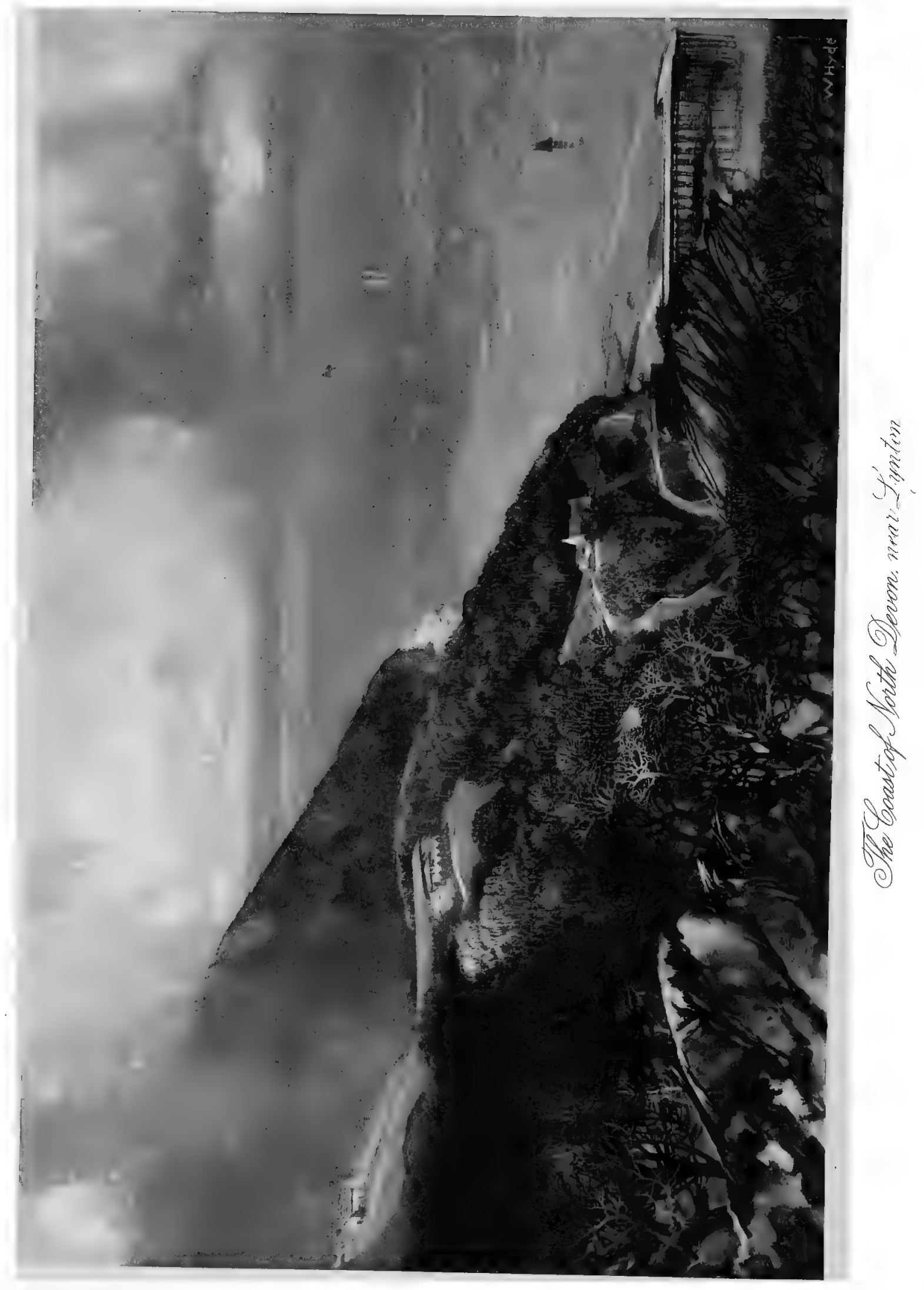






\section{THE}

\section{VICTORIA HISTORY}

OF THE COUNTY OF

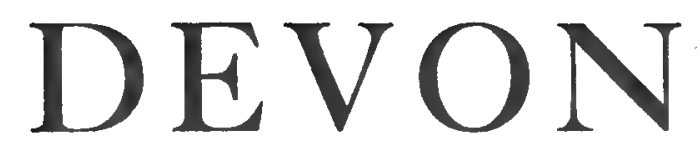

EDITED BY

WILLIAM PAGE, F.S.A.

VOLUME ONE

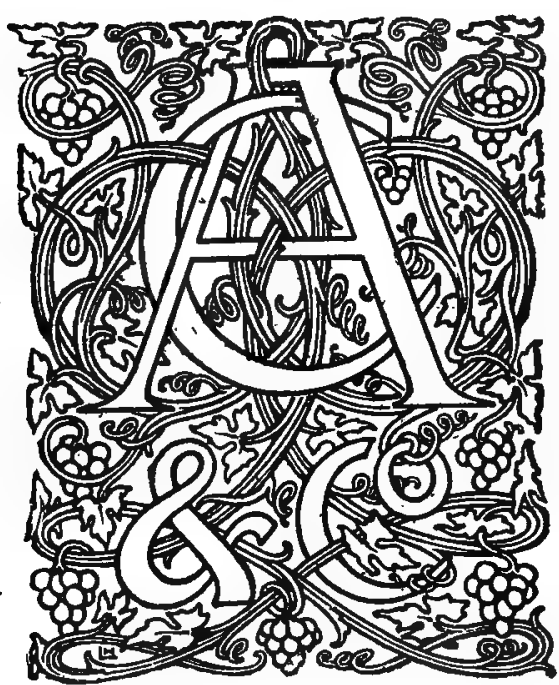

LONDON

JAMES STREET

HAYMARKET

I906 



\section{Counte Committee for Devongbire}

\section{THE RIGHT HON. THE EARL FORTESCUE \\ Lord Lieutenant, Chairman}

The Rt. Hon. The Earl of Mount Edgcumbe, P.C., G.C.V.O.

The Rt. Hon. The Viscount Sidmouth

The Rt. Hon. The Lord Auckland

The Rr. Hon. The Lord Poltimore, P.C.

The Rt. Hon. The Lord Coleridge

The Hon. W. F. D. SMith, M.P.

The Rt. Hon. Sir John H. Kennaway, Bart., P.C., C.B., M.P.

The Rev. The Hon. Graham Colborne, M.A.

Sir Robert S. Clifton, Bart.

Sir Henry Hoare, Bart.

Sir Frederick Pollock, Bart., LL.D., D.C.L., F.S.A.

Sir Charles D. Cave, Bart.

Sir George W. Kekewrch, K.C.B., M.P.

Col. Sir Robert T. White-Thomson, K.C.B.

Str Alfred W. Croft, K.C.I.E.

Sir Roper Lethbridge, K.C.I.E.

Maxwell Adams, Ese.

J. Sparke Amery, EsQ.

P. F. S. Amery, EsQ., J.P.

The Rev. S. Baring-Gould, M.A.

His Honour Judge Beresford

T. N. BRushFIELD, Eso., M.D., F.S.A.

D. W. R. Buchanan, Esq.

Robert Burnard, EsQ., F.S.A., J.P.

The Rev. Professor Chapman, M.A., LL.D.

R. Pearse Chope, Eso.

The Rev. W. H. Dallinger, D.Sc., D.C.L., LL.D., F.R.S.

George M. Doe, Esq.

Samuel Dordge, Esq.

The Rev. Canon Edmonds, B.D.

Edmund A. S. Elliot, Eso., M.R.C.S., M.B.O.U.
Frederick T. Elworthy, EsQ., F.S.A.

The Rev. J. Erskine-Risk, M.A.

H. M. Evans, EsQ.

A. T. Eve, EsQ., K.C., M.P., J.P.

Hardinge F. GrfFard, EsQ., F.S.A.

A. H. A. Hamiton, Eso., M.A., J.P.

The Rev. S. G. Harris, M.A.

James Hine, EsQ., F.R.I.B.A.

W. H. Hudleston, EsQ., M.A., F.R.S., F.G.S.

T. Cann Hughes, Esq., M.A., F.S.A.

John Jackson, EsQ., J.P.

The Rev. F. Emlyn Jones, M.A.

G. LAMBert, EsQ., M.P.

Arthur J. Lawman, EsQ.

W. A. Lindsay, Eso., K.C., M.A., J.P., D.L., F.S.A., Windsor Herald

G. B. Longstaff, EsQ., M.A., M.D., F.R.C.P., F.S.A.

Hugh C. F. Luttrell, EsQ., M.P.

John Y. A. Morshead, Ese.

J. S. Smyth Osbourne, EsQ., J.P.

W. Burd Pearse, Esq.

The Rev. J. B. Pearson, D.D.

J. D. Prickman, EsQ.

The Rev. Oswald J. Rerchel, M.A., B.C.L., F.S.A.

C. E. Robinson, Ese., M.Inst.C.E.

J. Brooking Rowe, EsQ., F.S.A., F.L.S.

Alexander Somervail, Eso.

George Stawell, Ese.

The Rev. T. R. R. Stebing, M.A., F.R.S., F.Z.S.

J. Stevens-Neck, Esq., J.P.

The Rev. W. H. Thornton, M.A.

T. W AINWRIGHT, EsQ.

EdWard Windeatt, EsQ.

H. B. S. Woodhouse, EsQ.

R. Hansford Worth, EsQ., C.E. 



\section{CONTENTS OF VOLUME ONE}

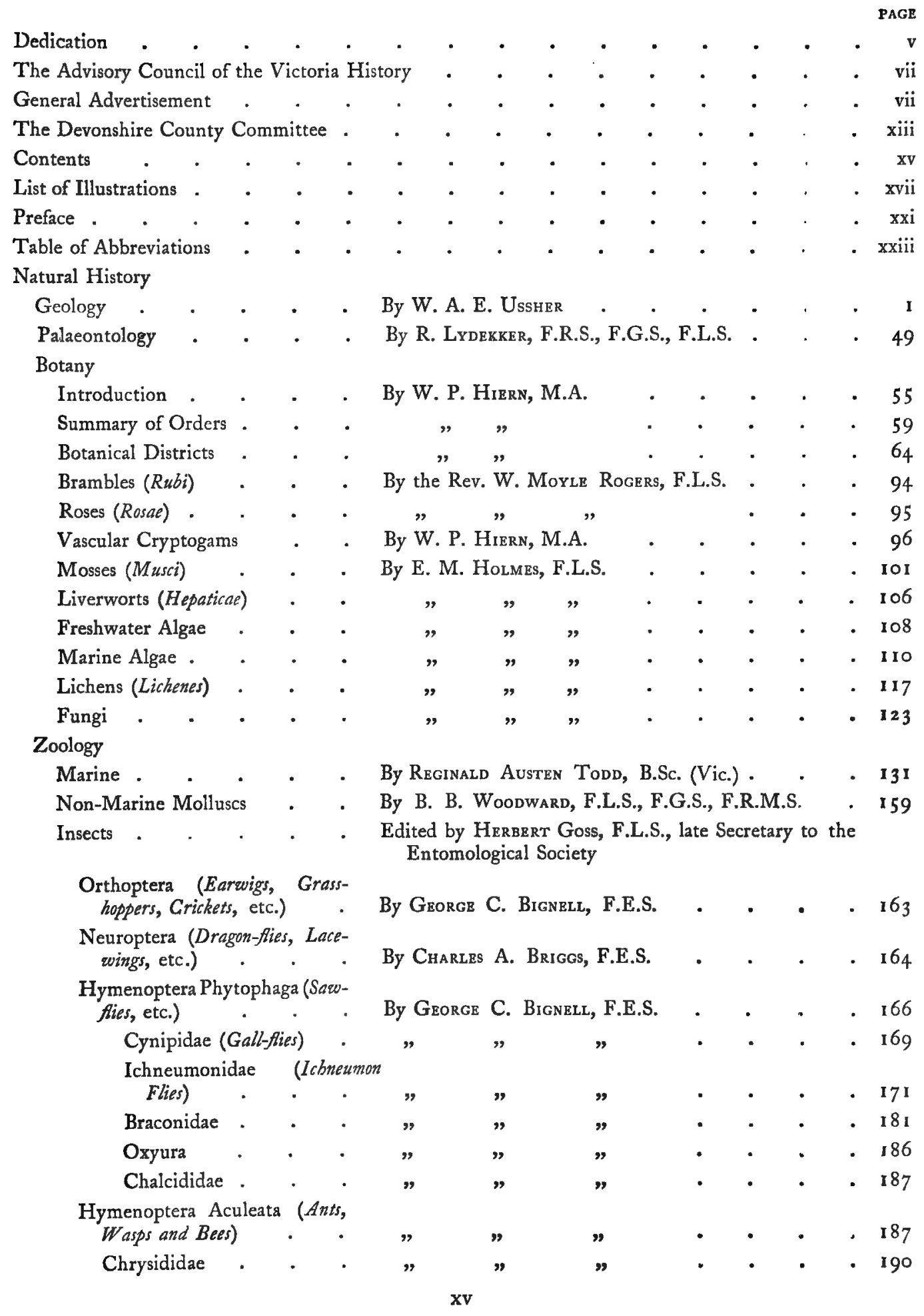




\section{CONTENTS OF VOLUME ONE}

Natural History (continued)

PAGB

Zoology (continued)

Insects (continued)

Coleoptera (Beetles) . By the Rev. Canon Fowrer, M.A., D.Sc., F.L.S. 190

Lepidoptera Rhopalocera (Butterfies) $\cdot$. . .

Lepidoptera Heterocera (Moths)

Diptera (Flies) • • •

Hemiptera Heteroptera (Bugs)

Hemiptera Homoptera .

Aphididae

Spiders . . . . .

Crustaceans

Reptiles and Batrachians . . By E. ERNEST Lowr, F.L.S. • • • • $\quad 288$

Birds . • • • . By William S. M. D’Urban • • • • . 291

Mammals . . • . By J. Brooking Rowz, F.S.A., F.L.S. • • 335

Early Man - . • • • By R. Burnard, F.S.A. • • • • • $34 \mathbf{I}$

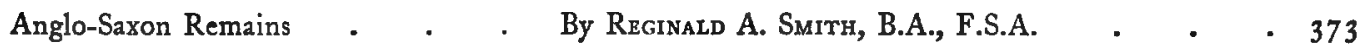

Introduction to the Devonshire
Domesday .

Translation of the Devonshire

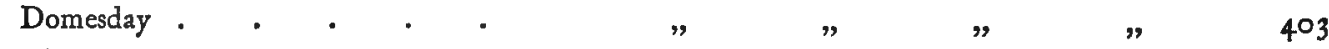

Feudal Baronage . . . . " " " " " $\quad 45$ I

Ancient Earthworks . • • • By J. Chardrs Wall . . . . . 573 


\section{LIST OF ILLUSTRATIONS}

The Coast of North Devon, Lynton. By William Hyde Sections of Kent's Cavern .

Plan and Sections, Cattedown Cave.

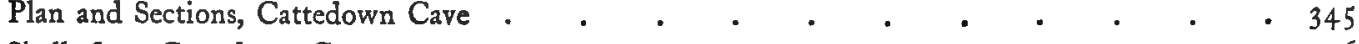

Skulls from Cattedown Cave . . . . . . . . . . . . . .

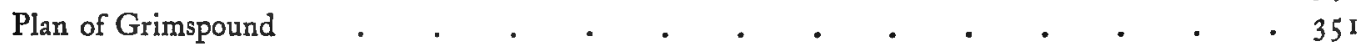

Legis Tor, plan of Hut 2, with Section of Entrance . . . . . . . . 353

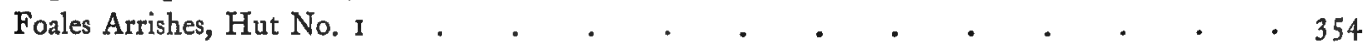

Group of Hut Circles on Standon Down . . . . . . . . . . 355

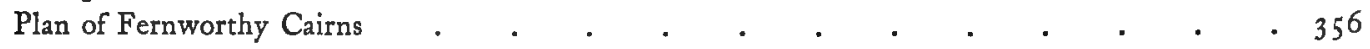

Ruined Kist yielding Burnt Bones and Cairn No. I, Fernworthy (sections) . . . . 357

Urn from Cairn No. 1, Fernworthy . . . . . . . . . . . 358

Dress-fastener from Cairn No. I, Fernworthy . . . . . . . . . 358

Flint Knife from Cairn No. I, Fernworthy . . . . . . . . . $\quad$. 359

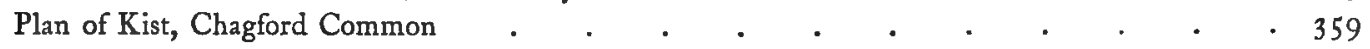

Plan of Barrow and Section, Chagford Common . . . . . . . . . 360

Food Vase or Beaker from Barrow, Chagford Common . . . . . . . 360

Section of Cairn, Hurston Ridge . . . . . . . . . . . . $36 \mathrm{I}$

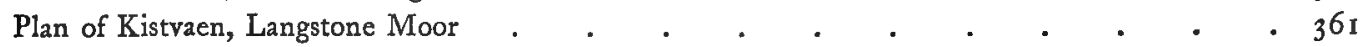

Flint Knives and Scrapers from Kistvaen on Lakehead Hill . . . . . . . . 362

Sections of Barrows on Broad Down and Gittisham Hill . . . . . . . $\quad$. 363

Bronze Objects . . . . . . . . . . . full-page plate, facing 364

Plan of Cranbrook Castle . . . . . . . . . " " " " $" 366$

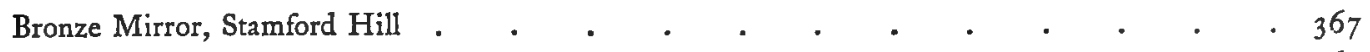

Stone Row and Mênhir at Maddocks Down . . . . . . . . . 367

Stone Row and Mênhir at Maddocks Down (Reconstructed) . . . . . . . 368

Triangle on Challacombe Common . . . . . . . . . . . . 368

Quadrilateral, Longstone Allotment, Chapman Barrows ․ . • • • • • $\quad 369$

Maddocks Down and Chapman Mênhirs. Views and Outlines . • . . . 370

Longstone, Chapman Barrows

Great Mênhir, Maddocks Down $\}$

full-page plate, facirig 370

Parallelogram and Triangle, Furzehill Common, over Hoaroak Water . . . . . $37 \mathrm{I}$

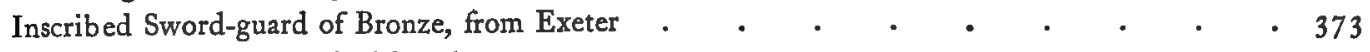

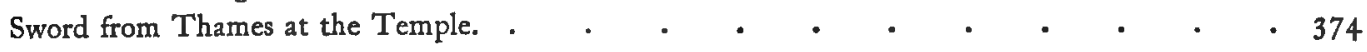

Ancient Earthworks-

Earthwork on Littlecombe Hill, Branscombe . . . . . . . 575

The Castle, Dunterton . . . . . . . . . . . . . . 576

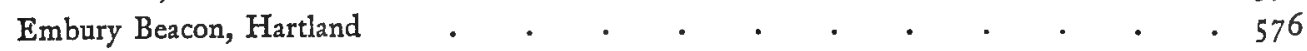

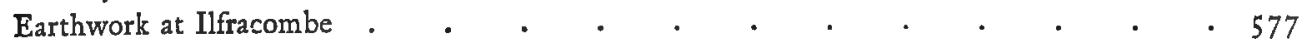

Marisco Castle, Lundy Island . . . . . . . . . . . . . $\quad .578$

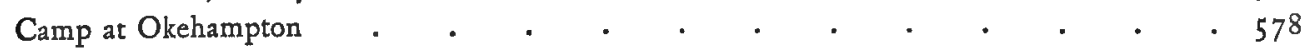

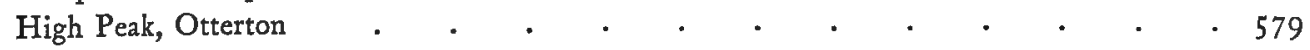

Camp at Parkham . . . . . . . . . . . . . . 579

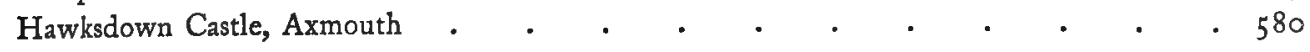

Hembury Castle, Buckland Brewer . . . . . . . . . . . $58 \mathrm{r}$

Cadbury Castle. 


\section{LIST OF ILLUSTRATIONS}

Ancient Earthworks (continued)-

Posbury Camp, Crediton .

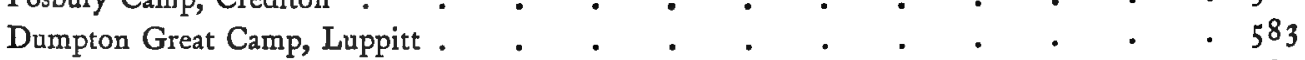

Membury Castle . . . . . . . . . . . . . . . 5483

Musbury Castle

Belbury Castle, Ottery St. Mary

Hembury Fort, Payhembury

Sidbury Castle .

Stockland Great Castle

Slapton Castle, Stokenham .

Denbury Camp, Torbryan

Woodbury Castle

Greenway Camp, Brixham .

Camp at Buckland Brewer .

Castle Dyke, Chudleigh

Clovelly Dykes .

Milber Down Camp, Coffinswell

Old Barrow Camp, Countisbury .

Prestonbury Castle, Drewsteignton

Shoulsbury Castle, High Bray

Holne Chase Castle .

Berry Castle Camp, Huntshaw

Camp at Lustlëigh

Cranbrook Castle, Moreton Hampstead

Wooston Castle, Moreton Hampstead .

Camp at Northcott

Blackbury Castle, Southleigh

Cranmore Castle, Tiverton

Castle Dyke, Ashcombe

Broadbury Castle, Beaworthy

The Castle, Berrynarbor

Camp at Bishop's Nympton

Camp at Charles

Camp at Chawleigh .

Camp at Chittlehampton

The Castle, Chulmleigh

Camp at Cornwood .

Cotleigh Castle, Dunsford .

Camp at Dunterton .

Farway Castle .

Halwell Castle .

Stanborough Camp, Halwell

The Castle, Knowstone

Roborough Castle, Lynton .

Stock Castle, Lynton .

Camp at Mamhead

The Beacon Martinhoe

Camp at Milton Abbot

Camp at Parracombe.

Voley Castle, Parracombe

Roborough Camp, Pilton

Camp at Roborough .

Camp at Shaugh Prior 


\section{LIST OF ILLUSTRATIONS}

Ancient Earthworks (continued) -

The Castle, Shirwell .

Burleigh Dolts, South Huish

Camp at South Molton

Camp at Stoke Canon

Woodbury Camp, Stoke Fleming

Camp at Stoke Rivers.

Camp at Stoke Rivers

Camp at Stowford

Camp at Tavistock

Higher Bury Camp, Tedburn

Huntsham Castle, Tiverton

Camp at Ugborough .

Berry Castle, Witheridge

Berry Castle, Woolfardisworthy

Barnstaple Castle

Castle Roborough, Loxhore

Kenwith Castle, Northam .

Castle Court, North Tawton

Croft Castle, Winkleigh

Bampton Castle

Camp at Bridestowe .

Hembury Castle, Buckfastleigh

The Rings, Loddiswell

Castle Dyke, Highweek

Lydford Castle .

Camp at Milton Damerel

Okehampton Castle .

Holwell Castle, Parracombe

Plympton Castle

Durpley Castle, Shebbear

Totnes Castle .

Camp at Wembworthy

Hemyock Castle

Banks at Braunton

Gallants Bower, Dartmouth

\section{LIST OF MAPS}

Geological Map of North Devon Geological Map of South Devon Orographical Map

Botanical Map.

Pre-Historical Map.

Domesday Map

Ancient Earthworks Map . 



\section{PREF A CE}

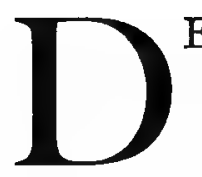

EVONSHIRE can scarcely be said, at the present time, to possess a county history according to the modern interpretation of that title. Antiquaries and topographers from the early part of the seventeenth century have devoted much time and study to its topography, but they have either claimed to do no more than make a general survey of the county or have specialized on some particular parish or district. Sir William Pole, who died in I 635 , left a large manuscript collection for the history of the county, much of which was unfortunately destroyed during the Civil Wars ; a portion, however, that survived, was published in $\mathrm{I} 79 \mathrm{I}$, under the title of Collections towards a description of the County of Devon. After him came Thomas Westcote, a less careful worker, whose View of Devonsbire and Pedigrees of Most of our Devonsbire Families, although completed about 1630, were not published till 1845 . Tristram Risdon owed much to his predecessors in his Chorograpbical Description or Survey of Devon, completed in 1630. This work was for some time circulated in manuscript, but in 17 I 4 an incomplete edition was published by Curll in two volumes, and in 18 I I a perfect edition with additions was published under the editorship of Rees, one of the publishers, John Taylor, F.R.S., William Woollcombe, M.D., and the Rev. John Swete.

The Rev. Richard Polwhele did not realize his more ambitious attempt at a History of Devonshire, the first part of which was published in 1793 . His scheme was somewhat on the lines of the present work, but his 'chorographical' descriptions of parishes, arranged under deaneries, are meagre and the general history of the county poor.

Since that date many valuable works bearing upon the history and antiquities of certain parishes and districts have been issued, while the papers in the Transactions of the Devonsbire Association are among the best of the publications of local archaeological societies.

The Editor wishes to acknowledge the great assistance he has received from Mr. J. Brooking Rowe, F.S.A., who has been ever ready with advice and help; and to express his obligations to $\mathrm{Mr}$. J. Horace Round, M.A., LL.D., for kindly reading the proofs of the Domesday Introduction; to Mr. I. Chalkley Gould, F.S.A., for his assistance and suggestions with regard to the article on Ancient Earthworks; and to the Dartmoor Exploration Committee, Mr. R. H. Worth, F.G.S. and the Rev. J. R. Anderson for the loan of blocks for illustrations of the article on Early Man. 



\section{TABLE OF ABBREVIATIONS}

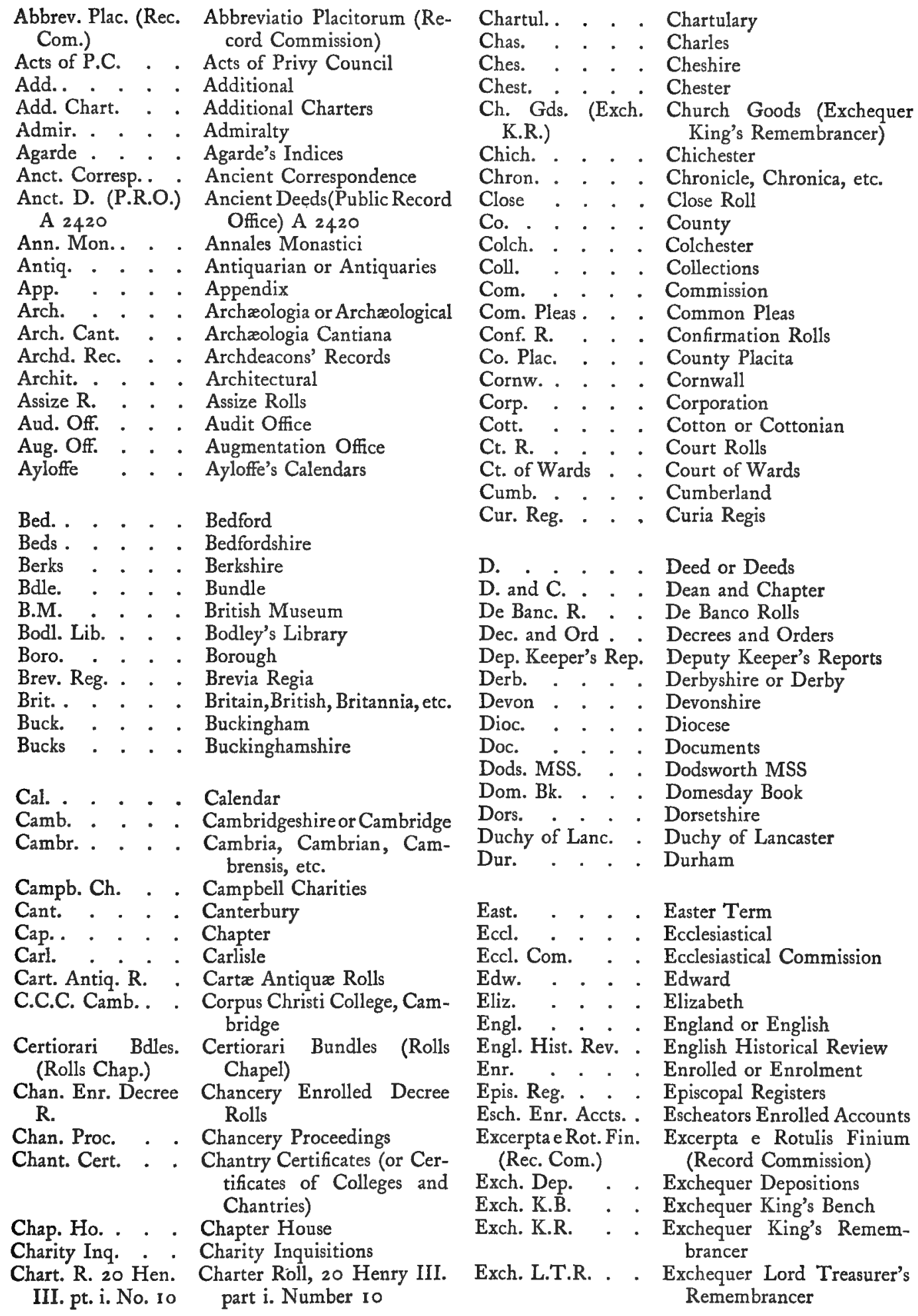




\section{TABLE OF ABBREVIATIONS}

Exch. of Pleas, Plea Exchequer of Pleas, Plea Roll $R$.

Exch. of Receipt . Exchequer of Receipt

Exch. Spec. Com. . Exchequer Special Commissions

Feet of F. . . Feet of Fines

Feod. Accts. (Ct. of Feodaries Accounts (Court of Wards) Wards)

Feod. Surv. (Ct. of Feodaries Surveys (Court of Wards)

Feud. Aids . . Feudal Aids

fol. . . . . Folio

Foreign R. . . . Foreign Rolls

Forest Proc. . . Forest Proceedings

Gaz. . . . Gazette or Gazetteer

Gen. . . . Genealogical, Genealogica,

Geo. . . . George

Glouc. - . . Gloucestershire or Gloucester

Guild Certif.(Chan.) Guild Certificates (Chancery)

Ric. II. Richard II.

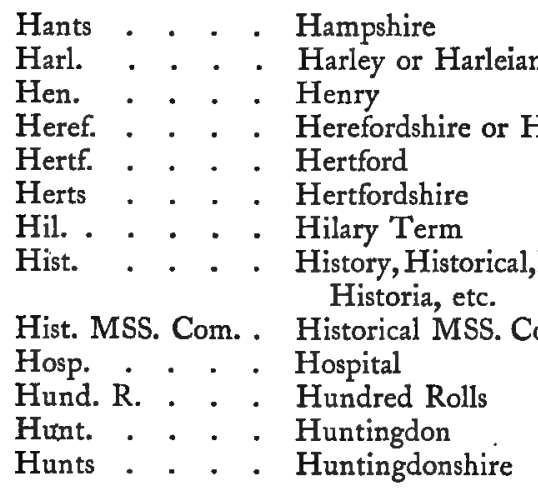

Inq. a.q.d. - . . Inquisitions ad quod damnum

Inq. p.m. . . . Inquisitions post mortem

Inst. . . . . Institute or Institution

Invent. . . . Inventory or Inventories

Ips. . . . . Ipswich

Itin. . . . . Itinerary

Jas. - . . . James

Journ. . . . Journal

Lamb. Lib. - . Lambeth Library

Lanc. . . . . Lancashire or Lancaster

L. and P. Hen. Letters and Papers, Hen. VIII. VIII.

Lansd. . . . Lansdowne

Ld. Rev. Rec. . . Land Revenue Records

Leic. . . . Leicestershire or Leicester

Le Neve's Ind. . Le Neve's Indices

Lib. . . . . Library

Lich. . . . Lichfield

Linc. . . . Lincolnshire or Lincoln

Lond. . . . London

m. . . . Membrane

Mem. . . . Memorials
Memo. R. . . Memoranda Rolls

Mich. . . . Michaelmas Term

Midd. . . . Middlesex

Mins. Accts. . . Ministers' Accounts

Misc. Bks. (Exch. Miscellaneous Books (Ex-

K.R., Exch. chequer King's Remem-

T.R. or Aug. brancer, Exchequer Trea-

Off.) sury of Receipt or Augmentation Office)

Mon. . . . Monastery, Monasticon

Monm. . . . Monmouth

Mun. . . . Muniments or Munimenta

Mus. . . . Museum

N. and Q. . . Notes and Queries

Norf. . . . Norfolk

Northampt. . . Northampton

Northants . . Northamptonshire

Northumb. . . Northumberland

Norw. . . . Norwich

Nott. . . . . Nottinghamshire or Nottingham

N.S. . . . New Style

Off. . . . . Office

Orig. R. - . Originalia Rolls

O.S. . . . . Ordnance Survey

Oxf. . . . . Oxfordshire or Oxford

p. . . . . Page

Palmer's Ind. - . Palmer's Indices

Pal. of Chest. - . Palatinate of Chester

Pal. of Dur. . . Palatinate of Durham

Pal. of Lanc. - . Palatinate of Lancaster

Par. . . . . Parish, parochial, etc.

Parl. . . . . Parliament or Parliamentary

Parl. R. . . . . Parliament Rolls

Parl. Surv. . . . Parliamentary Surveys

Partic. for Gts. - Particulars for Grants

Pat. . . . . Patent Roll or Letters Patent

P.C.C. - . - Prerogative Court of Canterbury

Pet. . . . . Petition

Peterb. . . . Peterborough

Phil. . . . Philip

Pipe R. . . . . Pipe Roll

Plea R. . . . . Plea Rolls

Pop. Ret. . . . Population Returns

Pope Nich. Tax. Pope Nicholas'Taxation(Re(Rec. Com.) cord Commission)

P.R.O. . . . . Public Record Office

Proc. . . . Proceedings

Proc. Soc. Antiq. - Proceedings of the Society of

pt. . . . . Part Antiquaries

Pub. . . . . Publications

R. . . . . Roll

Rec. . . . . Records

Recov. R. . . . Recovery Rolls

Rentals and Surv. . Rentals and Surveys

Rep. . . . . . Report

Rev. . . . . Review

Ric. . . . . Richard 


\section{TABLE OF ABBREVIATIONS}

Roff. . . . Rochester diocese

Rot. Cur. Reg. . Rotuli Curiz Regis

Rut. . . . . . Rutland

Sarum . . . Salisbury diocese

Ser. . . . Series

Sess. R. . . . . Sessions Rolls

Shrews. . . . Shrewsbury

Shrops . . . Shropshire

Soc. . . . . Society

Soc. Antiq. . . . Society of Antiquaries

Somers. . . . . Somerset

Somers. Ho. . . Somerset House

S.P. Dom. . . . State Papers Domestic

Staff. . . . Staffordshire

Star Chamb. Proc. Star Chamber Proceedings

Stat. . . . . . Statute

Steph. . . . . Stephen

Subs. R. . . . Subsidy Rolls

Suff. . . . . Suffolk

Surr. . . . . Surrey

Suss. . . . . . Sussex

Surv. of Ch. Liv- Surveys of Church Livings ings (Lamb.) or (Lambeth) or (Chancery)
Topog. . . . . Topography or Topographical

Trans. . . . Transactions

Transl. . . . Translation

Treas. . . . . Treasury or Treasurer

Trin. . . . Trinity Term

Univ. . . . University

Valor Eccl. (Rec. Valor Ecclesiasticus (Record Com.) Commission)

Vet. Mon. . . Vetusta Monumenta

V.C.H. . . . Victoria County History

Vic. . . . . Victoria

vol. . . . . Volume

Warw. . . . Warwickshire or Warwick

Westm. . . . Westminster

Westmld. . . Westmorland

Will. . . . William

Wilts . . . Wiltshire

Winton. . . Winchester diocese

Worc. . . . Worcestershire or Worcester

Yorks . . . Yorkshire 



\section{A HISTORY OF \\ D E V O N S H I R E}






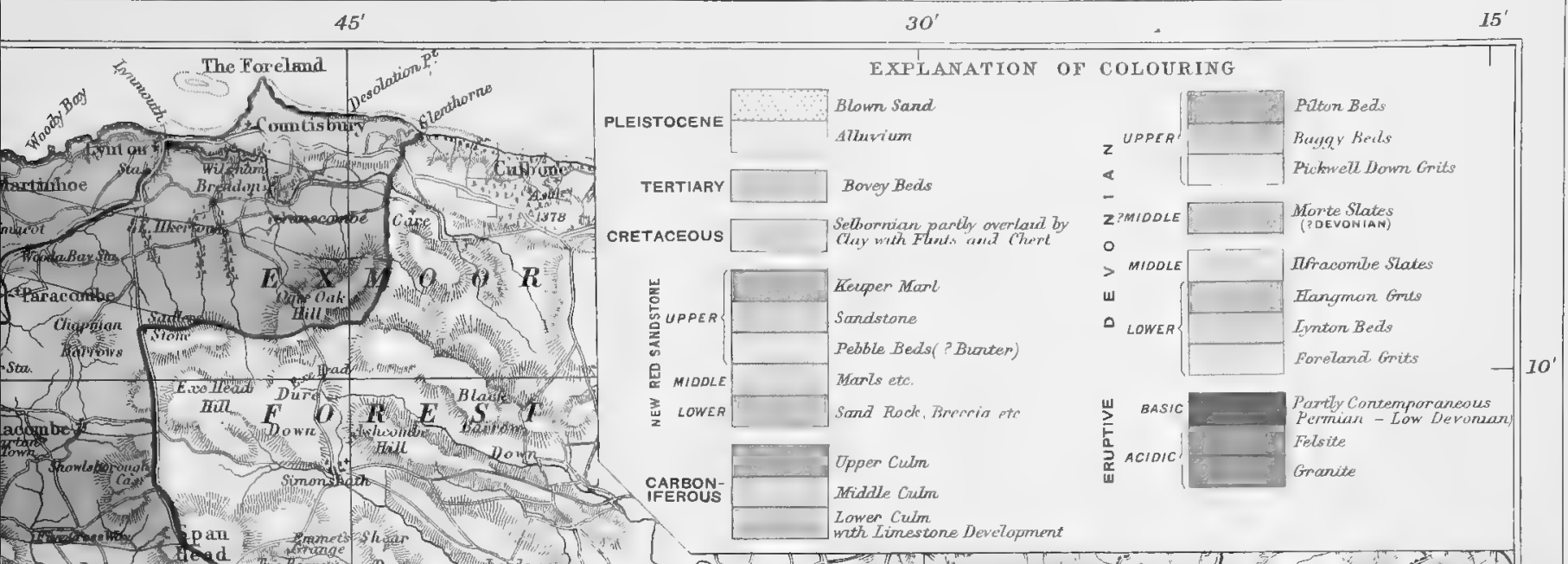

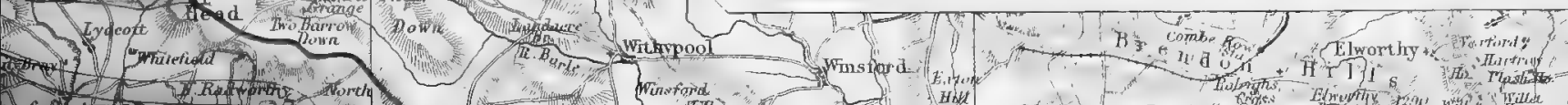

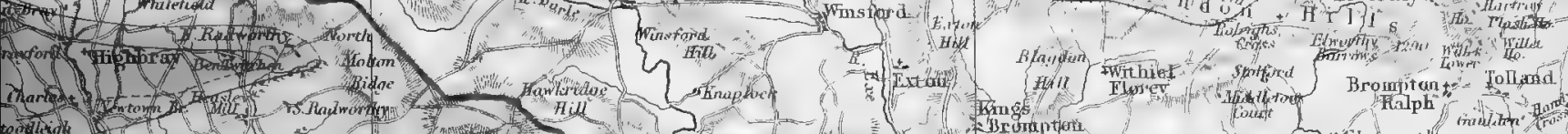

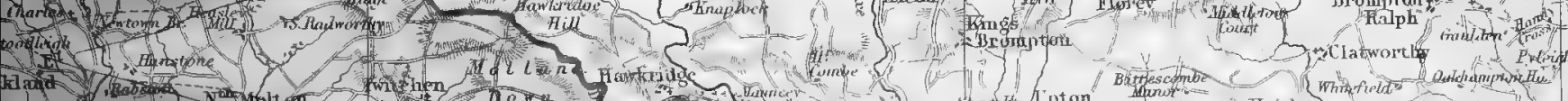

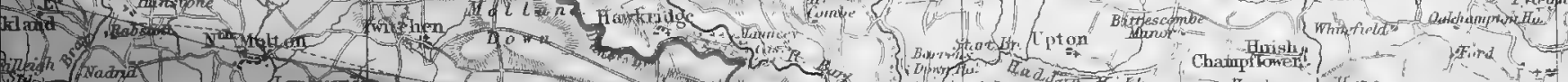

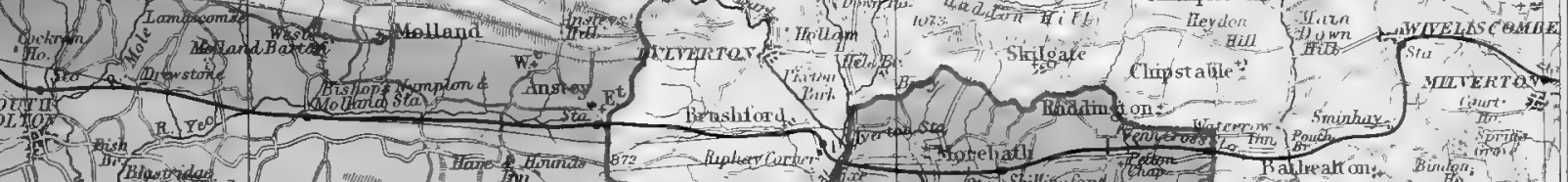

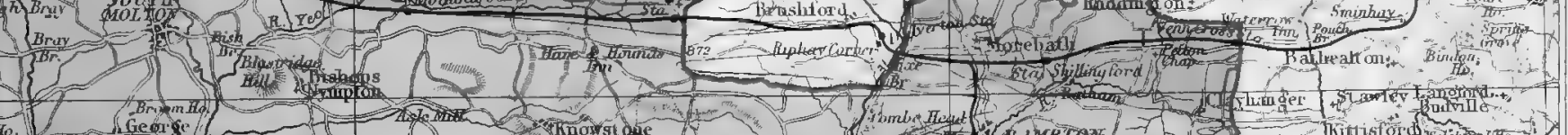

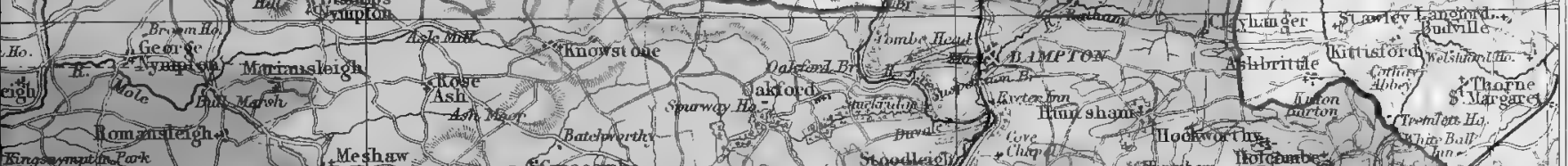

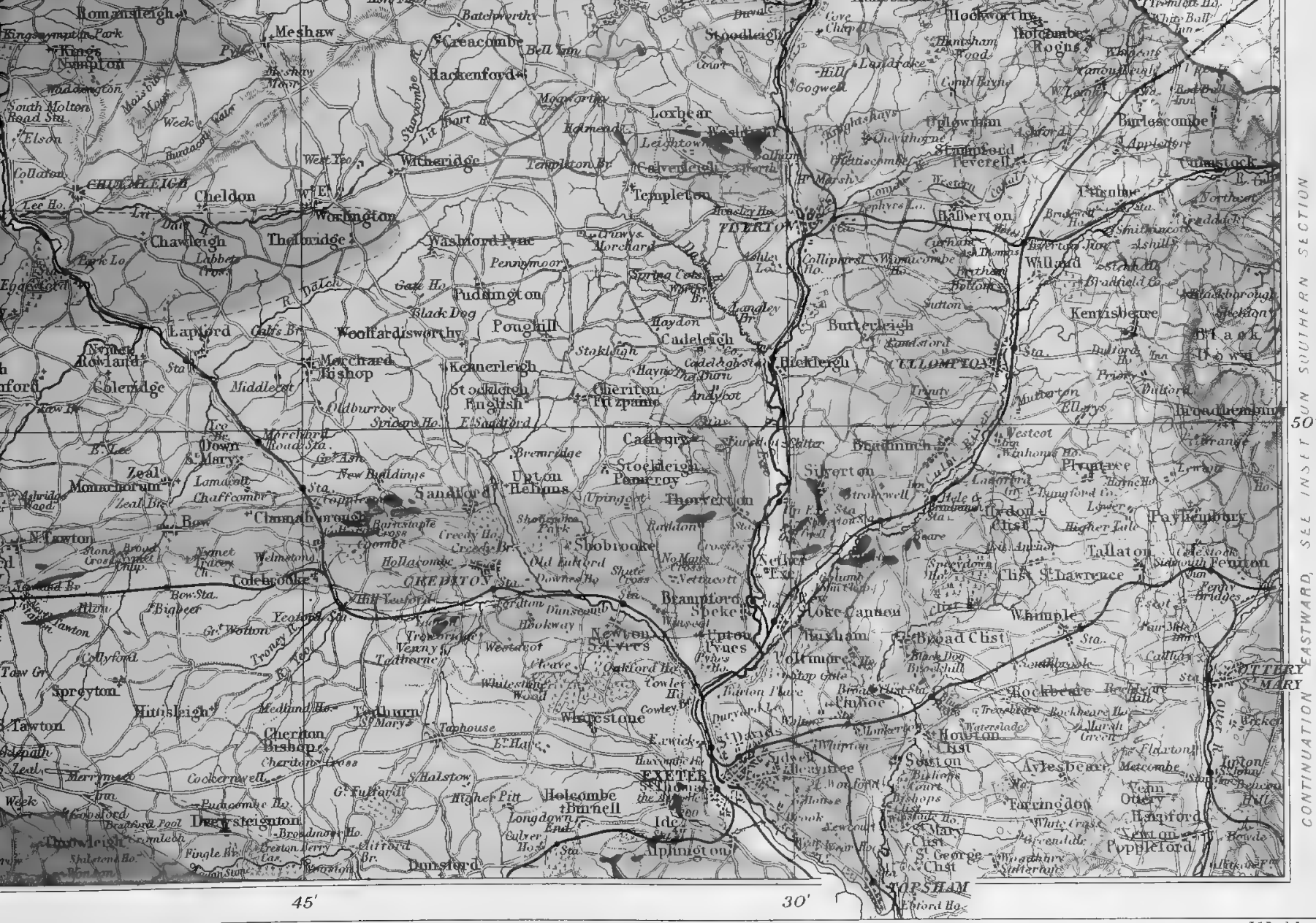





\section{GEOLOGY}

I

N Devon the south-western peninsula of England attains its greatest breadth from the Foreland on the shores of the Bristol Channel to Prawle Point on the English Channel, a distance of 75 miles. From this axis of greatest breadth the distance between the Channels decreases eastward to about 35 miles from Sidmouth northward. This expansion of the peninsula includes the highest land in England south of Derbyshire; the Exmoor highlands on the north, and the tor-crowned hills of Dartmoor on the south, being for the most part above the 1,000 feet contour. An orographical map distinguishing heights of, and over, $\mathrm{I}, 000$ feet brings these uplands of west Somerset and Devon in line with the mountainous areas of Wales.

The eastern border of the Welsh highlands if prolonged to Watchet and from thence southward across the Devon and Somerset boundary and by Exeter and Chudleigh to Newton Abbot and Torquay, divides Devon into two areas differing in their physical features and geological structure. The western area comprises north, central and south Devon. It is composed of older rocks than those in the eastern area, and exhibits a more diversified surface with bolder hills and narrower valleys.

The westerly trend of the rolling hill ranges of Exmoor is maintained throughout north Devon, the general summit-level declining southward and rising again toward Dartmoor.

To the south of Dartmoor the summits are much lower and the higher features run westward from Staddon Heights, near Plymouth, to Southdown Cliff and Scabbacombe Head on the coast south of Berry Head.

In the Start and Bolt districts the scenery is grander than on any other part of the Devon coast; this is due to the rugged character of the rocks rather than to altitude, as the height rarely exceeds 400 feet.

The western area is drained by the rivers Taw, East and West Okement, Teign, Dart, Avon, Erme and Yealm, all of which rise in the highlands of Dartmoor ; by the Torridge and Tamar with sources not far from Hartland Point on the north coast ; and near its eastern margin by the Exe and its tributaries.

In the eastern area the Blackdowns, from 500 to 800 feet in height, and the Haldons, from 700 to 800 feet, are the dominant elevations. They are disconnected portions of a great tableland, or peneplain, which once doubtless extended over the whole of the eastern area, and has been breached and cut back to its present limits by the river Exe and its tributaries the Culm and Clyst, and by the Otter, Sid and Axe. 


\section{A HISTORY OF DEVONSHIRE}

A minor feature forming an escarpment on Westdown Hill near Budleigh Salterton runs northward to Talaton, attaining a height of over 580 feet on Woodbury Common. Apart from these elevations the features of the eastern area are low and tame in comparison to those of the western, except near the border where the newer sediments mantle the slopes and cap the summits of the older rocks, attaining heights of from 700 to 800 feet. Tongues of the newer rocks with their accompanying features run westward through Tiverton and Crediton, causing the eastern area to dovetail irregularly into the western.

It will therefore be seen that the terms eastern and western area are synonymous with the extension of the newer and older rocks respectively. It may not be out of place here to give a brief general outline of the distribution of the rocks in each district and of their general structure, as these have so clear a connection with the physical features of the county, commencing with the older rocks or Palæozoic area.

The northernmost and most of the southernmost parts of this area are composed of Devonian rocks, the marine sediments of the epoch between the deposition of the Carboniferous and Silurian, during which the Old Red Sandstone was accumulated in lakes and estuaries.

The Devonian rocks of north Devon, consisting of great alternating masses of slate and sandstone, were deposited in shallower water and nearer to land than the corresponding rocks of the southern district. The latter consist principally of slates with local masses of limestone and contemporaneous volcanic rocks. Grits and silty intercalations are almost entirely confined to the lower horizons which crop out between Staddon Heights on the Plymouth coast and Southdown Cliff near Berry Head, reappearing on the north in the environs of Paignton and in the higher hills of the Torquay promontory. By fossils and by stratigraphical position the rocks of Upper, Middle and Lower Devonian age have been traced, both in the northern and southern outcrops. The strike, or outcrop, of the northern rocks is nearly east and west. The strike distribution of the southern Devonian rocks is in an easterly and westerly direction; except in parts of the district between Chudleigh and Ivybridge where it is deflected in rough parallelism to the Dartmoor granite boundary.

The Devonian areas are separated by rocks of Carboniferous age, extending from Tavistock, Okehampton and Ashburton over the great central district to Bideford Bay, Barnstaple and Morebath.

These rocks are of a type met with in Germany and are called Culm Measures; they consist of an assemblage of grits, shales and sandstones with plant remains, upon a series of fine grained sediments with beds of chert, calcareous bands and local limestone developments, containing marine fossils. The discovery of Radiolaria in the chert beds by Fox and Hinde ${ }^{1}$ led them to infer that the deposition of these Lower Culm rocks took place in deep water.

1 2uart. Fourn. Geol. Soc. (1895), vol. li. pp. 609-67. 


\section{GEOLOGY}

The general strike of the Culm Measures is east and west, but in the vicinity of the Dartmoor granite these rocks, as well as the Devonian, show in most cases deflections of strike in distinct accommodation to the irregularities of its boundary, ${ }^{1}$ a fact shown on De la Beche's map. Where unfaulted their lower beds are in contact with Upper Devonian rocks on the north and south.

The uppermost beds of the Culm Measures occur in two bands on the latitudes of Torrington and Eggesford, but they die out eastward toward Tiverton.

From the above it will be apparent that the Culm Measures form a broad synclinal curve, or trough, from beneath which the Devonian rocks emerge on the north and south.

That this structure is shallow is proved by the repetition of the upper beds in two synclinal undulations, troughing out eastward; by the innumerable small curves repeating the beds in the coast section, and in all exposures continuous across the strike for any distance; and by the repetition of the Lower Culm rocks by folds between Bampton and Burlescombe and on either side of Dartmoor. The Devonian rocks are also repeated by innumerable small folds, or contortions, and the strain has often been so great that the inverted folds have snapped, or yielded to the pressure to such an extent that their axes are lost on the shear of small thrust planes. There are also many faults. In north Devon, in spite of these structures, the general continuity of the subdivisions is unbroken and their features are well marked and continuous; but in south Devon the rocks are much displaced and disturbed and in consequence the surface is more irregular. In no part of the area is the evidence of these contractile movements more apparent than in the (? pre-Devonian) mica and quartz schists and altered basic rocks of the Start, Prawle and Bolt districts. These have had their original bedding planes gnarled, crumpled and broken so that it is generally impossible to trace the minor foldings, and strain cracks or planes which simulate bedding have been produced.

Throughout the Devonian area the cleavage planes appear to have resulted from the strains to which the bedding was subjected; in some rocks making, if developed at all, a very partial appearance; in others varying in direction and amount of dip, being most horizontal where gnarled, or zigzagged, bedding indications are very highly inclined or vertical. The latter character is well displayed in argillaceous Upper Devonian strata on the west of Dartmoor, by structures simulating even bedding with low dips (as in the altered basic rocks of the Prawle coast).

The prevalence of southerly cleavage dips and of bedding curves inverted toward the north has made the work of piecing together the complicated structures of south Devon one of great difficulty, whilst it proves that the earth movements were lessening northward. 


\section{A HISTORY OF DEVONSHIRE}

These movements were post-Carboniferous, and acting from south to north produced the east and west lines of folding so generally manifested throughout the Palæozoic area; but the obvious deflection of the strikes, as they sway round the discordant mass of Dartmoor granite, would certainly indicate the existence of that mass in its present site while the north and south contractile movements were in operation.

These movements had not only ceased prior to the deposition of the earliest sediments in the eastern part of the county, but an interval in time had elapsed of sufficient duration to permit of the denudation of an enormous amount of the crumpled strata of older rocks; so that the lower beds of the newer formations are found to rest on Lower Devonian rocks near Paignton and Cockington, on Middle and Upper Devonian at Torquay, Newton Abbot, etc., on Lower Culm Measures near Exeter and Burlescombe, and on Middle Culm Measures in the Crediton, Collumpton and Tiverton districts.

The old term New Red Sandstone has been applied to the earliest sediments of the newer rocks, because, although their lower beds have with reason been correlated with part of the German Permian and their uppermost subdivisions with the Trias, in lithological character and conditions of deposit their several members are linked together, and sharply contrasted from the much older strata beneath and the succeeding rocks into which they pass above.

Their lower beds, consisting of clays (local), conglomerates and breccia and soft sandstones, fill old creeks and valleys in the Carboniferous rocks in the Tiverton and Crediton districts, and, in the vicinity of these valleys, they occur as outliers at Hatherleigh, Stoodleigh and other places on higher ground. These strata dip at higher angles from the margin of the older rocks than elsewhere. Igneous rocks are plentiful in the breccias of the Crediton valley, of Exeter and of the Teignmouth coast, and contemporaneous lavas challenge comparison with the Melaphyr decken of the German Permian. These rocks are succeeded by Marls, which form comparatively low land rising westward to the scarp of the overlying Pebble beds. This Pebble-bed feature is conspicuous from West Down Hill, Budleigh Salterton, over the Common lands to the northward, and shows the north and south strike and easterly dip of the rocks. The Pebble beds pass under a series of Sandstones with irregular calcareous concretions, and these are in their turn overlain by the Keuper Marls. The succeeding Rhætic strata separating the New Red Sandstone from the Lower Lias clays and limestones are inconspicuous; both Rhætic and Lias being confined to the eastern margin of the county, where they are seldom well exposed owing to the overlap of the Cretaieous rocks of the Blackdown tableland.

The Cretaceous rocks, mainly consisting of Selbornian sands overlain near the eastern boundary of the county by Chalk, cover the older Secondary formations, resting in succession on Lower Lias, Rhætic beds, Keuper Marls, and the upper beds of the succeeding sandstones. They have been removed by denudation from the intermediate beds 


\section{GEOLOGY}

between the Blackdowns and the Haldons, and in the large outliers of Great and Little Haldon rest on the lower beds of the New Red Sandstone series.

The Cretaceous tableland is capped by an accumulation of clay with flints and chert and coarse gravel, which is considered to be of Eocene (Bagshot) age by Reid. In the broad depression of the valley of the river Bovey and between Milber Down, Kingsteignton, and Newton Abbot the Eocene clays, sands and lignite beds occur, and the sands and gravels often dip sharply under them from off the higher ground on their margin. A smaller flat at Petrockstow, north of Hatherleigh, is also probably an Eocene lake basin, as it contains similar white clay to a depth of over 80 feet and lignite veins were said to occur in it. Here also sands and gravel containing cretaceous fragments are found. Quartz gravel with flints occurs near Rivaton farm 4 miles to the west of the Petrockstow depression at about 500 feet above the sea level; and flint gravel at Orleigh Court $5 \frac{\mathrm{I}}{2}$ miles in a north-north-west direction from the Petrockstow depression. These occurrences, to which a patch of flint and quartz gravel at Colford, near Yeoford, may be added, suggest the extension of Bagshot sands and gravels, if not of Cretaceous rocks, over a considerable part of the Culm Measure area.

The presence of patches of New Red rock at Broad Sands (on the Paignton coast), at Durl Head (near Berry Head), at Slapton and Thurlestone, and at Portledge mouth on the northern coast, tends to show that a considerable part of the coast line of the older rocks has been determined by the old margin of the New Red rocks.

The inliers of older rock, such as some of the Lower Culm limestone hills (near Burlescombe), the Culm Measures of Spraydown (south of Bradninch) and the Devonian rocks of the Torquay promontory, are not far from the older rock margin; they prove that the floor of the older rocks on which the earlier New Red sediments were deposited was uneven or hummocky.

The Lower New Red breccias and sands appear to have been gravelly accumulations carried down by torrential rivers into broader channels or lakelets, where they passed out into and inosculated with sand. From vents in or bordering these channels trappean rocks were locally outpoured, and, overlapping the sediments already formed, encroached on the older rocks, as may be seen in the relations of the patches spared by denudation near Dunchideock, Poltimore, etc. There appear to have been masses or dykes of quartz-porphyry in the main areas of deposit in the Crediton valley and in the district south of Exeter, as the boulders of that rock are often large enough to forbid transport from a distance, and there is no source in the exposed area of older rocks from which they could have been derived. Felsitic rocks in line with and vicinity of the Crediton valley confirm this supposition. Ormerod called attention to the large percentage of felspar crystals of the variety known as Murchisonite in the Lower New Red breccias south of Exeter. To account for these phenomena R. N. 


\section{A HISTORY OF DEVONSHIRE}

Worth propounded the theory that the Dartmoor granite is the stump of an old volcano, the effusive materials from which were removed by denudation and incorporated in these breccias.

The presence of Upper Devonian fossils of north Devon types in some of the stones in the Lower New Red breccias of Tiverton, Silverton and Cadbury suggests a current from the north or north-west.

It seems therefore that the outliers of Lower New Red gravel at high levels are the relics of the valley bottoms of a contour which has been since so entirely changed by denudation that not a vestige of the higher land in their vicinity is left. The succeeding New Red subdivisions point to more tranquil deposition, except the well worn pebbles of the Budleigh Salterton beds, which, from the replacement of pebbles of extraneous derivation by those of local origin as they are traced northward, seem to indicate the pushing forward of a shingle beach during a period when the sea may have gained access to the lacustrine areas. The succeeding Sandstones contain Labyrinthodont remains, and underlie the Keuper Marls without any appearance of discordance.

There is no proof of the nature of the denudation of the palæozoic surface subsequent to the deposition of the Lower New Red beds until the Cretaceous period, when, after the earlier Secondary rocks by an uprise on the west, or a depression on the east, had been tilted, the Cretaceous sea cut a plane across them on which the earlier Cretaceous sediments were transgressively overlapped and the descending series of older formations overlain. In this and the subsequent deposition of the Eocene clays and gravels the older rock area was no doubt invaded, and the irregularities of its earlier contour planed and modified.

On the Cretaceous tableland the clay or loam with flints or chert fragments attains a thickness of 30 feet or more in places, filling pipes in the Greensand, and in the Chalk where present. It is very rare to find any extension of water-worn gravel on the Cretaceous summits, such as is found near Staple Hill on the Black Downs and on Great Haldon. This accumulation seems to be connected with the planing of the Cretaceous rocks about the period of their emergence in Tertiary times, and suggests the shorn remnants of argillaceous Tertiaries deposited on the relics of the Chalk, which had become mixed with flints and been carried down by subsequent percolation into pipes and potholes dissolved out of the subjacent rocks. The accumulation has been displaced with the Greensand on which it rests by a considerable north and south fault, repeating in Stockland Hill the escarpment above Honiton.

Between Haldon and Kingsteignton near Oldchard, Hestow farm and Ideford, saccharoid red-brown siliceous boulders are met with. They are probably greywethers and-coupled with the sands and gravels retained in potholes in the Devonian limestone and those fringing the Bovey valley - they point to a considerable amount of Eocene denudation and subsequent removal of resultant deposits. The lake basins of Bovey and Petrockstow do not appear to be due to local subsidences on fault lines, although the high dips of the sands on the south of the Bovey 


\section{GEOLOGY}

valley suggest such an explanation. In advocating a granite origin for the Bovey valley clays, and combating Maw's opinion to the contrary, Pengelly alluded to the occurrence of derivative crystals of felspar in one of the beds. From the relative positions of the gravels on the heights, and the gravels, sands and clays in the valleys, we may infer that they represent successive stages in the denudation effected in Bagshot times, subsequent to the Tertiary planing of the Cretaceous surface.

In Devon no proofs of Pliocene deposition have been found, although some of the gravels at high levels may belong to this period. The evidences of Pleistocene denudation are confined to the results of a long period of fluviatile erosion. Patches of gravel, marking stages in the excavation of the present river valleys, are of more frequent occurrence on the newer rocks than the older. The raised beaches rarely indicate a depression of more than 40 or 50 feet, but that they were elevated many feet above their present position is proved by the evidences of submerged forests on the foreshore in Torbay, Salcombe Estuary and Bigbury Bay on the south coast, and at Westward Ho on the north. Intermediate between the beach formation and the forest growth, probably at a time when the elevation of the beaches reached its maximum limit, a quantity of talus was shed from the high land over the landward margin of the raised beach platform. This old talus or Head is found in pinnacles on the old beach platform, insulated from the main cliffs by recent marine erosion in several places between Start Point and Plymouth Sound. In inland localities, especially on higher lands and near stream sources, there are flats or gentle slopes of marshy land with clay soil and fragments and boulders of local rock which may date to this period. We may also ascribe to it the 'head' or white surface clays of the Bovey valley, which contain Betula nana, Salix cinerea, $S$. myrtilloides, identified by Professor Heer.

The evidence of a colder climate furnished by this flora, and by the more rapid disintegration of exposed rock surfaces leading to the formation of talus fans or screes in favourable situations, is led up to by proofs of the prevalence of a somewhat colder climate during the formation of the old beaches. Hunt cites the shells Trophon truncatus and Pleurotoma turricula obtained from the raised beach on the Thatcher rock in evidence of this. An additional proof is afforded by the rare occurrence of large worn boulders of extraneous rocks on the old beach platform. Of this the granite boulder overlain by the raised beach at Saunton (Barnstaple Bay) is the familiar example. Two boulders, also of large dimensions, occur on the old beach platform on the east side of the Prawle. These boulders seem to indicate transport by floe ice, or by casual bergs drifted on shore many miles away from their sources. The forest growth must have flourished over a much more extended foreshore, when the raised beaches stood high above sea level, and their growth was circumscribed during the succeeding period of subsidence, which allowed the sea to gain access to its old cliff bounds in the older rock area and to encroach beyond them in the newer. 


\section{A HISTORY OF DEVONSHIRE}

The cavern accumulations and their fauna were coeval with these Pleistocene changes, and prove the co-existence of man with the extinct mammalia, besides affording evidence of his handiwork in Palæolithic, Neolithic and more recent times. Pengelly records the discovery of bones of Cervus elaphus, Bos longifrons and Elephas primigenius in the Torbay submerged forest; and two molars of $E$. primigenius, said to have been obtained from the submerged forest at Sidmouth by P. O. Hutchinson, were presented to the Exeter Museum.

The Exe has the most anomalous course of any of the Devonshire rivers, crossing the softer strata of the Tiverton and Crediton valleys without deflection, and traversing the Culm rocks across their strike between these valleys and on the north of Exeter. When the previous extension of the Lower New Red gravels over this part of the Culm area is taken into account the phenomenon is easily explained.

Devonshire has a voluminous geological literature commencing with the pages devoted to geology in Polwhele's History of Devonshire (1793I 806), which are well worthy of perusal.

The general distribution of the older and newer rocks and the extension of the granite were outlined by De la Beche, assisted in south Devon by Godwin-Austen. The results of their gratuitous labours are to be seen amongst the first maps published by the Geological Survey after its establishment in I 835 ; in De la Beche's Report on the Geology of Cornwall, Devon and West Somerset, published in 1839 ; and in 1842 in the article by him in Memoir I. of the Geological Survey. The grouping of the older rocks into Carboniferous and Devonian was effected by Sedgwick and Murchison in 1838. Amplifying the correlation of Lonsdale, they regarded the Devonian rocks as the marine representatives of the Old Red Sandstone. They gave the name Culm Measures to the Carboniferous rocks, from the local occurrence of seams of culm or anthracite in them in north Devon. In 1837,1838 and 1841 the Rev. D. Williams, Weaver, and Professor J. Phillips published classifications of the Culm rocks. The succession of the Devonian rocks of north Devon given by De la Beche in his Report, and endorsed by Professor Phillips, Hall and Etheridge, was disputed by Professor Jukes and Professor Hull, who claimed the uppermost horizons as Carboniferous and the lowermost as Silurian. Dr. Hicks, with the assistance of the Rev. G. F. Whidborne, relying on fossils discovered in what had been regarded as an unfossiliferous subdivision, advocated the faulted upthrust of pre-Devonian rocks in an otherwise unbroken Devonian succession.

Dr. Holl attempted to decipher the broken structures of the Devonian of south Devon, but more light was thrown upon the problem by Champernowne, who, from careful observations in the field, constructed a detailed map of the area between Chudleigh and Dartmouth. In consequence of the acceptance of this map by the Geological Survey, as set forth in the Report of the Director-General for the year 1887 , a detailed survey was begun in 1888 on 6-inch maps, during the progress of which the structural relations of the rocks were unravelled by Ussher. 


\section{GEOLOGY}

The subdivision of the New Red rocks, completed in 1880 , was not published till after the appearance of the new series $I$ inch maps (sheets 325,339 and $35^{\circ}$ ), to which the lines were transferred from the old ordnance sheets 21 and 22, part being still unpublished.

The mica-schists and altered igneous rocks of the Start, Prawle and Bolt districts were claimed as Archæan in 1874 by Professor Bonney, which opinion, subsequently endorsed by Miss Raisin, was combated by A. R. Hunt and other writers in favour of an extremely altered phase of the neighbouring Devonian rocks. The New Red succession on the coast given by Whitaker was amplified and extended throughout the county and the results published by Ussher. The Rev. A. Irving and Professor Hull propounded classifications of the series, in which the Budleigh pebble bed was taken as the base of the Trias. The whole series had been regarded as Keuper by Pengelly. The Cretaceous rocks have been investigated by Fitton, Meyer, the Rev. W. Downes and Jukes-Browne. The Bovey clays and lignites, described as Miocene by Pengelly and Professor Heer, were referred to the Eocene by Starkie Gardiner. The plastic clay of De la Beche and supra-cretaceous gravels were subsequently referred to the same general period (Bagshot) by C. Reid.

The cavern deposits of the Torquay and Brixham districts were exhaustively worked and described by Pengelly, those of the Plymouth district by R. N. Worth. The raised beaches and submerged forests and other Pleistocene phenomena were treated by Pengelly, Hunt and others. The study of recent marine action and of the submarine geology of the English Channel has been prosecuted by A. R. Hunt.

In the older rocks palæontological research is represented by the Tables of D'Archiac and De Verneuil in the Geological Society's Transactions; by Professor Phillips' Figures and Descriptions of Palæozoic fossils; and (specially relating to the faunas of the Torquay and Newton Abbot limestones, and of the Upper Devonian strata of north Devon) by Whidborne's Monographs in the Palæontographical.

As regards the igneous rocks of the county, the granite has been described by De la Beche and by many subsequent observers, including R. N. Worth and General MacMahon; the volcanic and intrusive rocks of the Palæozoic area by Champernowne, Rutley, MacMahon and by Watts and Teall; the volcanic rocks associated with the Lower New Red by Vicary, Bernard Hobson and Teall. In the following table of stratified rocks and deposits the highly altered rocks of the Start, Prawle and Bolt are given the subordinate position, and will be first described because they occupy the most southerly part of the county, have furnished no fossils, and are marked off by their greater alteration and compression from the Devonian rocks on the north, without prejudice to the probability that they may be an extremely altered phase of these rocks. 


\section{A HISTORY OF DEVONSHIRE}

\begin{tabular}{|c|c|c|}
\hline Recent & & $\begin{array}{l}\text { Alluvium and stream gravels. Peat or peaty soil } \\
\text { Sand and shingle beaches. Blown sand }\end{array}$ \\
\hline Pleistocene & & $\begin{array}{l}\text { Submerged forests } \\
\text { Head or stony loam exposed on the coasts, white } \\
\text { clays with Betula nana in the Bovey valley } \\
\text { Raised beaches ; old river gravels contempo- } \\
\text { raneous with raised beaches and marking prior } \\
\text { and subsequent stages in the excavation of the } \\
\text { present valley system } \\
\text { Cavern deposits ranging from recent to (?) early } \\
\text { Pleistocene }\end{array}$ \\
\hline Tertiary & $\begin{array}{l}\text { Bagshot. } \cdot \cdot \cdot \cdot \cdot \cdot \\
\text { Residue of pre-existent Cre- } \\
\text { taceous and Tertiary rocks }\end{array}$ & $\begin{array}{l}\text { Clays with lignite bands, sands and gravels of } \\
\text { the Bovey valley and of Petrockstow } \\
\text { Gravels at higher levels. Gravels on the Cre- } \\
\text { taceous tableland } \\
\text { Clay with flints and chert }\end{array}$ \\
\hline Cretaceous & $\left\{\begin{array}{l}\text { Sands, etc., represent- } \\
\text { ing Upper Greensand, } \\
\text { Malmstone, and Gault } \\
\text { Clay }\end{array}\right.$ & $\begin{array}{l}\text { Upper Chalk. Chalk with many flints on hard } \\
\text { nodular chalk, zones of Micrasters and Holaster } \\
\text { planus } \\
\text { Middle Chalk (Turonian). Chalk with layers of } \\
\text { flint (zone of Terebratulina). Hard chalk, in- } \\
\text { cluding Beerstone (zone of Rhynchonella cuvieri) } \\
\text { Lower Chalk (Cenomanian). Quartziferous } \\
\text { limestone and calcareous sandstone (chalk } \\
\text { present at Membury) } \\
\text { Chert beds on sands with shell bands and layers } \\
\text { of sandstone with concretions, used for whet- } \\
\text { stones in parts of the Blackdowns, on nearly } \\
\text { unfossiliferous sands } \\
\text { Sands with beds of clay in tunnel near Honiton } \\
\text { and at Whitecliff and Branscombe on the coast }\end{array}$ \\
\hline Liassic & Lower Lias . . . . & $\begin{array}{l}\text { Beds of limestone and dark shale. In the lower } \\
\text { part (zone of Ammonites planorbis) limestone } \\
\text { beds predominate }\end{array}$ \\
\hline Rhatic & $\begin{array}{l}\text { White Lias . . . . . } \\
\text { Black Shales . . . . . }\end{array}$ & $\begin{array}{l}\text { White or cream-coloured limestones and marly } \\
\text { beds } \\
\text { With Avicula contorta-and a bone bed-on grey } \\
\text { and greenish marls }\end{array}$ \\
\hline $\begin{array}{l}\text { New Red } \\
\text { Sandstone } \\
\text { Series }\end{array}$ & $\begin{array}{l}\text { Upper } \\
\text { New Red }\end{array}\left\{\begin{array}{l}\text { Keuper. } . \\
\begin{array}{c}\text { Lower Keuper or } \\
\text { Bunter }\end{array}\end{array}\right.$ & $\begin{array}{l}\text { Red and greenish cuboidal marls with veins of } \\
\text { gypsum, with sandy and calcareous layers, in } \\
\text { lower part more or less silty } \\
\text { Red sandstones, in parts with calcareous concre- } \\
\text { tions and two or three bands of a concretionary } \\
\text { brecciated character ; occasional pebbles, and } \\
\text { false-bedding in lower part } \\
\text { Pebble beds with foreign pebbles gradually giving } \\
\text { place to rocks of local derivation, northward } \\
\text { Red marls (cuboidal) without gypsum } \\
\text { Red marls with thick even beds of red and } \\
\text { whitish sandstone, developed at Straight Point, } \\
\text { where they contain local calcareous concre- } \\
\text { tionary beds and brecciated bands } \\
\text { Io }\end{array}$ \\
\hline
\end{tabular}




\section{GEOLOGY}

\begin{tabular}{|c|c|c|}
\hline $\begin{array}{l}\text { New Red } \\
\text { Sandstone } \\
\text { Series } \\
\text { (continued) }\end{array}$ & $\left.\begin{array}{c}\text { Lower } \\
\text { New Red }\end{array}\right\}$ Permian . . & $\begin{array}{l}\text { Red rock-sand, brecciated in the upper part and } \\
\text { with beds of breccia on the coast, and breccia } \\
\text { with sand-rock. Trap rocks of Silverton, } \\
\text { Killerton and Poltimore districts } \\
\text { Breccia with numerous igneous fragments, mur- } \\
\text { chisonite, etc., in a loamy sand matrix. North } \\
\text { of Crediton valley, gravels and gravelly breccia } \\
\text { with few igneous stones. Trap rocks of } \\
\text { Dunchideock, Exeter and Crediton districts. } \\
\text { Trap rocks of Tiverton (Washfield, etc.) } \\
\text { district } \\
\text { Breccio-conglomerates of Watcombe and Petitor } \\
\text { crags, Oddicombe, and Paignton, etc. ; also } \\
\text { sands and breccias } \\
\text { Loamy breccia and clays of Watcombe and } \\
\text { Petitor }\end{array}$ \\
\hline $\begin{array}{l}\text { Carboni- } \\
\text { ferous }\end{array}$ & $\begin{array}{l}\text { Upper Culm Measures . } \\
\text { Middle Culm Measures . } \\
\text { Lower Culm Measures. }\end{array}$ & $\begin{array}{l}\text { Hard, thick, even-bedded, grey grits, intercalated } \\
\text { with dark shales } \\
\text { Irregular and even grits, sandstones and shales, } \\
\text { in locally variable association } \\
\text { Hard shales with limestone bands locally develop- } \\
\text { ing into limestones with Posidonomya becheri } \\
\text { and Goniatites spiralis, etc., succeeded by hard } \\
\text { shales and chert beds (Radiolaria) based by } \\
\text { dense mudstones and dark shales. Contem- } \\
\text { poraneous volcanic rocks near the base of the } \\
\text { series in Tavistock and Ashton districts: }\end{array}$ \\
\hline $\begin{array}{l}\text { Devonian } \\
\text { Rocks of } \\
\text { North } \\
\text { Devon }\end{array}$ & $\begin{array}{l}\text { Upper Devonian } \cdot \cdot \cdot \\
\text { Middle Devonian, or Silurian } \\
\text { upthrust by faults (Hicks) }\end{array}$ & $\begin{array}{l}\text { Pilton beds. Argillaceous slates with beds of } \\
\text { grit and calcareous fossiliferous lenticles } \\
\text { Baggy beds. Slates with Lingula, brown sand- } \\
\text { stones with Cuculloea } \\
\text { Pickwell Down beds. Grits of various colours } \\
\text { and purple slates } \\
\text { Morte slates. Greenish and grey slates. Strick- } \\
\text { landinia lirata and Orthis rustica, etc., found } \\
\text { by Dr. Hicks; previously regarded as un- } \\
\text { fossiliferous } \\
\text { Ilfracombe slates. Silvery and grey slates with } \\
\text { limestone bands and lenticles, intercalated } \\
\text { with grits toward their base } \\
\text { Hangman grits. Red quartzose grits with Natica } \\
\text { and Myalina in upper part, grits thick and } \\
\text { thin bedded variously associated with, or in, } \\
\text { slates. (Middle Devonian of Etheridge) } \\
\text { Lynton beds. Irregular grey, partly calcareous, } \\
\text { slates intercalated with thick, even-bedded grits } \\
\text { Foreland grits. Shaly grits and thick-bedded } \\
\text { hard grits associated in places with red slates. } \\
\text { (Glengariff grits of Hull) }\end{array}$ \\
\hline $\begin{array}{l}\text { Devonian } \\
\text { Rocks of } \\
\text { North } \\
\text { Devon }\end{array}$ & nian . . & $\begin{array}{l}\text { Buff, greenish and grey slates ; purple-red, green- } \\
\text { mottled slates } \\
\text { In the limestone areas these slates contain } \\
\text { calcareous nodules, concretionary limestone } \\
\text { and thin, shaly, red-brown limestone, locally } \\
\text { with Budesheim Fauna }\end{array}$ \\
\hline & & \\
\hline
\end{tabular}




\section{A HISTORY OF DEVONSHIRE}

\begin{tabular}{|c|c|c|}
\hline $\begin{array}{l}\text { Devonian } \\
\text { Rocks of } \\
\text { South } \\
\text { Devon } \\
\text { (continued) }\end{array}$ & $\begin{array}{l}\text { Middle Devonian. . . } \\
\text { Lower Devonian . . . }\end{array}$ & $\begin{array}{l}\text { In the upper part of limestone masses the con- } \\
\text { tinental base of the Upper Devonian is repre- } \\
\text { sented by massive limestone, generally coralline } \\
\text { (as at Lummaton, Chudleigh, Ilsham, etc.); } \\
\text { by beds of limestone on shalstein (as at Black- } \\
\text { head and Goodrington) } \\
\text { Bedded limestones, generally coralline, shaly and } \\
\text { slaty limestones at base (Eifelian) on slates } \\
\text { South of Totnes volcanic rocks (Ashprington } \\
\text { development) on slates } \\
\text { Between Totnes and the Plymouth limestone } \\
\text { the series is represented by slates with volcanic } \\
\text { rocks and local patches of limestone } \\
\text { Staddon grits. Quartzose grits and sandstones } \\
\text { irregularly associated with slates and shales } \\
\text { Meadfoot, Looe and Ringmore beds. Irregular } \\
\text { slates with grit or siltstone beds, slates with } \\
\text { siliceous and calcareous films } \\
\text { Dartmouth slates. Reddish and buff slates, } \\
\text { purple-red and green glossy slates, with sili- } \\
\text { ceous films and occasional beds of hard grit } \\
\text { and quartzite }\end{array}$ \\
\hline $\begin{array}{l}\text { Archæan of } \\
\text { Bonney, etc. } \\
\text { Altered } \\
\text { Devonian } \\
\text { of Hunt, etc. }\end{array}$ & & $\begin{array}{l}\text { Hornblende-Epidote schists. An altered series } \\
\text { of basic rocks } \\
\text { Mica and quartz schists. Altered grits, slates } \\
\text { and shales, originally similar in composition } \\
\text { to Lower Devonian rocks on the north }\end{array}$ \\
\hline
\end{tabular}

\section{MICA AND QUARTZ SCHISTS AND HORNBLENDE EPIDOTE SCHISTS ${ }^{1}$}

The mica and quartz schists are a highly altered series of slates or shales, for the most part interbanded with harder siliceous materials (grits or silty bands) of the same types as are encountered in the Devonian area; their dark grey colour forms a marked contrast to the prevalent green hues of the hornblende-epidote schists in junction sections. The hornblende-epidote schists are a highly altered series of basic rocks, diabases and diabase tuffs similar to those in the Devonian area. They may be termed green schists, although locally exhibiting pronounced red, buff and brown colours, as in the section at Hope. These rocks are the newer group, as in the interfoldings with the mica schists at their junction, in such sections as the Portlemouth Coast and Southpool Creek, they decrease in thickness downward from the surface, the mica schists exhibiting the opposite tendency. At their junction there is often an appearance of interbanding of the green and dark grey schists.

The mica and quartz schists of the Start Point district are separated from those of the Bolt by a syncline of the green schists, which, running in a north-westerly direction across the Salcombe estuary, coalesces with a band of green schists prolonged westward from the Bickerton valley.

1 See Proc. Geol. Assoc. vol. xvii. pt. 3, pp. 119-35. 



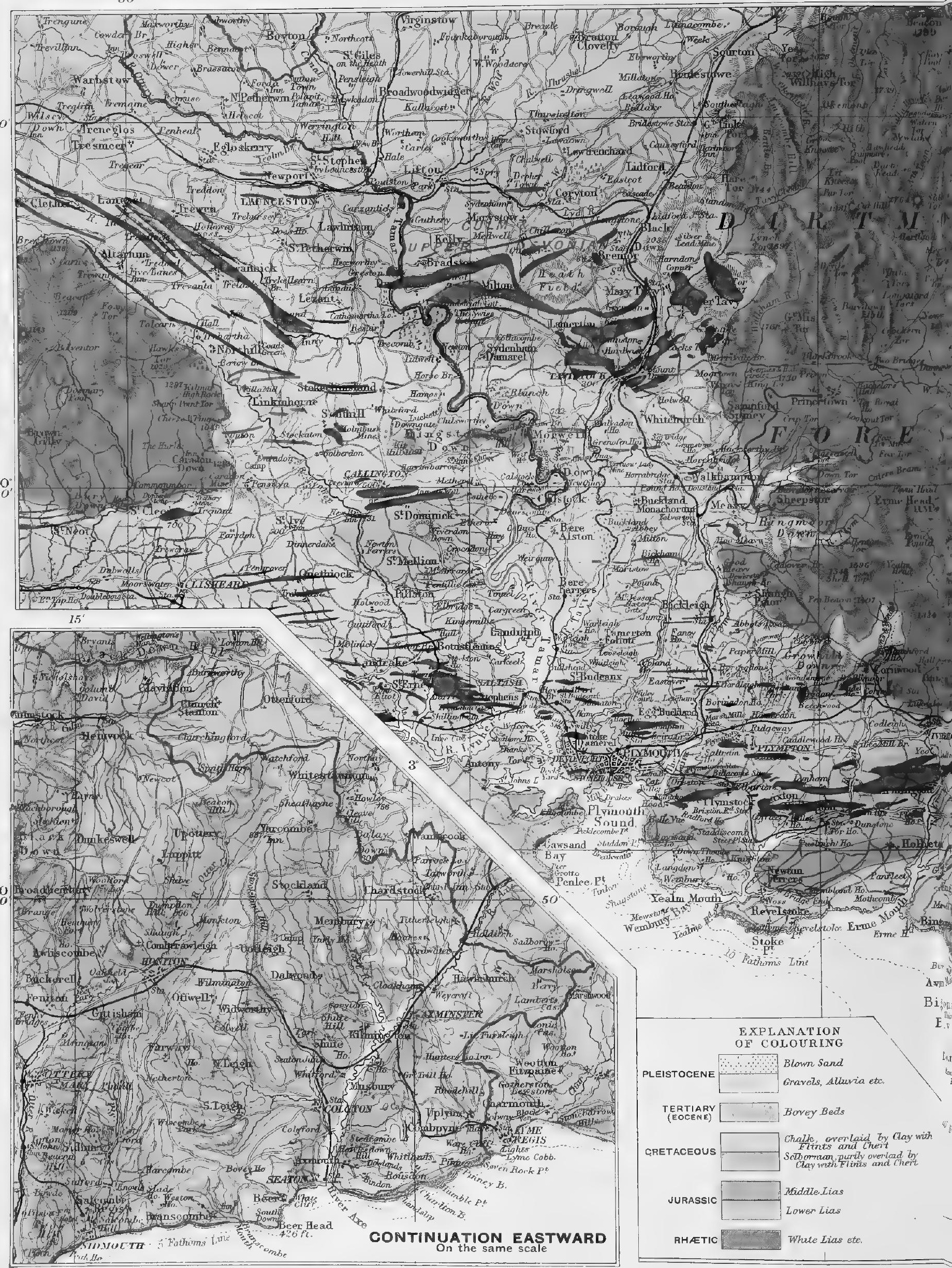




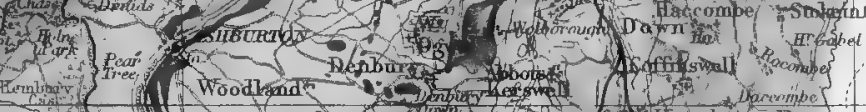

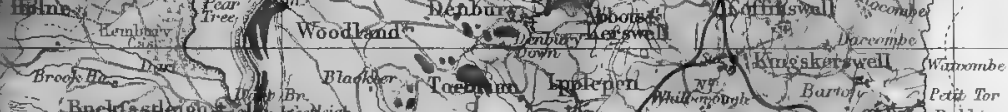

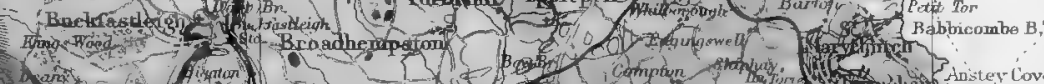

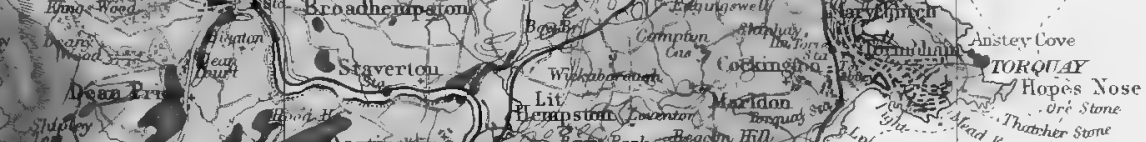

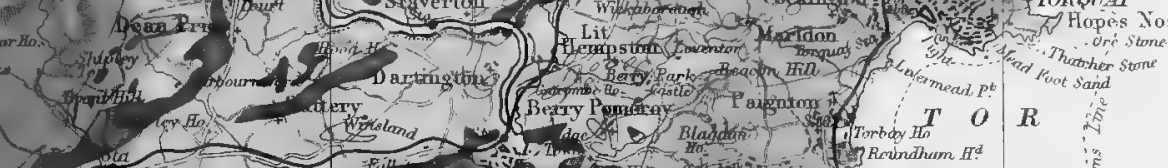

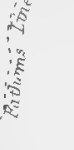
然

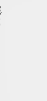





\section{GEOLOGY}

These bands on coalescing strike westward to the coast at Bolt Tail and Outer Hope, where they form a syncline separating the mica quartz schists of the Bolt from a narrower band of the same series on the north. The mica and quartz schists of the Start district in a complex anticline, therefore, separate the green schists of the Prawle from those of the Bickerton valley. These main structures are rendered shallow by an innumerable series of minor folds, accompanied by gnarling and small thrusts, as shown in the Hall Sands cliff. The minor folds are also indicated by joints, or strain planes, breaking the mica-quartz schists into vertical or horizontal masses, so well shown in the rugged outlines of Bolt Head; or by isolated synclinal masses of the green schists as at 'Spirit of the Ocean' Cove near Start Point. The green schists, where they occur in mass, are often intersected by even planes which give them an appearance of bedding, frequently seen to be at variance with the contorted foliation of the rock.

Although the distinction between these rocks and the grey, comparatively unaltered, Lower Devonian slates which bound them on the north is everywhere strongly marked, it is hardly possible to fix on any definite boundary in the numerous junction sections afforded by the coasts and the intermediate creeks of the Salcombe estuary. This is due to the presence of an intervening series of rusty brown rocks in which hard carbonates are generally present; these form a zone, of several chains in breadth, with which both the Devonian slates and the altered groups seem to be connected. The green schists are separated from the Devonian by these brown rocks from Malborough eastward, and from Malborough to Hope, where mica schists bound the Devonian slates, to judge from their presence at Hope Headland and Mouthwell ; though not exposed their occurrence may be assumed.

At Malborough the mica and quartz schists are peroxidated. At Lannacomb Mill, on the Start coast, they exhibit pinkish and grey tints, and quartz-albite (pegmatite) veins are plentiful. These however occur occasionally in the Lower Devonian rocks. The junction brown rocks seem to be a series of partly calcareous and siliceous materials associated with slates and volcanic rocks, but, apart from the hard carbonates in them, they are too rotten for petrographical investigation. Faults and thrusts occur in these junction rocks, but no evidence of a great thrust plane, or fault, separating the altered and unaltered rocks from east to west has been obtained.

The altered rocks conform in strike to the Devonian on the north, and there seems to be no proof of their having experienced a prior movement to that to which the latter were subjected.

\section{DEVONIAN ROCKS OF SOUTH DEVON}

The Devonian rocks of south Devon consist mainly of argillaceous sediments. These are associated in the Lower Devonian with more or less fine grained grits and silty rocks in very variable proportion. The Middle Devonian succession varies in different districts and in different 


\section{A HISTORY OF DEVONSHIRE}

parts of the same district. Slates invariably form the lower beds ; but in the area between Ashburton, Chudleigh and Brixham they are overlain by limestones mostly of coralline growth, interrupted here and there at various stages by incursions of volcanic materials; in the area south of Totnes volcanic eruptions put a stop to their extension. At Plymouth and Yealmpton limestones and volcanic rocks are also present on the slates, and between these limestone areas the general horizon of the limestones is marked by a mass of volcanic rocks in slates. Argillaceous slates form the bulk of the Upper Devonian, they also contain volcanic rocks in places, and, although they locally furnish fossils and in the limestone areas contain calcareous beds of marked character, their boundary with the Middle Devonian slates can seldom be ascertained.

Owing to the characters in common shared by different horizons, and to the variations in composition, or association, displayed by the same horizons traced along their strike, the Devonian subdivisions though generally distinct seldom admit of precise delimitation. The whole series has moreover been thrown into a number of small folds broken by thrusts.

Where rocks of different resisting power have been folded together, such as grits and slates, or limestones and slates, numerous faults have dislocated and displaced them.

Cleavage throughout the area is dependent on the relative powers of resistance of the rocks and on the nature of the local stresses to which they were subjected, consequently the same beds may be shales in one place and slates in another.

Lower Devonian.-The Lower Devonian rocks occupy the area between the highly altered rocks of the Start and Bolt districts and a line of outcrop extending from Staddon Heights (near Plymouth) to the vicinity of Sharkham Point (near Brixham), along which they rise in dominant features above the Middle Devonian districts. Further north they reappear in an anticline broken by faults which terminates some miles west of Paignton. Another anticline still more broken by faults forms the highest land in the Torquay promontory.

Coast Sections.-The relations of the Lower Devonian subdivisions are best shown by a comparison of the coast sections. The Plymouth coast section under Staddon Heights exhibits a series of red-brown grits, slates, shales and sandstones (the Staddon grits) in inverted and plicated junction with Middle Devonian slates. The Staddon grits pass down into the Meadfoot beds at Bovisand Bay, where grey slates with hard grit beds and irregular knubbly, partly calcareous, slates overlie dark grey slates and shales with one or two thin seams of igneous rock. These beds contain occasional crinoids, Zapbrentis, etc., and at the south end of Crownhill Bay give place to a slaty calcareous series with limestone bands containing Monticuliporoid corals, sandy decomposed red rocks with indeterminable brachiopods in abundance, and hard red grits, corresponding to the Looe rocks. These near Andurn Point are in faulted junction with glossy red and greenish Dartmouth slates. 


\section{GEOLOGY}

The Dartmouth slates, intercalated at intervals with beds of hard grit and quartzite (which on the Revelstoke coast display numerous contortions), extend to a little beyond Hoist Point. Near Piskeys Cove Pteraspis was found in them. Diabases and volcanic rocks occur in them at the mouth of the Erme and in other places.

Near Hoist Point the Dartmouth slates give place to dark grey, locally red stained, slates and shales with siliceous bands, crinoidal limestone films and a horizon of crushed brachiopods. These have been called the Ringmore beds; they are in plicated association with dark grey slates, locally red-stained, in which Pteraspis was found at Ayrmer Cove by Brook-Fox. At the bend in the Avon, near its mouth, the calcareous fossiliferous bands come in again, with (?) Monticuliporoid remains, near to an inlier of Dartmouth slates. From Avonmouth to Hope the section consists of dark grey slates with siliceous bands in places and occasional traces of fossils. Bands of igneous rock of the Torcross type occur in places. Interlaminated beds and slaty fine grits with calcareous and hard carbonate bands come on at Beacon Point and Woolman's Point. The Beacon Point rocks traced across the promontory exhibit developments of grit, most conspicuous north of Malborough and near Beeson; their horizon is everywhere separated from the altered rocks by dark grey slates without igneous bands. Throughout this section there is a constant repetition of horizons by plication, and there are numerous faults.

The eastern coast proceeding from the altered rocks northward begins with dark slates, as at Hope, overlying interlaminated beds which contain crinoidal limestone films, at Tinsey Head, and hard grit beds, at Beesands. The Tinsey Head and Beesands beds correspond to the Beacon Point and Woolman's Point beds. The only doubt as to the succession in these two sections is whether these rocks are a normal outcrop or an anticlinal repetition of the Ringmore beds. In either case, as every member of the series has its representative in the Looe area, the rocks must be regarded as parts of the Meadfoot series. On the east coast the Ringmore beds are not well shown. From Strete Gate to Scabbacombe Head the Dartmouth slates form the cliffs with igneous rocks here and there and grit beds.

Proceeding southward from their faulted junction with the Middle Devonian, south of Berry Head, the Lower Devonian section commences with Staddon grits, grits and sandstones associated with slates and shales, succeeded by dark slates with masses of dense fine grit, probably the top of the Meadfoot series. In Southdown cliffs these give place to slates, locally reddish, with very occasional crinoidal films and a seam of igneous rock. On the south of Man Sands dark slates and interlaminated beds with pale greenish igneous rocks of the Torcross type are in plicated association with dark grey and red shales and slates with seams of crinoidal limestone with (?) Monticuliporoid corals corresponding to the Ringmore beds. At Long Sands a mass of dense thick-bedded fine grit comes in. Whether this is a repetition by fault or fold of the grits in the 


\section{A HISTORY OF DEVONSHIRE}

north part of Southdown Cliff, or a local development, is not clear. The dark slates on either side of it contain hard black patches, in one of which on the north side Pteraspis structure was visible. At Scabbacombe Sands the Ringmore beds reappear-dark grey and red shales. and slates with siliceous bands, grits, and crinoidal films, also lenticular red limestone, identical with limestone on East Looe beach (which is characterized by irregular calc spar patches, probably Monticuliporoid corals). The junction of these beds with the Dartmouth slates is inaccessible, and probably a fault.

Though faults or thrusts have the effect of causing sections in the same rocks to exhibit differences, on the whole the correspondence of the beds on the north with those on the south side of the Dartmouth slates is too complete to be due to accidental similarity in different groups. The Dartmouth slates, so named by Sedgwick, are therefore the oldest Devonian rocks in south Devon and occur in an anticline. Their northern boundary is faulted between Plymouth and Modbury and also near Dartmouth, but near East Allington their persistence is rendered doubtful by the flattening out of the main structure in a series of folds. They consist of slates, shales often silty, with occasional hard grit beds, and exhibit pink, red, green and purple blended tints, but over a large part of their extension their colours are not to be clearly distinguished from those of higher horizons. Their connection with the Pteraspis beds of Polperro has been traced through the intervening area, but their continuity across Cornwall to Watergate Bay where Pteraspis was found in them by Howard Fox is doubtful. Pteraspis has been found at Higher Ford near Wembury, and obscure fish remains in Strete Cliffs. The occurrence of Pteraspis in dark slates on the north of Long Sands and near Ayrmer Cove renders the range of that fossil above the Dartmouth slates very indefinite. The Dartmouth slates are somewhat similar in character to the Gedinnien slates of the Rhine section and to the glossy Gedinnien slates of the Ardennes, and they appear to be homotaxeous with them.

Under the term Meadfoot beds are embraced rocks corresponding to the Taunus quartzite, etc., rocks of Lower Coblenzien, and possibly in part of Upper Coblenzien age, so unsatisfactory is their boundary with the Staddon grits, which are Upper Coblenzien. The types of the lower beds are taken from the Looe district in east Cornwall, which presents resemblances to all the varieties of rocks in natural or faulted contact with the Dartmouth slates in Devon. The Looe fauna is mainly characterized by such forms as Spirifer primarvus, Ortbis bipparionyx, Rbynchonella pengellyana, Streptorbynchus gigas and Pleurodictyum, and by irregular calcite patches in the lenticular limestones referred to in all the Devon sections as (?) Monticuliporoid corals. These rocks are cut out by the fault boundary of the Dartmouth slates between Membland and Wembury; they can in no case be separated from the Meadfoot beds. The most fossiliferous rocks, or rather the best preserved fossils, are found in the fault-broken Torquay anticline and in the larger Paignton 


\section{GEOLOGY}

anticline, in which districts the Dartmouth slates are not represented. Spirifer primavus and Homalonotus remeri (?) were found near Hope's Nose. Leptana looensis was doubtfully recognized at Hope farm, Ortbis bipparionyx in the New Cut and the New Drive, with spined Homalonoti. Rbynchonella pengellyana was doubtfully recognized with Leptana looensis by Davidson in the Saltern Cove railway cutting. Spirifer primaevus was found in the Ringmore beds. Amongst the Coblenzian fossils Stropbomena latecosta (= Tropidoleptus rbenanus), Chonetes sordida, C. plebeja are common, Renssellaria strigiceps occurs in Plymouth and Torquay districts. Spirifer macropterus is said to occur at Meadfoot beach. Bellerophon trilobatus, with spined Homalonoti, has been found near Halwell (south-south-east of Stanborough House), also at Torquay. Tentaculites occur in the higher beds near Plymouth, also on Warberry Hill, Torquay, where Beyricbia wilckensiana was found. Spirifer bystericus was obtained near Cockington, but the identification of Spirifer cultrijugatus near Kilmorey is very doubtful. Zaphrentid corals and crinoids are the most persistent forms throughout the extension of the Meadfoot beds, but are very seldom found in the Staddon grits. Pleurodictyum is not confined to any particular horizon above the Dartmouth slates, and ranges into the overlying Middle Devonian (Eifelian slates). Through decalcification and crushing the fossils of the Lower Devonian rocks between the Brixham and Plymouth coasts are seldom specifically determinable.

In their main outcrop the Staddon grits are so unfossiliferous that it is by no means certain whether the fossiliferous red beds of Lincombe Hill should be classed with them or regarded as red stained Meadfoot beds. The Meadfoot beds contain irregular, knubbly, partly calcareous slates which resemble those of the Lynton beds of north Devon, and the Staddon grits present many lithological similarities to grits in the Hangman group.

Middle Devonian.-The lower (or Eifelian) Middle Devonian slates present two types, of which the irregular slates of Berry Park (north of Totnes) and the more even slates of Mudstone Bay are examples. In the slate areas between Plymouth and Totnes limestone bands are locally encountered, as at Ugborough, at the base of the volcanic rocks. In the limestone areas thin-bedded and slaty, or shaly, limestones pass down into the slates by intercalation, as at Daddyhole and Hope's Nose. Sometimes these limestones are separated from the limestone above by slates and volcanic rocks, as at Batten Bay, Plymouth.

The characteristic fossil of these beds is Caleola sandalina. It is very scarce, having been found at Daddyhole, and at Paytoe, or Peloe (east of South Brent), where dark limestones have been locally developed in the slates-and it has been said to occur at Chircombe Bridge. On the borders of the great volcanic development (the Ashprington series of Champernowne) south of Totnes, limestones of this series, often crowded with rugose corals such as Cyatbopbyllum damnoniense, $C$. beliantboides, and Cystipbyllum vesiculosum, locally intervene between the volcanic rocks and the slates below. 


\section{A HISTORY OF DEVONSHIRE}

Amongst the fossils of the Eifelian limestones and slates are the following: Phacops latifrons, Gryphaus punctatus, Cyphaspis, Rbynchonella procuboides, Kayseria lens, Pentamerus galeatus, Productus subaculeatus, Spirifer speciosus, S. curvatus, Atrypa reticularis, A. aspera, A. desquamata, Cyrtina wbidbornei, Heliaster filiciformis and Heliolites porosus.

The Ashprington volcanic series occupies an area of about ten square miles, between Dittisham and Totnes, and consists of shalsteins (embracing tuffs and lavas), sheared diabases often vesicular, with intrusive masses of aphanitic and porphyritic diabase. The eruptions began during, and just prior to, the deposition of the Eifelian limestones and probably continued into the Upper Devonian period, as we find the limestone, which is mostly of coralline growth, interrupted by local vulcanicity in the upper part near Goodrington and at Black Head, Torquay, and at lower stages in different parts of the limestone districts, according to their proximity to local centres of vulcanicity in action at different times during the period represented by the volcanic rocks of the Ashprington series.

The limestone masses passed out irregularly on their borders into slates or slates with volcanic rocks. The Torquay and Babbacombe bedded limestones, partly represented by the Marldon, Little Hempston, and Dartington limestones, belong to the series immediately overlying the Eifelian, and above these come massive limestones such as those of Lummaton, Kingskerswell, the Daisons. In fact, where calcareous growth has been uninterrupted, the upper beds have a more or less massive character, and the lower a more or less evenly, or distinctly, bedded aspect. The south Devon limestones are locally dolomitic. They are rich in Stromatopora, favositid corals, and Alveolites. Brachiopods are most plentiful in the lower and upper horizons.

The Continental boundary between the basement (Rbynchonella cuboides) zone of the Upper Devonian and the Stringocephalus, or Middle Devonian, limestone should be taken in the upper massive limestones. In the Lummaton limestone the shelly material is very restricted but very prolific; in it Whidborne records the discovery of 106 specimens of Rhynchonella cuboides and 25 of Stringocepbalus burtini; at Woolborough 42 of the former, and 18 of the latter. Rbynchonella cuboides has also been found at Barton near Lummaton, at Langs Copse in the Bradley Woods, and at Lower Dunscombe. The Ramsleigh (with Acervularia pentagona), Petitor, Ilsham, Kersewell Down, upper part of Kingsteignton (north of), and upper part of the Brixham limestones belong to the same category, in which the Goodrington (Favosites cervicornis) bedded limestones must also be included, and perhaps part of the Plymouth limestone near Plymstock station.

Upper Devonian. - The relations of the Middle and Upper Devonian strata are rendered much more complex by the resistance offered by the Dartmoor granite to the forces which compressed and contorted them. Thus from Plymouth northward to Tavistock is a series of slates very unfossiliferous which from their characters seem mainly to belong to the 


\section{GEOLOGY}

Upper Devonian, although the existence in Plymouth Museum of a specimen of slate with Pleurodictyum, from Cann quarry (south of Bickleigh), renders it probable that Middle Devonian slates may be folded up with them, that fossil having been found in Eifelian slates at Bishopsteignton and near Marldon. Between the southern spur of Dartmoor, at Ivybridge, and the Middle Devonian shalsteins the occurrence of Upper Devonian is doubtful, as the slates are in line of deflected strike with those including the Eifelian limestone of Paytoe. To the north of this, and east of Buckfastleigh, there is no doubt as to the occurrence of Upper Devonian slates, in the area between the Buckfastleigh and Ashburton limestones and those of Ogwell, Denbury and Torbryan; imperfect indications of the Büdesheim fauna (small Goniatites and Bactrites) having been obtained at Wrigwell House, and Entomis serrato-striata, etc., near Highweek, at Knowles Hill, Newton Abbot, and also in the railway cutting near Kingsteignton. The Ashburton are, therefore, separated from the Newton Abbot limestones by a syncline. Ciardiola retrostriata and other Frasnien forms have been obtained in nodules in the slates (Knollen Kalk) by the Teign near Combe Cellars. Liver-coloured shaly limestones and concretionary limestone beds are found in contact with the massive limestones of Chudleigh, Kingsteignton, Petitor, Ilsham. Goniatites such as $G$. intumescens and $G$. sagittarius have been found in them at Lower Dunscombe; near Whiteway farm; and at Petitor (where they mark the sides of a syncline occupied by Upper Devonian slates). At Anstey's Cove the slates contain Entomis serratostriata and also at Goodrington. The Büdesheim fauna discovered at Saltern Cove by Mr. Lee is well known; the same fauna occurs in similar beds in contact with the Brixham limestone near Ivy Cove. Upper Devonian slates are faulted and folded up in the Lower Culm Measures north of Bickington. Entoms, Clymenia, etc., occur in them. At Druid and Holne Bridge near Ashburton Spirifer disjunctus is found in greenish Upper Devonian slates and mudstones, which have their counterparts near Beer Ferrers, on the Tavy, where the same fossil has been found. On the whole the Upper Devonian strata are very unfossiliferous-the prevalent tint is pale greenish grey but Indian red and green slates of the Mutley type are often present, especially in the Torquay, Newton and Paignton districts.

\section{DEVONIAN OF NORTH DEVON}

The succession of these strata is so well given in the section in De la Beche's Report, ${ }^{1}$ that it would hardly be necessary to refer to them after the classification given in the table, but for the older date assigned to the Morte slates.

Lower Devonian.-The thick and thin bedded quartzose grits of the Foreland with interbedded claret coloured slates form the lowest series and are homotaxeous with the Dartmouth slates. Seen from the sea these rocks pass under the Lynton beds, but their junction is a fault in the

\footnotetext{
1 Described on Pp. 46-53.
} 


\section{A HISTORY OF DEVONSHIRE}

Lynn valley, on the north side of which, near Oare (in west Somerset), a patch of Lynton beds rests upon them.

The Lynton beds consist of bluish-grey irregular slates, partly calcareous and interstratified with even bedded grits; they contain decomposed brown patches with fossil casts. Slates and grits of similar character occur in the Meadfoot beds with which the Lynton beds are homotaxeous. Amongst the badly preserved fossils recorded from these beds Valpy ${ }^{1}$ mentions Tentaculites scalaris, and numerous casts of Megalodon cucullatum as occurring at Woodabay, and Kayser ${ }^{2}$ obtained Spirifer bystericus and Cbonetes sarcinulata in the Valley of Rocks, Lynton. The Lynton beds pass under the Hangman group, the boundary runs from the coast through Martinhoe, to the south of Barbrick Mill and by Farley, crossing the Somerset boundary rather more than a mile south of Malmsmead.

The Hangman grits consist of brownish, green, buff and, locally, purplish-red mudstones and slates with grits and flaggy sandstones (the Trentishoe beds). Grits are prevalent in the upper beds, which contain types met with in the Staddon grits. Natica and Myalina have been found in red grits in the upper part of the series. They are homotaxeous with the Staddon grits, but it does not necessarily follow that they may not be in part of Eifelian age. Stringocepbalus burtini has been found in the slates with grit beds which overlie them in West Challacombe Bay and form the base of the Ilfracombe slates. The boundary runs on the north of Holstone and Parracombe to Oare Oak on the county boundary.

Middle Devonian.-The Ilfracombe slates are dull grey and silver grey with occasional seams, lenticles and beds of limestone, and with intercalated grits toward their base. The calcareous beds contain a characteristic Middle Devonian fauna. Valpy records however Renssellaria strigiceps, Tentaculites scalaris, and in his general list gives Steganodictyum cornubicum (of McCoy-now recognized as Pteraspis) and $R$ byncbonella cuboides (more probably $R$. procuboides) with such characteristic Middle Devonian forms as Cystipbyllum vesiculosum, Heliolites porosus and Stringocephalus burtini. In the Lynton railway cuttings no boundary could be drawn between the Ilfracombe slates and the Morte slates.

Morte Slates. - The lower beds of the Morte slates contain hard brown siliceous bands, and are succeeded by hard greenish slates and slaty mudstones. On the coast the slates make jagged reefs and are intersected by quartz veins. Gritty beds occur in the lower portion at Slade and Flat Point; these were regarded by Hicks as a repetition of the lower, or Challacombe Bay, beds of the Ilfracombe group, the in Tervening beds being treated as a syncline. Valpy was informed by a workman that fossil casts had been found at Slade. Hicks' fault boundaries have not been discovered by the upholders of the normal sequence, but, in view of the Lower Devonian age claimed for the

1 Notes on the Geology of Ilfracombe and the Neigbbourbood (Twiss \& Sons, Ilfracombe. Not dated).

2 Neu Fabrb. fïr Mineralogie (1889), vol. i. p. 182. 


\section{GEOLOGY}

fossils found at Treborough (in west Somerset) and recorded in the second part of his paper, it is quite possible that a detailed survey might justify his views. Stricklandinia lirata and Ortbis rustica with some new species including Lingula mortensis were found by him in Mullacot quarry, south of Ilfracombe. The Morte slates pass under the overlying Pickwell Down grits at Foxhunters Inn by the Ilfracombe railway. From the coast, on the south side of the Woolacombe valley, the junction can be easily traced by West Down to the vicinity of Arlington, where it is shifted by a south-easterly fault to Button farm, on the north of Bratton Fleming, and thence runs westward by Office farm to the Somerset boundary at Span Head. An intrusive felsite occurs in the Morte slates, near, or at, their junction with the Pickwell Down grits south of Bittadon," on the south of Hewish Down, at Smitha Park, Button Wood, and Office farm.

Upper Devonian.-The Pickwell Down grits consist of dull purple, red and greenish grits, sandstones, slates and mudstones, and have only yielded ligneous traces. The Baggy beds consist of greenish slates containing Lingula, overlain by brown micaceous sandstones and flags containing Cuculloa.

The Pilton beds consist of grey or dull greenish argillaceous slates, with intercalated beds and masses of brownish grit. They contain lenticles of fossiliferous limestone, now mostly represented by brown friable residua in which an abundant Upper Devonian fauna has been obtained by Professor Phillips, T. M. Hall and subsequent observers.

The Pickwell Down beds have been taken as the base of the Upper Devonian. At North Molton Ridge a synclinal in this series terminates, carrying the junction of the Pickwell Down and Baggy beds to East Buckland, whence (probably as a fault) it runs by Molland to Dulverton, crossing the county boundary near East Lipscomb farm.

The junction of the Pilton beds with the Culm Measures is seldom exposed in section; through the irregularity of the county boundary this line crosses it in three places, viz. near East Anstey, near Perry (east of Brushford), and finally near Clayhanger. The relations of the Culm and Upper Devonian are frequently faulted between Barnstaple and Clayhanger.

\section{CARBONIFEROUS}

As the relations of the Culm rocks, west of a line drawn from Hartland Point to Okehampton and nearly as far south as Tavistock, have never been systematically investigated, the lines on the map are generalized suggestions, especially in the Lower Culm area where Upper Devonian slates and volcanic rocks and Lower and Middle Culm Measures are known to be in plicated and faulted association. The absence of volcanic rocks in the Devonian and Lower Culm Measures of the northern outcrop, and their presence, together with masses of intrusive greenstone (on either side of Dartmoor, but chiefly on the west), in

1 Bonney, Geol. Mag. 1878 , p. 207. 


\section{A HISTORY OF DEVONSHIRE}

the southern outcrop of these rocks, render the relations of the Lower Culm in the latter area much more complex than in the former.

Lower Gulm.-In both areas where the succession is complete the upper beds consist of shales with hard mudstones with limestone bands. In the northern outcrop this subdivision sometimes mainly consists of shales with mudstones; sometimes of shales, mudstones and limestones; sometimes, as in the Westleigh quarries, chiefly of limestone, in which chert from the segregation of silex is locally met with, as well as dolomitic rock. In the southern outcrop, from the Tamar to Doddiscombsleigh, this horizon is distinguished by blackish shales with hard even mudstones and occasional developments of limestone ; it makes a plicated sinuous junction with the overlying grits and shales which carries it southward round Dartmoor. It is recognizable in the inliers in the Middle Culm rocks north of Kingsteignton and near Bickington. Posidonomya becheri is the characteristic fossil of these beds but Goniatites spiralis is even more plentiful in the Chudleigh district, especially near Waddon Barton.

Below the Posidonomya beds come the Coddon Hill chert beds; these and all the Lower Culm cherts of this horizon collected by Fox and examined by Dr. Hinde yielded Radiolaria. On the northern outcrop these beds are generally parti-coloured, grey, pale buff and white; on the southern outcrop they are pale grey, dark grey, or black. Near Bickington they form the base of the Culm, and occur as outliers on the Upper Devonian near Tamerton Folliott.

The thickness of the chert beds is variable, and there is reason to think that this horizon is locally represented by bands of chert in dark shales and mudstones. It is so plicated that it is difficult to believe that its maximum development attains to 100 feet.

The basement beds of the Culm consist of dark shales with occasional seams of chert or mudstone in the northern outcrop, and with hard even bedded, sometimes pale banded, mudstone in the upper part in south Devon, where present. Contemporaneous volcanic rocks seem to be associated with these beds in the Tavistock area. In the Ashton and Trusham district contemporaneous tuffs, etc., come in, in, or just above, the chert beds.

Middle Culm.-The Middle Culm Measures are a variable series; they contain interbedded (thin or thick) grits and shales; irregularly associated hard, even and irregular, developments of grit in shales; evenbedded sandstones and marly-splitting sandy shales; coarse and fine greywacke locally conglomeratic, associated with dark uneven shale in variable proportion.

Their lower beds vary considerably in different parts of the area. Between Crediton and Chudleigh they consist of hard, thin, even bedded grits intercalated in splintery shales; in the northern outcrop, of intercalations of even bedded grits (with anthracite seams near Bideford, Alverdiscot, etc.), sandstones and shales. On the south of Chudleigh the sandstone and greywacke type prevails. In Ugbrooke Park and 


\section{GEOLOGY}

the district south of Oldchard and Ideford, and in the faulted outliers between Newton Abbot and Ipplepen, beds of coarse and fine greywacke, associated with dark argillaceous shales, contain conglomerates in which fragments from the Lower Culm cherts and hard mudstones are distinguishable in association with materials derived from granitoid and volcanic rocks. These beds rest on the upper horizon of the Lower Culm, but sometimes on dark shales which may be locally their own lower horizon.

At Efford and Lower Compton (near Plymouth) even beds of greywacke with shaly partings and occasional small dark shale fragments rest on dark shales, upon Upper Devonian slates associated with volcanic rocks. The greywackes are generally coarse and are largely made up of materials derived from gneissose, and many varieties of volcanic rocks.

At Tavistock dark Middle Culm greywacke displays a similar derivation. At Beer Alston station a patch of grey chert underlies an outlier of plant-bearing greywacke and shales. This evidence is corroborated by the greywackes of Wearde (south of Saltash) which rest on Upper Devonian slates and volcanic rocks. It proves conclusively (unless we regard the unconformable greywacke as contemporaneous volcanic grits of Upper Devonian or Lower Culm age) that a considerable unconformity exists in part of south Devon and southern Cornwall between the Middle and Lower Culm, accounting for the complete destruction of the latter and the incorporation of its materials, together with volcanic rocks in abundance, in the newer sediments. Whilst the Middle and Upper Culm rocks contain plants, said to connect them with the calciferous sandstones of the north, the fine grained sediments and Radiolarian cherts of the Lower Culm denote deep water. It is therefore less remarkable that there should be a marked unconformity between them, than that their succession should be so uniform and regular over the greater part of the Culm area. Whether this is due to a gradual elevation not shared by those parts of the sea bed where the crust had been weakened by igneous intrusions and late Devonian and early Carboniferous volcanoes (such as Brent Tor), or to irregularities in the sea bed, due to the outpouring of igneous rocks from such volcanoes and to disturbance thereby of existing sediments, opens a wide field for conjecture.

Upper Culm.-The Upper Culm Measures, owing to their massive even bedded character, dark shale partings and pale grey colour, exhibit, in the coast section near Clovelly and in the exposures by the river Torridge, more beautifully than any other series of rocks in Devon the numerous curves that prevail everywhere throughout the Palæozoic area. This ability to undergo contortion without snapping makes their chief value as the index of the axis of the great Palæozoic trough and amply justifies their separation from the Middle Culm.

Although the term greywacke might be applied to most of the Culm and many of the Devonian grits its use is here restricted to rocks of a markedly felspathic character and betraying distinct derivation from volcanic as well as sedimentary materials. 


\section{A HISTORY OF DEVONSHIRE}

\section{IGNEOUS ROCKS IN THE PALÆOZOIC AREA}

The igneous rocks in the Palæozoic area may be divided into four classes: the contemporaneous volcanic rocks, rocks which may be either contemporaneous or intrusive, intrusive greenstones, intrusive felsites.

The first two classes are confined to south Devon.

There can be no doubt that many of the sheared rocks in the Dartmouth slates are contemporaneous; of this class a rhyolitic felsite at the south end of Blackpool Sands (near Stoke Fleming) and an amygdaloidal diabase at Muxham Point (Erme Mouth), both displaying well marked fluxion structure, are examples. In the Meadfoot group the Torcross type (apparently a modified diabase) is often of doubtful origin, but diabase porphyrite quarried south of East Charleton and many of the sheared bands in the Kingsbridge creeks and elsewhere are evidently contemporaneous. In the Middle Devonian and Upper Devonian (more locally) the contemporaneous rocks include tuffs, shalsteins, amygdaloidal and slaty diabase, or diabase tuffs. In the Ashton and Tavistock districts there are also volcanic rocks in the Lower Culm. Near Devonport Workhouse and south-east of Plympton there are examples of intrusive and contemporaneous basalts. Intrusive Greenstones are very numerous especially in the Middle and Upper Devonian, and in the Lower Culm locally, but the possibility of some of them being contemporaneous volcanic rocks must be admitted.

Examples of epidiorite occur among altered Lower Culm rocks on Cox Tor near Tavistock, east of Canonteign and south-west of Bagtor in the Chudleigh area.

Dolerites may be seen in the Middle Devonian in Borough quarry (west-north-west of Denbury), Yarner Beacon near Dartington, in the Lower Devonian at Capton near Morleigh ; ophitic dolerite in Lower Culm, north of Place Barton (near Ashton), south of Christow ; in Upper Devonian on Knowles Hill (Newton Abbot) ; in Middle Devonian near Lower Ware (Kingsteignton) and south of Dittisham corn mill, etc.

Leucophyre.-In Lower Culm east of Huish near Hennock, and railway cutting north of Ashton. ington.

Diorite.-In Lower Culm south of Standcombe farm near Bick-

Porphyritic diabase.-Dunhill quarry south of Brent, very common in Middle Devonian.

Aphanite is also very common in the Middle Devonian. Granular diabase is met with in Middle and Upper Devonian. An example occurs at Carey Arms (Babbacombe).

At Fremington Pill in north Devon in the Lower Culm there is a dyke of highly altered basalt (specimen identified by Teall).

Dr. Busz ${ }^{1}$ classes the Botter Rock near Hennock with the leucophyres. He also notes a Labrador porphyry allied to the leucophyres

2 Sitz d. Niederrbeinische Gesell. im Bonn. 1893, p. 83. 


\section{GEOLOGY}

on Babbacombe beach, and near the granite at South Brent a (quartzmica-diorite) kersantite.

Lamprophyres are met with in the Culm at Rose Ash in north Devon. In the Upper or Middle Devonian on the east of Ingsdon House.

Some dykes of decomposed rocks allied to the Exeter traps occur in the Lower Culm north of Kitton Barton (south of Ashbrittle).

Felsites.-Felsitic dykes occur between Tavistock and Plymouth in Cann Wood near Bickleigh, on Roborough Down near Walkhampton, at Horrabridge, Lopwell and Morwell Down, in Upper Devonian slates. Between Modbury and Kingston at Whympston Wood there is a spherulitic granophyre and several exposures of spherulitic banded and nodular felsites near Tor, Wastor and Shearlangston. Small exposures of felsite are met with in Putshill Copse and near Mounts east-north-east of Woodleigh (? of date of Permian eruptions). The Bittadon felsite in the Morte slates has been alluded to. Decomposed elvans occur in Middle Culm rocks on Itton Moor, at Gribbleford Bridge, etc., on the north of Dartmoor. At Meldon and in Cann quarry white granite dykes, or aplites, are visible. Near Christow there is a small mass of quartzporphyry. Near Woolley west of Bovey Tracey a rhyolitic rock borders the granite.

\section{GRANITE}

The Dartmoor granite is about 225 square miles in extent. It is, generally speaking, a coarsely porphyritic granite with large orthoclase felspars, quartz, biotite and muscovite, and is more or less schorlaceous especially towards the contact margin. At Trowlesworthy Tor a red granite, consisting of red felspar schorl and fluorspar, has been noted by Worth. ${ }^{1} \quad$ Finer grained granite is met with, here and there, in intrusive elvans very difficult to trace, sometimes in nearly horizontal bands, sometimes in small patches, and sometimes at the margin from which it passes into the coarser variety. MacMahon ${ }^{2}$ mentions the Tavy valley between Hill Town and White Tor as a good locality for studying the relations of the coarse and fine granites, which here suggest ' the imperfect mixing of two portions of the granite magma in different conditions of fluidity.' Rutley ${ }^{\frac{3}{3}}$ described a junction at Brazen Tor where amphibolite is in contact with schorl-spotted fine grained granite passing within 100 yards into porphyritic granite.

Dr. Busz noted three varieties of granite in contact with Culm hornfels in a quarry near Bovey Tracey-coarse granite with large orthoclase crystals, fine grained granite, and rock with large crystals of plagioclase yellowish orthoclase and large quartz grains in a fine grained grey matrix.

The presence of schorl, and of fluid inclusions in the quartzes, has been shown by Hunt to be characteristic of the Dartmoor granite, and

1 Rowe, A perambulation of Dartmoor (third edition revised, etc., 1896), p. 244.

2 Quart. Fourn. Geol. Soc. vol. xlix. p. 389.

3 Geol. Survey 'Memoir on Brent Tor,' p. 38.

- Geol. Mag. June, 1899, p. 256. 


\section{A HISTORY OF DEVONSHIRE}

in this and other respects it differs markedly from the granitoid fragments in the Middle Culm rocks. At Clasiwell Pool south of Princetown the granite is kaolinized, also at Shipley Bridge and Leftlake (north-west of Brent) where there were formerly china clay works.

West of Cornwood there are two isolated masses of granite; the smaller is Hemerdon Ball ; the larger, on Headon Down, is worked for china clay, also worked on the adjacent part of the main mass on Lee Moor, and near Cadover Bridge on the Plym.

The Dartmoor granite is divided laterally by impersistent planes which give it an appearance of stratification with dips toward the surrounding rocks. The tors are due to the intersection of these planes by more or less vertical or inclined joints.

Contact Alteration.-From its rather regular northern boundary and greatest axial breadth the granite extends southward with most irregular boundaries. Its longest axis is from north to south. The limit of alteration or contact zone seldom exceeds a mile in breadth, but is often less, the breadth being determined by the subterranean slope of the granite. Within this zone are Culm rocks, chiefly Lower Culm, from Tavistock round the north margin to Holne on the east. Upper Devonian slates occur on Wallaford Down and, except perhaps near Dousland, from Cornwood to Tavistock. The cleavage or schistosity of the rocks was affected by dynamic agencies before metamorphism.

MacMahon ${ }^{1}$ has described felsites and rhyolites at Sourton Tors, a mica diorite at South Down, and tuffs with matrix rendered similar by contact-metamorphism to the base of a rhyolite from Sourton Tors and from Meldon. On the flank of Cox Tor 'beds of fine grained volcanic dust' have been 'converted into a mixture of augite and felspar.' The dolerites have been converted into epidiorites. Banded dark green and creamcoloured rocks occur on Peak Hill near Dousland. These were examined by Teall, who considered their original character as probably decomposed igneous rocks containing carbonate. Similar banded rocks with a porcellanized appearance occur near Ivybridge, Wrangaton and South Brent ; and in their vicinity dark rocks with chiastolite are met with above Ivybridge and in the contact zone near South Brent. It is probable therefore that Culm and Upper Devonian rocks with associated volcanic rocks occur on the margin of the granite in these localities in which there are also intrusive greenstone masses. An example of the latter near Dousland was considered by Teall to have been originally an ophitic dolerite. Garnets are found at Shaugh and Peak Hill amongst other localities, axinite near Sourton, Walkhampton and South Brent, etc. Teall detected a foliated scapolite pyroxene rock amongst the altered rocks near Dousland. ${ }^{2}$ The Upper Devonian slates usually exhibit a spotted alteration on the west of Dartmoor and become mica schists.

Dr. Busz ${ }^{3}$ described the contact zone by the Avon above South Brent as composed of andalusite hornfels, andalusite schist, chiastolite

1 Quart. Fourn. Geol. Soc. vol. 1. p. 338.

2 Proc. Somerset Arch. and Nat. Hist. Soc. (1900), vol, xlvi. ${ }^{3}$ Op. cit, 


\section{GEOLOGY}

schist, knoten glimmerschiefer, actinolite hornfels and actinolite schist, with a dyke (gang), several metres in thickness, of garnet rock with actinolite and axinite.

Variously claimed as a post-carboniferous plutonic intrusion, a Permian volcano, ${ }^{1}$ an upthrust in the period before the deposition of the Middle Culm, ${ }^{2}$ or as an ancient granite ${ }^{3}$ modified amongst its surroundings at a comparatively late stage in the post-carboniferous movements (during a rise in the isogeotherms accompanied by the injection of elvan dykes), the Dartmoor granite, owing to its size and shape and the manifest effect its presence has had on the strikes and distribution of the surrounding rocks, remains the sphinx of Devon geology.

\section{NEW RED SANDSTONE SERIES}

The coast section exhibits numerous faults in the New Red rocks; these in the Lower breccias are often accompanied by high dips which become lower toward the east. In inland districts faults can only be traced by the displacement of lithologically distinguishable horizons.

The Lower New Red rocks show a remarkable attenuation toward Exeter, and further north their development is exceedingly irregular ; in rounding the spurs or inliers of the Culm rocks (at Pinhoe, Spraydowns near Collumpton, at Westleigh and Whipcots) being so feeble and inadequate as to suggest either a faulted or unconformable junction with the overlying Marls, rather than the outward replacement of coarser by finer sediment.

Watcombe Clays.-These consist of red rather silty finely micaceous clays or marls, with intercalated beds of comminuted slate in the lower part, exposed in Petitor Combe, and overlain by about 20 feet of similar material occasionally showing annelid tracks, where they pass under the conglomerates in Watcombe Combe. These clays lose their distinctive characters, becoming a loamy or clayey breccia, when traced inland. Their thickness on the coast may be about 150 feet.

Watcombe Conglomerate. - These beds are typically exposed in Watcombe and Petitor Crags. They are characterized by large well worn fragments of Devonian limestones associated with grit, quartz, and some igneous materials in a sandy matrix. Contemporaneous erosion is shown in them in Oddicombe Cliffs, where they contain thick beds of sand. If these rocks extend north of the Teign estuary they are represented by sand or sandstone about 20 or 30 feet thick at Bishopsteignton. On the coast they can scarcely be less than 500 feet. They seem to be lower in the series than the earliest evidences of contemporaneous trap. They pass under the next series at Mincombe and form the base of the cliff at the Ness.

Teignmouth or Boulder Breccias.-These beds form a brecciated and boulder-studded red sandy loam. The boulders are chiefly of quartz-

1 Worth, Trans. Plymouth Instit. (1 888-9).

2 Somervail, Report Brit. Assoc. Bristol (1898) and Geol. Mag. (Dec. 4), vol. v. p. 509.

3 Proc. Somerset Arcb. etc. (1892), vol. xxyviii. 


\section{A HISTORY OF DEVONSHIRE}

porphyry (of a type hitherto only met with near Christow on the border of Dartmoor). The stones include a great variety of altered and igneous rocks. The quartz-porphyry boulders are sometimes almost unworn and so large that they must have been derived from rocks in their immediate vicinity. These rubbly breccias locally contain harder beds of breccia or breccio-conglomerate with limestone pebbles, In 1887 a boring for water at Combe, near Teignmouth, was carried to a depth of $33^{2}$ feet in them. They overlie the trap (quartz basalt) patches of Dunchideock, Knowl and Westown.

Darvlish Breccia and Sands.-At Holcombe Tunnel the rubbly Boulder Breccias are succeeded by the Dawlish Breccias-the Murchisonite beds of Ormerod-beds of sand-rock thickly studded with rather small fragments of altered and igneous rocks and murchisonite, with occasional coarser bands of breccia. The breccias of the Crediton valley are of this type. These rocks contain intercalations of sandstone and vary from sand to breccia most irregularly along the coast from Dawlish to Exmouth. The materials become differentiated toward Exeter and form an upper series of sands at Topsham, upon the Heavitree breccias, the basement beds being brecciated loam and sand, which overlie the trap rocks of Pocombe and Northernhay. The sands of Broadclist and Poltimore seem to be the upper beds of the series, and there are several patches of trap associated with them.'

With the narrowing of the Crediton valley at Newton St. Cyres the sands give place to breccia full of igneous fragments, which overlies the trap patches of Posbury, Halse and Greenslade on the south, and of Knowle and Spencecombe on the north side of the valley. These trap patches rest on sand and breccia, which on the north side of the valley seems to be largely formed of tuffs. A similar phenomenon is observable under the Washfield traps.

The gravelly breccias occur over sands in the Spraydown Culm inlier, and are found to pass from the Culm highlands irregularly outward into sand, often through breccia or brecciated sands. They contain fossiliferous stones (Stropbalosia caperata, Rbyncbonella pleurodon), from the Upper Devonian area on the north, in the railway cuttings of the Exe valley line north of Tiverton. ${ }^{2}$ The Rev. W. Downes attributed these stones to volcanic ejectamenta, and pointed out the presence of fragments referable to the Washfield traps in the cuttings near Bolham. At Coleford Lodge near the margin of the Stoodleigh Beacon outlier a small patch of trap seems to rest on the gravel. The Upper Devonian fragments, also found in the gravels near Cadbury, Uplowman and Silverton, point to the explanation given in the introductory notes (see p. 5). The Halberton breccias seem to be the upper beds of the Lower New Red in that neighbourhood. The strikes of the Lower New Red rocks, as might be expected from the trend of the creeks, etc., in the older rocks, are more

1 For relations and petrology of trap rocks, see Geol. Survey Memoir on the country around Exeter (1902), chap. iv.

2 Downes, Trans. Devon Assoc. for 1881 , pp. 293-7. 


\section{GEOLOGY}

often east and west than north and south. The developments of breccia in the Crediton valley, and of the older boulder breccias south of Ide, are to be expected in areas of active vulcanicity, where deposition was taking place. The association of rhyolitic rock with andesite ${ }^{1}$ overlain by an outlier of Lower New Red at Horswell House near Thurlestone is confirmatory of this view.

Trap Rocks. - The trap rocks range in composition from trachytes or orthophyres to basalts or melaphyres (Teall, Exeter Memoir, p. 33). They occur in local clusters or as isolated patches, which are the relics of many sheets or flows of lava emitted from different eruptive foci.

The distribution of the traps with reference to the Lower New Red sands and breccias is such that it is impossible to regard them as the products of a single period of eruption, unless it be conceded that the coarse breccias with igneous fragments which overlie them at Dunchideock and many other places are higher in the series than the sands which underlie them in the Killerton district.

If we take the traps collectively as an horizon in the New Red rocks the relations of the lower sub-divisions of the group must be regarded as interchangeable to a much greater extent than can be proved to be the case. On this assumption the thick series of marls with beds of sandstone between Exmouth and Straight Point might indicate the outward passage of the coarser marginal deposits into finer sediments. The overlying marls would then be the upper beds of the Permian, and the Budleigh Salterton Pebble-beds the base of the Trias, as advocated by the Rev. A. Irving. ${ }^{2}$

Although contemporaneous trap rocks do not occur in the coast section, the horizon of the Dunchideock trap would appear to be below the boulder-bearing breccias of the Teignmouth and Labrador Inn coast, and above the breccio-conglomerates of Watcombe, Petitor, Oddicombe, etc., which die out northward from Newton Abbot. The acceptance of a definite trap horizon would therefore place these breccio-conglomerates on the same horizon as the sands underlying the Killerton trap rocks.

A volcanic agglomerate is often associated with the traps (at Pocombe, Culmjohn near Killerton, Posbury, etc.), identical with the ' thonstein porphyr' of Germany. The obvious correlation of the traps with the German Melaphyr decken, or Middle Sötern, renders the correspondence of the rocks above the Watcombe conglomerate with the Upper Sötern and Wadern beds of the Nahe sections very probable, as there are many points of resemblance. The Watcombe conglomerate, etc., would then be Lower Sötern.

T. M. Hall ${ }^{3}$ described the occurrence of a thin porphyritic granitoid vein in the New Red cliff at Portledge Mouth on the north coast. The breccia and sandstone outliers at this place are in line with the out-

\footnotetext{
1 Proc. Geol. Assoc. vol. xvii. pt. 3, p. 131.

2 2uart. Fourn. Geol. Soc. for May, I 888, p. 149.

3 Hall, Trans. Derion Assoc. I 879.
} 


\section{A HISTORY OF DEVONSHIRE}

liers on the north of the Tiverton valley. In the intervening distance of 30 miles the Minette dyke of Rose Ash ${ }^{1}$ occurs.

\section{MIDDLE NEW RED}

On the coast a series of red marls with thick even beds of red and whitish sandstone, displaced by numerous faults, are succeeded on the north of Straight Point by red marls (probably about 500 feet thick) with a bed or two of sandstone; these pass under the Budleigh Salterton. Pebble-beds on Westdown Hill. The Straight Point sandstones seem to be near the top of the intercalated series; in occasional brecciation they strongly resemble Lower New Red beds, but they also contain an irregular bed of dolomitic aspect, not found in the lower series. The marls in their cuboidal fracture resemble the Keuper marls, but do not contain gypsum or the thin calcareous and sandy bands found in that series. No continuous development of sandstones can be traced northward from the coast. Disconnected and frequently faulted patches of sandstone occur near Exmouth, Littleham, Withycombe, Lympstone, Woodbury, Whitecross, Aylesbeare, and east of Rockbeare. North of Whimple sandstones have not been detected in the marls. Faults preclude any certain estimate of the thickness of these beds which on the coast from Exmouth to Westdown Hill can scarcely be less than 800 feet, but they attenuate northward and at the Somerset boundary may not exceed 200 feet.

Their junction with the sandstones and breccias of the Lower New Red is so anomalous as to suggest unconformable overlap; whilst in their relation to the overlying Pebble-beds nothing of the kind has been proved. The slight irregularities in junction and occasional seams of marl in the base of the Pebble-beds ${ }^{2}$ are the least that could be expected from the change in physical conditions. Whether the marl series should be classed with the Nottingham Permian Marls (as suggested by Irving), the Upper Zechstein of the Harz, etc., or with the upper beds of the Upper Rothliegende of the Nahe sections, or whether they represent the whole or part of the Bunter, is open to question.

\section{UPPER NEW RED SANDSTONE}

Pebble Beds.-The Budleigh Pebble-bed is the thinnest member of the New Red series, rarely exceeding 80 feet in thickness. Its marked character enables the presence of faulted relations to the beds above or below to be easily detected. There are many breaches in its continuity owing to faults, especially between Sidmouth Junction and Kentisbeare.

The percentage of Silurian and Devonian quartzite pebbles," which forms so distinguishable a feature on the coast, decreases northward towards Uffculme. The pebbles from Talaton northward are on the

1 Downes, 'Mica Trap Rose Ash,' Trans. Devon Assoc. 1884.

2 Rev. A. Irving, 2uart. Journ. Geol. Soc. May, 1888, p. 156.

3 Vicary and Salter, ibid. August, 1864, p. 283 ; Davidson, ibid. Feb. I870, p. 70 ; Thomas, ibid. Nov. 1902, pp. 620-32. 


\section{GEOLOGY}

whole smaller than those further south and there is an increased percentage of quartz and grit pebbles. From a study of the minerals in the Pebble-bed Thomas also deduced the existence of a main current from the south, joined near Burlescombe by a confluent westerly current to which he ascribed a rise in the percentage of heavier materials north of Uffculm accompanied by the appearance of new minerals such as garnet and cassiterite.

The Pebble-beds display much false bedding, are irregularly intercalated with beds, seams and masses of sand, and are locally represented by sands with beds and seams of pebbles. There is no sharp line of demarcation between them and the overlying Sandstones in the lower part of which occasional pebbles are found.

Upper Sandstones.- The thick-bedded red sandstones which overlie the Pebble-beds often display false bedding. They are irregularly pervaded by calcareous matter which stands out in an irregular network from the softer sandstone, in many parts of the coast section, and obscures the bedding planes. Their outcrop at Sidmouth is cut off by fault letting down the Keuper marls at Chit rock. Thirty chains from this they crop out at the base of the cliff and at the surface near Ladram Bay, where faults repeat the base of the marls, so that their total thickness is represented between the large Otterton outlier and the outcrop of the pebble beds at Knowl near Budleigh Salterton and may not exceed $25^{\circ}$ feet. Irregular calcareous concretionary brecciated bands occur in this series, in several places ; they are well shown in the upper part, at Ladram Bay and at, and near, Otterton Point, where Irving ${ }^{1}$ noted subangular fragments of slate, vein quartz, trap, reddish granite, felspathic grit and quartzite in one band; the estimated position of the Otterton Point beds is about $r 00$ feet above the Pebble-beds. Whitaker ${ }^{2}$ discovered the jaw of Hyperodapedon in the Otterton Point beds in 1868 . Dr. Johnston Lavis ${ }^{3}$ discovered remains of Labyrinthodon in talus from the cliff on the west slope of High Peak Hill. The horizon is considered by Metcalfe ${ }^{4}$ to be a bed of lighter colour in the Sandstones, not far below their junction with the overlying Marls. Dr. Carter also discovered osseous structures in these localities. The junction of Sandstones and Marls is sometimes, as at Windygate, difficult to find (through the sandy nature of the base of the latter) and local intercalations are also met with, but otherwise there is no sign of passage beds.

The Sandstone outcrop is persistent but greatly attenuated through faults in places, as at Payhembury.

Keuper Marls.-These beds consist of red and green variegated, cuboidally splitting marls, less calcareous toward their base and locally sandy. They occasionally contain beds of sand-rock, I 5 feet of which caps the marl cliff at Seaton. Veins, filaments, and bands of gypsum are common between Branscombe Mouth and Salcombe Mouth. Calcareous or marlstone bands are also present, and in the lower beds shaly

1 Irving, 2uart. Fourn. Geal. Soc. 1902, p. 153.
3 Ibid. vol. xxxii. p. 274.
2 . Ibid. May, I 869, p. I 52.

Ibid. May, 1884, p. 257. 


\section{A HISTORY OF DEVONSHIRE}

sandstones and marlstone bands are prevalent. In these thin bands numerous pseudomorphs after rock salt were found at Windygate (the cliff between Peak and High Peak Hills). In one of these beds Hutchinson $^{1}$ found numerous casts of equisetoid plants.

In a boring put down near Lyme Regis in I 90 I the Keuper Marls were penetrated to a depth which may be estimated at about I, I 30 feet, without reaching their base. Jukes-Browne ${ }^{2}$ gives an abstract of their succession as follows :-

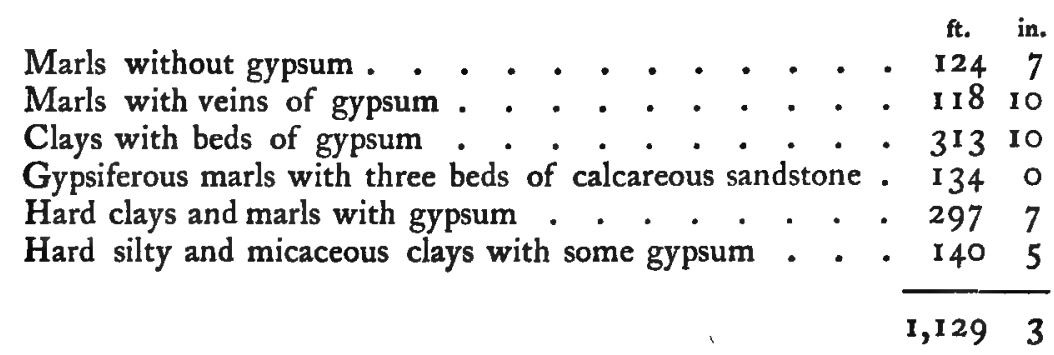

Teall $^{3}$ attributes the prevailing colour of the New Red rocks mainly to the (lateritic type of decomposition) 'subaerial decomposition of rocks containing ferriferous compounds' 'so common in tropical regions of the present day.' 'Under this mode of decomposition the iron becomes oxidized and deposited as a coating on the grains of quartz and other undecomposed minerals. The red material thus produced would mantle the slopes, fill up the hollows, or be spread out as flat fans over the low ground by torrential action. It would also be deposited in lakes, lagoons or seas. In the presence of decomposing organic matter the ferric oxide would be reduced, the red colour would disappear, and the iron would take the form of a sulphide or carbonate. Thus the change in the colour seen near Axmouth at the junction of the Rhætic and Keuper was directly connected with the absence of fossils from the latter and their abundance in the former deposit.'

\section{RHETIC BEDS}

Owing to the Cretaceous overlap the Rhætic beds cannot be traced continuously from the coast. They crop out, but are cut off by fault in Pinhay Bay. Their main outcrop in Dowlands Cliff is largely concealed by cretaceous debris from the landslip, but at Culverhole H. B. Woodward " gives their thickness as $6_{3} \mathrm{ft}$. 8 in., made up in descending order of 15 feet of thin bedded white lias limestones based by an impersistent bed of Cotham Stone, upon 18 feet of black shales containing Avicula contorta, Cardium rbaticum, Pecten valoniensis, etc., with a bone bed at their base containing Acrodus, Hybodus, etc. The succeeding passage beds to the Keuper consist of 10 feet of green marl upon 20 feet of alternating pale greenish and cream-coloured marls with hard

1 P. O. Hutchinson, Trans. Devon Assoc. vol. xi. p. 383.

2 Quart. Foum. Geol. Soc. May, 1902, p. 288.

3 Proc. Geol. Assoc. (1899), xvi. pt. 3, Pp. 141, 142 ; Exeter Memoir, 1902, p. 76.

4 Proc. Geol. Assoc. xvi. pt. 3, p. 135. 


\section{GEOLOGY}

marly limestone bands and blackish clays. In the boring east of Uplyme the black shales seem to be very much thicker, perhaps 40 feet, and the overlying white lias limestones are about the same thickness as at Culverhole. Rhætic beds are at the surface in the valleys north of Uplyme, bounding the Lias, and in three inliers in the valley of the Axe, and fringing the Cretaceous rocks of Dalwood and Membury.

\section{LOWER LIAS}

Through the denudation of the Greensand Lower Lias is at the surface in the valley of the Yarty north of Knap Cops ; in the valley of the Axe between Membury, Hawkchurch and Axminster; and in the Uplyme valley. Jukes-Browne estimates the thickness in the boring east of Uplyme as 'probably' $62 \mathrm{ft} .4$ in. From Pinhay Bay eastward the four zones, into which the interbedded limestones and shales of the Lower Lias have been divided, are encountered in ascending series. ${ }^{1}$ The basement zone of Ammonites planorbis estimated at from 22 to $24 \mathrm{feet}$ in thickness commences with thin shales full of spines of Echinoderms. The next zone characterized by $A$. angulatus is about 24 feet ; the overlying $A$. bucklandi zone about 38 feet, and the zone of $A$. semicostatus about 19 feet.

\section{CRETACEOUS}

The Greensands are capped by faulted masses of Chalk near Membury and Wilmington. The chief extension of Chalk is along the coast between Lyme Regis and Salcombe Mouth. Jukes-Browne prefers the term Selbornian for the so-called Upper Greensand of Devonshire, as this term includes Greensand, Malmstone and Gault clay, where typically developed in descending order, but clays or marl like Gault may occur in the highest stages.

The clayey beds found near the base of the formation at Culverhole, in the South-Western Railway tunnel east of Honiton (north of Offwell), and south-east of Dalwood Common, perhaps also on Shute Hill (where the chert-beds are exposed), may be equivalent to the Malmstone or Upper Gault (zone of $A$. rostratus) in Jukes-Browne's opinion. Five shafts were sunk in the construction of the tunnel east of Honiton. ${ }^{2}$ In the three westernmost of these clay with chert (resting on chert beds, broken up but perhaps partly in situ) from 33 to 54 feet in thickness, overlies the following succession :-

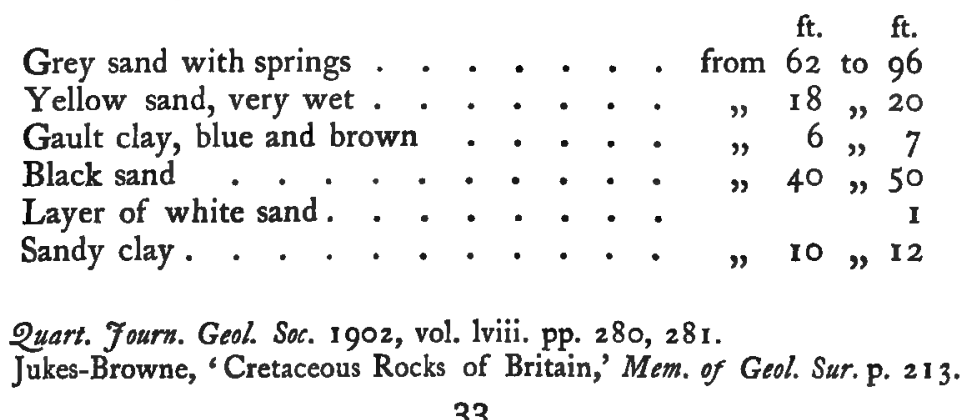




\section{A HISTORY OF DEVONSHIRE}

In the clay bed the Rev. W. Downes ${ }^{1}$ found Inoceramus concentricus, Pecten quadricostatus, Actzon affinis, and other shells.

The Rev. W. Downes divided the Greensands of the Blackdowns and the Haldons into 15 zones or beds, numbered from the base upward, as follows in brief abstract :-

Bed 15. Orbitolina chert, local, seen at Smallacombe Goyle, Little Haldon; in layers. 6 feet.

Bed 14. Schorlaceous sand not glauconitic. Exogyra occasional, Haldon. 8 feet.

Bed 13. 'Littoral concrete' of Prof. Duncan. Three shell beds in sand. Exogyra abundant. Trigonia vicaryana, Vermicularia. (? about 2 feet).

The above he considered to be higher than the Blackdown beds.

(Haldon. Sand with several layers of cherty sandstone. Pecten quadricostatus

Bed $12\left\{\begin{array}{l}\text { and Exogyra abundant. About } 35 \text { feet. } \\ \text { Blackdown. Sand with layers of cherty sandstone passing upwards into }\end{array}\right.$ chert. Pecten quadricostatus characteristic. About 25 feet.

Bed I I. Variegated fine sand with thin impersistent shell bands, fossils broken and waterworn. Pectunculus sublevis and Trigonia affinis abundant. 18 feet.

Bed IO. (Fitton's bed I) Very fossiliferous sandstone layers with sand partings. Cyprina cuneata, Exogyra conica, common; Trigonia scabricola, probably characteristic. About 3 feet.

Beds IO and II were thought to have a meagre representation at the base of the Greensand of the Haldons. Jukes-Browne ${ }^{2}$ considers bed 13 represented in bed 12 Blackdown; Haldon bed 12 to be $I I$, not 35 , feet; that bed 14 is above 15 , and that both are partly, or altogether, represented by the chert beds on the Devon coast.

Bed 9. (Fitton's bed 2) Thin layer of concretions used for scythestones. 'Hard fine vein.'

Bed 8. $\{$ ' Very fossiliferous bed in sand, Turitella granulata the prevailing fossil.

Bed 7. As above, Pectunculus umbonatus prevalent. Murex calcar often perfect.

In beds 7 and 8 (together corresponding to Fitton's bed 2) the fossils occur in clusters and have evidently been deposited in still water. The beds blend, though generally found in the order given. Their united thickness is about 4 feet.

Bed 6. (Fitton's bed 4) Sand with concretionary layers; few fossils including Inoceramus sulcatus, Pectunculus umbonatus ; known as 'Gutters.' About 5 feet thick.

Bed 5. Known as 'Burrows.' Concretionary beds divided by sand layers and used for building purposes, sometimes for whetstones. Few fossils, chiefly Inoceramus sulcatus and Trigonia aliformis. About 4 feet.

Bed 4. (Fitton's bed 6) 'Bottom stones,' concretions used for whetstones. Pectunculus, Inoceramus and Trigonia as above. From a few inches to 5 feet.

Bed 3. (Fitton's 'Rock-sand' bed 7) 'Bottom rock,' sand, few fossils including Trigonia aliformis. About 4 feet.

Bed 2. (Fitton's bed 8) Thin layer (a few inches) of concretions used for scythe stones. 'Soft fine vein.'

Beds 9 to 2, inclusive, have formerly been largely quarried for whetstones on Punchey Down.

1 Geol. Mag. July 1886, pp. 309, 310.

2 Op. cit. p. 225. 


\section{GEOLOGY}

Bed 1. Homogeneous whitey-brown sand, 'white rock', unfossiliferous and without current bedding. About 30 feet.

Jukes-Browne ${ }^{1}$ agrees in Downes' conclusion that the beds 9 to I inclusive have thinned out between Sidmouth and Haldon.

The sections on the north side of Great Haldon ${ }^{2}$ give a total thickness estimated at 66 feet, whilst total thicknesses exceeding 90 feet have been recorded on Little Haldon. ${ }^{3}$ The total maximum thickness of the Selbornian in Devon is about 200 feet.

Gbalk.-The Lower Chalk as seen on the coast and in the outlier between Offwell and Widworthy " (east of Honiton) consists of calcareous sandstones (containing Ammonites mantelli, Pecten asper, etc.), often very coarse and pebbly at the base, with an upper horizon of quartziferous limestone with glauconitic grains containing Scapbites aqualis, etc. The upper bed is sometimes absent. Jukes-Browne applies the term Cenomanien to this arenaceous equivalent of the Chalk Marl. The Lower Chalk of Membury is of a more ordinary type. The thickness of these beds varies, being in part of the outlier east of Honiton as much as 40 feet, of which the upper bed is only 2 feet. The Middle Chalk consists of white chalk with many flints, the zone of Terebratulina gracilis. It is about 80 feet thick in the coast near Beer, and forms the upper 52 feet of the large Beer quarry. The lower zone of hard chalk characterized by Rbyncbonella cuvieri is about 40 feet thick on the coast. It forms the lower part of the large quarry at Beer, of which the following downward succession is given by Jukes-Browne:-

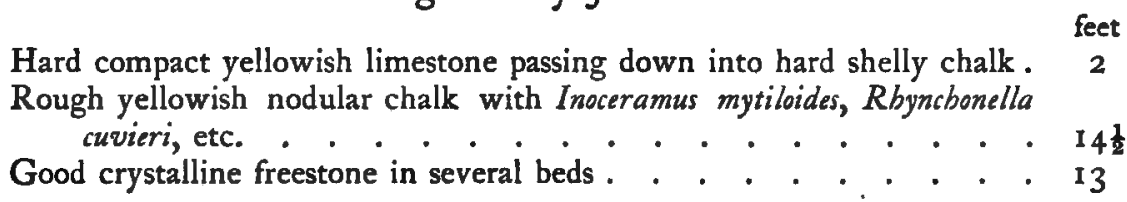

The Upper Chalk consists of chalk with many flints passing down into hard nodular chalk (chalk rock). The upper part is characterized as the zone of Micraster cortestudinarium, the lower part as the zone of Holaster planus. A prominent black flint band has been taken as the separating line at Whitecliff.

\section{TERTIARY AND TERTIARY REMANIES}

The clay with flints and chert varies from a red-brown clay with unworn and broken chalk flints, where it rests on the Chalk or in the vicinity of Chalk outliers, to a yellowish-brown clay or loam with, or without, broken chalk flints over, or mixed with, the chert fragments where it rests on Greensand. The Rev. W. Downes ${ }^{5}$ noted that the flinty clay on Blackdown, south of the latitude of Kentisbeare, capped the cherty accumulation without admixture. Quartz grains are occasionally met with in the clay, and any extraneous material, such as

1 Geol. Mag. July 1886, p. $225 . \quad 2$ Ibid. p. $220 . \quad{ }^{3}$ Ibid. pp. 224, 225.

- 2uart. Fourn. Geol. Soc. Aug. 1898, vol. liv. pp. 240-50. See also Woodward, Proc. Geol. Assor. 1899 , vol. xvi. pt. 3, pp. 1 34, $138-9$.

- 2uart. Fourm. Geol. Soc. Feb. 1882 , p. 83. 


\section{A HISTORY OF DEVONSHIRE}

boulders of flint breccia in the pits on the hills near Sidmouth, may be easily accounted for by gradual subsidence over the pipes which have been dissolved out of the subjacent Chalk or Greensand. In a quarry on Salcombe Hill one of these pipes was seen penetrating Greensand with beds of chert to a depth of nearly 20 feet; the narrow termination of the pipe for $5 \frac{\mathrm{I}}{2}$ feet was filled with sand, above this to the surface with clay with nodular chalk flints. In the same section are pipes of sand which do not extend more than 3 or 4 feet in the Selbornian.

The accumulation is confined to the plateaux; its thickness varies from a few to over 30 feet. Where the plateau has been broken by fault, as in the valley on the west side of Stockland Hill, the stony clay has been displaced with it. ${ }^{1}$ The upheaval and denudation of the Cretaceous sea bed, the deposition of argillaceous and loamy Tertiary materials, whether Woolwich and Reading beds or London Clay, and the subsequent solvent action of percolating water corroding the Chalk remnants or calcareous Selbornian beds, and substituting clay or loam derived by filtration from the overlying materials, explain the local variations of the accumulation.

There are some old marl pits on Brown Down (between Yarcombe and Otterford) which seem to have been opened in dark drab-brown clay without stones.

Between Otterford and Church Staunton small quartz pebbles occur in red clay, which was seen resting on reddish loamy sand with occasional pebbles and fragments of chert.

South of Stockland Hill, toward Dalwood Down, 5 to 6 feet of coarse brown and grey quartzose sand with whitish spots and a few chert stones occurs on the summit.

A patch of gravel rests on the Selbornian north of Burnworthy farm (near Staple Hill) ; its southern limits are within the Devon boundary. A pit gave the following downward section:-

Red-brown loam with irregular whitish streaks, with occasional quartz pebbles throughout, 3 to 4 feet.

Pale buff and red-brown sand with whitish mottling and an impersistent seam of fine quartz gravel, 2 to 3 feet.

Red-brown gravel, mostly of small irregular quartz pebbles, with angular fragments of siliceous rock and chert, close together in a clayey matrix; seams of finer gravel and coarse sand are present, 5 feet exposed.

Large pebbles of quartz, flint, and dark grey slaty rock occur in the lower part of the deposit.

This gravel was doubtfully ascribed to the Tertiary period in $1878 .^{2}$ The Haldon Gravels were pronounced to be Bagshot by Reid in $1898 .^{3}$ He describes the plateau gravel on Great Haldon as " mainly composed of large boulders of " annealed " or toughened chalk-flint weathered to a considerable depth. . . . Mixed with these is much Greensand chert

1 De la Beche's Report, p. 311 -faults near Wambrook.

2 Ussher, 2uart. Fourn. Geol. Soc. 1878, p. 452.

3 Reid, ibid. 1898, pp. 234-6. 


\section{GEOLOGY}

and veined Palæozoic grits like those so conspicuous further east. At Haldon Palæozoic rocks are somewhat more common in larger pieces, less worn, and Purbeck rocks are naturally absent; in other respects the Haldon and Dorset gravels are of identical composition and appearance, containing similar seams of white clay and rough quartz sand.'

At Upcott (or Lower Uppercott) limestone quarry, north-east of Chudleigh and about 200 feet lower than the Haldon gravels and clay, or loam, with flints, a mass of fine (? Selbornian) sand, subordinate to coarse sand and fine gravel with some worn chalk flints, has been preserved through the dissolution of the limestone, and forms the most northerly indication of the spread of the Milber Down and Kingsteignton, etc., sands and gravels. These gravels bound the lignitiferous clays and sands very irregularly. They exhibit high dips at Staple Hill, Woolborough and other places towards the depression occupied by the clays and sands. Near Lower Staple Hill Reid saw the gravels passing under the lower beds of the pipe clay. De la Beche observes, ' $U$ pon these [gravels] the clay and sands of the Bovey deposit may be seen to rest.' He however considered that the gravels overlaid ' undisturbed Greensand,' as a boring near the Bovey Heathfield Pottery ${ }^{1}$ was carried to a depth of 'nearly 300 feet through sands resembling those on Haldon" without reaching their base and without encountering lignite beds.

These marginal beds vary in composition, Culm fragments being most frequent toward the west. A patch of worn granite sand overlain by coarse gravel with granite boulders (and some white flint apparently) near Lustleigh, south of Alsford farm, points to the extension of the deposit in that direction.

About two miles north of Kingsteignton Reid notes a section of I 2 feet of 'coarse gravel of well rounded large chalk flints . . .; much small quartz; Palæozoic grits, coarse and fine; radiolarian chert (common) and red jasper (one pebble); greenstone and ash; Greensand chert (one large block)' on 3 feet of buff schorlaceous sand. Towards Haccombe he noted numerous large water-worn blocks of Greensand chert and large Chalk flints in the gravel. Near Staple Hill he found it ' largely composed of quartz, veined grit, radiolarian chert, and igneous rock' with 'several masses of Greensand chert and two rolled Chalk flints.'

The sand and gravel has been retained in hollows and potholes on the surface of the limestone north of Kingsteignton, and outliers are found on the Culm and New Red rocks. On the south-east of Ugbrooke Park, near Old Chard and Underhays, large reddish siliceous boulders, intensely hard and in places brecciated, accompany the deposit. At Hestow farm, south of Ideford, there is a mass of these boulders, which seem to be greywethers.

Pengelly ${ }^{2}$ gave detailed sections of the lignite pits of Bovey, the

1 Report on the Geology, etc. pp. 257, 248.

2 Pengelly and Heer, Phil. Trans. 1862, vol. clii. p. 1022, etc. 


\section{A HISTORY OF DEVONSHIRE}

deeper exposure exhibiting over 120 feet of alternating beds of sand and clay and of clay with 27 bands of lignite. At the Potteries near Heathfield station $\mathrm{H}$. B. Woodward ${ }^{1}$ summarizes the section as 'a variable series of grey and white clays, carbonaceous sands, and occasional lignite beds.' ' It was mentioned that a boring had been carried to a depth of 520 feet from the surface through clays, sands and lignites without reaching the base.' 'An excellent section of the lignite-series with bands of potters' clay' is visible 'in the "Great Plantation" east of Preston Manor Clay Works.' At Abbrooks gravel is interbedded with the clay.

Comparing the Bovey lignite flora to the fossil plants in the marine series of the Bournemouth beds, Starkie Gardiner says, ${ }^{2}$ ' when we compare the ferns as Osmunda (Pecopteris) lignita, Lastraa bunburyi, the cactus (Palmacites damonorops), the fruits, conifers or dicotyledons, it is seen that by far the larger proportion are not only specifically identical, but occur exactly in the same combinations and manner of preservation.' Reid ${ }^{3}$ however, pending a re-examination of the Bovey flora, does not regard the botanical evidence as conclusive. De la Beche reconciled the great difference in level between the Greensand said to occur beneath the Bovey clays and that of the Haldons by subsequent disturbance of the beds, or by previous erosion, in either case combined with faults."

'At Orleigh Court near Bideford, 43 $\frac{\mathrm{I}}{2}$ miles from the green sand of the Black Down Hills, and 36 miles from that of Great Haldon,' De la Beche ${ }^{5}$ referred to a patch of sand, 'a few acres in area,' resembling the fox mould of the Greensand near Lyme Regis, with ' superincumbent gravel' similar to 'that on the Haldons and Black Downs,' composed of flint and chert in which Galerites albogalerus, Spbatangus coranguinum, etc., were found. About twenty-five years ago no good sections of these deposits were visible ; red earthy clay with chalk flints and broken flints and chips was however exposed, and fragments of indurated coarse quartzose sand were met with in the vicinity.

South of Rivaton farm (on a common south-east of Stapledon), $5 \frac{1}{2}$ miles south-south-east of Orleigh Court, gravel of rather small angular and subangular, and occasional pebbly, quartz, chips and worn nodular pieces of blackish, red, and brown flint, with angular and subangular Culm grit stones in a sand of similar derivation, occurs at 500 feet above the sea, on the summit level of the Culm Measures of the vicinity. The Petrockstow depression (between Torrington and Hatherleigh) lies $2 \frac{\mathrm{I}}{2}$ miles east of this gravel. This depression is $4 \frac{\mathrm{I}}{2}$ miles in length from N. $30^{\circ} \mathrm{W}$. to S. $30^{\circ} \mathrm{E}$, and about three-quarters of a mile broad, but narrowing northward. The flattish surface consists of a Head of clay with slightly worn fragments of Culm grits and quartz and gravelly seams, from 5 to 10 feet thick; beneath this stiff unctuous white clay

\footnotetext{
1 Proc. Geol. Assoc. for 1900, pp. 426, 427.

2 Quart. Fourn. Geol. Soc. 1879, p. 227.

3 Reid, op. cit. p. 236.

- Op. cit. p. 236. See also Geological Manual, p. 200.

5 De la Beche, op. cit. pp. $236,249$.
} 


\section{GEOLOGY}

with drab bands has been encountered in a shaft sunk at the Clay Works between Lower Marland and Moor Hill farms to a depth of 80 feet without reaching its base. At 56 feet from the surface, veins (or irregular beds) of lignite, seldom exceeding a foot in thickness, were encountered. These clays were described and correlated with the Bovey clays in 1879..$^{1}$ About a quarter mile from Petrockstow church fine gravel (chiefly of worn quartz and occasionally bound in ferruginous cement) and coarse sand contains a few fragments of whitish sandy rock, in one of which a portion of Ecbinus was found, denoting derivation in part from Selbornian materials. Towards Huish gravel is also worked. These gravels may be marginal deposits of, or overlie, the clays. At about I I miles north-west of Haldon Belvidere and about I 4 miles from the Petrockstow depression, a patch of gravel rests on the Lower New Red breccia, at a quarter of a mile west of Colford (near Yeoford). A pit 5 feet deep showed (twenty-seven years ago) pale buff gravel of small subangular quartz stones with broken well-worn chalk flints and a few pieces of dark grey slaty rock.

This cordon of observations points to the north-westerly extension of Cretaceous rocks and of Tertiary deposits between Haldon and Bideford Bay, and it is not improbable that a detailed survey of the Culm area would afford further evidence.

The large tracts of disintegrated granite in the drainage depressions of Dartmoor may be, in part, the relics of Tertiary gravels and sands, dating to a period when the lakes of Petrockstow and Bovey were fed by streams rising in the granite.

The sloping surface of the Cretaceous plain from Black Down southward, coupled with the difficulty in distinguishing sand associated with Tertiary gravels from Greensand which may underlie them, in view of the great thickness of sand said to underlie the Bovey pipeclays in that depression, calls for additional and protracted investigations in the Bovey valley and on its borders, before we can reconstruct the former extension of the Cretaceous and Tertiary rocks, and assign a definite reason for discrepancies in level with reasonable hope of success. ${ }^{2}$ The simple explanation given in the prefatory notes of deposits left at different stages in the processes of Tertiary denudation has been provisionally adopted.

The planed surfaces of the Devonian limestone hills of the Torquay, Brixham and Plymouth area probably date from Permian times, as outliers of Lower New Red on the surface and in fissures are found near Waddeton and Brixham, etc.; but, as in the case of the potholes in the limestone north of Kingsteignton, these materials may have been removed during, or prior to, the deposition of Tertiary gravels and sands, and subsequently corroded hollows may have retained, very locally, traces of Tertiary deposition.

On the Hoe, Plymouth, averaging roo feet above mean tide,

1 Ussher, 'On the Deposits of Petrockstow in Devon,' Trans. Devon Assoc. for 1879.

2 Godwin-Austen attributed the discrepancies in level to subsequent movements (Quart. Fourn. Geol. Soc, vol. vi. p. 9i). 


\section{A HISTORY OF DEVONSHIRE}

Worth ${ }^{1}$ records the occurrence of clayey earth with scattered pebbles of quartz, schorl rock, grey grit, felspar, hornblende schist and brown pumiceous rock with iron ore and vein stuff. In this deposit patches of white clay, similar to Bovey clays, are present. The pebbles range up to over I $2 \mathrm{lb}$. in weight. The gravelly clay rests on white, drab, cream-coloured and red sand, fine and quartzose, and coarse and schorlaceous. These deposits also occur in hollows in the limestone of Cattedown.

On the northern slope of Cattedown, 60 to 70 feet above datum, Worth discovered a gravel deposit in clayey subsoil. The pebbles, running up to $3 \frac{\mathrm{I}}{2} \mathrm{lb}$. in maximum weight, betrayed derivation from fissures in the limestone, being partly encrusted with stalagmite. Flint (mostly chalk flint) pebbles, well rolled and up to $2 \mathrm{lb}$. in weight, formed $4 \circ$ per cent, and in another place 67 per cent, of the stones; next came schorl rock and quartz; amongst the limestone pebbles one of Lias limestone, two probably Culm limestone, and one of freestone like Beer stone, were found. Stones of grits of various kinds, of Culm chert (probably), Elvan and other granitoid rocks, shalstein, diabase, epidiorite, andesite, volcanic grit (probably from the Wearde-Efford beds =? Middle Culm) were also found. Whether the above are Tertiary or remaniés of Tertiary or early Pleistocene gravels is not apparent.

\section{PLEISTOCENE}

Gravels left at considerable heights above the adjacent or neighbouring lines of drainage, marking early stages in the elaboration of the existing contour, are met with in the watersheds of the Exe and its tributaries, and less frequently in the valleys of the Palæozoic area. These gravels are composed of materials derived from the catchment areas in which they occur. Flint and chert are usually more or less abundant in the old gravels on the cliffs near Dawlish and east of Exmouth.

In the Pebble-bed districts, west of Ottery St. Mary and near Budleigh Salterton, their materials have been so largely re-deposited that it is difficult to define their actual limits. A good instance of re-deposition of Lower New Red sands and breccia is shown in the railway cutting between Langstone Point and Dawlish, ${ }^{2}$ where it overlies an old gravel of the Exe, composed of well rolled flint, chert and derivatives from New Red breccia, which rests at 70 feet or more above datum, on sand and breccia (excepting inclined indications of bedding, very similar to the re-deposit). The coastline at the time the old gravels east and west of Exmouth were deposited was situated a considerable distance seaward of its present limits, and some of these gravels were probably contemporaneous with the raised beaches.

1 R. N. Worth, 'On the Geology of Plymouth,' Trans. Plymouth Instit. for 1875 ; 'Some Detrital Deposits associated with the Plymouth Limestone, Trans. Roy. Geol. Soc. Corm. for 1888 .

2 Ussher, 'On the Mouth of the River Exe,' Trans. Devon Assoc. 1878; 'The Geology of Dawlish,' Trans. Devon Assoc. I 881 . 



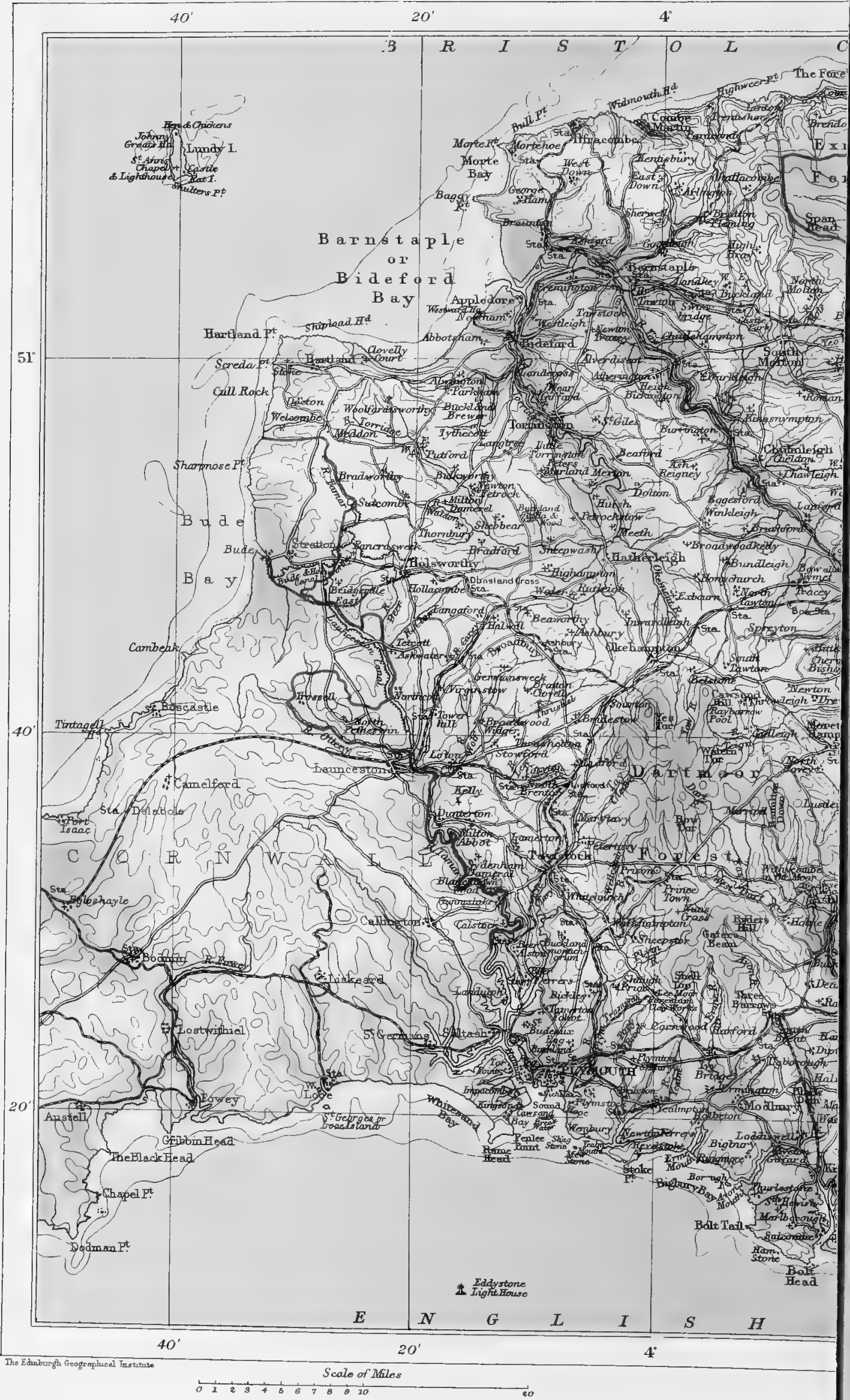


IICAL MAP.

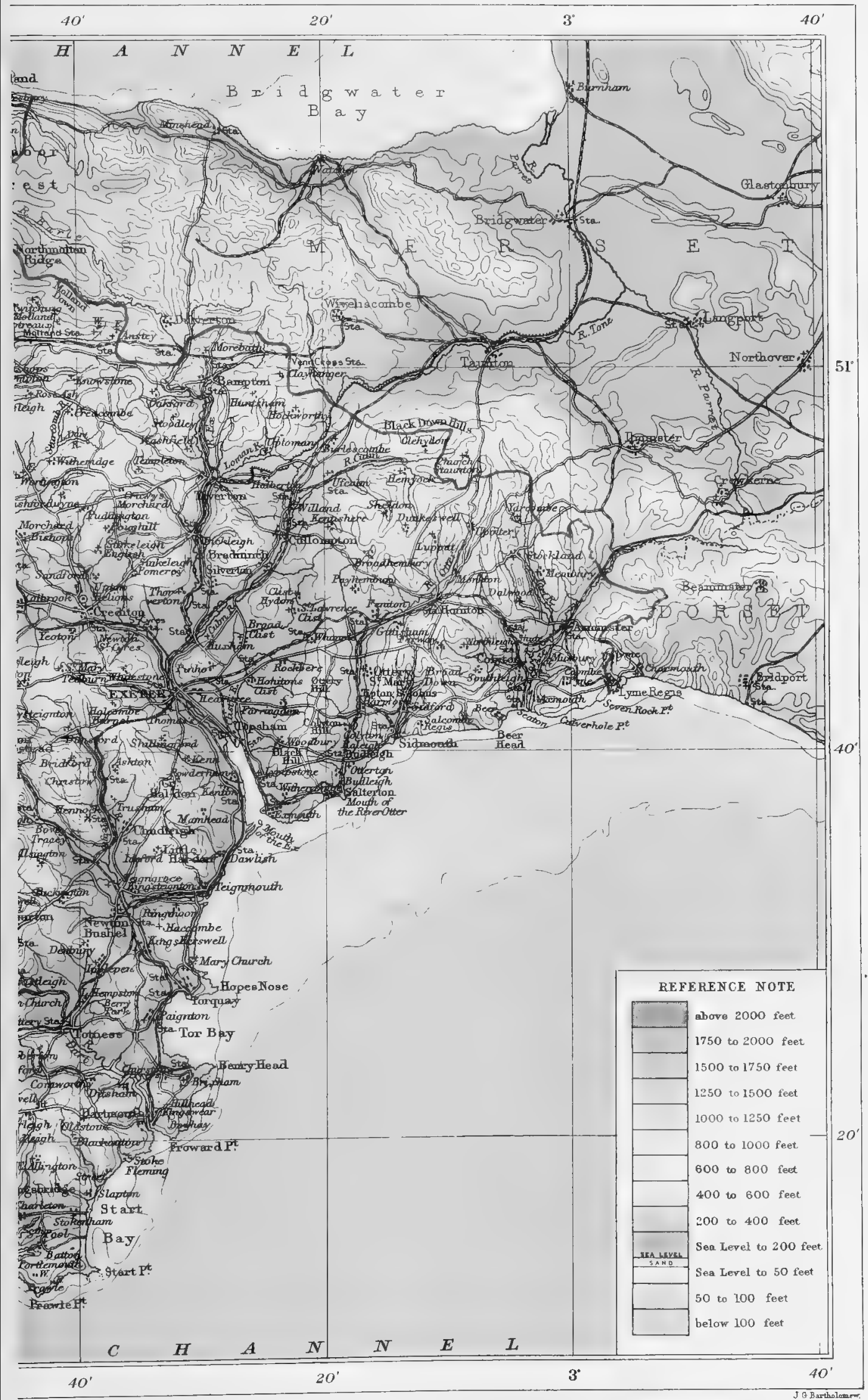





\section{GEOLOGY}

At Exeter remains of old gravels of the Exe rise to over roo feet above the alluvium. It is rare to find any more or less continuous extension of the older gravels in the Secondary area and still more so in the Palæozoic area. Good examples of gravels at various levels mark stages in the excavation of the Dart valley ${ }^{2}$ above Totnes. In the Teign valley many instances have been described. ${ }^{2}$ On a Culm sandstone quarry south of Bideford an old gravel of the Torridge was observed at about 70 feet above the adjacent alluvium. A good example of more recent gravel is visible at 10 feet above the alluvium of the same river at Beam Bridge, north-west of Torrington. The best terrace features of the Taw are in the vicinity of Umberleigh; of the Tamar at and north of Rumleigh House, opposite Calstock; of the Tavy north of Lopwell ; of the Plym between Shaugh Bridge and Bickleigh Bridge; of the Avon at Avonwick and South Brent; of the Erme between Keaton Bridge and Ivybridge. All these indicate a comparatively recent stage in the excavation of their respective valleys, although in some cases they occur at 50 to 100 feet above the adjacent alluvia. The stream valley deposits bear out the remark of Godwin-Austen. ${ }^{3}$ "There is not perhaps a single valley through which a river or even a brook at present takes its course, along which alluvia are not found at elevations such as the existing streams in their most swollen state never have attained.'

Raised Beaches. - The raised beaches rest on rock platforms at elevations which nowhere indicate a depression of 100 feet and seldom of more than 20 to 30 feet. The raised beach under the Hoe is described by Hennah ${ }^{4}$ as from 2 to 3 feet of cemented sand and water-worn pebbles from 15 to 18 feet above high water mark. Dr. Moore gives the height of the Hoe raised beach as 35 feet above high water.

Between Prawle and Start Points the steep slopes are fringed by a strip of ground, 40 to I 20 yards wide, composed of Head or stony clay, sloping gently seaward, from heights of 50 to 100 feet, to the cliff line which is formed by it for about 3 miles. The accumulation shows signs of admixture with old beach material in places at its base, which rests on a well-defined rock platform a few feet above high water mark. Upon this platform at Malcombe Point two large water-worn erratic boulders rest. In view of the boulders at the base of the Saunton raised beach it is more probable that these were stranded during the raised beach period than from the ballast of a wreck. The Head is 30 feet thick or more in the cliffs.

The raised beach at the south horn of Hope's Nose consists of stratified shelly sand, with pebbles and shells in the lower part, and its base, according to Godwin-Austen, ${ }^{5}$ is 3 I feet above high water mark. Old beach materials rich in shells are scattered over parts of reef platforms, at 10 to 20 feet above high water mark, on the Thatcher rock. Hunt ${ }^{6}$

1 Ussher, 'On some Old Gravels of the River Dart,' etc., Trans. Devon Assoc. 1876.

2 Ormerod, Quort. Fourn. Geol. Soc. vol. xxiii. p. 423 , etc.

3 Trans. Geol. Soc. ser. 2, vol. vi. p. $439 . \quad 4$ Op. cit. ser. I, vol. iv. p. 410.

S Op. cit. p. 44 I.

- Hunt, 'The Raised Beach of the Thatcher Rock,' etc., Trans. Devon Assoc. I 888, pp. $225-52$. 


\section{A HISTORY OF DEVONSHIRE}

concludes that the shells afford evidence of a different contour, of shelving shores and abundance of sand, whilst species such as Tropbon truncatus indicate colder conditions; Torbay being then, if hollowed out, not deeply indented. Raised beach materials, generally shelly, occur at io to 20 feet above high water in places on the Brixham coast.

Near Sharkham Point, the raised beach materials have been mixed with rubble from a hæmatite mine; they consist of small flint, slate and quartz pebbles, comminuted shells and sand.

On the north coast there are traces of raised beach at 8 to I 5 feet above high water mark, between Morthoe and Woolacombe, and at the south end of Woolacombe beach, the pebbles being mostly of local derivation, but small well worn flint are present and a few of granitoid rocks.

From the south side of Baggy Point to Braunton Burrows for about 2 miles raised beach materials are more or less continuously visible resting irregularly, at from 2 to 15 feet above high water mark, on a platform cut in Upper Devonian slates, and overlain by a variable thickness of Head (said by Pengelly to be in places Ioo feet). Toward Baggy Point, Sedgwick and Murchison ${ }^{1}$ state that the coarser shingles, in parts 19 feet thick, rise rapidly to the north to a height of 60 or 70 feet above high water mark. The upper part of the deposit consists as a rule of laminated consolidated sand upon coarse shelly concrete with pebbles, occasionally of flint. On the south side of Saunton promontory about 50 feet of laminated brownish sandstone, overlain by 8 feet of Head, rests on horizontal layers of cemented grit and quartz pebbles with shells, about ro feet thick, welded in the interstices of the slate reefs at about Io feet above high water mark. Toward Northam Burrows, south of Croyde, a cave worn in the consolidated beach discloses a large boulder of slaty rock behind which the red granite boulder is seen partly cemented in more or less consolidated beach material, false bedded in places, and containing pebbles. The boulders rest on the rock reef at 2 or 3 feet above high water mark.

There are traces of raised beach containing occasional flint pebbles between Appledore and Westward Ho, 5 to 10 feet above high water mark. From Westward Ho pier for half a mile the raised beach is more or less continuously visible; it consists of Culm grit pebbles, similar to those of the modern beach but rather smaller, associated with sand, from 6 to 20 feet thick and from 8 to 25 feet above high water mark.

North of Fremington old gravels, from io to 25 feet thick, occur at from 8 to I 5 feet above high water mark, and extend eastward through Muddlebridge and Hele to the Taw valley north of Tawstock Park on higher land which is flanked on the south by a flattish tract of clay. At Roundswell, in this tract, 100 feet above the sea, Maw ${ }^{2}$ describes a well section in which $7^{8}$ feet of smooth tough brown homogeneous clay,

1 Trans. Geol. Soc. ser. 2, vol. v. p. 28 I.

2 Quart. Fourn. Geol. Soc. vol. xx. p. 447. 


\section{GEOLOGY}

with stones in the upper I 2 feet, and driftwood at 40 feet from the surface, was penetrated and found to rest on a gravelly beach similar to that at Fremington and at the same level. He also records a well sinking at Roundswell hamlet mill in I 862, through 40 feet of smooth mostly unstratified brown clay to gravel beach. Near Upper Roundswell the clay is said to be 90 feet thick, thinning eastward and westward. The phenomena suggest an old channel of the Taw filled with gravel contemporary with the raised beaches, subsequently deepened during their elevation and filled with river mud overlain by $\mathrm{Head}$, or subaerial washes from the neighbouring slopes.

Submerged Rock Valleys, Head, etc.-If the old valley, above described, were re-excavated and submerged it would afford an apposite illustration of the submerged rock valleys of the Dart, Plym, Tamar and Tavy described by Codrington. ${ }^{1}$ Outside Kingswear jetty a stiff deposit with granite and quartz boulders was proved beneath the silt. The rock bed of the Tavy, in the deepest parts 25 to 68 feet below spring tide low water at the viaduct, was found to be covered by 2 to 4 feet of stiff yellow clay with small granite boulders, under the silt. Patches of clay with stones were found here and there on the rock sides or bottom under the silt which partly fills these submerged valleys. Codrington considered the stony clays to be relics of boulder clay, and that the valleys had been excavated to their present depth before the glacial period. The Yealm and Salcombe creeks may be also regarded as submerged rock valleys. At Puslinch by the Yealm a sinking of 40 feet was made without reaching rock. ${ }^{2}$

At Splat Cove a gravel deposit, probably contemporaneous with the raised beaches, occurs, at about 15 feet above high water mark, near the mouth of the Salcombe estuary.

These valleys were no doubt in existence during the raised beach period, but the argument against their excavation to their present depth at that time is rather favoured by the general absence of rock platforms and of contemporary gravels along their borders. The modifying and deepening of existing valleys, especially in their seaward extension, during the period of elevation which succeeded the raised beach formation, accords best with the phenomena described. At Rumleigh on the Tamar there are terraces marking successive steps in this erosion. The clayey gravel terrace with granite boulders, cited by Codrington, bounds the alluvium near Gawton Mine and belongs to the same series of accumulations as the Head on the coast which 'marks a time when the degradation of the surface proceeded much more rapidly than by the mere effects of decomposition, and when fragments of rock far exceeding the motive power of any rainfall were conveyed down slopes along which the minutest particles of matter, only, are now carried."

1 2uart. Fourn. Geol. Soc. vol. liv. pp. 26z-76.

2 Worth, 'The Geology of Plymouth,' Trans. Plym. Inst. 1875.

3 Godwin-Austin, 'On the Superficial Accumulations of the coasts of the English Channel,' 2uart. Fourn. Geol. Soc. vol. vii. 


\section{A HISTORY OF DEVONSHIRE}

It is not improbable that river ice may have assisted in this work of denudation and transport and even have aided during the closing stages of the elevation in the processes of excavation.

The Head on the coast and the fluviatile Head is often seen to pass into the detrital infillings of valley bottoms; of this there are good examples in the Bolt district, between the Start and Prawle, and in the deposit near Gawton Mine. The insulated pinnacles of coast Head near Peartree Point, on the old beach reef, face two of these valleys. At Venericks Cove (between the Prawle and Salcombe estuary) and on Ivy Island (Revelstoke coast) there are insulated patches of Head on the old beach reef. The relics of Head adhering to the cliffs in positions where no modern talus could rest testify at once to the antiquity of the accumulation and to the denudation it has undergone.

The Head of the Bovey valley contains here and there ' patches of fine potter's clay in which the clay diggers occasionally meet with stumps and roots of trees, the latter so ramifying as to indicate that they are in situ; in addition to these remains leaves have occasionally been met with, from which the dwarf birch (Betula nana) and three species of willow (Salix cinerea, S. repens, S. amygdalina) have been determined. These plants betoken a climate much colder than that which at present obtains in Devonshire." ${ }^{\prime \prime}$ Underlying the clay, which in one of the sections was Io feet thick and under 9 feet of unfossiliferous clay and sand, I 3 feet of sandy clay with angular and subangular stones was encountered.

The term 'Head' does not postulate more than the inclusion of the deposits to which it is applied in the same general period, in which the land attained its maximum elevation and began to subside. Of the vegetation of this period the dwarf birch and willows of the Bovey Head are the earliest examples of which we have evidence, the submerged forest traces are the latest.

Submerged Forests.-Between Saunton Down and the mouth of the Taw estuary Claypole ${ }^{2}$ observed Scrobicularia clay in which roots and rootlets were locally plentiful, projecting through the sands above low water mark.

Outside the south end of Northam Pebble Ridge for 200 yards along the shore, nearly as far out as low water, Ellis ${ }^{3}$ found peat with prostrate boughs and trunks of birch, alder and oak mixed with roots and acorns on a band, a few inches thick, of closely packed broken Culm grit pebbles upon blue clay. In the Exeter Museum the Westward Ho forest is described as peat 2 feet on-blue clay submerged 23 feet at high water, containing remains of wild boar, goat, long-fronted ox, wolf, roebuck, red deer, and bones of birds and fishes, 2 feet thick on-a kitchen midden, a dark line of mould containing numerous flints all of Palæolithic types, split bones, leg bones of ox and red deer, bits of pottery, a

\footnotetext{
1 Pengelly, Presidential Address, Trans. Devon Assoc. for I 867 ; Pbil. Trans. vol. clii. p. 1019.

2 Claypole, Proc. Bristol Nat. Soc. vol. vii. pt. i. p. 16.

Ellis, Trans. Devon Assoc. for 1866, p. 80 .
} 


\section{GEOLOGY}

stone pounder, charcoal, cockle and periwinkle shells. The position of the kitchen midden needs verification.

T. M. Hall, in I 864, recorded the exposure at Westward Ho of 4 feet of peat with trunks of between seventy and eighty large trees, apparently oak, birch and hazel, hazel nuts, flint flakes, bones of deer, ox, wild boar, wolf, etc. $\mathrm{He}$ found decomposed pointed stakes driven in a rude semicircle into a quantity of small perfectly angular stones which, in places, were observed under the peat.

In south Devon.-In Bigbury Bay, close to the north end of the Thurlestone Rock reefs, Pengelly ${ }^{2}$ records the exposure of dark blue clay with trunks of trees, including oak, at 150 feet seaward of high water mark.

At North Sands, in the Salcombe estuary, peat with remains of trees, including oak, is sometimes disclosed by gales.

At Blackpool near Stoke Fleming brown clay with twigs, nuts, leaves, tree trunks and rooted stumps, and bluish clays on the landward side, were exposed in $1869 .^{\circ}$ In 1881 the forest traces were again visible."

On Tor Abbey Sands ro feet of peat with trees has been seen, also on the foreshore at Paignton where the blue clay associated with the forest bed is often visible. In this Torbay Forest, at Tor Abbey Sands, Goodrington and Broad Sands, bones of red deer, wild boar, horse, long-fronted ox and mammoth are said to have been found. The peat passes under the alluvial clay of the Paignton and Goodrington marshes. Signs of submerged vegetation have been observed at Sidmouth. The extensive peat bogs on Dartmoor may be included in the same period of arboreal extension.

The slower rate of denudation during the later stages of the subsidence, which led to the advance of the sea to its present bounds and to the final submergence of the lower forest areas, is exemplified by the narrow waterways of the present streams and rivers excavated in their older and broader beds, by the silting up of estuaries and the formation of sandbanks like the Warren, or of gravel beaches as in the case of the Otter, Sid, and lesser streams across their mouths.

During this movement of subsidence oscillations seem to have taken place, and to this cause the occurrence of recent estuarine conditions in sites no longer affected by them, as in the case of the Alphington gravels, has been ascribed by Godwin-Austen. How far such oscillations may have contributed to the formation of such gravel beaches as Slapton Sands, and the Northam Pebble Ridge, is open to conjecture.

Caverns.-The bone caves and fissures of Devon are naturally confined to the limestone areas of Plymouth, Yealmpton and Torquay.

2 Hall, Proc. Soc. Antiq. for I Dec. 1864.

2 'On a Newly Discovered Submerged Forest, etc.' Trans. Devon Assoc. for 1866.

3 Pengelly, Trans. Devon Assoc. for 1869.

- Hunt, 'On Exposures, etc.' Trans. Devon Assoc. for 1881.

- Pengelly, 'The Submerged Forests of Torbay,' Trans. Devon Assoc. for 1865. 


\section{A HISTORY OF DEVONSHIRE}

Remains of extinct mammalia have been found in the osseous fissures of Oreston $;^{1}$ above a raised beach on Plymouth Hoe, 35 feet above high water, perhaps washed down from fissures in the limestone $;^{2}$ in Cattedown Quarry, in caverns with a connecting passage, the entrance to which seems to have been from above; in the Yealm Bridge Cavern, ${ }^{3}$ proved to have been an hyæna den; in Windmill Hill Cavern, Kent's Cavern and Chudleigh Cave.

Windmill Hill Cavern, Brixham (discovered in $185^{8}$ ), consists of a series of north and south galleries, in the direction of the jointing of the limestone, with an entrance about half way up the hill, at 94 feet above high-water mark. The deposits in descending order consisted of :-

Stalagmite not always present, up to over a foot in thickness.

Reddish Cave-earth, averaging 2 to 4 feet thick, with limestone fragments and occasional pieces of stalagmite, probably remains of an old floor.

For about 40 feet from the entrance a thin peaty layer rested on the Cave-earth. Animal remains occurred sparingly in the stalagmites; abundantly in the Cave-earth.

Waterworn shingle of pebbles of limestone, quartz, greenstone, grit and shale, 2 to 6 feet thick.

The formation of the cave is thought to have been carried on simultaneously with the excavation of the valley."

In Kent's Cavern, ${ }^{5}$ near the entrances (which overlook the valley between Anstey's Cove and Meadfoot beach) were found a layer of black leaf mould, from 3 inches to I foot thick, granular stalagmite up to 5 feet in thickness and below, a black band of 4 inches of charred wood. Under the above, Cave-earth with angular limestone fragments occurs, probably to a thickness of over 12 feet in places.

An older stalagmite floor, called the crystalline stalagmite, is found in parts of the cave, in places 12 feet thick and containing bones of bears. Beneath this is the oldest deposit-a breccia composed of subangular and rounded pieces of dark red grit, some quartz pebbles, and very rarely small angular limestone fragments with stalagmitic coating, in a sandy paste.

Happaway and Anstey's Cove and Buckfastleigh Caves call for no special reference.

Boulders, etc.-The evidences put forward in support of the prevalence of glacial conditions prior to the formation of the raised beaches are : The clay with flints on the cretaceous plain ${ }^{6}$; the stony clays on sides or bottom of submerged rock valleys; and the occurrence on high land of large boulders considered to have come from a distance. The

1 Worth, 'The Geology of Plymouth'' Trans. Plymoutb Inst. 1875, and 'The Bone Caves of the Plymouth District,' ibid. I 879 ; 'The Cattedown Bone Cave,' ibid. I887-8.

2 Dr. Moore, Rep. Brit. Assoc. 1841 ; Trans. of Sects. p. 62.

3 De la Beche, Report, etc. p. 414.

4 Report on the Exploration of Brixham Cave, Proc. Roy. Soc. No. 137 (1872), and Phil. Trans. 1873, Pp. $471-572$.

From papers by Pengelly; Foum. Plymouth Inst. 1875 ; Trans. Devon Assoc. 1882, p. $69 \mathrm{r}$.

6 Ussher, Quart. Fourn. Geol. Soc. 1878 , pp. 453,456 . 


\section{GEOLOGY}

first may be dismissed as a purely theoretical and unnecessary assumption. The second cannot be disproved, but can be equally well accounted for during the period of denudation and rapid accumulation subsequent to the raised beach formation. The felsitic boulders near Stapledon Common south-west of Torrington ${ }^{1}$ occur at intervals in a north and south line, just as the harder portions of a dyke would remain and withstand surface denudation. The large quartz rock blocks near Gawton ${ }^{2}$ and elsewhere are the portions of the local slates most interpenetrated by quartz veins which have survived the denudation of their less durable surroundings. The boulders of siliceous rock near Hestow farm and elsewhere between Chudleigh and Kingsteignton have been ascribed to greywethers from denuded Tertiaries, otherwise it would be difficult to explain their presence.

Economic.-The county is rich in materials for the manufacture of pottery, bricks and tiles. The Watcombe ware is made from the Lower New Red clays of that locality, and the Aller ware from the clays of the Bovey valley; these also furnish materials for bricks and tiles. China clay is worked in the decomposed granite of Headon Down, Lee Moor and Cadover Bridge at and near the south-western border of Dartmoor. Bricks are manufactured from alluvial clays and Head, as at Rumleigh and near West Looe; from the soil of the Keuper marls; from the Middle New Red marls; from the clays and brecciated clays of the Lower New Red at Exeter, Tiverton, etc.

The Cretaceous rocks furnish the best building stone, in the Beer stone, and whetstones on Blackdown. The Lower New Red breccias and breccia conglomerates, near Paignton, Torquay, Exeter and Halberton are quarried for building stone and wallstone.

The Permian trap rocks afford good building material, as may be seen in most of the churches in the districts in which they occur.

The Middle and Upper Culm rocks furnish grits and sandstones, locally used for building purposes. The seams of anthracite near Bideford, Alverdiscot, etc., are used in the manufacture of a pigment called 'Bideford black.'

The Coddon Hill beds are quarried for gravel and road metal.

The Westleigh limestones are quarried for lime-burning and building purposes. Interbanded Lower Culm rocks have been used with other materials in the construction of Tiverton church.

The Devonian limestones furnish ornamental and ordinary building stones, lime, and road metal. The Upper Devonian rocks have been quarried for slates near Buckfastleigh and elsewhere. The Eifelian have been more largely quarried for the same purpose, at Harbertonford for instance. The Lower Devonian grits are locally suitable for building purposes; the slates of the Meadfoot series are quarried for roofing slate in the Kingsbridge district, and often for building stone, where the

1 Rep. Brit. Assoc. 1876 ; Trans. of Sect. p. 110 ; and Ussher, 'On the Deposits of Petrockstow,' Trans. Devon Assoc. 1879.

2 Codrington, Quart. Journ. Geol. Soc. 1898, pp. 27 1-2. 


\section{A HISTORY OF DEVONSHIRE}

cohesion of the laminæ is pronounced, and for flags, ' lidstones,' or hedge stones.

The granite in places forms a good building stone, and where disintegrated furnishes material for gravelling walks, etc.

The hornblende-epidote schists are used for building purposes and road metal.

The diabases, dolerites, etc., are used for road metal. Near Trusham a hard greenstone has been dressed for building purposes, and here and there the diabases are quarried for building materials. Such felsites as the Roborough stone are suitable for building and for road metal. 


\section{PALÆONTOLOGY}

$7 \mathrm{HE}$ centre of interest in regard to the vertebrate palæontology of Devonshire is formed by the fossil remains obtained from the caves and cavernous fissures on the south coast : these cavern deposits being the first which were scientifically examined in Britain, and the first to afford evidence of the co-existence of man with the mammoth, woolly rhinoceros and other extinct Pleistocene mammals. Two of the most energetic workers in this field of research in the county were the late Rev. J. MacEnery and the late Mr. W. Pengelly, but many other palæontologists took a prominent part in this important investigation.

The earliest discovery of the occurrence of mammaliferous cave deposits in the county appears to have been made in 1816 by $\mathrm{Mr}$. Whidbey, at that time in charge of the works connected with the Plymouth breakwater. ${ }^{1}$ From the now well known cavernous fissures at Oreston near Plymouth that gentleman obtained numerous bones and teeth of large mammals, which were submitted to Sir Everard Home, by whom they were brought to the notice of the Royal Society in 1817 , four years previous to Dean Buckland's announcement of the discovery of fossil bones in Kirkdale Cave, Yorkshire. Other remains, including those of the lion, cave hyæna, Merck's rhinoceros and mammoth, were obtained from fissures in the neighbourhood at later dates, some of these and of the original specimens being described in Owen's British Fossil Mammals and Birds.

Other cavernous fissures near Yealmpton, about seven miles from Plymouth, were explored in the years 1835 and 1836 by Mr. Bellamy and Colonel Mudge, and yielded remains of similar animals. ${ }^{2}$ It has been thought probable that the bones were for the most part carried into both the Oreston and the Yealmpton fissures by the streams which flowed through them, although the suggestion has been made that the more cavernous portions were from time to time the resorts of hyænas, by which some of the bones may have been introduced.

Caves at Berry Head, Torbay, were explored at an early date by the Rev. Mr. Lyte.

'Kent's Hole,' the longest known and in some respects the most celebrated of all the Devon caves, was ascertained to contain mammalian remains in 1824 , and its strata were systematically excavated and the Hunting.

1 See Pengelly, 'Literature of the Oreston Cavern,' Trans. Devon Assoc. 1872; and Dawkins, Cave1870 .

See Pengelly, 'The Literature of the Caverns near Yealmpton, South Devon,' Trans. Devon Assoc. 


\section{A HISTORY OF DEVONSHIRE}

bones and teeth carefully examined and identified by Mr. MacEnery during the five years following. ${ }^{\text {. }}$ In these excavations it was definitely ascertained that flint implements of human manufacture occurred in association with the remains of the extinct mammals. Although the fact of this association was subsequently verified by other scientists, it was not generally accepted until a similar state of things was found to occur in Brixham Cave.

The fissures at Brixham, known as the Windmill Cave, were discovered accidentally in $185^{8}$, and their contents explored in that and the following year by an influential committee, of which Mr. Pengelly and the late Dr. Hugh Falconer were members. ${ }^{2}$ It was here, as already stated, that MacEnery's assertion as to the contemporaneity of the extinct mammalian remains with objects of human workmanship was fully and undisputably confirmed. Since however this is a subject connected more closely with the existence of prehistoric man in the county than with vertebrate palæontology, attention may here be restricted to the more important species of mammals obtained from Devonshire cavern deposits.

In the superficial layers of the floor of both Kent's Hole and Brixham Cave occur remains belonging to domesticated species, such as the Celtic shorthorn and the sheep or goat; and to these no further reference is necessary, except the bare mention that remains of the former animal have also been found in the county at Berry Head. It may be added that the remains of the rabbit recorded from Brixham Cave may also not improbably belong to a later epoch than the majority of those of the other mammals.

In the report of the committee for the exploration of Brixham Cave Professor Busk ${ }^{3}$ recorded twenty species or races of mammals (inclusive of the rabbit and Celtic shorthorn) as having been definitely identified from that cavern. Later investigations have somewhat enlarged the number, and the following list includes all the more important species known to occur in the deposits of Oreston, Kent's Hole and Brixham Cave. Most interesting of all are the canine and incisor teeth of the sabre-toothed tiger discovered by MacEnery and others in Kent's Hole, for it was upon the evidence of these that Owen named the species Macbarodus latidens. For many years these were the only remains of that species known from any British cavern, but this great tiger has been subsequently recorded from Creswell Crags, Derbyshire. Of these precious teeth one is in the museum of the Royal College of Surgeons, two are preserved in the British Museum, while a fourth is in the Albert Museum at Exeter. Teeth and other remains of the cave lion (Felis leo spelea), some of which are in the British Museum, have been obtained both from Brixham Cave and Kent's Hole, while those of the wild cat (F. catus) are known from the latter cave. Far more abundant

1 See Pengelly, 'Literature of Kent's Hole,' Trans. Devon Assoc. I 868-70.

2 See 'Report on the Exploration of Brixham Cave,' Proc. Royal Soc. xx. 5 I 4. ${ }^{3}$ Pbil. Trans. I 873, p. 517. 


\section{PALÆONTOLOGY}

are the jaws and teeth of the great cave hyæna (Hyana crocuta spelaa), an extinct race of the living spotted hyæna of south Africa, remains of this animal occurring in the Oreston fissures as well as in Brixham Cave and Kent's Hole. The wolf (Canis lupus) and the fox (C.vulpes) have likewise been recorded from the same three localities, but the badger (Meles meles) appears to be known only from Kent's Hole and Brixham, and the otter (Lutra lutra) from Kent's Hole alone. Remains of the polecat (Mustela putorius) have been identified from Brixham Cave and Berry Head, and those of the stoat (M. erminea) from Kent's Hole, Berry Head, a raised beach near Plymouth, and possibly Oreston. The polecat has been stated to occur also in the Plymouth raised beach, but it is not certain that the remains have been rightly identified. Of the brown bear (Ursus arctus) teeth and jaws occur in the Oreston, Brixham and Kent's Hole deposits ; and remains from the same three localities have been assigned to the North American race of the same species commonly known as the grizzly, but the identification is somewhat doubtful. The great extinct cave bear (U. spelaus) appears to have shared the accommodation afforded by Brixham Cave and Kent's Hole with the cave hyæna, or perhaps the bears may have used each of these caves as a den at one time and the hyænas at another.

Among the smaller mammals, jaws of the greater horseshoe bat (Rbinolophus ferrum-equinum) have been discovered in Kent's Hole, and those of the common shrew (Sorex araneus) in Kent's Hole and Brixham Cave. Remains of the field vole (Microtus agrestis) have been identified from Kent's Hole; those of the water vole (M. ampbibius) from Brixham, Kent's Hole and Oreston; and those of the bank vole (Evotomys glareolus) from Kent's Hole and Brixham Cave. The mouse (Mus musculus) has been recorded from Kent's Hole, but it does not seem certain that its remains are not of later date than those of the other mammals; and the same remark, as already said, will apply to those of the rabbit (Lepus cuniculus), which occur in Brixham Cave and Kent's Hole. The same two caves have yielded remains of the common hare ( $L$. europaus), and of the little pica or tailless hare (Ocbotona pusilla), a species now unknown in Britain.

Turning to the hoofed or ungulate mammals, of which the remains were probably in most cases dragged into the caves by hyænas and bears, although carried by water into the Oreston fissures, we have evidence of the great extinct wild ox or aurochs (Bos taurus primigenius) at Oreston, Brixham and in Kent's Hole. The Pleistocene bison (B. priscus) does not appear to have been recorded from either of the two caves named, although its remains are said to occur in some cavern deposits in the county. The so-called Bison minor, named by Owen in his British Fossil Mammals and Birds on the evidence of a leg-bone from Oreston, is identical with the Celtic shorthorn already mentioned. Deer are represented by the red deer (Cervus elapbus) and the extinct giant fallow deer or 'Irish elk' (C. giganteus) in Brixham Cave and Kent's Hole, by the roe (Capreolus capreolus) at Brixham, and the reindeer (Rangifer tarandus) 


\section{A HISTORY OF DEVONSHIRE}

in all the above-named caverns, as well as in the Oreston fissures. Remains of the Pleistocene race of the hippopotamus (Hippopotamus ampbibius major), always rare in cavern deposits, have been recorded from Kent's Hole, and those of the wild boar (Sus scrofa ferus) from the Oreston fissures.

Teeth and other remains of the wild horse (Equus caballus fossilis) occur in Brixham and Kent's Cave, as well as in the Oreston fissures; and two upper molar teeth from Oreston were regarded by Owen ${ }^{1}$ as indicating a fossil ass or zebra, which was named Asinus fossilis, but their right to distinction has not been subsequently recognized. The three common Pleistocene species of rhinoceros have been recorded from the county. Of these the woolly Siberian species (Rbinoceros antiquitatis), a near relative of the living Burchell's rhinoceros of south and central Africa, occurs in Brixham and Kent's Cave and at Oreston, as well as in another Pleistocene deposit near Plymouth. The leptorhine rhinoceros ( $R$. leptorbinus), another two-horned species, distinguished by the simpler form of its upper molar teeth, is recorded from Brixham Cave, and the closely allied Merck's rhinoceros ( $R$. mercki) from Oreston. The latter identification is important, since the species in question has been recorded elsewhere from British caves only at Gower in Glamorganshire. Finally, the mammoth (Elephas primigenius), a near relative of the existing Indian elephant, is abundantly represented by teeth and other remains in Brixham Cave, Kent's Hole and the Oreston fissures. It has also been recorded from Sidmouth.

The bird remains ${ }^{2}$ from Devonshire include a tarso-metatarsus of the snowy owl (Nyctea nyctea) from Kent's Hole, the bones of the buzzard (Buteo buteo) from Brixham Cave, a femur from Kent's Hole assigned to the blackcock (Tetrao tetrix), and a considerable series of bones from Brixham Cave referred to an undetermined species of sheldrake (Tadorna). All the above are in the British Museum. In addition to these there is said to be a falcon nearly allied to or identical with the peregrine (Falco peregrinus), the evidence resting on bones from a cave at Berry Head.

Passing on to the Mesozoic and Palæozoic formations of the county, we have first of all to refer to remains of that remarkable reptile Hyperodapedon gordoni from the Keuper of High Peak near Sidmouth. The reptile in question, which was first made known on the evidence of remains from the Trias of Elgin, is a relative of the tuatera, a lizard-like reptile now inhabitating two small islands near New Zealand, and the sole existing representative of a once numerous order. The Sidmouth specimen, a fragment of the upper jaw, is described by Huxley in vol. xxv. (p. I 46) of the 2uarterly Fournal of the Geological Society. The only other English county in which remains of Hyperodapedon have been discovered is Warwick. The Sidmouth Keuper has likewise yielded remains of the primeval salamanders, or labyrinthodonts, the

1 Brit. Foss. Mamm. and Birds.

2 See Lydekker, Cat. Foss. Birds Brit. Mus. I 190. 


\section{PALÆONTOLOGY}

majority of which are characterized by the sculptured bones of the skull and the curiously complicated internal structure of their teeth. A lower jaw of one of these creatures from Sidmouth has been made the type of a species by Professor H. G. Seeley ${ }^{1}$ under the name of Labyrintbodon lavisi, while various unnamed remains of the same group obtained from Sidmouth and Budleigh Salterton have been described by Mr. A. T. Metcalfe. ${ }^{2}$

From the Rhrtic formation of Axminster and Axmouth remains of a small number of species of fishes have been recorded, none of which however were named on the evidence of Devonshire specimens. They include two species of an extinct genus allied to the existing Port Jackson shark, namely Hybodus minor from Axminster and $H$. cloacinus from Axmouth. A fine specimen from the former locality belonging to the type commonly known as Nemacantbus monilifer is not improbably referable to Hybodus minor. ${ }^{3}$ A tooth of a ganoid or enamel-scaled fish known as Saurodon tomicus, belonging to the same family as the more common Lepidotus, has also been obtained from the Rhætic bone bed at Axminster. Another family of ganoids is represented in the last mentioned deposit by scales referable to a fish originally described from the Trias of Germany under the name of Gyrolepis albertii.

The Culm Measures of the county (like the Devonian formation) seem to be singularly poor in fish remains, although those of two species have been recorded from Instow. The first of these, Coelacantbus elegans, is typically an American species belonging to the group of fringe-finned ganoids, of which the sole survivors at the present day are the bishir and an allied type, both from Africa. The second, Elonichtbys aitkeni, is a species of a genus belonging to the same family (Palconiscida) as the above-mentioned Gyrolepis, and named on the evidence of Devonshire specimens obtained by Mr. J. Aitken.

In marked contrast to their comparative abundance in the freshwater Old Red Sandstone of Herefordshire is the scarcity in the approximately equivalent marine Devonian strata of Devonshire of remains of those extremely primitive Palæozoic fishes respectively classed as Ostracodermi and Artbrodira. From the Lower Devonian of Mudstone Bay there has however been obtained part of the head-shield of a pteraspid (Ostracodermi), although it is insufficient to allow of generic identification. This and similar remains from the Devonian of Polperro in Cornwall were originally described as fossil sponges, under the name of Steganodictyum cornubicum." The evidence for the presence of Artbrodira in the Upper Devonian of the county is afforded by an imperfect dorsal plate from Chudleigh, preserved in the collection of the British Museum, and probably referable to the typical genus Coccosteus. ${ }^{5}$ It is one of the numerous specimens from the west of England presented to the national collection by the late Mr. J. E. Lee of Caerleon.
1 2uart. Foum. Geol. Soc. xxxii. 278.
2 Ibid. x. 257.
3 See A. Smith Woodward, Cat. Foss. Fish. Brit. Mus. ii. I 16.
4 See E. R. Lankester, 2uart. Fourm. Geol. Soc. xxiv. 546, and Woodward, op. cit. 175.
$\checkmark$ See Woodward, op. cit. 293. 


BOTANICAL

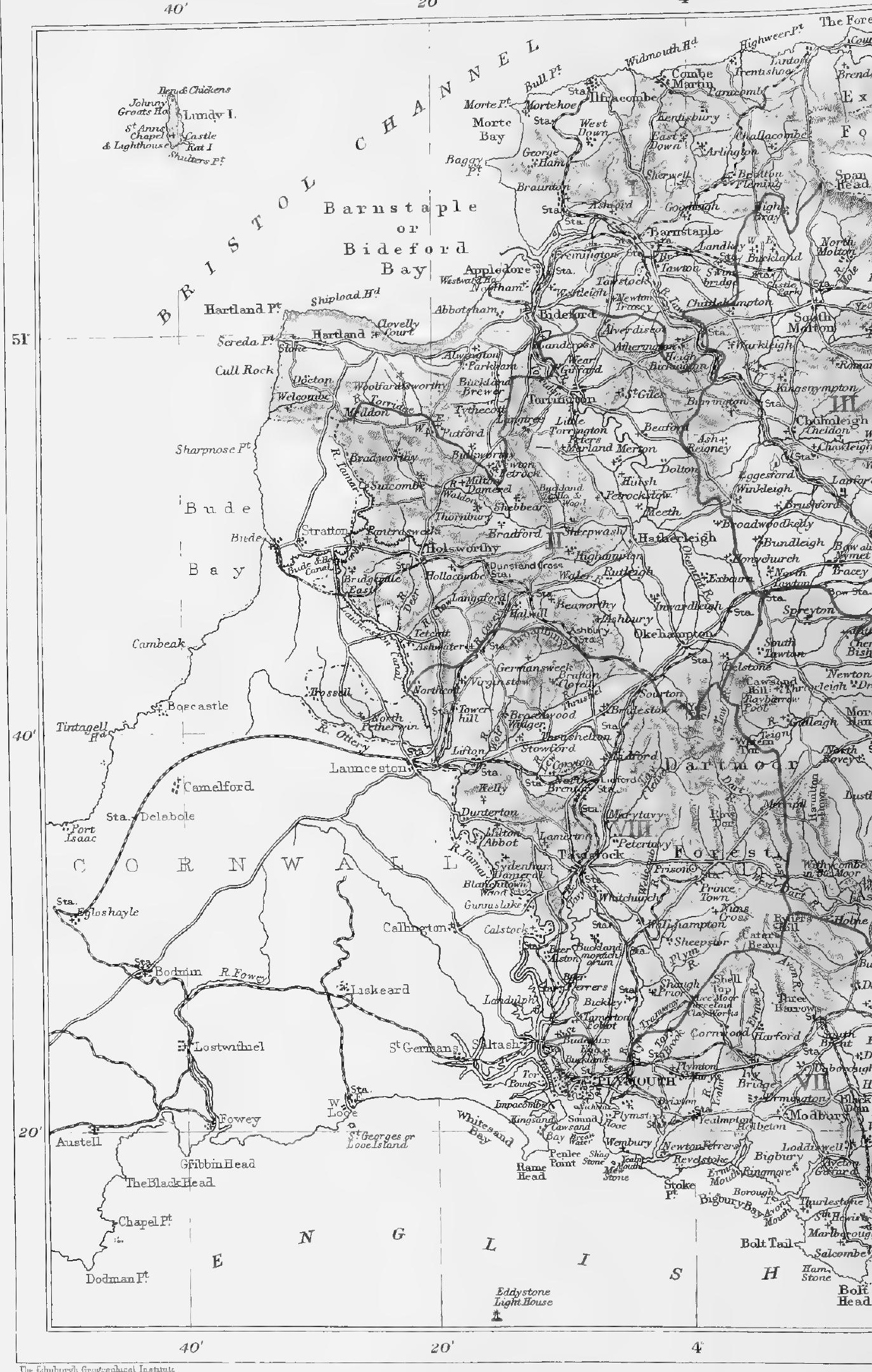


DISTRICTS.

$20^{\prime}$

2

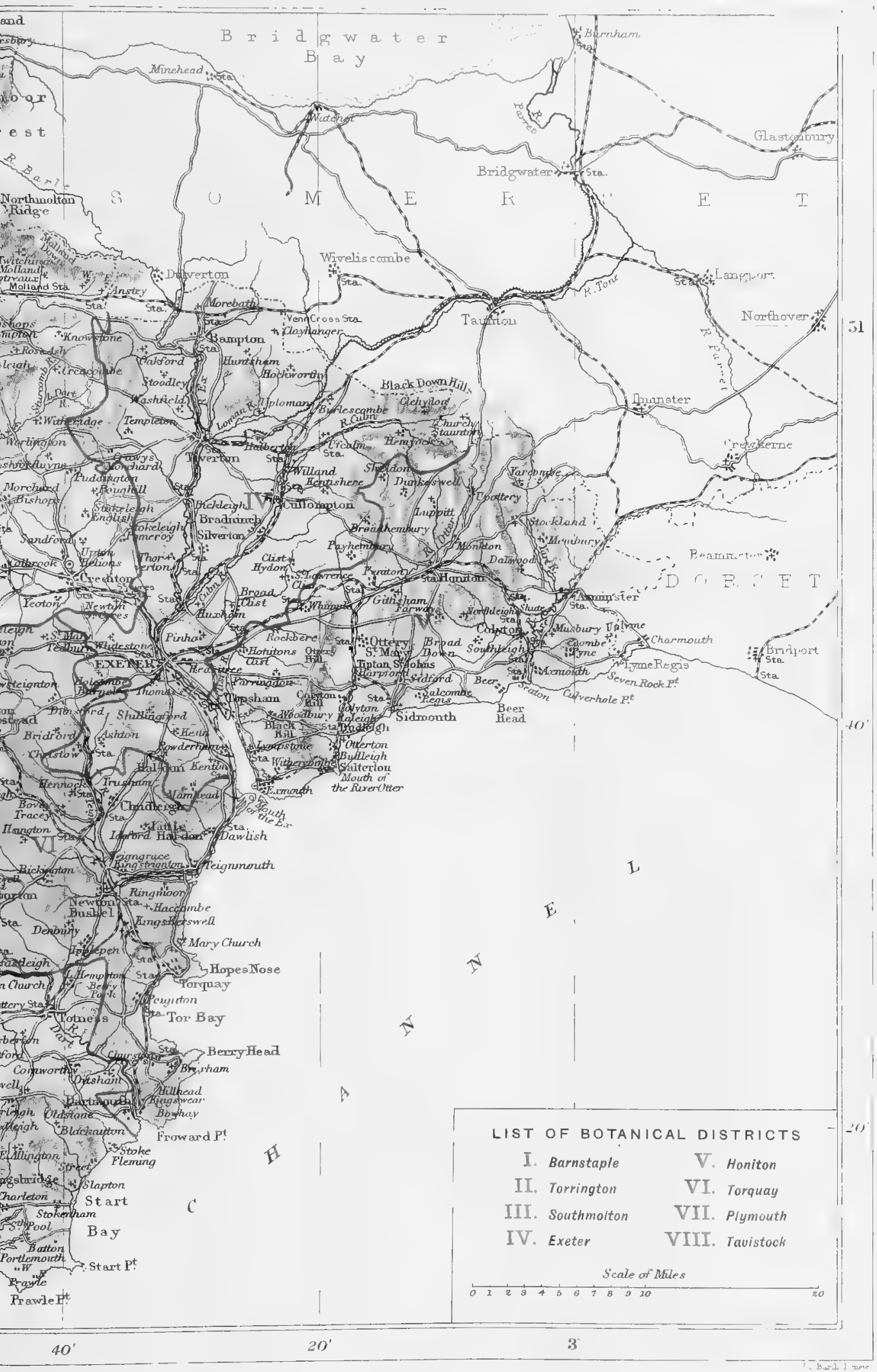

THE COUNTIES OF ENGLAND 



\section{BOTANY}

A

CONSPICUOUS feature of the county of Devon is the generally hilly nature of the surface, and this feature prevails in all districts; the hills vary in height from a moderate elevation to more than 2,000 feet above sea-level, and in a few cases almost deserve the name of mountains ; their shape is very various, in some cases being long and smooth about the top with gentle slopes and flowing outlines, in other cases rather conical and having precipitous sides with steep and narrow gorges; the tors of Dartmoor are striking piles of granite. The colours of the rocks in different regions show tints of pale greenish grey, silvery grey, steel grey, warm grey, pale reddish, red and buff.

The wooded valleys which abound in many parts produce pleasing diversities; the river scenery, especially in the valleys of south Devon, present very charming features; in north Devon the coast scenery is particularly grand.

According to Ordnance Survey figures the area of the whole is as follows :-

\begin{tabular}{rll}
$1,666,454.964$ & acres of land \\
$4,433.123$ & $\#$ & inland water \\
224.076 & $\Rightarrow$ & saltmarsh \\
$18,342.728$ & $\Rightarrow$ & foreshore \\
$3,061.521$ & & tidal water \\
\hline $1,692,516.412$ & & Total
\end{tabular}

The elimate is moist; the following table gives the monthly rainfall for three stations-one in the north part, the second in the south part, and the third in the west part of the county.

Monthly Average Rainfall of the Ten Years i8go-9, Depth in Inches, from Wallrs's British Rainfall, I899, p. 28 (1900)

\begin{tabular}{|c|c|c|c|c|c|c|c|c|c|c|c|c|c|c|}
\hline Stations & $\begin{array}{c}\text { Alti- } \\
\text { tudes } \\
\text { above } \\
\text { sea- } \\
\text { level }\end{array}$ & Jan. & Feb. & Mar. & April & May & June & July & Aug. & Sept. & Oct. & Nov. & Dec. & $\begin{array}{c}\text { An- } \\
\text { nual } \\
\text { total }\end{array}$ \\
\hline $\begin{array}{l}\text { Barnstaple, } \\
\text { Athenæum . } \\
\text { Ashburton, } \\
\text { Druid House } \\
\text { PolapitTamar, } \\
\text { Werrington }\end{array}$ & $\left|\begin{array}{c}\mathrm{ft} . \\
25 \\
572 \\
315\end{array}\right|$ & $\begin{array}{l}2 \cdot 83 \\
5 \cdot 25 \\
3 \cdot 34\end{array}$ & $\begin{array}{l}2 \cdot 23 \\
3 \cdot 40 \\
2 \cdot 33\end{array}$ & $\mid \begin{array}{c}2 \cdot 08 \\
3.59 \\
2 \cdot 18\end{array}$ & $\begin{array}{l}\mathrm{I} \cdot 88 \\
2 \cdot 9 \mathrm{I} \\
2 \cdot \mathrm{I} 5\end{array}$ & $\mid \begin{array}{l}1 \cdot 97 \\
2 \cdot 48 \\
2 \cdot 12\end{array}$ & $\begin{array}{l}1 \cdot 85 \\
2 \cdot 49 \\
1 \cdot 76\end{array}$ & $\begin{array}{l}2 \cdot 84 \\
2 \cdot 83 \\
2 \cdot 66\end{array}$ & $\mid \begin{array}{c}3 \cdot 30 \\
4.11 \\
3.64\end{array}$ & $\begin{array}{l}2 \cdot 88 \\
3 \cdot 19 \\
3 \cdot 09\end{array}$ & $\begin{array}{l}4.21 \\
6 \cdot 12 \\
4.96\end{array}$ & $\begin{array}{l}3.24 \\
6.08 \\
4.05\end{array}$ & $\mid \begin{array}{l}3.79 \\
7.52 \\
4.51\end{array}$ & $\begin{array}{l}33^{\circ} 10 \\
49^{\circ} 97 \\
3^{6} \cdot 79\end{array}$ \\
\hline Means & 304 & $3 \cdot 8 x$ & $2 \cdot 65$ & 2.62 & $2 \cdot 31$ & $|2 \cdot 19|$ & $2 \cdot 03 \mid$ & 2.78 & 3.68 & 3.05 & 5.10 & 4.46 & $5 \cdot 27$ & $39^{\circ} 95$ \\
\hline
\end{tabular}




\section{A HISTORY OF DEVONSHIRE}

In a paper contributed in 1899 to the Devonshire Association by Mr. A. Chandler, the temperature, rainfall and sunshine were investigated; he divided the county into the coast, the inner lands and the highlands, and showed that these zones exhibit three types of climate. First, on the coast there is an equable climate, warm in winter and cool in summer, and that in this zone the mean temperature is the highest, the mean range of temperature the least, the rainfall the smallest, and the sunshine the greatest. Secondly, in the zone of the inner lands the mean temperature, the rainfall and the amount of sunshine are intermediate between those of the other zones, while the mean range of temperature is greater than in the others. Thirdly, the highlands of Dartmoor have the lowest mean temperature, are intermediate in mean range of temperature, enjoy the greatest rainfall and, so far as cloud measurements prove, the least sunshine. The following table supplies the summary of the records :-

\begin{tabular}{|c|c|c|c|c|c|c|c|}
\hline & & $\begin{array}{c}\text { Mean } \\
\text { annual } \\
\text { tempera- } \\
\text { ture in } \\
\text { Fahrenheit } \\
\text { degrees }\end{array}$ & $\begin{array}{c}\text { Mean } \\
\text { annual } \\
\text { range of } \\
\text { tempera- } \\
\text { ture in } \\
\text { degrees }\end{array}$ & $\begin{array}{c}\text { Mean } \\
\text { annual } \\
\text { rainfall, } \\
\text { depth } \\
\text { in } \\
\text { inches }\end{array}$ & $\begin{array}{c}\text { Mean } \\
\text { annual } \\
\text { sunshine } \\
\text { in } \\
\text { hours }\end{array}$ & $\begin{array}{c}\text { Percentage } \\
\text { of } \\
\text { possible } \\
\text { sunshine }\end{array}$ & $\begin{array}{l}\text { Mean } \\
\text { daily hours } \\
\text { of } \\
\text { sunshine }\end{array}$ \\
\hline $\begin{array}{l}\text { Coast zone } . \\
\text { Inner land zone } \\
\text { Highland zone } \\
\text { Average for Devon }\end{array}$ & $\begin{array}{ll}\cdot & \cdot \\
\cdot & \cdot \\
\cdot & \cdot \\
\cdot & .\end{array}$ & $\begin{array}{l}50 \cdot 7 \\
49.6 \\
48 \cdot 6 \\
49.9\end{array}$ & $\begin{array}{l}10.9 \\
14.7 \\
12.5 \\
12.6\end{array}$ & $\begin{array}{l}35 \cdot 63 \\
42 \cdot 81 \\
57 \cdot 94 \\
44 \cdot 35\end{array}$ & $\begin{array}{l}1,671 \\
1,582 \\
1,491 \\
1,581\end{array}$ & $\begin{array}{l}38 \\
36 \\
34 \\
36\end{array}$ & $\begin{array}{l}4 \frac{1}{2} \\
4 \frac{1}{4} \\
4 \\
4 \frac{1}{4}\end{array}$ \\
\hline
\end{tabular}

Devonshire is remarkably well watered and has an immense number of streams; 'it has in it abundance of rivers, more perhaps than any other county in England' (Gibson's edition of Camden's Britannia, 1695).

The following is a list of principal rivers, mostly on the authority of De la Beche :-

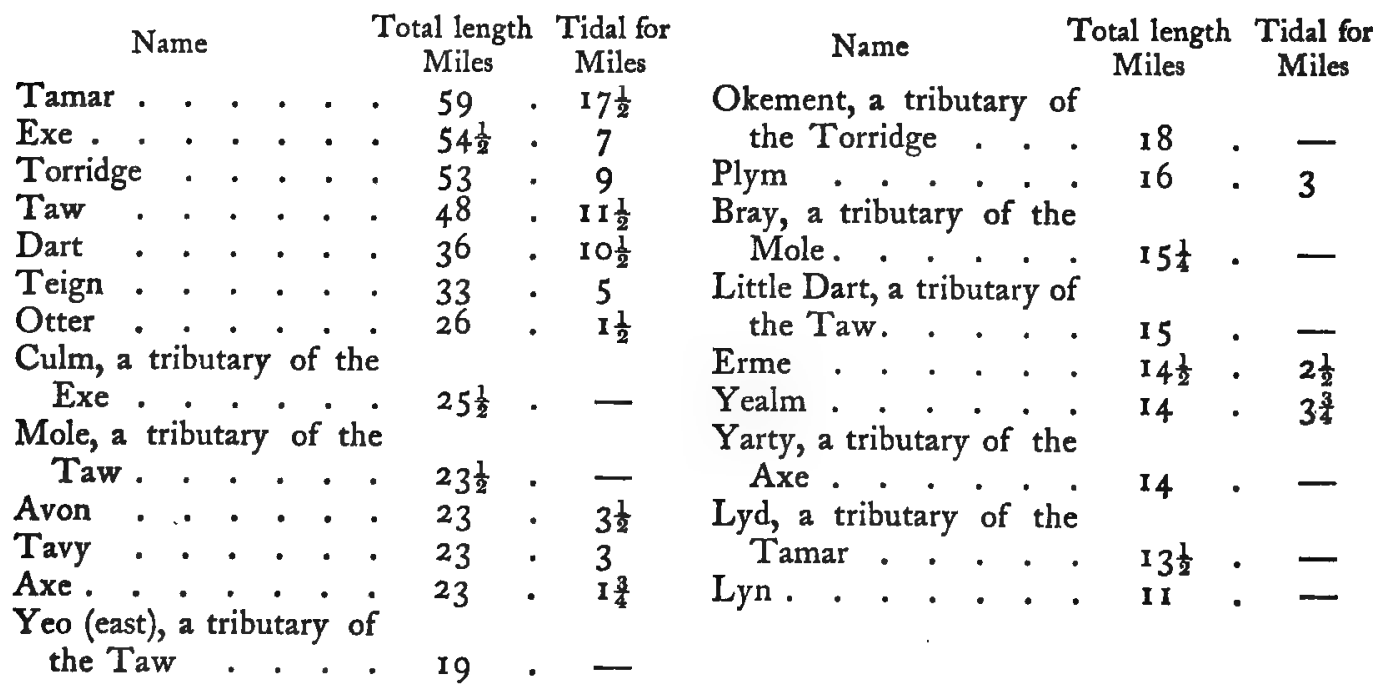

For botanical purposes a suitable and convenient division of the county into districts is required. This matter was discussed by Jones and 


\section{BOTANY}

Kingston in their Flora Devoniensis (1829) ; they pointed out that owing to the great variety of soil and inequality of surface there is probably no county in the kingdom that presents a more diversified appearance, and that although there are varying degrees of fertility and luxuriance accompanying the principal geological formations, there are no peculiar vegetable features applicable to the general distribution of the flowering plants by which it is easy to distinguish such formations from each other; the ploughman's spikenard (Inula Conyza) and a few other species affect the Devonian system; the old man's beard (Clematis Vitalba) also grows more luxuriantly among the crevices of those rocks than elsewhere; while the roast beef plant (Iris foetidissima) and the elm prevail mostly on the new red sandstone; but none of these plants is exclusively confined to any particular formation. Proximity to the seacoast produces an important botanical effect, and the same species may be traced along the coast and occur on various kinds of geological formations; 'thus the most characteristic botanical division of the county would be into the central inland portion and those on either side of it that border on the sea. That there is a great difference not only between the mean annual temperatures of these tracts, but also a much greater variation in the mean temperatures of the different months of a year in the former than in the latter, experience sufficiently proves. These differences arise from the elevation and exposure of the one, as well as the large extent of boggy and unreclaimed land it includes, and the sheltered and cultivated state of the other, together with its proximity to the ocean' (Jones and Kingston, Flora Devoniensis, ii. 203). In the result these authors did not form botanical districts for the purpose of their book. Subsequently H. C. Watson for his Topographical Botany divided the county into two vice-counties, south Devon and north Devon, separated by an imaginary line which was roughly adapted to the watershed; it began by the Cornish boundary at the river Tamar about midway between Tavistock and Launceston, thence passed over the ridge of Dartmoor, joined the Grand Western Canal at Tiverton and followed the course of that canal to the Somerset boundary in the parish of Holcombe Rogus ; these two divisions are manifestly insufficient for the present work.

In $188 \mathrm{I}$ a botanical record committee was formed at Barnstaple in connection with the Barnstaple Literary and Scientific Institution (the predecessor of the North Devon Athenæum) for collecting detailed and systematic records of the natural distribution of north Devon plants, that is of wild plants occurring in the part of the county drained by rivers and streams which ultimately empty their waters into the sea on the north coast; for this purpose the north Devon area of $15^{8}$ parishes and parts of parishes was divided into eleven districts, which were broadly based on the existing hundreds of the county but with some modifications. These districts are too small to be adopted in this work, though they were suitable for the objects of the local committee.

The division of the county into districts now made is designed to 


\section{A HISTORY OF DEVONSHIRE}

study botanical convenience; they are moderate in number, not very unequal in size, easy to be defined, and their limits can be traced on the ordnance maps; they are each named after a principal town therein, as follows: I, Barnstaple ; 2, Torrington; 3, South Molton; 4, Exeter ; 5, Honiton ; 6, Torquay ; 7, Plymouth ; 8, Tavistock.

The Barnstaple district contains the whole of the north coast; the Honiton, Torquay and Plymouth districts together contain the south coast; the Barnstaple district is entirely and the Torrington and South Molton districts are mostly north of the watershed; the Honiton and Plymouth districts are entirely, and the Exeter, Torquay and Tavistock districts are mostly south of the watershed.

The most fertile districts are those of Exeter, Honiton and Plymouth; those of Torquay, Barnstaple and South Molton are about the average of fertility; and those of Tavistock and Torrington are the most barren. Chalk is found only in the Honiton district over a limited area.

The summary on the opposite page, showing for each natural order the number of genera and species in the county, is drawn up generally in accordance with Hooker's Student's Flora of the British Islands (ed. 3), but the census of species is calculated on the scale of the London Catalogue of British Plants (ed. 9). The enumeration in the Catalogue included a considerable number of sub-species, colonists, aliens and casuals; it is therefore necessary for the sake of just comparison to count for the purpose of the census all such like plants occurring in Devonshire under circumstances similar to those which occasioned their inclusion in the Catalogue; but where the circumstances are substantially different they are not counted. The setter-wort (Helleborus fotidus) is regarded as native in the chalk pastures and thickets of southern and south-eastern England, but in Devon it occurs only as colonist or casual; it is therefore not counted in the census.

The American cress (Barbarea pracox) is an alien, but is well established in many parts of England and as such is mentioned in the Catalogue; it occurs in like manner and is spreading in Devon, and is therefore counted.

Barbarea intermedia is treated in the Student's Flora only as a subspecies occurring in cultivated fields, but in the Catalogue it is numbered as a species; in south Devon it occurs as a colonist, and under these circumstances it is counted in the census.

The cushion-pink or moss-campion (Silene acaulis) according to Ray was found in Devon by Mr. Gidley, and Hudson in 1778 recorded it as occurring on Dartmoor. There is no modern record of its occurrence there, and there is reason to suspect that some other plant was intended by the old authorities; it is therefore not counted.

The bryony (Bryonia dioica) has several times been reported as occurring in the county. Polwhele in his History of Devonsbire, i. 96 (1797), under the synonym of $B$. alba, stated that 'the beautiful berries of the bryony or wild vine very much ornament our hedges during the winter months, and the exprest juice is formed into an ointment as a 
BOTANY

SUMMARY OF ORDERS

\begin{tabular}{|c|c|c|c|c|c|c|}
\hline & & $\begin{array}{c}\text { Number of } \\
\text { Genera }\end{array}$ & $\begin{array}{l}\text { Number of } \\
\text { Species }\end{array}$ & & $\begin{array}{c}\text { Number of } \\
\text { Genera }\end{array}$ & $\begin{array}{l}\text { Number of } \\
\text { Species }\end{array}$ \\
\hline \multirow{5}{*}{\multicolumn{2}{|c|}{$\begin{array}{c}\text { PHANEROGAMIA } \\
\text { DICOTYLEDONES } \\
\text { ANGOOSPERMA } \\
\text { POLYPETALA } \\
\text { Thalamiflore }\end{array}$}} & & & 47. Boragineæ & 8 & 15 \\
\hline & & & & 48. Convolvulacex. & 3 & 6 \\
\hline & & & & 49. Solanacex . ' & 5 & 6 \\
\hline & & & & $\begin{array}{l}\text { 50. Plantaginex } \\
\text { 51. Scrophularineæ (ex- }\end{array}$ & & 0 \\
\hline & & & & cluding Lathraa) & 13 & 40 \\
\hline 1. Ranunculacex . & & 10 & 33 & $\begin{array}{l}\text { 52. Orobanchex (in- } \\
\text { cluding Latbraa) }\end{array}$ & & 6 \\
\hline 2. Berberidex. & & $\begin{array}{l}1 \\
2\end{array}$ & $\begin{array}{l}1 \\
2\end{array}$ & 53. Lentibularinez & 2 & $\begin{array}{l}0 \\
4\end{array}$ \\
\hline $\begin{array}{l}\text { 3. Pympnaacex } \\
\text { 4. Papaveracez. }\end{array}$ & & 4 & 8 & 54. Verbenacex . . & $\mathbf{I}$ & I \\
\hline 5. Fumariaceæ & & 2 & 7 & 55. Labiatz. . . & I9 & 46 \\
\hline 6. Cruciferz . & & 24 & 57 & INCOMPLET & & \\
\hline 7. Resedacex . & & $\mathbf{I}$ & 3 & 56. Illecebraceæ . . & 3 & 3 \\
\hline 8. Cistinex . . & & I & 2 & 57. Amaranthacex. & I & I \\
\hline 9. Violaceæ . . & - . & 1 & 10 & 58. Chenopodiaceæ . & 6 & 20 \\
\hline 10. Polygalex . & & I & 3 & 59. Polygonaceæ . . & 3 & $2 \mathrm{I}$ \\
\hline II. Caryophyllez . & - $\cdot$ & I I & 41 & 60. Aristolochiaceæ . & I & I \\
\hline 12. Portulacez. & $\cdot \cdot$ & $\mathbf{I}$ & 1 & 61. Thymelæacez. - & I & I \\
\hline 13. Tamariscinex & - $\cdot$. & $\mathbf{I}$ & $\mathbf{I}$ & 62. Loranthacex . . & 1 & I \\
\hline I4. Hypericinez & - $\cdot$ & $\mathbf{I}$ & 14 & 63. Santalacea. . . & $\mathbf{I}$ & I \\
\hline 15. Malvacex . & - $\cdot$ & 3 & 5 & 64. Euphorbiacex . . & 2 & II \\
\hline 16. Tiliaceæ . & & I & 2 & 65. Urticacez . . . & 4 & 6 \\
\hline 17. Linez · · & $\cdot \cdot$ & 2 & 4 & 66. Myricacex. . . & $\mathbf{I}$ & $\mathbf{I}$ \\
\hline 18. Geraniacex. & $\cdot \cdot \cdot$ & 3 & 18 & 67. Cupuliferæ. . . & 7 & 8 \\
\hline 19. Ilicinex - & - $\cdot$ & I & I & 68. Salicinex - . & 2 & I3 \\
\hline 20. Empetraceæ & & $\mathbf{I}$ & $\mathbf{I}$ & 69. Ceratophyllex. . & $\mathbf{I}$ & I \\
\hline Calyciftora & & & & GYMNOSPERMEE & & \\
\hline 21. Celastrinex. & $\cdot \cdot$ & I & $\mathbf{I}$ & 70. Coniferæ . . & 2 & 2 \\
\hline 22. Rhamnex - & - & I & I & MONOCOTYLEDONES & & \\
\hline $\begin{array}{l}\text { 23. Sapindaceæ . } \\
\text { 24. Leguminosæ }\end{array}$ & $\dot{.}$ & $\begin{array}{r}1 \\
15\end{array}$ & $\begin{array}{r}2 \\
64\end{array}$ & Microsperme & & \\
\hline 25. Rosacex. & . . & 12 & 102 & 7. Hydrocharidex . & 2 & 2 \\
\hline 26. Saxifrageæ . & $\cdot \cdot$ & 3 & 7 & 72. Orchidex . . & 8 & 22 \\
\hline 27. Crassulaceæ & 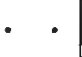 & 4 & I I & Epigynce & & \\
\hline 28. Droseracex. & $\cdot \cdot \cdot$ & 1 & 3 & 73. Iridez. . . . & & 5 \\
\hline 29. Lythracex . & $\cdot \cdot$ & 2 & $\begin{array}{l}2 \\
8\end{array}$ & 74. Amaryllideæ . . & 3 & 7 \\
\hline $\begin{array}{l}\text { 30. Haloragex. } \\
\text { 31. Onagrarieæ. }\end{array}$ & $\cdot \dot{ }$ & $\begin{array}{l}3 \\
3\end{array}$ & $\begin{array}{r}8 \\
12\end{array}$ & 75. Dioscoreæ . . & I & I \\
\hline 32. Umbelliferz & $\cdot \cdot$ & 29 & 49 & Coronaries & & \\
\hline 33. Araliacez - & - $\cdot$ & I & I & 76. Liliacex & I I & 16 \\
\hline 34. Cornaceæ . & - $\cdot$ & I & $\mathbf{I}$ & 77. Juncex • • • • & 2 & 19 \\
\hline Monopetale & & & & Nudiflore & & \\
\hline 35. Caprifoliacez . & - . & 4 & 6 & 78. Typhacez . · · & 2 & 5 \\
\hline 36. Rubiacez - & - $\cdot$ & 4 & 13 & 79. Aroidez • • • & 2 & 3 \\
\hline 37. Valerianex. . & $\cdot \cdot$ & 3 & 8 & 80. Lemnaceæ . . & $\mathbf{I}$ & 4 \\
\hline 38. Dipsacez . . & $\cdot \cdot$ & 2 & 5 & Apocarpe & & \\
\hline $\begin{array}{l}\text { 39. Compositz. } \\
\text { 40. Campanulacez. }\end{array}$ & : & $\begin{array}{r}42 \\
5\end{array}$ & $\begin{array}{r}96 \\
6\end{array}$ & 81. Alismacea . . & 3 & 4 \\
\hline 41. Ericacez . . & - . & 3 & 4 & Glumacee & 5 & 21 \\
\hline 42. Plumbaginea . & $\bullet$ & 2 & 4 & Grumacee & & \\
\hline 43. Primulacex. & $\cdot$ & $\begin{array}{l}7 \\
2\end{array}$ & $\begin{array}{r}13 \\
2\end{array}$ & $\begin{array}{l}\text { 83. Cyperacex . . . } \\
\text { 84. Graminex . . . }\end{array}$ & $\begin{array}{r}7 \\
4 \mathrm{I}\end{array}$ & $\begin{array}{r}62 \\
100\end{array}$ \\
\hline 45. Apocynaceæ . & $0^{\circ}$ & I & 2 & & & \\
\hline 46. Gentianex . & & 5 & 9 & Totals . . . & $4 \mathrm{II}$ & $1,13^{8}$ \\
\hline
\end{tabular}




\section{A HISTORY OF DEVONSHIRE}

specific for childblains.' There are other unsatisfactory records for its occurrence both in north and south Devon. Mr. Moyle Rogers found it as a casual on a garden wall at Teignmouth, but he does not on this account regard the species as a Devon plant. Mr. Ralph Morgan of Heavitree also about twenty years ago found it growing in the hedges of Stoke Wood about $2 \frac{1}{2}$ miles from Exeter, but on subsequent searches failed to find it again, and he agrees that without further evidence the species ought not to be included in the Devon list.

The sea-cudweed or cotton-weed (Diotis maritima) was reported by Withering (Bot. Arr. p. 707) to occur in the county, and in Croydon's Torquay Guide, p. $16_{3}$ (184I), it was recorded under the name of ' Gnaphalium maritimum (sea cotton-weed)' for Babbicombe. The old records are probably correct, but there is reason to fear that the plant is now extinct in the county, and it is therefore not included in the summary.

Altogether about 125 species, which are included in the London Catalogue, and of which the records for the county are considered on various grounds to be unsatisfactory, have been rejected and are thus excluded from the census.

The summary shows 867 Devon species of dicotyledons and 271 species of monocotyledons; these figures are nearly in the proportion of I 6 to 5. In Jones and Kingston's Flora Devoniensis (1 829 ) the number of dicotyledons mentioned is 605 and that of monocotyledons 193 ; these are approximately as 47 to 15. In Ravenshaw's List of Plants in Devon $(1872)$ the corresponding proportion is 60 to 19 . In Mansel-Pleydell's Flora of Dorsetsbire (ed. 2, 1895 ) the number of dicotyledons is to that of monocotyledons nearly as 19 to 6 ; this is a rather smaller proportion of dicotyledons than prevails in Devon. In Archer Brigg's Flora of Plymouth (1880) the proportion is nearly 7 to 2. The Student's Flora of the British Islands (ed. 3,1884 ) contains species of these groups, the numbers of which are approximately in the proportion of $3 \mathrm{I}$ to $\mathrm{I}$. The London Catalogue (ed. 9, 1895) contains corresponding figures which are nearly as 3 to $I$; this comparatively high proportion is due to the enumeration of the critical species of the brambles and hawkweeds.

A comparison of these sets of figures nicely confirm the general law arrived at by Robert Brown (Appendix iii. to Flinders' Voyage to Terra Australis, vol. 2, pp. 537, 538) in 1814 , "that from the equator to $30^{\circ}$ of latitude, in the northern hemisphere at least, the species of Dicotyledonous plants are to Monocotyledones as about 5 to $\mathrm{I}$; in some cases considerably exceeding, and in a very few falling somewhat short of this proportion; and that in the higher latitudes a gradual diminution of Dicotyledones takes place, until in about $60^{\circ} \mathrm{N}$. lat. and $55^{\circ} \mathrm{S}$. lat. they scarcely equal half their intratropical proportion.'

The larger dicotyledonous orders are comparatively more fully represented in Devon than are the monocotyledons. The following list shows in descending scale, for each order represented by at least as many species as Hypericinex, the percentage borne by the number of Devon species to that in the London Catalogue :- 


\section{BOTANY}

\begin{tabular}{|c|c|c|c|c|c|c|}
\hline $\begin{array}{l}\text { lypericineæ } \\
\text { reraniaceæ : } \\
\text { abiatæ : } \\
\text { henopodiaceæ } \\
\text { eguminosæ . }\end{array}$ & $\begin{array}{r}100 \\
\cdot \quad 78 \\
\cdot \quad 77 \\
\cdot \quad 74 \\
72\end{array}$ & $\begin{array}{l}\text { Scrophularineæ } \\
\text { Umbelliferæ . } \\
\text { Polygoneæ. . } \\
\text { Ranunculaceæ } \\
\text { Cruciferæ . . }\end{array}$ & $\begin{array}{l}71 \\
71 \\
70 \\
66 \\
66\end{array}$ & $\begin{array}{l}\text { Juncaceæ } \\
\text { Gramineæ } \\
\text { Boragineæ } \\
\text { Rosaceæ } \\
\text { Cyperaceæ }\end{array}$ & $\begin{array}{l}63 \\
60 \\
60 \\
60 \\
58\end{array}$ & $\begin{array}{l}\text { Caryophylleæ } \\
\text { Orchideæ. } \\
\text { Naiadaceæ } \\
\text { Liliaceæ . } \\
\text { Compositæ }\end{array}$ \\
\hline
\end{tabular}

The low place taken by Compositæ is mainly due to the exceedingly large number of species of Hieracium enumerated in the Catalogue, many of which are very local ; and Devonshire is not rich in number of species for this genus. The general percentage for all the flowering plants is rather greater than $6 \mathrm{I}$.

The general character of the county flora belongs to the Atlantic type of H.C. Watson; the majority of the species are of course members of the British type of that author, and many of them come under the head of the English type, but the type having the largest percentage of representation is the Atlantic. The extended distribution of several of the rarer species to the Channel Islands deserves notice, and conversely it appears that the rarer species of the Channel Islands are well represented in Devon; Marquand in his Flora of Guernsey (I gor) gives a list of thirty-four species which have a comital census below ten, that is, plants found in less than ten out of I I 2 counties and vice-counties into which England, Wales and Scotland are divided, according to the last edition of the London Catalogue; in this list are enumerated nineteen Devon species. Through this connection a relationship can be traced to the floras of Brittany and Normandy; in some instances however our species extend to the west of France, as also to Spain or Portugal, etc., without representation in the Channel Islands.

We have some plants occurring in no other English county : the gênotte of Guernsey (Romulea Columna) occurs on Dawlish Warren and nowhere else in Britain, and is absent from Ireland; it extends to the west of France, the south of Europe and the north of Africa. The round-headed club-rush (Scirpus Holoscbanus) grows plentifully on Braunton Burrows, the only other station for it in the British Isles being north Somerset, where it has been rediscovered apparently in small quantity; Sir J. D. Hooker gives the Channel Islands for the plant, but I know no other authority for the statement; the foreign distribution in Europe is from Belgium southwards, also in north Africa and Siberia. The Irish spurge (Euphorbia biberna) in Britain grows only in the Lynton neighbourhood ; besides the south and west of Ireland its foreign distribution is the west part of France and the north of Spain.

\section{PHENOLOGICAL OBSERVATIONS}

The following table, prepared from Mr. Edward Mawley's reports in the 2uarterly Fournal of the Royal Meteorological Society, exhibits the dates of the first flowering of thirteen common plants for Tiverton (in the Exeter botanical district, Miss M. E. Gill being the observer), Westward Ho (in the Barnstaple district, H. A. Evans and Miss Patterson being successively the observers), Barnstaple (in the latter district, Thomas 


\section{A HISTORY OF DEVONSHIRE}

Wainwright being the observer), and for the average of the British Isles, respectively, during each of the eight years I 894-I90I.

In the table each number in the columns under the names of the plants indicates the number of the day of the year on which the plants were first observed to have expanded flowers ; thus (except in the leap year I 896) I 3-3 I were in January, 32-59 in February, 60-90 in March, 9I-I 20 in April, $12 \mathrm{I}-5 \mathrm{I}$ in May, $152-8 \mathrm{I}$ in June, $182-2 \mathrm{I} 2$ in July, 21 3-43 in August, 244-73 in September, and 274-99 in October.

\begin{tabular}{|c|c|c|c|c|c|c|c|c|c|c|c|c|c|c|}
\hline Station & Year & 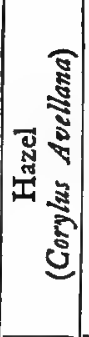 & 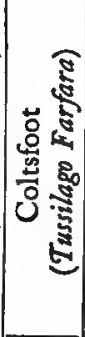 & 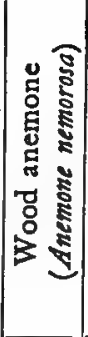 & 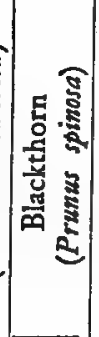 & 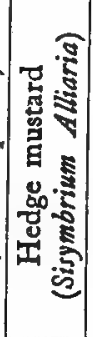 & 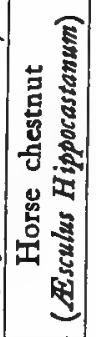 & 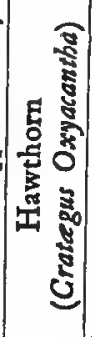 & 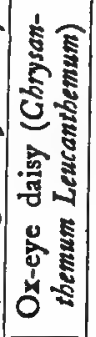 & 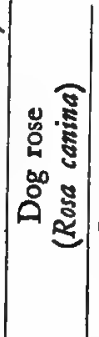 & . & 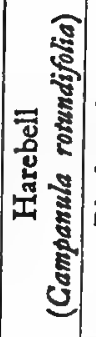 & 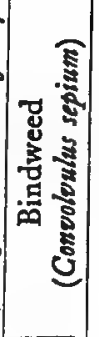 & 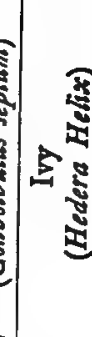 \\
\hline $\begin{array}{l}\text { iverton, } \\
270 \mathrm{ft} \text {. alt. }\end{array}$ & $\begin{array}{l}1894 \\
1895 \\
1896 \\
1897 \\
1898 \\
1899 \\
1900 \\
1901\end{array}$ & $\begin{array}{l}27 \\
28 \\
48 \\
17 \\
24 \\
51 \\
57\end{array}$ & $\begin{array}{l}\frac{44}{69} \\
58 \\
26 \\
59 \\
74 \\
49\end{array}$ & $\begin{array}{r}82 \\
91 \\
79 \\
78 \\
78 \\
85 \\
101 \\
97\end{array}$ & $\begin{array}{r}81 \\
\text { I I } \\
83 \\
83 \\
80 \\
88 \\
100 \\
103\end{array}$ & $\begin{array}{c}95 \\
997 \\
95 \\
111 \\
112 \\
113 \\
115\end{array}$ & \begin{tabular}{|l|l} 
I I I \\
I 20 \\
I I I \\
I 22 \\
128 \\
128 \\
I 29 \\
I 32 \\
I 24
\end{tabular} & 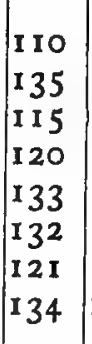 & $\begin{array}{l}135 \\
147 \\
142 \\
142 \\
153 \\
135 \\
141 \\
148\end{array}$ & $\mid \begin{array}{l}144 \\
156 \\
137 \\
150 \\
161 \\
156 \\
151 \\
156\end{array}$ & $\begin{array}{l}158 \\
166 \\
150 \\
165 \\
168 \\
169 \\
162 \\
159\end{array}$ & $\begin{array}{l}z \\
z \\
- \\
z \\
-\end{array}$ & $\begin{array}{c}Z \\
- \\
- \\
\frac{191}{197}\end{array}$ & $\begin{array}{l}\overline{268} \\
267 \\
263 \\
263 \\
256 \\
\frac{281}{281}\end{array}$ \\
\hline $\begin{array}{r}\text { West } \\
1\end{array}$ & $\begin{array}{l}1894 \\
1895 \\
1896 \\
1897 \\
1898 \\
1899 \\
1900 \\
1901\end{array}$ & $\begin{array}{l}- \\
27 \\
56 \\
71 \\
60 \\
40\end{array}$ & $\begin{array}{l}4^{6} \\
- \\
- \\
- \\
- \\
-\end{array}$ & $\begin{array}{l}- \\
- \\
- \\
- \\
-\end{array}$ & $\begin{array}{r}- \\
121 \\
84 \\
91 \\
93 \\
104 \\
111\end{array}$ & $\begin{array}{r}92 \\
121 \\
104 \\
104 \\
96 \\
138 \\
126 \\
116\end{array}$ & 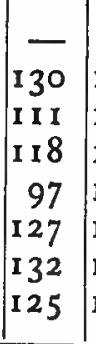 & $\begin{array}{l}-1 \\
141 \\
130 \\
124 \\
132 \\
144 \\
143 \\
139\end{array}$ & $\begin{array}{l}1 \overline{161} \\
142 \\
122 \\
136 \\
154 \\
161 \\
147\end{array}$ & \begin{tabular}{|}
$-\overline{1}$ \\
167 \\
125 \\
157 \\
157 \\
153 \\
168
\end{tabular} & $\begin{array}{l}186 \\
177 \\
194 \\
194 \\
198 \\
196 \\
203 \\
199\end{array}$ & $\begin{array}{l}- \\
E \\
z \\
-\end{array}$ & \begin{tabular}{|l|}
1 \\
187 \\
181 \\
190 \\
200 \\
187 \\
198
\end{tabular} & $\begin{array}{l}- \\
- \\
290 \\
278 \\
269 \\
297 \\
299\end{array}$ \\
\hline 9 & $\begin{array}{l}1894 \\
1895 \\
1896 \\
1897 \\
1898 \\
1899 \\
1900 \\
1901\end{array}$ & $\begin{array}{l}28 \\
76 \\
27 \\
14 \\
13 \\
22 \\
24 \\
37\end{array}$ & $\begin{array}{l}51 \\
81 \\
45 \\
46 \\
46 \\
54 \\
44 \\
56\end{array}$ & $\begin{array}{l}75 \\
93 \\
82 \\
83 \\
81 \\
85 \\
91 \\
97\end{array}$ & $\begin{array}{r}71 \\
108 \\
76 \\
78 \\
70 \\
78 \\
100 \\
102\end{array}$ & $\begin{array}{r}89 \\
109 \\
76 \\
85 \\
100 \\
106 \\
112 \\
111\end{array}$ & $\begin{array}{l}105 \\
121 \\
1115 \\
122 \\
124 \\
123 \\
123 \\
127\end{array}$ & 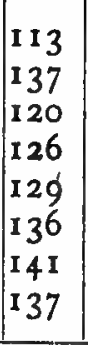 & $\begin{array}{l}140 \\
151 \\
138 \\
146 \\
148 \\
147 \\
151 \\
145\end{array}$ & $\begin{array}{l}154 \\
159 \\
138 \\
143 \\
145 \\
156 \\
162 \\
161\end{array}$ & \begin{tabular}{l|l|}
170 \\
178 \\
1788 \\
180 \\
18 \\
172 \\
170 \\
177 \\
178 \\
185
\end{tabular} & $\begin{array}{l}- \\
196 \\
195 \\
208 \\
208 \\
205 \\
204 \\
194\end{array}$ & $\begin{array}{l}169 \\
173 \\
163 \\
169 \\
183 \\
196 \\
189 \\
175\end{array}$ & $\begin{array}{l}277 \\
269 \\
254 \\
253 \\
255 \\
257 \\
264 \\
268\end{array}$ \\
\hline & $\begin{array}{l}1894 \\
1895 \\
1896 \\
1897 \\
1898 \\
1899 \\
1900 \\
1901\end{array}$ & $\begin{array}{l}30 \\
71 \\
32 \\
41 \\
27 \\
41 \\
54 \\
46\end{array}$ & $\begin{array}{l}58 \\
79 \\
58 \\
57 \\
49 \\
63 \\
78 \\
67\end{array}$ & $\begin{array}{r}85 \\
98 \\
83 \\
83 \\
84 \\
96 \\
101 \\
97\end{array}$ & $\begin{array}{r}86 \\
1117 \\
90 \\
97 \\
91 \\
101 \\
1112 \\
112 \\
112\end{array}$ & $\begin{array}{l}104 \\
120 \\
109 \\
114 \\
116 \\
118 \\
125 \\
122\end{array}$ & 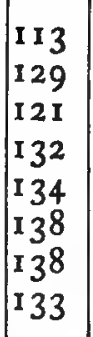 & $\begin{array}{l}120 \\
144 \\
125 \\
138 \\
1337 \\
142 \\
1444 \\
144 \\
14\end{array}$ & $\begin{array}{l}147 \\
149 \\
143 \\
154 \\
155 \\
157 \\
158 \\
158 \\
152\end{array}$ & $\begin{array}{l}164 \\
159 \\
148 \\
162 \\
165 \\
164 \\
166 \\
161\end{array}$ & $\begin{array}{l}190 \\
181 \\
179 \\
189 \\
193 \\
189 \\
191 \\
186\end{array}$ & $\begin{array}{l}192 \\
172 \\
186 \\
188 \\
194 \\
190 \\
193 \\
188\end{array}$ & $\begin{array}{l}195 \\
184 \\
181 \\
191 \\
197 \\
193 \\
198 \\
189\end{array}$ & $\begin{array}{l}274 \\
265 \\
266 \\
272 \\
273 \\
274 \\
280 \\
272\end{array}$ \\
\hline
\end{tabular}




\section{BOTANY}

Hybrids are supposed frequently to be produced among the species of several genera, such as Epilobium, Carduus, Cnicus, Verbascum, etc. ; in the case of the first of these genera, Mr. N. E. Brown in the third part of the supplement to the third edition of English Botany, gave in 1892 twenty-seven cases of hybrids, the following nine of which occurred in Devon :-

Epilobium aggregatum, Celakovsky, a supposed cross between E. montanum and E. obscurum

$E$. aschersonianum, Haussknecht, between $E$. lanceolatum and $E$. parviflorum

$E$. dacicum, Borbas, between $E$. obscurum and E. parviflorum

E. lamotteanum, Haussknecht, between E. lanceolatum and E. obscurum

$E$. neogradiense, Borbas, between E. lanceolatum and E. montanum

$E$. rivulare, Wahlenberg, between $E$. palustre and $E$. parviflorum

$E$. scbmidtianum, Rostkov., between E. obscurum and E. palustre

E. thuringianum, Haussknecht, between E. tetragonum (adnatum) and E. obscurum

E. weissenburgense, F. Schultz, between E. tetragonum (adnatum) and E. parviflorum

To this list may be added :-

E. ligulatum, Baker, between E. obscurum and E. palustre

a hybrid between $E$. birsutum and $E$. parviforum

a hybrid between $E$. birsutum and $E$. montanum

The literature dealing with the flowering plants of the county generally includes the following works :-

Camden's Britannia, edition by Edmund Gibson (1695). The catalogues of plants given at the end of each county were communicated by 'the great botanist of our age, Mr. Ray'; that for Devonshire, on page 42, consisted of ten species, nine of them being flowering plants.

John Ray : Synopsis methodica stirpium britannicarum, tum indigenis, tum in agris cultis locis suis dispositis; additis generum characteristicis specierum descriptionibus et virium epitome. Editio tertia (1 724$)$. Fourteen species of flowering plants are mentioned as occurring in Devonshire.

Camden's Britannia, edition by Richard Gough (1789). The list of 'rare plants found in Devonshire,' on page 4I, contains thirty-five flowering plants.

Richard Polwhele : The History of Devonshire vol. i. (1797), chap. 5, pp. 81-100, 'The indigenous plants of Devonshire.' 'From the eastern part of the county Mr. Welland, rector of Talaton, and Miss Burges of Tracey near Honiton' favoured the author "with botanical observations; from the neighbourhood of Exeter, Mr. Weston, late rector of Mamhead, and Mr. Swete of Oxton house [in Kenton]; from the north-east and the north of Devon Dr. Cruwys of Tiverton and Dr. Wavell of Barnstaple; and from the Southams Mr. Cornish of Totnes and Mr. Yonge of Puslinch [in Newton Ferrers].'

Dawson Turner and James Sowerby, in the Transactions of the Linnean Society of London (1800), v. 234-41; ' Catalogue of plants observed in a tour through the western counties of England made in June 1 799.' This catalogue mentions seventeen species including six cryptogams as occurring in Devon.

Dawson Turner and Lewis Weston Dillwyn: The Botanist's Guide through England and Wales (1805), i. 194-212, 'Devonshire.' This enumerates nearly a hundred flowering plants for the county.

John Pike Jones: $A$ botanical tour through various parts of the counties of Devon and Cornwall (1820), pp. viii., 74 . 


\section{A HISTORY OF DEVONSHIRE}

John Pike Jones and J. F. Kingston : Flora Devoniensis : or a descriptive catalogue of plants growing wild in the county of Devon, arranged both according to the Linnean and natural systems, with an account of their geographical distribution, etc. (1829), Pp. x. ; part i. pp. xi.-xlvii., 162 ; part ii. pp. lxvii., 218. This contains 798 species of flowering plants, besides cryptogams.

Hewett Cottrell Watson: The new Botanist's Guide to the localities of the rarer plants of Britain, 2 volumes (1835 and 1837). Vol. i. 12-27 (1835); 'Devon'; ii. 550-2 (1837), 'Devon supplement.'

The Phytologist, ser. i., conducted by George Luxford (1841-54); ser. ii., edited by Alexander Irvine ( $1855-63)$.

William Strong Hore, in the Phytologist, i. 160-2, 'List of plants found in Devonshire and Cornwall, not mentioned by Jones in the Flora Devoniensis, with remarks on the rarer species' (March, I842).

The Botanical Gazette, edited by Arthur Henfrey, 3 volumes (1 849-5I).

The reports of the Botanical Exchange Club (1859-1901).

Edited by John Gilbert Baker (1859-68, 1879).

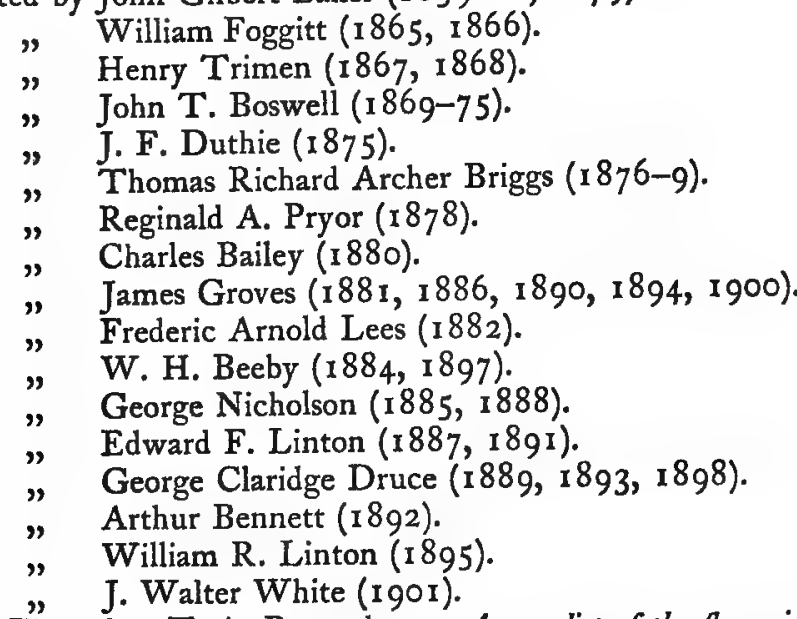

Thomas Fitzarthur Torin Ravenshaw : A new list of the flowering plants and ferns growing wild in the county of Devon (1860); and a second edition with supplement in 1872 .

The Fournal of Botany, British and Foreign, vols. I-9, edited by Berthold Seemann (186371); vols. 10-17, edited by Henry Trimen $(1872-9)$; vols. 18-41, edited by James Britten (1880-1903).

Isaiah Waterloo Keys, in the Transactions of the Plymoutb Institution and Devon and Cornwall Natural History Society (1866-7 I), 'Flora of Devon and Cornwall.'

The same Author in the Phytologist, 1850, iii. 1022-4, 'Plants found in Devonshire and Cornwall in addition to those contained in Jones' Flora Devoniensis and in the Phytologist.'

Hewett Cottrell Watson: Topographical Botany, 2 vols. (1873, 1874); also the second edition, edited by J. G. Baker and W. W. Newbould (1883).

The reports of the Botanical Record Club, edited by Frederic Arnold Lees (1 874-87).

William Philip Hiern, in Deacon \& Co.'s Devon and Cornwall Court Guide and County Blue Book (1896), pp. 97-129, 'The Botany of Devonshire.'

Frederick Hamilton Davey: A tentative list of the flowering plants, ferns, etc., known to occur in the county of Cornwall, including the Scilly Isles (1902), p. vii., 'Plants recorded for Devon but not known to occur in Cornwall.'

\section{BOTANICAL DISTRICTS}

\section{Barnstaple}

This district consists of the north-western or Barnstaple parliamentary division; it contains about 215,669 acres, being about 207,31,6 acres of land and inland water besides about 8,353 acres of foreshore and tidal water ; it includes Lundy Island. The mainland is bounded on the west by the Atlantic Ocean, on the north by the Bristol Channel, on the east by the county of Somerset, and on the south by the South Molton and Torrington botanical districts and for a small part by the county of Cornwall; it thus includes the whole of the 


\section{BOTANY}

northern sea coast of Devonshire, and no part of it is more than 13 miles distant from that coast ; its drainage is wholly to the north coast; it comprises fifty-seven civil parishes. The principal rivers are the Torridge with its tributary the (West) Yeo, the Taw (with its tributaries the Caen, the Brady and the North Yeo), the Heddon, and the West and East Lyn; it includes the most northerly (Foreland Point in Countisbury) and the most westerly (Knap Head in Hartland) parts of the county. It consists of the petty sessional divisions of Bideford and Braunton and the municipal boroughs of Bideford and Barnstaple; it comprises the seventeen parishes of the Bideford poor-law union and the forty parishes of the Barnstaple union; fifty-one of the parishes are rural and six are urban. The highest part is Chapman Barrows, I, 575 feet above sea-level, at a point about 4 miles distant from the coast. district :-

The following is a complete list in alphabetical order of the civil parishes in the

Abbotsham
Alwington
Arlington
Ashford
Atherington
Barnstaple (urban)
Berry Narbor
Bideford (urban)
Bishop's Tawton
Bittadon
Bratton Fleming
Braunton
Brendon
Buckland Brewer
Bulkworthy
Challacombe
Clovelly
Combmartin
Countisbury

East Down
East Putford
Fremington
Georgeham
Goodleigh
Hartland
Heanton Punchardon
High Bray
Horwood
Ilfracombe (urban)
Instow
Kentisbury
Landcross
Landkey
Littleham near Bideford
Loxhore
Lundy Island
Lynton (urban)
Martinhoe

Marwood
Monkleigh
Morte-Hoe
Newton St. Petrock
Newton Tracey
Northam (urban)
Parkham
Parracombe
Pilton, East (urban)
Pilton, West
Sherwill
Stoke Rivers
Swimbridge
Tawstock
Trentishoe
Welcombe
West Down
Westleigh
Woolfardisworthy near Bideford

Nearly the whole of the part of the district which lies north of Barnstaple town and on the Devon and Somerset branch of the Great Western Railway belongs to the Devonian period, the successive beds of which are arranged in nearly parallel strips. The Lower Devonian formation includes the Foreland sandstones and the Lynton beds; the Foreland sandstones occupy the north coast from Lynmouth to the Somerset county boundary, and are mainly limited on the south by a line about $4 \frac{1}{2}$ miles long from Lyndale to the county boundary about 2 miles inland from Glenthorne; the Lynton slates and grits come next, and are limited on the south by a line about 7 miles long, starting from the coast at Woody Bay, passing by Barbrook Mill and reaching the county boundary, the breadth of the area being about $I_{2} \frac{1}{2}$ miles opposite Lynmouth and about two-thirds of a mile near the county boundary. The Middle Devonian formation includes the Hangman grits, the Ilfracombe slates and limestones, and the Morte-Hoe slates. The Hangman grits run along the south side of the Lynton beds, and are limited on the south by a line about $9 \frac{1}{2}$ miles long, starting from Lester Cliff near Combmartin, passing by Parracombe rectory and reaching the county boundary on Thorn Hill in the south-eastern part of Lynton parish, the breadth of the area being mostly about 2 miles; the Ilfracombe slates and limestones follow next, and are limited on the south by a line about I $5 \frac{1}{4}$ miles long, starting from the north coast at Lee Bay near Ilfracombe, passing by Kentisbury Ford and reaching the county boundary near Moles Chamber, the breadth of the area being mostly $2 \frac{1}{4}-2 \frac{1}{2}$ miles; the Morte-Hoe slates form the next beds, and are limited on the south by a line about 17 miles long, starting from the coast at Woolacombe, passing by West Down village and Smithapark in Loxhore, and extending to the point where the South Molton botanical district reaches the county boundary near North Twitchen in the north part of North Molton, the breadth of the area being mostly about 2 miles.

The Upper Devonian formation includes in following order the Pickwell Down sandstones, the Cucullæa or Marwood or Baggy beds, and the Pilton beds. The Pickwell Down sandstones are limited on the south by a line about 17 miles long, starting from Bloodhill on the south side of Morte Bay, passing by Georgeham and Sherwill villages and nearly reaching Holswater Bridge in High Bray, after crossing about a mile and a half of the parish of Charles in the South Molton botanical district before entering the parish of High Bray, the breadth of the area being about 2 miles at the western end and $I-1 \frac{1}{3}$ mile towards the eastern end ; the 


\section{A HISTORY OF DEVONSHIRE}

Cucullæa beds are limited on the south by a line about 18 miles long, starting from the south end of the Baggy Point prominency, passing by Kingsheanton in Marwood and by Stoke Rivers village to Barton Wood, reaching High Bray after crossing about three quarters of a mile of Charles parish in the South Molton district, also throwing out a spur in the parish of Stoke Rivers from the village to Akeford about $\mathrm{I}_{\frac{1}{4}}$ miles long in a W.S.W. direction, the breadth of the main area being about half a mile and of the spur rather less; the Pilton beds begin at the coast at Croyde Bay, and their boundary on the south follows a line so as to include Santon, Braunton and Heanton Punchardon villages; it then crosses the river Taw, and just avoiding Bickington village recrosses the Taw a little above the town of Barnstaple; it passes on to Landkey Newland, strikes the Devon and Somerset Railway near Swimbridge station, and follows the course of the railway to the boundary of the South Molton district.

At the mouth of the Taw and Torridge estuary there is a delta, each of the three sides of which measures about $5 \frac{1}{2}$ miles; it includes a considerable area of blown sand outside the marshes; in this tract lies the famous botanical ground known as Braunton Burrows.

Nearly the whole of the remainder of the district belongs to the Carboniferous system, locally termed Culm Measures; the fertility of the soil over most of this system is much inferior to that of the Devonian formation.

The Radiolarian (Codden Hill) beds in the Lower Culm Series extend at intervals along a nearly straight line a short distance south of Barnstaple in narrow bands through the parishes of Fremington, Tawstock, Bishop's Tawton, Landkey and Swimbridge.

There is an isolated patch of New Red Sandstone by the coast of Bideford Bay, between Peppercombe and Portledge in the parish of Alwington; there is also a small piece of Greensand near Orleigh Court in Buckland Brewer.

The geological information for this and the other districts is mainly derived from sections of the original one-inch ordnance survey maps, coloured to indicate the different formations by Mr. Joseph Green Hamling, F.G.S., of Barnstaple, who has kindly lent them for the purpose; he states that the colourings were copied by him from similar ones made by the late Mr. Townshend Monckton Hall, F.G.S.

The climate of the district is illustrated by the following tables :-

TABLE I

Calculated for the decade $1890-9$ from the records given by Symons and Wallis in British Rainfall.

\begin{tabular}{|c|c|c|c|c|}
\hline Station & & $\begin{array}{l}\text { Altitude, } \\
\text { feet }\end{array}$ & $\begin{array}{c}\text { Mean annual rainfall, } \\
\text { inches }\end{array}$ & $\begin{array}{l}\text { Mean annual No. of days } \\
\text { on which or in. or } \\
\text { more of rain fell. }\end{array}$ \\
\hline $\begin{array}{l}\text { Parracombe . } \\
\text { Arlington Court } \\
\text { Barnstaple . } \\
\text { Horwood } \\
\text { Gammaton, Bideforc } \\
\text { Northam } \\
\text { Hartland Abbey }\end{array}$ & $\begin{array}{ll}\cdot & \cdot \\
\cdot & \cdot \\
\cdot & \cdot \\
\cdot & \cdot \\
\cdot & \cdot \\
\cdot & \cdot \\
\cdot & \cdot\end{array}$ & $\begin{array}{r}795 \\
613 \\
25 \\
288 \\
335 \\
173 \\
222\end{array}$ & $\begin{array}{l}45 \cdot 18 \\
51 \cdot 67 \\
33 \cdot 10 \\
33 \cdot 24 \\
37 \cdot 94 \\
34 \cdot 35 \\
39 \cdot 22\end{array}$ & $\begin{array}{l}179 \\
206 \\
171 \\
189 \\
172 \\
183 \\
165\end{array}$ \\
\hline
\end{tabular}

TABle 2

Partly taken from Mr. A. Chandler's paper above referred to, partly calculated from articles on the climate of Devon in various volumes of the Reports of the Devonsbire Association and partly calculated from figures furnished by Mr. Thomas Wainwright, secretary of the North Devon Athenæum, Barnstaple.

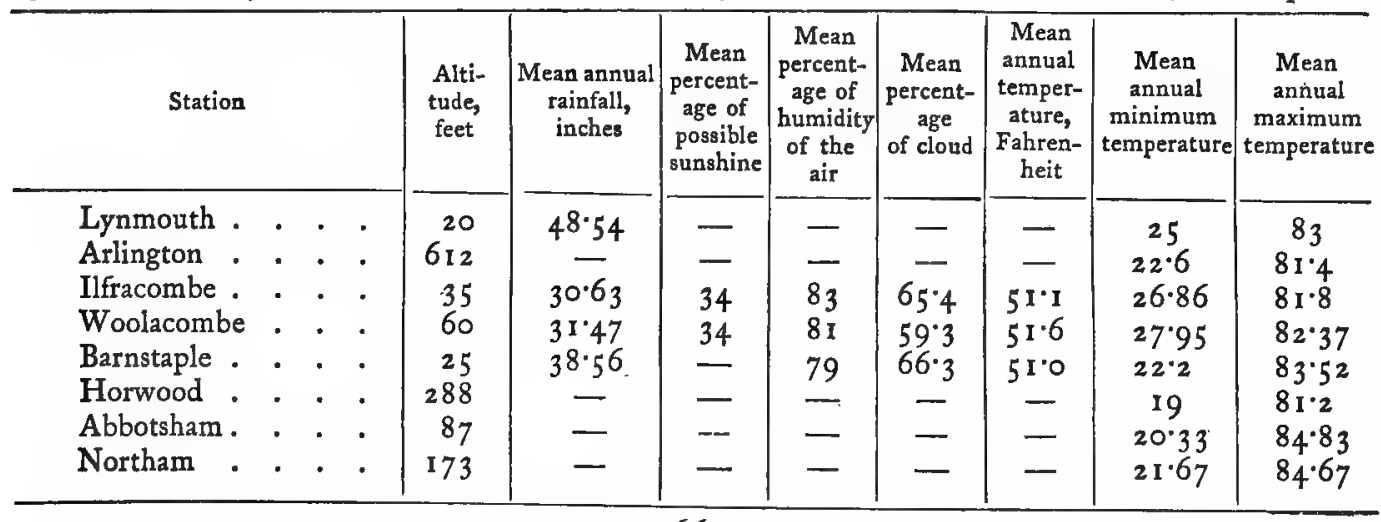




\section{BOTANY}

The district is abundantly supplied with the rampant fumitory (Fumaria capreolata); Curtis in his Flora Londinensis, fasc. 6, tab. 47, published in the latter part of the eighteenth century, gave a good figure of the species, and in the text accompanying his plate stated that it was plentiful about Barnstaple; the sub-species have been recently discussed and arranged by Mr. H. W. Pugsley in the Fournal of Botany for April and May, 1902, pp. 129-36, I 73-8I, tab. 436, where he enumerated six British sub-species, namely : I, $F$. capreolata proper (=F. pallidifora, Jord.); 2, F. speciosa, Jord. ; 3, F. purpurea, Pugsl. ; 4, F. muralis, Sond. ; $5, F$. Borai, Jord.; and 6, $F$. confusa, Jord.; all of which except the fourth occur in the Barnstaple district. Mr. Pugsley has kindly examined the specimens in my herbarium and has also favoured me with notes on the distribution of these plants in Devon; it thus appears that $F$. Borai is by far the commonest sub-species in the county, and in north Devon whole fields may sometimes be seen coloured purplish with its flowers, while the other sub-species are comparatively sporadic. Besides the typical form of $F$. Borcei four varieties are recognized : I, var. minor, Clavaud ; 2, var. ambigua, Pugsl. ; 3, var. serotina, Clav. ; and 4, var. muraliformis, Clav., the three latter of which are met with in the Barnstaple district, and the first at Dawlish in the Torquay district; the typical form is more prevalent in south Devon, and in north Devon the variation is rather in the direction of ambigua; the sub-species $F$. speciosa has been found at Lynmouth in this district and at Salcombe near Kingsbridge in the Plymouth district; and the sub-species to which Mr. Pugsley has given the name purpurea, and which so far as known is not found out of the British Isles, occurs at Lee near Ilfracombe and at Woolacombe in Morte-Hoe parish, both stations being in this district, in situations similar to those of $F$. capreolata (proper) and sometimes mixed with it.

The sea-stock (Mattbiola sinuata) was mentioned by Polwhele in 1797 as occurring on the rocks adjoining Braunton Burrows, and it still ornaments the maritime cliffs there.

Braunton Burrows is also noted as the classical station for Viola Curtisii, a sub-species of the wild pansy ( $V$. tricolor); the plant is very abundant, and the colours of the flowers are mostly different shades of yellow but occasionally a violet intermixture occurs.

The St. Peter's wort (Hypericum elodes) is frequent in boggy grounds and about shallow pools on the high moors, where with its pale yellow flowers it forms a conspicuous feature; it was recorded for the county in such places in Gibson's edition of Camden's Britannia of 1695.

The erect golden saxifrage (Cbrysosplenium alternifolium) grows in the valley of the North Yeo not far from Barnstaple, where it was recorded in 1797 ; the common golden saxifrage (C. oppositifolium) is much more common and plentiful, and in spring forms a beautiful decoration of wet banks and the sides of watercourses.

Epilobium tetragonum, sub-sp. E. obscurum, was first reported as a British plant in 1856 by C. C. Babington, who found it at Ilfracombe ; it is common in the district.

Wild madder (Rubia peregrina) was mentioned in Gibson's edition of Camden's Britannia of 1695 as growing on the rocks near the bridge at Bideford and all along the hedges on both sides of the road between Westleigh and Bideford, and in many other places; it still abounds in several parishes not far from the north coast; it was found on 7 July 1662, by Ray at Devil's Point, Stonehouse.

Senecio squalidus is thoroughly established on rocks and walls at Bideford; it was recorded by W. S. Hore in the Phytologist, 1842, i. 163, and by H. C. Watson, New Botanist's Guide (1835), i. 20 as collected by Hooker.

Hieracium murorum, sub-sp. (H. pallidum, var.) H. Scbmidtii, var. devoniense, a critical form of hawkweed, grows not far from the sea-coast east of Ilfracombe ; it is very local.

Mentba pubescens, a sub-species of $M$. aquatica, was recorded on the authority of Mr. Ley in the Botanical Record Club Report, 1883, p. 193, from 'streamside, on coast opposite Alvington [Alwington]'; an authentic specimen in the British Museum herbarium for this record belongs rather to $M$. aquatica proper; I am not aware of any other record for the sub-species $M$. pubescens in the county.

The garlic germander (Teucrium Scordium) is very plentiful on Braunton Burrows, more so probably than in any other part of England ; it formerly occurred in many counties, but is now limited to a few and has generally become very rare.

The Irish spurge (Eupborbia biberna) grows in the neighbourhood of Lynton, in the valley of the East Lyn river and its tributary the Badgeworthy water in north Devon and west Somerset and nowhere else in England; it was found there in 1840 and recorded by N. Ward in the Pbytologist, July $184 \mathrm{r}$, i. $2 \mathrm{r}$.

The mountain twayblade (Listera cordata) was discovered by $\mathrm{N}$. Ward and W. S. 


\section{A HISTORY OF DEVONSHIRE}

Hore on Codden Hill near Barnstaple in 1840-3; it was recorded in the Phytologist in I84I, but during recent years it has not been noticed there.

The round-headed bull-rush (Scirpus Holoscboenus) still grows in considerable quantity on Braunton Burrows, where it was recorded by Gibson in his edition of Camden's Britannia in I695; in England it is almost peculiar there and does not grow in any considerable quantity elsewhere in Britain. the district :-

The following are among the more remarkable and interesting species which occur in

Meconopsis cambrica, Vig.

Barbarea precox, R. Br.

Brassica oleracea, L.

Eriophila vulgaris, DC., sub-sp. E. brachycarpa, Jordan

Camelina sativa, Crantz

Cerastium quaternellum, Fenzl

- tetrandrum, Curtis

Spergularia rupestris, Lebel

Tamarix gallica, Sm. $($ not L.) $=$ T. anglica, Webb

Hypericum undulatum, Schousb.

Lavatera arborea, $L$.

Linum angustifolium, Huds.

Geranium rotundifolium, L.

Erodium maritimum, L'Herit.

Oxalis corniculata, $\mathrm{L}$.

- stricta, L.

Genista tinctoria, L., var. humifusa, Syme

Medicago maculata, Sibth.

Trifolium subterraneum, $\mathbf{L}$.

- maritimum, Huds.

Lotus hispidus, Desf.

Poterium muricatum, Spach.

Pyrus Aria, Sm., sub-sp. P. latifolia, Syme

Cotyledon Umbilicus, L.

Sedum Telephium, L. (S. purpurascens, Koch)

- rupestre, Huds.

Eryngium maritimum, L.

Carum Carui, L.

- segetum, Benth.

Sium angustifolium, L.

Pimpinella major, Huds.

Crithmum maritimum, L.

Valerianella carinata, Loisel.

Erigeron acris, L.

Onopordon Acanthium, L.

Crepis taraxacifolia, Thuill.

Hypochøris glabra, L.

Lactuca muralis, Fresen.

Campanula Trachelium, L.

Statice Limonium, L.

- auriculzfolia, Vahl

Primula veris, $L$.

Centunculus minimus, L.

Chlora perfoliata, L.

Anchusa sempervirens, L.

Myosotis palustris, L., var. M. strigulosa, Reichenb.
Convolvulus Soldanella, L.

Cuscuta Epithymum, Murr. (and a goldenyellow variety)

Hyoscyamus niger, $L$.

Verbascum Blattaria, L., sub-sp. V. virgatum, Stokes

Linaria spuria, Miller

Scrophularia Scorodonia, L.

Mimulus luteus, Pursh (not L.), M. Langsdorffi, Donn.

Sibthorpia europæa, L.

Bartsia viscosa, L.

Pinguicula lusitanica, L.

Utricularia vulgaris, $L$.

Nepeta Cataria, L.

Melittis Melissophyllum, L.

Scleranthus annuus, L.

Atriplex littoralis, L.

Polygonum maritimum, L.

Rumex rupestris, Le Gall

Daphne Laureola, L.

Mercurialis annua, $\mathrm{L}$.

Taxus baccata, $\mathrm{L}$.

Malaxis paludosa, Sw.

Epipactis latifolia, Sw., sub-sp. E. purpurata, Sm., var. E. media, Fries

- palustris, Crantz

Ophrys apifera, Huds.

Scilla autumnalis, $\mathrm{L}$.

Juncus acutus, L.

- obtusiflorus, Ehrh.

Sparganium ramosum, Huds. (S. neglectum, Beeby)

Alisma ranunculoides, L.

Potamogeton trichoides, Cham.

Ruppia maritima, L., sub-sp. R. rostellata, Koch

Carex stricta, Good.

- pendula, Huds.

- distans, L.

Phleum arenarium, $\mathrm{L}$.

Calamagrostis Epigejos, Roth

Gastridium lendigerum, Gaud.

Catabrosa aquatica, P. Beauv., and var. littoralis, Parn.

Glyceria distans, Wahlenb.

Bromus racemosus, $L$.

Agropyrum junceum, P. Beauv.

The literature dealing with the flowering plants of the district includes the following works :-

- Pike : A catalogue of plants which grow in and near the parish of Biddeford, 1759.

N. Ward, in the Phytologist, vol. i. part 2, July I 84I, List of rare plants found in Devonshire in the year 1840 , p. 21 , ' at or within six miles of Lynton.'

Banfield's Guide to Ilfracombe, Lynton, Clovelly, and the neigbbouring towns, etc., new edition (about 1850), 'Botany,' pp. 11 5-28. 


\title{
BOTANY
}

George Maw, in the Pbytologist, 1853, pp. 785-95, "Notes on the rare plants occurring in the neighbourhood of the estuary of the Taw and 'Torridge.'

T. F. Ravenshaw, in the Pbytologist, December, 1862, Pp. 355-9, 'Plants of Braunton Burrows, North Devon.'

The same Author : 'Botany of North Devon,' 1877 (extracted from Stewart's North Devon Handbook, ed. iv.).

Martin M. Bull, in the Fournal of Botany, 1875, p. 296, 'Hypericum bøticum, Boiss., in North Devon.'

Rev. W. Moyle Rogers, in the Foumal of Botany, 1877, pp. 36I-3, 'Notes on a few North Devon plants.'

The same Author in the same Fournal, 1879, pp. 330-3, and 1882, pp. 9-16, 'On some North Devon plants.'

Herbert Arthur Evans: A band-list of the plants occurring witbin seven miles of the United Services College, Westward Ho, and supplements $(188 \mathrm{I}-6)$. This list affects twenty-two parishes of this district and six of the Torrington district.

William Philip Hiern, in Twiss \& Son's Illustrated Guide to Ilfracombe and North Devon (1888), chap. I 2, pp. 86-90, 'Notes on the flora of Braunton parish, North Devon.'

C. E. Larter (Miss) : Notes on the botany of North Devon (1897).

\section{TORRINGTON}

This district consists of the Great Torrington petty sessional division (the smaller and western portion of the Northern or South Molton parliamentary division) and the Holsworthy and Hatherleigh petty sessional division (the northern part of the Western or Tavistock parliamentary division); it contains about 217,429 acres, being about 217,397 acres of land and inland water besides about 32 acres of foreshore and tidal water; it is wholly inland, but a small portion is washed with tidal water up the Torridge; it is bounded on the west by the county of Cornwall, on the north by the Barnstaple botanical district, on the east by the South Molton and Torquay botanical districts, and on the south by the Tavistock botanical district. It comprises 59 civil parishes, 42 of which are drained wholly to the north coast, 12 wholly to the south coast, and 5 partly to both coasts. It includes all the 21 parishes of the Holsworthy poor-law union, one parish (Northcott Hamlet) in the Launceston union, 16 out of the 28 parishes of the Okehampton union, and 21 out of the 23 parishes of the Torrington union; 56 of the parishes are rural and 3 are urban. The parish of Okehampton Hamlets includes a small piece of Dartmoor and contains High Willhays and Yes Tor, 2039.4 and $2029^{\circ} 6$ feet of altitude respectively, the highest points of southern England. The lowest part is at the north-west corner of the parish of Weare Giffard by the river Torridge, which is below high-water level of ordinary tides.

The principal rivers are the Torridge with its tributaries the West and East Okement and the Waldon, the Tamar (which forms the western border for some distance), and the Taw for short distances on the eastern border.

The following is a complete list in alphabetical order of the civil parishes in the district :-

$\begin{array}{ll}\text { Abbotsbickington } & \text { Great Torrington (urban) } \\ \text { Alverdiscott } & \text { Halwill } \\ \text { Ashbury } & \text { Hatherleigh } \\ \text { Ashwater } & \text { Highampton } \\ \text { Beaford } & \text { High Bickington } \\ \text { Beaworthy } & \text { Hollacombe } \\ \text { Belstone } & \text { Holsworthy (urban) } \\ \text { Blacktorrington } & \text { Holsworthy Hamlets } \\ \text { Bradford } & \text { Huish } \\ \text { Bradworthy } & \text { Huntshaw } \\ \text { Bridgerule, East } & \text { Iddesleigh } \\ \text { Bridgerule, West } & \text { Inwardleigh } \\ \text { Broadwoodkelly } & \text { Jacobstowe } \\ \text { Buckland Filleigh } & \text { Langtree } \\ \text { Clawton } & \text { Little Torrington } \\ \text { Cookbury } & \text { Luffincott } \\ \text { Dolton } & \text { Meeth } \\ \text { Dowland } & \text { Merton } \\ \text { Exbourne } & \text { Milton Damarell } \\ \text { Frithelstock } & \text { Monkokehampton }\end{array}$

\author{
Northcott Hamlet \\ Northlew \\ Okehampton (urban) \\ Okehampton Hamlets \\ Pancrasweek \\ Peters Marland \\ Petrockstow \\ Pyworthy \\ Roborough \\ St. Giles in the Wood \\ Sampford Courtenay \\ Shebbear \\ Sheepwash \\ Sutcombe \\ Tetcott \\ Thornbury \\ Weare Giffard \\ West Putford \\ Yarnscombe
}




\section{A HISTORY OF DEVONSHIRE}

Nearly the whole of the district belongs to the Carboniferous system, and a large proportion of the land is poor in quality. There is a patch of New Red Sandstone about the town of Hatherleigh, and a larger patch about a mile broad more or less stretching from Jacobstowe village to Exbourne and Sampford Courtenay villages and further eastwards.

There is a small patch of Greenstone about a mile south-west of Hatherleigh town; across Okehampton Park are some bands of Greenstone and a small patch of Limestone. In a quarry near Furzehill, not far from Hatherleigh, two Elvan bosses come up along the bedding planes of the Culm Measures and terminate abruptly at some feet from the surface (W. A. E. Ussher). The southern part of Okehampton Hamlets penetrates about 3 miles into Dartmoor and is granite.

The climate of the district is illustrated by the following tables, which have been obtained in the way explained under the Barnstaple district :-

TABLE I.-Decade 1890-9

\begin{tabular}{cc|c|c|c}
\hline Station & & $\begin{array}{c}\text { Altitude, } \\
\text { feet }\end{array}$ & $\begin{array}{c}\text { Mean annual rainfall, } \\
\text { inches }\end{array}$ & $\begin{array}{c}\text { Mean annual No. of days } \\
\text { on which or in. or } \\
\text { more of rain fell }\end{array}$ \\
\hline $\begin{array}{c}\text { Petrockstow . . . . . } \\
\text { Okehampton . . . . . }\end{array}$ & 332 & $\begin{array}{c}38.45 \\
45.54\end{array}$ & $\mathbf{I 8 9}$ \\
\hline
\end{tabular}

TABLE 2

\begin{tabular}{|c|c|c|c|c|}
\hline \multirow{2}{*}{ Station } & \multirow{2}{*}{$\begin{array}{l}\text { Altitude, } \\
\text { feet }\end{array}$} & \multirow{2}{*}{$\begin{array}{c}\text { Mean annual rainfall, } \\
\text { inches }\end{array}$} & \multicolumn{2}{|c|}{ Temperature in Fahrenheit degrees } \\
\hline & & & Mean minimum & Mean maximum \\
\hline $\begin{array}{l}\text { Alverdiscott } \\
\text { Great Torrington } \\
\text { St. Giles in the Wood } \\
\text { Okehampton . . }\end{array}$ & $\begin{array}{l}330 \\
336 \\
420 \\
521\end{array}$ & $\begin{array}{c}- \\
44^{\cdot 73} \\
49^{\cdot 26}\end{array}$ & $\begin{array}{l}19.25 \\
22.5 \\
18.8 \\
-\end{array}$ & $\begin{array}{l}85 \cdot 75 \\
85 \\
84 \cdot 8 \\
-\end{array}$ \\
\hline
\end{tabular}

A rare form of water-crowfoot (Ranunculus tripartitus $=R$. intermedius) occurs in the moorlands of the district ; south of Okehampton, according to Mr. Moyle Rogers, it grows usually on mud or in very shallow water, with its petals frequently 5-veined and as large as those of $R$. lenormandi, in company with which it is often found. The common scurvy grass (Cochlearia officinalis var. littoralis) grows near Holsworthy about 9 miles from the sea; the same botanist observes that the occurrence of this maritime plant in several inland places in this district (and a like observation applies to the Barnstaple district) points to its former cultivation in the neighbourhood. The mountain or English stonecrop (Sedum anglicum) is plentiful in rocky, stony and gravelly places, and is prevalent in the granite area ; it was recorded for such stations in the county by Hudson in 1778. The milk-thistle (Silybum marianum) has long been established at the base of the common at Great Torrington. The marsh gentian (Microcala filiformis) was discovered in Clawton parish i I September I883, by Mr. Archer Briggs and recorded in the fournal of Botany, xxi. 316 in the same year; it had been reported for the county, but without precise locality, by Hudson, in 1778. The stalked speedwell (Veronica montana) is fairly frequent in the hilly woods and thickets of the district; it was mentioned in 1797 by Polwhele on the authority of Dr. Wavell as a rare plant growing in a lane near Torrington leading towards Holsworthy. Red mint (Mentha sativa, sub-sp. $M$. gentilis) was reported from Winscott in Peters Marland by Polwhele in 1797 . The Devonshire or Dutch myrtle (Myrica Gale) has been found in good quantity by Mr. Moyle Rogers in Upper Bridgerule bog.

2uercus Robur.- 'On an estate of Dennis Rolle, Esq., near Great Torrington, an oak tree was cut through about 1776 , the body of which measured thirteen feet six inches diameter; and on the plane of the section two couples danced a country dance. . . In Okehampton Park is an oak which deserves attention for its girth and spread' (Polwhele in 1797). The haretail rush (Eriophorum vaginatum) is not uncommon in bogs on high moors; Polwhele recorded it near the road leading from Clovelly to Kilkhampton. The wood melic-grass (Melica nutans) was recorded by Polwhele in 1797 on the authority of Dr. Wavell as occurring in a wood near Dolton; this record has been doubted. Keys in his Flora of Devon and Cornwall wrote: 'As no later botanist has found it, it is scarcely worth while to insert it here. It is marked with an "o" for Devon in Cyb. Brit." The difficulty of accepting it is increased when it is remembered that Linnæus included both 


\section{BOTANY}

of our species of melic under his $M$. nutans, that the second species ( $M$. unifora) was separated by Retzius in 1779, that Hudson in the second edition of his Flora Anglica in 1778 in distinguishing our two species had kept the name of $M$. nutans for what is now called $M$. uniffora, and that Polwhele, who was familiar with Hudson's work, and perhaps knew nothing of that of Retzius, called his plant 'wood melic-grass,' which Hudson gave as the English name of his $M$. nutans (that is $M$. uniflora of Retzius), and did not mention any second species of the genus. On the other hand there is a specimen extant with a note purporting that it had been collected by Miss Griffiths in a 'wood near Dalton,' and the specimen belongs to the species now usually known as $M$. nutans, that is $M$. montana, Huds. Fl. Angl. ( 1778 ), ed. 2, p. 37 .

The following are among the more remarkable and interesting species which occur in the district :-

Berberis vulgaris, L.

Brassica campestris, L., sub-sp. B. Rapa, L., var sylvestris (B. Brigssii, Wats.)

Teesdalia nudicaulis, R. Br.

Viola canina, L., sub-sp. V. lactea, Sm.

Stellaria media, L., sub-sp. S. umbrosa, Opiz

Sagina apetala, L., sub-sp. S. ciliata, Fries

- nodosa, E. Mey.

Hypericum undulatum, Schousb.

Erodium moschatum, L'Herit.

Genista tinctoria, L.

Trifolium filiforme, $\mathrm{L}$.

Vicia Orobus, DC.

Potentilla Comarum, Nestl.

Agrimonia Eupatoria, L., sub-sp. A. odorata, Miller

Poterium officinale, Hook. f.

Pyrus communis, $L$.

- torminalis, Ehrh.

- Aria, Sm., sub-sp. P. latifolia, Syme

Drosera intermedia, Hayne

Myriophyllum alterniflorum, DC.

Callitriche verna, L., sub-sp. C. pedunculata, DC.

Carum verticillatum, Koch

Caucalis nodosa, Scop.

Galium uliginosum, L.

Valerianella Auricula, DC.

Gnaphalium sylvaticum, L.

Anthemis nobilis, L.

Artemisia Absinthium, L.

Arctium Lappa, L. (A. majus, Schkur), and sub-sp. A. minus, Schkur

Centaurea Cyanus, $\mathrm{L}$.

Carduus nutans, $L$.

Cnicus pratensis, Willd.

Picris echioides, L.

Leontodon hirtus, L.

- hispidus, L.

Wahlenbergia hederacea, Reichenb.

Campanula rotundifolia, $\mathrm{L}$.

Lysimachia vulgaris, $\mathrm{L}$.

Centunculus minimus, $\mathrm{L}$.

Menyanthes trifoliata, L.

Littorella lacustris, L.

Linaria Elatine, Miller

Antirrhinum Orontium, L.
Mimulus luteus, Pursh (not L.), M. Langsdorffii, Donn

Sibthorpia europæa, L.

Veronica scutellata, L., var. hirsuta, Weber Bartsia viscosa, $L$.

Pinguicula lusitanica, $L$.

Verbena officinalis, L.

Calamintha officinalis, Monch

Melissa officinalis, L.

Scutellaria minor, L.

Melittis Melissophyllum, L.

Stachys ambigua, Sm.

Lamium Galeobdolon, Crantz

Chenopodium Bonus-Henricus, L.

Polygonum Persicaria, L., var. P. nodosum, Pers.

Rumex acutus, L.

Euphorbia amygdaloides, L.

Salix triandra, L.

- alba, L., var. S. vitellina, L.

Spiranthes autumnalis, Rich.

Epipactis latifolia, All.

- palustris, Crantz

Habenaria viridis, R. Br.

- bifolia, R. Br.

Narcissus Pseudo-narcissus, L.

- biflorus, Curtis

Tamus communis, L.

Ruscus aculeatus, L.

Polygonatum multiflorum, All.

Potamogeton crispus, L.

Heliocharis multicaulis, Sm.

Scirpus sylvaticus, $L$.

- Savii, Seb. \& Maur.

- fluitans, L.

Rhynchospora alba, Vahl

Carex vulpina, L.

- pallescens, L.

- distans, L., and sub-sp. C. fulva, Good.

- lævigata, Sm.

- vesicaria, L.

Agrostis setacea, Curtis

Trisetum flavescens, P. Beauv.

Avena fatua, L.

- strigosa, Schreb.

Poa compressa, L. works :-

The literature dealing with the flowering plants of the district includes the following

Rev. W. Moyle Rogers in the Fournal of Botany, 1882, pp. 9-16, 'On some north Devon plants.' 


\section{A HISTORY OF DEVONSHIRE}

The same author in the same Foumal, I 883 , pp. I 6-20, 37-42, I0I-4, I 32-5, and I 886, pp. $8-14,78-82,104-10,144-8,176-81$, "On the flora of the upper Tamar and neighbouring districts.'

Thomas Richard Archer Briggs in the Foutnal of Botany, 1884, p. I70, 'On some Devonian stations of plants noted in the last century.'

Miss Helen Saunders in the Transactions of the Devonshire Association (1898), xxx. 200, 'Okehampton.'

The same author in the same Transactions (I gor), xxxiii. 470-2, 'Great Torrington.'

\section{South Molton}

This district consists of the petty sessional divisions of South Molton and Crediton and the municipal borough of South Molton; it forms the larger and eastern portion of the northern or South Molton parliamentary division, and contains about 233,41 5 acres of land and water; it is wholly inland, and is bounded on the west by the Torrington botanical district, on the north partly by the Barnstaple botanical district and partly by the county of Somerset, on the east by the Exeter botanical district, and on the south by the Torquay botanical district. It comprises 6I civil parishes, $3^{8}$ of which are drained wholly to the north coast, I I wholly to the south coast, and 12 partly to both coasts. It includes all the 29 parishes of the South Molton poor-law union, 28 out of the 30 parishes of the Crediton union, 2 (Ashreigney and Winkleigh) of the Torrington union, and 2 (Bondleigh and North Tawton) of the Okehampton union; 59 of the parishes are rural and 2 are urban.

The highest point is on Five Barrows Hill in North Molton parish, 1,618 feet above sealevel ; the lowest part is perhaps near Hawkridge bridge in Chittlehampton parish, about 33 feet altitude; another low part is by the river Creedy about 3 miles from Exeter. The principal rivers are the Taw with its tributaries the Bray, the Mole, the little Dart and the (South) Yeo, and the Creedy.

The following is a complete list in alphabetical order of the civil parishes in the district :-

Ashreigney
Bishopsnympton
Bondleigh
Bow
Brushford
Burrington
Charles
Chawleigh
Cheldon
Cheriton Fitzpaine
Chittlehamholt
Chittlehampton
Chulmleigh
Clannaborough
Coldridge
Colebrooke
Creacombe
Crediton Hamlets
Crediton Town (urban)
Down St. Mary
East Anstey

East Buckland
East Worlington
Eggesford
Filleigh
Georgenympton
Kennerleigh
Kingsnympton
Knowstone
Lapford
Mariansleigh
Meshaw
Molland
Morchard Bishop
Newton St. Cyres
North Molton
North Tawton
Nymet Rowland
Poughill
Puddington
Queensnympton

Rackenford

Romansleigh

Roseash

Sandford

Satterleigh and Warkleigh

Shobrooke

South Molton (urban)

Stockleigh English

Stockleigh Pomeroy

Thelbridge

Twitchen

Upton Hellions

Washford Pyne

Wembworthy

West Anstey

West Buckland

Winkleigh

Witheridge

Woolfardisworthy near Crediton

Zeal Monachorum

The greater part of the district belongs to the Carboniferous system; but, as in the Barnstaple district, nearly the whole of that part which lies to the north of the Devon and Somerset branch of the Great Western Railway is Devonian. The Upper Devonian beds run eastwards in continuation of those in the Barnstaple district on the west ; the Pickwell Down sandstones run across the parishes of Charles, North Molton, Twitchen, Molland, West Anstey and East Anstey, the breadth of the area varying from $\frac{1}{2}$ to $2 \frac{1}{4}$ miles; the Cucullæa beds run along the south side of the Pickwell Down sandstones, through the parishes of Charles and North Molton, the breadth of the area being mostly about half a mile; there are also three other patches of the Cucullæa beds which are surrounded by or adjoin the Pilton beds : one about $4 \frac{1}{2}$ miles long by about $\frac{1}{4}$ mile broad, extending from a point about a mile north of Castle Hill in Filleigh nearly to Limeslake in Bishopsnympton; another about $7 \frac{1}{2}$ miles long by two-thirds of a mile broad at the widest part, tapering towards each end, and extending 


\section{BOTANY}

from a point near Upcot in North Molton to the Somerset county boundary near East Lipscombe in East Anstey; and the third about half a mile long by about one-sixth of a mile broad at the eastern end, extending from West Lipscombe in East Anstey eastwards to the county boundary ; the Pilton beds extend for about I 5 miles from west to east through the parishes of Charles, West Buckland, East Buckland, Filleigh, South Molton, North Molton, Bishopsnympton, Twitchen, Molland, West Anstey and East Anstey.

There are several narrow patches of limestone within the areas of the Lower Culm Measures and Pilton beds in Filleigh and other parishes eastwards about the same latitude. There is a tract of New Red Sandstone measuring about $16 \frac{1}{2}$ miles from west to east, widening from I to $3^{\frac{3}{4}}$ miles from north to south, and extending through the parishes of North Tawton, Bow, Zeal Monachorum, Clannaborough, Down St. Mary, Colebrooke, Crediton Hamlets, Crediton Town, Sandford, Upton Hellions, Shobrooke, Newton St. Cyres and Stockleigh Pomeroy,

Mineral veins occur in the south part of the parish of Newton St. Cyres, within the areas of both the New Red Sandstone and the Culm Measures.

In the parish of West Buckland, in a quarry within the Lower Culm area and close to the southern boundary of the Pilton beds, the mineral called Wavellite occurs; it is a crystalline mineral, a phosphate of alumina.

With reference to the climate of the district calculations made from the records of British Rainfall show that the mean annual rainfall during the decade $1890-9$ at South Molton, 430 feet altitude, was 42.35 inches, and that the mean annual number of days on which $\circ \mathrm{Or}$ inch or more of rain fell was 198 . According to Mr. A. Chandler the mean annual temperature at South Molton is $484^{\circ} \mathrm{Fahr}$, and the percentage of possible sunshine is 36 . Calculations made from Mr. T. Wainwright's figures show that for the last few years at Romansleigh Rectory, 590 feet altitude, the mean annual minimum and maximum temperatures were $22.3^{\circ}$ Fahr. and $80.2^{\circ} \mathrm{Fahr}$. respectively; at Filleigh School, 368 feet altitude, the corresponding figures were $17.55^{\circ} \mathrm{Fahr}$. and $83^{\circ} 5^{\circ} \mathrm{Fahr}$. At the latter place the mean annual percentages of the humidity of the air and of cloud respectively during the years I894-I 900 were 84 and 70 .

The yellow vetchling or tare (Latbyrus Apbaca) is not common; it was recorded by Polwhele in 1797 as occurring in Chittlehampton, but it has not been recently reported thence.

The ivy bell-flower (Wablenbergia bederacea) was said by Gibson in 1695 to be no less common in this county than in Cornwall, in the like places; it is still plentiful in several parts of the district.

The Jacob's ladder (Polemonium caruleum) was found by Miss H. Saunders of South Molton on 23 July 1892, with white flowers in a wood in the parish of North Molton; it was probably only a casual there; the species is found also in the Plymouth district, and is not counted in the census of Devon plants.

The yellow monkey-flower (Mimulus Langsdorffii), a North American plant, is established in several places and increasing in quantity; it is the M. luteus, Pursh, not L. (see Fourn. Bot. 1895, p. 6).

The spurge laurel (Dapbne Laureola) is diminishing in the county; it occurs in the parish of Chittlehampton, and Dr. Wavell reported it from Raleigh Wood near Barnstaple.

The beech tree (Fagus sylvatica), according to Polwhele, is by far the most thriving of the trees in Rackenford, which is a parish with a clay soil south-east of South Molton; in I 797 there was a remarkably fine beech standing in the churchyard, the middle girth of which was $15^{\frac{3}{4}}$ feet, the least diameter of the spread of the branches being 67 feet; in the parish of Woolfardisworthy there were two widely spreading beech trees, so lofty as to be a seamark, growing on an estate called St. James' Tree (High Tree), adjoining the South Molton and Exeter road.

Robin's bastard daffodil (Narcissus eystettensis) has long been established in a field in the parish of Charles in this district ; it is considered to be a form of a double-flowered variety of the common Lent lily ( $N$. pseudo-narcissus); the perianth segments are repeated over and over again in regular order forming six rows, and no corona of the ordinary kind is developed; it was figured by Parkinson in I629 (Paradisus, p. 107, n. 4), who called it the 'lesser French double bastard daffodil.'

The Molland lily (Lilium pyrenaicum) is thoroughly established in the parish of Bishopsnympton, not far from Molland; it has maintained its position there for half a century.

Funcus tenuis was discovered by G. Don in Scotland in 1795 or 1796 , but for many 


\section{A HISTORY OF DEVONSHIRE}

years it was missed from the British flora until found in Herefordshire and recognized by Mr. Towndrow in 1884 ; in 1895 it was observed in moderate quantity in the parish of South Molton.

The moss-rush or gorse-corn ( $\mathcal{F}$. squarrosus) is plentiful on high moors in the district.

The following are among the more remarkable and interesting species which occur in the district :-

Ranunculus auricomus, $\mathrm{L}$.

Fumaria capreolata, L. (F. pallidiflora, Jord.) sub-sp. F. confusa, Jord.

Corydalis claviculata, DC.

Thlaspi arvense, $\mathrm{L}$.

Viola sylvestris, Fries, sub-sp. V. Reichenbachiana, Boreau

Stellaria aquatica, Scop.

Hypericum Androsæmum, L.

- quadrangulum, L. (H. dubium, Leers)

Geranium pratense, $L$.

- columbinum, L.

- lucidum, $\mathrm{L}$.

Euonymus europæus, L.

Rhamnus Frangula, L.

Genista tinctoria, L.

Melilotus altissima, Thuill.

Trifolium striatum, L.

- filiforme, L.

Vicia tetrasperma, Mœench

Geum intermedium, Ehrh.

Poterium Sanguisorba, L.

Chrysosplenium alternifolium, L.

Ribes Grossularia, L.

- rubrum, L.

- nigrum, L.

Sedum Telephium, L.

- rupestre, Huds., and sub-sp. S. Forsterianum, Sm.

- reflexum, $\mathrm{L}$.

Myriophyllum spicatum, L.

Epilobium angustifolium, L., form $\mathbf{E}$. brachycarpum, Leight.

- hirsutum, L.

Carum segetum, Benth.

Scandix Pecten-Veneris, L.

Viburnum Lantana, L.

Adoxa Moschatellina, L.

Valerianella Auricula, DC.

Inula Conyza, DC.

Anthemis tinctoria, L.

Artemisia Absinthium, L.

Centaurea nigra, L., var. C. decipiens, Tausch

Serratula tinctoria, $\mathrm{L}$.

Cnicus pratensis, Willd.

Cichorium Intybus, L.

Hieracium murorum, L. (H. sylvaticum, Gouan, not Sm.)

- murorum, L., sub-sp. H. pallidum, Fries)

Hieracium sylvaticum, Sm., not Gouan $(\mathrm{H}$. vulgatum, Fries)

Campanula rotundifolia, $\mathrm{L}$.

Lysimachia vulgaris, L.

Anagallis tenella, L.

Menyanthes trifoliata, $L$

Verbascum Blattaria, L.

Rhinanthus crista-galli, L., sub-sp. R. major, Ehrh.

Mentha viridis, L., var. M. paludosa, Sole.

Calamintha Acinos, Clairv.

Scutellaria minor, L.

Melittis Melissophyllum, L.

Stachys annua, $L$.

Galeopsis Tetrahit, L., var. G. bifida, Boenn.

Scleranthus annuus, L.

Polygonum Bistorta, L.

Rumex pulcher, $L$.

Humulus Lupulus, L.

Carpinus Betulus, $\mathbf{L}$.

Castanea vulgaris, Lam.

Populus alba, L.

Salix fragilis, $L$.

Neottia Nidus-avis, L.

Listera ovata, R. Br.

Spiranthes autumnalis, Rich.

Epipactis latifolia, All., and sub-sp. E. purpurata, Sm.

Orchis Morio, L.

Habenaria viridis, R. Br.

- bifolia, R. Br., and sub-sp. H. chlorantha, Bab.

Galanthus nivalis, L.

Polygonatum multiflorum, All.

Narthecium ossifragum, Huds.

Typha latifolia, L.

Potamogeton perfoliatus, $\mathrm{L}$.

Scirpus sylvaticus, $\mathrm{L}$.

- Savii, Seb. \& Maur.

Eriophorum vaginatum, L.

Carex præcox, Jacq.

Panicum Crus-galli, L.

Setaria viridis, P. Beauv.

Milium effusum, L.

Molinia cærulea, Mœnch

Catabrosa aquatica, P. Beauv.

Melica uniflora, Retz.

Bromus racemosus, $\mathrm{L}$.

The literature dealing with the flowering plants of the district includes the following works :-

Rev. W. Moyle Rogers in the Fournal of Botany, 1879, pp. 330-3, 'On some north Devon plants' (Zeal Monachorum, etc.).

Miss Helen Saunders in the Transactions of the Devonsbire Association, 1894, xxvi. 45 1-66, 'A list of plants growing wild in the parish of South Molton, and in the neigbouring parishes of North Molton, Filleigh, Chittlehampton, George Nympton, Satterleigh, King's Nympton, and Bishop's Nympton.'

The same author in the same Transactions, 1898, xxx. 198-200, 'Botanical Notes,' and 1901, voxxiii. 473 'Botanical Notes, No. ii.' 


\section{BOTANY}

The Rev. W. Moyle Rogers kindly sent to me for inspection a catalogue marked by him in June 1885 , for plants seen in the parishes of Bishopsnympton, Knowstone, Molland, Roseash and West Anstey.

\section{EXETER}

This district consists of the north-eastern or Tiverton parliamentary division and the parliamentary borough of Exeter; it contains about 21 5,652 acres, being about 21 4,439 acres of land, inland water and saltmarsh, besides about 1,213 acres of foreshore and tidal water; it is bounded on the west by the South Molton botanical district, on the north by the county of Somerset, on the east by the Honiton botanical district, and on the south by the Torquay botanical district; it consists of the three petty sessional divisions of Tiverton, Cullompton and Wonford and of the municipal borough of Tiverton; it is entirely inland, but parts of the parishes of Kenton, Powderham, Exminster, Topsham and Alphington are washed with tidal water. It comprises 65 civil parishes, all of which are drained to the south coast, except a fractional part of Cruwys Morchard that is drained by the small stream Dalch, a tributary of the river Taw, and except bits of the three parishes of Clayhanger, Hockworthy and Holcombe Rogus that are drained by the river Tone. It includes all the 26 parishes of the Tiverton poor-law union, the 5 Devonshire parishes in the Wellington (Somerset) union, 29 out of the 50 parishes of the St. Thomas union, 3 parishes (Broadhembury, Payhembury, and Plymtree) in the Honiton union, and the 2 parishes in the city of Exeter; 60 of the parishes are rural and 5 are urban.

The principal rivers are the Exe with its tributaries the Culm and the Clyst, the Tone for a short distance at the north-eastern boundary, and the Teign for a short extent on the south-west side.

The highest part is in the parish of Broadhembury, 930 feet above sea-level, and the lowest part is below high-water level of ordinary tides. district :-

The following is a complete list in alphabetical order of the civil parishes in the

\begin{tabular}{ll} 
Alphington & Dunchideock \\
Ashcombe & Exeter (urban) \\
Ashton & Exminster \\
Bampton (urban) & Halberton \\
Bickleigh near Tiverton & Heavitree (urban) \\
Bradninch & Hemyock \\
Brampford Speke & Hockworthy \\
Broadclist & Holcombe Burnell \\
Broadhembury & Holcombe Rogus \\
Burlescombe & Huntsham \\
Butterleigh & Huxham \\
Cadbury & Ide \\
Cadeleigh & Kenn \\
Christow & Kentisbeare \\
Clayhanger & Kenton \\
Clayhidon & Loxbeare (with Calverleigh) \\
Clysthydon & Mamhead \\
Clyst St. Laurence & Morebath \\
Cruwys Morchard & Netherexe \\
Cullompton & Oakford \\
Culmstock & Payhembury \\
Doddiscombsleigh & Pinhoe \\
\hline
\end{tabular}

Plymtree
Poltimore
Powderham
Rewe (with Upexe)
St. Leonard
St. Thomas (urban)
Sampford Peverell
Shillingford
Silverton
Stoke Canon
Stoodleigh
Templeton
Thorverton
Tiverton (urban)
Topsham
Uffculme
Uplowman
Upton Pyne
Washfield
Whitestone
Willand

The Carboniferous system occurs chiefly in the northern and western parts of the district, that is to say, more or less in 20 out of the 26 parishes of the Tiverton union; 2 in the Wellington union, 2 I out of the 50 parishes of St. Thomas union, also in parts of the city of Exeter.

Patches of Radiolarian (Codden Hill) beds occur about Bampton and Holcombe Rogus in the northern part of the district, and about Ashton in the southern part.

The New Red Sandstone formation occupies a considerable portion of the eastern and southern parts of the district.

Greensand occurs on the eastern side of the district, in the parishes of Culmstock, Hemyock, and Clayhidon, along the Blackdown Hills by the border of the county, also in 


\section{A HISTORY OF DEVONSHIRE}

Uffculme, Kentisbeare and Broadhembury ; the Haldon Hills at the south-west border belong to the Upper Greensand formation.

Patches of Greenstone occur north of Great Haldon, also between it and Exeter.

Limestone occurs near Hockworthy and Holcombe Rogus, and about Bampton.

Trappean rocks occur between Loxbeare and Washfield; there are also several patches about Silverton, and one at Exeter. The Upper Devonian system extends into the parishes of Morebath, Bampton and Clayhanger, about the most northerly part of the district.

The climate of the district is illustrated by the following tables, which have been obtained in the way explained under the Barnstaple district :-

Tabir 1.-Decade I8go-9

\begin{tabular}{|c|c|c|c|}
\hline Station & $\begin{array}{l}\text { Altitude, } \\
\text { feet }\end{array}$ & $\begin{array}{l}\text { Mean annual rainfall, } \\
\text { inches }\end{array}$ & $\begin{array}{l}\text { Mean annual number of days on } \\
\text { which or inch or more of rain fell }\end{array}$ \\
\hline $\begin{array}{l}\text { Huntsham . } . \cdot \cdot \cdot \cdot \cdot \\
\text { Cullompton . } \cdot \text {. } \\
\text { Exeter, Devon and Exeter Inst. } \\
\text { Exeter, Manston Terrace. } \\
\text { Christow, Kennick, Waterworks . }\end{array}$ & $\begin{array}{l}640 \\
202 \\
155 \\
166 \\
842\end{array}$ & $\begin{array}{l}45.28 \\
33 \cdot 72 \\
29.81 \\
29.14 \\
40.74\end{array}$ & $\begin{array}{l}203 \\
181 \\
160 \\
173 \\
199\end{array}$ \\
\hline
\end{tabular}

TABLE 2

\begin{tabular}{|c|c|c|c|c|c|c|c|c|c|c|c|}
\hline \multirow{2}{*}{ Station } & & \multirow{2}{*}{$\begin{array}{c}\text { Altitude, } \\
\text { feet } \\
\text { above } \\
\text { sea-level }\end{array}$} & \multirow{2}{*}{$\begin{array}{c}\text { Mean } \\
\text { annual } \\
\text { rainfall, } \\
\text { inches }\end{array}$} & \multirow{2}{*}{$\begin{array}{c}\text { Mean } \\
\text { annual } \\
\text { number } \\
\text { of } \\
\text { sunless } \\
\text { days }\end{array}$} & \multirow{2}{*}{$\begin{array}{c}\text { Mean } \\
\text { percentage } \\
\text { of } \\
\text { humidity } \\
\text { at } 9 \text { a.m. }\end{array}$} & \multirow{2}{*}{$\begin{array}{c}\text { Mean } \\
\text { annual } \\
\text { number } \\
\text { of hours } \\
\text { of sun- } \\
\text { shine }\end{array}$} & \multirow{2}{*}{$\begin{array}{l}\text { Mean } \\
\text { percent- } \\
\text { age of } \\
\text { possible } \\
\text { sunshine }\end{array}$} & \multirow{2}{*}{$\begin{array}{l}\text { Mean } \\
\text { percent- } \\
\text { age of } \\
\text { cloud } \\
\text { at } \\
9 \text { a.m. }\end{array}$} & \multicolumn{3}{|c|}{$\begin{array}{c}\text { Temperature in Fah. degrees, } \\
\text { average annual }\end{array}$} \\
\hline & & & & & & & & & $\begin{array}{c}\text { Mean at } \\
9 \text { a.m. }\end{array}$ & $\begin{array}{l}\text { Mini- } \\
\text { mum }\end{array}$ & $\begin{array}{l}\text { Maxi- } \\
\text { mum }\end{array}$ \\
\hline $\begin{array}{l}\text { Cullompton . } \\
\text { Exeter . }\end{array}$ & $\cdot$ & $\begin{array}{l}202 \\
155\end{array}$ & $\begin{array}{l}34 \cdot 26 \\
30 \cdot 23\end{array}$ & 69 & -7 & 1475 & $\begin{array}{l}34 \\
35\end{array}$ & 69 & $\begin{array}{l}49 \cdot 1 \\
50.7\end{array}$ & $\begin{array}{c}17.01 \\
22\end{array}$ & $\begin{array}{c}83 \cdot 46 \\
83\end{array}$ \\
\hline
\end{tabular}

The wild rocket or stinkweed (Diplotaxis muralis) was recorded by Hudson in 1778 as growing about Exeter. The procumbent pepperwort or wart-cress (Senebiera didyma) was also recorded by Hudson at the same time on the authority of Mr. W. Newbery as growing in waste places about Exeter; a peculiarity in the inflorescence was noticed at Plymouth and was the subject of a communication made to the Plymouth meeting of the British Association in 1878. The toadflax-leaved St. John's wort (Hypericum linarifolium) grows in great luxuriance and abundance on a rocky cleave in the parish of Christow more than a mile from the river Teign; it flowers from the middle of June to the beginning of October and attains 18 inches or more in height; the leaves are at first only very slightly or scarcely revolute, their midribs being very prominent beneath ; their hue is somewhat glaucous, and they are remarkably blunt, narrowing only a little from the base to the apex; their stems appear to be uniformly ascending, never erect or prostrate, and they are obscurely twoedged; there is a rigid look about the whole plant, and a regularity of outline in the leaves and in the especially bright yellow petals distinguishes the species from all other British ones; the peculiar resinous smell so characteristic of the tutsan is very strong in this species (Moyle Rogers). It was first recorded in Britain in 1840 by the Rev. Thomas Hincks as having occurred among granite rocks near the banks of the Teign in the summer of 1838 (Fourn. Bot. 1892, p. 275).

The yellow wood-sorrel (Oxalis corniculata) was first recorded as a British plant in 1795 as occurring in several places in the neighbourhood of Exeter, having been found by Mr. J. Turner (Fourn. Bot. 1892, p. 277); Mr. Moyle Rogers could not but regard it as truly indigenous at Trusham (a parish in the Torquay district and bordering this district), where it occurred not only in three lanes and in considerable quantities but also on an open furzy down where it looked quite as much native as Rumex Acetosella, Sedum anglicum and Geranium pusillum, which grew beside it.

The red valerian or drunken sailor (Centrantbus ruber) was recorded by Hudson in 1778 as plentiful in Devon on old walls and in waste places, and it was reported by Jones and Kingston as likewise occurring at Exeter; with white flowers it occurs on the cliffs at Dawlish (Jordan) and on the old city walls at Exeter (Parfitt); it is quite naturalized in several places. 


\section{BOTANY}

The sweet flag (Acorus Calamus) grows in the Exeter canal, where it has long been established and produces catkins. For a discussion on the question whether this plant is native in Britain see Fourn. Bot. $187 \mathrm{I}, \mathrm{p} .163$.

The deer's-foot grass (Agrostis setacea) was reported by Hudson in 1778 as occurring on the dry elevated heaths of Haldon near Exeter and elsewhere in Devon; it is locally abundant.

The following are among the more remarkable and interesting species which occur in the district :-

Ranunculus sceleratus, $L$.

Aquilegia vulgaris, $\mathrm{L}$.

Nuphar luteum, Sm.

Nasturtium palustre, DC.

Barbarea vulgaris, R. Br., sub-sp. B. intermedia, Boreau

Cardamine impatiens, L.

Erysimum cheiranthoides, L.

Diplotaxis tenuifolia, DC.

Viola hirta, L.

Linum angustifolium, Huds.

Geranium rotundifolium, L.

- Robertianum, L., var. G. modestum, Jordan

Genista anglica, L.

Medicago maculata, Sibth.

Melilotus officinalis, Desv.

Lotus angustissimus, $\mathrm{L}$.

Potentilla argentea, $\mathrm{L}$.

Alchemilla vulgaris, $\mathrm{L}$.

Poterium Sanguisorba, L.

- officinalis, Hook. f.

Pyrus torminalis, Ehrh.

Sedum Telephium, L.

- reflexum, $\mathrm{L}$.

Hippuris vulgaris, $\mathbf{L}$.

Callitriche verna, L., sub-sp. C. obtusangula, Leg.

Epilobium lanceolatum, Seb. \& Maur.

Carum segetum, Benth.

Sison Amomum, $\mathrm{L}$.

Anthriscus sylvestris, Hoffm.

Fœniculum officinale, All.

Enanthe pimpinelloides, L.

Silaus pratensis, Besser

Caucalis arvensis, Huds.

Rubia peregrina, L.

Galium tricorne, Stokes

Valerianella Auricula, DC.

Dipsacus pilosus, $\mathrm{L}$.

Inula Conyza, DC.

Bidens cernuus, L.

- tripartitus, $\mathrm{L}$.

Carduus tenuiflorus, Curtis

Picris hieracioides, $\mathrm{L}$.

Crepis biennis, $\mathrm{L}$.

Tragopogon pratensis, L.

Wahlenbergia hederacea, Reichenb.

Specularia hybrida, Alph. DC.

Lysimachia vulgaris, L.

Anagallis arvensis, L., var. A. cærulea, Schreb.

Anagallis tenella, L.

Samolus Valerandi, L.

Vinca minor, L.

Erythrza Centaurium, Pers., sub-sp. E. pulchella, Fries

Lithospermum arvense, $\mathrm{L}$.

Verbascum Blattaria, L., sub-sp. V. virgatum, Stokes

Linaria repens, Miller

Sibthorpia europæa, L.

Bartsia viscosa, L.

Orobanche major, $\mathrm{L}$.

- minor, Sutton

Pinguicula lusitanica, L.

Thymus Serpyllum, L., sub-sp. T. Chamzdrys, Fries

Calamintha Acinos, Clairv.

Salvia Verbenaca, L.

Scutellaria minor, L.

Melittis Melissophyllum, L.

Galeopsis Ladanum, L.

Leonurus Cardiaca, $L$.

Scleranthus annuus, $L$.

Chenopodium polyspermum, $\mathrm{L}$.

Hydrocharis Morsus-rana, $L$.

Malaxis paludosa, Sw.

Neottia Nidus-avis, L.

Spiranthes autumnalis, Rich.

Epipactis latifolia, All.

Galanthus nivalis, L.

Polygonatum multiflorum, All.

Luzula Forsteri, DC.

Sparganium simplex, Huds.

Lemna trisulca, L.

- polyrrhiza, L.

Butomus umbellatus, $\mathrm{L}$.

Scirpus sylvaticus, L.

Carex muricata, L., sub-sp. C. divulsa, Good.

- pallescens, L.

- Pseudo-cyperus, L.

Alopecurus geniculatus, L., sub-sp. A. bulbosus, Gouan

Calamagrostis lanceolata, Roth

Trisetum flavescens, P. Beauv.

Avena fatua, L.

Glyceria fluitans, L., var. G. plicata, Fries

Bromus erectus, Huds.

- madritensis, $\mathrm{L}$.

Hordeum pratense, Huds.

The literature dealing with the flowering plants of the district includes the following works :-

George Stacey Gibson in the Pbytologist, ii. 676-83, 'Notice of some localities of plants in Cornwall, etc., in the 8 th month, 1846, p. 683 , Exeter, 1846 (l). 


\section{A HISTORY OF DEVONSHIRE}

T. W. Gissing in the Pbytologist, June 1855 , 'Botanical notes from South Devon,' p. 29.

Fred. Mackenzie in the Transactions of the Devonsbire Association, I865, i. pt. 4, 68-71, 'On the flora of the neighbourhood of Tiverton.'

Rev. W. Moyle Rogers in the Fournal of Botany, 1878, pp. 15-25, 'Notes on some south-east Devon plants'; and I 879, pp. 9-13, 'On some south-east Devon plants.'

The same Author in the same Foumal, 1882, pp. 70-6, I 2 I-4, 133-6, I 77-83, 206-9, 239-43, 262-6, 'A contribution towards a Flora of the Teign Basin, S. Devon.'

W. S. M. D'Urban : 'A sketch of the Natural History of the neighbourhood of Exeter,' extracted from Besley's Book of Exeter (1 87 I), ed. ii. pp. 105-1 1, 'Botany'; this account included plants from the Honiton and Torquay besides the Exeter botanical district.

Miss Helen Saunders in the Transactions of the Devonshire Association, I901, xxxiii. 473, 'Botanical Notes, No. ii.'

Thomas Richard Archer Briggs in the Fournal of Botany, 1884, pp. 172, 174, ' On some Devonian stations of plants noted in the last century.'

\section{Honiton}

This district consists of the eastern or Honiton parliamentary division, excluding however the parish of Churchstanton (transferred in 1896 from Devon to Somerset) and including the parishes of Chardstock and Hawkchurch (transferred at the same time from Dorset to Devon); it contains about 173,178 acres, being about 171,078 acres of land, inland water and saltmarsh, besides about 2,100 acres of foreshore and tidal water. It is bounded on the west by the Exeter botanical district, on the north by the county of Somerset, on the east by the county of Dorset, and on the south by the English Channel; it consists of the four petty sessional divisions of Axminster, Honiton, Ottery and Woodbury; it comprises 60 civil parishes, all of which are drained to the south coast, 53 being rural and 7 urban; it contains all the 16 parishes of the Axminster poor-law union, 26 out of the 29 parishes of the Honiton union, and 18 out of the 50 parishes of the St. Thomas union. The most easterly part of the county is in the parish of Hawkchurch on the Dorset border.

The principal rivers are the Clyst, the Otter, the Sid, and the Axe with its tributary the Yarty ; the estuary of the Exe forms part of the western boundary.

The highest part of the district is near Sheldon, about 930 feet above sea-level. trict :-

The following is a complete list in alphabetical order of the civil parishes in the dis-

$\begin{array}{ll}\text { Awliscombe } & \text { East Budleigh } \\ \text { Axminster } & \text { Farringdon } \\ \text { Axmouth } & \text { Farway } \\ \text { Aylesbeare } & \text { Feniton } \\ \text { Beer } & \text { Gittisham } \\ \text { Bicton } & \text { Harpford } \\ \text { Branscombe } & \text { Hawkchurch } \\ \text { Buckerell } & \text { Honiton (urban) } \\ \text { Budleigh Salterton (urban) } & \text { Kilmington } \\ \text { Chardstock } & \text { Littleham and Exmouth (urban) } \\ \text { Clist Honiton } & \text { Luppitt } \\ \text { Clist St. George } & \text { Lympstone } \\ \text { Clist St. Mary } & \text { Membury } \\ \text { Colaton Raleigh } & \text { Monkton } \\ \text { Colyton } & \text { Musbury } \\ \text { Comberaleigh } & \text { Newton Poppleford } \\ \text { Combpyne } & \text { Northleigh } \\ \text { Cotleigh } & \text { Offwell } \\ \text { Dalwood } & \text { Otterton } \\ \text { Dunkeswell } & \text { Ottery St. Mary (urban) }\end{array}$

Rockbeare
Rousdon
Salcombe Regis (urban)
Seaton (urban)
Sheldon
Shute
Sidbury
Sidmouth (urban)
Southleigh
Sowton
Stockland
Talaton
Uplyme
Upottery
Ven Ottery
Whimple
Widworthy
Withycombe Raleigh
Woodbury
Yarcombe

The New Red Sandstone formation occupies most of the western portion of the district, and extends to Honiton as well as along the valley of the Otter to the Somerset county boundary; it also ascends the valleys of the Sid and the Axe and most of their tributary streams.

The Cretaceous beds occupy the chief part of the rest of the district, mostly on the eastern portion; there is also a patch of it at Blackhill near Withycombe Raleigh.

The Lias beds extend along the cliffs from Culverhole Point eastwards to the Dorset boundary, also inland about Uplyme, and around Axminster except the western side. 


\section{BOTANY}

Chalk occurs near the coast from Axmouth to Pinhay, also about Beer Head and Branscombe, with a small patch between Salcombe Regis and Weston Mouth, and there it is the most westerly example of chalk in England; small patches occur also near the Blackdown Hills.

The climate of the district is illustrated by the following tables, which have been obtained in the way explained under the Barnstaple district :-

TABLE 1.-Decade I $890-9$

\begin{tabular}{c|c|c|c|c}
\hline Station & $\begin{array}{c}\text { Altitude, } \\
\text { feet }\end{array}$ & $\begin{array}{c}\text { Mean annual rainfall, } \\
\text { inches }\end{array}$ & $\begin{array}{c}\text { Mean annual number of days on } \\
\text { which or inch or more of rain fell }\end{array}$ \\
\hline Clevelands, Axmouth . . . . & $\begin{array}{c}46 \\
\text { Rousdon. . . . . . . }\end{array}$ & 516 & $\begin{array}{l}33443 \\
31 \% 47\end{array}$ & 164 \\
\hline
\end{tabular}

$\mathrm{T}_{\mathrm{ABLE}} 2$

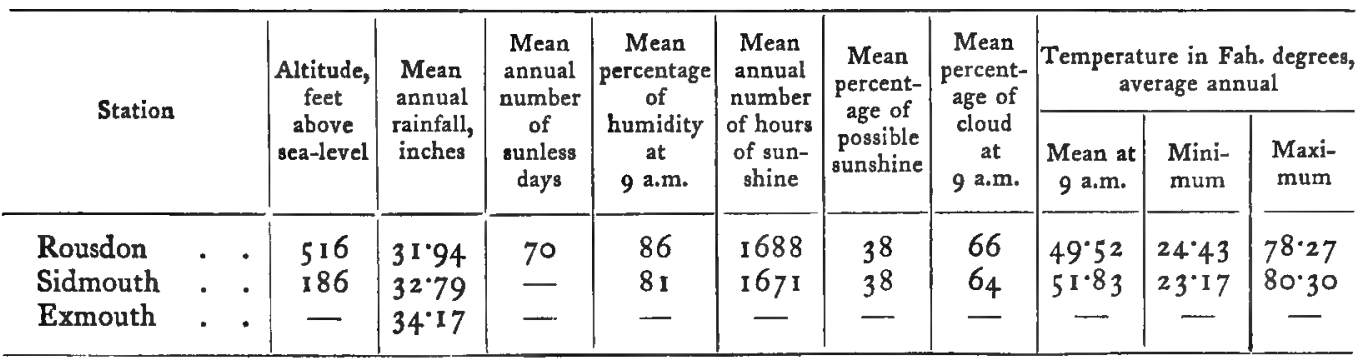

The meadow rue (Thalictrum flavum) was recorded by Jones and Kingston in 1829 as occurring by Bishop's Clyst bridge, and Mr. James Jermyn of Exeter tells me that it still exists there and has spread in the immediate neighbourhood. The all-seed (Polycarpon tetraphyllum) was also recorded by Jones and Kingston; it was found originally on some old walls at Lympstone by the Rev. William Newberry of Bushey, and subsequently by Miss Filmore.

The flower of the Axe (Lobelia urens) was first discovered in Britain by Mr. William Newbery of Stockland, Devon, on high ground in a boggy moor on Shute Common, between Axminster and Honiton, in October I768; it still maintains its place there, and has also been found in east Cornwall and on the borders of Dartmoor.

The tea-plant, a species of Lycium, often planted for ornament by cottagers, is a native of Greece and the Mediterranean region, etc., and is naturalized about Exmouth ; it is not the original L. barbarum of Linnæus, which belongs to hot dry tracts in north Africa and south Persia, and which differs from our plant by the corolla-tube being shorter and by the still shorter stamens; our plant is L. balimifolium, Miller, which is perhaps identical with L. chinense, Miller.

The twiny-leg (Bartsia viscosa) was recorded by Hudson in 1778 as occurring in Devon, a precise locality probably being on the side of a rivulet that runs by the bog nearest Budleigh Salterton, and plentifully in the marshy ground just at the end of the village (Fourn. Bot. 1884 , p. 172 ).

The spear-mint (Mentha viridis) was recorded by Hudson in 1778 as occurring in watery places and at the banks of streams near Exmouth; it is also reported from Sidford.

The shrubby stonecrop or glasswort (Suceda fruticosa) was recorded by Hudson in 1762 as inhabiting the sea-coast of Devon; it is also mentioned in Cullen's Flora Sidostiensis (1849) as indigenous to the vicinity of Sidmouth; Budleigh Salterton is given in Stewart's Torquay Flora on the authority of Miss A. Griffiths as a station for it, but specimens from that locality in that lady's herbarium at Torquay, labelled 'Chenopodium-? Budleigh Salterton,' seemed to Dr. Trimon to be a procumbent form of $S$. maritima (Keys); these records do not appear to justify the retention of $S$. fruticosa in our county flora.

The mistletoe (Viscum album) was recorded by Jones and Kingston in I 829 as occurring in an orchard at Holcombe Rogus on the borders of Somerset, on the authority of the Rev. A. Neck ; it was also reported in Flora Sidostiensis as occurring at Harpford, Larkbere, and as plentiful on Pin farm, Otterton; the last record has been confirmed during recent years.

The Portland spurge (Eupborbia portlandica) was recorded by Hudson in 1778 as occurring on the sands of the sea-shore near Exmouth, the precise locality apparently being near the 


\section{A HISTORY OF DEVONSHIRE}

passage on the sands at Exmouth (see Fourn. Bot. I884, p. 173); it has also been reported from Branscombe (Fl. Sidostiensis, p. 38).

The twig-rush (Cladium Mariscus) occurs in boggy ground at Hawkchurch Copse as recorded in the Flora of Dorset; Mr. Mansel-Pleydell verified this station, which contributes a good record for this genus to the Devon flora as a consequence of the transfer in 1896 of the parish of Hawkchurch from Dorset to Devon; there is however a less satisfactory record of this plant for Dartmoor.

The loose-flowered or reflected meadow-grass (Glyceria distans) was recorded by Hudson in 1778 as growing in sandy places near Exmouth; it was also reported in 1829 by Jones and Kingston on the authority of the Rev. Dr. John Jacob of Devonport as occurring near Topsham. The sea lyme-grass was recorded by Hudson in 1778 as inhabiting the seacoast near Exmouth; it was also reported from the same station by Jones and Kingston in 1829 on the authority of Miss Filmore, and by Ravenshaw in 1860 on the authority of Mr. Edward Parfitt, but according to a manuscript note in the British Museum herbarium copy of Ravenshaw's List, Mr. Parfitt's specimens are Triticum (Agropyrum) junceum; it has been also reported from Paignton and Goodrington sands in the Torquay district; Sir Joseph Hooker in his Student's Flora does not extend the distribution of the species to Devon, and Mr. Moyle Rogers has closely searched the Exmouth station and Dawlish Warren for this rare grass, and concludes that it is now absent from south Devon; recent observations however in other parts justify its retention in the county flora.

The following are among the more remarkable and interesting species which occur in the district :-

Clematis Vitalba, L.

Ranunculus Lingua, L.

Nuphar luteum, Sm.

Glaucium luteum, Scop.

Diplotaxis tenuifolia, DC.

Senebiera didyma, Pers.

Crambe maritima, L.

Reseda lutea, L.

Helianthemum vulgare, Gartn.

Viola palustris, $L$.

Silene nutans, $\mathrm{L}$.

Cerastium semidecandrum, L.

Hypericum montanum, L.

Malva rotundifolia, $L$.

Lavatera arborea, $\mathrm{L}$.

Erodium moschatum, L'Herit.

- maritimum, L'Herit.

Oxalis corniculata, $L$.

Trigonella ornithopodioides, DC.

Lotus angustissimus, $\mathrm{L}$.

Hippocrepis comosa, $\mathbf{L}$.

Vicia bithynica, L., var. latifolia, Syme, and var. angustifolia, Syme

Lathyrus Aphaca, L.

- Nissolia, L.

- sylvestris, L.

Drosera intermedia, Hayne

- anglica, Hudson

Hippuris vulgaris, $L$.

Carum segetum, Benth.

Sium latifolium, L.

Crithmum maritimum, L.

Daucus Carota, L., sub-sp. D. gummifer, Lam.

Cornus sanguinea, $L$.

Viburnum Lantana, L.

Sambucus Ebulus, L.

Rubia peregrina, $L$.

Galium Cruciata, Scop.

Asperula cynanchica, L.

Aster Tripolium, L., var. discoideus, Syme

Anthemis nobilis, L.
Artemisia maritima, L., and var. A. gallica, Willd.

Senecio erucifolius, L.

Centaurea Calcitrapa, L.

Carduus crispus, L., var. C. acanthoides, $L$.

Cnicus eriophorus, Roth

Cichorium Intybus, L.

Picris hieracioides, $\mathrm{L}$.

- echioides, L.

Hypochœris glabra, L.

Wahlenbergia hederacea, Reichenb.

Campanula Trachelium, L.

Statice Limonium, L.

Primula veris, $\mathrm{L}$.

Vinca major, L.

Chlora perfoliata, L.

Erythræa Centaurium, Pers., sub-sp. E. pulchella, Fries

Gentiana Amarella, L.

Lithospermum purpureo-cæruleum, L.

Plantago media, L.

Verbascum nigrum, L.

Veronica Anagallis, L.

Pinguicula Iusitanica, L.

Melittis Melissophyllum, L.

Chenopodium Vulvaria, L.

- murale, L.

Atriplex laciniata, L.

- portulacoides, L.

Daphne Laureola, L.

Euphorbia Paralias, L.

Populus alba, L., sub-sp. P. canescens, Sm.

Salix repens, L., var. S. fusca, $L$.

Ceratophyllum demersum, L.

Epipactis palustris, Crantz

Ophrys apifera, Hudson

Ruscus aculeatus, L.

Ornithogalum umbellatum, L.

Juncus obtusiflorus, Ehrh.

Luzula Forsteri, DC.

Alisma ranunculoides, $\mathrm{L}$. 


\section{BOTANY}

Sagittaria sagittifolia, L.

Butomus umbellatus, L.

Zostera marina, L., var. angustifolia, Fries

Scirpus Caricis, Retz.

Schœenus nigricans, L.
Alopecurus geniculatus, L., sub-sp. A. bulbosus, Gouan

Gastridium lendigerum, Gaud.

Glyceria Borreri, Bab.

Lepturus filiformis, Trin.

works :-

The literature dealing with the flowering plants of the district includes the following

Theodore H. Mogridge : A descriptive sketch of Sidmouth (1 836), pp. $65-86$.

R. C. R. Jordan in the Pbytologist, January 1844, p. 828, 'Exmouth.'

W. H. Cullen : Flora Sidostiensis; or a catalogue of the plants indigenous to the vicinity of Sidmouth (1 849 ).

T. W. Gissing in the Pbytologist, June 1855, 'Botanical notes from South Devon,' pp. 27, 28.

Zachary James Edwards: Ferns of the Axe and its thibutaries, also of Lyme, Cbarmouth, Uplyme, and Monkton Wyld, with an account of the flower Lobelia urens (1862).

George P. R. Pulman : Book of the Axe (1875), ed. 4.

W. Moyle Rogers in the Fournal of Botany, 1878, pp. 22-5, "Notes on some south-east Devon plants.'

Miss Helen Saunders in the Transactions of the Devonshire Association, I90r, xxxiii. 469, 470, 'Botanical Notes No. ii.'

John Clavell Mansel-Pleydell : The Flora of Dorsetshire (1895), ed. 2 ; for the parishes of Chardstock and Hawkchurch.

Thomas Richard Archer Briggs in the Foumal of Botany, 1884, pp. 168-74, 'On some Devonian stations of plants noted in the last century.'

\section{Torquay}

This district consists of the Mid or Ashburton and the Torquay parliamentary divisions; it contains about 206, 1 93 acres, being about 204,175 acres of land, inland water and saltmarsh, besides about 2,018 acres of foreshore and tidal water; it is bounded on the west by the Tavistock and Torrington botanical districts, on the north by the South Molton botanical district, on the east by the English Channel, and on the south by the Plymouth botanical district. It consists of the three petty sessional divisions of Crockernwell, Paignton and Teignbridge, and the municipal borough of Dartmouth; it comprises all the 38 parishes of the Newton Abbot poor-law union, 2 parishes (Cheriton Bishop and Hittisleigh) of the Crediton union, 9 parishes of the Totnes union, 6 parishes of the Okehampton union, and 3 parishes (Bridford, Dunsford and Tedburn St. Mary) of the St. Thomas union, altogether 58 civil parishes, of which 45 are rural and $\mathrm{r} 3$ are urban; its drainage is wholly to the south coast, except portions of South Tawton and Spreyton (in the Okehampton union), which are drained by the upper reaches of the river Taw and its tributary the (South) Yeo ; it contains part of Dartmoor.

The principal rivers are the Teign (with its tributaries the North Teign, the Waller brook on the west, and the Bovey), and on the south the Dart with its tributaries.

The highest part is Cawsand Beacon, 1,785 feet above sea-level. district :-

The following is a complete list in alphabetical order of the civil parishes in the

$\begin{array}{lll}\text { Abbotskerswell } & \text { Dartmouth (urban) } & \text { Kingskerswell } \\ \text { Ashburton (urban) } & \text { Dawlish, East (urban) } & \text { Kingsteignton } \\ \text { Bickington } & \text { Dawlish, West } & \text { Kingswear } \\ \text { Bishopsteignton } & \text { Drewsteignton } & \text { Lustleigh } \\ \text { Bovey Tracey } & \text { Dunsford } & \text { Manaton } \\ \text { Bridford } & \text { East Teignmouth (urban) } & \text { Marldon } \\ \text { Brixham (urban) } & \text { Gidleigh } & \text { Milber (urban) } \\ \text { Broadhempstone } & \text { Haccombe with Coombe } & \text { Moretonhampstead } \\ \text { Buckland in the Moor } & \text { Hennock } & \text { North Bovey } \\ \text { Chagford } & \text { Highweek (urban) } & \text { Ogwell } \\ \text { Cheriton Bishop } & \text { Hittisleigh } & \text { Paignton (urban) } \\ \text { Chudleigh } & \text { Holne } & \text { St. Mary Church } \\ \text { Churston Ferrers } & \text { Ideford } & \text { (urban) } \\ \text { Cockington } & \text { Ilsington } & \text { St. Nicholas (urban) } \\ \text { Coffinswell } & \text { Ipplepen } & \text { South Tawton }\end{array}$

\author{
Spreyton \\ Staverton \\ Stoke Gabriel \\ Stokeinteignhead \\ Tedburn St. Mary \\ Teigngrace \\ Throwleigh \\ Torbryan \\ Tormoham (urban) \\ Trusham \\ West Teignmouth \\ (urban)
}

Widdecombe in the Moor

Wolborough (urban)

Woodland

The Carboniferous system spreads across the northern portion of the district and passes round the granite to the central parts, extending south of Holne.

The New Red Sandstone formation prevails on the eastern side following the coast from Dawlish warren with a short interruption to Paignton and penetrating generally a few miles inland. 


\section{A HISTORY OF DEVONSHIRE}

Devonian rocks occur in the southern part of the district from Bickington and Newton Abbot to Ogwell, Ashburton, Broadhempstone, Woodland, and Staverton, on Warberry Hill, and at Barton near Torquay.

Granite occupies a considerable tract on the Dartmoor or western side of the district.

Patches of Greenstone are numerous and occur mostly in the central and southern parts of the district within the limits of the Carboniferous and Devonian systems.

Greensand occurs on Great Haldon along the border of the Exeter district and on Little Haldon; patches of it also occur north of Kingsteignton, near Newton Abbot, and on Milber Down.

Limestone occurs at Berry Head and on the south side of Torbay, reaching inland as far as Churston Ferrers village; also about Torquay between the Devonian rocks and the New Red Sandstones ; there are also inland patches irregular in size and outline on the eastern side of the district.

The miocene formation is represented by the Bovey beds, where a tract of lacustrine origin, about $8 \frac{3}{4}$ miles long by $3 \frac{2}{3}$ miles broad in the widest part, extending from Kingskerswell to a little beyond Bovey Tracey, contains a deposit of lignite, which has been used as fuel for two centuries at least, and which is of chief interest in the county geology.

There is a tin mine in the north part of the parish of Ashburton, and mineral veins and stream tin occur in the Dartmoor portion of the district.

The climate of the district is illustrated by the following tables, which have been obtained in the way explained under the Barnstaple district :-

Tabie 1.-Decade r890-9

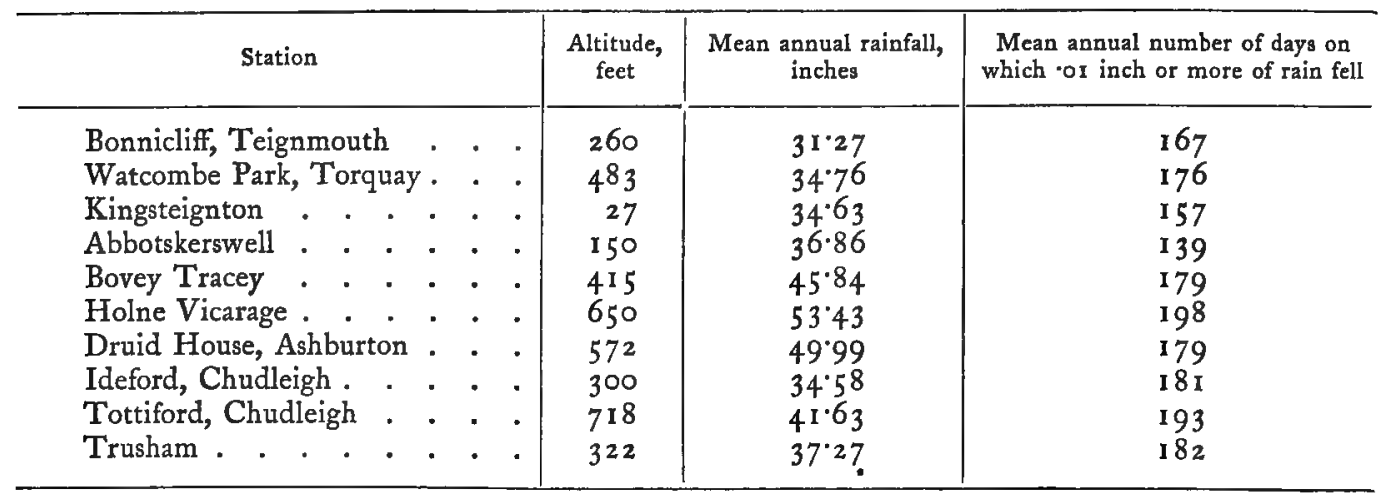

TABLE 2

\begin{tabular}{|c|c|c|c|c|c|c|c|c|c|c|}
\hline \multirow{2}{*}{ Station } & \multirow{2}{*}{$\begin{array}{c}\text { Altitude, } \\
\text { feet } \\
\text { above } \\
\text { sea-level }\end{array}$} & \multirow{2}{*}{$\begin{array}{l}\text { Mean } \\
\text { annual } \\
\text { rainfall, } \\
\text { inches }\end{array}$} & \multirow{2}{*}{$\begin{array}{c}\text { Mean } \\
\text { annual } \\
\text { number } \\
\text { of } \\
\text { sunless } \\
\text { days }\end{array}$} & \multirow{2}{*}{$\begin{array}{c}\text { Mean } \\
\text { percentage } \\
\text { of } \\
\text { humidity } \\
\text { at } \\
9 \text { a.m. }\end{array}$} & \multirow{2}{*}{$\begin{array}{c}\text { Mean } \\
\text { annual } \\
\text { number } \\
\text { of hours } \\
\text { of sun- } \\
\text { shine }\end{array}$} & \multirow{2}{*}{$\begin{array}{c}\text { Mean } \\
\text { percent- } \\
\text { age of } \\
\text { possible } \\
\text { sunshine }\end{array}$} & \multirow{2}{*}{$\begin{array}{c}\text { Mean } \\
\text { percent- } \\
\text { age of } \\
\text { cloud } \\
\text { at } \\
9 \text { a.m. }\end{array}$} & \multicolumn{3}{|c|}{$\begin{array}{c}\text { Temperature in Fah. degrees, } \\
\text { average annual }\end{array}$} \\
\hline & & & & & & & & $\begin{array}{c}\text { Mean at } \\
9 \text { a.m. }\end{array}$ & $\begin{array}{l}\text { Mini- } \\
\text { mum }\end{array}$ & $\begin{array}{l}\text { Maxi- } \\
\text { mum }\end{array}$ \\
\hline Dawlish. & - & $36 \cdot 13$ & - & - & - & - & - & - & - & 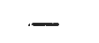 \\
\hline $\begin{array}{l}\text { Teignmouth } \cdot . \\
\text { Torquay, Cary }\end{array}$ & 70 & $34^{\circ} 99$ & - & 82 & - & 36 & 64 & $50 \cdot 6$ & 22.05 & 80.08 \\
\hline Green & 12 & - & 56 & 79 & 1897 & 39 & $6 I$ & $52 \cdot 04$ & $24^{\circ} 93$ & $77 \cdot 96$ \\
\hline Torquay, Chapel & & & & & & & & & & \\
\hline Hill . . . & 286 & $34 \cdot 24$ & 59 & 82 & 1712 & 39 & $6 I$ & $51 \cdot 0$ & $25 \cdot 17$ & 80.5 \\
\hline Brixham . & - & 43.04 & - & - & - & - & - & - & - & - \\
\hline Teignbridge . & 27 & $36 \cdot 13$ & - & - & 一 & - & - & - & - & - \\
\hline Highweek . & 一 & $40^{\circ} 00$ & - & - & - & - & - & - & - & - \\
\hline Bovey Tracey & 415 & $45 \cdot 18$ & - & - & - & - & - & - & 一 & - \\
\hline $\begin{array}{l}\text { Chagford } \\
\text { Tottiford, Chud- }\end{array}$ & & 53.20 & - & - & - & - & - & - & - & - \\
\hline leigh & 718 & $4 \mathrm{I} \cdot 10$ & - & - & - & - & - & - & - & - \\
\hline Ilsington & 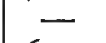 & $5 \times 105$ & - & - & - & - & - & - & - & - \\
\hline Holne Vicarage & 650 & $64 \cdot 4^{8}$ & - & - & - & - & - & - & - & - \\
\hline $\begin{array}{c}\text { Ashburton, Druld } \\
\text { House . }\end{array}$ & $5^{84}$ & 52.22 & - & 83 & 一 & 36 & 64 & $50 \cdot 3$ & 23.27 & 79.77 \\
\hline
\end{tabular}




\section{BOTANY}

The white mountain rock-rose (Heliantbemum polifolium) is very rare as a British plant, and along Torbay is one of the most striking features in its flora; it was recorded by Jones and Kingston in 1829 as occurring on the cliffs at Babbicombe and Torquay.

The tree mallow (Lavatera arborea) was recorded as a frequent Devon plant by Hudson in I 778 ; it was found on the Thatcher, Torbay, by the Rev. Aaron Neck as recorded by Jones and Kingston in 1829 .

The extreme abundance of Geranium lucidum in the Trusham neighbourhood is quite a feature in the flora, and one that can hardly fail to catch the eye of even non-botanical observers; it covers walls and hedge-banks and occupies favourable spots in the woods (Moyle Rogers). In the same vicinity, according to Moyle Rogers, Trifolium subterraneum is especially plentiful, as well as in south-east Devon generally; the turf wherever undisturbed is usually full of it, almost to the exclusion of the common clovers; in some of the more stony fallows and on the open downs $\mathcal{T}$. filiforme and $\mathcal{T}$.glomeratum dispute the ground with it. $\mathcal{T}$. striatum, $\tau$. arvense and $\mathcal{T}$. procumbens are also frequent; $\mathcal{T}$. minus is to be met with everywhere in the lanes and woods and along the borders of fields, while in one or two bare rocky spots at Trusham, and at the Chudleigh Rocks, $\mathcal{T}$. suffocatum is in as great quantity as almost any other species; T. scabrum Mr. Moyle Rogers found nowhere else in the neighbourhood than at the last mentioned station, where it is abundant together with most of the other species; T. bybridum was rapidly spreading throughout the region, and $\mathcal{T}$. medium was observed only in the neighbouring parish of Ashton in the Exeter district (Fourn. Bot. 1878, p. 18).

Lotus hispidus was found by $\mathrm{Mr}$. J. Woods near the castle at Dartmouth in June 1828 (Fourn. Bot. 1892, p. 308).

The great sundew (Drosera anglica) was recorded by Hudson in 1778 as inhabiting Devon marshes, a precise locality probably being on Bovey Heathfield, according to a manuscript note of Sir Francis Henry Drake (Fourn. Bot. 1 884, p. I69).

Bupleurum aristatum was first found in Devon by the Rev. Aaron Neck at Marble Rocks, Torquay, and sent to Sowerby I9 January I 802 ; it was figured in English Botany (tab. 2468); recent specimens from the same neighbourhood have been sent to me by Mr. Eden Phillpotts of Torquay:

The goldilocks (Aster Linosyris) was discovered in September I 812 , by the Rev. Charles Holbeach of Farnborough, Warwickshire, on the rocky cliff at Berry Head; it was figured in English Botany (tab. 2505) (see Fourn. Bot. 1893, p. 247).

The wild purslane or purple spurge (Euphorbia Peplis) was recorded by Hudson in 1788 as inhabiting the sands of the sea-coast near Exmouth, the precise locality probably being, according to a manuscript note of Sir Francis Henry Drake, among the sands near the first rocks between the Warren and Dawlish ; the species is apparently extinct at this station, for Mr. Moyle Rogers closely searched for it there without success (Fourn. Bot. 1878, p. 25 and 1884 , p. 173); there are however records for other stations in the county. The Devonshire or Dutch myrtle (Myrica Gale) was recorded by Hudson in 1778 , as inhabiting marshes in Devon, a precise locality probably being, according to a manuscript note of Sir Francis Henry Drake, about half a mile from the road following the hedge on the right hand as soon as Bovey Heathfield is reached from Chudleigh, in great plenty; it still grew on Bovey Heathfield in I 884 (Fourn. Bot. 1884, p. 173).

Romulea Columnee was discovered on 24 March, 1834, by Mr. W. C. Trevelyan and Mr. John Miford on Dawlish Warren and still maintains its place there (for a note on this plant see Dr. Shier in Report Devon Association, I 883, xv. 286-9).

The grape hyacinth (Muscari racemosum, Miller) was found many years ago at Paignton, and a few plants of it were seen 12 April 1900 at Goodrington by the Rev. H. Carr Smith.

Erica carnea, L., was recorded by Dr. H. F. Hance in Fourn. Bot. 1867, p. 136, as occurring in June I 852 on a heath near Newton Abbot; he distinguished this species from $E$. mediterranea, L.; there is no other authority for either of these plants in the county, and a search for Dr. Hance's specimen in the British Museum (Natural History), where his collection is deposited, has failed to find it.

Gladiolus communis, L., var. illyricus (Koch), was reported by W. G. Smith in Fourn. Bot. 1865, p. 220, on the authority of Mrs. Gulson, to occur sparingly at Bitton Wood near Teignmouth. The late Rev. Anson W. H. Cartwright of Teignmouth, 6 March 1902, kindly sent to me the following note: 'I made enquiries again yesterday of an old friend who has lived adjoining the Bitton property for more than forty years and in Teignmouth all his life, and he told me that he had never heard of Bitton Wood, but that his children in the 'sixties' 


\section{A HISTORY OF DEVONSHIRE}

and early 'seventies' used to call a certain portion of Bitton, near the Shaldon Bridge, the wood, and it is possible that the late Mrs. Gulson referred to this portion of the property in her communication to Mr. Smith. It may have been that she wrote to him the wood at Bitton and that he abbreviated her words into Bitton Wood. My friend, who is a retired medical man, had never heard of Gladiolus illyricus, and was surprised that the late Rev. R. Cresswell, who botanized extensively, had never come across it, if it were to be met with in the locality named.'

The fingered sedge (Carex digitata) is given in the Student's Flora as extending to Devon; the only precise locality for it in the county appears to be 'copse close by Forde Bog' on the authority of Stewart's Handbook of the Torquay Flora, p. I42; the late Dr. Trimen saw the specimen in C. Eyre Parker's herbarium at Torquay, and he considered it to be another species, namely $C$. ovalis. The evidence therefore for the retention of $C$. digitata in our county fiora is insufficient.

Mr. W. Botting Hemsley, F.R.S., the keeper of the Kew Herbarium, has favoured me with the following notes on the wild vegetation of the immediate neighbourhood of Dartmouth and Kingswear in July I902:-

'The lower Dart is not a rich district for the botanist, as there are neither open downs nor commons, and the woods are mostly artificial ; characteristic coast plants too are almost entirely wanting in consequence of the absence of conditions favourable to their existence ; but the hedgerow vegetation is perhaps unusually varied and is this season very luxuriant and beautiful, being in no way marred by dust.

' Conspicuous and abundant above all other herbaceous plants is Centrantbus ruber, locally named "Pride of Dartmouth"; it not only grows on waysides, wastes, banks, walls, railway cuttings and embankments, but it extends for miles along the hedgerows and is evidently on the increase; it was perhaps most effective in the old workings of the stone quarry at the junction of the roads on the hill above Kingswear church, where it covered the waste with a dense growth between 2 and 3 feet high and bore clusters of flowers from 6 inches to a foot in length; intermixed with the ordinary red there was sufficient of the white-flowered variety to form a very pleasing contrast; there was also a very striking edging of it overhanging a very considerable length of a terrace-wall on the road between the quarry and the cemetery. The red valerian is here a colonist of an exceedingly vigorous type, spreading and increasing with the same rapidity as Epilobium angustifolium does in the south-eastern counties, though it does not enter the woods; of the Epilobium I did not see a single plant in this part of Devonshire.

'Similarly the abundance of Tamus communis (black bryony) in the hedgerows caused one to miss Bryonia, with which it is commonly associated in some parts of England, Sussex for example.

'The small wood by the road leading down to Kingswear cemetery, at the top of the creek, is probably natural, and consists largely of stunted oak (2uercus sessilifiora), interspersed with large weather-beaten hollies of great age; ash, beech and hazel are also present, and elder abundant and conspicuous, being in full flower. The profusion and size of the honeysuckles on the shrubs and small trees are remarkable; bracken form the greater part of the undergrowth, and richly coloured foxgloves abound, especially in the half open spaces; seen from the road in varying lights these glades are very effective. The wood on the right bank of the creek, I was told, was partly planted, though it consists mainly of native trees ; I found no rare plants in it, but I was struck by the large size of such common things as Heracleum, Angelica, Cnicus palustris, Galium Aparine, Scrophularia nodosa and many others, and I never saw Cotyledon so large ; isolated plants of the latter were 3 feet high, forming a pyramid of branches supported by a dense tuft of unusually large radical leaves, with stalks from 9 inches to a foot long.

' I do not know the names of brambles, but I distinguished only three obviously distinct sorts; the commonest was a very ornamental kind, with robust habit and nearly orbicular abruptly acuminate leaflets, dark glossy green above and silvery beneath, and large pink flowers in very large broad clusters; the next in degree of predominance was a white-flowered sort, with obovate bright green leaflets something like what has been called corylifolius; the least common of the three was relatively small and slender, with small white flowers in elongated panicles and remarkable in having very long narrow, almost caudate, sepals. Incomplete specimens of these Rubi were submitted to the Rev. W. Moyle Rogers, who thought the first might be Rubus robustus, P. J. Muell.; the last a curious state or form of his own angustifolius (a variety of $R$. leucostachys, Schleich.); but the second one he would not venture to name from flowering specimens only. 


\section{BOTANY}

'Among plants of gregarious occurrence I particularly noted Ecbium, Origanum, Rubia and Erytbraa Centaurium; Clematis in more or less artificial plantations was observed climbing to a height of 30 to 40 feet, and Cuscuta Epithymum, on furze overhanging a terrace wall, was at least 6 or 7 feet long and very effective. Calystegia silvatica, a native of south-eastern Europe, having pure white flowers twice the size of those of $C$. sepium, has become established among the furze and brambles opposite "Fern Bank," Kingswear, and in several other places, where it forms a fine show from early morning until it closes its diurnal flowers about midday.'

The following are among the more remarkable and interesting species which occur in the district :-

Thalictrum minus, L.

Ranunculus trichophyllus, Chaix

- hirsutus, Curtis

- parviflorus, L.

Papaver Argemone, L.

Cardamine impatiens, L.

Barbarea vulgaris, R.Br., sub-sp. B. intermedia, Boreau

Raphanus maritimus, L.

Silene gallica, L., var. S. anglica, L.

Cerastium tetrandrum, Curtis, var. C. pumilum, Curtis

Sagina subulata, PresI

Hypericum calycinum, L.

- linarifolium, $V_{\text {ahl }}$

Geranium pusillum, L.

Oxalis corniculata, $\mathrm{L}$.

- stricta, L.

Ononis reclinata, L.

Trigonella ornithopodioides, DC.

Medicago maculata, Willd.

Lotus angustissimus, $L$.

Hippocrepis comosa, L.

Vicia tetrasperma, Mœnch, sub-sp. V. gracilis, Loisel.

- bithynica, $\mathrm{L}$.

Lathyrus Nissolia, L.

- hirsutus, L.

Spiræa Filipendula, L.

Potentilla argentea, L.

Pyrus torminalis, Ehrh.

Sedum reflexum, L., var. S. albescens, Haw.

Callitriche verna, L., sub-sp. C. obtusangula, Le Gall

Epilobium Ianceolatum, Seb. \& Maur.

Bupleurum tenuissimum, L.

Trinia vulgaris, $\mathrm{DC}$.

Crithmum maritimum, L.

Enanthe pimpinelloides, L.

Viburnum Lantana, L.

Rubia peregrina, L.

Galium tricorne, Stokes

Asperula cynanchica, L.

Valerianella carinata, Loisel.

Silybum marianum, Gærtn.

Hieracium murorum, L., sub-sp. H. pallidum, Fries

Hypochœris glabra, L.
Lobelia urens, L.

Wahlenbergia hederacea, Reichenb.

Centunculus minimus, $\mathrm{L}$.

Lithospermum purpureo-cæruleum, L.

Cuscuta europæa, L.

- epithymum, Murr., sub-sp. C. Trifolii, Bab.

Littorella lacustris, L.

Verbascum Blattaria, L., sub-sp. V. virgatum, Stokes

Bartsia viscosa, $\mathrm{L}$.

Lathræa squamaria, L.

Orobanche major, L.

- minor, Sutton, sub-sp. O. Hedera, Duby

Mentha sativa, L., sub-sp. M. gentilis, L.

Calamintha officinalis, Moench, sub-sp. C. sylvatica, Brompf.

Melittis Melissophyllum, L.

Salicornia radicans, Sm.

Thesium linophyllum, L.

Carpinus Betulus, $\mathrm{L}$.

Malaxis paludosa, $\mathrm{L}$.

Spiranthus autumnalis, Rich.

Orchis Morio, L.

- pyramidalis, L.

Ophrys apifera, Hudson

Scilla verna, Hudson

- autumnalis, L.

Luzula Forsteri, DC.

Lemna gibba, $L$.

- polyrrhiza, L.

Potamogeton trichoides, Cham.

Heliocharis acicularis, Sm.

Scirpus Savii, Seb. \& Maur.

Carex disticha, Hudson

- paludosa, Good.

- riparia, Curtis

Anthoxanthum Puelii, Lecoq. \& Lamotte

Alopecurus agrestis, L.

Agrostis setacea, Curtis

Calamagrostis lanceolata, Roth

Gastridium lendigerum, Gaud.

Poa compressa, L.

Glyceria Borreri, Bab.

Bromus erectus, Hudson

Agropyrum junceum, P. Beauv.

Lepturus filiformis, Trin.

Hordeum pratense, Huds.

The literature dealing with the flowering plants of the district includes the following works :--

John Pike Jones: A Botanical Tour through various parts of the Counties of Devon and Cornwall (1820), PP. $2-13,41-64,74$.

Nathaniel Bagshaw Ward' in the Phytologist, July 1841, i. pt. 2, pp. 20, 2I, 'List of Rare 


\section{A HISTORY OF DEVONSHIRE}

Plants found in Devonshire in the year $1840^{\prime}$-at or within 3 miles of Torquay; also near Holme Chase and on the borders of the river Dart.

Robert C. R. Jordan in the Pbytologist, January 1844, pp. 827, 828, "Rare Plants observed in the neighbourhood of 'Teignmouth, Devon.'

Robert Batterby, M.D., in the Pbytologist, September 1844, i. 1079, "Note on Iris foetidissima with double flowers.'

Samuel Hannaford: Flora Tottoniensis: A Catalogue of the Flowering Plants and Ferns growing Wild it the vicinity of Totnes (1851), and Supplement (1852).

Fraser Halle: Letters, Historical and Botanical; relating cbiefiy to places in the vale of Teign, and particularly to Chudleigh, Lustleigh, Canonteign and Bovey-Tracey (1851).

Thomas Waller Gissing in the Pbytologist, June 1855, 'Botanical Notes from South Devon,' pp. 25-8.

Robert Stewart: Handbook of the Torquay Flora (1860).

T. B. Flower in the Pbytologist, September 1860 , p. 288, 'Additional Devonshire Plants.'

Rev. W. Moyle Rogers in the foumal of Botany, 1878, pp. 15-25, 'Notes on some south-east Devon Plants'; and 1880, pp. 9-13, 'On some south-east Devon Plants'; the same author in the same Foumal, I 882 , pp. 70-6, I $21-4,133-6,177-83,206-9,239-43,262-6$, 'A Contribution towards a Flora of the Teign Basin, S. Devon.'

Miss Helen Saunders in the Transactions of the Devonshire Association, I 898, xxx. 201, 'Ashburton.'

Thomas Richard Archer Briggs in the Fournal of Botany, 1866, p. 288, 'Unrecorded Stations, mostly near Plymouth, of some uncommon Plants, etc.'; 1868 , p. 58, 'Ononis reclinata in Devonshire'; I879, p. 314, 'Devon Plants'; 1884, pp. I68-74, 'On some Devonian Stations of Plants noted in the last century.'

Edward Croydon: The Torquay Guide, etc., by several literary gentlemen (Torquay, 184r), ' Botany,' pp. 1 32-69; another edition (1852 ?), pp. 270-80, The Handbook for Torquay.

Edward and George Henry Croydon : The Teignmoutb Guide, ed. 16, 'Wild Flowers,' Pp. I37, 138 ; no date.

Cornelius's Guide: Dawlish, Historical and Topographical, ed. 2, 'The Botany of Dawlish,' pp. 7582 ; no date.

J. A. Walker in the Pbytologist, 1845, ii. 24, 25, 'On the Influence of the mild Climate of Torquay on Flowering Plants.'

Robert C. R. Jordan in the Pbytologist, 1845, ii. 338, 339, 'Occurrence of Alyssum calycinum and Narcissus biforus near Dawlish.'

Fenton J. A. Hort in the Pbytologist, 1848, iii. 321 , 322, 'Note on Alsine rubra, var. media, Bab.' ; p. 322 , Torquay and Dawlish.

C. E. Lartor (Miss): Manual of the Flora of Torquay (1900).

\section{Plymouth}

This district consists of the Southern or Totnes parliamentary division, the municipal boroughs of Plymouth and Devonport, and the parish of East Stonehouse; it contains about 200,874 acres, being about 195,068 acres of land, inland water and saltmarsh, besides about 5,806 acres of foreshore and tidal water; it consists of the three petty sessional divisions of Ermington and Plympton, Stanborough and Coleridge, and South Roborough, except the municipal borough of Dartmouth, but with the addition of those of Plymouth and Devonport; it comprises all the 26 parishes of the Kingsbridge poor-law union, 18 out of the 27 parishes of the Totnes union, and $I_{3}$ out of the 20 parishes of the Plympton union, besides the three towns of Plymouth, Devonport and Stonehouse, altogether 60 civil parishes, of which 51 are rural and 9 are urban ; the drainage is entirely to the south coast ; it includes part of Dartmoor. The principal rivers are the Dart, the Avon, the Erme, the Yealm, the Tory brook, and (on the western border) the Plym. The highest part is 1,692 feet above sea-level, at Petre's Bound Stone in the parish of Buckfastleigh West, where it joins the Torquay and Tavistock botanical districts. It includes Prawle Point, in the parish of Chivelstone, the most southern part of the county. It is bounded on the west by the county of Cornwall, on the north by the Tavistock and Torquay botanical districts, and on the east and south by the English Channel.

The following is a complete list in alphabetical order of the civil parishes in the district :-

Ashprington

Aveton Gifford

Berry Pomeroy

Bigbury

Rlackawton

Brixton
Buckfastleigh, East (urban)

Buckfastleigh, West

Buckland Tout Saints

Charleton

Chivelstone

Churchstow
Cornwood

Cornworthy

Dartington

Dean Prior

Devonport (urban)

Diptford 


\section{BOTANY}

$\begin{array}{lll}\text { Dittisham } & \text { Modbury } & \text { Slapton } \\ \text { East Allington } & \text { Morleigh } & \text { South Brent } \\ \text { East Stonehouse (urban) } & \text { Newton Ferrers } & \text { South Huish } \\ \text { Ermington } & \text { North Huish } & \text { South Milton } \\ \text { Halwell } & \text { Plymouth (urban) } & \text { South Pool } \\ \text { Harberton } & \text { Plympton Maurice } & \text { Stoke Fleming } \\ \text { Harford } & \text { Plympton St. Mary } & \text { Stokenham } \\ \text { Holbeton } & \text { Plymstock (urban) } & \text { Thurlestone } \\ \text { Ivybridge (urban) } & \text { Portlemouth } & \text { Totnes (urban) } \\ \text { Kingsbridge and Dodbrooke (urban) } & \text { Rattery } & \text { Ugborough } \\ \text { Kingston } & \text { Revelstoke } & \text { Wembury } \\ \text { Littlehempstone } & \text { Ringmore } & \text { West Alvington } \\ \text { Loddiswell } & \text { Salcombe (urban) } & \text { Woodleigh } \\ \text { Malborough } & \text { Sherford } & \text { Yealmpton }\end{array}$

By far the largest portion of the district belongs to the Devonian period. The southern part of the county from Malborough and South Pool villages to Bolt Head and East Prawle is Metamorphic or Altered Devonian, interspersed with patches of Greenstone ; there is Greenstone also about Prawle Point, and several patches of it are distributed within the Devonian area.

Limestone rocks occur in the southern parts of Devonport, East Stonehouse and Plymouth, extending about $1 \frac{3}{4}$ mile nearly due east of Plymstock church; there are also patches of limestone about Yealmpton, North Huish, Buckfastleigh, etc.

The part of the district which belongs to Dartmoor is granite. The Carboniferous system occurs to a limited extent round the granite area between it and the Devonian area.

Mineral veins occur near Buckfastleigh.

The climate of the district is illustrated by the following tables, which are obtained as explained under the Barnstaple district :-

Table 1.-Decade $1890-9$

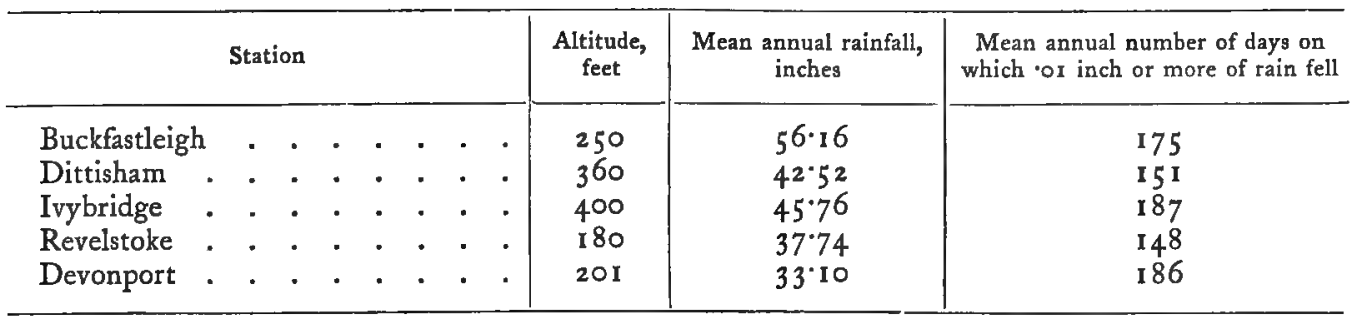

T

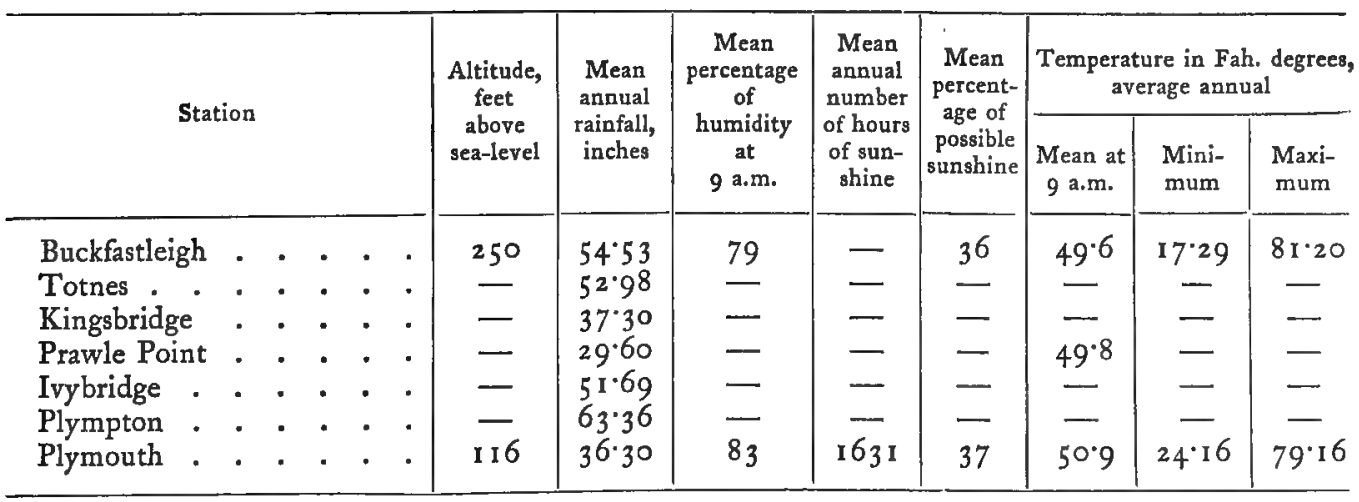

At Plymouth during the years 1895-1901 the average annual number of hours of sunshine was $1,762 \frac{1}{2}$, and the average number of sunless days was 59 in a year.

Archer Briggs in his Flora of Plymouth (1 880), p. xxi., gives the following average figures for the 15 years, I865-79, at Plymouth, N. lat. $50^{\circ} 22 \frac{1}{2}^{\prime}$, W. long. $4^{\circ} 7 \frac{1}{4}^{\prime}$ : -

Barometer, reduced to mean sea-level at $32^{\circ} \mathrm{Fahr}$. annual, in inches . $\mathbf{2 9} 945$

\begin{tabular}{|c|c|c|c|c|}
\hline$"$ & $"$ & $"$ & $\begin{array}{l}\text { monthly maximum } \\
, \quad \text { minimum }\end{array}$ & $"$ \\
\hline
\end{tabular}




\section{A HISTORY OF DEVONSHIRE}

Temperature in shade, annual, in Fahrenheit degrees ...+
"

The ramping fumitory (Fumaria capreolata) is frequent in the district; the sub-species $F$. pallidiflora was first recorded for Britain by C. C. Babington in 1860 , and the first locality for it given by him was Salcombe; the sub-species $F$. confusa was considered by Archer Briggs to be the one most common in the county.

Hypericum undulatum was first recorded as a British plant by Archer Briggs in 1864 from the neighbourhood of Plymouth; it has subsequently been noticed in the Barnstaple, Torrington and Tavistock districts; it occurs also in Cornwall.

The Plymouth strawberry (Fragaria muricata) is a monstrous state of the common strawberry $(F$. vesca) and was first found in a Plymouth garden; Parkinson in his Paradisus (1629) figured it on page 527 with the name of 'the prickly strawberry' and on page 528 referred to it as follows: "One Strawberry more I promised to shew you, which although it be a wilde kinde, and of no use for meate, yet I would not let this discourse passe, without giving you the knowledge of it. It is in leafe much like unto the ordinary, but differeth in that the flower, if it have any, is greene, or rather it beareth a small head of greene leaves, many set thicke together like unto a double ruffe, in the midst whereof standeth the fruit, which when it is ripe, sheweth to be soft and somewhat reddish, like unto a Strawberry, but with many small harmlesse prickles on them, which may be eaten and chewed in the mouth without any maner of offence, and is somewhat pleasant like a Strawberry ; it is no great bearer, but those it doth beare, are set at the toppes of the stalks close together, pleasant to behold, and fit for a Gentlewoman to weare on her arme, etc., as a raritie in stead of a flower.' A somewhat similar plant is figured by Barrelierus, Plant. Gall. Hispan. et Ital. (I 7 I 4), tab. 90, with the name of Fragaria spinoso fructu. For further particulars see Briggs, Fl. Plym. p. 108 and Fourn. Bot. 1898, p. 127, where a figure of it is reproduced from the Gardener's Chronicle for 1887 .

The crimson grass-vetch (Lathyrus Nissolia) was stated by Polwhele in 1797, on the authority of Mr. Cornish of Totnes, to be very common in the southern parts of the county, and (Polwhele added) in every other part; it appears during recent years not to be common, and about Plymouth to be spasmodic in its occurrence.

The sweet-scented agrimony (Agrimonia Eupatoria, sub-sp. A. odorata) was recorded in 1853 as occurring in Devon and Cornwall, and by Archer Briggs in 1866 as in the Plymouth district; it occurs also in the Torrington, South Molton, Torquay and Tavistock districts.

The field eryngo (Eryngium campestre) was found by Ray 7 July 1662, and recorded by him (Cat. Pl. Angl. [I670], ed. I, p. 105), ' on a rock which you descend to the Ferrey from Plymouth over into Cornwall'; Archer Briggs found it still there in 1880; but it was reported in $189 \mathrm{I}$ to be nearly extinct owing to the greater public use of the site.

Carum verticillatum was found by Archer Briggs about a mile from Cornwood railway station 3 August 1882 ; previous records of it for the county had been called in question. The moth mullein (Verbascum Blattaria) was recorded by Hudson in 1778 as inhabiting gravelly places about Plymouth and Ashburton; the sub-species $V$. virgatum also occurs in the district and may have been the plant intended by Hudson.

The abundance of the balm-leaved figwort (Scrophularia Scorodonia) about the town of Kingsbridge is a very noticeable feature in its botany; in 1878 there was plenty of it on the hedge banks all round the town and in many spots some miles beyond; also in greater or less quantities in a south-easterly direction a little beyond Charleton, at a station near East Down, by the lane ascending the hill from Frogmoor, and between the hamlet of Ford and Chivel- 


\section{BOTANY}

stone 3 or 4 miles from Kingsbridge up to 400 feet of altitude; in a southerly direction on the lower road to Salcombe as far as the little vale on the southern side of Callapit; and in a south-westerly direction at West Alvington, at a station within a quarter of a mile from Malborough village about 320 feet high nearly 4 miles from Kingsbridge, and in the parish of Thurlestone on the eastern bank of the Avon estuary close to the hamlet of Bantham ; the flowers of $S$. Scorodonia are rather brighter in colour than those of either $S$. nodosa or S. aquatica, the purple taking more of a reddish tint, and it is decidedly the prettiest of the three species; wasps are as partial to its flowers as to those of the other two species; it must not be supposed that the mica-slate formation has anything to do with the occurrence of this plant, since it is not on this rock but on the Devonian slate that it grows in such great quantity (Archer Briggs).

The Cornish money-wort (Sibtborpia europeca) was recorded by Bishop Gibson in 1695 as occurring in Devon no less frequently than in Cornwall and on the like watery banks, and Hudson in 1762 reported it as frequent in both counties by streams and springs; Jones and Kingston in 1829 on the authority of Sir Francis Drake and Mr. Hudson reported it about the springs in the village of Tor near Harford. Ray had placed this as a bastard species under Alsine, and some of the old botanists suspected that it was a hybrid between Cbrysosplenium alternifolium and Hydrocotyle vulgaris. It is on record that Linnæus, thinking that the English had erroneously multiplied the species, doubted the existence of the plant, until in 1750 Dr. Sibthorp of Oxford convinced him of its reality by sending to him a good specimen (Polwhele). It occurs also in the Barnstaple, Torrington, Exeter and Tavistock districts.

The round-leaved mint (Mentha rotundifolia) is by no means rare in the county, and in some spots of the extreme southern part of this district it is quite a common plant and apparently indigenous.

The bastard balm (Melittis Melissophyllum) was recorded in 1650 on the authority of Dr. Richard Heaton of Dublin as found near Totnes; it is more frequent in this county than elsewhere in England, and occurs in all the districts. $M$. grandifora, with a larger very handsome corolla, can scarcely be distinguished; it was reported from the neighbourhoods of Barnstaple and Ashburton.

The strap wort (Corrigiola littoralis) was recorded in 1787 by Stokes in his edition of Withering's Botanical Arrangement, p. 322, as found on Slapton Sands and near the Start Point.

Scirpus Savii is not uncommon in the district; it is probably the 'smooth sea rushgrass of Plimmouth' mentioned in Parkinson's Theatrum botdnicum (1640), p. I27 I and figured on p. 1270.

Sea kale (Crambe maritima) was cultivated in 1795 from plaris nriginally obtained from Slapton Sands (Phytologist, September I 860, p. 288).

The snow-flake (Leucojum astivum), until recently found on the banke of the Dart, is now apparently quite extinct (British Association Report for 1891, p. 362).

The following are among the more remarkable and interesting species which occur in the district:-

Ranunculus circinatus, Sibth.

Papaver hybridum, L.

Brassica alba, Boiss.

Diplotaxis tenuifolia, DC.

Raphanus maritimus, Sm.

Silene noctillora, L.

Sagina apetala, L., sub-sp. S. maritima, Don.

Spergularia salina, Presl, var. Lepigonum neglectum, Kindb.

— rupestris, Lebel

Polycarpon tetraphyllum, L.

Hypericum hirsutum, L.

Lavatera arborea, L.

Geranium sanguineum, L.

- perenne, Huds.

Medicago denticulata, Willd.

Trifolium fragiferum, $\mathrm{L}$.

- resupinatum, L.

Lotus hispidus, Desf.
Lotus angustissimus, L.

Vicia lutea, L.

Prunus Cerasus, L.

Pyrus germanica, Hook. f.

Myriophyllum spicatum, L.

Callitriche verna, L., sub-sp. C. obtusangula, Le Gall

Epilobium lanceolatum, Seb. \& Maur.

- roseum, Schreb.

Pimpinella major, Hudson

Crithmum maritimum, L.

Peucedanum sativum, Benth. \& Hook. f.

Daucus Carota, L., sub-sp. D. gummifer, Lam. Sambucus Ebulus, L.

Galium verum, L., var. ochroleucum, Syme

Dipsacus sylvestris, L.

Inula Conyza, DC.

- crithmoides, L.

Serratula tinctoria, L. 


\section{A HISTORY OF DEVONSHIRE}

Hieracium umbellatum, L., var, monticola (H. monticola, Jord.)

Lactuca virosa, L.

Wahlenbergia hederacea, Reichenb.

Specularia hybrida, Alph. DC.

Statice auriculæfolia, Vahl (S. binervosa, G. E. Sm.)

Centunculus minimus, $L$.

Erythræa Centaurium, Pers., sub-sp. E. pulchella, Fries

Convolvulus Soldanella, L.

Cuscuta Epithymum, Murr.

Hyoscyamus niger, L.

Linaria supina, Desf.

Mimulus luteus, Pursh. (not L.), M. Langsdorffii, Donn

Bartsia viscosa, $L$.

Orobanche minor, Sutton, sub-sp. O. amethystea, Thuill.

Pinguicula lusitanica, L.

Mentha Pulegium, L.

Calamintha officinalis, Mœnch, var. Briggsii, Syme

Chenopodium polyspermum, L.

- murale, L.

- rubrum, L., var. C. botryodes, Sm.

Polygonum aviculare, L., sub-sp. P. Roberti, Loisel.

Daphne Laureola, L.

The literature dealing with the flowering plants of the district include the following works :-

George Banks: The Plymouth and Devonport Flora, 8 parts (1830-2).

John Jacob: West Devon and Cornvoall Flora, 18 parts (1835-7).

Nathaniel Bagshaw Ward in the Pbytologist, July 1841, i. pt. 2, p. 21, 'List of rare plants found in Devonshire in the year $1840^{\circ}$ ' - at or within 8 miles of Devonport.

Samuel Gibson in the Phytologist, April 1842, i. 179, 'On British species and varieties of the genus Sagina.'

Samuel Hannaford: Flora Tottoniensis; a catalogue of the flowering plants and ferns, growing wild in the vicinity of Totnes ( 1851$)$, and supplement $(1852)$.

Thomas Rich:-1 Mrcher Briggs in the Pbytologist, August 1861, pp. 253, 254, 'Dates of flowering of plan's in the vicinity of Plymouth'; p. 256, 'Scrophularia vernalis'; December i 86I, pp. 368-7I, 'Localities of some uncommon plants and of varieties of common species within twelve miles of Plymouth'; July 1 862 , p. 223 , 'List of early flowering plants observed in flower near $\mathrm{Ply}$ mouth, with the date of their first appearance'; August 1862, Pp. 255, 256. 'Polygonatum mulifforum and Vibumum Lantana'; September 1862, pp. 286, 287, 'A new Epilobium ?'; October 1862, pp. 313, 314, Epilobium.

The same author in the Fournal of Botany, 1863, pp. 376-8, 'Stations of some uncommon plants in Devon and Cornwall'; 1866, pp. 287-90, "Unrecorded stations, mostly near Plymouth, of some uncommon plants, etc.' ; 1867, Pp. 309-1 I, 'Unrecorded stations of and notes respecting some Plymouth plants'; 1868, pp. 205-7, 327, 328, and I869, pp. 318-20, 'Notes respecting some Plymouth plants'; I870, pp. I90, 19I, 'On the fertilization of the primrose (Primula vulgaris, Huds.)'; 1870, pp. 356, 357, 'Viola odorata, L., and V. lactea, Sm., in S.W. England,' and 'Carex distans, L.' ; 187 I, pp. 241, 242, 'Stations of, and remarks on, some Plymouth plants'; I871, p. 306, 'Plants near Plymouth'; 1872, pp. I41-5, 'On some peculiarities in the Botany of the neighbourhood of Plymouth'; 1872 , p. 153 , 'Asarum europaum, L., not a Plymouth plant'; 1872, pp. 259-6I, 'Notes respecting some Plymouth plants'; 1873 , pp. 374,375 , 'Notes on some Plymouth plants with stations'; 1874 , pp. 327,328 , 'Notes on some plants of the neighbourhood of Plymouth, with stations'; 1875, pp. 266, 267, " Notes respecting some Plymouth plants, with a few unrecorded stations '; I 875 , pp. 294, 295, ‘Rumex rupestris, Le Gall, a British species'; i 875 , p. 297, 'Plymouth plants'; 1876, pp. 275-8, 280, 286, "Report of the curator of the Botanical Exchange Club for the year $1875^{\prime}$ '; 1878, pp. 292-9, 'Some notes on the flora of the extreme south of Devon'; 1879, pp. 18, I9, 'Scirpus parvulus, R. \& S. in S. Devon'; 1879, pp. 245, 246, 'Extracts from the report of the Botanical Exchange Club for I877-8'; I879, p. 3 I 4, 'Devon plants'; I88 I, Pp. 3 I 2, 313 , 'Leontodon bastilis, L.' ; 


\section{BOTANY}

1882 , p. 283 , 'Carum verticillatum in S. Devon'; 1884, Pp. 168-74, 'On some Devonian stations of plants noted in the last century'; and other papers on the brambles and roses.

The same author: Flora of Plymouth; an account of the fowering plants and ferns found witbin twelve miles of the town (1880).

Guilding in the Phytologist, 1847 , ii. 767 , 'Linaria supina near Plymouth.'

J. W. N. Keys in the Pbytologist, 1847 , ii. $938-40$, 'On the claims of Linario supina to a place in the British Flora.'

W. Pamplin in the Pbytologist, iii. 166-70, 'List of habitats of plants recorded in MS. in a copy of Blackstone's "Specimen botanicum," p. 167 (Plymouth, 1848).

Fenton J. A. Hort in the Pbytologist, iii. 321,322 , 'Note on Alsine rubra, var. media, Bab.' p. 322 (Plymouth, I 848).

J. W. N. Keys in the Pbytologist, I 849, iii. 459-61, 'Dates of the flowering of British plants,' and pp. $537-40$, 'On the flowering of British plants.'

\section{Tavistock}

This district consists of the three petty sessional divisions of Midland Roborough, Tavistock and Lifton, being the greater part of the southern portion of the western or Tavistock parliamentary division; it contains about 230,106 acres, being about 228,224 acres of land, inland water and saltmarsh, besides about $\mathrm{I}, 882$ acres of foreshore and tidal water ; it comprises all the 25 parishes of the Tavistock poor-law union, 5 out of the 6 Devonshire parishes of the Launceston union, 4 out of the 28 parishes of the Okehampton union, and 7 out of the 20 parishes of the Plympton union, altogether $4 \mathrm{I}$ civil parishes, one only of which is urban, and all of which are drained to the south coast except parts of Lydford, Bridestowe and Sourton (which parts are drained by the West Okement river towards the north coast); it is bounded on the west by the county of Cornwall, on the north by the Torrington botanical district, on the east by the Torquay botanical district, and on the south by the Plymouth botanical district. It is entirely inland, but part of the south-western corner is washed with tidal water ; it contains the chief part of Dartmoor Forest, including Cranmere Pool so-called, a centre on the moor not far from the sources of several of the principal rivers of the county, the West Okement, the East Okement, the Taw, the North Teign, the East Dart and the Tavy; other streams in the district are the South Teign, the West Dart, the Walkham, the Lyd, the Tamar (which forms the western border against Cornwall for a considerable distance) with its tributaries, the Carey, the Caudworthy Water, the Ottery (on the north-west), the Meavy and the Thrushel; about 2 miles south of Cranmere Pool the moor ascends at Cut Hill to 1,98 I feet above sealevel. district :-

The following is a complete list in alphabetical order of the civil parishes in the

Bere Ferrers
Bickleigh near Plymouth
Bradstone
Bratton Clovelly
Brentor
Bridestowe
Broadwood Widger
Buckland Monachorum
Compton Gifford
Coryton
Dunterton
Egg Buckland
Germansweek
Kelly

Lamerton
Lewtrenchard
Lifton
Lydford
Marystow
Mary Tavy
Meavy
Milton Abbot
North Petherwin
Peter Tavy
St. Budeaux
St. Giles in the Heath
Sampford Spiney
Shaugh Prior

Sheepstor
Sourton
Stowford
Sydenham Damarel
Tamerton Foliott
Tavistock Hamlets
Tavistock Town (urban)
Thruselton
Virginstowe
Walkhampton
Werrington
Weston Peverell
Whitchurch

The south-western part of the district belongs to the Upper and Middle Devonian system, the north-western to the Carboniferous, and the eastern part is chiefly Dartmoor granite; several Greenstone patches mostly running in lines from west to east traverse the Devonian part, and some others of an irregular outline traverse the Carboniferous part ; several mineral veins and stream tin occur in the granite part.

The climate of the district is illustrated by the following tables, which have been obtained as explained under the Barnstaple district :- 


\section{A HISTORY OF DEVONSHIRE}

\begin{tabular}{|c|c|c|c|c|}
\hline Station & & $\begin{array}{l}\text { Altitude, } \\
\text { feet }\end{array}$ & $\begin{array}{l}\text { Mean annual rainfall, } \\
\text { inches }\end{array}$ & $\begin{array}{l}\text { Mean annual number of days on } \\
\text { which } 01 \text { inch or more of rain fell }\end{array}$ \\
\hline $\begin{array}{l}\text { Bere Ferrers . . } \\
\text { Tavistock, Public Library. } \\
\text { Polapit Tamar, Werrington }\end{array}$ & $\begin{array}{lll}\cdot & \cdot & \cdot \\
\cdot & \cdot & \cdot \\
\cdot & \cdot & \cdot\end{array}$ & $\begin{array}{l}100 \\
283 \\
315\end{array}$ & $\begin{array}{l}41 \cdot 87 \\
43 \cdot 81 \\
36 \cdot 79\end{array}$ & $\begin{array}{l}185 \\
198 \\
\end{array}$ \\
\hline
\end{tabular}

TABLE 2

\begin{tabular}{|c|c|c|c|c|c|c|c|c|}
\hline \multirow{2}{*}{ Station } & \multirow{2}{*}{$\begin{array}{c}\text { Altitude, } \\
\text { feet } \\
\text { above } \\
\text { sea-level }\end{array}$} & \multirow{2}{*}{$\begin{array}{l}\text { Mean } \\
\text { annual } \\
\text { rainfall, } \\
\text { inches }\end{array}$} & \multirow{2}{*}{$\begin{array}{l}\text { Mean } \\
\text { annual num- } \\
\text { ber of days } \\
\text { on which } \\
\text { or inch or } \\
\text { more of } \\
\text { rain fell }\end{array}$} & \multirow{2}{*}{$\begin{array}{c}\text { Mean } \\
\text { percentage } \\
\text { of } \\
\text { humidity } \\
\text { at } \\
9 \text { a.m. }\end{array}$} & \multirow{2}{*}{$\begin{array}{c}\text { Mean } \\
\text { percent- } \\
\text { age of } \\
\text { possible } \\
\text { sunshine }\end{array}$} & \multicolumn{3}{|c|}{$\begin{array}{c}\text { Temperature in Fah. degrees, } \\
\text { average annual }\end{array}$} \\
\hline & & & & & & $\begin{array}{c}\text { Mean at } \\
9 \text { a.m. }\end{array}$ & $\begin{array}{l}\text { Mini- } \\
\text { mum }\end{array}$ & $\begin{array}{l}\text { Maxi- } \\
\text { mum }\end{array}$ \\
\hline $\begin{array}{l}\text { Lee Moor, Shaugh . } \\
\text { Head Weir, Plymouth Reservoir } \\
\text { Statsford, Whitchurch . } \\
\text { Beer Alston . } \\
\text { Princetown Prison . }\end{array}$ & $\begin{array}{r}720 \\
594 \\
124 \\
1359\end{array}$ & $\begin{array}{l}68 \cdot 01 \\
58 \cdot 25 \\
53 \cdot 00 \\
40 \cdot 48 \\
78 \cdot 59\end{array}$ & $\begin{array}{l}-174 \\
203 \\
196 \\
183\end{array}$ & $\overline{83}$ & $\frac{}{35}$ & $\begin{array}{l}- \\
- \\
49^{\cdot 8} \\
51 \\
45^{1} 8\end{array}$ & $\begin{array}{c}- \\
- \\
20.15 \\
23.67 \\
20.98\end{array}$ & $\begin{array}{c}- \\
- \\
82 \cdot 43 \\
8 \mathrm{I} \cdot 67 \\
76 \cdot 58\end{array}$ \\
\hline
\end{tabular}

The columbine (Aquilegia vulgaris) is frequent in many parts of the county, and appears to be native in some places, as for instance in the southern part of the district ; it is not confined to a calcareous soil.

The shepherd's cress (Teesdalia nudicaulis) is widely distributed over the district, with a preference for granite rocks; it also affects the neighbourhood of the coast in other districts.

The larch-leaved spurrey (Sagina subulata) is locally rather common on moors and downs; it was recorded by Jones and Kingston in 1829 .

The dwarf mallow (Malva rotundifolia) grows sparingly in several stations, preferring very dry spots.

The common flax (Linum usitatissimum) is not now cultivated in the district, but the plant occurs casually ; Polwhele in 1797 stated that every species of flax grew on Dartmoor and its cultivation might there be turned to good account.

The small-fruited pear (Pyrus communis, var. P. cordata) was first recorded for England by Dr. Boswell Syme in $187 \mathrm{r}$, on the authority of Archer Briggs; it was figured in the Fournal of Botany for 1876 , tab. 180 ; it occurs also in east Cornwall, and a nearly similar form is found in western France. Tillea muscosa was discovered by Archer Briggs on I3 May 186I, in Egg Buckland; in dry seasons it soon passes out of flower and dies. The smaller long-leafed sundew (Drosera intermedia) is native in several stations; it was reported by G. Banks in 1830 as occurring occasionally in company with the round-leaved sundew ( $D$. rotundifolia).

The profusion of Epilobium lanceolatum in many parts of the southern part of the district and elsewhere near Plymouth is a very striking fact; while abundant over a considerable extent of the country, it is rare in certain localities or altogether absent; it ascends above 500 feet near Roborough village, but mostly grows in low and warm situations especially where slaty or shaly soils prevail, though it is not confined to such places; it may often be seen growing abundantly on banks or railway cuttings; the flowers differ conspicuously in colour from those of $E$. montanum, being of a rosy or pinkish hue and not purplish; they are pure white at the time of opening, but soon assume colour (Archer Briggs).

The Cornish bladder-seed (Physospermum cornubiense) was first reported for Devon in a paper read at the Plymouth meeting of the British Association in 1841 as occurring in an oak coppice near Tavistock about a quarter of a mile from New Bridge; it is believed to exist still in that neighbourhood and ascends up to 400 feet altitude above sea level, favouring partially shaded woodland glades; it is rare and local.

The pale butterwort (Pinguicula lusitanica) was first recorded as a British plant in I666 as occurring in boggy meadows midway between Okehampton and Launceston; it is one of the common and characteristic plants of the peaty and moorland areas.

Arum italicum was detected by Archer Briggs in May I 884 in the parish of Egg 


\section{BOTANY}

Buckland in company with the common cuckoopint (A. maculatum); its presence there is possibly the result of ancient cultivation.

The cowberry (Vaccinium Vitis-idea, L.) has been recorded from Fur Tor, Dartmoor; its occurrence there requires confirmation.

The following are among the more remarkable and interesting species which occur in the district :-

Ranunculus marinus, Fries, var. R. confusus, Godr.

Meconopsis cambrica, Vig.

Nasturtium sylvestre, R. Br.

Viola canina, L. (V. flavicornis, Sm.)

Cerastium quaternellum, Fenzl

Spergularia salina, Pres 1

- media, Pers.

Hypericum undulatum, Schousb.

- linarifolium, Vahl

Geranium pusillum, L.

Empetrum nigrum, L.

Trigonella ornithopodioides, DC.

Medicago denticulata, Willd.

Melilotus alba, Desr.

Trifolium scabrum, L.

Pyrus torminalis, Ehrh.

Sedum Telephium, L.

- reflexum, L.

Pimpinella major, Hudson

Fœniculum officinale, All.

Viburnum Lantana, L.

Rubia peregrina, L.

Inula Conyza, DC.

Antennaria dioica, R. Br.

Anthemis nobilis, L.

Artemisia Absinthium, L.

Carlina vulgaris, $\mathrm{L}$.

Cichorium Intybus, L.

Crepis biennis, $L$.

Lactuca virosa, L.

Wahlenbergia hederacea, Reichenb.

Specularia hybrida, Alph. DC.

Primula veris, $L$.

Lysimachia vulgaris, $L$.

Centunculus minimus, $L$.

Anagallis tenella, L.

Erythræa Centaurium, Pers., sub-sp. E. pulchella, Fries

Gentiana campestris, L.

Cuscuta Epithymum, Murr., and sub-sp. C. Trifolii, Bab.

Littorella lacustris, L.

Linaria minor, Desf.

Sibthorpia europæa, L.

Orobanche major, $\mathrm{L}$.

Mentha sativa, L., sub-sp. M. rubra, Sm.
Calamintha Clinopodium, Benth.

Salvia Verbenaca, L.

Melittis Melissophyllum, L.

Lamium purpureum, L., sub-sp. L. hybridum, Vill.

Scleranthus annuus, L.

Chenopodium polyspermum, L.

Polygonum Bistorta, L.

- lapathifolium, L., sub-sp. P. maculatum, Trimen \& Dyer

Euphorbia Lathyris, L.

Myrica Gale, L.

Salix triandra, L.

- fragilis, L.

- alba, L.

Taxus baccata, L.

Spiranthes autumnalis, Rich.

Epipactis latifolia, All.

Habenaria conopsea, Benth.

— viridis, $\mathrm{R}$. Br.

- bifolia, R. Br., and sub-sp. H. chlorantha, Bab.

Iris fotidissima, $\mathrm{L}$.

Narcissus biflorus, Curtis

Galanthus nivalis, L.

Convallaria majalis, L.

Luzula Forsteri, DC.

Typha latifolia, L.

- angustifolia, $\mathrm{L}$.

Triglochin palustre, L.

- maritimum, $\mathrm{L}$.

Zostera nana, Roth

Scirpus lacustris, sub-sp. S. carinatus, Sm.

- triqueter, $\mathrm{L}$.

- Savii, Seb. \& Maur.

Carex montana, $\mathrm{L}$.

- extensa, Good

- distans, L.

Milium effusum, $\mathrm{L}$.

Agrostis setacea, Curtis

Poa compressa, L.

Glyceria fluitans, L., var. G. plicata, Fries

- maritima, Mert. \& Koch

- distans, Wahlenb.

Bromus erectus, Hudson

- racemosus, $\mathrm{L}$.

Lepturus filiformis, Trin.

The literature dealing with the flowering plants of the district includes the following works :-

George Banks: The Plymouth and Devonpart Flora, 8 parts (1830-2).

John Jacob : West Devon and Cornwall Flora, 18 parts (1835-7).

Thomas Richard Archer Briggs in the Pbytologist, August 1861, p. 256, 'Tillea muscosa'; December I $86 \mathrm{I}$, pp. 368-7I, 'Localities of some uncommon plants and of varieties of common species within twelve miles of Plymouth'; September 1862, pp. 286, 287, 'A new Epilobium ?'; December 1862, pp. 353-5, 'Botany of Dartmoor, Roborough Down, etc.'; February I 863 , p. 445 , 'Rhynchospora.'

The same author in the Fournal of Botany, r863, p. 377, 'Stations of some uncommon plants in 


\section{A HISTORY OF DEVONSHIRE}

Devon and Cornwall' ; 1864, pp. 45, 46, 'Hypericum undulatum, Schousb., a recent addition to the British Flora' ; I 866, Pp. 287-90, 'Unrecorded stations, mostly near Plymouth, of some uncommon plants, etc.'; I867, pp. 309-I I, 'Unrecorded stations of, and notes respecting some Plymouth plants'; I 868, pp. 205-7, 327, 328, and I869, pp. 318-20, 'Notes respecting some Plymouth plants'; 1870, pp. 22 3, 356, 'Gentiana campestris, Linn.' ; I 870, p. 357, 'Carex distons, L.' ; $187 \mathrm{r}$, Pp. 214 , 2 I 5,' Pyrus communis, Linn., var. Briggsii '(Syme, Rep. Lond. Bot. Ex. Club), 1870'; I871, Pp. 241, 242, 'Stations of, and remarks on, some Plymouth plants'; 1871, p. 306, 'Plants near Plymouth'; 1872, pp. 141-5, "On some peculiarities in the Botany of the neighbourhood of Plymouth'; I 872, pp. 259-61, ' Notes respecting some Plymouth plants'; 1873, pp. I72, I 73, 'Carex montana, Linn., in Devon'; 1873 , pp. 237, 238, 'A second station for Carex montana, Linn., in Devon' ; 1873 , pp. 374, 375, 'Notes on some Plymouth plants, with stations'; 1874 , pp. 327,328 , 'Notes on some plants of the neighbourhood of Plymouth, with stations'; 1875, pp. 266, 267, 'Notes respecting some Plymouth plants, with a few unrecorded stations'; 1875, p. 296, 'Seirpus triqueter, L., in Cornwall and Devon'; 1876, pp. 277, 282,286 , 'Report of the curator of the Botanical Exchange Club for the year $1875^{\prime}$; 1879 , p. 314, 'Devon plants'; 1881 , pp. 151 , 152, 'A state of Carex pilulifera, L., approaching var. Leesii'; 1884 , pp. 168-74, 'On some Devonian stations of plants noted in the last century'; 1884, pp. 2 I 2, 213 , 'Arum italicum, Mill., in Devon '; 1887, pp. 208, 209, 'Remarks on Pyrus communis v. cordata, Desv.'; I 888, pp. 236, 237, 'Remarks on Pyrus latifalia, Syme'; 1888, p. 378, 'Arum italicum, Mill., and A. maculatum, Linn.' ; 1889 , pp. 244 , 245, 'Orchis latifolio-maculata, Towns (?) in Devon'; 1889, pp. 270, 271, 'Hybrid thistles from Plymouth'; and other papers on the brambles and roses.

The same author: Florc of Plymouth; an account of the flowering plants and ferns found witbin twelve miles of the town (1880).

F. H. Goulding in the Pbytologist, 1849, iii. 643, 'New locality for Hypericum linariifolium.'

Samuel Rowe: A perambulation of the ancient and royal Forest of Dartmoor and the Venville precincts, ed. 3, by J. Brooking Rowe (1896); chap. xvii. pp. 350-98, 'The botany of Dartmoor and its borders,' furnished by Francis Brent.

\section{THE BRAMBLES (Rubi)}

There are very few, if any, districts of equal extent in Great Britain that have had their brambles so diligently studied as south Devon. This remark is especially true of as much of the county as lies within twelve miles of Plymouth, and so is included in the late T. R. Archer Briggs' Flora of Plymouth; but it is scarcely less applicable to such further parts of south Devon as intervene between that district and Exeter. The brambles of the western half of north Devon are also fairly well known. On the other hand very little has yet been done by students of the genus in the extreme east of the county extending from Exeter to the Dorset border in the south, and from Ilfracombe to the Somerset border in the north.

The total number of bramble forms already known for the county is 87 . Of these $5^{8}$ are classed as species, the remaining 29 taking rank as sub-species or varieties. Out of the aggregate number 87,66 are known to occur in south Devon, the number for north Devon being 63 ; while 49 only are common to both divisions. In the abundance of the individual bushes as well as in the number of distinct forms, the southern vice-county as a whole seems considerably richer than the northern; and further research is hardly likely to reverse this estimate. On the north coast the forms are comparatively few; and though many of great interest are found in the valley of the Upper Tamar and on parts of the moorland between that river and the town of Crediton, no part of the north seems likely to be found so full of different brambles as the country near Plymouth or the valley of the Teign. 


\section{BOTANY}

South Devon is especially rich in our rarer British forms; no less than $\mathrm{I} 6$ of these having been actually added to our present list, as new for the British Isles, from that vice-county. Fourteen were thus found and distinguished (though not all at once rightly named) by $\mathrm{Mr}$. Briggs before the publication of his Flora in 1880 , within 7 or 8 miles of Plymouth, viz. Rubus opacus Focke, R. affinis Wh. \& N., var. Briggsianus Rogers, $R$. erytbrinus Genev., $R$. dumnoniensis Bab., $R$. ramosus Briggs, $R$. micans Gren. \& Godr., $R$. Boraanus Genev., $R$. anglosaxonicus Gelert, $R$. Borreri Bell Salt., var. dentatifolius Briggs, $R$. radula Weihe, sub-sp. anglicanus Rogers, $R$. oigocladus Muell. \& Lefv., $R$. mutabilis Genev., var. nemorosus Genev., $R$. thyrsiger Bab., $R$. botryeros Focke. The remaining two were discovered a few years later : $R$. Rogersii Linton, near Moreton Hampstead in the Teign basin; and $R$. birtus W. \& K., var. rubiginosus (P. J. Muell.), in Bickleigh Vale, about 5 miles from Plymouth. Fifteen out of these sixteen well-marked bramble forms (i.e. all but $R$. nemorosus, Genev., which is still unknown away from the Plymouth neighbourhood) have now been found in other parts of England; but south Devon is still the chief home of about half of them, and especially of $R$. ramosus, $R$. Borcanus, $R$. thyrsiger and $R$. botryeros, all characteristic plants of more than one district in the immediate neighbourhood of Plymouth.

Other rare British brambles found in south Devon are $R$. integribasis P. J. Muell. (Chagford and Milber Down), R. bolerytbros Focke (Plymbridge Road, Egg Buckland; and the Kingston neighbourhood), $R$. Questierii Lefv. \& Muell. (Pamflete near Kingston), and $R$. divexiramus P. J. Muell. (between Beer Alston and Tavistock). Two more, the names of which may still be open to some doubt, are $R$. macrantbelos Marss. (so far found nowhere in the British Isles except in two localities near Plympton St. Mary and on the border of Dartmoor near Cholwichtown); and R. leucantbemus P. J. Muell. (near Fancy, Egg Buckland).

Several of these rarer south Devon brambles have not yet been detected in north Devon; but the latter vice-county is by no means badly furnished with rarities of its own. Of these the most interesting are $R$. sulcatus Vest., Holsworthy and Thornbury Road; $R$. cariensis Genev., neighbourhood of Lynton (its first recorded British station), and several localities from Pyworthy and Holsworthy to Barnstaple and Ilfracombe ; $R$. danicus Focke, Marnwood; $R$. mollissimus Rogers, between Holsworthy and Thornbury and Westward Ho!; R. setulosus Rogers, near Otterford, by the Somerset border ; R. Bloxamianus Colem., Bideford ; $R$. Griffitbianus Rogers, Lynton ; $R$. thyrsiger Bab., Clovelly; $R$. Kaltenbacbii Metsch., Heanton Punchardon and Molland; and $R$. ocbrodermis A. Ley, Marnwood. With the exception of $R$. thyrsiger these are all still unknown for south Devon.

\section{THE ROSES (Ros $Q$ )}

Though Devonshire can perhaps hardly claim the highest rank for the number of its species of wild roses, there is no other English county 


\section{A HISTORY OF DEVONSHIRE}

where our essentially southern forms are to be found in greater beauty or greater abundance; as there can be but few where the distribution of those forms has been so well ascertained. Rosa mollis Sm., $R$. sepium Thuill. and $R$. glauca Vill. are the only real species in our British list which are believed to be wholly wanting; and of these the very local $R$. sepium is alone at all likely to be found so far south. $R$. rubiginosa Sm. was considered by so good a judge as the late T. R. Archer Briggs to be native in 'bushy spots' in the Plymouth neighbourhood; but it occurs very sparingly in the county generally, and is no doubt usually only ' a garden escape' when its presence in a given locality is not due to the action of birds.

Our other species, seven in number, $R$. pimpinelloiaes Linn., $R$. tomentosa Sm., $R$. micrantba Sm., $R$. obtusifolia Desv., $R$. canina Linn., $R$. systyla Bast, and $R$. arvensis Huds., all occur abundantly; though $R$. pimpinelloides and possibly $R$. obtusifolia are more local than the rest, in Devon as in other English counties where they occur. $R$. pimpinelloides in both divisions of the county is found chiefly (perhaps exclusively) within a few miles of the sea, though often locally abundant there; while $R$. obtusifolia, though mostly common enough, may possibly be rather scarce in some parts of the county. It is certainly frequent in much of the country between Plymouth and Dartmoor and in the Teign basin in south Devon, and in the northern division between the Upper Tamar and Okehampton. It is however one of our least generally known roses, and so its exact distribution in the county as a whole is still but imperfectly ascertained.

$R$. micrantba $\mathrm{Sm}$. and the two aggregate species $R$. tomentosa $\mathrm{Sm}$. and $R$. canina Linn. are so generally distributed that no special localities need be named for them. The numerous varieties of the two latter are also exceedingly well represented. $R$. arvensis Huds. is quite common; and in almost every hedge one of the most strikingly beautiful of all our 'dog-roses' is the very luxuriant pink-flowered $R$. systyla Bast, which is chiefly southern, although it has been found as far north as Hertfordshire. Allied to this but easily distinguished from it are the very constant varieties or sub-species $R$. leucocbroa Desv. and $R$. pseudo-rusticana Crep. These seem more thoroughly at home in Devon than in any other English county, the former being common and the latter locally abundant especially in the Teign Valley, where it was first observed in 1877 . Both become rare in Dorset, and as yet are almost unknown further north.

\section{CRYPTOGAMIA}

\section{ACrogens. VAsculares}

\section{FILICES}

The county is remarkable for the luxuriance and beauty of the ferns which abound on hedgebanks and among the rocks, etc. With the object of protecting these and other interesting plants the Devon County 


\section{BOTANY}

Council resolved on $20 \mathrm{March}$, I902, 'that it is desirable that there should be added to the existing bye-laws a bye-law prohibiting the uprooting of ferns and flowering plants or shrubs on land within the County Council area to which the public have access; and that the subject be referred to the General Purposes Committee for consideration and report.' In accordance with this resolution the committee communicated the wishes of the council to the Home Secretary, whose approval to the proposed bye-law would be necessary. The reply from the Home Office contained the following remarks :-

'If it is confined to cases where serious damage and disfigurement is caused in public highways, etc., there may not be much difficulty from the legal point of view in framing the bye-law, but the Secretary of State would not be willing to allow a bye-law which would be likely to injure unsuspecting poor people residing in the district, or to lead to the punishment of young children. Possibly however the byelaw could be restricted in its operations so as not to involve any danger of this, e.g. by confining it to particular places to be indicated by notices. If however it is proposed that the bye-law should only apply to rare ferns or plants, the difficulties in framing it are likely to be greater. In any event a bye-law which would prevent any person from taking one or two common ferns or plants from the roadside for his own use, would in the opinion of the Secretary of State be inadmissible.'

Having regard to this view on the part of the Secretary of State the committee did not recommend the council to take any further action in the matter.

' Few of us accustomed to wander on our moors and take an interest in its flora can have failed to notice the diminution, it may be said destruction, of some of our rarer ferns. Osmunda regalis has been utterly eradicated from near Cornwood where

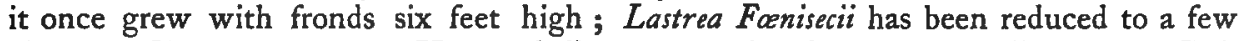
plants at Shaugh; the two Hymenophylla, once so abundant in the valley of the Cad, and on the rocks near the Meavy, can now scarcely be met with, and the little oak fern, always extremely rare, is entirely gone. Asplenium lanceolatum, Opbioglossum, and the mounwort, still linger on; the difficulty in finding them has conduced to their preservation, and it is to be hoped that those who are acquainted with their habitats will hesitate to divulge their localities to others than those who will carefully protect them. A few years since might be seen in the streets of Plymouth itinerant fern collectors who were exposing for sale large mats of Hymenophyllum, torn ruthlessly from their rocks, the scars on which remain in the Meavy and Cornwood valleys to the present day' (Francis Brent in Rowe's Perambulation of Dartmoor [18g6], ed. iii. p. 352).

The filmy ferns (Hymenopbyllum tunbridgense and $H$. unilaterale) are met with among mosses in damp and shady places in the Barnstaple, Torquay, Plymouth and Tavistock districts, but they are rather rare and local; the former was recorded by Hudson in 1778 as occurring on Dartmoor, but it is diminishing in consequence of the rapacity of collectors.

The maiden-hair fern (Adiantum Capillus-Veneris) was formerly plentiful on the coast of south Devon and occurred in several stations about Ilfracombe, Combmartin, etc., in north Devon; but it has been wantonly plundered and is now very scarce and is mostly restricted to spots difficult of access; on I4 May I902, at Ilfracombe, a boy of thirteen years of age in attempting to gather this fern fell down the Hillsborough cliff and died in consequence of the injury sustained. 


\section{A HISTORY OF DEVONSHIRE}

The bracken (Pteris aquilina) and the hard-fern (Lomaria Spicant) are common and abundant in all the districts; each is represented by several named varieties.

The parsley fern (Cryptogramme crispa) was found by N. Ward in I 840 within 6 miles of Lynton, but only one plant was then seen; it has also been recorded from the neighbourhood of Glenthorne about the same distance from Lynton on the authority of W. S. Hore and from Challacombe, all in the Barnstaple botanical district; in recent years it has been found about Chagford on the north-east side of Dartmoor.

Among the spleenworts the wall-rue (Asplenium Ruta-muraria), the English maiden-hair ( $A$. Trichomanes), the black spleenwort ( $A$. Adiantumnigrum), and the lady fern $(A$. Filix-foemina), with several varieties, are common and generally distributed; as varieties of the last $A$. rbaticum is recorded for Barnstaple, $A$. molle is said to be frequent, and $A$. incisum is reported from the parishes of Marwood and Bittadon. The forked spleenwort ( $A$. septentrionale) was found by $\mathbf{N}$. Ward in 1840 in tolerable quantity in the crevices of a loose stone wall facing the north at an elevation between I,000 and I, I 00 feet in Countisbury parish near Glenthorne, also according to Ravenshaw by Miss Hill in the same locality in I 856 , but it does not appear to have been seen there during recent years and may perhaps be extinct; Mr. Moyle Rogers has recorded it as having occurred in considerable plenty in $188 \mathrm{I}$ on some high rocks between Chudleigh and Dartmoor. The green spleenwort ( $A$. viride) is on record as having occurred more than thirty years ago on the moor near the head of the river above Petertavy; a specimen was seen and examined by C. C. Babington who did not dispute the identification. The sea spleenwort $(A$. marinum) is rather frequent along the maritime rocks of both north and south Devon, but it is becoming comparatively scarce through the greed of collectors; the variety acutum has been reported from Croyde in the parish of Georgeham. The spear spleenwort $(A$. lanceolatum) occurs in many stations in south Devon and less frequently in north Devon, but in several places where it was formerly quite plentiful it is now nearly or quite extinct through the action of collectors and dealers. The rusty-back fern (A.Ceteracb) is rather common about Plymouth and in some other parts of south Devon ; in north Devon it is local and rather rare, occurring in the parishes of Marwood, Kentisbury, Horwood, Fremington, Instow, Bideford, Buckland Brewer, Braunton and (in I 882) in Barnstaple, all these in the Barnstaple district; also in Bishopsnympton in the South Molton district, and in Okehampton in the Torrington district; it has also been reported from Lynmouth.

The hart's tongue (Scolopendrium vulgare) with numerous varieties is very common and abundant, producing handsome effects in many places.

The bladder fern (Cystopteris fragilis) is very rare in the county ; there are satisfactory records of it in the parish of Cornwood; it is also reported from Ilfracombe, Tiverton, St. Thomas, Lynton, Bickleigh and Woodside near Plymouth; the sub-species $C$. alpina has been reported 


\section{BOTANY}

from Diptford rectory in the Plymouth district, the specimen having been verified by Mr. Moore of Chelsea (Ravenshaw).

The prickly fern (Aspidium aculeatum) is widely distributed; the sub-sp. A. lobatum is reported from Barnstaple, and its variety loncbitidoides from Ilfracombe and Challacombe; the sub-sp. A. angulare is common, and numerous varieties are recorded from the neighbourhoods of Barnstaple, Ilfracombe and other places in the same district; the variety alatum is given in English Botany, ed. iii., for Ottery St. Mary, on the authority of Wollaston, and the variety gracile is stated to occur in Devon.

The male fern (Nepbrodium Filix-mas) is a common and conspicuous species; the var. affine is recorded by Moyle Rogers from Moretonhampstead and Trusham and is widely distributed; the var. Borreri (the golden male fern) is also frequent; and the var. abbreviatum is comparatively scarce, being reported from Hartland and Ilfracombe.

The crested shield fern ( $N$. spinulosum) is not very common in the typical form; the sub-species $N$. dilatatum however is frequent and is a very handsome fern, the var. nanum occurs at Ilfracombe, the var. Aspidium Boottii is reported from Ilfracombe, Challacombe and Hartland; the var. A. dumetorum is also reported from Ilfracombe and Challacombe; several other varieties occur. The hay-scented fern ( $N$. amulum) is widely distributed but appears to be very scarce in some districts; it is frequent in the upper Tamar country, not uncommon though local in the Barnstaple botanical district, rare according to Mr. Moyle Rogers in the area of the Teign basin and rather common within 12 miles of Plymouth. The marsh fern ( $N$. Thelypteris) is very rare; it occurs in Braunton Marsh in the Barnstaple district, and has been reported from Kingskerswell in the Torquay district. The sweet mountain fern $(N$. Oreopteris) is locally abundant and occurs in all the districts.

The common polypody (Polypodium vulgare) is plentiful in all districts; the variety with pinnatifid segments ( $P$. cambricum) is represented by the form crenatum which is reported as frequent in north Devon; other varieties are also recorded for north Devon.

The beech fern ( $P$. Phegopteris) is rare and very local ; it was recorded by Hudson in 1778 for the county, a precise locality probably being in the parish of Petertavy in the Tavistock district 'on the side of the hedge on the left of the road twixt Wilsworthy hamlet and Black Down Gate just beyond a lane that turns to the left hand' (Fourn. Bot. I 884, p. 174); there are several other stations for it on Dartmoor; in the Barnstaple botanical district it occurs in the parish of Challacombe near Exmoor ; it is said to have disappeared from the banks of the river Dart through the depredations of collectors. The oak fern ( $P$. Dryopteris) is also very rare and local; there are several recorded stations on Dartmoor ; in the Barnstaple district it occurs in the parishes of Parracombe, Bratton Fleming and Challacombe, and it is reported from Ilfracombe.

The flowering fern (Osmunda regalis) was formerly abundant in all districts, but is now rapidly disappearing from many places through the action of fern collectors and dealers. 


\section{A HISTORY OF DEVONSHIRE}

The adder's tongue (Ophioglossum vulgatum) is local, but is satisfactorily recorded for all the districts except that of South Molton; in some places it is locally plentiful.

The moon-wort (Botrycbium Lunaria) is local like the last and recorded for the same districts.

Nephrodium cristatum, Rich., was included by Jones and Kingston in their Flora Devoniensis, part ii. p. 108 (1829), but without precise locality; it was also recorded for Ilfracombe by Ravenshaw in 1860 ; also by the late Mr. Mansel-Pleydell, Flora of Dorsetsbire, ed. 2, p. 329, on the authority of $Z$. Edwards as occurring in the parish of Hawkchurch (now transferred to Devon), but the author of that Flora informed me that he could not guarantee the record; on the whole evidence it does not appear that the species should remain in our list of Devon plants.

A popular account of the ferns, especially in the northern part of the county, is given by Mrs. Charlotte Chanter, Ferny Combes (1 856 ). An account of the ferns in a portion of south-east Devon is given by the Rev. Zachary James Edwards: The Ferns of the Axe (1862).

The following is a complete list of the species of Devonshire ferns after the nomenclature adopted in the Student's Flora :-
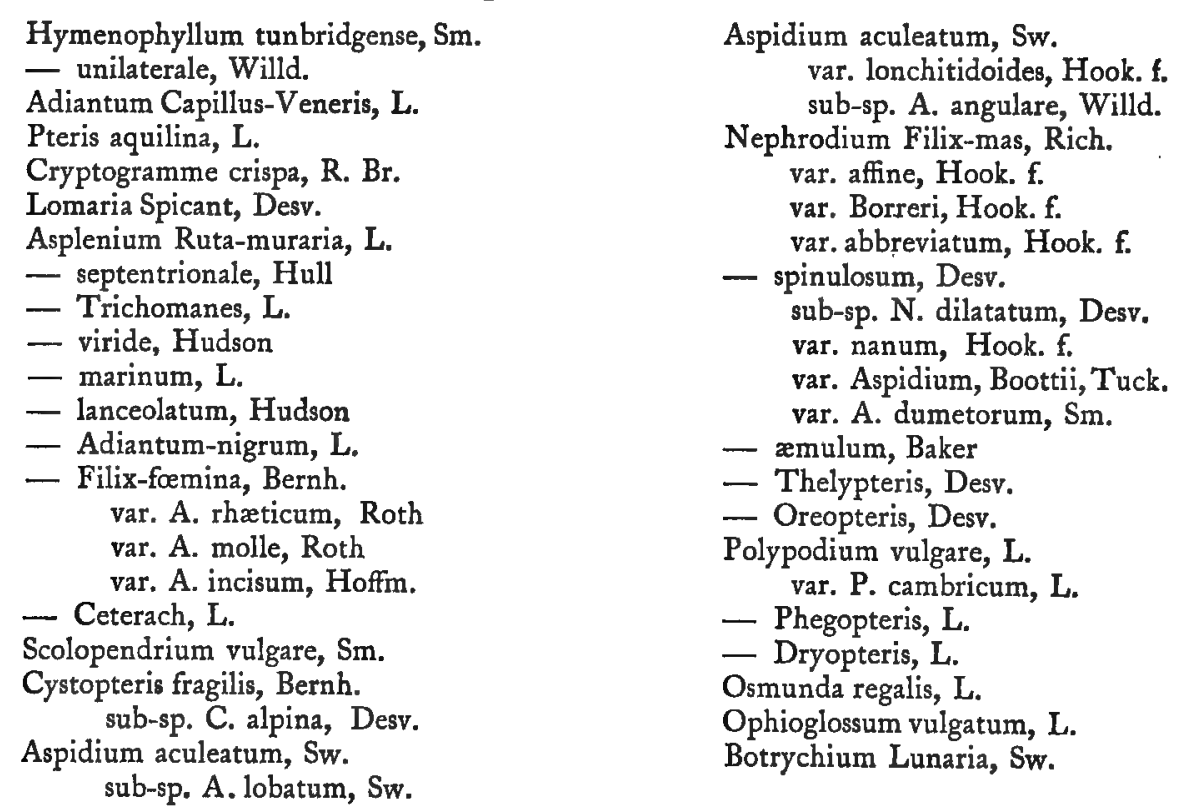

\section{EQUISETACE正}

The following is a complete list of the species occurring in the county :-

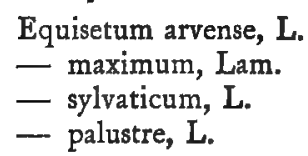

\author{
Equisetum limosum, L. \\ var. E. fluviatile, $L$. \\ - hyemale, L. (l) \\ - variegatum, Schleich.
}

The corn horsetail (E. arvense) and the marsh horsetail (E. palustre) are common and generally distributed. The great horsetail (E. maximum) is widely spread and has been reported from the Barnstaple, South 


\section{BOTANY}

Molton, Exeter, Honiton and Torquay botanical districts. The wood horsetail (E. sylvaticum) is not very common; it has been recorded from the South Molton, Honiton and Plymouth districts ; Moyle Rogers did not see it in the Devon parts of the upper Tamar country and remarked on its absence. The water horsetail ( $E$. limosum) is found in most of the districts; the variety $E$. fluviatile also occurs not less commonly. $E$. variegatum is rare in the county, but it occurs in the Barnstaple, Honiton and Torquay districts. The Dutch rush (E. byemale) has been reported from the Honiton district, but possibly in error.

\section{LYCOPODIACEE}

The following species occur in the county :-

Lycopodium clavatum, L. $\quad$ L. inundatum, L. $\quad$ L. Selago, L.

The common club-moss ( $L$. clavatum) is rather rare and local; it grows in a few stations in the northern parts of the Barnstaple and South Molton botanical districts ; it is reported also from Haldon, the Blackdown Hills, Ugborough Beacon and Dartmoor.

The marsh club-moss ( $L$. inundatum) is rare and local ; there are satisfactory records for it in the Barnstaple, Honiton, Torquay and Plymouth botanical districts.

The fir-moss is also local; it has however been found in all the botanical districts except Torrington.

\section{SELAGINELLACEÆ}

Selaginella selaginoides, Gray (the prickly club-moss) has been recorded from Dartmoor and from Trentishoe Down in the Barnstaple botanical district, but both these records require confirmation.

\section{MARSILEACEÆ}

The pillwort or pepper grass (Pilularia globulifera, L.) was recorded in 1797 by Polwhele as occurring on Blackdown; it has occurred also near Lympstone, according to Jones and Kingston (Fl. Dev. ii. I I I) on the authority of Miss Filmore.

\section{MUSCI (Mosses) ${ }^{1}$}

The Moss Flora of Devonshire differs from that of the neighbouring county of Cornwall, chiefly in the absence of a few species of a distinctly southern type, such as Pbilonotis rigida, Hookeria laetevirens, and Fissidens serrulatus, whilst on the other hand it includes many species of a distinctly subalpine type that have not been recorded for Cornwall. Of these the following may be mentioned:-Tetradontium Brownianum, Catbarinea crispa, Cynodontium polycarpum, Cynodontium virens, Blindia acuta, Dicranorweissia crispula, Dicranum spurium, Orthotrichum Hutcbinsiae, Grimmia Donniana, Grimmia Hartmanni, Grimmia montana, Grimmia ovata,

${ }^{1}$ This and the following orders, by E. M. Holmes, F.L.S. 


\section{A HISTORY OF DEVONSHIRE}

Glypbomitrium Daviesii, Bartramia itbyphylla, Webera cruda, Mnium serratum, Plagiothecium pulchellum, Habrodon Notarisii, and Hypnum eugyrium. The rare species Ditricbum subulatum appears to find its northern limit in Devon, and several other rare species, although found in N. Wales, Ireland, and warm localities in Scotland, do not, so far as is known, extend further eastward than Devon, such as Fissidens polyphyllus and Tortula canescens. Leptodontium gemmascens and Webera Tozeri, Eurbyncbium circinnatum and E. striatulum, are rare except in the southern counties. Only one species, Barbula cordata, recently detected in North Devon by Mr. E. M. Holmes, is found in no other county in Great Britain. Glypbomitrium Daviesii, which is usually confined to basaltic rocks, occurs only on Cocks Tor, which is remarkable as being the only tor of trap rock in the granitic expanse of Dartmoor. Two other rare species have lately been added by Miss C. E. Larter, viz. Barbula gracilis and Pottia commutata. Grimmia montana, so far as England is concerned, has been found only near Fingle Bridge, in Devon.

The rare Orthodontium gracile, local and rare on sandrocks, and rare on tree stumps, and known outside Britain only in France and California, was found by Mr. E. M. Holmes on a rotten tree stump near Shaugh Bridge. The usually mild winters allow of the development of fruit in many species rarely found in that state, such as Bryum roseum, Breutelia arcuata, Hypnum brevirostre, Bracbytbecium illecebrum, Heterocladium beteropterum, Antitricbia curtipendula, and Zygodon viridissimus. In the limestone districts around Plymouth and Torquay, Trichostomum nitidum forms a prominent feature of the dry stone walls, whilst Eurbyncbium circinnatum often covers the rocky banks for many yards. In the reddish soil round Totnes and Newton Abbot, and on the yellow clay slate on the confines of the Moor, Scbistostega osmundacea and Epipterygium Tozeri (the latter rarely in fruit), form a characteristic feature of the damp bare hollow portions of the hedge banks, and in similar situations Tortula cuneifolia and Pottia Starkeana often occur in quantity.

In Jones and Kingston's Flora of Devon, 1829, 175 species are recorded. In 'The Mosses of Devon and Cornwall,' by E. M. Holmes and F. Brent, published in the Transactions of the Plymoutb Literary and Scientific Institution in 1887 , the number was increased to 306 species, and in the Moss Flora of Devon, by E. Parfitt, a few more species are enumerated. The list now given includes 336 species, but probably many species will yet be added by subsequent observers. ${ }^{1}$

The species recently added are chiefly due to the investigations of Mr. W. Mitten, Miss C. E. Larter, and Mr. E. M. Holmes, in North Devon ; and to Mr. E. D. Marquand and Mr. L. J. Cocks on Dartmoor and the county generally, and their initials are placed after the rarest of the species. Mr. E. M. Holmes's collection of Devonshire and other mosses is now in the Herbarium of Cambridge University, and the collection of Mr. Parfitt's mosses and other cryptogams has recently been

${ }^{1}$ A list of mosses of the Lynmouth district was published in Science Gossip, Sept. 1900, Pp. 99-102, but it contained no species new to the county. 


\section{BOTANY}

presented to the Torquay Museum by Mrs. Vicary, of Newton Abbot, into whose possession it had come by purchase. The nomenclature adopted in this list is that of Dixon and Jameson, The Student's Handbook of British Mosses, ed. 2 (1 904), and Horrell, The European Spbagnaceae (1 901).

\section{MUSCI}

SPHAGNacear

Sphagnum acutifolium, Ehrh. I-8

- crassicladum, Warnst. I

- cuspidatum, R. and W. 8 var. submersum, Schimp.

- cymbifolium, Ehrh. 1,8

- fimbriatum, Wils. 6

- inundatum, Warnst. (L.J.C.)

- molluscum, Bruch. 4-6,8

- obesum, Warnst. 8

- papillosum, Lindb. 8 var. normale, Warnst. I

- recurvum, $R$. and $W$. var. mucronatum, Warnst. 8

- rigidum, Schimp. 1, 6-8

- rubellum, Wils. E.

- rufescens, Warnst. 1, 5

- squarrosum, Pers. 8 var. laxum, Braithw. 8

- subsecundum, Nees. I, 8 var. contortum, Schimp. 6,8 var. obesum, Wils. 8

- subnitens, R. and W. 5 var. violascens, Warnst. 5 var. flavo-rubellum, Warnst. (L.J.C.) var. purpurascens, Schlieph. (L.J.C.)

\section{Andrearaceae}

Andreaea crassinervia, Bruch. 8

- falcata, Schimp. 8

- petrophila, Ehrh. 6,8

*- Rothii, Web. and Mohr. 8

\section{Tetraphidackae}

Tetraphis pellucida, Hedw. $\quad 4,6,8$

Tetradontium *Brownianum, Grev. 8

\section{Buxbaumiacear}

Diphyscium foliosum, Mohr. I, 6, 8 var. acutifolium, Lindb. 8

\section{Polytrichaceaz}

"Catharinea crispa, James. 8

- undulata, W. and M. I, 4, 7

Polytrichum aloides, Hedw. I, 7,8

* alpinum, L. 8

- commune, Linn. I-8

var. perigoniale, B. and S. 8 var. fastigiatum, Braithw. 6,8

- formosum, Hedw. I, 6,8

- gracile, Dicks. 8

- juniperinum, Willd. I-8

- nanum, Brid. I-8 var. longisetum, Haw. 8

- piliferum, Schreb. I-8

- urnigerum, Brid. 1, 4,7
Dicranacrae

Pleuridium alternifolium, Rab. 4-8

- axillare, Lindb. 4, 6, 7

- subulatum, Br. and Schimp. I-8

Ditrichum homomallum, Hpe. I, 8

- - subulatum, Hpe. 7,8 (E.M.H.)

Ceratodon purpureus, Brid. I-8

Rhabdoweisia denticulata, B. and S. 8

- fugax, B. and S. 6,8

Cynodontium Bruntoni, B. and S. 8

* - polycarpum, Schp. 8

* - virens, Schp. 8

Dichodontium flavescens, Lindb. 6, 8

- pellucidum, Schp. I, 8

Dicranella cerviculata, Schp. 8

* - crispa, Schp. 7

- heteromalla, Schp. I-8

- rufescens, Schp. 7

* - secunda, Ldb. 6

- squarrosa, Schp. 1, 4, 8

- varia, Schp. I-8

Blindia acuta, B. and S. I, 8

Dicranoweisia cirrhata, Ldb. I-8

- crispula, Ldb. 8

Campylopus atrovirens, De Not. 8

- brevipilus, B. and S. 8

- flexuosus, Brid. 6,8 var. paradoxus, Husn. 8

- fragilis, B. and S. I, 5, 7

- pyriformis, Brid. I, 8

Dicranum Bonjeani, De Not. 1, 4, 5,7

- fuscescens, Turn. 4,7

- scoparium, Hedw. I-8

- Scottianum, Turn. 6,8 (L.J.C.)

*- spurium, Hedw. 8 (E.M.H.)

Leucobryum glaucum, Schimp. I, 4, 6

Fissidentacrae

Fissidens adiantoides, Hedw. 1, 4, 8

- bryoides, Hedw. I-8

- Curnowii, Mitt. 8

- decipiens, De Not. I, 8

-- exilis, Hedw. I, 6

- incurvus, Starke. 7

- osmundoides, Hedw. 8

- polyphyllus, Wils. 6, 7 (E.D.M.)

- pusillus, Wils. 7,8 var. Lyelli, Braithw. 7,8

- taxifolius, Hedw. I-8

- viridulus, Wahl. I, 7 . var. fontanus, Braithw. 7

\section{Grimmiacear}

Grimmia apocarpa, Hedw. I-8 var. gracilis, W. and M. 8 var. rivularis, W. and $M$. $\quad 1,6,8$

- decipiens, Lindb. 2, 7,8 var. robusta, Ferg. 7 


\section{A HISTORY OF DEVONSHIRE}

\section{Grimmiacear (continued)}

"Grimmia Doniana, Sm. I, 8 (E.M.H.)

- Hartmanni, Schp. 6 (E.D.M.)

* leucophaea, Grev. 5, 7,8

- maritima, Turn. 1, 6,7

* montana, B. and S. 6

"- orbicularis, Bruch. 1, 6-8

* - ovata, Schwgr. 1, 6

- patens, B. and S. 8

- pulvinata, Sm. I, 6,8

- trichophylla, Grev. 1, 6, 7

Racomitrium aciculare, Brid. I, 6, 8

- canescens, Brid. I, 6, 8 var. ericoides, B. and S. 8

- fasciculare, Brid. I, 6, 8

- heterostichum, Brid. 1, 8 var. alopecurum, Hub. 8 var. gracilescens, B. and S. 2, 6, 8 .

- lanuginosum, Brid. I, 8

- protensum, Braun. 8

- sudeticum, B. and S. I, 8

Ptychomitrium polyphyllum, Furnr.' I, 2, 6, 8

Glyphomitrium Daviesii, Brid. 8

Hedwigia ciliata, Ehrh. 1, 5, 6, 8 var. leucophaea, Schp. 8

\section{Tortulacrae}

Acaulon muticum, C.M. $\quad 5,6,7$ var. minus, Braithw. 6

* triquetrum, C.M. 6

- curvicolle, Ehrh. I, 7

Phascum cuspidatum, Schreb. I-8 var. piliferum, H. \& T. 6

Pottia commutata, Limpr. I (C.E.L.)

- crinita, Wils. 1, 6,8

- Heimii, Turn. 1, 6,7

- intermedia, Furnr. 1, 4, 5, 7 var. littoralis, Dixon 6

- lanceolata, C.M. 5, 6, 7

- minutula, Furnr. 4, 5,7

- recta, Mitt. I, 4, 6, 7

- Starkeana, C.M. 5, 6,7

- truncatula, Ldb. I-8

- Wilsoni, B \& S. I, 6 (E.M.H.)

Tortula aloides, De Not I-8

- ambigua, Angstr. I, 4, 7

- atrovirens, Lndb. 1, 6

*- canescens, Mont. 6 (E.M.H.)

- cuneifolia, Roth. I, 4-7

* - inclinata, Hedw. 7 (E.M.H.)

- intermedia, Berk. 6,7

- laevipila, Schwgr. 6,7

- Iamellata, Ldb. 6

- marginata, Spr. 5,6

- muralis, Hedw. I-8 var. rupestris, Wils. 6,7

- papillosa, Wils. 8

- rigida, Schrad. I, 4, 6, 7

- ruraliformis, Dixon. I, 6

- ruralis, Ehrh. 7

- subulata, Hedw. I, 2, 4, 6, 8

Barbula convoluta, Hedw. I -8 var. Sardoa, Bruch. 7

- cordata, Dixon. 6 (E.M.H.)

- cylindrica, Schimp. 1, 7,8
Tortulacrae (continued)

Barbula fallax, Hedw. I-8 var. brevifolia, Schultz. I

* gracilis, Schwaegr. I (C.E.L.)

*- Hornschuchiana, Schultz. 6, 7,8

"- Iurida, Ldb. I, 7

- revoluta, Mitt. I, 6, 7 .

- rigidula, Mitt. 1, 6,7

- rubella, Mitt. I, 4, 6,7

- sinuosa, Braithw. 6,7

- spadicea, Mitt. 6

- tophacea, Mitt. $1,6,7$

- unguiculata, Hedw. $\quad$ I-8 var. apiculata, Braithw. 7

- vinealis, Brid. I, 7

"Leptodontium flexifolium, Hpe. 2, 8

*- gemmascens, Braithw. 56

Weissia crispa, Mitt. 5, 6,7

- crispata, C.M. I (C.E.L.)

- microstoma, C.M. 1, 6, 7

*- tortilis, C.M. 7 var. subcylindrica, Schpr. 7

- verticillata, Brid. 1, 6,7

- viridula, Hedw. I-8 var. amblyodon, B. \& S. 1, 7 var. densifolia, B. \& S. I

Trichostomum crispulum, Bruch. I, 6,7 var. viridulum, Braithw. 6

- flavovirens, Bruch. I, 6, 7

- littorale, Dixon. 1, 7

- mutabile, Bruch. $\quad 1,6,7$

- nitidum, Schimp. 1, 6,7

- - tenuirustre, Lindb. 1, 8

- tortuosum, Dixon. 6,7

Pleurochaete squarrosum, Lindb. 6,7

"Cinclidotus Brebissoni, Husn. 4, 6,7

- fontinaloides, P. Beauv. 1, 4, 6

\section{Encalyptacear}

Encalypta streptocarpa, Hedw. I, 6, 8

- vulgaris, Hedw. 6,7

\section{Orthotrichaceae}

"Zygodon conoideus, H. \& T. 8

- Mougeotii, Brid. I, 8

- Stirtoni, Schp. 1, 6,7

- viridissimus, R. Br. I, 5-8 var. rupestris, Lindb. I subsp. Stirtoni, Schp. 7

Ulota Bruchii, Hornsch. i, 6, 8

- crispa, Brid. 7,8

- Hutchinsiae, Hamm. I, 6,8

- phyllantha, Brid. I, 6, 7,8

Orthotrichum affine, Schrad. I-8

- anomalum, Hedw. I, 6,7 var. cylindricum, Schimp. 7

- cupulatum, Hoffm. 2, 4,6

- diaphanum, Schrad. 1, 7

- leiocarpum, B. \& S. $\quad 5,6,7,8$

- Lyellii, H. and T. 1, 2, 6,8

*- pulchellum, Sm. 5,6,8

*- rivulare, Turn. 6

* - rupestre, Schleich. 4, 6

* - stramineum, Hornsch. 5

- tenellum, Bruch. I, 5, 7 


\section{BOTANY}

\section{Schistostegacear}

Schistostega osmundacea, Mohr. 6, 7, 8

\section{Splachnacear}

Splachnum ampullaceum, L. $5,6,8$ var. Turnerianum, Dicks. 8

- sphaericum, L. 8

\section{Funariaceae}

Physcomitrella patens, B. and S. 6, 7

Physcomitrium pyriforme, Brid. 1, 2, 7

Funaria calcarea, Wahl. 6, 7 var. patula, B. and S. 6,7

- ericetorum, Dixon. 4,8

- fascicularis, Schp. I, 5-8

- hygrometrica, Sibth. I-8

*- Templetoni, Sm. I, 4, 6

\section{Mersiactab}

Aulacomnium androgynum, Schwgr. 4,6 - palustre, Schwgr. I, 4, 6, 8

\section{Bartramiaceas}

Bartramia ithyphylla, Brid. I, 8 - pomiformis, Hedw. I, 4, 6, 7,8 var. crispa, B. and S. 6,8

-Philonotis calcarea, Schimp. I.

- fontana, Brid. I, 4, 6, 8 var. falcata, Brid. 6

Breutelia arcuata, Schp. I, 2, 6, 8

\section{Bryaceae}

*Orthodontium gracile, Schwgr. 8 (E.M.H.)

Leptobryum pyriforme, Wils. 6

Webera albicans, Schimp. I, 6, 8

- annotina, Schwgr. 1, 6

- carnea, Schimp. I, 4, 6, 7

- cruda, Schwgr. 8

- elongata, Schwgr. 8

- nutans, Hedw. I, 6, 8 var. longiseta, B. and S. 8

- - Tozeri, Schp. I, 6, 7

Bryum affine, Lindb. 7

- alpinum, Huds. $6,7,8$

- argenteum, Linn. I-8 var. majus, B. and S. 7

- atropurpureum, W. and M. 6,7

- bimum, Schreb. 7

- caespiticium, L. 1 and 8

- calophyllum, R.Br. I (W.M.)

- capillare, Linn. I-8

*- Donianum, Grev. I, 6

- erythrocarpum, Schwgr. 7,8

- filiforme, Dicks. 8

- inclinatum, Bland. 1,7

- intermedium, Brid. 7

*- Muhlenbeckii, B. and S. 8

- murale, Wils. 5, 7

- obconicum, Hornsch. I, 8

- pallens, Swartz. I, 6, 8

- pendulum, Schp. I

- pseudo-triquetrum, Schwg. 1, 6,8 var. compactum, B. and S. I

\section{BRYACEAR (continued)}

Bryum roseum, Schreb. I, 8

- torquescens, B. and S. 7

- uliginosum, B. and S. I

"Mnium affine, Bland. I, 8

- cuspidatum, Hedw. 2, 6,8

- hornum, Linn. I-8

- punctatum, Linn. $\mathbf{I}-8$

- rostratum, Schrad. I, 6, 7,8

- serratum, Schrad. 6,8

- stellare, Reich. I, 6, 8

- subglobosum, B. and S. 7

- undulatum, Linn. I, 6, 7,8

\section{Fontinalaceae}

Fontinalis antipyretica, Linn. I, 6, 7

- squamosa, L. 8 subsp. Dalecarlica, B. and S. 8

\section{Cryphazacear}

Cryphaea heteromalla, Mohr. 1,7 *var. aquatilis, Schp. 6

\section{Neckeraceae}

Neckera complanata, Hubn. $\quad$ I, 7

- crispa, Hedw. I, 6, 7

- pumila. Hedw. $\quad 1,4,6,7,8$ var. Phillippeana, Milde. 8

Homalia trichomanoides, Brid. I, 7

Pterygophyllum lucens, Brid. 1, 6, 7,8

\section{LeUCODONTACEAB}

Antitrichia curtipendula, Brid. 1, 2, 5, 6, 8

Habrodon Notarisii, Schp. I, 6

Leucodon sciuroides, Schwgr. I, 7

Porotrichum alopecurum, Mitt. I-8

Pterogonium gracile, Sw. I, 7, 8

\section{LESKEACEAB}

Leskea polycarpa, Ehrh. 4, 6, 7

Anomodon viticulosus, Hook. and Tayl. I, 6, 7,8

-Leptodon Smithii, Mohr. 6, 7

Heterocladium heteropterum, B. and S. 1, 7,8

Thuidium tamariscinum, B. and S. I, 8

\section{HypnacEar}

Climacium dendroides, W. and M. 6, 8

* Orthothecium intricatum, B. and S. I *var. robustum, B. and S. 8

Camptothecium lutescens, B. and S. I, 6, 7

- sericeum, Kindb. I-8

Brachythecium albicans, B. and S. 1, 6-8

* caespitosum, Diron. 7

- glareosum, B. and S. 6-8

- illecebrum, De Not. 4, 6, 7

- plumosum, B. and S. $1,6-8$

- populeum, B. and S. $1,7,8$

- purum, Dixon. I-8

- rivulare, B. and S. I, 6-8

- rutabulum, B. and S. $\quad$ I- 8 var. longisetum, B. and S. 7

- velutinum, B. and S. I-8 


\section{A HISTORY OF DEVONSHIRE}

Hypnacear (continued)

Hyocomium flagellare, B. and S. $1,7,8$

Eurhynchium abbreviatum, Schimp. 7

- circinnatum, B. and S. 6, 7

- confertum, Milde. I, 4, 6, 7

- crassinervium, B. and S. I, 6, 7

- megapolitanum, Milde. 7

- murale, Milde. 7

- myosuroides, Schmp. I, 7 var. rivulare, Holt.

- myurum, Dixon. I, 6, 8 var. robustum, B. and S. 8

- piliferum, B. and S. 6-8

- praelongum, Hobk. I-8 var. Stokesii, B. and S. I, 6-8

- pumilum, Schimp. I, 6, 7

- rusciforme, Milde. I, 6-8 var. Atlanticum, Brid. I, 5, 7, 8

* speciosum, Schimp, 4

- striatulum, B. and S. 7

- striatum, B. and S. I-8

- Swartzii, Hobk. I, 7 var. atrovirens, B. and S. 7

- tenellum, Milde. I, 6, 7 var. scabrellum, Dixon. 6,7

Plagiothecium denticulatum, B. and S. I-8 var. Donii, Lindl. 2, 8

- depressum, Dixon. I-8

- elegans, Sull. I, 8

- latebricola, B. and S. 7

- pulchellum, B. and S. 6

- sylvaticum, B. and S. 1, 7,8

- undulatum, B. and S. $1,7,8$

Amblystegium filicinum, De Not. I, 6, 8

* Auviatile, B. and S. 6

- irriguum, B. and S. I, 6

* - radicale, B. and S. 4

- serpens, B. and S. 1-8

Hypnum aduncum, Hedw. 7 var. Kneiffi, Schip. 7

- chrysophyllum, Brid. 6,7
Hrpnacear (continued)

Hypnum commutatum, Hedw. I, 8

*- cordifolium, Hedw. I, 4, 6

- cupressiforme, Linn. I-8 var. elatum, B. and S. I, 6-8 var. ericetorum, B. and S. 6,8 var. filiforme, Brid. I, 7,8 var. minus, Wils. 8 var. resupinatum, Schp. I, 6-8 var. tectorum, Brid. 8

- cuspidatum, Linn. I, 6, 7

- elodes, Spr. I var. Mackayi, Schp. I

* eugyrium, Schp. I

- exannulatum, Gumb. 4 var. purpurascens, Schp. 8

* _ falcatum, Brid. I var. gracilescens, Schp. I (C.E.L.)

- fluitans, Linn. 8 var. falcifolium, Ren. 8 (L.J.C.)

* _ imponens, Hedw. 7

- lycopodioides, Schwgr $\quad$ 1, 8

- molluscum, Hedw. 1, 6-8

- ochraceum, Turn. 8

- palustre, Huds. 1, 6-8

- Patientiae, Lindb. 6,8

- revolvens, Sw. I, 4, 6, 8 "var. Cossoni, Ren. 8

- riparium, L. 4-6

- scorpioides, L. 8.

- Schreberi, Willd. I, 6,8

- Sendtneri, Schp. 8

- stellatum, Schreb. 1, 6,8 var. protensum, B. and $S$. I, 6

- stramineum, Dicks. 1, 6,8

- uncinatum, Hedw. $\quad 4,6,8$

Hylocomium brevirostre, B. and S. 6

- loreum, B. and S. 8

- splendens, B. and S. $\mathrm{I}, 6,8$

- squarrosum, B. and S. I-8

- triquetrum, B. and S. I-8

\section{HEPATICAE (Liverworts)}

The county of Devon is peculiarly adapted for the growth of these moisture-loving plants, the stony streams and sheltered ravines of Dartmoor affording many species that are absent in less favoured counties. The number of species represented in the county amount to about half of those known to occur in Great Britain and Ireland. Those which are absent are chiefly of a northern character or which usually grow at an elevation of over $2,000 \mathrm{ft}$. Several also that occur on sandstone rocks in other counties do not occur on the less porous granite of Devon. The number of Hepaticae recorded from Devon exceeds that of Cornwall by twenty-five species, which are chiefly of such as occur on the higher parts of Dartmoor. But on the other hand Cornwall is richer in recorded species of Riccia, the following not having yet been detected in Devon, viz., Riccia bifurca, $R$. ciliifera, $R$. glaucescens, $R$. sorocarpa and $R$. tumida. On Braunton Burrows however, Riccia crystallina, which is not recorded for Cornwall, forms a marked feature of the dried-up ponds in the 


\section{BOTANY}

autumn. Targionia Michelii also, which is not recorded from Cornwall, is not unfrequent in Devon, although very restricted in its growth in the localities in which it occurs. In Jones and Kingston's Flora Devoniensis, published in 1829 , only thirty Devonian species of Hepaticae were recorded. This number was increased by Mr. E. M. Holmes to about seventy as enumerated in the "Scale Mosses and Liverworts of Devon and Cornwall,' published in vol. iii of the Annual Reports and Transactions of the Plymouth Institution and Devon and Cornwall Natural History Society in 1867 . The list of Hepaticae given in Ravenshaw's Botany of Nortb Devon (1876) contains three species not previously recorded for Devon, viz., Nardia byalina, Diplopbyllum obtusifolium, and Petalopbyllum Ralfsii. In I $885 \mathrm{Mr}$. E. Parfitt published a list of seventy-three species in the Transactions of the Devonsbire Association for the Advancement of Science, Literature, and Art, xvii, pp. 367-424. In this list two of the additional species are recorded by Mr. E. D. Marquand, F.L.S., viz., Nardia obovata and Lepidozia cupressina, and two others, viz., Aneura pinnatifida, and Riccia fluitans, by Mr. E. Parfitt. The nomenclature adopted in the following list of Devonshire Hepaticae is that of the Handbook of British Hepaticae, by Dr. C. Cooke, supplemented by McVicar's Key to the British Hepaticae (I 906). The rarer species are denoted by an asterisk.

\section{HEPATICAE}

JUNGERMANNIACEAE

Frullania dilatata, Dum. 6-8

* fragilifolia, Tayl. I, 8

- Tamarisci, Dum. I, 6, 8

* Jubula Hutchinsiae, Dum. 1, 8

* Lejeunea calcarea, Lib. I, 6

* - hamatifolia, Dum. I

- Mackaii, Dum. I, 6-8

- cavifolia, Lindb. I, 6,8

- ulicina, Spruce. I, 2, 8

Radula complanata, Dum. I-8

Porella laevigata, Carr. and Pears. I, 6, 8

* pinnata, Lindb. 6

- platyphylla, Carr. and Pears. I-8

* - Thuja, Carr. and Pears. 8

Ptilidium ciliare, Nees. I, 8

Trichocolea tomentella, Nees. I, 6-8

*Anthelia julacea, Dum. 6

* Pleurozia cochleariformis, Dum. 6,8

Bazzania trilobata, Carr. and Pears. I, 6-8

Kantia arguta, Carr. and Pears. 8

- Sprengelii, Carr. and Pears. I, 8

- Trichomanis, Carr. and Pears. I, 6-8

* Lepidozia cupressina, Sw. 8

- reptans, Dum. 1, 6, 8

Blepharostoma setacea, Mitt. 1, 7

- trichophylla, Dum. 8

Cephalozia bicuspidata, Dum. 6,8

- connivens, Spruce. 4, 7,8 var. minus, Mart. 7,8

- divaricata, Dum. 1, 7,8

- lunulaefolia, Spruce. 8

- sphagni, Spruce. 8

Adelanthus decipiens, Mitt. I

\section{JUngrRmanniacear (continued)}

Scapania compacta, Dum. I, 6, 8

- curta, Dum. 6, 7

- irrigua, Dum. 8

- nemorosa, Dum. 1, 6-8

- resupinata, Dum. 4, 7,8

* subalpina, Dum. 4

- uliginosa, Dum. 8

- umbrosa, Dum. 8

- undulata, Dum. 4-6,8

Diplophyllum albicans, Dum. I-8

- Dicksoni, Dum. 8

"- obtusifolium, Dum. 8

Lophocolea bidentata, Dum. I, 4, 8

- heterophylla, Dum. I, 6, 8

Chiloscyphus polyanthus, Dum. I, 6, 8

Plagiochila asplenioides, Dum. I-8

- spinulosa, Dum. I-8 f. Hagellifera, Carr. 8

Aplozia crenulata, Dum. I, 6-8

- gracillima, Dum. I, 4, 6-8

* - lanceolata, Dum. 4

* - Jurida, Dum. I, 8

- pumila, Dum. 4,6,8

* - riparia, Dum. I, 8

* - sphaerocarpa, Dum. 8

Jungermannia attenuata, Lindb. I

- barbata, Schrad. I, 6,8

- capitata, Hook. 8

- excisa, Dicks. var. Limprichtii, Lindb. 6

- exsecta, Schmidt. 6

- exsectaeformis, Steph. 8

- incisa, Schrad. 7, 8

- intermedia, Lindb. 8

*- Lyoni, Tayl. 8 


\section{A HISTORY OF DEVONSHIRE}

Jungrrmanniacras (continued)

Jungermannia turbinata, Raddi. I, 7

- ventricosa, Dicks. I, 4, 7,8

Mylia anomala, Carr. and Pears. I, 4

Gymnocolea affinis, Wils. 5, 6

- inflata, Dum. $5,6,8$

Saccogyna viticulosa, Dum. 1, 6-8

Nardia compressa, Carr. and Pears. I, 4

- emarginata, Carr. 1, 8

var. minor, Cooke. 8

var. aquatica, Ldg. 8

- hyalina, Spruce. 6,8

var. colorata, Nees. 8

obovata, Nees. 8

- Pearsoni, Schaeff. 8

- scalaris, Carr. I-8

Gymnomitrium crenulatum, Gottsche. 8

Fossombronia caespitiformis, De Not. 2

- Mittenii, Tindall. I

- pusilla, Dum. I, 4, 6, 7

Petalophyllum Ralfsii, Gottsche. I

Pellia calycina, Nees. 1, 8

- epiphylla, Corda. I, 8 var. endiviaefolia, Dicks. I

Pallavicinia Lyellii, Spruce.

* Blasia pusilla, Linn. 6, 8

Metzgeria conjugata, Lindb. 8
JungrRmanniaczar (continued)

Metzgeria furcata, Dum. I, 8

- pubescens, Raddi. 6

Aneura latifrons, Lindb. I, 7

- multifida, Dum. I, 6-8

- pimatifida, Nees. 4

-- pinguis, Dum. I, 4-8

\section{Marchantiacear}

Marchantia polymorpha, Lindb. I, 4, 8

Lunularia vulgaris, Mich. I-8

Reboulia hemisphaerica, Raddi. I, 6-8

Dumortiera irrigua, Nees. I, 6

Fegatella conica, Corda. I, 7, 8

Targionia hypophylla, Linn. I, 6, 7

\section{Ricciaceae}

Riccia ciliata, Hoffm.

*- crystallina, Linn. I

* - fluitans, Linn. 4

- glauca, Linn. 4, 6

*Anthoceros dichotomus, Raddi. 6

- laevis, Linn. 1, 8

- punctatus, Linn. I

\section{ALGAE (FRESH-WATER)}

This small group of Cryptogams has received very little attention from Devonshire botanists. Except a few species mentioned in Jones and Kingston's Flora Devoniensis, and about thirty-five species enumerated in Ravenshaw's Botany of Nortb Devon, there are only available the general records of Devonshire species in Smith's Synopsis of the British Diatomaceae, and in G. F. West's British Desmidiaceae. For these groups the nomenclature of these works has been followed, and that of Cooke's British Freshwater Algae (1882-4) for the remainder. Apparently the only botanist who has during recent years investigated the freshwater algae of the county is Mr. A. W. Bennett, F.L.S., who published a list of species found in Devonshire and Hampshire, in the Fournal of the Royal Microscopical Society in 1890, pp. I-I4, pl. I.

\section{CYANOPHYCEAE}

Nostochacrae

Nostoc commune, Vauch. 7

$\begin{array}{ll}\text { - pruniforme, C. Ag. } & 6 \\ \text { - verrucosum, Vauch. } & 8\end{array}$

Oscillatoriaceae

Lyngbya ochracea, Thur.

Oscillatoria limosa, C.Ag.

Scytongmacear

Scytonema Myochrous, C. Ag. I

Stigonema ocellatum, Thur. I

Rivulariacear

Rivularia calcarea, E.B. I

- dura, Kutz. 6

\section{CHLOROPHYCEAE}

Palmellaceae

Porphyridium cruentum, Näg.

Hydrurus penicellatus, C. Ag.

- Ducluzelii, Rabenh.

Tetraspora gelatinosa, Vauch.

Protococcaceae

Protococcus viridis, Ag. I, 7

\section{ZygnemaceaE}

Spirogyra porticalis, Vaulh. var. decimina, Cke. 6

- tenuissimum, Harv. f. inflata, C. Ag. 6

Zygogonium ericetorum, Kütz. var. terrestris, Cke. 


\section{BOTANY}

CHLOROPHYCEAE (continued)

VAUCHERIACEAE

Vaucheria dichotoma, Lyngb.

- Dillwynii, C. Ag.

- geminata, Walz.

- sessilis, Vauch.

var. ornithocephala, C. Ag.

Confervaceae

Cladophora crispata, Kutz.

- glomerata, Ktitz. I

\section{Oedogontaceas}

Oedogonium princeps, Harv. I

ULOTRICHACEAR

Hormiscia bicolor, Cke. I

\section{CHARACEAE}

Chara aspera, Willd.

- connivens, Braun.

- contraria, Kütz. I

- fragilis, Desv.

- obtusa, Desv.

- syncarpa, Chev.

- vulgare, L. 6

Nitella flexilis, C. Ag. 6

- opaca, C. Ag.

Tolypella glomerata, Leonh.

\section{DESMIDIACEAE}

Closterium abruptum, G. West

- acerosum, Ehr.

- aciculare, T. West

- angustum, Kutz

- Cornu, Ehr.

- costatum, Corda.

- Dianae, Ehr.

- didymotocum, Corda

- Ehrenbergii, Menegh.

- gracile, Breb.

- intermedium, Ralfs.

- Jenneri, Ralfs.

- Leiblinii, Kutz.

- lineatum, Ehr.

- Lunula, Nitzsch.

- moniliferum, Ehr.

- parvulum, Nag.

- rostratum, Ehr.

- setaceum, Ehr.

- striolatum, Ehr.

Cosmarium cucumis, Ralfs.

- granatum, Breb. var. subgranatum, Nordst.

- Hammeri, Reinsch.

- homalodermum, Nordst.

- pachydermum, Lund.

- Phaseolus, Bréb.

- pseudopyramidatum, Lund.

- pyramidatum, Bréb.

- Ralfsii, Bréb.

- tinctum, Ralfs.

Cylindrocystis diplospora, Lund.
DESMIDIACEAE (continued)

Euastrum affine, Ralfs.

- ansatum, Ralfs.

- bidentatum, Näg.

- binata, Ehr. f. hiascens, Gl. f. secta, Turn.

- crassum, Kutz.

- denticulatum, Gay

- Didelta, Ralfs.

- dubium, Näg.

- elegans, Kutz.

- erosum, Lund.

- gemmatum, Bréb.

- humerosum, Ralfs.

- inerme, Lund.

- insigne, Hass.

- insulare, Roy.

- pectinatum, Bréb.

- rostratum, Ralfs.

- sinuosum, Lenorm.

Gonatozygon Brebissoni, De Bary

- monotoenium, De Bary

Micrasterias denticulata, Bréb.

- oscitans, Ralfs. var. mucronata, Wille.

- papillifera, Bréb.

- rotata, Ralfs.

- truncata, Bréb.

Netrium Digitus, Itzig. and Rothe.

- oblongum, Latken

Penium curtum, Bréb.

- cylindrum, Bréb.

- didymocarpum, Lund.

- Libellula, Nordst.

- margaritaceum, Bréb.

- minutum, Cleve.

- navicula, Bréb.

- truncatum, Bréb.

Pleurotaenium Ehrenbergii, De Bary

- Trabecula, Nag.

Spirotaenia condensata, Bréb.

Staurastrum pileolatum, Bréb.

Tetmemorus Brebissoni, Ralfs.

- laevis, Ralfs.

\section{DIATOMACEAE}

Achnanthes brevipes, C. Ag.

- longipes, C. Ag.

Amphipleura implexa, Bréb.

Amphitetras antediluviana, Ehr.

Bacillaria paradoxa, Gmel.

Berkleya fragilis, Grev.

Biddulphia pulchella, Gray

Campylodiscus Ralfsii, W. Sm.

Diatoma vulgare, Berg.

Encyonema prostratum, Kütz.

Eupodiscus Ralfsii, W. Sm.

Exilaria fasciculata, Grev.

- fulgens, Grev.

Fragilaria capucina, Desn.

- hyemalis, Lyngb. I, 8

Gomphomena truncatum, Ehr. I

Grammatophora marina, Kütz. 1, 6

- serpentina, Kütz. I 


\section{A HISTORY OF DEVONSHIRE}

\section{DIATOMACEAE (continued)}

Homoeocladia martiana, C. Ag. 1, 6

Isthmia enervis, Ehr.

- nervosa, Kutz.

Licmophora flabellata, C. Ag. I

Mastogloia Danseii, Thw.

Melosira varians, C. Ag. I

Micromega comoides, C. Ag. I

- gracillimum, W. Sm. 6

- laciniatum, Harv. 6

- molle, W. Sm. 5

- obtusum, Grev. 6

- parasiticum, Harv. I

- ramosissimum, C. Ag. I, 6

- Smithii, C. Ag. I, 6

- torquatum, W. Sm. 6,7

Nitzschia Taenia, W. Sm. I

Odontidium hyemale, Kütz. I

Pleurosigma quadratum, W. Sm. I

- transversale, W. Sm.

Podosira Montagnei, Kütz. 6

Podosphaenia Jurgensii, Kütz. 6

\section{DIATOMACEAE (continued)}

Raphidospora paradoxa, Kutz.

- poliaeformis, Kutz. I

Rhabdonema adriaticum, Kutz. I

Schizonema Dillwynii, C. Ag. 1, 6

- dubium, Harv. 6

- fasciculatum, C. Ag. 6

- Grevillei, C. Ag. I, 6

- helminthosum, Chauv. 6

- implicatum, Harv. 7

- virescens, C. Ag. I

\section{R HODOPHYCEAE}

BATRACHOSPERMACEAE

Batrachospermum moniliforme, Roth. 7 var. confusum, Harv. 7

- vagum, C. Ag. 8

Lemaneaceae

Lemanea fluviatilis, C. Ag. $\quad \mathrm{r}, 4,6,7$

\section{ALGAE (MARINE)}

Few counties in Great Britain with a rocky coastline have been so thoroughly worked for marine algae as Devonshire. The earlier records are some of them very doubtful, as many of the species were not well understood at the end of the eighteenth century. Thus in Jones and Kingston's Flora Devoniensis the following species are recorded which are extremely unlikely to have occurred, and then can only have been in the form of drift weed, or detached from ships visiting the Devonshire ports from the north of England or the Mediterranean. Of these Odontbalia dentata, Lyngb., is recorded by Withering, and Fucus (Cystoseira) barbatus, Turn. by Hudson and Stackhouse. Specimens of the Gulf weed, Sargassum natans and $S$. bacciferam have undoubtedly been thrown up on the beach here and there after storms from the south-west, either brought by the Gulf Stream, or thrown overboard when nearing English shores, by sailors who had collected it as a curiosity. At the date of publication of the above-mentioned work in 1829 , 106 species of seaweed were recorded as natives of Devonshire, excluding the doubtful species.

Mrs. A. W. Griffiths, who died at Torquay in i 857 , was regarded by Professor Harvey as 'facile regina' of British algologists, and probably did more than any other algologist of her day in discovering new British species and recording accurate observations on the life history of these plants. She superintended the issue by Mary Wyatt of four fasciculi of Algae Devonienses, which are still extant in most of our national museums and in many private collections. Miss Cutler, of Sidmouth, who died in 1866 , contributed largely to the knowledge of the seaweeds of district 5 ; and Mrs. Gulson, of Exmouth, discovered the very rare Atractophora bypnoides near that town. Dr. J. Cocks, of Plymouth, who died at Devonport in I 86r, published at Plymouth a series of eighteen fasciculi of British seaweeds between I 855 and I 860 , illustrating the majority of the species found in that neighbourhood. He was the first to detect 


\section{BOTANY}

the rare Stenogramme interrupta in Britain. About the same date (186I) Mr. J. Boswarva, of Plymouth, published a Catalogue of the Marine Algae of Plymouth, including $22 \mathrm{I}$ species, or rather more than half of the then known British marine algal flora.

The Rev. W. S. Hore, of Plymouth, gathered the first plant of Bornetia secundiflora found in Great Britain near the Mewstone, in Plymouth Sound. His remarkably fine collection of twenty-one folio volumes, containing chiefly Devonshire seaweeds, was in 1906 presented to the North Devon Athenaeum at Barnstaple by his sister at the suggestion of Mr. W. P. Hiern, M.A., together with three folio volumes of mosses and lichens containing many Devonshire species. In i $888 \mathrm{Mr}$. E. Parfitt compiled a list of the marine algae of Devon (Trans. of the Devon Assoc. for the Advancement of Science and Literature, xxi, pp. 338-82), in which the number of Devonshire species was raised to 3 I8. Two of the species mentioned by him must, however, be omitted from the Devonshire flora, viz., Delesseria angustissima, Ag., which is a North British species not known to occur south of Yorkshire, and Ralfsia deusta, Berk., which is the $R$. verrucosa, Aresch., the true $R$. deusta, Ag., not occurring in Britain.

The list here given, in which the number of species occurring in Devon is raised to 468 , is probably the richest and most complete county list of marine algae, except possibly that of Dorsetshire. These additions are largely due to the investigations of Mr. E. M. Holmes, F.L.S., who for a long series of years has visited the shores of Devon. New species were also detected during the last forty years by the Rev. R. Cresswell, formerly of Teignmouth; Mr. J. Ralfs, chiefly at Ilfracombe; Mr. J. Brebner, F.L.S., at Plymouth; and Mr. E. A. L. Batters, F.L.S. The names of most of these algologists have been perpetuated in the British genera Battersia, Cutleria, Griffitbsia, Ralfsia, and the exotic genera (Gulsonia, Holmesia, Horea), and in the species Rbodocborton Brebneri, Scbizotbrix Cresswellii, and Nitophyllum Hilliae. The last mentioned was so named in honour of Miss Hill, of Plymouth, who died in 1830 , and who was described by Dawson Turner as a 'most indefatigable and faithful observer.'

The following species have as yet only been detected in Devon; four of them, indicated by a !, have been issued in Mr. Holmes's Fasciculi of Algae Britannica Rariores, of which eleven were issued between 1882 and 1905 ; these may be seen in most of our national and university herbaria.

Microcoleus tenerrimus, Gom.

Oscillatoria rosea, Batt.

Schizothrix Cresswellii, Harv. !

- lardacea, Gom. !

Rivularia australis, Born.

Halosphaeria viridis, Schm.

Codiolum gregarium, A. Br. !

Prasinocladius lubricus, Kuck.

Ulvella lens, Crn.

Endoderma leptochaete, $\mathrm{Hu}$ ber.

Ectocarpus Microspongium, Batt.

- Battersii, Born.
Ectocarpus ovatus, Kjellm. var. arachnoideus, Reinke. !

- Padinae, Sauv.

Sphacella subtillissima, Reinke.

Ralfsia disciformis, Crn.

Lithoderma simulans, Batt.

Acrochaetium entophyticum, Batt.

Gigartina Teedii, Lamour.

Polysiphonia pennata, Schm.

Rhodochorton Brebneri, Batt.

- pallens, Hauck.

Hymenoclonium serpens, Batt.

Grateloupia minima, Crn. 


\section{A HISTORY OF DEVONSHIRE}

\section{CYANOPHYCEAE}

Chroococcaceae

Anacystis parasitica, Kütz. 5

\section{Chamaesiphonaceae}

Dermocarpa Leibliniae, Born. 6

- violacea, Crn. 6,7

Hyella caespitosa, Born. and Flat. 6,7

- voluticola, Chod. 7

\section{LYNGBYACEAE}

Spirulina versicolor, Cohn. var. laxa, Gom. 7

\section{Oscillatoriaceae}

Oscillaria rosea, Batt. 7

Phormidium Ectocarpi, Gom. 7

- aestuarii, Lieb.

f. ferruginea, Gom. 7

Lyngbya Agardhii, Gom. 7

- majuscula, Harv. I, 5-7

Symploca hydnoides, Kuntz. var. genuina, Gom. 5,6

- atlantica, Gom. var. purpurea, Batt. 7

Plectonema Nostocorum, Born. 5, 6

- terebrans, Born. and Flah. 7

Microcoleus chthonoplastes, Thur. 5

- tenerrimus, Gom. 6

Hydrocoleum lyngbyaceum, Kutz. var. genuina, Gom. 5

Schizothrix Cresswellii, Harv. 5

- lardacea, Gom. 6

- vaginata, Gom. 5

\section{RivUlariacrae}

Calothrix aeruginea, Thur. 5, 6

- confervoides, Ag. 5-7

- parasitica, Thur. 7,8

- pulvinata, Ag. 5

- scopulorum, Ag. 5,7

Dichothrix gypsophila, Born. and Flah. 5

Brachytrichia Balani, Born. and Flah. 5

Isactis plana, Thur. 5

Rivularia atra, Roth. 5-7

- australis, Harv. 6

- Biassolettiana, Menegh. 1, 5,7

- bullata, Berk. 6

- mesenterica, Thur. 6

\section{CHLOROPHYCEAE}

Protococcacear

Chlorochytrium Cohnii, Wright. 6

\section{Palmellaceae}

Gloeocystis adnata, Näg. I, 5

Halosphoera viridis, Schm. 7

\section{Characiaceae}

Codiolum gregarium, A. Br. I, 5, 6 Prasinocladus lubricus, Kuch. 7

\section{CHLOROPHYCEAE (continued)}

Blastosporacear

Prasiola stipitata, Suhr. 6

ULVACEAE

Uvella Lens, Crn. 7

Monostroma laceratum, Thur. 7

- latissimum, With. 5

- Wittrockii, Born. 7

Enteromorpha clathrata, J. Ag. var. Linkiana, Batt. 5,6 var. procera, Hauck. 6 var. prostata, Le Jol. 6

- compressa, J. Ag. var. complanata, J.Ag. 5,6 var. nana. J. Ag. 5-7

- crinita, J. Ag. 6

- erecta, Hook. 6

- Hopkirkii, McCalla. 5

- intestinalis, Link. var. bullosa, Le Jol. I var. Cornucopia, Kutz. 7 var. flagelliformis, Le Jol. 7 var. ventricosa, Le Jol. 7

- lingulata, J. Ag. 6

- Linza, J. Ag. 4

- minima, Näg. 5

- paradoxa, Kuitz. 6

- prolifera, J. Ag. 6 var. tubulosa, Reinb. 6

- Ralfsii, Harv. 5

- ramulosa, Hook. 7

- torta, Reinb. 6

- usneoides, J. Ag. 6

Ulva Lactuca, Linn. var. latissima, DC. 5-7 var. myriotrema, Borr. 6 var. rigida, Le Jol. 6 [A]

\section{ULOTRICHACEAR}

Ulothrix flacca, Thur. 5-7

- implexa, Kutz. 5

\section{Chaetophoraceas}

Acrochaete parasitica, Oltm. 5 Endoderma viride, Lagerh. 6 - leptochaete, Huber. 6

Tellamia contorta, Batt. 5,7

\section{Cladophoraceas}

Urospora bangioides, Holm and Batt. 6,7 - isogona, Batt. 6

Chaetomorpha aerea, Kutz. I, 5, 6

- litorea, Cook. 7

- Melagonium, Kütz. 5,6

- tortuosa, Kutz. I, 5, 7

Rhizoclonium arenosum, Kutz. 6

- implexum, Batt. 1, 5, 6

- Kochianum, Kätz. 7

- riparium, Harv. 5, 6

Cladophora albida, Kittz. 6, 7 var. curvula, Kütz. I, 6

- falcata, Harv. 7

- flexuosa, Harv. 5-7 


\section{BOTANY}

\section{CHLOROPHYCEAE (continued)}

Cladophoraceae (continued)

Cladophora fracta, Kutz. 7 var. flavescens, Harv. 7

- glaucescens, Harv.

- gracilis, Kutz. 6,7

- hirta, Kutz. ;

- Hutchinsiae, Harv. 5-7

- Magdalenae, Harv.

- Neesiorum, Kutz. var. humilis, Batt.

- pellucida, Kutz. 6, 7

- rectangularis, Harv. 6

- refracta, Aresch. I, 6

- rupestris, Kutz. $\quad 5,6$

- sericea, Kutz. 5-7

- utriculosa, Kutz. 5-7

Acrosiphonia arcta, Kutz. 5-7 var. radicans, Batt. 6
var. centralis, Harv, 6

- uncialis, Kütz. I, 6, 7

- bombycina, Batt. 6

- lanosa, Kutz. I, 5, 6, 7

Gomontiacear

Gomontia polyrhiza, Born. and Flah. 5-7

Phyllosiphoniacear

Ostreobium Queketti, Born. and Flah. 5,7

Bryopsidaceae

Bryopsis hypnoides, Lam. I, 6

- plumosa, C. Ag. 6,7

Derbesia tenuissima, Crn. $5-7$

VAUCheriaceas

Vaucheria sphaerospora, Nordst. f. dioica, Rosenv. 5

SPongodiaceae

Codium adhaerens, Ag. 6,7

- Bursa, C. Ag. 6,7

- tomentosum, Stackh. I, 5, 6

\section{PHAEOPHYCEAE}

\section{Desmarestiacrae}

Desmarestia aculeata, Lamour. I, 5-7

- Dresnayi, Lamour. 7

- ligulata, Lamour. 5-7

- viridis, Lamour. $\quad 5-7$

Dictyosiphonacear

Dictyosiphon foeniculaceus, Grev. 5-7 Punctariaceae

Phaeostroma pustulosum, Ruck. 5

Litosiphon pusillus, Harv. 5

Phloeospora brachiata, Born. I, 6, 7

Stictyosiphon tortilis, Reinke. 7

Striaria attenuata, Grev. 1, 5-7

Punctaria latifolia, Grev. 5-7 var. laminarioides, Holm. and Batt. 6,7 var. lanceolata, Batt. 6

- plantaginea, Grev. I, 5-7

- tenuissima, Grev. 7
PHAEOPHYCEAE (continued)

SCYTOSIPHONACEAB

Phyllitis Fascia, Kutz. $\quad 5-7$

var. debilis, Hauck. 7

var. tenuissima, Batt. 7

Scytosiphon lomentarius, J. Ag. 5-7

Asperococcacrap

Asperococcus bullosus, Lamour. 1, 5-7

- compressus, Griff. I, 5-7

- fistulosus, Hook. 5-7

var. vermicularis, Batt. 5-7

Ectocarpacear

Streblonema aequale, Oltm. 9

- tenuissimum, Hauck. 6

- velutinum, Kutz. 5, 7

- volubile, Thur. 5-7

- Zanardinii, Crn. 6

Ectocarpus Battersii, Born. 5

- clandestina, Sauv. 5

- confervoides, Le Jol. $5-7$ var. arcta, Kjellm. 6 var. pencilliformis, Kuck. 5, 6

- Crouani, Thur. 5-7

- fasciculatus, Harv. 5-7 var. draparnoldioides, Crn. 5

- fenestratus, Berk. 6, 7

- globifer, Kitz. 5 var. rupestris, Batt. S

- granulosus, Ag. I, 5-7 var. refracta, Batt. 6

- Hincksiae, Harv. I, 6, 7

- Holmesii, Batt. I, 7,6

- Lebelii, Crn. 5

- Microspongium, Batt. 7

- Mitchellae, Harv. 6

- ovatus, Kjellm. var. arachnoides, Reinke. I

- Padinae, Sauv. 5

- penicellatus, J. Ag. 6,7

- repens, Reinke. 5

- Sandrianus, Zan. I

- secundus, Kutz. I, 6, 7

- siliculosus, Kutz. 5,6 var. hiemalis, Kuck. 6 var. spalatina, Kjellm. 5 var. subulata, Hauck. 7

- simplex, Crn. 5,6

- solitarius, Sauv. 5

- terminalis, Kutz. 5

- tomentosus, Lyngb. I, 6, 7

Pylaiella litoralis, Kjellm. 5-7

f. firma, Kjellm. 5-7

E. longifructus, Batt. 7

f. parvula, Kjellm. 5

f. subglobosa, Kuck. 6

Isthmoplea sphaerophora, Kjellm. I, 5-7

Myriotrichia clavaeformis, Harv. 5-7

- densa, Batt. 7

- filiformis, Harv. 6, 7 .

- repens, Batt. 7

\section{Arthrocladiacear}

Arthrocladia villosa, Duby. $\quad 5-7$ 


\section{A HISTORY OF DEVONSHIRE}

\section{PHAEOPHYCEAE (continued)}

Elachistaceae

Myriactis pulvinata, Kutz. 5-7

- stellulata, Batt. 6

Elachistea flaccida, Aresch. 5-7

- fuciola, Fries. 5-7

- scutulata, Duby. 6, 7

- stellaris, Aresch. 5,6

\section{Sphacelariaceat}

Sphacella subtilissima, Reinke. 7

Sphacelaria cirrhosa, J. Ag. var. aegagrophila, Griff. 5, 6 var. fusca, Holm. and Batt. I, 5, 6 var. nana, Griff. 6 var. pennata, Hauck. 5-7

- olivacea, Pringsh. 5, 7

- plumigera, Holmes. I

- radicans, Harv. 1, 6

Cladostephus spongiosus, J. Ag. 5-7

- verticillatus, J. Ag. 5-7

Halopteris filicina, Kütz. I, 5-7 var. sertularia, Batt. 6

Stypocaulon scoparium, Kutz. $\quad 5-7$

\section{Mrrionemaceas}

Myrionema aecidioides, Sauv. 5

- strangulans, Grev. 5-7 var. punctiformis, Holm. and Batt. 6,7

Ulonema rhizophorum, Fosl. 5

Hecatonema maculans, Sauv. 5

Chilonema Nathaliae, Sauv. 5-7

- ocellatum, Sauv. 5

Ascocyclus Hispanicus, Sauv. 5

- orbicularis, Magn. 6,7

- sphaerophorus, Sauv. 5

Ralfsia clavata, Farlow. 6

- disciformis, Crn. 7

- spongiocarpa, Batt. 7

- verrucosa, Aresch. 5-7

Lithoderma fatiscens, Aresch. 7

- simulans, Batt. 7

\section{Chordariacrae}

Spermatochnus paradoxus, Kiatz.

Stilophora rhizoides, J. Ag. 5-7

Chordaria flagelliformis, C. Ag. 5-7

Mesogloia Griffithsiana, Grev. 5-7

- vermiculata, Le Jol. 5-7

Castagnea contorta, Thur.

- virescens, Thur. 5-7

- Zosterae, Thur. 6

Petrospongium Berkeleyi, Näg. 5-7

Leathesia difformis, Aresch. 5-7

\section{Sporochinaceae}

Sporochnus pedunculatus, C. Ag. 5-7

Carpomitra Cabrerae, Kutz. 5-7

\section{Chordacear}

Chorda Filum, Stackh. 5-7 var. subtomentosa, Aresch. 5

- tomentosa, Lyngb. I, 6

\section{PHAEOPHYCEAE (continued)}

LAMINARIACEAE

Laminaria Cloustoni, Edm. 5-7

- digitata, Lamour. 5-7

- saccharina, Lamour. 5-7

Saccorhiza bulbosa, De la Pyi. 5-7

Alaria esculenta, Grev. I

\section{Cutleriacear}

Cutleria multifida, Grev. 5-7

Fucacear

Fucus Areschougii, Kjellm. 5, 6

- ceranoides, L. 6, 7

- platycarpus, Thur. 6,7

- serratus, L. 6, 7

f. angustifrons, Stackh. 5

f. integer, Turn. 5

Ascophyllum nodosum, Le Jol. 1, 5-7

Pelvetia canaliculata, Dene. \& Thur. 1, 5-7

Pycnophycus tuberculatus, Kütz. I, 6, 7

Himanthalia lorea, Lyngb. I, 6, 7

Halidrys siliquosa, Lyngb. I, 5-7 var. minor, Turn. 5,6

Cystoseira discors, C. Ag. I, 5-7

- ericoides, C. Ag. $\quad$ I, 5-7

- fibrosa, C. Ag. I, 5-7

\section{Tilopteridacear}

Tilopteris Mertensii, Kútz. I, 5-7

Akinetospora pusilla, Born. 1, 5-7 var. crinita, Batt. I, 6,7

\section{Dictyotaceae}

Dictyota dichotoma, Lamour. I, 5-7 var. implexa, J. Ag. 5-7

- ligulata, Kütz. 5, 6

Taonia atomaria, J. Ag. 1, 5-7 var. divaricata, Holm and Batt. 6

Padina Pavonia, Gaill. 5-7

Dictyopteris polypodioides, Lamx. I, 5-7

\section{RHODOPHYCEAE}

Porphyraceat

Conchocelis rosea, Batt. 5-7

Goniotrichum elegans, Le Jol. 5, 7

- cervicornis, Reinsch. 7

Erythrotrichia Boryana, Berth. 7

- carnea, J. Ag. 6

Bangia fuscopurpurea, Lyngb. I, 6, 7 var. Lejolisii, Holm. and Batt. 6

Porphyra coccinea, J. Ag. 7

- laciniata, C. Ag. I, 5-7 var. umbilicalis, J. Ag. 5,6

Helminthocladiaceas

Acrochaetium Chylocladiae, Batt. 6,7

- Daviesii, Näg. 1, 5-7

- entophyticum, Batt. 7

- microscopicum, Näg. 6

- secundatum, Näg. 5-7

- virgatulum, J. Ag. 5-7

Nemalion lubricum, Duby. 5,6

- multifidum, J. Ag. 6, 7

Helminthocladia purpurea, J. Ag. $\quad 5,6$

Helminthora divaricata, J. Ag. $5-7$ 


\section{BOTANY}

\section{RHODOPHYCEAE (continued)}

\section{Chaetangiacear}

Scinaia furcellata, Biv. 5-7

Choreocolax Polysiphoniae, Reinsch. 7 - tumidus, Reinsch.

\section{GrLidiaceas}

Harveyella mirabilis, Schmitz. and Reinke. 7 - pachyderma, Batt. 1, 5, 6 Atractophora hypnoides, Crn. 6 Naccaria Wiggii, Endl. 5-7 Pterocladia capillacea, Born. I, 5, 6

Gelidium aculeatum, Batt. I, 6 var. abnorme, Batt. I, 6

- attenuatum, Thur. 5,6 var. confertum, Batt. 5,6

- corneum, Lamour. 7

- crinale, J. Ag. I, 5,6 var. spathulatum, Hauck. 5

- latifolium, Born. 5,6 var. flexuosum, Batt. 6 var. laciniatum, Batt. 6

- pulchellum, Kutz. 6

- pusillum, Le Jol. 5-7

- sesquipedale, Thur. 5, 6

\section{Gigartinaceae}

Chondrus crispus, Stackh. I, 5-7 var. aequalis, Batt. 5,6 var. filiformis, Turn. 5,6 var. lacera, Turn. 5,6 var. patens, Turn. 5,6 var. plana, Turn. I, 5, 6 var. stellata, Batt. I, 5-7 var. virens, Turn. I, 5-7

Gigartina acicularis, Lam. I, 5-7. [Torquay, in fruit]

- mammillosa, J. Ag. I, 5-7

f. acuta, Good. and Woodw. 5 f. incurvata, Turn. ;

- pistillata, Stackh. 7

- Teedii,"Lamour. 6

Phyllophora Brodiaei, J. Ag. 6, 7

- membranifolia, J. Ag. 5-7

- palmettoides, J. Ag. 5-7

- rubens, J. Ag. 5-7

- Traillii, Holmes and Batt. 6, 7

Stenogramme interrupta, Mont. 1, 6

Gymnogongrus Griffithsiae, Mart. I, 5-7

- Norvegicus, J. Ag. I, 5-7

Ahnfeltia plicata, Fr. I, 5-7

Actinococcus aggregatus, Schm. 5, 6

- subcutaneus, Rosen.

- peltaeformis, Schm. 5, 6

Colacolepis incrustans, Schm. I, 5-7

Sterrocolax decipiens, Schm. I, 5-7

Callophyllis flabellata, Crn. I $1-7$

- laciniata, Kutz. 1, 5-7

Calloçolax neglectus, Schm. 1, 6, 7

Kallymenia Larteri, n. sp. I

— microphylla, J. Ag. I

\section{RHODOPHYLIIDACEAE}

Cystoclonium purpureum, Batt. I, 5-7 f. cirrhosa, J. Ag. I

Catenella repens, Batt.

\section{RHODOPHYCEAE (continued)}

RhoDophrulidacear (continued)

Rhodophyllis appendiculata, J. Ag. I, 6, 7

- bifida, Kutz. I, 5-7

\section{SPHAEROCOCCACEAE}

Sphaerococcus coronopifolius, Grev, 5-7

Gracilaria compressa, Grev. 4-7

- confervoides, Grev. I, 5-7 var. gracilis, Turn. 1, 6 var. procerrima, Turn. 5

- multipartita, J. Ag. 7

Calliblepharis ciliata, Kütz. I, 5-7

- jubata, J. Ag. I, 5-7
f. divaricata, Holm. and Batt. 6,7

f. dilatata, Holm. and Batt. 7

RHODYMENIACEAR

Rhodymenia nicaeensis, Holm. 5, 6

- palmata, Grev. I, 5-7

f. laciniata, Holm. and Batt. 6

f. marginifera, Harv. I, 5-7

f. sarniensis, Grev. 6

f. simplex, J. Ag. 6

f. sobolifera, J. Ag. 6,7

- palmetta, Grev. I, 5-7

f. flabelliformis, Kütz. 5,6

Cordylecladia erecta, J. Ag. 5-7

Lomentaria articulata, Lyngb. 5-7

- clavellosa, Gail. 5-7

- rosea, Thur. 5,7

Champia parvula, Harv. 5-7

Chylocladia kaliformis, Hook. I, 5-7

var. patens, Harv. 6,7

var. squarrosa, Harv. 6,7

- ovalis, Hook. I, 5-7 var. subarticulata, Kütz. 5, 6

- reflexa, Lamour. I, 5-7

Plocamium coccineum, Lyngb. I, 5-7 var. uncinatum, C. Ag. $\quad 5-7$

\section{Delesseriaceae}

Delesseria alata, Lamour. 1, 5-7

- Hypoglossum, Lamour. 1, 5-7 var. arborescens, J. Ag. 6 var. crispa, Crn. 6

- ruscifolia, Lamour. 1, 5-7

- sanguinea, Lamour. 1, 5-7

- sinuosa, Lam. I, 5-7

Gonimophyllum Bufthami, Batt. 5, 6

Nitophyllum Bonnemaisonii, Grev. I, 5-7 var. crassinervia, Batt. I

- Gmelini, Grev. 1, 5-7

- Hilliae, Grev. 1, 5-7

- laceratum, J. Ag. 1, 5-7 f. ciliifera, Kütz, 6 f. uncinata, Grev. I, 5

- litteratum, J. Ag. 6

- punctatum, Grev. var. crispata, Harv. 7 var. ocellata, J. Ag. I, 5-7

- reptans, Crn. I, 5

- Sandrianum, Zan. 6

- uncinatum, J. Ag. 6

- venulosum, Zan. 7

- versicolor, Harv. 1,7 


\section{A HISTORY OF DEVONSHIRE}

\section{RHODOPHYCEAE (continued)}

Bonnemaisoniaceae

Bonnemaisonia asparagoides, C. Ag. 6,7 - hamifera, Har. 6

\section{RHODOMELACEAE}

Bostrychia scorpioides, Mont. 1, 6, 7

Rhodomela subfusca, C. Ag. 1, 5-7 var. firmior, J. Ag. 6

Laurencia coespitosa, Lamour. I, 5-7

- obtusa, Lamour. 5-7

- pinnatifida, Lamour. 5-7

Halopithys pinastroides, Gmel. 5-7

Chondria dasyphylla, C. Ag. 5-7

- tenuissima, J. Ag. 6,7

Polysiphonia atrorubescens, Grev. I, 6, 7

- Brodiaei, Grev. 5-7 var. densa, Holm. and Batt. 5-7

- elongata, Grev. I, 5-7 var. rosea, J. Ag. I, 5-7

- elongella, Harv. 5-7

- fastigiata, Grev. I, 5-7

- fibrata, Harv. 1, 5-7

- fibrillosa, Grev. 5-7

- foetidissima, Cocks. 7

- fruticulosa, Spreng. 5-7

- furcellata. Harv. 5-7

- nigrescens, Grev. 1, 5-7

- obscura, Grev., J. Ag. 5

- Rhunensis, Thur. I

- simulans, Harv. 6,7

- subulata, J. Ag. var. Griffithsiana, J. Ag. 6

- subulifera, Harv. 6

- urceolata, Grev. I, 5-7 var. patens, J. Ag. I, 5-7 var. formosa, J. Ag. 6,7 var. sticta, Grev. 5-7

- variegata, Zan. 7

- violacea, Grev. 1, 5-7 var. allochroa, J. Ag. 6

Pterosiphonia complanata, Schm. 7

- parasitica, Schm. 6,7

- pennata, J. Ag. 5

- thuyoides, Schm. 1, 5-7

Brongniartella byssoides, Bury.

Dasya arbuscula, C. Ag. 6,7

- ocellata, Harv. 1, 5-7

Heterosiphonia coccinea, Falk. 1, 5-7 f. tenuis, Aresch. 6

\section{Ceramiaceae}

Spondylothamnion multifidum, Näg. I, 5-7 Spermothamnion barbatum, Nag. I, 7

- roseolum, Pringsh. 5

- Turneri, Aresch. 1, 5-7

Trailliella intricata, Batt. $5-7$

Ptilothamnion pluma, Thur. 1, 5, 7

Griffithsia corallina, C. Ag. I, 5-7

- Devoniensis, Harv. 6,7

- setacea, C. Ag. I, 5-7

Halurus equisetifolius, Kütz. I, 5-7

Bornetia secundiflora, Thur. 6,7

Monospora clavata, J. Ag. 5-7

Monospora pedicellata, Sol. $5-7$

\section{RHODOPHYCEAE (continued)}

Crramiacear (continued)

Pleonosporium Borreri, Näg. I , 5-7 var. fasciculatum, Holm. and Batt. 6

Rhodochorton Brebneri, Batt. 7

- floridulum, Näg. 5-7

- membranaceum, Magn. 5,7

- pallens, Hauck. 5

- Rothii, Näg. I, 5-7

Callithamnion Brodiaei, Harv. 6,7

- corymbosum, Lyngb. 1, 5-7

- Dudresnayi, Crn. 7

- granulatum, C. Ag. I, 6, 7

- Hookeri, C. Ag. I, 5-7

- polyspermum, C. Ag. 1, 5-7

- roseum, Harv. 6,7

- tenuissimum, Kutz. I, 7

- tetragonum, C. Ag. 1, 5-7 var. brachiatum, J. Ag. I, 5, 7

- tetricum, C. Ag. I, 5,7

Callithamnion bipinnatum, C. Ag. 6,7

Seirospora byssoides, Crn. 6, 7

- Griffithsiana, Harv. 7

Compsothamnion gracillimum, Schm. I, 5-7

- thuyoides, Schm. 1, 5-7

Plumaria elegans, Schm. I, 5-7

Antithamnion cruciatum, Näg. 6,7

- Plumula, Thur. I, 5-7

Hymenoclonjum serpens, Batt. 7

Crouania attenuata, J. Ag. 7

Spyridia filamentosa, Harv. 5-7

Ceramium acanthonotum, Carm. I, 5-7

- arborescens, J. Ag. 6

- botryocarpum, Griff. 6,7

- ciliatum, Ducluz. 1, 5-7

- circinnatum, J. Ag. 6,7

- Crouanianum, J. Ag. 6

- Deslongchampsii, Chauv. 1, 6,7

- diaphanum, Roth. I, 5-7

- echionotum, J. Ag. 6,7

- fastigiatum, Harv. 5-7

- flabelligerum, J. Ag. 6, 7

- fruticulosum, Kütz. 6

- gracillimum, Harv. 6,7

- pennatum, Crn. 7

- rubrum, C. Ag. I, 5-7

- strictum, Harv. 6,7 var. delicatum, J. Ag. 7

- tenuissimum, J. Ag. $\quad 5-7$

- vimineum, J. Ag.

Microcladia glandulosa, Grev. 5-7

\section{Cryptonemiaceae}

Gloiosiphonia capillaris, Carm.

\section{Grateloupiaceae}

Grateloupia dichotoma, J. Ag.

- minima, Crn. 1, 6

- filicina, C. Ag. 5-7 var. intermedia, Holm. and Batt. 1, 5, 6

Dumontiaceae

Dilsea edulis, Stackh. I, 5-7

Dumontia filiformis, Grev. 1, 5-7

Dudresnaya coccinea, Crn. $\quad 5-7$ 


\section{BOTANY}

RHODOPHYCEAE (continued)

Nemastomaceae

Schizymenia Dubyi, J. Ag. 7

Halarachnion ligulatum, Kutz. I, 5-7

Furcellaria fastigiata, Lamour. I, 5-7

\section{RHIZOPHYLLACEAI}

Polynoides rotundus, Grev. I, 5-7

\section{Squamariaceae}

Erythrodermis Alleni, Batt. 7

Rhododermis elegans, Crn. 7

- Georgii, Holmes. 6

- parasitica, Batt. 7

Rhododiscus pulcherrimus, Crn. 7

Petrocelis cruenta, J. Ag. I, 6

Cruoria pellita, Lyngb. 7

- rosea, Crn. 7

Cruoriopsis gracilis, Batt. 7

- Hauckii, Batt. 7

Cruoriella Dubyi, Schm. 5, 6

Peyssonelia Harveyana, Crn. 7

- Rosenvingii, Schm. 5, 7

- rubra, J. Ag. 7

- rupestris, Crn. 7

\section{RHODOPHYCEAE (continued)}

Hildganbandtiacear

Hildenbrandtia Crouani, J. Ag. 6

- prototypus, Nardo. I, 5-7

\section{Corallinacraz}

Schmitziella endophloea, Born. and Batt. 6

Choreonema Thureti, Schm. 5

Melobesia callithamnioides, Falk. 6,7

- Corallinae, Solms. 5-7

- farinosa, Lam. 1, 5-7

- Lejolisii, Rosenv. 7

- zonalis, Fosl. 7

Dermatolithon hapalidioides, Fosl. 5, 6

- Laminariae, Fosl. 6

- macrocarpum, Fosl. 5-7

- pustulatum, Fosl. 7

Lithophyllum corticiforme, Fosl. 1, 5-7

- incrustans, Fosl. 5-7

- Lenormandi, Fosl. 1, 5-7

- lichenoides, Fosl. 5-7

- membranaceum, Fosl. 1, 5-7

Phymatolithon polymorphum, Fosl. I, 5-7

Corallina officinalis, L. I, 5-7

- rubens, Ellis and Soland. I, 5-7 var. corniculata, Hauck. $\quad 5-7$

-- squamata, Ellis. 5-7

\section{LICHENES (Lichens)}

The county of Devon, like that of Cornwall, is especially suited to the growth of lichens, which require a moist atmosphere and pure air for their development. It has the advantage over Cornwall of a greater variety of geological strata, so that the species occurring on limestone are more numerous, and those occurring on the higher elevation of Dartmoor are more subalpine in character. In Jones and Kingston's Flora Devoniensis I 42 species are enumerated. In the Lichens of Devon and Cornwall by Mr. E. M. Holmes, published at Plymouth in I 869, the number was increased to 346 .

A few rare species have been detected by lichenologists visiting the county, of whom may be mentioned the Rev. J. M. Crombie, and the late Dr. H. B. Holl, Professor Dickie of Aberdeen, and Admiral Jones, but the majority of species added to the Flora since the publication of the Flora Devoniensis in 1829 have been detected by Mr. E. M. Holmes, Mr. E. Parfitt, Mr. E. D. Marquand, and Mr. W. H. Wilkinson.

Amongst the rarer species found on Dartmoor and its ravines may be mentioned Stictina Thouarsii, and S. crocata; Umbilicaria pustulata, the fruit of which is of very rare occurrence, but has been met with on Cosdon Beacon and Kis Tor; and Leptogium Burgessiz. The warmer portions of the coast line, including Torquay, Bolt Head, and Lynmouth, are remarkable for a large number of rare species that are almost unknown north of the Channel Islands and Cornwall. Of these may be mentioned Stigmatidium venosum, Parmelia olivetorum, Roccella phycopsis, and $R$. fuciformis, the last two apparently finding their northern limit at Lynton. At Torquay, Dirina repanda, Sticta aurata, Pbyscia cbrysopbtbalma, Placodium 


\section{A HISTORY OF DEVONSHIRE}

cirrocbroum, and $P$. xantbolytum are amongst the rarer southern species. At Bolt Head, Pbyscia leucomela and P. speciosa occur. The Grapbidiei are well represented in the county by rare species. Opegrapba involuta, not recorded for any other English county, occurs, as well as Graphis Ruiziana and O. Lentiginosa, at Ivybridge, and at Lynton O. grumulosa and Cbiodecton sarniense are met with. Lithographa dendrographa has been found in several localities, being commoner in the county than has been hitherto supposed ; it is usually found on elm trees in the same situations where the rare Lecidea carneo-lutea also occurs. Synalissa symphorea, which was first recorded from St. Vincent's rocks in Gloucestershire, has not recently been found there, and Torquay, and Portland in Dorset are now the only two British localities known for it. The Collemei are well represented in the limestone districts of Plymouth, Torquay, and Newton Abbot, but the Caliciei and the genus Pblyctis, which are more abundant on trees in drier counties, are of comparatively rare occurrence in Devon. On the other hand the species of the genus Pertusaria are more frequently found in fruit in the Dartmoor ravines than in any other county, including such species as $P$. globulifera, $P$. velata, $P$. dealbata, and $P$. amara, the fruit of the last, which occurred abundantly one year at Lydford, not having been detected elsewhere in Great Britain. The nomenclature followed in this list is that of Crombie, British Lichens (I 894) as far as Urceolaria, and Leighton's Lichen Flora of Great Britain (1879) for the remainder of the list. The rarer species are indicated by an asterisk.

\section{LICHENES}

\section{EPHEBACEI}

Ephebe pubescens, Nyl. 4, 6, 8

*Gonionema velutinum, Nyl. 6

\section{COLLEMACEI}

- Collema auriculatum, Hoffm. 7 subsp. granosum, Nyl. 6 [Chudleigh]

- cheileum, Ach. 4, 7 f. nudum, Nyl. 6,7

- concinnum, Flot. 7

- crispum, Ach. subsp. ceranoides, Nyl. 1, 4, 6

- cristatum, Hoffm. 6

- flaccidum, Ach. I-7

* - furvum, Ach. 7 var. tunaeforme, Nyl. 7

- glaucescens, Hoffm. 6

- granuliferum, Nyl. 6,7

- melaenum, Ach. f. marginale, Ach. 6

- microphyllum, Nyl. 4

- nigrescens, Ach. 5-7

- plicatile, Nyl. 6,7

- pulposum, Ach. 6,7 f. compactum, Nyl. 6

- tenax, Ach. 7 var. coronatum, Koerb. 7
COLLEMACEI (continued)

*Collemodium fluviatile, Nyl. I

- - fragile, Nyl. 6

- Schraderi, Nyl. 6,7

- turgidum, Nyl. 7

Dendriscocaulon bolacinum, Nyl. 6,8

*Leptogium Burgessii, Mont. 7,8

- lacerum, Gray. 6,7

f. fimbriatum, Nyl. 6,8 subsp. pulvinatum, $\mathrm{Nyl}$. $4,6,7$

- muscicolum, Fr. 6

* palmatum, Mont. 8

*- tenuissimum, Koerb. 6

*- tremelloides, Grey. 1, 6

- polyphyllum, Nyl. 6

Lichina confinis, C. Ag. 6, 7

- pygmaea, C. Ag. 6,7
-Synalissa symphorea, Nyl. 6

\section{LICHENACEI}

Caliciei

*Calicium curtum, Turn. and Borr. 6

* - hyperellum, Ach. 2

f. chlorodes, Nyl. 6

f. lenticulare, Nyl. 6

Sphinctrina turbinata, Leight. 4 
LICHENACEI (continued)

SPHAEROPHOREI

"Sphaerophoron compressus, Ach. 8

- coralloides, Pers. 6,8

- fragilis, Ach. 6,8

\section{BazomYCEI}

Baeomyces rufus, DC. 7 var. subsquamulosus, Nyl. 8

- roseus, Pers. I

\section{Strereocaulei}

Leprocaulon nanum, Nyl. I, 6, 7

"Stereocaulon coralloides, Fr. 8

- denudatum, Floerke. 8

- evolutum, Graewe. 6,8

* - tomentosum, Fr. 8

\section{Cladonier}

Cladina rangiferina, Nyl. I, 8

- sylvatica, Nyl. 8

- uncialis, Nyl. 6

f. adunca, Cromb. 8

f. bolacina, Cromb. 6

f. turgescens, Cromb. 8

Cladonia alcicornis, Floerke. $\quad 6,8$

- bacillaris, Floerke. var. subcoronata, Nyl 8

- bellidiflora, Floerke. 8

- caespititia, Floerke. 6

- cariosa, Flk. 6

* - cervicornis, Schoer. 6-8

- coccifera, Schoer. 8 subsp. pleurota, Cromb. 8

- cornuta, Fr. 8

- deformis, Hoffm. 8

- degenerans, Floerke. f. anomaea, Floerke. 8

- delicata, Floerke. I

- endiviaefolia, Fr. 5

- fimbriata, Fr. f. tubaeformis, Fr. 6 subsp. fibula, Nyl. 8

- firma, Nyl. 6

- Floerkeana, Fr. 8 f. trachypoda, Nyl. 8

- furcata, Hoffm. 6 subsp. racemosa, $\mathrm{Nyl}$. f. recurva, Floerke. 6,7

- gracilis, Hoffm. 8 f. spinulifera, Cromb. 6

- Lamarckii, Nyl. 6

- macilenta, Hoffm. 1, 6 var. coronata, Nyl. 6,7 f. carcata, Nyl. 8

- ochrochlora, Floerke. 6 f. ceratodes, Floerke. 6

- pityrea, Floerke. 8

- pungens, Floerke. 6 f. foliosa, Flk. 5

- pyxidata, Fr. 1, 6,7 f. chlorophaea, Floerke. 6

- scabriuscula, Nyl. 6

- sobolifera, Nyl. 6

\section{LICHENACEI (continued)}

Cladonier (continued)

Cladonia squamosa, Hoffm. 7 subsp. adspersa, Nyl. I

- subsquamosa, Nyl. 7,8

*- verticillata, Floerke. I

\section{ROCCELIRI}

"Rocella fuciformis, DC. I, 7

*- phycopsis, Ach. I, 7

\section{RamalineI}

Ramalina calicaris, Nyl. 6,7

- cuspidata, Nyl. 1, 6-8 f. minor, Nyl. 7 subsp. breviuscula, Nyl. 6

* - evernioides. Nyl. 6

- farinacea, Ach. 6, 7

- fastigiata, Ach. 1-8

- fraxinea, Ach. I-8 var. ampliata, Ach. 1, 8

- pollinaria, Ach. 7

- scopulorum, Ach. 7

- subfarinacea, Nyl. 7,8

\section{USNE1}

-Usnea articulata, Hoffm. 4, 6 f. intestiniformis, Cromb. 6

- ceratina, Ach. 6 var. scabrosa, Ach. 8 f. ferruginascens, Cromb. 6

- dasypoga, Nyl. 6,8

- florida, Ach. 6-8

- hirta, Hoffm. 7

\section{Alectoriei}

*Alectoria bicolor, Nyl. 6, 8

* - jubata, Nyl. 6 subsp. chalybeiformis, Nyl. 8

\section{Cetrarizi}

Cetraria aculeata, Fr. 8 f. hispida, Cromb. 8

* Platysma diffusum, Nyl. 6

* - Fahlunense, Nyl. 6

- glaucum, Nyl. 6,7 f. fallax, Nyl.

- ulophyllum, Nyl. $\quad 6,8$

\section{PARMeliet}

- Evernia furfuracea, Fr. 6,8 f. ceratea, Nyl. 6

- prunastri, Ach. I-8 "var. stictocera, Hook. 1, 6

Parmelia Borreri, Turn. 7

- caperata, Ach. 6,7

* - cetrarioides, Nyl. I, 6,8

- conspersa, Ach. 7,8 f. isidiata, Leight. 8

- exasperata, Nyl. 5

- fuliginosa, $\mathrm{Nyl} .7$

- laevigata, Ach. I, 6, 7 


\section{A HISTORY OF DEVONSHIRE}

\section{LICHENACEI (continued)}

Parmeligi (continued)

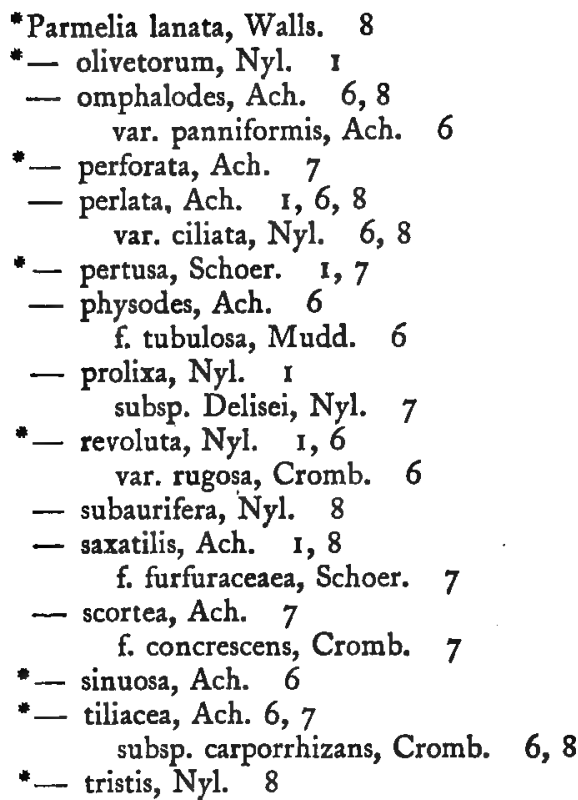

\section{STICTB1}

Lobaria pulmonaria, Hoffm. 6-8

Lobarina scrobiculata, Nyl. $5,7,8$

* Sticta aurata, Ach. 6

*Stictina crocata, Nyl. 7,8

*- Dufourei, Nyl. 1, 6,8

- fuliginosa, Nyl. I, 6 -8 f. ciliata, Holmes

- intricata, Nyl. 6 var. Thouarsii, Nyl.

- limbata, Nyl. 4, 6-8

- sylvatica, Nyl. 6-8

Ricasolia amplissima, Leight. $\quad 6-8$

- laetivirens, Leight. I , 6-8

\section{Peltigere}

* Peltidia aphthosa, Ach. 6 var. b. leucophlebia, Nyl. 8

Peltigera canina, Hoffm. I-8

- horizontalis, Hoffm. 6,8 f. muscorum, Schl. 6

- polydactyla, Hoffm. 1, 5-8

- rufescens, Hoffm. I, 6,8 f. praetextata, Floerke. 6,8

- scutata, Leight. 6-8

- spuria, Leight. 1, 6, 7

* Solorina saccata, Ach. 6

Nephromium Lusitanicum, Nyl. 6-8 var. Hibernicum, Nyl. 8

*- parile, Nyl. 5-8

Physcia adglutinata, Nyl. 7

- aipolia, Nyl. 6 var. anthelina, Cromb. 6 .

- aquila, Nyl. 6-8

- _ astroidea, Nyl. 7

- caesia, Nyl. 6

- - chrysophthalma, D.C. 6

\section{LICHENACEI (continued)}

Pritigerei (continued)

Physcia ciliaris, DC. 6,7

- - erosa, Leight. 6

*- flavicans, DC. 1, 6-8

- Leucomela, Mich. 1, 6, 7

- lychnea, Nyl. 6

- parietina, De Not. 7,8

f. cinerascens, Leight. 6

f. ectanea, Nyl. 6

- pulverulenta, Nyl. 6,7 var. angustata, Nyl. 6 subsp. venusta, Nyl. 6

* speciosa, Nyl. 7

- stellaris, Nyl. 5 var. leptalea, Nyl. 7 subsp. tenella, Nyl. 7

- ulothrix, Nyl. 6 var. virella, Cromb. 6

\section{GYROPHOREI}

Gyrophora cylindrica, Ach. 6,8 f. fimbriata, Ach. 8

- Hocculosa, Turn. and Borr. 8

- polyphylla, Turn. and Borr. 8 f. monophylla, Turn. and Borr. 6 f. congregata, Turn. and Borr. 8

- polyrrhiza, Krt. 6, 8

- torrefacta, Cromb. 2,8

- proboscidea, Ach. 6

Umbilicaria pustulata, Hoffm. 2, 6, 8

Coccocarpia plumbea, Nyl. 7 var. myriocarpa, Nyl. 2, 7

Leproloma lanuginosum, Nyl. $\quad 6-8$

Pannaria brunnea, Nyl. I, 6, 7

- nebulosa, Nyl. 6

- rubiginosa, Del. 8 var. caeruleo-badia, Mudd. 2, 6, 7

Pannularia nigra, Nyl. 6, 7

- triptophylla, Nyl. 1, 8

- carnosa, Cromb. I, 6, 7

Placodium callopismum, Mudd. 5-7 subsp. sympagea, Nyl. I, 6

- candicans, Mudd. 6

* cirrochroum, Cromb. 5,6

* - fulgens, Ach. I.

- lobulatum, Smrft. 6

- murorum, Leight. 6,7

* xantholytum, Nyl. 6,7

"Psoroma hypnorum, Ach. 6

Squamaria crassa, Huds. 6

Lecanora, aipospila, Wahlb. 6

- albella, Ach. 6

- allophana, Nyl. 6

- angulosa, Ach. 6 var. chondrotypa, Stiz. 6

- atra, Ach, 6, 7

- atrocinerea, Nyl. 7

- aurantiaca, Nyl. 7 subsp. erythrella, Nyl. 7

- badia, Ach. 7

- calcarea, Somm. 6, 7 var. contorta, Nyl. 6 var. Hoffmanni, Somm. 6 


\section{BOTANY}

LICHENACEI (continued)

GrRophoREI (continued)

Lecanora cerina, Ach. 7 var. stillicidiorum, Nyl. 8 subsp. haematites, Nyl. 6

- chalybea, Schaer. 6

- cinerea, Somm. 7

- citrina, Ach. 6 f. depauperata, Cromb. 6

- coccinea, Cromb. 8

- coilocarpa, Nyl.

- crenulata, Nyl. 6

- Dicksonii, Nyl. 6

* erysibe, Nyl. 6

- expallens, Ach. 6 var. lutescens, Nyl. 6,7

- ferruginea, Nyl. 1, 6, 7 var. festiva, Nyl. 7

- fuscata, Nyl. 7

- galactina, Ach. 6,7

- gibbosa, Nyl. I

- glaucoma, Ach. 1,7

- holophaea, Nyl. 1, 7

- intumescens, Koerb. 6

- irrubata, Nyl. 6 f. incrustans, Cromb. 6 subsp. calva, Nyl. 6

- lacustris, Fr. f. 7

- Nylanderiana, Nyl. 6

- ochracea, Nyl. 6,7

- orosthea, Ach. 6

- pallescens, Nyl. 6

- parella, Ach. I, 7 var. Turneri, Nyl.

- Parisiensis, Nyl.

- polytropa, Schaer. 7

- prosechoides, Nyl. I

- pruinosa, Nyl. I

- pyracea, Nyl. 6

- roboris, Nyl. 6

- rugosa, Nyl. 6

- simplex, Nyl. I, 6,7

- sophodes, Ach. 6

- squamulosa, Nyl. 6

- subcarnea, Ach. 6

- subfusca, Nyl. 6,7 var. campestris, Schaer. 6

- sulphurea, Ach. 6,7

- symmictera, Nyl. 6 var. aitema, Ach. 8

- syringea, Ach. 6

* - tartarea, Ach. 7,8 subsp. subtartarea, Nyl. $\quad 7,8$

* - tetrasticha, Nyl. 6,7

- varia, Ach. 6, 8

- variabilis, Ach. 6 var. ecrustacea, Nyl. 6

- ventosa, Ach. 8

- vitellinula, Nyl. 5

Pertusaria amara, Nyl. 6-8

- ceuthocarpa, T. and B. 6,7

- communis, DC. 5,6

- concreta, Nyl. I, 6

- dealbata, Nyl. 8

- globulifera, Nyl. $\quad 5,6,8$
LICHENACEI (continued)

GrRopHOREI (continued)

* Pertusaria lactea, Nyl. 6

- leioplaca, Schaer. 1, 6,8

f. hexaspora, Nyl.

*- melaleuca, Sm. 6

- multipunctata, Nyl. 1, 5, 6 f. laevigata, Cromb. 6,8

- - pustulata, Ach. 6

- velata, Nyl.

- Wulfenii, DC. I, 5,8

Thelotrema lepadinum, Ach. 6

Phlyctis agelaea, Koerb. 4-6

* - argena, Koerb. 6

Urceolaria scruposa, Ach. 6 subsp. bryophila, Ach. I, 6

- Lecidea abietina, Ach.

- alboatra, Leight. 4 f. epipolia, Leight.

- albocoerulescens, Leight. 6

- alocizoides, Leight. 6

- arceutina, Ach. 6

- aromatica, Sm. 5-7

- atroalba, Ach. 6

- calcarea, Leight. 6

- candida, Web. 6

- canescens, Dicks. 4,6

* - carneolutea, Leight. 5

- citrinella, Ach. 6, 8

- coarctata, Leight. 7 f. elachista, Leight. 1, 7

f. globulosa, Leight. 7

f. ornata, Leight. 7

- concentrica, Dav. 7

- confluens, Web. 6,7

- contigua, Fr. 4, 6, 8 f. limitata, Leight. 7

* corneola, Ach. 6

- crustulata, Ach. 4

- cupularis, Leight. 6

* - cyrtella, Ach. 6

- dealbatula, Nyl. 6

- decolorans, Flk. 8

- dilutiuscula, Nyl.

- disciformis, Fr. 5, 7

- discolor, Hepp. 6

- effusa, Sm. 6

var. fuscella, Fr. 6

var. inundata, Fr. 6

- Ehrhartiana, Ach.

- endoleuca, Nyl. 5,6

- enterochlora, Tayl. 6

- enteroleuca, Ach. 7

- - erysiboides, Nyl.

- exanthematica, Leight. 6

- Alexuosa, Fr. f. aeruginosa, Borr. 6

* Flotovii, Koerb. 4

- fuscoatra, Ach. 8

- geographica, Linn. 8

- grossa, Pers. 4-7

* herbarum, Hepp. I

- incompta, Borr. 4

- lucida, Ach. 4, 6,7

* lurida, Swartz. 6 


\section{A HISTORY OF DEVONSHIRE}

\section{LICHENACEI (continued)}

GYRoPHOREI (continued)

Lecidea lutea, Dicks. $\quad 2,4,6,8$

- lutosa, Mont. 6

- mammillaris, Gon. 6

- milliaria, Fr. 6

- myriocarpa, DC. 7

- obscurata, Schaer.

- Oederi, Web. 6

- oxyspora, Tul. 8

- pachycarpa, Duf. 4

- parasema, Ach. 7 var. elaeochrona, Ach. 7

- parasitica, Flk. 4, 7,8

- Parmeliarum, Sommf. 6-8

- petraea, Wulf. 5,6

- premea, Ach. I, 5,7 f. teichogena, Nyl. 3

- prominula, Borr. 4, 6

- protrusa, Fr. 6

*-_ pulverea, Borr. 5, 6,8

- quernea, Dicks. 5

- rivulosa, Ach. 6,8

- rubella, Ehrh. 4, 6, 7

- sabuletorum, Flk. 6

- Salweii, Borr. 1, 7

- sanguinaria, Linn. 6

- sphaeroides, Dicks. $\quad 4,6$

*- spododes, Nyl. 5

- squamulosa, Deak. 6

- stellulata, Tayl. 7

- subdisciformis, Leight. 6

- sylvicola, Flot. var. infidula, Nyl. 6

- Taylori, Mudd. 1, 6,7

- tenebrosa, Flot.

- tricolor, Leight.

* - truncigena, Ach. 5

- Turneri, Leight. 6

- uliginosa, Schrad. f. fuliginosa, Ach. 4

- verruculosa, Borr. I

- vesicularis, Hoffm. 1, 6, 7

- violacea, Crouan. I

- viridescens, Schrad. 4

\section{GraPHIDIEI}

Arthonia astroidea, Ach. 4,6

- cinnabarina, Wallr. 7,8 var. anerythrea, Nyl. 7 var. kermesina, Nyl. 4,6

* - proximella, Nyl.

- pruinosa, Ach. 5,7

- punctiformis, Ach. 6 f. galactina, Ach. 6

- spadicea, Leight. 4,6

- Swartziana, Ach. 6, 7

* trachylioides, Nyl. 6

- varians, Dav. I

* - vinosa, Leight. 6

*Chiodecton albidum, Tayl. var. Sarniense, Salw. 1, 6

Graphis dendritica, Ach. f. obtusa, Leight. 6 f. Smithii, Leight. 5-8
LICHENACEI (continued)

GraphidieI (continued)

Graphis elegans, Sm. 6,7

- inusta, Ach. 5, 7 f. vera, Leight.

* Lyellii, Sm. 5, 6, 8

- Ruiziana, Fée. 6

- scripta, Ach. 5, 7

f. diffusa, Leight. 4, 6

f. eutypa, Ach. 4

f. recta, Humb. I

f. minuta, Leight. 6

var. serpentina, Ach. 4,6

- sophistica, Nyl.

var. pulverulenta, Sm. 4,6

"Lithographa cerebrina, Schaer. 6

*_ dendrographa, Nyl. 1, 5-7

*- tesserata, Nyl.

Opegrapha atra, Pers. 7

f. arthonoidea, Leight. 5

f. denigrata, Ach. 4

f. nigrita, Leight. 4

f. parallela, Leight. 6

- confluens, Ach. I

* - grumulosa, Duf. I

- herpetica, Ach. 7,8

f. rubella, Pers. 4

f. vera, Leight, 4,7

f. rufescens, Pers. 4

* - involuta, Nyl. 7

- Leightonii, Cromb. 6

* - lentiginosa, Lyell. 7

- lyncea, Sm. 4-6

- saxicola, Ach. I, 7 var. Chevalieri, Leight. 5

f. gyrocarpa, $\mathrm{Zw}$. I

var. De Candollei, Stizb. 6

f. diaphora, Ach. 4,6

- varia, Pers. 7 f. notha, Ach. 4,6

f. pulicaris, Light. 4,7

f. rimalis, Fr.

- viridis, Pers. 4, 6

- vulgata, Ach. 4, 6, 7 f. stenocarpa, Ach. 6

Stigmatidium circumscriptum, Tayl. I, 7

- crassum, Dub. 4, 7

*- Hutchinsaie, Leight. I, 7

* - venosum, Ach. I

\section{Pyrenode}

Endocarpon fluviatile, DC. 6-8 var. euplocum, Ach. 7

- hepaticum, Ach. 7

- miniatum, Ach. 1, 6 var. complicatum, Sw. 6

- rufescens, Ach. 7

Melanotheca gelatinosa, Chev. 6

*Normandina laetevirens, Ach. 8

- pulchella, Borr. I-8

*Verrucaria antecellens, Nyl. 7,8

- biformis, Borr. 4, 6,7

- calciseda, DC. 6, 7 var. purpurascens, Hoffm. 


\section{BOTANY}

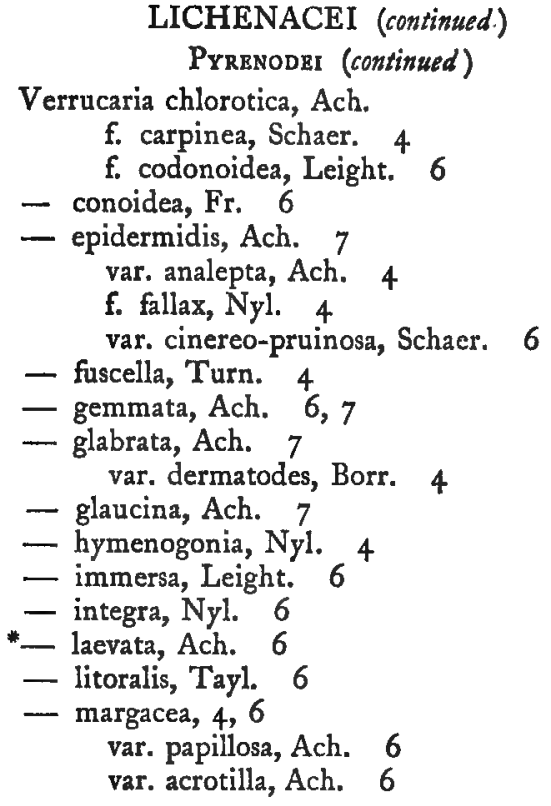

Verrucaria chlorotica, Ach.

f. carpinea, Schaer. 4

f. codonoidea, Leight. 6

- conoidea, Fr. 6

- epidermidis, Ach. 7

var. analepta, Ach. 4

f. fallax, Nyl. 4

var. cinereo-pruinosa, Schacr. 6

- fuscella, Turn. 4

- gemmata, Ach. 6,7

- glabrata, Ach. 7

var. dermatodes, Borr. 4

- glaucina, Ach. 7

- hymenogonia, Nyl. 4

- integra, Nyl. 6

* - laevata, Ach. 6

- litoralis, Tayl. 6

- margacea, 4, 6

var. papillosa, Ach. 6

var. acrotilla, Ach. 6

\section{LICHENACEI (continued)}

Prrenodei (continued)

Verrucaria marina, Deak. 6

- maura, Wahlb. 7

- mauroides, Schaer. 6

- murina, Leight. 4,6

- mutabilis, Borr. 5,6

- nigrescens, Pers. 6

- nitida, Weig. 1, 5

- perminuta, Deak. 6 f. nitidella, Deak. 5, 6

- platypyrenia, Nyl. 6

- polysticta, Borr. 4

- punctiformis, Ach. 4

- pyrenophora, Ach. 6

- rugulosa, Borr. 6

- rupestris, Schrad. 7 var. muralis, Ach. 4 var. subalbicans, Leight. 4,6

- Salweii, Leight. 1, 4

- sublitoralis, Leight. I, 5

\section{FUNGI}

The following list is compiled from the records published in Jones and Kingston's Flora Devoniensis (I 829) and Ravenshaw's Botany of North Devon. To these have been added a list from the Transactions of the British Mycological Seciety, made during a visit of the Society to Exeter and its neighbourhood, in $\mathrm{r} 90 \mathrm{r}$, for which the writer is indebted to the kindness of Mrs. A. Montague, of Crediton, a well-known and accurate mycologist. There has also been added a list of the fungi collected by the late Mr. E. Parfitt, of Exeter, which the writer has been permitted to see by the favour of Dr. H. Humphreys, of Torquay, the collection having recently been presented to the museum of that town by Mrs. Vicary, of Newton Abbot, into whose possession it had come some years ago. There were originally twelve folio volumes of drawings illustrating these fungi, made by Mr. Parfitt, but these cannot be traced as yet; and as many of the fleshy species are in bad condition, their identifications cannot be confirmed, and are in such cases omitted from the list. With the exception of districts 4 and 6 the fungal flora of Devon has been very little investigated, and the list here given is therefore probably far from an exhaustive one.

The general arrangement followed in this list is that of Cooke's Handbook of British Fungi (1871), supplemented by Massee's British Fungus Flora (1892-5), and Plowright's British Uredines and Ustilagineae (1 889).

\section{HYMENOMYCETES}

Agaricini

Amanita adnata, W. G. Smith. 5

- junquillea, Quél. 4

- mappa, Fr. 4

- muscaria, Fr. 1, 4,6

- pantherina, Fr. 4

- phalloides, Fr. 4

- rubescens, Fr. 4
HYMENOMYCETES (continued)

Agaricini (continued)

Amanita spissa, Fr. 4

- Vittadini, Morett. 4

Amanitopsis vaginata, Roze. 4

Lepiota amianthina, Scop. 4

- Badhami, Berk. 4

- cepaestipes, Sow. 4

- cristata, A. and L. 4 


\section{A HISTORY OF DEVONSHIRE}

\section{HYMENOMYCETES (continued)}

AgaricinI (continued)

Lepiota erminea, Fr. 4

- excoriata, Schaeff. 4

- granulosa, Batsch. I, 4

- holosericea, Fr. 4

* leucothites, Vitt. 4

- mastoides, Fr. 4

- metulaespora, B. and Br.

- permixta, Barla. 4

- procera, Scop. 1, 4

- rhacodes, Vitt. 4 var. puellaris, Fr. 4

Armillaria mellea, Vahi. I, 4, 6

- mucida, Schrad. 4

Tricholoma acerbum, Bull. 4

- albobrunneum, Pers. 4

- carneum, Bull. 4

- cuneifolium, Fr. 4

- flavobrunneum, Fr. 4

- lascivus, Fr. 4

- melaleucum, Pers. 4

- nudum, Bull. 4

- pessundatum, Fr.

- resplendens, Fr. 4

- rutilans, Schaeff. I

- saponaceum, Fr. 4

- scalpturatum, Fr. 6

- sejunctum, Sow. 4

- sordidum, Fr. 4

- terreum, Schaeff. 4, 6

- vaccinum, Fr. 4

- virgatum, Fr. 4

Clitocybe candicans, Pers. 4

- cerussata, Fr. 4

- dealbata, Sow. 4

- flaccida, Sow. 4

- fragrans, Sow. 4

- geotropa, Bull. 4

- gilva, Pers. 6, 8

- infundibuliformis, Schaeff. var. membranaceus, Fr. 6

- nebularis, Batsch. 4

- odora, Sow. 4, 6

- phyllophila, Fr. 4

- rivulosa, Pers. 4

Laccaria laccata, Scop. 4, 6

Collybia acervata, Fr. 4

- butyracea, Bull.

- confluens, Pers.

- dryophila, Bull. 1, 4

- esculenta, Wulf. 4, 6

- fusipes, Bull. 4

- maculata, A. and S. 4

- platyphylla, Fr.

- radicata, Relh. 4, 6

- tuberosa, Bull. 4, 6

- velutipes, Curt. 4

Mycena alcalina, Fr. 4

- amicta, Fr. 4

- ammoniaca, Fr. 4

- aurantio-marginata, Fr. 4

- clavicularis, Fr. 4

- corticola, Schum. 7

- debilis, Fr. 4

\section{HYMENOMYCETES (continued)}

Agaricini (continued)

Mycena epipterygia, Scop. 4

- filipes, Bull. 4

- galericulata, Scop. I, 4

- galopoda, Fr. 4

- haematopoda, Pers. 4

- leucogala, Cke.

- luteoalba, Bolton. 4

- pelianthina, Bolton. 4

- polygramma, Bull. 4

- pura, Pers. 4

- rugosa, Fr. 4

- sanguinolenta, A. and S. 4

- tenerrima, Berk. 4

Omphalia fibula, Bull. 4

- stellata, Fr. I

Pleurotus atrocoeruleus, Fr. 6

- ostreatus, Jacq. 4

- striatulus, Fr. [F\%. Dev.]

- subpalmatus, Fr. 6

"Volvaria bombycina, Schaeff. 4

- Taylori, Berk. 4

Pluteus cervinus, Schaeff. 4

Entoloma jubatum, Fr. 4

- nidorosum, Fr. 4

- repandum, Bull. I

- sericeum, Fr. 4

- sericellum, Fr. 4

Clitopilus carnea-albus, With:

- orcella, Bull. 4

- prunulus, Scop. 4

Leptonia chalybea, Pers. 4

- incana, Fr. 4

- lampropoda, Fr. 4

- serrulata, Fr. 4

Nolanea mammosa, Fr. 4

- pascua, Pers. 4

Claudopus euosmus, Berk. 7

- variabilis, Pers. 6, 7

Pholiota adiposa, Fr. 4

- - grandis, Rea. 4

- marginatus, Batsch. 4

- mutabilis, Schaeff. 4

- praecox, Pers. 4

- spectabilis, Fr. 4

- squarrosa, Mull. 4, 6

- terrigena, Fr. 4.

- togularis, Bull. 4

Inocybe asterospora, Quét.

- Bongardii, Weinm. 4

- eutheles, B. and Br. 4

- fastigiata, Schaeff. 4

- geophylla, Fr. 6

- pyriodora, Pers. 4

- rimosa, Bull. 4

Hebeloma crustuliniforme, Bull. 4

- fastibile, Fr.

- glutinosum, Lindgr. 4

- mesophaeum, Fr. 4

Flammula alnicola, Fr. 4

Naucoria escharoides, Fr. 4

- melinoides, Fr. 4

- semiorbicularis, Bull. 4

Galera hypnorum, Batsch. I, 4 


\section{BOTANY}

\section{HYMENOMYCETES (continued)}

\section{Agaricini (continued)}

Galera tenera, Schaeff. 4

Tubaria crobula, Fr. 4

- furfuracea, Pers. 4

Crepidotus alveolus, Lasch. 4

- mollis, Fr. 4

Psalliota arvensis, Schaeff. 4

- campestris, Linn. I, 4 var. sylvicola, Vitt. 4

- comptulus, Fr. 4

- cretaceus, Cke.

- haemorrhoidarius, Schulz. 4

- pratensis, Schaeff. 4

- xanthoderma, Genev. 4

Stropharia aeruginosa, Curt. I, 4

- semiglobata, Batch. I, 4

- squamosa, Fr.

Hypholoma appendiculatum, Bull. 4

- capnoides, Fr. 4

- fasciculare, Huds. 1, 4

- hydrophyllum, Bull. 4

- lachrymabundum, Fr.

- sublateritium, Schaeff. 4

- velutinum, Pers. 4

Psilocybe faenisecii, Pers. 4

- sarcocephala, Fr. 4

- semilanceata, Pers. 4

Psathyra corrugis, Pers. 4

- fibrillosa, Pers. 4

Anellaria separata, Karst. 4

- fimiputris, Karst. 4

Panaeolus campanulatus, Fr.

- cinctulus, Bolt. 4

- phalaenarum, Fr. 4

- separatus, Linn. 4

- sphinctrinus, Fr. 4

Psathyrella atomata, Fr. 4

- disseminata, Fr. 4

- gracilis, Fr. 4

Coprinus atramentarius, Fr. I, 4

- comatus, Fr. I

- fimetarius, Fr. [Fl. Dev. $]$

- lagopus, Fr. 4

- micaceus, Fr. I, 4

- picaceus, Fr. 4

- plicatilis, Fr. I, 4

Bolbitius tener, Berk. 4

\section{Cortinarius}

(Dermocybe) anomalus, Fr. 4

- caninus, Fr.

- cinnamomeus, Fr. 4, 6

- lepidopus, Cke. 4

- sanguineus, Fr. 4

(Hydrocybe) armeniaceus, Fr. 4

- subferrugineus, Fr.

(Inoloma) alboviolaceus, Fr. 4

- pholideus, Fr. 4

- violaceus, Fr. I, 4

[Myxacium) collinitus, Sow. 4

- delibutus, Fr. 4

- elatior, Fr. 4

- mucifluus, Fr. 4

- pluvius, Fr. 4
HYMENOMYCETES (continued)

Agaricini (continued)

Continarius (continued)

(Phlegmacium) calochrous, Fr. 4

- dibaphus, Fr. 4

- largus, Fr. 4

- multiformis, Fr. 4

- prasinus, Fr. 4

- purpurascens, Fr. 4 var. subpurpurascens, Fr. 4

- triumphans, Fr. 4

(Telamonia) hemitrichus, Fr. +

- hinnulens, Fr. 4

- torvus, Fr. 4

Gomphidius gracilis, Berk. 4

Paxillus involutus, Fr. 4

- panuoides, Fr. 6

Hygrophorus calyptraeformis, Berk. 4

- chlorophanus, Fr. 4

- chrysodon, Fr. 4

$\rightarrow$ coccineus, Fr. $I$

- conicus, Fr. I, 4

- eburneus, Fr. 6

- pratensis, Fr.

- psittacinus, Schaeff. I

- virgineus, Wulf.

Lactarius acris, Fr. I

- aurantiacus, Fr. 4

- blennius, Fr.

- circellatus, Fr. 4

- controversus, Fr. 4

- deliciosus, Fr. 4

- pallidus, Fr. 4

- piperatus, Fr. 6

- pyrogalus, Bull. 4

- quietus, Fr. $\quad$, 4

- rufus, Scop. 4

- serifluus, Fr. 4

- torminosus, Schaeff. 4

- turpis, Fr. 4

- uvidus, Fr. 4

- vellereus, Fr. 1, 4, 6

Russula adusta, Fr. I, 4

- armeniaca, Cke. 4

- caerulea, Fr. 4

- cyanoxantha, Schaeff. 4

- depallens, Fr. 4

- drimeia, Cke. 4

- emetica, Fr. 4

- fellea, Fr. 4

- fragilis, Fr. 4

- furcata, Fr. 4

- heterophylla, Fr. I

- integra, Fr. 4

- lepida, Fr. 4

- nigricans, Fr. 4

- nitida, Fr. 4

- ochroleuca, Fr. 4

- puellaris, Fr. 4

- vesca, Fr. 4

- virescens, Fr. 1

Cantharellus aurantiacus, Fr. $\quad 1,4$

- cibarius, Fr. 1, 4, 6,7

- Friesii, Quél. 4

- infundibuliformis, Fr. 4 


\section{A HISTORY OF DEVONSHIRE}

\section{HYMENOMYCETES (continued)}

Agaricini (continued)

Cantharellus retirugus, Fr. 4

- tubaeformis, Fr. 4

Nyctalis asterophora, Fr. 4

- parasitica, Fr. 4

Marasmius androsaceus, Fr. 4

- epiphyllus, Fr. 4, 6

- erythropus, Fr. 4

- Hudsoni, Pers. 4

- insititius, $\mathrm{Fr} .4$

- oreades, Fr. I, 4

- pernotatus, Fr. 4

- rotula, Fr. 4

- cochleatus, Fr. 4

Lentinus vulpinus, Fr. 6

Panus conchatus, Fr. 4

- stypticus, Fr. 4

Lenzites betulina, Fr. 4

\section{Polyporei}

Boletus aurantiporus, Howse. 4

- badius, Fr. 4

- bovinus, Linn. 6

- calopus, Fr. I

- chrysenteron, Fr. 4

- crassus, Mass. 4

- cyanescens, Bull. 6

- edulis, Bull. I, 4

- elegans, Schum. 4

- flavus, With. I, 4

- fragrans, Vitt.

- granulatus, Linn. 4

- laricinus, Berk. 4

- luridus, Schaeff. 1, 4, 6

- luteus, Linn. 4, 6

- piperatus, Bull. 4, 6

- radicans, Pers. 4

- Satanas, Lenz. 6

- scaber, Pers. 4, 6

- subtomentosus, Linn. 1, 4

- tenuipes, Cke. 4

Fistulina hepatica, Fr. $\quad$, 4,6

Merulius corium, Fr. 4

- lachrymans, Fr. 4

Daedalea quercina, Fr. 1, 4, 7

Poria blepharistoma, B. and $\mathrm{Br}$.

- medulla-panis, Fr.

- sanguinolenta, A. and S. 4

- vaporaria, Fr. 4

- vulgaris, Fr. 4

Polystictus abietinus, Fr. 4

- perennis, Fr. 1, 4,6

- radiatus, Fr. 4

- velutinus, Fr. 4

- versicolor, Fr. 4

Fomes annosus, Fr. 4

- applanatus, Wallr. 4 .

- fomentarius, Fr. 6

- igniarius, Fr. 6

- ulmarius, Fr. 4

Polyporus adustus, Fr. 4

- betulinus, Fr. 4

- caesius, Fr. 6

1 dryadeus, Fr. 4
HYMENOMYCETES (continued)

Polyporex (continued)

Polyporus fibula, Fr. 4

- giganteus, Fr. 4

- hispidus, Fr. I, 4

- intybaceus, Fr. 6

- rufescens, Fr. 4

- Schweinitzii, Fr. 4

- spumeus, Fr.

- squamosus, Fr. I, 4

- sulphureus, Fr. 4

Hyoner

Hydnum auriscalpium, Linn. 4

- farinaceum, Pers. 4

- repandum, Linn, I, 4 var. rufescens, Pers. 4

- udum, Fr. 4

- zonatum, Batsch. 4

Irpex obliquus, Fr. 4

Radulum fagineum, Fr. 4

Phlebia merismoides, Fr. 4

Grandinia granulosa, Fr. 4

Odontia fimbriata, Fr. 4

\section{Auricularini}

Craterellus cornucopioides, Pers. 4

- sinuosus, Fr. 4

Thelephora byssoides, Fr. 4

- caryophyllea, Pers. 4

- laciniata, Pers. 4, 7

- terrestris, Berk. 4

Hymenochaete rubiginosa, Lév. 7

Stereum hirsutum, Fr. 4

- ochroleucum, Fr. 4

- purpureum, Pers. 4

- rugosum, Fr. 4

- sanguinolentum, A. and S. 4

Corticium calceum, Fr. 4

- caeruleum, Fr. 4, 7

- comedens, Fr. 4

- giganteum, Fr. 4

- incarnatum, Fr. 4

- laeve, Fr. 4

- quercinum, Pers. 4, 8

- Sambuci, Fr. 4

Peniophora cinerea, Cke. 4

Auricularis mesenterica, Fr. I, 4, 6

\section{Clavariei}

Sparassis crispa, Fr. 4, 6

Clavariei amethystina, Bull. I

- cinerea, Bull. 4

- coralloides, Linn. [Fl. Dev.]

- cristata, Pers. 4

- fastigiata, Linn. I, 4

- flaccida, Fr. 4

- inaequalis. [Fl. Dev.]. 4

- muscoides, Linn. 4

- pistillaris, Linn. 4

- rugosa, Bull. 4

- vermiculata, Scop. 4

Typhula gyrans, Fr. var. Grevillei, Mass. 6

Calocera cornea, Fr. 4 


\section{BOTANY}

\section{HYMENOMYCETES (continued)}

Calocera stricta, Fr. 4

- viscosa, Fr. 4

Tremellini

Tremella albida, Huds. 4

- stricta, Fr. 4

- lutescens, Pers. 4

- mesenterica, Retz. 4

- Hirneola Auricula-Judae, Berk. 4

Femsjohnia luteo-alba, Fr. 4

Nematelia encephala, Fr. 4

Dacrymyces deliquescens, Dub. 4

- stillatus, Nees. 4

\section{GASTEROMYCETES}

Rhizopogon rubescens, Tul. 6 Hymenogaster decorus, Tul. 4 - tener, Berk. 4

\section{SClerodermeat}

Scleroderma verrucosum, Pers. 4 - vulgare, Tul. 4

\section{Nidulariea}

Crucibulum vulgare, Tul. 4,6 Cyathus striatus, Hoffm. 4

- vernicosus, DC. 4

\section{LYCOPERDEAE}

Lycoperdon Bovista, Linn. 4

- coelatum, Bull. 4

- excipuliforme, Scop. 4

- gemmatum, Batsch. I, 4

- giganteum, Batsch. I

- nigrescens, Vitt. 4

- perlatum, Pers. 4

- plumbeum, Pers. 4

- pyriforme, Schaeff. 4

- saccatum, Wahl. 4

Geaster fimbriatus, Fr. 4, 6

- hygrometricus, Pers. 4

Tulostoma mammosum, Fr. I

\section{Phalloidea}

Ithyphallus impudicus, Fisch. 4 Mutinus caninus, Fr. 4

Clathrus cancellatus, Tournef. 6

\section{CONIOMYCETES}

\section{SphaEronemei}

Asteroma Ulmi, K1. 4

Hendersonia cornu, Fckl. 4

Sphaeropsis Taxi, Berk. 6

\section{Melanconiei}

Cheirospora botryospora, Fr.

Coryneum microstictum, B. and Br. 4

Nemaspora crocea, Pers. 4

- Rosae, Desm. 6

Ramularia Petuniae, Cke. 7

\section{Torulace}

Helicosporium pulvinatum, Fr. 4

\section{CONIOMYCETES (continued)}

UREDINEAE

Uromyces Betae, Wint.

- dactylidis, Otth.

- Fabae, Pers.

- Geranii, DC.

- Poae, Rabenh.

Puccinia Asparagi, DC.

- Betonicae, Wint.

- Buxi, DC.

- Centaureae, Mart.

- fusca, Wint.

- Magnusiana, Korn.

- malvacearum, Mont.

- obscura, Schrot.

- poarum, Niel.

- polygonorum, Pers.

- Phragmitis, Schrot

- Primulae, Grev.

- pulverulenta, Grev.

- rubigo-vera, DC

- Saniculae, Grev.

- suaveolens, Wint.

- Trailii, Plowr.

- Umbilici, Guep.

- variabilis, Grev.

- Violae, Wint.

- Vincae, DC.

Gymnosporangium clavariaeforme, Plowr.

Triphragmium Ulmariae, Wint.

Phragmidium Fragrariastri, DC.

- Rubi, Pers.

- violaceum, Schultz.

Melampsora betulina, Pers.

- farinosa, Plowr.

- Helioscopiae, DC.

- hypericorum, DC.

Coleosporium Senecionis, Fr.

Cronartium flaccidum, Alb. and Schw.

- ribicolum, Dietr.

Caeomma Mercurialis, Plowr.

Aecidium Elatinum, Alb. and Schw.

Ustilago longissima, Wint.

- olivacea, Tul.

Cystopus candidus, Lev. 4, 7

\section{HYPHOMYCETES}

\section{Mucrdinesz}

Cylindrium flavovirens, Bon. Aspergillus glaucus, Link. 4 Penicillium glaucum, Link. 4

Rhinotrichum ramosissimum, B. and C. 4 Sporotrichum chlorinum, Link. 4

Botrytis fascicularis, Sacc. 4

Sepedoniei vulgaris, Fr. 4

Sepedonium agaricicola, Corda. 4

\section{Dematiel}

Acrothecium obovatum, Cooke. 4 - tenebrosum, Sace. 4

Arthrobotryum atrum, B. and Br. 4 Dendryphium comosum, Wallr. 4 Haplographium chlorocephalum, Grev. 


\section{A HISTORY OF DEVONSHIRE}

\section{HYPHOMYCETES (continued)}

DematreI (continued)

Helminthosporium fusiforme, Corda. 4

- obclavatum, Sace. 4

Hormiscium hysterioides, Sacc. 4

Zygodesmus fuscus, Corda. 4

Leptosporium bulbotrichum, Corda. 4

Sporochisma mirabile, B. and $\mathrm{Br}$. 4

Fumago vagans, Pers. 4

\section{Strilbeak}

Illosporium roseum, Fr. 7

Isaria farinosa, Fr. 4

Stysanus stemonitis, Corda. 4

Tubercularia vulgaris, Tode. 4

\section{Mucorini}

Mucor amethysteus, Berk. 4

\section{PYRENOMYCETES}

Hypocreaceae

Cordyceps militaris, Fr. 4

Epichloe typhina, Sacc. 4, 7

Hypocrea rufa, Fr. 4

Hypomyces aurantius, Tul. 4

Nectria aquifolia, Berk. 4

- cinnabarina, Tode. 4

- coccinea, Fr. 4

- ditissima, Tul. 4

- sanguinea, Fr. I

\section{Xylarieaz}

Daldinia concentrica, Sacc. 4 Hypoxylon coccineum, Bull. 4 - fuscum, Fr. 6

Ustulina vulgaris, Tul. 4

Xylaria hypoxylon, Grev. 4

\section{Dothideab}

Phyllacora graminis, Grev. 4

Rhytisma acerinum, Fr. 4

\section{Diatrypeas}

Quaternaria Persoonii, Tul. 4

\section{Valsear}

Valsa circumscripta, Mont. 4

- corylina, Tul. 4

- heteracantha, Sacc. 5

- leipheimia, Fr. 4

- prunastri, Fr. 4

- quaternata, Fr. 4

- stellulata, Fr. 4

\section{EUtXPEAr}

Diaporthe vepris, Nke. 4 Eutypa lata, Tul. 4

\section{Superficiales}

Bertia moriformis, Fckl. 4 Chaetosphaeria phaeostroma, Fckl. 4 Trichosphaeria pilosa, Fckl. 4
PYRENOMYCETES (continued)

Endoxyleae

Xylosphaeriz apiculata, Curr. 4

Obtectae

Didymosphaeria diplospora, Cke. 4

- epidermidis, Fckl. 6

- xylostei, Fckl.

Leptosphaeria fuscella, B. and $\mathrm{Br}$. 4

\section{Caulicolat}

Heptameria acuta, Mont. 4 Pleospora herbarum, Pers. 4 Raphidospora rubella, Fckd. 4

Rebenstischia unicaudata, B. and Br. 4

\section{ASCOMYCETES}

\section{Gymnoascacear}

Ascomyces pruni, B. and Br. 6

\section{HYSTERIACEAE}

Hysterium Fraxini, Pers. 4

- pinastri, Schrad. [Fl. Dev.]

- pulicare, Pers. 6

- virgultorum, DC. 4

Lophodermium pinastri, Chev. [Fl. Dev.]

Dichaena quercina, Fries. 7

\section{Discomycetres}

Coccomyces coronatus, Sacc. 6

- rubi, Karst. 6,8

Phacidium repandum, Fr. 4

Trochila Buxi, Capr. 4

- craterium, Fr. 4

- Ilicis, Crn. 4

Rhytisma acerinum, Fr. 7

Cryptomyces aureus, Mass. [Fl. Dev.]

Coryne sarcoides, Tul. 4

Ascobolus furfuraceus, Pers. 8

Mollisia cinerea, Karst. 4

- fusca, Mass. 4

Belonidium pruinosum, Mass. 4

Helotium citrinum, Fr. 4

- virgultorum, Karst. 4

Tapesia caesia, Fckl. 4

Lathnea hirta, Gillet. 4

- scutellata, Gillet. [Fl. Dev.].

Dasycypha calycina, Fckl. 4

- clandestina, Fckl. 4

- virginea, Fckl. 4

Geopyxis coccinea, Mass. 6, 7

Peziza repanda, Wahl. 6

- vesiculosa, Bull. 4

Otidea aurantia, Mass. 4

Helvella elastica, Bull. 4

- lacunosa, Afzel. 4, 6

Leotia lubrica, Pers. 4, 6

Mitrula phalloides, Chev. 4

Morchella esculenta, Pers. 7

- crassipes, Pers. 6

Geoglossum difforme, Fr. 4

- hirsutum, Pers. 4,6

- viscosum, Pers. 8 


\section{BOTANY}

ASCOMYCETES (continued)

Tureracei

Tuber dryophilum, Tul. 6

- puberulum, B. and Br. 6

\section{MYCETOZOA}

Ceratiomyzaceae

Ceratomyxa mucida, Schroet. 4

Phasarace

Badhamia hyalina, Berk. 4

- utricularis, Berk. 4

Physarum leucopus, Link. 4

- bivalve, Pers. 4

- cinereum, Pers. 4

- nutans, Pers. I

- verum, Sommf. 4

Fuligo septica, Gmel. 4

Craterium pedunculatum, Trente. 4

Leocarpus vernicosus, Link. 4

Chondrioderma Michelii, Rost. 4

- spumarioides, Rost. 4

\section{Didymiaceap}

Didymium clavus, Rost. 4

- effusum, Link. 4

- farinaceum, Schrad.

- squamulosum, A. and G. 4

Spumaria alba, DC. 4
MYCETOZOA (continued)

Didymiacear (continued)

Stemonitis fusca, Roth. 4

- Havozerita, Jahn. 4

Lamproderma irideum, Mass. 4

Amaurochaetacear

Brefeldia mixima, Rost. 4

Heterodermaceae

Cribraria argillacea, Pers. 4

Reticulariaceae

Reticularia Lycoperdon, Bull. 4

- umbrina, Tr. 6,7

Trichiacear

Trichia affinis, De Bary. 4

- Botrytis, Pers. 4

- fallax, Pers. 4

- persimilis, Kürst. 4

- scabra, Rost. 4

- varia, Pers. 4

Arcyriacear

Arcyria incarnata, Pers. 4

- punicea, Pers. 4

LYYOGALACEAE

Lycogala miniatum, Pers.

\section{ADDENDA}

Since the first portion of the article was written and printed, the following additional particulars have been collected.

The census of species, calculated on the scale of the ninth edition of the London Catalogue, must be slightly increased, so as to include eighteen extra species, namely, four belonging to the order Compositae, two to each of the two orders, Euphorbiaceae and Liliaceae, and one to each of the ten orders, Cruciferae, Leguminosae, Onagrarieae, Umbelliferae, Rubiaceae, Ericaceae, Scrophularineae, Labiatae, Salicineae, and Naiadaceae. Thus the total number of recognised Devon species of flowering plants becomes 1,156 , of which number 882 apply to dicotyledons and 274 to monocotyledons; the proportion of the numbers of these two groups is approximately as 3.22 to $I$; and the percentage of the number of Devon flowering plants, compared with that of the British Isles, is rather greater than $62 \cdot \mathrm{I}$.

The new literature dealing with Devon plants consists mainly of papers contributed to the Fournal of Botany. The lists of the more remarkable and interesting plants may be extended as follows :-

\section{Barnstaple District}

Ranunculus parviflorus, L.

Alyssum calycinum, $\mathrm{L}$.

Cochlearia anglica, L., var. Hortii, Syme

Reseda lutea, L.

Poterium Sanguisorba, $\mathrm{L}$.

Coriandrum sativum, L.

Hieracium dissimile, Lindeb., var. porrigens, Almq.
Verbascum pulvinatum, Thuill. (=V. Lychnitidi $x$ floccosum, Ziz.)

Mentha sylvestris, L., var. M. alopecuroides, Hull Nepeta Glechoma, Benth., var. parviflora, Benth.

Polygonum aviculare, L., subsp. P. Roberti, Loisel Ornithogalum umbellatum, L.

Juncus diffusus, Hoppe.

Glyceria distans, Wahlb., var. obtusa, Parn.

\section{Torrington District}

Impatiens glandulifera, Royle (a species of temperate Western Himalaya, abundant in 1905 near Torrington)

Trigonella ornithopodioides, DC.

Filago germanica, L., var. F. spathulata, Presl
Potamogeton salignus, A. Fryer (probably a hybrid ; differing from P. salicifolius, Wolfg. by the outermost veins of the leaves starting near the base of the midrib and not from the base of the margins of the leaves) 


\section{A HISTORY OF DEVONSHIRE}

Corydalis claviculata, DC. Barbarea praecox, $\mathrm{R}$. Br. Alyssum calycinum, $\mathrm{L}$. Teesdalia nudicaulis, R. Br. Stellaria aquatica, Scop.

\section{South Molton District}

Trifolium agrarium, L. (an alien in cornfields)

Epilobium roseum, Schreb. (a weed in cultivated ground)

Gnaphalium sylvaticum, L.

Hieracium crebridens, Dahlst.

\section{Exeter District}

Lathyrus hirsutus, L. Epilobium roseum, Schreb.

\section{Honiton District}

Ranunculus heterophyllus, Fries, form R. triphyllus, Wallr. Lotus corniculatus, L., subsp. L. tenuis, Waldst. and Kit. Centaurea Calcitrapa, L.

Elymus arenarius, L. (still found in an old station)

\section{Torquay District}

Sedum rupestre, $\mathrm{L}$.
Vaccinium Vitis-idaea, L.

Euphrasia pratensis, L., subsp. E. occidentalis, Wettst. Koeleria gracilis, Pers.

\section{Plymouth District}

Arenaria serpyllifolia, L., var. scabra, Fenzl (Druce) Medicago lupulina, L., var. Willdenowiana, Koch - leptoclados, Guss., var. scabra, Rouy and Euphrasia nemorosa, H. Mart. Fouc.

Malva rotundifolia, L., var. elachista, Beck, vel aff. Erodium cicutarium, L'Herit., var. micranthum, Beck
Mentha verticillata, L., var. paludosa, Druce

Urtica dioica, L., var. bispida, Gren. and Godr. Allium triquetrum, L. (at Salcombe, F. M. Ruddock) Typha angustifolia, L.

\section{Tavistock District}

Epilobium Lamyi, Schultz

Linaria vulgaris, Mill. (with double flowers)

Melampyrum pratense, L., var. hians, Druce
Mentha verticillata, L., var. subspicata, Druce Agropyrum repens, Beauv., var. lasiorachis, Hackel, fide Druce

The Linaria vulgaris with double flowers, occurred about three miles from Tavistock, and was figured in the Gardeners' Cbronicle, 9 November, 1895, p. 554, fig. 96. 'The calyx and corolla are mostly normal, and the stamens more or less petaloid; there is no pistil, but in its place there is a second corolla in a peloriate condition. The outer corolla is irregular with a single spur, but the supplementary corolla is regular and has a five-lobed limb and 5 spurs. The spike of inflorescence is about 9 in. long, the flowers being numerous and each, with the spur, $1 \frac{1}{6}$ in. long; the petaloid stamens are shortly exserted, and the included supplementary corolla is about half inch long.

\section{FILICES} district.

The bladder fern is still to be found in the parish of Challacombe in the Barnstaple

At the Linnean Society of London on 15 June, I899, Mr. W. Whitwell, F.L.S., exhibited a previously undescribed variety of a fern, regarded as belonging to Asplenium Rutamuraria, L. It had been obtained from an old wall on Dartmoor, about five miles from Plympton. Its chief peculiarities were stated to be the length, three inches, of the lamina, and its narrowness, half an inch. The pinnae were closely set, expanded, and flabellate, partially subdivided, and placed on short stalks on alternate sides of the rhachis; the contour of the whole thus differed entirely from that of the ordinary forms of $A$. Ruta-muraria.

\section{SELAGINELLACEAE}

Selaginella selaginoides, Gray, has been again reported on fairly satisfactory evidence as occurring in the neighbourhood of Lynton in the Barnstaple district. 


\section{ZOOLOGY}

\section{MARINE ZOOLOGY}

An account of the marine fauna of Devonshire is of necessity linked with the history of Marine Zoology in the British Isles. This connexion is probably due to the exceptional richness of a fauna which attracted the attention of many of the foremost naturalists of the nineteenth century. Among those who have published papers embodying information derived from the study of the marine fauna of Devonshire may be mentioned: Alder, Clarke, Couch, Cocks, Spence Bate, Gosse, Hincks, Montagu, Norman, and Parfitt. Many papers have also been published in the Association's Journal by workers at the Plymouth Laboratory of the Marine Biological Association.

The richness of the fauna being due chiefly to the very varied nature of the shore between tidemarks and of the bottom deposits in deeper water, a short sketch is given of the conditions prevailing in the more important estuaries and bays, and on the various trawling and dredging grounds off shore.

Of the former, Plymouth Sound, an arm of the sea, into which the estuaries of the Plym and Tamar open, is by far the most important and at the same time the most varied.

The shores round Plymouth Sound are for the most part rocky, with sand, mud, and gravel between the rocks. The latter vary in character and formation on different parts of the shore; being shale at Rum Bay, limestone under the Hoe, grit at Drake's Island, etc. Patches of sand are occasionally met with, the most productive being at Batten and Drake's Island, but there are no long stretches of sandy beach. In the estuaries of the Plym and Tamar the shore at low water is composed of mud, sand, or muddy gravel.

The bottom deposits of the Sound itself consist chiefly of fine muddy sand, with shoals of stones with mud, whilst honeycombed stones are to be found in Millbay Channel, and clean stones and shell gravel between Drake's Island and the west end of the breakwater. The latter is composed of large masses of limestone, which on the north side form a favourite collecting ground. The Church Reef in Wembury Bay, and the Reny Rocks between this and Bovisand Bay, are two reefs of rock with boulders. Rock pools are fairly numerous under the Hoe, in Wembury Bay, among the Reny Rocks, and on Drake's Island.

The shores of the Yealm River consist of mud, sand, and muddy gravel banks, with occasional patches of Zostera, whilst the bottom deposits in the channel are sand, with Zostera at the mouth, and stones and shells higher up. At Salcombe, the shore high up the estuary above the Salstone is fine mud ; from the Salstone to Snapes Point it is chiefly muddy gravel, whilst below Snapes Point it is almost entirely fine sand with several Zostera banks. The deposits in the channel above Snapes Point are chiefly fine sticky mud, with stones; round Snapes Point, clean shell gravel, and below this point stones and shells, whilst at the mouth of the harbour, on the bar, they are fine sand. 


\section{A HISTORY OF DEVONSHIRE}

Torbay is surrounded with rocks, with one or two sandy beaches, of which the largest is Paignton Sands. The bottom deposit in the bay is chiefly mud, with sand on the south side, and a few patches of rock, chief amongst which may be mentioned the 'Rough' off Elbury Cove.

In Teignmouth Bay the bottom deposits are sand inshore, down to 5 fms., outside this in I I-I 2 fms., mud, with large stones and shells.

In the estuary of the Exe low water exposes very large areas of mud, sand, and muddy gravel banks, with Ulva and occasional patches of Zostera, of which the largest is ' Greenlands,' situated high up the estuary ; the Zostera, however, is very stunted in growth compared with that found at Salcombe and in the Yealm. The Polesands, a large sandbank on the south side of the mouth of the estuary, consist of fine sand on the sea side, and coarse loose sand on the north side.

The conditions prevailing in the estuary of the Teign are somewhat similar to those in the Exe. Both have a particularly strong tide, which is said to run at 5 knots at Exmouth during spring tides.

On the north coast the shore is chiefly rocky, with a few sandy beaches, chief amongst which are those in Barnstaple and Morte Bays. The only important estuaries are those of the Taw and Torridge, both of which have a common outlet in Barnstaple Bay.

The bottom deposits in deeper water are no less varied. Mud is found in the east end of Start Bay, and from Berry Head to Teignmouth it extends in a south-east direction for IO-I 5 miles. With these exceptions most of the trawling grounds are of fine or coarse sand, with areas of various grades of gravel and stones, and an occasional outcrop of rock, especially close in shore. Of the rocky patches may be mentioned the Mewstone Ledge, off Yealm Head, the Eddystone Reef, the ledge in Teignmouth Bay, and the 'Rough' off Torcross.

Tidal differences are naturally of great importance to the shore collector. On the south coast they vary from $\mathrm{I} 8 \mathrm{ft}$. at springs and I $\mathrm{ft}$. at neaps at Plymouth to I 2 and $8 \frac{1}{2} \mathrm{ft}$. respectively at Exmouth.

The strength of the tidal currents in the estuaries is about 5 knots at spring tides at Exmouth and Teignmouth, 3 knots at Salcombe, and $\mathrm{I}-3$ in Plymouth Sound, whilst in the offshore area it varies between $\mathrm{I}$ and 2 knots.

According to Matthews ${ }^{1}$ the water in the channel during 1903 was derived chiefly from the Bay of Biscay in February and May, from the Irish Sea in August, and from both sources in November, its salinity varying between $35^{\circ} 26$ and $35^{\circ} 49$. Dickson ${ }^{2}$ gives the following as the mean monthly temperature of the surface of the sea at Salcombe, based on daily temperatures taken by the coastguards during i 7 years, I 880-86, I 888-97.

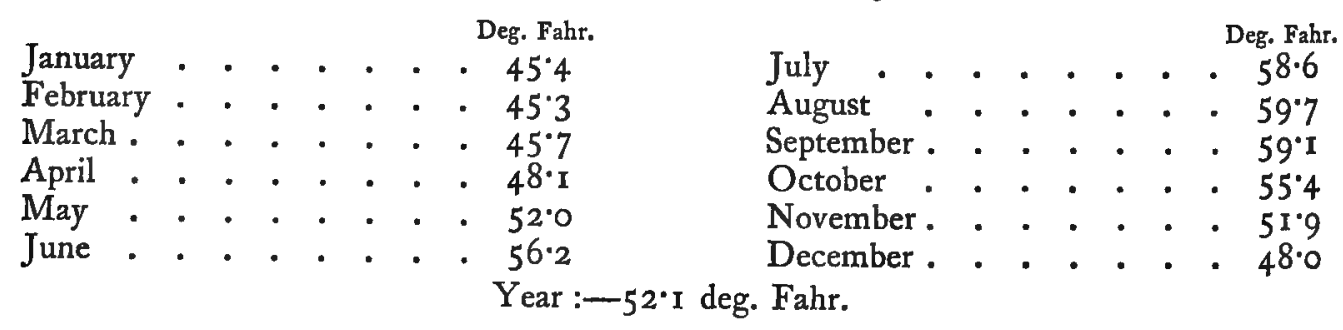

1 North Sea Fisheries Investigation Committee, Report 2, Southem Area.

The Mean Temperature of the Surface Waters of the Sea round the British Coast and its Relation to the Mean Temperature of the Air (Q.T.R. Met. Soc. Xxv.). 


\section{MARINE ZOOLOGY}

In the following list of the authorities which have been consulted, the nomenclature followed is that of the works marked with an asterisk.

Foraminifera: Brady, 'Synopsis of British Recent Foraminifera,'* J. R. M. S. I887 (Bdy.). Williamson, Recent Foraminifera of Great Britain (W.)

Porifera : Bowerbank, Monograph of British Spongiadae (B.). Hanitsch, 'Revision of the Generic

Nomenclature and Classification in Bowerbank's British Spongiadae, '* Trans. L. B. S. viii.

Hydrozoa: Hincks, A History of the British Hydroid Zoophytes * (H.)

Anthozoa : Gosse, British Sea Anemones and Corals* (G.)

Echinoderma : Bell, Catalogue of British Echinoderms in the British Museum.

Turbellaria: Gamble, 'British Marine Turbellaria,' * Q.J.M.S. xxxiv.

Nemertini : Burger, Nemertinen des Golfes von Neapel * (in general).

Polychaeta : St. Joseph, Les Annélides Polychètes des Côtes de Dinard * (in general).

Bryozoa : Hincks, $A$ History of the British Marine Polyzoa * (H.)

Mollusca: 'List of British Marine Mollusca and Brachiopoda,' * Journ. of Conch. vol. x. L. St. G.

Byne, 'A Contribution towards a List of the Marine Mollusca of Teignmouth.' 'Marine

Mollusca of Teignmouth Bay, additions,' Journ. of Conch. (L. B.). Forbes and Hanley, British

Mollusca (F. and H.). Alder and Hancock, A Monograph of the British Nudibranchiate Mollusca

(A. and H.). Jeffreys, British Concbology (J.G. J.)

Tunicata : Herdman, 'A Revised Classification of the Tunicata,'* Journ. Linn. Soc. Zoo. xxiii.

General : Journal of the Marine Biological Association (M. B. A.)

\section{FORAMINIFERA ${ }^{2}$}

\section{Miliolinas}

Biloculina ringens (Lam). Gen. dist. but c. only in certain localities (M.B.A.)

- depressa, D’Orb. Gen. dist. but 1.c. than B. ringens (M.B.A.)

- elongata, D'Orb. Plymouth (M.B.A.)

- tubulosa, D.C. Plymouth (?) (M.B.A.)

Spiroloculina planulata (Lam.). c. off Plymouth, r. off The Bolt (M.B.A.)

- limbata, D'Orb. Plymouth; Eddystone; Plymouth Sound shore (M.B.A.)

- tenuiseptata, Bdy. Plymouth, r. (M.B.A.)

- excavata, D'Orb. Plymouth ; Eddystone, gen. but r. (M.B.A.)

- fragilissima, Bdy. Off Eddystone (M.B.A.)

Miliolina trigonula (Lam.). Plymouth; Eddystone, gen. but not c. ; Bolt. occ. (M.B.A.); Brixbam ; Torbay; Plymouth Sound.

- tricarinata (D'Orb.). Plymouth; Eddystone, r. (M.B.A.)

- oblonga (M.). Plymouth; Eddystone, gen. dist. and c. ; Bolt Hd., l.c. (M.B.A.)
Miliolinae (continued)

Miliolina seminulum (L.). Plymouth ; Eddystone, gen. dist. and c. ; Bolt Hd., v.c. (M.B.A.)

- subrotunda (M.). Shore to 35 fms. ; gen. dist. ; v.c. in shallow water (M.B.A.)

- circularis (Born.). Plymouth ; Eddystone, f.c. ; off Bolt, occ. (M.B.A.)

- secans (D'Orb.) Gen. dist. in shallow water, especially on clean sand, c. on beaches (M.B.A.)

- bicornis (W. and J.). Gen. dist. and occ. plentiful (M.B.A.)

- boueana (D’Orb.). With M. bicornis, but l.c. (M.B.A.)

- agglutinans (D’Orb.). Gen. dist. and f.c. especially in shallow water (M.B.A.)

Peneroplidinae

Cornuspira foliacea (Phill.). Plymouth; Eddystone, gen. dist. but n.c. ; Exmouth, rr. (M.B.A.); Brixham; Torquay (W.)

- involvens (Reuss). Plymouth; Eddystone, gen. and f.c., often adhering to hydroids (M.B.A.)

1 Abbreviations used :-B. i, ii, etc. = Breeding Jan. Feb. etc. ; c. = common ; f.c. = fairly common; l.c. less common; m.c. $=$ moderately common; n.c. $=$ not common; occ. $=$ occasionally ; v.c. $=$ very common ; gen. dist. = generally distributed ; r. = rare ; rr. = very rare ; $d=$ dead ; h.w. = high water ; l.w. = low water ; s.t. $=$ spring tides.

List of abbreviations of names of authors of species:-A. Allmann; Ab. Abildgaard ; Ag. Agassiz; Ald. Alder; Aud. Audouin; A. and E. Audouin and M.-Edward; B. Bowerbank; Bdy. Brady ; Bgr. Burger; Bl. Blainville ; Bk. Busk ; Brug. Bruguiere ; Cant. Cantraine ; Ch. Chemnitz; Clpd. Claparede ; Cuv. Cuvier ; D’Orb. D'Orbigny ; D.C. Da Costa ; Dal. Dalyell ; Dfr. Defrance ; Dj. Dujardin ; Dl.C. Delle Chiaji ; Don. Donovan ; E. Ehrenberg; Eh. Ehlers; Esp. Esper ; E. and S. Ellis and Solander ; F. Fabricius ; Fb. Forbes ; Fl. Fleming ; Fr. Forskal ; F. and L. Frey u. Lenckart ; F. and M. Fictel and Moll ; G. Gosse ; Gb. Grube; Gm. Gmelin; Gr. Gaertner ; Gron. Gronovius ; H. Hincks ; Has. Hassall ; Hkl. Haeckel ; Ht. Hartlaut ; J. Johnston; Jn. Jensen ; K. Keferstein ; Koll. Kolliker ; L. Linnæus ; Lam. Lamarck ; Les. Lesson; Lv. Levinsen; L. B. L. St. G. Byne; M.-E. Milne-Edward ; M. Montagu ; Mcg. Macgillivray; McInt. MacIntosh; Mets. Metschnikoff; Mlg. Malgrem; M. and B. Marion et Bobretsky ; Mich. Michaud; N. Norman; O. Oersted; Ol. Olivi ; O. F. M. O. F. Muller ; P. Pallas ; Penn. Pennant ; Ph. Phillipi ; Pult. Pulteney; P. and J. Parker and Jones ; P. and L. Peron and Lesueur; Q. Quatrefages; Q. et G. Quoy et Gaimard; R. Riches; Rk. Rathke; R.L. R. Leuckart ; S. Sars ; Seg. Seguenza ; Sch. Schmidt ; Shz. Shultze ; St. Steenstrup; St. J. St. Joseph; Sb. Sowerby; Sv. Savigny ; T. Topsent ; Terq. Terquem; Th. Thompson; Turt. Turton; T.S. W. T.S. Wright; W. Williamson; W. and B. Walker and Boys; W. and J. Walker and Jacobs; v. B. van Beneden; v. G. von Graff ; v. M. von Marenzeller. 


\section{A HISTORY OF DEVONSHIRE}

\section{Astrorhizidab}

Haliphysema tumanowiczii, B. Plymouth ; Eddystone, gen. dist., c. near shore at times (M.B.A.); Torbay ; Budleigb Salterton (Bdy.)

- ramulosum, B. Budleigh Salterton (Bdy.)

\section{Lituolinae}

Haplophragmium canariense (D'Orb.). Plymouth; Eddystone, gen. dist., c. on silty areas (M.B.A.); Brixbam; Exmouth (W.)

- fontinense, Terq. Eddystone, r. (M.B.A.)

- globigerineforme (P. and J.). Plymouth; Eddystone, r. (M.B.A.)

Placopsilina varians, Carter. Budleigh Salterton (Bdy.)

\section{Trochammininae}

Ammodiscus incertus (D'Orb.). Eddystone, r. (M.B.A.) - gordialis (P. and J.). Eddystone, r. (M.B.A.)

Trochammina ochracea, W. Gen. dist. but n.c. (M.B.A.)

- inflata (M.). Estuaries to 35 fms. (M.B.A.)

\section{Textularinae}

Textularia sagittula, Dfr. Plymouth, n.c.; most typical specimens in estuarine waters (M.B.A.)

- trochus, D'Orb. Plymouth, n.c. (M.B.A.)

- agglutinans, D'Orb. Plymouth, gen. dist. but n.c. (M.B.A.)

var. porrecta, occ. (M.B.A.)

- gramen, D'Orb. Plymouth district, gen. dist. and c. Verneuilina polystropha (Reuss). Plymouth, gen. dist., but n.c. except in estuaries (M.B.A.)

\section{Bulimininas}

Bulimina pupoides, D'Orb. Plymoutb to Bolt Hd., c. within $25 \mathrm{fm}$. line, l.c. without ; Salcombe; Exmouth (M.B.A.) ; Torbay; Brixbam (W.)

- marginata, D'Orb. Plymouth to Bolt, half as numerous as B. pupoides (M.B.A.)

- aculeata, D'Orb. Plymouth to Bolt, about onetwentieth as numerous as B. pupoides (M.B.A.)

- elegans, D'Orb. Sparingly, with B. pupoides (M.B.A.)

- elegantissima, D'Orb. Plymoutb to Bolt Hd., gen.dist.but n.c.(M.B.A.); Torbay; Exmouth(W.)

Virgulina schreibersiana, Czjzek. Plymouth to Bolt, gen. dist. and c. (M.B.A.)

Bolivina punctata, D'Orb. Plymouth to Bolt, gen. dist. and c., even on muddy silt ; Salcombe, $\mathbf{r}$. (M.B.A.)

- textilaroides, Reuss. Plymouth to Bolt Hd., gen. dist. but n.c. ; Exmouth Dock (M.B.A.)

- difformis (W.). With B. textilaroides (M.B.A.)

- dilatata, Reuss. With B. punctata (M.B.A.)

- xnariensis (D.C.). Plymoutb to Bolt Hd., gen. dist. and c. (M.B.A.)

\section{Cassidulininaz}

Cassidulina crassa, D'Orb. Plymoutb to Bolt Hd., gen. dist. and f.c. (M.B.A.) ; Brixhom (W.)

- bradyi, N. With C. crassa, but l.c. (M.B.A.)

\section{LaGeninae}

Lagena globosa (M.). Plymouth to Bolt Hd., c. and gen. dist. (M.B.A.) ; Brixham; Exmouth (Bdy.)

- apiculata, Reuss. Plymouth, somewhat r.; Salcombe, r. (M.B.A.)
Lageninaz (continued)

Lagena botelliformis, Bdy. Plymouth, f.c. (M.B.A.)

- laevis (M.). Gen. but not v.c.

var. perlucida and striata, with the type. Plymouth to Exmouth

- clavata (D'Orb.). Gen. dist. but much l.c. than L. laevis

- gracillima (Seg.). Plymouth to Bolt Hd. f.c. (M.B.A.)

- aspera, Reuss. Plymouth to Bolt Hd., r., with a preference for shallower waters (M.B.A.)

- hispida, Reuss. Plymouth to Bolt Hd., somewhat r. (M.B.A.)

- lineata (W.). Plymouth to Eddystone, n.c. (M.B.A.); Exmoutb; Torbay, etc. (B.)

- distoma, P. and J. Plymouth to Bolt Hd., gen. dist. but scarce (M.B.A.)

- sulcata (W. and J.). Plymouth to Bolt, gen. and c. ; Salcombe, c. (M.B.A.)

- striata (D'Orb.). Plymouth to Bolt Hd., gen. and c. (M.B.A.)

- semistriata, W. Plymouth to Bolt Hd., gen. and c. (M.B.A.)

- gracilis, W. Plymouth to Bolt Hd., gen. and c. (M.B.A.)

- squamosa (M.). Plymouth to Bolt Hd., gen. and c. (M.B.A.) ; Torbay ; Exmouth (W.)

- hexagona (W.). Plymouth to Bolt, gen. and c.; Salcombe, r.; Exmouth, r. (M.B.A.)

- marginata, W. and B. Plymouth to Bolt Hd., gen. and $c$.

- Iucida, W. Brixbam; Plymouth Sound; Eddystone (W.)

- trigono-marginata (P. and J.). Plymouth to Eddystone, gen. dist. but r. (M.B.A.)

- quadrata (W.). Plymouth to Bolt Hd., gen. dist. but n.c. (M.B.A.) ; Brixbam (W.)

- orbignyana (Seg.). Plymouth to Bolt Hd., gen. dist. and commonest of the Lagenae ; Salcombe, f.c. ; Exmouth, r. (M.B.A.)

- lagenoides (W.). Plymouth to Bolt Hd., gen. but not v.c. ; Salcombe, f. (M.B.A.)

\section{Nodosarinae}

Nodosaria pyrgula, D'Orb. Plymouth to Bolt, gen. dist. but somewhat scarce (M.B.A.) ; Exmouth (W.)

- communis, D'Orb. Plymouth to Bolt, gen. dist. in moderate numbers, some very large (up to $4 \mathrm{~mm}$.) ; Exmouth (W.)

- scalaris (Batsch.). Plymouth to Bolt, gen. dist. but somewhat scarce in places (M.B.A.)

- calomorpha, Reuss. Plymouth to Bolt, f.c. (M.B.A.)

Lingulina carinata, D'Orb. Plymouth Sound, single specimen (W.)

Cristellaria crepidula (F. and M.). Gen. but n.c., Plymouth to Bolt (M.B.A.); Brixham (W.)

- rotulata (Lam.). Plymouth to Bolt, gen. and c. (M.B.A.)

- vortex (F. and M.). Plymouth, occ. (M.B.A.)

- italica (Dfr.). Plymoutb to Bolt, gen. but scarce (M.B.A.)

Amphicoryne falx (P. and J.). Plymoutb, r. (M.B.A.)

Polymorphina lactea (W. and J.) Plymoutb to Bolt, gen. but n.c.; Salcombe (M.B.A.); Brixham; Exmouth (W.) 


\section{MARINE ZOOLOGY}

\section{Nodosarinae (continued)}

Polymorphina gribba, D'Orb. Plymouth to Bolt Hd., gen. but n.c. (M.B.A.)

- concava (W.). Brixham (W.)

- problema, D'Orb. Plymouth Sound; Eddystone; Brixham, v.c. (W.)

- oblonga, W. Eddystone, occ. ; Plymoutb Sound, on Zostera (M.B.A.); v.c. near Eddystone in or before 1858 (Spence Bate)

- compressa, D'Orb. Plymouth, occ. ; Exmouth, r. (M.B.A.)

Uvigerina angulosa, W. Plymouth to Bolt, gen. but n.c. (M.B.A.) ; Torbay (W.)

\section{Grobigerinidar}

Globigerina bulloides, D'Orb. Plymouth to Bolt, gen. and f.c. ; Salcombe, f.c. (M.B.A.)

- inflata, D'Orb. Plymouth, r. (M.B.A.)

Orbulina universa, D'Orb. Plymouth, r. (M.B.A.) Eddystone ; Brixham (W.)

Sphaeroidina dehiscens, P. and J. Plymouth, r. (M.B.A.)

\section{SPIRILLININAB}

Spirillina vivipara, E. Plymouth to Eddystone, gen. but n.c.; Salcombe, f. (M.B.A.)

- margaritifera, W. Plymouth to Eddystone, occ. (M.B.A.)

- tuberculata, Bdy. Eddystone (Bdy.)

\section{Rotalinas}

Patellina corrugata, W. Plymouth to Bolt, gen. but n.c. (M.B.A.) ; Brixham (W.)

Discorbina globularis (D'Orb.). Plymouth to Bolt, gen. and in places c. (M.B.A.)

- rosacea (D'Orb.). Plymouth to Bolt, gen. and c. Salcombe (M.B.A.) ; Torbay ; Exmouth (W.)

- orbicularis (Terq.). Plymouth to Bolt, gen. and c. (M.B.A.)

- parisiensis (D'Orb.). Plymoutb to Bolt, f. gen. but scarce in places (M.B.A.)

- bertheloti (D'Orb.). Plymouth to Bolt, as D. parisiensis (M.B.A.)
Rotalinae (continued)

Planorbulina mediterraniensis, D'Orb. Plymouth to Bolt, gen. but in varying abundance; Salcombe, c. (M.B.A.) ; Brixham; Torquay; Exmouth (W.)

Truncatulina lobatula (W. and J.). Plymouth to Bolt gen., most c. where there are hydroids, especially Sertularella gayi ; Exmouth, c. (M.B.A.)

Pulvinulina repanda (F. and M.). Plymouth to Bolt, gen. but n.c. (M.B.A.)

- menardii (D'Orb.). Somewhat uncommon and doubtfully an inhabitant of the Plymouth district (M.B.A.)

Rotalia beccarii (L.). Gen. and v. c. especially on fine sand ; Io grains of bottom deposit from $20 \mathrm{fms}$. $4 \mathrm{~m}$. W. of Rame $\mathrm{Hd}$. are recorded as containing nearly $12,000 R$. beccarii, with an equal number of M. seminulum (M.B.A.)

- nitida, W. Plymouth to Bolt, gen. and c. (M.B.A.) Brixham; Torquay (W.)

\section{Polystomellinae}

Nonionina depressula (W. and J.). Plymouth to Exmouth, c. (M.B.A.)

- umbilicatula (M.). Plymouth to Bolt; estuaries to deep water, but chiefly in the latter (M.B.A.)

- turgida (W.). Plymouth to Bolt, gen. but n.c. (M.B.A.)

- scapha (F. and M.). With N. turgida

- stelligera, D'Orb. Plymouth to Exmouth, gen. with a preference for shallow water (M.B.A.)

Polystomella crispa (L.). Plymouth to Exmouth, gen. dist. inside 15 fms., $c$. inside 10 fms. but practically absent outside $20 \mathrm{fm}$. (M.B.A.).

- subnudosa (Munster). Plymouth, not infrequent outside 28 fms. (M.B.A.)

- macella (F. and M.). Plymouth Sound and probably everywhere with P. crispa (M.B.A.)

- striatopunctata (F. and M.). Gen. and c. to $30 \mathrm{fm}$. line, f.c. outside that limit (M.B.A.)

- arctica, P. and J. Plymouth to Bolt, gen. and $c$.

\section{PORIFERA}

\section{CALCAREA}

\section{HOMOCOELA}

Leucosolenia botryoides (E. and S.). Plymouth, shore, v.c. in certain places (M.B.A.)

- complicata (M.). Plymouth, f.c. in rock pools and deep water (M.B.A.)

- variabilis, Hkl. Plymouth, c. everywhere in rock pools (M.B.A.)

- lacunosa (J.). Plymouth, below tidemarks, n.c. (M.B.A.)

- coriacea (Fl.). Plymouth, between tidemarks, c. in places (M.B.A.)

\section{Heterocoela}

Sycon compressum (F1.). Plymouth, c. I.w. on rocky shores. Ova develop in Feb., larvae occur in June, and sponges $3 \mathrm{~mm}$. long in Sept. (M.B.A.)

\section{CALCAREA (continued)}

Heterocorla (continued)

Sycon coronatum, E. and S. Plymouth, c. on rocky stations at l.w. in less exposed situations than S. compressum (M.B.A.)

Leucandra gossei, B. Torquay; Budleigb Salterton (B.) - fistulosa, J. Eddystone Rk.; Budleigh Salterton (B.)

- nivea, B. Budleigh Salterton (B.)

\section{SILICEA}

Order Hexaceratina

Halisarca dujardini, J. Plymouth Sound (M.B.A.)

\section{Order Tetractinellida}

Dercitus bucklandi, B. Torqquay; Budleigh Salterton (B.)

Stellata collingsi, B. Paignton (B.)

Stryphrus ponderosus, B. Budleigh Salterton (B.)

Pachymatisma johnstonia, B. Torquay, Budleigh Salterton (B.) 


\section{A HISTORY OF DEVONSHIRE}

\section{SILICEA (continued)}

Order Monoxonida

Haplosclertdae

Chalina oculata (P.). Plymouth Sound; Wembury Bay (M.B.A.)

Pachychalina gracilenta, B. Torquay (B.)

- montagui, Fl. Devon (B.)

Halichondria albescens, J. Torbay (B.)

- coalita, Grant. Devon (B.)

- coccinea, B. Salcombe (B.)

- distorta, B. Brixbam trawlers (B.)

- fragilis, B. Dartmouth (B.)

- panicea, P. Plymouth Sound, c.; Exmouth, v.c. in channel between pier and Checkstone Ledge (M.B.A.)

Reniera anomala, B. Torbay (B.)

- cinerea, Grant. Budleigh Salterton (B.)

- indefinata, B. Ilfracombe (B.)

- indistincta, B. Exmouth (B.)

- pallida, B. Torquay (B.)

- ramuscula, B. Torbay (B.)

\section{Poeciloscleridae}

Leptolabis luciensis, T. Plymouth (M.B.A.)

Esperiopsis paupera, B. Torquay (B.)

Esperella macilenta, B. S. Devon (B.)

- lobata, M. Devon (B.)

Dendoryx incrustans, Esper. Budleigh Salterton (B.)

Stylostichon plumosum, M. Budleigh Salterton (B.)

Raspailia ramosa, M. Torquay; Brixbam trawlers (B.)

- hispida, M. Devon (B.)

\section{Axinellidar}

Hymeniacidon aureum, M. Kingsbridge Estuary (B.)

- carunculum, B. Salcombe (B.)

- fallax, B. Torbay (B.)

- pachydermum, B. Torquay (B.)

- perlevis, M. Devon (B.)

Tragosia infundibuliformis (J.). Plymouth (M.B.A.)

- polypoides, Schmidt. Mewstone Ledge (M.B.A.)

Axinella stuposa, M. Torbay (B.)

\section{Suberitidae}

Suberites carnosus, J. Plymouth (B.)

- domuncula, OI. Gen. (B.)

Polymastia mamillaris (M.) Plymouth Sound; Yealm R. ; off Merostone (M.B.A.)

Cliona celata, Grant. Universal, boring in shells, especially in oyster; also in limestone

\section{TEThyidae}

Tethya lyncurium, L. Plymouth (M.B.A.); Torquay; Budleigh Salterton (B.)

\section{ANTHOMEDUSAE}

(Hydroid Stage)

\section{Clavidae}

Clava leptostyla, Ag. Plymouth Sound. Gonophores, Mar. (M.B.A.)

- multicornis ( $\mathrm{Fr}$.). c. and gen. dist. in tidepools. Gon. Feb.-Apr., Nov. (M.B.A.)

- squamata (M.). Plymouth Sound, f.c. on shore. B. ii, v, ix (M.B.A.)

\section{ANTHOMEDUSAE (continued)}

Clavidar (continued)

Tubiclava cornucopiae (N.). Plymoutb district, m.c. on shells of Aporrhais, Dentalium, and Turritella, tenanted by Phascolion strombi. B. v, vi (M.B.A.)

- lucerna, A. Plymouth Sound (M.B.A.); Torquay (H.)

Turris neglecta, Les. Ilfracombe (H.)

\section{Hydractinimdae}

Hydractinia echinata, F1. Gen. dist. and c. on shells of Buccinum, etc., inhabited by Eupagurus Bernhardus. B. viii, $x$ (M.B.A.)

\section{Podocorynidae}

Podocoryne carnea, S. Plymouth, IO-20 fms., occ. on shells of living Nassa reticulata (M.B.A.); Torbay, on Nassa and Turritella (H.). Medusae liberated May, 1896. (M.B.A.)

- proboscidea, $\mathrm{H}$. Ilfracombe, $\mathrm{H}$.

\section{LARIDAE}

Lar sabellarum, G. Millbay Cbannel, r. on tubes of Potamilla torelli. Medusae (Willsia stellata) attached in Feb. (M.B.A.)

\section{Corynidar}

Coryne fruticosa, H. Exmouth (H.)

- pusilla, Gaer. Ilfracombe, rock pools (H.)

- vaginata, H. f.c. in tidepools, S. Devon, Ilfracombe (H.). B. iv-viii (M.B.A.)

Syncoryne eximia, A. I mile S. Merustone, single colony, May i 889 (M.B.A.)

- gravata (T.S.W.). Plymouth Sound, occ. with attached medusae, iii, iv

- pulchella, A. Ilfracombe, on roots of Laminaria $\left(\mathrm{H}_{\text {. }}\right)$

Zanclea implexa (Ald.). Plymouth, single colony, Aug. 1899 (M.B.A.)

\section{STAURIDIIDaE}

Cladonema radiatum, $\mathrm{Dj}$. Ilfracombe (G.)

Stauridium productum, T.S.W. Ilfracombe, v.c. (H.) ; Plymouth, in laboratory tank, June I 899 (M.B.A.)

\section{Clavatellidae}

Clavatella prolifera, H. Torquay ; Ilfracombe (H.)

\section{Mrriothelidae}

Myriothela phrygia, F. Plymouth, c. under stones, I.w. (M.B.A.); Torquay; Ilfracombe (H.). ripe Jan.-Aug. (M.B.A.)

\section{Eudendritdae}

Eudendrium album, Nutting. One of the most abundant hydroids in Plymouth Sound in the spring on stones from dredgings. Gonophores, Jan.-May (M.B.A.)

- capillare, Ald. Off Stoke Point on worm tubes, etc.; Eddystone Gnds. (M.B.A.); Torbay on Salicornaria, m.c. (H.)

- insigne, $\mathrm{H}$. Torquay, shore; Ilfracombe $(\mathrm{H}$.

- rameum (P.). Off the Eddystone, $30 \mathrm{fms}$., n.c. (M.B.A.)

- ramosum (L.). Gen. dist. to 30 fms. especially on sandy grounds. B. ii-v, xi (M.B.A.) 


\section{MARINE ZOOLOGY}

\section{ANTHOMEDUSAE (continued)}

\section{Atractylidar}

Perigonimus repens (T.S.W.). Plymouth Sound, and outside to $35 \mathrm{fms}$. on gastropod shells ; Cattewater, on abdomen of Carcinus ; Exmouth, on Donax vittatus (M.B.A.) ; Ilfracombe on stone (H.). B. viii, medusae iv (M.B.A.)

- serpens, A. Plymouth, on old rope, Oct. I 897 ; Eddystone Buoy, Apr. 1898 (M.B.A.); Torbay, on Halicornaria; Ilfracombe, on rocks and Laminaria (H.). Medusa buds, Oct. (M.B.A.)

Hydranthea margarica, $\mathrm{H}$. Ilfracombe, v.c. on Flustra foliacea, 7-10 fms. (H.)

Garveia nutans, T.S.W. Plymouth, shore-20 fms. m.c. B. ii-iv (M.B.A.)

Bimeria vestita, T.S.W. Torbay and Salcombe, m.c. on other zoophytes ( $\mathrm{H}$.)

Heterocordyle conybeari, A. Plymouth Sound, c. on living Nassa reticulata; outside the Sound on shells tenanted by Eupagurus Bernhardus. Gonophores, Aug., Oct. (M.B.A.)

Bougainvillia ramosa (V.B.). Plymouth shore-30 fms., f.c. gen. on polychaete tubes and other hydroids (M.B.A.); Torbay (H.). Medusae, Apr., May (M.B.A.)

- muscus, A. Torquay, v.c. in rock pool (H.)

\section{Tubularindae}

Tubularia indivisa, L. Plymouth, etc., shore-30 fms. m.c. especially on rough ground. B. ii-v (M.B.A.)

- humilis, A. Plymouth Sound, occ. B. iii (M.B.A.)

- larynx, E. and S. Plymouth Sound, v.c. on buoys, 1889, not taken in 1895 (M.B.A.); S. Devon, c. on stones between tidemarks $(H$.$) . B. i v-v$, xii (M.B.A.)

\section{Corymorphidae}

Corymorpha nutans, S. Plymouth Sound, May 1895, June 1904, r. Medusa buds, May (M.B.A.)

\section{Penarimdae}

Acharadria larynx, T.S.W. Ilfracombe, on stones (H.)

\section{(Medusa Stage) ${ }^{1}$}

\section{Codonidar}

Sarsia eximia, A. (Hydroid = Syncoryne). July - gemmifera, Fb. May-Sept. Medusa buds, July - prolifera, Fb. May-Aug., c. May I896, v.c. July 1899

- tubulosa (S.) Apr.-May occ. v.c.

Ectopleura dumortieri, Ag. Plymouth Sound, single specimen

Dipurena halterata, Fb. Sept. 1893, July 1899, r. - ophiogaster, Hkl. July I 897, single specimen

Perigonimus repens (T.S.W.). Budded off in tank in Mar.

Steenstrupia rubra, Fb. (Hydroid=Corymorpha nutans.) Abundant in spring and early summer

Hybocodon prolifer, Ag. Apr. I 894 r., Apr. I 898 m.c. Euphysa aurata, Fb. Sept. 1897, Apr.-June I 898, r.

$$
1 \text { M.B.A. records. }
$$

\section{ANTHOMEDUSAE (continued)}

\section{TIARIDAe}

Amphinema dinema (P. and L.). May-Sept. occ. c. Tiara pileata, Ag. May-June, Sept. occ.

\section{Margellidab}

Lizzia blondina, Fb. May-Sept., occ. v.c.

Podocoryne carnea, S. Mar., July-Aug.

Cyteandra areolata, Hkl. June, Sept. occ.

Margelis autumnalis, Ht. Sept. 1897 , I 898, r.

- bella, Ht. May, June $1898 \mathrm{r}$.

- britannica (Fb.). End of Apr.

- princeps, St. End of Apr.

Margellium octopunctatum (S.). Feb., Mar. 1893 v.c.; 1894 less abundant ; 1898 c. ; Apr., May, r.

\section{Cladonemidae}

Gemmaria implexa (Ald.). Aug., Sept. rr.

Willia stellata (Hydroid = Lar sabellarum), May-Sept. f.c. in Sept. 1897 .

\section{LEPTOMEDUSAE}

\section{(Hydroid Stage)}

\section{Campanulariidae}

Clytia Johnstoni, Ald. v.c. on other hydroids, etc., l.w.-35 fms. B. iii, iv, vii. Medusae with gonothecae, July (M.B.A.)

Obelia geniculata (L.). Gen. dist. and c. on Laminaria, Fucus, etc. Gonophores, Mar.-Sept. (M.B.A.)

- dichotoma (L.). Eddystone Gnds. on fine sand growing on other hydroids, etc. (M.B.A.). c. (H.)

- longissima (P.). S. of Eddystone; c. off Start Bay; c. (M.B.A.), v.c. in trawl refuse (H.)

Obelaria gelatinosa (P.). Plymouth Estuaries, often v.c. (M.B.A.) ; Exmouth, Ilfracombe (H.)

Campanularia angulata, H. Bovisand Bay (M.B.A.); Torbay (H.)

- caliculata, $\mathrm{H}$. Ilfracombe (H.)

- flexuosa (H.). c. and gen. dist. in littoral region

- hincksii, Ald. Plymouth, c. Io-30 fms. on hydroids, Cellaria, etc. (M.B.A.) ; Torbay, 8 fms. (H.)

- integra, Mcg. Ilfracombe, on red weed (H.)

- neglecta (Ald.). Plymouth Sound, on Tubularia indivisa (M.B.A.) ; Devonsbire, of large size (H.)

- raridentata, Ald. Torquay, on zoophytes from Brixham trawlers (H.)

- verticillata (L.). S. Devon, c. in trawl refuse

- volubilis (L.). Plymouth Sound, $5 \frac{1}{2}$ fms. c. (M.B.A.) ; Sonton, Smallmouth (H.)

Lovenella clausa (Loven). Torbay, 10 fms. (H.)

Gonothyraea loveni, A. Plymouth Sound, c. ; Dartmouth, Torquay (H.). B. iii, iv, ix, xi (M.B.A.)

\section{Campanulinidab}

Campanulina repens, A. Plymouth Sound, Mequstone Gnds. occ. Gonophores, July (M.B.A.)

Zygodactyla vitrina (G.). Ilfracombe (H.)

Opercularella hispida, Nutting. Plymouth, on stone with Clava multicornis (M.B.A.)

- lacerta (J.). Millbay Dock, on stems of Tubularia indivisa and Eudendrium (M.B.A.); Exmouth, on Bowerbankia; Ilfracombe (H.) 


\section{A HISTORY OF DEVONSHIRE}

\section{LEPTOMEDUSAE (continued)}

\section{LAFOËIDAB}

Lafoea dumosa (Fl.). c. and gen. dist. 15-35 fms., on shells, polychaete tubes, etc. ; var. robusta frequently with Myzomenia bangulensis (M.B.A.)

- fruticosa, S. Plymouth, m.c., I 5-30 fms. (M.B.A.); S. Devon (H.)

Calycella fastigiata (Ald.). Eddystone Gnds. (M.B.A.)

- syringa (L.). Plymouth, abundant on roots of Laminaria and on Tubularia indivisa and Sertularia (M.B.A.) ; gen. and c. (H.)

Cuspidella costata, $H$. Inner Eddystone trawling grounds (M.B.A.)

- grandis, H. Plymouth, on Halecium tenellum. (M.B.A.)

TRICHYDRIDAE

Trichydra pudica, T.S.W. Eddystone Gnds. on fine gravel ; Eddystone Buoy (M.B.A.)

\section{Coppinitidar}

Coppinia arcta (Dal.). c. and gen. dist., growing on other hydroids, especially Lafoea dumosa, var. robusta, and Sertularia abietina

\section{Haleciludae}

Halecium beanii (J.). f.c. and gen. dist. on shells, hydroids, Chàetoptertus tubes, etc. Gonophores, Jan., Mar., May, July, Oct. (M.B.A.)

- halecinum (L.). c. and gen. dist., growing on Chaetopterus tubes, shells, stones, etc. B. i-vii (M.B.A.)

- labrosum, Ald. Eddystone Gnds. (M.B.A.)

- tenellum, H. Plymouth, 18 fms. (M.B.A.) ; Salcombe, on Salicornaria (H.). B. iv (M.B.A.)

Haloikema lankesteri, Bourne. Plymouth Sound (M.B.A.)

Ophioides mirabilis, H. Ilfracombe, v.c. on Laminaria (H.)

\section{SERTULARIIDAE}

Sertularella fusiformis, H. S. Devon, shore; Ilfracombe (H.)

- gayi (Lam.). Gen. dist. v.c., especially on fine sand of Eddystone Gnds. B. ii, iii, viii, $\mathbf{x}$ (M.B.A.)

- polyzonias (L.). Gen. dist. and f.c. B. viii, ix (M.B.A).

- tenella, Ald. S. Devon, shore (H.)

Diphasia attenuata, H. Outer Eddystone Gnd., fine sand (M.B.A.) ; Ilfracombe (H.)

- pinaster (E. and S.). v.c. in trawl refuse S. of Eddystone (M.B.A.)

- pinnata (P.). 5 m. S. of Eddystone; Torbay (M.B.A.). B. iv, Gonophores v (M.B.A.)

- tamarisca (L.). Eddystone Gnds. (M.B.A.)

Sertularia abietina (L.). Gen. dist. and c., sometimes in great abundance inside the $30-\mathrm{fm}$. line

- argentea, E. and S. c. in trawl refuse and f.c. in estuaries. B. ii-iv (M.B.A.)

- cupressina, L. Eddystone, trawl refuse c. ; Plymoutb Sound, occ. (M.B.A.) ; Brixham, trawl refuse c. (H.)

- gracilis, Has. Ilfracombe (H.)

- operculata, L. f.c. in inshore waters, abundant Eddystone Rock, Apr. I 898 (M.B.A.)

- pumila, L. Gen. dist. and c. on rocks and Fucus. B. iii-vii (M.B.A.)

\section{LEPTOMEDUSAE (continued)}

Sertularindae (continued)

Hydrallmania falcata (L.) Gen. dist. and c., especially on stony grounds, attached to stones and shells. B. i-iv (M.B.A.)

Thuiaria articulata (P.). Wembury Bay; Merostone Gnds., etc. (M.B.A.) ; S. Devon, m.c. (H.)

- thuja (L.). S. Devon (H.)

\section{Plumularitdar}

Antennularia antennina (L.). c., especially on medium gravel and muddy sand, 15-30 fms. B. iv-vii (M.B.A.)

- ramosa (Lam.). With A. antennina but l.c. B. iv $-\mathrm{v}$ (M.B.A.)

Aglaophenia helleri, Marktanner-Turneretscher. Eddystone Rock, on roots of algae, May I 895 (M.B.A.)

- myriophyllum (L.). m.c. on fine sand and sand and gravel, 15-30 fms., often with Rhopalomenia aglaopheniae. B. iv-vi, viii (M.B.A.)

- pennatula (E. and S.). Torbay; Teignmouth, rr. (H.)

- pluma (L.). c. and gen. dist. in Laminarian zone on algae, especially Halidrys siliquosa. B. v, viii (M.B.A.)

- tubulifera (H). Plymouth, outside to $30 \mathrm{fms}$., AugOct. ; Salcombe, r. B. ix, x (M.B.A.)

Plumularia alleni, Nutting. Plymouth, on Antennularia ramosa. Gonophores, Apr., May (M.B.A.)

- catharina, J. c. I 5-35 fms. on other hydroids, on fine sand, also f.c. inshore, green var. often abundant on Chaetopterus tubes off Plymouth (M.B.A.) ; stemless var. Ilfracombe (H.). B. iv, v, viii (M.B.A.)

- echinulata, Lam. Plymouth Sound, on algae ; Yealm, m.c. on Delesseria (M.B.A.)

- frutescens (E. and S.). Off Plymouth, r. (M.B.A.)

- halecioides, Ald. Plymouth, parasitic on P. setacea and Antennularia (M:B.A.); Ilfracombe, in rock pools (H.)

- obliqua, Saunders. Ilfracombe; Capstone, abundant (H.)

- pinnata (L.). c. and gen. dist. 1.w.-30 fms., asexual stoloniferous reproduction observed in April. Gonophores, Apr.-June (M.B.A.)

- setacea (Ellis). 1.w.-30 fms. c. gen. on other hydroids and polychaete tubes, especially those of Chaetopterus; Hincks's branched var. Plymouth, v.c. gen. on Halichondria panicea. B. i-vi (M.B.A.)

- similis, H. S. Devon, abundant on weed in Laminarian zone (H.)

\section{(Medusa Stage)}

\section{Eucopidas}

Agastra mira (Ht.). (Hydroid=A. caliculata.) Aug. 1897 , single specimen

Obelia lucifera, Fb. v. plentiful, June 1892 ; most abundant Leptomedusan, Sept. 1897

- nigra, Browne. c. Apr.-June

Tiaropsis multicirrata (S.). Apr. 1895

Euchilota pilosella ( $F$ b.). c. every summer

Mitrocomella fulva, Browne. Off Eddystone, single specimen, May 1898

\footnotetext{
1 M.B.A. records.
} 


\section{MARINE ZOOLOGY}

\section{LEPTOMEDUSAE (continued)}

Eucopidar (continued)

Phialidium buskianum (G.). Sept. 1893, 1895, 1897, gen. present ; June 1898 , once only

- cymbaloideum (v. B.). (Hydroid=? Campanulina repens.) Apr.-Sept. gen. present, but n.c.

- temporarium, Browne. Spring to autumn

Entima insignis (K.). June-Oct., occ. single specimens Saphenia mirabilis (T.S.W.). Apr.-Sept., occ. c. Octorchis gegenbauri, Hk. July-Sept., v. occ.

Irene pellucida (Will.). Oct._Sept., occ.

\section{TRACHYMEDUSAE ${ }^{1}$}

\section{Geryonidar}

Liriantha appendiculata, Fb. Exc. abundant, Sept.Oct. I 893 ; absent, Sept. 1895 ; scarce, Sept. I 897, Jan. I 896

Solmaridae

Solmaris coronanthe, Hkl. Sept. 1895

\section{SIPHONOPHORA ${ }^{1}$}

MONOPHYIDAE

Muggiaea atlantica, Cunningham. Aug.-Dec., often in great abundance; occ. in Feb.

\section{Agalmidae}

Agalmopsis sarsi, Hkl. Mar. Igo2, two specimens

\section{STAUROMEDUSAE}

\section{LUCERNARIIDAR}

Lucernaria campanulata, Lam. Plymouth Sound; Reny Rocks, r.

Haliclystus auriculata (F.). Yealm Est., f.c. on Zostera ; Plymouth Sound

Depastrum cyathiforme, S. Plymouth Sound

\section{DISCOMEDUSAE ${ }^{1}$}

\section{Pelagindae}

Chrysaora isoceles (L.). Summer, occ. Planulae liberated in Aug.

\section{Cyaneidaz}

Cyanea capillata (L.)

- lamarckii, P. and L. Occ. every summer

\section{ULMARIDAE}

Aurelia aurita, Lam. c. in estuarine waters spring and summer; Ephyrae appear in Feb., metamorphose towards end of March, young medusae reappearing in May, and reaching their maximum abundance in June

\section{Pilimidar}

Rhizostuma octopus (L.). occ.

\section{ALCYONARIA}

\section{Cornulariidae}

Sarcodictyon catenata, Fb. Eddystone Grounds, generally on old shells; most plentiful on clean shell gravel (M.B.A).

\footnotetext{
1 M.B.A. record8.
}

\section{ALCYONARIA (continued)}

Alcyonidaz

Alcyonium digitatum, L. 1.w. to 35 fms., c. B. $x-i i$. (M.B.A.)

- glomeratum (Has.). Plymouth; Mewstone Ledge, rr. (M.B.A.)

- palmatum (P.). Plymouth; Merustone Ledge, occ. (M.B.A.)

Plexauridar

Eunicella cavolini, Koch. Gen. dist. and f.c. on rocky ground, IO-25 fms.

\section{VIRGULARIIDAE}

Virgularia mirabilis (O.F.M.). Near the Eddystone and off Stoke Point. Single specimens only (M.B.A.)

\section{ZOANTHARIA}

\section{SAGARTIADAE}

Actinoloba dianthus (Ellis). Plymoutb Promenade Pier, c. ; Torbay, m.c.; Teigmmouth Bay, v.c. on scruff (M.B.A.); Dart, up as far as Dittisham (G.)

Sogartia bellis (E. and S.). Gen. dist. and c. in muddy sand, sometimes in great profusion in estuaries, etc.

- miniata (G.). Plymouth Sound, r. (M.B.A.) ; Torquay; Dartmouth; Ilfracombe (G.)

- rosea (G.). Torbay; Teignmoutb (G.)

- ornata (Holdsworth). Dartmouth; Torquay (G.)

- icthystoma (G.). Orestone; Torbay (G.)

- venusta (G.). Torquay; Clovelly, on oysters from deep water (G.)

- nivea (G.). Plymouth Sound, r. (M.B.A.) ; Torquay; Dartmouth; Clovelly, Ilfracombe (G.)

- sphyrodeta, G. Dartmouth; Ilfracombe (G.). Plymouth Sound, occ. (M.B.A.)

- pallida, Holdsworth. Dartmouth; Torquay (G.)

- coccinea, G. Torbay, deep water, v.c. (G.)

- troglodytes, J. Teignmoutb; Torquay; Ilfracombe (G.) ; Plymouth, c. (M.B.A.)

- viduata (O.F.M.). Plymouth, l.w.-15 fms., f.c. Start Bay, occ. (M.B.A.); Torquay, v.c.; Dartmouth; Ilfracombe (G.)

- parasitica (Couch). Gen. dist. and c., 15-30 fms. on shells inhabited by Eupagurus Bernhardus

Adamsia palliata (Bohadsch.). Gen. dist. and c. 10-30 fms. on shells inhabited by Eupagurus prideauxi

Phellia murocincta, G. Torquay (G.)

- gausapata, G. Deep water off Torbay (G.)

Paraphellia expansa, Haddon. Eddystone Grounds, $\mathbf{r}$. (M.B.A.)

Gephyra dohrni, v. Koch. Mewstone Ledge, f.c. on Eunicella (M.B.A.)

\section{Antheadae}

Aiptasia couchii (Cocks). Plymouth Sound, occ. (M.B.A.)

Anthea cereus (E. and S.). Gen. dist. and c. on rocks between tidemarks

\section{Actiniadaz}

Actinia mesembryanthemum (E. and S.). Gen. dist. and $c$. on rocks between tidemarks. 


\section{A HISTORY OF DEVONSHIRE}

\section{ZOANTHARIA (continued)}

Bunodidae

Bunodes gemmacea (E. and S.) Plymouth Sound, f.c. (M.B.A.) Torquay; Paignton; Ilfracombe (G.) - thallia, G. Ilfracombe (G.)

- balli (Cocks). Plymoutb Breakwater, chiefly vars. dealbata and livida (M.B.A.) ; Torquay (G.)

- coronata, G. Off Berry Hd., 20 fms. ; Torbay ; off Teignmouth (G.); Plymouth, 20 fms., r. (M.B.A.)

Tealia digitata (O.F.M.). Trawling gnds. S. of $E$ ddystone, inside valves of Cardium echinatum, n.c.

- crassicornis (O.F.M.). Plymouth Sound shores, m.c.; outside in 10-40 fms. (M.B.A.). Gen. dist. (G.)

\section{ILYANTHIDAE}

Eloactis mazeli (Jourdan). Off Plymouth Mewstone, 20 fms. (M.B.A.)

Peachia hastata, G. Torbay, 1.w. (G.)

- triphylla (?), G. Start Bay, 6 fms. (M.B.A.)

Halcampa chrysanthellum (Peach). Yealm, f.c.; Salcombe, $r$. Larvae parasitic on medusae (Irene and Phialidium), c. in May (M.B.A.)

Halcampa microps, G. Oddicombe (G.)

Edwardsia callimorpha, G. Off Brixbam (G.)

- carnea, G. Plymouth, O-I 5 fms., rr. (M.B.A.); Torquay (G.)

- beautempsi, Q. realm, rr. (M.B.A.)

Cerianthus lloydii, G. Plymouth, rr.; Salcombe, f.c. on Zostera bed. Larvae (Arachnactis albida) c. in April (M.B.A.)

\section{CARYOPHYLLIACEA}

\section{Capneadae}

Aureliania angusta, G. Ilfracombe (G.)

Corynactis viridis, A. Gen. dist. and c., l.w. $-15 \mathrm{fm}$.

\section{ZOANTHIDAE}

Zoanthus couchi, J. Plymouth Sound, c. (M.B.A.); Torbay, near Orestone, 12 fms. (G.)

- sulcatus, G. Broadsands near Brixham (G.)

Epizoanthus incrustatus, Dub. et Kov. Mewstone Gnds., c. on shells inhabited by Anapagurus laevis; c. on Outer Eddystone trawling grounds (M.B.A.)

\section{Turbinoliadae}

Caryophyllia smithii, Stokes. f.c. everywhere on rocky ground, l.w. - $30 \mathrm{fms.,}$ often with Pyrgoma anglicum growing on the margin of the cup.

\section{Eupsammiadae}

Balanophyllia regia, G. Ilfracombe, c.; Plymoutb Sound, rr. (G.)

\section{Ctenophora}

Bolina infundibulum (F.). Plymouth, abundant at times (M.B.A.)

Beröe cucumis, F. Plymouth, occ. (M.B.A.)

Pleurobrachia pileus (F.). Always abundant at $P l y$ mouth towards the end of May; adults not seen after June, minute specimens appear Sept., Aug. (M.B.A.)

\section{ECHINODERMA}

\section{Synaptidar}

Synapta digitata (M.). Off Plymouth, occ. ; Teignmouth Bay (M.B.A.)

- inhaerens (O.F.M.). Sandy shores. Plymouth realm, occ. ; Salcombe, f.c. (M.B.A.)

\section{Cucumariidae}

Cucumaria brunnea (Th.) Plymouth, c., 1.w. -25 fms. - hyndmanni (Th.) Plymouth, m.c. (!) on coarse ground (M.B.A.)

- normani, Pace. Plymouth, l.w.-io fms., m.c. (M.B.A.)

- saxicola, Brad. and Rob. Plymouth, with C. normani ; Salcombe, r. (M.B.A.)

- frondosa (Gunn.) Plymouth (B.M.)

Thyone fusus (O.F.M.). Plymouth Sound and outside, to 35 fms. occ. (M.B.A.)

\section{HoLOTHURIDAE}

Holothuria nigra (Gray). Off Plymouth on clean gravel, v.c. in places, 99 having been taken in one haul with the Agassiz trawl $S$. of the Mewstone. Ripe, Apr., July, Dec. (M.B.A.)

\section{Antedonidae}

Antedon bifida (Penn.). Plymouth Sound; v.c. in Millbay Pit (23 fms.); occ. Merostone; Torbay (M.B.A.). Ripe, Oct.; Pentacrinoid larvae, Feb. July, Aug., abundant ; Myzostomum cirriferum c. on arms (M.B.A.)

\section{Astropectinidar}

Astropecten irregularis (Penn.) Gen. dist. and c. I 5-35 fims. on fine clean sand and clean gravel.

Luidia ciliaris (Phill.) Plymouth (B.M.)

- sarsi, D. and K. Occ. off Plymouth (M.B.A.)

\section{Gymnastremidas}

Porania pulvillus (O.F.M.). Occ. off Plymouth. B. ii-iv. (M.B.A.)

\section{Asterinidae}

Asterina gibbosa (Penn.) Under stones on rocky shores, c. B. v, vi (M.B.A.)

Palmipes placenta (Penn.) Off Plymouth, I 5-35 fms., m.c. (M.B.A.) ; Plymouth Sound (B.M.)

\section{STICHASTERIDAB}

Stichaster roseus (O.F.M.). Plymouth (B.M.)

\section{Solasteriddas}

Solaster papposus (F.). Plymouth, f.c. on coarse sand and gravel, I 5-35 fms.; Torbay; Teigmouth Bay (M.B.A.). Ripe, Mar. (M.B.A.)

\section{EchinasteriIDAB}

Henricia sanguinolenta (O.F.M.). Plymouth, 1.w.20 fims. on rocks and rough ground, f.c. ; Teigh mouth Bay (M.B.A.) 


\section{MARINE ZOOLOGY}

\section{ECHINODERMA (continued)}

\section{Asterindar}

Asterias glacialis, L. Plymouth, 1.w.-35 fms., f.c. Further east than Bolt Head specimens have only been taken very rarely; Salcombe (I); Teignmouth Bay (I) ; Brixbam (I) (M.B.A.) ; Ilfracombe (B.M.)

- rubens, L. l.w., 30 fms., f.c. everywhere, occ. abundant

\section{Ophioleridar}

Ophiura albida, Fb. Plymouth, 15-35 fms., f.c. on coarse grounds (M.B.A.)

- ciliaris (L.). Gen. dist. and c. on fine hard sand, I 5-35 fms. B. v, viii (M.B.A.)

\section{Amphiuridar}

Ophiocnida brachiata (M.). Plymouth f.c. and Salcombe c., in muddy sand between tidemarks (M.B.A.)

Amphiura chiaji, Fb. Plymouth, r..(M.B.A.)

- elegans (Leach). v.c., 1.w. -20 fms. B. v-ix (M.B.A.)

- filiformis (O.F.M.). Off the Eddystone (M.B.A.)

Ophiactis balli (Th.). Plymouth, I 5-35 fms., in crevices of rocks and on Chaetopterus tubes; Teigmmouth Bay (M.B.A.) Ripe, Sept. (M.B.A.)

\section{Ophiocomidar}

Ophiocoma nigra (O.F.M.). With, but l.c. than, Ophiothrix fragilis

Ophiopsila aranea, Fb. Merustone Ledge and Stoke Point grounds, f.c. in crevices in red sandstone, especially old Pholadidea borings. Ripe, Aug. (M.B.A.)

\section{OPHIOTHRICIDAE}

Ophiothrix fragilis (O.F.M.). Gen. dist. and c., l.w.- 35 fms., enormously abundant on certain grounds. Ripe, Mar.-June, Aug.-Sept.; spawning, Oct. (M.B.A.)

\section{Echinidae}

Echinus acutus, Lam. f.c., 15-35 fms. on fine sand, frequently with Siphonostoma affinis. B. vii (M.B.A)

- esculentus (L.) f.c. 15-35 fms., often with Scalisetosus assimilis. Ripe, Mar., May (M.B.A.)

- miliaris, L. 1.w.-35 fms., f.c. everywhere, largest specimens on the shore. B. v (M.B.A.)

Strongylocentrotus lividus (Lam.) Ilfracombe (B.M.)

\section{Clypeastridae}

Echinocyamus pusillus (O.F.M.). 10-35 fms. on gravel, f.c.

\section{SPatangidae}

Spatangus purpureus, O.F.M. I 5-35 fms., m.c. in places

Coarse ground,

Echinocardium cordatum (Penn.). Shore-35 fms. in sand, f.c.

- pennatifidum, N. Plymouth, 15-30 fms., f.c. (M.B.A.)

- flavescens (O.F.M.). Off the Eddystone (M.B.A)

\section{TURBELLARIA}

\section{Proporidae}

Proporus venosus (Sch.). m.c. Plymoutb Sound; Wembury Bay, l.w. (M.B.A.)

Monoporus rubropunctatus (Sch.). m.c. with $P$. venosus (M.B.A.)

\section{Aphanostomidae}

Aphanostoma diversicolor, O. Plymouth, f.c., l.w. (M.B.A.)

- elegans, Jn. Plymouth, single specimen (M.B.A.)

Convoluta saliens, v. G. Plymouth, r. among Zostera (M.B.A.)

- paradoxa, O. Plymouth, littoral zone, gen. dist. but n.c. (M.B.A.)

- flavibacillum, Jn. Plymouth, in sandy creeks (M.B.A.)

\section{Microstomidae}

Microstoma groenlandicum, Lv. Plymouth, among Ulva, r. (M.B.A.)

\section{Mesostomidae}

Promesostoma marmoratum (Shz.). Plymouth, f.c. in rockpools (M.B.A.)

- ovoideum (Sch.) Plymouth Sound, occ. in dredgings (M.B.A.)

- solea (Sch.). Plymoutb, abundant in dredgings (M.B.A.)

- agile (Lv.). Plymouth, among Zostera (M.B.A.)

Byrsophlebs graffi, Jn. Plymouth Sound, 1.w., r. (M.B.A.)

- intermedia, v. G. Plymourb Sound, l.w., r. (M.B.A.)

Proxenetes cochlear, v. G. Plymouth Sound (M.B.A.)

- flabellifer, Jn. Plymouth (M.B.A.)

Mesostoma neapolitanum, v. G. (?). Plymouth Breakwater, single specimen (M.B.A.)

\section{Proboscidae}

Pseudorhynchus bifidus (McInt.). Plymouth Sound (M.B.A.)

Acrorhynchus caledonicus (Clpd.). Plymouth tidepools, m.c. (M.B.A.)

Macrorhynchus naegeli (Koll.). Plymouth Breakwater, plentiful Aug. (M.B.A.)

- croceus (F.). Plymouth Sound, dredged once (M.B.A.)

- helgolandicus (Mets.). Plymouth Sound, once (M.B.A.)

Gyrator hermaphroditus, E. Plymouth Sound, tidepools, water at times brackish, early winter (M.B.A.)

Hyporhynchus armatus (Jn.). Plymouth, m.c. (M.B.A.)

- penicillatus (Sch.) Plymouth, once (M.B.A.)

\section{VorTICIDAE}

Provortex balticus (Shz.). Plymouth, between tide marks, f.c. (M.B.A.)

- affinis (Jn.). Plymouth, among algae (M.B.A.)

- rubrobacillus, Gamble. Plymouth Sound, dredged, r. (M.B.A.)

Fecampia erythrocephala, Giard. Plymouth, encysted stage, c. on all stony shores at 1.w. (M.B.A.)

\section{Plagiostomidae}

Plagiostoma dioicum (Mets.). Plymouth (M.B.A.)

- elongatum, Gamble. Wembury Bay; Plymouth Breakwater (M.B.A.) 


\section{A HISTORY OF DEVONSHIRE}

\section{TURBELLARIA (continued)}

Plagrostomidae (continued)

Plagiostoma pseudomaculatum, Gamble. Among weedtubes of Polydora caeca, Hamoaze (M.B.A.)

- sagitta, Uljanin. Plymouth, r. (M.B.A.)

- caudatum, Lv. Plymouth, once among Zostera (M.B.A.)

- vittatum (F. u. L.). Plymouth, abundant in littoral region. Egg capsules, Sept. (M.B.A.)

- koreni, Jn. Plymouth (M.B.A.)

(?) - siphonophorum (Sch.). Plymouth, once (M.B.A.)

- girardi (Sch.). Plymouth, f.c. (M.B.A.)

Vorticeros auriculatum (O.F.M.). Plymouth, abundant littoral species (M.B.A.)

- luteum, v. G. Plymouth, twice (M.B.A.)

Enterostoma austriacum, v. G. Plymouth Sound, c. below 5 fims. (M.B.A.)

- fingalianum, Clpd. Wembury Bay, among Florideae (M.B.A.)

Cylindrostoma quadrioculatum (Leuckart). With E. fingalianum (M.B.A.)

- inerme (Hallez). Plymoutb Sound; Hamoaze (M.B.A.)

- elongatum, Lv. Tidepools, Wembury Bay (M.B.A.)

Monoophorum striatum, v. G. Plymouth Sound, once (M.B.A.)

\section{Monotidab}

Monotus lineatus (O.F. M.). Plymouth, m.c. among Ulva (M.B.A.)

- fuscus (O.). Plymouth, littoral zone, c. especially among Balani and Ulva (M.B.A.)

- albus, Lv. Plymouth Sound, tidepools (M.B.A.)

Automolos unipunctatus (O.). Plymouth Sound, r. among algae from Duke Rock (M.B.A.)

- horridus, Gamble. Hamoaze, single specimen (M.B.A.)

(?)- ophiocephalus (Sch.). Plymouth Sound (M.B.A.)

\section{Planariddae}

Fovia affinis, Stimpson. Wembury Bay, etc. (M.B.A.)

\section{LEPTOPLANIDAE}

Cryptocelis alba, Lang. Millbay Cbannel, and off Mewstone, Nov. I 899 (M.B.A.)

Leptoplana tremellaris (O. F. M.). Plymouth, littoral zone to 15 fms., f.c. especially in July and Aug.; Exmouth (M.B.A.)

- draebachensis, O. Plymouth Sound (M.B.A.)

- fallax, Q. Plymouth Saund (M.B.A.)

\section{Plamoceridat}

Stylochoplana maculata, Q. Plymouth, c. In trawled Zostera (M.B.A.)

\section{EURYLEPTIDAE}

Prostheceraus vittatus (M.). Plymouth Sound; Yealm, f.c. and large. Off Stoke Point; Salcombe (Salstone) (M.B.A.)

Cycloporus papillosus. Lang. Plymoutbshore-30 fms., c. on sponges, ascidians, etc. (M.B.A.)

Eurylepta cornuta (O.F.M.). Plymouth shore-30 fms., m.c. (M.B.A.)

Oligocladus sanguinolentus (Q.). Plymouth Sound and outside, to 25 fms.; $r_{e a l m}$. c. (M.B.A.)

Stylostomum variabile, Lang. Plymouth Sound; realm (M.B.A.)

\section{TURBELLARIA (continued)}

Prosthiostomidae

Prosthiostomum sepunculus, (Dl.C.). Plymouth, rr., Salcombe (?), rr. (M.B.A.)

\section{NEMERTINI}

\section{Carinellidar}

Carinella linearis, Mc.Int. Plymoutb Sound, rr. (M.B.A.)

- polymorpha (Renier). Plymouth, 25 fms., rr.; Salcombe, on Salstone, rr. (M.B.A.)

- superba (Koll.). Plymouth shore, to $30 \mathrm{fms}$. r. ; realm; Salcombe, f.c. (M.B.A.)

- annulata (M.). Plymouth, 5-20 fms., m.c. ; realm; Salcombe (M.B.A.)

\section{Cephalothricidae}

Cephalothrix bioculata, O. Plymouth, c. in clean coarse sand on shore, and among corallines. B. iv-viii; pelagic larvae as late as xii (M.B.A.)

- linearis (Rathke). Plymouth Sound shore and outside. B. iii (M.B.A.)

\section{EUNEMERTIDAB}

Eunemertes neesi (O.). Plymouth Breakwater, c., occ. other localities. B. ii-x (M.B.A.)

- gracilis, J. Plymouth Breakwater, c. among Laminaria roots (M.B.A.)

- echinoderma (Marion). realm, 3 specimens (M.B.A.)

Nematopsis flavida (McInt.). Plymouth Sound, v.c. shore and dredge; realm, dredge (M.B.A.)

- tenuis, Burger. Plymouth Sound, v.c. shore and dredge ; realm, dredge (M.B.A.)

\section{Amphiporidar}

Amphiporus pulcher (J.). Eddystone Gnds., rr. (M.B.A.)

- lactifloreus (J.). Plymouth, c. between tidemarks under stones. B. iii (M.B.A.)

- dissimulans, Riches. Plymouth Sound, c. in certain localities; realm. B. spring, x (M.B.A.)

- bioculatus, McInt. Plymouth, single specimen from Millbay Cbannel (M.B.A.)

Drepanophorus spectabilis (Q.). Plymouth Sound and outside, to $30 \mathrm{fms}$. m.c. (M.B.A.)

\section{Tetrastemmidae}

Tetrastemma ambiguum, R. Plymouth, Nov. 1902, 5 specimens (M.B.A.)

- candidum (O. F.M.). Plymouth Sound, c. in

- vermiculatum (Q.). \}dredgings. T. candidum, c.

- melanocephalum (J.). ) outside to $30 \mathrm{fms}$. (M.B.A.)

- cephalophorum, Bgr. Plymoutb Sound, r. ; Yealm (M.B.A.)

Oerstedia dorsalis (Zool. Dan.). Plymouth, c. shore30 fms. ; var. cincta, abundant among Cellaria. B. ix-xi (M.B.A.)

- nigra (R.). Plymouth Sound, on Codium, etc., from Laminarian zone (M.B.A.)

- immutabilis (R.). Wembury Bay, c. among corallines ; Plymouth Sound (M.B.A.) 


\section{MARINE ZOOLOGY}

\section{NEMERTINI (continued)}

\section{Malacobdellidae}

Malacobdella grossa (O.F.M.). Plymouth, in branchial cavity of Cyprina islandica. Ripe in autumn (M.B.A.)

\section{Eupolitidae}

Eupolia curta, Hubrecht. Plymouth Sound and outside, r. (M.B.A.)

Oxypolia beaumontiana, Punnett. Two specimens dredged off Plymouth Mewstone in 1897 and I 899 (M.B.A.)

\section{Lineidae}

Micrella rufa, Punnett. realm shore, single specimen (M.B.A.)

Lineus longissimus, Gunn. Plymouth shore-30 fms., m.c. ; realm; Salcombe (M.B.A.)

- gesserensis (O.F.M.). Plymouth, c. on shore under stones. B. i-iii (M.B.A.)

- lacteus (Gb.). Plymouth Sound, r. (M.B.A.)

- bilineatus, McInt. Plymouth, c. 5-20 fms.; realm shore, v.c. (M.B.A.)

Micrura fasciolata, E. Plymouth, c.; Salcombe. B. x-xii (M.B.A.)

- purpurea (Dal.). Plymouth Sound, c. ; realm; Eddystone Gnds., etc. (M.B.A.)

- aurantiaca (Gb.). Plymouth Sound, r. ; Yealm; Mewstone Gnds., etc., r. (M.B.A.)

- lactea (Hubrecht). Off Stoke Point, one specimen, Io Nov. 1892 (M.B.A.)

Cerebratulus fuscus (McInt.). Plymouth Sound; Eddystone Gnds. (M.B.A.)

- pantherinus, Hubrecht. Off Stoke Point, single specimen (M.B.A.)

\section{Archtannelida}

Dinophilus taeniatus, Harmer. Plymouth Sound, rockpools far above h.w. in Mar. and Apr. (M.B.A.)

Polygordius (apogon (?) McInt.). Off Mewstone; Eddystone Gnds. (M.B.A.)

Protodrilus leuckarti, Hatschek. Plymouth, reared from tow-nettings taken in Sept. (M.B.A.)

Histriobdella homari, v. Ben. Plymouth, frequent on eggs of lobster (Homarus vulgaris). B. summer (M.B.A.)

\section{POLYCHAETA}

\section{SYLLIDAE}

Typosyllis prolifera, Krohn. Plymouth Sound, among weeds. realm, in red sponge

- alternosetosa, St. J. Eddystone $G$ nds.

Syllis hamata, CIpd. Salcombe

- cornuta, Rk. Eddystone Gnds.

- gracilis, Gb. Plymouth Sound

Odontosyllis ctenostoma, Clpd. Plymouth Sound shore.

- fulgurans, Clpd. Plymouth Sound

- gibba, Clpd. Plymouth Sound

Trypanosyllis zebra (Gb.). Plymouth Sound; realm, f.c.

Amblyosyllis spectabilis (J.). Plymouth Sound; Yealm, c. ; Eddystone Gnds.; Salcombe

Sphaerosyllis ovigera, Lang. Plymouth Sound, taken once

1 M.B.A. records.

\section{POLYCHAETA (continued)}

Syllidar (continued)

Autolytus pictus (Eh.). Plymouth Sound, c. among sponges and Alcyonidium

- rubropunctatus (Gb.). Plymouth Sound (Queen's Gnd.)

Myrianida pennigera (M.). Plymouth Sound and Docks, f.c. among ascidians and sponges; Salcombe, fine mud

\section{Hesionidae}

Kefersteinia cirrata (K.). Plymouth Sound

Castalia punctata (O.F.M.). Plymouth Sound, f.c.

Magalia perarmata, M. et B. Plymoutb Sound, f.c.

\section{Aphroditidae}

Aphrodite aculeata, L. Gen. dist. and f.c. especially on fine sand grounds.

Hermione hystrix (Sv.). Most c. on gravel grounds

Lepidonotus squamatus (L.). Gen. dist. and c. l.w.$30 \mathrm{fms}$., under stones, among weeds, hydroids, etc.

- clava (M.). C. among stones on shore, less so in deeper water

Gattyana cirrosa (P.). Off Eddystone; Yealm, and Salcombe, commensal with Amphitrite johnstoni

Eunoa nodosa (S.). Stony ground off Prawle, 30 fms.

Lagisca floccosa (Sv.). Everywhere 1.w.-30 fms.

- extenuata (Gb.). f.c. under Laminaria roots. Plymoutb Breakwater ; Eddystone Gnds.

- rarispina (S.). Eddystone and Mewstone Gnds.

Harmothoe imbricata (L.). Plymouth Breakwater; Eddystone Grds.

- spinifera (Eh.). Plymouth Sound, Yealm, c. ; Salcombe

- lunulata (D1. C.). Plymouth Breakwater; Salcombe

- setosissima (Sv.). Eddystone Grds., among Chaetopterus tubes and Cellaria; Salcombe, shore

- areolata (Gb.). Eddyszone Gnds, with H. setosis$\operatorname{sim} a$

Evarne impar (J.). Plymouth, c. 1.w.-35 fms. ; Salcombe ; Exmouth

Scalisetosus communis (D1. C.). Plymouth Sound

- assimilis (McInt.). Eddystone and Mewustone Gnds., among spines of Echinus esculentus

Malgremia castanea (McInt.). f.c. on spines of Spatangus, Plymouth

Halosydna gelatinosa (S.). 0-30 fms. m.c.

Polynoe scolopendrina, Sv. Plymouth Sound, commensal with Polymnia nebulosa, r. in dredgings; also from Eddystone $\mathrm{G} n d$.

Acholoe astericola (Dl. C.). Gen. dist. and f.c. in ambulacral grooves of Astropecten

Sthenelais boa (J.). Plymouth Sound, O-1 o fms. ; Salcombe; Exmouth

Phloe minuta (F.). Plymouth Sound, c.

Lepidasthenia argus, Hodgson. Commensal in tubes of Amphitrite edwardsi, Salcombe

\section{Amphinomidae}

Euphrosyne foliosa, A. and E. Plymouth Sound, Eddystone Gnds., Salcombe, Exmouth, n.c. 


\section{A HISTORY OF DEVONSHIRE}

\section{POLYCHAETA (continued)}

Phyllodocidae

Eulalia viridis (O.F.M.). Gen. dist. and f.c., especially on rocky shores

- punctifera (Gb.). Plymouth Sound, Yealm, Salcombe

- aurea, Gravier. Plymouth Sound, c. ; Yealm

- claparedii, St. J. Plymouth Sound, f.c.

- obtecta, Eh. Plymouth Sound; Mewustone Gnds., m.c.

- ornata, St. J. Plymouth Sound

- pallida, Clpd. Plymouth Sound, v.c. B. v-vii.

Pterocirrus macroceros (Gb.). Plymouth Sound, occ.

Phyllodoce laminosa, Sv. Plymouth Sound

- maculata (O.F.M.). Plymouth Sound, c.; realin; Exmouth (!) B. i, ii, iv, v.

- rubiginosa, St. J. Plymouth Sound, c.

- paretti, Bl. Mewstone Ledge, Stoke Point

- teres, Mlg. Exmouth and Teignmouth, in clean sand

Eteone picta, Q. Plymouth Sound, occ.

- pusilla, O. Exmouth, clean sand

\section{Tomopteridae}

Tomopteris helgolandica, Greef. In tow-nettings, Jan., June, Oct., Dec.

\section{Nereidar}

Nereis cultrifera, Gb. Gen. dist., c. on shore in muddy gravel

- diversicolor, O.F.M. c. in mud flats of estuaries where salinity of water is low

- dumerilii, A. and E. Plynouth Sound, c. in dredgings ; occ. on shore

- fucata, Sv. In shells of Buccinum inhabited by Eupagurus Bernhardus. f.c. B.v.

- irrorata, Mlg. Plymouth Sound, shore and dredgings ; Salcombe, c. in muddy sand; Exmoutb, rr.

- longissima, J. Plymouth Cattewater; realm; Salcombe, c. in muddy sand; $E x m o u t h$, rr.

- pelagica, L. Plymouth Sound, f.c. shore and dredgings ; realm; Exmouth, in sponge

\section{NePhThididaE}

Nephthys caeca (F.). Gen. dist. c. on sandy shores

- hombergi, A. and E. Plymouth Sound and outside, Solcombe, c. Exmouth, c.

- cirrosa, Eh. Tealm, sandbank ; Exmouth, c. in fine clean sand

\section{Eunicidae}

Eunice harassi, A. and E. Plymouth Sound to Eddystone

Onuphis conchilega, S. Off Stoke Point, Eddystone, etc., 20-30 fms. coarse shell gravel

Hyalinoecia tubicola (O.F.M.). c. in muddy gravel 20-30 fms. Off Eddystone, Mewstone, etc.

Marphysa sanguinea (M.). On shore in crevices of rocks. Plymoutb; Yealm; Salcombe; Exmouth

- belli, A. and E. Rum Bay, shore; Yealm est. in Zostera beds, c. ; Salcombe, $r$.

Lysidice ninetta, A. and .E. Plymouth Sound, c. : Wembury Bay, c. ; Yealm

Nematonereis unicornis (Eb.). Plymouth Sound

- impatiens, Clpd. Off Plymouth, coarse grounds ; Plymouth Sound; v. abundant on Queen's Gnd., spring 1903

\section{POLYCHAETA (continued)}

Eunictiab (continued)

Lumbriconereis latreillii, A. and E. Plymoutb Sound, and outside, shore and dredgings; Salcombe, shore, $c$.

Maclovia iricolor (M.). Plymouth Sound, occ. on shore; Salcombe, shore in muddy gravel, $r$.

Staurocephalus rubrovittatus, Gb. Plymouth Sound, c.

- ciliatus, K. Plymouth Sound, once

- pallidus, Lang. Plymouth Sound, once

Ophryotrocha puerilis, Clpd. et Meczn. Plymouth Sound. B. viii

\section{Glyceridae}

Glycera convoluta, K. Plymouth Sound, and outside; Salcombe, r. ; Exmouth, occ.

- capitata, O. Salcombe, rr.

- lapidum, Q. Wembury Bay. Off Mevustone and Plymouth Sound

- gigantea, Q. Plymouth Sound. Off Mewstone

Goniada maculata, O. Plymouth Breakwater, once; Salcombe, $\mathrm{r}$.

\section{SPHAERODORIDAE}

Ephesia gracilis, Rk. Plymouth Sound, f.c.

\section{Ariciidae}

Aricia cuvieri, A. and E. Off the Merustone once

- latreillii, A. and E. Exmouth Polesands, f.c. in hard sand

Scoloplos armiger (O.F.M.). In dirty sand. Hamoaze; Plymoutb Sound; Yealm; Salcombe; Exmouth

\section{SPIONIDAE}

Spio seticornis, F. Salcombe, v.c. in sand and muddy gravel on shore; Exmouth, c. in clean hard sand

Scolelepis vulgaris, J. On shore. Rum Bay; Wembury Bay, v.c. in mud

- giardi (Q.). Rum Bay, once in mud

Nerine cirratulus (Dl.C.). Plymouth Sound, shore, fine gravel ; Salcombe; Exmouth

- coniocephala, J. Plymouth Sound, shore, fine sand; Salcombe, rr. ; Exmouth, m.c.

- vulgaris, J. Salcombe, rr.

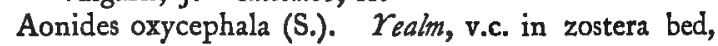
l.w.

Polydora ciliata (J.). Boring in limestone. Plymouth Breakwater, v.c.

- Alava, Clpd. Plymouth Sound; Hamoaze, in crevices of shale, c. ; realm. B. ii

- caeca, O. Eddystone Gnds.

- hoplura, Clpd. Boring in limestone. Plymouth Breakwater

\section{Disomidak}

Poecilochaetus serpens, Allen. In sand at 1.w. under Batten Castle; larvae m.c. in tow-nets in summer

\section{Chaetopteridae}

Chaetopterus variopedatus, Renier. c. in muddy gravel off Plymouth; occ. specs. in Sound; Start Bay; Teignmouth Bay; Salcombe, muddy gravel on the Salstone and in Zostera bed; larvae in tow-nettings, July-Oct. 


\section{MARINE ZOOLOGY}

\section{POLYCHAETA (continued)}

\section{Magelonidat}

Magelona papillicornis, O.F.M. Plymouth Sound and realm, fine sand, l.w. ; Salcombe, rr. ; larvae in tow-nettings, July-Sept.

\section{Ammocharidar}

Owenia fusiformis (DI.C.). Plymouth Sound, fine sand, l.w.; Salcombe, r.

\section{Cirratulidar}

Audouinia tentaculata (M.). Plymoutb Sound and Salcombe, etc. ; c. in sandy and muddy gravel just below h.w.m.

Dodecaceria concharum, O. Boring in limestone. Plymouth Breakwater, abundant

\section{TerebellidaA}

Amphitrite gracilis, Gb. Plymouth Sound, c. in sand, etc. ; Wembury Bay ; Yealm; Eddystone Gnds.

- edwardsi, Q. Salcombe, c. in Zostera bank

- johnstoni, Mlg. Yealm, in sand and gravel, 1.w.m.; Salcombe, v.c. on Salstone

Terebella lapidaria (Køhl.). Rum Bay, c. in crevices of shale

Polymnia nebulosa (M.). Plymouth to Exmouth, shore and dredge, c. in places

- nesidensis (DI.C.). Plymouth Sound

Lanice conchilega (P.). c. on all sandy shores, l.w. to moderate depths

Nicolea zostericola (O.). Plymouth Sound, shore (Reny Rocks etc.), c. ; Yealm

Thelepus setosus (Q.). Plymouth Sound; Eddystome Gnds.; Salcombe

Polycirrus aurantiacus, Gb. Plymouth Sound, c; Eddystone Gnds.; Yealm; Salcombe. B. iv, vii

- caliendrum, Clpd. Plymouth Sound, f.c.; Salcombe

Loimia medusa (Sv.). Queen's Ground, tubes only ; realm, shore

\section{Ampharetida}

Mellina adriatica, v. M. Plymouth Sound, c. in soft mud; Salcombe, upper parts of estuary in great abundance ; Exmouth, rr.

\section{Amphictenidae}

Pectinaria Koreni, Mlg. c. in sand under Batten Castle

- auricoma (O.F.M.). Eddystone Gnds.

- belgica (Pol.). Salcombe, shore, rr.

\section{Capitellidae}

Capitella capitata (F.). c. in black mud on shore; Plymouth Sound; Wembury Bay

Notomastus rubicundus, K. Yealm, c. on shore

- latericius, S. Yealm, shore; Salcombe, v.c. in mud and muddy gravel

Heteromastus filiformis, $\mathrm{Clpd}$. Exmouth, in sand

\section{OphrLIIDAB}

Ophelia bicornis, Sv. Exmouth Polesands, v.c. in coarse loose sand

Ammotrypane aulogaster, Rk. Plymouth Sound, rr.; Mewstone Gnds., rr.

Polyophthalmus pictus (Dj.). Shore, Wembury Bay and Reny Rocks

\section{POLYCHAETA (continued)}

Arenicolidat

Arenicola marina, L. c. on most fine sandy shores ; post larval Arenicola in tow-nettings, Feb.

- grubei, Clpd. Plymouth Sound, m.c. in sand with small stones at the base of rocks; Wembury Bay; Salcombe, $\mathrm{rr}$.

- ecaudata, J. Plymouth Sound, with A. grubei

\section{Scalibregmidae}

Scalibregma inflatum, Rk. realm, in muddy gravel l.w.m., two in 1900

Schlerocheilus minutus, Gb. Plymouth Sound, f.c.; Mewstone Gnds.

\section{Chlorharmidae}

Stylarioides plumosa (O.F.M.). Plymouth Sound, off Stoke Point, r.

Siphonostoma affinis, S. Plymoutb Sound; Wembury Bay; Merustone Grounds, among spines of Echinus acutus

\section{Sabelitidar}

Sabella pavonina (Sv.). Eddystone Gnds., f.c. ; realm, c.; Salcombe, c. on Salstone, etc. 1.w.; off Start Point, etc.

Branchiomma vesiculosum (M.). occ. Plymouth Sound and Yealm, shore; Salcombe, c. on shore in gravel

Dasychone bombyx (Dal.). Occ. in dredgings ; $P l y$ mouth Sound; Eddystone Gnds.; Torbay

Potamilla reniformis (O.F.M.). Boring in limestone, Plymouth Breakwater; also Jennycliff and Wembury Bays

- torelli, Mlg. c. Millbay Gb. and Yealm

- incertis, Lang. Tealm, dredged once

Bispira volutacornis (M.). In cracks in rocks, l.w.s.t. ; Reny Rocks ; Jennycliff Bay, $r$.

Myxicola infundibulum (Ren.). Plymoutb Sound, occ.; Salcombe, c. esp. S. of Pilevortby Point

\section{SERPULIDAE}

Serpula vermicularis, L. Occ. Plymouth Sound, outside to 35 fms.

Pomatoceros triqueter (L.). c. on shells and stones, shore to $35 \mathrm{fms}$.

Hydroides norvegica (Gunn). c. on shells and stones, $0-30 \mathrm{fms}$.

Filograna implexa (Berkeley). Plymouth Sound, c. on piles, occ. in dredgings to $35 \mathrm{fms}$.

Spirorbis borealis (Daudin). c. on weeds, esp. Fucus, and stones, on all shores

Protula tubularia (M.). Plymouth, 15-35 fms.; Teignmouth Bay, m.c.

\section{Hermelitidat}

Sabellaria alveolata (L.). Exmoutb; Orcombe Rks. v.c. - spinulosa, Leuck. f.c. attached to shells, to $35 \mathrm{fms}$. B. $v$, ix.

Pallasia murata, Allen. One specimen only, the type, has been taken; off Stoke Point, tubes, m.c.

\section{MYZOSTOMARIA}

Myzostomum cirriferum, R.L. c. on Antedon bifida ; Plymouth Sound 


\section{A HISTORY OF DEVONSHIRE}

\section{OLIGOCHAETA}

Clitellio arenarius, Clpd. Plymouth Sound, shores - ater, Clpd. Plymouth, shores, in mud

Pachydrilus, sp. c. in gravel Rum $B a y$ shore

\section{GEPHYREA}

Phascolion strombi, M. Plymouth, m.c. in shells of Apporrhais ; Salcombe, $\mathrm{r}$.

Phascolosoma vulgare, Bl. c. in stiff clay, gravel, etc. ; Salcombe

- pellucidum, K. Salcombe, c.

\section{GEPHYREA (continued)}

Thalasemma neptuni, Gt. Plymoutb Sound, shore and dredgings, f.c.

Phoronis hippocrepia, T.S.W. Abundant; Plymouth Sound

\section{HIRUDINEA}

Pontobdella muricata, L. Occ. parasitic on Rays. B. vii.

\section{CHAETOGNATHA}

Sagitta bipunctata (Q. et $G$.). In tow-nettings throughout the year

\section{BRYOZOA}

\section{ECTOPROCTA \\ CHEILOSTOMATA}

\section{Aeteidar}

Aetea anguinea (L.). S. Devon, v. abundant ; Ilfracombe (H.)

- recta (H.). Plymouth, 20-35 fms. on shells, etc. (M.B.A.) ; Torbay, v.c.; Salcombe (H.)

- truncata, Lansb. Salcombe (M.B.A.); Ilfracombe (H.)

\section{Eucrateidae}

Eucratea chelata (L.). S. Devon, v.c. ; Ilfracombe, 8-Io fms. Var. gracilis, N. Devon (H.)

Gemellaria loricata (L.). S. Devcn, n.c. ; Ilfracombe (H.)

\section{Celluluaridab}

Scrupocellaria reptans (L.). Plymouth (M.B.A.)

- scrupea, Busk. Plymouth, 25 fms. (M.B.A.); off Dartmouth, Berry Hd., Salcombe Bay (H.)

- scruposa (L.). gen. dist.

Caberea boryi (Aud.). Plymoutb (M.B.A.); Budleigh Salterton, on Eschara foliacea, and S. scruposa ; Ilfracombe (H.)

\section{BiCRLIARIIDAE}

Bicellaria ciliata (L.). Gen. dist. and f.c. on hydroids and polyzoa, 5-35 fms. Ovicells, Mar. (M.B.A.)

Bugula avicularia (L.). Off $P$ lymouth (M.B.A.)

- calathus, N. Off $S$. Devon on Eschara foliacea (H.)

- flabellata, Gray. S. Devon, c. inshore, 1.c. in deeper water. Ovicells, July, Sept. (M.B.A.)

- plumosa (P.). Plymouth, c.; Salcombe, m.c. (M.B.A.)

- turbinata (Ald.). Gen. dist. and c. $N$. and $S$. Devon, especially in sheltered positions on shore

Beania mirabilis, J. Ilfracombe, c.; Exmouth, Torbay, etc. $(\mathrm{H}$.)

\section{Notaminde}

Notamia bursaria (L.). S. Devon, on Hydrallmania (H.)

\section{Cellarindae}

Cellaria fistulosa (L.). S. Devon, gen. dist. and c. especially on fine sand, 15-30 fms.; Ilfracombe (H.)

- johnstoni, Lam. Eddystone Gnds. (M.B.A.)

- sinuosa (Hassall) S. Devon, c. with C. fistulosa

\section{CHEILOSTOMATA (continued)}

Flustridae

Flustra foliacea (L.)

- papyracea, E. and S. Plymouth Sound, occ. (M.B.A.); Salcombe; Torbay; Ilfracombe (H.)

- securifrons (P.) Plymouth Sound, occ. (M.B.A.)

\section{Membraniportdal}

Membranipora aurita, H. Off Plymouth Mewstone, 20 fms., rr. (M.B.A.)

- catenularia (Jameson). S. Devon, v.c. (H.)

- curvirostris, H. Eddystone Gnds. (M.B.A.)

- discreta, H. S. Devon (H.)

- dumerilii (Aud.). S. Devon, abund. on shells; Ilfracombe, on Laminaria (H.)

- flemingii, Busk. Eddystone Gnds. (M.B.A.)

- flustroides, H. Off Plymouth Mewstone, 25 fms. (M.B.A.)

- hexagona, Busk. Devon (H.)

- imbellis, H. Plymouth, a doubtful fragment (M.B.A.) ; Brixbam trawlers (H.)

- lacroixii, Aud. Plymoutb (M.B.A.); S. Devon, shore to moderate depths, c. (H.)

- lineata (L.). Plymouth Sound (M.B.A.) ; Ilfracombe; S. Devon (H.).

- membranacea (L.) Gen. dist and v.c. on Laminaria, etc.

- monostachys, Busk. Torquay; Ilfracombe (H.)

- nodulosa, $\mathrm{H}$. Off Brixbam (H.)

- pilosa (L.). c. everywhere between tidemarks on Fucus, etc.

- rosselii (Aud.). Plymouth Sound; Mewstone, $20 \mathrm{fms.}$ (M.B.A.) ; Torbay, on shell (H.)

- spiniferi (J.). Salcombe (H.)

\section{Microporidae}

Micropora coriacea (Esp.). Mewstone, 25 fms. (M.B.A.)

\section{Cribrilinidae}

Cribrilina figularis (J.). Eddystone Gnds., etc. Ovicells iv (M.B.A.)

- gattyae (Busk.). Sidmouth (H.)

- punctata (Has.). Mewstone, 25 fms. (M.B.A.); S. Devon; Ilfracombe (H.)

- radiata (Moll.). Off Plymouth Mewstone; Eddystone Gnds., v.c. (M.B.A.)

Membraniporella nitida (J.). S. Devon, c. on Lepralia foliacea, and on sponge and stones from shore (H.) 


\section{MARINE ZOOLOGY}

\section{CHEILOSTOMATA (continued)}

\section{MicroporkLLIDae}

Microporella ciliata (P.). Mewstone and Eddystone Gnds. on Pecten maximus (M.B.A.)

- impressa (Aud.). Plymouth, c. on red seaweed (M.B.A.); Ilfracombe (H.). Var. cornuta, Salcombe (H.)

- malusii (Aud.). Merustone Ledge, v.c.; Eddystone Gnds. (M.B.H.); Sidmouth, etc. (H.)

- violacea (J.). Mervstone Ledge; Eddystone Gnds. (M.B.A.); Torbay, etc. (H.)

Chorizopora brogniartii (Aud.). S. Devon, v.c.; Ilfiacombe on Laminaria (H.)

\section{Porinidas}

Lagenipora socialis, H. Off Plymouth Mewstone, 25 fms. (M.B.A.)

\section{Myriozoidae}

Schizoporella armata (H.). Mewstone, 25 fms. (M.B.A.)

- auriculata (Has.). Eddystone Gnds. (M.B.A.)

- cecilii (Aud.). Off Eddystone, 20 fms. (M.B.A.)

- cristata, H. Mewstone Ledge, on dead Pecten shell, rr. (M.B.A.)

- hyalina (L.). Plymouth Sound, c. ; Yealm, c. on red algae (M.B.A.)

- johnstoni, Quelch. Off the Mewstone, 25 frrs. (M.B.A.)

- linearis (Has.). S. Devon, c. ; Ilfracombe, 8-10 fms. (H.)

- simplex (J.). S. Devon, r. (H.)

- spinifera (J.). Plymouth (M.B.A.); Ilfracombe (H.)

- unicornis (J.). S. Devon, one of the commonest littoral species; Ilfracombe (H.); Plymouth, 25 fms. (M.B.A.)

Schizotheca fissa (Bk.). Eddystone Gnds. (M.B.A.); Exmouth, etc. (H.)

Hippothoa divaricata, Lam. Plymouth Sound and outside to $30 \mathrm{fms}$., f.c. on Pecten maximus, etc. (M.B.A.). Var. conferta, Sidmouth (H.)

- flagellum, Manzoni. Mewstone, 25 fms. (M.B.A.); S. Devon (H.)

\section{Escharidae}

Lepralia adpressa, Bk. Torbay (H.)

- edax, Bk. Plymouth Sound (H.)

- foliacea (E. and S.). Gen. dist. and c. on S. coast, especially rough ground, 10-30 fms.; Ilfracombe (H.)

- pallasiana (Moll.). S. Devon, most abundant littoral species (H.)

- pertusa (Esp.). S. Devon, v.c. (H.); realm, rr. ; Eddystone Gnds., rr. (M.B.A.)

Umbonula verrucosa (Esp.). Plymouth ; Salcombe, c. on rocks between tidemarks (M.B.A.); S. Devon, r.; Ilfracombe ( $\mathrm{H}$.)

Porella compressa (Sb.). Devon (H.)

- concinna (Bk.). Mequstone and Eddystone Gnds. (M.B.A.) ; Torbay (H.)

Escharoides quincuncialis (N.). Plymouth, single specimen, Âpr. I 889 (M.B.A.)

Smittia affinis (H.). Start. Bay, on shell (H.)

- cheilostoma (Manzoni). Eddystone Gnds. (M.B.A.)

- lansborovii (J.). Mewstone Ledge (M.B.A.)

- reticulata (Mcg.). S. Devon (H.)

- trispinosa (J.). S. Devon, v.c. (H.)

\section{CHEILOSTOMATA (continued)}

Escharidae (continued)

Phyllactella collaris (N.). Eddystone Gnds. (M.B.A.) Torbay (H.)

- labrosa (Bk.). S. Devon, n.c. (H.)

Mucronella coccinea (Ab.). S. Devon, c.; Ilfracombe (H.)

- peachii (J.). Eddystone Gnds. (M.B.A.)

- ventricosa (Has.). Start Point (H.); Eddystone Gnds., etc. (M.B.A.)

- variolosa (J.). Eddystone Gnds. (M.B.A.)

Palmicellaria skenei (E. and S.). Eddystone Gnds. (M.B.A.) ; Torbay (H.)

Rhynchopora bispinosum (J.). S. Devon, abund. (H.)

\section{Cemieporidae}

Cellepora avicularis (H.). Mezustone and Eddystone Gnds. (M.B.A.); S. Devon, c. on Sertularians, Gorgoniae, etc. (H.)

- costazii, Aud. S. Deqon; Ilfracombe (H.); Plymouth Sound (M.B.A.)

- dichotoma, H. Eddystone Gnds., 20-30 fms. (M.B.A.)

- pumicosa, L. c. and widely dist.

- ramulosa, L. Eddystone and Stoke Gnds. (M.B.A.); S. Devon (H.)

\section{CYCLOSTOMATA}

\section{Crisidar}

Crisia aculeata, Has. Plymouth, m.c. 4-5 fms. on red seaweeds, stones, sponges, etc. Ovicells, iv, v (M.B.A.)

- cornuta (L.). Plymouth, f.c., chiefly on red seaweeds. Ovicells commonest Apr., May (M.B.A.). S. Devon; Ilfracombe (H.)

- denticulata (Lam.). Plymouth Sound, m.c. at times (M.B.A.)

- eburnea (L.). Eddystone Gnds., c. almost always on red seaweeds. Ovicells Mar.-May (M.B.A.)

- geniculata, M.E. Plymouth, r. (M.B.A.)

- ramosa, Harmer. Plymouth, commonest species of Crisia, 4-30 fms.; Salcombe, c. Ovicells Feb.Aug. (M.B.A.)

\section{Tubuliporidar}

Stomatopora granulata, M.E. Eddystone Gnds.(M.B.A.); Lee near Ilfracombe (H.)

- fungia, Couch. Off Eddystone ; Torbay (H.)

- incrassata, Smitt. Salcombe, on Pecten maximus (H.)

- johnstoni, Heller. Eddystone Gnds. (M.B.A.)

- major, J. Eddystone Gnds. (M.B.A.)

Tubulipora flabellaris (F.). S. Devon, c. (H.)

- lobulata, Has. Torbay (H.)

Idmonea serpens (L.). Gen. dist. and m.c. I 5-35 fros. on Hydrallmania, etc.

Entalophora clavata (Bk.). Berry Head (H.)

Diastopora obelia, J. Eddystone Gnds. (M.B.A.)

- patina (Lam.). Gen dist. and c.; proliferous form ; Torbay (H.)

- sarniensis, N. Plymouth, c. 15-35 fms. (M.B.A.)

- suborbicularis. S. Devon, abundant (H.)

\section{LichenOPORIDAE}

Lichenopora hispida (Fl.). c. 15-30 fms. S. Devon; Ilfracombe (H.)

- radiata (Aud.). S. Devon; Brixbam; Salcombe, etc. $(\mathrm{H}$.) 


\section{A HISTORY OF DEVONSHIRE}

\section{CTENOSTOMATA}

Azcroniditdae

Alcyonidium albidum, Ald. Ilfracombe, m.c. (H.)

- gelatinosum (L.). Gen. dist. and f.c.

- hirsutum (Fl.). Plymouth Sound, r. (M.B.A.); S. Devon; Ilfracombe $(\mathrm{H}$.

- mytili, Dal. Plymouth (M.B.A.); Salcombe; Torbay (H.)

- parasiticum (Fl.). Plymouth, on Sertularia cupressina (M.B.A.); S. Devon, m.c. (H.)

\section{Flustrelitidas}

Flustrella hispida (F.). Abundant everywhere between tidemarks on Fucus, etc. B. ii-vii (M.B.A.)

\section{Arachiniditidat}

Arachnidium hippothooides, H. Torbay, on shell (H.)

VESICULARIIDAE

Vesicularia spinosa (L.). S. Devon; Ilfracombe (H.) Amathia lendigera (L.). c. on Halidrys, S. Devon; llfracombe (H.)

Bowerbankia caudata (H.). Cattewater (M.B.A.); Ilfracombe (H.)

- citrina (H.). Ilfracombe (H.)

- gracillima (H.). Ilfracombe; Salcombe; Torquay (H.)

- imbricata (Adams). Millbay Dock (M.B.A.); gen. and c. (H.). B. vii (M.B.A.)

- pustulosa (E. and S.). v.c. Plymouth; Salcombe (M.B.A.) ; Ilfracombe (H.)

Buskia nitens, Ald. Salcombe, on Lafoea dumosa (H.)

\section{CTENOSTOMATA (continued)}

\section{Cylindrorchidae}

Cylindroecium dilatatum (H.). Eddystone Gnds. (M.B.A.) ; S. Devon; Ilfracombe (H.)

- pusillum, H. Salcombe; Ilfracombe (H.)

Anguinella palmata, v. B. Ilfracombe (H.)

\section{HYPOPHORELLIDAR}

Hypophorella expansa, Eh. Plymouth, m.c. on tubes of Chaetopterus (M.B.A.)

\section{Triticellidat}

Triticella Boecki, S. Plymouth, on Gonoplax (M.B.A.)

\section{ValKeriIdar}

Valkeria uva (L.). Var. uva, S. Devon; Ilfracombe; var. cuscuta, Exmouth; Torquay ; Plymouth (H.)

- tremula, H. Salcombe; Ilfracombe (H.)

Mimosellidae

Mimosella gracilis, H. Salcombe Bay, c. ; Torbay, c.; Plymouth Sound (H.)

\section{ENTOPROCTA}

Pedecellinidae

Pedicillina cernua (P.). Plymouth, m.c. l.w. -35 fms. Salcombe, r. (M.B.A.) ; gen. (H.)

- gracilis, S. $5 \mathrm{~m}$. S. Mewstone, 27 fms. (M.B.A.); S. Devon, c. (H.)

\section{LOXOSOMIDAB}

Loxosoma phascolosomatum, Vogt. Salcombe and $E x$ mouth, c. on posterior end of Phascolosoma vulgare (M.B.A.)

\section{MOLLUSCA}

\section{AMPHINEURA}

\section{NEOMENIIDAB}

Rhopalomenia aglaopheniae (Kov. and Mar.). Plymouth, trawling grounds, 15-30 fms. c. on Aglaophenia myriophyllum (M.B.A.)

Myzomenia banyulensis (Pruvot). Plymouth, occ. on Lafoea dumosa. Gonads ripe in Aug. (M.B.A.)

\section{Chitonidae}

Lepidopleurus cancellatus (Sb.). S. Devon, n.c.; Teignmouth Bay, dead.

Hanleya hanleyi (Bean). Plymouth, trawl refuse, (J.G.J.)

Tonicella ruber (Lowe). S. Devon (J.G.J.)

Callochiton laevis (M.). Plymouth Sound, under stones, 1.w. (M.B.A.); Torquay and Exmouth (F. and $\mathrm{H}_{\text {.) }}$

Craspedochilus cinereus (L.). Gen. dist. on shore

- onyx (Spengl). Gen. dist. in 15-30 fims., and especially on muddy gravel

Acanthochites fascicularis (L.). Gen. on rocky shores, in crevices and empty barnacles, and in dredgings inshore

\section{PELECYPODA}

\section{Nuculidar}

Nucula nucleus (L.). Plymoutb; Salcombe; Teigrmouth $B a y$, I 5-30 fms., in mud or muddy gravel, c. Var. radiata, Torbay (F. and $\mathrm{H}$.)

- nitida, Sb. Not uncommon, S. Devon, in fine sand and mud, IO-27 fms. Var. radiata, Teignmouth Bay (L.B.)

Nuculana minuta (O.F.M.). Exmouth, v.r. in $20 \mathrm{fms}$. (F. and $\mathrm{H}$.)

\section{Anomiddar}

Anomia ephippium, L. f.c. everywhere, l.w. $30 \mathrm{fms}$., on stones, rocks, shells, etc.

- patelliformis, L. Gen. dist. below ro fms., but 1.c. than A. ephippium

\section{Arcidab}

Glycimeris glycimeris (L.). Sandy and muddy gravel, 7-30 fms., c. Plymouth; off Bolt Head, etc.

Barbatia lactea (L.). Plymouth, occ. on shore and in 15-30 fms., particularly in muddy gravel (M.B.A.) ; Ilfracombe; Exmouth (F. and H.) ; Teigmmouth Bay (L.B.) 


\section{MARINE ZOOLOGY}

\section{PELECYPODA (continued)}

Arcidae (continued)

Arca tetragona, Poli. occ. on all rocky shores, in crevices and among stones, 1.w.- 30 fins.; Teignmouth Bay, on O. edule (F. and H.)

\section{Mytilidar}

Mytilus edulis, L. Gen. dist. from h.w.m. to a few fms ; c. on piles and hulks moored in Plymouth Sound, etc. Large quantities taken annually for food and bait from large mussel beds in estuaries of Teign and Exe; young mussels $1 \mathrm{~mm}$. in length v.c. on Enteromorpha in the Exe during July and Aug., I 901 (M.B.A.)

Modiolus modiolus (L.). Salcombe, occ. (M.B.A.)

- barbatus (L.). Plymoutb Sound, occ. under stones on shore (M.B.A.) ; Torbay, r. ; Exmouth (F. and H.) ; Teignmouth Bay, r. (L.B.)

- adriaticus (Lam.). Dartmouth; Exmouth; Torquay (F. and H.) ; Teignmouth Bay (L.B.) n.c.

- phaseolinus ( $\mathrm{Ph}$.). f.c. I 5-30 fms.; occ. on shore. Plymouth (M.B.A.)

Modiolaria marmorata (Fb.). Gen. dist. in the tests of Ascidians

- costulata (Risso). Exmouth (F. and H.)

- discors (L.). (F. and H.)

Crenella rhombea (Berkeley). (F. and H.)

\section{Pteritdas}

Pteria hirundo (L.). v.r. in 35 fms. off Plymouth ; not recorded for some years

Pinna fragilis, Penn. Plymouth, 20-30 fms., small specimens occ. (M.B.A.); Torquay, very large (F. and H.); Teignmouth Bay, large living (L.B.) ; c. about 20 miles south of Start Point, on 'Caperlonger' ground (J. Tucker)

\section{Ostreidae}

Ostraea edulis, L. Gen. dist., n.c. in all estuaries. Beds in the Tamar and Yealm; c. in Teigmmouth $B a y$, where oyster dredging is carried on. Var. parasitica, Teigmmouth Bay (L.B.)

\section{Pectinidar}

Pecten maximus (L.). Gen. dist., n.c., in 1 5-35 fms.; v.c. on gravelly grounds; occ. on shore; Sal combe Estuary and realm c. in winter (M.B.A.)

Hinnites pusio (L.). f.c. on all rocky shores among stones, shells, etc., 1.w. - 30 fms.

Chlamys varius (L.). Plymouth Breakwater, f.c.; Plymouth Sound; Yealm; Salcombe; Start Bay (M.B.A.) ; Teigmmoutb Bay (L.B.)

Aequipecten opercularis (L.) Phymouth to Exmouth, f.c. everywhere, 15-30 fms., especially in certain localities ; Eddystone Grounds, etc., sometimes in great abundance in patches, occ. on the shore, Salcombe. Var. lineata; off Plymouth, Teignmouth, etc. Breeding, vi-viii (M.B.A.)

Palliolum tigerinus (O.F.M.). Plymouth, m.c. on coarse muddy or sandy gravel; occ. on Plymouth Breakwater (M.B.A.)

- incomparabilis (Risse). Exmouth (J.G.J.)

- striatus (O.F.M.). Plymouth (J.G.J.)

- similis (Laskey). Torbay: Plymouth, $25 \mathrm{fms}$.
PELECYPODA (continued) .

Limidae

Lima subauriculata (M.). Plymouth, 25 fms. (F. and H.)

- loscombii (Sb.). Plymoutb (M.B.A.); Exmouth (F. and $\mathrm{H}$.)

- hians (Gm.). Abundant in small patches of stones at l.w.s.t., on the north side of Plymouth Breakwater; occ. between tidemarks on the Reny Rocks (M.B.A.)

\section{KELLIELLIDAB}

Turtonia minuta (F.). Exmouth

\section{Astartidae}

Astarte sulcata (D.C.). Plymouth, f.c. in I 5-30 fms., especially on muddy or sandy gravel (M.B.A.)

Goodallia triangularis (M.). Plymouth; Exmouth (F. and $\mathrm{H}$.)

\section{ArCTICIDAB}

Arctica islandica (L.). Plymouth, occ. in $15-30 \mathrm{fms}$., gen. with Malacobdella grossa (M.B.A.); Teignmouth Bay, occ. Var. crassa, Jeff. ; Teggnmouth Bay (L.B.)

\section{Lucinidae}

Loripes lacteus (L.). Torbay (F. and H.)

Divaricella commutata (Ph.). Teignmouth Bay (J.G.J.)

Lucina spinifera (M.). Plymouth, I 5-30 fms., shells m.c. (M.B.A.); Dartmouth, 27 fms.; Plymouth, 28 fms.; Torquay, Salcombe (F. and H.)

- borealis (M.). Tealm sandbank occ.; Sakombe Zostera bed, f.c. (M.B.A.); Exmouth (F. and H.)

Cryptodon flexuosus (M.). realm sandbank occ.; Salcombe, f.c. in sand and Zostera (M.B.A.); Dartmouth, 9 fms.; Exmouth; Torbay (F. and H.) ; Teignmouth Bay, f.c. in mud (L.B.)

Montacuta substriata (M.). f.c. attached to spines of Spatangus purpureus (M.B.A.); deep water among corallines, Exmouth (F. and $\mathrm{H}$.)

- bidentata (M.). Salcombe (F. and H.); Teigmmouth Bay in mud (L.B.)

Tellimya ferruginosa (M.). Commensal with Echinocardium cordatum, realm and Salcombe (M.B.A.); Dartmouth, 7 fms. (F. and H.); Teigmouth Bay, dead (L.B.)

Diplodonta rotunda (M.). Shells f.c. Plymouth to Exmouth (M.B.A.); living specimens scarce, Exmouth; Plymouth (F. and H.) ; Teignmouth Bay (L.B.)

\section{LEPTONIDAE}

Kellia suborbicularis (M.). f.c. 1.w.- 30 fms., in rock crevices, Pholas borings, and dead bivalve shells, and especially where there is a certain amount of silt. Var. lactea, Exmouth, in Pholas borings with type (L.B.)

Lasaea rubra (M.). Abundant on all rocky shores, in crevices and amongst barnacles, Dartmouth, 7 fms. (F. and H.). Var. pallida, Teignmouth Bay (L.B.)

Lepton squamosum (M.). Salcombe, commensal with Gebia (N.) ; Plymouth, 28 fms.; Exmouth; Torbay (F. and $\mathrm{H}$.)

- nitidum (Turt.). Var. lineolata, Exmouth; var. pisidialis, Torbay (J.G.J.)

- clarkiae, Clarke. Exmouth; Plymouth; Torquay (J.G.J.) 


\section{A HISTORY OF DEVONSHIRE}

\section{PELECYPODA (continued)}

\section{Galeommidae}

Galeomma turtoni (Sb.). Exmouth, dredged (F. and H.)

\section{SCrobicularidDaE}

Syndosmya prismatica (M.). Exmouth (F. and H.)

- nitida (O.F.M.). Teignmouth Bay, c. in mud (L.B.)

- alba (Wood). Start Bay, Torbay, and Teigmmouth Bay, m.c.; v.c. in stomachs of plaice, etc. (M.B.A.)

- tenuis (M.). Torbay (F. and H.)

Scrobicularia plana (D.C.). c. in mud and muddy gravel in estuaries, especially so in that of the Exe, where it is the commonest bivalve after the mussel (M.B.A.)

\section{Tellinidae}

Tellina crassa (Gm.). Off Plymouth Mewstone, c. ; realm sandbank, occ. (M.B.A.); Exmouth; Torbay; Salcombe (F. and H.); Teignmouth Bay (L.B.)

— squalida, Pulteney. r. Salcombe (M.B.A.) ; Teignmouth beach (L.B.)

- donacina (L.). realm sandbank, occ. (M.B.A.); Teignmouth Bay, r. (L.B.); all along south coast of Devon (F. and $\mathrm{H}$.)

- pusilla (Ph.). Teignmouth Bay, r. (L.B.) ; Plymouth; Exmouth (J.G.J.)

- tenuis (D.C.). Exmoutb Polesands, v.c. (M.B.A.); Teignmoutb Bay, c. (L.B.)

- fabula, Gron. Plymouth Sound, in Zostera beds, m.c. ; Salcombe, rr. (M.B.A.)

Macoma balthica (L.). Kingsbridge estuary, shells c., living, r.; Exmouth, c. on surface of mud (M.B.A.) ; Teign, in mud (L.B.)

\section{Donacidar}

Donax vittatus (D.C.). Exmouth Polesands, v.c., gen. with Enteromorpha or other alga attached to the posterior end (M.B.A.) ; Teignmouth Bay, c. on beach (L.B.)

- variegatus (Gm.). Plymouth, shells only, rr. (M.B.A.); Exmouth; Dawlish; Milton, occ. (F. and H.)

\section{Mactridab}

Mactra stultorum, L. Shells c. West Channel, Plymouth ; Start Bay (M.B.A.); Teignmouth Bay, c. on the beach and in dredgings (L.B.)

Spisula solida (L.). c. gen. in sandy estuaries and bays; v.c. in Teignmouth Bay, where it is dredged for food (L.B.)

- elliptica (Brown). Plymouth, West Channel, abundant (M.B.A.)

- subtruncata (D.C.). Teignmoutb Bay (L.B.)

Lutraria elliptica (Lam.). Plymouth Sound, on sandy shores occ.; Yealm, occ.; Salcombe, c. in Zostera and sand (M.B.A.) ; Exmouth; Torbay (F. and H.) ; Teignmoutb Bay, chiefly on beach (L.B.). Gonads ripe, Mar.-May (M.B.A.)

- oblonga (Ch.). Exmouth; Torbay; Salcombe (F. and H.); Dawlish Warren, valve only (L.B.)

\section{PELECYPODA (continued)}

VENERIDAE

Lucinopsis undata (Penn). Plymouth to Exmouth, shells only dredged (M.B.A.); Exmouth; Dartmouth, 27 fms. (F. and H.); Teignmouth Bay, occ. thrown up on beach (L.B.)

Dosinia exoleta (L.). Plymouth Sound, realm sandbank, and off the south coast in $15-30 \mathrm{fms}$. occ. (M.B.A.); Exmouth (F. and H.); Teignmouth Bay, trawled (L.B.)

- lupina (L.). Exmouth; $P$ lymouth, in 25 fms.; Torbay (F. and H.); Teigmmouth Bay (L.B.)

Meretrix chione (L.) Rarely taken alive; shells c. in West Channel, Plymouth (M.B.A.); Teignmouth Bay, dead (L.B.)

Venus (Clausinella) fasciata (D.C.). c. in coarse muddy or sandy gravel, 6-30 fms., off $P l y$ mouth; occ. on Yealm sandbank and Salcombe in clean sand (M.B.A.); Teignmouth Bay, occ.(L.B.)

- (Ventricola) casina, L. Off Plymouth, in 10-30 fms., gravel occ. (M.B.A.) ; S. Devon (F. and H.)

- (Ventricola) verrucosa, L. Off Plymouth, occ. on mixed gravel grounds, I 5-30 fms. ; Plymouth Sound and Yealm sandbank, rr. (M.B.A.); S. Devon, f.c. (F. and H.)

- (Timoclea) ovata, Penn. Plymoutb Sound, m.c. in muddy sand and gravel, occ. outside in 15-30 fms.; Salcombe, rr. (M.B.A.)

- (Chamelaea) gallina, L. Plymouth, occ. 15-30

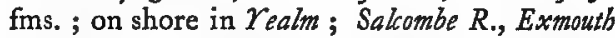
Polesands occ. (M.B.A.); Teignmouth Bay, c. Var. laminosa occ., dead c. (L.B.)

Tapes aureus (Gm.). Plymouth, shells only (M.B.A.)

- virgineus (L.).. c. Millbay Channel, Plymoutb, in gravel, occ. other parts of the Sound and outside in 15-30 fms.; Exmouth and Start Bay, rr. (M.B.A.); Teigmmouth Bay, type and var. sarniensis (L.B.)

- pullastra (M.). Gen. dist. on shore and in dredgings in estuaries. Var. perforans, c. in Pholas and Saxicava borings in limestone, etc.

- decussatus (L.). Laira, Yealm sandbank; Exmouth shore, in gravel, m.c.; Salcombe, rr. (M.B.A.) ; Torquay (F. and H.); Teignmouth Bay, c. Var. quadrangula, on mudflats behind Dazolish Warren (L.B.)

Gouldia minima (M.). Plymouth Sound and outside in 5-30 fms.; f.c. on fine and medium gravel (M.B.A.)

Irus irus (L.). Plymouth and other parts of Devonshire, in limestone (F. and H.); not recorded by the M.B.A.

\section{Carditdae}

Cardium aculeatum, L. Start Bay, dead ; Torbay, f.c. (M.B.A.) ; Teigntnouth Bay, f.c. in I 886 (L.B.); Paignton (J.G.J.) ; peculiar to S. Devon (F. and H.). Var. depressa, with the type, Teignmouth Bay (L.B.)

- echinatum, L. Plymouth, occ. in $15-30$ fms., dead shells c. on fine sand of the Eddystone trawling grounds; Teignmouth Bay, f.c.; Exmouth; Polesands, rr. (M.B.A.)

- tuberculatum, L. Paignton Sands, abundant at certain seasons (F. and H.) ; Teignmouth Bay, f.c. (L.B.)

- exiguum, Gm. Dartmouth; Torbay (F. and H.)

- fasciatum, M. Torbay; Exmouth, 7 miles from shore (F. and $H_{\text {. }}$ ) 


\section{MARINE ZOOLOGY}

\section{PELECYPODA (continued)}

\section{Cardidae (continued)}

Cardium nodosum, Turt. Exmouth; Torbay (F.and H.) - edule, L. Abundant locally; Hamoaze; realm R., below Steer Point; on Salstone, Salcombe; $E x-$ mouth (M.B.A.); Teign R., plentiful, especially just above Coombe Cellars (L.B.)

Laevicardium norvegicum (Spengler). Plymouth, c. on gravel, I 5-30 fms.; Exmouth Polesands (M.B.A.); Teignmouth Bay, c. ; var. gibba, Teignmouth Bay (L.B.)

\section{GARIDAE}

Psammobia tellinella, Lam. Plymouth Sound, Mewstone, and Stoke Point Grounds, $c$. in gravel and coarse sand; Eddystone Grounds, occ. (M.B.A.); Exmouth (F. and $\mathrm{H}$.)

- costulata, Turt. Eddystone Grounds, occ. with P. tellinella (M.B.A.); Torbay; Exmoutb (F. and H.)

- ferroensis (Chem.). Plymouth, I 5-30 fms., dead, c. (M.B.A.) ; Torquay, etc. (F. and H.) ; Teignmouth Bay, beach, and in dredgings, c. (L.B.)

- depressa, Penn. Tealm, shore, f.c. in coarse sand (M.B.A.) ; Exmouth; Torbay (F. and H.); Teignmouth Bay, scarce (L.B.)

\section{Myidar}

Mya arenaria, L. Exmouth (J.G.J.)

- truncata, L. occ. taken alive; realm shore (M.B.A.); Exmouth; Dartmouth, o-7 fins. (F. and H.); Teignmouth Bay (L.B.)

Sphenia binghami, Turt. Torquay, in limestone, Io fms. (F. and H.) ; Teignmouth Bay, in rock, rr. (L.B.)

Corbula gibba (Ol.). Plymouth Sound, occ. f.c. outside 1 5-30 fms. on muddy gravel ; Start Bay, occ. (M.B.A.) ; Exmouth, 10 fms. ; Torquay (F. and H.); Teignmouth Bay, in mud (L.B.); var. rosea, Teigmmouth Bay, with type (L.B.)

\section{SolenNidar}

Solecurtus scopula (Turt.). Eddystone Grounds, rr. (M.B.A.) ; Exmouth; Torbay (F. and H.)

- antiquatus (Pult.). Off Plymouth, occ. (M.B.A.); Exmouth; Plymouth, 25 fms. ; Dawlish; Torbay (F. and $\mathrm{H}$.)

Pharus legumen (L.). Bideford Bay, c.; Exmouth (F. and $\mathrm{H}$.)

Cultellus pellucidus (Penn). Plymouth Sound; Salcombe (shore) ; Start Bay; Teignmouth Bay; Torbay, f.c. in sandy mud (M.B.A.) ; S. Devon coast, 1027 fms. (F. and H.)

Ensis ensis (L.). Gen. dist. on sandy shores; v.c. in the realm in coarse sand ; Salcombe, r. (M.B.A.)

- siliqua (L.). Salcombe, f.c.; Polesands, m.c. (M.B.A.); Teignmoutb Bay, c. (L.B.)

Solen vagina, L. realm, sandbank, r.; Salcombe, c. in sand and Zostera beds; Exmouth, m.c. in firm muddy sand (M.B.A.) ; Teigrmouth Bay, rr. on beach (L.B.)

\section{SAXrcavidae}

Saxicavella plicata (M.). Plymouth, trawl refuse (J.G.J.)

Saxicava rugosa (L.). Gen. dist. and c., l.w.-20 fms., boring in limestone and sandstone

- arctica (L.). Everywhere, 10-30 fms., attached to shells, hydroids, etc. (M.B.A.); Plymouth, in limestone; Exmouth, in red sandstone (F. and $\mathrm{H}$.)

\section{PELECYPODA (continued)}

\section{Gastrochaenidae}

Gastrochaena dubia (Penn). Plymoutb Sound, occ. boring in limestone; $\mathrm{realm}_{\mathrm{R}} \mathrm{R}$., one in oyster shell (M.B.A.) ; Torbay, c. in limestone in Io fms.; Exmouth (F. and H.); Teignmoutb Bay, one in limestone (L.B.)

\section{Pholadidak}

Pholas dactylus, L. Rum Bay, c. in patches of shale rock ; Exmouth, c. in Orcombe Rks. (M.B.A.); Teignmouth Bay, in rock at 1.w.s.t., almost extinct (1892) (L.B.) ; var. gracilis, Exmouth, in pure sand (J.G.J.)

Barnea candida (L.). Torquay; Exmouth; Salcombe (F. and H.); Teignmouth Bay, l.c. than B. parva (L.B.) ; var. subovata, Exmouth, with the type, 1888 (L.B.)

- parva (Penn.). c. in Rum Bay; Orcombe Rks. (M.B.A.); Salcombe, in wood; Torquay, in red sandstone; Exmouth (F. and H.) ; Teignmouth Bay, formerly c., now found only at l.w.s.t. (I 892) (L.B.)

Pholadidea loscombiana, Goodall. Rum Bay, c. in shale; c. in red rock, $10-20$ fms. off Merostone and Stoke Point (M.B.A.); Exmouth; Teignmouth; Torquay, etc. in red sandstone (F. and $\mathrm{H}$.)

Xylophaga dorsalis (Turt.). Off Berry Head; Exmouth (F. and H.)

\section{Teredinidae}

Teredo norvegica, Spengler. Plymouth; Teignmouth; Torquay (F. and H.); Teignmouth Bay, in wreck (L.B.)

- navalis, L. Plymouth, f.c. in drift and submerged wood (M.B.A.)

- megotara, Hanley. Torbay; Exmouth, F. and H.; Dawlish Warren, in stranded timber (L.B.)

- malleolus, Turt. Torquay ; Exmouth; Plymouth (J.G.J.)

- bipinnata, Turt. Exmouth (J.G.J.)

Pandoridae

Pandora inaequivalvis (L.). Off Plymouth, occ. (M.B.A.); Dartmouth, 27 fms. ; Exmouth; Torbay (F. and H.) ; var. pinna, Teigmmouth Bay (L.B.)

\section{LYONSIIDAE}

Lyonsia norvegica (Chem.). Plymouth, occ. (M.B.A.)

\section{Anatinidai}

Cochlodesma praetenue (Pult.). Plymouth, dead (M.B.A.) ; Exmouth; Torbay (F. and H.)

Thracia fragilis, Penn. Plymouth Sound, shore, rr.; Salcombe (M.B.A.) ; Exmouth (F. and H.); Teignmouth Bay (L.B.)

- pubescens (Pult.). Dawlish; Plymouth, etc. (F. and H.) ; Teignmouth Bay (L.B.)

- convexa (Wood). Torbay; Teignmouth; Exmouth, n.c. (F. and $\mathrm{H}$.)

- distorta (M.). Torquay, Io fins., in limestone ; Exmouth (F. and H.); Teignmouth Bay, rr. (L.B.)

\section{SCAPHOPODA}

\section{Dentalindae}

Dentalium entalis, L. Plymouth, 15-30 fm., f.c. on fine sand, occ. on gravel (M.B.A.) ; Teigrmouth Bay, dead (L.B.) 


\section{A HISTORY OF DEVONSHIRE}

\section{GASTROPODA}

\section{PROSOBRANCHIA}

\section{Patellidak}

Patella vulgata, L. c. and gen. dist. on rocks between tidemarks ; var. picta, f.c. on stonework ; var. intermedia, c. on rocks at h.w. ; var. caerulea, f. with type, Teignmouth Bay (L.B.) ; var. elevata, N. Devon (J.G.J.)

Patina pellucida (L.). Gen. dist. and c. on stems of Laminaria

\section{Acmatidar}

Acmaea virginea (O. F. M.). Plymouth, 1.w. -5 fms., on rocky shores, under stones, etc., gregarious and f.c. ; Yealm R., m.c.; Salcombe, f.c. (M.B.A.) ; Teignmouth Bay, v.f. (L.B.)

\section{Fissurellidat}

Emarginula fissura (L.) Plymouth, f.c. o-3o f.ms. ; Salcombe, rr. (M.B.A.); Teigmmouth Bay, d. (L.B.)

- conica, Schumacher. Plymouth, 10-30 fms., m.c. (M.B.A.) ; Exmouth (F. and H.) ; Teignmouth Bay, rough ground (L.B.)

Fissurella graeca (L.). Plymouth, occ. on all rocky stations, 1.w.-1o fms.; Yealm R.; Salcombe, f.c. on ascidians and sponges (M.B.A.) ; Exmouth; Torbay (F. and H.) ; Teignmouth Bay (L.B.)

\section{Trochidak}

Eumargarita groenlandica (Chem.). Plymouth, single specimen, dead (M.B.A.)

Gibbula magus (L.). Plymouth, occ. on coarse shell sand and gravel ; Salcombe, f.c. ; Exmouth (M.B.A.); Teignmouth Bay, c. Var. conica, rr. with type (L.B.)

- tumida (M.). Plymouth, occ., I 5-30 fms. (M.B.A.)

- cineraria (L.). c. and gen. dist. under stones, on rocks, Zostera, Fucus, etc.

- umbilicata (M.). Gen. dist., h.w.-3 fms., l.c. than G. cineraria

Monodonta crassa (Montfort). Plymouth, c. in restricted areas at and above h.w. (M.B.A.) ; Devon, frequent (F. and H.); Teignmouth Bay, c. (L.B.)

Calliostoma montagui (Wood). Exmouth (F. and H.)

- striatum (L.). On Zostera ; Plymouth, occ.; Yealm Est., c.; Salcombe, v.c. (M.B.A.); Torbay; Exmouth (F. and H.)

- exasperatum (Penn.). Torquay (F. and H.); Teignmouth Bay, f.c. (L.B.)

- miliare (Brocchi). Var. pyramidata, Plymouth (J.G.J.)

- granulatum (Born). Gen. dist. on S. coast, on sandy gravel, 20-30 fms.

- zizyphinus (L.). C. and gen. dist. on all rocky shores; a well-marked variety, c. on the realm sandbank

\section{Cyclostrematidae}

Delphinoidea cutleriana (Clarke). Exmouth (F.and H.)

- nitens (Ph.). Coralline zone (J.G.J.)

- serpuloides (M.). Exmouth (F. and H.)

\section{PROSOBRANCHIA (continued)}

Turbinidae

Phasianella pullus (L.).. Gen. dist. and m.c. on Algae and Zostera, l.w. - 10 fms. Var. oblonga, Teignmouth Bay, f.c. at times on weeds (L.B.)

\section{LITTORINIDAE}

Lacuna crassior (M.). Teignmoutb Bay, on rough ground (L.B.)

- divaricata (F.). f.c. and gen. dist. on weeds, Zostera, and rocks, near l.w. Breeding ii-iv (M.B.A.)

- parva (D.C.). Plymouth Sound, occ. (M.B.A.); Teignmouth Bay, v.c. on weeds, l.w.s.t. ; var. clausa, Teignmouth Bay. Var. expansa, Teignmouth Bay (L.B.) ; Exmouth; Torbay (J.G.J.)

- pallidula (D.C.). Gen. dist. on Fucus, Chondrus crispus, etc. Breeding ii. (M.B.A.). Var. albescens, Teigmmouth Bay (L.B.)

Littorina obtusata (L.). c. and gen. dist. on Fucus, Zostera, etc. Breeding ii, iii. (M.B.A.)

- neritoides (L.). Gen. dist. and c. on rocks above h.w.

- rudis (Maton). Gen. dist. and c. on rocks near h.w. Vars. saxatilis, rr., jugosa, c., Teignmouth Bay (L.B.)

- littorea (L.). Gen. dist. and c. between tidemarks ; gathered in many places for food, and also to put on mussel beds to keep down Ulva. Breeding ii. (M.B.A.)

\section{Rissoidae}

Rissoa parva (D.C.). Gen. dist. on shore, on rocks and weeds, and occ. in dredgings. Var. interrupta, Teignmouth Bay, c. (L.B.)

- inconspicua, Ald. Torquay; Exmouth (F. and H.); Teignmouth Bay, r. (L.B.); Plymouth, trawl refuse, v.c. (J.G.J.). Var. variegata, Teignmouth Bay (L.B.) ; var. ventrosa, Exmouth (J.G.J.)

- albella, Lovén, var. sarsi. Teignmouth Bay, drift (L.B.)

- violacea, Desm. Teignmouth Bay, on weeds, s. (L.B.) ; Torquay

- guerini, Récluz. Exmouth; Torquay, c. (F. and H.); Teigmmouth Bay (L.B.)

Alvania lactea (Mich.). Barrycane, (J.G.J.)

- cancellata (D.C.). Plymouth, dead (M.B.A.); Torquay; Salcombe (F. and H.); Teignmouth Bay, rr. (L.B.)

- reticulata (M.). Plymouth, dead (M.B.A.); $E x$ mouth (F. and H.). Var. cimicoides; Exmouth (J.G.J.)

- punctura (M.). Plymouth; Exmouth, etc. (F. and H.) ; Teignmoutb Bay, occ. (L. and B.)

Manzonia zetlandica (M.). Exmouth; Barrycane (F. and $\mathrm{H}$.)

- costata (Adams). Gen. dist., but n.c.

Zippora membranacea (Adams). Gen. dist. and c., on Zostera, etc.

Onoba striata (Adams). Gen. dist. and c., 1.w.Io fms., particularly under stones with silt

Ceratia proxima, Ald. Teigmouth Bay, in shelly mud (L.B.); Exmouth, I 5 fms., shells and mud; Torbay and Plymouth, with H. vitrea (J.G.J.) 


\section{MARINE ZOOLOGY}

\section{PROSOBRANCHIA (continue.i)}

Rissoidar (continued)

Hyala vitrea (M.). Plymouth (J.G.J.); Exmouth (F. and H.); Teignmoutb Bay, with C. proxima (L.B.)

Setia fulgida (Adams). Torquay; Exmouth (F. and H.); Teignmouth Bay, drift, rr. (L.B.)

- obtusa (Cant.). Exmouth, F. and H. ; Teignmouth Bay, dead (L.B.)

Cingula semistriata (M.). Plymouth Sound, 23 fms. (M.B.A.); Torquay; Dartmouth, 7 fms. (F. and H.); Teigmmouth Bay, l.w. s.t., etc. (L.B.). Var. pura, under stones; Teignmouth Bay (L.B.)

- trifasciata, Adams. Gen. dist. and c. on rocky shores, under stones, in crevices, especially when there is silt; $80 \%$ of Teignmouth Bay specimens belong to var. graphica, Turton (L.B.)

Galeodina carinata (D.C.). Plymouth, dead (M.B.A.); Exmouth (F. and H.)

Barleeia rubra (M.). Teignmouth Bay, on weed, rr. (L.B.)

\section{Assinimeidar}

Paludinella littorina (Dl.C.). Exmouth (J.G.J.)

Paludestrina stagnalis (Basterot). c. in brackish water of estuaries, on mud, Zostera and under stones.

\section{Adeorbidat}

Adeorbis subcarinatus (M.). Plymouth, c, on rocks at Rams Cliff Point, I887; not taken since (M.B.A.); Dartmouth, 12 fms.; Exmouth (F. and H.); Teignmouth Bay, 30 spec. from rocks, 1888 (L.B.)

\section{SkEneidae}

Skenea planorbis (F.). Plymouth, c. among roots of seaweeds and corallines (M.B.A.); Teigmmoutb Bay, scarce (L.B.), etc.

\section{HOMOLOGYRIDAE}

Homologyra atomus (Ph.). Torbay (J.G.J.)

- rota (F. and H.). Exmouth (J.G.J.)

\section{Truncatellidar}

Truncatella truncata (M.). Salcombe; Plymouth, etc. (J.G.J.)

\section{Capulidar}

Capulus hungaricus (L.). On gravel grounds, I 5$35 \mathrm{fms}$., occ. attached to Pecten opercularis and Ostraea edulis. Breeding i-iii (M.B.A.)

Calyptraea chinensis (L.). In dredgings from $P$ lymouth Sound, c.; realm, c.; Salcombe Estuary, v.c. on stones and shells (M.B.A.); Dartmouth, 7 fms. (F. and H.). Breeding vii-ix (M.B.A.)

\section{Cypraeidae}

Trivia europaea (M.). Gen. dist. and f.c., l.w.$30 \mathrm{fms}$., on rocky and stony grounds

Ovula patula (Penn.). f.c., 10-30 fms., on Alcyonium digitatum. Plymouth-Exmouth. Breeding probably iv-vii (M.B.A.)

Erato laevis (Don.). Plymouth, m.c. on gravel, I 530 fms. (M.B.A.)

\section{PROSOBRANCHIA (continued)}

\section{Naticidae}

Natica sordida (Ph.). Exmouth, with N. catena (J.G.J.) - catena (D.C.). Plymouth, r. on sandy bottoms; Start to Exmouth, f.c. in sandy bays; Exmouth Polesands, f.c. Shells m.c. everywhere, inhabited by hermit crabs (M.B.A.)

- alderi (Fb.). Gen. dist. and f.c., l.w.-35 fms., on clean sand and gravel.

- montagui, Fb. Devonsbire, 30-35 fms., sand (J.G.J.)

\section{LaMELLARIIDAE}

Lamellaria perspicua (L.). Plymouth Sound; realm; Salcombe Estuary; m.c. shore and dredgings, occ. outside 15-30 fins. (M.B.A.); Exmouth (F. and H.); Teignmouth Bay, f.c. (L.B.). Spawn, Jan.-May (M.B.A.)

Velutina laevigata (Penn). Plymouth, on rough ground occ. (M.B.A.); Teignmouth Bay, rough ground occ. (L.B.)

\section{IANTHINIDAE}

Ianthina rotundata, Leach. Dawlish Warren (F. and H.)

\section{Cerithindar}

Bittium reticulatum (D.C.). Plymouth, m.c. on rocky shores, 1.w.- IO fms.; Salcombe, f.c. on Zostera (M.B.A.); Teignmouth Bay, occ. (L.B.)

Triforis perversa (L.). Plymouth, occ., 15-35 fm. (M.B.A.); Teigmouth Bay, 1.w. (L.B.)

Cerithiopsis tubercularis (M.). Plymouth Sound and outside 1 5-30 fms., m.c. on sponges; $Y_{e a l m, ~} \mathrm{c}$ on red sponge; Salcombe (M.B.A.); Exmouth (F. and H.); Teignmouth Bay, on and under stones, l.w. s.t., numerous in 1888 and 1889 (L.B.)

- sublenta (Wood). Teignmouth Bay, dredged, and under stones, r. 1894 (L.B.); Plymouth, trawl refuse (J.G.J.)

- concatenata, Conti. Plymouth, coralline zone (J.G.J.)

\section{SCAIIDAB}

Scala turtonis (Turt.). Plymouth (M.B.A.); Exmouth (F. and H.); Teigmmouth Bay, m.c. (L.B.)

- clathrus (L.) Occ. on both N. and S. coasts, on rough ground, shore and deeper water

- trevelyana (Leach). Plymouth (J.G.J.)

- clathratula (Adams). Plymouth, dead, (M.B.A.); Torbay (F. and H.); Teigrmouth Bay, rr. (L.B.)

Cioniscus albidus (Adams). Teignmouth Bay, drift (L.B.); Exmouth (J.G.J.)

Aclis ascaris (Turt.). Plymouth; Exmouth (J.G.J.)

- minor, Brown. Plymouth (J.G.J.)

Pherusina gulsonae (Clark). Exmouth (J.G.J.)

\section{Pyramidellidar}

Odostomia lukisi, J.G.J. S. Devon, on seaweeds at l.w. (J.G.J.)

- conoidea (Broc.). Exmoutb to Torquay (F. and H.). Var. australis, S. Devon (J.G.J.)

- umbilicaris (Mlg.). Torbay, etc., coralline zone (J.G.J.)

- acuta (J.G.J.). Torbay; Exmoutb (F. and H.); Teigmmouth Bay, mud (L.B.). Var. umbilicata, Teigmmouth Bay, muddy gravel (L.B.) 


\section{A HISTORY OF DEVONSHIRE}

\section{PROSOBRANCHIA (continued)}

Pyramidellidar (continued)

Odostomia conspicua (Ald.). Teignmouth Bay, rr. in muddy gravel (L.B.)

- unidentata, F. and $\mathrm{H}$. Torbay (F. and H.); Teignnouth Bay (L.B.)

- turrita, Hanley. Teignmouth Bay, 1.w. (L.B.). Var. striolata. Ilfracombe (J.G.J.)

- plicata (M.). Gen. dist. at l.w. on S. coast, f.c. sublittoral zone; Exmouth (J.G.J.)

Jordanula nivosa (M.). Teignmouth Bay (L.B.) ; Ilfracombe (J.G.J.)

- truncatula (J.G.J.). Plymouth, trawlers (J.G.J.)

Liostomia clavula (Loven). Teignmouth Bay (L.B.); Brixbam; Plymouth (J.G.J.)

Brachystomia rissoides (Hanley). v.c. among dwarf mussels, rocks at l.w.; Teignmouth Bay (L.B.). Vars. nitida and exilis, S. Devon (J.G.J.)

- ambigua (M. and R.). c. on S. coast, on 'ears' of Pecten opercularis and P. maximus. Var. crassa, Torquay

Ondina divisa (Adams). Teignmoutb Bay (L.B.); Torbay, etc. (F. and $\mathrm{H}$.)

- diaphana, J.G.J. Teigmmouth Bay, on trawled Zostera (L.B.); Exmouth (J.G.J.)

- obliqua (Ald.). Exmouth, deep water, rr. (F. and H.); Teignmouth Bay (L.B.)

- warreni (Th.). Burrow I., f.c., dead (F. and H.)

Oda dolioliformis (J.G.J.), v.r., dead; Exmouth (F. and H.) ; Teignmouth Bay, c. at times, 1.w. s.t. (L.B.); Torbay (J.G.J.)

Pyrgulina decussata (M.). Exmouth; Salcombe (F. and H.) Teignmouth Bay, drift (L.B.)

- indistincta (M.). Teignmouth Bay, in mud (L.B.). Var. brevior, Teignmouth Bay, on trawled Zostera (L.B.)

- interstincta (M.). Teignmouth Bay, rr. (L.B.)

- fenestrata (Fb.). Dartmouth; Torbay (F. and H.); Exmoutb (J.G.J.)

Spiralinella spiralis (M.). Exmouth (F. and $\mathrm{H}_{\text {.) }}$; Teignmouth Bay, dredged in mud, and under stones, l.w. (L.B.)

Miralda excavata (Ph.). Exmouth (J.G.J.)

Pyrgostelis scalaris (Ph.). Dartmouth; Exmouth (F. - and H.); Teignmouth Bay, dead (L.B.); Torquay (J.G.J.)

- interrupta ('Totten). Plymouth, occ. 15-35 fms. (M.B.A.); Exmouth (J.G.J.)

Turbonilla lactea (L.). Plymouth, occ., l.w.- 10 fms., stones with silt; Salcombe, v.c. on Zostera (M.B.A.); Teigmmouth Bay, f.c. (L.B.)

- pusilla (Ph.). Torbay; Barrycane; Ilfracombe (J.G.J.). Var. minuscula, Teignmouth Bay, with T. lactea (L.B.)

Eulimella commutata (Monter.). Dartmouth; Exmouth; Torbay; Burrow I. (F. and H.); Teignmouth Bay, dead (L.B.)

- nitidissima (M.). Exmouth (F. and H.); Teignmouth Bay, rr. (L.B.)

\section{EuLimide}

Eulima polita (L.). Plymouth, I 5-35 fms., m.c. on muddy gravel (M.B.A.); Teignmouth Bay (L.B.)

- intermedia, Cant. Exmouth; Plymouth (J.G.J.)

- incurva (Ren.). Plymouth Sound; realm $R$., c. among sponges (M.B.A.); Exmouth (F. and H.); Teignmouth Bay (L.B.)

\section{PROSOBRANCHIA (continued)}

Eulimidae (continued)

Leiostraca bilineata (Ald.). Plymouth, occ., 15-35 fms. (M.B.A.); Exmouth (F. and H.); Teignmouth Bay (L.B.)

- glabra (D.C.). Plymouth, occ. (M.B.A.); Teignmouth Bay, occ. (L.B.)

Stilifer stilifer (Turt.). Torbay, on Echinus esculenta (F. and H.); Plymouth, on E. miliaris and E. esculentus (J.G.J.), rr.

\section{Caecidar}

Caecum imperforatum (Adams). Devon, coralline zone, f.c. (J.G.J.)

- glabrum (M.). Plymouth (M.B.A.); Exmoutb (F. and H.); Teignmouth Bay, drift (L.B.)

\section{TURritellidae}

Turritella communis, Lam. c. in mud and muddy gravel, 15-30 fms. on S. coast. Var. nivea, Teignmouth Bay (L.B.); Exmouth (J.G.J.); var. gracilis, Torquay (J.G.J.)

\section{Aporrhaidar}

Aporrhais pes-pelecani (L.). f.c., 5-35 fms., on muddy gravel ; Teignmouth Bay, 4 specimens recorded with four spurs (L.B.)

\section{Buccinidae}

Buccinum undatum, L. f.c. everywhere, especially on gravel and coarse sand. Breeding $\mathrm{i}-\mathrm{iv}$. Hatching ii-iii (M.B.A.)

Liomesus dalei (Sb.). Torbay, may have come by Newfoundland fishermen (F. and $\mathrm{H}$.)

Donovania minima (M.). f.c., l.w.-20 fms., under stones, etc.

Tritonofusus gracilis (D.C.). Dead; Plymouth; Start Bay (M.B.A.); Teigmmouth Bay (L.B.)

Siphonophorus Jeffreysianus (Fisc.). Exmoutb; Torquay; Brixham; Plymouth (J.G.J.)

\section{Muricidae}

Ocinebra erinacea (L.). On rocks between tidemarks, gen. dist., c. only in places. Breeding, iv-v (M.B.A.)

Trophon muricatus (M.). Occ. 15-30 fms.

Purpura lapillus (L.). c. everywhere on rocks between tidemarks. Breeding, $i-v$, vii, ix (M.B.A.)

\section{NasSIDAB}

Nassa reticulata (L.). f.c. everywhere, 1.w.—5 fms., especially on muddy sand. Breeding, ii-ix (M.B.A.)

- incrassata (Strom). f.c. everywhere, 1.w.- I o fms., on rocky shores where there is silt. Breeding, ii-iv, vi, viii, ix (M.B.A.)

- pygmaea (Lam.). Torbay to Exmouth (F. and H.); Teignmouth Bay, plentiful in dredged material (L.B.)

\section{Pleurotomidae}

Bela rufa (M.). Teignmouth Bay, d. occ. (L.B.). Var. lactea and cranchii, $P$ lymouth; var. angusta, Exmouth (J.G.J.)

Haedropleura ecostata (D.C.); Salcombe, d. (M.B.A.); Torbay (F. and H.); Teignmouth Bay, rr. (L.B.) 


\section{MARINE ZOOLOGY}

\section{PROSOBRANCHIA (continued)}

Pleurotomidae (continued)

Mangilia striolata (Scac.). Torbay; Exmouth, r. (F. and H.); Teignmouth Bay, mud (L.B.)

- attenuata (M.). Occ. on muddy gravel, 20-30 fms.

- costata (Don.). Salcombe (M.B.A.); Teignmoutb Bay, mud (L.B.)

- brachystoma (Ph.). Torbay; Exmouth (F. and H.); Teignmouth Bay, mud (L.B.); Plymouth (J.G.J.)

- nebula (M.). Plymouth, r. (M.B.A.); Teignmouth Bay, f.c. (L.B.). Var. laevigata, Teigmmouth Bay, d.; var. vittata, Teignmouth Bay, occ. (L.B.) ; var. elongata, Plymouth; Exmouth (J.G.J.)

Teres anceps (Eichwald). Plymouth (J.G.J.)

Bellardiella gracilis (M.). Plymouth, m.c., muddy gravel, 20-35 fms.(M.B.A.); Exmouth, coralline zone, abundant; Torquay, c. (F. and H.); Teignmouth Bay, r. (L.B.)

Clathurella linearis (M.). Plymouth Sound, m.c. on clean shell gravel, and outside I 5-30 fms. occ. (M.B.A); Teigmouth Bay, r. (L.B.)

- reticulata (Ren.). Plymouth, occ. I5-30 fms. (M.B.A.)

- purpurea (M.). Salcombe, d. (M.B.A.); Teignmouth Bay, two taken in 1886 (L.B.)

\section{OPISTHOBRANCHIA}

Actazonidae

Actaeon tornatilis (L.). Plymouth, occ. d. (M.B.A.); Teignmouth Bay, d. (L.B.)

\section{Tornatinidae}

Tornatina mammillata (Ph.). Plymouth, d. occ. (M.B.A.) ; Exmouth (F. and H.)

- truncatula (Brug.). Plymouth, d. (M.B.A.); Teignmouth Bay, d. (L.B.)

Diaphana hyalina (Turt.). Devon (F. and H.)

\section{SCAPHaNDRIDaE}

Scaphander lignarius (L.). Plymouth trawling grounds, c. (M.B.A.) ; Teignmouth Bay (L.B.)

Volvulella acuminata (Brug.). Plymouth, trawl refuse (J.G.J.)

Bullinella cylindracea (Penn). Plymouth, d. (M.B.A.); Teignmouth Bay, shore and dredge (L.B.)

\section{Bullidae}

Haminaea hydatis (L.). Plymouth Sound, occ. ; realm, occ. ; Salcombe, f.c. on muddy and clean gravel at times (M.B.A.) ; Teigmmoutb Bay, occ. f.c. (L.B.) ; Exmouth (F. and H.) Var. globosa, Exmouth (J.G.J.)

Roxania utriculus (Broc.). Torbay ; Plymouth (J.G.J.)

\section{Phillinidas}

Philine scabra (O.F.M.). Devon (F. and H.)

- catena (M.). Plymouth, occ. (M.B.A.); Teignmouth Bay, occ. (L.B.); Exmouth (J.G.J.). Var. zona, Bigberry Bay (J.G.J.)

- punctata (Adams). Plymouth Sound, occ. ; plentiful in $20 \mathrm{fms}$. off Plymouth among Bugula, 1891 (M.B.A.) ; Torbay; Exmouth (F. and H.) ; Teignmouth Bay, r. (L.B.)

\section{OPISTHOBRANCHIA (continued)}

Phillinidae (continued)

Philine pruinosa (Clark). Exmouth (F. and H.); Plymouth; Budleigh Salierton (J.G.J.)

- nitida (J.G.J.). Teigmmouth Bay, dredged (L.B.)

- aperta (L.). c. on muddy bottoms, Plymouth Sound; Torbay; Teignmouth Bay, etc. ; occ. v. abundant. Breeding iv-vii (M.B.A.)

Colpodaspis pusilla (S.). Taken 2 miles S. of Plymouth Mewstone, Feb., 1893 (M.B.A.)

\section{Limacinidae}

Limacina retroversa (Fl.). Off the Eddystone 20 Sept., 1905; Plymouth Sound, 7 Sept. 1905 to 8 Nov.,c., due probably to a large influx of Atlantic water (L. H. Gough)

\section{Aplysimpar}

Aplysia punctata, Cuv. v.c. occ. on shore, with Zostera, etc., especially $\mathrm{v}-\mathrm{vi}$. B. iv-x, maximum $\mathrm{v}$ and vi (M.B.A.)

- depilans (L.). Torbay, c. 1875 and 1877 (A. R. Hunt)

\section{Pleurobranchidae}

Pleurobranchus plumula (M.). Plymouth and Salcombe, occ. (M.B.A.) ; Exmouth (F. and H.)

Oscanius membranaceus (M.). Plymouth Sound, v.c., 1893 , young specimens c. at surface Sept. 1892 (M.B.A.) ; Teigmmouth Bay, v.c., 1887-1889 (L.B.)

\section{Runcinidae}

Runcina coronata (Q.). Plymouth Sound, v.c. occ. on shore, realm (M.B.A.); Torbay, pools near h.w. (F. and H.)

\section{NUDIBRANCHIA}

\section{Hermeidae}

Hermaea bifida (M.). Plymouth Sound, v. occ. (M.B.A.) - dendritica (A. and H.). Plymouth Sound, v. occ on shore (M.B.A.) ; Torbay, on Codium tomentosus (F. and H.). Breeding vi (M.B.A.)

Stiliger bellulus, d'Orb. Plymouth Sound, single specimen (M.B.A.)

\section{Elysirdaz}

Elysia viridis (M.). Plymouth Sound, occ. ; Yealm, c. ; Salcombe, r.; Exmouth, m.c. Breeding $\mathbf{x}$ and probably earlier (M.B.A.)

\section{Limapontildae}

Limapontia capitata (O.F.M.). Plymouth Sound, occ. c. on shore in tidepools and among corallines (M.B.A.) ; Torbay (J.G.J.)

Acteonia corrugata, A. and H. Plymouth Sound, v. occ.

\section{EoLIDIDAB}

Eolis papillosa (L.). f.c. on all stony shores. Breeding ii-viii (M.B.A.)

Aeolidiella glauca (A. and H.). Plymouth, occ. (M.B.A.) ; off Berry Head (A. and H.)

- alderi (Cocks). On shore. Plymouth Sound; Wembury Bay ; Yealm. Breeding viii (M.B.A.)

Berghia caerulescens (Méneville). Taken off Plymouth, rr. (M.B.A.) 


\section{A HISTORY OF DEVONSHIRE}

\section{NUDIBRANCHIA (continued)}

Eolididae (continued)

Cuthona aurantia (A. and H.). Plymoutb Sound, occ. Breeding v. (M.B.A.)

Cratena amoena (A. and H.). Plymouth Sound, occ. on stony ground ; Salcombe, rr. (M.B.A.) ; Torbay (A. and $\mathrm{H}$.)

- viridis (Fb.). Plymouth Sound and Eddystone Grounds, occ. (M.B.A.)

- olivacea (A. and H.). Plymouth, on stony ground occ. ; realm R. (M.B.A.)

- peachi (A. and $H$.). Taken once $3-4 \mathrm{~m}$. S. of Plymouth Mezustone, on Hydractinia (M.B.A.)

- stipata (A. and H.). Torbay, on Sertularia (A. and $H$.)

Tergipes despectus (J.). Plymouth, occ.; Millbay Docks, c. on piles, Mar., I gor. Breeding iii-v (M.B.A.)

Embletonia pulchra (A. and H.). Plymouth Sound, once on Antennularia (M.B.A.)

Amphorina caerulea (M.): Plymouth Sound, and on Cellaria grounds outside m.c. (M.B.A.)

Galvina cingulata, A. and H. Plymouth Sound and Docks, on Plumularia ; realm, on Antennularia and Zostera. Breeding vii (M.B.A.)

- picta, A. and H. Plymouth Sound, m.c.; Yealm Est. on Zostera (M.B.A.) ; Torbay (A. and H.)

- tricolor (Fb.). f.c. on Plymoutb trawling grounds. 20-35 fms. Breeding v, xi (M.B.A.)

- exigua (A. and H.) Plymouth Sound, occ. Breeding iii (M.B.A.)

Coryphella rufibranchialis (J.). f.c., inner trawling grounds, Plymouth (M.B.A.)

- lineata (Lovén). Plymoutb and Eddystone, rr. (M.B.A.)

- landsburgi (A. and H.). Plymouth, occ. everywhere (M.B.A). ; Exmoutb (J.G.J.)

Favorinus albus (A. and H.). Plymouth Sound, m.c.; Yealm, f.c. on Zostera, I 897 (M.B.A.)

- carneus (A. and H.). Torquay (A. and H.); Salcombe (J.G.J.)

Facelina coronata, Fb. and Goodsir. Plymouth, m.c.

- drummondi (Th.). Plymouth Sound, r.; Yealm (M.B.A.)

- punctata (A. and H.). Plymouth Sound; Mewstone (M.B.A.) ; Torbay, deep water (A. and H.)

- elegans (A. and H.). Off Berry Head, 15 fms. (A. and $H$.)

Calma glaucoides (A. and H.). Plymouth Sound, among goby eggs; Mewstone Grounds, in Buccinum shells with eggs of Blennius ocellaris, occ. fifty in a shell; resembles the colour of the eggs with which it is associated (M.B.A.)

Fiona marina, Fr. Teignmouth $B a y$, two trawled (L. and B.)

Antiopa cristata (D1.C.). Plymouth, f.c. shore to 35 fms. ; Exmoutb Dock, m.c. (M.B.A.) ; Torbay (A. and H.). Breeding viii (M.B.A.)

- hyalina, A. and H. Plymouth Sound, occ. ; and off Mewstone and Eddystone (M.B.A.)

Hero formosa (Lovén). c. $6 \mathrm{~m}$. S. of Merustone, i-vi, I 895 ; occ. elsewhere; not recorded since 1898. Breeding iv (M.B.A.)

\section{LOMANOTIDAR}

Lomanotus genei, Vérany. Plymouth Sound, f.c. on Antennularia, occ. outside ; Salcombe (M.B.A.); Torbay (A. and H.). Breeding ix (M.B.A.)

\section{NUDIBRANCHIA (continued)}

Dotonidae

Doto fragilis (Fb.). c. on Antennularia from 15-35 frns. Breeding greater part of year (M.B.A.)

- pennatifida (M.). Plymouth, gen. dist., and c. on Antennularia. Breeding greater part of the year (M.B.A.)

- coronata (Gm.). Plymouth, c. on Antennularia (M.B.A.); Torbay; Salcombe (A. and H.). Breeding greater part of the year (M.B.A.)

Hancockia eudactylota, G. Plymoutb Sound and reaim R.; r. on Laminaria and Zostera (M.B.A.) ; Torbay, on Delesseria (A. R. Hunt)

\section{Dendronotidaz}

Dendronotus frondosus (Asc.). Gen. dist. on clean grounds, but n.c. ; usually small

\section{SCyllaEidar}

Scyllaea pelagica, L. Devon (A. and H.)

\section{Pleurophyllidiidar}

Pleurophyllidia loveni, Bergh. Single specimen $2 \mathrm{~m}$. N. of Eddystone (M.B.A.)

\section{Tritonimda}

Tritonia hombergi, Cuv. Occ. taken inside Plymouth Sound, Torbay, Teignmouth Bay, etc., but is more c. outside the 30-fm. line

Candiella plebeia, J. Plymouth Sound, etc., occ. ; pink variety is f.c. on Eunicella from the Mewstone Ledge (M.B.A.) ; Torbay (A. and H.); Teignmouth Bay (L.B.). Breeding viii, x, xi (M.B.A.)

- lineata, A. and H. Single specimen off Stoke Point (M.B.A.)

\section{Dorididas}

Archidoris tuberculata (Cuv.). f.c. shore-30 fms. B. i-vi (M.B.A.)

Archidoris flammea (A. and H.). Plymouth Sound, rr. (M.B.A.)

Rostanga coccinea (Fb.). Plymouth Sound, occ (M.B.A.)

Aldisa millegrana (A. and H.). Torbay (A. and $H$. .)

Jorunna Johnstoni (A. and H.). Plymouth Sound; realm, occ. (M.B.A.)

Platydoris testudinaria (Risso). Plymouth Sound; realm; Eddystone Grounds (M.B.A.)

\section{Polyceridae}

Aegirus punctilucens (D’Orb.). Plymouth Soutsd; Eddystone Grounds, occ. (M.B.A.)

Triopa clavigera (O.F.M.). Plymouth Sound, rr., occ. outside to 30 fms. (M.B.A.); Torbay, m.c., l.w.; Salcombe (A. and H.)

Thecacera pennigera (M.). Plymouth Sound, rr. Milton, rocks at l.w. (A. and $\mathrm{H}$.)

Palio lessoni (D'Orb.). Plymouth Southd, m.c. (M.B.A.); Torbay (A. and $\mathrm{H}$.)

Polycera quadrilineata (O.F.M.). Plymouth Sound; Yealm, etc. c. on Zostera most years, outside to 35 fms. B. vii-viii (M.B.A.)

Acanthodoris pilosa (O.F.M.). Plymouth Sound; realm. 2 m. N.E. of Eddystone, etc., m.c. B. $\nabla$ (M.B.A.)

- subquadrata (A. and H.). Torbay, deep water (A. and H.) 


\section{MARINE ZOOLOGY}

\section{NUDIBRANCHIA (continued)}

Polyceridae (continued)

Lamellidoris aspera (A. and H.). Plymoutb Sound; realm, rr. (M.B.A.)

- bilamellata (L). Plymouth Sound, c. ; realm; Eddystone; Salcombe (M.B.A.) ; Teignmouth Bay, c. under stones (L.B.). B. iii, iv (M.B.A.)

- diaphana, A. and H. Torbay (A. and $H$.)

- sparsa, A. and H. Taken once off Stoke Point (M.B.A.)

- depressa (A. and H.). Plymouth Breakwater; Mewstone Ledge, rr. (M.B.A.) ; Torbay, under stones, l.w. (A. and $H$.)

- pusilla (A. and H.). Plymouth (M.B.A.). Torquay (A. and H.). B. ii (M.B.A).

- maculata, Garstang. Plymouth Sound; Mewstone and Eddystone Grounds, r. (M.B.A.)

- oblonga, A. and H. Plymouth, v.c. at times on Cellaria (M.B.A.) ; deep water off Berry Hd. (A. and H.). B. iv (M.B.A.)

Goniodoris nodosa (M.). Plymoutb Sound, etc., v.c. B. i-v, ix, xii (M.B.A.)

- castanea, A. and H. Plymouth Sound; Yealm; Eddystone Grounds, occ. (M.B.A.); Salcombe (A. and H.). B. ii, v, vii, ix (M.B.A.)

Idalina elegans (R.L.). Devon (J.G.J.)

- leachi (A. and H.). Torbay (A. and H.)

Idaliella aspersa (A. and H.). Yealm Est., rr. (M.B.A.)

- quadricornis (M.). Devon (F. and H.)

Ancula cristata (Ald.). Plymouth Sound and Docks, f.c. (M.B.A.); Torbay, r. (A. and H.)

\section{PULMONATA}

\section{Otinidae}

Otina otis (Turt.). Plymouth Sound, v.c. in barnacle shells, crevices in rocks, near h.w. (M.B.A.) ; Teigmmouth Bay, in clefts in rocks (L.B.)

\section{Auriculidar}

Leuconia bidentata (M.). Plymouth, with $O$. otis (M.B.A.) ; Teignmouth Bay, under stones, etc. (L.B.)

Alexia myosotis (Drap.). Teignmouth Bay, d. (L.B.)

\section{CEPHALOPODA}

\section{OMMASTREPHIDAR}

Todaropsis eblanae (Ball). Single specimen off $P l y$ mouth, ca. I 890 (M.B.A.)

Sthenoteuthis pteropus (St.). Salcombe, ca. Jan. 1892 (M.B.A.)

\section{LOLIGINIDAR}

Loligo Forbesii, St. occ, taken everywhere. B. iv-ix (M.B.A.)

- media (L.). f.c. and gen. dist. B. iv-vi, late embryos x (M.B.A.)

- marmorae, Vérany. Plymouth Draystone, single specimen (M.B.A.)

\section{SEPIIDAR}

Sepia officinalis, L. Gen. dist. and f.c., comes into bays and estuaries to spawn, July, Aug. B. vii-ix, hatching $x$ (M.B.A.)

Sepia elegans, d'Orb. f.c. Plymouth trawling grounds ; Start Bay, etc. (M.B.A.)

\section{CEPHALOPODA (continued)}

SEPIOLIDAB

Sepiola atlantica, d'Orb. f.c. in bays and estuaries and occ. I 5-35 fms.

- scandica, St. Plymouth Sound, single specimen, Nov. I887; Merostone Gnds., Oct. 1899 (M.B.A.) ; Torbay (F. and H.)

Rossia macrosoma (Dl.C.). Plymouth trawling grounds occ. (M.B.A.)

\section{Octopodidar}

Polypus vulgaris (Lam.). Occ. specimens only were taken on the $S$. coast until 1900 , during the summer of which it became exceedingly plentiful all along the coast. In Sept. the depredations of the octopus caused large numbers of crabs (Cancer pagurus) 5 in. to 7 in. across to seek shelter between tidemarks in Plymouth Sound, and practically cleared the Sound of swimming crabs (Portunus depurator). Not content with the free crabs the octopus paid visits to the crab-pots, killing large numbers of crabs and lobsters, as the following, which represents the total catch of an average of 30 crab pots per day for 6 days in Plymouth Sound (Oct. I 5-20), will show: Crabs living, 3; lobsters living, 15 ; octopus, 64 . Crabs dead, 44 ; lobsters dead, $4 \mathrm{I}$. In Nov. the trawlers were catching large quantities of octopus, up to 100 per haul, 5 miles off Plymouth, and $20 \mathrm{~m}$. S.S.E. of Berry Hd. In 1901 the quantities of octopus caught were practically normal (M.B.A.)

Moschites cirrosa (Lam.). Plymouth trawling grounds frequent; also in lobster pots (M.B.A.)

\section{BRACHIOPODA}

Terebratulidab

Megathyris cistellula, Wood. Exmouth (J.G.J.)

Gwynia capsula (J.E.J.). Plymouth (J.G.J.)

\section{TUNICATA}

\section{Molgulidae}

Molgula oculata, Fb. f.c. on clean fine gravel off Plymouth (M.B.A.)

- simplex, A. and $H$. m.c. on Chaetopterus tubes from the Eddystone Gnds. (M.B.A.)

\section{Cynthimak}

Forbesella tessellata (Fb.). Eddystone Gnds. (M.B.A.) Styelopsis grossularia, v. B. Plymouth Sound, v.c. in places on rocks between tidemarks; small squat var. Eddystone Gnds.; Salcombe f.c. B. $v-x$ (M.B.A.)

Polycarpa pomaria (Sv.). Cattewater c. occ., probably from trawl refuse; Eddystone Gnds. v.c. (M.B.A.)

\section{Asciditidar}

Corella larvaeformis, Hancock. Off Eddystone (M.B.A.) - parallelogramma, O.F.M. Single specimens, Plymouth Sound and 2 miles S. of Mewstone (M.B.A.)

Phallusia mammillata, Cuv. Gen. dist. but n.c. 


\section{A HISTORY OF DEVONSHIRE}

\section{TUNICATA (continued)}

Ascidinas (continued)

Ascidiella aspersa (O.F.M.). Plymouth Sound; realm, c. ; Salcombe, v.c.; Exmouth, c. in Dock ; Teignmouth Bay, occ. (M.B.A.)

- scabra, O.F.M. Plymouth Sound ; Yealm, occ. ; Salcombe, c.; Eddystone Gnds. c., growing on stem of Sertularella gayi (M.B.A.)

- venosa, O.F.M. Eddystone Gnds. occ. (M.B.A.)

Ascidia depressa, Ald. Shell gravel off Bolt Head (M.B.A.)

- arachnoider, $\mathrm{Fb}$. Devon coast, m.c. (F. and $\mathrm{H}$.)

- mentula, O.F.M. Plymouth Sound occ., Mervstone Gnds. (M.B.A.) ; Torcross (A. and $\mathrm{H}_{\text {.) }}$

Ciona intestinalis (L.). Small specimens (4-5 in. long) n.c. shore-35 fms. off Plymouth, Torbay, Teignmouth Bay. In I90I became v.c. and of large size ( 12 in. in length) in Millbay Docks, Plymouth, and Exmouth Dock. It resumed its normal abundance the year after as far as Plymouth was concerned, but the writer has no record of Exmouth (M.B.A.). In 1905 it appeared in Millbay Dock again in great profusion (E. J. Allen). B. vi-ix. Those from Exmouth were often infested with Notopterophorus gibba (M.B.A.)

\section{Clavelinidae}

Diazona violacea, Sv. Eddystone and Stoke Point Grounds, n.c. (M.B.A.)

Perophora banyulensis, Lahille. Duke Rock, Plymouth Sound (M.B.A.)

- listeri, Wiegmann. Plymouth Sound, v.c.; Yealm; Salcombe (M.B.A.)

Pycnoclavella aurilucens, Garstang. Mewstone Ledge, 10-20 fms. on various objects; Plymouth Sound once on red weed (M.B.A.)

Clavelina lepadiformis (O.F.M.). Plymouth Sound, m.c.; Mewstone Ledge; on Salstone, Salcombe, v.c. B. vi-vii (M.B.A.)

\section{BotrYLLIDAB}

Botryllus violaceus, M. E. Gen. dist. and c. on rocks, stones, weeds, etc. at I.w. B. vi-viii (M.B.A.)

- bivitattus, M. E. Torquay (F. and H.)

Botrylloides rubrum, M.E. Plymouth Sound, c. under stones, l.w. B. viii- $-\mathrm{x}$ (M.B.A.)

\section{TUNICATA (continued)}

Distomidae

Distaplia rosea, Della Valle. Plymouth Sound, Duke Rk. on stones and shells, etc. (M.B.A.)

Archidistoma aggregatum, Garstang. Plymouth, Duke $R k$., v.c. on stones; $2 \mathrm{~m}$. S. of Merustone, rr. B. vi (M.B.A.)

\section{Polyclinidar}

Aplidium zostericola, Giard. Plymouth, on stalks of Bowerbankia or Amathia (M.B.A.)

Amaroucium albicans (M. E.). Plymouth Mewstone (M.B.A.)

- nordmanni (M. E.). Wembury Bay; Salcombe, c. on Salstone. B. vi (M.B.A.)

- punctum, Giard. $3 \mathrm{~m}$. S. of Mewstone (M.B.A.)

Morchellium argus, M. E. Plymouth Sound, c. ; Yealm, Salcombe (Salstone), v.c. on shore, occ. in dredgings. B. ix (M.B.A.)

Morchelloides alderi (Herdman). Plymouth Sound, on stones, shells ; Laminaria roots, m.c. (M.B.A.)

Fragarium elegans, Giard. Plymouth, Duke Rk. on stones (M.B.A.)

Circinalium concrescens, Giard. Plymouth Sound occ.; Wembury Bay (M.B.A.)

\section{Doliolidae}

Doliolum nationalis, Borgert. Plymouth, v.c., Aug., Sept., 1893 ; v.c., 1895 ; occ. Nov., 1905 (M.B.A.)

\section{SALPIDAE}

Salpa fusiformis, Cuv. Eddystone, 1904 (M.B.A.)

- mucronata, Fr. Plymouth, v.c. 1893 mid June to Ist week of July ; Nov. 1903. B. viii-x (M.B.A.)

\section{Appendicularindab}

Oikapleura dioica, Fol. Plymouth, gen. present in tow-nettings, occ. c. B. viii-x (M.B.A.)

Fritillaria borealis, Lohmann. Plymouth, Mar., Oct., Nov., I899; Aug., 1903 (M.B.A.) 


\section{MOLLUSCS}

\section{NON-MARINE}

Although limestones are abundant in the south of the county, there is, on the whole, a lack of calcareous soil in Devon, hence it is by no means a district highly favourable to molluscan life. Nevertheless, the moist climate and abundance of vegetation (save on the granite uplands of Dartmoor), with the diversified physical features, enable a large number of different molluscs to dwell there. So that, out of some 140 species known to inhabit the British Islands, Iog have been met with in the area.

Two records, namely those of Helicella itala (the heath snail) and Helix pisana, both cited for Ilfracombe in a local guide to that place, have been rejected as being too doubtful, careful collectors working recently over that district having failed to find them. The latter is a very local shell, which occurs at Tenby and at Swansea on the other side of the Bristol Channel, as well as at Falmouth, Whitesand Bay, and St. Ives, in Cornwall, so that the locality at Woolacombe Sands given for it in the Guide was not an unlikely one, though since it was said to be rare, while the white banded-snail (Helicella virgata) was common on the spot, it is probable that fine specimens of the latter were mistaken for it, for where Helix pisana does occur it is always present in considerable quantities.

The lack of any reliable record of the presence of the heath snail (Helicella itala) in the county is remarkable. This mollusc should be sought for especially on the chalk patches between Beer Head and Dorsetshire. Another snail one would expect might be added to the list is Vitrea radiatula, which may be looked for in moss and under decaying logs in woods. Of freshwater species noteworthy absentees are Limnea stagnalis, Planorbis corneus, and Bithynia leacbii.

Two importations are worth mention. A single specimen of Helix pomatia, familiarly known as the Roman snail, was picked up beside the Exeter Canal: probably it had been thrown away from some French boat. While the pretty little Opeas goodalli, frequently introduced into greenhouses with plants from the West Indies, was very common in Pince's Exeter Nursery in $185^{\circ}$, but according to Parfitt had died out by 1874 .

The most interesting mollusc of those chronicled as inhabiting the county is Hygromia revelata. Like Helix pisana and the Kerry slug (Geomalacus maculosus) it is one of the rarer and more local of those western species, of which Helicella barbara and $H$. virgata are more widely distributed examples, that came to these shores from the Portuguese region along the now submerged plateau connecting these islands with the Continent, which plateau was dry land during a part of the Pleistocene period.

Another species, Vertigo moulinsiana, is one that had a wider range in Pleistocene times, but has since been dying out, and is now confined to 


\section{A HISTORY OF DEVONSHIRE}

limited spots in such widely-separated localities as the Isle of Arran, west Galway, Derby, Notts., Herts., Hants, and Dorset, as well as its recently discovered habitat near Ilfracombe.

Literature on the non-marine molluscan fauna of Devon is not plentiful. Only one memoir, which includes the marine species as well, deals with the county as a whole, namely, that by Mr. E. Parfitt, in the Transactions of the Devonsbire Association for 1874 (vol. vi. pp. 567-640). The author appears, however, to have been unacquainted with an interesting paper on the non-marine shells of the Plymouth district, by Mr. J. C. Bellamy, in the Edinburgh Fournal of Natural History for October, I 837 (vol. i. p. I I 5). A list of the species to be found in south Devon was published by Mr. E. D. Marquand, in the Fournal of Concbology for October, I889 (vol. vi. pp. I 36-140). For the Ilfracombe district there is an antiquated list in a local Guide to Ilfracombe, which dates back to I 845 ; and a list by Mr. J. Le B. Tomlin, supplemented recently by a paper by Mr. H. Beeston and Mr. C. E. Wright, both which communications appear in the Fournal of Conchology (vol. v. I887, pp. I 8 II 83 ; and vol. xi. 1904, pp. 72-82).

Most of the remaining records are scattered references of isolated occurrences. From these and the Census of the Conchological Society the following list has been compiled.

For the sake of uniformity the same names are employed as in previously published volumes of this series of County Histories, but for a later and more correct nomenclature recourse should be had to the List of British Non-marine Mollusca published by the Conchological Society.

\section{A. GASTROPODA}

\section{PULMONATA \\ a. StYlommatophora}

Testacella haliotidea, Drap. The Castle, Tiverton; Hele Bay, near Ilfracombe

- scutulum, Sby. Plymouth; Bideford

Limax maximus, Linn.

- flowus, Linn.

- arborum, Bouch.-Chant. Stoke Wood; Haldon Woods

Agriolimax agrestis (Linn.)

- hevis (Mull.). A single specimen was found at Exmouth

Amalia sowerbii (Fér.)

- gagates (Drap.). Topsham

Vitrina pellucida (Müll.)

Vitrea crystallina (Müll.)

- lucida (Drap.). Topsham ; Torquay; Torcross ; Ermington ; Ilfracombe

- alliaria (Miller)

- glabra (Brit. Auct.). ${ }^{1}$ Exeter; Plymouth

- cellaria (Mull.)

- mitidula (Drap.)

- pura (Ald.)

1 Now known as Vitrea rogersi, B. B. Woodw.
Vitrea excavato (Bean). Ivybridge

- nitida (Milll.). Topsham; Ilfracombe

- fulva (Mull.). Stoke Woods; Haldon Woods ; Plymouth ; Ilfracombe

Arion ater (Linn.)

- hortensis, (Fér.) Common : two albinos have been taken at $\mathrm{Hele}$, near Ilfracombe

- circumscriptus, John. North Devon

- intermedius, Norm. A single specimen found at Exmouth

- subfuscus (Drap.). Topsham.

Punctum pygmeeum (Drap.). Stoke Woods; Haldon Woods

Pyramidula rupestris (Drap.). Torbay ; Ivybridge; Plymouth ; Ilfracombe [Guide]

- rotundata (Mull.). Common : the white variety has been met with at Plymouth, and predominated at Hele, near Ilfracombe

Helicella virgata ( $\mathrm{Da} \mathrm{C}$.)

- caperata (Mont.)

- barbara (Linn.)

- cantiana (Mont.). Exeter; Plymouth

Hygromia fusca (Mont.). Exmouth Warren ; Plymouth; near Ilfracombe; Combe Martin (rare) 


\section{NON-MARINE MOLLUSCA}

Hygromia granulata (Ald.)

- bispida (Linn.)

- revelate (Fér.). Axminster; Torquay; Torcross ; Plymouth

- rufescens (Penn.)

Acanthinula aculeata (Mull.). Exeter; Kingsbridge; Plymouth; near Ilfracombe

Vallonia pulchella (Mull.). Exeter; Torcross; Plymouth; Ilfracombe

Helicigona lapicida (Linn.). Newton Abbot; Goodrington Sands ; Ashburton; Lynton

- arbustorum (Linn.). Exeter; Newton Abbot; Barnstaple

Helix aspersa, Mull.

- nemoralis, Linn.

- bortensis, Müll.

Buliminus montanus (Drap.). Axminster; Plymouth

- obscurus (Mull.)

Cochlicopa lubrica (Mull.)

Azeca tridens (Pult.). Tawstock Woods, near Barnstaple

Cacilianella acicula (Mull.). Torquay ; Ilfracombe [Guide]

Pupa secale, Drap. Axminster

- cylindracea ( $\mathrm{Da} \mathrm{C}$.

- muscorum (Linn.). Plymouth ; Ilfracombe

Spbyradium edentulum (Drap.). Haldon Woods; Plymouth

Vertigo antivertigo (Drap.). Plymouth [Bellamy]

- substriata (Jeff.). 'Devon' [Jeffreys]

- pygmea (Drap.). Bovisand, near Plymouth; Braunton Burrows, near Barnstaple

- moulinsiana (Dup.). Braunton Burrows

- pusilla, Mull. 'Devon' [Jeffreys]

Balea perversa (Linn.)

Clausilia laminata (Mont.). Sidmouth; Torquay; Ilfracombe

- bidentata (Ström.)

- rolphii, Gray. Newton Abbot

Succinea putris (Linn.)

- elegans, Risso. Exeter
Succinea oblonga, Drap. Braunton Burrows, near Barnstaple, including one white specimen

\section{b. BasomMatophora}

Carycbium minimum, Mull.

Melampus denticulatus (Mont.). Ilfracombe [Guide] Alexia myosotis (Drap.). Exmouth Warren; Torbay

Leuconia bidentata (Mont.)

Ancylus fuviatilis, Mull.

Velletia lacustris (Linn.). Exminster marshes

Limnea auricularia (Linn.)

- pereger (Mull.)

- palustris (Mull.)

- truncatula (Müll.)

Planorbis albus, Mull.

- glaber, Jeff. South Devon

- nautileus (Linn.) Exeter canal; Exminster marshes

- carinatus, Müll. Near Exeter

- marginatus, Drap.

- vortex (Linn.)

- spirorbis, Mull.

- contortus (Linn.)

- fontanus (Lightf.). Exminster marshes

Physa fontinalis (Linn.)

- byprorum (Linn.)

\section{PROSOBRANCHIA}

Paludestrine jenkinsi (Smith). Topsham; River Yeo, near Barnstaple

- ventrosa (Mont.). Plymouth [Bellamy]

- stagnalis (Bast.). Occurs round the coast

Bitbynia tentaculata (Linn.)

Vivipara vivipara (Linn.)

- contecta, (Millett)

Exeter

Valvata piscinalis (Mull.)

- cristata, Mull. Topsham

Pomatias elegans (Mull.)

Acicula lineata (Drap.). Exeter

Neritina fuviatilis (Linn.). South Devon

\section{B. PELECYPODA}

Dreissensia polymorpha (Pall.). Exeter canal, near Topsham

Unio pictorum (Linn.). South Devon

- margaritifer (Linn.). River Teign at Dunsford; Plymouth district [Bellamy]; River Taw, near Bishops Tawton

Anodonta cygnea (Linn.)

Sphecrium corneum (Linn.)

- ovale (Fér.). Exmouth

- lacustre (Mül1.). Exmouth; Plymouth; Braunton Burrows, near Barnstaple
Pisidium amnicum (Mull.)

- pusillum (Gmel.). Exmouth; Plymouth; Braunton Burrows, near Barnstaple

- nitidum, Jenyns. Wonford, near Exeter

- fontinale (Drap.). Exminster Marshes, where also the variety, by most now considered a separate species, $P$. Henslowionum is found; Braunton Burrows

- milium (Held.). Exminster Marshes; Braunton Burrows 



\section{INSECTS}

With the exception of York and Lincoln, Devon is the largest county in England, containing about $1,660,000$ acres. Although the greater part of the county is arable or pasture land, it contains over 86,000 acres of woods and plantations in addition to more than 157,000 acres of forest, moorland, and wild pasture. From its extensive coast-line, both on the north and south of the county, its varied geological formations and its numerous moors, woods, and plantations, its insect fauna is a rich one, though probably not equal to that of such counties as Kent, Sussex, or Hampshire.

Through the exigencies of space it has been found necessary to adopt the following symbols in the lists throughout this article; viz. :-* denotes common; ** very common or abundant; $\$$ not common; † rare or scarce; + very rare, and the abbreviation 'gen. dist.' is used for generally distributed.

\section{ORTHOPTERA ${ }^{2}$}

\section{Earwigs, Cockroaches, Grasshoppers, and Crickets}

With the exception of Kent and possibly Hampshire, no county has a better list of species of this order than Devon. The county possesses an earwig not known elsewhere-Anisolabis annulipes, Lucas-first brought to notice by Mr. Swale, who discovered it in an old bakehouse at Tavistock in I 894 ; on inquiry he found it had been established there for a considerable period. Since then it has been discovered at Queenborough, Kent, and several specimens have been imported into Kew Gardens from abroad.

\section{FORFICULARIA}

Earwigs

Anisolabis annulipes, Lucas. Taken at Tavistock, first noticed by Mr. Swale in 1894

Labia minor, Linn. Abundant in summer, flying round heaps of manure, in S. Devon; also

observed at Lynmouth, N. Devon (C. A. Briggs)

* Forficula auricularia, Linn.

- lesnei, Finot. Sidmouth (C. W. Dale)

\section{BLATTODEA}

\section{Cockroaches}

Ectobidar

Ectobia lapponica, Linn. A specimen taken in the vaults of the City Bank, Exeter (Parfitt)

- panzeri, Steph. Cliffs, Wembury (Bignell); Whitsand Bay (Keys); Dawolisb Warren (W. J. Lucas); Kingsbridge and Plymouth (J. F. Stephens)

- livida, Fabr. Devonshire (Stephens)
Ectobidae (continued)

Phyllodromia germanica, Linn. Observed in many houses in Plymouth (Bignell and Keys). It is interesting to note that forty young emerged in September, 1895 , from one egg capsule of a specimen of this species, the female was obtained from a Plymouth restaurant by Mr. James Keys. The capsule of

1 The sequence of the orders here followed is that adopted by Dr. D. Sharp, F.R.S., in the Cambridge Natural History, $1889-92 .-\mathrm{H}$. G. As the lists of species in this article have been contributed by various writers, it has been difficult to maintain uniformity with regard to the names of authorities, etc., but it is presumed that the abbreviations used will be familiar to all entomologists.

I have to express my thanks for valuable assistance received to Mr. Ernest E. Austen ; Mr. George Carter Bignell, F.E.S. ; the late Mr. Charles G. Barrett, F.E.S. ; Mr. Charles A. Briggs, F.E.S.; Mr. Malcolm Burr, B.A., F.L.S. ; the Rev. Alfred E. Eaton, M.A., F.E.S.; the Rev. Canon Fowler, M.A., D.Sc., F.L.S.; Mr. William J. Lucas, B.A., F.E.S. ; the late Mr. Robert McLachlan, F.R.S., F.L.S. ; the late Rev. Thomas A. Marshall, M.A., F.E.S. ; Mr. George T. Porritt, F.L.S. ; and Mr. Edward Saunders, F.R.S. 


\section{A HISTORY OF DEVONSHIRE}

Ectoridar (continued)

the large cockroach (Blatta orientalis) generally contains sixteen eggs. The latter does not begin to reproduce her species until the fourth year. When germanica begins to reproduce is not known, but is presumed to do so at the same age

Blatta orientalis, Linn. Common cockroach, too well known as a household pest

Periplaneta americana, Linn. About twelve years since Mr. G. C. Bignell observed a great number of these cockroaches in the streets of Plynouth, evidently flown from a ship then

\section{Ectobidar (continued)}

in the Great Western Dock. Since that time they have established themselves in several bakehouses in Plymouth. Periplaneta americana has driven out Orientalis from more than one bakehouse in Plymouth; the two do not appear to be able to live in harmony

\section{Panchloridaz}

Rhyparobia madeirae, Fabr. Several specimens observed by the same gentleman in the streets of $P$ lymouth, about twenty years since, having flown from a ship in the Great Western Railway Docks

\section{ACRIDIODEA}

\section{Grasshoppers}

\section{'TrYXaLidae}

Stenobothrus lineatus, Panzer. Wembury Cliffs (Bignell) ; Braunton Burrozus, N. Devon (Parfitt)

- - viridulus, Linn.

\$- rufipes, Zetterstedt. On the coast (Bignell); Lynton (Briggs)

* - bicolor, Charpentier. Very variable in colour

* - parallelus, Zett. In fields

Gomphocerus rufus, Linn. Wembury, Bolt Head (Bignell). Sandhills, Exmouth and Dawlish (Parfitt)

- maculatus, Thunb. Gen. dist.
Oedipodidae

Pachytylus migratorius, Linn.
- cinerascens, Fabr.

AcrididaE

Schistocerca peregrina, Oliv. About thirty of this species of locust were found in and around Plymouth, 9 October, 1869; Mr. Bignell secured about a dozen; they extended along the south coast

Trtrigidar

Tettix bipunctatus, Linn. Gen, dist.

- subulatus, Linn. Taken in Bickleigh Woods, 22 April (Bignell)

\section{LOCUSTODEA}

\section{Grasshoppers}

\section{Phankroptrridar}

Leptophyes punctatissima, Bose. Frequently met with in Cann and Bickleigh Woods. Obtained by beating (Bignell); Lynmouth (Briggs)

\section{Meconemidae}

"Meconema varium, Fabr. Bickleigh and Cann Woods (Bignell) ; Lynmouth and Lynn River (Briggs); Totnes (Lucas); Stoke Woods, Exeter (A. O. Rowden); at sugar in S. Devon (G. Porritt)

\section{Conocerhalidae}

Xiphidium dorsale, Latr. In a marsh, $S$. Devon (Porritt)

\section{Locustidar}

Locusta viridissima, Linn. Bickleigh; Horrabridge; Bovisand (Bignell); Torquay (A. H. Hamm);

Decticidae Teignmouth; Newton Abbot (Parfitt)

Thamnotrizon cinereus, Linn. Irybridge, out of bramble (Bignell); Lynmouth (Briggs);

\section{Decticidae (continued)}

Haldon (Parfitt) ; Stoke Wood nr. Exeter; Sidmouth (Rowden); Bovey Tracey (F. C. Summerson)

Platycleis grisea, Fabr. Dawlish (C. Babington)

- brachyptera, Linn. Haldon; Woodbury Common, August (Parfitt)

\section{Gryllidae}

Gryllus campestris, Linn. Devon (Stephens)

- domesticus, Linn. House cricket, gen. dist.

\section{Gryllotalpidae}

Gryllotalpa gryllotalpa, Linn. Mole cricket, occurs rather frequently in the neighbourhood of Exeter. A few years since several were found in the streets early in the morning ( Parfitt). Mr. Bignell has seen three found in a potato patch, each had consumed a great portion of the potato under which they were found in September

\section{NEUROPTERA}

\section{Stone-fies, May-fies, Dragon-fies, Caddis-fies, etc.}

Owing to the very varied nature of the country and to the fact that the southern portion has been carefully worked by such keen observers as Mr. Parfitt of Exeter, the Revd. A. E. Eaton, Mr. G. C. Bignell of Plymouth, and, in earlier days, by Dr. Leach, while the northern portion has received attention from Mr. MacLachlan, Mr. Stanley Edwards, and myself, the Devonshire list of Neuroptera is, even now, a fairly lengthy one. 


\section{INSECTS}

Much, however, remains to be done; but when the central and western portions of the county, hitherto but little worked, have received due attention, there can be but little doubt that Devonshire will take a very high position among the counties of England as regards Neuroptera.

The arrangement followed is that of The Catalogue of British Neuroptera by MacLachlan and Eaton, published by the Entomological Society of London in 1870 .

In a few instances where there has been no recent capture, and there seems to be a possibility of error, I have added the name of the recorder in brackets.

\section{NEUROPTERA BRITANNICA}

\section{PSEUDO-NEUROPTERA}

\section{Psocidar}

Atropos divinatoria, Müll.

Clothilla pulsatoria, Lin.

- picea, Mots.

Hyperetes guestfalicus, Kolbe.

Bertkauia prisca, Kolbe.

Psocus longicornis, Fab.

- nebulosus, Steph.

- variegatus, Fab.

- fasciatus, Fab.

- sexpunctatus, Lin.

- bifasciatus, Latr.

- quadrimaculatus, Latr.

- bipunctatus, Lin. (Parfitt)

Stenopsocus immaculatus, Steph.

- cruciatus, Lin.

Caecilius pedicularis, Lin.

- flavidus, Steph.

- obsoletus, Steph.

- Dalii, McLach.

- vittatus, Latr.

- perlatus, Kolbe.

- piceus, Kolbe.

Ectopsocus Briggsii, McLach.

Peripsocus phoeopterus, Steph.

Elipsocus unipunctatus, Mull.

- cyanops, Rostock

- Westwoodii, McLach. var. abietis, Kolbe.

- hyalinus, Steph.

- flaviceps, Steph.

\section{Perlidae}

Dictyopteryx microcephala, Pict. - rectangulata, Pict. (Stephens)

Perla marginata, Panz. (Parfitt)

- maxima, Scop.

\section{PSEUDO-NEUROPTERA (cont.)}

Perlidae (cont.)

Perla cephalotes, Curt.

Isopteryx torrentium, Pict.

- tripunctata, Scop.

Taeniopteryx nebulosa, Lin.

- Risi, Morton

Leuctra geniculata, Steph.

- hippopus, Kemp

- klapaleki, Kemp

Nemoura variegata, Oliv.

- meyeri, Pict.

- cinerea, Oliv.

- marginata, Pict.

- inconspicua, Pict.

\section{Ephrmeridat}

Ephemera vulgata, Lin.

- danica, Lin.

- lineata, Eaton

Leptophlebia submarginata, Steph.

Habrophlebia fusca, Curt.

Ephemerella ignita, Poda.

Coenis halterata, Fab.

- dimidiata, Steph.

Baetis binoculatus, Lin.

- scambus, Eaton

- niger, Lin.

- pumilus, Burm.

- Rhodani, Pict.

- vernus, Curt.

Cloëon dipterum, Lin.

Centroptilum luteolum, Eaton

Rhithogenia semicolorata, Curt.

Heptagenia sulphurea, Mull.

Ecdyurus venosus, Fab.

- insignis, Eaton

- volitans, Eaton

\section{ODONATA}

LiBgLLULIDAE

Sympetrum striolatum, Charp.

-- vulgatum, Lin.

- flaveolum, Lin.

- sanguineum, Mull.

- scoticum, Don.

Platetrum depressum, Lin.

Libellula quadrimaculata, Lin.

Orthetrum cancellatum, Lin.

- coerulescens, Fab.

\section{Cordulimase}

Oxygastrii curtisii, Dale (Curtis)

\section{GOMPHIDOR}

Cordulegaster annulatus, Latr.

\section{Aeschnidae}

Brachytron pratense, Mull.

Aeschna cyanea, Múll,

- mixta, Latr.

- juncea, Lin.

- grandis, Lin.

\section{Calopterygidae}

Calopteryx virgo, Lin.

— splendens, Lin.

\section{Agrionidar}

Lestes sponsa, Hans.

Platycnemia pennipes, Pall.

Pyrrhosoma nymphula, Sulz.

- tenellum, Vill.

Ischnura elegans, Lind.

Agrion pulchellum, Lind.

- puella, Lin.

Enallagma cyathigerum, Charp

\section{NEUROPTERA PLANIPENNIA}

\section{SIALIDAE}

Sialis lutaria, Lin.

— fuliginaria, Pict.

\section{RaphididDa}

Raphidia notata, Fab. (Parfitt)

- xanthostigma, Steph. (Parfitt)

\section{Osmylidae}

Osmylus chrysops, Lin.

Sisyra fuscata, Fab.

- terminalis, Curt.

\section{SIALIDAE (cont.)}

Hemerobidae

Micromus variegatus, Fab.

- paganus, Lin.

Hemerobius pellucidus, Walker

- inconspicuus, McLach.

- nitidulus, $\mathrm{Fab}$.

- micans, Oliv. var. fuscinervis, Schn.

- humuli, Lin.

- lutescens, Fab.

- marginatus, Steph.

\section{SIALIDAE (cont.)}

Hemerobidar (cont.)

Hemerobius stigma, Steph.

- atrifrons, McLach.

- pini, Steph.

- subnebulosus, Steph.

Megalomus hirtus, Lin. (Parfitt)

\section{Chrysopidas}

Chrysopa flava, Scop.

- vittata, Wesm.

- alba, Lin. 


\section{A HISTORY OF DEVONSHIRE}

SIALIDAE (cont.)

Chrysopidae (cont.)

Chrysupa flavifrons, Brauer

- vulgaris, Schr.

- septempunctata, Wesm.

- ventralis, Curt.

- aspersa, Wesm.

- perla, Lin.

- abbreviata, Curt. (Curtis)

Nothochrysa fulviceps, Steph. (Stephens)

- capitata, Fab. (Stephens)

\section{Coniopterygidae}

Coniopteryx tineiformis, Curt.

- psociformis, Curt.

- aleyrodiformis, Steph.

\section{Panorpidae}

Panorpa communis, Lin.

- germanica, Lin.

- cognata, Ram. (Parfitt)

\section{TRICHOPTERA \\ INAEQUIPALPIA}

Phryganidae

Phrygania grandis, Lin.

- varia, Fab.

\section{LYMNOPHILIDAE}

Colpotanlius incisus, Curt.

Grammotaulius atomarius, Fab.

Glyphotoelius pellucidus, Oliv.

Limnophilus rhombicus, Lin.

- flavicornis, Fab.

- marmoratus, Curt.

- lunatus, Curt.

- griseus, Lin.

- bipunctatus, Curt.

- affinis, Curt.

- centralis, Curt.

- vittatus, Fab.

- auricula, Curt.

- luridus, Curt.

- sparsus, Curt.

- fuscicornis, Ram.

Anabolia nervosa, Curt.

\section{INAEQUIPALPIA (cont.)}

LyMNOPHILIDAE (cont.)

Stenophyllax stellatus, Curt.

- concentricus, Zell

- vibex, Curt.

Micropterna sequax, McLach.

- lateralis, Steph.

Halesus radiatus, Curt.

- digitatus, Schr.

Drusus annulatus, Steph.

Choetopteryx villosa, Fab.

Apatania muliebris, McLach.

\section{Seriscostomidae}

Seriscostoma personatum, Steph.

Notidobia ciliaris, Lin.

Goèra pilosa, Fab.

Silo pallipes, Fab.

- nigricornis, Pict.

Brachycentrus subnubilus, Curt.

Crunvecia irrorata, Curt.

Lepidostoma hirtum, Fab.

Lasciocephala basalis, Kol.

\section{AEQUIPALPIA}

Leptoceridae
Deroea pullata, Curt.
- maurus, Curt.
- articularis, Pict.
Molanna angustata, Curt. (Ste-
phens)

Odontocerum albicorne, Scop.

Leptocerus nigronervosus, Retz.

- alboguttatus, Hag.

- annulicornis, Steph.

- aterrimus, Steph.

- cinereus, Curt.

- albifrons, Lin.

- commutatus, McLach.

- bilineatus, Lin.

- dissimilis, Steph.

Mystacides Azurea, Pict.

- nigra, Lin.

-C longicornis, Lin.

Trioenodes bicolor, Curt.

- conspersa, Ramb.

Adicella reducta, McLach.
AEQUIPALPIA (cont.)

Leptoceridak (cont.)

Oecetes testacea, Curt.

Setodes tineiformis, Curt.

- interrupta, Fab.

\section{HYDROPSYCHIDAR}

Hydropsyche pellucidula, Pict.

- instabilis, Curt.

- angustipennis, Curt.

- guttata, Pict.

- lepida, Pict.

Diplectrona felix, Westwood

Philopotamus montanus, Don. var. insularis, McLach. var. chrysopterus, Morton

Wormaldia occipitalis, Pict.

- subnigra, McLach.

Plectrocnemia conspersa, Curt.

- geniculata, McLach.

- brevis, McLach.

Polycentropus flavomaculatus, Pict.

- Kingi, McLach.

- multiguttatus, Curt.

Holocentropus dubius, Ramb

Ecnomus tenellus, Ramb.

Tinodes woeneri, Lin.

- unicolor, Pict.

- aureola, Zett.

Lype phoeopa, Steph.

Psycromia pusilla, Fab.

\section{RHYACOPHILIDAE}

Chimarra marginata, Lin.

Rhyacophila dorsalis, Curt.

- obliterata, McLach.

- munda, McLach.

Glossoma Boltoni, Curt.

- vernale, Pict.

Agapetus fuscipes, Curt.

- comatus, Pict.

\section{HYDROPTILIDAE}

Agraylea multipunctata, Curt. Hydroptila tineoides, Dalm.

- McLachlani, Klap.

- forcipata, Eaton

Orthotrichia angustella, McLach.

Oxythira falcata, Morton

\section{HYMENOPTERA}

\section{PHYTOPHAGA}

The Phytophagous Hymenoptera, better known by the name of Sawflies, from the fact that each possesses an instrument for the purpose of making incisions in the selected food plant, whether in leaves or branches; the reason for so doing is to deposit their eggs. Each species has a different form of cutting power adapted to its special requirements. Trees, shrubs, their leaves and bark, and low-growing vegetation are laid under contribution; each species has its own particular tree, shrub, or plant. One of the most conspicuous of these insects forms the gall found on willow-leaves (Pontania gallicola), with large red blotches. These galls are formed before the larvae emerge from the eggs. This is in direct opposition to the procedure of the Cynipidae-galls of the oak; the oakgalls are not formed until the larvae have emerged from their eggs. 


\section{INSECTS}

Sawfly larvae feeding exposed on trees, shrubs, etc., may be very easily mistaken for the larvae of Lepidoptera (Butterflies and Moths). As a general rule they can be separated by the number of legs; lepidopterous larvae never have more than sixteen (six proper and ten pro-legs), while the larvae of the sawfly may have twenty-two (six proper and sixteen pro-legs); but like everything in nature there is no hard-and-fast line, therefore a great diversity will be found among the different species in colour, form, and method of feeding. Several species may be mentioned to illustrate these remarks, Leptocercus luridiventris for instance; the larvae of this species is flat, and lies stretched out on the underside of the leaves of the elder, and resembles a scale insect rather than a caterpillar; while that of Eriocampoides limacina is more like a slug than a caterpillar in appearance and manner of feeding, which is on the upper epidermis of the leaf at first; as they grow they commence feeding on the lower side of the leaf until every particle of green is consumed, leaving only a brown skeleton, which shortly drops to the ground. Pear, apple, and plum trees often suffer from these 'slugworms.' At the last change the slug-like skin is thrown off, then it appears as an ordinary caterpillar.

Another form of larva is that of Pampbilius depressus, which has no pro-legs on the abdominal part for holding, consequently it has to spin a series of threads across the leaf, a sort of ladder arrangement, wherever it desires to travel, sometimes connecting the outer edges of the leaves of the alder for protection, or fastening two leaves together.

The sawfly caterpillar which forces itself mostly on our notice is that of Pteronus ribesii, the great destructor of the foliage of our gooseberry and currant bushes, eating the leaves, thereby destroying the fruit; the crop, however, may be saved by hand-picking when they first make their appearance, which is indicated by numerous small holes in the leaves. The farmer is sometimes troubled with Athalia spinarum, better known to him by the name of the ' nigger,' 'black army,' etc., from the black colour of the larvae; these often attack the turnip crop, eating the leaves, causing immense damage.

Another sawfly the farmer dreads is Cephus pygmaeus, which attacks the stems of wheat and barley, depositing its egg in the stem near the ground, the larva, eating upwards, consuming the vital part of the stem; when full fed it returns to the root, therein changes to a pupa, and where it remains through the winter, ready to again commence the cycle of life after escaping from its winter quarters. The larva eating the inside of the stem causes the plant to become too weak to bear fruit, and the stem is usually blown down by the first storm. Any indication of an attack should be noted, and to get rid of the pest the stubble after harvest should be gathered and burnt.

Many of the sawfly larvae are conspicuous and feed exposed; when this is the case the majority are able to protect themselves by emitting a very disagreeable odour; this no doubt suggests an objectionable taste which would prevent a bird from attacking them a second time should it at first sight have thought the larva a tempting morsel. This, however, does not prevent the ichneumon fly from depositing its egg in the larva.

Among the sawflies many species are known to be reproduced by virgin females; in some instances the offspring are all females, in another species all males, in a third they may be of both sexes. In some species the males are so few and far between that many collections are without them.

The writer does not know of any naturalist in the county who has devoted his entire energy to this branch (Sawflies), therefore the list of captures, about $15 \mathrm{I}$, is only a portion of what might be expected to occur in a county which has such a variety of vegetation on its moors, heaths, and hedgerows.

The list is arranged according to Pastor Konow's system of nomenclature.

\section{LIST OF THE PHYTOPHAGOUS HYMENOPTERA OCCURRING IN THE COUNTY}

\begin{abstract}
Neurotoma flaviventris, Ratz., Bickleigh

Pamphilius sylvarum, Kirby, Ivybridge, 26 April

- betulae, Lin., Devon (Stephens)

- depressus, Cam. Gen. dist.

- sylvaticus, Lin., N. Devon (Parfitt)

— inanitus, Vill., Ivybridge

Janus cynosbati, Fab., Horrabridge; Bickleigh
\end{abstract}

Macrocephus linearis, Kirby, Horrabridge

Cephus pygmaeus, Lin., Bickleigh, 26 June

Sirex gigas, Lin., Plymbridge; Cann Wood

- noctilio, Fab. ${ }^{1}$ Several bred from pine trees

1 This until 1904 was supposed to be juvencus, $F$. The true juvencus has not been observed in England.
Cimbex femorata, Lin., Exeter $\times^{3}$ - connata, Vell., Plymouth District Trichiosoma lucorum, Lin., Crabtree, Plymouth

- betuleti, Klg., Church Lane, Heavitree $x$

Abia sericea, Lin., Bickleigh, 22 April

2 Marked thus ( $x$ ) have been recorded by the late Edward Parfitt for the Exeter district. 


\section{A HISTORY OF DEVONSHIRE}

\section{LIST OF THE PHYTOPHAGOUS HYMENOPTERA OCCURRING IN \\ THE COUNTY (continued)}

Abia nigricornis, Leach, Plymouth (Parfitt)

- fasciata, Lin., Avon Valley

Arge caerulescens, Geoff., Holne (Yerbury)

- ustulata, Lin., Torcross

- atrata, Först., Bickleigh

- cyaneo-crocea, Först., Torcross $x$

Lophyrus sertiferus, Cam., $P l y-$ mouth District

Trichiocampus pecticornis, Eourcroy, Starcross $x$

- viminalis, Voll., Longbridge, Bickleigh

- ulmi, Lin., Horrabridgex

Pricphorus padi, Lin. Bred from sallow $x$

Leptocercus luridiventris, Fallen. $x$

Dineura stilata, Htg., Walkbam Valley

- despecta, Htg. $X$

Cryptocampus pentandrae, Cam., Walkbam Valley

一 angusta, Cam., Plymouth District

- saliceti, Cam., Plymoutb District

Pontania xanthogaster, Foer., $L y d$ ford

- nigrolineatus, Cam., Plymouth District

- baccarum, Cam., Plymouth District

- salicis-cinereae, Thoms., $P l y-$ moutb $x$

- bellus, Zaddach, $x$

- gallicola, West. Bred in June and November $X$

Pteronus melanaspis, Htg., $P l y-$ mouth $\times$

- microcercus. Bred from sallow

- myosotidis, Htg., Bickleigh $\times$

- salicis, Lep. Bred 21 May from larva found at Longbridge in the previous year

- melanocephalus, Htg. $X$

- ribesii, Dbm. Common garden pest on gooseberries throughout the county. Bred 18 July; often two broods in a year

- bipartitus, Lep. $X$

Amauronematus histrio. Bred from a decayed branch of apple

- fallax, Lep., Plymouth District $x$

Croesus septentrionalis, Lin. $x$

- varus, Vill., N. Devon (Parfitt)

Holcocneme lucida, Panz., Horrabridge $x$

Nematus abdominalis, Dbm., $P l y$ mouth District
Nematus luteus, Panz. $X$

Pachynematus rumicis, Fallen. $X$

- capreae, Panz., Crabtree near Plymouth; Exmouth (Parfitt)

- obductus, Htg., Plymouth $\times$

- conductus, Ruthe., Plymouth X

Pristiphora ruficornis, Olivier $\times$

- pallipes, Lep., Bickleigh

Eriocampoides annulipes, Thoms., Bickleigb

- limacina, Retz, Laira, Plympton $X$

Hoplocampa pectoralis, Thoms. $x$ - crataegi, Klug., Shaugb-bridge

Periclista melanocephala, Fab. Bred 12 May from larva found feeding on oak the previous year

Pareophora luridiventris, Klug. $\times$

Ardis sulcata, Cam., $X$

Phymatoceros aterrima, Klug., Pentillie on the Tamar

Tomostethus nigritus, Fab. $x$

- fuscipennis, Fall., Bickleigh; Ivybridge $X$

- dubius, Gmel., Walkbam Valley; Starcross $X$

Scolioneura betuleti, Klug., Bickleigh

Monophadus albipes, Gmel., Bickleigh

- ruficruris, Brullé $x$

Harpiphorus lepidus, Klug. Bred from an old Cynips kollarigall

Athalia glabricollis, Thoms. $x$

- spinarum, Fab. $x$

- lineolata, Lep., Bickleigh; Ivybridge $x$

- lugens, Klug., Bickleigh

Selandria serva, Ste., Torcross; Shaugb-bridge $x$

- sixii, Voll. $x$

- stramineipes, Ste., Bickleigh$V a l e$; and Ieybridge $X$

- morio, Ste., Bickleigh-Vale ; Bovisand X

Stromboceros delicatulus, Thoms., Cann Wood, Plympton

Strongylogaster cingulatus, Horrabridge and Shougb-bridge $\times$

Eriocampa ovata, Lin. $\times$

- annulipes, Klug., Bickleigh $\times$

- limacina, Retz. $x$

Poecilosoma pulverata, Ratz. $X$

- longicornis, Thoms. $X$

- liturata, Gm., Horrabridge

- excisa, Thoms., Exmouth X

Emphytus succinctus, Klug. $X$

- cinctus, Klug., Walkbam Valley $x$

- melanarius, Klug., $x$

- rufocinctus, Retz., Bickleigh $\times$

- calceatus, Klug., Bickleigh
Emphytus serotinus, Klug., Shaughbridge $\times$

- carpini, Htg., Plymouth District

- pallipes, Spinola, Horrabridge

- tener, Fallen, Bickleigh

Taxonus agrorum, Fall., Devon (Stephens)

- equiseti, Fall., Shipley-bridge, Brent (Keys)

- glabratus, Fall. Bred from old bramble stem $X$

Dolerus pratensis, Fall., Seaton; Bickleigh $\times$

- anticus, Thoms. Slade

- palustris, Klug., Seaton $X$

- gonager, Klug., Bickleigh $\times$

- puncticollis, Thoms., Plymouth District

- haematodis, Klug. $x$

- anthracinus, Klug., Bickleigh

- nigratus, Mull., IqybridgeX

- niger, Klug., Horrabridge ; Ivybridge $x$

* - aeneus, Klug.

Scioptery $x$ costalis, Klug. $x$

Rhogogastera punctulata, Klug. $x$

- viridis, Lin., Cann Wood, Plympton $\times$

- lateralis, Fab., Bickleigh $\times$

- aucupariae, Klug., Plymbridge

Tenthredopsis litterata, Geoff. Bickleigh $\times$

* - coqueberti, Klug.

- campestris, Lin. $X$

- dorsalis, Lep., Bickleigb

* Pachyprotasis rapae, Thoms.

- antennata, Thoms., Bickleigh $\times$

- variegata, Thoms., Devon (Stephens)

Macrophya blanda, Evers., Bickleigh $\mathrm{X}$

* - annulata, Geoff.

* - rustica, Evers.

- rufipes, Thoms, Torcross $x$

- punctum-album, Thoms., $x$

Allantus scrophulariae, Brischke, Bickleigh $\times$

- marginellus, Rudow, Bickleigh

- arcuatus, Step., Ivybridge, June and August $X$

- maculatus, Fourc., Bickleigh and Ivybridge $X$

- temulus, Scop., Bickleig $\mathrm{X}$

Tenthredo flava, Scop., Plymouth District

- livida, Lin., var. Maura, Fab., Ivybridge; Bickleigh, June $\times$

- ferruginea, Schr., Camn Wood, Plympton $\mathrm{X}$

- balteata, Klug. $x$

- atra, Lin., Bickleigh

- mesomela, Lin., Bickleigh and Horrabridge $x$ 


\section{INSECTS}

\section{Y N I P I D A E}

The Cynipidae are small insects; in size they vary from 2 to $6 \mathrm{~mm}$. Their mode of living may be divided into three divisions: Gall-makers (vegetable feeders); inquilines; or lodgers in galls ; the remainder are parasites and hyperparasites (parasites on parasites). The inquilines adapt themselves to circumstances with regard to food. When the victim (the gallfly-larva) is consumed and more food is required they turn their attention to the vegetable matter within the gall. Although they are called lodgers, it is only about 3 per cent. of the gall-makers escape if the gall is attacked by them, and then it is only in the large galls (Cynips Kollari). The different groups will be dealt with separately.

\section{PARASITIC GROUP}

These were all captured; very few have been bred.

*Anacharis typica, Walker

$\$$ - tincta, Walk.

$\$$ - ensifera, Walk.

tAnolytus rufipes, Foerster

*Aegilips rufipes, Westwood

$\S$ - nitidula, Dal.
$\S$ Eucoela crassinerva, West.

$\$$ - nigricornis, Cameron

§- Marshalli, Cam.

- rapae, West. Taken at $P$ lymbridge, 7 Aug. I 882 . Westwood bred it from tumours on turnips formed by Ocyptera brassicaria

$\S$ Kleditoma Marshalli, Cam.

$\$$ - longicornis, Cam.

\$- filicornis, Cam.

*- subaptera, Walk.

\section{HYPERPARASITES ON THE APHIDIIDES}

Allotria minuta, Htg. Bred from Praon volucre, Haliday; Aphidius sonchi, Marshall ; and $\mathrm{A}$. ribis, $\mathrm{Hal}$.

- flavicornis, Htg. Bred from Aphidius cerasi, Marsh

- victrix, Wesm. Bred from Aphidius pascuorum,Marsh.; and $\mathrm{A}$. rosae, $\mathrm{Hal}$.
Allotria Ullrichi, Giraud. Bred from Praon volucre, Hal.

- cursor, Htg. Bred from Aphidius urticae, Hal.; and $\mathrm{A}$. avenae, $\mathrm{Hal}$.

- erythrocephala, Jurine. Bred from Aphidius pascuorum, Marsh.
Allotria ancylocera, Cam. Bred from Aphidius brassicae, Marsh.

- Tscheki, Gir. Bred from Aphidius ervi, $\mathrm{Hal}$.

- basimaculata, Cam. Bred from Monoctonus caricis, $\mathrm{Hal}$.

Diastrophus aphidivorus, Cam. Bred from Siphonophora urticae, Kalt.

\section{SYNERGI}

These inquilines, or lodgers in galls, as a general rule destroy the gall-makers, and are so much like the gall-makers that breeders of gall-flies and early writers on the subject have often mistaken one for the other. The variation in size in the same species is remarkable, and is dependent on the quantity of food. In some large species from six to twelve may be found to have existed on one victim, whereas one or two would consume the same sized larva, and would then only produce normal sized flies.

In small galls (spangle galls) two small flies often appear from a single gall, because the victim is only food enough for one.

Each larva at an early stage provides itself with a separate cell, no matter what number of parasitic larvae the gall may contain. As many as fifty-one have emerged from one Kollari gall. These made twenty-seven holes for their exit. Many of these fed on the vegetable matter composing the gall.

Synergus albipes, Htg. Bred from Andricus curvator; Dryophanta divisa ; D. disticha ; Neuroterus baccarum and $\mathrm{N}$. tricolor

- apicalis, Htg. Bred from Neuroterus albipes; $N$. vesicatrix; and Andricus trilineatus

- facialis, Htg. Bred from Biorhiza terminalis and Neuroterus baccarum

- incrassatus, Htg. Bred from Andricus radicis, A. corticis, and A. Sieboldii

- melanopus, Htg. Bred from Cynips Kollari
Synergus nervosus, Htg. Bred from Andricus Malpighii ; A. glandulae; A. quadrilineatus ; A. autumnalis, A. callidoma ; and Dryophanta Tashenbergi

- pallicornis, Htg. Bred from Neuroterus baccarum ; Andricus callidoma; Dryophanta folii; D. longiventris ; and D. divisa

- radiatus, Mayr. Bred from Andricus glandulae and albopunctata

- Reinhardi, Mayr. Bred from Cynips Kollari
Synergus tristis, Mayr. Bred from Andricus ostreus

- Tscheki, Mayr. Bred from Neuroterus lenticularis; $N$. numismatis ; $N$. fumipennis : Andricus ostreus; Dryophanta folii ; and D. divisa

- vulgaris, Htg. Bred from Andricus fecundatrix

Sapholytus connatus, Htg. Bred from Andricus trilineatus and Neuroterus punctatus

Ceroptres arator, Hitg. Bred from Andricus trilineatus

\section{INQUILINES IN THE GALLS ON ROSES}

Periclistus Brandti, Ratz. Bred from galls of Rhodites rosae Periclistus caninae, Htg. Bred from galls of Rhodites eglanteriae 


\section{A HISTORY OF DEVONSHIRE}

\section{GALL MAKERS}

The life histories of the gall-makers are the best known of the Cynipidae group, and the majority are found on the oak. The cycle of life of nearly all the gall-makers has been carefully and ably worked our by Drs. Mayr and Adler, and their observations confirmed by others.

The oak gall-flies are very remarkable in having an alternation of generations, i.e. the autumnal galls are all females, reproducing their species without the assistance of the male. Nearly the whole of the galls observed on oak trees in spring or early summer produce males and females. Another great feature is that each generation is never like that which it proceeded from, but always like its grandparents. In two instances, the winged sexes produce apterous (wingless) generations, all of which are females. These deposit their eggs and produce winged males and females. The galls of the two generations are never alike in any way, nor are the flies, in size, form, or colour. The spring generation is often three times smaller than its parent. The spring galls, as a rule, from the time the egg is deposited to the appearance of the flies, are within six months, while many of the autumnal galls do not produce flies until the third, and sometimes the fourth year.

Oak gall-flies are not alone in being able to reproduce their species by virgin females. Dr. Adler has proved that the bedeguar-rose or robin's-pincushion gall fly (Rhodites rosae) is able to continue her race without being fertilized, and no doubt several others are able to do the same. Among the saw-flies (Tentbredinidae) several cases have been known by experiment, one virgin female producing all males, another species all females, while a third would produce males and females.

Among the oak gall-flies there are several whose bisexual form is not known, if we presume that there is one, for instance that of the common marble gall (Cynips Kollari), which is so conspicuous in winter, when the trees are leafless. All these galls produce female flies, and all efforts to produce flies in confinement from these females have so far been fruitless. The writer has tried during the past thirty years to accomplish this object by placing flies on oaks of all ages, from one year old, up to branches on trees of sixty years. All these experiments were made by placing the several young trees or branches under a muslin frame to prevent the escape of the flies and to exclude intruders. Cynips Kollari is not the only oak gall-fly that is supposed to be parthenogenetic. Dr. Adler says that he has bred Andricus albopunctata from flies of albopunctata. These flies are always females, consequently parthenogenetic reproduction appears to be proved in the case of A. albopunctata.

Andricus solitarius, glandulae, quadrilineatus, seminationis, Dryophanta agama, and disticha all produce females only; parthenogenesis may exist with all of them. The writer is not of that opinion. Take the case of glandulae. The gall is found commonly in October; the gall-fly emerges in April. The question is, how is the time passed from April to August or'September? Dr. Adler says 'Embryonic development begins immediately after the egg is laid; absolute rest in the evolution of the egg never occurs, for even if the temperature should be very low, the formation of the blastoderm begins at once. Naturally this proceeds more slowly in a cold than in a warm season.' Consequently there must be a connecting link to keep up the continuity of this species.

It was at one time supposed that the marble-gall was produced at the expense of the acorn. Botanists well know that the fruit-producing flowers appear on the oak in April, at the same time as the catkins; consequently after the female flower is fertilized, the future acorn very gradually commences its growth; in fact, the embryo acorn is not seen until after the fourth or fifth leaf is produced. The eggs producing Kollari galls are not deposited on buds of old wood, but on twigs that have grown some time after the growth of the acorn commenced; or, in other words, the female flowers were produced, fertilized, and the acorn started on its journey of life, while the twig which the parent of Kollari gall would be likely to select to deposit her eggs was still within the bud.

It is only within the last two years that the writer has had an opportunity to experiment with gall-flies that have not a known bisexual form, to try if the flowering buds would prove the condition the flies required; the experiment so far has certainly proved a failure.

Unfortunately some of these gall-flies do not emerge until the third year, and require during that period much attention. They must not be kept in the sun or allowed to get too dry, and must be exposed to all weathers.

The inquilines or lodgers (Synergi) are more often parasites than they are generally supposed to be. One hundred Cynips Kollari galls gathered in March and April, I gor, produced only eighteen gall-makers, 495 Synergus Reinbardi, forty-seven $S$. melanopus, and fifteen Cbalcids. Only one gall produced the maker and inquiline.

Of the following list of oak gall-files all, with one exception, Andricus agama, have been obtained in the valley of the Plym. 


\section{INSECTS}

\section{OAK GALL-FLIES}

Galls producing females only (Agamic)

Andricus fecundatrix, Hartig

- globuli, Hartig

- radicis, Fab.

- Sieboldii, Hartig .

- corticis, Lin.

- collaris, Harti

- autumnalis, Hartig

- callidoma, Adler.

- Malpighii, Adler.

Trigonaspis renum, Giraud .

Biorhiza aptera, Fab.

Dryophanta folii, Lin.

- longiventris, Hartig

- divisa, Hartig

Neuroterus lenticularis, Olivier

- fumipennis, Hartig

- laeviusculis, Schenck.

- numismatis, Olivier

- Schlechtendali, Mayr

- punctatus, Bignell (females only were bred).

Andricus quadrilineatus, Hartig

- marginalis, Schlech ${ }^{1}$

- ostreus, Giraud

- albopunctata, Schlech

- seminationis, Giraud

- glandulae, Schenck

- solitarius, Fonscol

Cynips Kollari, Hartig

Dryophanta agama, Hartig

- disticha, Hartig

The agamic form unknown
Galls producing males and females (Sexual)

The next generation is

Andricus pilosus, Adler

- inflator, Hartig

- trilineatus, Hartig

- testaceipes, Hartig

- gemmatus, Adler

- curvator, Hartig

- ramuli, Lin.

- cirratus, Adler

- nudus, Adler

Trigonaspis megaptera, Panzer

Biorhiza terminalis, Fab.

Dryophanta Taschenbergi, Schlec

- similis, Adler

- verrucosa, Schlec

Neuroterus baccarum, Lin.

- tricolor, Hartig

- albipes, Schenck

- vesicatrix, Schlec.

- aprilinus, Giraud

Sexual forms unknown, if there are any

\section{ROSE GALL FLIES AND OTHERS}

Rhodites eglanteriae, Htg. Gen. dist.

- rosae, Linn. Gen. dist.

- spinosissimae, Gir. Nr. the sea on Rosa spinosissima

Aulax hypochaeridis. Around Plymoutb on $\mathrm{H}$ ypochoeris radicata
Xestophanes potentillae, Linn. Egg Buckland on Potentilla reptans (T. R. Archer Briggs)

Diastrophus rubi, Htg. Nr. Plymouth on Rubus fruticosus

\section{ICHNEUMONIDAE}

\section{PARASITIC HYMENOPTERA}

The Ichneumonidae are such a neglected order that some of their life histories, it is trusted, may prove interesting to those who have not given them much time or thought, is the excuse for making the introduction rather long, but possibly not tedious.

The Ichneumonidae are readily distinguished from all other flies (if we except the wasps and bees) by the distinct constriction of their abdomen at its juncture with the thorax, their long, slender bodies, peculiar veined wings, all having a stigma and exserted ovipositor; but like everything in nature there is no hard and fast line, for there are females whose wings are no longer than their eyes, Aptesis nigrocincta, Gr.; others entirely without wings, genus Pezomacbus, Gr.; while the males in the majority of cases have ample wings reaching to the end of the abdomen.

So it is with the ovipositor; in the greater number of females of the genus Ichneumon and others, the ovipositor is hidden, while that of the Rbyssa persuasoria, Linn., is an inch and a half in length, longer than the fly (head, thorax, and abdomen). Others shorten in their respective families until they are hidden when looking at them from above. The Ichneumonidae are parasites on butterflies and moths (Lepidoptera), sawflies (Tenthredinidae), flies (Diptera) (two-winged flies), and other orders. 


\section{A HISTORY OF DEVONSHIRE}

The eggs of the Ichneumonidae vary greatly in shape, size, and colour; the great majority, about 95 per cent., cannot be seen, from their being deposited beneath the skin; an indication when recently pierced is often observed by a black dot on the caterpillar : should it change its skin the mark is removed. Eggs laid on the surface of the caterpillar before the last moult are removed with the change of skin, and leave no ill effect. Those laid after the last moult are carried by the larva into its resting place; the larva is then consumed. Notwithstanding the aggressive nature of the work allotted in nature to these parasites, it is worthy of remark that in their earlier stages of existence they are exceedingly delicate and cannot stand the slightest injury; an abrasion of the surface of the skin sufficient to allow the least portion of the fluid to escape would prove fatal ; yet these very larvae, 'having passed unhurt through this scarcely other than foetal condition, acquire a perfection of organization, a degree of activity and power, and an acuteness of instinct fully equal, or perhaps superior, to the organic and the functional endowments of other tribes of insects.' One egg only of the larger species is usually deposited in each caterpillar. If, however, two should be laid either by the same parent, or by a separate female of the same or of an allied species, both eggs may be hatched, but the weaker would fall a victim to the stronger, that is, supposing the food to be only sufficient for one; but with the smaller parasites of the Braconidae group, I 80 have been known to emerge from one caterpillar, Arctia caia, Linn., whereas the same larva would only sustain one of the larger species (Pimpla instigator, Fab.). The larvae of the Ichneumonidae without exception are legless and entomophagous, and when they leave the egg are quite incapable of locomotion.

The eggs deposited in the body of the caterpillar soon mature; the larvae finding plenty of food do not move, but lie perfectly motionless, embedded in the fatty tissue between them and the alimentary canal, always on the dorsal surface, and usually with their heads in the direction of the head of the caterpillar, and are nourished entirely by suction.

The general form of the body and the construction of the digestive organs at the earlier periods of the growth, are almost, if not precisely, the same in most of these parasites. The special development of each is regulated by the same laws; they cast their skins at succeeding stages of growth as certainly as do the larvae of butterflies and moths.

The protection of the larva during its change to the pupa stage, and from that to the perfect $\mathrm{Aly}$, is very variable. Many make cocoons after the manner of ordinary silkworms; but in the genus Ichneumon and many others, they do not make cocoons in any form, but remain in the pupa-case of their host for final transformation to the perfect insect.

In the genus Opbion and many others, the parasitic larvae construct cocoons for themselves. Opbion on or below the surface of the earth. The Limneria, however, remain on the twig, leaf, or branch, on which the caterpillar was resting, the parasitic larva issuing from its host on the underside; the empty skin of the caterpillar is often found attached to the cocoon (Limneria ruficincta, Gravenhorst). Others do not leave the body of their victim, but make use of the skin of the caterpillar as an outer covering; the commonest met with is that of Limneria vulgaris, Tschek., a parasite on the Brimstone butterfly larva (Gonopteryx rbamni, Linn.). One of the most wonderful forms of protection is that manifested by the larva of Limneria Kriecbbaumeri, Bridgman. It makes a very curious oval cocoon about the size of a large hempseed on its leaving its host, the common quaker (Taeniocampa stabilis, Vieweg.). Directly after leaving, the maggot-like form suspends itself by a silken thread, about two inches in length, previously attached to the tree on which the caterpillar was feeding, and commences to make its future abode, in which it has to pass the winter. The form is that of an oblong sphere $6 \mathrm{~mm}$. by 4 , chocolate colour, with a whity-brown zone in the middle. It is after the cocoon is made that the wonder and agility of the larva, within the cocoon, must be looked upon by all who have seen it with the greatest astonishment. Shortly after the aerial cocoon is finished it is blown down by the wind; but supposing there is no wind, or but little, not sufficiently strong to blow it down, it begins to bound about with a view to breaking the suspending cord. When that is accomplished, and it feels itself on the ground, the cocoon begins to jump about, making leaps 300 times its own length, until it has jumped or bounded into a place whence it cannot extricate itself. It then commences to roll, first on one side and then on the other, until it finds itself fixed, unable to move any way. It is then satisfied, and no further attempt is made; but remove it from the spot after some days, and it will again go through the same performance, and repeat it, as often as it is removed, until it is exhausted. The manner of jumping at least 300 times its own length, when confined within the walls of its cocoon, is one of the greatest curiosities in nature; yet it is done by the larva within the cocoon bringing its head and tail together and suddenly straightening itself out, as would a piece of steel, if the two ends were brought together and suddenly released. The maggot of the dipterous fly often found in old cheese does the same, and it is generally known by the name of cheesehopper (Piophila casei, Linn.) from its hopping propensity; but this larva has no impediment; the other is enclosed in its cocoon. 


\section{INSECTS}

The larva of the drinker moth (Odonestis potatoria, Linn.) when about half-grown may be often seen resting on a twig of some sort, apparently waiting for the time when its old clothes will be rent asunder, to appear in its new dress; but sometimes we find a stop to the progress has been made by the larva of an ichneumon fly (Rhogas geniculator, Nees) within, attacking some vital part and causing its death ; there it remains, and in the course of a week or two the lodger within will cut an opening out of its back and escape, ready to repeat the same tactics on another unfortunate larva; probably Arctia villica, Linn., may be selected, for the same species of parasite destroys both.

The eggs that are deposited externally on nearly or quite full-fed caterpillars as a rule do not hatch until the victim has prepared a place in which it intends to complete its metamorphosis; if, on the other hand, they should have hatched, the development is very slow to enable the caterpillar to proceed as if it were not attacked. The puss moth (Dicranura vinula, Linn.) is often attacked by these external parasites, Paniscus cephalotes, Holmgren, and P. testaceus, Gravenhorst. The caterpillar of the puss moth when full-fed makes its cocoon generally on the bark of a tree, excavating a portion. 'The bark that is removed in the excavation is utilized by mixing it in with the silk first produced to form an outer covering ; subsequently the spun silk is rubbed over by the larva with formic acid, the fluid secreted in the prothoracic gland, which changes the fibrous character of the silk to the tough, hardened, gelatinous material of the ordinary cocoon of this species. In this cocoon the parasites just mentioned lie side by side in their black envelopes until they are ready to emerge. In the genus Lissonota the cocoons made by the larvae are long oval, measuring $44 \mathrm{~mm}$. long and 4 broad with a bright polished surface. Those of the genus $O$ pbion are much stouter and contain several distinct layers in their manufacture, the outer one composed mostly of flossy silk; the remainder are like so many layers of 'gold-beaters'-skin,' each layer appears to be put on in a fluid state, or the silk woven, afterwards covered or smeared over with formic acid, the acid dissolving the silk and giving it the appearance of 'gold-beaters'-skin.' It is insoluble in hot or cold water, alcohol, ether, or chloroform. It is soluble in strong acids, being most readily dissolved in hot nitric acid, also in strong alkaline solution, preferably caustic potash. It contains moisture, nitrogen, a trace of sulphur, and on destructive distillation leaves about half its weight of carbon; would, therefore, contain $\mathrm{C}, \mathrm{H}, \mathrm{O}, \mathrm{N}$, and $\mathrm{S}$ in certain proportions.

Stems of plants, cow-parsnip (Heracleum sphondylium), also afford protection for the cocoons of Lepidoptera (Depressaria beracleana), consequently they also afford security for the parasites infesting the larvae-Icbneumon heracliana, Bridgman, with a short ovipositor and Pimpla strigipleuris, Thoms, with a long ovipositor.

Even caterpillars that are hidden deep in the solid wood of growing trees are not free from the attacks of the ichneumon fly, any more than those that are exposed on the surface of the leaf. They are certainly more difficult to get at. But once the caterpillar is discovered by the inquisitive fly, Rbyssa persuasoria, or its allies, so sure is the attempt made to deposit the egg in the hidden larva ; this is done by forcing her delicate ovipositor, not so large as the finest needle made for domestic use, through the solid wood into the caterpillar below. It is almost beyond belief that a fly with an ovipositor nearly two inches in length can thrust it into the solid wood of a growing tree the whole length, if necessary, to reach its victim ; yet she does it. The genus Perithous, Holmgr., has also a long ovipositor, and is assigned by nature to attack those little bees and wasps that make the decayed stems of bramble and rose their nidus, in which they pass the winter in their larva or maggot stage ; while those wasps and bees that make their home in old walls or earth banks have the attention of a very curiously-formed parasitic fly, by name Foenus jaculator, Linn., whose abdomen seems to proceed from the middle of its back; that and its long ovipositor, also abnormally hind legs, with the apex of the tibia flattened or spatulated, giving it a very grotesque appearance. Spiders also receive attention from the Ichneumonidae; Hemiteles fragilis and similis, Gravenhorst, are common parasites in the egg bags of our 'domestic spiders,' Zilla $x$-notata, Clerck, and others. 'Hunting spiders,' Ocyale mirabilis, Clk., Lycosa pullata, Clk., and others of the group are kept under by Hemimachus fasciatus, Fabricus, Pezomachus micrurus, Foerster, and P. zonatus, Fourcroy, attacking the egg cocoons. Orb-weavers, Epeira cornuta, Clk., whose habitation is beautifully concealed, in which the spider makes her egg cocoon, do not escape the prying eyes of Pimpla epeira, Bignell. The eggs are not touched, it is the young spiderlings that are consumed by the remorseless Pimpla.

It seems an anomaly to write about flies attacking spiders, and yet at the proper season it must be a daily occurrence. The writer perhaps is the only living naturalist who has ever been an eyewitness of an attack, and it may be interesting to here record how the ichneumon fly obtains its end. Having selected a spider sufficiently large in which it has some confidence, she approaches it carefully, but the spider, objecting to the confidence trick, drops from its hiding-place on the bough of the tree by the usual silken cord. The ichneumon fly does not appear in the least troubled on that account, no doubt having had previous experience. Taking advantage of the spider's rope, she very leisurely walks down to the spider and apparently coaxingly touches it with her antennae. 


\section{A HISTORY OF DEVONSHIRE}

But the spider objects to the patronage and makes a further drop; in a few seconds the fly follows the object of her adoration and again touches the spider, and she, no doubt by instinct, knowing her foe, resigns herself to her fate, and does not move. The fly turns round, walks backward until within striking distance, and then thrusts her ovipositor into the thorax and deposits her egg. The ichneumon egg is hatched in about forty-six hours, the larva taking about ten days in consuming the unfortunate spider by suction. When feeding, it lies sack-like across the spider's back till it has almost consumed its victim; when the larva finds the spider is getting inconveniently small for it to rest upon, it attaches itself by the hooked tubercles with which its back is provided, to the web the spider had previously made, so as to feed on the last remains of its victim, and then make its cocoon. When the spider is emptied of nourishment, the legs and skin drop to the ground.

The larva is footless, it has in place of legs eight sucking discs, and on its back eight tubercles furnished with moveable hooks which can be withdrawn into the tubercles, the discs are for attaching itself to the body of the spider, and the tubercles for attaching itself to the web.

The larva, suspended by the back, has now to make itself a cocoon in which to pass the pupa stage. The larva takes about three days to do this work. It is during this time the eight tubercles on the back of the larva have to play such prominent parts, having to perform the work of the claspers of an ordinary caterpillar. When the silken cord is to be released by the tubercle attached to it, it is done by withdrawing the hooklets into the tubercle, which at once becomes disengaged and ready to make another attachment. The anal segment has important duties to perform while the cocoon is making, by carrying the silken thread from the mouth into the corners, where the blunt head of the caterpillar could not carry it, or adjust it to its satisfaction. The anal segment was seen to be used, while the caterpillar was feeding, by bringing it to its mouth, for the purpose of removing some muscular fibre that had got entangled about its mandible. When full-fed, the larva is about three-eighths of an inch in length. The fly, Polyspbincta tuberosa, Gravenhorst, is mature and escapes from the cocoon in about twenty days.

Parasitic flies after they are mature remain within their pupa-cases a few days, to strengthen themselves to combat with life in the outer world. When sufficiently strong they proceed to make an opening in the cocoon with their mandibles; when completed they make their exit, and are able to at once take flight; there is no drying and stretching of wings as is the case with butterflies and moths.

If it is absolutely necessary, which it surely is, that we should have parasites to keep down the superabundance of insects, then it is also necessary to keep these parasites in check to counterbalance the destruction constantly going on in the insect world, and these we have under the designation of hyperparasites; and they are not confined to one genus alone, although the whole of the genus Mesochorus are hyperparasites. To carry on this work the hyperparasitic flies have to discover larvae adapted to their several requirements; that is, a lepidopterous or other larva must contain parasitic larvae living within its body before the hyperparasite is able to keep up the continuity of its race ; and, as previously mentioned, the parasitic larva lives and feeds in what one may say is the centre of its victim, with not the slightest outward visible sign of its existence within. Therefore one might naturally presume that it would be safe from external attack; but such is not the case : the possession of some specialized sense on the part of the hyperparasites enables them to discover the parasitic larva concealed within the body of the caterpillar, and the egg is quickly deposited. But it does not follow that the egg deposited always enters the parasitic larva within, although the parent fly did its best to make it do so; if not, the hyperparasitic egg, if hatched, not having its proper food, cannot exist.

In confirmation of this latter assertion, the writer desires to mention that on $30 \mathrm{May}, 1880$, he observed a hyperparasite flitting about from leaf to leaf, keeping the antennae in constant motion, evidently hunting about for a suitable place in which to perpetuate her race. After waiting and watching the lady for some time, she came up to the object of her search, which was a caterpillar of the magpie moth (Abraxas grossulariata, Linn.). After passing round it several times to make sure everything was correct, she suddenly sprang on the unfortunate caterpillar and thrust her ovipositor into the second segment, just below the dorsal line. The caterpillar, during the operation, strongly objected to the proceeding, and painfully twisted itself from side to side to get rid of the foe. The hyperparasite (Mesochorus olerum, Curtis) then alighted on the leaf of a currant bush close by, she was duly boxed (captured), the caterpillar was removed, fed up, and in due time the ichneumon larva left the caterpillar and spun up as do ordinary larvae of the parasite (Casinari vidua, Gravenhorst), whose peculiar cocoon cannot be mistaken for any other; this was naturally expected; but the surprise was that the maker of the cocoon appeared instead of the hyperparasite $M$. olerum. By these remarks it will be perceived the hyperparasite was correct in its knowledge that the magpie moth caterpillar contained, hidden within, the ichneumon larva of which it was in search, but the egg failed to reach the larva it was intended for. $M$. olerum is a common hyperparasite on $C$. vidua, and has often been bred from the cocoon made by $C$. vidua. 


\section{INSECTS}

The duration of life of the ichneumon fly depends on the species; those which emerge in the autumn may live through the winter. A great number that confine their attacks to one species do not leave their habitation until the caterpillar is ready to receive the egg of the parasite, passing at least ten months in the larva stage; others pass through two or three cycles of existence during the year, and do not confine their attacks to one or two species.

There formerly existed, and perhaps still exists, among entomologists an idea that each species of parasite had its own particular host, each to each, and no more. This, however, is fallacious; in breeding a great number it has been proved that they infest different species of Lepidoptera. Thirteen parasites and hyperparasites the writer has obtained from Abraxas grossulariata, Linn.

When an Ichneumon is said to be bred from a butterfly or moth, it is from the larva or pupa, that should have produced the perfect insect; a few beetles, (Coleoptera), and other orders are however attacked by parasitic flies when in the adult stage.

\section{PUPIVORA ${ }^{1}$}

\section{ICHNEUMONIDAE}

\section{OXYPYGI}

Genus, Chasmodes, Wesmael - motatorius, Fab., Bickleigh, 8 Sept. I 882

- lugens, Gr., Ivybridge, 4 June, 1893

Genus, Ichneumon, Linné

- bilineatus, Gmel. Bred I4, 18 , and 19 June, 1886 , from pupae of Bryophila muralis and Abraxas grossulariata

- sugillatorius, Lin., Exeter, Ivybridge, I 2 May, I 896

- cyaniventris, Wesm. Bred I7 June, I 883, from pupa of Odontopera bidentata

- leucocerus, Gr. Captured at Bickleigh, 2 September, 188 I

- lineator, Fab. Bred 27 May, I88I, from a pupa of an unknown larva

- impressor, Zett. Bred II June, 1885 , from Gortyna ochracea

- comitator, Lin.

- castaneiventris, Gr.

$\$$ - fuscipes, Gmel.

$\$-$ pistorius, Gr.

$\$$ - culpator, Schr.

- trilineatus, Gmel. Bred from Abraxas grossulariata and Bryophila muralis

- multiannulatus, Gr. Bred from Noctua brunnea

$\$-$ molitorius, Lin.

$\$$ - punctus, Gr.

* - computatorius, Mull.

* - languidus, Wesm.

* - vaginatorius, Lin.

*- xanthorius, Först.

\section{OXYPYGI (cont.)}

Genus, Ichneumon, Linné

$\$$ - confusorius, Gr.

- tempestivus, Holmgr., Bickleigh, 28 July, 1886

- gracilentus, Wesm. Bred June from Noctua festiva

$\$$ - melanotis, Holmgr.

- bisignatus, Gr. Bred July from Vanessa urticae

*- luctatorius, Lin.

*- minutorius, Desv.

* l latrator, Fab.

- pulchellatus, Bridg. Bred from Eupithecia pulchellata 4 May

- multipictus, Gr.

- primatorius, Forst. Bred 22 June from Triphaena fimbria

- - gracilicornis, Gr.

$\$$ - caloscelus, Wesm.

- quaesitorius, Lin. Bred 3 Sept. from Nonagria cannae

\$- cessator, Múll.

$\$$ - submarginatus, Gr.

- saturatorius, Lin. Bred 3 September from Nonagria cannae and arundinis

- nigritarius, Gr. Bred from Abraxas grossulariata

- - fabricator, Fab.

§- curvinervis, Holmgr. Bickleigh, I6 June, 1886

\$- maculifrons, Ste.

* - corruscator, Lin.

* - varipes, Gr.

- jugatus, Gr. Bred from Tephrosia luridata

*- pallidatorius, $\delta$ Gr.; ruffifrons 으.

* lanius, Gr.

*- albilarvatus, Gr.

\section{OXYPYGI (cont.)}

Genus, Ichneumon, Linné

- heracliana, Bridg. Bred Aug. from Depressaria heracleana

$\$$ - incubitor, Lin.

t- inquinatus, Wesm.

*- fugitivus, Gr. = ruthus, Holmgr.

$\S$ - dumeticola, Gr.

- leucomelas, Gmel. Bred 22 June from Noctua brunnea

- oscillator, Wesm.

- - vestigator, Wesm.

" - chionomus, Wesm.

\$- anator, $\mathrm{Kab}$.

* derogator, Wesm

- - bilunulatus, Gr.

- - ruficeps, Gr.

$\$$ - ochropus, Gmel.

* ridibundus, Gr.

- albicinctus, Gr. Bred May, I 885, from Hypsipetes ruberata, and 9 September, 1885 , from Eupithecia rectangulata

\$- erythraeus, Gr.

$\S$ - bipunctorius, Ste.

$\$$ - cingulipes, Ste.

\$- gasterator, Ste.

$\$$ - rufator, Ste.

Genus, Hepiopelmus, Wesmae

$\dagger$ - leucostigmus, Gr.

Genus, Exophanes, Wesmael

$\$-$ exulans, Gr.

$\$$ - occupator, Gr.

Genus, Amblyteles, Wesmael

- - palliatorius, Gr.

- homocerus, Wesm. Bred July from Argynnis paphia

- armatorius, Forst. Bred July from Triphaena comes and from Agrotis Ashworthii

${ }^{1}$ Abbreviations of authors' names :-Bignell, Bignell, G. C.; Boie, Boie, F.; Bridg. Bridgman, J. B. ; Christ, Christ, J. L. ; Curt. Curtis, J.; Desv. Desvignes, T.; Fab. Fabricius, J. C.; Forst. Furster, A. ; Forst. Forster, J. R.; Fourc. Fourcroy, A. E.; Gmel. Gmelin, J. F.; Gr. Gravenhorst, J. L. C. ; Hal. Haliday, A. H.; Holmgr. Holmgrén, A. E.; Kriech. Kriechbaumer, J.; Lin. Linné, C. von ; Lat. Latreille, P. A.; Müll. Müller, O. F.; Panz. Panzer, G. W. F.; Parfitt, Parfitt, Edward; Ratz. Ratzeburg, J. T. C. ; Rossi, Rossi, P.; Schiödte, Schiödte, J. G.; Schr. Schrank, F. von P.; Shuck. Shuckard, W. E.; Ste. Stephens, J. F.; Tasch. Taschenberg, E. L. ; Thom. Thomson, C. G. ; Tschek, Tschek, C. ; Vill. Villers, C. J. de ; Wesm. Wesmael, C.; Zett. Zetterstedt, J. W. 


\section{A HISTORY OF DEVONSHIRE}

\section{OXYPYGI (cont.)}

Genus, Amblyteles, Wesmael

$\S-$ infractorius, Panz.

$\$$ - oratorius, Fab.

- margineguttatus, Gr. Bred May from Noctua brunnea

- notatorius, Fab. Bred June from Triphaena fimbria

$\$$ - subsericans, Gr.

$\$$ - crispatorius, Lin.

$\$$ - glaucatorius, Fab.

$\$$ - vadatorius, Rossi

$\$$ - Gravenhorstii, Wesm.

$\S$ - negatorius, Fab.

- castanopygus, Ste. Bred from Xanthia fulvago

- castigator, Fab. Bred from Argynnis paphia, August

$\S$ - fossorius, Gr.

$\$$ - funereus, Fourc.

- Panzeri, Gr. Bred July from from Agrotis exclamationis

- Proteus, Christ. Bred from Choerocampa elpenor

- fuscipennis, Wesm. Bred June from Choerocampa porcellus

- alticola, Gr. Bred August from Taeniocampa stabilis

Genus, Trogus, Panzer

- lutorius, Fab. Bred from Smerinthus ocellatus

- alboguttatus, Gr. Bred from Dasychira pudibunda

- exaltatorius, Panz. Bred July from Sphinx ligustri. This is the largest fly of the genus

Genus, Listrodromus, Wesmael - quinqueguttatus, Gr. Bred July from Lycaena argiolus

Genus, Hypomecus, Wesmael

$\S$ - albitarsis, Wesm.

Genus, Eurylabus, Wesmael

- dirus, Wesm. Bred from Eriøgaster lanestris

- tristis, Gr. Bred from Dianthoecia capsincola

Genus, Platylabus, Wesmael

§- rufus, Wesm.

- nigrocyaneus, Gr. Bred June from a Noctua pupa

$\S$ - Thedenii, Holmgr.

* - decipiens, Gr.

- pedatorius, Fab. Bred Sept. from Eupithecia subnotata

†- orbitalis, Gr.

- tricingulatus, Gr. Bred June from Eupithecia pulchellata

- dimidiatus, Gr. Bred May from Melanippe montanata

\$- nigricollis, Wesm.

$\$$ - errabundus, Gr.

Genus, Gnathoxys, Wesmael

$\S$ - marginellus, Holmgr.

Genus, Herpestomus, Wesmael

- brunnicornis, Gr. Bred from Hyponomeuta padellus

* - intermedius, Wesm.

\section{OXYPYGI (cont.)}

Genus, Colpognathus, Wesmael

* - celerator, Gr.

Genus, Dicoelotus, Wesmael

$\$$ - pumilus, Gr.

$\$$ - ruficoxatus, Gr.

* _ parvulus, Gr.

$\S$ - pusillator, Gr.

Genus, Centeterus, Wesmacl

- - opprimator, Gr.

Genus, Phaeogenes, Wesmael

t- semivulpinus, Gr.

§- melanogonus, Gmel.

$\$$ - scutellaris, Wesm.

- stimulator, Gr.

- calopus, Wesm. Bred July from Eriogaster lanestris

* - suspicax, Wesm.

$\S$ fuscicornis, Wesm.

* fulvitarsis, Wesm.

$\$$ - ischiomelinus, Gr.

$\S$ - cephalotes, Wesm.

* - troglodytes, Gr.

* _ jucundus, Wesm.

- candidatus, Gr. Bred from Tortrix viridana

$\S$ - trepidus, Wesm.

$\$$ - tetricus, Wesm.

$\$$ - varicolor, Wesm.

Genus, Aethecerus, Wesmael

$\S$ - nitidus, Wesm.

Genus, Alomyia, Panzer

$\S$ - debellator, Fab.

\section{CRYPTIDES ,}

Genus, Stilpnus, Gravenhorst

*- gagates, Gr.

$\S$ pavoniae, Scop.

- blandus, Gr.

Genus, Phygadeuon, Gravenhorst

t- caliginosus, $\mathrm{Gr}$.

†- dumetorum, Gr.

$\$$ - variabilis, $\mathrm{Gr}$

$\S$ - probus, Tasch.

*- fumator, Gr.

$\$$ - troglodytes, Gr.

* - jejunator, Gr.

\$- aereus, Gr.

$\$$ - nitidus, $\mathrm{Gr}$.

§- vagans, Gr.

$\$$ - brachyurus, Thom.

$\$$ - improbus, Gr.

* profligator, Fab.

$\$$ - semipolitus, Tasch

$\$$ vagabundus, Gr.

\$- graminicola, Gr.

\$- abdominator, Gr.

$\S$ - jucundus, $\mathrm{Gr}$.

$\$$ - arridens, Gr.

\$- nycthemerus, Gr.

*- hortulana, Gr.

$\$$ - lacteator, Gr.

$\S$ - sperator, Múll.

$\$$ - erythrinus, Gr

$\$$ - galactinus, Gr.

$\$$ - cinctorius, Fab.
CRYPTIDES (cont.)

Genus, Cryptus, Fabricius

†- lugubris, Gr.

t- spiralis, Fourc.

t- moschator, Fab.

- larvatus, Gr.

$\$$ - bitinctus, Gmel.

$\S$ - ovatus, Gr.

$\$$ - anatorius, $\mathrm{Gr}$.

- - stomaticus, Gr.

- - leucopsis, Gr.

* titillator, Gr.

\$- minator, Gr.

\$ albatorius, Vill.

†- Dianae, Gr.

- obscurus, Gr. Bred 22 May from Smerinthus populi

$\S-$ arrogans, Gr.

$\S-$ hostilis, Gr.

- porrectorius, Fab.

* analis, Gr.

- rufiventris, Gr.

$\$$ - alternator, Gr.

$\$$ - peregrinator, Lin.

- tricolor, Gr. Bred from Simyra venosa

- ornatus, Gr. Bred from Oden. estis potatoria

*_- cimbices, Tschek.

- migrator, Fab. Bred from Bombyx quercus and trefolli, and several others

$\$-$ pygoleucus, Gr. The females of this species are almost wingless, and have until recently gone under the name of Agrothereutes Hopei decayed bramble stem, con

- signatorius, Fab. Bred from larvae of a small wasp, Spilomena troglodytes

† - tarsoleucus, Schr.

Genus, Mesostenus, Gravenhorst

- - obnoxius, Gr. Bred from Zygaena filipendulae

Genus, Nematopodius, Gr.

†- ater, Brischke

Genus, Hemiteles, Gravenhorst

- furcatus, Tasch. Bred from Dianthaecia cucubali, May

* - tenebricosus, Gr.

\$- imbecillus, Gr. Captured at Dousland, 23 August

* - micator, Gr.

$\$$ - necator, Gr.

- oxyphimus, Gr.

- gyrini, Parfitt. Bred 6 July from pupae of Gyrinus natator (the whirligig beetle)

t- crassicornis, Gr.

- similis, Gr. Bred from the egg-bag of house spider

- fulvipes, Gr. Bred April from $V$ anessa atalanta

$\$$ - decipiens, Gr.

- formosus, Desv. Bred Aug. from egg-bag of spider, Agelena brunnea 


\section{INSECTS}

\section{CRYPTIDES (cont.)}

Genus, Hemiteles, Gravenhorst - vicinus 9, Gr. I Bred July

- melanarius ठ,Gr. $\}$ from one Argynnis paphia pupa 2 males and 16 females, also from a pupa of Pieris rapae, 8 males and 5 females.

- tristator, Gr.

$\$$ - apertus, Thom.

$\$$ - incisus, Bridg.

$\$$ - conformis, Gr.

* bicolorinus, Gr.

* - ridibundus, $\mathrm{Gr}$.

* - distinctus, Bridg.

* - politus, Bridg.

\$- meridionalis, Gr.

- submarginatus, Bridg. Hyperparasite, bred Aug. from Apanteles nothus; the latter was parasitic on a grassfeeding larva

- - aestivalis, Gr.

- - areator, Panz.

- castaneus, Gr. Bred April from sawfly cocoon, Trichiosoma betuleti

- - inimicus, Gr.

- floricolator, Gr. Bred August from Depressaria nervosa

$\$$ - tenerrimus, Gr.

\$- dissimilis, Gr.

4- cingulator, Gr.

* - gracilis, Thom.

$\$$ - furcatus, Tasch.

* capreolus, Thom.

§- melangonus, Gr.

$\$$ - scrupulosus, Gr.

Genus, Orthopelma, Taschenberg

- Iuteolator, Gr.

Genus, Stibeutes, Forster (A.)

\$- Heinemanni, Först.

Genus, Cremnodes, Förster

$\$$ - atricapillus, $\mathrm{Gr}$.

Genus, Aptesis, Förster. The females of this genus are almost wingless

†- nigrocincta, Gr.

- hemiptera, Fab. Bred July from Euzophera cinerosella.

$\S$ - brachyptera, Panz.

- - stenoptera, Marsh.

Genus, Theroscopus, Förster

†- Esenbeckii, Gr. Wbitsand Bay, a female with wings almost microscopic

Genus, Hemimachus, Ratzeburg

- instabilis, Gr. 9 Bred July

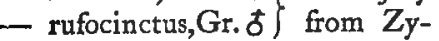
gaena filipendulae. Females perfectly wingless; the males winged

- fasciatus, Fab. Bred July from egg-bags of spiders of Lycosa pullata

$\$$ - annulicornis, Marsh.

\section{CRYPTIDES (cont.)}

Genus, Pezomachus, Gravenhorst $^{1}$

$\$$ - tener, Gr.

- zonatus, Fourc. Bred July from egg-bag of spider, Agelena brunnea

\$- procursorius, Först.

$\$$ - nigricornis, Forst.

$\$$ - carnifex, Forst.

* - rufulus, Först.

- corruptor, Forst.

* - agilis, Först.

- transfuga, Forst. Bred from spiders' nests, Agelena labyrinthica

* faunus, Först.

* incertus, Forst.

* - distinctus, Forst.

*- micropterus, $\mathrm{Gr}$.

*- costatus, Bridg.

\$- vagantiformis, Bridg.

$\$$ - brevis, Bridg.

- insolens, Forst.

- micrurus, Först. Bred from egg-bag of spider, Ocyale mirabilis

* - insidiosus, Forst.

$\$$ - dysalotus, Forrst.

- tristis, Först. Bred, hyperparasite on Apanteles ruficoxis, the host being Arctia villica. Lin.

- hieracii, Bridg. Bred from Aulax hieracii galls

$\S$ - furax, Forrst.

$\$$ - vagans, Oliver

\$- xylochopilus, Forst.

\section{OPHIONIDES}

Genus, Henicospilus, Stephens

- merdarius, Gr. Bred from Hecatera serena

- ramidulus, Lin. Bred from Hadena pisi

Genus, Ophion, Fabricius

- obscurum, Fab. Bred from Hadena protea

- luteum, Lin. Bred May from Miselia oxyacanthae, and other pupae

* minutum, Kriech.

- distans, Thom. Bred from Dianthaecia irregularis

- longigena, Thom. Bred from Cucullia scrophulariae

Genus, Schizoloma, Wesmael

- amicta, Fab. Bred from Eupithecia linariata

1 The females of this genus are all wingless, and look more like ants than ichneumons. The majority of the known males are winged; many, no doubt, when the two sexes are bred from the same host, will be found located in another genus where there are males without females.

\section{OPHIONIDES (cont.)}

Genus, Exochilum, Wesmael

- circumflexum, Lin.

Genus, Anomalon, Jurine

* - xanthopus, Schr.

"- ruficorne, Gr. This has been bred from the half-grown larvae of the drinker moth, Odonestis potatoria

* _ bellicosum, Wesm.

- cerinops, Gr.

- arquatum, Gr. Bred from Taeniocampa gothica

- perspicillator, Gr. Bred from Acronycta menyanthidis

- clandestinum, Gr. Bred from Eupithecia castigata

- perspicuum, Wesm. Bred from Cleora lichenaria in June

Genus, Agrypon, Forster

* flaveolatum, Gr.

* - tenuicorne, Gr.

- septentrionale, Holmgr. Bred from Eupithecia campanulata

Genus, Trichomma, Wesmael

- enecator, Rossi. Bickleigh

Genus, Opheltes, Holmgrén

†- glaucopterus, Lin.

Genus, Paniscus, Schrank

$\S$ - cephalotes, Holmgr.

* testaceus, Gr.

$\dagger$ - inquinatus, Gr.

†— fuscicornis, Gr.

Genus, Parabatus, Förster

* tarsatus, Brischke

- virgatus, Fourc.

Genus, Absyrtus, Holmgr.

- luteus, Holmgr. Bickleigh

Genus, Campoplex, Gravenhorst

- _ mixtus, Gr.

* pugillator, Lin

†- nobilitatus, Holmgr.

- carbonara, Ratz. Bred from Notodonta chaonia

- ebeninus, Gr. Bred from Orgyia fascelina

† - anceps, Holmgr.

- nitidulator, Holmgr. Bred from Eupithecia venosata

$\S$ - carinifrons, $\mathrm{Gr}$.

$\$$ - eurynotus, Först.

- oxyacanthae, Boie. Bred from Himera pennaria

- falcator, Thunb. Bred from Notodonta ziczac

- erythrogaster, Forst. Bred from Hybernia rupicapraria

- confusus, Forst. Bred from Taeniocampa populeti

- femorator, Bridg. Bred 24 May, host not known

- juvenilis, Gr. Bred 16 June from Eupithecia nanata

Genus, Cymodusa, Holmgrén $\$$ - leucocera, Holmgr. 


\section{A HISTORY OF DEVONSHIRE}

\section{OPHIONIDES (cont.)}

Genus, Cymodusa, Holmgrén

- cruentata, Gr. Bred from Anisopteryx aescularia

Genus, Sagaritis, Holmgrén

†- declinator, Gr.

- zonata, Gr. Bred from Hecatera serana

- latrator, Gr.

Genus, Casinaria, Holmgrén

- vidua, Gr. Bred from Abraxas grossulariata

- tenuiventris, Gr. Bred from Hemithea strigata

Genus, Limneria, Holmgrén

\$- alternans, Gr.

$\$$ - alticloa, Gr.

$\$$ - argentata, Gr.

$\$$ - armillata, Gr.

* auctor, Gr.

- bicingulata, Gr. Bred from Hybernia marginaria

$\$$ - brevicornis, Gr.

* - cerophaga, Gr.

- chrysosticta, Gr. Bred from Hyponomeuta evonymellus and padellus

* - crassicornis, Gr.

$\$$ - annulata, Gr.

$\$$ - deficiens, Gr.

$\$$ - auriculatus, Först.

- lugubrina, Holmgr. Bred Aug. from Eidophasia messingiella

t - concinna, Holmgr.

- cursitans, Holmgr. Bred from Vanessa atalanta

†- robusta, Wolstedt

* - ovata, Brischke

- Kriechbaumeri, ${ }^{1}$ Bridg. Bred from half-grown larva of Taeniocampa stabilis

* - crassiuscula, Gr.

* - difformis, Gmel.

- dispar, Gr. Bred September from Symaethis oxyacanthella

- ensator, Gr. Bred from Butalis grandipennis

- erucator, Gr. Bred from Hybernia marginaria

- exareolata, Ratz. Bred from Vanessa cardui

* - exigua, Gr.

* Faunus, Gr.

- femoralis, Gr. Bred from Depressaria nervosella

- fenestralis, Holmgr. Bred from Tortrix costana

$\$$ - fulviventris, Gmel.

\$- geniculata, Gr.

* - gracilis, Gr.

* - hydropota, Holmgr.

- carbonaria, Brischke. Bred from Boarmia roboraria

1 This fly is mentioned in the introduction and only obtainable by collecting half-grown laryae in May on the chance of getting the parasite.

\section{OPHIONIDES (cont.)}

Genus, Limneria, Holmgrén

- vulgaris, Tschek. Bred 4 July, I897, from Gonopteryx rhamni (Brimstone butterfly). More than one-half of the larvae of this butterfly are destroyed by this parasite

- clausa, Brishcke. Bred from Hybernia marginaria

- interrupta, Holmgr. Bred 4 July, 1882, from Taeniocampa stabilis

* combinata, Holmgr.

- rufa, Bridg. Bred from halfgrown larva of Bombyx quercus

t- arvensis, Gr.

- rufata, Bridg. Bred from Choreutes scintillulana

- vestigialis, Ratz. Bred from Symaethis oxyacanthella

- borealis, Holmgr. Not common

* - erythropyga, Holmgr.

* - pedella, Holmgr.

*- volubilis, Holmgr.

- insidiator, Gr.

* - lateralis, Gr.

* - longipes, Müll.

*- majalis, Gr.

- moesta, Gr. Bred from $\mathrm{Hy}$ berni defoliaria

- mutabilis, Holmgr. Bred from Ephippiphora nigricostana

- notata, Gr. Bred from Gnophos obscuraria

- obscurella, Holmgr. Bred from Hemithea strigata

- rapax, Gr. Not common

- curvicauda, Gr. Bred from a sawfly pupa, Nematus gallicola

$\$$ - pagana, Holmgr.

* - assimilis, Holmgr.

- ruficincta, Gr. Bred from Agrotis agathina, and from several other larvae

- rufipes, Gr. Bred from Eupithecia castigata

\$ - sericea, Gr.

* - sordida, Gr.

$\$-$ transfuga, Gr.

* - tristis, Gr.

\$- tumidula, Gr. Captured at Bickleigh, 5 August, 1884

- Brischkei, Bridg. Bred from Noctua triangulum

* - dumetorum, Holmgr.

*- immolator, Gr.

* - floricola, Gr.

- unicincta, Gr. Bred during April, 1 882, from Vanessa urticae and other larvae

\$- viennensis, Gr.

- teucrii, Bridg. Bred from Oxyptilus teucrii I 78
OPHIONIDES (cont.)

Genus, Canidia, Holmgrén

$\S$ - pusilla, Ratz.

Genus, Nemeritis, Holmgrén

\$- macrocentra, Gr.

- cremastoides, Holmgr. Bred May, from fir cones containing Coccyx strobilella

Genus, Cremastus, Gravenhorst

$\S$ - interruptor, Gr.

$\$$ - spectator, Gr.

$\$$ - infirmus, Gr.

Genus, Atractodes, Gravenhorst

\$- gravidus, Gr.

* vestalis, $\mathrm{Hal}$.

$\$$ - albovinctus, $\mathrm{Hal}$.

$\S$ - exilis, $\mathrm{Hal}$.

$\$$ - bicolor, Gr.

$\$$ - gilvipes, Holmgr.

§- fumatus, $\mathrm{Hal}$.

$\$$ - citator, $\mathrm{Hal}$.

$\$$ - properator, $\mathrm{Hal}$.

Genus, Exolytus, Holmgrén

* - laevigatus, Gr.

Genus, Mesochorus, ${ }^{2}$ Gravenhorst

t- splendidulus, $\mathrm{Gr}$.

- strenuus, Holmgr. Bred from Limneria Kriechbaumeri, out of half-grown larva of Taeniocampa stabilis

$\$$ - thoracicus, Gr.

- confusus, Holmgr. Bred from Microplitis fumipennis, the latter a parasite on Symaethis oxyacanthella, and several other pupae

†— gibbulus, Holmgr.

- gracilentus, Brischke. Bred from Limneria vulgaris, the latter a parasite on Gonopteryx rhamni

- fuscicornis, Brischke. Bred from Apanteles nothus, the latter a parasite on Abraxas grossulariata

- aciculatus, Bridg. Bred from Apanteles glomeratus, a parasite on Pieris brassicae

- facialis, Bridg. Bred from Apanteles popularis, out of Euchelia Jacobaeae

- graniger, Thom. Bred from Exorista vulgaris, out of Abraxas grossulariata

- temporalis, Thom. Bred from Zygaena filipendulae

- tetricus, Holmgr. Bred 4 August from Apanteles octonarius, out of Notodonta dromedarius

- formosus, Bridg. Bred from Apanteles fulvipes, out of Asteroscopus sphinx and others

\$- dorsalis, Holmgr.

This genus are hyperparasites in the larvae stage, living within the bodies of other larvae. 


\section{INSECTS}

\section{OPHIONIDES (cont.)}

Genus, Mesochorus, Gravenhorst

$\S$ - politus, Gr.

- sericans, Curt. Bred from a dipterous pupa,Exorista vulgaris, out of Abraxas grossulariata

- vittator, Zett. Bred from Limneria chrysosticta, out of Hyponomeuta evonymellus

- fulgurans, Hal. Bred from Casinaria vidua, out of Abraxas grossulariata

- semirufus, Holmgr.

- sylvarum, Hal. Bred from Microgaster subcompletus, out of Vanessa atalanta

- pictilis, Holmgr. Bred from an Apanteles cocoon, out of Iodis lactearia

- olerum, Curt. Bred from Casinaria vidua, out of Abraxas grossulariata

$\$-$ arenarius, Hal. Captured at Longbridge, 27 June

- anomalus, Holmgr. Bred from Apanteles popularis, out of Euchelia Jacobaeae

Genus, Miomeris, Förster

†— aequisgranensis, Först.

Genus, Dicolus

$\S$ - pectoralis, Först.

\$- subtiliventris, Först.

Genus, Plectiscus, Gravenhorst

$\$$ - canaliculatus, Forst.

$\$$ - zonatus, Gr.

Genus, Porizon, Fallen

$\$$ - harpurus, Schr.

$\$$ - erythrostomus, Gr.

* minator, Gr.

Genus, Thersilochus, Holmgrén

- - jocator, Fab.

- saltator, Fab. Bred from Micropteryx unimaculella

\$- marginatus, Bridg.

$\$$ - triangularis, $\mathrm{Gr}$

$\$$ - microcephalus, Gr.

\$- truncorum, Holmgr.

Genus, Collyria, Schiödte

t- calcitrator, Lin.

Genus, Pristomerus, Curtis

- vulnerator, Panz. Captured Budleigh Salterton, I 5 June, 1897, by Mr. C. W. Dale

Genus, Exetastes, Gravenhorst

\$- fornicator, Fab.

- cinctipes, Ratz. Bred during June from Mamestra brassicae and others

\$- calobatus, Gr. Captured at Trew, near Crediton

- nigripes, Gr. Bred from Mamestra brassicae

\$- illusor, Gr.

$\$$ - albitarsus, Gr.

Genus, Arotes, Gravenhorst

†- albicinctus, Gr.

\section{OPHIONIDES (cont.)}

Genus, Banchus, Fabricius

$\S$ - variegator, Fab.

- pictus, Fab. Bred from Selenia bilunaria

$\$$ - falcator, Fab. Captured in Bickleigh Wood

- moniliatus, Gr. Bred from Anarta myrtilli

\section{TRYPHONIDES}

\section{HomalopI}

Genus, Mesoleptus, Gravenhorst

t- typhae, Gr.

†- melanocephalus, Gr.

- testaceus, Fab. Bred from Eupithecia castigata

$\dagger$ - fugax, Gr.

$\$$ - similis, Brischke.

$\$$ - leptocerus, Gr.

$\$$ - laevigatus, Gr.

$\$$ - paludicola, $\mathrm{Gr}$.

$\$$ - indefessus, Gr.

* facialis, Gr.

$\$$ - ventralis, Curt.

Genus, Catoglyptus, Holmgrén

* - fortipes, Gr.

$\$$ - fuscicornis, Gmel.

Genus, Euryproctus, Holmgrén

- nigriceps, Gr. Bred II June from a sawfly, Trichiosoma betuleti

\$- atomator, Gr.

\$- sinister, Brischke

†- mundus, $\mathrm{Gr}$.

Genus, Perilissus, Holmgrén

- - filicornis, Gr.

$\$$ - lutescens, Gr.

$\$$ - subcinctus, Holmgr.

*- praerogator, $\mathrm{Gr}$.

$\$$ - vernalis, Gr.

Genus, Eclytus, Holmgrén

- ornatus, Holmgr. Bred May from Totrix heparana

* fontinalis, Holmgr.

Genus, Megastylus, Schiodte

$\$$ - conformis, Forst.

$\$$ - cruentator, Schiodte

* - mediator, Schiodte

Genus, Idioxenus

- - borealis, Holmgr.

Genus, Mesoleius, Holmgrén

* - ruficornis, Gr.

* - Bignellii, Bridg.

\$- aulicus, Gr.

- dubius, Gr. Bred August from a sawfly, Nematus ribesii

$\$$ - caligatus, Gr.

- sanguinicollis, Gr. Bred September from sawfly galls on Salix caprea

\$- haemotodes, Gr.

$\S$ - armillatorius, Gr.

$\$$ - molestus, Holmgr.

$\$$ - rufoleptus, Holmgr.

$\S$ - insolens, Gr.

$\S$ - hamulus, Gr.

\section{TRYPHONIDES (cont)}

Homalopi (cont.)

Genus, Mesoleius, Holmgrén

$\$$ - lateralis, Gr.

- fallax, Holmgr.

Genus, Trematopygus, Holmgrén

- vellicans, Gr.

$\$$ - rubiginosus, Gr.

Genus, Tryphon, Fallén

\$- brachyacanthus, Gmel.

\$- trochanteratus, Holmgr.

* signator, Gr.

\$- albipes, Gr.

$\$$ - rutilator, Lin.

Genus, Eumesius, Westwood

* - albitarsus, Curt.

Genus, Polyblastus, Hartig

\$- varitarsus, Gr.

- Westringi, Holmgr.

\$- pyramidatus, Holmgr.

$\$$ - rivalis, Holmgr.

Genus, Acrotomus, Holmgrén

$\S$ - Iucidulus, Gr.

Genus, Cteniscus, Haliday

- triangularis, Gr. Not common

\$- gnathoxanthus, Gr.

§- lituratorius, Lin.

$\$$ - sexcinctus, Gr.

\$- succinctus, Gr.

$\$$ - ustulatus, Holmgr.

§- bimaculatus. Holmgr.

$\$$ - pictus, Gr.

\$- exstirpatorius, Gr.

Genus, Exyston, Schiödte

- cinctulum, Gr.

Genus, Sphecophaga, Westwood

- vesparum, Curt. Bred from wasp nests in autumn, and from old nests in spring

Prosors

Genus, Colpotrochia, Holmgrén

- elegantula, Schr.

Genus, Exochus, Gravenhorst

- mansuetor, Gr. Bred July from Pyralis farinalis

- alpinus, Zett. Bred May from a Tortrix feeding on honeysuckle

\$- flavomarginatus, Holmgr.

- squalidus, Holmgr. Bred July from Botys terrealis

$\$$ - curvator, Fab.

- podagricus, Gr. Bred September from Symaethis oxyacanthella

- gravis, Gr.

- pictus, Holmgr.

\$- procerus, Holmgr.

\$- nigripalpis, Thom.

Genus, Chorinaeus, Holmgrén

- cristator, Gr.

- funebris, Gr. Bred February from Depressaria heracleana

Genus, Orthocentrus, Gravenhorst

$\$$ - anomalus, Gr.

$\$$ - confinis, Holmgr.

§- fulvipes, Gr. 


\section{A HISTORY OF DEVONSHIRE}

TRYPHONIDES (cont.)

Prosopi (cont.)

Genus, Orthocentrus, Gravenhorst

$\S$ - intermedius, Holmgr.

$\$$ - vittatus, Holmgr.

$\$$ - affinis, Zett.

$\$$ - agilis, Holmgr.

\$- marginatus, Holmgr.

$\$$ - cognatus, Holmgr.

$\$$ - ventralis, Holmgr.

Genus, Zootrephus, Thomson

- Holmgreni, Bridg.

Genus, Bassus, Fabricius

- albosignatus, Gr.

- laetatorius, Fab. Bred, a common parasite on the larvae of the 'hovering fly' (Syrphus), the larvae of which feed exclusively on different species of Aphis

- multicolor, Gr.

Genus, Homoporus, Thomson

- cinctus, Gr.

- graculus, Gr.

- biguttatus, Gr.

- abdominator, Bridg.

- flavolineatus, Gr.

- fissorius, Gr.

- Sundevalli, Holmgr.

- strigator, Fab.

- elegans, Gr.

- pallidipes, Gr.

- signatus, Gr.

Genus, Promethus, Thomson

- scutellaris, Bridg.

- sulcator, Gr.

- cognatus, Holmgr.

- pulchellus, Holmgr.

- festivus, Fab.

- dorsalis, Holmgr.

- laticarpus, Thoms.

\section{Aspidopi}

Genus, Metopius, Panzer

$\S$ - micratorius, Fab.

- dentatus, Fab. Bred from pupae of Lasiocampa quercifolia

†- dissectorius, Panz.

\section{Pimpuides}

Genus, Acaenitus, Latreille †- fulvicornis, Gr.

Genus, Rhyssa, Gravenhorst

- persuasoria, Lin.

t- leucographa, Gr.

Genus, Ephialtes, Gravenhorst

\$- imperator, Kriech.

$\S-$ tuberculatus, Fourc.

\$- rex, Kriech.

Genus, Perithous, Holmgrén

- mediator, Fab. Bred from old bramble stems containing small wasps
TRYPHONIDES (cont.)

Pimplides (cont.)

Genus, Perithous, Holmgrén

- divinator, Rossi. Bred from old bramble stems containing small wasps

- varius, Gr. Bred from old bramble stems containing small wasps

Genus, Pimpla, Fabricius

- - instigator, Fab.

- examinator, Fab.

$\$$ - pomorum, Ratz.

†- epeira, Bignell. Bred from the egg-bag of a spider, Epeira cornuta, taken out of a bramble bush at Ivybridge

†- Bridgmanii, Bignell. Captured in the act of attacking a spider, Drassus lapidicolens, in which she was desirous of depositing her egg

- turionellae, Lin. Bred July from Rhodophae consociella

" rufata, Gmel.

- flavonotata, Holmgr. Bred 29 March from Tortrix viridana and several other species

- scanica, Vill.

- ruficollis, Gr. Bred November from Retinia resinella

* oculatoria, Fab.

- detrita, Holmgr. Bred July from Chilo phragmitellus

- rufipleura, Bignell. Bred April from Pygaera curtula

* - graminellae, Schr.

*- didyma, Gr.

$\$$ - calobata, Gr.

- stercorator, Fab. Bred I4 March from Eupithecia linariata

- brevicornis, Gr. Bred August from Dianthaecia cucubali

- nucum, Ratz.

Genus, Polysphincta, Gravenhorst ${ }^{1}$

- varipes, Gr.

- gracilis, Holmgr. Bred June; an external parasite on Meta segmentata

- multicolor, Gr. Bred August; host of which was Meta merianae

- tuberosa, Gr. Bred June ; host, Epeira cucurbitina

Genus, Acrodactyla, Haliday

* - madida, Hal.

*- degener, Hal.

Genus, Clistopyga, Gravenhorst

- incitator, Fab.

Genus, Glypta, Gravenhorst

- - teres, Gr.

- monoceros, Gr. Bred June from Tortrix costana

1 The genus Polysphincta are external parasites on adult spiders ; the method of attack will be found in the introduction.
TRYPHONIDES (cont.)

Pimplides (cont.)

Genus, Glypta, Gravenhorst

*- fronticornis, Gr.

- ceratites, Gr. Bred June from Ephippiphora nigricostana

- haesitator, Gr. Bred June from Hedya ocellana

- - scalaris, Gr.

-- bifoveolata, Gr.

- - flavolineata, Gr.

- filicornis, Thom.

Genus, Lycorina, Holmgrén

$\S$ - triangulifera, Holmgr.

Genus, Schizopyga, Gravenhorst

- podagrica, Gr.

$\$$ - circulator, Panz.

Genus, Lampronota, Haliday

$\$$ - nigra, Gr.

$\$$ - caligata, Gr.

Genus, Lissonota, Gravenhorst

* - variabilis, Gr.

- brachycentra, Gr. Bred 16 June from Anticlea badiata

$\$$ - varicoxa, Thom.

— anomala, Holmgr. Bred Aug. from Larentia flavicinctata

$\$$ - parallela, Gr.

$\$$ - deversor, Gr.

\$- lineata, Gr.

\$- notabilis, Desv.

- decimator, Gr. Bred from Gortyna flavago

* - bellator, Gmel.

* - commixta, Gr.

* - cylindrator, Vill.

* - insignita, Gr.

* - segmentator, Fab.

* _- rufomedia, Bridg.

* - sulphurifera, Gr.

- impressor, Gr.

Genus, Meniscus, Schiodte

- murinus, Gr. Bred from Chrysis neglectus, a parasite on a wasp, Odynerus spinipes

- setosus, Gr. Bred from halfgrown larvae of Cossus ligniperda

* catenator, Panz.

Genus, Phytodiaetus, Gravenhorst

- segmentator, Gr. Bred January from Peronea hastiana

- coryphaeus, Gr. Bred July from Tortrix viridana

* - vetulus, Gr.

Genus, Oedimopsis

5- scabriculus, $\mathrm{Gr}$.

Genus,Cryptopimpla, Taschenberg

- plantarius, Gr. Bred from Taeniocampa populeti

Genus, Mitroboris

$\$$ - cornuta, Ratz

Genus, Phthinodes, Tschek.

- hecticus, Gr. Bred May from decaying apple-tree containing wasps and beetles 


\section{INSECTS}

\section{B R A C O ID AE}

A brief introduction to the Braconidae may be interesting, to show how or why they are separated from the true Ichneumonidae. It is by the neuration of the fore wings; these have the exterior discoidal transverse nervure wanting, and by having the first cubital areolet generally, though not always, separated from the praediscoidal, and the entire absence of the small areolet. The Braconidae perform the same function in nature as the Ichneumonidae, and are related to them in structure and habits, and like them the ovipositor may be of any length; that is, be as long or longer than the body, or it may be hidden.

The larvae vary in their habits when preparing for the change to the pupa stage, as much as, if not more than, the Ichneumons. Some attack small half-grown larvae, others the largest larvae ; some attack adult beetles, some the beetle larvae; in fact nothing appears too large or too small in the insect world to escape their attention.

Many species do not leave their host to make their change to the pupa, but remain within the skin of the larva, until the fly is ready to take flight, when it cuts a hole and escapes. The habits of the larvae of the Microgasteridae are quite the reverse of those just mentioned. Without any exception they leave their host and spin silken cocoons, very much like that of the silkworm moths of commerce, of course in miniature.

Some larvae are solitary in their habits, while others are gregarious; as many as I 80 may emerge from a single caterpillar, each one making a separate cocoon, or make an alveariform mass. One particular species, Apanteles formosus, builds up a stem, on the top of which the cocoon is made. The way the alveariform mass is made may be worth recording. The writer was present on one occasion when ninety-five larvae of Microgaster flavipes had just commenced to emerge from an unfortunate caterpillar of Boarmia gemmaria, each one making a hole for its exit, all trying to escape at the same moment. This wriggling mass of maggots at once commenced to spin silken cords. The caterpillar at this time was stretched out full length on a privet twig; the spinning of the maggots continued, and it was three hours before any form or order could be detected, whether they were going to make a round mass or the usual semi-pyramid of cocoons. From that time it began to get into the final shape, and was completed, to all external appearance, in about seven hours. The mass was formed under the twig. The caterpillar by that time had twisted itself half round, apparently trying to protect its destroyers; by the next morning it had taken up a position over the cocoons, just as a hen would over a brood of chickens.

The Apbidiides are solitary parasites. The larvae generally remain in the body of the Aphis, 'green fly,' the whole of the changes taking place within it ; when the fly is ready to escape, it cuts a circular hole in the body of the dead Aphis, and flies off.

In the genus Praon the parasite, when full grown, emerges from the body of the Apbis on the underside, and spins a tent-like cocoon; the dead Apbis remains on the top long after the parasitic fly has escaped from the cocoon.

Small as these must be when the whole of their sustenance was obtained while living within the body of an Aphis, few would imagine that the continuity of the hyperparasite depends on the female (Allotria) discovering an Apbis already infested with a parasite wherein to deposit her eggs.

It may be interesting to know how this last observation was obtained. One bright sunny afternoon, while the writer was on the search for Apbides infested with parasites, his attention was attracted to the actions of some small flies, who evidently were on the same business as himself. The infested Aphis are readily detected by their brown, hard, dead-looking bodies. These small flies were paying great attention to the infested Aphides by constantly applying their antennae to several parts of the body of the Aphis. When one of the flies had ascertained it was a suitable subject, she commenced depositing her eggs within it. Having watched these manouvres for some time, one was selected that was seen to deposit eggs, she was brought home, also the Aphis that the eggs were deposited in. A week or two after two flies emerged, the offspring of the parent that deposited the eggs (Allotria victrix). The larvae of the two flies that were bred fed on the single larva that had consumed the Aphis.

\section{CYCLOSTOMI}

Braconidgs

Genus, Bracon

- erythrostictus, Marsh. Bred from gall on Lyme grass, Psamma arenaria

- fulvipes, Nees. Bickleigh, relverton

- longicollis, Wesm. Bickleigh, July; Oreston Quarry, August

- variegator, Nees. Bred 7 July from Gelechia pinguinella

\section{CYCLOSTOMI (continued)}

BRACONides (continued)

Genus, Bracon

- nigratus, Wesm. Horrabridge, June; Bickleigh, July

— stabilis, Wesm. Bred July from Sciaphila chrysantheana

- erraticus, Wesm. Plym Bridge, Bickleigh, August

- Roberti, Wesm. Braunton Burrows, T. A. Marshall 


\section{A HISTORY OF DEVONSHIRE}

\section{CYCLOSTOMI (continued)}

BRACONIDES (continued)

Genus, Bracon

- laevigatus, Ratz. Bred 22 August from Nematus salicis-cinereae and gallicola galls on willows

- mediator, Nees. Bred 26 June from an osier stem ; a parasite on Trochilium crabroniforme

- fuscicoxis, Wesm. Bickleigh, August

- tornator, Marsh. Bickleigh, July

- Satanas, Wesm. Plym Bridge, June

- epitriptus, Marsh. Bred from galls of Hormomyia capreae, 23 August and 3 Sept. I 884

- larvicida, Wesm. Bickleigh, August

- praetermissus, Marsh., Bickleigh, August ; Exeter, September

- discoideus, Wesm. Bred 5 July, 1886, from Anticlea badiata, and from Nematus gallicola galls, I 5 June, collected the previous autumn

- regularis, Wesm. Yelverton, 4 August, I885

- variator, Nees. Bred 25 August from a Geometra larva found feeding on yarrow, Achillea millefolium

- osculator, Nees. Bred from Coleophoro solitariella, caespititiella, and discordella in June

- obscurator, Nees. Bred 28 May, from roots of Plantago lanceolata, containing larvae of Homaeosoma sinuella, Fab.

- anthracinus, Nees. Bowisand, June

\section{ExoTheCIDEs}

Genus, Phanomeris, Först.

- fragilis, Hal. Exeter, August

\section{RhYSSALIDES}

Genus, Rhyssalus, Hal.

- clavator, Hal. Barnstaple

- indagator, Hal. Vinstone, nr. Plymouth ; Bishopsteignton

Genus, Colastes, Hal.

- decorator, Hal. Bred from fallen oak leaves gathered in the winter

- hariolator, Hal. Bickleigh

- braconius, Hal. Bred from fallen oak leaves, and from dipterous larvae mining leaves of laburnum and Verbascum thapsus

Genus, Oncophanes, Först.

- lanceolator Nees. Bred from Lithocolletis trifasciella and Tortrix viridana

\section{Spathindes}

Genus, Spathius, Nees.

- exarator, L. Exeter; Laira, Plymouth

\section{Doryctides}

Genus, Histeromerus, Wesm.

- mystacinus, Wesm. Three specimens of this rare species were discovered by $\mathrm{Mr}$. J. H. Keys on 14 August, I90I, while hunting for Sinodendron cylindricum (a beetle) in 2 decayed oak in the Tary Valley

\section{Hormines}

Genus, Chremylus, Hal.

- rubiginosus, Nees. Exeter

\section{Rogadides}

Genus, Heterogamus, Wesm.

- dispar, Cur. Bickleigh; Lydford

\section{CYCLOSTOMI (continued)}

Rogadides (continued)

Genus, Rhogas, Nees.

- dissector, Nees. Exeter

- dimidiatus, Spin. Bred from Agrotis tritici, July

- gasterator, Jurines Yelverton, August

- geniculator, Nees. Bred from young larvae of Odonestis potatoria and from Arctia villica

- tristis, Wesm. Bred from Dianthaecia cucubali, May; eight months in cocoon

- circumscriptus, Nees. Bred from half-grown larva of Ebulea crocealis, June, and several other larvae

- bicolor, Spin. Exeter

\section{CRYPTOGASTRES}

\section{SigalPHides}

Genus, Allodorus, Forst.

- lepidus, Hal. Kingsteignton, Marshall

Genus, Sigalphus, Latr.

- caudatus, Nees. Exeter

- striatulus, Nees. Barnstaple, Marshall

- obscurellus, Nees. Bred from Gymnetron collinus, a beetle larva feeding on the unripe seeds of the toad-flax, Linaria vulgaris, September

\section{Chrlonide}

Genus, Chelonus, Jur.

* - inanitus, L.

- carbonator, Marsh. Oreston Quarry, August

- corvulus, Marsh. Barnstaple, Marshall

- dispar, Marsh. Bickleigh, August

- parcicornis, Schaff. Bickleigh, July

- sulcatus, Nees. Langstone Cliff, Parfitt

Genus, Ascogaster, Wesm.

- instabilis, Wesm. Bickleigh, June

- annularis, Nees. Bred from decaying furze-sticks containing larvae of Oecophora lambdella

- rufipes, Latr. Bred from a Tortrix caterpilar on birch

- rufidens, Wesm. Bred from Tortrix ribeana and xylosteana

- canifrons, Wesm. Bickleigh, 9 July, 1885 ; first recorded capture in England

- quadridentatus, Wesm. Bred from larva on wormwood

Genus, Acampsis, Wesm.

- alternipes, Wesm. Captured at Bickleigb

\section{AREOLARII}

\section{Microgasterides}

Genus, Mirax, Hal.

- spartii, Hal. Bred from Nepticula septembrella,Aug. Genus, Acoelius, Hal.

- subfasciatus, Hal. Bred from three species of Nepticula

Genus, Apanteles, Först.

- solitarius, Ratz. Bred from Taeniocampa miniosa and several other larvae

- salebrosus, Marsh. Bred from Oporabia dilutata and from Hybernia defoliaria

- tetricus, Reinh. Bred from Epinephele ianira; two broods of six and seven respectively

- ruficrus, Hal. Bred from Spilosoma menthastri ; a gregarious lot of twenty-four cocoons 


\section{AREOLARII (continued)}

Microgasterides (continued)

Genus, Apanteles, Forst.

- ordinarius, Ratz. Exeter

- congestus, Nees. Bred from Hadena pisa, and at least a dozen other noctua. The cocoons are often mistaken for a spider's nest

- Bignellii, Marsh. Bred 26 July, 1883, from Melitaea aurinia

- limbatus, Marsh. Bred from Abraxas grossulariata

- rubripes, Hal. Bred from Geometra papilionaria

- rubecula, Marsh. Bred from half-grown larvae of Pieris rapae, June

- glomeratus, L. The well-known parasite of both species of the white cabbage butterflies, Pieris brassicae and rapae

- sericeus, Nees. A solitary parasite bred from eight different species of Lepidopterous larvae

- spurius, Wesm. Bred gregariously from Melitaea aurinia and several other larvae

- Geryonis, Marsh. Bred from Ino geryon, June

- zygaenarum, Marsh. Bred from Zygaena filipendulae, July

- jucundus, Marsh. Bred 24 June from a larva found on oak

- caiae, Bouché. Bred from Arctia caia, 4 July; the caterpillar was found at Torcross, 20 June, and produced I 80 parasitic larvae a few days after

- juniperatae, Bouche. Bred from Odontoptera bidentata, April, and from several other larvae

- nothus, Reinh. Bred from Anticlea badiata and other larvae

- difficilis, Nees. Bred from Bombyx rubi, August, and other larvae

- punctiger, Wesm. A female captured, 24 June, at Bickleigh

- lictorius, Reinh. Exeter (Parfitt)

- falcatus, Nees. Bred from Leioptilus microdactylus, June

- dilectus, Hal. Bred from Gracilaria syringella, July

- xanthostigmus, Hal. Bred from Swammerdammia caesiella, and from Diurnea fagella and other larvae

- praetor, Marsh. Oreston Quarry

- cmarginatus, Nees. Bred from Depressaria nervosa, August, from half-grown larvae

- obscurus, Nees. Bred from Ebulea crocealis; a solitary parasite

- viminetorum, Wesm. Bred from Elachista magnificella, June ; singly

- sodalis, Hal. Exeter; Teigmmouth; larvae gregarious

- albipennis, Nees. Bred from Leioptilus microdactylus; a solitary parasite, recorded from a dozen other species

- impurus, Nees. Bred from Tortrix forsterana; gregarious

- naso, Marsh. 'On the sandhills at Starcross' (Marshall)

- gagates, Nees. Yelverton, 2 I August

- Halidaii, Marsh. Bred from Hedya neglectana

- longicaudis, Wesm. Bred 18 August, from Laverna subbristrigella

- fuliginosus, Wesm. Bred 16 July, from Gracilaria syringella; a solitary parasite

- sicarius, Marsh. Bred from Sericoris littoralis, 19 June

- octonarius, Ratz. Bred from Cleora lichenaria and other larvae, June and August

\section{AREOLARII (continued)}

Microgasterides (continued)

Genus, Apanteles, Forst.

- astrarches, Marsh. Bred from Lycaena astrarche, I 3 May

- abjectus, Marsh. Bred from Lophopteryx camelina ; larvae gregarious

- immunis, Hal. Bred from Pseudoterpna pruinata and other species; solitary parasite

- caberae, Marsh. Bred from Cabera pusaria and other larvae, April and June ; solitary parasite

- popularis, Hal. Bred from Euchelia jacobaeae and other larvae; gregarious. July

- fraternus, Reinh. Bred from Aspilates ochrearia, September ; larvae, gregarious, forming an alveariform mass of cocoons

- triangulator, Wesm. Bred from Dasychira puddibunda, August

- pallidipes, Reinh. Bred from Plusia gamma and other larvae ; gregarious

- bicolor, Nees. Bred from Gnophos obscuraria and other larvae; solitary when host is small

- formosus, Wesm. Bred several times from Uropteryx sambucaria in May. The larva on emerging from its host builds uparigid peduncle, at the top of which the cocoon is formed

- callidus, Hal. Bred from Abraxas grossulariata and other larvae; gregarious

- lateralis, Hal. Bred from Eupithecia assimilata and other larvae; gregarious

- vitripennis, Hal. Bred from Boarmia repandata ; the caterpillar not half-grown

- fulvipes, Hal. Bred from Miselia oxyacanthae, June, and from at least a dozen other species; larvae gregarious

- cleoceridis, Marsh. Bred from Cleoceris viminalis, June; gregarious

- gonopterygis, Marsh. Bred from half-grown larvae of Gonopteryx rhamni ; solitary para. site. Cocoon, bright orange-colour, firmly attached to a twig

Genus, Microplitis, Först.

- Spinolae, Nees. Bred from half-grown larva of Acronycta alni, August. Cocoon, pearly green

- ocellatae, Bouché. Bred from Smerinthus ocellatus, populi, and tiliae, in May. Cocoons, greyish brown, rough, closely agglutinated together

- vidua, Ruthe. Bred from Taeniocampa incerta ; solitary

- tristis, Nees. Bred from Dianthaecia cucubali, May ; gregarious

- spectabilis, Hal. Bred by D'Orville, Exeter, from Dianthaecia capsincola

- mediator, Hal. Bred from Cerastis spadicea, June, and several other larvae ; gregarious

- mediana, Ruthe. Bred singly from not half-grown caterpillars of Taeniocampa stabilis and others

- tuberculifera, Wesm. Bred singly from young larvae of Taeniocampa miniosa, June

Genus, Microgaster, Latr.

- Alvearius, Fab. ${ }^{1}$ Bred seventy out of Boarmia repandata, 26 June

— posticus, Nees. Bred from Porthesia similis, July

- Glavipes, Hal. ${ }^{1}$ Bred from Boarmia gemmaria and Amphipyra pyramidea, July

- minutus, Reinh. ' 1 Bred from Cleora glabraria

1 Cocoons arranged in an alveariform mass; how they are constructed is described in the introduction to the Braconidae. 


\section{A HISTORY OF DEVONSHIRE}

\section{AREOLARII (continued)}

Microgasterides (continued)

Genus, Microgaster, Latr.

- marginatus, Nees. Bred from Larentia viridaria

- calceatus, Hal. Bred from Thera variata and other larvae, singly

- connexus, Nees. Bred from Porthesia similis, Aug.

- subcompletus, Nees. Bred from Vanessa atalanta and Hypena proboscidalis

- globatus, L. Bred from Lithosia complanula, I 2 May

- tibialis, Nees. A solitary parasite, bred from Emmelesia decolorata and Eupithecia campanulata

- spretus, Marsh. Bred from Rhodophaea consociella, July

\section{Agathidides}

Genus, Agathis, Latr.

- brevisetis, Nees. Bred from Coleophora troglodytella and Nannodia hermannella

- nigra, Nees. Bred August from Apodia bifractella

Genus, Earinus, Wesm.

- gloriatorius, Panz. Bred from Stigmonota germarana, July

- nitidulus, Nees. Bickleigh, to May

Genus, Microdus, Nees.

- cingulipes, Nees. Bred froma Coleophora glauci, 2I July

Genus, Orgilus, Hal.

- obscurator, Nees. Bred 1 I July from Retinia buoliana in tips of branches of fir trees

\section{POLYMORPHI}

\section{EUPHORIDES}

Genus, Euphorus, Nees.

- pallidipes, Cur. Marsh Mills; Plymbridge

- picipes, Hal. Plymbridge, 27 May

- coactus, Marsh. Taken near Barnstaple by Marshall

- apicalis, Cur. Bickleigh

- ornatus, Marsh. A single specimen beaten out of holly at Irybridge, 28 May, 1898. Only one previous capture of this insect is recorded

- fulvipes, Cur. Teignmouth (Marshall)

Genus, Wesmaelia, Först.

- cremasta, Marsh. N. Devon (Marshall)

Genus, Perilitus, Nees.

- rutilus, Nees. Osier bed near Longbridge, June

- falciger, Ruthe. Bred from a beetle, Timarcha laevigata, $7 \mathrm{May}^{1}$

- aethiops, Nees. Oreston Quarry, August

- brevicollis, Hal. Plymbridge, July

Genus, Microctonus, Wesm.

- cultus, Marsh. Nr. Barnstaple (Marshall)

\section{Metrorides}

Genus, Meteorus, Hal.

- albiditarsis, Cur. Bred 13 May from Anarta myrtilli. Cocoon, felted, straw-colour

- chrysophthalmus, Nees. Bred from Odontoptera bidentata. Cocoon much like the preceding

- deceptor, Wesm. Bred from Crocallis elinguaria and several other larvae. Cocoons, white, felted, fusiform

1 It is not usual for an imago to be attacked by parasites.

\section{POLYMORPHI (continued)}

Metrorides (continued)

Genus, Meteorus, Hal.

- caligatus, Hal. Bred from Eupithecia expallidata. Cocoon, white, oval, felted

- ictericus, Nees. Ivybridge; Bickleigh. Cocoon, white and very thin

- pallidipes, Wesm. Bovisland and Bickleigh

- facialis, Ruthe. Barnstaple, Marshall

- obfuscatus, Nees. Bred from Orchesia micans, feeding in fungus Boletus

* - punctiventris, Ruthe

- atrator, Cur. Plymouth District (Parfitt)

- albicornis, Ruthe. Bickleigh and Exeter

* - abdominator, Nees.

- pulchricornis, Wesm. Bred from Agrotis agathina in June ; a solitary parasite

- scutellator, Nees. Bred from Taeniocampa stabilis and several other larvae

- unicolor, Wesm. Bred from Tethea retusa and Orthosia lota

- versicolor, Wesm. Bred from Geometra papilionari ; a solitary parasite

(Bred from larvae of Cis boleti, - filator, Hal. $\left\{\begin{array}{l}\text { a small beetle found feed- } \\ \text { ing }\end{array}\right.$

- profligator, Hal. $\left\{\begin{array}{l}\text { ing in a fungus (Polyporus } \\ \text { - }\end{array}\right.$

- cinctellus, Nees. Bolt Head and Bickleigh

- tenellus, Marsh. Bred from Peronea hastiana,Nov.

- leviventris, Wesm. Bickleigh; Yelverton

- rubens, Nees. Bred from Agrotis tritici, May; larvae gregarious

- fragilis, Wesm. Bred from Taeniocampa stabilis. When the larva leaves its host it spins a single thread about 2 inches long; thus suspended it weaves its brown cocoon ; soon after it falls to the ground

- luridus, Ruthe. Bred from Noctua brunnea. Cocoons, brown, heaped together, attached by a few threads

\section{Calyptides}

Genus, Eubadizon, Nees.

- extensor, L. Bred out of alder catkins gathered 25 February

- flavipes, Hal. Bred from a pupa found on a wall at Paignton

Genus, Calyptus, Hal.

- fasciatus, Nees. Taken by Marshall at Barnstaple

- tibialis, Hal. Bickleigh, June

\section{Blacides}

Genus, Pygostolus, Hal.

- sticticus, Fab. Bred from a cocoon found on blackthorn ; solitary parasite

Genus, Blacus, Nees.

- ruficornis, Nees. Bred from fallen oak leaves gathered in the winter

- tuberculatus, Wesm. Bickleigh and Plymbridge

- tripudians, Hal. Yelverton and Bickleigh

\section{LIOPHRONIDES}

Genus, Liophron, Nees.

- ater, Nees. Plymbridge

- lituratus. Clearbrook

Genus, Centistes, Hal. $\left.\begin{array}{l}\text { - lucidator, Nees. } \\ \text { - fuscipes, Nees. }\end{array}\right\} \begin{gathered}\text { Captured at Bickleigh and } \\ \text { Yelverton }\end{gathered}$ 


\section{INSECTS}

\section{POLYMORPHI (continued)}

Macrocentrides

Genus, Macrocentrus, Curt.

* - abdominalis, Fab. Gregarious

* pruinata. Gregarious

- marginator, Nees. Bred from several species of Sesia; larva solitary

" - thoracicus, Nees.

- infirmus, Nees. Bred 174 females from a single caterpillar of $\mathrm{Hydraecia}$ petasitis

- collaris, Spin. Bred eighty from Noctua triangulum

Genus, Zele, Curt.

- testaceator, Curt. Bickleigh and Bisbopsteignton

- chlorophthalma, Nees. Bred from Cidaria immanata

— discolor, Wesm. Bred from Cabera pusaria

\section{Diospilides}

Genus, Diospilus, Hal.

- oleraceus, Hal. Gen. dist.

* capito, Nees.

$\$$ - morosus, Reinh.

Genus, Dolops, Marsh.

- hastifer, Marsh. Yelverton, August

- aculeator, Marsh. Teignmouth and Cornworthy

\section{Oprides}

Genus, Hedylus, Marsh.

†- habilis, Marsh.

Genus, Opius, Wesm.

- apiculator(?) Nees. Stoke Wood, nr. Exeter (Parfitt)

- spretus, Hal. Marsh Mills; Bishopsteigmton

- tacitus, Hal. Bred from Dipterous larvae

- analis, Wesm. Bred from oak leaves gathered from the ground in winter

†- saevus, Hal.

\$- maculipes, Wesm.

$\$$ - cingulatus, Wesm.

†- irregularis, Wesm.

Parvulus, Wesm. Bred from Oecophoro fulviguttella $\$$ - zelotes, Marsh.

†- nitidulator, Nees.

- reconditor, Wesm. Bickleigh

- caelatus, Hal. Barnstaple (Marshall)

- rufipes, Wesm. Cornworthy (Marshall)

- comatus, Wesm. Plympton

Genus, Biosteres, Först.

- carbonarius, Nees. Ivybridge

- rusticus, Hal. Beesands

- impressus, Wesm. Cormworthy (Marshall)

Genus, Diachasma, Först.

- cephalotes, Wesm. Slapton

\section{EXODONTES}

\section{Alysindes}

Genus, Chasmodon, Hal.

t- apterus, Nees.

Genus, Alloea, Hal.

- contracta, Hal. Bred from fallen oak leaves

Genus, Alysia, Latreille

- manducator, Panz. Bred from larva feeding on carrion

- tripulae, Scop. Exeter; Yelverton

t- incongrua, Nees. Nr. Plymouzh

- Iucicola, Hal. Devon (Marshall)

†- truncator, Nees. Exeter (Hellins)

\section{EXODONTES (continued)}

Arysidess (continued)

Genus, Pentapleura, Först.

- pumilio, Nees.

Genus, Aphaereta, Först.

- cephalotes, Hal.

Genus, Phaenocarpa, Först.

-

†- pullata, Hal. ${ }^{1}$

- picinervis, Hal.

†- Eugenia, Hal. Seaton; C. W. Dale

- conspurcator, Hal. ${ }^{1}$

Genus, Adelura, Forst.

†- Isabella, Hal.

- - apii, Cur. A parasite on the larva of the celeryfly

Genus, Mesocrina, Furst.

- pugnatrix, Marsh. Cormworthy (Marshall)

Genus Aspilota, Först.

- ruficornis, Nees.

- concinna, Hal. (Bred from old wasps' nests

- nervosa, Hal. $\{$ infested with a dipteron,

- fuscicornis, Hal. Bickleigh

- distracta, Nees. Vinstone Nursery

\section{Dacnusides}

Genus, Oenone, Hal.

- hians, Nees.

- ringens, $\mathrm{Hal}$

Genus, Dacnusa, Hal.

- adducta, Hal. Bishopsteignton (Marshall)

- senilis, Nees.

$\$$ - petiolata, Nees.

- lepida, Marsh. Bishopsteignton (Marshall)

- aphanta, Marsh. Nr. Plymouth (Marshall)

$\$$ - stramineipes, Hal

- _ areolaris, Nees.

†- leptogaster, Hal.

Genus, Gyrocampa, Först.

$\S$ - foveola, Hal.

Genus, Chorebus, Hal.

t- limoniadum, Marsh.

Genus, Chaenusa, Hal.

- conjungens, Nees. Not uncommon in swampy places

Genus, Coelinius, Nees.

†- viduus, Cur.

- niger, Nees.

- - elegans, Hal.

Genus, Chaenon, Curtis

$\$$ - anceps, Cur.

Genus, Polemon, Giraud

- liparae, Giraud. Bred from a dipteron, Lipara lucens

\section{FLEXILIVENTRES}

\section{Aphididides}

The following groups are interesting on account of their parasitism ; the whole of them live within the body of the several species of Aphis, commonly called green-fly.

1 Parasites on the larvae of dipterous flies that feed in manure and other refuse heaps. 


\section{A HISTORY OF DEVONSHIRE}

\section{FLEXILIVENTRES (continued)}

Aphidides (continued)

Genus, Praon, Hal.

The larva of this genus, when full-fed, leaves its host and forms a tent-like structure under the body.

* - abjectum, Hal.

* - longicorne, Marsh.

* flavinode, Hal.

* - volucre, Hal.

Genus, Ephedrus, Hal.

The following do not leave the host, like the preceding, the metamorphosis taking place within the body.

* validus, $\mathrm{Hal}$.

- lacertosus, Hal.

- plagiator, Nees.

Genus, Toxares, Hal

* - deltiger, Hal.

Genus, Monoctonus, Hal.

* - caricis, $\mathrm{Hal}$.

Genus, Trioxys, Hal.

- betulae, Marsh. Not common

* - angelicae, $\mathrm{Hal}$.

Genus, Aphidius, Nees.

$\S$ - abietis, Marsh.

- pini, Hal.

* - urticae, Hal.

- gregarius, Marsh.

* - rosae, Hal.

* lonicerae, Marsh.

* - avenae, Hal.

- - ervi, Hal.

* - ulmi, Marsh.

- pascuorum, Marsh.

- pterocommae, Marsh.

- granarius, Marsh.

* - crepidis, Hal.

\section{FLEXILIVENTRES (continued)}

ApHididess (continued)

Genus, Aphidius, Nees.

* - pseudoplatani, Marsh.

- - sonchi, Marsh.

- ribis, Hal.

* _ cirsii, Hal.

* - hortensis, Marsh.

- chrysanthemi, Marsh.

* - matricariae, Hal.

* - cardui, Marsh.

* - salicis, Hal.

* - scabiosae, Marsh.

* - brassicae, Marsh.

* fabarum, Marsh.

* dauci, Marsh.

- polygoni, Marsh.

In the following eight species of Aphidius the females are unknown.

$\S$ - silenes, Marsh.

— crithmi, Marsh.

* - absinthii, Marsh.

* - euphorbiae, Marsh.

* lychnidis, Marsh.

* - cerasi, Marsh.

†- acalephae, Marsh.

t- callipteri, Marsh.

Genus, Dyscritus, Marsh.

†- planiceps, Marsh.

Evamindae

Genus, Hyptia, Illiger.

†- minuta, Fab.

Genus, Foenus, Fabricius

* - jaculator, Lin.

*_ assectator, Lin.

\section{OXYURA}

This group of parasitic flies has been much neglected since the days of Haliday, no doubt from their small size ; many are not so large as an ordinary Aphis, and being parasites on small insects, the opportunity of breeding does not often occur.

This list contains only forty-one species, these are in the writer's collection, out of 373 enumerated in the Rev. T. A. Marshall's 'Catalogue of the Oxyura,' published by the Entomological Society of London, in 1873 , and it is impossible to say whether certain species are common or not.

\section{Proctotrypidar}

Proctotrypes gravidator, Lin.

- niger, Panz.

- ater, Nees.

- longicornis, Nees.

- pallidipes, Jurine

- viator, $\mathrm{Hal}$.

- calcar, Hal.

- aculeator, Hal.

\section{Ceraphronidat}

Lygocerus Carpenteri, Cur.

Megaspilus abdominalis, Boh.

- alutaceus, Thoms.

- thoracicus, Boh.

\section{BethyLIDAR}

Cephalanomoea formiciformis, Westw. ${ }^{1}$

Perisemus triareolatus, Walk.

\section{Dryinidae}

Chelogynus ephippiger, Dalm.

- frontalis, Dalm.

Antaeon flavicornis, Dalm.

- infectus, Walk.

Aphelopus melanoleucus, Dalm.

1 Bred from beetles Pentarthrum Huttonl, or Anobium domesticum, probably from both, from one piece of decayed wood in which both beetles were plentiful, October.

\section{EMbolimidae}

Embolimus Ruddi, Wesm.

\section{HrLoridar}

Helorus anomalipes, Panz. - coruscus, Först.

\section{Belytidae}

Belyta sanguinolenta, Nees.

- nigriventris, Thoms.

— validicornis, Thoms.

- depressa, Thoms.

Cinetus filicornis, Thoms. 


\title{
INSECTS
}

Diaprildar Diaprildae (cont.)

Platymischus dilatatus, Westw. Paramesius rufipes, Westw. Taken under seaweed at - belytoides, Marsh.

Plymouth, abundant on rocks, - claviscapus, Thoms.

Torquay, September

Galesus fuscipennis, Cur.

- clypeatus, Cur.

Aneurrhynchus oviventris, Thoms.
- elongatus, Thoms.

Spilomicrus nigripes, Thoms.

Diapria conica, Fab.

\author{
Diaprindar (cont.) \\ Basalys fumipennis, Westw. \\ Loxotropa tritoma, Thoms. Cap- \\ tured I I May, I89I, on the \\ coast, under a stone, in the \\ nest of a small ant, Tetra- \\ morium caespitum. \\ - parva, Thoms.
}

\section{CHALCIDIDAE}

The Chalcididae consist chiefly of beautiful metallic coloured insects, green and golden-green predominating. Those obtained from galls are certainly hyperparasites. Those attacking pupae are no doubt direct parasites. Chalcids have been bred from all orders of insecta, and it may be safely said that there are hundreds if not thousands in collections awaiting identification.

Syntomaspis caudatus, Brischke. Bred from galls of of Biorhiza terminalis and Cynips Kollari

- cynaeus, Bris. Bred from galls of Dryophanta verrucosa

Pteromalus tibialis, West. Bred from galls of Andricus nudus; A. ostreus; A. pilosus ; Neuroterus tricolor; N. albipes; $N$. vesicatrix ; and Dryophanta divisa

- incrassatus, Ratz. Bred from galls of Dryophanta divisa; Cynips Kollari; and Neuroterus tricolor

- puparum, Lin. Bred from a pupa of the small tor toise-shell butterfly ( $V$ anessa urticae), and from pupa of the small white butterfly (Pieris rapae)

Pteromalus omnivorium, Walker. Bred from pupa of small white butterfly (Pieris rapae), and from an unknown noctua pupa, found at Loddiswell, 25 July, I 896

- (unnamed) hyperparasite. Bred from pupae of the whirligig beetle, Gyrinus natator, Io Jaly, I 884 ; pupae found attached to the common rush (Juncus conglomeratus) on the bank of the Exeter Canal by the late Rev. J. Hellins

Torymus abdominalis, Fons. Bred from Biorhiza terminalis; Cynips Kollari ; Andricus curvator; A.ostreus; Neuroterus lenticularis; Dryophanta longiventris ; and $\mathrm{D}$. divisa

- regius, Nees. Bred from Biorhiza terminalis ; Cynips Kollari; Dryophanta folii; and D. longiventris

- auratus, Fons. Bred from Neuroterus baccarum; N. tricolor; Andricus ostreus; A. curvator; and $A$. quadrilineatus

- erucarum, Gir. Bred from Andricus radicis

- corticis, Gir. Bred from Andricus corticis

- bedeguaris, L. Bred from Rhodites rosae

Callimome dauci, Curtis. Bred from galls on wild carrot
Megastigmus dorsalis, Fab. Bred from Andricus corticis and A. fecundatrix

- stigmaticans, Fab. Bred from Cynips Kollari

- giganticus, Kollar. Bred from Cynips Kollari

Platymesopus tibialis, West. Bred from Neuroterus aprilinus

Eulophus gallarum, Nees. Bred from Biorhiza terminalis; Andricus cirratus; and A. ramuli

- euedoreschus, Walker. Bred from Andricus fecundatrix. Not appearing until March or April of the third year

Eurytoma rosae, Nees. Bred from Cynips Kollari; Andricus Sieboldi; A. corticis; Neuroterus tricolor; Dryophanta divisa ; and D. longiventris - aethiops, Ratz. Bred from Rhodites rosae

- diastrophi, Gir. Bred from Diastrophus rubi, 22 June, 1885

- tristis, Mayr. Bred from Urophora cardui

Eutyrtus scaurus, Walker. Bred from pupa of Depressaria nervosa

Copidosoma chalconotum, Dalm. Bred seventy-three from pupa of Depressaria nervosa

Decatoma biguttata, Swederus. Bred from Cynips Kollari and Dryophanta folii

Perilampus pallipes, Curtis. Captured 5 May, 1897, in Bickleigh Wood

Tridymus salicis, Nees. Bred from a hovering fly (Syrphus); larva or pupa found on lime tree, Io August, $188_{4}$

Cerapterocerus mirabilis, West. Bred 13 September, 1884, from coccus Eriopeltis festucae, found on fescue-grass ; has been mistaken for the cocoon of an Apanteles

Caratomus megacephalus, Fab. Taken 29 July, 1882, on cobwall at Exeter, where several species of bees and wasps found a home

Encyrtus clavellatus, Dalm. Bred from Cecidomyia salicis

\section{HYMENOPTERA ACULEATA}

\author{
Ants, Wasps, Bees, Etc.
}

The climate and geological formation of the county of Devon should without doubt make it a grand hunting ground for the lovers of this section of insect life. Although in and around Plymouth, the residence of the writer, the limestone formation and the granite on the moors do not contribute towards the requirements of these insects, yet the neighbourhood of Exeter must be an ideal place, also the Starcross and Dawlish sand dunes. As the burrowing wasps and bees form the great majority of these insects, it must be a grand home for them, 


\section{A HISTORY OF DEVONSHIRE}

and as the locality is well represented with horticulturists they have the next essential thing, food. A choice variety of flowers is grown in abundance for those requiring honey or pollen, and where there is abundance of flowers it follows that an abundance of Aphides will be found; those using these green flies for food will have a good supply, and so will others find the food they require, whether it be caterpillars, beetles, flies, or spiders.

Unfortunately there is no one in the county that I am aware of who has devoted his whole attention to this order, consequently it cannot be said to have been properly and systematically worked. Devonshire was one of the favoured counties of the older entomologists, Leach, Curtis, and Stephens, for these names we often meet in old records. As Leach resided at Spichwick, which is only a short distance from Exeter, I would suggest that the three worked together occasionally during their holidays.

A few of Leach's bees have not been observed since his time, and there is some doubt about the correctness of these records, as he was known to have collected on the Continent.

\section{HETEROGYNA}

FORMICIDAE Ants

Formica, Linn.

* - rufa, Linn. Race congerens, occasionally found in the same localities

$\$$ - exsecta, Nyl.

*- fusca, Linn.

$\$$ - do. race cunicularia, Latr.

"Lasius, fuliginosus, Latr.

"- niger, Linn.

*- do. race alienus, Först.

- - umbratus, Nyl.

\$- do. race mixtus

**- flavus, De Geer.

§Tapinoma erraticum, Latr.

\section{Poneridae}

†Ponera contracta, Latr.

\section{Myrmicidar}

$\$$ Myrmecina Latreillei, Curt.

*Tetramorium caespitum, Linn.

†Stenamma Westwoodi, West.

\$Leptothorax acervorum, Fab.

$\$$ - tuberum, Fab.

- do. race unifasciatus, Latr.

**Myrmica rubra, Linn. Its races are gen. dis.

- race I, sulcinodis, Nyl.

- „ 2, ruginodis, Nyl.

- " 3, laevinodis, Nyl.

- " 4, scabrinodis, Nyl.

- " 5, lobicornis, Nyl.

\section{Introduced Species}

Prenolepis vividula, Nyl. Found at the root of an imported palm, July, 1895 (a colony; no female was found when search was made, but eggs were observed) ${ }^{1}$

Monomorium Pharaonis, Linn. Too plentiful to be pleasant in bakehouses and private residences in and around $P$ lymouth

1 Workers have been known to produce eggs when the female has been destroyed; these would produce males only. G.C.B.

\section{HETEROGYNA (cont.) \\ MYrMicidae (cont.)}

†Tetramorium simillimum, Smith. In a hothouse at $\mathrm{Mr}$. Parkin's, Union Road, Exeter. (Parfitt)

Cremastogaster scutellaris, Oliv. Found I Nov., 1892, in imported apples

\section{FOSSORES}

\section{Mutillidar}

†Mutilla europaea, Linn. \$Myrmosa melanocephala, Fab. †Methoca ichneumonides, Latr.

\section{Tiphindar}

$\S$ Tiphia femorata, Fab.

*- minuta, V.d. Lind.

\section{SAPYGIDAB}

\$Sapyga 5-punctata, Fab. $\$$ - clavicornis, Linn.

\section{PoMpILIDAE}

$\S$ Pompilus unicolor, Spin.

\$- bicolor, Lep.

*- rufipes, Linn.

$\$$ - cinctellus, Spin.

$\$$ - plumbeus, Fab.

- niger, Fab.

- - viaticus, Linn.

\$- minutulus, Dahlb.

$\S$ - spissus, Schiödte

$\$$ - chalybeatus, Schiodte

- gibbus, Fab.

$\$$ - pectinipes, Linn.

"Salius fuscus, Linn.

\$- affinis, V. de Lind.

- - exaltatus, Fab.

- notatulus, Saund.

* - pusillus, Schiodte

$\$$ - parvulus, Dahlb.

$\$$ Calicurgus hyalinatus, Fab.

Ceropales maculatus, $\mathrm{Fab}$.

\section{Sphegidae}

\$Astatus boops, Schr.

"Tachytes pectinipes, Linn. †- unicolor, Panz.
FOSSORES (cont.)

SPHEGIDAE (cont.)

§rypoxylon figulus, Linn.

- clavicerum, Lep.

- attenuatum, Sm.

*Ammophila sabulosa, Linn.

$\$$ - campestris, Latr.

* - hirsuta, Scop.

"Spilomena troglodytes, V. de Lind.

§emphredon lugubris, Fab.

\$- Shuckardi, Mor.

$\$$ - Wesmaeli, Mor.

- morio, V. de Lind.

- - lethifer, Shuck.

§Diodontus minutus, Fab.

\$- luperus, Shuck.

*- tristis, V. de Lind.

§assaloecus corniger, Shuck.

$\$$ - gracilis, Curt.

$\$$ Mimesa equestris, Fab.

$\$$ - bicolor, Jur.

$\$$ - unicolor, V. de Lind.

$\$$ Psen pallipes, Panz.

*Gorytes mystaceus, Linn.

$\$$ - campestris, Linn.

$\$$ - bicinctus, Rossi.

"Nysson spinosus, Fab.

- interruptus, Fab. Not rare

\$ trimaculatus, Rossi.

$\$$ - dimidiatus, Jur.

*Mellinus arvensis, Linn.

Cerceris Rybyensis, Linn.

- - arenaria, Linn. Portlemouth, on slopes towards the sea, storing its cells with a beetle (Otiorynchus sukatus)

- 5-fasciata, Linn.

- L labiata, Fab.

*Oxybelus uniglumis, Linn.

$\$$ - mandibularis, Dahlb.

t- nigripes, Oliv.

t- mucronatus, Fab.

"Crabro tibialis, Fab.

- clavipes, Linn.

- - leucostomus, Linn.

$\$$ - cetratus, Shuck.

*- podagricus, V. de Lind.

†- palmarius, Schreb.

t- palmipes, Linn.

- varius, Lep. 


\section{INSECTS}

FOSSORES (cont.)

Sphrgidar (comt.)

†Crabro anxius, Wesm.

$\$$ - Wesmaeli, V. de Lind.

- elongatus, V. de Lind.

*- 4-maculatus, Fab.

*- dimidiatus, Fab.

"- vagabundus, Panz.

*- cavifrons, Thoms.

\$ chrysostomus, Lep.

*- vagus, Linn.

- cribrarius, Linn.

- peltarius, Schreb.

$\$$ - lituratus, Panz.

*- interruptus, De Geer.

†Entomognathus brevis, V. de Lind.

\section{DIPLOPTERA}

VESPIDAB

-Vespa crabro, Linn. Local

*"- vulgaris, Linn.

* - germanica, Fab.

- - rufa, Linn.

$\$$ - sylvestris, Scop. Gen. dis.

- norvegica, Fab. Occasionally throughout Devon ${ }^{1}$

\section{Eumenidar}

-Odynerus spinipes, Linn.

t- melanocephalus, Gmel.

$\$$ - laevipes, Shuck.

\$- reniformis, Gmel.

* callosus, Thoms.

- parietum

* pictus, Curt.

\$ - trimarginatus, Zett.

$\$$ - parietinus, Linn.

\$- Antilope, Panz.

\$- gracilis, Brullé

- sinuatus, Fab.

Eumenes coarctata, Linn. Local

\section{ANTHOPHILA}

Subdivision I-OBTUSILINGUES

\section{Collgtidar}

*Colletes succincta, Linn.

*- fodiens, Kirb.

*- picistigma, Thoms.

- marginata, Sm.

- Daviesana, Sm.

§rosopis communis, Kirb.

$\$$ - signata, Panz.

$\$$ - punctulatissima, Sm.

* - hyalinata, Sm.

- confusa, Nyl.

*- brevicornis, Nyl.

t- pictipes, Nyl.

1 Nests generally built in gooseberry bushes in this county, in Scotland in the furze. The writer removed one from a gooseberry bush : the nest was rebuilt, to all outward appearance, in seven days. G.C.B.

\section{ANTHOPHILA (cont.)}

Subdivision II-ACUTILINGUES

\section{Andrenida}

Sphecodes gibbus, Linn.

- subquadratus, $\mathrm{Sm}$.

$\$$ - spinulosus, V. Hag.

$\$$ - puncticeps, Thoms.

* pilifrons, Thoms.

- - similis, Wesm.

t- ferruginatus, Schk.

- affinis, V. Hag.

- hyalinatus, Schk. Sidmouth (Perkins)

- variegatus, V. Hag. Sidmoutb (Perkins)

"Halictus rubicundus, Chr.

§- xanthopus, Kirb.

*- leucozonius, Schr.

- zonulus, Sm.

- quadrinotatus, Kirb.

*- laevigatus, $\mathrm{Kirb}$.

t- sexnotatus, Kirb.

"- cylindricus, Fab.

- albipes, Kirb.

t- subfasciatus, Nyl.

*- villosulus, Kirb.

- breviceps, Saund.

$\S$ - punctatissimus, Schk.

- nitidiusculus, Kirb.

- minutus, Kirb.

$\$$ - minutissimus, Kirb.

* tumulorum, Linn.

†- gramineus, Smith

* Smeathmanellus, Kirb.

- morio, Fab.

t- leucopus, Kirb. Local

*Andrena albicans, Kirb.

- pilipes, Fab.

\$- tibialis, Kirb.

f— bimaculata, Kirb.

$\$$ - rosae, Panz.

\$- florea, Fab.

*- thoracica, Fab.

* nitida, Fourc.

- cineraria, Linn.

\$- fulva, Schr.

"- nigroaenea, Kirb.

- - Gwynana, Kirb.

- angustior, Kirb.

†— praecox, Scop.

*- varians, Rossi

\$- helvola, Linn.

\$- fucata, Sm.

\$- nigriceps, Kirb.

S- simillima, Sm.

t- fuscipes, Kirb.

- denticulata, Kirb.

*- fulvicrus, Kirb.

†- fasciata, Nyl.

t- bucephala, Steph

*- Hattorfiana, Fab.

\$- Cetii, Sehr.

$\$$ - cingulata, Fab.

$\$$ - humilis, Imh.

$\$$ - labialis, Kirb.

$\$$ - chrysosceles, Kirb.

t-Coitana, Kirb.
ANTHOPHILA (cont.)

Subdivision II-ACUTILINGUES (cont.)

Andremidar (comt.)

-Andrena fulvago, Chr.

* albicrus, Kirb.

- minutula, Kirb.

* - nana, Kirb.

$\S$ - proxima, Kirb.

*- dorsata, Kirb.

$\$-$ similis, Sm.

- Wilkella, Kirb.

- Afzeliella, Kirb.

*Dasypoda hirtipes, Latr.

†Cilissa haemorrhoidalis, Feb.

$\$$ - leporina, Panz.

- Panurgus calcaratus, Scop.

- - ursinus, Gmel.

†Nomada obtusifrons, Nyl.

- solidaginis, Panz.

- succincta, Panz.

\$ lineola, Panz.

- - alternata, Kirb.

- - jacobaeae, Panz.

t- Lathburiana, Kirb.

- - ruficornis, Linn.

- - bifida, Thoms.

- borealis, Zett.

- Iateralis, Panz.

- ochrostoma, Kirb.

* armata, H.-S.

- ferruginata, Kirb.

* Fabriciana, Linn.

"- flavoguttata, Kirb.

- furva, Panz.

\section{Apidae}

-Epeolus rufipes, Thoms.

- productus, Thoms.

\$Ceratina cyanea, Fab.

Chelostoma florisomne, Linn. Local, not scarce

†Coelioxys conoidea, Illig.

†- quadridentata, Linn.

\$- rufescens, Lep.

\$- elongata, Lep.

$\S$ - acuminata, Nyl.

Megachile maritima, Kirb.

- Willughbiella, Kirb.

- circumcincta, Lep.

\$- ligniseca, Kirb.

* - centuncularis, Linn.

†- versicolor, Sm.

$\$$ - argentata, Fab.

- Osmia rufa, Linn.

$\$$ - pilicornis, Sm.

\$- xanthomelana, Kirb.

$\$$ - parietina, Curt.

$\$$ - coerulescens, Linn.

- Leaiana, Kirb.

- aurulenta, Panz. Local, bred from whelk shells

*- leucomelana, Kirb.

§- spinulosa, Kirb.

§Stelis aterrima, Panz.

*- phoeoptera, Kirb.

\$- 8-maculata, Sm. 


\section{A HISTORY OF DEVONSHIRE}

ANTHOPHILA (cont.)

Subdivision II-ACUTILINGUES
(cont.)
ApIDAE (cont.)
\$Anthidium manicatum, Linn.
\$Eucera longicornis, Linn.
\$Melecta luctuosa, Scop.
"- armata, Panz.
"Anthophora pilipes, Fab.
*- furcata, Panz.
\$— quadrimaculata, Panz.
"Saropoda bimaculata, Panz.
"Psithyrus rupestris, Fab.
\$- vestalis, Fourc.

ANTHOPHILA (cont.)

Subdivision $I I-A C U T I L I N G U E S$

Apidae (cont.)

*Psithyrus Barbutellus, Kirb.

* - campestris, Panz.

* Bombus venustus, Sm.

* agrorum, Fab.

- Latreillellus, Kirb.

*- hortorum, Linn.

$\S$ - Jonellus, Kirb.

- pratorum, Linn.

- lapponicus, Fab. Not rare

- - sylvarum, Linn.
ANTHOPHILA (cont.)

Subdivision II-ACUTILINGUES (cont.)

Apidar (cont.)

*Bombus Derhamellus, Kirb.

†- soroensis, Fab.

* - lapidarius, Linn.

* - terrestris, Linn.

Apis mellifica, Linn. Honey-bee'

1 On very rare occasions they have been known to suspend their comb from a branch of a shrub growing in the open garden; such did occur at Tavistock in I gor; the deserted comb was about three inches in length. G. C. B.

\section{CHRYSIDIDAE}

\section{Hymenoptera Tubulifera}

There are only about twenty-five species in England, and only six are recorded for the county; two others, however, occur just within the Cornish boundary in the north. These beautiful flies with their brilliant metallic colours do not appear to have been collected-or are these parasitic flies comparatively scarce or rare in the districts visited by collectors? The bees are well represented; consequently it is surprising to find so few in the collections of the entomologists that have travelled over the country.

Specimens of Hedycbridium minutum are to be obtained in July by visiting the cliffs near Bovisand; roseum was observed at Rickham, near Portlemouth, II July; Chrysis neglecta and viridula were bred from the nests of Odynerus spinipes; Cbrysis cyanea have been obtained at Exminster and Loddiswell; Ruddii and pustulosa were obtained at Millhook in North Cornwall, a short distance out of the county; while ignita is generally distributed, infesting several species of bees and wasps. These beautiful coloured flies are parasites, the parent depositing her egg on the food of the bee or wasp. The eggs of the latter hatch ; the larvae consume the food stored by the parent, and when full fed the egg of the parasitic fly is developed; it then attacks the aculeate larva, and very soon sucks its victim dry ; consequently the cell is then tenanted by the parasitic larva; it changes to pupa in the following spring, and ultimately emerges a perfect fly from the cell that should have produced a wasp or bee. These changes can be observed by placing the aculeate larva, with parasitic egg, in a small glass cylinder of the same size as the bee or wasp cell (should be kept cool and from the light).

\section{COLEOPTERA}

\section{Beetles}

The county of Devon is decidedly rich in Coleoptera, and as large tracts of country have not yet been worked, a considerable number of species will certainly in time be added to the list. The high ground of Dartmoor, the rich park-land and woods of the interior, and the double stretch of coast-line north and south, including the so called 'Burrows,' especially Northam Burrows (now best known as Westward Ho!) and Braunton Burrows, are all very productive, and the lake and marshy ground at Slapton Ley are well known as the habitat of several scarce species. Bidessus minutissimus and Scopaeus rubidus have only occurred in Britain in this locality, and Pbilonthus punctus has only been taken in two or three other places. The chief Devon species is perhaps Carabus intricatus, which may still be found in its old locality, as is proved by the capture of two specimens in 1898 by Mr. J. H. Keys. Mr. Keys has added a large number of good species to the Devon list, and I am much indebted to him for a list of his captures and for other information. I have made considerable use of Mr. Parfitt's catalogue of the Coleoptera of Devon, which, I think, is fairly accurate, as so many of his species have since been confirmed, and my thanks are also due to the Rev. A. Thornley and Mr. Thouless. Dr. Power's captures at Seaton and in the Lee valley are of great interest, and the late Mr. Vernon Wollaston and Mr. F. Smith did much good work at Slapton Ley and in other localities. It is of course well known that Dr. Leach resided in Devonshire, and that Stephens quotes many of his records. Many of these have been considered doubtful, but in the face of recent captures I am inclined to think that most of them are right, and that even Agabus brunneus will again be discovered 


\section{INSECTS}

somewhere in South Devon. Several of the beetles which have been recorded from Plymouth have really occurred at Whitsand Bay, which is only just over the border line. The chief of these I have noted in footnotes. Lundy Island possesses a fair number of species; of these Psylliodes luridipennis and Melanopbthalma distinguenta appear to be peculiar to the island.

Among the beetles especially worth recording may be mentioned the following: Calosoma sycopbanta and Diacbromus germanus (both doubtfully indigenous), Dinarda dentata, Actocharis Readingi, Tacbinus rufipennis, Quedius longicornis, Medon ripicola, Emus birtus, Pbilonthus astutus, some very good species of Colon, Meligethes, and Melö̈, Gnorimus nobilis, Microrrbagus pygmaeus, Agelastica alni, Strangalia aurulenta, Coenopsis fissirostris, Exomias pyrenaeus, Gymnetron rostellum, etc. At Seaton Dr. Power captured Scydmaenus poweri, a quite new species described by myself, and recently Mr. Frank Bouskell has recorded the occurrence of Apbodius sturmi, an insect new to the British list, near Plymouth, where it was taken by Mr. J. H. Keys.

Mr. Keys has published a very interesting paper in the transactions of the Plymouth Institution, in which he refers to the northern and hill country species which he has found in the Plymouth district, chiefly on or near Dartmoor. The insects noted in his paper are the following: Hydroporus septentrionalis, Hydraena atricapilla, Coccinella 5-punctata (a very remarkable capture), Barynotus Scbönherri, Pterosticbus aetbiops, 2uedius auricomus and its variety quadriguttatus and Corymbites aeneus, and to these he has added Perileptus areolatus, Gnypeta caerulea, Silpha nigrita, and quite recently Homalota eremita: the occurrence of these northern forms in the extreme south is very interesting, being even more striking than the capture of many of the northern forms in the midlands by the late Mr. W. G. Blatch. Doubtless, as Mr. Keys remarks, many of the northern beetles which tenanted Dartmoor have become extinct; at the present time Pterostichus aethiops only appears to occur in a limited space on the top of one particular hill. Observers therefore are much needed in these localities.

\section{CICINDELIDAE}

Cicindela campestris, $\mathrm{L}$.

- hybrida, L. var. maritima, Dej. Brouncon Burrows

- germanica, L Seaton, June, 1895 (P. de la Garde, R.N.)

\section{CARABIDAE}

Cychrus rostratus, L. Woods near Plymouth, Dartmoor

Carabus intricatus, $L$. In woods under moss and lichens, on trunks of old oaks or under bark or logs, Tavistock, Holswortby, Asbburton, Bickleigh Vale, Torquay district. A large number of specimens were taken by Mr. Reading some forty or fifty years ago, and Mr. Wollaston captured a fine example on 18 April, 1872, about two miles from Newton Abbot on the road to Torquay. In May, I898, Mr. Keys took two specimens in the Plymouth district. It is one of the most characteristic of the Devon insects.

- catenulatus, Scop.

- nemoralis, Múil.

- violaceus, $L$.

var. exasperatus, Duft. Asbburton

- auratus, L. Recorded taken at Exmouth and 'on the coast at Torquay about I 852' (R. Lyddeker)

- granulatus, L.

- arvensis, F. Dartmoor (Thouless)

Calosoma inquisitor, L. Irybridge (J. H. Keys)

- sycophanta, L. Nr. Dartmouth (Leach); Ugborough Beacon, an elytron in a roadway $2 \mathrm{I} \mathrm{July,}$ 1862 (Reading). The following note by Mr. J. Brooking Rowe appears in the Entomologists' Monthly Magazine, October, I872: -A fine specimen of Calosoma sycophanta was taken by a friend of mine, Dr. Harper, last week. He saw it flying in Exeter Street, $P$ lymouth, and on its alighting on a wall he caught it in his hand (receiving a nip from the insect), and brought it home in his glove.

\section{CARABIDAE (continued)}

Notiophilus bigattatus, $\mathbf{F}$.

- substriatus, Wat.

- quadripunctatus, Dej. Plymouth, Exeter

- aquaticus, $\mathrm{L}$.

- palustris, Duft.

Leistus spinibarbis, $F$.

- fulvibarbis, Dej.

- ferrugineus, L.

Nebria complanata, L. Barnstaple, Bideford, Woolecombe sands near Ilfracombe (Power)

- brevicollis, $\mathrm{F}$.

Blethisa multipunctata, L. Slapton Ley (Wollaston)

Elaphrus riparius, L.

- cupreus, Duft.

- uliginosus, F. Shapton Ley (Wollaston)

Clivina fossor, Linn.

- collaris, Herbst.

Dyschirius thoracicus, Rossi. Exmouth Warren

- impunctipennis, Daws. Dawlish, Braunton Burrows

- salinus, Schaum. Exmouth Warren

- aeneus, Dej. Exmouth Warren

- politus, Dej. Braunton Burrows

- globosus, Herbst.

Broscus cephalotes, L. Braunton Burrows

Panagaeus crux-major. Bamstaple, under moss, etc., on alders (W. Matthews)

- quadripustulatus, Sturm. Recorded by Parfitt from near Plymouth, but ' whether actually in the county or in Cornwall' he is not sure

Badister unipustulatus, Bon. Barnstaple

- bipustulatus, $\mathrm{F}$.

- sodalis, Duft.

Chlaenius vestitus, Payk. Plymouth, Barnstaple, Sidmouth, Banks of Exe, Slapton Ley

- nigricornis, $F$.

var. melanocornis, Dej. Valley of the Tavy (Thurlestone)

- Oodes helopioides, F. Under moss, Barmstaple (Matthews) 


\section{A HISTORY OF DEVONSHIRE}

\section{CARABIDAE (continued)}

Acupalpus exiguus, Dej. ; var. luridus, Dej.

- meridianus, $\mathrm{L}$.

Bradycellus distinctus, Dej.

- verbasci, Duft.

- harpalinus, Dej.

- similis, Dej.

Harpalus sabulicola, Panz. Exmoutb (Thouless)

$\ddagger$ - punctatulus, Duft. Exeter (Parfitt)

- azureus, F. N. Devon, Plymouth

- puncticollis, Payk.

- ruficornis, $F$.

- aeneus, $F$.

- consentaneus, Dej. (attenuatus, Steph.)

- tenebrosus, Dej. Slapton Ley (Wollaston), Avonmouth

- rubripes, Duft.

†- discoideus, F. P多mouth

- latus, L.

- tardus, Panz.

- serripes, Schön. Blackdown

- ignavus, Duft. (honestus, Duft.)

- neglectus, Dej. Torrington (J. L. Henderson)

- pubescens, Payk.

Dichirotrichus obsoletus, Dej.

- pubescens, Payk.

Anisodactylus binotatus, $\mathrm{F}$. Ivar. atricornis, Steph. Bovey Heathfeld

- poeciloides, Steph.

Diachromus germanus, Er. Recorded many years ago by Dr. Leach as from Kingsbridge

Stomis pumicatus, Panz.

Pterostichus cupreus, $\mathrm{L}$. var. affinis, Sturm. Okehampton

- versicolor, Sturm.

- dimidiatus, Ol. Formerly not rare on Woodbury Common

- madidus, F.

- aetiops, Panz. Dartmoor

†- oblongo-punctatus, F. N. Devon (Matthews)

- cristatus, Duft. (parumpunctatus, Germ.). Woodbury Common

- niger, Schall.

- vulgaris, $\mathrm{L}$.

- anthracinus, III. Exeter.

- nigrita, $F$.

- minor, Gyll.

- strenuus, Panz.

- diligens, Sturm.

- inaequalis, Marsh. Batten, Bovey Heatbfield

- vernalis, Gyll.

- striola, F.

Amara apricaria, $F$.

— aulica, Panz. (spinipes auct. nec. L.)

- convexiuscula, Marsh

- livida, F. (bifrons, Gyll.)

- ovata, F.

- similata, Gyll.

- acuminata, Payk.

- tibialis, Payk.

- lunicollis, Schiödte

- familiaris, Duft.

- lucida, Duft.

- trivialis, Gyll.

- communis, Panz.

- plebeia, Gyll. ${ }^{1}$

1 Amara tibialis and $A$. continua have been recorded from Whitsand Bay.

\section{CARABIDAE (continued)}

Calathus cisteloides, Ill.

- fuscus, $F$.

- mollis, Marsh.

- melanocephalus, $L$.

- micropterus, Duft. Recorded by Parfitt from Exmouth Warren; it probably occurs on Dartmoor

- piceus, Marsh

Taphria nivalis, Panz.

Pristonychus terricola, Herbst.

Laemostenus complanatus, Dej. Plymouth (J. H. Keys)

Sphodrus leucophthalmus, L. Plymoutb

†Anchomenus augusticollis, F. Barnstaple (Matthews). The only record I can find for this usually common beetle, so it is evidently very scarce in Devon.

- dorsalis, Mull.

- albipes, $\mathrm{F}$.

- oblongus, $\mathrm{F}$ :

- marginatus, $L$.

- sexpunctatus, L. Barnstaple

- parumpunctatus, $\mathrm{F}$.

- atratus, Duft.

- viduus, Panz. var. moestus, Duft.

- scitulus, Dej. Bozly Heathfeld, not common

- fuliginosus, Panz.

- gracilis, Gyll.

- piceus, L. Slapton Ley

Olisthopus rotundatus, Payk.

Lymnaeum nigropiceum, Marsh. Batten, Phymoutb (J. H. Keys)

Tachys parvulus, Dej. Plymouth (J. H. Keys)

Cillenus lateralis, Sam.

Bembidium rufescens, Guér.

t- quinquestriatum, Gyll. Sioke Wood (Parfitt)

- obtusum, Sturm.

- guttula, F.

- mannerheimi, Sahl.

- biguttatum, F.

- riparium, Ol. (iricolor Bedel.)

- assimile, Gyll.

- minimum, F. Exmouth

- normannum, Dej.

- lampros, Herbst.

- tibiale, Duft. var. velox, Er.

- atrocoeruleum, Steph.

- decorum, Panz.

- nitidulum, Marsh.

- affine, Steph.

- monticola, Sturm. Valley of the Meary

- quadriguttatum, $\mathrm{F}$.

- quadrimaculatum, Gyll.

t- testaceum, Duft. Banks of the Teign

- concinnum, Steph. Yealmpton

- femoratum, Sturm.

- littorale, Ol.

- punctulatum, Drap. Valleys of the Meavy and Avon

I- varium, O1. (ustulatum, Sturm.), Banks of the Exe (Parfitt)

†- obliquum, Sturm. Exeter district

2 Tachys bistriatus, Duft., will probably be found, as it has occurred on the shores of the Bristol Channel. 


\section{INSECTS}

\section{CARABIDAE (continued)}

†Tachypus pallipes, Duft. Stoke Wood (Parfitt)

- flavipes, L.

Perileptus areolatus, Creutz. Valley of the Tary

Aepus marinus, Ström. Plymouth district, etc.

- robinii, Lab. Plymouth district

Trechus lapidosus, Daws. Lundy Island (Wollaston) ${ }^{1}$

t- rubens, F., Woodbury Common (Parfitt)

- minutus, $\mathrm{F}$.

- obtusus, Er.

Pogonus littoralis, Duft.

- chalceus, Marsh.

Cymindis axillaris, F. Sidmoutb (Thouless)

tLebia cyanocephala, L. Exeter district (Parfitt)

- chlorocephala, Hoff. Seaton (Power)

- crux-minor, L. High Buckington, Devon (Steph. Man. 33) ; said to have been taken in Devon by Mr. T. Vernon Wollaston

- haemorrhoidalis, F. Devon (Steph. Man, 35)

Demetrias unipunctatus, Germ.

- atricapillus, L.

Dromius linearis, $\mathrm{Ol}$.

- agilis, F.

- meridionalis, Dej.

- quadrimaculatus, L.

-- quadrinotatus, Panz.

- quadrisignatus, Dej. Slapton Ley (J. H. Keys)

- melanocephalus, Dej.

- nigriventris, Thoms.

- vectensis, Rye. Seaton (Power)

Blechrus maurus, Sturm.

Metabletus foveola, Gyll.

- obscuro-guttatus, Duft. Slapton Ley

Lionychus quadrillum, Duft. Slapion Ley; where an immaculate form occurs, quite different in appearance from the type (J.H. Keys)

Brachinus crepitans, L.

- sclopeta, F. Devon (Leach). Very doubtfully indigenous

\section{HALIPLIDAE}

Brychius elevatus, Panz.

Haliplus obliquus, $\mathrm{F}$.

- confinis, Steph.

- flavicollis, Sturm.

- fulvus, F.

- ruficollis, De G.

- fuviatilis, Aubé.

- lineatocollis, Marsh.

tCnemidotus impressus. Exminster Marsbes (Parfitt)

\section{DYTISCIDAE}

Noterus sparsus, Marsh.

Laccophilus interruptus, Panz.

- obscurus, Panz. (hyalinus, auct.)

Bidessus unistriatus, Schr. Devon (Stephens)

- minutissimus, Germ. Slapion Ley

- geminus, F. Dartmoor

Hyphydrus ovatus, $L$.

Caelambus versicolor, Schall. (reticulatus, F.)

- inaequalis, $\mathrm{F}$.

- confluens, $\mathrm{F}$.

- impressopunctatus, Schall. (picipes, F.)

1 As I have taken Trechus lapidosus, Sam., plentifully at Charmouth, just outside the eastern boundary of Devon, and Mr. Keys has taken it at Whitsand Bay just outside the western boundary, I have no doubt that it occurs all along the Devonshire coast, as well as on Lundy Island.

\section{DYTISCIDAE (continued)}

Deronectes latus, Steph. River Plym (J. H. Keys)

- assimilis, Payk. River Plym (Leach)

- depressus, $\mathrm{F}$.

- duodecim-pustulatus, F.

Hydroporus pictus, F. Exmouth; not common

- Havipes, Ol.

- lepidus, Ol. The Canal, Exeter

- rivalis, Gyll. Recorded by Dr. Leach (Steph. Mand., 5I 2). Taken by Mr. J. H. Keys nr. $P$ lym Bridge in 1894 (one example), and since then in some numbers in the Meavy, Tavy and West Dart by the same collector

- lineatus, $\mathrm{F}$.

- angustatus, Sturm.

- gyllenhalii, Schiodte

- palustris, L.

- incognitus, Sharp. Dartmoor (Sharp)

- erythrocephalus, L.

- rufifrons, Duft. Lundy Island

- longulus, Muils. (celatus, Clark). Iqybridge (J. H. Keys)

- memnonius, Nic. Lundy Island

- melanarius, Sturm. Dartmoor

- nigrita, F.

- discretus, Fairm. Exmouth (Thouless)

- pubescens, Gyll. (melanocephalus, Brit. Coll.)

- planus, $\mathrm{F}$.

- lituratus, F. Ivybridge, Plymouth, etc.

- marginatus, Duft. Marsh at Totbill, Plymoutb (J. H. Keys)

- ferrugineus, Steph. Once only, River Tavy (J. H. Keys)

- obsoletus, Aub. Dartmoor (J. H. Keys)

- oblongus, Steph. Once only, River Avon, Dartmoor (J. H. Keys)

Agabus guttatus, Payk.

_- biguttatus, Ol. Dartmoor, etc.

- paludosus, $\mathrm{F}$.

- brunneus, F. Taken originally, according to Stephens, by Dr. Leach in S. Devon. The record is very probably correct

- unguicularis, Thoms.

- didymus, Ol.

- nebulosus, Först. Lundy Island

- abbreviatus, F. Nr. Exeter (Parfitt)

Agabus sturmii, Gyll.

- chalconotus, Panz.

- bipustulatus, L.

Platambus maculatus, L.

Ilybius fuliginosus, $\mathrm{F}$.

- ater, De G.

Copelatus agilis, F.

Rhantus pulverosus, Steph. Exeter district

- bistriatus, Berg.

Colymbetes fuscus, $\mathrm{L}$.

Dytiscus punctulatus, F.

- marginalis, $L$.

Hydaticus transversalis, Berg. Recorded doubtfully Acilius sulcatus, $L$.

\section{GYRINIDAE}

Gyrinus urinator, Ill. Slapton Ley (Stephens), River Plym (J. H. Keys)

- bicolor, Payk. Exmouth, somewhat doubtful; perhaps in error for G. elongatus

- natator, Scop. 


\section{A HISTORY OF DEVONSHIRE}

\section{GYRINIDAE (continuedi)}

Gyrinus elongatus, Aub. Slapton

- marinus, Gyll.

Orectochilus villosus, Mull. Dartmoor

\section{HYDROPHILIDAE}

Hydrobius fuscipes, L.

Philhydrus testaceus, $\mathrm{F}$.

- melanocephalus, Ol.

- minutus, $\mathrm{F}$.

Enochrus bicolor, Gyll. Slapton Ley

Paracymus nigroaeneus, Sahl. Mary Tazy, Dartmoor

Anacaena globulus, Payk. (limbata, Sharp)

- limbata, F. (variabilis, Sharp). Lundy Island

Helochares lividus, Forst.

- punctatus, Sharp

Laccobius sinuatus, Mots (nigriceps, Thoms.)

- alutaceus, Thoms. Plymouth

Berosus signaticollis, Sharp (aericeps, Curt.)

- affinis, Brullé.

Limnebius truncatellus, Thoms.

- papposus, Muls.

- nitidus, Marsh.

Chaetarthria seminulum, Herbst.

Helophorus rugosus, $\mathrm{Ol}$.

- nubilus, $\mathrm{F}$.

- aquaticus, L.

- dorsalis, Marsh. Exeter district (Parfitt)

- affinis, Marsh.

- brevipalpis, Bedel. ${ }^{1}$

- nanus, Sturm. Exeter; very rare (!) (Parfitt)

Hydrochus elongatus, $F$.

- angustatus, Germ.

Henicocerus exsculptus, Germ. Dartmoor

Octhebius pygmaeus, $\mathrm{F}$.

- bicolon, Germ.

- Poweri, Rye. One specimen taken by Dr. Power at Seaton

Hydraena testacea, Curt. Dartmoor; Exminster

- nigrita, Germ.

- gracilis, Germ.

- atricapilla, Wat. Valley of the Meavy

- pygmaea, Wat. Valley of the Meavy

Cyclonotum orbiculare, $\mathrm{F}$.

Sphaeridium scarabaeoides, F.

- bipustulatum, $\mathrm{F}$. var. marginatum, $\mathrm{F}$.

Cercyon littoralis, Gyll.

- depressus, Steph. N. Devon (Power)

- haemorrhous, Gyll.

- haemorrhoidalis, Herbst.

- obsoletus, Gyll.

- aquaticus, Muls.

- flavipes, F.

- lateralis, Marsh.

- melanocephalus, $\mathrm{L}$.

- unipunctatus, L.

- quisquilius, L.

- nigriceps, Marsh.

- pygmaeus, Ill.

- terminatus, Marsh. Exmouth, in refuse on the sand (Parfitt), and Lundy Island

1 This species is not actually recorded from Devon, but it is the very common species which is sometimes named $H$ : griseus and sometimes $\mathrm{H}_{i}$ granularis. $\mathrm{H}$. affinis is the $\mathrm{H}$. griseus of Thomson and much scarcer than $\mathrm{H}$. brevipalpis, which is the $\mathrm{H}$. griseus of Erichson and the $\mathrm{H}$. granularis of Thomson.

\section{HYDROPHILIDAE (continued)}

Cercyon analis, Payk.

- lugubris, Payk.

- granarius, Thoms.

- minutus, Muls.

Megasternum boletophagum, Marsh.

Cryptopleurum atomarium, Muls.

\section{STAPHYLINIDAE}

Aleochara ruficornis, Grav. Devon (Stephens); under the name of Ceranota Daltoni (Kirby)

- fuscipes, $\mathrm{F}$.

- lata, Grav.

†- brevipennis, Grav. Dawvlish (Parfitt)

- tristis, Grav. Somewhat doubtful as a Devon insect

- bipunctata, OI.

- cuniculorum, Kr. Lundy Island

- lanuginosa, Grav.

- moesta, Grav.

- succicola, Thoms.

- nitida, Grav.

- morion, Grav.

- grisea, $\mathrm{Kr}$. Lundy Island (Wollaston)

- algarum, Fauv. (Kirbyi, Steph.)

- obscurella, Grav.

Microglossa suturalis, Mann.

- pulla, Gyll.

Oxypoda spectabilis, Märk. Ivybridge

- lividipennis, Mann.

- vittata, Märk.

- opaca, Grav.

- alternans, Grav.

- longiuscula, Er.

- formiceticola, Märk.

- haemorrhoa, Sahl.

- brachytera, Steph.

Thiasophila angulata, Er.

Ischnoglossa prolixa, Grav. relverton

Phloeopora reptans, Grav.

- corticalis, Grav.

Ocalea castanea, Er.

- latipennis, Sharp. Brickleigh Vale (J. H. Keys)

- badia, Er.

Ilyobates nigricollis, Payk.

$\ddagger$ - forticornis, Lac. Taken in Polyporus versicolor, Fordlands (Parfitt)

Calodera aethiops, Grav. Exeter district

- riparia, Er. Dartmoor

- umbrosa, Er. Plymoutb

Chilopora longitarsis, Er.

Dinarda dentata, Grav. In nests of Formica fusca and F. sanguinea. First found at $P$ lymouth by Mr. J. J. Reading, and afterwards recorded from Exeter as taken with $\mathrm{F}$. rufa

Atemeles emarginatus, Payk. Plymouth, in nests of Formica rufa (Reading)

† Myrmedonia humeralis, Grav. In nests of Formica rufa, Stoke Wood (Parfitt)

Astilbus canaliculatus, F.

Thamiaraea cinnamomea, Grav.

- hospita, Märk.

Notothecta flavipes, Grav.

- anceps, Er.

Alianta incanta, Er. Slapton Lev and Woodbury Common - plumbea, Wat. 


\section{INSECTS}

\section{STAPHYLINIDAE (continued)}

Homalota currax, Kr. Bickleigh Vale

- insecta, Thoms. Valley of the Tavy

- pavens, Er. Bickleigh Vale

- cambrica, Woll. Bickleigh Vale

t- planifrons, Wat. Watermouth, North Devon (Power)

- gregaria, Er.

- longula, Heer. Slapton Ley (Wollaston)

- luteipes, Er. Plymouth

- fallax, Kr.

- volans, Scriba.

- vestita, Grav.

- silvicola, Fuss. Ivybridge

- vicina, Steph. Mr. Keys has also taken at Plymouth the bituberculate form of the male

- graminicola, Gyll.

- halobrëctha, Sharp.

- algae, Hardy. (puncticeps, Thoms.)

- fungivora, Thoms.

- angustula, Gyll. Buckfast

- debilis, Er.

- circellaris, Grav.

- elegantula, Bris. Plymouth, South Brent

- aegra, Heer. Slapton Ley; somewhat doubtful

- cuspidata, Er.

- eremita, Rye. Dartmoor, valley of the Plym, nr. Ditsworthy Warren (J. H. Keys)

- analis, Grav.

- decipiens, Sharp

- depressa, Gyll.

- aquatica, Thoms. (subaenea, Sharp)

- zanthoptera, Steph.

- incognita, Sharp. Lee Valley, N. Devon (Power)

- euryptera, Kirby

- trinotata, $\mathrm{Kr}$.

- triangulum, $\mathrm{Kr}$.

- fungicola, Thoms.

- coriaria, $\mathrm{Kr}$.

— sodalis, Er.

- divisa, Marrk.

- nigricornis, Thoms.

- palustris, Kies.

$\ddagger$ - puberula, Sharp. Watermouth, North Devon (Power)

-- oblita, Er.

- sericea, Muls.

t- indubia, Sharp. Seaton (Power)

一 nigra, Kr.

- germana, Sharp

- cauta, Er. (parvula, Mann. ; parva, Brit. Coll. nec. Sahl.)

- villosula, Kr.

- macrocern, Thoms. Dartmoor; one specimen (J. H. Keys)

- atramentaria, Gyll.

- marcida, Er.

- longicornis, Grav.

- sordida, Marsh.

- testudinea, Er.

- aterrima, Grav.

- laticollis, Steph. (fusca, Sharp)

†- orbata, Er. Barrucane Bay, N. Devon

- fungi, Grav. var. dubia, Sharp var. clientula, Er.

- orphana, Er. Seaton Beach (Power)

\section{STAPHYLINIDAE (continued)}

Gnypeta labilis, Er.

- coerulea, Sahlb. Ivybridge (J. H. Keys) ${ }^{1}$

Tachyusa atra, Grav. Slapton Ley

Myrmecopra uvida, Er.

- sulcata, Kies.

Falagria sulcata, Payk.

†- sulcatula, Grav. Exeter; in the nest of Formica rufa (Parfitt)

- thoracica, Curt.

- obscura, Grav.

Autalia impressa, O1.

- rivularis, Grav.

Encephalus complicans, West. Lundy Island

Gyrophaena affiinis, Mann.

- fasciata, Marsh.

Agaricochara laevicollis, $\mathrm{Kr}$.

Epipeda plana, Gyll. Barnstaple

Actocharis Readingii, Sharp. Under seaweed, etc., usually below high-water mark. Plymouth (Reading and Wollaston). Mr. Keys has only taken it on the Cornish side, and also Phytosus spinifer and balticus, both of which probably occur in Devon

Leptusa analis, Gyll. Stowford Woods, Ivybridge, Bickleigh Vale, Mary Tavy

- fumida, Er.

Sipalia ruficollis, Er.

- testacea, Bris. Batten, Plymouth (J. H. Keys)

Bolitochara bella, Märk.

Diglossa mersa, Hal. Batten, Plymouth

Oligota inflata, Mann.

- pusillima, Heer. (ruficornis, Sharp)

- granaria, Er.

- apicata, Er.

Myllaena kraatzii, Sharp

- brevicornis, Matth.

Gymnusa brevicollis, Payk. Dartmoor

Hypocyptus longicornis, Payk.

- laevinsculus, Marsh.

- ovulum, Heer. Exmoor

- seminulum, Er.

- punctum, Mots (seminulum, Er. var.), Seaton

Conosoma littoreum, $\mathrm{L}$.

- pubescens, Grav.

- immaculatum, Steph.

- lividum, Er.

Tachyporus obtusus, L.

- formosus, Mat. Lundy Island

- solutus, Er.

- pallidus, Sharp

- chrysomelinus, L.

- humerosus, Er.

- tersus, Er.

- hypnorum, F.

- pusillus, Grav.

- brunneus, $\mathbf{F}$.

Cilea silphoides, $\mathrm{L}$.

$\ddagger$ Tachinus flavipes, F. Haldon (Parfitt)

I- rufipennis, Gyll. Barnstaple (E. Saunders), Dartmoor (J. H. Keys)

- humeralis, Grav.

- scapularis, Steph. Stoke Wood; in the tubes of Boleti (Parfitt)

1 Mr. Keys' specimens of G. coerulea have been examined by M. Fauvel, and he has pronounced them to belong to the species which occurs chiefly in Finland and Scandinavia. He regards G. coerulea as distinct from G. ripicola, with which it has been regarded as synonymous. 


\section{A HISTORY OF DEVONSHIRE}

\section{STAPHYLINIDAE (continued)}

Tachinus bipustulatus, $\mathrm{F}$.

- rufipes, L.

- subterraneus, L.

var. bicolor, Grav.

- marginellus, $\mathrm{F}$.

- laticollis, Grav. ${ }^{1}$

Megacronus cingulatus, Mann. Barnstaple, etc., Braunton Burrows, under refuse on shore (Hudson-Beare)

- analis, $F$.

- inclinans, Grav. Haldon

Bolitobius lunulatus, L. (atricapillus, F.)

- trinotatus, Er.

- exoletus, Er.

- pygmaeus, $\mathbf{F}$.

Mycetoporus splendens, Marsh. Barnstaple, South Brent

- lepidus, Grav.

- longulus, Mann.

- splendidus, Grav.

Habrocerus capillaricornis, Grav.

Trichophya pilicornis, Gyll.

Heterothops binotata, Er. Braunton Burrows, etc.

- dissimilis, Grav.

Quedius longicornis, $\mathrm{Kr}$. One specimen among hedge clippings in a lane leading to Dartmoor (J. H. Keys)

- mesomelinus, Marsh.

- fulgidus, $\mathrm{F}$. var. fageti, Thoms

- cinctus, Payk. (impressus, Panz.)

- brevis, Er. Buckfast

- fuliginosus, Grav.

- tristis, Grav.

- molochinus, Grav.

- picipes, Mann.

- nigriceps, $\mathrm{Kr}$.

- fumatus, Steph. (peltatus, Er.)

- maurorufus, Er.

- obliteratus, Er.

- umbrinus, Er. Plymouth district

F- suturalis, Kies. (humeralis, Steph.) Stoke Wood, Exeter (Parfitt)

- scintillans, Grav. Efford, nr. Plymouth, etc.

- auricomus, Kies. Barrucane Bay, Dawlish Warren, Dartmoor, Ivybridge

- rufipes, Grav.

- attenuatus, Gyll. Plymouth, Dartmoor, Barnstaple

- semiaeneus, Steph.

- boops, Grav.

Creophilus maxillosus, $\mathrm{L}$.

Emus hirtus, L. Formerly recorded by Dr. Leach from Devon

Leistotrophus nebulosus, $\mathrm{F}^{2}$

- murinus, L.

Staphylinus pubescens, De G. Brent Tor, nr. Tavistock (Thornley)

- fulvipes, Scop. Buckfast (P. de la Garde)

- stercorarius, O1. Croyde, nr. Barnstaple, etc.

- erythropterus, L. As gen. dist. (Parfitt)

1 Parfitt records 'T. cellaris,' Grav. as a Devon insect, probably in error for T. collaris, Grav. In any case I think he is mistaken, as I know of no record further south than Repton, Derbyshire.

2 The Rev. A. Matthews (Ent. Monthly Mag. xiv, 35) records a specimen of Leistotrophus cingulatus, Grav, as taken in Devonshire in August, I 874, by his brother the Rev. H. Matthews, but this was probably in error.

\section{STAPHYLINIDAE (continued)}

Staphylinus caesareus, Ceder. Exeter, Instow, etc. (Staphylinus latebricola, Grav., must be omitted from the Devon list. I recorded a specimen from Plymouth in my work on the British Coleoptera (ii, 252), on the authority of Mr. C. G. Bignell, but Mr. Keys informs me that he has the insect in his possession and it is only S. stercorarius)

Ocypus olens, Müll.

- brunnipes, $\mathrm{F}$.

- cupreus, Rossi.

- pedator, Grav. Dawlish, Spitcbwick (Stephens)

- ater, Grav. Lympston, Exmouth, Ilfracombe, etc. I have found it rather commonly on the links at Westward Ho! Lundy Island

- morio, Grav.

- compressus, Marsh. Exeter, Brent Tor, Lundy Island

Philonthus splendens, F.

- intermedius, Boisd.

- laminatus, Creutz.

- aeneus, Rossi.

- proximus, $\mathrm{Kr}$.

- succicola, Thoms.

- carbonarius, Gyll.

- atratus, Grav. Sidmouth, Exmouth, Newton, etc. (Parfitt) $^{3}$

- decorus, Grav.

- politus, F.

- varius, Gyll. var. bimaculatus, Grav. Plymouth, etc.

- marginatus, F.

- albipes, Grav.

- umbratilis, Grav.

- cephalotes, Grav.

- fimetarius, Grav.

- sordidus, Grav.

- ebeninus, Er.

- corvinus, Er. Valley of the Meavy

- fumigatus, $\mathrm{Er}$

- debilis, Grav.

- sanguinolentus, Grav.

- cruentatus, Gmel. (bipustulatus, Panz.)

- longicornis, Steph. (scybalarius, Nord.)

- varians, Payk.

- agilis, Grav. Brent, Dartmoor. Three specımens (J. H. Keys)

- ventralis, Grav.

- discoideus, Grav.

- quisquiliarius, Gyll. var. dimidiatus, Er. Slapton Ley

- nigrita, Nord. Dortmoor (J. H. Keys)

- micans, Grav.

$\ddagger$ astutus, Er. Mr. Keys has taken four examples at different times on the wall of a house in Plymouth, but has failed to discover its native habitat

- nigritulus, Grav. Recorded by Parfitt from Newton, Exeter, and the north of the Exe, but probably in error for the next species, which he does not mention

- trossulus, Nord.

- fulvipes, F. Barnstaple district (Matthews)

- punctus, Grav. This rare Philonthus is one of the characteristic insects of Slapton Ley. It seems of late to have become very scarce

I am inclined to think that Parfitt has recorded this in error for $P$, carbonarius, as the latter species does not appear in his list, and there is no other Devon record for P. atratus. 


\section{INSECTS}

\section{STAPHYLINIDAE (continued)}

Cafius fucicola, Curt. Batten nr. Plymouth, in numbers - xantholoma, Grav. var. variolosus, Sharp.

- sericeus, Holme.

Actobius cinerascens, Grav.

- signaticornis, Rey. Valley of the Meavy

- procerulus, Grav. Batten

Xantholinus fulgidus, $F$.

- glabratus, Grav.

- punctulatus, Payk.

- ochraceus, Gyll.

- tricolor, Payk.

- linearis, 01 .

- longiventris, Heer.

Leptacinus parumpunctatus, Gyll.

- batychrus, Gyll.

- linearis, Grav.

- formicetorum, Märk.

Baptolinus alternans, Grav.

Othius fulvipennis, $\mathrm{F}$.

- laeviusculus, Steph.

- melanocephalus, Grav.

- myrmecophilus, Kies.

Lathrobium elongatum, L.

- boreale, Hoch.

- fulvipenne, Grav.

†- rufipenne, Gyll. Exeter (Parfitt)

- angustatum, Lac.

- brunnipes, $\mathrm{F}$.

- longulum, Grav.

- punctatum, Zett. Slapton Ley and Marsh Mills

- quadratum, Payk. Slapton Ley, Marsh Mills, and Valley of the Tavy

- terminatum, Grav. var. immaculatum, Fowler

- multipunctum, Grav.

Cryptobium glaberrimum, Herbst (fracticorne, Payk.)

Stilicus rufipes, Germ.

- orbiculatus, Er.

- similis, Er. Bickleigh Vale

- affinis, Er. ${ }^{1}$

Scopaeus Erichsoni, Kol. Slapton Ley

- rubidus, Rey. (Ryei Woll.). Slapton Ley. Taken sparingly by Wollaston in May 1869, and 1872, under stones in dry places, also in sandy refuse at the side of the Ley (HudsonBeare)

- sulcicollis, Steph. (minutus, Er.)

Medon pocofer, Peyr. (Lithocaris maritima, Aubé.), Batten and Slapton

- brunneus, Er.

- fusculus, Mann.

- ripicola, $\mathrm{Kr}$. Batten, Plymouth, in some numbers (J. H. Keys)

- propinquus, Bris.

- melanocephalus, $\mathrm{F}$.

Lithocharis ochracea, Grav.

Sunius angustatus, Payk.

— intermedius, Er. S. Devon (Joy)

Paederus littoralis, Grav.

— riparius, $\mathrm{L}$.

1 Parfitt records S. geniculatus, Er., in the Devon list, but his reference to Stephens (Manual, 3252 ) applies to S. punctipennis, Kirby, which insect, as far as Stephens is concerned, Waterhouse (Catalogue, 27) assigns doubtfully to $\mathrm{S}$. geniculatus, Er.; the latter insect has only been recorded from the London counties, and once from Ireland.

2 Sunius filiformis has occurred at Whitsand Bay.

\section{STAPHYLINIDAE (continued)}

Paederus fuscipes, Curt. Taken recently in thousands at Slapton Ley with the two commoner species by Professor Hudson-Beare. Exeter, very rare (Parfitt)

Evaesthetus scaber, Thoms.

- ruficapillus, Lac.

- laeviusculus, Mann.

Dianous coerulescens, Gyll.

Stenus biguttatus, L. Banks of the Exe

- guttula, Muill.

- bimaculatus, Gyll.

- juno, F.

- guynemeri, Duv.

- speculator, Er.

- providus, $\mathrm{Kr}$.

- Iustrator, Er.

- buphthalmus, Grav.

- melanopus, Marsh.

- canaliculatus, Gyll.

- nitens, Steph. (aemulus, Er.)

- fuscipes, Grav. Exeter

- vafellus, Er. (submarginatus, Steph.)

- declaratus, Er. (nanus, Steph.)

- crassus, Steph. (crassiventris, Thoms.)

- opticus, Grav. Plymouth (J. H. Keys)

- brunnipes, Steph.

- ossium, Steph. (impressipennis, Duv.) var. insularis, Joy. Lundy Island

- geniculatus, Grav.

- impressus, Germ.

- aerosus, Er. (annulatus, Crotch.)

- flavipes, Steph. (filum, Er.)

- pubescens, Steph. Stoke Wood, scarce (Parfitt)

- pallitarsis, Steph.

- bifoveolatus, Gyll. (nitidus, Steph.)

- nitidiusculus, Steph. (tempestivus, Er.)

- picipennis, Er.

- picipes, Steph. (rusticus, Er.)

- cicindeloides, Grav.

— similis, Herbst.

- tarsalis, Ljungh.

- paganus, Er.

- latifrons, Er. Lundy Island

Oxyporus rufus, L. Bickleigh Vale

Bledius spectabilis, Kr. Dawlish

- tricornis, Herbst. Exmouth Warren

- unicornis, Germ. Dawlish, Exmouth Warren

- arenarius, Payk.

Platystethus arenarius, Fourc.

- cornutus, Gyll.

- alutaceus, Thoms. Slapion Ley (J. H. Keys)

- capito, Heer. Stoke Wood (Parfitt)

Oxytelus rugosus, Grav. var, terrestris, Lac.

- insecatus, Grav.

- sculptus, Grav.

- laqueatus, Marsh.

- piceus, Grav. Lympston, under sea-weed (Parfitt)

- inustus, Grav. Exmouth, Barnstaple

- sculpturatus, Grav.3

- nitidulus, Grav.

- complanatus, Er.

- tetracarinatus, Block. (depressus, Grav.)

- O. maritimus certainly occurs in Devon. It has been found at Whitsand Bay, and is very common at times in the Isle of Wight, and probably inhabits the whole southern coast. 


\section{A HISTORY OF DEVONSHIRE}

\section{STAPHYLINIDAE (continued)}

Ancyrophorus omalinus, Er. Bickleigh Vale, Valleys of the Meavy and Tavy (J. H. Keys)

- aureus, Fauv.

Trogophloeus arcuatus, Steph. Valley of the Meavy

- bilineatus, Steph.

- elongatulus, Er.

- fuliginosus, Grav.

- corticinus, Grav.

- pusillus, Grav.

Syntomium aeneum, Mull.

Lesteva longelytrata, Goeze.

- sicula, Er. (punctata, Brit. Cat.)

Acidota crenata, F. Valley of the Plym nr. Ditsworthy Warren (J. H. Keys)

Olophrum piceum, Gyll.

Lathrimaeum atrocephalum, Gyll.

- unicolor, Steph.

Micralymma brevipenne, Gyll. South Devon, Kingsbridge, etc.

Philorhinum sordidum, Steph.

Homalium rivulare, Payk.

- laeviusculum, Gyll.

- riparium, Thoms.

- allardi, Fairm. Lundy Islond

- oxyacanthae, Grav.

- excavatum, Steph. (fossulatum, Er.)

- caesum, Grav.

- punctipenne, Thoms.

- rufipes, Fourc. (florale, Payk.)

- vile, Er.

- iopterum, Steph.

- planum, Payk.

- concinnum, Marsh.

- deplanatum, Gyll.

- striatum, Grav. Plymouth, Exeter, Lundy Island

Anthobium minutum, $F$.

- ophthalmicum, Payk.

- torquatum, Marsh.

- sorbi, Gyll. Haldon, Fordlands

Proteinus ovalis, Steph. (brevicollis, Er.)

- brachypterus, F.

- macropterus, Gyll.

- atomarius, Er. Dartmoor (J. H. Keys)

Megarthrus denticollis, Beck.

- affinis, Mill.

- depressus, Payk.

- sinuatocollis, Lac.

- hemipterus, Ill.

Phloeobium clypeatum, Mull.

Phloeocharis subtilissima, Mann.

Prognatha quadricornis, Lac. Exeter district

\section{LEPTINIDAE}

Leptinus testaceus, Mull. Ivybridge, Bickleigh Vale, etc. (J. H. Keys)

\section{SILPHIDAE}

Calyptomerus dubius, Marsh.

Clambus pubescens, Redt.

- armadillo, De G.

- minutus, Sturm. Exeter

Agathidium nigripenne, Kug. Plymouth

- atrum, Payk.

- seminulum, L.

- laevigatum, Er.

\section{SILPHIDAE (continued)}

Agathidium marginatum, Sturm.

- rotundatum, Gyll. Cann Woods

Liodes humeralis, Kug.

Cyrtusa pauxilla, Schmidt.

Anisotoma cinnamomea, Panz. Devon (Stephens)

- dubia, Kug.

- punctulata, Gyll. (litura, Steph.)

- calcarata, Er.

- nigrita, Schmidt.

- parvula, Sahl. Plymouth

- furva, Er. (ferruginea, Steph. coll.), Devon (Stephens)

- ciliaris, Schm. Braunton Burrozus

Colenis dentipes, Gyll.

Necrophorus humator, Goeze

- mortuorum, $\mathrm{F}$.

- vestigator, Hersch. Dawlish

- ruspator, Er. Parfitt records this species as common

- interruptus, Steph., Bickleigh Vale (J. H. Keys) var. gallicus, Duv. Bickleigh Vale (J. H. Keys)

- vespillo, L.

Necrodes littoralis, $L$.

Silpha tristis, IIl.

- nigrita, Creutz. Dartmoor, Izybridge

- obscura, L.

- quadripunctata, L. Plymouth

- opaca, L.

- thoracica, L.

- rugosa, L.

- sinuata, F.

- laevigata, $\mathrm{F}$.

- atrata, L. var. subrotuntata, Leach. Cbristorb, in a

Choleva angustata, $\mathrm{F}$. dead rook, rare (Parfitt)

- cisteloides, Frohl.

- spadicea, Sturm. Bickleigh Vale, Ivybridge, Valley of the Tavy

- velox, Spence.

- wilkini, Spence.

- fusca, Panz.

- nigricans, Spence.

- morio, $F$.

- grandicollis, Er.

- nigrita, Er.

- tristis, Panz.

- kirbyi, Spence.

- chrysomeloides, Panz.

- fumata, Spence.

- watsoni, Spence.

Catops sericeus, F.

Colon viennense, Herbst. Ilfracombe (E. Saunders)

- dentipes, Duft. Ilfracombe (E. Saunders) var. zebei, Kr. Ilfracombe (E. Saunders)

- brunneum, Latr.

Bathyscia wollastoni, Jans. Nr. Plymout t'

\section{SCYDMAENIDAE}

Neuraphes elongatulus, Mull.

- sparshalli, Den.

Scydmaenus collaris, Müll.

- pusillus, Müll. In the nests of Formica rufa, Stoke Wood, rare (Parfitt); Slapton Ley (Wollaston) 


\section{INSECTS}

\section{SCYDMAENIDAE (continued)}

Scydmaenus poweri, Fowler. Seaton (Power)

Eumicrus tarsatus, Mull.

Euthia scydmaenoides, Steph.

Cephennium thoracicum, Mull.

\section{CLAVIGERIDAE}

Claviger testaceus, Preyss. (foveolatus, Mull.). Plympton

\section{PSELAPHIDAE}

Pselaphus heisei, Herbst.

Tychus niger, Payk.

Bythinus puncticollis, Denny

- validus, Aubé

- bulbifer, Reich

- curtisii, Leach. Haldon

Rybaxis sanguinea, L.

Bryazis fossulata, Reich

- haematica, Reich. Barnstaple district, with Formica flava and Myrmica rubra (Parfitt)

- juncorum, Leach

Euplectus signatus, Reich

- nanus, Reich (Kirbyi, Denny)

- sanguineus, Denny

\section{TRICHOPTERYGIDAE}

Ptinella denticollis, Fairm.

Truchopteryx atomaria, De G.

- angusta, Matth. Radford nr. Plymouth, two specimens (J. H. Keys) ${ }^{1}$

- grandicollis, Mann. Recorded doubtfully by Parfitt. It certainly occurs and can always be known at once by the long outstanding setae at the sides of the body

- lata, Mots.

- fascicularis, Herbst.

- laetitia, Matth.

- sericans, Heer. Exeter

- bovina, Mots.

- variolosa, Muls. (Boeocrara littoralis, Thoms.). Taken on Dartmoor by Mr. T. V. Wollaston

Ptenidium punctatum, Gyll.

- nitidum, Heer. (pusillum, Er.)

†- laevigatum, Gyll. Exeter district (Parfitt)

- evanescens, Marsh. (apicale, Er.)

\section{CORYLOPHIDAE}

Corylophus cassidioides, Marsh.

- lateralis, Gyll.

\section{PHALACRIDAE}

Phalacrus corruscus, Payk.

- caricis, Sturm.

Olibrus corticalis, Panz.

- aeneus, $\mathrm{F}$.

- millefolii, Payk. Exmouth

Stilbus testaceus, Panz. (geminus, III.)

- atomarius, L. (piceus, Steph.). Stoke Wood, on sallows

1 Vide Fowler, Brit. Coll, vol. iii, 388.

\section{COCCINELLIDAE}

Subcoccinella 24-punctata, L. (Lasia globosa, Sch.)

Hippodamia variegata, Goeze (mutabilis, Scriba.). Valley of the Meavy

Anisosticta I9-punctata, L. Starcross. Taken by sweeping Carex maritima

Adalia obliterata, L.

- bipunctata, L.

Mysia olongoguttata, L. Stoke Wood

Anatis ocellata, L.

Coccinella ro-punctata, L. (variabilis, III.)

- I I -punctata, L.

- 5-punctata, L. Valley of the Meavy

- 7-punctata, L.

Halyzia I6-guttata, L.

- I4-guttata, L.

- conglobata, L. (14-punctata, L.)

- 22-punctata, L.

Micraspis 16-punctata, L.

Hyperaspis reppensis, Hbst. Lundy Island (Wollaston) Seymnus redtenbacheri, Muls. Tavistock. Mr. Keys has taken a Scymnus in the locality which must apparently be referred to this species

- pygmaeus, Fourc.

- frontalis, $\mathrm{F}$.

- suturalis, Thunb. (discoideus, Ill.). Exmouth var. limbatus, Steph. Topsham, Lundy Island

- testaceus, Mots. (Mulsanti, Wat.)

- haemorrhoidalis, Herbst.

- capitatus, F.

Chilocorus similis, Rossi (renipustulatus, Scriba.)

- bipustulatus, L.

Exochomus quadripustulatus, $\mathrm{L}$.

Rhizobius litura, F.

Coccidula rufa, Herbst.

\section{ENDOMYCHIDAE}

Mycetaea hirta, Marsh.

Alexia pilifera, Múll.

Lycoperdina bovistae, F. Plympton

Endomychus coccineus, L. Torquay. By sweeping (Bignell). Mr. Keys thinks that Parfitt's record from Devon is probably an error

\section{EROTYLIDAE}

Dacne humeralis, F. Tealmpton

- rufifrons, F. Exeter, etc.

Triplax russica, L. Killerton Park

\section{COLYDIIDAE}

Orthocerus muticus, L. (Sarrotrium clavicorne, L.), Dawlish (Thouless). The above is the only representative of the Colydiidae that has been recorded from Devon. Probably several others occur. It seems strange that even the very common Cerylon histeroides has not yet been noticed. Lundy Island (Wollaston)

\section{HISTERIDAE}

Hister unicolor, $\mathrm{L}$.

- merdarius, Hoff. Exeter, Fordlands. Rare (Parfitt)

- cadaverinus, Hoff.

- stercorarius, Hoff. Exmouth. Scarce (Parfitt). 


\section{A HISTORY OF DEVONSHIRE}

\section{HISTERIDAE (continued)}

Hister sinuatus, Ill. Duubtfully indigenous. Recorded by Stephens from Devonshire (Kingsbridge, etc.)

- I 2-striatus, Schr.

- bimaculatus, $\mathrm{L}$.

Carcinops minima, Aubé. Slapton Ley

Dendrophilus pygmaeus, L. Buckfast

Myrmetes piceus, Payk. Buckfast

Saprinus nitidulus, Payk.

- aeneus, F.

- maritimus, Steph. Plymouth

A braeus globosus, Hoff.

Acritus minutus, Herbst.

Onthophilus globulosus, Ol. (sulcatus, F.). Recorded by Stephens from Kingsbridge

- striatus, $\mathrm{F}$.

\section{MICROPEPLIDAE}

Micropeplus porcatus, Payk.

- staphylinoides, Marsh.

- margaritae, Duv.

\section{NITIDULIDAE}

Brachypterus pubescens, Er.

- urticae, Kug.

Cercus pedicularius, $\mathrm{L}$.

†- bipustulatus, Payk. Haldon

- rufilabris, Latr.

Carpophilus hemipterus, L.

Epuraea aestiva, L.

- florea, Er.

- deleta, Er.

Omosiphora limbata, F.

Nitidula bipustulata, L.

t- quadripustulata, F. Topsbam (Parfitt)

- rufipes, L. South Brent

Soronia punctatissima, Ill.

- grisea, L.

Omosita depressa, L.

- colon, L.

- discoidea, $\mathrm{F}$.

Pocadius ferrugineus, F.

Pria dulcamarae, Scop. Brixham

Meligethes rufipes, Gyll.

- fulvipes, Bris. N. Devon

- subrugosus, Gyll. Wrangaton. Two specimens (J. H. Keys)

- coracinus, Sturm. Seaton. One specimen (Major J. N. Still)

- aeneus, $F$.

- viridescens, $F$.

- difficilis, Heer. Nr. Totnes

- memnonius, Er.

- brunnicornis, Sturm. Nr. Tomes

- pedicularius, Gyll. Exmouth, Dawlish

- bidens, Bris. Nr. Totnes

- umbrosus, Sturm. Nr. Totnes

- ovatus, Sturm. Lee (N. Devon)

- picipes, Sturm.

- symphyti, Heer. Nr. Totnes

- murinus, Er. (seniculus, Er., female)

- lugubris, Sturm. Lundy Island, nr. Totnes, etc.

- obscurus, Er. Lundy Island

- erythropus, Gyll. Exeter, Instow

- exilis, Sturm. Braunton Burrows, nr. Instow, on Echium vulgare (Mason)

\section{NITIDULIDAE (continued)}

Cychramus luteus, $\mathrm{F}$.

Cryptarcha strigata, F.

Ips quadriguttata, $F$.

- quadripustulata, F. Topsbam

Rhizophagus cribratus, Gyll. Mount Edgecumbe, $P$ lymouth (Wollaston)

- depressus, F.

- perforatus, Payk.

- parallelocollis, Er.

- ferrugineus, Payk.

- bipustulatus, $\mathrm{F}$.

\section{TROGOSITIDAE}

Tenebrioides mauritanicus, $\mathrm{L}$.

\section{MONOTOMIDAE}

Monotoma conicicollis, Aubé. Plymouth. In ants' nests (Reading)

- picipes, Herbst.

- sub-4-foveolata, Wat. relverton nr. Plymouth

\section{LATHRIDIIDAE}

Anommatus 12-striatus. Teigmmouth (Wollaston), Exeter (Parfitt)

Lathridius lardarius, De G.

Coninomus nodifer, Westw.

Enicmus minutus, L.

- transversus, $\mathrm{Ol}$.

Cartodere ruficollis, Marsh.

- elongata, Curt.

- filiformis, Gyll. Bred in a fungus shut up in a box in Coaver garden; the first time it was discovered in Britain; 20 Jan. 1856 (Parfitt)

Corticaria pubescens, Gyll. (punctulata, Marsh.)

- crenulata, Gyll.

- denticulata, Gyll.

- fulva, Com.

- elongata, Humm.

Melanophthalma gibbosa, Herbst.

- transversalis, v. Wollastoni, Wat. Lundy Island, etc.

- distinguenda, Com. Lundy Island

- fuscula, Humm.

Laemophloeus pusillus, Schön. Plymouth

- ferrugineus, Steph.

Psammoechus bipunctatus, F.

Silvanus surinamenis, $L$.

- unidentatus, F. Exeter

\section{BYTURIDAE}

Byturus sambuci, Scop.

- tomentosus, F.

\section{CRYPTOPHAGIDAE}

Diphyllus lunatus, F. Plymouth, Bickleigh Vale

Telmatophilus caricis, $\mathrm{Ol}$.

Antherophagus nigricornis, F.

- pallens, Ol. ${ }^{1}$

Cryptophagus lycoperdi, Herbst.

- setulosus, Sturm.

I A. silaceus has been recorded by Commander J. J. Walker from Whitsand Bay, Plymouth, just over the Cornish border. 


\section{CRYPTOPHAGIDAE (continued)}

Cryptophagus pilosus, Gyll.

- ruficornis, Steph. Mount Edgecumbe (Wollaston)

- scanicus, L. var. patruelis, Sturm.

- dentatus, Herbst.

- acutangulus, Gyll.

- cellaris, Scop.

- affinis, Sturm.

- pubescens, Sturm. Ivybridge

- bicolor, Sturm. Exeter district. Rare (Parfitt)

Micrambe vini, Panz.

Paramecosoma melanocephalum, Herbst.

Atomaria linearis, Steph.

- fuscipes, Gyll.

- munda, Er. Lundy Island

- fuscata, Schön.

- atra, Herbst. Stoke Wood. In fungi, rare (Parfitt), Slapton Ley (Wollaston)

- pusilla, Payk.

- atricapilla, Steph.

- berolinensis, Kr. Lundy Island

- mesomelas, Herbst.

- gutta, Steph. Blackdown and Topsham (Parfitt)

- apicalis, Er. Lundy Island

- analis, Er.

Ephistemus globosus, Waltl.

- gyrinoides, Marsh.

\section{SCAPHIDIIDAE}

Scaphidium quadrimaculatum, $\mathrm{Ol}$.

Scaphisoma agaricinum, L.

- boleti, Panz

\section{MYCETOPHAGIDAE}

Typhaea fumata, L.

†Mycetophagus quadripustulatus, L. Exeter

- piceus, F. Mary Tavy

- quadriguttatus, Mull. Plymouth, Bickleigh

- multipunctatus, Hellw.

\section{DERMESTIDAE}

Dermestes vulpinus, $F$.

- Frischii, Kug. One specimen in the street, Plymouth (J. H. Keys)

- murinus, $\mathrm{L}$.

- undulatus, Brahm. (tessellatus, W.C.), Slapton Ley (J. H. Keys), Seaton (Power)

- lardarius, $L$.

Attagenus pellio, L.

Tiresias serra, F. Bred from larvae found under bark in Killerton Park; scarce (Parfitt)

Anthrenus pimpinellae, F. Devon (Stephens)

- varius, Fab. Exeter. On flowers of Portugal laurel

- musaeorum, L.

- claviger, Er.

Trinodes hirtus, F. Nr. Exeter (Stephens)

\section{BYRRHIDAE}

Syncalypta hirsuta, Sharp.

Byrrhus pilula, L.

- fasciatus, F. Blackdown

Cytilus varius, F. Dartmoor, etc.

\section{BYRRHIDAE (continued)}

Simplocaria semistriata, $F$.

Limnichus pygmaeus, Sturm. Seaton (Power)

Aspidiphorus orbiculatus, Gyll. Teigrmouth (Wollaston), Plymouth (J. H. Keys)

\section{PARNIDAE}

Elmis aeneus, Mull.

- volkmari, Panz.

- parallelopipedus, Mull. Exmoutb and Exeter

- nitens, Müll. Exmouth

Limnius tuberculatus, Gyll. Slapton Ley (Wollaston)

- troglodytes, Gyll. Slapton Ley (Wollaston)

Parnus prolifericornis, $F$.

- auriculatus, Panz.

\section{HETEROCERIDAE}

Heterocerus femoralis, Kies. Exmoutb

- laevigatus, Panz. Slapson Ley

\section{LUCANIDAE}

Lucanus cervus, L.

Dorcus parallelopipedus, L.

Sinodendron cylindricum, $\mathrm{L}$.

\section{SCARABAEIDAE}

Onthophagus taurus, L. Exmouth. Rare (Parfitt), doubtfully indigenous

- ovatus, L.

- coenobita, Herbst.

- vacca, L.

- fracticornis, Payk. Exmouth, Northam Burrows

- nuchicornis, L.

Aphodius erraticus, L.

- subterraneus, L. Exmouth, Dawlish

- fossor, $\mathrm{L}$.

- haemorrhoidalis, L.

- foetens, F.

- fimetarius, L.

- ater, De G.

- constans, Duft.

- granarius, L.

- nitidulus, F. Dawolish

- sturmi, Harold. Nr. Plymouth (J. H. Keys) ${ }^{1}$

- sordidus, F. Fordlands, Dartmoor

- rufescens, F.

- porcus, $\mathrm{F}$.

- pusillus, Herbst.

- merdarius, $\mathrm{F}$.

- sticticus, Panz.

- punctato-sulcatus, Sturm.

- prodromus, Brahm.

- contaminatus, Herbst.

- luridus, $\mathrm{F}$.

- rufipes, L.

- depressus, Kug.

Heptaulacus sus, Herbst. Dartmoor

Psammobius sulcicollis, M. Westraard $\mathrm{Ho}_{0}$.

Aegialia arenaria, F.

Geotrupes typhaeus, L.

- spinger, Marsh.

- stercorarius, $L$.

1 Recorded by Mr. Frank Bouskell as a new British species. Entom. Record, xv, 92.

1 Psammobius porcicollis occurs at Whitsand Bay. 


\section{A HISTORY OF DEVONSHIRE}

\section{SCARABAEIDAE (continued)}

Geotrupes mutator, Marsh.

- sylvaticus, Panz.

- vernalis, $\mathrm{L}$.

- pyrenaeus, Charp. Devon (Stephens)

Trox sabulosus, L. Spitchwick (Stephens)

- scaber, L. Plymouth, Braunton Burrows

Hoplia philanthus, Fuss.

Serica brunnea, L.

Rhizotrogus solstitialis, L.

Melolontha vulgaris, $\mathrm{F}$.

Phyllopertha horticola, L.

Anomala Frischii, F. Braunton Burrows, Exmoutb. The very rare unicolorous green variety has been found at Braunton Burrows or in the district

Cetonia aurata, L. Torquay, Lurdy Island (Wollaston)

Gnorimus nobilis, L. Devon (Stephens)

Trichius fasciatus, L. Taken nr. Exeter by Mr. Harris (Parfitt). This record is probably correct as the species has occurred in numbers in $S$. Wales

\section{BUPRESTIDAE}

Aphanisticus pusillus, $\mathrm{Ol}$. Kingsbridgo

\section{THROSCIDAE}

Throscus dermestoides, $L$.

Microrrhagus pygmaeus, F. Bickleigh Vale (J. H. Keys). A very interesting capture in Devon

\section{ELATERIDAE}

Lacon murinus, $\mathrm{L}$.

Cryptohypnus riparius, $\mathrm{F}$.

- quadripustulatus, F. Barnstaple

- dermestoides, Herbst. Banks of the Meavy var. quadriguttatus, Lap. Banks of the Meavy

Elater sanguineus, L. Spitchwick (Stephens). Perhaps in error

- balteatus, $\mathrm{L}$.

Melanotus rufipes, Herbst. (Parfitt records Cratonychus niger, F., Ectinus aterrimus, Stephens, Melanotus punctolineatus, Pel., as from 'Stoke Wood, very rare in old oaks.' This is certainly in error, as $\mathbf{M}$. punctolineatus is only found in damp places at the roots of grass, etc., chiefly on the south-east coast)

Athous niger, $\mathrm{L}$.

- longicollis, O1. Exmouth, etc.

- difformis, Lac. Newtown

- haemorrhoidalis, $\mathrm{F}$.

- vittatus, $\mathrm{F}$.

Limonius cylindricus, Payk.

- minutus, $\mathrm{L}$.

Adrastus limbatus, F.

Agriotes sputator, L.

- obscurus, L.

- lineatus, $\mathrm{L}$.

- sobrinus, Kies.

- pallidulus, Ill.

Dolopius marginatus, L.

Corymbites cupreus, F. Dartmoor var. aeruginosus, $\mathrm{F}$.

- tessellatus, F.

- quercus, Gyll. var. ochropterus, Steph.

\section{ELATERIDAE (continued)}

Corymbites holosericeus, O1. Dartmoor, etc. ; Lundy Island

- aeneus, L. On high ground, Dartmoor, Drewsteignton, Blackdown, etc.

- bipustulatus, L. Killerton Park, Exeter

Campylus linearis, $\mathrm{L}$.

\section{DASCILLIDAE}

Dascillus cervinus, L.

Helodes minuta, L.

- marginata, $\mathrm{F}$.

Microcara livida, F.

Cyphon coarctatus, Payk.

- nitidulus, Thoms.

- variabilis, Thunb.

- pallidulus, Boh.

- padi, L.

Hydrocyphon deflexicollis, Mull. Ivybridge, Bickleigh Vale

Scirtes hemisphaericus, L.

- orbicularis, Panz. Spitchwick Park (Stephens)

\section{LYCIDAE}

Platycis minutus, F. Woodland, Devon (Leach)

\section{LAMPYRIDAE}

Lampyris noctiluca, L. Torquay, Haldon, Dartmoor, Stoke Wood, Torrington, etc. Parfitt says that this insect is not so abundant in Devon as in the eastern counties, but I have never noticed it in such abundance anywhere as I have seen it on a hot summer evening at Torrington, N. Devon

\section{TELEPHORIDAE}

Podabrus alpinus, Payk. Lydford (Thouless), etc. Telephorus rusticus, Fall.

- lividus, L.

- pellucidus, $\mathrm{F}$.

- nigricans, Mull.

- bicolor, F.

- haemorrhoidalis, F. Lundy Island (Wollaston)

- oralis, Germ. (lateralis, L.)

- flavilabris, Fall.

- thoracicus, Ol. Valley of the Walkham

Rhagonycha unicolor, Curt. Parfitt records this species as generally distributed in watery places, but as a rule it is very scarce

- fulva, Scop.

- testacea, L.

- limbata, Thoms.

- pallida, $F$.

Malthinus punctatus, Fourc.

- fasciatus, $\mathrm{Ol}$.

- balteatus, Suff.

Malthodes marginatus, Latr.

- mysticus, Kies.

- dispar, Germ.

- minimus, L. (sanguinolentus, Fall.)

- atomus, Thoms. (brevicollis, Payk.). Devon (Stephens) 


\section{INSECTS}

\section{MELYRIDAE}

Malachius aeneus, L. Exminster Marshes, Barnstaple

- bipustulatus, L.

- viridis, F. Powderbam Park

- marginellus, $\mathrm{Ol}$.

Anthocomus fasciatus, $\mathrm{L}$.

Dasytes flavipes, $\mathrm{F}$.

- aerosus, Kies. (plumbeo-niger, Goeze)

Psilothrix nobilis, Ill.

Dolichosoma lineare, Rossi.

Haplocnemus nigricornis, F. Ivybridge and Bickleigh Vale (J. H. Keys)

Phloeophilus edwardsi, Steph. Yelverton

\section{CLERIDAE}

Tillus elongatus, L. Stoke Wood

Necrobia ruficollis, $\mathrm{F}$.

- violacea, $\mathrm{L}$.

- rufipes, De G.

\section{PTINIDAE}

Ptinus germanus, F., Devon (Stephens)

- fur, L.

- sexpunctatus, Panz. In houses, not uncommon, Exeter; in a nest of humble bees in some numbers in 1858 , Teignmouth, etc.

Niptus hololeucus, Fabr.

- crenatus, $\mathrm{F}$.

Hedobia imperialis, L.

Dryophilus pusillus, Gyll. Buckfast

Priobium castaneum, $F$.

Anobium domesticum, Fourc.

- paniceum, L.

Xestobium tessellatum, F.

Ernobius mollis, L.

Ptilinus pectinicornis, L.

Ochina hederae, Mull.

Xyletinus ater, Panz. Spitchwick, in moss (Leach)

Coenocara bovistae, Hoff. Spitchwick (Leach)

\section{LYCTIDAE}

Lyctus canaliculatus, $\mathrm{F}$.

\section{CISSIDAE}

Cis boleti, Scop.

- micans, F. S. Devon

- hispidus, Payk.

- bidentatus, Ol. Okehampion

- alni, Gyll. Bickleigh Vale, Mount Edgecumbe, Plymouth

- nitidus, Herbst.

- jacquemarti, Mel. Ivybridge (Dr. M. Cameron)

- festivus, Panz. S. Devon

Octotemnus glabriculus, Gyll.

\section{PRIONIDAE}

Prionus coriarius, L. Parfitt records this species as not unconmmon at the roots of old oaks in winter

\section{CERAMBYCIDAE}

Aromia moschata, $\mathrm{L}$.

Callidium violaceum, L. Newport, nr. Topsbam

- sanguineum, L. Exeter (Stephens)

\section{CERAMBYCIDAE (continued)}

Clytus arietis, L

Gracilia pygmaea, F.

Leptidea brevipennis, Muls. Plymouth. Evidently an importation, but it appears to be becoming naturalized

Cerambyx heros, Scop. (Recorded from Devon, but not indigenous)

Khagium inquisitor, $\mathrm{F}$.

- bifasciatum, $\mathrm{F}$.

Toxotus meridianus, L.

Pachyta cerambyciformis, Schrank (8-maculata, Schall). Fordlands, Barnstaple; Common, $\mathrm{nr}$. Exeter (Thouless)

- collaris, L. Devon (Stephens)

Leptura sanguinolenta, L. Devon (Stephens)

- fulva, De G. (tomentosa, F.). Kingsbridge

- livida, F.

Strangalia aurulenta, F. North coast of Devon, between Barmstaple and Bideford (Stephens); Bickleigh Vale (J. H. Keys)

- quadrifasciata, L. Dunsford, Walkbam Valley, Bickleigh Vale

- armata, Herbst.

- nigra, L.

- melanura, L.

Grammoptera tabacicolor, De G. (laevis, F.)

- ruficornis, $\mathrm{F}$.

\section{LAMIIDAE}

Acanthocinus aedilis, L. Imported in timber Leiopus nebulosus, L.

Pogonochaerus bidentatus, Thoms. (hispidus, Laich.) - dentatus, Fourc. (pilosus, F.)

Monochammus sartor, F. Devon (Stephens), one specimen found crawling on the wall outside a carpenter's shop in Plymouth (J. H. Keys)

Tetrops praeusta, L.

Phytoecia cylindrica, L. Nr. Ide ; Exeter

\section{BRUCHIDAE}

Bruchus cisti, F. Powderbam, on broom

- rufimanus, Boh.

- atomarius, L. (seminarius, Brit. Coll.)

- loti, Payk.

\section{EUPODA}

Donacia versicolorea, Brahm (bidens, Ol.). Tamerton - dentipes, $F$.

- limbata, F. (lemnae, F.). Meadows below Stoke Wood, Exeter

- bicolora, Zsch. (sagittariae, F.). Seaton (Rev. T. Hardy)

- simplex, F. (linearis, Hoppe)

- semicuprea, Panz. (simplex, F.)

- sericea, L.

- discolor, Panz. (comari, Suffr.). Dartmoor

- affinis, Kunze

Lema cyanella, L. (puncticollis, Curt.)

- lichenis, Voet (cyanella, Suff.)

- erichsoni, Suff. Slapton Ley

- melanopa, L.

Crioceris asparagi, L. Plymouth, Dartmoor 


\section{A HISTORY OF DEVONSHIRE}

\section{CAMPTOSOMATA}

Clythra quadripunctata, L.

Cryptocephalus aureolus. Valley of the Walkham

- hypochaeridis, L. (sericeus, F.). Fordlands, on Crepis tectorum

- ochrostoma, Har. (nitidulus, Gyll.). Devon (Stephens), perhaps in error

- moraei, L. Bickleigh Vale

- fulvus, Goeze (minutus, F.)

- pusillus, $\mathrm{F}$.

- labiatus, L.

- querceti, Suffr. (geminus, Wat. Cat.). If the synonymy is right this species has been recorded as found in Devon on the common barberry

\section{CYCLICA}

Lamprosoma concolor, Sturm. Haldon, Seaton, Brent Tor, etc.

Timarcha tenebricosa, F. (laevigata, Duft.)

- violaceonigra, De G. (coriaria, Laich.)

Chrysomela banksi, F. Brent Tor, etc., Lundy Island

- staphylaea, L.

- polita, L.

- haemoptera, L. I have taken this very abundantly on rushes on the golf links at Westward Ho! Abundant at Brent Tor, nr. Tavistock (Thornley)

- varians, Schall. Exeter, Brent Tor.

- goettingensis, L. Under stones on slopes at the mouth of the Meary

- fastuosa, Scop. Dartmoor

- didymata, Scriba.

- hyperici, Forst.

Melasoma aeneum, L. Exeter

- populi, L.

Phytodecta olivacea, Forst. var. litura, F. Powderham, Exmouth, Exeter

Gastroidea viridula, De G. (raphani, Herbst.), Haldon

- polygoni, L.

Phaedon tumidulus, Germ.

- armoraciae, L. (betulae, Küst.)

- cochleariae, E.

- concinnus, Steph. Exmoutb (Thouless); Stoke Wood, scarce (Parfitt)

Phyllodecta vulgatissima, $L$.

- cavifrons, Thoms.

- vitellinae, L.

Hydrothassa aucta, F.

- marginella, L.

Prasocuris junci, Brahm (beccabungae, IIl.)

— phellandrii, L.

Agelastica alni, L. Several specimens were captured in June near Exeter by Stephens

Phyllobrotica quadrimaculata, L. Tavy Valley

Luperus nigrofasciatus, Goeze

- rufipes, Scop.

- flavipes, $L$.

Lochmaea capreae, L.

- suturalis, Thoms.

- crataegi, Forst.

Galerucella sagittariae, Gyll.

- lineola, $\mathrm{F}$.

- calmariensis, L.

- tenella, L.

Adimonia tanaceti, $L$.

\section{CYCLICA (continued)}

Sermyla halensis, L.

Longitarsus ater, F. (parvulus, Payk.). Seaton Down Devon (Power)

- holsaticus, L.

- luridus, Scop.

- suturellus, Duft. (fuscicollis, var. Steph.)

- atricillus, L.

- melanocephalus, All.

- atriceps, Kuts.

- suturalis, Marsh.

- piciceps, Steph. (atricapillus, Redt.)

- membranaceus, Foudr. (tencrii, All.), Lee Valley, N. Devon (Power)

- Alavicornis, Steph. Lee Valley, N. Devon (Power)

- exoletus, L. Lundy Island

- pusillus, Gyll.

- tabidus, F. (verbasci, Panz.)

- jacobacae, Wat.

- ochroleucus, Marsh.

- gracilis, Kuts. Lee Valley, Lundy Islond

- laevis, Duft.

Haltica lythri, Aubé.

- oleracea, L.

- pusilla, Duft.

Hermaeophaga mercurialis, F. Nr. Totnes

Phyllotreta nodicornis, Marsh. Torquay

- nigripes, F. (lepidii, Koch)

- consobrina, Curt. (melaena, Ill.). Seaton Downs (Power); Torquay (Thouless)

- punctulata, Marsh. Woodbury Common

- atra, Payk. Woodbury, Exmouth

- vittula, Redt.

- undulata, Kuts.

- nemorum, L.

- ochripes, Curt. Powderbam Marshes

- exclamationis, Thunb. (brassicae, F.)

Aphthona lutescens, Gyll. Lee Valley (Power)

- nonstriata, Goeze (coerulea, Payk.)

- venustula, Kuts. (euphorbiae, All.)

- atro-coerulea, Steph. (cyanella, Redt.)

- virescens, Foudr. Seaton Down (Power)

- herbigrada, Curt. Exeter

Batophila rubi, Payk.

- aerata, Marsh

Sphaeroderma testaceum, F.

- cardui, Gyll.

Apteropeda orbiculata, Marsh (graminis, Koch)

Mniophila muscorum, Koch

Podagrica fuscipes, $\mathrm{L}$.

- fuscicornis, $\mathrm{L}$.

Mantura rustica, $\mathrm{L}$.

- chrysanthemi, Koch. Meavy Valley and St. Budeaux, nr. Plymouth, Lundy Island (Wollaston)

Crepidodera transversa, Marsh.

- ferruginea, Scop.

- rufipes, L.

- ventralis, Ill. Seaton (Power)

- nitidula, L. Powderbam (Parfitt)

- helxines, $\mathrm{L}$.

- chloris, Foudr.

- aurata, Marsh.

Hippuriphila modeeri, L

Chaetocnema hortensis, Fourc. (aridella, Payk.)

- confusa, Boh. Thurlestone

- sahlbergi, Gyll. Blackdown, very rare (Parfitt). I found one specimen of the aeneous green variety at Westruood $\mathrm{Ho} !$ in 1883 


\section{INSECTS}

\section{CYCLICA (continued)}

Plectroscelis concinna, Marsh.

Dibolia cynoglossi, Hal. Fordlands, Dawlish Warren, on Cynoglossum vulgare, rare (Parfitt). This species has been omitted from our lists, but in the face of its recent capture by $\mathrm{Mr}$. Donisthorpe it must also be reinstated as a Devon insect

Psylliodes luridipennis, Kuts. Lundy Island (Wollaston) ${ }^{1}$

- napi, Koch

- cuprea, Koch (cupronitens, Forst.). Brent Tor, nr. Tovistock

- affinis, Payk. (atricilla, W.C.)

- dulcamarae, Koch. Lundy Island (Wollaston)

- chalcomera, Ill.

\section{CRYPTOSTOMATA}

Cassida murraea, L. Newton, Ivybridge

- vibex, F.

- sanguinolenta, F. Dunsford,' Meavy Valley

- vittata, Vill. (oblonga, Ill.). Exmouth and Barnstaple

- nobilis, L. Dawlish Warren, among Salicornia

†- subferruginea, Schrank. (ferruginea, F.). On Achillea millefolium and other low plants, somewhat doubtfully indigenous. Recorded by Stephens from Devon

- viridis, $\mathrm{F}$.

- hemisphaerica, Herbst. Taken amongst tall marsh plants in Powderbam Park, very rare (Parfitt); Braunton Burrows (Blandford)

\section{TENEBRIONIDAE}

Blaps mucronata, Latr.

- mortisaga, L. One specimen from a grain store in the docks, Plymouth (J. H. Keys). Parfitt's record of this insect as common in houses in Exeter must be referred to the preceding species

Heliopathes gibbus, F. Dawlish and Exmouth beach, Woolacombe

Opatrum sabulosum, L.

Phaleria cadaverina, F. Dawlish, Barnstaple

Tenebrio molitor, $L$.

- obscurus, $\mathrm{F}$.

Gnathocerus cornutus, F.

Tribolium ferrugineum, $F$.

- confusum, Duv.

Palorus melinus, Herbst. (depressus, F.)

Hypophloeus castaneus, F. Plymouth

Helops striatus, Fourc.

\section{LAGRIIDAE}

Lagria hirta, L.

\section{CISTELIDAE}

Cistela murina, L.

Cteniopus sulphureus, L. Very abundant nr. Torquay, etc.; Lundy Island (Wollaston)

1 This is probably an extreme and local variety of P. chrysocephala, L. It has recently (1905) been taken in some numbers on the island by Dr. Joy.

$2 \mathrm{Mr}$. Thouless, who records two specimens from this locality, says that he believes that they must be referred to the form which has been recorded as C. chloris.

\section{MELANDRYIDAE}

Clinocara tetratoma, Thoms. (minor, Walk.). Cann Woods

- undulata, Kr. Cann Woods

Melandrya caraboides, L.

Abdera bifasciata, Marsh. Devon (Wollaston)

\section{PYTHIDAE}

Salpingus castaneus, Panz.

- aeratus, Muls.

Rhinosimus ruficollis, Steph.

- viridipennis, Steph.

- planirostris, $\mathrm{F}$.

Mycterus curculionoides, F. Kingsbridge (Stephens. Very doubtfully indigenous)

\section{OEDEMERIDAE}

Oedemera nobilis, Scop. (coerulea, L.)

- lurida, Marsh.

Oncomera femorata, F. Seaton; Brixham; Exeter; realmpton

Nacerdes melanura, Schmidt.

Ischnomera coerulea, $\mathrm{L}$.

\section{PYROCHROIDAE}

Pyrochroa serraticornis, Scop. (rubens, Schall)

\section{SCRAPTIIDAE}

IScraptia fuscula, Mull (nigricans Steph.). Taken by sweeping ivy at Exwick. July, 1863 (Parfitt)

\section{MORDELLIDAE}

Mordella fasciata, F. Exeter

Mordellistena pumila, Gyll.

- brunnea, F. Devon (Wollaston)

Anaspis frontalis, L.

- garneysi, Fowler. Bred from wood (probably found in the Plymouth district (Bignell))

- pulicaria, Costa, (forcipata, Muls.)

- rufilabris, Gyll.

- geoffroyi, Müll. (fasciata, Forst.)

- ruficollis, $F$.

- flava, L var, thoracica, L.

- subtestacea, Steph.

- maculata, Fourc.

\section{ANTHICIDAE}

Anthicus floralis, L. var. quisquilius, Thoms.

- angustatus, Curt. Slapton Ley

\section{XYLOPHILIDAE}

Xylophilus oculatus, Duv.

\section{MELOIDAE}

Meloe proscarabaeus, L

- violaceus, Marsh. Bickleigh Vale; Walkbam Valley, etc.

- autumnalis, O1. Exmouth; Tovistock

- rugosus. Valley of the Tavy (J.H. Keys); Exmouth.

- brevicollis. Valley of the Tavy (J. H. Keys); Plymouth (Power, Spiers, and Bignell) ; Dartmoor (Thouless)

Sitaris muralis, Forst. (humeralis, F.) 


\section{A HISTORY OF DEVONSHIRE}

\section{PLATYRRHINIDAE}

Platyrrhinus latirostris, F. Ugbrooke Park nr. Chudleigh (Bowring)

\section{CURCULIONIDAE}

Apoderus coryli, L. Fordlands; Barnstaple; in the latter locality occasionally on a birch (Rev. $\mathrm{H}$. Matthews)

Attelabus curculionoides, L. Stoke Wood

Byctiscus populi, L. Fordlands

Rhynchites aequatus, L.

\section{- aeneovirens, Marsh.}

var. fragariae, Gyll. Bickleigh Vale, one specimen (J. H. Keys)

- minutus, Herbst. (germanicus, Aubé.)

- pubescens, F. Fordlands; Bickleigh Vale

Deporaủs betulae, $\mathrm{L}$.

Apion pomonae, $\mathrm{F}$.

- craccae, L. Exeter district, scarce (Parfitt)

- subulatum, Kirby. Seaton land-slip (Power)

- ulicis, Först.

- malvae, $F$.

- miniatum, Germ.

- cruentatum, Walt. Plymouth; Lundy Island

- haematodes, Kirby (frumentarium, Payk.)

- rubens, Steph. Blackdown, etc.

- rufirostre, $\mathrm{F}$.

- viciae, Payk. Exeter; Bideford

- difforme, Germ. Seaton land-slip (Power)

- varipes, Germ. Seaton land-slip ; Exeter, etc.

- apricans, Herbst. (fagi, Kirby ; assimile, Kirby)

- bohemani, Thoms. (ononidis, Gyil). Torquay

- trifolii, L.

- dichroum, Bedel (Havipes, Brit. Cat.)

- nigritarse, Kirby. Seaton land-slip (Power) ; Slapton Ley (Wollaston)

- hookeri, Kirby. Larvae feeding in the heads of Matricaria inodora, v. maritima

- aeneum, $\mathrm{F}$.

— radiolus, Kirby

- onopordi, Kirby

- carduorum, Kirby

- virens, Herbst.

- pisi, F.

- aethiops, Herbst.

- ebeninum, Kirby

— filirostre, Kirby.

- immune, Kirby

- ononis, Kirby

- spencei, Kirby

- ervi, Kirby

- vorax, Herbst.

- scutellare, Kirby. Newton; Exeter; BrentTor, etc.

- loti, Kirby ${ }^{1}$

- seniculum, Kirby

- tenue, Kirby

- simile, Kirby. Torquay

- pubescens, Kirby

- curtisi, Walt.

- marchicum, Herbst. Lundy Island (F. Smith)

- violaceum, Kirby

- hydrolapathi, Kirby

- humile, Germ.

1 Commander J. J. Walker has taken A. waltoni, Steph., at IV hitsand Bay, Plymouth.

\section{CURCULIONIDAE (continued)}

Otiorrhynchus tenebricosus, Herbst.

- tuscipes, Walt. Tavistock

- atroapterus, De G. Exmouth, on brambles; Dawlish

- scabrosus, Marsh.

- ligneus, $\mathrm{Ol}$.

- picipes, F.

- sulcatus, $\mathrm{F}$.

- rugifrons, Gyll. Woolacombe Sands; Batten, nr. Plymouth; Lundy Island

- ovatus, L.

var. pabulinus, Panz.

- muscorum, Bris. Chagford

Trachyphloeus scaber, $\mathrm{L}$.

- scabriculus, L.

Coenopsis fissirostris, Walt. Valley of the Meavy (J. H. Keys)

- Waltoni, Schon. Woods nr. Plymouth (J. H. Keys)

Strophosomus coryli, F.

- capitatus, De G. (obesus, Marsh.)

- retusus, Marsh.'

Exomias araneiformis, Schr. (brunnipes, Ol.)

- pellucidus, Boh. Ivybridge

- pyrenaeus, Seidl. Plymouth district, in several localities. Taken at roots of grass, in faggots, under bark, and by beating hawthorn, by Mr. J. H. Keys, who first discovered the insect in Britain. Mr. Champion has since found it in Cornwall

Sciaphilus muricatus, F.

Tropiphorus tomentosus, Marsh. (mercurialis, Brit. Cat.)

Barypeithes sulcifrons, Boh. Batten nr. Plymouth (Keys); Mount Edgecumbe (Wollaston); Killerton (Gorham)

Liophloeus nubilus, F.

Polydrusus tereticollis, De G. (undatus, F.)

- pterygomalis, Boh.

- cervinus, $L$.

- confluens, Steph. Meavy Valley

- chrysomela, Ol. Lundy Island (Wollaston)

Phyllobius oblongus, L.

- calcaratus, F.

- urticae, De G. (alneti, F.)

- pyri, L.

- argentatus, L.

- maculicornis, Germ.

- pomonae, Ol.

- viridiaeris, Laich. (uniformis, Marsh.)

Philopedon geminatus, $F$.

Atactogenus exaratus, Marsh. I do not feel quite certain as to this record, but it is probably correct

Barynotus obscurus, $F$.

- schönherri, Zett. Once only nr. Tavistock (J.H. Keys). I know of no other record further south than Lancaster

Alophus triguttatus, F. Exeter; Barnstaple; Ilfracombe Sitones griseus, $F$.

- cambricus, Steph. Plymoutb; Exeter; Valley of the Meavy

- regensteinensis, Herbst. Parfitt records this species as found in gardens on beans. As a rule it only occurs on broom or furze

2 Commander J. J. Walker has taken S. faber at Whitsand Bay. 


\section{INSECTS}

\section{CURCULIONIDAE (continued)}

Sitones waterhousei, Walt. Batten nr. Plymouth (Keys); Seaton, 3-10 January, I 865 (Power). It has also been found at Whitsand Bay and at intervals all along the southern coast of England

- crinitus, $\mathrm{Ol}$.

- tibialis, Herbst.

- hispidulus, F.

- humeralis, Steph.

- flavescens, Marsh.

- puncticollis, Steph.

- suturalis, Steph.

- lineatus, L.

- sulcifrons, Germ.

Gronops lunatus, L. I once found this species abundantly on Northam Burrows, but I know of no other Devon record ${ }^{2}$

Limobius mixtus. Exeter; very rare (Parfitt)

Hypera punctata, $F$.

- fasciculata, Herbst. Recorded by Parfitt in his catalogue without locality

- arundinis, Payk. Taken in marshy places near Exeter (Stephens)

- rumicis, $\mathrm{L}$.

- pollux, $\mathbf{F}$.

- polygoni, L.

- suspiciosa, Herbst.

- murina, F. Exeter district (Parfitt)

- plantaginis, De G.

- trilineata, Marsh.

- nigrirostris, $\mathrm{F}$.

Rhinocyllus latirostris, Latr. Seaton (Major J. N. Still)

Lixus bicolor, Ol. Plymouth (?) (Parfitt). A very doubtful record, but may be correct, as the insect has been taken by $\mathrm{Mr}$. Moncreaff singly near Portsmoutb

Larinus carlinae. Valleys of the Meavy and Tavy (J. H. Keys)

Liosoma ovatulum, Clairv. var. collaris, Rye. Bickleigh Vale

Curculio abietis, L. Plymouth

Trachodes hispidus, L. Ioybridge; Bickleigh Vale (J. H. Keys)

Orchestes quercus, L.

- alni, L. var. ferrugineus, Marsh (melanocephalus, Ol.)

- ilicis, F. Ivybridge; Woodbury Common

- avellanae, Don.

- lonicerae, Herbst. Recorded by Stephens as found on Lonicera xylosteum (Fly Honeysuckle) at Spitchwick; the record is very probably correct

- fagi, L.

- pratensis, Germ. Fordlands and Woodbury

- rusci, Herbst.

- salicis, L.

Rhamphus flavicornis, Clairv.

Orthocaetes setiger, Beck. Bickleigh Vale

Erirrhinus scirpi, F. Slapton Ley

- acridulus, L.

Dorytomus vorax, F. Exeter

- tremulae, F. Fordlands

- tortrix, L. Blackdown; Exeter, etc.

- maculatus, Marsh.

- pectoralis, Gyll.

Smicronyx reichei, Gyll. Newton Abbot (Dale)

I In my work on The Coleoptere of the British Islands, vol. v. 227, 'Seaford, Devon' is in error for 'Seaford, Sussex.'

\section{CURCULIONIDAE (continued)}

Tanysphyrus lemnae, F. Slapton Ley

Bagous alismatis, Marsh.

- claudicans, Naez.

Elleschus bipunctatus, L. Valley of the Meavy (J. H. Keys)

Tychius venustus, F. Bickleigb Vale (J. H. Keys)

- schneideri, Herbst. Slapton Ley

- lineatulus, Steph. Slapton Ley; Lundy Island

- junceus, Reich.

- tomentosus, Herbst. Lundy Island

Miccotrogus picirostris, F.

Sibinia arenariae, Steph.

- primita, Herbst.

Gymnetron beccabungae, L. var. veronicae, Germ.

- rostellum, Herbst. Degon (Wollaston)

- pascuorum, Gyll.

- antirrhini, Payk. (noctis, Herbst.)

Mecinus pyraster, Herbst.

- circulatus, Marsh.

Anthonomus ulmi, De G.

- rosinae, Des Gozis

- pedicularius, $\mathrm{L}$.

- pomorum, L.

- rubi, Herbst.

- comari, Crotch. Lundy Island

Nanophyes lythri, F.

Cionus scrophulariae, L.

- blattariae, $\mathrm{F}$.

- pulchellus, Herbst.

Orobitis cyaneus, L.

Cryptorrhynchus lapathi, L.

Acalles roboris, Curt.

- ptinoides, Marsh.

- turbatus, Boh.

Mononychus pseudacori, F. Torquay (Curtis) a somewhat doubtful record

Coeliodes quercus, $\mathrm{F}$.

- ruber, Marsh.

- cardui, Herbst. (fuliginosus, Marsh.)

- quadrimaculatus, $\mathrm{L}$.

- exiguus, Ol.

Poophagus nasturtii, Germ. Exminster marshes; South Brent; Dartmoor

Ceuthorrhynchus assimilis, Payk.

- setosus, Boh. Plymouth

- cochleariae, Gyll.

- ericae, Gyll.

- erysimi, F.

- contractus, Marsh. var. pallipes, Crotch. Lundy Island

I- pilosellus, Gyll. Seaton, two specimens, I-5 January, I 866 (Power)

- quadridens, Panz. Lundy Island, etc.

- pollinarius, Forst.

- viduatus, Gyll. Bickleigh Vale (J. H. Keys)

- pleurostigma, Marsh. (sulcicollis, Gyll.)

- verrucatus, Gyll. (biguttatus, Boh.) On the Horned Poppy or Sea Poppy (Glaucium luteum); very local; Seaton beach, in abundance (Power)

- punctiger, Gyll. South Brent

- rugulosus, Herbst. (melanostigma, Marsh.). Seaton; Plymouth

- melanostictus, Marsh. Slapton Ley

- asperifoliarum, Gyll. Seaton

- litura, F. (ovalis, L ?) 


\section{A HISTORY OF DEVONSHIRE}

\section{CURCULIONIDAE (continued)}

Ceuthorrhynchidius floralis, Payk.

- nigrinus, Marsh. Exmouth

- melanarius, Steph. Plymouth

- terminatus, Herbst. Batten nr. Plymouth

- horridus, F. Kingsbridge

- distinctus, Bris. South Brent

- quercicola, Payl. Exeter

- troglodytes, F.

- Dawsoni, Bris. Batten nr. Plymouth; Landy $1 s$ land. Formerly abundant at Wbitsand Bay, and I have taken it at Ventnor, I. of Wight. It occurs on Plantago coronopus

Rhinoncus pericarpius, L.

- gramineus, F. (inconspectus, Aubé). Exeter, Slapton Ley

- perpendicularis, Reich. (subfasciatus, Gyll.)

- castor, $\mathrm{F}$.

- bruchoides, Herbst.

Eubrychius velatus, Beck

Litodactylus leucogaster, Marsh.

Phytobius waltoni, Boh.

- quadrituberculatus, $F$.

- canaliculatus, Fähr.

Baris lepidii, Germ. Seaton Down (Power)

Balaninus venosus, Grav. (glandium, Brit. Cat.)

- nucum, L.

- betulae, Steph. (cerasorum, Herbst.). Exeter; Bickleigh Vale

- villosus, Herbst.

- salicivorus, Payk. (brassicae, Brit. Cat.)

- pyrrhoceras, Marsh.

Calandra granaria, $L$.

- oryzae, L.

\section{CURCULIONIDAE (continued)}

Pentarthrum huttoni, Woll. Alphington; Plymouth (J. H. Keys)

Cossonus ferrugineus, Clairv. Exeter; Phymouth

* Rhopalomesites tardyi, Curt. Mount Edgecumbe Park nr. Plymouth; Ilfracombe (J. J. Walker)

Rhyncolus lignarius, Marsh. (cylindrirostris, Ol.)

Caulotrypis aeneopiceus, Boh. Plymouth (in old oak beams in house); Batten (in old tree); Ilfracombe

Codiosoma spadix, Herbst. Batten

Magdalis armigera, Fourc. (atramentaria, Marsh.)

- pruni, L.

\section{SCOLYTIDAE}

Scolytus destructor, $\mathrm{Ol}$.

- multistriatus, Marsh.

Hylastes ater, Payk. Exeter

- opacus, Er. Bickleigh Vale

Hylastinus obscurus, Marsh. Batten; Plymouth

Hylesinus crenatus, F. Exeter district

- fraxini, Panz.

- vittatus, F.

Myelophilus piniperda, L.

Phloeophthorus rhododactylus, Marsh.

Pityophthorus pubescens, Marsh. (micrographus, Brit. Cat.)

Xylocleptes bispinus, Duft.

Dryocaetes villosus, $F$.

Tomicus laricis, $\mathbf{F}$.

Trypodendron domesticum, L. Bickleigh Vale, Mount Edgecumbe

\section{E P I D O P T E R A}

\section{Butterflies and Moths}

For Lepidoptera the county of Devon has been a favoured hunting-ground from the earliest times during which we have any knowledge of the study of this order. A. H. Haworth, in his Lepidoptera Britannica (1803) recorded the captures of Dr. Leach, Mr. Fracillon, and Mr. Raddon, in the previous century ; and those of Captain Blomer are constantly referred to by J. F. Stephens, in his Illustrations of British Entomology (1827) and by other writers.

From that date till the present our knowledge of the subject in this county has constantly been kept up by a succession of earnest workers. The late Dr. R. C. R. Jordan and his brothers at Teignmouth; Messrs. J. J. Reading and J. S. Dell in the Plymouth district; Dr. Battersby at Torquay ; Mr. G. F. Mathew, R.N., F.L.S. at Barnstaple ; and Messrs. J. D'Orville and Edward Parfitt at Exeter, brought the subject into a very advanced stage of knowledge, strengthened by the publication of valuable catalogues.

At Exeter the late Rev. John Hellins, M.A., devoted his spare hours for many years to the working out of the preparatory stages, and collaborated most effectively with Mr. W. Buckler in the next county, in advancing our knowledge of the larvae. At Plymouth my friend Mr. G. C. Bignell has spent a large portion of his valuable life enriching our stores of knowledge in other orders as well as the present, and it is to his unwearied assistance that I personally owe much of my own knowledge of this the county of my birth. Here also good work has been done by Mr. W. H. Basden-Smith. Among the many others who have left records of their work in the contemporary magazines, may be mentioned Mr. E. S. Norcombe, of Heavitree ; Dr. W. S. Riding of Honiton ; Dr. Longstaff for the district of Morthoe; Miss K. M. Hinckliff of Instow; the late Major Still, who did good work at Seaton and on Dartmoor ; Mr. F. G. Briggs of Egg Buckland; Major C. M. Wells of Sidmouth ; and Mr. E. F. Studd of Oxton, near Exeter.

Visitors have done a large share of the entomological work since the county began to be distinguished as the principal point of immigration for conspicuous European species not previously recognized as British residents; and the published and privately furnished records of the late Mr. H. T. 
Stainton at Chudleigh and Great Haldon ; of Mr. George T. Porritt of Huddersfield ; Mr. W. F. de Visme Kane of Monaghan; the late Major-General George Carden of Surbiton; the Rev. Canon Cruttwell of Ewelme; Mr. Herbert Goss of Surbiton; Mr. J. Jaeger of London; Mr. Richard South, editor of the Entomologist; and many others have been consulted and used.

\section{RHOPALOCERA}

\section{Pieridar}

Aporia crataegi, Linn. (Black-veıned White butterfly)

The late Canon Tristram, writing to Mr. G. T. Bethune Baker, says, "I was at Torquay in ill-health in the spring and summer of 1854 . In the late spring or early summer I came across numbers of this butterfly in one field, a grassy slope, with rather steep banks, adjoining a wood. I think it was what they call the Castle Hill, but I have never been there since. I never saw the insects anywhere else. All that I captured were in the same field; I filled a store box, and for some years supplied my friends from it.' Another locality in which it was reported to have been taken is Moreton Hampstead; but there is no reason to believe that it has occurred in the county for at least half a century. During that period it has disappeared from all parts of England in which it formerly existed, except from the south-eastern corner.

- Pieris brassicae, Linn. (Large White)

* - rapae, Linn. (Small White)

* - napi, Linn. (Green-veined White)

- daplidice, Linn. Recorded at Rosebery Wood nr. Exeter in I 843 - one specimen only-and another at Culver in 1877

Anthocharis cardamines, Linn. (Orange Tip). Widely dist., sometimes scarce on the north coast, and on the wing in some years as late as June.

Leucophasia sinapis, Linn. (Wood White). Found near Exeter in I 855 ; at Stoke near Barnstaple in 1859 ; and in various subsequent years at Torquay; Honiton; Tiverton; Bickleigh Vale; Newnham Park; Woodbury; Catshill; Holsworthy; Instow; and Bridestowe. Still to be found on the south coast in abundance three or four miles east of Sidmouth and between this locality and Bere-Regis flying on broken slopes of the cliffs, and even over the shingle down to the sea-margin. The late Maj.-Gen. Carden found it in 1890 near Teigmmouth. -H. G.

Colias hyale, Linn. (Pale Clouded Yellow). Plymouth; Dartmouth; Teignmouth; Rame Head; Sidmouth; Tomes; Torquay; Babbington; Honiton; Morthoe; always casual and uncertain

- edusa, Linn. (Clouded Yellow). Plymouth; Exeter; Exmouth; Dawlish; Torquay; Sidmouth; Dartmouth; Teignmouth; Honiton; Barnstaple; Morthoe; Ilfracombe; perhaps everywhere in certain years, and then abundant. The pale variety - helice-is not rare, and a small form sometimes called var. chrysotheme is occasionally met with

*Gonepteryx rhamni, Linn. (Brimstone)

\section{LYCAENIDAE}

Thecla betulae, Linn. (Brown Hair-streak). Plymouth; Sidmouth; Dawlish; Dartmouth; Honiton: the glens of Dartmoor; Axminster; and various localities around Exeter. A very local species

I

\section{LYCAENIDAE (continued)}

Thecla quercus, Linn.(Purple Hair-streak). Ilfracombe and Chambercombe Woods; Barnstaple; Instow; Morthoe; Bridestowe and all the oak woods around Dartmoor; Stoke; Honiton; Starcross; Plymouth district and Sidmouth

- rubi, Linn. (Green Hair-streak). Sidmouth; Salcombe; Honiton; Stoke; Bickleigh Vale; Great Haldon; Chagford; Bideford; Barnstaple; Morthoe; on moors among genista and even furze, and often settling freely among cotton-grass

*Chrysophanus phlaeas, Lind. (Common Copper butterfly)

- circe, $\mathrm{Hb}$. dorilis, $\mathrm{Hfn}$. At a meeting of the Ent. Soc. London, in August, 1890, Prof. Meldola exhibited a male specimen captured at Ilfracombe, August, 1887. Nothing further is known of this specimen, nor has any other been recorded here. Probably it was accidentally introduced with plants or otherwise, from abroad

Polyommatus aegon, Sihiff. (Silver-studded Blue). Teignmouth; Torquay; Bovey Tracey; Bolt Head; Pinhay Cliffs

- agestis, Hb., medon, Esp. (Brown Argus). Plymouth; Dawlish; Teignmouth; Salcombe; Newton Abbot ; Starcross; Exeter; Braunton Burrows; Morthoe

* - alexis, Hb., incarus, Esp. (Common Blue)

- adonis, Hb., bellargus, St. C. (Clifden Blue). Formerly locally common at Torquay; Seaton and Sidmouth, on calcareous formations on the south coast. Still found nr. Torquay, as reported by Mr. G. T. Porritt

- argiolus, Linn. (Holly Blue). Plymouth; Teignmouth; Dawlish; Paignton; Sidmouth; Dartmouth; Exeter district, Woodbury; Stoke; Barnstaple; Instow; Morthoe; in two generations

- alsus, Schiff., minima, St. C. (Bedford Blue). Plymouth; Dartmouth; Teignmouth; Seaton; Torquay; Tinhay Cliff and Mecoy Vale; in calcareous districts

- aron, Linn. (Large Blue). Formerly plentiful on the cliffs of Bolt Head and Bolt Tail near Salcombe; now very nearly exterminated there from frequent burning of the furze; also at one time nr. Ashburton; and very rarely nr. Plymouth

†Nemeobius lucina, Linn. Dartmouth; also once taken by Mr. Parfitt in Dunsford Wood

\section{NYMPHALIDAE}

Apatura iris, Linn. Newnham Park; Dunsford Wood; Starcross; rarely nr. Tiverton. Seldom captured, though sometimes seen flying about the summits of oaks

Danais plexippus, Linn., archippus, Fab. During about fifteen years, from $1876-90$, a gallant and noble attempt was made by this grand 


\section{A HISTORY OF DEVONSHIRE}

\section{NYMPHALIDAE (continued)}

American butterfly to effect a settlement in the south of England. Flocks were observed in the course of their passage across the Atlantic-even resting on outward-bound ships on the ocean-and of those which made good their passage three at last were captured at Devon-one at Exmouth in 1885 ; another at $P$ lymouth; also, in 1886 , one at Dartmouth. The migratory impulse was at that time so powerful in this species that it is known to have made its way from one group of islands in the Pacific Ocean to another, becoming very common in Samoa and elsewhere, and reaching Australia-where it is plentiful-New Guinea, Tasmania, and New Zealand. Yet no effective settlement has been made by it in Great Britain, or, so far as is known, in any part of Europe

Vanessa polychloros, Linn. (Large Tortorseshell). Exeter; Bickleigh Vale; Teignmouth; Dawlish; Seaton; Topsham; Ivybridge; Barnstaple; Ilfracombe

* - urticae, Linn. (Small Tortoiseshell). Including Lundy Island

*- io, Linn. (Peacock B.). In I 899, and some previous years comparatively scarce

t- antiopa, Linn. (Camberwell Beauty). Specimens on record from Plymouth; Plympton; Torquay; Brixbam; Honiton; Ilfracombe; Bideford; Barnstaple; and East Ogwell. Some taken at ivy-bloom in the sunshine

*- atalanta, Linn. Including Lundy Island. Has also been taken at night, attracted by a stray light

- cardui, Linn. (Painted Lady). Common in every part of the county in some seasons, in others scarce; but in the most favoured seasons sometimes to be seen in hundreds upon blossoming ivy

Argynnis paphia, Linn. Sidmouth; Honiton; Barnstaple; Martinboe; Mortboe; Instow; Avonwick; Harpford Woods; Stoke; Fordlands; Dunsford; Bickleigh Vale and other woods skirting Dartmoor; and in all large woods

- adippe, Linn. Exeter; Stoke; Bickleigh Vale; Honiton; Barnstaple; Instow, and the Dartmoor woodlands, but far less common than the preceding

- aglaia, Linn. Exeter; Stoke; the Plymouth district ; Sidmouth; Salcombe; Honiton; Bridestowe; Instow; Martinboe; Mortboe. Most plentiful on the coast

†- lathonia, Linn. Single specimens recorded from Oxton and Roseberry Wood nr. Exeter; Braunton Burrows nr. Barnstaple; and at Bovey Tracey

- euphrosyne, Linn. Gen. in woods

- selene, Schiff. Rather more local ; found nr. Torrington; Bideford; Morthoe; Martinboe; Barnstaple; Modbury; Stoke; Haldonsometimes double-brooded - Bickleigh and Dawlish

Melitaea athalia, Esp. Bickleigh Vale; Horrabridge; and elsewhere on Dartmoor ; formerly in Fordlands Woods nr. Exreter; Buckland

\section{NYMPHALIDAE (continued)}

Woods; Newton Bushel; Barnstaple; Instow; Torrington; and Exmoor. Devon seems to have always been specially favoured by this very local and beautiful butterfly

Melitaea artemis, Hb., aurinia, St. C. Fordlands nr. Exeter; Torquay; Ivybridge; Axminster; Dartmoor; Instorw; Barnstaple; Bideford; Torrington. It has now disappeared from most of these places

\section{SATYRIDAE}

Melanargia galathea, Linn. In the Exeter district nr. Fordlands; Stoke Canon; and Kenton; along the south coast nr. Sidmouth; Teignmouth; Dawlish; Dartmouth; and Berry Pomeroy; northward at Holswortby; Instow; Mortboe, and Bridestowe; also nr. Axminster and Homer Down. Always very local

*Satjrus semele, Linn. On hilly heaths and coast cliffs

* Pararge aegerı, Linn. In shaded lanes and the borders of woods

- megaera, Linn. About all road-sides

**Epinephile janira, Linn. In fields, including Lundy Island

**- tithonus, Linn. About brambles when in bloom

- hyperanthus, Linn. Plymoutb; Dawlish; Topsham; Haldon; Haugh Moor; Bridestorie; Barnstaple; Instow; Mortboe. Abundant in some woods. Varieties with diminished numbers of spots are found, and also that called arete, in which these all are minute or absent

** Coenonympha pamphilus, Linn.

\section{HESPERIIDAE}

Syrichthus alveolus, Hb., malvae, St. C. Bickleigh Vale; Plymouth; Stoke; Haldon; Woodbury; Sidmouth; Bideford; Barnstaple; Instow; not scarce; the pretty var. taros also occasionally

*Hesperia linea, Fab., thaumas, St. C. Plymoutb; Salcombe; Stoke; Tavistock; Cattedown; Barnstaple; Martinboe; Morthoe; Instow

- actaeon, Esp. On the cliffs and in the chines near Sidmouth; Bere Regis; Torquay, and Exmouth. This is a portion of the only locality for the species in the United Kingdom; the remainder extends in Dorset to Weymouth and 3 miles east of Swanage. Also common to the west of Swanage for 2 miles.

* - sylvanus, Esp. Sidmouth; Salcombe ; Plymouth; Exwick; Stoke Woods; Barnstaple; Martinboe: Mortboe

- comma, Linn. Plymouth; Exmouth; Dartmouth; Sidmouth; Stoke. Very local, and confined to the calcareous districts

Nisoniades tages, Linn. Bickleigh Vale; Sidmouth; Bideford; Barnstaple; Instow; Mortboe; common in Fordlands and Stoke Woods. Said by Mr. E. Parfitt to be double-brooded in Devon 


\section{INSECTS}

\section{H E T E ROCER A}

\section{SPHINGIDAE}

Smerinthus ocellatus, Linn. Plymouth; Topsham; Sidmouth; Dartmoor; Barnstaple

- populi, Linn. Exeter; Stoke; Plymouth; Sidmouth; Dartmoor; Barnstaple; Morthoe. About poplars, not very common

\$- tiliae, Linn. Exeter; Starcross; Plymouth; Ashburton; Spitchwick. About elms

Acherontia atropus, Linn. (Death's Head). In some seasons the larva is found in potato fields in all parts of the county

Sphinx convolvuli, Linn. Quite uncertain, yet seen or taken in some part of the county almost every year ; in some favoured years, such as $1859,1868,1887,1900$, and 1901 , occurs all over the county, sometimes in numbers, yet most frequently on the north and south coasts. There is little doubt that the vast majority seen are immigrants from the Continent, but in Igor the larva was frequently found, and numbers of genuine native specimens must have occurred

- pinastri, Linn. The capture of a single specimen in a garden nr. Plympton, September, 1861 , is recorded in the Entomologist for 1872

- lingustri, Linn. Has been found here to feed upon holly, laurustinus, ash, guelder-rose, both wild and cultivated, as well as on its ordinary foods-privet and lilac

Deilephila euphorbiae, Linn. In the Aurelian, by Moses Harris, is the following :- Mr. Raddon, the celebrated engraver, detected this species in considerable numbers, on Euphorbia paralias, growing in great abundance on the extensive sandhills at Appledore and Braunton Burrows nr. Barnstaple. See for further details the Entomological Magazine, No. 9, in illustration of two beautiful plates figuring the entire history of the insect by Mr. Raddon himself. In the following number of the same work Mr. Raddon has supplied some additional particulars showing its occasional rarity or abundance; thus in I 814 he would not capture any of the caterpillars which were not full-fed, and after one day's pursuits, having forgotten to take any food for his caterpillars, and it being nearly dark, he cut an armful of the spurge and at night put it into water ; next night he found this food covered with, he thinks, not less than a hundred minute larvae not above a day or two old. Since that period the surface of the valley in which the insects were found has been completely altered by the action of the winds, and the insect has been almost exterminated.' Apparently it became quite so, for although the most frequent and thorough search has been made in many subsequent years, during the greater part of a century, in the same locality, no further capture of this fine moth has, in any stage, there been made

†- galii, Schiff. On the coast ; recorded from Plymouth; Devonport; Kingsbridge; Starcross; Alphington; Exeter, and Ilfracombe. Usually singly

\section{SPHINGIDAE (continued)}

tDellephila livornica, Esp. Recorded from Plymouth and Mount Edgecumbe; Horrabridge; Plympton; Kingsbridge; Starcross; Sbeldon; Torquay; Dartmouth; Exeter, and Tiverton. At Starcross the larva has been found and recorded by $\mathrm{Mr}$. Jaeger

tChaerocampa celerio, Linn. One recorded at Exeter in 1871; one at Teignmouth in 1865 ; one at $P$ lymouth about the same time; another at the same place; and one at Ilfracombe in 1885

§- porcellus, Linn. Plymouth; Dartmoor; Horrabridge; Westward Ho

- elpenor, Linn. More frequent, and found in almost all parts of the county

†— nerii, Linn. There is an old record that Mr. Raddon took a specimen near Barnstaple more than a century ago; one was taken at Teignmouth by Capt. Blomer in 1832 ; one at Stoke Henning, nr. Dartmouth, by Master Owen in 1892 ; and a second at Teignmouth in 1900

"Macroglossa stellatarum, Linn. (Humming-bird). Gen. dis.; and in some years common. Usually seen hovering at flowers

$\S$ - fuciformis, Linn. (Broad-bordered Bee Hawk). Budleigh Vale; Stoke; Fordlands ; Torquay

§- bombyliformis, Esp. (Narrow-bordered Bee Hawk). Barnstaple; Woodbury ; Plymouth

\section{SESIJDAE}

Sesia tipuliformis, Linn. Exeter; Plymouth ; Barnstaple. In gardens about currant trees

- cynipiformis, Esp. Devonport; Radford Wood nr. Plymouth; Stoke; Topsham. On oak and elm trunks

- myopaeformis, Bkh. Exeter; Plymouth; Barnstaple. Among apple trees

- formiciformis, Esp. Recorded only from Stoke

- ichneumoniformis, Schiff. Plymouth; Bickleigh Vale; Stoke; Teignmouth. Mr. Parfitt says on all grassy slopes near the sea on the south coast, and also on the north to Lynton. It feeds on bird's-foot trefoil

- philanthiformis, Linn. Torquay, where it was first taken in England in 1870 ; Salcombe; Lynton; Plymouth. Generally on rocky coasts among thrift

- chrysidiformis, Esp. Dr. Longstaff states that he saw a specimen on the cliffs at Woolacombe, 7 September, 1888

Sphaecia bembeciformis, Hb. Exeter; Plymouth; Plympton; Teignmouth; Sidmouth; Barnstaple. Among sallows and poplars

†- apiformis, Linn. Bickleigh Vale; Barnstaple. Among poplars

\section{ZYGAENIDAE}

Procris statices, Linn. Exeter district; Southdown, Plymouth; Musbury; Axminster; Berry Pomeroy; Newton. Rather scarce in this county 


\section{A HISTORY OF DEVONSHIRE}

\section{ZYGAENIDAE (continued)}

Zygaena trifolii, Esp. Bideford; Barnstaple; $A x$ minster; Plymouth. In marshy places

\$- lonicerae, Esp. Barnstaple; Cattedown nr. Plymouth; Southdown; Teignmouth; Torquay

- - filipendulae, Linn. Gen. dis. and often abundant

\section{ZEUZERIDAE}

†Zeuzera aesculi. Linn. (Wood Leopard). Plymouth; Fordlands Wood; Heavitree; Saltram; Barnstaple

Cossus ligniperda, Fab. (Goat Moth). Exeter; Plymouth; Mount Edgcumbe ; Axmouth ; Topsham; Dunsford; Tavistock; Barnstaple. Usually found in the larva state

$\S$ Hepialus hectus, Ochs. Plymouth; Torquay ; Exeter; Stoke Wood; Cann Wood; Ivybridge; Barnstaple; Lynton. Among fern

*- lupulinus, Linn. In meadows

- sylvinus, Linn. Plymouth; Exeter; Braunton Butrows; Instow; Morthoe; Lynton.

- velleda, Esp. Dartmoor, local; also taken rarely at Ivybridge and at Torquay many years ago

- humuli, Linn. Exeter; Plymouth; Barnstaple; Lynton; Morthoe. Moving at dusk in meadows

\section{LIMACODIDAE}

Heterogenea asella, Schiff. Recorded at Bickleigh Vale by Mr. J. Basden-Smith, and in the Plym Valley by Mr. F. J. Briggs

\section{CHLOEPHORIDAE}

Halias prasinana, Linn. Exeter; Plymouth; Dartmoor; Bridestowe ; Lynton; Martinhoe. About oaks; common in the north of the county ; and there the male may be heard, when flying, to make a buzzing noise. This has been particularly noticed by Mr. R. South, editor of the Entomologist

†- quercana, Schiff, bicolorana, St. C. Nr. Exeter; taken at light

\section{NOLIDAE}

Nola cucullatella, Linn. Also taken at light at Exeter and Dortmouth

- strigula, Schiff. Topsham; Starcross

* - confusalis, $\mathrm{Hb}$. Tiverton. On apple trees; Exeter; Plymouth; Bickleigh Vale, on oak trees ; Seaton

- albulalis, Hb. Mr. G. T. Porritt writes in the Entomologist for January, 1902 : 'I have had one or two specimens sent me for determination, captured near Paignton, in South Devon, during the past season, but am asked at present not to state the exact locality. It is a fine and well-marked example.' This warrants a hope that this pretty species, hitherto almost confined to Kent, may now be extending its range

\section{LITHOSIDAE}

Nudaria senex, Hb. Sixty years ago the late Dr. R. C. R. Jordan used to take this species in a small alder swamp at Teignmouth. This locality is long since drained and ploughed. A single specimen was taken in the Exminster marshes by Mr. E. Parfitt

- mundana, Linn. Plymouth; Exminster; Exeter; Lynton; Martinhoe. About lichen-covered walls and rocks

Calligenia miniata, Forst. Exeter ; Stoke; Starcross; Plymouth; Babbacombe; Paignton; Dartmouth; and elsewhere along the sea coast

Setina irrorella, Linn. Mount Edgcumbe ; Whitsand Bay; Banpool; Seaton; Babbington. Very scarce

\$- mesomella, Linn. Fordlands and Stoke Wood nr. Exeter; Cann Wood; Plymouth; Topsham; Torquay; Dartmoor

Lithosia aureola, $\mathrm{Hb}$., sororcula, St. C. Taken at light at Exeter; also at Sidmouth, but scarce

- caniola, Hb. Torquay; Babbacombe; Boll Head. Only on the extreme south coast, frequenting the cliffs, especially at the brow and face of a precipice

- complana, Linn. Plymouth district; Fordlands and Stoke Wood nr. Exeter; Barnstaple; Morthoe. On open heaths and on the coast

$\$$ - complanula, Bdv., lurideola, St. C. Gen. dis.

- griseola, Hb. Plymouth; Mount Edgrumbe; Bickleigh Vale; Exeter; Barnstaple. In marshy woods

— rubricollis, Linn. Plymouth ; Ivybridge; Kingsbridge; Torquay; Axminster; Dartmoor. Uncommon around Exeter; Barnstaple. Somewhat irregular and uncertain in appearances

Oenistis quadra, Linn. Exeter; Stoke Woods; Dartmouth; Torquay; Shaugh Bridge. Scarce

\section{ARCTIIDAE}

Deiopeia pulchella, Linn. Several have been taken at Babbacombe; Paignton; Dartmouth; Slapton; Kingsands, and Stonehouse; but it is only casual and very rare

Eughelea jacobaeae, Linn. Gen. dis. among ragwort

Callimorpha dominula, Linn. Plymouth; Bickleigh Vale; Chagford, and other valleys bordering Dartmoor; Axminster; Torquay; Sidmouth; also Stoke and other places round Exeter, and formerly not uncommon near Barnstaple

- hera, Linn. This magnificent species seems to have been quite unknown, as British, up to the middle of the past century, though well known in the Channel Islands and abundant on many parts of the Continent. From that time casual specimens, very rarely and at long intervals, appeared on different parts of the English south coast. One such was captured at Alphington nr. Exeter, August, 1871, by the late Mr. D'Orville, well known as the curator at the Exeter Museum. In I 873 two were taken at Hazelzoood nr. Loddiswell. By $188 \mathrm{I}$ and 1882 it had become well established, having been taken at Dawolish and at Starcross, where it 'excited 


\section{ARCTIIDAE (continued)}

no surprise, as the inhabitants had seen the species before.' From that time to the present its headquarters seem to have been around these two places, but it has spread abroad over the south of the county, so that it may be beaten out of the hedges, or even seen flying in bright sunshine over the fields in July and August, almost all over that district; and it indeed forms the principal inducement to the many enterprising entomologists who in recent years have spent their well-deserved and hard-earned holidays in South Devon. Varieties having the hind wings orange or yellow are frequent. It does not seem as yet to have extended its range beyond the limits of this county, but has been freely reared elsewhere from eggs deposited by females captured here

Euthemonia russula, Linn. Gen. dist. upon heaths, and has even been known to come to a strong light, nr. Exeter

Nemeophila plantaginis, Linn. Taken on the hills of Dartmoor and also at Lynton, but not gen. dist.

*Arctia caja, Linn. In gardens

- villica, Linn. Exeter; Torcross; Topsham; Seaton; Sidmouth; Plymouth; Bickleigh Vale; Stoke; Barnstaple; Instow; Torrington; Morthoe. Well dist. in lanes, rough ground, and woods - a most attractive insect

- Phragmatobia fuliginosa, Linn. On hills and at the edges of woods

$\$$ Spilosoma mendica, Linn. Exeter; Stoke; Plymouth; Dartmoor; Topsham; Dartmouth. Usually taken flying in the sunshine

*- lubricepeda, Linn., and S. menthastri, Schiff.

\section{LIPARIDAE}

Porthesia chrysorrhoea, Linn. Mr. Parfitt says that it has been taken at Topsham and Torquay. It seems to have become here, as in many parts of England, extinct

†- auriflua, Fab., similis, St. C. Torquay and nr. Exeter

Liparis salicis, Linn. Rare at Exeter, also formerly found at Torquay and Tomes

$\$$ Psilura monacha, Linn. Plymouth; Bickleigh Vale; Ivybridge; Dartmouth; Paignion; Exeter; Barnstaple; Mortboe; Hartland

Dasychira pudibunda, Linn. Exeter; Plymoutb; Seaton; Sidmouth; Honiton; Barnstaple; Morthoe

* Demas coryli, Linn. Very widely distributed, especially on the hills; the larva sometimes common on beech and hazel bushes

†Orgyia gonostigma, Fab. Mr. Parfitt found larvae in Stoke Wood nr. Exeter

- antiqua, Linn. Gen. abundant and even mischievous, defoliating some of the shrubs in the gardens in certain years; in others, scarce

\section{PSYCHIDAE}

tPsyche villosella, Och. On heaths in the south

- fusca, Haw. Recorded by Mr. J. S. Dell as rare nr. Plymoutb

\section{PSYCHIDAE (continued)}

tEpichnopteryx reticella, Newn. Captured on the coast (Parfitt)

Fumea roboricolella, Br., nitidella, Stn. Wonford Marshes; Exeter; Staddon Heights; Plymouth; Torquay. Its case, resembling a little bundle of bits of dried grass, is often to be seen

\section{BOMBYCIDAE}

Poecilocampa populi, Linn. Plymouth; Sidmouth; Seaton; Honiton; Excter; and elsewhere; frequently found about gas lamps, at nights, in the winter

Trichiura crataegi, Linn. Exeter; Plymouth; Torquay; Lustleigh; Dartmouth; Egg Buckland; Dartmoor. Scarce

Eriogaster lanestris, Linn. Barnstaple; Braunton; Seaton. Excessively local

*Clisiocampa neustria, Linn.

-- castrensis, Linn. J. F. Stephens records this in his Illustrations $(1828)$ as found on the coast of Devon, at the mouth of the Exe; and $M r$. E. Parfitt stated in 1853 that he had taken one specimen nr. Exeter, but omitted it from his List (1878). Now almost restricted to the Kent and Essex salt-marshes

Lasiocampa trifolii, Esp. Formerly on the coast at Bovisand; Wbitsatt, and elsewhere near Plymouth. There abundant till about I 860 , but from this time it began to be scarce, and was gradually, it is to be feared, exterminated by excessive collecting. It has also been found at Torquay; Bolt Head; Bolt Tail; Dartmoor; Alpbington; Stoke, and Bovey Heatbfield; but not now apparently existing in any one of these localities

- quercus, Linn. Gen. dist.

- rubi, Linn. Widely dist., especially frequenting moorlands and hills

Odonestis potatoria, Linn. Exeter; Plymouth; Dartmoor; Barnstaple; Morthoe. Common in lanes; its conspicuous larva feeding on rough grasses on hedge-banks

Gastropacha quercifolia, Linn. Countess $W$ eir and Alphington, nr. Exeter; Sidmouth; Torquay; Plymouth. Not rare

- ilicifolia, Linn. A larva of this very rare species was found nr. Lynton, in a wood abounding with bilberry, Aug. 1864, by the Rev. E. Horton of Worcester. Unfortunately it was sickly, and died; but there appears to be no doubt as to the species. No other record seems to exist for this county

\section{ENDROMIDAE}

Endromis versicolor, Ochs. Mr. J. F. Stephens in his Illustrations has this record: "Taken near Plymouth, Dr. Leach.' Mr. Parfitt says that it has been taken once at Torquay and once at Barnstaple; also that Mr. G. F. Mathew reported that the males had been seen flying wildly in Lustleigh Cleve, where birch was common. It does not seem to have been noticed recently 


\section{A HISTORY OF DEVONSHIRE}

\section{SATURNIDAE}

Saturnı carpini, Schiff,, pavonia, Linn. Frequently on heaths and hills. Stoke; Haugh; Roborough Down; Woodbury Common; Barnstaple; Haldon; Yes Tor and other parts of Dartmoor; Torquay; Sidmouth; Exmouth; Bickleigh Vale; Plymouth. Mr. G. C. Bignell found a colony of half-grown larvae feeding on a most unusual plant, for them-Rhamnus frangula

\section{DREPANULIDAE}

Drepana falcataria, Linn. Plymouth; Bickleigh Vale; Exeter; Barnstaple. Among birch

- hamula, Schiff., binaria, St. C. Stoke Wood and Fordlands, nr. Exeter; Honiton; $A x$ minster; Torquay. Among oak

$\$$ - unguicula, Hb., cultraria, St. C. Bickleigh Vale; Greenbank, nr. Plymouth; Dartmouth; Exeter; Honiton. Among beech

Platypteryx lacertinaria, Linn. Exeter; Axminster; Torquay; Bickleigh Vale. Scarce

Cilix spinula, Schiff. Gen. dist. in lanes and about hawthorn edges

\section{NOTODONTIDAE}

Cerura bicuspis, Bkh. Three larvae of this rare species were found feeding on alder in Walkham Valley, nr. Plymouth, Aug. I 894, by $\mathrm{Mr} . \mathrm{H}$. W. Basden Smith; and of these one was reared

- furcula, Linn. Plymouth district; Dartmouth; Torquay; Dartmoor; Landulph; Sidmouth; Barnstaple; Ilfracombe; and Stoke Wood; Exeter: About sallows

- bifida, Hb. Frequent among poplars. Nr. Exeter; Plymouth; Dartmouth; Torguay; Sidmouth; Dartmoor; and Barnstaple

- vinula, Linn. Gen. dist. among poplar

tStauropus fagi, Linn. Mount Edgecumbe; Antony Park, Plymouth; Ivybridge; Bickleigh Vale; Exeter; Sidmouth; Basnstaple

Notodonta dictoea, Linn.; Plymouth; Dartmouth; Seaton; Teignmouth; Torquay; Sidmouth; Stoke Wood, nr. Exeter; Barnstaple. Among poplars

- dictaeoides, Esp. Plymouth; Egg Buckland; twice taken at Oxton; Exeter; Plymouth; Seaton. Scarce among birch

$\$$ - ziczac, Linn. Exeter; Plymouth; Seaton; Torquay; Sidmouth; Barnstople; Instow

\section{NOTODONTIDAE (continued)}

†Notodonta tritophus, Fab. A larva was beaten out of alder nr. Exeter, by the Rev. J. Hellins, Aug. 1870, but, unfortunately, was not reared. No other record seems to exist here, and not more than five or six in the United Kingdom.

- trepida, Esp. Killerton Park and elsewhere in the Exeter district ; Seaton; Plymouth; Dartmouth ; Bickleigh Vale; Dartmoor; Bernstaple; Instow. Scarce ; among large oaks

§dromedarius, Linn. Exeter; Plymouth; Bickleigh Wood; Seaton; Sidmouth; Axminster; Tavistock; Tiverton; Dartmoor; Barnstaple. Among oak

- bicolora, Hb. A single specimen captured in the Exeter district (1880) is recorded by the late Maj. J. N. Still in the Entomologist's Monthly Magazine, May, 1892

Drymonia chaonia, Hb. Exeter; Plymouth; Bickleigh Vale; Dartmoor; Seaton; Topsham; Dartmouth; Torquay. Scarce among oaks, but very strongly attracted by a bright light

- dodonaea, Schift. ; trimacula, St. C. Exeter; Honiton; Bickleigh Vale; Plymouth; Seaton; Dartmouth. Among oak ; scarce

"Lophopteryx camelina, Linn. Gen. dist. about woodlands, but not plentiful

- cucullina, Schiff. In 1857 the Rev. E. Horton recorded the capture by himself of seven larvae in July, at Martinboe, near Lynton. A single specimen also was taken nr. Exeter by the Rev. J. Hellins

§ Pterostoma palpina, Linn. Gen. dist. among poplars

Ptilophora plumigera, Esp. Torquay. Formerly not rare in some seasons; may be met with on the gas-lamps in the public grounds (E. Parfitt)

Petasia cassinea, Schiff. Widely dist., but not very common. Mr. Parfitt says that it may be found sticking on old ports and sails, resembling in colour the greyish lichens which grow on them. It is more frequently captured at gas-lamps. In $1896 \mathrm{Mr}$. Studd found it in plenty at light at Oxton, one specimen having a male antenna on the right side, female on the left

- Pygaera bucephala, Linn. Its larvae sometimes partially defoliating various trees

Diloba coeruleocephala, Linn. Exeter; Plymouth; Teignmouth; Honiton; Barnstaple. Not very local

\section{NOCTUINA}

\section{CYMATOPORIDAE}

Gonophora derasa, Linn. Gen. dist. in woods Thyatira batis, Linn. Also widely dist. in woods Cymatophora duplaris,Linn. Exeter; Plymouth; Dartmoor; Barnstaple. Probably in most large woods

- or, Schiff. Recorded in Stephens's Illustrations Asphalia diluta, Schiff. Mr. Parfitt says also in woods all over the county, but more frequent in those of the northern portion

\section{CYMATOPORIDAE (continued)}

Asphelia flavicornis, Linn. Fordlands, nr. Exeter; Wembury, nr. Plymouth; Stoke; Instow. On birch bushes

- ridens, Fab. Stoke Wood; Exeter; Bickligh Vale; Plymouth ; Plymbridge; Honiton; Dartmoor; Exwick Wood; Great Torrington. Usually scarce; said to have, at one time, been common at Camborme 


\section{INSECTS}

\section{TRIFIDAE}

†Diphthera orion, Esp. Bickleigh Vale and other woods on the borders of Dartmoor; Wembury; Great Torrington; Shaugb

Acronycta leporina, Linn. Mr. Parfitt says not uncommon round Plymoutb-at Greenbank; Nortb Hill; Milebouse; and Devonport-also taken on Dartmoor; Egg Buckland; Haldon; Torquay; and Teignmouth

†- aceris, Linn. Mount Edgcumbe; Saltram Woods; Sidmouth; and Torquay.

$\$$-megacephala, Schiff. Plymoutb; Seaton; Torquay; Barnstaple; Stoke; Instow

- alni, Linn. Plymouth; Avonwick; Axminster; Bickleigh Vale; Seaton; Honiton; the Exeter district; Barnstaple; Tavistock. Always rare, and usually only found as a larva

†- tridens, Schiff. Plymouth district; Dartmoor; Barnstaple.

* - psi, Linn.

* - rumicis, Linn. In woods

- ligustri, Schiff. Exeter; Stoke; Honiton; Plymouth; Plymbridge; Cann Wood; Dartmoor; Paignton; Barnstaple; Lynton; Mortboe. Among ash trees

Agrotis valligera, $\mathrm{Hb}$. On all the sandhills of the north and south coasts

- suffusa, $\mathrm{Hb}$. Widely dist. ; often common at ivy-bloom in autumn

* - segetum, Schiff. Most mischievous in the larval state

- corticea, Hb. Plymouth; Teignmouth; Dart mouth; Torquay; Topsham; and Instow

- puta, Hb. Gen. dist.; usually taken at light

- Agrotis exclamationis, Linn.

- lunigera, Steph. Cliffs at Torquay; Bolt Head; Seaton; and Morthoe. A specimen flew into Mr. Basden Smith's house at Hillsborough, $P$ lymouth, in 1888

- ripae, Hb. On coast sands below Exeter, and at Instow and Bideford

- cursoria, Bkh. On the sands at Dawlish Warren and at Mortboe

$\$$ - nigricans, Linn. Exeter; Plymouth; Bickleigh Vale; Torquay; Mortboe

- tritici, Linn. Exeter; Plymouth; Dawlish Warren; Morthoe. Most frequent on the coast

†- aquilina, Schiff. Plymouth; Cann Wood; Torquay; Ilfracombe; Braunton

- obelisca, Hb. On coast cliffs. Mount Edgecumbe; Stonebouse; Teignmouth; Torquay; and Dr. Longstaff reports three specimens at Morthoe in 1904

t- agathina, Dup. Alpbington; Cann Wood; Honiton; Lynton; Morthoe. On heaths

*- porphyrea, $\mathrm{Hb}$. On heaths

- praecox, Linn. Coast below Exeter; Dawlish Warren; Braunton Burrows. Only on sea sandhills

- saucia, Hb. Very irregular; usually not common, but in some seasons abundant all over the county

- pyrophila, Fab. The capture of a specimen at Morthoe is reported by Dr. Longstaff

- ravida, $\mathrm{Hb}$. Alpbington and Torquay

- lucernea, Linn. Torquay; Dartmouth; Stonebouse, and elsewhere on the south coast, among rocks; also at Lynton

\section{TRIFIDAE (continued)}

*Axylia putris, Linn.

Triphaena fimbria, Linn. Exeter; Stoke; Plymouth; Dartmouth, and elsewhere on the south coast; also at Ilfracombe ; Lynton; Mortboe; and on Dartmoor

- janthina, Esp. Gen. dist.

$\$$ - interjecta, Hb. Plymouth; Torquay; Sidmouth; Morthoe; widely dist.

- orbona, Fab.

* - pronuba, Linn.

\$Noctua glareosa, Esp. Plymouth; Ivybridge; Daswlish; Dartmouth; Bolt Head; Torquay; Ugborough Beacon; Ilfracombe; Morthoe. Some specimens are flanked with beautiful pink colouring

- augur, Fab. Taken nr. Exeter at light ; also rarely in both the north and south of the county

** - plecta, Linn.

†- depuncta, Linn. Exeter; Yealmpton; Alpbington; Harford Bridge; Bridgerule. Usually a northern species

\$- triangulum, Tr. Exeter; Plymouth; Plymbridge; Dartmoor; Teigrmouth; Sidmouth; Torquay ; Instow

* c-nigrum, Linn. Gen. dist.

†- ditrapezium, Bkh. Devonport; Plymbridge; Bickleigh; Torquay; Barnstaple

$\$$ - brunnea, Fab. Gen. dist.

\$- dahlii, Hb. Stoke Wood; Exeter; Shaugh Woods; Plymbridge; Torquay; Bamstaple; Morthoe

- festiva, Hb.

- baja, Fab.

- rubi, View.

- umbrosa, $\mathrm{Hb}$.

Gen. dist. in woods and usually common

* - xanthographa, Fab. In woods

\$- neglecta, Hb. Exeter; Honiton; Dartmoor Plymouth ; Teignmouth

Eurois herbida, $\mathrm{Hb}$. Plymouth district and Dartmoor Woods, about Lynton. Mr. Parfitt says that it is scarce in the county and rare at Exeter; also that it flies in Aug. and Sept. This is singular since it is plentiful in woods in many parts of the south of England in June and July

- occulta, Linn. A single specimen secured on the south coast nr. Dartmouth in 1900 by Mr. G. T. Porritt

- adusta, Esp. Gen. dist. in woods

\$- Charaeas graminis, Linn. Devonport; Hillsborough; Starcross; Exeter; Honiton; Morthoe

Heliophobus popularis, Fab. Gen. dist. and often common

§- cespitis, Fab. Exeter; Exmouth; Darmouth; Plymouth; Honiton; Barnstaple; Morthoe. In some years in plenty on Dartmoor

$\$$ Neuria saponariae, Bkh. Exeter; Seaton; Crabtree; Plymouth district; Slapton; Torquay; Chag ford

Aplecta advena, Fab. Plymouth district ; Topsham; Barnstaple. Rather scarce

- tincta, Hb. Once taken at Egg Buckland by Mr. F. J. Briggs; in the Plymouth district (E. Parfitt)

\$- nebulosa, Hufn. Exeter; Plymouth; Sidmouth; Darimoor; Barnstaple; Lynton

†Hadena contigua, Vill. Exeter district

§- thalassina, Rott. Gen. dist. in woods 


\section{A HISTORY OF DEVONSHIRE}

\section{TRIFIDAE (continued)}

†Hadena suasa, Bkh., dissimilis, St. C. Exeter ; Starcross; Dawlish Warren; Topsham; Winslade

- pisi, Linn. Gen. dist. ; common on the slopes of the tors of Dartmoor

* * oleracea, Linn. In gardens

- genistae, Steph. Seaton; Barnstaple; Instow. Rather scarce

"- dentina, Esp. Exeter district; Tiverton; Instow; Morthoe

†- chenopodii, Fab.; tripolii, St. C. Alphington; Millbrook; Barnstaple

- glauca, Hb. Recorded by Mr. Parfitt at Alphington, Exeter; but probably in error. It is distinctly a northern insect

Mamestra albicolon, Hb. Taken by Mr. G. F. Mathew, R.N., on the coast nr. Barnstaple

"*- brassicae, Linn.

- persicariae, Linn. Gen. dist.

Hecatera serena, Fab. Exeter; Plymouth; Salcombe; Teignmouth; Torquay; Dartmoor; Morthoe

Dianthecia carpophaga, Bkh. Frequent on the coast

- cucubali, Fues. Gen. dist. on the coast and inland

$\$$ - capsincola, $\mathrm{Hb}$. Gen. dist.

$\dagger$ - albimacula, Bkh. Taken at Seaton by the late Major Still

- conspersa, Esp. Torquay ; Bolt Head; Dartmouth; Seaton; Teigmmouth; Barnstaple; Morthoe. Sometimes not scarce

- luteago, Hb. A single specimen taken nr. Ilfracombe by Mr. W. C. Boyd, July, 1879 . This was the first specimen known to have been taken in England, those previously known having occurred in Ireland and Wales; since then a few have been found in Cornwall

Ulochlaena hispida, Tr. Plymouth; Dartmouth ; Exmouth; Teignmouth; Babbacombe; Torquay. Confined to the cliffs of the south coast

Aporophyla lutulenta, Bkh. Alphington; Stoke; Dartmouth; Brixham; Plymouth; Morthoe. Rather scarce

-- nigra, Haw. Frequent all along the south coast ; also nr. Exeter, on Dartmoor, and at Newton Abbot. In the north only recorded at Mortboe

- australis, Bdv. Formerly abundant at Torquay; also found at Teignmouth, Dartmouth, and Bolt Head; always on the coast cliffs

Epunda lichenea, Hb. Gen. dist. on both coasts

Dasypolia templi, Thumb. Exeter; Plymouth; Teignmouth; Torquay; Sidmouth. Usually at gas-lamps at night

*Polia flavicincta, Fab. Exeter; Egg Buckland; Dartmouth; Plymouth; Teigmmouth; Torquay

- xanthomystas, Hb. ; nigrocincta, Tr. Several specimens were taken at Morthoe by Dr. Riding in 1883 and it has since been found there and near Ilfracombe by Dr. Longstaff and Prof. Dixey. Otherwise it seems almost confined to the Isle of Man

- chi, Linn. Dartmoor; Plymouth; Avonwick; and common nr. Dartmouth. Usually a northern insect

-Dryobota protea, Bkh. Among oaks

\section{TRIFIDAE (continued)}

Cleoceris viminalis, Fab. Sparsely distributed in woody districts (E. Parfitt). Mr. W. H. Basden-Smith records it at Plymouth

Chariptera aprilina, Linn. Gen. dist. among oak

- Miselia oxyacanthae, Linn. In late autumn

- "Luperina testacea, Hb.

Cerigo cytherea, Fab., matura, St. C. Alphington Berry Pomeroy; Wembury; Torquay; Brixham ; Morthoe

Hama abjecta, $\mathrm{Hb}$. Taken at Dau'lish Warren by Mr. E. F. Studd

- anceps, Hb. ; sordida, St. C. Widely dist. but scarce

- furva, Hb. Recorded nr. Honiton by Dr. Riding ; also once taken at Keyham; Devonport

"Xylophasia lithoxylea, Fab.

- sublustris, Esp. Sidmouth; Harford Bridge; Dartmoor

**- polyodon, Linn., monoglypha, St. C.

* - rurea, Fab.

- hepatica, Hb. In all woods

- scolopacina, Esp. Exeter, rare ; Plymoxth district; Barnstaple; near Ilfracombe. Very local

* Apamea basilinea, Fab.

\$- gemina, Hb. Widely dist.

- unanimis, Fr. Exeter; Torquay; Ilfracombe. Not at all common

** oculea, Gn., didyma, St. C.

Miana literosa, Haw. Gen. dist.

** - strigilis, $\mathrm{Cl}$.

\$- fasciuncula, Haw. Exeter; Plymouth; Babbacombe; Barnstaple; Morthoe

*- furuncula, Tr. On both coasts and in great variety

Eremobia ochroleuca, Esp. Recorded by Mr. H. Jenner Fust, but without locality

†Dipterygia pinastri, Linn. Stoke Wood, nr. Exeter Torquay ; Bickleigh Vale

Trachaea atriplicis, Linn. Bred from larvae taken nr. Plymouth by Mr. H. S. Bishop (E. Parfitt). Probably some error occurred; this species is mainly confined to the eastern counties, and is rare there

Euplesia lucipara, Linn. Exeter; Plymouth; Horrabridge; Stoke; Barnstaple; Morthoe; Instow. Common in $S$. Devon

* Phloyoptera meticulosa, Linn.

Hydraecia nictitans, Bkh. Exeter and various parts of the south coast to Sidmouth; also Dartmoor and Morthoe

- micacea, Esp. Plymouth; Dartmoor and Bridestowe; Morthoe; Exeter; Paignton. Scarce

Gortyna flavago, Esp. Gen. dist., the larvae feeding usually in the stem of the foxglove

Nonagria cannae, Ochs. Mr. Parfitt records this species from Torquay and Teignmouth

- sparganii, Esp. This, which has not been known as British until comparatively recent years, and is probably an immigrant, has now been found by Mr. G. T. Porritt nr. Dartmouth

- typhae, Esp. Exeter; Starcross; Torquay; Barnstaple. Local

Coenobia rufa, Haw.; despecta, Tr. Recorded in Devon by Mr. H. Jenner Fust, but without locality. A marsh insect 


\section{INSECTS}

\section{TRIFIDAE (continued)}

Tapinistola fulva, $\mathrm{Hb}$. Exeter; Cann Wood, nr. Plymouth; Teigmmouth; Honiton, common; rare at Morthoe

- bondii, Knaggs. ${ }^{1}$ This extremely local species occurs in the extreme south-eastern corner of the county towards Lyme Regis, among Festuca arundinacea

Calamia lutosa, $\mathrm{Hb}$. Recorded, though without locality, by Mr. H. Jenner Fust. Doubtless to be found in the county

Leucania pudorina, Hb. Recorded at Sidmouth in I8g I by Mr. W. R. S. Majendie

- - impura, Hb.

- pallens, Linn. Scarce at Morthoe

- straminea, $\mathrm{Tr}$. Recorded in the county by Mr. H. Jenner Fust, but without locality. One specimen found nr. Dartmouth, recorded by Mr. G. T. Porritt

- putrescens, Hb. Torquay and Babbacombe ; Sidmouth; Teignmouth; Dartmouth. Discovered at Torquay in 1859 by Mr. R. M. Stewart and Dr. Battersby. Still existing on the cliffs $\mathrm{nr}$. the sea at this and other localities along the coast; but excepting on a portion of the coast of South Wales, not known elsewhere in Great Britain

\$- comma, Linn. Exeter; Tiverton; Barnstaple; Instow; Honiton; Torquay; Bickleigh Vale

- littoralis, Curt. Dawlish Warren; Dartmouth district; Instow; Morthoe. Only found on the coast sandhills

t- unipuncta, Haw. ; extranea, Gn. Has occurred twice at Torquay and once at Morthoe

†- vitellina, Hb. Found from Torquay along the south coast to Dartmouth; and here more frequently than in any other part of the United Kingdom

- conigera, Fab. Gen. dist.

†- albipuncta, Fab. Found occasionally along the south coast, from Torquay to Dartmouth; also once at Exeter

* - lithargyria, Esp..

- turca, Linn. Recorded by Parfitt (I 878) at Alphington; Kingsbridge and Werrington Park; Torquay; apparently not noticed more recently

Panolis piniperda, Panz. Exeter; Plymouth; Plybridge, S. Devon; also at Morthoe in I 90 I

*Taeniocampa gothica, Linn. At sallow bloom in the spring

* - miniosa, Fab. To be found at the same attractive bait, and nr. Plymouth abundant

- cruda, Tr.

** stabilis, View.

- populeti, Tr. Woodside, Plymouth; Trews Weir, Exeter; scarce

- instabilis, Esp. Stoke Wood; Exeter ; Plymouth; Barnstaple; Morthoe. Exceedingly variable

- munda, Esp. ) Gen. dist. at sallow bloom in - gracilis, $\mathrm{Fab}$. $\}$ the spring

†Pachnobia leucographa, $\mathrm{Hb}$. Taken at Exeter and Barnstaple at sallows

- rubricosa, Fab. Plymouth district; Bickleigh Vale; Dartmouth; Paignton; Torguay; Sidmouth; Exeter; Barnstaple and Lynton. At sallows

1 I have collected the species in abundance near Lyme Regis, but I think the locality is in Dorset, not Devon, though it is not far from the border of Devon.-H.G.

\section{TRIFIDAE (continued)}

Rusina tenebrosa, Hb. Gen. dist. ; scarce in the northern parts

Mania maura, Linn. Widely dist. in moist places

- Naenia typica, Linn.

Amphypyra pyramidea, Linn. Gen. dist. in woods ; abundant in some years

* - tragopogonis, Linn.

Toxocampa pastinum, Tr. Torquay; Topsham; Membury; Kingsbridge Road, Teignmouth; Iffracombe; Barnstaple; Martinboe. Very local

- craccae, Fab. Discovered on the north coast of Devon in July, I $86 \mathrm{I}$, by the late Rev. J. Hirton, and taken in the Lynton and Lynmouth districts in many subsequent seasons up to the present time. Not known to occur in any other part of the United Kingdom. Its larva feeds on Vicia sylvatica

\$yydrilla arcuosa, Haw. Exeter; Bickleigh Vale; Lympston; Torquay; Sidmouth; Morthoe

tLaphygma exigua, $\mathrm{Hb}$. On the south coast from Torquay to Dartmouth rather more frequently than anywhere else in the United Kingdom; also taken at Lynmouth and Bideford

Stilbia anomala, Haw. Stoke, nr. Exeter; Budleigh Salterton; Honiton; Bickleigh Vale; Plymbridge; Dartmoor; Teignnouth; Dartmouth; Torquay; Sidmouth; Wembury; Haldon; Morthoe. Widely dist. and frequent on heaths

Caradrina morpheus, Tr. Gen. dist.

- ambigua, Fab. This species furnishes a most interesting history of probable immigration, but certain recent extension of the range of a species. In the year 1879 it was first noticed as an inhabitant of the United Kingdom. This was in Sussex; in a very few years it had spread into Kent and the Isle of Wight, then to the Isle of Purbeck, and in I 896-7 it had reached Paignton and Torquay; here it increased and multiplied so as to become a comparatively common species, and by 1900 had reached Dartmouth and almost the whole south coast of Devon, and had also become so common that hundreds might be seen at sugar on a favourable night; yet it does not seem to have found its way inland anywhere

- alsines, Bork. Exeter; Lynton; Instow. Still scarce though recorded from Devon by J. E. Stephens (1828)

- Caradrina blanda, Tr.; taraxaci, St. C.

* - cubicularis, Bkh. Sometimes very destructive, the larvae feeding in multitudes on grain in the ricks of wheat

- Grammesia trilinea, Bkh.

Dyschorysta ypsilon, Bkh. Common in woods nr. Exeter; also found at Plymouth, but generally rare in the county

Dicycla oo, Linn. Taken at Exeter, as a larva, in 1882 by the Rev. J. Hellins

"Calymnia trapezina, Linn. In woods in the more sheltered districts

- pyralina, Schiff. Alphington; Modbury; Honiton; Sidmouth; Lynton. Scarce

§- diffinis, Linn. Exeter; Sidmouth; and Mr. Parfitt says along the south coast; about elms 


\section{A HISTORY OF DEVONSHIRE}

\section{TRIFIDAE (continued)}

Calymnia affinis, Linn. Exeter; Honiton; Barnstaple; Dartmouth; Torquay; Paignton; Sidmouth. Commoner than the last named; also among elms

Tethea subtusa, Schiff. Exeter; Plymoutb; Dartmouth; Teignmouth; Bolt Head; Torquay. Scarce

- retusa, Linn. Plymbridge; Cann Wood; Dartmouth; Exeter; Bridgerule. Very local, among willow

* Orthosia rufina, Linn. In woods

** - ferruginea, Schiff.

** pistacina, Schiff.

- litura, Linn. Stoke Wood, nr. Exeter; Totnes. Rather rare here

- lunosa, Haw. Exeter; Egg Buckland; Dartmoor; Plymouth; Teignmouth; Barnstaple; Morthoe. Sometimes abundant at ivybloom, often found on gas lamps

** lota, Linn.

* macilenta, $\mathrm{Hb}$. In woods and at ivy-bloom

Cirraedia xerampelina, $\mathrm{Hb}$. Torquay; Bickleigh Vale; Clovelly. About ash trees

Xanthia citrago, Linn. Torquay and Babbacombe; Exeter; Plymouth; Barnstaple. Among lime

- cerago, Schiff, fulvago, St. C. Exeter; $P$ bymouth; Dartmoor; Barnstaple; Mortboe

- silago, Hb., flavago, St. C. Plymouth; Egg Buckland; Plymbridge. Rare at Exeter; Dartmoor; Barnstaple; Morthoe

- aurago, Schiff. Exeter; Plymouth; Honiton; Teignmouth. Scarce.

\$Hoporina croceago, Schiff. Exeter; Plymouth; Bickleigh Vale; Dartmoor; Egg Buckland; Dartmouth ; Paigmion; Barnstaple

Dasycampa rubiginea, Schiff. Topsham; Torquay; Paignton; Dartmouth; Plymouth; Honiton; Exeter; Alphington; Stoke Wood; Barnstaple. The south of this county is always the most reliable district in England for obtaining this scarce moth

†Cerastis erythrocephala, Schiff. One specimen taken at Ivybridge, and one at Honiton

* - vaccinii, Linn. In woods

- ligula, Esq., spadicea, Stn. Teignmouth; Honiton; Plymouth; Egg Buckland; Dartmoor; Barmstaple. A fine variety having a yellow subterminal line is found about Topsham

*Scopelosoma satellitia, Linn. In woods in autumn

Xylinai semibrunnea, Haw. Exeter; Dartmouth; Teignmouth; Torquay. At ivy-bloom in autumn

- petrificata, Schiff. Gen. dist.

- rhizolitha, Fab., ornithopus, St. C. Gen. in woods

Xylocampa lithoriza, Bkh., areola, St. C. Rather common nr. Exeter, and widely dist.

Calocampa exoleta, Linn. Alphington; Stoke Wood; Plymouth; Dartmoor; Barnstaple; Morthoe. Sometimes plentiful

*- vetusta, $\mathrm{Hb}$. Exeter; Plymouth. In woods in S. Devon

Cucullia verbasci, Linn. Plentiful about mullein

- lychnitis, Ramb. Recorded nr. Exeter by Mr. E. S. Norcombe

- absynthii, Linn. Exeter; Alphington; Wembury; Slapton Ley; Teignmouth; High Bickington; Dartmouth; Clinnicott, nr. Yealmpton; Morthoe. Among wormwood

\section{TRIFIDAE (continued)}

†Cucullia abrotani, Fab., artemesiae, St. C. The capture of two specimens at Starcross, August, I 885 , recorded by $\mathrm{Mr}$. W. Brooks

- chamomillae, Schiff. Plymouth; Devonport; Dartmoor; Dartmouth. Frequent in waste places along the south coast among Pyrethrum inodorum, and $P$. maritimum

- umbratica, Linn. Gen. dist., but only common in the Exeter district

Plusia chryson, Esq., orichalcea, Hn. The capture of two specimens at Plymouth is recorded by Mr. Parfitt.

* - chrysitis, Linn. Gen. dist.

- festucae, Linn. Alphington; Exeter; Dortmouth; Plymouth district. Rather scarce

"- pulchrina, Haw., v-aureum, Gn. Bickleigh Vale; Plymouth; Dartmoor; Sidmouth; Honiton: Martinhoe

- icta, Linn. Gen. dist.

* - gamma, Linn. Gen. dist.

$\ddagger$ ni, $\mathrm{Hb}$. The only specimen known to have been taken in this county-which also was the first captured in the British Isles-was taken at flower of red valerian at Alphington, Exeter, by Mr. H. D'Orville, in 1868

- interrogationis, Linn. Mr. Parfitt states that two specimens of this northern species have been captured, one at Torquay, the other at Cann Quarry, Plymouth

$\S$ Halrostola urticae, Hb., tripartita, St. C. Gen. dist.

- triplasia, Linn. Widely dist. and not scarce

Chariclea marginata, Fab., umbra, St. C. Dartmouth; Torquay; Sidmouth; Instow. Very local

Heliothis armigera, $\mathrm{Hb}$. A rare species here, but has been taken, usually singly, at Exeter; Dartmouth; Torquay; Babbacombe; Sidmouth; Barnstaple, and Westward Ho

- peltigera, Schiff. Mr. Parfitt says that it is found from Plymouth Hoe to Exmouth Warren, its larva feeding on Ononis and Hyoscyamus ; it has also been taken at Exeter and Morthoe; probably more frequent in this county than anywhere else in the United Kingdom

†- scutosa, Schiff. A single specimen was captured nr. Dartmouth, September, I900, by Mr. F. Capel Hanbury

Heliodes arbuti, Fab. Honiton; Wonford; Stoke; Dartmoor, and Barnstaple. Flying among flowers in sunshine

Acontia luctuosa, Esp. Torquay ; Babbacombe; Sidmouth; Dawlish; Exmouth; High Bickington; Dartmouth. Sometimes common, but very local

Hydrelia uncana, Linn. Only known in one locality-a bog nr. Great Mis Tor, Dartmoor.

Thalpochares ostrina, $\mathrm{Hb}$. The first specimen recorded in the British Isles was captured in June, 1825 , nr. Bideford by Captain Blomer. More than thirty years after a specimen was secured by Miss Battersby on the coast nr. Torquay. This led to a search, when several more were found by Dr. Battersby and his family. No more seem to have occurred in this county, and very few in others 


\section{INSECTS}

TRIFIDAE (continued)

Thalpochares parva, $\mathrm{Hb}$. A single specimen-the first known in Great Britain-was caught at Teignmouth in July, I844, by Dr. R. C. R. Jordan. It has been stated that two were taken at Torquay by Dr. Battersby, but these were, I think, small examples of the last species

Erastria fuscula, Bkh. Cann Wood, nr. Plymouth; Dartmoor; Barnstaple. Locally common

Bryophila glandifera, Hb. Torquay; Sidmouth; Exeter; Stoke; Barnstaple; Hartlond; Mortboe. Plentiful and very beautifully variable at Plymouth and Starcross. On old walls

* perla, Fab. On old walls

Phytometra aenea, $\mathrm{Hb}$. Gen. dist.

Anarta myrtilli, Linn. Bickleigh Down; Brent Hill, nr. Tavistock, and other parts of Dartmoor; Plymouth; Honiton; Haldon, and Torquay. On heaths

\section{SARROTHRIPIDAE}

Sarrothripa revayana, Tr. Exeter; Lympston; Lynton. Among oak; singularly variable

\section{GONOPTERIDAE}

Gonoptera libatrix, Linn. Gen. dist.

\section{QUADRIFIDAE}

Catocala fraxini, Linn. The capture of a single specimen of this magnificent species, at Westward Ho, September, 1895 , recorded by Mr. O. F. E. Cooke

\section{QUADRIFIDAE (continued)}

Catocala nupta, Linn. Exeter; Alphington; Topsham; Teignmouth; Torquay; Sidmouth. Among willows, and on walls and tree trunks

- promissa, Esp. The Rev. J. Hellins is stated to have captured a specimen on the trunk of an oak tree at Chagford, August, $1877^{1}$

Euclidia glyphica, Linn. Widely dist., and not scarce

- mi, Cl. Gen. dist. in flowering meadows.

\section{DELTOIDES}

Aventia flexula, Schiff. Exeter; Oxton, and Stoke Wood. Several times taken at light by $\mathrm{Mr}$. E. F. Studd

Aethia emortualis, Schiff. Mr. J. F. Stephen's specimen was captured in Devonshire

Herminia barbalis, Linn. Mr. Parfitt records this species but without locality-it can hardly be absent

- tarsipennalis, Tr. Exeter; Barnstaple. Rather common; local

- grisealis, $\mathrm{Hb}$. Gen. dist.

Hypenodes albistrigalis, Haw. Plymouth ; Dortmouth; Teignmouth ; Exmouth; Honiton; Exeter; Barnstaple. Local

$\S$ costaestrigalis, Steph. Lynton; Morthoe; Barnstaple: Dartmouth

Bomolocha crassalis, Fab., fontis, St. C. Spitchwick; Dunsford; Clovelly; Lynton; Dartmoor; Topsham. Among whortleberry

§ypena rostralis, Linn. Exeter district; Plymouth * - proboscidalis, Linn. Among nettles

Rivula sericealis, Scop. Exeter; South Devon; Barmstaple; Martinboe. Not scarce

\section{GEOMETRINA}

\section{BOARMIIDAE}

Ourapteryx sambucata, Linn. Gen. dist.

Angerona prunaria, Linn. Stoke Wood; Plymouth district; Dartmoor; Teignmouth; Paignton; Barnstaple. In large woods and grassy borders of woods

* Rumia crataegata, Linn. Among hawthorn.

Venilia maculata, Linn. Gen. dist. in woods and in heaths

* Cabera pusaria, Linn. Apparently rare in some places on the north coast

- exanthemaria, Scop.

Bapta temerata, Schiff. To be found in all woods, yet not commonly

- taminata, Schiff. Exeter; Plymouth; Paignton; Barnstaple. In woods, but scarce

Aleucis pictaria, Curt. Recorded as occurring in Stoke Wood, by Mr. Parfitt. This is not confirmed, and indeed is probably a mistake

\$Macaria alternata, Schiff. Exeter; Plymouth; the road from Plympion to Shaugh; Egg Buckland; Tiverton; Honiton

- notata, Linn. Plymouth district; Bickleigh Vale; Compton, and nr. Exeter. Scarce ; among birch

- liturata, Linn. Exeter; Cann Wood; Dartmoor; Honiton. In fir woods; very local

*Halia wavaria, Linn. In gardens

* Panagra petraria, Hb. Among bracken on heaths

\section{BOARMIIDAE (continued)}

Strenı clathrata, Linn. Mr. Parfitt records this species as occurring on the borders of Dartmoor. It generally frequents clover fields

* Fidonia atomaria, Linn. On heaths

- piniaria, Linn. Redhills, nr. Exeter; Stoke Wood; Plymouth district ; Honiton ; Paignton; Barnstaple. About fir woods

- conspicuata, Schiff. Mr. Parfitt records the capture of a single specimen nr. Bickleigh. This, however, is so improbable that one is forced to the conclusion that a variety of F. atomaria has been mistaken for this eastern species

Numeria pulveraria, Linn. Fordlands and Stoke Wood, nr.Exeter; Wattles Down; Bovisand ; Bickleigh Bridge; Honiton; Seaton; Dartmouth; Woodbury; Paignton; Sidmouth. Apparently very local

\$Aspilotes strigillaria, $\mathrm{Hb}$. Haldon and heathy places in Plymouth district

- citraria, Hb. Plymouth cliffs ; Starcross; Dartmouth; Stoke ; Yealm; Babbacombe; Dartmoor; Morthoe; Braunton; Ilfracombe. On the coast

- gilvaria, Schiff. Braunton; Ilfracombe. Scarce

1 This species is almost confined to the New Forest, Hants, and $I$ doubt its occurrence in Devon.-H.G. 


\section{A HISTORY OF DEVONSHIRE}

\section{BOARMIIDAE (continued)}

Eurymene dolobraria, Linn. Exeter district; Cann Wood; Bickleigh Vale; Topsham; Honiton; Tiverton; Dartmoor; Instow; Lynmouth; Morthoe. Scarce in woods

Odontoptera bidentaria, Linn. Gen. dist. in woods

Ennomos alniaria, Linn., tiliaria, St. M. Exeter district; Exminster; Plymouth; Dartmoor; Tiverton. Infrequent

- fuscantaria, Haw. Exeter; Statcross; Plymouth; Teignmouth; Paignton; Torquay; Sidmouth; Barnstaple. Among oak

- erosaria, Schiff. Exeter; Plymouth; Dartmouth; Torquay; Tiverton; Barnstaple; Shaugh, and Haldon. Very common

- ungularia, Schiff. Gen. dist. in wooded districts

Crocallis elinguaria, Linn. Woods and hedgebanks generally

"Himera pennaria, Linn. Exeter; Cann Wood; Plymouth; Bickleigh Vale; Tothill; Torquay; Sidmouth; Barnstaple; S. Devon

\$Selenia illustraria, Hb. Exeter; Compion; Saltram; Bickleigh; Avonswick; Dartmouth; Plymouth; Batnstaple; Bideford

- lunaria, Schiff. Exeter district ; Compton; Saltram; Bickleigh; Honiton; Tiverton; Barnstaple; Instow

* - illunaria, Hb. About hawthorn hedges

$\S$ Pericallia syringaria, Linn. Widely dist.

Epione advenaria, Hb. Haldon. Among whortleberry

- apiciaria, Schiff. Gen. dist. in damp lanes and marshes

"Metrocampa margaritata, Linn. In woods

Ellopia fasciaria, Linn. In all fir woods

§Biston hirtarius, Linn. Exeter; Topsham; Barnstaple

- prodromarus, Schiff., stratarius, St. C. $P l y-$ mouth; Stoke; Exeter; Honiton; Sidmouth; Barnstaple. Widely dist.

- - betularius, Linn. In all woods and among trees

Phigalia pilosaria, Schiff. Gen. dist.

Nyssia hispidaria, Schiff. Mr. Studd has taken several males by means of his illuminated moth-traps at Oxton; Exeter. Otherwise this moth-which sits on oak trunks by day, and the female of which is apterous-does not seem to have been noticed here

Gnophos obscurata, Schiff. Gen. dist. on both north and south coasts, also found on heaths inland

Cleora lichenaria, Schiff. Widely dist., frequenting lichen-covered tree trunks, walls, and rocks

- glabraria, Hb. Plymouth district; Avonswick; Exeter district; Egg Buckland; Ashburton; Honiton; Clovelly; Lynton. Local, and rather scarce

- viduaria, Schiff. Recorded by Mr. Parfitt; but this is doubtless erroneous, referring perhaps to dark varieties of the last species

Tephrosia consonaria, Hüb. Bickleigh and Cann Woods

- biundularia, Esp., and its probable varietycrepuscularia, Schiff. Both widely dist.

\section{BOARMIIDAE (continued)}

Tephrosia extersaria, Hüb., luridata, St. C. Ivybridge; Cann Quarry and Woods; Maristow; Dartmoor ; Tiverton. Local

$\S$ - punctularia, Schiff. Plymouth; Bickleigh Vale; Dartmoor; Boringdon Wood

+Boarnica roboraria, Schiff. Mr. Parfitt records its capture in Cann Woods

- cinctaria, Schiff. Also recorded by Mr. Parfitt, who says that it was taken by Mr. D'Orville, but gives no locality

- abietaria, Schiff. High Bickington; Egg, Buckland; Oxton; Exeter; Bickleigh Vale. The Rev. J. Hellins reported the rearing of a specimen from a larva found on whortleberry

- - repandata, Linn. The very handsome banded variety called conversuria is frequently taken in the north of this county

*Boarmia rhomboidaria, Schiff., gemmaria, St. C. In gardens as well as in woods

Hemerophila abruptaria, Thumb. Exeter; Plymouth; Seaton; Sidmouth. Often found in gardens

Hibernia aurantiaria, Hb. Exeter; Bickleigh Vale; Cann Wood; Shaugh; Stoke Wood; Barnstaple. Scarce

- defoliaria, Linn. Gen. dist. Very variable

- - progemmaria, $\mathrm{Hb}$.

- leucophaearia, Schiff. Gen. dist. in oak woods

* - rupicapraria, Schiff. In hedges and orchards

Anisopteryx aescularia, Schiff. Gen. dist.

- Abraxas grossulariata, Linn. Gen. dist. and in Lundy Island

\$-ulmata, Fab. Barnstaple and Lymmouth. Has also been taken nr. Exeter, at light

Ligdia adustata, Schiff. Gen. dist. in woods and hedges where the spindle tree is found

*Lomaspilis marginata, Linn. Among sallow

\section{GEOMETRIDAE}

Pseudoterpna cytisaria, Schiff, pruinata, St. C. Gen. dist. among furze and broom

Geometra papilionaria, Linn. Dist. all over the county among alder and birch, yet scarce

Iodis vernaria, Linn. Mr. Parfitt records it in Stoke Wood

*- lactearia, Linn. In woods

Nemoria viridata, Linn. Woodbury. Very local

Phorodesma bajularia, Schiff. Stoke Woods, and elsewhere in woods in the Exeter district

* Hemithea thymiaria, Linn. In woods and hedges

\section{ACIDALIIDAE}

Ephyra porata, Linn. Starcross; Woodbury; Topsham; Dartmouth ; Honiton; Tiverton; Barnstaple; Hartford; Instow. Among oak bushes

- punctaria, Linn. Stoke Wood, nr. Exeter; Cann Wood; Bickleigh; Plymbridge; Topsham; Dartmoor; Sidmouth

- trilinearia, Bkh. Bickleigh; Stoke Woods; Dartmouth; Sidmouth; Barnstaple. Among beech

- omicronaria, Schiff., annulata, St. C. Exeter district; Honiton; Teignmouth; Sidmouth. Among maple

- orbicularia, $\mathrm{Hb}$. Recorded at Tiverton by Mr. C. M. Major 
ACIDALIIDAE (continued)

Ephyra pendularia, Linn. Exeter, taken at light ; Starcross. Scarce

Acidalia scutulata, Schiff. Gen. dist. in hedges

* - bisetata, Bkh. In woods

- trigeminata, Haw. This has, I think, been recorded in error in some cases for a variety of the last species; but the Rev. C. T. Cruttwell assures me of its occurrence between Lynten and Porlock on the county border

- rusticata, Schiff. Said to have been taken at Rame Head; but quite recently Mr. C. Grenville Clutterbuck has taken several specimens at Torquay

- dilutaria, Hb., interjectaria, Bdv. Gen. dist.

- - incanaria, $\mathrm{Hb}$.

* - promutata, Gn., marginepunctata, Stn. c. north and south coasts, and found on the hills of Dartmoor

- straminata, Tr. Taken by the late Major Still on Dartmoor

- subsericeata, Hw. Exeter; Honiton; Dartmouth. Abundant at Sidmoush. Its smaller variety is recorded in Mr. Parfitt's lists under the names of pinguidenata and mancuniata

- ornata, Scop. Recorded here in Stephen's Illustrations

- immutata, Linn. Cann Wood; Bickleigh Vale. In moist and marshy spots

- remutata, $\mathrm{Hb}$.

* - aversata, Linn.

- fumata, Steph. Found by Mr. W. H. BasdenSmith, on Dartmoor

- inornata, Haw. Said by Mr. Parfitt to have been taken in Stoke Wood. Not confirmed

*Timandra imitaria, Hb. Both coasts

Bradyepetes amataria, Linn. Exeter; Plymouth; Barnstaple. Sometimes rather common in lanes

Ania emarginata, Linn. Exeter; Exwick; Starcross; Shaldon; Topsham; Barnstaple. Rather local

\section{LARENTIDAE}

Melanippe tristata, Linn. Crabtree; Blackdown, nr. Kingsbridge; Lynmouth; Chagford, and other borders of Dartmoor. Usually a northern mountain-frequenting species

* - rivata, Haw., sociata, St. C.

- galiata, Schiff. Widely dist. ; especially c. upon the coast

Melanthia rubiginata, Schiff.
- ocellata, Linn.

- albicillata, Linn. Stoke Wood, nr. Exeter; Honiton; Dortmoor; Barnstaple; Lynton. Rather local

- procellata, Schiff. Sidmouth; Torquay; Dartmouth; Plymouth; Excter. Usually found in chalky districts

- unangulata, Haw. Gen. dist. in woods and lanes

Anticlea sinuata, Schiff. Exeter; Egg Buckland; Buckfastleigh ; Bickleigh Bridge; Slapton Ley; Totnes; Dartmoor; Dartmouth; Plymbridge. Usually a very scarce species. Among Galium verum

\section{LARENTIDAE (continued)}

Anticlea rubidata, Schiff. Widely dist. in S. Devon, especially on the coast

- badiata, Schiff. Widely dist.

- derivata, Schiff. Excter district; Cann Quarry; Laira; Mutley, and elsewhere nr. Plymouth; Barnstaple. In borders of woods

- Coremia montanata, Schiff.

- fluctuata, Linn.

- propugnata, Schiff. Gen. dist. in woods

* - ferrugata, Linn.

- unidentaria, Haw. Gen. dist., but less common than the last

- pectinitaria, Feussl., viridaria, St. C. Gen. dist. in woods and woody heaths

- salicata, Hb. Taken by Mr. A. Chandler in a sand pit on Exmoor

- didymata, Linn.

- multistrigaria, Haw. Stokenham Quarry; Exeter; Plymoutb district; Instow. Very local

Larentia olivata, Schiff. Exeter; Starcross; Honiton; Topsham; Sidmouth; Barmstaple; Hartland; Lynton. Sometimes plentiful

Asthena luteata, Schiff. Exeter; Honiton; Barnstaple; Bickleigh; Plymouth. Gen. dist. (E. Parfitt)

- candidata, Schiff. In the open portions and margins of woods

- sylvata, Schiff. Stoke Wood; Exeter; Plymbridge; Cann Quarry; Bickleigh; Dartmoor; High Bickington; Dartmouth; Barnstaple; Mortboe. Local in woods

- blomeri, Curt. Recorded by Mr. Parfitt at Teignmouth, and by Major Still at Tiverton

Eupisteria leparata, Schiff. Gen. dist. among alder

Sterrha sacraria, Linn. The fourth recorded British specimen was taken on a gas lamp at Plymouth in September, 1857, by Mr. Charles Rogers. Two years later one was obtained at Bartstaple. Specimens since taken at Mount Batten; Devonport; Exmouth; Alpbington; Babbacombe. Still a very rare species in the United Kingdom

Emmelesia affinitata, Steph. Widely dist. in lanes and at the edges of woods among Lychnis dioica.

* - alchemillata, Linn. In similar places among Galiopsis tetrahit

- albulata, Schiff. Generally in pastures among yellow rattle

- decolorata, Hb. Widely dist. in lanes among Lychnis vespertina

- unifasciata, Haw. Exeter; Honiton; Dartmoor; Lynton; Hartland. Among Bartsia adontites

Cidaria psittacata, Schiff. Gen. dist. Frequenting ivy-bloom in the autumn

- miata, Linn. Widely dist., but much less common, having similar habits

- corylata, Thumb. In all woods; often common

- picata, Hb. Exeter; Starcross; Honiton; Bickleigh; Plymouth district; Topsham; Sidmouth; Barnstaple; Tiverton; Martinboe. On the borders of woods

* - russata, Schiff., truncata, St. C.

- immanata, Haw. Widely dist. in woods 


\section{A HISTORY OF DEVONSHIRE}

\section{LARENTIDAE (continued)}

Cidaria suffumata, Schiff. Gen. dist. in lanes and the margins of woods

- silaceata, Schiff. In all woods where Epilobium flourishes

- prunata, Linn. Generally in gardens and hedges among currant and blackthorn

- dotata, Linn., associata, St. C. Exeter and Stoke Wood; Plymouth; Dartmoor; Sidmouth. Among currant

* - fulvata, Forst. Among wild rose

- pyraliata, Bkh., dotata, St. C. Gen dist. in lanes, woods, and gardens, especially among cleavers

- populata, Linn. Stoke; Bickleigh; Cann Wood; Bridestowe; Lynton; Martinboe ; Barnstaple. Among whortleberry

- testata, Linn. Ilfracombe; Barnstaple; Morthoe; Exeter and Plymouth districts; Sidmouth. Among sallow and on heaths

Pelurga comitata, Linn. Found on Dartmoor by Major Still among Chenopodium

Scotosia undulata, Linn. Exeter; Horrabridge; Bickleigh Vale, and other parts of Dartmoor; Cann Wood; Lynton. Scarce

- dubitata, Linn. Exeter; Seaton; Iffracombe; Mortboe; Lynton. Not common

- certata, Hb. Alphington; Sidmouth; Lynton. Scarce. Among Berberis

Camptogramma fluviata, Hb. Exeter; Sidmouth; Torquay; Dartmouth; Plymoutb-where in I857 it was almost common-Exmouth; Croyde; Brautiton Burrows. Always a scarce insect, but more frequent here than in other parts of England

- lignata, Hb. Starcross; Topsbam; Dartmoutb district. In marshy meadows common

- tersata, Schiff. Recorded at Torquay by Mr. Parfitt

Thera firmata, Hb. Exeter; Dartmoor; Topsham; Torquay; Instow. Among Scotch fir

- - variata, Schiff. Among fir

- juniperata, Linn. A single specimen recorded by Mr. Mathew at Barnstaple

Hypsipetes ruberata, Frey. Stoke Wood; Honiton; Barnstaple; Lynton. Scarce

- impluviata, Schiff. Plymouth; Plymbridge; Plympton; Exeter; Sidmouth; Barnstaple

- - elutata, Schiff. As in more northern districts it is found that specimens reared in Vaccinium are smaller and more brightly coloured than those fed in sallow

*Aporabia dilutata, Schiff. In woods and lanes

Cheimatobia boreata, $\mathrm{Hb}$. Exeter. Males have been attracted by Mr. Studd's illuminated moth-trap

*- brumata, Linn.

\$Lobophora lobulata, Hb. Exeter; Seaton; Cann Wood and Quarry; Dartmoor; Lynton

- hexapterata, Schiff. Exeter district; Topsham; Sidmouth. Very local, among aspen

- sexalata, $\mathrm{Hb}$. Exeter district; Honiton; Sidmouth. Among sallows

- viretata, Hb. Plymouth; Plymbridge; Cann Wood; Dartmouth; Torquay; Starcross; Exeter; Barnstaple

- polycommata, Schiff. Recorded at Sidmouth by Mr. Majendie

\section{LARENTIDAE (continued)}

\$Chesias spartiata, Schiff. Dunsford; Plymbridge and Marsh Mills, nr. Plymouth

- obliquaria, Schiff. Dartmoor. Also taken by Mr. Studd at Exeter by means of light

-Anaitis plagiata, Lınn. Particularly in rocky places

Mesotype lineolata, Schiff. Exmouth and Dawlish Warrens; Woodbury, nr. Starcross. Always in coast-sands

*Eubolia palumbaria, Schiff. On heaths and cliffis

*"- bipunctaria, Schiff. Exeter; Bovisand; Crabtree and elsewhere in the Plymouth district; Sidmouth; Dartmouth; Babbacombe ; Barnstaple; Ilfracombe

- cervinata, Schiff. Efford; Stoke; Milebouse, and elsewhere in the Plymouth and Exeter districts. Also occasionally nr. Barnstaple

* - mensurarıa, Schiff.

Eupithecia togata,. Hb. Taken nr. Exeter by Mr. E. F. C. Studd

- venosata, Fab. Exeter; Plymouth; Seaton; Salcombe; Barnstaple; Instow. Among Silene

- consignata, Bkh. Taken at Seaton by Maj. Still

- pulchellata, Steph. Exeter; Dunsford; Plymouth; Dartmouth; Barnstaple; Instow; Morthoe; Lynton. Among foxglove

- linariata, Schiff. Gen. dist. among Linaria vulgaris

* - centaureata, Schiff. In gardens

- succenturiata, Linn. Exeter. Among Artemisia vulgaris

- subfulvata, Haw. Exeter; Exmouth; Torquay; Dartmoor; Barnstaple; Morthoe. Among ragwort

- subumbrata, Schiff. Stoke Wood; Tiverton. Scarce

$\$$ - irriguata, Hb. Boringdon Wood; Exeter district ; Tiverton

- pusillata, Schiff. Exeter; Phymouth. Among spruce fir

* - nanata, $\mathrm{Hb}$. On all heaths

- indigata, $\mathrm{Hb}$. Exeter. Among fir trees

- constrictata, Gn. Barnstaple. Among thyme

- lariciata, Frr. Exeter; Plymouth district; Ilfracombe; Lynton; Heddon; Dartmoor. Among larch

- castigata, Haw.

- virgaureata, Dbld. Barnstaple; Tiverton; Seaton; Sidmouth; Plymouth

- albipunctata, Haw. Exeter; Babbacombe; Plymbridge; Plymouth; Bickleigh Vale. Among Heracleum

- pimpinellata, $\mathrm{Hb}$. Exeter district

- plumbeolata, Haw. Exeter district; Barnstaple; Lynton. Among Melampyrum pratense

- isogrammata, H.S. Exeter; Newton; Torquay; Morthoe. About clematis

- pygmaeata, Hb. Barnstaple. Among stitchwort

- trisignata, H. S. Bickleigh Vale. Among Angelica

- valerianata, Hb. Exeter district. Among Valeriana officinalis

- fraxinata, Crewe. Exeter. On ash trees, and at light

- innotata, $\mathrm{Hb}$. Larvae beaten from mugwort by the Rev. J. Hellins, nr. Exeter, proved to be of this rare species 


\section{INSECTS}

\section{LARENTIDAE (continued)}

Eupithecia subnotata, Hb. Seaton; Braunton. Also recorded by Mr. Parfitt, but without locality. Among Chenopodium

- vulgata, Haw.

- expallidata, Gn. Exeter district. Among golden-rod

- absynthiata, Linn. Exeter; Plymouth; Wembury; Shaldon Cliffs; Barnstaple; Morthoe

- minutata, Hb. Exeter and Plymouth districts. On heaths among heather or ling

- assimillata, Dbld. Alphington; Plymouth; Barnstaple; Morthoe. In gardens among currant, also about wild hops

- jasioneata, Crewe. Morthoe; Lynton. Discovered in $N$. Devon in 1881 , the larva feeding on seeds of Jasione montana

- tenuiata, Hb. Exeter; Plymouth. Among sallows

- subciliata, Gn. Exeter; Babbacombe. Among maple

\section{LARENTIDAE (continued)}

Eupithecia dodonaeata, Gn. Borington Wood, nr. Exeter

- abbreviata, Steph. Gen. dist. in oak woods

- exiguata, Hb. Ivybridge; Maker, nr. Plymouth; Exeter; Seaton; Tiverton; Dartmouth. About hawthorn

- debiliata, Hb. Dunsford; Plymbridge; Dartmoor; Martinboe. Among Vaccinium myrtillus

$\$-$ coronata, Hb. Exeter; Tiverton; Topsham; Seaton; Plymbridge; Morthoe; Lynton

- - rectangulata, Linn. Among apple trees

- pumilata, $\mathrm{Hb}$. Widely dist. and sometimes abundant

\section{OENOCHROMIDAE}

Tanagra chaerophyllata, Linn., atrata, St. C. $P l y-$ moutb; Cann Wood; Horrabridge. Abundant on Dartmoor and at Bolt Head; Barnstaple; Martinhoe. In damp meadows among Bunium flaxuosum

\section{PYRALIDINA}

\section{PYRAUSTIDAE}

Odonteas dentalis, Schiff. Stephens says 'not far from Tavistock'

Pyrausta purpuralis, Linn. Widely dist.

- ostrinalis, Hb. Exmouth; Dartmouth; Dartmoor; Morthoe. On sloping cliffs and in pastures

- punicealis, Schiff. Barnstaple. Usually in chalky places. Mr. Parfitt has evidently confused this species

-Pyrausta cespitalis, Schiff. On wastes and cliffs

- cingulalis, Linn. Plymouth; Bolt Head; Salcombe; Stoke; Barnstaple; Lynton; Mortboe

- octomaculalis, Linn. Exeter district. Among golden-rod

*Botys fuscalis, Schiff. Exeter; Barnstaple. In meadows among yellow-rattle

- terrealis, Tr. Teignmouth Cliffs; Lynmouth; Lynton; Clovelly; Martinboe. Among golden-rod; extremely local

- esinalis, $\mathrm{Hb}$. On both north and south coasts, wherever Rubia peregrina is common

- repandalis, Schiff. The only known British specimens were reared by the late Rev. Henry Burney from larvae found by him feeding in the spikes of Verbascum nigrum, upon the south coast of Devon more than thirty-five years ago

- sambucalis, Schiff. Plymouth; Sidmouth; Exeter; Mortboe. In gardens among alder

- crocealis, Tr. Exeter; Sidmouth; Barnstaple. Among Inula dysenterica

- ferrugalis, $\mathrm{Hb}$. Very irregular, but on all parts of the coast, and occasionally in great abundance

** - forficalis, Linn. In gardens

"- lutealis, Haw. Ilfracombe; Braunton; Mortboe. Among thistles

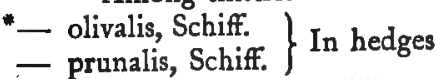

Perinephile lancealis, Schift. Exeter; Dartmoor; Colyton; Dartmouth; Barnstaple. In woods among hemp-agrimony

\section{PYRAUSTIDAE (continued)}

Spilodes striticalis, Linn. Dartmouth ; Ilfracombe; Instow. Very scarce

- palealis, Schiff. Sidmouth. One in 1857

- cinctalis, Tr. Exeter; Dartmouth; Barnstaple. In clover fields

*- verticalis, Schiff., ruralis, St. C. Among nettles * - - urticalis, Linn.

Margarodes unionalis, Hb. Torquay; Dartmouth. One specimen each - a great rarity

Diasemia literalis, Scop. High Bickington; Exeter and Plymouth districts. In rough pastures

" Nomophila hybridalis, $\mathrm{Hb}$. In pastures; especially on coast-sands and cliffs

\section{PYRALIDAE}

Cledeobia angustalis, Schiff. Torquay ; Teignmouth; Salcombe; Exmouth Warren; Dartmouth; Exeter (at light); Barnstaple; Martinhoe

Pyralis farivalis, Linn. Everywhere in stables and corn stores

— costalis, Schiff. Recorded nr. Exeter by Mr.

- glaucinalis, Linn. $\}^{\text {Rarfitt }}$

Aglossa pinguinalis, Linn. Everywhere about stables, outhouses, and corn and meal stores

- cuprealis, Hb. Taken rarely in the Exeter district (E. Parfitt)

\section{HYDROCAMPIDAE}

Cataclysta lemnalis, Linn. Abundant about ponds, ditches, and streams

Paraponyx stratiotalis, Linn. Exeter district. About water

-Hydrocampa nymphaealis, Linn.) About ponds and - stagnalis, Don.

Stenia punctalis, Schiff. Torquay; Teignmouth; Exmouth Warren; Sidmouth; Braunton Cliffs; Baggy Point. Very local 


\section{A HISTORY OF DEVONSHIRE}

\section{ENDOTRICHIDAE}

Endotricha flammealis, Schiff. Torquay; Dartmouth; Lynton; Martinhoe. Locally common

\section{SCOPARIIDAE}

Scoparia cembrae, Haw. Lynton; Morthoe; Hartland. Common at Teignmouth

* - ambigualis, Tr. About trees

* - dubitalis, Haw.

- muralis, Curt. Has repeatedly been recorded in the county. It may exist on the Tors of Dartmoor and at the Valley of Rocks. Its usual home is in the north of England and Scotland

- truncieolalis, Stn. In fir woods nr. Exeter and in $N$. Devon

- resinea, Haw. Alphington; Stoke Wood; also at light at Exeter: Morthoe. Local

- crataegella, Hb. Exeter; Honiton; Barnstaple

- mercurella, Linn. Gen. dist.

- lineola, Curt. Exeter district ; Morthoe. Very local

- angusta, Gurt. Plymouth and Drake's Islandwhere it is distinctly double-brooded; also at Teignmouth; Sidmouth; Dartmouth, and elsewhere near the coast

* pallida, Curt. Braunton Burrows ; Teignmouth; Dartmouth. In marshy places

\section{PTEROPHORIDAE}

Platyptilia isodactylus, Zell. Formerly found in some numbers in a marsh nr. Teignmouth by the late Dr. R. C. R. Jordan

- gonodactylus, Schiff. Sidmouth; Chudleigh; Seaton; Teignmouth. Among coltsfoot

- zetterstedtii,Zell. Lynton. Taken by Mr. R. South

*Amblyptilla acanthodactylus, Hb. Exmoutb; Salcombe; Teignmouth; Exeter ; Honiton; Morthoe; Hartland. Among Ononis, but the larva has been found in gardens feeding on the buds of scarlet geraniums

\$- punctidactylus, Haw. Stoke Wood; Plymouth; Honiton; Hartland

Mimaescoptilus phaeodactylus, Hb. Torquay; Teignmouth; Exmouth; Plymouth. Among rest-harrow

- bipunctidactylus, Steph. Common among meadow scabious everywhere

- fuscus, Rtg., pterodactylus, Linn. Stoke Wood; Morthoe. Among Veronica chamaedrys

Aedematophorus lithodactylus, Tr. Morthoe. Among Inula

*Pterophorus monodactylus, Linn.

Leioptilus tephradactylus, $\mathrm{Hb}$. Lynton; Heddon. Among golden-rod

- lienigianus, Zett. Taken in a lane between Teigmmouth and Haldon, in 1892 , by Mr. B. A. Bower

- osteodactylus, Zek. Exeter; Lynton; Martinhoe. Among golden-rod

- microdactylus, Hb. Plymouth; Seaton; Teignmouth. Among hemp-agrimony

Aciptilia baliodactylus, Zett. Torquay. Among marjoram

- - pentadactylus, Linn. Among bindweed

\section{ORNEODIDAE}

Orneodes polydactyla, $\mathrm{Hb}$. Common among honeysuckle

\section{PHYCITIDAE}

Phycis spissicella, Fab., roborella, Schiff. Stoke Wood; almost Exeter, at light

Dioryctria abietella. Recorded without locality by Mr. Parfitt, but doubtless to be found in fir woods

- palumbella, Schiff. Taken at light nr. Exeter by $\mathrm{Mr}$. Studd ; doubtless to be found on the heaths

Salebria carbonariella, Fisch. Dattmouth; Lynton

- carnella, Linn. Torquay and Babbacombe

Hypochalcia ahenella, Schiff. Exeter district

Crytoblabes bistriga, Haw. In Mr. Parfitt's lists, without locality

Acrobasis consociella, Hb. Stoke Wood, nr. Exeter; Lynton; Heddon. Among oak

- tumidella, Zinck. Exeter district ; Heddon. Among oak

Rhodophaea advenella, Zinck. Taken at light at Exeter

- marmarea, Haw. Exeter district, among blackthorn

Euzophera cinerosella, Zell., artemesiella, Stn. Alphington. Taken in Mr. D'Orville's garden. Feeds on wormwood

Pempelia ornatella, Schiff. Mr. Parfitt records the capture of two specimens at Cocver, but these may belong to the next species

- dilectella, Steph. Valley of Rocks; Lynton; Heddon; Hartland. Among thyme

Alispa angustella, $\mathrm{Hb}$. Recorded in Devon by Mr. Parfitt. No known locality

Homaeosoma sinuella, Fab. Teignmouth; Exmouth. On the coast among plantain

- nebulella, Gn. Exeter district. Among musk thistle

- binaevella, Steph. Exeter district. Very scarce

- nimbella, Zell. Exmouth; Morthoe. Among camomile

Ephestia kuhniella, Zell. Exeter. In flour mills

*- elutella, Hb. Exeter. In a chemist's shopdoubtless in dried fruit stores everywhere

Plodia interpunctella, $\mathrm{Hb}$. In similar places

\section{ANERASTIIDAE}

Anerastia lotella, Hb. Exmouth Warren, Morthoe. On coast sand hills

\section{CRAMBIDAE}

Platytes cerusellus, Schiff. Dunsford; Plymouth; Bridestoove. Locally abundant

Crambus falsellus, Schiff. Torquay. Scarce

- pratellus, Linn. In every grassy place

- hamellus, Thumb. Exmoutb Watren

* - pascuellus, Linn. In meadows

- uliginosellus, Zert. Mr. Porritt records its capture by $\mathrm{Mr}$. D'Orville, without locality

- margaritellus, $\mathrm{Hb}$. Taken on the cliffs, among coarse herbage, at Exmouth, towards Budleigh Salterton, in June (E. Parfitt). It is difficult to understand how he could be mistaken in so definite a statement ; yet this species is notoriously restricted to the moors and mosses of Scotland and the north of England 


\section{INSECTS}

\section{CRAMBIDAE (continued)}

Crambus pinetellus, Linn. Exeter; Bridestowe; Lynton; Morthoe; Martinhoe

- latistrius, Haw. Exeter district ; Teignmouth

- perlellus, Scop. Exeter district; Torquay; Valley of Rocks; Martinboe. Abundant on Lundy Island

* - tristellus, Schiff.

- selasellus, Haw. Barnstaple

- inquinatellus, Schiff. Torquay

- geniculeus, Haw. Exeter district; Dartmouth ; Morthoe. In dry fields and on sea sand-hills

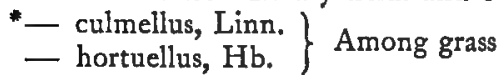

†Schaenobius forficellus, Thumb. Exeter

\section{GALLERIIDAE}

Aphomia sociella, Linn. Exeter; Starcross; Dartmoor; Morthoe; Instow. About humblebees' nests

Galleria mellonella, Linn., cereana, L. Exeter. About bee-hives

\section{TORTRICIDAE}

"Tortrix podana, Sc., pyrastrana, $\mathrm{Hb}$.

- crataeana, $\mathrm{Hb}$. Exeter district; Dartmoor. Among oak

- xylosteana, Linn. Gen. dist.

\& sorbiana, Hb. Heddon

* - rosana, Linn.

- diversana, Hb. Mr. Parfitt records it at Stoke Wood, Exeter

- cinnamomeana, Tr. Recorded in Stephens's Illustrations

* - heparana, Schiff.

- ribeana, $\mathrm{Hb}$.

- corylana, Hb. Exeter; Martinhoe. Widely dist.

- - unifasciana, Dap. In gardens and woods

- viburnana, Schiff. Widely dist. on heaths and coasts

- - icterana, Frol. Found on the north coast by Mr. R. South

* - viridana, Linn. Among oaks

* - ministrana, Linn. In woods

- - forsterana, Fab. About ivy

Dichelia grotiana, Fab. Exeter district ; Dartmoor; Lynton; Martinhoe. In woody places

Oenectra pilleriana, Schiff. Scarce, in the Exeter district (E. Parfitt). This is not confirmed, and is most improbable

Leptogramma literana, Linn. Exeter district ; Barnstaple; Morthoe. In wooded places

- scabrana, Steph. Exeter; Haldon. Among elm. The insect recorded by Mr. Parfitt as 'boscoma' is also this species-an earlier brood

Peronea sponsana, Fab., favillaceana, $\mathrm{Hb}$. Exeter; Morthoe; Hartland. Among beech

- mixtana, Hb. Haldon; Broad Clyst, Exeter, at light

- perplexana, Bar. Taken at Hartland Point by Canon Cruttwell

- schalleriana, Linn. Exeter; Dunsford; Morthoe; Hartland

- comparana, Hb. Exeter; Morthoe

\section{TORTRICIDAE (continued)}

Peronea permutana, Dab. Morthoe

- - varieganas, Schiff. In hedges

- cristana, Linn. Exeter Woods, and also taken at Mr. Studd's attracting lamp

- hastiana, Linn. Exeter; Morthoe. Among sallow

- ferrugana, Schiff. Excter. In woods among oak and birch

- tristana, Hb. Mortboe; Dartmoor ; Haldon

- espersana, Hb. Exeter district. Scarce

Teras caudana, Fab. Gen. dist.

*- contaminana, $\mathrm{Hb}$. In hedges

* Dictyopteryx loeflingiana, Linn. Among oaks

- holniana, Linn. Widely dist. In hedges

* - bergmanniana, Linn. Among rose

- forskaleana, Linn. Exeter; Morthoe; Lynton

Argyrotoza conwayana, Fab. Exeter; Braunton; Lynton

Ptycholoma lecheana, Linn. Exeter district; Lynton

Ditula semifasciana, Haw. Martinboe

- hortmanniana, Linn. Recorded in Devon by Westwood

Perthina picana, Frol., corticava, $\mathrm{Hb}$.

- betuletana, Haw. Exeter; Morthoe

- - pruniana, $\mathrm{Hb}$. In hedges and blackthorn

- ochroleucana, Hb. Gen. dist. Among rose

- cynosliana, Linn. Gen. dist.

$\dagger$ - carbonana, Dbld. Taken in $N$. Devon

†- marginana, Haw. Exeter district

Spilonota ocellana, Schiff. Gen. dist.

- lariciana, Zell. Nr. Lynton. Among larch

- dealbana, Frol. Recorded in N. Devon by Mr. South

- neglectana, Dup. Exeter ; Lynton

- suffusana, Koll. Gen. dist. About hawthorn

- rosaecolana, Dbld. Exeter. In gardens among rose

- roborana, Schiff. Widely dist. Among rose

Pardia tripunctana, Linn. Exeter; Heddon. Among rose

Sideria achatana, Schiff. Recorded in Devon by Professor Westwood

Aspis udmanniana, Linn. Gen. dist. Among bramble

Sericoris littorana, Curt. Torquay; Budleigh Salterton; Salcombe; Morthoe; Ilfracombe ; Lynton; Hartland. Among thrift

- cespitana, Hb. Recorded in Devon by Professor Westwood

* *- lacunana, Schiff.

- urticana, Hb. Plentiful among whortleberry in $N$. Devon

Orthotaenia striana, Schiff. Exeter; Christow; Lynmouth; Morthoe. In rough fields

- ericetana, Bent. Morthoe. Taken by Dr. Longstaff

Cnephasia pylitana, Haw. Exeter district. In heaths

§- musculana, Hb. Exeter; Exmouth; Teignmouth; Tiverton; Lynton

Sciaphila nubilana, Hb. Exeter. In hedges

- conspersana, Dgl. Hartland. Taken by Canon Cruttwell

- - subjectana, Gn.

- virgaureana, $\mathrm{Tr}$.

$\S$ - alternana, Schiff., Chrysantheana, Dup. Morthoe : Exeter district

- hybridana, $\mathrm{Hb}$. Exeter district 


\section{A HISTORY OF DEVONSHIRE}

\section{TORTRICIDAE (continued)}

Capua ochraceana, Steph. Redbills, nr. Exeter; Heddon

- Bactra lanceolana, Hb. Among rushes

- furfurana, Haw. Slapton Ley. Very local

†Phoxopteryx siculana, Hb. Bovey Heatbfield

- unguicana, Linn. Heddon, and elsewhere among heath

- comptana, Frol. Beer Head. In chalky places

- Iundana, Fab. Redbills, nr. Exeter

- myrtillana, Tr. Nr. Exeter. Among whortleberry

- derasana, Hb. Exeter district; Spitchwick. In woods

- uncana, Hb. Heddon. In heathy places

- mitterbacheriana, Schiff. Exeter; Heddon. Among oak

Grapholitha paykulliana, Fab. Morthoe

- nisana, Linn. Exeter district. Among sallow

- nigromaculana, Haw. Morthoe; Heddon. Among ragwort

- campoliliana, Schiff. Stoke Wood; Tiverton; Heddon

*- trimaculana, Don. Exeter. Among elm

- penkleriana, Schiff. Morthoe. Among alder

- obtusana, Haw. Chudleigh; Stoke Hill. Scarce

* naevana, $\mathrm{Hb}$. Dunsford

- geminana, Steph. Exeter ; Lynton. Among whortleberry

Phlaeodes tetraquetrana, Haw. Morthoe

- immundana, Fisch. Exeter; Stoke Wood; Morthoe

Hyperinacia angustana, $\mathrm{Hb}$. Taken in N. Devon by Mr. South

* cruciana, Linn.

* "Batodes angustiorana, Haw.

Poedisca rufimitrana, $H$. S. Taken at light at Oxton, Exeter, by Mr. Studd ; and at Hartland, among fir, by Canon Cruttwell

* * certicana, Schiff. On tree trunks in woods

- profundana, Schiff. Exeter; Honiton; Hartland; Heddon. Among oaks

-- occultana, Dgl. Exeter; Lynmouth; Heddon. Among larch

$\S$ - solandriana, Linn. Exeter; Morthoe

- semifuscana, Haw. Exeter district. Among sallow

- sordidana, $\mathrm{Hb}$. Lynmouth. Among alder

Halonota cirsiana, Zell. Tiverton; Chudleigh

§- scutulana, Schiff. Redhills, nr. Exeter; Lynton; Tiverton

- brunnichiana, Schiff. Teignmouth; Morthoe; Lynton. Among coltsfoot

- turbidana, Tr. Chudleigh; Lynton. Very local. Among Petasites vulgaris

- toeneana, Linn. Exeter. Among mugwort

- trigeminana, Steph. Morthoe. Among ragwort

- tetrogenana, Steph. Martinboe; Lynton; Lynn Vale

- signatana, Dgl. Lynmouth

- obscurana, Steph. Taken nr. Lynton by Mr. South

Olindia ulmana, Hb. Chudleigb; Heddon; Martixboe; Lynton. In woods and hedges

Semasia spiniana, Fisch. Exeter district. In hedges - janthinana, Dup. Heavitree. Among hawthorn - woeberana, Schiff. Exeter; Morthoe. Among fruit trees

\section{TORTRICIDAE (continued)}

Semasia populana, Fab. Hartland. Taken by Canon Cruttwell

Coccyx splendidulana, Gn. Exeter

- argyrana, Hb. $\}$ Exeter district

- vacciniana, Fisch. Taken among bilberry nr. Ashburton by Mr. Chant

Retinia buoliana, Schiff. Exeter. Very destructive to young pines in nursery grounds

- pinisolana, Dbl. Taken at light at Exeter

Carpocapsa splendana, Hb. Exeter district; Morthoe. Larva in acorns

- juliana, Curt. Exeter district. Among oak

- pernonana, Linn. In orchards and gardens. Mr. Parfitt says that this species, which is most destructive to apples, has here two broods in the season-May and Aug.

Opadia funebrana, Tr. Heddon, N. Devon. Among plum-trees

Endopisa nebritana, Tr. Gen. dist.; in pea fields

Stigmonsta interruptana, H. S. Recorded by Mr. Stainton, but without locality

- composana, Fab. Exeter. In clover fields

- redimitana, Gn. Exeter; Lynmouth; Heddon

- regiana, Zett. Exeter. On sycamores

Dicrorampha politana, Schiff. Exeter. Among yarrow

- alpinana, Tr. (flavidorsana). Exeter ; Starcross; Dartmouth; Lynton; Hartland. Among tansy

- plumbagana, Tr. Lynton

- sequana, Hb. Chudleigh

- - petinnana, Linn.

- plumbana, Scop. Exeter; Chudleigh

- tanacetana, Stn., saturnana, Gn. Exminster ; Heddon

- simpliciana, Haw. Morthoe

- acuminatana, Zell. Exeter ; Morthoe; Lynton

Pyrodes rhediana, Linn. Exeter. About hawthorn

* Cateptria ulicetana, Haw. Among furze

- cana, Haw. Exeter. Among thistles

- modestana, H.S. Near Lykton. Among goldenrod

- pupillana, Linn. South coast, Mt. Stainton

Tricheris mediana, Schiff. Exeter; Lynton; Morthoe. In the flowers of large Umbelliferae

Lobesia permistana, Hb. Fordlands, nr. Exeter

Eupoecilia maculosona, Haw. Exeter; Lynton; Chudleigh

- atricapitana, Steph. Morthoe

- angustana, Hb. Stoke Wood; Chudleigh; Dartmouth; Lynton; Hartland. Probably gen. dist.

- curvistrigana, Wilk. Barnstaple; Lynton

- notulana, Zell. Chudleigh; Exeter

- rupicola, Curt. StokeWood; Lynmouth; Heddon

- mussehliana, Tr. Taken many years ago in Devonshire (H. T. Stainton)

- affinitana, Dgl. Barnstaple. On salt marshes

- ciliella, Hb. Morthoe. Among primrose

Xanthosetia zoegana, Linn. Exeter; Dartmoor; Hartland; Morthoe; Lynton

* hamana, Linn.

Chrosis tesserana, Schiff. Exeter district ; Heddon

Argyrolepia conicana, Dbl. Lynmouth

- badiana, Hub. Widely dist.

Conchylis francillonana, Fab. Exeter; Wonford

- smesthmanniana, Fab. Lynmouth 
TORTRICIDAE (continued)

Conchylis stramineana, Haw. Lynton; Martinhoe; Morthoe; Hartland. Among Centaurea nigra - inopiana, Haw. Stoke Wood; Barnstaple. Among fleabane

"Tortricodes hyemana. Amongst oaks

\section{CHORENTIDAE}

Choreutes scintillulana, $\mathrm{Hb}$. Devont (Westwood and Humphrey)

"*Simaethis fabriciana, Linn. Among nettles and over umbelliferous flowers

- pariana, Linn. Teignmouth; Exeter. Among apple

\section{TINEINA}

*Lemnatophila phryganella, Hb. Exeter; Stoke Wood

*Diurnea fagella, Schiff. About trees

§Epigraphia steinkillneriana, Schiff. Exeter

Talaeporia pseudolomlycella, Hb. Exeter. Among trees

Psychoides verhuellella, Heyd. Exeter; Ashburton; Bideford. Among maiden-hair, spleenwort, and hartstongue ferns

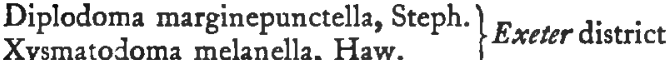

- argentimaculella, Stn. Taken at Hartland Point by the Rev. C. T. Cruttwell

Scardia granella, Linn. Exeter. In stables and granaries

* cloacella, Haw.

- ruricolella, Stn. Exeter district

- arcella, Fab. Hartland Point; Exeter

Tinea ferruginella, $\mathrm{Hb}$. Exeter district

- - rusticella, Hb. Exmouth

- tapetzella, Linn. Exeter. Probably everywhere among saddlery

*- pellionella, Linn. In houses

* - fuscipunctella, Haw. About stables and houses

- lapella, Str. Exeter district

- biselliella, Haw. Exeter. Destructive to furniture in houses

- nigripunctella, Haw. Redhills; Coaver; Exeter

- semifulvella, Haw. Exeter; Sidmouth; Hartiand Point

Phylloporia bistrigella, Haw. Lynmouth. Among birch

Lampronia quadripunctella, Fab. Coaver; Exeter

- luzella, Hb. Exeter; Tiverton; Teignmouth; Heddon

- praelatella, Schiff. Stoke Wood. Among wild strawberry

- rubiella, Bjerk. Exeter. Among raspberry

Incurvaria masculella, Fab.

- pectinea, Haw.

- oehlmanniella, $\mathrm{Hb}$.

Nemophoria swammerdamella, Linn. Exeter; Redhills; Tiverton; Heddon. In woods

- schwarziella, Stn. Exeter; Stoke Wood; Heddon

\$ - metaxella, Hb. Fordlands; Stoke Wood

Adela fibulella, Schiff. Fordlands; Exeter; Chudleigh; Lynton. In flowers of Cardamine pratensis

- rufimitrella, Stn. Exeter; Stoke Wood; Chudleigh; Lynton. On flowers of Cardamine pratensis

\section{TINEINA (continued)}

Adela sulzella, Schiff. Exeter; Heddon. Scarce

- degeerella, Linn. Dunsford; Chudleigh. In woods

* - viridella, Linn. Among oaks

- cuprella, Fab. Recorded on Dartmoor by the late Major Still

Nemotois scabiosella, Scop. Barnstaple

- minimella, Zell. Recorded at Oxton, Exeter. by Capt. B. B. Thompson

Swammerdamia apicella, Don., comptella, Hb. Exeter; Ilfracombe

- oxyacanthella, Zell. Exeter district

- griseocapitella, Stn. Reared at Exeter by Mr. Parfitt

- lutarea, Haw. Recorded by Stoke Wood by Mr. Parfitt

- pyrella, Vill. Exeter; Dunsford. In hedges

Scythropia crataegella, Linn. Exeter district

Hyponomeuta cognatella, Hb. Exeter; Hartland. Mr. Parfitt apparently records this species under the name of plumbellus

- padella, Linn. Abundant in hawthorn hedges

Anesychia decemguttella, $\mathrm{Hb}$. Braunton Burrows, Exeter

Prays curtisella, Don. Fordlands ; Stoke Wood ; Dunsford

- Plutella cruciferarum, Stn.

$\S-$ porrectella, Linn. Exeter

tCerostoma sequella, Linn. Redhills

- vittella, Linn. Exeter district. Among elms

* radiatella, Don.

- costella, Fab. Gen. dist. in woods

Hypsolopha sylvella, Linn. Widely dist. in woods

- alpella, Schiff. Dunsford

- lucella, Fab. Mr. Parfitt says in various parts of the county

Harpipteryx scabrella, Linn. Stoke Wood

- nemorella, Linn. Dursford Woods

- harpella, Schiff. Among honeysuckle

Pteroxia caudella, Linn. Honiton; Alphington; Stoke Wood. Among spindle

Orthotaelia sparganiella, Thunb. Teignmouth; Exeter district. Among burr-reed

Henicostoma lobella, Schiff. Cowley Bridge Road, Exeter

Phibalocera quercana, Fab. In oak woods gen.

* Depressaria costosa, Haw. Among furze

- umbellana, Steph. Exeter; Morthoe; Hartland

- assimilella, Tr. Exeter district

- nanatella, Stn. Torquay. Among carline thistle

- arenella, Schiff. Exeter; Teignmouth; Morthoe

- propinquella, Tr. Exeter; Fordlands; Barnstaple; Hartland

- subpropinquella, Stn. Morthoe

- rhodochrella, H.S. J Morthoe

- alstroemeriana, Linn. Exater; Plymouth; Dartmouth; Teignmouth. Among hemlock

- purpurea, Haw. Stoke Wood; Fordlands; Teignmouth

†- carduella, Hb. Dartmouth; Lynmouth

- ocellana, Fab. Exeter; Morthoe; Lynmouth

- yeatiana, Fab. Exeter; Barnstaple

**- applana, Fab.

- ciliella, Stn. Exeter district

- rodundella, Dgl. Dartmouth district

- pimpinellae, Zell. Plymouth; Bickleigh; Teign mouth 


\section{A HISTORY OF DEVONSHIRE}

\section{TINEINA (continued)}

Depressaria albipunctella, Hb. Exeter; Alphington; Teignmouth; Morthoe; Barnstaple

- chaerophylli, Zell. Exeter; Teignmouth

- douglasella, Stn. Dartmouth district. Taken by Mr. Porritt

- ultimella, Stn. Exeter; Teignmouth

- nervosa, Haw. Exeter; Dacolish; Teignmouth; Warcombe; Barnstaple. Among Oenanthe crocata

- badiella, Hb. Bideford; Morthoe; Hartland; Exeter

- pastinacella, Dup. Plymouth; Teignmouth

- heracleana, De Geer

Psoricoptera gibbosella, Zell. Bickleigh Vale. On oaks

Gelechia celerella, Haw. Morthoe; Hartland

- rufescens, Haw. Fordlands, nr. Exeter

- gerronella, Zell. Taken at Hartland by Canon Cruttwell

- vilella, Zell. Exeter district

- populella, Linn. Hartland

- velocella, Fisch. Exeter; Exmouth Warren

- fumatella, Dgl. Dawlish Warren (Stainton's Manual)

- mulinella, Fisch. Dartmouth district

- diffinis, Haw. Dawulish and Exmouth Warrens

- desertella, Stn. Exmouth Warren

- acuminatella, Stn.)

— artemisiella, Tr. $\}$ Dawlish Warren

- affinis, Haw. Exwick

- senectella, Zell. Hartland; Dawlish Warren

- mundella, Dgl. Abundant at Davolish Warren

- domestica, Haw. Exeter district. On old walls

- umbrosella, Zell. Dawlish Warren

- rhombella, Hb. Stoke $W_{\text {ood }}$

- notatella, Hb. Morthoe

- humeralis, Zell. Exeter; Dunsford

- vulgella, Hb. Old Stoke Road, Exeter

- luculella, Hb. Heddon. On oaks

- scriptella, Hb. Recorded by Mr. Parfitt, without locality

- fugitivella, Zell. Hartland. Among elm

- celerella, Dgl. Exmouth and Dawlish Warrens; Starcross

- leucomelanella, Zell. Hartland Point. Taken by Canon Cruttwell

- maculea, Haw. Exeter district ; Hartland

- tricolorella, Haw. Ide; Hartland. Among stitchwort

- fraternella, Dgl. On the cliffs at Plymouth

- viscariella, Stn. Bideford

- maculiferella, Mn. Exeter district

- semidecandriella, Thnb. Dartmouth district

* - marmorea, Haw. Dawlish Warren

- instabilella, Dgl. Barnstaple

†- leucatella, Linn. Exeter district

- nanella, Hb. Exmouth. About pear trees

- dodecella, Linn. Recorded by Mr. Parfitt, without locality

- triparella, Zell. Dunsford

- tenebrella, Hb. Coaver; Exeter

- ligulella, Zell. Stoke Wood; Exeter

- taeniolella, Tr. Exeter district

- anthyllidella, $\mathrm{Hb}$. Langstone Cliff; Dawlish

- naeviferella, Zell. Shaldon; Fordlands; Exmouth

- brizella, Tisch. Dawlish. Among thrift

\section{TINEINA (continued)}

Gelechia inopella, Zell. Shaldon Cliffs; Teignmouth; Bideford. Among fleabane

- subocellea, Steph. Teignmouth. Among marjoram

Cleodora cytisella, Curt. Taken by Canon Cruttwell at Hartland Point

Chelaria huebnerella, Don. Stoke Wood; Hartland; Morthoe

"Harpella geoffrella, Linn. In lanes

Dasycera sulphurella, Fab. Exeter; Tiverton. Probably everywhere among rotten wood

Oecophora minutella, Linn. Exeter

- tripuncta. Alphington and Fordlands, nr. Exeter

- augustella, $\mathrm{Hb}$. Exeter; Fordlands

- lionaris, Haw. Exeter; Exroick

- lambdella, Don. Exeter district; Plymouth coast ; Lynton; Hartland Point. Among dead furze sticks

- tinetella, Tr. Exeter district

- unitella, Stn. Exeter woods

- flavifrontella, Hb. Taken at light at Oxton, Exeter

- fuscescens, Haw. Exeter district

- - pseudospretella, Stn. In houses

Oecogenia quadripuncta, Haw. Exeter. In warehouses

Endrosis fenestrella, Scop. In every house

Butalis grandipennis. Exeter; Torrington; Great Haldon. About furze bushes. Its larvae obscuring them with their webs

Acrolepia granitella, Tr. Chudleigh ; Exeter ; Lynton. About fleabane

- pygmaeana, Haw. Exeter district. Among Solanum dulcamara

-"Glyphipteryx fuscovirideila, Haw.

- thrasmella, Scop.

- haworthana, Steph. Lynton Moors. Among cotton-grass

- equitella, Scop. Heddon. Among stonecrop

* - fischerella, Zell.

Aechmia dentella, Zell. Chudleigh; Budleigh Salterton

Perittia obscuripunctella, Stn. Exeter; Stoke Wood

Tinagma sericiella, Haw. Stoke Wood. Flying in sunshine

- stanneella, Fisch. Fordlands

Argyresthia nitidella, Fab. Exeter district

- conjugella, Zell. Heddon

- spiniella, Zell. Gen. dist.

* - albistria, Haw. In hedges

- glaucinella, Zell. Exeter

- mendica, Haw. Between St. Thomas, Exeter and Redhills

- andereggiella, Fisch. Morthoe; Martinhoe. Among apple trees

- curvella, Linn. Exeter; St. Mary's Clist. Among apple

- pygmecella, Hb. Stoke Wood. Among sallow

*- goedartella

- brockeella, $\mathrm{Hb}$. $\}$ Among alders

Zelleria insignipennella, Stn. Stoke Wood, nr. Exeter

Gracilaria swederella, Thunb. Exeter; Teignmouth

- stigmatella, Fab. Stoke Wood; Dutnsford; Haldon 


\section{INSECTS}

\section{TINEINA (continued)}

Gracilaria semifascia, Haw. Teignmouth. Among maple

- elongella, Linn. Dunsford

- tringipennella, Zell. Exeter; Dawulish; Shaldon Cliffs

- syringella, Fab. Common in gardens and woods

Coriscium brongniardellum, Fab. Exeter district

- cuculipennellum, Hb. Exeter; Starcross; High Bickington; Shaldon Cliffs. Among privet

- sulphurellum, Haw. Fordlands; Stoke Wood; Teignmouth

Ornix avellanella, Stn. Exeter; Teigmmouth

- devoniella, Stn. Dasulish. Taken by Mr. Stainton

- - anglicella, Stn.

- fagivora, Frey. Recorded by Mr. Parfitt

- torquillella, Stn. Haldon

- betulae, Stn. Taken at light at Exeter

- guttea, Haw. Exeter district. In orchards

Coleophora fabriciella, Vill. Chudleigh ; Stoke Hill ; Exeter

- deauratella, Linn. Exeter district

- alcyonipennella, Koll. Chudleigh; Stoke Wood

- fuscocuprella, Stn. Stoke Wood

- albicosta, Haw. Exeter district. Among furze

- anatipennella, $\mathrm{Hb}$. Sidmouth

- palliatella, Zell. Stoke Wood; Exeter

- ibipennella, Hayd. Exeter

- currucipennella, Fisch. Nr. Exeter (Mr. Parfitt)

- discordella, Zell. Dawlish; Shaldon Cliff

- troglodytella, Stn. Shaldon Cliffs

- therinella, Stn. Powderham Marshes

- lineolea, Haw. Redhills, nr. Exeter

- murinipennella, Fisch. Stoke Wood

" - caespititiella, Zell. On rushes.

- alticolella, Zell. Chudleigh. Taken by Mr. Stainton

- apicella, Stn. Exeter; Hartland

- argentula, Zell. Exeter district; Dawlish. On yarrow

- virgaureae, Stn. Stoke Wood; Bideford

- albitarsella, Zell. Exeter. About ground-ivy

- nigricella, Steph.

- fuscedinella, Zell.

- gryphipennella, Bouché. Wonford; Redhills

- siccifolia, Stn. Exeter

- viminetella, Zell. Exeter; Bovey Heathfield

- solitariella, Zell. Exeter district. On Stellaria

- lutipennella, Zell. Stoke Wood; Fordlands

- badiipennella, Fisch. Stoke Wood; Redhills

- limosipennella, Fisch. Exeterdistrict. Among elm

Bedellia somnulentella, Zell. Exeter; Bideford; Teignmouth

Batrachedra praeangusta, Haw. Stoke Wood

Chauliodus illigerellus, $\mathrm{Hb}$. Exeter. In marshy places

Laverna lacteella, Steph. Chudleigh

- epilobiella, Fab., longiella, Hb. Exeter district; Teignmouth. Among enchanter's nightshade

- fulvescens, Haw. Exeter district. Among Epilobium

- ochraceella, Curt. Chudleigh

- decorella, Steph. Exeter; Chudleigh; Teignmouth
TINEINA (continued)

Laverna subbistrigella, Haw. Chudleigh

- rhamniella, Zell. Fordlands

Chrysoclista linneella, Stn. Exeter. On trunks of limes

- schrankella, Hb. Exeter district ; Dumsford

- flavicaput, Haw. Exeter. In hedges

Asychna modestella, Dup. Exeter district

Antispila pfeifferella, Fab. Countess Weir. Among dogwood

Elachisia magnificella, Tengs. Hartland Point. Taken by Canon Cruttwell

- atricomella, Stn.

- luticomella, Zell. All taken in the Exeter

- nigrella, $\mathrm{Hb}$.

- subnigrella, Dgl.

- bedellella, Sirc. Hartland Point (Canon Cruttwell)

- obscurella, Stn.

- megerlella, Stn.

- adscitella, Stn. All taken in the Exeter district

- rhynchosporella, Stn. Haldon. Reared from Eleocharis

* - rufocinerea, Haw.

- cygnipennella, Hb. Exmouth; Dawlish; Dartmoor

Tischeria marginea, Haw. Exeter; Teignmouth

Lithocolletis lantanella, Schr. Exeter; Haldon; Teignmouth

- lautella, Zell. Exeter; Teigmmouth. On oak

- bremiella, Zell. Newton; Chudleigh. In Vicia sepium bark

- pomifoliella, Zell. On hawthorn

- coryli, Nic. On nut

- spinicolella, Zell. On sloe

- faginella, Mann. On beech; gen. dist.

- torminella, Frey. Exeter. Reared from Morella cherry

- salicicolella, Sirc. Chudleigh; Stoke Wood

- viminetorum, Stn.

- ulmifoliella, $\mathrm{Hb}$. Exeter district

- spinolella, Dup. Chudleigh

* quercifoliella, Fisch.

- messaniella, Zell. Exeter. Among evergreen oak

- corylifoliella, Haw. Teigmmouth; Haldon

- viminiella, Sirc. Exeter; Teigrmouth; Dawlish; Lynmouth

- alniliella, Hb. Teignmouth; Shaldon; Exeter district

*- cramerella, Fab. Stoke Wood; Exeter; Teignmouth

- sylvella, Haw. Alphington; Redhills; Teignmouth

- emberizaepennella, Bouché. Exeter; Chudleigh; Haldon

- frolichiella, Zell. Shaldon

— stettinensis, Nic. Dawlish

- nicellii, Zell. Exeter; Haldon; Teignmouth

- schreberella, Fab. Exmouth; Stoke Wood; Haldon: Teignmouth

- tristrigella, Haw. Exeter; Teignmouth; Haldon

- trifasciella, Haw. Exeter; Hartland; Haldon; Teigrmouth

Lyonetia clerkella, Linn. Exeter; Teignnouth. Among apple 


\section{A HISTORY OF DEVONSHIRE}

TINEINA (continued)

Phyllocnistis suffusella, Zell. Mount Pleasant; Exeter; Exmouth Warren

- saligna, Zell. Stoke Wood; Exeter

Cemiostoma laburnella, Hayd. Exeter. Blotching laburnum leaves

$\$$ scitella, Zell. Stoke Wood

tOpostega crepusculella, Fisch. Exeter

Bucculatrix cidarella, Tisch. Chudleigh

- ulmella, Mann.

- crataegi, Dup. Nr. Exeter

- maritima, Stn. Barnstaple. In salt marshes

- frangulella, Goe. Exeter. Among Rhamnus

Nepticula atricapitella, Haw.

- ruficapitella, Haw. Exeter. Among oak

- anomalella, Geo. Coaver Garden; Exeter

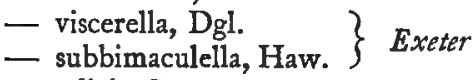

- salicis, Stn.

- microtheriella, Wing.

- ignobilella, Stn.

- plagicolella, Stn.

- prunetorum, Stn.

- tityrella, Dgl.

All taken in the Exeter district

\section{TINEINA (continued)}

Nepticula angulifasciella, Stn. Teignmouth

- marginicolella, Stn. Exeter; Haldon. Among elm

- alnetella, Stn. Exeter district. About alder

* aurella, Fab. Among bramble

\section{MICROPTERYGIDAE}

Eriocephala calthella, Linn. Fordlands Wood; Ide ; Heddon. In flowers of Caltha

- seppella, Fab. Fordlands; Chudleigh; Lynton. In flowers of Veronica chamaedrys

- aruncella, Scop. Taken in N. Devon by Mr. R. South

- mansuetella, Zell. Exwick

- allionella, Fab. Chudleigh; Lynton

- thunbergella, Fab. Stoke Wood; Exeter; ChudLeigh; Lynton

Micropteryx purpurella, Steph. Fordlands. On birch twigs

- semipurpurella, Steph. Exeter district

- sparmanella, Borc. Fordlands. In a meadow

- subpurpurella, Haw. Abundant at Stoke Wood, and taken at Exeter at light.

\section{DIPTERA}

Flies

The following list contains the names of some five hundred species, or roughly one-sixth of the total number of Diptera recorded up to the present time as occurring in the British Islands. Unless otherwise stated, the specimens upon which the list is based are in the British Museum, and have been collected in South Devonshire by Lieut.-Colonel J. W. Yerbury, and determined by the present writer. ${ }^{1}$

The names and particulars of the species of Tipulidae (sens. lat.) distinguished with a cross $(X)$ have kindly been furnished by Mr. G. C. Bignell, by whom they have been collected and determined. The Psychodidae have been collected, determined, and presented to the British Museum by the Rev. A. E. Eaton.

Colonel Yerbury, to whose exertions our knowledge, such as it is, of the Dipterous fauna of Devonshire is mainly due, has kindly supplied the following field-notes:-_'As a collecting-ground for Diptera Devonshire is without doubt one of the richest districts in the British Isles. For, in addition to the rich valleys running down from Dartmoor, the county possesses a considerable coast line. My own collecting has been done entirely in South Devonshire, where I have found the valleys of the Erme, Walkham, Plym, Tavy, etc., especially productive. Dartmoor itself, however, always proved a failure, but many a sheltered valley lying under its shoulder, e.g. Holne, turned out most remunerative. The following are some of the many interesting captures made in the county :Microdon mutabilis, Ivybridge ; Eristalis cryptarum, Ivybridge and Shaugh; Theria muscaria, Ivybridge ; Spilographa abrotani, Ivybridge ; Chaetostoma curvinervis, Bickleigh ; etc., etc. On the other hand many Families, as for instance the Stratiomyidae are badly represented; in fact, speaking generally, none of the marsh-loving insects are at all common. Similarly, the lack (in South Devonshire at any rate) of sandhills may account for the absence of such insects as Phtbiria pulicaria, and the sand-loving Anthomyinae. So far as my own experience went, the sandhills at the mouth of both the Exe and the Avon turned out unproductive; still I am inclined to think that Bantham (in spite of the dearth of good insects in the Avon Valley) will prove to be a rich district, for, in addition to the sandhills, there is a large marsh close at hand. Collecting in Devonshire has a great charm, for, apart from the presence of a rich insect faur $\mathrm{z}$, there is the additional attraction of beautiful scenery; whilst one's zoological interests may be varied by watching a fox or an otter, a dipper or a curlew. Certainly the naturalist will find no lack of subjects for study, though he will encounter one drawback in the hosts of biting flies, such as Haematopota, Simulium, Stomoxys, and others of their kin.'

${ }^{1}$ Other collectors or writers are indicated by their initials, as follows :-G. H.V., G. H. Verrall ; C. J. W., C. J. Wainwright ; C. M., Coryndon Matthews ; C. P., C. Pratt ; G. C. B., G. C. Bignell ; A. E. E., Rev. A. E. Eaton. 


\section{INSECTS}

\section{ORTHORRHAPHA}

\section{NEMATOCERA}

\section{Mrcetophilidae}

Sciara thomae, Linn. Budshead Wood, I July, 1889 ; Crownkill Fort, 7 July, 1889; Tamerton Foliot,, 8 Aug. I 889

Allodia ornaticollis, Mg. Ivybridge, 22 Aug. 1887 (G. H. V.)

\section{Bibionidae}

Dilophus febrilis, Linn. Cremyll, I 8 Apr. I889; Crownhill, I 5 Aug. 1889

Bibio venosus, $\mathrm{Mg}$. Ivybridge, 8, 20, and $30 \mathrm{Apr}$. 1893 ; Beer Ferris, II Apr. I893; Lydford, 17 Apr. 1893; Cormwood, 23 Apr. 1893; Bickleigh Vale, 25 Apr. 1893

- laniger, Mg. Walkham Valley, 4 Apr. I890; Bickleigh Vale, 2 I Apr. 1889

- johannis, Linn. Walkham Valley, 2 I Mar. 1893 ; Torpoint, I9 Apr. 1889

- ? sp. (nr. B. laniger, Mg.) Bickleigh, 24 Apr. 1893 ; Cornwood, 23 Apr. 1893

\section{Chironomidae}

Chironomus dorsalis, Mg. Slapton, 8 Sept. 1888 (G. H. V.)

— viridis, Macq. Slapton, 7 Sept. 1888 (G.H.V.)

- brevitibialis, Ztt. Slapton, 7 Sept. 1888 (G. H. V.)

— nigrimanus, Staeg. Slapton, 10 Sept. 1888 (G. H.V.)

Cricotopus sylvestris, Fabr. Slapton, 8 and Io Sept. I 888 (G. H. V.)

- pilitarsis, Ztt. Slapton, 7 Sept. 1888 (G. H.V.)

- trifasciatus, Pz. Slapton, ro Sept. 1888 (G. H. V.)

Tanypus varius, Fabr. Slapton, 8 Sept. 1888 (G. H. V.)

- nebulosus, Mg. Slapton, 8 Sept. I 888 (G. H. V.)

- phatta, Egg. Slapton, 24 Aug. 1885 (G. H. V.)

- melanops, Mg. Slapton, 8 Sept. 1888 (G. H. V.)

- griseipennis, v. d. Wulp. Slapton, ro Sept. I 888 (G. H. V.)

Ceratopogon fuscus, $\mathrm{Mg}$. Slapton, 9 Sept. 1888 (G. H.V.)

\section{Psychodidae}

Pericoma nubila, Mg. Exwick, 24 July, 189 I

- trivialis, Eaton. Seaton, 25 June; Aylesbeare Common, I 7 July ; Exwick, 2 I July, I891

- pulchra, Eaton. Seaton, 26 June, 1891

- ocellaris, Mg. Aylesbeare Common, I 4 July, I 891

- ambigua, Eaton. Exwick, 7, 9 July, I891; Aylesbeare Common, I 5, I 7 July, I 891

- decipiens, Eaton. Seaton, 26, 29 June, 1891

- labeculosa, Eaton. Aylesbeare Common, 14, 17 July, i 89 I

- caliginosa, Eaton. Seaton, 29 June, 1891

- fusca, Macq. Seaton, 29 June, $189 \mathrm{I}$

Psychoda phalaenoides, Linn. Exwick, 9 July, I 89 I

Trichomyia urbica, Hal. Exeter, 9 July, I891

\section{Disidak}

Dixa nebulosa, Mg. Slapton, 8 Sept. I 888 (G. H. V.) - aprilina, Mg.X Bickleigh, I4 Sept. 1884

\section{NEMATOCERA (continued)}

Ptychopteridae

Ptychoptera lacustris, Mg. Warleigh Marsh, 6 June, I 889

- albimana, Fabr. Tamerton Foliott, 21 May, 1889

- contaminata, Linn. $\times$ Seaton, 9 May, 1894 , Torcross, 28 May, I 893

\section{LimNoBidaE}

Limnobia nubeculosa, Mg. $\times$ Cann Wood, 24 May, 1893; 9 Aug. 1894

- analis, Mg. (Syn. nitida, Verr.) $\times$ Ivybridge, 7 May, 4 Oct. I 894

- macrostigma, Schum. $x$ leybridge, 30 Apr. 1893

Dicranomyia pilipennis, Egg. $x$ Ivybridge, 29 June, 1893

- modesta, Mg. $\times \quad$ Horrabridge, I 3 Oct. 1894

- " chorea, $\mathrm{Mg} \cdot \times$

- didyma, Mg. $\times$ Shipley Bridge, Brent, 29 May 1896

Geranomyia unicolor, Hal. $\times$ Bovisand, 5 May ; Salcombe, 23 May, 1893 ; Whitsand Bay, 5 Oct. 1894

Rhamphidia longirostris, $\mathrm{Mg} \times \times$ MarshMills, 6 June, I 893

Empeda nubila, Schum. $\times$ Irybridge, Io May, I 897

Goniomyia tenella, Mg. Princetown, 6 Sept. I 886 (G. H. V.)

Acyphona maculata, Mg. $\times$ Bovisand, 14 Apr. 1894 ; Yelverton, 29 Apr. 1895 ; Cann Wood, 6 June, 1893; 2 Sept. 1894; Ivybridge, 19 June, 1894

Molophilus appendiculatus, Staeg. $\times$ Grenofen Wood, I 1 June, 1894 ; Cann Wood, 8 Oct. I 893

- bifilatus, Verr. $\times$ Ivybridge, Io May, 1897

- murinus, $\mathrm{Mg} . \times \quad$ Iqybridge, $20 \mathrm{May}, 1893$

Rhypholophus varius, Mg. Ivybridge, 10 Sept.; Grenofen, 15 Sept.; Horrabridge, 18 Sept. I 894

- nodulosus, Macq. $\times$ Clearbrook, 28 Apr. 1893

- haemorrhoidalis, Ztt. $\times$ Plymbridge, 8 Oct. 1893

Erioptera taenionota, Mg. $\times$ Bovisand, I 4 Apr. 1894; Jenny Cliff Gave, I 8 Oct. ; Grenofen and Cann Wood, 29 Oct. 1895

- fuscipennis, Mg.x relverton, 25 Apr. 1896; Ivybridge, I 4 May, I 894

- trivialis, Mg. $\times$ Gratton Bridge; Yelverton, $25 \mathrm{Apr}$. 1896

Symplecta stictica, Mg. $\times$ Hallsands, Torcross, 18 Aug. 1895 . Ovipositing in trodden wet ground

Ephelia apicata, Lw. $\times$ Grenofen, 5 July, 1894

- submarmorata, Verr. $\times$ Grenofen Wood, I I June, 1894

*Poecilostola punctata, Schrk. $\times \quad B i c k l e i g h, 22$ Apr.; Gratton Bridge, 25 Apr. I 893

Limnophila meigenii, Verr. $X$ Clearbrook, 28 Apr.; Horrabridge, 18 Sept. I 894

- dispar, Mg.X Ivybridge, 30 Apr.; 4 and 17 May, 1893 ; Shaugh Bridge, 21 May, 1895

- lineola, Mg.X Bickleigh, 25 Apr.; Ivybridge, I 7 May, I 893

- lineolella, Verr. X Iqybridge, 14 and 27 May; Bickleigh, 22 May ; CannWood, 6 June, 1893 ; Marsh Mills, 6 June, 1896 


\section{A HISTORY OF DEVONSHIRE}

\section{NEMATOCERA (continued)}

Limnobidas (continued)

Limnophila aperta, Verr. $\times$ Ivybridge, 20 May, 3 June, I893; Bickleigh, 22 May; Cann Wood, 6 June 1894

- ferruginea, Mg. $\times$ Ivybridge, 2 May; Shaugh, 3 May; Grenofen, I I May, I 893

- discicollis, Mg. X Marsh Mills, 6 June, 1893

- lucorum, Mg.X Ivybridge, 30 Apr.; Bickleigh, 4 Aug. 1893

- nemoralis, $\mathrm{Mg} \times \times$ Ivybridge, 3 June ; Cornwood, 6 June ; Fernworthy, 29 July, 1894

- filata, Walk. $\times \quad$ Ioybridge, 3 June, 1894

Ula pilosa, Schum. $\times$ Bickleigh, 22 May; Ivybridge, 27 May, I 893

Dicranota pavida, Hal. $\times$ Bickleigh, 22 May, 1893 ; Horrabridge, 4 Oct. I 892

- bimaculata, Schum. $\times$ Bovisand, 18 Apr. 1892

Amalopis immaculata, Mg. $\times$ Bickleigh, 22 Apr. 1893 ; Cann Wood, 29 Oct. 1892

- claripennis, Verr. $\times$ Yealm, 3 Apr.; Walkham Valley, 28 Apr.; Bickleigh, 4 Aug. 1893

- littoralis, Mg. $\times$ Bickleigh, I 5 May; Torcross, 29 May ; Horrabridge, I 3 Oct. I 894

- occulta, Mg. Bickleigh, 22 Apr. 1889 ; Stowford Cleve, 6 June, 1888 (C. M.)

Cylindrotoma distinctissima, Mg. $\times$ Bickleigh, 25 Apr., 6 May, 1893; Shaugh Bridge, 6 May I 894

Phalacrocera replicata, Linn.X Meazy, Dartmoor, 23 Apr. 1893

Pedicia rivosa, Linn. Axmouth, $20 \mathrm{July,} \mathrm{I900}$ (A. E. E.)

\section{Tiputidae}

Dolichopeza sylvicola, Curt. $X$ Walkham Valley, 25 Apr. 1896; Ivybridge, I I, 17 May, 9 Sept. 1894

Nephrotoma dorsalis, Fabr. $\times$ Cann Wood, Io Aug. I 894

Pachyrrhina crocata, Linn. Walkham Valley, 2 I June, 1889 ; Grenofen Viaduct, July ; Ivybridge, I Aug. 1896 (G. C. B.)

- imperialis, Mg.X Ivybridge, 7 Aug.; Cann Wood, 10 Aug. 1896

- histrio, Fabr. $\times$ Torcross, 29 May; Ivybridge, 3 June, 8 Aug. ; Cann Wood, 6 June, 9 Aug. I 893

- maculosa, Mg. Bickleigh, 25 Apr., 28 May, I 893 (G. C. B.) ; Tamerton Foliott, 2 I May, 1889

- quadrifaria, Mg. $\times$ Seaton, 2 I May

- annulicornis, Mg. $\times$ Grenofen Wood, I I May; Ivybridge, 14, 18, $20 \mathrm{May,} \mathrm{I893;} \mathrm{Hazelwood,}$ 14 June, I 896

Tipula pagana, Mg. $\times$ Horrabridge, 18 Sept. I 894

- confusa, v. d. Wulp. $X$ Horrabridge, 18 Sept. 1894

- rufina, Mg. Tamerton Foliott, 5 May, I 889 Crownhill Fort, 14 May, 1889

- longicornis, Schum. $x$ Ivybridge, 3 June, 1893 ; Morley Marsh, 28 June, 1894

- pabulina, Mg.x Lydford, I 7 Apr.; Cann Wood, 23 Apr. 1894

- varipennis, Mg. $\times$ Grenofen Wood, II May ; Ivybridge, 22 May, I 894

- scripta, Mg. Grenofen Wood, II May, 1893 ; Iqybridge, I 8 May, I 893

\section{NEMATOCERA (continued)}

Tipulidar (continued)

Tipula flavolineata, $\mathrm{Mg} \times \quad$ Irybridge, 27 May, 1894 - lunata, Linn. Walkham Valley, 9 Apr., 9 May

- lateralis, Mg. Ivybridge, 28 Apr. 1889; Horrabridge, 19 Aug. 1893 (G. C. B.)

- vernalis, Mg. $\times$ Bred 29 Apr. and 4 May, 1897. Larvae obtained at Slapton on 12 Apr. at the edge of the Lee

- vittata, Mg. Shaugh Bridge, I Apr. 1893 ; Cornwood, 2 Apr. 1893; Walkham Valley, 3 and 8 Apr. 1893

- gigantea, Schrk. $\times \quad$ Ivybridge, 3 June, 1894

- lutescens, Fabr. Ivybridge, 28 June; Fernworthy, $29 \mathrm{July,} 1897$ (G. C. B.) ; Crownhill, 27 July, 1889

- oleracea, Linn. Gen. dist.

- paludosa, Mg. Devonport, 24 Sept. 1888

- fascipennis, Mg.X Ivybridge, 3 June, 1893

- ochracea, Mg.X Cann W.ood, 6 June, 1893

Dictenidia bimaculata, Linn. $x$ Shaugh Bridge, I 5 May, 1893 . I walking up the trunk of a tree

\section{RHYPHIDAE}

Rhyphus fenestralis, Scop. Bickleigh, 12 Apr. 1893 ; Plymouth, 26 Apr. 1893 ; Torcross, 25 May, 1893; Holne, 2 I June, 1896; Exmouth, Sept. $189^{\circ}$ (C. P.)

- punctatus, Fabr. Salterton, 27 Apr. 1898; Crownkill Fort, 6, I1, 17 May and 17 July, I889; Ivybridge, 15 July, I 889

\section{BRACHYCERA}

\section{Stratiomyidae}

Pachygaster atra, Pz. Avon Valley, I 9 June, 1896 - leachii, Curt. Torcross

Nemotelus pantherinus, Linn. Bantham, 26 June, I 896; Axmouth, I 7 July, r 900 (A. E. E.)

Oxycera pulchella, Mg. Salcombe, I4 July, I887, (C. M).

- trilineata, Fabr. Bantham, 29 May, 1896

Chrysonotus bipunctatus, Scop. Crownhill, I3, 2 I Sept. 1889

Sargus flavipes, Mg. Avon Valley, Io June, 7 July I 896 ; Holne, 7 July, 1896

- iridatus, Scop. Shaugh, I5 May, 1893; Avon Valley, 23, 25, 28 May, 1896

Chloromyia formosa, Scop. Shaugh; Salcombe; Avon Valley, 23, 25, 28 May, 1896

Microchrysa polita, Linn. Marsh Mills, 16 May, 1893

- flavicornis, Mg. Avon Valley, 28 June, 1893

Beris vallata, Forst. Tamerton Foliott, 6 June, I889; Grownhill Fort, II June, I889; Bantham, 26 June, 1896

- chalybeata, Forst. Torcross, 27 May, 1893

- nigra, Mg. Crownhill Fort, 8 July, 1889

Chorisops tibialis, Mg. Torcress

\section{Tabanidae}

Haematopota pluvialis, Linn. Crownhill; Ivybridge; Whitleigh Wood, June, 1889

- crassicornis, Whlbg. Iqybridge, r 6 June, I 889

Therioplectes solstitialis, Schin. (? Mg.). Walkham $V$ alley, 2 I July, I 889 


\section{INSECTS}

\section{BRACHYCERA (continued)}

Tabanidae (continued)

Tabanus sudeticus, Zlr. Budshead Wood, I July, 1889

- bovinus, Lw. (Schiner, pp.) Irybridge, 26 July, I 889

- maculicornis, Ztt. Walkham Valley, 2 I June ; Ioybridge, 23 June, 1889

- bromius, Linn. Bickleigh; Ioybridge; Cornwood; Warleigh Marsh; Crownhill Fort, June-July, 1889

- autumnalis, Linn. Warkeigh Marsh, 26 June, 1889 ; Tamerton Foliott, 29 June, I 889

- cordiger, Wied. Walkham Valley, 2 I July, I 889

Chrysops caecutiens, Linn. Torcross; Plymbridge; Ivybridge; Walkham Valley; Crownhill Fort; Bickleigh Vale, May-July, I 889

- quadrata, Mg. Holne, Dartmoor, $6 \mathrm{July,}, 896$

- relicta, Mg. Torcross, 24-26 May, 1893

\section{LEPTIDAE}

Leptis scolopacea, Linn. Crozonhill Fort; Bovisand; Torcross, May, June, 1889, 1893

- tringaria, Linn. Crownhill Fort; Bickleigh Vale, May-July, r 889

- nigriventris, Lw. Avon Valley, 24 May, 1896 ; The Dewerstone, 7 June, I 889

- lineola, Fabr. Holne; Dartmoor, 16 July, I896 ; Bickleigh Vale, 18, 28 July, 1889; Walkham Valley, 2 I July, 1889; Crownhill, I 5 Aug. I889; Ivybridge, I Sept. I 889

Chrysopilus aureus, Mg. Crownhill Fort, 4 July, I 889 ; Bickleigh Vale, i 8 July I 889

- atratus, Fabr. (Syn. auratus, Fabr.) Avon Valley; Torcross; Whitleigh Wood; Tamerton Foliott, 22 May-29 June.

Atherix marginata, Fabr. Avon Valley, 22 May, 1896 ; Plymbridge, 8 June, 1889 ; Ivybridge, 23, 30 June, 1889; Bickleigh Vale, 18-20 July, 1889

- ibis, Fabr. Shaugh, 5 May, 1893 ; Plymbridge, 18 May, 1889 ; Avon Valley, 22, 25 May, 1896

Xylophagus ater, Fabr. Ivybridge, 12 , 17 May, 1893 ; Plymbridge, 28 May, I889; Avon Valley, I 2 July, 1896

\section{Asilidae}

Dioctria oelandica, Linn. Avon Valley, 23 May, I 896; Walkham Valley, 2 I June, 1889

- rufipes, Deg. Shaugh, 8 May, 1893; Bovisand, I6 May, 1896 ; Salconbe, 22 May, 1893; Grownhill Fort, 4 July, 1889

- baumhaueri, Mg. Salcombe; Avon Valley; Dartmoor; Tamerton Foliots; Crownhill Fort, $20 \mathrm{May}-$ 4 July

- linearis, Fabr. Avon Valley, I I, 28 June, 1896; Plymbridge, 15 June, 1894

Isopogon brevirostris, Mg. Shaugh, I 5 May, I 893 ; Ivybridge, 17, 18 May, I893; Salcombe, 21 May, I 893 ; Avon Valley, 24-28 May, I 896; Yelverton, 7 June, I 889 ; Walkham Valley, 2 I June, I 889 ; Ivybridge, 16 June, 1889; 21 June, 1896 ; Dartmoor, 24 June, 1896

Asilus crabroniformis, Linn. Hoine, Dartmoor, $5 \mathrm{July,}$ I 896 ; Walkham Valley, 21 July, I 889 ; Ivybridge, 26 July, 1889 ; Crownhill, 5, 7 Aug. 1889 ; Tamerton Folioti, 8 Aug., Io Sept. 1889

\section{BRACHYCERA (continued)}

Asilidae (continued)

Pamponerus germanicus, Linn. Curtis, British Entomology

Epitriptus cingulatus, Fabr. Walkham Valley, 2 I, 3 I July, 1889; Holne; Dartmoor, 21, 23 July, 1896

Neoitamus cyanurus, Lw. Ivybridge, $18 \mathrm{May,} 1893$; 26 July, I889; Avon Valley, 24 May, I 896

Machimus atricapillus, Fln. Holne; Dartmoor, I9-22 July, I 896 ; Ieybridge, I Aug. I 896 ; I I Aug. 2 I Sept. 1889

Dysmachus trigonus, Mg. Walkham Valley, I 3 May, 1896; Bantham, 29 May, 1896 ; Salcombe, I 5 June-1o July, 1896

\section{Bombytidae}

Anthrax paniscus, Rossi. Salcombe, $7 \mathrm{July,} 1889$; Bovey Tracy, I6 Aug. I 899 ; A. H. Hamm (in Oxford Museum ; determined by E. E. A.)

- hottentota, Linn. Holne, Dartmoor, 28 July, 1896

Bombylius discolor, Mik. Walkham Valley, 28 Mar., 6 Apr. 1893; Beer Alston, 31 Mar. I893; Salterton, 26 Apr. 1898

- major, Linn. Beer Alston, 6 Apr. 1893

- canescens, Mik. Walkham Valley, 28 Apr., 9 May, 1893 ; Avon Valley, 25 May, 10, 25 June, 1896 ; Ivybridge, I 2 May, I 893 ; Brent Moor, Dartmoor, 24 June, 1896

\section{Therevidae}

Thereva annulata, Fabr. Bantham, 29 May, 1896

\section{Cyrtidae}

Acrocera globulus, Pz. Aylesbeare Common, nr. Exeter, 14. 25 July, 1891 (A.E.E.)

\section{EMPIDAB}

Rhamphomyia tarsata, Mg. Lynton, I7 May, I883 (G. H. V.)

Empis tessellata, Fabr. Teignmouth, 2 I Apr. 1883 (G. H. V.)

-- pennipes, Linn. Dawlish, 7 June, 1883 (G.H.V.)

Pachymeria femorata, Fabr. Iqybridge, I 3 June, 1883 (G. H. V.)

Clinocera bipunctata, Hal. Iqybridge, I4 June I 883 (G. H.V.)

- nigra, Mg. Lynton, 20 May, I 883 (G. H. V.)

Trichopeza longicornis, Mg. Ivybridge, I 4 June, 1883 (G.H.V.)

Lepidomyia melanocephala, Fabr. Ivybridge, I 3 June, 1883 (G. H. V.)

Chersodromia cursitans, Ztt. Torcross, 18, 21, 23 Aug. I903. On seaweed

- hirta, Walk. Torcross, 1 8, 28 Aug. 1903. On seaweed and running over the sand

\section{Dolichopodidae}

Psilopus platypterus, Fabr. Avon Valley, 10 June, 9 July, 1896

Eutarsus aulicus, Mg. Torcross, 1 2, 17, 23, Aug. 1903

Dolichopus atratus, Mg. Cornwood, 2 June, I 889

- scotti, Verr. Slapton, 6 Sept. 1889 (G.H.V.) 


\section{A HISTORY OF DEVONSHIRE}

\section{BRACHYCERA (continued)}

Dolichopodidar (continued)

Poecilobothrus nobilitatus, Linn. Crownkill Fort, 9 June, 1889 ; Budshead Wood, 27 June, I 889 : Tamerton Foliott, I I July, 1889

Chrysotus blepharosceles, Kow. Teigmmouth, I I June, I $88_{3}$ (G. H. V.)

Argyra diaphana, Fabr. Tomerton Foliott, 2 1, 23 May, 1889; Plymbridge, 28 May, 1889; Crownhill Fort, 30, 3 I May, I 889; Avon Valley, 22 May, 6 June, 1896

- leucocephala, Mg. Tamerton Foliott, 2 I May, 29 June, I889; Avon Valley, I7 June, I 896 ; Cornwood, 8 Sept. 1889

Porphyrops crassipes, Mg. Lynton, I 9 June, I 883 (G. H.V.)

\section{BRACHYCERA (continued) \\ Dolichopodidae (continued)}

Hydrophorus bipunctatus, Lehm. Crownhill, 1 2, 15 Aug. 1889

Liancalus virens, Scop. Whitsand Bay, 29 Apr. I 893 ; Torcross, 26 May, I893; Avon Valley, 17 June, I 896 ; Walkham Valley, 2 I July, I 889 ; Crownhill, I 5 Aug. 1889 ; Plymbridge, 2o Oct. I 889 Sympycnus annulipes, Mg. Torcross, 12 Aug. 1903 Chrysotimus molliculus, Fln. Torcross, I 5 Aug. 1903 Aphrosylus raptor, Hal. Seaton, 24 June, 1890 , (G. H. V.) ; Bovisand, $3 \mathrm{July,} 1896$; Torcross. 23, 24 Aug., 6 Sept. 1903

- ferox, Hal. Plymouth, 3 Sept. I888 (G.H.V.); Torcross, 16 Aug. 1903 ; Prowle Point, 18 Aug. 1903. Obtained by sweeping seaweed

\section{CYCLORRHAPHA}

\section{PROBOSCIDEA}

Platypezidae

Platypeza dorsalis, Mg. Torcross, I 5 Aug., 3, 9 Sept. 1903; Slapton (G. H.V.) ${ }^{1}$

\section{Pipunculidae}

Verrallia villosa, von Roser. (G. H. V.) ${ }^{3}$

Pipunculus zonatus, Ztt. Avon Valley, 19 June, I 896

- modestus, Hal. (G. H. V.) ${ }^{3}$

- varipes, Mg. Shaugh, 5 May, 1893

- campestris, Latr. Shaugh, 3 May, I893; Holne, Dartmoor, 26 July, 1896

- geniculatus, Mg. Exeter (G. H.V.) ${ }^{4}$

\section{SYRPHIDAR}

Paragus tibialis, Fln. Torcross, 8 Aug. 1903

Pipizella virens, Fabr. Crownhill Fort, 29 May, 1889 Pipiza noctiluca, Linn. Cornwood, 8 Sept. I889

-- lugubris, Fabr. Ivybridge, I6 June, 1889; Warleigh Marsh, 24 June, I 889 ; Crownhill, 30 Aug. 1889

Orthoneura nobilis, Fln. Ivybridge, 4 May, 1893; Crownhill Fort, 17 June, I 889

Liogaster metallina, Fabr. Tamerton Foliott, 2 I May, 1889 ; Crownhill, I9 July, 7, I 5 Aug. I 889

Chrysogaster splendens, Mg. Salcombe, 2 I May, I893; Tamerton Foliott, 5 June ; Plymbridge, 15 June, I889; Ivybridge, I Sept. I889; Torcross, 9 Sept. 1903; Cornwood, 14 Oct. I 888

- hirtella, Lw. Plymbridge, $28 \mathrm{May,} 1889$; Tamerton Foliott, 9 June; Crownhill Fort, I I June, 1889

- solstitialis, Fln. Shaugh Bridge, 7 June ; Crownhill Fort and Tamerton Foliott, 1 I June ; Plymbridge, I 5 June, 1889

Chilosia sparsa, Lw. Iqybridge, 9 May, 1889

-- antiqua, Mg. Bickleigh, 5, I 2, 21 Apr. ; Cornwood, 23 Apr. 1893

- scutellata, Fln. Iqybridge, 8 July, 1888 (C. M.); I I Aug. I 889

- soror, Ztt. Ivybridge, 30 June, I 889

1 Brit. Flies, vol. viii, p. 48.

8 Op. cit. F. 8 g.
2 Op. cit. p. 74.

${ }^{4}$ Op. cit. p. 121 .

\section{PROBOSCIDEA (continued)}

SYRPHIDAz (continued)

Chilosia pulchripes, Lw. Tamerton Foliott, 6 May, Io Sept. I889; Crownhill Fort, 23 May, I889; Bickleigh, 30 July, I 889; Torcross, 25 Aug. 1903

- variabilis, $\mathrm{Pz}$. Bickleigh Vale, 18 June ; Crownhill Fort, I9 June; Warleigh Marsh, 24 June; Ivybridge, 30 June, I 889

- intonsa, Lw. Tamerton Foliott, 5 May ; Wistman's Wood, Dartmoor, 6 May; Ivybridge, I Sept. I 889; Torcross, 3 Sept. I 903; Exmouth, I 4 Sept. 1890 (C. P.)

- illustrata, Harr. Salcombe, 2 May, 1893; Ivybridge, 24 June, 1888 ; Bickleigh, 30 July ; Tor Royal, Dartmoor, 4 Aug. 1889

- grossa, Fln. Walkham Valley, 2 I Mar. ; Bovisand, 23 Mar. I 893

- albipila, Mg. Bovisand, 23 Mar.; Ivybridge, 20 Apr. 1893

- nebulosa, Verr. Bickleigh, 5 Apr.; Walkham Valley, 6 Apr. ; Ivybridge, 8 Apr. 1893

- (?) impressa, Lw. Ivybridge, I Sept. I889

- albitarsis, Mg. Tamerton Foliott, 2 I, 23 May, 1889

- fraterna, Mg. Cornwood, 2 June; Tamerton Foliott, I 5 Sept. 1889

- praecox, Ztt. Torcross, 25 Aug. I903

- vernalis, Fln. Ioybridge, II Aug. ; Crownhill, 12, 15,30 Aug. 1889

- modesta, Egg. Ivybridge, Io May, I891; 17 May, 1890 (C. M.)

Platychirus manicatus, Mg. Gen. dist.

- discimanus, Lw. Bickleigh, 5 Apr.; Ivybridge, 15 Apr. 1893

- peltatus, Mg. Crownhill Fort, I7 May; Warleigh Marsh and Tamerton Foliott, 6 June, 1889

- albimanus, Fabr. Salterton, 23, 26 Apr. 1898

-- immarginatus, Ztt. Warleigh Maish, II July ; Tamerton Foliott, 12 July, 1889

- clypeatus, Mg. Cornwood, 3 I July, 1889 . Gen. dist.

- angustatus, Ztt. Ivybridge, 3 I July, I 887 (C. M.)

- sticticus, Mg. Ivybridge, I5 May, 1887 (C. M.)

Pyrophaena granditarsa, Forst. Shaugh, 8 May; Torcross, 25 May, 1893 ; Crovonhill Fort, 30 May, I 7 June ; Whitleigh Marsh, I 3, 24 June, 1889 


\section{INSECTS}

\section{PROBOSCIDEA (continued)}

\section{Syrphidae (continued)}

Pyrophaena rosarum, Fabr. Ivybridge, $7 \mathrm{May,} 1893$; Tamerton Foliott, I9 June, 1889; Holne, Dartmoor, 4 July, 1896; Crownhill Fort, 8, 19 July; Ivybridge, 26 July; Tamerton Foliott, 8 Aug. 1889

Melanostoma ambiguum, Fln. Yealm Mouth, 3 Apr., Io May; Beer Ferris, i I Apr. 1893

- mellinum, Linn. Gen. dist.

- scalare, Fabr. Warleigh Marsh, I I July ; Crownhill, I9 July, 1889

Leucozona lucorum, Linn. Irybridge, 19 May; Tamerton Foliott, 23 May, I, 5 June, 1889

Ischyrosyrphus glaucius, Linn. Ivybridge, I July, I I Aug. I Sept. ; Bickleigh Vale, I 7 July, I 889

- laternarius, Mull. Ioybridge, 10 July, I892, I I Aug. I 889

Didea fasciata, Macq. Shaugh, 8 May, I 893 ; Holne, Dartmoor, 3, I 8, 28 July, I 896

Catabomba pyrastri, Linn. Bickleigh, 7 June ; Walkham Valley, 2 I June; Budshead Wood, 27 June ; Tamerton Foliott, 29 June, I 2 July; Plymbridge, I July, I 889

- pyrastri, var. unicolor, Curt. Tamerton Foliott, 27 June ; Bickleigh, 30 July ; Crownhill, 15 Aug. 1889

- selenitica, Mg. Cornwood, 3 July, 1889

Syrphus albostriatus, Fln. Shaugh, 3 May; Walkham Valley, 9 May ; Salcombe, 20 May, I 893

- tricinctus, Fln. Ivybridge, I I Aug. 1889

- venustus, Mg. Ivybridge, $18 \mathrm{May}, 1890$ (G.H.V.)

- annulipes, Ztt. Lynton (C. J. W.) ${ }^{1}$

- annulatus, $Z_{\mathrm{tt}}$ Ivybridge (G. H. V.)

- grossulariae, Mg. Ieybridge, I 6 June; Cornwood, $3 \mathrm{July,} 1889$

- ribesii, Linn. Ivybridge, I 2 May, I893; Holne, Dartmoor, 4 July, 1896. Gen. dist.

- vitripennis, Mg. Ioybridge, 30 Apr. I893. Gen. dist.

- latifasciatus, Macq. Bickleigh, 2 I Apr.; Shaugh, 8 May; Torcross, 28 May, 1893; Budshead Wood, I July ; Fox Tor Mire, Dartmoor, 4 Aug.; Crownhill, 7 Aug. 1889 ; Bickleigh, 4 Sept. 1884 (G. C. B.)

- nitidicollis, Mg. Shaugh, 20 May, I8g6; Salcombe, 2 I May, I 893

- nitens, Ztt. Ivybridge, $24 \mathrm{July,} 1887^{3}$

- corollae, Fabr. Torcross, 24, 25 May, 1893

- luniger, Mg. Torcross, 26 May, 1893

- bifasciatus, Fabr. Ivybridge, 8 Apr.; Bickleigh, 12 May, 1893 ; Tamerton Foliott, 5 June, 1889 ; Dawlish, 8 June, I 893 (G. H.V.)

- balteatus, Deg. Gen. dist.

- cinctellus, Ztt. Bickleigh Vale, I 2, 25 Apr. ; Ivybridge, I 5 Apr. I I Aug. I Sept. I 893

- cinctus, Fln. Shaugh, I5 May, 1893 ; Iuybridge, 14 June, I 883 (G. H. V.)

- auricollis, Mg. Budshead Wood, 28 June; Tamerton Foliott, 29 June, 1889

var. maculicornis, $Z_{\text {tt. }}$ Ivybridge, 8, 15 Apr. 26 June, 1893 ; Salterton, 26 Apr. 1898

- euchromus, Kow. Beer Alston, I 3 Mar. ; Bickleigh Vale, 25 Apr. 1893

- punctulatus, Verr. Bickleigh, 12 Apr. 1893

1 Cf. G. H. V. Brit. Flies, vol. viii, p. 356.

2 Op. cit. p. $359 . \quad$ B. G. H. V. op. cit. p. 379.

\section{PROBOSCIDEA (continued)}

SXrPHIDAR (continued)

Syrphus guttatus, Fln. Ivybridge, II June, 1893 - compositarum, Verr. Ivybridge (G. H.V.) - lasiophthalmus, $Z$ tt. Ivybridge, 26, 30 Mar. 8, I 5 Apr.; Bickleigh, 5, I 2 Apr. 1893

Sphaerophoria scripta, Linn. Exeter, 17 July, 1871 (G. H. V.) ${ }^{5}$

- menthastri, Linn. BudsheadWood, I July ; Crownhill, 15, 25, July, 1889

var. picta, Mg. Ivybridge, $30 \mathrm{Apr} .1893$; Crownhill, I 7, I9 July, I 2 Aug.; Wralkham Valley, 2 I July; Fox Tor Mire, Dartmoor, 8 Aug. 1889

Xanthogramma ornatum, Mg. Ioybridge, 12 May Shough, I 5 May; Torcross, 27 May, I893; Tomerton Foliott, I June; Crownhill, 4 July, 7,30 Aug. I 889

Baccha obscuripennis, Mg. Torcross, 14 August, 1903

- elongata, Fabr. Tamerton Foliott, 6, 23 May, 19, 27 June, 1889; Ivybridge, I 8 May, I 893

Sphegina clunipes, Fln. Ivybridge, 25 Sept. 1887 (C. M.)

Ascia podagrica, Fabr. Beer Ferris, II Apr.; Iqybridge, 12 Apr.; Bovisand, 14 Apr. ; Cornwood, 23 Apr.; Torcross, 27 May, 1893

- dispar, Mg. Ivybridge, 30 Apr. 1893 ; Wistman's Wood, Dartmoor, 6 Sept. I 889

Brachyopa bicolor, Fln. Walkham Valley, 13 May, I 896 ; Seaton, 6 June, 1902 (A. E. E.)

Rhingia campestris, Mg. Gen. dist., Apr.-June

Volucella bombylans, Linn. Typical form and var. plumata, Deg. Gen. dist.

- inanis, Linn. Holne, Dartmoor, 17, 19, 22 July; Walkham Valley, 31 July, 1896 ; Ivybridge, I Sept. 1889

- inflata, Fabr. Avon Valley, 27 May, 10, 25 June, I 896

- pellucens, Linn. Salcombe, 21 May, I 893 ; Avon Valley, 25 May, 1896

Eristalis sepulchralis, Linn. Torcross, 23, 25 May, 1893 ; Crownhill, 24 June, 1889

- aeneus, Scop. Yealm Mouth, 3 Apr.; Bovisand, 14 Apr., 6 May ; Torcross, 26 May, 1893

- cryptarum, Fabr. Ivybridge, 26, 30 Apr., 4 May, I 893; 19 May, 1889; 30 Apr. I 893 (C. M.)

- tenax, Linn.

- intricarius, Linn.

- arbustorum, Linn.

- nemorum, Linn.

- pertinax, Scop.

- horticola, Deg.

Gen, dist.

Myiatropa florea, Linn. Ivybridge, 30 June, I 5 July, I Sept. ; Tamerton Foliott, 2 I May, 1889

Helophilus trivittatus, Fabr. Crownhill, I I, 1 2, 30 July, 5 Sept. ; Tamerton Foliott, 10 Sept. 1889; Torcross, I 2 Sept. 1903

- hybridus, Lw. Torcross, t,

- pendulus, Linn. Gen. dist.

- versicolor, Fabr. Torcross, 23, 25 May, 1893

- transfugus, Linn. Torcross, 23, 25, 26 May, 1893

-- lineatus, Fabr. Torcross, 23, 25 May, 1893

Tropidia scita, Harr. Paignton (C. J.W.) ${ }^{6}$

4 Op. cit. p. 414. Op. cit. p. 434.

${ }^{6}$ Cf. Entomologist's Montbly Mag. 2nd ser. vol. xiii (1 902), p. 70. 


\section{A HISTORY OF DEVONSHIRE}

\section{PROBOSCIDEA (continued)}

SYRPHIDAE (continued)

Criorrhina berberina, Fabr. Ivybridge, 25 May, 1896

- oxyacanthae, Mg. Ivybridge, 7, I9 May, I889, I9 May, I896; Shaugh, 8 May, I893; Avon Valley, Io June, 1896

- floccosa, Mg. Bovisand, 18 Apr. 1893

Xylota segnis, Linn. Ivybridge, $26 \mathrm{July}$; Comzwood, 8 Sept. I 889

- lenta, Mg. Avon Valley, 25 May, 1896

- sylvarum, Linn. Irybridge, 15, 23 July; Bickleigh Vale, 18, 28 July, 1889

Syritta pipiens, Linn. Gen. dist. Apr.-Sept.

Eumerus sabulonum, Fln. Salcombe, 7 July, I 889, captured in the burrow of a Fossorial Hymenopteron; Salcombe and Bovisand, on earthy cliffs Holne, Dartmoor (G. H. V.)

- strigatus, Fln. Woodbury Hill, $15 \mathrm{July,} \mathrm{I89} \mathrm{I}$ (A. E. E.)

Chrysochlamys cuprea, Scop. Bickleigh, 12 Apr. Bovisand, 18 Apr.; Salcombe, 20, 22 May, I893; The Dewerstone, 7 June; Tamerton Foliott, 15 Sept. ; Ivybridge, 22 Sept. 1889

Arctophila mussitans, Fabr. Cornwood, 8 Sept.; Tamerton Foliott, 27 Sept. 1889 ; Cornworthy, Aug.-Sept., the Rev. T. A. Marshall ; N. Devon (G. H. V.)

Sericomyia borealis, Fln. Ivybridge, 26 June, 1 5, 26 July, I Sept. ; Cornwood, 3 July ; Fox Tor Mire, Dartmoor, 4 Aug.; Tamerton Foliott, Io Sept. 1889

- lappona, Linn. Ivybridge, 19, 26 May, 16 June Cornwood, 2 June, 1889

Chrysotoxum cautum, Harr. Shaugh, I 5 May ; Torcross, 26 May; Salcombe, 27 May, 1893 Tamerton Foliott, 9 June, 1889

- octomaculatum, Curt. Salcombe, 21, 22 May, I 889

- elegans, Lw. Salcombe, 7 Sept. 1889

- festivum, Linn. Salcombe, 22 May, 1893 ; Cornwood, 3 July; Tamerton Foliott, 10 Sept. 1889

- bicinctum, Linn. Ivybridge, 23 June, 1889

Microdon mutabilis, Linn. Iqybridge, I 2, $18 \mathrm{May,}$ I 893, 12 June, 1889; Ioybridge, 2 I June, I89I (G. C. B.) ; Shute Hill, nr. Axminster, 30 June, I 900 (A. E. E.)

\section{Conopidar}

Conops flavipes, Linn. Irybridge, 14 June, 21, 26 July, I I Aug. 1889

Physocephala rufipes, Fabr. Bickleigh Vale, 28 July; Crownhill, i 5 Aug. I 889

Oncomyia atra, Fabr. Torcross

- (?) pusilla, Mg. Bovisand, 6 May, 1893

Myopa buccata, Linn. Walkham Valley, 9 May; Shough, I5 May, I893; Ioybridge, I9 May, I889 (C. M.) ; Yelverton, 6 June, I 889

- testacea, Linn. Ivybridge, I9 May; Tamerton Foliott, r Trne 1889

- polystigma, Ko d." "Salterton, 23 Apr. 1898

\section{Orstridar}

Gastrophilus equi, Fabr. Cullompton, pupae found 14 July, I 898, flies emerged I 5-2 5 Aug. I 898 , F. Milton

- nasalis, Linn. Torcross

$$
1 \text { Op. cit. p. 619. } \quad \text { Op. cit. p. } 633 .
$$

\section{PROBOSCIDEA (continued)}

Oestridas (contitued)

Hypoderma lineatum, Vill. Bickleigh Vale, 21, 25 Apr.; Horrabridge, 28 April, 1893; Walkham Valley, 8 May, 1895, 13 May, I 896 ; Salcombe, 22 May ; Torcross, 25 May, 1893

- bovis, Deg. Avon Valley, 25 June, 1896 ; Cann Wood; Bickleigh Vale, 13 Aug. (G. C. B.)

\section{Muscidae \\ Muscinas}

Meigenia bisignata, Mg. Torcross, 3, 6 Sept. 1903

- floralis, Mg. Crownhill, 14 July, I889; Torcross, $6,9,28,29$ Aug. 1903

Ceromasia sp. Torcross, 6 Sept. 1903

Gymnochaeta viridis, Fln. Ivybridge, 30 Mar. 8 Apr. 19 May; Cormwood, 2 Apr. ; Bickleigh, 12 Apr. 1893

Blepharidea vulgaris, Fln. Crownhill, 12 Aug. 1889

Epicampocera succincta, Mg. Torcross, 29 Aug. 1903

Sisyropa angusta, Br. and von Berg. Cornwood, 23 Apr. 1893

Eutachina rustica, Mg. Torcross, 23 May, 1893 ; Ioybridge, I I Aug., I Sept. I 889

Gonia ornata, Mg. (capitata, Deg., Schin.) Salterton, 23, 25 Apr. 1898 ; Torcross, 25 May, 1893; Kingsbridge (? Dr. Leach)

Brachychaeta spinigera, Rond. Walkham. Valley, 4. Apr. 1890

Aporomyia dubia, Fln. Iaybridge, 4 May, 1893

Pelatachina tibialis, Fln. Shaugh, 18 May; Avon Valley, 23 May, 1896

Macquartia flavipes, Mg. Ivybridge, 8, I 5 Apr. 1893 - dispar, Fln. Bovisand, 6 May, I893; Tamerton Foliott, 23 May, I 889

- grisea, Fln. Cornwood, 6 Sept. I889

- sp. Torcross, 6, 25 Aug., I Sept. 1903

Degecria (l) convexifrons, Ztt. Holne Dartmoor, $22 \mathrm{July,}, 1896$

Thelaira leucozona, Pz. Salcombe, 22 May, 1893 ; Bickleigh Vale, 18 July, 1889

var. nigripes, Fabr. Walkham Valley, 9 May ; Torcross, 25 May, 1893 ; Cornevood, 3 June, 1889

Demoticus plebejus, Fln. Crownhill, 22 June, 19 July, 1889

- frontatus, Boh. Ivybridge, 26 July ; Wistman's Wood, Dartmoor, 6 Sept.; Tamerton Foliott, Io Sept. 1889

Myobia fenestrata, Mg. Avon Valley, I I June;

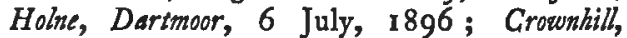
5 Aug. I 889 ; Torcross, I 6 Aug. 1903

- inanis, Fln. Holne, Dartmoor, 20 July, 1896; Cornwood, 8 Sept. I 889

- (?) pacifica (Mg.) Schin. Budshead Wood, 22 July, I 889

Olivieria lateralis, Fabr. Budshead Wood; Crownhill; Ermington, I July-9 Sept.

Micropalpus vulpinus, Fln. Walkham Valley, 2 I July, 1889

Ernestia (Erigone) radicum, Fabr. Tamerton Foliott, 8 Aug. ; Ivybridge, i I Aug. 1889; Holne, Dartmoor, 22 July, 1896

- rudis, Fln. (strenua, Mg.) Shaugh, 8 May, 1893; $18 \mathrm{May}, 1896$

- sp. Ivybridge, 19 May, I 896 


\section{INSECTS}

\section{PROBOSCIDEA (continued)}

\section{Muscidae (continued) \\ Muscinax (continued)}

Tachina grossa, Linn. Salcombe, 7 July, I889 ; Ivybridge, I3, 31 July, I 887 (C. M.), I 5, 26 July, I 889

- fera, Linn. Gen. dist. Apr.-Sept.

Fabricia ferox, $\mathrm{Pz}$. Holne, Dartmoor, 5, 17 July ; Walkham Valley, 31 July, 1896; Exmouth, Sept. I 889 (C. P.)

Servillia lurida, Fabr. Bickleigh, 5, 2 I Apr.; Walkham Valley, 9 Apr. 1893

var. leucocoma, $\mathrm{Mg}$. Shaugh, $8 \mathrm{May}, \mathrm{I} 893$; Ieybridge, 30 June, I 888 (C. M.)

— ursina, Mg. Walkham Valley, 2 1, 28 Mar., 6 Apr. ; Bickleigh, 5 Apr. 1893

Plagia ruralis, Fln. Torcross, 25 May, 1893

Thryptocera (?) pilipennis, Fln. Salcombe, $10 \mathrm{July,}$ 1896

- (?) minutissima, Ztt. Avon Valley, 27 June, 1896

Siphona cristata, Fabr. Plympton, 27 Apr. 1893

- geniculata, Deg. Gen. dist. Aug.-Sept.

Alophora hemiptera, Fabr. Ivybridge, 8, I 4, 22 July, I 888 (C. M.) ; 26 July, I 889

- pusilla, Mg. Torcross, 6, 15, 25 Aug. 1903

Phyto melanocephala, Mg. Bantham, 26 June, 1896

Stevenia maculata, Fln. Crownhill, 4 July, 1889

Clista lepida, Mg. Torcross

Sarcophaga carnaria, Linn. Gen. dist.

- muscaria, Mg. Ivybridge, 30 June, I July, 1888 (C. M.)

Cynomyia mortuorum, Linn. Ottery Hill, 17 July, 1891

Onesia sepulchralis, Mg. Bickleigh, 2 I Apr. I 893 ; Walkham Valley, I 8 May, 1895; Cormwood, 2 June, 8 Sept.; Ivybridge, I I Aug. 22 Sept.; Tamerton Foliott, 1o Sept. ; Crownhill, 2 I Sept. 1889

- cognata, Mg. Crownhill, I 7 July, I 889

Nyctia halterata, Pz. Bovisand, 6 May; Torcross, 25, $26 \mathrm{May}, 1893$

Metopia leucocephala, Rossi. Ivybridge, 2 May, 1893

Dexiosoma caninum, Fabr. Bickleigh Vale, 18, 28 July, 1889

Dexia rustica, Fabr. Bickleigh Vale, 18 July, 1889

- vacua, Fln. Walkham Valley, 2 I July, 1889

Myiocera carinifrons, Fln. Ivybridge, I I Aug. 1889

Stomoxys calcitrans, Linn. Torcross, 25 Aug. 1903. Gen. dist.

Haematobia stimulans, Mg. Gen. dist.

- irritans, Linn. Torcross

Pollenia rudis, Fabr.

- vespillo, Fabr.

Musca domestica, Linn.

Gen. dist.

- corvina, Fabr.

Graphomyia maculata, Scop. Tor Royal; Dartmoor, 4 Aug. ; Crownhill, 7, 30 Aug. 1889 ; Ivybridge, I I, 25 Sept. 1889 (C. M.)

Myiospila meditabunda, Fabr. Tamerton Foliott, 6 May, 1889; Dawlish, 8 May, I 888 (G. H.V.)

Cyrtoneura (Morellia) hortorum, Fln. Plymbridge, I 8 May; Tor Royal, Dartmoor, 4 Aug. 1889

- simplex, Lw. Plymbridge, 18 May; Ivybridge, 30 June ; Crownhill, I I July ; Wistman's Wood, Dartmoor, 6 Sept.; Tamerton Foliot, Io Sept. 1889

\section{PROBOSCIDEA (continued) \\ Muscidae (continued) \\ Muscinar (continued)}

Mesembrina meridiana, Linn. Tamerton Foliott; Crownhill; Ivybridge, May-Aug. (Col. Yerbury, C.M.)

Protocalliphora azurea, Fln. Exeter, 17 July, 1871 (G. H. V.)

Calliphora erythocephala, Mg. Gen. dist.

- vomitoria, Linn. Ivybridge, 3 Aug. 1889 (C. M.) ; Tor Royal, Dartmoor, 4 Aug. 1889

Pyrellia cyanicolor, Ztt. Walkham Valley, 9 Apr. 1893 ; Cornwood, 8 Sept. ; Crownhill, 13 Sept. I 889

$\left.\begin{array}{l}\text { Euphoria cornicina, Fabr. } \\ \text { Lucilia caesar, Linn. }\end{array}\right\}$ Gen. dist.

Anthomyinar

Hyetodesia semicinerea, Wied. Dawlish, 8 June, 1883 (G. H. V.)

- umbratica, Mg. Doone Valley, 6 June, 1883 (G. H. V.)

- signata, Mg. $\}$ Dawlish, 8 June, 1883 (G. H. V.)

Spilogaster nigrinervis, Ztt. West Dunsford, 9 June, 1883 (G. H.V.)

- maculosa, Mg. Exeter, 6 June; Irybridge, 12 June, 1883 (G. H.V.)

- trigonalis, Mg. Lynton, I 9 June, I 883 (G. H. V.)

Hydrotaea parva, Meade

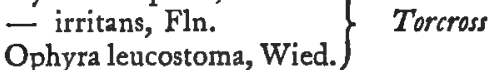

Anthomyia sulciventris, Ztt. Irybridge, 12 June, I 883

Phorbia ignota, Rond. Dawlish, 8 June ; Doone Valley, I 8 June, I 883 (G. H.V.)

- cilicrura, Rond. Doone Valley, 18 June, 1883 (G. H. V.)

Homalomyia hamata, Macq. Irybridge, I 3 June, I 883 (G. H.V.)

- pallitibia, Rond. Slapton, 25 Aug. 1885 (G. H.V.)

Azelia macquarti, Staeg. Lynton, I7 June, 1883 (G. H.V.)

- cilipes, Hal. Dawlish, 8 June, 1883 (G. H.V.)

Lispe tentaculata, Deg. Crownhill, 2-15 Aug. I889; Slapton, 7 Sept. 1889 (G. H.V.)

Fucellia maritima, $\left.\mathrm{H}_{21}\right\}$ Torcross

\section{Cordyluridae}

Amaurosoma fasciatum, $\mathrm{Mg}$. Torcross

Norellia spinimana, Fln. Tamerton Foliott, 2 I May, 1889

Trichopalpus fraternus, Mg. Torcross

Spathiophora hydromyzina, Fln. Torcross

Scatophaga scybalaria, Linn. Salterton, 27 Apr. 1898

- stercoraria, Linn. Gen. dist.

- squalida, Mg. Ivybridge, 19 May, I889

- litorea, Fln. Beer Ferris, II Apr. I893; Salterton, 23-27 Apr. I 898

\section{Phycodromidal}

Orygma luctuosum, Mg. Salcombe, 7 July. Under seaweed

Phycodroma sciomyzina, Hal. Thurlestone

Caelopa pilipes, Hal. Thurlestone 


\section{A HISTORY OF DEVONSHIRE}

\section{PROBOSCIDEA (continued)}

\section{HELOMYZIDAE}

Helomyza rufa, Fln. Torcross

- affinis, Mg. (Lw.) Crownhill, 3 I May, 1889

- similis, Mg. Bickleigh Vale, 18 July; Crownhill, I 5 Aug. 1889

Blepharoptera caesia, Mg. Crownhill Fort, 18 May, 1889

- modesta, Mg. Crownbill Fort, 29 May, 1889

Heteromyza commixta, Coll. Bovisand and Slapton Ley (Collin, Ent. Monthly Mag., Apr. 190I)

"Tephrochlamys rufiventris, Mg. Crownhill Fort, 2I Mar. 6 June, $189^{\circ}$

\section{Heteroneuridae}

Heteroneura albimana, $\mathrm{Mg}$. Torcross

\section{ScIomyzidaz}

Oedoparea buccata, Fln. Dawolish, 25 Feb. 1893

Dryomyza flaveola, Fabr. Ivybridge, 30 Apr. I 893 ; Tamerton Foliott, 2 I May, I June ; Budshead Wood, 6 June ; Crownhill, 13 Sept. 1889

Neuroctena anilis, Fin. Ivybridge, I4 July, 8 Sept. I 888 (C. M.) ; II Aug. I, 22 Sept. 1889

Sciomyza pallida, Fln. Crownhill, 9 Sept. 1889

- nana, Fln. Torcross

- schoenherri, Fln. Salterton, 24 Apr. 1898

-- cinerella, Fln. Torcross

- dorsata, Ztt. Torcross

Pelidnoptera nigripennis, Fbr. Ivybridge, 17 May 1893 ; Chagford (Dr. Meade, Ent. Monthly Mag., I 899, p. I02)

Tetanocera elata, Fabr. Ivybridge, 7 May, 1893 ; I Aug. 1896 ; Holne, Dartmoor, 3 June, I 896

- laevifrons, Lw. Torcross

- sylvatica, Mg. Avon Valley, 23 May, 1896

- ferruginea, Fln. Salterton, 27 Apr. 1898 ; Plymton, 27 Apr. I 893 ; Bovisand, I 6 May, 1896 ; Tamerton Foliott, 25 May, 1889; Torcross, 26 May, 1893 ; Crownhill, I 5 Aug. 1889 ; Wistman's Wood, Dartmoor, 6 Sept. 1889

- robusta, Lw. Shaugh Bridge, 23 Apr. 1893 ; Plymbridge, 8, I 5 June, I889

- punctata, Fabr. Salterton, 24 Apr. 1898

- coryleti, Scop. Plymouth, I7 June, 1884 (A. Beaumont); Bickleigh Vale, 6 July, 1896 ; Crownhill, 7 Sept. 1889

- punctulata, Scop. Shaugh, 8 May, 1893 ; Bickleigh Vale, 10 July, 1893 (A. Beaumont)

Limnia marginata, Fabr. Bantham, 26 June, 1896

- unguicornis, Scop. Torcross

-- obliterata, Fabr. Crownhill, 16 Sept. 1889

Elgiva albiseta, Scop. Torcross, 25 May, 1893 ; Bickleigh Vale, i 8 June, 1889

- dorsalis, Fabr. Avon Valley, I 2 May, 1896

Sepedon spinipes, Scop. Torcross

\section{Psilidar}

Psila fimetaria, Linn. Shaugh, $8 \mathrm{May}, \mathrm{I} 893$; Avon Valley, 6 June, 1896; Tamerton Foliott, 19 June; Ivybridge, 26 July, 1889

Chyliza atriseta, Mg. Salcombe, 20, 22 May, 1893 - leptogaster, Pz. Kingsbridge, 23 May, I 893

Loxocera albiseta, Schrk. Bantham, 26 June, 1896 ; Warleigh Marsh, 5 Aug. I889; Crownhill, I 5 Aug. 1889

\section{PROBOSCIDEA (continued)}

Psilidar (continued)

Loxocera sylvatica, Mg. Ivybridge, 20, $30 \mathrm{Apr}$. 19 May; Bickleigh Vale, 2 1, 25 Apr.; Shaugh Bridge, 22 Apr. 1893

Lissa loxocerina, Fln. Kingsbridge

\section{Micropezidar}

Micropeza corrigiolata, Linn. Seaton, 26 June, 1891 (A. E. E.)

\section{Ortalidar}

Pteropaectria frondescentiae, Linn. Whitleigh Wood, I 3 June ; Ivybridge, I 9 June, I889 ; Bantham, 26 June ; Avon Valley, 28 June, 1896 ; Aylesbeare Common, nr. Exeter, 14 July, I891 (A. E. E.)

\section{TRYPETIDAE}

Acidia heraclei, Linn. Branscombe, 23 May, I 902 (A. E. E.)

Chaetostoma curvinervis, Rond. Bickleigh Vale, 1 Apr. 1893

Spilographa abrotani, Mg. Ivybridge (C. M.)

Trypeta onotrophes, Lw. Holne, Dartmoor, 28 July, 1896

- tussilaginis, Fabr. Bickleigh Vale, 18 July, 1899

Urophora solstitialis, Linn. Avon Valley, 25 June, I 896

Sphenella marginata, Fln. Torcross

Tephritis miliaria, Schrk. Avon Valley, 23 June; Holne, Dartmoor, 23 July, 1896

- vespertina, Lw. Tavistock, Io Mar.; Shaugh, 8 May, 1893

\section{LONCHAEIDAR}

Palloptera angelicae, von Roser (Lw., Becker). Ivybridge, I Sept. 1889

- umbellatarum, Fabr. Torcross

\section{SAPROMYZIDAE}

Peplomyza wiedemanni, Lw. Torcross

Sapromyza longipennis, Fabr. Ivybridge, 20 May, I888; 2 I June, I 887 ; 22 July, I 888 ; (C. M.) ; Torcross

- inusta, Mg. Ivybridge, 14 May, 1893 ; Cormwood, 3 July; Crownhill, 15 Aug. I889; Ivybridge, 9, 14 July, 1888 (C. M.)

- decempunctata, Fln. Plymouth, I 7 June, I 894 (A. Beaumont)

- notata, Fln. Torcross

- pallidiventris, Fln. Ivybridge, 22 Aug. 1888 (C. M.)

- quadripunctata, Linn. Torcross

Lauxania aenea, Fln. Torcross; Ivybridge, 9 July, I 887 (C. M.)

\section{OPOMYZIDAE}

Balioptera combinata, Linn. Torcross

- venusta, Mg. Torcross

Opomyza germinationis, Linn. Bickleigh (G. C. B.)

- florum, Fabr. Torcross

Pelethophila flava, Linn. Seaton, 2 Aug. 1902 (A. E. E.) 


\section{INSECTS}

PROBOSCIDEA (continued)

\section{SEPSidae}

Sepsis punctum, Fabr. Avon Valley, 18 June, 1896 Nemopoda cylindrica, Fabr. Crownhill, 18 May, 7 Sept. 1889 ; Avon Valley, 18 June, 1896 ; Ivybridge, 17 Sept. 1887 (C. M.)

Henicita annulipes, $\mathrm{Mg}$. Torcross

Themira putris, Linn. S. Devon

Gromyzidae

Diastata unipunctata, $Z_{\mathrm{tt}}$. Torcross

Drosophilidae

Noterophila glabra, Fln. Torcross, 12 Aug. 9 Sept. I 903

\section{Chloropidae}

Meromyza laeta, Mg. Salcombe, 15 June, 1896

Diplotoxa messoria, Fln. Torcross
PROBOSCIDEA (continued)

\section{Agromyzidae}

Leucopis griseola, Fln. Irybridge, I 8 July, I 887 (C. M.) ; bred from Aphis on thistle; Torcross, 25 Aug. 1903

Ochthiphila flavipalpis, $\mathrm{Hal}$. Bantham, 26 June, 1896

Schoenomyza litorella, Fln. Torcross

\section{EPROBOSCIDEA}

\section{NyctrRibidae}

Nycteribia hermanni, Leach. Barnstaple, 2 I Oct. 1898 (Oxley Grabham); from Lesser Horseshoe Bat (Rhinolophus hipposiderus, Bechst.), found in a disused iron-working; no fewer than eighteen specimens were taken from a single bat

\section{HEMIPTERA HETEROPTERA}

\section{Bugs}

In this section of the Hemiptera 230 out of 454 British species have been recorded as occurring in Devonshire.

The small number tabulated for the county is not surprising, when it is taken into consideration that no individual has concentrated his thoughts solely on this section of the fauna. The writer is confident that one hundred could be added to the list within two years, and another fifty with little extra exertion.

The great majority of these are to be found on trees, shrubs, low-growing plants and grasses; the greater number are vegetarians, others are not particular when they find some soft bodied insect suitable to their requirements. A few, however, are found in running streams, stagnant pools, brackish water, and in the sea. Many of these are carnivorous, sucking the juices of the body of their victims; some are to be found skimming over the surface of the water, waiting for an unlucky insect that might drop in ; others traverse the mud bottoms; whilst some pass most of their lives swimming about on their backs. One species has been found in the sea; this appears to feed upon decaying matter, living under stones from about half-tide to low water; many of the stones under which it has been found were only uncovered when the tides were extremely low.

Very few persons are aware of the affection shown by one species for its young (Acanthosoma interstinctum). It was first mentioned by De Geer and verified by the late Edward Parfitt and the late Rev. J. Hellins, both of Exeter. The latter records the fact in the Entomologist's Montbly Magazine, vol. vii, p. 53 (August, I870); a further record in vol. xi, p. 42 (July, I 874), gives the following account of his interesting observations. He says, 'On the 4th of June she began by depositing one egg, then a row of two or three, then about five, till at the widest row there must have been seven or eight; then she diminished the rows again till she came to a point, the whole mass, in number between thirty and forty, forming a rough diamond figure just about the size of her own body. The outer eggs were laid on their sides, the inner ones stood up on end. The mother now took her stand over these eggs, but I do not think her body touched them; towards the end of June (29th) I found the young bugs all hatched, and clustered under their mother. On July 3rd I found them showing a red streak down the middle of the abdomen, and, on the 6th they had moved from among the egg-shells, and were got together by themselves. On the gth I found them moulting; their colours were bright at first, yellow with vermilion stripe, and they soon began to move about freely. I do not think the female feeds whilst brooding over her eggs or young.'

There is one species in this group of insects (the bed-bug) that is a great terror to the careful mistress of the house : but when we hear of a house being pestered with these disgusting things, it is not wise to condemn the housekeeper for their presence, especially if it is a newly-built house, for on the sap of the pine-boards these insects feed.

Mr. Parfitt records that a newly-built cottage which had never been inhabited was swarming with them (Cimex lectularius). They were traced to the pine wood that had been imported from America with which the cottage had been built. It may not be out of place here to say that half a pint of carbolic acid to one gallon of water is one of the best remedies known, the floor, and especially behind the skirting boards, being well wetted, a mop being used for that purpose ; it is also a grand remedy for destroying other obnoxious insects. 


\section{A HISTORY OF DEVONSHIRE}

\section{PENTATOMINA}

SCUTELLERIDAE

Corimelaena, White

- scarabaeoides, Lin. Cathedral Close, Exeter (Parfitt)

Eurygaster, Lap.

$\$$ - maura, Lin.

Podops, Lap.

$\S$ - inuncta, Fab.

\section{Cydnidas}

Sehirus, Am. S.

$\S$ - bicolor, Lin.

* _ biguttatus, Lin.

§- morio, Lin.

Gnathoconus, Fieb.

$\S-$ albomarginatus, Fab.

\section{Pentatomidae}

\section{Neottiglossa, Curt.}

$\S-$ inflexa, Wolff

Pentatoma, Oliv.

$\dagger$ - fuscispina, Boh.

* - baccarum, Lin.

* prasina, Lin.

Strachia, Hahn.

†- festiva, Lin.

Pizodorus, Fieb.

* - lituratus, Fab.

Tropicoris, Hahn.

$\S$ - rufipes, Lin.

\section{Asopidar}

Picromerus, Am. S.

$\$$ - bidens, Lin.

Podisus, H. S.

$\S$ - luridus, Fab.

Asopus, Burm.

$\$$ - punctatus, Lin.

Zicrona, Am. S.

†- caerulea, Lin.

\section{Acanthosomidab}

Acanthosoma, Curt.

* - haemorrhoidale, Lin.

- interstinctum, Lin. Protects its young as mentioned in the introduction

†- tristriatum, Lin.

\section{COREINA}

\section{Coreidar}

Enoplops, Am. S.

* - scapha, Fab.

Syromastes, Latr.

- marginatus, Lin.

Verlusia, Spin,

$\$$ - rhombea, Lin.

Pseudophlaeus, Burm.

$\S$ - Fallenii, Schill.

Coreus, Fab.

* - denticulatus, Scop.

Alydus, Fab.

- calcaratus, Lin.

\section{GYMNOCERATA}

\section{COREINA (cont.)}

STENocephalidar

Stenocephalus, Latr.

- agilis, Scop. Gen. dist.

*- neglectus, H. Schiff.

\section{Corizidaz}

Therapha, Am. S.

$\$$ - hyoscyami, Lin.

Corizus, Fall.

$\S-$ capitatus, Fab.

\section{Berytidae}

Neides, Latr.

t- tipularius, Lin. (Parfitt)

Berytus, Fab.

$\S-$ minor, H. Schiff.

- Signoreti, Fieb. Gen. dist.

t- montivagus, Fieb. Coast of N. Devon (Stainton)

\section{Metacanthidae}

Metacanthus, Costa

$\$$ - punctipes, Germ.

\section{LYGAEINA \\ LYGAEIDAE}

Nysius, Dall.

t- thymi, Wolff.

\section{Cymidae}

Cymus, Hahn.

$\$$ - glandicolor, Hahn.

†- claviculus, Fall.

Ischnorhynchus, Fieb.

* - resedae, Panz. On heaths

$\$$ - geminatus, Fieb.

\section{Henestaridae}

Henestaris, Spin.

- laticeps, Curt. On the coast (Keys)

\section{Heterogastridae}

Heterogaster, Schill.

$\S$ - urticae, Fab.

\section{Pachymeridae}

Rhyparochromus, Curt.

$\S$ - dilatatus, H. Schft.

\$ - chiragra, Fab.

- antennatus, Schill. Manaton (Douglas)

t- praetextatus, H. Schft.

Tropistethus, Fieb.

- holosericeus, Schltz. Seaton (Dr. Power)

Ischnocoris, Eieb.

- angustulus, Boh. Warren Starcross

Macrodema, Fieb.

- micropterum, Curt. Dawlish Warten

\section{LYGAEINA (cont.)}

Pachymgridaz (cont.)

Plinthisus, Fieb.

- brevipennis, Latr. Daswlish Warren

Stygnus, Fieb.

- rusticus, Fall. Bickleigh

$$
\text { Woods }
$$

* - pedestris, Fall.

* - arenarius, Hahn.

Peritrechus, Fieb.

- luniger, Schill.

- nubilus, Fall. Exeter district

$\dagger-$ geniculatus, Hahn.

Trapezonotus, Fieb.

*- agrestis, Panz. Local

Aphanus, Lap.

$\$$ - Rolandri, Lin.

$\S$ - pedestris, Panz.

Beosus, Am. S.

- luscus, Fab. Torcross

Eremocoris, Fieb.

- podagricus, Fab. Nr. Okehampton

Scolopostethus, Fieb.

*_- affinis, Schill.

* - neglectus, Edw.

*- decoratus, Hahn.

Notochilus, Fieb.

†- contractus, H.S.

Drymus, Fieb.

$\$$ - sylvaticus, Fab.

$\$$ - brunneus, Sahlb.

Gastrodes, Wesm.

$\S-$ ferrugineus, Lin.

PyrRhocoridae

Pyrrhocoris, Fall.

- apterus, Lin. Oreston Rock; Torquay. Abundant in 1865 , and as plentiful on this barren rock in 1898

\section{TINGIDINA}

Piesmidae

Piesma, Lep.

* - quadrata, Fieb. From moss on oak trees

- capitata, Wolff. Ivybridge. Out of moss on oak trees

\section{Tingididar}

Orthostira, Fieb.

- brunnea, Germ. Ivybridge. Out of moss on oak trees

- parvula, Fall. Exmouth Warren

Dictyonota, Curt.

- crassicornis, Fall. Gen. dist.

- strichnocera, Fieb. Bovey Heathfield

Derephysia, Spin.

* - foliacea, Fall.

Monanthia, Lep. Out of moss

- ampliata, Fieb. $\left\{\begin{array}{l}\text { on trees } \\ \text { - }\end{array}\right.$ 


\section{INSECTS}

\section{TINGIDINA (cont.)}

Tingididae (cont.)

Monanthia, Lep.

$\$$ - quadrimaculata, Wolff.

$\$$ - dumetorum, H. Schff.

t- humuli, Fab.

\section{ARADINA}

Aradus, Fab.

* - depressus, Fab.

Aneurus, Curt.

$\S$ - laevis, Fab.

\section{HYDROMETRINA}

\section{Ä̈POPHILIDAE}

Aëpophilus, Sign.

- Bonnairei, Sign. Not rare. Batten Bay ${ }^{1}$

Hydrometra, Latr.

** - stagnorum, Lin.

Velia, Latr.

- - currens, Fab.

Gerris, Fab.

- najas, De G.

- thoracica, Schum.

* - gibbifera, Schum.

* - lacustris, Lin.

$\S$ - argentata, Schum.

\section{REDUVIINA}

Emesidae

Ploiaria, Scop.

$\S$ - vagabunda, Lin.

\section{NabidaE}

Nabis, Latr.

*- lativentris, Boh.

- major, Cost.

- _ ferus, Lin.

- rugosus, Lin.

\section{SALDINA}

Salda, Fab.

- lateralis, Fall. Topshom (Parfitt)

-- orthochila, Fieb. Local on the sea coast

$\$$ - scotica, Curt.

* C-album, Fieb.

* - saltatoria

- Cocksii, Curt. Meavy Valley. Out of Sparganium (Keys)

- cincta, H. Schff. Slapton Ley

\section{CIMICINA}

\section{Cimicidae}

Cimex, Lin.

* - lectularius, Lin. Bed-bug

- columbarius, Jen. In old pigeon cots

- hirundinis, Jen. In martins' nests

- pipistrelli, Jen. In the homes of bats

1 Found in the sea under stones at low water; mature in winter (Keys).
CIMICINA (cont.)

Anthocoridae

Lyctocoris, Hahn.

- campestris, Fall. Exeter district (Parfitt)

Piezostethus, Fieb.

$\uparrow$ - galactinus, Fieb.

- cursitans, Fall. Not rare

Temnostethus, Fieb.

$\dagger$ - pusillus, Schff.

Anthocoris, Fall.

- - confusus, Reut.

* - nemoralis, Fab.

* - gallarum-ulmi, De G.

- - sylvestris, Lin.

Tetraphleps, Fieb.

- vittata, Fieb. (Keys)

Triphleps, Fieb.

- majuscula, Reut. Plymouth district

*- minuta, Lin.

Xylocoris, Duf.

†- ater, Duf. Stonehouse. Alighted on newspaper

\section{Microphysidae}

Microphysa, Westw.

- elegantula, Baer. Not rare, but local

\section{CAPSINA}

Pithanus, Fieb.

*- Maerkeli, H. Schff.

Miris, Fab.

* holsatus, Fab.

$\$$ - laevigatus, Lin.

* - calcaratus, Fall.

Megaloceraea, Feib.

$\$$ - erratica, Lin.

- ruficornis, Fourc.

Leptopterna, Fieb.

* - ferrugata, Fall.

- dolobrata, Lin.

Bryocoris, Fall.

$\$$ - pteridis, Fall.

Monalocoris, Dahlb.

- filicis, Lin.

Pantilius, Curt.

* - tunicatus, Fab.

Lopus, Hahn.

- sulcatus, Fieb. Rather local (on the coast)

Miridius, Fieb.

$\S$ - quadrivirigatus, Costa

Phytocoris, Fall.

* - populi, Lin.

* - Tiliae, Fab.

* longipennis, Flor.

-. ulmi, Lin.

- varipes, Boh. Not rare

Calocoris, Fieb.

* - striatellus, Fab.

* fulvomaculatus, De G.

* - sexguttatus, Fab.
CAPSINA (cont.)

Calocoris, Fieb. (cont.)

* - roseomaculatus, De G.

$\$$ - ticinensis, Mey.

* Chenopodii, Fall.

* _ bipunctatus, Fab.

* - striatus, Lin.

Oncognathus, Fieb.

* binotatus, Fab.

Plesiocoris, Fieb.

$\S$ - rugicollis, Fab.

Lygus, Hahn.

*- pabulinus, Lin.

* - viridis, Fall.

* lucorum, Mey.

\$- Spinolae, Mey.

- pratensis, Fab.

- - pastinacae, Fall.

- Kalmii, Lin.

$\S-$ cervinus, H. Schff.

- rubricatus, Fall. Not rare

Poeciloscytus, Fieb.

- Gyllenhalii, Fall.

$\S-$ nigritus, Fall.

$\$$ - unifasciatus, Fab.

Liocoris, Fieb.

- tripustulatus, Fab.

Capsus, Fab.

* Laniarius, Lin.

* - scutellaris, Fab.

Rhopalotomus, Fieb.

- ater, Lin.

Labops, Burm.

*- saltator, Hahn.

Dicyphus, Fieb.

-_ epilobii, Reut.

$\$-$ globulifer, Fall.

Campyloneura, Fieb.

$\$$ - virgula, H. Schff.

Cyllocoris, Hahn.

*- histrionicus, Lin.

* - flavonotatus, Boh.

Aetorhinus, Fieb.

* - angulatus, Fab.

Globiceps, Latr.

*- flavomaculatus, Fab.

Mecomma, Fieb.

- ambulans, Fall. Cyrtorrhinus, Fieb.

$\S$ - caricis, Fall.

Orthotylus, Fieb.

$\$$ - nassatus, Fab.

$\$$-- flavinervis, $\mathrm{Kbm}$.

$\S$ - chloropterus, Kbm.

$\S$ - flavosparsus, Sahlb.

* - rubidus, Put.

* - ericetorum, Fall.

Hypsitylus, Fieb.

- bicolor, Douglas and Scott. Shaugh Bridge, Aug. (Keys)

Malacocoris, Fieb.

- chlorizans, Fall.

Loxops, Fieb.

* - coccineus, Mey.

Heterotoma, Lat.

* - merioptera, Scop.

Heterocordylus, Fieb.

$\S$ - tibialis, Hahn. 


\section{A HISTORY OF DEVONSHIRE}

\section{CAPSINA (cont.)}

Onychumenus, Reut.

$\$$ - decolor, Fall.

Macrotylus, Fieb.

$\S-$ Paykulli, Fall.

Hoplomachus, Fieb.

*- Thunbergi, Fall.

Macrocoleus, Fieb.

- molliculus, Fall. Local

- tanaceti, Fall. Local

Harpocera, Curt.

*- thoracica, Fall.

\section{NAUCORIDINA}

Naucoris, Geoffr.

* - cimicoides, Lin.

\section{NEPINA}

Nepa, Lin.

*- cinerea, Lin.

Ranatra, Fab.

† - linearis, Lin.

\section{NOTONECTINA}

Notonecta, Lin.

* - glauca, Lin.

\section{CAPSINA (cont.)}

Phylus, Hahn.

$\$$ - palliceps, Fieb.

* melanocephalus, Lin.

- coryli, Lin.

Atractotomus, Fieb.

\$- mali, Mey.

Psallus, Fieb.

*- betuleti, Fall.

* - ambiguus, Fall.

* - variabilis, Fall.

§- Fallenii, Reut.

\section{CRYPTOCERATA}

\section{NOTONECTINA (cont.)}

Plea, Leach.

* - minutissima, Fab.

\section{CORIXINA}

Corixa, Geoffr.

*- Geoffroyi, Leach.

* - atomaria, Illig.

* - lugubris, Fieb.

* - hieroglyphica, Duf.

* - Sahlbergi, Fieb.

* - striata, Lin.
CAPSINA (cont.)

Psallus, Fieb.

$\S$ - lepidus, Fieb.

$\$$ - varians, H. Schff.

$\S$ - sanguineus, $F_{a b}$.

†- salicellus, Mey.

Plagiognathus, Fieb.

-_albipennis, Fall.

* arbustorum, Fab.

*- viridulus, Fall.

$\S-$ Bohemanni, Fall.

\section{CORIXINA (cont.)}

Corixa, Geoffr.

*- distincta, Fieb.

* Fallenii, Fieb.

*- moesta, Fieb.

* - limitata, Fieb.

$\S$ - semistriata, Fieb.

t- venusta, Douglas and Scott

* Fabricii, Fieb.

* - fossarum, Leach

- Scotti, Fieb. Not rare

*- coleoptrata, Fab.

Sigara, Fab.

* - minutissima, Lin.

\section{HEMIPTERA HOMOPTERA}

\section{MEMBRACIDAE}

Centrotus, Fab.

*-_ cornutus, Lin.

\section{TETTIGOMETRIDAE}

Tettigometra, Latr.

§- impressopunctata, Sign.

ISSIDAE

Issus, Fab.

* - coleoptratus, Geoff.

\section{CIXIIDAE}

Cixius, Làtr.

$\S-$ pilosus, Ol.

*- cunicularis, Lin.

* - nervosus, Lin.

$\S$ - stigmaticus, Germ.

\section{DELPHACIDAE}

Liburnia, Stål.

t- Ineola, Germ.

§- fuscovittata, Stål.

$\$$ - lepida, Boh.

$\$$ - pellucida, Fab.

*_ difficilis, Edw.

$\S$ - striatella, Fall.

* - limbata, Fab.

Stiroma, Fieb.

*- pteridis, Boh.

\section{CERCOPIDAE}

Triecphora, Am. et Serv. * - vulnerata, Illig.

\section{CERCOPIDAE (cont.)}

Aphrophora, Germ.

*- alni, Fall

Philaenus, Stål.

- spumarius, Lin. ${ }^{1}$

$\S-$ exclamationis, Thunb.

* lineatus, Lin.

\section{LEDRIDAE}

Ledra, Fab.

- aurita,Lin. Gen. dist. Hibernates in the larva stage; found mature in Sept. (Bignell)

\section{PAROPIIDAE}

Magophthalmus, Curt.

$\S-$ scanicus, Fall.

\section{BYTHOSCOPIDAE}

Macropsis, Lewis

* - lanio, Linn.

Bythoscopus, Germ.

- alni, Schr.

$\$$ - rufusculus, Fieb.

* - flavicollis, Lin.

Pediopsis, Burm.

$\$$ - scutellatus, Boh.

$\S$ - virescens, Fal.

1 The larva of this insect is the maker of the 'Cuckoo Spit' frequently seen in the spring ; it is for the protection of its soft body.
BYTHOSCOPIDAE (cont.)

Idiocerus, Lewis

$\S-$ distinguendus, $\mathrm{Kbm}$.

$\$$ - tremulae, Estl.

$\S$ - laminatus, Flor.

$\S$ - lituratus, Fall.

$\S-$ populi, Lin.

$\S$ - confusus, Flor.

Agallia, Curt.

- puncticeps, Germ.

$\$$ - venosa, Fall.

\section{TETTIGONIDAE}

Evacanthus, Lep. et. Serv.

- interruptus, Lin.

$\$-$ acuminatus, Fab.

Tettigonia, Geoffr.

* - viridus, Lin.

\section{ACOCEPHALIDAE}

Acocephalus, Germ.

- nervosus, Schr. Sparsely dist.

†- bifasciatus, Lin.

*- albifrons, Lin.

Eupelix, Germ.

- cuspidata, Fab.

Platymetopius, Burm.

† undatus, De G. ${ }^{2}$

2 Captured for the first time in England, Aug. 1882 , in a meadow between Plymbridge and Bickleigh Vale, on the common bracken fern. None seen since 1884. The locality now, Igoz, is a fir plantation. (Bignell. 


\section{INSECTS}

JASSIDAE

Athysanus, Burm.

\$- grisescens, Zett.

" - communis, J. Sahl.

* - obscurellus, Kbm.

- obsoletus, Kbm.

Deltocephalus, Burm.

- socialis, Flor. Local

- striatus, Lin. Local

t- punctum, Flor. Stonehouse; Plymouth. Flew into the house (Bignell)

- pulicaris, Fall. Local

Allygus, Fieb.

\$ - modestus, Fieb.

*- mixtus, Fab.

Thamnotettix, Zett.

$\S$ - subfuscula, Fall.

* - splendidula, Fab.

* - crocea, H. S.

Limotettix, J. Sahl.

$\$$ - quadrinotata, Fab.

t- nigricornis, J. Sahl.

Cicadula, Fieb.

* - sexnotata, Fall.

Gnathodus, Fieb.

$\S$ - punctatus, Thunb.
TYPHLOCYBIDAE

Alebra, Fieb.

$\dagger$ albostriella, Fall.

Kybos, Fieb.

$\S-$ smaragdulus, Fall.

Chlorita, Fieb.

$\$$ - viridula, Fall.

Eupteryx, Curt.

$\$$ - vittatus, Lin.

* - urticae, Fab.

$\S$ - stachydearum, Hardy

*- melissae, Curt.

* - auratus, Lin.

- filicum, Newm. On ferns, in a fernery, Plymouth (Bignell)

*_ pulchellus, Fall.

* - concinna, Germ.

Typhlocyba, Germ.

$\S$ - sexpunctata, Fall.

* - ulmi, Lin.

* - tenerrima, H. S.

* aurovittata, Dougl.

*- Lethierryi, Edw.

*- rosae, Lin.

*- quercus, Fab.

* - geometrica, Schr.
APHALARIDAE

Rhinocola, Först.

* - ericae, Curt.

PSYLLIDAE

Psyllopsis, F. Lơw.

- fraxini, Lin.

Psylla, F. Löw.

$\$$ - mali, Schbdg.

*- pineti, Flor.

* - salicicola, Först.

- Forsteri, Flor.

- alni, Lin.

TRIOZIDAE

Trichopsylla, Thoms.

$\$$ - Walkeri, Först.

Trioza, Först.

- - urticae, Lin.

- - crithmi, F. Löw

- remota, Först.

- albiventris, Först.

3 Stonehouse; Plymouth. Abundant on the rocks, where the Samphire (Crithmum maritimum) grows freely. During August they may be seen in all stages, eggs, larvae, pupae, and the perfect insect. (Bignell)

\section{APHIDIDAE}

The increase of Apbides (generally known as blight) in a few days on certain plants appears marvellous; but when the life history of an aphis is known it does not remain so great a wonder. It may, therefore, be interesting to know something of its life history. As a general rule the first aphis in the spring proceeds from an egg, and it requires about twenty days to arrive at maturity; it then commences to bring forth its young at the rate of from eight to twenty a day; one old writer says twenty-five a day; that must have been a very exceptional case; the number depends entirely on heat and the food supply. Viviparous females, without the intervention of the male, will again under favourable conditions begin to reproduce their species in five or six days. Males and oviparous females are not produced until the end of the breeding season, which is generally with the first indication of the autumnal change, i.e. by those that continued breeding viviparously: for some species may have only a few broods. There is no hard-and-fast line in nature, for what a hundred may do, the remainder do not do ; both, however, attain the same end, but in different ways.

Kyber remarks that although the duration of the state of viviparousness is in a certain sense almost unlimited, yet in great measure it is controlled and determined by insufficient food and reduced temperature. Further, he says that certain species, feeding on herbaceous plants which fruit early in the year and then wither, produce males and apterous oviparous females in the middle of the summer. Also, that the same thing occurs when the food plants rapidly become woody in texture. On the contrary, where Aphides get their nourishment from plants persistent throughout the year, the males and oviparous females as a rule do not appear until late autumn or early winter. $\mathrm{He}$ found that the rose aphis might be kept in its viviparous condition for years if the habitat were artificially warmed and a good supply of succulent food provided. He kept them for four consecutive years; at the expiration of that time the energy of the power of the agamic reproduction was undiminished. The rose aphis may live a month or longer under favourable circumstances in the garden.

De Geer's most important deductions may be thus summarized :-

First.-That the oviparous forms never produced living young.

Second.-That the viviparous female never deposited eggs.

Third. - That at the end of the autumn the viviparous females in most cases produced males and females, which through oviposition provided the species for the ensuing year, the egg retaining its vitality throughout the rigour of winter.

Fourth.-That if cold weather did not supervene, the male and the oviparous females did not occur at all.

By natural law where the power of reproduction is great there must be a number of enemies to keep it in check; no insect is so productive as the Aphis, and no insect has so many enemies, or of such various kinds. The larvae of fifty-eight species of hovering flies (Syrpbidae). 


\section{A HISTORY OF DEVONSHIRE}

Diptera are known to feed on them, and the larvae of the lace-winged flies (Neuroptera). Many solitary wasps store them for food for their young. The larvae of the lady-birds and the lady-birds themselves (Coleoptera) live exclusively on them. The ichneumon flies (Aphidiidae) deposit their eggs in the body of Aphis in order to perpetuate their race. The small birds, tits, etc, often obtain a 'tit-bit' from them; in fact the limit of their foes is not known, they are so numerous.

A great number of the Aphides have been noted in the county. Localities have not been mentioned in consequence of their general distribution, with one exception. The writer has devoted much time to them, with a view of obtaining the parasitic ichneumon fly ('Tribe Apbidiidae), which he has succeeded in obtaining from forty different species of Apbis. During his wanderings in search of the infected Apbides he discovered one new species, Apbis Critbmi, which was found on Samphire (Critbmum maritimum) growing on the rocks at the hend of the Salcome estuary near Kingsbridge.

\section{APHIDES}

Siphonophora rosae, Réaumer. $X$ On roses and sweetbriar

- scabiosae, Schr. $\times$ Scabious

- granaria, Kirby. $\times$ Wheat, oats, barley

- menthae, Buckton. Common broom

- chelidonii, Kalt. $\times$ Raspberry

- polygoni, Buckton. Persicaria

- alliariae, Koch. $\times$ Nipple wort

- fragariae, Koch. Strawberry

- hieracii, Kalt. Hawkweeds

- pisi, Kalt. $\times$ Bladder campion

- millefolii, Fab. Yarrow

- circumflexa, Buckton. Cineraria in greenhouses

- dirhoda, Walk. Oats and grasses

- pelargonii, Kalt. Mallow, garden chrysanthemum

- scrophulariae, Buckton. Figwort

- lactucae, Kalt. X Gardenlettuce

- rubi, Kalt. $X$ Blackberry.

- urticae, Kalt. $\times$ Nettle

- lorigipennis, Buckton. $\times$ Grass

- convolvuli, Kalt. Convolvulus

- avellanae, Schr. Hazel

- tanaceti, Lin. Tansy

- absinthii, Lin. $X$ Wormwood

- artemisiae, Koch. Mugwort

- solidaginis, Fab. Golden-rod

- tussilaginis, Walk. Colt's-foot

- sisymbrii, Buckton. Hedgemustard

- sonchi, Lin. $\times$ Black knapweed

- olivata, Buckton. X Spearthistle

Phorodon humili, Schr. Sloe

- galeopsidis, Kalt. Hemp-nettle

Myzus cerasi, Fab. $\times$ Garden cherry, curling the leaves

- gracilis, Buckton. Sycamore

- ribis,Lin. $\times$ Red currant,causing the leaves to form red blotches

Drepanosiphum platanoides, Schr. Maple

- acerina, Walk. $\times$ Sycamore

Rhopalosiphum ribis, Lin. Black currant, forming red and brown blotches

- ligustri, Kalt. Privet

- dianthi, Schr. Carnation, peach, and other plants

Melanoxanthus salicis, Linn. $x$ Common osier

\section{APHIDES (cont.)}

Siphocoryne pastinaceae, Lin. $x$ Carrot

- xylostei, Schr. $\times$ Honeysuckle

- foeniculi, Pass. $X$ Fennel

- capreae, Fab. $\times$ Willows

Aphis brassicae, Lin. $\times$ Cabbage, charlock

- crataegi, Kalt. Hawthorn leaves on top shoots

- malvae, Walk. Willow herbs, yarrow, etc.

- abietina, Walk. Spruce fir.

- mali, Fab. Apple trees in curled leaves

- pyraria, Pass. $\times$ Pear trees

- scabiosae, Kalt. $\times$ Pellitory

- sorbi, Kalt. Mountain ash

- tanacetina, Walk. $\times$ Tansy

- pruni, Réaum. Plum, peach, and other trees

- crithmi, Buck. $\times$ Samphire, growing on rocks near Kingsbridge

- hieracii, Kalt. $\times$ Cow-parsnip

- petasitidis, Buckton. Butterbur

- euonymi, Fab. Spindle tree

- epilobii, Kalt. $\times$ Willow herb

- lychnidis, Lin. $x$ Red-robins

- hederae, Kalt. Young shoots of ivy

- viburni, Schr. Guelder-rose distorted leaves

- jacobaeae, Schr. $\times$ Ragwort

- rumicis, Lin. $\times$ Dock, thistle, broad beans, etc.

- papaveris, Fab. Red poppy

- cardui, Lin. $\times$ Thistles

- instabilis, Buckton. $X$ Feverfew

- sambucaria, Pass, Elder tree, under the leaves

- pyri, Boyer de Fonscolombe. Crab-apple

- bellis, Buckton. Daisy.

- sambuci, Lin. Elder on young shoots

- opima, Buckton. Cinerarias in greenhouses

- myosotidis, Koch. $X$ Forgetme-not

Hyalopterus pruni, Fab. Plums and blackthorn, forming hoary masses under the leaves

- dilineatus, Buckton. Moss-rose

\section{APHIDES (cont.)}

Hyalopterus melanocephalus, Buckton. Bladder campion

Pterocomma pilosa, Buckton. $X$ Common osier

Cryptosiphum artemisiae, Buckton. Mugwort,distorting theleaves

Callipterus coryli, Goetze. Hazel

- betularius, Kalt. $\times$ Birch

- quercus, Kalt. $X$ Oak

Pterocallis alni, Fab. Alder

- tiliae, Lin. $x$ Lime

Phyllaphis fagi, Lin. Beech

Lachnus agilis, Kait. Scotch fir

- pini, Lin. $x$ Scotch fir

- viminalis, Fonsc. Willow bark

- longipes, Dufour. Oak

Stomaphis quercus, Réaum. Oak, on the bark of branches

Schizoneura lanigera, Hausman. Apple trees. 'American blight'

- ulmi, Lin. $\times$ Elm, causing the leaves to blister and curl

Pemphigus bursarius, Hart. Black poplar, forming galls

- spirothecae, Koch. Black poplar, forming galls

-- pallidus, Haliday. 'Elm, form ing galls at base of mid-rib

Tetraneura ulmi, De Geer. Elm, forming galls on upper side of leaf

Giyphina betulae, Heyd. Birch, ends of twigs

Chermesabietis,Lin. Sprucefir,causing swelling very like fir cones

- laricis, Hartig. Larch, covered with tufts of white silky fibre

- pini, Koch. Scotch fir, covered with long silky filaments

Phylloxera punctata, Licht. Oak leaves

- quercus, Boyer de Fonsc. Oak, -the parent deposits her ova in a circle around her on the underside of the leaf.

Forda formicaria, Heyd. At roots of grasses, often protected by ants

- viridana, Buckton. At roots of grasses, often protected by ants

Tychea phaseoli, Pass. Scarletrunner beans, at the roots

From those marked with $x$ an ichneu. mon fly (Tribe Apbididae) has been bred. 


\title{
SPIDERS
}

\section{ARACHNIDA}

\author{
Spiders
}

The county of Devon should, owing to its warm climate and humid atmospheric conditions, be very suitable for the support of spider-life. There is, moreover, no doubt that this is indeed the case, although owing to lack of collectors the actual number of species placed on record is comparatively insignificant.

Amongst those who have contributed to the following list are the late Rev. Hamlet Clark, Mr. G. C. Bignell of Stonehouse, Plymouth, the Rev. T. R. R. Stebbing of Tunbridge Wells, the Rev. O. Pickard-Cambridge, and the present author.

Of the species of spiders (over 500) regarded as indigenous to Great Britain and Ireland, II4 only have been recorded from this county, seven species of pseudo-scorpions, and two harvestmen.

Among the rarer species may be noted Atypus affinis, Dysdera crocota, Philodromus fallax, Diaea devoniensis, Oxyptila sanctuaria, Euophrys petrensis, Lycosa trabalis, Meta Menardi, Araneus angulatus, Araneus marmoreus, Tmeticus reprobus, Leptorboptrum butbwaitii, Steatoda riparium, Asagena phalerata, Teutana nobilis and Dictyna variabilis.

\section{ARANEAE ${ }^{1}$ \\ $M$ YGALOMORPHAE ATYPIDAE}

Spiders with eight eyes, four lung books, and three tarsal claws.

\section{Atypus affinis, Eichwald}

Cleanbrook, Plymouth (G. C. B.). Adult in May, June, and October.

This is the only example of the Mygalomorphoe found in the British Islands. Though belonging to the same sub-order as the well-known trap-door spiders of the south of Europe and other tropical and sub-tropical regions, distinguished from the Arachnomorphae by the possession of two pairs of pulmonary organs, or lung books, and by the vertical movement of the mandibles, these spiders make no trap-door at all.

The retreat consists of a long tunnel, half an inch in diameter and from seven to nine inches long, burrowed in the soil, and lined throughout with white silk, terminating at the lower end in a slightly enlarged cell, where the egg-sac is formed and the young are hatched and tended by the female. The upper end of the silk lining is prolonged for about three inches beyond the extremity of the burrow, forming a loose tube, closed at the end, and either lying on the surface of the soil, woven amongst the roots of heather and herbage, or hanging down free, according to the nature of the surroundings.

Mr. Enock reports that the spider does not leave this retreat in search of prey, but waits in the slack portion of the tube lying outside the burrow until some insect sets foot upon this silken, purse-like structure. Instantly the fangs of the spider's mandibles are struck through the walls of the tube, the insect seized and dropped into the burrow through a rent in the silk, which is afterwards mended from within. The male is smaller, almost black, and may sometimes be found moving slowly about in the sunshine in the neighbourhood of the colony. The species occurs all along the coast from Hastings to the Land's End, and in various localities inland. The spider has also been recorded under the names $\mathcal{A}$. sulzeri and $A$. piceus by English authors.

\section{DYSDERIDAE}

Spiders with six eyes and two pairs of stigmatic openings, situated close together on the genital rima, the anterior pair communicating with lung books, the posterior with tracheal tubes. Tarsal claws, two in Dysdera, three in Harpactes and Segestria.

\section{Dysdera crocota, C. L. Koch.}

Salcombe (T. R. R. S.).

Larger than the last species, with a deep orangepink carapace, orange legs and abdomen with a delicate rosy-pink flush. The palpal bulb of the male has a cross-piece at the apex. This spider is known also as D. rubicunda, Blackwall.

\section{Dysdera cambridgii, Thorell.}

\section{Stonehouse (G. C. B.).}

Not uncommon under stones and bark of trees, where it lurks within a tubular retreat. The spider is easily recognizable by its elongate form, orange legs, dark mahogany carapace and pale clay-yellow abdomen.
The palpal bulb of the male has no cross-piece at the apex. The spider is also known as $D$. erythrina, Blackwall.

\section{Harpactes hombergii, Scopoli.}

Langstone; Mount Batten; Plymouth (G.C. B.). Rare under bark of trees, and recognizable by its linear ant-like form, black carapace, and pale clay-yellow abdomen and three tarsal claws.

\section{Segestria senoculata, Linnaeus.}

Stonehouse; Horrabridge (G. C. B.).

Not common; under bark of trees, in the crevices of loose stones walls and amongst detached rocks. Recognizable by its linear form and the black diamondshaped blotches on the dorsal surface of the abdomen.

6. Segestria forentina, Rossi.

Plymouth (Dr. Leach ; O. P.-C.).

Known also as Segestria perfida, Walckenaer. A very rare species indeed.

1 This list has been revised and corrected by the Rev. O. Pickard-Cambridge, M.A., F.R.S. 


\section{A HISTORY OF DEVONSHIRE}

\section{DRASSIDAE}

Spiders with eight eyes, situated in two transverse rows. The tracheal openings are just in front of the spinners. The tarsal claws are two in number, the anterior pair of spinners are set wide apart at the base, and the maxillae are more or less impressed across the middle.

7. Drassodes lapidosus, Walckenaer.

Mount Batten, Plymouth (G. C. B.); Salcombe (T. R. R. S.).

Very common under stones. Also known as Drassus lapidicolens.

8. Prosthesima petiverii, Scopoli. Salcombe (T. R. R. S.).

9. Prosthesime pedestris, C. L. Koch. (O. P.-C.).

\section{CLUBIONIDAE}

Spiders with eight eyes, situated in two transverse rows. The tracheal openings are immediately in front of the spinners. The tarsal claws are two in number, but the anterior pair of spinners are set close together at the base ; the maxillae are convex and not impressed across the middle.

\section{Clubiona terrestris, Westring.}

\section{Bickleigh (G. C. B.).}

Not uncommon in the summer time, when it may be found wandering about at night on the walls of outhouses, palings, etc. Known also as $C$. amarantha, Blackwall.

11. Clubiona recluse, O. P.-Cambridge. Beer Ferris (G. C. B.).

A rarer species than the last; usually beaten from foliage and bushes in the summer time.
12. Clubiona brevipes, Blackwall.

Ivybridge ; Langstone (G. C. B.) ; Ilfracombe (T. R. R. S.).

Not uncommon amongst foliage in the summer time.

13. Clubiona compta, C. I. Koch.

Langstone; Bickleigh; Horrabridge (G. C. B.).

A very small species, whose abdomen is striped diagonally on each side, similarly to that of $C$. corticalis. Not uncommon amongst the foliage of bushes and shrubs in the summer time.

14. Micaria pulicaria, Sundevall.

Salcombe (T. R. R. S.).

A brilliant little spider with iridescent scales on its body. Not uncommon, running about in the hot sunshine. Known also as Drassus nitens and D. micans, Blackwall.

\section{ANYPHAENIDAE}

\section{5. Anyphaena accentuata, Walckenaer. Ivybridge (G. C. B.)}

Often common on the foliage of oak and other trees. This spider is remarkable for the position of the spira- cular opening, which is situated in the middle of the ventral surface of the abdomen between the genital rima and the spinners, and not, as in the last family immediately in front of the spinners. Known also under Clubiona.

\section{THOMISIDAE}

Spiders with eight eyes, situated in two transverse rows, two tarsal claws, and anterior spinners close together at the base. Maxillae not impressed. The crab-like shape and side-long movements of the spiders are their chief characteristics, enabling them to be easily distinguished, as a rule, from the more elongate Drassidae and Clubionidae.

\section{Philodromus margaritatus, Clerck.}

Bickleigh (G. C. B.).

This magnificient species, by far the largest of the genus found in Great Britain, is not uncommon in many parts of England. Its mottled white, black and grey hues blend admirably with the lichencovered trees which they frequent, and unless the spiders move it is almost impossible to see them. The beautiful variety $P$. tigrinus, De Geer, also named $P$. jejunus, C. L. Koch, almost pure white, with distinct spots and blotches, is also not uncommon.

\section{Philodromus dispar, Walckenaer.}

Bickleigh (G. C. B.); Salcombe (T. R. R. S.).

This species with its shiny black abdomen is not uncommon in the summer time amongst foliage, and can sometimes be observed crouching flat upon palings. It is very swift in its movements.

\section{Pbilodromus aureolus, Clerck.}

Salcombe (T. R. R. S.).

A very abundant species with usually a dull red abdomen, with yellowish central pattern. It frequents the foliage of trees of all kinds, and especially in the immature condition will outnumber all other species which fall into the umbrella beneath the beating-stick.

\section{Pbilodromus caespiticolis, Walckenaer.}

Exeter (O. P.-C.).

The species is possibly only a variety of the last named, and frequents similar situations. Known also as $P$. cespiticolens, Blackwall.

\section{Philodromus fallax, Sundevall.}

North Devon (T. R. R. S.)

This beautiful spider lives amongst the sand-hills and runs swiftly, squatting when at rest quite flat on the sand, which it so exactly resembles in tint as to be impossible to detect from its surroundings.

\section{Xysticus cristatus, Clerck.}

(O. P.-C.)

This is by far the commonest of the 'crabspiders,' and is found abundantly on foliage or 


\section{SPIDERS}

crouching on bare places in fields and commons. Known also under Thomisus.

22. Xysticus luctuosus, Blackwall.

Cann Wood; Bickleigh (G. C. B.).

Known also as Thomisus luctuosus.

23. Xysticus erraticus, Blackwall.

(O. P.-C.)

Known as Thomisus erraticus, Blackwall.

24. Xysticus pini, Hahn.

(O. P.-C.)

Known as Thomisus audax, Blackwall.

25. Misumena vatia, Clerck.

Ivybridge (G. C. B.) ; (O. P.-C.)

This species, one of the largest of the Thomisidae, can be found in the blossoms of various woodland plants, where it crouches amongst the petals on the watch for its prey. The colour of the female is yellow, with red lateral slashes, the male being much smaller and almost black. Known also as Thomisus citreus, Blackwall.
26. Diaea devoniensis, O. P.-Cambridge.

Torquay (Hamlet Clark ; O. P.-C.).

A single immature female taken many years ago.

27. Diaea dorsata, Fabricius.

Bickleigh ; Shaugh (G. C.-B).

A bright green spider with a large dull red-brown central patch on the abdomen; not uncommon on herbage and amongst flowers. Known also as Thomisus foricolens, Blackwall.

28. Tibellus oblongus, Walckenaer.

Woolacombe (T. R. R. S.).

A long, very narrow, dull white or straw-coloured spider, often common amongst dry grass in many different localities. They attain, however, their largest size amongst the sedge grass and rushes in swamps and bogs. The elongate form assists in their concealment from foes as they squat close to the pale rush stems and slender dry blades. Known also as Philodromus or Thanatus oblongus.

29. Oxyptila sanctuaria, O. P.-Cambridge.

Mount Batten, Plymouth (G. C. B.).

$A$ very rare and minute species.

\section{SALTICIDAE}

The spiders of this family may be recognized in a general way by their mode of progression, consisting of a series of leaps, often many times their own length. More particularly they may be known by the square shape of the cephalic region, and the fact that the eyes are arranged in three rows of $4,2,2$, the centrals of the anterior row being much the largest and usually iridescent. Those of the second row are the smallest, while the posterior pair is placed well back and helps to give the quadrate character to the carapace. Otherwise these spiders are simply specialized Clubionids, with two tarsal claws and other minor characters possessed in common with members of this latter family.

They can be beaten from foliage or found amongst herbage or under stones. The commonest, Saliticus scenicus, will be well known to all observers, running and leaping on the walls of houses in the bright sunshine.

30. Salticus cingulatus, Panzer.

(O. P.-C.)

Known also as Epiblemum cingulatum.

3I. Salticus scenicus, Clerck.

Langstone; Exeter (G. C. B.) ; Salcombe (T. R. R. S.).

A black species with white lateral stripes. Known also under Epiblemum and Calliethera.
32. Euophrys frontalis, Walckenaer.

Salcombe ('T. R. R. S.); Exeter (O. P.-C.).

Known also under Salticus.

33. Euophrys petrensis, C. L. Koch.

Bickleigh (G. C. B).

34. Ergane falcata, Clerck.

Bickleigh ; Langstone ; Clearbrook (G. C. B.).

Common amongst dry leaves in woods. Known also as Salticus or Hasarius falcatus.

35. Heliopbanus cupreus, Walckenaer.

Bickleigh (G. C. B.) ; Salcombe (T. R. R. S.).

A shining black and coppery spider, found in some abundance on the coast. Known also under Salticus.

36. Heliophanus favipes, C. L. Koch.

Salcombe (T. R. R. S.).

37. Attus saltator, Simon.

Woolacombe (T. R. R. S.).

Known also as Salticus foricola, C. L. KochBlackwall.

38. Attus pubescens, Fabricius.

(O. P.-C.)

Known also as Salticus sparsus, Blackwall.

\section{PISAURIDAE}

Spiders with eight eyes in three rows of $4,2,2$, the small anterior eyes being sometimes in a straight line, sometimes recurved, and sometimes procurved. Those of the other two rows are situated in the form of a rectangle, and are much larger than the eyes of the anterior row. The tarsal claws are three in number.
Pisaura runs freely over the herbage, carrying its egg-sac beneath the sternum, while Dolomedes (an allied genus) is a dweller in marshes and swamps.

\section{Pisaura mirabilis, Clerck.}

Exeter (O. P.-C.) ; Salcombe (T. R. R. S.).

Known also a Dolomedes or Ocyale mirabilis. 


\section{A HISTORY OF DEVONSHIRE}

\section{LYCOSIDAE}

The members of this family are to be found running freely over the ground, and carrying the eggsac attached to the spinners. Many of the larger species make a short burrow in the soil and there keep guard over the egg-sac. Eyes and tarsal claws as in the Pisauridae, with slight differences.

40. Lycosa perita, Latreille.

Wembury Bay (G.C. B.) ; Paignton (T. R. R.S.).

A very beautiful species usually frequenting sandy places, its colours assimilating admirably with its surroundings. It constructs a shallow burrow in the sand, often having a narrow lid-like roof over the entrance. Known also as Lycosa picta.

4I. Lycosa ruricola, De Geer.

Ilfracombe (T. R. R. S.) ; (O. P.-C.).

Very similar in general appearance to the next species, but the male has a claw on the palpus and the female a much smaller vulva. Known also as Trochosa ruricola and as Lycosa campestris, Blackwall.

42. Lycosa terricola, Thorell.

Ilfracombe ; Salcombe (T. R. R. S.).

Both this and the last species make a small celllike hollow in the damp earth beneath a stone and there nurse the large silken egg-sac into maturity. The present species may be generally recognized by its red-clay tint, while $L$. ruricola is olive-brown. Known also as Lycosa agretyca, Blackwall, and as Trochosa terricola.
43. Lycosa pulverulenta, Clerck.

Exeter (O. P.-C.) ; Salcombe (T. R. R. S.).

Known also as Tarentula pulverulenta and Lycosa rapax, Blackwall.

44. Lycosa trabalis, Clerck.

Exeter (Edward Parfitt ; O. P.-C.).

A somewhat similar spider to the above, but larger. A single immature female is the only British example yet taken. Known also as Tarentula trabalis.

45. Pardosa pullata, Clerck.

Ilfracombe (T. R. R. S.).

Known also as Lycosa pullata and Lycosa exigua, Blackwall (in part).

46. Pardosa amentata, Clerck.

(O. P.-C.) ; Ilfracombe; Woolacombe (T. R. R. S.).

A very abundant species on logs of wood or hatchways in meadows and by the riverside; but also otherwise almost ubiquitous. It is one of our largest Pardosas, and is known also as Lycosa amentata and Lycosa saccata, Blackwall.

47. Pardosa nigriceps, Thorell.

Salcombe (T. R. R. S.; O. P.-C.).

A very distinctly marked spider; clear greenish yellow with black head and a dark stripe down each of the mandibles in front. Occurring not uncommonly in all heathy and moorland districts.

\section{AGELENIDAE}

Spiders with eight eyes, situated in two transverse rows. Legs with three tarsal claws. The species of this family spin a large sheet-like web, and construct a tubular retreat at the back of it, which leads to some crevice amongst the rocks, or the herbage, or the chinks in the walls of outhouses, wherever the various species may happen to be found. The posterior pair of spinners is usually much longer than the other two pairs.

\section{Amaurobius atropos, Walckenaer.}

Stonebottom ; Dartmoor (G. C. B.); Ilfracombe (T. R. R. S.).

This fine species spins a tube-like retreat beneath a stone or in the crannies of a loose stone wall, laying a flat broad sheet of webbing outside in the usual agelenoid fashion. Abundant where it occurs, on moors and mountains. Known also as Coelotes saxatilis, Blackwall, and Coclotes atropos.

49. Tegenaria atrica, C. L. Koch.

Ilfracombe (T. R. R. S.).

A very large spider with long legs, not uncommon in cellars and outhouses, where it spins a huge sheetlike web having a tubular retreat running into some place of safety. It is also found in the open country in rabbit burrows, holes in banks, and on the sandhills, etc., etc.
50. Tegenaria derhamii, Scopoli.

Salcombe (T. R. R. S.).

A smaller, paler, and more common spider than the last named; almost entirely confined to houses and outbuildings. Known also as T. civilis.

51. Tegenaria sileestris, L. Koch.

Princetown ; Dartmoor (G. C. B.).

A still smaller species, sometimes not uncommon amongst rockeries in greenhouses and gardens. Known also as $T$. campestris.

52. Agelena labyrinthica, Clerck.

Star Cross (G. C. B.); Ilfracombe ; Salcombe (T. R. R. S.).

A common spider, large when full grown, spinning a huge sheet-like white web over the herbage, with a funnel-shaped tubular retreat. Like others of the family the posterior pair of spinners is formed of two distinct segments.

53. Textrix denticulata, Olivier.

Langstone (G. C. B.).

A common spider where it occurs, found under rocks and stones on the undercliff, or beneath the coping stones of walls across the moors. It may be recognized by its swift movements, and the red, sometimes almost white, dentate band on the abdomen. Known also as Textrix lycosina, Sundevall.

\section{ARGYOPIDAE}

The spiders included in this family have eight eyes, situated in two rows, the lateral eyes of both rows being usually adjacent if not in actual contact, while the central eyes form a quadrangle. The tarsal claws are three, often with other supernumerary claws. The web is either an orbicular snare, or consists of a sheet of webbing beneath which the spiders hang and capture the prey as it falls upon the sheet. This 


\section{SPIDERS}

immense family includes those usually separated under the names Eperridae and Linyphiidae.

\section{Meto segmentata, Clerck.}

Bickleigh (G. C. B.); North Devon (T. R. R. S.).

$A$ very abundant spider in the summer and autumn amongst nettles and other herbage along hedgerows. The spiders vary very much in size, and spin an orbicular web having a clear space in the centre as do others of the genus and also Tetragnatha, thus differing from the genus Araneus (Epeira). Known also as Epeira inclinata, Blackwall.

\section{Meta merianae, Scopoli.}

Bickleigh ; Clearbrook; Beer Ferris (G. C. B.);

Ilfracombe (T. R. R. S.); Exeter (O. P.-C.).

A larger species found in cellars and damp places. Known also as Epeire antriada, Blackwall, and a variety with a white band down the centre of the abdomen as $E$. celata, Blackwall.

\section{Meto menordi, Latreille.}

Bickleigh ; Shaugh Bridge (G. C. B.).

This fine species is usually found in caves and cellars. It spins a large orb-web like others of the genus. Known also as Epeira fusca, Blackwall.

\section{Tetragnatha extensa, Linnaeus.}

Ivybridge; Bickleigh; Horrabridge.

A very common species of elongate form which sits in the centre of its web with legs stretched in front and behind. Not so entirely confined to marshy localities as the next species, and easily recognized by the silvery white bands under the abdomen. The jaws in the males of this genus are very large and conspicuous.

\section{Pachygnatha listeri, Sundevall.}

Ilfracombe (T. R. R. S.).

A rarer species than the following; found amongst grass and dry leaves in woods.

\section{Pachygnatha clerkii, Sundevall.}

Lipstone; Plymouth ; Beer Ferris (G. C. B.); Ilfracombe (T. R. R. S.).

Resembles a Tetragnatha in the possession of very large mandibles, but is not elongate, and spins no web to speak of. Found under leaves and at the roots of herbage, especially in marshy places.

\section{6o. Pachygnatha degeerii, Sundevall.}

Mount Batten; Beer Ferris; Clearbrook (G. C. B.); Ilfracombe (T. R. R. S.).

Smaller and commoner than the last species. Found at the roots of herbage.

\section{Cyclosa conica, Pallas.}

Bickleigh ; Langstone (G. C. B.).

A rare but easily recognized species, having a triangular abdomen, and sits in the centre of its web on a band of white transparent silk, possibly from a distance appearing like the dung of a small bird upon the surrounding foliage. Known also under Epeira.

62. Zilla $x$-notata, Clerck.

Ivybridge; Stonehouse (G. C. B.); Salcombe (T. R. R. S.).

A very common spider, usually spinning its web on or in the proximity of buildings. The web has a vacant, wedge-shaped piece with a single free ray from the centre. Known also as Epeira similis, Blackwall.

63. Zilla atrica, C. L. Koch.

Beer Ferris (G. C. B.).

Almost as common as the above, but more usually confined to the foliage of trees and bushes. The males have a very long palpus, while in $x$-notata these are very short. Known also as Epeira callophylla, Blackwall.

\section{Araneus cucurbititus, Clerck.}

Ivybridge (G. C. B.); Exeter (O. P.-C.).

A beautiful green spider with bright red tips to the tail end, rendering it like the bud of a flower. Known also under Epeira.

\section{Araneus diadematus, Clerck.}

(O. P.-C.); Ilfracombe (T. R. R. S.).

By far the commonest of our spiders, being known as the 'garden spider'; of large size, usually redbrown with white lozenge-shaped spots, spinning an orb-web. Known also under Epeira.

\section{Araneus cornutus, Clerck.}

Ivybridge (G. C. B.).

Abundant in the rush-beds, etc., near streams or in swampy places. Known also as Epeira apoclisa, Blackwall.

\section{Araneus umbraticus, Clerck.}

Bickleigh (G. C. B.).

A large species very like the last in general appearance but much more flattened, for it lives under the bark of trees and posts, spinning a strong orb-web and venturing out of its lurking place only at nightfall. Known also under Epeira.

\section{Araneus redii, Scopoli.}

(O. P.-C.) ; Salcombe (T. R. R. S.).

Known also as Epeira Redii, Epeira solers, and Epeira sollers.

69. Aroneus triguttatus, Fabricius.

Bickleigh ; Cann Wood (G. C. B.).

Not uncommon on iron palings, where the rustred patch on the forepart of the abdomen resembles a spot of iron-rust, and thus favours concealment. It can also be beaten from the foliage of trees. Known also as Epeira agalena, Epeira triguttata, and Epeira youngii.

\section{Araneus angulatus, Clerck.}

Bickleigh ; Shaugh Bridge (G. C. B.) ; Bovey Tracey (O. P.-C.).

This fine species has been taken in many parts of the south of England, notably in the New Forest. It can be recognized by its large size and the two stout pointed cones on the shoulders of the abdomen.

\section{Araneus gibbosus, Walckenaer.}

Bickleigh (G. C. B.).

This species somewhat resembles the last, but is much smaller. Known also as Epeira arbustorum and Epeira bicornis.

\section{Araneus marmoreus, Clerck.}

Bradleigh Down, Tiverton (G. C. B.).

Known also as Epeira scalaris and Epeira pyramidata. 


\section{A HISTORY OF DEVONSHIRE}

73. Araneus adiantus, Walckenaer.

Ilfracombe (T. R. R. S.).

Known also as Epeira adianta.

74. Linyphia triangularis, Clerck.

Laughton; Bickleigh (G. C. B.) ; Ilfracombe ; Salcombe (T. R. R. S.).

A very abundant species in autumn, whose sheet-like snares glistening with dewdrops form a conspicuous feature on the hedges and bushes in the early mornings. The mandibles in the male are very long, resembling those in Tetragnatha.

75. Linyphia insignis, Blackwall.

Ilfracombe (T. R. R. S.).

76. Linyphia pusilla, Sundevall. Ivybridge (G. C. B.).

A smaller species than the last, with deep black ventral regions. The palpus in the male sex has a long spiral spine. It spins its web near the ground amongst herbage. Known also as $L$. fuliginea, Blackwall.

77. Linyphia montana, Clerck.

Ivybridge (G. C. B.).

A large species whose habits are similar to those of triangularis. It is however often found in conservatories and outhouses. Known also as $L$. marginata, Blackwall.

78. Linyphia hortensis, Sundevall.

Exeter (O. P.-C.) ; Ivybridge ; Stonehouse ; Bickleigh (G. C. B.).

Not a common species; somewhat similar to pusilla in general appearance and habits. Known also as $L$. pratensis, Blackwall.

79. Linyphia clathrata, Sundevall.

Exeter (O. P.-C.) ; Ilfracombe (T. R. R. S.).

Resembles montana, but is smaller. Very common amongst herbage. Known also as Neriene marginata, Blackwall.

80. Labulla thoracica, Wider.

Exeter (O. P.-C.).

Not uncommon in outhouses or under overhanging banks and rocks. The male is remarkable for the enormously long spiral spine on the palpal bulb. Known also as Linyphia cauta, Blackwall.

\section{Leptbyphantes leprosus, Chlert.}

Exeter (O. P.-C.).

A very common species in stables, hay-lofts, and outhouses. Known under Linyphia.

82. Lepthyphantes minutus, Blackwall.

Exeter (O. P.-C.).

Known also under Linyphia.

83. Lepthyphantes blackwallii, Kulczynski.

Ilfracombe (T. R. R. S.).

Known also as Lepthyphantes zebrinus and Linyphic zebrina.
84. Lepthyphantes fawipes, Blackwall. Horrabridge (G. C. B.).

85. Bathyphantes concolor, Wider.

(O. P.-C.).

Known also as Theridion flipes, Blackwall, and under Linyphia.

86. Bathyphantes dorsalis, Wider.

Ivybridge; Horrabridge (G. C. B.).

Common on the foliage of trees and bushes in the summer time. Known also under Linyphia and as L. claytoniae, Blackwall.

87. Tmeticus reprobus, O. P.-Cambridge.

Mount Batten (G. C. B.).

$A$ rare spider found among seaweeds at high-water mark. Known also as Neriene reproba.

88. Leptorhoptrum huthwaitii, O. P.-Cambridge. (O. P.-C.)

Known also as Neriene butbwaitii, Neriene formidabilis, O. P.-Cambridge, Neriene lapidicola, Thorell and Tmeticus huthwaitii.

89. Poeciloneta variegata, Blackwall.

Ivybridge ; Langstone ; Mount Batten ; Stonehouse (G. C. B.).

Known also under Linyphia and Neriene.

90. Erigone atra, Blackwall.

Clearbrook (G. C. B.).

Known also under Neriene. Often abundant on railings in the spring.

\section{Erigone longipalpis, Sundevall.}

(O. P.-C.)

Known also as Neriene longipalpis.

92. Erigone dentipalpis, Wider. (O. P.-C.)

Known also as Neriene dentipalpis.

93. Hypomma bituberculatum, Blackwall.

Chelson Meadow, Plymouth (G. C. B.).

Known also under Neriene.

94. Hypomma cornuta, Blackwall.

(O. P.-C.)

Known also as Neriene cornuta.

95. Trachygnatha dentata, Wider.

Chelson Meadow, Plymouth (G. C. B.).

Known also as Neriene dentato and Gongylidium dentatum.

96. Gonatium rubens, Blackwall.

Salcombe (T. R. R. S.).

Not a rare species. Known as Neriene rubens.

97. Gonatium isabellinum, C. L. Koch.

Salcombe (T. R. R. S.).

Very similar to the last species in general appearance, but quite distinct. Known as Neriene isabellina and Neriene rubella, Blackwall. 


\section{SPIDERS}

\section{THE R I D I D A E}

The members of this family have eight eyes situated very much like those of the Argyopidae, but the mandibles are usually weak, the maxillae are inclined over the labium, and the posterior legs have a comb of stiff curved spines beneath the tarsi. The web consists of a tangle of crossing lines, and the spider often constructs a tent-like retreat where the egg-sac is hung up.

\section{Theridion lineatum, Linnaeus.}

Salcombe (T. R. R. S.).

Known also as Phyllonethis lineato and under the specific name ovata.

\section{Steatoda riparia, Blackwall.}

Clearbrook (G. C. B.).

Rare and local. This interesting species has been found in several parts of England, and as its name implies it is usually to be found under overhanging banks. Here it weaves the usual tangled web peculiar to the family, having, as has also its near kinsman formosa, a long tent-like retreat, beautifully constructed of silk, sand, dry twigs, etc., often much resembling the case of the caddis-worm in general appearance. A detailed account of its habits has been given in Science Gossip. Known also under Theridion.

\section{Steatoda tepidariorum, C. L. Koch.}

$$
\text { (O. P.-C.) }
$$

This large species is one of the commonest spiders in conservatories and greenhouses, where the curious triangular-shaped female may be seen hanging with legs closely gathered to the body in the middle of the tangled web. Sometimes, but not often, a few chips of dry leaf fallen into the web may be utilized as a sort of apology for a tent-like retreat, constructed in the case of $T$. formosum with such elaborate skill. When prey of any kind falls into the toils the spider hurries down, and with the tarsal comb on the fourth pair of legs commences kicking out from the spinners a silken fluid, often quite moist like treacle, which strikes against and hardens on the victims. In this way very large spiders, beetles, and wood-lice are ensnared and converted into food. With a rapid and irritable movement of the fore-legs also small tufts of fine silk are gathered and flung promiscuously over the web. The male, a much smaller spider, may often also be seen hanging near at hand in the web, and the one or more brown pear-shaped egg-sacs also hanging in the upper part of the toil. Sometimes these spiders are found outside the houses, but rarely, if ever, amongst the shrubs in the open garden. Known also under Theridion.

101. Steatoda varians, Hahn.

Bickleigh ; Cann Wood (G. C. B.).

A very much smaller species, varying considerably in colour, found abundantly in greenhouses and also amongst shrubs in the open garden. This species makes no tent-like retreat, but sits close to the one or more pale rounded egg-sacs usually spun up against a beam or window sill. Known also under Theridion.

\section{Steatoda denticulatum, Walckenaer.}

\section{Bickleigh (G. C. B.)}

Also a very small and abundant species, occurring on the outside of windows and outhouses and also on walls and palings. It makes no tent-like retreat, and the habits are very similar to those of the last species. Known also under Theridion.

\section{Steatoda sisypbium, Clerck.}

Ivybridge ; Horrabridge ; Langstone (G. C. B.).

Very common on gurse and holly bushes, where they construct a tent-like domicile and spin up within its shelter the small greenish egg-sacs. The young when hatched pass also their earlier days within the tent, but on the death of the mother spider they scatter, taking up positions for themselves among the neighbouring foliage. Known also as Theridion nervosum, Blackwall.

\section{Steatoda bimaculata, Linnaeus.}

Wembury (G. C. B.); Salcombe (T. R. R. S.)

Known also as Theridion carolinum, Blackwall, and $T$. bimaculatum. The males can be recognized by the sharp spur on the coxa of the fourth pair of legs.

105. Steatoda pallens, Blackwall.

Horrabridge; Bickleigh (G. C. B.).

This minute Theridioid, pale yellow in colour, with often a dark or paler dorsal spot on the abdomen, lives beneath the leaves of shrubs and trees, laurel, elm, lime, etc., where it spins its minute pear-shaped purewhite egg-sac, which rests on its larger end, and has several other small cusps towards the small sharppointed stalk. Known also under Theridion.

\section{Steatoda vittata.}

Bickleigh (G. C. B).

Known also as Theridion pulchellum, Walckenacr.

107. Episinus truncatus, Walckenaer.

$$
\text { Starcross (G. C. B.). }
$$

Known also as Theridion angulatum, Blackwall.

\section{I08. Teutana nobilis, Thorell.}

Torquay (Hamlet Clark, O. P.-C.).

Known also as Steatoda clarkii.

Iog. Asagena phalerata, Panzer.

$$
\text { (O. P.-C.). }
$$

Known also as Theridion signatum, Blackwall.

\section{DICTYNIDAE}

The spiders belonging to this family possess three tarsal claws, and the eyes, eight in number, are situated in two transverse rows, the lateral being in contact. The cribellum (or extra pair of spinning organs) and the calamistrum (a row of curving bristles on the protarsi of the fourth pair of legs) are present in all members of the family. They construct a tubular retreat with an outer sheet of webbing, which is covered with a 


\section{A HISTORY OF DEVONSHIRE}

flocculent silk made with the calamistrum from threads furnished by the cribellum.

1 10. Cinifo similis, Blackwall.

(O. P.-C.) ; Ilfracombe (T.R. R. S.).

$A$ very common species in greenhouses, stables and other outhouses. The males may often be found wandering about the walls of dwelling-houses after nightfall. Known also under the name Amaurobius.

I I I. Cinifo ferox, Walckenaer.

(O. P.-C.) ; Salcombe (T. R. R. S.).

A much larger species, shiny black with pale markings, found in cellars and also beneath rocks and stones on the coast or in crevices of banks in the open country. Known also under the name of Amaurobius.
I 12. Dictyna arundinacea, Linnaeus. Ivybridge (G. C. B.).

A common species spinning its nests in the heads of rushes and grass.

I 1 3. Dictyna variabilis, C. L. Koch.

Exeter (Mr. Parfitt ; O. P.-C.).

A very rare species, pale with a pink dorsal spot on the abdomen. It has hitherto only been taken on four or five occasions in England. Known also as Ergatis pallens, Blackwall.

I 14. Lathys bumilis, Blackwall.

Bickleigh (G. C. B.).

Known also as Lethia bumilis and Cinifo bumilis.

\section{OPILIONES}

The harvestmen are spider-like creatures with eight long legs, the tarsi long and very flexible. Eyes simple, two in number, situated on each side of an eye-eminence. Body not divided into two distinct regions by a narrow pedicle, as in spiders. Abdomen segmentate; breathing apparatus consisting of tracheal tubes connected with external stigmata beneath.

I I . Platybunus comiger, Hermann. Plymouth (G. C. B.).

116. Megabunus insignis, Meade. Plymouth (G. C. B.).

\section{PSEUDO-SCORPIONES}

117. Gbthonius rayi, L. Koch. Plymouth (G. C. B.).

I 8 . Obisium muscorum, Leach. Plymouth (G. C. B.).

I 19. Obisium maritimum, Leach. Plymouth (G. C. B.).

Found under stones below high-water mark on the sea shore.
I 20. Roncus cambridgii, L. Koch. Dartmoor (O. P.-C.).

121. Chelifer subruber, Simon. Plymouth, (G. C. B.).

122. Chernes nodosus, Schrank. Stonehouse (G. C. B.).

123. Chernes cimicoides, Fabricius. Stonehouse (G. C. B.). 


\section{CRUSTACEANS}

As a representative of British carcinology Devonshire may claim to be the premier county. If it be conceded that Thomas Pennant outside its boundaries already in the eighteenth century had something of the modern spirit in his treatment of crustaceans, he was still devoid of initiative, and never rose to the level of contemporary continental progress. It was reserved for Devonshire in the opening years of the nineteenth century to give scientific dignity to this branch of the English fauna. An example was set of adequately describing new species. Improvements in classification were originated. A zeal for collecting specimens and observing their habits was inspired, and interest in the whole subject was astonishingly quickened and sustained.

For this class of animals Devon shares with Cornwall a favourable situation in the south-western peninsula of Great Britain. With the Bristol Channel forming its northern and the English Channel its southern border, it might have been expected to derive some advantage from a double coast-line, but the diversity of aspect has not at present yielded any marked divergence between the northern and southern groups of species. Comparative paucity in the north may naturally be explained by lessened salinity in that channel which is continually receiving a vast admixture of fresh water from the Severn. The maritime parts of Devon, like those of Cornwall, are diversified by sheltered bays, by harbours of varying importance, and tidal estuaries. They are much frequented by shipping, which plays its own unconscious part in the distribution of crustacea. They give occupation to fleets of trawlers and other agencies of the fishing trade, by which again the branch of zoological science with which we are here concerned is materially assisted. The scalloped and indented outline of the coast, as seen on a map, prepares the naturalist for what he actually finds. There are not only wave-beaten headlands and pebble beaches, which brittle-coated animals not unnaturally mislike, but at intervals there are low rocky ledges coated with seaweed and pitted with clear but weed-fringed pools wherein many quaint sea creatures love to dwell, and at other intervals come long stretches of sand, barren to the untutored eye, though teeming with life for those who know how to search it out.

Owing, perhaps, to the attractive richness of its marine fauna, the inland waters of the county have been comparatively neglected. These, however, are not likely to be less prolific in freshwater Entomostraca than those of our counties in general. In that, as in other respects, the diligence of research may in time bring about a far greater equality in our faunistic catalogue than at present exists, at least wherever the conditions are reasonably comparable. It is a question which cannot at present be answered with assurance, whether the crustacean population of marshes, ponds, ditches, rivulets, rivers, and lakes, differs essentially in various parts of the country. Under exceptional conditions, such as those presented by brine-pools, large lakes, and highperched mountain tarns, species may be found with a very limited distribution; but this limitation will most likely be traceable to the needful environment, not to the latitude or longitude of any particular county.

Marine crustaceans have their own great and obvious facilities for dispersion. There can be but few, if any, of the species which would be daunted by the journey round the coast of England. Many of them are well equipped with apparatus for walking or swimming. Much of the travelling can be performed by hugging the shore or launching out into the deep. They have at command assisted passages on vessels steering a course or wreckage in a current, on slowly-floating weed or swiftly-swimming fishes. The course of a species from point to point need not be accomplished within the lifetime of an individual. It is open to patient, gradual achievement by an indefinite succession of generations. Still, from this view of the matter one cannot infer anything like complete uniformity of distribution. It is possible that a close observer, by spending years over the quest, might in the least favoured of our sea-board counties come across examples of almost all the English marine crustaceans, whereas probably the same investigator would in Devon attain the same result with a tenth part of the time and labour which he had had to bestow elsewhere. It will easily 


\section{A HISTORY OF DEVONSHIRE}

be understood that an important institution such as "The Marine Biological Association of the United Kingdom' fixed its headquarters at Plymouth not by any random choice, but for the solid reason that no other locality was more promising for its intended researches. Scientific literature had already spoken with no uncertain sound in this behalf. Especially in this branch of it the men who must be regarded as the chief pioneers of British carcinology either had their residence in Devonshire or resorted to it eagerly for material. Foremost must be mentioned Colonel George Montagu (175I-I8I5), who was born at Lackham in Wiltshire, but settled late in life at Knowle House, near Kingsbridge, from which point of vantage he made the neighbourhood of Salcombe notable by his discoveries among marine invertebrates. So identified did he become with the region that Dr. Edward Moore, writing in 1838 on the Malacostracous Crustacea of South Devon, says: 'I am unwilling that in the county of Montagu and Leach the subject should appear to be altogether neglected, possessing, as it does, such remarkable advantages for the pursuit.' ${ }^{1}$ The William Elford Leach (1790-1836) to whom Moore is referring was born at Plymouth, and has therefore an indigenous claim to rank among the worthies of Devon. His precocious genius inspired him at the age of twenty-three to undertake the task of reforming classification in the three extensive groups of crustaceans, myriapods, and arachnids. His first efforts at arranging this assemblage were contained in an article to which he gave the barbarous title 'Crustaceology.' We speak of efforts in the plural, because the article has an appendix containing additions and alterations. It was published in the seventh volume of Brewster's Edinburgh Encyclopaedia, which appeared in 1813. Similar treatises from his pen were soon afterwards published in The Transactions of the Linnean Saciety and in the fifth edition of the Encyclopaedia Britannica. The merit and capacity of the young naturalist were speedily recognized by his election into the Royal Society in 1817 , and by his employment in the following year to write on his special subject for the Dictionnaire des Sciences Naturelles, at an epoch when French zoologists might well have deemed themselves self-sufficing for such a task. To the success of Leach's studies contribution was gladly made by the veteran Montagu, by Cranch, and by Prideaux. The last of these, Mr. Charles Prideaux of Hatch Arundel, near Kingsbridge, is mentioned by Moore as chief benefactor in providing the museum of the Plymouth Institution with a very excellent collection of British Malacostraca. In the second half of the nineteenth century public attention was directed scarcely less prominently than in the first half to Devonian carcinology. In this case the leading spirit was Charles Spence Bate (1819-89), a Cornishman by birth, but from I 85 I onwards resident at Plymouth. He had abilities and ambition, and the confidence in himself which those qualities are apt to generate. His numerous and extensive writings created in Great Britain a livelier interest in the subject of crustacea than it could otherwise at the time have enjoyed. In regard to some groups he had the merit and excuse of being to some extent a pioneer, a road-maker. But through too frequent miscalculation of his own powers he built his roads at many points so faultily as to earn anything but blessings from those who used them. The names of other zoologists who have concerned themselves with the crustacea of Devon are in this field less conspicuous than Spence Bate's, because for the most part they have extended their survey to many other branches of marine zoology. This was the case with Philip Henry Gosse (1810-88), long resident at St. Mary Church and well known for his Rambles on the Devonshire Coast and many other scientific works both popular and technical. It was the case also with Edward Parfitt (I820-93), a most industrious naturalist, for many years curator of the Devon and Exeter Institution. In the same category may be placed many who on occasional visits or during more or less protracted periods of residence have worked on the fauna of Devon, such as Charles Kingsley (1819-75), the Rev. Dr. Norman, F.R.S., Walter Garstang, F.Z.S., and others whose names will be cited in the course of this chapter. The united efforts of so many eager and accomplished naturalists have concentrated upon this county a mass of information which can only here be dealt with by severe compression. In the lists which follow it should be understood that the generic and specific names beginning a line are those considered by the present writer to be valid for the species in question. ${ }^{2}$ They are followed by the name of the author who established the species, such author's name being in parenthesis when the species no longer stands in the genus to which he at first assigned it. The synonymy of rejected names is added for the convenience of students who may wish to seek further information in such works as Bell's History of British Stalk-eyed Crustacea, the Histoire Naturelle des Crustacés by H. Milne-Edwards, or elsewhere. Verification of the occurrence of species in Devonshire will be found largely in the Fournal of the Marine Biological Association of the United Kingdom (new series), vol. vii, No. 2, pp. 234-258, I904; in Parfitt's Catalogues contributed to the Devonshire Association; and in various other works to which general or special references will be given later on. The division of the class Crustacea here adopted is into four sub-classesMalacostraca, Leptostraca, Entomostraca, Thyrostraca.

${ }^{1}$ Charlesworth's Mag. Nat. Hist. (new ser.), iii, 284 (1839).

${ }^{2}$ See Hist. of Crustacea (Inter. Scientific Ser.), vol. lxxiv (1893) for the general system of nomenclature here adopted. 


\section{CRUSTACEANS}

\section{Sub-Ciass MALACOSTRACA}

Order Brachyura. Division Brachyura genuina

Tribe Cyclometopa (carapace with arched anterior margin)

\section{Family XanthidaE}

Xantho hydrophilus (Herbst). Cancer h. Herbst ; Xantho rivulosus, Risso, Milne-Edwards ; X. rivulosa, Bell

- poressa (Olivi). Cancer p., Olivi ; C. floridus, Montagu ; C. incisus, Leach; Xantho incisa, Leach, Bell

Xanthias couchii (Couch). Xantho couchii (Bell MS.), Couch ; ${ }^{1} X$. tuberculato (Couch MS.), Bell. ${ }^{2}$ Xanthodes tuberculatus, Bouvier. ${ }^{3}$

Pilumnus hirtellus (Linn.). Bristly crab; the furry Pilumnus

\section{Family Portunidae}

Portunus puber (Linn.). The velvet swimming crab

- depurator (Linn.). The cleanser swimming crab 'The Flying Crab of fishermen' (Moore)

- corrugatus (Pennant). The wrinkled swimming crab

- pusillus, Leach. The dwarf swimming crab

- emarginatus, Leach. $\quad P$. arcuatus, Leach, is probably the same species

- holsatus, Fabricius. P. marmoreus, Leach, is doubtfully distinct

Bathynectes longipes (Risso). Portunus longipes, Risso, Bell, Parfitt

Portumnus latipes (Pennant). Cancer latipes, Pennant ; Portumnus variegatus, Leach, Bell ; Platyonichus latipes, Latreille

- biguttatus (Risso). Portunus b., Risso ; Platyonycbus nasutus, Heller. ${ }^{5}$

Polybius henslowii, Leach. Henslow's swimming crab. The Nipper crab of fishermen

Carcinus maenas (Linn.). The common shore crab

\section{Family Cancridar}

Cancer pagurus, Linn. The great eatable crab

Pirimela denticulata (Montagu). Cancer denticulatus, Montagu ${ }^{6}$

Atelecyclus septemdentatus (Montagu). Cancer Hippa septemdentatus, Montagu; ${ }^{7}$ Atelecyclus s., Leach; $A$. heterodon, Leach, Bell. 'Old-man's face crab' of fishermen (Leach)

\section{Family Corystidae}

Corystes cassivelaunus (Pennant). The masked crab

1 Rep. Penzance Nat. Hist. Soc. (1851), p. 13.

a Brit. Stalk-eyed Crust. p. 359.

- Feuille des Yeunes Naturalistes, ser. 3, No. 332 (1898). For the change of the preoccupied Xanthodes to Xanthias, see Miss M. J. Rathbun, Proc. Biol. Soc. Washington, xi, I65 (1897).

4 Crustacés de Nice, p. 3I, pl, i, fig. 2 (not fig. I as given by

Risso himself and others), (1816).

5 Crust. Südl. Europa, p. 94 (1863).

- Trans. Linn. Soc. Lond. ix, 87, pl. ii, fig. 3 (1808).

7 Ibid. xi, 1, pl. i, fig. I (1813).

\section{SuB-Class MALACOSTRACA (continued)}

Order Brachyura (contimued)

Division Brachyura genuina (continued)

Tribe Catometopa (inter-orbital space or 'front' bent downwards)

\section{Family Goneplacidar}

Goneplax rhomboides (Linn.). Cancer angulatus, Pennant; Ocypoda angulata $+O$. rhomboides, Bosc; Ocypode angulata, Latreille, Leach ; Goneplax bispinosa, Leach; Gonoplax b., Leach; G. angulata, Bell

\section{Family Grapsidat}

Planes minutus (Linn.). Planes linnaeana, Leach, Bell, Parfitt, Sowerby ; Nautilograpsus minutus, Milne-Edwards. The turtle crab (Patrick Browne)

\section{Family Pinnotheridae}

Pinnotheres pisum (Linn.). Common Pea crab (White)

- veterum, Bosc. Pinna Pea crab (White)

Tribe Oxyrrhyncha (carapace anteriorly narrowed, often forming a 'sharp beak')

\section{Family INAchidaB}

Inachus dorsettensis (Pennant). Scorpion spider crab (White)

- dorynchus, Leach. Leach's Spider crab (White)

- leptochirus, Leach. Slender-armed Spider crab (White)

Macropodia rostrata (Linn.). Cancer rostratus, Linn.; C. phalangium, Pennant; Maia p., Leach; Leptopodia ${ }^{8}$ p., Leach ; Macropodia p., Leach; Stenorynchus p., Milne-Edwards, Bell

- tenuirostris (Leach). Leptopodia t., Leach; Macro. podio t., Leach; Stenoryncbus t., Bell (not Inacbus longirostris, Fabricius)

- egyptia (Milne-Edwards). Stenorynchus phalangium. Audouin; S. egyptius, Milne-Edwards; Macropodia egyptia, Garstang ${ }^{9}$

Achaeus cranchii, Leach. Cranch's Spider crab (White)

\section{Family Hradidae}

Hyas aranea (Linn.). Great Spider crab (White). Harper crab (Sibbald); Sea toad (Gordon) ${ }^{10}$

- coarctata, Leach. Contracted crab (White)

Blastus tribulus (Linn.). Cancer t., Linn.; ? Arctopsis lanata, Lamarck; Cancer biaculeatus, Montagu ; ${ }^{11}$ Pisa biaculeata, Leach ; $P$. gibbsii, Leach, Bell, Parfitt

- tetraodon (Pennant). Cancer t., Pennant; Blastus t., Leach; Pisa t., Leach, Bell

Eurynome aspera (Pennant). Cancer asper, Pennant ; Eurynome aspera, Leach, Bell

8 See Rathbun in Proc. Biol. Soc. Washington, xi, 155 (1897)

9 fourn. Mar. Biol. Assoc. vii, 254 (1904).

10 White, Popular Hist. Brit. Crust. (1857), p. 22.

11 Trans. Linn. Soc. Lond. xi, 2, pl. i, fig. 2. 


\section{A HISTORY OF DEVONSHIRE}

SuB-Class MALACOSTRACA (continued)

Order Brachyura (continued)

Division Brachyura genUina (continued)

Tribe Oxyrrhyncha (carapace anteriorly narrowed, often forming a 'sharp beak') (continued)

Family Mamairdaz

Mamaia squinado (Herbst). Cancer squinado, Herbst; Maia s., Leach, Bell ; Mamaia s., Stebbing ${ }^{1}$

Tribe Oxystomata (having the oral frame narrowed anteriorly)

Family Levcosimae

Ebalia tuberosa (Pennant). Cancer tuberosus, Pennant; Ebalia Pennantii, Leach, Bell
Sub-Class MALACOSTRACA (continued)

Order Brachyura (continued)

Division Brachyura genuina (continued)

Tribe Oxystomata (having the oral frame narrowed anteriorly) (continued)

Family Leucosinae (continued)

Ebalia tumefacta (Montagu). Cancer tumefactus, Montagu ; Ebalia Bryerii, Leach, Bell

- cranchii, Leach. Cranch's Ebalia (Bell)

\section{Division Brachyura anomala or Dromiacea}

\section{Family Dromirdae}

? Dromia vulgaris, Milne-Edwards. A conjectural member of the Devon fauna

It appears that at least a dozen of the species above enumerated were introduced to science by Montagu and Leach. On these it is fitting that some remarks should be offered. But there are others on which our Devonshire authorities have thrown illuminating gleams. The species Xantho bydropbilus and $X$. poressa are nearly allied. Bell explains that in the former the fingers of the chelipeds are brown and the movable finger is grooved above, whereas in the latter the fingers are black, without grooves. Parfitt, however, describes a link between the two, a female specimen of $X$. poressa, in which 'the movable finger is grooved above, having a line of punctures in the centre of the groove.' This might perchance have been a hybrid. The Italian naturalist, O. G. Costa, discussing $X$. poressa in the Fauna del Regno di Napoli, is sarcastic on the making of species out of simple varieties, which he thinks might be almost indefinitely multiplied. Leach founded the genus Pilumnus in 1815 . He found the species $P$. birtellus extremely abundant all along the rocky shores from Plymouth to Berry Head, and noted that sometimes the left, at others the right cheliped is the larger, besides which, he says, 'the wrists and hands are sometimes quite smooth, without the slightest appearance of that granulated surface which Linné considered as the essential mark of this species.' ${ }^{2}$ Portunus pusillus, when established by Leach in 1815, was attributed first of all to the waters of Devon, only with appended acknowledgement of another locality. In the following year he writes: 'This beautiful little species is found occasionally in the Firth of Forth ; and is very common in some parts of the southern coast of Devon, where it is taken by dredging in deep water. The fingers are generally annulated with red, and whitish, and the back is not unfrequently ornamented with a red streak or spot.' ${ }^{3}$ Portunus emarginatus, Leach, is another truly Devonian species, having been first 'discovered at 'Torcross.' The author might well say, ' it is extremely rare,' since, except the original specimen which he received from Mr. Gibbs and deposited in the British Museum and that noted as rare by Cocks at Falmouth in 1849 , no other appears to be on record. Its emarginate 'front' may be merely an occasional variation. To the name founded upon this feature Leach unluckily gave what is called page precedence over his better named Portunus arcuatus. The latter designation must therefore lapse as a synonym. The species $P$. bolsatus, Fabricius, and $P$. marmoreus, Leach, though reckoned as independent members of our fauna by good authorities, are doubtfully distinct. Of the latter Leach writes: 'This elegant species, which derives its name from its colour, was discovered by G. Montagu, Esq. It is very common on the sandy shores of southern Devon, from Torcross to the mouth of the river Exe, and is frequently found entangled in the shore-nets of the fishermen, or thrown on the shore after storms. It is distinguished from every other discovered species by the rounded dentations of the front, the very slight elevation of the lines on the hands, and by the convexity, remarkable smoothness, and marbled appearance of the shell.' 4 It must, however, be remembered that Leach does not notice $P$. bolsatus, except indirectly by the description of a faded specimen, which he called $P$. lividus. With regard also to the extreme smoothness of $P$. marmoreus, it may be noticed thr $\mathrm{O}$. G. Costa calls $P$. bolsatus in Italian Portuno rasato, the crab all shaven and shorn.

Portumnus latipes (Pennant) is discussed by Leach, Bell, and Parfitt under the later name given it by Leach, who writes: 'Portumnus variegatus is one of the most beautiful, as it is the most

${ }^{1}$ South African Crust. (1905), pt. iii, p. 22.

2 Malacostraca. Podophthalmata Britanniae, text to pl. xii (I March, I816).

${ }^{3}$ Op. cit. text to pl. ix (1 Sept. 1816). ${ }^{2}$ Encycl. Brit. (ed. 5), supplement, p. 4 I I (1816). 256 


\section{CRUSTACEANS}

common, species of the Malacostracous animals that inhabit our coasts; it is taken by digging beneath the sand at low-water mark, and is found thrown on all the sandy shores of Great Britain in great abundance, especially during storms. The very fine specimens presented in the annexed plate were given to me by my indefatigable friend, C. Prideaux, Esq., along with a vast number of rare and curious Crustacea, taken by himself on the southern coast of Devonshire.' ${ }^{1}$ It occurs also on the northern coast. Bell and Parfitt deny that this species is by any means common within their experience, and the Marine Biological Association omits it entirely without comment from its list in 1904. The Association, however, adds to the fauna of Devon Portumnus biguttatus, described and figured by Risso in 1816, and later recorded by several authors as Platyonichus (or Platyonychus) nasutus, the specific name alluding to its very prominent interorbital 'front." Risso states that the carapace is yellowish white, with two great blotches of coral red, while O. G. Costa supplies the useful warning that these distinctive blotches vanish after death, and Garstang thinks 'it is possible that these spots are only to be observed in the breeding season, and that they are due to the colour of the reproductive glands showing through the carapace." 2 The Association record speaks of occasional specimens burrowing in fine gravel at Drake's Island; females in berry, and the megalops stage being observed in August. The same trustworthy register says of Polybius benslowii that 'the $t$ is not uncommon at times on the shrimp-trawling grounds, particularly in Cawsand B., also swimming near the surface at the deep water stations, but the t has only twice been recorded at the Laboratory.' Females were observed in berry, and the young were hatched, in September.

Pirimela denticulata, though first described by Montagu, was not first found in Devonshire. The genus in which it now stands was established by Leach, and must not be confounded with the botanical Primula, as the preliminary list of the Marine Biological Association in 1888 might lead the unwary to suppose. ${ }^{3}$ Leach says of this species, 'Dead examples were found at Bantham on the south coast of Devon, by C. Prideaux, Esq., who, with his usual liberality, supplied my cabinet with a fine series, exhibiting considerable variation in colour; but the finest individual that I have seen, and which is figured in the adjoining plate, was found amongst the rubbish of some trawl-fishers at Torquay by that accurate observer of nature, Dr. Goodall, Provost of Eton, who gave it to me with a number of other marine productions that now enrich my cabinet.' ${ }^{4}$

Atelecyclus septemdentatus hails originally from this county. Like the preceding species it owes its generic name to Leach, and the specific to Montagu, who first described, as he was also the first to find it. His description of it was read at the Linnean Society on 7 April, 1807 , and begins with a statement explanatory of its name, 'Thorax suborbicular, smooth, with a slightly embossed, urn-shaped impression : front obtuse, tridentate, the sides serrated with seven denticulations each, besides those which guard the eyes.' ${ }^{3}$ The author must have felt somewhat aggrieved that his paper should not have been published till six years after it had been read. His species indeed ran a narrow chance of forfeiting its name, since Leach, who accepted septemdentatus in his earlier writings, in 1815 took the liberty of changing it without adequate reason into beterodon.

The intelligent reader no doubt accepts in a general way the aphorism that nature does nothing in vain. Nevertheless this vague faith has allowed thousands of naturalists to contemplate the marginal teeth of the carapace in cyclometopous crabs, and to use them freely for generic and specific discrimination, without going on to inquire what meaning or functional value these 'dents, as Dana has called them, with all their diversity in number, width, slope, and acuteness, may have for the crabs themselves. Mr. Walter Garstang, however, working at the Plymouth station, has redeemed the investigation from the reproach of its long neglect. In his exceedingly interesting paper he says, "So far as I am aware no one has hitherto elucidated the remarkable constancy of antero-lateral serrations of the carapace in this group of crabs. I here present evidence which tends to show that the presence of conspicuous serrations on these margins of the carapace is functionally related to the exigencies of respiration when these animals are buried in sand.' ${ }^{6}$ For the method by which representative species of Batbynectes, Atelecyclus, and Portumnus, were induced to give up the intimate secret of how to breathe in a situation seemingly so inconvenient, recourse must be had to Mr. Garstang's own ingenious exposition. The same writer has thrown instructive light upon the habits and respiratory mechanism of Corystes cassivelaunus, a species in which the elongate, hairfringed, geniculating second antennae are so notable a feature.

Concerning Goneplax rbomboides Leach records an observation by Cranch, that these crabs ' live in excavations formed in the hardened mud, and that their habitations, at the extremities of which they live, are open at each end. ${ }^{7}$ The long-armed males of this species taken at Torquay,

1 Malac. Pod. Brit. text to pl. iv (1 March, i 815).

Fourn. Mar. Biol. Assoc. (new ser.), iv, 404 (1895-7).

4 Malac. Pod. Brit. text to pl. iii (1 March, 1816).

${ }^{3}$ Ibid. No. 2, p. 170 (1 888 ).

- Trans. Linn. Soc. Lond. xi, I (1813).

7 Malac. Pod. Brit. text to pl. xiii (I March, 1816). 


\section{A HISTORY OF DEVONSHIRE}

when kept in captivity, seemed to indicate an angry impatience by the frequent noisy clashing of their chelipeds. Bell says 'it was not until this species was obtained by Montagu in the estuary of Kingsbridge, Devon, that it was ascertained to be British.' But Leach more correctly gives the credit to Pennant. ${ }^{1}$

As to Planes minutus, Parfitt remarks that 'there are three specimens of this species in the British Museum collection, believed to have been taken on the coast of Devon; but this species can only be looked upon as an occasional visitor, being drifted upon our coast, from the Gulf-stream on the Sargosso [Sargassum] bacciferum, and other weeds. It cannot claim to be a native.' 2 Bell quotes from Sloane's Natural History of Jamaica, that 'Columbus finding it alive on the sargasso floating in the sea, concluded himself not far from some land, in the first voyage he made, on the discovery of the West Indies.' In Harris's Collection of Voyages and Travels there is a different story. There we read that Columbus and his little fleet left the Canaries on 6 September, 1492. 'September the 7 th they lost sight of land; and with that, their courages too; a great many of them taking their leaves of this, and expecting the next time to land in the other world. Columbus comforted these cowards as well as he could; and to do it effectually, was fain to cheat them in his reckoning. . . . On Sunday, the 16 th, they saw grass and herbs floating on the water, and some small animals (grasshoppers) alive amongst them; which made some of them (now come to a little better hope) believe, they should see dry Land once more, and that quickly, too.' 3

There is still some doubt as to the number of genuine British species assignable to the genus Pinnotberes. According to various authorities examples have been found in the shells of Pinnae, Ostrea edulis, Mytilus modiolus, Glycimeris, Cardium edule, and Cardium norvegicum. The variety of habitat may or may not correspond with some variation in the tenants. Besides the two species accepted in our list, Leach at one time or another distinguished $P$. varians (Olivi); $P$. mytilorum, Latreille; and introduced on his own account $P$. mytili, with the remark that 'this interesting species was discovered by a most zealous and enlightened collector, Mr. Cranch, in Mytilus modiolus, from the Kingsbridge estuary, dredged from the oyster-bed near Gerston Point'; $P$. pinnae, 'discovered by Montagu in Pinna ingens, from the Salcombe estuary, since which Mr. Cranch has taken two females out of the same shell from the same situation'; $P$. modioli, 'discovered. by Montagu in Mytilus modiolus, from the Kingsbridge estuary: female unknown.' 4 He subsequently thought himself warranted in making several changes in the names, for $P$. mytilorum substituting $P$. latreillii, for $P$. mytili and $P$. modioli adopting respectively the names of their reputed discoverers, $P$. crancbii and $P$. montagui, but dropping $P$. pinnae as a synonym of $P$. veterum.

The Oxyrrhyncha, all familiarly known as spider crabs, which dress themselves 'some in rags, and some in tags, and some in velvet gowns,' are a fascinating set of creatures. In recent times their manners and customs have been closely observed. The quaint villosity of their shells, the shapes and positions of their varied hairs and spines, have been shown to be no caprice of nature, but furniture in the right place and necessary to the owner's welfare. Parfitt, in a long and interesting note on Blastus tribulus, compares some of its hairs to the ambulacral spines of sea-urchins, by others he is reminded of the crockets in Gothic architecture. Of a third set, which are very conspicuous to the naked eye, he says, ' as far as their use is concerned, I conceive they are only for ornamentation; but,' as he prudently adds, 'in this I may be mistaken:"5

Inacbus doryncbus 'was discovered by Dr. Leach, whilst he was washing some specimens of I. Dorsettensis, sent him by Mr. Prideaux and Mr. Cranch from the Kingsbridge estuary.' ${ }^{6}$ Like the companion species, he says, 'it is generally overgrown with marine matter, which circumstance has doubtless concealed it from notice." 7 In establishing Inachus leptocbirus Leach says, 'Mr. John Cranch discovered this species of Inachus on the western coast of Devon or Cornwall; and it has since been taken from a crab-pot in Bigbury Bay by C. Prideaux, Esq. It is easily distinguished from Inacbus Dorbyncbus by its more slender arms, and by the number and dispositions of the spines on the back. The tubercle also on the breast of the male is a very striking and curious character.' ${ }^{3}$ Whether the altered spelling of doryncbus in the last quotation was accidental or corrective must be left to conjecture. Our readers may remember that in the interval between 1815 and 1817 Leach had become acquainted with a great scholar, Joseph Goodall, then provost and previously head master of Eton, who may have expressed a horror of an unaspirated rhynchus. Concerning Macropodia tenuirostris Leach says, 'I first observed this species amongst some crustacea collected at Torquay, in southern Devon, by Hooker, and have since found it a very common inhabitant of all the deep water off the coast of that county, especially in the Sound of Plymouth.' ${ }^{\prime}$. egyptia, first figured in Savigny's fine plates of Egyptian crustacea, was not known as British to Leach or Bell or Parfitt.

${ }^{1}$ Edinb. Encycl. vii, 393.

${ }^{3}$ Op. cit. p. 5 (1705).

- Trans. Devon. Assoc. iv, 192 (1870).

7 Malac. Pod. Brit. text to pl. xxii, figs. 7,8 (1 Jan. 18 I 5).

${ }^{9}$ Op. cit. text to pl. xxiii (Sept. I 8 I 5 ).
Trans. Devon. Assoc. iv, pt. i, p. 187 (1870).

'Edinb. Encycl. vii, 430,431 .

${ }^{6}$ Edinb. Encycl. vii, 431 .

${ }^{8}$ Op. cit. text to pl. xxii в (I July, I 817 ). 


\section{CRUSTACEANS}

In recent years it has been found both in Devon and Sussex. Its rostrum is nearly as long as the peduncle of the second antennae, instead of being much shorter as in $M$. rostrata, or decidedly longer as in $M$. tenuirostris. Whether Hyas coarctata was obtained by Leach first from Scottish or Devonian waters cannot certainly be determined. The order in which he gives his localities is Firth of Forth, Plymouth Sound, and Salcombe. ${ }^{1}$ Since the Hyades were feminine stars, the name of this species must by rule be $H$. coarctata, but whether its companion should be $H$. araneus or $H$. aranea depends on a point which only a necromancer can decide, namely, whether Linnaeus in calling it Cancer araneus was minded to use araneus as an adjective or as a substantive. In the former case it must be altered into agreement with the new generic name, but in the latter it should remain unchanged. Mamaia squinado, for the number of organisms that have been found settled or roaming about upon its back, might almost be described as a common lodging house. Adam White in his Popular History of British Crustacea ${ }^{2}$ quotes at great length, from Glaucus, or The Wonders of the Shore, Charles Kingsley's vivacious account of this creature, so accommodating, so ungainly, so truly serviceable even to mankind. White also borrows Gosse's description of the exuviation or process of sloughing, which he had observed at Ilfracombe in 1852 , in the case of a large spider crab. Gosse did not observe ' any of the struggling that is sometimes spoken of; it seemed to be a very easy and simple matter. The new integuments were perfected, though soft, before the old were thrown off, and the immediate cause of the separation of the crust appears to be the sudden growth of the animal within, forcing asunder the upper and lower crusts at the posterior margin; then the pulling out of the limbs presents no more difficulty than what depends on the enfeebled condition of the muscular energy.' On this, however, it must be remarked that the spider crabs are without the great distal distension of the claws which makes the act of 'casting' hazardous and probably more or less painful for the great eatable crab and the common lobster.

In the tribe Oxystomata, Ebalia tumefacta, though first described and figured by Montagu, was not first taken in Devonshire. That distinction belongs to $E$. crancbii, of which Leach tells us, ' this species was discovered by that enterprising naturalist, Mr. J. Cranch (whose death in the late expedition to Congo has been so much lamented by naturalists), in the Sound of Plymouth, where Mr. C. Prideaux has likewise observed it, in considerable plenty, and has supplied my collection with a complete series.' 3

\section{ORDER MACRURA}

\section{Division Macrura anomala}

Tribe Paguridea

Family Paguridae

Eupagurus bernhardus (Linn.). Cancer b., Linn.; Astacus b., de Geer, Pennant ; Pagurus b., Fabricius, Leach, Bell ; Eupagurus b., Brandt ; Pagurus streblonyx, Leach

- prideaux (Leach). Pagurus prideaux, Leach; $P$. pridauxii, Milne-Edwards ; $P$. pridecuxii, Bell

- cuanensis (W. Thompson). Pagurus c., Thompson, Bell

- sculptimanus (Lucas). Pagurus forbesit, Bell; ? Anapagurus $f_{\text {f. }}$, Bouvier

Anapagurus laevis (W. Thompson). Pagurus l., Thompson, Bell

- hyndmanni (W. Thompson). Pagurus b., Thompson, Bell, Parfitt

Diogenes pugilator (Roux). Pagurus $p$. Roux; Diogenes varians, Costa; Pagurus dillwynii, Bate

\section{Tribe Galatheidra}

\section{Family Porcellanidae}

Porcellana longicornis (Linn.). Cancer l., (Linn.); Porcellana l., Leach, Moore. Minute porcelain crab (Bell)

- platycheles (Pennant). Hairy porcelain crab (Bell)

1 Trans. Linn. Soc. Lond. xi, 329. 2 Op. cit. p. 24. s Malac. Pod. Brit. text to pl. xxv, figs. 7-1 I (1 April, i8 i 7 ),

\section{ORDER MACRURA (continued)}

Division Macrura anomala (continued)

Tribe Galatheidea (continued)

\section{Family GaLatheidae}

Galathea strigosa (Linn.). Cancer strigosus, Linn.; Galathea strigosa, Fabricius, Leach, Bell ; $G$. spinigera, Leach ;" Galatea s., Leach

- squamifera, Leach, G. s. ${ }^{\circ}+$ G. Fabricii, Leach ; Galatea $F .{ }^{6}{ }^{6}$ Leach ; Galatea digitidistans, Bate

- dispersa, Bate ${ }^{\gamma}$

- intermedia, Lilljeborg. G. squamifera (partly), Leach ; G. intermedia, Lilljeborg, Norman ${ }^{8}$

Munida rugosa (Fabricius). Pagurus rugosus, Fabricius; Astacus Bamfius, Pennant; Galatbea rugosa, Fabricius; G. Bamfia, Leach ; Munida rugosa, Leach ; $M$. Rondeletii, Bell

\section{Division Macrura genuina}

Tribe Thalassinidea

Family Calitanassidae

Callianassa subterranea (Montagu). Cancer Astacus subterraneus, Montagu ;' Callianassa subterranea, Leach. ${ }^{10}$ Burying shrimp (Couch)

Op. cit. pl. xxviii B (1 May, x 815).

5 Op. cit. pl. xxviii A (1 May, 1815).

6 Encycl. Brit. (ed. 5), Suppl. p. 419, pl. 21.

7 Fourn. Proc. Linn. Soc. $(1858)$, iii, 3 .

8 Norman and Scott, Crust. of Devon and Cornw. (1 906), p. I I.

9 Trans. Linn. Soc. Lond. ix, 88, pl. iii, figs 1, 2, (1808).

10 Edinb. Encycl. vii, 400 (1813). 


\section{A HISTORY OF DEVONSHIRE}

\section{Order MACRURA (continued)}

Division Macrura genuina (continued)

Tribe Thalassinidea (continued)

Family Callianassidae (continued)

Upogebia stellata (Montagu). Cancer Astacus stellatus, Montagu ; Upogebia stellata, Leach ; ${ }^{2}$ Gebia s., Leach, Bell ; G. deliaura, Leach ; G. deltura, Leach

\section{Family Axirdae}

Axius stirynchus, Leach. Slow shrimp (Couch)

Jaxea nocturna, Nardo. J.n., Nardo; Calliaxis adriatica, Heller ; larva (Trachelifer, Brook), (off Salcombe) R. Gurney, Norman

\section{Family Thaumastochelidae}

Calocaris macandreae, Bell. G. m., Bell, R. Gurney ; G. Macandrei, White

Tribe Scyllaridea

Family ScYlLaridae

Arctus ursus, Dana. Cancer arctus, Linn. ; Scyllarus a., Fabricius, Milne-Edwards, White, Sowerby

\section{Family Palinuridar}

Palinurus vulgaris, Latreille. 'Long Oyster' (Borlase), 'Thorny Lobster' (Leach), Crawfish or Sea crayfish. Phyllosoma stage, young of the Palinurus $^{3}$

\section{Tribe Astacidea}

Family Nephropsidae

Astacus gammarus (Linn.). Cancer gammarus, Linn.; Astacus verus, Borlase; Astacus marinus, Fabricius ; $A$. gammarus, Pennant, Leach, White, Sowerby ; Homarus vulgaris, Milne-Edwards, Bell, Bate

Nephrops norwegicus (Linn.). Cancer n., Linn.; Astacus n., Fabricius; Nephrops norvegicus, Leach

\section{Tribe Penaeidea}

Family Penaeidae

Penaeus kerathurus (Forskal). Cancer k., Forskal ; Alpheus caramote, Risso; Penaeus trisulcatus, Leach ; $P$. caramote, Bell

\section{Tribe Caridea}

\section{Family Crangonidar}

Crangon vulgaris, Fabricius. The common sand Shrimp

- allmanni, Kinahan. Steiracrangon a., Kinahan

Pontophilus spinosus, Leach. Crangon s., Leach, Bell ; Pontopbilus s., Leach ; Cherapbilus s., Kinahan

Philocheras fasciatus (Risso). Crangon $f$., Risso, Bell ; Aegeon $f$., Kinahan ;" Pbilocberas f., Kemp"

1 Trans. Linn. Soc. Lond. ix, 89, pl. iii, fig. 3

2 Edinb. Encycl. vii, 400.

- J. T. Cunningham, Fourn. Mar. Biol. Assoc. (new ser.), ii, 141, pls. viii, ix, (1889-9o)

4 Descriptiones animalium in itinere orientali, p. 95 (1775).

5 Trans. Royal Irisb Acad. xxiv, 80 (1871).

6 Ann. Nat. Hist. (ser, 7), xvii, 298 (1 go6).

\section{ORDRR MACRURA (continued)}

Division Macrura genuina (continued)

Tribe Caridea (continued)

Family Crangonidaz (continued)

Philocheras sculptus (Bell). Aegeon s., A. M. Norman;' Pbilocheras s., Kemp

- bispinosus (Westwood). Pontophilus b., Westwood ; Crangon b., Bell ; Cherapbilus b., Kinahan ; Crangon nanus, Kröyer; Pbilocberas n., Stebbing ${ }^{8}$

- trispinosus (Hailstone), Pontopbilus t., Hailstone; Crangon t., Bell ; Cherapbilus t., Kinahan

\section{Family Procrssidar}

Processa canaliculata, Leach. P. c., Leach ; ${ }^{9}$ Nika edulis, Risso, ${ }^{10}$ Bell, Norman ; Processa e., Latreille, Guérin ; Nike e. $+N$. conaliculata, Desmarest

\section{Family. Alpheidae}

Alpheus ruber, Milne-Edwards

- macrocheles (Hailstone). Hippolyte m., Hailstone; Alpheus megacheles, Norman; $A$. macrocheles, Norman

Athanas nitescens (Leach). Palaemon $n$. , Leach; Athanas $n$., Leach ${ }^{11}$

\section{Family HupPolytidae}

Hippolyte varians, Leach. H. $v$., Leach ; $H$. fascigera, Gosse

- prideauxiana, Leach. H.P., Leach, Bell, Norman ; Alpheus viridis, Otto ; (i) Bellidia Huntii, Gosse ${ }^{12}$

Spirontocaris cranchii (Leach). Hippolyte c., Leach

- pusiola (Kröyer). Hippolyte p., Kröyer; Spirontocaris p., Norman

\section{Family Pandalidar}

Pandalus montagui, Leach. $P$. $m$., Leach, White, Calman ; $P$. annulicornis, Leach, Bell

Pandalina brevirostris (Rathke). Pandalus b., Rathke, Heller ; $P$. Thompsoni, Bell, Norman; $P$. Jef freysi, Bate; $P$. Rathkii, Heller; Pandalino brevirostris, Calman, ${ }^{13}$ Norman

\section{Family Pontonidaa}

Typton spongicola, O. G. Costa. T. s., Costa, Norman, Borradaile; $\mathcal{T}$. spongiosum, Bate

\section{Family Palaemonidas}

Leander serratus (Pennant). Astacus s., Pennant; Palaemon s., Fabricius, Leach, Bell

- squilla (Linn.). Cancer s., Linn.; Palaemon s., Fabricius, Leach, Bell

Palaemonetes varians (Leach). Palaemon v., Leach, Bell ; Palaemonetes v., Heller, Weldon ${ }^{14}$

7 Norman and Scott, Crust. of Devon and Cornqu. p. 15 (1906).

8 Soush African Crustacea, pt. i, p. 48 (1900)

- Malac. Pod. Brit. (1 July, 1815) text to pl. xli.

10 Crustacés de Nice, p. 84, pl. iii, fig. 3 (1816)

11 Edinb. Encycl. vii, 401, 432 .

12 Cf. Ann. Nat. Hist. (1877), ser. 4, xx, 313 ; ibid. (1878),

ser. 5, ii, 135; Crust. of Devon and Cornw. (1906), p. 18 .

${ }_{18}$ Ann. Nat. Hist. (1899), ser. 7, iii, 37, pls. i-iv, fig. 4.

14 On variations in the rostrum, Fourn. Mar. Biol. Assoc. (new 


\section{CRUSTACEANS}

In this order, as in the Brachyura, we find our list containing a dozen species, here certainly as there doubtfully thirteen to the dozen, which owe their initiative to Montagu and Leach. Of Eupagurus prideaux Leach tells us that 'it inhabits the sea on the southern coast of Devon, and is most common in the Sound of Plymouth,' as also that 'Mr. Prideaux has observed it in a vast variety of habitations, even in the tubes of the Dentalia and in the shell of Scaphander lignarius." ${ }^{1}$ In accordance with the above statement, my friend Mr. Rupert Vallentin has sent me a very small, perfectly straight specimen, lodged in a Dentalium shell. Mr. Holt records a specimen which had an Adamsia polypus upon its shell as well as the normal $A$. palliata, and Mr. Pace remarks that occasionally the shell is invested with a colony of Hydractinia ecbinata in place of the Adamsia palliata, and very rarely both are present upon the same shell. ${ }^{2} \quad$ Mr. Parfitt informs us that Anapagurus Hyndmanni was dredged by Mr. Boswarva near Plymouth : 'spawn silver green.' 3 The same writer, discussing Porcellana longicornis, says, - Amongst some annelids dredged off Salcombe in the coralline zone by Mr. Walker (Sept. I 865), was a minute specimen of this species; the carapace was only one line in length. Notwithstanding its diminutive size, its abdomen was full of eggs! The hairs fringing the hands of this species are strong yellowish bristles longitudinally striate, and thickly set with short, red, stiffish seta [setae], directed upwards towards the point of the bristle." Concerning Galatbea squamifera, Leach, who adopts Montagu's manuscript name for it, explains that 'This elegant species of Galathea was discovered by Montagu on the southern coast of Devon, where it occurs in very great abundance. Young specimens generally have a white line running down their back, and in this state they are frequently taken by the dredge in deep water. It may be found under stones at low tide on all the rocky shores of Southern Devon from Plymouth to Torquay." F From this species Leach thought himself obliged to separate a specimen of uncertain habitat, which he named Galatbea fabricii, noting especially that 'the fingers of the fore-feet are more bent than in Galathea

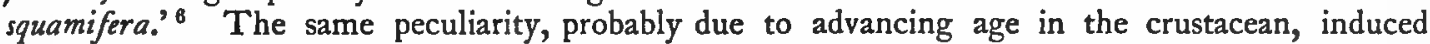
Spence Bate to give this species yet another synonym in the significant name $G$. digitidistans.

Among Montagu's numerous discoveries few have excited greater interest than that of Callianassa subterranea. After a full description, which is too long for quotation here, he remarks, 'This new and curious species of crab was discovered in digging for Solen Vagina, at the depth of nearly two feet beneath the surface, on a sand bank in the estuary of Kingsbridge. It is by no means plentiful ; but a sufficient number has been taken, with much trouble, to learn that the large arm is not constant to one side, nor always so very disproportionate as in that from which the figure was taken. The crustaceous covering of the body is very thin, and not far remote from membranaceous. The females, as in most of the Cancer tribe, more rarely occur; the ova of one taken were fixed under the tail, and of a red colour.' ${ }^{7}$ He uses the terms crab and Cancer in the old unrestricted sense, and partly for that reason his dictum as to the comparative rarity of female crustaceans eludes criticism; but with regard to the difficulty of procuring specimens of Callianassa in the Kingsbridge or Salcombe estuary many naturalists have found cause for regretful sympathy with his statement. After describing Upogebia stellata, he says, 'This species, which I am inclined to believe is also a nondescript, was taken with the preceding; it is more rare, but appears, like that crab, to inhabit the subterraneous passages made by the Solenes.' 8 Subsequent research has found this species not more but less rare than its companion. Leach in one place, probably by inadvertence, robs Montagu of the credit of first finding this species, saying, 'Gebia stellata was discovered by Mr. Gibbs in the Kingsbridge estuary; it has likewise been taken on some of the shores of Plymouth sound, under the mud in which it makes winding horizontal passages, often of an hundred feet or more in length.' He also establishes a species Gebia deltaura, concerning which he observes, "This species lives with Gebia stellata, with which it was confounded until the distinctions were discovered by Mr. J. D. C. Sowerby.' 9 The length of the galleries and Sowerby's distinctions alike await corroboration. Mr. R. A. Todd says, 'none of those we followed were more than two or three feet long.' (Journ. Mar. Biol. Assoc. (new ser.), vi. 201.) Norman, however, speaks of the 'long galleries' in which he observed the curious commensalism between this crustacean and the little flattened mollusc Lepton squamosum (Montagu). ${ }^{10}$ Parfitt follows Leach in making Gibbs the discoverer of Upogebia, and mistakenly asserts that 'Colonel Montagu does not say where he obtained'Callianassa subterranea, but he himself supplies the following information of interest about it: "In the original drawing of this species now before me there is a quadrate black spot on the base of the third abdominal segment above, and there are

1 Malac. Pod. Brit. text to pl. xxvi, figs. 5, 6 (1 Nov. 1815).

- Journ. Mar. Biol. Assoc. vii, $253 . \quad 3$ Trans. Devon. Assoc. iv, 186

- Ibid.

6 Trans. Linn. Soc. Lond. xi, 340.

${ }^{8}$ Ibid. 9o.

10 Ann. Nat. Hist. (1891), Ser. 6, vii, 277; Ser. 7, iv, 289 (1899). 


\section{A HISTORY OF DEVONSHIRE}

also two brownish rusty blotches on the large anterior foot; the sides of the thorax and tail appendages ochraceous yellow ; the rest pale flesh colour.' ${ }^{1}$ As to another rare species Leach writes, 'Axius stirynchus was first obtained by myself at Sidmouth, where it was taken amongst prawns on the shore. Montagu afterwards procured near Plymouth another specimen, which forms part of his collection of British Zoology, lately purchased by the trustees of the British Museum." ${ }^{2}$ Bell observes that the occurrence among prawns on the shore must have been purely accidental, as this, like the two preceding species, is essentially fossorial in habit. For the occurrence in Devonian waters of Bell's purblind Calocaris macandreae we have Mr. Robert Gurney's authority. But he only claims to have observed the 'Zoaea stage.," ${ }^{3}$ Of the adult Bell writes that 'it occasionally inhabits a depth of no less than one hundred and eighty fathoms, in which situation it is fossorial in sandy mud.' 4 With such a moat around its fortress-burrow it may well escape any frequent capture even by man's ingenuity. Messrs. J. T. Cunningham and R. A. Todd report rare occurrences of Arctus ursus at Plymouth stations. ${ }^{5}$

For Palinurus vulgaris, the crawfish, and Astacus gammarus, the common lobster, as members of the Devonian fauna, we might adopt the expression of Gerarde the herbalist, and give the testimony of 'what our eies have seene and handes have touched' both at Torquay and Salcombe. For the occurrence of the pretty Norway lobster, Nephrops norwegicus, in these waters, we have to rely on the authority of Dr. Moore, whose remark upon it is: 'Rare; we have two excellent Devon specimens in our museum.' This sort of record, without specification of precise locality, is far from satisfactory, since an enthusiastic collector might easily purchase specimens in the county without thinking to inquire whether they had been imported from a distance or otherwise. The river crayfish, Potamobius pallipes (Lereboullet), is for this reason under much suspicion. Mr. Moore says, "I am doubtful if this be common in our rivers, as on trying to procure specimens we are sure to have Palinurus sent. We have, however, a good Devon specimen in our museum from Mr. Prideaux.' ${ }^{\text {C }}$ On the distribution of this species Mr. Parfitt contributes the following important observations: ' I have made inquiries in every direction where I thought to obtain reliable information respecting this species as indigenous in Devonshire, but I cannot hear from any one that it is so. Mr. Paulman, author of The Book of the Axe, informs me that he does not know it in any stream west of the Frome which flows by Dorchester. They were formerly, and he believes still are, very plentiful in the meadows between Dorchester and Maiden Newton. And, he says, "I remember years ago making inquiries on the Otter, but the people whom I saw never heard of the "craytur." They are found plentifully at Salisbury, and they are also taken in the Tone at Taunton, as I have had specimens from both places. Now, if we draw a line across from the British to the Bristol Channel, beginning in the meadows at Maiden Newton, the line would cut Taunton at an angle of about 45 degrees west, and terminate in Wachet [Watchet] Bay, and so far as I am able to ascertain this species is not found west of this line. This crayfish is considered by some to be almost a necessary adjunct to a dish of trout, by supplying a delicious sauce nearly equal to lobster; and some streams supply both the fish and the sauce ; but although we got [get] a tolerable supply of trout, the sauce appears to be wanting in our Devonshire streams. It would be an interesting inquiry to ascertain the reason of the absence of this species in Devon, and particularly as it comes so near without stepping over the boundary.' 7

These remarks are very likely to be overlooked, as they occur not in an essay on distribution, but in one entitled Crustacea Podothalmata, and the Histology of their Shells. Among the numerous shrimps and prawns of the tribe Caridea there are many which call for comment, but in order to leave space for the several remaining divisions of our subject, notice will only be taken of one which has a rather exceptional interest. Leach, when establishing the genus Processa in I 815, writes : The discovery of the species constituting this most curious and interesting genus is the result of Montagu's indefatigable labours. The circumstance of the legs on different sides of the same animal being different in length is not an uncommon occurrence in several crustaceous genera, but this is the first instance in which the legs of opposite sides have been found to differ in essential structure.' He is referring to the fact that in Processa canaliculata the first pair of legs are not a proper pair, as a rule one of them being chelate, the other simple or monudactyle, as it is sometimes called, while also in the second pair one is very long, having both its wrist and forearm many-jointed, the other much shorter, with the wrist alone many-jointed. To his description of it Leach appends the remark that 'Montagu discovered a single specimen of the above species at Torcross in the southern coast of Devon, and with his usual liberality sent it to me for this work.' In a footnote he says : 'Since writing the above I have received intelligence of the death of this most zealous zoologist, in whom science has lost an able supporter, the world an accomplished gentleman, and his friends a

1 Trans. Devon. Assoc. iv, 184,185 , with a reference to Mont. MSS. t. 24 , f. I, A.

2 Malac. Pod. Brit. (1 Sept. I816), text to pl.xxxiii. ${ }^{3}$ Journ. Mar. Biol. Assoc. vii, 252.

4 Brit. Stalk-eyed Crust. 235.

${ }^{6}$ Charlesworth, Mag. Nat. Hist. (new ser.) (1 839), iii. $289 . \quad{ }^{7}$ Trans. Devon. Assoc. iv. 179. 


\section{CRUSTACEANS}

most valuable companion and correspondent. His Testacea Britannica and Ornitbological Dictionary, not to mention his numerous papers published in the Transactions of the Linnean Society of London, leave proofs of his industry and research that will cause his name ever to be remembered and respected by British zoologists.' 1

\section{OrDER SCHIZOPODA}

\section{Division Euphausiacea}

Family Euphausildar

Nyctiphanes couchii (Bell). Thysanopoda c., Bell - norvegica (Michael Sars).

\section{Division Mrsidacea \\ Family Mrsidae \\ Sub-family Mrsinae}

Praunus flexuosus (Müller). Cancer Alexuosus, O. F. Muller, Herbst ; Cancer astacus multipes, Montagu ; Praunus flexuosus, Leach, Stebbing ; Mysis spinulosus, Leach, Sowerby; Mysis chamaeleon, Vaughan Thompson, Bell ; Synmysis flexuosa, Czerniavsky ; Macromysis f., Norman

- neglectus (Sars). Mysis neglecta, Sars; Synmysis n., Czerniavsky ; Macromysis n., Norman

- inermis (Rathke). Mysis i., H. Rathke, Sars ; Kesslerella i., Czerniavsky; Macromysis i., Norman, Beaumont

Schistomysis spiritus (Norman). Mysis s., Norman, Sars ; Synmysis s., Czerniavsky ; Schistomysis s., Norman, Holt and Beaumont

- ornata (Sars). Mysis 0., Sars ; Synmysis o., Czerniavsky ; Scbistomysis o., Norman, Beaumont ; Mysis kervillei, Sars

- arenosa (Sars). Mysis a., Sars ; Austromysis a., Czerniavsky ; Scbistomysis a., Norman, Beaumont

- parkeri, Norman. S. p., Norman, Hodgson, Holt

- helleri (Sars). Mysis b., Sars ; Austromysis k., Czerniavsky; Scbistomysis h., Norman

Neomysis vulgaris (Vaughan Thompson). Mysis थ., Thompson; Neomysis v., Czerniavsky, Holt, Beaumont

Acanthomysis longicornis (Milne-Edwards). Mysis l., M. E., Sars ; Aconthomysis l., Czerniavsky, Norman; Dasymysis l., Holt and Beaumont

Hemimysis lamornae (Couch). Mysis l., Couch ; Hemimysis pontica, Czerniavsky; $H$. lamornae, Norman, Todd

Mesopodopsis slabberi (van Beneden). Podopsis s., van B. ; Macropsis s., Sars, Norman, Beaumont, Holt, Pace ; Mesopodopsis s., Norman (1906)

Sub-family Heteromysinae

Heteromysis formosa (S. I. Smith). H. f., Smith, Norman, Garstang, Beaumont.

\author{
Order SCHIZOPODA (continued) \\ Division Mysidacea (continued) \\ Family Mysrdae (continued) \\ Sub-family LeptomysinaE
}

Leptomysis lingvura (Sars). (?) Cynthia Flemingii, Goodsir, Bell ; Mysis lingvura, Sars ; Leptomysis l., Sars, Norman, Beaumont, Todd; (at Ilfracombe) Stebbing

- gracilis (Sars). Mysis g., Sars; Leptomysis g., Sars, Norman, Holt, Beaumont, Garstang

- mediterranea, Sars. L. m., Sars, Norman, Beaumont

Mysidopsis gibbosa, Sars. M. g., Sars, Norman, Hoit, Beaumont, Gamble, Garstang

- angusta, Sars. M. a., Sars, Norman, Todd, Beaumont

Erythrops elegans (Sars). Nematopus e., Sars ; N. pygmaeus, Sars ; Erythrops pygmaea, Sars ; E. elegans, Norman, Holt, Beaumont

\section{Sub-family Sirrellinae}

Siriella rostrata (Guérin). Mysis rostratus, Guérin ; M. armata, Milne-Edwards ; Cynthio a., M.E., Siriella a., Sars, Norman, Holt and Beaumont ; Mysis Grifitbsiae, Bell ; Cyntbilia frontalis, Norman (not M.E)

- clausii, Sars. S. c., Sars, Norman, Beaumont

- jaltensis, Czerniavsky. S. j., Czerniavsky, Norman, Garstang, Gamble, Beaumont; S. crassipes, Sars

- norvegica, Sars. In Ilfracombe harbour (Stebbing)

\section{Sub-family Acanthocarinae}

Acanthocaris sancta (van Beneden). Mysis s., v. B. ; Gastrosaccus sanctus, Sars, Norman, Holt, Beaumont ; Pontomysis caucasica, Czerniavsky

- spinifera (Goës). Mysis s., Goës ; Gastrosaccus sanctus, Norman; Acanthocaris Livingstoniana, Sim ; Gastrosaccus spinifer, Stebbing, Norman

Haplostylus normani (Sars). Gastrosaccus $n$., Sars, Norman ; Haplostylus n., Kossmann, Garstang, Beaumont

Anchialina agilis (Sars). Anchialus a., Sars, Norman, Holt and Beaumont; Anchialina a., Norman ${ }^{3}$

The Schizopoda owe their name to what is, perhaps, comparatively a primitive feature in the crustacean stock. Their legs are cleft terminally into two branches, one of which is natatory. The tribe Euphausiacea is distinguished by having its graceful branchiae unusually displayed, while

'Malac. Pod. Brit. (I July, I 8 I 5), text to pl. xli.

Hist. of Crustacea (Internat. Sci. Ser.), lxxiv, 283.

3 The principal references for this list are papers by Canon Norman, Ann. Nat. Hist. Ser. 6 (1892), ix, 459 ; x, I 43 ; by Holt and Beaumont, Trans. Royal Dublin Soc. Ser. 2 (1900), vii, pt. 7, 221 ; the Joum. Mar. Biol. Assoc. (new ser.) (I 904$)$, vii, 245 ; and the Crust. of Devon and Cornw., by A. M. Norman and T. Scott, a work not issued until after this chapter had been sent to the press, so that the use made of it has been less ample than would have been otherwise desirable. 


\section{A HISTORY OF DEVONSHIRE}

the Mysidacea have no proper branchiae to display. For a long time this order was mixed up with the Squillidae under the common name of Stomapoda. Mr. Parfitt in 1870 no doubt intended to accept this nomenclature, although he misprints the title as 'Stampoda.' In the preliminary report on the fauna of Plymouth Sound in I 888 the word is adopted in its improved form 'Stomatopoda,' but the Marine Biological Association having entrusted the list of crustacea to Spence Bate, he not only maintained the discarded union of the Squillidae with the Schizopoda, but mixed up with them still a third order then known as the Cumacea, and threw in a Phyllosoma apparently just for the fun of the thing. In these matters there are plenty of pitfalls even for the wary. The synonymy of Praunus flexuosus will be some indication of this. It is a pretty little multitudinous species which no collector fails to observe. How to choose its right name is the puzzle. The genus Mysis was established by Latreille in $\mathbf{1} 802$ for Cancer pedatus of $\mathrm{O}$. Fabricius, with a suggestion that Cancer oculatus of the same author was a nearly related species. This ill-defined genus was subsequently made the recipient of many other species, which in turn have been redistributed to several genera. In 1813 Leach established the genus Praunus for P. flexuosus apart from Mysis, and although he afterwards rejected the name flexuosus in favour of Mysis spinulosa, that procedure was not in accord with modern views of propriety. Since Muller's fexuosus has been by general consent removed from Mysis, it is obvious that it must now be restored to Praunus. Even so we are not clear of our difficulties. For in the Plymouth catalogue of 1904 Mr. Beaumont writes as follows :-

'Macromysis flexuosa (Müler) and M. nigra: F. Keeble and Gamble, Pbil. Trans. Roy. Soc., (Ser. B), vol. cxcvi, pp. 332, etc.

'Together these two species include, but they do not respectively coincide with, the Macromysis flexuosa and M. neglecta of Sars and of Norman. Both are known to occur in the Plymouth area, but they have only been quite lately differentiated, and consequently their distribution has not yet been fully determined. The evidence at present available points to the conclusion that $M$. flexuosa is essentially an estuarine form, and $M$. nigra more strictly marine. This is in agreement with the experience of Gamble. The Macromysis which swarms about the shores of the Tamar at Saltash (especially in the summer months) appears to be exclusively $M$. flexuosa. In Plymouth Sound $M$. nigra is the predominant form. . . .'1

It should be mentioned that Schistomysis parkeri was described by Norman from specimens first found at Starcross by Mr. C. Parker.

On the synonymy of Dasymysis longicornis the reader must be referred to the elaborate discussion by Messrs. Holt and Beaumont. ${ }^{2}$ They reject the earlier genus Acantbomysis of Czerniavsky on the ground of misdescription. Whether the reason is adequate seems open to question. Siriella rostrata (Guérin) has a right of priority over S. armata, if we believe Guérin's statement that his figure of the species was published long before the description by Milne-Edwards. ${ }^{3}$ Gastrosaccus appears to have been used for a fossil crab some years before it was applied to an existing schizopod. It must therefore give way to Acanthocaris, the. generic name applied by Sim in 1872 .

\section{ORDER STOMATOPODA}

Family SQuillidar

Squilla desmarestii, Risso. S. d., Risso, Couch, Bell, Todd

Mr. R. A. Todd reports a single specimen of this species as having been obtained 'near New Ground Buoy, Dec. I900,' and Dr. Allen remarks that 'The larvae are occasionally met with in tow-nettings.'

\section{ORDER SYMPODA (formerly CUMACEA)}

\section{Fạmily Bodotrindae}

Bodotria scorpioides (Montagu). Cancer s., Montagu ; ${ }^{5}$ Cuma Edevardsii, Goodsir, Bell (with figures of C.E. and C. Audouinii transposed); Bodotria scorpioides, T. Scott, Calman

1 See further, Holt and Beaumont, Trans. Royal Dublin Soc. (Ser. 2). vii, pt. 7, 239.

8 Iconographie, Crustacts, p. 17, pl. xxiii, fig. 2.

+ See Stebbing in Willey's Zoological Results, pt. 5, p. 609 (1 g00).

5 Trans, Linn. Soc. L.ond. (1 804), vii, 70, pl. 6, fig. 5.

\section{ORDER SYMPODA (formerly CUMACEA) (continued)}

Family Bodotrirdae (continued)

Iphinoe trispinosa (Goodsir). Cuma t., Goodsir, Bell ; Halia t., Bate; Iphinoe t., Bate, Sars, Stebbing, Garstang, Norman; Iphithoe t., Norman, Bate ; Venilia gracilis, Bate; Cyrianassa g., Bate

Cumopsis longipes (Dohrn). Cuma l., Dohrn; Cumopsis laevis, Sars ; Cumopsis longipes, Stebbing, Norman

- goodsir (van Beneden). Cuma edwuardsii, Bate; Bodothria goodsir + Bodotria goodsizit, v. B.; Cumopsis g., Sars ; (from stomach of Asterias, at Paignton) D. Pidgeon

\section{Family Leuconidar}

Eudorella truncatula (Bate). Eudora t., Bate; Eudorella t., Norman, Sars, Stebbing; $E$. inermis, Meinert 


\section{CRUSTACEANS}

ORDER SYMPODA (formerly CUMACEA) (continued)

Family Nannastacidar

Nannastacus unguiculatus (Bate). Cuma unguiculata, Bate ; Nannastacus binoculoides, Bate ; N. unguiculatus, Sars ; (Ilfracombe Harbour) Stebbing Cumella pygmaea, Sars. C.p., Sars, Norman; C. agilis, Norman

\section{Family Pseudocumidar}

Pseudocuma longicorne (Bate). Cyrianassa longicorris, Bate; Leucon cercaria, van Beneden; Pseudocuma c., Sars, Norman; P. longicornis, Stebbing; $P$. bistriata, Sars; Cuma bella, Meinert
ORDER SYMPODA (formerly CUMACEA) (continued)

Family Diastruidae

Diastylis rathkii (Kröyer). Cuma R. C. angulata, Kröyer ; Diastylis Rathkei, Sars, Norman; D. Rathkii, Norman, Stebbing; Alauna rostrata, Goodsir ${ }^{1}$

- sculpta, Sars. D. s., Sars, 1871; (dredged in Torbay) Stebbing

- laevis, Norman. D. l., Norman; D. rostrata, Sars

- bradyi, Norman. D.B., Norman, Sars

- lucifera (Kröyer). Cuma l., Kröyer ; Diastylis l., Sars, Norman

- rugosa, Sars. D. r., Sars, Norman ; D. strigata, Norman

This interesting order of small sessile-eyed Malacostraca is now regarded as having closer affinity to the stalk-eyed than to those which agree with it in having immovable eyes. So little had it come into notice until recently that Parfitt in his Sessile-eyed Crustacea of Devon in 1873 does not allude to it at all. ${ }^{2}$ Even in 1904 the Marine Biological Association only mentions the occurrence of six species, to which I have added Bodotria scorpioides taken at Meadfoot, Torquay, and Cumopsis longipes from the stretches of sand at Woolacombe and Saunton in North Devon. In Bate's Plymouth list of the year 1888 we find the additional species, Cuma unguiculata, Bate, which should be Nannastacus unguiculatus (Bate), Vaunthomsonia edwardsii (Kröyer), which belongs to Cumopsis, and Vaunthomsonia cristata (Bate), for which, according to Dr. Calman, Bate's original spelling was Vauntompsonia cristata; but as no references or special localities are given for the occurrence of these species in Devonian waters, it would, in my opinion, be very unsafe to accept them as members of the fauna without some corroborative testimony. On the other hand, Dr. Norman's personal researches have added to my list as originally written the species Cumella pygmaea, Diastylis lucifera, and D. rugosa.

\section{Order ISOPODA}

Division Anomala

Tribe Chelifera or Tanaldacea

Family Tanaidae

Tanais cavolinii, Milne-Edwards. T.c., M.-E., Dollfus ; T. tomentosus, Kröyer, Stebbing, Todd; Crossurus vittatus, Rathke ; Tanais v., Lilljeborg, Bate and Westwood

Leptochelia dubia, Kroyer. Tanais dubius, Kröyer; Leptochelia dubia, Norman

Paratanais batei, Sars. P. forcipatus, Bate and Westwood (not Tanais $f_{\text {., Lilljeborg) }}$

Tanaissus lilljeborgi (Stebbing). Leptognathia $L$., Stebbing $^{3}$; Tanaissus Lilljeborgii, Norman and Scott ${ }^{4}$

Leptognathia breviremis (Lilljeborg). Tanais b., Lilljeborg ; Leptogmathia b., Sars, Norman

\section{Family Apseudidae}

Apseudes talpa (Montagu). Cancer Gammarus t., Montagu ; 3 Apseudes t., Leach, Norman and Stebbing, Todd

1 As to whether Goodsir's species should stand here or should supersede D. laevis, see Norman, Crust. of Dewon and Cornw. p. 3 I. Trans, Devon. Assoc. vi, 236.

Ann. Nat. Hist. (Ser. 6, 189 I), viii, 328, pl. xvi.

1 Norman and Scott, Crust. of Devon and Cornw. p. 34, pl. i, figs. $1-7$.

5 Trans. Linn. Soc. Lond. (1808), ix, 98, pl. iv, fig. 6.

\section{Order ISOPODA (continued) \\ Division Anomala (continued)}

Tribe Chelifera or Tanaidacea (continued)

Family Arseudidar (continued)

Apseudes latreillii (Milne-Edwards). Rhoea l., M.-E.; Apseudes l., Bate and Westwood, Sars, Norman and Stebbing ; A. latreillei, Pace

\section{Division Isopoda Genuina}

Tribe Flabellifera

Family Anthuridar

Anthura gracilis (Montagu). Oniscus g., Montagu ; ${ }^{6}$ Anthura g., Leach, Bate and Westwood, Norman and Stebbing, Todd, Garstang ${ }^{7}$

\section{Family Gnathindar}

Gnathia maxillaris (Montagu). Cancer $m$., Montagu ;' Gnathic termitoides, Leach ; Anceus maxillaris, Bate and Westwood, Parfitt ; Gnothio m., Leach, Stebbing, Sars, Todd; Oniscus caeruleatus, Montagu

6 Ibid. p. I03, pl. v, fig. 6.

7 Trans. Devon. Assoc. (1892), xxiv, 385 .

8 Trans. Linn. Soc, Lond. (1804), vii, 65, pl. vi, fig. 2. 


\section{A HISTORY OF DEVONSHIRE}

\section{ORDER ISOPODA (continued)}

Division Isopoda Grevina (continued)

Tribe Flabeluifera (continued)

Family Gnathirdaz (continued)

Gnathia oxyuraea (Lilljeborg). Anceus oxyuraeus, Lilljeborg ; Gnathia oxyuraea, Norman

\section{Family Eurydicidae}

Eurydice achata (Slabber). Oniscus achatus, Slabber; Eurydice pulchra, Leach, Bate and Westwood, Hansen

- truncata (Norman). Cirolana t., Norman; Eurydice t., Hansen, Norman

- spinigera, H. J. Hansen. E. s., Hansen, Stebbing ${ }^{1}$

- inermis, H. J. Hansen. E. i., Hansen, Norman ${ }^{2}$

Cirolana cranchii, Leach. C. c., Leach, Bate and Westwood, Hansen; Nelocira Swainsoni, Leach; Conilera grampoides, Gourret

- borealis, Lilljeborg. C. b., Lilljeborg, Norman; C. spinipes, Bate and Westwood

- hirtipes, Milne-Edwards. C. l., M.-E., A. O. Walker

Conilera cylindracea (Montagu). Oniscus cylindraceus, Montagu ; Conilera montagui, Leach ; C. cylindracea, White, Bate and Westwood, Hansen, Todd, Pace

\section{Family AgGrdae}

Rocinela danmoniensis, Leach. $R . d$., Leach, Bate and Westwood, Schiödte and Meinert, Stebbing; $R$. damnoniensis, Todd, Pace

- dumerilii (Lucas). Acherusia D., Lucas ; Rocinela $D$., Schiödte and Meinert ; R. Dumerili, Todd, Pace

Aega rosacea (Risso). ${ }^{3}$ Cymotboa r., Risso ; Cirolana r., Risso ; Aega bicarinata, Leach, Bate and Westwood (partly); Aega rosacea, Schiödte and Meinert ${ }^{4}$

\section{Family Crmothoidae}

Nerocila neapolitana, Schiödte and Meinert. $N . n$., A. M. Norman

\section{Family Sphaeromidar}

Sphaeroma serratum (Fabricius). ? Oniscus conglobator, Pallas ; O. serratus, Fabricius ; Cymothoa serrata, Fabr.; Sphaeroma cinerea, Bosc.; S. 'serrata, Leach ; S. serratum, Leach

- (l) rugicauda, Leach. S. r., Leach, Bate and Westwood, Parfitt ; (?) S. Hookeri, Leach, Bate and Westwood, Parfitt

Dynamene bidentata (Adams). Oniscus bidentatus, Adams; Nesaea bidentata, Leach; Naesa b., Leach, Bate and Westwood, Parfitt, Todd, Pace ; Dynamene Montagui $+D$. rubra $+D$. viridis, Leach, Bate and Westwood, Parfitt ; Dynamene varians, Stebbing; D. bidentata, Stebbing ${ }^{6}$

1 Ann. Nat. Hist. Ser. 6 (1895), xv, 24 .

Fourn. Linn. Soc. Lond. (1905), xxix, 370.

${ }^{3}$ Cruseacés de Nice, 140, pl. iii, fig. 9.

- Naturbist. Tidsskrift, ser. 3 (1879), xii, 353, 354 .

5 Fourn. Mar. Biol. Assoc. (new ser.), vii. 343.

6 Stebbing, in Herdman's Pearl Fisbery Rep. Isopoda, Rep. 23 p. 29 (1905).

\section{ORDER ISOPODA (continued)}

Division Isopoda Genuina (continued)

Tribe Flabellifera (continued)

Sub-Family Spharromidae (continued)

Cymodoce truncata (Leach). C. t. and C. emarginata, Leach ; Cymodice t., Leach ; Cymodocea t., and C. emarginata, Leach, Bate and Westwood, Parfitt; Sphaeroma curtum and S. Prideauxianum, Leach $^{7}$

Campecopea hirsuta (Montagu). Oniscus hirsutus, Montagu $;{ }^{8}$ Campecopea hirsuta, Leach, Bate and Westwood, Parfitt, Stebbing ; ${ }^{9}$ C. cranchii, Leach

\section{Family LumnorindaE}

Limnoria lignorum (J. Rathke). Cymothoa l., Rathke; Limnoric terebrans, Leach ; L. lignorum, White, Bate and Westwood, Stebbing

\section{Tribe $V_{\text {ALVIFERA }}$}

Family Idoteidae

Idotea balthica (Pallas). Oniscus baltbicus, Pallas; Idotea marina, Fabricius : I. entomon, Leach ; 1 . tricuspidata, Desmarest, Bate and Westwood; Idothea baltica, Sars, Pace, H. Richardson; Idotea balthica, Norman

- pelagica, Leach. I. p., Leach, Bate and Westwood ; Idothea p., Sars, J. T. Cunningham

- emarginata (Fabricius). Cymothoa e., Fabr.; Idotea e., Fabr., Bate and Westwood, Norman; Idothea e., Sars, Hodgson, Todd, Pace; Oniscus oestrum, Pennant; Idotea oe., Leach

- linearis (Linn.). Oniscus l., Linn., Pennant; Idotea l., Fabricius, Bate and Westwood, Parfitt, Norman; Idothea l., Pace; Idotea hecticus + Stenosoma bectica + S. lineare, Leach

- neglecta, Sars. Idothea n., Sars, Pace ; Idotea n., Norman ${ }^{10}$

Stenosoma acuminatum, Leach. S. acuminata, Leach ; S. acuminatum, Leach, Dollfus; Idotea acuminata, White, Bate and Westwood, Parfitt ; I. (? Leptosoma) a., Stebbing

- lanciforme (Risso). Idotea lanciformis, Risso; I. appendiculata, Bate and Westwood; (? Leptosoma appendiculata, Risso); Stenosoma lanciferum, Norman.

Zenobiana prismatica (Risso). Zenobia prismatica+ Z. mediterranea, Risso; Z. p., Dollfus; Idotea p., Heller ; I. chelipes, Costa ; I. parallela, Bate and Westwood, Garstang; " Zenobiana prismatica, Stebbing, ${ }^{12}$ Norman.

\section{Family Astacillidae}

Astacilla longicornis (Sowerby). Oniscus l., Sowerby; Arturus l., Westwood; Astacilla l., Fleming, Stebbing, Sars, Todd, Pace; Leacia lacertosa, Johnston; Leacia gracilis, Goodsir

7 See interesting discussion of this synonymy in Crust. of Devon and Cornw. p. 44 .

8 Trans. Linn. Soc. Lond. (1804), vii, 71, pl. vi, fig. 7.

y Trans. Devon. Assoc. (1874), vi, 773 .

10 Ann. Nat. Hist. Ser. 7 (1904), xiv, 442 .

11 Trans. Devon Assoc. (1892), xxiv, 384

12 Ann. Nat. Hist. Ser. 6 (1895), xv, 24 . 


\section{CRUSTACEANS}

\section{ORDER ISOPODA (continued)}

Division Isopoda Genurna (continued)

Tribe Valvifera (continued)

Family Astacillidae (continued)

Astacilla intermedia (Goodsir). Leacia i., Goodsir ; Arcturus intermedius, Bate and Westwood; Astacilla intermedia, Stebbing, W. Heape, T. V. HodgBon

- deshayesii (Lucas). Arcturus d., Lucas, Stebbing; Arcturus gracilis $+A$. linearis, Stebbing; Astacilla d., Stebbing, Norman

Arcturellin damnoniensis (Stebbing). Arcturus d., Stebbing $;^{1}$ Astacilla d., Stebbing; ${ }^{2}$ Arcturella d., Norman ${ }^{3}$

\section{Tribe Asellota}

\section{Family Asellidae}

Asellus aquaticus (Linn.). Oniscus a., Linn.; Asellus a., Leach, Bate and Westwood, Sars, Parfitt ; A. vulgaris, Latreille, Leach, Moore

\section{- Family Janiridae}

Janira maculosa, Leach. J.m., Leach, Bate and Westwood, Stebbing, Todd; Ianira m., Sars

Jaera marina (O. Fabricius). Oniscus marinus, $O$. Fabr.; Jaera albifrons, Leach, Bate and Westwood, Parfitt, Stebbing; Icera marina, Sars; Jaera m., Todd, Pace; J. maculata, Parfitt

- nordmanni (H. Rathke). Janira n., Rathke; Jaera n., Bate and Westwood, Stebbing ${ }^{4}$

\section{Family Munnidae}

Munna kroyeri, Goodsir. M.k., Goodsir, Bate and Westwood, Stebbing, Sars, Todd ; $M$. Whiteana, Bate and Westwood; M. Fabricii, Kröyer (partly)

- limicola, Sars. M. l., Sars, Norman

- fabricii, Sars. M. f., Krơyer, Sars, Norman

\section{Tribe Epicaridea}

Family Cryptoniscidae

Cryptothir balani (Bate). Liriope b., Bate; Hemioniscus 6., Buchholz, Bonnier ; ${ }^{5}$ Cryptothiria b., Bate and Westwood; Cryptothir b., Stebbing, Sars, Todd; Balanus balanoides oै, Goodsir. On Balanus balanoides

\section{Family BOPYRIDAE}

Bopyrina Giardi, Bonnier. B. virbii, Stebbing (not Walz) ; B. giardi, Bonnier, ${ }^{6}$ Norman

Bopyrus squillarum, Latreille. Oniscus s., Montagu ; B. s., Latr., Bate and Westwood, Sars, Todd; Monoculus crangorum, Fabricius; Bopyrus fougerouxi, Giard and Bonnier. On Leander serratus

1 Anr. Nat. Hiss. Ser. 4 (1874), iv, 13, pl. xv, and Trans. Devor. Assoc. (1879), xi, 523 .

2 Hists of Crustacea, P. 37 i.

B Crust. of Devon. and Cornw. p. 48

4 Ann. Nat. Hist. (Ser. 4), xvii, 79, pl. v, fig. 7.

- Les Bopyridae, Travaux Zool. de Wimereux (1 goo), viii, 38.

"Cf. Histo of Crustacea, p. 417, and Les Bopyridae, Travaux de Wimereux, p. 365 .

\section{ORDER ISOPODA (continued)}

Division Isopoda Genuina (continued)

Tribe Epicaridia (continued)

Family Bopyridar (continued)

Gyge branchialis, Cornalia and Panceri. G. galatheae, Bate and Westwood; G. branchialis, Norman. On Upogebia stellata

Pseudione sp.? Gyge galatheae, Todd. On Galathea squamifera

- hyndmanni (Bate and Westwood). Phryxus H., B. and W.; Pseudione H., Bonnier, Normán. On Eupagurus bernhardus

Pleurocrypta galatheae, Hesse. P. g., Hesse, Norman. On Galathea squamifera

- longibranchiata (Bate and Westwood). Phryxus longibranchiatus, B. and W.; Pleurocrypta longibranchiata, Sars, Norman. On Galathea squamifera

Athelgue paguri (Rathke). Phryxus p., Bate and Westwood; Athelges p., Sars, Norman. On Eupagurus bernhardus

Ione thoracica (Montagu). Oniscus thoracicus, Montagu, $^{8}$ Leach; Ione t., Latreille, Milne-Edwards; I. thoracica, Bate and Westwood; Bonnier. On Callianassa subterranea

\section{Tribe Oniscidea}

Family Ligndae

Ligia oceanica (Linn.). Oniscus oceanicus, Linn. Ligia oceanica, Fabricius, Leach; L. scopulorum, Leach

\section{Family Trichoniscidae}

Trichoniscus pusillus, Brandt. T.p., Brandt, Sars ; Itea riparia, Koch; Philougria r., Bate and Westwood, Parfitt

- roseus (Koch). Itea rosea, Koch ; Philougria r., Kinahan, Bate and Westwood, Parfitt, Stebbing; Trichoniscus roseus, Budde-Lund, Sars, Stebbing ${ }^{9}$

\section{Family Oniscidae}

Oniscus asellus, Linn. O. a., Linn., Leach, Bate and Westwood ; O. murarius, Cuvier, Budde-Lund; ? O. fossor, Koch, Parfitt

Philoscia muscorum (Scopoli). Oniscus m., Scopoli ; Philoscia m., Latreille, Bate and Westwood, Parfitt

- couchii, Kinahan. P. c., Kinahan, Bate and Westwood, Stebbing ${ }^{10}$

Platyarthrus hoffmannseggii, Brandt. P.h., Brandt, Bate and Westwood, Parfitt, Stebbing

Porcellio scaber, Latreille. P.s., Latr., Bate and Westwood

- pictus, Brandt and Ratzeburg. P.p., B. and R., Bate and Westwood, Parfitt

7 See Norman, Ann. Nat. Hist. Ser. 7, rvi, 86, on the erroneous establishment of Gyge galatheae.

8 Trans. Linn. Soc. Lond. (1 808), 1x, 103, pl. iii, figs. 3, 4.

9 Trans. Devon. Assoc. (1879), xi, 523.

10 Trans. Devon. Assoc. $(1874)$, vi, 773 . 


\section{A HISTORY OF DEVONSHIRE}

Order ISOPODA (continued)

Division Isopoda Genuina (continued)

Tribe Oniscidea (continued)

Family Oniscidae (continued)

- laevis, Latreille. P. l., Latr., Leach, Bate and Westwood, Budde-Lund

Porcellio dilatatus, Brandt and Ratzeburg. P. d., B. and R., Bate and Westwood, Stebbing

Metoponorthus cingendus (Kinahan). Porcellio c., Kinahan, Bate and Westwond; Metoponorthusc., Dollfus, Stebbing ${ }^{1}$
ORDER ISOPODA (continued)

Division Isopoda Genuina (continued)

Tribe Oniscidas (continued)

Family Omiscidae (continued)

Metoponorthus pruinosus (Brandt). Porcellio p., Brandt, Bate and Westwood, Parfitt; Metoponorthus p., Budde-Lund, Sars, Stebbing

\section{Family Armadilidididar}

Armadillidium vulgare (Latreille). Armadillo vulgaris, Latr., Bate and Westwood, Parfitt ; Armadillidium vulgare, Milne-Edwards, Budde-Lund

It is impossible here to deal with the numerous points of interest that suggest themselves in connexion with this great group. Reference must be made to the authorities whose names have been cited. Their writings in most cases will be familiar to students of the Isopoda. The valuable list supplied by the Fournal of the Marine Biological Association for 1904 depends on the combined exertions of many workers, to whom the fauna of Devon is deeply indebted in regard to a large number of groups. My own researches, so far as the carcinology of Devon is concerned, have been conducted almost exclusively in the neighbourhood of Torquay, Salcombe, and Ilfracombe. Among less recent papers one that seems to deserve particular commemoration is that by Dr. E. Moore, F.L.S., in Charleswortb's Magazine of Natural History, ' $\mathrm{On}$ the occurrence of the 'Teredo navalis and Limnoria terebrans in Plymouth harbour.' 'This essay supplies a very striking picture of the damage that can be done to unprotected submerged timber by an animal so insignificant in size as the so-called Gribble, and one so sluggish in its habits, except apparently in the use of its jaws. Montagu remains the envy of English collectors in regard to his capture of Callianassa subterranea with its parasite Ione thoracica at Salcombe. Of the latter he says, "This curious species of Oniscus inhabits the thoracic plate of Cancer subterraneus, concealing itself between the fleshy part and the shell, and forming a tumour on one side. From this situation I have extracted it alive, and have kept it in that state for several days in a glass of sea water. In the few of that rare species of crab I have obtained, two or three of these parasitical insects have occurred, and have always been attended by the male, who attaches himself firmly by his claws to the ventral fins, or appendages.'

Idotea neglecta has only recently been added to the English fauna. The opinion of Sars that it had long been confused with other species is confirmed by Norman, who speaks of having received hundreds of specimens of it from the Plymouth marine station. Leach knew of only one occurrence of Porcellio laevis in Britain, and that was in Devonshire. Budde-Lund gives it a vast list of synonyms, and gives it the whole world for its country, Britain being one of the lands over which it is generally distributed. The distribution of Porcellio dilatatus is being gradually extended. Salcombe may be added to the localities at which it has been found.

\section{ORDER AMPHIPODA}

\section{Tribe Gammaridea}

Family Lysianassidae

Acidostoma obesum (Bate). Anonyx obesus, Bate; Acidostoma obesum, Lilljeborg, Stebbing ${ }^{3}$

Nannonyx goěsii (Boeck). Orchomene g., Boeck; Nannonyx g., Sars, Norman," A. O. Walker

Lysianassa plumosa, Boeck. L. p., Boeck, Stebbing; $L$. costar, Bate and Westwood (not MilneEdwards); Lysianax septentrionalis, Della Valle, Norman

\footnotetext{
1 Hist. of Crustacea, 428, and Ann. Nat. Hist. (Ser. 6), $\mathbf{x v}, 22$.

Op. cit. (new ser.), ii, 206 (1838).

- Trans. Devon. Assoc. xi, 518 .

- Ann. Nar. Hist. (Ser. 7), v, 207 (1900).
}

\section{OrDER AMPHIPODA (continued)}

Tribe Gammaridea (continued)

Family Lysianassidar (continued)

Perrierella audouiniana (Bate). Lysianassa a., Bate; Perrierella a., Bonnier; Pararistias audouinianus, Robertson; Perrierella crassipes, Chevreux and Bouvier

Orchomene batei, Sars. (?) Lysianassa bumilis, A. Costa; Orchomene b., Norman; Lysianassa longicornis (part) + Anonyx edwardsi, Bate and Westwood; Anonyx serratus, Stebbing; Orchomene batei, Sars, Hodgson, Stebbing

Scopelocheirus crenatus, Bate. S. c., Bate; Callisoma crenata, Bate ; C. Hopei, Norman

Orchomenella nanus (Kroyer). Anonyx n., Kroyer; Tryphosa n., Boeck, Bonnier; T. ciliata, Sars; Orchomenella $c .$, Sars ; O. nana, Sars, Hodgson 


\section{CRUSTACEANS}

\section{ORDBR AMPHIPODA (continued)}

Tribe Gammaridea (continued)

\section{Family Ampeliscidae}

Ampelisca brevicornis (A. Costa). Arameops b., Costa ; Ampelisce b., Della Valle, Norman ; $A$. laevigata, Lilljeborg, Sars; Tetromatus bellianus, Bate; Ampelisca belliana, Bate and Westwood

- spinipes, Boeck. A. s., Boeck, Sars, Norman, Todd

- typica (Bate). Tetromatus typicus, Bate ; Ampelisca typica, Boeck, Sars, Norman; A. gaimardit (part), Bate

- tenuicornis, Lilljeborg. A.t., Lilljeborg, Sars, A. C. Walker, Norman

\section{Family Haustorildae}

Bathyporeia guilliamsoniana, Bate. B. g., Bate; B. pilosa, Bate, Stebbing (not Lindstrom)

- pelagica, Bate. B. p., Bate, Sars, Hodgson

Haustorius arenarius (Slabber). Oniscus a., Slabber ; Haustorius a., Statius Muller, Stebbing

Urothoe brevicornis, Bate. U. b., Bate, Stebbing

- elegans, Bate. U. e., Bate, Stebbing ; U. norvegica, Boeck, Sars

- marina (Bate). Sulcator marinus, Bate; Urothoe m., Stebbing ; U. marina, Bate, Norman

\section{Family Phoxocrphalidar}

Phoxocephalus holbolli (Kroyer). Phoxus h., Kröyer, Bate; Phoxocephalus h., Stebbing, Sars, Norman

- simplex (Bate). Phoxus kröyerii, Bate (not Stimpson); $P$. simplex, Bate ; Phoxocephalus s., Stebbing, Della Valle, A. O. Walker

Metaphoxus fultoni (T. Scott). Phoxocephalus f., T. Scott, Norman, ${ }^{1}$ Calman

Harpinia antennaria, Meinert. Phoxus plumosus, Bate (not Kroyer); Harpinia plumosa (part), Boeck; $H$. antennaria, Meinert ; $H$. neglecta, Sars, Norman ${ }^{2}$

\section{Family Amphilochidae}

Amphilochus manudens, Bate. A. m., Bate, Sars, Norman, A. O. Walker ; A. manuidens, Boeck; A. concinna + Callimerus acudigitata, Stebbing

- spencebatei (Stebbing). Probolium spence-batei, Stebbing ' ; Amphilochus anomalus, Chevreux

Peltocoxa marioni, Catta. P.m., Catta; Cyproidia damnoniensis, Stebbing; Peltocoxa d. + P. marionis, Della Valle

\section{Family LeucothoIdat}

Leucothoe spinicarpa (Abildgaard). Gammarus spinicarpus, Abildg.; Cancer articulosus, Montagu ; Leucothoe articulosa, Leach, Bate ; L. spinicarpa, Stebbing, Sars, Norman, Todd

\section{Family Mztopidar}

Metopa alderii (Bate). Montagua a., Bate; Metopa a., Boeck, Della Valle; Probolium alderi, Stebbing ${ }^{s}$

1 Ann. Nat. Hist. (Ser. 7), v, 336

2 Ibid. p. 337

8 Ibid. (Ser. 4), xvii, 344, pl. xix, fig. 4

1 Campagnes Sci. de Monaco, fasc. 16, p. 48, pl. vii, fig. 2 (1 goo).

- Trans. Devon. Assor xi, 518.

\section{ORDER AMPHIPODA (continued}

Tribe Gammaridea (continued)

Family Cressidar

Cressa dubia (Bate). Danaia d., Bate, Stebbing; Cressa d., Stebbing, Sars

\section{Family Stenothoidae}

Stenothoe monoculoides (Montagu). Cancer Gammarus m., Montagu ; Montagua m., Bate; Probolium m., Norman ; Stenothoe m., Boeck, Sars, Norman

- marina (Bate). Montagua m., Bate; Probolium marinum, Heller; Stenotboe marina, Boeck, Sars, A. O. Walker

- setosa, Norman. S. s., Norman ${ }^{6}$

\section{Family Phliantidae}

Pereionotus testudo (Montagu). Oniscus t., Montagu ; Pereionotus t., Bate and Westwood, Norman ${ }^{8}$

\section{Family Colomastigidae}

Colomastix pusilla, Grube. C. p., Grube ; Cratippus tenuipes, Bate, Stebbing; Exunguia stilipes, Norman

\section{Family Acanthonotozomatidae}

Panoploea minuta (Sars). Iphimedia eblanae, Stebbing (not Bate) ; I. minuta, Sars, Hodgson, Todd

Iphimedia obesa, Rathke. I. o., Rathke, Bate, Boeck, Stebbing, Sars, Hodgson

\section{Family Lirjeborgirdar}

Liljeborgia pallida (Bate). Gammarus ? pallidus, Bate; Liljeborgia pallida, Bate, Stebbing

\section{Family Ordicerotidar}

Perioculodes longimanus (Bate and Westwood). Monoculodes l., B. and W. ; Perioculodes l., Sars, Norman

Pontocrates arenarius (Bate). Kroyera arenaria, Bate; Pontocrates arenarius, Stebbing, Norman

- altamarinus (Bate and Westwood). Kroyera altamarina, $B$. and $W$.

Westwoodilla caecula (Bate). Westruoodia c., Bate; Westwoodilla c. and $W$. hyalina, Bate; Oediceros parvimanus, Bate and Westwood; Halimedon p., Norman, Della Valle; H. Milleri, Boeck, Sars

Monoculodes carinatus (Bate). Kroyera carinata, Bate ; Monoculodes carinatus and $M$. Stimpsoni, Bate; M. c., Norman, Sars

\section{Family Tironidar}

Argissa hamatipes (Norman). Syrrboe b., Norman; Tiron b., Stebbing ; Argissa b., T. Scott, Norman

\section{Family Calliopindae}

Calliopius laeviusculus (Kroyer). Ampbitboe laeviuscula, Kroyer ; Calliope l., Bate ; Calliopius laeviusculus, Boeck

6 Ann. Nat. Hist. (Ser. 7), vi, 39, pl, iii, figs, 2-4.

7 Trans, Linn. Soc. Lond, ix, 102.

8 Ann. Nat. Hist. (Ser. 7), xvi, 8 I (1905). 


\section{A HISTORY OF DEVONSHIRE}

\section{ORDER AMPHIPODA (continued)}

Tribe Gammaridea (continued)

Family Calliopirdar (continued)

Apherusa cirrus (Bate). Pherusa c., Bate ; P. bicuspis, Bate and Westwood, Stebbing ; ${ }^{1}$ Apherusa $b$., Sars

-- bispinosa (Bate). Dexamine b., Bate; Atylus bispinosus, Bate ; Apherusa bispinosa, Sars, Todd

- jurinei (Milne-Edwards). Ampbithoe j., M.-E.; Pherusa jurinit, A. O. Walker; Apherusa j., Sars; Pberusa fucicola (not Leach) +? Gossea microdeutopa, Bate and Westwood

- ovalipes, Norman. A. o., Norman ${ }^{2}$

\section{Family Atruidae}

Nototropis swammerdamei (Milne-Edwards). Amphithoe s., M.-E. ; Atylus swammerdamii, Bate and Westwood; Paratylus swammerdami, Sars, Todd, Hodgson

\section{Family Gammaridae}

Gammarellus homari (Fabricius). Astacus b., Fabr.; Gammarus b., Fabr.; Cancer Gammarellus b., Herbst; Amatbilla b., Stebbing, Norman; Gammarellus b., Stebbing; Amatbilla sabini + Grayia imbricata, Bate and Westwood

Niphargus aquilex, Schiödte. N. a., Schiödte, Bate and Westwood, Parfitt; ${ }^{3}$ ? Gammarus subterraneus, Leach, Norman

Cheirocratus sundevallii, Rathke. Gammarus s., Rathke; Cbeirocratus s., Stebbing, Della Valle; Liljeborgia sbetlandica, Bate and Westwood; L. normanni, Stebbing; Protomedeia wbitei, Bate

Megaluropus agilis, Norman. M. a., Norman, Hoek Melita palmata (Montagu). Cancerpalmatus, Montagu;" Astacus p., Pennant ; Melita palmata, Leach

- obtusata (Montagu). Cancer Gammarus obtusatus, Montagu; Melita obtusata $+M$. proxima $+M e-$ gamoera Alderi, Bate

-- gladiosa, Bate. M. g., Bate, Stebbing, Norman

Ceradocus semiserratus (Bate). Megamoera semiserrata, Bate; Ceradocus semiserratus, Stebbing, Norman

Maera grossimana (Montagu). Cancer Gammarus grossimamus, Montagu ; Maera grossimana, Leach

- othonis (Milne-Edwards). Gammarus o., M-E.; Megamoera o. $+M$. longimanus, Bate; Maera o., Norman, Stebbing, Sars, Todd, Hodgson; Gammarus longimanus, W. Thompson

Elasmopus rapax, A. Costa. E. r., Costa, Della Valle, Norman; Megamoera brevicaudata, Bate

Pherusa fucicola, Leach. P. f., Leach (not Bate and Westwood); Gammarella brevicaudata + G. normanni, Bate and Westwood ; G. b., Stebbing ${ }^{5}$

Gammarus marinus, Leach. G. m., Leach

- pulex, Linn. Cancer p. (part), Linn. ; Squilla p., de Geer ; Gammarus p., Fabricius ; Cancer Gammarus pulex, Montagu.

-- locusta (Linn.). Cancer l., Linn. ; Gammarus l., Fabricius ; Cancer Gammarus l., Montagu ${ }^{7}$

1 Trans. Devon. Assoc. vi, 766.

Crust. of Devon and Cornw. p. 75, pl. viii, figs. I-8.

B Trans. Devon. Assoc. vi, pt. i, 238, 245 (1873)

4 Trans. Linn. Soc. Lond. vii, 69, pl. vi, fig. 4.

5 Ann. Nat. Hist. (Ser. 4), xiv, I3, pl. ii, fig8. 3, 3g. (1874);

and $A . O$. Walker, ibid. (Ser. 6), vii, 418 (189I).

6 Trans. Linn. Soc. Lond. ix, 93, pl. iv, fig. 2.

7 Ibid. g2, pl. iv, fig. I.

\section{Order AMPHIPODA (continued)}

Tribe Gammaridea (continued)

Family Dexaminidar

Dexamine spinosa (Montagu). Cancer Gammarus spinosus, Montagu ; ${ }^{8}$ Dexamine spinosa, Leach

Tritaeta gibbosa (Bate). Atylus gibbosus, Bate and Westwood, Stebbing ; ${ }^{9}$ Tritaeta gibbosa, Boeck

\section{Family Talitridae}

Talitrus saltator (Montagu). Oniscus locusta, Pallus; Cancer Gammarus saltator, Montagu ;0 Talitrus l., Latreille, Stebbing, Norman ; Talitrus saltator, Milne-Edwards

Orchestia mediterranea, A. Costa. O.m., Costa, Bate, Parfitt, Hodgson

- gammarellus (Pallas). Oniscus g., Pallas ; Cancer Gammarus littoreus, Montagu; Orchestia littorea, Leach, Bate and Westwood; O. gam. marellus, Bate, Stebbing

Talorchestia deshayesii (Audouin). Orchestia d., Audouin ; Talorchestia d., Chevreux, Stebbing; ${ }^{11}$ Orchestoidea d., Norman ${ }^{13}$

- brito, Stebbing. T. b., Stebbing, ${ }^{13}$ Chevreux; Orchestoidea b., Norman ${ }^{14}$

Hyale prevostii (Milne-Edwards). Ampbitboe $p$ M.E.; Allorchestes nilssonii, Rathke, Bate and Westwood; Hyale prevostii, Stebbing, Della Valle (part)

- pontica, Rathke. H. p., Rathke, Stebbing; Allorchestes imbricatus + Nicea lubbockiana, Bate and Westwood

\section{Family Aoridar}

Aora typica, Kroyer. A. t., Kröyer ; A. gracilis, Bate, Sars

Microdeutopus chelifer (Bate). Stimpsonia chelifera, Bate and Westwood, Stebbing ; Stimpsonella c., Della Valle, Norman

- damnoniensis (Bate). Lembos d., Bate; Microdeutopus gryllotalpa, Bate (not Costa)

Coremapus versiculatus (Bate). Lembos ø., Bate; Microdeutopus v., Bate, Walker; Microdeuteropus v., Norman, Stebbing ; Coremapus थ., Norman ${ }^{15}$

Lembos websterii, Bate. L. w., Bate, Stebbing, Norman; Microdeutopus w., Bate and Westwood; Microdeuteropus bidentatus, Stebbing

\section{Family Photidar}

Microprotopus maculatus, Norman. M. m., Norman, Stebbing

Photis longicaudata (Bate and Westwood). Eiscladus longicaudatus, B. and W. ; Photis longicaudata, Meinert

Eurystheus maculatus (Johnston). Gammarus m., Johnston ; Gammaropsis m., Stebbing ; Gammarus (Gammaropsis) erythrophthalmus, Liljeborg; Eurystheus e. + Gammarus maculatus, Bate and Westwood

8 Ibid. xi, 3, pl. ii, fig. I. 9 Trans. Devon. Assoc. xi, 519.

10 Trans. Linn. Soc. Lond. ix, 94, pl. iv, fig. 3 .

11 Ibid. (Ser. 2), vii, pt. 2, 400, pl. xxxa (1899).

12 Ann. Nat. Hist. (Ser. 7), v, 39 (1 goo).

18 Ibid. (Ser. 6), viii, 324, pl. xv (1891).

14 Ibid. (Ser. 7), v, 140 (1900).

15 Ibid. (Ser. 7), xvi, 78 (1905). 


\section{CRUSTACEANS}

\section{ORDER AMPHIPODA (continued)}

Tribe Gammaridea (continued)

Family Photrdaz (continued)

Podoceropsis sophia, Boeck. P. s., Boeck; Noenia tuberculosa, Bate and Westwood

- nitida (Stimpson). Podocerus nitidus, Stimpson; Noenia rimapalmata $+N$. excavata, Bate and Westwood; Podoceropsis e., Sars, Norman

Megamphopus cornutus, Norman. $M$. c., Norman ; Podoceropsis intermedia, Stebbing

\section{Family Isaeidas}

Isaea montagui, Milne-Edwards. 1.m., M.-E., Bate and Westwood ; on Mamaia squinado

\section{Family Ampithoidae}

Ampithoe rubricata (Montagu). Cancer Gammarus rubricatus, Montagu ; Ampitboe rubricata, Leach; Ampbithoe r. + A. littorina, Bate

Pleonexes gammaroides, Bate. P.g., Bate, Sars ; Sunamphitbö̈ g., Stebbing

Sunamphitoe pelagica (Milne-Edwards). Amphithoep., M.-E., Chevreux; Sunampbitoe bamulus (? l) + S. conformata (ठ), Bate

Biancolina cuniculus (Stebbing). Ampbitboe c., Stebbing, Della Valle; Biancolina algicola, Della Valle

\section{Family Jassidae}

Parajassa pelagica (Leach). Jassa p., Leach, Norman ; Parajassap., Stebbing; Podocerus capillatus, Rathke, Bate and Westwood

Jassa pulchella, Leach ; $J . p$. ., Leach, Stebbing ; Podocerus pulchellus, Milne-Edwards, Bate and Westwood ; Bruzeliella falcata, Norman

- ocius (Bate). Podocerus ocius, Bate; Bruzeliella ocia, Norman

- falcata (Montagu). Cancer Gammarus falcatus, Montagu. Involved in doubt

\section{Family Corophindat}

Ericthonius difformis, Milne-Edwards. E. d., M.-E. ; Cerapus d. + Dercothoe (Cerapus + ) punctatus, Bate

Unciola crenatipalma (Bate). Dryope c., Bate, Stebbing; D. crenatipalmato, Bate and Westwood; Unciola c., Bonnier, Chevreux and Bouvier; Unciola irrorata, ${ }^{1}$ Gosse, Bate, Norman (but (?) not Say)

Corophium volutator (Pallas). Oniscus v., Pallas ; Cancer grossipes, Linn.; Corophium longicorne, Latreille
Order AMPHIPODA (continued)

Tribe Gammaridea (continued)

Family Corophindar (continued)

Corophium crassicorne, Bruzelius. C. c., Bruzelius, Todd, Hodgson

- bonellii (Milne-Edwards). Corophia Bonellii, M. E.; Corophium Bonnelli, M. E. ; C. bonellii, Hodgson

Family Cheluridae

Chelura terebrans, Philippi. C. t., Philippi, Stebbing, Sars, Todd, Pace

\section{Family Podocraidae}

Podocerus variegatus, Leach. $P$. $v$., Leach, MilneEdwards, Stebbing; Cyrtophium darwinit, Bate ; Platophium d., Stebbing; $P$. variegatum, Norman

\section{Tribe Caprellidea \\ Family Caprellidae}

Phtisica marina, Slabber. P. m., Slabber, Stebbing, Norman, Allen ; Gammaruspedatus, Abildgaard ; Cancer Gammarus p., Montagu ; Proto pedata, Leach, Bate; $P$. Goodsirii, Bate

Pseudoprotella phasma (Montagu). Cancer P., Montagu ; Caprella p., Leach ; Protella p., Bate, Stebbing; Pseudoprotello p., Paul Mayer, Norman

Caprella acanthifera, Leach. C. a., Leach, Bate and Westwood, Stebbing, Hodgson

- linearis (Linn.) Cancer l., Linn.; Caprella l., Leach, Bate and Westwood, Cunningham, Parfitt ; C. lobata, Bate and Westwood

- equilibra, Say. C. e., Say ; C. aequilibra, Bate, Todd

- septentrionalis, Kroyer. C. s., Kröyer ; C. s. var. nodifera, Mayer, Norman; C. bystrix, Bate and Westwood, Parfitt

- tuberculata, Guérin. C. t., Guérin, Bate and Westwood, Todd

- penantis, Leach. C.p., Leach, ${ }^{2}$ Moore, Stebbing; C. acutifrons, Latreille, Bate, Mayer, Norman

- fretensis, Stebbing. C. $f$., Stebbing, Mayer

Pariambus typicus (Kröyer). Podalirius t., Kröyer, Mayer; Caprella typica, Bate ; Pariombus typicus, Stebbing, Sars, Norman, Hodgson

\section{Tribe Hyperindea}

\section{Family Hyperidae}

Hyperia galba (Montagu). Cancer Gammarus $g$., Montagu; Hyperia g., Bate, Sars, Norman, Todd Parathemisto oblivia (Kröyer). Hyperia o., Kröyer); Parathemisto 0., Sars, Todd

The Devonshire Amphipoda, it will be seen, already include more than a hundred species. As during a prolonged residence in the county and numerous subsequent visits this order engaged my particular attention, I should naturally be disposed to write at considerable length on the observations which opportunity enabled me to make. The very wealth of the material precludes my discussing either my own researches or those which could claim more attention as having been carried out by Montagu, Leach, Gosse, Bate, Norman, and the numerous workers at the Plymouth

${ }^{1}$ For distinction between U. irrorata, Say, and U. crenatipalma (Bate), see A. O. Walker and Bateson in Fourn. Mar. Biol. Assoc. (new ser.) iii, I I9 (1893-5).

${ }^{2}$ Edin. Encycl. vii, 404 (1 813). 


\section{A HISTORY OF DEVONSHIRE}

station. I have endeavoured to indicate generally the sources from which information and verification may be obtained, and I may be permitted to explain that, for the great tribe of the Gammaridea, the arrangement and nomenclature are followed which will appear in the Amphipoda of Das Tierreich.

\section{SUB-CLASS LEPTOSTRACA}

Family Nebalmidab

Nebalia bipes (O. Fabricius). Cancer b., O. Fabricius ; Monoculus rostratus, Montagu ; ' Nebalia Herbstii, Leach ; N. Montagui, Thompson ; N. bipes, Krbyer, Baird, Sars, Allen. This species which, among other places; occurs on the shore at Salcombe, was long the only known representative of this sub-class. Its position in classification is still in dispute. The accessions to our knowledge will be found well discussed in 'Die Leptostraken,' by Dr. Joh. Thiele ${ }^{2}$

\section{Sub-Class ENTOMOSTRACA}

Order Branchiopoda

Sub-order Phyllopoda

Family Branchipodidar

Chirocephalus diaphanus, Prevost. Mr. A. K. Coomaraswamy, F.L.S., has favoured me with the following records for the occurrence of the 'Fairy Shrimp' in Devonshire, from Mr. F. J. Partridge of Barnstaple, who found it 'in a small roadside pool, which is dry in the hot season, between Braunton and Saunton, N. Devon, in the spring season of 1900 ; also in the same kind of pool between Morte Station and Lee, near Ilfracombe, very numerous at Christmas, 1901, a thin sheet of ice over the pool.' The species has been found in Surrey also at Christmas-tide under ice

\section{Sub-order Cladocerra}

\section{Tribe Onychopoda}

Family Polyphemidar

Evadne nordmanni, Loven. Observed at the Plymouth station from March to October; carrying ova in August (Hodgson); winter eggs produced about mid-September (E. J. Bles)

Podon intermedius, Liljeborg. Plymouth neighbourhood, April to September; carrying ova in August (Hodgson)

- leuckarti, Sars. Plymouth neighbourhood, April I 904, rare (L. H. Gough)

This sub-order comprises two sections, the Calyptomera and Gymnomera, the latter divided into two tribes, the Onychopoda and Haplopoda, the former also into two tribes, the Ctenopoda and Anomopoda. In the work just published by Dr. Norman and Dr. T. Scott on the Crustacea of Devon and Cornwall, between twenty and thirty species of Calyptomera have been recorded for Devonshire, most of them forms well known and widely distributed.

1 Trans. Linn. Soc. Lond. xi, 14, pl. ii, fig. 5 (1813).

2 Results of the 'Valdivia' Expedition, vili (1 904$)$.
Sub-CLASS ENTOMOSTRACA (continued)

\section{Order Ostracoda}

Tribe Mrodocopa

Family Asteropidae

Asterope mariae (Baird). Plymouth (Norman); Ilfracombe (Stebbing)

\section{Family Cypridinidae}

Philomedes interpuncta (Baird). Plymouth (Norman) - brenda (Baird). Ilfacombe (Stebbing)

\section{Family Polycopidas}

Polycopsis compressa (Brady and Robertson). Dredged off the Eddystone lighthouse (B. and $R$.)

\section{Tribe Podocopa}

\section{Family Cyprididae}

Pontocypris mytiloides (Norman). Plymouth (Spence Bate) ${ }^{3}$

- trigonella, Sars. Plymouth (Bate)

Anchistrocheles acerosa (Brady). Off the Eddystone (Brady); Plymouth (Norman) ${ }^{5}$

Argilloecia cylindrica, Sars. Off the Eddystone (Brady and Robertson); Salcombe (Norman)

\section{Family Bairdudar}

Bairdia inflata (Norman). Devonshire coast, $60 \mathrm{fms.}$ (Bate)

- acanthigera, Brady. Devonshire coast, $60 \mathrm{fms}$. (Bate); Plymouth from trawlers (Barlee)

\section{Family Crtheridae}

Cythere pellucida, Baird. Devonshire coast, $60 \mathrm{fms}$. (Bate); Plymouth (Norman)

- porcellanea, Brady. Devonshire (H. B. Brady, 1870)

- (?) semipunctata, Brady. Off the Eddystone and Ilfracombe (Brady and Robertson)

- teres, Brady. Dartmouth Harbour (Norman)

- oblonga, Brady. Salcombe and Plymouth (Norman)

- albomaculata, Baird. Exmouth (Norman)

- convexa, Baird. Devonshire coast, $60 \mathrm{fms}$. (Bate); Torquay, in sand (W. C. Williamson)

- marginata, Norman. Salcombe (Norman)

- navicula (Norman). Salcombe (Norman)

- pulchella, Brady. Off Ilfracombe (Brady and Robertson); Dartmouth Harbour (Norman)

- quadridentata, Baird. Plymouth (Norman)

- emaciata, Brady. Off Ilfracombe and Eddystone (Brady and Robertson); Plymouth (Norman)

\footnotetext{
${ }^{3}$ Brady, Trans. Linn. Soc. Lond. xxvi, pt. 2, 386 (1868).

4 Ibid. 387.

- Trans. Roy. Dublin Soc. (Ser. 2), iv, 110 (1889).
} 


\section{CRUSTACEANS}

\section{Sub-CLAsS ENTOMOSTRACA (continued)}

\author{
Order Ostracoda (continued) \\ Tribe Podocopa (continued) \\ Family Crtheridae (continued)
}

Cythere runcinata, Baird. Dredged in Dartmouth harbour and Plymouth Sound (Norman), at present (1889) the only known British localities

Cythereis villosa (Sars). Devonshire coast (Bate)

- finmarchica, Sars. Devonshire coast (Bate); off Ilfracombe and the Eddystone (Brady and Robertson)

- tuberculata, Sars. Exmouth (Norman); Devonshire coast (Bate)

- antiquata, Baird. Plymouth and Dartmouth Harbour (Norman); off Eddystone (Brady and Robertson)

- whiteii, Baird. Torquay (W. A. Williamson); Dartmouth Harbour (Norman)

- jonesii, Baird. Off the Eddystone (Brady and Robertson)

Cytheridea elongata, Brady. Devonshire coast (Bate); Exmouth (Norman)

Loxoconcha impressa, Baird. Devonshire coast, 60 fms. (Bate)

- guttata (Norman). Plymouth (Norman)

- viridis (Muller). Dartmouth Harbour (Norman)

- multifora (Norman). Off Eddystone and Ilfracombe (Brady and Robertson); Start Bay (Norman)

- tamarindus (Rupert Jones). Devonshire coast 5-6o fms. (Bate)

Xestoleberis aurantia (Baird). Off Eddystone and Ilfracombe (Brady and Robertson); Start Bay, Salcombe, and off Berry Head (Norman)

- depressa, Sars. Devonshire coast, $60 \mathrm{fms}$. (Bate)

- labiata, Brady and Robertson. Salcombe (Norman)

Cytherura gibba (Muller). Dartmouth Harbour (Norman)

- cornuta, Brady. Salcombe (Norman)

- acuticostata, Sars. Plymouth (Rev. G. Barlee)

- striata, Sars. Plymouth (Barlee)

- angulata, Brady. Plymouth (Norman)

- producta, Brady. Off Eddystone (Brady and Robertson)

- concentrica, Brady, Crosskey, and Robertson. Salcombe (Norman)

- fulva, Brady and Robertson. Off Eddystone (Brady); Salcombe (Norman)

Cytheropteron nodosum, Brady. Off Ilfracombe and Eddystone (Brady and Robertson); Salcombe (Norman)

- depressum, Brady and Norman. Off Eddystone (Brady and Robertson); Dartmouth Harbour (Norman)

Bythocythere constricta, Sars. Off Ilfracombe and Eddystone (Brady and Robertson)

- turgida, Sars. Off Eddystone (Brady and Robertson)

Pseudocythere caudata, Sars. Off Eddystone (Brady and Robertson)

Sclerochilus contortus (Norman). Devonshire (Bate); Plymouth (Norman)

Cytherideis subulata, Brady. Devonshire coast (Bate) Cytherois fischeri (Sars). Devonshire (H. B. Brady, 1870)
SUB-CLASS ENTOMOSTRACA (continued)

Order Ostracoda (continued)

Tribe Podocopa (continued)

Family Paradoxostomatidae

Paradoxostoma ensiforme, Brady. Plymouth (Norman)

- abbreviatum, Sars. Plymouth Sound, 5 fms. (Bate)

- obliquum, Sars. Off Ilfracombe (Brady and Robertson)

- normani, Brady. Plymouth and Dartmouth Harbour (Norman).

- hibernicum, Brady. Devonshire (H. B. Brady)

- arcuatum, Brady. Dartmouth Harbour (Norman)

- flexuosum, Brady. Off Eddystone (Brady and Robertson); Dartmouth Harbour (Norman).

\section{Order Copepoda \\ Tribe Calanidea \\ Family Calanidas}

Calanus finmarchicus (Gunner). Plymouth (Professor P. T. Cleve, L. H. Gough)

\section{Family Paracalanidas}

Paracalanus parvus (Claus). Plymouth (G. C. Bourne, Cleve, Gough)

Family Pseudocalanidas

Pseudocalanus elongatus (Boeck). Plymouth (Bourne, Cleve, Gough)

\section{Family Centropagidar}

Centropages typicus, Kröyer. Plymouth (Bourne, Cleve, Gough)

Isias clavipes, Boeck. Plymouth (Cleve, Gough)

\section{Family Temoridar}

Temora longicornis (O. F. Muller). Plymouth (Bourne, Cleve, Gough)

\section{Family Pontellidas}

Anomalocera patersonii, Templeton. Plymouth (Bourne, Gough)

Labidocera wollastoni (Lubbock). Plymouth (Gough); off Eddystone (Bourne)

\section{Family Parapontellidar}

Parapontella brevicornis (Lubbock). Near Eddystone, the Cattewater (Bourne); Plymouth (Gough)

\section{Family Acartimdae}

Acartia longiremis, Lilljeborg. Plymouth (Bourne) - clausii, Giesbrecht. Plymouth (Cleve, Gough)

1 It will easily be seen by anyone in the least acquainted with the subject how fully the above catalogue is indebted to the Monograpb of recent Britisb Ostracoda published by Professor G. S. Brady in 1868 , and to his subsequent work in collaboration with the Rev. Canon Norman in 1889 and 1896 . 


\section{A HISTORY OF DEVONSHIRE}

\section{Sur-CLAss ENTOMOSTRACA (continued)}

\section{Order Copepoda (continued)}

Tribe Arpacticidea

Family Longipedidae

Longipedia scotti, Sars. L. coronata, Brady (not Claus); L. scotti, Sars. Plymouth (Bourne)

\section{Family Ectrinosomatidas}

Microsetella norvegica (Boeck). Plymouth (Cleve)

\section{Family Arpacticidae}

Arpacticus chelifer (O. F. Muller). Torquay (Stebbing)

\section{Family Peltidinae}

Peltidium purpureum, Philippi. Ilfracombe Harbour (Stebbing)

Alteutha depressa, Baird. Plymouth (Bate)

\section{Family Idyidas}

Psamathe longicauda, Philippi. Scutellidium tisboides, Claus. Ilfracombe (Stebbing)

\section{Family Thalestridae}

Phyllothalestris mysis (Claus). Thalestris m., Claus ; Pbyllotbalestris m., Sars, 1905 . The Cattewater (Bourne)

Dactylopusia valida, Norman and T. Scott. Near Beggar's Island ; Plymouth (Norman)

Vallentinia ornata (Norman and T. Scott). Dactylopusia 0., N. and S. Dredged, coast of Devon (Norman)

Megarthrum purpurocinctum (Norman and T. Scott). Dactylopusia purpurocincta, N. and S. Dredged, Salcombe (Norman)

\section{Family Drosaccidar}

Diosaccus tenuicornis (Claus). Starcross (Parker)

At this point the new classification of the Copepoda by Professor G. O. Sars for the moment ends ; and since he rearranges both families and genera, it would be useless, even if one had the requisite skill and presumption, to attempt to forestall its future developments. The following species of the Arpacticidea are therefore not allotted to distinctive families.

Euterpina acutifrons (Dana). Euterpe gracilis, Claus. Plymouth (Bourne, Cleve)

\section{Sub-Class ENTOMOSTRACA (continued)}

Order Copepoda (continued)

Tribe Arpacticidea (continued)

Family Drosaccidae (continued)

Stenhelia pygmaea, Norman and T. Scott. Off Eddystone (Norman)

- simulans, Norman and T. Scott. Near Chequer Buoy, Plymouth (Norman)

- neglecta, Norman and T. Scott. Salcombe, Mil Bay, Plymouth (Norman)

- longirostris, Norman and T. Scott. Salcombe (Norman)

Ameira simplex, Norman and T. Scott. Estuary of the Exe, near Starcross (Norman)

\section{Tribe Crclopidea}

Family Crclopidae

Oithona plumifera, Baird. Plymouth (Bourne)

- similis, Claus. Plymouth (Cleve)

Cyclopina littoralis (Brady). Plymouth (Bourne)

\section{Tribe Monstrillidea (? Thaumatorssidea)}

Thaumatoessa longispinosa (Bourne). Monstrilla l., Bourne; Thaumaleus longispinosus, Giesbrecht. Plymouth (Bourne)

- claparedii (Giesbrecht). ? Cymbasoma rigidum, J. C. Thompson; Thaumaleus claparedii, Giesbrecht. Ilfracombe (Stebbing)

- thompsonii (Giesbrecht). i Monstrilla rigida, Bourne. Thaumaleus thompsonii, Giesbrecht. Plymouth (Bourne)

\section{Family Nicothoidar}

Nicothoe astaci, Audouin and Milne-Edwards. On branchiae of Astacus gammarus. Common at Ilfracombe (Stebbing)

\section{Family Corycaridar}

Corycaeus anglicus, Lubbock. Plymouth (Bourne, Cleve, Hodgson)

- venustus, Dana. Plymouth (Cleve)

\section{Family Oncaetdae}

Oncaea mediterranea (Claus). Plymouth (Bourne, Cleve)

- minuta, Giesbrecht. Plymouth (Cleve)

- subtilis, Giesbrecht. Plymouth (Cleve)

The remaining species will be named without apportionment to tribes, although obviously many of them will belong to the Caligidea and Lernaeidea.

\section{Family Lichomolgidae}

Hermannella parva, Norman and T. Scott. In Plymouth Sound, among Hydrozoa, etc. (Norman)
Family Astrrocheridae (' Ascomyzontidat)

Mesocheres anglicus, Norman and Scott. Dredged in Plymouth Sound (Norman) 


\section{CRUSTACEANS}

Sur-cLass ENTOMOSTRACA (continued)

Order Coprpoda (continued)

Family Caligidar ${ }^{1}$

Caligus rapax, Milne-Edwards. i C. elongatus, Nordmann, 1832. On salmon, cod, grey mullet. Plymouth (P. W. Bassett-Smith)

- diaphanus, Nordmann (not Baird). On Trigla hirundo and T. cuculus. Plymouth (B.-S.)

- pelamydis, Kröyer, G.p., Kroyer, Wilson, Norman and Scott; C. scomberi, Bassett-Smith. On mackerel, Plymouth (B.-S.)

- minimus, Otto. On Labrax lupus. Plymouth (B.-S.)

- curtus, O. F. Maller. C. mulleri, Leach. On cod, poor-cod, and Trigla gurnardus. Plymouth (B.-S.)

- gurnardi, Kroyer. On Trigla cuculus. Plymouth (B.-S.)

- (Sciaenophilus) tenuis, van Beneden. On Sciaena umbra. Plymouth (B.-S.)

Pseudocaligus brevipedis (Bassett-Smith). Caligus b., B.-S. On Motella tricirrata. Plymouth (B.-S.)

Lepeophtheirus salmonis (Kröyer). L. stromii (Baird). On salmon. Plymouth (B.-S.); Ilfracombe, Salcombe (Stebbing)

- pollachius, Bassett-Smith. On Gadus pollachius and Molva vulgaris. Plymouth (B.-S.)

- thompsoni, Baird. ${ }^{2}$ ? L. rhombi, Kröyer. On turbot and brill. Plymouth (B.-S.) ; Ilfracombe (Stebbing)

- pectoralis (O. F. Muller). Lermea p., Muller. On plaice, flounder, dab. Plymouth (B.-S.)

Elytrophora brachyptera, Gerstaecker. I Caligeria bella, Dana. On tunny. Outside Plymouth (B.-S.)

Trebius caudatus, Kroyer. On Raia batis. Plymouth (B.-S.)

Echthrogaleus coleoptratus (Guérin). Torbay (Stebbing)

Cecrops latreillii, Leach. On Orthagoriscus mola. Plymouth (B.-S.)

Pandarus bicolor, Leach. On Scyllium catulus. Torcross (Leach); Plymouth (B.-S.); Torbay (Stebbing)

\section{Family Dichelestridae}

Anthosoma crassum (Abildgaard). A. Smitbii, Leach. - Discovered sticking to a shark which was thrown ashore on the coast of Exmouth in Devon, by T. Smith, Esq., of Paper Buildings, Temple."3

Lernanthropus kroyeri, van Beneden. On Labrax lupus. Plymouth (B.-S.)

Hatschekia " mulli (van Beneden). Clavella m., van Beneden. On red mullet. Plymouth (B.-S.)

Congericola ${ }^{5}$ pallida, van Beneden. C. p., van B.; Cycuus pallidus, B.-S. On conger. Plymouth (B.-S.)

\section{Family Lernabidas}

Pennella ${ }^{6}$ sagitta (?) (Linn.). Penella s. (?) B.-S. On unknown fish. Plymouth (B.-S.)

1 See Bassett-Smith in Proc. Zool. Soc. London, 1899, Pp.

438-507. xxviii, 6rg (1905).

8 Encycl. Brit. (ed. 5), Supp. 406 (1816).

4 Poche, Zool. Anzeiger, xxvi, I6.

5 Stebbing, Willey's Zool. Results, pt. $\nabla, 672$ (1 900)

6 Stebbing, Soutb African Crustacea, pt. iil, 1 I 7 (1905).
Sub-CLAss ENTOMOSTRACA (continued)

Order Copepoda (continued)

Family Lernaejdar (continued)

Lernaeenicus spratta (Sowerby). Lernaconema monillaris, Milne-Edwards, B.-S. On young herring. Plymouth (B.-S.)

Lernaea branchialis, Linn. On cod, bass, whiting, haddock. Plymouth (B.-S.)

- lusci, Bassett-Smith. On Gadus luscus. Plymouth (B.-S.)

\section{Family Chondracanthidat}

Chondracanthus cornutus (O. F. Muller). On plaice, soles, and flounders. Plymouth (B.-S.)

- elongatus, Bassett-Smith. On Pleuronectes microcephalus. Plymouth (B.-S.)

- merluccii, Holten. On hake. Plymouth (B.-S.)

- zei, Delaroche. On John Dory. Plymouth (B.-S.)

- lophii, Johnston. On the angler fish. Plymouth (B.-S.)

Oralien asellinus (Linn.). On various species of gurnard. Plymouth (B.-S.)

\section{Family Splanchnotrophidar}

Splanchnotrophus gracilis, Hancock and Norman. Parasite of the nudibranch mollusc, Doris pilosa, obtained on the coast of Devonshire (H. and N.) ${ }^{7}$

\section{Family Lernaropodidae}

Lernaeopoda salmonea (Linn.). On Salmo salar. Plymouth (B.-S.)

- galei. Kröyer. On Mustelus vulgaris, Galeus vulgaris, Acanthias vulgaris. Plymouth (B.-S.)

Brachiella thynni, Cuvier. On tunny. Plymouth (B.-S.)

- insidiosa, Heller. On hake. Plymouth (B.-S.)

- bispinosa, Nordmann. On gurnards. Plymouth (B.-S.)

- triglae, Claus. On gurnards. Plymouth (B.-S)

- merluccii, Bassett-Smith. On hake. Plymouth (B.-S.)

Thysanote impudica (Nordmann). Brachiella i., Nordmann, B.-S. On gurnards. Plymouth (B.-S.)

Anchorella emarginata, Kröyer. On Clupea alosa. Plymouth (B.-S.)

- paradoxa, van Beneden. On mackerel. Plymouth (B.-S.)

- uncinata (O. F. Muller). On cod, haddock, pollock, whiting, whiting-pout. Plymouth (B.-S.)

- quadrata, Bassett-Smith. On the dragonet, Callionymus lyra. Plymouth (B.S.)

With regard to the Copepoda parasitic on fish, it will be easily seen that this chapter is principally indebted to Mr. Bassett-Smith's researches. The remarkable parasite Splancbnotropbus gracilis will, however, be a warning to the student that the parasitic Copepoda do not confine their attentions to fishes, though it is upon these hosts that they have been most easily and frequently observed. Their forms and habits are fraught with varied interest, involving as they do

7 Trans. Linn. Soc. London, xxiv, pt. ii, 49 ; pls. xv, xvi, figs. 7-10 (1863). 


\section{A HISTORY OF DEVONSHIRE}

\section{SuB-cLAss ENTOMOSTRACA (continued) \\ Order Coprpoda (continued)}

strange methods for obtaining food and lodging and other domestic advantages, any further allusion to which must be reluctantly relinquished. On the whole subject of the Copepoda of Devon a large fund of information has been supplied in the work already mentioned from the pens of Canon Norman, F.R.S., and Dr. Thomas Scott, LL.D. Conditions of time and space are in league to prevent any adequate use now and here of this important contribution. But attention may be called to the fact that in the family Thalestridae the authors have established two new genera, one called Megarthrum, with reference to an exceptionally large joint in the first foot, the other $V$ allentinia, as a tribute of well-deserved respect to Mr. Rupert Vallentin, F.L.S.

\section{SUB-CLASS THYROSTRACA}

\section{Family Balanidar}

Balanus tintinnabulum (Linn.). On ships. Torbay (Mrs. Griffiths) ${ }^{1}$

- spongicola, Brown. On upper valve of Pecten opercularis. Rame-Eddystone grounds (Pace)

- perforatus, Bruguière. Of this species Parfitt mentions also var. angustus (Gmelin) on rocks between Seaton and Beer; var. Cranchii, Leach, between Exmouth and Budleigh Salterton; and describes var. depressus on rocks at Torquay; and var. D., very much like $B$. laevis, from back of Corystes cassivelaunus

- porcatus, da Costa. Teignmouth (Parfitt). Dredged off the coast (Mrs. Griffiths)

- crenatus, Bruguière. Torbay (Mrs. Griffiths). On a large lobster taken off Torbay (Parfitt)

- balanoides (Linn.). On old shells (Parfitt), rocks between tidemarks in the Sound (Pace), Darwin's var. (a) "on the shore about three miles to the right of Shaldon (Parfitt)

Acasta spongites (Poli). Acasta Montagui, Leach. In sponges, Torbay (Walker) : in Halichondria panicea, Exmouth (Parfitt)

\section{Family Creusindae}

Pyrgoma anglicum, G. B. Sowerby. On Caryophyllia smithii (invariably, Parfitt); in Plymouth Sound (Holesworth, Todd, Pace); Barricane Bay, N. Devon (Parfitt)

\section{Sub-class THYROSTRACA. (continued)}

Family Chthamalidas

Chthamalus stellatus (Poli). Rocks between tidemarks, N. and S. Devon (Parfitt, Pace, Garstang, Stebbing)

\section{Family Verructdat}

Verruca strömia (O. F. Muller). On roots of Laminaria, upper valve of Pecten maximus, etc. (Parfitt, Pace)

\section{Family LePAdidar}

Lepas anatifera, Linn. On floating timber (Parfitt, Todd, Pace); at Ilfracombe an enormous number surrounding an old floating bottle (Stebbing)

- anserifera, Linn. On ships ; 'taken on driftwood by Col. Montagu, on the south coast' (Parfitt)

- pectinata, Spengler. On various floating objects. Torbay (Mrs. Griffiths)

- fascicularis, Ellis and Solander. On floating objects, the peduncle often surrounded by a vesicular ball of cement tissue. Bovisand (Miss H. Bellamy)

Conchoderma auritum (Linn.). Lepas cornuta, Montagu. Stranded vessel, coast of Devon (Montagu)

- virgatum (Spengler). Lepas membranacea, Montagu. On stranded vessel, S. Devon (Montagu); on wreck, Torbay (Mrs. Griffiths) ; Exmouth (Mrs. Luscombe); Teignmouth, alive on ship's bottom (Parfitt)

\section{Family Pollicipedidar}

Scalpellum vulgare, Leach. On various Hydroids, Plymouth, Torbay (Parfitt, Todd, Pace)

Pollicipes cornucopia, Leach. On vessel towed into Dartmouth Harbour (Montagu); Goodrington, Torbay (Mrs. Griffiths)

\section{Family Trypetraidar}

Trypetesa lampas (Hancock). Alippe l., Hancock ; Trypetesa l., Norman. Taken, embedded in Fusus antiquus and Buccinum undatum, off the Eddystone Lighthouse, by Mr. Spence Bate

\section{Family Peltogastridae}

Peltogaster paguri, Rathke. On Eupagurus bernhardus. Saunton sands, N. Devon (Stebbing) Pachybdella carcini, Thompson. Sacculina c., Thompson. On Carcinus maenas, Starcross (Norman), Plymouth (Pace, Garstang)

Considering the extensive material with which this chapter has been forced to deal it is perhaps fortunate that the freshwater Cladocera, Ostracoda, and Copepoda of Devon have been so long neglected. They will in course of time tend largely to inflate the record of Devonian carcinology. The most liberal allowance of space for this subject has still made it necessary to offer the reader rather a sort of scientific pemmican than anything that can assume the guise of literature. Those who remember the fascinating diffuseness of Gosse and Kingsley will be inclined to frown and then to smile at the contrast between that recollection and the uninspiring columns here presented of technical names and recurring references. None the less, the eager student will find in these names and notes finger-posts to guide him through a country, difficult but delightful to explore, one which has been opened up by men of energy and genius, yet one no doubt still full of hidden treasures and unrevealed beauties.

${ }^{1}$ Parfitt in Trans. Devon. Assoc. iv, pt. 2, 548 (1871); to this paper and to the list in the Journ. Mar. Biol. Assoc. for 1904, these records of Cirripedes are almost entirely due. 


\section{F I S H ES}

The list of Devonshire fishes is rather a long one, a number of southern species, many of which have their headquarters in the Mediterranean, occurring either as regular or occasional visitors. A number of species recently added to the British fauna have been found off the Devonshire coasts in the course of the collecting work of the Plymouth Laboratory, and many of the records given in the list below are taken from the fournal of the Marine Biological Association, to which the Laboratory belongs. The writer was a member of the staff of the Laboratory from 1887 to 1895 .

These recent discoveries include Zeugopterus norvegicus, Arnoglossus grobmanni, Callionymus maculatus, Syngnatbus rostellatus, and Gadus esmarkii.

Among the characteristic southern forms which are plentiful are the red mullet, the tub or latchet among the gurnards, the sole, turbot and brill, the pilchard and the mackerel. There is an extensive pilchard fishery at Plymouth, but few are taken east of that district. The question whether the pilchard is the same as the sardine is often asked and seldom answered correctly. The French sardine of commerce is prepared on the west coast of France between Brest and Bordeaux, and the fish are caught in summer and autumn. They are small fish from 5 to 7 inches in length, and are the young of the same species as the pilchard. Pilchards of the same size occur off the coast of Devon and Cornwall, but only in comparatively small numbers, or at irregular intervals, while on the other hand the larger pilchards, or sardines, 7 to 10 inches long, are caught on the coast of France in winter, as well as on the coasts of Devon and Cornwall. Both in Devon and in France the larger fish are caught with ordinary drift nets, but in French waters the small summer fish are caught by a somewhat different method, a single net being shot at a time and bait, consisting of salted cod's roe, being thrown into the water to attract the fish. Sardines also occur in the Mediterranean, but there they are a smaller race, though of the same species, and do not exceed 7 or 8 inches in length when mature.

Many northern species are either scarce or absent from Devon waters, or, if present, do not reach so large a size or such fine condition for the table. The plaice are smaller than those of the northern part of the North Sea, cod are not plentiful and not so fine, the haddock is rare. The whiting, on the other hand, is abundant and well developed.

An asterisk prefixed to a name denotes a freshwater species; two asterisks indicate occurrence in both fresh and salt water. 


\section{A HISTORY OF DEVONSHIRE}

\section{TELEOSTEANS}

\section{ACANTHOPTERYGII}

* I. Perch. Perca Auviatilis, Linn.

Common.

**2. Sea Bass. Morone labrax, Linn. (Labrax lupus, Day).

Fairly common, and grows to a large size. It enters the estuary of the Tamar, and ascends a considerable distance from the sea. Fine specimens are usually to be seen in the Plymouth Aquarium. It is mostly taken by seine, or by hook and line.

3. Comber. Serranus cabrilla, Linn.

According to Day it is common at Plymouth. There are specimens from that port in the British Museum (Natural History).

4. Stone Bass. Polyprion americanus, Bl. Schn. ( $P$. cernium, Day)

According to Holdsworth, not uncommon. A specimen, $20 \frac{1}{2}$ inches long, was taken by hook and line off Plymouth in 1892 (Fourn. $M . B . A$. vol. ii.). It is sometimes called wreck-fish from its habit of following floating wreckage, especially timbers covered with barnacles, which appear to be the attraction to the fish.

\section{Shade-fish. Sciana aquila, Lacép.}

Occasional specimens; one over roo lb. weight, was taken in 1850 at Brixham; others have been captured at Teignmouth, at Beer, at Dartmouth, etc. (Day).

\section{Black Sea Bream. Cantharus lineatus,} Montagu.

One of the occasional visitors. A young specimen only $6 \frac{1}{4}$ inches long was taken by Mr. Holt in July 1897 at the mouth of the Lynher in the Hamoaze.

7. Bogue. Box vulgaris, Cuv, and Val.

A rare visitor. One was taken near Plymouth in 1872 (Day).

8. Common Sea Bream. Pagellus centrodontus, De la Roche.

Very common, taken both in trawls and on hooks. The young are called chad, and abound in summer and autumn. In these the black shoulder spot is not developed, and many suppose them to be of a distinct species.

9. Spanish Bream. Pagellus bogaraveo, Brunn.

As it is not uncommon off the Cornish coast, it probably occurs in Devon waters occasionally.
I0. Axillary Bream. Pagellus owenii, Gunther. Has been recorded (Day).

I I. Pandora. Pagellus erytbrinus, Linn. Common in summer and autumn.

12. Gilt-head. Pagrus auratus, Linn.

Another rare visitor. Two specimens obtained by Montagu, in 1802, at Torcross.

I3. Red Mullet. Mullus barbatus, Linn., var. surmuletus, Linn.

Fairly common, taken in seines and trawls. The striped variety is the one which occurs. The writer does not recollect seeing the plain form (barbatus) at Plymouth, though it is said to have been taken in Berwickshire and in Cornwall. The evidence seems to prove that the plain variety is the Mediterianean form, the striped that of the Atlantic. It is interesting to watch these fish in the Plymouth Aquarium using their two stiff barbels to probe the gravel in search of shrimps or other prey.

I4. Ballan Wrasse. Labrus maculatus, Bloch.

Very common on weedy ground. It is very variable in colour, one variety, called the comber, having a white band along the side from the eye to the tail, while the Labrus lineatus of Donovan is green with yellow streaks. Mr. Holt (Fourn. M.B.A. v. 193) has shown that the colour of the same individual is highly variable. A specimen taken in Zostera was uniformly green, then changed to olive grey, with dark transverse bars, and afterwards showed a pale round spot on several of the scales. The most frequent coloration is brown or green with a bright spot on every scale.

15. Cook or Cuckoo Wrasse. Labrus mixtus, Linn.

Fairly common. The colours of the male are very brilliant, red with blue stripes. The female is yellow with three black spots on the hinder part of the back.

16. Conner. Crenilabrus melops, Linn.

Very common.

17. Jago's Goldsinny. Ctenolabrus rupestris, Linn.

Common.

18. Rock Cook. Centrolabrus exoletus, Linn.

Not uncommon. In Salcombe estuary.

19. Rainbow Wrasse. Coris julis, Linn.

A Mediterranean species. Specimens obtained by Leach from south Devon are in the British Museum. 


\section{FISHES}

*20. Miller's Thumb. Cottus gobio, Linn.

Occurs in streams and brooks.

21. Father-lasher, Short-spined Cottus. Cottus scorpius, Linn.

Much less common than Cottus bubalis, if it occurs at all.

22. Long-spined Bull-head, or Cottus. Cottus bubalis, Euphr.

Very common on the shore, and in shrimptrawls. Specimens from deep water are often of a beautiful carmine colour with white spots.

23. Grey Gurnard. Trigla gurnardus, Linn. Common on the trawling grounds.

24. Piper. Trigla lyra, Linn.

Not so common. Reaches a larger size.

25. Tub, Latchet, or Sapphirine Gurnard. Trigla birundo, Linn.

Fairly abundant, and much more valuable in the market than the common gurnard. The latter is sold as inferior or offal fish, the tub as prime.

26. Streaked Gurnard. Trigla lineata, Gmel. Not uncommon.

27. Red Gurnard or Elleck. Trigla cuculus, Linn.

Common.

28. Lanthorn Gurnard. T rigla obscura, Linn.

Parnell obtained several from the Brixham trawlers, to whom it was known as the longfinned captain. It is not abundant. A number of specimens in the Plymouth market in March 1897, were said to have been caught on rough ground off the Start (Fourn. M.B.A. vol. v.)

29. Pogge, or Armed Bull-head. Agonus cataphractus, Linn.

Not so common as on the east coast.

30. Armed Gurnard. Peristetbus cataphractus, Gmel.

A Mediterranean species. One specimen was taken in 1836 in a trawl between $\mathrm{Ply}$ mouth and the Eddystone.

31. Lumpsucker. Cyclopterus lumpus, Linn.

Abundant on the east coast of Scotland, comparatively rare on the coasts of Devon.

32. Sea Snail. Liparis vulgaris, Flem.

Has been taken at Exmouth and Plymouth.
33. Montagu's Sucker. Liparis montagui, Cuv.

Originally discovered by Colonel Montagu at Milton on the south coast of Devon.

34. Two-spotted Goby. Gobius ruthensparri, Euphr.

Abundant in Zostera beds in the estuaries.

35. Willughby's Goby. Gobius paganellus, Gmel.

Under rocks and stones on the shore.

36. Rock Goby. Gobius niger, Linn.

Common in estuaries.

37. Freckled Goby. Gobius minutus, Gmel.

Common on sandy ground and taken abundantly in shrimp nets. The breeding habits of this species have been fully described by Guitel (Arch. Zool. Exp. 1892, 1895). The male scoops out a cavity beneath a pecten shell, and guards the eggs which the female attaches to the lower surface of the shell.

38. Parnell's Goby. Gobius parnelli, Day.

Parnell stated that his species occurred plentifully on the south coast. According to Holt it is the estuarine race of Gobius minutus, Gmel.

39. Painted Goby. Gobius pictus, Malm.

Mr. E. W. L. Holt found this species common in Plymouth Sound, although it had only been previously recorded from British waters at Colwyn Bay in Wales.

40. Jeffreys' Goby. Gobius jeffreysii, Günther.

A rather deep-water species taken by $\mathrm{Mr}$. Holt in about 23 fathoms south by east of the Mewstone.

4I. Transparent Goby. Apbia pellucida, Nardo.

Abundant in Cawsand Bay in July. An adult male $2 \frac{1}{4}$ inches long was taken at the surface in a tow-net south of the Mewstone in June 1894, but the species lives usually near the bottom. According to Collett it is an annual, reaching maturity in one year, and dying after breeding.

42. Crystal Goby. Crystallogobius nilssonii, Dub. and Kor.

Discovered by the writer on the Eddystone trawling grounds. It had previously been described from Norwegian waters by Collett, and found in Ballinskellig's Bay, Ireland, by Holt. Also an annual according to Collett. The writer took $20 \mathrm{r}$ specimens in a single haul of a fine meshed trawl (mosquito netting) 


\section{A HISTORY OF DEVONSHIRE}

at night on 9 July 1891 ; depth 28 fathoms, bottom sand. Only thirteen were adult males.

\section{John Dory. Zeus faber, Linn.}

Common. Fine specimens are taken by the trawlers, and small ones by the shrimpers in summer.

\section{Boar-fish. Capros aper, Linn.}

In summer time is often very abundant on the trawling grounds. It seems to have been rare or at least less common before the year I 840, when it was noted by Mr. Gatcombe in the Zoologist that the Plymouth trawlers were throwing overboard hundreds of them. It is probable that they were known to the fishermen before but not to naturalists.

45. Mackerel. Scomber scombrus, Linn.

There is a large fishery for mackerel off Plymouth, both in summer and winter. The Cornish boats fish there in the latter season until the middle of January. After the spawning season, from about the end of June to the end of September, mackerel are taken on hooks by whiffing, and at this season they approach the shore and enter Plymouth Sound. At this time they are often taken in seines.

46. Spanish Mackerel. Scomber colias, Gmel.

Occurs in small numbers. The Spanish mackerel in America is a different species, namely Cybium maculatum.

47. Tunny. Thunnus thynnus, Linn.

A specimen 9 feet long was taken near Plymouth in 1873 , another $8 \mathrm{ft} .9$ in. long at Dawlish in 1868 (Day). A specimen 3 feet long was brought to the Plymouth Laboratory in September I894. It was a female with the ovaries small and immature. There were a large number of Trematodes on the gills, and specimens of Brachilla thynni behind the pectoral fins.

48. Germon or Long-finned Tunny. Thunnus germo, Lacépède.

A few specimens recorded. In August, 1865 , one 24 inches long was killed a little way up the river Exe.

49. Bonito. Thunnus pelamys, Linn.

A tropical oceanic species of which specimens have wandered to the coast of Devon. In September 1876 one $20 \frac{1}{2}$ inches long was taken in a trammel in the Cattewater at Plymouth, and a few larger specimens have been taken there (Day).

50. Pelamid. Pelamys sarda, Bloch.

Probably occurs occasionally, as it has been taken on the south coast.
51. Plain Bonito. Auxis rocbei, Risso.

Occurs occasionally. A specimen was obtained at the Plymouth Laboratory in August 1894, having been taken with mackerel in a seine at Mount Batten. It was a female 16 inches in length.

52. Scabbard-fish. Lepidopus caudatus, Euphr.

The first recorded British specimen was captured at Dawlish in 1787 ; another $5 \frac{1}{2}$ feet long was taken at Salcombe (Day).

\section{Silvery Hair-tail. Tricbiurus lepturus, Linn.}

Occasional specimens recorded; one $2 \mathrm{ft}$. 8 in. long in January 1876 , in the Hamoaze.

54. Sword-fish. Xiphias gladius, Linn.

A specimen over 7 feet long was taken in 1863 in pilchard drift-nets off Plymouth (Day).

\section{Ray's Bream. Brama raii, Bl. Schn.}

A deep-water form thrown on shore or found in shallow water after storms. Has been recorded from Devonshire by Montagu.

56. Opah or King-fish. Lampris luna, Gmel.

An occasional straggler from deep water. One $4 \mathrm{ft} .5 \mathrm{in}$. long and weighing $140 \mathrm{lb}$. recorded as taken at Brixham in 1772 .

57. Scad or Horse-Mackerel. Caranx trachurus, Linn.

Common, taken in mackerel nets.

58. Pilot-fish. Naucrates ductor, Linn.

Was recorded from Dartmouth harbour in I818. Many have accompanied ships into the Cattewater, Plymouth (Day).

59. Blackfish. Centrolophus pompilus, Linn.

A specimen taken in June 1877 in a seine at Babbicombe. Is observed to accompany sharks.

60. Greater Weever. Tracbinus draco, Linn.

Common on sandy ground, but the writer has not seen it in such numbers at Plymouth as in the produce of the trawl at Lowestoft.

\section{Lesser Weever, Viper Weever. Trach-} inus vipera, Cuv, and Val.

Common in shrimp trawls. The weevers bury themselves vertically in the sand and erect the spinous dorsal fin when alarmed. It is however the spine at the top of the operculum which is poisonous, and the writer knows from experience that the pain caused by a prick from this spine is very intense for a few minutes, extending up the arm to the 


\section{FISHES}

shoulder. After a while the pain subsides and the wound heals without further trouble.

62. Dragonet or Skulpin. Callionymus lyra, Linn.

Very common. An interesting description of the habits and behaviour of this fish in courtship has been published by Holt (Proc. Zool. Soc. 1898).

63. Spotted Dragonet. Callionymus maculatus, Bonap.

One specimen trawled off the Mewstone in 1898 , three specimens off Plymouth later in the same year. It is common in the Mediterranean.

64. Cornish Sucker. Lepadogaster gouanii, Lacép.

Common.

65. Double-spotted Sucker.

Lepadogaster bimaculatus, Flem.

Not uncommon.

66. Cat-fish or Wolf-fish. Anarrbichas lupus, Linn.

Parfitt states that it has been taken in Plymouth Sound, but no specimen has been recorded at the Plymouth Laboratory.

67. Gattorugine. Blennius gattorugine, Bloch. Not uncommon in rather deep water.

68. Montagu's Blenny. Blennius galerita, Linn.

Fairly common, in rock pools.

69. Butterfly Blenny. Blennius ocellaris, Linn.

Not uncommon in rather deep water. Several times shells have been dredged in which a male of this species was guarding the eggs, and once the nursery was a hollow beef bone.

70. Shanny. Blennius pholis, Linn.

Common in rock pools and under stones between tide-marks.

71. Butterfish or Gunnel. Centronotus gunnellus, Bl. Schn.

Common between tide-marks.

72. Vivaparous Blenny. Zoarces viviparus, Linn.

A northern species; only one specimen recorded from the coasts of Devon.

73. Red Band-fish. Cepola rubescens, Linn.

Not uncommon. Has been taken at Salcombe, in the Exe, at Brixham, and at Plymouth. Two specimens were caught in
Plymouth Sound in December 1897, 10 $\frac{3}{4}$ and $12 \frac{1}{8}$ inches long, both females.

74. Angler, Fishing-frog, Monk-fish, or Seadevil. Lophius piscatorius, Linn.

Common. The spawn of this fish is shed in the form of a gelatinous band several feet in length; it has been found floating at the surface off Mevagissey, but is also occasionally taken in the trawl.

\section{ANACANTHINI}

75. Cod. Gadus morrbua, Linn.

A considerable number of cod are taken both by trawl and by hook, but they are not usually so large nor of so fine a quality as those from the North Sea.

76. Haddock. Gadus aglefinus, Linn.

Seldom caught, though said to have been abundant before 1870 off the coast of Cornwall.

77. Bib, Pout, Whiting-pout. Gadus luscus, Linn.

Very common. In the aquarium this fish very often develops a peculiar affection of the eyes; the membrane over the cornea becomes distended and bulges out. The same thing is observed to some extent in the dead fish after capture.

78. Power, or Poor Cod. Gadus minutus, Linn.

Equally common with the bib, to which it is at first sight very similar.

\section{Whiting. Gadus merlangus, Linn.}

This fish is as characteristic of the south coast as the haddock of the east. It affords good fishing with hook and line both to professionals and amateurs, and is also largely taken by the trawlers.

8o. Coal-fish. Gadus virens, Linn.

A northern species of extensive range, not uncommon off the Devon coast.

81. Pollack. Gadus pollachius, Linn.

A characteristic Devon fish, abundant and of large size.

82. Norway Pout. Gadus esmarkii, Nilsson.

A specimen taken in a ground seine in the Hamoaze in August I900; it was actually landed on the Cornish shore between Saltash and the river Lynher. Originally discovered on the Norwegian coast, the species was round in considerable numbers on the west coast of Scotland in 1888 , on the west of Ireland in 1890 and 1891 . In 1889 speci- 


\section{A HISTORY OF DEVONSHIRE}

mens were taken from the stomach of a pollack caught 40 miles north-west of St. Ives. The Hamoaze specimen is the first recorded from the English Channel, and the first obtained in Britain from the shallow water of an estuary.

83. Hake. Merluccius vulgaris, Cuv.

A characteristic southern gadoid. Not so abundant now as formerly. Accounts agree in stating that 40 or 50 years ago it was plentiful in the inshore waters of Devon and Cornwall, where now it is comparatively scarce. Large numbers are landed at Milford Haven by the deep-sea steam trawlers.

84. Greater Fork-beard. Pbycis blennoides, Bl. Schn.

Rare, and usually a deep-water fish; one however was trawled in Cawsand Bay according to Mr. Holt (Fourn. M.B.A. v. I47).

85. Ling. Molva vulgaris, Fleming.

Common; large specimens are taken in deep water.

86. Five-bearded Rockling. Motella mustela, Linn.

Not uncommon.

87. Four-bearded Rockling. Motella cimbria, Linn.

According to Holt certainly occurs in the Plymouth district. A small species not known to exceed 14 inches in length.

88. Three-bearded Rockling. Motella tricirrata, Bl.

Quite common. The young are found at the surface in June; they are silvery with elongated pectoral fins, at the end of which are black spots. At a later stage up to 6 or 7 inches in length the fish are of a uniform dark colour and are found under stones between tide marks. The adults which reach 20 inches in length are light chestnut in colour with a number of black spots or blotches. These are found in deep water, and the variegated colouring is very striking. The young in the surface-swimming stage are known as mackerel midges and were described as a distinct species under various names.

89. Motella maculata, Risso.

Occurs as a distinct species according to Holt. Day considers it ' in part' a synonym of tricirrata.

9o. Lesser Fork-beard. Raniceps raninus, Linn.

Occasional specimens occur. One is recorded from Plymouth (Day).

\section{PLEURONECTOIDEI}

91. Halibut. Hippoglossus vulgaris, Flem.

A northern deep-water fish, which is but rarely taken on the coast of Devon.

92. Long Rough Dab. Hippoglossoides limandoides, $\mathrm{Bl}$.

Day says he received specimens from Brixham, and it is certainly landed by the trawlers occasionally both at Brixham and Plymouth, but in these cases it may be caught on distant grounds. It is a northern species.

\section{Turbot. Rbombus maximus, Linn.}

Common, and reaches a large size. The young in pelagic condition and in process of metamorphosis have often been taken in Sutton Pool and other inlets of Plymouth Sound, and taken alive to the Plymouth Laboratory.

94. Brill. Rhombus leevis, Gottsche.

Taken with the turbot by trawlers. The young in pelagic condition are more common than those of the turbot.

\section{Common Topknot. Zeugopterus punctatus,} Bl. (Rbombus punctatus, Gunther).

Not uncommon in Plymouth Sound and outside it.

\section{One-spotted Topknot. Zeugopterus uni- maculatus, Risso.}

Three specimens, one male and two females, trawled by Holt in June 1897 in Teignmouth Bay. One specimen, a male, 4 miles south of Mewstone, Plymouth, in May I 898 .

97. Norway Topknot. Zeugopterus norvegicus, Günther.

Five specimens trawled in summer in $189 \mathrm{I}$ and 1892 a few miles south of Plymouth Breakwater.

98. Sail-fluke or Megrim. Lepidorhombus megastoma, Donov.

This species is taken in considerable numbers by the Plymouth trawlers. The first name was given to it from its alleged habit of floating to the shore in the Orkneys with its tail erected like a sail. It is called megrim by the Plymouth fishermen, a name given by Couch to Arnoglossus laterna.

99. Scald-fish or Scald-back. Arnoglossus laterna, Walb.

This species is very common off the coast, the young in shallow waters on sandy ground, the adults on the trawling grounds. The adult male is distinguished by the elongation of the anterior rays of the dorsal fin, and of 


\section{FISHES}

the rays of the pelvic fins. This was originally described as a distinct species, Arnoglossus lophotes, Gunther. The young are sometimes mistaken for young soles.

100. Broad Scald-fish. Arnoglossus grobmanni, Bonap.

One specimen was obtained by the writer at Plymouth; six specimens were obtained by Holt in the same neighbourhood in 1897 and 1898 .

101. Plaice. Pleuronectes platessa, Linn.

Fairly abundant. The plaice of the south coast are smaller than those of the northern part of the North Sea, but about the same size as those trawled off Lowestoft. The males are smaller than the females.

102. Lemon Dab. Pleuronectes microcephalus, Donovan.

This fish is usually known as the lemon sole in the fish trade, but, as it is not a species of sole, but allied to the dab and plaice, the above name is more appropriate. It is caught in considerable numbers on the Devon trawling grounds.

\section{Dab. Pleuronectes limanda, Linn. Common.}

104. Witch or Pole Dab. Pleuronectes cynoglossus, Linn.

A northern deep-water species which is rare on Devon coasts, if it occurs at all. Day states that he has obtained it from Brixham, but as mentioned above the trawlers there go to distant grounds.

105. Flounder. Pleuronectes flesus, Linn.

Common in all the estuaries, and in the spawning season, February to May, on the trawling grounds up to a depth of 30 fathoms.

106. Sole. Solea vulgaris, Quensel.

Common and of fine size and quality.

107. Lemon Sole, Sand Sole, French Sole. Solea lascaris, Risso.

This is never called lemon sole by the fishermen or dealers, but usually sand sole. It is distinguished by the dilated and fringed nostril on the blind side. Only occasional specimens are captured on the trawling grounds.

108. Thickback. Solea variegata, Donovan.

A very characteristic Devon species. Although small it is excellent for the table, but is consumed locally, not being valuable enough to repay carriage to distant markets.
I09. Solenette. Solea lutea, Risso.

Very common. Half-grown specimens are usually taken in shrimp trawls in Plymouth Sound, and are often believed by the fishermen to be the young of the common sole.

\section{PLECTOGNATHI}

110. Sun-fish. Orthagoriscus mola, Linn.

Specimens are occasionally taken on the south coast during summer and autumn. One was captured some miles up the Exe in 1877 .

\section{PERCESOCES}

I I . Thick-lipped Grey Mullet. Mugil cbelo, Cuv.

Very common in estuaries and docks. When the dry dock at Plymouth was pumped out numbers of fine specimens were often taken. The young are found as 'brit' at the surface outside Plymouth breakwater. Living specimens are usually kept in the Plymouth Aquarium.

\section{2. Thin-lipped Grey Mullet. Mugil} capito, Cuv.

Day states that he examined many specimens obtained from Brixham, but the writer never recognized it at $\mathrm{Plymouth}$.

I 13. Sand Smelt or Atherine. Atherina presbyter, Jenyns.

Abundant in creeks and estuaries.

1 4. Larger Launce or Sand-Eel. Ammodytes lanceolatus, Lesauvage.

Common on sandy shores.

I 15. Lesser Launce or Sand-Eel. Ammodytes tobianus, Linn.

More common than the larger species. Specimens can be obtained from the sand at low water by digging with a walking stick or other implement, but they are caught in numbers with a small meshed seine. Sandeels caught at Slapton Sands are sold in Dartmouth for food. They are also largely used as bait.

\section{6. Garfish. Belone vulgaris, Fleming.}

Taken frequently in summer in mackerel nets, and at times in seines. Young specimens about 3 inches long were taken with sprats in a seine in Hamoaze in September I 894 .

I 7 . Saury Pike or Skipper. Scombresox saurus, Bl. Schn.

The writer has not seen it, but Parfitt states 


\section{A HISTORY OF DEVONSHIRE}

that a number were taken in a seine at Seaton in 1870 (Day).

I 8 . Greater Flying-Fish. Exocatus volitans, Linn.

In I 849 according to Day a specimen was left by the tide in Stonehouse Pool, and Couch states that another was thrown on the quay at Plymouth.

\section{HEMIBRANCHII}

**I I g. Three-spined Stickleback. Gasterosteus aculeatus, Linn.

Common. A specimen taken in the dock at Exmouth with grey mullet in Igor.

*I20. Ten-spined Stickleback. Gasterosteus pungitius, Linn.

Occasional.

12 I. Fifteen-spined Stickleback. Gasterosteus spinacbia, Linn.

Common in Plymouth Sound and the other estuaries.

\section{LOPHOBRANCHII}

122. Broad-nosed Pipe-fish. Siphonostoma typhle, Linn.

Common in Zostera beds, where its upright attitude, slow waving movements and green colour, give it a resemblance to the fronds of the weed.

123. Greater Pipe-fish. Syngnatbus acus, Linn.

Common among Fucus and other brown seaweeds. Taken in shrimp trawls.

124. Syngnatbus rostellatus, Nilsson.

Common in the Exe estuary, the river Yealm, and probably other localities. The species has only recently been distinguished in British waters, having doubtless been previously confounded with Syngnatbus acus. In the latter the number of preanal bony rings is $19-20$, in rostellatus $13-15$.

125. Snake Pipe-fish. Nerophis equoreus, Linn.

Common.

I 26. Straight-nosed Pipe-fish. Nerophis ophidion, Linn.

Common.

127. Worm Pipe-fish. Nerophis lumbriciformis, Yarr.

Common under stones between tide-marks.

128. Sea-horse. Hippocampus antiquorum, Leach.

One specimen recorded from Devon by
Parfitt, but none have been obtained at the Plymouth Laboratory.

\section{HAPLOMI}

129. Pike. Esox lucius, Linn.

In Slapton Ley and the Exe.

\section{OSTARIOPHYSI}

* I 40. Carp. Cyprinus carpio, Linn.

Occurs in lakes and ponds.

*131. Crucian Carp. Cyprinus carassius, Linn.

Gold-fish (var, auratus).

In ornamental ponds, etc.

*132. Gudgeon. Gobio fluviatilis, Flem.

Introduced according to Day, who mentions that a specimen $7 \frac{1}{2}$ inches long was taken among herrings at Clovelly.

*133. Roach. Leuciscus rutilus, Linn.

In Slapton Ley, the river Axe, the Exeter canal and railway ponds.

"134. Chub. Leuciscus cepbalus, Linn.

Rare according to Day.

* 35. Dace. Leuciscus dobula, Linn.

Abundant in rivers.

* 1 36. Rudd. Leuciscus erythrophtbalmus, Linn.

Abundant in Slapton Ley.

*137. Minnow. Leuciscus phoxinus, Linn.

Common.

*138. Tench. Tinca vulgaris, Cuv.

In the Exeter Canal, and some ponds of the South Devon Railway (Day).

*139. Loach. Nemacbilus barbatulus, Linn. Occurs.

\section{MALACOPTERYGII}

**140. Salmon. Salmo salar, Linn.

In the Exe, Teign, etc. There is a regular salmon fishery in the Exe which is carried on by several large rowing boats each manned by four to six men, and each working one seine. The net is about Ioo fathoms long, 3 to 4 fathoms deep in the middle, and I fathom deep at the ends, with a mesh of $4 \frac{1}{4}$ inches. The net is shot across the stream, then towed down with the tide two or three hundred yards and hauled.

**141. Peal. Salmo trutta, Linn., var. cambricus, Donovan.

Common, ascending the estuaries of the Tamar, Tavy, Exe and other rivers. 


\section{FISHES}

*142. Trout. Salmo fario, Linn.

In the rivers, e.g. the Dart.

143. Anchovy. Engraulis encrasicbolus, Linn. Appears to be present in the Channel off the coast of Devon every year in autumn and winter. In November and December I 889 , considerable numbers were taken with sprats in the sprat seines in Torbay ; one-fifth of the whole catch was stated to consist of anchovies. The fishermen of Plymouth catch a few anchovies from time to time every year in winter, both in pilchard and mackerel nets. In November 1890, 584 anchovies were caught at one time by a pilchard boat, and on another occasion 500. There is a regular fishery for anchovies in summer in the Zuyder Zee in Holland, and there is every reason to believe that these fish migrate in autumn from there into or through the English Channel. Small-meshed drift nets were obtained and used by the staff of the Plymouth Laboratory in 1891 and 1892 , but only a few anchovies were caught with these. It would seem that the route of migration of these fishes is in most years some distance to the south of the Devon coast, so that only stragglers are caught.

\section{Herring. Clupea harengus, Linn.}

Herrings visit the coast of Devon only at one season of the year, namely the winter. They spawn regularly in Bigbury Bay from the beginning of January onwards to the middle of March, and ripe fish are sometimes caught in the Channel even later. The fish enter Plymouth Sound and the Hamoaze as early as September, and fishing is carried on in the Sound with drift-nets from small boats. They are also caught with moored nets in Cawsand Bay. The naturalists of the Plymouth Laboratory did not succeed in dredging the spawn, but the young fish, not long hatched, were frequently taken at the surface in January and February.

\section{Sprat. Clupea sprattus, Linn.}

Caught in large numbers in the autumn and winter in Torbay and in the Hamoaze. Many were taken in small-meshed nets by the employes of the Plymouth Laboratory between September 1891 and January 1892, off Plymouth.

The egg of this species, like that of the pilchard, is pelagic, and is taken off Plymouth from January to April. The fish migrate from the coast to the open sea in the spawning season, and are not caught in breeding condition by the fisherman. The few spawn- ing specimens that have been captured were taken in the trawl.

146. Pilchard. Clupea pilcbardus, Walb., Artedi.

There is a regular fishery for pilchards at Plymouth from about August to Christmas, many Cornish boats making this port their headquarters for this fishery. The proof that the eggs of this fish are buoyant and pelagic was obtained at the Plymouth Laboratory by the present writer in the years 1887 to 1894. The spawning fish are seldom taken in the ordinary course of the fishery, but in mackerel nets in June it often happens that a few ripe pilchards are taken. In the writer's experience only the females are caught in this way, the distended abdomen being too large to pass through the mackerel mesh. Ripe fish of both sexes were however obtained on one occasion in a pilchard net at the beginning of September 10 miles south of the Eddystone.

The sardines prepared in tins on the west coast of France are young pilchards, 5 to 7 inches in length. Fish of this size have been taken off Plymouth, but not in numbers suffciently large to be commercially important. In November I 891 several hundred pilchards of this size were taken in small-meshed nets belonging to the Plymouth Laboratory and shot at various positions south of Plymouth Breakwater. Still smaller specimens, some less than 3 inches in length, have been taken in seines in the Hamoaze in September.

147. Allis Shad. Clupea alosa, Linn.

Eight specimens 13 or 14 inches long taken in a mackerel seine in Plymouth Sound in August 1893. Four more in September from a seine in the Hamoaze.

148. Twaite Shad. Clupea finta, Cuv.

Caught by whiffing with a slice of mackerel at the mouth of the Dart according to Holdsworth.

149. Maurolicus pennantii, Cuv. and Val.

A single specimen has been taken off Devonshire, as recorded by Walcott (Yarrell).

\section{APODES}

**150. Eel. Anguilla vulgaris, Turton.

Common. The eggs and larvæ have been proved in Sicily to be developed in the sea, but have not been identified yet in British waters. The young eels just after their metamorphosis, from 2 inches in length upwards, are common under stones on the shore from February onwards. 


\section{A HISTORY OF DEVONSHIRE}

I 51. Conger. Conger vulgaris, Cuv.

Very common, often abundant. Usually taken on long lines baited with pilchard or squid. Small specimens from 18 inches to about $2 \frac{1}{2}$ feet long are called straps by the fishermen.

Specimens are regularly kept alive in the Plymouth Aquarium. The present writer made observations and experiments upon them there in order to study their breeding and life history. Males were found not to exceed $2 \frac{1}{2}$ feet in length, and several became sexually mature in the aquarium. They could be distinguished from females of the same size by the blunter snout and larger eyes. They lived for about six months without feeding, gradually getting thinner and feebler, till they died. Females also ceased to feed when the roes were developing, and lived for about six months in a fasting condition. Then they died with the abdomen much distended by the roes, but the eggs were not quite ripe, and were not spawned.

A specimen of the larva formerly known as Leptocephalus morrisii was caught on the shore in front of the Plymouth Laboratory in June, 1895, and is described in the Fourn. $M . B . A$. of that year (iv. 73).

152. Muraena belena, Linn.

A specimen $44^{\circ} 6$ inches long was brought to the Plymouth Laboratory in March 1897 , with the information that it was trawled off the Eddystone and was just alive when brought on deck. It proved to be a male with ripe testes. The whole body was very soft and flabby (Fourn. M.B.A. v. 91).

\section{GANOIDS}

**153. Sturgeon. Acipenser sturio, Linn. Occasionally taken on the Devonshire coast, according to Parfitt.

\section{CHONDROPTERYGIANS}

154. Blue Shark. Carcharias glaucus, Linn.

On the mackerel grounds off the Cornish coast this species is decidedly common, often damaging the drift-nets, but it is seldom seen near the shore. It occurs also off the Devon coast. One 8 feet long was taken off Plymouth in 1876 (Gatcombe, Zool. 1877) and another a little smaller in 1883 .

155. Tope. Galeus vulgaris, Flem.

Not uncommon on the trawling grounds.

I56. Hammer-head. Zygrena malleus, Risso.

A specimen $13 \mathrm{ft}$. 7 in. long was taken at Ilfracombe in 1865 . It was found floundering among the rocks and was secured by means of ropes.

157. Rough Hound, or Small-spotted Dogfish. Scyllium canicula, Linn.

Very common. Taken both in the trawl and on long lines.

158. Nurse Hound, or Large-spotted Dogfish. Scyllium catulus, Cuv.

Common, but not so abundant as the last species. Both these species are oviparous, producing rectangular eggs with tendrils at the corners of the shell. In Falmouth Harbour catulus attaches its eggs to stems of Laminaria which are uncovered at low water in spring tides. The eggs of canicula are sometimes dredged, attached to growths of Gorgonia.
159. Smooth Hound. Mustelus vulgaris,
Müller and Henle. Occurs frequently.

160. Porbeagle. Lamna cornubica, Gmel.

Two or three taken at Clovelly in 1871, and one at Plymouth in 1835 .

16r. Thrasher. Alopias vulpes, Gmel.

Recorded at Teignmouth Bay, off the Start, at Dawlish and Plymouth; specimens from $4 \mathrm{ft}$. 7 in. to $14 \mathrm{ft}$. in length. The writer has seen it leap clear above the surface of the sea in the early morning.

162. Basking Shark. Selache maxima, Gunn.

Occasional stragglers occur. Yarrell mentions a specimen in Devon. It is common off the west coast of Ireland, where it is regularly hunted.

163. Picked Dog-fish, or Spur Dog. Acanthias vulgaris, Risso.

Very common. Often taken in the trawl, on long lines, and sometimes swarms about mackerel nets and pilchard nets in thousands. It is viviparous.

164. Spinous Shark. Echinorbinus spinosus, Gmel.

Several specimens recorded as captured off the Devon coast, most of them trawled off Brixham and Berry Head. A specimen of the female sex, $6 \mathrm{ft}$. 6 in. in length, was 


\section{FISHES}

taken on a long line forty miles south of the Mewstone in 1896 , and is described in the Fourn. M.B.A. iv. 264 .

I65. Angel Fish, or Monk Fish. Rbina squatina, Linn.

Common. Frequently taken in trawls and in seines.

166. Torpedo. Torpedo nobiliana, Bonap.

Several specimens have been taken. One was recorded from Beer in 1879 , and specimens have been obtained from time to time at the Plymouth Laboratory.

167. Common Skate. Raia batis, Linn.

Common.

168. Flapper Skate. Raia macrorbynchus, Raf.

Day states that he has obtained it at Plymouth.

169. White Skate, Burton Skate, or Bordered Ray. Raia alba, Lacép.

A specimen over 6 feet in length and $5 \mathrm{ft}$. $\mathrm{I}$ in. across the wings was obtained at Plymouth in February 1892 .

170. Long-nosed Skate. Raia oxyrbyncbus, Linn.

Probably occurs.

17 I. Shagreen Ray. Raia fullonica, Linn.

Montagu recorded several from the coast of Devon.
I72. Thornback Ray. Raia clavata, Linn. Common.

173. Spotted Ray, or Homelyn Ray. Raia maculata, Montagu.

Common.

174. Blonde. Raia blanda, Holt and Calderwood.

A species formerly confounded with Raia maculata.

175. Painted Ray. Raia microcellata, Montagu.

Probably occurs.

176. Cuckoo Ray or Sandy Ray. Raia circularis, Couch.

Occurs at Plymouth.

177. Sting Ray. Trygon pastinaca, Linn.

Two small specimens were trawled off Plymouth in January 1898 , several off Salcombe a few days previously. The fish was well known to the local fishermen.

178. Eagle Ray, or Whip Ray. Myliobatis aquila, Linn.

Specimens recorded as taken at Torbay and Plymouth. A specimen was taken with the sting rays above mentioned off Plymouth in 1898. A specimen was also obtained in November 1894, which measured $3 \mathrm{ft} .6$ in. in extreme length, and $2 \mathrm{ft}$. $I$ in. across the pectoral fins. The species is viviparous, the 'purse' attributed to it by Couch really belonging to a species of Raia.

\section{CYCLOSTOMES}

179. Sea Lamprey. Petromyzon marinus, Linn.

Day suggests that it occurs in the Torridge in north Devon. Has been recorded from the county of a length of 3 feet.

180. Lampern, or River Lamprey. Petromyzon fuviatilis, Linn.

According to Parfitt they were common in the Exe, Axe and Plym.
181. Planer's Lamprey, or Mud Lamprey. Petromyzon branchialis, Linn.

Probably common.

\section{CEPHALOCHORDA}

182. Lancelet. Brancbiostoma lanceolatum, Pall.

Undoubtedly occurs in sandy localities. Small specimens were taken at the surface near Plymouth in 1895. 


\section{REPTILES}

\section{AND BATRACHIANS}

The reptiles and batrachians of Devonshire have formed the subject of several local lists, but of these all the writer has been able to consult are of an uncritical nature. They are as follows : Turton, W., and Kingston, J. F., Guide to Watering Places; Teignmouth, Darolish and Torquay, part ii, Natural History of the District, I 830 . Bellamy, J. C., The Natural History of South Devon (1839), p. 236. Brooking Rowe, J., 'Reptiles and Amphibians of Devon' (Transactions of the Plymoutb Institution, I 863, p. 86). D'Urban, W. S. M., 'Reptiles,' in Besley's Handbook of Devonsbire, Exeter, I 875 (?). Parfitt, E., 'Fauna of Devon : Reptiles' (Transactions of the Devonsbire Association, 1877, ix, p. 236).

Parfitt concludes his introduction to the last-named essay with the words 'Our Devonshire reptilian fauna contains all the British species with the exception of the two turtles, which cannot be called indigenous, but only stragglers from warmer seas.' Since his list contains no mention of the Smooth Snake (Coronella austriaca), which, though British, does not occur in Devonshire, and includes at least three species whose claim to membership of the Devonshire fauna is of the most doubtful character, this statement is obviously incorrect and quite unwarranted.

The natural result, therefore, of a critical survey extending over several years has been a reduction in the number of species regarded as indigenous, and as shown hereafter only nine are considered entitled to rank as members of the county fauna.

With our present limited knowledge it is not possible to write a general account of the distribution of these interesting animals within the county borders, and it is sincerely hoped that the meagreness of the present list may be an inducement to observers to search carefully for additions, both in the direction of new species and of new facts concerning the species already recorded.

It only remains to acknowledge the great and valued assistance which has ever been received from Mr. G. A. Boulenger, F.R.S., of the British Museum, and to express sincere thanks to the friends who have helped with specimens and personal experiences.

\section{REPTILES}

\section{LACERTILIA}

1. Common Lizard. Lacerta vivipara, Jacq. Cadover Bridge, Dartmoor, I3 March, I903. Cann Quarry, Bickleigh Vale, near Plymouth, I4 May, 1905. "Common throughout the county' (Brooking Rowe).
This species and the Slow-worm are the only lizards of which the occurrence in Devonshire is established beyond doubt. Both the Sand Lizard and the Green Lizard have been recorded, but the latter is, of course, not even a native of Britain, and the records of the former are in general insufficiently critical to establish a claim to its 


\section{REPTILES}

AND

inclusion in the Devonshire fauna, and in one case quite incorrect. The circumstances are discussed below under the names of the species :

[Sand Lizard. Lacerta agilis, Linn.

'Scarce. I have seen it near Weston Mills, Plymouth ; and Mr. Reading on Roborough Down' (Brooking Rowe). 'I have seen it on Haldon, and Woodbury Common' (Parfitt).

No specimens caught within the county are extant to the writer's knowledge, and since the species has such a peculiar and limited distribution in England there would seem to be strong probability that the above records are cases of mistaken identity. Parfitt did not distinguish between the Common and the Sand Lizard even on close examination, for the specimen described in his list in the Trans. Devon Assoc., 1877 , as a variety of the present species, and preserved in the Albert Memorial Museum, Exeter, proved on examination by the writer (through the kindness of Mr. F. R. Rowley) to be merely the Common Lizard (Lacerta vivipara).

The 'Lacerta agilis' referred to by Bellamy as ' 2 common animal on heaths and commons, and occasionally even in gardens' is undoubtedly the common Lacerta vivipara, the specific name agilis being often applied to this species by the older writers.

The Sand Lizard has been authentically recorded from Hampshire, Dorsetshire, Berkshire, Surrey, Sussex, parts of the Lancashire coast, and (with a slight doubt) parts of the Cheshire coast. ${ }^{1}$ There is, however, no reason known to the writer why it should not occur in Devonshire, and a look-out should be kept for it. It may be distinguished from the Common Lizard by its larger size (length about eight inches), bulkier form, more massive head, and the larger size of the flat scales on the temples-in particular of those bordering the parietal shields.]

[Green Lizard. Lacerta viridis, Dum. and Bibr.

This species is recorded by Parfitt, and though it certainly has been taken in the district there is no doubt that all the specimens were 'escapes,' the Green Lizard having no claim to be considered a native of Britain. By the courtesy of $\mathrm{Mr}$. A. Somervail the writer has been able to examine three caught in Torquay and preserved in the museum of the Torquay Natural History Society. They are undoubtedly referable to this species, but are as undoubtedly escaped specimens. One of them formed the subject of a note in the Zoologist for June, I 869 , p. 1724 .]

\section{BATRACHIANS}

2. Slow-worm or Blind-worm. Anguis fragilis, Linn.

Fairly common in the Plymouth district, where it is usually found under stones on dry and warm hedge-banks. The largest local specimen the writer has seen is $15 \frac{1}{2}$ in. long, and is preserved in the Plymouth Municipal Museum (No. 100'05). It was brought in alive from Radford Woods, near Plymouth, in April, 1903.

\section{OPHIDIA}

\section{Common, Ringed or Grass Snake. Tropidonotus} natrix, Linn.

\section{Locally, Long-cripple (Bellamy)}

All the local lists cited in the introduction speak of this species as generally common. There does not appear to be any doubt however that on Dartmoor it is by no means so frequent as the Viper, though in the lowlands it is probably more plentiful and the commoner species. The only living specimen the writer has seen in the district is one brought to the Plymouth Museum from Newley Coombe, Dartmoor, in May, 1903, by Mr. H. P. Hearder.

\section{Viper or Adder. Vipera berus, Linn.}

Seen by the writer on Dartmoor, where it is evidently still abundant, many specimens having been brought from there to the museum during the last four years by Mr. H. P. Hearder of Plymouth, who has always generously supplied information, specimens, and practical assistance. Both blackish and red forms occur as well as the type, and Mr. Hearder says of the first that those he has caught (about half a dozen) have always been distinctly aggressive. Whether the variations of colour are sexual or not the writer is unable to say, but Mr. Boulenger states that red vipers are females. ${ }^{2}$ The Rev. G. C. Bateman, in Leighton's British Serpents (1901), p. 255 , connects this reddish colour with the red land of certain parts of Devonshire, and Leighton, in a footnote, speaks of it as the most definite example of colour protection he has heard of in British adders. Mr. Hearder however has seen red specimens all over the moor, showing no special connexion with reddish land. A specimen measuring $3 \mathrm{ft} .2$ in. in length was found by Mr. Fred. Holme of Penzance in the road near Holsworthy in August, $1884^{\circ}$ As the largest viper caught in recent years appears to be that recorded in Leighton's British Serpents (1901), p. 76, $28 \frac{1}{2}$ in. in length, from the Monnow Valley in South Herefordshire, the Holsworthy record is distinctly noteworthy.

\section{BATRACHIANS}

\section{ECAUDATA}

5. Common Frog. Rana temporaria, Linn.

Common. Breeds very early in the season in

1 Also recorded from Buckinghamshire, Warwickshire, and Worcestershire in the Victoria History of those Counties. [Editor V.C.H.]

Devonshire; in 1903, for example, well developed spawn was present in pools near Cadover Bridge, Dartmoor, $700 \mathrm{ft}$. above sea level, on 14 February, the frogs themselves having left the water. The spawn was probably deposited in the latter part of January.

2 V.C.H. Hants, i, 206. $\quad{ }^{8}$ Zoologist, 1884, p. 768. 


\section{A HISTORY OF DEVONSHIRE}

[Edible Frog. Rana esculenta, Linn.

Turton and Kingston in the work named in the introduction have the following remark: ' $R$. esculenta. The Edible Frog. Has protuberance on the middle of the back, and margined sides-is less common than the preceding, but by no means of unfrequent occurrence,' and, no doubt influenced by this statement, all the other writers cited in the introduction have included the species. There is no doubt, however, that their statements are erroneous, and Messrs. Turton and Kingston's brief description shows them to have been unacquainted with the features (absence of the dark blotch from eye to angle of mouth, etc.) which distinguished the Edible Frog from the common species. In Britain the Edible Frog is only known to have established itself in Cambridgeshire and Norfolk. It has in the writer's opinion no claim whatever to a place in the Devonshire fauna.]

6. Common Toad. Bufo vulgaris, Laur.

Common : more plentiful than the common frog in the writer's experience. On 10 June, 1903, tadpoles fully developed and just leaving the water were observed in rocky pools immediately alongside the River Tavy, near Denham Bridge.

\section{[Natterjack Toad. Bufo calamita, Laur.}

Introduced to the Devonshire list by Mr. J. Brooking Rowe on the strength of a specimen . . . observed . . . by Mr. Reading, near Wrangerton' (Trans. Plymouth Inst. 1 863, p. 88). There is at present no other evidence, and the above is insufficient to justify giving the Natterjack a place in the Devonshire list. The species may be most readily distinguished from the common toad by having almost invariably a yellowish line or stripe down the middle of the back. The hind legs are also very short, proportionately shorter than those of the common toad, with the consequence that the Natterjack cannot hop-a deficiency counterbalanced, however, by good running powers. An outlook should be kept for the species in May and June, especially among such sandhills as occur on the Devonshire coast.]

\section{CAUDATA}

7. Great Crested Newt. Molge cristata, Laur.

All the local lists cited describe this newt as more or less common in Devonshire, but the writer can find no recent evidence of its existence. In four years' collecting in south Devonshire and south-east Cornwall it has not been met with, nor can any reliable records be found. There is a specimen from Exeter in the British Museum however, presented by Dr. Leach, and on the strength of this the species is here given a place in the county list.

8. Common or Smooth Newt. Molge vulgaris, Linn. (Triton punctatus, Latr.)

As in the case of the Great Crested Newt the local lists speak of this species as common throughout the county, but it has not been met with in recent years, nor is there a county specimen in existence to the writer's knowledge ; neither is our highest authority, Mr. G. A. Boulenger, F.R.S., aware of its ever having occurred in Devonshire. It appears to be replaced entirely by the Palmated Newt, which is abundant, at any rate in many places in south Devonshire, and probably some of the statements in the local lists are due to mistaking this species for the Smooth Newt.

Two old specimens in the Torquay Museum, from Berry Pomeroy, said to belong to this species, are too faded and in too bad a condition generally for identification, but they resemble the Palmated rather than the Smooth Newt.

The writer would have been inclined to exclude the Smooth Newt from the list but for an observation contained in a recent letter ( 14 February, 1906) from Mr. J. Brooking Rowe, in which he says, "As to the Smooth Newt ( $T$. punctatus) I am surprised at what you say [i.e. that it apparently does not occur in the county]. It was without question some years ago the common species, and found everywhere. I was the first to observe $T$. palmatus, and found it in a pond not far from here [Plympton].'

The Smooth Newt should be carefully searched for: it may be distinguished from the Palmated Newt by the spotted throat, and from the Great Crested Newt by the long continuous wavy crest of the breeding male extending from head to tip of tail without interruption.

\section{Palmated Newt. Molge palmata, Schneid.}

Occurs in several places in the Plymouth district, and in April, 1903, 1904, and 1905, numbers were breeding in a small pit partly filled with builders' rubbish, etc., in the western part of the town. There are two specimens (male and female) from this pit, 20 April, 1903, in the Plymouth Municipal Museum (No. 68'05).

On I4 February, 1903, numbers of larvae of the Palmated Newt were taken by the writer from ponds near Cadover Bridge, Dartmoor, about frog-spawn. They were about $30 \mathrm{~mm}$. long and still possessed external gills, having evidently passed the winter in this stage. Six of these are in the Plymouth Museum (No. I I'03).

The species is mentioned by Mr. Brooking Rowe as ' not uncommon in many localities,' and Parfitt gives Exminster marshes, Wonford, and Dartmouth, as places where it has occurred.

The Palmated Newt may be most readily distinguished from the other two species, at all times of the year, by its pale yellowish throat, without markings or spots. Its gradual increase in numbers, apparently with the result of ousting the Smooth Newt, as evidenced by the note from Mr. Brooking Rowe given under that species, is a remarkable and interesting phenomenon. 


\section{BIRDS}

Devonshire is a well defined and natural ornithological district, differing in many respects from any other part of the British Islands, and its fauna possesses distinctive features which distinguish it from that of Somerset adjoining it on the north-east and Dorset on the east, and in a minor degree from that of Cornwall on the west. On the whole however, Cornwall, Devon and the western part of Somerset are closely allied in character, and as they together form the south-western peninsula of England they all partake of the peculiar conditions which their unique geographical position confers upon them, lying as they do between the Bristol and English Channels, and jutting out into the Atlantic Ocean. Devonshire forms the central and broadest part of this peninsula and with Cornwall possesses both a north and south coast, a conformation that has a great influence on its avifauna, as its coasts receive visitors arriving by several of the great routes of migration from the north, south and east, whilst its proximity to the continent on the south, and to the Atlantic on the west, adds many rare stragglers to swell the number of species which can be claimed as occurring within its limits.

Devonshire has moreover a most diversified surface : bleak, barren, elevated moorlands; steep, rugged sea-coasts; sand-hills; extensive muddy, tidal estuaries; fresh-water ' leys' ; deep, sheltered 'combes' and wooded valleys ; cultivated fields surrounded by timbered and leafy hedgerows, and much pasture land. Attractions are thus afforded to birds of varied habits, and though modern improvements and increased population have driven away from some parts many birds which a century ago abounded, others have increased in numbers, and some species formerly unknown have been recognized as visiting us more or less frequently.

In the subjoined list of Devonshire birds, the arrangement and nomenclature followed are those adopted by Mr. Howard Saunders in the second edition of his Manual of British Birds. Of the 294 species enumerated 83 may be classed as residents and $3_{1}$ as regular summer migrants, giving a total of II 4 annual breeders within the limits of the county, including Lundy Island, where some species of sea birds breed that are not found nesting elsewhere in Devonshire. Of winter visitors there are 38 , and visitors on migration in autumn and spring 17 . Of these 9 species are occasional breeders. Birds of casual occurrence number 67 , and of them 9 are occasional breeders, viz. lesser whitethroat, bearded tit, golden oriole, pied flycatcher, crossbill, hooded crow, hoopoe, wryneck and Montagu's harrier; whilst 8 others that formerly bred in Devon do so no longer, viz.: Dartford warbler, marsh-harrier, hen-harrier, white-tailed eagle, kite, osprey, little bittern 


\section{A HISTORY OF DEVONSHIRE}

and night heron. Of birds of accidental occurrence there are 56 species, about two-thirds of which have only occurred once or twice. The American white-winged crossbill and yellow-billed cuckoo have been found dead on the coast, each on a single occasion. They are not admitted into the British list by Mr. H. Saunders, and are therefore not enumerated. Three introduced species are included, viz. pheasant, red-legged partridge and mute swan, but the Egyptian and Canada geese and the summer or wood duck, not being completely naturalized, are excluded, as is also the collared turtle dove, which has bred in a wild state near Plymouth.

The following 24 species have been recorded as occurring in the county either altogether erroneously or upon insufficient evidence, or were introduced and escaped, and are therefore not admitted :-

Great Reed-Warbler
Savi's Warbler
Pine Grosbeak
Calandra Lark
Short-toed Lark
Great Black Woodpecker
Eagle Owl
Golden Eagle

Iceland Falcon
Goshawk
Great White Heron
Buffle-headed Duck
Harlequin Duck
Hooded Merganser
Red Grouse
Ptarmigan

Baillon's Crake
Little Ringed Plover
Killdeer Plover
Spotted Sandpiper
Yellowshank
Roseate Tern
Sooty Tern
Great Auk

There is a considerable difference between the ornis of the northern and southern portions of Devon, probably, in great measure, owing to the obstacle which the high land of Dartmoor interposes to the progress northwards of stragglers from the continent. No less than 46 species have occurred in south Devon which have not yet been observed in north Devon, but many of these birds were accidental visitors, and have occurred only once or twice. Some species amongst the geese, ducks, terns and skuas are of more frequent occurrence in south than in north Devon. On the other hand 9 species which have been observed in north Devon and Lundy Island have not yet been noticed in the south of the county, viz. twite, alpine swift, spotted eagle, ruddy sheld-duck, redcrested pochard, cream-coloured courser, and three American sandpipers; and in some localities bird-life is much more in evidence than in the south. In the eastern portion of the county a few species occur that are little known in the west, like the redstart and nightingale, and some 25 or $3 \circ$ Atlantic birds and wanderers from the south have only been obtained in the south-western part of the county, and others are much more plentiful there than in eastern Devon.

Unusually fine dry summers and autumns are always distinguished by the number of wading birds occurring, both as species and individuals. Severe winters bring us many aquatic species, such as wild swans, never seen at other times. Many birds come in flights at more or less distant intervals of time, such as the crossbill, sand-grouse, quail, bittern, snowy owl, rough-legged buzzard, osprey, ruddy sheld-duck, swans, godwits, grey phalarope, terns, skuas, etc., their occurrence at other times being rare. 


\section{BIRDS}

In the late summer and early autumn there is often a great influx of warblers, supposed to come from Ireland, and the great gatherings of mistle thrushes, ring ouzels, wheatears, willow warblers, wagtails (pied, white, yellow and grey), swallows, house martins, spotted flycatchers, linnets, sky-larks, wood-larks, land-rails, etc., on the south coast, prior to their departure in autumn, are often most remarkable. Mr. W. Eagle Clarke observed many species passing the Eddystone lighthouse, in September and October I90I, in great numbers going south. In spring warblers of various kinds come in great 'rushes' to our south coast, and for a short time are very plentiful, but as a rule Devonshire is remarkable for the scarcity of warblers. The stone curlew, which in other parts of England is a summer migrant, is a winter visitor to Devonshire, finding the extreme northern limit of its winter range in this county and west Somerset.

Numerous sea-fowl frequent the indentations of the south coast, viz. Plymouth Sound and harbours, Bigbury Bay, Start Bay and Torbay, especially during the autumnal migration, when large numbers of scoters, phalaropes, terns, gulls, skuas, divers, grebes and shearwaters are sometimes met with. In severe winters flocks of ducks, geese and swans visit them, and in early spring gannets, puffins, razorbills and guillemots are plentiful.

The estuaries of the large rivers the Tamar and Tavy at Plymouth, the arm of the sea at Kingsbridge, and the estuaries of the Dart, Teign and Exe on the south coast, and of the Taw and Torridge on the north coast, with their extensive mud-flats and sand-banks, are visited by many waders and aquatic birds, especially during the spring and autumn migrations.

At several points along the south coast, and at Baggy Point on the north coast, where the cliffs are precipitous, there are considerable breeding places for sea-fowl and other birds.

The principal breeding place for sea birds is at Lundy Island, off the north coast of the county. This mass of granite, rising some 300 to 800 feet above the sea, is flat-topped, contains about I, 200 acres, and is nearly three miles long, and about a mile wide at its broadest part. Politically it does not belong to Devon, nor to any other county, but as it lies out in the Bristol Channel only I 4 miles away from our coast, and is 30 miles from Wales, it can fairly be claimed as naturally appertaining to this county. No less than 140 species of birds have been observed on the island, of which 27 are residents, 3 I summer migrants, 23 winter visitors, and 59 occasional visitors. About a dozen other species have been seen but not obtained. It is worthy of note that no tits are found on the island, and as there are no trees woodpeckers are, of course, absent. The jackdaw and rook are only occasional visitors. The house sparrow, formerly only a visitor, has established itself of late years. A spotted eagle was shot in the winter of 1858 , but lost over the cliffs, and an example of the American buff-breasted sandpiper was killed in the same year. Neither of these birds has occurred on the mainland of Devon. 


\section{A HISTORY OF DEVONSHIRE}

Amongst the $6 \mathrm{r}$ species of birds that formerly bred more or less regularly on the island, the Cornish chough, once numerous, is now exterminated, and the quail and snipe no longer breed there. A few pairs of ravens and two pairs of peregrine falcons still breed on the steep cliffs. The osprey had an eyrie here as late as $183^{8}$. Lundy was formerly remarkable for the flights of quail, landrail, woodcock and snipe that visited it in autumn and winter. The rose-coloured starling and hoopoe used to be frequently seen in spring, and probably reached it by coming up the Channel from the south and keeping along the north coast of Cornwall. The common starling resorts to the island in immense flocks in winter. Both partridges and grouse have been introduced at various times, but were destroyed by the rats which swarm all over the island. The cliffs are highest and most precipitous on the western side, and here are the breeding places of the cliff birds for which Lundy is so famous. They come in from the sea early in May and remain until August. Immense numbers of ' murres' or guillemots, razorbills, puffins, kittiwakes, shags and cormorants still frequent the cliffs. The gannets that in former times inhabited an islet off the north-east end, still called the ' Gannet Rock,' have abandoned it, and only a few pairs now breed on Lundy itself. The herring gull, lesser and greater black-backed gulls also breed sparingly on some of the cliffs and rocky islets. The Manx shearwater and stormy petrel are common, and no doubt breed underground in deep holes. Tradition asserts that the great auk, known as the 'king murre,' at one time frequented the rocky shores of the island.

Slapton Ley is a remarkable sheet of fresh water, running parallel with the sea, and only separated from it by a bank of sand and shingle some two hundred yards wide. It is situated in the centre of the indentation of the south coast, between the entrance to Dartmouth Harbour and the Start Point, known as Start Bay. The 'ley' or lake is long and narrow in shape, and about 300 acres in extent, stretching for more than two miles along the coast. The higher or eastern part is nearly filled up with dense beds of tall reeds, and forms the great stronghold for the coots which breed there plentifully, and in winter are reinforced by vast numbers of 'foreigners.' The wild duck and teal nest annually, the former plentifully, and the tufted duck and pochard are believed to do so. There is strong presumptive evidence that the wigeon has occasionally bred here. Vast flights of the latter bird frequent the ley in winter as well as many other kinds of ducks, swans, geese, bitterns, divers and grebes. Many of the rarest of our birds have occurred in this neighbourhood, and the reed warbler breeds freely amongst the reeds of Slapton, Thurlestone and Milton Leys.

Little is known of the ornithology of the county till the close of the eighteenth century. About the earliest allusion to the birds of Devon will be found in Synopsis Cborograpbia, or an Historical Record of the Province of Devon, by John Vowell, alias Hoker (born at Exeter in I 524, died I60I), of which a MS. copy (Portledge) exists in the 294 


\section{BIRDS}

Harleian library at the British Museum. He gives a quaint list of some of the birds, including domestic species, found in Devon. It is quoted by Polwhele in his History of Devonshire. In the Gentleman's Magazine for I 795 and 1796, Captain Laskey gives an account of a 'Three days' excursion on Dartmoor,' in which he makes some allusions to the ornithology, and describes Slapton Ley, mentioning the great abundance of coots and wildfowl there. The Rev. Richard Polwhele's History of Devonshire was published between 1793 and 1806 . The first volume, published in 1797 (after the 2nd vol.), contains a chapter on the 'Land Birds and Water-Fowl,' deriving his information from various friends in the neighbourhoods of Plymouth and Ashburton. Next we have the important papers by Colonel George Montagu of Kingsbridge, published in the Transactions of the Linnean Society for $1804-8$, containing his observations on the cirl bunting, Montagu's harrier, hen-harrier, Dartford warbler, and 'little white heron.' Colonel Montagu published his Ornitbological Dictionary in I 802 (a supplement was printed in Exeter in ${ }_{1} 8 I_{3}$ ), and in it makes mention of many birds obtained, some for the first time, in south Devon. Dr. Andrew Tucker of Ashburton commenced a quarto work in I809, entitled Ornitbologia Danmoniensis, of which, unfortunately, but two parts, containing a general introduction to the study of ornithology only, were published. Prof. A. Newton considers 'the two parts printed show the author to have been a physiologist, anatomist, and outdoor-observer far beyond most men of his time.' Dr. Tucker was a correspondent of Montagu, and assisted him in his researches. Dr. William Turton and J. F. Kingston published a 'Natural History of the District' (Torquay, Dawlish, Teignmouth, and inland to Dartmoor), in a Guide to the Watering Places (Teignmouth, I $83^{\circ}$ ), with a list of birds arranged under three divisions-land birds, waders and anseres. Dr. Edward Moore of Plymouth was the first to attempt a systematic catalogue of Devonshire birds arranged according to modern ideas, and published a treatise 'On the Ornithology of the South of Devon,' in the Transactions of the Plymouth Institution for 1830 , and one 'On the Birds of Devonshire,' in The Magazine of Natural History for 1837 . The Rev. Thomas Johnes, rector of Bradstone, contributed an excellent account of the birds found in the neighbourhood of Dartmoor in the form of a letter, dated 1832 , to Mrs. Bray, who published it in 1836 in her work, Borders of the Tamar and Tavy. In 1837 the Rev. W. F. Cornish printed at Exeter a little treatise on British Migrants, which was put forth as a feeler, prior to the proposed publication of a larger work, ' the labour of thirteen years,' but which never saw the light. In I 839 appeared a very interesting work, The Natural History of South Devon, by J. C. Bellamy, surgeon, of Plymouth, which contained much information about the birds of the southern portion of the county, but is not free from the usual errors of the day. The Rev. Samuel Rowe, vicar of Crediton, produced in 1848 The Perambulation of Dartmoor, to which Dr. Edward Moore contributed a list of the birds, which perpetuated numerous mistakes and did not add 


\section{A HISTORY OF DEVONSHIRE}

much to the correct knowledge of the moorland fauna. In the third edition of this work, 1896 , is a chapter on the 'Birds of the Moor and its Borders.'

The pursuit of British ornithology had by this time become more systematic, and Yarrell's British Birds, published from i 837 to I 843 , gave a great impetus to the study and became the standard work on the subject. The Rev. Robert Holdsworth, rector of Brixham, who had been a correspondent of Montagu's, furnished much valuable information on Devonshire birds in a series of letters to Mr. Yarrell, and is repeatedly mentioned by him in his book. For more than forty years however the only publications relating to the ornithology of the county were the notes and articles contributed by a host of observers, which appeared in various natural history periodicals, the transactions of local societies and the lists in guide books. In the Transactions of the Plymoutb Institution (vol i. 1862-3), appeared 'A Catalogue of the Mammals, Birds, etc., indigenous to or observed in the County of Devon,' by J. Brooking Rowe, F.L.S., who received much assistance from the late Mr. John Gatcombe of Stonehouse. The notes contributed by the latter from I 85 I to I 855 to the Naturalist, and from I 855 to the time of his decease in 1887 to the Zoologist, were most valuable, and furnish a mine of information respecting Devonshire birds in general, and to those occurring near Plymouth in particular. John Gatcombe probably did more to elucidate the avifauna of the county than any other ornithologist since the time of Montagu. In I89 I Mr. W. E. Pidsley and the late Rev. H. A. Macpherson published the Birds of Devonsbire, which was followed in 1892 by the larger work, The Birds of Devon, by W. S. M. D'Urban and the Rev. M. A. Mathew. A supplement to the latter work was published in 1895 , and contains some information contributed by Mr. E. A. S. Elliot of Kingsbridge, who has done much for the ornithology of his most interesting district, and has published numerous valuable papers in the Transactions of the Devonsbire Association and other scientific periodicals. The information he has afforded has been of great service to the writer in compiling the following list of Devonshire birds.

Note.-As the example of the black-headed warbler enumerated under No. 18 of the following list was only seen, and not actually obtained, and as that species has not yet been admitted to the British list, it should perhaps not have been numbered.

x. Mistle-Thrush. Turdus viscivorus, Linn. Locally, Holm Screech.

A common resident, breeding freely in orchards and gardens. In August there is a considerable influx of migrants from the north and east, and they appear to congregate in the extreme south-west of the county, prior to their departure for the south. Mr. W. Eagle Clarke observed a great passage of mistle-thrushes on the night of 12 October, I 901 , at the Eddystone Lighthouse. They were seen in great numbers in the Kingsbridge district at the end of September 1902, by Mr. E. A. S. Elliot. In Montagu's time this species appears to have been much less plentiful than it is at present. A severe winter however diminishes its numbers for a time. 


\section{BIRDS}

2. Song-Thrush. Turdus musicus, Linn.

Locally, Grey Bird, Grey Drush, Trossel, Drostle or Throstle.

An abundant resident, and generally distributed over the county, excepting the moors. Our summer resident thrushes are to a great extent replaced in autumn by immigrants from the eastward. These birds are much darker in plumage, and the spots on the breast are much more intense in colour, than in our native birds. Great numbers of song-thrushes have been seen passing to the south at the Eddystone Lighthouse in September and October. In severe weather thrushes assemble in south Devon in surprising numbers. This was especially noticeable in February 1900.

\section{Redwing. Turdus iliacus, Linn. \\ Locally, Windle, Winnard.}

A winter visitor, generally very abundant, but in some years few are seen. Arrives from the middle of October to the beginning of November, and remains to the middle or end of March. Large numbers pass the Eddystone Lighthouse in October going south. In severe seasons great numbers perish. White and buff-coloured specimens have been obtained. A mottled specimen occurred at Kingsbridge in January I 899 , with yellow beak and flesh-coloured legs.

\section{Fieldfare. Turdus pilaris, Linn.}

Locally, Blue-bird, Vole-vier or Vilveer, Felt.

A winter visitor, arriving in October and remaining till March, but only seen on the lowlands in severe weather. At such times it congregates. in great numbers in gardens, even in the large towns, to feed on holly berries. At ordinary times this bird keeps to the borders of the moors where abundance of mountain ash and hawthorn berries can be procured. In February 1895 vast numbers of fieldfares visited Kingsbridge, and again in February I 900 .

\section{White's Thrush. Turdus varius, Pallas}

An example of this eastern species was shot by Mr. E. Studdy in Dene Wood on the edge of Dartmoor near Ashburton, II January 1881. It was in company with three or four other birds apparently of the same species.

\section{Blackbird. Turdus merula, Linn. \\ Locally, Black Drush, Colly (A.-S. col= black).}

An abundant resident throughout the county excepting the moors. There appears to be a considerable emigration to the south in autumn. Parti-coloured individuals are not uncommon, and albinos occur occasion- ally. The late Mr. J. Gatcombe met with an albino having a black tail at Plymouth in May 1885 .

7. Ring-Ouzel. Turdus torquatus, Linn.

Locally, Tor-Ouzel, Moor Blackbird, Mountain Colly, Michaelmas Blackbird.

A summer migrant frequenting many of the rocky tors of Dartmoor especially when the whortleberries are ripe in July and August. Being very sensitive to cold winds, it is always found on the lee side of the tor. It is also to be met with on most of the other parts of the moor being almost the only denizen of the central swamps. It breeds on Dartmoor and on some of the moors in north Devon in May. A few pairs inhabit the Valley of Rocks near Lynton, and it has been seen on Lundy Island. During the period of migration in autumn, flocks visit the south coast for about a fortnight previous to their departure for the south. Stragglers occasionally occur in the more cultivated districts in spring and autumn, and in a few instances have been seen in winter on the south coast. An albino specimen shot by Mr. F. R. Wolfe, on Leighon Tor in the parish of Manaton, 9 October, 1890, is in the Torquay Museum.

\section{Wheatear. Saxicola cenantbe (I,inn.) Locally, Chickell.}

A summer migrant. Breeds freely on Dartmoor, and in small numbers about Plymouth Sound, the Bolt Head and Thurlestone. Numerous especially in spring and autumn on some parts of our sea-coasts, as at Plymouth, between Bolt Tail and Start Point, Exmouth and near Axmouth in south Devon, and Morte Point in north Devon. Also on the moors and other uncultivated parts of the county in summer. Usually arrives about the middle of March, though sometimes at the beginning of that month, the males preceding the females by about a fortnight or three weeks. Towards the end of September wheatears assemble on the coasts, and take their departure during October, early in which month they have been observed passing the Eddystone Lighthouse. The writer saw one at Exmouth as late as 4 November I 894 . Examples of the large race of wheatear have been obtained at Kingsbridge, Exmouth and other places.

\section{Whinchat. Pratincola rubetra (Linn.) Locally, Furze-chat.}

A summer migrant, arriving about the middle or end of April and departing in October. Though not a numerous species in 


\section{A HISTORY OF DEVONSHIRE}

south Devon, it is met with on the southwestern borders of Dartmoor (where it is known to breed), about the various 'leys' in the Kingsbridge district in spring, near Plymouth, Totnes, Teignmouth, Torquay, Exmouth, Woodbury Common and Axmouth. In north Devon it is a scarce species. It has been observed on Lundy Island. On 24 and 26 April 1891 whinchats were observed on several parts of the south coast in unusual numbers.

\section{Stonechat. Pratincola rubicola (Linn.) Locally, Furze-chat.}

Resident. Frequents moors, heaths, marshlands, and the cliffs of the sea-coasts. It is nowhere plentiful, but is generally to be found in suitable localities. Many have been observed to pass the Eddystone Lighthouse in October going south.

\section{Redstart. Ruticilla phoenicurus (Linn.) Locally, Fire-tail.}

A well known summer migrant around Exeter and in sheltered places between that city and Totnes, but scarce in the extreme west of the county and in the southern promontory, being only seen on migration in spring and autumn in the Kingsbridge district. It is very common in the leafy lanes about Chagford and breeds there, but is said to have been unknown in that neighbourhood less than twenty years ago. In 1860 however it was observed and fairly common. It is now not uncommon in north Devon. It usually arrives about the third week of April, but sometimes earlier, and departs in September, on the night of the 23rd of which month, in 1901, Mr. Eagle Clarke obtained some examples at the Eddystone Lighthouse.

\section{Black Redstart. Ruticilla titys (Scopoli)}

A winter visitor, arriving sometimes as early as the end of September or the beginning of October, but generally about the last week of November and remaining until the end of March or the beginning of April.

The black redstart is frequently observed on the south-west coast of the county, especially near Plymouth, at Thurlestone, around Torbay and at Teignmouth. East of the Exe it is much more rarely seen. A few examples have occurred near Exeter and Exmouth. It is rare in north Devon but has been seen at Barnstaple, Instow and Ilfracombe. Bellamy says it has been known to breed near Exeter (Nat. Hist. of S. Devon, p. 206).

13. Bluethroat. Cyanecula suecica (Linn.)

An accidental visitor of very rare occur- rence. An example is recorded in the Zoologist for 1852 as having been shot near Whimple, about eight miles to the east of Exeter, in September of that year. Another is said to have been seen close to Exeter about 1869. A doubtful occurrence in Devonshire is given by M. C. Cooke in the Naturalist for 1853 .

$$
\begin{aligned}
& \text { I4. Redbreast. Eritbacus rubecula (Linn.) } \\
& \text { Locally, Robin, Redocke, Ruddocke (A.-S. } \\
& \text { rudduc). }
\end{aligned}
$$

An abundant resident. White and buffcoloured specimens have occurred.

\section{Nightingale. Daulias luscinia (Linn.)}

A summer migrant occurring sparingly every year in some part of the county, but principally in that portion lying to the east of Dartmoor. In the South Hams the nightingale is only seen occasionally on migration in spring. Though certainly this delightful songster is by no means plentiful in Devonshire, it is frequently heard, especially near Ashburton and in the Teign valley, where it has bred regularly for many years, a fact established by Mr. A. E. S. Elliott (Trans. Devon Assoc. 1 902). It is rare in north Devon.

16. Whitethroat. Sylvia cinerea, Bechstein Locally, Whittybeard, White Drot.

A summer migrant abundant throughout the county in suitable localities. Arrives about the middle of April, and is seldom seen after August. A very common breeder.

\section{Lesser Whitethroat. Sylvia curruca (Linn.)}

This warbler is decidedly very rare in Devonshire, but appears to have been obtained once or twice near Plymouth. The records of its occurrence are few, and some of them are doubtful. It has been reported as breeding in the South Hams, and near Dawlish and Tiverton, but probably erroneously.

\section{[18. Black-headed Warbler. Sylvia melano- cephala (Gmelin)}

On 16 April I890, whilst the writer was watching a male blackcap which with many others had just arrived, and was feeding on ivy-berries in a garden at Exmouth, another bird with a jet black head, but with a pure white throat and under parts, settled on a twig close to it, and he was enabled to compare the two birds. The stranger was certainly smaller than the blackcap, and it had a more slender beak, longer, and larger in proportion, than in that bird. It answers to the description of Sylvia melanocephala, a com- 


\section{BIRDS}

mon species in the south of France and which might easily visit our south coast.]

\section{Blackcap. Sylvia atricapilla (Linn.)}

A summer migrant generally distributed and abundant in all wooded localities, and breeds freely. Usually arriving some time about the middle of April, it stays with us very late in the season, and not unfrequently individuals remain throughout the winter.

\section{Garden-Warbler. Sylvia bortensis, Bech- stein}

A summer migrant numerous in some localities. It arrives in April and occasionally remains up to October. It is frequently seen near Exeter and Exmouth, and it has been found to be common in the valley of the Plym. Montagu met with it on the banks of the Avon a few miles from the sea in 1806 , but at present it appears to be only seen in the South Hams during its autumnal migration. It is not common in north Devon. Owing to its inconspicuous colouration and retiring habits it is frequently overlooked, and it is probably more generally distributed than it is supposed to be.

\section{Dartford Warbler. Sylvia undata (Bod- daert)}

Formerly a resident in the southern parts of the county. It is now rarely met with, and it is supposed that numbers perished in the severe snowstorms of the memorable winters of $1880-1$ and $1886-7$. Mr. E. A. S. Elliot sometimes notices it in turnip fields near Kingsbridge in the autumn, perhaps on migration. The breeding habits of this interesting little bird were first discovered by Colonel Montagu in this county. He first met with it in the southern parts of Devon on 8 September 1802 , but he had previously made its acquaintance in Cornwall. He found nests, eggs and young on a large furze common near Kingsbridge, 16 July 1806 . It has not been observed nesting in the county since that date. This little warbler has been reported from north Devon, and has been obtained at Budleigh Salterton, Lympstone, Bickleigh Vale and Mount Batten, Plymouth.

22. Golden-crested. Regulus cristatus, K. L. Koch

Locally, Tidley-Goldfinch.

Resident, frequenting fir plantations. It is most numerous in winter, when its numbers are recruited by immigration commencing in October. White and cream-coloured specimens have occurred in the county.
23. Fire-crested Wren. Regulus ignicapillus (C. L. Brehm)

A casual visitor of very rare occurrence. A female example obtained at Torquay, 6 March I873, was seen by Mr. J. H. Gurney in the possession of Mr. Shopland, to whom it had been brought in the flesh for preservation. One is said to have been picked up dead at Torre in I874. Several occurrences have been reported from Devonport and Axminster, but must be considered doubtful. It is also said to have visited Lundy Island.

24. Yellow-browed Warbler. Pbylloscopus
superciliosus (J. F. Gmelin)

According to Mr. Frohawk four individuals of this rare straggler to England were seen at West Buckland in south Devon on 1 October I 895 .

\section{Chiffchaff. Phylloscopus rufus (Bechstein) Localy, Choice and cheap (around Totnes).}

A summer migrant, generally distributed, and sometimes very numerous on its first arrival in early spring, and on its autumnal passage. It usually appears at the end of March, departing at the end of September or in October. A few however occasionally remain all through the winter.

\section{Willow-Wren. Pbylloscopus trocbilus} (Linn.)

Locally, Hay-bird, Ground Isaac (from O. E. beisugge).

A summer migrant, common and generally distributed. Arrives usually about the middle of April, sometimes in a great 'rush' across the Channel. Such an occasion was observed by Mr. E. A. S. Elliot at Kingsbridge during the week between 12 and 18 April 1890 . There is sometimes a great influx of birds of the year into south Devon in August. A few willow-wrens sometimes remain till as late as October on the south coast.

\section{Wood-Wren. Pbylloscopus sibilatrix (Bechstein)}

A summer migrant, local, frequenting oak woods, but sometimes abundant where it occurs. At Plymouth this wren arrives in April and departs in October. It is common in Bickleigh Vale, and in all large woods with tall trees, but is very rarely observed in brushwood. It also occurs at Ivybridge, Tavistock, Newton Abbot and Berry Pomeroy. It is seen rarely near Torquay and Topsham late in the summer. In September I 900 Mr. E. A. S. Elliot saw some wood-wrens in. 


\section{A HISTORY OF DEVONSHIRE}

company with many other birds of various species at Kingsbridge, evidently on migration. In north Devon it is common in the oak woods near Clovelly, and near Dulverton in Somerset, which is close to the north-eastern border of Devon.

\section{Rufous Warbler. Aëdon galactodes (Temminck)}

An accidental visitor from southern Europe. The same district of south Devon has afforded two instances of the occurrence of this species. The first was shot near the Start by Mr. W. Dillwyn Llewellyn in September 1859 , after a strong south wind had been blowing for some time, and was so much injured that it could not be mounted. It had lost its tail and was very thin. The second bird occurred near Kingsbridge on 12 October I876. The bird was first noticed by the late $\mathrm{Mr}$. W. Bastard of Slapton, whose attention was attracted to it by its colour and its strange antics. When flushed in a turnip field it flew to a hedge, from which it made short flights into the air, descending each time with its broad, fan-shaped tail expanded. It was shot, and proved to be a young bird of the year. It is now in the possession of Mrs. Bastard.

\section{Melodious Warbler. Hypolais polyglotta (Vieillot)}

Probably a regular summer migrant to the extreme south-eastern corner of the county. Bellamy in his Natural History of South Devon gives a description of an unknown warbler, which appears to have been a species of $\mathrm{Hypo-}$ lais, and possibly this one, the most western of the genus, inhabiting France and the Iberian Peninsula. It was shot at Whiteford, in the neighbourhood of Plymouth, by Pincombe, a taxidermist of Devonport. No further occurrence is recorded until early in May 1897 the Rev. Murray A. Mathew visited Lyme Regis, and in the wooded undercliff at Ware, about a mile to the west of the town, and on the Devonshire side of it, heard two birds singing which he believed at the time to be icterine warblers ( $H$. icterina). The next year $(1898)$ at the same time he once more visited the spot, and was fortunate enough to again meet with many individuals of the warblers, which he was enabled to identify as belonging to this species as far as was possible without having them in his hand. As there were so many of the birds it is probable that those heard the previous year had nested and successfully brought off their brood (Zool. iv. 2, p. 265).

\section{Reed-Warbler. Acrocephalus streperus (Vieillot)}

A summer migrant, very local in its distribution, but in most years abundant where it occurs, and breeds freely. It was formerly supposed to be of very rare occurrence in Devonshire, and was not even suspected of being found in the county by Colonel Montagu, who states it was unknown in the southwestern counties. At the beginning of May I 87 I Mr. J. H. Gurney visited Slapton Ley and saw several reed-warblers there, and frequently heard them singing during the rest of the month. In the Zoologist for 1892 (p. 408) Mr. E. A. S. Elliot drew attention to a statement made by the late Mr. W. V. Toll, who resided close to Slapton Ley, that hundreds of reed-warblers bred every year amongst the reeds in the Ley. In the second week of May 1895 the Rev. Murray A. Mathew and the writer paid a visit to this interesting piece of water, and saw great numbers of reed-warblers in the hedges around the Ley, and some were engaged in carrying materials for their nests, although the reeds were not yet grown up. Mr. Elliot has since ascertained that the reedwarbler is a regular summer visitor to Thurlestone and Milton Leys. It is also stated to frequent reed beds on the Dart near Totnes. It has not been detected on the Exe.

\section{Sedge-Warbler. Acrocephalus phragmitis} (Bechstein)

A summer migrant, generally distributed and numerous in suitable localities, such as sedgy banks of rivers, ponds, marsh-ditches and withy-beds. Arrives about the end of April and disappears about Michaelmas. Mr. Eagle Clarke obtained examples on the night of 23 September 1901, at the Eddystone Lighthouse.

\section{Grasshopper-Warbler. Locustella nevia (Boddaert)}

A summer migrant of very local distribution. It is rare in the Plymouth district, but is more numerous about Kingsbridge and Exeter. The late Lord Lilford found six nests of this warbler in a small patch of ground on the northern slopes of Dartmoor. The only place in north Devon at which its occurrence has been recorded is Westward Ho! where it is met with in early spring.

33. Hedge - Sparrow. Accentor modularis (Linn.)

Locally, Dinnick, Segge (O.E. beisugge)

An abundant resident, generally distributed throughout the county, except the central 


\section{BIRDS}

parts of Dartmoor. Albinos have occurred near Exeter.

\section{Alpine Accentor. Accentor collaris (Scopoli)}

An accidental visitor to the south-western coast of the county. Three specimens have been obtained near Plymouth, one at Berry Head, and one at Teignmouth, in the months of December and January.

\section{Dipper. Cinclus aquaticus, Bechstein Locally, Water-Ouzel, Water-Colly.}

The dipper was formerly a common resident on all our rocky streams, and although it may still be seen pretty frequently on the higher parts of the rivers issuing from the moors, it has been greatly reduced in numbers. A pair used to breed at Exwick Weir on the Exe, close to Exeter. It is fairly common in the Kingsbridge district, frequently constructing its beautiful nest under the arches of bridges. It is occasionally seen in the southeast of Devon and sometimes on the sea coast.

\section{Bearded Titmouse. Panurus biarmicus (Linn.)}

A casual visitor of very rare occurrence at the present day, but according to Dr. E. Moore was formerly a resident near Topsham and Thorverton on the Exe. Mr. H. Saunders in his Manual of British Birds (2nd ed.) seems to credit certain information he had received that this bird still breeds in one locality in the county. It was occasionally met with near Bovey Tracey, according to Turton and Kingston (1 830). The late Mr. Bower Scott of Chudleigh stated that he had seen a bearded tit at Slapton Ley about $\mathrm{I} 872$, and the Rev. M. A. Mathew once saw a small flock in a marsh near Barnstaple. There were two specimens in the collection of the late Mr. Cecil Smith stated to have come from 'Devonshire.'

\section{Long-tailed Titmouse. Acredula caudata (Linn.)}

Locally, Bottle-Tit, Long-tailed Pie, Longtailed Cap'n.

Resident, generally distributed and abundant in wooded districts. Its beautiful nest is known to every bird-nesting boy. It appears to be more abundant in winter than in summer, and probably its numbers are recruited by immigration during the former season. Small flocks are often seen mingled with other species of tit and various small birds, especially frequenting white poplar trees by the side of rivers and ponds.
38. Great Titmouse. Parus major, Linn.

Locally, Hackmall, Heckymal, Big Hickmal, Ox-eye.

Resident and generally distributed in wooded districts. It is one of the most abundant species of tit about Exeter and Chagford. In winter it flocks with other small birds.

\section{Coal-Titmouse. Parus ater, Linn.}

Resident and tolerably common in the south of the county, being frequently seen in suburban gardens around Exeter and Exmouth, especially in autumn and winter, but it usually frequents fir plantations. It is not a common species in north Devon.

40. Marsh-Titmouse. Parus palustris, Linn.

Resident and common in some localities. About Exeter this species is not so common as the coal-tit. In the Kingsbridge district it is by far the most numerous of all the tits. It is also common at Chagford and in the northern part of the county. It is sometimes gregarious with the other species of tit. Examples closely resembling the Siberian marsh-titmouse have been obtained by $\mathrm{Mr}$. Elliot at Kingsbridge in autumn and winter, and the writer saw one at Chagford in 1896 . This bird was larger and very different in appearance from the ordinary dingy-coloured race of the English marsh-tit. The crown of the head was intense black, the upper parts of a delicate lavender-gray, and the lower parts white, the three colours being strongly contrasted. It seems probable it was an example of $P$. camchatkensis.

41. Blue Titmouse. Parus caruleus, Linn. Locally, Billy Biter, Blüthpecker, Blue Cap, Bluespick, Titmal, Hackmal.

A common resident, generally distributed. Probably the most abundant species of tit in Devon. In winter it is gregarious, and large flocks, mingled with other small birds, are met with.

42. Crested Titmouse. Parus cristatus, Linn.

The only recorded occurrence of this species of titmouse in Devon rests upon the authority of Baron A. von Hugel, who observed one in Chelston Lane, Torquay, on 26 March 1874. The bird he saw was so tame that he almost touched it with his walking-stick (Zool. 1874, p. 4065). In the second edition of Mr. Howard Saunders's Manual of British Birds the locality given for this occurrence is 'Bournemouth,' probably through inadvertence. Mr. J. H. Gurney 


\section{A HISTORY OF DEVONSHIRE}

however, in his paper 'On the Occasional Appearance in England of the Crested Tit,' (Zool. 1880, p. 211 ) gives the locality as above.

\section{Nuthatch. Sitta ceesia, Wolf.}

Resident in wooded districts throughout the county. It is nowhere numerous, but is occasionally seen, especially in the autumn and winter months. Mr. Elliot considers that it is becoming increasingly common in the Kingsbridge district.

\section{Wren. Troglodytes parvulus, K. L.} Koch.

Locally, Jinty (E. Devon), Cuddy Vran (W. Devon), Cutty, Kitty Tope, Titty Todger, Tidley Tope, Cracky and Crackil (N. Devon).

Resident and generally distributed. There is a large influx to the southern part of the county in October, at which time also wrens are seen migrating at the Eddystone Lighthouse. In winter it is often very numerous amongst sedges in marshy places. A white specimen was in the collection of $\mathrm{Mr}$. J. Comyns.

45. Tree-Creeper. Certbia familiaris, Linn. Locally, Climb-tree, Tree-Climber, Hoodpecker.

Resident and tolerably numerous in wooded districts throughout the county.

\section{Pied Wagtail. Motacilla lugubris, Tem- minck.}

Locally, Dish or Ditch-washer, Lady White Dishes.

Resident, generally distributed and abundant. The pied wagtail is seen throughout the year, but is especially numerous in spring and autumn. At the latter season there is a great influx of birds of the year, and large flocks are observed arriving on the north coast. It also congregates in great numbers with grey and yellow wagtails in August and September in the neighbourhood of the sea, and immense numbers roost where there are reed-beds in the estuaries of the larger rivers, and on the leys along the south coast. Numbers pass the Eddystone Lighthouse in October going south. The late Mr. Henry Nicholls of Kingsbridge had a pure white specimen.

\section{White Wagtail. Motacilla alba, Linn.}

A summer migrant arriving in March and April, when it may be frequently seen singly or in small flocks in the neighbourhood of the sea coasts. It has been observed in considerable numbers early in September on the south coast previous to its departure for the continent. Mr. Eagle Clarke obtained young birds in their winter plumage at the Eddystone Lighthouse on the nights of $I_{3}$ and $I_{4}$ October I90I. There is reason to believe that this wagtail has bred both in the north and south of the county.

\section{Grey Wagtail. Motacilla melanope, Pallas}

Resident and common. Most numerous on the low lands in winter, though it breeds throughout the county. In summer it is very commonly seen by the sides of the moorland streams. It places its nest under the arches of small bridges or in holes in stone walls by the sides of mill-dams, etc. The cuckoo has been observed to deposit its eggs in the nest of this wagtail near Honiton by Mr. J. R. Earle, and at Kingsbridge by Mr. Elliot.

\section{Blue-headed Yellow Wagtail. Motacilla} flava, Linn.

A casual visitor of rare occurrence. A few examples have been obtained in southwestern Devon, near Plymouth and Kingsbridge, generally in the month of May. The late Mr. J. Gatcombe however saw a pair close to Plymouth in August 1874.

\section{Yellow Wagtail. Motacilla raii (Bona- parte)}

A summer migrant, but breeds rarely in Devon. Generally seen singly or in small parties in April and early in May, principally in the extreme south-western part of the county. In autumn it congregates in numbers mixed with other kinds of wagtails all along the south coast, and many were seen passing the Eddystone Lighthouse at the end of September I 90 I by Mr. E. Clarke. It was observed breeding at Plymouth by $\mathrm{Mr}$. Gatcombe; at Tor Point by Mr. T. R. A. Briggs; and in Huish Marshes by Mr. Elliot. It is also reported to breed occasionally on Lundy Island.

\section{1. Meadow-Pipit. Anthus pratensis (Linn.)}

Locally, Mountain Lark, Tit Lark, Titty Lark.

Resident, generally distributed, and very abundant on heaths, moors and marsh lands. There is a great immigration in autumn, and large flocks congregate in the neighbourhood of Plymouth about the beginning of September, remaining only a few days. Vast numbers were observed on emigration in the early mornings on sixteen days in October I 901 by $\mathrm{Mr}$. Eagle Clarke at the Eddystone Lighthouse, passing south. The cuckoo is particularly partial to the nest of this bird for depositing its eggs. 


\section{BIRDS}

52. Tree-Pipit. Anthus trivialis (Linn.)

A summer migrant common throughout the county in wooded districts. Arrives about the middle of April and remains up to the end of September, or even far into October.

53. Richard's Pipit. Anthus richardi, Vieillot.

A casual visitor of occasional occurrence, both in the north and south of the county, generally during the winter months. Mr. J. Gatcombe met with nine examples near Plymouth between 1841 and 1878 , and three were obtained on Braunton Burrows by $\mathrm{Mr}$. G. F. Mathew, R.N., in I864 and I869.

\section{Water-Pipit. Anthus spipoletta (Linn.)}

An accidental visitor in early spring of very rare occurrence. The late Mr. J. Gatcombe was positive that he saw a pipit of this species near Plymouth on 8 March 1873 (Zool. I 873, p. 3062), and one was seen by the writer on I 8 March 1883, on the quay at Exeter. The bird was rather tame, though restless, and allowed close approach. Southeasterly winds had prevailed for a fortnight previously.

\section{Rock-Pipit. Antbus obscurus (Latham) Locally, Rock Lark, Sand Lark.}

Resident, and common all along the coasts, especially in autumn, when large flocks arrive on passage southwards. The Scandinavian variety rupestris is sometimes seen on the cliffs of the south coast in spring.

\section{Golden Oriole. Oriolus galbula, Linn.}

A casual visitor in spring and summer of not very infrequent occurrence, usually in the months of April and May, especially in the South Hams. In July 1887 the Rev. H. Haden observed a single bird in an orchard at Stokenham, and the late Rev. Dr. Kerr saw one in August and September I 890 , close to the rectory at St. Mary Clyst. It is known to have bred in the grounds of Pilton Abbey near Barnstaple (Naturalist, I 866, p. 358). The last occasion on which a specimen of this bird was actually obtained in Devon was early in May 1900, when a female flew against the window of a house at Exmouth, and was picked up in a dying state. When the body was examined by the bird-stuffer who mounted the specimen, she was found to contain eight well-developed eggs. $\mathrm{Mr}$. Arnold Taylor, writing to Knowledge for September 1900 , states that at least one pair had been seen in Devonshire at intervals for several years in June and July.

\author{
57. Great Grey Shrike. Lanius excubitor, \\ Linn.
}

An occasional autumn and winter visitor. A few records of its occurrence in spring and summer may perhaps apply to the next species to be enumerated. About twenty specimens have been recorded from south Devon and seven from north Devon.

\section{Lesser Grey Shrike. Lanius minor, J. F. Gmelin.}

An accidental visitor of extremely rare occurrence. An immature example was obtained near Plymouth, 23 September 1876. It was taken alive by a bird-catcher, and passed into the hands of a bird-stuffer in whose possession it was recognized by the late $\mathrm{Mr}$. J. Gatcombe. A bird probably of this species was seen by Mr. F. C. Shaw near Budleigh Salterton, 22 July 1894. It was catching and devouring humble bees that flew past its perch on a burnt stump of gorse.

59. Red-backed Shrike. Lanius collurio, Linn. Locally, Butcher Bird.

A summer migrant, not very numerous but frequently met with in the neighbourhood of the sea-coast, both in the north and south of the county. It arrives early in May and usually departs about the middle of August. Its nest and eggs are frequently obtained, especially on the north coast.

6o. Woodchat. Lanius pomeranus, Sparrman.

An accidental visitor to the south coast of very rare occurrence. A female is said to have been captured by means of birdlime in the autumn of I866 at Plymouth, and was detected by the late Mr. J. Gatcombe in a bird-stuffer's shop. A male in full adult plumage was seen at Lyme Regis on the eastern border of Devon, 22 June 1876. Mr. E. A. S. Elliot shot a young female near Bantham, at the mouth of the river Avon, on 2 September 1892 . Two other records of its occurrence, one at Plymouth and the other near Kingsbridge, are doubtful.

61. Waxwing. Ampelis garrulus, Linn.

A casual visitor during the winter months, appearing at long intervals of time. Examples were obtained in I 790 near Totnes and Ashburton, and it has occurred near Plymouth. One was shot near Kingkerswell on 20 January I 829, and shortly afterwards a male at Ashburton. None seem to have occurred in Devonshire since January 1850 , when a male was obtained in the parish of Blackawton near Dartmouth, and two or three were shot near Exeter and Crediton. 


\section{A HISTORY OF DEVONSHIRE}

62. Pied Flycatcher. Muscicapa atricapilla, Linn.

A casual summer visitor more frequently met with in the northern part of Devon than in the south, where it is rarely seen. It is believed to have bred in north Devon, two old birds and four young ones having frequented Pottington Marsh near Barnstaple in August 1 859. Old birds were seen by the Rev. Murray Mathew haunting some low bushes in the vicinity of a boggy part of Huish Down near Combmartin at the end of July 1858 , and they probably bred there. A pair were shot on Lundy Island in May I86r. Examples have been obtained at Plymouth, Tavistock and Torquay. There is no record of this species having occurred in the country east of the Teign. Mr. W. Eagle Clarke obtained specimens on the night of the 22-3 September I 901 , at the Eddystone Lighthouse, when a great migratory movement of birds to the south took place.

63. Spotted Flycatcher. Muscicapa grisola, Linn.

Locally, Wall-plat (plat, a flat beam lying on the top of a wall; a favourite situation for the nest).

A summer migrant, generally distributed over the county, and numerous. Arrives about the middle of May, and remains till late in September. In some years it is not seen before the beginning of June. A greyish buff variety was seen by Mr. Gatcombe at Plymouth on 5 August 1884. It showed no trace of the normal dark markings on the head and breast.

64. Swallow. Hirundo rustica, Linn. Locally, Chimney Swallow, Barn Swallow.

A summer migrant, usually abundant. Stragglers are sometimes seen as early as the first week in March (one was picked up dead in a garden at Torquay, 22 February 1898 ) but the second week of April is the usual time of arrival. In the autumn great numbers congregate on the south coast previous to their departure, many not leaving until the end of October, and young birds have been seen in November. White examples frequently occur, and a whole brood of buffcoloured birds were hatched at Honiton.

\section{House-Martin. Chelidon urbica (Linn.)}

A summer migrant, generally abundant about towns, breeding under the eaves of the houses. Some also breed on the cliffs of the south coast. The martin usually arrives towards the end of April, though stragglers are sometimes seen much earlier. In some years none are noticed till far into May. It disappears generally before the last week of October. Young birds are however often seen late in November, or even in December. Immense numbers congregate about houses near the sea on the south coast in September and October. White martins are not uncommon.

\section{Sand-Martin. Cotile riparia (Linn.) Locally, Bank-Martin.}

A summer migrant, locally abundant wherever there are suitable sandpits, soft cliffs, or river banks, in which it can excavate its nesting holes. The vast number of sandmartins seen flying over the Exe near Topsham in the evenings of May I 900 were very remarkable. This is the first of the swallow family to arrive in spring, being often seen in March. Mr. E. A. S. Elliot had a white sand-martin brought to him in August 1900.

67. Greenfinch. Ligurinus chloris (Linn.) Locally, Green Linnet.

Resident and generally distributed. Its numbers are largely recruited in autumn, when large flocks arrive and remain throughout the winter, congregating on the borders of fields around wheat-ricks, the dockyards at Devonport, and wherever grain is to be found.

68. Hawfinch. Coccotbraustes vulgaris, Pallas.

An occasional visitor to all parts of the county, including Lundy Island, principally in the winter months, but is sometimes seen in pairs and in breeding plumage near Exeter. It has bred near Kingsbridge. This fine finch is but little known in Devonshire, and has been mistaken for the pine grosbeak.

\section{Goldfinch. Carduelis elegans, Stephens Locally, Gool Finch, Blossom Bird.}

Resident and formerly numerous, but at present occurs only sparingly in most localities. A few pairs are said to breed in private grounds in the neighbourhood of Exeter. Great numbers perished in the severe winters of $1845-6,1853^{-4}$ and $1880-1$. Single birds are sometimes seen in spring in the vicinity of the south coast, and in October small flocks move along its shores, probably on passage. At this time many were formerly captured by bird-catchers in their nets.

\section{Siskin. Carduelis spinus (Linn.)}

A winter visitor of uncertain appearance. Occurring in small flocks between Michael- 


\section{BIRDS}

mas and April in some years. The siskin is generally found extracting the seeds from the fruits of the alder, or picking up seeds amongst the debris left by floods on the banks of streams, and is very tame and easily captured. None have been met with for many years in the neighbourhood of Exeter, and Mr. Elliot has not seen any since February I 886 near Kingsbridge. The siskin has occurred near Plymouth, Ashburton, Newton Abbot, Kingsbridge, Starcross and around Exeter. It has been rarely seen in north Devon. In 1828 three were caught in summer in the marshes near Newton, according to Turton and Kingston. Mr. Gatcombe states that young birds were taken with goldfinches near Plymouth on 24 September 188 o.

71. Serin. Serinus bortulanus, K. L. Koch.

According to Mr. W. E. Pidsley (Zool. I 892, p. I I 4), an example of this finch was captured by a bird-catcher between Exmouth and Budleigh Salterton on 29 November I89I. It was possibly an escaped one from an aviary in Exmouth.

\section{House-Sparrow. Passer domesticus (Linn.)}

Resident, extremely abundant and generally distributed throughout the county. Although of course absent from the uninhabited central parts of Dartmoor, it is plentiful enough at times in all the towns, villages and farms on and around the moor. It was formerly only an occasional visitor to Lundy Island, but a colony has established itself there within the last few years. Albinos and parti-coloured birds frequently occur.

73. Tree-Sparrow. Passer montanus (Linn.)

A casual visitor to the southern parts of the county, of irregular and rather rare occurrence in the autumn and winter months (October to March). It appears to be most numerous in the Kingsbridge district, where it has been met with in small parties from autumn to late spring. A small flock was noticed one winter frequenting the borders of reed beds and some waste ground near Torquay by Mr. C. Dixon. It has occurred at Plymouth, near Totnes and East Budleigh.

74. Chaffinch. Fringilla coelebs, Linn.

Locally, Maze Finch, Copper Finch, Daffinch, White Finch or Winch, Silver Winch, Chink, Pink and Wink.

Resident, generally distributed and very abundant. Large flocks assemble in winter, and probably mainly consist of immigrants.
Mr. W. Eagle Clarke observed numbers passing the Eddystone Lighthouse in October IgOI.

75. Brambling. Fringilla montifringilla, Linn.

A winter visitor of irregular appearance depending on the character of the season; in some years, after severe weather, occurring in large numbers, whilst in others it is very scarce or altogether absent. An adult male occurred at Landkey near Barnstaple on 24 July 1856 , in company with yellow buntings and greenfinches.

\section{Linnet. Linota cannabina (Linn.)}

Locally, Brown Linnet (in winter), Rose Linnet, Fiery and Red Linnet (in spring).

Resident, generally distributed and abundant. Immense flocks are seen on the stubblefields in autumn and winter, and are probably mainly composed of immigrants from the north and east. Flocks also appear on the south coast in spring for a few days, and consist of very dark coloured birds. Examples of the male in full red plumage in spring are not very common in this county, and it has been observed that the brightest plumaged birds are met with on the sea coast. White varieties have occurred.

\section{Mealy Redpoll. Linota linaria (Linn.)}

The reported instances of the occurrence of this northern species in Devon are not well authenticated. The specimen in Pincombe's collection at Plymouth, mentioned by Dr. E. Moore and Bellamy, was considered to be very doubtful by the late Mr. J. Gatcombe. There was an example in the collection of the late Mr. Cecil Smith labelled 'North Devon.' It passed into the possession of the Rev. Murray A. Mathew.

\section{Lesser Redpoll. Linota rufescens (Vieillot)}

An autumn and winter visitor of irregular appearance. From September to March inclusive, small flocks of this little finch may be met with in some years feeding on the seeds of birch and alder trees. Sometimes they frequent the margins of rivers and leys to feed on the seeds of reeds, etc., washed up on the shores. It has been observed near Dulverton in June, and the late Rev. H. A. Macpherson saw an old redpoll feeding a newly-fledged nestling on the branch of an ash tree near Lynton, north Devon, in July 1879. It is said by Mr. C. Dixon to have nested at Torquay. The last recorded occurrence of the lesser redpoll in Devonshire was in December 1899, when one was picked up dead in a field near Kingsbridge. Some 


\section{A HISTORY OF DEVONSHIRE}

had been seen in the previous March on alder trees along the banks of the Avon near that town (E. A. S. Elliot).

\section{Twite. Linota favirostris (Linn.)}

A casual autumn and winter visitor to north Devon, but unknown in the south of the county. The Rev. M. A. Mathew used to meet with small flocks on Northam Burrows, and on the side of the north walk in the town of Barnstaple, and obtained specimens near that town.

\section{8o. Bullfinch. Pyrrbula europeea, Vieillot.}

Locally, Hoop, Bud Finch, Bud-picker, Coalhood.

Resident and tolerably numerous in some localities. In early spring small parties visit gardens just as the fruit trees and bushes are budding and work havoc on them, especially on pears and gooseberries. Rewards were formerly paid for their destruction in many parishes in Devon. At Ashburton between I 761 and $I 820$ inclusive, I, 66I 'hoops' were destroyed at an expenditure of $£_{3}$ IOs. $4 \frac{1}{2} d$. At Dulverton as much as $3 d$. per head appears to have been paid for them (Dr. Brushfield in Trans. Devon Assoc. 1897). In summer and autumn it keeps to the woodlands and copses. Black and white specimens have been obtained. In the latter the breast retains some of its roseate hue.

[The pine grosbeak (Pyrrbula enucleator) has been recorded as a Devonshire bird, but the evidence of its occurrence is not satisfactory. In some instances the hawfinch and crossbill have been mistaken for it.]

\section{Crossbill. Loxia curvirostra, Linn. \\ Locally, Shell-apple.}

A casual visitor of very irregular occurrence, but appearing at all times of the year. Flocks were seen in July 1810, I831, October 1835 ; June 1837 ; in the winter of 1 838-9; June 1848; Nov. I861 ; March 1869 ; July to October I868; January to March 1869; January 1888; September to November 1889; March 1895; and in the winter of $1898-9$.

In July 1868 there was a simultaneous immigration of crossbills into Ireland, Cornwall, Devon, Somerset and Gloucestershire. In November 1869 crossbills and snow buntings mixed together were seen flying across Lundy Island and some of both were shot.

The crossbill has bred several times in Devon. Mr. W. R. Hall Jordan saw a nest in a spruce fir tree at Ogwell House on IO April I839. The male was shot, but the female bird still continued to attend the nest. A pair bred in Mr. C. Pearson's garden at Hatherleigh in north Devon in June 1894. The nest was placed in a Scotch fir. A young bird in striped plumage was shot at Budleigh Salterton in September I 889.

In January I 888 a flock of the large northern race of this bird, known as the parrot crossbill (Loxia pityopsittacus), visited the fir plantations at Marley near Exmouth, and remained for some weeks. Many were shot and examined by the writer, who ascertained that they were examples of the stout-billed race of crossbill.

[A male example of the American whitewinged crossbill (Loxia leucoptera) in the red plumage of the second year was found dead on the beach at Exmouth, apparently just washed ashore, by Mr. E. B. Fitton, I 7 September 1845. A strong south-west wind was blowing and had continued for several days. It had probably been conveyed across the Atlantic in the rigging of some passing vessel, and had fallen into the sea from exhaustion.]

\section{Corn-Bunting. Emberiza miliaria, Linn.} Locally, Horse Lark, Bunting Lark.

Resident, but local, frequenting corn-lands. It is sometimes abundant on the coast near Plymouth, Kingsbridge, Berry Head, etc., during migration in August and September. It is tolerably common in the South Hams, breeding near Kingsbridge and along the coasts. It is not numerous near Exeter, according to the experience of the writer, but Mr. Pidsley appears to have found it abundant at Broad Clyst. In north Devon it is very local; a few frequent the high ground above Ilfracombe, and it occurs on Braunton Burrows and at Instow. A buff-coloured variety was obtained at Alphington near Exeter in February 1875 .

\section{Yellow Bunting. Emberiza citrinella, Linn. \\ Locally, Gladdy (A.-S. gladde= bright), Yellow Yowley (A.-S. geolu=yellow) \\ Resident, generally distributed and abundant.} Lutino varieties occur.

\section{Cirl Bunting. Emberiza cirlus, Linn.} Locally, French Yellow Hammer.

Resident, rather local, but at times common in many places both in the north and south of the county.

The cirl bunting was first observed as a British bird by Colonel Montagu at Kingsbridge, south Devon, in 1800 . In the following year he found the nest in Devonshire. It is now well known as a breeding bird in the 


\section{BIRDS}

county. In winter the cirl bunting associates with other small birds about farmyards. At that time of the year it wanders about in small flocks, in some years being abundant, and in others scarce in the same locality.

85. Reed-Bunting. Emberiza scboeniclus, Linn.

Resident, and not uncommon on the banks of rivers, leys and ponds, osier-beds and marshes, where reeds, sedges and rushes grow, throughout the county. It is said to be a winter visitor to Lundy Island. Generally seen in pairs or small parties and seldom in flocks. It is most frequently noticed in the winter months, when it seems more numerous and probably migratory accessions arrive at that season. A fawn-coloured variety has been obtained.

\section{Snow-Bunting. Plectrophenax nivalis (Linn.)}

Locally, White Lark.

A winter visitor of irregular appearance, but not uncommon, usually singly or in small parties, and sometimes met with as early as September, remaining rarely as late as April and May. It occurs most frequently near the coasts, more especially in the north of the county. It is met with occasionally on Dartmoor and the Haldon Hills. On Lundy Island it is sometimes seen in considerable numbers in winter.

\section{Starling. Sturnus vulgaris, Linn.}

Locally, Stare or Steer, from its cry.

An extremely abundant resident throughout the county excepting Dartmoor and its borders, where it is an autumn and winter visitor. Its numbers have greatly increased in Devonshire since about 1844 , and it now breeds freely in north Devon and the southwestern portion of the county, where less than fifty years ago it was only known as a winter visitor. There are enormous migratory accessions in autumn and winter, and vast flocks then assemble to roost in plantations where they become an insufferable nuisance owing to the strong effluvium from their droppings. They are also partial to reed-beds as roosting places. A famous locality for such an assemblage in autumn and winter is at Slapton Ley, where the aerial evolutions of the large flocks, before alighting on the reeds, excite great interest. White and cream-coloured specimens occur occasionally, and the late Mr. Gatcombe saw a perfectly black and unspotted individual feeding with a flock of ordinary starlings. In October I $901 \mathrm{Mr}$. W. Eagle Clarke saw vast numbers of starlings passing the Eddy- stone Lighthouse, and many struck against the lantern and were captured. They all belonged to the purple-headed continental variety with green ear-coverts.

88. Rose-coloured Starling. Pastor roseus (Linn.)

A casual visitor during the spring and summer months (May to October). Many specimens of this beautiful bird have been obtained in the county, especially in the South Hams and on Lundy Island, where it was formerly a regular spring visitor. A female full of eggs was shot at Berry Head, I2 July $185 \mathrm{I}$.

89. Chough. Pyrrbocorax graculus (Linn.) Locally, Cornish Chough, Red-legged Crow, Killigrew (obs.)

Resident in small numbers in some spots on the north coast of the county. It is now unfortunately extinct on Lundy Island, where it was formerly numerous. The peregrine falcons have been the main agents in exterminating this interesting bird, but doubtless the island being now so much frequented by tourists is no longer a desirable place of residence for it. On the south coast it is now but a casual visitor, though it has been known to breed once or twice within the last thirty years. Probably the jackdaws which have increased so greatly of late years have destroyed the eggs and driven the choughs away from their former breeding places on the cliffs.

\section{Nutcracker. Nucifraga caryocatactes (Linn.)}

An accidental visitor of rare occurrence. One was shot in north Devon in August 1808 ; another at Washford Pyne Moor, near Tiverton, by Mr. W. Tucker of Dawlish, in I 829. An example was seen on the banks of Hooe Lake, in the parish of Plymstock, by the late Mr. Thomas Bulteel; and Mr. J. Brooking Rowe thinks that he saw one in the woods at Saltram in October 1862 . A nutcracker was seen by Mr. M. H. Rotherham on 14 July 1880 , in Huntsham Woods near Bideford; and another was seen two years previously at Instow by Mr. C. F. Hinchliff. A specimen in the possession of Mr. L. Sparrow of Stroode near Ivybridge is believed by that gentleman to have been obtained in the neighbourhood by his father.

\section{Jay. Garrulus glandarius (Linn.) Locally, Jay Pie.}

Persecution has caused the disappearance of 


\section{A HISTORY OF DEVONSHIRE}

this beautiful bird from many parts of the county where once it was numerous and bred freely. It is still rather common in the more wooded and secluded districts, especially in the South Hams.

\section{Magpie. Pica rustica (Scopoli) \\ Locally, Pie, Mock-a-pie, Piannet.}

Like the jay, the magpie is gradually disappearing from the more thickly inhabited districts, though still a plentiful resident in some parts, as, for instance, in the South Hams and on the south-eastern borders of Dartmoor, where as many as ten to twenty are frequently seen together in autumn and winter. Mr. Gervaise F. Mathew, R.N., saw a magpie with a yellow beak near Buckfastleigh in July I 867 . A brood of young magpies with all the parts usually white of a buff colour occurred near Buckland-tout-Saints.

\section{Jackdaw. Corvus monedula, Linn.}

Locally, Daw, Chauk, Chofe, Chuffe, Chow.

This is one of those birds that are increasing in numbers throughout the county. It is resident and very numerous, breeding in a great variety of situations, e.g. the towers of Exeter Cathedral, in holes in railway viaducts, in thick ivy against the walls of houses, in chimneys, in holes in trees, and in company with rooks on the tops of lofty trees, in rabbitburrows as at Vitifer tin mine on Dartmoor, in clefts of rocks as at the Dewerstone, and in great numbers on the cliffs of the sea-coasts. It has become a serious nuisance in some places, and destructive fires have been occasioned through the large quantities of sticks, feathers, horse-hair, sheep's wool and other materials collected in the chimneys of country houses. Large flocks may be seen on winter evenings winging their way back to their roosting places. The jackdaw was formerly unknown on Lundy Island, but has now become an occasional visitor there. Albinos occur at Exeter, Torquay, Berry Pomeroy Castle and Plymouth. At Otterton, late in the eighteenth century, $6 d$. per dozen was paid by the parish for the destruction of 'chofes' and jays.

\section{Raven. Corvus corax, Linn.}

The raven is decidedly on the increase in the South Hams, where it has become a perfect pest to the sheep farmers. Many pairs breed on the cliffs both of the north and south coasts, on some of the rocky tors of Dartmoor, and in trees in some woods in north Devon. A pair or two nest on Lundy Island.

\section{Carrion Crow. Corvus corone, Linn. Locally, Black-bill.}

Although considerable numbers may be seen in winter feeding at low-water on the sands along the coasts, this species appears to be decreasing generally as a resident, and it is no longer seen in some places where it used to breed annually. It is still however numerous in wooded districts in the south of the county, but is not common in north Devon. It is found on Lundy Island, where it breeds. White, cream-coloured and other varieties occur.

\section{Hooded Crow. Corvus cornix, Linn. Locally, Mussel-Crow, Grey Crow.}

At the present day the hooded crow is a rare straggler to Devon in the autumn and winter months. It appears to have been formerly numerous in winter at Plymouth and on the coast of north Devon. Pennant was misled by Gilbert White in stating that this bird bred on Dartmoor, and according to Polwhele it was never seen there in summer. It is however stated to have once bred at Youlston Old Park in north Devon, and it is thought to have interbred with the carrion crow on Lundy Island.

\section{Rook. Corvus frugilegus, Linn.}

\section{A.-S. $b r a b c=a$ croaker.}

A very abundant resident increasing in numbers. There are many large rookeries in the county. The immense flocks seen in winter are perhaps partly formed by immigrants from the north and east.

\section{Sky-Lark. Alauda arvensis, Linn.}

Although an abundant resident, the numbers of those sky-larks that remain with us all the year are as nothing compared to the vast hosts which visit us in winter, especially in severe seasons, when thousands perish. Mr. W. Eagle Clarke saw great numbers passing the Eddystone Lighthouse in October I 901 . White, buff and black varieties occur.

\section{Wood-Lark. Alauda arborea, Linn.}

In some woodland districts in the county this delightful songster is rather common, but it must be considered very local in the breeding season. It nests in the neighbourhood of Kingsbridge and of Plymouth. In autumn and winter large numbers arrive, and may be met with in small parties on high rough ground or near the coast, according to the severity of the season. A buff-coloured variety occurred at Crediton in August 1851. 


\section{BIRDS}

100. Crested Lark. Alauda cristata, Linn.

The only specimen of this continental species that has been obtained in Devonshire was shot about the year 1851 , on Braunton Burrows, by the late Mr. Clevland of Tapley Park near Bideford. The late Lord Lilford thought he had seen a pair close to the Sands Hotel at Slapton Ley in July 1852.

[Both the short-toed lark ( $A$. bracbydactyla) and the calandra lark ( $A$. calandra), natives of southern Europe, have been reported from Devonshire. The examples obtained of the first are known to have been imported, and the two specimens of the latter, said to have been killed near Plymouth and Exeter respectively, were obtained from dealers.]

\section{IO1. Shore-Lark. Otocorys alpestris (Linn.)}

Although this lark has occurred on several occasions on the neighbouring coast of Dorset, and twice in Cornwall, the only properly substantiated occurrence in Devonshire is that recorded by Mr. Hutchinson of Bideford, who saw a flock of shore-larks on Northam Burrows on 2 January 1875, and shot one. An adult specimen, formerly in the collection of the late Mr. Cecil Smith, is said to have been shot at Paignton. Two specimens are said to have been killed on Dawlish Warren, but there is some doubt as to their origin, as they were obtained from a dealer. It is however by no means improbable that this pretty species should have occurred at that locality. It is apparently extending its range of winter migration westward, and is likely to visit Devonshire more frequently in the future.

102. Swift. Cypselus apus (Linn.)

Locally, Devil's Screecher, Skeer Devil.

A summer migrant usually very abundant throughout the county, especially around towns and villages. Arrives generally early in May, but sometimes during the last week of April. It ordinarily takes its departure in the second week of August. Instances however have occurred in which individuals have lingered until October and November. This well known bird breeds freely under the roofs of houses in Exeter and other towns, in holes in trees in woods, in the crevices of granite tors and stone walls on Dartmoor, and on cliffs of the sea-coast at Beer and Berry Head and elsewhere.

103. Alpine Swift. Cypselus melba (Linn.)

An immature example was shot, and two others seen, near Ilfracombe, 4 October I 876 , by Mr. H. E. Rawson. They were flying in company with about a score of common swifts.
104. Nightjar. Caprimulgus europaus, Linn.

Locally, Fern-Owl, Night-Crow, Dor-Hawk.

A summer migrant very numcrous, in suitable localities, throughout the county, especially on the borders of Dartmoor. Usually arrives in April or early in May, and departs in September. Individuals have been known to remain as late as November. It is a summer visitor and common breeder on Lundy Island. Mr. Comyns of Mount Pleasant near Dawlish possessed a white specimen.

105. Wryneck. I ynx torquilla, Linn.

A casual visitor of rare occurrence in spring and autumn, principally in the south of the county. It was stated to breed near Dawlish by Mr. Comyns, according to Dr. E. Moore (Trans. Plymouth Inst. I830).

106. Green Woodpecker. Gecinus viridis (Linn.)

Locally, Woodawl, Woodmaul, Woodwalf, Parrot Woodpecker.

This beautiful bird is a common resident throughout the county, excepting the moors. It would be very numerous but for the starlings that appropriate its nesting holes in dead trees. There appears to be a considerable immigration of green woodpeckers into the western counties in severe winters.

[A specimen of the great black woodpecker (Picus martius) is said to have been killed at Crediton, and was in the collection of the late $\mathrm{Mr}$. John Newton of Millaton near Bridestowe. It is highly improbable that this large woodpecker, an inhabitant of the great pine forests of Scandinavia, should visit our county. Several other supposed occurrences have been recorded, but there is little doubt that the great spotted woodpecker has been confused with this bird.]

107. Great Spotted Woodpecker. Dendrocopus major (Linn.)

Locally, French Pie, Pied Woodpecker, Magpic Woodpecker.

A resident but not common anywhere in Devonshire, though occasionally met with in wooded districts throughout the county. It is most often seen in winter and early spring. It is occasionally found breeding in woods, and would probably be more numerous but for the starlings which appropriate the nesting holes of this as well as of the green woodpecker. Orchards of small cherries, called ' mazzards,' offer a great attraction to this bird, which feeds on them when ripe. 


\section{A HISTORY OF DEVONSHIRE}

108. Lesser Spotted Woodpecker. Dendroi copus minor (Linn.)

Resident and not uncommon in woods in some localities, both in the north and south of the county. It is most numerous in winter and spring, perhaps because it is most readily seen when the trees are bare of leaves. It often frequents old elm trees in gardens even in the towns.

ro9. Kingfisher. Alcedo ispida, Linn.

A common resident in the greater part of the county, but rare in the more central portion. An extensive immigration takes place in September, and kingfishers are sometimes plentiful in severe winters, when many perish. It breeds in many localities.

I I . Roller. Coracias garrulus, Linn.

An accidental visitor of very rare occurrence in autumn, and almost entirely confined to the southern portion of the county, in which three specimens have been obtained; only one example being recorded from north Devon.

\section{I. Bee-eater. Merops apiaster, Linn.}

An accidental visitor, during the spring and summer months, of rare occurrence, and only to the southern part of the county. About seven examples have been observed.

\section{I12. Hoopoe. Upupa epops, Linn.}

A summer migrant, of rather irregular appearance, principally in spring and autumn, most frequently occurring in the South Hams, especially in the Plymouth and Kingsbridge districts, and formerly on Lundy Island. A nest with four young was taken close to the house at Morwell in the parish of Tavistock before 1836 . No other instance of its breeding in Devonshire is known, though individuals were observed frequenting a certain locality in the extreme south-western part of the county all through the summer of 1899 . An example (the first Devonshire specimen recorded) was shot in December 1790 , near Little Dartmouth. A hoopoe was killed about 23 March 1897, near Kingsbridge, and one was seen on I March 1900 , in a garden at Modbury, and remained about there for some time.

\section{13. Cuckoo. Cuculus canorus, Linn.}

Locally, Gowk, Gookoo.

An abundant summer migrant. Arrives usually in the third or fourth week of April, but has several times been reported as having been seen and heard in March. The adults leave us in July; young birds remain till late in the autumn. Polwhele records that a cuckoo was heard on Io January I 778, near Mount Edgcumbe, and in that month in several parts of the South Hams, it being an extraordinarily mild season. Early in December 1898 an adult cuckoo is said to have been twice seen in the neighbourhood of Dunsford, the temperature prevailing being abnormally high for the time of the year.

[A specimen of the American yellow-billed cuckoo (Coccyzus americanus) is said to have been picked up dead beneath the lighthouse on Lundy Island in October I 874.]

\section{Barn Owl. Strix flammea, Linn.}

Locally, Screech Owl, White Owl.

Resident, generally distributed, and still fairly numerous, though its numbers have been greatly reduced by persecution.

\section{Long-eared Owl. Asio otus (Linn.)}

A winter visitor, rather irregular in its appearance, and rarely numerous, though sometimes large flights have visited the county, as in the winter of $1873-4$; from September to December 1879 ; and in January 1886. It has occurred sometimes in spring in the south-western part of the county, and is said to have bred on Haldon in 1863 . The nest has also been found in north Devon, but this owl is rare there. It is not recorded from Lundy Island.

116. Short-eared Owl. Asio accipitrinus (Pallas)

Locally, Woodcock Owl.

A winter visitor, varying greatly in numbers in different years. It was abundant throughout Devonshire in $1875-6$ and in 1878 . This owl arrives sometimes as early as September and occasionally remains as late as March. It is considered as the precursor of the woodcock on Lundy Island, where in some winters it has been very abundant. Mr. F. H. Carruthers Gould found its nest near Braunton, containing three young birds and an infertile egg, in the spring of 1893 .

\section{Tawny Owl. Syrnium aluco (Linn.)}

Locally, Brown Owl, Wood Owl.

Resident and generally distributed in wooded districts throughout the county, in spite of keepers and their cruel pole-traps.

\section{8. Little Owl. Athene noctua (Scopoli)}

An accidental visitor of very rare and doubtful occurrence in a really wild state, being often imported and kept in captivity as an amusing pet, and sometimes turned loose. 


\section{BIRDS}

An example is mentioned in the supplement to Montagu's Ornitbological Dictionary as having been seen and shot at, in north Devon, in 1808 . Dr. E. Moore says that one procured at Plymouth was sent to Mr. Drew for preservation. Another occurred at Ashburton in 1809 , according to Dr. Tucker. Bellamy mentions one that was shot not far from Yealmpton. Specimens from Downes, near Crediton, in October I85I, and Torquay about September 1890, were probably imported birds.

I 19. Snowy Owl. Nyctea scandiaca (Linn.)

An accidental visitor of very rare occurrence. A specimen was killed near Plymouth in December 1838 , and was originally in the collection of Dr. Cornelius Tripe of Devonport, and at the sale of his collection in 1860 passed into the possession of the Rev. W. Hore, who bequeathed it with the rest of his mounted birds to Mrs. Connop of Bradfield Hall. On 13 March I876, the weather being very cold, a snowy owl was seen at Ditsworthy rabbit warren, and was shot by a little boy eight years of age, the grandson of the warrener. Another snowy owl occurred about ten days later on Exmoor and was trapped. A specimen in the Albert Memorial Museum at Exeter is said to have been obtained at Exmouth some time prior to I $85 \mathrm{I}$. The tail feathers are much soiled and worn. A tarso-metatarsal bone of this owl was obtained from Kent's Cavern, Torquay.

[A hawk owl (Surnia funerea) is supposed to have been seen by Captain J. N. Still of Slade, Knoyle, Wilts, at Musbury, in east Devon at the end of August or the beginning of September 1869.]

[Dr. Edward Moore includes the eagle owl (Bubo ignavus) in his lists of Devonshire birds, and states that he had seen one in a living state near Honiton in 1820 , but does not mention whether it was in captivity or wild.]

120. Marsh - Harrier. Circus aruginosus (Linn.)

Locally, Moor-Buzzard.

At one time a very common resident, especially on Dartmoor where it formerly nested, but now a very rare casual visitor in autumn. Up to 1850 it was not uncommon about rabbit warrens on Dartmoor, but soon after that year it became almost extinct in Devonshire. One frequented the Barnstaple river about 1857. A male in the second year's plumage was killed at Slapton Ley on 1 November 1875 , whilst in the act of taking a coot. A pair frequented a valley near
Musbury in September 1876. An adult male was shot on Braunton Marshes on 3 November 1890 . It is said to have been an occasional visitor to Lundy Island.

121. Hen-Harrier. Circus cyaneus (Linn.)

Locally, Blue Hawk, Vuzz Kit or Furze Kite.

This is another species that was formerly an abundant resident, breeding commonly in the neighbourhood of the moors, but it is now like the preceding species only a rare casual visitor, generally in the autumn and winter. Adult males are very seldom met with. $\mathrm{Mr}$. Bragg of Furlong near Chagford has in his possession a female hen-harrier, together with her nest and four eggs, which he obtained from Tor Hill in the parish of Throwleigh about 1861. This is probably the last instance of this hawk breeding in Devonshire, though a pair were killed near Chagford in I87 I. The most recent instances of the occurrence of the hen-harrier in the county are a female on Black Hill near Exmouth in January 1888; another female near Exmouth in 1900 ; and one at Heanton near Barnstaple in December I889.

122. Montagu's Harrier. Circus cineraceus (Montagu)

A casual visitor, occurring not unfrequently in all parts of the county, but more especially in the south-western part of Dartmoor and the Kingsbridge district. It is usually seen in spring and summer, and has been known to breed in the county on several occasions. It does not appear to have been met with in Devonshire later in the year than the month of October. Its food consists principally of lizards and small birds' eggs. A black variety has occurred several times in Devon. Colonel Montagu first discriminated between this species and the hen-harrier in 1802 .

\section{Buzzard. Buteo vulgaris, Leach.}

Locally, Kit, Kitt or Keat, Black Kite, Eagle.

Resident, and still not uncommon about the cliffs of the sea-coasts, in wild uncultivated localities, and in wooded districts throughout the county. It is however most plentiful in autumn and spring, when there are migratory movements from other countries, small parties of three to five being often seen in the neighbourhood of the south coast, Dartmoor and Exmoor. It nests annually in several localities in the county, both on the coasts and inland. Pure white, buff-coloured and entirely black varieties have occurred.

124. Rough-legged Buzzard. Buteo lagopus (J. F. Gmelin)

A casual visitor during the winter months 


\section{A HISTORY OF DEVONSHIRE}

of rare occurrence. In some years an unusually large immigration of this bird takes place to the eastern parts of England, and then some few stragglers reach the south-western counties, and frequent the moors for a time. Examples occurred in Devon, principally on Dartmoor, in November, 1836,1848 , October, $1875,1876,1877$, and March, I883. A black variety was killed in north Devon about Christmas, 1875 .

125. Spotted Eagle. Aquila maculata (J. F. Gmelin)

An eagle believed to have been one of this species was shot in the winter of 1858 on Lundy Island by Mr. Spencer Heaven. It rolled over the cliffs and was lost. Two examples have occurred in Cornwall, one on 4 December, 1860 , not far from the Devonshire boundary.

[The golden eagle (Aquila chrysaëtus) was formerly supposed to visit Dartmoor, and to have bred on the Dewerstone on the Plym near Plymouth. There is however little doubt that the eagles that had an eyrie there belonged to the next species.]

\section{White-tailed Eagle. Haliaztus albicilla (Linn.)}

A casual visitor of occasional occurrence. It formerly nested at the Dewerstone, on the Plym, and on Lundy Island. It does not now breed anywhere in the county. Most of the examples met with at the present day occur in the autumn and winter months, and are young birds of the year on passage south. The most recent instances of its occurrence in Devonshire were as follows: An eagle probably of this species was seen on Dartmoor in May or June 1891 , and an immature female was trapped at Dartmouth in the autumn of that year. Another immature bird weighing $8 \mathrm{lb}$. was shot at Bude Haven in November 1893; and an example, also immature, at Stokeley in November 1899.

[The occurrence of the goshawk (Astur palumbarius) in Devonshire is extremely doubtful. It appears to have been admitted to the county fauna on the authority of Dr. Andrew Tucker of Ashburton, who included it in a 'List of some rare Land Birds which have been discovered in the neighbourhood of Ashburton' in Jones' Guide to the Scenery, 1823. A reported occurrence at South Tawton in 1830 is not worthy of credence.]

127. Sparrow-Hawk. Accipiter nisus (Linn.)

The sparrow-hawk is still, as formerly, the most abundant species of hawk in Devonshire, and is rather a common resident in all parts of the county that are well wooded. There is sometimes a considerable influx of this species into western Devon in autumn, as in September 1873, and September and November 1874. An albino was killed at Castle Hill, north Devon, on 28 June 1849 . An old male shot on 21 November I89I, between Modbury and Ivybridge, was of a smoky tint all over.

\section{Kite. Milvus ictinus, Savigny. \\ Locally, Fork-tailed Kite.}

A casual visitor of very rare occurrence, but said to have been common in the neighbourhood of Dartmoor at the end of the eighteenth century, and to have bred at Holne Chase. Montagu however, writing in 1813 (Supp. Orn. Dict.) stated that in twelve years he had only seen one kite in the southern district of the county, and in a MS. notebook for I 798 he observes that he had met with ' the kite rarely' in Devon. In all probability the ' kitts' or ' keets' so often mentioned in the churchwardens' accounts in rural parishes, and for whose destruction rewards were paid, were only buzzards, then, as now, known by those names. About ten examples of the 'fork-tailed kite' have occurred in Devon within the last fifty years, generally in spring and autumn. They were mostly obtained in the south-western part of the county. The last occurrence however was a fine male shot at West Down near Ilfracombe in October I 886.

1 29. Honey-Buzzard. Pernis apivorus (Linn.)

A casual visitor of very rare occurrence during the spring and autumn migration. Seven specimens have been obtained in Devon since 1813. A very fine male bird was caught in a gin on Lord Morley's property near Plymouth in July 1850 . It was observed by a keeper sitting on a pheasant's nest, and had been feeding on the eggs. The last that occurred was an immature bird shot at Spriddlestone near Plymouth, I2 October I881. It has only occurred once in north Devon.

130. Greenland Falcon. Falco candicans, J. F. Gmelin.

Three or four examples of this fine falcon are known to have occurred in Devon. One was winged and taken alive on the Lynher river near Plymouth, 7 February 1834 . Another was shot on Lundy Island in the month of November, many years ago, by $\mathrm{Mr}$. Philip Wathen. A Greenland falcon is said to have been seen by Mr. Henry Swaysland, 


\section{BIRDS}

junr., in June I882, at Rousdon near Lyme Regis, and one, apparently an immature specimen, was shot on Lundy Island $2 \mathrm{I}$ March I903.

\section{Peregrine Falcon. Falco peregrinus, Tunstall.}

Locally, Cliff-Hawk, Blue Hawk.

A well known resident, not uncommon and rather on the increase, though subject to unceasing persecution. It is met with throughout the county, especially on the seacoasts, and a celebrated white-chested race has occupied Lundy Island from time immemorial. There are many eyries known both on the north and south coasts.

\section{Hobby. Falco subbuteo, Linn.}

A summer migrant now unfortunately not often seen. A few pairs probably still nest annually in the woods on the borders of Dartmoor, but the young are ruthlessly destroyed by gamekeepers, and there seems to be but little chance of this beautiful little falcon ever increasing in numbers again. It has been known to occur as early as March, and to remain as late as October in the southwestern part of the county.

\section{I33. Merlin. Falco asalon, Tunstall. Lccally, Little Blue Hawk.}

An autumn and winter visitor not of very frequent occurrence. Arrives generally about the middle of September, remaining as late as March. It is rarely seen in summer, but one was observed on Dartmoor near the North Teign on 30 July 1895 . The merlin is believed to have nested on Exmoor.

\section{Red-footed Falcon. Falco vespertinus (Linn.)}

Two adult examples, both males, of this beautiful African falcon are said to have been procured near Plymouth, one in March 1837, and the other about i 862. Both specimens were in the collection of J. Pincombe, the well known dealer in birds at Devonport. The first was brought to him by a sailor, and was said to have been taken on board a vessel near the Plymouth breakwater. The other was stated to have been shot at Wembury Cliffs, where a female was also seen. Another specimen said to have been killed in Devon was in the collection of the late Mr. MarshDunn of Teignmouth. None of these occurrences are satisfactorily authenticated. This falcon has however occurred several times in Cornwall.
135. Kestrel. Falco tinnunculus, Linn.

Locally, Wind-fanner, Windhover, Criss or Crasset Hawk.

Next to the sparrow-hawk, the 'windhover' is the commonest of our birds of prey. In the autumn many arrive on passage south. Our resident birds nest on the cliffs all along the sea-coasts, on the sides of quarries and on some of the Dartmoor tors.

\section{Osprey. Pandion baliaettus (Linn.)}

Locally, Herriot (according to Polwhele).

No example of this fine bird appears to have occurred since the autumn of 1875 , when quite a flight seems to have visited Devonshire, and specimens were obtained on the Teign, Dart, Avon, Tamar and Taw. Up to the middle of the last century the osprey was well known on the estuaries of the larger rivers, especially in the south of the county, being seen at all times of the year, but generally in spring and autumn. It is stated by Polwhele to have bred on a pinnacle of the cliffs at Beer in the eighteenth century, and also on the cliffs of the north Devon coast. There was an eyrie at Gannet's Combe on Lundy Island, where a pair bred as lately as 1838 , in which year the male was shot and the female disappeared never to return.

137. Cormorant. Phalacrocorax carbo (Linn.) Locally, Shag, Topsham Pilot, Isle of Wight Parson

An abundant resident on all the Devonshire coasts, breeding on the cliffs of the coasts and frequenting the estuaries of the larger rivers, and often following the course of the streams far inland. Up till very recently thirty or more cormorants might be seen at a time on the sandbanks in the estuary of the Exe, drying their half-open wings in the wind. The Exe Board of Conservators have however lately (February 1902) offered $6 d$. (since increased to Is.) a head for their destruction, and their numbers have been greatly reduced, about 250 having. been returned as killed within the first ten months in the Report of the Conservators. The old birds were ruthlessly destroyed on their nests on the cliffs between Exmouth and Ladram Bay. The Dart Fishery Board are about to follow suit, and it seems probable that this species and the next will soon be driven away from the south coast. They are specially excluded from the provisions of the Wild Birds Protection Acts, and may be killed at any time of the year owing to the great destruction of fish they accomplish. On 15 June 1883 


\section{A HISTORY OF DEVONSHIRE}

a cormorant, with a conspicuous white collar round its neck, was seen on the rocks at Wembury near Plymouth. 138. Shag or Green Cormorant. Pbalacro-
corax graculus (Linn.)

Locally, Green Cormorant, Crested Shag, Shoalster (N. Devon).

On the south coast the shag is not so plentiful as the cormorant to the east of the Teign, but to the west of that river it is even more numerous. At Plymouth it is not seen in any numbers before the commencement of the usual November gales. On the Exe it is rarely seen and only after great storms. It ascends the Taw as far as the tide flows, as high as Barnstaple. The shag never visits the inland streams like the cormorant, though it is sometimes driven ashore by stormy weather. It breeds freely on Lundy Island, and in large numbers on the cliffs between the Bolt Head and Bolt Tail in April and May.

139. Gannet or Solan Goose. Sula bassana (Linn.)

Locally, Channel Goose (N. Devon).

Lundy Island was formerly the great breeding place of this fine bird, being the only one in England. Gannets had bred there from time immemorial on the 'Gannet Rock,' but having been constantly harried by the channel pilots, who plundered their nests in the breeding season, they migrated to Lundy itself, where however they did not escape persecution, and there are now but a few pairs left. Herring gulls are said to have largely contributed to their decrease by destroying their eggs. Adult birds are common off the coasts during the greater part of the year, sometimes approaching the shore in large numbers in the winter months in pursuit of pilchards, herrings and sprats. Immature birds in their dark-grey, white-spotted plumage are sometimes driven into the Kingsbridge estuary by stormy weather, and are picked up in an exhausted and dying condition. Two nestling birds in the Albert Memorial Museum at Exeter are said to have been taken from the cliffs between Exmouth and Budleigh Salterton about $186 \mathrm{I}$.

140. Common Heron. Ardea cinerea, Linn. Locally, Herne, Erne, Crane.

Fairly numerous, frequenting the estuaries of our rivers, inland ponds and streams, the sea coasts and occasionally the Dartmoor bogs. There are heronries at Shute Park near Axminster, Killerton near Exeter, Powderham Park on the Exe, Sharpham on the Dart, Totnes, Bellever on Dartmoor, Halwell Woods and Ilton Castle on the Kingsbridge estuary, Aunemouth on the Avon, Little Orcherton Wood at the mouth of the Erme, Warleigh on the Tamar, Kitley near Yealmpton, Fremington and Arlington Court near Barnstaple, and Pixton Park near Dulverton.

141. Purple Heron. Ardea purpurea, Linn.

The African Heron of Montagu.

An accidental visitor of very rare occurrence. Four or five examples, all in immature plumage, are reported to have been obtained, and two others seen in southwestern Devon. One was shot near Aveton Gifford, and was figured by Bewick in his work on British Birds. Another was knocked down by a waggoner with his whip, when he was passing over Whitechurch Down, in January 1832 . Yarrell mentions a specimen shot near Plymouth in February 1839. The Rev. W. S. Hore obtained a female from Pincombe of Devonport, and stated that he had 'closely inspected the body immediately after the bird was skinned.' The last and best authenticated occurrence is that recorded by the late Mr. J. Gatcombe, who purchased a specimen he saw hanging up in a poulterer's shop in Stonehouse, and which had been shot on the Tamar 30 October 1857 .

[Montagu mentions in the supplement to his Ornithological Dictionary that the Rev. Kerr Vaughan, rector of Aveton Gifford, thought he had seen a great white heron $(A . a l b a)$ on the Avon in the autumn of 1805 . It continued within the range of a few miles for two months, but its extreme wariness disappointed the many attempts made to shoot it.]

\section{Little Egret. Ardea garzetta, Linn.}

An accidental visitor of extremely rare occurrence. An adult male was shot on the Exe near Topsham in May 1870, and Mr. Howard Saunders says in his Manual of British Birds that, as far as he can learn, it is 'the only example about which there can be no doubt' that it has occurred in the British Islands. Yarrell mentions an immature bird shot on the Dart in 1816 , and Dr. E. Moore another, said to have been obtained near Crediton. Mr. J. Brooking Rowe speaks of 'one or two on the Tamar,' on the authority of Dr. Moore.

\section{Buff-backed Heron. Ardea bubulcus, Audouin.}

The little white heron of Montagu was this bird in immature plumage. This is another species of heron of which as far as is known but one well authenticated Britishkilled example exists. An immature female 


\section{BIRDS}

was obtained towards the end of October 1805, in Devonshire. It was shot by $\mathrm{Mr}$. W. F. Cornish at South Allington in the parish of Chivelstone, near the Start Point. Montagu recorded the specimen as "the little white heron' in the Transactions of the Linnean Society, and it is also mentioned by him in his Ornitbological Dictionary. The specimen still exists in the Natural History Museum at South Kensington. In the Zoologist for 1851 (p. 3116) Mr. Clevland of Tapley Park, Bideford, states that he had received a very fine specimen of the little white heron $(A$. russata), which had been shot in the south of Devon in the April of that year. Colonel Irby however assured Mr. Howard Saunders that 'the bird came from a well known dealer, and that no reliance can be placed on the date or locality assigned.'

144. Squacco Heron. Ardea ralloides, Scopoli.

An accidental visitor, of occasional occurrence both in the north and south of the county. A nearly adult specimen was killed by a gamekeeper, in the employ of Sir Frederick Rogers, by the side of a large pond close to the house at Blachford near Ivybridge, in June or July I 840. Mr. Brooking Rowe mentions one or two having been killed on the Tamar, and there was a specimen in the collection of the late Mr. Marsh-Dunn which he obtained from Bolitho of Plymouth, and which was said to have been shot at Kingsbridge. An adult male was killed on Braunton Marsh 10 June 1878 .

I 45. Night Heron. Nycticorax griseus (Linn.)

This beautiful heron has been frequently obtained in Devonshire, principally in the southern part, no less than eight (four adult males and four females) having been shot at Flete near Holbeton, and Erme Bridge in May and June 1849. Several examples have been killed in the Kingsbridge district. It has also occurred near Axminster, Exeter and Plymouth, and an adult male was shot on the Taw, two miles above Barnstaple, in May 1869, several others being seen there the same summer. An adult male was shot near Countess Wear in June I897. The last specimen that has occurred was a very fine male with four plumes, shot near Kingsbridge in April I 899.

146. Little Bittern. Ardetta minuta (Linn.)

About twenty examples are recorded as having occurred in Devonshire, most of them in the extreme south-western portion of the county and generally in the spring. The neighbourhood of Crediton has produced several specimens. A female shot there in May 1808, contained forty eggs, some considerably enlarged, and as a male was afterwards shot which had been previously observed near the same place, it is probable the pair would have brought out a brood if they had not been destroyed. The last examples obtained were one shot near Newton St. Cyres in April I90I, and another at Hatherleigh, North Devon, at the beginning of April 1903. On several occasions little bitterns, in an exhausted condition, have been captured by hand on the south coast.

I47. Common Bittern. Botaurus stellaris (Linn.)

A winter visitor of rather irregular appearance, coming in flights at long intervals, generally in severe seasons, when numbers are seen. The last great flights were in the winters of $1890-1$ and in that of $1899-1900$. Bitterns are principally met with where beds of tall reeds, known in Devonshire as 'spires,' grow in river estuaries and leys. In most seasons a few examples are obtained in the county, especially in north Devon. One was shot at Monk Okehampton in June 1889 ; Dr. E. Moore mentions two specimens obtained on the Exe in May; and the Rev. G. C. Green of Modbury saw one on the Erme on I4 April I 887. With these exceptions the bittern has only been met with during the winter months (November to March).

\section{American Bittern. Botaurus lentiginosus} (Montagu)

The Freckled Heron of Montagu.

Two examples only of this American species have occurred in Devonshire : one at Mothecombe near Plymouth 22 December I 829 ; another, a young bird of the year, was shot towards the end of October 1875 by the Rev. Marcus Rickards on some high ground near Parracombe, north Devon.

149. White Stork. Ciconia alba, Bechstein.

A casual visitor of rare occurrence. Dr. E. Moore mentions three examples obtained at Slapton Ley between 1820 and 1830 . In Morris's British Birds it is stated on the authority of Mr. N. Rowe that one was shot at Topsham on the estuary of the Exe, 28 July 1852 . A specimen in the Albert Memorial Museum, Exeter, is said to have been shot near Clyst St. George, probably at the junction of the Clyst with the Exe, a little below Topsham, in January 1856. At this spot also, at the beginning of September 1898 , a white stork was seen, and on the 18 th of the same month one was 


\section{A HISTORY OF DEVONSHIRE}

shot at Aveton Gifford, and two had been seen for a week previously on the Kingsbridge estuary. These birds are supposed to have strayed from Chequers Court, Bucks, the seat of the Rev. Hubert D. Astley, who had kept six birds in a state of semi-captivity on his grounds, and some of them had flown away not long before.

\section{50. Black Stork. Ciconia nigra (Linn.)}

It is somewhat doubtful if this rare accidental straggler to England can be reckoned amongst Devonshire birds, accounts differing as to the exact place where the only example was shot on 5 November I 831. According to some authorities it was obtained on the Tamar, whilst others state that it was on the Lynher, a tributary of that river on the Cornish side, but not far from the borders of Devon. This specimen was in the splendid collection of the late Mr. E. H. Rodd of Penzance, who records it in his Birds of Cornwall. The late Mr. Marsh-Dunn of 'Teignmouth also had a specimen which purported to have been killed on the Tamar, but was obtained from a dealer. A black stork is supposed to have been seen near Topsham in February 1855 , during very severe weather; and two are reported to have been observed on 3 April 1894 standing on the rocks at Salcombe Cove near Sidmouth.

151. Glossy Ibis. Plegadis falcinellus (Linn.)

An accidental visitor in autumn. About a dozen examples have been obtained, nearly all immature birds, and all but one in south Devon. Two of them occurred far inland at Holsworthy and Bridestowe. The last was obtained on the Dart, not far from Totnes, 26 September 1869.

152. Spoonbill. Platalea leucorodia, Linn.

A casual visitor during the autumn and winter months to both the north and south of the county. About thirty spoonbills are known to have occurred during the past century, mostly immature birds. Montagu obtained an adult I6 March I807, and Bolitho of Plymouth had one from the St. German's river in Cornwall, not far from the borders of Devon, 6 April 1867. One was seen on Dawlish Warren Io May, I900. All the other occurrences were from September to December. The last example was shot on the Kingsbridge estuary I9 November I 901 .

153. Grey Lag-Goose. Anser cinereus, Meyer.

A casual visitor of very rare occurrence. A few geese of this species were brought to the Plymouth market in January 1829 and January I 830 . The late Mr. J. Gatcombe obtained one in the Plymouth market in January 1858 ; and another in the Devonport market, 25 November I 858 , the last having been shot on the Tamar. It is now in the Albert Memorial Museum at Exeter. One was killed near Barnstaple many years ago. A grey lag-goose was observed for several days feeding on the ley at Thurlestone in company with four tame geese, and was shot 26 September I886. The last occurrence of this species was at Slapton Ley, 25 October 1889 , when one was shot.

154. White-fronted Goose. Anser albifrons (Scopoli)

Locally, Laughing Goose (N. Devon).

A winter visitor which has been in some severe seasons rather plentiful, both inland and on the marshlands of the river estuaries. Next to the Brent goose this species may be considered the commonest of all the wild geese which visit Devonshire. The last seen and identified were observed by Mr. E. A. S. Elliot in Charlton Marsh near Kingsbridge in March I900. Five wild geese seen near Crediton in November 1902 were probably of this species.

\section{Bean-Goose. Anser segetum (J. F. Gmelin)}

A casual winter visitor. There have been few occurrences of this goose since 1830 , when many were shot and brought to Plymouth market. Some were seen there by Mr. Gatcombe in February $1855 ; 22$ December 1874 ; December 1879 ; and January $188 \mathrm{r}$. Three were shot on Slapton Ley on 7 February 1888. An adult male was shot by Mr. E. A. S. Elliot on the Kingsbridge estuary 29 December I 890 , another bird being seen in company with it. This species used to frequent the Braunton marshes in north Devon in severe winters, and a flock was seen on Lundy Island in 1860.

\section{56. Pink-footed Goose. Anser bracbyrbynchus, Baillon.}

A casual visitor of very rare occurrence. The late Mr. Henry Nicholls saw three or four birds of this species hanging up in a poulterer's shop in Kingsbridge many years ago. They had been shot on the estuary there. On 8 February 1896 , in very severe weather, one of these geese was shot in Aveton Gifford Marsh. About the same time three geese had been seen in the Kingsbridge estuary, and afterwards at Slapton Ley, which probably belonged to this species. 


\section{BIRDS}

157. Red-breasted Goose. Bernicla ruficollis (Pallas)

Only two examples of this handsome species are recorded as having been obtained in Devonshire. One was killed on Dawlish (or Kenton) Warren at the mouth of the Exe in 1828; another was shot on the Teign marshes 21 February 1 837. It is only an accidental wanderer to the British Islands from Siberia.

\section{Bernacle Goose. Bernicla leucopsis (Bechstein)}

This species is decidedly rare in Devonshire at the present day, though Montagu says that a large flock was observed on Slapton Ley in the winter of 1801 . Two pairs have been killed at that place, one before 1856 , the other in 1860 . One was shot at Thurlestone in the autumn of 1882 , and $\mathrm{Mr}$. E. A. S. Elliot saw four there in January I891. An example is said to have been shot near Axminster early in January 1892. Bernacle geese have occurred occasionally on Dawlish Warren, near Plymouth and in the north of the county.

\section{Brent Goose. Bernicla brenta (Pallas)}

Locally, Brant (A.-S. brante = burnt, from its colour).

A winter visitor, flocks, sometimes of considerable size, being always seen on the estuaries during cold spells of weather. They sometimes feed on the cockle-sands quite close to the railway station at Exmouth. In some years this goose appears as early as September and October, remaining till the end of April or even to May. There are two forms of this species visiting the British Islands; one having a dark breast and abdomen, from northern Europe and Asia, is most plentiful on the east coast of England ; and the other, with the under-parts white or light coloured, comes from the Atlantic coast of America and Greenland. The latter form has occurred several times on the south coast of Devon, and specimens obtained by Mr. E. A. S. Elliot near Kingsbridge are, he considers, intermediate between the two forms. He records an immature female that was taken alive on Borough Island in Bigbury Bay on 28 October I 900 , which had sustained some injury to its back. It belonged to the whitebreasted Arctic American and Greenland form.

[Two other species of geese, the Egyptian goose (Chenalopex agyptiaca) and the Canada goose (Bernicla canadensis), have not unfrequently occurred in an apparently wild state, but the examples observed were no doubt escapes trom ornamental waters. Up to the present no examples of the lesser whitefronted goose (Anser erytbropus) nor of the American white-fronted goose (A. gambeli) have been identified, though the latter is not unlikely to be met with on our south-western coast. All the bean geese that have been obtained are believed to be true $A$. segetum and not $A$. arvensis.]

160. Whooper Swan. Cygnus musicus, Bechstein.

A casual visitor now of very rare occurrence, though up to the year 1830 large numbers used to visit the estuaries of the county in severe winters. The last recorded occurrences of this fine swan were in January 1891, when seven were seen in Torbay, one being killed about the same time at Slapton Ley, and several on the Barnstaple river; and an immature female shot in the bight off Exmouth on 23 January 1893 , and now in the possession of the writer.

16r. Bewick's Swan. Cygnus berwicki, Yarrell.

Like the last species this small swan is a casual visitor in severe winters, now of very rare occurrence. In January 1838 a flock of fifteen visited the Exe and Clyst, and were nearly all killed. A pair was seen 14 November 1876 on the Kingsbridge estuary, and the female was shot. Another was also shot a few days afterwards. Only one or two other occurrences are known. The last occurrence was on the Kingsbridge estuary, when one was shot out of a flock on 9 December 1902. There are no records of any examples having been obtained in north Devon.

162. Mute Swan. Cygnus olor (J.F. Gmelin)

Principally an introduced species in Devonshire, a few pairs being kept in a semi-domesticated state between Exeter and Topsham, and on many ponds and ornamental waters throughout the county. Some swans are in the habit of flying from the great swannery at Abbotsbury in Dorsetshire to the mouth of the Axe near Seaton, Devon. Some of the wild swans which have been shot from time to time in the county may have possibly been feral examples of this species.

163. Common Sheld-Duck. Tadorna cornuta (S. G. Gmelin)

Locally, Bar-Duck, Burrow-Duck (N. Devon). This beautiful duck is now resident in limited numbers only at certain spots on both the north and south coasts, and is mainly a casual visitor in winter and early spring, 


\section{A HISTORY OF DEVONSHIRE}

especially during or after severe weather. On the whole this bird seems to be on the increase, and if the eggs were not taken it would soon be a common bird again. The nest is usually placed in a rabbit burrow, amongst sand hills on the coast.

I64. Ruddy Sheld-Duck. Tadorna casarca (Linn.)

An example of this rare duck was shot at Braunton, north Devon, in April 1 882, and is preserved in the United Service College Museum at Westward Ho! Devonshire participated with the rest of England in the remarkable visitation of ruddy sheld-ducks in the summer of 1892 , three having been obtained at the mouth of the $T a w$ in June of that year. It is not known to have occurred in south Devon.

\section{Mallard. Anas boscas, Linn. Locally, Wild Duck.}

The mallard is now not nearly so generally abundant in Devonshire, as it was at the beginning of the eighteenth century, when many decoys were in operation in various parts of the county. It is now principally a winter visitor. A few pairs nest annually, in some retired places, throughout the county, and it seems to be rather on the increase again as a breeding species. It is at Slapton and Thurlestone Leys however that the greatest number of our resident wild ducks are to be found. At the former stronghold for wildfowl scores of the drake mallards may be seen rising from the reeds when disturbed during the breeding season, the ducks nesting in hedges in the fields around the ley. In winter large flights arrive on the south-west coast, immense flocks being seen in Bigbury Bay, especially under the Bolt Tail, where they rest by day, flying inland to feed at night. White and other varieties are very frequent, probably through interbreeding with domestic ducks, and hybrids between the mallard and wigeon and mallard and teal have been obtained.

\section{Gadwall. Anas strepera, Linn.}

A casual visitor of rare occurrence. It has been met with only on the south coast. A female killed at Slapton Ley in January I 885 is believed to be the first recorded example shot there. A nearly adult male was also obtained there on 2 January I 893. The gadwall has occurred several times at Plymouth, at Kingsbridge and at Seaton. One or two have been obtained on the Exe estuary and one on the Dart.
167. Shoveler. Spatula clypeata (Linn.)

This beautiful duck is not uncommon in some winters. Large flocks were seen at Slapton Ley in January 1899 and January 1893 , and some were obtained there in the winter of $1894-5$. The shoveler used to occur almost every winter on the Barnstaple river in small parties or singly. It has frequently been obtained at Plymouth, on the Exe and on the Dart. It usually occurs from September to April, but Montagu speaks of one shot on 5 August I807, ' on a freshwater lake on the south coast of Devon'; and the late Mr. W. V. Toll killed one on 6 August 1891 on Slapton Ley, and he believed it had been hatched there. A young shoveler was shot on Thurlestone Ley on I August I 899 , but, according to Mr. Elliot, could not have been bred there, as he had the Ley under constant observation, and saw no breeding birds. These young birds may have possibly come from the south of Ireland, where the shoveler is known to breed.

\section{Pintail. Dafila acuta (Linn.)}

Locally, Sea-Pheasant, Pheasant-Duck.

A winter visitor to our bays, estuaries and rivers, principally to those in the south of the county. It is very rare in north Devon at present, though formerly well known to the boatmen at Instow. It visits Lundy Island. A few examples have been obtained at Plymouth and on the Exe. On Slapton and Torcross Leys pintails are shot nearly every winter, mostly immature birds, but some fine full-plumaged males have been obtained. A flock of some thirty pintails visited the Kingsbridge estuary 6 February 1895 , and $\mathrm{Mr}$. Elliot shot two male birds in full breeding dress out of it. A pair were shot on the rocks under the cliffs on the estuary, January I 897 .

\section{I69. Teal. Nettion crecca (Linn.)}

Partially a resident, and a common winter visitor, arriving in September and remaining till late in the spring. Many pairs breed in the marshy meadows bordering the streams that run into Slapton Ley, and probably in the Dartmoor bogs, and in other suitable situations in the county. A male bird with a white ring round the neck was seen by $\mathrm{Mr}$. Gatcombe at Ilfracombe.

\section{70. American Green-winged Teal. Nettion carolinense (J. F. Gmelin)}

The only example of this accidental wanderer from America that has occurred in Devonshire was obtained by Mr. R. P. Nicholls of Kingsbridge, 23 November 1879. 


\section{BIRDS}

It was shot out of a flock by a local gunner on an arm of the Kingsbridge estuary. Only two other instances of its occurrence in the British Islands are known.

[The North American summer duck (Aix sponsa), often kept on ponds, has been killed at Plymouth and at Slapton Ley.]

\section{Garganey. 2uerquedula circia (Linn.)}

Locally, Summer Teal.

This beautiful little duck is now very rare indeed in Devonshire, and no occurrences within the last twenty years have been recorded. The spring of 1870 was remarkable for the number of examples shot. It has been obtained at Plymouth, Modbury, Kingsbridge, Newton Abbot, Topsham and Barnstaple. The garganey usually occurred in pairs in the months of March and April, but on 17 April 1875 a flock of eight was seen at Strode near Modbury, and a pair was shot.

172. Wigeon. Mareca penelope (Linn.)

Locally, Yellow-poll.

A regular winter visitor, though greatly reduced in numbers, and few are now seen in some parts of the county where formerly it was the most abundant species of wild duck. It still continues to be fairly plentiful in the extreme southern portion of Devon, its great places of resort being Slapton and Thurlestone Leys, where sometimes thousands are seen in winter. The wigeon frequently arrives in September, and has been shot as early as 6 August (1871) on Slapton Ley, where a few pairs sometimes remain all the summer. Although it has not yet been actually proved that the wigeon nests on the shores of that most interesting sheet of fresh water, there seems great probability that it does do so in small numbers. No place could be better adapted to its requirements as a breeding station. 'Flight shooting' by moonlight is not so much pursued by gunners as formerly, as places that were once favourable for it are now too much frequented and disturbed to allow of much sport being obtained.

173. Red-crested Pochard. Netta rufina (Pallas)

The only instance of the occurrence of this rare species of duck in Devonshire was in December 1867 , when a fine adult drake was shot on Braunton Burrows. It was purchased by the late Mr. Marsh-Dunn of Teignmouth for his collection.

174. Pochard. Fuligula ferina (Linn.)

Locally, Frosty-back (male), Dun Curre, Cur Wigeon (female and young).

A winter visitor, most numerous in severe seasons. It is most frequently met with at Slapton Ley, where a few pairs remain during the breeding season, and most probably nest. Elsewhere in Devon it is by no means a common species. It has occurred occasionally near Barnstaple, at Plymouth, rarely on the Kingsbridge estuary, in Torbay, and sometimes on the Exe estuary, where a small party of adult males and females appeared in January 1900 . These birds were very tame and most of them were shot.

\section{Ferruginous Duck. Fuligula nyraca (Guldenstädt)}

An accidental visitor of very rare occurrence. An immature bird is recorded by Dr. E. Moore from Plymouth. A female was shot on Slapton Ley about the middle of November 1874, and another example occurred there 8 November 1897 , being shot out of a small flock of ducks that were not identified.

176. Tufted Duck. Fuligula cristata (Leach) Locally, Black Wigeon, Pied Wigeon.

A winter visitor, arriving in October and not uncommon in some seasons on our larger rivers. It is especially numerous on Slapton and Torcross Leys, where some often remain all the year and very probably breed. Immense numbers of tufted ducks appeared in the winter of 1894-5 on these leys. A brood of very young birds was seen at the end of July, 1859, by the Rev. M. A. Mathew on the Barnstaple river, which he thought might have been reared somewhere close at hand.

\section{Scaup-Duck. Fuligula marila (Linn.) \\ Locally, Blue-bill.}

A winter visitor, generally arriving in October. The scaup is one of the most numerous of the wild fowl visiting Devon. As a rule the large flocks keep out at sea, some miles from the shore, but after heavy gales in autumn small parties ascend the estuaries of some of the rivers, and, being then very tame, are easily approached and shot. One was killed on the Exe I I August I876, and Mr. Elliot met with some scaups on the Kingsbridge estuary in June 1899 . This duck sometimes visits Slapton Ley in severe winters. There is a variety of the female scaup in the Albert Memorial Museum at Exeter which has a white collar round the neck. It was shot on the Exe in 1847 .

178. Goldeneye. Clangula glaucion (Linn.)
Locally, Diving Wigeon, Cur Wigeon (female
and young).
A common winter visitor, generally seen in 


\section{A HISTORY OF DEVONSHIRE}

small parties consisting of females and immature birds on our river estuaries and leys. Adult males occur singly and are much less common.

In November 1893 a young male of unusually large size occurred on the Kingsbridge estuary, which Mr. Elliot thinks must have been either an immature Barrow's goldeneye (C. islandica) or one of the American form of goldeneye, which is larger than our bird. This specimen was unfortunately not preserved.

[An example of the American buffel-headed duck (C. albeola) is said to have been killed on the West Mud near Devonport in the winter of 1841 , and was in the collection of the late Rev. W. S. Hore, who obtained it from Mr. Cornelius Tripe of Devonport, who had other American birds in his collection, and therefore a mistake may have been made. Mr. Tripe thought the bird was a goldeneye, and had no idea of its value if a British-killed specimen.]

\section{Long-tailed Duck. Harelda glacialis} (Linn.)

A winter visitor of occasional occurrence on our bays and estuaries, chiefly on the south coast of the county. It arrives in October, and has been seen as late as March. Only a few long-tailed ducks have been obtained at Plymouth, but many have been killed on the Kingsbridge and Exe estuaries, Slapton Ley and Torbay. It is rare in north Devon. An example in full summer plumage was killed on the Exe in 1847. Adults in full winter plumage are sometimes obtained, but immature birds are most frequently met with. Mr. E. A. S. Elliot has observed that the iris of the eye in this duck undergoes a seasonal change of colour, being pale straw-colour in winter and hazel in summer.

[It is very doubtful if the harlequin duck (Cosmonetta bistrionica) can be included amongst the species that have occurred in Devonshire. Dr. E. Moore says a female was shot off the Hamoaze near Plymouth in the winter of 1830 , but it was in all probability a longtailed duck, as were the two examples recorded in the Zoologist for 1847 , as having been killed in Torbay, and which proved to be immature birds of that species.]

\section{Eider Duck. Somateria mollissima (Linn.)}

A casual winter visitor of rare occurrence. About a dozen examples, mostly females and immature birds, have been obtained in Plymouth Sound, in Torbay and at the mouth of the Exe. The last killed in south Devon was shot in the winter of 1875 . Only two specimens are recorded from north Devon; one of them was shot on the Taw in December $189 \mathrm{I}$, and the other, a young male, on the mudflats at Appledore in November 1893.

181. King Eider. Somateria spectabilis (Linn.)

The only occurrence of this duck in Devonshire rests upon the authority of the late Mr. J. Gatcombe, who saw an immature example in the flesh at a bird-stuffer's shop, which was said to have been killed near Plymouth.

\section{Common Scoter. Edemia nigra (Linn.)} Locally, Black Duck.

Principally a winter visitor, large flocks arriving on our south coast in August and September, remaining till April. It is not so numerous on the north coast. Scoters usually keep well out to sea, and rarely ascend the estuaries, and only through stress of weather. Some, probably non-breeding birds, remain all the summer on the south coast, which fact seems to have led Dr. Moore to suspect that some might breed in Devonshire.

\section{Velvet Scoter. Edemia fusca (Linn.)}

A casual winter visitor, not often obtained, because it seldom approaches the shore, being exclusively a sea-duck and keeping far out in the channels. Examples have occurred in Plymouth Sound, the Kingsbridge estuary, Torbay, at the mouth of the Exe and in Barnstaple Bay.

184. Surf Scoter. Edemia perspicillata (Linn.)

This American duck has occurred three times on our south coast. An immature bird was shot in Torbay in 1860, one on Slapton Ley in or about 1862, and an immature male near Kingsbridge 20 October I 89 I.

185. Goosander. Mergus merganser, Linn.

A winter visitor of occasional occurrence (up to 1891 ), either singly or in small parties, on the bays, leys and estuaries of the south coast, and rarely on the north coast. The examples that have been met with were mostly immature birds or else females, adult males in full plumage being rare.

186. Red-breasted Merganser. Mergus serrator, Linn.

Locally, Spike-billed Wigeon (on the Taw), Saw-bill (on the Exe).

A winter visitor of rather frequent occurrence on the coasts, and in the river estuaries, appearing sometimes in October, and has been 


\section{BIRDS}

seen as late as May in north Devon. The adults are often met with in pairs in early spring.

187. Smew. Mergus albellus, Linn.

Locally, White Wigeon (adult male), Vare-

Wigeon (female and young male).

A winter visitor, most abundant in severe seasons, and occasionally met with from November to March on the coasts, sometimes penetrating far inland along the courses of our larger rivers. Immature birds are most often seen on the south coast, adults being usually scarce, but in January I 891 many occurred all over the county. In north Devon adult males are said to be the commonest. When in full plumage this beautiful little merganser generally occurs in pairs, but in some instances several adult males have been seen in company. On 2 August $1897 \mathrm{Mr}$. E. A. S. Elliot saw a pair close to the shore at Thurlestone. The male was in the eclipse stage of plumage.

188. Ring-Dove. Columba palumbus, Linn. Locally, Culver, Wild or Wood-Pigeon.

An abundant resident, generally distributed in all wooded parts throughout the county. It is increasing in numbers to a serious extent in some places. Being a most voracious bird, and terribly destructive to the crops of grain, tares and turnips, as well as to garden produce, it is a perfect pest to the agriculturist. Large flocks appear in winter, especially when beech-mast and acorns are abundant.

\section{Stock-Dove. Columba anas, Linn.}

Formerly this pigeon was but little known in Devonshire, being only an irregular winter visitor, appearing in those years in which beech-mast was plentiful. Since about the year 1876 it has become a common resident, and may now be seen at all times of the year. It breeds in a great diversity of situations ; in hollow trees, in holes in walls, in ivy against the walls of houses, on the sea cliffs, and in rabbit burrows on sandhills. It is very numerous on some parts of the south coast, and is often mistaken for the rock-dove.

I90. Rock-Dove. Columba livia, J. F. Gmelin. Locally, Cliff-Culver.

'The 'cliff-culver' appears to have been formerly much more abundant than it is at present, and it is no longer found in some localities, such as Lundy Island, where once it was well known. It is now little more than an occasional visitor to some suitable spots, though a few pairs are said to breed in caves and fissures of the sea-cliffs. The common 'blue rock' of the farmyard frequently reverts to a wild state, and it is then difficult to distinguish it from the genuine wild rockdove. Great numbers of pigeons, probably domestic birds gone wild, or their descendants, inhabit the white cliffs at Beer, where freestone has been quarried for many centuries, and which are pierced by tunnels or galleries branching in all directions.

I9I. Turtle Dove. Turtur communis, Selby.

A summer migrant, visiting suitable localities throughout the county, but is usually far from numerous. It arrives at the end of April or early in May, and leaves about Michaelmas. Some pairs breed annually in the Kingsbridge district, in the woods at Kelly on the Tamar, and on Braunton Burrows. Although usually seen singly, in pairs, or small parties, flocks have been seen on Dawlish Warren in July, and on the borders of Dartmoor in August. Mr. W. Eagle Clarke observed many turtle doves passing the Eddystone Lighthouse on the nights of 22-23 September and 13-14 October $190 \mathrm{I}$.

[The collared turtle ( $T$. risorius) has occasionally been killed in a wild state near Plymouth, and it is said to have bred in some of the large woods and plantation in that neighbourhood. It is often kept in confinement.]

\section{Pallas's Sand-Grouse. Syrrbaptes para- doxus (Pallas)}

An accidental visitor of very rare occurrence. Like the rest of England, Devonshire was visited by flocks of this curious bird in $186_{3}$ and 1888. A pair was shot out of a flock of thirteen on the sands at Slapton Ley in June I 863 ; an old female was killed by a gamekeeper at Heanton Court near Barnstaple I I December I 863; a flock appeared on Lundy Island in May, remaining for several weeks, and several were obtained at Hartland about 3 June 1888 .

\section{Black Grouse. Tetrao tetrix, Linn. \\ Locally, Heath Poult, Moor Fowl, Black Cock and Grey Hen.}

At the beginning of the last century this fine species of grouse was abundant on most of the moors of the county. Some few still exist on Dartmoor, on the Haldons, and Blackdown Hills, on the high ground in the extreme eastern part of the county, and on the moorlands in north Devon. A stray bird now and then occurs in the Kingsbridge district, especially on the sands at Strete, close to Slapton Ley. Hybrids between the black cock and hen pheasant, and cock pheasant and grey hen, have been obtained several times at Plymouth. 


\section{A HISTORY OF DEVONSHIRE}

[The red grouse (Lagopus scoticus) is said to have occurred on Dartmoor and at Stokenham in the South Hams. Possibly stragglers may have reached this county from south Wales. About 1820 or 1825 several pairs of red grouse were turned out on Exmoor, and the Devonshire examples (if they were really that species) might have strayed from thence.]

[The occurrence of the ptarmigan ( $L$. mutus) on Dartmoor is so very improbable that no credence can be given to the statement made by Dr. E. Moore in Rowe's Perambulation of Dartmoor, published in 1848 , that a specimen in summer plumage was shot there in the month of October by Mr. Newton of Millaton near Bridestowe.]

\section{Pheasant. Pbasianus colcbicus (Linn.)}

It is probable that the pheasant was introduced into Devonshire during the Roman occupation, as a metatarsal bone of a gallinaceous bird, either a domestic cock or a cock pheasant, was found amongst the bones deposited in the ancient camp on the summit of Peak Hill near Sidmouth, and a similar specimen is in the Exeter Museum from the Bench Cavern near Brixham. In September I625 it is recorded that at two banquets, provided on the occasion of a visit from Charles I. to Plymouth, amongst the poultry placed on the tables were seven pheasants at the first and five at the second. At the end of the eighteenth century, according to Polwhele, the pheasant had been almost annihilated in Devonshire, but was beginning to increase again where carefully preserved. Other authorities state that pheasants were introduced into Devonshire about that time. They were probably the Chinese ring-necked pheasant $(P$. torquatus), whose introduction to England then began. By 1830 pheasants were common in the neighbourhood of Mamhead, Kenn, and other preserves. The Duke of Bedford and Sir W. P. Coll appear to have introduced the ring-necked, as that species was the most common in the extreme western part of the county in 1832 . It is now diffcult to find a pure-bred 'red pheasant' of the old breed. Nearly all the birds in our preserves are more or less marked with the white collar round the neck. Another species, the Japanese $P$. versicolor, has been occasionally turned down, and will perhaps still further modify the plumage of the Devonshire pheasants. Many varieties occur; white-tailed, pied, mottled, and buff-coloured or 'Bohemian' are frequently met with, besides pure white albinos. Hybrids between the pheasant and black grouse have been already mentioned, and they have also occurred between the domestic fowl and pheasant in the southwestern part of the county.

\section{Partridge. Perdix cinerea, Latham.}

Resident, generally distributed throughout the county, excepting the wilder parts of Dartmoor, and in some years fairly plentiful. Bags of from fifty to sixty-five brace have been made in both the north and south of the county. White, cream-coloured, silvery grey and buff-coloured varieties occur. Females are frequently marked with a white 'horseshoe' on the breast in the Kingsbridge district. In the west of Devon a large dark variety without the horse-shoe mark is met with. The variety ferrugineus of Gould has been obtained on several occasions near Tavistock and Plymouth.

\section{Red-legged Partridge. Caccabis rufa (Linn.)}

Locally, Frenchman.

This handsome bird was first introduced into Devonshire probably about 1840 , and at different times since then some have been turned down in several parts of the county, but although a few examples are met with from time to time, it has never thoroughly established itself, presumably owing to the climate being unsuited to it. $A$ hen bird was seen to drop into the water when flying over the Exe estuary from the Starcross side towards Exmouth on 15 April 1892 . She was found to contain thirty well developed eggs. The cock bird in company with her was afterwards seen about the docks at Exmouth. Strangely enough an exactly similar occurrence took place in the spring of 1900 , another female full of eggs being picked up in the river near Starcross in a dying state. A male was found dead near Woodbury about the same time. It is difficult to account for these birds crossing the river in this manner, and it is not known where they come from.

\section{Quail. Coturnix communis, Bonnaterre.}

A summer migrant of irregular appearance, arriving in spring and frequently remaining throughout the winter. A few generally occur every year, but in a good 'quail year,' such as 1870 and 1892 , many visit us, and numerous pairs breed in various parts of the county, especially in the South Hams, and formerly on Lundy Island. During the autumnal migration in some years quails are plentiful, both in north and south Devon. At one time Lundy Island was annually visited by them, and good bags have been made there in September. 


\section{BIRDS}

198. Land-Rail. Crex pratensis, Bechstein. Locally, Barley Drake, Barley Hen, Corn Crake.

A summer migrant, arriving at the end of April or in the first week in May, and departing at the end of October. A few remain with us during the winter months. Landrails sometimes congregrate in a remarkable manner in the extreme southern promontory of the county in autumn, just before leaving for the south. As many as forty-two brace were shot by two guns in one morning, 23 September I 86I, in the parish of Malborough. The land-rail is also sometimes plentiful in north Devon, and was formerly especially abundant on Lundy Island during the autumnal migration. It frequently breeds in north Devon, but not often in the southern part of the county. A specimen of a dingy white colour was shot at Pinhoe near Exeter 3 May I $88 \mathrm{r}$.

\section{Spotted Crake. Porzana maruetta (Leach)}

Locally, Silver Rail (on the Exe), Spotted Skitty (N. Devon).

A passing visitor in spring and autumn, most frequently met with at the latter season, i.e. from August to the middle of November. It is much less frequently seen than formerly. It used to be found on Braunton Burrows in winter, and has occasionally visited Lundy Island. The only specimen recorded as having been noticed on Dartmoor was shot near Post Bridge in 1862.

200. Little Crake. Porzana parva (Scopoli)

A casual visitor occurring at all seasons of the year, but rarely seen on account of its skulking habits. The first example of this species made known in Britain was shot near Ashburton in 1809, and Colonel Montagu received it from Dr. A. Tucker of that place. The specimen was figured and described in the supplement to the Ornithological Dictionary under the name of 'little gallinule.' Dr. E. Moore calls it the 'olivaceous gallinule.' Six examples have been obtained, occurring both in the north and south of the county, and others have been seen.

[There is no properly authenticated occurrence of Baillon's crake (P. bailloni) in Devonshire. All that have been recorded proved to be little crakes.]

201. Water-Rail. Rallus aquaticus, Linn. Locally, Skitty Cock, Grey Skitty (A.-S. skit $=$ to glide), Gutter Cock, Ore Cock.

Common and partially resident, breeding in some localities, but most frequently seen at the end of September and the beginning of October, when there seems to be an arrival of immigrants from other parts.

202. Moor-Hen. Gallinula cbloropus (Linn.) Locally, Water Hen, Cuddy (i.e. shorttailed).

Resident, generally distributed and abundant in all suitable localities. It appears to be increasing in numbers.

\section{Coot. Fulica atra, Linn. \\ Locally, Bald Coot.}

As a resident the coot is of very local distribution, being almost entirely confined to Slapton, Thurlestone and Milton Leys on the south-west coast of the county, breeding on the first in considerable numbers. In winter, especially if the weather is severe, great flocks of 'foreigners' arrive on the leys and elsewhere on the south coast and on the Barnstaple river in the north. On a day usually in the month of January, an annual battue, or public shoot, known as 'Ley Day,' takes place on Slapton Ley, when large numbers of coots are slaughtered.

\section{Crane. Grus communis, Bechstein.}

There are only two recorded occurrences of this fine bird in Devonshire. The first was killed at Buckland Monachorum on the borders of Dartmoor in the autumn of I 826 . It had frequented the banks of the Tamar for several days, and was at last shot near its tributary, the Tavy. The second was seen for five or six days on the high level fields near the Start Lighthouse in September I 869. It always kept out of gunshot and was not secured.

205. Great Bustard. Otis tarda, Linn.

A casual visitor of very rare occurrence in winter. Montagu mentions four specimens obtained near Plymouth. A female was shot at Bratton Clovelly on 31 December 1851 ; and two, both females, were killed and a third wounded out of a flock of seven or eight at Croyde and Braunton 3I December 1870.

\section{Little Bustard. Otis tetrax, Linn.}

At least a dozen little bustards, all females or immature birds, have been recorded from Devonshire, occurring during the winter months, generally in December. Five of them occurred in the South Hams, and the rest in north Devon. The last occurrence was in November 1893, when three were seen on Braunton Burrows. 


\section{A HISTORY OF DEVONSHIRE}

207. Stone-Curlew. CEdicnemus scolopax (S.G. Gmelin)

Locally, Great Plover.

A casual visitor of occasional occurrence, usually in the autumn and winter months, i.e. October to February, and mostly on the south coast and its neighbourhood. It has occurred only once in the month of March (1867), and twice in May (1889 and 1897). In the winter of 1889 one was killed out of a flock, but this bird usually occurs either singly or in pairs in Devonshire.

\section{Pratincole. Glareola pratincola (Linn.)}

An accidental visitor of extremely rare occurrence. Two pratincoles are reported to have been seen on Dawlish Warren on 7 September 1851 by the late Mr. W. W. Buller; and two at the same place by the late Mr. F. W. L. Ross. One is said to have been seen near the banks of the Erme in Flete Park on 14 August 1885.

209. Cream-coloured Courser. Cursorius gallicus (J. F. Gmelin)

A very rare accidental visitor in spring and autumn. It has occurred twice on Braunton Burrows. Two were seen there and one shot in the last week of October 1856, the other being killed a few days afterwards, and two were seen in March 1860 .

2 Io. Dotterel. Eudromias morinellus (Linn.)

The dotterel rarely visits Devonshire, and generally only on passage in spring and autumn, but a few have occurred in the winter months. It is usually met with either singly or in small trips, at rare intervals, on the south-western side of Dartmoor, in the Kingsbridge district, on Dawlish Warren, on Whitestone Hill near Exeter, and near Barnstaple. Mr. Gatcombe saw one on the top of Great Hangman's Hill near Combe Martin 22 October 1875.

\section{II. Ringed Plover. Egialitis biaticula (Linn.) \\ Locally, Cat-poll, Cat-head, Sand-Lark, Sea- Lark.}

An abundant resident on our coasts and estuaries, breeding wherever there is a pebbly beach. Large numbers arrive from the north in autumn and flock with dunlins and other shore birds. A smaller race than our resident birds arrives in spring on our shores, and has been mistaken for the little ringed plover ( $\mathbb{E}$. curonica), which has not yet been obtained in Devonshire, though one was shot at Trescoe in the Scilly Islands.

\section{Kentish Plover. Egialitis cantiana (Latham)}

Only three examples of this little plover are recorded from Devonshire, viz. a pair on the Plymouth breakwater 7 May I86r, and an immature bird on the Hamoaze in the autumn of 1875 .

[According to Mr. C. Dixon an example of the kildeer plover ( $\boldsymbol{E}$. vocifera) was flushed from Paignton Sands on 7 September 1898 . It is a common American species and has occurred in Hampshire and at the Scilly Islands.]

\section{Golden Plover. Charadrius pluvialis,} Linn.

Chiefly known in Devonshire as a regular autumn visitor from the north, flocks making their appearance at that time of the year on all our moorlands, whence in severe frosts they descend into the marshes and meadows, and are then also found on the coasts. Large flocks remain until late in the spring in south Devon, and the birds are then in full breeding plumage, but it is uncertain whether any golden plovers now nest on Dartmoor, as they were reported to do formerly. Dr. E. Moore records 'a brood of six' being obtained on the banks of the Tamar in 1827 , and two young ones were found on Roborough Down in 1829 .

2 4 . Grey Plover. Squatarola belvetica (Linn.) Locally, Silver Plover.

A winter visitor to our coasts and estuaries, but never numerous, occurring singly or in pairs, and rarely in small flocks. It arrives in September and October, sometimes prolonging its stay till the middle of May. Examples are occasionally obtained in full breeding plumage.

Many 'grey plovers' are reported passing the Eddystone Lighthouse in autumn.

215. Lapwing. Vanellus vulgaris, Bechstein.

Locally, Lapwink, Horniwink, Green Plover, Peewit.

Increasing as a resident breeding species, but those that remain all the year with us on the moors and marshlands are few compared to the vast flocks that arrive from the eastward in autumn and during severe weather in winter.

216. Turnstone. Strepsilas interpres (Linn.)

A passing visitor in April and May, and again in August and September, a few occasionally remaining through the winter. Flocks composed of turnstones, ringed plovers, knots and dunlins, mingled together, are some- 


\section{BIRDS}

times met with, but this species is more often seen in small parties on the sea shore and in the estuaries of our larger rivers. Occasionally large flocks have occurred at Instow in autumn. In spring the birds obtained are often in their rich breeding plumage. Mr. Howard Saunders came across a pair, in a favourable situation for breeding, on Lundy Island 28 May 1861 , which might have had a nest, but one of the two was unfortunately shot.

217. Oyster-Catcher. Hamatopus ostralegus, Linn.

Locally, Sea-Pie.

Partially a resident, but principally only a passing visitor in spring and autumn. It formerly bred amongst the rocks near the Start Point, and does so still at one spot on the south coast. A few pairs breed on Lundy Island. It is rarely common at any time on the south coast, though on the increase, but is most frequently met with in small parties, from August to October, on the sea shore and in the river estuaries, and is then sometimes in adult winter plumage, but more generally examples met with at that season are immature birds. Large flocks visit the mussel beds off the Bideford Lighthouse, especially in winter.

218. Avocet. Recurvirostra avocetta, Linn.

This remarkable bird is now a very rare visitor to Devonshire. It usually occurs in autumn and winter, seldom in spring. Examples have been obtained on the Tamar and Tavy (3), on the Kingsbridge estuary (4 or 5), on the Exe estuary (12), on the Dart (2), and on the Taw and Torridge (5). The last occurrences were in 1888 , when two were killed at Exmouth at the end of October, one near Instow on 13 November, and one on the Taw, the next day.

2 19. Black-winged Stilt. Himantopus candidus, Bonnaterre.

About 1720 a small flight of this curious bird seems to have visited the western counties, as no less than five were killed at a shot near Penzance in Cornwall, and at about the same time one was shot at Brampton (query Braunton) 'on the north sea in Devonshire' (The Works of Walter Moyle, Esq., 2 vols., London, I 726). Dr. Edward Moore records a specimen obtained at Slapton Ley, and one in Mr. Comyn's collection. No example has occurred in Devonshire within the last sixty or seventy years.

220. Grey Phalarope. Phalaropus fulicarius (Linn.)

An irregular autumnal visitor, only approach- ing our shores during heavy south-westerly gales in September, October and November. It rarely occurs as early as August or as late as December and January, and very seldom in spring. In some years, such as 1845,1846 , $1866,1870,1875$ and $189 \mathrm{r}$, great numbers appear on our coasts, especially the extreme south-western part, and many are driven by stormy weather inland, when they are often seen on freshwater ponds. They are generally very tame and easily approached, being sometimes so worn out as to allow themselves to be caught by hand. The examples obtained on our shores are almost always in immature or else winter plumage. The late $\mathrm{Mr}$. Henry Nicholls of Kingsbridge obtained a fine male in summer plumage in May I844; and a bird in moult, with some orange feathers of the summer dress still remaining, was captured alive by a boy in Stonehouse Creek, on 5 August 1876 . There was also a specimen in full summer plumage in the collection of the late Mr. Bond which was said to have been obtained in Devonshire.

\section{Red-necked Phalarope. Phalaropus by- perboreus (Linn.)}

Two specimens only of this pretty species have been procured in Devon, viz. an immature bird at Plymouth in 1831 , and an adult in summer plumage on the Hamoaze 7 June I869. Two were seen singly about the Eddystone Lighthouse, 21 September and I October I901, by Mr. W. Eagle Clarke.

\section{Woodcock. Scolopax rusticula, Linn.}

Locally, Muff Cock.

Principally a winter visitor, but some few pairs nest in the county and probably furnish the "early woodcocks' recorded from time to time by the local press in August and September. The average date of the first arrival is between October 6 and Io, but the main body of immigrants seldom appears before the middle of that month, and may continue to arrive up to the middle of December. The time of departure is the beginning of March.

Large flights of woodcocks used to arrive at Bindon and Dowlands near Axmouth on the undercliffs, on moonlight nights in October, and they are sometimes abundant at Shute Park. They are also plentiful at times in Woodleigh Woods near Kingsbridge. It is remarkable that woodcocks rarely strike the lantern of the Start Lighthouse, and it does not appear that any have been reported from the Eddystone. In former days before the island had become cultivated, great num- 


\section{A HISTORY OF DEVONSHIRE}

bers used to resort to Lundy in severe winters. They are still sometimes fairly plentiful in the covers on the mainland of north Devon. White, cream-coloured and buff varieties are not very rare. Parti-coloured examples also occur. Devonshire woodcocks belong to the red race, and specimens of the grey race are rarely seen.

\section{Great Snipe. Gallinago major (J. F. Gmelin) \\ Locally, Double Snipe, Solitary Snipe.}

A casual visitor occasionally met with in autumn on the moors. In September and October 1868 no less than seven examples occurred in Devonshire. Specimens obtained in this county are nearly always in immature plumage, but the late Mr. Gatcombe records an adult killed on Dartmoor on 23 August I 876 . Only one great snipe has occurred on Lundy Island.

\section{Common Snipe. Gallinago coelestis (Frenzel)}

Locally, Full Snipe, Whole Snipe, Heather Bleater.

Resident locally, breeding in small numbers on Dartmoor and on the neighbouring commons, in some marshes on the south-west coast, on all the moors, and in many marshy meadows in north Devon. The snipe is however mainly a winter visitor, arriving continuously from the end of August throughout the autumn and winter, and remaining until the end of March or even to the middle of April, the great bulk of the foreign birds appearing early in October, and being followed in succession by smaller flights. The common snipe was formerly a most abundant species in Devonshire, but a great diminution in its numbers has taken place during the last halfcentury, and it has now become rather scarce in most places. A large race, weighing as much as $7 \frac{1}{2}$ oz., occurs occasionally, and many varieties of plumage have been met with.

\section{Jack Snipe. Gallinago gallinula (Linn.) Locally, Jack, Half-Snipe, Atterflitter (N. Devon).}

A winter visitor tolerably numerous in suitable localities. It is seen early in September on Dartmoor, but does not appear on the marshlands in our estuaries until November, or even December in mild and open seasons. Jack snipe remain as late as March on Dartmoor, and have been known to linger till the middle of April in north Devon.

\section{American Pectoral Sandpiper. Tringa maculata, Vieillot.}

Two were shot by the Rev. Marcus Rickard on Braunton Burrows 12 September 187 I. Bellamy mentions specimens having been shot on the Tamar (probably about 1 839) and preserved by Pincombe of Devonport.

\section{Bonaparte's Sandpiper. Tringa fusci- collis, Vieillot.}

Four specimens of this American sandpiper were shot on the Taw near Instow in November 1870 .

\section{Dunlin. Tringa alpina, Linn.}

Locally, Sanderling, Stint, Purre, Sea-Lark, Summer Lark (N. Devon).

The most abundant of our shore birds, and some may be seen on the coasts and the estuaries of our larger rivers nearly all the year round, but especially in spring and early autumn, when large numbers arrive in flocks from other parts. These flocks consist of small dark-coloured birds, but later in the autumn and in winter a larger and lightercoloured race visits us. This large grey form is seldom obtained in summer plumage, but when it does occur in that state it is noticed in pairs, or in twos and threes in April, and are newcomers and not those that have remained throughout the winter. The smaller and darker form, in full breeding plumage, arrives in May in small companies and remains a few weeks. In July they return again, still in summer plumage, and remain until October, when they give place to the larger form. A few of the small race breed on Dartmoor, where they have been occasionally seen in June and July. Some unusually small dunlins, apparently answering to the description of Tringa schinzi of Brehm, were killed at Plymouth in August 1870.

\section{Little Stint. Tringa minuta, Leisler.}

A passing visitor of occasional occurrence in May, and more frequently in August, September and October. Flocks of considerable size are sometimes seen both on the north and south coasts, wherever the shores are sandy. One killed at Plymouth 9 December, 1875, in full winter plumage, is the only specimen known to have occurred at that season of the year. The little stint sometimes occurs singly or in pairs mixed with dunlins and curlew sandpipers, but its presence can always be detected by its note. 


\section{BIRDS}

230. American Stint. Tringa minutilla, Vieillot.

Only three specimens of the American stint have occurred in the British Islands, one of which was shot on 10 October 1853 on the shore of Mount's Bay in Cornwall. The other two were obtained in north Devon, the first of them on 22 September 1869 on Northam Burrows, and the second on 22 August 1892 at the same place.

\section{Temminck's Stint. Tringa temmincki, Leisler.}

Three specimens of this small stint were obtained in Devonshire early in the last century, but none recently. Colonel Montagu had a specimen which was shot in November on a salt marsh near the sea in south Devon. Two were shot 28 June 1837, near Stonehouse Bridge, and were in Bolitho's collection when Dr. E. Moore wrote his 'Catalogue of the Wading Birds of Devonshire' (Mag. Nat. Hist. 1837, p. 320). The Rev. Murray Mathew felt certain he had twice seen this stint in winter on Braunton Burrows.

\section{Curlew-Sandpiper. Tringa subarquata (Güldenstädt)}

A passing visitor in spring and autumn, of frequent occurrence in small flocks, at the latter season, on the mud banks of our larger estuaries and on the sandy shores of the sea coast, both in the north and south of the county. It used to be not uncommon on the estuary of the Exe in September, and was especially numerous on the Taw. It is occasionally plentiful on the Kingsbridge estuary from August to September, and is not very rare at Plymouth in September and October, where it sometimes remains throughout the winter in small numbers. Specimens in full breeding plumage have been frequently obtained, and a pair in their bright-red summer dress was shot on Lundy Island in June. A few are sometimes seen at the end of May by the side of the Bude Reservoir.

233. Purple Sandpiper. Tringa striata, Linn.

A winter visitor in small parties, frequenting the rocky parts of the south coast, arriving annually in October or the beginning of November and remaining until the end of May, when it is just beginning to assume its breeding dress. It is not often met with in north Devon, but is said to have occurred on Lundy Island occasionally.

234. Knot. Tringa canutus, Linn. Locally, Silver Plover (on the Exe).

A passing visitor in spring and autumn, most numerous at the latter season when flocks visit our estuaries from August to October, the greater part remaining only for a few weeks, but some continue throughout the winter on the south coast. The specimens obtained in autumn are generally in immature plumage, but examples in the rich red summer dress are sometimes met with both in spring and autumn. The late Mr. Henry Nicholls shot a white-plumaged knot on the Kingsbridge estuary on 16 September 1863 .

\section{Sanderling. Calidris arenaria (Linn.)}

Principally a passing visitor to the coasts and estuaries in spring and autumn, though large flocks are sometimes seen in winter, and on the sands at Bantham and Thurlestone sanderlings have been obtained in every month of the year. It occurs in every stage of plumage.

\section{Ruff. Machetes pugnax (Linn.)}

A casual visitor of occasional occurrence, generally in autumn and winter, and rarely in spring. A few ruffs and reeves have been obtained in full breeding plumage, but the greater part of those shot in Devonshire are either birds of the year or adults in winter garb. Examples have been obtained on Dartmoor, but it is on the estuaries or in the neighbourhood of the sea coast that the ruff is most frequently met with. A small flock consisting of birds of both sexes visited Thurlestone Ley in the autumn of 1899 , and again in May 1900.

\section{Buff-breasted Sandpiper. Tringites rufescens (Vieillot)}

The only undoubted Devonshire example of this American sandpiper was shot on Lundy Island in the autumn of 1858 .

\section{Common Sandpiper. Totanus bypoleucus} (Linn.)

Locally, Summer Snipe, Otterling (on the Exe, perhaps in allusion to its diving habits when wounded).

A common summer migrant arriving in April and departing about the middle of October. A few stragglers occasionally remain throughout the winter. On their first arrival small flocks frequent our estuaries, but soon leave for the higher ground, and may be seen in pairs on the upper waters of the Okement, Avon and other Dartmoor streams during the summer. The eggs are laid in May, and the nest has been frequently found. In July it forsakes the moors and descends to the creeks and shores of tidal estuaries, where small flocks of old and young remain till their departure for the season. 


\section{A HISTORY OF DEVONSHIRE}

239. Wood - Sandpiper. Totanus glareola (J. F. Gmelin)

This sandpiper is very scarce in Devonshire, and but few records exist of its occurrence. It was first discovered as a British bird by Colonel Montagu, who had a specimen which was shot on the south coast of the county in the month of August. Two have occurred at Slapton Ley; the last of them was seen on I 8 July 1895. Two immature birds have been obtained near Barnstaple, and one on Braunton Burrows in the months of August and September. A specimen of this sandpiper in the Natural History Museum at South Kensington is labelled 'Torquay.'

\section{Green Sandpiper. Totanus ochropus (Linn.)}

In Devonshire the green sandpiper occurs but sparingly, and generally singly or in pairs, rarely in small flocks, in spring, summer and autumn. Occasionally it is met with in winter, and indeed in almost every month in the year. It is probably partially a resident breeding species, as very young birds have been seen in both the north and south of the county in July and August. It frequents inland waters as well as the sea shore and estuaries.

241. Common Redshank. Totanus calidris (Linn.)

Locally, Pill-cock (N. Devon). A 'pill' is a little stream of water which has cut for itself a deep channel in the mud.

The redshank was formerly a resident species, and one of the most abundant of the shore birds on some of our estuaries. In some years, when the summer and autumn have been fine and warm, it still occurs in considerable numbers in small flocks on migration, during August and September. It is sometimes seen in the summer months, and probably a few pairs breed at Slapton Ley, where young birds were seen in June 1894 , and both at Bantham and on the Kingsbridge estuary in June, 1900. Some occasionally remain throughout the winter on the south coast, and Mr. Gatcombe thought the redshank had become a regular winter visitor to the Plymouth estuaries.

242. Spotted Redshank. Totanus fuscus

A rare autumnal straggler principally to the south coast of the county. About a dozen examples have been obtained on the Plymouth, Kingsbridge and Exe estuaries in August, and one was shot on the Hamoaze in November 1829. Mr. Gatcombe mentions one killed in
February 1874 at the mouth of the Erme in perfect winter plumage. The only recorded north Devon specimen was obtained in October I8gI.

\section{Greenshank. Totanus canescens (J. F. Gmelin)}

A passing visitor, principally in autumn. Small flocks occasionally visit our estuaries in August and September, in fine seasons, associating with redshanks, dunlins and other shore birds. It is rarely seen in spring, and only now and then in winter, and is by no means a common species at any time.

\section{Red-breasted Snipe. Macrorbamphus griseus (J. F. Gmelin)}

Colonel Montagu, who first made this American species known as a British bird, records under the name of 'brown snipe' a male obtained on the coast of Devon in October of some year about I80 I. Dr. E. Moore mentions another which was in the collection of $\mathrm{Mr}$. Drewe of Devonport. A male in winter plumage was shot in the parish of South Huish in the winter of 1855 , and one is said to have been killed at Hatherleigh.

\section{Bar-tailed Godwit. Limosa lapponica} (Linn.)

Locally, Sea-Woodcock, Goddin (N. Devon), Cinereous Godwit and Red-breasted Snipe of Montagu.

A passing visitor in spring and autumn, sometimes numerous for a few weeks on the mud-flats of our estuaries, both in the north and south of the county. The number arriving depends on the prevailing winds. On the south coast they are most numerous in spring in those years in which there has been a long continuance of easterly winds. Such good 'godwit years' occurred in 1876 and I 895, when in May great numbers visited the Plymouth and Kingsbridge estuaries. In 1856 on 26 and 27 September large flocks were driven into the Barnstaple river by severe storms. Some of those that arrive in spring, probably non-breeding birds, remain during the summer, and sometimes flocks are seen on the south coast in winter. Specimens in the rich red dress of the breeding season are often obtained both in the spring and autumn, but most of the birds at the latter season are in immature plumage.

\section{Black-tailed Godwit. Limosa belgica} (J. F. Gmelin)

A casual visitor of occasional occurrence in spring and autumn, principally on the 


\section{BIRDS}

south coast of the county, where several have been shot in breeding plumage. It is very rare in north Devon, only two specimens in immature plumage having been obtained on the Taw.

\section{Common Curlew. Numenius arquata (Linn.)}

Locally, Whistling Curlew.

A common resident, considerable numbers breeding in April in some parts of Dartmoor, and also in many places on the rough high ground in the north-east and north-west of north Devon. After the adults have left for the moors in spring a few non-breeding or immature birds may be seen feeding on the mud-flats in the estuaries throughout the summer. At the end of August and the beginning of September large flocks appear on the marsh lands on their return from the moors, and pass the winter on the sea coasts and estuaries.

\section{Whimbrel. Numenius phacopus (Linn.)}

Locally, Cuckoo Curlew, Half Curlew, Jack Curlew, May-bird, Seven Whistlers.

A passing visitor, arriving in April and May on our coasts. After a short stay with us in spring, it leaves for its breeding places, returning in July and August, remaining until the end of September, and frequenting the mud-flats and marshes in our estuaries. Some occasionally remain with us all the winter.

249. Black Tern. Hydrochelidon nigra (Linn.)

A spring and autumn visitor, usually in small numbers, but at long intervals vast numbers have appeared at the latter season on our shores and estuaries. In 1849 a large flight of immature birds came up the Exe as far as Exeter, and numbers were knocked down by boys with their caps and sticks. In 1859 large flights appeared on the sand-flats at Barnstaple. Young birds were plentiful at $\mathrm{Ply}-$ mouth and Exmouth in September 1866, and in I 868 many came up the Exe as far as Stoke Canon. The black tern has rarely been seen in winter in Devonshire. Adult examples are sometimes obtained in April on the south coast.

250. White-winged Black Tern. Hydrocbelidon leucoptera (Schinz)

An example, in full moult, was shot in the harbour at Ilfracombe on 2 or 3 November 1870. A specimen stated to have been obtained at Kingsbridge was in the collection of the late Mr. Marsh-Dunn of Teignmouth ; and another in Pincombe's collection at Plymouth was said to have been killed on the breakwater there. An immature specimen in the Albert Memorial Museum at Exeter was probably killed on the Exe.

25 I. Whiskered Tern. Hydrochelidon bybrida (Pallas)

An adult example was picked up on the water by some fishermen, alive, but apparently in an exhausted state, off Plymouth, Io May I 865. The late Mr. J. Gatcombe presented this bird to Mr. Howard Saunders, and it is now in the Natural History Museum at South Kensington.

252. Gull-billed Tern. Sterna anglica, Montagu.

An immature bird was killed near Plymouth in October 1 866, and is in the possession of Mr. J. Brooking Rowe of Plympton.

253. Caspian Tern. Sterna caspia, Pallas.

A specimen was shot on the Exe near Topsham by Mr. Rudd, a well known sportsman, and was figured by the late Mr. F. W. L. Ross in his MS. 'British Laridæ,' now in the Exeter Free Library. An immature bird occurred at Teignmouth in October I 86I. Another is said to have been shot on Torbay, 28 September 1873 .

254. Sandwich Tern. Sterna cantiaca, J. F. Gmelin.

An occasional spring, summer and autumn visitor to our shores and estuaries. The specimens obtained in autumn are usually immature. Adult birds occur in spring and summer, but are rare.

255. Common Tern. Sterna fuviatilis, Naumann.

Locally, Miret, Mackerel-Bird, Sea-Swallow, Gull-Teaser (N. Devon).

A passing visitor in spring and autumn, most frequently seen at the latter season, especially after storms, when it ascends the river estuaries. Extraordinary numbers are sometimes met with on our coasts in a weak and exhausted condition. It has occurred on the Exe in July and August, and rarely in winter. There is a large breeding-place of this tern on the Chesil Bank on the coast of Dorset, and it also breeds on the Scilly Islands and the Cornish coast, but nowhere in Devon.

256. Arctic Tern. Sterna macrura, Naumann.

Locally, Pearl Gull.

Like the common tern, with which it is often confused, the arctic tern is a common spring and autumn visitor to our coasts, much 


\section{A HISTORY OF DEVONSHIRE}

more abundant in some years than in others. After gales in spring and autumn great flights have appeared on our estuaries in a weak and exhausted condition.

\section{Little Tern. Sterna minuta, Linn.}

Adults of this small tern are rarely seen in breeding plumage, and generally occur singly in the summer months, whilst adults in winter plumage and young birds of the year are met with in small flocks in the autumn on the south coast. It is a scarce species on the north Devon coast. Many pairs breed on the Chesil Bank in the neighbouring county of Dorset.

\section{Sabine's Gull. Xema sabinii (Joseph Sabine)}

Less than a dozen examples of this gull, all in immature plumage, have occurred on the south coast of Devon in the month of October. It has not yet been met with on the north coast, though it has occurred on the neighbouring coast of Somerset. An adult assuming winter dress was seen near the Eddystone Lighthouse on 29 September x 90 I by Mr. Eagle Clarke.

259. Little Gull. Larus minutus, Pallas.

This small gull has frequently been obtained on the coasts of Devonshire in autumn and winter, i.e. August to February. The examples met with are generally in immature plumage. The little gull occasionally wanders inland, following the course of some river. It is much less frequently seen on the north coast than on the south.

260. Black-headed Gull. Larus ridibundus, Linn.

Locally, Peewit Gull, Maddrick Gull, Redlegged Gull.

Although there is no 'gullery' in Devonshire, this species is extremely plentiful on our coasts and estuaries, from which it is seldom altogether absent, except in severe frost. Towards the end of July large flocks of old and young birds arrive from the breeding places (the most western one of which is on the north of Poole Harbour in Dorset) and remain all the winter on our estuaries, at times visiting the ploughed fields far inland. When the marshes have been flooded, great flocks of this gull assemble to feed on the drowned worms. Numbers may always be seen fluttering over the sewage outfalls of our sea coast towns. About the middle of April, on the Exe estuary, the greater part leave for nesting purposes, but many non-breeding birds remain all the summer there. At Plymouth most of the black-headed gulls leave by the middle of March, and until 26 July $1882 \mathrm{Mr}$. Gatcombe had never seen any there in summer. They also quit the Kingsbridge estuary early in the spring.

\section{6r. Great Black-headed Gull. Larus ich- thyaëtus, Pallas.}

The only example of this large gull which has occurred in the British Islanids was shot at Exmouth at the end of May or the beginning of June 1859 . The specimen is now in the Albert Memorial Museum at Exeter.

\section{Common Gull. Larus canus, Linn.}

Locally, Sea Mew, Winter Mew, Barley-Bird, Coddy Noddy (young).

An autumn and winter visitor to our coasts and estuaries from August to the middle of April, when for the most part the common gulls leave for their breeding places, a few remaining on the coasts as late as May, and rarely to June and July. The 'sea mew' is most familiar to us in its winter plumage, only a few examples being met with in the full summer dress late in the spring. At Plymouth it is most plentiful in March, and is then seen in pairs. It is not improbable that some pairs may occasionally nest in suitable spots both on the north and south coasts of the county. For some reason this gull has disappeared from the upper part of the estuary of the Exe, where it was formerly to be constantly seen in winter, feeding in flocks on the shingle at low water, its place having been taken by the black-headed gull. It is known as the 'barley bird' in some parts of the county, as it visits the freshly ploughed fields about the time of barley sowing, seeking food turned up by the plough. It goes as far inland in spring as the centre of Dartmoor, where solitary pairs may sometimes be seen.

263. Herring Gull. Larus argentatus, J. F.
Gmelin.
Locally, Ladram Gull (on the Exe), Wagel
(young).
A very abundant species breeding in conA very abundant species breeding in con-
siderable numbers on the cliffs along the south coast from Sidmouth to Plymouth. One of these nesting places is at Ladram Bay (between Sidmouth and Budleigh Salterton); another at Berry Head; and one at Wembury at the mouth of the Yealm. On the north coast the great breeding stations are at Baggy Point and Lundy Island. During the winter great numbers visit the freshly ploughed fields inland during the day, returning at dusk to the sea-cliffs to roost. 


\section{BIRDS}

264. Lesser Black-backed Gull. Larus fuscus, Linn.

Although this gull is a resident throughout the year on some parts of the Devonshire coasts it is never numerous, and usually keeps to the sea-cliffs and salt water. It is most frequently seen on the south coast in winter and spring after stormy weather, when some enter the estuaries, sometimes penetrating far inland and feeding in water meadows. It is a spring visitor to Plymouth Sound, appearing usually at the end of March, and keeping in pairs. Adults leave the Plymouth harbours by I May, but immature nonbreeding birds are seen in flocks as late as June. The breeding birds return early in August with their young. Flocks are also seen at that time off the coast of north Devon. Small numbers breed on the cliffs in the neighbourhood of Lynmouth, and a few pairs on Lundy Island. Adults in pairs are seen at breeding time about the south coast between Bolt Head and Bolt Tail, where there are suitable grassy slopes for them to nest upon.

265. Great Black-backed Gull. Larus marinus, Linn.

Locally, Saddle-back (Exe), Cobb (N. Devon).

Partially a resident, some few pairs breeding on Lundy Island, and it is said to have formerly nested on the cliffs at Bolt Head. It is principally a winter visitor to our south coast and estuaries, appearing after storms or during severe weather, singly or in pairs. On the north coast a few are seen all the year round.

\section{Glaucous Gull. Larus glaucus, O. Fabricius.}

An occasional winter visitor to the harbours of the south coast from January to March, rarely to April, or later. After severe gales it is sometimes numerous in Plymouth Sound, as in the early months of 1872 and 1873. The specimens obtained are usually immature, but adults are not very rare. One in winter plumage occurred at Exmouth in February 1903. It has been met with twice only on the north coast.

267. Iceland Gull. Larus leucopterus, Faber.

Examples of this species occur occasionally in Plymouth Sound and in Torbay, from October to February, sometimes lingering to April and even May. It was especially numerous in the winter of $1874-5$. Those obtained are usually immature birds; adults are very rare. Since 1855 it has become a more frequent visitor to Plymouth Sound than previously.

268. Kittiwake. Rissa tridactyla (Linn.)

Locally, Hacket, or Hacklet Gull, Tarrock (young), Petrel.

Breeds in large numbers on Lundy Island, where it is a summer visitor. On the south coast it is most numerous in autumn and winter, appearing in large flocks in October and November, especially when sprats are on the coast. It leaves Plymouth Sound at the end of February. In 1873 the late Lord Lilford found a pair with young, nearly full fledged, on the Cod Rock off Berry Head, and Dr. A. Tucker of Ashburton states (MS. notes) that 'it used to breed in abundance on the Orestone, near 'Torbay,' but as he says the eggs were generally four and sometimes five, and that no nest was made, there seems to be some doubt as to whether it was this gull that laid them, as the kittiwake only lays two or three eggs, and makes a large nest. The kittiwake is an oceanic species and keeps out at sea, only frequenting our estuaries in stormy weather when it is in a starving condition. Great numbers are thrown up dead on the seashore after heavy gales, and some are often driven far inland.

269. Ivory Gull. Pagophila eburnea (Phipps)

The only Devonshire example known was shot when in an exhausted state at Livermead near Torquay on 18 January 1853 . It is in nearly adult plumage, and is preserved in the Torquay Museum.

270. Great Skua. Megalestris catarrbactes (Linn.)

Locally, Tom Harry, Old Hen.

This powerful species of parasitic gull is rare on the Devonshire coasts, as it seldom approaches the shore or enters the estuaries. When obtained the examples are usually in an exhausted condition, and have been sometimes driven far inland by severe gales and found dead in the fields. Mr. Eagle Clarke observed some great skuas near Eddystone Lighthouse in September and October 1901, and it is probably not uncommon in the Channel in autumn, winter and spring. It sometimes accompanies fishing boats on the fishing grounds, plundering the gulls congregating around them of such fishes as they may chance to pick up.

271. Pomatorhine Skua. Stercorarius pomatorbinus (Temminck)

Locally, Irish Lord, Lords and Captains, Tom Harry, Boatswain Gull.

A passing visitor in spring and autumn, 


\section{A HISTORY OF DEVONSHIRE}

being most numerous at the latter season, when large flights appear off the south coast at intervals of about ten years (as in October and November 1879 and November 1890), and sometimes small numbers are seen in the Bristol Channel. This species is the least rare of all the skuas, and is generally to be met with in Torbay and Plymouth Sound in October, and sometimes in winter, but it is not so numerous on the north coast of the county. It was very abundant in September and October I goI off the Eddystone Lighthouse, according to Mr. Eagle Clarke.

272. Arctic or Richardson's Skua. Stercorarius crepidatus (J. F. Gmelin)

Locally, Robber Gull, Irish Lord, Tom Harry.

A passing visitor, sometimes not uncommon in autumn but rarely seen in spring, as it generally keeps well out to sea on its migration at that season. The young or immature birds are most frequently met with in Torbay in October. It occurs occasionally all along the south coast, and also on the north Devon coast, where one was shot as early as August in I 872. It was common off the Eddystone Lighthouse in September and October 190I, according to Mr. E. Clarke, but was not so numerous as the pomatorhine skua. An adult in full plumage was caught on a baited fishhook at Instow in Fune. An extremely black-plumaged bird was obtained at Budleigh Salterton, I November $189 \mathrm{I}$.

273. Long-tailed or Buffon's Skua. Stercorarius parasiticus (Linn.)

An extraordinary flight of this species, usually extremely rare in Devonshire, visited our coasts in October I89I, after severe gales. Many specimens both of adults and immature birds were obtained in various stages of plumage.

274. Razorbill. Alca torda, Linn.

Locally, Razor-billed Murre.

An abundant resident, breeding on Lundy Island in some numbers and also sparingly on some of the cliffs of the north and south coasts, but on the latter it is most numerous in winter. It is sometimes very plentiful at Plymouth, large flocks of adult birds assembling outside the breakwater in January and February, and some are brought in by the mackerel boats. Numbers are destroyed by south-west gales in early spring, as was the case in February 1872 , and some, principally young birds, are driven inland by stormy weather and are then picked up in most unlikely places. Great numbers are also frequently found dead on the shore in autumn after heavy gales, especially on the north coast. The birds that breed on Lundy arrive there about the end of April or early in May.

[A great auk (Alca impennis) is stated to have been picked up dead on Lundy Island in the year 1829. The original account, which appeared in Dr. E. Moore's 'Catalogue of the Web-footed Birds of Devonshire,' published in the Magazine of Natural History (1837), is as follows: 'Great Auk. Mr. Gosling of Leigham informed me that a specimen of this bird was picked up dead near Lundy Island in the year 1829 , and Professor Jameson suggests that it might have been one which had been obtained by $\mathrm{Mr}$. Stevenson at St. Kilda, and had escaped from the lighthouse keeper at Pladda, about that time, when on its way to Edinburgh.' Tradition handed down from early inhabitants of Lundy seem to point to the great auk having formerly nested upon the island, and about 1838 or 1839 an enormous egg was brought to the Rev. H. G. Heaven, the proprietor of the island, by one of his men in the egging season, which the men affirmed to be the egg of the 'king and queen murr,' and his description of the birds coincided with the appearance and habits of the great auk.]

275. Common Guillemot. Uria troile (Linn.) Locally, Mers, Mairs, Murre, Pick-billed Murre, Baron-bill, Willock.

Resident and numerous all along the coast in spring and summer, but usually keeps out at sea in winter. Breeds on Lundy Island in immense numbers, on the north coast of the county at Baggy Point, where there are a few, and on some of the cliffs of the south coast. Great numbers are sometimes thrown up dead on the sea shore after heavy gales in autumn, and individuals are occasionally driven inland. White specimens have been killed in Torbay, and the variety known as the bridled or ringed guillemot is not uncommon.

\section{Black Guillemot. Uria grylle (Linn.)}

About nine specimens of this pretty species, mostly birds of the year, have occurred, all but one (picked up dead on the Taw) on the south coast of the county. The black guillemot is only met with in autumn and winter, and the few adults that have been obtained were in winter plumage, with one exception, a specimen in black summer plumage mentioned by Dr. E. Moore.

\section{Little Auk. Mergulus alle (Linn.)}

This small species frequently occurs in the autumn and winter months, being usually 


\section{BIRDS}

found dead or in an exhausted condition after storms on both the north and south coasts of the county. It was very numerous in 1868 and again in 1873 .

\section{Puffin. Fratercula arctica (Linn.) \\ Locally, Sea Parrot, Lundy Parrot, Coulter- neb, Nath, Pope.}

A resident bird, examples being found on some part of our coasts at all times of the year, but excepting during the breeding season keeps far out at sea. Vast numbers breed in burrows on the slopes of Lundy Island, arriving early in May and leaving again in August. This island is supposed to derive its name from the Scandinavian lunde, a puffin, and $e y$, an island. On the south coast adults occasionally occur in summer as well as in winter, but they are most numerous in February, when examples are sometimes picked up dead on the shore. Young birds have also been obtained several times in summer on the south coast.

279. Great Northern Diver. Colymbus glacialis (Linn.)

Locally, Loon, Imber (young).

A winter visitor of frequent occurrence both on the north and south coasts, and it has been observed a few times in summer. The specimens obtained are usually either immature birds or adults in winter plumage, but old birds in partial summer dress are not unfrequent, examples in full breeding plumage being rather rare. Although this fine diver is usually found in our bays and estuaries, individuals occasionally make their way far up our larger rivers, and even visit inland ponds. It is sometimes very numerous (from November to March) in Plymouth Sound, where the late Mr. Gatcombe once saw towards the end of winter between twenty and thirty birds apparently in pairs.

280. Black-throated Diver. Colymbus arcticus, Linn.

A casual visitor of occasional occurrence, from October to March, in Plymouth Sound, Kingsbridge estuary, Torbay, the estuary of the Taw, etc. The specimens met with are nearly always immature birds. One in full plumage was shot on Slapton Ley 8 November 1865 .

281. Red-throated Diver. Colymbus septentrionalis, Linn.

Locally, Herring-bone, Sprat Loon, Speckled Diver, Wabble (young).

A winter visitor, arriving about the end of October and remaining till May; sometimes numerous on the estuaries of our larger rivers, in the bays and along the sea coasts, following the shoals of sprats. It is more frequently seen on the south than on the north coast. The specimens obtained are usually in immature plumage, but adult birds have not uncommonly occurred, and some have been killed in spring in full breeding dress.

282. Great Crested Grebe. Podicipes cristatus (Linn.)

Locally, Tippet Grebe, Great Dabchick.

A winter visitor of frequent occurrence off the coasts and in the estuaries, but rarely seen inland and never numerous, but generally met with singly or in small parties. It is seldom obtained in full breeding plumage and does not often remain until spring.

\section{Red-necked Grebe. Podicipes griseigena} (Boddaert)

A casual winter visitor, of occasional occurrence in late autumn and winter, in the bays and estuaries of our south coast, usually in immature plumage. It is rare in north Devon. Some adults have been obtained on Slapton and other leys, and on the Kingsbridge estuary. A very young bird with striped neck occurred in July I 892 at Kingsbridge.

284. Slavonian or Horned Grebe. Podicipes auritus (Linn.)

A winter visitor from the north, of not uncommon occurrence in bays and estuaries, almost always in immature plumage. The only adult that has been obtained in full breeding plumage was shot in Torbay.

285. Black-necked or Eared Grebe. Podicipes nigricollis (C. L. Brehm.)

A casual visitor to our bays and estuaries in the winter and spring months, coming from the south, and specimens in full summer dress are occasionally obtained in spring. A party of five was seen flying over the river Exe between Exmouth and Starcross on 10 April I 903, and having been fired at three were killed, all of which were in adult breeding plumage.

286. Little Grebe. Podicipes furviatilis (Tunstall)

Locally, Dabchick, Dipchiek.

This grebe, the smallest of the family, is also the most common, being the only resident species throughout the county. It breeds on quiet ponds, leys and sluggish streams, but is most frequently seen in small parties in winter (October to March), when probably immigrants arrive from other parts 


\section{A HISTORY OF DEVONSHIRE}

and its numbers are greatly increased. It then frequents the estuaries, canals and leys in the south of the county. It occasionally visits Lundy Island.

287. Storm-Petrel. Procellaria pelagica, Linn.

This small petrel generally keeps well out at sea, and is rarely met with near the shore except in the breeding season and after violent gales, especially in autumn and winter, when examples are not unfrequently picked up dead or in an exhausted state, even far inland and in the streets of the towns, having been driven ashore by the violence of the wind. Numbers are killed every winter by flying against the lanterns of the lighthouses on the south coast. A few pairs probably breed on Lundy Island and, it is said, on the Thatcher Rock in Torbay.

\section{Leach's Fork-tailed Petrel. Oceano- droma leucorrboa (Vieillot)}

A casual visitor during the autumn and winter months, when individuals are driven inland by violent south-westerly gales from the Atlantic. It has occurred all along the south coast of the county on numerous occasions, and several times on the north coast.

289. Wilson's Petrel. Oceanites oceanicus (Kuhl)

In some seasons this oceanic species comes further north in the Atlantic than usual and approaches the entrance to the English Channel. Rarely a bird is driven ashore by storms. One or two examples have occurred on our south coast ; one, said to have been obtained at Sidmouth in December 1870 , and one at Exmouth I 3 November 1887 .

290. Great Shearwater. Puffinus gravis (O'Reilly)

Locally, Hackbolt.

An irregular autumnal and winter visitor from the Atlantic to the south coast of Devon, sometimes in large numbers. It generally keeps far out at sea, but early in November 1874 numbers were seen on the south coast after an almost total disappearance for many years. Mr. W. Eagle Clarke found it common in September and October 1901 about the immense shoals of pilchards in the vicinity of the Eddystone Lighthouse. The great shearwater is sometimes taken with a baited hook and line, and examples thus caught are occasionally brought in by fishermen at Plymouth and in Torbay. Now and then individuals are captured in an exhausted con- dition. An adult bird was killed off Plymouth at the end of July 1876 , and Mr. E. A. S. Elliot saw two large shearwaters in the Channel off the south coast in July 1895 .

29I. Sooty Shearwater. Puffinus griseus (J. F. Gmelin)

Mr. J. H. Gurney saw a specimen of this species in Mr. Gould's collection labelled 'Plymouth,' which was sent to him by Mr. Gatcombe, and other specimens seem to have occurred in the same locality. Mr. E. A. S. Elliot saw a sooty shearwater off Burrow Island in Bigbury Bay in May I900, and Mr. W. Eagle Clarke saw single examples about the Eddystone lighthouse on 23 September and 12, 14 and 19 October 1901 .

292. Manx Shearwater. Puffinus anglorum, (Temminck)

Locally, Mackerel Cock (Torbay), Cuckle or Cockle (Lundy Island).

This shearwater is believed to breed on Lundy Island, where it is very numerous in summer. It is often seen in the Channel off the south-west coast in March and April in great abundance, and early in July I $895 \mathrm{Mr}$. Elliot saw hundreds feeding on mackerel 'brit' a few miles out at sea off the Bolt Tail. The Manx shearwater frequently visits Torbay in autumn in pursuit of herrings and sprats, and is not uncommon about the Eddystone. One was killed near Kingsbridge in 1874 , but this species very rarely enters the estuaries.

\section{Levantine Shearwater. Puffinus yelkou- anus (Acerbi)}

Two examples of the Levantine shearwater are in the British Museum from Devon, and Mr. J. H. Gurney has another Devonshire specimen, according to Mr. Howard Saunders.

\section{Fulmar. Fulmarus glacialis (Linn.)}

Locally, Mollymew, Mollymauk.

The mollymew, so well known to sailors, is only a waif and stray of the ocean in the western counties, but occasionally in winter an example in an exhausted condition wanders to the south coast of Devon, where several have been obtained, only one instance (an immature bird, in bluish-grey plumage, at Barnstaple) being known from north Devon. This specimen had a bony tumour on the neck, and one killed with an oar on the Exe estuary had a calcareous concretion in the vent. The last specimen that has occurred was found dead at Thurlestone in November 1898 . 


\section{MAMMALS}

Hooker' tells us that 'Devonshire doth yeild great abundance and varietye of all manner of thinges which the earth or water doth yeild and afford for the use of man. First, horses of all sorts, geldinges, mares, moyles, asses, pigges and hoggs, goate, sheepe, foxe, wolfe, beare, brocke, hares, conye, bull, oxe, cowe, calfe, and all kindes of roother, ${ }^{2}$ otter, wants, doggs of all sortes-the mastiffes, greyhounds, hounds, spaniells, currs, deere, as the stagg and hinde, the buck, and the doe, roe, deare, gray, hedgehoggs, weasel, ferrett, polecat, veyre, ${ }^{3}$ rats, mice.' The old chamberlain of Exeter is not scientific, but his list is not unworthy of notice. He was acquainted, not only with the domestic animals and beasts of the chase, but with the 'small deer' of Shakespeare," then and now in common parlance known as vermin.

Mammals are well represented in this county, for although there are no forests within its area, there are many small woods, copses and plantations in which the few remaining Carnivora and Rodentia find shelter, and there are some parts where the conditions are favourable for the preservation of other families. The coast-line, north and south, and the channels beyond, give us seals and cetaceans, and we include in our list altogether forty-seven species.

Polwhele, in that wonderful work of his, The History of Devonsbire (3 vols. Exeter I793-1 806)-for it must be remembered that it was the work of one man, and he left nothing untouched, imperfect as much of it is-was the first writer to deal with the natural history of the county, and his chapters, more especially those relating to the botany, the birds, and the quadrupeds are interesting, and in some respects, notwithstanding the progress of knowledge, valuable. John Cremer Bellamy in his Natural History of South Devon, W. S. M. D'Urban, in his Sketch of the Natural History of Soutb Devon, and J. Brooking-Rowe, in his Catalogue of the Mammals, Birds, Reptiles and Ampbibia of Devon, brought the history of the Mammalia of the county down to a recent period, and Edward Parfit, and others, from time to time contributed to the Zoologist, the Transactions of the Plymouth Institution, and the Devonshire Association Transactions, notes of facts which had come under their observation.

1 Discourse of Devonshire and Cornwall, Harl. MS. 5827 , circa 1580.

2 Roother, i.e. rother, a horned beast (Halliwell).

$s$ Probably meaning the marten.

4 But mice and rats, and such small deer, Have been Tom's food for seven long year.

King Lear, act iii. sc. 4. 


\section{A HISTORY OF DEVONSHIRE}

\section{CHEIROPTERA}

Polwhele evidently thought that there was but one species of bat. 'The Bat,' he says, 'was placed by Pliny, Gesner and other naturalists among the birds; it is introduced by naturalists of the present day among quadrupeds. Its flitting in the air has certainly the appearance of flight. In this respect it resembles a bird, but it approaches nearer to a quadruped in possessing teeth, in being viviparous and in suckling its young. In this chapter therefore on the quadrupeds of Devon I shall begin with the bat as a sort of link between the birds and the beasts." ${ }^{1}$ Old Richard Polwhele would have been astonished to know, if there had been any one to tell him, that at the end of the nineteenth century between four and five hundred species of 'the Bat' would be described. ${ }^{2}$ Of these but few are British, and only eight have been noted as occurring in Devon.

1. Greater Horse-shoe Bat. Rbinolopbus ferrum-equinum, Schreber.

It is stated in both editions of Bell's History of British 2uadrupeds that the greater horseshoe bat was first discovered as a British species in this county, and this statement has been followed by all writers on the subject since. But an attempt to verify the quotation, and a reference to the fourth edition of Pennant's Zoology, i. 147, proves this to be a mistake, and that the place where this animal was found by Latham was not Dartmouth in Devon, but Dartford in Kent.

With us it has been noticed only along the south coast. I have met with it at Hooe near Plymouth, in the immediate neighbourhood of Plymouth, and at Plympton, and Montagu found it in Kent's Cavern, Torquay, a place it still frequents. Dr. Edward Moore states that it has been found on Dartmoor, but there is no evidence in support of this, and it is not likely to be correct, there being on Dartmoor no places suitable for the habitat of members of this curious genus. Parfit also mentions Chudleigh.

\section{Lesser Horse-shoe Bat. Rbinolophus bippo-} siderus, Bechstein.

The general distribution of this species is much the same as that of $R$. ferrum-equinum. In this county it appears to be rarer than its larger relative. Like it, it has only been

1 Polwhele's Devon, i. 125.

2 Vide British Museum Catalogue of Cbeiroptera (G. E. Dobson, 1878 ). noticed in the south of the county, and in almost the same localities. In Kent's Cavern Montagu found both species in company. I have only noticed it once in the neighbourhood of Plymouth, a specimen being caught in a cave under the Hoe and kept alive by its captor for some days; this was as long ago as April 1863.

\section{Long-eared Bat. Plecotus auritus, Linn.}

A common species throughout the county. Early or late in the year, depending upon the warmth of the season, it recovers from its hibernation, and it continues on the wing as late as November. In 1902 it was apparently in flight the whole of the year.

\section{Barbastelle. Barbastella barbastellus, Schre- ber. \\ Bell-Barbastellus daubentonii.}

Rare. It was first noticed by Colonel George Montagu at Milton Abbot, and afterwards he found it at Kingsbridge near his own residence. But very few examples have since been recorded.

\section{Parti-coloured Bat. Vespertilio murinus,} Linn.

An individual of this species captured at Plymouth early in the nineteenth century was sent by Dr. William Alfred Leach to the British Museum where it now is. This is the only notice of its occurrence in England. It is a common European species.

6. Great or White's Bat (Noctule). Pipistrellus noctula, Schreber.

Bell-Scotopbilus noctula.

White-Vespertilio altivolans.

Generally common from May to October. In some years not observed at all or very sparsely. This with the long-eared bat and the pipistrelle may be said to be the three indigenous species of the county. The longeared bat is the most common, but the noctule is not so often met with as the others.

\section{Pipistrelle or Common Bat. Pipistrellus pipistrellus, Schreber. \\ Bell-Scotopbilus pipistrellus.}

Common throughout Devon except in severe weather. The specimen (Vespertilio pygmaus) found by. Leach at Spitchwick is a young example of this species.

7a. Natterer's Bat. Myotis nattereri, Kuhl.

Recorded by J. E. Harting (Zool. I 889 , 24I). 


\section{MAMMALS}

8. Whiskered Bat. Myotis mystacinus, Leisler.

Bell-Vespertilio mystacinus.

Some specimens were obtained by Montagu from south Devon-probably from Kings- bridge where he was then living-or the neighbourhood, and sent by him to the British Museum. There is no further record; but it has probably been overlooked, as it is by no means rare in other parts of England.

\section{INSECTIVORA}

\section{Hedgehog. Erinaceus europaeus, Linn.}

A common animal in all parts of the county and including the borders of Dartmoor, but not on the moor itself for obvious reasons. Often found under accumulated heaps of leaves in the winter. I witnessed a pretty sight one early autumn some years ago, an old hedgehog shortly before dusk leading out her brood of six young ones.

10. Mole. Talpa europea, Linn.

Common in all parts of the county except on the higher moors.

I I. Common Shrew. Sorex araneus, Linn.

Generally common.
12. Pigmy Shrew. Sorex minutus, Palias. Bell-Sorex pygmaus.

First recorded by the Rev. R. Douglas in I 877 as occurring at Manadon. Mr. W. B. Stark has noticed the species close to the moor. I have found examples at Plympton, and I have no doubt this little quadruped is more common than is supposed.

13. Water Shrew. Neomys fodiens, Pallas. Bell-Crossopus fodiens.

Certainly not so frequently met with as the common shrew, but by no means scarce. Mr. W. B. Stark has found both species near the moor over 800 feet above the sea level.

\section{CARNIVORA}

\section{Wild Cat. Felis catus, Linn.}

Formerly a common inhabitant of the woods-more numerous and more extended than now-the wild cat has long ceased to exist in the west of England. It is mentioned in the charter, now in the Cathedral Library, Exeter, of King John, then Earl of Mortain, circa I 194, to the free tenants of the forest of Dartmoor. It was extinct in Devon when Polwhele wrote.

\section{Fox. Vulpes vulpes, Linn.} Bell-Vulpes vulgaris.

Extermination in a very brief period would be the fate of the fox were it not for foxhunting. The packs of hounds and other matters relating to this animal will be found fully dealt with in the chapter on sport.

\section{Pine Marten. Mustela martes, Linn.} Bell-Martes abietum.

When the woods within the limits of the county were quiet and little visited, and before the plough and the habitations of man had invaded localities, martens were often met with. Specimens of this species and of the Beech Marten ( $M$. foina) are in the museum of the Plymouth Institution at the Athenæum. The latter is said to exist in North Devon, but we have no particulars.
I 7. Polecat. Putorius putorius, Linn.

$$
\text { Bell-Mustela putorius. }
$$

Like the marten this animal was formerly found in woods and other suitable habitats. Independently of its not being able to secure the seclusion it likes the polecat is too mischievous an animal to be allowed to have its own way in the world. The farmer and the sportsman both waged war against it, for it not only robbed the hen-roost and made free with the poultry, but partridges, hares and rabbits fell before it. The last instance of its having been met was in 1887 , when Mr. J. D. Prickman and Mr. Williams of Rewe killed one in the Taw Valley.

\section{Stoat. Putorius ermineus, Linn.}

$$
\text { Bell-Mustela erminea. }
$$

Common, more especially in the neighbourhood of rabbit warrens. They were very abundant in the winter of 1879 . One I saw was quite white, in the ermine state, another very white but with some brown marks still remaining about the head. I have seen some with light-coloured fur.

\section{Weasel. Putorius nivalis, Linn. Bell-Mustela vulgaris.}

Common in all parts of the county. 


\section{A HISTORY OF DEVONSHIRE}

20. Badger. Metes meles, Linn.

Bell-Meles taxus.

Though frequently met with, this harmless creature is not, as it is just now in the north of Cornwall, anywhere common in this county. Where a landowner protects it, it flourishes, but if it has no friend at court the poor animal is persecuted and captured, and too often, we fear, in spite of legal enactments, done to death, after inflicting serious injury upon the dogs set to draw it.

21. Otter. Lutra lutra, Linn.

Bell-Lutra vulgaris.

Common on all rivers of any size. Otter hunting will be found dealt with in the chapter on sport.
22. Common Seal. Phoco vitulina, Linn.

On the north coast this seal is often observed. On the south coast stragglers only are met with. The last we have heard of was killed in the Teign river in March 1903 .

\section{Grey Seal. Halicherus grypus, Fabricius.} Bell-Halicherus gryphus.

A fine female of this species, measuring about $7 \frac{1}{2}$ feet from the tip of the snout to the end of the tail, was caught in the trawl net of the Electric, off the Eddystone 24 September 1891. It was purchased for the British Museum, where the skin and skeleton are preserved.

\section{RODENTIA}

24. Squirrel. Sciurus leucourus, Kerr. Bell-Sciurus vulgaris.

Common in all parts of the county where there are woods or plantations. Although squirrels dislike damp more than cold, they do not approach the higher regions near the moor. Individuals with white tails are often noticed, and these have also white tips to their ears.

25. Dormouse. Muscardinus avellanarius, Linn.

Bell-Myoxus avellanarius.

Not common in the south of the county. Said to be abundant in the neighbourhood of Exeter (W. S. M. D’Urban). My impression is that it is much scarcer than formerly. Several were noticed in the autumn of 1879 .

26. Harvest Mouse. Mus minutus, Pallas.

Generally distributed in all parts of the county, but not common.

27. Wood Mouse or Long-tailed Field Mouse. Mus sylvaticus, Linn.

Common everywhere.

28. House Mouse. Mus musculus, Linn.

Polwhele says that some years before the date of his writing several hundreds of white mice presumably of this species were found in a mow at Whiteway, Kingsteignton. Albinos of all the small mammals occur.

29. Black Rat. Mus rattus, Linn.

Polwhele (Hist. Devon, i. 129) says that before the grey, or as we now call it the brown rat (M. decumanus), was known in England, the black rat had overrun Lundy Island, and that the craggy pyramidical rock there had obtained from the frequency of this animal the name of Rat Island. He also says that the 'introduction of the present destructive race of grey rats into this country is within the memory of many old men. An old carpenter of the neighbourhood of Plympton who was born in the year (17) 23 informed me that when he was a lad and worked with his father, there were none but black rats known, but that about this time a ship which arrived at Oreston in the river Plym, and was there broken up, brought this kind of rat.' ${ }^{1}$ She was called the Elizabeth, and the master's name was Henley.

This rat is now very scarce. I have no note of its having been observed anywhere in the county recently. I have the skin of a parti-coloured individual.

30. Brown Rat. Mus decumanus, Pallas.

Devon is by no means free from this destructive little animal.

31. Field Vole. Microtus agrestis, Linn. Bell-Arvicola agrestis.

Common everywhere.

32. Bank Vole. Evotomys glareolus, Schreber. Bell-Arvicola glareolus.

I have no knowledge of this species in Devon. The Rev. W. S. Hore was a good naturalist and observer, and he stated (Rowe's Perambulation of Dartmoor, ed. 1848, p. 236) that he had met with it. In March 1879 a living specimen captured at Ide near Exeter was brought to Mr. W. S. M. D'Urban.

33. Water Vole. Microtus ampbibius, Linn. Bell-Arvicola ampbibius.

Common everywhere.

1 Polwhele's Devon, i. 129. 


\section{MAMMALS}

34. Common Hare. Lepus europacus, Pallas. Bell-Lepus timidus.

As elsewhere much scarcer than formerly, and its numbers are diminishing yearly. An account of the packs of harriers in the county will be found in the chapter on Sport.
35. Rabbit. Lepus cuniculus, Linn.

Warrens are numerous, and the animal is abundant everywhere. Enormous numbers are killed and brought to the markets. Rabbit warrens will be referred to in the chapter on Sport.

\section{UNGULATA}

\section{Red Deer. Cervus elaphus, Linn.}

The history of the red deer and of the chase in Devon and on its borders will be found in the chapter on sport.

\section{CETACEA}

37. Common Rorqual. Balenoptera musculus, Linn. (B. physalis, True, Proc. U.S. Nat. Mus. xxi. 6I 7 )

The body of one of these animals was found off the Eddystone in October I 831 and towed into Plymouth. It was preserved, and is now in the British Museum. In 1863 a dead whale, which proved to be a rorqual, was found in the Channel and taken to Falmouth, and it is now I believe at the Alexandra Palace, and in 1875 in the month of January another was washed upon the shore near Teignmouth. In January 1879 another was found floating near the same place and it was brought into Looe. It was a male $6_{4}$ feet 7 inches long, and its circumference behind the pectoral fins $3^{8}$ feet. It was seized for the Crown and sold for $£$ io. In October 1885 two dead rorquals were brought into Plymouth and exhibited. One was found near the Start Point, and the other near the Eddystone. They were male and female about the same size, the former just 60 feet long, the female a little larger. They were on show for some days. ${ }^{1} \quad$ Besides these there have been other instances of the occurrence of these great animals on the coasts of the county.

\section{Lesser Rorqual. Balenoptera rostrata,} Fabricius. (B. acuto-rostra, Lacép.)

On 18 May I 887 the Lowestoft fishing smack, the Bluebell, landed on the Barbican, Plymouth, what the captain described as ' $a$ big fish with his mouth full of hair.' This proved to be a young female of the lesser rorqual which had been caught in the nets in the Channel 60 miles south-west of the Scilly Islands. Its length was 12 feet $7 \frac{1}{2}$ inches. It was sold by the boat's crew for $£ 3$ I 7 s. $6 d$. and carried round the town on a pony-cart for exhibition. The skull was obtained for the Museum of the Plymouth Institution.

$$
3 \text { Vide Trans. Plym. Inst. ix. } 314 .
$$

According to Hooker the roe deer (Caprealus caprea) in his time was found in Devon.

\section{Balcenoptera (Eschrichtius) robustus (Lillje- borg)}

Bones of this species were some years since washed up on the beaches of Babbicombe and Petitor, Torbay; these and some bones cast ashore on the coasts of Sweden belonging to two or at most three individuals, are the only remains of $B$. robustus known to science. ${ }^{2}$

\section{Sperm Whale. Physeter macrocephalus, Linn.}

Bellamy states that the carcase of a whale of this species was cast ashore some years before the time of his writing at the mouth of the Erme. ${ }^{3}$ Another was found off Ilfracombe in the month of February $1845^{4}$

\section{White Whale. Delphinapterus leucas, Pallas.}

The late Mr. Philip Henry Gosse in a letter received by me through a relative states that he saw a white whale off Berry Head in the summer of 1832 . The following is an extract from Mr. Gosse's letter :-

On August 5 th 1832 I was returning from Newfoundland to England, and was sailing up the British Channel close to the land, when just off Berry Head I saw just under the ship's bow a large cetacean of a milky white hue, but appearing slightly tinged with green from the intervening stratum of clear water. It was about 16 feet long with a round bluff head. It continued to swim along before the vessel's head a few yards beneath the surface for about ten minutes maintaining our rate of speed which was five knots an hour, all which time I enjoyed from the bowsprit a very good view of it. It could have been no other than the white whale Beluga albicans of Lesson. ${ }^{5}$

The late John Gatcombe (Zoologist, I886, p. $3^{64}$ ) states that ' $\mathrm{Mr}$. J. C. Willcocks, a

2 Trans. Dev. Assoc. vol. i. pt. 4, p. 65 ; Ann. Nat. Hist. xv. 492.

${ }^{3}$ Natural History of South Devon (1839), p. 197.

4 Zoologist, 1845, p. 912.

5 Trans. Plym. Inst. iv. 154. 


\section{A HISTORY OF DEVONSHIRE}

good authority on such matters assured me that one day at the end of July he had a good view of a beluga or white whale, Delphinapterus leucas, as it rose off the Bolt Head, close to the steam tug Perseverance, in which vessel he was a passenger.' Commenting on this Mr. Thomas Southwell says that 'this essentially arctic species has undoubtedly been met with several times in Scotch waters, and one so late as 18 June 1902 at the mouth of the Tyne, and strangely enough considering its northern habitat generally in the summer months. But where the record is not substantiated by the production of the specimen, I think such statements should be received with caution, as several examples of pure white porpoises have been met with which might readily be mistaken for this species by the casual observer.'

42. Porpoise. Phocona communis, Lesson.

Common on both north and south coasts.

43. Pilot Whale or Black Fish. Globicephalus melas, Traill.

This species is also known as the pilot whale and the black fish. Bellamy mentions one captured off Plymouth in $1839 .^{1}$ Another was found in a dead or a dying state on a reef of rocks called Hook Ebb, east of Sidmouth, after a severe gale in February $1883 .{ }^{2}$ The skin was preserved and it is now in the Exeter Albert Museum.

44. Grampus. Orca gladiator. Lacépède. Often observed in the channel.

\section{Risso's Grampus. Grampus griseus, Cuvier.}

On 28 February 1870 I was asked to go to the Plymouth Railway Station to see a large fish which had been taken in the mackerel nets. Unfortunately the train with the truck containing the animal was just leaving, but I was enabled to see enough of it to decide it was a cetacean and an uncommon species. I wrote Dr. Gray by the evening post telling him that a cetacean of a rare species would be in the Columbia market on the following morning, and asking him to send some one from the museum to look after

\footnotetext{
1 Natural History South Devon, p. 190.

2 Zoologist, 1883 , p. 173.
}

it and ascertain the species. Although Dr. Gray acknowledged the receipt of my letter he did nothing more, and the carcase was bought by an enterprising individual who, fitting up a hand cart, enclosed it in it, and exhibited it in the streets of London, loudly proclaiming the capture of the sea-monster, ' $a$ cross between a shark and a whale.' Fortunately the show was encountered by $\mathrm{Mr}$. Gerrard, who made a bargain with the owner and secured it for the British Museum, where it now is. It proved to be a Risso's grampus, captured about 20 miles south-west of the Eddystone. It was a female about II feet long, and she weighed about $7 \mathrm{cwt}^{3}$ In February I 888 some fishermen captured in their herring-nets about 8 miles south of the Eddystone another specimen, also a female, in beautiful condition. Its length was 9 feet, the greatest girth 5 feet 2 inches. The skeleton is in the museum of the Plymouth Institution, and the skin in the Exeter Albert Memorial Museum. A full account with an engraving will be found in the Transactions of the Plymouth Institution, ix. 3 I 4 .

\section{Common Dolphin. Delpbinus delpbis, Linn.}

Frequently visits our coasts. One killed in Plymouth Sound 1883 .

\section{Bottle-nosed Dolphin. Tursiops tursio, Fabricius. Bell-Delphinus tursio.}

Montagu mentions one killed in Duncannon Pool, in the Dart river, 5 miles from its mouth, and the specimen is described in Wernerian Memoirs, iii. 75. The skull is in the British Museum. Mr. Southwell says: 'The late John Gatcombe (Zoologist, I 884, p. 65) describes two immature specimens of $D$. tursio, each about 8 feet in length, which he saw on 15 December 1883 at Hooe Lake near Plymouth, where they had been captured on the mud banks in shallow water; he also mentions that the only other instance which had come to his knowledge of the occurrence of this animal was an adult male 12 feet long driven on shore under Plymouth Hoe a few years previous.'

\footnotetext{
3 Trans. Dev. Assoc. xviii. 69 ; Trans. Plym. Inst. iv. 154 .
} 



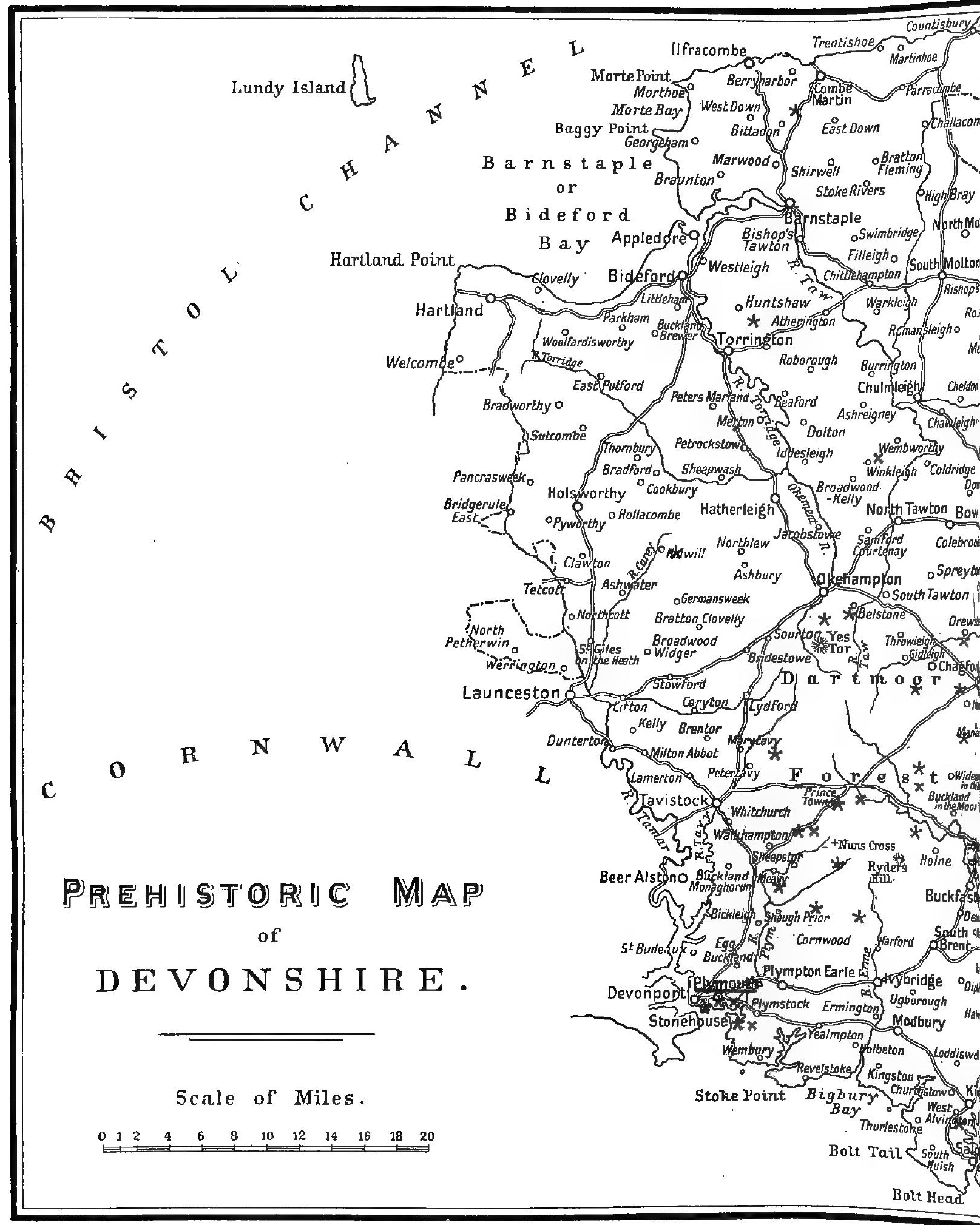





\section{EARLY MAN}

The Palaeolithic Age

7 HE examination of bone-caves in Devon during the past century is the main source of our knowledge of early man in the county. Devonshire has the distinction of at one time possessing at Oreston, Plymouth, a bone-cave in the Devonian limestone which was systematically explored as long ago as 1816 , and of still having within its borders, in Kent's Cavern or Kent's Hole, the most important cave dwelling of primitive man known in the country.

Oreston.-The quarries at Oreston, from which the stone was obtained for the construction of Plymouth breakwater, were opened in 18 I 2 under the superintendence of Mr. Whidbey, who was requested by Sir Joseph Banks, then President of the Royal Society, to narrowly examine any caverns his workmen might break into for bones or fossil remains, and if such were found, steps were to be taken for their preservation and examination.

This request was responded to by $\mathrm{Mr}$. Whidbey in an efficient manner, for he discovered several caverns and ossiferous fissures containing fossil bones, which were deposited in the Museum of the Royal College of Surgeons, and reported on by $\mathrm{Mr}$. Clift, the curator.

Colonel Hamilton Smith ${ }^{1}$ states that prior to 1833 , and repeatedly since, caves have been opened at Oreston, near Plymouth, several of which had in them bones of extinct animals and abundant hyaena coprolite, denoting that they had been dens of carnivora. Among these remains the upper portion of a humerus of man was recognized. On this being pointed out to the possessor it was immediately thrown away. Colonel Hamilton Smith further states that this is not the only instance of the kind.

Shortly after the exploration of Oreston came the researches of Dean Buckland in the cave at Kirkdale, in Yorkshire, and the famous examination of Kent's Cavern, Torquay.

Kent's Cavern, Windmill. Hill Cavern.-Devonians may congratulate themselves that their county yielded not only the first-fruits of the systematic investigation of bone-caves and the antiquity of man in Britain, but the heaviest crop of all was garnered from Kent's Cavern by the Rev. J. McEnery, Mr. Godwin Austen, the Torquay Natural History Society, and a committee of the British Association, which from I 865 to I 880 persevered with a scientific and thorough investigation, mainly carried out and reported on by the late Mr. Pengelly, F.R.S., of Torquay.

Mr. Pengelly describes the cavern ${ }^{2}$ as situated in a small wooded hill of Devonian limestone, which rises a little more than $200 \mathrm{ft}$. above sea level, and is about one mile east of Torquay Harbour. On the eastern side of the hill there is a small vertical cliff in which are the two apertures leading into the cavern. These are nearly on the same level, $54 \mathrm{ft}$. apart, about I90 $\mathrm{ft}$. above the level of mean tide, and about $60 \mathrm{ft}$. to $70 \mathrm{ft}$. above the bottom of the valley in the same vertical plane. The cavern consists of two parallel divisions, an eastern and a western, each containing a series of chambers and passages, and throwing off lateral branches, some of which are of considerable length and very tortuous. The divisions are united near their northern and their southern ends. The connecting passage at the latter extremity is completely filled with various deposits, whilst the northern appears to have always been a comparatively lofty open chamber. The eastern division, into which the two apertures or entrances directly open, and which has been completely explored by the committee, is $285 \mathrm{ft}$. long, $90 \mathrm{ft}$. in greatest breadth, and, when measured from the bottom of the excavation made by the explorers, $22 \mathrm{ft}$. in maximum height. The western division is probably of greater length, and is at a considerably lower level than the eastern.

1 Natural History of the Human Species, 95-96.

Trans. Plymoutb Inst, i, 345-346. 


\section{A HISTORY OF DEVONSHIRE}

Kent's Cavern seems to have been known from time immemorial, and it has been resorted to by man from palaeolithic times to the present day. There are abundant indications that it was very much used during the Romano-British period, and it was frequented as late as the early part of the fifteenth century, probably as a place of refuge. It has been a well-known show place from the eighteenth century, and must have been visited long before that by the curious, for Mr. McEnery records inscriptions of the seventeenth century incised on the stalagmite, the earliest being I6I5.

The earlier explorers clearly demonstrated the fact that rude stone implements fashioned by the hand of man were found in red loam under a covering of stalagmite blended with the bones of extinct cave mammalia, such as tichorhine (or woolly) rhinoceros, hippopotamus, cavebear, etc. The inference was therefore drawn that man and such mammals must have been contemporaneous.

This pushed back the antiquity of man so far that grave doubts were raised by many, not as to the human origin of the implements, but rather as to their being contemporaneous with the remains of extinct mammalia.

The accidental discovery, however, in 1858 , of Windmill Hill Cavern at Brixham, set these doubts at rest, and that the matter might be carefully investigated, the cavern was handed over to a committee appointed by the Royal and Geological Societies, under the superintendence of Mr. Pengelly. In twelve months this comparatively small cave was exhausted, and the result of the researches amply bore out the evidence obtained from Kent's Cavern by the earlier explorers, for flint tools were found unmistakably blended with the remains of extinct cave mammalia, and in such a manner as to preclude any of the objections previously raised.

The bone-bearing loam, of an ochreous red colour, was covered with stalagmite varying from $I$ in. to 15 in. in thickness. No human bones were found, but flint implements were disinterred from the lowest part of the bone-bearing loam. Man resorted to the cavern before the whole of this loam was deposited, and evidently preceded the cavebear, for in the upper part of the red loam the remains of bears and their cubs were numerous. After the stalagmite had begun to form, the cave was evidently

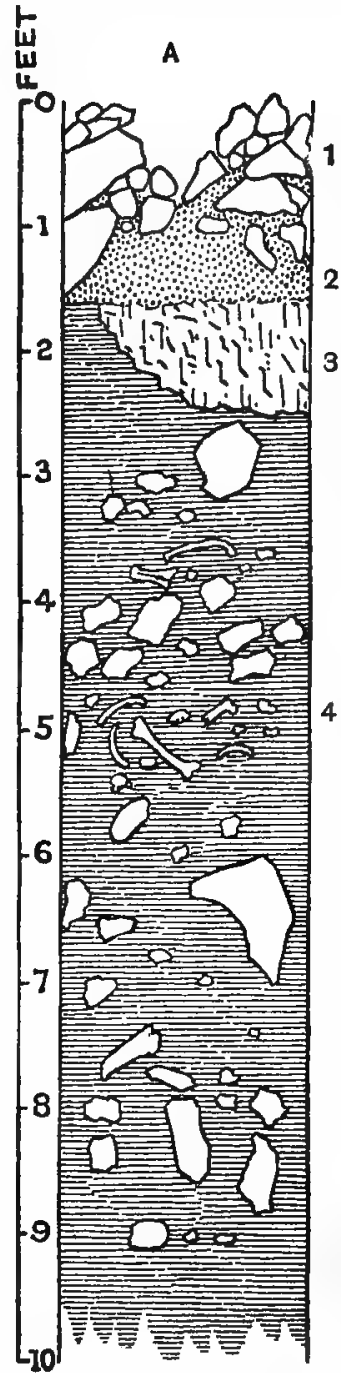

Fig. 1.-Srctions or Kent's Cavern ${ }^{1}$ from Diagrams in British Museum

A, 1865 Report $\quad$ B, 1872 Report c

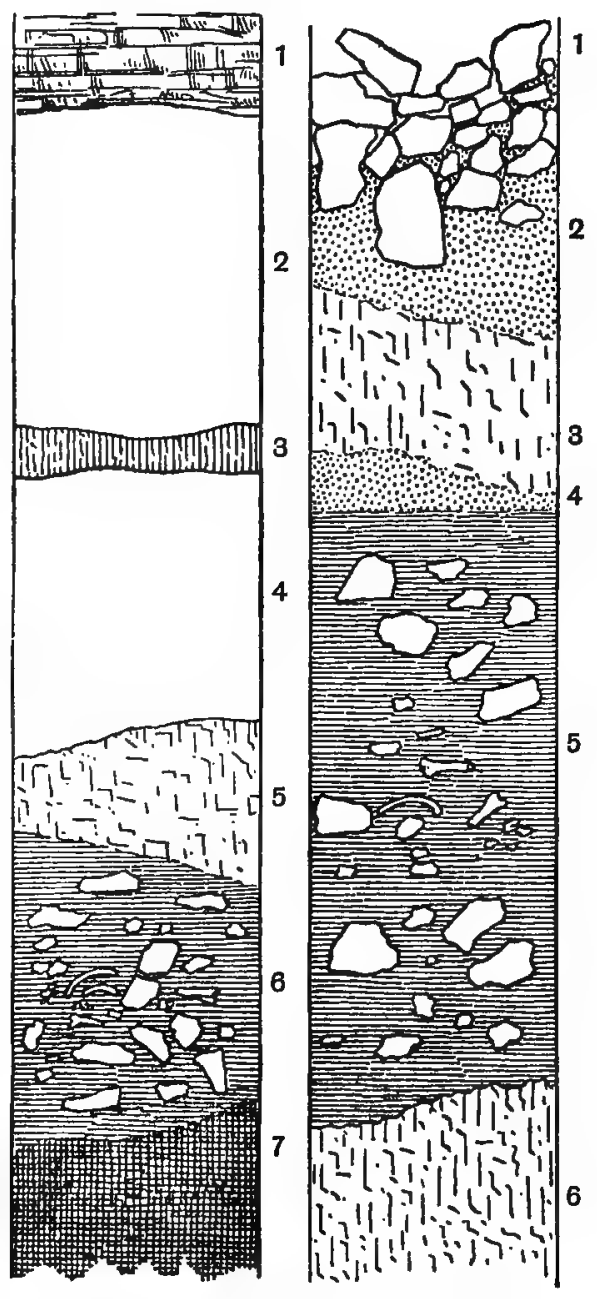

C, 1867 Report

still occasionally resorted to by beasts of prey and other animals, for the remains of bear, rhinoceros, and reindeer, were found in the floor of this material.

The most ancient deposit in Kent's Cavern was a dark red grit described as the breccia, containing rounded pieces of quartz, flint implements of palaeolithic type, fashioned from the nodule,

1 The deposits in Kent's Cavern are as follows :-A, Limestone blocks fallen from the roof $(1)$, in some parts leaving the black mould (2) exposed, floor of granular stalagmite (3), cave-earth (4) with limestone blocks and various remains. B, Roof of cavern (I), unoccupied (2), floor of crystalline stalagmite (3), unoccupied (4), floor of granular stalagmite (5), cave-earth (6), dark red breccia (7). C, Limestone blocks (1), black mould (2), floor of granular stalagmite (3), black band (4), cave-earth (5), crystalline stalagmite (6) 10 to $12 \mathrm{ft}$. thick with dark red breccia below. 


\section{EARLY MAN}

not flakes struck off from the same, and the animal remains found were exclusively those of the bear. This deposit was sealed up by an enormous floor of stalagmite (carbonate of lime) of crystalline texture, as much as Io ft. or $\mathrm{I} 2 \mathrm{ft}$. thick, which, like the breccia, contained remains of the bear. Resting on the top of this was the cave-earth of light red clay mixed up with fragments of limestone covered with films of stalagmite. This bed was not present in all parts of the cave explored, and its maximum thickness was $4 \mathrm{ft}$. Here the flint and chert implements were flakes struck off from the nodule or core, and exhibited a decided advance in manufacture compared with the more rudely shaped palaeoliths found in the deposit underneath. The caveearth yielded a bone needle, spear-heads of bone or horn, hammer-stones, whet-stones, a perforated badger's tooth, and a hare's leg-bone with holes neatly drilled in it, suggesting its use as a whistle.

The top of the cave-earth was partially covered with wood charcoal about 4 in. thick, described as the black band, and on this, still ascending through the strata, was a granular floor of stalagmite varying from $I$ in. to nearly $5 \mathrm{ft}$. in thickness.

Water containing carbonic acid derived from organic matter slowly dissolves limestone, and with this in solution it percolated through the roof and sides of the cavern. The dissolved limestone (carbonate of lime) either crystallized, or became deposited in the granular form. Even supposing an abundance of drip water extra charged with carbonic acid, in fact all the conditions favourable for speedy deposit, a very long period of time must have elapsed before such thickness of stalagmite could possibly be formed.

We have not yet done with the ascending strata, for resting on the granular stalagmitic floor was a layer of dark, decayed vegetable matter, 3 in. to 12 in. thick, which for the sake of distinction was described as the black mould

In this were found remains of the Bronze Age, weapons and implements of bronze, a cake of smelted copper, succeeded by spindle-whorls, bone combs, and ornamented pottery of Early British down to Romano-British times, the bones of animals, and also those of man.

All these strata were finally covered with blocks of limestone, some small, others weighing scores of tons, which had from time to time become detached from the roof of the cavern. These were partially cemented by calcareous drip, and the whole formed a natural sarcophagus, underneath which lay buried the story of the primitive men of Devon. ${ }^{1}$

Man was evidently living in or near Kent's Cavern during the formation of the breccia, preceding the hyaena.

It was noticed during Mr. Pengelly's exploration that while no animal relics were found below $7 \mathrm{ft}$. from the cave floor, three palaeolithic tools made from flint nodules were found in the eighth-foot level, and several flint chips, struck off in implement making, occurred in the ninth or lowest foot level. Man's handiwork was the oldest of all.

The history as revealed by the strata may be summed up as follows:-The palaeolithic breccia men had massive tools made of nodules of flint, roughly chipped and irregular in outline, but no delicate flakes, or implements of bone. They left no trace of fire behind them. The later men of the cave-earth possessed carefully chipped and symmetrically formed but unpolished flakes of flint ; they had bone needles, could make a fire, and adorned their persons with necklaces or bracelets strung with the perforated teeth of mammals. They had no pottery, metal, or spindle-whorls. These were, however, possessed by the succeeding men of the black mould, who were advanced enough in civilization to smelt and alloy metals and wear amber beads.

The Torbryan Caves near Denbury.-These are variously described as caves, rock shelters, tunnels, or fissures in the limestone at Torbryan. They were mostly explored by the unaided efforts of Mr. J. L. Widger, and were reported on by the late Mr. J. L. Lee. ${ }^{2}$ They contained a bone bed with the usual cave fauna, including teeth of hyaena, bear, and rhinoceros. A flint implement described as of the older type was found under two thick floors of stalagmite. The cave was evidently also occupied by a neolithic race, for it yielded implements of that period and much charcoal.

Happaway Cavern, Torquay.-This was explored by Mr. Pengelly and his son with their own hands, and to avoid any possibility of mistakes, or worse, the entrance was blocked and a door inserted. When the explorers were not in the cavern the door was kept locked, and the key remained in the possession of the careful and indefatigable explorer.

The general character of the zoological remains was that of a recent fauna, but a decided air of antiquity was imparted by the discovery of a few relics of the hyaena and rhinoceros. It seemed apparently not improbable that the cavern was more or less filled in palaeolithic times, was subsequently almost emptied (by water), and then was nearly completely refilled in times long subsequent to the British hyaena and his extinct contemporaries. The absence of a separating floor of stalagmite allowed the occasional commingling of the new deposits with the old, and thus deprived the cavern of anthropological value, and caused the enthusiastic labour bestowed on it to become of but little value.

\footnotetext{
${ }^{1}$ See Section C for sequence of strata. N.B.-Breccia not shown. $\quad$ 'Proc. Soc. Antig. (ser. 2), viii, 247.
} 


\section{A HISTORY OF DEVONSHIRE}

The Cattedown Bone-Cave.-Mr. McEnery found human bones in Kent's Cavern beneath the stalagmite, but under such circumstances as to lead him to form the opinion that they had been placed there after the remains of extinct mammalia had been deposited. As we have previously noticed, on the authority of Colonel Hamilton Smith, human bones were observed, and disregarded in one of the Oreston Caves, some fifty-seven years since. In the first instance we are compelled to accept the doubt of Mr. McEnery, and the second is open to criticism, apparently for want of confirmation; we must therefore admit that up to 1886 there existed no reliable evidence that the bones of man and extinct mammalia had been found in any of the Devonshire bone-caves in undoubted original association.

In the autumn of the above-mentioned year this uncertainty disappeared, for bones of the cavemen of Devonshire were discovered at Cattedown, Plymouth, in juxtaposition with the remains of the cave hyaena. The Cattedown Bone-Cave was accidentally discovered by some workmen who were employed by Messrs. Burnard \& Alger to remove limestone from an old quarry at Cattedown for the purpose of providing stone for quay extension on the northern shore of the Cattewater. It was situated-for it is now destroyed-about 150 yds. north of the old shore of the Cattewater, in a line with No. 7 warehouse, and Messrs. Burnard \& Alger's office.

This old quarry had been excavated many years since down to some $60 \mathrm{ft}$. below the cliff surface, and the old upper floor of a portion of the excavation was being worked down to a lower level when a fissure was broken into. This was seen to be filled with soil and small stones, the débris of the former workers of the quarry, and on clearing it out a few ox bones were observed which, from their condition, were indicative of considerable antiquity. Recognizing the possibility of more finds being made underneath, Messrs. Burnard \& Alger bore the cost of an exploration which extended over several months, and placed its conduct in the hands of the late Mr. R. N. Worth, F.G.S., his son, Mr. R. Hansford Worth, C.E., and the writer.

Before briefly dealing with the contents of the cavern, it may be stated that it consisted of a gallery $54 \mathrm{ft}$. long, pointing north and south in the direction of a natural jointing of the rock. This gallery was only 4 to $5 \mathrm{ft}$. in width, but expanded into a chamber at each end. Both these chambers had a length of $20 \mathrm{ft}$. That of the south end was about $5 \mathrm{ft}$. wide and descended to a depth of $9 \mathrm{ft}$. below the recent quarry level.

The greatest width of the northern chamber was $8 \mathrm{ft}$., with a depth of $\mathrm{r} 3 \mathrm{ft}$.- deep enough to admit the tide at high water. The gallery connecting the chambers did not descend more than $2 \mathrm{ft}$, so that the lowest depths of these chambers were separated by nearly $20 \mathrm{ft}$. of rock (see Fig. 2).

The original height of the cavern, or whether it led into other fissures, is unknown, for the rock above it was removed many years ago. It is probable that the cave was the nethermost of a series of caverns, for the limestone at Cattedown is honeycombed with cavities and fissures of varying dimensions. Where these cavities occurred in the operations of quarrying they formed convenient receptacles for 'spoil,' and the modern filling of the Cattedown Bone-Cave may be thus accounted for. This 'spoil ' could be easily distinguished from the natural deposits on which it rested, and when it was removed the strata of stalagmitic floor, breccia, granular stalagmite and caveearth were clearly distinct. In the northern chamber the total depth of material excavated was $27 \mathrm{ft}$, and of this $20 \mathrm{ft}$. was more or less ossiferous.

The bones in the stalagmitic breccia represented the remains of man, hyaena, wolf and deer, the latter predominating. Portions of skeletons were found more or less complete, and indicated that when deposited they were clothed with flesh, and that there had been no subsequent disturbance. The lowest human skull was found at $7 \mathrm{ft}$. below the stalagmitic breccia, but human bones and teeth occurred at the lowest point reached. The feature of the concrete floor was that it contained a great many remains of the hog, whilst the most important finds in the cave-earth were humeri and teeth of the cave-lion, a radius and vertebra of rhinoceros, associated with teeth and bones of man.

Compared with the breccia the remains in the cave-earth were neither so numerous nor so complete in partial skeleton form, and suggested a more gradual accumulation.

The concrete floor and cave-earth were probably produced slowly by the drainage of water into the cave, and this may also have been the vehicle for the introduction of the fragmentary animal remains. The stalagmitic breccia, on the other hand, suggests that its component parts mixed up with the bodies of human beings and animals were deposited by some sudden and violent rushing of water into the cave. This apparent connexion, either with the recesses of other then existing caverns or the surface, suggests some cataclysm which swept the bodies in a confused mass into the cave, and piled them up at the very end of the fissure. This disaster must have affected a considerable local area, for it collected human beings, hyaenas, wolves and deer, and buried them pell-mell in a common grave, deep in the recesses of the limestone.

A few fragments of charcoal were found in the breccia, and fewer still in the concrete floor, and traces were observed in the cave-earth. There occurred in the cave-earth of the northern chamber a natural flint pebble $4 \frac{5}{8}$ in. long and $2 \frac{3}{4}$ in. wide, of which a portion had been broken off 


\section{EARLY MAN}

longitudinally, and one end removed in a slanting direction. With the exception of this flint nodule, which may have been a hammer-stone, and a few fragments of slate, all the stone contents of the cave were immediately local. The cave yielded a few gnawed bones, a little coprolite, and the lower jaw of a very young hyaena with its first set of teeth incomplete. It is possible that the cave, remote as it was in recesses of the rock, was resorted to by human beings at one period, and by hyaenas at another, before it was partially filled, but the slight evidence of human occupation is obscured by the theory of the sudden rush of water into the cave carrying with it the sweepings of

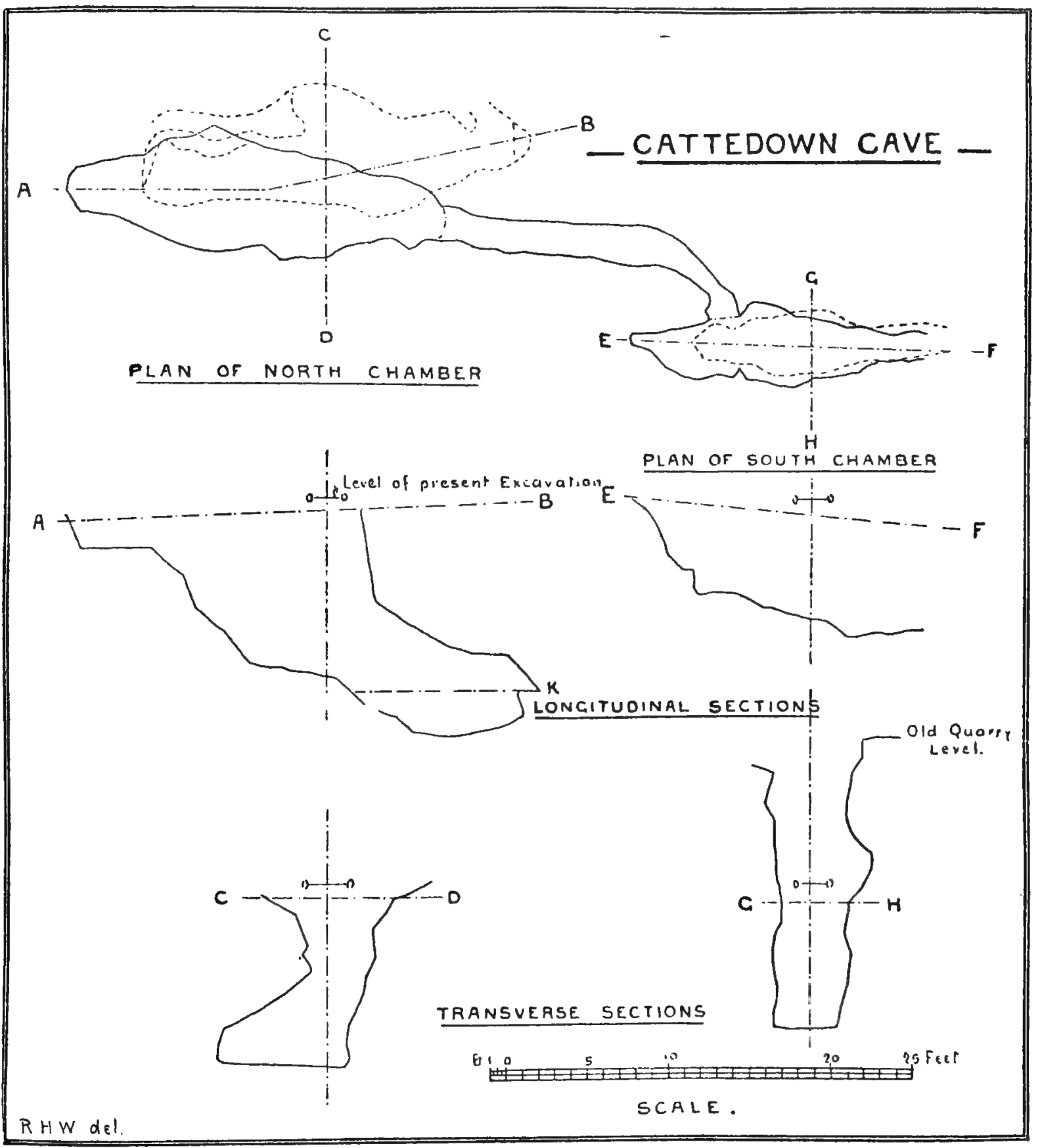

Fig. 2.-Plan and Sections, Cattedown Cave

other caverns which may have communicated with the surface. Avoiding all speculation, one fact stands out clear and distinct, and that is, that human beings and hyaenas lived in the flesh at one and the same time in the neighbourhood of Cattedown. This alone invests the human remains with an extraordinary amount of interest, for their discovery presents an opportunity of actually studying the physical characteristics of men who were certainly contemporary with these long-extinct animals, and probably also (with) the rhinoceros and cave-lion. The human bones represent the remains of some 


\section{A HISTORY OF DEVONSHIRE}

fifteen or sixteen individuals of both sexes ranging from childhood to old age. Two facially perfect skulls are represented in the accompanying illustration (see Fig. 3), and from the measurements calculated from these portions $\mathrm{Mr}$. R. N. Worth reported that they appeared to him to be orthocephalic in type, i.e. midway between the long head and the round. They are orthognathous, and some of the powerful lower jaws discovered have prominent chins. Some of the skulls were exceptionally thick, and others again very thin. The teeth generally are massive, and however much worn show but little traces of decay. One of the humeri is perforated, and the tibiae are distinctly platyknemic. The race was a short one, various calculations making the average slightly over five feet.

Axminster. - Although the principal evidence of the existence of palaeolithic man in Devonshire is rendered by certain of its caverns, we have in the valley of the Axe a deposit of river drift implements. These have been found in a ballast pit at Broom, near Axminster, and close to the River Axe. Some of the implements are of large size and of chert, some water-worn, and others

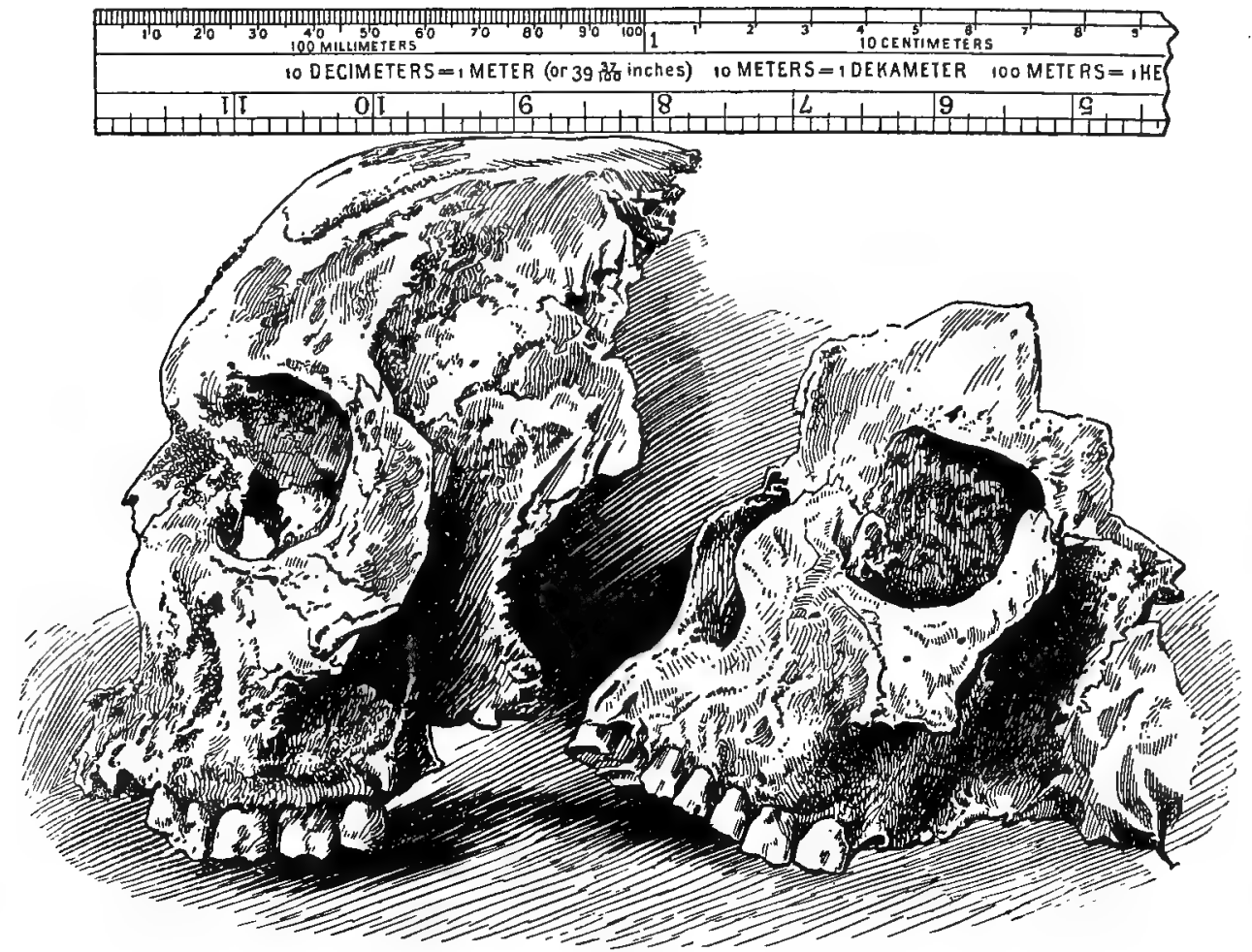

Fig. 3.- Skulls from Cattrdown Cave

again quite sharp and uninjured. The ovate type seems to predominate, ${ }^{1}$ but the pointed forms are not scarce. There is a fine series of palaeolithic implements from Broom at the Albert Memorial Museum at Exeter.

\section{The Neolithic Age}

The records of primitive man in Devonshire, as revealed by the exploration of Kent's Cavern, are plain and distinct. The formation of the breccia and the infilling of the cave with red earth, both containing relics of human handiwork, must have taken a long period of time. During this, as we have previously realized, man and predaceous animals, in turn, were at least visitors if not actual occupants of the cave. Over this again was the black band, where charcoal, the débris of ancient fires, indicated a more continuous human occupation. Above all was the floor of thick stalagmite, and this also must have taken, measured by years, a very long time to form. This stalag-

1 For a typical example figured see Evans, Stone Imp. 638. 


\section{EARLY MAN}

mite contained but few remains, human or otherwise, and it is surmised that these few may have fallen in from higher levels during the formation of the deposit. There is thus apparently a great break of unknown dimensions represented by the interposition of this stalagmitic floor between the earlier forms of animal life and implements below, and the later forms and more advanced tools above. Beneath are all the phases of the Palaeolithic Age, and above we are suddenly brought face to face with a superior culture and the incoming of existing forms of mammalian life. There is no transition stage recorded in the annals of the cavern. Whether generally there was an absolute and lengthened break between the disappearance of the cave-men and the arrival of the new-comers is at present unknown. Such breach of continuity may have been local, for whilst the bulk of the cave-men followed the retreating reindeer north, it is not unreasonable to conjecture that some remained behind and became amalgamated with the new race which had migrated from the East into Britain. Evidence may yet be forthcoming, especially from the Continent, that there was an actual transitional period, but up to the present nothing is furnished by Devonshire. Between the disappearance of the cave-men and the advent of these human beings of more advanced culture great changes had come over the face of Western Europe. The climate had become more temperate, and there was a greater rainfall; Great Britain had become an island, and Devonshire possessed a coast line. The waters of this coast line are often found to cover submerged forests, as at Torbay; Blackpool, nr. Dartmouth; Bigbury, and Northam, nr. Bideford. At Torbay there are considerable accumulations of vegetable matter, with remains of trees projecting from a bluish clay, and there is reason to believe that this extends beyond the five-fathom line. In this, and in similar deposits in other localities in Devonshire, the bones of the red deer, horse, hog, and long-fronted ox have been found. In addition to these some fishermen dredged up the tooth of a mammoth, about I860, some four miles out in Torbay, and in 1842 a piercing tool, fashioned from a red deer antler by the hands of man, was found in the sub-aërial portion of the forest in Tor Abbey Sands, twelve feet under the surface, during extensive drainage-excavations. ${ }^{1}$ These objects were found in varying circumstances, and too far apart to establish contemporaneity between man and the mammoth, but they illustrate the great antiquity of some of the submerged forests of Devonshire.

The better-equipped man possessed domestic animals, cultivated cereals, and had learnt the art of weaving, and of making rude vessels of pottery. His implements and weapons were often hafted and of greater variety of form and effectiveness. His stone axe-heads were sometimes ground and polished, and he brought down his quarry with bow and arrow-sticks tipped with heads of flint. He practised inhumation, and later on cremation, in the disposal of the dead, placing the carnal remains in stone chambers covered with long barrows. The cremated remains are mostly found in round barrows. Although there is a sharp and well-defined break of apparently long duration between the palaeolithic and the later periods of human culture, such is not the case between what we term the neolithic and bronze periods (convenient terms for indicating an age of stone and an age of bronze), for the former overlaps the latter, and both actually run into that period when iron first

\footnotetext{
${ }^{1}$ Trans. Devon. Assoc. (1865), pt. iv.
} 


\section{A HISTORY OF DEVONSHIRE}

appears in Britain. Not only is this the case with implements of stone and of metal, but the same overlapping may be observed in the study of pottery.

With all this overlapping of periods it is difficult to separate the purely neolithic in Devonshire, and all the more so because there are no known long barrows in the county. These may have existed in the low lands near the neolithic clearings, and have been ploughed down during many centuries of agricultural operations, but this does not account for their absence on some of the great uplands of Devonshire-vast stretches of uncultivated moorland.

Neolithic men visited Dartmoor, for they have left their tools and weapons behind them as silent witnesses of the fact, but there are no traces of their houses or their graves, with perhaps one possible exception, for the nearest approach to a grave of the Neolithic Age is the only Devonshire dolmen in the parish of Drewsteignton, on the borders of the Moor. This was a family or tribal ossuary in use during the Stone Age, and even probably continued down to the time of the early use of bronze. It lies two miles west of the village of Drewsteignton, in a field adjoining Shilstone Farmhouse. It is locally known as the Spinster's Rock. Three vertical stones about $6 \mathrm{ft}$. high carry the cap or quoit, which is $12 \mathrm{ft}$. long, $9 \mathrm{ft}$. wide in the broadest part, and just $2 \mathrm{ft}$. thick, and weighs about 16 tons. It unfortunately fell on 3 I January, I 862 , but was replaced by the late Mrs. Bragg, of Furlong, some ten months after.

Close to the dolmen there formerly existed remains of stone circles and stone rows (avenues). Some of these were noticed by Polwhele in I793, and others mapped by Mr. Grey in 1832 . One stone row led towards the dolmen, just as we find in other districts that these often lead to a kistvaen, which is but a later type of dolmen on a smaller scale. Although this is the only dolmen now standing in Devonshire, there is reason to believe that formerly others stood in various parts of the county, for the oft-occurring name of Shilstone, or Shelfstone, is indicative of their existence.

Polished implements, such as celts or perforated axes, some of which may belong to a later age, ${ }^{1}$ have been found near the head of the Walkham. One each has been reported from nr. Princetown, Tavistock, Cosdon Beacon, Bere Alston, North Bovey, Berry Cross nr. Great Torrington, Brentor, Ashbury, Holsworthy, Runnage nr. Post Bridge, Withycombe Raleigh, Hope's Nose, and Chelson, near Torquay, Houndiscombe and Compton Gifford, Plymouth.

There is thus evidence of the presence of neolithic man over consideraable portions of the area of Devonshire. He evidently lived in Kent's Cavern and Torbryan, but all traces of his dwellings or places of burial elsewhere, excepting the dolmen grave at Drewsteignton, have disappeared. We have, therefore, no opportunity of examining the physical characteristics of neolithic skeletons in Devonshire, but we may take it, from investigations in other counties, that they were long-headed, in contradistinction to the short or round heads of the Bronze Age.

\section{The Bronze Age}

We fortunately possess abundance of evidence of the existence of a late stone and early metal-using people in Devonshire. Few counties possess such

I These probably belong to the Bronze Age. 
open and large tracts of uncultivated land, and Dartmoor is unique. The surface of much of this moorland area has never been disturbed by the plough-it has remained a purely pastoral grazing district from time immemorial.

The aspect of the heights and slopes of Dartmoor during the prehistoric period was much as it is now, but many of the valleys we should hardly recognize. Some of these were marshes studded with bulrushes, a good example of which is Broad Marsh on the East Dart running up to the foot of Cut Hill. This was drained by the 'old men,' i.e. the ancient tinners, who dug through solid rock and lowered the bed of the river to such an extent that the marsh was relieved of the water, thus enabling them to stream the surface for tin. It still remains boggy, and bulrushes may yet be seen in the wettest portions.

Other valleys again were densely wooded thickets, containing oak, alder, hazel, and furze. Gawler Bottom near Post Bridge is a type of the once wooded valley, and Wistmans Wood is an example of an existing specimen. Gawler Bottom, now a bog, was apparently formed by the Gawler Brook becoming choked in the lower end of the valley, and the resulting formation of the peat killed the trees. When the turf-cutters are at work, remains of black oak and even hedge-nuts may be seen lying deep in the bog four to five feet under the surface. Without multiplying instances we can easily imagine the thickets in some of the valleys and the marshes in others-the former harboured the wolf and the latter teemed with wild fowl.

The slopes sweeping up to the tors were generally bare as they are now, and studding these, in favoured positions, were the dwellings of a primitive pastoral people. Sometimes these occurred in clusters, and were surrounded by a massive wall-in other cases the villages were encircled by a less substantial wall which seems not to have been erected for defensive purposes, but more for the purpose of corralling cattle-in others again the villages were quite open with corrals attached to the huts and forming quite a network of enclosures.

In addition to these villages there are hut circles scattered over the dry and best favoured areas of the moor. The foundations of these circular huts are composed of such massive granite blocks that they have withstood the ravages of time in a remarkable manner, and many of them stand to-day much as they must have been soon after they ceased to serve as habitations for the particular people who used them. They are to be found in hundreds, and must have formerly existed in even greater numbers, for a great number have been destroyed by the newtake wall builder and the road-mender.

Like the other stone monuments of Dartmoor the hut circles have furnished abundant material for conjecture of a haphazard character, and to settle this the investigation of these remarkable relics was systematically and scientifically undertaken in 1893 by the Rev. S. Baring Gould and the writer, with whom some other gentlemen were afterwards associated. ${ }^{1}$

Broadun Ring and Broadun Post Bridge.-The evidence yielded by the diggings disclosed a stone-using people, and the explorers were under the impression that the finds indicated a very primitive people living in a purely neolithic condition; but in the light of subsequent and numerous explorations in similar ruins of circular dwellings in other parts of Dartmoor they are now of opinion that

${ }^{1}$ Dartmoor Exploration Com. Devon. Assoc. 


\section{A HISTORY OF DEVONSHIRE}

probably the great bulk of the hut-circle occupation of the moor was during a period when stone implements and weapons were still in general use, but bronze was known and employed to a limited extent. So far this alloy has only been found in the graves. It is not to be wondered at that it is wanting in the hut circles, for it was at this period a precious commodity, and was only placed in the graves as an offering to the honoured dead in a sparing manner.

GRIMSPOUND.-This is the best example of a protected village with a very substantial defensive wall and a unique example of a prehistoric settlement. It presented exceptional advantages for examination, for there had been no pillaging of the wall nor of the hut circles, and it was taken in hand by the Dartmoor Exploration Committee in the spring of 1894 . The wall encloses about four acres, and within this are the ruins of twenty-four hut circles, half of which had evidently been human habitations, for these had fire-holes or hearths, and the floors were strewn with charcoal. The manner of the construction of these circular huts seems to have been as follows :Slabs of granite were collected and set up in a more or less perfect ring, such slabs when erected being about three feet high. As these slabs often terminate in a point at the top they give the extreme height of the original walls, and they agree within an inch or so of the height of the upper surface of the capstones of the doorways. If slabs which would stand vertically were scarce, smaller stones were laid in horizontal courses, small stones were used as fillers, and the wall was then backed up outside with earth and turf. The doorways, which were generally two and a half to three feet high, were formed of two jambs of granite with a lintel on the top. The entrances were generally paved, and sometimes protected with curving walls so as to cut off the prevailing winds. The huts usually faced south-west.

It was ascertained that the roofs could not have been of stone constructed in bee-hive form, but were probably of rush-thatch laid on poles wigwam fashion; it would have required several cartloads of stone to have domed one of these huts, but in no single instance has enough been found on the spot sufficient for the purpose. In nine out of twenty huts examined there were stone platforms which were raised from eight inches to a foot above the floor of the hut. These platforms are not common in the hut circles, for thus far they have only been observed at Broadun, Langstone Moor, Shapley Common, with traces at Crapp's Ring near Post Bridge, near Ger Tor Tavy Cleave, and Har Tor near Princetown.

The floors of the huts were of the 'calm' or clay sub-soil beaten hard, with paving-stones in places. On the floor in some cases was a hearth-stone and in others a fire-hole.

The objects found in the huts were a few flint implements, a rubber stone, a few fragments of rude pottery, and some 'cooking' stones-i.e. pebble stones which were heated in a fire and then placed hot in pits or pots so as to cook meat or boil water after the manner of existing savage races.

By referring to the plan of Grimspound (fig. 4) it will be observed that there are now three entrances, but that labelled as such is the original entry into the pound. The other two on the east and west sides are comparatively modern, and are due to a trackway leading from Headland Warren in the direction of Widecombe. The original entrance is paved, and is $7 \mathrm{ft}$. wide, and the wall at this point is $14 \mathrm{ft}$. thick. This paving of the entrance to Grimspound was a matter of necessity to the occupants, for the gateway faces the steep slope of Hameldon, and without it the soil would be speedily worked into an impassable slough by the passage of cattle into and out of the enclosure. As the descent is rapid, the paving is on the slope.

The enclosing wall of Grimspound is in a condition of sad, and, at first sight, inexplicable ruin; but the wrecked condition of the wall presents some significant and instructive features. In several places two faces, one outer, the other inner, remain comparatively perfect, showing that the wall originally did not consist of a congeries of stones piled together at random, but was composed, like the hut walls, of stones some set on edge and others laid in courses.

Twenty-five yards north of the western exit of the track which runs through the pound the wall has both faces perfect. The width is here $9 \mathrm{ft}$. from face to face. The outer face is still $4 \frac{1}{2} \mathrm{ft}$. high, the inner $1 \frac{1}{2} \mathrm{ft}$. A little further on the wall is $10 \mathrm{ft}$. thick. The wall by hut No. vii is $9 \frac{1}{2} \mathrm{ft}$. thick. The inner face is there $4 \mathrm{ft} .4 \mathrm{in}$. high. From this examination, and the condition of the walls, it is difficult to account for the height of the wall being originally more than $5 \mathrm{ft}$. It seems absurd that a wall of huge stones Io $\mathrm{ft}$. thick should be only $5 \mathrm{ft}$. high ; but if the wall was solid, and of this width, it is clear that there were not enough stones to account for even this height, and as there are no newtake walls near, it is certain that there has been no pillaging of stone to provide for their construction. The complete state of ruin of the wall is quite inexplicable if it was constructed solid.

It is observable that very generally the wall faces have collapsed towards each other, and that between them are to be found either large slabs tilted inwards or else confused masses of small stones. On a scrutiny of the wall on the NW. side, it was made abundantly clear that here there had been a double wall with a space between. Each wall is $3 \mathrm{ft}$. wide at the top and about $3 \mathrm{ft} .6 \mathrm{in}$. at the bottom. The space between them is about $3 \mathrm{ft}$. wide at the bottom and $3 \mathrm{ft}$. $6 \mathrm{in}$. at the 


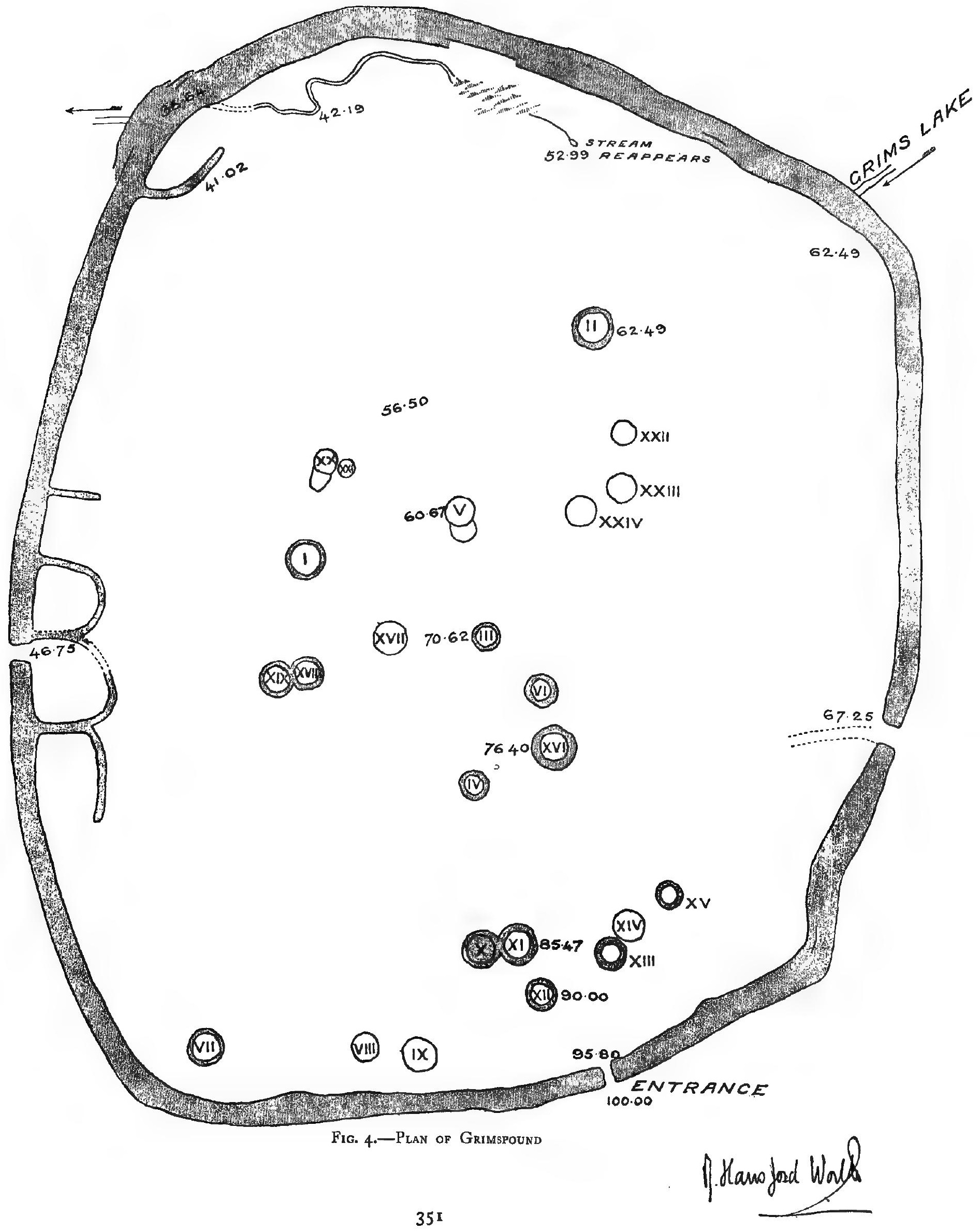




\section{A HISTORY OF DEVONSHIRE}

top. When the stones were removed from this space and replaced on the walls, it was apparent that these latter had never been more than $5 \mathrm{ft}$. high or $5 \frac{1}{2} \mathrm{ft}$. at the very outside.

We thus have a double wall with a space between. This space, it would seem, is too narrow to serve any useful purpose. There is apparently an entrance into it from the interior of the pound on the NW. side, but with one most doubtful exception no others were observed. The balance of evidence is rather against this presumed entrance being a work of design. It is clear that the wall has never been built solid throughout, except at the flanks of the gateway; a vacant zone of irregular width being everywhere else left between the inner and outer facings, which are of very varying thickness. The most reasonable supposition is that this space between the two facing walls was to have been filled with a core of earth which in its turn might have carried a wooden fence or palisade. But there is no trace of such earth-filling, and if it ever existed it could not have utterly disappeared. It could not possibly have been washed away, for the walls would have retained it, and vegetation would have grown up and held it fast. It seems not unlikely that the work was never finished, for it is impossible to conceive that the builders should have spent immense labour, and rested content on raising a double wall with a space between and a total thickness of some $10 \mathrm{ft}$. and height of only about $5 \mathrm{ft}$.- too low to keep out either a hungry wolf or active human assailants. A single solid wall carried to a greater height, with a banquette, would have been more easily erected and much more effective. The unfinished character of the wall is strongly supported by Mr. C. W. Dymond, F.S.A., who subjected it to a rigorous examination at the request of the writer.

We are not familiar with the conditions of warfare existing at the time when Grimspound was designed and built, but it strikes one at once, on viewing the site, that it is commanded on all sides but one by the fire of archers and slingers, and that the entrance is so placed that it is in the most favourable position for a rush of assailants down the slope of Hameldon.

However unsuitable the position may appear to us to-day in a strategic sense, we must not conclude that this cyclopean defence was erected with no object other than to be a fence to fold cattle. Some of the stones employed in the wall are of great size. On the west is a huge slab set on edge measuring $10 \mathrm{ft}$. by $5 \mathrm{ft}$. and 9 in. to $1 \mathrm{ft}$. in thickness, and weighing from 3 to 4 tons. Other stones laid in courses, if not so long, are not of less weight. Such a wall was not erected by the occupants of a dozen huts and for the protection or convenience of a limited number of persons.

Presumably Grimspound was not a fortified village any more than it was merely a cattle pound, but was more probably the unfinished oppidum, the place of refuge for the scattered population in the neighbourhood, and the dozen householders were the oppidani, the caretakers or guardians. The lunette arrangements on the western side were probably cattle-pens.

Grimslake, a small stream that dries up in very hot summers only, flows through the enclosure at its northern extremity. It passes under the wall, flows through it for some way, and then emerges three-quarters of the way down. It has been confidently asserted that the stream was diverted from its proper course by the hands of man to force it to enter the pound. This, however, is not the case. There is an outcrop of feldspathic granite in the midst of the furrow between Hameldon and Hookner Tor, so that the drainage of these hills cannot unite, but flows parallel in independent channels, and meets below the road from Chagford to Widecombe, at a distance of 400 yards from the enclosure. There is at present very little drainage from Hookner Tor: nevertheless, it must have been greater in former times, as the groove of the channel and its stream is not only distinctly visible, but is more important than that of Grimslake.

Raddick Hill near Princetown (O.S. cvi, SE).-This is a good example of a pound enclosing hut circles. The wall of the enclosure is in an imperfect condition, but enough remains to show that this was not a massive defensive wall like Grimspound, but was probably a mere corral for harbouring cattle by night. The hut circles within the pound are eleven in number, and of these seven gave unmistakable evidence of having served as habitations. They varied in internal diameter from ' $10 \mathrm{ft}$. to $25 \mathrm{ft}$.

The most interesting of the series is one which has a diameter of a little over $15 \mathrm{ft}$. It is paved throughout, the entrance ill-defined, but apparently facing SSW. Nearly opposite this supposed entrance is the hearth, slightly raised, backed by a large stone in the wall. To the right of this hearth is the cooking or fire-hole, under a large slab that has fallen forward out of the wall. This slab, in falling, protected from destruction a fine vessel of rude hand-made pottery, which occupied the cooking or fire-hole, and was fairly intact. It had the peculiar shape of the cinerary urns used in the early Bronze Age, with a rib running as a hoop round it $2 \frac{1}{2}$ in. from the lip, and the portion above this was ornamented by rude chevrons. The bottom of the pot was rounded. This was distinctly seen when it was in situ, but when taken out the bottom, which was very rotten, went to pieces. The vessel measures $10 \frac{1}{2}$ in. in height and was $10 \mathrm{in}$. in diameter at the top. The rib was $I$ in. thick.

Although the utmost care was taken, considerable portions of the wall of the vessel crumbled on air-drying, but these were restored, and this interesting specimen of a cooking pot was deposited 


\section{EARLY MAN}

in the Municipal Museum at Plymouth. The same hut yielded much charcoal, numerous cooking stones, a rubber stone, and an oval sparry river-pebble that had both its ends bruised and broken
by use.

LEGrs Tor (O.S. cxii, SE).- There is an important series of hut circles and enclosures lying above the River Plym on the south slope of Legis Tor. Many of these were explored in 1 895-6 by Mr. R. Hansford Worth. The remains are very extensive, consisting of a series of irregularlyshaped enclosures, with lesser boundaries subdividing in rectangular or sub-rectangular patches, and a number of hut circles of varying dimensions, mostly within the enclosures. An evident settlement scription of hut No. 2 will suffice as an illustration of these prehistoric dwellings (see fig. 5).

This hut circle measures along its least internal diameter about $12 \frac{1}{2} \mathrm{ft}$., and along its greatest internal diameter, at right angles to the last, about $\mathrm{I} 3 \frac{1}{2} \mathrm{ft}$. It has a well-defined entrance facing almost due south. A considerable portion of its floor was found to be paved with flat granite stones. There was a hearth-stone at or near the centre, cracked as if by fire, and a cooking or fire hole filled with ashes was found immediately adjacent to the hearthstone and to the north of it. At the entrance to this circle there were two steps leading down from the outer ground level. The north-western half of the floor was unpaved, and consisted of natural hard 'calm' or sub-soil.

The raised hearth was the first indication of human habitation. Immediately over the actual floor of the hut were found some thirty rounded cooking-stones, mainly of elvan rock, many of which had been splintered by the action of fire. One flint flake was found in the soil within a few inches of the floor.

The cooking or fire hole to the north of the hearth was of a curved oval or kidney shape, being very nearly $2 \mathrm{ft}$. in length and about Io in. in breadth. It was found full of charcoal and ash. On the western side of the entrance, from a pit sunk below the general level of the floor, was

\section{LEGIS TOR}
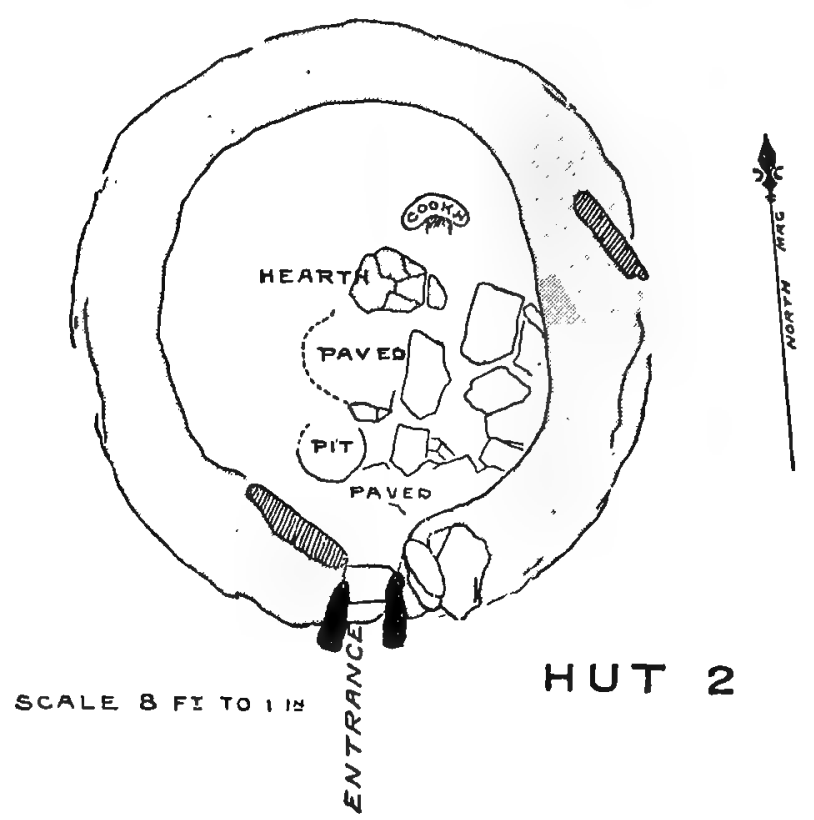

$s$

SECTION OF ENTRANCE

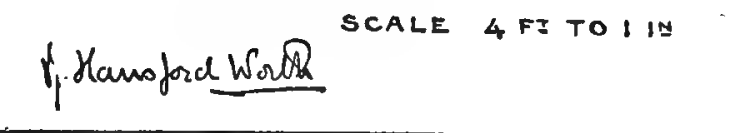

Fig. 5.-Plan of Hut 2 with Section of Entrancr excavated a quantity of rough pottery, being fragments of a pot, the exterior of which was of a red colour, and the interior had been blackened by charred organic matter. From all appearance this pot had been crushed where found by the weight of some of the wall stones which had fallen within the circle. From the point near the hearth a broken fired clay spindle-whorl was excavated from the joint between two of the paving-stones, the only example yet found in a Dartmoor hut circle.

Another hut circle in this group yielded a broken cooking-pot of unornamented pottery which was found set in the 'calm' below the level of the floor. In diameter it measured 10 in. at its widest point. Its total depth, including the rim, which was found inside it, was a little over 


\section{A HISTORY OF DEVONSHIRE}

I 2 in. Two cooking-stones were found in it, and curiously enough the bottom of this pot, which was cracked, had been mended in situ with white china clay, a mass of clay having been pressed into the bottom to fill up cracks and cement together the broken fragments. This pot had a hemispherical bottom, and could not have stood on a plane surface. Judging from the fragments it was handmade, but not of such primitive shape as that found at Raddick Hill.

Other finds in this interesting group consisted of many fragments of rudely ornamented pottery, flint implements, and a wedge-shaped rubber stone which had been pierced from both sides with a hole for suspension, but never completely perforated.

Blackslade Common (O.S. cviii, NW). - There is a considerable collection of hut circles in and about some remains of enclosures known as Foales Arrishes. The ancient name of this group of hut circles is Torr Town or Torr Hill. It probably received its modern name from some squatter who seems to have hazarded its cultivation, for attempts appear to have been made to gather the surface stone in heaps, so that a scanty tillage might be carried on between them.

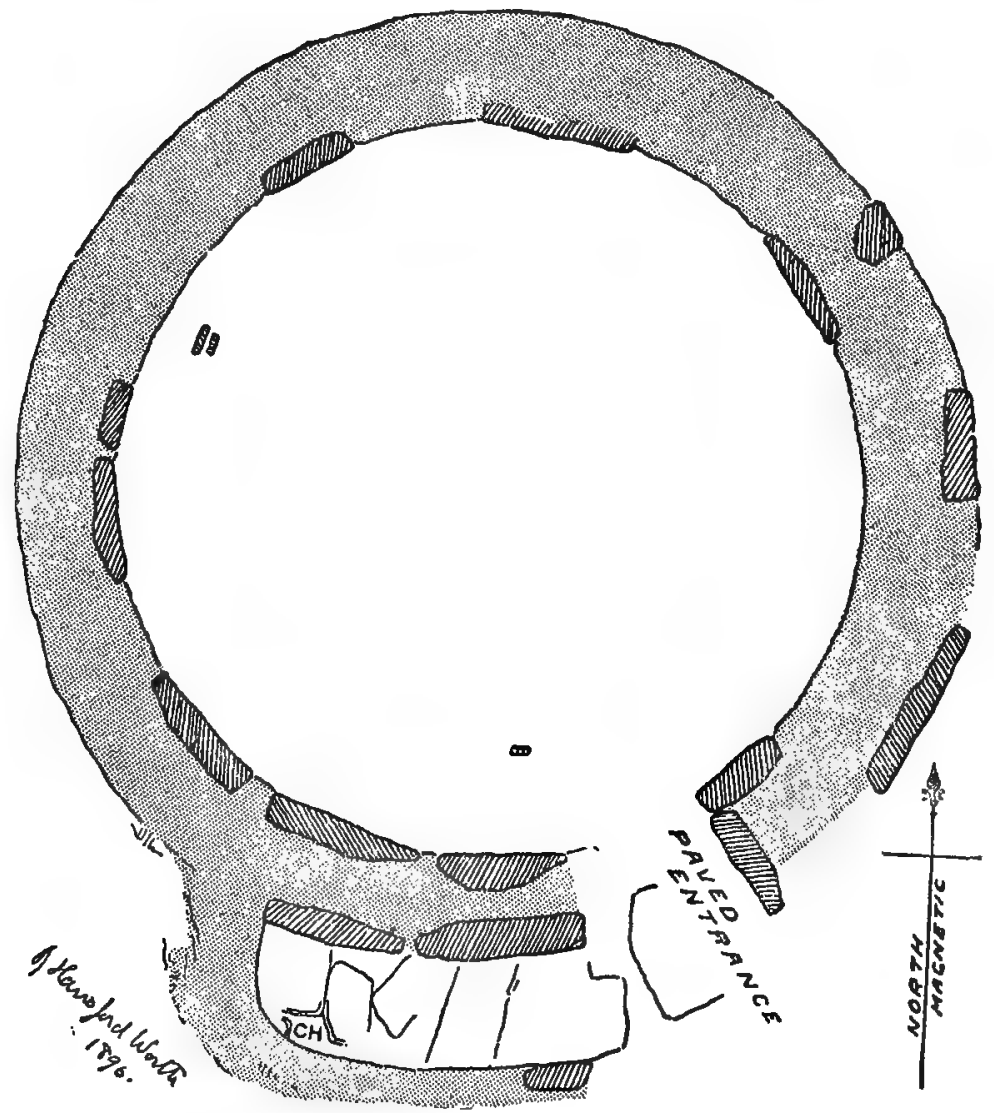

FOALES ARRISHES, hUT No/.

$$
\text { - Scale-10 feel lol inch }
$$

Fig. 6. - Foales Arrishes, Hut No. I

Croker ${ }^{1}$ states that this place possessed a circular pound almost as large as Grimspound enclosing hut circles. The road-menders have been busy since $185 \mathrm{I}$, for no trace of the enclosure can now be recognized.

One of the still existing hut circles (No. I) merits a brief description, for the cooking-place is outside the hut in a semilunar shaped erection (see fig. 6). It contained a cooking or fire hole and a hearth with much charcoal and some fragments of thin pottery. The whole of the floor of this kitchen was paved.

Considering the size of the hut circle, from 30 to $3 \mathrm{It}$. in diameter, involving a large roof, which would be most difficult to keep weathertight in the winter, and the position of the main fire and cooking-place, the reasonable supposition is that this hut represents a summer habitation, and if this surmise is correct it is an interesting illustration of the great antiquity of the summering of cattle on Dartmoor, for these huts and enclosures on the moor generally are the dwell-

ings and paddocks of a primitive people, whose chief support was evidently obtained from their flocks and herds. Some of the smaller huts were doubtless permanent habitations, but the large examples were probably only occupied in the summer, when herbage is plentiful, and when a far greater head of stock could be supported than in the winter.

The exploration of the hut itself yielded fragments of blackened pottery and a flint knife. A curious feature was a small stone standing 6 in. above the floor, just inside the western side of the entrance, and two more standing side by side 15 in. above the floor near the north-western circumference. These stones seemed to have been fixed so as to follow the sweep of the circle, and were firmly earth-fast. They were not observed between these points. They had a surface length of only 8 in. or 10 in., and were merely thin slabs of granite let into the ground for some unknown purpose. They were not suitable for supporting planks of wood to form seats, nor could they be

${ }^{1}$ Guide to Eastern Escartment of Dartmoor (1 851 ). 


\section{EARLY MAN}

portions of a stone dais or platform, for they occur in the part of the hut which shows the greatest signs of occupation.

Other hut circles in this group yielded fragments of ornamented pottery of material and workmanship superior to the rude example found at Raddick Hill, but as far as can be judged from the fragments they are portions of hand-made vessels. One sherd has distinct impressions of the potter's thumb.

Standon, commonly known as Stannon Down (O.S. xcviii, NE.).-This is crowned by Standon Hill, and this again is dominated by high ground rising to over 1,700 ft., lying between the great loop of the Tavy, which, rising not far from Walkham Head, flows almost due north, then turning toward the west trends away in a southerly direction through the romantic gorge of Tavy Cleave.

On the left bank of the Tavy, on the north-west slope of Standon, is a level plateau about $200 \mathrm{ft}$. above the bed of the river. This is wet and boggy, but where it slopes gently towards the

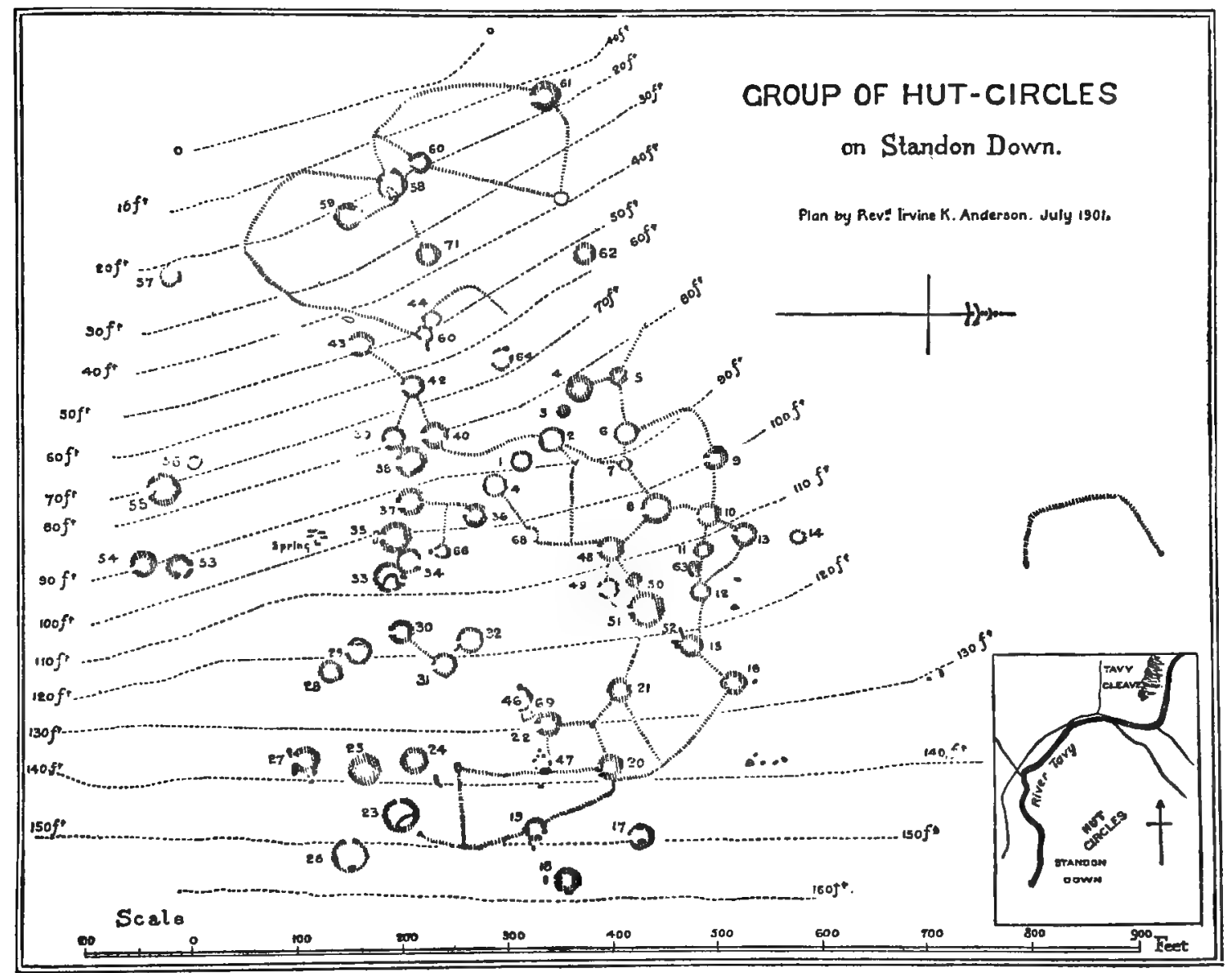

Fig. 7.-Group of Hut Circles on Standon Down

west the ground is dry and boulder-strewn. On this favourable surface are the remains of over seventy hut circles in a fair state of preservation. In some the walls are massive, 3 to $4 \mathrm{ft}$. high, built up with large stones placed vertically; others again appear to have been rebuilt so as to form modern shelters for sheep and shepherds.

None of the huts explored - forty-three in number-contained raised platforms of stone as at Grimspound, but some of the circles had interior walls forming inner compartments. The floors of many of the huts were regularly and wholly paved with flat stones, and nearly all had some portion thus treated. Owing to this there were but few finds of potsherds and other articles, for these could get no further than the paving. Where the floor is of 'calm' or sub-soil they have a chance of preservation, for they work down into this medium, and are thus ensured some manner of protection.

A reference to the accompanying plan (see fig. 7) will show the great extent and importance of this prehistoric village. Many of the huts appear to have been connected by walls forming a 


\section{A HISTORY OF DEVONSHIRE}

complicated system of cattle and sheep pens. There is no suggestion in the construction and capacity of the walls of anything but protection from the weather, and for night corralling of cattle.

The little pottery found in the huts appeared to be hand-made, and the ornamentation of the Bronze Age type. No cooking or fire holes were observed, but fires were evidently made on the paved floors, for many of these were strewn with charcoal and ashes. Pebbles, which had been fired and then used for cooking purposes, were numerous, and many large river-pebbles were found which had evidently been used as anvils and pounders. Flint objects were few and far between, and those which turned up were unimportant.

Such then in brief is a description of some of the villages and dwellings of the early pastoral race who migrated from the lowlands as each summer came round, and squatted with their flocks and herds on the breezy uplands of Dartmoor.

One of the striking peculiarities of the examination of the hut circles is the almost entire absence in them of means of grinding grain; one only, on Whiten Ridge, yielded a muller, oval in shape, and with a grinding surface of 12 by 9 in. It must, however, be borne in mind that parched grain could easily have been reduced to meal by many of the flat pebbles and pounders found in the hut circles without their appearance specially suggesting an occasional use for this purpose.

Up to the present no metallic objects have been discovered in the hut circles, indeed the whole surface of Dartmoor, including the graves, has yielded but few implements or weapons made of bronze. No middens have yet been found in any of the hut settlements. This is not surprising, for this primitive folk had little to throw away which would endure; potsherds, broken pebbles,

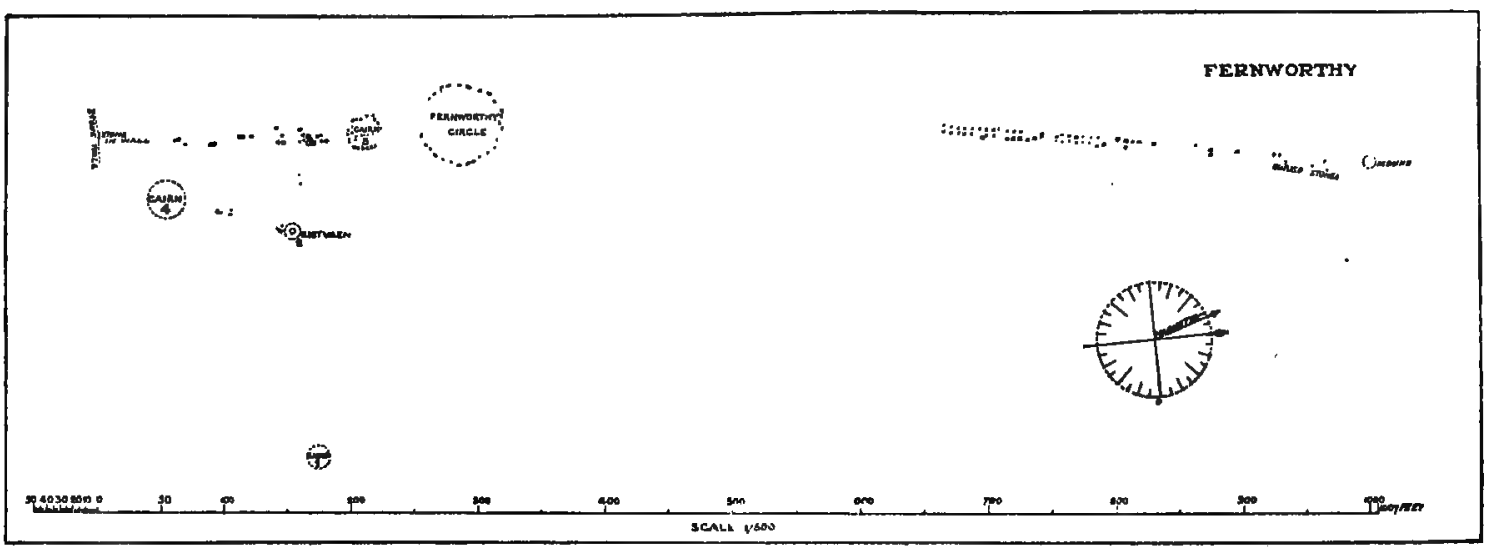

Fig. 8.-Pian of Frrnworthy Cairns

and spalls of flint exhaust the list suggested to one's mind. These thrown outside the door of the hut would be speedily trodden in and disappear in the peaty soil. A few of these were fortunately allowed to remain in the huts, and we are thus enabled to form some idea of the utensils employed. We are also able from these and from the ornamentation and nature of the pottery to gauge the state of culture in which these people existed. They must have led peaceful lives, for only one arrowhead of flint has been found in a hut circle, and but few outside. These people existed at a time when bronze was known in Devonshire, but thus far no trace of early tin-smelting has been discovered in any of the hut circles.

YEs Tor BotTom (O.S. cvi, SE.).-One hut circle, just beyond the twentieth milestone on the Princetown Railway, yielded tin slag, and the remains of an earthenware mug or jug, of fourteenth or early fifteenth century type, 6 in. under the surface; but this was clearly a comparatively modern intrusion, for the true floor of the hut circle was not reached until 20 in. had been removed, and this prehistoric level yielded the rim and two fragments of a hand-made cooking pot, with chevron ornamentation and one fragment of flint, and a flake of the same material. Later tin streamers had adapted the hut for their requirements in the same manner as a hut had been used on Shapley Common-probably by a shepherd-who had left behind him his eighteenth-century tobacco pipe.

That a stone-using people existed on Dartmoor is amply demonstrated, irrespective of the evidence obtained from the hut circles, from the fact that its surface or sub-surface has in almost all directions yielded large quantities of flint spalls, amongst which cores, flakes, and implements exist in considerable numbers. They are found in profusion at Post Bridge, Brownberry, and Huccaby-examples of the few cultivated spots on the moor. There is no doubt that in many 


\section{EARLY MAN}

of the fields of these farms hut circles formerly existed; in some, traces of these primitive dwellings still exist, and in others they have been removed within recent recollection.

The flints are turned up in the process of cultivation, and some fields have yielded several thousand specimens, mostly spalls, with occasional finelyworked implements or weapons. The flints are mostly from the chalk, with here and there specimens of chert. As a rule, the spalls have been struck from flint pebbles of no great size, but a few of the larger flakes and some of the finer implements have evidently been fabricated from nodules of flint of considerable dimensions. The ancient workers in flint seem to have somewhat neglected the greensand of Devonshire, and to have preferred to go farther afield for their raw material. The superior chalk flint found east of Devonshire was imported into Dartmoor, and was largely used by its prehistoric folk.

Implements struck from the nodule are chisel - shaped implements or fabricators, borers, saws, knives, flakes of uncertain use, a few arrow and spear heads, and scrapers in profusion.

\section{Graves}

We must now turn to the graves of this pre-

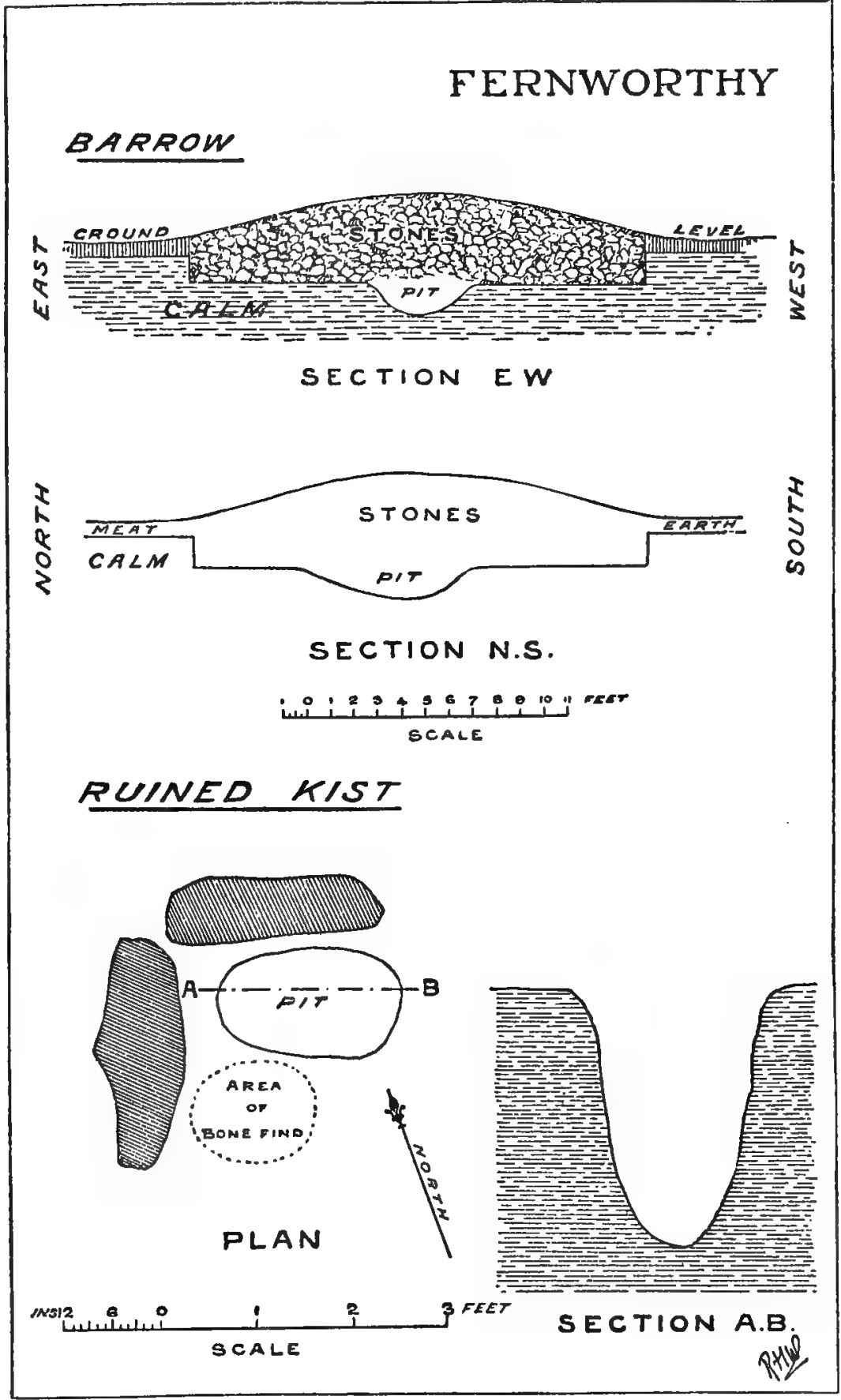

Fig. 9.-Ruined Kist yielding Burnt Bones and Cairn No. I Fernworthy (Sections)

historic people, and describe a few examples on Dartmoor and in other parts of Devonshire. Some of these are associated with menhirs. The finest examples of these high standing stones are at Drizzlecombe, near Sheepstor, Merivale, near Princetown, Gidleigh Common, and Langstone Moor, near White Tor. Beardown Man is not associated with sepulchral remains. ${ }^{1}$

FERNWORTHY.-This very important group of antiquities (see fig. 8) consists of the stone or socalled 'sacred' circle, a cairn (No. 3) standing at the head of a short stone row running south, a

${ }^{1}$ See note on the Stone Rows of Dartmoor, p. 372. 


\section{A HISTORY OF DEVONSHIRE}

kistvaen (No. 2) with remains of a stone row connecting with a large cairn (No. 4). About $80 \mathrm{yds}$. ESE. of the stone circle is a small cairn standing on a slight rise in the ground (No. I), whilst farther north is a double stone row terminating with a

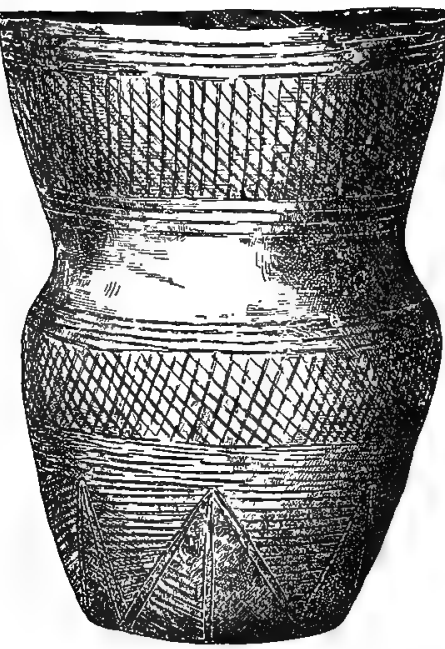

Fig. 10.-UURn from $\mathrm{C}_{\mathrm{AI} \text { RN }}$ No. 1, FERNWORTHY mound. This stone row points to the stone circle, but whether it was ever actually joined to it is doubtful.

Cairns Nos. 2, 3, and 4 have all been disturbed at some unknown time. No. 2 (see fig. 9) contained a kistvaen; and although previously rifled masses of burnt bones mixed with 'calm' or sub-soil and peaty earth weighing $4 \frac{1}{2} \mathrm{lb}$. were found in the bottom of the kist, the burnt bones could not positively be identified as they were too fragmentary and consumed by fire, but they corresponded with the characteristics of human remains. Cairns Nos. 3 and 4 gave no results, with the exception of a tiny flint chip in No. 4 .

The leading idea of this group of antiquities was evidently sepulchral, and as No. I cairn appeared to have escaped previous disturbance it was thoroughly explored in 1897. It had a diameter of $19 \mathrm{ft}$., was $2 \mathrm{ft}$. above ground-level in the centre, but was concealed by a growth of heather, and short furze and bracken, the roots of the two latter being found deep down in the structure. The cairn was originally surrounded by standing stones; three of these were in position, 9 to $10 \mathrm{ft}$. from the existing foot of the slope of the barrow.

The accompanying sections (see fig. 9) explain the structure of the cairn. It was evidently made by clearing away a circular area of ground, with a diameter of about $19 \mathrm{ft}$., and after excavating about 14 in. of the 'calm,' or sub-soil, a pit was sunk in the centre to a depth of $\mathrm{I} 8 \mathrm{in}$. This pit was $4 \mathrm{ft}$. wide from east to west, whilst from north to south it was $7 \mathrm{ft}$. The depth of this pit from original ground-level was $3 \mathrm{ft}$. 3 in., so that the total depth from the highest barrow surface was $5 \mathrm{ft} .3$ in. A trench was dug from west to east, and subsequently another was cut from north to south, so that almost the whole of the interior of the cairn was exposed. These trenches disclosed the area occupied by the pit. The portions of the cairn left intact were subsequently examined, but nothing was found in them.

On removing the turf of the cairn it was found to be built of handy-sized stones, gathered from the surface. Some were large enough to require two hands to lift them. Not only was the cairn piled up with these stones, but the entire pit was filled up or packed with them, so that some little difficulty was experienced in getting them out. In the central pit at a depth of about $4 \frac{1}{2} \mathrm{ft}$. from the surface a small piece of oxidized bronze was found, with fragments of some fibrous wood attached to it. The bronze object was $I \frac{1}{2}$ in. long, with a greatest width of $\frac{3}{4}$ in., and weighed $\frac{1}{2} \mathrm{oz}$; ; it is apparently either the remains of a small knife or spear-head, most likely the former. Near this were two or three fragments of pottery, and close under them a small urn was discovered (see fig. 10), which had been crushed by the subsidence of the cairn stones. A large dress-fastener or button of horn was found on the same level as the bronze (see fig. I I) and distant $2 \mathrm{ft}$. towards the NW. The upper surface is polished, and has a brown lustre. The bottom of the urn was resting on the 'calm,' or subsoil, and a flint knife was lying amongst the sherds in such a manner as to suggest that it might have been placed in the urn (see fig. I IA).

Not a trace of bone, burnt or unburnt, could be detected anywhere in the pit of the
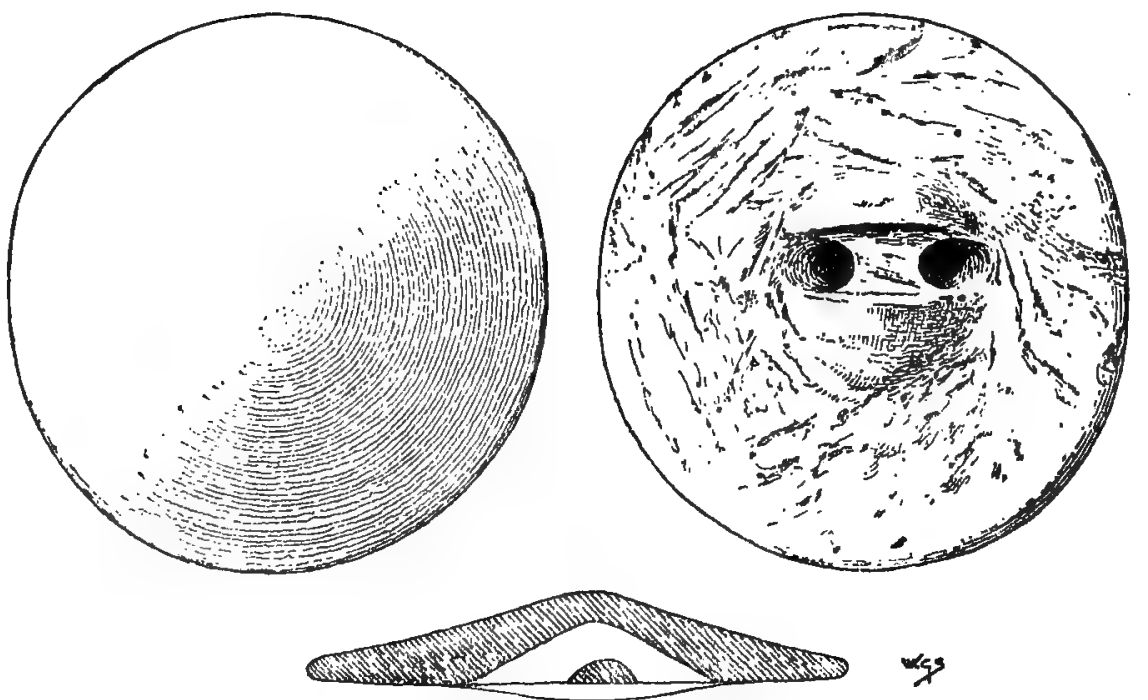

Fig. il.-Horn Dress-Fastener from Cairn No. i, Fernworthy 


\section{EARLY MAN}

cairn, nor were there any of the larger-sized urns or remains of same for containing a cinerary interment. This absence of bone may seem surprising, but when it is remembered that the roots of the furze, etc., penetrated down to the lowest depths of the cairn, it is not difficult to realize that these plants growing for an unknown period had assimilated, and thus entirely removed, any signs of phosphatic matter.

The vase was evidently placed in the bottom of the pit on the 'calm' or sub-soil, and some of the cairn stones were roughly built around it, two flat stones forming the cover of this rude receptacle. The bronze object was found lying on the upper cover.

Although no bone ashes were recognized, the Exploration Committee had no hesitation in considering this to be an interment after incineration, for wood charcoal was discovered in the bottom of the pit.

The dress-fastener or button may have been deposited in the cairn alone, or it may have been attached to the dress, and the whole placed therein with the ashes of the dead, the vase, the bronze object, and the flint knife at the time of interment. This most interesting and important exploration settles the period of the interment as that of the period of
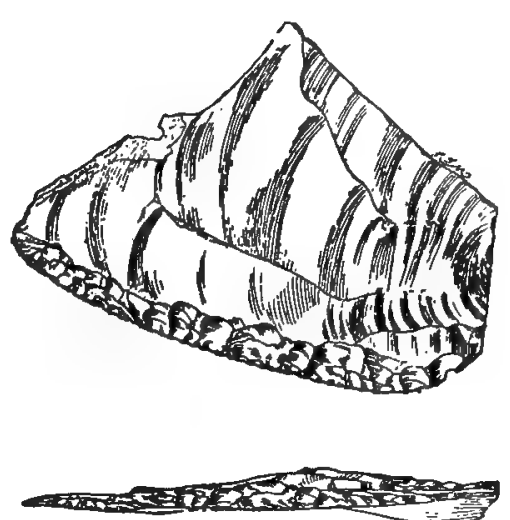

Fig. II A. - Flint Knife from Cairn No. I, FERNwORThY culture known as the late Neolithic and early Bronze Age. The bronze object above the vase, and the flint knife below, links the Stone and Early Metal Ages in a most satisfactory manner, and in addition to this there is the decoration on the vase and the evidence of the dress-fastener. ${ }^{1}$

The vase contained fine soil, which gave distinct traces of phosphoric acid, and may have contained food, but the shape suggests a class of vessels known as drinking cups or beakers which, regarded as a class, is considered by some eminent authorities to be the oldest Bronze Age ceramic in Great Britain. The dimensions of the Fernworthy vase are as follows:-Extreme height $7 \frac{1}{2}$ in., diameter of bottom 3 in., at mouth (internal) 5 in., thickness of rim $\frac{1}{8}$ in. The dress-fastener has a diameter of $2 \frac{1}{4}$ in.

The next step taken was to examine the interior of the stone circle, and this was done by driving a trench north and south and another east and west. These were cut through peat, locally known as 'ven,' forming a layer 18 in. thick, which rested on the 'calm' or sub-soil. There was no ' meat' earth. The trenches were driven right through the circle with a width of $2 \mathrm{ft}$., and from end to end it was observed that the floor of the 'calm' or sub-soil was strewn with small pieces

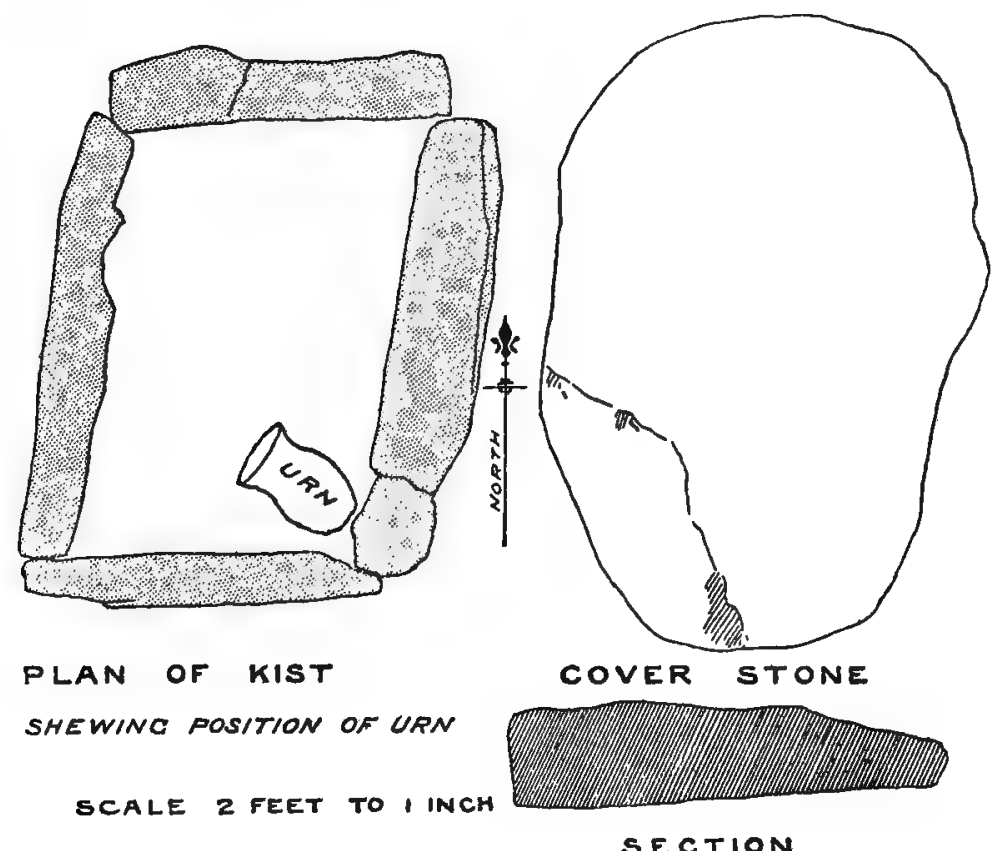

Fig. I 2.-Plan of Kist, Chagford Common of wood charcoal. There was no charcoal in the 'calm' or sub-soil ; it was all existing on it, and the floor was as definite as those found in the hut circles.

Three pits were dug between the trenches, and another at the foot of one of the largest stones of the circle. The whole of these gave the same indications of charcoal-the remnants of fires of wood; in fact, fires seem to have been kindled all over the circle, for every scoop of the pick and shovel which was removed from the floor displayed charcoal. This is a very interesting and important discovery, for it may unravel the mystery of the so-called 'sacred" circles.

1 For further particulars of buttons or dress-fasteners see Evans, Stone Imp. and Greenwell, British Barrozes. 


\section{A HISTORY OF DEVONSHIRE}

Fernworthy stone circle is the important and predominant feature of a group of sepulchral remains, and it is very probable that we can now see in this the crematorium or the site of the funeral feasts, or both.

Chagrord Common.-This common is a continuation towards the east of Watern Down . (O.S. xcix, NE). In 1897 the writer found an unopened barrow lying close to the north side of
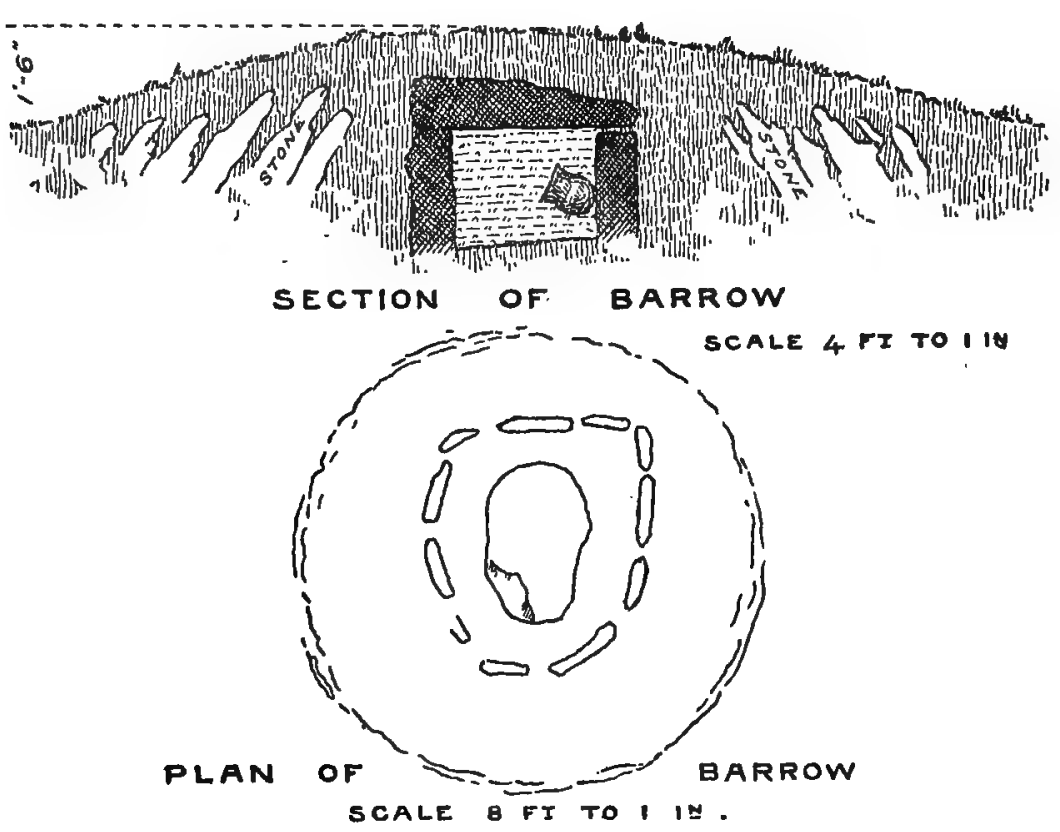

Fig. 13.-Section and Plan of Barrow, Chagford Common the grassy trackway which leads from Willandhead and Hurston to King's Oven, and about 400 yds. NE of the Stone Row. It was not very distinguishable, for it was not surrounded by a circle of stones. The barrow was small, $15 \mathrm{ft}$. in diameter, with a central height of only $18 \mathrm{in.} \mathrm{It}$ disclosed on exploration a small kistvaen, which was surrounded by an oblong enclosure formed of a series of stones lying inwards layer on layer. The kistvaen measured $3 \frac{1}{2} \mathrm{ft}$. long and from $2 \mathrm{ft}$. 3 in. to $2 \frac{1}{2} \mathrm{ft}$. wide (see fig. 12). It was full of soil. The top was evidently black peaty earth, which had washed in; but below this thin layer the kist was filled with compact "calm or sub-soil, in which could be seen here and there small fragments of wood charcoal. (For section and plan of Barrow see fig. 13.)

The filling was carefully removed and sifted, and as the work proceeded a small urn was found lying on its side in the $\mathrm{SE}$ angle of the kist with its mouth pointing to the $\mathrm{NW}$ angle. It was lying 4 in. above the floor of the kist, which was $2 \mathrm{ft}$. deep, and had evidently been placed as found, for its bottom was set close into the angle, and it was resting on a bed of 'calm' or sub-soil. The urn is hand-made, Io in. high, with the following diameters : Mouth 7 in., neck $5 \frac{1}{2}$ in., body 7 in. The wall of the vessel is $\frac{3}{8}$ in. thick. The paste, of a light chocolate-brown colour, is clay mixed with a small proportion of apparently small fragments of partially decomposed granite. Very little quartz is visible. The ornamentation consists of a series of impressions forming short $\mathbf{U}$-shaped dots arranged in parallel and diagonal lines, produced by some instrument-the half of a split bird bone would do admirably.

Nothing was found in the soil filling the kist except a little wood charcoal. If it had ever contained bone ash as well as charcoal, all traces of such had utterly disappeared. The disappearance in this instance cannot be ascribed to vegetation, for there was no appearance of the penetration of the joints of the kist by the roots of the heather which covered the barrow.

The urn itself contained from 3 to $4 \mathrm{lb}$. of light brown soil flecked with a little black matter. The contents were analysed, and it was found to be 'calm' or sub-soil with carbonaceous matter adhering to fragments of quartz. The amount of phosphoric acid $\left(\mathrm{P}_{2} \mathrm{O}_{5}\right)$ present, viz. $\mathrm{O}^{\circ} \mathrm{I} 9$ per cent., and lime $(\mathrm{CaO}) 0^{\circ} 13$ per cent., is only suggestive of the decomposition of the granitic matter or of food which may have been placed in the urn. No bone ashes were ever placed within it, and it must therefore be accepted either as a food vase or a beaker (see fig. 14)

HuRston RIDGE.-A stump of a pillaged cairn $26 \mathrm{ft}$. in diameter was observed on Hurston Ridge (O.S. xcix, NE), and this on exploration by the writer in 1900 yielded a large portion of a broken urn with a circumference of $4 \mathrm{ft}$. 5 in., resting mouth downwards on a flat stone which covered a pit filled with wood charcoal. The urn was partially protected by a leaning

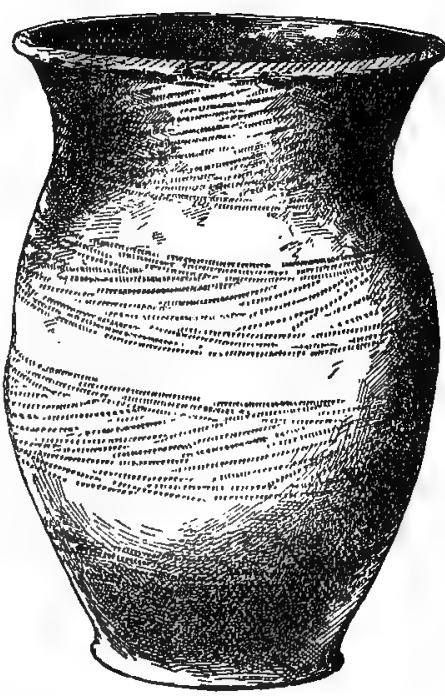

Fig. 14.-Food Vase or Beaker from Barrow, Chagford Common 


\section{EARLY MAN}

stone kept in a suitable position by means of trigger stones. The remains of the urn are highly ornamented. The pottery is dark and friable, and contains but little clay. For section of cairn from east to west see fig. 15. There was no surrounding stone circle.

Langstone Moor.- Three small cairns lying close to the stone circle on Langstone Moor were discovered in 1897 , and explored the same year. Two gave no sign of interments, not even a

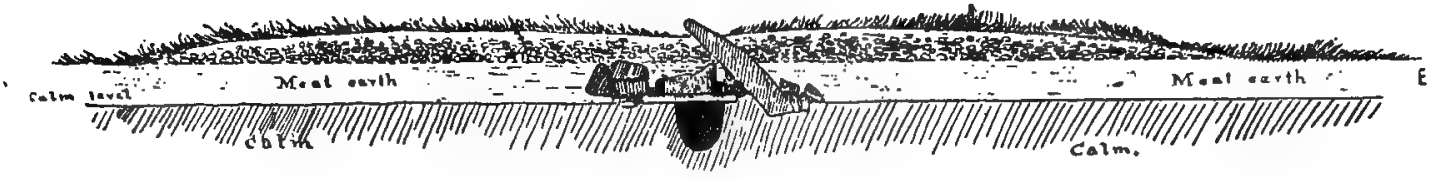

Section $E$ to W of ruined Cairn Hurston Ridge. Diameter of cairn 26 feet, leaning stone 30 inches long, 2 inches wide at top, 6 inches at bottom. At 14 inches diameter, 14 inches deep. This was filled with charcoal.

$$
\text { Fig. 15.-Section of Cairn, Hurston Ridgr }
$$

trace of charcoal ; but the third, with a diameter of I I $\mathrm{ft}$. and with the centre of the cairn I $\mathrm{ft}$. above the level of the ground, contained a small kistvaen, which was packed with 'calm' containing small pieces of wood charcoal and a little bone ash. It did not contain any pottery or implements. The bottom of the kistvaen was paved. There were traces of a surrounding stone circle. This is the smallest and best-made kistvaen yet found on Dartmoor, and with the consent of his Grace the Duke of Bedford it has been removed from the cairn (where it would have lain invisible) to the Municipal Museum at Plymouth. It is only 21 in. long, I 3 in. wide, and 14 in. deep (see fig. 16).

Hameldon (O.S. c, SW).-One of the barrows on Hameldon was explored in or about the year 1872 by the late Mr. Spence Bate, F.R.S. (For section see fig I 8.)

The main portion of the barrow was of earth, with a small central cairn and margins of stone. The exploration yielded portions of a bronze dagger (see fig. I9 and fig. 2 on same), an amber pommel ornamented with gold pins (probably the pommel of the hilt of this dagger), and some burnt bones, which were undoubtedly human.

Kistvaen on Lakehead Hill (O.S. xcix, SW).-A small kist denuded of its covering of stones or earth. It had previously been rifled, but the writer found on re-examination in 1897 fragments of pottery and three finely-worked and apparently unused scrapers, and the same number of knives, all of flint. These were embedded in the floor of the kist close to the stones, and had escaped the attention of the previous explorers (see fig. 17).

Kistvaen in Archerton NewTake (O.S. xcix, SW).-A small irregularly shaped kist close to and on the south side of the Powder Mills leat and about 350 yds. due south of Archerton House. It stands in the centre of a small wasted cairn with a present diameter of $14 \mathrm{ft}$. The bottom of the kist contained a small pit sunk below this level. On clearing this out some wood charcoal was observed together with a worked flint flake. A thorough search under the eastern side stone resulted in the discovery of an archer's wrist-guard, fashioned from a fine gritty stone and rubbed down perfectly smooth. It is perforated at each corner; two of the holes on the reverse side are countersunk to accommodate the knots of the thongs which bound the guard to the wrist. The obverse is slightly convex and the reverse concave.

These bracers or guards are not uncommonly found in barrows and kistvaens, mostly of the Bronze Age. Examples have been furnished by Scotland, Denmark, and Germany, and in several counties of England, both in stone and bone.

Cairn in Stannon Bottom, Post Bridge.-In the enclosure

\section{LANGSTONE MOOR}

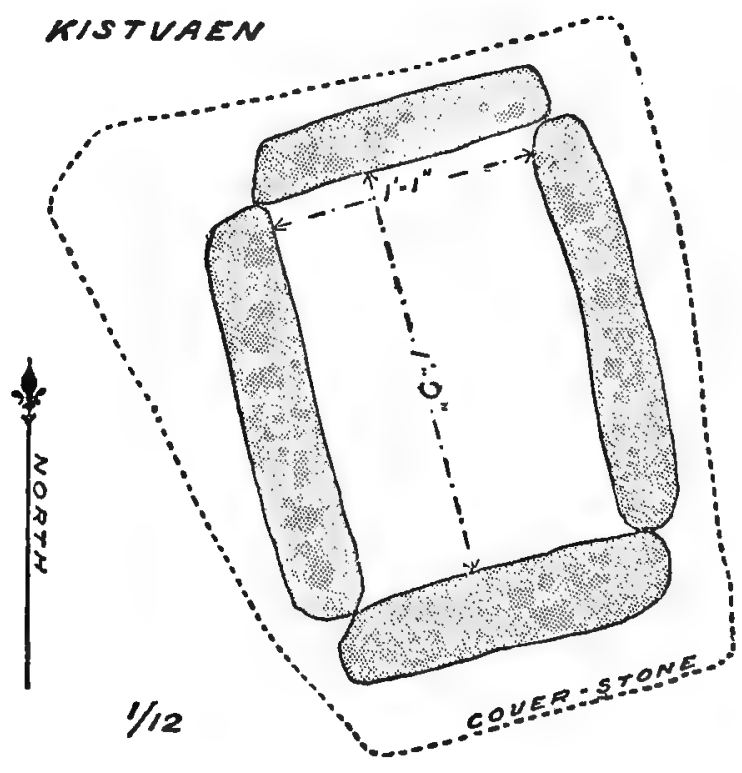

Fig. 16. - Plan of Kistvaen, Langstone Moor 361 


\section{A HISTORY OF DEVONSHIRE}

north of Stannon is a kistvaen marked in O.S. xcix, NE; $440 \mathrm{ft}$. WSW of this in the direction of Stannon Tor is a small cairn $15 \mathrm{ft}$. in diameter. This cairn had been previously opened in the centre. The writer drove trenches through it in 1895 , and found the interment in a circular hole dug in the 'calm' or sub-soil. This urn-shaped cavity was filled with wood charcoal, ashes, and calcined bones, and also yielded a finely-pointed yellowish translucent fint.

The previous explorers looked for the interment in the centre, but did not find it as the cavity was near the NE circumference.

Kistvaens in the Plym Valley.-A kistraen at Calveslake yielded a flint arrow-head and three flakes, and another at Langcombe, Deadman's Bottom, three arrow-heads and fragments of an urn.

Many of the districts of Devonshire outside the Dartmoor area are rich in barrows, and a considerable number of these monuments have been opened during the past thirty-five years by competent persons and the results recorded. The finds have not been in every case of much consequence, for some barrows turned out to be barren, having been opened in search of treasure in an irregular manner and at an unknown period. In confirmation of this there is a curious document in the Patent Rolls of 17 Edw. II. It secures to one Robert Beaupel ${ }^{1}$ the privilege of excavating six
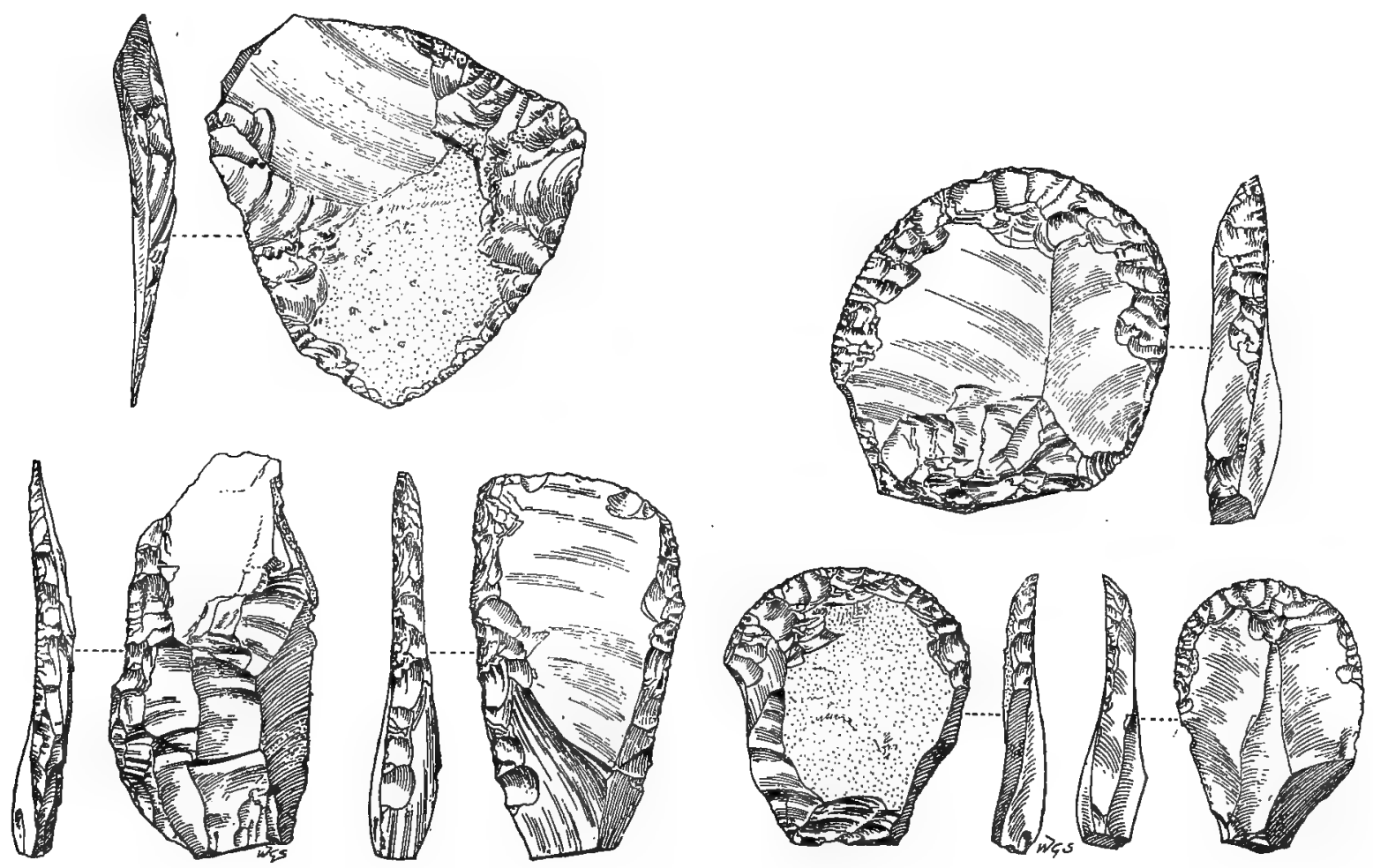

Fig. 17.-Flint Knives and Scrapers from Kistvaen on Lakehead Hill.

barrows in Devonshire, on condition that the search is made in the open day and in the presence of the sheriff and other responsible officers.

When previously unopened barrows have been explored under proper supervision some valuable evidence has been obtained.

Honiton.- There are many barrows in the neighbourhood of Honiton, and some of these on Broad Down and Gittisham Hill have been examined, first by the Rev. R. Kirwan and recently by Mr. R. H. Worth. All interments discovered were cinerary. (See fig. I8).

The important finds were as follows :-

Two drinking vessels of Kimmeridge coal with a capacity of about a gill, both ornamented with incised lines of such regularity as to suggest that these vessels were turned on a lathe; a bronze spear-head (see fig. I9 and fig. 3 on same), and supposed fragments of another, and a celt of the socket type (see fig. I 9 and fig. 5 on same); hand-made urns, including a very small, highly ornamented example, filled with burnt bones. It is only 2 in. high and 3 in. wide at the mouth, with

1 The Beaupels held lands in Brendon, North Devon, on the borders of Somerset, and it has been suggested that the six barrows in respect of which the grant was made may have been Chapman Barrows, a short distance to the south-west. This immediate group originally consisted of at least five tumuli, and there are still remaining many others in the vicinity. Trans. Devon. Assoc. xviii, 107. 


\section{EARLY MAN}

an average thickness of $\frac{1}{2}$ in. One side of the vessel has two small perforations. This and similar vessels have been termed ' incense cups '-an imaginative description, at any rate in this case, for the cup served the purpose of a diminutive cinerary urn.

UPTON PYNE, NEAR EXeTER.-A barrow here was opened by Mr. Kirwan in 1870 and yielded a bronze dagger and pin which were resting on a deposit of charred wood and burnt bones; an even smaller ornamented vessel of hand-made pottery of the so-called 'incense cup' type, containing a snuff-coloured coarse-grained dust, the nature of which was apparently not ascertained; the component parts of an amulet or necklace, consisting of flat beads of perforated shale, with a fusiform central bead of the same material ; portion of the stalk of an encrinite which apparently did duty as a bead; two beads of shale ornamented with chevron patterns, and a red bead of fired clay.

Berry Down Barrows,

BERRYNARBor. - In one of these barrows an inverted broken urn was found with the large diameter of 19 in., together with some of its fragments. The mouth of the vessel downward for about onethird of its original height was ornamented with a chevron pattern enclosed within two sets of triple incised lines running around the circumference of the urn, which is furnished with two lugs. The vessel was hand-made, of coarse ware, and, as usual, imperfectly fired.

In May, $1883, \mathrm{Mr}$. George Doe and others examined two barrows situated in a field which was once part of Berry Down. In one a deposit of fine clay streaked with charcoal was observed, and the other yielded another inverted sepulchral urn covering a deposit of burnt bonessome of these were recognized as human.

Plymouth.-A kistraen containing a large urn, mouth upwards, was found in Stillman Street, and reported by Mr. Francis Brent in $1881{ }^{1}$ It was supposed to have contained bone ashes.

Halwill. - A barrow in this parish was examined by the writer in 1895 . It is

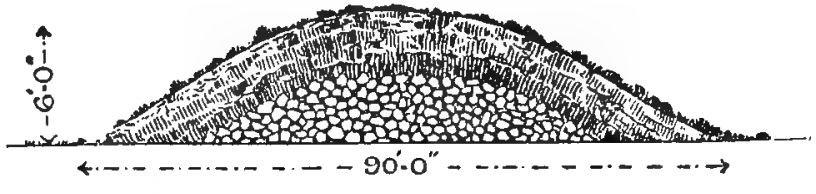

BROAD DOWN

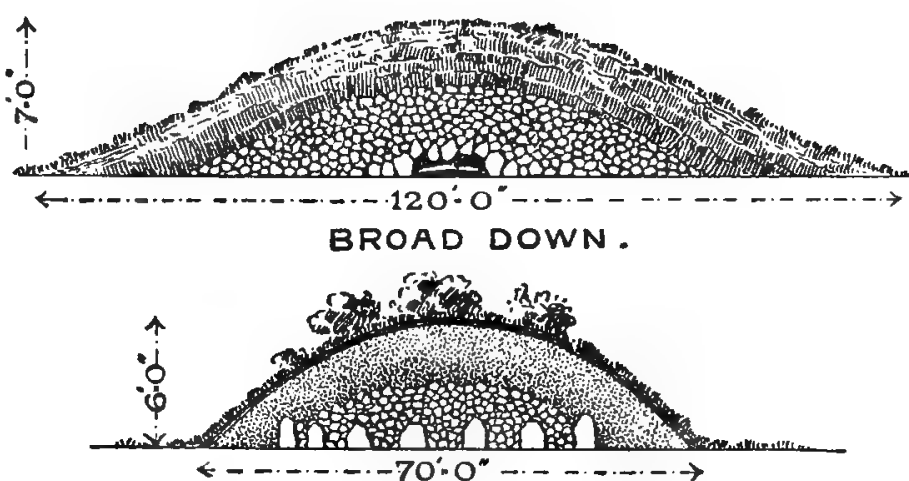

BROAD DOWN.

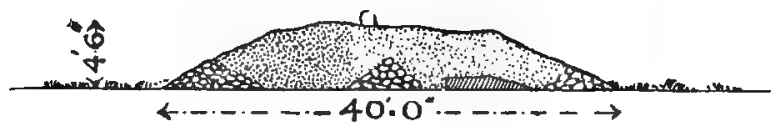

HAMELDON. (NOT TO SCALE)

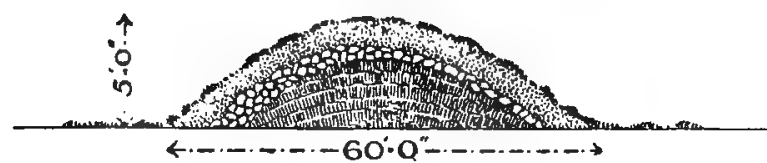

CITTISHAM HILL.

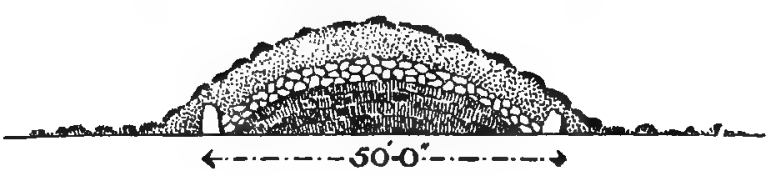

CITTISHAM HILL.

RERRAWN by R.MWORTH

VERTICAL SCALE THRICE NORIZONTAL.

Fig. I 8.-Sections of Barrows on Broad Down and Gittisham Hill. (See page 361 for reference to Hameldon.) situated in a field known as Burrow Park Tolly, and lies close to the road leading from Halwill Railway Station to the Manor House. A quarry has been opened (now disused) on the eastern side of the barrow, taking a considerable piece out of the circumference, but without in any way disturbing the central portion of the monument which stands $5 \mathrm{ft}$. high in the centre above ground-level. A trench was driven, $20 \mathrm{ft}$. long, I $2 \mathrm{ft}$. wide, and $5 \mathrm{ft}$. deep, in a westerly direction and to the centre of the barrow. This disclosed a platform in the centre of small flat stones $12 \mathrm{ft}$. long, $6 \mathrm{ft}$. wide, and I ft.

1 Trans. Devon. Assoc. xiii, 99-101. 


\section{A HISTORY OF DEVONSHIRE}

thick, resting on the 'meat' earth. It was strewn with fragments of wood charcoal and covered with wood ashes, and the stones showed signs of having been subject to heat. Close to this platform was a pit, $4 \frac{1}{2} \mathrm{ft}$. by $3 \mathrm{ft}$. and $4 \mathrm{ft}$. deep, dug out of the sub-soil and full of 'meat' earth mixed with fragments of calcined bones. The latter on examination did not appear to be human, but bones of some animal. The barrow was made of stiff clay, and some of this near the platform was burnt so hard as to resemble pottery. It was impossible to sift the material removed, for the clay was too heavy and plastic, but careful scrutiny disclosed a pear-shaped amber pendant $\frac{3}{4}$ in. long, some pottery of the early Bronze Age, and a little calcined bone. This was found $15 \mathrm{in}$. below the surface of the centre of the barrow, which rose $5 \mathrm{ft}$. above the surface of the ground. More pottery and calcined bones were found at a depth of $5 \mathrm{ft}$. The pottery is hand-made, $\frac{3}{4}$ in. thick, and black on the inner surface. The paste is well made, hard burnt, and of the clay of the district with a little sand to temper it.

\section{Miscelianeous}

We have thus briefly shown that Devonshire is rich in evidence of the Bronze Age, possessing as it does the ruins of the huts, together with a great number of the graves, of the people of that period.

The domestic pottery of the huts, with the exception of some of the pots used for cooking, differs but little in make or ornamentation from the vessels found in the graves. They are sufficiently akin to form a connexion, and to satisfy the expert that the people occupying the huts on Dartmoor were buried in the cairns which studded both that area and other parts of Devonshire as well.

It is hardly to be wondered at that no bronze has been found in the hut circles. It was precious, great care was taken of it, and on rare occasions only was it placed in graves with the honoured dead. There are sixteen records of finds of bronze in graves in Devonshire, but one of these (Lovehayne Farm) may be considered to be unconnected with the interment.

Considering the small number of graves which have been opened under efficient superintendence during recent years, and which were previously undisturbed, this is not a small proportion, for according to Canon Greenwell articles such as bronze swords, spear-heads, and celts appear only on the rarest occasions to have been interred with the dead.

There are records of finds of bronze in Devonshire not connected with interment.

Gawler Bottom, Post Bridge.-A bronze ferrule of the shaft of a spear was found here in I892, $4 \mathrm{ft}$. deep in the peat, and is now in the Municipal Museum, Plymouth; the remains of the blade of a dagger were also found in the peat at the same depth at Broadhole near the head waters of the Plym. The bronze ferrule is interesting, for it is not of common occurrence in Great Britain. Only some forty examples have been thus far recorded, and of these only three or four are in the British Museum. Four specimens about 7 in. long were found with bronze spear-heads at Bloody Pool, South Brent. The latter were barbed and 14 in. long, but, unfortunately, they were all broken.

Teigngrace.-A spear-head I I in. long was discovered here. It has a delicate bead, which runs down each side of the midrib, and is continued as a square projection below the blade.

W ASHFIELD.-Leaf-shaped spear-heads were found in an ancient entrenchment at Worth in this parish. With these were also found a leaf-shaped sword 14 in. long, with rivet notches at the side of the base and a flat plate of bronze, apparently hammered out, 5 in. by $5 \frac{1}{2}$ in. and $1 \frac{1}{8}$ in. thick.

Escot.-Several bronze swords were discovered in a field near here.

Talaton.-Six sword blades were found here. They were of the rapier type, and from 12 to 22 in. in length.

WinkLEIGH, NEAR CREDITON.-Another blade of $I \eta$ in. was found in this place.

Sidmouth. - A flat celt $4 \frac{1}{2}$ in. long, having the faces ornamented with a number of longitudinal cuts, was found near here. 


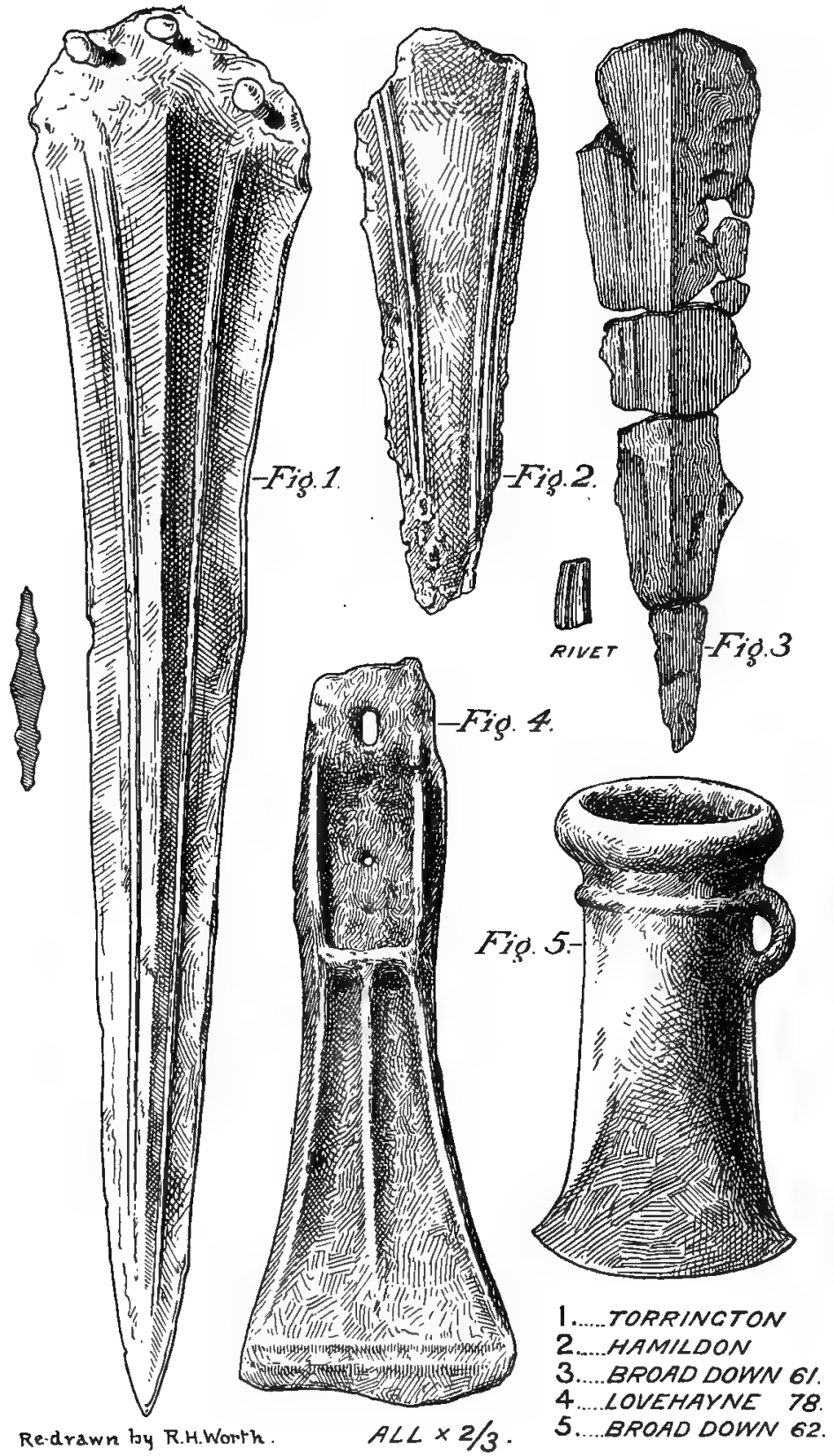

Bronze Objects. Fig. I 9 



\section{EARLY MAN}

Huntshaw, near Great Torrington.-A bronze dagger found in a barrow with burnt bones, ashes, and charcoal. ${ }^{1}$ (See fig. I 9 and fig. I on same).

Farway Castre, near Honiton.-A palstave was picked up in this immediate vicinity. Other examples have also occurred at Morebarton, in a field near Subhill between Clyst St. Lawrence and Rockbeare, at Chagford, Drewsteignton, and at Rumby, Bovey Tracey.

Honiton.-A socketed celt is recorded as having been found here. ${ }^{2}$

Hennnock, near Chudleigh.-Moulds for bronze castings have also been found in Devonshire. Two are figured by Evans ${ }^{8}$ which were found in this parish; they are of a light greenish micaceous schist. One mould is a trifle over $2 \mathrm{ft}$. in length and $3 \mathrm{in}$. wide-the other $21 \frac{1}{2}$ in. long and the same width. These moulds produced castings of rapier-shaped blades.

Lovehayne Farm, Colyton.-A large number of celts and spear-heads 'half filling a wheelbarrow' were found by road-makers in removing a cairn in I770. The bulk of these were sold as old metal in the neighbouring town of Honiton. One celt from this find, a palstave, was in existence in 1868, and is figured in Trans. Devon. Assoc. ii, 647. (See fig. 19 and fig. 4 on same).

PLymstock.-In I 868 there was a remarkable discovery of bronze implements here consisting of sixteen celts, a spear-head or dagger, ${ }^{4}$ and a narrow chisel. They lay beneath a flat stone at a depth of about $2 \mathrm{ft}$. below the surface. The celts were of the flat and flanged variety; the chisel was 4 in. long with a cutting edge of $\frac{1}{6}$ in. square in section in the upper part, and gradually tapering to an edge at the lower end. ${ }^{5}$

Plymoutr.-In the early part of $188_{4}$ a perfect and an imperfect palstave, together with a celt with a square socket, were found on removing an old hedge at Torr Lane.

W HITE ToR.- This (perhaps more generally known as Whittor) is a fine tor, I,526 ft. above the level of the sea, on Cudlipptown Down, overlooking Marytavy and Petertavy. The summit of the tor is surrounded by the ruins of two walls with a space between of about $10 \mathrm{ft}$. or more in places. Both the walls appear to have been 10 to I I ft. thick, and judging from the débris the inner wall must have been about 4 to $4 \frac{1}{2} \mathrm{ft}$. high, whilst the outer reached a height of 6 to $7 \mathrm{ft}$. The area enclosed is $\mathrm{I} \frac{1}{2}$ acres.

The supposed entrances are to the east, where access is easy. The other approaches to the summit of the tor are steep and covered with 'clatters' of rock. Within the enclosure are the remains of half a dozen hut circles or huts of a semi-circular character, which were built up under the lee of large boulders of igneous rock. These and other portions of the enclosure were explored in 1898 and yielded charcoal, a little hand-made pottery, a flint scraper, a trimmed flake which might have served as an arrow-point, and a great number of chips. These chips occur under the turf surface all over the enclosure.

The large cairn was explored right down to the surface on which it stands, and this also was dug into in search of a possible interment, but nothing was found except two small flint flakes. No charcoal was seen and nothing to indicate the use of this great heap of stones, amounting to hundreds of cartloads. All the stones are of handy size, and it has been suggested that they may have been intended for use as missiles or for repairing the walls of the enclosure. The summit of the cairn, which is about Io ft. high, has never been used as a beacon-for, as previously stated, not a trace of charcoal was noticed in the exploration. There is no trace of water within the protecting walls, nor is there any now available within a distance of several hundred yards.

The exploration yielded no sign of the place having been assailed or occupied by a fighting force, for no weapons, with one doubtful exception, or sling-stones were observed.

There was just enough evidence found to indicate that this defensive place apparently appertained to the same period as the hut circles, the potsherds and the prevalence of flint chips being the principal evidence.

Cranbrook Castre.-Excavations were made in many places on this site, including the moat, south-west, and south, and rendered many sling-stones and large pieces of charred wood; in many other trenches no results were obtained. The principal finds were in excavations numbered 1,2 , 3 , and 4 .

At No. I the site of a hut or dwelling of some kind was found, and this yielded, about I ft. below the grass surface (the soil at Cranbrook is very shallow), some small fragments of pottery, and the bottom stone of a granite quern 13 in. in diameter with a central hole of 2 in. in diameter. There was much wood charcoal, and two fire-holes were observed.

At No. 2 another site of a dwelling or shelter was opened out-this also had a fire-hole scooped out of the sub-soil. There was a good deal of charcoal strewn over the site, and some potsherds also were found.

Trans. Devon. Assoc. vii, 102-5.

Ibid. 434 .
Evans, Bronze Imp., Trans. Devon. Assoc. iv, 298-9. 4 Arch. Journ. xxvi. 


\section{A HISTORY OF DEVONSHIRE}

Excavations 3 and 4 yielded charcoal but no pottery. Sling-stones were found in nearly all the excavations, and some of these were battered and broken as if by impact.

The interior of the camp is studded with small round stumps of stone of about 4 to $5 \mathrm{ft}$. in diameter, and these are continued over the north slope of the hill, where they are dotted about in dozens. Several of them were examined both inside and outside the camp, and were found to be little heaps of stones placed on the grass surface. No information could be obtained regarding them, and their occurrence is inexplicable. No metal of any kind was observed in any of the excavations.

The ornamentation of the pottery is similar to that of the Bronze Age, and the sherds appeared to be portions of a hand-made vessel. But as we now know that the style of ornamentation usually associated with the period of bronze survived down to the Prehistoric Iron Age, and as rudely made vessels which may apparently be described as 'hand-made' were also in use at the same time as wheel-made pots, it is not safe to assume that the pottery found on the floors of the hut or shelter sites in Cranbrook Castle indicates that this defensive place appertained to the Bronze Age; in fact the quern and the elaborate system of fortification on the southern portion of the camp favour the assumption that it was erected and occupied not earlier than during the Prehistoric Iron Age. The discovery of the remains of iron objects would have settled this doubt, but, as previously stated, no metal of any kind was seen.

SidBury Castre.-A hoard of sling-stones was found here in March, 1864. ${ }^{1}$ They were found in a cavity which was situated on the outside slope of the inner rampart, and would have filled one or two wheelbarrows.

HIGH PEAK.-Relics have also been found in and about the remains of a camp which formerly existed at High Peak about $I \frac{1}{2}$ miles west of Sidmouth. ${ }^{2}$ The eroding action of the sea has practically caused it to disappear. The Rev. R. Kirwan and Mr. P. O. Hutchinson found some thirty-three years since that only a small portion remained, and that the falls of the cliff - the site of the camp was some $500 \mathrm{ft}$. above sea level-had exposed a kitchen midden, which yielded much charcoal, bones of hog, deer, and ox, sling-stones, flint chips, and cores, rude bone implements, and potsherds, both hand and wheel-made. Some of the sherds were plain, others were ornamented with incised lines made with a toothed instrument, some had circular indented lines and bands impressed upon the clay before firing and others had raised hoop-like marks or ridges formed either by the hand or by the wheel. All this domestic debris was presumed to be the accumulation of the dwellers in the camp. If this assumption be correct it indicates a prolonged occupancy.

There is again evidence of the overlapping of the hand-made Bronze Age type of vessels with the wheel-made pots, and of the continuation of the earlier form of ornamentation with that described as 'Circular indented lines,' a characteristic of the Prehistoric Iron Age.

Ashburton.-A spindle-whorl was found in the wall of Boro Wood Camp, near Ashburton. ${ }^{3}$

It will be observed that all the graves which have been examined indicate cremation; in fact there are no reliable records of carnal interments in Devonshire barrows. It is possible that such may yet be discovered, for a contracted interment was found in a kistvaen in Sheviock parish, just over the border in Cornwall. In this kistvaen was a small urn similar to the pottery of the Bronze Age period. From the examination elsewhere of osseous remains of the people of this period, it is evident that their skulls were generally broader than those of the Neolithic folk who were at home in Devonshire when these Celtic roundheads arrived. These first Celtic invaders overran the fertile and temperate portions of Britain, dispossessing the Neolithic people, who retired to less favoured districts, or remained and gradually blended with the conquering race.

Sir John Evans is of opinion that the Bronze Age in Britain commenced about 1200 to I 400 B.C. and continued down to about 500 B.c. To this period must be assigned the dawn of decorative art. Weapons, implements, and vessels of daily use were more effective and of greater variety and symmetry, and were often ornamented in a lavish and comparatively elegant manner.

\footnotetext{
Trans. Devon. Assoc. ii, 376.

${ }^{3}$ Ibid. vi, 263.
}

I Ibid. iv, 647-65 I. 


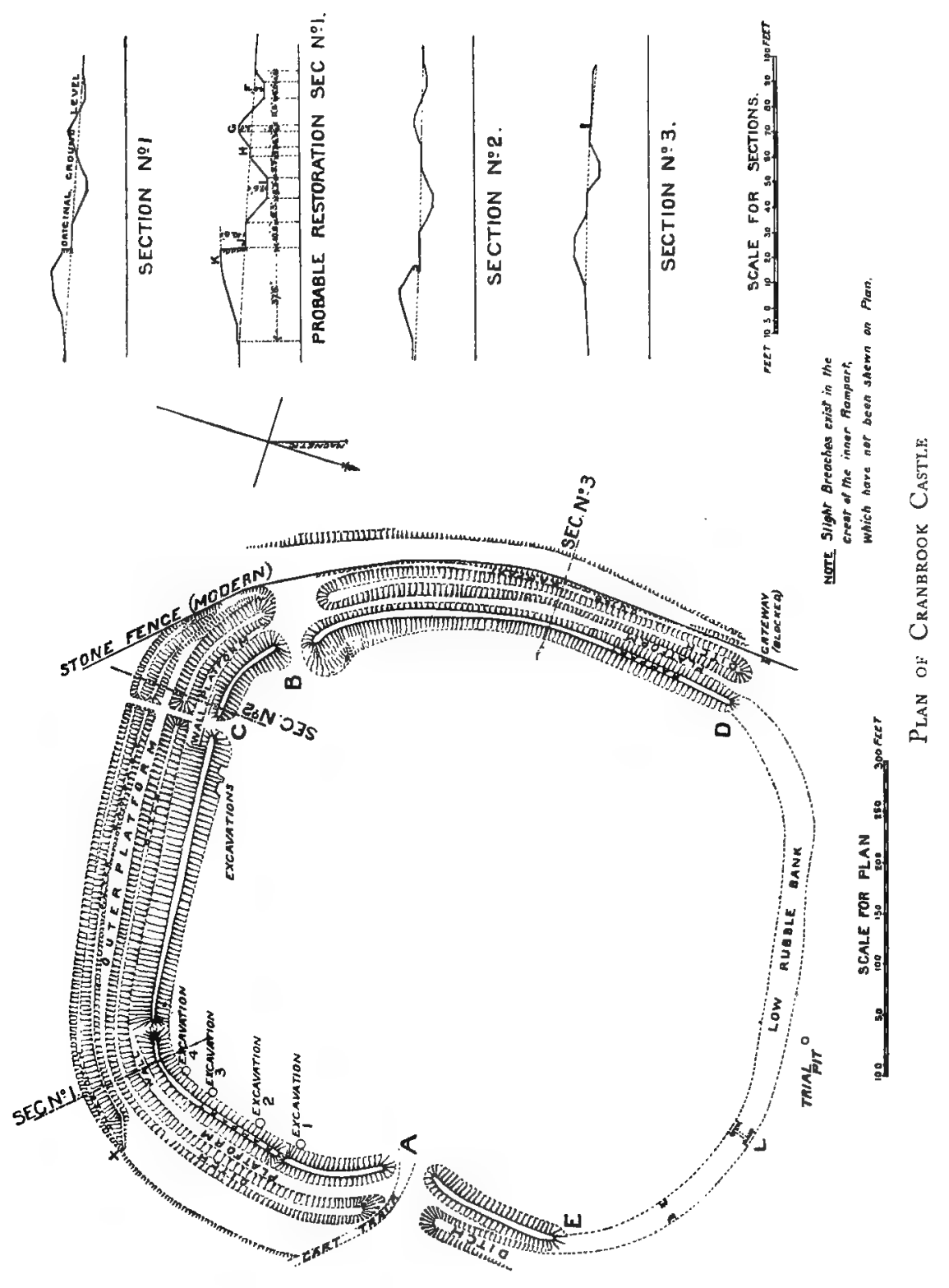

To face page $366^{\circ}$ 



\section{EARLY MAN}

\section{The Prehistoric Iron Age}

As the use of stone implements overlapped the period when bronze came into use, so objects of bronze continued in use in what is known as the Prehistoric Iron Age.

The knowledge of the preparation of iron was either brought to Britain through the peaceful channels of improved communication with the Continent and adopted by the Britons, or, as some think, the new metal (i.e. new to this country) formed portions of the equipment of another wave of Celtic invaders who swarmed into Britain somewhere about 400 to 500 B.c.

Finds of early iron are rare, for iron oxidizes rapidly, leaving but traces of rust in the soil. But notwithstanding this perishable nature of the metal, it is curious that so far none of the Dartmoor hut circles have rendered signs either of the remains of iron objects or of pottery characteristic of the Prehistoric Iron Age. The later hut circles, if such exist, may yet disclose such evidence, for it is not quite reasonable to suppose that all the numerous hut circles on Dartmoor were occupied only during the Bronze Age, or that people of a later period did not resort to the moors for the summering of their

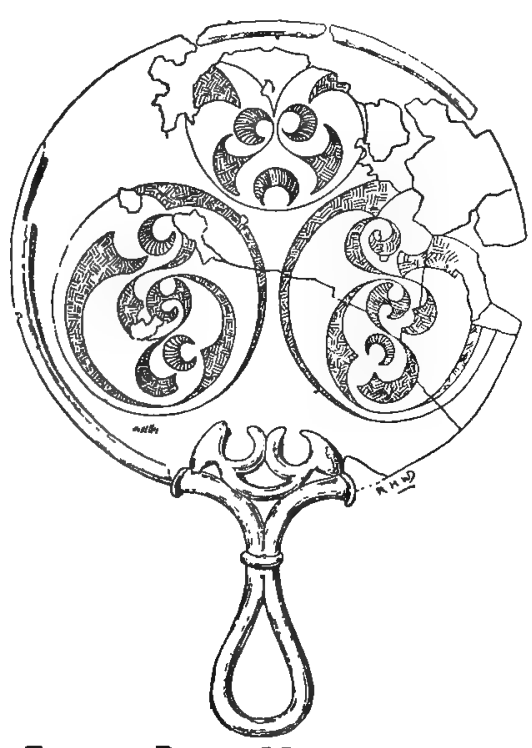

Fig. 20-Bronze Mirror, Stamford HILL flocks and herds. We do not know when the circular form of dwelling was superseded by the rectangular, but we do know that what are known as the 'ancient tenements' have been fixed abodes for many centuries, and it is possible that in the sites of these homesteads we have the very early moorland farms, which followed the earlier settlements. If this be the case there is little wonder that relics of the Prehistoric Iron Age are so rare, for objects of iron have long since gone to complete decay, and potsherds in and about a place in continuous occupation have broken up and disappeared. Kitchen middens on farms are not likely to exist, for their contents would be a fertile aid to the cultivator.

This paucity of relics of the Prehistoric Iron Age is general over Devonshire, and but for a cemetery of the period discovered on Stamford Hill near Plymouth in 1864 the county would be almost barren in this respect.

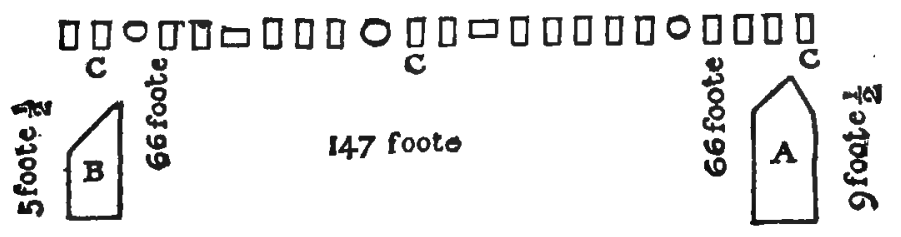

A the great Jone 9 foot 6 inches $B$ the other great fine 5 foot 6 inches $C C C$ the row of 23 itones

Fig. 21.-Stone Row and Mênhir at Maddocks Down $3^{6} 7$
STAMFORd Hill.-In cutting away the slope of the hill so that the guns on the new fort which was being erected here might have no interference with their range seawards an ancient burying-place was disclosed. The relics were found in pits, generally about 4 or $4 \frac{1}{2} \mathrm{ft}$. deep, I ft. of which consisted of soil, the remaining 3 having been excavated in the 


\section{A HISTORY OF DEVONSHIRE}

partially disintegrated rock (slate). These graves were mere hollow excavations, the walls sometimes sharply cut. This appears to have been the more evident where the soft slaty rock was firmest. The bottoms of the excavations were deepest towards the centre, and they were filled in with the débris which had been taken out of them, together with numerous large, rough, worn blocks of
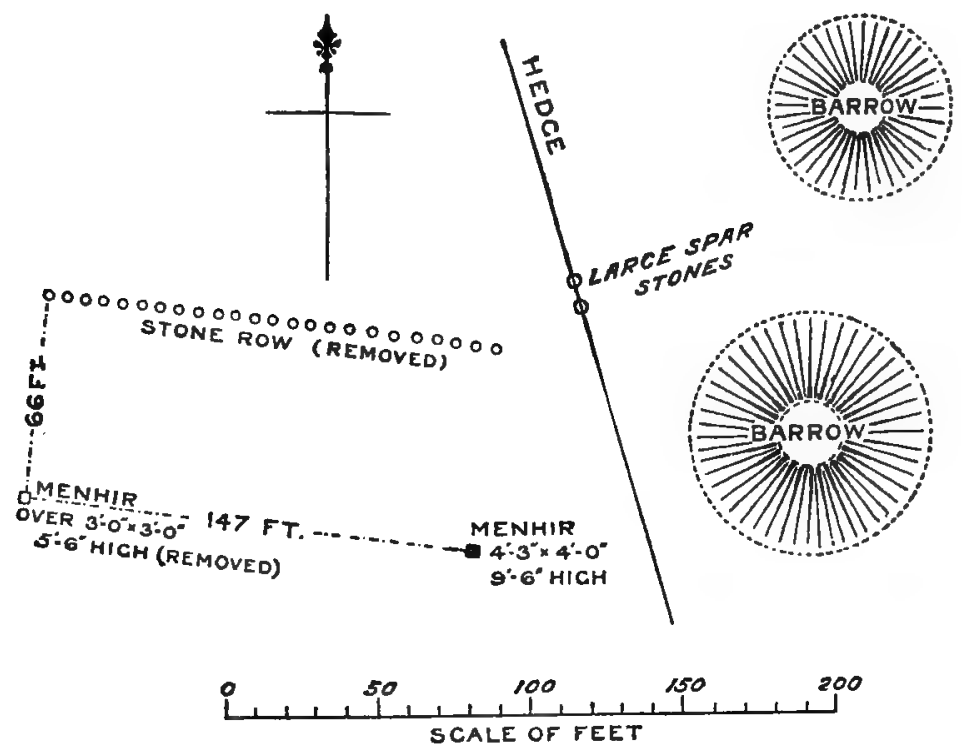

Fig. 22.-Stone Row and miênhir at Maddocks Down (Reconstructed) limestone, that must have been purposely brought from one of the neighbouring limestone hills. These stones, according to the late Mr. Spence Bate, were used as walling to the graves and as coverings for the body, which was placed in the grave in a sitting posture. The graves were very numerous, but, unfortunately, they were not explored in the interests of science. All that Mr. Spence Bate could do was to follow the excavations of the contractor's workmen, and rescue and note all he could whilst the work rapidly proceeded. The longitudinal axis of many of the graves was east and west, but this was not invariably the case.

Human bones were disinterred together with the following objects of bronze:-Portions of mirrors (see fig. 20), a small broken cup or pot, bracelets, fibulae, dagger sheath, and finger rings. The circular and running scroll ornamentation of these are characteristically Late Celtic.

The objects that were found made of iron were too decomposed to identify with confidence. They consisted generally of irregularly-shaped nodules of rusted iron from which a point or sharp tongue projected-others again appeared to be the remains of a pair of scissors and blades of knives and of the tongues of knives that were driven into handles. Some of the latter had remains of wood still attached to them.

The black, yellow, and red pottery consisted of remains of bowls, jugs, a vase and drinking cups.

In addition to the fragmentary vase a small perfect example was rescued from the destruction wrought by the excavators. The bottom is flat, and about $1 \frac{1}{2}$ in. in diameter, from which it gradually rises outwardly until just above the middle, from which point it rounds more suddenly inwards, to form a constricted ring just beneath the edge of the mouth, which turns outwards. The diameter at the mouth is 3 in., at the broadest part $3 \frac{1}{2}$ in., and the height is about 4 in.

The fragments of a low, broad, glass bowl or basin were thrown out of one grave ; these were of a beautiful amber colour with iridescent surfaces. The lower portion of the vase was ornamented by a series of raised lines radiating from the base, but instead of passing directly to the circumference, flowed diagonally outward, as if they were formed as the plastic material was revolved by the Celtic glass blower.

Holne Chase Castle (O.S. cviii, SW).-A man digging out a rabbit near this ancient camp in

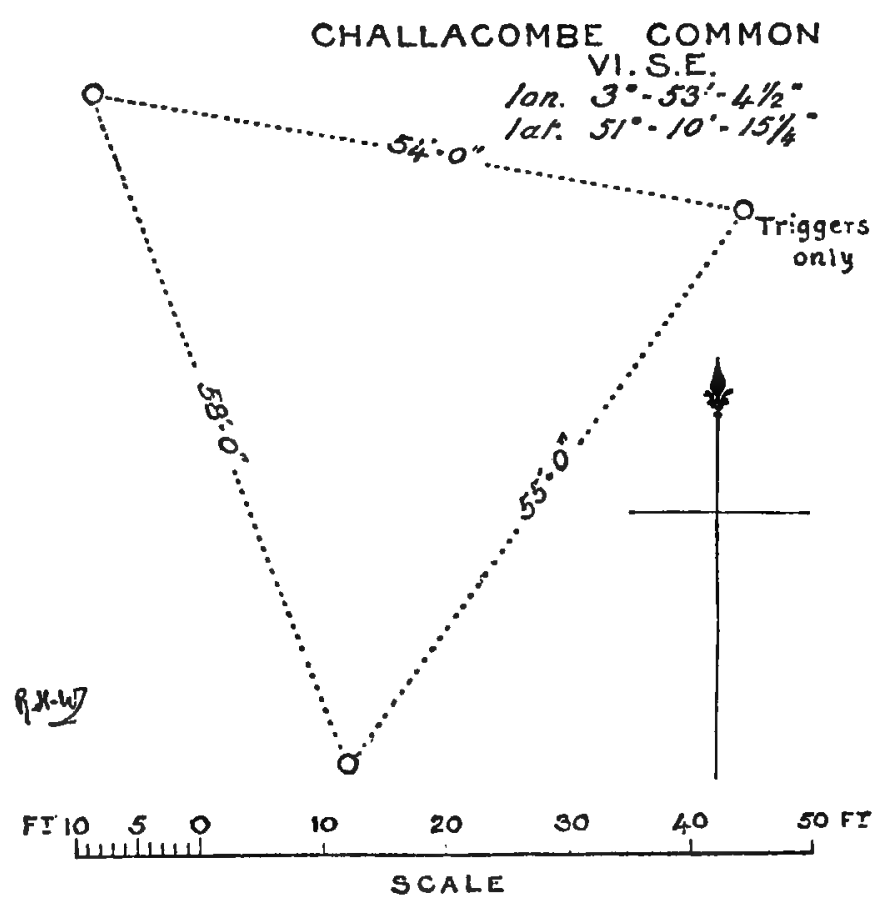

Fig. 23.-Triangle on Challacombe Common 368 


\section{EARLY MAN}

I 870 found about a dozen rusty flat iron objects about $4 \mathrm{ft}$. below the surface. These were carelessly thrown out, and being very brittle, broke into fragments. Two or three of the most perfect pieces were preserved and handed to Mr. P. F. S. Amery of Ashburton, who communicated the find to the members of the Devonshire Association in $1873^{1}$

The most perfect specimen was a flat bar of iron 24 in. long by $1 \frac{8}{4}$ in. wide and $\frac{1}{4}$ in. thickrather tapering towards one end, which was, however, much decayed. The other end possessed a rude handle formed by turning up the edges so as to meet one another. 'This fold was 3 in. long, but being of thinner metal suffered greatly from rust.

The edges of the bars were quite square and appear never to have been sharpened. The objects were presumed to be unfinished weapons. This find attracted the attention of Mr. Reginald A. Smith, F.S.A., who recognized them as 'currency bars,' a form of the monetary system of the Britons, in use before and during Caesar's visits to these shores. ${ }^{2}$

In a communication to the Society of Antiquaries, entitled 'British Iron Currency' 3 this gentleman described finds of similar iron bars in Dorsetshire, Hants, Somersetshire, Gloucestershire, Worcestershire, Berkshire and the Isle of Wight. They have generally been found in or near ancient camps, lying together in considerable numbers, and evidently intentionally concealed. In 1824 a large number-394-were found deposited in the middle of the ancient camp on Meon Hill, Gloucestershire. In $1856 \quad 150$ specimens were found near Malvern, and in the following year a further deposit of a similar number was discovered 3 or $4 \mathrm{yds}$. distant from the first.

The bars were often mistaken for unfinished swordblades, but the similarity vanishes on examination, for the swordblade of the period did not contain so much metal, and had a thin blade with a slender rounded tang for a wooden handle.

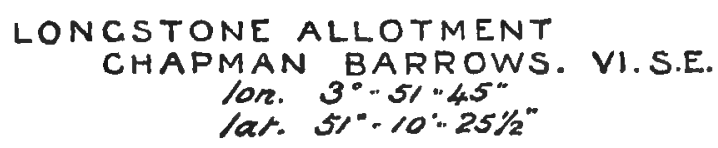

LONCSTONE ALLOTMENT

CHAPMAN BARROWS. VI.S.E.

lon. $3^{\circ}-51.4 .5^{\circ}$

lar. 5 " $^{\circ} 10$ ". $25 \% "$

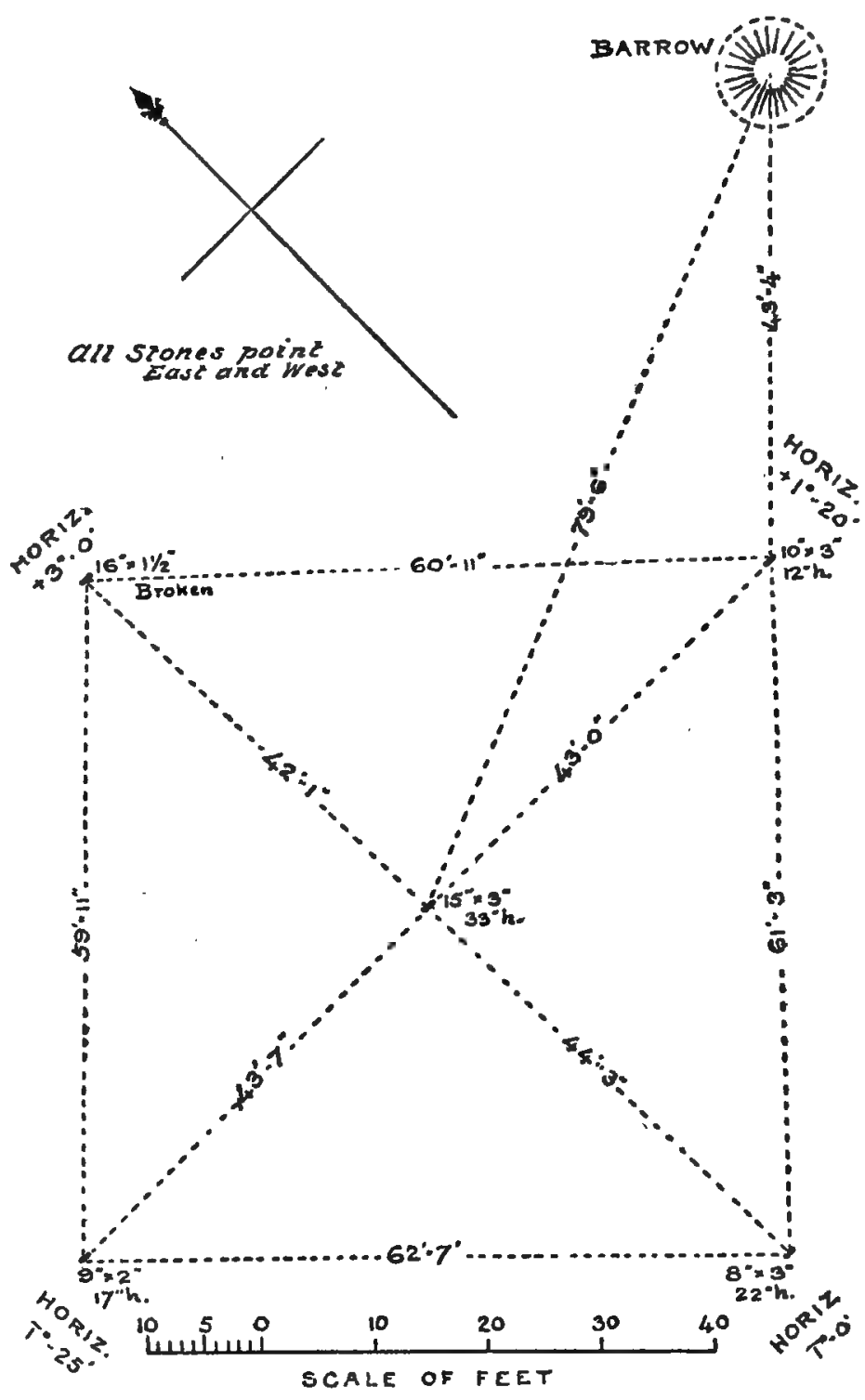

Fig. 24.-Quadrilaterai, Longstone Allotment, Chapman Barrows

The bars hitherto found

are hoarded like coins, and as money were much more likely to be concealed in a British camp at a time of commotion than half-made sword-blades. The smith who made a blade would finish it, there was probably no such division of labour in pre-Roman times involving an artificer for one stage, and a finisher for the second.

1 Trans. Devon. Assoc. vi, 264.

${ }^{2}$ Caesar, Commentaries (5th Book).

"Proc, Soc, Antig. vol xx, No. I I, pp. 179-95. 


\section{A HISTORY OF DEVONSHIRE}

In addition to this the bars, which have been found in various places, conform to a series of sizes and weights, for amongst the recorded discoveries is a smaller set representing half the value of the ordinary specimens, and a larger series four times the weight of the smallest.

Exmoor.-A part of this moor lies in Devonshire, and is ill provided with surface blocks of stone suitable for rude stone monuments. Some of these, however, were sufficiently prominent in the seventeenth century to attract the notice of William Camden, Tristram Risdon, and Thomas Westcote, and were described by the first as 'stones pitched in order, some triangle-wise and some in round compasse,' whilst the last gives a detailed description and a diagram (fig. 2I) of a stone row with two mênhirs associated therewith. The row of stones and one mênhir situated on Maddocks Down have disappeared. The remaining mênhir of quartz is still standing, its dimensions and reconstruction of the whole monument by Mr. R. H. Worth are indicated on the accompanying plan (fig. 22).

In addition to the destruction caused by the formation of enclosures there has been a gradual decay of the Exmoor stone monuments owing to the perishable nature of the slate employed, but enough remains to indicate a considerable amount of primitive work.

Some of these remnants have lately been examined and described, ${ }^{1}$ and it would seem that, like the Dartmoor examples, the stone rows and mênhirs are contiguous to and apparently connected with interments. This same association applies to stones forming an isolated triangle, for it is

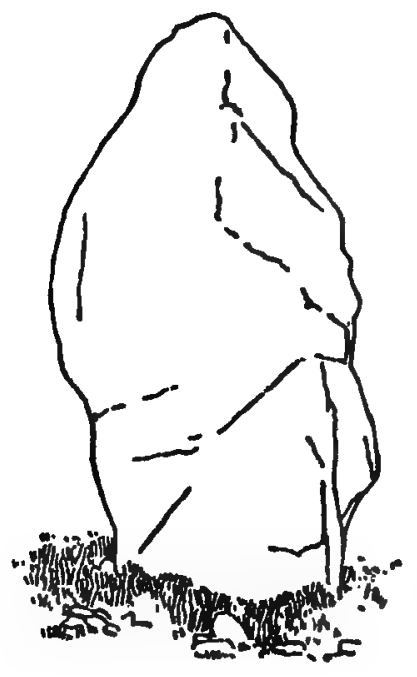

MADDOCKS DOWN looking sbyE\%E

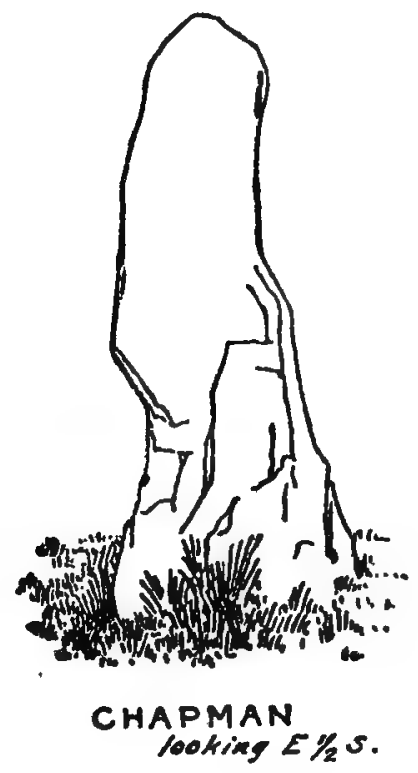

Figs. 25, 26.-Maddocks Down and Chapuan Menhirs. adjacent to a group of barrows on Challacombe Common (now enclosed) O. S. vi, SE. This triangle possesses two of its stones intact, whilst the third is represented by the trigging stones which supported it. It is not quite equilateral, being north $54 \mathrm{ft}$., south-east $55 \mathrm{ft}$., and south-west $58 \mathrm{ft}$. (fig. 23).

In Longstone Allotment, Chapman Barrows (O.S. vi, SE) is an example of what Messrs. Chanter and Worth describe as a quadrilateral, the definition being four stones placed on or near the circumference of a circle so as to form an approximately rectangular figure; at the intersection of the diagonals of this figure, and hence approximately at the centre, there stands a fifth stone. A reference to the accompanying plan (fig. 24) gives details and position of adjacent barrow. The figures appended to each stone are dimensions in inches-the first gives the width, the second the thickness, and the third the height above ground. 'The word 'Horiz." accompanied by an angle plus indicates the elevation of the visible horizon in the alignment of the stones.

To the north of this quadrilateral and $500 \mathrm{ft}$. distant from its centre, a line drawn practically parallel to the east and west diagonal would pass through seven barrows of the Chapman group (O.S. vi, SE); other barrows lie south and east, and the Longstone itself is companion to this quadrilateral, although $2,440 \mathrm{ft}$. distant (see figs. 25 and 26 for illustrations of Maddocks Down and Chapman mênhirs). 7 I $3 \mathrm{ft}$. distant, in a direction approximately 30 degrees south of east, is a large barrow which was opened in 1904, and which yielded a covered interment-pit with charcoal and bone-ash. Fourteen hundred and fifty feet from the centre of the quadrilateral, and in a direction approximately 21 degrees north of west, is a barrow which was opened in 1885 , and which yielded an inverted urn and burnt bone.

Near Woodbarrow Arms (O.S. vii, SW, described in O.S. as Woodbarrow Hangings) is an example of the quadrilateral in combination with a triangle; and on Furzehill Common, over Hoaroak Water (O. S. vii, NW), is an instance of a parallelogram associated with a triangle (fig. 27). Originally it consisted of three rows of three members each; now the central pillar is represented by its triggers only, while the southern centre stone is broken off at ground-level. There are con-

1 Trans. Devon. Assoc. vol. xxxvii, p. $375-397$. 

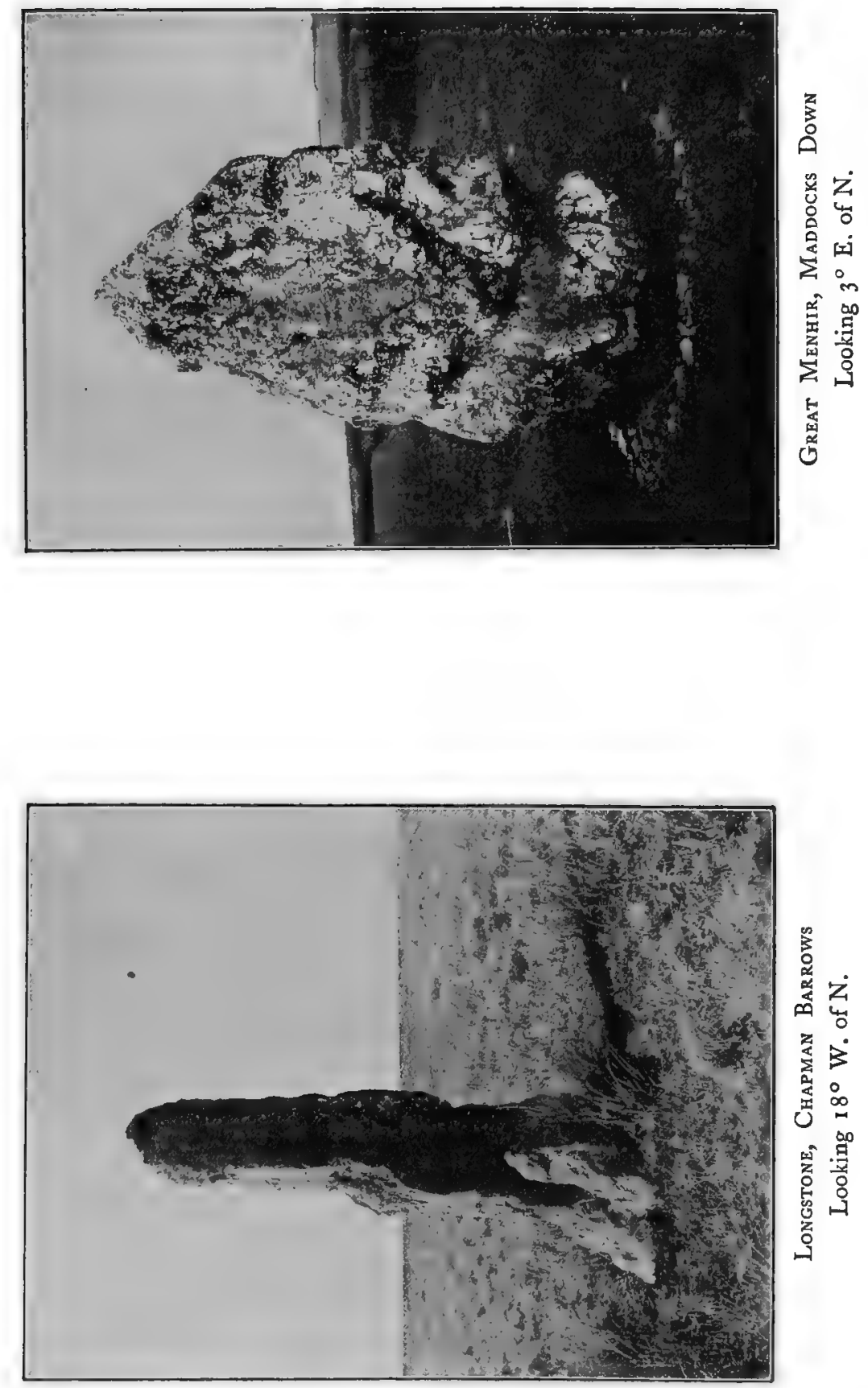



\section{EARLY MAN}

siderable irregularities in the spacing, but through it all the diameters and diagonals intersect with great exactitude at the point where the centre-stone has been.

It is not necessary to dwell on the unequal distances of the stones, as the figure is fully dimensioned. The parallelogram is approximately $69 \mathrm{ft}$. long by $47 \mathrm{ft}$. broad. The tallest stone is 13 in. wide, 6 in. thick, and 24 in. high. All stones point one way, along the length of the figure (approximately north and south), except the north-west and southwest corner stones, which lie at right angles to this. To the south, and about $30 \mathrm{ft}$. distant, lies a triangle, which makes no attempt at equilateral symmetry, its north side being $17 \mathrm{ft}$. 9 in., west side $24 \mathrm{ft}$. 6 in., and south-east side $28 \mathrm{ft} .2$ in. There is no parallelism or precise alignment between the members of the triangle and of the parallelogram. Were it not for the exact accuracy of the work where it was evidently intended to be precise it might be suggested that the south-east side of the triangle was designed to point to the southeast corner of the parallelogram and the west side of the triangle to the south-west corner. ${ }^{1}$

\section{Ancient Roads}

Devonshire in preRoman times evidently contained a numerous and comparatively cultured population. They were skilled workers in bronze and iron, and efficient potters. We know from Caesar that, like the Veneti, they built better ships than the Romans.

These people had roads or trackways linking their villages and camps, and in addition to this, main roads connecting them with other parts of Britain. The minor roads are now difficult to trace or recognize, but here and there trackways of great antiquity may be seen. Some

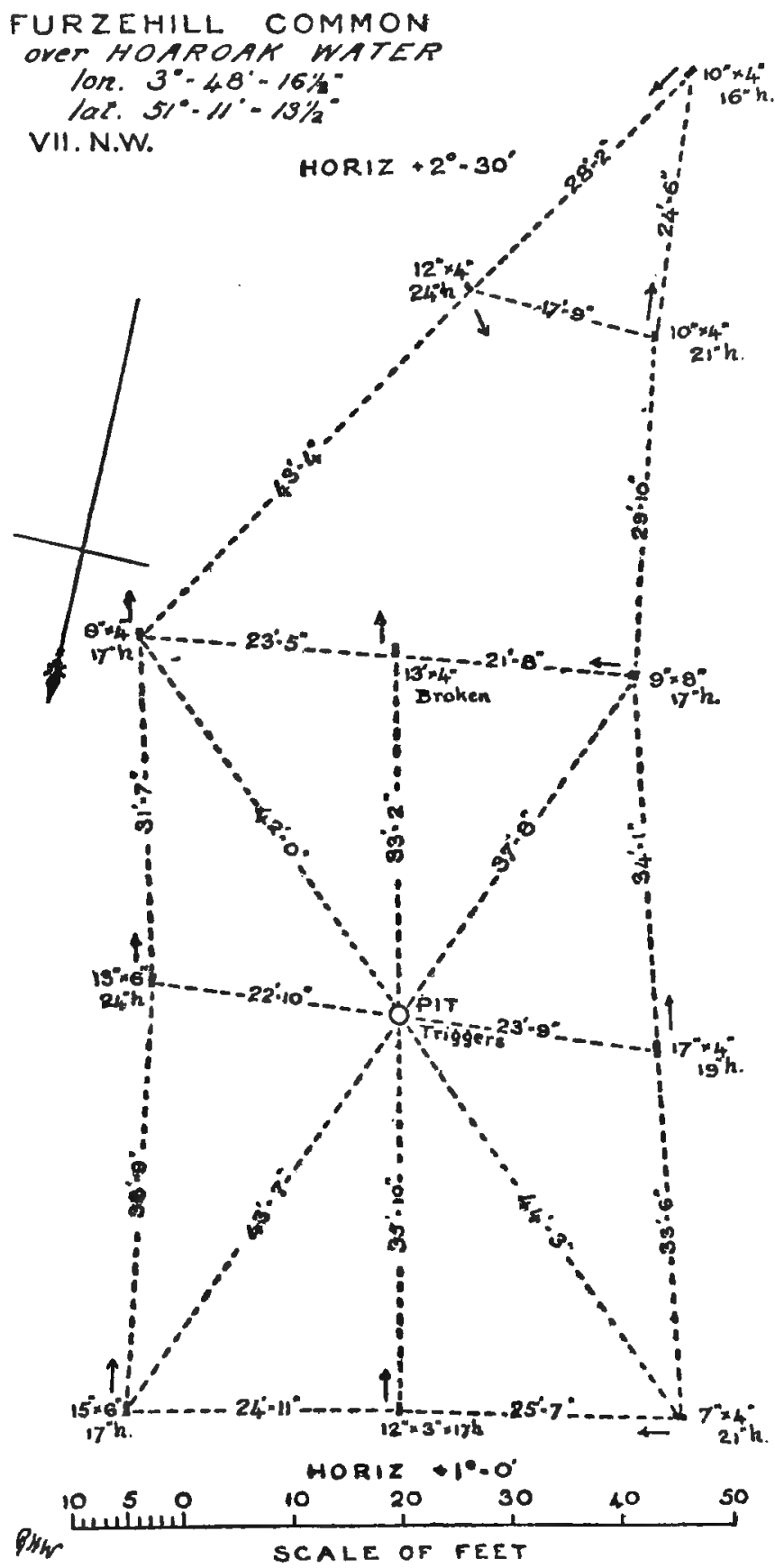

Fig. 27.-Parallelogram and Triangle, Furzehill Common, OVER Hoaroak Water branched from Exeter and others traversed the northern and southern regions of the county.

The very ancient roads entered Devonshire from the eastward. There was the coast road, which the Romans used as their route between Durnono-

1 These curious monuments, presumably coeval with the barrows, are being subjected to further examination, and it is to be hoped some additional light will be thrown on their meaning. 


\section{A HISTORY OF DEVONSHIRE}

varia (Dorchester) and Isca Dunmoniorum (Exeter) via Moridunumsupposed by some authorities to be either Honiton or Hembury Castle near by ; and there was the main line of the Fosse Way joining the other road somewhere near Moridunum. The united road passed through Exeter, continued in a direct line over Haldon to the ford of the Teign, near Chudleigh, crossed Dartmoor as the great central trackway to the vicinity of Tavistock, took the lowest ford on the Tamar, and finally passed along the backbone of Cornwall to Mount's Bay. ${ }^{1}$

The Romans doubtless greatly improved the old British trackways, but they appear to have done but little road-making, for the only evidence of this west of Exeter was rendered by the rebuilding of Teign Bridge in 1815 , when Roman foundations of a previous structure were discovered. This may mean either a new road tapping South Devonshire from Exeter to this point, or the improvement of a British route.

The Roman bridge over the Teign is supposed to be the furthest point in a westerly direction to which the Romans carried their strictly Roman road, or improved British road. Beyond this the road followed the devious winding and irregular course which characterizes a British trackway.

Lists of tumuli, hut circles, kistvaens, stone circles, etc., will be found appended to the article on the Ancient Earthworks of the county.

\section{Note on the Stone Rows of Dartmoor}

Small menhirs set in single, double, and even up to eight lines of stones, and usually starting from a circle of upright stones or from a cairn or kistvaen, and often ending in a similar manner, form the well-known Stone Avenues or Stone Rows. They are of varying lengths and point in no settled direction. The longest is a single row on Stalldon Moor (O.S. I I 3 SW.) which, starting from a stone circle, is clearly visible for a mile and a half-it probably extended for another three-quarters of a mile and terminated at a kistvaen. There are some fifty of these stone rows on Dartmoor. ${ }^{2}$ Although the particular meaning of these monuments is at present not definitely known, the recent attempt to invest them with an astronomical use is speculative and can hardly be borne out by a close study of these alignments.

\footnotetext{
${ }^{1}$ R. N. Worth in Trans. Devon. Assoc. xxiii, 59.

' For list of the more important stone rows see Rowe's Perambulation of Dartmoor, 1896, pp. 409-1 3.
} 


\section{ANGLO-SAXON REMAINS}

$7 \mathrm{HE}$ history of the county in pre-Norman days is detailed enough to explain the absence of any Anglo-Saxon remains dating from the pagan period. After pointing out that the West Saxons were already Christians when they reached the west country, Prof. Freeman in 1873 said of Exeter that whatever the exact date at which the city first became an English possession, it was with the driving out of the Welsh inhabitants under Athelstan (926) that it first became a purely English city. ${ }^{1} \quad$ Later in the same century this stronghold on the Exe was the chief bulwark of western England during the renewed Danish invasions of Athelred's reign, and it is no doubt to our Danish period that the only relic to be noticed here must be referred.

In 1833 the bronze sword-guard here illustrated was found below the foundations of the house of a plumber named Downe, in South Street, Exeter. It has been inadequately published, ${ }^{2}$ and deserves special notice, not only as the only relic of its kind from the county, but also for its intrinsic interest. The design, a pleasing variety of the fret or key-pattern, is somewhat different on the two faces, one of which has in the centre an interlaced triangle that is of frequent occurrence in the Viking period, both here and in Scandinavia. In itself the ornamentation can only fix the date within somewhat wide limits, as it is one of the commonest motives on stone monuments ${ }^{3}$ and illuminated
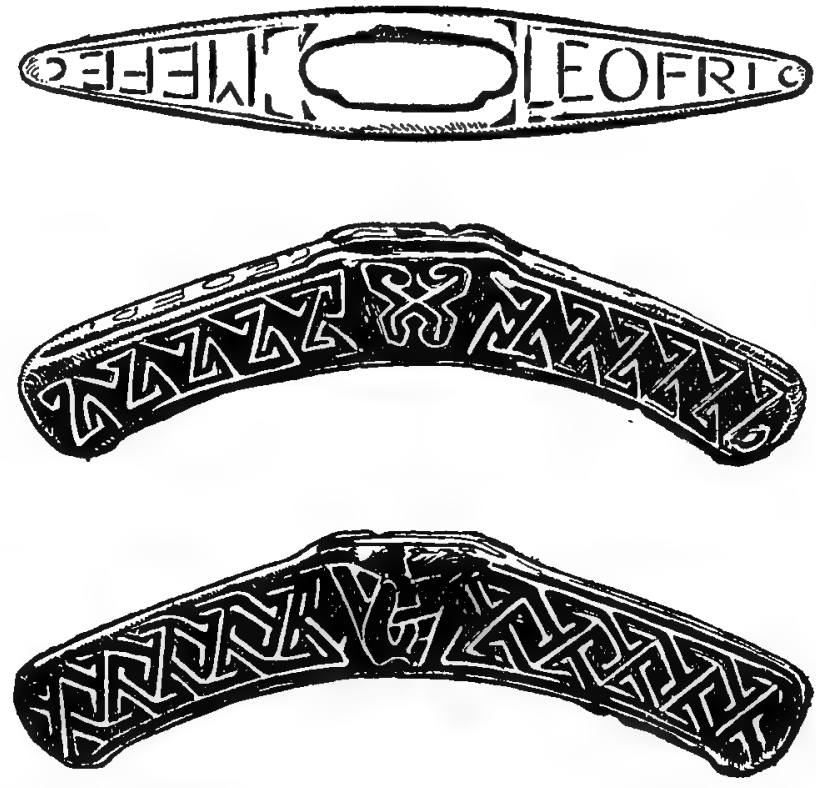

Inscribed Sword-guard of Bronze, from Exeter $\left(\frac{1}{1}\right)$. manuscripts in our islands, from the beginning of the eighth century-the date of the Lindisfarne Gospels-to the Norman Conquest. More precision might be expected

'Arch. Journ. xxx, 309.
W. T. P. Shortt, Syloa Antiqua Iscana, frontispiece No. 5 (inaccurate drawing) : a grotesque interpretation of the inscription is given on P. I 43.

${ }^{3}$ Allen and Anderson, Early Christion Monuments of Scotland, pp. 340-359. 


\section{A HISTORY OF DEVONSHIRE}

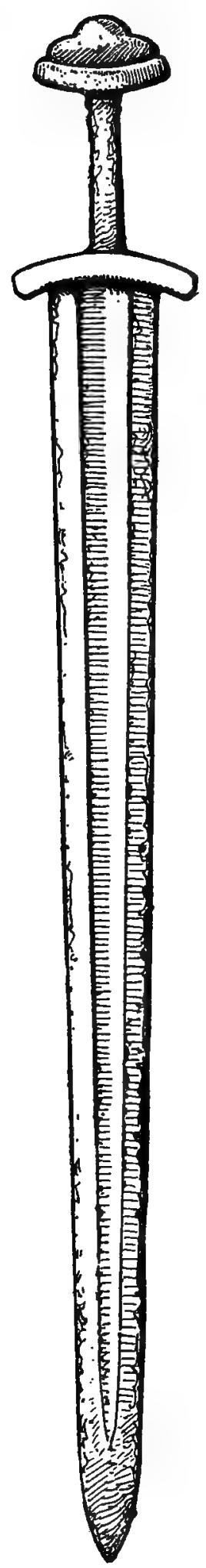

SWORD FROM

Thames at THE

Temple (1)

from the inscription, which is lightly engraved along the top of the guard. Though now somewhat obliterated by use, it was evidently intended for LEOFRIC ME FECIT (Leofric made me) ; and though the form of the legend is usual, the use of Latin for such a purpose is somewhat unexpected. It is possible to regard Leofric as the sword-smith or as the owner who had ordered the sword to be made; but the balance of probability is in favour of the former view. Thus on a sword-knife ${ }^{1}$ found at Sittingbourne occurs the owner's name on one side, with the maker's on the other, the two appearing as of equal importance; whereas on the famous Alfred jewel ${ }^{2}$ the name of the craftsman is suppressed. These and several other relics of the later Anglo-Saxon period have the inscription in Old English, and the difference of language suggests that the Exeter sword belonged to a religious foundation. Before the appointment of Leofric to the new see of Exeter in 1050, there was a monastery in the city, and one of its abbots (about A.D. 970) bore that name, ${ }^{3}$ which was indeed common enough; but, supposing the sword to have belonged to the abbot or to one of his followers, we should have expected a cross to precede the inscription," for such was the rule even on objects of secular use, and in the attestations of charters.

The original form of Leofric's sword may be deduced from an extant example of which the metal portion is complete, the bone or wooden grip alone having perished. It was found in the Thames off the Temple, and is now in the British Museum : the blade tapers and is $27 \frac{1}{2}$ in. long, with a wide groove down the centre of both sides. Its guard ( 4 in.) is slightly longer than the Exeter specimen $(3.3$ in.), the opening $(0.8$ in.) for the tang also being a little longer. The blade being 21 in. broad at the base, a heavy pommel was necessary as a counterpoise, and this generally took the form of three lobes, the base measuring in the present instance $3 \frac{1}{8}$ in. It is therefore permissible to regard the sword of Leofric as a specimen of one of the two leading patterns of the pre-Norman period, the other having a straight guard and pommel like those of pagan Anglo-Saxon times; but though we may fix the date between the establishment of the West Saxons ${ }^{6}$ in the west and the siege of Exeter by the Normans, there is at present insufficient evidence for a more precise chronology.

Arch. xliv, pl. xil, p. 332.

3 F. E. Warren, Leofric Missal, p. Ix.

2V. C. H. Somerset, i, 376.

- The leather sheath of Charlemagne's hunting-knife (so-called) has the cross and Latin inscription: Franz Bock, Karl's des Grossen Pfalzkapelle, p. 46; see also coinbrooch from Canterbury, with inscriptions Noming Domins and + probman FECIE (late 1oth century). Proc. Soc. Antiq. Lond. xix, 210.

${ }^{5}$ A.D. $74^{1-785}$, according to Journ. Brit. Arch. Assoc. xxxix, 259. 


\section{DOMESDAY SURVEY}

Account of the Exeter Book-The commissioners and their decisions on questions of title-The hundreds-Booklands and manors-Low level of assessment-Domesday measures-Lands of the king-Lands of churches and clerical tenants-The lesser lay tenants-Tenants in serjeanty-English tenants-Exeter-Other boroughs-The forest-Mills-Salterns and fisheries.

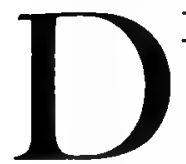

EVONSHIRE is one of the five western shires, the Domesday Survey of which is epitomized not only in the Exchequer Book but also in another volume known as the Exeter Book. The latter contains, besides a fuller abstract of the returns made by the undred juries, also a copy of the returns made by the commissioners appointed to collect the land-tax in $1084 .^{1}$ Neither of these books in describing the Devonshire estates mentions the hundreds to which they belong. In such circumstances identification becomes difficult. In some cases it would probably be impossible were it not for the Exeter Book, which, placing together all the estates held by the same owner in each hundred and adopting a regular rotation in the entries of the hundreds, and further grouping together the military squires and each of the three classes of the king's officials and treating their estates in a similar manner, supplies a clue to the hundred in which each should be sought. The importance of the Exeter Book therefore for purposes of identification cannot be overrated.

It is for this reason that the editors of the Victoria History resolved to make the text of the Exeter Book the groundwork for their translation in the case of the county of Devon, only supplementing it by the Exchequer where the pages have been lost and in places where there are important variants of phraseology or nomenclature. The Devonshire Association, it is true, published the Exeter version along with the Exchequer in its reprint of Domesday, which is now out of print ; but this was only done after cutting it up into paragraphs to make the entries correspond with the Exchequer entries, whereby its value for purposes of identification was in great measure lost. Hitherto the Exeter Book has been only available in the Record Commissioners' folio reprint, vol. iv, Additamenta, i 8 I 6.

The book itself, which is preserved in the Chapter Library at Exeter, is a quarto volume of 532 pages, made up of 103 separate booklets of various sizes, no one of them containing more than eight leaves, besides a number of single and a few double leaves." These booklets were originally unbound, one booklet or more being used for each of the larger tenants and a new page for each of the lesser ones. For a long time these booklets continued separate and were kept together with copies of the returns of the land-tax collectors of 1084, and of portions of the Exchequer Domesday referring to Wiltshire and Dorsetshire; but about the beginning of the fifteenth century they, or at least such of them as had escaped loss, were bound up in two volumes and paged. So carelessly, however, was the work

1 Freeman, Norman Conquest, iv, 683.

'On this subject see Mr, Whale's article in Trans. Devon Assoc. xxxvii, 246 seq. (1905). 


\section{A HISTORY OF DEVONSHIRE}

done that single leaves belonging to one tenant were in several cases misplaced among the booklets belonging to other tenants. Before the binding took place some whole booklets and leaves containing the fiefs of Robert Bastard, Richard son of Torolf, Hervei de Helion's widow, and Alvred the Breton, had entirely disappeared, as also a leaf containing the last five manors and a house in Exeter belonging to Ruald Adobed (Exch. fol. I I 5). In addition a leaf containing six of Walter de Dowai's estates (fol. 347) was long missing, having apparently been annexed previous to I 508 by Dean Willoughby. This leaf at the beginning of the nineteenth century was accidentally discovered among the papers of Sir John Trevelyan, bart. (in whose family it had found a home since at least 1656 , when the heiress of the Willoughbys married a Trevelyan), and was by him restored. But whatever may have been lost, Sir Henry Ellis was wrong in saying that the lands of Godbold, Nicholas the Crossbowman, Fulcher and Haimeric, together with eleven manors of the king's serjeants (servientes regis), are omitted in the Exeter Book. There are no such omissions; only instead of appearing under separate headings as in the Exchequer Book the entries referring to these officials are all grouped together in the Exeter Book. On the other hand the Exeter Book names 'Sotrebroc,' the land of Floher (fol. 459), which the Exchequer omits to mention. In 18 I 6 the binding of the two volumes was broken up ; the booklets were rearranged and rebound in one volume which received a fresh numbering of the pages. Unfortunately in the process one leaf got misplaced. It is now numbered fol. $40 \mathrm{r}$, but it ought to come before fol. 400.

As at present arranged the volume begins with the returns of the landtax collectors for Wiltshire, of which it contains no fewer than three copies (fols. I, 7, I3). A description of Shaftesbury, Dorchester, Bridport, and Wareham in Dorsetshire follows on fol. I I, Shaftesbury not being called by that name, but 'the town of St. Edward'; otherwise the description is given in the exact terms of the Exchequer Domesday. On fol. I 7 come the landtax returns for Dorsetshire and a description of a few manors in that county; but no fewer than forty titles of tenants-in-chief are absent. The returns of the landtax collectors for Devonshire follow (fol. 65), for Cornwall (fol. 72), and for Somersetshire (fol. 75), preceded by two distinct lists of Devonshire hundreds (fol. 63), neither of which observes the same order as that followed in the text. After a detailed description of the estates of these three counties on fols. 83 to 494 comes a list of 'Lands entered upon' (terrae occupatae) in Devonshire (fol. 495), in Cornwall (fol. 507), and in Somersetshire (fol. 508). On fol. 526 is the land-tax collector's return for two Somersetshire hundreds which had been omitted in the earlier portion and an account of the tax paid by the manors of 'Torna' and 'Torleberge' and by Mauger de Cartraio. At the end is a summary of the property of Glastonbury Abbey in Wiltshire, Dorsetshire, Devonshire, and Cornwall, and of the property of St. Petroc in the county of Cornwall ; the like of the lands of Ralf de Mortuo mari and Miles Crispin in Wales, of Robert son of Girold in Wiltshire, Dursetshire, and Somersetshire, and of the count of Mortain in Wiltshire, Dorsetshire, Devonshire, and Cornwall. An analysis of the sections relating to Devonshire has been printed by Mr. Whale in the Trans. of the Devon Assoc. (I896), xxviii, $402-63$, but a few of the identifications need reviewing. 


\section{DOMESDAY SURVEY}

It thus appears that the book is a composite work containing (I) a copy of the returns made by the land-tax commissioners in 1084 for the five western counties, commonly known as the Geldroll (Inquisitio geldi); (2) a digest from the actual returns of the commissioners appointed to take the survey (descriptio) of the kingdom for each hundred in the counties of Devon, Cornwall, and Somerset; and (3) a copy of the digest made by the Exchequer authorities from similar returns for the counties of Wilts and Dorset. Sir Henry Ellis considered it certain from the evidence of the record that the time when the tax was raised was the time of the survey; ${ }^{1}$ and it is clear that both the Exeter and the Exchequer digests must have been made after 1083 . For both of them show the king in possession of Queen Matilda's lands, and she died 2 November, ro83. Moreover, we have it on the evidence of the colophon at the end of the Exchequer volume containing the account of the eastern counties that the survey was made in I086, ${ }^{2}$ which is further supported by the famous passage in the AngloSaxon Chronicle of this year recording the execution and compilation of the survey. There is every reason for concluding that the Exeter Book was compiled in, or not earlier than, 1086, and probably not much, if at all, before the returns were sent off to Winchester.

The work of the king's commissioners was concluded when they sent in the original returns from the hundreds and their decisions thereon. The task of digesting these materials was work for other hands to do. The returns for Wiltshire and Dorsetshire were probably at first lodged with the bishop of Sherborne and then sent on to Winchester, where they were tabulated in the Exchequer form. The resting-place of the returns from Devonshire, Cornwall, and Somersetshire was with the bishop at Exeter, where no doubt the Exeter Book was compiled. The compilation probably took place under the direction of either the sheriff, Baldwin, or possibly Reginald de Valletorta, if we may accept the entry (fol. 97b) as correctly rendered: 'I Reginald have been paying $£ 24$ for Ordulf's land,' which clearly points to Reginald de Valletorta, who had Ordulf's land of Broad Clyst in charge for the king, and was paying for it $£_{24}$ a year (fol. 95). It is, however, more than doubtful if the use of the first person in this entry is intended, and it is far more likely that the 'ređđi' of the text is not intended for 'reddidi ' but for 'reddit.' It seems a reasonable conjecture that the original returns for Wiltshire and Dorsetshire not being at Exeter, the compiler of the Exeter Book, after entering the geld

\footnotetext{
1 The language used in reference to the withholding of headrents from Axminster manor (fol. $84 b$ ) suggests at first sight that the survey was antecedent to the tax. Honiton, it is there stated, has ceased to pay its headrent of 30 pence 'since the count of Mortain had it'; Charton has done the same 'since the bishop of Coutances had it'; Smallridge has not paid 'for the last I 2 years' or, as it is put under terre occupate, 'since Ralf Pomeray has had it,' nor Membury 'for the last 12 years.' From these statements it would seem that the survey for Devonshire was held twelve years after the spoils of conquest had been divided, unless Ralf did not receive his manors until 1074. Further, for the last eighteen years the canons of Rouen have withheld the headrent due from Rawridge. Now Ottery and Rawridge were given to the canons in I061 (Cal. of Doc. France, I), and eighteen years counting from 1061 tally with twelve years counting from 1067, and give 1079 or 1080 as the date of the survey, which incidentally agrees with Swereford's statement in the Red Book that the survey was taken in King William's fourteenth year (Red Book of Exch. Rolls Ser. i, 4 ; where, however, it is noted that it is uncertain whether Swereford intended to write xiiii or xxiiii) : on the other hand eighteen years from 1067 brings us to 1085 . The passage, though puzzling, cannot be regarded as sufficien: 'proof of the earlier date of the survey. See Whale in Trans. Devon Assoc. xxxv. 156.

2 'Anno milesimo octogesimo sexto ab Incarnatione Domini, vigesimo vero regni Willelmi, facta est ista descriptio non solum per hos tres conitatus [Essex, Norfolk, Suffolk], sed jam per alios.' Freeman, Norman Conquest, iv, 691 .
} 


\section{A HISTORY OF DEVONSHIRE}

collectors' accounts, had copies made of the already completed Exchequer digests for these two counties, while for Devonshire, Cornwall, and Somersetshire he had his digest made from the original returns. If Reginald de Valletorta was the official employed, it is probable that being a chief-tenant of the count of Mortain, he made it at the count's instance, and that it was made for reference in the west before the returns were dispatched to Winchester.

In tabulating the returns from the counties of Devon, Cornwall, and Somerset, several assistants were evidently employed. One of these, Richard by name, has in several places put himself in evidence. At the foot of fol. 316 he has written: 'Richard wrote the above' (b. scripsit Ricardus). On fol. 4I 4, between 'Ruald has a manor called' and 'Hanecheforda' he has inserted: 'Thus far R. has written' (Usq : buc scr psit $R$.). Several times at the end of a fief the word 'Finished' (Consummatum est) is found, as on fols. $209 b, 370 b, 3^{8} 7 b, 474 b$, and $494 b$. Once (fol. 490): 'Finished up to here'; but there is nothing to indicate that all these notes were made by the same hand, for it is certain that more than one scribe was employed. One uses $\mathcal{Y}$, the Runic contraction for et; another writes it $\&$, and even writes 'hab\&'; and hence it has been inferred, though apparently without sufficient reason, that one was a Saxon and another a Norman. There is also evidence of a supervisor who has added the word 'Verification' (Probatio) in the margin of fol. 317 , and to whose care is no doubt due the occasional belated entry of a manor which had been accidentally omitted in dealing with the hundred to which it belonged, and the not infrequent correction of names and numbers. In working, the first scribe would take the roll of one hundred, say for instance Hairidge, and extract from it all the estates belonging to one baron, say Baldwin the sheriff, whilst another scribe would take the roll returned from another hundred, say Wonford, and extract from it the estates belonging to another baron, say the count of Mortain. This done they would then exchange rolls, and thus the sequence of these two hundreds would be interchanged in the booklets of these two barons. The same interchange of the hundred sequence would again be in evidence whenever two scribes were simultaneously at work upon any two hundreds. Judging therefore by the sequence of hundreds we infer that the extracting was carried on by two scribes or by two or more sets of two each. ${ }^{1}$ In the case of Coldridge, Ermington, and Stanborough hundreds, as also in the case of Hairidge, Wonford, and Hemyock, ${ }^{2}$ the variations of sequence are more complicated. Perhaps a third scribe or the supervisor took a turn in the work of extracting. The handwriting and colour of the ink on fol. $153^{b}$ and $43^{6 b}$ are different from the rest of the MS.

It has been generally taken for granted that when the returns from the hundreds had been digested in the Exeter Book, that volume was forwarded to Winchester together with the original returns, where a further process of editing took place, resulting in the Exchequer text as we now have it. But for this supposition I cannot find a particle of evidence; whereas it is clear that parts of the Exeter Book were taken from the Exchequer Book. These parts of the latter must therefore have been made first. No doubt the

${ }^{1}$ See Mr. Whale's paper in Trans. Devon Assoc. xxviii, 391, and remarks thereon, ibid. xxxiii, $5^{83}$.

Trans. Devon Assoc. xxxiii, 583. 


\section{DOMESDAY SURVEY}

returns from the hundreds were forwarded from Exeter to Winchester, and from these returns the Exchequer Book was made direct; but they were not forwarded, it would seem, until the portions of the Exeter Book relating to Devonshire, Cornwall, and Somersetshire had been first compiled from them. Internal evidence goes to show that excepting the counties of Wilts and Dorset, which were incorporated in the Exeter Book from the Exchequer, the two books are entirely independent compilations.

The Exeter Book, for instance, has many items of detail which are entirely wanting in the Exchequer record, and reflects local pronunciation in different parts of the county as it appeared in the original returns ${ }^{1}$ in such variations of spelling as ferding, ferting, ferling ; or Alwius, Alwidus, Alivet for Alwi or Ælfwig; or Fitel, Vitalis, Vithelet for Vidley or Videlig; or Torgic, Torgis, Turgis, and Turginus for Torgey or Torgig. In two places it writes $\mathrm{P}$ for $\mathrm{W}$, Pillanda and Pediccheswella (fol. I 27b), where the Exchequer (fol. I02b) has Welland and Wedicheswelle, owing to the Exeter scribe's misreading the old English form of W as P in the Shirwell return. The Exeter scribe was clearly not a native of the county, for wherever he can he uses a hard instead of a soft sound. He writes Herchenbold for Erchenbold (fol. $2 \mathrm{r} \circ b$ ), Hermentona for Ermentona (fol. 85b), Hedric for Edric (fol. 294b), Halebrix for Alebric (fol. 33 rb), Hluperiga for Luperiga (fol. 397), harare for arare (fol. I 21 ), and hunius for unius (fol. 3 I 10 ).

He writes Melefort (fol. I 2 Ib), Kagefort (fol. I 3 I), Hetfelt (fol. I 83), Sanfort (fol. 290), Bradefort (fol. 291) for Meleford, Chageford, Hethfeld, Sanford, and Bradeford; Editda (fol. 93) and Eideta (fol. 946) for Eddida; and Guitda (fol. 93, 966) or Gutda (fol. 194b) for Githa, though the Devonshire pronunciation Geda and Gida has remained to tell its tale in two places (fols. 94, 96). Another peculiarity of the western scribe is that $O$ everywhere usurps the place of $U$ in proper names, Olpessa (fol. 1 32 ) for Ulpesse; Olwardesdona (fol. I 109 ) for Ulwardesdone; Olfaldeshodes (fol. 416b) for Ulfaldeshodes; Olveberia (fol. 3 I 3) for Ulveberie ; Olurintona (fol. 379) for Ulurintone ; Olveleia (fol. 294b) for Ulvelie ; Olmar (fol. 409b) for Ulmar; Ordolf (fol. 4I 2) for Ordulf; Aiolf (fol. 319) for Aiulf; Torstin (fol. 340b) for Turstan; Torgic (fol. 326b) for Turgis. Evidently the dictating scribe was a Norman, who like the Frenchman of to-day reads sunt as though it were written sont. Dr. Round has shown that Alfred was a common name in Brittany, but hardened into Alfrit or Alvrit. ${ }^{2}$

Whilst on the subject of pronunciation it may be well to point out that the names encountered in the pages of the record, when once the rules of pronunciation are grasped, become the familiar names of everyday life. Thus $c$ before $e$ and $i$, and occasionally before $a$, is used to express the sound now written $c b$, so that 'Cilletona,' 'Cedelintona,' 'Calvelia,' and 'Cagefort' should be read Chilton, Chillington, Chawleigh, and Chagford. Chon the other hand is used to express our $k$, so that 'Chentona,' ' Chenemetona,' and 'Chemeworda' should be read Kenton, Kilmington, Kimworthy. $S$, or more frequently double $s$ or $s c$, and at the beginning of a word $E s$, are used to express

${ }^{1} \mathrm{Mr}$. Whale, in Trans. Devon Assoc. xxxii, 523 , considers these variations, which he calls errors, due to the want of distinctions in the rolls.

${ }^{2}$ Round, Feudal Engl. 327, n, 26. 


\section{A HISTORY OF DEVONSHIRE}

our sh, so that 'Aissa' and 'Essa,' 'Selvestan,' 'Scage,' 'Scapelie,' and 'Eselingeforde' should be read Ash, Shilstone, Shaugh, Shapley, and Shelingford.' The initial $r$, so common in Devonshire names, is either omitted altogether, or an $H$ is substituted, so that 'Erticoma,' 'Aeidestan,'" 'Hernescoma' should be read Yarcombe, Yardlestone, Yarnscombe. 'Alwineclancauele's resolves itself into Yalwin's or Alwin's Lan Goel, now called Yeowellston. The common termination -wortby is written -orda or -urde, so that 'Begeurda,' 'Hocoorda,' ' Fereurdi,' 'Colteshorda,' 'Olfereordi' should be read Beaworthy, Hockworthy, [Al] fardisworthy, Coltsworthy., Woolfardisworthy, but in a few cases -worthy has since been softened into-ford. For instance, 'Blacheorde' is now Blatchford, 'Odeordi' now Woodford, ' Waleurda' now Wallaford, 'Hochaorda' now Hockford, 'Wiborda ' now Womberford. Conversely, 'Chileforda' is now Chilsworthy. $\mathcal{T}$ also is used not only to express $t$, but the hard $t$, so that 'Teweberie,' 'Torna,' 'Torlestan,' 'Torneberie,' 'Tresetona' should be read Theoborough, Thorn, Thurlestone, Thornbury, and Thrushelton. G, too, represents the soft sound of $y$, just as 'leap-gates ' was formerly written ' leap-yeats."

Changes in the names of places have also been frequent in Devon, not only to distinguish neighbouring estates which originally bore the same name, ${ }^{5}$ but also by custom and use where no such necessity existed, ${ }^{6}$ or from the places where churches were built. ${ }^{7}$

Glancing at the work of the commissioners appointed to inquire concerning the lands of England, it would appear from the abbot of Tavistock being disseised by them of Werrington that they had power not only to inquire but also to determine subject to a right of appeal to the king. ${ }^{8}$ In the case of Werrington the appeal resulted in the abbot's reinstatement by William Rufus. But it was otherwise in the case of Newton St. Cyres (fols. I I 7, 483), which Donne claimed to hold of the king. The bishop produced his charters proving that it belonged to his church in King Edward's time; more than that, he alleged that his claim to it had been allowed in court in King William's time. But for all that the commissioners entered it as held of the king, and their decision seems not to have been set aside on appeal.

Two other instances may be referred to in which the commissioners determined a question of title. They are interesting because they incidentally show that William de Walvil was at some period most probably steward of the royal manors in the county of Devon. On fol. 1266 it is stated that

1 Trans. Devon Assoc. xxvi, 149.

'The Record Commissioners' text has twice misread the initial $A$ as $D$, viz., in Aeidestan (fol. $403 b$ ), and Aisa (fol. 394).

${ }^{3}$ Trans. Devon Assoc. xxviii, $465, n .3$.

'Ibid. $473, n, 18$.

${ }^{5}$ Thus there are three Putfords in the record (fols. 2 I 1, 335b, 399), which now bear the names of East Putford, Churston, and Cory respectively ; two Doltons (fols. 295, 462), one now called Iddlecot, after its tenant Edric ; two Poltimores (fols. $469 b, 307 b$ ), one now called Cutton, after its tenant Cota ; three Tedburns (fols. $305 b, 306,403$ ), now called Town Barton, Colley, and Farwood respectively ; many nimets or intakes which have taken their owner's name, such as Hampson from Hermer (fol. $389 b$ ), Merdis nimet from Merta (fol. 390b) ; many Otris, now distinguished as Ottery St. Mary (fol. 195), Upottery (fol. 342), Raplinghayes (fol. 340), Warin's town or Weringston (fol. 400), Doda's town or Dotton (fol. 308b), Ivedon (fol. 405 ), etc.

'The most remarkable instance of this custom is 'Colebroca' (fol. 216 ), which appears in the fee-lists as Holbrook Grindham. In the official records of the Duchy of Lancaster as Withien Furze, and at a later time as Holbrook Moor, but is now called Denbow after its thirteenth-century tenant Denebaud (Trans. Devon Assoc. xxxv, 290).

7 'Thus 'Dunewinesdona' is now called St. Pancras; 'Notona' is now called Churchstow ; ' Bredefort' is now Christow.

${ }^{8}$ Round, in V.C.H. Essex, i, 411 , states the same as his conclusion. 


\section{DOMESDAY SURVEY}

Bristric, son of Camme, or Cammesons, had a virgate of land which T.R.E. had become independent of the king's manor called Braunton, but 'William de Walvil restored it to Braunton in King William's time.' The other passage is on fol. $85^{b}$, where it is stated in reference to Diptford, "To this manor another manor called Farley was added in the time of William de Walvil.'

Excepting churches, which had to show that they were in possession of the estates claimed by them on the day on which King Edward was alive and dead, all claimants to property were required to establish their title either by naming the person who had put them in possession (liberator), such as the sheriff, or appealing to some other person as voucher (advocatus) who had the king's authority, or else by producing the king's writ (breve). ${ }^{1}$ But there were two ways by which it was attempted to circumvent this requirement ; the first was by entering upon (occupare) and claiming as appurtenant to some manor of which the claimant was in lawful possession an estate which in King Edward's time had been held independently (pariter or in paragio). The oft-recurring phrase used of land that it was held pariter, which is occasionally equated with pro manerio or libere, means no more than that the land to which it applies was not village or peasants' land subject to services, but an independent holding or bookland. Whenever by usurpation an independent bookland had been entered upon and made dependent on some other estate, it figures in Domesday as 'an estate entered upon' (terra occupata). Besides noting the fact in the body of the text, the Exeter Domesday gives a complete separate list of 'estates entered upon.' The right to these was no doubt specially reserved for the king to settle, and was usually a matter of payment.

The other method-which, as Dr. Round has stated, ${ }^{2}$ was resorted to, to conceal a defective title-was the allegation of exchange. Some exchanges were no doubt quite genuine transactions, such as the acquisition by the crown of Ermington and Blackawton (fol. $85^{b}$ ) by exchange with Walter de Dowai for Bampton (fol. $345^{b}$ ), or the acquisition by the count of Mortain of the castle of Cornwall (fol. I08) from the bishop of Exeter in exchange for Haxon and Benton, though in this case the exchange may not have been altogether voluntary. But when Ruald Adobed is stated to hold West Panson (fol. 343) by exchange, one may suspect that possibly Ralf de Pomeray made the exchange because his title was not flawless. ${ }^{3}$ And when of two of the king's principal officials it is stated that Nicholas the crossbowman has acquired Greenslinch (fol. 469b) and Bramleigh (fol. 473) by exchange, and William the usher has acquired Cocktree and Crook Burnel (fol. 475), Cadeleigh, East Raddon, Blackborough Boty, Bolham, Ilesham, and Sutton Lucy (fol. 475 seq.) all by exchange, we may well ask whether all these exchanges were genuine, or not set up to conceal irregular possession by force. Was not Raddon, for instance, filched from a church tenant ? ${ }^{4}$

Passing from general remarks to particular points, the first to deserve attention is the position of the hundreds in this shire. With one exception

${ }^{1}$ Freeman, Norman Conquest, v, 758 . In Exeter Domesday, fol. 346, Walscin calls the king to vouch for his holding Diptford of the queen.

V.C.H. Essex, i, 386.

3 Exeter Domesday, fol. 497, Ralf de Pomeria entered upon a manor called Panson, and gave it to Ruald in exchange for 'Brochelande' and 'Radiz.'

'It was held T.R.E. by Edward the priest. 


\section{A HISTORY OF DEVONSHIRE}

these are the same to-day as they were at the time of the survey, but the names of several have been changed owing to the place of meeting being changed. 'Walchetona' has become Roborough, 'Sulfretona' Hairidge, 'Alleriga' Ermington, 'Dippeforda' Stanborough, 'Cedelintona' Colridge, 'Mertona' Shebbear, and 'Carsewilla' Haytor. 'The one exception to entire identity is the hundred of Bampton, which appears to have included several estates to the west of the River Exe which now belong to the hundred of Witheridge. The grounds for saying so are, first, that otherwise the total number of hides in Witheridge hundred would exceed the total named in the Geldroll by $6 \frac{1}{4}$ hides, whilst the total number in Bampton hundred would fall short of the total named in the Geldroll by the same amount; and, further, that the Geldroll shows the bishop of Coutances as a landholder in Bampton hundred with an allowance of I hide $3^{\frac{1}{2}}$ ferlings for his demesne in that hundred (Geldroll, fol. 69, A, 3), whereas not a single one of the bishop of Coutances' estates lies in the present hundred of Bampton. It is suggested ${ }^{1}$ that six of the bishop of Coutances' estates (fol. I 33), as also divers estates of others now forming the parishes of Oakford, Stoodleigh, and part of Washfield, belonged to Bampton hundred, and were separated from it in consequence of the exchange with Walter de Dowai by the crown." Mr. Whale has accounted for the shortage in the assessment of Bampton hundred by suggesting that an older hundred called 'Hertesberie' was then united with it. But apart from the fact that the older hundred next Bampton was called Molland, ${ }^{3}$ and that it contained at least I 5 hides, which would make the united hundred err more by excess than it now does by shortage, the exemption allowed to Walter de Dowai in Braunton and Shirwell hundred (Geldroll, fol. 66, A, 4) requires 'Hurtesberie' (fol. 345) or Berrynarbor to be in Braunton, not in Bampton hundred. Besides, his explanation leaves the difficulty of the bishop of Coutances' estates in Bampton hundred untouched."

Although with the exception named the general outline of the hundreds in Devon has remained unaltered since the time of the survey, ${ }^{5}$ yet hundreds have been in several cases grouped together, and in one case a hundred has been split into two. South Tawton hundred is now united with Wonford, Werrington with Black Torrington, Bampton with Uffculme, South Molton with North Molton and Molland, East and West Budleigh with Ottery, Tiverton with Halberton, Axminster with Axmouth. Even before the time of the Geldroll Shirwell had been combined with Braunton, and North Tawton with Winkleigh. Since then Moreton and Carsewell have been united to form Haytor hundred, whilst on the other hand the Domesday hundred of 'Listona' has had the new hundred of Tavistock severed from it since II $4 \cdot^{6}$

The hundred assessments show a curious combination of minute assessment on the one hand and 'a haughty treatment of small numbers' ${ }^{7}$ on the other. Thus we have assessments recorded of Smitham (fol. 2 I I $b$ ), 'Liteltrorilande' (fol. 2 I 2), although only $\frac{1}{3}$ ferling each ; those of 'Lidefort' (fol. 235), Newton (fol. $3^{89 b}$ ), Hill and Combe (fol. $47^{\text {I } b}$ ), although only

1 Trans. Devon Assoc. xxx, 454. 'See fol. $85 b$.

4 Ibid. xxxvi, 366.

7 Maitland, Domesday and Beyond, 476.

\footnotetext{
${ }^{3}$ Trans. Devon Assoc. xxxiii, 591.

${ }^{6}$ Oliver, Mon. 95.
} 


\section{DOMESDAY SURVEY}

$\frac{1}{2}$ ferling each. But the military tenants of Tavistock, who really had I $\frac{1}{2}$ hides and $\frac{1}{8}$ ferling, ${ }^{1}$ are said to have only I $1 \frac{1}{2}$ hides (fol. 176 ). Woolfardisworthy Spenser (fol. $4 \mathrm{I} 66$ ) is said to pay geld for I virgate $3 \frac{1}{2}$ ferlings, but the contributions of the lord and villagers together come to 2 virgates. Farworthy (fol. 388 ) is said to pay geld for I virgate, whereas the combined assessment of its parts is only 3 辛 ferlings. The military tenants of Bampton (fol. $23 \mathrm{Ib}$ ) are stated to hold I hide, but the particulars given show that they held only $3^{\frac{3}{4}}$ virgates. The same contempt for small fractions shows itself in the hundred totals. It tells both ways. The I hide allowed to the feegatherers must have been intended to provide against loss.

The above data seem to indicate that the position of the hundreds in Devon was somewhat different from what it was in other counties. Devonshire was won by the Saxons after they had become Christians by a series of peaceful acts of penetration varied by occasional outbreaks of hostilities, each link in the series being the acquisition of some new but undefined territory as an additional lordship of the crown. To each tract of territory thus won a fixed quota of hides seems to have been allotted as its assessment value, and the amount to have been afterwards distributed by subpartitionment ${ }^{2}$ as booklands were granted out to individual owners. To the booklands thus granted out, or rather to the owners of such booklands when grouped together for military, judicial, and administrative purposes, the term 'hundred' was applied, whilst the portions still cultivated as folkland under the king were known as the king's demesne or lordship (dominicatus). ${ }^{3}$ The hundred was therefore an aggregate of disconnected booklands often separated from one another by uncultivated areas called the forest, or by booklands reclaimed from other centres. This circumstance will explain how Delworthy and Yarnscombe, although locally situated in the midst of Fremington hundred, belong as outliers to Hartland hundred; how Butterleigh in the midst of Hairidge hundred belongs to Cliston hundred; how Bratton Fleming in the midst of Shirwell hundred belongs to Braunton, and conversely how Pickwell in the midst of Braunton hundred belongs to Shirwell hundred; how East and West Buckland lying between Shirwell and South Molton hundreds belong to Braunton; how the Teignhide district containing Combe and Stokeinteignhead, although lying on the south side of Exminster and Teignbridge hundreds with outliers of its own, is nevertheless part of Wonford hundred, as are also its outliers, the two Ogwells and Hobbin, and also Sigford, Bagtor, and Horridge in Ilsington, situated in the very middle of Teignbridge hundred; how Worth and Washfield which lie between Tiverton and Witheridge hundreds belong to West Budleigh; how Emlet in Woolfardisworthy Spenser in the midst of Witheridge hundred belongs to North Tawton hundred; and how Thorncombe and Holditch lying in the county of Dorset belong to the hundred of Axminster in Devon. ${ }^{4}$

Passing from the hundreds as units of settlement to the sub-units, the land booked or bestowed by charter ${ }^{5}$ upon individual owners, it is of supreme

'Trans. Devon Assoc. xxvii, 193, n. 48.

2 Round, Feudal England, 97.

3 Trans. Devon Assoc. xxxiii, 571 .

' Ibid. 575.

'In fol. 135 'bookland' is written as the equivalent or substitute for 'mansio.' In Maitland, Domesday and Beyond, I $54, n .1$, 'bocland ' = 'terra hereditaria,' ' terra testimentalis,' ' terra libera,' and even 'feudum.' Round, in $V$. C. H. Essex, i, 410 , shows that 'terra' is often used as a substitute for 'manerium.' 


\section{A HISTORY OF DEVONSHIRE}

moment to remember that in Devon these booklands consisted of clearly defined areas sometimes adjoining one another, but more often separated by waste lands ${ }^{1}$ which formed part of the royal forest until the men of Devon in I 204 paid 5,000 marks to have the county disafforested up to 'the regards of Dartmoor and Exmoor." These booklands were by no means manors in the legal acceptation of the term, ${ }^{3}$ only very few of them having free tenants; indeed the original grants were rather grants of townships or tithings which were subsequently split up into sections, the maneria or homesteads ${ }^{4}$ of the record. We see, however, manors in the making, as when lands which had been free booklands (held pariter or in paragio) before the Conquest were added to other homesteads as dependencies. Some of the booklands were very small, not more than 30 acres. But whatever the size might be the basis of a bookland or squire's estate, or Rittergut, as the Germans would call it, was the lord's hall, sele, ${ }^{5}$ or homestead (manerium), called also the court (curia), with its appurtenant rickyard or barton and a certain quantity of land for the support of the sitting tenant, known as the lordship 'inland' or demesne. With this hall was combined as the second element in a bookland another definite area of land known as the village land, 'outland,' peasants' land, or Bauergut, as the Germans would call it, occupied by the ancient cultivators of the soil upon condition of cultivating the lord's land for him. ${ }^{6}$ This second element supplied as it were the capital for cultivating the lord's land. These two features are present in all the Devonshire manors, though in two cases the demesne has disappeared because the whole was farmed by the villagers, ${ }^{7}$ and in three others the lord has absorbed the villagers' land into the demesne. ${ }^{8}$ In one case a villein has the whole in demesne.

One feature is, however, peculiar to the Devonshire booklands. The lord's land is not as in other parts of England intermixed with the villagers' land, the lord having strips in the open field where the villagers have strips also; but the lord's land is apart and often at some distance from the villagers' land. Thus Cottle's barton, the lordship, lies more than half a mile from North Tawton township and village. Totleigh barton, the lordship of Black Torrington, is an outlier of the parish. The north manor and town of Collompton, though detached from Silverton, is the demesne manor of Silverton hundred. Knightshayes lies away from Bolham, Colcombe from Colyton, Delamore from Cornwood, Chumwell from Bratton Fleming, Hulham barton from Hulham village, ${ }^{10}$ and Newton and Ford the demesne from the village and land of Drewsteignton. Plympton Earl and Dodbrook are demesnes to

1 Trans. Devon Assoc. xxvi, 143. T Pipe R. 6 John. The charter in Trans. Devon Assoc. xxxiii, 605.

3 Maitland, Domesday and Beyond, 64, 91.

'Called 'Knystesmetehom' in an old English document in Dugdale, Mon. ii, 477.

3 Maitland, op. cit. 108. 'Then there were two halls, now it is one manor.' Thus Sele alias Zeal Monachorum, i.e. the Monks' Hall, is the present name for the monks' nimet or intake of Domesday, fol. 182 . In Poillei's gift to the monastery of Séez (Cal. of Doc. France, 235) we hear of a certain manor called Harrowbear (Baraberga), part or member of Buckland (monachorum), with its hall in that township (villa). In the Berkshire Domesday, fol. 63, complaint is made of the removal of the hall. Freeman, Norman Conquest, v, 771 ; Maitland, Domesday and Beyond, rog.

- Trans. Devon Assoc. xxvi, I40; Maitland, op. cit. 58.

7 At Gorhuish, fol. 292, and Culbeer, fol. 3146 .

${ }^{8}$ At Endscott, fol. $122 b$, Cheldon, fol. $310 b$, and Loviston, fol. 388b. In a lease of Ottery St. Mary to the men of the place in 1145 (Cal. of Doc. France, No. I I) one stipulation is that 'they will cause to be restored to the lordship whatever belonged to it of ancient right.'

${ }^{9}$ At Woodscombe, fol. 404 .

${ }^{10}$ Trans. Devon Assoc. xxvii, 404. 


\section{DOMESDAY SURVEY}

which Herdwick and Portlemouth respectively belong as outlands. Another peculiarity is the small assessment laid on the Devonshire booklands. Not only does the virgate take the place of the hide elsewhere, ${ }^{1}$ so that manors are assessed at I hide I virgate, ${ }^{8}$ or 5 virgates, which would elsewhere be assessed at 5 hides, but manors of unusual size are assessed at a single virgate, and ordinary manors at a ferling, $\frac{1}{2}$ ferling, or $\frac{1}{3}$ ferling. Thus Chittlehampton with some 2,700 acres is assessed at I virgate; Stoke Rivers with about I,200 acres at 3 virgates; Sampford Spiney with nearly 1,200 acres at half a hide; Dartington with over I,400 acres at I virgate; Pyworthy with roughly I, 500 acres at 3 virgates; and among demesne manors of the crown North Tawton with approximately 2,500 acres at $\frac{1}{2}$ virgate; South Molton with close on 3,600 acres at $\mathrm{I} \frac{1}{2}$ virgates; Hemyock with just over $\mathrm{I}, 400$ acres at I virgate. On the other hand one or two exceptionally high assessments are met with in the east of the county. Woodbury with about 3,200 acres is assessed at ro hides; Broad Clyst with some 3,000 acres at $9 \frac{1}{2}$ hides; Holcombe with something like 2,000 acres at 9 hides; Standon with apparently only 30 acres at I virgate; an Otterland of about 40 acres at I virgate; and Clyst William with little more than 40 acres at half a hide.

It is now generally admitted that whatever the hide may have originally connoted as being the land of one family, yet as ordinarily used in the pages of our record it denotes simply a unit of assessment. The hide is the unit which paid 2 shillings towards the normal king's geld, the virgate is a quarter of that unit, the ferling is a quarter of a quarter or one-sixteenth of the unit. ${ }^{3}$ What is more, there is no necessary connexion between area and assessment, the assessments having been originally fixed by partitioning among those liable to contribute an assessment levied on the hundred." Once only is the acre met with as a measure of assessment, having the value of one-thirtieth of a virgate," namely, at Sherwood (Haiserstona, fol. I 82b), where the abbot of Bucfast was assessed for $\mathrm{I} \frac{1}{2}$ ferlings and 3 acres. On the rare occasions when hide and virgate are not used to describe assessment the question arises, what is the area they denote? Thus in Ottery St. Mary (fol. I95) there are stated to be ' 200 acres of meadow and 6 hides of pasture'; in Combe Raleigh (fol. 3486) there are ' 50 acres of wood(land) and I hide of pasture' ; in Smallridge (fol. 343), ' $3 \mathrm{I}$ acres of pasture and $\frac{1}{2}$ hide of wood(land).' Similarly in Lipson (fol. 222) there is ' I acre of meadow and I virgate of pasture'; in Little Cadbury (fol. 4I 5b), ' 4 acres of meadow and 3 virgates of pasture'; in Doddiscombsleigh (fol. 468b), 'I virgate of wood(land)'; in Middle Washburton (fol. 396), ' I virgate of pasture'; and in Lowly (fol. 4686) there are' 20 acres of pasture and I ferling of coppice.'

1 In Trans. Devon Assoc. xxxiii, 601, it is shown that Ipplepen, assessed in Domesday at $15 \frac{1}{2}$ virgates, is described in a charter of 956 as $15 \frac{1}{2}$ hides of land (Cart. Sax. iii, 132). Eyton, Key to Domesday, Dorset, 14; Round, Feudal England, 93.

${ }^{2}$ For instance Lympstone, Blackborough Boty, Pirzwell, and at half that quantity Fenacre, Magnelege, Church Putford.

${ }^{3}$ For proof refer to Filleigh (fol. 300b), where the Exeter Book says it was assessed for 4 virgates, the Exchequer for I hide; to Riddlecombe (fol. 389 ), where 6 ferlings $+2 \frac{7}{2}$ virgates are stated to make up I hide; to Church Putford (fol. 4I 2 ), where $\frac{1}{2}$ hide $+\frac{1}{2}$ virgate are equated with I virgate and I ferling + I virgate and I ferling; to Alverdiscott (fol. 2 I $2 b$ ), where 6 virgates $=1 \frac{1}{2}$ hides.

Maitland, Domesday and Beyond, I 20, 206, 451; Trans. Devon Assoc. xxxiii, 573.

${ }^{5}$ Round, Feudal England, 38. 


\section{A HISTORY OF DEVONSHIRE}

There is ample evidence to show that in post-Domesday times a ferling of land in Devon contained 16 acres or a quarter of a plough-land, and an early survey of Berry Pomeroy in I 292, ${ }^{1}$ when compared with the Domesday description, shows that it contained the same area at the Conquest. The demesne is there stated to have consisted of ' 16 ferlings of land of which each one contains 16 acres, each acre being worth 3 pence yearly, and so each ferling is worth 4 shillings yearly.' The i 6 ferlings of I 292 represent the 4 plough-lands of 1086 , so that 4 ferlings of 16 acres each went to the plough-land. ${ }^{2}$ In Exminster again (fol. 83) a certain priest was allowed to hold a ferling of land which must be the vicarial glebe of Exminster, exactly I 6 acres, for the 100 acres of Kenbury represent the $\frac{1}{2}$ hide held by the priests of Exminster (Geldroll, fol. 69, A I I). If the areal virgate was equivalent to 4 areal ferlings, 64 acres should represent the virgate of woodland or pasture used as a measure of area, and 256 acres the hide of woodland or pasture when also used as a measure.

It has been too readily assumed that because in some parts of England the plough-land (carucata terrae) or land for one plough (terra ad unam carucam) represents the arable land of a hide, therefore the plough-land must necessarily always represent 120 acres. ${ }^{3}$ For Devon, the facts wherever they can be tested point to a different conclusion. In the case of Berry Pomeroy already referred to 4 plough-lands of Domesday are represented in 1292 by $16 \times 16$ or 256 acres, i.e. 64 acres to a plough-land. ${ }^{4}$ The cultivated area of Stockleigh Pomeroy was, again, $35^{\circ}$ acres in I 292, ${ }^{5}$ while in our record it is described as land for 6 ploughs, giving $58 \frac{1}{3}$ acres as the extent of a plough-land there. Again, the record describes Beenleigh (fol. $42 \mathrm{I}$ ) as land for 4 ploughs; but the hundred jury in 1243 describe it as 14 ferlings, ${ }^{6}$ equivalent to 224 acres, giving 56 acres to the plough-land. Newton Tracey (fol. 3886 ), a manor hemmed in by estates belonging to other honours, contains 336 acres. The record describes it as consisting of 4 plough-lands and $\mathrm{I} 3$ acres of meadow, wood(land), and pasture. This gives 323 acres for 4 plough-lands, or $8 \mathrm{I}$ acres each, supposing every acre to be cultivated, and there to be neither roads, hedges, nor waste. Clannaborough contains 874 acres, and includes the two Domesday estates of 'Cloenesberga' (fol. 295b), and Walson and Thorn ('Nimet,' fol. 296). The two contained ro plough-lands and 54 acres of meadow, pasture, and wood(land). In this case the division gives exactly 82 acres to the plough-land, including roads, merebalks and linches. Ringmore and Shaldon form the parish of St. Nicholas in Teignhide, containing 580 acres. The Domesday 'Rumor' (fol. 304b) contains 8 plough-lands and 18 acres of meadow. This gives 562 acres for 8 plough-lands, or 701 acres each. Other examples might be quoted, ${ }^{7}$ showing that whatever the size of the plough-lands may have been in other counties, in Devon it was roughly 64 acres, or 80 acres including

1 Testa de Nevill (Rec. Com.), p. I $85 a$; Trans. Devon Assoc. xxviii, 367.

2 Mr. Chope, in Trans. Devon Assoc. xxxiv, 420, has quite independently arrived at the conclusion that the plough-land was about 60 acres.

${ }^{3}$ Mr. Whale, in Trans. Devon Assoc. xxxv, 663. Plough-lands and ploughs do not always correspond. In North Tawton the king has 3 plough-lands and 3 ploughs. In Heavitree there are 2 plough-lands and 2 ploughs. But at Chittlehampton Godwin has 5 plough-lands, but only 3 ploughs.

Trans. Devon Assoc. xxxiii, 598.

- Testa de Nevill (Rec. Com.), I92a: Richard de Benlegh has I fee in Benlegh, whereof Nic. de Molis has 5 ferlings of land and himself 9 ferlings.

${ }^{7}$ Trans. Devon Assoc. xxxiii, 599. 


\section{DOMESDAY SURVEY}

roads, hedges, and waste. On this basis the total acreage accounted for in the Domesday Survey of Devon is, roughly, 743,320 acres, or about half the present total. The present total, however, includes not only a large number of manors of post-Domesday creation, which were waste and part of the forest in 1085 , but considerable tracts which are still untilled, Dartmoor Forest and other commons, river beds, marshlands, and the foreshore. Advocates of a larger area for the plough-land are apt to forget that if they are right no place remains for the forest which extended over all Devon until King John's time, the Domesday booklands being only clearances of limited areas.

There is no reason to think that the acre in Domesday times differed from the acre as fixed by the Statute 33 Edw. I, st. 6: "When an acre of land containeth 40 poles [i.e. 220 yards] in length, then it shall be in breadth 4 poles [i.e. 22 yards].' "In the open field, where the lie of the ground allowed it, the acreman first measured off 220 yards for the furrowlong and then 22 yards in breadth for the villager entitled to I acre, I I yards for one entitled to $\frac{1}{2}$ acre, and $5 \frac{1}{2}$ yards for one entitled to $\frac{1}{4}$ acre. Hence when used as a long and not a square measure the acre was 22 yards, and this is the Domesday use. At Abbots Kerswell there is ' pasture 5 furlongs in length by 30 acres in breadth,' i.e. 30 times 22 yards in breadth; at Cullicombe is ' woodland 20 acres in length by 2 in breadth,' and at Throwleigh is ' pasture $\frac{1}{2}$ league in length by 4 acres in breadth.'

Placing Io acre strips side by side a perfect square is obtained, measuring 220 yards every way. This is the quarantine or furlong of land. Twelve quarantines or square furlongs whether placed in a single row or in two rows of six each or in three rows of four each, described in Domesday phraseology as 'taking length and breadth into account' (inter longitudinem et latitudinem), make a league (leuca) or I 20 acres, the normal length of which is therefore I 2 times 220 yards or I $\frac{1}{2}$ miles, whilst the normal width is I furlong or 220 yards. $^{2}$ Mr. Eyton ${ }^{3}$ has correctly observed that where the phrase 'taking length and breadth' is employed, areal leagues of 120 acres each are intended; but that when wood(land) or pasture is stated to be so many leagues long and so many leagues broad, the league must be reckoned as $I_{2} \frac{1}{2}$ miles. He further endeavoured to deduce the exact acreage of the woodland and pasture by multiplying the length by the breadth; but it is clear that where so large a unit is used the estimates of the Domesday commissioners must have been extremely rough; and moreover, while the product of the average length and breadth would yield a total representing the acreage sufficiently accurately, it is obvious that if the measures given were the greatest length and breadth of irregular blocks the product would be greatly in excess of the true area. It is therefore inadvisable to attempt any calculation of the amounts of woodland and pasture assigned to the several manors.

In one place the seam (summa) or sumpter horse-load is met with (fol. 408) as a measure of quantity. A seam of hay according to present use

1 Trans. Devon Assoc. xxvi, 308.

With this view agrees Mr. Whale in Trans. Devon Assoc. xxxii, 524.

${ }^{3}$ Key to Domesday, Dorset (1878), 31. Dr. Round, in V.C.H. Worc. i, 27 1, suggests the possibility that the league contained only 4 furlongs, which would certainly make a more workable unit, but lacks the support of evidence. 


\section{A HISTORY OF DEVONSHIRE}

in Devonshire is $336 \mathrm{lb}$. or $3 \mathrm{cwt}$; a seam of straw is $224 \mathrm{lb}$. or $2 \mathrm{cwt}$. A seam or pack of wool was I 7 stone and $2 \mathrm{lb}^{1}$ The Domesday seam of salt or fish was probably the last-named quantity or $240 \mathrm{lb}^{2}$

The holders of land in Domesday may be divided into three classes: first the king, who was regarded as the ultimate owner of all the land in the kingdom, ${ }^{3}$ but who also held certain estates which had never been booked to subjects as his inland or demesne, either for the support of the crown or for county purposes, besides a few which had formerly been booked to subjects but had come into his hands either by forfeiture or escheat. Next stand the great and small landholders to whom estates had been booked or given by charter, churches, barons, and knights, all holding. by military service or else in free alms, and tenants by special service about the king's court or person (servientes), whether military, household, or estate service. Then thirdly comes the peasant class, the agricultural workers of different sorts, villeins with their one or two ferlings each, bordars * with their tenements and single acre or half-acre plots, ${ }^{5}$ landless coscets, cottagers (cotarii), and slaves employed chiefly in labour on the lord's demesne, besides boors (buri) or half-freemen (coliberti) $^{6}$ on a few estates, and in one single case a bondwoman. ${ }^{7}$ The king's lands and the estates of his barons, knights, and officials are described in full in the pages of the survey. But there is no detailed description of the lands cultivated by the peasant class. It is only stated what part of the assessment they bear. Occupying as they do at the lord's will, they are not said to hold land at all (tenere), but only to be settled upon it (manere in). ${ }^{8}$

The foremost place among the king's lands is occupied by a list of nineteen estates described in the heading as "The King's demesne (dominicatus) in Devonshire belonging to the kingdom.' It includes nearly half of the original centres of Saxon settlement in the county, the demesne manors to which the manors of the hundred formed the fee. ${ }^{9}$ They are usually called ancient demesnes of the crown. On these estates the villagers and other occupiers held their tenements as folkland, not as bookland, and subject to fixed and heavy payments. ${ }^{10}$ The commissioners do not value these estates, but specify the amounts which were actually paid by them towards the king's supply service (firma). Compared with other counties the amounts are small. Nineteen manors together paid $£_{2} 232$ a year. If the value of Bampton is added the total comes to $£_{2} 53$, not more than was paid in some places as supplies for two and a half nights. It must, however, be borne in mind that

1 Phillips, in 1 706, and Simmonds, Dictionary of Trade Weights ; ex inform. Mr. Elworthy.

${ }^{2}$ Mr. E. T. Elworthy informs me that 'a pack or horse-load (seam) of wool is properly $2401 \mathrm{~b}$., but in certain parts and of certain kinds is sometimes sold as $244 \mathrm{lb}$, and was once as much as $252 \mathrm{lb}$.' 'The same authority tells me that in old Somerset leases which provided that so many seams of rotten dung were to be expended it was usually stipulated that such seams should be 12 score lb. and no more, i.e. 2401 lb.

${ }^{3}$ Freeman, Norman Conquest, v, 20, 45 ; iv, 27.

'Bordars = those having a 'bordellum' (Liber Niger, 382 ).

5 Trans. Devon Assoc. xxviii, 369 .

'Buri" are only once mentioned in Devon at Burrington (fol. 179), where they come after slaves. Maitland (Domesday and Beyond, 36) and Round (V.C.H. Warw. i, 384) say that 'buri '= 'coliberti.' 'Coliberti' are named at Werrington (fol. 98) and Broad Clyst (fol. 95).

${ }^{7}$ At Uppacot in North Tawton (fol. 475).

${ }^{8}$ At Witheridge (fol. 96): "These ferlings I plough can till and upon them 3 villeins are settled (in istis manent).' At Tossel's barton in East Buckland (fol. $129 b$ ): 'Drogo has 1 ferling and I plough(land) in demesne and upon the other ferling a serf is settled (monet).' At Bovey 'Tracey (fol. I 35): 'To, this manor the land of 15 thanes has been added. One is called On-down-Bovey, and there 4 thanes were settled.'

${ }^{2}$ Trans. Devon Assoc. xxvii, 198, n. 56.

${ }^{10}$ Ibid. xxx, 298. 


\section{DOMESDAY SURVEY}

Devonshire was never other than an agriculturally poor county. Before the disafforestation it must have been poorer still; for a large share of what grew must have been devoured by the extensive herds of wild deer which once flourished there.

Next to the ancient demesnes comes a second set of royal estates to which the Exeter Book prefixes the heading: 'The King's demesne (dominicatus) in Devonshire.' It consists partly of ancient centres of settlement, partly of portions of such centres set apart for county purposes, the support of the queen and royal family and the earl. Mr. Eyton has applied to these estates the name of 'comital ' or county lands. Professor Maitland ${ }^{1}$ and Dr. Round ${ }^{2}$ have followed him. The Exeter Book treats them as one class, and describes them in the order in which the hundred returns were dealt with, whereas the Exchequer Book groups them according to their holders T.R.E., first describing four held aforetime by Eadgyth or Eddida the Confessor's queen, daughter of Earl Godwin and sister of Harold, next the eight held by 'Githa the mother of Harold,' followed by the fourteen held by Earl Harold himself and the five held by Earl Leofwine his brother, and last of all Ordulf's manor of Broad Clyst, making a total of thirty-two. With the exception of Witheridge and Broad Clyst, which are valued at $\oint_{0}^{6}$ and $£_{2} 24$ respectively, the return specifies the amount they pay to the king, and even in these two cases the amount valued must have been the amount paid (fol. 976). Baldwin paid $£_{375}$ for the lands of the earls, i.e. for the lands of Countess Githa and Earls Lewin and Harold, excepting it would seem King's Nympton (fol. 98), ${ }^{3}$ whilst Goscelin paid $\oint_{0}$ ro8 for Queen Eddida's (fol. 976) four estates, and Reginald de Valletorta $£_{0} 24$ for Ordulf's land of Broad Clyst. Among the ancient centres of settlement set apart for county purposes we find Lifton, North Molton, Wonford, South Tawton, Hartland, Witheridge, Chillington, Tiverton, Werrington, Black Torrington, Shebbear, Molland, and Moreton. Why ancient demesnes should have been assigned to this purpose it is at present impossible to say, but it is worthy of remark that there are thirteen of them, exactly one-third of the thirty-nine original hundred centres.

In addition to the two above-named classes of royal estates the king also held estates which had come to him by forfeiture or escheat. In Devonshire this class was confined to a group of sixteen, of which fifteen had formerly belonged to Brictric son of Ælfgar or Algar, ${ }^{4}$ and on his forfeiture had been bestowed on Queen Matilda. One, however, had previously belonged to Boia, possibly the clerk of that name at Bodmin who held the small estate of Pendavid in Gluston in Cornwall by favour of the count of Mortain, as recorded in Domesday. ${ }^{6}$ Queen Matilda died 2 November, I $083,{ }^{6}$ when all her estates reverted to the king. With the exception of Ashprington, which was given to Nonant, the whole of them were farmed by Goscelin and Walter de Clavil, ${ }^{7}$ and were included in the dowry

${ }^{1}$ Domesday and Beyond, $167-8$.

" 'Domesday,' V. C. H. Somers. i.

3 The list given in Trans. Devon Assoc. xxix, 458,n. IO, is faulty because it includes the booklands of subjects, Ash, Blackpool, and Nimet, and omits Hartland, Shebbear, and Kilmington. Mr. Whale's list in Trans. Devon Assoc. xxxii, 529 , is also at fault because it includes the abbot of Battle's 'Sirefort,' names a wrong payment from Woodbury, and entirely omits Werrington. Yet Werrington, Hartland, and Woodbury are the only three whose value is stated 'before Baldwin' had them.

- Freeman, Norman Conquest, 1v, 165, 763 ; Round, Feudal Engl. 424.

5 Exeter Book, fol. 507 .

Trans. Devon Assoc, xxxiv, 721.

${ }^{7}$ Exeter Book, fols. 82, 84, 88, 92. 


\section{A HISTORY OF DEVONSHIRE}

which Henry I bestowed on his natural son Robert with Maud, eldest daughter of Robert son of Hamon. ${ }^{1}$ In I24I these manors, together with Muxbear, Swetton (fol. 46I), and Iddlecot (fol. 462), which had been wrongfully united with Brictric's lands, constituted the fifteen fees belonging to the honour of Gloucester which are described as 'Earl Richard's share.'

Not all lands which were held by the church in Domesday were held by it in alms. St. Peter's of Exeter, the abbots of Glastonbury, Tavistock, and Horton, held their lands by military service. St. Mary of Bucfast, however, appears to have held hers in alms, as there is no mention of military tenants holding of Bucfast Abbey. The Exeter Book gives a special list of ' lands which have been given to the saints in alms.' The phrase is even used of lay tenants, as Professor Freeman remarks, to indicate the restoration of property which had been held by the grantee before. ${ }^{3}$ The bishop of Coutances, however, did not hold in alms at all. His was a secular barony the holder of which happened to be bishop of St. Lo. Slight differences of arrangement are here met with between the Exeter and the Exchequer Books. In the Exeter Book only the estates of the greater church tenants are arranged in groups. The 'lands given to the saints in alms' follow one another without grouping in the regular sequence of hundreds. In the Exchequer copy the grouping extends to all churches. Again, the Exeter Book describes one group as 'lands of St. Peter's Church at Exeter in Devonshire,' whereas the Exchequer Book calls them 'land of the bishop of Exeter.' Conversely the Exeter Book has ' Lands of the abbot of Tavistock Church in Devonshire,' whereas the Exchequer has 'Land of the Church of Tavistock.' The Exeter Book begins the list of the bishop of Exeter's estates with Bury in Lapford, because the return from North Tawton hundred was the first which the scribe extracted; the Exchequer Book begins with St. Stephen's Church, Exeter, because St. Stephen's was the head (caput) of the bishop's barony. The Exeter Book describes each of the four manors allotted for the canons' maintenance in its place in the order of hundreds, adding a note each time as to the purpose for which it was assigned; the Exchequer places the four together, and after Combe Pafford in St. Mary's Church, adds: 'The four above-named townships (villae) are for the canons' maintenance.' The note was of some importance, as implying that these estates, being held in alms, were free from the claims of military tenure.

It has been stated on what seems satisfactory authority that at the time of the coniquest of Devon one-tenth of the conquered land was given to God. This statement by no means asserts that parochial endowments were then set up-as a fact most of the parochial endowments in Devon date from the twelfth century-but probably implies that the tenth part of the land was devoted to

1 Trans. Devon Assoc. xxxiii, 623. See Feudal Baronage.

- Testa de Nevill (Rec. Com.), pp. $177 a$ and b, so describes Ashreigny 1, Hollowcombe in Winkleigh $\frac{1}{2}$, Winkleigh Kaynes $\frac{1}{2}$, Winkleigh Tracy I, Birch and Southcot $\frac{1}{2}$, North Lew and Halwill $\mathrm{I} \frac{1}{2}$, Bideford $\frac{1}{2}$, Bulworthy $\frac{1}{8}$, Langtree I, Iddesleigh 2, Holcombe Burnell I, Halberton I, Moorston $\frac{1}{2}$, Wombernford in Halberton I, Muxbear and Swetton I, Clovelly I, and Calcot $\frac{7}{4}$, but does not name Iddlecot $\frac{8}{4}$ (Feudal Aids, p. 370 , says $\frac{4}{5}$, but p. $\left.422, \frac{1}{2}\right)$. I take them to be the fifteen fees for which William de Redvers accounted in I John. See Trans. Devon Assoc. xxxiii, 365.

${ }^{3}$ Freeman, Norman Conquest, v, 804. Instance Undercleave (fol. 85), Braunton Dean (fol. I 94b), Little Dartmouth (fol. 327), and Ashton (fol. 483).

' 'Anecdota Oxoniensia,' vii, 106, in Trans. Devon Assoc. xxx, 296, has a letter of Archbishop Dunstan's (960-988). 'The King (Ethelred) then fared thither and subdued them (the West Welsh of the Horn) and gave a tenth of his land to God.' 


\section{DOMESDAY SURVEY}

religious purposes, of which the principal one was the care of the poor, under the administration of the bishop and his clergy. Side by side with this provision for the living other gifts to monastic and collegiate churches had been made on behalf of the dead, for prayers for their souls. For this purpose 44 hides, representing some 36,000 acres, ${ }^{1}$ had been given to various churches before the Confessor's time, followed by 35 hides more in the Confessor's time. ${ }^{2}$ The gifts of the Conqueror and his companions to the saints in alms amount to 34 hides. $^{3} \quad$ Comparatively little of these land gifts was available for what we should call diocesan purposes, and many of the estates given to God were administered by and for the benefit of monasteries outside the county; nearly all the Conqueror's gifts were to alien establishments.

Out of the 15 hides and 185 plough-lands in Crediton manor the squires or knights (milites) of the bishop of Exeter and his peasants had among them 9 hides and 172 plough-lands. Two centuries later we meet with Umfravil, fitz Stephen, the earl of Devon, Hydon, Peverel, Knighton, Dourish and Lancels ${ }^{4}$ holding under the bishop lands in Crediton. Most of them were probably successors to his Domesday squires.

More information is available as to the abbot of Tavistock's under-tenants, who, according to his return in I I 66, held $18 \frac{1}{2}$ fees, viz., i 7 of old feoffment and $I_{2} \frac{1}{2}$ of new. ${ }^{5}$ The names of Ermenald, Ralf, Hugh, Robert, Ralf de Tili and Geoffrey are given in the record as under-tenants at Tavistock; of Walter, Geoffrey, Ralf and a Frankish knight, possibly de Tili, at Hatherleigh ; of Nigel and Robert at Romansleigh. It is probable that Hugh's 2 virgates $2 \frac{1}{3}$ ferlings represent Wick Dabernon in Milton Abbot $\mathrm{I}_{\mathrm{I}} \mathrm{fee}^{6}$ held by Hugh de Wicha in I I $66 ;{ }^{7}$ and that Ralf's $\frac{1}{2}$ virgate is Notley in Tavistock $\frac{1}{3}$ fee $;{ }^{8}$ also that Walter's $2 \frac{1}{2}$ virgates at Hatherleigh represent Broomford and Jacobstowe, Walter being the predecessor in title of Geoffrey de Lega who, in I I 66, held $1 \frac{1}{2}$ fees of the abbot and 'extorted an additional $\frac{1}{2}$ fee from his church."

The detailed consideration of the great lay tenants in chief, whose possessions formed the numerous baronies or honours which are found in this county at a later date, and of their sub-tenants, is reserved for our section on the Feudal Baronage. Suffice it here to remark that the estates of Hugh earl of Chester passed by his forfeiture to the crown, Stowford and Houndbear continuing to be held of the king, ${ }^{10}$ whilst East Anstey went to the honour of Cardinan. Those of the count of Mortain, after being resumed by Henry I and held by the king or some member of the royal family for two centuries, were in I 337 settled on the prince of Wales. The estates of

1 Trans. Devon Assoc. xxx, 290.

Ibid. 294.

${ }^{3}$ Ibid. $x x x, 282,286$.

- Feud. Aids, i, 337. Trans. Devon Assoc. xxxvi, 356 ; xxxiii, 627. The Red Book (Rolls Ser.), 556, names in 12 ז 2 Drogo of Teignton, Robert son of Geoffrey, Roger the archbishop, William de Mesthale, Henry the Earl's son, John Malherbe, William Botreaux, Hugh Peverel of Sampford, Richard de Hokeweye and Baldwin de Reddon as holding of the bishop by knights' service.

${ }^{5} \mathrm{Lib}$. Nig. 118 . Red Bk. of Exch. (Rolls Ser.), $55^{8}$ in 1212 says $17 \frac{1}{2}$ fees.

${ }^{6}$ Feud. Aids, i, 322. ${ }^{7}$ Lib. Nig. I 18.

${ }^{8}$ In Feud. Aids, pp. 354, 402, Notley is said to have been held aforetime by William Cornu. William Cornu also held Thornbury for $\frac{2}{3}$ fee (Ibid. 327). Now since Ralf was predecessor of W. Cornu at 'Thornbury, it is probable that he also preceded him elsewhere as at Notley (Trans. Devon Assoc. xxxvi, 350). Besides Roger Cornutus is stated to have held 2 knights' fees of the abbot in I 66 (Lib. Nig. I I 8 ).

${ }^{9}$ Lib. Nig. I I 8 . Trans. Devon Assoc. xxxvi, 352.

${ }^{10}$ Testa de Nevill (Rec. Com.), 1 79a, 190b, 191b. 


\section{A HISTORY OF DEVONSHIRE}

Geoffrey bishop of Coutances, with additions and exceptions, went to form the honour of Barnstaple; those of Baldwin the sheriff the honour of Okehampton; those of Juhel of Totnes the honours of Hurberton and Totton; Ralf de Pomeray's the honour of Berry; those of William his brother, better known as William Capra, the honour of Braneys or Bradninch. Walter de Dowai's were divided between the honours of Bampton and Marshwood. William de Faleise's constituted the honour of Dartington; those of Odo son of Gamelin, together with his father-in-law Tetbald's, the honour of Torington, whilst Goscelin's and Clavil's and Queen Matilda's went to the honour of Gloucester. The rest, with the exception of a few which went to out-county honours or were held of the king in chief, constituted the great honour of Plympton. ${ }^{1}$ As to those tenants whose Devonshire estates were subsequently held of honours of which the seats were in other counties, the following notes will not be out of place.

Among the count of Mortain's under-tenants who became tenants in chief when that honour fell into the hands of the crown was Richard son of Torolf or Turold, who, besides being an under-tenant of the count of Mortain, and an under-tenant of Baldwin the sheriff (at Martin, fol. 205), was also a tenant in chief of the crown in respect of four manors assessed at 4 hides, and with a cultivated area of roughly 2,000 acres. $^{2}$ These four, together with the manors which he held as under-tenant, constituted the Cornish honour of Cardinan. ${ }^{3}$

William de Moione or de Mohun was another tenant in chief who held Clayhanger, assessed at I hide, with about 400 acres essarted from the forest, as part of his own Somersetshire honour of Dunster. ${ }^{4}$ A third was Alvred 'de Ispania,' of Spain, or, as Dr. Round surmises, of Epaignes, ${ }^{5}$ who held in chief the estates of the dispossessed Alwi Bannesons, ${ }^{\circ}$ King Edward's reeve, viz., Arlington and Orway, assessed at I 2,000 acres under cultivation, as part of his own honour of Stowey. Alvred's line vanished in an heiress, Isabel, the wife of Robert de Chandos, who died in I I 20 ; and Chandos's heiress, Maud, brought them before I 66 to her husband, Philip de Columbers. ${ }^{7}$

A fourth tenant in chief whose Devonshire estates went to a distant honour was Torstin the son of Rolf, whose manor of Churchstanton, assessed at 3 hides, with approximately 2,000 acres essarted from the forest, was afterwards held of the honour of Kerlihun. ${ }^{8}$ This Torstin is probably the Turstin who bore the Conqueror's standard at the battle of Hastings. ${ }^{8}$ Before I095 his land had passed by gift of William Rufus to Wynebald de Ballon, whom Rufus brought over with him. ${ }^{10}$ Wynebald had two sons, who succeeded him, and a daughter, his eventual heiress, who married Newmarch. His grandson, Henry de Newmarch, in Henry II's reign confirmed his grandfather's grants to religious houses. ${ }^{11}$ Towards the close of John's reign the two co-heiresses

${ }^{1}$ Feud. Aids, 235.

${ }^{4}$ Liber Niger, p. 9.
${ }^{6}$ Exeter Domesday, fols. 162 and $37 \mathrm{I} b$.

Exch. Domesday, fol. 1 I 3.

${ }^{s}$ See Feudal Baronage.

${ }^{7}$ Liber Niger, p. 97. After the death of King Henry one knight was enfeoffed on the demesne of Arlington (Alurington), viz. Hugh de Ralege.

${ }^{8}$ Testa de Nevill (Rec. Com.), p. $183 b$.

9 Eyton, Key to Domesday, Dorset, 76. Round, Peerage Studies, 189.

${ }^{10}$ Round, Peerage Studies, p. 19o. His name de Ballon is taken from his home near Le Mans in Maine.

"Dugdale, Mon. ii, 73. 


\section{DOMESDAY SURVEY}

of Newmarch married Ralf Russell, of Kingston Russell, and Nicolas de Moelis respectively, ${ }^{1}$ the latter obtaining Churchstanton.

Yet another tenant in chief whose tenure was not connected with the county was William de $\mathrm{Ou}$ or de Eu. $\mathrm{He}$ is grouped with the Frank squires and held Powderham and Church Whitestone. He was the son of Robert de Eu and Lescellina his wife, and had two brothers, Hugh bishop of Lisieux (I050-77), and William Busac. ${ }^{2}$ He married a sister of Hugh earl of Chester, by whom he left a son, but he forfeited his estates for treason under William Rufus in $1096,{ }^{8}$ and they were given to a family who took their name from Powderham." On the attainder of John de Powderham they were given to Humphrey de Bohun, earl of Hereford, who gave them in marriage with his daughter Margaret to Hugh earl of Devon. They were held of the honour sometimes called Swale, ${ }^{5}$ sometimes the castle of Haye. ${ }^{6}$

Two lands, Blackpool and Warkleigh, which at the time of the survey had been added to the hundred manor of Molland, Speechwick, one of the king's manors, as also the manor of West Bray, which was held by the Englishman Alnod, went with the honour of William Meschin, ${ }^{7}$ which was united with that of Stoke Curcy before II 66 by the marriage of an heiress, and were held of the honour of Stoke Curcy, ${ }^{8}$ to which honour were further added the manors of Colyton Raleigh, Bystock, and Clyst William. ${ }^{9}$ East Down and Bradwell, Ralf de Limesei's estates, went to the honour of Odingesels, ${ }^{10}$ Molland Botreaux to the Herefordshire honour of Wigmore. ${ }^{11}$

Besides the tenants who held lands by ordinary military service, there existed a number of tenants in chief of the crown who held lands by some special service about the king's person or court, which are now known as grand or petty serjeanties. The Exeter Book says nothing about the terms of service, but the headings ' The land of Nicolas the crossbowman,' 'The King's servants,' 'English thanes,' for which the Exchequer writes 'The King's thanes,' differentiate these tenants from the rest. Their estates did not as a matter of course go from father to son, unless the office also went from father to son, but could be given by the king to whomsoever he pleased. ${ }^{12}$ From subsequent sources the services are mostly known in consideration of which the lands were held, but in many cases they were commuted into ordinary military service. ${ }^{18}$

The first to be mentioned among the king's tenants by special service are his military officials, introduced by the heading 'The land of Nicolas the crossbowman' (arcbibalistarius or arbalestarius). They include Nicolas himself the crossbowman or ordnance-master, Godbold another crossbowman (arbalestarius), a third, Fulcher (arcbibalestarius), William the gatekeeper (portitor, ${ }^{14}$

${ }^{1}$ Round, Peerage, 198.

${ }^{9}$ Freeman, Norman Conquest, iii, I 18.

${ }^{3}$ Round, Peerage, 187.

- Polwhele, ii, I70. Lysons, ii, $422 . \quad{ }^{5}$ Hund. R. (Rec. Com.), No. 43, p. 86. Feud. Aids, 316.

- Inq. p.m. 2 Hen. V, No. 49 ; 2 I Edw. III, No. 2.

7 Liber Niger, 91.

- Testa de Nevill (Rec. Com.), i 79a. Trans. Devon Assoc. xxix, 506.

${ }^{10}$ Testa de Nevill (Rec. Com.), i 83b, 196. Trans. Devon Assoc. xxix, 506. Feudal Aids, 326. Dugdale, Baronage, i, 4 I 4 .

${ }^{11}$ Feud. Aids, 3 I 6.

12 The jury so find in regard to Chilsworthy held by the king's drysalter; Cal. Inq. p. m. Hen. III, p. 282 .

${ }^{13}$ Testa de Nevill (Rec. Com.), p. 197a, 198b. Red Book of Exch. (Rolls Ser.), ccxxix, and 452, names five special service holdings, and Testa de Nevill (Rec. Com.), p. I95a.

If Exeter Domesday, fol. 472. 


\section{A HISTORY OF DEVONSHIRE}

portarius, ${ }^{2}$ janitor ${ }^{2}$ ), and Haimeric the bowyer (de arcba, fol. 472b, or de arcis, fol. $469 b)$. Dr. Round has identified Nicolas with Nicolas de la Pole. ${ }^{8}$ His successor in most of his holdings in 1286 was Robert, son of Pagan. ${ }^{4}$ Godbold had Doddescombe for his successor at the same date. ${ }^{5}$ Fulcher had Briwere, represented by Chaworth and Mohun in $1286 .^{6}$ William the gatekeeper, who held Bicton by the service of keeping the gate of Exeter gaol, had Ralf de Normant in $125^{8} .^{8}$ Subsequent tenants of Bicton bore the name of Arblaster or le Balister.' Haimeric the bowyer had William de Toriton for successor. ${ }^{10}$ Altogether the king's military officials had thirty-nine manors assessed at $27 \frac{1}{2}$ hides containing some I 2,500 acres, most of which went to the honour of Plympton.

Of the king's household officials only two had estates in Devon, William the usher (bostiarius), and Ansger, either the king's kitchen-master (coquus), or his hearth-keeper (focarius). ${ }^{11}$ William had eleven manors, some of them probably held by the doubtful title of an alleged exchange, assessed at 9 hides with a cultivated area of over 5,000 acres, besides which he was also undertenant of the abbot of Tavistock at East Raddon, and of Baldwin the sheriff at Hele Poer and Woodington. In all these manors Briwere represented him at a later date, ${ }^{18}$ and two of them, Mariansleigh and Tormoham, were given by Briwere to religious houses. William's undertenant Richard is represented by Speccot ${ }^{13}$ at Sutton Lacy; Ralf Boty (Botinus) is represented at Blackborough Boty by the family of Boty. All William's estates were afterwards held of the honour of Plympton. Ansger's single estate of Gatcombe passed to Prowse, who held it of Pomeray. ${ }^{14}$ It was assessed at I hide, and had an area of approximately 350 acres.

The English thegns in Devon were a fairly numerous class, twenty-one in all, holding among them fifty-two manors assessed at 27 hides, with an area of more than 20,000 acres essarted from the forest. They were either Englishmen who, having possibly rendered some signal service to the king, were allowed to continue in possession of their estates, such as perhaps Godwin, Donne, and Odo Edric's son, or else Englishmen whose services were required in estate-management or as attendants on the king when hunting. Colvin was a reeve, or bailiff; Alvric, who held Skeridon and Hockner farm, was probably a huntsman, as he had to find a pair of arrows for the king whenever he came to hunt in the forest of Dartmoor. ${ }^{15}$ Their estates are also found held of divers honours. Colvin, Alward Merta, Godwin, and Godric of Calverleigh held manors which were afterwards incorporated in the honour of Gloucester. ${ }^{16}$ Ansgod's estate of Meddon, Adret's at Manaton, and Saulf's at Mowlish went to the honor of Berry, ${ }^{17}$ Godeva's at Torbryan and

${ }^{1}$ Geldroll, fol. 68, A. 12.

2 Testa de Nevill (Rec. Com.), p. 1989.

${ }^{3}$ V.C.H. Warw. i, 280 .

${ }^{4}$ Feud. Aids, i, 31 3, 31 8, 339.

${ }^{5}$ Ibid. pp. 31 2, 346, 348. Trans. Devon Assoc. xxix, 266, n. 49.

${ }^{6}$ Ibid. Pp. 31 2, $319,339 . \quad{ }_{7}$ Testa de Nevill (Rec. Com.), p. 198a.

${ }^{8}$ Inq. p.m. 43 Hen. III, No. I, 18 ; Cal. Inq. p. m. Hen. III, No. 420.

${ }^{9}$ Inq. p. m. I Edw. I, No. 3. Feud. Aids, i, $325,365$.

11 Exeter Domesday, Somerset, fol. $477 b$, names Ansger ' focarius.'

${ }^{12}$ Trans. Devon Assoc. xxx, 405. Feud. Aids, i, 321.

${ }^{10}$ Feud. Aids, i, 3 I 3.

${ }^{14}$ Ibid. i, 330.

15 This, at least, was the tenure by which David be Seyredun subsequently held, 'et ita tenuerunt antecessores sui post conquestum': Testa de Nevill (Rec. Com.), p. I95a.

${ }^{16}$ Ibid. $177 b, 178 a$.

${ }^{17}$ Ibid. $182 b$. 


\section{DOMESDAY SURVEY}

Dodbrooke, and Saulf's at Sowton to the honour of Okehampton. ${ }^{1}$ Adret's holdings at Wick and Goodamoor (in Bicheforda, fol. 488b), Saulf's at Ashford Peverel (Mannebeua, fol. 490), Alward's at Kismilton, and all Edwin's, Odo Edric's son's, Alveva's, Aldric's, and Leuric's estates to the honour of Plympton. ${ }^{2}$ Alnod's to the honour of Stoke Curcy. ${ }^{3}$ Alwin's, Alvric's, Algar's, Alfilla's, Ulf's, and those of the priests of Bodmin do not appear at all in the fee lists.

Adret or Eldred held North Wick, Manaton, and Godamoor (Bicheforda), and the Geldroll shows that one Aderet the forester, presumably the same, held some other estate in Exminster hundred," possibly the detached section of Kenton manor. ${ }^{5}$ Ansgod was another Englishman who was allowed to retain his manor of Meddon and Diptford, and received in addition Farley, the estate of the dispossessed Done, which in $125 \mathrm{I}$ was Jordan de Esse's. ${ }^{6} \quad$ Alward Merta, who held Kismilton (Colsouenescota, fol. 48r), besides $\frac{1}{2}$ virgate in Ashreigney given to him by the queen in alms, was probably the Alward, described as a freeman, whose second name survives in Merdisnymet, alias Wolfsnymet (fol. 390b), of which he was unfortunate enough to be dispossessed in favour of Walter de Clavil.

Donne, who held Newton St. Cyres and Nutwell before the Conquest, was one of those who contrived to keep his estates under William. Holding Newton of the bishop he tendered his fee-farm rent to the crown, and although the bishop produced his charters proving that he had been seised thereof before the Confessor's reign, and brought forward Normans (francigenae) in support of his claim, ${ }^{7}$ Donne was entered by the commissioners among tenants of the crown. Alwi or Alwin, who had the small estate of Middlecot, may possibly be the dispossessed Alwi Bannesons whose lands Alvred of Spain or Epaignes enjoyed. ${ }^{8}$ Odo son of Edric, Edrit, or Adret, who held Southwood and Uggarton, besides other manors, may possibly be the son of Adret the forester, but his successors took their name from Down Ralf, and were known as de Dun. Alveva, the lady who held St. James's Church (fol. 487), may have been the same Alveva who held Withycombe Raleigh before the Conquest, and it is possible that there was a church there also, if the tradition is of any value that before the settlement of parishes in this county the inhabitants of what are now four parishes used to resort to a church at Withycombe with the invocation of St. John in the Wilderness of Egypt. She is described as a freewoman, and had property also at Ash Thomas, assuming the Alveva at Ash to be the same. Godeva, who held Torbryan, together with Dodbrooke and Portlemouth, was Brictric's widow. ${ }^{9}$ She had estates in Teignbridge, ${ }^{10}$ Haytor, ${ }^{11}$ and Coldridge ${ }^{12}$ hundreds.

Outside the general system of land tenure, ${ }^{18}$ and independent of the regular divisions of hundreds, tithings, and manors, are the county and market boroughs and the land reserved for the king's pleasure, the forest. Only four

1 Testa de Nevill (Rec. Com.), 1806.

- Geldroll, fol. 69, A Io.

Ibid. 181a, 182 .

5 Polwhele, ii, $165 n$

${ }^{3}$ Feud. Aids, 326.

${ }^{6}$ Held of the honour of Berry, Teste de Nevill (Rec. Com.), p. 182b. Feud. Aids, p. 342, says that it was held of Mohun, Mohun being one of Briwere's heirs, and Briwere being the purchaser of the North Devon section of Pomeray's honour.

${ }^{7}$ Exeter Domesday, fol. I 17.

${ }^{9}$ Geldroll, fol. 69, A 10.

${ }^{12}$ Ibid. fol. 69, A 4 .

${ }^{8}$ Freeman, Norman Conquest, iv, 31.
${ }^{11}$ Geldroll, fol. 69b, A 12.
${ }^{13}$ Maitland, Domesday Book and Beyond, p. I 78.

395 


\section{A HISTORY OF DEVONSHIRE}

boroughs are described in the record of Devon. A fifth is incidentally mentioned, but it was never of the same kind as the other four. Of the four one lay in the eastern part of the county, viz. Exeter, the three others lay in the western part situated respectively north, middle, and south, viz., Barnstaple, Lidford, and Totnes.

It would be superfluous here to dwell on Professor Freeman's graphic description of the resistance offered to the Conqueror by the city of Exeter, of its unconditional surrender, and of the hard terms imposed on it by King William, ${ }^{1}$ for Dr. Round has already disposed of all this as pure fiction. ${ }^{2}$ But there are some points with which Dr. Round has not dealt. As Professor Maitland has pointed out, the revenue which the king would receive from a borough before the Conquest would usually be threefold: (I) the groundrent from tenements belonging to the king and rented by the burgesses, the house or land gavel; (2) the profits of the market and the borough court, for which the citizens usually paid a fixed sum; and (3) the Danegeld as an occasional tax. ${ }^{3}$ Under the first heading we learn that in Exeter were 285 houses paying their customary dues to the king besides forty-eight lying in ruins (fol. 88). This was the king's share in the city revenue, and quite distinct from the shares of the queen, the sheriff, or the king's barons.

From several notices we gather that this due was usually $8 d$. a house, but there were exceptions. One house which the abbot of Tavistock held in pledge from a burgess paid $8 d$. (fol. I $80 b$ ). One which Juhel held paid $8 d$. (fol. $334 b$ ). One which Richard son of Torolf held (fol. 5066 ), one held by Ruald (Exch. fol. I I 5), one held by Tedbald (fol. 4I 06 ), each paid 8d. Seven which Battle Abbey held paid together 4s. 8d. (fol. 196). Ten of the bishop's, however, paid Ios. Iod. (fol. I 20b), or at the rate of Is. Id. each, and six of Pomeray's paid $40 d$., or at the rate of $6 \frac{2}{3} d$. each. Allowing for these exceptions the revenue from this source comes to $f_{0} 9 \mathrm{I} 3^{s}$. $6 \mathrm{~d}$., and this I take to be the origin of the payment made by the city to the earl of Cornwall after the king had given him the city, amounting to $\oint_{1} 29$ s. in 1274,4 and to £ 13 ros. in $1290.0^{\circ}$

The second source of revenue which the king derived from the city arose from the profits of the market and the borough court. For these before the Conquest a sum of $\oint_{1} 8$ was paid, of which two-thirds went to the king, one-third to the earl. The king's share had been from of old assigned to the queen and was held by Eddida at the time of the Conquest, and the Conqueror did not interfere with this arrangement. ${ }^{\circ}$ This share was afterwards enjoyed by Henry I's queen, when it had increased to $f_{0} 25$, and with the king's consent was bestowed on the canons of the Holy 'Trinity in London. ${ }^{7}$

With regard to the third source of revenue " the city did not pay geld in King Edward's time except when London, York, and Winchester paid, and then only half a mark for the men in the king's pay (solidarii),' i.e. as a gratuity

${ }^{1}$ Freeman, Norman Conquest, iv, 162. Exeter (1887), p. 32.

2 Round, Feudal Engl. p. 446.

3 Maitland, Domesday Book and Beyond, p. 204.

${ }^{4}$ Hund. R. (Rec. Com), No. I 5, p. 70, in Trans. Devon Assoc. xxix, n.3.

${ }^{5}$ Izacke, Mem. of Exeter, p. 28.

${ }^{6}$ Round, Feudal Engl. p. 448 : the burdens to which Exeter was subject were not raised at all, but remained precisely the same as had been paid to former kings.

${ }^{7}$ Anct. D. A 231. Trans. Devon Assoc. xxix, 455 I. Round, Commune of London, 85-7. 


\section{DOMESDAY SURVEY}

to the house-carls.' "When an expedition fared forth the city of Exeter did the same service as five hides of land' (fol. 88), i.e. it found one mounted squire (miles) ${ }^{2}$ to go forth with the host.

Of the 285 houses which paid ground rent to the king, and the five which were exempt from payment, five were appurtenant to the royal manor of Tawstock and I 2 I belonged to the king's barons. Of these the bishop of Exeter had forty-eight besides two in ruins (fol. I 206) and nine appurtenant to his manor of Bishopsteignton (fol. I I7). The bishop of Coutances had nine besides one in ruins, but four of these were discharged from all payment (fol. I 36). Battle Abbey had eight, of which one was discharged (fol. I 96); the count of Mortain had one (fol. 222b); Baldwin had nineteen, of which eleven belonged to Kenn (fol. 297); Juhel had one (fol. 334b); Walter de Dowai ten (fol. 349b); William Capra two (fol. 406); Richard son of Torolf one (fol. 506b); Ralf Paynel one (fol. 460); Pomeray six (fol. 343b); Ruald one (Exch. I I 5); Tedbald one (fol. 410); Alvred the Breton one (Exch. fol. I I 5); Godbold two (fol. 473); and Osbern de Salceid one (fol. 462b). In only one case are we informed of the rack-rent value of these houses. Ralf Paynel received from his ros. a year (fol. 460). This is presumably the house which figures in the Pipe Rolls after an escheat as William de Heliun's, from which a sum of Ios. was annually paid into the Exchequer. ${ }^{3}$ It must also be noticed that 'in the Devon Survey the houses in Exeter held by each tenant in chief appear not in the separate survey of the city, but at either the beginning or the end of the list of his rural properties, as if the commissioners had noted them down when entering the rural properties, added them up, and entered them in one total."

The three other Devonshire boroughs show little of the co-operation principle unless they are taken together as three links in one chain of defenceworks against the West Wealla. 'This township,' says the record, speaking of Totnes, 'did not pay geld except when Exeter paid it, and when it did pay the amount was 4 od. (or 1 mark), and whenever an expedition fares forth Barnstaple, Totnes, and Lydford between them do the same service as Exeter' (fol. 334). An interesting fact may also be gathered from the ' Burghal Hidage,' viz. that the borough in South Devon was originally not at Totnes, but at Halwell, where a camp is still to be seen, and that the borough in North Devon was originally not at Barnstaple, but at Pilton, ${ }^{6}$ at a place now called Roborough Camp. This was in the days when defence was the first consideration. The removal to Totnes and Barnstaple was no doubt made to secure the advantage of water carriage for the markets held there; but it left the old camps undefended, and so was a source of danger to the county.

At Barnstaple there were forty burgesses within the borough, and nine without, besides twenty-three houses lying in ruins. ${ }^{7}$ At Lydford there were twenty-eight burgesses within the borough and forty-one without. ${ }^{8}$ At

${ }^{1}$ Freeman, Norman Conquest, iv, 147,162 .

- Maitland, Domesday Book and Beyond, p. 156. Trans. Devon Assoc. xxxiii, 574.

${ }^{3}$ Pipe R. I 2 Hen. II, in Trans. Devon Assoc. xxix, 489.

- Ballard, The Domesday Boroughs, 27.

5 Maitland, Domesday Book and Beyond, p. 187, 503 ; Halgan wille, alias Halgan Wylle.

${ }^{6}$ Ibid. P. 503 ; Wiltone Wisbearstaple, alias Piltone wid Bearstaple.

${ }^{7}$ Exeter Domesday, fol. 876.

${ }^{8}$ Ibid. \{ol. 876 . 


\section{A HISTORY OF DEVONSHIRE}

Totnes there were ninety-five within the borough and fifteen employed as labourers without. ${ }^{1}$ At Barnstaple the bishop of Coutances had ten burgesses who paid him 3 s. $9 d$., besides seven houses in ruins, ${ }^{2}$ Baldwin had seven burgesses besides six houses in ruins which between them paid him 7 s. $6 d .{ }^{8}{ }^{8}$ and Drogo had a fruit-garden (ortus) which went with the manor of East Bray. ${ }^{4}$ At Totnes all the burgesses and labourers were Juhel's. ${ }^{5}$ At Lydford the burgesses without were half as many again as those within the borough. ${ }^{6}$ Among their holdings must be included Fernworthy near Chagford, which though many miles off still belongs to the borough of Lydford. ${ }^{7}$ Each of these three boroughs paid $f_{0} 3$ annually to the king. At Barnstaple he kept $\oint_{0} 2$ out of the $\oint_{3} 3$ for himself, giving $£_{0}$ t to the bishop of Coutances as lord of the honour. ${ }^{8}$ At Lydford he kept the whole amount, and at Totnes he gave the whole amount to Juhel, who soon raised it to $f_{0} 8$.

When after the Conquest the castle took the place of the co-operative stronghold, boroughs ceased to be strongholds and became places of privileged government and market centres only. Perhaps the earliest borough of this kind was that at Okehampton. 'On that land,' says the record, 'stands the castle of Okehampton. There Baldwin has four burgesses and a market paying 4s. a year' (fol. 288). The Okehampton burgesses therefore paid Is. apiece for the market privilege. Only one other market is mentioned in Devonshire. 'At Otterton is a market on Sundays' (fol. I 94b). Judging by the large number of thirty-three saltworkers there, may we infer that Otterton market was chiefly a market for salt, and that the market was held at Sidmouth?

The record only mentions one castle in Devonshire, that of Okehampton, but it is well known that at the time of the survey a castle also existed at Exeter, for the canons of St. Mary (within the castle) are named as estateholders (fol. 3076). Mr. Freeman also finds reference to Exeter Castle in the mention of ruined houses there (fol. 88). These, he says, had been pulled down to provide a site for the castle. ${ }^{10}$ A castle also perhaps existed at Totnes, and soon after at Plympton and Tiverton. The record further mentions the castle of Cornwall as having been given by the bishop to the count of Mortain in exchange for Haxon and Benton (fol. I I 8), but this was outside our county.

The whole of Devonshire, excepting the boroughs of Barnstaple and Totnes, was prior to the year 1204 set apart for the royal sport, the inhabitants being allowed to cultivate such portions of it as had been essarted or brought into cultivation subject to the forest law. ${ }^{11}$ These essarted portions being alone a source of revenue to the crown, are the only portions described in the record. Not a word is said about the great forest; and its very existence is ignored except as a matter of inference. In one place we are told (fol. 95) that ' to Molland manor belongs the third penny of the hundreds of Northmolton, Bampton, and Braunton, and [the agistment dues of] every third animal [lying out] on the moors'; also that 'with Moreton

1 Exeter Domesday, fol. 334 .

Ibid. fol. 128.

Ibid. fol. 136.

7 Dartmor Preservation Society's Publications, fol. 334 .

${ }^{10}$ Freeman, Norman Conquest, iv, I62.
${ }^{2}$ Ibid. fol. 334 .

ications, i, 65,91 . particular body of law is enforced, having for its object the preservation of certain animals ferae naturae.'
3 Ibid. fol. 3 I 5 .

${ }^{6}$ Ibid. fol. $87 b$.

8 Exeter Domesday, fol. 876 .

876 .

en Soc.), is 'a tract of land within which a 398 


\section{DOMESDAY SURVEY}

manor goes the third penny of the hundred of Teignbridge' (fol. 96). Who would gather from these bald statements that from time immemorial all the landholders in the county, with the exception of those in the two boroughs above named, had the right of depasturing their cattle by day in a forest nearly as large as the cultivated area, and could also by paying agistment or lying-out dues leave their cattle in the forest after sunset, and that whilst the king had two-thirds of these dues one-third of them in the north went with the comital manor of Molland and one-third of those in the south with that of Moreton Hampstead? Yet so it was, and the right survives to the present day in all that remains of the forest of Devonshire, the Forest of Dartmoor. ${ }^{1}$

To obtain freedom from the oppressive forest-law the men of Devonshire in I 204 paid King John 5,000marks, and received from him a charter of disafforestation. ${ }^{2}$ This charter was, however, not put in force until I 242 (27 Hen. III), when the boundaries of the forest were delimited, Dartmoor and Exmoor being still left forests. Round the Forest of Dartmoor lie 'the Devonshire Commons,' on which, however, only the manors of the adjoining township now have rights. The tenants enjoying these rights are termed Venvil tenants, ${ }^{3}$ and pay a fixed fine or commutation-rent instead of agistment dues for the trespassing of their cattle on the forest. The forest of Exmoor is mostly in Somerset, but its 'regard' extends into Devonshire."

Although the record says nothing about the forest, yet it frequently mentions the forest ponies (equae silvestres) kept for breeding purposes. There were five at Colyton Raleigh, ten at North Lew, eighteen at Ottery St. Mary, eight at Bratton Clovelly, seventy-two at Lynton, four at Combe Raleigh, two at Ashford, six at Sourapple, and six at Villavin. At several other places, as Cornworthy, Musbury, Bradworthy, and Brendon, unbroken mares (equae indomitae) are mentioned, by which Dartmoor and Exmoor ponies are doubtless meant, as many as 104 occurring at Brendon.

A mill is an unfailing mark of a manor, but not every manor has a mill, because for a water-mill water is necessary, and wind-mills were unknown in England at the time of the survey. No fewer than seventy-seven manors are mentioned as possessing mills, and the number of mills in them amounts to over ninety-nine. The number is comparatively small, seeing that the estates which had to be served by them numbered I,266. In the hundred manor of Hairidge were three mills, and the same number at Ottery St. Mary and Otterton. At Axminster, Tiverton, Halberton, Holcombe Rògus, Uffculme, Weycroft, and Lamerton there were two each, but Buckerell (fol. $33^{8}$ )

${ }^{1}$ Mr. Birkett, Dartmoor Preservation Association's Report, p. xv, observes: The perambulation [of 1224 ] establishes three important facts-(1) that the moor was originally part of a royal forest, (2) that the commons of Devon were once part of the forest, and (3) that the moor is not waste of any manor in particular. Trans. Devon Assoc. xxxiii, $585, n .57$.

${ }^{2}$ Pipe R. 6 John, m. 7.

${ }^{3}$ 'The term 'Venvil' is a corruption of Fines villarum or township fines. A list of these commutation fines is in Trans. Devon Assoc. xxii, I 85 ; xxiv, 418 ; xxxiii, $585, n .57$ and $591, n .61$. Dartmoor Preservation Society's Publications, i, 63, 66.

4 At an inquisition held at Wells 2 July, I 366 (Devon N. and Q. ii, 189), it was found that 'R.A. and W.T. had taken and killed a young stag within the hundred of Witheridge within the regard of the Forest of Exmoor, which regard is continuous (unum est) and extends as well within the county of Somerset as of Devon. And they say that all the officials of the aforesaid forest belonging to the county of Somerset, although the aforesaid regard is partly situate in Devon, have entry (intromittunt se).' Exmoor Forest was enclosed and the boundaries defined in 1815 by 55 Geo. III, c. I38. 


\section{A HISTORY OF DEVONSHIRE}

is said to have half a mill, probably because Feniton had an equal share in it, though the record does not say so. Of two mills, one at Tavistock (fol. I 76), the other at Stoke Fleming (fol. 3486), it is said that they are 'for the use of the house,' but most of them must have been for the use of outsiders also, which probably accounts for their difference in value. The most valuable mill was one at Columb John, which is returned as worth $25^{s}$. Three others, those at Broad Clyst, Barnstaple, and Hazel Barton, are stated to be worth 20s. each. One at Cornworthy was worth I5s.; those at Cowick, Exwick, Bampton, Ottery Mohun, Gittisham, and Broadhembury were worth Ios, each. One at Upton Pyne was worth 8s.; several were worth 7 s. $6 d$., viz. those at Woodbury, Colyton Raleigh, Honiton, Denson. The three at Hairidge and one at Okehampton were worth 6s. 8d. each. The greater number are worth $5^{s}$. or less, for instance the two at Axminster, the two at Holcombe Rogus, the two at Uffculme, and the one each at Kilmington, Culmstock, Bovey Tracey, Bickleigh, Kentisbeare, Dotton, Wolborough, Musbury, Bradninch, Tale, Buckerell, Huxham, Smallridge, Culm Davy, and Widworthy; while others are returned as only worth $50 d$. (Kenton, Talaton, Powderham), 4od. (Willand, Colyton, Bishop's Nympton, Payhembury), 30d. (Crediton, Kenn, Clayhidon, Culm Pyne, Oakford, Ogwell Malston, Newton St. Cyres), 20d. (Nymet Rowland, Upottery), I $5 d$. (Cliston Hayes), 8d. (Petton, Diptford), and one at Hatherleigh only ${ }^{\circ} 6 d$. That at Lyn is stated to be a new mill.

The salt industry was an important one. Before the discovery of modern methods it was carried on by evaporating brine in sheds called salterns (salinae). The late Mr. Davidson explained the process as carried on in Devon in the eleventh century. ${ }^{1}$ More recently Dr. Round has supplied a Scotchman's account of a somewhat similar process. ${ }^{2} \mathrm{Mr}$. Davidson showed from documentary evidence the existence of salterns at Teignmouth some twenty years before the Conquest. ${ }^{3}$ These, no doubt, are the twenty-four salterns at the bishop's 'Teintona,' which the record refers to as paying Ios. Salterns are also mentioned in twenty-three other manors. At Ermington and Blackawton, at Ashprington, Woodford, Netherton, Buckland Monachorum, and South Lob, were salterns, but their value is not stated. At Northam were two paying ros. ; appendant to Honiton, and probably situate at Beer by Seaton, were four worked by two saltworkers. At Beer Ferris and Alston were seven paying IOs.; at Orcherton one paying 5s.; at Tamerton Foliott one paying $5^{\text {s. }}$ At Hollacombe were three saltworkers paying $3^{\text {s. }} 9 d$. besides five seams of salt; at Otterton thirty-three saltworkers; at Lower Holcombe four paying $6 s$. $5 d$; at Kenton eight paying 20s. a year. At Sidmouth was one saltern belonging to Ottery St. Mary, at Bigbury one, at Saunton one, each paying $30 d$.; at Egg Buckland one paying $2 s$. ; at Whiteway one paying I $2 d$.; at Seaton eleven paying I $1 d$; and at Paignton one paying $10 d$.

There is only a single notice of bee-keeping in Devon. At Lustleigh (Sutreworde, fol. 347) were five honeyers (mellitarii) who paid seven sestiers (sextarii) of honey. No certain conclusion can be arrived at from this entry. Either bee-keeping was so common and taken such small account of

1 Trans. Devon Assoc. xiii, I 13.

${ }^{2}$ V.C.H. Essex, i, 38 I.

- Trans. Devon Assoc. xiii, rog. 


\section{DOMESDAY SURVEY}

as not to deserve mention, or bee-keeping was not practised at all, except at Lustleigh on the borders of Dartmoor.

Nowhere is there a notice of a vineyard in Devon, but there is mention of an orchard (bortus) in Barnstaple belonging to East Bray, and of another at Sidmouth belonging to Ottery St. Mary. The count of Mortain had a fruit-garden (virgultum) in Exeter, and there was a park (parcus bestiarum) at Winkleigh.

Fisheries are mentioned in thirteen places. Some of these must have been sea-fisheries in tidal waters, but others were river fisheries. Probably in all cases the method was the wasteful one of fishing by weirs and seahedges. ${ }^{1}$ There was a fishery (pescaria) at Loddiswell which paid thirty salmon a year to its owner; another (piscatoria) at Cornworthy which paid the same. At Dartington two fishermen paid eighty salmon. Fisheries are mentioned at Woodford and Netherton, the value of which is not stated. The most valuable fishery (piscatio) was that at Bideford, worth 25s. That at Exminster came next, worth 20s. One at Buckland Monachorum was worth Ios., one at Bickleigh 5s., one at Heanton Punchardon 2s. Half a fishery (piscatura) at Wear Gifford was worth $40 d^{\text {. ; }}$ it is not stated who had the other half, perhaps the lord of Torington. At Northam the fishery is returned as worth 30d. ; at Efford I $2 d$.

In concluding this survey the author wishes to express his obligations to the Dean and Chapter of Exeter for the facilities of access to their precious MS. accorded to him, and to their most obliging assistant librarian, the Rev. E. T. Foweraker, for his ready help.

${ }^{1}$ Round, in V.C.H. Essex, i, 424 . 


\section{NOTE}

The translation here given is taken from the text of the Exeter MS. and collated with the Exchequer MS.

In the translation the ever-recurring phrase 'on the day on which King Edward was alive and dead - in contemporary English 'On pam timan pe Eadward cing waes cucu and dead' (Freeman, Norman Conquest, v, 743)-is expressed for the sake of brevity by the letters T.R.E. (tempore Regis Edwardi), that being the formula used throughout the Exchequer Domesday.

For brevity's sake the 'ands' which superabound in the text have been mostly omitted.

Usually where a place-name ends in $a$ in the Exeter book, an $e$ is found in the Exchequer copy.

There is some difference in the order in which the fiefs follow one another between the Exeter and the Exchequer books, and slight differences in the arrangement of the crown manors, which are noted from time to time.

It should be borne in mind that the date of the Domesday Survey is I 086 , that the day on which King Edward was alive and dead was 5 January, 1066, that the hide was the unit of assessment on which the (Dane)geld was paid, that the virgate was a quarter of a hide and a ferling a quarter of a virgate. A plough-land consisted of as much land as 8 oxen could cultivate; in this county it contained 4 ferlings of land; and a ferling of land is by later authorities constantly identified with 16 acres. The Domesday manor consists of demesne and villagers' land. Demesne is the lord's home farm, villagers' land is the land occupied by his dependents on condition of cultivating the lord's home farm for him.

As regards identifications it should be noted that the modern manor or parish, of which the name is given in square brackets, is not necessarily co-extensive with its Domesday equivalent. The latter in many cases included many subsequent sub-infeudations, and was therefore of considerably greater extent than the later manor. In other cases the Domesday manor was only a portion of the estate of which it bore the name, and many of these portions at a later date became known by other names. The determination of the exact identity and extent of each Domesday estate is reserved for treatment in the topographical section of this History. 



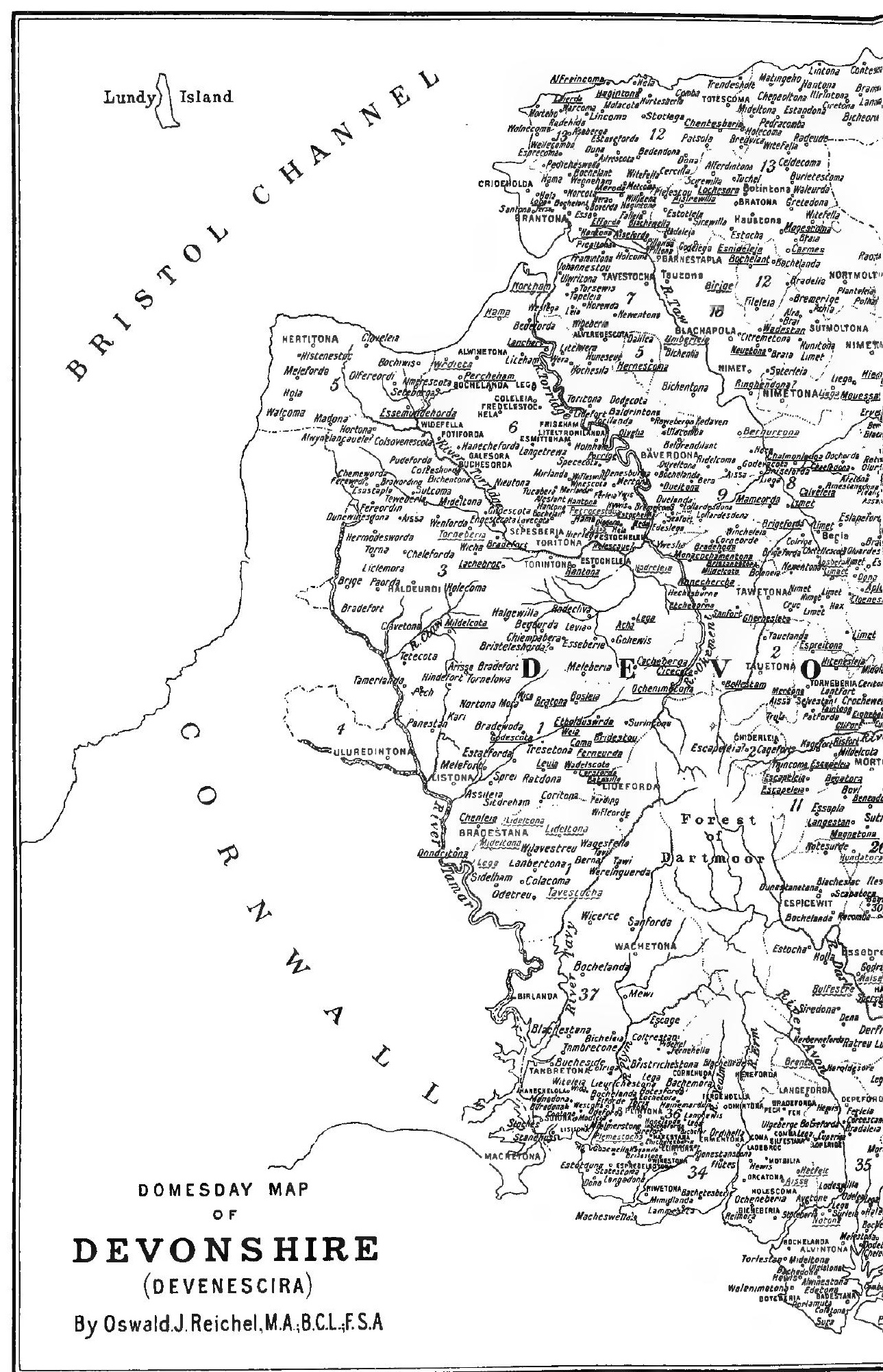





\section{TEXT OF THE EXETER BOOK}

fol. 83.

\section{THE KING'S DEMESNE BELONGING} TO THE KINGDOM IN DEVENESCIRA ${ }^{1}$

The king has a manor called Tawetona [Tawton] ${ }^{2}$ which paid geld for $\frac{1}{2}$ virgate in the time of King Edward. This 30 ploughs can till. There the king has 3 ploughlands (carucatas) and 3 ploughs (carucas) in the demesne, and the villeins have 27 ploughs. There the king has 3 I villeins, 28 bordars, 3 serfs, Io beasts (animalia), 90 sheep, 40 acres of wood(land) (nemus), 30 of coppice (nemusculum), 40 acres of meadow and 5 of pasture. It pays 15 pounds a year by weight.

The king has a manor called Aexeministra (Exch. Axeminstre) [Exminster $]^{3}$ which King Edward held on the day on which he was alive and dead, and it was assessed (defendit se) at I hide. This 20 ploughs can till. Thereof the king has in demesne I virgate of land all but a ferling and 2 ploughs; and the villeins have $\frac{1}{2}$ hide and I 6 ploughs. There the king has 18 villeins, 7 bordars, 6 serfs, 12 cottagers (quotarios), 4 beasts, 120 sheep, and I fishery paying 20 shillings a year, also I league of wood(land) taking length and breadth, I 8 acres of meadow, $3 \frac{1}{2}$ leagues of pasture taking length and breadth, and coppice I 2 furlongs in length by 6 in breadth. It pays 8 pounds a year and paid the same when Baldwin first got it. Of the above manor William de $\mathrm{Ou}$ holds $\frac{1}{2}$ virgate ${ }^{4}$ which belonged to the aforesaid manor T.R.E. Of the same manor the monks of Battle (Batailla ; Exch. Labatailge) hold I ferding ${ }^{5}$ of which Eccha the reeve allowed a

'Instead of the heading 'The King's demesne belonging to the Kingdom,' the Exchequer Book begins with the cities of Exeter, Barnstaple and Lydford. Then comes a heading 'The King's land 'followed by the various hundred-manors in the same order as the Exeter Book as far as Maker, except that the two manors got by exchange for Bampton, viz. Ermington and Blackawton, are placed at the end after Maker, and immediately before them is the note; "The above 12 manors were in the demesne of King Edward and belong to the king.'

${ }^{2}$ The hundred-manor of North Tawton.

${ }^{3}$ The hundred-manor of Exminster. Exminster was one of the manors bequeathed by King Alfred to his younger son. Kemble, Cod. Dipl. ii, I 1 2, No. 3 I 4 .

4 Perhaps Exwell in Powderham parish in the hundred of Exminster. See fol. $456 b$.

${ }^{5}$ Either Kenbury in Exminster in the hundred of Exminster, or more probably the 16 acres of Exminster glebe. See Trans. Devon Assoc. xxxvi, 379. The assessments on the demesne, the villeins, William de $\mathrm{Ou}$, and certain priest the benefit (accomodavit) T.R.E. This half a plough can till.

rol. 8gb.

The king has a manor called BRANTona [Braunton] $^{6}$ which King Edward himself held T.R.E., and it was assessed at I hide. This 40 ploughs can till. Thereof the king has I ploughland (carucata terrae) and I plough, and the villeins have 30 ploughs. There the king has 40 villeins, 30 bordars, 4 serfs, I00 sheep, 40 acres of coppice, 2 acres of meadow, and 40 of pasture. It pays 16 pounds a year by weight, and paid the same when Baldwin the sheriff, who farms it of the king, first received it. To this manor has been added I virgate of land 7 which belonged to Fileleia [Filleigh], a manor of Baldwin the sheriff, T.R.E. It is worth 20 shillings a year. From the same Bractona [Braunton] I virgate ${ }^{8}$ has been taken away which belonged to it T.R.E. ; now Robert de Ponte Cardonis holds it. It used to contribute 20 shillings to the farm rent when it was (included) in the king's manor.

The king has a manor called Sut (Exch. Sud) Moltona [South Molton] ${ }^{9}$ which King Edward held T.R.E. In that manor are $I_{2} \frac{1}{2}$ virgates of land. This 40 ploughs can till. There the king has I plough and the villeins have 20 . There the king has 12 villeins, 4 bordars, 2 serfs, I 2 swineherds, and 9 beasts, also wood(land) I league in length and 3 furlongs in breadth, Io acres of meadow and 30 of pasture. It pays Io pounds a year by weight. To this manor has been added $\frac{1}{2}$ virgate of land called RINGEDONA, ${ }^{10}$ which is worth 5 shillings a year.

The king has a manor called Sulfretona [Silverton] ${ }^{11}$ which King Edward held T.R.E. In this manor it is not known how many hides there are, because it never paid geld. This $4 \mathrm{I}$

Battle Abbey, together only amount to $3 \frac{1}{2}$ virgates. It is suggested that the missing half-virgate may be Matford Speke, fol. 40I. Trans. Devon Assoc. xxvii, 178 ; xxx, 281 .

- The hundred-manor of Braunton.

7 North Lob in Braunton; see fol. 3006.

8 Blakewell in Marwood, in Braunton hundred ; see fol. 2996 .

${ }^{9}$ The hundred-manor of South Molton.

${ }_{10}$ Possibly represented by Frenchstone alias Freynstone, an outlier of South Molton parish next George Nympton, in the hundred of South Molton.

in The hundred-manor of Silverton or Hairidge. 


\section{A HISTORY OF DEVONSHIRE}

ploughs can till with all that belongs thereto. fol. 84.

There the king has in demesne 3 ploughs and the villeins 33 ploughs. On it (In ea) the king has 45 villeins, 3 I bordars, I 5 serfs; also 20 sheep, 3 mills paying 20 shillings a year, 4 acres of wood(land), 50 of meadow, 200 of pasture. It is worth 40 pounds a year weighed and assayed (ad pondus et arsuram).

The king has a manor called Haminoc [Hemyock] ${ }^{1}$ which King Edward held T.R.E., and it paid geld for I virgate. This I 2 ploughs can till. There the king has $I$ plough in demesne and the villeins 9. There he has 12 villeins, 12 bordars, 7 serfs, also 2 beasts, 40 sheep, 8 furlongs of wood(land), 16 acres of meadow, and pasture 2 leagues in length by $1 \frac{1}{2}$ in breadth. It pays 6 pounds by weight. When Baldwin received it it was paying the same.

The king has a manor called BoDeleia (Exch. Bodelie) [Budleigh] ${ }^{2}$ which King Edward held T:R.E., and it paid geld for $\frac{1}{2}$ hide. This 13 ploughs can till. There the king has 16 villeins who have 12 ploughs; there also he has 20 bordars, Io swineherds, 4 serfs, 2 beasts, 12 sheep, 20 acres of wood(land), 5 of meadow, and roo of pasture. It pays Io pounds by weight; when Baldwin received it the same.

The king has a manor called Texntona [Teignton] ${ }^{3}$ which King Edward held T.R.E., and it paid geld for I hide and I virgate. This I6 ploughs can till. Thereof the king has I virgate and $I$ plough in demesne, and the villeins I hide and 12 ploughs. There the king has I 4 villeins, 30 bordars, 3 serfs, 30 sheep, fol. $84 \mathrm{~b}$.

I 5 acres of wood(land), and 4 of meadow. It pays 14 pounds by weight and Io shillings by tale. When Baldwin received it it was paying Io pounds by weight.

The king has a manor called Alseministra [Axminster] ${ }^{4}$ which King Edward held T.R.E. How many hides are there is not known because it never paid geld, but the land can be tilled by 40 ploughs. Thereof the king has 2 ploughs in demesne and the villeins 18 . There the king has 30 villeins, 20 bordars, 4 serfs, 2 beasts, 50 sheep, 2 mills paying 10 shillings, I00 acres of coppice, 30 of meadow, and 100 of pasture. It pays 26 pounds a year weighed and assayed (ad pondus et ad arsuram). When Baldwin received it the same.

1 The hundred-manor of Hemyock.

- The hundred-manor of East and West Budleigh.

3 The hundred-manor of Teignbridge.

1 The hundred-manor of Axminster. Axminster was one of the manors bequeathed by King Alfred to his younger son. Kemble, Cod. Dipl. ii, I 12 , No. 314.
The count of Mortain has a manor called Honetona [Honiton] which T.R.E. paid $3^{\circ}$ pence a year to the king's manor of Axminster, but since the count has had it it has ceased to pay. Ralf de Pomeria has a manor called Esmaurige [Smallridge] which T.R.E. paid 30 pence a year to the aforesaid manor, but for the last twelve years Ralf has withheld them. William Capra has a manor called Manberia [Membury] which likewise paid 30 pence a year to the same royal manor, but William has withheld them for the last twelve years. The bishop of Coutances has a manor called Cherletona [Charton] which paid I 5 pence a year to the aforesaid manor T.R.E., but since the bishop has held it it has never paid. The canons of St. Mary of Rouen have a manor called Rouerige [Rawridge] which T.R.E. paid 30 pence a year to the aforenamed royal manor, but for the last eighteen years they have withheld this customary due. And to this manor has been added a certain other manor called DENEORD [Deanworth], which Ailrich (Exch. Alric) held T.R.E., and it paid geld for 2 virgates. These $I \frac{1}{2}$ ploughs can till. There the king has 2 villeins and 1 bordar. It contributes Io shillings a year to the abovenamed manor. Also in Axminster itself is another manor called OdEscLiva [Undercleave], ${ }^{6}$ which Eddric the cripple (mancus) held in alms of King Edward, and now the same Eddric's son Edward holds it of King William, and it pays geld for I virgate. This I plough can till. There Edward has 2 villeins and I bordar who have I plough. Worth 5 shillings a year.

To the church of Axminster $\frac{1}{2}$ hide of the land of this manor belongs. ${ }^{7}$ This 2 ploughs can till, and they are there, as also 12 bordars. Worth 20 shillings a year.

fol. 85 .

The king has a manor called CArsewilla ${ }^{8}$ (Exch. Carsewella) [Kerswell] which King Edward held T.R.E., and it paid geld for $1 \frac{1}{2}$ hides. This 17 ploughs can till. Thereof the king has $I \frac{1}{2}$ virgates and I plough in demesne, and the villeins I hide and 16 ploughs. There the king has I 6 villeins, 33 bordars, 3 serfs, I 20 sheep, 20 acres of wood(land), and 9 of meadow. It pays 14 pounds by weight; when Baldwin received it the same. Also to the church of this township (villa) belongs $\frac{1}{2}$ virgate of land.

\section{The king has a manor called Culitona [Coly-}

- Probably the $\frac{1}{2}$ hide given to the abbey of Bec and held by the prior of Goldclive in 1338 , i.e. Membury Goldclive in Membury. See Add. MSS. 28649, p. 491 ; Inq. p.m. I I Edw. III, No. 26 (O.J.R.); or Uphay (T.W.W.). See fol. $5 \circ 3$.

${ }^{6}$ Hodesclive alias Undercleave in Axminster and Membury. See fol. 503 .

7 Priestaller in Axminster.

${ }^{8}$ The hundred-manor of Kerswell or Haytor.

${ }^{\circ}$ Kingskerswell Church manor. 


\section{HOLDERS OF LANDS}

ton] ${ }^{1}$ which King Edward held T.R.E., and it paid geld for I hide. This I6 ploughs tan till. Thereof the king has I virgate in demesne and I plough, and the villeins have 3 virgates and 14 ploughs. There the king has $2 \mathrm{I}$ villeins, I 0 bordars, 3 serfs, 6 beasts, and I mill paying 40 pence a year, ro acres of coppice, 36 of meadow, and 200 acres of pasture. It pays yearly II pounds and Io shillings weighed and assayed (ad combustionem). The aforesaid manor has a church to which belongs $\frac{1}{2}$ virgate. ${ }^{2}$ Worth 5 shillings.

The king has a manor called Alsemuda [Axmouth] ${ }^{3}$ which King Edward held T.R.E. How many hides are there is not known because it never paid geld. This 12 ploughs can till. Thereof the king has in demesne half a plough and the villeins 6 ploughs. There the king has 8 villeins, I 2 bordars, 4 serfs, 18 sheep, 16 goats, 8 acres of coppice, 16 acres of meadow, and pasture $I$ league in length by 4 furlongs in breadth. fol. $85 \mathrm{~b}$.

It pays yearly 8 pounds by weight and I mark of silver.

The king has a manor called ERmentona [Ermington] $]^{4}$ which Ansger (Exch. Asgar) the hunchback (contractus) held T.R.E., and it paid geld for 3 hides. This 20 ploughs can till. Thereof the king has I hide and 3 ploughs in demesne, and the villeins have 2 hides and 17 ploughs. There the king has 16 villeins, 36 bordars, Io serfs, 50 sheep, I 2 goats, I saltern, wood(land) 2 leagues in lengih by $\frac{1}{2}$ in breadth, and 8 acres of meadow. It pays is pounds and to shillings yearly weighed and assayed (ad combustionem).

This is by way of exchange for Badendone [Bampton].

The count of Mortain has a manor called FERDENDEL [Fardle] which Godfrey holds of him, which T.R.E. paid by custom 30 pence to the

- king's manor of Hermentona [Ermington], besides other customary dues payable to the hundred; but since King William has reigned the aforesaid customary dues have been withheld from the king's manor. Reginald holds a manor of the count of Mortain called Dunitona [Dinnaton] which T.R.E. used to pay 30 pence by custom to the king's manor of Ermentone [Ermington], besides the other customary dues to the hundred, but they have been kept back

1 The hundred-manor of Colyton. Geldroll, fol. 68b, A, $\mathrm{x}$.

2 Possibly Tudhayes in Colyton, afterwards held by Polsloe Priory, and described in Inq. p.m. 34 Edw. I, No. 147 , as consisting of I messuage and 3 ferlings of land.

${ }^{3}$ The hundred-manor of Axmouth. Geldroll, fol. $68 b$.

"Ermington manor in the hundred of 'Alleriga' or Ermington. Geldroll, fol. 70. since King William got the land. Reginald holds a manor of the count of Mortain called Dunitona [Dinnaton] which T.R.E. paid 30 pence by custom to the king's manor of Ermentona [Ermington], besides the other customary dues to the hundred; but since King William received the kingdom both the customary dues and the pence have been withheld. Also from BRADEFORDA [Broadford], one of the count's manors which Reginald (Raginal) holds of the count, the customary dues as well as the 30 pence have been withheld from the aforesaid king's manor. And from LuDEBroca [South Ludbrook], which Reginald holds of the count, the 30 pence and the customary dues to the hundred have been withheld from the aforesaid king's manor. Raginal (sic).

The king has a manor called Auetona $[\text { Awton }]^{5}$ which Ansgar (Exch. Asgar) the hunchback held T.R.E., and it paid geld for 6 hides. These 24 ploughs can till. Thereof the king has $2 \frac{1}{2}$ hides and 2 ploughs in demesne, and the villeins have $3 \frac{1}{2}$ hides and 24 ploughs. Thereof (sic) There the king has 25 villeins, 22 bordars, 9 serfs, I saltern, I 20 sheep, 20 goats, 30 acres of wood(land), 5 acres of meadow and 30 of pasture. It pays 14 pounds and ro shillings a year.

And these two aforesaid manors are (held) in exchange for Badentone [Bampton].

The king has a manor called Depeforda [Diptford] ${ }^{6}$ which King Edward held T.R.E., and it paid geld for 3 virgates. These ro ploughs can till. Thereof the king has I virgate and I plough in demesne, and the villeins have 2 virgates and 8 ploughs. There the king has 8 villeins, 7 bordars, 4 serfs, 8 beasts, 3I sheep, 2 acres of meadow and 12 of pasture. It pays 7 pounds and 5 shillings by weight; when Baldwin received it 100 shillings by weight. fol. 86.

To this manor a manor called FerLeIA (Exch. Ferlie) $\left[\right.$ Farley] ${ }^{7}$ was added in the time of William de Walville, which a certain thane held in parage (pariter; Exch. libere) T.R.E., and he was free to go to whomsoever he would. It paid geld for I virgate. There he has 2 villeins and 5 bordars who have I plough. It contributes yearly to the above-named farm-rent Io shillings by weight.

The king has a manor called Alvintona [Alvington] ${ }^{8}$ which King Edward held T.R.E.,

${ }^{5}$ Blackawton in the hundred of Colridge. Geldroll, fol. $69 b$.

${ }^{6}$ The hundred-manor of Diptford or Stanborough. See Geldroll, fol. $69 b$.

${ }^{7}$ Farley in Diptford, in Stanborough hundred.

${ }^{8}$ West Alvington in the hundred of Stanborough. The assessment on the king and the villeins together falls short of the total by I virgate, which probably represents Combe Royal, an outlier separated by the borough of Kingsbridge. (O. J. R.) 


\section{A HISTORY OF DEVONSHIRE}

and it paid geld for I hide. This 10 ploughs can till. Thereof the king has I virgate and I plough in demesne, and the villeins $\frac{1}{2}$ hide and 5 ploughs. There the king has ro villeins, 5 bordars, 4 serfs, 42 sheep, and 2 furlongs of wood(land). It pays 7 pounds and 5 shillings by weight; when Baldwin received it the same.

The king has a manor called Plintona [Plympton] ${ }^{2}$ which King Edward held T.R.E., and it paid geld for $2 \frac{1}{2}$ hides. These 20 ploughs can till. Thereof the king has I hide and 2 ploughs in demesne, and the villeins $\mathrm{I} \frac{1}{2}$ hides and 12 ploughs. There the king has 15 villeins, I 2 bordars, 6 serfs, 5 beasts, 50 sheep, wood(land) I league in length by $\frac{1}{2}$ league in width, 6 acres of meadow and 20 acres of pasture. It pays 13 pounds and 10 shillings by weight; when Baldwin received it 12 pounds and 10 shillings by weight.

Independently of these $2 \frac{1}{2}$ hides ( $E x c h$. Over and above this land) the canons of St. Peter of Plympton hold 2 hides. ${ }^{2}$ These 6 ploughs can till. There the canons have $\mathrm{I} 2$ villeins, who have 4 ploughs. They (the hides) are worth 35 shillings a year.

fol. $86 \mathrm{~b}$.

The king has a manor called EuIntona [Yealmpton] ${ }^{3}$ which King Edward held T.R.E., and it paid geld for $2 \frac{1}{2}$ hides. These 20 ploughs can till. Thereof the king has I hide and I plough in demesne, and the villeins $x \frac{1}{2}$ hides and 12 ploughs. There the king has 16 villeins, 9 boŕdars, 1o serfs, wood(land) 3 furlongs in length and $\frac{1}{2}$ in width, 6 acres of meadow and 10 acres of pasture. It pays 12 pounds and Io shillings by weight; when Baldwin received it the same.

Also the priests (sacerdotes; Exch., clerici) of that township hold I hide of land, ${ }^{4}$ whereof they have 3 virgates and I plough in demesne, and the villeins I virgate and I plough. (They have) 3 villeins, 4 bordars, 2 serfs, 4 beasts, Io swine, 31 sheep, 2 acres of meadow and Io acres of pasture. It is worth 10 shillings, which the king allows them in alms.

The king has a manor called W AcheToNa [Walkhampton] $^{5}$ which King Edward held

1 The hundred-manor of Plymholme, including the manor and castle of Plympton Earl and Herdwick in Plympton St. Mary. See Geldroll, fol. 70, A, I.

'Colbrook and Boringdon in Plympton St. Mary, and Wembury Church manor (Bronescombe Reg. 477; Trans. Devon Assoc. xxx, 290; xxxiv, 290). Geldroll, fol. 70, A, 4 .

${ }^{3}$ The manor of Yealmpton, in the hundred of Plympton. Geldroll, fol. 70, A, I.

'Lyneham in Yealmpton ; in the Geldroll, fol. 70, A, 3, 'held by St. Mary of Yealmpton (Alentona)," and administered by the canons of St. Mary (of Old Sarum). Feudal Aids, i, 334 .

${ }^{5}$ The hundred-manor of Walkhampton or Roborough.
T.R.E., and it paid geld for $\frac{1}{2}$ virgate. This 4 ploughs can till. Thereof the king has I ferling in demesne, and the villeins I ferling and 4 ploughs. There the king has 6 villeins, 4 bordars, 2 serfs, 3 beasts, 50 sheep, wood(land) I league in length by $\frac{1}{2}$ league in breadth, I acre of meadow and 100 acres of pasture. It pays 3 pounds a year by weight.

The king has a manor called Sutona [Sutton] which King Edward held T.R.E., and it paid geld for I virgate. This 6 ploughs can till. fol. 8 .

Thereof the king has $\frac{1}{2}$ virgate and $\frac{1}{2}$ plough in demesne, and the villeins $\frac{1}{2}$ virgate and 5 ploughs. There the king has 4 villeins, 2 bordars, I serf, 15 sheep, 2 acres of meadow and 20 acres of pasture. It pays 20 shillings a year by weight.

The king has a manor called TAnbretona [Tamarton] ${ }^{7}$ which King Edward held T.R.E., and it paid geld for I virgate. This 6 ploughs can till. Thereof the king has $\frac{1}{2}$ virgate in demesne, and the villeins $\frac{1}{2}$ virgate and $4 \frac{1}{2}$ ploughs. There the king has 6 villeins, 2 bordars, I serf, wood(land) 3 furlongs in length by $I$ in breadth. It pays 20 shillings a year by weight. These 3 manors used to contribute one night's supplies ${ }^{8}$ (frmam bunius noctis), with all that that involves (cum suis appendiciis).

From the manor of WACHETONA [Walkhampton] has been taken MACRETONa [Maker], ${ }^{9}$ which King Edward held T.R.E., and it paid geld for 1 virgate. This ro ploughs can till. And this manor used to contribute 6 pounds all but 30 pence a year to Wachetona (Exch. towards the king's farm (firma)) T.R.E.

And these 3 manors are appurtenant to Wachetona [Walkhampton].

fol. 87b.

The king has a borough called BARNESTAPLE [Barnstaple] ${ }^{10}$ which King Edward held T.R.E. There the king has 40 burgesses within the borough and 9 without, and they pay 40 shil-

${ }^{6}$ Sutton-on-Plym, otherwise Sutton Lake, including Sutton Vautort (Ancient Deeds, D. 1,097), and Sutton Prior, in the hundred of Roborough, which together with Pennycross (alias Weston Peverel) and Stonehouse formed the ancient parish of St. Andrews, Plymouth. Trans. Devon Assoc. xxxvi, 170 ; Geldroll, fol. $70, \mathrm{~A}, \mathrm{I}$.

7 King's Tamarton, the chief manor in St. Budeaux, in the hundred of Roborough.

${ }^{8}$ Valued in Northamptonshire at $£ 30$, in Oxfordshire at $\oint_{150}$, in Hampshire and Dorset at $\oint_{106}$ The supplies were wheat, honey, and malt. See Round, in V.C. H. Nortbants, i, 274.

${ }^{9}$ Vaultersholme tithing, better known as Mount Edgecumbe, in the parish of Maker, in the hundred of Roborough, the rest of Maker being in Cornwall.

${ }^{10}$ The borough of Barnstaple. 


\section{HOLDERS OF LANDS}

lings by weight to the king, and 20 shillings by tale to the bishop of Coutances. There also 23 houses have been laid in ruins (vastatae) since King William has had England.

The king has a borough called LIDEForda [Lydford] ${ }^{1}$ which King Edward held T.R.E. There the king has 28 burgesses within the borough and 4I without, and they pay 3 pounds a year ( $E x c h$. 60 shillings) by weight to the king. There also 40 houses have been laid in ruins (vastatae; Exch, vastae) since King William has had England. The aforesaid burgesses have land for 2 ploughs outside the city (foras civitatem). If an expedition goes forth by land or by sea this borough renders the same service as Totenais [Totnes] or Barnstaple. fol. 88.

In the city of Essecestra (Exch. Exonia)

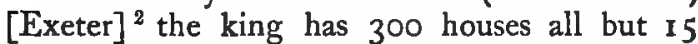
which pay the customary due. (The city) pays I 8 pounds a year, out of which Baldwin the sheriff has 6 pounds weighed and assayed and Colvin has 12 pounds in currency for the behoofs of Queen Eddida. This city did not pay geld T.R.E. except when London, York, and Winchester paid it, and that was half a mark of silver for the hired troops (ad solidarios; Exch., ad opus militum). ${ }^{3}$ And whenever an expedition went forth by land or by sea, the city did the same service as 5 hides of land, whilst Toteneis [Totnes], Lidford, and Barnestabla [Barnstaple] together did the same service as the aforesaid city. In this (city), however, 48 houses have been laid in ruins ${ }^{4}$ since King William has had England. And the aforesaid burgesses have land for I 2 ploughs outside the city subject to no customary payment save to the city itself. ${ }^{5}$

\section{o․ 93. \\ THE DEMESNE OF THE KING IN DEVENESIRA [DEVONSHIRE] ${ }^{6}$}

The king has a manor called Bradestana

'Lydford castle, with the borough and village of Lydford.

${ }^{2}$ The city of Exeter, including Exe Island without the city and St. Leonard's, Trans. Devon Assoc. xxxiv, 718. The customary due for a house here and elsewhere was 8 pence. See Introduction, p. 396

${ }^{3}$ For the king's housecarls; Freeman, Norman Conquest, iv, 147.

"To make room for the castle; Freeman, op. cit. iv, 162 .

'Duryard manor, conterminous with St. David's parish other than Hoopern. Trans. Devon Assoc. xxxiv, 718.

${ }^{6}$ Instead of this general heading, the Exchequer book prefixes separate headings to each group of estates, arranging them according to their tenants T.R.E. First-with the heading, "The underwritten lands Queen Eddida held; now the king has them '-
[Bradstone], ${ }^{7}$ which Earl Harold held T.R.E., and it paid geld for $\frac{1}{2}$ hide. This 8 ploughs can till. Thereof the king has $\frac{1}{2}$ virgate and I plough in demesne, and the villeins $I_{2} \frac{1}{2}$ virgates and 6 ploughs. There the king has 17 villeins, 4 bordars, 3 serfs, 4 beasts, 40 sheep, I 5 acres of wood(land), and 1 acre of meadow. This manor pays 60 shillings by weight; when Baldwin the sheriff first had it to farm it was paying the same.

The king has a manor called Lrstona [Lifton] ${ }^{8}$ which Queen Editda held T.R.E., and it paid geld for $3 \frac{1}{2}$ virgates. This 25 ploughs can till. Thereof the king has $\frac{1}{2}$ virgate and 5 ploughs in demesne, and the villeins $1 \frac{1}{2}$ virgates and I4 ploughs. There the king has 26 villeins, 24 bordars, I 2 serfs, 55 beasts, I 7 swine, 250 sheep, 50 goats, also 40 acres of wood(land), 40 acres of meadow, and 42 acres of pasture. This manor pays 15 pounds a year. Colvin farms it of the king. 'To this manor belonged two (other) manors T.R.E. One is called LAMLiner (Exch. Lanliner) [Lanliner] and the other $\mathrm{T}_{\mathrm{RE}-}$ BICHEN [Trebichen]. The count of Mortain now holds both. ${ }^{9}$

The king has a manor called TAUETona [South Tawton] ${ }^{10}$ which Guitda [Githa] held T.R.E., and it paid geld for 3 hides and $I$ virgate. These 50 ploughs can till. Thereof the king has $\frac{1}{2}$ hide and 8 ploughs in demesne and the villeins $I_{\frac{1}{2}}$ hides and 1 ferding and 36 ploughs. There the king has 50 villeins, 30 bordars, 12 serfs, 100 beasts, 400 sheep, 70 goats, wood(land) 2 leagues in length by 2 furlongs in breadth, 6o acres of meadow, and pasture

come in the following order Listone, Chentone, Nortmoltone, and Wenfort. Next-with the heading, 'The following manors Gytha the mother of Earl Herald held'-come (South) Tauetone, Hertitone, Toritone, Wirige, Wodeberie, Cedelintone, Sireford, and Tovretone. Then-after the heading, "The following 14 lands Earl Herald held'-are enumerated Bradestone, (Black) Torintone, Haldeword, Sepesberie, Tauestoche, Mollande, Morbade, Alfintone, Topeshant, Mortone, Coletone (Raleigh), Hamistone, Spicewite, and (King's) Nimetone. Uluredintone comes afterwards without any heading. It had belonged to Tavistock. Lastly-with the heading, "The underwritten lands Earl Lewin held'-come Baverdone, Pinnoch, Chenemetone, Witeford, and Langeford Lestre. And then without any heading at all comes Clistone, described as "aforetime the land of Ordulf.'

${ }^{7}$ Bradstone in Lifton hundred.

8 The hundred-manor of Lifton.

${ }^{9}$ Lanliner and Trebichen in Cornwall.

${ }^{10}$ The hundred-manor of South Tawton, now united with Wonford hundred. The shortage of 1 hide 3 ferlings between the total assessments and the assessments of the king and the villeins together is probably represented by Ash I $\frac{1}{2}$ virgates, Parford 1 virgate, Escapeleia I virgate I ferling, and Cocktree I virgate. See Trans. Devon Assoc. xxxiv, 588. 


\section{A HISTORY OF DEVONSHIRE}

4 leagues in length by 4 leagues in breadth. This manor pays 48 pounds by weight; when fol. 93b.

Baldwin received it it paid the same. Within this manor is a manor called AIssa [Ash ${ }^{1}$ which Ulvric (Exch. Ulfric) held T.R.E. in parage (pariter; Exch., pro manerio), and it paid geld for $I \frac{1}{2}$ virgates. This 3 ploughs can till. There the king has 6 villeins, who have 3 ploughs; there also the king has I serf, Io acres of meadow, and 8 acres of pasture. It is worth 30 shillings, and was entered upon as part of (occupata in) the aforesaid manor in King William's time.

The king has a manor called TorintonA [Torington] ${ }^{2}$ which Earl Harold held T.R.E., and it paid geld for 2 hides all but 1 virgate. These 3 I ploughs can till. Thereof the king has $\frac{1}{2}$ hide and 6 ploughs in demesne, and the villeins I hide I virgate and 25 ploughs. There the king has 20 villeins, 22 bordars, Io swineherds, I 5 serfs, 80 beasts all but 2, 30 swine 200 sheep, 50 goats, also wood(land) 1 league in length by $\frac{1}{2}$ league in breadth. This manor pays I 8 pounds by weight; when Baldwin the sheriff received it it was paying the same. The manor of Podiforda [West Putford $]^{3}$ pays 30 pence yearly to this manor.

The king has a manor called HaLdeurd (Exch. Haldeword) [Holsworthy] ${ }^{4}$ which Earl Harold held T.R.E., and it paid geld for 2 hides all but $I_{\frac{1}{2}}$ virgates. These 30 ploughs can till. Thereof the king has I virgate and 8 ploughs in demesne, and the villeins $I_{2} \frac{1}{2}$ hides and 20 ploughs. There the king has 40 villeins, 20 bordars, I 5 serfs, 100 beasts all but 10,30 swine, 300 sheep, 20 goats, I furlong (Exch. I acre) of wood(land), 80 acres of meadow, also pasture I league in length and another in breadth. This manor pays 12 pounds by weight. When Baldwin the sheriff received it it paid the same.

The king has a manor called Hertitona [Hartland] ${ }^{5}$ which Guitda [Githa] held T.R.E., and it paid geld for 9 hides. These I Io ploughs

1 Ash in South Tawton in Wonford hundred.

The hundred-manor of Blacktorington. Geldroll, fol. $65, \mathrm{~A}, \mathrm{I}$.

3 West Putford in Blacktorington hundred, fol. 4 I 2.

- Holsworthy in the hundred of Blacktorington. The excess of the king's and the villeins' assessments (which amount together to I hide 3 virgates) over the total assessment of $\mathrm{I}$ hide $2 \frac{1}{2}$ virgates, suggests that the $\frac{1}{2}$ virgate of Chilsworthy (fol. $48 \mathrm{I}$ ) had been taken out of the villeins' assessment. On the other hand, the king's exemption being $3 \frac{1}{2}$ hides in this hundred (Geldroll, fol. 65, A, I), whereas the assessments of 'Toritona' $1 \frac{3}{4}$ hides, and 'Haldeurdi' I hide $2 \frac{1}{2}$ virgates, together only amount to 3 hides $I_{\frac{1}{2}}$ virgates, suggests that the half-virgate of Chilsworthy must have been there treated as royal demesne.

The hundred-manor of Hartland. Geldroll, fol. $65, \mathrm{~A}, \mathrm{I}$. can till. Thereof the king has 1 hide and I 5 ploughs in demesne, and the villeins 8 hides and 30 ploughs. There the king has 60 villeins, 45 bordars, 30 serfs, 137 beasts, 50 swine, 700 fol. 94 .

sheep, I 00 goats, also I 2 acres of wood(land), IO acres of meadow and pasture I league in length by another in breadth. This manor pays 48 pounds by weight; when Baldwin the sheriff received it it was paying 23 pounds by weight.

The king has a manor called SePEsBeria [Shebbear ${ }^{6}$ which Earl Herald held T.R.E., and it paid geld for 5 hides. These 37 ploughs can till. Thereof the king has 1 hide and 10 ploughs in demesne, and the villeins have 4 hides and 23 ploughs. There the king has 36 villeins, I 4 bordars, 20 serfs, 6 swineherds, 100 beasts all but Io, 30 swine, 500 sheep, 50 goats, wood(land) I league in length by as much in breadth, 30 acres of meadow, by pasture I league in length and as much in breadth. It pays yearly I 8 pounds by weight.

The king has a manor called Toritona [Torington] ${ }^{7}$ which Geda [Githa] held T.R.E., and it paid geld for I virgate and I ferling. This 7 ploughs can till. Thereof the king has I ferding and I plough in demesne, and the villeins have I virgate and 5 ploughs. There the king has 6 villeins, 4 bordars, 3 serfs, 3 beasts, 50 sheep, 2 acres of wood(land), I acre of meadow and 8 acres of pasture. It pays 40 shillings a year by weight.

The king has a manor called Baverdona [Beaford] ${ }^{8}$ which Earl Lewyn held T.R.E., and it paid geld for I hide $I \frac{1}{2}$ virgates. This 18 ploughs can till. Thereof the king has $1 \frac{1}{2}$ virgates and 2 ploughs in demesne, and the villeins have I hide and 9 ploughs. There the king has I 6 villeins, 5 bordars, 5 serfs, I 4 beasts, 73 sheep, 28 goats, also 50 acres of wood(land), 5 acres of meadow, and 50 acres of pasture. It pays yearly 8 pounds by weight.

fol. $94 \mathrm{~b}$.

The king has a manor called Tauestocha [Tawstock] ${ }^{9}$ which Earl Harold held T.R.E., and it paid geld for 5 hides. These 80 ploughs can till. Thereof the king has 2 virgates and ro ploughs in demesne, and the villeins $4 \frac{1}{2}$ hides and 2 I ploughs. There the king has 60 villeins, and 5 houses in Exeter, also 7 swineherds who render 35 swine, 18 serfs, 67 beasts, 28 swine, 500 sheep, 20 acres of wood(land), I 2 acres of

6 The hundred-manor of Merton or Shebbear.

${ }^{7}$ Little Torington (T.W.W.) ; or Upcot on the Toridge in Sheepwash (O.J.R.), still held with Hartland.

${ }^{8}$ Beaford in the hundred of Shebbear.

- Tawstock in the hundred of Fremington. Geldroll, fol. $65 b, A, 1$. 


\section{HOLDERS OF LANDS}

meadow and pasture I league in length and another in breadth. It pays 24 pounds by weight. When Baldwin the sheriff received it it paid the same.

From the aforesaid manor called Tauestoca [Tawstock] a manor has been taken away called Bichenelia [Bickingleigh] ${ }^{1}$ which belonged there T.R.E., and it paid geld for $1 \frac{1}{2}$ virgates. These ro ploughs can till. Twelve villeins are there, also 20 acres of meadow and 100 acres of pasture. It pays 4 pounds yearly. Now it is wrongfully included in the manor called Bichentona [High Bickington].

The king has a manor called Chentona [Kenton] ${ }^{2}$ which Queen Eideta (Eddida) held T.R.E., and it paid geld for 3 hides and I ferding. These 20 ploughs can till. Thereof the king has in demesne 1 hide and 4 ploughs, and the villeins 2 hides I ferding and 15 ploughs. There the king has 30 villeins, 10 bordars, 6 serfs, 4 swineherds paying 20 shillings a year, 8 saltworkers paying 20 shillings a year, 20 beasts, 200 sheep, 20 goats, also I mill paying 50 pence a year, wood(land) $\frac{1}{2}$ league in length by 4 furlongs in breadth, IO acres of meadow and I 50 acres of pasture. It is worth 30 pounds a year.

The king has a manor called Nortmontona [North Molton] ${ }^{3}$ which the queen held T.R.E., and it paid geld for $I \frac{1}{2}$ hides. These Ioo ploughs can till. Thereof the king has in demesne I virgate and 7 ploughs, and the villeins $3 \frac{1}{2}$ virgates and 40 ploughs. There the king has 44 villeins, 50 bordars, 4 ironworkers (ferruarii), I 5 swineherds, I I serfs, 30 beasts, I 70 sheep, 30 goats, I league of coppice taking length and breadth, 2 leagues of meadow and 2 leagues of pasture. It pays 45 pounds a year.

sol. 95.

The king has a manor called Mollanda [Molland] ${ }^{4}$ which Harold held T.R.E., and it paid geld for 4 hides and $I$ ferding. These 40 ploughs can till. Thereof the king has in demesne I hide and 3 ploughs, and the villeins 3 hides I ferding and 16 ploughs. There the king has 30 villeins, 20 bordars, I o serfs, 30 beasts, 70 sheep, 15 acres of wood(land), 12 acres of meadow and 3 leagues of pasture taking length and breadth. It pays yearly 24 pounds by weight. And to that manor of Molland has

' Now called Langleigh, an outlier of High Bickington in North Tawton hundred (O.J.R.).

Kenton manor in Exminster hundred.

3 The hundred-manor of North Molton now united with South Molton hundred. Pulham and Praunsley in 'Twitchin, assessed at $1 \frac{1}{2}$ virgates, represent the shortage between the total assessment and the king's and villagers' assessments together.

1 The hundred-manor of Molland Botreaux, now united with South Molton hundred. been added a manor called Blacapola [Blackpool] ${ }^{5}$ which Edward held T.R.E., and it paid geld for $\frac{1}{2}$ hide. This 2 ploughs can till. There he has 5 villeins and $\mathrm{I}$ serf. It is worth 20 shillings a year weighed and assayed (ad pondus et arsuram). Also to that manor has been wrongfully added a certain manor called Nimeta [an Intake $]^{6}$ which is worth 15 shillings a year. ${ }^{7}$

The king has a manor called Morbatha [Morbath] ${ }^{8}$ which Harold held T.R.E., and it paid geld for 3 hides. This 20 ploughs can till. Thereof the king has in demesne I hide and I plough, and the villeins 2 hides and I 5 ploughs. There the king has 20 villeins, 13 bordars, I 2 serfs, 40 acres of wood(land), IO acres of meadow, and 20 of pasture. It pays 7 pounds a year.

The king has a manor called Clistona [Broad Clyst ${ }^{9}$, which Ordulf held T.R.E., and it paid geld for $9 \frac{1}{2}$ hides. These 35 ploughs can till. Thereof the king has in demesne 2 hides and I plough, and the villeins $6 \frac{1}{2}$ hides and 26 ploughs. There the king has 35 villeins, 7 colberts, 30 bordars, I I serfs, I rouncey (runcinus), Io beasts, 4 swine, Ioo sheep, I mill paying 20 shillings a year, 150 acres of wood(land), 40 acres of meadow and $\frac{1}{2}$ league of pasture. Worth 24 pounds a year, by weight.

To the manor called Molland belongs the third penny of the hundreds of Northmolton, Bampton, and Braunton, and (the dues of every) third beast agisted on the moors (tercium animal pascue morarum). These customary dues the king has not had since he has had England. ${ }^{10}$

${ }^{5}$ Blackpool in the parish of Chittlehampton in the hundred of South Molton (O. J. R.), Feud. Aids, i, 326 ; or Blackpool in South Molton (T.W.W.).

- Warkleigh in the hundred of South Molton, held together with Blackpool of the honour of Stoke Curcy (O. J. R.), Testa de Nevill, p. $183 b$; or Twitchin (T. W. W.).

${ }^{7}$ Fol. $499^{b}$ says $\frac{1}{2}$ virgate held by two sisters in parage.

a Morbath manor in Bampton hundred.

The hundred-manor of Broad Clyst. There is seemingly a shortage of I hide between the sum of the king's and villagers' and the total assessment, but there is a doubt whether $6 \frac{1}{2}$ should not be read as $7 \frac{1}{2}$.

${ }^{10}$ See fol. $499 b$. The third penny of the profits of jurisdiction called the pleas of the shire and that of the issues of the county town were the normal perquisites of the earl, the final remnant of the earl's judicial functions. See the Dialogus de Scaccario, Oxford University Press (1902), p. 205. From time immemorial all the landholders of Devon, excepting those in the boroughs of Totnes and Barnstaple, enjoyed the right of depasturing the cattle levant and couchant on their holdings in the forest by day. For keeping them in the forest by night they paid lying-out or agistment dues, the existing stone huts being used by those looking after the cattle from distant parts of the county. When the rest of the county was dis- 
fol. 95b.

\section{A HISTORY OF DEVONSHIRE}

The king has a manor called WENFORT [Wonford] ${ }^{1}$ which Queen Edida held T.R.E., and it was assessed as half a hide. This 20 ploughs can till. Thereof the king has in demesne I virgate and 2 ploughs; the villeins have 8 ploughs. There the king has 18 villeins, Io bordars, 10 serfs, 45 sheep, 14 goats, 60 acres of wood(land), 30 acres of meadow, also pasture half a league in length and the same in breadth. It pays 18 pounds a year, at 20 pence to the ounce (ore). ${ }^{2}$

The king has a manor called Pinnoc [Pinhoe ${ }^{3}$ which Earl Liwin held T.R.E., and it paid geld for 2 hides all but a virgate $(E x c h$. I hide and 3 virgates). This Io ploughs can till. Thereof the king has 3 virgates and I plough in demesne, and the villeins have 7 ploughs. There the king has 8 villeins, 6 bordars, I serf, ro beasts, IOO acres of wood(land), 20 acres of meadow and 100 acres of pasture. It pays 6 pounds a year by weight. Under this manor the abbot of Battle holds the church, to which belongs I virgate of the aforesaid land. ${ }^{4}$ Worth 5 shillings a year.

The king has a manor called Alfintona [Alphington $]^{5}$ which Earl Harald held T.R.E. It paid geld for $2 \frac{1}{2}$ hides. These 16 ploughs can till. Thereof the king has $\frac{1}{2}$ hide and 2 ploughs in demesne, and the villeins have 14 ploughs. There the king has 32 villeins, 5 bordars, 6 serfs, I rouncey, 5 beasts, 40 sheep, 20 acres of wood(land), coppice $x$ league in length by $\frac{1}{2}$ in breadth, and 3 acres of meadow. It pays 6 pounds a year by weight.

The king has a manor called Toprshant [Topsham] ${ }^{6}$ which Earl Harald held T.R.E., and it paid geld for I hide. This 12 ploughs can till. Thereof the king has $\frac{1}{2}$ hide and

afforested in 1204 , this right continued in Dartmoor Forest, where agistment dues are still payable, but the looking after is done by "moormen" appointed by the Duchy of Cornwall at a fixed charge. See Dartmoor Preservation Society's Publications.

1 The hundred-manor of East and West Wonford. Probably Pocombe in Alphington represents the I virgate held by Owen the king's reeve in Geldroll, fol. 7 I, B, I I ; see Testa de Nevill (Rec. Com.), 198a; Trans. Devon Assoc. xxxvi, I 7 I.

${ }^{2}$ As the pennies struck by the moneyers contained $\frac{1}{21}$ instead of $\frac{1}{20}$ ounce of silver, these pounds were short weight or currency pounds (numero). See Trans. Devon Assoc. xxix, $454 n .2$; and Round, in V.C.H. Worcs. i, 294.

${ }^{3}$ Pinhoe manor in Wonford hundred.

- Monkerton alias Monkenland in Pinhoe.

${ }^{5}$ Alphington in Wonford hundred.

${ }^{6}$ Topsham in Wonford hundred. Topsham had been given by Aedelstan in 937 to the monastery at Exeter. Kemble, Cod. Dipl. ii, 204, No. 369, and iii, 4I I, and Birch, Cart. Saxon. ii, 428, give the boundaries.
I plough, and the villeins have 12 ploughs. There the king has 16 villeins, 12 bordars, 5 serfs, I rouncey, 5 beasts, 50 sheep, Io acres fol. 96.

of meadow, and 60 acres of pasture. It pays 6 pounds a year by weight.

The king has a manor called WIRIGA [Witheridge] ${ }^{7}$ which Gida held T.R.E., and it paid geld for I virgate. This 3 ploughs can till. Thereof the king has $\frac{1}{3}$ virgate and I plough in demesne, and the villeins have $\frac{2}{3}$ of that virgate and 3 ploughs. There the king has 3 villeins, 3 bordars, 2 serfs, 26 sheep, 12 acres of wood(land), 6 acres of meadow and 40 of pasture. It pays 6 pounds a year, and was worth the same when he received it. And to this manor has been united the land of two thanes ${ }^{8}$ who held in parage (pariter; Exch., libere) T.R.E., and it paid geld for 3 ferdings. These I plough can till, and upon them also 3 villeins are settled (adbuc manent) paying 5 shillings a year to the aforesaid manor.

The king has a manor called Mortona [Moreton] ${ }^{9}$ which Herald held T.R.E., and it paid geld for 3 hides. These 20 ploughs can till. Thereof the king has in demesne 1 hide and 3 ploughs, and the villeins 2 hides and 8 ploughs. There the king has 16 villeins, 6 bordars, 6 serfs, 20 beasts, 130 sheep, wood(land) I league in length by $I$ furlong in breadth, 20 acres of meadow and 60 of pasture. It pays 12 pounds weighed and assayed (ad pondus et ad arsuram), and paid the same when Baldwin first received it. With the aforesaid manor goes (adjacet) the third penny of the hundred of fol. $96 \mathrm{~b}$.

Taignebrige [Teignbridge].

The king has a manor called WodererIa [Woodbury] $^{10}$ which Guitda held T.R.E., and it paid geld for Io hides. These 35 ploughs can till. Thereof the king has 5 hides and 2 ploughs in demesne, and the villeins 5 hides and 20 ploughs. There the king has 30 villeins, 22 bordars, 6 serfs, 2 rounceys, 15 beasts, 4 swine, 60 sheep, I mill paying 7 shillings and 6 pence, wood(land) I league in length by $\frac{1}{2}$ in breadth, 30 acres of meadow, and 300 acres of pasture. It pays 23 pounds by weight; when Baldwin received it 18 pounds. Thereof the abbot (Exch. the church) of St. Michael of the Mount holds the church and the land which the priest (sacerdos) held T.R.E., that is $\frac{1}{2}$ hide (Exch.

${ }^{7}$ The hundred-manor of Witheridge.

8 Probably Yeatheridge and Burridge, two outliers of Witheridge between Worlington and Lapford. Trans. Devon Assoc. xxxiv, 620 .

${ }^{9}$ Moreton Hampstead in the hundred of Teignbridge.

${ }_{10}$ Woodbury in the hundred of East Budleigh. 


\section{HOLDERS OF LANDS}

I hide) and I virgate and $\frac{1}{2}$ ferling. ${ }^{1}$ Worth 20 shillings with common of pasture.

The king has a manor called Coletona [Colyton Raleigh] ${ }^{2}$ which Earl Harold held T.R.E., and it paid geld for 3 hides. These I6 ploughs can till. Thereof the king has $1 \frac{1}{2}$ hides and 2 ploughs in demesne, and the villeins $1 \frac{1}{2}$ hides and 12 ploughs. There the king has 20 villeins, 8 bordars, 6 serfs, 5 forest mares (equas silvestraes), ${ }^{3} 4$ beasts, 50 sheep, 9 goats, I mill paying 7 shillings and 6 pence, 40 acres (of woodland), 16 acres of meadow and 400 acres of pasture. It pays 8 pounds by weight, the same as when Baldwin first received it. To this manor half a virgate of land has been added which a certain thane held in parage (pariter; Exch., libere) T.R.E. and he could go to any lord he liked. Worth 4 shillings a year.

The king has a manor called Hamistona [Hempston] $^{6}$ which Earl Harold held T.R.E., and it paid geld for 2 hides. These 6 ploughs can till. Thereof he has I hide and I plough in demesne and the villeins I hide and 5 ploughs. fol. 97.

There the king has 8 villeins, 7 bordars, 4 serfs, 48 sheep, 40 goats, 24 acres of wood(land), 4 of meadow and 24 of pasture. This manor pays 40 shillings by weight; when Baldwin received it the same.

The king has a manor called EsPicewita (Exch. Spicewite) [Speechwick] ${ }^{b}$ which Earl Harold held T.R.E., and it paid geld for I hide. This 8 ploughs can till. Thereof the king has $\frac{1}{2}$ hide in demesne and the villeins $\frac{1}{2}$ hide and 4 ploughs. There the king has 8 villeins, 4 bordars, 5 serfs, wood(land) I league in length by I furlong in breadth and 100 acres of pasture. It pays 60 shillings by weight; when Baldwin received it the same.

The king has a manor called Chienemetona (Exch. Chenemetone) [Kilmington] ${ }^{7}$ which Earl Lewin held T.R.E., and it paid geld for 2 hides. These Io ploughs can till. Thereof the king has in demesne $\frac{1}{2}$ hide and I plough, and the villeins $1 \frac{1}{2}$ hides and 6 ploughs. There the king has 12 villeins, 8 bordars, 2 serfs, 3 beasts,

${ }^{1}$ Halstow manor in Woodbury.

2 Colyton Raleigh in East Budleigh hundred.

3'Equus silvestris' $=$ forest pony in Pipe Roll of Bishopric of Winchester, p. xxxi.

${ }^{4}$ Bystock an outlier of Colyton Raleigh, locally in Withycombe Raleigh.

${ }^{3}$ Little Hempston alias Hempston Arundel in Haytor hundred (O. J. R.) ; or Broad Hempston (T.W.W.).

'Speechwick the chief manor of Widecombe-inthe-Moor in Haytor hundred.

${ }^{7}$ Kilmington in Axminster hundred the parish.
I mill paying 5 shillings, 6 acres of wood(land), 30 acres of meadow, and 12 furlongs and 12 acres of pasture. It pays 7 pounds a year weighed and assayed (ad pondus et combustionem).

The king has a manor called WITEFoRT (Exch. Witeford) [Whitford $]^{8}$ which Earl Lewin held T.R.E., and it paid geld for 5 hides. These 16 ploughs can till. Thereof the king has in demesne I hide and I plough, and the villeins 4 hides and 12 ploughs. There the king has I 4 villeins, 13 bordars, 2 serfs, I rouncey, 6 beasts, 23 sheep, I mill paying 5 shillings a year, 5 furlongs of wood(land), 27 acres of meadow, and 3 furlongs of pasture. It pays I I pounds weighed and assayed, the same as when Baldwin received it.

The king has a manor called Cedelintona [Chillington] ${ }^{9}$ which Guitda held T.R.E., and it paid geld for 7 hides. These 53 ploughs can till. Thereof the king has in demesne 3 hides and 2 ploughs and the villeins 4 hides and fol. $97 \mathrm{~b}$.

36 ploughs. There the king has 43 villeins, 48 bordars, ro serfs, I 57 sheep, wood(land) 20 furlongs in length by $\frac{1}{2}$ league in breadth, 12 acres of meadow and I 50 acres of pasture. It pays 24 pounds weighed and assayed (ad pondus et ad combustionem); when Baldwin received it the same. With the aforesaid manor went (adjacebat) another manor in the time of King Edward called Sirefort [Sherford] ${ }^{10}$ which the abbot of Battle (Batailla ; Exch., Labatailge) now holds, and it pays geld for $\frac{1}{2}$ hide. This 8 ploughs can till. Thereof the abbot has in demesne I virgate and I plough; and the villeins I virgate and 6 ploughs. There the abbot has 4 villeins, I I bordars, 2 serfs, 37 sheep, 6 acres of coppice and 4 of meadow. Worth 3 pounds a year; when the abbot received it the same.

The king has a manor called LANGEForda [Langford] ${ }^{11}$ which Earl Lewin held T.R.E., and it paid geld for 3 hides. These 20 ploughs can till. Thereof the king has in demesne I hide and I plough; and the villeins have 2 hides and 13 ploughs. There the king has I 6 villeins, Io bordars, 6 serfs, 8 beasts, 50 sheep, 7 acres of wood(land), 4 of meadow, and 200 of pasture. It pays I I pounds a year by weight; when Baldwin received it the same.

From this manor the king gave to Judhel 20 shillings (a year) which the borough of Totnes (burgum Tbotonensium; Exch., burgum

${ }^{8}$ Whitford manor in Shute in Colyton hundred.

- The hundred-manor of Chillington, or Colridge.

${ }^{10}$ Sherford in the hundred of Colridge, a chapelry of Stokenham.

${ }^{11}$ Langford Lestre in Ugborough in the hundreds of Ermington and Stanborough. Trans. Devon Assoc. xxxi, 390 ; xxxiii, 622. 


\section{A HISTORY OF DEVONSHIRE}

Totbeneis $)^{1}$ paid to this manor by way of farm (ad firmam).

Baldwin pays to the king 375 pounds from the estates (de terris) of the earls by way of farm yearly. ${ }^{2}$

Gotselin pays yearly to the king for the estates of Queen Edida 108 pounds. ${ }^{3}$ I Raginald ${ }^{4}$ have been paying yearly towards the king's farm 24 pounds for the estate of Ordulf. ${ }^{5}$

fol. 98 .

The king has a manor called Nrmetona [Nympton] $^{6}$ which Earl Herald held T.R.E., and it paid geld for 3 hides. These 50 ploughs can till. Thereof the king has I hide and 3 ploughs in demesne, and the villeins have 2 hides and 16 ploughs. There the king has 40 villeins, 4 bordars, 7 serfs, 6 swineherds, I 6 beasts, Ioo sheep all but 8, wood(land) I league in length and breadth, 8 acres of meadow, and I league of pasture every way. It pays I 8 pounds a year by weight. To this manor has been united a manor of half a virgate ${ }^{7}$ which I thane held T.R.E., and he could go with his land to what lord he liked (Exch. freely). It pays 7 shillings towards the king's farm.

The king has a manor called Tovretona [Tiverton] ${ }^{8}$ which Gida held T.R.E., and it paid geld for $3 \frac{1}{2}$ hides. These 36 ploughs can till. Thereof the king has in demesne $1 \frac{1}{2}$ hides, and the villeins 2 hides and 30 ploughs. There the king has 35 villeins, 24 bordars, 19 serfs, 3 swineherds paying Io swine, 28 sheep, 2 mills paying 5 shillings and 6 pence, coppice $I$ league in length by $6 \frac{1}{2}$ furlongs in breadth, 4 furlongs of wood(land), 14 acres of meadow, and 40 acres of common pasture. It is worth I 8 pounds a year weighed and assayed (ad pondus et arsuram). ${ }^{9}$

The king has a manor called ULUREDINTONA [Werrington] $^{10}$ which Earl Harald's mother

1 Totnes in Colridge hundred. See fol. 334.

2 i.e. the 8 estates held by Gytha, Harold's mother, the I4 estates of Earl Harold, and the 5 estates of Earl Lewin as enumerated above. In making up f. 375 the added estates are not brought into account. (T. W. W. in Trans. Devon Assoc. xxxii, 529.)

${ }^{3}$ i.e. the 4 manors which Edida held, enumerated above.

"See Introduction, p. 377.

'viz. 'Clistone ' which follows ' Langeford' without any heading in the Exchequer book, but is there described as Ordulf's land.

${ }^{6}$ King's Nympton in Witheridge hundred.

'Probably Garland in King's Nympton in Witheridge hundred.

${ }^{8}$ Tiverton manor in Tiverton hundred. Tiverton was one of the manors bequeathed by King Alfred to his younger son. Kemble, Cod. Dipl. ii, 112 , No. 314. Trans. Devon Assoc. ix, 214.

${ }^{9}$ This entry is again repeated, fol. $110 b$.

${ }^{10}$ (Written Olwritona below, fol. 178, and in Geldroll, fol. 65, A, 9 Oluritona.) The hundredmanor of Werrington.
(Exch. Gytha) held T.R.E. and it paid geld for $6 \frac{1}{2}$ hides. This 86 ploughs can till. Of these (De bis) the king has in demesne I hide and 6 ploughs, and the villeins the rest of the land and 27 ploughs. There the king has I 6 (Exch. 16) villeins, 25 bordars, 25 colberts (quolibertos), 20 serfs, 25 beasts, 150 sheep, 20 swine, 20 acres of wood(land), I00 acres of meadow, and 500 acres of pasture. It pays 20 pounds by tale and in King Edward's time it used to pay 24 pounds. Of these $6 \frac{1}{2}$ hides the count of Mortain has $\frac{1}{2}$ hide which belonged there T.R.E. ${ }^{11}$

\section{LAND OF QUEEN MAHILLA IN DEVENESIRA [DEVONSHIRE] ${ }^{12}$} fol. 208.

The king has a manor called Levia [Lew] ${ }^{13}$ which Bristric held T.R.E., and it was part of the land of Queen M[ahilla] and paid geld for I hide, I virgate, and I ferling. This 12 ploughs can till. Thereof the king has I virgate and 4 ploughs in demesne, and the villeins $I$ hide and 10 ploughs. There the king has 20 villeins, 7 bordars, 7 serfs, 10 forest mares, 50 beasts, Io swine, I 00 sheep, 25 goats, 20 acres of wood(land), 30 of meadow, and pasture 8 furlongs in length by 4 in breadth. This manor Goscelm holds at farm, and pays for it 9 pounds yearly by tale.

The king has a manor called HaLgewirla $\left[\right.$ Halwill ${ }^{14}$ which Bristric held T.R.E., and it paid geld for I virgate. This 5 ploughs can till. Thereof the king has 1 ferling and 2 ploughs in demesne, and the villeins 3 ferlings and 5 ploughs. There the king has 10 villeins, I bordar, and 6 serfs, 33 beasts, 20 swine, 50 sheep, 2 acres of wood(land), 40 of meadow, and pasture I league in length by 2 furlongs in breadth. This manor Goscelm holds at a farm-rent, paying for it 70 shillings by tale.

The king has a manor called Cloveleia [Clovelly] ${ }^{15}$ which Bristric held T.R.E., and it paid geld for 3 hides. These I 2 ploughs can till. Thereof the king has I hide and 5 ploughs

"Bullapit Tamar in Werrington (O. J. R.). Boyton in Cornwall (T. W. W.).

${ }^{12}$ Instead of this heading the Exchequer has : 'The underwritten lands were held by Brictric and afterwards by Queen Mathilda.' Moreover, since ' Oluardesdona' was held by Boia and not by Brictric, it was placed at the end after those held by Brictric in the Exchequer copy. As to Bristric or Brictric, son of Algar, see Freeman, Norman Conquest, iv, 762 .

${ }^{13}$ North Lew in the hundred of Blacktorington. The ferling unaccounted for probably represents Gorhuish.

${ }^{14}$ Halwill in the hundred of Blacktorington.

${ }^{15}$ Clovelly in the hundred of Hartland. 


\section{HOLDERS OF LANDS}

in demesne, and the villeins 2 hides and 7 ploughs. fol. ro8b.

There the king has 16 villeins, 1 I bordars, Io serfs, 45 beasts, I 5 swine, Ioo sheep, and I 8 goats, also 40 acres of wood(land), 30 of meadow, and pasture I league in length by $\frac{1}{2}$ in breadth. This manor pays I 2 pounds by tale. Goscelm holds it at a farm-rent; when he received it it was paying 6 pounds.

The king has a manor called BEDIFoRdA [Bideford] ${ }^{1}$ which Bristric held T.R.E., and it paid geld for 3 hides. These 26 ploughs can till. Thereof the king has $\frac{1}{2}$ hide and 4 ploughs in demesne, and the villeins $2 \frac{1}{2}$ hides and 20 ploughs. There the king has 30 villeins, 8 bordars, I 4 serfs, I 8 beasts, 300 sheep, I 50 acres of wood(land), 10 acres of meadow, and 20 of pasture. It pays 16 pounds yearly. To this manor was appurtenant (adjacebat) a certain fishery T.R.E. which pays 25 shillings yearly.

The king has a manor called Liteham [Littleham] ${ }^{2}$ which Bristric held T.R.E., and it paid geld for I hide. This 8 ploughs can till. Thereof the king has I virgate and I plough, and the villeins 3 virgates and 4 ploughs. There the king has I 2 villeins, 3 bordars, 7 serfs, 7 beasts, 100 sheep, 60 acres of wood(land), Io acres of meadow, and 20 acres of pasture. It pays 3 pounds a year. These two manors Goselm holds at farm of the king.

The king has a manor called LANGTREwa [Langtree $]^{3}$ which Bristric held T.R.E., and it paid geld for 2 hides all but $\frac{1}{2}$ virgate. These 20 ploughs can till. Thereof the king has $\frac{1}{2}$ hide and 2 ploughs in demesne, and the villeins have $I$ hide, $1 \frac{1}{2}$ virgates, and 16 ploughs. There the king has 24 villeins, 2 bordars, 8 serfs, fol. $\mathbf{z o g}$.

2:swineherds, 6 beasts, 60 sheep, wood(land) I league in length and the same in breadth, also I 5 acres of meadow. It pays 7 pounds and 5 shillings yearly.

The king has a manor called EDEsLega [Iddesleigh] ${ }^{4}$ which Bristric held T.R.E., and it paid geld for 3 hides. These 22 ploughs can till. Thereof the king has $I$ hide and 4 ploughs in demesne, and the villeins have 2 hides all but I virgate and 16 ploughs. There the king has 24 villeins, 15 serfs, I 5 beasts, 60 sheep, wood(land) 2 leagues in length by $I$ in breadth, and 15

${ }^{1}$ Bideford in the hundred of Shebbear.

'Littleham in the hundred of Shebbear.

3 Langtree in the hundred of Shebbear, the parish with the outlier of Alscot next Buckland Filleigh.

"Iddesleigh in the hundreds of Shebbear and North Tawton. acres of meadow. It pays 14 pounds yearly. Of this land Walter [de Clavil] has I virgate which he holds of the king. ${ }^{5}$ Alwatet (Exch. Alware) held it T.R.E., and he could not become independent (separari) of Bristric with his land. This land 3 ploughs can till, and it is worth 20 shillings yearly. To this manor belong $\frac{1}{2}$ hide and $\frac{1}{2}$ virgate which lie in the hundred of Tawton.

The king has a manor called Wincheleia [Winkleigh] ${ }^{6}$ which Bristric held T.R.E., and it paid geld for $5 \frac{1}{2}$ hides. These 40 ploughs can till. Thereof the king has 2 hides and 8 ploughs in demesne, and the villeins have $3 \frac{1}{2}$ hides and 40 ploughs. There the king has 60 villeins, 16 serfs, 10 swineherds, also 38 beasts, I 5 swine, I60 sheep, 500 acres of wood(land), 80 acres of meadow, and pasture I league in length and another in breadth. In this manor is also a park for beasts. It pays 30 pounds a year by tale. Of the aforesaid land Norman the park-keeper (custos parci) has $I \frac{1}{2}$ virgates worth I 2 shillings and 6 pence a year. ${ }^{7}$ Goscelm has it at farm.

fol. rogb.

The king has a manor called Aissa [Ash] ${ }^{8}$ which Bristric held T.R.E., and it paid geld for 2 hides less $\frac{1}{2}$ virgate. These 15 ploughs can till. Thereof the king has $\frac{1}{2}$ hide and 2 ploughs in demesne, and the villeins I hide, I virgate, and 10 ploughs. There the king has 14 villeins, 6 bordars, 10 serfs, 2 swineherds paying Io swine, I 7 beasts, 14 swine, 80 sheep, 35 goats, 200 acres of wood(land), 20 acres of meadow and pasture $\frac{1}{2}$ league in length by $\frac{1}{2}$ league in width. This manor pays 7 pounds in currency. Goscelm has it in charge.

The king has a manor which was Queen M[ahilda]'s called EsLAPAForda [Lapford] which Bristric held T.R.E., and it paid geld for $2 \frac{1}{2}$ hides. These I I ploughs can till. Thereof the king has in demesne $\frac{1}{2}$ hide and 3 ploughs and the villeins 2 hides and 8 ploughs. There the king has 18 villeins, I 2 bordars, 7 swineherds, 6 serfs, I rouncey, 30 beasts, I 8 swine, I 80 sheep, I 30 acres of wood(land), 20 acres of meadow, Io of pasture. It is worth yearly I 2 pounds and I 2 shillings. To this manor is appurtenant (adjacet) and included in the afore-

\footnotetext{
${ }^{3}$ Ash and Hill in Iddesleigh in Shebbear hundred, elsewhere written 'Iweslei,' where the tenant is called Aleuesclef (Exch. Aleuesdef)

${ }^{6}$ The hundred-manor of Winkleigh.

${ }^{7}$ Hollocombe in Winkleigh.

${ }^{8}$ Ashreigny alias Ringsash in the hundred of North Tawton. The shortage between the total assessments and the sum of the king's and villeins' assessment probably represents Alward Merta's half virgate of Arson (fol. 483).

${ }^{\circ}$ Lapford in North Tawton hundred.
} 


\section{A HISTORY OF DEVONSHIRE}

said value $\frac{1}{2}$ virgate of land which is called Eruescoma [Irishcombe]. ${ }^{1}$

The king has a manor called OLUARDESDONA (Exch. Ulwardesdone) [ $]^{2}$ which Boia held T.R.E., and itpaid geld for $1 \frac{1}{2}$ virgates. These 2 ploughs can till. Hadolf holds it of the king. There Hadulf has in demesne I virgate and I plough, and the villeins 2 ferlings and I plough. There Hadulf has 3 villeins, 2 serfs, 5 beasts, fol. rro.

2 swine, ro sheep, wood(land) 2 furlongs in length by $I$ in breadth, 3 acres of meadow and 2 furlongs of pasture. It pays to the king 10 shillings a year.

The king has a manor called Bichentona [Bickington] ${ }^{3}$ which Bristric held T.R.E., when it paid geld for $1 \frac{1}{2}$ hides and $\frac{1}{2}$ virgate. These 16 ploughs can till. Thereof the king has in demesne I virgate and 2 ploughs, and the villeins $5 \frac{1}{2}$ virgates and seven ploughs. There the king has I 4 villeins, 2 bordars, 3 serfs, 2 swineherds paying yearly I 6 swine, I 2 beasts, 50 sheep, I0O acres of wood(land), 8 acres of meadow, and 100 acres of pasture. It pays 12 pounds a year. To the aforesaid manor has been added another manor called Bicheneleia [Bickingleigh] ${ }^{4}$ which T.R.E. belonged to Tauestoca [Tawstock] and it pays to Bickington 4 pounds a year.

The king has a manor called MoRcheT [Morchard] ${ }^{5}$ which Bristric held T.R.E., and it paid geld for $\frac{1}{2}$ hide. This 8 ploughs can till. Thereof the king has I virgate and 2 ploughs in demesne, and the villeins have I virgate and 3 ploughs. There the king has 8 villeins, 2 serfs, 5 beasts, 40 sheep, 40 acres of $\operatorname{wood}($ land), and 2 acres of meadow. It pays yearly 4 pounds by tale. This manor was Queen Mathilda's.

fol. n1ob.

The king has a manor called Holecumba [Holcombe] ${ }^{6}$ which Bristric held T.R.E., and it paid geld for I hide. This 7 ploughs can till. Thereof the king has in demesne $\frac{1}{2}$ virgate and 2 ploughs and the villeins $3 \frac{1}{2}$ virgates and 5 ploughs. There the king has ro

' Irishcombe, an outlier of Lapford in North Tawton hundred.

${ }^{2}$ Probably in Down St. Mary in North Tawton hundred.

${ }^{3}$ High Bickington manor alias Womberleghe in the hundred of North Tawton.

- Langleigh alias 'Westera Langeleye next Womberleghe' (Pedes Fin. Index, 7I), an outlier of High Bickington next Tawstock (Trans. Devon Assoc. xxxiv, 727). Atherington (T. W. W.). See above, fol. $94 b$.

${ }_{5}^{3}$ Morchard Bishop in North Tawton hundred.

${ }^{6}$ Holcombe Burnel in Wonford hundred. villeins, 8 bordars, 4 serfs, 5 beasts, 50 sheep, 50 goats, and 1 ro acres of wood(land). It pays 8 pounds 15 shillings a year.

The king has a manor called Tovretona [Tiverton], \&c. ${ }^{7}$

The king has a manor called HaLsBretona $[\text { Halberton }]^{8}$ which Bristric held T.R.E., and it paid geld for 5 hides. These 28 ploughs can till. Thereof the king has in demesne $I_{2} \frac{1}{2}$ hides and 4 ploughs, and the villeins $3 \frac{1}{2}$ hides and 22 ploughs. There the king has 43 villeins, Io bordars, 8 serfs, 5 swineherds paying 30 swine; fol. rix.

also the king has 2 rounceys there, II beasts, I I swine, 150 sheep, 2 mills paying 10 shillings, wood(land) 16 furlongs in length by 13 in breadth, 36 acres of meadow, and pasture 5 furlongs in length by 3 in breadth. It pays 27 pounds yearly. Of those 5 hides Goscelm has I virgate of land, ${ }^{9}$ namely (part) of the villeins' land, where he has I plough, I bordar, and I serf. It paid 10 shillings towards the farmrent of Halberton when Goscelm received it.

The king has a manor called Aisbertona $[\text { Washburton }]^{10}$ which Bristric held T.R.E., and it paid geld for 3 hides. This Io ploughs can till. Thereof the king has in demesne I hide and 2 ploughs, and the villeins 2 hides and 3 ploughs. There the king has 7 villeins, 8 bordars, 4 serfs, I rouncey, 2 beasts, I00 sheep, 2 fisheries, I saltern, wood(land) I league in length by $\frac{1}{2}$ league in breadth, 3 acres of meadow, and 40 of pasture. It pays 4 pounds a year. This is the manor which Juhel held of the queen.

fol. IIy.

\section{LANDS OF ST. PETER OF THE CHURCH OF EXETER IN DEVENESCIRA ${ }^{11}$}

Bishop O[sbern] has a manor called Beria

${ }^{7}$ Repeated word for word as on fol. 98 .

8 The hundred-manor of Halberton. Of Halberton manor 3 virgates lie in Tiverton hundred (Geldroll, fol. $67, \mathrm{~B}, 6)$.

${ }^{9}$ Probably Woburnford alias Oburnford alias Pit Farm (O.J.R.).

${ }^{10}$ The manor of Washburton in Ashprington in the hundred of Colridge.

"11 The Exchequer Book reads : 'Land of the bishop of Exeter,' and begins with the bishop's holding in Exeter, the church of which was the head of his barony, followed by Crediton his principal manor. Then in the third place come Bury and Teignton followed by Dawlish, Ide, Staverton, and St. Mary Church, after which is a note: 'The above 4 manors are assigned for the support of the canons.' The rest follow in the order of the Exeter Book, omitting Staverton and St. Mary Church. 


\section{HOLDERS OF LANDS}

[Bury] ${ }^{1}$ which T.R.E. paid geld for I virgate. This 3 ploughs can till. There the bishop has 3 (Exch. 4) villeins who have 2 ploughs; there the bishop has also 3 bordars and 5 acres of meadow. It pays (Exch. is worth) 7 shillings and 6 pence a year.

The bishop has a manor called Chriterona [Crediton] $^{2}$ which T.R.E. paid geld for 15 hides. These 185 ploughs can till. Thereof the bishop has 6 hides and 13 ploughs in demesne and his knights (milites) and villeins have between them 9 hides and 172 ploughs. There the bishop has 264 villeins, 73 bordars, 40 serfs, 30 swineherds paying 150 swine yearly, 4 rounceys, 64 beasts, 57 swine, 400 sheep all but I2, I I 5 goats, I mill paying 30 pence a year, wood(land) 5 leagues in length and $\frac{1}{2}$ league in breadth, 80 acres of meadow and 200 acres of pasture. It is worth 75 pounds a year. When the bishop first received it it was paying 21 pounds.

Along with this manor the bishop claims (corrected into 'has') a manor called NIWENTONa [Newton], ${ }^{3}$ held by Domne, which paid geld for 3 hides. As to this manor Bishop Osbern produced his charters showing that his church was seised thereof before King Edward's reign; and besides, he asserts (adbuc dicit) that in King William's time he had a suit concerning this land, and proved his right to it (disraisnavit esse suam) by the witness of Frenchmen (francigenarum). It is worth 3 pounds.

The bishop has a manor called TAINTONa (Exch. Tantone) [Teignton] ${ }^{4}$ which T.R.E. paid geld for 18 hides. These 55 ploughs can till. Thereof the bishop has 5 hides and 4 ploughs in demesne, and the villeins have 13 hides and $5 \mathrm{I}$ ploughs. There the bishop has 57 villeins, 36 bordars, 14 serfs, 10 swineherds paying 35 swine yearly, 2 rounceys, 37 beasts, 7 swine, 400 sheep, 10 and 40 goats, also 9 houses

${ }^{1}$ Bury in Lapford in the hundred of North Tawton, which in Norden's Survey of 1598 is described as held of the manor of Crediton; the gift of Athelstan, Trans. Devon Assoc. xiii, I 19 (O.J.R.)

2 The hundred-manor of Crediton alias Kirton.

${ }^{3}$ Newton St. Cyres in Crediton hundred. See fol. 483. The Exchequer reads here: 'Moreover in King William's time he proved his right to it (diratiocinavit esse suam) before the king's barons.' Round, Cal. of Docts. France, 147, says : ' proved his right to it, a translation supported by a passage in his Feud. Engl. p. 138. But since Newton, notwithstanding the bishop's argument, is stated, fol. 483, to be held by Domne of the king, Mr. Whale, in Trans. Devon Assoc. xxxii, 524, suggests that this is an early instance of a transfer by means of a fictitious suit.

"The manor of Bishop's Teignton in the hundred of Exminster. fol. nxzb.

in the borough of Essecestre [Exeter] paying yearly 3 shillings, 24 salterns paying yearly 10 shillings, wood(land) I league in length by the same in breadth, IO acres of meadow, and pasture 4 leagues in length by $\frac{1}{2}$ league in breadth. It is worth 24 pounds a year; when the bishop received it it was worth $\$ 4$ pounds.

The bishop has a manor called DoukuIs [Dawlish ${ }^{5}$ which T.R.E. paid geld for 7 hides. These 30 ploughs can till. Thereof the bishop has I hide and 2 ploughs in demesne, and the villeins have 6 hides and 24 ploughs. This manor is assigned for the support (est de victu) of the canons. There the bishop has 30 villeins, 8 bordars, 3 serfs, 3 cows, 2 swine, roo sheep, coppice 3 furlongs in length and $I$ in breadth, 6 acres of meadow, and 12 acres of pasture. It is worth 8 pounds a year; when the bishop received it it was worth 7 pounds.

The bishop has a manor called IDA [Ide] ${ }^{6}$ which T.R.E. paid geld for 2 hides. These 6 ploughs can till. Thereof the bishop has I hide and I plough in demesne, and the villeins have I hide and 5 ploughs. There the bishop has II villeins, as many (Exch. II) bordars, 2 serfs, I rouncey, 3 beasts, 33 sheep, and 4 acres of coppice. Worth 40 shillings a year; when the bishop received it it was worth the same. This is assigned for the support of the canons.

The bishop has a manor called Haustona [Haxon] ${ }^{7}$ which Ordulf held T.R.E., and it paid geld for 2 virgates. These 5 ploughs can till. There the bishop has 6 villeins who have 3 ploughs, also 2 acres of meadow and common pasture at Bratona [Bratton Fleming]. It is worth 25 shillings; when the bishop received it it was worth the same.

The bishop has a manor called Botintona [Button] ${ }^{8}$ which Ednod held in parage ( $E x c h$., libere), and he could go to what lord he liked T.R.E. It has been added to the above-named manor, and paid geld for I virgate. This 2 ploughs can till. There the bishop has I villein and common pasture at Bratona [Bratton]. It is worth 5 shillings; when the bishop received it it was worth the same.

fol. Ir8.

These two above-named manors the count of Mortain gave to the bishop in exchange for the castle of Cornwall.

${ }^{5}$ Dawlish manor in the hundred of Exminster. Given to Bishop Leofric by the Confessor in 1044 . Trans. Devon Assoc, vi, $38 \mathrm{I}$; xiii, I 16.

'Ide in the hundred of Exminster. Trans. Devon Assoc. Xxx, 305 ; the gift of King Ethelstan in 937. Trans. Devon Assoc. xiii, I 19.

${ }^{7}$ Haxon in Bratton Fleming in Braunton hundred.

${ }^{8}$ Button in Bratton Fleming in Braunton hundred held with Haxon in I 303 , Feudal Aids, i, 360 . 


\section{A HISTORY OF DEVONSHIRE}

Bishop O[sbern] has a manor called TAUTONA [Tawton]. ${ }^{1}$ In it are 12 hides, of which 9 paid geld in the time of King $\mathrm{E}$ [dward], and the remaining 3 the bishop held in demesne, and they never paid. These 12 hides 150 ploughs can till. Thereof the bishop has 3 hides and 6 ploughs in demesne, and the villeins have 100 ploughs. There the bishop has 80 villeins, I I bordars, 18 serfs, 22 swineherds paying 100 swine yearly, I rouncey, I 5 beasts, 153 sheep, 12 acres of coppice, 24 of meadow, and 100 acres of pasture. It is worth 40 pounds a year ; when the bishop received it it was worth 50 pounds.

Bishop O[sbern] has a manor called Culmesтосна [Culmstock] ${ }^{2}$ which paid geld for 5 hides. These I 5 ploughs can till. Thereof the bishop has I hide and 2 ploughs in demesne, and the villeins have 4 hides and II ploughs. There the bishop has 24 villeins, 8 bordars, 5 serfs, 40 sheep, I mill paying 5 shillings, wood(land) 7 furlongs in length by $I$ in breadth, 50 acres of meadow, pasture I leaguc in length by as much in breadth. It pays 6 pounds; when the bishop received it it was worth 4 pounds.

The bishop has a manor called Sтосна [Stoke $]^{3}$ which in King E[dward]'s time paid geld for I hide. This 6 ploughs can till. Thereof the bishop has I virgate in demesne and I plough, and the villeins have 6 ploughs. There the bishop has 4 villeins, 8 bordars, 2 serfs, 14 beasts, 12 swine, 2 mills paying 30 fol. $x \times 8 \mathrm{~b}$.

shillings a year, 4 acres of coppice, 8 acres of meadow, and 50 acres of pasture. Worth 50 shillings a year.

Bishop O[sbern] has a (manor) called TALETONA [Talaton] ${ }^{4}$ which Bishop Leuric held T.R.E., and it paid geld for 7 hides. These I6 ploughs can till. Robert holds it of the bishop. Thereof R[obert] has 3 hides and I plough; and the villeins 4 hides and 9 ploughs. There R[obert] has 15 villeins, 12 bordars, 6 serfs, 3 beasts, 60 sheep, I mill paying 50 pence a year, 6 acres of coppice, 20 acres of meadow and $I 50$ acres of pasture. Worth 6 pounds a year; when he received it 100 shillings.

${ }^{1}$ Bishop's Tawton manor in South Molton hundred.

${ }^{2}$ Culmstock manor in Hemyock hundred. The boundaries are given in a charter purporting to be by Ethelstan, but dated 670. Ethelstan's charter dated 938 in Cart. Sax. ii, 432 ; Kemble, Cod. Dipl. ii, 209, No. 373 , and iii, 412 .

${ }^{3}$ Stoke Canon in Wonford hundred, written ' $\mathrm{Hro}$ castoc' and 'Brocastoc' in charters of Ethelstan 938 and Cnut 103r. See Cart. Sax. ii, 43r; Kemble, Cod. Dipl. ii, 207, No. $37 \mathrm{r}$, and iii, $4 \mathrm{Ir}$.

- Talaton in the hundred of Hairidge.
Bishop O[sbern] has a manor called SiDEberIa [Sidbury] ${ }^{5}$ which Alwin and Godwin held in parage T.R.E., and it paid geld for 3 (Exch. 5) hides. These 20 (Exch. 30) ploughs can till. Thereof the bishop has I hide and 2 ploughs, and the villeins 2 hides and 18 (Exch. 25) ploughs. There the bishop has 32 villeins, 5 bordars, 2 serfs, 10 beasts, 3 swine, I 40 sheep, 14 goats, 300 acres of wood(land), 12 acres of meadow, and 100 acres of pasture. Worth 6 pounds; when the bishop received it, the same.

Bishop O[sbern] has a manor called Selcoma [Salcombe] $^{6}$ which Bishop Leuric held T.R.E., and it paid geld for 3 hides. These 6 ploughs can till. Thereof the bishop has $I$ hide and I plough in demesne and the villeins 2 hides and 7 ploughs. There the bishop has 16 villeins, 7 bordars, 2 serfs, 80 sheep, 14 acres of fol. xyg.

wood(land), 6 acres of meadow, and pasture I league in length by 4 furlongs in width. It is worth 60 shillings; when the bishop received it, the same.

Bishop O[sbern] has a manor called BRETRICESTAN (Exch. Bedricestan) [ $]^{7}$ which Bishop Leuric held T.R.E., and it paid geld for I hide. This 5 ploughs can till. Thereof the bishop has $\frac{1}{2}$ hide and I plough in demesne, and the villeins $\frac{1}{2}$ hide and 3 ploughs. There the bishop has 3 villeins, 6 bordars, 2 beasts, 42 sheep, 40 acres of meadow, and 50 acres of pasture. Worth 20 shillings; when the bishop received it, the same.

Bishop O [sbern] has a manor called Peintona [Paignton] ${ }^{8}$ which Bishop Leuric held T.R.E., and it paid geld for 20 hides. These 60 ploughs can till. Thereof the bishop has in demesne 6 hides and 8 ploughs, and the villeins I 4 hides and 42 ploughs. There the bishop has 52 villeins, 40 bordars, 36 serfs, 5 swineherds paying 50 swine, 4 rounceys, I saltern paying Io pence, 20 beasts, 16 swine, 350 sheep, 4I acres of wood(land), I8 acres of meadow, and 40 of pasture. Worth 50 pounds a year; when he received it it was worth I 3 pounds.

\section{Bishop O[sbern] has a manor called Essebre-}

${ }^{5}$ Sidbury in East Budleigh hundred.

${ }^{6}$ Salcombe Regis in East Budleigh hundred.

7 This seems to be the 'Bryghteston juxta Honiton Clift' of Inq. P.m. 22 Ric. II, No. 34, and the 'Brichtestone' of the Taxation of Pope Nicholas, i.e. Traysbere, in the parish of Honiton Clyst in East Budleigh hundred. Trans. Devon Assoc. xiii, I 28 ; xxxv, 297.

${ }^{8}$ Paignton manor in Haytor hundred. 


\section{HOLDERS OF LANDS}

TONA [Ashburton] ${ }^{1}$ which Bishop Leuric held T.R.E., and it paid geld for 6 hides. These 20 ploughs can till. Thereof he has in demesne 2 hides and 2 ploughs, and the villeins 4 hides and 16 ploughs. There the bishop has 34 villeins, 16 bordars, Io serfs, I rouncey, 5 fol. $13 \mathrm{~g}$ b.

beasts, 76 sheep, I league of wood(land), 3 acres of meadow, and I league of pasture. It is worth 20 pounds a year; when he received it it was worth 8 pounds.

Bishop O[sbern] has a manor called ChenrSTETONA [Knighton] ${ }^{2}$ which Bishop Leuric held T.R.E., and it paid geld for $\frac{1}{2}$ hide. This 3 ploughs can till. Roger holds it under the bishop. Thereof $R$ [oger] has in demesne $\mathrm{I} \frac{1}{2}$ virgates and 2 ploughs and the villeins $\frac{1}{2}$ virgate and I plough. There R[oger] has 4 villeins, 3 serfs, I rouncey, 6 beasts, 24 sheep, 3 acres of meadow, and 6 acres of pasture. It is worth Io shillings a year and was worth the same when he received it.

Bishop O[sbern] has a manor called NIMETONA [Nympton] ${ }^{3}$ which Bishop Leuric held T.R.E., and it paid geld for 3 hides. These 52 ploughs can till. Thereof the bishop has in demesne I hide and 4 ploughs, and the villeins 2 hides and 44 ploughs. There the bishop has 56 villeins, 25 bordars, 17 swineherds paying 90 swine, 14 serfs, 6 beasts, 3 I sheep, I mill paying 40 pence, 200 acres of wood(land), 150 acres of meadow, and 140 acres of pasture. It is worth 16 pounds a year and was worth 7 pounds when he received it.

fol. 120 .

Bishop O[sbern] has a manor called StovreTONA (Exch. Sovretone) [Staverton] ${ }^{4}$ which Bishop Leuric held T.R.E., and it paid geld for $2 \frac{1}{2}$ hides. These 20 ploughs can till. Thereof the bishop has in demesne $\frac{1}{2}$ hide and I plough and the villeins 2 hides and 16 ploughs. There the bishop has 20 villeins, 12 bordars, 9 serfs, 5 beasts, IOI sheep, 4I acres of coppice, I 2 acres of meadow, and 30 furlongs of pasture. It is worth 7 pounds a year. This is allotted for the support of the canons.

Bishop O[sbern] has a manor called St. MARY $\mathrm{CHURCH}^{5}{ }^{5}$ which Bishop Leuric held T.R.E., and

1 Ashburton manor in Teignbridge hundred.

${ }^{3}$ Knighton in Hennock (O.J.R.) in Teignbridge hundred.

${ }^{3}$ The manor of Bishop's Nympton in the hundred of Witheridge, given by King Eadgar to the bishop in 974. Trans. Devon Assoc. viii, 352. Cart. Sax. iii, 624 gives the boundaries.

* The manor of Staverton in Haytor hundred.

${ }^{5}$ Combe Pafford in St. Mary Church in Haytor hundred. Trans. Devom Assoc, xviii, 153. it paid geld for 2 virgates. These 3 ploughs can till. Thereof the bishop has in demesne I virgate and I plough and the villeins I virgate and 2 ploughs. There the bishop has 4 villeins, 4 bordars, and 64 sheep. It is worth 15 shillings a year. This is allotted for the support of the canons.

Bishop O[sbern] has a manor called BRANchescoma [Branscombe] ${ }^{6}$ which Bishop Leuric held T.R.E., and it paid g[eld] for 5 hides. These 16 ploughs can till. It is allotted for the support of the canons. Thereof the $c$ [anons] have I hide in demesne and I plough, and the villeins have 4 hides and 15 ploughs. There the c[anons] have 22 villeins, 5 bordars, I serf, I beast, I 50 sheep, I 2 acres of fol. rzob.

coppice and 2 acres of meadow. It is worth 6 pounds a year.

Bishop O[sbern] has a manor called Didasam $\left.{ }^{[D i t t i s h a m}\right]^{7}$ which Bishop Leuric held T.R.E. and it paid geld for 3 hides. These 16 ploughs can till. Baldwin holds it of the bishop. Thereof Baldwin has in demesne 1 hide and 2 ploughs, and the villeins 2 hides and 10 ploughs. There $B$ [aldwin] has 22 villeins, ro bordars, 2 serfs, I rouncey, 20 swine, 60 sheep, 40 goats, 40 acres of coppice, and 60 acres of pasture. It is worth 100 shillings a year; when the bishop received it it was worth 3 pounds (Exch. 60 shillings).

The bishop has a manor called Stadona [Slapton] ${ }^{8}$ which paid geld for 6 hides. These 26 ploughs can till. Baldwin the sheriff holds it of the bishop. Thereof $\mathrm{B}$ [aldwin] has in demesne 3 hides and 2 ploughs, and the villeins 3 hides and $2 \mathrm{I}$ ploughs. There $\mathrm{B}$ [aldwin] has 26 villeins, and 2 I coscets, 6 serfs, 9 beasts, 12 swine, Ioo sheep, 31 acres of wood(land), Io acres of meadow, and 50 acres of pasture. It is worth 12 pounds a year; when the bishop received it 10 pounds.

The bishop has a church in Exeter [St. Stephen's] ${ }^{9}$ which pays yearly I mark of silver, also 48 houses. Of these houses 10 pay 10 shillings and Io pence by way of customary due (Exch. 47 houses paying Io shillings and Io

\footnotetext{
${ }^{6}$ Branscombe manor in Colyton hundred; in 857 the property of the crown, and mentioned in Edelwulf's will; given by King Alfred to his younger son in 901. Cart. Sax. ii, I82; Kemble, Cod. Dipl. ii, 1 I 2, No. 1067 ; Trans. Devon Assoc. ix, 214.

7 Dittisham manor in Colridge hundred. Oliver was sub-tenant (Geldroll, fol. 696, B, 3).

${ }^{8}$ Slapton manor in Colridge hundred. Robert son of Gervin was sub-tenant (Geldroll, fol. 6gb, B, 2).

'St. Stephen's church, accounted the 'head' or chief seat of the bishop's barony.
} 


\section{A HISTORY OF DEVONSHIRE}

pence) and 2 have been destroyed (vastate) by fire. Also he has $2 \frac{1}{2}$ acres of land ${ }^{1}$ which lie intermixed with the land of the burgesses, and belong to the church.

\section{LAND OF THE BISHOP OF COU- TANCES IN DEVRESCIRA ${ }^{2}$}

fol. 223.

The bishop of Coutances has a manor called Coritona [Coryton] ${ }^{3}$ which Alwin held T.R.E., and it paid geld for $\frac{1}{2}$ hide. This 8 ploughs can till. This Drogo son of Malger holds of the bishop. Of this ( $D e b a c$ ) Drogo has in demesne $\frac{1}{2}$ virgate and $I$ plough, and the villeins $I_{2} \frac{1}{2}$ virgates and I plough. There $\mathrm{D}$ [rogo] has 8 villeins, 7 coscets, 6 serfs, 2 swine, 40 sheep, 12 goats, 30 acres of wood(land), 12 acres of meadow, and pasture $\frac{1}{2}$ league in length by 2 furlongs in breadth. Worth 3 pounds; when the bishop received it it was worth 4 pounds.

The bishop of Coutances has a manor called Surintona [Sourton] " which Alwin held T.R.E., and it paid geld for 3 virgates. These 12 ploughs can till. Engebald's (Exch. Ingelbald's) wife (widow) holds it of the bishop. In demesne she has $\frac{1}{2}$ virgate and 3 ploughs, and the villeins hold the rest of the land and have $4 \frac{1}{2}$ ploughs. There are there $\mathrm{I}_{4}$ villeins, 7 coscets (quosceti), 2 swinefol. 12xb.

herds, 7 serfs, I rouncey, I 8 beasts, 9 swine, I 00 sheep, 50 goats, 12 acres of coppice, 60 acres of meadow, and pasture I league in length by $\frac{1}{2}$ league in breadth. Worth 7 pounds yearly; when the bishop received it it was worth I I pounds.

The bishop of Coutances has a manor called Melefort [Milford] ${ }^{5}$ which Alwin held T.R.E., and it paid geld for I virgate T.R.E. This 5 ploughs can till. Engelbald's wife (widow)

\footnotetext{
${ }^{1}$ Probably this means $2 \frac{1}{2}$ acres in each of the 120 shots into which the three fields were divided, these 300 acres being now represented by St. Sidwell's manor (Trans. Devon Assoc. xxxiv, 7 1 8), as the canons' land is stated to be 'intermixed with the land of the burgesses' in the suit in the thirteenth century (Jenkins, Exeter, 42; Freeman, Exeter, 157 ; Trans. Devon Assoc. xxxiv, 9r).

${ }^{2}$ The Exchequer book follows a slightly different order. Beginning with the bishop's holding in Exeter, it first enumerates his demesne manors, Ashwater, Merton, Fremington, Clyst Satchvil, and Bovey Tracy. Then follow the manors held by Drogo, with a note at the end: "The above 73 estates are held by Drogo of the bishop.' Last of all come the manors of other tenants, Ingebald's widow, the bishop's niece, Olwi, Edric, Osbern, Geoffrey, Edmer, Eddilla, Robert, Geoffrey de Tralei, Leuric, and Ansger.

${ }^{3}$ Coryton manor in Lifton hundred.

${ }^{4}$ Sourton in Lifton hundred.

${ }^{3}$ Milford in Stowford in Lifton hundred.
}

holds it of the bishop, and has thereof in demesne I ferting and I plough; the villeins have 3 ferdings and 3 oxen. There the lady has 3 villeins, I coscet, 3 serfs, I 5 acres of wood(land), 6 acres of meadow, and 6 acres of pasture. Worth 20 shillings a year; when the bishop received it it was worth 40 shillings.

The bishop of Coutances has a manor called Arssa $[\mathrm{Ash}]^{6}$ which Alwin held T.R.E., and it paid geld for I hide. This 20 ploughs can till. Thereof the bishop has I virgate and 2 ploughs in demesne, and the villeins have 3 virgates and 17 ploughs. There the bishop has 40 villeins, 12 bordars, 6 serfs, 42 beasts, 3 swine, I6I sheep, 30 goats, 15 acres of coppice, 100 acres of meadow, and 200 acres of pasture. It pays 7 pounds and ro shillings a year; when the bishop received it the same.

The bishop has a manor called Sutecoma [Sutecombe] $^{7}$ which Edrit (Exch. Edric) held T.R.E., and it paid geld for $\frac{1}{2}$ hide. This 7 ploughs can till. Now Edrit holds it of the bishop. There the bishop has $\frac{1}{2}$ virgate and I plough in demesne, and the villeins have $I_{2}^{\frac{1}{2}}$ virfol. I22.

gates and 6 ploughs; also 9 villeins, 6 coscets, 4 serfs, 18 beasts, 12 swine, 80 sheep, 6 acres of wood(land), I 4 acres of meadow, and 12 acres of pasture. Worth 40 shillings a year; when the bishop received it it was worth 50 shillings.

The bishop has a manor called Torna [Thorn $]^{8}$ which Sawin held T.R.E., and it paid geld for $\frac{1}{2}$ virgate. This 3 ploughs can till. Engelbald's wife (i.e. widow) holds it of the bishop. Thereof Engelbald's wife has I ferling and I plough in demesne, and the villeins have the other ferling and $\mathbf{I}$ ox. There E[ngelbald's] wife has 4 villeins, 4 bordars, 3 cows, 12 acres of meadow, and pasture $\frac{1}{2}$ league in length by 2 furlongs in breadth. Worth Io shillings a year; when the bishop received it 15 shillings.

The bishop has a manor called HermondeswORDA (Exch. Hermodesword) [Hamsworthy] ${ }^{9}$ which Alward held T.R.E., and it paid geld for $\frac{1}{2}$ virgate. This 2 ploughs can till. Drogo holds it of the bishop. There Drogo has I plough, 2 serfs, 20 acres of meadow, and pasture 5 furlongs in length by 2 in breadth. Worth 5 shillings; when the bishop received it it was worth 2 shillings.

'Ash Walter alias Ashwater in Blacktorington hundred.

${ }^{7}$ Sutcombe in Blacktorington hundred.

${ }^{8}$ Thorn in Holsworthy in Blacktorington hundred.

${ }^{9}$ Hamsworthy in Pancrasweek in Blacktorington hundred. 


\section{HOLDERS OF LANDS}

The bishop has a manor called CHemeworda [Kimworthy] ${ }^{1}$ which Edwin held T.R.E., and it paid geld for I virgate. This 3 ploughs can till. Now the bishop's niece holds it of him. Thereof the bishop's niece has $\frac{1}{2}$ virgate and I plough in demesne, and the villeins $\frac{1}{2}$ virgate and I plough. There the bishop's niece has 2 villeins, 2 serfs, 15 beasts, 40 sheep, 20 acres of meadow, and 30 acres of pasture. Worth 20 shillings; when the bishop received it it was worth 5 shillings.

fol. r2ab.

The bishop has a manor called Hortona [Horton] ${ }^{2}$ which Osulf held T.R.E., and it paid geld for I virgate. This 2 ploughs can till. Drogo holds it of the bishop. Thereof he has $\frac{1}{2}$ virgate in demesne and I plough, and the villeins $\frac{1}{2}$ virgate. There Drogo has 2 serfs, 20 acres of meadow, and roo acres of pasture. The aforesaid thane could go with his land to what lord he liked. The bishop holds this united with Horwode [Horwood] manor, but it did not belong to that manor 'T.R.E. Worth 5 shillings a year.

The bishop has a manor called EngEstecota [Endscot] ${ }^{3}$ which 3 thanes, Gola, Hedric, and Godric, held T.R.E. Now the bishop holds it as one manor, and it paid geld for $\frac{1}{2}$ virgate. This 3 ploughs can till. Drogo holds it of the bishop. Drogo has it in demesne, and has on it 2 villeins, 4 serfs, 20 beasts, 60 sheep, 20 goats, 60 acres of wood(land), to acres of meadow, and 2 furlongs of pasture. Worth 15 shillings a year; when he received it it was worth 20 shillings.

The bishop has a manor called W ALcoma (Exch. Walcome) [Welcombe ${ }^{4}$ which Ansgot (Exch. Osgot) held T.R.E., and it paid geld for I hide and $\frac{1}{2}$ virgate. This I 5 ploughs can till. fol. .23.

Olwi holds it of the bishop. There Olwi has in demesne I virgate and 2 ploughs, and the villeins have $I$ hide all but 2 ferdings, and 7 ploughs. Upon it Olwi has 15 villeins, 7 bordars, 3 serfs, 8 beasts, 25 swine, 100 sheep, I league of wood(land), 10 acres of meadow, and 20 of pasture. Worth 60 shillings a year; when he received it it was worth 100 shillings.

The bishop has a manor called Mertona [Merton] ${ }^{5}$ which Torquil (Exch. Torchil) held T.R.E. It paid geld for $\frac{1}{2}$ hide, $\frac{1}{2}$ virgate, and $\frac{1}{2}$ ferding. These to ploughs can till. Of

${ }^{1}$ Kimworthy in Bradworthy in Blacktorington hundred.

2 Horton in Bradworthy in Blacktorington hundred.

${ }^{3}$ Endscot alias Henscot in Bradford Dabernon in Blacktorington hundred.

${ }^{4}$ Welcombe in the hundred of Hartland.

${ }^{5}$ Merton in Shebbear hundred. these (De his) the bishop has in demesne $\frac{1}{2}$ virgate, $\frac{1}{2}$ ferding, and 3 ploughs, and the villeins $\frac{1}{2}$ hide and 6 ploughs. There the bishop has 12 villeins, 8 bordars, 9 swineherds paying 25 swine a year, 3 serfs, I rouncey, I 2 beasts, 9 swine, 50 sheep, 60 acres of wood(land), 15 acres of meadow, and 30 acres of pasture. Worth 8 pounds a year; when the bishop received it it was worth 6 pounds.

The bishop has a manor called Bochelan [Buckland]. ${ }^{6}$ It paid geld for I hide, I virgate, and $\mathrm{I}$ ferding. Ulveva held it T.R.E. This land 8 ploughs can till. Drogo son of Malger holds it of the bishop. Thereof Drogo has $I$ virgate and 2 ploughs in his demesne, and the villeins I hide, and I ferding, and 5 ploughs. There Drogo has Io villeins, 4 bordars, 5 serfs, fol. rasb.

I 6 beasts, Io swine, 60 sheep, I 5 goats, wood(land) $\frac{1}{2}$ league in length by 3 furlongs in breadth, 8 acres of meadow, and $I$ league of pasture counting length and breadth. Worth 40 shillings a year; when the bishop received it it was worth the same.

The bishop has a manor called Hierlega [Hartleigh] $]^{7}$ which Oliveva (Exch. Ulveva) held T.R.E., and it paid geld for $\frac{1}{2}$ virgate. This 5 ploughs can till. Drogo son of Malger holds it of the bishop. Thereof $\mathrm{D}[\mathrm{rogo}]$ has in demesne I ferding and I plough, and the villeins I ferding and 3 ploughs. There $D$ [rogo] has 2 villeins, I bordar, 3 serfs, 3 swineherds paying II swine a year, 4 beasts, 65 sheep, wood(land) 4 furlongs in length by 2 furlongs in breadth, 3 acres of meadow, and 15 acres of pasture. Worth 20 shillings a year, and it was worth the same when the bishop received it.

The bishop has a manor called BELDRENDILANT [Barlington ?] ${ }^{8}$ which Elward (Exch. Alward) held T.R.E, and it paid geld for $1 \frac{1}{2}$ virgates. This 3 ploughs can till. Drogo holds it of the bishop. There Drogo has 3 villeins, 2 bordars, and 5 acres of wood(land). Worth 10 shillings a year.

The bishop has a manor called Framintona [Fremington] ${ }^{9}$ which Earl Harold held T.R.E., and it paid geld for 3 hides. These 30 ploughs can till. Thereof the bishop has $\frac{1}{2}$ hide and 3 ploughs in demesne, and the villeins $2 \frac{1}{2}$ hides and 27 ploughs. There the bishop has 40 vilfol. ז24.

leins, 30 bordars, 6 serfs, I burgess in Barnstaple

${ }^{6}$ Buckland Filleigh in the hundred of Shebbear.

${ }^{7}$ Hartleigh in Buckland Filleigh in Shebbear hundred. dred.

West Barlington in Roborough in Shebbear hun-

${ }^{9}$ The hundred manor of Fremington alias Lovecote. 


\section{A HISTORY OF DEVONSHIRE}

who pays I 5 pence, I 3 swineherds paying 2 I swine, I 8 beasts, I 50 sheep, ro acres of wood(land), 20 acres of meadow, and 100 acres of pasture. This manor pays 22 pounds; when the bishop received it it was paying 12 pounds.

The bishop has a manor called ToRsewIs [Torridge Huish $]^{1}$ which Ulveva held T.R.E., and it paid geld for $x$ hide. This 6 ploughs can till. $\mathrm{D}$ [rogo] son of Matelger [Malger] holds it of the bishop. Thereof $\mathrm{D}$ [rogo] has I virgate and I plough in demesne, and the villeins 3 virgates and 2 ploughs. There Drogo has 4 villeins, 2 serfs, 5 beasts, 7 swine, 50 sheep, 8 acres of wood(land), 4 acres of meadow, and 30 acres of pasture. Worth 30 shillings; when the bishop received it it was worth the same.

The bishop has a manor called Horewda [Horwood] ${ }^{2}$ which Osulf held T.R.E., and it paid geld for 3 virgates. These 5 ploughs can till. Drogo holds it of the bishop. Thereof $\mathrm{D}$ [rogo] has I virgate and I plough in demesne, and the villeins 2 virgates and $\frac{1}{2}$ plough. There $\mathrm{D}$ [rogo] has 6 villeins, 4 serfs, 3 beasts, 30 sheep, and 3 acres of meadow. Worth 30 shillings; when the bishop received it it was worth the same.

The bishop has a manor called Tapeleia [Tapleigh] $^{3}$ which Ulveia (Exch. Ulveva) held T.R.E., and it paid geld in KingEdward's time for I hide. This 8 ploughs can till. Now Osbern holds it of the bishop. Thereof Osbern has $\mathrm{I} \frac{1}{2}$ virgates and 2 ploughs in demesne, and the villeins have $I \frac{1}{2}$ virgates and I plough. There $\mathrm{O}$ [sbern] has 3 villeins, 2 bordars, 2 serfs, Io beasts, I 5 swine, 50 sheep, 20 goats, 3 acres of wood(land), 3 acres of meadow, and 10 acres of pasture. Worth 30 shillings a year. From the aforesaid hide I virgate ${ }^{4}$ has been taken away which Roger holds of the bishop. It belonged to the aforesaid manor T.R.E.

fol. 124b.

The bishop has a manor called ULwRIToNa (Exch. Uluretona) [Worlington] ${ }^{5}$ which Dodo held T.R.E., and it paid geld for $\frac{1}{2}$ hide. This 5 ploughs can till. Now Drogo holds it of the bishop. Thereof $\mathrm{D}$ [rogo] has I virgate and $\mathbf{I}$ plough in demesne, and the villeins have the other virgate and $2 \frac{1}{2}$ ploughs. There $\mathrm{D}$ [rogo] has 4 villeins, 3 bordars, I serf, 20 sheep, Io acres of wood(land), 2 acres of meadow, and 12 of pasture. Worth 20 shillings a year; when the bishop received it it was worth 25 shillings.

1 Huish Beaupel, an outlier of Westleigh in Fremington hundred, locally in Instow parish.

3 West or Church Horwood in Fremington hundred.

${ }^{3}$ Tapleigh in Westleigh in Fremington hundred.

4 Perhaps now the glebe of Westleigh.

- Worlington in Instow in Fremington hundred.
The bishop has a manor called RawEBERGA [Roborough] ${ }^{6}$ which Ulwevia (Exch. Ulveva) held T.R.E., and it paid geld for $I \frac{1}{2}$ hides. This I 4 ploughs can till. Now Drogo holds it of the bishop. Thereof Drogo has $1 \frac{1}{2}$ virgates in demesne and I plough; and the villeins have I hide, and $\frac{1}{2}$ virgate, and 8 ploughs. Drogo has there I 5 villeins, 3 bordars, 5 serfs, 15 beasts, 9 swine, 60 sheep, 20 acres of wood(land), I 6 acres of meadow, and 20 acres of pasture. Worth 3 pounds a year; when the bishop received it it was worth 4 pounds. To this manor has been added a manor called Baldrintona [Barlington] ${ }^{7}$ which Alvred held T.R.E., and he could go to what lord he liked. It paid geld for I virgate. This 3 ploughs can till. It pays ro shillings; when the bishop received it it was worth the same.

The bishop has a manor called Bolenela [Bondleigh] $^{8}$ which Alwin held T.R.E., and it paid geld for I hide all but $\frac{1}{2}$ virgate. This I 2 ploughs can till. $\mathrm{D}$ [rogo] holds it of the bishop. Thereof Drogo has I virgate and 2 ploughs in demesne, and the villeins $2 \frac{1}{2}$ virgates and 2 ploughs. There $\mathrm{D}$ [rogo] has 6 villeins, 8 serfs, 3 swineherds paying is swine, 7 beasts, 30 sheep, 30 acres of wood(land), 12 acres of meadow, and 30 acres of pasture. Worth 40 shillings; when the bishop received it it was worth 30 shillings.

fol. 125 .

The bishop of Coutances has a manor called LIMET [Nimet or Intake ${ }^{9}$ which Alwin held T.R.E., and it paid geld for $\mathrm{I} \frac{1}{2}$ hides. This $\mathrm{I} 2$ ploughs can till. Now Drogo holds it of the bishop. Thereof $\mathrm{D}$ [rogo] has $\frac{1}{2}$ hide and I plough in demesne, and the villeins have I hide and 6 ploughs. There $\mathrm{D}$ [rogo] has I I villeins, 8 bordars, 6 serfs, I rouncey, 4 beasts, I pig, 52 sheep, wood(land) $\frac{1}{2}$ a league in length by 6 furlongs in breadth, 6 acres of meadow, and pasture I league in length by 3 furlongs in breadth. Worth 4 pounds; when the bishop received it it was worth 4 pounds.

The bishop of Coutances has a manor called Colriga [Coldridge] ${ }^{10}$ which 2 thanes held in parage (pariter; Exch., libere) T.R.E., and it paid geld for I hide. This 8 ploughs can till. Now D[rogo] holds it of the bishop. Thereof Drogo has I virgate and 2 ploughs in demesne, and the villeins have 2 virgates and 6 ploughs.

- Roborough in the hundred of Fremington.

7 Possibly Wansley and Ebberley in Roborough in Fremington hundred, which seem to have been once part of Barlington.

8 Bondleigh in the hundred of North Tawton.

9 Nymet Tracy alias Nymet Bow (Assize R. 9 Edw. I, No. I8I, m. 8), the chief manor of Bow in North Tawton hundred.

${ }^{10}$ Coldridge in North Tawton hundred. 


\section{HOLDERS OF LANDS}

There D[rogo] has II villeins, I bordar, 2 serfs, 3 swineherds paying 50 swine a year, 3 beasts, 36 sheep, wood(land) I league in length by $\frac{1}{2}$ league in breadth, 6 acres of meadow, and 30 acres of pasture. Worth 4 pounds a year; when the bishop received it it was worih 100 shillings: Of the aforesaid hide Ingelbald holds I virgate ${ }^{1}$ under Drogo, which Algar held T.R.E., and be could go with his land to what lord he liked. Worth Io shillings a year ; when Drogo received it it was worth 5 shillings.

The bishop has a manor called Matingero [Martinhoe] $^{2}$ which Dodo held T.R.E., and it paid geld for I hide. This 8 ploughs can till. Drogo holds it of the bishop. Thereof D[rogo] fol. 125b.

has I virgate and I plough in demesne, and the villeins 3 virgates and 3 ploughs. There $D$ [rogo] has 8 villeins, 4 bordars, I serf, 3 beasts, 14 swine, Io sheep, I 7 goats, 50 acres of wood(land), and I league of pasture. Worth 30 shillings; when the bishop received it it was worth 5 shillings.

The bishop of Coutances has a manor called Pillanda [Pilland] ${ }^{3}$ which Edmer held T.R.E., and it paid geld for $\frac{1}{2}$ hide. This 5 ploughs can till. Drogo holds it of the bishop. Thereof $\mathrm{D}$ [rogo] has $\frac{1}{2}$ virgate and 2 ploughs in demesne, and the villeins $I \frac{1}{2}$ virgates and 3 ploughs. There $\mathrm{D}$ [rogo] has 3 villeins, 5 bordars, 5 serfs, I rouncey, 80 sheep, 8 acres of wood(land), 4 acres of meadow, and 40 acres of pasture. Worth 40 shillings; when the bishop received it it was worth 50 shillings.

The bishop has a manor called PI[LTO]NA (Exch. Wiltone) [Pilton] ${ }^{4}$ which Dodo held T.R.E. and it paid geld for $\frac{1}{2}$ hide. This 5 ploughs can till. Drogo holds it of the bishop. Thereof $\mathrm{D}$ [rogo] has $\frac{1}{2}$ virgate and I plough in demesne, and the villeins $I \frac{1}{2}$ virgates and 3 ploughs. There $\mathrm{D}$ [rogo] has 6 villeins, 7 bordars, 4 serfs, I rouncey, 3 beasts, Io sheep, 60 acres of wood(land), 8 acres of meadow, and 40 acres of pasture. Worth 30 shillings; when the bishop received it it was worth 40 shillings.

The bishop has a manor called Duna (Exch. Dune) [Down $]^{5}$ which Algar held T.R.E., and it paid geld for $\frac{1}{2}$ hide. This 5 ploughs can till. Drogo holds it of the bishop. Thereof D[rogo] has $\frac{1}{2}$ virgate and I plough in demesne, and the fol. 126 .

villeins $\mathrm{I} \frac{1}{2}$ virgates and 7 ploughs. There

1 Possibly Clotworthy in Coldridge in North Tawton hundred. Testa de Nevill (Rec. Com.), I 846.

- Martinhoe in Shirwell hundred.

$s$ Pilland (T.W.W.), or Pulcress manor in Pilton in Braunton hundred (O.J.R.), Trans. Devon Assoc. xxxiv, 730 .

1 Pilton in Braunton hundred.

- West Down in Braunton hundred.
$\mathrm{D}$ [rogo] has I 5 villeins, 3 bordars, 4 serfs, 3 beasts, 26 sheep, I 8 goats, also wood(land) I league in length by $\frac{1}{2}$ league in breadth, and 3 acres of meadow. Worth 40 shillings; when the bishop received it it was worth the same. To this manor have been added 3 lands which 3 thanes held in parage (pariter; Exch. libere) T.R E., and they could go to what lord they liked, and they paid geld for $\frac{1}{2}$ hide. This 4 ploughs can till. There Drogo has 3 villeins who have 2 ploughs. Worth 20 shillings; when the bishop received it it was worth the same.

The bishop has a manor called HaGINTONA [Heanton] ${ }^{6}$ which Ulfert held T.R.E., and it paid geld for I virgate. This 2 ploughs can till. Drogo holds it of the bishop. There $\mathrm{D}$ [rogo] has 2 villeins who have 2 ploughs, I bordar, and 20 acres of pasture. It pays 6 shillings ; when the bishop received it it was worth 10 shillings.

The bishop has a manor called RadeleIA $\left[\right.$ Raleigh] ${ }^{7}$ which Bristric held T.R.E., and it paid geld for $\frac{1}{2}$ hide. This 4 ploughs can (till). Drogo holds it of the bishop. Thereof $\mathrm{D}$ [rogo] has $I$ virgate and $I \frac{1}{2}$ ploughs in demesne, and the villeins I virgate and I plough. There $\mathrm{D}$ [rogo] has 4 villeins, 4 bordars, 4 serfs, 2 beasts, 30 sheeep, 16 goats, 30 acres of wood(land), 2 acres of meadow, and 5 acres of pasture. Worth 30 shillings; when the bishop received it it was worth the same.

The bishop has a manor called Mrtcoma [Metcombe] ${ }^{8}$ which Bristric held T.R.E., and it paid geld for $\frac{1}{2}$ virgate. This I plough can till. Drogo holds it of the bishop. There $\mathrm{D}$ [rogo] has 2 villeins who have I plough, I serf, 4 acres of meadow, and 20 acres of pasture. It pays 10 shillings; when the bishop received it it was worth the same. fol. $226 \mathrm{~b}$.

The bishop has a manor called BraIA [Bray] ${ }^{9}$ which Alwin held T.R.E., and it paid geld for $\mathbf{I} \frac{1}{2}$ virgates. Drogo holds it of the bishop. This 6 ploughs can till. Thereof $D$ [rogo] has $\frac{1}{2}$ virgate and I plough in demesne, and the villeins I virgate and 3 ploughs. There $\mathrm{D}$ [rogo] has 6 villeins, 2 bordars, I serf, also 3 beasts, I 3 sheep, 20 acres of wood(land), 30 acres of meadow, and pasture 2 leagues in length by $\frac{1}{2}$ league in breadth. Worth 20 shillings; when the bishop received it it was worth the same.

To this manor has been added a manor called Witefella [Whitfield $]^{10}$ which Sawin held

${ }^{6}$ Kingsheanton in Marwood in Braunton hundred.

${ }^{7}$ Raleigh in Pilton in Braunton hundred.

${ }^{8}$ Metcombe in Marwood in Braunton hundred.

${ }^{9}$ High Bray in Shirwell hundred. Geldroll, fol. $66, A, 2$.

${ }^{10}$ Whitfield in High Bray in Shirwell hundred. 


\section{A HISTORY OF DEVONSHIRE}

T.R.E., and it paid geld for I virgate. This 2 ploughs can till. Drogo holds it of the bishop. Thereof $\mathrm{D}$ [rogo] has $\frac{1}{2}$ virgate and I plough in demesne, and the villeins $\frac{1}{2}$ virgate. There $\mathrm{D}$ [rogo] has 2 villeins, $\mathrm{I}$ bordar, $\mathrm{I} 5$ goats, and Io acres of wood(land). Worth ro shillings; when the bishop received it it was worth the same.

The bishop has a manor called Bera [Beer ${ }^{1}$ which Bristric held T.R.E., and it paid geld for $\frac{1}{2}$ hide. This 5 ploughs can till. Drogo holds it of the bishop. Thereof $\mathrm{D}$ [rogo] has $\frac{1}{2}$ virgate and I plough in demesne, and the villeins $I \frac{1}{2}$ virgates and 2 ploughs. There $D$ [rogo] has 4 villeins, I bordar, I serf, 2 beasts, 6 sheep, Io goats, 6 acres of wood(land), and 2 acres of meadow. Worth 20 shillings; when the bishop received it it was worth the same. [Bri]stric son of Camme ${ }^{2}$ had I virgate of land called BoEURDA [ $]^{3}$ T.R.E., and before the death of King Edward it had been obtained from (fuit accepta de) the king's manor called Brantona [Braunton], but William [de W]alville restored it to Braunton in King William's time. This the bishop of Coutances claims, but the thanes cannot say how Bristric became possessed of it.

The bishop has a manor called Celdecomba [Challacombe] ${ }^{4}$ which Ulmer held T.R.E., and it paid geld for I virgate. This 3 ploughs can till. Drogo holds it of the bishop. Thereof $\mathrm{D}$ [rogo] has $\frac{1}{2}$ virgate and 1 plough in demesne, and the villeins have $\frac{1}{2}$ virgate and $\frac{1}{2}$ plough. There $\mathrm{D}$ [rogo] has 3 villeins, 2 bordars, $I$ serf, I I beasts, Io swine, 55 sheep, 19 goats, also 7 acres of meadow, and 10 acres of pasture. fol. x27.

Worth 20 shillings; when the bishop received it it was worth the same.

The bishop has a manor called WITEFELla [Whitfield $]^{5}$ which Bristric held T.R.E., and it paid geld for $\frac{1}{2}$ virgate. This 2 ploughs can till. Drogo holds it of the bishop. Worth 6 shillings; when the bishop received it it was out of cultivation (vastata).

The bishop has a manor called BuRIETEscoma [Burscombe] $^{6}$ which Alvric held T.R.E., and it paid geld for $\frac{1}{2}$ virgate. This 2 ploughs can till. Drogo holds it of the bishop. There D[rogo] has I villein who has $\frac{1}{2}$ plough, 7 acres of meadow, and 40 acres of pasture. Worth 2 shillings; when the bishop received it it was out of cultivation (vastata).

${ }^{1}$ Beer Charter in Braunton in Braunton hundred.

'See fol. 3176 .

${ }^{3}$ Possibly Bude alias Boode in Braunton in Braunton hundred.

${ }^{4}$ Challacombe in Shirwell hundred.

${ }^{5}$ Whitfield in Challacombe in Shirwell hundred.

${ }^{6}$ Burscombe in Challacombe in Shirwell hundred.
The bishop has a manor called Patsola [Patehole] $^{7}$ which Ednod held T.R.E., and it paid geld for $\frac{1}{2}$ hide. This 5 ploughs can till. Drogo holds it of the bishop. Thereof $\mathrm{D}$ [rogo] has I virgate and I plough in demesne, and the villeins I virgate and $3 \frac{1}{2}$ ploughs. There $\mathrm{D}$ [rogo] has 9 villeins, 2 bordars, 2 serfs, 8 beasts, 2 swine, 90 sheep, 16 goats, and 3 acres of wood(land). Worth 40 shillings; when the bishop received it the same.

The bishop has a manor called Cheneoltona [Kellaton] ${ }^{8}$ which Algar held T.R.E., and it paid geld for $\frac{1}{2}$ virgate. This 2 ploughs can till. Drogo holds it of the bishop. Thereof D[rogo] has I ferling and I plough in demesne, and the villeins $\mathrm{I}$ ferling and 6 ploughing oxen (boves in carr'). There $\mathrm{D}$ [rogo] has I villein, $\mathrm{I}$ bordar, 2 serfs, 5 beasts, 5 swine, 18 sheep, 30 goats, 6 acres of wood(land), I acre of meadow, and 30 acres of pasture. Worth 15 shillings; when the bishop received it it was worth the same.

fol zajb.

The bishop has a manor called Pullanda (Exch. Welland) [Pilland] ${ }^{9}$ which Alvric held T.R.E., and it paid geld for $\frac{1}{2}$ hide. This 4 ploughs can till. Drogo holds it of the bishop. Thereof $\mathrm{D}[\mathrm{rogo}]$ has $\frac{1}{2}$ virgate and $1 \frac{1}{2}$ ploughs in demesne, and the villeins $1 \frac{1}{2}$ virgates and $1 \frac{1}{2}$ ploughs. There $\mathrm{D}[\mathrm{rogo}]$ has 6 villeins, 4 serfs, 8 beasts, 15 swine, 67 sheep, I I goats, 3 acres of wood(land), I acre of meadow, and 30 acres of pasture. Worth 20 shillings; when the bishop received it it was worth 10 shillings.

The bishop has a manor called PediccheswELLa (Exch. Wedicheswelle) [Pickwell] ${ }^{10}$ which Ulf held T.R.E., and it paid geld for $\frac{1}{2}$ hide. This 5 ploughs can till. Drogo holds it of the bishop. Thereof $\mathrm{D}$ [rogo] has $\frac{1}{2}$ virgate and I plough in demesne, and the villeins $I \frac{1}{2}$ virgates and $I \frac{1}{2}$ ploughs. There $D[$ rogo] has 4 villeins, 5 bordars, 1 serf, 13 swine, 12 acres of meadow, and 100 acres of pasture. Worth 20 shillings; when the bishop received it it was worth 10 shillings.

The bishop has a manor called Arresvescota (Exch. Eilvescote) [Aylscot] ${ }^{11}$ which two brothers held in parage (pariter; Exch., libere) T.R.E., and they could go to what lord they liked. It paid geld for $\frac{1}{2}$ hide. This 5 ploughs can till. Drogo holds it of the bishop. Thereof $\mathrm{D}$ [rogo] has $\frac{1}{2}$ virgate and I plough in demesne, and the villeins $I \frac{1}{2}$ virgates and $I \frac{1}{2}$ ploughs.

${ }^{7}$ Patehole in Kentisbury in Braunton hundred.

${ }^{8}$ Kellaton alias Killington in Martinhoe in Shirwell hundred, called Kynewelton in 1303, Feudal Aids, $36 \mathrm{I}$.

${ }^{9}$ The Priory manor in Pilton in Braunton hundred.

${ }^{10}$ Pickwell in Georgeham in Shirwell hundred.

${ }^{11}$ Aylscot in West Down in Braunton hundred. 


\section{HOLDERS OF LANDS}

There $\mathrm{D}$ [rogo] has 5 villeins, 3 bordars, I serf, 8 beasts, 2 swine, 88 sheep, Io goats, 20 acres of meadow, and roo acres of pasture. Worth 20 shillings; when the bishop received it it was worth Io shillings.

The bishop has a manor called ESTAVEForda (Exch. Staveford) [Stowford] ${ }^{1}$ which Edric held T.R.E., and it paid geld for $\frac{1}{2}$ virgate. This 2 ploughs can till. Drogo holds it of the bishop. Thereof $\mathrm{D}$ [rogo] has I ferling in demesne, and fol. 128.

the villeins (have) I ferling. There $D$ [rogo] has 2 villeins, 6 acres of meadow, and 50 acres of pasture. Worth 5 shillings; when the bishop received it it was worth the same.

The bishop has a manor called Gretedona [Gratton] ${ }^{2}$ which Alcerl held T.R.E., and it paid geld for $1 \frac{1}{2}$ virgates. This 8 ploughs can till. Drogo holds it of the bishop. Thereof $\mathrm{D}$ [rogo] has $\frac{1}{2}$ virgate in demesne and the villeins I virgate and 2 ploughs. There $\mathrm{D}$ [rogo] has 6 villeins, 3 bordars, 1 serf, 30 acres of wood(land), 12 acres of meadow and 200 acres of pasture. Worth 15 shillings; when the bishop received it it was worth 20 shillings.

The bishop has a manor called WennehaM

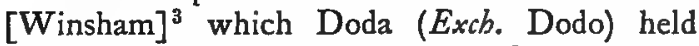
T.R.E., and it paid geld for $1 \frac{1}{2}$ hides and I $\frac{1}{2}$ ferlings. These 8 ploughs can till. Drogo holds it of the bishop. Thereof $D$ [rogo] has in demesne $\frac{1}{2}$ hide and $I_{\frac{1}{2}}$ ferlings and 1 plough, and the villeins (have) I hide and I plough. There $\mathrm{D}$ [rogo] has 7 villeins, 2 serfs, I I swine, 20 sheep, 12 goats, 20 acres of wood(land), 2 acres of meadow, and 100 acres of pasture. Worth 20 shillings; when the bishop received it it was worth 20 shillings.

The bishop has a manor called HrLa [Hele] which Edwi held T.R.E., and it paid geld for $\frac{1}{2}$ virgate. This 3 ploughs can till. Drogo holds it of the bishop. Thereof $\mathrm{D}$ [rogo] has I ferling and I plough in demesne and a villein I ferling and 2 ploughing oxen (boves in carr'). There D[rogo] has I villein, I bordar, I serf, 5 beasts, 7 swine, 20 sheep, Io goats, 20 acres of wood(land) and 30 acres of pasture. Worth Io shillings; when the bishop received it it was worth 5 shillings.

The bishop has a manor called BRAI [Bray] ${ }^{5}$ which Ailward held T.R.E., and it paid geld fol.x28b.

for $\frac{1}{2}$ virgate. This 4 ploughs can till. Drogo

\footnotetext{
1 Stowford in West Down in Braunton hundred.

${ }^{8}$ Gratton in High Bray in Shirwell hundred.

3 Winsham in Braunton in Braunton hundred.

${ }^{4}$ Hele in Ilfracombe in Braunton hundred.

${ }^{5}$ East Bray in South Molton in South Molton hundred (O.J.R.), Trans. Devon Assoc. xxxvi, 355 ; or Little Bray in Charles in Shirwell hundred (T.W W.).
}

holds it of the bishop. Thereof $D$ [rogo] has $I$ ferling and $I$ plough in demesne and the villeins I ferling and I plough. There $D$ [rogo] has 8 villeins and I orchard (ortum) in Barnstaple paying 4 pence, 3 beasts, 4 swine, 23 sheep, 20 goats, 30 acres of wood(land), 5 acres of meadow, and 30 of pasture. Worth 20 shillings; when the bishop received it it was worth Io shillings.

The bishop has a manor called Norcota [Northcot] ${ }^{8}$ which Ulf held T.R.E., and it paid geld for $2 \frac{1}{2}$ ferlings. These 4 ploughs can till. Drogo holds it of the bishop. Thereof $\mathrm{D}$ [rogo] has I ferlings and I plough in demesne, and the villeins have the rest of the land. There $\mathrm{D}$ [rogo] has 2 villeins, I serf, 7 beasts, Io swine, I I sheep, 12 acres of wood(land), 3 acres of meadow, and I 00 acres of pasture. Worth 15 shillings; when the bishop received it it was worth 5 shillings.

The bishop has a manor called Breduica [Burdick] $^{7}$ which Ailmar held T.R.E., and it paid geld for I virgate. This 3 ploughs can till. Drogo holds it of the bishop. Thereof $\mathrm{D}$ [rogo] has $\frac{1}{2}$ virgate and $I$ plough in demesne, and the villeins $\frac{1}{2}$ virgate and I plough. There $D$ [rogo] has 5 villeins, 5 bordars, I 3 beasts, I 5 swine, 50 sheep, 13 goats, 3 acres of meadow, and 60 acres of pasture. Worth 20 shillings; when the bishop received it it was worth 10 shillings.

The bishop has a manor called TrendesholT [Trentishoe] $^{8}$ which Bricsi (Excb. Brixi) held T.R.E., and it paid geld for $\frac{1}{2}$ hide. This 5 ploughs can till. Drogo holds it of the bishop. Thereof $\mathrm{D}$ [rogo] has 2 virgates and $\frac{1}{2}$ plough in demesne and the villeins 2 virgates and $\frac{1}{2}$ plough. There $\mathrm{D}$ [rogo] has 4 villeins, 4 bordars, I serf, Io swine, 15 sheep, 22 goats, 50 acres of fol. 129.

wood(land), and 60 acres of pasture. Worth Io shillings, and was worth the same when the bishop received it.

The bishop has a manor called Norcota [Northcote] ${ }^{9}$ which Ailmar held T.R.E., and it paid geld for $\frac{1}{2}$ hide. This 6 ploughs can till. Drogo holds it of the bishop. Thereof $\mathrm{D}$ [rogo] has I virgate and I plough in demesne and the villeins I virgate and $2 \frac{1}{2}$ ploughs. There $\mathrm{D}$ [rogo] has 5 villeins, 9 bordars, 3 serfs, 8 beasts, 7 swine, 40 sheep, I 7 goats, 4 acres of wood(land), 4 acres of meadow, and 40 acres of pasture. Worth 25 shillings, and was worth Io shillings when the bishop received it.

\footnotetext{
${ }^{6}$ Northcot in Braunton parish and hundred.

${ }^{7}$ Breadwick alias Burdick in Kentisbury in Braunton hundred.

8 Trentishoe in Braunton hundred. The particulars, which are double the total assessment, probably include Patehole which was held with it. Trans. Devon Assoc. xxxiii, 635 .
}

${ }^{9}$ Northcot in East Down in Braunton hundred. 


\section{A HISTORY OF DEVONSHIRE}

The bishop has a manor called WALEURDA [Wallaford] ${ }^{1}$ which Leugar held T.R.E., and it paid geld for I virgate. This 3 ploughs can till. Drogo holds it of the bishop. Thereof $\mathrm{D}$ [rogo] has 2 ferlings in demesne, and the villeins (have) 2 ferlings and 1 plough. There $\mathrm{D}$ [rogo] has 2 villeins, 2 bordars, 13 swine, and 60 acres of pasture. Worth 6 shillings, and was worth the same when the bishop received it.

The bishop has a manor called Warcoma [Warcombe] ${ }^{2}$ which Bristric (Exch. Brictric) held T.R.E., and it paid geld for I virgate. This Drogo holds of the bishop. Thereof $\mathrm{D}$ [rogo] has $\frac{1}{2}$ virgate and I plough in demesne, and the villeins $\frac{1}{2}$ virgate and I plough. There $\mathrm{D}$ [rogo] has 4 villeins, I bordar, I serf, 40 sheep, 4 acres of meadow and 50 of pasture. Worth Io shillings, and was worth 5 shillings when the bishop received it.

The bishop has a manor called Mideltona [Middleton] ${ }^{3}$ which Edmer held T.R.E., and it paid geld for $\frac{1}{2}$ virgate. This 4 ploughs can till. Drogo holds it of the bishop. Thereof fol. ragb.

$D[$ rogo $]$ has I ferling and I plough in demesne, and the villeins I ferling and I plough. There $\mathrm{D}$ [rogo] has 2 villeins, 15 swine, 3 acres of wood(land), 6 acres of meadow, and 30 acres of pasture. Worth I 2 shillings, and was worth 8 shillings when the bishop received it.

The bishop has a manor called Estotreia (Exch. Stodlei) [Stoly] ${ }^{4}$ which Bristuold (Exch. Brictuold) held T.R.E., and it paid geld for $\frac{1}{2}$ virgate. This 3 ploughs can till. Drogo holds it of the bishop. Thereof $\mathrm{D}$ [rogo] has I ferling and I plough in demesne and the villeins I ferling and I plough. There $D$ [rogo] has I villein, I serf, 5 beasts, 5 swine, 20 sheep, I 6 goats, 30 acres of wood(land), I acre of meadow, and 20 acres of pasture. Worth Io shillings, and was worth 5 shillings when the bishop received it.

The bishop has a manor called Bochelanda [Buckland] ${ }^{5}$ which Ulmer held T.R.E., and it paid geld for $\frac{1}{2}$ virgate. This 2 ploughs can till. $\mathrm{D}[\mathrm{rogo}]$ holds it of the bishop. Thereof $\mathrm{D}$ [rogo] has I ferling and I plough in demesne, and on the other ferling a certain serf is settled $(E x c h$. There is I plough in demesne, and 2 serfs). There $\mathrm{D}[$ rogo $]$ has 4 beasts, 6 sheep,

${ }^{1}$ Wallover alias Wallaford in Challacombe in Shirwell hundred.

Higher and Lower Warcombe in Ilfracombe in Braunton hundred.

${ }^{3}$ Middleton in Parracombe in Shirwell hundred.

- Stoly in Shirwell in Shirwell hundred.

${ }^{5}$ Part of East Buckland in Braunton hundred afterwards called Tossel's barton (O. J. R.), Trans. Devon Assoc. xxxvi, 354.
6 acres of wood(land), 6 acres of meadow, and 12 of pasture. Worth 5 shillings, and was worth 3 shillings when the bishop received it.

The bishop has a manor called BochelaNDA [Buckland] ${ }^{6}$ which Ulf held T.R.E., and it paid geld for I ferling. This (Hunc) (ferling) 2 ploughs can till. Drogo holds it of the bishop. Thereof $D$ [rogo] has $\frac{1}{2}$ ferling in demesne and the villeins $\frac{1}{2}$ ferling and 1 plough. There $\mathrm{D}$ [rogo] has I villein, 2 serfs, 3 acres of meadow and 3 of pasture. Worth 5 shillings, and was worth 3 shillings when the bishop received it.

The bishop has a manor called Bremerige [Bremridge] $^{7}$ which Edmer held T.R.E., and it paid geld for $\frac{1}{2}$ hide. This 6 ploughs can till. Drogo holds it of the bishop. Thereof D[rogo] fol. 130.

has I virgate and 2 ploughs in demesne; and the villeins I virgate and I plough. There $\mathrm{D}$ [rogo] has 3 villeins, 3 bordars, I serf, 5 beasts, Io swine, 60 sheep, 22 goats, I 5 acres of wood(land), and 30 of pasture. Worth 20 shillings, and was worth Io shillings when the bishop received it.

The bishop has a manor called Alra [Aller] ${ }^{8}$ which Edmer held T.R.E., and it paid geld for $\frac{1}{2}$ virgate. This 2 ploughs can till. Drogo holds it of the bishop. Thereof $D[$ rogo] has I ferling and I plough in demesne, and the villeins I ferling. There $\mathrm{D}[\mathrm{rogo}]$ has I villein, I serf, 6 acres of meadow, and 12 acres of pasture. Worth ro shillings, and was worth 5 shillings when the bishop received it.

The bishop has a manor called Pleirstou (Exch. Pleistou) [Plaistow ${ }^{9}$ which Bristuold (Exch. Brictuold) held T.R.E., and it paid geld for I virgate. This 4 ploughs can till. Drogo holds it of the bishop. Thereof $\mathrm{D}[\mathrm{rogo}]$ has $\frac{1}{2}$ virgate and $\mathrm{I}$ plough in demesne, and the villeins $\frac{1}{2}$ virgate and 2 ploughs. There (Inde) $\mathrm{D}$ [rogo] has 5 villeins, 3 bordars, 2 serfs, 8 beasts, 3 swine, 48 sheep, ro goats, 6 acres of meadow, and 30 of pasture. Worth 20 shillings, and was worth ro shillings when the bishop received it.

The bishop has a manor called FalleIA [Fairleigh] ${ }^{10}$ which Bristuold (Exch. Brictuold) held T.R.E., and it paid geld for I ferling. This (Hunc) (ferling) 2 ploughs can till. Drogo

${ }^{6}$ In East Buckland in Braunton hundred. Trans. Devon Assoc. xxxiv, 731 ; afterwards called Middlecot.

${ }^{7}$ Bremridge in South Molton in South Molton hundred.

${ }^{8}$ South Aller in South Molton in South Molton hundred.

${ }^{9}$ Plaistow in Shirwell in Shirwell hundred.

${ }^{10}$ Fairleigh in Marwood in Braunton hundred. 


\section{HOLDERS OF LANDS}

holds it of the bishop. Thereof Drogo has $\frac{1}{2}$ ferling and $\mathbf{I}$ plough in demesne, and the villeins $\frac{1}{2}$ ferling and $\frac{1}{2}$ plough. There $D$ [rogo] has I villein, 2 bordars, I serf, 3 beasts, 4 swine, 6 sheep, Io goats, 4 acres of wood (land), and 6 acres of meadow. Worth 6 shillings, and was worth the same when the bishop received it.

fol. xзob.

The bishop has a manor called Bedendona [Bittadon] ${ }^{1}$ which Ailmer held T.R.E., and it paid geld for $\frac{1}{2}$ hide. This 6 ploughs can till. Drogo holds it of the bishop. Thereof D[rogo] has I virgate and $I \frac{1}{2}$ ploughs in demesne, and the villeins I virgate and 2 ploughs. There $\mathrm{D}$ [rogo] has 4 villeins, 3 bordars, 3 serfs, 6 beasts, 3 swine, 135 sheep, 48 goats, I acre of wood (land), 5 acres of meadow and 60 of pasture Worth 25 shillings, and was worth 15 shillings when the bishop received it.

The bishop has a manor called Mollanda [Molland] ${ }^{2}$ which Ulwena held T.R.E., and it paid geld for $\frac{1}{2}$ hide. This 4 ploughs can till. Drogo holds it of the bishop. Thereof D[rogo] has I virgate and I plough in demesne, and the villeins I virgate and I plough. There $D$ [rogo] has 3 villeins, 4 bordars, 2 serfs, 8 beasts, IO swine, 40 sheep, 30 acres of wood(land), and $1 \frac{1}{2}$ acres of meadow. Worth 25 shillings, and was worth the same when the bishop received it.

The bishop has a manor called AnEstiga [Ansty] ${ }^{3}$ which Algar held T.R.E., and it paid geld for $\frac{1}{2}$ hide all but I ferling. This 3 ploughs can till. Drogo holds it of the bishop. Thereof $\mathrm{D}$ [rogo] has $2 \frac{1}{2}$ ferlings and 2 ploughs in demesne, and the villeins $3 \frac{1}{2}$ ferlings and I plough. There $\mathrm{D}$ [rogo] has 3 villeins, 6 serfs, 6 beasts, 4 swine, 30 sheep, 16 goats, 20 acres of wood(land), I acre of meadow, and pasture I league in length by $\frac{1}{2}$ in breadth. Worth 20 shillings; when the bishop received it it was worth I 5 shillings.

fol. r3r.

The bishop has a manor called Bochelanda [Buckland] ${ }^{4}$ which Alcerl held T.R.E., and it paid geld for I virgate. This 3 ploughs can till. Drogo holds it of the bishop. Thereof D[rogo] has $\frac{1}{2}$ virgate and I plough in demesne, and the villeins $\frac{1}{2}$ virgate and I plough. There $D$ [rogo] has 2 bordars, 2 serfs, 4 beasts, 40 sheep, 15 goats, 20 acres of wood(land), 12 acres of meadow, and 20 acres of pasture. Worth IO shillings;

${ }^{1}$ Bittadon in Braunton hundred, the parish.

Molland Champeaux alias Champeston in Molland in South Molton hundred.

'Anstey Moyne in West Anstey' in South Molton hundred. Unless we assume that Drogo's $2 \frac{1}{2}$ ferlings is a copyist's error for $3 \frac{1}{2}$, there is a shortage of I ferling. Trans. Devon Assoc. xxxiiii, 637.

- East Buckland manor in Braunton hundred. when the bishop received it it was worth 20 shillings.

The bishop has a manor called Rodelera [Rowley] ${ }^{5}$ which Aluard held T.R.E., and it paid geld for $\frac{1}{2}$ virgate. This 2 ploughs can till. Drogo holds it of the bishop. Thereof Drogo has I ferling in demesne, and the villeins I ferling and I plough. There $D$ [rogo] has 3 villeins, 2 bordars, 6 beasts, 12 sheep, 24 goats, 10 acres of wood(land), I acre of meadow, and 30 acres of pasture. Worth 7 shillings and 6 pence; when the bishop received it it was worth ro shillings.

The bishop of Coutances has a manor called KAGEFort (Exch. Chageford) [Chagford] ${ }^{6}$ which Dodo held T.R.E., and it paid geld for I hide. This 6 ploughs can till. Drogo holds it of the bishop. Thereof Drogo has I virgate and I plough in demesne, and the villeins have I $\frac{1}{2}$ ploughs. There Drogo has 8 villeins, 5 bordars, 4 serfs, 5 beasts, 4 I sheep, I 5 acres of wood(land), 18 of meadow, and 60 of pasture. Worth 30 shillings a year; when the bishop received it it was worth 20 shillings.

The bishop has a manor called TAIncoma [Teigncombe] $^{\boldsymbol{T}}$ which Alric held T.R.E., and it paid geld for $\frac{1}{2}$ hide. This 4 ploughs can till. Now Drogo holds it of the bishop. Thereof fol. rzsz.

$\mathrm{D}$ [rogo] has $\mathrm{I} \frac{1}{2}$ ferlings and I plough in demesne, and the villeins have $1 \frac{1}{2}$ ploughs. There $D$ [rogo] has 6 villeins, 2 bordars, 2 serfs, 6 beasts, 5 swine, 20 sheep, 14 goats, 4 acres of wood(land), 4 acres of meadow, pasture 3 leagues in length by I league in breadth. Worth 20 shillings a year; when the bishop received it it was worth I 5 shillings.

The bishop has a manor called Clis $\left[\right.$ Clyst] ${ }^{8}$ which Edrit (Exch. Edric) held T.R.E., and it paid geld for I hide. This 5 ploughs can till. Now Gosfrid holds it of the bishop. Thereof Gosfrid has $\frac{1}{2}$ hide in demesne and 2 ploughs, and the villeins have $3 \frac{1}{2}$ ploughs. There G[osfrid] has 9 villeins, 7 serfs, 2 rounceys, 8 beasts, 4 swine, roo sheep, 20 acres of meadow, and 12 acres of pasture. Worth 4 pounds a year; when the bishop received it it was worth 50 shillings.

\section{Rowley in Parracombe in Shirwell hundred.}

${ }^{6}$ Chagford manor in Wonford hundred (Trans. Devon Assoc. xxv, $5 \times 5$ ).

${ }^{7}$ Teigncombe manor in Chagford in Wonford hundred.

${ }^{8}$ Clyst Fomison alias Clyst St. Michael and Clyst Gabriel alias Bishop's Clyst, i.e. the parish of Sowton in Wonford hundred. Trans. Devon Assoc. xxxiii, 636 . Osbern appears to have been under-tenant in Geldroll, fol. $70 b, \mathrm{~B}, \mathrm{I}$. 


\section{A HISTORY OF DEVONSHIRE}

The bishop has a manor called Branfortuna [Brampford], ${ }^{1}$ which Wlnot (Exch. Ulnod) held T.R.E., and it paid geld for $\mathrm{I}$ hide. This 8 ploughs can till. Now Drogro holds it of the bishop. Thereof $\mathrm{D}$ [rogo] has $\mathbf{1} \frac{1}{2}$ virgates in demesne and I plough; and the villeins have 5 ploughs. There $\mathrm{D}$ [rogo] has Io villeins, 8 bordars, 6 serfs, I rouncey, 5 beasts, 32 sheep, 8 acres of wood(land), and 60 acres of pasture. Worth 60 shillings a year, and was worth the same when the bishop received it.

The bishop has a manor called REUwA [Rewe] ${ }^{2}$ which Wlnot ( $E x c h$. Ulnod) held T.R.E., and it paid geld for I hide. This 5 ploughs can till. Now Drogo holds it of the bishop. Thereof $\mathrm{D}$ [rogo] has $\frac{1}{2}$ hide in demesne. There $\mathrm{D}$ [rogo $]$ has 5 villeins, 3 bordars, 2 serfs, Io acres of wood(land), and 7 acres of meadow. Worth fol. I32. $^{2}$

20 shillings a year, and was worth 60 shillings when the bishop received it.

The bishop has a manor called Niressa $[\text { Netherexe }]^{3}$ which Wlnot (Exch. Ulnod) held T.R.E., and it paid geld for I hide and I virgate. This 8 ploughs can till. Now Drogo holds it of the bishop. Thereof $\mathrm{D}$ [rogo] has I virgate in demesne and I plough, and the villeins have 6 ploughs. There $\mathrm{D}[$ rogo $]$ has Io villeins, 8 bordars, 6 serfs, I cow, 4 swine, 60 sheep, 4 acres of meadow, and 12 acres of pasture. Worth 3 pounds a year; when the bishop received it it was worth 70 shillings.

The bishop has a manor called Olpessa (Exch. Ulpesse) [Upex] ${ }^{4}$ which Olnot (Exch. Ulnod) held T.R.E., and it paid geld for I virgate. This 3 ploughs can till. Drogo holds it of the bishop, and Humphrey (Unfridus) of Drogo. Thereof Humphrey has in demesne $\frac{1}{2}$ virgate, and the villeins $\frac{1}{2}$ virgate and I plough. There Humphrey has 7 villeins, 7 bordars, 3 serfs, 4 beasts, I mill paying I 2 shillings, 6 acres of wood(land), ro of meadow, and 12 of pasture. Worth 2 pounds a year; when he received it it was worth 3 pounds.

The bishop has I hide of land ${ }^{5}$ which Olnot (Exch. Ulnod) held T.R.E. There the bishop has I villein and I mill paying 20 shillings. This hide is worth 30 shillings a year.

fol. xzab.

The bishop has a manor called Cursta [Clyst $^{6}$ which Wigo (Exch. Wigod) the priest held T.R.E., and it paid geld for 3 virgates.

'Brampford Speke in Wonford hundred.

${ }^{2}$ Rewe in Wonford hundred.

${ }^{3}$ Netherexe in Wonford hundred.

4 Upex in Rewe in Hairidge hundred.

${ }^{5}$ Hazel Barton in Rewe with Pound Mill.

${ }^{6}$ Clyst Satchvil alias Bishop's Court alias Clist House, an outlier of Faringdon in East Budleigh hundred.
These 6 ploughs can till. Thereof the bishop has $I \frac{1}{2}$ virgates and 2 ploughs in demesne, and the villeins $1 \frac{1}{2}$ virgates and 5 ploughs. There the bishop has 9 villeins, 5 bordars, 5 serfs, I rouncey, Io beasts, 3 swine, 63 sheep, 20 acres of meadow, and I2 acres of pasture. Worth 60 shillings; when the bishop received it it was worth 20 shillings.

The bishop has a manor called CRauelec [Crayleigh] $^{7}$ which Alwin the server (dapifer) held of Alwin, and could not make independent (separare) of him T.R.E., and it paid geld for I virgate. This 2 ploughs can till. Edmer holds it of the bishop. Thereof $\mathrm{E}$ [dmer] has 2 ferlings and I plough in demesne, and the villeins 2 ferlings and I plough. There $E$ [dmer] has 2 villeins, I bordar, I serf, and 8 acres of meadow. Worth ro shillings; when the bishop received it 5 shillings.

The bishop has a manor called CRrdia [Creedy $^{8}$ which Goda held T.R.E., and it paid geld for I virgate. This 2 ploughs can till. Drogo holds it of the bishop. There $\mathrm{D}$ [rogo] has 4 villeins who have $\frac{1}{2}$ plough. Worth 5 shillings; when the bishop received it 12 shillings and 6 pence. It belongs to the manor called Morceta [Morchard].

The bishop has a manor called Morceta [Morchard] ${ }^{9}$ which Algar held T.R.E., and it paid geld for I virgate and I ferling. These 4 ploughs can till. Drogo holds it of the bishop. Thereof Drogo has in demesne ( $\frac{1}{2}$ virgate . . .) and the villeins $\frac{1}{2}$ virgate and $I$ ferling and $I$ fol. . 33 .

plough. There Drogo has 4 villeins, 4 bordars, I serf, 13 beasts, 13 swine, 40 sheep, 36 goats, ro acres of wood(land), 6 acres of meadow, and 100 acres of pasture. Worth 12 shillings and 6 pence a year; when the bishop received it it was worth 5 shillings.

The bishop has a manor called SpREwEA [Spurway $]^{10}$ which Algar held T.R.E., and it paid geld for I virgate. This 3 ploughs can till. Now Drogo holds it of the bishop. Thereof Drogo has in demesne $\frac{1}{2}$ virgate and 2 ploughs, and the villeins $\frac{1}{2}$ virgate and 2 oxen. There Drogo has 2 villeins, 2 bordars, 2 serfs, Io beasts, ro swine, 40 sheep, ro acres of wood(land), 3 acres of meadow and 20 acres of pasture. Worth Io shillings a year; when the bishop received it it was worth 5 shillings.

'Crayleigh alias Crealey in Faringdon in East Budleigh hundred.

${ }^{8}$ Probably Hassok alias Haske on the Creedy in Upton Helion in West Budleigh hundred.

${ }_{9}$ That part of Morchard Bishop which is in Witheridge hundred, i.e. Rowlstone.

${ }^{10}$ East Spurway in Oakford in Witheridge hundred. 


\section{HOLDERS OF LANDS}

The bishop has a manor called Coma [Combe] ${ }^{1}$ which Ailward held T.R.E., and it paid geld for $\frac{1}{2}$ hide. This 3 ploughs can till. Drogo holds it of the bishop. Thereof Drogo has in demesne I virgate and I plough, and the villeins I virgate and I plough. There Drogo has 3 villeins, I serf, Io beasts, 5 swine, ro sheep, 8 goats, 6 acres of wood(land), 3 acres of meadow, and 20 acres of pasture. Worth Io shillings a year, and was worth the same when the bishop received it.

The bishop has a manor called CerlvertesBERIA [ $]^{2}$ which Ailwin (Exch. Alwin) held T.R.E., and it paid geld for I hide all but a ferling. This 6 ploughs can till. Now Drogo holds it of the bishop. Thereof $\mathrm{D}$ [rogo] has in demesne $\frac{1}{2}$ hide and I plough, and the villeins 3 fertings I virgate and 2 oxen. There $D$ [rogo] has 3 villeins, 2 bordars, 2 serfs, 6 beasts, I 5 swine, 27 sheep, 6 acres of coppice, 15 acres of fol. xagb.

meadow and 100 acres of pasture. Worth 15 shillings; when the bishop received it it was worth 5 shillings.

To this manor has been added a manor called Coma $[\text { Combe }]^{3}$ which Weland held T.R.E., and it paid geld for $\mathbf{I}$ virgate and I ferling. This I plough can till, and it is unoccupied (vacua).

The bishop has a manor called Estolleia (Exch. Stollei) [Stoodleigh] ${ }^{4}$ which Alwin held T.R.E., and it paid geld for I hide. This Io ploughs can till. Now Drogo holds it of the bishop. Thereof Drogo has in demesne I ferting and 2 ploughs, and the villeins 3 virgates, 3 fertings, and 2 ploughs. There Drogo has 5 villeins, 9 bordars, 4 serfs, 6 beasts, 7 swine, 40 sheep, 33 goats, 40 acres of wood(land), 2 acres of meadow and 16 of pasture. Worth 40 shillings a year; when the bishop received it it was worth 20 shillings.

The bishop has a manor called Coma [Combe] ${ }^{5}$ which Brongar (Exch. Brungar) held T.R.E., and it paid geld for I virgate. This I plough can till. Drogo holds it of the bishop. Thereof he has I plough; and he has there I serf, Io beasts, 8 swine, 30 sheep, 2 acres of coppice, and 3 acres of meadow. Worth 5 shillings a year, and was worth 3 shillings when the bishop received it.

\footnotetext{
${ }^{1}$ Combe Temple alias Templeton in Witheridge hundred.

' Possibly Coulson alias Colston in Templeton in Witheridge hundred. Trans. Devon Assoc. xxxv, 685.

${ }^{3}$ Combe, appurtenant to Colston in Templeton. Trans. Devon Assoc. xxx, 4I 3.

${ }^{4}$ East Stoodleigh in Stoodleigh in Witheridge hundred.

${ }^{5}$ Combe Monceaux in Templeton in Witheridge hundred
}

The bishop has a manor called Bradaleia

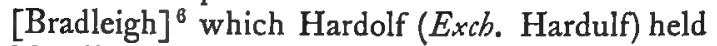
T.R.E., and it paid geld for $\frac{1}{2}$ hide. This 4 ploughs can till. Drogo holds it of the bishop. Thereof Drogo has in demesne $I$ virgate and $\frac{1}{2}$ plough, and the villeins I virgate and $\frac{1}{2}$ plough. There Drogo has I villein, I serf, 12 sheep, and 5 acres of wood(land). Worth 5 shillings a year, and was worth 10 shillings when the bishop received it.

fol. 134 .

The bishop has a manor called TalebruA (Exch. Talebrige) [Thelbridge] ${ }^{7}$ which Olveva (Exch. Ulveva) held T.R.E., and it paid geld for $\frac{1}{2}$ hide and $\frac{1}{2}$ virgate. These ro ploughs can till. Drogo holds it of the bishop. Thereof Drogo has in demesne I virgate and I plough, and the villeins $I \frac{1}{2}$ virgates and $2 \frac{1}{2}$ ploughs. There Drogo has I 5 villeins, 6 serfs, 3 beasts, 5 swine, 27 sheep, 29 goats, 16 acres of wood(land), 12 acres of meadow, and 12 acres of pasture. Worth 40 shillings a year, and was worth 50 shillings when the bishop received it. And to the manor has been added the manor known as Wicca $\left[\right.$ Wick ${ }^{8}$ which Brismar held T.R.E., and it paid geld for $\frac{1}{2}$ virgate. This 2 ploughs can till. Drogo holds it of the bishop. There Drogo has 3 villeins and 6 acres of meadow. Worth 5 shillings a year.

The bishop has a manor called OluRIDINTONA (Exch. Uluredintune) [Worlington] ${ }^{9}$ which Olveva (Exch. Ulveva) held T.R.E., and it paid geld for I virgate. This 3 ploughs can till. Drogo holds it of the bishop. Thereof he has in demesne 2 ferdings and 1 plough; and the villeins (have) 2 ferdings and $1 \frac{1}{2}$ ploughs. There Drogo has 7 villeins, 1 serf, 3 acres of wood(land), 3 of coppice, 4 acres of meadow, and ro of pasture. Worth 7 shillings and 6 pence a year; when the bishop received it it was worth 20 shillings.

fol. $x_{34} \mathrm{~b}$.

The bishop has a manor called RinestaneDoNa [Ringstonedown] ${ }^{10}$ which Abbet held T.R.E., and it paid geld for I ferding. This I plough can till. Etdilla (Excb. Eddilla) holds it of the bishop. Thereof Etdilla has in demesne $\frac{1}{2}$ ferding and $\frac{1}{2}$ plough; and a villein $\frac{1}{2}$ ferding and $\frac{1}{2}$ plough. There $E$ [tdilla] has $I$ villein, I bordar, I serf, 7 beasts, 30 sheep, and 6 acres of meadow. Worth 5 shillings a year, and was worth the same when he received it.

\footnotetext{
${ }^{6}$ Great Bradleigh in Tiverton in Tiverton hundred.

7 Thelbridge in Witheridge hundred.

${ }^{8}$ Middlewick in Thelbridge in Witheridge hundred.

9 East Worlington in Witheridge hundred.

${ }_{10}$ Ringstonedown alias Russen in East Worlington in Witheridge hundred.
} 


\section{A HISTORY OF DEVONSHIRE}

The bishop has a manor called BRADELEIA [Bradleigh] ${ }^{1}$ which Algar held T.R.E., and it paid geld for I ferting. This I plough can till. Now Drogo holds it of the bishop. On it (In ea) Drogo has I bordar, and it pays 30 pence a year.

The bishop has a manor called LochesBera [Loxbear] $^{3}$ which Algar held T.R.E., and it paid geld for 3 virgates. These 4 ploughs can till. Now Drogo holds it of the bishop. Thereof Drogo has I ferting and I plough in demesne, and the villeins have $\frac{1}{2}$ hide, and 3 fertings, and I plough. There Drogo has 2 villeins, 4 bordars, 2 acres of coppice, and 3 acres of meadow. Worth I 5 shillings a year, and was worth 10 shillings when the bishop received it.

The bishop has a manor called Pedehael [Peadhill] $^{3}$ which Algar held T.R.E., and it paid geld for 3 virgates. These 4 ploughs can till. Drogo holds it of the bishop. Thereof $D$ [rogo] has I virgate and I plough in demesne, and the villeins $\frac{1}{2}$ (a plough). There $D$ [rogo] has 3 men (bomines), 3 swine, ro sheep, 5 acres of wood(land), and 3 of meadow. Worth I 5 shillings a year, and was worth the same when the bishop received it.

fol. 135 .

The bishop has a manor called Teigna [Teign] ${ }^{4}$ which Edric held T.R.E., and it paid geld for I hide. This 7 ploughs can till. Gauffrid de Trailei holds it of the bishop. Thereof G[auffrid] has I virgate and I plough in demesne, and the villeins 3 virgates and 6 ploughs. There G[auffrid] has 16 villeins, 5 serfs, I rouncey, 7 beasts, 4 swine, 64 sheep, 12 acres of wood(land), I acre of meadow, and 60 of pasture. Worth 100 shillings, and when the bishop received it it was worth the same.

The bishop has a manor called FAREweIA [Farway $]^{5}$ which Ailmar held T.R.E., and it paid geld for $\frac{1}{2}$ hide. This 4 ploughs can till. Drogo holds it of the bishop. Thereof $\mathrm{D}$ [rogo] has in demesne $\frac{1}{2}$ virgate and I plough, and the villeins $1 \frac{1}{2}$ virgates and 3 ploughs. There $\mathrm{D}$ [rogo] has 5 villeins, 4 bordars, 2 serfs, 8 beasts, 5 acres of meadow, and 40 acres of pasture. Worth 20 shillings a year, and was worth 10 shillings when the bishop received it.

The bishop has a manor called Cheletona (Exch. Cherleton) [Charleton $]^{6}$ which Edric held

1 West Bradleigh alias Little Bradleigh in Tiverton in Tiverton hundred.

2 Loxbear in Tiverton hundred.

3 Peadhill in Tiverton in Tiverton hundred.

- Canon Teign in Christow in Teignbridge hundred.

${ }^{3}$ Farway in Colyton in Colyton hundred.

6 Charlton Luggeston alias Luxen and Chipplehays in Upottery in Axminster hundred (O.J.R.), or Charlton in Axmouth (T.W.W.).
T.R.E., and it paid geld for 3 virgates. These 3 ploughs can till. Robert holds it of him. Thereof $\mathrm{R}$ [obert] has I virgate and I plough in demesne, and a villein 3 virgates and $\frac{1}{2}$ plough. There $\mathbf{R}$ [obert] has I villein, 2 bordars, 6 acres of wood(land), 6 acres of meadow, and 12 of pasture. It pays Io shillings a year (de firma), and paid the same when Robert received it.

The bishop has a manor called Bovr [Bovey] ${ }^{7}$ which Eddric held T.R.E., and it paid geld for 2 hides. These ro ploughs can till. Thereof the bishop has in demesne $\frac{1}{2}$ hide and 3 ploughs, and the villeins $I \frac{1}{2}$ hides and 10 ploughs. There the bishop has 16 villeins, 8 bordars, 8 serfs, I rouncey, 30 beasts, 7 swine, 85 sheep, 5 goats, 1 fol. 335 b.

mill paying 5 shillings, 60 acres of wood(land), 5 acres of meadow, and 50 of pasture. It pays Io pounds a year all but 2 shillings and 6 pence. To this manor has been added the land of 15 thanes. ${ }^{8}$ One of these booklands ${ }^{9}$ is called AnoNeBovi ${ }^{10}$ [Down Bovey], and there 4 thanes were settled. Another is called Wermenri [Warmhill $], 11$ and there 1 thane was settled. A third is called SCABATORA [Scabator], ${ }^{12}$ and there 2 thanes were settled. Another is called Brungarstona [ $]^{13}$ and there 2 thanes were settled. Another AIlavesfort (Exch. Eilavesford) [Aylsford] ${ }^{14}$; another Oluesera (Exch. Ulvelei) [Woolley]. ${ }^{16}$ These two I thane held, and with that thane there was another thane in Olueleia. Yet another is HaUocmora [Hawkmoor], ${ }^{16}$ and there I thane was settled. Another HaRLEIA [Harley], ${ }^{17}$ and there $I$ thane was settled. Another Polebroc [Pulbrook $]_{3}^{18}$ and there I thane was settled. And these 15 hold together 2 hides and $\frac{1}{2}$ virgate, and they held their land in parage (pariter) T.R.E. These 8 ploughs can till, but only 7 are there; and these thanes pay yearly to Bovi [Bovey] 4 pounds 2 shillings and 6 pence by way of rent (de censu) over and above the above-named 10 pounds.

7 South Bovey alias Bovey Tracy in Teignbridge hundred.

8 But only 14 are enumerated.

9 'Mans[ionibus]' is altered to 'bochelandis.'

10 Down Bovey or Little Bovey in Bovey Tracy in Teignbridge hundred.

"Warmhill in Hennock in Teignbridge hundred.

12 Scabator in Widdecombe-in-the-Moor in Haytor hundred.

${ }_{13}$ Perhaps Bawtor in Hennock in Teignbridge hundred.

14 Aylsford in Bovey Tracy in Teignbridge hundred.

15 Woolley in Bovey Tracy in Teignbridge hundred.

${ }^{16}$ Hawkmoor in Bovey Tracy in Teignbridge hundred.

17 Hatherly alias Harley in Bovey Tracy in Teignbridge hundred.

${ }_{18}$ Pulbrook in Bovey Tracy in Teignbridge hundred. 


\section{HOLDERS OF LANDS}

The bishop has a manor called LANGEHEwis [Lang Hewish] ${ }^{1}$ which Sawin held T.R.E., and it paid geld for $\frac{1}{2}$ hide all but I ferling. This I ploughs can till. He could go to any lord he liked. Leuric holds it of the bishop. There L[euric] has I plough in demesne, 2 bordars, 10 acres of coppice, and 2 acres of meadow. Worth Io shillings; when the bishop received it it was worth 5 shillings.

The bishop has I ferling of land in Polesseuia (Exch. Poleslewe) [Polsloe] ${ }^{2}$ which Alwin held T.R.E. This I plough can till. Anger $\left(E_{x x c b}\right.$. Ansger) holds it of the bishop. There A[nger] has 1 plough in demesne, 2 serfs, 20 sheep, 6 goats, 2 acres of meadow, and 16 of pasture. Worth ro shillings a year; when the bishop received it 4 shillings.

fol. 136 .

The bishop has Io burgesses in BARNESTAPLA [Barnstaple $]^{3}$ who pay 3 shillings and 9 pence; 7 houses lying in ruins, $\frac{1}{2}$ virgate of land and I mill paying 20 shillings a year, beside 20 shillings out of the customary dues of the king's burgesses.

The bishop has in Essecestra (Exch. Execestre) $\left[\right.$ Exeter ${ }^{4} 3$ houses and $I$ lying in ruins which were in the demesne of King $E$ [dward] T.R.E. and paid customary dues.

The bishop has 6 houses in Essecestra [Exeter] which Drogo holds of him. Four of these were quit of customary dues T.R.E. and 2 paid dues. Drogo now withholds them, viz., I 6 pence.

LAND OF THE GLASTONBURY (GLASTINGHEBERIENSIS) ABBOT (Exch. OF THE CHURCH OF GLASTINBERY) IN DEVENESIRA

fol. x6r.

The abbot (Exch. The church) of Glastonbury has a manor called LIM [Uplyme] ${ }^{5}$ and it paid geld for 6 hides. These 7 ploughs can till. Thereof the a[bbot] has 3 hides and 2 ploughs in demesne, and the villeins 3 hides and 5 ploughs. There the a[bbot] has 16 villeins, 4 bordars, 4 serfs, 5 beasts, 7 swine, 26 sheep, 4 goats, wood(land) 20 furlongs in length by I furlong in breadth, 8 acres of meadow, pasture 2 leagues in length by I league in breadth. Worth 3 (Exch. 4 as also Exon. fol.

${ }^{1}$ Lang Hewish alias Langage in Plympton St. Mary in Plympton hundred.

Polsloe in Heavitree in Wonford hundred. Devon $N$. and $Q$. ii, 24, 188 .

${ }^{3}$ Barnstaple locally in Fremington hundred.

- Exeter locally in Wonford hundred.

5 Uplyme in Axminster hundred. Cars. Sax. i, 313. $\left.527^{b}\right)$ pounds a year, and was worth the same when he received it.

\section{LANDS OF THE ABBOT OF TAVE- STOCH CHURCH IN DEVENESIRA ${ }^{6}$ fol. 177 .}

The abbot (Exch. The church) of Tavestoch [Tavistock] has a manor called Tavestocha [Tavistock] ${ }^{7}$ which paid geld in the time of King Edward for $3 \frac{1}{2}$ hides. These 40 ploughs can till. Thereof the abbot has $\frac{1}{2}$ hide and 5 ploughs in demesne, and the villeins have thereof $1 \frac{1}{2}$ hides and 14 ploughs. There the abbot has I 7 villeins, 20 bordars, I 2 serfs, I rouncey, 26 beasts, 12 swine, 200 sheep, 30 goats, I mill for the service of the abbey $\left(E_{x c h}\right.$. the court), wood(land) 2 leagues in length by $I$ in breadth, I6 acres of meadow, and pasture 10 furlongs in length by a like amount in breadth.

Of these $3 \frac{1}{2}$ hides 6 knights (milites) hold $1 \frac{1}{2}$ hides, ${ }^{8}$ which 4 thanes held of the abbot without being able to become independent of (separari) the church T.R.E.

Thereof Ermenald has $\frac{1}{2}$ virgate. ${ }^{9}$ There Ermenald has I plough, and his villeins I plough, 7 beasts, and 40 sheep.

Ralf has thereof $\frac{1}{2}$ virgate, ${ }^{10}$ a villein, and 3 coscets, and they have $\frac{1}{2}$ plough.

Hugh has $\frac{1}{2}$ hide, $\frac{1}{3}$ virgate, and I ferling, and has in demesne 2 ploughs. $\mathrm{He}$ has there I villein, 6 bordars, and 2 serfs, who have 7 ploughing oxen (boves ad caruc'). Hugh has there 10 beasts, 12 swine, and 60 sheep.

Rotbert has I virgate and 2 ferlings, ${ }^{12}$ and $I \frac{1}{2}$ ploughs in demesne, and the villeins $I \frac{1}{2}$ ploughs.

'Exch. reads 'Land of the Church of 'Tavestoch.' See Dugdale, Mon. Angl. ii, 494, for a list of estates given to the church by the founders, and the bull of Pope Celestine III, in Oliver, Mon. p. 93, for those confirmed to it in 1193.

7 Tavistock alias Hurdwick manor (Trans. Devon Assoc. xxviii, 478; Ancient Deeds, A 7080) in the hundreds of Tavistock and Roborough, extending over Tavistock parish, together with part of Milton Abbot and Cudlip in Petertavy. Before I I I4 Tavistock hundred formed part of Lifton hundred.

${ }_{8}^{8}$ The assessments of the $6 \mathrm{knights}$ amount together to I hide, 2 virgates, of ferling (Trans. Devon Assoc. xxíii, $192, n .4^{8}$ ), and their area to 850 acres, lying in Tavistock and Milton Abbot.

${ }^{9}$ Perhaps Cudlip in Petertavy in Roborough hundred (O.J.R.). Feud. Aids, p. $354: \frac{1}{3}$ fee.

${ }^{10}$ Perhaps Notley in Tavistock in Roborough hundred (O.J.R.). Feud. Aids, p. 354: $\frac{1}{12}$ fee.

${ }^{11}$ Wick Amadas alias Dabernon in Milton Abbot in Tavistock hundred (O.J.R.). Feud. Aids, p. 322 : in Wyke, Hasworthy, and Okbere I fee.

${ }_{18}$ Hundecot alias Youngcot in Milton Abbot in Tavistock hundred (O.J.R.). - Robert is probably Robert de Albemarle, ancestor of William de Albemarle, who held Hundcot in 1283. Feud. Aids, p. 322 . 


\section{A HISTORY OF DEVONSHIRE}

There $\mathrm{R}$ [otbert] has 3 villeins, 6 bordars, 2 serfs, 12 beasts, 60 sheep, and 20 goats.

Ralf de Tilio has $\frac{3}{4}$ virgate $^{1}$ and I plough, I villein, and 4 bordars, who have 2 oxen, 7 beasts, 30 sheep, and ro goats.

Gosfrid has I ferting, ${ }^{2}$ and he has there I plough, I bordar, 6 beasts, and 30 sheep.

fol. rz7b.

This manor is worth to the abbot (ad opus abbatis) 12 pounds a year, to the knights 100 shillings. When the abbot and the knights received it it was worth 14 pounds and 8 pounds respectively. (Exch. Formerly it was worth 22 pounds in all.)

The abbot of Tavistock (Exch. The church itself) has a manor called Mideltona [Milton] ${ }^{3}$ which paid geld for $\frac{1}{2}$ hide in King E[dward]'s time. This I 5 ploughs can till. Thereof the abbot has I virgate and 5 ploughs in demesne; and the villeins have I virgate and 10 ploughs. There the abbot has 14 villeins, 12 bordars, I 2 serfs, 22 beasts, 12 swine, I 70 sheep, 30 goats, Io acres of wood, 20 of meadow, and 400 acres of pasture. It is worth 8 pounds, and was worth 4 pounds when the abbot received it.

Along with this manor the abbot holds 2 manors ( $E x c h .2$ lands) which 2 thanes held in parage (pariter; Exch. as 2 manors) T.R.E., one called LEGA [Leigh], ${ }^{4}$ the other Lideltona [Liddaton], ${ }^{5}$ and they paid geld for $\frac{1}{2}$ hide. This 15 ploughs can till. Thereof the abbot has I ferting and I plough in demesne, and the villeins have I virgate, 3 fertings, and 4 ploughs. There the abbot has 4 villeins, 3 serfs, 5 bordars, 4 beasts, and 30 sheep. They are worth 60 shillings a year; when the abbot received them 30 shillings.

The abbot has a manor called Lideltona [Liddaton] ${ }^{6}$ which paid geld T.R.E. for $\frac{1}{2}$ hide. This 3 ploughs can till. Gosfrid holds it of the fal. $x y 8$.

abbot and it is part of the abbot's demesne. Thereof Gosfrid has $\frac{1}{2}$ virgate and I plough in

1 Perhaps Tavyton in Tavistock in Roborough hundred (O.J.R.). Feud. Aids, pp. 340, 355 : Tavyton $\frac{1}{2}$ fee.

${ }^{2}$ Probably Crebar in Tavistock in Tavistock hundred, held together with Fishleigh and Hannaborough in Hatherleigh for $\frac{1}{2}$ fee by William de Crievebere in I 166 (Lib. Nig. p. I I 8), and by Robert Hereward in I 241 (Testa de Nevill (Rec. Com.) I 78b) ; Gosfrid held also in Hatherleigh, see below. Trans. Devon Assoc. xxxvi, 350.

${ }^{3}$ Milton Abbot, the chief manor of Milton Abbot in Tavistock hundred.

4 Leigh Champeaux in Milton Abbot in Tavistock hundred.

${ }^{5}$ This and the next entry probably represent West Liddaton and Chilliton in Milton Abbot in Tavistock hundred. Trans. Devon Assoc. xxxvi, 350.

${ }^{6}$ See last note. demesne, and the villeins $\frac{1}{2}$ virgate and 2 ploughs. There G[osfrid] has 5 villeins, 2 bordars, 3 serfs, 24 beasts, I 0 swine, I00 sheep, 50 goats, I 2 acres of wood, Io acres of meadow, and 80 of pasture. Worth 30 shillings a year; when the abbot received it it was worth 20 shillings.

The abbot (Exch. The church itself) has a manor called HadRELEIA ( $E x c h$. Adrelie) [Hatherleigh $]^{7}$ which in the time of King $E$ [dward] paid geld for 3 hides. These 30 ploughs can till. Thereof the abbot has $\frac{1}{2}$ hide in demesne and 3 ploughs, and the villeins $1 \frac{1}{2}$ hides and 10 ploughs.

Nigel a French knight (francus miles) has thereof $\frac{1}{2}$ virgate all but $\frac{1}{2}$ ferling. ${ }^{8} \quad$ On it (In ea) Nigel has I plough in demesne and the villeins another plough. He has there 5 coscets, I serf, 6 beasts, 4 swine, and 40 sheep.

Walter has thereof $\frac{1}{2}$ hide and $\frac{1}{2}$ virgate, ${ }^{9}$ of which Walter has $\frac{1}{2}$ virgate and I plough in demesne and the villeins $\frac{1}{2}$ hide, 4 ploughs, and I 2 beasts. On it Walter has 10 swine, 50 sheep, I mill paying 6 pence yearly, 7 villeins, 2 bordars, and I serf.

Gosfrid has $\frac{1}{2}$ virgate and $\frac{1}{2}$ ferling. ${ }^{10}$ On it Gosfrid has I plough and the villeins another; also 4 villeins, I serf, I cow, and 5 goats $(E x c h$. adds, a mill paying 6 pence on Gosfrid's land).

Ralf has $\frac{1}{2}$ virgate ${ }^{11}$ and I plough, I villein, 4 bordars, 7 beasts, 3 swine, 12 sheep, and 6 goats.

On this manor the abbot has 26 villeins, 6 coscets, 6 serfs, I 5 beasts, 4 swine, 44 sheep, 24 goats, wood(land) $2 \frac{1}{2}$ leagues in length by $\frac{1}{2}$ league in width, 100 acres of meadow, pasture 3 leagues in length by $\frac{1}{2}$ league in width. This manor is worth yearly to the abbot (ad opus abbatis) 10 pounds, to the knights 60 shillings; when the abbot received it it was worth 4 pounds, when the abbot received the land of the knights it was worth 40 shillings. (Exch. Formerly it was worth 9 pounds in all.) fol. 1786 .

The abbot has a manor called Torneberia [Thornbury] ${ }^{12}$ which in King E[dward]'s time paid geld for I hide. This Io ploughs can till.

\footnotetext{
${ }^{7}$ Hatherleigh manor, including the parishes of Hatherleigh and Jacobstow in Blacktorington hundred.

${ }^{8}$ Probably Pulworthy in Hatherleigh, subsequently held for $\&$ fee (O.J.R.). Trans. Devion Assoc. xxviii, 488 .

${ }^{9}$ Probably Broomford and Jacobstow tithing with Shelston in Exbourne in Blacktorington hundred, subsequently held for $\frac{1}{2}$ fee (O.J.R.).

${ }_{10}$ Probably Fishleigh and Hannaborough in Hatherleigh in Blacktorington hundred, afterwards held together with Crebar for $\frac{1}{2}$ fee (O.J.R.); see note 2 above.

${ }_{11}$ Probably Langbear and Marshford in Hatherleigh, afterwards held for $\frac{1}{4}$ fee (O.J.R.). Trans. Devon Assoc. xxxvi, 350 .

12 Thornbury manor in Blacktorington hundred,
} 


\section{HOLDERS OF LANDS}

Ralf holds it of the abbot. Thereof Ralf has I virgate and 2 ploughs in demesne, and the villeins 3 virgates and 4 ploughs. There Ralf has 10 villeins, 6 bordars, 3 serfs, I 4 beasts, I 5 swine, 50 sheep, 30 goats, 20 acres of wood(land), 100 acres of meadow, and $\mathrm{r} 20$ of pasture. Worth 60 shillings ( $E x c h .3$ pounds); when the abbot received it it was worth less.

The abbot of Tavistock was seised of OLWRITONA [Werrington] ${ }^{1}$ on the day on which King William sent his barons to inquire as to the lands of England, and his predecessor before him had been seised thereof; but by the king's barons he was disseised thereof on the ground that according to the Englishmen's testimony it did not belong to the abbey T.R.E.

The abbot (Exch. The church itself) has a manor called HaMA [Ham] ${ }^{2}$ which paid geld for 2 hides. These 20 ploughs can till. Thereof the abbot has 1 virgate and 2 ploughs in demesne, and the villeins I hide, 3 virgates, and I 5 ploughs. There the abbot has 2 I villeins, 6 bordars, 4 serfs, 6 beasts, 4 swine, I 18 sheep, 2 acres of wood(land), 6 acres of meadow, and 60 acres of pasture. This manor pays 100 shillings a year; when the abbot received it it was worth 60 shillings.

The abbot (Exch. The church itself) has a manor called WRDIETA [Worthyate] ${ }^{3}$ which in King E[dward]'s time paid geld for $\frac{1}{2}$ hide. This 5 ploughs can till. Thereof the abbot has $\frac{1}{2}$ virgate and $x$ plough in demesne, and the fol. 179 .

villeins $I \frac{1}{2}$ virgates and $I$ plough. There the abbot has 6 villeins, 5 bordars, I serf, 20 sheep, 6 goats, 12 acres of wood(land), and 8 acres of meadow. Worth 20 shillings; when the abbot received it it was worth 10 shillings.

Abbot Gosfrid (Exch. The church itself) has a manor called Bernurtona (Exch. Bernintone) [Burrington] ${ }^{4}$ which Abbot Sitric held T.R.E., and it paid geld for 4 hides (Exch. 3 hides). This 24 ploughs can till. Thereof the abbot has $\frac{1}{2}$ hide and 3 ploughs in demesne, and the villeins have $2 \frac{1}{2}$ hides and $8 \frac{1}{2}$ ploughs. On this (manor) the abbot has 15 villeins, I I bordars,

${ }^{1}$ Werrington in Blacktorington hundred. This entry is absent from the Exch. copy. It only appears as the king's 'Uluredintone' on fol. 98. Freeman, Norman Conquest, v, 747, states that it was regranted to the abbey in 1096 .

"Combe the demesne with the manor of Abbotsham in Shebbear hundred, the parish.

${ }^{3}$ Worthgate in Parkham in Shebbear hundred.

4 Burrington manor in North Tawton hundred. From the particulars as well as from the Exch. book 4 hides seems an error for 3 hides.
4 serfs, 4 boors (buri), 4 swineherds paying 40 swine yearly, 8 beasts, 6 swine, 46 sheep, 28 goats, 60 acres of wood(land) and coppice, 8 of meadow, and I league of pasture. It pays 7 pounds a year; when he received it it was worth 100 shillings.

To this manor (mansio) 2 manors (maneria; Exch. 2 estates [terre]) have been added, the land of 2 thanes who held it in parage (pariter; Exch. as 2 manors) on King E[dward]'s day. ${ }^{5}$ Of these lands William Capra has $1 \frac{1}{2}$ virgates ${ }^{6}$ where there is land for 6 ploughs. There W[illiam] has $I \frac{1}{2}$ ploughs, 3 villeins, and I bordar. Worth 20 shillings a year and was worth 30 shillings when he received it. This W[illiam] holds of the abbot.

Of that same manor Gosfrid holds a manor which paid geld for I virgate. ${ }^{7}$ There Gosfrid has 3 ploughs, and land which 5 ploughs can till, 3 villeins, 3 bordars, 6 acres of coppice, 3 acres of meadow and common pasture. Worth I 5 shillings a year; when he received it it was worth 30 shillings.

fol. $x$ rgb.

The abbot has a manor called RADDONA [Raddon $]^{8}$ which Olmer (Exch. Ulmer) held T.R.E., and it paid geld for I virgate. This 2 ploughs can till. William holds it of the abbot. Thereof $W$ [illiam] has in demesne 3 ferdings, and the villeins $\mathrm{I}$ ferding and $\frac{1}{2}$ plough. There $W$ [illiam] has I villein and I serf, I 3 acres of meadow, and 50 of pasture. Worth 5 shillings yearly.

The abbot has a manor called LIEGA [Leigh] ${ }^{9}$ which paid geld for I hide T.R.E. This Io ploughs can till. Nigel and Robert hold it of the abbot. Thereof $\mathbf{N}$ [igel] has in demesne $2 \frac{1}{2}$ ferdings and 2 ploughs, and his villeins 2 virgates all but $\frac{1}{3}$ ferding, and three ploughs. There N[igel] has 7 villeins, 6 bordars, 4 serfs, 6 beasts, 10 swine, 6o sheep, 17 goats, 26 acres of wood(land), 20 of meadow, and 40 of pasture. This part is worth 25 shillings a year. Robert has thereof in demesne $1 \frac{1}{3}$ ferling ${ }^{10}$ and I plough, also 3 villeins, 4 bordars, 3 serfs, 6 beasts, 6 swine, 40 sheep, Io goats, I 3 acres of wood(land),

' After 'King Edward's day,' the Exch. substitutes: 'and they paid geld for $\frac{1}{2}$ hide. Arable for 11 ploughs. In demesne are $4 \frac{1}{2}$ ploughs, 6 villeins, and 4 bordars; 3 acres of meadow are there; 6 acres of coppice and common pasture. Formerly 60 shillings; now it is worth 35 shillings. William Capra and Goisfrid now hold it of the abbot.'

${ }^{6}$ Capra's manor is Northcot in Burrington.

7 Probably Halsbury in Burrington.

${ }^{-}$Chapel Raddon in Thorverton in Hairidge hundred, now part of East Raddon (Lysons, ii, 504) Seemingly William is William the usher who held East Raddon.

${ }^{9}$ Romansleigh manor in Witheridge hundred.

${ }^{10}$ Probably Wodham alias Odam in Romansleigh in Witheridge hundred. 


\section{A HISTORY OF DEVONSHIRE}

IO acres of meadow, and 20 of pasture. Worth I5 shillings a year; when he received it it was worth 12 shillings.

The abbot has a manor called Hundatora [Houndtor] ${ }^{1}$ which Abbot Sistric held T.R.E., and it paid geld for $\frac{1}{2}$ hide. This 4 ploughs can till. Rainald holds it of the abbot. Thereof $\mathrm{R}$ [ainald] has I virgate and I plough in demesne, and the villeins $I$ virgate and I plough. There fol. 180 .

R[ainald] has 2 villeins, 4 bordars, 2 serfs, 7 beasts, 28 sheep, 18 goats, 2 acres of wood(land), 9 acres of meadow, and I league of pasture. Worth 20 shillings a year.

The abbot (Exch. The church itself) has a manor called Devenaberia [Denbury] ${ }^{2}$ which Archbishop Aldret held T.R.E., and it paid geld for $\frac{1}{2}$ hide. This 5 ploughs can till. Thereof the abbot has $\frac{1}{2}$ virgate and I plough in demesne, and the villeins $I \frac{1}{2}$ virgates and 4 ploughs. There the abbot has 4 villeins, 5 bordars, 2 serfs, 4 beasts, 70 sheep, I 5 goats, 25 acres of wood(land), 2 of meadow, and 15 of pasture. Worth 40 shillings; when the abbot received it it was worth 10 shillings.

The abbot has a manor called WILLA (Exch. Welle) [Well] ${ }^{3}$ which Archbishop Aldret (Exch. Eldred) held T.R.E., and it paid geld for 2 hides. These 8 ploughs can till. A certain knight Grento holds it of the abbot. Thereof G[rento] has $\frac{1}{2}$ hide and 2 ploughs in demesne, and the villeins $1 \frac{1}{2}$ hides and 5 ploughs. There $G$ [rento] has Io villeins, I 2 bordars, 4 serfs, 5 beasts, 40 sheep, 4 acres of meadow, and 20 acres of pasture. Worth 4 pounds; when the abbot received it, 40 shillings.

The abbot (Exch. The church itself) has a manor called Plemestocha [Plymstock] ${ }^{4}$ which Abbot Sitric held T.R.E., and it paid geld for $\frac{1}{2}$ hide. This 4 ploughs can till. Thereof the abbot has $\frac{1}{2}$ virgate and I plough in demesne, and the villeins $1 \frac{1}{2}$ virgates and 3 ploughs. There the abbot has 4 villeins, 9 bordars, 5 serfs, 4 beasts, 70 sheep, 34 goats, $\frac{1}{2}$ acre of meadow, and 30 acres of pasture. Worth 40 shillings; when the abbot received it, 20 shillings.

fol. x8ob.

The abbot has in Essecestra (Exch. Exonia) [Exeter] I house, ${ }^{5}$ which he held in pledge from a burgess, which was wont to pay the king's customary due, viz. 8 pence.

\footnotetext{
${ }^{1}$ Houndtor in Manaton in Teignbridge hundred.

${ }^{2}$ Denbury in Haytor hundred.

${ }^{3}$ Coffinswell in the hundred of Haytor.

- Plymstock in the hundred of Plympton.

${ }^{5}$ Now the Bear Inn, in South Street, Exeter.
}

LANDS OF THE ABBOT OF BUL-

FESTRE (Exch. BUCFESTRE) [BUCFAST] CHURCH IN DEVENESCIRA fol. 182 .

The abbot (Exch. Bucfestre church) has a manor called Petrocestoua [Petrockstow] ${ }^{B}$ which Abbot Alwin held T.R.E., and it paid geld for $I \frac{1}{2}$ virgates. These 5 ploughs can till. Thereof the abbot has in demesne $\frac{1}{2}$ virgate and I plough, and the villeins I virgate and 2 ploughs. There the abbot has 6 villeins, I bordar, 2 serfs, 4 beasts, 20 sheep, wood(land) 3 furlongs in length by $I_{2} \frac{1}{2}$ in breadth, 6 acres of meadow, pasture 8 furlongs in length by 5 furlongs in breadth. Worth $\mathrm{r} 5$ shillings, and was worth the same when he received it.

The abbot (Exch. The church itself) has a manor called Arssa [Ash] ${ }^{7}$ which paid geld for $\mathrm{I} \frac{1}{2}$ virgates T.R.E. These 3 ploughs can till. Thereof the abbot has in demesne $\frac{1}{2}$ virgate and I plough, and the villeins I virgate and 2 ploughs. There the abbot has 5 villeins, 3 bordars, 3 serfs, Io beasts, 40 sheep, 6 acres of wood(land), 6 acres of meadow, and 3 furlongs of pasture, taking length and breadth. Worth 20 shillings a year, and was worth ro shillings when he received it.

The abbot (Exch. The church itself) has a manor called Limae (Exch. Limet) [Nymet or Intake] ${ }^{8}$ which paid geld for I hide T.R.E. This 7 ( $E_{x<c}$. 8) ploughs can till. Thereof the abbot has I virgate and I plough in demesne, and the villeins have 3 virgates and 6 ploughs. There the abbot has Io villeins, I4 bordars, 4 serfs, 9 beasts, 4 swine, 72 sheep, 4 acres of coppice, and 3 acres of meadow. Worth 50 shillings a year.

The abbot (Exch. The church itself) has a manor called Dona [Down] ${ }^{9}$ which paid geld for 2 hides T.R.E. This Io ploughs can till. fol. $\mathrm{r} 82 \mathrm{~b}$.

Thereof the abbot has in demesne $\frac{1}{2}$ hide and I plough ; and the villeins $\mathrm{I} \frac{1}{2}$ hides and 5 ploughs. There the abbot has I2 villeins, 9 bordars, 7 serfs, 6 beasts, 4 swine, 66 sheep, 8 furlongs of coppice, 8 acres of meadow and 12 acres of pasture. Worth 3 pounds a year.

The abbot (Exch. The church itself) has a manor called Trisma [Trusham] ${ }^{10}$ which paid geld for I hide T.R.E. This 4 ploughs can till. Thereof the abbot has in demesne I virgate and I plough, and the villeins 3 virgates and

${ }^{6}$ Petrockstow manor in the hundred of Shebbear.

${ }^{7}$ Ash in Petrockstow in Shebbear hundred.

${ }^{8}$ The monks' intake, hall, or sele, commonly called Zeal Monachorum in North Tawton hundred.

${ }^{9}$ Down St. Mary in Crediton hundred.

${ }^{10}$ Trusham manor in Exminster hundred, conterminous with the parish. 


\section{HOLDERS OF LANDS}

3 ploughs. There the abbot has 4 villeins, 9 bordars, Io serfs, 6 beasts, 9 swine, 103 sheep, 22 goats, I 6 acres of wood(land), 3 acres of meadow and 10 of pasture. Worth 30 shillings a year, and was worth 25 shillings when he received it.

The abbot ( $E_{x c h}$. The church itself) has a manor called Haiserstona (Exch. Aiserstone) [Ashburton ?] ${ }^{1}$ which paid geld for $1 \frac{1}{2}$ ferdings and 3 acres T.R.E. There the abbot has I villein who pays 40 pence a year.

The abbot (Exch. The church itself) has a manor called AIssa [Ash] ${ }^{2}$ which Abbot Alwin held T.R.E., and it paid geld for $I \frac{1}{2}$ hides. This Io ploughs can till. Thereof the abbot has $I \frac{1}{2}$ virgates in demesne and I plough, and the villeins have I hide, $\frac{1}{2}$ virgate and 5 ploughs. There the abbot has 8 villeins, 9 bordars, 6 serfs, 9 beasts, 68 sheep, II goats; wood(land) 3 furlongs in length by $I$ in breadth, 4 acres of meadow, and pasture $I$ league in length by $\frac{1}{2}$ league in breadth. Worth 30 shillings a year, and was worth the same when the abbot received it.

The abbot (Exch. The church itself) has a manor called HetFelt (Exch. Hetfold) [Heathfield $]^{3}$ which abbot Alwin held T.R.E., and it paid geld for 2 hides. These 12 ploughs can till. Thereof the abbot has in demesne $\frac{1}{2}$ fol. 183 .

hide and I plough, and the villeins have $I \frac{1}{2}$ hides and 5 ploughs. There the abbot has ro villeins, 9 bordars, 6 serfs, I I beasts, 5 swine, 60 sheep, 16 goats, 2 acres of coppice and 40 acres of pasture. Worth 40 shillings a year; when the abbot received it it was worth 30 shillings.

The abbot has a manor called Bulfestra (Exch. Bucfestre) [Bucfast] ${ }^{4}$ and it is the seat (caput) of the abbey, and never paid geld. There the abbot has I smith and ro serfs who have 2 ploughs; there the abbot has also 3 swine and and wood(land) I league in length by $\frac{1}{2}$ league in width.

The abbot (Exch. The church itself) has a manor called Notona [Norton] ${ }^{5}$ which Abbot Alwin held T.R.E., and it paid geld for 2 hides. These ro ploughs can till. Thereof he has

1 In Ashburton in Teignbridge hundred. Geldroll, fol. 69, A, 8. Trans. Devon Assoc. xxix, 228.

${ }^{2}$ Abbot's Ash alias Ashford in Aveton Giffard in Ermington hundred (Testa de Nevill I 94a) (O.J.R.).

"Heathfield in Aveton Giffard in Ermington hundred.

'Bucfast Abbey and the manor of Bucfast, the parish of Buckfastleigh in Stanborough hundred. Trans. Devon Assoc. xxxiv, 725. In Exch. this entry comes last of all.

${ }^{5}$ Norton in Churchstow in Stanborough hundred. $\frac{1}{2}$ hide and I plough in demesne, and the villeins I $\frac{1}{2}$ hides and 5 ploughs. There the abbot has 9 villeins, I 2 bordars, 6 serfs, 4 beasts, 3 swine, 70 sheep, wood(land) 2 furlongs in length by $I$ in breadth, 2 acres of meadow, and 20 acres of pasture. Worth 40 shillings; when the abbot received it, 30 shillings.

The abbot (Exch. The church itself) has a manor called Cherrforda [Charford] ${ }^{6}$ which Abbot Alwin held T.R.E., and it paid geld for I hide. This 8 ploughs can till. Thereof the abbot has I virgate and I plough in demesne, and the villeins 3 virgates and 3 ploughs. There the abbot has 7 villeins, 6 bordars, 4 serfs, fol. $183 \mathrm{~b}$.

6 beasts, 44 sheep, 2 acres of meadow, and 20 acres of pasture. Worth 30 shillings, and was worth 20 shillings when the abbot received it.

The abbot (Exch. The church itself) has a manor called BRENTA [Brent] ${ }^{7}$ which Abbot Alwin held T.R.E., and it paid geld for 2 hides. These Io ploughs can till. Thereof the Abbot has $\frac{1}{2}$ hide and I plough in demesne, and the villeins $I_{2} \frac{1}{2}$ hides and 5 ploughs. There the abbot has Io villeins, 8 bordars, 5 serfs, I 4 beasts, 55 sheep, 5 acres of wood(land), 4 of meadow and 30 of pasture. Worth 40 shillings, and was worth 30 shillings when the abbot received it.

The abbot (Exch. The church itself) has a manor called Brenta [Brent] ${ }^{8}$ which Abbot Alwin held T.R.E., and it paid geld for 2 hides. These 6 ploughs can till. Thereof the abbot has $\frac{1}{2}$ hide and $\frac{1}{2}$ plough in demesne, and the villeins $I_{\frac{1}{2}}$ hides and 3 ploughs. There the abbot has 8 villeins, 6 bordars, 4 serfs, II beasts, 70 sheep, 30 goats, wood(land) I league in length by I furlong in breadth. Worth 30 shillings; and when the abbot received it 20 shillings.

\section{fol. 184.}

\section{LAND OF THE ABBOT (Excb. CHURCH) OF HORTHON [HORTON] IN DE- VENESCIRA}

The abbot (Exch. Hortun church) has a manor called Liteham [Littleham] ${ }^{9}$ which in King E[dward]'s time paid geld for $\frac{1}{2}$ hide. This 8 ploughs can till. Thereof the abbot has $\frac{1}{2}$ virgate and I plough in demesne (Exch. omits I plough) and the villeins have 8 ploughs. There the abbot has 15 villeins, 20 bordars, I rouncey, 8 beasts, 139 sheep, 5 acres of coppice, 6 acres of meadow and 6 furlongs of pasture

\footnotetext{
${ }^{6}$ Charford in South Brent in Stanborough hundred.

${ }^{7}$ South Brent manor in Stanborough hundred.

${ }^{8}$ Great Aish in South Brent in Stanborough hundred.

${ }^{9}$ Littleham Abbot in East Budleigh hundred.
} 


\section{A HISTORY OF DEVONSHIRE}

taking length and breadth. Worth 40 shillings a year.

The abbot (Exch. The church) has a manor called Carsuelia ${ }^{1}$ [Kerswell] which in King $E\left[d\right.$ ward]'s time paid geld for $1 \frac{1}{2}$ hides. This 8 ploughs can till. Thereof the abbot has $\frac{1}{2}$ hide in demesne and 2 ploughs; and the villeins have I hide and 4 ploughs. There the abbot has ro villeins, 9 bordars, 2 serfs, I rouncey, 6 beasts, I 00 sheep, 30 goats, I 2 furlongs of wood(land) counting length and breadth, I 2 acres of meadow and pasture 5 furlongs in length by 30 acres in breadth. Worth 40 shillings a year.

The abbot (Exch. The church) has a manor called FLUTA ${ }^{2}$ which he held himself T.R.E., and it paid geld for $\frac{1}{2}$ hide. This 6 ploughs can till. Thereof the abbot has $\frac{1}{2}$ virgate in demesne and I plough; and the villeins have $3 \frac{1}{2}$ virgates and 3 ploughs. There the abbot has 6 villeins, I 9 bordars, 2 serfs, I rouncey, 2 beasts, 46 sheep, 8 acres of meadow, pasture 5 furlongs in length by a like amount in breadth, I I salterns paying I I pence yearly. Worth 40 shillings a year.

The abbot (Exch. The church) has a manor called BERA [Beer] ${ }^{3}$ which he held himself T.R.E., and it paid geld for $\frac{1}{2}$ hide. This 7 ploughs can till. Thereof the abbot has $\frac{1}{2}$ virfol. 184b.

gate in demesne and I plough, and the villeins have $3 \frac{1}{2}$ virgates and 5 ploughs. There the abbot has 7 villeins, 20 bordars, 2 serfs, I cow, roo sheep all but 7,7 acres of meadow and pasture I league in length by $\frac{1}{2}$ in breadth. Worth 4 pounds a year. From this manor I ferting of land ${ }^{4}$ and 4 salterns have been taken away, which Drogo now holds of the earl of Mortain.

fol. 194 .

\section{LANDS OF CHURCHES WHICH HAVE BEEN GIVEN TO THE SAINTS IN ALMS ${ }^{6}$}

The abbot of Caen (Abbas Cadomensis) (Exch. The church of St. Stefan of Caen) has a manor

1 Abbot's Kerswell in Haytor hundred.

2 Probably the present Seaton in the parish of Beer and Seaton in Colyton hundred.

${ }^{3}$ Beer in the parish of Beer and Seaton in Colyton hundred. The assessments of the demesne and villeinage of these two estates probably treat the two estates as one. Otherwise the quantities are in each case double of the total assessments.

- Probably near Whitecliff on the streamlet in Beer and worked by the two saltworkers belonging to Honiton (fol. 2 I $6 b$ ) which Drogo held.

${ }^{5}$ Instead of following the sequence of the hundreds the Exchequer Book here follows the seniority or importance of the churches to which the estates belonged. called Northam [Northam] ${ }^{6}$ which Bristric (Exch. Brictric) held T.R.E., and it paid geld for 2 hides and $\frac{1}{2}$ virgate. Now the abbot of St. Stephen holds it by gift of the queen in alms. This 20 ploughs can till. Thereof the abbot has 1 virgate and 3 ploughs in demesne, and the villeins have $1 \frac{1}{2}$ hides and $\frac{1}{2}$ virgate and 14 ploughs. There the abbot has 23 villeins, 5 bordars, 8 serfs, I swineherd, 23 beasts, 345 sheep, I fishery paying yearly 30 pence, 2 salterns paying Io shillings a year, 24 acres of wood(land), 30 acres of coppice, I 5 acres of meadow, and 15 of pasture. It pays 12 pounds a year and paid the same when the abbot received it.

The abbess of the Holy Trinity at Caen (Exch. The church of the Holy Trinity of Caen) has a manor called UMBERLEIA [Umberleigh] ${ }^{7}$ which Bristric (Exch. Brictric) held T.R.E., and it paid geld for I hide and I virgate. This 12 ploughs can till. Thereof the abbess has I virgate and 2 ploughs in demesne, and the villeins $I$ hide and 8 ploughs. There the abbess has 12 villeins, 2 bordars, 3 swineherds paying 30 swine, 6 serfs, 16 beasts, 80 sheep, wood(land) 6 furlongs in length by 2 in breadth and 100 acres of pasture. It pays 11 pounds; when the abbess received it it was worth 10 pounds.

The abbot of Creneburn Church (Exch. Cranborne Church) has a manor called LosBerA [Losebear] $^{8}$ which in King E[dward]'s time paid geld for $\frac{1}{2}$ hide. This 6 ploughs can till. Thereof the abbot has 1 virgate and 2 ploughs fol. rg4b.

in demesne, and the villeins 1 virgate and 4 ploughs. There the abbot has 8 villeins, 7 bordars, 4 serfs, 2 beasts, 60 sheep, 6 acres of wood(land), 3 acres of meadow, and 30 acres of pasture. Worth 60 shillings.

Sawin the queen's priest has a manor called BIRIGE [Bridge] ${ }^{9}$ which Brisfert (Exch. Bricfert) his uncle (avunculus) held in parage (pariter) and he could go to what lord he liked T.R.E. It paid geld for 3 virgates. These 4 ploughs can till. Thereof S[awin] has I virgate and $\frac{1}{2}$ plough in demesne, and the villeins 2 virgates and I plough. There S[awin] has 3 villeins, I serf,

6 Northam in Shebbear hundred, Cal. Docts. France, Nos. 452, 459, p. I62 ; Trans. Devon Assoc. xxv, 5 I 5 . In the villeins' assessment I virgate has been omitted, as the Geldroll (fol. $65 b, \mathrm{~B} 3$ ) shows, which probably represents Ashridge, making the total right.

I Umberleigh manor in the parish of Atherington in North Tawton hundred.

${ }^{8}$ Loosebear in Zeal Monachorum in Winkleigh hundred. Trans. Devon Assoc. xxix, 271.

' Swymbridge in Swymbridge parish in South Molton hundred, or Bridge in Lapford (Feud. Aids, i, index). 


\section{HOLDERS OF LANDS}

7 beasts, 30 sheep, and 2 acres of meadow. Worth Io shillings; when $S[$ awin] received it it was worth 5 shillings. This land Queen $M$ [athilda] gave to him in alms.

Algar the priest has a hide of land in the king's manor of BRANTONa [Braunton] ${ }^{1}$ which he holds in alms of the king. This 8 ploughs can till. Thereof the priest has I virgate and 3 ploughs in demesne, and the villeins have 3 virgates and 5 ploughs. There the priest has 3 villeins, 23 bordars, 5 beasts, 12 swine, I00 sheep, 10 goats, and 20 acres of pasture. Worth 50 shillings a year.

In the king's manor of Sut Moltona [South Molton] 4 priests (sacerdotes; Exch. presbyteri) hold I virgate of land in alms of the king. ${ }^{2}$ Worth 20 shillings a year.

The abbot (Exch. The church) of St. Michael of the Mount has a manor called Otritona [Otterton] ${ }^{3}$ - a market is held there on Sundays, -which Countess Githa (Gutda) held T.R.E., fo1. 195 .

and it paid geld for $\mathrm{I}_{4}$ hides. These 25 ploughs can till. Thereof the abbot has 2 hides and 6 ploughs in demesne, and the villeins 12 hides and 40 ploughs. There the abbot has 50 villeins, 20 bordars, 33 saltworkers (salinarios), 4 rounceys, I 8 beasts, 60 swine, 300 sheep, 22 goats, 3 mills paying 40 shillings, I 50 acres of wood(land), 45 acres of meadow, and $\mathrm{I} \frac{1}{2}$ leagues of pasture. This manor is worth 18 pounds; when the abbot received it it was worth io pounds.

The abbot of Battle (Abbas de Praelio; Exch. Ecclesia de Labatailage) has I hide of land and I church in Colitona [Collumpton] ${ }^{4}$ which Torbert held T.R.E. There the abbot has in demesne I virgate and I plough, and the villeins 3 virgates and 3 ploughs. There the abbot has 6 villeins, 4 bordars, 8 beasts, 2 acres of wood(land), and 8 of meadow. Worth 30 shillings a year.

${ }^{1}$ Braunton Dean alias the church manor in Braunton in Braunton hundred. See Geldroll, $x x$, A 13 .

2 Church manor of South Molton in South Molton hundred, of which a part lay in Braunton hundred (Geldroll, fol. 66, A I 3 ; and South Molton, fol. 66, A 7).

${ }^{3}$ Place Barton the demesne with the manors of Otterton alias Nether, or Monks' Ottery, and Hetherland in East Budleigh hundred, extending over the parishes of Otterton and Sidmouth together with Yattington in Bicton. Devon Notes and Queries, i, 245 ; Trans. Devon Assoc. xxxiv, 715.

" The church manor in Collumpton alias Upton, Weaver, and Ash in Hairidge hundred with the outlier of Henland locally in Kentisbeare. Testa de Nevill (Rec. Com.), 194b, $197 b$.
The canons (Exch. The church) of St. Mary of Rouen hold a manor called OTRI [Ottery] ${ }^{5}$ and also an orchard (ortum) and a saltern paying 30 pence a year in the land of St. Michael of Sedemuda [Sidmouth] which they held in King $\mathrm{E}$ [dward]'s time, when it paid geld for 25 hides. These 46 ploughs can till. Thereof the canons have in demesne 5 hides and 3 ploughs, and the villeins 20 hides and 40 ploughs. There the canons have 55 villeins, 24 bordars, 17 serfs, 5 swineherds paying $3 I$ shillings and 3 pence, also I rouncey, 24 beasts, 6 swine, 130 sheep, 18 forest mares, 3 mills paying 30 shillings a year, 20 acres of wood(land), 200 acres of meadow, and 8 hides of pasture.

The same canons (Exch. The church itself) have another manor called Rourige [Rawridge] ${ }^{6}$ fol. rgsb.

which Olvieva (Exch. Ulveva) held T.R.E., and that (manor) King William gave to the canons ( $E x c h$. to St. Mary) and it paid geld for 3 hides. This I 2 ploughs can till. Thereof they have themselves in demesne 1 hide and 2 ploughs, and the villeins 2 hides and Io ploughs. There the canons have 20 villeins, 8 bordars, 4 serfs, 20 beasts, 72 sheep, 30 goats, half a hide of wood(land), and 20 acres of meadow. These 2 manors pay yearly 70 pounds of Rouen money (rotbomagensium denariorum).

St. Michael (Exch. The church itself) has a manor called ERTicoma (Exch. Herticome) [Yarticombe $]^{7}$ which Earl Herrold held T.R.E., and it paid geld for 3 hides. These 12 ploughs can till. Thereof the abbot of St. Michael has I hide and I plough in demesne, and the villeins 2 hides and 10 ploughs. There the abbot has 16 villeins, 8 bordars, 4 serfs, I rouncey, 7 beasts, I mill worth 6 shillings a year, I swineherd paying Io swine a year, 40 acres of wood(land), 4 acres of meadow, and 50 of pasture. It pays 60 shillings a year; when he received it it was paying the same.

The abbot of St. Michael has a manor called Donitona [Denningon] ${ }^{8}$ which Earl Herrold held T.R.E., and it paid geld for 3 hides. 'This

5 Ottery St. Mary manor in East Budleigh hundred, the parish including salterns at Sidmouth, given to the canons by the Confessor in 1061. Kemble, Codex Diplomaticus, iii, 339 ; Cal. Docts. in France, No. I.

${ }^{6}$ Rawridge in Upottery in Axminster hundred. Rouen pence were, at a later date, worth half the value of English pence.

${ }^{7}$ Yarticombe alias Yarcombe in Axminster hundred. Cal. Docts. France, No. 734.

${ }^{8}$ Dennington with Stout Mill in Yarcombe in Axminster hundred. Trans. Devon Assoc. xii, 464; xxxv, 694. Bull of Adrian IV in Cal. Docts. in France, 269, confirms to St. Michael besides 'Erticombe,' 'Estelleia, and Wiscombe.' Devon Notes and Queries, i, 253. 


\section{A HISTORY OF DEVONSHIRE}

Io ploughs can till. Thereof the a[bbot] has I hide and I plough in demesne, and the villeins 2 hides and 2 ploughs. There the a[bbot] has 9 villeins, 4 bordars, 3 serfs, I rouncey, I6 beasts, 200 sheep, 23 goats, 100 acres of wood(land), and 200 of pasture. It pays 40 shillings fol. $\mathbf{x g 6 .}$

a year ; when he received it it paid the same.

The abbot of Battle (Abbas Bataillie; Exch. The church itself) has in Essecestra (Exch. Excestre) [Exeter] a church, ${ }^{1}$ St. Oilaf's (Exch. St. Olaf's), and 7 houses which paid the customary dues, i.e. 4 s. $8 d$. a year, and one that did not pay them. ${ }^{2}$

fol. 210 .

LAND OF THE COUNT OF MORTAIN $[D E$ MORITONIO; Exch. MORITONIENSIS] IN DEVENESCIRA ${ }^{3}$

The count has a manor called EstocheleIA (Exch. Stochelie) [Stockleigh] ${ }^{4}$ which Alward

1 Church of St. Olave and the site of St. Nicholas's priory, a dependency of Battle Abbey. See Testa de Nevill (Rec. Com.), 194b; Oliver, Mon. p. 115.

${ }^{2}$ Here follows in the Exch. copy : The land of Earl Hugo.

${ }^{3}$ Instead of following the order of the hundreds the Exchequer Book enumerates first the manors which the count had in demesne, beginning with Exeter, followed by Stockleigh, Wick, Shobrook, and Raddon. Next come the tenants' manors, beginning with Stockleigh, in the same order as the Exeter Book down to Bulkworthy and Smytham belonging to Edmeratorius' honour, after which follow the other manors held with that honour, 'Liteltroriland,' Stockleigh Luccombe, Sutton Satchvil, Poughill, Holbrook Grindham, Little Faringdon, Honiton, Wombernford, Northleigh, North Ludbrook, Luperidge, Edmerston, Higher Hearston, Winston, and Densham, and a memorandum at the foot: 'The aforesaid 17 lands the count of Mortain holds with the land of Edmeratorius which was delivered to him ; for T.R.E. the aforesaid thanes held them independently.' Then comes a group of manors formerly held by Edmer, viz. Frizenham, Wedfield, Feniton, Cotleigh, Cornwood, Newton Ferrers, Great Bolbury, and Buckland; another group formerly held by Ordulf, Alverdiscot, Bratton Fleming, Croyde, St. Mary Church, Broad Hempston, Bigbury, Harford, and Beer Ferrers ; and a group of seven with the heading: 'The underwritten seven lands the count held along with Ordulf's honour,' the seven being Hele Satchvil, Stockleigh English, Little Modbury, Torridge, Hearston, Spriddleston, and 'Wederige.' The rest follow in order, viz. Stockleigh Frances, Matford, Tatscombe, Denson, Petton, Clyst St. Laurence, Thornbury, Chitterleigh, Bickleigh, Corscombe, Charlton, Modbury, Orcherton, Luscombe, Fardle, Venn, Bittaford, Dennaton, Torpeake, South Ludbrook, Little Bolbury, Batson, Bawcombe, Shilston, Spriddlecombe, Honicknowl, and Lipson.

"Stockleigh in Highampton in Blacktorington hundred (O.J.R.) ; Lee in Hartland (T.W.W.). held T.R.E., and it paid geld for I ferling. Now Alward holds it of the count. This I plough can till. There Alward has I plough in demesne, 3 bordars, 2 serfs, 4 beasts, 24 sheep, and 24 goats. This manor is worth 10 shillings a year.

The count has a manor called GHiderreit ${ }^{5}$ (Exch. Chiderleia) [Gidleigh] which Godwin the priest held T.R.E., and it paid geld for 3 ferlings. This I plough can till, and Godwin now holds it of the count (consule). This manor is worth 5 shillings a year.

The count has a manor called Alwinetona [Alwington $]^{6}$ which Ordulf held T.R.E., and it paid geld for I hide and 3 virgates. This land 20 ploughs can till. Now Hamelin holds it of the count. Thereof Hamelin has $\frac{1}{2}$ hide and 2 ploughs in demesne, and the villeins I hide, I virgate, and 9 ploughs. There $\mathrm{H}$ [amelin] has I 5 villeins, I 5 bordars, Io serfs, 2 I beasts, Io swine, 120 sheep, 12 goats, 30 acres of wood(land), 3 acres of meadow, and 60 of pasture. This manor is worth 4 pounds, and was worth 30 shillings when the $c$ [ount] received it.

The count has a manor called LEGA [Leigh] ${ }^{7}$ which Ordulf held T.R.E., and it paid geld for I hide. This Io ploughs can till. Alvered holds it of the count. Thereof A[lvered] has I virgate and 2 ploughs in demesne, and the villeins 3 virgates and 6 ploughs. There A[lvered] has 15 villeins, 6 bordars, 4 serfs, 9 beasts, I00 sheep, 4 acres of wood(land), Io acres of meadow, and 6o acres of pasture. fol. 2rob.

It is worth 4 pounds, and was worth the same when the count received it.

The count has a manor called Fredeletestoc (Exch. Fredelestoch) [Frithelstock ${ }^{8}$ which Ordulf held T.R.E., and it paid geld for 3 hides. These 20 ploughs can till. Rotbert son of Ivon holds it of the count. Thereof $R$ [otbert] has $2 \frac{1}{2}$ virgates and 4 ploughs in demesne, and the villeins 2 hides $\frac{1}{2}$ virgate and I I ploughs. There R [otbert] has 30 villeins, 12 bordars, 10 serfs, 20 beasts, I o swine, 80 sheep, 16 goats, 60 acres of wood(land), 20 acres of meadow, and 60 acres of pasture. This manor is worth 8 pounds, and was worth the same when the count received it.

The count has a manor called Colreria (Exch. Colelie)[Culleigh $]^{9}$ which Chepin held in parage

${ }^{5}$ Gidleigh Church manor in the hundred of South Tawton (O.J.R.).

${ }^{6}$ Alwington manor in Shebbear hundred.

7 Monkleigh in Shebbear hundred.

8 Frithelstock in Shebbear hundred. The total assessment exceeds the sum of the lord's and villeins' assessment by I virgate, which is probably the I virgate added to Bulkworthy, fol. $497 b$. Trans. Devon Assoc. xxxii, 53 I.

9 Culleigh in Frithelstock in Shebbear hundred. 


\section{HOLDERS OF LANDS}

(pariter) T.R.E., and the men of the count of Mortain (Moritaniae) hold it as of (tenent ad) Ordulf's honour, but that thane could go to what lord he liked without Ordulf's leave; and it paid geld for I virgate. This 2 ploughs can till, and now Herchenbold (Exch. Erchenbald) holds it of the count. Thereof $\mathrm{H}$ [erchenbold] has $\frac{1}{2}$ virgate and I plough in demesne, and the villeins $\frac{1}{2}$ vir. gate and $\frac{1}{2}$ plough. There $\mathrm{H}$ [erchenbold] has 2 villeins, 3 bordars, I serf, 4 beasts, 20 sheep, I 6 goats, 4 acres of wood(land), and 2 acres of meadow. It pays 12 shillings, and was worth 4 shillings when the count received it.

The count has a manor called Bochelanda [Buckland] ${ }^{1}$ which Edmaratorus (Exch. Edmer) held T.R.E., and it paid geld for 3 hides all but $\frac{1}{2}$ virgate. These 20 ploughs can till. Now Ansger the Breton holds it of the count. Thereof $A$ [nsger] has $\frac{1}{2}$ hide and 3 ploughs in demesne, fol. 2rI.

and the villeins 2 hides $I \frac{1}{2}$ virgates and $8 \frac{1}{2}$ ploughs. There $A$ [nsger] has 42 villeins, 5 bordars, 3 swineherds, 7 serfs, I 9 beasts, I 50 sheep, 50 goats, 100 acres of wood(land), 40 acres of meadow, and pasture $I$ league in length by $\frac{1}{2}$ league in breadth. Worth 7 pounds 10 shillings, and was worth the same when the count received it.

To this manor has been added a manor called Galeshora [Galsworthy] ${ }^{2}$ which Edwi held T.R.E., and he could go to what lord he liked (Exch. held it as a manor), and it paid geld for $\frac{1}{2}$ virgate. This I plough can till. Ansger holds it of the count. There $A$ [nsger] has 3 villeins, who have I plough and 20 acres of pasture. Worth Io shillings, and was worth the same when the count received it.

The count has a manor called Potrforda ${ }^{3}$ [Putford] which Edmaratorius (Exch.Edmer) held T.R.E., and it paid geld for $\mathrm{I}$ hide. This 6 ploughs can till. Ansger the Breton holds it of the count. Thereof $A\left[n\right.$ ngger] has I virgate and $1 \frac{1}{2}$ ploughs in demesne, and the villeins 2 virgates and I plough. There $A[$ nsger] has 9 villeins, I bordar, 7 serfs, 23 beasts, I 50 sheep, 30 acres of meadow, and pasture $I$ league in length by $\frac{1}{2}$ league in breadth. Worth 30 shillings, and was worth the same when the count received it.

The count has a manor (called) Buchesurda [Bulkworthy] ${ }^{4}$ which Iric held T.R.E., and he could go with that land to what lord he liked, and it paid geld for $\mathrm{I}$ virgate. This 4 ploughs can till. Ansger holds it of the count. Thereof $A$ [nsger] has $\frac{1}{2}$ ferling and 7 oxen in demesne,

1 Buckland Brewer in Shebbear hundred.

2 Galsworthy in Buckland Brewer in Shebbear hundred.

${ }^{3}$ East Putford in Shebbear hundred; Wedfield probably represents the I virgate unaccounted for.

4 Bulkworthy in Shebbear hundred. and the villeins have the rest of the land and I plough. There A[nsger] has 6 villeins, 3 bordars, I serf, 3 beasts, 2 swine, 100 sheep all but 3,5 acres of meadow and 30 of pasture. Worth fol, 2rrb.

20 shillings, and was worth the same when the count received it. And this land the count holds along with the honour of Edmeratorius.

The count has a manor called Esmitteram [Smytham] ${ }^{5}$ which Alvric held T.R.E., and he could go to what lord he liked with that land, and it paid geld for $\frac{1}{3}$ ferling. This I plough can till. Anger (Exch. Ansger) holds it of the count. There $A[n(s) g e r]$ has I villein, I bordar, and I serf, who have I plough, also 2 acres of meadow and 4 of pasture. Worth 5 shillings, and was worth the same when the count received it. And that land also the count holds with the honour of Edmeratorius.

The count has a manor called Friseram [Frizenham] ${ }^{6}$ which Edmeratorius held T.R.E., and it paid geld for I virgate. This 3 ploughs can till. Alvered the cup-bearer (pincerna) holds it of the count. Thereof $A$ [lvered] has $\frac{1}{2}$ virgate and I plough in demesne, and the villeins $\frac{1}{2}$ virgate and 3 ploughs. There A[lvered] has 5 villeins, 3 bordars, 2 serfs, $I \frac{1}{2}$ acres of meadow and 20 of pasture. Worth 20 shillings, and was worth the same when the count received it.

The count has a manor called W IDEFELLA [Wedfield] $^{7}$ which Almar (Excb. Aedmaer) the Red (rufus) held T.R.E. of Edmeratorius, and he could not become independent of him with that land. It paid geld for I virgate. This 2 ploughs can till. Alvered the cup-bearer holds it of the count. Thereof $A\left[\right.$ lvered] has $\frac{1}{2}$ virgate and I plough in demesne, and the villeins $\frac{1}{2}$ virgate, I plough, and 2 oxen. There A[lvered] has 3 villeins, 2 bordars, I serf, 8 beasts, 29 sheep, ro acres of meadow, and 30 acres of pasture. fol. 212.

Worth Io shillings, and was worth the same when the count received it.

The count has a manor called LiteltroriLANDA [Little Toridge land] ${ }^{8}$ which Elward the Red (rufus) held T.R.E., and it paid geld for $\frac{1}{3}$ ferling, and he could go to what lord he liked with that land. Alvered the cup-bearer holds it of the count. There $A[$ lvered] has I villein who has I plough; there A[lvered] also has I bordar, 2 serfs, and 4 acres of pasture. Worth 5 shillings and was worth the same when the

5 Smytham in Little Torington in Shebbear hundred.

${ }^{6}$ Frizenham in Little Torington in Shebbear hundred.

7 Wedfield in West Putford in Shebbear hundred.

\& Perhaps Woodland in Little Torington in Shebbear hundred. 


\section{A HISTORY OF DEVONSHIRE}

count received it. That land the count holds along with the honour of Edmeratorius.

The count has a manor called HELA [Hele] ${ }^{1}$ which 2 thanes held in parage (pariter; Exch. libere) T.R.E., and they could go to what lord they liked with that land. Thereof Almar Estrege had 3 ferlings and Frawin I ferling, and it paid geld for I virgate. This 4 ploughs can till. Erchenbold ( $E x c h$. Erchenbald) holds it of the count. Thereof E[rchenbold] has I ferling and $I$ plough in demesne, and the villeins 3 ferlings and I plough. There E[rchenbold] has 6 villeins, I bordar, I serf, 6 beasts, Io sheep, 3 acres of wood(land), Io acres of meadow, and 20 of pasture. Worth Io shillings, and when the count received it it was worth 20 shillings. This land the count holds along with Ordulf's honour.

The count has a manor called Estocheleia (Exch. Stochelie) [Stockleigh] ${ }^{2}$ which Alsi held T.R.E., and it paid geld for 3 ferlings. These 2 ploughs can till. Erchenbold holds it of the count. Thereof E[rchenbold] has I ferling and I plough in demesne, and the villeins 2 ferlings and I plough. There Erchenbold has 3 villeins, 2 serfs, 2 acres of wood(land), 8 acres of meadow, and 30 of pasture. Worth 10 shillings; when the count received it it was worth 20 shillings, and he (who held it) could go to what lord he liked.

fol. 212b.

Robert, count of Mortain, has a manor called Alveredescota [Alverdiscot] ${ }^{3}$ which Ordulf held T.R.E., and it paid geld for $1 \frac{1}{2}$ hides. This ro ploughs can till. Erchenbold holds it of the count. Thereof E[rchenbold] has $\frac{1}{2}$ hide and 3 ploughs in demesne; and the villeins $I$ hide and 6 ploughs. There E[rchenbold] has 8 villeins, 7 bordars, 6 serfs, 6 beasts, 4 swine, 50 sheep, I 2 goats, 5 acres of meadow, and 20 of pasture. Worth 60 shillings, and was worth 6 pounds when the count received it.

Of these 6 virgates a certain thane held 1 virgate ${ }^{4}$ who could not become independent of (separare de) Ordulf.

The count has a manor called MADForT [Matford] ${ }^{5}$ which Alward Tabe (Exch. Alwi)

1 Hele Satchvil in Buckland Brewer in Shebbear hundred (O. J. R.), in Littleham (T. W. W.), Trans. of Devon. Assoc. xxxvi, 353.

Stockleigh Frances in Meeth in Shebbear hundred (O.J. R.).

${ }^{3}$ Alverdiscot in Fremington hundred.

- Perhaps Nethercot in Alverdiscot (O. J. R.), or Wear in Westleigh (T.W.W). Trans. Devon Assoc. xxxvi, 159.

- Matford Dinham alias Butter alias Inner alias Lower Matford in Alphington in Exminster hundred. See Feud. Aids, p. 346, Trans. Devon Assoc. xxxi, I 24. The lord's and villeins' assessments together only amount to half a virgate. Probably the missing half a virgate is Matford Speke. held T.R.E., and it paid geld for I virgate. This 2 ploughs can till. Alvered the cupbearer holds it of the count. Thereof $A$ [lvered] has in demesne I ferding and I plough, and the villeins I ferding and $\frac{1}{2}$ plough. There $A$ [lvered] has I villein, 4 serfs, and 4 acres of meadow. Worth Io shillings a year, and was worth the same when the count received it.

The count has a manor called Totrescoma [Tattiscombe] ${ }^{6}$ which Edric held T.R.E., and it paid geld for $\frac{1}{2}$ hide. This 6 ploughs can till. William de Lestra holds it of the count. Thereof W[illiam] has $I$ virgate and I plough in demesne, and the villeins I virgate and 2 ploughs. There W[illiam] has 5 villeins, 3 bordars, 2 serfs, 10 beasts, 20 swine, 100 sheep, 25 goats, 8 acres of wood(land), 7 acres of meadow, and pasture $\frac{1}{2}$ league in length by 2 furlongs in breadth. Worth 20 shillings, and was worth 40 shillings when the count received it.

fol. 233 .

Count Robert has a manor called Bratona (Exch. Brotone) [Bratton] ${ }^{7}$ which Ordulf held T.R.E., and it paid geld for I hide. This 12 ploughs can till. Erchenbold holds it of the count. Thereof $\mathrm{E}$ [rchenbold] has $\frac{1}{2}$ hide and 4 ploughs in demesne, and the villeins $\frac{1}{2}$ hide and Io ploughs. There E[rchenbold] has $I_{4}$ villeins, 4 bordars, 8 serfs, I I beasts, 40 swine, Ioo sheep, 35 goats, 60 acres of wood(land), 4 acres of meadow, and 100 acres of pasture. Worth 8 pounds, and was worth the same when the count received it.

To this manor 3 manors ${ }^{8}$ have been added which 3 thanes held in parage (pariter; Exch., libere), and they could go to what lord they liked. Two of these were Ordulf's liegemen and the third was not his man. Those 3 manors paid geld for 3 virgates; 7 ploughs can till them. There Erchenbold has 5 villeins who have there 5 ploughs, 3 serfs, 10 acres of wood(land), 8 acres of meadow, and 50 of pasture. The 3 manors are worth $2 \mathrm{I}$ shillings and 7 pence $(E x c b .22$ shillings).

The count has a manor called CRIDEholda [Credhow] ${ }^{9}$ which Ordulf held T.R.E., and it paid geld for I hide. This ro ploughs can till. Erchenbold holds it of the count. Thereof $E$ [rchenbold] has I virgate and 3 ploughs in demesne, and the villeins 3 virgates and 6 ploughs. There E[rchenbold] has 10 villeins, 9 bordars, 2 serfs, I I beasts, I 2 swine, Ioo sheep, and 5 acres of meadow. Worth 4 pounds,

6 Tattiscombe alias Tottescomb in Trentishoe in Braunton hundred.

${ }^{7}$ Bratton Fleming in Braunton hundred.

${ }^{8}$ Perhaps Stowford, Honicot and Spreycot in Bratton Fleming.

${ }^{2}$ Credhow alias Croyde in Georgeham in Braunton hundred. 


\section{HOLDERS OF LANDS}

and was worth the same when the count received it. A sister of Ordulf held I virgate ${ }^{1}$ thereof, which she could not make independent of Ordulf.

fol. 213b.

The count has a manor called Donicestona [Dyningston] ${ }^{2}$ which Donin (Exch. Donninc) held T.R.E., and it paid geld for $3 \frac{1}{2}$ virgates and $\frac{1}{2}$ ferling. These 5 ploughs can till. Mauger de Cartreo holds them of the count. Thereof $M$ [auger] has a moiety in demesne and I plough, and the villeins the other moiety and 4 ploughs. There [Mauger] has 12 villeins, 6 bordars, $3^{6}$ sheep, 1 mill worth 7 shillings and 6 pence yearly, 12 acres of wood(land), I 3 acres of meadow, and 20 acres of pasture. Worth 40 shillings a year, and was worth the same when the count received it.

To the aforesaid manor has been added a certain manor called ALwinestona ${ }^{3}$ which did not belong to that manor in King $\mathrm{E}$ [dward]'s time, but Alwin held it T.R.E. and it paid geld for 3 virgates. These 5 ploughs can till. Mauger holds them of the count. Thereof $M$ [auger] has in demesne I virgate 3 ferdings and I plough, and the villeins I virgate and I ferding. There $M$ [auger] has 4 villeins, 2 bordars, 2 serfs, 20 sheep, Io goats, I mill worth 8 pence yearly, 7 acres of wood(land), 6 acres of meadow, and 30 acres of pasture. Worth I 5 shillings a year, and was worth 40 shillings when the count received it.

The count has a manor called CiIst [Clyst] which Alward held T.R.E., and it paid geld for 4 hides. These 7 ploughs can till, Alward holds it of the count. Thereof $A[$ lward] has in demesne $2 \frac{1}{2}$ hides, $\frac{1}{2}$ virgate, and 2 ploughs, and the villeins $I \frac{1}{2}$ hides, $\frac{1}{2}$ virgate, and 3 ploughs. There $A$ [lward] has 17 villeins, 9 bordars, 4 serfs, 7 beasts, 20 swine, I 9 sheep, $3^{8}$ goats, 150 acres of wood(land), 50 acres of meadow, and I 30 acres of pasture. Worth 3 pounds a year, fol. 2x4.

and was worth 30 shillings when the count received it.

1 Perhaps Middleborough or Puttsborough in Georgeham.

- Dyningston alias Denson in Clayhanger in Bampton hundred.

${ }^{3}$ Apparently Little Bampton alias Petton in Rampton in Bampton hundred. Trans. Devon Assoc. xxx, $44^{1}, 450$.

${ }^{4}$ Clyst St. Laurence in Cliston hundred. The sum of the lord's and villeins' assessments amount to 4 hides I virgate. The virgate in excess probably represents Eveleigh. Eveleigh was taken out of the villeins' land and pays a chief rent of 6 pence a year to Clyst St. Laurence (Exeter Charities, p. 543).
The count of Mortain (de Moritonio) has a manor called Torneberia [Thornbury] ${ }^{5}$ which Alcerlo held T.R.E., and it paid geld for I virgate. This 3 ploughs can till. Alvered holds it of the count. There Alvered has 3 villeins who have I plough, I serf, 2 acres of meadow, and 40 of pasture. Worth Io shillings a year, and was worth 7 shillings when the count received it.

The count has a manor called Chederlia

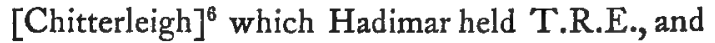
it paid geld for 3 virgates. These 4 ploughs can till. Alvered holds it of the count. Thereof $A$ [lvered] has in demesne $I \frac{1}{2}$ virgates and $I$ plough, and the villeins $1 \frac{1}{2}$ virgates and 3 ploughs. There A[lvered] has 4 villeins, 2 bordars, I serf, 2 beasts, 9 acres of meadow, and 16 of pasture. Worth 40 shillings a year, and was worth 30 shillings when the count received it.

The count has a manor called Estochelia ${ }^{7}$ (Exch. Stochelie) [Stockleigh] which Hadimar held T.R.E., and it paid geld for I virgate. This 2 ploughs can till. Thereof the count has in demesne $\frac{1}{2}$ virgate and $\frac{1}{2}$ plough, and the villeins $\frac{1}{2}$ virgate and $\frac{1}{2}$ plough. There the count has 2 villeins, 3 bordars, 3 beasts, and 8 sheep. Worth 1o shillings a year, and was worth 5 shillings when the count received it.

The count has a manor called Bichelia [Bickleigh] $]^{8}$ which Alward held T.R.E. and it paid geld for I hide. This 8 ploughs can till. Alward holds it of the count. Thereof A[lward] has in demesne I virgate and 2 ploughs, and the villeins 3 virgates and 6 ploughs. There fol. 2I4b.

A[lward] has I I villeins, I 3 bordars, 6 serfs, 3 beasts, 60 sheep, 8 swine, 59 acres of wood(land), 13 acres of meadow, and 20 acres of pasture, besides I mill paying 5 shillings a year. Worth 3 pounds, and was worth 40 shillings when the count received it.

The count has a manor called Finatona [Feniton] $^{9}$ which Etmar (Exch. Edmaer) held T.R.E., and it paid geld for 3 hides. These 6 ploughs can till. Drogo holds them of the count. Thereof Drogo has in demesne $\frac{1}{2}$ hide and $\frac{1}{2}$ plough, and the villeins $2 \frac{1}{2}$ hides. There

- Thornbury an outlier of Hittisleigh in Wonford hundred (O. J. R.). Trans. Devon Assoc. xxxvi, 367 , and xxvii, 165.

${ }^{6}$ Chitterleigh alias Chederleigh in Bickleigh in Hairidge hundred.

${ }^{7}$ Stockleigh in Hairidge hundred.

${ }^{8}$ Bickleigh in Hairidge hundred.

${ }^{9}$ Feniton Malherb in Hairidge hundred. 


\section{A HISTORY OF DEVONSHIRE}

Drogo has 8 villeins, 4 bordars, 2 serfs, 5 swine, I 5 sheep, 6 acres of wood(land), I 5 of meadow, and 100 acres of pasture. Worth 40 shillings a year, and was worth 4 pounds when he received it.

The count has a manor called Cochalescoma [Corscombe] ${ }^{1}$ which Ineguar (Exch. Inewar) held T.R.E., and it paid geld for $\mathrm{I}$ hide. This 4 ploughs can till. Drogo holds it of the count. Thereof $D$ [rogo] has in demesne $\frac{1}{2}$ hide, and the villeins $\frac{1}{2}$ hide and $1 \frac{1}{2}$ ploughs. There $\mathrm{D}$ [rogo] has 4 villeins, 4 bordars, I serf, 2 acres of coppice, and 2 acres of meadow. Worth 20 shillings a year, and was worth 40 shillings when he received it.

The count has a manor called Cherletona [Charlton] $^{2}$ which Semar held T.R.E., and it paid geld for 1 hide. This 2 ploughs can till. Bretel holds it of the count. Thereof Bretel has in demesne 3 virgates and $I$ ferding and $\frac{1}{2}$ plough, and the villeins 3 ferdings and $\frac{1}{2}$ plough. There $B$ [retel] has 3 villeins, I bordar, I 8 sheep, 8 acres of wood(land), 4 of meadow, and 15 of pasture. Worth 15 shillings a year, and was worth 15 shillings when he received it. fol. 255 .

The count of Mortain has a manor called Estochelera (Exch. Stochelie) [Stockleigh] ${ }^{3}$ which Orgar held T.R.E. He was the man of Edmeratorius, but could go with that land to what lord he liked. It paid geld for I hide. This ro ploughs can till. Alvered the cupbearer (pincerna) holds it of the count. Thereof $A$ [lvered] has I virgate and 2 ploughs in demesne, and the villeins 3 virgates and 7 ploughs. There $A$ [lvered] has 8 villeins, 4 bordars, 5 serfs, 5 beasts, I 9 sheep, 6 acres of wood(land), 7 acres of meadow, and 40 acres of pasture. Worth 50 shillings, and was worth 60 shillings when the count received it.

The count has a manor called EstochereIA (Exch. Stochelie) [Stockleigh] ${ }^{4}$ which Haimer held T.R.E. He was the man of Edmeratorius, but could go to what lord he liked with that land. This Alvered the cupbearer holds of the count, and it pays geld for I hide. This 7 ploughs can till. Thereof $A$ [lvered] has $I \frac{1}{2}$ virgates and 2 ploughs in demesne, and the villeins $2 \frac{1}{2}$ virgates and 5 ploughs. There $A$ [lvered] has 4 villeins, 4 bordars, 5 serfs, 4 beasts, 7 swine, 20 sheep, 15 goats, 12 acres of wood(land), 6 acres of meadow, and 4 of pasture. Worth 30 shillings,

1 Corscombe in Feniton in Hairidge hundred.

- Charlton in Plymtree in Hairidge hundred.

${ }^{3}$ South Stockleigh alias Sutton Satchvil with Upcot in Cheriton Fitzpaine in West Budleigh hundred. Trans. Devon Assoc. xxxy, 300.

"Stockleigh Luccombe alias Little Stockleigh in Cheriton Fitzpaine in West Budleigh hundred. Trans. Devon Assoc. xxxvi, 363. and was worth 40 shillings when the count received it.

The count has a manor called Pocherlua [Poughill] $^{5}$ which 2 thanes held in parage (pariter; Exch., libere) T. R.E. They were the men of Edmeratorius, but could go with that land to what lord they liked. It paid geld for $\frac{1}{2}$ hide and $\frac{2}{3}$ virgate. This 4 ploughs can till. Alvered the cupbearer holds it of the count. Thereof $A[$ lvered] has I virgate and 2 ploughs in demesne, and the villeins have the rest of the land and 2 ploughs. There A[lvered] has 3 villeins, 4 bordars, 3 serfs, and 2 acres of meadow. Worth 25 shillings, and was worth Io shillings when the count received it. These 3 manors the count holds along with the honour of Edmeratorius.

fol. $215 b$.

The count has a manor called WrCA [Wick ${ }^{6}$ which Ordulf held T.R.E., and it paid geld for I hide. This 4 ploughs can till. Thereof the count has $\frac{1}{2}$ hide and 2 ploughs in demesne, and the villeins $\frac{1}{2}$ hide and 2 ploughs. There the count has 5 villeins, 3 bordars, 6 serfs, 80 sheep, I 4 goats, 4 acres of wood(land), and 2 acres of meadow. It pays 60 shillings; when the count received it ro shillings.

The count has a manor called Sotebroca (Exch. Sotebroch) [Shobrook] ${ }^{7}$ which Ordulf held T.R.E., and it paid geld for 2 hides. These 8 ploughs can till. Thereof the count has I hide and 2 ploughs in demesne, and the villeins I hide and 4 ploughs. There the count has 7 villeins, 4 bordars, 6 serfs, I cow, 40 sheep, 8 swine, 4 acres of meadow and 30 acres of pasture. It pays 4 pounds Io shillings; when the count received it, 20 shillings.

The count has a manor called RatDona [Raddon] $^{8}$ which Ordulf held T.R.E., and it paid geld for 1 hide and 3 virgates. This 7 ploughs can till. Thereof the count has 3 virgates and 2 ploughs in demesne, and the villeins I hide and 4 ploughs. There the count has 8 villeins, 4 bordars, 6 serfs, 7 beasts, 2 swine, 40 sheep, 40 goats, 4 acres of wood(land), 4 acres of meadow, and ro acres of pasture. It pays 4 pounds Io shillings; when the count received it it was worth 20 shillings.

The count has a manor called Estocheleia (Exch. Stochelie) [Stockleigh $]^{9}$ which Saiulf $(E x c h$. Saulf) held in parage (pariter) T.R.E., and he

- Poughill in West Budleigh hundred.

6 Wick in Shobrook in West Budleigh hundred.

7 Shobrook in West Budleigh hundred.

8 West Raddon in Shobrook in West Budleigh hundred.

${ }^{9}$ Stockleigh English alias West Stockleigh in West Budleigh hundred. 


\section{HOLDERS OF LANDS}

could go to what lord he liked. It paid geld for I hide. This 5 ploughs can till. Rainald holds it of the count. Thereof $R$ [ainald] has $\frac{1}{2}$ hide and $I$ plough in demesne; and the villeins $\frac{1}{2}$ hide and 4 ploughs. There $R$ [ainald] fol. 216.

has 6 villeins, 4 bordars, 3 serfs, 2 beasts, 4 swine, 20 sheep, 20 acres of wood(land), 2 of meadow and 32 of pasture. Worth 25 shillings, when the count received it 15 shillings. This the count holds along with Ordulf's honour.

The count has a manor called Ferentona [Faringdon] ${ }^{1}$ which 2 brothers held in parage (pariter) T.R.E., and they could go to what lord they liked with that land; it paid geld for I virgate. This 2 ploughs can till. Bretel holds it of the count. There Bretel has I plough and 2 cottagers, 6 cows, 4 swine, 20 sheep, 3 acres of wood(land), 8 acres of meadow, and 12 acres of pasture. Worth 7 shillings, and was worth the same when the count received it.

The count has a manor called Colebroca [Holbrook] ${ }^{2}$ which Samer (Exch. Semer) held in parage (pariter) T.R.E., and he could go to what lord he liked; it paid geld for 3 virgates. These 3 ploughs can till. Bretel holds it of the count. Thereof $B$ [retel] has 2 virgates and I ferling, and I plough in demesne, and the villeins 3 ferlings and I plough. There $B$ [retel] has 2 villeins, I bordar, I serf, 8 acres of wood(land), and 4 acres of meadow. Worth 15 shillings, and was worth the same when the count received it. The aforesaid 3 manors the count holds along with the honour of Edmeratorius, and those who held them were the men of Edmeratorius.

The count has a manor called Rochebera [Rockbear] ${ }^{3}$ which Sawin held T.R.E. He was the man of Edmeratorius, but could go with that land to what lord he liked. It paid geld for I virgate. This 2 ploughs can till. Alward holds it of the count. Thereof A[lward] has $\frac{1}{2}$ virgate and $I$ plough in demesne, and the villeins $\frac{1}{2}$ virgate and $\mathrm{I}$ ox. There $A$ [lward] fol. 2a6b.

has 2 villeins, 2 bordars, 2 serfs, 4 acres of wood(land), 8 acres of meadow, and 25 acres of pasture. Worth 7 shillings and 6 pence; when the count received it it was worth Io pence. This land the count holds along with the honour of Edmeratorius.

1 Little Faringdon in Faringdon in East Budleigh hundred.

${ }^{3}$ Holbrook Grindham alias Withien Furse (Trans. Devon Assoc. xxxii, 409) alias Mora Holbrokes (Feud. Aids, p. 365) alias Denbow in Faringdon in East Budleigh hundred. Testa de Nevill (Rec. Com.), 191 $a$, in Trans. Devon Assoc. xxxv, 290; xxxvi, 166.

3 Rockbear alias Hockland in Colyton Raleigh in East Budleigh hundred. See Testa de Nevill (Rec. Com.), 191a, in Trans. Devon Assoc. xxxv, 294.
The count has a manor called DonevoldeHAMME [Densham $]^{4}$ which Ademar held T.R.E., and that land has been united with the land of Etmaratolus ; it paid geld for I virgate and I ferling. These 3 ploughs can till. Alvered holds it of the count. Thereof A[lvered] has in demesne I ferding, and the villeins I virgate and 3 ploughs. There A[lvered] has 6 bordars, I serf, 5 acres of wood(land), and 4 acres of meadow. Worth ro shillings a year, when he received it it was worth 5 shillings.

The count has a manor called ST. Mary's Cherche (Exch. Cerce) [St. Mary Church] ${ }^{5}$ which Ordulf held T.R.E., and it paid geld for I hide. This 4 ploughs can till. Richard son of Torolv holds it. Thereof R[ichard] has in demesne 2 virgates and 2 ploughs, and the villeins 2 virgates and $1 \frac{1}{2}$ ploughs. There $R$ [ichard] has 5 villeins, 8 bordars, 3 serfs, I rouncey, 4 beasts, 5 swine, I IO sheep, and I acre of meadow. Worth 40 shillings a year, and was worth 20 shillings when the count received it.

The count has a manor called Honetona [Honiton] $^{6}$ which Elmer held T.R.E., and it paid geld for 5 hides. These 18 ploughs can till. Drew (Exch. Drogo) holds it of the count. There he has in demesne 2 hides, and the villeins (have) 3 hides and 16 ploughs. There $D[$ rew] has 24 villeins, 6 bordars, 3 serfs, 2 beasts, 4 swine, 100 sheep all but 20, 50 acres of wood(land), I 8 acres of meadow, pasture I league in length by 5 furlongs in breadth, I mill fol. 217.

paying $7 \frac{1}{2}$ shillings, and 2 saltworkers paying 5 shillings a year by way of rent (de firma). Worth 6 pounds a year, and was worth the same when he received it. This manor was free T.R.E. The manor has been added to the land of Elmeratorius.

The count has a manor called WIBORDA (Exch. Wiburde) [Wombernford $]^{7}$ which Olward (Exch. Ulward) held T.R.E., and it paid geld for I virgate. This 3 ploughs can till. Drew (Exch. Drogo) holds it of him. There D[rew] has in demesne I ferling and I plough, and the villeins 3 ferlings and 2 ploughs. There $D[$ rew $]$ has 6 villeins, I serf, 7 beasts, 50 sheep, 40 acres of wood(land), 4 acres of meadow, and 40 acres of pasture. Worth 5 shillings a year, and was worth 3 shillings when he received it.

- East Densham in Woolfardisworthy Spenser in Witheridge hundred.

${ }^{5}$ St. Mary Church in Haytor hundred.

${ }^{6}$ Honiton in Axminster hundred. Probably the 2 saltworkers had their salterns at Beer. See fol. $184 b$.

${ }^{7}$ Wombernford in Cotleigh in Colyton hundred. 


\section{A HISTORY OF DEVONSHIRE}

The count has a manor called LEGA [Leigh] ${ }^{1}$ which Salwin (Exch. Sawin) held T.R.E., and it paid geld for $\frac{1}{2}$ hide. This 5 ploughs can till. Alward holds it of him. There A[lward] has in demesne I ferling and 2 ploughs, and the villeins 3 ferlings and 3 ploughs. There A[lward] has 7 villeins, 4 bordars, 2 serfs, I 6 beasts, 10 sheep, I 0 goats, 30 acres of wood(land), 8 acres of meadow, and 40 of pasture. Worth 20 shillings a year, and was worth 5 shillings when he received it. These two manors were held freely (liberate) T.R.E. Now they have been added to the land of Elmeratorius.

The count has a manor called Hamistona [Hempston] $^{2}$ which Ordulf held T.R.E., and it paid geld for 2 hides. These ro ploughs can till. Hamelin holds it of the count. Thereof $\mathrm{H}$ [amelin] has $\mathrm{I}$ hide and 2 ploughs in demesne, and the villeins 1 hide and 6 ploughs. There fol. 2rzb.

$\mathrm{H}$ [amelin] has ro villeins, 9 bordars, 3 serfs, 9 beasts, 9 swine, 68 sheep, I2 acres of wood(land), 4 acres of meadow, and Io acres of pasture. Worth 60 shillings, and was worth 40 shillings when the count received it.

The count of Mortain (de Moritonio) has a manor called CoteleIA [Cotleigh] ${ }^{3}$ which Edmar (Exch. Edmaer) held T.R.E., and it paid geld for 2 hides. These 8 ploughs can till. Now Richard holds it of the count. Thereof $\mathrm{R}$ [ichard] has I hide and $\frac{1}{2}$ virgate in demesne and 2 ploughs, and the villeins have $I$ hide all but $\frac{1}{2}$ virgate and 6 ploughs. There R[ichard] has I 7 villeins, 4 bordars, I serf, 8 swine, and 60 sheep. Worth 40 shillings a year, and was worth 20 shillings when the count received it.

The count has a manor called Motbinia [Modbury] $^{4}$ which Ordrit (Exch. Ordric) held T.R.E., and it paid geld for 4 hides. These 23 ploughs can till. Now Raginald holds it of the count. Thereof $R$ [aginald] has I hide in demesne and 3 ploughs, and the villeins have 3 hides and II ploughs. There R[aginald] has 22 villeins, I 6 bordars, 7 serfs, I rouncey, 4 beasts, 7 sheep, I swineherd, wood(land) 5 furlongs in length (by) $I$ in breadth, and I (acre) of meadow. Worth 6 pounds a year; it was worth 12 pounds when the count received it.

\footnotetext{
${ }^{1}$ Northleigh in Colyton hundred. The missing virgate is probably Hockland in Budleigh hundred, held with it in 1241 (Testa, p. 183b). Sawin held both before Domesday.

'Broadhempston alias Hempston Borard alias Great Hempstone alias Hempstone Cantelo in Haytor hundred (O.J.R.) ; or Little Hempstone (T. W. W.), Trans. Devon Assoc. xxxvi, 369.

${ }^{3}$ Cotleigh in Colyton hundred.

" Modbury in Ermington hundred.
}

The count has a manor called Orcartona [Orcherton] $^{5}$ which Norman held T.R.E., and it paid geld for I hide. This 4 ploughs can till. Raginald holds it of the count. Thereof $R$ [aginald] has $\frac{1}{2}$ hide in demesne and $\mathbf{I}$ plough, and the villeins $\frac{1}{2}$ hide and I plough. There $\mathrm{R}$ [aginald] has 3 villeins, 5 bordars, 3 beasts, 22 sheep, 4 goats, 6 acres of wood(land), I acre of meadow and I saltern worth 5 shillings a year. This manor is worth 20 shillings a year, and was worth 40 shillings when the count received it.

fol. 218.

The count has a manor called Holescoma [Luscombe] ${ }^{6}$ which Levegar held T.R.E., and it paid geld for $\frac{1}{2}$ hide. This 4 ploughs can till. Now Ralf de Pomaria holds it of the count. Thereof $\mathrm{R}$ [alf] has $\mathrm{I}$ virgate and 2 ploughs in demesne, and the villeins I virgate and 2 ploughs. There $R$ [alf] has 5 villeins, 3 bordars, I serf, 4 beasts, 2 swine, 20 sheep, 4 acres of wood(land), ro acres of meadow, and 4 salterns paying 40 pence a year, and 2 seams (saginas) of salt. Worth 40 shillings, and was worth 30 shillings when the count received it.

The count has a manor called BrcheBeria

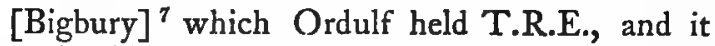
paid geld for 2 hides. These 12 ploughs can till. Raginald de Valletorta holds it of the count. Thereof $R$ [aginald] has $\frac{1}{2}$ hide in demesne and 2 ploughs, and the villeins have $1 \frac{1}{2}$ hides and $3 \frac{1}{2}$ ploughs. There $R$ [aginald] has 12 villeins and as many bordars, I rouncey, Io beasts, 9 swine, 107 sheep, 9 goats, I saltern worth 30 pence a year, wood(land) $\mathbb{I}$ league in length by I furlong in breadth, 5 acres of meadow, and 30 of pasture. Worth 6o shillings a year, and was worth 7 pounds when the count got it.

The count has a manor called Ferdenderra [Fardle] ${ }^{8}$ which Domne (Exch. Donno) held T.R.E., and it paid g[eld] for I hide. This 4 ploughs can till. Now Raginald holds it of the count. Thereof $R$ [aginald] has $I \frac{1}{2}$ virgates in demesne and I plough, and the villeins $\frac{1}{2}$ hide and $\frac{1}{2}$ virgate and $1 \frac{1}{2}$ ploughs. There $\mathrm{R}$ [aginald] has 5 villeins, 4 bordars, 4 serfs, I rouncey, I I beasts, I 8 swine, 75 sheep, 30 goats, wood(land) 4 furlongs in length by 1 in breadth, IO acres of meadow and 300 acres of pasture. Worth 30 shillings a year, and was worth 40 shillings when the count received it.

From this manor the hundredmen and the king's reeve claim 30 pence and the customary court-dues towards the farm of Ermington, the king's manor.

${ }^{5}$ Orcherton in Modbury in Ermington hundred.

${ }^{6}$ Luscombe alias Halwell's combe alias Jeclescombe (Feud. Aids, p. 351) alias Julescombe in Bigbury in Ermington hundred.

${ }^{7}$ Bigbury manor in Ermington hundred.

${ }^{8}$ Fardle in Cornwood in Ermington hundred. 
fol. $2 x 8 b$.

\section{HOLDERS OF LANDS}

The count has a manor called CoRneHuda (Exch. Cornehode) [Cornwood] ${ }^{1}$ which Edmar (Exch. Edmaer) held T.R.E., and it paid geld for I hide. This 5 ploughs can till. Raginald (holds it) of the count. Thereof $\mathrm{R}$ [aginald] has I virgate in demesne and $I \frac{1}{2}$ ploughs; and the villeins have 3 virgates and 2 ploughs. There $\mathrm{R}$ [aginald] has 8 villeins, 7 (Exch. 8) bordars, 8 serfs, 3 unbroken mares (indomitas), to beasts, 3 swine, 22 goats, wood(land) 2 leagues in length by $\frac{1}{2}$ in width, and pasture $I$ league in length by $\frac{1}{2}$ in width. Worth 40 shillings, and was worth the same when the count received it.

The count has a manor called Niwetona [Newton] ${ }^{2}$ which Edmarr (Exch. Edmaer) held T.R.E., and it paid geld for 2 hides. These Io ploughs can till. Raginald holds it of the count. Thereof $R$ [aginald] has $\frac{1}{2}$ hide in demesne and 4 oxen, and the villeins $I \frac{1}{2}$ hides and 3 ploughs. There $R$ [aginald] has 16 villeins, 5 bordars, 12 serfs, 6 beasts, 3 swine, 24 sheep, Io goats, wood(land) I league in length by I furlong in breadth, 2 acres of meadow, and 60 acres of pasture. Worth 3 pounds ro shillings a year; when the count received it it was worth 6 pounds.

The count has a manor called Hereforda [Harford $]^{3}$ which Ordulf held T.R.E., and it paid geld for I hide. This 5 ploughs can till. Raginald holds it of the count. Thereof $R$ [aginald] has $\frac{1}{2}$ hide in demesne and I plough, and the villeins (have) $\frac{1}{2}$ hide and 2 ploughs. There $\mathrm{R}$ [aginald] (has) 6 villeins, 6 bordars, 2 serfs, 5 beasts, 5 swine, 20 sheep, I I goats, and pasture I league in length by $\frac{1}{2}$ in breadth. Worth 30 shillings a year, and was worth 3 pounds when the count received it.

The count has a manor called Fen [Fenton] ${ }^{4}$ which Norman held T.R.E., and it paid geld for I virgate. This 3 ploughs can till. Raginald holds it of the count. Thereof R[aginald] has $\frac{1}{2}$ virgate and the villeins have ( ) ploughs. There $R$ [aginald] has 4 villeins, 2 bordars, and 7 acres of pasture. Worth 10 shillings a year; when fol. 2rg.

the count received it it was worth 20 shillings.

The count has a manor called BRADEForda [Bradford] ${ }^{5}$ which Edrit (Exch. Edric) held T.R.E., and it paid geld for I virgate. This 2 ploughs can till. Raginald holds it of the count. Worth 5 shillings a year; when the count received it ro shillings.

1 Cornwood in Ermington hundred.

${ }^{2}$ Newton Ferrers in Ermington hundred.

3 East and West Harford in Ermington hundred.

4 Venn alias Fenton in Ugborough in Ermington hundred.

${ }^{5}$ Bradford alias Broadaford in Ugborough in Ermington hundred.
The count has a manor called Dinintona [Dennaton] ${ }^{6}$ which Donna (Exch. Donno) held T.R.E., and it paid geld for I hide. This 4 ploughs can till. Now Raginald holds it of the count. Thereof R[aginald] has $\frac{1}{2}$ hide in demesne and I plough, and the villeins have $\frac{1}{2}$ hide and I plough. There $\mathrm{R}$ [aginald] has 4 villeins, 3 (Exch. 4 ) bordars, 4 serfs, 4 beasts, 3 swine, 60 sheep, 20 goats, wood(land) 4 furlongs in length by 2 furlongs in breadth, $I$ acre of meadow and $\frac{1}{2}$ league of pasture. Worth 20 shillings a year, and was worth 30 shillings when the count received it.

The count has a manor called PEch [Peak] which Alcherl held T.R.E., and it paid geld for I virgate. This 2 ploughs can till. Raignald holds it of the count. Thereof R[aignald] has in demesne 2 ferdings and 1 plough, and the villeins 2 ferdings. There R[aignald] has 3 villeins, 2 bordars, I serf, I beast, 50 sheep, 20 goats, 2 acres of coppice and I league of pasture. Worth 20 shillings a year, and was worth 15 shillings when the count received it.

The count has a manor called LODEBRoc [Ludbrook $^{8}$ which Alwin held T.R.E., and it paid $g[$ eld] for 3 virgates. These 3 ploughs can till. Rainald holds them of the count. Thereof $R$ [ainald] has in demesne $I \frac{1}{2}$ virgates and $I$ plough, and the villeins $1 \frac{1}{2}$ virgates and $1 \frac{1}{2}$ ploughs. There $\mathrm{R}$ [ainald] has 3 villeins, 5 bordars, 6 serfs, I furlong of wood(land), and I fol. 2rgb.

acre of meadow. Worth 20 shillings a year, and was worth 30 shillings when the count received it.

The count has a manor called LODEBroc [Ludbrook ${ }^{9}$ which Colbert held T.R.E., and it paid geld for $\frac{1}{2}$ hide. This 3 ploughs can till. Rainald holds it of the count. Thereof $\mathrm{R}$ [ainald] has in demesne I virgate and I plough, and the villeins $I$ virgate and $1 \frac{1}{2}$ ploughs. There $R$ [ainald] has 5 villeins, 3 bordars, 2 serfs, 5 beasts, I 5 swine, 80 sheep, I mill paying 2 shillings a year, and $\frac{1}{2}$ acre of meadow. Worth 20 shillings a year, and was worth 25 shillings a year when the count received it. This land Colbert held in parage (pariter; Exch., libere). Now it has been added to the honour of Edmeratorius, which the count holds.

The count has a manor called LOPERIGE [Luperidge] ${ }^{10}$ which Eddric (Exch. Edric) held T.R.E., and it paid geld for I virgate. This

6 Dennaton in Cornwood in Ermington hundred.

7 Torpeak in Ugborough in Ermington hundred.

${ }^{8}$ South Ludbrook alias Ludbrook House, an outlier of Modbury in Ermington hundred. See fol. $504 b$, and 856 .

${ }^{9}$ North Ludbrook in Ugborough in Ermington hundred. The mill identifies it.

${ }^{10}$ Luperidge in North Huish in Ermington hundred. 


\section{A HISTORY OF DEVONSHIRE}

3 ploughs can till. Rainald holds it of the count. Thereof $R$ [ainald] has in demesne $\frac{1}{2}$ virgate and I plough, and the villeins $\frac{1}{2}$ virgate and $I \frac{1}{2}$ ploughs. There $R$ [ainald] has 3 villeins, 2 bordars, 2 serfs, 4 beasts, 6 swine, and 66 sheep. Worth I 5 shillings a year, and was worth 19 shillings when the count received it. This land Edric held in parage (pariter; Exch., libere) in King E[dward]'s time; but now it has been added to the honour of Edmeratorius.

\section{fol. 220.}

The count has a manor called BoteBERIA [Bolbury] ${ }^{1}$ which Wado held T.R.E., and it paid geld for $\frac{1}{2}$ hide. This 4 ploughs can till. Richards holds it of the count. Thereof $\mathrm{R}$ [ichard] has $\mathbf{I}$ virgate and $\mathbf{I}$ plough in demesne, and the villeins I virgate and $\frac{1}{2}$ plough. There $R$ [ichard] has 3 villeins, I bordar, and I serf. Worth 7 shillings and 6 pence; when the count received it 27 shillings.

The count has a manor called Boltesberia [Bolbury] ${ }^{2}$ which Edmer held T.R.E., and it paid geld for I hide. This 4 ploughs can till. Hugh (de Valletorta) ${ }^{3}$ holds it of the count. Thereof $\mathrm{H}$ [ugh] has $\frac{1}{2}$ virgate and I plough in demesne, and the villeins $\frac{1}{2}$ hide and $I$ plough. There $\mathrm{H}$ [ugh] has I villein, 3 bordars, I serf, I pig, and 9 sheep. Worth ro shillings; when the count received it 30 shillings.

To this manor has been added a manor called Bochelanda [Buckland] ${ }^{4}$ which Edeva (Exch. Eddena) held in parage (pariter; Exch., libere) T.R.E., and he could go to what lord he liked. It paid geld for I virgate. This $I_{2} \frac{1}{2}$ ploughs can till. There $\mathrm{H}$ [ugh] has 2 villeins and 2 bordars who have I plough. Worth 4 shillings, and was worth the same when the count received it.

The count has a manor called Badestana [Batson] ${ }^{5}$ which Uluric held T.R.E., and it paid geld for $I \frac{1}{2}$ hides. This 4 ploughs can till. Hugh holds it of the count. Thereof H[ugh] has $\frac{1}{2}$ hide and I plough in demesne, and the villeins I hide and I plough. There $\mathrm{H}$ [ugh] fol. $220 \mathrm{~b}$.

has 3 villeins, 3 bordars, I serf, 6 swine, 30 sheep, 6 goats, 1o acres of wood(land), 3 acres of meadow, and 50 of pasture. Worth 15 shillings, and was worth 35 shillings when the count received it.

'Little Bolbury alias North Bolbury alias Bolbury Allen in Malborough in Stanborough hundred. Geldroll, fol. $69 b, \mathrm{~B} 3$, shows Odo as sub-tenant.

${ }^{2}$ Great Bolbury alias Bolbury Beauchamp alias Much Bolbury in Malborough in Stanborough hundred.

${ }^{3}$ Geldroll, fol. 69b, B 2.

- Buckland in Thurleston in Stanborough hundred.

B Batson in Salcombe in Stanborough hundred.
The count has a manor called Comma (Exch. Cume) [Combe] ${ }^{6}$ which Alvric held T.R.E., and it paid geld for $\frac{1}{2}$ hide. This 4 ploughs can till. Rainald holds it of the count. Thereof $\mathrm{R}$ [ainald] has I virgate and I plough in demesne, and the villeins I virgate and I plough. There $\mathrm{R}$ [ainald] has 3 villeins, 3 bordars, $\mathrm{I}$ serf, 4 beasts, 80 sheep, and I acre of meadow. Worth Io shillings; when the count received it 20 shillings.

The count has a manor called Silfrestana

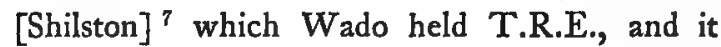
paid geld for $\frac{1}{2}$ hide. This 3 ploughs can till. Richard holds it of the count. Thereof $\mathrm{R}$ [ichard] has I virgate and I plough in demesne, and the villeins I virgate and I plough. There $R$ [ichard] has 4 villeins, 3 bordars, 2 serfs, 1 beast, I 4 sheep, and $\frac{1}{2}$ acre of meadow. Worth Io shillings; when the count received it 20 shillings.

The count has a manor called Hewis ${ }^{8}$ which Edric held T.R.E., and he could go to what lord he liked. It paid geld for I virgate. This 2 ploughs can till. Rainald holds it of the count. 'There $R$ [ainald] has 2 villeins who have I plough. It pays 10 shillings; when the count received it the same. This the count holds along with the honour of Edmeratorius.

The count has a manor called CoмBA [Combe] ${ }^{9}$ which Ansgot (Exch. Osgot) held T.R.E., and it paid geld for I virgate. This fol. 221 .

2 ploughs can till. Donne (Exch. Donno) holds it of the count. Thereof $\mathrm{D}$ [onne] has $\frac{1}{2}$ virgate and I plough in demesne and the villeins I virgate. There $\mathrm{D}$ [onne] has I villein, 2 bordars, 4 beasts, I 5 sheep, $\frac{1}{2}$ acre of wood(land), and I acre of meadow. Worth 7 shillings; when the count received it the same.

The count has a manor called Motberia [Modbury] ${ }^{10}$ which $W$ ado held in parage (pariter; Exch., libere) T.R.E., and he could go to what lord he liked. It paid geld for I hide. This 5 ploughs can till. Richard holds it of the count. Thereof $R$ [ichard] has $\frac{1}{2}$ hide and $I$ plough in demesne and the villeins $\frac{1}{2}$ hide and a virgate, which virgate Wado held of Ordulf, ${ }^{11}$

- Bawcombe in Ugborough in Ermington hundred.

7 Shilston in Modbury in Ermington hundred.

${ }^{-}$Possibly Yedmerston in Modbury, see fol. 505.

- Spriddlecombe in Modbury in Ermington hundred. Probably the missing $\frac{1}{2}$ virgate in the total assessment may be the unnamed 'Lodderton contributary with Littlecomb $\frac{1}{6}$ fee' (Feud. Aids, p. 352), - where Littlecomb is written in error for Spriddlecomb-i.e. Lutton in Cornwood.

${ }^{10}$ Little Modbury in Modbury in Ermington hundred. Geldroll, fol. 706, B 2 .

${ }^{11}$ Perhaps Stubston in Modbury. 


\section{HOLDERS OF LANDS}

and with that virgate he could not be independent of Ordulf, but with the aforesaid hide he could. Those villeins have 3 ploughs. There $R$ [ichard] has 4 villeins, 2 bordars, I serf, 6 beasts, 6 swine, 60 sheep, 30 goats, and 2 acres of wood(land). The aforesaid hide is worth 30 shillings, and was worth the same when the count received it. And this hide the count wrongfully holds along with Ordulf's honour. The aforesaid virgate is worth 2 shillings, and was worth the same when the count received it.

The count has a manor called TORI ${ }^{1}$ which Wado held in parage (pariter; Exch., libere) T.R.E., and he could go to what lord he liked. It paid geld for I hide. This 4 ploughs can till. Rainald holds it of the count. Thereof $\mathrm{R}$ [ainald] has $\frac{1}{2}$ hide and I plough in demesne and the villeins $\frac{1}{2}$ hide and $\frac{1}{2}$ plough. There R[ainald] has 3 villeins, 5 bordars, 2 serfs, 2 beasts, 6 swine, 5 acres of coppice, 3 acres of meadow and 5 acres of pasture. Worth 15 shillings, and was worth 20 shillings when the count received it. This also has been united with the honour of Ordulf. fol. $22 x b$.

The count has a manor called Harestana [Hearston] ${ }^{2}$ which Suet held in parage T.R.E., and he could go to what lord he liked. It paid geld for 3 virgates. This 2 ploughs can till. Rainald holds it of the count. Thereof $R$ [ainald] has $\mathbf{I} \frac{1}{2}$ virgates and $I$ plough in demesne, and the villeins $I \frac{1}{2}$ virgates. There $\mathrm{R}$ [ainald] has 3 villeins, 3 serfs, 3 beasts, 35 sheep, wood(land) $\frac{1}{2}$ league in length by $x$ furlong in breadth. Worth Io shillings, and was worth is shillings when the count received it. This has been united with the honour of Ordulf.

The count has a manor called Harestana [Hearston] ${ }^{3}$ which Edric held in parage T.R.E., and he could (go) to what lord he liked. It paid geld for $\frac{1}{2}$ hide. This 2 ploughs can till. Rainald holds it of the count. Thereof [Rainald] has I virgate and $\frac{1}{2}$ plough, and the villeins I virgate and 4 ploughing oxen (boves in carr'). There [Rainald] has 2 villeins, I bordar, 2 beasts, and 2

1 Possibly Ridge on the Tory in Plympton St. Mary in Plympton hundred (Feud. Aids, p. 334) : (O. J. R.) Trans. Devon Assoc. xxxvi, 373 ; or Torr in Newton Ferrers in Ermington hundred. (T.W.W.) Geldroll, fol. $70, \mathrm{~B} 2$, shows Serlo sub-tenant under Godfrey de Valletorta, Reginald's predecessor in title here as well as at Fardle. See Exon. Domesday, fol. $85 b$ and fol. 218.

${ }^{2}$ Hearston alias Hareston alias Areston (Feud. Aids, i, 200) alias Asseton (Ibid. 206) alias Haston (Ibid. 212 ) in Brixton in Plympton hundred. See Trans. Devon Assoc. xxxiv, 293. Geldroll, fol. 70, B 3, shows Adzo sub-tenant.

${ }^{3}$ Higher Hearston alias Hareston in Brixton in Plympton hundred. acres of meadow. Worth ro shillings, and was worth I 5 shillings when the count received it; and it has been united with the honour of Edmeratorius.

The count has a manor called Winestona [Winston] ${ }^{4}$ which Edwin held in parage T.R.E., and he could go to what lord he liked. It paid geld for $\frac{1}{2}$ hide. 'This I $\frac{1}{2}$ ploughs can till. Rainald holds it of the count. Thereof R[ainald] has I virgate and $\frac{1}{2}$ plough, and the villeins I virgate and $\frac{1}{2}$ plough. There $R$ [ainald] has 3 villeins, 2 bordars, I serf, 2 beasts, 20 sheep, and I acre of meadow. Worth 8 shillings, and was worth fol. 222.

20 shillings when the count received it. This has been united with the honour of Edmeratorius.

The count has a manor called EspredeleSTONA ${ }^{5}$ (Exch.Spredelestone) [Spriddlestone] which 4 thanes held in parage (pariter; Exch., libere) T.R.E., and they could go to what lord they liked; each one of them held $\mathrm{r}$ ferling, and it paid geld for I virgate. This 2 ploughs can till. Rainald holds it of the count. Thereof R[ainald] has $\frac{1}{2}$ virgate and $\frac{1}{2}$ plough in demesne, and the villeins $\frac{1}{2}$ virgate and $\frac{1}{2}$ plough. There $R$ [ainald] has 4 villeins, 3 bordars, $I$ acre of meadow and Io acres of pasture. Worth Io shillings, and was worth 20 shillings when the count received it. This has been united with Ordulf's honour.

The count has a manor called Hanenchelola [Honicknowl] $^{6}$ which Wadel (Excb. Wado) held T.R.E., and it paid geld for $\frac{1}{2}$ hide. This 2 ploughs can till. Rainald holds it of the count. There $R$ [ainald] has I virgate in demesne, and the villeins I virgate. There $R$ [ainald] has 2 villeins, 2 serfs, 3 acres of wood(land), I acre of meadow, and pasture 3 furlongs in length by I furlong in breadth. Worth to shillings, and was worth the same when he received it.

The count has a manor called LIsIsTonA [Lipson] $^{7}$ which Gowin (Exch. Godwin) held T.R.E., and it paid geld for $\frac{1}{2}$ hide. This 3 ploughs can till. Rainald holds it of the count. Thereof $\mathrm{R}$ [ainald] has I virgate in demesne, and the villeins I virgate and I plough. There R[ainald] has 4 villeins, 3 bordars, I serf, fol. 222b.

6 beasts, 28 sheep, 30 goats, 6 acres of coppice, $I$ acre of meadow, and I virgate of pasture. Worth 1o shillings, and was worth 20 shillings when the count received it. This Lisistona

- Winston in Brixton in Plympton hundred.

5 Spriddleston and Fen in Brixton in Plympton. Geldroll, fol. 70, B 4, shows Frotmund sub-tenant.

6 Honicknowle in St. Budeaux in Roborough hundred.

${ }^{7}$ Lipson in the parish of St. Charles the Martyr, Plymouth, in Roborough hundred. 


\section{A HISTORY OF DEVONSHIRE}

[Lipson] Godwin held in parage (pariter; Exch., libere); now it has been added to Algar's lands.

The count has a manor called WEDRERIGA (Exch. Wederige) which Otre held T.R.E., and it paid geld for I ferling. This $\frac{1}{2}$ plough can till. Rainald holds it of the count. There $R$ [ainald] has I serf, 2 acres of wood(land) and 6 acres of pasture. It pays 3 shillings a year. The above-named Otre held his land in parage (pariter); now it has been added to the land of Ordulf.

The count has a manor called BirLanda [Beerland $]^{1}$ which Ordulf held T.R.E., and it paid geld for 4 hides. These 15 ploughs can till. Rainald holds it of the count. Thereof $R$ [ainald] has $I$ hide in demesne, and the villeins 3 hides and 6 ploughs. There R [ainald] has I 6 villeins, 5 bordars, 5 serfs, 3 swineherds paying I 5 swine a year, and 7 salterns paying 10 shillings a year. There $\mathrm{R}$ [ainald] has I rouncey, 5 beasts, 3 swine, 30 goats, wood(land) $\mathbf{I} \frac{1}{2}$ leagues in length by I furlong in breadth, and pasture 5 furlongs in length by $I$ furlong in breadth. Worth 100 shillings, and was worth 3 pounds when he received it.

The count of Mortain (de Moritonio) has in ECCECESTRA (Exch.Execestre) [Exeter] i church, ${ }^{2}$ fol. 223.

I house, and a fruit-garden (virgultum) which were in the demesne of King Edward on the day on which he was alive and dead.

\section{LAND OF EARL HUGH IN DEVENESCIRA ${ }^{3}$}

fol. 286 ,

Earl Hugh has a manor called ANEstinga [Anstey] ${ }^{4}$ which Alnod held T.R.E., and it paid geld for $\frac{1}{2}$ hide. This 6 ploughs can till. Thereof the earl has $\frac{1}{2}$ virgate in demesne, and the villeins have $1 \frac{1}{2}$ virgates and 3 ploughs. There the earl has 7 villeins, I bordar, 4 serfs, 48 acres of wood(land), 12 acres of meadow, and pasture $I$ league in length by $\frac{1}{2}$ league in breadth. Worth 20 shillings, and was worth the same when the earl received it.

The earl has another manor called Anestiga [Anstey] $^{5}$ which Norman held T.R.E., and it

${ }^{1}$ The manor of Beerland in Roborough hundred.

2 St. Laurence in Fore Street.

${ }^{3}$ Hugh of Avranches, earl of Chester. In the Exchequer Book Earl Hugh's lands come before those of the count of Mortain.

4 East Anstey alias Anstcy Dinham alias Anstey Crewes (Feud. Aids, i. 325) in South Molton hundred.

${ }^{5}$ In East Anstey, probably Lilliscombe alias Liscombe in South Molton hundred. paid geld for $\frac{1}{2}$ hide. This 6 ploughs can till. On it the earl has 8 villeins, who have 2 ploughs, I bordar, 2 serfs, 40 acres of wood(land), and pasture $\frac{1}{2}$ league in length by 3 furlongs in breadth. Worth 20 shillings, and was worth the same when the earl received it.

Earl Hugh has a manor called Estaforda (Exch. Staford) [Stowford] ${ }^{6}$ which Ednod (Exch. Alnod) held T.R.E., and it paid geld for I hide. This 3 ploughs can till. Worth Io shillings, and was worth the same when the earl received it.

The earl has a manor called LANDEshers ( $E x c b$. Landesherg $)^{7}$ ] ] which Alnod held T.R.E., and it paid geld for $\frac{1}{2}$ virgate. This 2 ploughs can till. Worth 1o shillings, and was worth 3 shillings when the earl received it.

fol. 288.

THE LAND OF BALDWIN THE SHERIFF IN DEVENESIRA [DEVONSHIRE]. ${ }^{8}$

Baldwin the sheriff (vicecomes) has a manor called Ochenemitona [Okehampton] ${ }^{9}$ which Osfers (Exch. Offers) held T.R.E., and it paid geld for 3 virgates and $I$ ferding. This 30 ploughs can till. Thereof $B$ [aldwin] has I virgate and 1 ferding and 4 ploughs in demesne, and the villeins 2 virgates and 20 ploughs. There B[aldwin] has 3 I villeins, I I bordars, I 8 serfs, 6 swineherds, 1 rouncey, 52 beasts, 80 sheep, I mill paying 6 shillings and 8 pence a year, wood(land) 3 leagues in length by $I$ in breadth, 5 acres of meadow, pasture I league in length by $\frac{1}{2}$ league in breadth. On this land stands the castle of Ochenemitona [Okehampton]. There $B$ [aldwin] has 4 burgesses and a market paying 4 shillings a year. This manor is worth with appurtenances ro pounds. When Baldwin received it it was worth 8 pounds.

Baldwin has a manor called Cicecota [Chidecot] ${ }^{10}$ which Brismar held T.R.E., and it paid

6 Stowford in Colyton Raleigh in East Budleigh hundred.

7 Prabably identical with Houndbear, an outlier of Woodbutry in East Budleigh hundred (O. J. R.) Trans. Devon Assoc. xxxiv, 299 ; xxxvi, 362 ; or Hawkerland in Colyton Raleigh (T.W.W.).

${ }^{8}$ The Exchequer Book begins the list of Baldwin's estates with Exeter, followed by Barnstaple. It then pursues the same order as the Exeter Book up to Clysthydon, after which come Middle Marwood and Snedleigh, and then the 4 estates of the canons of Exeter Castle, viz. Ash Clist, Cutton, Hoopern, and West Clist, and the I estate of the monks of Mont St. Michel, Forsham. With Whimple the order in the Exeter Book is resumed, and, omitting the church estates already named, is continued to the end.

'The castle, borough, and manor of Okehampton in Lifton hundred.

${ }^{10}$ Chidacot alias Chichacot in Okehampton in Lifton hundred. 


\section{HOLDERS OF LANDS}

geld for $\frac{1}{2}$ virgate. This 3 ploughs can till. Now Roger holds it of Baldwin. There R[oger] has $I$ plough in demesne, and the villeins till with 2 oxen. There R[oger] has 4 villeins, I bordar, I serf, I 3 beasts, 3 swine, 32 sheep, 30 acres of wood(land), 3 acres of meadow, and 3 acres of pasture. Worth 15 shillings a year, and was worth the same when Baldwin received it.

fol. 288b.

Baldwin has a manor called BRATONA [Bratton ${ }^{1}$ which Bristric ( $E x c h$. Brictric) held T.R.E., and it paid geld for I virgate. This I 5 ploughs can till. Thereof Baldwin has $\frac{1}{3}$ virgate and 3 ploughs in demesne, and the villeins the other $\frac{2}{3}$ and 6 ploughs. There $B$ [aldwin] has 12 villeins, I 2 serfs, 8 forest mares, 18 beasts, 60 sheep, 5 acres of wood(land), 20 of meadow, and 200 of pasture. Worth 7 pounds, and was worth 9 pounds when $B$ [aldwin] received it. Within this manor were 2 thanes in King E[dward]'s time who held $\frac{1}{2}$ virgate of land $^{2}$ in parage (pariter; Exch., libere), and they could go with that land to what lord they liked. This land is worth 30 shillings, and was worth 60 shillings when $B$ [aldwin] received it.

Baldwin has a manor called Bosleia [Bowsleigh] ${ }^{3}$ which Bristric (Exch. Brictric) held T.R.E., and it paid geld for I virgate. This 8 ploughs can till. Now Roff (Exch. Rolf) holds it of Baldwin. Thereof R[off] has I ferding and $I \frac{1}{2}$ ploughs in demesne and the villeins the rest of the land, and they have there I plough. There $\mathrm{R}$ [off] has 7 serfs, I 2 beasts, 40 sheep, I 2 goats, 2 acres of coppice, 60 acres of meadow, and 60 acres of pasture. Worth 40 shillings, and was worth 30 shillings when Baldwin received it.

Baldwin has a manor called BRIDEsTou [Bridestow $]^{4}$ which Edmer held T.R.E., and it paid geld for $\frac{1}{2}$ hide and $\frac{1}{2}$ ferling. This 6 ploughs can till. Now Ralf de Pomaria [Pomeroy] holds it of Baldwin. There $\mathrm{R}$ [alf] has 2 ploughs in demesne and the villeins 4 . There $R$ [alf] has 9 villeins, 4 bordars, 8 serfs, I rouncey, 6 beasts, 10 swine, 135 sheep, 20 goats, 40 acres of wood(land), I 2 acres of meadow, and 30 acres of fol. 289.

pasture. Worth 4 pounds, and was worth 60 shillings when $B$ [aldwin] received it.

Along with this manor Baldwin holds the land of 6 thanes which did not belong to the aforesaid manor T.R.E. In King E[dward]'s

\footnotetext{
${ }^{1}$ Bratton Clovelly alias Bratton Clavil in Lifton hundred.

${ }^{2}$ Perhaps Ellacot in Bratton Clovelly in Lifton hundred.

${ }^{3}$ Bowsleigh alias Boasley in Bratton Clovelly in Lifton hundred.

${ }^{4}$ Bridestow in Lifton hundred.
}

time it paid geld for $\frac{1}{2}$ hide and $1 \frac{1}{2}$ ferlings. This 6 ploughs can till. One of these 6 lands is called Carsforda [Causeyford ${ }^{5}$ which Sawin Topa held. Another is called Batésilla [Battishill ${ }^{6}$ which Dodo held. A third ComBA [Combe] ${ }^{7}$ which Dodo held. A fourth ETBoldus WRDA [Ebbsworthy] ${ }^{8}$ which Godwin held. A fifth FrRneurda [Fernworthy ${ }^{9}$ which Godwin held. And the sixth WEIA [WAY] ${ }^{10}$ which Abbot Suatric held. These thanes could go with that land to any lord they liked. That land is worth 60 shillings all but 20 pence, and was worth 30 shillings when $B$ [aldwin] received it.

Baldwin has a manor called WrcA (Exch. Wiche) [Week $]^{11}$ which Ednod held T.R.E., and it paid geld for $\frac{1}{2}$ virgate. This 5 ploughs can till. Now Rainer holds it of Baldwin. There $\mathrm{R}$ [ainer] has 2 ploughs in demesne and the villeins $I$ plough and 2 oxen. There $\mathrm{R}$ [ainer] has 5 villeins, 8 bordars, 4 serfs, 5 beasts, 3 swine, 48 sheep, 20 acres of coppice, IO acres of meadow, and pasture $\frac{1}{2}$ league in length by 3 furlongs in breadth. Worth 30 shillings, and was worth 40 shillings when $\mathrm{B}$ [aldwin] received it.

Baldwin has a manor called LeuYa (Exch. Lewe) [Lew] ${ }^{12}$ which Bristric (Exch. Brictric) held T.R.E., and it paid geld for $\frac{1}{2}$ hide. This 7 ploughs can till. Now Roger de Molis holds it of Baldwin. There $\mathrm{R}$ [oger] has I plough in fol. $289 \mathrm{~b}$.

demesne and the villeins 6 ploughs. There Roger has 12 villeins, 8 bordars, 6 serfs, 18 beasts, 50 sheep, 30 acres of wood(land), 20 acres of meadow, and 60 acres of pasture. Worth 4 pounds; when $B$ [aldwin] received it it was worth 60 shillings.

Baldwin has a manor called WADELscota [Wadlescot] ${ }^{13}$ which Wadell held T.R.E., and it paid geld for I virgate. This 2 ploughs can till. Now Roger de Molis holds it of Baldwin. The villeins have there I plough. There $\mathrm{R}$ [oger] has 3 villeins, 2 swineherds, I bordar, $I$ acre of wood(land), 5 acres of meadow, and 40 acres of pasture. Worth 20 shillings; when $\mathrm{B}$ [aldwin] received it it was worth 15 shillings. That (Wadel) could go to any lord he liked.

${ }^{5}$ Causeyford in Bridestow in Lifton hundred.

${ }^{6}$ Battishill in Bridestow in Lifton hundred.

7 Combe in Bridestow.

${ }^{8}$ Ebbsworthy in Bridestow.

${ }^{9}$ Fernworthy in Bridestow.

${ }^{10}$ Way in Bridestow.

${ }^{11}$ Week Langford in Germansweek in Lifton hundred, the parish other than South Wick.

${ }^{12}$ Lew Trenchard in Lifton hundred.

${ }^{13}$ Waddlescot alias Waddleston alias Warstrong in Lew Trenchard in Lifton hundred. 


\section{A HISTORY OF DEVONSHIRE}

Baldwin has a manor called Chenleis [Kelly] which Osfers (Exch. Offers) held T.R.E., and it paid geld for $\frac{1}{2}$ hide. This 8 ploughs can till. Now Motbert holds it of B[aldwin]. There $M$ [otbert] has 2 ploughs in demesne and the villeins 5 ploughs. There $M$ [otbert] has 7 villeins, I 5 bordars, Io serfs, II beasts, 8 swine, 80 sheep, wood(land) 2 furlongs in length by $I$ in breadth, 5 acres of meadow, and 8 acres of pasture. Worth 4 pounds; when B[aldwin] received it it was worth 60 shillings.

Baldwin has a manor called DondRITONa [Dunterton] $^{2}$ which Brismer (Exch. Brismar) held T.R.E., and it paid geld for $\frac{1}{2}$ hide. This 6 ploughs can till. Now Ralf de Brueria holds it of $\mathrm{B}$ [aldwin]. There $\mathrm{R}$ [alf] has 2 ferlings and 2 ploughs in demesne, and the villeins $3 \frac{1}{2}$ ploughs. There $R[$ alf] has 4 villeins, 8 bordars, 6 serfs, II beasts, 33 swine, I 00 sheep, 30 goats, 160 (Exch. 60) acres of wood(land), $\mathrm{I}$ acre of meadow, and 40 pasture. Worth 40 shillings; when $B$ [aldwin] received it it was fol. 290 .

worth 20 shillings.

Baldwin has a manor called Godescota [Gus$\cot ^{3}$ which Bristric (Exch. Brictric) held T.R.E., and it paid geld for I virgate. This 4 ploughs can till. Now Colvin holds it of Baldwin. There C[olvin] has I plough in demesne and the villeins 2 ploughs. There $\mathrm{C}$ [olvin] has 6 villeins, 4 bordars, 3 serfs, 30 sheep, and wood(land) 3 furlongs in length by $I$ in breadth. Worth 25 shillings, and was worth 20 shillings when $B$ [aldwin] received it.

Baldwin has a manor called SANForT [Sampford $]^{4}$ which Norman held T.R.E., and it paid geld for $2 \frac{1}{2}$ hides. This 40 ploughs can till. Of these $B$ [aldwin] has I hide and 4 ploughs in demesne, and the villeins $I \frac{1}{2}$ hides and 20 ploughs. There $B$ [aldwin] has 40 villeins, 30 bordars, 8 serfs, 1 rouncey, 28 beasts, 200 sheep, 40 goats, 40 acres of wood(land), 60 acres of meadow, and pasture 2 leagues in length by $I$ in breadth. Worth 12 pounds a year, and was worth 9 pounds when he received it.

Baldwin has a manor called Beilestam [Be]stone ${ }^{5}$ which Osfers held T.R.E., and it was assessed (se defendit) for $\frac{1}{2}$ hide. This 6 ploughs can till. Richard holds it of $\mathrm{B}$ [aldwin]. There $R$ [ichard] has in demesne $\frac{1}{2}$ virgate and 2 ploughs; and the villeins have $1 \frac{1}{2}$ virgates and 3

${ }^{1}$ Kelly in Lifton hundred.

Dunterton in Lifton hundred.

${ }^{3}$ Guscot, an outlier of Bratton Clovelly in Lifton hundred.

"Sampford Courtney in Blacktorington hundred, the parish.

${ }^{5}$ Belstone in Blacktorington hundred. ploughs. There $R$ [ichard] has 8 villeins, 5 bordars, 4 serfs, 4 beasts, 40 sheep, Io goats, $\frac{1}{2}$ league of wood(land), 8 acres of meadow and I league of pasture. Worth 30 shillings a year, and was worth the same when Baldwin received it.

fol. 2906 .

Baldwin has a manor called Doneslanda [Dunsland] ${ }^{6}$ which Oluric (Exch. Uluric) held T.R.E., and it paid geld for $\frac{1}{2}$ virgate. This 4 ploughs can till. Cadio holds it of Baldwin. Thereof he has in demesne I ferding of land and I plough, and the villeins $I$ ferding and 3 ploughs. There Cadio has 6 villeins, 3 bordars, I serf, I 5 beasts, 4 acres of wood(land), 20 acres of meadow, and as many of pasture. Worth 25 shillings a year; when $B$ [aldwin] received it it was worth 30 shillings.

Baldwin has a manor called MonacochamenTONA (Exch. Monuchementone) [Monk-Okehampton] ${ }^{7}$ which Olnot (Exch. Ulnod) held T.R.E., and it paid geld for $\frac{1}{2}$ hide. This 6 ploughs can till. There $\mathrm{B}$ [aldwin] has in demesne $x \frac{1}{2}$ ferdings and 2 ploughs, and the villeins have $6 \frac{1}{2}$ ferdings and 8 ploughs. There $B$ [aldwin] has I 2 villeins, 6 bordars, 5 serfs, I rouncey, 18 beasts, 60 sheep, 20 acres of wood(land), 12 acres of meadow and IO acres of pasture. Worth 3 pounds a year; when B[aldwin] received it it was worth 20 shillings.

Baldwin has a manor called ETcheborna (Exch. Echeburne) [Exbourne ${ }^{8}$ which Almer (Exch. Elmer) held T.R.E., and it paid geld for $\frac{1}{2}$ hide. This 9 ploughs can till. Roger holds it of $\mathrm{B}$ [aldwin]. There Roger has $\frac{1}{2}$ virgate of land and 2 ploughs and the villeins have $I \frac{1}{2}$ virgates and 5 ploughs. There Roger has ro villeins, 5 bordars, 13 serfs, I 8 beasts, 3 swine, 60 sheep, wood(land) 3 furlongs in length by 2 furlongs in breadth, and 20 acres of meadow. fol. 29 r.

Worth 4 pounds a year; when he received it it was worth 3 pounds.

Baldwin has a manor called Hantona (Exch. Hanitone) [Hampton] ${ }^{9}$ which Brismar held T.R.E., and it paid geld for 3 virgates. This ro ploughs can till. Roger holds it of $B$ [aldwin]. There Roger has in demesne $I$ virgate and 2 ploughs, and the villeins have 2 virgates and 7 ploughs. There Roger has I9 villeins, 3 bordars, 9 serfs, I swineherd, 1 rouncey, 17 beasts, 50 sheep, wood(land) $\frac{1}{2}$ league in length by the same in breadth, 60 acres of meadow, and 4

\footnotetext{
${ }^{6}$ Dunsland in Bradford Dabernon in Blacktorington hundred.

${ }^{7}$ Monk-Okehampton in Blacktorington hundred.

${ }^{8}$ Exbourne in Blacktorington hundred.

${ }^{9}$ Highampton in Blacktorington hundred.
} 


\section{HOLDERS OF LANDS}

furlongs of pasture. Worth 3 pounds a year ; when Baldwin received it it was worth the same.

Baldwin has a manor called LACHEBRoc ${ }^{1}(E x c h$. Lochebroc) [Lashbrook] which Algar the tall (longus) held T.R.E., and it paid geld for I virgate. This 8 ploughs can till. Roger holds it of $B$ [aldwin]. There Roger has in demesne $I \frac{1}{2}$ ferdings and 2 ploughs, and the villeins have $2 \frac{1}{2}$ ferdings and 3 ploughs. There Roger has Io villeins, 6 bordars, IO serfs, 17 beasts, 45 sheep, 2 I goats, 15 acres of wood(land), 50 acres of pasture and 50 acres of meadow. Worth 50 shillings a year; when Baldwin received it it was worth the same.

Baldwin has a manor called BRADEFORT ${ }^{2}$ (Exch. Bradeford) [Bradford] which Algar the tall held T.R.E. and it paid geld for $\frac{1}{2}$ hide. This 6 ploughs can till. There B[aldwin] has in demesne $\frac{1}{2}$ virgate and I plough, and the villeins have $I \frac{1}{2}$ virgates and 2 ploughs. There $\mathrm{B}$ [aldwin] has 5 villeins, 2 bordars, 6 serfs, 9 beasts, 6 swine, 50 sheep, 16 goats, 60 acres of fol agrb.

wood(land), 12 acres of meadow, and 30 acres of pasture. Worth 40 shillings a year; when he received it it was worth 20 shillings.

Baldwin has a manor called CAcheberga [Keckbear $^{3}$ which Sewin (Exch. Sawin) held T.R.E., and it paid geld for I virgate. This 5 ploughs can till. Raner ( $E x c h$. Rainer) holds it of Baldwin and has in demesne $I$ ferding and $I$ plough, and the villeins have 3 ferdings and 3 ploughs. There Raner has 6 villeins, 5 bordars, I serf, 5 beasts, 30 sheep, 3 swine, 2 acres of wood(land), 12 acres of meadow, and 20 acres of pasture. Worth 30 shillings a year, and was worth 20 shillings when he received it.

Baldwin has a manor called LEGA [Leigh] ${ }^{4}$ which Inwar ( $E x c h$. Inewar) held T.R.E., and it paid geld for I hide. This Io ploughs can till. Now Otelin (Exch. Othelin) holds it of Baldwin. Thereof $O$ [telin] has $\frac{1}{2}$ virgate and I plough in demesne; and the villeins $3 \frac{1}{2}$ virgates and 9 ploughs. There $\mathrm{O}$ [telin] has I 2 villeins, 8 bordars, 6 serfs, I swineherd, 7 beasts, 2 swine, 40 sheep, Io goats, 60 acres of wood(land), 30 acres of meadow, and 50 acres of pasture. Worth 3 pounds 10 shillings; when he received it the same.

Baldwin has a manor called AснA [Oak] ${ }^{5}$ which Ansgot (Exch. Osgot) held T.R.E., and it

${ }^{1}$ Lashbrook Mules in Bradford Dabernon in Blacktorington hundred (O.J.R.).

${ }^{2}$ Bradford ${ }^{\natural}$ Dabermon in Blacktorington hundred.

3 Keckbear alias $\mathrm{X}$ igbear in Okehampton in Blacktorington hundred.

" Inwardleigh'in Blacktorington hundred.

- Oak in Invardleigh in Blacktorington hundred. I 449 paid geld for 3 ferdings. This 5 ploughs can till. Now Richard holds it of Baldwin. There $\mathrm{R}$ [ichard] has in demesne I ferding and I plough, and the villeins 2 ferdings and 2 ploughs. fol. 292.

There R[ichard] has 6 villeins, 3 bordars, I serf, 8 beasts, 8 sheep, 8 acres of wood(land), 20 acres of meadow, and 60 acres of pasture. It pays 40 shillings a year; when he received it 30 shillings.

Baldwin has a manor called GoHewis [Gorhuish] ${ }^{6}$ which Alnot (Exch. Alnod) held T.R.E., and it paid geld for $I$ ferding. This I plough can till. Bernard holds it of $B$ [aldwin]. His villeins have that ferding of land and $\frac{1}{2}$ plough. There Bernard has 2 villeins and I bordar, also 22 beasts, I I sheep, 7 goats, 2 acres of coppice, 6 acres of meadow, and 40 acres of pasture. Worth 5 shillings a year.

Baldwin has a manor called BradeHoda [Bradwood] ${ }^{7}$ which Leuric held T.R.E., and it paid geld for I virgate of land. This 4 ploughs can till. Now Motbert (Exch. Modbert) holds it of B[aldwin]. There Motbert has I ferding of land and $\mathrm{I}$ plough, and the villeins 3 ferdings and $I \frac{1}{2}$ ploughs. On it Motbert has 3 villeins, 5 bordars, 2 serfs, I 3 beasts, 14 swine, 50 sheep, wood(land) $\frac{1}{2}$ league in length by 2 furlongs in breadth, 8 acres of meadow, and $\frac{1}{2}$ league of pasture. Worth 20 shillings a year, and was worth the same when he received it.

Baldwin has a manor called HoNechercha (Exch. Honecherde) [Honeychurch ${ }^{8}$ which Alwin Black (Niger) held T.R.E., and it paid geld for $\frac{1}{2}$ hide. This 5 ploughs can till. Now Walter holds it of B[aldwin]. On it Walter fol. 2gab.

has in demesne I virgate of land and 2 ploughs, and the villeins I virgate and I plough. There Walter has 4 villeins, 4 serfs, 30 beasts, 8 swine, 42 sheep, 19 goats, and 2 acres of coppice, 4 acres of meadow, and Io acres of pasture. Worth 30 shillings a year, and was worth 15 shillings when he received it.

Baldwin has a manor called Mizdelcota [Middlecot] $^{9}$ which Alwold held T.R.E., and it paid geld for I virgate. This 2 ploughs can till. Rannulf holds it of B[aldwin]. There Rannulf has in demesne I ferding and I plough,

${ }^{6}$ Gorhuish in North Lew in Blacktorington hundred.

'Bradwood alias Broadwood Kelly in Blacktorington hundred.

${ }^{8}$ Honeychurch in Blacktorington hundred.

${ }^{9}$ Middlecot in Bradwood Kelly (T.W.W.); in Ashwater (O.J.R.) in Blacktorington hundred. There is a ferding missing unless 3 ferdings for 2 ought to be read as the villeins' assessment. 


\section{A HISTORY OF DEVONSHIRE}

and the villeins 2 ferdings and $I$ plough. On it Rannulf has 2 villeins, 4 bordars, I serf, and 40 sheep. Worth 20 shillings a year; when he received it 10 shillings.

Baldwin has a manor called Bristanestona

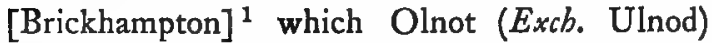
held T.R.E., and it paid geld for I virgate of land. This 4 ploughs can till. Richard holds it of $\mathrm{B}$ [aldwin]. There Richard has in demesne 2 ferdings and $\mathrm{I} \frac{1}{2}$ ploughs, and the villeins 2 ferdings and I plough. On it Richard has 4 villeins, 3 bordars, 2 serfs, 14 beasts, I I sheep, wood (land), 6 furlongs in length by $I$ in breadth, 15 acres of meadow, and 10 acres of pasture. Worth 20 shillings a year; when he received it it was worth $12 \frac{1}{2}$ shillings.

Baldwin has a manor called Mildelcota [Middlecot] ${ }^{2}$ which Alwod (Exch. Alwold) held T.R.E., and it paid geld for $\frac{1}{2}$ virgate. This 2 ploughs can till. Richard holds it of $\mathrm{B}$ [aldwin]. There Richard has in demesne $I$ ferding and fol. 293.

I plough, and the villeins I ferding and $\frac{1}{2}$ plough. On this (land) Richard has 2 villeins, I bordar, I serf, 6 beasts, wood(land) 4 furlongs in length by one in width, ro acres of meadow, and I 5 acres of pasture. Worth 20 shillings a year; when he received it to shillings.

Baldwin has a manor called ESSEMUNDEHORDA [Ashmansworthy $]^{3}$ which Brismar held T.R.E., and it paid geld for $\frac{1}{2}$ hide of land. This 5 ploughs can till. Gislebert holds it of B[aldwin]. On it G[islebert] has in demesne 2 ferdings and I plough, and the villeins I virgate, 2 ferdings, and 3 ploughs. There G[islebert] has 6 villeins, 4 bordars, 4 serfs, I forest mare, 25 beasts, Ioo sheep, 60 acres of meadow, and pasture I league in length by $\frac{1}{2}$ league in breadth. Worth 30 shillings a year; when he received it ro shillings.

Baldwin has a manor called Hernescoma [Yarnscombe] ${ }^{4}$ which Godvin held T.R.E., and it paid geld for $\frac{1}{2}$ hide. This 10 ploughs can till. Robert holds it of B[aldwin]. There Robert has in demesne 2 ferdings and I plough,

\footnotetext{
1 Brickhampton alias Brixton in Bradwood Kelly in Blacktorington hundred.

2 Possibly identical with Corbynston alias Corston in Bradwood Kelly in Blacktorington hundred. Richard de Noville held ' Bristanestona,' 'Midelcota,' and ' Mameorde.' Inq. p.m. I Rich. II, No. 12 , shows 'Brighteneston, Corpyston, and Wemworth' were held together.

${ }^{3}$ Ashmansworthy in East Woolfardisworthy in Hartland hundred.

"Little Yarnscombe in Yarnscombe in Hartland hundred. The shortage of I virgate may be Coxleigh in Shirwell hundred, not otherwise accounted for in Domesday. See Feud. Aids, i, 416 (O.J.R.) ; or Much Yarnscombe (T.W.W.).
}

and the villeins 2 ferdings and $\frac{1}{2}$ (a plough). On it Robert has 3 villeins, I serf, 5 swine, 28 sheep, Io goats, 100 acres of wood(land), Io of meadow, and I league of pasture. Worth 20 shillings a year, and was worth 40 shillings when he received it.

Baldwin has a manor called Perchemam

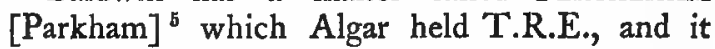
paid geld for 2 hides. This ro ploughs can till. Richard holds it of B[aldwin]. Here R[ichard] has in demesne 1 virgate of land and 2 ploughs; and the villeins 7 virgates and 5 ploughs. Upon it R[ichard] has 9 villeins, 3 bordars, 4 serfs, fol. 293b.

4 beasts, 5 swine, 60 sheep, $\frac{1}{2}$ league of wood(land), ro acres of meadow, pasture 3 furlongs in length by $I$ in width. Worth 40 shillings a year; when he received it it was worth 30 shillings.

To the aforesaid manor called Percheham was appurtenant SetLeberga ( $E x c h$. Seteberge) [Sedborough] which Brismar held T.R.E. It paid geld for I virgate. ${ }^{6}$

Baldwin has a manor called ToRintona [Torington] ${ }^{7}$ which Etmar ( $E_{x c h}$. Edmar) held T.R.E., and it paid geld for I hide and I virgate. This 8 ploughs can till. Richard holds it of $B$ [aldwin]. There R[ichard] has in demesne I virgate and 2 ploughs, and the villeins 3 virgates and 5 ploughs. There R[ichard] has 6 villeins, 5 bordars, 5 serfs, 16 beasts, 50 sheep, I 5 goats, $\frac{1}{2}$ league of wood(land), 8 acres of meadow, and I league of pasture. Worth 40 shillings a year; when he received it it was worth 20 shillings.

Baldwin has a manor called Hantona [Heanton $]^{8}$ which Edwin held T.R.E., and it paid geld for $\frac{1}{2}$ hide, $\frac{1}{2}$ virgate, and I ferding. This 5 ploughs can till. Ralf de Brueria holds it of $\mathrm{B}$ [aldwin]. There $\mathrm{R}$ [alf] has in demesne I virgate and I plough, and the villeins $I$ virgate, 3 ferdings, and 3 ploughs. On it R[alf] has 5 villeins, 4 bordars, I serf, 8 beasts, I 3 swine, 20 sheep, 2 acres of coppice, 10 of meadow, and 30 of pasture. Worth 20 shillings a year, and was worth 10 shillings when he received it.

${ }^{5}$ Parkham in Shebbear hundred.

${ }^{6}$ Sedborough in Parkham in Shebbear hundred, described below, fol. 482 .

${ }^{7} \mathrm{~A}$ vill on the Torridge in Shebbear hundred, probably Little Torington. Probably the virgate unaccounted for represents Frizenham. Both 'Torintona' and 'Friseham' were held before the Conquest by Edmeratorius (O.J.R.), Trans. Devon Assoc. xxvii, 193.

${ }^{8}$ Heanton Satchvil in Petrockstow in Shebbear hundred. 


\section{HOLDERS OF LANDS}

Baldwin has a manor called Porriga [Potheridge ${ }^{1}$ which Olf (Exch. Ulf) held T.R.E., and it paid geld for 2 virgates all but $\frac{1}{2}$ ferding. This 7 ploughs can till. Alberid (Exch. Alberi) holds it of $\mathrm{B}$ [aldwin]. There A[lberid] has in demesne 3 ferdings and 2 ploughs, and the villeins $\frac{1}{2}$ ferding, I virgate, and 2 ploughs. There $A$ [lberid] has 6 villeins, 5 bordars, 3 serfs, I cottager (quat[arium]), 5 beasts, 9 swine, 30 sheep, I 5 goats, wood(land) 5 furlongs in length by 2 in breadth, 3 acres of meadow and 4 furfol. 294 .

longs of pasture. Worth 40 shillings, and when he received it the like (similiter).

Baldwin has a manor called Estochelia (Exch. Stochelie) [Stockleigh] ${ }^{2}$ which Colvin held T.R.E., and it paid geld for I virgate. This 3 ploughs can till. Now Alberid (Exch. Alberic) holds it of B[aldwin]. There A[1berid] has in demesne $\frac{1}{2}$ virgate and I plough and the villeins $\frac{1}{2}$ virgate and $\frac{1}{2}$ plough. On it A [lberid] has 3 villeins, 3 bordars, I serf, I beast, 5 swine, 20 sheep, 2 acres of wood (land), 9 acres of meadow, and of common pasture there is enough there (satis ibi babetur). Worth 15 shillings a year.

Baldwin has a manor called Oladona [Wooladon] ${ }^{3}$ which Sewin held T.R.E., and it paid geld for I virgate. This $I_{\frac{1}{2}}$ ploughs can till. Now Alberid (Exch. Alberi) holds it of B[aldwin]. There $A$ [lberid] has I villein, 2 bordars, I serf, and 9 acres of meadow. Worth 5 shillings a year.

Baldwin has a manor called MEDA [Meeth] ${ }^{4}$ which Alnot (Exch. Alnod) held T.R.E., and it paid geld for $\frac{1}{2}$ hide. This 4 ploughs can till. Bernard holds it of $B$ [aldwin]. There $B$ [ernard] has in demesne I virgate and I plough, and the villeins I virgate and I plough. On it $\mathrm{B}$ [ernard] has 2 villeins, 3 bordars, 13 beasts, 12 swine, 22 sheep, 18 goats, 2 furlongs of coppice, 4 acres of meadow and common pasture. Worth 20 shillings; when he received it Io shillings.

Baldwin has a manor called LANcHERs [Lancross] $^{5}$ which Alveva held T.R.E., and it paid geld for I virgate. This 3 ploughs can till. Rotbert holds it of $\mathrm{B}$ [aldwin]. There R[obert] has in demesne $\frac{1}{2}$ virgate and I plough, and the fol. 294b.

villeins $\frac{1}{2}$ virgate and $\frac{1}{2}$ plough. On it $\mathrm{R}$ [obert] has 3 villeins, 2 serfs, 5 beasts, 3 swine, 70 sheep,

${ }^{1}$ Great and Little Potheridge in Merton in Shebbear hundred.

Stockleigh Barton alias Stockleigh Dabernon and Slew Park in Meeth in Shebbear hundred.

${ }^{3}$ Woolladon alias Welton in Petrockstow and Meeth in Shebbear hundred.

- Meeth in Shebbear hundred.

s Lancross in Shebbear hundred.
30 goats, 20 acres of wood(land), 3 of meadow and 20 of pasture. Worth 20 shillings a year; when he received it 30 shillings.

Baldwin has a manor called Olublia (Exch. Ulvelie) [Woolley] ${ }^{6}$ which Alsi held T.R.E., and it paid geld for I virgate. This 4 ploughs can till. Colvin holds it of $\mathrm{B}$ [aldwin]. There Colvin has in demesne I ferding and I plough, and the villeins have 3 ferdings and 3 ploughs. On it Colvin has 3 villeins, 3 bordars, I serf, 5 acres of coppice, and 1 of meadow. Worth I 5 shillings a year; when he received it ro shillings.

Baldwin has a manor called Helescaue [Hele Poure [] ${ }^{7}$ which Hedric (Exch. Edric) held T.R.E., and it paid geld for I virgate. This 8 ploughs can till. There William who holds it of $B$ [aldwin] has in demesne $\frac{1}{2}$ virgate and $I$ plough; and the villeins $\frac{1}{2}$ virgate and 2 ploughs. Upon it W[illiam] has 4 villeins, 4 bordars, 3 acres of wood(land), 2 acres of meadow and 6 of pasture. Worth 40 shillings a year; when he received it it was worth 30 shillings.

Baldwin has a manor called Calveleta [Chawleigh $]^{8}$ which Sedward (Exch. Siward) held T.R.E., and it paid geld for 3 hides. These 30 ploughs can till. Thereof $\mathrm{B}$ [aldwin] has in demesne $I$ hide and 6 ploughs, and the villeins 2 hides and 10 ploughs. There $\mathrm{B}$ [aldwin] has 30 villeins, 6 bordars, 12 serfs, 3 swineherds paying 15 swine a year, 20 beasts, 10 swine, 200 sheep, 20 acres of wood(land), Io acres of meadow, and pasture I league in length by fol. 295.

$\frac{1}{2}$ league in breadth. Worth $\mathrm{I} 2$ pounds a year; when $B$ [aldwin] received it it was worth Io pounds.

Baldwin has a manor called Dueltona (Exch. Oueltone) [Dolton] ${ }^{8}$ which Olf ( $E_{x c h}$. Ulf) held T.R.E., and it paid geld for I hide. This 18 ploughs can till. Baldwin gave it to William son of Wimund with his daughter in marriage. Thereof W[illiam] has in demesne I virgate and 6 ploughs, and the villeins 3 virgates and 12 ploughs. There $W$ [illiam] has 19 villeins, 9 bordars, 2 swineherds paying 10 swine a year, 7 serfs, 30 beasts, 8 swine, 82 sheep, 25 goats, 150 acres of wood(land), Io acres of meadow and 50 acres of pasture. Worth 8 pounds a year, and was worth the same when $\mathrm{B}$ [aldwin] received it.

'Woolley in Beaford in Shebbear hundred

'Hele Poure alias Crocker's, Blinch's and Frye's alias Giffard's Hele in Meeth in Shebbear hundred (O.J.R.), Trans. Devon Assoc. xxix, 264; xxxvi, 353 ; or Huntshaw Wood (T.W.W.), formerly 'Henshaw, held of Okehampton (Feud. Aids, 358) are suggested.

${ }^{8}$ Chawleigh in North Tawton hundred.

${ }^{9}$ Dolton in North Tawton hundred. 


\section{A HISTORY OF DEVONSHIRE}

Baldwin has a manor called LIMET [Nymet or Intake] ${ }^{1}$ which Ailric held T.R.E., and it paid geld for $\frac{1}{2}$ hide. This 6 ploughs can till. Walter holds it of $\mathrm{B}$ [aldwin]. There $\mathrm{W}$ [alter] has in demesne $\frac{1}{2}$ virgate and I plough, and the villeins I virgate, 2 ferdings, and 3 ploughs. There $\mathrm{W}$ [alter] has 4 villeins, 5 bordars, 2 serfs, 2 swineherds paying ro swine, 5 beasts, 20 sheep, I mill paying 20 pence a year, 5 acres of wood(land), 4 acres of meadow and 3 acres of pasture. Worth 30 shillings a year ; when $B$ [aldwin] received it it was worth 20 shillings.

Baldwin has a manor called LIEGA [Leigh] ${ }^{2}$ which Ailric held T.R.E. and it paid geld for 3 virgates. These 6 ploughs can till. Walter holds it of $B$ [aldwin]. Thereof $W$ [alter] has in demesne I virgate and 2 ploughs, and the villeins 2 virgates and 4 ploughs. There $W$ [alter] has 5 villeins, 5 bordars, 3 serfs, 2 swineherds paying I 5 swine, 4 beasts, 27 sheep, 3 acres of wood (land), 5 acres of meadow and 5 acres of pasture. Worth 40 shillings a year; when $\mathrm{B}$ [aldwin] received it 30 shillings.

Baldwin has a manor called Bera [Bear] ${ }^{3}$ which Ailnod (Exch.Alnod) held T.R.E., and it paid sol. 295b.

geld for I virgate and $\frac{1}{2}$ ferding. This 4 ploughs can till. Walter holds it of $B$ [aldwin]. Thereof W [alter] has in demesne $\frac{1}{2}$ ferding and I plough; and the villeins $I$ virgate and 2 ploughs. There W[alter] has 3 villeins, 3 bordars, I serf, 10 beasts, 26 sheep, 27 goats, 4 acres of wood (land), 4 acres of meadow and 10 acres of pasture. Worth 20 shillings a year; when Baldwin received it it was worth ro shillings.

Baldwin has a manor called Limet [Nymet or Intake] ${ }^{4}$ which Walo (Exch. Wado) held T.R.E., and it paid geld for 3 virgates. These 6 ploughs can till. Ralf de Brueria holds them of $\mathrm{B}$ [aldwin]. There $\mathrm{R}$ [alf] has in demesne I virgate and I plough, and the villeins 2 virgates and $3 \frac{1}{2}$ ploughs. There $R$ [alf] has 5 villeins, 7 bordars, 5 serfs, 5 beasts, 30 sheep, 10 acres of meadow and 50 acres of pasture. Worth 25 shillings a year; when $\mathrm{B}$ [aldwin] received it 20 shillings.

Baldwin has a manor called Apledoa [Appledore $]^{5}$ which Lievenot (Exch. Levenot) held

\footnotetext{
1 Nymet Rowland in North Tawton hundred.

3 Rowland's Leigh alias Rashley in Wembworthy in North Tawton hundred (O.J.R.); or Leigh in Coldridge (T.W.W.).

${ }^{3}$ Cherrybear in Dolton in North Tawton hundred (O.J.R.). Inq. p.m. 19 Hen. VI, No. 40.

- Broad Nymet in Bow in North Tawton hundred.

- Apledore an outlier of Bow in North Tawton hundred.
}

T.R.E., and it paid geld for I virgate. This I $\frac{1}{2}$ ploughs can till. Ralf de Brueria holds it of $\mathrm{B}$ [aldwin]. Thereof $\mathrm{R}$ [alf] has $\frac{1}{2}$ virgate and 5 oxen in demesne, and the villeins $\frac{1}{2}$ virgate and I plough. There $R$ [alf] has 2 villeins, I bordar, I serf, 3 beasts, 25 sheep, and 2 acres of meadow. Worth ro shillings a year; when $\mathrm{B}$ [aldwin] received it 5 shillings.

Baldwin has a manor called $\mathrm{HAX}_{\mathrm{AX}}$ [Halse] ${ }^{6}$ which Aimar (Exch. Almaer) held T.R.E., and it paid geld for I virgate. This 2 ploughs can till. Motbert son of Lambert holds it of B[aldwin]. Thereof $M$ [otbert] has in demesne 3 ferdings and I plough, and the villeins I ferding. There $M$ [otbert] has 2 bordars, 2 serfs, 1 beast, 20 sheep, an acre of meadow and 40 acres of pasture. Worth 13 shillings a year; when $B$ [aldwin] received it ro shillings.

Baldwin has a (manor) called Cloenesberga

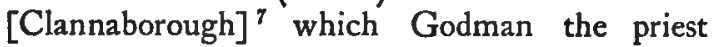
held T.R.E., and it paid geld for 3 virgates. These 6 ploughs can till. Ralf de Pomeria holds them of $B$ [aldwin]. Thereof $R$ [alf] has fol. 296

in demesne 2 virgates and 2 ploughs, and the villeins I virgate and $\frac{1}{2}$ plough. There $R$ [alf] has 2 villeins, 5 bordars, 3 serfs, 8 beasts, 60 sheep, I 5 acres of wood(land), 5 acres of meadow, and 4 of pasture. Worth 25 shillings; it was worth the same when $B$ [aldwin] received it.

Baldwin has a manor ralled Limer [Nymet or Intake] ${ }^{8}$ which Walo $\left(E_{x c h}\right.$. Wado) held T.R.E., and it paid geld for I virgate. This 4 ploughs can till. Ralf de Pomeria holds it of B[aldwin]. Thereof $\mathrm{R}$ [alf] has in demesne $\frac{1}{2}$ virgate and $I \frac{1}{2}$ ploughs, and the villeins $\frac{1}{2}$ virgate and $I \frac{1}{2}$ ploughs. There $R$ [alf] has 4 villeins, I bordar, 2 serfs, I rouncey, 6 beasts, 34 sheep, 12 acres of wood(land), 4 acres of meadow and 12 of pasture. Worth 25 shillings a year; when $\mathrm{B}$ [aldwin] received it $\mathrm{I} 5$ shillings.

Baldwin has a manor called BRIGEForda

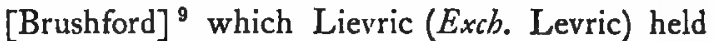
T.R.E., and it paid geld for $\frac{1}{2}$ hide. This 3 ploughs can till. Godefrid the Chamberlain holds it of B[aldwin]. Thereof G[odfrey] has in demesne I virgate and $\frac{1}{2}$ plough, and the villeins 1 virgate and $\frac{1}{2}$ plough. There G[odfrey] has 4 villeins, 4 serfs, 2 beasts, 4 swine, 8 acres of wood(land), 4 acres of meadow and I 2 acres of pasture. Worth 20 shillings a year; when $\mathrm{B}$ [aldwin] received it it was worth 7 shillings and 6 pence.

${ }^{6}$ Halse in Bow in North Tawton hundred.

${ }^{7}$ Clannaborough in North Tawton hundred.

8 The intake called Walson and Thorn in Clannaborough in North Tawton hundred.

${ }^{9}$ East Brushford alias Church Brushford in Brushford Speke in North Tawton hundred. 


\section{HOLDERS OF LANDS}

Baldwin has a manor called BRIGeForda [Brushford] ${ }^{1}$ which Lievric (Exch. Leuric) held T.R.E., and it paid geld for $\frac{1}{2}$ hide. This 3 ploughs can till. Godefric the chamberlain holds it of B[aldwin]. Thereof G[odfrey] has fol. $296 \mathrm{~b}$.

in demesne I virgate and $\frac{1}{2}$ plough, and the villeins I virgate and $\frac{1}{2}$ plough. There G[odfrey] has I villein, 4 bordars, I beast, 8 acres of wood(land), 3 acres of meadow, and 6 acres of pasture. Worth Io shillings a year; when $B$ [aldwin] received it 7 shillings and 6 pence.

Baldwin has a manor called Limet [Nymet or Intake] ${ }^{2}$ which Osfers held T.R.E., and it paid geld for I hide. This 4 ploughs can till. Otley (Otelinus; Exch.,Otbelinus) holds it of B[aldwin]. Thereof $\mathrm{O}$ [tley] has in demesne I virgate and 2 ploughs, and the villeins 3 virgates and 4 ploughs. There $\mathrm{O}$ [tley] has 6 villeins, 5 bordars, 3 serfs, 5 beasts, 20 sheep, 6 acres of wood(land), and 8 acres of meadow. It is worth 40 shillings a year; when $B$ [aldwin] received it it was worth the same.

Baldwin has a manor called GHERnesLeta [Greenslade] $^{3}$ which Walo (Exch. Wado) held T.R.E., and it paid geld for $\frac{1}{2}$ virgate. This 3 ploughs can till. Rainer the server (dapifer) holds it of $B$ [aldwin]. Thereof $R$ [ainer] has in demesne $\frac{1}{2}$ ferding and $I$ plough, and the villeins $\frac{1}{2}$ ferding and $\frac{1}{2}$ plough. There $R$ [ainer] has 2 villeins, 2 bordars, 2 beasts, 2 swine, 20 sheep, I acre of meadow, and Io acres of pasture. This part is worth 10 shillings a year, and was worth 7 shillings and 6 pence when $\mathrm{B}$ [aldwin] received it. A moiety of the aforesaid $\frac{1}{2}$ virgate has been added to the king's demesne manor called Tauetona [North Tawton].

Baldwin has a manor called MAMEorda [Wembworthy] $^{4}$ which Lieuric held T.R.E., and it paid geld for I hide. This I 2 ploughs can till. Richard de Novilla holds it of B[aldwin]. Thereof $R$ [ichard] has in demesne 2 virsol. 297.

gates and $2 \frac{1}{2}$ ploughs, and the villeins 2 virgates and $\mathrm{I} \frac{1}{2}$ ploughs. There $R$ [ichard] has 5 villeins, 5 bordars, 3 serfs, 12 beasts, 16 swine, 25 sheep, 30 goats, wood(land) $\frac{1}{2}$ league in length by $\frac{1}{2}$ (a league) in breadth, and 10 acres of meadow. Worth 40 shillings a year; when B[aldwin] received it it was worth 20 shillings.

\footnotetext{
1 West Brushford in Brushford Speke in North Tawton hundred.

${ }^{2}$ An intake representing Great Burston alias Bordevileston in Zeal Monachorum, Eggesford, Partridge Wall, and Hayes in North Tawton hundred, all held by Hidon, successor in title to Otley.

${ }^{3}$ Greenslade in North Tawton parish and hundred.

+ Wembworthy in North Tawton hundred.
}

Baldwin has a manor called Chent [Kenn] ${ }^{5}$ which Brismer (Exch. Brismar) held T.R.E., and it paid geld for 6 hides. These 25 ploughs can till. Thereof $B$ [aldwin] has 3 hides and the villeins 3 hides and 25 ploughs. There $B$ [aldwin] has among villeins and bordars 42 in all, 20 serfs, 5 swineherds paying 44 swine, $\mathrm{I}$ rouncey, 5 beasts, 150 sheep, I mill paying 30 pence, Io acres of wood(land), 30 acres of meadow and I 00 of pasture. This manor is worth Io pounds ; when $B$ [aldwin] received it it was worth 12 pounds. To this manor are appurtenant I I burgesses who are settled in Exeter and pay 4 shillings and 5 pence. They are included in the above-given value.

Baldwin has a manor called Tergna [Teign] ${ }^{6}$ which Ustret (Exch. Uctred) held T.R.E., and it paid geld for 3 virgates. These 4 ploughs can till. Now Roger de Molis holds it of Baldwin. Thereof $R$ [oger] has $\frac{1}{2}$ virgate and $I$ plough in demesne, and the villeins $2 \frac{1}{2}$ virgates and 3 ploughs. There R[oger] has 4 villeins, 8 bordars, 3 serfs, 4 beasts, 1 pig, 40 sheep, wood (land) $4 \frac{1}{2}$ furlongs in length by $2 \frac{1}{2}$ in breadth, 3 acres of meadow, pasture 5 furlongs in length by 2 in breadth. Worth 20 shillings; when $\mathrm{B}$ [aldwin] received it it was worth the same.

fol. $297 \mathrm{~b}$.

Baldwin has a manor called BEGATORA [Beetor] which Erdulf (Exch. Eddulf) held T.R.E., and it paid geld for I virgate. This 4 ploughs can till. Anger (Exch. Ansger) holds it of B[aldwin]. Thereof $A$ [nsger] has I ferding and I plough in demesne, and the villeins 3 ferdings and 2 ploughs. There $A$ [nsger] has 4 villeins, I bordar, I serf, and 8 acres of meadow. It pays 25 shillings; when $B$ [aldwin] received it it was worth 20 shillings.

Baldwin has a manor called EscapeleIA (Exch. Scapelie) [Shapleigh $]^{8}$ which Aret held T.R.E., and it paid geld for I virgate. This 2 ploughs can till. Rotbert holds it of Baldwin. Thereof $\mathrm{R}$ [obert] has $\frac{1}{2}$ virgate and I plough in demesne, and the villeins $\frac{1}{2}$ virgate and I plough. There R[obert] has 4 villeins, $\mathrm{I}$ serf, 2 acres of wood(land), and 5 acres of meadow. Worth 12 shillings and 6 pence; when B[aldwin] received it it was worth the same.

Baldwin has a manor called EscapeIEIA (Exch. Scapelie) [Shapleigh] ${ }^{9}$ which Edwy held T.R.E., and it paid geld for $\frac{1}{2}$ hide. This

${ }^{5}$ Kenn in Exminster hundred.

${ }^{6}$ George Teign in Ashton in Exminster hundred.

7 Beetor in North Bovey in Exminster hundred.

${ }^{8}$ Lower Shapleigh in Chagford in Exminster hundred.

${ }^{9}$ Higher Shapleigh in Chagford in Exminster hundred. This and the preceding manor together are called Shapleigh Helion (O.J.R.). 


\section{A HISTORY OF DEVONSHIRE}

3 ploughs can till. Rotbert holds it of Baldwin. Thereof R[obert] has I virgate in demesne and the villeins I virgate. There R[obert] has 5 acres of meadow. Worth 7 shillings and 6 pence; when $B$ [aldwin] received it it was worth the same.

Baldwin has a manor called Mammenetua (Excb. Mammeheue) [Mamhead] ${ }^{1}$ which Algar held T.R.E., and it paid geld for 3 virgates. These 5 ploughs can till. Ralf de Pomeria holds it of Baldwin. Thereof $\mathrm{R}$ [alf] has $\mathrm{I} \frac{1}{2}$ virgates and 2 ploughs in demesne, and the villeins $\mathrm{I} \frac{1}{2}$ virgates and 4 ploughs. There $\mathrm{R}$ [alf] has 6 villeins, 4 bordars, 3 serfs, I rouncey, 2 beasts, 70 sheep, 27 goats, 2 acres of wood(land), 6 acres of meadow, and 50 of pasture. Worth 30 shillings; when $B$ [aldwin] received it it was worth 20 shillings.

Baldwin has a manor called Escapeleia (Exch. Scapelie) [Shapleigh] ${ }^{2}$ which Uluric held T.R.E., and it paid geld for I virgate. This 3 ploughs can till. Godwin holds it of Baldwin. Thereof G[odwin] has $\frac{1}{2}$ virgate in demesne and the villeins $\frac{1}{2}$ virgate and $I_{2} \frac{1}{2}$ ploughs. There G[odwin] has 4 villeins, I serf, and 5 acres of meadow. Worth 15 shillings; when $B$ [aldwin] received it it was worth 10 shillings.

fol. 298.

Baldwin has a manor called Aiscirewilla (Exch. Ascerewelle) [Shirwell] ${ }^{3}$ which Brismar held T.R.E., and it paid geld for I hide. This 12 ploughs can till. Rotbert de Bello Monte (Beaumont) holds it of the sheriff. Thereof $\mathrm{R}$ [obert] has I virgate and 2 ploughs in demesne, and the villeins have 3 virgates and $4 \frac{1}{2}$ ploughs. There R[obert] has 9 villeins, 6 bordars, 2 serfs, 2 beasts, 8 swine, Ioo sheep, 28 goats, 2 dwelling houses (mansurae) in the borough of Barnstaple paying 2 shillings a year, 300 acres of coppice, 3 acres of meadow, and pasture I league in length by $\frac{1}{2}$ league in width. Worth 3 pounds a year; when $\mathrm{B}$ [aldwin] the sheriff received it it was worth the same.

$\mathrm{B}$ [aldwin] the sheriff has a manor called AisEFORDA [Ashford] ${ }^{4}$ which Ailmar held T.R.E., and it paid geld for $\frac{1}{2}$ hide. This 5 ploughs can till. Now Rotbert holds it of B[aldwin]. Thereof Rotbert has I virgate and I plough in demesne, and the villeins have I virgate and I plough. There $\mathrm{R}$ [obert] has 9 villeins, 2 bordars, I serf, 40 sheep, 5 goats, 4 acres of coppice, 4 acres of meadow and 30 of pasture. Worth 20 shillings a year; when $\mathrm{B}$ [aldwin] received it it was worth 30 shillings.

${ }^{1}$ Mamhead in Exminster hundred.

${ }^{2}$ Part of Shapleigh in Chagford in Exminster hundred.

${ }^{3}$ Shirwell in Shirwell hundred.

4 Ashford alias East Ashford in Braunton hundred.
$B$ [aldwin] has a manor called Lochesora [Loxhore $^{3}$ which Doleswif held T.R.E., and it paid geld for $\frac{1}{2}$ hide. This 3 ploughs can till. Now Rotbert de Bello Monte (Beaumont) holds it of $\mathrm{B}$ [aldwin]. Thereof $\mathrm{R}$ [obert] has I virgate and $I$ plough in demesne, and the villeins have I virgate and I plough. There [Robert] has 3 villeins, 2 bordars, I serf, 5 beasts, 3 swine, 60 sheep, 24 goats, 100 acres of wood(land), and 200 acres of pasture. Worth 20 shillings a year; when $B$ [aldwin] received it it was worth the same.

fol. $298 \mathrm{~b}$.

$\mathrm{B}$ [aldwin] the sheriff has a manor called LOCHESSORA [Loxhore] ${ }^{6}$ which Ulward held T.R.E., and it paid geld for $\frac{1}{2}$ hide. This 5 ploughs can till. Now Rotbert de Bello Monte (Beaumont) holds it of $\mathrm{B}$ [aldwin]. Thereof $\mathrm{R}$ [obert] has I virgate in demesne and I plough, and the villeins have the other virgate and 2 ploughs. There $R$ [obert] has 7 villeins, 2 bordars, 2 serfs, I 5 beasts, 10 swine, 40 sheep, 4 acres of alder (alneti), and 100 acres of pasture. Worth 20 shillings a year, and was worth the same when $B$ [aldwin] received it.

$\mathrm{B}$ [aldwin] has a manor called Hantona [Heanton] $^{7}$ which Brismar held T.R.E., and it paid geld for 2 hides. These 12 ploughs can till. Now Rotbert de Ponte Cardonis [Punchardon] holds it of $\mathrm{B}$ [aldwin]. There $R$ [obert] has 24 villeins and II serfs, and thereof $R$ [obert] has $\frac{1}{2}$ hide in demesne and 3 ploughs, and the villeins have $1 \frac{1}{2}$ hides and 5 ploughs. There R[obert] has II beasts, 9 swine, I 63 sheep, I mill paying 4 shillings a year, I fishery worth 2 shillings a year, 100 acres of wood(land), Io acres of meadow, and 100 acres of pasture. Worth 4 pounds a year, and was worth the same when B[aldwin] received it.

$\mathrm{B}$ [aldwin] has a manor called HaGintona (Exch. Haintone) [Haggington $]^{8}$ which Ulf held T.R.E., and it paid geld for 1 hide. This 10 ploughs can till. Now R[obert] holds it of Baldwin. Thereof $R$ [obert] has I virgate and 2 ploughs in demesne, and the villeins have 3 virgates and 5 ploughs. There R[obert] has 12 villeins, 4 bordars, 2 serfs, 2 beasts, 4 swine, 100 sheep, and 50 acres of pasture. Worth 3 pounds a year, and was worth the same when B[aldwin] received it.

\footnotetext{
${ }^{5}$ Over Loxhore alias Laxford in Loxhore in Shirwell hundred.

${ }^{6}$ Nether Loxhore alias Loxford in Loxhore in Shirwell hundred.

${ }^{7}$ Heanton Punchardon in Braunton hundred.

${ }^{8}$ West Haggington in Ilfracombe in Braunton hundred.
} 
fol. 29g.

\section{HOLDERS OF LANDS}

$B$ [aldwin] has a manor called CARMEs [Charles] $^{1}$ which Brismar held T.R.E., and it paid geld for I virgate. This 12 ploughs can till. Now Rotbert holds it of $B$ [aldwin]. Thereof $R$ [obert] has I ferting and 2 ploughs in demesne, and the villeins have 3 fertings and 5 ploughs. There R[obert] has 6 villeins, 6 bordars, 3 serfs, 9 beasts, I 3 swine, 57 sheep, 100 acres of wood(land), and 53 acres of pasture. Worth 40 shillings a year; when $B$ [aldwin] received it the same.

$B$ [aldwin] has a manor called Mogrscoma [Mockham] ${ }^{2}$ which Alcherlo (Exch. Alcherl) held T.R.E., and it paid geld along with the above-named manor for I virgate, so that these two manors acquitted themselves to the king's geld (by paying) for I virgate. Now they are reckoned as (computantur) 2 virgates. The virgate which lies in Mogescoma 3 ploughs can till, and now Rotbert de Ponte Cardonis (Punchardon) holds it of $\mathrm{B}$ [aldwin]. Thereof R[obert] has I ferting and I plough in demesne, and the villeins have 2 ploughs. There R[obert] has 2 villeins, I serf, 12 beasts, 2 swine, 80 sheep, 16 goats, and 20 acres of pasture. Worth 20 shillings a year, and was worth 15 shillings when the sheriff received it.

B[aldwin] has a manor called Bochelant [Buckland] $^{3}$ which Alnot (Exch. Alnod) held T.R.E., and it paid geld for $\mathbf{I}$ virgate. This 6 ploughs can till. Now Ansger holds it of Baldwin. Thereof $A$ [nsger] has $I$ ferding and 2 ploughs in demesne, and the villeins have 4 ploughs. There $A$ [nsger] has 6 villeins, 4 bordars, 2 serfs, 12 beasts, 35 sheep, 36 goats, 24 acres of wood(land), and 4 acres of meadow. Worth 40 shilling a year, and was worth the fol. aggb.

same when Baldwin received it.

$\mathrm{B}$ [aldwin] has a manor called BLAchewiLlA $[\text { Blakewell }]^{4}$ which was assessed (se defendit) for I virgate T.R.E. 'This 4 ploughs can till. Now Rotbert de Ponte Cardonis (Punchardon) holds it of $B$ [aldwin]. Thereof $R$ [obert] has I ferting and I plough in demesne, and the villeins have I plough. There $R$ [obert] has 3 villeins, I serf, 5 beasts, 20 sheep, 60 acres of coppice, and as many of pasture. Worth 20 shillings. It was worth the same when $B$ [aldwin] received it. This virgate the men of the hundred say belongs to the king's manor of Bractona [Braunton]. ${ }^{5}$

B[aldwin] has a manor called Chentesberia $[\text { Kentisbury }]^{6}$ which Almar held T.R.E., and it paid geld for 2 hides. These 20 ploughs can

1 Charles in Shirwell hundred ; called 'Charmys' in 1303 , Feud. Aids, 36r.

${ }^{2}$ Mockham in Charles in Shirwell hundred.

${ }^{3}$ West Buckland in Braunton hundred.

- Blakewell in Marwood in Braunton hundred.

${ }^{5}$ See fol. $83 b$.

${ }^{6}$ Kentisbury in Braunton hundred. till. There $B$ [aldwin] has $\frac{1}{2}$ hide and I plough in demesne, and the villeins have 5 ploughs. There B[aldwin] has in villeins, 5 serfs, 20 (acres) of wood(land), and 100 acres of pasture. Worth 2 pounds a year.

$\mathrm{B}$ [aldwin] has a manor called HoLecoma $[\text { Holcombe }]^{7}$ which Ulf (corrected to Seward; Exih. Seward) held T.R.E., and it paid geld for 9 hides. These 22 ploughs can till. Rogo holds them of $\mathrm{B}$ [aldwin]. Thereof $\mathrm{R}$ [ogo] has 4 hides in demesne and 2 ploughs, and the villeins (have) 5 hides and 7 ploughs. There R[ogo] has 30 villeins, 6 bordars, 8 serfs, I rouncey, 5 beasts, 22 swine, 60 sheep, 12 goats, 2 mills fol. 300 .

paying 10 , a year, 50 acres of coppice, 24 acres of meadow, and 200 acres of pasture. Worth 6 pounds a year. It was worth 10 pounds when $\mathrm{B}$ [aldwin] received it.

$\mathrm{B}$ [aldwin] has a manor called Hocoorda [Hockworthy] $^{8}$ which Colebran (Exch. Colbrand) held T.R.E., and it paid geld for 2 virgates $2 \frac{1}{2}$ ferdings. These 3 ploughs can till. Rogo holds them of $B$ [aldwin]. Thereof $R$ [ogo] has in demesne 2 virgates and $\frac{1}{2}$ ferding, and the villeins 2 ferdings and $\frac{1}{2}$ plough. There $R$ [ogo] has 4 villeins, 2 bordars, 2 serfs, 30 acres of wood(land), 2 acres of meadow, and 40 of pasture. Worth 12 shillings and 6 pence a year. It was worth 20 shillings when $B$ [aldwin] received it.

$\mathrm{B}$ [aldwin] has a manor called Anestinga [Anstey $^{9}{ }^{3}$ which Godwin held T.R.E., and it paid geld for 1 hide. This 9 ploughs can till. Ansger holds it of $\mathrm{B}$ [aldwin]. Thereof $\mathrm{A}$ [nsger] has in demesne $I$ virgate and 2 ploughs, and the villeins 3 virgates and 4 ploughs. There $A$ [nsger] has 7 villeins, 5 bordars, 7 serfs, I swineherd paying 6 swine, 25 beasts, 60 sheep, 30 goats, 120 acres of thicket (silva), 6 acres of meadow, and a league of pasture taking length and breadth. Worth 40 shillings a year. It was worth 30 shillings when $\mathrm{B}$ [aldwin] received it.

To the aforesaid manor has been added a manor called Ringhendona [ $]^{10}$ which Chepin (Exch. Cheping) held T.R.E., and it paid geld for I virgate. This 2 ploughs can till. Ansger holds it of Baldwin. There A[nsger] has fol. $300 \mathrm{~b}$.

2 villeins who have $\frac{1}{2}$ plough. Worth 3 shillings a year, and was worth 12 pence when $\mathrm{B}$ [aldwin] received it.

\footnotetext{
${ }^{7}$ Holcombe Rogus in Bampton hundred.

${ }^{8}$ Hockworthy in Bampton hundred.

${ }^{8}$ West Anstey and Anstey Reigny in West Anstey in South Molton hundred.

${ }^{10}$ Probably identical with Fraynes and Westacot in South Molton hundred, an outlier of Chittlehampton.
} 


\section{A HISTORY OF DEVONSHIRE}

$\mathrm{B}$ [aldwin] has a manor called Filelexa [Filleigh $]^{1}$ which Osfers held T.R.E., and it paid geld for 4 virgates (Exch. I hide). These 8 ploughs can till. Thereof $B$ [aldwin] has in demesne I virgate and 3 ploughs, and the villeins 2 virgates and 6 ploughs. There $B$ [aldwin] has 9 villeins, 6 bordars, 3 serfs, 3 swineherds paying 15 swine, 14 beasts, Io swine, 60 sheep, Io acres of wood(land), 7 acres of meadow, and 30 acres of pasture. Worth 3 pounds, and was worth the same (corrected to 40 shillings; Exch. 40 shillings) when he received it.

From the aforesaid manor has been taken away a manor called LOBA [Lob]. ${ }^{2}$ It has been added to the king's demesne manor of Brantona [Braunton]. It paid geld for I virgate. This 3 ploughs can till. Four villeins are there, and it is worth 20 shillings a year.

B [aldwin] has a manor called Neuetona [Newton] ${ }^{3}$ which Alwoud (Exch. Alwold) held T.R.E., and it paid geld for I virgate. This 2 ploughs can till. Ansger holds it of $B$ [aldwin]. There $A$ [nsger] has 2 villeins and $I$ bordar, who have I plough, also $\frac{1}{2}$ acre of meadow and 5 acres of pasture. Worth ro shillings a year, and was worth (the same) when $\mathrm{B}$ [aldwin] received it.

$\mathrm{B}$ [aldwin] has a manor called WADESTAN [Whitestone] ${ }^{4}$ which Edmar held T.R.E., and it paid geld for $\frac{1}{2}$ virgate. This 2 ploughs can till. Ansger holds it of B[aldwin]. Thereof $A$ [nsger] has in demesne I ferding and 6 oxen, and the villeins $I$ ferding and 2 oxen. There A [nsger] has 2 villeins, I bordar, ro beasts, 8 swine, 20 sheep, 3 acres of meadow, and 5 acres of pasture. Worth 10 shillings a year; when fol. 3or.

$B$ [aldwin] received it the same.

$\mathrm{B}$ [aldwin] has a manor called Lincoma [Lincombe] ${ }^{5}$ which Brismer (Exch. Brismar) held T.R.E., and it paid geld for 2 hides. These 8 ploughs can till. Robert holds them of B[aldwin]. Thereof $R$ [obert] has in demesne 2 virgates and 2 ploughs, and the villeins I hide, 2 virgates, and 5 ploughs. There $R$ [obert] has 8 villeins, 9 bordars, 3 serfs, I rouncey, 8 beasts, I 2 swine, 100 sheep, 15 goats, $x \frac{1}{2}$ acres of meadow, and 100 acres of pasture. Worth 3 pounds a year ; when $B$ [aldwin] received it it was worth the same.

1 Filleigh manor in Braunton hundred.

2 North alias Higher Lob in Braunton manor and hundred. See fol. 836 .

${ }^{3}$ Newton in Chittlehampton in South Molton hundred.

"Whitestone alias Weston in Chittlehampton in South Molton hundred.

${ }^{5}$ Lincombe in Ilfracombe in Braunton hundred.
To the above-named manor has been added a manor called LaIerda [La Yard] ${ }^{6}$ which Godric held in parage (pariter) T.R.E., and it paid geld for I virgate. This 2 ploughs can till. Worth Io shillings a year; when $B$ [aldwin] received it 20 shillings.

B[aldwin] has a manor called Alfreincoma [Ilfracombe] ${ }^{7}$ which Almar held T.R.E., and it paid geld for I hide. This 9 ploughs can till. Rotbert holds it of $B$ [aldwin]. Thereof $R$ [obert] has in demesne 1 virgate and 2 ploughs, and the villeins 3 virgates and 9 ploughs. There $\mathrm{R}$ [obert] has $\mathrm{I} 2$ villeins, $\mathrm{I} 2$ bordars, 5 serfs, I rouncey, 5 beasts, 15 swine, 133 sheep, 5 acres of meadow, and 100 acres of pasture. Worth 4 pounds a year; when he received it the same.

$\mathrm{B}$ [aldwin] has a manor called EFForda (Exch. Esforda) [Ashford] ${ }^{8}$ which Brismar held T.R.E., and it paid geld for $\frac{1}{2}$ hide and $x$ ferding. This 4 ploughs can till. Rotbert holds it of $B$ [aldwin]. Thereof $\mathrm{R}$ [obert] has in demesne I virgate and 2 ploughs, and the villeins I virgate, I ferding, and I plough. There $R$ [obert] has 3 villeins, 3 bordars, 2 serfs, 2 beasts, 2 swine, 30 sheep, and 15 acres of pasture. Worth 20 shillings a fol. gorb.

year; when he received it the same.

$\mathrm{B}$ [aldwin] has a manor called CLIST [Clyst] ${ }^{9}$ which Boughered (Exch. Bughered) held T.R.E., and it paid geld for 3 hides. These 8 ploughs can till. Otley (Otelin; Exch., Othelin) holds them of $B$ [aldwin]. Thereof $O$ [tley] has in demesne I hide and 2 ploughs, and the villeins 2 hides and 5 ploughs. There $\mathrm{O}$ [tley] has 9 villeins, 5 bordars, 8 serfs, 5 beasts, 32 sheep, 80 acres of wood(land), 44 acres of meadow and 20 of pasture. Worth 3 pounds, and was worth the same when $B$ [aldwin] received it.

$\mathrm{B}$ [aldwin] has a manor called Cuist [Clyst] ${ }^{10}$ which 4 thanes held in parage (pariter) T.R.E., and it paid geld for I hide and $\frac{1}{2}$ virgate. These 9 ploughs can till. The canons of St. Mary hold it of $\mathrm{B}$ [aldwin]. Thereof they have in demesne $\frac{1}{2}$ hide and 2 ploughs, and the villeins $\frac{1}{2}$ hide, $\frac{1}{2}$ virgate, and I plough. There the canons have Io villeins, 4 bordars, I serf, 20 beasts, 5 acres of wood(land), 17 acres of meadow, and 50 acres of pasture. Worth 40 shillings a year, and was worth the same when B[aldwin] received it.

${ }^{6} \mathrm{La}$ Yard in Ilfracombe in Braunton hundred (O.J.R.).

7 Ilfracombe in Braunton hundred.

${ }^{8}$ West Ashford in Heanton Punchardon in Braunton hundred.

${ }^{9}$ Clysthydon in Cliston hundred. Otley is predecessor in title of $\mathrm{Hydon}$.

${ }^{30}$ Ash Clyst alias East Clyst in Broad Clyst in Cliston hundred, held by the canons of St. Mary of the castle of Exeter. 


\section{HOLDERS OF LANDS}

B [aldwin] has a manor called MERODA [Marwood] ${ }^{1}$ which Alwin held T.R.E., and it paid geld for I virgate. This 2 ploughs can till. Robert holds it of $\mathrm{B}$ [aldwin]. Thereof R[obert] has in demesne $\frac{1}{2}$ virgate and I plough, and the villeins $\frac{1}{2}$ virgate and $\frac{1}{2}$ plough. There $R$ [obert] has 2 villeins, I bordar, 2 serfs, 4 beasts, 5 swine, 28 sheep, 8 goats, 16 acres of wood(land), 2 acres of meadow, and 20 of pasture. Worth 15 shillings a year, and was worth the same when $B$ [aldwin] received it.

fol. зo2.

$\mathrm{B}$ [aldwin] has a manor called WINPLA [Whimple] ${ }^{2}$ which Almer (Exch. Almar) held T.R.E., and it paid geld for 2 hides and 1 virgate. These Io ploughs can till. The wife of the same B[aldwin] holds it of him. Thereof $\mathrm{B}$ [aldwin] has in demesne I hide, I virgate, and 2 ploughs, and the villeins I hide and 6 ploughs. There $\mathrm{B}$ [aldwin] has $\mathrm{I} 3$ villeins, 8 bordars, 9 serfs, 18 beasts, 6 swine, 55 sheep, 24 goats, 50 acres of coppice, 50 acres of meadow, and 100 acres of pasture. Worth 10 pounds a year, and was worth 6 pounds when he received it.

To the above-named manor $\frac{1}{2}$ hide of land was appurtenant in King $E[d$ ward]'s time called LAUROCHEBERA (Exch. Liurochebere) [Larkbear] ${ }^{3}$ which the same Almer (Exch. Almar) held. This 2 ploughs can till. There is $\frac{1}{2}$ plough, I serf, 12 acres of wood(land), 8 acres of meadow, and 13 acres of pasture. Worth 5 shillings a year. This $\frac{1}{2}$ hide Alvered the Breton holds along with another manor called Laurochebera.

Baldwin has a manor called Esnideleia (Exch. Smidelie) [Snedleigh] ${ }^{4}$ which Edric held T.R.E., and it paid geld for I virgate. This 3 ploughs can till. Gislebert holds it of B[aldwin]. Thereof $G$ [islebert] has in demesne I ferding and I plough, and the villeins 3 ferdings and I plough. There G[islebert] has 3 villeins, 2 bordars, 2 serfs, 5 beasts, 48 sheep, 4 acres of meadow, and 40 acres of pasture. Worth I 5 shillings a year ; when G[islebert] received it it was worth 10 shillings.

Baldwin has a manor called HAMBERIA [Hembury] ${ }^{5}$ which Alnod held T.R.E., and it paid geld for 2 hides. These 8 ploughs can till. Rainer holds them of B[aldwin]. Thereof $\mathrm{R}$ [ainer] has in demesne $\frac{1}{2}$ hide and 1 plough, fol. goab.

and the villeins $I \frac{1}{2}$ hides and 5 ploughs. There $\mathrm{R}$ [ainer] has 12 villeins, 8 bordars, I serf, I pack dred.

${ }^{2}$ Middle Marwood in Marwood in Braunton hun-

${ }^{2}$ Whimple in Cliston hundred.

${ }^{3}$ Larkbear in Talaton in Cliston hundred.

4 Snedleigh alias Stoodleigh in West Buckland in Braunton hundred.

${ }^{5}$ Payhembury in Hairidge hundred, the parish other than Higher and Lower Tale and Uggarton. horse, 4 beasts, 4 swine, 80 sheep, I mill paying 40 pence a year, 14 acres of meadow and 40 acres of pasture. Worth 3 pounds a year; when $B$ [aldwin] received it it was worth 2 pounds.

Baldwin has a manor called LANGAForT (Exch. Langeford) [Langford] ${ }^{6}$ which Brismer (Exch. Brismar) held T.R.E., and it paid geld for I hide and 3 virgates. These 6 ploughs can till. Rainer holds it of $B$ [aldwin]. Thereof R[ainer] has in demesne $\frac{1}{2}$ hide and $\mathrm{I}$ virgate and 2 ploughs, and the villeins $\frac{1}{2}$ hide, I virgate, and 3 ploughs. There $R$ [ainer] has ro villeins, 7 bordars, 2 serfs, I rouncey, 3 beasts, 3 swine, 27 sheep, 2 acres of coppice, and 12 acres of meadow. Worth 40 shillings a year, and was worth the same when $B$ [aldwin] received it.

Baldwin has a manor called Pantresfort (Exch. Pantesford) [Pondsford] ${ }^{7}$ which Edwin held T.R.E., and it paid geld for $\frac{1}{2}$ hide. This 2 ploughs can till. William Black (Niger) holds it of B[aldwin]. Thereof $\mathrm{W}$ [illiam] has in demesne I virgate and I plough, and the villeins I virgate and I plough. There W[illiam] has 3 villeins, 4 bordars, 1 serf, 4 beasts, 82 sheep, 8 acres of meadow, and 60 acres of pasture. Worth 15 shillings a year, and was worth 30 shillings when $\mathrm{B}$ [aldwin] received it.

$\mathrm{B}$ [aldwin] has a manor called PANTesfort

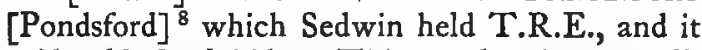
paid geld for $\frac{1}{2}$ hide. This 2 ploughs can till. William holds it of B[aldwin]. There W[illiam] has 4 bordars, 4 acres of meadow, and 60 acres of fol. зоз.

pasture. Worth 10 shillings a year; when $B$ [aldwin] received it the same.

$\mathrm{B}$ [aldwin] has a manor called CHINNEsForT (Exch. Chinnesford) [Kentisford] ${ }^{9}$ which Ezi held T.R.E., and it paid geld for $\frac{1}{2}$ virgate. William holds it of $B$ [aldwin]. There W[illiam] has $\frac{1}{2}$ plough, 2 bordars, I serf, 4 swine, IO sheep, 4 acres of wood(land) and 6 acres of meadow. Worth 5 shillings a year, and was worth the same when $\mathrm{B}$ [aldwin] received it.

Baldwin has a manor called Chentesbera [Kentisbeare] ${ }^{10}$ which Edwy held T.R.E., and it paid geld for I virgate. This 2 ploughs can till. William holds it of $\mathrm{B}$ [aldwin]. Thereof $\mathrm{W}$ [illiam] has in demesne 2 ferdings and I plough, and the villeins 2 ferdings. There W[illiam] has 4 bordars, I serf, Io acres of wood(land), and

${ }^{6}$ Langford in Collumpton in Hairidge hundred. The missing virgate probably represents Whiteheathfield on the Culm (O.J.R.).

7 Pondsford in Cullompton in Hairidge hundred.

${ }^{8}$ Ibid.

${ }^{9}$ Kentisford in Kentisbeare in Hairidge hundred.

${ }^{10}$ Kentisbeare Mauger or Treasurer's Kentisbeare (Feud. Aids, 425, 483) in Kentisbeare in Hairidge hundred. 


\section{A HISTORY OF DEVONSHIRE}

I 0 acres of meadow. Worth Io shillings a year, and was worth 5 shillings when $B$ [aldwin] received it.

$\mathrm{B}$ [aldwin] has a manor called BLACHEBERIA [Blackborough] ${ }^{1}$ which Godric held T. R. E., and it paid geld for $\frac{1}{2}$ hide. This I plough can till. William holds it of $\mathrm{B}$ [aldwin]. Thereof $\mathrm{W}$ [illiam] has in demesne I virgate and I plough, and the villeins I virgate. There W[illiam] has I villein, 4 bordars, I serf, 5 acres of meadow, and 20 acres of pasture. Worth Io shillings a year ; when $B$ [aldwin] received it 5 shillings.

Baldwin has a manor called Chentesbera [Kentisbeare $]^{2}$ which Norman held T. R. E., and it paid geld for $\frac{1}{2}$ hide. This 4 ploughs can till. William holds it of $\mathrm{B}$ [aldwin]. Thereof W[illiam] has in demesne I virgate and 2 ploughs, and the villeins I virgate and 2 ploughs. There W[illiam] has 3 villeins, 5 bordars, 2 serfs, 5 beasts, IO swine, 40 sheep, 1 mill paying 5 shillings a year, Io acres of wood(land), and Io acres of meadow. Worth 30 shillings a year; when $\mathrm{B}$ [aldwin] received it 40 shillings.

fol. 3ogb.

Baldwin has a manor called AvrRa ${ }^{3}$ (Exch. Avra) [Aller] ${ }^{4}$ which Ailward held T.R. E., and it paid geld for I virgate. This 2 ploughs can till. William holds it of B[aldwin]. Thereof W[illiam] has in demesne 3 ferdings and $I$ plough, and the villeins 1 ferding. There W [illiam] has I villein, 5 bordars, I serf, 3 swine, IO acres of wood(land), and 10 acres of meadow. Worth Io shillings a year; when $\mathrm{B}$ [aldwin] received it the same.

$\mathrm{B}$ [aldwin] has a manor called Conunp (Exch. Colun) [Collumpton] ${ }^{5}$ which Brismer $(E x c h$. Brismar) held T.R. E., and it paid geld for I hide. This 4 ploughs can till. Rogo holds it of B[aldwin]. Thereof Rogo has in demesne $2 \frac{1}{2}$ virgates and $I$ plough, and the villeins $I \frac{1}{2}$ virgates and $I$ plough. There R[ogo] has 3 villeins, $x$ bordar, 2 serfs, I rouncey, 7 beasts, 60 sheep, 15 goats, 12 acres of wood(land), 8 acres of meadow, and I6 acres of pasture. Worth 30 shillings a year; when $B$ [aldwin] received it it was worth 20 shillings.

B[aldwin] has a manor called BernardesMORA $^{6}$ which Almer held T.R.E., and it

${ }^{1}$ Blackborough in Kentisbeare in Hairidge hundred.

2 Kentisbeare Prior in Kentisbeare.

3 The $\mathrm{v}$ above the line after an erasure.

- Aller Farm, Kentisbeare (T. W. W.).

${ }^{5}$ Part of Collumpton in Hairidge hundred; or Hele in Bradninch on the Culm (O. J. R.), see Feud. Aids, 322,368 ; Montacute Cbartul. (Somers. Rec. Soc.), I 78.

- Possibly East Culm with Longmoor in Collumpton in Hairidge hundred. William son of Rogo gave 'Colne et $\mathrm{x}$ solidatas de Culintona' to Montacute Priory (Dugdale, Mon. v, I67) (O. J. R.). Dunmore, Silverton (T. W. W.). paid geld for $\frac{1}{2}$ hide. This 3 ploughs can till. Rogo holds it of B[aldwin]. Thereof R[ogo] has in demesne I virgate and I plough, and the villeins I virgate and 2 ploughs. There R[ogo] has 6 villeins, 2 bordars, I serf, 5 swine, 30 sheep, Io goats, I mill paying 5 shillings a year, 6 acres of meadow, and 6 acres of pasture. Worth 20 shillings a year; when $B$ [aldwin] received it 15 shillings.

Baldwin has a manor called Corc [Cowick] ${ }^{7}$ which Ailmar (Exch. Almar) held T. R. E., and it paid geld for I hide. This 8 ploughs can till. Thereof $B$ [aldwin] has in demesne $\frac{1}{2}$ hide and fol. 304.

2 ploughs, and the villeins $\frac{1}{2}$ hide and 6 ploughs. There B [aldwin] has 8 villeins, 3 bordars, 2 serfs, I rouncey, 3 beasts, 40 sheep, I mill paying Io shillings a year, 3 acres of wood(land), and 3 acres of meadow. Worth 30 shillings; when $B$ [aldwin] received it it was worth 20 shillings.

Baldwin has a manor called Taintona [Teignton] $^{8}$ which Osfers held T.R.E., and it paid geld for 2 hides. These 12 ploughs can till. Thereof $\mathrm{B}$ [aldwin] has in demesne $\frac{1}{2}$ hide and $I$ virgate and 2 ploughs, and the villeins I hide and $I$ virgate and 6 ploughs. There $B$ [aldwin] has 12 villeins, 8 bordars, 4 serfs, I 4 beasts, I oo sheep, 3I goats, wood(land) I league in length by 3 furlongs in breadth, 6 acres of meadow, and 60 acres of pasture. Worth 50 shillings a year, and was worth 40 shillings when $B$ [aldwin] received it.

Baldwin has a manor called EsPREITONa (Exch. Spreitone) [Spreyton] ${ }^{9}$ which Osfers held T.R.E., and it paid geld for 2 hides. These I 2 ploughs can till. Thereof $\mathrm{B}$ [aldwin] has in demesne 3 virgates and 2 ploughs, and the villeins I hide, $I$ virgate, and 6 ploughs. There $B$ [aldwin] has I 4 villeins, 10 bordars, 3 serfs, I I beasts, 60 sheep, 35 goats, wood(land) I league in length by 2 furlongs in breadth, 3 acres of meadow, and 10 acres of pasture. Worth 60 shillings a year; when $\mathrm{B}$ [aldwin] received it it was worth 50 shillings.

Baldwin has a manor called Essorc [Exwick] ${ }^{\text {to }}$ which Eurewac (Exch. Eurewacre) held T.R.E., and it paid geld for I hide. This 8 ploughs can till. Thereof $\mathrm{B}$ [aldwin] has in demesne I virgate and I plough, and the villeins 3 virgates and 6 ploughs. There $\mathrm{B}$ [aldwin] has 9 villeins, 5 serfs, 40 sheep, I mill paying 10 shillings, 3 acres of coppice, 3 acres of meadow, and

7 Cowick in Wonford hundred alias St. Thomas, Exeter. The mill is probably Calabear Mill.

${ }^{8}$ Teignton Drew alias Dabernon in Drewsteignton in Wonford hundred.

${ }^{9}$ Spreyton in Wonford hundred.

${ }^{10}$ Exwick in St. Thomas, Exeter, in Wonford hundred. Dugdale, Mon. vi, 1068. 


\section{HOLDERS OF LANDS}

50 acres of pasture. Worth 30 shillings a year, fol. 304b.

and was worth 20 shillings when $B$ [aldwin] received it.

Baldwin has a manor called CliforT (Exch. Cliford) [Clifford $]^{1}$ which Brismer held T.R.E., and it paid geld for I hide. This 8 ploughs can till. Stephen (Exch. Stefan) holds it of B[aldwin]. Thereof S[tephen] has in demesne 2 virgates and 3 ploughs, and the villeins 2 virgates and $4 \frac{1}{2}$ ploughs. There S[tephen] has 10 villeins, 6 bordars, 4 serfs, 6 beasts, I I swine, 82 sheep, 4 acres of wood(land), 3 acres of meadow, and 6 furlongs of pasture. Worth 40 shillings a year; it was worth 20 shillings when $B$ [aldwin] received it.

Baldwin has a manor called Hidova [Hidon] ${ }^{2}$ which Godwin held T.R.E., and it paid geld for 3 hides. These I 2 ploughs can till. Otelin holds them of $\mathrm{B}$ [aldwin]. Thereof $\mathrm{O}$ [telin] has in demesne I hide, 2 virgates, and 3 ploughs, and the villeins I hide, 2 virgates, and 5 ploughs. There $O[$ telin] has I 4 villeins, 6 bordars, 6 serfs, 22 beasts, 12 swine, 60 sheep, 27 goats, I mill paying 30 pence, wood(land) I league in length by $\frac{1}{2}$ league in breadth, 26 acres of meadow, and pasture 3 furlongs in length by $I$ furlong in breadth. Worth 4 pounds a year; when $B$ [aldwin] received it 4 pounds.

$\mathrm{B}$ [aldwin] has a manor called Rumor [Ringmore $]^{3}$ which Bristric (Exch. Brictric) held T.R.E., and it paid geld for I hide and I virgate. These 8 ploughs can till. Stephen holds them of B[aldwin]. Thereof S[tephen] has in demesne 2 virgates and 2 ploughs, and the villeins 3 virgates and 4 ploughs. There S[tephen] has 8 villeins, 6 bordars, 4 serfs, I rouncey, 6 beasts, fol. 305 .

roo sheep, 3 acres of meadow, and I 5 acres of pasture. Worth 30 shillings a year; when $\mathrm{B}$ [aldwin] received it it was worth 20 shillings.

$\mathrm{B}$ [aldwin] has a manor called RISFORT (Exch. Risford) [Rushford] " which Edwy held T.R.E., and it paid geld for $I$ hide. This 5 ploughs can till. Edwy holds it of B[aldwin]. Thereof $\mathrm{E}[\mathrm{dwy}]$ has in demesne $1 \frac{1}{2}$ virgates and $\mathrm{I}$ plough, and the villeins $2 \frac{1}{2}$ virgates and 3 ploughs. There $\mathrm{E}$ [dwy] has 8 villeins, 5 bordars, I serf, 9 beasts, 59 sheep, 4 acres of coppice, 5 acres of meadow, and 4 acres of pasture. Worth 30 shillings a year, and was worth the same when $B$ [aldwin] received it.

${ }^{1}$ West Clifford alias Combe Hall in Drewsteignton in Wonford hundred; Trans. Devon Assoc. xxxvii, $33 \mathrm{I}$.

'Clayhidon in Hemyock hundred.

${ }^{3}$ Ringmore, forming with Shaldon the parish of St. Nicolas in Teignhead in Wonford hundred.

' Rushford Barton in Chagford in Wonford hundred.
B[aldwin] has a manor called Hitenesteia [Hittisleigh] ${ }^{5}$ which Doda (Exch. Dode) held T.R.E., and it paid geld for $\frac{1}{2}$ hide. This 7 ploughs can till. Ralf de Pomeria holds it of $\mathrm{B}$ [aldwin]. Thereof R[alf] has in demesne I virgate and 2 ploughs, and the villeins $I$ virgate and 3 ploughs. There $\mathrm{R}$ [alf] has 7 villeins, 2 serfs, 6 beasts, 6 swine, 74 sheep, wood(land) I league in length by $\frac{1}{2}$ league in breadth, and 6 acres of meadow. Worth 30 shillings a year, and was worth 20 shillings when $B$ [aldwin] received it.

B[aldwin] has a manor called Mertona [Mar$\operatorname{tin}]^{6}$ which Iadolf (Exch. Iadulf) held T.R.E., and it paid geld for I virgate. This 3 ploughs can till. Richard son of Torolv holds it of B[aldwin]. There $R$ [ichard] has in demesne 2 ferdings and $I$ plough, and the villeins 2 ferdings and $\frac{1}{2}$ plough. There $\mathrm{R}$ [ichard] has 2 villeins, I serf, 5 acres of wood(land), and 2 acres of meadow. Worth Io shillings a year; when $\mathrm{B}$ [aldwin] received it it was worth the same.

$\mathrm{B}$ [aldwin] has a manor called MELEw Is [Melhuish $]^{7}$ which Brismer (Exch, Brismar) held T.R.E., and it paid geld for $\frac{1}{2}$ hide. This 7 ploughs can till. Hugh of Rennes (redonensis) fol. 3o5b.

holds it of $\mathrm{B}$ [aldwin]. Thereof $\mathrm{H}$ [ugh] has in demesne I virgate and I plough, and the villeins I virgate and 6 ploughs. There $\mathrm{H}$ [ugh] has 8 villeins, 4 bordars, 4 serfs, 6 beasts, 24 goats, 24 acres of wood(land), 3 acres of meadow, and I 5 acres of pasture. Worth 30 shillings a year; when $\mathrm{B}$ [aldwin] received it it was worth 20 shillings.

$\mathrm{B}$ [aldwin] has a manor called TaIgna (Exch. Taine) [Teign $]^{8}$ which Alvric held T.R.E., and it paid geld for 2 virgates. These 4 ploughs can till. Hugh holds them of B[aldwin]. Thereof $\mathrm{H}$ [ugh] has in demesne I virgate and 2 ploughs, and the villeins I virgate and I plough. There $\mathrm{H}$ [ugh] has 4 villeins, I bordar, 2 serfs, 9 beasts, 44 sheep, 2 acres of meadow, and 20 acres of pasture. Worth 15 shillings a year; when $\mathrm{B}$ [aldwin] received it it was worth $\mathrm{ro}$ shillings.

$\mathrm{B}$ [aldwin] has a manor called WALDERIGE [Oldridge] ${ }^{9}$ which Doda (Exch. Dode) held T.R.E., and it paid geld for $\frac{1}{2}$ virgate. This 6

${ }^{5}$ Hittisleigh in Wonford hundred.

${ }^{6}$ Martin alias Mardon, an outlier of Drewsteignton in Wonford hundred.

${ }^{7}$ Melhuish Barton in Wonford hundred, principally in Tedburn St. Mary, partly in Dunsford.

${ }^{8}$ Teign Harvey in Stoke-in-Teignhead in Wonford hundred.

${ }^{9}$ Oldridge tithing, an outlier of St. Thomas, Exeter, in Wonford hundred, including Oldridge Cadiho and Oldridge Grant. Here the sum of the lord's and villeins' assessments amounts to I virgate, whereas Oldridge is returned as $\frac{1}{2}$ virgate. Probably the details refer to the two Oldridges, the total to one only. 


\section{A HISTORY OF DEVONSHIRE}

ploughs can till. Goscelin Bervin (Exch. Gozelin) holds it of B[aldwin]. Thereof G[oscelin] has in demesne $I \frac{1}{2}$ ferdings and I plough, and the villeins $2 \frac{1}{2}$ ferdings and 4 ploughs. There G[oscelin] has 6 villeins, 3 bordars, 3 serfs, 2 beasts, 24 sheep, 62 goats, 60 acres of wood(land), 6 acres of meadow, and 100 acres of pasture. Worth 20 shillings a year; and was worth the same when $\mathrm{B}$ [aldwin] received it.

$\mathrm{B}$ [aldwin] has a manor called Teteborna (Exch. Teteburne) [Tedburn] ${ }^{1}$ which Elmer held T.R.E., and it paid geld for I virgate. This 4 ploughs can till. Rainer holds it of Baldwin. Thereof $\mathrm{R}$ [ainer] has in demesne $\mathrm{I} \frac{1}{2}$ ferdings and I plough, and the villeins $3 \frac{1}{2}$ ferdings and I plough. There R[ainer] has 2 villeins, 3 bordars, $r$ serf, 40 sheep, 45 acres of wood(land), 3 acres of fol. 306.

meadow, and 40 acres of pasture. Worth ro shillings a year; when $\mathrm{B}$ [aldwin] received it it was worth 15 shillings.

$\mathrm{B}$ [aldwin] has a manor called TETEBoRna [Tedburn] ${ }^{2}$ which Doda (Exch. Dode) held T.R.E., and it paid geld for I virgate. This 4 ploughs can till. Ralf de Pomeria holds it of $\mathrm{B}$ [aldwin]. Thereof $\mathrm{R}$ [alf] has in demesne $\mathrm{I}$ ferding and $I$ plough, and the villeins 3 ferdings and 2 ploughs. There $R$ [alf] has 4 villeins, 2 bordars, 2 serfs, I beast, 22 sheep, 45 acres of wood(land), 3 acres of meadow, and 45 acres of pasture. Worth ro shillings a year, and was worth the same when $B$ [aldwin] received it.

$\mathrm{B}$ [aldwin] has a manor called Boleham [Bolham $]^{3}$ which Almer (Exch. Elmer) held T.R.E., and it paid geld for 2 hides and a half $\left(E_{x c h}\right.$. 2 hides). These 8 (Exch. 6) ploughs can till. Otelin (Exch. Othelin) holds them of B[aldwin]. Thereof $O$ [telin] has in demesne I hide, I virgate (marked for omission), and 3 ploughs; and the villeins I hide, I virgate (marked for omission), and 2 ploughs. There $\mathrm{O}$ [telin] has 8 villeins, 6 bordars, 2 serfs, Io beasts, 50 sheep, Io goats, wood(land) $\frac{1}{2}$ league in length by I furlong in breadth, 24 acres of meadow, and pasture 1 league in length by $\frac{1}{2}$ league in breadth. Worth 35 shillings, when B[aldwin] received it 20 shillings.

$B$ [aldwin] has a manor called CoLun [Culm] ${ }^{4}$ which Godwin held T.R.E., and it paid geld

1 Tedburn St. Mary in Wonford hundred. Probably $3 \frac{1}{2}$ ferdings in the villeins' assessment is a mistake for $2 \frac{1}{2}$ ferdings ; otherwise the total is a ferding too much.

'In Tedburn St. Mary in Wonford hundred.

${ }^{3}$ Bolham in Clayhidon in Hemyock hundred. The 2 virgates marked for omission probably represent the $\frac{1}{2}$ hide of Newcourt and Hole which was held with Bolham as one manor.

' Culm Pyne, an outlier of Clayhidon in Hemyock hundred, locally in Hemyock parish. for $\frac{1}{2}$ hide and I ferding. This 4 ploughs can till. Otelin (Exch. Othelin) holds it of B[aldwin]. Thereof $O$ [telin] has in demesne I virgate, I ferding, and $2 \frac{1}{2}$ ploughs, and the villeins I virgate and I plough. There $\mathrm{O}$ [telin] has 4 villeins, 4 bordars, 2 serfs, 9 beasts, 12 swine, 40 sheep, 20 goats, I mill paying 30 pence fol. $306 \mathrm{~b}$.

a year, 6 acres of wood(land), 4 acres of meadow, and pasture 2 furlongs in length by $I$ furlong in breadth. Worth 20 shillings a year, and was worth 10 shillings when $\mathrm{B}$ [aldwin] received it.

$\mathrm{B}$ [aldwin] has a manor called BRENForT (Exch. Brenford) [Brampford $]^{5}$ which Brungar held T.R.E., and it paid geld for $\frac{1}{2}$ hide. This 2 ploughs can till. Walter holds it of B[aldwin]. Thereof $W$ [alter] has in demesne I virgate and I plough, and the villeins I virgate and $\frac{1}{2}$ plough. There $W$ [alter] has 2 villeins, 2 bordars, I serf, 5 beasts, 40 sheep, 20 goats, 3 acres of meadow, and 6 acres of pasture. Worth I 5 shillings a year, and was worth 10 shillings when $B$ [aldwin] received it.

$\mathrm{B}$ [aldwin] has a manor called HoLNa [Hole] ${ }^{6}$ which Edolf (Exch. Eddulf) held T.R.E., and it paid geld for $\frac{1}{2}$ hide. This 2 ploughs can till. Otelin (Exch. Othelin) holds it of B[aldwin]. Thereof $O$ [telin] has in demesne I virgate and I plough, and the villeins I virgate and I plough. There $\mathrm{O}$ [telin] has 2 villeins and 5 acres of pasture. Worth 5 shillings a year; when $\mathrm{B}$ [aldwin] received it it was out of cultivation.

$\mathrm{B}$ [aldwin] has a manor called WITESTAN

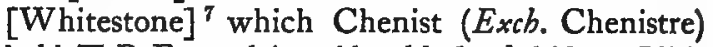
held T.R.E., and it paid geld for $\frac{1}{2}$ hide. This 4 ploughs can till. Bernard the Napeless (sine napa) holds it of $\mathrm{B}$ [aldwin]. Thereof $\mathrm{B}$ [ernard] has in demesne I virgate and $\frac{1}{2}$ plough, and the villeins $I$ virgate and I plough. There $\mathrm{B}[\mathrm{er}-$ nard] has 4 villeins, 2 bordars, I serf, 4 beasts, 12 sheep, 3 acres of coppice, and 2 acres of meadow. Worth ro shillings a year; when Bal[dwin] received it it was worth 5 shillings.

$\mathrm{B}$ [aldwin] has a manor called Medenecoma [Maidencombe] ${ }^{8}$ which Chenist $\left(E_{x e c h}\right.$. Chenistre) held T.R.E., and it paid geld for $\frac{1}{2}$ hide and $\frac{1}{2}$ ferding. This 3 ploughs can till. Bernard fol. $3 \%$.

holds it of $\mathrm{B}$ [aldwin]. Thereof $\mathrm{B}$ [ernard] has in demesne $I$ virgate and $\frac{1}{2}$ plough, and the villeins I virgate, $\frac{1}{2}$ ferding, and $\frac{1}{2}$ plough.

s Brampford Pyne in Wonford hundred (O. J. R.). Geldroll, fol. 70b, B 7, calls Walter Baldwin's cupbearer.

${ }^{6}$ Hole in Clayhidon in Hemyock hundred.

${ }^{7}$ Now Weston with Hackadown in Whitestone in Wonford hundred.

${ }^{8}$ Maidencombe alias Minnicomb in Stoke-in-Teignhead in Wonford hundred. 


\section{HOLDERS OF LANDS}

There $\mathbf{B}$ [ernard] has I villein, 2 bordars, I serf, and 19 sheep. Worth 5 shillings a year; when Bal[dwin] received it 30 pence.

$\mathrm{B}$ [aldwin] has a manor called Racoma [Rocombe ${ }^{1}$ which Osmer held T.R.E., and it paid geld for $\frac{1}{2}$ hide. This 3 ploughs can till. Bernard holds it of $\mathrm{B}$ [aldwin]. Thereof $\mathrm{B}$ [ernard] has in demesne I virgate and $\frac{1}{2}$ plough, and the villeins $I$ virgate and $\frac{1}{2}$ plough. There $B$ [ernard] has I villein, 2 bordars, and 2 serfs. Worth 5 shillings a year; when $\mathrm{Bal}[\mathrm{dwin}]$ received it it was worth 30 pence.

B[aldwin] has a manor called BREDEForT (Exch. Bradeford) [Bridford] ${ }^{2}$ which Bristric (Exch. Brictric) held T.R.E. and it paid geld for 2 hides. These 14 ploughs can till. Emma his wife holds them of Baldwin. Thereof $E[\mathrm{mma}]$ has in demesne $\frac{1}{2}$ hide and 3 ploughs and the villeins $I \frac{1}{2}$ hides and 7 ploughs. There $\mathrm{E}$ [mma] has $\mathrm{I}_{3}$ villeins, 8 bordars, 6 serfs, 14 beasts, 100 sheep, 18 goats, 24 acres of wood(land), 12 acres of meadow, and 30 of pasture. Worth 40 shillings a year, and was worth 30 shillings when $\mathrm{B}$ [aldwin] received it.

B[aldwin] has a manor called Firrseham

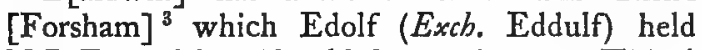
T.R.E., and it paid geld for I virgate. This 6 ploughs can till. The monks of St. Michael hold it of B[aldwin]. Thereof the monks have in demesne $\frac{1}{2}$ virgate and I plougb and the villeins $\frac{1}{2}$ virgate and 2 ploughs. There the monks have 5 villeins, 2 bordars, I serf, 4 sheep, IO acres of wood(land), 3 acres of meadow, and 20 acres of pasture. Worth 30 shillings a year, and was worth 20 shillings when $B$ [aldwin] received it.

fol. 307b.

Baldwin has a manor called BRENFORT (Exch. Brenford) [Brampford] ${ }^{4}$ which Godman the priest held T.R.E., and it paid geld for I hide. This 9 ploughs can till. Vitalis of Colintona [Collumpton] holds it of $\mathrm{B}$ [aldwin]. Thereof V [italis] has in demesne I virgate and 2 ploughs, and the villeins 3 virgates and 6 ploughs. There $\mathrm{V}$ [italis] has $\mathrm{I} 6$ villeins, 8 bordars, 4 serfs, 2 rounceys, 23 beasts, 23 swine, 65 sheep, I mill paying 8 shillings, 40 acres of wood(land), 5 acres of meadow, and 20 acres of pasture. Worth 45 shillings a year; when $B$ [aldwin] received it it was worth 20 shillings.

${ }^{1}$ Rocombe Cadiho (Feud. Aids, i, 3 I 3) alias Hugh (Ibid. 3 I 2) alias Middle Rocombe, an outlier of Combe-in-Teignhead in Wonford hundred, locally in Stoke-in-Teignhead (Ibid. 387).

'Afterwards called Christow in Wonford hundred.

${ }^{3}$ Forsham in Drewsteignton in Wonford hundred. dred.

' Brampford alias Upton Pyne in Wonford hun-
$\mathrm{B}$ [aldwin] has a manor called Pontrmora [Poltimore $]^{5}$ which Olmer (Exch. Ulmer) Cota held T.R.E., and it paid geld for $\frac{1}{2}$ hide. This 2 ploughs can till. The canons of St. Mary hold it of B[aldwin]. Thereof the canons have $I \frac{1}{2}$ virgates in demesne and I plough, and the villeins $\frac{1}{2}$ virgate. There the canons have 2 bordars, 5 serfs, 14 beasts, 3 swine, 92 sheep, 6 acres of meadow, and 80 acres of pasture. Worth Io shillings a year; when $B$ [aldwin] received it the same.

$B$ [aldwin] has a manor called Polesseruga [Polsloe] ${ }^{6}$ which Alvric held T.R.E., and it paid geld for $\frac{1}{2}$ virgate. This 2 ploughs can till. The canons of St. Mary hold it of B[aldwin]. There the canons have 2 ploughs, I bordar, 4 serfs, 4 beasts, and 2 acres of meadow. Worth Io shillings a year; when $\mathrm{B}$ [aldwin] received it 5 shillings.

$\mathrm{B}$ [aldwin] has a manor called Erghebera (Exch. Eigebere) [Eggbear $]^{7}$ which Lieueger (Exch. Leuegar) held T.R.E., and it paid geld for $\frac{1}{2}$ hide and $\frac{1}{2}$ virgate. These 6 ploughs can till. Motbert holds them of $\mathrm{B}$ [aldwin]. Thereof fol. 308 .

$M$ [otbert] has in demesne $\mathbf{I}$ virgate and 2 ploughs, and the villeins $1 \frac{1}{2}$ virgates and $2 \frac{1}{2}$ ploughs. There M[otbert] has 4 villeins, 4 bordars, 2 serfs, 3 beasts, 4 swine, 36 sheep, 4 goats, 6 acres of wood(land), to acres of meadow, and 30 acres of pasture. Worth 20 shillings a year; when $B$ [aldwin] received it $I 5$ shillings.

Baldwin has a manor called Opecota [Upcot ${ }^{8}$ which Elmer held T.R.E., and it paid geld for I virgate. This 5 ploughs can till. $\mathrm{M}$ [otbert] holds it of $\mathrm{B}$ [aldwin]. Thereof $M$ [otbert] has in demesne 2 ferdings and $I$ plough, and the villeins 2 ferdings and I plough. There M[otbert] has 2 villeins, 3 bordars, I serf, 3 beasts, 35 sheep, I acre of wood(land), 6 acres of meadow and 5 acres of pasture. Worth ro shillings a year and was worth the same when $B$ [aldwin] received it.

B[aldwin] has a manor called Folefort (Exch. Foleford) [Fulford ${ }^{9}$ which Arit $\left(E_{x c b}\right.$. Aric) held T.R.E., and it paid geld for I virgate. This 3 ploughs can till. Now Motbert holds it of $\mathrm{B}\left[\right.$ aldwin]. Thereof Motbert has $\frac{1}{2}$ virgate in demesne and I plough, and the villeins have 2 ploughs. There $M$ [otbert] has 3 villeins, 4 bordars, I serf, 3 beasts, 25 sheep, 4 acres of

'Cota's town alias Cutton in Poltimore in Wonford hundred, held by the canons of St. Mary in the Castle of Exeter. Trans. Devon Assoc. xxx, 283. dred.

${ }^{6}$ Polsloe in St. David's, Exeter, in Wonford hun-

${ }^{7}$ Eggbear in Cheriton Bishop in Wonford hundred.

${ }^{8}$ Upcot in Tedburn in Wonford hundred.

${ }^{9}$ Great Fulford in Dunsford in Wonford hundred. 


\section{A HISTORY OF DEVONSHIRE}

meadow and 20 acres of pasture. Worth 15 shillings a year ; when $B$ [aldwin] received it it was worth 7 shillings.

$\mathrm{B}$ [aldwin] has a manor called RoceBera ( $E x c h$. Rochebere) [Rockbear] ${ }^{1}$ which Edmar (Exch. Edmer) held T.R.E., and it paid geld for $\frac{1}{2}$ hide. This 4 ploughs can till. Now Rainer holds it of $\mathrm{B}$ [aldwin]. Thereof $\mathrm{R}$ [ainer] has I virgate and 2 ploughs in demesne, and the villeins have I plough. There $\mathrm{R}$ [ainer] has 6 villeins, 4 serfs, fol. 308b.

8 beasts, 60 sheep, 6 acres of wood(land), Io acres of meadow and 40 acres (of pasture). Worth I 5 shillings a year, when $B$ [aldwin] received it it was worth Io shillings.

$\mathrm{B}$ [aldwin] has a manor called ROCHEBERA [Rockbear] $^{2}$ which Sawin held T.R.E., and it paid geld for 3 fertings. These I plough can till. Now Rainer holds it of B[aldwin]. There $\mathrm{R}$ [ainer] has I villein who has I plough and pays 5 shillings a year. It was worth the same when $B$ [aldwin] received it.

$\mathrm{B}$ [aldwin] has a manor called OTRIT [Otter $]^{3}$ which Dodo (Exch. Dode) the priest held T.R.E., and it paid geld for I virgate and 3 fertings. This 2 ploughs can till. Now Rainer holds it of $\mathrm{B}$ [aldwin]. $\mathrm{On}$ it $\mathrm{R}$ [ainer] has $\mathrm{I} \frac{1}{2}$ ploughs, 2 bordars, I serf, 2 swine, I5 sheep, I mill paying 5 shillings a year, 2 acres of meadow and 10 acres of pasture. Worth 7 shillings a year, and was worth the same when $B$ [aldwin] received it.

$\mathrm{B}$ [aldwin] has a manor called Ailesberga (Exch. Eilesberge) [Aylesbeare] ${ }^{4}$ which Ailmar (Exch. Almer) held T.R.E., and it paid geld for $2 \frac{1}{2}$ hides. This 8 ploughs can till. Thereof $\mathrm{B}$ [aldwin] has $\mathrm{I}$ hide in demesne and 2 ploughs, and the villeins have 5 ploughs. There $\mathrm{B}$ [aldwin] has 8 villeins, 3 bordars, 4 serfs, I 2 beasts, I00 sheep, 50 acres of coppice, and 6 acres of meadow. Worth 40 shillings a year; when $B$ [aldwin] received it it was worth the same.

$\mathrm{B}$ [aldwin] has a manor called WITEsTAN [Whitestone] ${ }^{5}$ which Edrit (Exch. Edric) held T.R.E., and it paid geld for I virgate. This 2 ploughs can till. Now Robert de Beaumont (de bello monte) holds it of B[aldwin]. Thereof fol. 3og.

$\mathrm{R}$ [obert] has $\frac{1}{2}$ virgate and I plough in demesne,

\footnotetext{
${ }^{1}$ Rockbear Baldwin alias Marsh in Rockbear in East Budleigh hundred.

- Upcot in Rockbear in East Budleigh hundred.

3 Dode's town alias Dotton Mill alias Datton, on the Otter in East Budleigh hundred, an extra-parochial liberty. Trans. Devon Assoc. xii, 464 ; xxx, 306.

1 Aylesbeare in East Budleigh hundred.

${ }^{5}$ Now Heath Barton in Whitestone in Wonford hundred.
}

and the villeins have I plough. There R[obert] has 2 villeins, I bordar, I serf, 2 beasts, 40 sheep, 10 goats, 8 acres of wood(land) and 4 acres of meadow. Worth Io shillings a year, and was worth the same when he received it.

B[aldwin] has a manor called RocheberA [Rockbear] ${ }^{6}$ which Ulmar held T.R.E., and it paid geld for 3 hides all but a virgate. This 6 ploughs can till. Now Goselm holds it of B[aldwin]. Thereof G[oselm] had I hide, I ferting, and 2 ploughs in demesne; and the villeins have $3 \frac{1}{2}$ ploughs. There G[oselm] has 9 villeins, 5 bordars, 4 serfs, 4 beasts, I 6 swine, 50 sheep, 50 acres of wood(land), 55 acres of meadow and 50 acres of pasture. Worth 40 shillings a year; when $B$ [aldwin] received it it was worth I 5 shillings.

$\mathrm{B}$ [aldwin] has a manor called CLISTA [Clyst] ${ }^{7}$ which Ulvevia (Exch. Ulveva) held T.R.E., and it paid geld for $2 \frac{1}{2}$ virgates. This 3 ploughs can till. Now the canons of St. Mary hold it of $\mathrm{B}$ [aldwin]. Thereof the canons have $I \frac{1}{2}$ virgates in demesne and. I plough, and the villeins have $2 \frac{1}{2}$ ploughs. There the canons have 3 villeins, 3 bordars, 3 serfs, I cow, 5 sheep, 10 acres of meadow and 3 acres of pasture. Worth I 5 shillings a year, and was worth is shillings (Exch. 20 shillings) when B[aldwin] received it.

From this manor I ferding of land has been taken away which rightfully belonged there in King E[dward]'s time, and has been added to Odo's manor called Ponrimora. ${ }^{8}$ Worth 12 pence a year.

B[aldwin] has a manor called Petecota [Pennycot $]^{9}$ which Alwold held T.R.E., and it paid geld for $\frac{1}{2}$ hide. This 3 ploughs can till. Roger de Molis holds it of B[aldwin]. Thereot $\mathrm{R}$ [oger] has I virgate and I plough in demesne, fol 39gb.

and the villeins I plough. There $\mathrm{R}$ [oger] has 3 villeins, 3 bordars, 2 serfs, 5 acres of meadow and 5 acres of pasture. Worth 20 shillings a year, and was worth the same when $B$ [aldwin] received it.

$\mathrm{B}$ [aldwin] has a manor called Chalmonleuga (Exch. Calmonleuga) [Chulmleigh] ${ }^{10}$ which Bris-

${ }^{6}$ Rockbear Burnell alias John alias Giffard alias Rockbear Court in East Budleigh hundred.

${ }^{7}$ Clyst Mois alias West Clyst (Trans. Devon Assoc. xxvi, 167) in Broad Clyst in Wonford hundred.

${ }^{8}$ Neither Odo son of Gamelin, nor Odo son of Edric the Englishman, nor Odo Juhel's tenant, had any manor called 'Pontimore.' Presumably Haimeric's manor of Poltimore, fol. $469 b$, must be the manor to which a portion of West Clyst was added, and Odo was under-tenant of Cutton.

${ }^{9}$ Pennycot in Shobrook in West Budleigh hundred.

${ }^{10}$ Chulmleigh in Witheridge hundred. 


\section{HOLDERS OF LANDS}

mer (Exch. Brismar) held T.R.E., and it paid geld for 5 hides. These 40 ploughs can till. Thereof $\mathrm{B}$ [aldwin] has in demesne 2 hides and 10 ploughs, and the villeins 3 hides and 20 ploughs. There $\mathrm{B}$ [aldwin] has 30 villeins, 20 bordars, 20 serfs, 5 swineherds paying 30 swine, 60 beasts, 400 sheep, 10 goats, 40 acres of wood(land), 30 acres of meadow and I league of pasture taking length and breadth. It is worth $I 3$ pounds; when $B$ [aldwin] received it it was worth 12 pounds.

B[aldwin] has a manor called Maukssart [Meshaw] $^{1}$ which Alueret (Exch. Aluert) held T.R.E., and it paid geld for 3 virgates. These ro ploughs can till. Gislebert holds them of $\mathrm{B}$ [aldwin]. Thereof $\mathrm{G}$ [islebert] has in demesne $\frac{1}{2}$ virgate and $\frac{1}{2}$ plough, and the villeins $2 \frac{1}{2}$ virgates and 6 ploughs. There G[islebert] has I 2 villeins, 9 bordars, 5 serfs, and 2 swineherds paying 15 swine a year. Gislebert has also there II beasts, 6 swine, 66 sheep, I 9 goats, 40 acres of wood(land), 40 acres of meadow, and pasture $I$ league in length by $\frac{1}{2}$ (a league) in breadth. Worth 30 shillings; when he received it 20 shillings.

fol. 3 ro.

B [aldwin] has a manor called Hirrda [Yard] ${ }^{2}$ which Almar held T.R.E., and it paid geld for 3 virgates. These 6 ploughs can till. Ansger holds them of $B$ [aldwin]. Thereof Ansger has in demesne $\frac{1}{2}$ virgate and 2 ploughs, and the villeins $2 \frac{1}{2}$ virgates and 3 ploughs. There A[nsger] has 8 villeins, 4 bordars, 5 serfs, 12 beasts, 8 swine, $4 \mathrm{I}$ sheep, 80 acres of wood, 25 acres of meadow and IOO acres of pasture. Worth 30 shillings a year; when B[aldwin] received it it was worth Io shillings.

$\mathrm{B}$ [aldwin] has a manor called AIssa $\left[\right.$ Ash ${ }^{3}$ which Seward (Exch. Siward) held T.R.E., and it paid geld for I hide and I virgate. These 18 ploughs can till. Ansger holds them of $B$ [aldwin]. Thereof $A$ [nsger] has in demesne I virgate and 4 ploughs, and the villeins I hide and 10 ploughs. There $A$ [nsger] has 20 villeins, 12 bordars, 8 serfs, 30 beasts, 6 swine, 80 sheep, 150 acres of wood(land), 60 acres of meadow and pasture $\frac{1}{2}$ league in length by $\frac{1}{2}$ league in breadth. Worth I 00 shillings; when $\mathrm{B}$ [aldwin] received it 60 shillings.

B[aldwin] has a manor called CRAHECome (Exch. Crawecome) [Creacombe ${ }^{4}$ which Seward (Exch. Siward) held T.R.E., and it paid geld for 3 ferlings all but $\frac{1}{4}$ ferling (iiiia parte bunius ferlini minus). This 2 ploughs can till. The

${ }^{1}$ Meshaw in Witheridge hundred.

2 Yard in Roseash in Witheridge hundred.

${ }^{3}$ Ash Ralf otherwise Roseash in Witheridge hundred.

- Creacombe in Witheridge hundred. same Ansger ${ }^{5}$ (Exch. Ansger) holds it of B[aldwin]. There A[nsger] has $\frac{3}{4}$ ferling and $I$ plough, and the villeins 2 ferdings and I plough. There $A$ [nsger] has 3 villeins, 2 serfs, 10 beasts, 40 sheep, 2 acres of wood(land), 6 acres of meadow and 7 acres of pasture. Worth 10 shillings a year; when $\mathrm{B}$ [aldwin] received it the same.

fol. эrob.

Along with the aforesaid manor Baldwin has another manor called CRAWEComa [Creacombe] ${ }^{6}$ which Lieuegar (Exch. Leuegar) held in parage (pariter; Exch., libere) T.R.E., and it paid geld for $\mathrm{I} \frac{1}{4}$ ferdings. This $\mathrm{I} \frac{1}{2}$ ploughs can till. Ansger holds it of $B$ [aldwin]. There $A$ [nsger] has I villein, and he has with him $\frac{1}{2}$ plough, I bordar, I serf, 3 beasts, Io sheep, I acre of coppice, 6 acres of meadow and 7 acres of pasture. Worth 5 shillings a year; when he received it it was worth 2 shillings.

$\mathrm{B}$ [aldwin] has a manor called Ordia [Worthy] $^{7}$ which Almer held T.R.E., and it paid geld for I virgate. This I plough can till. Anschitil holds it of $\mathrm{B}$ [aldwin]. There A[nschitil] has $\frac{1}{2}$ virgate and I plough in demesne, and the villeins $\frac{1}{2}$ virgate. There A[nschitil] has I villein, I serf, 8 sheep, 6 acres of meadow, and 8 acres of pasture. Worth 5 shillings a year; when he received it 2 shillings.

$\mathrm{B}$ [aldwin] has a manor called WeLisEdiNGA (Exch. Welingedinge) [ ] which Topic held T.R.E., and it paid geld for I virgate. This I plough can till. William holds it of $B$ [aldwin]. There $W$ [illiam] has $\frac{1}{2}$ virgate and I plough in demesne, and the villeins $\frac{1}{2}$ virgate. There $W$ [illiam] has I villein, I serf, 8 beasts, I 2 acres of meadow, and Io acres of pasture. Worth 5 shillings a year; when he received it it was worth 3 shillings.

$\mathrm{B}$ [aldwin] has a manor called Chaeledona (Exch. Cheledone) [Cheldon] ${ }^{8}$ which Elmerd (Exch. Elmar) held T.R.E., and it paid geld for 3 ferlings. This I plough can till. Walter holds it of B[aldwin]. There $W$ [alter] has the whole land and I plough in demesne, also I serf, 2 acres of wood(land), 6 acres of meadow, fol. 3ir.

and 60 acres of pasture. Worth 3 shillings a year; when he received it it was worth 2 shillings.

B[aldwin] has a manor called RACHENEFODA $[\text { Rackenford }]^{9}$ which Seward (Exch. Siward) held

${ }^{5}$ Ansgerid, no doubt for Ansger idem.

- Creacombe in Witheridge hundred.

${ }^{7}$ Worthy in Rackenford in Witheridge hundred.

${ }^{8}$ East Cheldon alias Cheldon Farm in Cheldon in Witheridge hundred.

${ }^{2}$ Much alias Great Rackenford in Witheridge hundred. The shortage of half a virgate probably represents Nedcot. 


\section{A HISTORY OF DEVONSHIRE}

T.R.E., and it paid geld for $\frac{1}{2}$ hide and $\frac{1}{2}$ virgate. This 6 ploughs can till. Joselin (Exch. Goselin) holds it of B[aldwin]. There J[oselin] has $\frac{1}{2}$ virgate and I plough in demesne and the villeins $\mathrm{I} \frac{1}{2}$ virgates and 2 ploughs. There J[oselin] has 4 villeins, 4 bordars, 2 serfs, 12 beasts, 2 swine, 60 sheep, 25 goats, 3 acres of wood(land), 20 acres of meadow, and 200 acres of pasture. Worth I 5 shillings a year; when he received it it was worth 10 shillings.

B[aldwin] has a manor called Eltemetona ${ }^{1}$ which Elmer held T.R.E., and it paid geld for $\frac{1}{2}$ virgate. This 2 ploughs can till. Rainald holds it of B[aldwin]. There [Rainald] has $\frac{1}{2}$ ferding in demesne, and the villeins $1 \frac{1}{2}$ ferdings and $\frac{1}{2}$ plough. There $\mathrm{R}$ [ainald] has 3 villeins, 3 bordars, I pig, 40 acres of coppice, 6 acres of meadow and 100 acres of pasture. Worth 5 shillings a year, and was worth the same when he received it.

$\mathrm{B}$ [aldwin] has a manor called Ausa (Exch. Avse) $\left[\right.$ Apps] ${ }^{2}$ which Alebric held T.R.E., and it paid geld for I virgate. This 3 ploughs can till. Rogus holds it of B[aldwin]. There $\mathrm{R}$ [ogus] has $\frac{1}{2}$ virgate and I plough in demesne, and the villeins $\frac{1}{2}$ virgate and I plough. There $\mathrm{R}$ [ogus] has 2 villeins, 2 bordars, 2 serfs, 5 swine, I 5 sheep; Io acres of coppice and 30 acres of pasture. Worth Io shillings; when $B$ [aldwin] received it it was worth 2 shillings.

fol. 3rixb.

B[aldwin] has a manor called Odeborna [Woodburn] $^{3}$ which Aldred (Exch. Eldred) held T.R.E., and it paid geld for 3 ferdings. These 2 ploughs can till. Oswic holds it of $\mathrm{B}$ [aldwin]. There $O$ [swic] has I ferding in demesne, and the villeins 2 ferdings and I plough. There $O$ [swic] has I villein, 4 bordars, 15 acres of meadow, and Io acres of pasture. Worth 5 shillings a year; when he received it it was worth the same.

B[aldwin] has a manor called HAcoma [Haccombe] ${ }^{4}$ which Ulf held T.R.E., and it paid geld for $\frac{1}{2}$ hide. This 5 ploughs can till. Stephen (Exch. Stefan) holds it of B[aldwin]. Thereof S[tephen] has in demesne I virgate and I plough, and the villeins I virgate and 3 ploughs. There S[tephen] has 8 villeins, 4 bordars, 3 serfs, 6 beasts, 8 swine, 40 sheep, 30 goats, 4 furlongs of coppice, and 2 acres of meadow. Worth 20 shillings a year; when $\mathrm{B}$ [aldwin] received it it was worth the same.

1 Unknown, possibly part of Rackenford in Witheridge hundred. Trans. Devon Assoc. xxx, 418,n.2 r (O. J. R.).

West Apse alias West Tapps in Okeford in Witheridge hundred.

${ }^{3}$ Woodburn in Oakford in Witheridge hundred.

- Haccombe in Haytor hundred, with part of Combe-in-Teignhead.
$\mathrm{B}$ [aldwin] has a manor called Taigna [Teign $]^{3}$ which Ulf held T.R.E., and it paid geld for 2 hides. These 5 ploughs can till. Ralf de Brueria holds them of B[aldwin]. Thereof Ralf has in demesne $I$ hide and I plough, and the villeins $\mathrm{I}$ hide and 3 ploughs. There $R$ [alf] has 4 villeins, 7 bordars, 4 serfs, I rouncey, I 2 beasts, 2 swine, 35 goats, 35 acres of wood(land), 20 acres of meadow, and pasture I league in length by 4 furlongs in breadth. Worth 20 shillings a year; when B[aldwin] received it it was worth 10 shillings.

B[aldwin] has a manor called LANGHestan (Exch. Langestan) [Langstone] ${ }^{6}$ which Edwy held T.R.E., and it paid geld for I virgate. This 2 ploughs can till. Hugh holds it of $\mathrm{B}$ [aldwin]. Thereof $\mathrm{H}$ [ugh] has in demesne $\mathrm{I}$ ferding and I plough, and the villeins 3 ferdings and I plough. There $\mathrm{H}[\mathrm{ugh}]$ has 4 villeins, 4 bordars, I serf, 2 beasts, 3 swine, 13 sheep, II goats, II acres of meadow and I 2 acres of pasture. Worth Io shillings a year; when fol. 3r2.

$\mathrm{B}$ [aldwin] received it it was worth 5 shillings.

B[aldwin] has a manor called HaINoc [Hennock $]^{7}$ which Alnod held T.R.E., and it paid geld for 1 hide. This 13 ploughs can till. Roger son of Pagan holds it of B[aldwin]. Thereof R[oger] has in demesne 3 ferdings and 2 ploughs, and the villeins 3 virgates, 1 ferding, and 6 ploughs. There $\mathrm{R}$ [oger] has 9 villeins, 6 bordars, 5 serfs, I rouncey, and 60 sheep. Worth 30 shillings a year; when B[aldwin] received it it was worth 10 shillings.

B [aldwin] has a manor called Beneadona [? by Neadon $]^{8}$ which Adret held T.R.E., and it paid geld for I virgate. This I plough can till. Hervei's wife (widow) holds it of B[aldwin]. There she has 2 bordars and with them she has I plough, I serf, 4 acres of meadow and 5 acres of pasture. Worth 5 shillings a year; when $\mathrm{B}$ [aldwin] received it it was worth 12 pence.

$\mathrm{B}$ [aldwin] has a manor called WITEWEIA [Whiteway] $]^{9}$ which Osferd (Exch. Offers) held T.R.E., and it paid geld for $\frac{1}{2}$ hide. This 2 ploughs can till. Now Rainulf (Exch. Rannulf) holds it of $\mathrm{B}$ [aldwin]. Thereof $\mathrm{R}$ [ainulf] has I virgate and I plough in demesne, and the villeins have I virgate and I plough. There $\mathrm{R}$ [ainulf] has I villein, 4 bordars, I serf, 33 sheep,

${ }^{5}$ Teign Grace alias Teign Bruer in Teignbridge hundred.

${ }^{6}$ Langstone in Manaton in Teignbridge hundred.

${ }^{7}$ Hennock in Teignbridge hundred. Geldroll, fol. $70 b$, A II.

${ }^{8}$ Neadon in Lustleigh in Teignbridge hundred.

${ }^{9}$ Whiteway Barton in Kingsteignton in Teignbridge hundred. 


\section{HOLDERS OF LANDS}

I saltern paying 12 pence a year, and $I$ acre of wood(land). Worth 15 shillings a year ; when $B$ [aldwin] received it it was worth Io shillings.

$\mathrm{B}$ [aldwin] the sheriff has a manor called Chiveorna [Chevithorn] ${ }^{1}$ which Almer held T.R.E., and it paid geld for I virgate. This 5 ploughs can till. Rogro (Exch. Rogo) holds it of $B$ [aldwin]. Thereof $R$ [ogro] has in demesne $\frac{1}{2}$ virgate and I plough, and the villeins $\frac{1}{2}$ virgate and $\frac{1}{2}$ plough. There $R$ [ogro] has 3 villeins, 3 bordars, 3 serfs, 5 beasts, 16 swine, 18 sheep, 6 goats, IOO acres of wood(land), I I acres of meadow and 12 acres of pasture. Worth 20 shillings a year; and was worth the same fol. 3rab.

when he received it.

$\mathrm{B}$ [aldwin] the sheriff (vice-consul) has a manor called Chetellescoma [Chettiscombe] ${ }^{2}$ which Olmar (Exch. Ulmer) held T.R.E., and it paid geld for $\mathrm{I}$ hide. This 6 ploughs can till. Ansger holds it of B[aldwin]. Thereof Ansger has in demesne $I_{2} \frac{1}{2}$ virgates and 2 ploughs, and the villeins $2 \frac{1}{2}$ virgates and 3 ploughs. There $A$ [nsger] has 6 villeins, 5 bordars, 4 serfs, I mill paying 2 shillings, 6 beasts, 60 acres of wood(land), Io acres of meadow and 40 acres of pasture. Worth 40 shillings a year; when $\mathrm{B}$ [aldwin] received it it was worth Io shillings.

B[aldwin] has a manor called Magnetona [Manaton] ${ }^{3}$ which Edwi held T.R.E., and it paid geld for I virgate. This I plough can till. Edwi holds it of $\mathrm{B}$ [aldwin]. There E[dwi] has $\frac{1}{2}$ virgate and I plough in demesne, and the villeins $\frac{1}{2}$ virgate. There $\mathrm{E}$ [dwi] has 3 villeins, 3 bordars, I serf, ro beasts, 30 sheep, 25 goats, wood(land) $\frac{1}{2}$ league in length by $\frac{1}{2}$ furlong in breadth, 5 acres of meadow and 10 acres of pasture. Worth ro shillings a year.

$B$ [aldwin] has a manor called Sriac [Sallake] ${ }^{4}$ which Brismer held T.R.E., and it paid geld for $\frac{1}{2}$ hide. This 2 ploughs can till. Goscem (Exch. Goscelm) holds it of B[aldwin]. There G[oscem] has in demesne $x$ virgate and 3 ferlings and I plough, and the villeins I ferling and $\frac{1}{2}$ plough. There G[oscem] has 2 villeins and 2 bordars, 6 acres of wood(land) and 2 acres of meadow. Worth Io shillings a year; when $\mathrm{B}$ [aldwin] received it it was worth 5 shillings.

$\mathrm{B}$ [aldwin] has a manor called SPERCHEwILla [Sparkwell] $^{\mathbf{5}}$ which Bristric (Exch. Brictric) held

'East Chevithorn in Tiverton in Tiverton hundred.

2 Chettiscombe in Tiverton in Tiverton hundred.

${ }^{8}$ Little Manaton in Manaton in Teignbridge hundred. Trans. Devon Assoc. xxxvi, 358.

"Sallake in Halberton in Halberton hundred.

${ }^{5}$ Sparkwell in Staverton in Haytor hundred.
T.R.E., and it paid geld for $\frac{1}{2}$ hide. This fol. 3 r3.

4 ploughs can till. Richard holds it of $B$ [aldwin]. There $R$ [ichard] has in demesne $I$ virgate and I plough, and the villeins I virgate and I plough. There he has 4 villeins, 2 bordars, 2 serfs, 30 sheep, 8 acres of wood(land), 4 acres of meadow and 20 acres of pasture. Worth I5 shillings a year, and was worth ro shillings when he received it.

B [aldwin] has a manor called OLveberRIa $(E x c b$. Ulveberie) [Wolborough] ${ }^{8}$ which Seward (Exch. Siward) held T.R.E., and it paid geld for I hide. This 8 ploughs can till. Ralf de Brueria holds it of $B$ [aldwin]. Thereof $R$ [alf] has in demesne I virgate and 2 ploughs, and the villeins 3 virgates and 4 ploughs. There $R$ [alf] has 6 villeins, 7 bordars, 4 serfs, 12 beasts, I00 sheep, I mill paying 5 shillings, 30 acres of coppice, 15 acres of meadow and 30 acres of pasture. Worth 40 shillings; when $B$ [aldwin] received it it was worth 20 shillings.

$\mathrm{B}$ [aldwin] the sheriff has a manor called MusBERIA [Musbury] ${ }^{7}$ which Almer held T.R.E., and it paid geld for 7 hides. These 16 ploughs can till. Thereof the sheriff has in demesne 4 hides and 3 ploughs, and the villeins 3 hides and 6 ploughs. There the sheriff has 16 villeins, 4 bordars, 8 serfs, 18 unbroken mares (indomitas), I 6 beasts, 16 swine, 100 sheep, 30 goats, 40 acres of wood(land), x mill paying 5 shillings, 43 acres of meadow and 50 acres of pasture. Worth 4 pounds; when $B$ [aldwin] received it it was worth 60 shillings.

$B$ [aldwin] has a manor called Tornecoma [Thorncombe ${ }^{8}$ which Etward (Exch. Edward) held T.R.E., and it paid geld for 2 hides. These I 2 ploughs can (till). Thereof the sheriff (vicecomes) has in demesne I hide and 2 ploughs, and the villeins $I$ hide and Io ploughs. There the sheriff (vice-consul) has I 6 villeins, 8 bordars, 2 serfs, 10 beasts, 66 sheep, I 5 acres of wood (land), I 8 acres of meadow and 30 of pasture. fol. 3rgb.

Worth 100 shillings a year; when he received it 4 pounds.

$\mathrm{B}$ [aldwin] has a manor called FordA [Ford] ${ }^{9}$ which Alveva held T.R.E., and it paid geld for $\frac{1}{2}$ hide. This 4 ploughs can till. Ranulf (Exch. Rannulf) holds it of $\mathrm{B}$ [aldwin]. Thereof Ranulf has in demesne I virgate and 2 ploughs,

${ }^{6}$ Wolborough manor in Haytor hundred.

${ }^{7}$ Musbury Courtney, 2 hides of which are in Axminster (Add. MSS. 28,649, fol. 49I), the rest in Axmouth hundred.

${ }^{8}$ Thorncombe in Axminster hundred, since 1842 included in Dorset.

${ }^{8}$ Ford next Trill with Ash in Musbury in Axminster hundred. 


\section{A HISTORY OF DEVONSHIRE}

and the villeins 1 virgate and 2 ploughs. There $R$ [anulf] has 2 villeins, 5 bordars, $I$ serf, 12 beasts, 12 swine, 30 sheep, 30 goats, I mill paying 30 pence, 15 acres of wood(land), I 2 acres of meadow and 50 acres of pasture. Worth 25 shillings a year; when he received it it was worth 20 shillings.

$B$ [aldwin] the sheriff has a manor called ALraFORDA [Allerford] ${ }^{1}$ which Juin held T.R.E., and it paid geld for I virgate. This $1 \frac{1}{2}$ ploughs can till. Ranulf (Exch. Rannulf) holds it of $\mathrm{B}$ [aldwin]. There R[anulf] has $\frac{1}{2}$ plough and 3 bordars, 4 beasts, 3 swine, 4 sheep, 3 acres of meadow and 20 acres of pasture. Worth 5 shillings a year, and was worth the same when he received it.

B [aldwin] has a manor called Hesmalacoma (Exch. Smelecome) [Smallicombe] ${ }^{2}$ which Godwin held T.R.E., and it paid geld for $\frac{1}{2}$ hide. This I plough can till. Roger holds it of $\mathrm{B}$ [aldwin]. There [Roger] has I plough, I serf, 3 acres of meadow and 6 acres of pasture. Worth 5 shillings a year, and was worth the same when he received it.

$\mathrm{B}$ [aldwin] has a manor called Estotacoma (Exch. Stotecoma) [Stedcombe] ${ }^{3}$ which Ulf held T.R.E., and it paid geld for I hide. This 4 ploughs can till. Morin holds it of B[aldwin]. Thereof Morin has in demesne 2 virgates, and fol. $3 \times 4$.

the villeins 2 virgates. There Morin has 3 serfs and 6 acres of coppice, 6 acres of meadow and Io acres of pasture. Worth 12 shillings a year.

$B$ [aldwin] has a manor called Colewilla [Colwell] ${ }^{4}$ which Almer held T.R.E., and it paid geld for $\mathrm{I} \frac{1}{2}$ hides. These 8 ploughs can till. Rogro (Exch. Rogo) holds it of B[aldwin]. Thereof Rogro has in demesne $\frac{1}{2}$ hide and I plough, and the villeins I hide and I $1 \frac{1}{2}$ ploughs. There R[ogro] has 6 villeins, 2 bordars, 2 serfs, 5 beasts, 8 swine, 57 sheep, 20 goats, 80 acres of wood(land) and 300 acres of pasture. Worth 20 shillings a year; when he received it it was worth 10 shillings.

$\mathrm{B}$ [aldwin] has a manor called Coma [Combe ${ }^{5}$ which Gotman (Exch. Godman) held T.R.E., and it paid geld for I hide. This 4 ploughs can till. Odo holds it of B[aldwin]. Thereof Odo has in demesne 2 virgates and I plough, and the

'Allerford in Axminster parish and hundred; or perhaps Rosamondford in East Budleigh hundred (O.J.R.).

'Smallicombe, an outlier of Offwell in Northleigh.

${ }^{3}$ Stedcombe in Axmouth parish and hundred.

4 East and West Colwell in Offwell in Colyton hundred. This Rogro appears to be identical with Roger de Roeric of the Geldroll (fol. 68b, B 3).

${ }^{5}$ Combe Pyne in Axmouth hundred. Feud. Aids, i, 328 . villeins 2 virgates and $\frac{1}{2}$ plough. There Odo has 4 villeins, 2 bordars, 3 serfs, 3 beasts, 75 sheep, 9 acres of meadow and 30 acres of pasture. Worth 20 shillings a year; when he received it it was worth the same.

$\mathrm{B}$ [aldwin] has a manor called OfFawiLla [Offwell] $^{6}$ which Borgaret (Exch. Borgeret) held T.R.E., and it paid geld for I virgate. This 2 ploughs can till. Raginald holds it of $B$ [aldwin]. Thereof $R$ [aginald] has in demesne $\frac{1}{2}$ virgate and I plough, and the villeins $\frac{1}{2}$ virgate and 2 ploughs. There R[aginald] has 6 villeins and 2 bordars, ro beasts, wood(land) 5 furlongs in length by 20 perches in breadth, and 80 acres of pasture. Worth 13 shillings a year; when he received it it was worth 12 pence.

fol. 3r4b.

$\mathrm{B}$ [aldwin] the sheriff has a manor called Wilelmitona [Wilmington $]^{7}$ which Etmer (Exch.Edmer) held T.R.E., and it paid geld for I virgate. This 2 ploughs can till. Morin holds it of B [aldwin]. Thereof Morin has in demesne $\frac{1}{2}$ virgate and I plough, and the villeins $\frac{1}{2}$ virgate and $\frac{1}{2}$ plough. There $M$ [orin] has 2 villeins, 2 bordars, I serf, 3 sheep, I 5 acres of wood(land) and 7 acres of meadow. Worth $7 \frac{1}{2}$ shillings; when he received it it was worth 5 shillings.

$\mathrm{B}$ [aldwin] has a manor called Collabera (Excb. Collebere) [Culbeer ${ }^{8}$ which Adelwold (Exch. Alwold) held T.R.E., and it paid geld for 3 virgates. These 2 ploughs can till. There Morin who holds it of $\mathrm{B}$ [aldwin] has nothing (in demesne). Worth 3 shillings a year.

B[aldwin] has a manor called Engleborna [Inglebourn] ${ }^{9}$ which Brismar held T.R.E., and it paid geld for $\frac{1}{2}$ hide. This 5 ploughs can till. W- holds it of $\mathrm{B}$ [aldwin]. Thereof W- has in demesne I virgate and I plough, and the villeins I virgate. There $W$ - has 3 villeins, I bordar, I serf, 3 beasts, 4 swine, 32 sheep, 13 goats, 13 acres of meadow and 40 acres of pasture. Worth I 5 shillings a year; when he received it it was worth the same.

$\mathrm{B}$ [aldwin] has a manor called Prenla [Prawle] ${ }^{10}$ which Osfern (Exch. Osfers) held T.R.E., and it paid geld for $I$ hide. This 6 ploughs can till. Otley (Otelinus; Exch., Othelinus) holds it' of $\mathrm{B}$ [aldwin]. Thereof $\mathrm{O}$ [tley] has in demesne $I$ virgate and 2 ploughs, and the villeins 3 virgates

- Offwell in Colyton hundred.

${ }^{7}$ North Wilmington and Cleave, an outlier of Offwell in Colyton hundred next Cotleigh. Ancient

Deeds, C 2,3 10 .

${ }^{9}$ Culbear in Offwell in Colyton hundred.

${ }^{9}$ Inglebourn Abbots in Harberton in Colridge hundred. Trans. Devon Assoc. viii, 882.

${ }^{10}$ West Prawle in Portlemouth in Colridge hundred. 


\section{HOLDERS OF LANDS}

and 4 ploughs. There $\mathrm{O}$ [tley] has Io villeins, 8 bordars, 4 serfs, 4 beasts, I 5 swine, 40 sheep, 15 goats, 60 acres of wood(land), 4 acres of fol. 355 .

meadow and 63 (Exch. 64) acres of pasture. Worth 20 shillings a year; when $\mathrm{B}$ [aldwin] received it it was worth Io shillings.

Baldwin has 7 burgesses in BARNestapLE [Barnstaple $]^{1}$ and 6 houses lying in ruins, and those burgesses and the houses lying in ruins pay 7 shillings and 6 pence a year.

Baldwin has in Essecestra ${\text { [Exeter }]^{2}}^{12}$ (fol. 297 says II) houses which belonged to his manor called Chent [Kenn] T.R.E. And he has there 7 other houses by gift of King $\mathrm{W}$ [illiam], which were in the demesne of King $E$ [dward] on the day on which he was alive and dead.

\section{THE LAND OF JUHEL (Exch. JUDHEL) IN DEVENESIRA ${ }^{3}$ \\ fol. 376 .}

Juhel (Exch. Judhel) has a manor called Tresetone [Thrushelton] ${ }^{4}$ which Grim held T.R.E. This in King E[dward]'s time paid geld for I hide. This I4 ploughs can till. Thereof J[uhel] has $\frac{1}{2}$ virgate and 3 ploughs in demesne, and the villeins $3 \frac{1}{2}$ virgates and II ploughs. There J[uhel] has 20 villeins, 20 bordars, I I serfs, 17 beasts, 60 sheep, 20 acres of wood(land), 100 acres of meadow and 100 acres of pasture. This manor pays Io pounds a year; when J[uhel] received it it was worth the same.

Juhel has a manor called RATDona [Raddon] ${ }^{5}$ which Osulf held T.R.E., and it paid geld for I hide. This 15 ploughs can till. Nigel holds it of Juhel. Thereof Nigel has $I_{2} \frac{1}{2}$ virgates and 3 ploughs in demesne, and the villeins $2 \frac{1}{2}$ virgates and 2 ploughs. There Nigel has 8 villeins, I 5 bordars, 5 serfs, 20 beasts, 140 sheep, wood(land) $\frac{1}{2}$ league in length by $I$ furlong in breadth, IO acres of meadow, pasture $\frac{1}{2}$ league in length by $I$ furlong in breadth. This manor is worth roo shillings (and 5 interlined; Exch. 100); when Juhel received it it was worth 7 pounds and Io shillings.

1 Barnstaple locally in Fremington hundred.

Exeter locally in Wonford hundred.

${ }^{3}$ The Exchequer book follows the same order as the Exeter book in describing Juhel's manors, except that it places Totnes and Exeter first, whereas the Exeter book places them last.

"Thrushelton in Lifton hundred.

s Raddon in Maristow in Lifton hundred.
Juhel has a manor called BRADEwODA [Bradwood] ${ }^{6}$ which Chenestan (Exch. Chinestan) held T.R.E., and it paid geld for $\frac{1}{2}$ hide all but $1 \frac{1}{2}$ ferlings. This 6 ploughs can till. Thereof fol. $356 \mathrm{~b}$.

Nigel has $\frac{1}{2}$ virgate and $I \frac{1}{2}$ ploughs in demesne, and the villeins I virgate, $\frac{1}{2}$ ferling, and $2 \frac{1}{2}$ ploughs. There Nigel has Io villeins, I I bordars, 8 serfs, 3 I beasts, 4 swine, 200 sheep, 8 goats, wood(land) I league in length by I furlong in breadth, 50 acres of meadow, and pasture I league in length by $\mathrm{I}$ furlong in breadth. Worth 4 pounds a year; when Juhel received it it was worth 6 pounds.

Juhel has a manor called Nortona [Norton] ${ }^{7}$ which Bristrict (Exch. Brictric) held T.R.E., and it paid geld for I virgate. This 6 ploughs can till. Now William holds it of Juhel. Thereof William has I ferting and I plough in demesne, and the villeins 3 ferlings and 5 ploughs. There William has 7 villeins, 4 bordars, 3 serfs, I 7 beasts, 40 sheep, wood(land) 3 furlongs in length by $I$ in breadth, 70 acres of meadow, and pasture $\frac{1}{2}$ league in length by 3 furlongs in breadth. Worth 60 shillings a year, when Juhel received it the same.

Juhel has a manor called $\mathrm{K}_{\text {ARI }}[\mathrm{Cary}]^{8}$ which Chenestan held T.R.E., and it paid geld for $I_{\frac{1}{2}}$ virgates. This 6 ploughs can till. Waldin now holds it of Juhel. Thereof Waldin has $\frac{1}{2}$ virgate and I plough in demesne, and the villeins I virgate and $3 \frac{1}{2}$ ploughs. There Waldin has 8 villeins, 6 coscets, 3 serfs, 8 beasts, 42 sheep, 20 goats, 20 acres of wood(land), 40 acres of meadow, and pasture 2 furlongs in length fol. 317.

by $I$ in breadth. Worth 40 shillings a year; when Juhel received it it was worth 50 shillings.

Juhel has a manor called Sidreham [Sydenham $]^{9}$ which Bristric (Exch. Brictric) held T.R.E., and it paid geld for I virgate. This 5 ploughs can till. William holds it of Juhel. Thereof William has $\frac{1}{8}$ virgate and I plough in demesne, and the villeins $\frac{2}{3}$ virgate and 4 ploughs. There W[illiam] has 5 villeins, 7 coscets, 5 serfs, 2 beasts, II swine, 40 sheep, wood(land) 3 furlongs in length by I (furlong) in breadth, and Io acres of meadow. Worth 40 shillings a year; when Juhel received it it was worth 30 shillings.

${ }^{6}$ Bradwood alias Broadwood Widger alias Vipunt (Trans. Devon Assoc. xvi, 175) in Lifton hundred.

"Norton Bauzan in Bradwood Widger in Lifton hundred.

${ }^{8}$ Downacarey in Bradwood Widger in Lifton hundred.

${ }^{9}$ Little Sydenham in Maristow in Lifton hundred. 


\section{A HISTORY OF DEVONSHIRE}

Juhel has a manor called Assineria [Ashleigh] ${ }^{1}$ which Grin (Exch. Grim) held T.R.E., and it paid geld for I $\frac{1}{2}$ virgates. These 5 ploughs can till. Ralf holds it of Juhel. Thereof $R$ [alf] has $\frac{1}{2}$ virgate and I plough in demesne, and the villeins I virgate, 3 ploughs and I ox. R[alf] has there 7 villeins, 3 bordars, 25 sheep, 2 acres of alder (alneti) and 8 acres of meadow. Worth 25 shillings a year; when Juhel received it 30 shillings.

Juhel has a manor called Mora [Moor]. ${ }^{2}$ This Aldret (Exch. Eldred) held T.R.E., and it paid geld for I virgate. This 5 ploughs can till. Nigel holds it of Juhel. A third part of the aforesaid virgate Nigel has in demesne and I plough, and the villeins have $\frac{2}{3}$ virgate and 2 ploughs. There Nigel has 8 villeins, I coscet, 6 serfs, 7 beasts, wood(land) 2 furlongs in length by 12 perches in breadth, 40 acres of meadow, fol. 3 rgb.

and pasture $\mathrm{I}$ league in length by 4 furlongs in breadth. Worth 30 shillings a year; when Johel received it 40 shillings.

Juhel has a manor called BRADEForda [Bradford $]^{3}$ which Sawin held T.R.E., and it paid geld for $\frac{1}{2}$ virgate. This 2 ploughs can till. Now Nigel holds it of Juhel. There Nigel has I villein, ro acres of wood(land), 8 acres of meadow and I acre of pasture. Worth 30 pence a year; when Juhel received it it was worth the same.

Juhel has a manor called ToRnelowa [Tilleslowe ${ }^{4}$ which Sawin held T.R.E., and it paid geld for $\frac{1}{2}$ virgate. This 2 ploughs can till. Nigel now holds it of Juhel. Thereof Nigel has I ferling and I plough in demesne, and the villeins have the other ferling and 3 oxen. There Nigel has I villein, 2 bordars, I serf, 8 beasts, 28 sheep, ro acres of wood(land), 7 acres of meadow and 2 acres of pasture. Worth Io shillings a year; when Juhel received it it was worth the same. The thane who held the two above-named manors could go with his land to whatever lord he liked T.R.E.

Juhel has a manor called Tavi [Tavy] ${ }^{5}$ which Bristuit (Exch. Bricstuit) Camesone held T.R.E., and it paid geld for I virgate. This 4 ploughs can till. Nigel holds it of Johel. Thereof Nigel has $\frac{1}{2}$ virgate and $\mathrm{I} \frac{1}{2}$ ploughs in demesne,

${ }^{1}$ Ashleigh in Lifton in Lifton hundred.

2 Temple Moor and Moor fitz-Stephen in Bradwood Widger in Lifton hundred.

${ }^{3}$ Bradford Heriz in Virginstow in Lifton hundred. See fol. $500 b$.

"Tilleslowe alias Twoslough in Virginstow in Lifton hundred.

${ }^{5}$ Tavy St. Mary alias Mary Tavy in Lifton. and the villeins $\frac{1}{2}$ virgate and I plough. There fol. 318.

Nigel has 6 villeins, 2 bordars, 6 serfs, 16 beasts, 4 swine, 120 sheep, 12 acres of meadow, and pasture $\frac{1}{2}$ league in length by 6 furlongs in breadth. Worth 40 shillings; when Juhel received it it was worth 30 shillings.

Along with this manor Juhel has the land of three thanes which did not belong to the abovenamed manor in King E[dward]'s time. ${ }^{6}$ One of them is called Wagesferla [Warn] ${ }^{7}$ which Alvric held. Another of them is called Berna [Burntown $]^{8}$ which Alwin held. And the third is called WeringurRda [W ringworthy] ${ }^{\theta}$ which Adulf held. These 3 thanes could go to what lord they liked with that land, and it paid geld in King E[dward]'s time for I virgate. This 3 ploughs can till. Nigel holds in demesne of Juhel, and he has there $\frac{1}{2}$ plough, 2 villeins, 5 bordars, I serf, 12 beasts, 30 sheep, 30 goats, 12 acres of meadow, and pasture $I$ league in length by $\frac{1}{2}$ league in breadth. Those 3 townships (villae) are worth 25 shillings a year; when Juhel received them the value was the same.

Juhel has a manor called Sidelham [Sydenham] ${ }^{10}$ which 4 thanes held in parage (pariter; Exch., libere) T.R.E., and it paid geld for $\frac{1}{2}$ hide. This 8 ploughs can till. Now Nigel holds it of Juhel. Thereof $\mathbf{N}$ [igel] has $I$ virgate and 2 ploughs in demesne, and the villeins I virgate and I plough. There N[igel] has 5 villeins, 8 bordars, 2 serfs, 2 beasts, 40 sheep, 20 acres of fol. ar8b.

wood(land), 5 acres of meadow and 20 acres of pasture. Worth 30 shillings; when J[uhel] received it it was worth 40 shillings.

Juhel has a manor called Crauetona [Clawton] ${ }^{11}$ which Alvered (Exch. Alvred) held T.R.E., and it paid geld for I hide all but I ferling. This 20 ploughs can till. Of this Juhel has in demesne I virgate and 4 ploughs, and the villeins have 3 virgates all but a ferling and II ploughs. There Juhel has 28 villeins, 6 bordars, 16 serfs, 2 swineherds, 47 beasts, 9 swine, 200 sheep all but IO, I league of wood(land) (nemusculum corrected into nemus; Exch., silva) taking length and breadth, meadow $x$ league in length by $\frac{1}{2}$ league in breadth, and I league of pasture taking length and breadth. Worth 8 pounds a year; when $\mathrm{J}$ [uhel] received it it was worth ro pounds.

${ }^{6}$ See fol. $495 b$.

7 Warn in Mary Tavy in Lifton hundred.

${ }^{8}$ Burntown in Mary Tavy in Lifton hundred.

${ }^{\circ}$ Wringworthy in Mary Tavy in Lifton hundred.

${ }^{10}$ Sydenham Damarel alias South Sydenham in Lifton hundred. See fol. 495 b.

"Clawton in Blacktorington hundred. Geldroll, fol. 65, A 8 . 


\section{HOLDERS OF LANDS}

Juhel has a manor called PAORDA [Pyworthy] ${ }^{1}$ which Alvered (Exch. Alvred) held T.R.E., and it paid geld for 3 virgates. These 16 ploughs can till. Of these Ju[hel] has in demesne I virgate and 5 ploughs, and the villeins 2 virgates and 5 ploughs. There Ju[hel] has 30 villeins, 5 bordars, 8 serfs, 2 swine, 35 beasts, 200 sheep all but IO, wood(land) 4 furlongs in length by 20 perches in breadth, 1 league of meadow taking length and breadth, and I league of pasture taking length and breadth. Worth 8 pounds; fol. 3rg.

when he received it it was worth I I pounds.

Juhel has a manor called Tetecota [Tetcot] ${ }^{2}$ which Aldret held T.R.E., and it paid geld for $\frac{1}{2}$ hide. This 7 ploughs can till. Of this $(D e$ bac) J[uhel] has in demesne $\frac{1}{2}$ virgate, I plough, and 2 oxen, and the villeins $1 \frac{1}{2}$ virgates and $2 \frac{1}{2}$ ploughs. There J[uhel] has I 2 villeins, 4 bordars, 6 serfs, I swineherd, 8 beasts, 2 swine, 36 sheep, 40 acres of wood(land), 30 acres of meadow, and pasture $\frac{1}{2}$ league in length by 4 furlongs in breadth. Worth 4 pounds a year; when he received it it was worth 100 shillings.

Along with this manor Juhel has a ferding of land ${ }^{3}$ which Elwin held in parage (pariter; Exch., libere) T.R.E. when it did not belong to the above-named manor. This I plough can till. There J[uhel] has I (Exch. 2) villeins and I pig, and they have there I plough. Worth IO shillings a year, and was worth the same when he received it.

Juhel has a manor called LiclemoRE ${ }^{4}$ which Aiulf held T.R.E., and it paid geld for I ferding. This $2 \frac{1}{2}$ ploughs can till, and those ploughs are there. Aiolf holds it of J[uhel] and pays to him therefrom 20 shillings a year. It was worth 25 shillings when J[uhel] received it.

Juhel has a manor called BRADEFORT [Bradaford] ${ }^{5}$ which Aileva held T.R.E., and it paid geld for I ferding. This 2 ploughs can till. William holds it of J[uhel], and has there fol. 3rgb.

I plough in demesne and 2 serfs, 3 beasts, meadow 3 furlongs in length by I in breadth, and pasture 2 furlongs in length by $I$ in breadth. Worth ro shillings a year, and was worth the same when J[uhel] received it.

Juhel has a manor called Hindefort [Henford] ${ }^{6}$ which Brore (Exch. Brodre) held T.R.E.,

${ }^{1}$ Pyworthy in Blacktorington hundred.

2 Tetcot in Blacktorington hundred. See fol. 495 .

${ }^{8}$ East Peak, an outlier of Tetcot in Blacktorington hundred (O. J. R.). Fernhill, Tetcot (T.W.W.).

'Possibly More Killatree in Pyworthy in Blacktorington hundred.

${ }^{5}$ Bradaford in Pyworthy in Blacktorington hundred.

${ }^{6}$ Henford in Ashwater in Blacktorington hundred. and it paid geld for I virgate. This Ralf de Pomeria holds of J[uhel], and 3 ploughs can till it. R[alf] has (there) of $\frac{1}{2}$ virgate in demesne and I plough, and the villeins the other moiety of a virgate and $\mathrm{I}$ plough. There $\mathrm{R}$ [alf] has I villein, 8 bordars, I serf, 5 beasts, 2 swine, 18 sheep, 15 goats, 15 acres of wood(land), 15 acres of meadow, and $\frac{1}{2}$ league of pasture taking length and breadth. This manor is worth 15 shillings a year, and was worth 12 shillings when J[uhel] received it.

Juhel has a manor called BRIDEForda [Bridford] ${ }^{7}$ which Alwin held T.R.E., and it paid geld for 2 hides. These I 2 ploughs can till. Thereof J[uhel] has in demesne 3 virgates and 2 ploughs, and the villeins I hide I virgate and 9 ploughs. There Juhel has 20 villeins, 7 bordars, 4 serfs, I swineherd paying Io swine, I beast, I rouncey, 80 sheep, 20 goats, wood(land) and coppice I league in length by I furlong in breadth, 14 acres of meadow and 70 acres of pasture. Worth 3 pounds a year, and was worth the same when he received it.

fol. 320.

Juhel has a manor called Bovi [Bovey $]^{8}$ which John held T.R.E., and it paid geld for I hide and 3 virgates. This 8 ploughs can till. Turgis holds it of Juhel. Thereof T[urgis] has 3 virgates and $I$ plough in demesne, and the villeins I hide and 5 ploughs. There T[urgis] has I I villeins, 6 bordars, 5 serfs, 8 beasts, 50 sheep, 10 acres of wood(land), 20 acres of meadow, and I league of pasture. Worth 40 shillings, when J[uhel] received it the same.

Juhel has a manor called Comba [Combe] ${ }^{9}$ which Alwold held T.R.E., and it paid geld for $\frac{1}{2}$ hide. This 3 ploughs can till. Ralf de Pomaria holds it of Juhel. Thereof R[alf] has $I$ virgate and $\frac{1}{2}$ plough in demesne, and the villeins $I$ virgate and $I$ plough. There $R$ [alf] has 3 villeins, 2 bordars, 26 sheep, 3 acres of meadow and I acre of pasture. Worth 15 shillings, and was worth the same when J[uhel] received it.

Juhel has a manor called Comba [Combe] ${ }^{10}$ which Alvric held T.R.E., and it paid geld for I virgate. This I plough can till. Ralf holds it of J[uhel]. Thereof R[alf] has $\frac{1}{2}$ virgate and $\frac{1}{2}$ plough in demesne, and the villeins $\frac{1}{2}$ virgate and $\frac{1}{2}$ plough. There $R$ [alf] has 2 villeins, A. 3 .

${ }^{7}$ Bridford in Wonford hundred. Geldroll, fol. $70 b$,

${ }^{8}$ North Bovey in Teignbridge hundred. Geldroll, fol. 69, A. 4 .

${ }^{9}$ Combe Fishacre alias Punchardon in Ipplepen in Haytor hundred.

${ }^{10}$ Longcombe in Berry Pomeroy in Haytor hundred (O.J.R.). Combe Fishacre rlias Punchardon (T.W.W.). 


\section{A HISTORY OF DEVONSHIRE}

I bordar, and $1 \frac{1}{2}$ acres of meadow. Worth 10 shillings; when $J[$ uhel] received it the same.

fol. $320 \mathrm{~b}$.

Juhel has a manor called Hamistona [Hempston] ${ }^{1}$ which Algar held T.R.E., and it paid geld for I virgate. This I plough can till. $\mathrm{R}$ [alf] de Pomaria holds it of J[uhel]. There $\mathrm{R}$ [alf] has $\mathrm{I}$ plough in demesne and 4 bordars. Worth 5 shillings; when J[uhel] received it 30 pence.

Juhel has a manor called LovenETORNA [Loventor] $^{2}$ which Otro (Exch. Otre) held T.R.E., and it paid geld for $\frac{1}{2}$ hide. This 3 ploughs can till. R[alf] de Pomaria holds it of J[uhel]. Thereof $R$ [alf] has I virgate and I plough in demesne, and the villeins I virgate and 2 ploughing beasts (animalia in carr'). There R[alf] has 3 bordars, I serf, I beast, 29 sheep, 2 acres of wood(land), 2 acres of meadow and 5 acres of pasture. Worth Io shillings; when $\mathrm{J}[\mathrm{uhel}]$ received it 5 shillings.

Juhel has a manor called Coletona [Collaton] ${ }^{3}$ which Alvric held T.R.E., and it paid geld for I virgate. This 2 ploughs can till. $R$ [alf] de Pomaria holds it of J[uhel]. Thereof R[alf] has $\frac{1}{2}$ virgate and I plough in demesne, and the villeins $\frac{1}{2}$ virgate and I plough. There $R$ [alf] has I villein, 5 bordars, I serf, 29 sheep, 5 acres of meadow and 2 acres of pasture. Worth 10 shillings; when J[uhel] received it 5 shillings.

Juhel has a manorcalled Loche'ToNA [Lupton] ${ }^{4}$ which Otro (Exch. Otre) held T.R.E., and it paid geld for $I$ virgate. This 3 ploughs can till. $\mathbf{R}$ [alf] de Pomeria holds it of J[uhel]. Thereof $\mathrm{R}$ [alf] has $\frac{1}{2}$ virgate and I plough in demesne, and the villeins $\frac{1}{2}$ virgate and 6 ploughing beasts (animalia in carr'). There R[alf] has 2 villeins, fol. 32r.

2 bordars, I serf, 2 beasts, I 3 swine, 57 sheep, and 4 acres of wood(land). Worth Io shillings; when J[uhel] received it 5 shillings.

Juhel has a manor called BRISEHAM [Brixham] which Ulf held T.R.E., and it paid geld for $2 \frac{1}{2}$ hides. This ro ploughs can till. Thereof J[uhel] has $\frac{1}{2}$ hide and 2 ploughs in demesne, and the villeins 2 hides and 8 ploughs. There

${ }^{1}$ Hempston Chatard alias Uphempston in Little Hempston in Haytor hundred.

' Loventor in Berry Pomeroy in Haytor hundred.

${ }^{3}$ Collaton Sheephay in St. Mary Church in Haytor hundred (O.J.R.); or part of Coleton, Brixham (T.W.W.). Oliver, Mon. Pp. 174, 1 76, shows that Collaton Sheephay was purchased by William Briwere from Pomeray, and given by him together with Ilesham to Torre Abbey.

4 Lupton in Brixham in Haytor hundred.

${ }^{5}$ Brixham in Haytor hundred. Geldroll, fol. $69 b$, A. 4 .
J[uhel] has I 5 villeins, I 2 bordars, 5 cottagers, 12 serfs, 2 rounceys, 4 beasts, Io swine, 200 sheep all but 20, 12 acres of wood(land), 4 acres of meadow and 12 acres of pasture. Worth 4 pounds; when $J[$ uhel] received it 60 shillings.

Juhel has a manor called Cercitona [Churston ${ }^{6}$ which Ulf held T.R.E., and it paid geld for $I \frac{1}{2}$ hides. This 6 ploughs can till. There J[uhel] has $\frac{1}{2}$ hide and 2 ploughs in demesne, and the villeins $I$ hide and 4 ploughs. There J[uhel] has 8 villeins, 7 bordars, 3 cottagers, 7 serfs, I rouncey, 6 beasts, 8 swine, I 20 sheep, 15 acres of wood(land) and 12 acres of pasture. Worth 40 shillings; when J[uhel] received it the same.

Juhel has a manor called Coletona [Collaton] ${ }^{7}$ which Alward held T.R.E., and it paid geld for $\frac{1}{2}$ hide. This 2 ploughs can till. Warin holds it of Juhel. Thereof $W$ [arin] has I virgate and I plough in demesne, and the villeins I virgate and I plough. There $W$ [arin] has 5 villeins, 2 bordars, I serf, 6 beasts, I 4 swine, 70 sheep, 6 goats, and 4 acres of pasture. Worth 15 shillings; when J[uhel] received it 5 shillings.

Juhel has a manor called LoDEswrLLA [Loddiswell $]^{8}$ which Hece (Exch. Heche) held T.R.E., and it paid geld for 2 hides. These 12 ploughs fol. 32rb.

can till. Thereof J[uhel] has I hide and 2 ploughs in demesne, and the villeins $I$ hide and 8 ploughs. There J[uhel] has 20 villeins, Io bordars, 6 cottagers, 8 serfs, I rouncey, 4 beasts, 6 swine, 42 sheep, I I goats, I fishery paying 30 salmon, I league of wood(land), 13 acres of meadow and $\frac{1}{2}$ league of pasture. Worth 100 shillings; when $J[$ uhel] received it the same.

Juhel has a manor called ToRLEsTan [Thurlstone $]^{9}$ which John held T.R.E., and it paid geld for 2 hides. These 6 ploughs can till. Thereof J[uhel] has I hide and 2 ploughs in demesne, and the villeins. I hide and 6 ploughs. There J[uhel] has 15 villeins, 6 bordars, 4 cottagers, 5 serfs, I rouncey, 16 swine, I 50 sheep, 2 acres of meadow and 2 acres of pasture. Worth 60 shillings ; when J[uhel] received it 4 pounds.

A certain thane held $\mathbf{I}$ virgate ${ }^{10}$ parcel of the above-named manor T.R.E., but he could not become independent of (separare a) John. Now a certain knight (miles) holds it of J[uhel]. There that knight has $\frac{1}{2}$ virgate and $I$ plough in

- Churston Ferrers in Haytor hundred.

${ }^{7}$ Collaton Fishacre in Brixham in Haytor hundred.

${ }^{8}$ Loddiswell in Stanborough hundred. Geldroll, fol. $69 b$, A. 5 :

9 Thurlstone in Stanborough hundred.

${ }^{10}$ North Upton in Thurlestone (Feud. Aids, i. 397)

in Stanborough hundred (O.J.R.), or Bere Island (T.W.W.). 


\section{HOLDERS OF LANDS}

demesne, and the villeins $\frac{1}{2}$ virgate and $\frac{1}{2}$ plough, also 2 villeins, 3 bordars, and 2 beasts. Worth Io shillings; when J[uhel] received it 5 shillings. fol. 322.

Juhel has a manor called BAchedon A [Bagton] ${ }^{1}$ which Algar held T.R.E., and it paid geld for $\frac{1}{2}$ hide. This 2 ploughs can till. Osbert holds it of Juhel. Thereof he has I virgate and I plough in demesne, and the villeins I virgate and I plough. There $O$ [sbert] has 3 villeins, 3 bordars, 2 serfs, 8 beasts, 15 swine, 80 sheep, 2 acres of meadow and 2 acres of pasture. Worth I 5 shillings; when J[uhel] received it the same.

Juhel has a manor called CoLetona [Collaton] ${ }^{2}$ which Colo (Exch. Cole) held T.R.E., and it paid (geld) for $\frac{1}{2}$ hide. This 3 ploughs can till. Turgis holds it of Juhel. Thereof T[urgis] has I virgate and $\frac{1}{2}$ plough in demesne, and the villeins I virgate and $\frac{1}{2}$ plough. There $T$ [urgis] has 3 villeins, I bordar, 3 beasts, I I sheep, 6 acres of wood(land), $\frac{1}{2}$ acre of meadow and 2 acres of pasture. Worth 5 shillings; when J[uhel] received it 20 shillings.

Juhel has a manor called Heurs [Huish] ${ }^{3}$ which Algar held T.R.E., and it paid geld for I hide. This 4 ploughs can till. Ralf holds it of J[uhel]. Thereof $\mathrm{R}$ [alf] has $\frac{1}{2}$ hide and $\mathrm{I}$ plough in demesne, and the villeins $\frac{1}{2}$ hide and 3 ploughs. There R[alf] has 6 villeins, 4 bordars, 2 serfs, 4 beasts, 143 sheep, 5 goats, 6 acres of meadow and 20 acres of pasture. Worth 25 shillings, when J[uhel] received it the same.

Juhel has a manor called WALENimtona (Exch. Walementone) [Galmpton] $]^{4}$ which Alward held T.R.E., and it paid geld for I hide. This 5 ploughs can till. Ralf holds it of J[uhel]. Thereof $R$ [alf] has $\frac{1}{2}$ hide and $I$ plough in demesne, and the villeins $\frac{1}{2}$ hide and 4 ploughs. There R[alf] has 8 villeins, 6 bordars, I serf, and fol. 322b.

2 acres of meadow. Worth 30 shillings; when $\mathrm{J}$ [uhel] received it 40 shillings.

Juhel has a manor called Porlamuta [Portlemouth $]^{5}$ which Hece (Exch. Heche) the sheriff held T.R.E., and it paid geld for I hide. This 4 ploughs can till. Fulco ${ }^{8}$ holds it of J[uhel]. Thereof $\mathrm{F}[\mathrm{ulco}]$ has $\frac{1}{2}$ hide and I plough in dred.

${ }^{1}$ Bagton in West Alvington in Stanborough hun-

"Collaton Prawle in Malborough in Stanborough hundred.

${ }^{3}$ South Huish in Stanborough hundred.

- Galmpton in South Huish in Stanborough hundred.

${ }^{s}$ West Portlemouth in Malborough in Stanborough hundred

${ }^{6}$ In this and the two following entries written Fulge, corrected to Fulco. demesne, and the villeins $\frac{1}{2}$ hide and 2 ploughing beasts (animalia in carr'). There F[ulco] has 3 bordars, I serf, wood(land) 3 furlongs in length by $I$ in breadth, 2 acres of meadow and 60 acres of pasture. Worth ro shillings; when J[uhel] received it 40 shillings.

Juhel has a manor called EDETONA (Exch. Eddetone) [Ilton] ${ }^{7}$ which Algar held T.R.E., and it paid geld for $\frac{1}{2}$ hide. This 3 ploughs can till. Fulco holds it of J[uhel]. Thereof F[ulco] has I virgate in demesne, and the villeins I virgate and 6 ploughing beasts (animalia in carr'). There F[ulco] has I villein, 4 bordars, I serf, and 2 acres of meadow. Worth 5 shillings; when $J[$ uhel] received it 20 shillings.

Juhel has a manor called Alwinestona [Alwinston ${ }^{8}$ which Alwin held T.R.E., and it paid geld for $\frac{1}{2}$ hide. This 2 ploughs can till. Fulco holds it of Juhel. Thereof F[ulco] has I virgate and I plough in demesne, and the villeins I virgate and $\frac{1}{2}$ plough. There $F[$ ulco] has 3 bordars, 2 acres of meadow, and 3 acres of pasture. Worth Io shillings; when [Juhel] received it 20 shillings.

Juhel has a manor called SuRa [Sewer] ${ }^{9}$ which Algar held T.R.E., and it paid geld for I hide. This 4 ploughs can till. Odo holds it of Juhel. Thereof $O\left[d o\right.$ ] has $I \frac{1}{2}$ virgates and I plough in demesne, and the villeins $\frac{1}{2}$ hide and $\frac{1}{2}$ virgate fol. 323.

and $1 \frac{1}{2}$ ploughs. There Odo has 1 villein, 3 bordars, 2 serfs, I 5 beasts, and of (inter) sheep and goats 240 (in all), 4 acres of coppice, I acre of meadow, and 100 acres of pasture. Worth 20 shillings; when J[uhel] received it 40 shillings.

The above-named 9 manors have been laid waste by Irishmen ${ }^{10}$ (per irlandinos bomines).

Juhel has a manor called SuRleia [Sowleigh] ${ }^{11}$ which Algar held T.R.E., and it paid geld for $\frac{1}{2}$ hide. This 3 ploughs can till. Fulco holds it of J[uhel]. Thereof F[ulco] has I virgate and I plough in demesne, and the villeins I virgate and 2 ploughs all but 2 oxen. There F[ulco] has 3 villeins, 4 bordars, I serf, I 8 beasts, I2 swine, 125 sheep, and I acre of meadow. Worth I 5 shillings; when J[uhel] received it Io shillings.

'Ilton alias Edilton alias Yedeston (Feud. Aids, i, 396) in Malborough in Stanborough hundred.

${ }_{8}$ Alston in Malborough in Stanborough hundred.

${ }^{9}$ Sewer in Malborough in Stanborough hundred.

${ }^{10}$ In the raid made by Harold's sons in 1069 , who had taken shelter in Ireland and Denmark (Freeman, Norman Conquest, iv, 789).

1 Sowleigh in the northern outlier of West Alvington in Stanborough hundred. 


\section{A HISTORY OF DEVONSHIRE}

Juhel has a manor called Polochestona [Polson] ${ }^{1}$ which Bristuold held T.R.E., and it paid geld for I virgate. This $\mathrm{I} \frac{1}{2}$ ploughs can till. Rotbert holds it of J[uhel]. Thereof $\mathrm{R}$ [obert] has $\frac{1}{2}$ virgate and I plough in demesne, and the villeins $\frac{1}{2}$ virgate and $\frac{1}{2}$ plough. There $\mathrm{R}$ [obert] has 2 villeins, 2 bordars, 3 serfs, Io beasts, 14 swine, 122 sheep, 33 goats, and $\frac{1}{2}$ acre of meadow. Worth ro shillings; when J[uhel] received it 5 shillings.

Juhel has a manor called CoRTescanola [Curtis Knowl] ' which Alwin held T.R.E., and it paid geld for $\frac{1}{2}$ hide. This 3 ploughs can till. Ralf de Pomaria holds it of Juhel. Thereof $\mathrm{R}$ [alf] has I virgate and I plough in demesne, and the villeins I virgate and $\frac{1}{2}$ plough. There $\mathrm{R}$ [alf] has 2 villeins, 5 bordars, 2 beasts, 50 sheep, 5 goats, 5 acres of wood(land), $\frac{1}{2}$ acre of meadow, and 5 acres of pasture. Worth 15 shillings; when J[uhel] received it 10 shillings.

fol. 323b.

Juhel has a manor called BRADELEIA [Broadley] ${ }^{3}$ which Ailwin (Exch. Alwin) held T.R.E., and it paid geld for I virgate. This 2 ploughs can till. Ralf holds it of Juhel. Thereof R[alf] has $\frac{1}{2}$ virgate and I plough in demesne, and the villeins $\frac{1}{2}$ virgate and $\mathrm{I} \frac{1}{2}$ ploughs. There $\mathrm{R}$ [alf] has 3 villeins, 4 bordars, I serf, and I acre of coppice. Worth I5 shillings; when J[uhel] received it ro shillings.

Juhel has a manor called Hewis [Hewish] ${ }^{4}$ which Ailwin (Exch. Alwin) held T.R.E., and it paid geld for 3 virgates. These 6 ploughs can till. Ralf holds it of J[uhel]. Thereof $\mathrm{R}$ [alf] has I virgate and I plough in demesne, and the villeins 2 virgates and 4 ploughs. There $\mathrm{R}$ [alf] has 5 villeins, 7 bordars, 3 serfs, 5 beasts, 50 sheep, and 2 acres of meadow. Worth 30 shillings; when J[uhel] received it the same.

Juhel has a manor called Uisistona [Woolstone $]^{\mathrm{b}}$ which Ustret (Exch. Uctred) held T.R.E., and it paid geld for $\frac{1}{2}$ hide. This 3 ploughs can till. Colbert holds it of J[uhel]. Thereof $\mathrm{C}$ [olbert] has $\frac{1}{2}$ virgate and I plough in demesne, and the villeins $I \frac{1}{2}$ virgates and 2 ploughs. There $\mathrm{C}$ [olbert] has 3 villeins, 3 bordars, 3 serfs, and 2 acres of meadow. Worth 15 shillings; when $\mathrm{J}$ [uhel] received it to shillings.

Juhel has a manor called CORNEORDA [Cornworthy $]^{6}$ which Ulf held T.R.E., and it paid

${ }^{1}$ Polson in Halwell in Stanborough hundred.

${ }^{2}$ Curtisknowl in Diptford in Stanborough hundred.

${ }^{3}$ Broadley in North Huish in Stanborough hundred.

${ }^{4}$ North Huish in Stanborough hundred.

${ }^{5}$ Woolston in West Alvington in Stanborough hundred.

${ }^{6}$ Cornworthy in Colridge hundred. Geldroll, fol. 696, A. 2 . geld for 3 hides. These 13 ploughs can till. fol. 324.

Thereof J[uhel] has in demesne $I$ hide and 2 ploughs, and the villeins 2 hides and 8 ploughs. There J[uhel] has 20 villeins, 15 bordars, 8 serfs, I rouncey, 5 beasts, I I swine, I40 sheep, I mill paying I 5 shillings a year, I fishery (piscatoria) paying 30 salmon, 30 acres of wood(land), 3 score and 10 acres of coppice, and roo acres of pasture. Worth 60 shillings a year, and was worth the same when J[uhel] received it.

Juhel has a manor called Chrletona [Charleton] ${ }^{7}$ which Hece (Exch. Heche) held T.R.E., and it paid geld for 5 hides. These 15 ploughs can till. Thereof J[uhel] has in demesne 2 hides and 2 ploughs, and the villeins 3 hides and 8 ploughs. There J[uhel] has 20 villeins, I 5 bordars, 12 serfs, 2 rounceys, $I$ beast, 27 swine, 60 sheep, 4 goats, 5 acres of meadow, and 2 of pasture. Worth IOO shillings a year, and was worth the same when J[uhel] received it.

Juhel has a manor called LEGA [Leigh $]^{8}$ which Osmer held T.R.E., and it paid geld for $\frac{1}{2}$ hide. This 2 ploughs can till. William holds it of him. Thereof W[illiam] has in demesne I virgate and I plough, and the villeins I virgate and fol. 324b.

I plough. There $W$ [illiam] has 3 villeins, 3 bordars, 2 serfs, 8 sheep, I acre of wood(land), and Io acres of pasture. Worth 15 shillings, and was worth the same when J[uhel] received it.

Juhel has a certain manor called Pola [Pool] ${ }^{9}$ which Algar held T.R.E., and it paid geld for 2 hides. These 4 ploughs can till. Will[iam] holds it of Juhel. Thereof Will[iam] has I hide and $I$ plough in demesne, and the villeins $I$ hide and 3 ploughs. There $W$ ill [iam] has 6 villeins, 7 bordars, 3 serfs, I rouncey, 2 beasts, 40 sheep, Io acres of coppice, and 4 acres of meadow. Worth 20 shillings a year; it was worth the same when he received it.

Juhel has a manor called ConBa (Exch. Cumbe) $[\text { Combe }]^{10}$ which Alrist held T.R.E., and it paid geld for $\frac{1}{2}$ hide. This 2 ploughs can till. William holds it of J[uhel]. There W[illiam] has this (bec) $\frac{1}{2}$ hide, 6 villeins, 4 bordars, I serf, and 2 ploughs in demesne, also 2 acres of coppice and $\frac{1}{2}$ acre of meadow. Worth 10 shillings; it was worth the same when J[uhel] received it.

7 Charleton in Colridge hundred. Geldroll, fol. 696, A. 2 .

${ }^{8}$ East and West Leigh alias Thurisleigh in Harberton in Colridge hundred.

'South Pool in Colridge hundred.

${ }^{10}$ Combe in South Pool in Colridge hundred. 


\section{HOLDERS OF LANDS}

Juhel has a manor called Aurntona [Allington] ${ }^{1}$ which Gode held T.R.E., and it paid geld for I hide. This 2 ploughs can till. Turgin (Exch. Turgis) holds it of J[uhel]. Thereof T[urgin] has $\frac{1}{2}$ hide and $x$ plough in demesne, fol. 325.

and the villeins $\frac{1}{2}$ hide, I plough and 2 beasts. There T[urgin] has 4 villeins, 4 bordars, 2 serfs, 50 sheep, I $\frac{1}{2}$ acres of meadow, and 15 acres of pasture. Worth 15 shillings a year, and was worth the same when $J[$ uhel] received it.

Juhel has a manor called Stancoma ${ }^{2}$ [Stancombe] which Esnot (Exch. Snot) held T.R.E., and it paid geld for $\frac{1}{2}$ hide. This 2 ploughs can till. Turgin (Exch. Turgis) holds it of J[uhel]. There $T$ [urgin] has in demesne $I$ virgate and I plough, and the villeins I virgate and I plough. There $T$ [urgin] has 2 villeins, 3 bordars, 7 beasts, 13 swine, 60 sheep, and 1 acre of meadow. Worth 10 shillings a year; and was worth the same when he received it.

Juhel has a manor called MELLEstona [Malston] $]^{3}$ which Bristric (Exch. Brictric) held T.R.E., and it paid geld for I hide. This 4 ploughs can till. Ralf holds it of J[uhel]. Thereof $R$ [alf] has $\frac{1}{2}$ hide and I plough in demesne, and the villeins, $\frac{1}{2}$ hide and 3 ploughs. There R[alf] has 6 villeins, 4 bordars, I serf, 2 swine, 40 sheep, 5 acres of coppice, and 2 acres of meadow. Worth 40 shillings a year; and was worth the same when J[uhel] received it.

fol. $335 \mathrm{~b}$.

Juhel has a manor called Forda [Ford] ${ }^{4}$ which Otre held T.R.E., and it paid geld for $\mathbf{I}$ hide. This 2 ploughs can till. Ralf holds it of J[uhel]. Thereof R[alf] has $\frac{1}{2}$ hide and $\mathbf{x}$ plough in demesne, and the villeins $\frac{1}{2}$ hide and 2 ploughs. There $R$ [alf] has 2 villeins, $I$ bordar, 33 sheep, I 5 acres of coppice, 4 acres of meadow and 6 acres of pasture. Worth ro shillings a year; and was worth the same when $\mathrm{J}$ [uhel] received it.

Juhel has a manor called Cheveletona [Chivelstone] ${ }^{6}$ which Alvric held T.R.E., and it paid geld for $\mathrm{I}$ hide. This 3 ploughs can till. Ralf holds it of J[uhel]. Thereof R[alf] has $\frac{1}{2}$ hide and I plough in demesne, and the villeins $\frac{1}{2}$ hide and 2 ploughs. There $R$ [alf] has 3 villeins, 5 bordars, I 3 swine, 33 sheep, Io goats, 3 acres of meadow and 6 acres of pasture. Worth 15 shillings a year; when J[uhel] received it the same.

${ }^{1}$ South Allington alias Allington Crespyn and Burrow in Chivelstone in Colridge hundred.

Stancombe in Sherford in Colridge hundred.

${ }^{3}$ Malston in Sherford in Colridge hundred.

4 Ford in Chivelstone in Colridge hundred.

${ }^{5}$ Chivelstone in Colridge huindred.
Juhel has a manor called Foletona [Follaton] ${ }^{6}$ which Alvric held T.R.E. and it paid geld for $\mathbf{I}$ virgate. This 2 ploughs can till. J[uhel] gave it to St. Mary for the queen's soul. Thereof St. Mary has $\frac{1}{2}$ virgate and I plough in demesne, fol. 326 .

and the villeins $\frac{1}{2}$ virgate. There St. Mary has I villein, I serf, I acre of coppice and I acre of meadow. Worth 10 shillings a year; when he received it it was worth 8 shillings.

Juhel has a manor called ORDIHELLA [Worthill] ${ }^{7}$ which Alwin held T.R.E., and it paid geld for I hide. This 5 ploughs can till. Thereof J[uhel] has $\frac{1}{2}$ hide and 2 ploughs in demesne, and the villeins $\frac{1}{2}$ hide and 4 ploughs. There J[uhel] has 7 villeins, 7 bordars, 5 serfs, 4 beasts, 50 sheep, 10 goats, 8 acres of coppice, ro acres of meadow and 100 acres of pasture. It pays 6 pounds a year, and (paid) the same when he received it.

Juhel has a manor called LEGA [Leigh] ${ }^{8}$ which Alwin held T.R.E., and it paid geld for I hide. This 5 ploughs can till. Thereof J[uhel] has $\frac{1}{2}$ hide and 2 ploughs in demesne, and the villeins $\frac{1}{2}$ hide and 3 ploughs. There J[uhel] has 7 villeins, 2 bordars, 5 serfs, 40 sheep, 12 acres of coppice, 3 acres of meadow and 12 acres of pasture. It pays 6 pounds a year; when he received it the same.

fol. $326 \mathrm{~b}$.

Juhel has a manor called BOTREFoRdA [Butterford $]^{9}$ which Alric held T.R.E., and it paid geld for $\frac{1}{2}$ hide. This 3 ploughs can till. Torgic (Exch. Torgis) holds it of J[uhel]. Thereof $T$ [orgic] has in demesne I virgate and 6 oxen, and the villeins 1 virgate and $I \frac{1}{2}$ ploughs. There T[orgic] has 4 villeins, 2 bordars, 2 serfs, I beast, I 7 sheep, 2 acres of coppice, and I acre of meadow. Worth 15 shillings a year; when $\mathrm{J}$ [uhel] received it it was worth 20 shillings.

Juhel has a manor called BOTREFoRDA [Butterford $]^{10}$ which Tovi held T.R.E., and it paid geld for $\frac{1}{2}$ hide. This 2 ploughs can till. Torgic (Exch. Torgis) holds it of J[uhel]. Thereof $T$ [orgic] has in demesne I virgate, and the villeins I virgate and $\frac{1}{2}$ plough. There $T$ [orgic] has 2 villeins, 2 acres of coppice and $\frac{1}{2}$ acre of meadow. Worth 5 shillings a year; when $J$ [uhel] received it it was worth 10 shillings.

${ }^{6}$ Follaton next Totnes in Colridge hundred.

${ }^{7}$ Worthill in Ermington in Ermington hundred.

${ }^{8}$ Leigh Britnell alias Bretwill in Modbury in Ermington hundred.

${ }^{9}$ Butterford in North Huish in Ermington hundred.

${ }^{10}$ Butterford in North Huish in Ermington hundred. 


\section{A HISTORY OF DEVONSHIRE}

Juhel has a manor called STotBERIA [Stotbury] which Tovi held T.R.E., and it paid geld for I virgate. This 3 ploughs can till. Ralf holds it of J[uhel]. Thereof R[alf] has in demesne $\frac{1}{2}$ virgate and I plough, and the villeins $\frac{1}{2}$ virgate and 2 ploughs. There $\mathrm{R}$ [alf] has 5 villeins, 4 bordars, 2 serfs, 6 swine, 26 sheep, 6 goats, and 20 acres of wood(land). Worth 15 shillings a year; when J[uhel] received it it was worth the same.

fol. 327 .

Juhel has a manor called REIMORA [Ringmore] ${ }^{2}$ which Hece (Exch. Heche) held T.R.E., and it paid geld for $1 \frac{1}{2}$ hides. These 6 ploughs can till. Ralf holds them of J[uhel]. Thereof $\mathbf{R}$ [alf ] has in demesne $\frac{1}{2}$ hide and 2 ploughs, and the villeins I hide and 3 ploughs. There [Ralf] has 6 villeins, 6 bordars, 2 serfs, 67 sheep, 6 acres of meadow and 2 acres of pasture. Worth 30 shillings a year; when $J[$ uhel] received it it was worth the same.

Juhel has a manor called OcheneBeria [Okenbury $]^{3}$ which Tovi held T.R.E., and it paid geld for $\frac{1}{2}$ hide. This 3 ploughs can till. Ralf holds it of J[uhel]. Thereof R[alf] has in demesne I virgate and I plough, and the villeins I virgate and 2 ploughs. There $R$ [alf] has 5 villeins, 2 bordars, I serf, 40 sheep, and 2 acres of meadow. It pays 40 shillings a year, and (paid) the same when J[uhel] received it.

Juhel has a manor called BLACHEORDA [Blatchworthy $]^{4}$ which Ailwin (Exch. Alwin) held T.R.E., and it paid geld for I virgate. This 3 ploughs can till. Ralf holds it of J[uhel]. Thereof R[alf] has in demesne $\frac{1}{2}$ virgate and 6 oxen, and the villeins $\frac{1}{2}$ virgate and 2 ploughs. There R[alf] has 4 villeins, 3 serfs, I 5 sheep, 20 acres of wood(land), 2 of meadow and $I \frac{1}{2}$ leagues of pasture. Worth Io shillings a year; when $J[u h e l]$ received it it was worth the same.

fol. $327 \mathrm{~b}$.

Juhel has a manor called LAmmeseta [Lamside] ${ }^{5}$ which Tovi held T.R.E., and it paid geld for $1 \frac{1}{2}$ hides. These 6 ploughs can till. Ralf holds it of J[uhel]. Thereof [Ralf] has in demesne 3 virgates and I plough, and the villeins 3 virgates and 3 ploughs. There $R$ [alf] has 6 villeins, 12 bordars, 3 serfs, 40 sheep, and 2 acres of meadow. Worth 30 shillings a year; when J[uhel] received it it was worth the same.

\footnotetext{
1 Stadbury in Aveton Giffard in Ermington hundred.

${ }^{2}$ Ringmore in Ermington hundred.

${ }^{3}$ Okenbury in Ringmore in Ermington hundred.

- Over Blatchworthy alias Blatchford in Cornwood in Ermington hundred.

${ }^{5}$ Lamside in Holbeton in Ermington hundred.
}

Juhel has a manor called MimidLanda [Membland $]^{6}$ which Alebric (Exch. Alberic) held T.R.E., and it paid geld for I hide. This 5 ploughs can till. Waldin holds it of J[uhel]. Thereof W[aldin] has in demesne $\frac{1}{2}$ hide and I plough, and the villeins $\frac{1}{2}$ hide and $I \frac{1}{2}$ ploughs. There W[aldin] has 5 villeins, 4 bordars, I serf, 6 beasts, 7 swine, 42 sheep, I acre of coppice and 4 acres of meadow. Worth 20 shillings a year ; and when J[uhel] received it it was worth the same.

Juhel has a manor called BochrLANDA [Buckland $]^{7}$ which Hece $(E x c b$. Heche) held T.R.E., and it paid g(eld) for I $\frac{1}{2}$ hides (Exch. I hide). These 6 ploughs can till. Thereof [Juhel] has in demesne 3 virgates and 2 ploughs, and the villeins 3 virgates and 6 ploughs. There J[uhel] has Io villeins, 8 bordars, Io sheep, I rouncey, 3 beasts, 70 sheep, I saltern paying 2 shillings a year, 200 acres of wood(land), I acre of meadow, and pasture $\frac{1}{2}$ league in length by 2 furlongs in breadth. Worth 50 shillings a year ; fol. 328.

when he received it the same.

Juhel has a manor called Modizia [Mutley] which Godwin held T.R.E., and it paid g(eld) for I virgate. This I plough can till. Odo holds it of J[uhel]. There $\mathrm{O}[\mathrm{do}]$ has 2 villeins who have I plough, 2 acres of wood(land), and 3 acres of pasture. Worth 5 shillings a year; and was worth the same when J[uhel] received it.

Juhel has a manor called Modiera [Mutley] ${ }^{9}$ which Alwin held T.R.E., and it paid geld for I virgate. This I plough can till. $\mathrm{O}[\mathrm{do}]$ holds it of J[uhel]. Thereof $\mathrm{O}$ [do] has in demesne 3 ferdings and $\frac{1}{2}$ plough, and the villeins $I$ ferding. There $O[\mathrm{do}]$ has $\mathrm{I}$ villein, 2 bordars, ro sheep, 3 acres of coppice and I acre of meadow. Worth 5 shillings a year; when J[uhel] received it the same.

Juhel has a manor called Ligurichestona (Exch. Levricestona) [Leigham ?] ${ }^{10}$ which Saolf

${ }^{6}$ Membland in Holbeton in Ermington hundred.

' Eggbuckland in Roborough hundred. The Exchequer Book reads I hide, but the particulars support the reading of the Exeter Book $\mathrm{I} \frac{1}{2}$ hides. Perhaps Harrowbeer (Devon Notes and Queries, i, 209) may have been looked upon as distinct from the manor. In Round's Calendar of Documents of France, 235 , it is described in ro93 as "Baraberga a part or member of Bocheland with its own hall in that township.' Geldroll, fol. 70, A. 2.

${ }^{B}$ Mutley in Weston Peverel in Roborough hundred.

${ }^{9}$ Mutley in Weston Peverel in Roborough hundred.

${ }^{10}$ Leigham in Eggbuckland in Roborough hundred. Buzun is successor in title to Odo and held $\frac{1}{4}$ fee in Leigham and Manadon. Testa de Nevill (Rec. Com.), $176 b$ (O.J.R.); or Laira Green, Eggbuckland (T.W.W.). 


\section{HOLDERS OF LANDS}

(Exch. Saulf) held T.R.E., and it paid geld for I virgate. This 2 ploughs can till. Odo holds it of J[uhel]. Thereof $O[d o]$ has in demesne $\frac{1}{2}$ virgate and I plough, and the villeins $\frac{1}{2}$ virgate and I plough. There $\mathrm{O}$ [do] has 2 villeins, I serf, 8 acres of wood(land), and 3 acres of meadow. Worth Io shillings; when J[uhel] received it the same.

Juhel has a manor called Westona [Weston] ${ }^{1}$ which Olnod (Exch. Ulnod) held T.R.E., and it paid $g$ (eld) for 3 virgates. These 4 ploughs can till. Odo holds it of J[uhel]. Thereof $\mathrm{O}[\mathrm{do}]$ has in demesne $2 \frac{1}{2}$ virgates and $2 \frac{1}{2}$ ploughs, and the villeins $\frac{1}{2}$ virgate and $\frac{1}{2}$ plough. There $\mathrm{O}[\mathrm{do}]$ has I villein, I bordar, 3 serfs, 15 fol. $328 \mathrm{~b}$.

beasts, Io swine, 180 sheep, 60 goats, 4 acres of wood(land), 3 acres of meadow and 4 acres of pasture. Worth 30 shillings; when Juhel received it it was worth ro shillings.

Juhel has a manor called BuRETona [Burrington $]^{2}$ which Alwin held T.R.E., and it paid geld for I ferding. This $I \frac{1}{2}$ ploughs can till. Odo holds it of J[uhel]. Thereof O [do] has in demesne $\frac{1}{2}$ ferding and 1 plough, and the villeins $\frac{1}{2}$ ferding and $\mathrm{I}$ plough. There $\mathrm{O}[\mathrm{do}]$ has 3 villeins, 2 serfs, and 3 acres of meadow. Worth Io shillings a year; when J[uhel] received it the same.

Juhel has a manor called Manedona [Manadon $]^{3}$ which Colbert held T.R.E., and it paid geld for $\frac{1}{2}$ hide. This 3 ploughs can till. Odo holds it of $\mathrm{J}[$ uhel]. Thereof $\mathrm{O}[\mathrm{do}]$ has in demesne I virgate and I plough, and the villeins I virgate and I plough. There $\mathrm{O}[\mathrm{do}]$ has 3 villeins, I bordar, I serf, 30 acres of wood(land), and 2 acres of meadow. Worth 12 shillings and 6 pence a year; when J[uhel] received it it was worth 20 shillings.

Juhel has a manor called WItreria [Whitley] ${ }^{4}$ which Godwin held T.R.E., and it paid geld for $\frac{1}{2}$ hide. This 2 ploughs can till. Odo holds it of J[uhel]. Thereof $\mathrm{O}[\mathrm{jo}$ ] has in demesne $\mathbf{I}$ virgate and $\frac{1}{2}$ plough, and the villeins $I$ virgate and $\frac{1}{2}$ plough. There $O[\mathrm{do}]$ has 2 villeins, $\mathbf{I}$ bordar, I serf, I beast, 27 sheep, 16 goats, and I5 acres of coppice. Worth 7 shillings and fol. 329 .

6 pence a year; when $J[$ uhel] received it it was worth 2 shillings.

${ }^{1}$ Weston Peverel alias Pennycross in Roborough hundred.

${ }^{2}$ Burrington in Weston Peverel in Roborough hundred.

${ }^{\mathrm{S}}$ Manadon in Weston Peverel in Roborough hundred.

West Whitley in St. Budeaux in Roborough hundred.
Juhel has a manor called Colrigr [Colridge ${ }^{5}$ which Edmer held T.R.E., and it paid g(eld) for $\frac{1}{2}$ virgate. This 2 ploughs can till. Odo holds it of J[uhel]. Thereof $\mathrm{O}[\mathrm{do}]$ has in demesne I ferding, and the villeins I ferding and I ploughing beast (animal in carr'). There $\mathrm{O}$ [do] has 2 bordars and 30 acres of wood(land). Worth 15 pence a year; when J[uhel] received it it was lying uncultivated.

Juhel has a manor called Contons [Compton $^{6}$ which Osolf (Exch. Osulf) held T.R.E., and it paid geld for I hide and I virgate. These 4 ploughs can till. Stephen (Exch. Stefan) holds them of J[uhel]. There S[tephen] has in demesne 3 virgates and 2 ploughs, and the villeins 2 virgates and 2 ploughs. There S[tephen] has 6 villeins, 4 bordars, 2 serfs, 5 beasts, 2 swine, 52 sheep, 20 acres of coppice, and I acre of meadow. Worth 30 shillings a year; when $\mathrm{J}[\mathrm{uhel}]$ received it it was worth the same.

Juhel has a manor called Mewi [Meavy] ${ }^{7}$ which Alward held T.R.E., and it paid g(eld) for $\frac{1}{2}$ hide. This 2 ploughs can till. William holds it of J[uhel]. Thereof W[illiam] has in demesne I virgate and I plough, and the villeins I virgate. There $W$ [illiam] has 2 bordars, I serf, 9 beasts, Io swine, 20 sheep, $\frac{1}{2}$ league of coppice, 2 acres of meadow, and pasture $\frac{1}{2}$ league in length by 2 furlongs in breadth. Worth 7 shillings and 6 pence a year; when $J[u h e l]$ received it 5 shillings.

Juhel has a manor called Mrwi [Meavy] ${ }^{8}$ which Alwin held T.R.E., and it paid geld for I virgate. This 2 ploughs can till. Torgic $(E x c h$. Torgis) holds it of J[uhel]. Thereof T[orgic] has in demesne $\frac{1}{2}$ virgate, and the villeins $\frac{1}{2}$ virgate and I plough. There T[orgic] has 3 villeins, 2 bordars, I serf 7 (swine, corrected into) sheep, 4 furlongs of wood(land), and 5 furlongs of pasture. Worth ro shillings a year; when J[uhel] received it it was worth 5 shillings.

fol. 3296.

Juhel has a manor called Mrwi [Meavy] ${ }^{9}$ which Edward held T.R.E., and it paid g(eld) for $1 \frac{1}{2}$ virgates. These 4 ploughs can till. Torgic (Exch. Turgis) holds them of J[uhel]. Thereof T[orgic] has in demesne $\frac{1}{2}$ virgate, and the villeins I virgate and $I_{2} \frac{1}{2}$ ploughs. There $T$ [orgic] has 4 villeins, 2 bordars, 2 serfs, 20 sheep, wood(land) 5 furlongs in length by 2 furlongs in

\footnotetext{
${ }^{5}$ Colridge alias Colwell Barton in Eggbuckland in Roborough hundred.

'Compton Giffard in St. Charles' the Martyr, Plymouth, in Roborough hundred. See fol. 222.

${ }^{7}$ Meavy in Roborough hundred.

${ }^{8}$ Good-a-Meavy Pomeroy in Meavy in Roborough hundred (Feud. Aids, i, 341). De la Pole is successor in title to Turgis in Good-a-Meavy and Church Meavy (Ibid. 321).

${ }^{9}$ Church Meavy in Roborough hundred.
} 


\section{A HISTORY OF DEVONSHIRE}

breadth, 3 acres of meadow, and $\frac{1}{2}$ league of pasture taking length and breadth. It pays 30 shillings a year; when $J[$ uhel] received it it was worth 15 shillings.

Juhel has a manor called MEwI [Meavy] ${ }^{1}$ which Osolf (Exch.) Osulf) held T.R.E., and it paid g(eld) for I virgate. This I plough can till. Nigel holds it of J[uhel]. Thereof N[igel] has in demesne $\frac{1}{2}$ virgate, and the villeins $\frac{1}{2}$ virgate and $\frac{1}{2}$ plough. There $\mathrm{N}$ [igel] has I villein, I bordar, and 3 acres of meadow. It pays 10 shillings a year; when J[uhel] received it it was worth 5 shillings.

Juhel has a manor called Sirefort (Exch. Sireford) $\left[\right.$ Sherford] ${ }^{2}$ which Alebric held T.R.E., fol. 330 .

and it paid geld for $\frac{1}{2}$ hide. This 3 ploughs can till. William holds it of J[uhel]. Thereof W [illiam] has in demesne I virgate and I plough, and the villeins I virgate and I plough. There W[illiam] has 4 villeins, 6 bordars, 2 serfs, I beast, 60 sheep, 2 acres of meadow, and $I$ acre of peat (mora). Worth 20 shillings a year; when J[uhel] received it it was worth the same.

Juhel has a manor called Chichelesberia

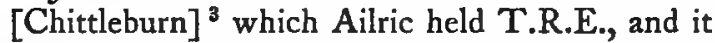
paid g(eld) for $\frac{1}{2}$ hide. This 2 ploughs can till. William holds it of J[uhel]. There W[illiam] has coppice 2 furlongs in length by $\frac{1}{2}$ furlong in breadth. Worth 12 pence a year; when J[uhel] received it 5 shillings.

Juhel has a manor called Olvievetona (Exch. Ulvevetone) [Wollaton] ${ }^{4}$ which Alvric held T.R.E., and it paid g(eld) for $\frac{1}{2}$ hide. This 2 ploughs can till. Will[iam] holds it of J[uhel]. Thereof W[illiam] has in demesne I virgate, and the villeins $I$ virgate and $\frac{1}{2}$ plough. There $\mathrm{W}$ [illiam] has 3 villeins, 4 bordars, $\frac{1}{2}$ acre of meadow, and 2 furlongs of pasture taking length and breadth. Worth 5 shillings a year; when $\mathrm{J}$ [uhel] received it it was worth 4 shillings.

Juhel has a manor called Brisestona [Brixton] ${ }^{b}$ which Sedwin held T.R.E., and it paid g(eld) for $\frac{1}{2}$ hide. This 2 ploughs can till. Will[iam] holds it of Juhel. Thereof W[illiam] has in demesne I virgate and I plough, and the villeins I virgate and I plough. There W[illiam] has 4 villeins, 5 bordars, $I$ beast, 80 sheep, and fol. 33ob.

I acre of meadow. Worth 15 shillings a year; when J[uhel] received it it was worth the same.

${ }^{1}$ Hoo Meavy in Meavy in Roborough hundred.

2 Sherford Prior in Brixton in Plympton hundred.

3 Chittleburn in Brixton in Plympton hundred. Trans. Devon Assoc. xxxv, 708.

- Wollaton in Brixton in Plympton hundred.

${ }^{5}$ Brixton English alias East Brixton in Plympton hundred.
Juhel has a manor called BRisestona [Brixton $]^{6}$ which Alvric held T.R.E., and it paid g(eld) for $\frac{1}{2}$ hide. This 2 ploughs can till. Will[iam] holds it of J[uhel]. Thereof W[illiam] has in demesne I virgate, and the villeins I virgate and I plough. There W[illiam] has 4 villeins, 5 bordars, and I acre of meadow. Worth 5 shillings a year; when $J[u h e l]$ received it it was worth the same.

Juhel has a manor called Dova [Down] which Alebric (Exch. Alvric) held T.R.E., and it paid $g($ eld $)$ for 3 ferdings. These 3 ploughs can till. Will[iam] holds it of J[uhel]. Thereof $W$ [illiam] has in demesne $I \frac{1}{2}$ ferdings and I plough, and the villeins $I \frac{1}{2}$ ferdings and I plough. There W[illiam] has 4 villeins, I serf, 90 sheep, and 12 acres of pasture. Worth I 5 shillings a year; when J[uhel] received it it was worth the same.

Juhel has a manor called Stotrscoma [Staddiscombe $]^{8}$ which Alebric ( $E x c h$. Alvric) held T.R.E., and it paid g(eld) for I virgate. This 2 ploughs can till. Will[iam] holds it of $J[u h e l]$. Thereof $W$ [illiam] has in demesne $\frac{1}{2}$ virgate and $\frac{1}{2}$ plough, and the villeins $\frac{1}{2}$ virgate. There W[illiam] has I villein, I bordar, 2 beasts, I pig, I 2 sheep, wood(land) 2 furlongs in length by I furlong in breadth, $\frac{1}{2}$ acre of meadow, and 20 acres of pasture. Worth 7 shillings and 6 pence a year; when J[uhel] received it it was worth 5 shillings.

fol. з3т.

Juhel has a manor called Estotdona (Exch. Stotdone) [Stoddon] ${ }^{9}$ which Alwin held T.R.E., and it paid g(eld) for I virgate. This 2 ploughs can till. Will[iam] holds it of J[uhel]. Thereof W[illiam] has in demesne $\frac{1}{2}$ virgate, and the villeins (have) $\frac{1}{2}$ virgate. There $W$ [illiam] has I bordar, wood(land) I furlong in length by $\frac{1}{2}$ furlong in breadth, and 20 acres of pasture. Worth 5 shillings a year; when J[uhel] received it it was worth the same.

Juhel has a manor called Bristrichestona (Exch. Brictricestone) [Brixton] ${ }^{10}$ which Elmer held T.R.E., and it paid g(eld) for I virgate. This 3 ploughs can till. Ralf holds it of J[uhel]. Thereof $R$ [alf] has in demesne $\frac{1}{2}$ virgate and $I$ plough, and the villeins $\frac{1}{2}$ virgate and I plough. There R[alf] has 4 villeins, 2 bordars, 7 swine,

- Brixton Reigny alias Church Brixton in Brixton in Plympton hundred. Possibly, however, the identifications of this and the preceding entry should be reversed.

${ }^{7}$ Down Thomas in Wembury in Plympton hundred.

${ }^{8}$ Staddiscombe Brett alias Stodscombe in Plymstock in Plympton hundred.

${ }^{9}$ Staddon alias Stoddon in Plymstock in Plympton hundred.

${ }^{10}$ Brixton Farm alias North Brixton in Shaugh in Plympton hundred. 


\section{HOLDERS OF LANDS}

$3^{1}$ sheep, 2 acres of meadow, and 10 acres of pasture. Worth 10 shillings a year; when he received it the same.

Juhel has a manor called BachEMora [Baccamoor ${ }^{1}$ which Ealous (Exch. Elous) held T.R.E., and it paid geld for $\frac{1}{2}$ hide. This 4 ploughs can till. Ralf holds it of J[uhel]. Thereof R[alf] has in demesne I virgate and I plough, and the villeins I virgate and 2 ploughs. There $R$ [alf] has 4 villeins, 3 bordars, 2 beasts, 28 sheep, 20 acres of coppice, 4 acres of meadow, and pasture I league in length by $\frac{1}{2}$ league in breadth.

To this manor has been added another called Bachemora [Baccamoor] ${ }^{2}$ which Seric held T.R.E. The two manors paid geld for $\frac{1}{2}$ hide and are worth 20 shillings a year.

fol. 33rb.

Juhel has a manor called W ALEFORDA (Exch. Waliforde) [Walford] ${ }^{3}$ which Helebrix (Exch. Alebric) held T.R.E., and it paid geld for $\frac{1}{2}$ virgate. This I plough can till. W[illiam] holds it of J[uhel]. Thereof W[illiam] has in demesne I ferling and $\frac{1}{2}$ plough, and the villeins I ferling. There $W$ [illiam] has I villein, I bordar, 2 swine, and wood(land) 2 furlongs in length by $I$ furlong in breadth. Worth 5 shillings a year; when $\mathrm{J}$ [uhel] received it the same.

Juhel has a manor called Honelanda [Holland] ${ }^{4}$ which 2 thanes Helebrix (Exch. Alebrix) and Algard (Exch. Algar) held T.R.E., and it paid geld for I virgate. This 2 ploughs can till. Ralf holds it of J[uhel]. Thereof R[alf] has $\frac{1}{2}$ virgate in demesne, and the villeins (have) $\frac{1}{2}$ virgate. There $\mathrm{R}$ [alf] has I villein, I bordar, 8 acres of wood(land), and 2 acres of meadow. Worth 5 shillings a year; when J[uhel] received it it was worth Io shillings.

Juhel has a manor called Langadona (Exch. Langedone) [Langdon] ${ }^{6}$ which Hece (Exch. Heche) held T.R.E., and it paid geld for $\frac{1}{2}$ hide. fol. 332.

This 2 ploughs can till. Waldin holds it of $\mathrm{J}$ [uhel]. Thereof W[aldin] has in demesne I virgate and I plough, and the villeins I virgate and I plough. There W[aldin] has 4 villeins, 3 bordars, I serf, 2 beasts, I pig, 84 sheep, 2 acres of meadow, and 4 acres of pasture.

\footnotetext{
${ }^{1}$ Baccamoor in Plympton St. Mary in Plympton hundred.

2 Baccamoor in Plympton St. Mary in Plympton hundred. See fol. $505 b$.

'Walford, written Wyleford and Walesford (Feud. Aids, i, 305, 401), said to be Collaford (Trans. Devon Assoc. xix, 373) alias Collard in Shaugh Prior in Plympton hundred (O.J.R.).

"Holland in Plympton St. Mary in Plympton hundred.

${ }^{3}$ Langdon in Wembury in Plympton hundred.
}

Worth Io shillings a year; when J[uhel] received it the same.

Juhel has a manor called LANGaDONa (Exch. Langedone) [Langdon] ${ }^{\circ}$ which Goda (Exch. Gode) held T.R.E., and it paid g(eld) for $\frac{1}{2}$ hide. This 2 ploughs can till. Waldin holds it of J[uhel]. Thereof $W$ [aldin] has in demesne I virgate, and the villeins I virgate and I plough. There W[aldin] has 4 villeins, 3 bordars, 2 acres of meadow, and 6 acres of pasture. Worth 10 shillings a year; when J[uhel] received it the same.

Juhel has a manor called Coltrestan [Collardstone ${ }^{7}$ which Alvric held T.R.E., and it paid g(eld) for $\frac{1}{2}$ hide. This 3 ploughs can till. Torgic (Exch. Torgis) holds it of J[uhel]. Thereof T[orgic] has in demesne I virgate and I plough, and the villeins $I$ virgate and I plough. There W. (sic) has 3 villeins, 2 bordars, I serf, 5 beasts, ro swine, 40 sheep, 22 goats, 14 acres of wood(land), 4 acres of meadow, and pasture $\frac{1}{2}$ league in length by 2 furlongs in breadth. Worth Io shillings a year; when [Juhel] received it the same.

Juhel has a manor called FrRnEHELLA [Fernhili ${ }^{8}$ which Alwin held T.R.E., and it paid fol. 33ab.

geld for I ferding. This I plough can till. Torgic (Exch. The same Turgis) holds it of J[uhel]. Thereof $T$ [orgic] has in demesne two-thirds of that ferding and the villeins onethird ferding. There T[orgic] has 1 villein who has I ploughing ox (bovem in carr'), Io acres of wood(land), I acre of meadow, and pasture $\frac{1}{2}$ league in length by 2 furlongs in breadth. Worth 3 shillings; when J[uhel] received it the same.

Juhel has a manor called PiDenel [Pithill] ${ }^{9}$ which Alebric held T.R.E., and it paid g(eld) for I ferding. This I plough can till. Torgic (Exch. Turgis) holds it of J[uhel]. Thereof T[orgic] has in demesne $\frac{1}{2}$ ferding and the villeins $\frac{1}{2}$ ferding and $\frac{1}{2}$ plough. There $T$ [orgic] has 2 villeins, wood(land) 2 furlongs in length by $\frac{1}{2}$ furlong in width, and $\frac{1}{2}$ acre of meadow. Worth 4 shillings a year; when J[uhel] received it it was worth 2 shillings.

Juhel has a manor called Escaga (Exch. Scage) [Shaugh] ${ }^{10}$ which Alebric held T.R.E., and it paid g(eld) for I virgate. This $1 \frac{1}{2}$ ploughs can till. Torgic (Exch. The same Turgis) holds it

${ }^{6}$ Langdon in Wembury in Plympton hundred.

' Collardstone alias Coldstone in Shaugh in Plympton hundred.

${ }^{8}$ Fernhill in Shaugh in Plympton hundred.

- Pithill in Shaugh in Plympton hundred.

10 Shaugh Prior in Plympton hundred, united with the following manor. 


\section{A HISTORY OF DEVONSHIRE}

of J[uhel]. Thereof $\mathrm{T}$ [orgic] has in demesne $\frac{1}{2}$ virgate, and the villeins $\frac{1}{2}$ virgate and I plough. There T[orgic] has 3 villeins, I bordar, I serf, 38 sheep, $\frac{1}{2}$ acre of meadow, and pasture $\frac{1}{2}$ league in length by 4 furlongs in breadth. Worth 10 shillings; when J[uhel] received it the same.

Juhel has a manor called Escaga (Exch. Scage) $\left[\right.$ Shaugh] ${ }^{1}$ which Alebric held T.R.E., and it paid g(eld) for I virgate. This I $\frac{1}{2}$ ploughs can till. Torgic (Exch. The same Turgis) holds it of J[uhel]. Thereof $\mathrm{T}$ [orgic] has in demesne $\frac{1}{2}$ virgate and the villeins $\frac{1}{2}$ virgate and I plough. There T[orgic] has 4 villeins, I bordar, 5 acres of wood(land), and pasture $\frac{1}{2}$ league in length by 4 furlongs in breadth. Worth 10 shillings a year; when J[uhel] received it the same.

fol. 333.

Juhel has a manor called Torix [Torridge] ${ }^{2}$ which Alebrix held T.R.E., and it paid geld for I virgate. This I plough can till. Ralf holds it of J[uhel]. Thereof R[alf] has $\frac{1}{2}$ virgate and I plough in demesne, and the villeins $\frac{1}{2}$ virgate and $\frac{1}{2}$ plough. There R[alf] has 2 villeins, I bordar, I serf, 5 beasts, 6 swine, Io sheep, 18 goats, 8 acres of coppice, $I$ acre of meadow, and $I 2$ acres of pasture. Worth Io shillings a year; when $\mathrm{J}[\mathrm{uhel}]$ received it the same.

Juhel has a manor called LocheTora [Loughtor $]^{3}$ which Alebrix held T.R.E., and it paid geld for I virgate. This 2 ploughs can till. Ralf holds it of J[uhel]. Thereof $\mathrm{R}$ [alf] has $\frac{1}{2}$ virgate and I plough in demesne, and the villeins $\frac{1}{2}$ virgate and $\frac{1}{2}$ plough. There $R$ [alf] has 5 villeins, 3 bordars, I serf, 3 beasts, 7 sheep, 5 acres of wood(land), and 30 acres of pasture. Worth I2 shillings a year; when J[uhel] received it the same.

Juhel has a manor called LEGA [Leigh] ${ }^{4}$ which Alebrix held T.R.E., and it paid geld for I ferling. This $\mathrm{I} \frac{1}{2}$ ploughs can till. R[alf] holds it of J[uhel]. There R[alf] has $\frac{1}{2}$ ferling in demesne, and the villeins $\frac{1}{2}$ ferling and $\frac{1}{2}$ plough.

fol. 33зb.

There $R$ [alf] has 3 villeins, Io acres of coppice, $I \frac{1}{2}$ acres of meadow, and 30 acres of pasture. Worth three shillings a year; when he received it the same.

Juhel has a manor called OdeForda [Wood-' ford] ${ }^{5}$ which Elmer held T.R.E., and it paid

${ }^{1}$ Shaugh Prior in Plympton hundred.

${ }^{2}$ Little Torridge, probably Old Newnham, in Plympton St. Mary in Plympton hundred.

${ }^{3}$ Loughtor, now Newnham Park in Plympton St. Mary in Plymouth hundred.

' Elfordleigh in Plympton St. Mary in Plympton hundred (O.J.R.).

${ }^{5}$ Lower Woodford in Plympton St. Mary in Plympton hundred. The saltern settles the locality. geld for $\frac{1}{2}$ hide. This 2 ploughs can till. $\mathrm{R}$ [alf] holds it of J[uhel]. Thereof R[alf] has $I$ virgate and I plough in demesne, and the villeins I virgate. There $R$ [alf] has 2 villeins, 2 bordars, I serf, I saltern, I fishery, wood(land) 3 furlongs in length by $I$ furlong in breadth, and $I$ acre of meadow.

To this manor has been added another called OdeForda [Woodford] ${ }^{6}$ which Albrix (Exch. Alebrix) held T.R.E., and it paid geld for $\frac{1}{2}$ hide. This 2 ploughs can till. R[alf] holds it of $\mathrm{J}$ [uhel]. Thereof $\mathrm{R}$ [alf] has in demesne $\mathrm{I}$ virgate, and the villeins $I$ virgate and I plough. There $R$ [alf] has 3 villeins, 2 bordars, I serf, and pasture $I$ league in length by $\frac{1}{2}$ league in breadth. These manors are worth 40 shillings a year.

fol. 334 .

Juhel has a manor called Ho [Hooe $]^{7}$ which Alebrix held T.R.E., and it paid geld for $\mathbf{I}$ virgate. This $2 \frac{1}{2}$ ploughs can till. Stephen (Exch. Stefan) holds it of J[uhel]. Thereof S[tephen] has in demesne $\frac{1}{2}$ virgate and I plough, and the villeins $\frac{1}{2}$ virgate and I plough. There $\mathrm{S}$ [tephen] has 6 villeins, 2 bordars, I serf, 4 beasts, 40 sheep, and 5 acres of pasture. Worth 20 shillings a year; when J[uhel] received it the same.

Juhel has a manor called HAGAwILA [Halwell] ${ }^{8}$ which Alvric held T.R.E., and it paid geld for $\frac{1}{2}$ hide. This 2 ploughs can till. William holds it of J[uhel]. Thereof $\mathrm{W}$ [illiam] has I virgate and $\frac{1}{2}$ plough in demesne, and the villeins have I virgate. There W[illiam] has I villein, 3 bordars, I serf, 20 sheep, 2 acres of wood(land), and 2 acres of meadow. Worth 10 shillings a year ; when J[uhel] received it it was worth 12 shillings.

Juhel has a borough called Toteners [Totnes] ${ }^{9}$ which King E[dward] held on the day on which he was alive and dead. There J[uhel] has Ioo burgesses all but 5 within the borough, and 15 without the borough who work the land. All these together pay 8 pounds a year by tale (ad numerum); when J[uhel] received it they fol. 334b.

were paying 60 shillings weighed and assayed (ad pensum et ad arsuram). This township (villa) does not pay geld unless Exeter (Exonia) pays. When it did use to pay geld, the payment was 40 pence. If an expedition goes forth by land or sea, Barnestable [Barnstaple], Toteneis [Totnes], and Lideford [Lydford] between them pay the same service as Exeter pays.

' Higher Woodford in Plympton St. Mary in Plympton hundred,

${ }^{7}$ Hooe alias East Hooe in Plymstock in Plympton hundred. Feud. Aids, i, 353.

${ }^{8}$ Halwell Bret in Brixton in Plympton hundred.

${ }^{9}$ Totnes in Colridge hundred. See fol. 88. Trans.

Devon. Assoc. xii, I 62, 455, and xvi, 173. 


\section{HOLDERS OF LANDS}

Juhel has a house in EssecEstra [Exeter] ${ }^{1}$ which pays the customary due of the time of King Edward, that is, 8 pence a year.

fol. 335 .

\section{THE LAND OF RALF DE POMARIA IN DEVENESIRA ${ }^{2}$}

Ralf de Pomaria has a manor called Lidefor'T (Exch. Tideford) [Lidford] ${ }^{3}$ which Wadels held T.R.E., and it paid geld for $\frac{1}{2}$ ferding. One plough can till it and it pays 3 shillings a year. Roger holds it of Ralf.

Ralf has a manor called WYca (Exch. Wiche) [Week ${ }^{4}$ which Alward held T.R.E., and it paid geld for $\frac{1}{2}$ virgate. This 5 ploughs can till. Thereof R[alf] has I ferling and I plough in demesne, and the villeins the other ferling and 2 ploughs. There $\mathrm{R}$ [alf] has 4 villeins, 3 bordars, 2 serfs, 30 beasts, 2 swine, 38 sheep, 2 acres of wood(land), 30 acres of meadow and 12 acres of pasture. Worth 50 shillings a year; when [Ralf] received it it was worth 40 shillings.

Ralf has a manor called Dunewinusdona $(E x c h$. Dunewinesdone) [Dunsdon $]^{5}$ which Tovi held T.R.E., and it paid geld for 3 virgates. These I 2 ploughs can till. Of 2 (of these) virgates Ralf has $I$ in demesne and 2 ploughs, and the villeins have I virgate and 3 ploughs. On these 2 virgates $R$ [alf] has 6 villeins, 7 bordars, 9 serfs, 35 beasts, Io swine, 40 sheep, Io goats, 40 acres of meadow and 60 of pasture.

And the third aforesaid virgate which belonged to the aforesaid manor T.R.E. has been taken away. The count of Mortain holds it.. ${ }^{8}$ This

1 The Tabard Inn, Exeter, Trans. Devon. Assoc. xii, 196.

'In the Exchequer copy the following barons' lands come before Pomeray's in the order named:-No. I8, William de Moion; I9, William Chieure; 20, William de Faleise; 2I, William de Poilgi; 22, William de $\mathrm{Ow} ; 23$, Walter de Dowai ; 24, Walter de Clavile ; 25 , Goscelm ; 26, Richard son of Coun Gislebert; 27, Roger de Busli ; 28, Rubert de Albemarle ; 29, Robert Bastard ; 30, Richard son of Torulf; 31, Ralf de Limesi ; 32, Ralf Pagenel ; 33, Ralf de Felgheres. The present position of Pomeray's section in the Exon book is probably due to the binder. The sequence of Pomeray's estates is the same in both copies with the exception of 'Tidefort' coming third in the Exchequer instead of first, as in the Exon book.

${ }^{3}$ Probably Lidford in Lifton hundred, but see Trans. Devon Assoc. xxxvi, 350.

' South Week in Germansweek in Lifton hundred.

${ }^{5}$ Dunsdon in Blacktorington hundred. See fol. $496 b$.

${ }^{6}$ Hallacot alias Aylescot in Panscrasweek. See Testa, I 84 a (O.J.R.). Dexboro' and Hudson (T.W.W.). third virgate 2 ploughs can till. This manor is worth IOO shillings without the virgate which fol. 335b.

the count of Mortain holds; when Ralf received it it was worth 4 pounds.

Ralf has a manor called Alywynelancautle (Exch. Alwineclancauele) [ $]^{7}$ which Letwyn (Exch. Leduin) held T.R.E., and it paid geld for $\frac{1}{2}$ virgate and $\frac{1}{2}$ ferting. 'This 2 ploughs can till. There $\mathrm{R}$ [alf] has I plough, and the villeins I ; also 2 villeins, 4 bordars, I serf, IO beasts, 12 sheep, 20 acres of meadow, and 30 acres of pasture. Worth 10 shillings a year; when he received it it was worth 15 shillings.

Ralf has a manor called AIssa $[A s h]^{8}$ which Letmar (Exch. Leimar) held T.R.E. and he could go with that land to what lord he liked. It paid geld for I virgate. This 3 ploughs can till. There $\mathrm{R}$ [alf] has 4 villeins and 2 bordars, who have 2 ploughs, also 10 acres of meadow and the like of pasture. Worth ro shillings a year; when he received it it was worth 20 shillings. Afterwards Ralf seized upon this (Hanc $p$ [ostea] occupavit; Exch. Hanc invasit), as the Frenchmen (franci) and English bear witness.

Ralf has a manor called Bravordina (Exch. Brawardine). [Bradworthy] ${ }^{9}$ which Tovi ${ }^{10}$ held T.R.E., and it paid geld for 3 hides I virgate. This 12 ploughs can till. Thereof R[alf] has $I$ hide, I virgate and 3 ploughs in demesne, and the villeins 2 hides and 7 ploughs. There R [alf] has 20 villeins, to bordars, 9 serfs, 40 beasts, I0 swine, 30 unbroken mares (indomitas), 120 sheep, 5 goats, 40 acres of meadow, and pasture 3 leagues long by $I$ broad. Worth 8 pounds a year; when he received it it was worth 100 shillings.

fol. 336 .

Ralf has a manor called Pudeforda [Putford] ${ }^{11}$ which Letmar (Exch. Ledmar) held T.R.E., and it paid geld for $\frac{1}{2}$ virgate. This 2 ploughs can till. Now Roger holds of Ralf. Thereof Roger has I ferting in demesne and I plough, and the villeins the other ferting and I plough. There Roger has 2 villeins, 4 bordars, I serf,

"May possibly be East Yeowelston in Bradworthy in Blacktorington hundred (O.J.R.); see Trans. Devon Assoc. xxviii, 4 n. 5 .

${ }^{8}$ Ash in Bradworthy in Blacktorington hundred. See fol. 497 .

${ }^{9}$ Bradworthy honour (Feud. Aids, i, 340) and manor in Blacktorington hundred. Geldroll, fol. 65 , A 4 .

${ }^{10}$ Substituted in Exon Book for 'Alward'; Exch. reads 'Tovi.'

11 Julian Putford in West Putford in Blacktorington hundred. See fol. 479. 


\section{A HISTORY OF DEVONSHIRE}

2 beasts, I pig, 2 acres of wood(land), 12 acres of meadow, and pasture $\frac{1}{2}$ league long by 2 furlongs broad. Worth ro shillings a year ; when he received it it was worth 15 shillings.

Ralf has a manor called Horewoda [Horwood] ${ }^{1}$ which Alward held T.R.E. It paid geld for 3 virgates. These 5 ploughs can till. Thereof $\mathrm{R}$ [alf] has 2 virgates and 3 ploughs in demesne, and the villeins (have) I virgate and 2 ploughs. There $\mathrm{R}$ [alf] has 2 villeins, 4 bordars, 4 serfs, I cow, 7 swine, 60 sheep, I acre of wood(land) and 8 acres of meadow. It pays ${ }^{2} 60$ shillings; when $R[$ alf] received it it was worth 40 shillings.

Ralf has a manor called Torilanda [Toridgeland $]^{3}$ which Almer ( $E x c b$. Almar) held T.R.E., and it paid geld for $\frac{1}{3}$ ferling. This 1 plough can till. Roger holds it of Ralf. There R[oger] and his men (bomines) have I plough. There $\mathrm{R}$ [oger] has 3 bordars and I serf. Worth 5 shillings; when Ralf received it the same.

Ralf has a manor called Arssecoma [Ashcombe ${ }^{4}$ which Alvric piga held T.R.E. To this 3 manors have been added which 3 thanes held in parage (pariter; Exch. in paragio) and these 4 manors paid geld for 2 hides. These 9 ploughs can till. Thereof $\mathrm{R}$ [alf] has in demesne $\frac{1}{2}$ hide and 4 ploughs, and the villeins $\frac{1}{2}$ hide and 7 ploughs. There $R$ [alf] has 8 villeins, 4 bordars, 4 serfs, 42 beasts, 24 swine, fol. $336 \mathrm{~b}$.

I 50 sheep, 40 goats, 4 acres of wood(land), 5 acres of meadow, and 150 acres of pasture. It pays Io pounds a year; 9 pounds when he received it. ${ }^{5}$

Ralf has a virgate called HoLcomma [Holcombe $]^{6}$ which Aimar (Exch. Almaer) held T.R.E. This I plough can till. There $R$ [alf] has 4 salterns paying 6 shillings and 5 pence a year.

Ralf has a manor called Peumera [Peamore] ${ }^{7}$ which Wichin held T.R.E., and it paid geld for I virgate of land. This 3 ploughs can till. Roger son of Pagan holds it of R[alf]. There-

1 East Horwood and Pin Horwood in Horwood in Fremington hundred. Geldroll, fol. $65 b, A_{3}$.

' 'Reddit' substituted for 'valet.'

${ }^{3}$ Probably the 'Dodecota' of Inq. p.m. 7 Edw. No. 13, i.e. Yelland or Kingscot next Dodscot in St. Giles' in the Wood in Fremington hundred. Trans. Dev. Assoc. xxxvi, 35 I.

- Ashcombe in Exminster hundred. See fol. 4986. Geldroll, fol. 68b, A 9.

${ }^{5}$ In four cases in this entry the figures originally entered in the Exeter book have been subsequently corrected to agree with the Exchequer MS.

${ }^{6}$ Lower Holcombe in Dawlish in Exminster hundred.

${ }^{7}$ Peamore in Exminster parish and hundred. of $\mathrm{R}$ [oger] has in demesne $\frac{1}{2}$ virgate and $I$ plough and the villeins $\frac{1}{2}$ virgate and 2 ploughs. There R[oger] has 4 villeins, 3 bordars, 2 serfs, 36 sheep, and 3 acres of meadow. Worth I 5 shillings a year; when $R$ [alf] received it Io shillings.

Ralf has a manor called Bolewis [? Moulish] ${ }^{8}$ which Lieuegar (Exch. Leuegar) held T.R.E., and it paid geld for I virgate. This I plough can till. Richard holds it of $\mathrm{R}$ [alf]. The whole of this Ri[chard] has in demesne excepting 1 ferding which 2 villeins and 2 serfs hold. Worth 5 shillings; when $R$ [alf] received it it was worth the same.

fol. 337.

Ralf has a manor called BRANDONa [Brendon] ${ }^{9}$ which Alward Tocheson held T.R.E., and it paid geld for I hide. This 8 ploughs can till. Thereof $R$ [alf] has in demesne $\frac{1}{2}$ hide and 2 ploughs; and the villeins have $\frac{1}{2}$ hide and 6 ploughs. There $R$ [alf] has 7 villeins, 6 bordars, 5 serfs, I rouncey, 104 unbroken mares, 25 beasts, 8 swine, 100 sheep, 30 goats, 30 acres of wood(land), and 2 leagues of pasture. Worth roo shillings; when he received it it was worth 30 shillings.

To this manor has been added LANcoma [Lank Combe] ${ }^{10}$ which Edwin held T.R.E., and it paid geld for I ferding. This I plough can till ; and there is a villein who pays 3 shillings yearly. That Edwin could go where he liked.

Ralf has a manor called Ciretona [Cherriton] ${ }^{11}$ which Chitell (Exch. Chetel) held T.R.E., and it paid geld for $\frac{1}{2}$ virgate. This 2 ploughs can till. Thereof $\mathbf{R}$ [alf] has in demesne I ferding and I plough, and the villeins (have) I ferding and I plough. There $R$ [alf] has 4 villeins, 2 bordars, 2 serfs, 27 beasts, 8 swine, 30 sheep, 20 acres of wood(land), and 2 leagues of pasture. Worth 40 shillings a year; when he received it it was worth ro shillings.

Ralf has a manor called HaNToNa [Heanton] which Ulf held T.R.E., and it paid geld for I virgate. This 3 ploughs can till. Helgod holds it of $\mathrm{R}$ [alf]. Thereof $\mathrm{H}$ [elgod] has $\frac{1}{2}$ virgate in demesne and I plough, and the villeins $\frac{1}{2}$ virgate and I plough. There Helgod has 3 villeins, 2 serfs, I 3 beasts, 8 swine, 50 sheep, 20 goats, I 2 fol. 337b.

acres of wood(land), and 30 acres of pasture.

${ }^{8}$ Moulish in Kenton in Exminster hundred.

'Brendon in Shirwell hundred. Geldroll, fol. 66, A 7 .

${ }^{10}$ Lank Combe in Brendon in Shirwell hundred. See fol. 499.

${ }^{11}$ Cherriton in Brendon in Shirwell hundred.

12 Heanton Coffin alias Foryn in Lynton in Shirwell hundred. 


\section{HOLDERS OF LANDS}

Worth 37 shillings a year ; when he received it it was worth 20 shillings.

Ralf has a manor called Estandona (Exch. Standone) []$^{1}$ which Algar the priest held T.R.E., and it paid geld for $I$ virgate. There $R$ [alf] has $I$ villein and 30 acres of pasture. Worth 3 shillings a year.

Ralf has a manor called Hane (Exch. Hanc) [Ank] ${ }^{2}$ which Burgered held T.R.E., and it paid geld for 3 hides. These 7 ploughs can till. Thereof Ralf has $I_{\frac{1}{2}}$ hides and 3 ploughs in demesne, and the villeins have 3 ploughs. There $R$ [alf] has 12 villeins, 6 bordars, 6 serfs, I rouncey, I I beasts, I 2 swine, I00 sheep, 20 acres of meadow, and 23 acres of pasture. Worth 3 pounds a year; when Ralf received it it was worth the same.

Ralf has a manor called Sildenna (Exch. Sildene [Sheldon] ${ }^{3}$ which Alric held T.R.E., and it paid geld for 1 hide. This 6 ploughs can till. Thereof $\mathrm{R}$ [alf] has in demesne $\mathrm{I}$ virgate and 1 plough, and the villeins 3 virgates and 6 ploughs. There R[alf] has 9 villeins, 2 bordars, 2 serfs, ro beasts, 45 sheep, 30 acres of coppice, and 300 acres of pasture. Worth 3 pounds and Io shillings a year; when he received it it was worth 2 pounds.

Ralf has a manor called BlachaBERGA (Exch. Blackeberge) [Blackborough] ${ }^{4}$ which Alnotd (Exch. Alnod) held T.R.E., and it paid geld for $\frac{1}{2}$ hide. This 2 ploughs can till. There R[alf] has in demesne I virgate and I plough, and the villeins I virgate and I plough. There $\mathbf{R}$ [alf] has 2 villeins, 3 bordars, I 3 beasts, 4 swine, 30 sheep, 8 goats, 3 acres of meadow and roo acres of pasture. Worth 20 shillings a year; when he received it ro shillings.

fol. 338 .

Ralf has a manor called TaLA [Tale] ${ }^{5}$ which Aldnotd (Exch. Alnod) held T.R.E., and it paid geld for $\mathrm{I} \frac{1}{2}$ hides all but $\mathrm{I}$ ferding. These 3 ploughs can till. Thereof $\mathrm{R}$ [alf] has in demesne I hide and I plough, and the villeins $\frac{1}{2}$ hide all but I ferding and $\frac{1}{2}$ plough. There $R$ [alf] has 4 villeins, 3 bordars, 15 acres of coppice, 2 acres of meadow and 50 acres of pasture. Worth 20 shillings a year; when he received it ro shillings.

\footnotetext{
1 There is said to be an 'Eastanton Down' in Lynton (T.W.W.).

${ }^{2}$ Anke in Clyst Hydon in Cliston hundred. See fol. 501. Geldroll, fol. 67, A 3 .

A 7.

${ }^{3}$ Sheldon in Hairidge hundred. Geldroll, fol. $67 b$,

${ }^{4}$ Blackborough Bolhay alias All Hallows in Hairidge hundred.

${ }^{5}$ Higher Tale in Payhembury in Hairidge hundred, given by Joselin de Pomeray to Ford Abbey (Oliver, Mon. 346). Geldroll, fol. 67b, A 7 .
}

Ralf has a manor called TALA [Tale] ${ }^{6}$ which Borgaret held T.R.E., and it paid geld for I hide. This 4 ploughs can till. Thereof $R$ [alf] has in demesne $\frac{1}{2}$ hide and 2 ploughs, and the villeins $\frac{1}{2}$ hide and I plough. There R[alf] has 5 villeins, I bordar, 3 serfs, I rouncey, I 5 beasts, I pig, 30 sheep, 50 goats, I mill paying 5 shillings a year, 20 acres of coppice, 20 acres of meadow and 50 of pasture. Worth 40 shillings a year; when he received it 20 shillings.

fol. 338b.

Ralf de Pomaria has a manor called Orescomma [Awliscombe] ${ }^{7}$ which Edmer (Exch. Edmaer) held T.R.E., and it paid geld for $\frac{1}{2}$ hide. This $4 \frac{1}{2}$ ploughs can till. Thereof $R$ [alf] has $I$ virgate and 2 ploughs in demesne, and the villeins I virgate and $2 \frac{1}{2}$ ploughs. There $R$ [alf] has 5 villeins, 5 bordars, 3 serfs, I rouncey, 4 beasts, 5 swine, 23 sheep, half a mill paying 5 shillings, 2 furlongs of wood(land), and 5 acres of meadow. Worth 40 shillings; when Ralf received it it was worth the same.

Ralf has a manor called OTERI (Exch. Otri) [Otter ${ }^{8}$ which Godric held T.R.E., and it paid geld for $\frac{1}{2}$ hide. This 2 ploughs can till. Roscelin holds it of Ralf. Thereof R[oscelin] has $I_{\frac{1}{2}}$ virgates and $\mathbf{I}$ plough, and the villeins $\frac{1}{2}$ virgate. There $\mathrm{R}$ [oscelin] has $\mathrm{I}$ villein, 3 bordars, I serf, 8 beasts, I 6 swine, 78 sheep, 5 acres of meadow and 2 furlongs of pasture. Worth ro shillings; when $R$ [alf] received it it was worth the same.

Ralf has a manor called Doduceswilla (Exch. Doducheswelle) [Dunkswell] ${ }^{9}$ which Almer held T.R.E., and it paid geld for $I \frac{1}{2}$ hides and I virgate ( $E x c h$. omits I virgate). This Io ploughs can till. Thereof R[alf] has $\frac{1}{2}$ hide and I plough in demesne, and the villeins I hide I virgate and 5 ploughs. There $R$ [alf] has 6 beasts, Io goats, and 50 sheep, also I I villeins, 3 serfs, 8 acres of coppice, 4 acres of meadow and 10 furlongs of pasture. Worth 50 shillings; when he received it it was worth the same.

Ralf has a manor called Orescomme (Exch. Orescome) [Awliscombe $]^{10}$ which Aldwin held T.R.E., and it paid geld for $\frac{1}{2}$ hide and $\frac{1}{2}$ virgate. fol. 339 .

This 3 ploughs can till. Hegot holds it of him.

${ }^{6}$ Lower Tale in Payhembury in Hairidge hundred.

${ }^{7}$ Old Awliscombe, or Oulescombe, now the village of Buckerel in Hemyock hundred. See fol. $502 b$.

${ }^{8}$ Waringston on the Otter in Buckerel in Hemyock hundred. Warin and Roscelin held together. Geldroll, fol. 66b, B 3 ; Trans. Devon. Assoc. xxxvi, 362.

${ }^{9}$ Dunkswell in Hemyock hundred. Geldroll, fol. $676, \mathrm{~A}_{5}$.

${ }^{10}$ Awliscombe Giffard in Awliscombe in Hemyock hundred (O.J.R.) ; or Harcombe, Buckerel (T.W.W.). $48 \mathrm{I}$ 


\section{A HISTORY OF DEVONSHIRE}

There $\mathrm{H}$ [egot] has $\mathrm{I}_{2} \frac{1}{2}$ virgates and $\mathrm{I}$ plough in demesne, and the villeins I virgate and 2 ploughs. There $\mathrm{H}$ [egot] has 4 villeins, 2 bordars, I serf, 16 beasts, I pig, 20 sheep, 17 goats, 6 acres of wood(land), I I acres of coppice, 8 acres of meadow and 4 furlongs of pasture. Worth 20 shillings a year; when he received it it was worth the same.

Ralf has a manor called Oghawillae [Ogwell ${ }^{1}$ which Alvric held T.R.E., and it paid geld for 3 virgates. These 4 ploughs can till. Of these William the Poictevin (Pitavensis) has 2 virgates and Robert I virgate. There W[illiam] has in demesne I virgate and I plough, and his villeins I virgate and I plough. There W[illiam] has 2 villeins, 3 bordars, I serf, 6 beasts, 30 sheep, 12 acres of coppice, 1 of meadow and 12 of pasture. Worth 20 shillings a year; when he received it it was worth Io shillings. Also $\mathrm{R}$ [obert] has there 4 villeins and 3 bordars. Worth ro shillings a year; when he received it the same.

Ralf has a manor called OgHawirls [Ogwell] ${ }^{2}$ which Edric held T.R.E., and it paid geld for $\frac{1}{2}$ hide. This 3 ploughs can till. William the Poictevin (pictavensis) holds it of him. There $\mathrm{W}$ [illiam] has $\frac{1}{2}$ virgate and $x$ plough in demesne, and the villeins $I$ virgate and I plough. There fol. 339b.

W[illiam] has 2 villeins, 3 bordars, I serf, 3 swine, 25 sheep, 15 goats, 6 acres of wood(land), 1 acre of meadow and 10 acres of pasture. Worth Io shillings a year; when he received it it was worth 5 shillings.

Ralf has a manor called Hochesam (Exch. Hochesham) [Huxham] ${ }^{3}$ which Wichin held T.R.E., and it paid geld for $3 \frac{1}{2}$ virgates. These 6 ploughs can till. Roger holds it of him. Thereof $R$ [oger] has $I \frac{1}{2}$ virgates and $I$ plough in demesne, and the villeins 2 virgates and 5 ploughs. There $R$ [oger] has Io villeins, 3 bordars, $\mathrm{I}$ serf, I rouncey, 3 beasts, 4I sheep, I mill worth 5 shillings a year, 8 acres of meadow and 20 acres of pasture. Worth 25 shillings a year; when he received it 10 shillings.

Ralf has a manor called Clisewic [Clystwick] ${ }^{4}$ which Wichim (Exch. Wichin) held T.R.E., and it paid geld for I virgate (vilg'). This 6 ploughs can till. Roger holds it of him. There R[oger] has $\frac{1}{2}$ virgate and I plough in demesne, and the villeins $\frac{1}{2}$ virgate and 5 ploughs. There $R$ [oger] has 3 villeins, I 3 bordars, I serf, 3 beasts, I 4 sheep,

${ }^{1}$ This and the manor next entered constitute Ogwell Peytevin in Wonford hundred.

See last note; $\frac{1}{2}$ a virgate is unaccounted for.

${ }^{3}$ Huxham in Wonford hundred. Geldroll, fol. 70b, A 9, calls the tenant Roger Flavus.

'Now Clyst St. George alias Clyst Champernown (Ancient Deeds, A 8,921) in East Budleigh hundred. and 8 acres of meadow. Worth 40 shillings a year; - when he received it it was worth 10 shillings.

Ralf has a manor called Estocheneia (Exch. Stochelie) [Stockleigh] ${ }^{5}$ which Alveva held T.R.E., and it paid geld for $I$ hide. This 6 ploughs can till. Thereof $R$ [alf] has $\frac{1}{2}$ hide and 3 ploughs in demesne, and the villeins $\frac{1}{2}$ hide and 3 ploughs. fol. 340.

There R[alf] has 7 villeins, I 8 bordars, 3 serfs, I rouncey, 5 beasts, I 4 swine, IO3 sheep, 23 goats, 8 acres of wood(land), 3 acres of meadow and 10 acres of pasture. Worth 40 shillings; when $R[$ alf] received it the same.

Ralf has $\frac{1}{2}$ hide of land in OTERI [on the Otter $]^{6}$ which Godric held T.R.E. This Roscelin holds of Ralf. One plough can till it. There $R$ [oscelin] has 3 villeins who have 3 ploughing oxen (boves in carr') (Exch. There are 3 villeins with $\frac{1}{2}$ plough) 1 acre of wood(land), 5 acres of meadow, and pasture I league in length by $\frac{1}{2}$ in breadth. Worth 30 pence; when Ralf received it 12 pence.

Ralf has a manor called Danescoma [Dunscombe $]^{7}$ which Sagar held T.R.E., and it paid geld for $\frac{1}{2}$ hide. This 3 ploughs can till. William holds it of Ralf. Thereof W[illiam] has I virgate and 2 ploughs in demesne, and the villeins I virgate and I plough. There W[illiam] has 3 bordars, 2 serfs, 8 beasts, 17 swine, 45 sheep, 17 goats, 3 acres of meadow and 30 acres of pasture. Worth 20 shillings; when $R$ [alf] received it the same.

Ralf has a manor called HEPPASTEBA ${ }^{8}$ which Ralf entered upon (saisivit) along with his manor called Hanca [Ank]. Oluard (Exch. Ulward) held it T.R.E., and it paid geld for I virgate. This I plough can till. There $R$ [alf] has I serf and 5 acres of meadow. Worth 5 shillings a year.

Ralf has a manor called CRIDia [Creedy] ${ }^{9}$ which Goda held T.R.E., and it paid geld for I virgate. This 2 ploughs can till. William holds it of Ralf. Thereof $W$ [illiam] has $\frac{1}{2}$ virgate fol. $34 \mathrm{ab}$.

and I plough in demesne, and the villeins $\frac{1}{2}$ virgate

${ }^{5}$ Stockleigh Pomeroy in East Budleigh hundred. Geldroll, fol. 67b, A 9. Trans. Devon Assoc. xxviii, 385 .

${ }^{6}$ Raplinghayes in Gittisham in East Budleigh hundred. Geldroll, fol. $67 b, \mathrm{~B} 3$.

'Dunscombe in Cheriton Fitzpaine in West Budleigh hundred (O.J.R.).

${ }^{8}$ This may perhaps be Oxeton alias Hill and Exton in Faringdon in East Budleigh hundred. See Trans. Devon Assoc. xxv, 291.

${ }^{9}$ Creedy Peytevin alias Widger alias Creedy Farm in Upton Helions in West Budleigh hundred. Trans. Devon Assoc. xiv, 266. 


\section{HOLDERS OF LANDS}

and $I$ plough and 7 oxen towards a(nother) plough (Exch. There are 2 ploughs). There W[illiam] has 4 bordars, 4 serfs, 4 beasts, 37 sheep, 5 acres of meadow and 30 acres of pasture. Worth Io shillings; when [Ralf] received it 5 shillings.

Ralf has a manor called ADDEBERIA [Yeadbury $]^{1}$ which Saric held T.R.E., and it paid geld for $\frac{1}{2}$ virgate. This I plough can till. William holds it of Ralf. There W[illiam] has 2 serfs and 30 acres of pasture. Worth 3 shillings; when Ralf received it the same.

Ralf has I ferling of land in CRedA

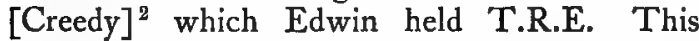
I plough can till. William holds it of Ralf. There [William] has an acre of meadow and the rest of the land has so gone out of cultivation (est ita vastata) that it is not worth more than 2 shillings; when $R$ [alf] received it the same.

Ralf has a manor called Estreta [Street] ${ }^{3}$ which Ailmer held T.R.E., and it paid geld for $\frac{1}{2}$ hide. This 2 ploughs can till. Torstin (Exch. Turstan) holds it of Ralf. Thereof $T$ [orstin] has $I$ virgate and $\mathbf{I} \frac{1}{2}$ ploughs in demesne, and the villeins I virgate and $\frac{1}{2}$ plough. There T[orstin] has 2 villeins, 6 bordars, 3 serfs, 4 beasts, 3 swine, 30 sheep, 60 goats, 12 acres of wood(land), 8 acres of meadow and 60 acres of pasture. Worth Io shillings; when R[alf] received it the same.

fol. 34 I.

Ralf has a manor called W ASFELIA [Washfield] ${ }^{4}$ which Goda held T.R.E., and it paid geld for 3 virgates. These 4 ploughs can till. Thereof $\mathrm{R}$ [alf] has $\mathrm{I} \frac{1}{2}$ virgates and 2 ploughs in demesne, and the villeins $I \frac{1}{2}$ virgates and 2 ploughs. There $\mathrm{R}$ [alf] has 7 villeins, 4 bordars, 2 serfs, 4 beasts, 3 swine, 32 sheep, 7 goats, 40 acres of wood(land), 4 acres of meadow and 15 acres of pasture. It pays 30 shillings; when $R$ [alf] received it 6 shillings.

Ralf has a manor called Estodleia (Exch. Stodlei) [Stoodleigh] ${ }^{5}$ which Almer held T.R.E., and it paid geld for 3 virgates. These ro ploughs can till. Ro[bert] holds them of Ralf. Thereof Ro[bert] has in demesne 1 virgate and 3 ploughs; and the villeins 2 virgates and 3 ploughs. There Ro[bert] has 12 villeins, I bordar, 6 serfs,

${ }^{1}$ Yeadbury in Cruwys Morchard in West Budleigh hundred.

${ }^{2}$ Now Merrifield (O.J.R.); or Upper Creedy (T.W.W.) in Upton Helions in West Budleigh hundred.

${ }^{3}$ Street Raleigh in Whimple in East Budleigh hundred.

${ }^{4}$ Washfield in West Budleigh hundred including Hatherland. Geldroll, fol. $67 b, \mathrm{~B} 3$.

${ }^{5}$ West Stoodleigh in Stoodleigh in Witheridge hundred.
I 8 beasts, I 8 swine, Ioo sheep, wood(land) I league in length by $\frac{1}{2}$ in width, and 2 acres of meadow. Worth 40 shillings a year; when $R$ [alf] received it it was worth 30 shillings.

Ralf has a manor called Henleia [Highleigh] ${ }^{6}$ which Brismer held T.R.E., and it paid geld for I virgate. This 3 ploughs can till. Thereof $\mathrm{R}$ [alf] has in demesne $\frac{1}{2}$ virgate and I plough, and the villeins $\frac{1}{2}$ virgate and I plough. There R[alf] has I villein, 2 bordars, 3 beasts, and 2 sheep. Worth Io shillings a year; when he received it the same.

Ralf has a manor called ODEORDI [Woodford $]^{7}$ which Seric held T.R.E., and it paid geld fol. 34xb.

for I ferding. This $\frac{1}{2}$ plough can till. William holds it of $R[a l f]$. There $W[i l l i a m]$ has $\frac{1}{2}$ plough, I serf, and 2 acres of meadow. Worth 30 pence a year.

Ralf has a manor called Chevetorna (Exch. Chenetorne) [Chevithorn] ${ }^{8}$ which Almar held T.R.E., and it paid geld for I virgate. This 4 ploughs can till. Beatrix holds it of R[alf]. Thereof Beatrix has in demesne $\frac{1}{2}$ virgate and 2 ploughs, and the villeins $\frac{1}{2}$ virgate and 2 ploughs. There Beatrix has 4 villeins, 2 bordars, 8 serfs, Io beasts, 60 sheep, 3 acres of wood(land), 8 acres of meadow and 15 acres of pasture. Worth 40 shillings a year; when he received it it was worth the same.

To this manor has been added a manor called Oppaluma (Exch. Oppelaume) [Uplowman] ${ }^{9}$ which Alwin held T.R.E., and it paid geld for I virgate and I ferding. This I plough can till. Alwin himself holds it of Ralf. Worth 5 shillings a year.

Ralf has a manor called Gatepada [Gappah] ${ }^{10}$ which 5 thanes held in parage (pariter) T.R.E., and they could go to what lord they liked. It paid geld for 3 virgates. These 4 ploughs can till. Ralf holds them as I manor, and Roger holds it of Ralf. Thereof R[oger] has I virgate and 3 ploughs in demesne, and the villeins 2 virgates and I plough. There R[oger] has Io bordars, 2 serfs, I 2 beasts, I 5 swine, IOO sheep, 60 goats, 14 acres of wood(land), 6 acres of

${ }^{6}$ Highleigh St. Mary alias St. Maryleigh (Testa de Nevill, Rec. Com., 190a), called 'Henlega cum pertinentiis' in Liber Niger, p. I29 ; 'Bylidleghe' and 'Heleghe' in Taxation of Pope Nicholas (Bronescombe Reg. 476), an extra parochial chapelry in Witheridge hundred.

${ }^{7}$ Woodford in Thelbridge in Witheridge hundred.

${ }^{8}$ West Chevithorn in Tiverton parish and hundred. See fol. $502 b$.

${ }^{9}$ Uplowman in Tiverton hundred.

${ }^{10}$ Gappah in Kingsteignton in Teignbridge hun. dred. See fol. $502 b$. 


\section{A HISTORY OF DEVONSHIRE}

meadow and 4 furlongs of pasture. Worth 30 shillings; when Ralf received it 5 shillings. fol. 342 .

Ralf has a manor called Otria [Otter $]^{1}$ which Semar held T.R.E., and it paid geld for I virgate. This I plough can till. There Ralf has $\frac{1}{2}$ villein who has I plough. Otria is worth 30 pence a year. Ralf has added this manor to the manor called Holescomba (Exch. Holescumbe) [Awlescombe].

Ralf has a manor called Dunestanetuna [Dunston] ${ }^{2}$ which Edwin held T.R.E., and it paid geld for $\frac{1}{2}$ virgate. This I plough can till. Roger holds it of Ralf. On it Ro[ger] has 3 villeins and 4 bordars who have $\frac{1}{2}$ plough, also 5 beasts, 3 sheep, 3 acres of meadow and 30 acres of pasture. Worth 7 shillings and 6 pence a year; when he received it 30 pence.

Along with this manor Ralf has a manor called BLACKESTAC (Exch. Blackestach) [Blackslade] ${ }^{3}$ which Edwit (Exch. Eduin) held T.R.E., and it paid geld for I virgate. This $\mathrm{I}$ plough can till. On it are 2 villeins and 3 bordars, also 2 acres of meadow. Worth 3 shillings a year.

Ralf has a manor called OTRI [Otter ${ }^{4}$ which Lewrit (Exch. Leuric) held T.R.E., and it paid geld for $I \frac{1}{2}$ virgates and $I$ ferting. This I plough can till. Now Roselin holds it of R[alf]. There R[oselin] has I plough and I serf. Worth 3 shillings a year.

Ralf has a manor called BERI [Berry $]^{5}$ which Alric held T.R.E., and it paid geld for 2 hides. These 25 ploughs can till. Thereof $\mathrm{R}$ [alf] has I hide and 4 ploughs in demesne and the villeins I hide and 17 ploughs. There $R$ [alf] has 45 villeins, 17 bordars, 16 serfs, 8 beasts, I7 swine, fol. $342 \mathrm{~b}$.

560 sheep, roo acres of wood(land), ro acres of meadow and 40 acres of pasture. Worth 12 pounds; when $R$ [alf] received it $I 6$ pounds.

' An estate on the Otter called 'Holescoma,' i.e. Buckerel in Hemyock hundred, added to Old Awlescombe. The other half villein belonged to Capra's manor of 'Otri,' fol. 405 (O. J. R.), Trans. Devon Assoc. xxxvi, $36 r$.

${ }^{2}$ Dunston in Widdecombe-in-the-Moor in Haytor hundred. See fol. 502.

${ }^{3}$ Blackslade in Widdecombe-in-the-Moor in Haytor hundred.

${ }^{4}$ Possibly part of Ivedon on the Otter in Awlescombe in Tiverton hundred. Warin, who held Ivedon, and Roselin were in arrears in Tiverton hundred. Geldroll, fol. 67, B 3. (O. J. R.) Trans. Devon Assoc. xxxvi, 358. Hembury Fort, Buckerel (T. W. W.).

${ }^{5}$ Berry Pomeray Castle and park, in Haytor hundred. Geldroll, fol.6gb, A 7. Trans. Devon Assoc. xxviii, 363 .
Ralf has a manor called AfETona [Arton] ${ }^{6}$ which Ailsi held T.R.E., and it paid geld for 3 virgates. These 5 ploughs can till. Thereof $\mathrm{R}$ [alf] has $I_{\frac{1}{2}}$ virgates and I plough in demesne, and the villeins the like amount and 3 ploughs. There R[alf] has 6 villeins, 3 bordars, 3 serfs, I rouncey, I o beasts, 9 swine, 23 r sheep, ro acres of wood(land), 1 acre of meadow and 8 acres of pasture. Worth 25 shillings; when R[alf] received it 15 shillings.

To this manor has been added I virgate of land ${ }^{7}$ which a certain woman held in parage (pariter) T.R.E., and she could go to what lord she liked. This I plough can till. There $\mathrm{R}$ [alf] has I villein who has $\frac{1}{2}$ plough. Worth 5 shillings; when $R$ [alf] received it the same.

Ralf has a manor called OTRI [Ottery $]^{8}$ which Alric the priest held T.R.E., and it paid geld for 4 hides. These I 5 ploughs can till. Thereof $\mathrm{R}$ [alf] has $\mathrm{I}$ hide and 3 ploughs in demesne, and the villeins 3 hides and 8 ploughs. There $\mathrm{R}$ [alf] has $\mathrm{I} 8$ villeins, 4 bordars, 8 serfs, I mill paying 20 pence a year, I rouncey, 7 beasts, 8 swine, 62 sheep, 13 goats, 60 acres of wood(land), 20 acres of meadow and 500 acres of pasture. Worth 5 pounds a year; when $R$ [alf] received it it was worth the same.

fol. 343 .

Ralf has a manor called Esmarige (Exch. Smarige) [Smalridge] ${ }^{9}$ which Olnod (Exch. Ulnod) held T.R.E., and it paid geld for I hide. This 4 ploughs can till. Thereof $R$ [alf] has $I$ virgate and 2 ploughs in demesne, and the villeins 3 virgates and $2 \frac{1}{2}$ ploughs. There $R$ [alf] has 8 villeins, 5 bordars, 5 serfs, 15 beasts, 8 swine, 57 sheep, 32 goats, I mill paying 5 shillings a year, $\frac{1}{2}$ hide of wood(land), I 5 acres of meadow and 31 acres of pasture. Worth 40 shillings a year; when $R$ [alf] received it it was worth the same.

Ralf has a manor called Wigacrosta [Weycroft ${ }^{10}$ which Witchim (Exch. Wichin) held T.R.E., and it paid geld for I hide. This 4 ploughs can till. Roger (de Corcella) ${ }^{11}$ holds it of him. Thereof $\mathrm{R}$ [oger] has I virgate and I plough in demesne, and the villeins 3 virgates and $\mathrm{I} \frac{1}{2}$ ploughs. There $\mathrm{R}$ [oger] has 3 villeins,

${ }^{6}$ Arton in Berry Pomeray in Haytor hundred. See fol. $503 b$.

${ }^{7}$ Wickaborough in Berry Pomeray in Haytor hundred.

${ }^{8}$ Upottery in Axminster hundred. Geldroll, fol. 68, A 7. See Kemble, Codex Dipl. iii, 339, No. 7 I 4.

Smalridge in Axminster parish and hundred. See fol. $503 b$.

${ }^{10}$ Weycroft in Axminster parish and hundred. Robert was under-tenant of Roger de Curcelles. Geldroll, fol. 68, B I.

"Geldroll, fol. 68, B 1 . 


\section{HOLDERS OF LANDS}

6 bordars, 2 serfs, 7 beasts, 7 swine, 25 goats, 2 mills paying 7 shillings and 6 pence a year, 25 acres of wood(land), and 3 acres of meadow. Worth 20 shillings a year; when $R$ [alf] received it Io shillings.

Ralf has a manor called BROCHELANDA [Brockland $]^{1}$ which Ailard the monk held T.R.E., and it paid geld for I hide. This 4 ploughs can till. Gosfred (Exch. Goiffrid) holds it of R[alf]. fol. $343 \mathrm{~b}$.

Thereof G[osfred] has 3 bordars, 4 acres of meadow and 4 acres of pasture. Worth 12 pence a year.

Ralf has a manor called RedDrx (Exch. Redic) [Rayrish] ${ }^{2}$ which 2 thanes held T.R.E., and it paid geld for $\frac{1}{2}$ hide. This two ploughs can till. Gosfred (Exch. Goiffrid) holds it of Ralf. There G[osfred] has 15 acres of coppice. Worth 3 shillings a year; when he received it the same.

The above 3 manors were given to Ralf in exchange for I manor of I virgate.

Ralf has a manor called Chenighedona (Exch. Chenigedone) [Keynedon] ${ }^{3}$ which Edwi held T.R.E., and it paid geld for $\frac{1}{2}$ hide. This 3 ploughs can till. Roger holds it of $R$ [alph]. There $R$ [oger] has in demesne $\mathbf{I}$ plough, 4 villeins, Io bordars, 2 serfs, 10 beasts, Io swine, ro6 sheep, 2 furlongs of wood(land), 2 acres of meadow and 8 acres of pasture. Worth Io shillings a year; when he received it it was worth 7 shillings.

To this manor has been added a manor called Pola [Pool $]^{4}$ which Edwi held in parage (pariter; Exch., libere) T.R.E., and it paid geld for $\frac{1}{2}$ hide. This 2 ploughs can till. Roger holds it of R[alf]. Worth ro shillings a year.

Ralf has a manor called Hevetrowa (Exch. Hevetrove) $[\text { Heavitree }]^{5}$ which Wichin held T.R.E. Roger holds it of R[alf]. On it are 2 ploughlands (carucatae terrae) and 2 ploughs; one is the demesne land of $\mathrm{R}$ [alf] (una dominica est $R$.), and the other is the villeins'. There is I villein and 2 serfs. Worth 20 shillings a year.

Ralf has $\frac{1}{2}$ virgate of land ${ }^{6}$ which $I$ plough can till. Roger holds it of R[alf]. On it is

1 Brockland Trill in Axmouth parish and hundred.

${ }^{2}$ Rayrish, an outlier of Colyton in Colyton hundred. Trill is successor in title to Gosfred (Feud. Aids, i, 33 I). Geldroll, fol. 68b, B 2. Trans. Devon Assoc. xxxvi, 366.

${ }^{3}$ Keynedon in Sherford in Colridge hundred. See fol. 504 .

"Pool in Colridge hundred.

${ }^{5}$ Heavitree manor with the church in Heavitree parish in Wonford hundred.

${ }^{6}$ Perhaps Pinney Wood in Axminster parish and hundred. See fol. $505 b$.
I villein and I mill. Worth 30 pence a year. This Alwrit (Exch. Aluric) held in parage fol. 344 .

T.R.E. Now it has been added to Ralf's manor called Willecrosta [Weycroft].

Ralf has in Essecestra (Exch. Execestre) [Exeter] ${ }^{7} 6$ houses, from which he has kept back the king's customary dues, that is 3 shillings and 4 pence.

fol. 345 .

\section{THE LAND OF VALSCIN DE DUACO (Exch. WALTER DE DOWAI) IN DEVENESIRA \&}

Walter has a manor called HolEcoma [Hollacombe] ${ }^{9}$ which Alwin held T.R.E., and it paid geld for $\frac{1}{2}$ virgate. In demesne he has I ferding and $\frac{1}{2}$ plough, and the villeins (have) I ferding and $\frac{1}{2}$ plough. There Alwin has 1 villein, I serf, 3 beasts, 20 sheep, I furlong of wood(land), 6 acres of meadow and 10 acres of pasture. Worth 5 shillings a year.

Walscin de Duaco (Exch. Walter himself) has a manor called Hurtesberia [ $]^{10}$ which Editda (Exch. Eddida) held T.R.E., and it paid geld for 2 hides. These 17 ploughs can till. Thereof W[alscin] has $\frac{1}{2}$ hide and 4 ploughs in demesne, and the villeins $1 \frac{1}{2}$ hides and 7 ploughs. There $W$ [alscin] has 20 villeins, Io bordars, 6 serfs, 2 rounceys, II beasts, 30 swine, 200 sheep, 83 goats, 100 acres of wood(land), I acre of meadow and 200 acres of pasture. Worth 6 pounds; when W[alscin] received it it was worth the same.

Walter ${ }^{11}$ has a manor called Hagitona [Haggington] ${ }^{12}$ which Ulmar and Godrit (Exch. Godric) held in parage (pariter ; Exch., paragio) T.R.E. and it paid geld for 3 virgates. These 5 ploughs can till. Now Ulwric holds it of Walscin. Thereof Ulwrit has I virgate and 2 ploughs in demesne, and the villeins 2 virgates and I plough. There Ulwrit has ro villeins, 2 bordars, 3 serfs, ro beasts, 9 swine, 50 sheep, 37 goats, 20 acres of wood(land) and 100 acres of pasture. The manor which Vlmar held is

${ }^{7}$ Exeter, locally in Wonford hundred.

${ }^{8}$ Walter de Dowai is No. 23 in the Exchequer copy

${ }^{9}$ Hollacombe in Kentisbury in Braunton hundred. (O. J. R.) ; or Holcombe in Bampton (T. W. W.).

10 Possibly Berry Narbor in Braunton hundred. Geldroll, fol. 66, A 4. Trans. Devon Assoc. xxxvi, 366.

II 'Soinus' is interlined above the last three letters of 'Walter.'

${ }^{12}$ East Haggington in Berry Narbor in Braunton hundred. .See fol. $498 b$. 


\section{A HISTORY OF DEVONSHIRE}

worth 20 shillings; and the other which Godrit held is worth Io shillings; when Walscin received it it was worth 15 shillings.

fol. $345 \mathrm{~b}$.

Walscin has a manor called Stotrega ${ }^{1}$ which Algar held T.R.E., and it paid geld for $\frac{1}{2}$ virgate. This 2 ploughs can till. Now Arnald (Exch. Ernald) holds it of Walcin. On it A[rnald] has I plough and 2 villeins who have $\frac{1}{2}$ plough, also 2 bordars, 3 serfs, 3 beasts, 25 sheep, 6 acres of wood(land), and 20 acres of pasture. Worth Io shillings a year; when $\mathrm{W}$ [alscin] received it it was worth 5 shillings.

Walscin has a manor called Baentona [Bampton] ${ }^{2}$ which King Edward held on the day on which he was alive and dead. How many hides there are in that land we know not, because it never paid geld. This land 25 ploughs can till. Thereof $W[a I s c i n]$ has land for 4 ploughs in demesne, and there he has 2 ploughs, and the villeins have land for 18 ploughs. There also $\mathrm{W}$ [alscin] has $3 \mathrm{I}$ villeins, 20 bordars, 2 serfs, I 5 swineherds paying $106 \frac{1}{2}$ swine, 2 rounceys, I mill paying Io shillings, 2 beasts, 6 swine, 23 sheep, 50 goats, and 320 acres of wood(land).

Along with that land goes (jacet) I hide of land which 5 thanes held in parage (pariter); (Exch., in paragio ${ }^{3}$ as 5 manors. This 4 ploughs can till. Of it Renuall ( $E_{x c h}$. Rademer) has $2 \frac{1}{2}$ virgates. There Renuall has I plough and 7 villeins who have 2 ploughs, also 3 bordars, 2 serfs, I beast, I pig, 2 acres of wood(land) and 3 acres of meadow. And of that same land Rademar has I virgate. ${ }^{4}$ There R[ademar] has I plough, 3 villeins, $1 \frac{1}{2}$ acres of meadow and Io acres of pasture. Likewise Girard has thereof $\frac{1}{2}$ ferling. $^{5}$ And of that same hide $\mathrm{W}$ [illiam] de Moion has $\frac{1}{2}$ ferling ${ }^{6}$ which he got wrongfully in fraud of (injuste super) Walscin. fol. 346 .

Including this estate (cum ista terra) Baentona [Bampton] is worth I 8 pounds a year; when Walscin received it it was worth $2 \mathrm{I}$ pounds by weight.

1 Probably in Barry Narbor in Brainton hundred (O.J.R.), Trans. Devon Assoc. xxx, 452 ; or part of Stoodleigh in Witheridge hundred but close to Bampton.

${ }^{2}$ The hundred manor of Bampton. - Geldroll, fol. 686, A 2.

${ }^{3}$ Dadscombe and Hayne in Bampton $\frac{1}{2}$ fee (O. J. R.). Duvale (T. W. W.).

"Poulton and Leigh in Bampton 1 fee (O. J. R.). Hele, Clayhanger (T.W.W.). Rademar the clerk was brother to Walter de Douai (Eyton, Somerset, p. 62).

${ }^{5}$ Duvale in Bampton $\frac{1}{40}$ fee (O. J. R.). Part of Hockworthy (T.W.W.). Girard was Walter de Douai's steward (Eyton, Somerset, p. 62).

${ }^{6}$ Rose Farm in Bampton $\frac{1}{4}$ of $\frac{1}{20}$ fee (O. J. R.). Thornland Hockworthy (T. W. W.).
Wálscin holds 2 manors which 2 thanes held in parage (pariter; Exch., in paragio) called DePPAFORDA [Diptford] ${ }^{7}$ T.R.E., and it paid geld for $\frac{1}{2}$ hide. This 6 ploughs can till. Oluric (Exch. Uluric) holds it of W[alscin]: Thereof $\mathrm{O}$ [luric] has in demesne I virgate and 2 ploughs, and the villeins $I$ virgate and 3 ploughs. There O[luric] has 6 villeins, 4 bordars, 3 serfs, 2 asses, 4 beasts, 12 swine, Io9 sheep, 54 goats, IO acres of wood(land), $2 \frac{1}{2}$ acres of meadow, 10 acres of pasture and $x$ mill paying 8 pence a year. This manor is worth 30 shillings a year; when he received it it was worth 20 shillings. This manor Walscin holds of the queen, and for that he calls the king to vouch (vocat regem advocatum). ${ }^{8}$

Walscin (Exch. Walter himself) has a manor called Hochaorda [Hockford] ${ }^{9}$ which Etnot (Exch. Ednod) held T.R.E. It paid geld for 3 virgates of land. This 5 ploughs can till, Girard holds it of W[alscin]. Thereof G[irard] has in demesne I virgate and I plough, and the villeins 2 virgates and 3 ploughs. There G[irard] has 7 villeins, 6 bordars, 2 serfs, 6 beasts, I 4 swine, I2 sheep, 3 furlongs of wood(land), 2 acres of meadow and 50 acres of pasture. Worth 35 shillings a year; when he received it it was worth 30 shillings.

Walscin has a manor called CREssewalla [Kerswell $]^{10}$ which Ordric held T.R.E. It paid geld for $\frac{1}{2}$ hide. This I plough can till. Girard holds it of W[alscin]. Thereof G[irard] has I serf and 2 acres of meadow. Worth 5 shillings yearly.

fol. $346 \mathrm{~b}$.

Walscin has a manor called Offrecoma [Ufculm] ${ }^{11}$ which Etdeva held T.R.E. It paid geld for 14 hides. These 30 ploughs can till. Thereof W[alscin] has in demesne 5 hides and 2 ploughs, and the villeins 9 hides and 15 ploughs. There $W[a l s c i n]$ has 45 villeins, 6 bordars, 6 serfs, I rouncey, 14 beasts, 2 swineherds paying 15 swine, 220 sheep, Io goats, 2 mills paying 10 shillings a year, 25 acres of coppice, 25 acres of meadow and 60 acres of pasture. Worth 12 pounds a year; when he received it 10 pounds.

Walscin has a manor called Chenuestan [Knowstone] ${ }^{12}$ which Algar held T.R.E., and it

${ }^{7}$ Diptford in Bampton.

${ }^{8}$ All lands were held to be lands wrongfully entered upon (invasiones) for which the owner could not show the king's writ or name the man who had put him in possession (liberator) or voucher (advocatus) acting for the king. Freeman, Norman Conquest, v, 758.

${ }^{9}$ Hockford in Hockworthy in Bampton hundred.

${ }^{10}$ Kerswell in Hockworthy in Bampton hundred.

"The hundred manor of Ufculm. Geldroll, fol. 68 .

${ }^{12}$ Knowstone Beaupel alias Church or West Knowstone in South Molton hundred (O.J.R.). 


\section{HOLDERS OF LANDS}

paid geld for $\frac{1}{2}$ hide. This 7 ploughs can till. Now Rolf holds it of Walscin. Thereof Rolf has I virgate in desmesne and I plough; and the villeins have I plough. There R[olf] has 6 villeins, I bordar, I serf, 3 beasts, I 5 swine, 80 sheep, 27 goats, wood(land) 5 furlongs in length by $\mathrm{I}$ in breadth, 12 acres of meadow and 60 acres of pasture. Worth 20 shillings a year; when W[alscin] received it it was worth 30 shillings.

Walscin has a manor called Chenuestan [Knowstone $]^{1}$ which Lewin held T.R.E., and it paid geld for 3 fertings. These 4 ploughs can till. Now Rolf holds it of W[alscin]. Thereof R[olf] has I ferting in demesne and I plough, and the villeins have I plough. There $\mathrm{R}$ [olf] has 3 villeins, I bordar, I serf, 9 beasts, 3 swine, 22 sheep, 18 goats, wood(land) 4 furlongs in length by 1 furlong in breadth, 16 acres of meadow and 30 acres of pasture. Worth ro shillings a year.

fol. 347.

Walscin has a manor called Dunzsforda [Dunsford] ${ }^{2}$ which Elsi held T.R.E., and it paid geld for $I \frac{1}{2}$ hides. These Io ploughs can till. Now Gerard holds it of Walscin. Thereof G[erard] has $I$ virgate in demesne and I plough, and the villeins 5 ploughs. There G[erard] has 12 villeins, 8 bordars, 7 serfs, 8 beasts, 75 sheep, 4 goats, Io furlongs of wood(land), 20 acres of meadow, and pasture 5 furlongs in length by 2 furlongs in breadth. Worth 50 shillings a year; when Walscin received it 40 shillings.

Walscin has a manor called LitrL RACHENEFORDA [Little Rackenford] ${ }^{3}$ which Godric held T.R.E., and it paid geld for $\frac{1}{2}$ virgate. This I plough can till. Ludo holds it of Walscin. There L[udo] has I plough, 2 serfs, 2 acres of meadow and 20 acres of pasture. Worth 5 shillings; when $\mathrm{W}$ [alscin] received it 30 pence.

Walscin has a manor called EsPREUWEIA [Spurway] ${ }^{4}$ which Oluric (Exch. Uluric) held T.R.E., and it paid geld for I hide. This 6 ploughs can till. Hermer holds it of W[alscin]. Thereof $\mathrm{H}[\mathrm{ermer}]$ has in demesne I virgate and 2 ploughs; and the villeins 3 virgates and 3 ploughs. There $\mathrm{H}$ [ermer] has 9 villeins, I serf, 6 beasts, 17 sheep, 5 acres of wood(land), 5 acres of meadow and 40 acres of pasture. Worth 20 shillings a year, and was worth 15 shillings when $W$ [alscin] received it.

' Knowstone Botreaux alias East Barton in Molland in South Molton hundred (O. J. R.).

'Dunsford in Wonford hundred. Geldroll, fol. 7ob, A. 10.

${ }^{3}$ Little Rackenford in Witheridge hundred.

${ }^{4}$ West Spurway alias Spurway House in Oakford in Witheridge hundred.
Walscin has a manor called SUTREWORDA ${ }^{5}$ which Esgar (Exch. Asgar) held T.R.E., and it paid geld for I virgate. This 12 ploughs fol. 347b.

can till. Now Ansger holds it of Walscin. Thereof $A$ [nsger] has I ferting and 2 ploughs in demesne, and the villeins have 3 fertings and 8 ploughs. There $A$ [nsger] has 21 villeins, 14 bordars, 5 serfs, 5 swineherds paying 60 swine a year, 5 bee-keepers (mellitarii) paying 7 sestiers of honey, ${ }^{6} 25$ beasts, 100 sheep, and wood(land) I league in length by $\frac{1}{2}$ league in breadth ; 23 acres of meadow and Io furlongs of pasture taking length and breadth. Worth 8 pounds a year; when he received it it was worth the same.

Walscin has a manor called GodRIntona [Goodrington] ${ }^{7}$ which Ansgar (Exch. Asgar) held T.R.E., and it paid geld for 2 hides. These 8 ploughs can till. A certain knight, Ralf, holds it of W[alscin]. Thereof R[alf] has $\frac{1}{2}$ hide and 2 ploughs in demesne, and the villeins $I \frac{1}{2}$ hides and 6 ploughs. There $R$ [alf] has 9 villeins, 6 bordars, 4 serfs, I rouncey, 16 beasts, 15 swine, 166 sheep, and 20 acres of wood(land). Worth 50 shillings; when Walscin received it 25 shillings.

Walscin has a manor called HeTfella ${ }^{8}$ which Ailsi (Exch. Alsi) held T.R.E., and it paid geld for 3 hides. These 8 ploughs can till. Ludo holds them of $\mathrm{W}$ [alscin]. Thereof $\mathrm{L}$ [udo] has in demesne I hide and I plough; and the villeins 2 hides and 4 ploughs. There $\mathrm{L}$ [udo] has 8 villeins, 6 bordars, 2 serfs, I rouncey, 36 beasts, 20 swine, Io acres of wood(land), 20 acres of meadow, and I00 acres of pasture. Worth 40 shillings a year; when W[alscin] received it it was worth the same.

fol. 348 .

Walscin has a manor called OTRI [Ottery] ${ }^{9}$ which Alsi held T.R.E., and it paid geld for 5 hides. These 12 ploughs can till. Ludo holds them of W[alscin]. Thereof L[udo] has in demesne I hide and 3 ploughs; and the villeins 4 hides and 7 ploughs. There L[udo] has 9 villeins, 9 bordars, 7 serfs, I swineherd paying Io swine, 2 rounceys, 26 beasts, 30 swine, 100 sheep, 50 goats, 20 forest mares, 1 mill paying 10 shillings a year, 100 acres of wood(land), 20 acres of

${ }^{5}$ Apparently Lustleigh in Teignbridge hundred. Trans. Devon Assoc. xxix, 236 .

${ }^{6}$ In Wiltshire one shilling, in Warwickshire 15 pence is named as the money value of a sestier of honey in Domesday.

${ }^{7}$ Gurrington alias Goodrington in Woodland in Haytor hundred.

${ }^{8}$ Possibly Holditch in Thorncombe in Axminster hundred, since 1842 transferred to Dorset. Ludo, called a knight of Walter de Douai's. See fol. 500.

${ }^{9}$ Ottery Mohun alias Fleming in Luppit in Axminster hundred. 


\section{A HISTORY OF DEVONSHIRE}

meadow, and 150 acres of pasture. Worth 100 shillings a year; when $W$ [alscin] received it 4 pounds.

Walscin has a manor called Lovapir [Luppit] ${ }^{1}$ which Alsi held T.R.E., and it paid geld for 2 hides. These 6 ploughs can till. Ludo holds them of $\mathrm{W}$ [alscin]. Thereof $\mathrm{L}[\mathrm{udo}$ ] has in demesne I hide and 2 ploughs; and the villeins I hide and 2 ploughs. There L[udo] has 3 villeins, $x$ bordar, 3 serfs, 26 beasts, 30 goats, IO acres of coppice, IO acres of meadow and I 5 acres of pasture. Worth 20 shillings a year; when $W[$ alscin] received it the same.

Walscin has a manor called Grenowria [Greenway] ${ }^{2}$ which Ailsi (Exch. Alsi) held T.R.E., and it paid geld for 1 hide. This 5 ploughs can till. Ludo holds it of W[alscin]. Thereof $L$ [udo] has in demesne $\frac{1}{2}$ hide and 3 ploughs; and the villeins $\frac{1}{2}$ hide and $I$ plough. There L[udo] has 3 villeins, 4 bordars, 4 serfs, 40 beasts, I 2 swine, 60 sheep, 40 goats, Io acres of coppice, Io acres of meadow and Io acres of pasture. Worth 40 shillings a year; when W[alscin] received it 30 shillings.

fol. $348 \mathrm{~b}$.

To the aforesaid manor has been added another manor called Escoвecoma [Shapcombe ${ }^{3}$ which in King E[dward's] time went with (jacuit in) Hamberia [Hembury], i.e. with Bristric's ( $E_{x c h}$. Brictric's) land, and it paid geld for I hide. This 4 ploughs can till. Ludo holds it of W[alscin]. Thereof $L$ [udo] has in demesne $I$ virgate, I ferding, and I plough; and the villeins 3 virgates all but I ferding and I plough. There L[udo] has 3 villeins, I bordar, I serf, 20 acres of wood(land), 12 acres of meadow and 15 acres of pasture. Worth 20 shillings a year; when he received it the same.

Walscin has a manor called OTRI [Otter] ${ }^{4}$ which Ailsi (Exch. Alsi) held T.R.E. It paid geld for 4 hides. These 8 ploughs can till. Hubert holds them of W[alscin]. Thereof $H$ [ubert] has in demesne 3 hides, I virgate, and 3 ploughs; and the villeins 3 virgates and 2 ploughs. There $\mathrm{H}$ [ubert] has 5 villeins, 6 bordars, 4 serfs, $I$ rouncey, 38 beasts, 28 swine, 200 sheep, 50 goats, 4 forest mares, 50 acres of wood(land), 24 acres of meadow, and $I$ hide of pasture. Worth 40 shillings a year; when he received it, 4 pounds.

Walscin has a manor called SToc [Stoke] ${ }^{5}$ which Ansgar (Exch. Asgar) held T.R.E. It

${ }^{1}$ Luppit in Axminster hundred.

' Greenway in Luppit in Axminster hundred.

${ }^{3}$ Shapcombe in Luppit in Axminster hundred. See fols. 378,500 , and 5036 .

${ }^{4}$ Probably Combe Raleigh alias Baunton alias Coffin on the Otter in Axminster hundred.

${ }^{5}$ Stoke Fleming in Colridge hundred. paid geld for 5 hides. These 24 ploughs can till. Of these Ludo holds 4 hides of W[alscin]. Thereof $\mathrm{L}$ [udo] has in demesne $\mathrm{I}$ hide and 4 ploughs; and the villeins 3 hides and 12 ploughs. There L[udo] has 27 villeins, 16 bordars, 12 serfs, 2 rounceys, 16 beasts, 5 swine, 260 sheep, 30 goats, I mill which now only serves his mansion fol. 349.

(domui suae), 30 acres of coppice and 4 acres of meadow. Worth 100 shillings a year; when he received it the same.

Of these 5 hides Ralf has $\frac{1}{2}$ hide. $^{6}$ He has there I plough and the villeins I plough. There $\mathrm{R}$ [alf] has 2 villeins, 3 bordars; I serf, I beast, 30 sheep, and 4 acres of wood(land). Worth I 5 shillings a year; when $\mathrm{W}$ [alscin] received it Io shillings.

Of these a certain woman also has $\frac{1}{2}$ hide $^{7}$ which W[alscin] gave her in alms. She has there I plough and the villeins I plough. There she has 6 villeins, I bordar, I serf, 7 beasts, 5 swine, and 50 sheep. Worth ro shillings a year; when she received it the same.

Walscin has a manor called Colridge [Colridge ${ }^{8}$ which Biche held T.R.E., and it paid geld for I virgate. This 2 ploughs can till. Alric holds it of W[alscin]. Thereof A[lric] has in demesne $I$ ferding and $\frac{1}{2}$ plough, and the villeins 1 ferding and $\frac{1}{2}$ plough. There Alric has 3 bordars, 2 beasts, I pig, 35 sheep, 6 goats, and 6 acres of coppice. Worth 5 shillings a year; when $W$ [alscin] received it the same. This manor in King E[dward's] time was a thane's, free to go wherever he liked (ad pergendum quocunque voluit), and it has been added to Ansgar's honour (Exch. to the lands of Ansgar).

Walscin has a manor called Wodiacomma [Widdecombe] ${ }^{9}$ which Eddric held T.R.E. It paid geld for $\frac{1}{2}$ hide. This 2 ploughs can till. Ailric holds it of W[alscin]. There A[ilric] has I $\frac{1}{2}$ ploughs, also 4 villeins, 4 beasts, 5 swine, 45 sheep, and I acre of meadow. Worth 15 shillings. This manor also was in King E[dward's] fol. $349 \mathrm{~b}$.

time a thane's, free to go wherever he liked, and has been added to Ansgar's honour (Exch. to the lands of Asgar).

Walscin has a manor called Colriga [Colridge] ${ }^{10}$ which Alvieva (Exch. Alveva) held T.R.E., and it paid geld for I virgate. This

'Probably Norton fitz-Stephen alias Dawnay in Townstal in Colridge hundred.

${ }^{7}$ Possibly Little Dartmouth alias St. Petrock's Dartmouth in Colridge Hundred.

${ }^{s}$ Colridge in Stokenham in Colridge hundred. See fol. 504 .

${ }^{9}$ Widdecombe in Stokenham in Colridge hundred. See fol. 504 .

${ }^{10}$ Colridge in Stokenham in Colridge hundred. 


\section{HOLDERS OF LANDS}

I plough can till. Alvieva holds it of W[alscin]. There A[lvieva] has I plough, 6 sheep, and 7 acres of coppice. Worth 40 pence a year, when $W[$ alscin] received it the same.

Walscin has a manor called Dunkstal [Townstal ${ }^{1}$ which Ansger held T.R.E., and it paid geld for $\frac{1}{2}$ hide. This 2 ploughs can till. Ralf holds it of Walscin. Thereof $\mathrm{R}$ [alf] has $I$ ferling and I plough in demesne; and the villeins 3 ferlings and $x$ plough. There $R$ [alf] has 5 villeins, 4 bordars, 2 serfs, 6 beasts, 40 sheep, 15 goats, and 6 acres of wood(land). Worth Io shillings; when $W$ [alscin] received it the same.

Walscin has in Essecestra (Exch. Execestre) [Exeter] Io houses which Ansger (Exch. Asgar) held T.R.E., and he has I other house in pledge from a burgess whence the customary due is withheld.

\section{THE LAND OF WILLIAM DE MOION \\ fol. 356. IN DEVENESIRA ${ }^{2}$}

William has a manor called Clemangra [Clayhanger] $^{3}$ which Oltred (Exch. Uctred) held T.R.E., and it paid geld for 3 virgates and $3 \frac{1}{2}$ ferdings. These 5 ploughs can till. Robert holds them of $W$ [illiam]. Thereof R[obert] has in demesne 2 virgates and 2 ploughs; and the villeins I virgate, $3 \frac{1}{2}$ ferdings, and 2 ploughs. There R[obert] has 5 villeins, 3 bordars, 2 serfs, 7 swine, 65 sheep, 3 acres of coppice, 12 acres of meadow and 12 acres of pasture. Worth 20 shillings a year; when $W[$ illiam] received it it was worth 30 shillings.

\section{THE LAND OF WILLIAM DE}

\section{FALESIA IN DEVENESIRA 4} fol. 366 .

William de Falesia has a manor called Comba [Combe] $^{5}$ which Bristric (Exch. Brictric) and Edvin (Exch. Edwi) held in parage (pariter; Exch., libere) T.R.E., and it paid g(eld) for 2 hides and $\mathrm{I}$ virgate. These 20 ploughs can till. Thereof W[illiam] has 3 virgates and 3 ploughs in demesne, and the villeins $I \frac{1}{2}$ hides and I4 ploughs. There $W$ [illiam] has 18 villeins,

\footnotetext{
1 Townstal alias Hardnes alias St. Saviour's Dartmouth in Colridge hundred.

'In the Exchequer copy William de Moion alias Mohun is No. 18.

${ }^{3}$ Clayhanger in Bampton hundred.

4 In the Exchequer copy William de Faleise is No. 20.

${ }^{5}$ Combe Martin in Braunton hundred. Geldroll, fol. 66, A 9.
}

ro bordars, 9 serfs, 2 I beasts, 9 swine, 140 sheep, 19 goats, 5 acres of wood(land), and pasture $I$ league in length by $I$ league in breadth. Worth 100 shillings a year; when $W$ [illiam] received it the same.

William has a manor called Fersa [Foss] which Alcerl ( $E_{x c b}$. Alcherl) held T.R.E., and it paid geld for $\frac{1}{2}$ virgate. This 3 ploughs can till. It is altogether out of cultivation (penitus vastata).

William has a manor called Pedracomba

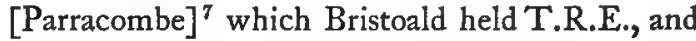
it paid geld for $\frac{1}{2}$ hide. This 8 ploughs can till. Thereof W[illiam] has I virgate and 2 ploughs in demesne; and the villeins I virgate and 4 ploughs. There $W[$ illiam] has 5 villeins, 8 bordars, 5 serfs, ro beasts, 27 sheep, 27 goats, 30 acres of wood(land), 8 acres of meadow, and I league of pasture. Worth 40 shillings a year; when William received it it was worth the same.

fol. $366 \mathrm{~b}$.

W[illiam] has a manor called Cercilla [Churchill] $^{8}$ which Bristuold (Exch. Brictuold) held T.R.E., and it paid geld for I virgate. This 8 ploughs can till. Norman holds it of William. Thereof Norman has I virgate and I plough in demesne, and the villeins 2 virgates and 4 ploughs. There Norman has 8 villeins, 4 bordars, 5 serfs, roo sheep, 40 acres of wood(land) and I league of pasture. Worth 40 shillings. When W[illiam] received it it was worth the same.

William has a manor called BERA $[\mathrm{Bar}]^{9}$ which Bristuold (Exch. Brictuold) held T.R.E., and it paid geld for I virgate. This 2 ploughs can till. Roger holds it of William. Thereof $\boldsymbol{R}$ [oger] has in demesne $\frac{1}{2}$ virgate and $\frac{1}{2}$ plough, and the villeins $\frac{1}{2}$ virgate and $\frac{1}{2}$ plough. There Roger has 2 bordars, I serf, I00 sheep, 3 acres of meadow and I league of pasture. It pays I 5 shillings, and was worth the same when W[illiam] received it.

fol. 367 .

William de Falesia has a manor called W [Washford] ${ }^{10}$ which Chenegar held T.R.E., and it paid geld for I virgate. This 2 ploughs can till. Now Peter holds it of William. Thereof

\footnotetext{
- Foss alias Fairlinch in Braunton manor and hundred.

${ }^{7}$ Parracombe in Shirwell hundred.

${ }^{8}$ Churchill in Fast Down in Braunton hundred. The missing virgate is probably Twitchin held by the same tenant Bridewold before the Conquest.

${ }^{9}$ Bar alias Beare in East Worlington in Witheridge hundred.

${ }^{10}$ Part of Little Washford, south of Witheridge, now Stewarton (O.J.R.) ; Trans. Devon Assoc. xxxvi, 357 ; or Washford Moor in Washford Pyne (T.W.W.).
} 


\section{A HISTORY OF DEVONSHIRE}

Peter has 3 fertings in demesne and $\mathbf{I}$ plough, and 3 bordars of his have 1 ferting. There Peter has 5 beasts, 4 acres of meadow and I 5 acres of pasture. Worth 5 shillings a year; when William received it the same.

William has a manor called ULWRINTONA (Exch. Olurintone) [Worlington $]^{1}$ which 2 thanes held in parage (pariter; Exch., libere) T.R.E., and it paid geld for I ferting. This I plough can till. Now Hugh de Dol holds it of W[illiam], and his villeins have $\mathbf{I}$ plough on that land. There Hugh has 2 villeins, $\frac{1}{2}$ acre of wood (land), and 2 acres of meadow. It pays ro shillings a year; and when William received it it was worth the same.

William has a manor called BRADEFORDA [Bradford] ${ }^{2}$ which Brungar held T.R.E., and it paid geld for $I \frac{1}{2}$ fertings. This $\mathbf{I} \frac{1}{2}$ fertings I plough can till. There William has I villein who has $\mathrm{I}$ plough besides 2 acres of meadow. Worth 5 shillings a year; when William received it the same.

William has a manor called Dimewoldesham

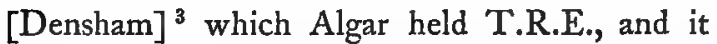
paid geld for $\frac{1}{2}$ virgate. This I plough can till. Now a certain Englishman holds it of W[illiam] and has on it $\mathbf{I}$ plough. Worth 5 shillings a year ; when William received it the same.

fol. 367b.

William de Faleisa has a manor called CHochintona [Cockington] ${ }^{4}$ which Alric held T.R.E., and it paid geld for 3 hides. These I3 ploughs can till. Thereof W[illiam] has I hide and 5 ploughs in demesne and the villeins 2 hides and 7 ploughs. There $W$ [illiam] has 18 villeins, 6 bordars, I 4 serfs, I rouncey, 8 beasts, 159 sheep, 42 goats, 50 acres of wood(land), I5 acres of meadow and 50 acres of pasture. Worth 50 shillings; when W[illiam] received it the same.

And as parcel of $(d e)$ the aforesaid 3 hides the same Alric held a manor called Depdona [Deandon] ${ }^{5}$ T.R.E. which paid geld for I virgate and has been added to the aforesaid manor. Worth Io shillings; when $W[i l l i a m]$ received it the same. W[illiam] holds the two as one manor.

${ }^{1}$ In East Worlington in Witheridge hundred. See fol. $501 b$.

2 Bradford in Cruwys Morchard in Witheridge hundred (O.J.R.).

${ }^{3}$ Minikinland alias 'Munecheneland' (Testa de Nevill, Rec. Com. 190a) or the nuns' land at Densham in Wolfardisworthy Spenser in Witheridge hundred. Trans. Devon Assoc. xxix, 258.

4 Cockington in Haytor hundred. See fol. 5026 . Geldroll, fol. 696 , A 10 .

${ }^{5}$ Deandon in Widdecombe-in-the-Moor in Haytor hundred. Trans. Devon Assoc. xxvii, 382.
William has a manor called Holla [Holne] ${ }^{6}$ which Alwin held T.R.E., and it paid geld for I $\frac{1}{2}$ hides. This $x 2$ ploughs can till. Thereof W[illiam] has $\frac{1}{2}$ hide and I plough in demesne, and the villeins $I$ hide and 5 ploughs. There W[illiam] has I 3 villeins, 7 bordars, 8 serfs, I rouncey, 6 beasts, 6 swine, 52 sheep, wood(land) I league in length by $I$ in breadth, and I league of pasture taking length and breadth. Worth 60 shillings; when $W$ [illiam] received it the same.

William has a manor called Estocha (Exch. Stoche) [Stock] ${ }^{7}$ which Ulveva held T.R.E., and it paid geld for $\frac{1}{2}$ hide. This 2 ploughs can fol. 368 .

till. Winemer holds it of William. Thereof W[inemer] has I virgate and I plough in demesne, and the villeins I virgate. There $\mathrm{W}$ [inemer] has 4 villeins, 2 bordars, I serf, I beast, 7 swine, 40 sheep, wood(land) I league in length by $I$ furlong in breadth, and $\frac{1}{2}$ league of pasture. Worth 10 shillings; when $\mathrm{W}$ [illiam] received it the same.

William has a manor called Dena [Dean ${ }^{8}$ which Ulwin held T.R.E., and it paid geld for 3 hides. These 12 ploughs can till. Four knights (milites) hold it of William. Thereof the knights have I hide and 3 ploughs in demesne, and the villeins 2 hides and 6 ploughs. There the knights have 19 villeins, 15 bordars, 2 serfs, I 6 beasts, I 3 swine, 70 sheep, 50 goats, 6 acres of wood(land), Io acres of meadow, and I league of pasture. Worth 4 pounds; when W[illiam]. received it the same.

Of the aforesaid manor a certain Englishman holds (land) enough whence to pay William Io shillings a year. ${ }^{9}$ He held it T.R.E. but could not be independent of (separari) his lord.

William has a manor called RATREU [Rattery] ${ }^{10}$ which Alwin held T.R.E., and it paid geld for 3 hides. These 12 ploughs can till. Thereof $\mathrm{W}$ [illiam] has $\mathrm{I}$ hide and 2 ploughs in demesne ; and the villeins $I$ hide $I \frac{1}{2}$ virgates and 5 ploughs. There W[illiam] has I 3 villeins, 4 bordars, 6 serfs, 2 swineherds paying 8 swine, 8 beasts and 72 sheep. Worth 40 shillings; when W[illiam] received it the same.

Thereof 2 knights (milites) have $\mathrm{I}_{2}^{\frac{1}{2}}$ virgates ${ }^{11}$ and 2 ploughs in demesne, also 8 villeins, 7 bordars, 2 serfs, 19 beasts, I 2 swine, I I I sheep, 40

${ }^{6}$ North Holne alias Holne Bauzan and South Holne in Stanborough hundred. Geldroll, fol. 69b, A 2.

${ }^{7}$ Stock in Holne in Stanborough hundred.

${ }^{8}$ Dean Prior alias Church in Stanborough hundred. Geldroll, fol. $69 b, A 2$.

${ }^{9}$ Perhaps Whitoxden or Wallaford in Dean Prior.

${ }_{10}$ Rattery in Stanborough hundred.

1I Possibly Hood and Luscombe Barnage in Rattery in Stanborough hundred. 


\section{HOLDERS}

goats, $\frac{1}{2}$ acre of meadow, and 5 acres of pasture. Worth 20 shillings; when $W$ [illiam] received it the same.

Also a certain Englishman holds thereof I virgate $^{1}$ which he held himself T.R.E., but without being able to be independent of his lord. Thence he pays William Io shillings a year.

fol. $368 \mathrm{~b}$.

William has a manor called Dertrintona [Dartington] $^{2}$ which Alwin held T.R.E., and it paid geld for I virgate. This I 5 ploughs can till. Thereof W[illiam] has I ferling and 2 ploughs in demesne; and the villeins 2 ferlings and $8 \frac{1}{2}$ ploughs. There W[illiam] has I 3 villeins, 7 bordars, 9 serfs, 2 swineherds paying I9 swine, 2 fishermen paying 80 salmon, I rouncey, I I beasts, I pig, 72 sheep, wood (land) $\frac{1}{2}$ league in length by $2 \frac{1}{2}$ furlongs in breadth, 30 acres of coppice, and 100 acres of pasture. Worth 4 pounds 5 shillings; when W[illiam] received it the same.

And as part of $(d e)$ the aforesaid virgate a certain thane held a manor called Loscumma [Luscombe $]^{3}$ T.R.E. which paid geld for I ferling, but he could be independent of his lord. Now Anschetil holds it of W[illiam]. Thereof A[nschetil] has $\frac{1}{2}$ ferling and I plough in demesne, and the villeins $\frac{1}{2}$ ferling and $I \frac{1}{2}$ ploughs. There A[nschetil] has 4 villeins, I serf, 4 beasts, and $\frac{1}{2}$ acre of meadow. Worth 15 shillings; when $\mathrm{W}$ [illiam] received it $\mathrm{I} 0$ shillings.

William has a manor called Herberneforda (Exch. Erberneforde) [Harbournford] ${ }^{4}$ which Alric held T.R.E., and it paid geld for $\frac{1}{2}$ hide. This 3 ploughs can till. Rainald holds it of W[illiam]. Thereof R[ainald] has I virgate in demesne, and the villeins $I$ virgate and I plough. There $\mathrm{R}$ [ainald] has 3 villeins, 2 bordars, I serf, 4 acres of wood(land) and 2 acres of meadow. Worth ro shillings; when $\mathrm{W}$ [illiam] received it 8 shillings.

W[illiam] has a manor called ENGLEBORNA [Inglebourne] $^{5}$ which Alrix (Exch. Alric) held T.R.E., and it paid geld for $\frac{1}{2}$ hide. This 3 ploughs can till. Rainald holds it of W[illiam]. Thereof $R$ [ainald] has in demesne I virgate and 3 ferlings and 2 ploughs; and the villeins $I$ ferling and $\frac{1}{2}$ plough. There R[ainald] has 4 bordars, I serf, I rouncey, I beast, 62 sheep, and 10 acres of meadow. Worth 20 shillings a year; when $W$ [illiam] received it it was worth the same.

\footnotetext{
${ }^{1}$ Possibly Marley in Rattery in Stanborough hundred.

${ }^{2}$ Dartington in Stanborough hundred.

${ }^{3}$ Luscombe alias West Combe in Dartington in Stanborough hundred.

"North Harbournford in South Brent in Stanborough hundred.

${ }^{5}$ Little Inglebourn in Harberton in Colridge hundred.
}

\section{fol. 37 r. \\ THE LAND OF ALVRED OF EPAIGNES ${ }^{6}$}

\section{(ISPANIENSIS) IN DEVENESIRA}

Alvered has a manor called Alferdintona [Arlington] $^{7}$ which Ailwid (Exch. Alwi) ${ }^{8}$ held T.R.E., and it paid geld for I hide. This 15 ploughs can till. Thereof $A$ [lvered] has in demesne I virgate and 2 ploughs, and the villeins 3 virgates and 5 ploughs. There A[lvered] has II villeins, 3 serfs, 5 beasts, 100 sheep, 4 acres of wood(land), 3 acres of meadow and $\frac{1}{2}$ league of pasture taking length and breadth. Worth 3 pounds a year; when A[lvered] received it 8 pounds.

To the aforesaid manor a certain manor has been added called Tuchel [Twitchin] ${ }^{9}$ which Bridewold held T.R.E., and it paid geld for I virgate. This 3 ploughs can till. Thereof $A$ [lvered] has in demesne $\frac{1}{2}$ virgate, and the villeins $\frac{1}{2}$ virgate and I plough. There A[lvered] has 4 villeins and 30 acres of wood(land). Worth 5 shillings a year; when A[lvered] received it it was worth 20 shillings.

Alvered has a manor called OrRawia (Exch. Orrewai) [Orway] ${ }^{10}$ which Alwi held T.R.E., and it paid geld for $\frac{1}{2}$ hide. This 3 ploughs can till. Thereof $A[$ lvered] has in demesne I virgate and I plough, and the villeins. I virgate. There A[lvered] has 8 villeins, 6 bordars, I serf, 4 acres of wood(land), 5 acres of meadow, and roo acres of pasture. Worth 30 shillings a year; when he received it the same.

fol. 376.

\section{THE LAND OF ODO}

\section{SON OF GAMELIN IN DEVENESIRA ${ }^{11}$}

Odo has a manor called EstatForda (Exch. Staford) [Stowford] ${ }^{12}$ which Sawin held T.R.E., and it paid geld for $\frac{1}{2}$ hide all but $\frac{1}{2}$ ferding. This 6 ploughs can till. Now Ralf Vitalis holds it of Odo. Thereof $R$ [alf] has $\frac{1}{2}$ virgate and 2 ploughs in demesne, and the villeins have

${ }^{6}$ Alvred the Épaigniard is No. 39 in the Exchequer copy. Mr. Round suggests that he should be called the Epaigniard as coming from the village of Epaignes, formerly Latinised as 'Ispania' (Cal. of Docs. France, I 12 ). Freeman, Norman Conquest, v, 737, observes that he is called Alfred Aspania in Cod. Dipl. vi, 211 .

${ }^{7}$ Arlington in Shirwell hundred. Geldroll, fol. 66, A 10.

${ }^{8}$ Alwi Bannesone, fol. 162 .

9 Twitchin in Arlington in Shirwell hundred. See fol. 499.

${ }^{10}$ Orway in Kentisbeare in Hairidge hundred.

11 Odo son of Gamelin, son-in-law of Tetbald (fol. 497), is No. 43 in the Exchequer copy, with 'Dalilei" first in order of his estates, 'Staford" following.

${ }^{12}$ Stowford in Lifton hundred. 


\section{A HISTORY OF DEVONSHIRE}

the rest of the land and 2 ploughs and 3 oxen. There $\mathrm{R}$ [alf] has Io villeins, 3 bordars, 2 serfs, Io beasts, 6o sheep, 20 goats, I 2 acres of wood(land), 10 acres of meadow, and 15 acres of pasture. Worth 40 shillings; when $O$ [do] received it 50 shillings.

Odo has a manor called Dalilea (Exch. Dalilei) [Dalley] $^{1}$ which Bristric (Exch. Brictric) held T.R.E., and it paid geld for I virgate. This 4 ploughs can till. Thereof $\mathrm{O}[\mathrm{do}]$ has $\frac{1}{2}$ virgate and I plough in demesne, and the villeins $\frac{1}{2}$ virgate and I plough. There $O[$ do] has 4 villeins, I serf, I rouncey, 5 beasts, 50 sheep, 4 acres of wood(land), 2 acres of meadow and 10 acres of pasture. This manor is worth 20 shillings; it was worth 10 shillings when $O$ [do] received it.

Odo has a manor called Almerescota [Almiston $]^{2}$ which Leuesgar (Exch. Leuegar) held T.R.E., and it paid geld for I virgate. This 3 ploughs can till. Now Colvin holds it of Odo. Thereof he has $\mathbf{I}$ ferling and $\mathbf{I}$ plough in demesne, and the villeins 3 ferlings and 2 ploughs. There C[olvin] has 6 villeins, I serf, I 5 beasts, 60 sheep, 20 goats, Io acres of meadow and 40 acres of pasture. Worth 20 shillings; when Odo received it it was worth 3 shillings.

fol. $376 \mathrm{~b}$.

Odo has a manor called YwIs (Excb. Iwis)

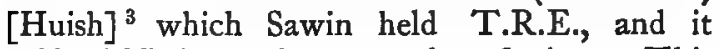
paid $\mathrm{g}(\mathrm{eld})$ for 3 virgates and I ferting. This 7 ploughs can till. Now Ralf Vitalis holds it of Odo. Thereof R[alf] has I virgate and 3 ploughs in demesne; and the villeins have 2 virgates and 2 ploughs. There R[alf] has 7 villeins, 3 bordars, I serf, 24 beasts, 7 swine, I02 sheep, 30 acres of wood(land), 35 goats, 12 acres of meadow and 50 acres of pasture. The aforesaid ferting one thane held T.R.E., who with that ferting could be independent of (separari) Sawin (Exch. could go where he liked). This manor is worth 40 shillings; when $R$ [alf] received it it was worth 60 shillings.

Odo has I virgate of land called Litelwera

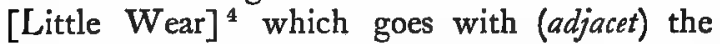
manor called Toritona [Torington]. Bristric held it T.R.E., and it paid geld for I virgate. This 4 ploughs can till. Thereof $\mathrm{O}$ [do] has in demesne $\frac{1}{2}$ virgate, and the villeins $\frac{1}{2}$ virgate and

1 Dalley alias Tyle and Delworthy in Yarnscombe in Hartland hundred. Geldroll, fol. $65, \mathrm{~A}_{4}$.

${ }^{2}$ Almiston alias Almereston (Feud. Aids, i, 342) in Woolfardisworthy in Hartland hundred. Geldroll, fol. $65, \mathrm{~B}$ I.

${ }^{3}$ Huish in Shebbear hundred. See fol. 497. Geldroll, fol. $656, A_{4}$. I ferling is not accounted for here, but see fol. 497 .

"Littlewear in Wear Giffard in Fremington hundred.
I plough. There $\mathrm{O}[\mathrm{do}]$ has 4 villeins, 2 bordars, and 4 acres of pasture. Worth Io shillings a year; when he received it the same.

Odo has a manor called ToRITONA [Torington $]^{5}$ which Bristrit (Exch. Brictric) held T.R.E., and it paid geld for $3^{\frac{1}{2}}$ hides. These 40 ploughs can till. Thereof $O$ [do] has 3 virgates in demesne and 4 ploughs, and the villeins have 2 hides and 26 ploughs. There $O[$ do] has 45 villeins, Io bordars, 7 serfs, 12 beasts, Io swine, I 46 sheep, 300 acres of wood(land), 20 acres of meadow, pasture 2 leagues long by I league wide, also 25 swineherds paying I Io swine yearly. Worth I5 pounds; when $O[d o]$ received it 24 pounds.

Of the aforesaid $3 \frac{1}{2}$ hides, 3 Frenchmen (francigene) have 3 virgates. Goselm holds thereof I virgate $^{6}$ worth I 5 shillings a year, Walter holds another virgate ${ }^{7}$ worth 15 shillings a year, and Ansger holds the third virgate ${ }^{8}$ worth I 5 shillings a year.

fol. 377 .

Odo has a manor called Bocchelanda [Buckland $]^{9}$ which Godo (Exch. Gode) held T.R.E., and it paid geld for $\mathrm{I} \frac{1}{2}$ virgates. This 4 ploughs can till. Alviet (Exch. Almer) holds it of Odo. There Alviet has 2 ploughs in demesne, and the villeins $1 \frac{1}{2}$ ploughs. There Alviet has 6 villeins, 3 bordars, 2 serfs, I 4 beasts, 55 sheep, I 2 acres of wood(land), 2 acres of meadow and 30 acres of pasture. Worth 20 shillings a year; when he received it 15 shillings.

Odo has a manor called WiLledenNa [Widdin] ${ }^{10}$ which Norman held T.R.E., and it paid geld for I virgate. This 2 ploughs can till. Ralf holds it of Odo. There R[alf] has in demesne $\frac{1}{2}$ virgate and I plough, and the villeins $\frac{1}{2}$ virgate and $\frac{1}{2}$ plough. There $R$ [alf] has 2 villeins, I bordar, I serf, 4 beasts, 8 swine, 18 sheep, 3 goats, 5 acres of coppice, $\frac{1}{2}$ acre of meadow and 40 of pasture. Worth Io shillings a year; when he received it the same.

Odo has a manor called SrREwILIA [Shirwell] ${ }^{11}$ which Fitel (Exch. Vitalis) held T.R.E., and it paid geld for I virgate. This two ploughs can till. Gislebert holds it of $\mathrm{O}[\mathrm{do}]$. Thereof

${ }^{5}$ Great Torington alias Cheping Torington in Fremington hundred. Geldroll, fol. $65 b, \mathrm{~A} 8, \mathrm{~B} 2$. Trans. Devon Assoc. vii, 90.

${ }^{6}$ Hereward's Dodscot alias Hairsham (Testa de Nevill, Rec. Com. 176a), in St. Giles'-in-the-Wood in Fremington hundred.

'Stevenstone in St. Giles'-in-the-Wood in Fremington hundred.

${ }^{8}$ Winscot in St. Giles'-in-the-Wood in Fremington hundred.

${ }^{9}$ Buckland in Dolton in North Tawton hundred.

${ }^{10}$ Widdin alias Wydedon (Feud. Aids, i, 359) in Marwood in Braunton hundred.

"Shirwell in Shirwell hundred. 


\section{HOLDERS OF LANDS}

$\mathrm{G}$ [islebert] has in demesne $\frac{1}{2}$ virgate and $\frac{1}{2}$ plough, and the villeins $\frac{1}{2}$ virgate and $\frac{1}{2}$ plough. There $G$ [islebert] has I villein, I serf, 5 sheep, and I acre of meadow. Worth 5 shillings a year.

Odo has a manor called LIMET [Nymet or Intake ${ }^{1}$ which 4 thanes held in parage (pariter: Exch., in paragio) T.R.E., and it paid geld for I hide. This Io ploughs can till. Thereof Odo has $\mathrm{I} \frac{1}{2}$ virgates and 4 ploughs in demesne, and the villeins have 4 ploughs. There Odo has 10 villeins, 5 bordars, 5 serfs, 12 beasts, fol. $377 \mathrm{~b}$.

I rouncey, 3 swine, 36 sheep, Io acres of wood(land) and 10 of meadow. Worth 40 shillings a year; when $\mathrm{O}[\mathrm{do}]$ received it it was worth ro shillings.

Odo has a manor called HuNITONA [Honiton] ${ }^{2}$ which Alwald (Exch. Alwold) held T.R.E., and it paid geld for $\frac{1}{2}$ virgate. This 2 ploughs can till. On it Odo has I plough in demesne and the villeins another plough. There Odo has 3 villeins, 2 bordars, and 7 acres of meadow. Worth 5 shillings a year; when Odo received it it was worth 2 shillings.

Odo has a manor called Alra [Aller ${ }^{3}$ which Godeva held T.R.E., and it paid geld for $\frac{1}{2}$ virgate. This 2 ploughs can till. On it Odo has 2 ploughs and the villeins have I plough. There $O$ [do] has 4 villeins, I bordar, I serf, 6 beasts, $3^{6}$ sheep, 4 acres of meadow and 30 acres of pasture. Worth Io shillings a year.

Of the aforesaid $\frac{1}{2}$ virgate Eda (Exch. Edda) held I ferding ${ }^{4}$ and he could go with his land to what lord he liked T.R.E. This I plough can till.

Odo has a manor called Acria [Hacche] ${ }^{5}$ which Vithelet (Exch. Vitalis) held T.R.E., and it paid geld for $\frac{1}{2}$ hide. This 4 ploughs can till. Now Alwis (Exch. Alwi) holds it of Odo. Thereof Alwis has I virgate and 2 ploughs in demesne, and the villeins have I plough. There A[lwis] has 4 villeins, 4 bordars, 3 serfs, I 4 beasts, 3 swine, 38 sheep, 25 goats, Io acres of wood(land), 4 acres of meadow and 20 acres of pasture. Worth 20 shillings a year; when $\mathrm{O}[\mathrm{do}]$ received it it was worth the same.

\section{fol. 378.}

Odo has a manor called STANLINz [Stallenge] ${ }^{6}$ which Alric the priest held T.R.E., and it

1 George Nympton in South Molton hundred, the parish. See fol. 499b. ' Geldroll, fol. 66, A 3.

${ }^{3}$ Honiton in South Molton parish and hundred.

${ }^{3}$ North Aller in South Molton parish and hundred.

4 Probably Ford in South Molton parish and hundred. See fol. $499 b$.

${ }^{5}$ Hacche in South Molton parish and hundred.

'Stallenge, formerly 'Stanlynch' (Feud. Aids, 381) in Hockworthy in Bampton hundred. Geldroll, fol. 69, A 5 . paid geld for I hide. This 2 ploughs can till. Thereof $O[d o]$ has $\frac{1}{2}$ hide and 1 plough in demesne, and the villeins $\frac{1}{2}$ hide and $x$ plough. There $\mathrm{O}[\mathrm{do}]$ has I villein, 4 bordars, 8 beasts, I pig, 80 sheep, 2 acres of coppice and 2 acres of meadow. Worth ro shillings a year. It forms part of (est de) Honessam [Huntsham] manor.

Odo has a manor called Bredelia [Brayley] which Olwena (Exch. Ulwene) held T.R.E., and it paid geld for I virgate. This 6 ploughs can till. Rainald holds it of $\mathrm{O}[\mathrm{do}]$. Thereof $\mathrm{R}$ [ainald] has in demesne $\frac{1}{2}$ virgate and I plough, and the villeins $\frac{1}{2}$ virgate and $\frac{1}{2}$ plough. There $\mathrm{R}$ [ainald] has 2 villeins, I bordar, 4 serfs, Io acres of coppice, 2 acres (of meadow) ${ }^{8}$ and $\frac{1}{2}$ league of pasture. Worth 20 shillings a year.

Odo has a manor called Hankeria [Hembury $]^{9}$ which Bristric (Exch. Brictric) held T.R.E., and it paid geld for 4 hides. These 18 ploughs can till. Thereof Odo has in demesne I hide and 2 ploughs, and the villeins 3 hides and ro ploughs. There Odo has 29 villeins, I I bordars, 2 swineherds paying Io swine a year, 6 serfs, I rouncey, 7 beasts, 5 swine, roo sheep, I mill paying Io shillings a year, 8o acres of wood(land), IO acres of meadow and 50 acres of pasture. Worth 8 pounds a year; when he received it I I pounds.

With that manor I hide used to go (In ista mansione jacebat) T.R.E. called SсовассомA (Exch. Cobecume) [Shapcombe]. ${ }^{10}$ This 3 ploughs can till. Ludo holds it along with Walter's land wrongfully. Thereof Ludo has 2 ploughs and 6 villeins. Worth 30 shillings a year.

fol. $378 \mathrm{~b}$.

Odo has a manor (called) Plumtrei [Plymtree $]^{11}$ which Bristric (Exch. Brictric) held T.R.E., and it paid geld for 2 hides and I virgate. These 5 ploughs can till. Of these $\mathrm{O}[\mathrm{do}]$ has in demesne I hide and 2 ploughs; and the villeins I hide, I virgate, and 3 ploughs. There $O$ [do] has I 5 villeins, 4 bordars, 4 serfs, I rouncey, I 3 beasts, 20 sheep, 20 acres of wood(land), and 20 acres of meadow. Worth 100 shillings; when he received it the same.

${ }^{7}$ Brayley in East Buckland in Braunton hundred.

8 'Prati' omitted in Exeter text, supplied by Exchequer.

${ }^{9}$ Broad Hembury in Hairidge hundred. Geldroll, fol. $67 b, A_{5}$ and $B$

${ }^{10}$ Shapcombe in Luppit in Axminster hundred. See above, fol. $348 b$.

Il Plymtree in Hairidge hundred. Geldroll, fol. $67 b, A$ 5. Given by Odo to St. Peter's, Gloucester (Ghartulary, Rolls Series, i, 74) in 1095, and acquired by Nicolas de la Pole before II60 in exchange for Aylestone in Warwickshire (V.C.H. Warwick, i, 280). 


\section{A HISTORY OF DEVONSHIRE}

Odo has a manor called Hillesdona [Hillersdon] ${ }^{1}$ which Siruwald (Exch. Sirewald) held T.R.E., and it paid geld for $\frac{1}{2}$ hide. This 3 ploughs can till. Rainald holds it of $O[d o]$. Thereof R[ainald] has I virgate and I plough, and the villeins I virgate. There $\mathrm{R}$ [ainald] has 5 villeins, 7 bordars, I serf, 3 beasts, 2 swine, 86 sheep, 3 acres of meadow, and 7 furlongs of pasture. Worth ro shillings a year; when he received it the same.

Odo has a manor called LABera [ $]^{2}$ which Etmar (Exch. Edmar) held T.R.E., and it paid geld for I virgate. This $\frac{1}{2}$ plough can till. Hubert holds it of $\mathrm{O}$ [do]. There $\mathrm{H}$ [ubert] has $\frac{1}{2}$ plough, I 5 sheep, 5 goats, and 4 acres of meadow. Worth 3 shillings a year; when he received it the same.

Odo has a manor called DerTa [Dart] ${ }^{3}$ which Bristic (Exch. Brictric) held T.R.E., and it paid geld for $1 \frac{1}{2}$ virgates. These 4 ploughs can till. Thereof $\mathrm{O}[\mathrm{do}]$ has $\frac{1}{2}$ virgate and $\mathrm{I}$ plough in demesne, and the villeins I virgate and I plough. fol. 379 .

There $O[$ do] has 4 villeins, 3 bordars, 3 serfs, 5 beasts, 5 acres of meadow, and 40 acres of pasture. Worth 30 shillings; when $O[d o]$ received it 20 shillings.

O[do] has a manor called Olurintona (Exch. Ulurintone) [Worlington] ${ }^{4}$ which Aluric held T.R.E., and it paid geld for I ferding. This $\frac{1}{2}$ plough can till. Alwi holds it of $\mathrm{O}$ [do]. There $A\left[\right.$ lwi] has $\frac{1}{2}$ plough. Worth 30 pence a year.

To this manor 9 thanes have been added who held their lands in parage (pariter; Exch., libere in paragio) T.R.E., and paid geld for 3 virgates. ${ }^{5}$ These 6 ploughs can till. There Alwi has I virgate and $\frac{1}{2}$ ferding and 2 ploughs; and the villeins (have) 2 virgates all but $\frac{1}{2}$ ferding and $I \frac{1}{2}$ ploughs. On these (lands) A[lwi] has I 2 villeins, 3 bordars, I serf, 27 beasts, 3 swine, 45 sheep, 7 acres of coppice, 12 acres of meadow and 30 acres of common pasture. Worth 30 shillings; when he received it it was worth the same.

With these two other manors have been combined (conjuncte), which 2 thanes held in parage (pariter; Exch., in paragio) ${ }^{6}$ T.R.E. They paid

${ }^{1}$ Hillersdon in Collumpton in Hairidge hundred.

2 Probably Ashbear in Witheridge parish and hundred.

${ }^{3}$ Dart Tracy alias East Dart in Witheridge parish and hundred. Feud. Aids, i, 344 .

"West Worlington in Witheridge hundred.

${ }^{5}$ Probably Afton in West Worlington, see fol. 50r.

${ }^{6}$ Probably Halse, an outlier of West Worlington in Witheridge hundred, and Batchwood alias Badekesworth alias Batakaworthy (Feud. Aids, i, 433) in Halberton in Tiverton hundred (T.W.W. in Trans Devon Assoc. xxviii, 401). Testa, p. I 766 , shows Worlington, Hacche, and Batchworthy in the same tenant's hands. See fol. 501, and Geldroll, fol. 67, B 5 . geld for 2 virgates. These 3 ploughs can till. Worth Io shillings a year.

Odo has a manor called Cilletona [Chilton] ${ }^{7}$ which Ailmar held T.R.E., and it paid geld for $\frac{1}{2}$ virgate. This 2 thanes can till. Thereof $\mathrm{O}[\mathrm{do}]$ has I ferling and I plough in demesne, and the villeins $\mathrm{I}$ ferling and $\mathrm{I}$ plough. There $\mathrm{O}$ [do] has 2 villeins, 2 bordars, 3 serfs, I rouncey, 3 beasts, $3^{8}$ sheep, 5 acres of wood(land), I acre of meadow, and 5 acres of pasture. Worth 10 shillings; when $O[\mathrm{do}]$ received it 5 shillings.

fol. 37gb.

Odo has a manor called Honessam [Huntsham $]^{8}$ which Alric the priest held T.R.E., and it paid geld for $\mathbf{r}$ hide. This 8 ploughs can till. Thereof he has in demesne 1 virgate and 2 ploughs, and the villeins 3 virgates and 5 ploughs. There $O[\mathrm{do}]$ has 20 villeins, 4 bordars, 2 serfs, I swineherd paying 5 swine, Io beasts, 5 swine, 75 sheep, 6 goats, 50 acres of wood(land), 6 acres of meadow, and 15 acres of pasture. Worth 40 shillings a year; when he received it 20 shillings.

Odo has a manor called WILLEIANDA [Willand] ${ }^{9}$ which Etmar (Exch. Edmer) held T.R.E., and it paid geld for 1 hide. This 6 ploughs can till. Vitalis holds it of $\mathrm{O}[\mathrm{do}]$. Thereof Vitalis has in demesne I virgate and I plough, and the villeins 3 virgates and I plough. There Vitalis has 8 villeins, 3 bordars, I serf, 4 beasts, 2 swine, 33 sheep, I mill paying 40 pence a year, wood(land) 4 furlongs in length by $I$ in breadth, meadows 3 furlongs in length by $\mathrm{I}_{2}^{\frac{1}{2}}$ in breadth, and pasture 8 furlongs in length by 3 in breadth. Worth 40 shillings a year; when he received it the same.

fol. 382.

\section{THE LAND OF TORSTIN SON OF ROF (Excb. ROLF) IN DEVENESIRA ${ }^{10}$}

Torstin (Exch. Turstin) son of Rof (Exch. Rolf) has a manor called Estantona (Exch. Stantone) [Staunton] ${ }^{11}$ which Alwena (Exch. Alveva) held T.R.E. and it paid geld for 3 hides. These 20 ploughs can till. Gereon holds it of Torstin. Thereof G[ereon] has I hide and I plough in demesne, and the villeins 2 hides and $9 \frac{1}{2}$ ploughs. There G[ereon] has 24 villeins, 8 bordars, 4 serfs, 7 beasts, 27 swine,

\footnotetext{
${ }^{7}$ Chilton in Cheriton Fitzpaine in Budleigh hundred.

${ }^{8}$ Huntsham in Tiverton hundred. Geldroll, fol. $67, \mathrm{~A} 4$.

${ }^{9}$ Willand in Halberton hundred.

${ }^{10}$ Turstin son of Rolf is No. 38 in Exchequer
} Book.

${ }^{11}$ Church Staunton alias Staunton 'Tuddenham in Hemyock hundred. Geldroll, 67b, A 1 . 


\section{HOLDERS OF LANDS}

I 30 sheep, 23 goats, wood(land) 5 furlongs long by 4 broad, 7 acres of meadow, and pasture $I \frac{1}{2}$ leagues long by I broad. Worth IoO shillings; when $T$ [orstin] received it ro pounds.

fol. 388.

\section{THE LAND OF GOSCELM AND WALTER IN DEVENESIRA ${ }^{1}$}

Goscelm and Walter have a manor called Fereordin [Farworthy] ${ }^{2}$ which Edwin held T.R.E., and it paid geld for I virgate. This 3 ploughs can till. Ricolf (Exch. Riculf) holds it of G[oscelm] and W[alter]. Thereof R[icolf] has in demesne $\frac{1}{2}$ ferding and I plough, and the villeins 3 ferdings and 2 ploughs. There R[icolf] has 4 villeins, 3 bordars, 2 serfs, 2 I beasts, IO swine, 20 sheep, 20 acres of meadow, and pasture $\frac{1}{2}$ league in length by 3 furlongs in breadth. Worth 20 shillings a year; when he received it I 5 shillings.

Walter has I virgate of land which belonged to YwesleIa (Exch. Iweslei) [Iddesleigh] ${ }^{3}$ which Aleuesclef (Exch. Aleuesdef $)^{4}$ held T.R.E. without being able to be independent of (separari) that manor. This 3 ploughs can till. There William (sic) has I plough in demesne, and his villeins 2 ploughs. There W[alter] has 4 villeins, 2 bordars, 20 beasts, I 3 swine, 40 sheep, I 2 goats, 8 acres of wood(land), and I 5 acres of meadow. Worth 20 shillings a year; when Walter received it it was worth 15 shillings.

Goscelm has a manor called FedAven [Villavin ${ }^{5}$ which Edlouedief and Eidieva ( $E x c h$. Eddeva) held T.R.E., and it paid geld for $\frac{1}{2}$ hide. This 4 ploughs can till. Walter the Burgundian (Borgundiensis) holds it of G[oscelm]. Thereof W[alter] has in demesne 3 ferdings and 1 plough, and the villeins 5 ferdings and 4 ploughs. There W[alter] has 5 villeins, 3 serfs, 3 bordars,

\footnotetext{
1 The lands of Walter de Clavil and Goscelm are taken separately in the Exchequer Book, Walter being No. 24, Goscelm No. 25. Walter's estates begin with Bywood, and follow in order to Combe Payne, then Bewhill to Bere, Buckland, North Pool, Colemore, All Hallow's Leigh, followed by Ash and Hill, Dowland, West Leusdon, Upcot, Instow to Shobrook, then Berlescombe, Ciclet, last of all Farworthy. Goscelm's begin with Villawin and follow in order to Newton, then Goodcot, Fenacre to Marlecombe, Gittisham, Chillowman to Witenage, Farway to Buckland, Luperidge and Ulverstone.

' Farworthy, an outlier of Sutcombe in Blacktorington hundred. There is a shortage of $\frac{1}{2}$ ferling which may represent Midcot in Sutcombe.

${ }^{3}$ Ash and Hill in Iddlesleigh in Shebbear hundred.

${ }^{4}$ Called Alwatet, fol. 84.

${ }^{5}$ Villavin alies Feldefen in Roborough in Shebbear hundred.
}

20 beasts, 5 swine, 89 sheep, 16 forest-mares, 3 fol. $388 \mathrm{~b}$.

acres of coppice, 8 acres of meadow, and $\frac{1}{2}$ league of pasture taking length and breadth. Worth 20 shillings a year; when G[oscelm] received it 10 shillings.

Goscelm has a manor called Hyw Is [Huish] ${ }^{6}$ which Alwid (Exch. Alwin) held T.R.E., and it paid geld for $\frac{1}{2}$ virgate. This I plough can till, and Goscelm has the whole in demesne. There he has I plough, I bordar, I serf, 8 beasts, 2 swine, ro sheep, Io goats, wood(land) 2 furlongs long by $\frac{1}{2}$ furlong broad, and 2 acres of meadow. Worth 5 shillings a year; and was worth the same when he received it.

Goscelm has a manor called Newentona [Newton] ${ }^{7}$ which Elward held T.R.E., and it paid geld for $\frac{1}{2}$ hide. This 4 ploughs can till. Walter the Burgundian holds it of Goscelm. Thereof $W$ [alter] has I virgate and $I \frac{1}{2}$ ploughs in demesne, and the villeins I virgate and $2 \frac{1}{2}$ ploughs. There W[alter] has 4 villeins, 3 bordars, 2 serfs, 3 swine, 2 acres of wood, 6 acres of meadow, and 5 acres of pasture. Worth 20 shillings; when G[oscelm] received it ro shillings.

From this manor Golsuen ( $E x c h$. Colsuen) the bishop of Coutances' man withholds (aufert) the common pasture which belonged to the township [Exch. which went with it (ibi adjacebat)] in King E[dward's] time, which Goscelm held undisturbed for five years after King W[illiam] obtained England (Anglicam).

Goscelm has a manor called Dodecota [Dodscot $]^{8}$ which Dodo (Exch. Dode) held T.R.E., and it paid geld for I virgate. This $\mathrm{I} \frac{1}{2}$ ploughs can till. Walter the Burgundian holds it of Goscelm. Thereof $W$ [alter] has $2 \frac{1}{2}$ ferlings and I plough in demesne, and the villeins $I \frac{1}{2}$ ferlings and $\frac{1}{2}$ plough. There W[alter] has 3 villeins, I bordar, 2 serfs, 20 beasts, 6 swine, 76 sheep, 6 acres of meadow, and 20 acres of pasture. Worth I 5 shillings; when G[oscelm] fol. 389 .

received it 7 shillings and 6 pence.

Goscelm has a manor called RIDELCOMA [Riddlecombe] ${ }^{9}$ which Algar held T.R.E., and it paid geld for I hide. This 5 ploughs can till. There G[oscelm] has in demesne 6 ferlings and 2 ploughs, and the villeins $2 \frac{1}{2}$ virgates and 3 ploughs. On it G[oscelm] has 7 villeins, 4 bordars, 3 serfs, I rouncey, 20 beasts, I 8 swine, II 4 sheep, I 40 acres of wood(land),

\footnotetext{
${ }^{6}$ Loviston in Huish in Shebbear hundred.

7 Newton Tracy in Fremingțon hundred.

'Dodscot in St. Giles' in Fremington hundred.

${ }^{9}$ Riddlecombe in Ashreigny in North Tawton hundred.
} 


\section{A HISTORY OF DEVONSHIRE}

10 acres of meadow and 150 acres of pasture. Worth 40 shillings a year; when he received it 30 shillings.

Goscelm has a manor called Lollardesdona [Leusdon] ${ }^{1}$ which Alvric held T.R.E., and it paid geld for $\frac{1}{2}$ hide. This 4 ploughs can till. There G[oscelm] has in demesne I virgate and 2 ploughs, and the villeins I virgate and 2 ploughs. On it G[oscelm] has 5 villeins, 3 bordars, 2 serfs, Io beasts, Io acres of wood(land), 8 of meadow, and 50 of pasture. Worth 20 shillings a year; when he received it 10 shillings.

Goscelm has a manor called Brigrforda [Brushford] ${ }^{2}$ which Aloiss (Exch. Alouf) held T.R.E., and it paid geld for I virgate. This 2 ploughs can till. Godefrid holds it of Goscelm. There Godefrid has in demesne $I \frac{1}{2}$ ferdings fol. $38 \mathrm{gb}$.

and I plough, and the villeins $2 \frac{1}{2}$ ferdings and I plough. On it Godefrid has 3 villeins, 2 bordars, I serf, 7 beasts, 36 sheep, 8 goats, 2 acres of coppice, and I of meadow. Worth Io shillings a year; when he received it 5 shillings.

Goscelm has a manor called Nimet [Intake] ${ }^{3}$ which Aluold (Exch. Alwold) held T.R.E., and it paid geld for I virgate and $\frac{1}{2}$ ferling. This 2 ploughs can till. Hermer holds it of G[oscelm]. There Hermer has in demesne $\frac{1}{2}$ virgate and I plough; and the villeins $\frac{1}{2}$ virgate and $\frac{1}{2}$ ferling and $I$ plough. On it Hermer has 3 villeins, 5 bordars, 9 beasts, and 6 acres of meadow. Worth 12 shillings a year; when he received it 9 shillings.

Goscelm has a manor called NIMET [Intake] which Alward held T.R.E., and it paid geld for I hide. This 6 ploughs can till. Osmund holds it of G[oscelm]. There Osmund has in demesne $\frac{1}{2}$ hide and I plough, and the villeins $\frac{1}{2}$ hide and 4 ploughs. On it $\mathrm{O}$ [smund] has 6 villeins, 3 bordars, 2 serfs, 10 beasts, 29 sheep, I 6 goats, 6 acres of wood(land), 5 of meadow, and 3 of pasture. Worth 30 shillings a year; when he received it Io shillings.

Goscelm has a manor called Nieutona (Exch. Niwetone) [Newton] ${ }^{5}$ which Bristuin (Exch. Brictuin) held T.R.E., and it paid geld for $\frac{1}{2}$ ferding. This I plough can till. Osmund holds it of

${ }^{1}$ Leusdon in Winkleigh parish and hundred; Geldroll, fol. 66b, B 3 .

${ }^{2}$ Brushford in Winkleigh parish and hundred.

3 Hermer's town alias Hampston in Bow in North Tawton hundred; formerly 'Hermaneston' (Feud. Aids, i, 370).

"Nicol's Nymet in North Tawton parish and hundred. Trans. Devon Assoc. xxix, 253.

${ }^{5}$ South Newton in Zeal Monachorum in North Tawton hundred.
$\mathrm{G}$ [oscelm]. There $\mathrm{O}$ [smund] has 2 villeins and $\frac{1}{2}$ plough, also 3 acres of coppice, 2 acres of meadow, and 4 of pasture. Worth 7 shillings a fol. 390.

year; when he received it 30 pence.

Goscelm has a manor called Duvelanda [Dowland] ${ }^{6}$ which Award (Exch. Alward) Merta held T.R.E., and it paid geld for $\frac{1}{2}$ hide. Alward could go with his land to whatever lord he liked. This 4 ploughs can till. There W[alter] has in demesne I virgate and I plough, and the villeins $I$ virgate and 2 ploughs. On it W[alter] has 3 villeins, 7 bordars, 4 serfs, I rouncey, 23 beasts, I 16 sheep, I 5 swine, 2 furlongs of wood(land), 3 acres of meadow, and 4 furlongs of pasture. Worth 30 shillings a year; when he received it 20 shillings.

Walter has a manor called LoLlardesdona [Lewsdon] ${ }^{7}$ which Alward Merta held of Bristric (Exch. Brictric) T.R.E., and it paid geld for $\frac{1}{2}$ hide. This 3 ploughs can till. There W [alter] has 5 villeins, 3 bordars and $2 \frac{1}{2}$ ploughs, also wood(land) 5 furlongs long by $\frac{1}{2}$ furlong broad, 6 acres of meadow, and 2 furlongs of pasture. Worth I 5 shillings a year; when he received it 20 shillings.

In the aforesaid manor called Duvelanda [Dowland] Walter has I virgate of land ${ }^{8}$ which 2 thanes held in parage (pariter) T.R.E., and it paid geld for I virgate. This 3 ploughs can till. $\mathrm{On}$ it $W$ [alter] has in demesne 3 ferdings and I plough; and the villeins I ferding and I plough. There W[alter] has I villein, 2 bordars, 2 serfs, I rouncey, Io beasts, I furlong of coppice, and 2 acres of meadow. Worth 12 shillings and 6 pence; when he received it the same. fol. 39ob.

Walter has a manor called Johannesto (Exch. Johannestou) [Instow $]^{9}$ which Alward held T.R.E., and it paid geld for I virgate of land. This 2 ploughs can till. There W[alter] has 7 bordars and I priest ; also 3 serfs, 7 beasts, I rouncey, 36 sheep, 4 acres of wood(land), 2 acres of meadow, and 60 acres of pasture. Worth I5 shillings a year; when he received it the same.

Walter has a manor called Chetellescota (Exch. Chetelscote) [Gilscot] ${ }^{10}$ which Chetel held T.R.E., and it paid geld for I virgate of land. This 2 ploughs can till. Riculf holds it

${ }^{6}$ Dowland in North Tawton hundred.

${ }^{7}$ West Lewsdon alias Pewson in Dowland in North Tawton hundred.

${ }^{8}$ Upcot in Dowland in North Tawton hundred.

${ }^{9}$ Instow in Fremington hundred. Geldroll, fol. $65 b$, A 5 .

${ }_{10}$ Gilscot in Colridge in Winkleigh hundred (O. J.R. in Trans. Devon. Assoc. xxxvi, 356). 


\section{HOLDERS OF LANDS}

of W[alter]. Riculf has there in demesne $\frac{1}{2}$ virgate and I plough, and the villeins $\frac{1}{2}$ virgate and $\frac{1}{2}$ plough. On it $R$ [icult] has 2 villeins, 5 bordars, I serf, 12 beasts, 50 sheep, I0 acres of meadow and common pasture. Worth ro shillings a year; when he received it the same.

Walter has a manor called Nimet [Intake] ${ }^{1}$ which Alward Merta (Exch. Alward a freeman) held T.R.E., and it paid geld for I virgate of land. This I plough can till. Walter holds it of W[alter]. Thereof W[alter] has in demesne 3 ferlings and I plough, and the villeins 1 ferding and I plough. Upon it W[alter] has I villein, 3 bordars, 2 serfs, I rouncey, 18 beasts, 3 swine, 6o sheep, 3 acres of meadow, and 20 acres of pasture. Worth 15 shillings a year; when he received it 5 shillings. This Alward could go with his land to what lord he liked.

fol. 39 r.

Walter has a manor called EschIPABRoca (Exch. Schipebroc) [Shobrook] ${ }^{2}$ which Bristric (Exch. Brictric a freeman) held T.R.E., and it paid geld for I virgate. This 2 ploughs can till. Walter holds it of W[alter]. Thereof $W$ [alter] has in demesne I ferding, and the villeins 3 ferdings and 2 ploughs. There $W$ [alter] has 4 villeins, 5 bordars, I acre of meadow, and 6 acres of pasture. Worth Io shillings a year; when he received it the same. Bristric could go with that land where he was minded.

Goscelm has a manor called Godevacota ( $E x c h$. Godevecota) [Goodcot] ${ }^{3}$ which Godeva (Exch. Godeve) held T.R.E., and it paid geld for I virgate. This 2 ploughs can till. Thereof $\mathrm{G}\left[\right.$ oscelm] has $\frac{1}{2}$ virgate and I plough in demesne, and the villeins $\frac{1}{2}$ virgate and 1 plough. There $\mathrm{G}$ [oscelm] has 2 villeins, I serf, 7 beasts, Io swine, 30 sheep, and 2 acres of meadow. Worth Io shillings; when G[oscelm] received it it was worth 5 shillings.

Walter has a manor called BerLescoma [Burlescombe] ${ }^{4}$ which Olviet (Exch. Ulivet) held T. R. E., and it paid geld for I hide, $\mathbf{r} \frac{1}{2}$ virgates. These 4 ploughs can till. Thereof W[alter] has in demesne 2 virgates, I ferding, and 2 ploughs, and the villeins 3 virgates, $I$ ferding, and 3 ploughs. There W[alter] has 2 villeins, 4 bordars, 2 serfs, I rouncey, 22 beasts, 70 acres of wood(land), 2

'Merta's Nymet alias Merdesnymet alias Wolfsnymet alias Wolfin in Down St. Mary in North Tawton hundred. See fol. 498. Trans. Devon. Assoc. xroxvi, 356.

${ }^{2}$ Shobrook in Morchard Bishop in North Tawton hundred. See fol. 498.

${ }^{3}$ Goodcot in Ashreigny in North Tawton hundred.

- Burlescombe in Bampton hundred. Geldroll, fol. 69, A 4 . acres of meadow and 50 acres of pasture. Worth 25 shillings a year; when he received it 20 shillings.

fol. $39 \mathrm{rb}$.

Walter has a manor called CicLet [ which Gonner (Exch. Connar) held T. R. E., and it paid geld for $\frac{1}{2}$ hide. This 4 ploughs can till. Walter the woodman (silvestris) holds it of W[alter]. There $W$ [alter] has 4 villeins who have 2 ploughs, 3 bordars, 24 acres of wood(land), 2 acres of meadow and 85 acres of pasture. Worth 20 shillings a year; when $W$ [alter] received it 10 shillings.

Goscelm has a manor called VENNACRE (Exch. Wennacre) $[\text { Fenacre }]^{5}$ which Olviet (Exch. Ulviet) held T. R. E., and it paid geld for $\frac{1}{2}$ hide and $\frac{1}{2}$ virgate. These $I \frac{1}{2}$ ploughs can till. Osmund holds them of G[oscelm]. Thereof $\mathrm{O}$ [smund] has in demesne $\frac{1}{2}$ hide and I plough, and the villeins $\frac{1}{2}$ virgate and I plough. There $O$ [smund] has 2 villeins, I serf, 5 beasts, 6 acres of coppice, 2 acres of meadow and 5 of pasture. Worth 20 shillings a year; when G[oscelm] received it it was worth 15 shillings.

Goscelm has a manor salled WIDEBERIA [Woodbear ${ }^{6}$ which Winemer (Exch. Winemar) held T.R. E., and it paid geld for $\mathrm{I}$ hide. This 4 ploughs can till. Godefrid holds of Goscelm. Thereof G[odefrid] has $I \frac{1}{2}$ virgates and 2 ploughs in demesne, and the villeins $2 \frac{1}{2}$ virgates and $I \frac{1}{2}$ ploughs. There G[odefrid] has 6 villeins, 3 bordars, 3 serfs, 6 beasts, 2 swine, 58 sheep, 30 goats, Io acres of wood(land), 2 acres of meadow and 50 acres of pasture. Worth 25 shillings; when G[oscelm] received it it was worth Io shillings.

Goscelm has a manor called Aulescoma [Aulescombe] ${ }^{7}$ which Chenias held T. R. E., and it paid geld for $\mathrm{I}$ hide. This 3 ploughs can till. Thereof G[oscelm] has $\frac{1}{2}$ hide and I plough in demesne, and the villeins $\frac{1}{2}$ hide and 2 ploughs. There fol. 392 .

G[oscelm] has 5 villeins, I bordar, 5 serfs, I rouncey, II beasts, Ioo sheep, 80 goats, I 4 acres of wood(land) and 18 of meadow. Worth 20 shillings; when G[oscelm] received it I 5 shillings.

Walter de Clavilla has a manor called Biuda [Bywood] $^{8}$ which Mastilda (Exch. Matilda) held T.R. E., and it paid geld for $3 \frac{1}{2}$ virgates. These 3 ploughs can till. Thereof W[alter] has $2 \frac{1}{2}$ virgates and I plough in demesne, and the vil-

${ }^{5}$ Fenacre in Burlescombe in Bampton hundred.

${ }^{6}$ Woodbear in Plymtree in Hairidge hundred.

${ }^{7}$ Aulescomb in Hemyock hundred. Geldroll, fol. $67 b$, A 6.

${ }^{8}$ Bywood in Dunkeswell in Hemyock hundred. Geldroll, $67 b, \mathrm{~A}_{4}$.
63 


\section{A HISTORY OF DEVONSHIRE}

leins I virgate and $\frac{1}{2}$ plough. There $W$ [alter] has 4 villeins and 150 acres of pasture. Worth Io shillings; when Walter received it it was worth the same.

Walter has a manor called Branfort [Brampford] ${ }^{1}$ which Alvevia ( $E x c h$. Alveva) held T. R. E. and it paid g(eld) for $\frac{1}{2}$ hide. This 2 ploughs can till. Thereof Walter has I virgate and I plough in demesne, and the villeins have I plough. There $W$ [alter] has 2 villeins, 2 bordars, I 2 beasts, 1 rouncey, 67 sheep, I 3 goats, 20 acres of wood(land), 5 acres of meadow and 50 acres of pasture. Worth 15 shillings a year; when $\mathrm{W}$ [alter] received it 5 shillings.

Goscelm has a manor called Grdesam (Exch. Gidesham) [Gittisham] ${ }^{2}$ which Chinias held T. R.E., and it paid geld for $4 \frac{1}{2}$ hides. These 10 ploughs can till. Thereof G[oscelm] has I $\frac{1}{2}$ hides and 3 ploughs in demesne, and the villeins 3 hides and 5 ploughs. There G[oscelm] has 28 villeins, I bordar, 5 serfs, 2 rounceys, 7 beasts, fol. 392b.

4 swine, 77 sheep, I mill paying Io shillings, 2 acres of wood(land), 40 acres of meadow and pasture 9 furlongs in length by 8 furlongs in breadth. Worth 60 shillings; when G[oscelm] received it the same.

Walter de Clavilla has a manor called WIDEcoma [Withecombe] ${ }^{3}$ which Alveva (Exch. Elveva) held T. R. E. and it paid geld for I hide. This 5 ploughs can till. There $\mathrm{W}$ [alter] has $\frac{1}{2}$ hide and I plough in demesne, and the villeins $\frac{1}{2}$ hide and 3 ploughs. There $W$ [alter] has $8(E x c h .3)$ villeins, 6 bordars, 2 serfs, I rouncey, 16 beasts, 68 sheep, 4 acres of wood(land), 5 acres of meadow and 30 acres of pasture. Worth 20 shillings ; when $\mathrm{W}$ [alter] received it the same.

Walter has a manor called RATDONA [Raddon] ${ }^{4}$ which Alveva held T.R.E. and it paid geld for two thirds of a virgate. This I plough can till. Walter the sewer (dapifer) holds it of W[alter]. There W[alter] has I plough in demesne, I villein, I bordar, I serf, and 2 acres of meadow. Worth 6 shillings; when W[alter] received it the same.

Walter has a manor called W AFForda (Exch. Wasforde) [Washford] ${ }^{5}$ which 2 thanes held in

${ }^{1}$ In Brampford Pyne in Wonford hundred. Geldroll, fol. 70b, A Io, if Walter is Walter de Clavil.

Gittisham in East Budleigh hundred. Geldroll, fol. 68, A 6 .

${ }^{3}$ Withycombe Clavil alias Raleigh. Geldroll, fol. 68, A 7 .

"Raddon, i.e. West Yeo alias Fremanscot in Witheridge (O.J.R.), Feud. Aids, i, 343; Trans. Devon Assoc. xxx, 399; or part of West Raddon in Shobrook (T. W. W.).

${ }^{5}$ Washford Pyne in Witheridge hundred, the parish. See fol. 5 106. Geldroll, fol. 666, A 5 . parage T.R.E., and it paid geld for $\mathbf{I}$ hide all but I ferling. This 6 ploughs can till. Thereof Walter (Exch. the sewer) has in demesne $\frac{1}{2}$ hide all but $I$ ferling and 2 ploughs; and the villeins have $2 \frac{1}{2}$ ploughs. There $W[$ alter $]$ has 7 villeins, 4 bordars, 4 serfs, I rouncey, 30 beasts, 4 swine, 80 sheep, wood(land) 4 furlongs long by I broad, fol. 393 .

5 acres of meadow, and pasture $\frac{1}{2}$ league in length by I furlong in breadth. Worth 40 shillings a year; when he received it 25 shillings. To this manor I ferding of land ${ }^{6}$ has been added. Worth 3 shillings a year.

Walter has a manor called DrahrFora [Drayford] ${ }^{7}$ which Alverona (Exch. Alveron) held T.R.E. and it paid geld for 3 virgates all but $\frac{1}{2}$ ferling. This 3 ploughs can till. Thereof W[alter] has I virgate and I plough in demesne, and the villeins have 2 ploughs. There $W$ [alter] has 2 villeins, 3 bordars, 3 beasts, I 8 sheep, wood(land) 2 furlongs in length by $I$ in breadth, 4 acres of meadow and 12 acres of pasture. Worth 15 shillings a year; when Walter received it it was worth 10 shillings.

Walter has a manor called Sideham [Sidedown $]^{8}$ which Alverona (Exch. Alvern) held T.R.E. and it paid geld for I virgate. This 2 ploughs can till. Now Osbert (Exch. Osbern) holds it of Walter. Thereof $\mathrm{O}$ [sbert] has $\frac{1}{2}$ virgate and I plough in demesne, and the villeins have I plough. There $\mathrm{O}$ [sbert] has I villein, 3 bordars, I serf, 4 beasts, 2 swine, 20 sheep, wood(land) 3 furlongs in length by $\mathbf{I}$ in breadth, 8 acres of meadow and 10 acres of pasture. Worth Io shillings a year; when Walter received it it was worth the same.

Walter has a manor called Lonmera [Lowman $]^{9}$ which Alveron held T.R.E. and it paid geld for 3 virgates. This 3 ploughs can till. Thereof $W$ [alter] has in demesne $I \frac{1}{2}$ virgates and 2 ploughs, and the villeins $1 \frac{1}{2}$ virgates and 2 ploughs. There $\mathrm{W}$ [alter] has 3 villeins, 2 bordats, 5 serfs, I rouncey, 20 beasts, 2 swine, 8 sheep, 30 acres of wood(land), 6 acres of meadow and 6 acres of pasture. Worth 30 shillings a year; when he received it it was fol. $393 \mathrm{~b}$.

worth the same.

To this manor has been added another manor called Cadewila [Kidwell] ${ }^{10}$ which Alveron

${ }^{6}$ Hele next Upcot Squire in Little Washford the outlier of Witheridge (O. J. R.), Feud. Aids, i, 342 ; Trans. Devon Assoc. xxx, 399 ; Wonham in Washford Pyne (T. W. W.).

${ }^{7}$ Drayford in Witheridge parish and hundred.

${ }^{8}$ Sidedown in Rackenford in Witheridge hundred.

${ }^{9}$ Lowman Clavil alias Windhayes in Uplowman in Tiverton hundred. See fol. 502b. Geldroll, fol. $67, \mathrm{~A}_{3}$.

${ }^{10} \mathrm{Kidwell}$ in Uplowman in Tiverton hundred. 


\section{HOLDERS OF LANDS}

held T.R.E. and it paid geld for I virgate. This I plough can till. Thereof $\mathrm{W}$ [alter] has I ferling in demesne, and the villeins 3 ferlings and I plough. There $W$ [alter] has 3 villeins, 2 bordars, 8 acres of coppice and 2 acres of meadow. Worth Io shillings a year.

Walter has a manor called MoRLEia [Marley] ${ }^{1}$ which Alnod held T.R.E. and it paid geld for $\frac{1}{2}$ hide. This 2 ploughs can till. Walter the woodman (salvagius corrected into silvestris) holds it of W[alter]. Thereof W[alter] has I virgate, I ferling, and I plough in demesne, and the villeins $\frac{1}{2}$ virgate, I ferling, and I plough. There $W$ [alter] has 2 villeins, I bordar, 2 serfs, 7 beasts, 9 swine, 34 sheep, 9 acres of wood(land), I acre of meadow, and I perch and 30 acres of pasture. Worth 15 shillings a year; when he received it it was worth 5 shillings.

Walter has a manor called Coma [Combe] ${ }^{2}$ which Gonerd (Exch. Gonhard) held T.R.E., and it paid geld for $\frac{1}{2}$ hide. This 3 ploughs can till. Walter the woodman holds it of him. Thereof $W$ [alter] has in demesne I virgate, I ferling, and I plough, and the villeins $\frac{1}{2}$ virgate, I ferling, and $2 \frac{1}{2}$ ploughs. There $W$ [alter] has fol. 394.

3 villeins, 2 bordars, 4 serfs, 9 beasts, I pig, 5 I sheep, 4 goats, 6 acres of meadow and 30 of pasture. Worth 15 shillings a year; when he received it the same.

Goscelm has a manor called Lonmina [Lowman $]^{3}$ which Alnod held T.R.E. and it paid geld for $\frac{1}{2}$ hide. This 6 ploughs can till. Thereof G[oscelm] has in demesne I virgate and 2 ploughs, and the villeins I virgate and 4 ploughs. There G[oscelm] has 8 villeins, 5 bordars, 2 serfs, I rouncey, I 4 beasts, 45 sheep, 30 acres of wood(land), 8 acres of meadow and IO acres of pasture. Worth 30 shillings a year; when he received it 20 shillings.

Goscelm has a manor called Conma (Exch. Come) [Combe] ${ }^{4}$ which Alnod held T.R.E. and it paid geld for $\frac{1}{2}$ hide. This 2 ploughs can till. Thereof G[oscelm] has in demesne I virgate, 3 ferlings, and I plough, and the villeins I ferling. There G[oscelm] has 4 bordars, I serf, I mill paying 4 shillings a year, and 4 acres of meadow. Worth ro shillings a year; when he received it 5 shillings.

Goscelm has a manor called Lotelanda [ ] which Soteman held T.R.E., and it paid geld for I virgate. This I plough can till.

${ }^{2}$ Marley in Uplowman in Tiverton hundred.

2 Combe Payne in Uplowman in Tiverton hundred.

${ }^{3}$ Chillowman an outlier of Halberton in Tiverton hundred. Geldroll, fol. 67, A 2.

4 Combe Payne in Uplowman in Tiverton hundred.
There G[oscelm] has in demesne $I$ villein and $\frac{1}{2}$ plough, also 4 beasts. It pays 5 shillings a year; when he received it the same.

Goscelm has a manor called Oplomia [Uplowman $]^{5}$ which Elmer (Exch. Almer) held T.R.E. and it paid geld for 3 ferlings. These $\frac{1}{2}$ plough can till. Almer the priest holds it of fol. 394b.

G[oscelm]. Worth 3 shillings a year.

Goscelm has a manor called Aisa [Ash] ${ }^{6}$ which Alviva (Exch. Alveva a freewoman) held T.R.E. and it paid geld for $\frac{1}{2}$ hide. This $I \frac{1}{2}$ ploughs can till. Godefrid holds it of him. Thereof G[odefrid] has in demesne I virgate, I ferling, and I plough, and the villeins 3 ferlings and $\frac{1}{2}$ plough. There G[odefrid] has 2 villeins, 2 bordars, 2 serfs, 9 beasts, 62 sheep, 9 acres of wood(land), 4 acres of meadow and 25 acres of pasture. Worth Io shillings a year; when he received it the same. This woman was a freewoman; now it goes with (est $a d)$ the land of Bristrix (Excb. Brictric).

Goscelm has a manor called Magnelega [Manley] $^{7}$ which Alward held T.R.E. and it paid geld for $\frac{1}{2}$ hide and $\frac{1}{2}$ virgate (Exch. marks $\frac{1}{2}$ for erasure). This 3 ploughs can till. Thereof $\mathrm{G}$ [oscelm] has I virgate and I plough in demesne, and the villeins $I \frac{1}{2}$ virgates and 2 ploughs. There G[oscelm] has 5 villeins, 2 bordars, I serf, 7 beasts, 5 acres of meadow and 60 acres of pasture. Worth 15 shillings a year; when he received it the same.

Goscelm has a manor called Witenes [Whitnage ${ }^{8}$ which Olmer (Exch. Ulmer) held T.R.E. and it paid geld for I hide. This 3 ploughs can till. Thereof $G$ [oscelm] has $\frac{1}{2}$ hide and 3 ferlings in demesne, and the villeins I virgate and I ferling. There G[oscelm] has 4 villeins, 3 bordars, 2 serfs, I rouncey, 2 beasts, 23 sheep, 5 acres of wood(land), 2 acres of meadow and 40 acres of pasture. Worth 15 shillings a year ; fol. 395 .

when he received it the same.

Walter has a manor called BiHede [Bewhead] ${ }^{8}$ which Olueron (Exch. Ulueron) held T.R.E. and it paid geld for $\frac{1}{2}$ hide. This

${ }^{5}$ Uplowman glebe in Tiverton hundred.

${ }^{6}$ Ash Thomas in Halberton parish and hundred. See fol. $502 b$.

${ }^{7}$ East Manley in Halberton parish and hundred, with West Manley in Tiverton parish and hundred. The $\frac{1}{2}$ virgate marked for erasure probably represents West Manley in Tiverton hundred (O. J. R.).

${ }^{8}$ Whitnage in Uplowman in Halberton hundred. Geldroll, fol. 68, A 4 .

${ }^{9}$ Now Bewhill in Burlescombe in Halberton hundred. 


\section{A HISTORY OF DEVONSHIRE}

I plough can till. There W[alter] has 4 bordars, IO acres of coppice, 5 acres of meadow and 20 acres of pasture. It pays 10 shillings a year.

Walter has a manor called Bihede [Bewhead] which Liured (Exch. Leuret) held T.R.E. and it paid geld for 3 virgates, $I \frac{1}{2}$ ferlings. This 2 ploughs can till. There W[alter] has 2 villeins, 4 bordars, I serf, I 2 acres of coppice, 2 acres of meadow and 35 acres of pasture. It pays 10 shillings a year.

Walter has a manor called AIseforda (Exch. Aisseford) [Ashford ${ }^{1}$ which Oluarda (Exch. Ulward) held T.R.E. and it paid geld for I hide. This 3 ploughs can till. Walter the sewer (dapifer) holds it of him. There W[alter] has $\frac{1}{2}$ hide and I plough in demesne, and the villeins $\frac{1}{2}$ hide and 2 ploughs. There $W$ [alter] has 4 villeins, 7 bordars, 3 serfs, I rouncey, I 4 beasts, 2 swine, 33 goats, 2 forest mares, 12 acres of wood(land), I2 acres of meadow, and 60 acres of pasture. Worth 20 shillings a year ; when he received it to shillings.

Walter has a manor called SuRapta [Sour Appledore] ${ }^{2}$ which Olwiet (Exch. Ulwi) held T.R.E. and it paid geld for 2 hides. This 6 ploughs can till. Thereof $\mathrm{W}$ [alter] has in fol. 395b.

demesne $\frac{1}{2}$ hide and 2 ploughs, and the villeins $I \frac{1}{2}$ hides and 4 ploughs. There W[alter] has I 4 villeins, 5 bordars, 3 serfs, I rouncey, Io beasts, 7 swine, I 2 I sheep, I 2 goats, 6 forest mares, 40 acres of wood(land), 8 acres of meadow and 50 acres of pasture. Worth 40 shillings a year; when he received it 20 shillings, and he could go to what lord he liked.

Walter has a manor called LEIGA [Leigh] ${ }^{3}$ which Alveron held T.R.E. and it paid geld for $I \frac{1}{2}$ virgates. These I plough can till. Thereof W [alter] has I virgate and 1 ferling in demesne, and I villein who holds I ferling; also 40 acres of wood(land), 2 acres of meadow and 20 acres of pasture. Worth 7 shillings and 6 pence a year ; when he received it the same.

Walter has a manor called LANNOR [Leonard] ${ }^{4}$ which Semer held T.R.E. and it paid geld for 3 virgates. These 2 ploughs can till. There W[alter] has 3 villeins who have I plough and 3 bordars, also 5 acres of meadow and 12 of pasture. It pays io shillings a year.

${ }^{1}$ Ashford in Burlescombe in Halberton hundred.

${ }^{2}$ Sour Appledore in Burlescombe in Halberton hundred. Geldroll, fol. 68, A 3 .

${ }^{3}$ Canonsleigh in Burlescombe in Halberton hundred. Trans. Devon Assoc., xxx, 444, n. I I.

"Leonard alias Lynor Abbot in Halberton parish and hundred Walter has a manor called BERA [ $]^{\mathrm{s}}$
which Wordrou held T.R.E. and it paid geld for 3 virgates (Exch. $\frac{1}{2}$ hide). This 4 ploughs can till. Thereof W[alter] has in demesne I virgate and I plough; and the villeins I virgate and 4 ploughs. There W[alter] has 4 villeins, fol. 396 .

2 bordars, I serf, I rouncey, Io beasts, 3 swine, 26 sheep, 12 goats, 20 acres of coppice and 4 acres of meadow. Worth 20 shillings a year ; when he received it the same.

Goscelm has a manor called FarewEIA [Farway $]^{6}$ which Chenias held T.R.E. and it paid geld for I virgate. This 4 ploughs can till. Ludo holds it of G[oscelm]. Thereof L[udo] has in demesne $\frac{1}{2}$ virgate and I plough, and the villeins $\frac{1}{2}$ virgate and 2 ploughs. There L[udo] has 5 villeins, 3 bordars, I serf, 12 beasts, 20 sheep, 30 goats, 20 acres of wood(land) and 5 acres of meadow. Worth 20 shillings a year; when $G$ [oscelm] received it it was worth the same.

Goscelm has a manor called W ASEBorna [Washbourn] ${ }^{7}$ which Algard (Exch. Algar) held T.R.E. and it paid geld for I hide. This 3 ploughs can till. Hermer holds it of G[oscelm]. Thereof $\mathrm{H}$ [ermer] has in demesne 2 virgates and 2 ploughs; and the villeins 2 virgates and $I \frac{1}{2}$ ploughs. There $\mathrm{H}$ [ermer] has 2 villeins, 5 bordars, I serf, I beast, 16 swine, 24 sheep, I I goats, 2 acres of coppice, 1 acre of meadow and $I$ virgate of pasture. Worth 20 shillings a year; when $G[0 s c e l m]$ received it 10 shillings.

Goscelm has a manor called Bochelanda [Buckland] ${ }^{8}$ which Alvrix (Exch. Alvric) held T.R.E. and it paid geld for $\frac{1}{2}$ virgate. This $\mathrm{I} \frac{1}{2}$ ploughs can till. Baldwin holds it. Thereof $\mathrm{B}$ [aldwin] has I ferling and I plough in demesne, fol. $396 b$.

and the villeins I ferling and I plough. There B[aldwin] has 4 villeins, 2 bordars, 6 beasts, I 2 swine, 56 sheep, 26 goats, $\frac{1}{2}$ acre of coppice and 2 acres of meadow. Worth Io shillings a year; when $G$ [oscelm] received it the same. This manor one thane held as a free land T.R.E. Now it has been added to Bristric's (Exch. Brictric's) lands.

Walter has a manor called Bochelanda [Buckland] ${ }^{2}$ which Odeman held T.R.E. and it

5 Probably Clavil's manor of Netherton in Farway in Colyton hundred(O.J.R.). Geldroll, fol. 686, A 4 . Trans. Devon Assoc. xxxvi, 366.

${ }^{6}$ Farway in Colyton hundred.

${ }^{7}$ Middle Washburton in Halwell in Colridge hundred, the Cold Ash or Wash coupled with Hermaneston or Hampson in Feud. Aids, i, 370.

${ }^{8}$ Buckland Toutsaints in Colridge hundred.

9 Woodmason alias 'Wodemaneston' in Buckland Toutsaints. 


\section{HOLDERS OF LANDS}

paid geld for $\frac{1}{2}$ virgate. This $1 \frac{1}{2}$ ploughs can till. There $W$ [alter] has $I \frac{1}{2}$ ploughs, also 5 bordars and 2 acres of meadow. It pays ro shillings a year. This was a free manor T.R.E. Now it has been added to Bristric's lands.

Walter has a manor called PoLA [Pool] ${ }^{1}$ which Bristric (Exch. Brictric) held T.R.E. and it paid geld for I hide. This 4 ploughs can till. Ansfred ( $E x c h$. Ansfrid) holds it of W [alter]. Thereof $A$ [nsfred] has $\frac{1}{2}$ hide and $I \frac{1}{2}$ ploughs in demesne, and the villeins $\frac{1}{2}$ hide and $1 \frac{1}{2}$ ploughs. There $A$ [nsfred] has 4 villeins, 4 bordars, I serf, I 2 swine, I 5 sheep, 5 acres of coppice, 4 acres of meadow and 10 acres of pasture. Worth 25 shillings; when $W[$ alter] received it it was worth Io shillings.

fol. 397 .

Gotselm (Exch. Goscelm) has a manor called LuPERIGA [Luperidge] ${ }^{2}$ which Estnota (Exch. Snode) held T.R.E. and it paid geld for $\frac{1}{2}$ hide. This 4 ploughs can till. Baldwin holds it of Goselm. On it B[aldwin] has I plough and 2 bordars, also coppice 2 furlongs in length by I furlong in breadth, and 2 furlongs of pasture taking length and breadth. Worth 7 shillings a year.

Walter has a manor called KLUPERIGA [Luperidge $]^{3}$ which Cola $(E x c h$. Cole) held T.R.E. and it paid geld for $I \frac{1}{2}$ virgates. These 2 ploughs can till. There W[alter] has I villein who has I plough with I burgess, also wood(land) I furlong in length by $\frac{1}{2}$ furlong in breadth, I acre of meadow, and of pasture I furlong taking length and breadth. Worth 5 shillings a year; when $\mathrm{W}$ [alter] received it it was out of cultivation.

Walter has a manor called LEGA [Leigh ${ }^{4}$ which 2 thanes held of Bristric (Exch. Brictric) son of Algar without being able to be independent of him (separari $a b e 0$ ) in King E[dward's] time ; and it paid geld for $I$ hide all but $\frac{1}{2}$ virgate. This 4 ploughs can till. Thereof W[alter] has in demesne $\frac{1}{2}$ hide and 2 ploughs; and the villeins $I \frac{1}{2}$ virgates and 3 ploughs. There $W$ [alter] has 7 villeins, 6 bordars, 4 serfs, I rouncey, 2 beasts, 80 sheep, 4 acres of wood(land) and 4 acres of meadow. Worth 30 shillings a year; when $W$ [alter] received it it was worth the same. These (two lands) W[alter] holds as one manor.

Gotselm has a manor called Wolestanecota [Ulverstone ?] $]^{5}$ which (Bristric corrected into)

${ }^{1}$ North Pool in South Pool in Colridge hundred.

${ }^{2}$ Luperidge in North Huish in Ermington hundred.

${ }^{3}$ Colemore alias Comers next Luperidge in North Huish in Ermington hundred. Testa de Nevill (Rec. Com.), I 78 a.

'All Hallows' Leigh in Churchstow in Stanborough hundred. Geldroll, fol. $69 b, \mathrm{~A}_{4}$.

${ }^{5}$ Ulverstone in Awlescombe in Hemyock hundred (O. J. R.), Trans. Devon Assoc. xxxvi, 362; Wilson in Cheriton (T. W. W.).
Ulestan held T.R.E. and it paid for I virgate. This I plough can till. There Gotselm has I serf, and $\mathrm{I}$ bordar, and 3 acres of meadow. Worth Io shillings a year; when Gotselm received it the same. That thane used to hold his land freely of Bristric in King $\mathrm{E}$ [dward's] time, but he could not become independent of the lands of Bristrit.

fol. 398.

\section{THE LAND OF GOSCELM OF ESSICESTRA [EXETER] ${ }^{6}$}

Goscelm has a manor called Herstanahaia ${ }^{7}$ which Canister (Exch. Canistre) held T.R.E. and he could go to what lord he liked. It paid geld for $I_{2} \frac{1}{2}$ hides. These 3 ploughs can till. There G[oscelm'] has 6 villeins who hold these $I_{\frac{1}{2}}$ hides and 3 ploughs at farm (ad firmam), and I mill which pays 15 pence a year besides 5 acres of meadow and pasture 2 furlongs in length by $I$ furlong in breadth. It pays 20 shillings a year.

\section{THE LAND OF WILLIAM CAPRA ${ }^{8}$} (Exch. CHIEVRE) IN DEVENESIRA

fol. зg9.

William Capra has a manor called Fereurd [Alfardisworthy] ${ }^{9}$ which Godric held T.R.E. and it paid geld for $\frac{1}{2}$ virgate. This I plough can till, and it is there. There W[illiam] has 2 serfs, 10 beasts, 40 sheep, 10 acres of meadow and 20 acres of pasture. This manor paid 20 shillings a year; when $W$ [illiam] received it it was worth Io shillings.

William has a manor called EsAstapla [Enstaple] ${ }^{10}$ which Godric held T.R.E. and it paid geld for $\frac{1}{2}$ virgate. This I plough can till. Now Anschetil (Exch. Anschitil) holds it of William. Worth 5 shillings a year; when W[illiam] received it it was worth 7 shillings.

William has a manor called Poteforda (Exch. Potsforde) [Putford] ${ }^{11}$ which 2 thanes held in parage T.R.E. and it paid geld for I virgate.

${ }^{6}$ Geldroll, fol. 67, A 7; Gotselin the canon.

${ }^{7}$ Hayes alias Cliston Hayes (Pole I 72 ) alias Blewet or Bluehayes in Broad Clyst in Cliston hundred. Geldroll, fol. 67, A 7 .

${ }^{8}$ Brother of Ralf de Pomeray, Geldroll, fol. $65 b$, A 4. William Chievre is No. I9 in the Exchequer Book, and his holding in Exeter comes first in that copy instead of last as in the Exeter Book.

${ }^{9}$ Alfardisworthy in Bradworthy in Blacktorington hundred.

${ }^{10}$ Enstaple in Bradworthy in Blacktorington hundred. See fol. 4966.

${ }^{11}$ In West Putford in Blacktorington hundred; afterward called Sessacot or Cory. See fol. 4966 . 


\section{A HISTORY OF DEVONSHIRE}

Godric had thereof $\frac{1}{2}$ virgate and Rewruin $\frac{1}{2}$ virgate. This land 4 ploughs can till. Now Anschetil (Exch. Anschitil) holds it of William. There A[nschetil] has I plough in demesne and I villein and I serf, also 8 beasts, 5 swine, 20 sheep, 10 acres of meadow and 10 acres of pasture. Worth 7 shillings and 6 pence; when Will $[\mathrm{iam}]$ received it it was worth 20 shillings.

William has a manor called Huneseua [Hunshaw ${ }^{1}$ which Alward held T.R.E. and it paid geld for I hide. This Io ploughs can till. Thereof W[illiam] has I virgate and 3 ploughs in demesne; and the villeins 3 virgates and 4 ploughs. There $W$ [illiam] has I2, villeins, 5 fol. $39 g \mathrm{~b}$.

serfs, 2 swineherds paying 20 swine, ro beasts, Ioo sheep, 30 goats, 30 acres of wood(land), IO acres of meadow, and pasture $I$ league in length by $\frac{1}{2}$ league in breadth. Worth 4 pounds; when W[illiam] received it it was worth the same.

William has a manor called LEIA [Leigh] ${ }^{2}$ which Alward held T.R.E. and it paid geld for $\frac{1}{2}$ hide. This 5 ploughs can till. Anschetil holds it of William. Thereof $A[$ nschetil] has $\frac{1}{2}$ virgate and 2 ploughs in demesne, and the villeins $\mathrm{I}_{\frac{1}{2}}$ virgates and $\mathrm{I}_{\frac{1}{2}}$ ploughs. There A[nschetil] has 6 villeins, 2 serfs, 8 beasts, 7 swine, 50 sheep, $\mathbf{I}$ acre of wood(land), 2 acres of meadow and 40 acres of pasture. Worth 25 shillings; when $\mathrm{W}$ [illiam] received it it was worth the same. With this manor goes (adjacet) $\frac{1}{2}$ virgate of land, which has been so concealed from the king that the king has not had his geld therefrom. ${ }^{3}$

William has a manor called SELINGEForda [Shillingford] ${ }^{4}$ which Edmar held T.R.E. and it paid geld for 2 hides all but a virgate. This 8 ploughs can till. Thereof W[illiam] has in demesne I hide, I virgate and 2 ploughs, and the villeins I (? $\left.\frac{1}{2}\right)$ hide and 3 ploughs. There W[illiam] has 6 villeins, 9 bordars, 4 serfs, I I beasts, 80 sheep, 6 acres of coppice and 4 acres of meadow. Worth 4 pounds a year; when he received it it was worth 3 pounds.

William has a manor called Esseministra [Exminster]. ${ }^{5}$ This Wichin held T.R.E. and it

${ }^{1}$ Huntshaw in Shebbear hundred. Geldroll, fol. $65 b$, A 4 .

${ }^{2}$ Eastleigh in Westleigh in Fremington hundred. Geldroll, fol. 656, B 3 .

${ }^{3}$ Weach Barton in Westleigh in Fremington hundred (T.W.W.).

"North Shillingford alias Shillingford Abbot with Pengelly in Exminster parish and hundred. Geldroll, fol. $68 \mathrm{~b}, \mathrm{~A} 4$, requires I hide, I virgate for the demesne. See Trans. Devon Assoc. xxvii, 178.

${ }^{6}$ Exminster parish and hundred. paid geld for I hide. This 5 ploughs can till. Thereof $W$ [illiam] has in demesne $\frac{1}{2}$ hide and 2 ploughs, and the villeins $\frac{1}{2}$ hide and 2 ploughs. There $W$ [illiam] has 6 villeins, 2 bordars, 4 serfs, I rouncey, roo sheep, 6 acres of meadow and 6 fol. 4or.

acres of pasture. Worth 30 shillings a year; when he received it 20 shillings.

William has a manor called MATFORDA [Matford $]^{6}$ which Wichin held T.R.E. and it paid geld for $\frac{1}{2}$ virgate. This I plough can till. Ralf holds it of W[illiam]. Thereof Ralf has in demesne $I \frac{1}{2}$ ferdings and $I$ plough, and the villeins $\frac{1}{2}$ ferding. There $R[a l f]$ has I bordar, I serf, 2 beasts, 62 sheep, 3 acres of meadow and 4 acres of pasture. Worth 5 shillings a year; when he received it the same.

William has a manor called Hacoma [Haccombe $]^{7}$ which Otre held T.R.E. and it paid geld for $\frac{1}{2}$ hide. This 4 ploughs can till. Robert holds it of W[illiam]. Thereof R[obert] has in demesne I virgate and 2 ploughs, and the villeins I virgate and I plough. There $\mathrm{R}$ [obert] has 4 villeins, 3 bordars, 2 serfs, 6 beasts, 6 swine, 40 sheep, 20 goats, I saltern, I fishery, 2 acres of meadow and 20 acres of pasture. Worth 30 shillings a year; when William received it it was worth 20 shillings.

William has a manor called WoLNecoma [Wollacombe] ${ }^{8}$ which Edwin held T.R.E. and it paid geld for 2 hides. These ro ploughs can till. Thereof $W$ [illiam] has in demesne $x$ hide and 4 ploughs, and the villeins $\mathrm{I}$ hide and 6 ploughs. There $W$ [illiam] has $\mathrm{I} 2$ villeins, 7 serfs, 16 beasts, I 2 swine, 300 sheep, 5 goats, 20 acres of coppice, 9 acres of meadow, and 30 acres of pasture. Worth 13 pounds and I5 shillings a year; when he received it the same.

William has a manor called BocheLANT [Buckland] ${ }^{9}$ which Brestic (Exch. Brictric) Camesone held T.R.E. and it paid geld for fol. 4orb.

I hide. This 6 ploughs can till. Thereof $\mathrm{W}$ [illiam] has in demesne $\frac{1}{2}$ hide and 3 ploughs, and the villeins $\frac{1}{2}$ hide and 3 ploughs. There $\mathrm{W}$ [illiam] has 10 villeins, 2 bordars, 5 serfs, I rouncey, 12 beasts, 5 swine, roo sheep,

${ }^{6}$ Matford Speke alias Higher Matford in Exminster parish and hundred.

${ }^{7}$ Nether Haccombe alias Netherton in Combe-inTeignhead in Exminster hundred. Geldroll, fol. 68b, B 3 ; Devon Notes and Queries, i, ro7.

${ }^{8}$ Wollacombe Tracy in Morthoe in Shirwell hundred. Geldroll, fol. 66, A 5.

${ }^{9}$ Buckland Dinham alias North Buckland (Feud. Aids, $\mathrm{i}, 360,415,466)$ in Georgeham in Braunton hundred. 


\section{HOLDERS OF LANDS}

20 goats, 15 acres of wood(land), I acre of meadow and 60 acres of pasture. Worth 3 pounds a year; when he received it it was worth (the same corrected into) 30 shillings.

William has a manor called Bochelant [Buckland] ${ }^{1}$ which Ulf held T.R.E. and it paid geld for I virgate. This 3 ploughs can till. Godefric (Exch. Godefrid) holds it of William. Thereof G[odefric] has in demesne $\frac{1}{2}$ virgate and $I$ plough, and the villeins $\frac{1}{2}$ virgate and 2 ploughs. There G[odefric] has 4 villeins, I serf, 4 beasts, 9 sheep, 26 goats, 5 acres of wood(land), I acre of meadow and 50 acres of pasture. Worth 20 shillings a year; when he received it the same.

William has a manor called Essa [Ash ${ }^{2}$ which Elward held T.R.E. and it paid geld for I virgate. This 3 ploughs can till. Ralf holds it of W[illiam]. Thereof $\mathrm{R}$ [alf] has in demesne $\frac{1}{2}$ virgate and 2 ploughs, and the villeins $\frac{1}{2}$ virgate and I plough. There R[alf] has 3 villeins, I bordar, 2 serfs, 7 beasts, 9 swine, 26 sheep, 4 goats, 40 acres of coppice, 3 acres of meadow and 40 acres of pasture. Worth 20 shillings a year; when he received it the same.

William has a manor called Contesberia [Countesbury] $^{3}$ which Ailmer held T.R.E. and fol. 402.

it paid geld for $\frac{1}{2}$ hide. This ro ploughs can till. Thereof W[illiam] has in demesne I virgate and 4 ploughs, and the villeins I virgate and 6 ploughs. There $W$ [illiam] has I 2 villeins, 6 bordars, I 5 serfs, I swineherd paying Io swine a year, I rouncey, 32 beasts, 24 swine, 300 sheep all but I 3,35 goats, 50 acres of wood(land), 2 acres of meadow, pasture I league in length by $\mathrm{I}$ furlong in breadth. Worth 4 pounds a year; when he received it it was worth 20 shillings.

William has a manor called Lintona [Lynton ${ }^{4}$ which Ailward Tochesone (Exch. Alward) held T.R.E. and to (cum) that manor has been added a certain other called INCRINTONA (Exch. Crintone) [Ilkerton] which Algar held. These $\mathrm{W}$ [illiam] holds as one manor, and they paid geld for I hide. This I 2 ploughs can till. Thereof W[illiam] has in demesne $\frac{1}{2}$ hide and 5 ploughs, and the villeins $\frac{1}{2}$ hide and 7 ploughs. There $\mathrm{W}$ [illiam] has $\mathrm{I}_{3}$ villeins, I bordar, I 2 serfs, 58 beasts, 22 swine, 200 sheep, 75 goats, 72 forest mares, 7 furlongs of wood(land), and pasture 2 leagues in length by $\frac{1}{2}$ league in

${ }^{1}$ Buckland Challons alias Little Buckland in Braunton parish and hundred.

- Ash Rogus in Braunton parish and hundred.

${ }^{3}$ Countisbury in Shirwell hundred.

${ }^{4}$ Lynton in Shirwell hundred. breadth. Lintona [Lynton] is worth 4 pounds a year and Incrintona [Ilkerton] 3 pounds; when he received them Lintona [Lynton] was worth 20 shillings and Incrintona [Ilkerton] I5 shillings.

William has a manor called Lina [Lyn] ${ }^{5}$ which Algar held T.R.E. and it paid geld for 3 virgates. These 7 ploughs can till. Thereof W[illiam] has in demesne $1 \frac{1}{2}$ virgates and 2 ploughs, and the villeins $I \frac{1}{2}$ virgates and 5 ploughs. fol. $402 \mathrm{~b}$.

There W[illiam] has 9 villeins, 5 bordars, 5 serfs, 2 swineherds paying 20 swine a year, 34 beasts, 7 swine, 50 sheep, I 2 goats, I new mill, wood(land) $\frac{1}{2}$ league in length by 2 furlongs in breadth and pasture 2 leagues in length by $\frac{1}{2}$ league in breadth. Worth 4 pounds a year; when he received it 40 shillings.

William has a manor called Bicheordin [Badgeworthy] $^{6}$ which has been added to the aforesaid manor. It paid geld for I virgate. This 2 ploughs can till. Fulcoid (Exch. Fulcold) holds it of W[illiam]. Thereof F[ulcoid] has in demesne $\frac{1}{2}$ virgate and I plough, and the villeins $\frac{1}{2}$ virgate and I plough. There F[ulcoid] has I villein, I serf, I beast, 3 swine, 24 sheep, 8 goats, 5 acres of meadow and 30 acres of pasture. Worth Io shillings a year; when he received it it was out of cultivation (vastata). These W[illiam] holds as one manor.

William has a manor called RAORDIN [Radworthy $]^{7}$ which Ailward Tochesone (Exch. Alward) held T.R.E. and it paid geld for I virgate. This 3 ploughs can till. Thereof $\mathrm{W}$ [illiam] has in demesne $\frac{1}{2}$ virgate and 2 ploughs, and the villeins $\frac{1}{2}$ virgate and I plough. There W[illiam] has 8 villeins, 4 serfs, to beasts, 4 swine, 50 sheep, wood(land) I league long by I furlong broad, I acre of meadow and 40 acres of pasture. Worth 3 pounds a year; when he received it 15 shillings.

William has a manor called Winpla [Whimple] $^{8}$ which Ultret (Exch. Uctred) held T.R.E. and it paid geld for $\mathbf{I}$ hide and $\frac{1}{2}$ virgate. These 4 ploughs can till. Ralf holds it of W[illiam]. Thereof Ralf has in demesne 3 virgates and $I \frac{1}{2}$ ploughs, and the villeins $I \frac{1}{2}$ virgates and $I \frac{1}{2}$ ploughs. There $R$ [alf] has 2 villeins, fol. 400 .

3 bordars, I serf, I 8 sheep, 9 acres of coppice,

${ }^{5}$ East and West Lyn in Lynton in Shirwell hundred. See fol. 499 .

${ }^{6}$ Badgeworthy alias Bickworthy and Fen in Brendon in Shirwell hundred.

' South Radworthy in North Molton parish and hundred.

${ }^{8}$ In Whimple in Cliston hundred. 


\section{A HISTORY OF DEVONSHIRE}

19 acres of meadow and 40 acres of pasture. Worth 30 shillings a year; when he received it it was worth 15 shillings.

William has a manor called Pissewilla (Exch. Pissewelle) [Piswell ${ }^{1}$ which Alvric held T.R.E. and it paid geld for I hide I virgate. These 4 ploughs can till. Hamo holds it of W[illiam]. There Hamo has in demesne $2 \frac{1}{2}$ virgates and 2 ploughs, and the villeins $2 \frac{1}{2}$ virgates and 2 ploughs. There Hamo has 7 villeins, 4 bordars, 5 serfs, 9 swine, 44 sheep, I 4 acres of coppice, 8 acres of meadow and 30 acres of pasture. Worth 40 shillings a year; when he received it it was worth 30 shillings.

William has a manor called Hewisa ${ }^{2}$ which Wichin held T.R.E. and it paid geld for $\frac{1}{2}$ hide. This one plough can till. Hamo holds it of W[illiam]. There Hamo has 7 acres of meadow. Worth 5 shillings a year; when he received it the same.

William has a manor called Colebroca [Colbrook $]^{3}$ which Almer (Excb. Almær) held T.R.E. and it paid geld for $\frac{1}{2}$ hide. This 2 ploughs can till. Mainfred holds it of W[illiam]. Thereof Mainfred has in demesne I $\frac{1}{2}$ virgates and I plough, and the villeins $\frac{1}{2}$ virgate and $\frac{1}{2}$ plough. There Mainfred has 2 villeins, 2 bordars, 2 serfs, 7 beasts, 50 sheep, 25 goats, and 4 acres of meadow. Worth 10 shillings a year; when he received it the same.

William has a manor called CAdelia [Cadeleigh $]^{4}$ which Alward held T.R.E. and it paid geld for 3 virgates. These 3 ploughs can till. Thereof W[illiam] has in demesne 2 virgates fol. 40ob.

and 2 ploughs, and the villeins $I$ virgate and I plough. There $\mathrm{W}$ [illiam] has 2 villeins, 2 bordars, 3 serfs and a rouncey, also 4 beasts, 50 sheep, 4 acres of wood(land), 2 acres of meadow and 8 acres of pasture. Worth 30 shillings a year; when he received it 20 shillings.

William has a manor called Aulescoma [Awliscombe] ${ }^{5}$ which Ailward held T.R.E. and it paid geld for $\frac{1}{2}$ hide. This 2 ploughs can till. Ralf holds it of Will[iam]. Thereof

\footnotetext{
${ }^{1}$ Piswell in Kentisbeare in Hairidge hundred.

2 Possibly Orway in Kentisbeare in Hairidge hundred (O.J.R.), Trans. Devon Assoc. xxxvi, 36 I.

${ }^{3}$ Colbrook in Collumpton in Hairidge hundred. Geldroll, fol. $67 b$, A 8.

" Little Cadleigh alias Cadleigh Court in Cadeleigh in Hairidge hundred.

${ }_{5}^{5}$ Awlescomb Tremanet in Hemyock hundred. Speke appears to be Ralf's successor here as well as at Matford and Cobbaton (i.e. 'Winpla').
}

$R$ [alf] has I virgate, I ferling, and I plough in demesne, and the villeins 3 ferlings and $\frac{1}{2}$ plough. There $R$ [alf] has 2 villeins, I bordar, I serf, 2 beasts, 84 sheep, Io acres of wood(land), IO acres of meadow and $I 2$ acres of pasture. Worth 15 shillings; when $W$ [illiam] received it it was worth the same.

William has a manor called Aulescoma [Awliscombe] ${ }^{B}$ which Wichin held T.R.E. and it paid geld for I hide. This 5 ploughs can till. Hamo holds it of Will[iam]. Thereof Hamo has $I \frac{1}{2}$ virgates and 2 ploughs, and the villeins $2 \frac{1}{2}$ virgates and $I \frac{1}{2}$ ploughs. There $\mathrm{H}$ [amo] has 5 villeins, 4 bordars, 2 serfs, 6 beasts, 60 sheep, 7 acres of meadow and 15 acres of pasture. Worth 25 shillings; when W[illiam] received it it was worth the same.

William has a manor called OTERI [Otter] ${ }^{7}$ which Bristric (Exch. Brictric) held T.R.E. and it paid geld for $\frac{1}{2}$ hide and I virgate and $I \frac{1}{2}$ ferlings. ( $I$ virgate $\mathbf{I} \frac{1}{2}$ ferlings are an after insertion). This 2 ploughs can till. Warin holds it fol. 403.

of Will[iam]. Thereof W[arin] has $I_{2}^{\frac{1}{2}}$ virgates and I plough in demesne, and the villeins $\frac{1}{2}$ virgate and $\frac{1}{2}$ plough. There $W[$ arin] has I villein, 3 bordars, 2 serfs, Io beasts, 20 swine, 80 sheep, 5 acres of meadow and 2 furlongs of pasture. Worth I 2 shillings; when W[illiam] received it it was worth the same.

William Capra has a manor called Comba (Exch. Cumbe) [Combe ${ }^{8}$ which Alvric held T.R.E. and it paid geld for $\frac{1}{2}$ hide. This 4 ploughs can till. Thereof W[illiam] has I virgate and I plough in demesne, and the villeins have I virgate and 2 ploughs. There $\mathrm{W}$ [illiam] has I villein, 6 bordars, 2 serfs, Ioo sheep, and 5 acres of meadow. Worth 20 shillings; when W[illiam] received it it was worth 10 shillings.

William Capra has a manor called RaconBA (Exch. Racumbe) [Rocombe] ${ }^{9}$ which Edric held T.R.E. and it paid geld for $\frac{1}{2}$ hide. This 3 ploughs can till. Thereof $W$ [illiam] has $\mathbf{I} \frac{1}{2}$ virgates and $\frac{1}{2}$ (ferling) and 2 ploughs in demesne, and the villeins $I \frac{1}{2}$ ferlings and $I$ plough. There W[illiam] has 2 villeins, 3 bordars, 2 serfs, I rouncey, I 3 beasts, 6o sheep, ro acres of coppice and I acre of meadow. Worth 30 shil-

${ }^{6}$ In Awlescombe in Hemyock hundred.

7 Doubtless Waringston alias Weringston on the Otter in Buckerel in Hemyock hundred. The missing I virgate, $\mathbf{I} \frac{1}{2}$ ferlings may represent Hockederys alias Hockerigg alias Hookedrise, possibly Cockenhays. See Feud. Aids, i, 338.

${ }^{8}$ Combe-in-Teignhead in Wonford hundred. Geldroll, fol. $7 \circ b, \mathrm{~A} 5$.

${ }^{9}$ Higher Rocombe in Wonford hundred (T.W.W.); or Horridge in Ilsington (O.J.R.), Feud. Aids, 316. 


\section{HOLDERS OF LANDS}

lings a year; when he received it it was worth Io shillings.

William Capra has a manor called Teteborna ['Tedburn] ${ }^{1}$ which Bogred (Exch. Bugered) held T.R.E. and it paid geld for 1 hide. This 5 ploughs can till. Thereof W[illiam] has $\frac{1}{2}$ hide and I plough, and the villeins $\frac{1}{2}$ hide and 3 ploughs. There W[illiam] has 8 villeins, 7 bordars, 2 serfs, I rouncey, 12 beasts, 100 sheep all but 6,40 acres of wood(land), 6 acres fol. $403 \mathrm{~b}$.

of meadow and 40 acres of pasture. Worth 40 shillings; when he received it 20 shillings.

W[illiam] Capra has a manor called BraDENESA [Bradninch] ${ }^{2}$ which Bristold (Exch. Brictuold) held T.R.E. and it paid geld for $2 \frac{1}{2}$ hides. This 20 ploughs can till. Thereof $W$ [illiam] has in demesne $\frac{1}{2}$ hide and 5 ploughs, and the villeins $I \frac{1}{2}$ hides and 15 ploughs. There $W$ [illiam] has 42 villeins, 16 bordars, 7 serfs, 3 rounceys, 34 beasts, 8 swine, 324 sheep, 30 acres of wood(land), 30 of coppice, 33 of meadow, 200 acres of pasture and I mill paying 5 shillings. This manor is worth 14 pounds; when he received it I 00 shillings.

William has $\frac{1}{2}$ virgate of land which goes with (jacet in) Horescoma [Awlescombe] ${ }^{3}$ and has there (Exch. 2 villeins who have) I plough. Worth 4 shillings.

W[illiam] Capra has a manor called AEIDEstan (Exch. Aedelstan) [Yardlestone] ${ }^{4}$ which Etzi (Exch. Ezi) held T.R.E. and it paid geld for I hide. Thereof Will[iam] Capra holds one half and Ralf de Pomaria the other half. ... Will[iam's] (moiety) I plough can till. A certain knight Ralf holds it of W[illiam]. There $\mathrm{R}$ [alf] has I plough, 2 serfs, 4 beasts, 6 swine, 40 sheep, I 5 goats, 6 acres of wood(land), 4 acres of meadow and 12 acres of pasture. This (moiety) is worth 7 shillings and 6 pence; when $\mathrm{W}$ [illiam] received it $\mathrm{I} 2$ pence.

Will[iam] Capra has $\frac{1}{2}$ hide of land in OTERI [on the Otter $]^{5}$ which Bristric held T.R.E. Now Warin holds it of Will[iam]. There W[arin] has 3 villeins who have 3 ploughing oxen (boves in carr'), I acre of wood(land), 5 acres of

1 Tedburn St. Mary in Wonford hundred.

3 Bradninch alias Braneys in Hairidge hundred. Geldroll, fol. $67 b$.

3 Probably Owlacombe or Garlandshayes in Bradninch in Hairidge hundred. See fol. $501 b$.

- Yardlestone in Tiverton in West Budleigh hundred (O. J. R.). Healstone alias Yowleston in Puddington in West Budleigh hundred (T.W.W.)

${ }^{5}$ Raplinghays on the Otter in Gittisham in East Budleigh hundred. Testa de Nevill, p. 190b; Trans. Devion Assoc. xxxv, 286. meadow, pasture a furlong long by $\frac{1}{2}$ furlong fol. 404 .

broad. Worth 30 pence; when W[illiam] received it the same.

William Capra has a manor called MORCHET [Morchard] $^{6}$ which Almerd (Exch. Almer) held T.R.E. This Alward Toqisone (Exch. Tochisone) forcibly took from him since King W[illiam] has held England (Anglicam). ${ }^{7}$ It paid geld for I hide; 20 ploughs can till it. Thereof W[illiam] has $\frac{1}{2}$ hide and 4 ploughs in demesne, and the villeins $\frac{1}{2}$ hide and 7 ploughs. There W[illiam] has 20 villeins, 4 bordars, 7 serfs, I smith, 24 beasts, 6 swine, 16o sheep, 30 acres of wood(land), (Exch. 40) acres of meadow and 200 acres of pasture. Worth

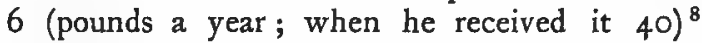
shillings. This W[illiam] holds along with Alward's honour.

[William] Capra has a manor called MAdEsCAMA [Woodscombe] ${ }^{\theta}$ which Almer (Exch. Elmer) held T.R.E. and it paid geld for $\frac{1}{2}$ virgate. (This) I plough (can) till. Hamond (Exch. Hamon) holds it of W[illiam]. There (Hamond has) I villein who has all the land in demesne. Worth 30 pence a year. It has been added to Orescoma [Awlescombe].

W[illiam] Capra has a manor called Alforda [Oakford] ${ }^{10}$ which Brismet (Exch. Brismer) held T.R.E. (and it paid) geld for I hide. This I 4 ploughs can till. Thereof (William has $\frac{1}{2}$ ) hide and 4 ploughs in demesne, and the villeins $\frac{1}{2}$ hide and 4 ploughs. There $W$ [illiam] has 20 villeins, 7 bordars, 2 serfs, .. . beasts, I5 swine, 200 sheep, I00 goats, ... I mill worth 30 pence a year, I00 acres of wood(land), fol $404 \mathrm{~b}$.

12 acres of meadow and 8 acres of pasture. Worth 6 pounds a year; when he received it it was worth 3 pounds.

To this manor has been added another manor called Mildedona [Milton] ${ }^{11}$ which Edibda (Exch. Eddida) held T.R.E. and it paid geld for I virgate. This 3 ploughs can till. There W[illiam] has 8 villeins, also 2 ploughs and

${ }^{6}$ Cruwys Morchard in Witheridge hundred. See fol. 50 r $b$. Geldroll, fol. $66 b, A_{4}$. Freeman, Normans Conquest, $\mathrm{v}, 750$.

${ }^{7}$ Exch. reads: This he took from Alward Tochisone (abstulit Alwardo) after King William came to England.

'The Exon MS. being damaged at this point the words in brackets are supplied from the Exchequer text.

'Woodscombe in Cruwys Morchard in Witheridge hundred.

${ }^{10}$ Oakford in Witheridge hundred. See fol. 502.

${ }^{11}$ Milton alias Meldon in Oakford in Witheridge hundred. 


\section{A HISTORY OF DEVONSHIRE}

3 acres of meadow. Worth ro shillings a year; when he received it 3 shillings.

W[illiam] Capra has a manor called WIPLETONA [Whipton] ${ }^{1}$ which Wichin held T.R.E. and it paid geld for I hide. This 8 ploughs can till. Thereof W[illiam] has in demesne $\frac{1}{2}$ hide and 2 ploughs, and the villeins 4 ploughs and $\frac{1}{2}$ hide. There $W[$ illiam] has 5 villeins, 3 bordars, 7 serfs, I rouncey, 12 beasts, 60 sheep, ... 12 acres of meadow and 100 acres of pasture. Worth (Exch. 6 pounds); when he received it 20 shillings.

W[illiam] Capra has (a manor called) PotIToNA [Puddington $]^{2}$ which Alward Tochesone held T.R.E. and it paid geld for I hide. This 8 ploughs can till. Ralf (holds it) of W[illiam]. There $R$ [alf] has $I$ virgate and 2 ploughs in demesne, and the villeins $\frac{1}{2}$ hide, I virgate, and 4 ploughs. There R[alf] has 8 villeins, ... (Exch. 6 bordars), 3 serfs, I4 beasts, 69 sheep, ... (Exch. 6 acres) of wood(land), 9 acres of meadow and 30 of pasture. Worth 40 shillings; when he received it 20 shillings.

W[illiam] Capra (has a manor) called BRADEFORDA [Bradford] ${ }^{3}$ which Almer held T.R.E. and it paid geld for I virgate. This ... (Exch. 4 ploughs) can (till. Now) Biatrix (Exch. Beafol. 405 .

trix) holds it of $W[$ illiam]. There $B$ [iatrix] has $\frac{1}{2}$ virgate and $I$ plough in desmesne, and the villeins $\frac{1}{2}$ virgate and 2 ploughs. There $B$ [iatrix] has 6 villeins, 6 bordars, 2 serfs, 7 beasts, 5 swine, 20 sheep, I I goats, 6 acres of meadow and 6 acres of pasture. Worth 20 shillings a year; when he received it the same.

To this manor another manor has been added called Toredona ${ }^{4}$ which Alvric Colim held in parage (pariter; Exch. libere) T.R.E. and it paid geld for $\frac{1}{2}$ virgate. This I plough can till, and it pays 40 pence a year.

William Capra has a manor called BochrLANDA [Buckland] ${ }^{5}$ which Alric held T.R.E. and it paid geld for I virgate. This 2 ploughs can till. (Excb. Robert holds) it (of William). Thereof R[obert] has $\frac{1}{2}$ virgate and I plough in demesne, and the villeins ( $\frac{1}{2}$ virgate and I plough. There) $\mathrm{R}$ [obert] has 3 villeins, 3 bordars, 2 serfs, 3 (beasts ?), . . . 9 sheep, 25 goats, I acre of meadow and 25 acres of pasture. (Exch. worth 10) shillings; when W[illiam] received it 6 shillings.

${ }^{1}$ Whipton in Heavitree in Wonford hundred.

' Puddington in Witheridge hundred.

${ }^{3}$ Bradford Tracy in Witheridge parish and hundred.

${ }^{4}$ Possibly Down in Witheridge parish and hundred.

${ }^{3}$ Buckland Baron in Combe-in-Teignhead in Haytor hundred.
[William Capra] has a manor called OTRIA [Otter] ${ }^{b}$ which Olnot (Exch. Ulnod) held [T.R.E.] and it paid geld for 2 hides. These 4 ploughs can till. Warin (holds it) of William. There Warin has I plough, I villein, I bordar, . . . also 12 acres of meadow and 2 furlongs of pasture. Worth (Exch. Io shillings); when he received it the same.

William has a manor called .... (Exch. Otri) $[\mathrm{Otter}]^{7}$ which Samer (Exch. Semar) held T.R.E. and it paid geld (Exch. for I virgate of land). This $\frac{1}{2}$ plough can till. Ralf son of Pagan holds that half-virgate of William. fol. $405 \mathrm{~b}$.

There Ralf has $\frac{1}{2}$ villein who has $\frac{1}{2}$ plough. William has added this manor to his manor called Holescoma [Awlescombe]. It pays 30 pence a year.

William has a manor called MAABERIA [Membury $]^{8}$ which Eldill ( $E_{x c h}$. Eldille) held T.R.E. and it paid geld for I hide. This 3 ploughs can till. Warin holds it of him. Thereof W[arin] has $\frac{1}{2}$ hide and I plough in demesne, and the villeins $\frac{1}{2}$ hide and I plough. There $W$ [arin] has 4 villeins, 2 bordars, I serf, 6 beasts, 5 swine, I 4 sheep, I 4 goats, wood(land) 6 furlongs long by 3 broad, 6 acres of meadow and $(E x c h$. pasture) 7 furlongs long by 2 broad. (Exch. worth Io shillings); when $W$ [illiam] received it the same.

W[illiam] Capra has a manor called ALsEMinistra [Axminster] ${ }^{9}$ (Exch. which Wichin held T.)R.E. and it paid geld for I (Exch. virgate of land. This) 2 ploughs can till. Ulf (Exch. Eddulf) holds it of (the earl corrected into) W[illiam], . . . (There Ulf has) $\mathrm{I}_{\frac{1}{2}}$ ploughs, I 2 bordars, 4 serfs, I rouncey, 5 beasts, 20 goats, 8 acres of meadow and com(mon pas)ture. Worth 20 shillings a year; when he received it 20 shillings.

William has a manor called LEgA [Leigh] 10 which EI[mer held T.]R.E. and it paid geld for 2 hides. These 10 ploughs can till. Beatrix his sister holds it of him. Thereof Beatrix has I hide and 2 ploughs in demesne and the villeins I hide and 2 (ploughs). There Beatrix has 7

6 Probably Ivedon in Awlescombe on the Otter in Tiverton hundred. Geldroll; fol. $67, \mathrm{~B}_{3}$.

${ }^{7}$ An estate of 40 acres on the Otter, called ' Holescoma,' fol. 502b, added to Awlescombe in Hemyock hundred. Trans. Devon Assoc. xxxvi, $35^{8}$; the other half of the villein, fol. 342 , went to Pomeray's 'Holescoma.'

${ }^{8}$ East Membury in Membury in Axminster hundred. See fol. 503b. Geldroll, fol. $68 b, \mathrm{~B} 2$.

${ }^{9}$ In Axminster parish and hundred, "Haccombe fee.'

${ }^{10}$ Southleigh in Colyton hundred. Geldroll, fol. $686, \mathrm{~B}$ I. 


\section{HOLDERS OF LANDS}

villeins, 6 bordars, 3 serfs, Io beasts, 20 sheep, fol. 406.

I 8 goats, 40 acres of wood(land), 15 acres of meadow and 200 acres of pasture. Worth 40 shillings a year; when he received it it was worth 50 shillings.

Will[iam] has in Essecestra [Exeter $]^{1}{ }_{2}$ houses which paid in King E[dward]'s time I6 pence a year by way of customary due.

fol. $40 \%$

\section{THE LAND OF TETBALD SON OF BERNER IN DEVENESIRA ?}

Tetbald has a manor called Herlescoma [Yarnscombe] ${ }^{3}$ which two thanes held in parage (pariter; Exch. in paragio) T.R.E. and they could go with their land to what lord they liked. It paid geld for I virgate. This 8 ploughs can till. Thereof $T$ [etbald] has I ferling and I plough in demesne, and the villeins 3 ferlings and 3 ploughs. There T[etbald] has 53 villeins, 5 bordars, 2 serfs, 12 acres of wood(land), 5 acres of meadow and 60 acres of pasture. Worth 20 shillings; when he received it it was worth I 00 shillings.

Tetbald has a manor called Bochrywis [Bukish $]^{4}$ which 3 thanes held in parage (pariter; Exch. in paragio) T.R.E. and they could go with their land to what lord they liked. It paid geld for $\frac{1}{2}$ hide. This 4 ploughs can till. Thereof T[etbald] has in demesne $\frac{1}{2}$ virgate. There T[etbald] has 4 villeins, 20 acres of coppice, 5 acres of meadow and 10 acres of pasture. Worth 12 shillings and 6 pence a year; when Tetbald received it 5 shillings.

Tetbald has a manor called HoLA [Hole] ${ }^{5}$ which Sawin held T.R.E. and it paid geld for 5 virgates. These 4 ploughs can till. Now Gosbert (Exch. Goisbert) holds it of T[etbald]. Thereof Gausbert (sic) has a hide and I plough in demesne, and the villeins $I$ virgate and $\frac{1}{2}$ plough. There G[ausbert] has 7 bordars, I serf, 5 beasts, 27 sheep, to goats, 5 acres of meadow and 10 acres of pasture. Worth 25 shillings a year; when $T$ [etbald] received it 10 shillings.

Tetbald has a manor called Melerorda [Milford $]^{6}$ which Sawin held T.R.E. and it paid geld

${ }^{1}$ Exeter dwelling-houses.

2 Tetbald son of Berner, father-in-law of Odo, is No. 37 in Exchequer copy.

${ }^{3}$ Much Yarnscombe in Hartland hundred. Geldroll, fol. 65, A 3 .

${ }^{4}$ Bukish in Woolfardisworthy in Hartland hundred. See fol. 497.

${ }^{5}$ Hole in Hartland parish and hundred.

- Milford in Hartland parish and hundred. Geldroll, fol. 65, B 2 . for $\frac{1}{2}$ hide. This 3 ploughs can till. Gausbert (Exch. Goisbert) holds it of T[etbald]. Thereof fol. $407 \mathrm{~b}$.

$G$ [ausbert] has $I$ virgate in demesne and $I \frac{1}{2}$ ploughs, and the villeins have the other virgate and I plough. There G[ausbert] has 5 villeins, 2 serfs, 5 swine, 37 sheep, 5 goats, 2 acres of meadow and 10 acres of pasture. Worth 25 shillings; when $T$ [etbald] received it it was worth I 5 shillings.

Tetbald has a manor called Spececota [Spec$\cot ]^{7}$ which Ailaf (Exch. Goislebert) held T.R.E. and it paid geld for I virgate. This 3 ploughs can till. Now Gausbert (Exch. Goislebert) holds it of T[etbald]. There Gausbert has I villein, 15 acres of meadow and 40 acres of pasture. Worth 7 shillings and 6 pence a year; when Tetbald received it it was worth the same.

Tetbald has a manor called Merlanda [Marland] $^{8}$ which Leuegar held T.R.E. and it paid geld for I virgate and I ferling. This 3 ploughs can till. Now Gausbert (Exch. Goisbert) holds it of T[etbald]. Thereof G[ausbert] has 3 fertings and 1 plough in demesne. There Gausbert has 3 villeins, I serf, coppice 3 ferlings long by I wide, Io acres of meadow and 20 acres of pasture. Worth Io shillings a year ; when Tetbald received it 5 shillings.

Tetbald has a manor called ULACOMBA (Exch. Olecumbe) [Owlacombe] ${ }^{9}$ which Alvevia (Exch. Alveva) held T.R.E. and it paid geld for $\frac{1}{2}$ hide. This 4 ploughs can till. Now Goselm (Exch. Goscelin) holds it of Tetbald. Thereof Goselm has I virgate and I plough in demesne, and the villeins the other virgate and 2 ploughs. There G[oselm] has 2 villeins, I bordar, 5 beasts, 20 sheep, 20 acres of coppice, I acre of meadow and 60 acres of pasture. Worth 20 shillings a year; when $T$ [etbald] received it it was worth 25 shillings.

Tetbald has a manor called Picaltona [Bickleton $]^{10}$ which Godewin held T.R.E. and it paid geld for $\frac{1}{2}$ hide. This 4 ploughs can till. Now Bernard holds it of Tetbald. There Bernard has I plough, I serf, I beast, $\frac{1}{2}$ acre of fol. 408 .

wood(land) and 3 acres of meadow. Worth 7 shillings and 6 pence a year; when T[etbald] received it it was worth 20 pence.

Tetbald has I virgate of land called Horcomma (Exch. Holecome) [Hollowcombe] $]^{11}$ which

${ }^{7}$ Speccot in Merton in Shebbear hundred.

${ }^{8}$ Little Marland alias Marland Pye (Feud. Aids, $i, 359$ ) in Petrockstow in Shebbear hundred.

9 Owlacombe in Roborough in Shebbear hundred.

${ }^{10}$ Bickleton in Instow in Fremington hundred.

${ }^{11}$ Hollowcombe in Fremington parish and hundred. 


\section{A HISTORY OF DEVONSHIRE}

Aimar held T.R.E. This I plough can till. There $T$ [etbald] has 3 saltworkers paying 4 shillings and 9 pence a year, besides 5 seams (summas) of salt and I seam of fish.

Tetbald has a manor called SANTona [Saunton] ${ }^{1}$ which Doda (Exch. Dode) held T.R.E. and it paid geld for 2 hides. These ro ploughs can till. Thereof $T$ [etbald] has in demesne I hide and $I$ plough, and the villeins $I$ hide and 6 ploughs. There T[etbald] has 12 villeins, Io bordars, 2 serfs, I saltern paying 30 pence, 20 sheep, I6 acres of (wood corrected into) meadow, and 60 acres of pasture. Worth 3 pounds a year; when he received it the same.

Tetbald has a manor called Hola [Hole] ${ }^{2}$ which Etmar (Exch. Edmar) held T.R.E. and it paid geld for $\frac{1}{2}$ virgate of land. This 2 ploughs can till. There T[etbald] has 2 villeins who have I plough and pay 5 shillings a year.

Tetbald has a manor called Hama [Ham] ${ }^{3}$ which Etmar (Exch. Edmar) held T.R.E. and it paid geld for 3 virgates of land. These 6 ploughs can till. Thereof T[etbald] has in demesne $I_{2} \frac{1}{2}$ virgates and $I$ plough, and the villeins $I_{2}^{\frac{1}{2}}$ virgates and 2 ploughs. There T[etbald] has 5 villeins, 2 serfs, I acre of meadow and 20 acres of pasture. Worth 20 shillings a year; when he received it the same.

Tetbald has a manor called Esprecoma (Exch. Sprecome) [Spreacombe] which Fitel (Exch. Vitalis) held T.R.E. and it paid geld for I virgate. This 3 ploughs can till. Thereof T[etbald] has in demesne $\frac{1}{2}$ virgate and I plough, and the villeins $\frac{1}{2}$ virgate and $I \frac{1}{2}$ ploughs. There $T$ [etbald] has 2 villeins, 2 bordars, 1 serf, 7 acres of fol. $408 \mathrm{~b}$.

coppice, 1 acre of meadow, and 40 acres of pasture. Worth 20 shillings a year; when he received it it was worth 3 pounds.

Tetbald has a manor called Asaberga ( $E x c b$. Aseberge) [Osborough] ${ }^{5}$ which Wadel held T.R.E. and it paid geld for I virgate. This 3 ploughs can till. Thereof $T$ [etbald] has in demesne $\frac{1}{2}$ virgate and the villeins $\frac{1}{2}$ virgate and I plough. There T[etbald] has I villein, 2 bordars, I serf, 2 acres of wood(land), I acre of meadow and 30 acres of pasture. Worth 5 shillings a year; when he received it it was worth 15 shillings.

1 Saunton in Braunton parish and hundred. Geldroll, fol. 66 , A 6.

${ }^{2}$ South Hole in Georgeham in Shirwell hundred (Feud. Aids, i, 360, 416).

${ }^{3}$ Georgeham in Braunton hundred.

"Spreacombe in Morthoe in Shirwell hundred (Feud. Aids, i, 360, 416).

${ }^{5}$ Osborough in Morthoe in Braunton hundred.
Tetbald has a manor called Weldecoma [Wallacombe] ${ }^{6}$ which Brisid held T.R.E. and it paid geld for I virgate. This 2 ploughs can till. Goselin (Exch. Gozelin) holds it of Tetbald. Thereof Goscel[in] has in demesne $\frac{1}{2}$ virgate and I plough, and the villeins $\frac{1}{2}$ virgate and $\frac{1}{2}$ plough. There G[oscelin] has I villein, 5 beasts, 5 swine, 30 sheep, I acre of meadow and 20 acres of pasture. Worth 10 shillings a year; when he received it 10 shillings.

Tetbald has a manor called Merrhoda $\left[\right.$ Marwood] ${ }^{7}$ which Bristric (Exch. Brictric) held T.R.E. and it paid geld for I vi-gate. This 2 ploughs can till. Oliver holds it of $T$ [etbald]. Thereof Oliver has in demesne $\frac{1}{2}$ virgate and I plough, and the villeins $\frac{1}{2}$ virgate and I plough. There Oliver has I villein, 2 bordars, I serf, 4 beasts, 4 swine, 20 sheep, 20 acres of wood(land), 6 acres of meadow and 60 acres of pasture. Worth 15 shillings a year; when he received it the same.

fol, 409.

Tetbald has a manor called Mollanda [Molland] ${ }^{8}$ which Norman held T.R.E. and it paid geld for I virgate. This 2 ploughs can till. Bernard holds it of $T$ [etbald]. Thereof $B$ [ernard] has in demesne $\frac{1}{2}$ virgate and 3 oxen. The other moiety of the virgate has wholly gone out of cultivation (est tota vastata). There $\mathrm{B}$ [ernard] has I serf, 2 beasts, 5 swine, 15 sheep, 12 goats, I2 acres of wood(land) and 3 acres of meadow. Worth 7 shillings and 6 pence a year; when $T$ [etbald] received it it was worth 3 shillings.

Tet[bald] has a manor called ComBa [Combe $]^{9}$ which Colbrand held T.R.E. and it paid geld for I hide. This 4 ploughs can till. Oliver holds them of Tetbald. Thereof O[liver] has $\frac{1}{2}$ hide all but $I$ ferling and 2 ploughs in demesne, and the villeins 2 virgates, I ferling, and I plough. There O[liver] has 6 villeins, 6 bordars, 2 serfs, 20 beasts, 18 goats, I mill paying 5 shillings, 48 acres of wood(land), I 2 acres of meadow and 4 furlongs of pasture. Worth 50 shillings; when $T$ [etbald] received it it was worth 70 shillings.

Along with the manor called Comba (goes) a manor called .... (Exch. Gerwille) [Gorwell] ${ }^{10}$ which the same Colbrand (held), but it did not belong to C[ombe] T.R.E. (Now) Oliver holds it of (Tetbald). Worth ro shillings a year, and it paid $g[$ eld $]$ for $\frac{1}{2}$ virgate.

- Over Wallacombe in Morthoe in Shirwell hundred.(Feud. Aids, i, 336).

'In Marwood in Braunton hundred, the present Westcote.

${ }^{B}$ Molland Sarazen in North Molton in South Molton hundred.

? Combe David alias Culm Davey in Hemyock parish and hundred. See fol. $500 b$.

${ }^{10}$ Gorwell in Hemyock parish and hundred. Geldroll, fol. $67 b, \mathrm{~B} 2$. 


\section{HOLDERS}

Tetbald has a manor called Cerintona [Cheriton] $^{1}$ which Almer held T.R.E. and it paid geld for 1 hide all but $\frac{1}{2}$ virgate. This 5 ploughs can till. Thereof $\mathrm{T}$ [etbaild] has $\frac{1}{2}$ hide and I ferling and I plough in demesne, and the villeins $\frac{1}{2}$ hide and $\mathrm{I}$ ferling and 3 ploughs. There $T$ [etbald] has 5 villeins, 10 bordars, 2 serfs, 6 acres (Exch. of wood) and 3 acres of meadow. Worth 20 shillings; when $T$ [etbald] received it it was worth 40 shillings.

fol. $409 \mathrm{~b}$.

Tetbald has a manor called Comba (Exch. Cumbe) ${ }^{2}$ which Ailmer held T.R.E. and it paid geld for $\frac{1}{2}$ hide. This 3 ploughs can till. Jagelin holds it of Tetbald. Thereof J[agelin] has I virgate and 2 ploughs in demesne, and the villeins $I$ virgate and $I \frac{1}{2}$ ploughs. There J[agelin] has 2 villeins, 2 bordars, II serfs, 9 beasts, 2 swine, 7 I sheep, 28 acres of wood(land), 6 acres of meadow and 60 acres of pasture. Worth I5 shillings; when T[etbald] received it it was worth the same.

Tetbald has a manor called Wesfort [Washford] $^{3}$ which Colbert held T.R.E. and it paid geld for I virgate. This I plough can till. There Tetbald has 2 villeins who have $\frac{1}{2}$ plough upon it, 2 bordars, and 2 acres of meadow. It pays Io shillings; when he received it the same.

Tetbald has a manor called W ESFORDA [Washford $]^{4}$ which 4 thanes held in parage (pariter; Exch. in paragio) T.R.E. and it paid geld for I virgate. This $I \frac{1}{2}$ ploughs can till. There $T$ [etbald] has I plough and 2 bordars and I acre of meadow. Worth 5 shillings a year; when he received it it was worth the same.

Tetbald has a manor called WESFORDA [Washford ${ }^{s}$ which Ulmar held T.R.E. and it paid geld for $\frac{1}{2}$ hide. This 3 ploughs can till. Now Alwald (Exch. Alwald) holds it of Tetbald. Upon it Alwold has I virgate and I plough in demesne, and the villeins have I plough. There A[lwald] has 3 villeins, 2 bordars, 5 beasts, 10 swine, 20 sheep, 2 acres of wood(land) and 5 acres of meadow. Worth 12 shillings and 6 pence a year. The three above-named manors Tetbald holds as I manor. Six thanes held them in parage T.R.E.

${ }^{1}$ Cheriton Fitzpaine in West Budleigh hundred. Geldroll, fol. 68, A I I.

${ }^{3}$ Combe an outlier of Cheriton Fitzpaine in West Budleigh hundred.

${ }^{3}$ Little Washford, an outlier of Witheridge parish and hundred. See fol. $501 b$.

"Little Washford, an outlier of Witheridge parish and hundred.

${ }^{5}$ Little Washford, an outlier of Witheridge parish and hundred. Trans. Devon Assoc. xxx, 405 ; xxxvi, 357. These 3 represent Stretch, Upcot and Westcot, Feud. Aids, 344.

\section{OF LANDS}

fol. 4 to.

Tetbald has a manor called Dertera (Exch. Dertre) $[\mathrm{Dart}]^{6}$ which Alwald (Exch. the same Alwald) held T.R.E. and it paid geld for I virgate. This 3 ploughs can till. Now the aforesaid Alward holds it of Tetbald. Thereof Alward has $\frac{1}{2}$ virgate and I plough in demesne, and the villeins $\frac{1}{2}$ plough. There $A[$ lward $]$ has 3 villeins, 2 serfs, 3 beasts, 2 swine, 20 sheep, 25 goats, 7 acres of meadow, and 12 acres of pasture. Worth 12 shillings and sixpence a year; when $T$ [etbald] received it it was worth 20 shillings.

Tetbald has a manor called Restona [Rifton] ${ }^{7}$ which Ailmar held T.R.E. and it paid geld for $\frac{1}{2}$ hide. This 3 ploughs can till. Now Albric holds it of Tetbald. Thereof Albric has I virgate in demesne and I plough, and the villeins have $\frac{1}{2}$ plough. There Albric has 3 villeins, 2 bordars, 6 beasts, 30 sheep, 5 acres of wood(land), 5 acres of meadow and 10 acres of pasture. Worth 10 shillings a year.

To this manor has been added I virgate of land ${ }^{8}$ which I thane held, who could go with his land to what lord he liked. Worth 4 shillings a year.

Tetbald has a manor called WIDEWORDA

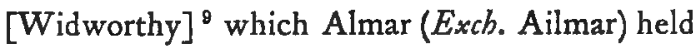
T.R.E. and it paid geld for I hide. This 5 ploughs can till. Now Oliver holds it of Tetbald. Thereof O[liver] has $\frac{1}{2}$ hide and I plough in demesne, and the villeins $\frac{1}{2}$ hide and I plough. There O[liver] has 7 villeins, 4 bordars, 3 serfs, 12 beasts, 6 swine, 80 sheep, I mill (paying) 5 shillings, I 55 acres of wood(land), I acre of meadow and 30 acres of pasture. Worth 30 shillings a year; when Tetbald received it it was fol. 4 rob.

worth the same.

To this manor has been added another manor called Willecmatoma [Wilmington] ${ }^{10}$ which Alward held T.R.E. and it paid geld for $\frac{1}{2}$ hide. This 2 ploughs can till. Worth 5 shillings a year.

Tetbald has a house in Essecestra (Exch. Excestre) [Exeter] which paid T.R.E. (Exch. to the king) 8 pence a year by way of customary due.

- Queen Dart in Witheridge parish and hundred. Feud. Aids, i, 344, 364 .

${ }^{7}$ Rifton in Stoodleigh in Witheridge hundred (O. J. R.) ; Reston in Cheldon (T.W.W.). See fol. 5016.

${ }^{8}$ Perhaps Diptford in Stoodleigh in Witheridge hundred. Trans. Devon Assoc, xxx, 423.

${ }^{9}$ Widworthy in Colyton hundred. Geldroll, fol.

\section{$68 b, \mathrm{~B} 4$}

${ }^{10}$ South Wilmington in Widworthy in Colyton hundred. See fol. 503. 
THE LAND OF RUALD ADOBAT (Exch. ADOBED) IN DEVENESIRA ${ }^{1}$

Ruald has a manor called LANBRETONa [Lamerton] ${ }^{2}$ which Ordulf held T.R.E. and it paid geld for $I \frac{1}{2}$ hides. This 17 ploughs can till. Thereof R[uald] has $\frac{1}{2}$ hide and 5 ploughs in demesne, and the villeins I hide and 12 ploughs. There R[uald] has 34 villeins, 16 serfs, 5 swineherds, 4 beasts, 20 swine, 75 sheep, 2 mills paying 9 shillings a year, wood(land) 7 furlongs long by 3 broad, 40 acres of meadow, pasture $I \frac{1}{2}$ leagues long by $\mathrm{I}$ broad. Worth I 2 pounds; when $R$ [uald] received it it was worth 9 pounds.

Ruald has a manor called Brige [Bridge] ${ }^{3}$ which Frawin held T.R.E. and it paid geld for I $\frac{1}{2}$ virgates. These 8 ploughs can till. Of these $\mathrm{R}$ [uald] has in demesne $\frac{1}{2}$ virgate and 2 ploughs, and the villeins 1 virgate and 6 ploughs. There $\mathrm{R}$ [uald] has $\mathrm{I} 4$ villeins, 8 bordars, 3 serfs, 5 beasts, 6 swine, 68 sheep, I mill paying 4 shillings, meadow 7 furlongs long by 2 broad, and pasture I league long by $\frac{1}{2}$ broad. Worth 50 shillings a year; when $R$ [uald] received it it was worth the same.

fol. 4 rrb.

Ruald has a manor called WeNForda [Wanford $]^{4}$ which Edmer held T.R.E. and it paid geld for $\frac{1}{2}$ virgate. This 2 ploughs can till. Walter de Osmundvil holds it of Ruald. Of this $W$ [alter] has in demesne I ferding and I plough, and the villeins $I$ ferding and $I$ plough. There $\mathrm{W}$ [alter] has 2 villeins, 2 serfs, 20 beasts, 8 swine, 30 sheep, Io goats, coppice I furlong long by $\frac{1}{2}$ furlong broad, 50 acres of meadow, and 2 furlongs of pasture taking length and breadth. Worth 15 shillings a year; when R[uald] received it it was worth 5 shillings.

Ruald has a manor called PANEstan [Panson] ${ }^{5}$ which Leivegar (Exch. Leuegar) held T.R.E. and it paid geld for I virgate. This 4 ploughs can till. Aluid (Excb. Alvered) the Breton holds it of $\mathrm{R}$ [uald]. Thereof $A$ [luid] has in demesne $\frac{1}{2}$ virgate and I plough, and the villeins $\frac{1}{2}$ virgate and 2 ploughs. There A[luid] has 5 villeins, 2 serfs, 3 beasts, I pig, I 2 sheep, 4 acres of wood(land), 12 acres of meadow, and pasture 4 furlongs in length by $I$ in breadth. Worth

${ }^{1}$ Ruald comes No. 35 in the Exchequer copy. 'Adobatus' is a Latin form of the Norman 'adobé,' one who has been 'dubbed' a knight. Trans. Devon Assoc. xvi, 445.

${ }^{2}$ Lamerton in Lifton hundred.

${ }^{3}$ Bridgerule in Blacktorington hundred. Geldroll, fol. 65, A 7 .

4 West Wanford in Milton Damarel in Blacktorington hundred.

${ }^{5}$ East Panson with Cary in St. Giles'-in-theHeath in Werrington hundred. See fol. 497.
20 shillings a year; when $R$ uald received it it was worth 30 shillings. This manor R[uald] holds by way of exchange for Brochelanda [Brockland] and Radix (Exch. Redix) [Rayrish].

Ruald has a manor called TamerLande [Tamarland] ${ }^{6}$ which Sedwin held T.R.E. and it paid geld for I virgate. This 3 ploughs can till. Walter de Osmundvil holds it of R[uald]. Thereof W[alter] has in demesne I ferding and I plough, and the villeins 3 ferdings and I plough. There W[alter] has I villein, I serf, 5 beasts, 4 fol. 4I2.

swine, I I sheep, 9 goats, 3 acres of coppice, I 2 acres of meadow, and pasture 2 furlongs in length by $\mathrm{I}$ in breadth. Worth Io shillings a year ; when $R$ [uald] received it it was worth 40 shillings.

$\mathrm{R}$ [uald] has a manor called Pech [Peek] ${ }^{7}$ which Edwin held in parage (pariter; Excb. in paragio) T.R.E. and it paid geld for $\frac{1}{2}$ virgate. This Walter de Osmundvil holds of R[uald]. There W[alter] has in demesne $I$ ferding. There also W[alter] has 2 villeins, 6 acres of meadow, and pasture 4 furlongs in length by 2 in breadth. Worth 5 shillings a year; when R[uald] received it it was worth Io shillings.

Ruald has a manor called Chiempabera (Exch. Chempebere) [Kimber ${ }^{8}$ which Aiolf (Exch. Aiulf) held T.R.E. and it paid geld for I virgate. This 3 ploughs can till. Roger of Flanders holds it of $\mathrm{R}$ [uald]. There R[oger] has in demesne I ferding, and the villeins I ferding and I plough and 3 oxen. There R[oger] has 3 villeins, 2 bordars, I serf, 9 beasts, 2 acres of coppice, 20 acres of meadow, and pasture 3 furlongs in length by 2 in breadth. Worth ro shillings a year; when $R$ [uald] received it it was worth 5 shillings.

Ruald has a manor called RADEcLIVA [Redcliff $]^{9}$ which Alric held in parage (pariter; Exch. in paragio) T.R.E. and it paid geld for I ferling. This 2 ploughs can till. Roger of Flanders (Exch. the same Roger) holds it of $\mathrm{R}$ [uald]. There $\mathrm{R}$ [oger] has in demesne $\frac{1}{2}$ 'ferling and $\frac{1}{2}$ plough, and the villeins $\frac{1}{2}$ ferling. There R[oger] has 3 bordars, 5 beasts, 6 swine, 20 sheep, I goat, Io acres of wood(land), I 5 acres of meadow and 15 acres of pasture. Worth 5 shillings a year; when $R$ [uald] received it it was fol. 4 r2b.

worth the same.

${ }^{6}$ Luffincot, on the Tamar, in Blacktorington hundred. See fol. 496.

${ }^{7}$ West Peek in Luffincot in Blacktorington hundred.

${ }^{8}$ West Kimber in North Lew in Blacktorington hundred. See fol. 496b. Half a virgate is not accounted for.

${ }^{9}$ Redcliff in North Lew in Blacktorington hundred. 


\section{HOLDERS OF LANDS}

Ruald has a manor called Potafort (Exch. Poteford) [Putford] ${ }^{1}$ which 2 thanes held in parage (pariter; Exch. in paragio) T.R.E. and it paid geld for $\frac{1}{2}$ hide and $\frac{1}{2}$ virgate. One of them, Alwold, had thereof $\frac{1}{2}$ hide, and the other, Lewin, $\frac{1}{2}$ virgate. These 6 ploughs can till. Rainald holds it of $\mathrm{R}$ [uald]. There $\mathrm{R}$ [ainald] has in demesne I virgate, I ferding, and 2 ploughs, and the villeins I virgate, I ferding, and I plough. There R[ainald] has 3 villeins, 3 bordars, 2 serfs, 20 beasts, 30 sheep, 30 acres of meadow, pasture I furlong in length by $I$ in breadth. Worth 20 shillings a year; when $R$ [uald] received it it was worth Io shillings.

Ruald has a manor called WeRA [Wear] ${ }^{2}$ which Ordolf ( $E x c h$. Ordulf) held T.R.E. and it paid geld for I hide. This 7 ploughs can till. Thereof $R$ [uald] has in demesne I virgate and 2 ploughs, and the villeins $2 \frac{1}{2}$ virgates and 5 ploughs. There R[uald] has I 6 villeins, 9 serfs, 3 beasts, 3 swine, 60 sheep, half a fishery worth 40 pence a year, 15 acres of wood(land), 10 acres of meadow and 20 acres of pasture. Worth 40 shillings a year, and was worth the same when $R$ [uald] received it.

The count of Mortain has $\frac{1}{2}$ virgate, part of the same manor, ${ }^{3}$ which Erchenbald holds of him. This I plough can till. There E[rchenbald] has 2 serfs, 5 beasts, and 8 sheep. Worth 5 shillings a year, and was worth the same when $\mathrm{E}$ [rchenbald] received it.

Ruald has a manor called Hochesila [Huxwell $]^{4}$ which Alward (Exch. Elward) held T.R.E. and it paid geld for I virgate. This 2 ploughs can till. There R[uald] has 3 villeins who have I plough, 3 acres of coppice, I acre fol. 4 ז3.

of meadow and 4 acres of pasture. Worth 5 shillings a year; when $R$ [uald] received it it was worth 30 pence.

Ruald has a manor called Holnham [Holham $]^{5}$ which Edmar held T.R.E. and it paid geld for I ferding. This 3 ploughs can till. Gislebert holds it of R[uald]. Thereof G[islebert] has in demesne $\frac{1}{2}$ ferding and I plough, and the villeins $\frac{1}{2}$ ferding and $\frac{1}{2}$ plough. There G[islebert] has 4 villeins, 2 bordars, I serf, 6 beasts, II swine, 50 sheep, 2 acres of coppice, 2 acres of meadow and 4 acres of pasture. Worth 10 shillings a year; when R[uald] received it it was worth 5 shillings.

${ }^{1}$ Church Putford alias Churston in West Putford in Blacktorington hundred. See fol. 4966.

${ }^{2}$ Wear Giffard in Shebbear hundred.

${ }^{3}$ Lower Wear in Wear Giffard in Shebbear hundred (O. J. R.). Little Wear (T.W.W.). Geldroll, fol. $656, \mathrm{~A}_{3}$.

${ }^{4}$ Huxwell in Wear Giffard in Shebbear hundred.

${ }^{5}$ Holham in Little Torrington in Shebbear hundred.
Ruald has a manor called Mrrianda [Marland] ${ }^{6}$ which Elric held T.R.E. and it paid geld for 3 virgates. These 9 ploughs can till. Rainald holds them of $\mathrm{R}$ [uald]. Thereof $\mathrm{R}$ [ainald] has in demesne $\mathbf{I}$ virgate and I plough, and the villeins 2 virgates and 5 ploughs. There $R$ [ainald] has 7 villeins, 4 serfs, 7 beasts, 40 sheep, 6 acres of coppice, IO acres of meadow, and pasture 2 furlongs long by I broad. Worth 30 shillings a year; when $R$ [uald] received it it was worth 20 shillings.

Ruald has a manor called Tucabera (Exch. Tuchebere) [Twigbeare] ${ }^{7}$ which Ailsi (Exch. Alsi) held T.R.E. and it paid geld for I virgate. This 3 ploughs can till. Thereof R[uald] has in demesne $\frac{1}{2}$ virgate and 2 ploughs, and the villeins $\frac{1}{2}$ virgate and 1 plough. There $R$ [uald] has 5 villeins, 3 serfs, 6 beasts, 4 swine, 50 sheep, 20 goats, 4 acres of wood(land), 5 acres of fol. 4 rab.

meadow and 6 acres of pasture. Worth I 5 shillings a year; when $R$ [uald] received it it was worth Io shillings.

Ruald has a manor called Winescota [Winscot $]^{8}$ which Alfer held T.R.E. and it paid geld for I virgate. This 3 ploughs can till. Thereof $R$ [uald] has in demesne $\frac{1}{2}$ virgate and $I$ plough, and the villeins $\frac{1}{2}$ virgate and I plough. There R[uald] has 5 villeins, I serf, 80 sheep, 4 acres of wood(land), 4 acres of meadow and 4 acres of pasture. Worth 15 shillings a year ; when $R$ [uald] received it it was worth Io shillings.

Ruald has a manor called W IVLeswilla (Exch. Wifleswille) [Winswell] ${ }^{9}$ which Alwin held T.R.E. and it paid geld for I virgate. This 2 ploughs can till. Thereof $R$ [uald] has in demesne $\frac{1}{2}$ virgate and I plough, and the villeins $\frac{1}{2}$ virgate and I plough. There R[uald] has 3 villeins, 6 beasts, 2 acres of coppice, 4 acres of meadow and 6 acres of pasture. Worth 10 shillings a year; when he received it 5 shillings.

Ruald has a manor called Lovecota [Lovecot] $]^{10}$ which Love (Exch. Lofe) held T.R.E. and it paid geld for $\frac{1}{2}$ virgate. This 2 ploughs can till. There R[uald] has in demesne I ferding and the villeins I ferding. There R[uald] has 2 villeins, 4 acres of meadow and 12 acres of pasture. Worth 4 shillings and 2 pence a year, when he received it 30 pence.

Ruald has a manor called TUICABERIa (Exch. Tuichebere) [Twigbear] ${ }^{11}$ which Alward held

${ }^{6}$ Peter's Marland in Shebbear hundred.

7 Twigbear in Peter's Marland in Shebbear hundred.

${ }^{8}$ Winscot in Peter's Marland in Shebbear hundred.

${ }^{9}$ Winswell in Peter's Marland in Shebbear hundred.

${ }^{10}$ Lovecot in Buckland Filleigh in Shebbear hundred.

11 Twigbear in Peter's Marland in Shebbear hundred. 


\section{A HISTORY OF DEVONSHIRE}

T.R.E. and it paid geld for $\frac{1}{2}$ virgate. This 2 ploughs can till. Thereof $\mathrm{R}$ [uald] has in fol. 414.

demesne I ferding and the villeins $I$ ferding. There R[uald] has 3 villeins, I serf, 6 acres of wood(land), 2 acres of meadow and 5 acres of pasture. Worth 4 shillings a year; when he received it it was worth 30 pence.

Ruald has a manor called

(in the margin) 'Thus far R[ichard] wrote.'

HANECHEFORDA

[Hankford] ${ }^{1}$ which Edmær held T.R.E. and it paid geld for I ferding. This I plough can till. Rainald holds it of $\mathrm{R}$ [uald]. There $\mathrm{Ra}$ [inald] has I villein and I bordar who have I plough, also 4 acres of meadow and 6 of pasture. Worth 3 shillings and 4 pence a year; when $\mathrm{Ru}$ [ald] received it the same.

Ruald has a manor called LOBA [Lob] ${ }^{2}$ which Ulf held T.R.E. and it paid geld for $\frac{1}{2}$ hide. This 4 ploughs can till. Rainald holds it of Ruald. Thereof $R$ [ainald] has I virgate and $I$ plough in demesne, and the villeins I virgate. There R[ainald] has 3 (Exch. 4) villeins, 2 bordars, 'I saltern, 2 serfs, 3 beasts, 28 sheep, 7 acres of meadow and 30 acres of pasture. Worth 20 shillings; when $R$ [uald] received it it was worth 30 shillings.

Ruald has a manor called Crochewerla [Crockernwell] $^{3}$ which Ulf held T.R.E. and it paid geld for I virgate. This 4 ploughs can till. Rainald holds it of Ruald. Thereof $\mathrm{R}$ [ainald] has $\frac{1}{2}$ virgate and I plough in demesne, and the villeins $\frac{1}{2}$ virgate and I plough. There R[ainald] has 2 villeins, 2 bordars, I serf, 8 beasts, 56 sheep, 4 goats, 4 acres of meadow and 30 acres of pasture. Worth $x 5$ shillings; when $\mathrm{R}$ [uald] received it it was worth the same.

fol. $424 \mathrm{~b}$.

Ruald has a manor called Polmam [Polham] ${ }^{4}$ which 2 thanes held in parage (pariter; Exch. in paragio) T.R.E. and it paid geld for $I_{\frac{1}{2}}$ virgates. This 3 ploughs can till. Now Raginald (Exch. Rainald) holds it of Ruald. Thereof Raginald has $\frac{1}{2}$ virgate and I plough in demesne. There Raginald has I villein, 2 bordars, 2 beasts, 27 sheep, 6 acres of wood(land) and 5 acres of meadow. Worth 13 shillings a year.

${ }^{1}$ Hankford in Bulkworthy in Shebbear hundred.

- Lob Phelip (Feud. Aids, i, 375) alias South or Lower Lob in Braunton parish and hundred. The saltern settles the locality.

${ }^{3}$ Crockernwell in Cheriton Bishop in Wonford hundred. Geldroll, fol. 70b, B 8, calls the tenant Godfrey de Tortavalle, predecessor in title to Rainald de Valletort here and at Fardle.

4 Polham alias Pulham in North Molton parish and hundred. See fol. $499 b$.
The (manor) which could be put under (ire ad) any lord (its tenant) liked is called Planteleia [Praunsley]. ${ }^{5}$ In it is $\frac{1}{2}$ virgate and it is worth 3 shillings a year.

Ruald has a manor called Srgerorda [Sigford] ${ }^{6}$ which Bristrit (Exch. Brictric) held T.R.E. and it paid geld for I virgate. This I $\frac{1}{2}$ ploughs can till. Now Salomon holds of Ruald. On it Salomon has 4 oxen, 5 sheep, Io goats, I cow, 6 acres of wood(land), and 8 acres of pasture. Worth 5 shillings a year; when Ruald received it it was worth 3 shillings.

Ruald has a manor called Pochenilua [Poughill] ${ }^{7}$ which Edmar held T.R.E. and it paid geld for I virgate and I ferling. This 2 ploughs can till. Uponitare . . . (Exch. 2) ploughs. Worth 5 shillings; when Ruald received it 3 shillings.

Ruald has a manor called Dochorda [Dockworthy $]^{8}$ which Alfleta (Exch. Alflet) held T.R.E. and it paid geld for I virgate. This 2 ploughs can till. Walter holds it of him. There W[alter] has I ferling and I (Excb. ${ }^{9}$ plough, I serf, 2 bordars, 3 acres of meadow, 4 acres of wood(land), and pasture I furlong in length by $\frac{1}{2}$ furlong in breadth. Worth 5 shillings; formerly 3 shillings.)

Ruald himself holds Avetone [Aveton].10 Sawin held it T.R.E. and it paid geld for 3 hides. Arable (terra) for 12 ploughs. In demesne are 3 ploughs and 8 serfs, also 16 villeins, and 17 bordars with 4 ploughs, 20 acres of meadow are there, 30 acres of pasture, and 44 acres of coppice (silva minuta). Formerly worth 20 shillings; now worth 3 pounds.

Rainald holds Alfelmestone [Yealmpstone] ${ }^{11}$ of Ruald. Offerd held it T.R.E. and it paid geld for $\frac{1}{2}$ hide. Arable for 4 ploughs, $I \frac{1}{2}$ ploughs are there, also 5 villeins, 3 bordars, I saltern, 4 acres of meadow and 20 acres of coppice. Formerly worth 10 shillings, now worth 15 shillings.

Walter holds Hainemardun [Hemmerdon] ${ }^{19}$ of Ruald. Godo held it T.R.E. and it paid

${ }^{5}$ Praunsley in North Molton parish and hundred. This seems to mean that Polham and Praunsley were held as separate manors by two thanes T.R.E. but were united under Ruald.

${ }^{6}$ Sigford in Ilsington in the Teignhide section of Wonford hundred. Trans. Devon Assoc. xxxii, 544.

7 Poughill in West Budleigh hundred.

${ }^{8}$ Dockworthy in Cheldon in Witheridge hundred.

- The leaf completing this entry and containing the following 6 entries is lost. The missing parts are supplied from the Exchequer copy.

${ }^{10}$ Aveton alias Awton Giffard in Ermington hundred. Geidroll, fol. 70, A 5 .

"Yealmpstone in Plympton parish and hundred.

${ }^{12}$ Hemerdon in Plympton parish and hundred. 


\section{HOLDERS OF LANDS}

geld for I hide. Arable for 4 ploughs. There 2 villeins and 5 bordars have $\frac{1}{2}$ plough. Sixty acres of pasture are there. Formerly and now worth ro shillings.

Ruald himself holds W ICERCE [Whitchurch]. ${ }^{1}$ Sawin held it T.R.E. and it paid geld for $\mathbf{I}$ hide. Arable for 12 ploughs. In demesne are 3 ploughs and 8 serfs; also 20 villeins and 15 bordars with 5 ploughs. Twenty acres of meadow are there, pasture I league in length by 4 furlongs in breadth, wood(land) 2 leagues in length by 4 furlongs in width. Formerly worth 30 shillings, now 70 shillings.

Rainald holds of Ruald MAcheswelie [ ]. ${ }^{2}$ Sawin held it T.R.E. and it paid geld for I ferling. Arable for I plough. Half a plough is there with I bordar and I serf. Formerly worth 2 shillings, now 3 shillings.

Ruald himself has in Execestre [Exeter] $^{3}$ I house which pays the king's customary due.

fol. 415 .

\section{THE LAND OF WILLIAM DE POILLEIO IN DEVENESIRA. ${ }^{4}$}

William de Poilleio has a manor called RADEUDA (Exch. Radeode) [Radworthy] ${ }^{5}$ which Alric held T.R.E. and it paid geld for I virgate. This 6 ploughs can till. Rainald holds it of $\mathrm{W}$ [illiam]. Thereof $\mathrm{R}$ [ainald] has $\frac{1}{2}$ virgate and $I$ plough in demesne, and the villeins $\frac{1}{2}$ virgate and 2 ploughing oxen (boves in carr'). There $\mathrm{R}$ [ainald] has 3 villeins, $\mathrm{I}$ serf, 6 beasts, 7 swine, I 7 sheep, I I goats, 20 acres of meadow and 2 leagues of pasture. Worth ro shillings; when W[illiam] received it 6 shillings.

William de Poilleio has a manor called Sirewilla (Exch. Sirewelle) [Shirwell] ${ }^{6}$ which Ulward held T.R.E. and it paid geld for 3 virgates. These 8 ploughs can till. Thereof W[illiam] has I virgate and 2 ploughs in demesne, and the villeins I virgate and 3 ploughs. There he has 8 villeins, 4 serfs, 1 cow, 2 swine, 30 sheep, I 00 acres of wood(land), I acre of meadow and 2 leagues of pasture. Worth 40

${ }^{1}$ Whitchurch in Roborough hundred. Geldroll, fol. $70, \mathrm{~A} 7$.

${ }^{2}$ Unknown, possibly part of Maker in Roborough hundred (T.W.W.).

${ }^{3}$ Exeter dwelling-house.

4 William de Poilleio is No. 21 in Exchequer Book. All his estates are named in two grants of tithes in 1093 and rog6. Cal. Docts. France, 235 ; Trans. Devon Assoc, xxxvi, 363 .

${ }^{5}$ Radworthy in Challacombe in Shirwell hundred (O. J. R.). Testa de Nevill (Rec. Com.), 200.

In Shirwell parish and hundred. Geldroll, fol. 66, A 11 .

shillings; when W[illiam] received it it was worth 20 shillings. Of the aforesaid 3 virgates 2 knights (milites) hold I virgate of Will[iam] and have there 2 ploughs in demesne. They are included in the aforesaid value (pretium). ${ }^{7}$

William has a manor called Estocha (Exch. Stoche) $[\text { Stoke }]^{8}$ which Alric held T.R.E. and it paid geld for 3 virgates. These 12 ploughs can till. Thereof $W$ [illiam] has I virgate and 2 ploughs in demesne, and the villeins 2 virgates and 3 ploughs. There $W$ [illiam] has 6 villeins, 3 bordars, 7 serfs, 6 beasts, Ioo sheep, 4 goats, fol. $415 \mathrm{~b}$.

30 acres of wood(land), I acre of meadow and 40 acres of pasture. Worth 40 shillings; when $\mathrm{W}$ [illiam] received it it was worth 20 shillings.

William has a manor called Begeurda [Beaworthy ${ }^{9}$ which Leuric held T.R.E. and it paid geld for $\mathrm{I} \frac{1}{2}$ virgates. This 8 ploughs can till. Thereof W[illiam] has $\frac{1}{2}$ virgate and I plough in demesne, and the villeins $\frac{1}{2}$ virgate and I plough. There $W$ [illiam] has 3 villeins, 3 serfs, 20 beasts, 5 swine, 50 sheep, 20 acres of meadow, and pasture $I$ league in length by $\frac{1}{2}$ in breadth. Worth 20 shillings; when W[illiam] received it it was worth the same.

Will[iam] has a manor called Meleberia [Milbury $]^{10}$ which Ulnod held T.R.E. and it paid geld for I virgate. It is wholly out of cultivation (penitus vastata).

William has a manor called Derta [Dart ${ }^{11}$ which Alric held T.R.E. and it paid geld for I hide. This 6 ploughs can till. Ralf ${ }^{12}$ holds it of W[illiam]. Thereof Ralf has I virgate and I plough in demesne, and the villeins 3 virgates and 2 ploughs. There Ralf has 5 villeins, 7 bordars, 2 serfs, I mill, 20 acres of wood(land), 20 acres of meadow and 20 acres of pasture. Worth 30 shillings; when $W$ [illiam] received it it was worth the same.

W[illiam] has a manor called CADABIRIA [Cadbury] ${ }^{13}$ which Inguar (Exch. Inewar) held

${ }^{7}$ Sebrightston and Sipscot in Shirwell parish and hundred. Inq. p.m. I Ric. II, No. 12, shows Shirwell and Brightlicot held of the honour of Okhampton and Shirwell with Sebrightston and Sipscot held of the honour of Plympton; Pole, 407.

${ }^{8}$ Stoke Rivers alias Stoke St. Mary (Inq. p.m. I Ric. II, No. 12) in Shirwell hundred, the parish.

${ }^{2}$ Beaworthy in Blacktorington hundred. Geldroll, fol. 65, A 6.

${ }^{10}$ Milbury in Beaworthy in Blacktorington hundred.

"On the Dart in Hairidge hundred, perhaps Wellton in Cadeleigh (O. J. R.); Dart farm, Silverton, (T.W.W.).

${ }^{13}$ Ralf son of Goscelin, Cal. Docts. France, 235.

${ }^{13}$ Cadbury in Hairidge hundred. Geldroll, 676 , A 9 . 


\section{A HISTORY OF DEVONSHIRE}

T.P.E. and it paid geld for 3 virgates. These 5 ploughs can till. Thereof W[illiam] has in demesne I virgate and 2 ploughs, and the fol 416 .

villeins 2 virgates and 3 ploughs. There W[illiam] has 7 villeins, I bordar, 3 serfs, I rouncey, 60 sheep, 7 goats, 4 acres of meadow and 3 virgates of pasture. Worth 30 shillings a year; when he received it it was worth 20 shillings.

William has a manor called Bovelia [Bowley] ${ }^{1}$ which Olmar $\left(E_{x c b}\right.$. Ulmar) held T.R.E. and it paid geld for $\frac{1}{2}$ hide. This 2 ploughs can till. Thereof W[illiam] has in demesne I virgate and I plough, and the villeins I virgate and I plough. There $W$ [illiam] has 4 villeins, 3 serfs, 80 sheep, and 4 acres of meadow. Worth 20 shillings a year; when he received it I 5 shillings.

W[illiam] has a manor caled Blacagrava [Blackgrave] ${ }^{2}$ which Haca (Exch. Hache) held T.R.E. and it paid geld for I virgate. This 6 ploughs can till. Thereof $\mathrm{W}$ [illiam] has in demesne I ferding and I plough, and the villeins 3 ferdings and I plough. There W[illiam] has 8 villeins, 4 bordars, I rouncey, 27 beasts, 40 sheep, 15 goats, 12 acres of meadow and 60 acres of pasture. Worth 20 shillings a year; when he received it it was worth 5 shillings.

W[illiam] has a manor called Prdaliga [Pidley $]^{3}$ which Hacca (Exch. Hache) held T.R.E. and it paid geld for $2 \frac{1}{2}$ ferdings. These 2 ploughs can till. Ralph holds it of W[illiam]. Thereof $R[a l f]$ has in demesne $I \frac{1}{2}$ ferdings and I plough, and the villeins I ferding. There $\mathrm{R}$ [alf] has I villein, I bordar, 2 beasts, 2 swine, I 5 sheep, 4 acres of wood(land) and 6 acres of meadow. Worth 10 shillings a year; when he received it 5 shillings.

fol. $456 \mathrm{~b}$.

W[illiam] has a manor called Assacota [ $]^{4}$ which Almar held T.R.E. and it paid geld for $\frac{1}{2}$ ferding. This $\frac{1}{2}$ plough can till. Holduin (Exch. Elduin) holds it of W[illiam]. There $\mathrm{H}$ [olduin] has I villein paying 30 pence.

These two manors have been united with Blacagrava, i.e. Pideliga and Assacota, and pay 12 shillings and 6 pence.

W[illiam] has a manor called Olfaldeshodes (Exch. Ulfaldeshodes) [Woolfardisworthy] ${ }^{5}$ which

1 Bowley in Cadbury in Hairidge hundred.

2 Blackgrave in East Worlington in Witheridge hundred. See fol. 502 .

${ }^{3}$ Pidley in East Worlington in Witheridge hundred. See fol. 502 .

${ }^{4}$ Possibly identical with Wood, an outlier of East Worlington in Witheridge hundred. See fol. 502.

${ }^{5}$ Woolfardisworthy Spenser in Witheridge hundred. Geldroll, fol. $67, \mathrm{~B} 3$.
Olnot (Exch. Ulnod) held T.R.E. and it paid geld for $\frac{1}{2}$ hide all but $\frac{1}{2}$ ferding. These 4 ploughs can till. Thereof W[illiam] has in demesne $\frac{1}{2}$ virgate and I plough, and the villeins $\mathrm{I} \frac{1}{2}$ virgates and 3 ploughs. There $W$ [illiam] has 6 villeins, I bordar, I serf, 4 beasts, 20 acres of wood(land) and 4 acres of meadow. Worth I 5 shillings a year; when he received it 5 shillings.

W[illiam] has a manor called DERTa [Dart] ${ }^{6}$ which Leuegar held T.R.E. and it paid geld for I virgate. This 2 ploughs can till. Ralf holds it of W[illiam]. Thereof $\mathrm{R}$ [alf] has in demesne I ferding, and the villeins 3 ferdings and they have I plough. There $\mathrm{R}$ [alf] has 3 villeins, I serf, 3 acres of wood(land), 2 of meadow and 9 of pasture. Worth 7 shillings a year; when he received it ro shillings.

Will[iam] has a manor called WRDA (Exch. Worde) $[\text { Worth }]^{7}$ which Saward held T.R.E. and it paid geld for $\frac{1}{2}$ hide. This 5 ploughs can till. Ralf holds it of Will[iam]. Thereof $\mathrm{R}$ [alf] has 2 furlongs and I plough in demesne, and the villeins 2 furlongs and I plough. There Ralf has 5 villeins, 2 serfs, 3 beasts, 40 sheep fol. $\mathbf{1 4 7}$.

and 2 acres of meadow. Worth ro shillings; when $W[$ illiam] received it the same.

William has a manor called Forohoda (Exch. Forhode) [Farwood] ${ }^{8}$ which Leumer (Exch. Lesmer) held T.R.E. and it paid geld for $\frac{1}{2}$ hide. This 5 ploughs can till. Herbert ${ }^{\theta}$ holds it of $\mathrm{W}$ [illiam]. Thereof $\mathrm{H}$ [erbert] has I virgate and I plough in demesne, and the villeins I virgate and 2 ploughs. There $H$ [erbert] has 4 villeins, 2 bordars, 4 sheep, $\mathrm{I} 6$ acres of wood(land), 4 acres of meadow and 30 acres of pasture. Worth $2 \mathrm{C}$ shillings a year; when he received it it was worth Io shillings.

William de Poileio has a manor called LEGA [Leigh] ${ }^{10}$ which Osferd ( $E x c h$. Offers) held T.R.E. and it paid geld for 2 hides. These 8 ploughs can till. Thereof W[illiam] has 1 hide and 2 ploughs in demesne, and the villeins I hide and 2 ploughs. There $W$ [illiam] has 9 villeins, 7 serfs, 6 beasts, I 10 sheep, wood(land) 4 furlongs long by 2 broad, 2 acres of meadow, and pasture 4 furlongs in length by 2 in breadth. Worth 40 shillings; when W[illiam] received it it was worth 20 shillings.

\footnotetext{
${ }^{6}$ Dart Ralph (Feud. Aids, i, 364) in Witheridge parish and hundred.

${ }^{7}$ Worth in Washfield in West Budleigh hundred.

${ }^{8}$ Probably Fardown, an outlier of Colyton in Colyton hundred. Trans. Devon. Assoc. xxxvi, 364.

${ }^{\circ}$ Herbert the seneschal. Cal. Docts. France, 235.

${ }^{10}$ Challonsleigh in Plympton parish and hundred. Geldroll, fol. 70, A 2.
} 


\section{HOLDERS OF LANDS}

William has a manor called BoTESFORDA [Battisford] ${ }^{1}$ which Alwin held T.R.E. and it paid geld for $\frac{1}{2}$ hide. These 3 ploughs can till. Thereof W[illiam] has I virgate and I plough in demesne, and the villeins I virgate and I plough. There $\mathrm{W}$ [illiam] has 3 villeins, I bordar, and 3 acres of meadow. Worth ro shillings; when W[illiam] received it it was worth 5 shillings.

William has a manor called Gosewella [Goosewell] ${ }^{2}$ which Heccha (Exch. Heche) held T.R.E. and it paid geld for $\frac{1}{2}$ hide. This 2 fol. $4 \mathrm{xz}$ b.

ploughs can till. Rotbert holds it of W[illiam]. Thereof $\mathrm{R}$ [obert] has I virgate in demesne and I plough, and the villeins have I $(d . i$.$) virgate$ and 2 oxen. There $R$ [obert] has $I$ villein, 3 bordars, I cow, 30 sheep, 5 swine, and 2 acres of wood. Worth 5 shillings a year.

W[illiam] has a manor called BicheleiA [Bickleigh] $^{3}$ which Brismar held T.R.E. and it paid geld for I hide. This 8 ploughs can till. Thereof $W[$ illiam] has I virgate in demesne and 2 ploughs, and the villeins 3 virgates and 3 ploughs. There $W$ [illiam] has 7 villeins, 4 bordars, 7 serfs, 6 beasts, 5 swine, 146 sheep, I 4 goats, I fishery worth 5 shillings a year, wood(land) I league in length by $I$ in breadth, 4 acres of meadow, and pasture $I$ league in length by 4 in breadth. Worth 40 shillings a year; when William received it it was worth 20 shillings.

$\mathrm{W}$ [illiam] has a manor called Bochelanda [Buckland] ${ }^{4}$ which Brismar held T.R.E. and it paid geld for 3 hides $\mathbf{I} \frac{1}{2}$ virgates. These 15 ploughs can till. Thereof W[illiam] has $\mathrm{I} \frac{1}{2}$ hides in demesne and 3 ploughs, and the villeins have 2 hides all but $\frac{1}{2}$ virgate and 7 ploughs. There $W$ [illiam] has 24 villeins, Io bordars, I 2 serfs, 20 beasts, 40 swine, 130 sheep, I fishery worth Io shillings a year, I saltern, wood(land) 4 leagues in length by 2 furlongs in breadth, 8 acres of meadow, and pasture I league in length by $I$ in breadth. Worth 100 shillings a year; when W[illiam] received it it was worth 50 shillings.

William has a manor called SANFORDA [Sampford $]^{5}$ which Brismar held T.R.E. and it paid geld for $\frac{1}{2}$ hide. This 8 ploughs can till. Rotbert holds it of $\mathrm{W}$ [illiam]. Thereof $\mathrm{R}$ [obert] has $\frac{1}{2}$ fol. 4 I8.

virgate in demesne and I plough, and the villeins

${ }^{1}$ Battisford in Plympton.

"Goosewell in Plymstock in Plympton hundred.

8 Bickleigh in Roborough hundred. Geldroll, fol. 70, A 4. Trans. Devon. Assoc. xxxvi, 170.

'Buckland Monachorum in Roborough hundred. Geldroll, fol. 70, A 4 .

${ }^{5}$ Sampford Spiney in Roborough hundred. have $\mathrm{I} \frac{1}{2}$ virgates and 2 ploughs. There $\mathrm{R}$ [obert] has I 2 villeins, I bordar, I serf, 4 sheep, wood(land) I league in length by 4 furlongs in breadth, and pasture $\frac{1}{2}$ league in length by $\mathbf{I}$ furlong in breadth. Worth 20 shillings; when he (ipse) received it it was worth ro shillings.

fol. 4 Ig.

THE LAND OF ROTBERT DE ALBAMARLA (Exch. ALBEMARLE) IN
DEVENESIRA

Rotbert has land in 3 townships (villae) which 3 thanes held as 4 manors ${ }^{7}$ and Robert holds them as I manor. In King E[dward]'s time it paid geld for 3 virgates. These 8 ploughs can till. Thereof $\mathrm{R}$ [obert] has $\frac{1}{2}$ virgate and I plough in demesne, and the villeins have the rest of the land and 5 ploughs. There R[obert] has 12 villeins, 8 bordars, 8 serfs, I 4 beasts, IO swine, 80 sheep, 20 goats, wood(land) 4 furlongs and (Exch. omits 4 furlongs and) 20 acres in length by 2 in breadth, ro acres of meadow, and pasture $\frac{1}{2}$ league in length by $\frac{1}{2}$ in breadth. Two of the above manors are worth 20 shillings, the other two 40 shillings. One of the four is called Onetreu [Ottery] which Oslac and Bulgeret held; the third Colacoma [Cullicombe] which Oslac held, and the fourth Wilavestreu [Willestrew] which Bulgeret held.

Rotbert has a manor called Mideltona [Milton ${ }^{8}$ which Wlwena (Exch. Ulveva) held T.R.E. and it paid geld for $\frac{1}{2}$ hide. This 10 ploughs can till. Thereof $R$ [otbert] has $\frac{1}{2}$ virgate and 2 ploughs in demesne, and the villeins I virgate and $\mathrm{I} \frac{1}{2}$ ferdings, and 5 ploughs. There R[otbert] has Io villeins, I I bordars, 7 bordars (Exch. serfs), 30 beasts, 23 swine, 75 sheep, 25 goats, 50 acres fol. $4 \mathrm{ggb}$.

of wood(land), 15 acres of meadow, pasture $I$ league in length by $\frac{1}{2}$ league in breadth. Worth 50 shillings; when $\mathrm{R}$ [otbert] received it it was worth 100 shillings.

Rotbert has a manor called Wicha [Week] ${ }^{9}$ which a woman, namely Uluerona $(E x c h$. Ulueron), held T.R.E. and she could go to what lord she liked. It paid geld for $1 \frac{1}{2}$ virgates. This

- In the Exchequer copy Robert de Albemarle is No. 28 on the list and follows Roger de Busli. Among his estates 'Mideltone' holds the first place; the rest follow as in the Exeter Book.

"Ottery, Cullicombe, and Willestrew alias Willeshay in Milton Abbot in Lifton hundred. See Geldroll, fol. $65, \mathrm{~A}_{4}$.

${ }^{8}$ Milton Damarel in Blacktorington hundred. Geldroll, fol. 65, A 5.

${ }^{9}$ Cookbury alias Week Damarel in Blacktorington hundred. 


\section{A HISTORY OF DEVONSHIRE}

ro ploughs can till. Thereof Rotbert has $\frac{1}{2}$ virgate and 2 ploughs in demesne and the villeins I virgate and 4 ploughs. There Rotbert has 9 villeins, 7 bordars, 5 serfs, 20 beasts, 7 swine, 50 sheep, I 2 acres of coppice, 50 acres of meadow, and pasture $I$ league in length by the same in breadth. Worth 3 pounds a year; when Rotbert received it it was worth 4 pounds.

Rotbert has a manor called TEWEBERIA [Theoborough] ${ }^{1}$ which Bristold (Exch. Bristuold) held T.R.E. and it paid geld for 1 virgate. This 5 ploughs can till. Now Franco holds it of Rotbert. Thereof Franco has $\frac{1}{2}$ virgate and 2 ploughs in demesne, and the villeins $\frac{1}{2}$ virgate and I plough. There Franco has 4 villeins, 6 bordars, I serf, 30 beasts, 23 swine, 100 sheep all but 5, 10 goats, 2 acres of coppice, 30 acres of meadow and 40 acres of pasture. Worth 35 shillings a year; when Rotbert received it it was worth the same.

Rotbert has a manor called Gir.Descota [Gatecot ? ${ }^{2}$ which Ulward held T.R.E. and it paid geld for I virgate. This 4 ploughs can till. Now Gislebert holds it of Rotbert. There Gislebert has I plough, also 2 villeins and 2 serfs who have fol. 420 .

another plough, 15 beasts, 4 swine, 5 sheep, 13 goats, 20 acres of coppice, 4 acres of meadow and 50 acres of pasture. Worth Io shillings a year; when Rotbert received it it was worth the same.

Rotbert has a manor called WESLEGA [Westleigh] ${ }^{3}$ which Ulward held T.R.E. and it paid geld for $\frac{1}{2}$ hide. This 5 ploughs can till. Thereof $\mathrm{R}$ [otbert] has $\frac{1}{2}$ virgate and 2 ploughs in demesne, and the villeins have $\mathrm{I} \frac{1}{2}$ virgates and 2 ploughs. There R[otbert] has 4 villeins, 5 bordars, 4 serfs, 7 beasts, 3 swine, 50 sheep, 2 acres of wood(land), 2 acres of meadow and 3 acres of pasture. Worth 30 shillings a year ; when $R$ [otbert] received it it was worth 40 shillings.

Rotbert has a manor called GodelEga [Goodleigh $]^{4}$ which Ulward held T.R.E. and it paid geld for I hide. This ro ploughs can till. Therof $\mathrm{R}$ [otbert] has I virgate and 3 ploughs in demesne, and the villeins 3 virgates and 7 ploughs. There $\mathrm{R}$ [otbert] has I 3 villeins, 5 bordars, 8 serfs, Io swine, 60 sheep, 5 goats, 20 acres of wood(land), $I$ acre of meadow and 20 acres of pasture. Worth 60 shillings; when $R$ [otbert] received it it was worth the same.

\footnotetext{
1 Theoborough in Sutcombe in Blacktorington hundred.

${ }^{2}$ Gatecot alias Gitcot in Milton Damarel in Blacktorington hundred.

${ }^{3}$ Westleigh in Fremington hundred.

1 Goodleigh in Braunton hundred. Geldroll, fol. 66, A I 4 .
}

Rotbert has a manor called MEReuda [Marwood $]^{5}$ which Ulward held T.R.E. and it paid geld for I virgate. This 4 ploughs can till. Two knights (milites) hold it of Rotbert. Thereof the two knights have $\frac{1}{2}$ virgate and I plough in demesne, and the villeins $\frac{1}{2}$ hide (? virgate) and I $\frac{1}{2}$ ploughs. They have there 3 villeins, 2 borfol. $420 \mathrm{~b}$.

dars, 2 serfs, 8 beasts, II swine, 80 sheep, 9 goats, wood(land) $\frac{1}{2}$ league in length by 2 furlongs in breadth, and 4 acres of meadow. Worth I 5 shillings; when $R$ [otbert] received it it was worth the same.

Rotbert has a manor called WITEFELLA [Whitfield] $^{6}$ which Alwin held T.R.E. and it paid geld for I virgate. This 5 ploughs can till. Two knights hold it of Rotbert. Thereof the two knights have $\frac{1}{2}$ virgate and I plough in demesne and the villeins $\frac{1}{2}$ virgate and 4 ploughs. There they have 4 villeins, 7 bordars, 3 serfs, 5 beasts, 3 swine, Ioo sheep, Io goats, wood(land) $\frac{1}{2}$ league in length by 2 furlongs in breadth, 5 acres of meadow and pasture $\frac{1}{2}$ league in length by 2 furlongs in breadth. Worth 30 shillings; when $\mathrm{R}$ [otbert] received it it was worth the same.

Rotbert (has) a manor called Brснecoma [Bickcombe] $^{7}$ which Ailmar held T.R.E and it paid geld for 4 virgates. These 4 ploughs can till. Now Rotbert de Herrefort holds it of $\mathrm{R}$ [otbert] de Albamarula. Thereof R[otbert] has $I \frac{1}{2}$ virgates and I plough in demesne, and the villeins have $2 \frac{1}{2}$ virgates and 4 ploughing oxen (boves in carr'). There R[otbert] has 2 villeins, I bordar, I serf, 7 beasts, 5 swine, 20 sheep, Io goats, 20 acres of wood(land), 10 acres of meadow fol. 42x, .

and 40 acres of pasture. Worth 15 shillings a year; when Rotbert de Albamarula received it it was worth 5 shillings.

Rotbert has a manor called FuUtes [Fleet] ${ }^{8}$ which Bristuald (Exch. Brictuald) held T.R.E. and it paid geld for I hide. This 6 ploughs can till. Thereof R[otbert] has in demesne I virgate and I plough, and the villeins 3 virgates and 3 ploughs. There $R$ [otbert] has 8 villeins, 2 bordars, 2 serfs, 42 sheep, Io acres of coppice, 4 acres of meadow and 10 acres of pasture. Worth 20 shillings a year; when R[otbert] received it it was worth the same.

Rotbert has a manor called Benleia [Beenleigh ${ }^{9}$ which Ailward held T.R.E. and it paid

${ }^{5}$ Marwood in Braunton hundred.

${ }^{6}$ Whitefield in Marwood in Braunton hundred.

7 Bickham in Oakford in Witheridge hundred.

${ }^{8}$ Fleet Damarel in Holbeton in Ermington hundred. Geldroll, fol. 70, A 7 .

${ }^{9}$ Beenleigh in Harbertonford in Stanborough hundred (O. J. R.) ; or in Diptford (T.W.W.). 


\section{HOLDERS OF LANDS}

geld for $\frac{1}{2}$ hide. This 4 ploughs can till. Gislebert holds it of Rotbert. Thereof G[islebert] has I virgate and $\frac{1}{2}$ plough in demesne, and the villeins $I$ virgate and $I_{\frac{1}{2}}$ ploughs. There G[islebert] has 3 villeins, 3 bordars, I beast, 75 sheep, and ro acres of pasture. Worth I 2 shillings; when $R$ [otbert] received it 8 shillings.

Rotbert has a manor called Odelea [Woodleigh] ${ }^{1}$ which Alvrix Pic (Exch. Alvric) held T.R.E. and it paid geld for I hide I virgate. This I 2 ploughs can till. Thereof $R$ [otbert] has $I$ virgate and 3 ploughs in demesne, and the villeins I hide and 9 ploughs. There R[otbert] has I 5 villeins, 8 bordars, 7 serfs, 2 swineherds paying 16 swine (porcarios for porcos) a year. There $R$ [otbert] has 15 beasts, 16 swine, fol. 42Ib.

I 00 sheep, Io goats, I fishery, Ioo acres of wood(land), Io acres of coppice, 2 acres of meadow and 30 acres of pasture. Worth 60 shillings a year; when $R$ [otbert] received it it was worth 40 shillings.

Rotbert has a manor called HALETRou [Halstow $]^{2}$ which Bristrix (Exch. Brictric) and Alward held T.R.E. and it paid geld for $\frac{1}{2}$ virgate. This I plough can till. There $\mathrm{R}$ [otbert] has I villein who pays 30 pence a year; when $R$ [otbert] received it he was paying 5 shillings.

Rotbert has a manor called SToches [Stoke] ${ }^{3}$ which Brismar held T.R.E. and it paid geld for 2 hides. These 12 ploughs can till. Thereof $\mathrm{R}$ [otbert] has $\frac{1}{2}$ hide in demesne and 2 ploughs, and the villeins have $\mathrm{I} \frac{1}{2}$ hides and 8 ploughs. There $\mathrm{R}$ [otbert] has $\mathrm{I} 6$ villeins, 4 bordars, 5 serfs, I 5 beasts, I 2 swine, I 60 sheep, 40 goats, 2 acres of wood(land) and 12 acres of pasture. Worth 70 shillings a year; when R[otbert] received it 40 shillings.

Rotbert has a manor called WIDA [Widey] which Wadelo held T.R.E. and it paid geld for $\frac{2}{2}$ hide. This 4 ploughs can till. Now Ossulf (Exch. Osulf) holds it of R[otbert]. Thereof $\mathrm{O}$ [ssulf] has I virgate and I plough in demesne, and the villeins $I$ virgate and $\frac{1}{2}$ plough. There $\mathrm{O}$ [ssulf] has I villein, 3 serfs, 7 beasts, 2 swine, 40 sheep, 18 goats, wood(land) $\frac{1}{2}$ league in length by 3 furlongs in breadth, and 40 acres of pasture. Worth ro shillings a year; when $\mathrm{R}$ [otbert] received it 30 (Exch. omits past value).

${ }^{1}$ Woodleigh in Stanborough hundred.

2 Halstow, an outlier of Woodleigh in Stanborough hundred.

${ }^{3}$ Stoke Damarel in Roborough hundred. Geldroll, fol. $70, \mathrm{~A}_{3}$

Widey Court in Egg Buckland in Roborough hundred.
The following two entries are found in the Exchequer book only :-

To this manor has been added WITELIE [Whitleigh]. ${ }^{5}$ Wadelo held it T.R.E. and it paid geld for $\frac{1}{2}$ hide. Arable for 3 ploughs. In demesne is I plough, and 3 bordars have I plough. There are 30 acres of pasture and 9 acres of wood. Formerly worth 5 shillings, now ro shillings.

Robert himself has in BARNESTAPLE [Barnstaple] 2 houses lying in ruins, paying 4 pence.

fol. 455.

LANDS OF THE FRENCH KNIGHTS

\section{(FRANCORUM MILITUM) IN DE- VENESIRA ${ }^{\circ}$}

(At the foot of the page is added) Osbern, Girald, R[alf] Paganel, William de $\mathrm{Ou}$ and Ansger de monteacuto in Devenesira.

Osbern de Salceit (Exch. Salceid) has a manor called Patforda [Parford] ${ }^{7}$ which Godric held T.R.E. and it paid geld for I virgate. This 5 ploughs can till. Thereof $\mathrm{O}$ [sbern] has $\frac{1}{2}$ virgate in demesne, and the villeins $\frac{1}{2}$ virgate and I plough. There $O$ [sbern] has 6 villeins, I serf, 12 acres of meadow and 20 acres of pasture. Worth 20 shillings; when $O$ [sbern] received it the same. This manor owes to the king's demesne-manor called Tauetona [Tawton] either 30 pence a year or I ox by way of customary due.

Girold the chaplain has a manor called Escapeleia (Exch. Scapelie) [Shapleigh] ${ }^{8}$ which a thane held T.R.E. and it paid geld for I virgate and I ferling. This manor owes to the king's demesne-manor called Tauetona [Tawton] Io shillings a year by way of customary due.

Girold the chaplain has a manor called BICATONA [Bickington] ${ }^{9}$ which Godo (Exch. Gode)

${ }^{5}$ East Whitleigh alias Witley in St. Budeaux in Roborough hundred. See fol. 505 .

${ }^{6}$ The lands of the French knights are given separately in the Exchequer copy. Osbern de Salceid is No. 44; Girold is No. 46; Ralf Paganel is No. 32 ; William de Ow is No. 22 ; Ansger de Montagud is No. 4 I (among Ansger's estates 'Staford' comes first, then I virgate in 'Toritone,' then Brimcombe, and the rest in order); Ralf de Limesi, is No. 3 I ; Girard is No. 47 ; Richard, son of Count Gislebert, is No. 26; Roger de Busli No. 27; Aiulf No. 42; Morey is among the king's servants No. 52 ; Ralf de Felgeres is No. 33 ; and Floher is not named at all. Freeman, Norman Conquest, v, 766 .

${ }^{7}$ Parford in Drewsteignton in South Tawton hundred (O. J. R.) ; Batworthy, Gidleigh (T.W.W.) See fol. 496 ; Trans. Devon Assoc. xxxvi, 377.

${ }^{8}$ Shapleigh in South Tawton hundred.

${ }^{9}$ Abbots Bickington in Blacktorington hundred. 


\section{A HISTORY OF DEVONSHIRE}

the priest held T.R.E. and it paid geld for $\frac{2}{3}$ virgate. This land 3 ploughs can till. There G[odo] has 8 bordars, 2 serfs, and I plough in demesne, and the villeins 2 ploughs. There G[odo] has 20 beasts, 60 sheep, wood(land) 2 furlongs in length by $\mathrm{I}$ in breadth, 5 acres of meadow, and pasture 2 furlongs in length by $I$ in breadth. It pays 20 shillings; when G[odo] received it it was worth the same.

Girold has a manor called Nistenestoc [Nectan's Stoke] ${ }^{1}$ which the canons of the same manor held T.R.E. and it paid geld for 2 hides. fol. 456b.

These I 2 ploughs can till, which (ploughs) are there. These the I 2 canons hold of G[erold], who themselves held them before. (Worth altered into) They pay for them to G[erold] 40 shillings a year.

Ansger holds a virgate of land in Toritona [ $]^{2}$ which Alwrit (Exch. Aluric) held T.R.E. This 2 ploughs can till. Thereof Ansger has I ferting and I plough in demesne, and the villeins have 3 fertings and $x$ plough. There Ansger has I villein, 3 bordars, 6 beasts, 5 swine, 30 sheep, 8 goats, and 4 acres of meadow. Worth 15 shillings a year; when An[sger] received it the same.

Ansger $^{3}$ ( $E x c h$. of Montagud) has a manor called STAForT [Stafford] ${ }^{4}$ which Aluric held T.R.E. and he could go with that land to any lord he liked. It paid geld for $\frac{1}{2}$ virgate. This $\mathbf{I} \frac{1}{2}$ (Exch. I) ploughs can till. Thereof $\mathrm{A}$ [nsger] has in demesne $I$ ferding and $\frac{1}{2}$ plough, and the villeins $I$ ferding and $\frac{1}{2}$ plough. There $A$ [nsger] has 5 bordars and I serf, 2 acres of wood(land), 4 acres of meadow, and pasture 2 furlongs in length by $I$ in breadth. Worth $I 2$ shillings and 6 pence a year; when $A[$ nsger] received it it was worth 7 shillings and 6 pence.

Ansger has a manor called Bremelcombe [Brimcombe] $^{5}$ which Algher (Exch. Alcher) held T.R.E. and he could go with that land to what lord he liked. It paid geld for $\frac{1}{2}$ virgate. This $I \frac{1}{2}$ ploughs can till. Thereof $A$ [nsger] has in demesne $I$ ferding and $\frac{1}{2}$ plough, and the villeins I ferding and $\frac{1}{2}$ plough. There $A$ [nsger]

'Stoke St. Nectan alias Hartland Abbey manor in Hartland parish and hundred. Geldroll, fol. 65, A 2.

2 Probably Whitsleigh in St. Giles'-in-the-Wood on the Toridge in Fremington hundred.

${ }^{3}$ De ponte Senardi. See fol. 462 , and Geldroll, fol. $66 b$, A 5 .

'Stafford in Dolton in North Tawton hundred. See fol. $498 b$.

${ }^{5}$ Bremelcombe alias Brimcombe in Dowland in North Tawton hundred. See fol. 4986 . has 2 bordars. Worth 7 shillings and 6 pence a year; when he received it it was worth 5 shillings.

fol. 457.

William de Hou (Exch. de Ow) has a manor called Poldreham [Powderham] ${ }^{6}$ which Torssus held T.R.E. and it paid geld for $\frac{1}{2}$ hide. This I 2 ploughs can till. Rannulf holds it of W[illiam]. Thereof Rannulf has in demesne I virgate and 3 ploughs, and the villeins I virgate and 8 ploughs. There R[annulf] has I 2 villeins, I 2 bordars, 8 serfs, I rouncey, I mill paying 50 pence a year, 8 beasts, I 7 swine, I 50 sheep, 40 goats, I league and 4 acres of coppice, 20 acres of meadow and 50 acres of pasture. Worth 6 pounds a year; when he received it it was worth the same.

Included in that manor is $\frac{1}{2}$ virgate of land ${ }^{7}$ which went with Exminster (jacebat in Esseminestra) T.R.E. There is a plough, and it pays 5 shillings a year.

Ralf Pagan (Exch. Pagenel) has a manor called Donsedoc [Dunchideock ${ }^{8}$ which Merlesuein (Exch. Merlesuain) held T.R.E. and it paid geld for 1 hide. This 5 ploughs can till. Thereof $\mathrm{R}$ [alf] has $\frac{1}{2}$ hide and I plough in demesne, and the villeins $\frac{1}{2}$ hide and 4 ploughs. There R[alf] has 8 villeins, 4 bordars, 2 serfs, 20 acres of wood(land) and 2 acres of meadow. It pays I oo shillings; when $R$ [alf] received it it was worth 3 pounds.

Ralf de Limeseio (Exch. Limesi) has a manor called Duna [Down] ${ }^{9}$ which Edric held T.R.E. and it paid geld for 5 virgates. These 12 ploughs can till. Thereof $R$ [alf] has 2 virgates in demesne and $I$ plough, and the villeins have Io ploughs. There $\mathrm{R}$ [alf] has I4 villeins, 6 bordars, 9 serfs, 4 beasts, 3 swine, I I o sheep, 20 acres of coppice and 20 acres of pasture. Worth 4 pounds a year; when $R$ [alf] received it the same.

fol. $457 \mathrm{~b}$.

Ralf de Limeseio has a manor called Bradeuilla (Exch. Bradewelle) [Bradwell $]^{10}$ which Edric held T.R.E. and it paid geld for I hide. This ro ploughs can till. Thereof $\mathrm{R}$ [alf] has $\frac{1}{2}$ hide and 2 ploughs in demesne, and the villeins have 4 ploughs. There $R$ [alf] has I I villeins, 5 bordars, 8 serfs, 2 beasts, 65 sheep, 9 goats, 23 acres of meadow and 50 acres of pasture. Worth 60 shillings a year; when $R$ [alf] received it it was worth 4 pounds.

6 Powderham in Exminster hundred.

7 Exwell in Powderham in Exminster hundred. See fol. 498.

${ }^{8}$ Dunchideock in Exminster hundred. Geldroll, fol. $68 b, \mathrm{~B} 2$.

${ }^{9}$ East Down in Braunton hundred. Geldroll, fol. 66, A 8.

${ }^{10}$ Bradwell in West Down in Braunton hundred. 


\section{HOLDERS OF LANDS}

Ralf de Limeseio (Exch. the same Ralf) has a manor called RADEHIDA [Roadway] ${ }^{1}$ which Edrit (Exch. Edric) held T.R.E. and it paid geld for I virgate. This 2 ploughs can till. Upon it R[alf] has 2 villeins and ro acres of coppice. Worth 30 pence a year.

Osbern (Exch. Osbern de Salceid himself) has a manor called CLIST [Clyst] ${ }^{2}$ which Ultret (Exch. Uctred) held T.R.E. and it paid geld for 3 hides and 1 virgate. These 8 ploughs can till. Thereof $\mathrm{O}$ [sbern] has in demesne $\mathrm{I} \frac{1}{2}$ hides and I plough, and the villeins I hide 3 virgates and 3 ploughs. There $\mathrm{O}$ [sbern] has 3 villeins, 6 bordars, 2 serfs, 8 beasts, 2 swine, 6 I sheep, 27 goats, 26 acres of wood(land), 40 acres of meadow and 60 acres of pasture. Worth 40 shillings a year; when he received it it was worth 15 shillings.

fol. 458 .

Ralf de Limeseio has a manor called Morteno [Morthoe] ${ }^{3}$ which Edrit (Exch. Edric) held T.R.E. and it paid geld for $\frac{1}{2}$ hide. This 3 ploughs can till. Now Alwrit (Exch. Aluric) holds it of R[alf]. Thereof Alwrit has I virgate in demesne and I plough, and the villeins I plough. There Alwrit has 2 villeins and I serf. Worth Io shillings; when $R$ [alf] received it the same.

Osbern de Salceio ( $E_{x c h}$. Osbern himself) has a manor called Selvestan [Shilston] ${ }^{4}$ which Edrit (Exch. Edric) held T.R.E. and it paid geld for I virgate. This 3 ploughs can till. Thereof Osbern has $\frac{1}{2}$ virgate and 2 ploughs, and his villeins have I plough. There O[sbern] has 2 villeins, 2 bordars, 2 serfs, 14 beasts, II swine, ro goats, 8 acres of meadow and 40 acres of pasture. Worth 20 shillings a year; when Osbern received it it was worth (the same corrected into) ro shillings.

With this manor went (adjacebat) I virgate of land T.R.E. ${ }^{5}$ No one holds it now, but upon it 2 villeins are settled. Worth 3 shillings a year.

Osbern de Salceio (Exch. Osbern himself) has a manor called LANFORDA [Lamford] ${ }^{6}$ which Leuegar held T.R.E. and it paid geld for I virgate. This 2 ploughs can till. One plough is there, 2 villeins, and 1 bordar; also 5 acres of wood(land), 5 acres of meadow and 20 acres of

\footnotetext{
1 Roadway in Morthoe in Braunton hundred.

- Clyst Gerard in Broad Clyst in Cliston hundred. Geldroll, fol. $67, \mathrm{~A}_{4}$.

${ }^{3}$ Morthoe in Braunton hundred.

4 Shilston in Drewsteignton in Wonford hundred. See fol. 500. Geldroll, fol. 70b, A I 3 .

${ }^{5}$ Lower Shilston in Drewsteignton in Wonford hundred.

${ }^{6}$ Lamford alias Lambert in Cheriton Bishop in Wonford hundred.
}

pasture. Worth ro shillings a year; when $O$ [sbern] received it it was worth 5 shillings.

fol. $458 \mathrm{~b}$.

Ralf Paganel has a manor called Carseuilia (Exch. Carsewelle) [Keshill] ${ }^{7}$ which Merlesoan held T.R.E. and it paid geld for 2 hides. These 8 ploughs can till. Now Gonher holds it of $\mathrm{R}$ [alf]. Thereof G[onher] has in demesne $\frac{1}{2}$ hide and I plough, and the villeins $I_{2}^{\frac{1}{2}}$ hides and $2 \frac{1}{2}$ ploughs. There G[onher] has 8 villeins, 5 bordars, 4 serfs, 5 beasts, 28 sheep, I 5 goats, I mill paying 5 shillings, 30 acres of wood(land), Io of meadow and IOO of pasture. Worth 60 shillings a year; when he received it 40 shillings.

Ralf Paganel has a manor called Arra [Aller] ${ }^{8}$ which Merlesoan held T.R.E. and it paid geld for I hide. This ro ploughs can till. Thereof $\mathrm{R}$ [alf] has in demesne I virgate and I plough, and the villeins 3 virgates and 7 ploughs. There $\mathrm{R}$ [alf] has I 4 villeins, 9 bordars, 5 serfs, I beast, 66 sheep, 20 acres of wood(land), 20 acres of meadow and 50 of pasture. Worth 5 pounds a year; when he received it 60 shillings.

Ralf has a manor called TRULA [Throwleigh] ${ }^{\ominus}$ which Merlosuin held T.R.E. and it paid geld for I hide. This 8 ploughs can till. Thereof $R$ [alf] has I virgate and 2 ploughs, and the villeins have 6 ploughs. There $R$ [alf] has 10 villeins, I bordar, 3 serfs, 4 beasts, 32 sheep, 12 acres of wood(land), 8 acres of meadow, and pasture $\frac{1}{2}$ league in length by 4 acres in breadth. (Worth corrected into) It pays 4 pounds.

fol. 459.

Ralf Pagan has a manor called CAGEForT [Chagford] ${ }^{10}$ which Merlosuan held T.R.E. and it paid geld for $\frac{1}{2}$ hide. This 4 ploughs can till. Thereof $R$ [alf] has $\frac{1}{3}$ hide in demesne and I plough, and the villeins have 3 ploughs. There $R$ [alf] has 6 villeins, 3 serfs, Io swine, 30 sheep, 6 acres of wood(land), 8 acres of meadow and 4 acres of pasture. Worth 3 pounds a year; when $R[$ alf] received it it was worth the same.

William de $\mathrm{Ou}$ has a manor called WrTESTANI [Whitstone] ${ }^{11}$ which Toli held T.R.E. and it paid geld for I virgate. This 2 ploughs can till.

${ }^{7}$ Keshill alias Kerswell Peverel and Dulford in Broadhembury in Hairidge hundred. Geldroll, fol. $67 b, \mathrm{~B} 2$.

${ }^{8}$ Aller Peverel alias Bullaller in Collumpton in Hairidge hundred. Geldroll, fol. 67b, A $\mathrm{I}_{4}$ and B 3 .

${ }_{9}^{9}$ Throwleigh in Wonford hundred. Geldroll, fol. 706, A 7 .

${ }^{10}$ Chagford in Throwleigh in Wonford hundred.

"Church Whitestone in Wonford hundred. 


\section{A HISTORY OF DEVONSHIRE}

There Rannulf, who holds the land of W[illiam], has 6 villeins who have upon it. 2 ploughs, 2 serfs, 4 acres of wood(land) and 2 acres of meadow. Worth ro shillings a year; when William received it it was worth 6 shillings.

Floher has a manor called Sotrebroc [Shytternbrook $]^{1}$ which Alviet held T.R.E. and it paid geld for $\frac{1}{2}$ virgate. This 4 oxen can till. Worth 2 shillings a year. ${ }^{2}$

Girard has a manor called Manelia [Manley] ${ }^{3}$ which Alestantilia held T.R.E. and it paid geld for I virgate. This 2 ploughs can till. Thereof Girard has in demesne $\frac{1}{2}$ virgate and I plough, and the villeins $\frac{1}{2}$ virgate and I plough. There Girard has I villein, 3 bordars, 7 swine, 40 sheep, fol. 459b.

4 acres of wood(land) and 5 acres of meadow. Worth Io shillings a year; when he received it 5 shillings.

Girard has a manor called Nochecota [Noggacot ${ }^{4}$ which Alestilla (Exch. Alestan) held T.R.E. and it paid geld for I virgate. This 2 ploughs can till. Thereof G[irard] has in demesne $\frac{1}{2}$ virgate and I plough, and the villeins $\frac{1}{2}$ virgate and (I plough). There G[irard] has I villein, 4 bordars, I serf, 50 sheep, I 5 goats, 5 acres of coppice and 5 acres of meadow. Worth Io shillings a year; when he received it 5 shillings.

Ansger has a manor called Chadeledona (Exch. Cadeledone) [Cheldon] ${ }^{5}$ which Mathildis held T.R.E. and it paid geld for $I \frac{1}{2}$ virgates. This 2 ploughs can till. There $A$ [nsger] has $\frac{1}{2}$ virgate and I plough in demesne, and the villeins $I$ virgate and 2 ploughs. There $A$ [nsger] has 4 villeins, 4 bordars, 1 serf, 4 beasts, 6 swine, 42 sheep, 7 acres of wood(land), 2 acres of meadow and 10 acres of pasture.

To this manor has been added another called Chadeledona [Cheldon] ${ }^{6}$ which Brismer held T.R.E. (Exch. as a manor) and it paid geld for $\frac{1}{2}$ virgate and $\frac{1}{2}$ ferling. This $1 \frac{1}{2}$ ploughs can till. (These 2 pay 50 shillings a year.)

1 Shytternbrook alias Floyer's Hayes next Exe Island in Wonford hundred. Trans. Devon Assoc. xxx, 505.

3 This entry is not in the Exchequer book.

${ }^{3}$ Now called Pole Antony next West Manley in Tiverton parish and hundred. Feud. Aids, p. 319, shows Pole Antony and Noggacot both held by Briwer's heirs, Chaworth and Mohun, Briwer being successor to Girard at Little Tiverton and Little Washfield after an escheat (O. J. R.). West Manley, Tiverton (T.W.W.).

Noggacot alias Northcote in Tiverton parish and hundred.

${ }^{5}$ Church Cheldon in Witheridge hundred.

- Included in Church Cheldon in Witheridge hundred. See fol. 502 . fol. 460 .

Richard son of Count Gislebert has a inanor called Leurstona [Lympstone] ${ }^{7}$ which Saward held T.R.E. and it paid geld for I hide and I virgate. This 8 ploughs can till. William Capra holds it of Richard. From it Io villeins, 6 bordars, and 2 serfs pay 8 pounds to William by way of farm (ad firmam). When Richard received this manor it was worth Io pounds.

Osbern de Salceit has a manor called AInechesdona [Ingsdon] ${ }^{8}$ which Frawin held T.R.E. and it paid geld for $1 \frac{1}{2}$ hides. This 6 ploughs can till. Thereof $\mathrm{O}$ [sbern] has $\frac{1}{2}$ hide and I plough in demesne, and the villeins $I$ hide and 5 ploughs. There $O$ [sbern] has I 5 villeins, 4 bordars, 2 serfs, 5 beasts, I 25 wethers (berbices), 12 acres of wood(land), 8 acres of meadow and 40 acres of pasture. Worth 40 shillings; when $O$ [sbern] received it 20 shillings.

Ralf Pagan (Exch. Pagenel) has a manor called Ilestintona (Exch. Lestintone) [Ilsington] ${ }^{9}$ which Merlesuen held T.R.E. and it paid geld for 2 hides. These 12 ploughs can till. Thereof $\mathrm{R}$ [alf] has $\frac{1}{2}$ hide and I plough in demesne, and the villeins $1 \frac{1}{2}$ hides and 7 ploughs. There $R$ [alf] has 22 villeins, 6 bordars, 7 serfs, 5 beasts, 40 sheep, 23 goats, 2 10 acres of wood(land), I acre of meadow, and 2 leagues 8 furlongs of pasture taking length and breadth. It pays 9 pounds; when he received it the same.

Also 1 house in Essrcestra [Exeter] ${ }^{10}$ paying Io shillings.

The same Ralf has a manor called AinzchesDoNA [Ingsdon] ${ }^{11}$ which Merlesuen held T.R.E. and it paid geld for 2 hides. These 9 ploughs can till. Thereof $R$ [alf] has $\frac{1}{2}$ hide and 2 ploughs in demesne, and the villeins $I \frac{1}{2}$ hides and 5 ploughs. There $R$ [alf] has 20 villeins, 8 bordars, 5 serfs, I rouncey, 3 beasts, IOO sheep, 70 acres of wood(land), I acre of meadow and 4 acres of pasture. It pays 9 pounds; when $R[\mathrm{alf}]$ received it the same.

Ralf Pagan has a manor called Tovretona [Tiverton] ${ }^{12}$ which Merlesoan held T.R.E. and it paid geld for I virgate. This 2 ploughs can till. Girard holds it of Ralf. There Girard has 4 villeins, I serf, and 2 acres of meadow. Worth 30 shillings a year; when Ralf received it it was worth 40 shillings.

7 Lympstone in East Budleigh hundred.

${ }^{8}$ Ingsdon in Isington in Teignbridge hundred. Geldroll, fol. 69, A 7 .

${ }^{9}$ Ilsington in Teignbridge hundred. Geldroll, fol. $69, \mathrm{~A}_{3}$.

${ }^{10}$ Exeter dwelling-house.

11 Ingsdon in Ilsington in Teignbridge hundred.

${ }^{18}$ Little Tiverton alias West Exe in Tiverton parish and hundred. (O. J. R.) Trans. Devon Assoc. xxix, 495 ; xxxiii, 397. 


\section{HOLDERS OF LANDS}

The aforesaid Ralf has a manor called WASFELTA [Washfield] ${ }^{1}$ which Merlesoan held T.R.E. and it paid geld for I virgate. This 2 ploughs can till. Girard holds it of Ralf. Thereof Girard has in demesne $\frac{1}{2}$ virgate, and the villeins $\frac{1}{2}$ virgate and I plough. There G[irard] has I villein, 3 bordars, I acre of meadow, and I of wood(land). Worth 20 shillings; when $R$ [alf] received it 10 shillings.

Roger de Busleio (Exch. Busli) has a manor called SANForda [Sampford] ${ }^{2}$ which Bristrit (Exch. Brictric) held T.R.E. and it paid geld for $3 \frac{1}{2}$ hides. This 12 ploughs can till. Thereof Roger has in demesne $\mathrm{I} \frac{1}{2}$ hides and $\frac{1}{2}$ ferling and I plough, and the villeins have 2 hides all but $\frac{1}{2}$ ferling and 8 ploughs. There Roger has 20 villeins, 8 bordars, 6 serfs, 8 swine, $8_{4}$ sheep, 50 goats, 80 acres of wood(land), 30 acres of meadow, and I 50 acres of pasture. (Worth corrected into) It pays ro pounds a year; when he fol. $46 \mathrm{r}$.

received it it was paying 100 shillings. This manor Queen Mathildis gave to Roger de Busleio with his wife.

Ansger (de Senarpont) has a manor called Mochelesberia [Mucksbear] ${ }^{3}$ which 5 thanes held in parage (pariter; Exch. libere) under Bristrit (Exch. Brictric), and they could not become independent of him (separari $a b$ eo) T.R.E. It paid geld for I hide. This 5 ploughs can till. Thereof Ansger has $\frac{1}{2}$ hide in demesne and $I$ plough, and the villeins have $\frac{1}{2}$ hide and $\frac{1}{2}$ plough. There Ansger has 9 villeins, I bordar, 7 beasts, 24 sheep, I 8 goats, I mill paying 60 pence, 6 acres of wood(land), 26 acres of meadow, and pasture 4 furlongs in length by 2 in breadth. Worth 30 shillings a year; when he received it 40 shillings. These lands of the aforesaid 5 thanes Ansger holds as I manor.

Ansger (de Senarpont) has a manor called Suntatona [Swetton] ${ }^{4}$ which Godric held T.R.E. and it paid geld for $\frac{1}{2}$ hide. This $I \frac{1}{2}$ ploughs can till. Thereof Ansger has in demesne $I \frac{1}{2}$ virgates and I plough, and the villeins $\frac{1}{2}$ virgate and I plough. There $A$ [nsger] has 3 villeins, I bordar, I serf, I rouncey, I 7 beasts, 2 swine, ro acres of coppice, 8 acres of meadow and 10 acres of pasture. Worth Io shillings a year; when he received it it was worth the same. The aforesaid land Godric and his

' Little Washfield in Washfield in Witheridge hundred (O. J. R.). Testa de Nevill (Rec. Com.), 190a, shows it held in socage of William Briwer's heirs Trans. Devon Assoc. xxxv, 3 I 3 ; xxxvi, 358.

${ }^{2}$ Samford Peverel in Tiverton and Halberton hundreds. Geldroll, fol. 67, B 2, and fol. 68, A 2.

${ }^{3}$ Muxbear in Halberton parish and hundred. See fol. $502 b$.

- Swetton in Halberton parish and hundred. See fol. $5 \circ 3$.

I brother held in parage (pariter), and that land has been wrongfully united with Bristric's land.

Aiulf ${ }^{5}$ has a manor called Suetretona [Swetton] ${ }^{B}$ which Etdric (Exch. Edric) held T.R.E. and it paid geld for $\frac{1}{2}$ hide. This $I \frac{1}{2}$ ploughs can till. Floher holds it of Aiulf. Thereof Floher has in demesne $\frac{1}{2}$ virgate and $\frac{1}{2}$ fol, $46 \mathrm{rb}$.

plough, and the villeins $I \frac{1}{2}$ virgates and I plough. There Floher has 4 villeins, I bordar, 3 acres of meadow, and 10 acres of pasture. Worth Io shillings a year; when he received it it was worth the same.

Aiulf has a manor called LEGA [Leigh] ${ }^{7}$ which Etmar (Exch. Edmar) held T.R.E. and it paid geld for $\frac{1}{2}$ hide and $\frac{1}{2}$ virgate. These $I_{2} \frac{1}{2}$ ploughs can till. William holds it of A[iulf]. Thereof W[illiam] has in demesne I virgate, I ferding and I plough, and the villeins I virgate, I ferding, and I plough. There $W[$ illiam] has 2 villeins, 5 bordars, 2 serfs, 5 beasts, 6 swine, 24 sheep, 30 goats, I mill paying $3^{6}$ pence, 25 acres of wood(land), 6 acres of meadow and $3^{8}$ acres of pasture. Worth 20 shillings a year; when he received Io shillings.

Morin $^{8}$ has a manor called LINOR (Exch. Limor) [Leonard] ${ }^{9}$ which Frawin held T.R.E. and it paid geld for 3 virgates. These 2 ploughs can till. Upon it $\mathrm{M}$ [orin] has I plough, I villein, 3 bordars, 2 serfs, 7 beasts, 29 sheep, 16 acres of wood(land), 8 of meadow and 60 acres of pasture. Worth 15 shillings a year; when he received it it was worth 40 shillings. Now Maurin holds it of the king.

Ralf Pagan has a manor called WILLA [Well] ${ }^{10}$ which Merlesuen held T.R.E. and it paid geld for 2 hides. These 5 ploughs can till. Thereof $R$ [alf] has I hide and 2 ploughs in demesne, and the villeins $I$ hide and 4 fol. 462.

ploughs. There $R$ [alf] has 16 villeins, 2 bordars, 7 serfs, I beast, Io swine, 6 sheep, 56 acres of wood(land), 12 acres of meadow and 32 acres of pasture. It pays 105 shillings; when he received it 6 pounds.

Ansger has a manor called Duveltona [Dolton] ${ }^{11}$ which Edric held T.R.E. and it

${ }^{3}$ Aiulf was sheriff of Dorset.

Swetton in Halberton parish and hundred.

${ }^{7}$ Leigh Boty alias Besley in Holcombe Rogus in Halberton hundred.

${ }^{8}$ Morey of Caen. Geldroll, fol. 68, A 5 .

${ }^{9}$ Moorston next Leonard in Halberton parish and hundred.

${ }^{10}$ Edginswell in St. Mary Church in Haytor hundred. Geldroll, 69b, A II.

"Iddlecot alias Edrichescot in Dolton in North Tawton hundred. See fol. $503 b$; Geldroll, fol. $66 b, \mathrm{~A} 5$. 


\section{A HISTORY OF DEVONSHIRE}

paid geld for 2 virgates. These 6 ploughs can till. Thereof Ansger has in demesne $\frac{1}{2}$ virgate and I plough, and the villeins $I \frac{1}{2}$ virgates and 2 ploughs. There Ansger has 4 villeins, 4 borbars, I serf, 4 beasts, 24 sheep, II goats, 5 acres of coppice, 4 acres of meadow and 10 acres of pasture. Worth 50 shillings a year.

This manor has been added (Exch. wrongfully) to Bristric's land, which a certain thane held, who was able to go (potens eundi) to whatsoever lord he might like. (Exch. He who held it in King Edward's time was able to go where he liked.)

Ralf de Felgeriis (Exch. Felgheres) has a manor called IpLePena [Ipplepen] ${ }^{1}$ which Goda held T.R.E. and it paid geld for 4 hides all but $\frac{1}{2}$ virgate. These 20 ploughs can till. Thereof $\mathrm{R}$ [alf] has in demesne $\mathrm{I}$ hide and 3 ploughs, and the villeins 3 hides all but $\frac{1}{2}$ virgate and I 2 ploughs. There $R$ [alf] has 37 villeins, I 6 bordars, 3 serfs, I rouncey, 5 beasts, 5 swine, 250 sheep, $\frac{1}{2}$ league of coppice taking length and breadth, 30 acres of meadow and 10 acres of pasture.

fol. $462 \mathrm{~b}$.

Ralf has a manor called GaLmentona [Galmpton] ${ }^{2}$ which Goda held T.R.E. and it paid geld for 2 hides. These 6 ploughs can till. Thereof $\mathrm{R}$ [alf] has in demesne 1 hide and 2 ploughs, and the villeins I hide, 5 ploughs, and 4 oxen. There R[alf] has 14 villeins, 2 bordars, 2 serfs, I rouncey, 2 swine, I oo sheep, coppice I league in length by 12 perches in breadth, I acre of meadow and 4 acres of pasture. This manor, along with the aforesaid Iplepena [Ipplepen], is worth 30 pounds a year.

Osbern de Salcet has I house in Essecestra [Exeter] ${ }^{3}$ which used to pay 8 pence yearly by way of customary due in King E[dward's] time; and $O$ [sbern] withholds them.

\section{fol. 468 .}

THE LAND OF NICOLAS THE CROSSBOWMAN (ARBALESTARIUS) IN DEVENESIRA. ${ }^{4}$

Godebold has a manor called HeLA [Hele] ${ }^{5}$ which Olf (Exch. Ulf) held T.R.E. and it paid A 3 .

${ }^{1}$ Ipplepen in Haytor hundred. Geldroll, fol. $69 b$,

${ }^{2}$ Galmpton in Churston Ferrers in Haytor hundred.

3 Exeter dwelling-house.

- In the Exchequer copy these lands are given separately ; those of Godebold are No. 48 ; those of Nicolas the Crossbowman, No. 49 ; those of Fulcher, No. 50 ; and those of Haimeric, No. 51 ; William the gatekeeper is among the king's servants, No. 52 .

${ }^{5}$ Hele Golding alias Maundevil in Petrockstow in Shebbear hundred. Geldroll, fol. $65 b$, A 8 . geld for $I \frac{1}{2}$ virgates. This 5 ploughs can till. There G[odebold] has in demesne $\frac{1}{2}$ virgate and I plough; and the villeins I virgate and 2 ploughs. Upon it G[odebold] has 4 villeins, 4 bordars, 6 beasts, $I$ acre of meadow, and 2 acres of pasture. Worth Io shillings a year; when he received it the same.

Nicholas the crossbowman ${ }^{6}$ (arcbibalistarius) has a manor called Wibeberia (Exch. Wiberie) $[\text { Webworthy }]^{7}$ which Odrit (Exch. Ordric) held T.R.E. and it paid geld for $\frac{1}{2}$ hide. This 3 ploughs can till. Now Reger (sic) Aculeus ${ }^{8}$ holds it of Nicholas. Thereof (Nicholas corrected into) $\mathrm{R}$ [oger] has $\mathrm{I} \frac{1}{2}$ virgates in demesne and I plough. There N[icholas] has 4 bordars, 30 sheep, 4 acres of wood(land), and 20 acres of pasture. Worth I 5 shillings a year; when $\mathrm{N}$ [icholas] received it it was worth 12 pence.

Godebold the crossbowman (arbalestarius) has a manor called HocA. [Hook] ${ }^{9}$ which Ulf held T.R.E. and it paid geld for I virgate. This 2 ploughs can till. There G[odebold] has 6 acres of meadow and 80 acres of pasture. Worth 4 shillings; when $\mathrm{G}$ [odebold] received it it was worth Io shillings.

Godebold has a manor called BRIsFordA [Brushford] ${ }^{10}$ which Alvric held T.R.E. and it paid geld for I virgate. This 3 ploughs can till. There G[odebold] has 2 bordars, I serf, 27 sheep, 6 acres of wood(land), and 6 acres of meadow. It pays 20 shillings; when G[odebold] received it it was worth Io shillings.

The same Godebold has a manor called Newentona [Newton] ${ }^{11}$ which Alvric held T.R.E. and it paid geld for I virgate. This 3 ploughs can till. There G[odebold] has 2 vilfol. $468 \mathrm{~b}$.

leins who have I plough. There also G[odebold] has $I$ acre of meadow, and $I 0$ acres of pasture. It pays 7 shillings and 6 pence a year; when G[odebold] received it it was worth 5 shillings.

Godebold has a manor called LEuGa [Leigh $]^{12}$ which paid geld for 3 virgates. Alsti (Exch.

6 Dr. Round identifies him as Nicholas de la Pole, V. C. H. Warwick, i, 280.

${ }^{7}$ Webworthy in Alverdiscot in Fremington hundred. Geldroll, fol. $65 b$, A 6 .

8 'Aculeus' means 'a dart' or similar missile; possibly a crossbow bolt. Lebaudé d'Anisy identifies Roger 'Aculeus' as Roger d'Aguilon.

${ }^{9}$ Hook in Ashreigny in North Tawton hundred.

${ }^{10}$ Brushford in North Tawton hundred, but see Trans. Devon. Assoc. xxix, $253, n .35$.

11 Newton with Voley alias 'Foldehegh' (Testa de Nevill (Rec. Com.), 181) in Zeal Monachorum in North Tawton hundred.

${ }^{12}$ Doddescombsieigh in Exminster hundred. There is a deficiency of I virgate and I ferling. Geldroll, fol. $68 b, \mathrm{~A} 8$. 


\section{HOLDERS OF LANDS}

Alsi) held it T.R.E. This 8 ploughs can till. Thereof Godebold has in demesne $\frac{1}{2}$ virgate and 2 ploughs; and the villeins I virgate and I ferding and 4 ploughs. There G[odebold] has ro villeins, 5 bordars, 2 cottagers, 2 serfs, 15 beasts, 6 swine, 120 sheep, 50 goats, I virgate of wood(land), Io acres of meadow and 30 acres of pasture. Worth 3 pounds a year; when he received it it was worth 40 shillings.

Godebold has a manor called LEUALIGA (Exch. Levelege) [Lowly] ${ }^{1}$ which Alsi held T.R.E. and it paid geld for I virgate. This 4 ploughs can till. Thereof G[odebold] has in demesne I ferding and I plough, and the villeins $\frac{1}{2}$ virgate and $\mathrm{I} \frac{1}{2}$ ploughs. Upon it G[odebold] has 2 villeins, 4 bordars, 2 serfs, I60 sheep, 20 goats, I ferding of coppice, 2 acres of meadow, and 20 acres of pasture. Worth 20 shillings a year; when he received it to shillings.

Fulcer (Exch. Fulcher) has a manor called Esselingaforda [Shillingford] ${ }^{2}$ which Brismar held T.R.E. and it paid geld for I hide. This 3 ploughs can till. Thereof Fulcer has in demesne $\frac{1}{2}$ hide and 2 ploughs, and the villeins $\frac{1}{2}$ hide and $3 \frac{1}{2}$ ploughs. There Fulcer has 3 villeins, 6 bordars, 3 serfs, I beast, and 3 acres of meadow. Worth 30 shillings a year; when he received it it was worth 20 shillings.

fol. 469 .

Godebold has a manor called Molacota [Mullacot] $^{3}$ which Alger (Exch. Alcher) held T.R.E. and it paid geld for $\frac{1}{2}$ hide. This 4 ploughs can till. Thereof G[odebold] has $I$ virgate and I plough in demesne, and the villeins I virgate and I plough. There [Godebold] has 5 villeins, 2 bordars, 2 serfs, 4 beasts, 60 sheep, I acre of meadow, and ro acres of pasture. Worth 20 shillings; when G[odebold] received it it was worth ro shillings.

One ferling ${ }^{4}$ of the above-named $\frac{1}{2}$ hide a certain thane held T.R.E. and he could go to what lord he liked. There G[odebold] has I bordar. The rest of the land lies uncultivated for pasture (jacet vastata ad pasturam).

Godebold has a manor called SATERLEIa [Saterleigh] $]^{5}$ which Olnod (Exch. Ulnod) held T.R.E. and it paid geld for I virgate. This 5 ploughs can till. Thereof G[odebold] has in demesne $\frac{1}{2}$ virgate and 2 ploughs, and the villeins $\frac{1}{2}$ virgate and I plough. There G[odebold] has 6 villeins, 2 bordars, 2 serfs, 3 beasts, 57

\footnotetext{
${ }^{1}$ Lowly in Doddescombsleigh in Exminster hundrcd; I ferling is not accounted for.

'Shillingford St. George in Exminster hundred, the parish. Geldroll, fol. 68b, A 5 .

${ }^{3}$ Mullacot Corbyn in Ilfracombe in Braunton hundred. See fol. $498 b$. Geldroll, fol. 66, A I 2.

- Perhaps Foley in Ilfracombe in Braunton hundred.

3 Saterleigh in South Molton hundred. Geldroll,

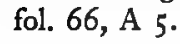

sheep, to acres of wood(land), 4 acres of meadow, and IO acres of pasture. Worth 25 shillings a year; when he received it 20 shillings.

Fulcher has a manor called Colum [Columb] ${ }^{6}$ which Brismar held T.R.E. and it paid geld for 3 virgates. These 3 ploughs can till. Thereof Fulcher has in demesne I virgate, I ferding, and I plough, and the villeins 2 virgates all but I ferding. There F[ulcher] has 4 villeins, 4 bordars, 3 serfs, ro beasts, 40 sheep, I mill paying 25 shillings a year, 6 acres of coppice, 7 acres of meadow, and 36 acres of pasture. Worth 45 shillings a year; when he received it it was worth 3 pounds.

fol. $469 \mathrm{~b}$.

Fulcher has a manor called Ivereia [Eveleigh] ${ }^{7}$ which Brismar held T.R.E. and it paid geld for I virgate. This I plough can till. There F[ulcher] has 2 villeins who have I plough and 3 bordars, 100 acres of wood(land), 2 acres of meadow, and 40 acres of pasture. Worth 15 shillings a year; when he received it it was worth the same.

Godebold has a manor called BORNA [Burn $]^{8}$ which Chippin (Exch. Chiping) held T.R.E. and it paid geld for I virgate. This $\frac{1}{2}$ plough can till. There G[odebold] has $\frac{1}{2}$ plough and 4 serfs. Worth 6 shillings a year.

Godebold has a manor called HEIERDA [Yard] ${ }^{8}$ which Chippin (Exch. Chiping) held T.R.E. and it paid geld for I virgate. This $\frac{1}{2}$ plough can till. Richard holds it of G[odebold]. There $R$ [ichard] has 2 villeins who have $\frac{1}{2}$ plough. Worth 5 shillings a year.

Nicholas has a manor called Grenneliza [Gransleigh] ${ }^{10}$ which 4 thanes held in parage (pariter; Exch. in paragio) T.R.E. and it paid geld for 2 virgates. These 3 ploughs can till. Thereof Nicholas has in demesne I virgate and I plough, and the villeins 2 virgates and 2 ploughs. There N[icholas] has 4 villeins, 3 bordars, I serf, and 2 acres of meadow. Worth 20 shillings a year ; when he received it it was worth Io shillings. Nicholas holds this manor by exchange.

Haimeric de Arcis has a manor called PultiMORA [Poltimore] ${ }^{11}$ which Bertrit (Exch. Bric-

\footnotetext{
${ }^{6}$ Columb John in Broad Clyst in Cliston hundred. Geldrroll, fol. 67, A 5 .

7 Eveleigh in Faringdon in Cliston hundred.

${ }^{8}$ Burn in Silverton in Hairidge hundred.

${ }^{9}$ Yard Down in Silverton in Hairidge hundred.

${ }^{10}$ Gransleigh alias Greenslinch in Silverton in $\mathrm{Hai}$ ridge hundred. Geldroll, fol. 67b, A 10.

${ }^{11}$ Poltimore in Wonford hundred. The I virgate interlined makes a shortage of $3 \frac{1}{2}$ ferlings, of which three possibly represent Rockham, Hill, and Combe. See fol. $499 b$. Geldroll, fol. 706, A 8.
} 


\section{A HISTORY OF DEVONSHIRE}

tric) and Scirewald (Exch. Scirewold) held in parage (pariter; Exch. in paragio) T.R.E. and it paid geld for 3 hides and $x$ virgate and 3 ferlings. This 9 ploughs can till. Thereof Haimeric has I hide, 2 virgates, and $2 \frac{1}{2}$ fertings, fol. 470 .

and the villeins have $I$ hide, 2 virgates, and $I$ ferting, and 4 ploughs. There Haimeric has 22 villeins, 3 bordars, 4 serfs, I rouncey, 20 beasts, 8 swine, 30 sheep, roo acres of wood(land), 47 acres of meadow, and 53 acres of pasture. Worth 50 shillings a year; when he received it 20 shillings.

Godebold has a manor called Cliforda [Clifford] ${ }^{1}$ which Adred (Exch. Edred) held T.R.E. and it paid geld for $\frac{1}{2}$ hide. This 6 ploughs can till. Thereof G[odebold] has in demesne I virgate and 2 ploughs, and the villeins I virgate and 2 ploughs. There G[odebold] bas 5 villeins, 3 bordars, 3 serfs, 6o sheep, 20 acres of wood(land), 3 acres of meadow, and 20 acres of pasture. Worth 20 shillings a year; when he received it it was worth the same.

Godebold has a manor called Halestou (Exch. Alestou) [Halstow ${ }^{2}$ which Alred (Exch. Aldred) held T.R.E. and it paid geld for $\frac{1}{2}$ hide. This 6 ploughs can till. Thereof G[odebold] has in demesne I virgate and I plough, (and) the villeins I virgate and I plough. There he has 5 villeins, 5 bordars, 2 serfs, 7 beasts, 63 sheep, 3 acres of wood(land), 4 of meadow, and 20 of pasture. Worth 15 shillings a year; when he received it it was worth the same.

Fulcer has I virgate of land in Colum [on the $\mathrm{Culm}]{ }^{3}$ and I villein who pays to shillings 2 year.

fol. $470 \mathrm{~b}$.

Nicholas has a manor called Stoches [Stoke] ${ }^{4}$ which Ordrit (Exch. Ordric) held T.R.E. and it paid geld for $\frac{1}{2}$ hide and $\frac{1}{2}$ virgate. This 5 ploughs can till. Thereof Nicholas has in demesne I virgate and 2 ploughs, and the villeins have 2 ploughs. There Nicholas has 6 villeins, 7 bordars, 5 serfs, 2 beasts, 60 sheep, 3 acres of meadow, and 20 acres of pasture. Worth 30 shillings a year; when Nicholas received it it was worth the same.

Nicholas the crossbowman (arcbibalistarius) has a manor called Racum (Exch. Rachum) [Ro-

\footnotetext{
1 East Clifford alias Clifford Corbyn in Dunsford in Wonford hundred. Geldroll, fol. 70b, A 6.

${ }^{2}$ Halstow in Dunsford in Wonford hundred.

- Possibly Culm Vale in Stoke Canon in Wonford hundred; or Whiteheathfield in Collumpton in Hairidge hundred (O.J.R.), see Trans. Devon Assoc. xxxvi, 359 ; part of Culm John (T.W.W.).

1 Stoke-in-Teignhead in Wonford hundred. Geldroll, fol. $706, \mathrm{~A}$ I 2.
}

combe ${ }^{5}$ which Ordrit (Exch. Ordric) held T.R.E. and it paid geld for 1 hide. This 5 ploughs can till. Now Ralf holds it of Nicholas. Thereof (Nicholas corrected into) R [alf] has $\frac{1}{2}$ hide and 2 ploughs in demesne, and the villeins have I plough. There Nicholas has 2 villeins, 3 bordars, I serf, Io beasts, 80 sheep, I 4 goats, 2 acres of meadow, and 15 acres of pasture. Worth 20 shillings a year; when Nicholas received it ro shillings.

$\mathrm{N}$ [icholas] the crossbowman has a manor called Woguwer (Exch. Wogwel) [Ogwell] ${ }^{6}$ which Ordrit (Exch. Ordric) held T.R.E. and it paid geld for $\frac{1}{2}$ hide and $\frac{1}{2}$ virgate. This 5 ploughs can till. Thereof Nicholas has I virgate in demesne and 2 ploughs, and the villeins have 2 ploughs. There Nicholas has 4 villeins, 7 bordars, 3 serfs, I cow, I 24 sheep, I mill paying 30 pence a year, 6 acres of coppice, 3 acres of meadow, and 10 acres of pasture. Worth 30 shillings a year; when he received it himself it was worth 20 shillings.

fol, 47.

Nicholas the crossbowman has a manor called Holebema [Hobbin] ${ }^{7}$ which Ordrit (Exch. Ordric) held T.R.E. and it paid geld for I virgate. This 3 ploughs can till. Now Roger Aculeus holds it of Nicholas. There (Nicholas corrected into) R[oger] has I plough in demesne, and the villeins have I plough. There Nicholas (has) 2 villeins, I bordar, 2 serfs, besides ro acres of wood(land), and I acre of wood(land) (Exch. meadow). Worth 20 shillings; when Nicholas received it to shillings.

Nicholas the crossbowman has a manor called Bagathora (Exch. Bagetore) [Bagtor] ${ }^{8}$ which Ordrit (Exch. Ordric) held T.R.E. and it paid geld for 1 virgate. This 5 ploughs can till. Roger holds it of Nicholas (a later insertion). Thereof (Nicholas corrected into) R[oger] has I ferting and I plough in demesne, and the villeins have 4 ploughs. There Nicholas has 6 villeins, 2 bordars, I serf, 5 beasts, 3 swine, 35 sheep, 15 goats, 3 acres of wood(land), and pasture $I$ league in length by $\frac{1}{2}$ in breadth. Worth 20 shillings a year; when Nicholas received it it was worth 15 shillings.

Fulcher the crossbowman has a manor called Chiwarthiwis [ $]^{9}$ which 2 thanes held in

${ }^{5}$ Higher and Lower Rocombe alias Rocombe Blaumoster (Feud. Aids, i, 345, 388) in Stoke-inTeignhead in Wonford hundred.

${ }^{6}$ Ogwell Malston, or the North manor to which the mill belongs, in East Ogwell in Wonford hundred. Trans. Devon Assoc. xxxvi, 375 .

7 Holbeam alias Hobbin in East Ogwell in Wonford hundred.

${ }^{8}$ Bagtor in Ilsington, an outlier of the Teignhide section of Wonford hundred.

${ }^{9}$ Huish Tremanet in Tedburn St. Mary in Wonford hundred. See fol. 500 . 


\section{HOLDERS OF LANDS}

parage (pariter ; Exch. in paragio) T.R.E. and it paid geld for $\frac{1}{2}$ hide and $\frac{1}{2}$ virgate. This 5 ploughs can till. Now Helgot holds it of Fulcer. Thereof Helgot has I virgate and I plough in demesne, and the villeins have 4 ploughs. There Helgot has 4 villeins, 4 bordars, I serf (Exch. has ' 3 bordars' and omits the serf), I rouncey, 6 beasts, 3 acres of wood(land), 4 acres of meadow, and 20 acres of pasture. Worth 30 shillings a year; when Fulcher received it it was worth I 5 shillings.

fol. 47xb.

Godebold has a manor called WithechenolLA (Exch. Withechenolle) [Whitenhole] ${ }^{1}$ which Chipin (Exch. Chiping) held T.R.E. and it paid geld for $1 \frac{1}{2}$ virgates. These 2 ploughs can till. Jachelin holds it of G[odebold]. Thereof $\mathrm{J}$ [achelin] has in demesne I virgate and I plough, and the villeins $\frac{1}{2}$ virgate and 2 ploughs. There Jachelin has 2 villeins, 3 bordars, I serf, I 5 beasts, 4 acres of coppice, 5 acres of meadow, and 3 acres of pasture. Worth 10 shillings a year; when he received it 30 pence.

Haimeric de Arcis has a manor called RoueComma [Rockham] ${ }^{2}$ which Almar held T.R.E. and it paid geld for $\frac{1}{2}$ virgate. This I plough can till. There Haimeric has I plough and I serf, also 4 acres of wood(land), I of meadow and common pasture. Worth 5 shillings a year.

Haimeric has a manor called HILLA $[\mathrm{Hill}]^{3}$ which Etmar (Exch. Edmar) held T.R.E. and it paid geld for $\frac{1}{2}$ ferding. There $\mathrm{H}$ [aimeric] has $\frac{1}{2}$ plough and I serf, also 20 acres of meadow and 100 acres of pasture. Worth 40 pence a year.

Haimeric has a manor called Couba (Exch. Cumbe) [Combe] ${ }^{4}$ which Etmar (Exch. Edmar) held T.R.E. and it paid geld for $\frac{1}{2}$ ferding. This $\frac{1}{2}$ plough can till. There Haimeric has $\frac{1}{2}$ plough and I villein, besides I acre of meadow. Worth 30 pence a year.

Fulcer has a manor called Ferhendona [Faringdon $]^{5}$ which Alric held T.R.E. and it paid geld for I virgate. This 3 ploughs can till. Thereof F[ulcer] has in demesne I ferding and I plough, and the villeins 3 ferdings and 2 ploughs. There F[ulcer] has 3 villeins, I bordar, 3 serfs, 5 beasts, 8 acres of wood(land), 4 acres of meadow and 8 acres of pasture. Worth dred.

'Rockham in Cruwys Morchard in Witheridge hundred. dred.

${ }^{3}$ Hill in Cruwys Morchard in Witheridge hun-

' ( 'Thorn)combe in Cruwys Morchard in Witheridge hundred.

${ }_{5}^{5}$ Faringdon in Aylesbeare in East Budleigh hundred. Geldroll, fol. 68, A 13 .
20 shillings a year; when he received it, it was worth 10 shillings.

fol. 472 .

William the gate-keeper (portitor) has a manor called Bechatona [Bicton] ${ }^{6}$ which Ailsi (Exch. Alsi) held T.R.E. and it paid geld for I hide. This 4 ploughs can till. Thereof W[illiam] has $\frac{1}{2}$ hide and 2 ploughs in demesne, and the villeins $\frac{1}{2}$ hide and 3 ploughs. There W[illiam] has 4 villeins, 8 bordars, I serf, I rouncey, 9 beasts, 72 sheep, 8 acres of wood(land) and 16 acres of meadow. It pays roo shillings; when W[illiam] received it 50 shillings.

Nicholas the crossbowman has a manor called YUDAFORDA [Ideford] ${ }^{7}$ which Ordric held T.R.E. and it paid geld for $3 \frac{1}{2}$ hides. These Io ploughs can till. Thereof $\mathrm{N}$ [icholas] has $I \frac{1}{2}$ hides and 2 ploughs in demesne, and the villeins 2 hides and 4 ploughs. There $N$ [icholas] has 8 villeins, 7 bordars, 4 serfs, 5 beasts, 120 sheep, I 5 goats, 3 acres of wood(land), 8 acres of meadow and I league of pasture. Worth 40 shillings, when $\mathrm{N}$ [icholas] received it the same.

Nicholas has a manor named Estapeleia (Exch. Stapelie) [Staple Hill] ${ }^{8}$ which Bristuold (Exch. Brictuold) held T.R.E. and it paid geld for $\frac{1}{2}$ hide. This 2 ploughs can till. Thereof $\mathrm{N}$ [icholas] has I virgate in demesne, and the villeins I virgate and 3 ploughing oxen (boves in carr'). There N[icholas] has 3 villeins, 4 bordars, 6 acres of wood(land), 6 acres of meadow and I 2 acres of pasture. Worth 5 shillings; when $\mathrm{N}$ [icholas] received it the same.

fol. $472 b$.

Nicholas has a manor called Bochelanda [Buckland] ${ }^{9}$ which Ailsi (Exch. Alsi) held T.R.E. and it paid geld for 3 virgates. These 4 ploughs can till. Thereof $\mathrm{N}$ [icholas] has I virgate and $1 \frac{1}{2}$ ploughs in demesne, and the villeins 2 virgates and I plough. There N[icholas] has 8 villeins, 2 bordars, 3 serfs, 2 beasts, 60 sheep, 20 goats, wood(land) $\frac{1}{2}$ league in length by $\mathbf{I}$ furlong in breadth, 4 acres of meadow and 4 furlongs of pasture. Worth 10 shillings ; when $\mathrm{N}$ [icholas] received it the same.

Thereof a certain knight Roger holds I virgate of Nicholas. ${ }^{10}$ Worth 7 shillings a year ; when $\mathrm{N}$ [icholas] received it the same.

${ }^{6}$ Bicton in East Budleigh hundred, the parish. Geldroll, fol. 68, A I 2 . Bicton was held by the service of keeping the gate of the castle of Exeter (Testa de Nevill (Rec. Com.), 194a).

${ }^{7}$ Ideford in Teignbridge hundred. Geldroll, fol. 69, A 5 .

${ }^{8}$ Staplehill, stated to be in Ashton (Lysons, ii, 17), probably in Ilsington, an outlier of the Teignhide section of Wonford hundred.

${ }^{9}$ Buckland-in-the-Moor in Haytor hundred.

${ }^{10}$ Cherlecombe alias Challermoor in Buckland-inthe-Moor in Haytor hundred. 


\section{A HISTORY OF DEVONSHIRE}

Godebold has a manor called Lewendona [Livaton] ${ }^{1}$ which 2 thanes held in parage (pariter; Exch. in paragio) T.R.E. and it paid geld for $\frac{1}{2}$ hide. This 4 ploughs can till. Rainer holds it of Gudebald. Thereof R[ainer] has $\frac{1}{2}$ virgate in demesne, and the villeins $\mathrm{I} \frac{1}{2}$ virgates and I plough. There R[ainer] has 4 villeins, 2 bordars, I serf, 4 acres of meadow and 20 acres of pasture. Worth ro shillings; when G[odebold] received it the same.

Haimeric de Archa has a manor called BRADELEIA [Bradleigh] ${ }^{2}$ which Edmar ( $E x c h$. Edmer) held T.R.E. and it paid geld for I virgate. This I plough can till. Thereof $\mathrm{H}$ [aimeric] has 3 ferlings and 2 ploughs in demesne and the villeins I ferling. There $\mathrm{H}$ [aimeric] has 3 bordars, 2 serfs, 3 beasts, 2 swine, 20 sheep, 12 acres of wood(land) and 3 acres of pasture. Worth 18 shillings; when $\mathrm{H}$ [aimeric] received it 5 shillings. fol. 473 .

Fulcer has a manor called LEGA [Leigh $]^{3}$ which Goduin held T.R.E. and it paid geld for 3 virgates. These 5 ploughs can till. Roger holds the same of Fulcer. Thereof Roger has in demesne I virgate, I ferding and 2 ploughs, and the villeins 2 virgates all but $\mathrm{I}$ ferding, and I plough. There Roger has 4 villeins, 2 bordars, 4 serfs, 8 acres of meadow and 30 acres of pasture. Worth 30 shillings a year; when he received it it was worth 40 shillings.

Nicholas the crossbowman (arcbibalistarius) has a manor called Alra [Aller] ${ }^{4}$ which Edmer held T.R.E. and it paid geld for $\frac{1}{2}$ virgate. This 2 ploughs can till. John holds it of Nicholas. Thereof J[ohn] has I ferling and I plough in demesne and the villeins $I$ ferling and 2 oxen towards a plough. There J[ohn] has 2 villeins, 5 bordars, Io beasts, 24 goats, 3 acres of wood(land), IO acres of meadow and 30 acres of pasture. Worth ro shillings; when $\mathrm{N}$ [icholas] received it 5 shillings.

Nicholas has a manor called LEGA [Leigh] ${ }^{5}$ which Summerlet held T.R.E. and it paid geld for I virgate. This $\frac{1}{2}$ plough can till. There Nicholas (has) I bordar, 3 acres of wood(land), $\mathrm{r} \frac{1}{2}$ acres of meadow, and 20 acres of pasture. Worth 3 shillings a year. This manor is (one) of the exchanges of Nicholas.

${ }^{1}$ Livaton alias Lounston Peveral in Ilsington in Teignbridge hundred. See fol. 502. Trans. Devon Assoc. xxix, 238.

${ }^{2}$ East Bradleigh in Tiverton parish and hundred. Geldroll, fol. 67, A 6.

${ }^{3}$ Leigh in Loxbear in Tiverton hundred. Trans. Devon Assoc. xxxv, 3 I 3.

- Aller alias Over Aller in Abbot's Kerswell in Haytor hundred. Feud. Aids, i, 345 .

${ }^{5}$ Bramleigh in Farway in Colyton hundred (O.J.R.). Whitleigh (T.W.W.). Geldroll, fol. 68b, A 5 .
Godebold has 2 houses in EssECESTRA [Exeter] ${ }^{6}$ which paid by custom T.R.E. I 6 pence a year.

fol. 475 .

\section{THE LAND OF THE KING'S SER- VANTS (SERVIENTIUM) IN DEVENESIRA ?}

William the (king's) usher (bostiarius) has a manor called Tauelanda [Tawland] $^{8}$ which Godric held T.R.E. and it paid geld for I virgate. This 3 ploughs can till. Now Robert holds it of William. Thereof $R$ [obert] has I ferling and I plough in demesne, and the villeins 3 ferlings and $I \frac{1}{2}$ ploughs. There $R$ [obert] has 3 villeins and 2 serfs, 12 beasts, 6 swine, 30 sheep, 10 acres of wood(land), 8 acres of meadow and 50 acres of pasture. Worth 20 shillings; when $\mathrm{W}$ [illiam] received it it was worth Io shillings This manor paid to the king's demesne-manor called Tauetona [Tawton] by way of customary due either $I$ ox or 30 pence a year.

William has a manor called CRUc [Crook] ${ }^{9}$ which Ailward (Exch. Alward) held T.R.E. and it paid geld for 3 virgates. This 6 ploughs can till. There $W$ [illiam] has in demesne I virgate and 2 ploughs, and the villeins 2 virgates and 4 ploughs. There $W[$ illiam] has 10 villeins, 3 bordars, 6 serfs, I rouncey, 9 beasts, 5 swine, 50 sheep, $23 \frac{1}{2}$ acres of meadow and 200 acres of pasture. Worth 30 shillings a year; when he received it 1o shillings.

These 2 manors are amongst William's lands by exchange.

William has I virgate of land ${ }^{10}$ which 2 ploughs can till. Ralf holds it of W[illiam]. There Ralf has I serf and I bondwoman (ancilla). It pays 5 shillings. This also is amongst W[illiam's] exchanges.

W[illiam] has a manor called CAdeLia [Cadeleigh] $^{{ }_{11}}$ which 2 thanes held in parage

${ }^{6}$ See fol. $506 b$.

${ }^{7}$ The king's servants are grouped together as No. 52 in the Exchequer book. First among them is enumerated William the gate-keeper, whom the Exeter book places among military tenants, and last among them are Morin, whom the Exeter book places among the French knights, and the priests of Bodmin, whom the Exeter book places among English thanes. In point of order 'Lege' and 'Torre' are placed at the end of William the usher's estates. Gatcombe is followed by Limor, Holecome, and Newetona.

${ }^{8}$ Probably Cocktree with Taw Green in South Tawton parish and hundred. See fol. 496.

${ }^{9}$ Crook Burnel in North Tawton parish and hundred.

${ }^{10}$ Uppacot in North Tawton parish and hundred (O.J.R.), Trans. Devon Assoc. xxxvi, 357 ; or Netacot, Uplowman (T.W.W.).

il Cadeleigh in Hairidge hundred. Geldroll, fol. $676, A$ I 2 . 


\section{HOLDERS OF LANDS}

(pariter; Exch. libere corrected into in paragio) T.R.E. and it paid geld for I hide. This 7 ploughs can till. Thereof W[illiam] has in demesne I virgate and 3 ploughs, and the villeins 3 virgates and 6 ploughs. There $W[$ illiam] has fol. 475 b.

I4 villeins, 5 bordars, 3 serfs, 4 swine, 80 sheep, 44 acres of wood(land), I I acres of meadow, 20 acres of pasture, and I mill paying 4 shillings a year. This manor is worth 50 shillings a year ; when he received it 15 shillings. This manor is amongst William's exchanges.

W[illiam] the (king's) usher (hostiarius) has a manor called REDDONA [Raddon] ${ }^{1}$ which Etward (Exch. Edward) the priest held T.R.E. and it paid geld for $\frac{1}{2}$ hide and $\frac{1}{2}$ virgate. These 3 ploughs can till. Thereof W[illiam] has in demesne I virgate and I plough, and the villeins $\mathrm{I} \frac{1}{2}$ virgates and 2 ploughs. There W[illiam] has 3 villeins, 2 bordars, I rouncey, I I beasts, 65 sheep, 6 acres of meadow and II acres of pasture. Worth 25 shillings a year; when he received it it was worth 10 shillings. The manor is one of William's exchanges.

W[illiam] the usher has a manor called BlaCABERGA [Blackborough] ${ }^{2}$ which Lewir Socca held T.R.E. and it paid geld for $I$ hide and I virgate. These 3 ploughs can till. Ralf Botin holds it of William. Thereof R[alf] has in demesne $\frac{1}{2}$ hide and $\frac{1}{2}$ virgate, and the villeins $\frac{1}{2}$ hide and $\frac{1}{2}$ virgate. There $\mathrm{R}$ [alf] has 9 villeins, 2 serfs, 2 beasts, I 2 swine, 40 sheep, 30 goats, 2 acres of coppice, 4 acres of meadow and 100 acres of pasture. Worth 20 shillings a year; when he received it 10 shillings. This manor also is one of William's exchanges.

W[illiam] the usher has a manor called Lerga (Exch. Lege) [Leigh] ${ }^{3}$ which Aldred held T.R.E. and it paid geld for I virgate. This 9 ploughs can till. Thereof William has I ferling and 2 ploughs in demesne and the villeins 3 ferlings and 5 ploughs. There W[illiam] has I 2 villeins, 5 bordars, 4 serfs, 7 fol. 476 .

beasts, 4 swine, 86 sheep, I00 acres of wood(land), 24 acres of meadow and 30 acres of pasture. Worth 40 shillings a year; when he received it 20 shillings.

W[illiam] the usher (bostiarius) has a manor called Bolmham $[\text { Bolham }]^{4}$ which Bristric

\footnotetext{
${ }^{1}$ East Raddon in Thorverton in Hairidge hundred.

' Blackborough Boty in Kentisbeare in Hairidge hundred.

${ }^{3}$ Mariansleigh in Witheridge hundred (Geldroll, fol. 666, A 6), the parish. Trans. Devon Assoc. xxx, 408.

${ }^{4}$ Bolham in Tiverton parish and hundred. Geldroll, fol. $67, \mathrm{~A}_{5}$.
}

(Brictric) held T.R.E. and it paid geld for 3 virgates. These 5 ploughs can till. Thereof $W$ [illiam] has in demesne $I$ virgate and I plough, and the villeins 2 virgates and 4 ploughs. There W[illiam] has I I villeins, 6 bordars, 3 serfs, I rouncey, I beast, 7 swine, I mill paying 7 shillings, 4 acres of wood (land), 6 acres of meadow and 20 of pasture. Worth 40 shillings a year; when he received it it was worth 50 shillings. This manor also is one of William's exchanges.

William the usher has a manor called TorRA [Tor] ${ }^{6}$ which Alfric (Exch. Alfric) held T.R.E. and it paid geld for 2 hides. These 7 ploughs can till. Thereof $W$ [illiam] has $\frac{1}{2}$ hide and 2 ploughs in demesne, and the villeins $1 \frac{1}{2}$ hides and 4 ploughs. There he has 16 villeins, 12 bordars, 4 serfs, 2 rounceys, 3 beasts, 7 swine, 145 sheep, 12 acres of wood(land), 24 of meadow and 200 acres of pasture. Worth 60 shillings; when $W[i l l i a m]$ received it the same.

W[illiam] the usher has a manor called Ilesam (Excb. Ilesham) [Ilesham $]^{6}$ which Bere held T.R.E. and it paid geld for $I$ hide. This 3 ploughs can till. Roger holds it of Will[iam]. Thereof R[oger] has $\frac{1}{2}$ hide and $I$ plough in demesne, and the villeins $\frac{1}{2}$ hide and $\frac{1}{2}$ plough. fol, $476 \mathrm{~b}$.

There R[oger] has 2 villeins, 2 bordars, 2 serfs, 2 beasts, 2 swine, 40 sheep, 10 goats and 20 acres of pasture. Worth Io shillings; when W[illiam] received it the same. This also is one of William's exchanges.

William the usher has a manor called Sutuna (Exch. Sutona) [Sutton] $]^{7}$ which Ulwin held T.R.E. and it paid geld for I hide. This 7 ploughs can till. Now Richard holds it of W[illiam]. Thereof he has I virgate and I plough, and the villeins have 3 virgates and 4 ploughs. There $R$ [ichard] has 7 villeins, 4 bordars, 2 serfs, 3 beasts and 64 sheep. Worth 20 shillings a year; when he received it Io shillings. This manor is one of William's exchanges.

Ansger the king's servant ${ }^{8}$ has a manor called Gatcumba [Gatcombe] ${ }^{2}$ which Burgered held T.R.E. and it paid geld for I hide. This 4 ploughs can till. Thereof Ansger has I virgate

${ }^{6}$ Tor Mohun alias Brewer in Haytor hundred, the south-west part of Torquay. Geldroll, fol. $69 b$, A 6

${ }^{6}$ Ilesham alias Eylesham in St. Mary Church in Haytor hundred, the east part of Torquay.

7 Sutton Lucy in Widworthy in Colyton hundred.

${ }^{8}$ Either the kitchener (coquus), fol. $477 b$, or the fower or hearth-keeper (focarius), fol. $478 b$.

${ }^{9}$ Gatcombe in Colyton parish and hundred. Geldroll, xxxiv, A 6. 


\section{A HISTORY OF DEVONSHIRE}

and I plough in demesne and the villeins have 3 virgates and 3 ploughs. There Ansger has 6 villeins, 3 bordars, 3 serfs, 8 beasts, roo sheep, 3 acres of coppice, 2 acres of meadow and 27 acres of pasture. Worth 20 shillings a year; when Ansger received it it was worth Io shillings.

fol. $4^{8 x}$.

\section{LANDS OF ENGLISH THANES IN DEVENESIRA ${ }^{1}$}

Aldret (Exch. Eldred) has a manor called WICA (Exch. Wiche) [Week ${ }^{2}$ which paid geld in King $\mathrm{E}\left[\mathrm{d}\right.$ ward]'s time for $\frac{1}{2}(E x c h$. I) virgate. This 3 ploughs can till. Thereof he has I ferding in demesne and I plough, and the villeins I ferding and I plough. There A[ldret] has 3 villeins, 3 serfs, 8 beasts, 30 wethers (berbices), Io goats, 5 acres of wood(land), Io acres (acbros) of meadow and 15 acres of pasture. Worth 20 shillings a year.

Alward has a manor called Colsovenescota [Kismilton ? $]^{3}$ which A[lward] himself held T. R. E. and it paid geld for $\frac{1}{2}$ virgate. This 2 ploughs can till. He has therefore in demesne I ferding and I plough, and the villeins I ferding and I plough. There A[lward] has I villein, 3 bordars, 1 serf, 8 beasts, 4 swine, 25 sheep and 30 acres of pasture. Worth 10 shillings a year.

Colvin has a manor called Chelesorda (Exch. Chelesworde) [Chilsworthy $]^{4}$ which Oseva (Exch. Odeva] held T. R. E. and it paid geld for $\frac{1}{2}$ vi[r]gate. This 3 ploughs can till. Thereof he has in demesne one third of $\frac{1}{2}$ virgate and I

1 In the Exchequer book the estates of each one of the king's English thanes are placed together as No. 53 with the heading The King's Thanes, and are grouped together in the following order :-Colvin; Godvin ; Godric; Odo son of Edric; Eldred; Alward; Ansgot; Donne; Alnod; Alwin; Edvin; Ulf; Algar ; Alric; Aluric; Leuric; Saulf; Alveva; Alfhilla; Godeva, the last three being ladies. In the Exeter book they follow the order of the hundreds.

${ }^{2}$ Northweek in Thornbury in Blacktorington hundred (O. J. R.) ; or Week, Peter's Marland in Shebbear hundred (T.W.W.).

${ }^{3}$ Kismilton in West Putford in Blacktorington hundred. The $W$ has become an $M$ as in Lympstone for Lewenston, and the suffix ton has been substituted for cot, as in Elmiston.

${ }^{4}$ Chilsworthy in Holsworthy in Blacktorington hundred. Colvin, who was Queen Eddida's steward (fol. $87 b$ ), and is called the reeve or manor-steward (prepositus) in Geldroll, fol. 65, A 5, probably held this and the following estates as the endowment of office in divers manors. The office for which Chilsworthy was subsequently held was that of drysalter or baconcurer (salsatius) to the king. Cal. Inq. Hen. III, No. 8 I 5 . plough, and the villeins have two thirds of that land and 2 ploughs. Upon it Colvin has 6 bordars, and I serf, I 5 beasts, 40 sheep, 20 acres of meadow and 20 acres of pasture. Worth 20 fol. 48xb.

shillings a year.

Colvin has a manor Bristeleshorda (Exch. Bristelsworde) [Brexworthy $]^{5}$ which he held himself T.R.E. and it paid geld for $I \frac{1}{2}$ ferlings. This I plough can till. There $\mathrm{C}$ [olvin] has I villein and 3 serfs, also 15 acres of meadow and 30 acres of pasture. Worth 5 shillings a year.

Colvin has a manor called Coltrshorda (Exch. Coltesworde) [Coltesworth] ${ }^{6}$ which he held himself T.R. E. and it paid geld for $J \frac{7}{3}$ ferlings. This 2 ploughs can till. There C[olvin] has 1 villein and 3 bordars, 5 acres of meadow and 20 of pasture. Worth 7 shillings and 6 pence a year.

The priests (sacerdotes; Exch. presbiteri) of Bomine [Bodmin] have a manor called Holecoma

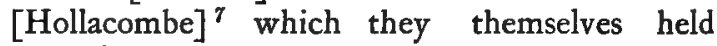
T.R.E. and it paid geld for I virgate. This 3 ploughs can till. There the aforesaid priests have in demesne 1 plough and the villeins 2 ploughs. Upon it the priests have 6 villeins, 2 serfs, 12 beasts, 60 sheep, 30 acres of meadow, and pasture 4 furlongs in length by 2 in breadth. Worth 20 shillings a year.

Colvin has a manor called OlFEREORd (Exch. Olvereword) [Woolfardisworthy $]^{8}$ which Godric held T.R.E. and it paid geld for $\frac{1}{2}$ hide. This 4 ploughs can till. There C[olvin] has in demesne $I$ ferding and $I$ plough, and the villeins 3 ferdings and 3 ploughs. There C[olvin] has 2 villeins, 4 bordars, 2 serfs, fol. 482 .

20 beasts, 60 sheep, 5 acres of meadow and IO acres of pasture. Worth 40 shillings a year; when C[olvin] received it it was worth 20 shillings.

Ansgod (Exch. Ansgot) has a manor called Madona [Meddon] ${ }^{9}$ which Ansgod himself held T.R.E. and it paid geld for $\frac{1}{2}$ hide of land.

${ }^{5}$ Possibly Brexworthy in Bradworthy in Blacktorington hundred (T. W.W.).

${ }^{6}$ Coltsworthy in Clawton in Blacktorington hundred (O. J. R.).

${ }^{7}$ Hollacombe in Blacktorington hundred. Geldroll, fol. $65, \mathrm{~B}$.

${ }^{8}$ East Woolfardisworthy in Hartland hundred. The missing virgate probably represents Dunsbear, in the same ownership before Domesday. Geldroll, fol. 65, A 5 .

${ }^{9}$ Meddon in Hartland parish and hundred. Geldroll, fol. $65, \mathrm{~A} 6$. 


\section{HOLDERS OF LANDS}

This $2 \frac{1}{2}$ ploughs can till. Thereof $A[$ nsgod] has in demesne $\frac{1}{2}$ virgate of land and I plough, and the villeins $I \frac{1}{2}$ virgates and $I \frac{1}{2}$ ploughs. There $A$ [nsgod] has 3 villeins, 3 bordars, I serf, 7 beasts, 25 sheep, wood(land) 2 furlongs in length by $I$ in breadth, 60 acres of meadow and 8 acres of pasture. Worth 20 shillings a year.

Colvin has a manor called Denesberga [Dunsbeare] ${ }^{1}$ which Godric held T.R.E. and it paid geld for I virgate. This 4 ploughs can till. Colvin holds it of the king. There $\mathrm{Col}[\mathrm{vin}]$ has in demesne I ferding and I plough, and the villeins 3 ferdings and 3 ploughs. Upon it Col[vin] has 4 bordars and 2 serfs, also Io beasts, 50 sheep, 5 acres of meadow and 10 of pasture. Worth 20 shillings a year; when he received it the like (similiter).

Colvin has a manor called Arrslant (Exch. Alesland) [Hasland $]^{2}$ which Godric held T.R.E. and it paid geld for $1 \frac{1}{2}$ virgates. This 6 ploughs can till. Thereof Col[vin] has in demesne $\frac{1}{2}$ virgate and I plough, and the villeins have I virgate and 3 ploughs. There $\mathrm{Col}[\mathrm{vin}]$ has 4 villeins, 3 bordars, 2 serfs, 12 beasts, fol. $482 \mathrm{~b}$.

30 sheep, 5 acres of meadow and 12 of pasture. Worth 30 shillings a year; when he received it the like.

Colvin has a manor called Hama [ which Godric held T.R.E. and it paid geld for $\frac{1}{2}$ virgate. This I plough can till. There $\mathrm{Col}[\mathrm{vin}]$ has I villein. Worth 5 shillings.

Colvin has a manor called Hantona [Heanton] ${ }^{4}$ which Bristeva (Exch. Bricteva) held T.R.E. and it paid geld for I virgate. This 2 ploughs can till. There Col[vin] has I villein and 2 bordars, also 5 acres of wood(land), 5 of meadow, and Io of pasture. Worth 5 shillings.

Ansgot has a manor called FerLeia [Farley] ${ }^{5}$ which Dona (Exch. Done) held T.R.E. and it paid geld for $\frac{1}{2}$ virgate. This I plough can till. There $A$ [nsgot] has I bordar, 2 serfs, Io beasts, I5 sheep, 30 goats, 12 acres of wood(land), I2 of meadow and 15 of pasture. Worth 5 shillings a year.

${ }^{1}$ Dunsbear in Merton in Shebbear hundred. Geldroll, fol. $65 b$, A 5 .

${ }^{2}$ Alesland alias Hasland in Buckland Filleigh (O.J.R.); or in Petrockstow (T.W.W.) in Shebbear hundred.

${ }^{3}$ Suggested identifications are Embury in Buckland Filleigh in Shebbear hundred (O.J.R.), Chilsdon in Petrockstow (T.W.W.).

${ }^{4}$ West Heanton in Buckland Filleigh in Shebbear hundred. Geldroll, fol. 65b, A 9 .

${ }^{5}$ Farley in Petrockstow in Shebbear hundred. I
Ansgot has a manor called SEDEBorga [Sedborough ${ }^{6}$ which Brismar held T.R.E. and it paid geld for I virgate. This I plough can till. There Ansgot has I bordar, 3 serfs, 6 beasts, 30 sheep, 12 goats, 6 acres of meadow and 12 of pasture. Worth 5 shillings a year.

fol. 483 .

This Sedberga [Sedborough] went with (jacebat in) $\mathrm{B}$ [aldwin] the sheriff's manor called Percheham [Parkham] T.R.E.

The priests (sacerdotes: Excb. Ipsi presbiteri) of Bomine [Bodmin] have a manor called NIETONA [Newton] $]^{7}$ and it paid geld for $\mathbf{I}$ hide. This 7 ploughs can till. They have in demesne I virgate of land and 2 ploughs, and the villeins 3 virgates and 5 ploughs. There the priests have 8 villeins, 4 bordars, 2 serfs, 8 beasts, 40 sheep, wood(land) $\frac{1}{2}$ league in length by 3 furlongs in breadth, 8 acres of meadow and 20 acres of pasture. Worth 20 shillings a year.

Alward Merta has half a virgate of land ${ }^{8}$ and has there I plough. Two ploughs can till it and it is worth 5 shillings a year. The queen gave it to him in alms.

Godwin ( $E x c h$. Godvin) has a manor called LimeTA [Nymet or Intake] ${ }^{9}$ which Alestan held T.R.E. and it paid geld for I virgate. This 2 ploughs can till. Upon it are 2 ploughs, also 2 villeins and I bordar, 5 acres of meadow and 30 acres of pasture. Worth ro shillings a year; when Godwin received it it was worth I 5 shillings.

Domne (Exch. Donne) has a manor called Newentona [Newton] ${ }^{10}$ which he held himself T.R.E. and it paid geld for 3 hides. These 30 ploughs can till. Thereof Domne has I hide, I virgate, and I plough in demesne, and the villeins have 2 hides all but I virgate and 20 ploughs. There Domne has 21 villeins, 8 bordars, 6 serfs, I mill paying 32 pence yearly, coppice I league in length by $\frac{1}{2}$ in fol. $483 b$.

breadth, 20 acres of meadow, and 28 acres of pasture. Worth 6 pounds a year. This land Domne held of King Edward, and now he says that he holds it of King William.

${ }^{6}$ Sedborough in Parkham in Shebbear hundred.

${ }^{7}$ Newton St. Petrock in Shebbear hundred. Geldroll, fol. $65 b, \mathrm{~B}$ 2. See Cal. Ghart. Rolls, $36 \mathrm{Hen}$. III, p. 75, m. 18 .

${ }^{8}$ Arson alias Ashton in Ashreigny in North Tawton hundred.

${ }^{9}$ Perhaps Natson alias Notceston in Bow in North Tawton hundred.

${ }^{10}$ Newton St. Cyres in Crediton hundred. Geldroll, fol. 66b, B 2. See fol. I I 7 . 


\section{A HISTORY OF DEVONSHIRE}

Alnod has a manor called BraIA [Bray] which Ulwin held T.R.E. and it paid geld for $\frac{1}{2}$ hide. This 6 ploughs can till. Thereof Alnod has $\frac{1}{2}$ virgate and I plough in demesne, and the villeins have 3 ploughs. There $A[\operatorname{lnod}]$ has 4 villeins, 4 bordars, 4 serfs, 6 beasts, 30 sheep, wood(land) I 2 furlongs in length by I $\frac{1}{2}$ in breadth, 4 acres of meadow and pasture $\frac{1}{2}$ league ( $E_{x c h}$. in length) by $\mathrm{I}$ furlong in breadth. Worth 30 shillings a year.

Alwin has a manor called Mrdelcota [Middlecot] $^{2}$ which he held himself T.R.E. and it paid geld for $\frac{1}{2}$ ferding. This I plough can till. Upon it Alwin has I plough, I serf, 4 beasts, 34 sheep, 10 goats and 3 (Exch. 2) acres of coppice. Worth 5 shillings a year.

Edwin (Exch. Edvin) has a manor called Buterdera [Butterleigh] ${ }^{3}$ which E[dwin] himself held T.R.E. and it paid geld for I virgate. This 2 ploughs can till. Thereof Edwin has in demesne $\frac{1}{2}$ virgate and I plough, and the villeins $\frac{1}{2}$ virgate and 2 ploughs. There $\mathrm{E}$ [dwin] has 4 villeins, I bordar, I serf, I beast, ro swine, 40 sheep, and 8 acres of wood(land). fol. 484 .

Worth 15 shillings a year.

Godwin (Exch. Godvin) has a manor called Citremetona [Chittlehampton] ${ }^{4}$ which he held himself T.R.E. and it paid geld for I virgate. This 30 ploughs can till. Of it Godwin has 5 ploughlands in demesne and 3 ploughs, and the villeins have ro ploughs. There Godwin has 7 villeins, 8 serfs, 22 swineherds paying 64 swine a year, 4 beasts, 3 swine, 60 sheep, wood(land) I $\frac{1}{2}$ leagues in length by $\frac{1}{2}$ league in breadth, 12 acres of meadow and pasture 9 furlongs in length by 3 furlongs in breadth. Worth 7 pounds a year.

Godwin has a manor called Cerritona [Cheriton $^{5}{ }^{5}$ which Alestan held T.R.E. and it paid geld for I virgate. This 3 ploughs can till. Thereof Godwin has 2 fertings and I plough in demesne, and the villeins have $\mathbf{I}$ plough. There G[odwin] has 3 villeins, 2 serfs, 15 sheep, and I furlong of wood(land). Worth 10 shillings a year; when he received it it was worth 20 shillings.

${ }^{1}$ West Bray in Chittlehampton in South Molton hundred (O.J.R.), and South Bray (T.W.W.). Geldroll, fol. 66, B 3 .

${ }^{2}$ Middlecot in Chagford in Wonford hundred (O.J.R.), Trans.Devon Assoc. xxxvi, 376; Middle Blackpole, Chittlehampton in South Molton hundred (T.W.W.), Devon Notes and Queries, iii, 159.

${ }^{3}$ Butterleigh an outlier of Cliston hundred. Geldroll, fol. 67, A 8.

${ }^{4}$ Chittlehampton in South Molton hundred.

- Cheriton Bishop in Wonford hundred. Geldroll, 70b, A 14.
Godwin has a manor called LANTroRT [Lamford $]^{6}$ which Alestan held T.R.E. and it paid geld for I virgate. This 12 ploughs can till. Thereof G[odwin] has 2 fertings and I plough in demesne and the villeins have 5 ploughs. There G[odwin] has I5 villeins, 5 serfs, 4 beasts, 30 sheep, 15 acres of fol. $484 \mathrm{~b}$.

wood(land), 3 acres of meadow and 4 acres of pasture. Worth 20 shillings a year; when G[odwin] received it 40 shillings.

Godwin has a manor called Mrdelanda [Medland] ${ }^{7}$ which Alestan held T.R.E. and it paid geld for I hide. This 8 ploughs can till. Thereof G[odwin] has I virgate in demesne and I plough, and the villeins have 3 ploughs. There G[odwin] has I 3 villeins, I serf, 20 sheep, 3 acres of wood(land) and Io acres of pasture. Worth 20 shillings a year; when G[odwin] received it it was worth 40 shillings.

Godwin has a manor called WogrwIL [Ogwell ${ }^{8}$ which Alestan held T.R.E. and it paid geld for $\frac{1}{2}$ hide. This 3 ploughs can till. Thereof G[odwin] has I virgate in demesne and I plough, and the villeins have I plough. There G[odwin] has 3 villeins, 3 bordars, 2 serfs, 3 beasts, $3^{\circ}$ sheep and 6 acres of pasture. Worth Io shillings a year; when $G$ [odwin] received it it was worth 20 shillings.

Godwin has a manor called Cuma [Combe] ${ }^{9}$ which Alestan held T.R.E. and it paid geld for I virgate. This 2 ploughs can till. On it Godwin has I plough and 6 bordars. Worth 5 shillings a year; the same when G[odwin] received it.

fol. 485 .

Godwin has a manor called WereIA (Exch. Wergi) [Wray] ${ }^{10}$ which Adestan (Exch. Alestan) held T.R.E. and it paid geld for I hide. This 6 ploughs can till. Thereof G[odwin] has I virgate and 2 ploughs in demesne, and the villeins 3 virgates and 4 ploughs. There G[odwin] has I I villeins, 3 bordars, 4 serfs, 8 beasts, 4 swine, 30 sheep, 8 acres of meadow and 5 acres of pasture. Worth 30 shillings; when G[odwin] received it 60 shillings.

fol. $486 \mathrm{~b}$

Ulf has a manor called W ADEHAM [Wadham] ${ }^{11}$ which he held himself T.R.E. and it paid geld

${ }^{6}$ Little Lamford alias Forder in Cheriton Bishop in Wonford hundred. Testa de Nevill (Rec. Com.), I78a; Feud. Aids, i, 31 5,345 .

${ }^{7}$ Medland in Cheriton Bishop in Wonford hundred.

${ }^{8}$ Church Ogwell in West Ogwell in Wonford hundred. Trans. Devon Assoc. xxvi, 375 ; Feud. Aids, i, 315.

${ }^{9}$ Combe Borough alias Comberew in Drewsteignton in Wonford hundred. Feud. Aids, i, 315.

${ }^{10}$ Wray in Moreton Hampstead in Teignbridge hundred. Geldroll, fol. 69, A 9.

"Wadham in Knowstone in South Molton hundred. Geldroll, fol. 66, A 6. 


\section{HOLDERS OF LANDS}

for I virgate. This 3 ploughs can till. Now he holds the same of the king. Thereof Ulf has I ferting and I plough in demesne, and the villeins have 2 ploughs. There Ulf has 4 villeins, 3 serfs, I 2 beasts, 8 forest mares, wood(land) 4 furlongs in length by 3 in breadth, 6 acres of meadow and 50 acres of pasture. Worth 20 shillings a year.

Godwin ${ }^{1}$ has a manor called Colum [Culm] ${ }^{2}$ which Adestan (Exch. Alestan) held T.R.E. and it paid geld for I hide. This 3 ploughs can till. Thereof G[odwin] has in demesne I virgate and I plough, and the villeins 3 virgates and 2 ploughs. There G[odwin] has 6 villeins, 3 bordars, 3 serfs, 9 sheep and 6 acres of meadow. Worth 20 shillings a year; when (he received it) 40 shillings.

Odo has a manor called Hemberia [Hembury] ${ }^{3}$ which Edric held T.R.E. and it paid geld for I hide. This 2 ploughs can till. Thereof Odo has in demesne 3 virgates and I plough, and the villeins I virgate and I plough. There Odo has 2 villeins, 2 bordars, 3 serfs, I rouncey, 2 beasts, I6 sheep, 7 acres of meadow and 40 acres of pasture. Worth 15 shillings a year; when he received it it was worth the same.

Odo has a manor called Codaforda [Codaford] ${ }^{4}$ which Etdric (Exch. Edric) held T.R.E. and it paid geld for I virgate. This 2 ploughs can till. Thereof $\mathrm{O}[\mathrm{do}]$ has in demesne 3 ferdings and $\frac{1}{2}$ plough, and the villeins I ferding and $\frac{1}{2}$ plough. There O $[\mathrm{do}]$ has I villein, 2 bordars, fol. $48 \%$.

I serf, 7 sheep, 3 acres of meadow and 2 acres of pasture. Worth 6 shillings a year; when he received it it was worth the same.

Etdwin (Exch. Edvin) has a manor called Curst [Clyst] ${ }^{5}$ which Alwin held T.R.E. and it paid geld for $\frac{1}{2}$ hide. This $\frac{1}{2}$ plough can till. There Etduwin has I villein, 2 serfs, 2 acres of coppice, 3 acres of meadow and 2 acres of pasture. Worth 6 shillings a year.

Alvevia (Exch. Alveva) has a manor called JACOBESCHERCA (Exch. Jacobescherche) [St. James' Church] ${ }^{6}$ which she (ipsa) held herself T.R.E. and it paid geld for $\mathrm{I} \frac{1}{2}$ virgates. This

${ }^{1}$ Of Chittlehampton (Cillemetona). Geldroll, fol. $67 b, \mathrm{~A}_{13}$.

${ }^{2}$ Culm Reigny alics Combe Sachvil alias Silverton Park in Silverton in Hairidge hundred. Geldroll, fol. $67 b, \mathrm{~A}_{3} 3$.

${ }^{3}$ Broad Hembury, or possibly Payhembury, in Hairidge hundred. Geldroll, fol. 6, $b$, A I 5 .

${ }^{4}$ Codford in Payhembury in Hairidge hundred.

Trans. Devon Assoc. xxxiv, 361 .

${ }^{5}$ Clyst William in Plymtree in Hairidge hundred. Geldroll, fol. $67 b$, A I 1 .

${ }^{6}$ 'Trew St. Jacob alias St. James' Church alias Old Abbey in Heavitree in Wonford hundred. Devon Notes and Queries, i, 4I.
I plough can till. Upon it Alvevia has I plough, 2 cottagers, I serf, I 4 sheep. Worth 40 pence a year.

Algar has a manor called Chenutdestana [Knightstone] ${ }^{7}$ which Algar himself held T.R.E. and it paid geld for I virgate. This 3 ploughs can till. Thereof $A[\operatorname{lgar}]$ has in demesne $\frac{1}{2}$ virgate and I plough, and the villeins $\frac{1}{2}$ virgate and I plough. There A[lgar] has 2 villeins, 3 bordars, 2 serfs, 7 beasts, Io swine, 30 sheep, I acre of coppice, 5 acres of meadow and 30 acres of pasture. Worth 7 shillings and 6 pence a year.

Alsfilla (Exch. Alfhilla) has a manor called Chenutdestana [Knightstone] ${ }^{8}$ which she held herself (ipsa) T.R.E. and it paid geld for $\frac{1}{2}$ virgate. fol. $487 \mathrm{~b}$.

This I plough can till. There Alsfilla has $\frac{1}{2}$ plough and 2 serfs, also 5 beasts, IO sheep, I acre of coppice, 2 acres of meadow and I5 acres of pasture. Worth 30 pence a year.

Godric has a manor called Bolehorda [Bulworthy $]^{8}$ which Almar held T.R.E. and it paid geld for I virgate and I ferding. This 2 ploughs can till. Thereof G[odric] has in demesne I ferding and I plough, and the villeins I virgate and I plough. There G[odric] has 3 villeins, I bordar, 2 ( $E x$ xch. ) serfs, 4 beasts, 24 sheep, 40 goats (Exch. I acre) of coppice, 20 acres of meadow and 20 acres of pasture. Worth 10 shillings a a year; when he received it it was worth 5 shillings.

Donne has a manor called Noteswilla [Nutwell] ${ }^{10}$ which he held himself T.R.E. and it paid geld for $\mathrm{I} \frac{1}{2}$ hides. This 6 ploughs can till. Thereof $D$ [onne] has $I$ hide and I plough in demesne, and the villeins $\frac{1}{2}$ hide and 2 ploughs. There $\mathrm{D}$ [onne] has 7 villeins, 4 bordars, 2 serfs 100 sheep all but 7,4 acres of meadow and 5 acres of pasture. Worth 20 shillings.

Godwin (Exch. the same Godvin) has a manor called Holebroca [Holbrook] ${ }^{11}$ which he held himself T.R.E. and it paid geld for $\frac{1}{2}$ hide. This 3 ploughs can till. Thereof G[odwin] has I virgate and I plough in demesne, and the villeins I virgate. There G[odwin] has 2 villeins, 4 bordars, I serf, 7 sheep and 6 acres of meadow. Worth 10 shillings.

\footnotetext{
${ }^{7}$ East Knowstone in South Molton hundred (O. J. R.).

${ }^{8}$ Part of Knowstone in South Molton hundred afterwards called Harpsen (O. J. R.).

${ }^{9}$ Bulworthy in Rackenford in Witheridge hundred (O. J. R.). Trans. Devon Assoc. xxxvi, 357.

${ }^{10}$ Nutwell in Woodbury in East Budleigh hundred. Geldroll, fol. 68, A I 5 .

${ }^{11}$ Holbrook in Honiton Clist in East Budleigh hundred. Geldroll, fol. 68, A 14 .
} 
fol. 488 .

\section{A HISTORY OF DEVONSHIRE}

Alric has a manor called WIRLbesLiga ( $E_{x c} b$. Wasberlege) [Warsbrightly ${ }^{1}$ which he held himself T.R.E. and it paid geld for $\frac{1}{2}$ hide. This 4 ploughs can till. Thereof he has in demesne $\frac{1}{2}$ virgate and $\mathrm{r}$ plough, and the villeins 3 ferlings and 2 ploughs. There Alric has 4 villeins, 2 bordars, I serf, 6 beasts, . . . sheep, 6 acres (Exch. of wood), 5 of meadow and 30 acres of pasture. (Exch. worth 20 shillings); when he received it it was worth the same.

Godric has a manor called Calodelia [Calverleigh ${ }^{2}$ which Almar held T.R.E. and it paid geld for $\frac{1}{2}$ hide. This 4 ploughs can till. Thereof Godric has in demesne $\frac{1}{2}$ virgate and I plough, and the villeins $I \frac{1}{2}$ virgates and $I \frac{1}{2}$ ploughs. There G[odric] has 3 villeins, 5 bordars, 5 serfs, I pig, 40 acres of wood(land) and 7 acres of meadow. Worth 30 shillings a year; when he received it it was worth Io shillings.

Alvric has a manor called Essapla [Shapleigh] ${ }^{3}$ which he held himself T.R.E. and it paid geld for $\frac{1}{2}$ virgate. This 2 ploughs can till. Thereof $A$ [lvric] has I ferling and I plough in demesne, and the villeins $I$ ferling and I plough. There A[lvric] has 3 villeins, I bordar, I serf, 6 beasts, I 6 sheep, and ro acres of pasture. Worth 8 shillings.

fol. $488 b$.

Adret (Exch. Eldred) ${ }^{4}$ has a manor called Manitona [Manaton] ${ }^{5}$ which he held himself T.R.E. and it paid geld for I virgate. This 2 ploughs can till. Thereof $A\left[\right.$ dret] has $\frac{1}{2}$ virgate and I plough in demesne, and the villeins $\frac{1}{2}$ virgate and $I$ plough. There $A[$ dret] has 3 villeins, 2 bordars, I serf, 3 beasts, 10 sheep, 5 acres of wood(land), 5 acres of meadow, and 2 acres of pasture. Worth 20 shillings.

Leuric has a manor called Betunia [by Twyney ? $]^{6}$ which he held himself T.R.E. and it paid geld for I virgate. This I plough can till. Thereof $L$ [euric] has $\frac{1}{2}$ virgate in demesne, and the villeins $\frac{1}{2}$ virgate and 3 oxen towards a plough. There L[euric] has 2 villeins, I bordar, and 2 acres of meadow. Worth 30 pence.

${ }^{1}$ Warsbrightly in Stoodleigh in Witheridge hundred. Half a virgate and I ferting are missing. Perhaps they represent Blatchworthy held with Warsbrightly and Whitenhole. Testa de Nevill, 1 $81 a$, 190a; Trans. Devon Assoc. xxx, 404.

${ }^{2}$ Calverleigh in Tiverton hundred, a chapelry of Loxbeare. Geldroll, fol. 67, A 7 .

${ }^{3}$ Shapleigh in North Bovey in Teignbridge hundred. Trans. Devon Assoc. xxxv, 700 ; xxxvii, 432.

The forester. Geldroll, fol. 68b, A 10.

${ }^{5}$ Manaton in Teignbridge hundred (Feud. Aids, i, 339). Trans. Devon Assoc. xxix, 238.

${ }^{6}$ Twyney in Kingsteignton in Teignbridge hundred. Trans. Devon Assoc. xxix, 239, Conf. "by Neadon,' fol. 312.
Godeva ${ }^{7}$ has a manor called ToRra [Torre ${ }^{8}$ which Bristric (Exch. Brictric) held T.R.E. and it paid geld for 3 hides. These 12 ploughs can till. Thereof G[odeva] has I hide and 4 ploughs in demesne, and the villeins 2 hides and 8 (ploughs). There G[odeva] has 16 villeins, I 2 bordars, 4 serfs, I 20 sheep, I00 acres of wood(land), 6 acres of meadow and 20 acres of pasture. Worth 100 shillings.

Adret (Exch. Edred) has I ferling of land in BICHEFORDA [Bickford] ${ }^{9}$ which he held himself T.R.E. This I plough can till. Thereof $A$ [dret] has $\frac{1}{2}$ ferling and $\frac{1}{2}$ plough in demesne, and the villeins the like quantity and $\frac{1}{2}$ plough. There $A$ [dret] has 2 villeins, I serf, 6 beasts, I fol. 489 .

furlong of wood(land), I acre of meadow, and I2 acres of pasture. Worth 15 shillings.

Odo $^{10}$ has a manor called Wrcca (Exch. Wiche) [Wick] ${ }^{11}$ which Bristric (Exch. Brictric) held T.R.E. and it paid geld for I virgate (Exch. of land). This I plough can till. There Odo has I plough, also I villein, 3 bordars, 2 serfs, 4 beasts, 3 swine, 20 sheep, 5 goats, 6 acres of wood(land), 6 of meadow and 5 of pasture. Worth 8 shillings a year; when he received it it was worth 10 shillings.

Godwin has a manor called Duna [Down] $]^{12}$ which he held himself T.R.E. and it paid geld for I hide. This 6 ploughs can till. Thereof Godwin has in demesne $\frac{1}{2}$ hide and 2 ploughs, and the villeins $\frac{1}{2}$ hide and $I$ plough. There Godwin has 7 villeins, 4 bordars, 2 serfs, 30 sheep, 6 acres of coppice, 3 acres of meadow and 10 acres of pasture. Worth 20 shillings a year.

Odo has a manor called Dona [Down] ${ }^{13}$ which Mathil[d]a held T.R.E. and it paid geld for I virgate. This 2 ploughs can till. Thereof Odo has in demesne $\frac{1}{2}$ virgate and $I$ plough, and the villeins $\frac{1}{2}$ virgate and $x$ plough. There Odo has I villein, 6 bordars, 2 serfs, I 5 sheep, II goats, 3 (acres) of coppice, 2 acres of meadow and 3 acres of pasture. Worth 20 shillings a year; when he received it it was worth 20 shillings.

'Brictric's widow. Geldroll, fol. 69, A 10.

${ }^{8}$ Torbryan in Haytor hundred, the parish including the outlier of Moorleigh. Geldroll, 69b, A 12.

9 Bickford in Plympton parish and hundred.

${ }^{10}$ Son of Edric (Geldroll, fol. $67 b, A \times 5$ ), or the Englishman (ibid. fol. 68, A 8).

${ }^{11}$ Wyke Ralph alias North Wick in Axminster parish and hundred. Geldroll, 68b, A 8.

${ }^{13}$ Down Umfravil alias Charton in Axmouth parish and nundred. Geldroll, fol. 686, A 3 .

${ }^{13}$ Down Ralph alias Rousdon alias St. Pancras in Axmouth hundred. Geldroll, fol. 68b, A 4. 
Godeva has a manor called DoDErRoca (Exch. Dodebroch) [Dodbrook] ${ }^{1}$ which Bristric (Exch. Brictric) held T.R.E. and it paid geld for 2 hides. These 12 ploughs can till. Thereof G[odeva] has I hide and 4 ploughs in demesne, and the villeins $I$ hide and 12 ploughs. There G[odeva] has 16 villeins, I 8 bordars, 8 serfs, I rouncey, 16 beasts, 9 swine, I 88 sheep, 27 goats, coppice 8 furlongs in length by $I$ in breadth, and 4 acres of meadow. Worth 5 pounds a year.

Algar has a manor called Donestantona (Exch. Donestanestone) [Dunston] ${ }^{2}$ which Algar himself held T.R.E. and it paid geld for $\frac{1}{2}$ hide. This 3 ploughs can till. Thereof A[lgar] has in demesne $I$ virgate and $I$ plough, and the villeins I virgate and I plough. There A[lgar] has I villein, I bordar, 2 serfs, 30 sheep, I 2 goats, and 4 acres of wood(land). Worth I0 shillings a year.

Alvric has a manor called Siredona [Skerraton $]^{3}$ which he held himself T.R.E. and it paid geld for I virgate. This 2 ploughs can till. Thereof A[lvric] has I ferling and I plough in demesne, and the villeins 3 ferlings and I plough. There $A[$ lvric] has 3 villeins, 3 bordars, I serf, 6 beasts, 2 swine, 24 sheep, Io goats, 20 acres of wood(land), and 60 acres of pasture. Worth 7 shillings and 6 pence.

fol. 490 .

Saulf has a manor called Dunnesforda [Dunsford] ${ }^{4}$ which he held himself T.R.E. and it paid geld for I virgate. This I plough can till. Upon it Saulf has 3 bordars and 20 acres of pasture. Worth 40 pence a year.

Saulf has a manor called ManneHeva [Mamhead] ${ }^{5}$ which he held himself T.R.E. and it paid geld for I virgate. This I plough can till. Upon it is I villein, and it pays 45 pence yearly.

Saulf has a manor called Melehyvis (Exch. Milchewis) [Mowlish] ${ }^{6}$ which he held himself T.R.E. and it paid geld for I virgate. This I plough can till. Upon it Saulf has 2 villeins, I bordar, and I cow. Worth 50 pence a year. ${ }^{7}$

${ }^{1}$ Dodbrook in Colridge hundred. Geldroll, fol. $696, \mathrm{~A}_{4}$.

'Dunstone in Yealmpton hundred in Ermington hundred. Geldroll, fol. 70b, A ı. Trans. Devon Assoc. xxxv, 709 .

${ }^{3}$ Skerraton in Dean Prior in Stanborough hundred. Testa de Nevill (Rec. Com.), 195a, 1970 ; Inq. p.m. 33 Hen. III, No. 67.

4 Little Dunsford in Dunsford in Wonford hundred.

${ }^{5}$ Ashford Peverel alias Newhouse in Mamhead in

Exminster hundred. Geldroll, fol. 68b, A 12 . Feud. Aids, i, 347 .

${ }^{6}$ Mowlish in Kenton in Exminster hundred. Geldroll, fol. 68b, A 12.

' (In margin) 'finished up to here.'

\section{THE LAND OF ROBERT}

\section{BASTARD $^{8}$}

Robert Bastard holds BAcHEsTANE [Backstone $]^{9}$ of the king. Alvric held it T.R.E. and it paid geld for 3 ferlings of land. Arable (terra) for I plough, which is there, also 2 serfs, and 2 bordars with $\frac{1}{2}$ plough. Ten acres of meadow are there, and 4 score acres of pasture. Formerly worth 5 shillings, now worth Io shillings.

Robert himself holds Haroldesore [Hazard].10 Alvric held it T.R.E. and it paid geld for I virgate of land. Arable for 5 ploughs. In demesne are 2 ploughs with 1 serf, also 6 villeins and 3 bordars with 3 ploughs. Two acres of meadow are there and 40 acres of pasture. Formerly worth 10 shillings, now worth 20 shillings.

Osbern holds Cumbe [Combe] ${ }^{11}$ of Robert. Alvric held it T.R.E. and it paid geld for I virgate of land. Arable for 3 ploughs. In demesne is I plough with I serf, also 2 villeins and 2 bordars with I plough. Two acres of meadow are there and 6 acres of wood(land). Formerly worth 3 shillings, now worth Io shillings.

Rannulf. holds Dunestantona [Dunstone] ${ }^{12}$ of Robert. Alwin held it T.R.E. and it paid geld for $\frac{1}{2}$ hide. Arable for 3 ploughs. In demesne is I plough, also 2 villeins and 3 serfs with 2 ploughs and 4 acres of wood(land). Formerly worth 5 shillings, now worth 20 shillings.

Robert himself has BLAcHEURDE [Blackworthy].13 Alwin held it T.R.E. and it paid geld for I virgate of land. Arable for 3 ploughs. In demesne is I plough with I serf, also 3 villeins and 3 bordars with I plough. Three acres of meadow are there, and 20 acres of wood(land). Formerly worth 5 shillings, now worth Io shillings.

Robert himself has ELFORDE [Efford]. ${ }^{14}$ Alwin held it T.R.E. and it paid geld for $\frac{1}{2}$ hide. Arable for 4 ploughs. In demesne are 2 ploughs and 2 serfs, also 3 villeins with $I$ bordar and I plough. There is a fishery paying 12 pence, also 2 acres of meadow and 20 acres of wood-

${ }^{8}$ The pages containing the next thirty-eight entries have been lost from the Exeter book, and are supplied from the Exchequer copy.

Backstone in Rackenford in Witheridge hundred.

${ }^{10}$ Hazard in Harberton in Stanborough hundred.

${ }^{11}$ Combe Royal, an outlier of West Alvington in Stanborough hundred.

${ }^{12}$ Dunstone in Stokenham in Colridge hundred.

${ }^{13}$ Nether Blackworthy alias Glebe of St. Michael's Cornwood in Ermington hundred. Geldroll, fol. 70, A 6. Feud. Aids, i, 353.

14 Efford, part of Plymouth, in Egg Buckland in Roborough hundred. Geldroll, fol. 70, A 5 . 


\section{A HISTORY OF DEVONSHIRE}

(land). Formerly worth Io shillings, now worth 20 shillings.

Robert himself has Stanehus [Stonehouse]. ${ }^{1}$ Alwin held it T.R.E. and it paid geld for I ferling. Arable for I plough. One villein is there who pays 5 shillings.

Robert himself has Bicheror [Bickford].2 Alwin held it T.R.E. and it paid geld for I virgate of land. Arable for 2 ploughs. One plough is there with I serf, I villein, I bordar, also 4 acres of meadow and 60 acres of pasture. Formerly worth 5 shillings. now worth 10 shillings.

Robert himself holds METwi [Meavy]. ${ }^{3}$ Alwin held it T.R.E. and it paid geld for I virgate of land. Arable for 2 ploughs which are there with I serf, and 5 villeins. Three acres of meadow are there, Ioo acres of pasture, and wood(land) 3 furlongs long by I furlong broad. Formerly worth 10 shillings; now worth 20 shillings.

Robert himself has 2 villeins on the land of St. Peter of Plintone [Plympton] ${ }^{4}$ which Alwin held T.R.E. They pay 5 shillings.

\section{THE LAND OF RICHARD, SON OF TUROLD}

Richard son of Torulf holds ODEHIwIs [Woodhuish $^{5}$ of the king. Ordulf held it T.R.E. and it paid geld for I hide. Arable (terra) for 5 ploughs. In demesne are 2 ploughs and 5 serfs, also 6 villeins and 6 bordars with $2 \frac{1}{2}$ ploughs. Two acres of meadow are there and 6 acres of wood(land). Formerly worth 20 shillings, now worth 40 shillings.

Richard himself holds Noteswrde [Natsworthy]. ${ }^{6}$ Edward held it T.R.E. and it paid geld for I ferling. Arable for 2 ploughs which are there with I serf, 2 villeins, and 2 bordars. Five acres of meadow are there and 6 acres of coppice. Formerly worth 5 shillings, now worth 15 shillings.

Richard himself holds Alintone [Allington]. ${ }^{7}$ Ulnod held it T.R.E. and it paid geld for 3 hides.

${ }^{1}$ West Stonehouse, part of Plymouth, in Roborough hundred.

${ }^{2}$ Bickford in Plympton parish and hundred.

3 Loveton Bastard in Meavy in Roborough hundred.

4 At Sutton Prior, i.e. St. Charles, Plymouth, in Roborough hundred.

${ }^{5}$ Woodhuish in Brixham in Haytor hundred. Geldroll, fol. $69 b$, A 5 .

${ }^{6}$ Natsworthy in Widdecombe-in-the-Moor in Haytor hundred.

East Allington in Stanborough hundred. Geldroll, fol. 696, A 6 .
Arable for 16 ploughs. In demesne are 2 ploughs and 10 serfs, also 17 villeins and 15 bordars with Io ploughs, 5 acres of meadow and 8 acres of coppice. Formerly and now worth IOC shillings.

Richard himself has in ExECEstRe [Exeter] ${ }^{8}$ I house from which he withholds the king's customary due, that is 8 pence.

\section{THE LAND OF HERVEI DE HELION}

The wife (i.e. widow) of Hervei de Helion holds Essestone [Ashton] ${ }^{9}$ of the king. Almer held it T.R.E. and it paid geld for I hide and 2 ferlings. Arable for 3 ploughs. Four villeins are there and 4 bordars with one serf, who have 2 ploughs. There also are 2 acres of meadow, 40 acres of pasture and as many of wood(land). Formerly and now worth 20 shillings.

To this manor have been added 2 virgates of land and $\frac{1}{2}$ ferling. Two thanes held them in parage (in paragio) T.R.E. as 2 manors. Arable for 3 ploughs. Three villeins are there and 2 bordars having I plough, also I swineherd paying 4 swine. There are 2 acres of meadow, Io acres of wood(land) and 40 acres of pasture. Formerly and now worth Io shillings.

These 3 estates (terras) Harvei's wife holds as one manor.

She herself holds Hacheurde [Hackworthy]. ${ }^{10}$ Edric held it T.R.E. and it paid geld for I virgate of land. Arable for 2 ploughs which are there with 3 villeins, 2 bordars, and 2 serfs. Four acres of meadow are there and 24 acres of pasture. Formerly and now worth Io shillings.

\section{THE LAND OF ALVRED THE BRETON}

Alvred the Breton holds WIFLEuRde [Willsworthy $]^{11}$ of the king. Siward held it T.R.E. and it paid geld for I virgate of land. Arable for 4 ploughs. In demesne is I plough and 4 serfs, also I2 acres of wood(land). Pasture 2 leagues in length by $I$ league in breadth. Formerly and now worth 30 shillings.

${ }^{8}$ Exeter dwelling-house.

${ }^{9}$ Ashton in Exminster hundred. See fol. $498 b$. Geldroll, fol. 68b, A 6.

${ }^{10}$ Hackworthy in Tedburn St. Mary in Wonford hundred.

${ }^{11}$ Willsworthy an outlier of Peter Tavy in Lifton hundred. Geldroll, fol. 65, A 3. 


\section{HOLDERS OF LANDS}

Wihuenech holds SPREI [Sprey] ${ }^{1}$ of Alvr[ed]. Alcher held it T.R.E. and it paid geld for I virgate of land. Arable for 2 ploughs. One and a half ploughs are there with I serf and 4 bordars. Three acres of meadow are there and 5 acres of wood(land). Formerly and now worth I 0 shillings.

Fulco holds Ferding ${ }^{2}$ [? a ferding of land] of Al[vred]. Sawin held it T.R.E. and it paid geld for I ferling of land. Arable for $1 \frac{1}{2}$ ploughs. There 5 bordars with 1 serf have $\frac{1}{2}$ plough. Ten acres of meadow are there. Formerly and now worth ro shillings.

Alvred himself holds Genelie [Innerleigh]. ${ }^{3}$ Godric held it T.R.E. and it paid geld for I virgate of land. Arable for 6 ploughs. In demesne is I plough with I serf, also 4 villeins and 4 bordars with I plough. Eight acres of meadow are there and the like of wood(land) and 10 acres of pasture. Formerly worth 30 shillings, now worth 20 shillings.

Alvred himself holds Hechesburne [Exbourn]. ${ }^{4}$ Ulnod held it T.R.E. and it paid geld for 1 virgate of land. Arable for 6 ploughs. In demesne is I plough with I serf, also 2 villeins and 2 bordars with $I$ plough. Six acres of meadow are there, 8 acres of pasture and ro acres of wood(land). Formerly worth 30 shillings, now worth 20 shillings.

Three thanes hold Corneurde [Curworthy] ${ }^{5}$ of Alvr[ed]. They held it themselves T.R.E. and it paid geld for I virgate of land. Arable for 3 ploughs. One villein is there and I serf, also 12 acres of meadow, the like of wood(land) and 300 acres of pasture. Formerly and now worth 10 shillings and 6 pence. There were 3 manors.

Wihuenec holds EsseberIe [Ashbury] ${ }^{6}$ of Al[vred]. Leuric held it T.R.E. and it paid geld for I virgate of land. Arable for 5 ploughs. Two ploughs are there and 2 serfs, also 5 villeins, 4 bordars, 10 acres of meadow, 6 acres of wood(land), and 200 acres of pasture. Formerly worth 5 shillings, now worth 20 shillings.

\footnotetext{
${ }^{1}$ Spryton, alias Spry in Stowford, in Lifton hundred.

'Possibly Beardon member of Willsworthy in Lifton hundred (O. J. R.). Trans. Devon Assoc. sxviii, 478 ; xxxvi, $35 \mathrm{I}$.

${ }^{3}$ Innerleigh, alias Ingley in Bradwood Kelly, in Blacktorington hundred.

-Now Coxhole, alias Coxwell in Exbourne, in Blacktorington hundred.

${ }^{5}$ Curworthy with Whitefield in Inwardleigh in

Blacktorington hundred. See fol. 496.

${ }^{6}$ Ashbury in Blacktorington hundred.
}

The same Wihuenec holds Hirie [Hele] ${ }^{7}$ of Alvred. Aiolf held it T.R.E. and it paid geld for $\frac{1}{2}$ hide. Arable for 5 ploughs. Four ploughs are there and 3 serfs, also 5 villeins, 2 bordars, 6 acres of meadow, 30 acres of pasture and $20^{\circ}$ acres of wood(land). Formerly worth 20 shillings, now worth 25 shillings.

William holds RADEWEI [Redway] ${ }^{8}$ of Alvred. Godric held it T.R.E. and it paid geld for $\frac{1}{2}$ virgate of land. Arable for 2 ploughs. One plough is there with I serf and I villein, also 2 acres of meadow and 20 acres of pasture. Formerly and now worth ro shillings.

Alvred himself holds Lavrochebere [Larkbear]. ${ }^{9} \quad$ Ulfheld it T.R.E. and it paid geld for $\frac{1}{2}$ hide. Arable for 2 ploughs. One plough is there and 2 serfs, also 5 villeins with $I$ bordar. Twenty acres of meadow are there, the like of wood(land), and 200 acres of pasture all but 7 . Formerly and now worth 20 shillings.

William holds BACHEIEFoRd [Battleford] ${ }^{10}$ of Al[vred]. Saulf held it T.R.E. and it paid geld for I hide. Arable for 3 ploughs. One and a half ploughs are there with I serf, also 3 villeins with I bordar. Seven acres of meadow are there, 20 acres of pasture and $\frac{1}{2}$ acre of coppice. Formerly worth 20 shillings, now worth I 5 shillings.

The same William holds Grismetone [Grimpston $]^{11}$ of Al[vred]. Alnod held it T.R.E. and it paid geld for $\frac{1}{2}$ hide. Arable for 2 ploughs. There, nevertheless, is I plough in demesne with I serf, also 2 villeins, and 2 bordars with 2 ploughs and 15 acres of pasture. Formerly worth Io shillings, now worth I 5 shillings.

The same William holds Lrge [Leigh] ${ }^{12}$ of Al[vred]. Alnod held it T.R.E. and it paid geld for I virgate of land. Arable for 3 ploughs which are there with I serf also 3 villeins, and Io acres of pasture. Formerly worth Io shillings, now worth 20 shillings.

Alvred himself holds BACHETESBERIA [Battisborough]. ${ }^{13}$ Almar held it T.R.E. and it paid geld for I hide. Arable for 8 ploughs. In demesne is I plough with I serf, also 6 villeins

${ }^{7}$ Hele, alias Luttock's Hele in Collumpton, in Hairidge hundred (O.J.R.). Geldroll, fol. $67 b$, A. 6 , Trans. Devon Assoc. xxviii, 477 ; xxix, 265; xxxvi, 36r ; Fryer's Hele, Meeth (T.W.W.).

${ }^{8}$ Redway in Rew in Hairidge hundred.

${ }^{9}$ Larkbear in Talaton in Cliston hundred. Geldroll, fol. 67, A. 6 .

${ }^{10}$ Battleford in Ipplepen in Haytor hundred.

${ }^{11}$ Grimpstone in Blackawton in Colridge hundred. Geldroll, fol. 69b, A 3, says ' an orphan' held it.

${ }^{12}$ Grimpstonleigh, alias Leigh Arthur, an outlier of Morleigh in Colridge hundred.

${ }^{13}$ Battisborough, the chief manor in Holbeton, in Ermington hundred. 


\section{A HISTORY OF DEVONSHIRE}

and 5 bordars with 2 ploughs. Fifteen acres of pasture are there, and 4 acres of coppice. Formerly and now worth 30 shillings.

To this manor Crawecome [Creacombe $]^{1}$ has been added. Almar held it as a manor in parage (in paragio) T.R.E. and it paid geld for $\frac{1}{2}$ hide. Arable for 2 ploughs. Half a plough is there. Worth Io shillings.

Tovi holds Mideltona [Milton] ${ }^{2}$ of Al[vred]. Two thanes held it freely in parage (libere; in the margin, inparagio) T.R.E. and it paid geld for 2 hides. Arable for 12 ploughs. In demesne are 2 ploughs with I serf, also 8 villeins and 6 bordars with 3 ploughs. Six acres of meadow are there. Formerly and now worth 60 shillings.

William holds MoRtex [Morleigh] ${ }^{3}$ of Al[vred]. Alvric held it T.R.E. and it paid geld for $\frac{1}{2}$ hide. Arable for 4 ploughs. Three ploughs are there and 4 serfs, also 5 villeins, 4 acres of meadow, 40 acres of pasture, and 7 acres of wood(land). Formerly and now worth 20 shillings. This land has been added to Lege [Leigh] manor.

Alvred himself holds ULGEBERGE [Ugborough]. ${ }^{4}$ Alwin held it T.R.E. and it paid geld for 3 hides and I virgate of land. Arable for I5 ploughs. In demesne are 2 ploughs and 5 serfs, also 9 villeins and 9 bordars with 6 ploughs. Twelve acres of meadow are there, 50 acres of pasture, and 15 acres of coppice. Worth 60 shillings.

Alvred himself holds Buchesine [Butshead]. ${ }^{5}$ Alwin held it T.R.E. and it paid geld for $\frac{1}{2}$ hide. Arable for 5 ploughs. In demesne are 2 ploughs and 4 serfs, also 5 villeins with I plough. Four acres of meadow are there, coppice $\frac{1}{2}$ league in length by 2 furlongs in breadth. Formerly and now worth 30 shillings.

Alvred himself holds Tambretone [Tamerton]. ${ }^{B}$ Inewar held it T.R.E. and it paid geld for 2 hides. Arable for 10 ploughs. In demesne are 3 ploughs and 7 serfs, also I 6 villeins and 6 bordars with 5 ploughs. A saltern is there paying 5 shillings and 3 acres of meadow;

${ }^{1}$ Creacombe in Holbeton in Ermington hundred.

${ }^{2}$ South Milton, alias Middleton Foliot, in Stanborough hundred. See fol. 504b. Geldroll, fol. 69b, A 7 .

${ }^{3}$ Morleigh in Stanborough hundred. See fol. $504 b$.

"Ugborough in Ermington hundred. Geldroll, fol. $70, \mathrm{~A}_{4}$.

5 Butshead in St. Budeaux in Roborough hundred. Geldroll, fol. 70, A. 6.

6 Tamerton Foliot in Roborough hundred. Geldroll, fol. 70, A 6 . pasture 3 furlongs in length by $I$ furlong in breadth, and wood(land) $\frac{1}{2}$ league in length by 3 furlongs in breadth. Formerly worth 60 shillings, now worth roo shillings.

Alvred himself holds Blachestane [Blackstone]. ${ }^{7} \quad$ Inewar held it T.R.E. and it paid geld for I hide. Arable for 4 ploughs. In demesne are 2 ploughs and 5 serfs, also 4 villeins with $I$ plough, I swineherd paying 5 swine, and a saltern paying 30 pence ; wood(land) I league in length by 3 furlongs in breadth; pasture 3 furlongs in length by $I$ in breadth. Formerly worth 20 shillings, now worth 50 shillings.

Alvred himself holds TAwI [Tavy]. ${ }^{8}$ Siward held it T.R.E. and it paid geld for I hide. Arable for 7 ploughs. In demesne are 2 ploughs and 9 serfs, also 5 villeins and 6 bordars with 2 ploughs. Four acres of meadow are there; pasture 16 furlongs in length by 9 furlongs in breadth; wood(land) 3 furlongs in length by 1 in breadth, and 16 acres of coppice. Formerly worth 20 shillings, now worth 60 shillings.

Alvred himself has a house in ExCESTRE [Exeter $]^{9}$ which pays 8 pence by way of customary due.

fol. 495 .

\section{LANDS ENTERED UPON (occupatae) ${ }^{10}$ IN DEVENESCIRA}

Baldwin the sheriff has $\frac{1}{2}$ virgate of land in the manor called BRATONA [Bratton] ${ }^{11}$ which 2 thanes held independently T.R.E., and they could go with their lands to what lord they liked. This $7 \frac{1}{2}$ ploughs can till. Worth 30 shillings a year; when he received it it was worth 60 shillings.

Baldwin the sheriff has 6 manors in the manor called BRIDESTOU [Bridestow] ${ }^{12}$ which 6 thanes held in parage T.R.E. The names of the manors are these: CASForda [Causeyford] which Sawin Topa held; Batesilla [Battishill] which Dodo held; CumBa [Combe] which Dodo held; BOLDEsworda [Ebbsworthy] which Godwin held; FERnewrda [Fenworthy] which Godwin held; WEIA [Way] which Abbot Suetric held. These manors in King Edward's time paid geld for $\frac{1}{2}$ hide, $\mathrm{I} \frac{1}{2}$ ferlings. This 6 ploughs can till.

7 Blackstone in Tamerton in Roborough hundred.

${ }^{8}$ Peter Tavy in Roborough hundred.

${ }^{9}$ Exeter dwelling-house.

${ }^{10}$ Without the king's writ or possession given by the sheriff. Freeman, Norman Conquest, v, 756.

${ }^{11}$ In Bratton Clovelly in Lifton hundred. 'See fol. 2886 .

${ }^{12}$ In Bridestow in Lifton hundred. See fol. 2886 . 


\section{HOLDERS OF LANDS}

Worth 3 pounds a year, and when Baldwin received them the value was 30 shillings.

Baldwin has a manor in LEUYA [Lew ${ }^{1}$ which did not belong to that manor T.R.E. It is called Wadelescota [Waddleston]. Wadell held it T.R.E. and could go with his land to what lord he liked. It paid geld for I virgate. This Roger de Molis holds of Baldwin. It can be tilled by 2 ploughs, and is worth 20 shillings a year.

Robert de Albamarla has a manor called Odetrewa [Ottery]. ${ }^{2}$ To this manor three other manors have been added which 2 thanes held in parage (pariter) T.R.E. Worth 50 shillings a year.

Nigel holds a manor of Juhel called TAvi [Tavy]. ${ }^{3} \quad$ To it have been added three manors which did not belong there in King Edward's time -Wagesfella [Warn] Berna [Burntown], and Weringheorda [W ringworthy] - which 3 thanes held in parage (pariter) T.R.E. They paid geld fol. $495 \mathrm{~b}$.

for I virgate. This 3 ploughs can till. The 3 manors are worth 25 shillings a year.

Juhel has a manor called Sidreham [Sydenham.$^{4} \quad$ Nigel holds it of Juhel. To this have been added 3 manors which did not belong there T.R.E., and these manors 3 thanes held in parage T.R.E. and they paid geld for I virgate and 2 ferdings. This 4 ploughs can till, and they are worth 20 shillings a year.

The abbot of Stavestoch [Tavistock] has $\frac{1}{2}$ hide of land with a manor called Milneltona [Milton] $^{5}$ which did not belong there in King Edward's time, but 2 thanes held it in parage (pariter). This 15 ploughs can till, and it is worth 6 pounds a year.

The count of Mortain (de Moritonio) has 2 manors, Lanliner [Lanliner] and Tribichen [Trebichen], which belonged to the royal demesne-manor of Listona [Lifton] ${ }^{6}$ T.R.E., and there the count has $I \frac{1}{2}$ virgates of land. These 6 ploughs can till. They are worth 12 shillings and 6 pence a year; when he received them they were worth 60 shillings.

${ }^{1}$ In Lew Trenchard, Waddleston, in Lifton hundred. See fol. $289 b$.

'Ottery, in Lamerton, in Lifton hundred. See fol. 419 .

In Mary Tavy; Warn, Burntown, and Wringworthy, in Lifton hundred. See fol. $317 b$.

${ }^{4}$ In South Sydenham, in Lifton hundred. See fol. 318.

${ }^{5}$ In Milton Abbot, in Lifton (now Tavistock) hundred. See fol. $177 b$.

${ }^{6}$ In Lifton manor, but lying in Cornwall. See fol. 93 .

I
Along with a certain manor called TETEcota [Tetcot] which Juhel has, he holds I ferding of land ${ }^{7}$ which I thane held in parage (pariter) T.R.E. It did not then belong to the aforesaid manor, and can be tilled by I plough. Worth Io shillings a year; when he received it the same.

William the (king's) usher (bostiarius) has a manor called Tauelanda [Tawland] ${ }^{8}$ which I thane held in parage (pariter) T.R.E., and it paid to the king's demesne-manor called TAuetona [Tawton] either I ox or 30 pence a year. Since fol. 496.

W [illiam] the usher has held it the king has not had therefrom this customary payment.

Osbern de Salciet has a manor called PatFORDA [Parford] ${ }^{9}$ which $I$ thane held in parage (pariter) T.R.E., and it paid to the king's demesne-manor called Tauetona [Tawton] either I ox or 30 pence a year by custom. Since Osbern has held it the king has not had therefrom his customary due.

Girold the chaplain has a manor called EscaPELEIA [Shapleigh] ${ }^{10}$ which $I$ thane held in parage (pariter) T.R.E. Nevertheless it used to pay to the king's demesne-manor called TAUETONA ['Tawton] Io shillings a year by custom. but since G[irold] has held it the king has not had his customary due therefrom.

Juhel and Alvered hold $I$ hide of land in BRADroda [Bradwood] ${ }^{11}$ which by custom used to pay to the king's demesne-manor called LIsTONA [Lifton] 7 shillings and 6 pence a year by way of customary due, but since these two have had it the king has not had his customary due.

Ruald has a manor called TAmerLanda [on the Tamar]. To this has been added another manor called Pech [Peak] ${ }^{12}$ which did not belong to this manor T.R.E. Worth 5 shillings a year; when $R$ [uald] received it it was worth Io shillings.

Alvered the Breton has a manor called Corneorda [Curworthy], ${ }^{13}$ to which have been added 2 other manors that did not belong to this

7 Probably East Peak in Tetcot, in Blacktorington hundred. See fol. 319 .

${ }^{8}$ Probably Cocktree, in South Tawton parish and hundred. See fol. 475 .

${ }^{9}$ Probably Parford in Drewsteignton, in Wonford hundred. See fol. 456 .

${ }^{10}$ In Shapleigh, in South Tawton hundred. See fol. 456 ,

${ }^{11}$ In Bradwood Widger, in Lifton hundred. See fol. 316

${ }_{12}$ West Peak, in Blacktorington hundred. See fol. 412.

${ }^{13}$ Curworthy and Whitefield in Inwardleigh, in Blacktorington hundred. See P. I 33. 


\section{A HISTORY OF DEVONSHIRE}

manor T.R.E. One of these is called CoRnEORDA [Curworthy] and the other WITEFELDA [Whitefield]. Worth 7 shillings and 6 pence. Two thanes held them in parage (pariter).

Ruald has a manor called ChIEMPabera [Kimber]. ${ }^{1}$ To this has been added a certain manor called RADECLIVA [Redcliff] which fol. $496 \mathrm{~b}$.

I thane held in parage (pariter) T.R.E. It did not belong to the aforesaid manor, and is worth 5 shillings a year.

The bishop of Coutances has a manor called HaREODA [Horwood], to which a certain manor has been added called HorTona [Horton] ${ }^{2}$ that did not belong to the aforesaid manor T.R.E. This I thane held in parage (pariter). Drogo holds it of the bishop, and it is worth 5 shillings a year.

William Capra has a manor called Eszstapla $[\text { Enstaple }]^{3}$ which he and his brother added to the manor called BraORDina [Bradworthy]. This a certain thane held in parage (pariter) T.R.E. and it did not belong to the aforesaid manor, i.e. Braord(ina). Worth 5 shillings a year; when he received it it was worth 7 shillings.

Ruald has a manor called Potaforda [Putford].: To this $\frac{1}{2}$ virgate of land has been added which I thane held in parage (pariter) T.R.E. and it did not belong to the aforesaid manor. Rainald holds it of Ruald. Worth Io shillings a year; when he received it 5 shillings.

William Capra has a manor called Potaforda [Putford], to which has been added $\frac{1}{2}$ virgate of land ${ }^{5}$ that $I$ thane held in parage (pariter) T.R.E., and it did not belong to the aforesaid manor. Anschetil holds it of William. Worth 3 shillings and 9 pence a year; when W[illiam] received it it was worth 10 shillings.

The count of Mortain has I virgate of land ${ }^{6}$ which has been taken away from a manor of Ralf de Pomaria called Denewynesdone [Duns-

1 West Kimber in North Lew, in Blacktorington hundred. See fol. $4^{12}$.

${ }^{2}$ Horton in Bradworthy in Blacktorington hundred, held with Horwood, in Fremington hundred. See fol. $122 b$.

${ }^{3}$ Enstaple in Bradworthy, in Blacktorington hundred. See fol. 399.

${ }^{4}$ In West Putford, in Blacktorington hundred. See fol. $4 \mathrm{I} 2$.

'Scotworthy, in Cory manor, in West Putford, in Blacktorington hundred. See fol. 399.

${ }^{6}$ Aylescot in Pancras Week, in Blacktorington hundred (O. J. R.) ; or Dexboro' (T. W. W.). See fol. 335 . don]. This Tovi held T.R.E. and it is worth 30 shillings a year.

fol. 497 .

The bishop of Coutances has a manor called Hainghestecota [Endscot]. To this have been added 2 manors ${ }^{7}$ which 2 thanes held in parage, and they did not belong to the aforesaid manor T.R.E. Worth ro shillings a year; when he received them they were worth 15 shillings.

Ralf de Pomeria entered upon (occupavit) a manor which is called PANESTAN [Panson], ${ }^{8}$ and gave it to Ruald in exchange for Brochelande and Radiz. Worth 20 shillings a year; when $R$ [alf] received it it was worth 30 shillings.

The same Ralf entered upon a manor called EissA [Ash ${ }^{9}$ which $I$ thane held in parage (pariter) T.R.E. Worth 10 shillings a year; when he entered upon it it was worth 20 shillings.

Ralf has a manor called Puteforda [Putford] ${ }^{10}$ which paid by custom 30 pence a year to the king's demesne-manor called Torintona [Torington]. Since Ralf has held this land the king has not had thence his customary due.

Tetbald has a manor called Bochewys [Bukish]. To this has been added I virgate of land ${ }^{11}$ which 2 thanes held in parage (pariter) T.R.E. It did not belong to the aforesaid manor. Worth 6 shillings and 3 pence.

The count of Mortain has a manor called Colenera [Culleigh] ${ }^{12}$ which I thane held in parage (pariter) T.R.E. It paid geld for I virgate. In King E[dward]'s time this did not belong to Ordulf's lands which the count holds (now). Erchenbald holds it of the count. Worth I 2 shillings; when he received it it was worth 4 shillings.

Odo has a manor called YwIs [Huish], along with which Tetbald his father-in-law entered upon I ferding of land ${ }^{13}$ that I thane held in parage (pariter) T.R.E. Worth I 5 shillings a year; when $T$ [etbald] entered upon it it was worth ro shillings.

${ }^{7}$ Endscot in Bradford Dabernon, in Blacktorington hundred. See fol. $122 b$.

${ }^{8}$ East Panson in St. Giles'-in-the-Heath, in Blacktorington hundred. See fol. 4 I $1 b$.

${ }^{9}$ Ash in Bradworthy, in Blacktorington hundred. See fol. $335^{b}$.

${ }^{10}$ Julian Putford in West Putford, in Blacktorington hundred. See fol. 3356 .

${ }^{11}$ Walland next Bukish in Woolfardisworthy in Hartland hundred. See fol. 407.

${ }^{12}$ Culleigh in Frithelstock in Shebbear hundred See fol. 2 s $0 b$.

${ }^{13}$ In Huish in Shebbear hundred. See fol. 3766 . 


\section{HOLDERS OF LANDS}

The count of Mortain has a manor called Bochelanda [Buckland]. To this has been added $\frac{1}{2}$ virgate of land called Galeshora [Galsworthy] ${ }^{1}$ which I thane held in parage (pariter), and it did not belong to the aforesaid manor fol. $497 \mathrm{~b}$.

T.R.E. Ansger the Breton holds it of the count. Worth io shillings a year, and was worth the same when he received it.

The count has a manor called Bochesorda [Bulkworthy] ${ }^{2}$ which Irich held in parage (pariter). It did not belong T.R.E. to the lands of Edmeratorius which the count (now) holds, to which this manor has been wrongfully added. Ansger the Breton holds it of the count. Worth 20 shillings a year, and was worth as much when the count received it.

The count has a manor called Esmitemam [Smytham] $^{3}$ which $I$ thane held in parage (pariter) T.R.E. This has been wrongfully added to the lands of Edmeratorius the predecessor of the count which the count holds. Ansger the Breton holds it of the count. Worth 5 shillings a year, and was worth the same when the count received it.

The count has a manor called LiteltroRILANDA [Little Torington] ${ }^{4}$ which I thane held in parage (pariter) T.R.E. This has been added to the lands of Edmeratorius. Alvred the cup-bearer holds it of the count. Worth 5 shillings a year, and was worth the same when the count received it.

The count has a manor called $E_{L A}[\mathrm{Hele}]^{5}$ which 2 thanes held in parage (pariter) T.R.E. This manor has been added to the lands of Ordulf which the count holds. Erchenbald holds it of the count. Worth Io shillings; when he received it it was worth 20 shillings.

The king has a manor called SLAPEForda [Lapford], to which have been added 4 manors $^{6}$ which 4 thanes held in parage (pariter) T.R.E. and they did not belong to the aforesaid manor in King E[dward]'s time. These Walter de Clavil holds (included) in the farm of Slapeford. They pay towards the farm rent 20 shillings a year.

The abbot of Tovestoch [Tavistock] has a manor called Bernintona [Burrington]. To

\footnotetext{
${ }^{1}$ Galsworthy in Buckland Brewer in Shebbear hundred. See fol. 2 Iob.

${ }^{2}$ Bulkworthy in Shebbear hundred. See fol. 21 I.

${ }^{3}$ Smytham in Little Torington in Shebbear hundred. See fol. 2116 .

${ }^{4}$ In Little Torington in Shebbear hundred. See fol. 212.

${ }^{5}$ Hele Satchvil in Shebbear hundred. See fol. 2 I 2.

- In Lapford manor in North Tawton hundred. See fol. $109 b$.
}

this 2 manors $^{7}$ have been added which did not belong to the aforesaid manor T.R.E. These 2 thanes held in parage (pariter). William Capra and Goisfrid hold them of the abbot. Of these lands William's share is worth 20 shillings; when the abbot received it 30 shillings; Goisfrid's share is worth I 5 shillings; when the abbot received it 30 shillings.

fol. 498.

Walter de Clavil has a manor called LoluaRDESDONA [Leusdon] which was part of Bristric's land. To this has been added a certain manor called Duvelanda [Dowland] ${ }^{8}$ which 2 thanes held in parage (pariter). It did not belong to the aforesaid manor T.R.E. Worth 12 shillings and 6 pence a year; when $W$ [alter] received it the same.

In the aforesaid Duvelanda [Dowland] Walter has I virgate of land ${ }^{9}$ which 2 thanes held in parage (pariter) T.R.E. without belonging to the aforesaid manor. Worth I 2 shillings and 6 pence a year; worth the same when he received it.

The king has a manor called Tauntona [Tawton]. To this has been added a certain manor called AIssa [Ash] ${ }^{10}$ which Olwric held in parage (pariter) T.R.E. This was entered upon along with the aforesaid manor in King W[illiam's] time. Worth 30 shillings a year.

The bishop of Coutances has a manor called Colriga [Colridge]. To this has been added I virgate of land ${ }^{11}$ which $I$ thane held in parage (pariter) T.R.E. It did not belong to the aforesaid manor in King E[dward]'s time. Drogo holds it of the bishop. Worth Io shillings a year.

Walter de Clavil has a manor called NIMETH [Nymet] ${ }^{12}$ which Alward a thane held, who could go with that land to what lord he liked T.R.E. Worth 15 shillings; when he received it worth 5 shillings.

The same W[alter] has a manor called EschipenRocha [Shobrook $]^{13}$ which Bristric held, who could go with his land to what lord he liked T.R.E. Worth io shillings a year.

${ }^{7}$ Northcot and Halsbury in Burrington in North Tawton hundred. See fol. I79.

${ }^{8}$ Dowland in North Tawton hundred. Fol. 390.

${ }^{9}$ Upcot in Dowland in North Tawton hundred. See fol. 390.

${ }^{10}$ Ash in South Tawton manor and hundred. See fol. $93 b$.

${ }^{11}$ Clatworthy in Colridge in North Tawton hundred. See fol. I 25.

${ }^{12}$ Wolfsnymet, alias Wolfin in Down St. Mary, in North Tawton hundred. See fol. 3906 .

${ }^{13}$ Shobrook in Morchard Bishop in North Tawton hundred. See fol. 391. 


\section{A HISTORY OF DEVONSHIRE}

Sawin the priest has a manor called BIRIGr [Bridge] ${ }^{1}$ which his uncle held, who could go with his land to what lord he liked T.R.E. Worth ro shillings a year. This Queen $M$ [atilda] gave to this priest in alms.

$W$ [illiam] de Ou has $\frac{1}{2}$ virgate of land ${ }^{2}$ which belonged to the king's demesne manor called AxEministra [Exminster] T.R.E. Worth 5 shillings a year.

Ralf de Pomaria has a manor called Arssecoma [Ashcombe]. To this have been added 3 manors $^{3}$ which 3 thanes held in parage (pariter) T.R.E. They did not belong to the aforesaid manor in King E[dward]'s time. Worth 7 pounds fol. $498 \mathrm{~b}$.

Io shillings a year.

The monks of Battle (batailla) have I ferding of land, part of the king's demesne manor called Arxeministra [Exminster]. ${ }^{4}$ This Eccha the king's reeve allowed a certain priest the benefit of in King E[dward]'s time. Worth 2 shillings a year.

Hervei's wife (i.e. widow) has a manor called Essetona [Ashton]. To this have been added 2 other manors ${ }^{5}$ which did not belong to the aforesaid manor in King E[dward]'s time, but 2 thanes held them in parage (pariter) T.R.E. Worth 20 shillings a year.

Ansger has a manor called STADForda [Stafford ${ }^{6}$ which did not belong in King E[dward]'s time to the lands of Bristric that he holds, but a certain thane held it in parage (pariter) T.R.E. Worth 12 shillings and 6 pence; when A[nsger] received it it was worth 7 shillings and 6 pence.

The same Ansger has a manor called BremeLComa [Brimcombe ${ }^{7}$ which in King E[dward]'s time did not belong to the lands of Bristric that he holds. This a certain thane held in parage (pariter) T.R.E. Worth 7 shillings and 6 pence a year.

Godebold has a manor called MaLAcota [Mullacot]. To this has been added I ferding ${ }^{8}$

${ }^{1}$ Swymbridge in South Molton hundred. See fol. $194 b$.

${ }^{2}$ Exwell in Powderham in Exminster hundred. See fol. 457 .

${ }^{3}$ In Ashcombe in Exminster hundred. See fol. 336 .

${ }^{4}$ The glebe of 16 acres in Exminster parish and hundred. See fol. 83 .

${ }^{5}$ Ashton in Exminster hundred. See Exch. fol. I I 3 .

${ }^{6}$ Stafford in Dolton in North Tawton hundred. See fol. $456 b$.

${ }^{7}$ Brimcombe in Dowland in North Tawton hundred. See fol. 4566 .

${ }^{8}$ Foley added to Mullacot in Ilfracombe in Braunton hundred. See fol. 469 . which did not belong to the aforesaid manor T.R.E. This $I$ thane held in parage (pariter) and it has altogether gone out of cultivation.

The king has a manor called Brantona [Braunton]. To this has been added $\mathbf{I}$ virgate of land ${ }^{9}$ which belonged to Filileia [Filleigh], a manor of Bal[dwin] the sheriff, T.R.E. Worth 20 shillings a year. From the same Brantona has been taken away I virgate of land ${ }^{10}$ which belonged there T.R.E. Now Robert de Ponte Cardonis holds it. It paid 20 shillings towards the farm rent of King Edward's manor.

Walcin has a manor called Hagitona [Haggington]. To this has been added a manor ${ }^{11}$ which a certain thane held in parage (pariter) T.R.E. Worth Io shillings a year.

W[illiam] Capra has a manor called LINTONA [Lynton]. To this has been added a certain manor called INCRINTONA [Ilkerton] ${ }^{12}$ which I thane held in parage (pariter) T.R.E. Worth 3 pounds a year; when $W[$ illiam] received it I 5 shillings.

fol. 499.

W[illiam] Capra has a manor called LrNa [Lyn]. To this has been added a manor called BICHEORDIN ${ }^{13}$ [Badgeworthy] which I thane held in parage (pariter) T.R.E. Fulco holds it of W[illiam]. Worth Io shillings a year; when he received it it had altogether gone out of cultivation.

Ralf de Pomaria has a manor called BRANDONA [Brendon]. To this has been added Lancoma [Lank Combe] ${ }^{14}$ which Edwin held T.R.E. Worth 3 shillings a year.

The count of Mortain has a manor called Bratona [Bratton] which Ordulf held. To this have been added 3 manors ${ }^{15}$ which 3 thanes held in parage (pariter) T.R.E. Worth 20 shillings and 8 pence.

The bishop of Exeter has a manor called Chrietona [Crediton]. In it his canons hold a manor called Chefecoma [Shircombe] $]^{16}$

${ }^{9}$ Lob in Braunton parish and hundred. See fol. $83 b$.

${ }^{10}$ Blakewell in Marwood in Braunton hundred. ? See fol. $83 b, 299 b$.

${ }^{11}$ Part of East Hagginton in Berry Narbor in Braunton hundred. See fol. 345 .

${ }^{12}$ Ilkerton in Lynton in Shirwell hundred. See fol. 402 .

is Badgeworthy in Brendon in Shirwell hundred. See fol. 402 .

${ }^{14}$ Lank Combe in Brendon in Shirwell hundred. See

fol. 337 .

${ }^{15}$ In Bratton Fleming in Braunton hundred. See fol. 213 .

${ }^{16}$ Shircombe in Down St. Mary in Crediton hundred. 


\section{HOLDERS OF LANDS}

which I thane held in parage (pariter) T.R.E. Worth Io shillings a year.

The king has a manor called Moldanda [Molland]. To this has been added a certain manor called Blacapola [Blackpool] ${ }^{1}$ which I thane held in parage T.R.E. Worth 20 shillings a year.

The king has a manor called Tauestocha [Tawstock]. From this I manor has been taken away called Bicheneleia [Bickingleigh] ${ }^{2}$ which belonged to Tauestoc [Tavistock] T.R.E. Now it has been wrongfully added to the king's manor called Bichentona [Bickington], one of Bristric's lands. It pays 4 pounds a year.

The bishop of Coutances has a manor called Dune [Down]. To this have been added 3 manors ${ }^{3}$ which 3 thanes held in parage (pariter) T.R.E. Now Drogo holds them of the bishop. Worth 20 shillings a year.

Alvered de Ispania has a manor called AlfrerDinTona [Arlington]. To this has been added a manor called TUCHeL [Twitchin] ${ }^{4}$ which I thane held in parage T.R.E. Worth 5 shillings a year; when Alvered received it it was worth 20 shillings.

The bishop (of Coutances) has a manor called BraIA [Bray]. To this has been added a manor called W ITEFELLA [Whitfield] ${ }^{5}$ which I thane held in parage T.R.E. Worth 10 shillings a year, and was worth the same when the bishop received it. Drogo holds it of the bishop.

fol. $499 \mathrm{~b}$.

Ruald has a manor called Polham [Polham]. To this has been added $\frac{1}{2}$ virgate, ${ }^{6}$ the land of $I$ thane who held it in parage (pariter) T.R.E. It is called Plateleia [Praunsley]. Rainald holds it of Ruald. Worth 3 shillings a year.

The count of Mortain has a manor called Donicestona [Denson]. To this has been added I manor called Alwinestona [ $]^{7}$ which I thane held in parage T.R.E. Malger holds it of the count. Worth 15 shillings a year when he received it it was worth 40 shillings.

${ }^{1}$ Blackpool in Chittlehampton added to Molland Botreaux manor and hundred. See fol. 95.

${ }^{2}$ Langleigh in High Bickington in North Tawton hundred. See fol. II $\mathrm{O}$.

${ }^{3}$ In West Down in Braunton hundred. See fol. $125 b$.

Twitchin in Arlington in Shirwell hundred. See fol. $37 \mathrm{I}$.

${ }^{5}$ Whitefield in Highbray in Shirwell hundred. see fol. I 266 .

${ }^{6}$ Praunsley in North Molton parish and hundred. See fol. $4 \mathrm{I} 3 b$.

${ }^{7}$ Petton in Bampton parish and hundred. See fol. $2 \mathrm{I} 3 b$.
The king has a manor called Sur Moltona [South Molton]. To this has been added $\frac{1}{2}$ virgate of land called Ringhendona. ${ }^{8}$ Worth 5 shillings a year.

The king has a manor called Morlanda [Molland]. To this has been added $\frac{1}{2}$ virgate of land called Nimet $[\mathrm{Nymet}]^{9}$ which 2 sisters held in parage (pariter) T.R.E. Worth I5 shillings a year.

Odo the son of Gamelin has a manor called Limet [Nymet] ${ }^{10}$ which 4 thanes held in parage T.R.E. Worth 40 shillings.

The bishop of Exeter has a manor called Haistona [Haxon] which Ordulf held. To this has been added a manor called BonTINTONA [Button] ${ }^{11}$ that $I$ thane held in parage T.R.E. Worth 5 shillings a year.

Baldwin the sheriff has a manor called ANEsTINGHEs [Anstey]. To this has been added a manor called Ringendona [ $]^{12}$ which I thane held in parage T.R.E. Worth 3 shillings a year.

Odo son of Gamelin has a manor called Alra [Aller]. To this has been added I ferling of land ${ }^{13}$ which a certain woman held in parage (pariter) T.R.E. Worth 12 pence a year.

To Molland manor belongs since King Edward's time the third penny of the hundreds of Normoltone [Northmolton], Badentone [Bampton] and Brantona [Braunton], and (dues of every) third beast agisted on the moors (tercium animal pascue morarum). ${ }^{14}$ These customary dues the king has not had since he held England.

Baldwin has a manor called LiNcoma [Lincombe]. To this has been added a certain manor called LAIERdA ${ }^{15}$ [La Yard] which I thane held in parage T.R.E. Worth 10 shillings a year.

\footnotetext{
${ }^{8}$ Perhaps Frencheston, afias Freynstone, an outlier of South Molton. See fol. $83 b$.

${ }^{9}$ Warkleigh in South Molton hundred. See fol. 95.

${ }^{10}$ George Nymet in South Molton hundred. See fol. 377 .

"11 Button in Bratton Fleming in Braunton hundred. See fol. 118 .

${ }_{13}$ To West Anstey, Fraynes and Westacot in Chittlehampton in South Molton hundred. See fol. 300 .

is Ford next Aller in South Molton parish and hundred. See fol. $377 b$.

${ }^{14}$ See above, fol. 95 .

${ }^{15}$ Yard in Ilfracombe in Braunton hundred. See fol. 401 .
} 


\section{A HISTORY OF DEVONSHIRE}

Alvered the Breton has 1 hide of land called LAUROCHEBERA [Larkbear], ${ }^{1}$ which by right fol. 500 .

belongs to a certain manor of Baldwin called WINPLA [Whimple]. Worth 5 shillings a year.

The bishop of Coutances has a manor called TAPELEIA [Tapleigh] from which I virgate of land ${ }^{2}$ has been taken that Roger holds of the bishop. Worth Io shillings.

The bishop of Coutances has a manor called RaWEBERga [Roborough]. To this has been added a manor called BALDRINTONA [Barlington] which Alvered held, who could go with that land to what lord he liked T.R.E. Drogo holds it of the bishop. Worth 10 shillings a year; when the bishop received it the same.

The abbot of Tavestoch [Tavistock] has a manor called RADONa [Raddon] ${ }^{4}$ which $I$ thane held in parage T.R.E. and it did not belong to the abbey T.R.E. This Will[iam] the (king's) usher (bostiarius) holds of the abbot. Worth 10 shillings a year.

In the hundred of Moltone is $I$ ferding of land ${ }^{5}$ which I plough could till. It is altogether out of cultivation. No one claims it.

The bishop of Coutances has a manor called Allevescota [Aylscot]. To this has been added I virgate of land ${ }^{6}$ which I thane held, who could go with his land to what lord he liked T.R.E. Worth 10 shillings a year. This he holds of the bishop.

Ludo a knight (miles) of Walcin has a hide of land called Scoвacoma [Shapcombe] ${ }^{7}$ which by right. belongs to the manor of Odo the son of Gamelin called Hamberia [Broad Hembury]. Worth 30 shillings a year.

Hameric de Arcis has a manor called PultiMORA [Poltimore]. To this have been added $\mathrm{I} \frac{1}{2}$ hides and 3 fertings. ${ }^{8}$ Worth 30 shillings a year.

${ }^{1}$ Larkbear in Talaton in Cliston hundred. See fol. 402.

${ }^{2}$ Perhaps now the glebe of Westleigh in Fremington hundred. See fol. 124 .

${ }^{3}$ Now Wansley and Ebberly in Roborough in Fremington hundred. See fol. $124 b$.

${ }^{4}$ Chapel Raddon in Thorverton in Hairidge hundred. See fol. $\mathbf{1 7 9 b}$.

${ }^{5}$ Perhaps Cesterig alias Shortridge in Warkleigh in South Molton hundred. Feud. Aids, pp. 326, 376.

${ }^{6}$ Lower Aylscot in West Down in Braunton hundred. See fol. $127 b$.

${ }^{7}$ Shapcombe in Luppit in Arminster hundred. See fol. $348 b$.

${ }^{8}$ Poltimore in Wonford hundred. See fol. 4696.
Osbert de Salceio has a manor called SeLvesTAN [Shilston]. To this belonged I virgate of land T.R.E. which now nobody holds. ${ }^{9}$ Worth 3 shillings a year.

Fulcher the crossbowman (archibalistarius) has a manor called Chiwarthrwis [Huish]. To this has been added $I$ virgate and I ferding ${ }^{10}$ which I thane held in parage (pariter) T.R.E. Heldgold holds it of F[ulcher]. Worth I5 shillings a year.

The count of Mortain has a manor called EstocheleIA [Stockleigh] ${ }^{11}$ which Orgar held, who could go with his land to what lord he liked. This the count holds along with the lands of Edmeratorius, and Alvered the cupbearer holds it of the count. Worth 50 shillings a year; when he received it 60 shillings.

\section{fol. 50ob.}

The count of Mortain has a manor called Pocheella [Poughill] ${ }^{12}$ which 2 thanes held in parage (pariter) T.R.E. This the count holds with the lands of Edmeratorius. Alvered the cup-bearer holds it of him as one manor. Worth 25 shillings a year, and when the count received it to shillings.

The count of Mortain has a manor called Estocheleia [Stockleigh] ${ }^{13}$ which Haimer held in parage (pariter) T.R.E. This the count holds along with the lands of Edmeratorius. Alvered holds it of him. Worth 30 shillings a year; when the count received it it was worth 40 shillings.

Juhel has a manor called BRADEFORDA [Bradford] which Sawin held. To this has been added another manor called ToRnelowa [Tornelow] ${ }^{14}$ which the same Sawin held, who could go with that land to what lord he liked T.R.E. Nigel holds it of Juhel. Worth 10 shillings a year; when Juhel received it the same.

Tetbald has a manor called Comba [Combe]. To this has been added a certain manor called Gorwilla [Gorwell] ${ }^{16}$ which Colbrand who held

${ }^{8}$ Lower Shilston in Drewsteignton in Wonford hundred. See fol. 458.

${ }^{10}$ West Huish in Tedburn St. Mary in Wonford hundred. See fol. 47 I.

${ }^{11}$ South Stockleigh alias Sutton Satchvil in Cheriton Fitzpaine in West Budleigh hundred. See fol. 215.

${ }_{13}$ Poughill in West Budleigh hundred. See fol. 215.

${ }^{13}$ Stockleigh Luccombe in Cheriton Fitzpaine in West Budleigh hundred. See fol. 215.

14 Tilleslow, in Virginstow, in Lifton hundred. See fol. $317 b$.

${ }^{15}$ Gorwell in Culm Davy, in Hemyock parish and hundred. See fol. 409. 


\section{HOLDERS OF LANDS}

Comba [Combe] also held T.R.E. Oliver holds it of Tetbald and it is worth Io shillings a year. It did not belong to Comba [Combe] in King E[dward]'s time.

The king has a manor called W IRIGA [Witheridge] which Ghitda held. To this has been added the land of 2 thanes, ${ }^{1}$ who could go with their lands to what lord they liked T.R.E. On this thaneland (teglanda) are 3 villeins who pay 5 shillings to the aforesaid manor.

Baldwin has a manor called CiIsta [Clyst]. From this has been taken I ferding of land ${ }^{2}$ which by right belonged to it in King E[dward]'s time. This has been added to Odo's manor called Pontimora [Poltimore]. Worth 12 pence a year.

The count of Mortain has a manor called EstocheleIA [Stockleigh] ${ }^{3}$ which Saulf held, who could go with that land to what lord he liked T.R.E. This the count holds along with Ordulf's honour. Rainald holds it of the count. Worth 25 shillings a year; when the count received it 15 shillings.

fol. 501.

The count of Mortain has a manor called Ferentona [Faringdon] ${ }^{4}$ which 2 brothers held in parage T.R.E. This the count holds along with the honour of Edmeratorius. Bretell holds it of the count. Worth 7 shillings a year; when the count received it the same.

The count of Mortain has a manor called Colebroca [Holbrook] ${ }^{5}$ which Samer held, who could go with that land to what lord he liked T.R.E. This the count holds along with the honour of Edmeratorius. Bretell holds it of the count. Worth 15 shillings a year; when the count received it it was worth the same.

The count of Mortain has a manor called RocheBera [Rockbear] ${ }^{6}$ which Sawin held, who could go with that land to what lord he liked T.R.E. This the count holds along with the honour of Edmeratorius. Alward holds it of the count. Worth 7 shillings and 6 pence a year.

Odo has a manor called Olurintona [Worlington]. To this have been added the lands of

1 Yeatheridge and Burridge, 2 outliers in Witheridge parish and hundred. See fol. 96.

The land taken from West Clyst in Broad Clyst in Cliston hundred, added to Cutton in Poltimore. See fol. 309 .

${ }^{3}$ Stockleigh English in West Budleigh hundred. See fol. $215 b$.

' Little Faringdon in Faringdon in East Budleigh hundred. See fol. 216.

${ }^{5}$ Holbrook Grindham alias Denbow in Faringdon in East Budleigh hundred. See fol. 216.

${ }^{6}$ Rockbear in Colyton Raleigh in East Budleigh hundred. See fol. 2 r 6.
9 thanes, ${ }^{7}$ who could go with their lands to what lord they liked T.R.E. Alvei holds them of $\mathrm{O}[\mathrm{do}]$. Worth 30 shillings a year. Along with these have been added 2 other manors ${ }^{8}$ which 2 thanes held, who could go with their lands to what lord they liked T.R.E. Worth Io shillings a year.

Ralf has a manor called HaNca [Anke]. To this has been added a certain manor called Heppasteba [ $]^{9}$ which I thane held, who could go with that land to what lord he liked T.R.E. Worth 5 shillings a year.

The count of Mortain has a manor called Donevoldehamma [Densham ${ }^{10}$ which Ademar held, who could go with his land to what lord he liked T.R.E. This the count holds along with the honour of Edmeratorius. Alvered holds it of the count. Worth ro shillings a year.

fol. 5orb.

Hervei's wife (widow) has a manor called EssEorda [Exeworthy]. From this manor $\frac{1}{2}$ ferding of land 11 has been taken away which belonged there T.R.E. and added to the king's manor called SuFfertona [Silverton]. Worth 5 shillings a year.

William de Falesia has a manor called Olurintona [Worlington]. To this has been added $\frac{1}{2}$ ferding of land 12 which I thane held in parage (pariter) T.R.E. Worth 5 shillings a year.

William Capra has a manor called Orescoma [Owlacomie]. To this has been added a manor called Madescama [Woodscombe] ${ }^{13}$ which Elmer held in parage T.R.E. Hamond holds it of W[illiam]. Worth 30 pence a year.

Tetbald has a manor called WEsForda [Washford] ${ }^{14}$ which Colbert held. To this has been added another manor called WESFORDA [Washford] which 4 thanes held in parage

7 In West Worlington, possibly Afton in Witheridge hundred. See fol. 379.

${ }^{8}$ Halse in West Worlington, in Witheridge hundred, and Batchwood in Halberton in Tiverton hundred. See fol. 379 .

${ }^{9}$ Hill and Oxeton in Faringdon in East Budleigh hundred. See fol. 340 .

${ }^{10}$ Densham in Woolfardisworthy Spenser in Witheridge hundred. See fol. 2166 .

11 Probably Underleigh taken from Exeland in Bickleigh in Hairidge hundred and added to Silverton (O. J. R.) Trans. Devon. Assoc. xxxvi, 378.

12. In East Worlington in Witheridge hundred. See fol. 367 .

${ }^{13}$ Woodscombe in Cruwys Morchard in Witheridge hundred, added to Owlacombe in Bradninch. See fol. $403 b$.

14 Little Washford, an outlier of Witheridge parish in Witheridge hundred, including Upcot, Westcot, Stratton, and Westway. See fol. $409 b$. 


\section{A HISTORY OF DEVONSHIRE}

T.R.E. Worth 5 shillings a year. Along with the aforesaid Wesforda Tetbald has a manor similarly called WESFORDA [Washford] which Ulmer held in parage T.R.E. Alward holds it of Tetbald. Worth I 2 shillings and 6 pence a year. These Tetbald holds as one manor.

William Capra has a manor called Morcer [Morchard] ${ }^{1}$ which Almer held in parage T.R.E. This Alward son of Tochi took away from Almer by force (per in = per vim) since King [William] has held England. W[illiam] holds it along with Alward's honour. Worth 6 pounds a year.

Walter de Clavil has a manor called WAsFORDA [Washford]. To this has been added I ferding of land ${ }^{2}$ which I thane held in parage (pariter) T.R.E. Worth 3 shillings a year.

Tetbald has a manor called Restona [Rifton] which Ailmar held. To this has been added I virgate of land ${ }^{3}$ which $I$ thane held in parage (pariter) T.R.E. Worth 4 shillings a year.

fol. 502:

The bishop of Coutances has a manor called Chelvertesberia [? Colston] which Alwin held. To this has been added I virgate of land and $I$ ferding. ${ }^{4}$ This land $I$ thane held in parage (pariter) T.R.E. Now it lies out of cultivation.

William Capra has a manor called Alforda [Oakford] which Brismar held. To this another manor has been added called Mịnedona [Milton] ${ }^{5}$ which Editda held in parage (pariter) T.R.E. Worth Io shillings a year.

The king has a manor Coletona [Colyton] which Earl Harold held. To this has been added half a virgate of land ${ }^{6}$ which I thane held in parage (pariter) T.R.E. Worth 4 shillings.

The King has a manor called Nimetona [Nympton] which Earl Harold held. To this has been added a certain manor of half a virgate ${ }^{7}$ which I thane held in parage (pariter) T.R.E. It paid 7 shillings towards the king's farm.

William de Poilelo has a manor called BLAcHAgava [Blackgrave]. To this have been added

${ }^{1}$ Cruwys Morchard in Witheridge hundred. See fol. 404 .

${ }^{2}$ Hill town, in Witheridge parish and hundred, added to Washford Pyne. See fol. $392 b$.

${ }^{3}$ Perhaps Diptford next Rifton in Stoodleigh in Witheridge hundred. See fol. 410.

${ }^{4}$ Combe added to Colston in Templeton in -Witheridge hundred. See fol. 133 .

${ }^{5}$ Milton in Oakford, in Witheridge hundred. See fol. $404 b$.

6 Bystock locally in Withycombe Raleigh added to Colyton Raleigh in East Budleigh hundred. See fol. 966 .

7 Garland added to King's Nympton in Witheridge hundred, Feud. Aids, i, 363 . See fol. 98 .
2 manors Piedelega [Pidley] and Assacota ${ }^{8}$ which 2 thanes held in parage (pariter) T.R.E. Worth 12 shillings and 6 pence a year.

Godebold the crossbowman (archibalistarius) has a manor called Lewendona [Livaton]. To this has been added I virgate of land ${ }^{9}$ which I thane held in parage (pariter) T.R.E. Rainer holds it of G[odebold]. Worth 5 shillings a year.

William Capra has a manor called BradeFORDA [Bradford] which Almer held. To this has been added a certain land called TOREDONA ${ }^{10}$ which Alvric Colin held in parage T.R.E. Beatrix W[illiam's] sister holds it of him. Worth 40 pence a year.

Ansger de Senarpont has a manor called Chadeledona [Cheldon] which Matilda held. To this has been added another manor called Chadeledona [Cheldon] 11 that Brismer held in parage T.R.E. It paid geld for $\frac{1}{2}$ virgate and $\frac{1}{2}$ ferding. Worth 25 shillings a year.

fol. $502 \mathrm{~b}$.

William de Falesia has a manor called Chochintona [Cockington] which Alric held. To this has been added a certain manor called Depdona [Deandon] ${ }^{12}$ which the same Alric held in parage (pariter) T.R.E. It paid geld for I virgate. Worth Io shillings.

Ralf de Pomeria has a manor called CHeveToRna [Chevithorn] which Almar held. To this has been added a manor called Oppaluma [Uplowman] ${ }^{18}$ which Alwin held in parage (pariter) T.R.E. This Alwin himself holds of $\mathrm{R}$ [alf]. It paid geld for I virgate and I ferding. Worth 5 shillings.

Walter de Clavil has a manor called LoNNeLla [Lowman] which Alveron held. To this has been added a manor called Chadewilla [Kidwell] ${ }^{14}$ which Alveron held in parage (pariter) T.R.E. It paid geld for I virgate. Worth Io shillings a year.

Ralf de Pomeria has a manor called GatePADA [Gatepath]. To this have been added the

${ }^{8}$ Pidley in East Worlington and perhaps [Ash] Wood in East Worlington in Witheridge hundred added to Blackgrave. Trans. Devon. Assoc. $\mathrm{xxx}, 420$. See fol. 416.

9 Perhaps Coalsworthy in Ilsington in Teignbridge hundred added to Livaton Peverel. See fol. $472 b$.

${ }^{10}$ Down in Witheridge parish and hundred added to Bradford Tracy. See fol. $405, n .875$.

${ }^{11}$ Cheldon in Witheridge hundred. See fol. $459 b$.

12 Deandon in Widecombe-in-the-Moor in Haytor hundred added to Cockington. See fol. 3766 .

${ }^{13}$ Uplowman added to Chevithorn in Tiverton parish and hundred. See fol. $34 \mathrm{I} b$.

$14 \mathrm{Kidwell}$ added to Lowman Clavil in Uplowman in Tiverton hundred. See fol. 393. 


\section{HOLDERS OF LANDS}

lands of 4 thanes, ${ }^{1}$ who held them in parage (pariter) T.R.E. Worth 24 shillings a year.

Goselm entered upon I virgate of the villeins' land ${ }^{2}$ in the king's manor called HALSBRETONA [Halberton], and has there I plough also I bordar and I serf. It paid ro shillings towards the farm of Halsbretona [Halberton].

Goselm has $\frac{1}{2}$ hide of land ${ }^{3}$ which has been added to the lands of Bristrit. This $\frac{1}{2}$ hide a certain woman held in parage (pariter) T.R.E. Worth ro shillings a year.

To William Capra's manor called OrREA [Otterland] another manor has been added called Holescoma [Awlescombe] ${ }^{4}$ which pays 30 pence a year.

To the manor called Otria [Otterland] Ralf de Pomaria has added I manor called Holescoma [Oulescombe] $^{5}$ which pays 30 pence a year.

To Ralf de Pomaria's manor called DunestaNAETUNA [Dunston] has been added another manor of $\mathbf{I}$ virgate called BAcHEsLAC [Blackslade $]^{6}$ which Edwin held in parage (pariter) T.R.E. Worth 3 shillings a year.

Ansger de Senarpont has the lands of 5 thanes in the manor called Mochelesberia [Muxbear]. Worth 30 shillings a year.

fol. 503.

Ansger de Senarpont has a manor called Suetatona [Swetton] ${ }^{8}$ which 2 thanes held in parage (pariter) T.R.E., and that manor has been wrongfully added to Bristrit's lands. Worth Io shillings a year.

The king has a manor called Alseministra [Axminster], to which has been added another manor called Deneford [ $\quad{ }^{9}{ }^{9}$ which Ailrich held T.R.E. and it paid geld for 2 virgates. There the king has 2 villeins and I bordar. It pays Io shillings to the aforesaid manor.

${ }^{1}$ Belmarsh and Babcombe in Kingsteignton in Teignbridge hundred, added to Gappah. See fol. $34 \mathrm{I} b$.

${ }^{2}$ Oburnford, alias Pitt, in Halberton parish and hundred. See fol. I rob.

${ }^{3}$ Ash Thomas in Halberton parish and hundred. See fol. 394 .

"The land added to Awlescombe in Hemyock hundred. See fol. 405 b.

${ }^{5}$ The land added to Old Oulescombe alias Buckerel in Hemyock hundred. See fol. 342.

${ }^{6}$ Blackslade added to Dunston in Widecombe-inthe-Moor in Haytor hundred. See fol. $34^{2}$.

${ }^{7}$ Muxbear in Halberton parish and hundred. See fol. 461 .

${ }^{8}$ Swetton in Halberton parish and hundred. See fol. $46 \mathrm{I}$.

${ }^{9}$ Possibly Membury Goldclive in Membury in Axminster hundred. See fol. 85 .

I
Also in the king's manor called Arseministra [Axminster] another manor has been included called Odescliva [Undercleave] ${ }^{10}$ which Edrit the cripple (mancus) held in alms of King Edward T.R.E. Now Edward son of the same Edrit holds it of King W[illiam]. It paid geld for I virgate. This I plough can till. Worth 5 shillings a year.

To the church of Alseministra [Axminster] belongs $\frac{1}{2}$ hide ${ }^{11}$ of the land of that manor. This 2 ploughs can till, which are there, as also 12 bordars. Worth 20 shillings a year.

To Tetbald's manor called INUDEBORDA [Wideworthy] has been added a manor called Willemitona [Wilmington] ${ }^{12}$ which Alward held in parage (pariter) T.R.E. It paid geld for $\frac{1}{2}$ hide and was worth 5 shillings a year.

The count of Mortain has a manor called HonETONa [Honiton] ${ }^{13}$ which Elmer held freely T.R.E. Now it has been added to the lands of Elmeratorius. Worth 6 pounds a year.

The count of Mortain has a manor called WIBORDA [Womberneford] ${ }^{14}$ which Olward held in parage T.R.E. Worth 5 shillings a year. It has been added to the lands of Elmeratorius.

The count of Mortain has a manor called LEGA [Leigh] ${ }^{15}$ which Sawin held T.R.E. in parage. It has been added to the lands of Almeratorius. Worth 20 shillings a year. These 3 aforesaid manors Drogo holds of the count.

Hervei's wife has a manor called Witrleia [Witley] to which has been added $\frac{1}{2}$ hide of land ${ }^{16}$ that Edmar held in parage T.R.E. Worth 7 shillings a year.

fol. $503 \mathrm{~b}$.

The count of Mortain has a manor called HONETONA [Honiton] which by custom paid 30 pence a year to the king's manor called Axeministra [Axminster] T.R.E., but since the count received this land to farm from the king (the pence) have not been paid. This Drogo holds of the count.

10 Undercleave in Membury and Axminster in Axminster hundred. See fol. 85 .

11 Priestaller in Axminster parish and hundred. See fol. 85 .

12 South Wilmington in Widworthy in Colyton hundred. See fol. 410 .

${ }^{13}$ Honiton in Axminster hundred. See fol. $216 b$.

14 Womberford in Cotleigh in Colyton hundred. See fol. 217.

15 Northleigh in Colyton hundred. See fol. 217.

${ }^{16}$ Whitley in Farway, in Colyton hundred, not mentioned before. Geldroll, fol. 686, A 7 ; Trans. Devon Assoc. xxxiii, 588 ; xxxvi, 378.
69 


\section{A HISTORY OF DEVONSHIRE}

From the manor of the abbot of Horton I ferding of land and 4 salterns ${ }^{1}$ have been taken away, which Drogo holds of the count of Mortain.

To the manor of Walscin of Duaco [Douay] called Grenoweia [Greenway], which Ludo holds of him, another manor has been added Escoвecoma [Shapcombe] ${ }^{2}$ which T.R.E. belonged to HaInberia [Broad Hembury], included in Bristrit's land. Worth 20 shillings a year.

Ansger de Senardi ponte has a manor called Duveltona [Dolton] ${ }^{3}$ which Bristrit held T.R.E. in parage (pariter). It has been added to the lands of Bristrit and is worth 50 shillings a year.

Ralf de Pomaria has a manor called Afretona [Arton]. To this has been added I virgate of land ${ }^{4}$ which a certain woman held in parage T.R.E. Worth 5 shillings a year.

Ralf de Pomaria has a manor called EsMaURIGE [Smalridge] ${ }^{5}$ which T.R.E. paid by custom 30 pence towards the farm-rent of Axeministre [Axminster], a royal manor; but since $\mathrm{R}$ [alf] has had the land the king has not had the customary due.

William Capra has a manor called Manberia

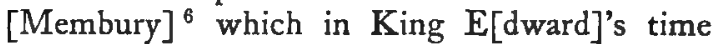
paid by custom 30 pence to Axeministre, a royal manor; but for (the last) 12 years W[illiam] has withheld that customary due.

The bishop of Coutances has a manor called Cherletona [Cherlton] ${ }^{7}$ which in King $\mathrm{E}$ [dward's] time paid by custom 30 pence to Axeministre [Axminster], a royal manor; but since the bishop has had the land, he has not paid this customary due.

The canons of Rouen have a manor called ROUERIGE [Rawridge] ${ }^{8}$ which paid 30 pence to the royal manor called Axeministra [Axminster], by custom in King $\mathrm{E}$ [dward's] time; but for a long time the canons have withheld this customary due.

The king has a manor called Cadelintona [Chillington] which Giutda held. To this

1 Salterns at Beer in Colyton hundred. See fol. $184 b$.

'Shapcombe in Luppit, taken from Broadembury, added to Greenway in Axminster hundred. See fol. 3486 .

${ }^{3}$ Dolton in North Tawton hundred. See fol. 462 .

4 Wickaborough next Arton in Berry Pomeray in Haytor hundred. See fol. $342 b$.

${ }_{5}^{5}$ Smallridge in Arminster hundred. See fol. 343.

${ }^{6}$ East Membury in Axminster hundred. See fol. $4 \circ 5 b$.

Charlton Luxon in Upottery in Axminster hundred. See fol. I35.

${ }^{8}$ Rawridge in Upottery in Axminster hundred. See fol. 195 . another manor was appurtenant in King [Edward's] time called SirEForda [Sherford] ${ }^{9}$ which the abbot of Battle [Bataille] now holds. Worth 3 pounds a year; was worth the same when the abbot received it.

fol. 504.

Walscin has a manor called Colriga [Colridge $]^{10}$ which Biche held in parage (pariter) T.R.E. This Ailric holds of W[alscin], and it has been added to the honour of Ansger which W[alscin] holds. Worth 5 shillings a year.

The same $W$ [alscin] has a manor called Wodiacoma [Widdecombe] ${ }^{11}$ which Eddric held in parage (pariter) T.R.E. This Ailric holds of $W[$ alscin], and it has been added to Ansger's honour that W[alscin] holds. Worth 15 shillings a year.

The king has a manor called DEPEForda [Diptford]. To this has been added a certain manor in the time of William de Walvilla, called FERLEIA [Farley] ${ }^{12}$ which $I$ thane held in parage (pariter) T.R.E. It pays annually to this aforesaid manor by way of farm rent ro shillings by weight.

Goscelm has a manor called BochelandA [Buckland] ${ }^{13}$ which Alvric held in parage (pariter) T.R.E. This Baldwin holds of G[oscelm], and it has been wrongfully added to Bristric's lands. Worth Io shillings a year ; when he received it the same.

Walter de Clavil has a manor called BocheLANDA [Buckland] ${ }^{14}$ which Odoman held in parage T.R.E. It has wrongfully been added to Bristric's lands. Worth Io shillings a year.

Ralf de Pomaria has a manor called CheningHEDONA [Keynedon] which Edwi held. To this has been added a manor called PoLA [Pool] $]^{15}$ which the same Edwi held in parage T.R.E. Roger holds it of $\mathrm{R}$ [alf]. Worth 10 shillings a year.

The count of Mortain has a manor called Ferdendel [Fardle] ${ }^{16}$ which Godefrid (de Valletorta) holds of him. This in King

${ }^{9}$ Sherford next Stokenham in Colridge hundred. See fol. 97.

10 Colridge in Stokenham in Colridge hundred. See fol. 349.

11 Widdecombe in Stokenham in Colridge hundred. See fol. 349 .

${ }^{12}$ Farley in Diptford in Stanborough hundred. See fol. $85 b$.

13 Buckland Toutsaints in Colridge hundred. See fol. 396 .

14 Woodmason in Buckland Toutsaints in Colridge hundred. See fol. $396 b$.

I5 Ranscombe next Pool added to Keynedon in Sherford in Colridge hundred. See fol. $343 b$.

16 Fardle in Cornwood in Ermington hundred. See fols. 85,218 . 


\section{HOLDERS OF LANDS}

$\mathrm{E}$ [dward's] time paid 30 pence by custom to Hermentona [Ermington], a royal manor, besides other customary dues belonging to the hundred; but since W[illiam] has held England all the aforesaid customary dues have been withdrawn (ablate) from the king's manor.

Raignald holds a manor of the count of Mortain called Dunitona [Dennaton] ${ }^{1}$ which in King E[dward's] time paid to Ermentone [Ermington], the king's manor, 30 pence by custom and other customary dues belonging to fol. $504 \mathrm{~b}$.

the hundred; but they have been withheld from the time that $W[i l l i a m]$ got the land.

Raignald holds a manor of the count of Mortain called Dunitona [Dennaton] ${ }^{2}$ which in King $\mathrm{E}$ [dward's] time paid 30 pence by custom to Ermentona a royal manor, besides other customary dues to the hundred; but since W[illiam] obtained the kingdom both the customary dues and the pence have been withheld therefrom.

Also from BRADEFORD [Broadaford], ${ }^{3}$ one of the count's manors which Raignald [de Valletorta] holds of the count, the customary dues and 30 pence have been withheld from the aforesaid royal manor.

Also from Ludebroca [Ludbrook] ${ }^{4}$ which Raignald holds of the count the 30 pence and customary dues of the hundred have been withheld from the aforesaid royal manor.

Alvred the Breton has a manor called BACETESBERIA [Battisborough] which Almar held. To this has been added another manor called CRAwrComa [Creacombe] ${ }^{5}$ which Almar held in parage (pariter) T.R.E. Alvred holds the two as one manor. Worth Io shillings a year.

The bishop of Coutances has a manor called Bovr [Bovey], to which have been added the lands of 15 thanes ${ }^{6}$ who were so free that they could go with their lands to what lord they liked T.R.E. They pay 4 pounds 2 shillings and 6 pence a year to the farm of Bovi [Bovey].

\footnotetext{
${ }^{1}$ Dues from Dinnaton in Cornwood in Ermington hundred. See fol. 219.

? Probably identified with Langham in Cornwood in Ermington hundred, not described in the text.

${ }^{3}$ Broadaford in Ugborough in Ermington hundred. See fol. 2 I 9 .

4South Ludbrook in Modbury in Ermington hundred. See fol. 2 I 9.

${ }^{5}$ Creacombe added to Battisborough in Holbeton in Ermington hundred. See Exch. fol. I I 5 .

6 'Thanes' lands added to Bovey 'Tracy in Teignbridge hundred. See fol. I 35 .
}

To the manor of Alvered the Breton called Morleia [Morleigh] another manor has been added called LrGa [Leigh] ${ }^{7}$ which Alwald held in parage T.R.E. and it paid geld for $\frac{1}{2}$ hide. Worth 20 shillings a year.

Along with the manor of Alvred the Breton called Miltetona [Milton] has been included $\frac{1}{2}$ hide and $\frac{1}{2}$ virgate $^{8}$ which I thane held in parage (pariter) T.R.E. Now Alvred holds the two as I manor. Worth 20 shillings a year; when Alvred received it the same.

fol. 505 .

The bishop of Coutances has a manor called Hortona [Horton] ${ }^{9}$ which Osulf held in parage (pariter) T.R.E. This has been added to the manor of HOREODE [Horwood] which the bishop himself holds. Drogo holds it of the bishop. Worth 5 shillings.

The king has a manor called W Achetona [Walkhampton] from which a manor has been taken away called Macretona [Maker]. ${ }^{10}$ King E[dward] held it T.R.E. and it paid 6 pounds all but 30 pence to this Wachetona [Walkhampton].

To the manor of Robert de Albamarula called WIDA [Widey] another manor has been added called Witeleia [Whitley] ${ }^{11}$ which Wadolo held in parage T.R.E. Worth Io shillings a year.

The count of Mortain has a manor called LODREBROC [Ludbrook] ${ }^{12}$ which Raginald holds of him. It has been added to the lands of Edmeratorius. Worth 25 shillings a year.

The count of Mortain has a manor called OlPERIGA [Lupridge ${ }^{13}$ which Raginald holds of him. It has been added to the lands of Edmeratorius. Worth 15 shillings a year.

The count of Mortain has a manor called Botestesberia [Bolbury] which Hugh [de Valletorta] holds of him. To this has been

${ }^{7}$ Grimstonleigh in Colridge hundred added to Morleigh in Stanborough hundred. See Exch. fol. 115.

Horsewell added to South Milton in Stanborough hundred. See $E x c h$. fol. I I 5 .

${ }^{9}$ Horton in Bradworthy in Blacktorington hundred added to Horwood in Fremington hundred. See fol. 122.

${ }^{10}$ Maker taken from Walkhampton in Roborough hundred. See fol. 87.

${ }^{11}$ East Whitley in St. Budeaux added to Widey in Eggbuckland in Roborough hundred. See fol. $421 b$.

${ }_{13}$ North Ludbrook in Ugborough in Ermington hundred. See fol. 2 I $g b$.

${ }^{13}$ Luperidge in North Huish in Ermington hundred. See fol. $219 b$. 


\section{A HISTORY OF DEVONSHIRE}

added another manor called BochELANDA [Buckland] ${ }^{1}$ which Eddeva held in parage T.R.E. Worth 4 shillings a year.

The count of Mortain has a manor called HEwIs [Huish] ${ }^{2}$ which Raginald holds of him. It has been added to the lands of Edmeratorius. Worth Io shillings a year.

The count has a manor called MotBeria [Modbury $]^{3}$ which Richard holds of the count. It has been added to the lands of Ordulf. Worth 30 shillings a year.

The count of Mortain has a manor called TORr [Toridge]. ${ }^{4}$ This Raginald holds of the count and it has been added to the lands of Ordulf. Worth 15 shillings a year.

The count of Mortain has a manor called Harestana [Hearston] ${ }^{5}$ which Raginald holds of him and it has been added to the lands of Ordulf. Worth Io shillings a year.

The count has a manor called Harestana [Hearston $]^{6}$ which Raginald holds of the count and it has been added to the lands of Edmeratorius. Worth Io shillings a year.

The count has a manor called Winestona

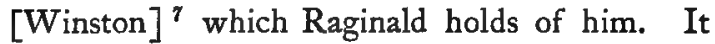
has been added to his lands. Worth 10 shillings.

fol. $505 \mathrm{~b}$.

The count of Mortain has a manor called EsPREDELeTONa [Spriddleston] ${ }^{8}$ which Raginald holds of him. It has been added to Ordulf's lands. Worth to shillings a year.

Ralf de Pomaria has a manor called WrLLeCROsTA [Weycroft] which Roger holds of R[alf], to which has been added $\frac{1}{2}$ virgate of land.9 Worth 20 pence a year.

Juhel has a manor called BAcHAMORE [Baccamoor] which Ralf holds of him. To this has been added another manor called

1 Buckland in Thurlstone added to Great Bolbury in Malborough in Stanborough hundred. See fol. 220 .

${ }^{2}$ Possibly Yedmerston in Modbury in Ermington hundred. See fol. $220 b$.

${ }^{3}$ Little Modbury in Modbury in Ermington hundred. See fol. 221 .

'Toridge in Plympton parish and huńdred. See fol. 221 .

${ }^{5}$ Lower Hearston in Brixton in Plympton hundred. See fol. 22 I $b$.

${ }^{6}$ Higher Hearston in Brixton in Plympton hundred. See fol. $221 b$.

${ }^{7}$ Winston in Brixton in Plympton hundred.

\& Spriddleston in Brixton in Plympton hundred. See fol. 222.

${ }^{9}$ Perhaps Pinney Wood in Axminster parish and hundred. See fol. $343 b$.
BACHEMORA [Baccamoor] ${ }^{10}$ that Siric held in parage T.R.E. Worth IO shillings a year.

The count of Mortain has a manor called Lisitona [Lipson] ${ }^{11}$ which Raginald holds of him. It has been added to Algar's lands. Worth $\mathrm{I} 0$ shillings a year.

The count of Mortain has a manor called WeDRERIGa [ $]^{12}$ which Raginald holds of him. It has now been added to Ordulf's lands. Worth 3 shillings a year.

Juhel has a manor called ODgForda [Woodford] which Elmar held. Now Ralf holds it of him. To this has been added another manor called Odeforda [Woodford] ${ }^{13}$ which Albrit held T.R.E. Worth 20 shillings a year.

Out of Ruald's manor called WerA [Wear] the count of Mortain has $\frac{1}{2}$ virgate, ${ }^{14}$ which Erchenbold holds of him. Worth 5 shillings a year.

The abbot of Bataillie [Battle] has in Exeter (Exonia) a church, St. Olave's (de sancto Olavo), ${ }^{15}$ and 7 houses which paid by way of customary due 4 shillings and 8 pence a year in King $E$ [dward's] time and I house quit, but he has withheld (payment).

William Capra has 2 houses $^{16}$ in Exeter which paid by way of customary due in King $\mathrm{E}$ [dward's] time $\mathrm{r} 6$ pence a year. These W[illiam] has withheld since he has had the houses.

Juhel has a house ${ }^{17}$ in Exeter which paid by way of customary due in King $E$ [dward's] time 8 pence, but he has withheld (payment).

Alvered the Breton has a house ${ }^{18}$ in Exeter which paid in King E[dward's] time 8 pence a fol. 506 .

year by way of customary due. These he has withheld since he received it.

Ralf de Pomeria has in Exeter 6 houses ${ }^{19}$ from which he has withheld the king's

${ }^{10}$ Lower Baccamoor in Plympton parish and hundred. See fol. 331 .

${ }^{11}$ Lipson part of Plymouth. See fol. 222.

${ }^{12}$ Said to be Withyhedge in Plymstock in Plympton hundred. See fol. 222b.

${ }^{13}$ Higher Woodford in Plympton parish and hundred. See fol. $333 b$.

${ }^{14}$ Lower Wear in Wear Giffard in Shebbear hundred. See fol. 4I 2 .

${ }^{15}$ St. Olave's in Fore Street. See fol. 196.

${ }^{16}$ See fol. 406.

${ }^{17}$ See fol. 334 .

${ }^{18}$ See Exch. fol. I 15.

${ }^{10}$ See fol. 3436 . 


\section{HOLDERS OF LANDS}

customary due since he has had them, i.e. 3 shillings and 4 pence.

Ruald Adobat has a house in Exeter ${ }^{1}$ which paid by way of customary due 8 pence in King E[dward's] time. These King W[illiam] never has had.

Godebold the crossbowman has 2 houses in Exeter $^{2}$ which paid by way of customary due I 6 pence a year in King E[dward's] time. These (pence) King W[illiam] never has had.

The bishop of Coutances has 6 houses in Exeter $^{3}$ which Drogo holds of him; 4 of these were quit of customary due in King E[dward's] time, and 2 paid 16 pence which Drogo keeps back.

Osbern de Salceio has a house in Exeter ${ }^{4}$ which paid by way of customary due 8 pence a year in King E[dward's] time. These he withholds.

Tetbald has a house in Exeter ${ }^{5}$ which paid by way of customary due 8 pence a year in King $\mathrm{E}$ [dward's] time. These he withholds.

The Abbot of Tavistock has a house in Exeter in mortgage (in vadimonio) from a burgess. It paid by way of customary due 8 pence in King E[dward's] time which he withholds.

William Capra has a manor called LEIA

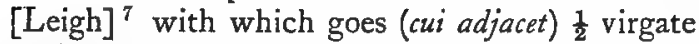
of land, which has been so kept from notice (celata) that the king has not had his geld.

Robert son of Ivo holds a virgate of land ${ }^{8}$ of the count of Mortain in the manor called

${ }^{1}$ See Exch. fol. I $16 . \quad{ }^{2}$ See fol. 473.

See fol. $462 b$

${ }^{5}$ See fol. 4 Iol. $\quad{ }^{6}$ The Bear Inn. See fol. I $80 b$.

${ }^{7}$ Eastleigh in Westleigh in Fremington hundred. See fol. 3996 .

${ }^{8}$ Perhaps the outlier belonging to Bulkworthy in East Putford in Shebbear hundred. See fol. 2 II.
Bochesurde [Bulkworthy] wlich has hitherto been kept from notice. From this he has withheld the geld.

The bishop of Exeter has a manor called SIdeberia [Sidbury] ${ }^{8}$ which 2 thanes held in parage (pariter), namely Alwin and Godvin. It was worth 6 pounds a year; when the bishop received it the same.

The bishop of Coutances has a manor called CRIDIA ${ }^{10}$ [Creedy] which Goda held in parage (pariter) T.R.E. Worth 5 shillings a year.

The same bishop has a manor called TALEBREIA [Thelbridge]. To this has been added a manor called WICA [Wick ${ }^{\text {Il }}$ that did not belong to the above-named manor. Worth 5 shillings.

Richard son of Turulf has a house in Exeter ${ }^{12}$ from which he withholds the king's customary due, namely 8 pence a year.

fol. $506 \mathrm{~b}$.

Walcin has a house in Exeter ${ }^{13}$ in mortgage from a burgess, from which the king's customary due has not been paid, namely 8 pence.

Baldwin has a manor called CRABEComa [Creacombe] ${ }^{14}$ which Seiward held. To this has been added a manor called CRAwEComa [Creacombe] which Leuegar held in parage T.R.E. Worth 5 shillings a year; when Baldwin received it it was worth 2 shillings.

${ }^{9}$ Sidbury in East Budleigh hundred. See fol. I I $8 b$.

${ }^{10}$ Haske on the Creedy in Upton Helion in West Budleigh hundred. See fol. 132b.

"Middlewick in Thelbridge in Witheridge hundred. See fol. I 34 .

12 See Exch. fol. I 13.

${ }_{13}$ Perhaps one of the 10 named, fol. 3496.

${ }^{14}$ Creacombe in Witheridge hundred. See fol. $310 b$. 


\section{FEUDAL BARONAGE}

\section{THE HONOUR OF PLYMPTON AND THE EARLDOM OF}

DEVON

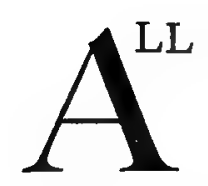

the manors of tenants-in-chief and their knights which did not belong to other honours in or out of the county, most of the manors held by the king's military and household officers, and many of those held by his English thanes, together with the majority of the royal estates which had been granted out after the date of Domesday, and a few manors taken from other honours, went to make up the great honour of Plympton, second only in extent to that of Okehampton, and superior to it by virtue of its connexion with the earldom, it having been bestowed on the Redvers family in the twelfth century, together with the earldom of Devon. It consisted of eighty-nine fees, ${ }^{1}$ and was held of the crown for two and a half fees. ${ }^{2} \quad$ It is usually stated that Henry I created the earldom of Devon in favour of Richard de Redvers, lord of Néhou, when he bestowed upon him the castles of Plympton and Tiverton, ${ }^{3}$ but Mr. Round, not without reason, asserts that Richard's son Baldwin was the first earl of Devon, and was advanced to the honour by the Empress Maud shortly before June, I I $1{ }^{4}{ }^{4}$ being previously styled (as in I I $23^{5}$ ) Baldwin de Redvers simply. Baldwin's son Richard, who succeeded him in I I 55, married Hawise, daughter of Reginald, earl of Cornwall, but died in I I $62,{ }^{6}$ when his father-in-law was put into possession, ${ }^{7}$ probably as guardian of his own grandson Baldwin. From this Baldwin it passed to his brother Richard, and from Richard to his uncle, William de Vernon. When William died, on Io September, I2I7, it came to his grandson, another Baldwin, and on his death, in $1244,{ }^{8}$ to his infant son Baldwin, born in 1235 , who died 28 August, I 262. His sister, Isabella de Fortibus, countess of Albemarle, was then found to be his next heir, ${ }^{9}$ and succeeded as such. She survived her husband and all her children, and died in I 293, when Hugh Courteney, the second of that name, great-grandson of Mary, daughter of William de Vernon, obtained the honour, and in I 335 was authorized to assume the title of earl of Devon, ${ }^{10}$ as the heir of the de Redvers family, and in possession of their

${ }^{1}$ Testa de Nevill (Rec. Com.), p. 1876 in Trans. Devon. Assoc. xxix. 500. A list will be found in Testa de Nevill (Rec. Com.), p. I 81 , also in Inq. p.m. I Ric. II, No. 1 2, and I Hen. VI, No. 63. See also Cal." Inq. Hen. III, pp. I 75-6.

"Trans. Devon. Assoc. xxxii, 540.

3 Trans. Devon. Assoc. xxix, 457, 540; xix, 556 ; vii, 363 ; Dugdale, Baronage, i, 255 ; Risdon's Notebook, 56 .

${ }^{4}$ Geoffrey de Mandeville, 271-2.

${ }^{5}$ Feudal England, 486.

${ }^{6}$ Pipe R. 8 Hen. II, in Trans. Devon. Assoc. xxix, 478.

${ }^{7}$ Pipe R. 14 Hen. II, ibid. xxxiii, 366.

${ }^{8}$ Cal. Inq. Hen. III, No. 50.

${ }^{9}$ Inq. p.m. 47, Hon. III, No. 23. Cal. Inq. Hen. III, No. 564. See also, for her heirship, Round, Genealogical Mag. i, 4 .

${ }^{10}$ In 9 Edw. III. See case in the House of Lords, 1832. 


\section{A HISTORY OF DEVONSHIRE}

estates. $^{1}$ In his person the honours of Plympton and Okehampton were united.

Foremost among the estates held of the honour were those enjoyed at the time of the Domesday Survey by 'Ruald Adobed' (Rhiwallon the dubbed knight), 2 consisting of thirty-two manors, assessed at I4 hides, and containing more than I 3,000 acres under cultivation. When Ruald entered religion these estates reverted to the crown, excepting the church-manor of Poughill, which he gave with himself to St. Nicholas' Priory. ${ }^{3}$ Apparently Roger, son of Stephen de Mandevil, then held them at pleasure, but before I 160 thirteen and a half fees were held of the honour by Walter Giffard and Eustace his son, ${ }^{5}$ and continued in that family until 1276 , when Emma, daughter of Walter Giffard and Alice his wife, died a lunatic, leaving, by Hugh de Widworthy, her husband, an only daughter, Emma, who married Robert de Dinham, and died without issue. ${ }^{\circ}$

Another group, consisting of twenty-one manors, formerly held by the Saxons Alric, Brismer, Heche, Ulnod, and Ulward, assessed at I $4 \frac{1}{4}$ hides, with about I 3,000 acres under cultivation, was held at the time of the survey by William de Poillei. By two charters, dated respectively 1093 and Iog6, he gave the tithe of them all to St. Martin and the monastery of Sées. ${ }^{7} \mathrm{He}$ had a son Robert, ${ }^{7}$ but his estates were at a very early time dispersed. Apparently Poillei's estates formed a group of ten fees. ${ }^{8}$

Another group of estates, afterwards held of the honour of Plympton, were the sixteen manors acquired from the dispossessed Saxons Ulward, Bristold, and Wadelo, assessed at I I hides, with some I0,500 acres under cultivation, which Robert de Albemarle held at the time of the survey. They formed a group of fourteen fees, which continued for many generations in his family, ${ }^{\theta}$ but eleven were quit-claimed to his overlord by Ralph Damerleigh. ${ }^{10}$

Robert Bastard held at the time of the survey nine manors, assessed at $2 \frac{1}{2}$ hides, with about 2,000 acres, acquired from the Saxons Alvric and Alwey. Goislen Bastard held them in $1160,{ }^{11}$ Nicolas Bastard in $1243,{ }^{12}$ Baldwin Bastard in $1286 .^{13}$ They formed a group of three and a half fees. ${ }^{14}$

${ }^{1}$ According to ' the case in the House of Lords, 1832,' Hugh II de Courteney, the first earl of the new line, died 14 Edw. III. His son, Hugh III, second earl of Devon, died 5 I Edw. III, and was succeeded by his grandson, Sir Edward Courteney, Sir Edward's uncle being Sir Philip of Powderham. On the death of the third earl in $7 \mathrm{Hen} . \mathrm{V}$, his son, Sir Hugh, succeeded as fourth earl, but died $10 \mathrm{Hen}$. V. Thomas Courteney, his son, succeeded as fifth earl, and died $36 \mathrm{Hen}$. VI. The sixth earl was his grandson, Thomas Courteney, who was attainted, and beheaded I Edw. IV. In I Hen. VII the earldom was revived in favour of Sir Edward Courteney, who died in 1509 . His son William and grandson Henry having both been attainted, a new grant of the earldom was made by patent on 3 September, 1553 , to his great-grandson, Sir Edward Courteney, without reversal of the attainder of his ancestor. On his death, on 18 September, 1556, the four daughters of Sir Hugh Courteney of Boconnoc in Cornwall, married respectively to W. Mohun, J. Arundel, J. Trethurfe, and J. Trelawny, were found to be his next of kin, but the attainder barred his and their succession to the lands anciently held with the earldom.

${ }^{2}$ Freeman, Norman Conquest, iv, 173 ; iii, $233 . \quad{ }^{3}$ Oliver, Mon. I 9.

${ }^{1}$ He gave the church of Aveton Giffard, one of Ruald's estates, to Plympton, ibid. I 35.

' Ibid. shows that in that year Walter Giffard gave to Plympton Priory lands formerly Ruald's at Yealmpstone, Awton Giffard, also North and South Brentor, Longstone, Rawdon, and Waterfall in Lamerton parish. Cal. Inq. Her. ILoI, p. 176, gives Giffard's fees as fifteen.

${ }^{6}$ Wrottesley, The Giffards, I 3.

${ }^{8}$ Cal. Inq. Hen. III, p. I76; ten (unspecified) fees held by the heirs of William de Brywere, and 'they ought to find a crossbowman in Plumpton Castle at their own cost.'

${ }^{9}$ Trans. Devon. Assoc. xxix, 465, n. 28 ; Cal. Inq. Hen. III, p. 176.

${ }^{10}$ Trans. Devon. Assoc. xxxii, 541 .

13 Testa de Nevill (Rec. Com.), pp. $189 b, 1916,192 b, 194 a$.

${ }^{13}$ Feud. Aids, pp. 340,341, 350 .

${ }_{14}$ Cal. Inq. Hen. III, p. 176. 


\section{FEUDAL BARONAGE}

Of the estates subsequently held of the honour of Plympton, Ralf Paynel, had in 1086 ten manors, ${ }^{1}$ all previously held by Merlesuein, sheriff of Lincolnshire, assessed at I2. hides, with over 6,000 acres at the time of the survey. These estates had been given to his father, William Paynel, in 1069 , when Merlesuein was dispossessed of them after his rebellion ; ${ }^{2}$ the same William Paynel subscribed a charter to Bayeux Cathedral in 1073, and died in 1084. ${ }^{\circ}$ Ralf Paynel married Beatrice, daughter and heiress of William, son of Ansculf, and had a son, Fulk Paynel, but his Devonshire estates, as well as Roger de Buslei's, passed at an early date to William Peverel and his sister Maud, ${ }^{4}$ who bestowed Sampford Peverel, Aller Peverel, and Karswell upon Hugh Peverel in the time of Henry I, and suffered a forfeiture in the reign of Henry II for supporting Stephen against the Empress Maud. ${ }^{5}$ Throwleigh and Edginswell then passed to Fulk de Ferrers, ${ }^{8}$ Ilsington and Ingsdon ${ }^{7}$ to Beaumont.

A second, part of whose estates were afterwards held of the honour of Plympton, was Osbern de Salceio, or de Salceid. He held five manors, assessed at 5 hides, with rather more than 2,000 acres, which had belonged to five different Saxons. Two at least of these were afterwards $\mathrm{Hugh}$ de Sausey's, ${ }^{8}$ but Clyst Gerard went to Mandevil. ${ }^{\circ}$

A third baron was Roger de Buslei. He had only one manor in Devon, Sampford Peverel, which had been Brictric's, with an assessment of $3 \frac{1}{2}$ hides and about $\mathrm{I}, 000$ acres under cultivation. It went to Peverel, being given to Hugh Peverel, by whose descendant it was held in $1286 .{ }^{10}$ A fourth, whose estates went to the honour of Plympton, was Ralf de Fougères. He held two manors assessed at 6 hides all but half a virgate, with some 2,000 acres under cultivation at the time of the survey from which the Saxon Goda had been dispossessed. Ralf is stated to have been the youngest and only surviving son of Alfred, son of Maen II, baron of Fougères in Brittany. He succeeded his father in 1084 , and by his wife Alice, daughter of Richard de Bienfaite, had seven sons. ${ }^{11}$ One of these was ancestor of Ralf de Meulent or Mullond, ${ }^{12}$ who forfeited Ipplepen and Galmpton in King John's time, ${ }^{13}$ when they were bestowed successively on Henry, the earl's son, ${ }^{14}$ Nicholas del Estre, ${ }^{15}$ and Americ de St. Amand. ${ }^{16}$

The fifth and last of the 'French knights' who held of the honour of Plympton was Girard, who held two manors in chief, Pole Antony (the 'Manelia' of Domesday) and Northcot, with an assessment of half a hide and rather more than 300 acres under cultivation. He held besides two other manors, Little Tiverton and Little Washfield, as under-tenant of Ralf Paynel.

\footnotetext{
${ }^{1}$ In Dunchideock (fol. 457), Karswell and Dulford, Aller Peverel, Throwleigh, Wonston (fol. 458b), Ilsington, Ingsdon, Little Tiverton, Little Washfield (fol.460), and Edginswell (fol. 461 $b$ ).

${ }^{2}$ Planche, The Conqueror and bis Companions, ii, 235.

4 Ibid. Testa de Nevill (Rec. Com.), 194b; Trans. Devon. Assoc. xxxii, 4 I 6.

${ }^{5}$ Planché, The Conqueror and bis Companions, ii, 235 ; Dugdale, Bar. i, 437.

${ }^{5}$ Feud. Aids, 313,317 ; Cal. Inq. Hen. 111, p. 176.

'Feud. Aids, 339. Cal. Inq. Hen. III, p. I76; 'Elstinton' and Shirwell, two fees held by Philip de Beaumont.

${ }^{8}$ Ingsdon and Shilston, Cal. Inq. Hen.III, p. I76. ${ }^{9}$ Feud. Aids, 333.

${ }^{10}$ Testa de Nevill (Rec. Com.), p. 194a. Feud. Aids, 338.

11 Planché, The Conqueror and bis Companions, ii, 225. ${ }^{12}$ Testa de Nevill (Rec. Com.), pp. 195a, 197b.

${ }^{13}$ Ibid. p. $197 b$.

${ }^{15}$ In 1219. Testa de Nevill (Rec. Com.), p. I 976.

${ }^{16}$ In I234. Cal. Charter $R$. I9 Hen. III, m. 3 . 


\section{A HISTORY OF DEVONSHIRE}

The two last named passed to the crown by escheat in $1162^{1}$ and were given to William Briwere; the two former were also held of Briwere's heirs in I 286, Chaworth holding Pole Antony, ${ }^{2}$ and Mohun holding Northcot. ${ }^{8}$

With the honour of Plympton also went thirty-nine manors, held in 1086 by the king's military officers, with an assessment of $27 \frac{1}{2}$ hides and about 12,500 acres under cultivation, eleven manors held by his household officers, with an assessment of 9 hides and some 5,000 acres, and fourteen manors held by the Englishmen Adret, Alward and Saulf, Edwin, Ulf, Odo son of Edric, Alveva, Alric, and Leuric, ${ }^{4}$ with an assessment of $4 \frac{1}{2}$ hides and rather less than 2,500 acres essarted from the forest, making a total for the honour of ${ }_{1} 85$ manors assessed at $125^{\frac{1}{2}}$ hides, with a cultivated area of roughly 86,000 acres. To the above must be added the royal estates which passed by grant after the date of the survey into the hands of subjects, and were held of this honour, viz. Wonford and Halsford, ${ }^{5}$ North Tawton, ${ }^{6}$ Exminster, ${ }^{7}$ Ermington Peverel, ${ }^{8}$ Walkhampton ${ }^{2}$ including Sheepstor, ${ }^{10}$ Bradstone, ${ }^{11}$ Witheridge, ${ }^{12}$ Stokenham, ${ }^{13}$ Herdwick and Plympton, ${ }^{14}$ and Tiverton $;{ }^{15}$ also Honiton, ${ }^{16}$ which had been the count of Mortain's, besides six manors, which at the time of the survey were Baldwin's, viz. Middlecot, Torington, Hele Poer, Woodington, Whiteway, and 'Alraford'; three which were Juhel's, viz. Eggbuckland, Compton Giffard, and Hooe; and one which was Odo's, viz. Plymtree.

\section{THE HONOUR OF OKEHAMPTON}

The largest landholder in Devon at the time of the Domesday Survey was Baldwin de Brionis, son of Count Gilbert de Brionne, in Normandy, called also Baldwin de Molis, from the castle of Meules where he was born, or de Clare ${ }^{17}$ but usually, Baldwin the sheriff. He owned 177 manors assessed at 146 hides, and comprising roughly 100,000 acres under cultivation in the county. With six exceptions, viz. Middlecot, Whiteway, and 'Alraford,' of which three Rannulf was under-tenant, ${ }^{18}$ Hele Poer and Woodington, of which William the usher was under-tenant, and Torington, of which Richard was under-tenant, all Baldwin's estates are subsequently found held of the honour of Okehampton. That honour included in addition three fees held of the bishop of Exeter, ${ }^{19}$ at Yeo in Crediton, ${ }^{20}$ at Dittisham and at Slapton, ${ }^{21}$ one of the bishop of Coutances' estates, viz. Brampford Speke, ${ }^{22}$ one of William Capra's, viz. Hunshaw, ${ }^{88}$ two estates held by Godeva, Brictric's widow, viz.

${ }^{1}$ Whale in Trons. Devon. Assoc. xxxiii, 397.

${ }^{8}$ Feud. Aids, 2 I 9.

${ }^{3}$ Ibid.

4i.e. North Wick, Kismilton, Butterleigh, Wadham, Southwood, Codaford, Clyst William, St. James' Church, Warsbrightleigh, Twyney, Goodameavy, Northweek, Rousdon, and Ashford Peverel. See above, Domesday Survey.

${ }^{5}$ Feud. Aids, 3 1 3. ${ }^{6}$ Cal. Inq. Hen. III, p. I76. Inq. p.m. I Ric. II, No. I 2.

7 The submanors of Exminster, Breynton, Haldon, and Southwood are all held of Plympton in Cal. Inq. Hen. III, p. 176 and Feud. Aids, 389 .
${ }^{8}$ Cal. Inq. Hen. III, p. 175 .
${ }^{12}$ Feud. Aids, 342.
${ }^{9}$ Ibid. p. 176.
10 Ibid.
11 Ibid.
Stokes Herbert, Cal. Inq. Hen. III, p. 175.

- Cal. Ing. Hen. III, p. I 2, in 29 Hen. III, Baldwin de Ripariis, sometime earl of Devon, died, seised of 'Erdewik and Bottesford,' with the hundred court and borough of Plympton. Ibid. p. 176; 'Herdewik' manor with lands in Hysley, co. Cornwall.

${ }_{15}$ Cal. Inq. Hen. III, p. I2; 'Thuverton' manor and borough with the hundred court.

${ }^{16}$ Ibid. p. 176.

${ }^{17}$ Round, Feudal Engl. 473.

${ }^{18}$ Presuming that 'Alraforde' is Rosamondford, Briwere was successor to Rannulf in all three.

${ }^{19}$ Lib. Niger, 115.

${ }^{21}$ Ibid. 331 . Trans. Devon. Assoc. xxxii, 542.

${ }^{23}$ Trans. Devon. Assoc. xxxvi, 353.

${ }^{22}$ Inq. p.m. I Ric. II, No. I 2. 


\section{FEUDAL BARONAGE}

Torbryan ${ }^{1}$ and Dodbrook, ${ }^{2}$ and Saulf's Little Dunsford or Sowton ${ }^{8}$ in all $92 \frac{3}{4}$ fees $;{ }^{4}$ and it was held by the service of three knights. ${ }^{5}$

Baldwin was twice married, (I) to Albreda the Conqueror's niece ${ }^{6}$ or cousin, ${ }^{7}$ and (2) to Emma, ${ }^{8}$ and had issue three sons, William, Robert, and Richard, ${ }^{9}$ and a daughter Adeliza, and possibly another, Emma. Mr. Round gives the three sons to Emma, though he is doubtful about William. ${ }^{10}$ Planché gives her only the two daughters. ${ }^{11}$ William succeeded his father as sheriff ${ }^{12}$ in $1090,{ }^{13}$ and was succeeded by his brother Richard, who held that office together with the honour of Okehampton in II 29. ${ }^{14}$ Richard died without issue on 25 June I $37,{ }^{15}$ when the sisters became his heirs. The elder one Adeliza was married to William, son of Wimund, ${ }^{16}$ and died in I 142 after founding Ford Abbey. ${ }^{17}$ The exact descent of the honour now becomes involved in considerable doubt, but it appears to have passed through the Avenel family to Maud d'Avranches, whose second husband, Robert, a natural son of Henry I, was in possession of it in I I 66. ${ }^{18}$ Upon the death of Robert in II 72 and that of his widow in the following year ${ }^{19}$ the honour came into the hands of Reginald de Courteney, who appears to have availed himself of a grant of the wardship of the two daughters to marry his son William to Hawise, daughter of Maud d'Avranches by her first husband, and to himself marry Maud's namesake and daughter by her second husband, Robert the king's son. ${ }^{20}$ The honour of Okehampton thus came to the Courteney family, in which it continued until Thomas Courteney was attainted in the first year of Edward IV.

As we glance through the list of the Okehampton retainers in the Black $B o o k,{ }^{21}$ the feature to attract attention is their number rather than the size of their holdings. The Domesday Rogo is represented by Simon son of Rogo, who held five fees, which continued in his family for many generations. ${ }^{22}$ Hugh of Rennes has given place to Guy de Brionna or Bryan, who held five fees which the Bryans continued to possess up to the end of the thirteenth century. ${ }^{23}$ Otley has been succeeded by Hidon the holder of $6 \frac{1}{2}$ fees, which after several generations passed to the Dinhams. ${ }^{2 *}$ Ralf de Brueria is represented by Antony de Bruiera the holder of five fees, and the latter by William de la Bruere in I 24I, ${ }^{25}$ after whom they passed by co-heiresses to Gras and

${ }^{1}$ Feud. Aids, 317.

2 Ibid. 332.

${ }^{3}$ Ibid. 314.

${ }^{4}$ Red Bk. of Exch. (Rolls Ser.), p. $55^{8}$; Trans. Devon. Assoc. xxvii, 99 seq.

${ }^{5}$ Authority quoted by Whale in Trans. Devon. Assoc. xxxii, 542 .

6 Oliver, Mon. 338. Trans. Devon. Assoc. xxx, 506.

7 Planché, The Conqueror and his Companions, ii, 43.

${ }^{9}$ Cal. of Doc. France, 524.

${ }^{8}$ Feud. Engl. 473. Exeter Domesday, fol. 307.

${ }^{10}$ Feud. Engl. 473 .

Planché, The Conqueror and his Companions, ii, 44

${ }^{12}$ Charters in Oliver, Mon. 1 I 7, I 53 ; Round, Feud. Engl. 330, n. 37.

${ }^{13}$ Planché, loc. cit. 43.

${ }^{15}$ Oliver, Mon. $338 .{ }^{16}$ Exeter Domesday, fol. 295.

${ }^{18}$ Round, Feud. Engl. 266 ; Lib. Niger, 1 I 9.

${ }^{17}$ Oliver, Mon. 342.

${ }^{19}$ Dugdale, Mon. v, 381 .

${ }^{20}$ Close $8 \mathrm{Hen}$. III, m. 8. There appears to be some doubt as to the identity of Maud's first husband. $\mathrm{He}$ is usually supposed to have been Robert d'Avranches (Courthope, Historic Peerage, I 28), but is called in Bracton's Notebook (ed. Maitland, case I 70) 'Geoffrey de Crimes' and (case 1,569) 'William de Curcy.'

${ }^{21}$ Lib. Niger, I 19. Red Bk. of Exch. (Rolls Ser.), 25 1-4. Compare therewith a list given by Mr. Whale in Trans. Devon. Assoc. xxxii, 543, and the Testa de Nevill.

${ }^{22}$ Whale in Trans. Devon. Assoc. xxxii, 543.

${ }^{23}$ In 1286 Guy Bryan held Melhuish and Teign Harvey (Feud. Aids, 3 I 3).

24 Trans. Devon. Assoc. xxxii, 543 .

${ }^{25}$ Feud. Aids, 318. Ralf de Brueria must not be confounded with the family of Briwere, to which the famous judge belonged, the founder of Tor Abbey. 


\section{A HISTORY OF DEVONSHIRE}

Courteney. ${ }^{1}$ Ansger is represented at Roseash and elsewhere by Baldwin de Esse, the holder of two fees, which continued in that family for several generations, ${ }^{2}$ but at West Buckland by Hugh de Raleigh the holder of half a fee ; Robert de Beaumont, by Thomas de Beaumont, whose heiress in the time of Henry VII carried four fees to the family of Chichester ; Roger de Molis, by Joel de Molis, whose family held four fees till the reign of Edward III, when they passed to Prous and Chudleigh ; Rainer, Baldwin's house-steward, by Roger de Langford, whose family remained in possession of four fees until John de Langford, ${ }^{3}$ the last of that name, disposed of them piecemeal. Modbert son of Lambert had been succeeded by Nicholas de Kelly, whose three fees still remain with the family of that name. Of the two Richards, Baldwin's undertenants, Richard son of Torolf, who held Belstone, had been succeeded by Baldwin de Belstone the holder of three fees, whose co-heiresses in the reign of Henry III carried them to Speccot, Chamberlain, and Fulford; whilst Richard de Novil, who held Wembworthy as his head manor, has given place to Richard Speke, also the holder of three fees, whose family continued in possession of them to the time of Charles I. " To Robert de Punchardon, the Domesday tenant of Heanton Punchardon, succeeded William de Punchardon, the holder of four fees, which his descendants continued to hold until co-heiresses brought them to Beaumont and Basset; but at Ilfracombe and elsewhere he had for successor Henry de Champernown, also the holder of four fees, from whose family they passed after several generations to Polglass and chief-justice Herle. To Walter, Baldwin's butler, succeeded Roland de Nimet, the holder of one fee at Nymet Roland, Rolandsleigh and Beer, which was afterwards in the family of Wolrington and passed to Hach and Buckington. Morey, the tenant of Stedcombe, has been succeeded by Robert de Ver and Robert Poer. Stephen, the tenant of West Clifford, Ringmore, and Haccombe, has been succeeded by William son of Stephen, who held one fee of Okehampton, which continued in his family until an heiress carried it to the Ercedekne family. Bernard 'the napeless' is represented by Robert son of Bernard, who held $2 \frac{1}{2}$ fees, but these were soon distributed between Ralf son of William, ${ }^{5}$ Alan son of Rouald, ${ }^{6}$ Tregony, ${ }^{7}$ and Speke; ${ }^{8}$ and Goscelin Bervin is succeeded by William Bervin, who held two fees, which, before the end of the thirteenth century had passed to Tirel ${ }^{9}$ and Giffard. ${ }^{10}$ Excepting at Clannaborough, Walson, and Thorn, Ralf de Pomeray as under-tenant of Baldwin is represented by Punchardon and Bolley. ${ }^{11}$

1 Trans. Devon. Assoc. xxxii, 543 .

3 Inq. p. m. 17 Edw. III, No. 18.

${ }^{2}$ Ralf de Esse was in possession in 1286 (Feud. Aids, 325).

${ }^{4}$ Richard le Espek, also Quespec, Espet, or Speke, held also two fees of the honour of Barnstaple (Lib. Niger, 123), of which Brampford Speke was doubtless a part (Feud. Aids, 3 I 5, 385, 486). These latter fees he got through his wife, Margaret, daughter and heiress of William 'de tribus minutis' or 'treimenettis,' granddaughter of Walter 'de tribus minutis.' He was succeeded by his son, Robert de Spec, and he by his brother William (Notes and Qu. 14 January 1905, p. 30). To William succeeded Richard, whose heir William (Feud. Aids, 370) held Wembworthy and Brampford in $124 \mathrm{I}$ (Testa de Nevill, pp. 179b, I 75b), but Little Torington was then Champernown's. Pole, 424; Lysons, 55 I.

${ }^{5}$ Feud. Aids, 3 I 3, Rocombe Cadio.

${ }^{7}$ Ibid. 3 13 , Minnicombe.

Ibid. 314, 329, Westtown and Meeth.

${ }^{9}$ Ibid. 317,343 , Oldridge and Rackenford.

${ }^{10}$ Ibid. 368 ; Trans. Devon. Assoc. xxxvi, 292, Rockbear, Selake.

${ }^{11}$ Feud. Aids. 3 I 4, shows Hittisleigh half a fee held by John de Punchardon of Jacob de Bollei ; Colhey, in Tedburn, half a fee held of John de Punchardon. Testa de Nevill, pp. 180b, I 886 , shows Bridestow held by Muriel de Bollei, but does not name the mesne lord of either Clannaborough, described as one fee, or of Mamhead, one fee. See below p. 562 . 


\section{FEUDAL BARONAGE}

\section{THE HONOUR OF BARNSTAPLE}

The bishop of Coutances was entered in Domesday among church tenants because he was a bishop, but his barony was a lay rather than an ecclesiastical fief. On his death in 1093 it passed to his nephew, Robert de Mowbray, earl of Northumberland, and on Robert's rebellion and forfeiture two years later it was disintegrated. The Somersetshire estates, together with Clyst Fomison or Sowton ${ }^{1}$ and Canonteign in Devon, were given by William Rufus to Robert son of Hamon ${ }^{2}$ and passed with his daughter to Robert first earl of Gloucester ; ${ }^{3}$ Welcombe the king retained in his own hands ; ${ }^{4}$ Highbray, with Bray and Bratton Fleming, was attached to the honour of Launceston, ${ }^{5}$ Brampford Speke to the honour of Okehampton, ${ }^{6}$ whilst the rest of the bishop's estates in Devon, together with Ralf de Limesei's Roadway and Morthoe, ${ }^{7}$ and Walter de Dowai's Hockford ${ }^{8}$ (fol. 346), constituring the honour of Barnstaple, were given to Juhel of Totnes in extreme old age, ${ }^{9}$ and from him passed to his son Alfred. ${ }^{10}$ In I 166 the honour was held in two moieties, one moiety by the second William de Braose, who succeeded his father, Philip, in I I 55, and was the son of Juhel's daughter; ${ }^{11}$ the other moiety by Oliver de Tracy, who succeeded his father, Henry de Tracy, in I I 65 , and was also a descendant of Juhel. ${ }^{18}$ William de Braose's fees then amounted to $28,{ }^{14}$ those of Oliver de Tracy to $23 \frac{1}{2} .{ }^{15}$ Those of the whole barony amounted to 56 in $1234{ }^{16}$ to 52 in $124 \mathrm{I} .{ }^{17}$

In I I 78 Oliver de Tracy was succeeded in his moiety by his son, Oliver, the younger ${ }_{,}^{18}$ from whom, in I I 94 , William de Braose sought acknowledgement of his right to the other moiety. ${ }^{19}$ The result was an agreement whereby Oliver acquired all the estates belonging to the honour of Barnstaple, excepting Fremington and 5 fees. ${ }^{20}$ In 12 IO Oliver was succeeded by his

1 Testa de Nevill (Rec. Com.), I 78a; Feud. Aids, 315.

- Eyton, Somerset, i, 56 ; Trans. Devon. Assoc. xxxiii, 631 .

8 Round, in V.C.H. Northants, i, 288.

1 Pole, 37 I, and Lysons, ii, 549, say that it was bestowed on fitz-Richard, confounding it with Capra's Woolacombe Tracy. Testa de Nevill (Rec. Com.), 179a, shows it held of the king in chief appurtenant to the Somersetshire manor of Orchardley (fol. 147). Orchardley was given by Hen. II to Henry de Cultura (Lib. Niger, 97 ; Collinson, Somerset, ii, 222), whose successor in $124 \mathrm{I}$ held both it and Welcombe.

${ }^{5}$ Feud. Aids, i, 437 ; Trans. Devon. Assoc. xxxiv, 567.

${ }^{6}$ Feud. Aids, $315 . \quad{ }^{7}$ Testa de Nevill (Rec. Com.), I75a. ${ }^{8}$ Ibid. I76a.

${ }^{9}$ So say the Hundred Rolls of 3 Edw. I (Rec. Com.), p. 70. Round, Feud. Engl. 486, quotes an authority to show that Juhel held the honour in 1113 ; see below, p. 559 .

${ }^{10}$ Oliver, Mon. 199 ; Pipe R. 3 I Hen. I, Alvred son of Johel accounts for fil 10 for relief of his father's land.

" Round, Cal. Doc. France, 36o. Lib. Niger, 127 ; Dugdale, Bar. i, 414 ; Trans. Devon. Assoc. xxxvii, 422, n. 12 ; Oliver, Mon. 200; Testa de Nevill (Rec. Com.), 195a, states that Henry II also gave him the crown manor of Tawstock; Trans. Devon. Assoc. xxxvii, 422.

${ }_{12}$ Lib. Niger. I 2 2, Pipe R. I I Hen. II in Trans. Devon. Assoc. xxix, 488, 490; Dugdale, Bar. i, 62 I.

${ }^{13}$ This was the Henry de Tracy who put down William de Mohun's insurrection in I 149 (Trans. Devon. Assoc. vi, 183), and in 1146 confirmed the donation of 'Johel son of Alvred' (Oliver, Mon. I98), and the donations of 'Alvred of Totnes' to Barnstaple Priory (ibid. 199). Possibly he may have married a daughter of Alvred.

${ }^{14}$ Lib. Niger, 127 ; Trans. Devon. Assoc. xxxiii, 381. ${ }^{15}$ Lib. Niger, 122 ; Trans. Devon. Assoc. xxxiii, 380.

${ }_{16}$ Testa de Nevill (Rec. Com.), 1876,$1954 ;$ Trans. Devon. Assoc. xxix, 500.

17 Testa de Nevill (Rec. Com.), I $75 b$, gives the number as $52 \frac{1}{4}+\frac{1}{20}$; Hund. R. (Rec. Com.), p. 63, n. 52.

${ }^{18}$ Pipe Rolls, 24 Hen. II ; Trons. Devon. Assoc. xxxvii, 428, n. 22.

19 Abbrev. Placit. 6 Ric. I, Devon, Michaelmas, Rot. 5 ; Wills. de Brewes petit versus Oliverum de Tracy ut teneat finem suum de medietate baronie de Bernstable. Trans. Devon. Assoc. xxxiii, 729.

${ }_{20}$ Abbrev. Placit. I John, Easter and Trinity, Rot. 5 ; Dugdale, Bar. i, 4I $5,622$. 


\section{A HISTORY OF DEVONSHIRE}

son, Henry de Tracy, ${ }^{1}$ upon whom King John, on the forfeiture of the third William de Braose, ${ }^{2}$ bestowed the entire honour in $12 \mathrm{I} 3,{ }^{3}$ all but the borough of Barnstaple and I 5 of Braose's fees, which he had given to Peter son of Herbert. ${ }^{4}$ To Henry de Tracy succeeded his son of like name, ${ }^{5}$ who married Maud, daughter of Reginald de Braose, and died in $1273,{ }^{6}$ when his granddaughter, Maud, daughter of Eva de Tracy, by her husband, Guy de Bryane, was found to be his next heir. ${ }^{7}$ This Maud was twice married, first to Nicholas Martin, the younger son of Nicholas Martin, lord of Dartington, and secondly, to Geoffrey de Camvil. In right of Maud, Geoffrey de Camvil held the honour in 1285 by the courtesy of England, ${ }^{8}$ and was succeeded by Maud's son and heir, William Martin. ${ }^{9}$ On the death of William Martin in $1325,{ }^{10}$ and of his son, Nicholas, two years later, ${ }^{11}$ the honour came to his sister Joan, wife of James, Lord Audley, in whose family it continued until the fifteenth year of Richard II. On the death of Nicholas, Lord Audley, in $1392,{ }^{12}$ it reverted to the crown.

\section{THE HONOURS OF HURBERTON AND TOTTON}

Juhel's fief at the time of the Domesday Survey consisted of I 3 manors, assessed at 70 hides, and comprising over 40,000 acres under cultivation. It was made up of the estates of 39 dispossessed Saxon owners, Alwy's I 3, Alvric's I 2, and Alebric's IO, being the most numerous ; but there were also 7 of Algar's, 6 of Hecche's, who has left his name in Hecche's Buckland, now called Eggbuckland, 4 each of Brictric Camesons' ${ }^{13}$ and Tovi's, 3 each of Osulf, Alvred, Ulf, and Alward, 2 each of 9 others, and of 17, I each. All those estates, not excepting Eggbuckland, Compton Giffard, and Hooe, which were held by Stephen in Domesday and afterwards of the honour of Plympton, ${ }^{15}$ are found forming part 'of the tenement of Totnes, ${ }^{16}$ or of one of its constituent parts, the honours of Hurberton and Totton ${ }^{17}$; but to these honours also belonged ${ }^{18}$ the crown demesne-manors of Broadclyst ${ }^{19}$ and Hurberton, ${ }^{20}$ besides Queen Matilda's Ashprington, ${ }^{21}$ Pomeray's Washfield, ${ }^{22}$ and the count of Mortain's Broadhempston. ${ }^{23}$

${ }^{1}$ Testa de Nevill (Rec. Com.), 175 a.

${ }^{2}$ Lib. Niger, 377 ; Trans. Deron. Assoc. xxxiii, 368.

${ }^{3}$ Rot. Claus. (Rec. Com.), I 5 John, pt. I, m. 4 ; Dugdale, Bar. i, 622 ; Risdon, Notebook, 63.

$T$ Testa de Nevill (Rec. Com.), 195a.

5 Editors of Risdon, Notebook, 63 note.

${ }^{6}$ Inq. p. m. 2 Edw. I, No. 32 ; Trans. Devon. Assoc, xxxiii, 729.

7 Eva died before her father (Dugdale, Bar. i, 622'; Risdon, Notebook, 63). Maud was found to be 3I years of age in I 273, and she had then recently married Geoffrey de Camvil. Her father, Guy de Bryane, married again Sibilla, and died in 1307 (Inq. p. m. 35 Edw. I, No. 32 ; Roberts, Cal. Ger. No. 32, p. 213$)$.

${ }^{8}$ Hund. Rolls (Rec. Com.), p. 63 ; Feud. Aids; Galfridus de Kamvill qui tenet per legem Angliae. Geoffrey died I 308 (Inq. p. m. 2 Edw. II, No. 75).

${ }_{9}^{9}$ Dugdale, Bar. i, 729 ; Risdon, Notebook, 63. $\quad{ }^{10}$ Inq. p. m. I9 Edw. II, No. Ioo.

${ }^{11}$ Inq. p. m. I Edw. III, No. 40.

${ }^{13}$ See Exeter Domesday under Mary Tavy, fol. 3176.

${ }^{12}$ Inq. p. m. I 5 Ric. II, No. I.

${ }^{14}$ Inq. p. m. 22 Edw. I, No. 21 , describes Hooe as held of Hurberton.

${ }^{15}$ Testa de Nevill (Rec. Com.), $182 a . \quad{ }^{16}$ Lib. Niger, 125. Totton.

${ }_{17}$ Testa de Nevill (Rec. Com.), 1 766 , has a list of fees held of Hurberton, and p. $183 b$ of those held of

${ }^{18}$ Lib. Niger, 125 , and Trans. Devon. Assoc. xxxiii, 381 , enumerate 75 fees in all.

${ }^{19}$ Testa de Nevill (Rec. Com.), $177 a$ and $195 a .{ }^{20}$ Ibid. $177 a$; Feud. Aids, 331.

${ }^{21}$ Feud. Aids, 427 . 2 rans. Devon. Assoc. xxxiv, $289 . \quad{ }^{22}$ Trans. Devon. Assoc. xvi, 175 ; xxxvi, 369. 


\section{FEUDAL BARONAGE}

Juhel, Johel, or Judhel, of Totnes, was himself the son of an Alfred, and was succeeded by his son Alfred," who joined Baldwin de Redvers in his defence of the castle of Exeter in I 136.

An early authority ${ }^{2}$ alleges that after the death of the Conqueror, William Rufus expelled Juhel from Totnes and gave his inheritance to Roger de Nonant ; but Juhel was certainly lord of both Barnstaple and Totnes in I I I $3,{ }^{3}$ though it is possible that Nonant may have been in possession of Totnes under him; for in I I 23, whilst Juhel still held Barnstaple, Guy de Nonant apparently held Totnes." Henry I must, therefore, have given Totnes or approved its transfer to Roger de Nonant some time before I I $23,{ }^{5}$ but not until he had previously granted the castle, together with the manors of Cornworthy and Loddiswell, to Reginald de Braose. ${ }^{6}$ Roger de Nonant was succeeded by his sons Guy, Henry, and Roger II, in succession, ${ }^{7}$ then by Guy's son, Roger III, ${ }^{8}$ and Roger III was followed by his son Guy, who held

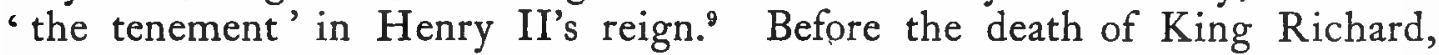
Henry de Nonant, who married Isabel Bulbek, was in possession, ${ }^{10}$ and was deeply in debt to the Jews. ${ }^{11}$ A claim was then put forward to "the tenement ' by William de Braose, ${ }^{12}$ a descendant of Juhel's daughter, resulting in a partition effected in $1206 .^{13}$ One moiety called specifically the honour of Totton was awarded to William de Braose ${ }_{,}^{14}$ together with twenty-eight fees, ${ }^{15}$ whilst Henry de Nonant retained the other moiety, including Hurberton, Broad Clyst, and Ashprington, specifically described as the honour of Hurberton, also with twenty-eight fees. ${ }^{16}$ On the death of Henry de Nonant ${ }^{17}$ the honour of Hurberton was acquired by Roger de Valletort, probably by

${ }^{1}$ Oliver, Mon. 241 ; Trans. Devon. Assoc. xii, 162 ; xxix, 233,n. 16.

${ }^{2}$ Dugdale, Bar. i, 414; Risdon, Notebook, 60.

${ }^{3}$ Round, Feud. Engl. 486,n. 4, draws attention to the fact that in IOgI Roger de Nonant attested the foundation charter of Old Sarum Cathedral, and that the monks of Laon reported, in I I I 3 : ' We came to the castle called Bannistaple, where dwelt a lord called Joel of 'Toteneis' (Herman, ii, 17) ; adding that they afterwards visited Totnes, "the castle of the aforesaid lord.'

"Charter of Henry I in Round, Feud. Engl. p. 483, has Johel of Berdestaple and Guy of Totnes as witnesses.

${ }^{5}$ Testa de Nevill (Rec. Com.), 195a, in Trans. Devon. Assoc. xxxvii, 424 : "The manor of Hurberton, King Henry I gave to Roger de Nunhaut, the elder, by what service is not known.' Hund. R. (Rec. Com.), No. 29, p. 83 : 'Henry I granted the borough of Totton to Roger de Nonant. Many fees were then alienated, but no one knows how, or when.'

${ }^{6}$ Testa de Nevill (Rec. Com.), i95a; Dugdale, Bar. i, 6 ro.

${ }^{7}$ Cartulary of Bucfast Abbey in Hingeston-Randolph, Exeter Episc. Reg. Grandisson, pp. I 570-3, 1594-7, shews that Roger de Nonant's wife was called Alice, that Guy, Henry, and Roger II were their sons; that Henry's wife was called Elizabeth, and Guy's Mabel ; that Guy's son, Roger III, succeeded his uncle, Roger II, and had to wife, Alice, who held Dartington, Langacre alias Borough House in Broad Clyst, and Holne (Pipe R. 22 Hen. II), and that she had a son called Guy.

${ }^{8}$ Cartulary of Bucfast Abbey, in Hingeston-Randolph's Grandisson, p. $1572 . \quad{ }^{\circ}$ Oliver, Mon. I 35.

${ }^{10}$ Pipe R. I John : Robert son of John was disseised of Brixton by Henry de Nonant, before the death of King Richard.

11 Testa de Nevill (Rec. Com.), I 950 ; Trans. Devon. Assoc. xxxvii, 427.

12 According to Dugdale, Bar. i, 418, William de Braose died in 1212 , leaving by Maud de Haia, commonly called Maud de St. Walerie, two sons, Giles, bishop of Hereford, and Reginald, and four daughters, Joanna, wife of Richard, Lord Percy, Loretta, wife of Robert, earl of Leicester, Margaret, wife of Walter de Lucy, and Maud, wife of Griffith, prince of Wales. His eldest son and his widow were starved to death at Windsor by command of King John. For a correction of Dugdale see Trans. Devon. Assoc. xxxvii, 422, $\pi$. I 2. ${ }^{13}$ Rot. Lit. Claus. (Rec. Com.), 8 John, m. 2. ${ }^{14}$ Red Book, 594 ; Dugdale, Baronage, i, 6 I o.

${ }^{15}$ Red Book, 594, gives $28 \frac{1}{2}+\frac{1}{6}+\frac{1}{20}$ as William de Braose's share in I III, but in 1234 Eva de Braose paid on twenty-eight fees, Testa de Nevill (Rec. Com.), i 87b, 195a, Trans. Devon. Assoc. xxix, 500.

${ }^{16}$ Red Book, 594, in Trans. Devon. Assoc. xxxiii, 384, gives $27 \frac{3}{4}+\frac{1}{5}+\frac{1}{6}+\frac{1}{40}$ fees as Henry de Nonant's share in 1211 , but in 1234 Reginald de Valletort paid on 28 (Testa de Nevill (Rec. Com.), I87b, 195a; Trans. Devon. Assoc. xxix, 500), and in 1286 they were said to be 32 (Feud. Aids, 331 ).

17 Henry's widow, Isabella, remarried Robert de Ver, earl of Oxford, who held her dower-land in 12 I 7 (Testa de Nevill (Rec. Com.), 195a). 


\section{A HISTORY OF DEVONSHIRE}

purchase, and continued with the Valletorts until 1275. In I 30 I it was claimed by Henry de Pomeray and Peter Corbet as next heirs, ${ }^{1}$ but in I 3 I 5 judgement-was given against them, ${ }^{2}$ and the Hurberton moiety was granted to the holder of the Totton moiety, probably by purchase from the crown.

The other moiety constituting the barony of Totton, after being awarded to William de Braose, ${ }^{3}$ passed first to his son Giles, bishop of Hereford, and then to his third son, Reginald de Braose, the husband of Graecia, daughter of William Briwere." Reginald died in $122 \mathrm{I}$ and was succeeded by his son, William de Braose, who married Eva, sister and coheiress of Walter Marshall, earl of Pembroke, ${ }^{5}$ and died in 1229 , when, by the marriage of his daughter Eva, ${ }^{6}$ this moiety passed to William de Canteloup, and on the latter's death in $1253^{7}$ to his son, George Canteloup. George having died without issue on $1 \mathrm{March}, \mathrm{I} 273,{ }^{8}$ it fell to the share of the younger of his two sisters, Milicent, wife of Eudo or Ivo de la Zouche, who on Ivo's death remarried John de Montalt or Monhaut. ${ }^{9}$ The Zouches shortly after I 3 I 5 acquired the other moiety or honour of Hurberton, and held the united honours until John, Lord Zouche, sustained a forfeiture in 1485 . The barony then came to an end, but most of the estates passed by grant of Henry VII to the family of Edgecombe. ${ }^{10}$ By far the largest under-tenant of Juhel was Ralf de Pomeray, but nowhere did the Pomeray family continue to be under-tenants of Totnes. In one group, consisting of seven fees, Richard son of Stephen had taken their place in I $286,{ }^{11}$ and in another group of seven fees, Jacob or Avice de Bolley. ${ }^{12}$

\section{THE HONOURS OF BERRY POMEROY AND BRANEYS alias BRADNINCH}

The honours of Berry and Bradninch, held in 1086 by the two brothers ${ }^{13}$ Ralf de Pomeray and William Capra, contained another large slice of the

${ }^{1}$ Roberts, Cal. Gen. (Rec. Com.), No. 32, p. 56 ; Inq. p. m. I 5 Edw. II, No. 34, Henry de Pomeray was son of Joan, the daughter of Hawise de Valletort, and Peter Corbet was the husband of Joan's sister Beatrice. See below, p. 20. They claimed as next of kin on the ground that Roger de Valletort was insane at the time he made it over to the earl of Cornwall and Alexander de Okeston.

${ }^{2}$ Abbrev. Plac. (Rec. Com.), 9 Edw. II, Easter, Rot. I 23.

${ }^{3}$ Pipe Rolls of I I John have an 'Account of Wm. de Braose and Reginald his son,' for $\frac{1}{4}$ year before they were delivered to Henry, son of the earl, of Totnes, Cornworthy, Barnstaple, Lapford, Chilworthy, and ' Trekineyerht.'

${ }^{4}$ Reginald had livery of his father's lands in 1216 (Rot. Lit. Claus. I Hen. III, m. I 5 d.), and on 12 November, 1218 , obtained the king's writ for delivery of those knight services of the honour of Totton which were then in the king's hand (Rot. Lit. Claus. 3 Hen. III, m. I3), Dugdale, Bar. i, 4 I9.

${ }^{5}$ Roberts, Cal. Ger. 227.

${ }^{6}$ Rot. Fin. 35 Hen. III, m. 19; Abbrev. Plac. (Rec. Com.), 37 Hen. III, Mich. Rot. 12, p. 132 ; and 38 Hen. III, Hil. Rot. I4, p. 143.

${ }^{7}$ Inq. p. m. 38 Hen. III, No. 46 in Roberts, Cal. Gen. p. 6 I.

${ }^{8}$ Inq. p. m. I Edw. I, No. 16, in Roberts, Cal. Gen. (Rec. Com.), p.' 197. He died seised of Cornworthy and Loddiswell manors, of Totton barony and borough, of Dartmouth market, besides Woodford, Ringmore, North Bovey, Brixton, Raddon in Maristow, and Tidworthy (Trans. Devon. Assoc. xii, 197).

${ }^{9}$ Dugdale, Bar. i, 527. In Oliver, Mon. 239, is a deed in which Milicent, daughter and heiress of William de Cantilupe, lady of Totnes and relict of Eudo de la Zouche, confirms a deed of her grandfather, William de Braose. Inq. p. m. 27 Edw. I, No. I, shows that she died in 1299 , seised of Totnes borough, Cornworthy manor, and revenues in Loddiswell and Dartmouth.

${ }^{10}$ Lysons, i, cxxiii $a$.

${ }^{11}$ At Ashleigh (Feud. Aids, 321 ), South Huish and Galmpton (p. 323), Chivelstone (p. 33 I), Ringmore (p. 32 1), Brixton (p. 335), Baccamoor and Holland (p. 334).

${ }_{12}$ At Combe Fishacre (Feud. Aids, 317 ), Lovenetor (p. 3 17), Lupton (p. 31 6), Curtisknowle, North Huish, Bradleigh (p. 324), Malston (p. 332), Loughtor, Woodford (p. 333), Lamside and Adston (Testa de Nevill (Rec. Com.), 193b), and Blatchford (Ibid. 194a). See above, p. 556, n. II.

${ }^{13}$ Geldroll, Fremington, fol. $65 b, \mathrm{~A} 4$. 


\section{FEUDAL BARONAGE}

county, comprising I06 manors assessed at 75 hides, with a cultivated area of over 45,000 acres. Ralf's honour, sometimes called the honour of Bradworthy, ${ }^{1}$ because Bradworthy was the head of its North Devon section, as Berry Pomeroy was of its South Devon section, included sixty manors, of which six had been held by two or more Saxons of the name of Alvric or Alric, one called Alvrix Pic, ${ }^{2}$ or Alvric Piga, ${ }^{3}$ the other Alric the priest ${ }^{4}$; six had been held by Almer, Almar or Ailmer, ${ }^{5}$ five by Wichin, ${ }^{6}$ three by Edwin, three by Alward Tochesons, ${ }^{7}$ two each by Tovy, ${ }^{8}$ Alwey, Edwy, Borgaret, Alnod, Godric, Goda, and Saric, and one each by eighteen others, amongst whom was Algar the priest, ${ }^{9}$ besides two held by several thanes in common, ${ }^{10}$ and one by a woman. ${ }^{11}$ In addition to Pomeray's estates, the honour of Berry included Gatcombe, ${ }^{12}$ which Ansger, the king's cook or kitchen-master, held at the time of the survey; Meddon and Diptford, held by the English thane Ansgod; Manaton, held by the English forester Adret; and Mowlish, held by the Englishman Saulf. ${ }^{13}$ In I 66 the honour consisted of thirty-two fees, ${ }^{14}$ in I 234 of twenty-one. ${ }^{15}$ From Ralf de Pomeray the honour descended to his son Joslin alias Gollin alias Johel de Pomeray, who in I 25 gave the manor of Canonteign and the tithe of Berry and Upottery to St. Mary du $\mathrm{Val}_{,}{ }^{16}$ and it then came to his grandson Henry, who is said to have married Rohesia, sister of Reginald, earl of Cornwall. ${ }^{17}$ After several generations it passed, on the death of Sir Henry Pomeray, the tenth of that name, in I 374, to the latter's niece, Joan, the wife of James Chudleigh, ${ }^{18}$ but it had previously been considerably reduced by the sale of the northern section to Briwere about I I $99 .{ }^{19}$

Among Pomeray's under-tenants the two whose names occur most frequently are Roger and William, but there were apparently two Rogers, one Roger son of Payn of Putford, ${ }^{20}$ the other Roger 'Aculeus,' called also Roger the Sandy (flavus). ${ }^{\text {.1 }}$ The former was tenant of Julian Putford and Peamore and was represented in I 66 by Richard son of Payn de Putteford. ${ }^{22}$ The latter held Huxham and other manors ${ }^{23}$ in the south and east of the county,

1 Feud. Aids, 340.

Exeter Domesday, fol. 421.

Ibid. fol. $325 b$.

$4 \mathrm{He}$ held Upottery (fol. 342b), probably also Berry Pomeroy (fol. 342), and Sheldon (fol. 3376 ), and certainly Stanlinch (fol. 354), and Huntsham (fol. 356), the two last-named of the honour of Toriton.

${ }^{5}$ His principal estates were Dunkswell (fol. 338b), Street Raleigh (fol. 340b), and West Stoodleigh (fol. 341 ).

6 Peamore (fol. 336b), Huxham (fol. 339b), Clyst St. George (fol. 339b), Weycroft (fol. 343), and Heavitree (fol. 3436 ).

${ }^{7}$ Brendon (fol. 337), South Week in Germansweek (fol. 335), and Horwood (fol. 336).

${ }^{8}$ Pancras Week (Dunewinesdona, fol. 335), and Bradworthy (fol. $335 b$ ).

'He held 'Estandona' (fol. $337 b$ ), and he, or one of the same name, also held of the king at Braunton (fol. 194b), and was apparently the provost of a college of clergy at Braunton (Trans. Devon. Assoc. xxx, 286).

${ }^{10}$ Gappah (fol. 34 I $b$ ), Borcombe (Redix, fol. 343).

12 Testa de Nevill (Rec. Com.), $182 b$. ${ }^{11}$ Wickaborough (fol. 342b).

1s Lib. Niger, I 28 ; Red Book, 558 , gives thirty-two as the number in 1212.

15 Testa de Nevill (Rec. Com.), i87b, 195a, in Trans. Devon. Assoc. xxix, 500.

${ }^{16}$ Cal. of Doc. France, 536.

${ }_{17}$ Risdon, Notebook, 68; Pole, pp. 17, 281, states that Johel de Pomeray married the sister of Herbert, ' and it seemeth that she was base-daughter of King Henry I, and sister of Reginald, earl of Cornwall.'

${ }^{28}$ Inq. p. m. 48 Edw. III, No. 5 I.

${ }^{20}$ So described in Exeter Domesday, fol. 3366.

${ }^{21}$ Geldroll, fol. 7ob, A 9. Trans. Devont. Assoc. xxvii, 182.

${ }^{2}$ Lib. Niger, 129. Richard de Putford held them in 1241 (Teste de Nevill, 182b), and Walter Pollard in 1303 (Feud. Aids, 358).

${ }^{23}$ Clyst St. George, Gappah, Dunston, Blackslade in Widdicombe-in-the-Moor, Weycroft, Kennedon and Ranscombe, and Heavitree. 


\section{A HISTORY OF DEVONSHIRE}

which were dispersed among the families of Geoffrey de Pomeray, ${ }^{1}$ Henry de Pomeray of Buckerel, ${ }^{2}$ and those of Huxham, ${ }^{3}$ Bolley, ${ }^{4}$ and Prawle. ${ }^{5}$ 'William' is the ancestor of William le Peytevin or William of Poitou, who held four fees of Pomeray in I i66, including Ogwell Peytevin, West Ogwell, Dunscombe, Creedy Peytevin, Yeadbury in Morchard, and Woodford in Thelbridge, and of Robert Peytevin, who held the same in $1243 .^{\circ}$ Beatrice, who held West Chevithorn in Tiverton and Uplowman, was Pomeray's sister; she also held Southleigh and Bradford Tracy of William Capra, and is called his sister. ${ }^{7}$ Roscelin was Pomeray's under-tenant, as Warin was William Capra's, but the two appear to have held their estates jointly at Waringston, ${ }^{8}$ Raplinghays, ${ }^{9}$ and Ivedon, ${ }^{10}$ in Hemyock, Tiverton, and Budleigh hundreds. ${ }^{11}$

William Capra's honour of Bradninch consisted of fewer manors than Pomeray's, 46 compared with 60, of lower assessment, $34 \frac{1}{2}$ hides as against $40 \frac{1}{2}$, but its acreage was nearly equal, 22,000 odd acres as compared with something like 23,700. It included seven manors which had belonged to the Saxon Alward Tochesons, six which had been Wichin's, six which had been Ailmer's, four which had been Alvric's, three Brictric's, two each of Godric's and Algar's, and the remaining four single estates of different Saxon owners. Its earlier history is somewhat obscure. According to Risdon it followed the earldom of Cornwall. ${ }^{12}$ Mr. Whale associates it with the honour of Plympton. ${ }^{13}$ It does not appear as Earl Reginald's in his return of 1 I 66, ${ }^{14}$ but appears there as William Tracy's. Besides William Capra's estates the honour included Clyst St. George, which was held of it by Pomeray, ${ }^{15}$ and Lympstone. ${ }^{16}$ And since Lympstone, which at the date of the Domesday Survey was held by Capra under Richard son of Gilbert, count of Eu and Brionne, was granted out in serjeanty by Henry $\mathrm{I},{ }^{17}$ it is evident that the honour must at the time have been in the king's hand either by escheat or forfeiture. Henry I seems to have bestowed it on William Tracy, with whose daughter and heiress it passed to John de Sudely and to his second son William, who took his mother's name of 'Tracy and was the murderer of Archbishop Thomas of Canterbury. With this William's daughter Eva it passed in II 74 to William Courteney, who also called himself Tracy. Divers estates were bestowed on religious houses by the archbishop's murderer, and others were sold to

${ }^{1}$ He had Clyst St. George (Testa de Nevill (Rec. Com.), 191 $b$; Trans. Devion. Assoc. xxxv, 299), also Street Ralegh (Testa de Nevill, I91a), in Domesday Torstan's.

${ }^{2} \mathrm{He}$ had Weycroft (Feud. Aids, 320), Brockland (p.328), and Borcombe (p. 331). At the two last named he succeeded the Domesday Geoffrey.

${ }^{3} \mathrm{He}$ had Huxham and Heavitree (Feud. Aids, 316 ), also Hill and Exeton (Trans. Devon. Assoc. xxxv, 291 ; Testa de Nevill, I 91 (a).

"He had Gappah, Belmarsh, and Babcombe (Testa de Nevill. (Rec. Com.), 1826), held in I166 by Hamelin de Boileia (Lib. Niger), I 29.

${ }^{6}$ Testa de Nevill (Réc. Com.), i $82 b$, i $90 b$.

7 Exeter Domesday, fol. 406, $404 b, 341 b$.

8 'Oteri' (Exeter Domesday, fol. 3386 ), and 'Oteri' (fol. 400b), both in Buckerel.

' In 'Oteri' (Exeter Domesday, fol. 340b), and in 'Oteri' (fol. 403b), both in Gittisham.

10 ' Otri' (Exeter Domesday, fol. 342), and 'Otria' (fol. 405), both in Awlescombe.

11 Geldroll, fol. 67, B 3 .

${ }^{12}$ Risdon, Notebook, 74. List of fees belonging to it in Inq. p. m. 2 Ric. II, No. 57.

${ }_{13}$ Trans. Devon. Assoc. xxxiii, 365 .

${ }^{14}$ Ibid. xxxiv, 5 I I.

${ }^{16}$ Exeter Domesday, fol. 460.

${ }^{15}$ Testa de Nevill (Rec. Com.), 1916.

${ }_{17}$ Trans. Devon. Assoc. xxxv, 299. Lympstone appears to have been held by Pomeray as under-tenant, because Reginald de Albemarle in 1243 held it of Muriel de Bolley (Testa de Nevill, I 9 I $b$ ), and de Bolley constantly represents Pomeray wherever he was under-tenant. See Trans. Devon. Assoc. xxxv, 284. 


\section{FEUDAL BARONAGE}

Briwere by his successor, ${ }^{1}$ so that the twenty-one and a quarter fees of $1166^{2}$ were reduced to sixteen in $1209,{ }^{8}$ though in 1234 Henry de Turbevil paid on twenty-one. ${ }^{4} \quad$ On the death of Eva, Henry the son of Earl Reginald purchased the honour for 1,200 marks ${ }^{5}$ and held it for sixteen fees. ${ }^{6}$ On his rebellion Henry III resumed it, and after holding it for some years ${ }^{7}$ gave it to his brother Richard, earl of Cornwall, with the Lady Sanchia in free marriage. ${ }^{8}$ On the death of Richard's son and successor, Edmund, in $1300^{\circ}$ it was again in the king's hands and eventually, on I7 March, 1337, was incorporated in the duchy of Cornwall. ${ }^{10}$

\section{THE HONOURS OF BAMPTON AND MARSHWOOD}

Walter de Dowai, called also Walscin, had for his share of the spoils of the Conquest in Devonshire twenty-six manors assessed at $47 \frac{1}{2}$ hides and comprising about 20,000 acres under cultivation. The Saxons dispossessed in his favour were Alsi from five manors, Asgar ${ }^{11}$ from four, Algar and Eddida each from two, and twelve others from one each. One of these manors, Bampton, had been King Edward's demesne-manor, and was obtained by Walter in exchange for Blackawton and Ermington, which had been Asgar's. All Walter's Devonshire estates with the exception of East Haggington, which was held of the honour of Dartington, ${ }^{12}$ Diptford, held of the honour of Torington, ${ }^{13}$ and Hockford, held of the honour of Barnstaple, ${ }^{12}$ are found held of the two honours of Bampton and Marshwood. ${ }^{14}$ Stenhall, however, in Uffculm is stated to be held of the honour of Worle in Somerset, ${ }^{15}$ and Dunsford, one of the Marshwood fees, is in one place said to be held of the honour of 'Iwode,' ${ }^{16}$ and in another to be held of the honour of Swale ; ${ }^{17}$ but these seem only variant names of Marshwood. In addition to half of Walter de Dowai's estates, the honour of Marshwood included West Raddon in Shobrook, ${ }^{18}$ which at the time of the Domesday Survey belonged to the count of Mortain, and the English thane Saulf's manor of Mowlish. ${ }^{18}$

Walter de Dowai was succeeded in the honour of Bampton and in one half of his Devonshire estates, ${ }^{19}$ by his son ${ }^{20}$ Robert de Bampton. Robert's daughter

${ }^{1}$ Devon N. and Q. ii, 24 ; Dugdale, Bar. i, 622.

'Lib. Niger, I 2 I. Pipe R. of I I Hen. II (printed in Trans. Devon. Assoc. xxix, 486) indicates 30 fees.

${ }^{3}$ Trans. Devon. Assoc. xxxiii, 369. In Inq. p. m. 28 Hen. III, No. 5, and in Cal. Inq. Hen. III, No. 23 , Braneys manor to which pertain the homages of $16 \frac{1}{2}+\frac{1}{5}+\frac{1}{8}$ fees, of all which Hawise late the wife of Henry de Turbevil holds a third part in dower.

Testa de Nevill (Rec. Com.), 1876 , in Trans. Devon. Assoc. xxix, 500.

${ }^{5}$ Dugdale, Bar. i, 610; Trans. Devon. Assoc. xxix, 486, n. 47. In 1207 Henry confirmed to Ford Abbey 'all the lands which it held of the fee of Braneys before he recovered his inheritance.' (Oliver, Mon. 347). Pipe R. 3 Hen. III : Henry the 'Earl's son accounts for $40 \frac{1}{2}$ marks for the fee of Braeneis.'

${ }^{6}$ Red Book, 558.

${ }^{7} \mathrm{He}$ first granted it to Henry de Turbevil, who died seised of it in 1243 (Inq. p. m. 28 Hen. III, No. 5, Cal. of Ing. No. 23), and then William de la Londe held it as bailiff (Testa de Nevill (Rec. Com.), I 82b)

${ }^{8}$ Hund. R. (Rec. Com.), No. 7, p. 65 ; Trans. Devon. Assoc. xxvii, 198, $n 56$.

${ }^{9}$ Inq. p. m. $28 \mathrm{Edw}$. I, No. 48.

${ }^{10}$ Charter R. I I Edw. III.

11 The honour of Ansgar or Asgar is named in Exeter Domesday (fol. 504).

${ }^{12}$ Testa de Nevill (Rec. Com.), p. $178 a$.

14 Ibid. p. $183 a$.

${ }^{16}$ Feud. Aids, p. 316 , perhaps intended for 'Mersiwode.

Ibid. p. I79a; Collinson, Somerset, iii, 6 I 4

${ }^{17}$ Hund. R. (Rec. Com.), No. 43, p. 85

${ }^{18}$ Testa de Nevill (Rec. Com.), p. $183 a$

19 Trans. Devon. Assoc. xxx, 257. He confirmed the tithes of Bampton to Bath Abbey.

${ }^{30}$ Abbrev. Plac. (Rec. Com.), 92 ; Cbartul. of Bath (Somers. Rec. Soc.), vii, 39, where Emma, mother of Robert 'de Bahentona,' is mentioned as well as his father Walter. 


\section{A HISTORY OF DEVONSHIRE}

and heiress Juliana ${ }^{1}$ married (I) Fulk Paynel, a grandson of Ralf Paynel the Conqueror's companion, ${ }^{2}$ by whom she had three sons, William, ${ }^{8}$ Fulk, and Hasculf Paynel, and (2) Warin de la Haule or de Aula. ${ }^{5}$ Juliana's first husband had seisin of the honour of Bampton in I I64, but in I I66 Warin de Aula was in possession. ${ }^{6}$ In I I 80 Fulk Paynel her son ${ }^{7}$ accounted for I,, 00 marks for a fine of the honour of Bampton, ${ }^{8}$ but six years later he was put out of possession for being in arrear. ${ }^{2}$ After the honour had been held for a time by Hugh Bard ${ }^{10}$ and William Briwere ${ }^{11}$ Fulk Paynel recovered it in 1 I $99,{ }^{12}$ and died in $1216,{ }^{13}$ leaving a son called William and a daughter Christina, the wife of Miles Cogan. ${ }^{14}$ Through this daughter it, in I 274, passed to the Cogan family. ${ }^{15}$

The honour continued in the Cogan family till the 2 Ist year of Richard II, first in the person of John Cogan the younger, who died in I 30 I ; then of his son Thomas, who died before I3 15 ; next of his grandson Sir Richard Cogan, who came of age in 1327 and died in 1367, and finally of his great grandson Sir William Cogan. On Sir William Cogan's death in I 382 , without issue, ${ }^{16}$ it came to his sister Elizabeth, wife of Sir Fulk Fitzwarren, ${ }^{17}$ whose daughter Elizabeth, after her brother Fulk's death without issue in $1408,{ }^{18}$ brought it to Richard Hankford, ${ }^{19}$ whose daughter Catherine, after the death of Richard Hankford the younger in $143{ }^{1},{ }^{20}$ carried it to William Bourchier Lord Fitzwarren. ${ }^{21}$ From him it passed to his son Fulk, Lord Fitzwarren, father of John Bourchier, earl of Bath, and thus was united with the earldom of Bath.

The other portion of Walter de Dowai's Devonshire estates went to the Somersetshire honour of Marshwood apparently by gift of Henry I to Geoffrey de Mandevil, ${ }^{22}$ possibly uncle of the earl of Essex. ${ }^{23}$ On Geoffrey's death in I 44 it passed to his younger son Ralf, and with Ralf's daughter Dionisia ${ }^{24}$

1 Abbrev. Plac. (Rec. Com.), I 5 John, Mich. Rot. 14 in dorso.

3 Abbrev. Plac. (Rec. Com.), I 5 John, Mich. Rot. I 4 in dorso, p. 92.

3 Abbrev. Plac. (Rec. Com.) 9 John, Rot. 12 in dorso, p. 98.

'Lib. Niger, 124 ; Trans. Devon. Assoc. xxxiii, 365.

7 Abbrev. Plac. (Rec. Com.), 98, where he is found in possession of the manor of Rainham in Essex, held in 1086 by Walter de Dowai.

${ }^{8}$ Pipe R. 26 Hen. II, m. 7.

${ }^{9}$ Ibid. 32 Hen. II, m. 14, shows it in the sheriff's hand, Fulc Painel owing f.359 8s. 9d.

10 Ibid. 33 Hen. II, m. I I.

12 Rot. de Obl. (Rec. Com.), I John, m. 22 and 21 in dorso.

"Abbrev. Plac. (Rec. Com.), I 5 John, Mich. Rot. 14 in dorso.

${ }_{15}$ William had married Alice (Rot. Fin. 12 Hen. III, m. 7), sister and one of the co-heiresses of William Briwere the younger and widow of Reginald de Mohun of Dunster, and died in 1228 (Rot. Fin. 1 3 Hen. III, m. I 3). His son William succeeded, who in 1241 was in the wardship of Herbert son of Mathew (Testa de Nevill, $183 a$ ), and came of age in 1244 (Rot. Fin. 29 Hen. III, m. I), but this William having died without issue in 1248 , his sister Auda, wife of John de Balun, succeeded (Inq. p. m. 33 Hen. III, No. 23 ; Cal. Inq. Hen. III, No. 139). She also died without issue in 1274 (Inq. p. m. 3 Edw. I, No. 19), when the honour devolved on John Cogan the younger, great-grandson of Miles Cogan and Christina.

${ }^{16}$ Inq. p. m. 6 Ric. II, No. 22 ; Editors of Risdon's Nosebook, 72.

${ }^{17}$ She died in 1399 (Inq. p. m. 2 I Ric. II, No. 23).

${ }^{13}$ Inq. p. m. 9 Hen. IV, No. $36 .{ }^{19} \mathrm{He}$ died in 1420 (Inq. p. m. 7 Hen. V, No. 70).

${ }^{30}$ Inq. p. m. 9 Hen. VI, No. 54.

2 She died in 1468 (Inq. p. m. 7 Edw. IV, 43); Lysons, xcviii.

${ }^{22}$ Testa de Nevill (Rec. Com.), p. 194b, only mentions the gift of Wonford. Marshwood is in Dorset, but it is called a Somersetshire honour in Testa de Nevill (Rec. Com), p. $183 a$.

${ }^{2}$ Batten, South Somerset, 120.

${ }^{24}$ Testa de Nevill (Rec. Com.), p. 1946, calls her daughter of Geoffrey, but Batten (South Somerset (1894), p. 117) shows that she was Geoffrey's granddaughter. Trans. Devon Assoc. xxxvi, 427; Devon. N. and $Q$. iii, $21-58$. 


\section{FEUDAL BARONAGE}

to her husband, William son of John. ${ }^{1}$ On the death of William son of John in I I 94 it was claimed by Geoffrey de Mandevil of Coker, descended from Geoffrey's elder son," notwithstanding which William's son Henry de Tilli obtained it by the king's writ in $1200 .^{8}$ On Henry de Tilli's death, however, in I 208, the claim to it of Robert de Mandevil of the Coker line was successfully asserted. ${ }^{4} \quad$ The Devonshire section consisted of eleven fees. ${ }^{5}$

\section{THE HONOUR OF DARTINGTON}

To William de Faleise's share fell, when the spoils of the Conquest were divided, nineteen manors within the county of Devon, assessed at $16 \frac{1}{2}$ hides and comprising some 12,000 acres under cultivation. Of these manors Cockington, North Harbournford, and Little Ingleborn had belonged to the Saxon Alric ; Holne, Dean, Rattery, and Dartington had been Alwin's; Combe Martin had belonged to two thanes, Bristric and Edwy; whilst the rest had been the property each of some individual freeman in Saxon times. All William's estates are found in aftertimes held of the honour of Dartington, sometimes called the honour of 'Blakedon ${ }^{8}$ from Blagdon in Somersetshire, which at the time of the survey was held by Serlo de Burcei, ${ }^{7}$ William de Faleise's father-in-law. ${ }^{8}$ In I I6 I this honour, which included also Walter de Dowai's estate of East Haggington, ${ }^{9}$ was held by Robert son of Martin, ${ }^{10}$ the first of that name to hold lands in Devon, ${ }^{11}$ but Dartington and Holne were Alice de Nonant's in $1176,{ }^{12}$ and were seized into the king's hands because she had married without leave. ${ }^{18}$ On Robert's death in I 207, his son William son of Martin ${ }^{14}$ succeeded, who died in 1208, leaving by Avice his wife ${ }^{16}$ a son also called William son of Martin, who died in 1216 . The latter William left two sons, Nicholas and Robert, the custody of whom during minority was committed first to Fulk de Breauté ${ }^{16}$ and afterwards to Henry de Trubevil. ${ }^{17}$ Nicholas Martin was succeeded by his grandson, William, Lord Martin, ${ }^{18}$ who by his wife Elinora, widow of Lord John Mohun, ${ }^{19}$ had issue a son William, second Lord Martin, and two daughters, Eleanor wife of Philip de Columbers ${ }^{20}$ and Joan wife of Nicholas de Audelegh. ${ }^{21}$ On the death of

1 Cal. Rot. Cart. p. 75 ; Lib. Niger, 84.

'Charter of John, in Batten, op. cit. I 20.

In I 200, Trans. Devon. Assoc. xxxvi, 427.

- Batten, South Somerset, I 17. This Robert was a grandson of Robert the eldest son of Geoffrey the original grantee. His father, Geoffrey, in 1194 , 'fined in 100 marks to have the judgment of the King's Court touching his inheritance in England and Normandy, whereof he had been deforced by Henry de Tilli,' Trans. Devon. Assoc. xxxvii, 427. Dugdale, Bar. i, 206.

${ }^{5}$ Testa de Nevill (Rec. Com.), p. 188a. Trans. Devon. Assoc. xxix, 501.

${ }^{6}$ Feud. Aids, 415 : Combe Martin held of the honour of Blakedon. Inq. p. m. I9 Edw. II, No. 100.

7 Exeter Domesday, fol. 452. ${ }^{8}$ Ibid. fol. $369 b$.

- Testa de Nevill (Rec. Com.), p. $178 a$.

${ }^{10}$ Pipe R 8 Hen. II. in Trans. Devon. Assoc. xxix, 478, 48 I. Dugdale, Bar. i, 729.

" $\mathrm{He}$ was the son of Robert son of Martin, who witnessed a charter of the Empress Maud in 1 I 42 (Pipe R. Soc. x, p. 43, No. 26) and died before 1158, when his Somerset estates were in the king's hand (Pipe R. Soc. i, 21, 22; ii, 19, 41, 58), and the grandson of Robert son of Martin (Risdon, Notebook, 65), who was grandson of Martin of Tours.

${ }_{12}$ Pipe R. 22 Hen. II ; 23,28 Hen. II.

${ }^{14}$ Rot. Fin. 9 John, m. I 3. Trans. Devon. Assoc. xxxiii, 365.

${ }^{15}$ Rot. Lit. Claus. I7 John, pt. I, m. 4 .

${ }^{13}$ Ibid. 33 Hen. II.

${ }_{17}$ Rot. Fin. 8 Hen. III, m. 2.

${ }^{18} \mathrm{He}$ was son of Nicholas Martin the younger, who died before his father, after marrying Maud, daughter and heiress of Guy de Bryan, by Eva, daughter and heiress of Henry de Tracy, lord of Barnstaple. Rot. Fin. Io Edw. I, m. I 6 and 15 .
${ }^{19}$ Oliver, Mon. 304.
${ }_{20}$ Inq. p. m. I Edw. III, No. 82.
${ }^{21}$ Risdon, Notebook, p. 66. 


\section{A HISTORY OF DEVONSHIRE}

William second Lord Martin in $1325,{ }^{1}$ and of his son Nicholas two years afterwards, ${ }^{2}$ the honour fell into abeyance, and the estates were divided between Philip de Columbers ${ }^{3}$ and James Audley, ${ }^{4}$ the infant son of Nicholas Audley.

\section{THE HONOUR OF TORINGTON}

Odo son of Gamelin held at the time of the Domesday Survey twenty-four manors in Devon with an assessment of 20 hides and some I 4,000 acres of cultivated land. Tedbald son of Berner had twenty-six with an assessment of $14 \frac{1}{2}$ hides, and about 9,000 acres of cultivated land. Odo's consisted of six held in Saxon times by Bristric or Brictric, two each held by Sawin, Edmar, Alric the priest, and Vidley or Vitalis, and of ten others held by individual Saxons ; Tedbald's consisted of five held by Ailmar, Almer or Aimar, three held by groups of thanes, two each held by Sawin and Edmer, and one each held by fourteen other Saxons, among them being Eilaf or Ailaf, Bristric or Brictric, Vidley, Godwin, Alwold, and Dode. Odo was son-in-law to Tedbald and eventually his heir. ${ }^{5}$ All of these estates, with the exception of Plymtree, which was held of Plympton, ${ }^{6}$ are subsequently found constituting the Devonshire honour of Toriton or Torington, ${ }^{7}$ which in addition included also the royal estate of Kilmington, originally a member of Axminster, ${ }^{8}$ and held of Plympton, ${ }^{9}$ Diptford, ${ }^{10}$ which belonged to the queen before the royal estate of Bampton was changed away, West Ruckham, Hill, and Combe, ${ }^{11}$ held by Haimeric de Arcis, one of the Conqueror's ordnance-masters, and seven fees held of the honour of Gloucester, ${ }^{12}$ in all twenty-nine fees. ${ }^{13}$

Mr. Round is of opinion that William son of Odo, who attests a charter of Henry I in II 23 as a great feudatory, was the son of Odo the son of Gamelin, ${ }^{14}$ in which case he cannot have been father, but may have been uncle to Robert de Toriton, who afterwards held the honour, since Robert de Toriton's father seems to have been called Roger. ${ }^{16}$ To Robert succeeded his son William de Toriton, who was in possession in $1166,{ }^{16}$ and to William his two sons John ${ }^{17}$ and Mathew, ${ }^{18}$ with a childless son of John

${ }^{1}$ Inq. p. m. $19 \mathrm{Edw}$. II, No. 100.

Inq. p.m. I Edw. III, No. 4 O.

${ }^{3} \mathrm{He}$ had Combe Martin, Barnstaple, Holsworthy, South Molton, and rents in East and West Anstey. Inq. p. m. I Edw. III, No. 13 .

'He had Kilmington, North Lew, Tackbear, Staunton, Warkleigh, Nymet Tracy, Bovey Tracy, and Raddon, whilst Dartington went to William's widow for life. Rot. Orig. Ig Edw. II, Rot. 23.

${ }^{5}$ Exeter Domesday, fol. 497.

${ }^{7}$ List of the fees held of it in Testa de Nevill (Rec. Com.), p. I $76 a$.

${ }^{8}$ Pole, 116.

${ }_{10}$ Testa de Nevill (Rec. Com.), p. I76a.

11 Ibid. I goa ; Red Book, 558 .

${ }^{12}$ Red Book of Exch. (Rolls Ser.), 558 ; Trans. Devon. Assoc. xxxiii, 367, 382.

${ }^{13}$ Lib. Niger, 1 24, says 30 fees. Testa de Nevill (Rec. Com.), p. $188 a$ in Trans. Devon. Assoc. xxix, 500.

14 Round, Feud. Engl. 487.

${ }^{15}$ Presumably William son of Robert, son of Roger, who held ten fees of the honour of Gloucester in 1 I 66 (Lib. Niger, 16I) and held seven fees in Devon of that honour (Red Book, 559), Iddesleigh being one of them (Feud. Aids, 328), was his son.

${ }_{16}$ Lib. Niger, I 24 . Pipe R. 2 Hen. II : William, son of Robert de Torintun.

17 John was in possession in 1194 , when he had a dispute with the abbess and convent of St. Edward's as to the advowson of Torington church (Abbrev. Plac. 6 Ric. I, m. I5, Mich. Rot. 8), also in 1201, when he paid 50 marks for a licence to go beyond sea (Rot. de Obl. 3 John, m. 8).

${ }_{18} \mathrm{He}$ paid $f_{100}$ in 1223 for the relief of his lands (Rot. Fin. $8 \mathrm{Hen}$. III, m. 9) on the death of his nephew William. 


\section{FEUDAL BARONAGE}

called William ${ }^{1}$ between them. On Mathew's death in 1226 the honour fell into abeyance and the estates were distributed among his five sisters, co-heiresses. ${ }^{2}$

One other 'honour' - that of Briwere--deserves mention, although not an honour in the same sense as those already described, but only a huge agglomeration of estates in the hands of a single individual to which by analogy the term honour is applied. Some of these estates were bestowed by the crown, such as Axminster, ${ }^{3}$ and were held of it in chief; others were acquired by purchase and were held of the various honours of Mortain, Barnstaple, Okehampton, Totton, Berry, and Bradninch, to which they belonged. ${ }^{4}$ William Briwere, the judge who created the original honour of Briwere in Richard I's time-his name is variously written Briewerre, Briegwere, Briguere, Briwere, Briwarr, and Bruere ${ }^{5}$ does not appear to have been connected with the county before $1179^{\circ}$; for his family must not be confounded with that of Ralf de Brueria ${ }^{7}$ in Domesday, an under-tenant of Baldwin, although in the twelfth century the two families were connected by marriage. ${ }^{8}$ Sheriff of Devon from I I 79 to I I 89, of Cornwall in I202-3, of Dorset in $1209-10,{ }^{9}$ William Briwere ${ }^{10}$ acquired a large number of manors in these counties, which on his death in 1226 passed to his son William Briwere the younger, and on his death, without issue, 12 February, 1232, were divided among his four daughters, co-heiresses. ${ }^{11}$

One of these daughters, Margaret, was married to William de la Ferte or de Affertis, and had by him an only daughter, Gundreda, who brought

1 William was in possession in succession to his father, John, in 1214 (Lit. Claus. 16 John, pt. ii, m. 24 in dorso) and died in 1223 , leaving a widow, Avice, who married again and enjoyed her dower up to 1230 (Lit. Claus. 8 Hen. III, pt. 1, m. IO ; Rot. Fin. 15 Hen. III, m. 7).

${ }^{2}$ Rot. Lit. Claus. (Rec. Com.), i I Hen. III, m. I6. (1) Emma, wife of (Thomas ?) Merton and mother of Henry de Merton, who did homage for her share in 1227 (Rot. Fin. 12 Hen. III, m. 9) ; (2) Dionisia, wife of Henry, son of William, who did homage for her share in 1227 (Rot. Fin. I 2 Hen. III, m. 7), and then sold it to Henry de Merton, who died, seised of $\frac{2}{5}$ in 1245 (Inq. p. m. 30 Hen. III, No. 16) ; (3) of Richard Tremenet, who sold her share to Henry de Tracy, from whom it passed to Geoffrey de Camvil, in right of his wife, Maud de Bryan, great granddaughter of the said Henry de Tracy (Hund. R., Rec. Com., 3 Edw. I, No. I7, p. 70) ; (4) Sybil, the wife of Richard Umfravil and mother of Gilbert de Umfravil; and (5) Mabel, the wife of Walter de Sully and mother of Raymund de Sully, who in I 232 paid f49 9s. 3d. for the issue of his lands (Pipe R. I 7 Hen. III, Rot. 6) and died in I241, after having given his share with Sybil his daughter to Guy de Bryan.

${ }^{3}$ Testa de Nevill (Rec. Com.), 194b, 196b, 197b; Trans. Devon. Assoc. xxxvii, 419, 427, 437 ; Dugdale, Bar. i, 701.

A list in Testa de Nevill (Rec. Com.), 199a, and Trans. Devon. Assoc. xxxvii, 446.

${ }^{5}$ Bruerre in Red Book of Exch. (Rolls Ser.), 232. As witness to two charters in I 190 and 198 (Cal. Doc. France, 119,462 ), William is called Briguere. In the foundation deed of Tor Abbey (Oliver, Mon. 173), he attests for himself as Briewere, and his son as Briegwere. Testa de Nevill (Rec. Com.), 194b, writes Bruere, 1966 Briwarr, $197 b$ and $199 a$ Briwere.

${ }^{6}$ In which year he purchased Ilesham. Dugdale, Bar. i, 700.

7 Ralf de Brueria is probably connected with Geoffrey de Bruere, a witness to charters in 1092 and II47 (Cal. Doc. France, I67, 518), and Gofer (Geoffrey) de Boueria, a witness in 1152 (Ibid. 375, 419).

${ }^{8}$ William Briwere held Greendale in Woodbury by grant of Reginald de Albemarle, his uncle (Oliver, Mon. 180), and gave it in free-marriage with his sister Englesia to William de la Brueria (Ibid. I 87). Englesia and her husband gave it back to William Briwere in exchange for Holbeton, whereupon William Briwere gave it to Tor Abbey (Oliver, Mon. 187 ; Trans. Devon. Assor. xxxv, 289). Another estate, Wolborough or Newton Abbot, which William Briwere gave to Tor Abbey, he obtained before I 196 from William, son of Antony de Bruera (Oliver, Mon. 186).

9 Maclean's Trigg Minor, iii, 148.

${ }_{10}$ William Briewere married Maud de Vaux (de Vallibus) (Oliver, Mon. 173), the mother by Reginald, earl of Cornwall, of Henry the earl's son. His eldest son, Richard, met his death before 1196 fighting against the Welsh.

${ }^{11}$ Testa de Nevill (Rec. Com.), I99a; Risdon, Notebook, 74. 


\section{A HISTORY OF DEVONSHIRE}

her share of William Briwere's honour to her husband, Payn de Chaworth. ${ }^{1}$ From Payn it descended to his son Patrick de Chaworth, ${ }^{2}$ who died in 1257. Patrick was succeeded by his two sons successively, Payn who died without issue in $1278^{8}$ and Patrick who died in $1315,{ }^{4}$ leaving an only daughter, Maud, who became the wife of Henry Plantagenet, duke of Lancaster. Henry IV incorporated all the estates which she inherited, some thirty fees, known as the honour of Chaworth, in the duchy of Lancaster. ${ }^{5}$

Of William Briwere's other daughters, Graecia was married to Reginald de Braose; Isabel was twice married, Baldwin Wake being her second husband ; Alice married Reginald de Mohun, and Joan William de Percy, ${ }^{6}$ to whose families they brought their respective interests in Briwere's honour.

To complete the survey of the Feudal Baronage of Devonshire it is necessary to briefly notice a few groups of fees which were held under honours of which the seats were in other counties. The descents of these honours will be treated in the counties to which they belong, and the consideration of the smaller groups of Devonshire fees will be given in detail in the topographical section of this History.

\section{THE HONOUR OF GLOUCESTER}

The honour of Gloucester, which Henry I bestowed upon his natural son Robert with the hand of Maud, the eldest of the four daughters. of Robert son of Hamon, ${ }^{7}$ included among its Devonshire constituents not only fifteen estates formerly held by Queen Matilda, ${ }^{8}$ assessed at thirty-one hides, with about 25,000 acres of land under cultivation, but also all Walter de Clavil's and Goscelin's estates, consisting of sixty-one manors assessed at thirty-seven hides, with some 17,000 acres under cultivation. In addition, it included two of the bishop of Coutances' estates, viz. Clist Fomison or Sowton and Canonteign, the estates of the three French knights Ansger de Montacute, or de ponte Senardi, ${ }^{9}$ Aiulf, and Morey of Caen, one of the estates held in chief by the English thane Alward Merta, and all those held by the three English thanes, Colvin, Godric, and Godwin, excepting Chilsworthy. ${ }^{10}$

The estates held by Clavil of the honour of Gloucester are returned in I 66 as ' 10 fees in Devon and elsewhere.' 11 Burlescombe was Clavil's head manor. After continuing for many generations in the Clavil family these fees were the subject of litigation in I 370 on the extinction of the elder branch, and were dispersed. ${ }^{12}$ Goscelin's estates came to Champernown. In

${ }^{1}$ Dugdale, Bar. i, 5 17. Testa de Nevill (Rec. Com.), 200a, gives a list of her estates. The Devonshire ones in Trans. Devon. Assoc. xxxvii, 450. Editors of Risdon, Notebook 74, call her the eldest daughter. m. 5 .

${ }^{2}$ Hund. R. (Rec. Com.), No. 32, p. 79 ; Trans. Devon. Assoc. xxxvii, 428, n. 22 ; Rot. Fin. 23 Hen. III,

${ }^{3}$ Risdon, Notebook 75 .

${ }^{5}$ Risdon, Notebook 75 ; Inq. p.m. 35 Edw. III, No. 122 . in Devon.

${ }^{6}$ Dugdale Bar. i, 702. Testa de Nevill (Rec. Com.), 200, mentions the estates of all the daughters

'Dugdale, Bar. i, 534 ; Planché, The Conqueror and his Companions, ii, 242 ;. Trans. Devon. Assoc. xxxiv, 201, 205 ; Lib. Niger, 161; Risdon, Notebook, 59.

${ }^{8}$ See Dom. Surv. 16.

- Geldroll, fol. 66b, A 5 .

"Red Book, 559.

${ }^{10}$ Trans. Devon. Assoc. xxxiii, 623 ; xxix, 465, $\pi .27$.

${ }^{12}$ Lysons, ii, 90. 


\section{FEUDAL BARONAGE}

I 66 they were held by Jordan de Champernown, ${ }^{1}$ but were most of them entered in the name of his under-tenant as ' 9 fees held of the same by William the son of Baldwin,' Book calls William de Lovayne. ${ }^{8}$ Goscelin's under-tenant Baldwin was, therefore, ancestor of the Loman family. The heiress of Champernown carried them to Wellington, ${ }^{4}$ from whom they passed to Beaumont and Basset. ${ }^{5}$ Walter of Burgundy is represented in I 303 by Henry de Tracy's heir at Dodscot and Newton Tracy, ${ }^{6}$ at Villavin by Nicolas Burnell. ${ }^{7}$ Osmund was succeeded by Lamprey, ${ }^{8}$ Ludo, Walter de Dowai's squire, ${ }^{\circ}$ by Fleming, and then by Mohun. ${ }^{10}$

Of the three French knights whose estates went to the honour of Gloucester Ansger de Montacute or de Senarpont (de ponte Senardi) had seven manors assessed at 3 hides and $\frac{1}{2}$ virgate with 2,000 acres essarted from the forest. Aiulf, probably the sheriff of Dorset and brother of Humfrey, Queen Matilda's chamberlain, ${ }^{11}$ had two assessed at I hide and $\frac{1}{2}$ virgate with about 300 acres under cultivation, and Morey (Morinus) of Caen had one assessed at $\frac{8}{4}$ hide with some $25^{\circ}$ acres under cultivation. Of the estates of all three it is stated that they did not belong to but had been grouped with Brictric's lands, and in Testa de Nevill, like Brictric's lands, they are described as 'belonging to Earl Richard's share' of the honour of Gloucester. ${ }^{12}$

\section{THE MORTAIN FEES}

Excepting Baldwin the sheriff and the bishop of Exeter, Robert count of Mortain, son of Herlwin and Herleva, the Conqueror's half-brother, held the largest fief in Devon. Already possessed of the comté of Mortain, he received after the Conquest lands in this country in no fewer than twenty counties. In the four counties of Wilts, Dorset, Devon, and Cornwall he possessed ' 623 manors assessed at 833 hides all but $2 \frac{1}{2}$ virgates,' with 'arable land for I,480 ploughs,' of a 'value of $£_{1}, 409$ all but 6 shillings and Io pence.' Of these hides 'he had 200 all but 2 in demesne,' 'worth to him $£_{4} 400$ and a mark of silver'; and his 'liegemen had 655 hides all but $\frac{1}{2}$ virgate, worth to them $\oint_{0} \mathrm{r}, 000$ all but 6 shillings and ro pence.' ${ }^{13} \mathrm{His}$ Devonshire estates included eighty-one manors assessed at $79 \frac{1}{2}$ hides with something like 40,000 acres under cultivation, including the honours of the Saxons Ordulf (excepting Broad Clyst), Edmeratorius, ${ }^{14}$ and Alward the

${ }^{1}$ Lib. Niger, pp. 161, 164: "Jordan de Champernown (Capnun) for Umberleigh and Betinton 2 fees; and his other knights have been already set down in the aforesaid roll." The Red Book, p. 559, in I 2 I 2 gives to Roes de Campo Ernulfi only one fee in Devon, but sets down nine as William de Lomene's. Trans. Devon. Assoc. xxxv, 285 .

Lib. Niger, 161. Baldwin was under-tenant of Goscelin in Domesday times at Buckland Toutsaints and Luperidge in North Huish.

${ }^{3}$ Trans. Devort. Assoc. xxxv, 285.

'Feud. Aids, $37 \mathbf{I}$.

1 Lysons, ii, I8.

"Ibid. 359, 4 iा.

${ }^{8}$ Ibid. 370,369 , at Nicols Nimet and Fenacre. ${ }^{9}$ Exeter Domesday, fol. 500.

${ }^{10}$ At Farway (Feud. Aids, 330) and also at Holditch (Testa de Nevill (Rec. Com.), 197b; Trans. Devon. Assoc. xxxvii, 437).

in Freeman, Norman Conquest, iv, 163 .

${ }^{12}$ Testa de Nevill (Rec. Com.), $177 b$.

${ }^{13}$ The figures are quoted as given in the Exeter Book, fol. 531 , without attempting to harmonize them.

"His name is variously written Edmer, Edmaer, Edmer Atre, Edmeratorius, Elmeratorius, Almeratorius, and he was possibly identified with Edmer 'Atule,' a pre-Conquest tenant in Herts, V.C.H. Herts, i, 281. 


\section{A HISTORY OF DEVONSHIRE}

Englishman. His name constantly crops up as a land-grabber of the most valuable portions of manors bestowed on others, none daring to dispute the acts of one so closely allied to the king.

On the Mortain fief merging in the crown on John's accession in I I 99, the Mortain vavassours became tenants-in-chief and their fiefs became baronies or honours. In $1227^{1}$ Henry III bestowed the Mortain fief on his brother Richard earl of Cornwall and it descended to his son Edmund, who died without issue in $1300,{ }^{2}$ when it reverted to the crown. In I 307 Edward II gave it to his favourite, Peter de Gaveston, who had married Edmund's widow. Gaveston met his death I9 May, I3 I 2. Ultimately it was constituted an appanage of the heir to the throne, and as the duchy of Cornwall it still retains certain quasi-royal privileges. It is characteristic of all the honours which grew out of this fief that they consist of small or Mortain fees, a Mortain fee being roughly described as $\frac{2}{3}$ of an ordinary fee, ${ }^{3}$ but being really 5. of such a fee." With the honour of Mortain went also the estates held in chief by Richard son of Torolf ${ }^{5}$ and the royal demesne-manor of South Tawton. ${ }^{6}$

The chief vavassour of the count of Mortain in Devonshire was Reginald de Valletort, whose fief is known as the Honour of TREMAToN, from Trematon Castle, its chief seat in Cornwall. It is described in I 66 as consisting of 59 small fees $^{7}$ and included, besides the estates held in Devonshire and Cornwall by Reginald de Valletort at the time of Domesday, the ancient demesnes of Maker, Sutton and Kingstamerton, ${ }^{8}$ also one estate then held by Hugh de Valletort under the count, ${ }^{9}$ viz. Batson, and one, Spriddlecombe, held by Donne-probably the same Donne who held in chief Newton St. Cyres and Nutwell. It continued in the Valletort family until I270, when Roger granted it to Richard, king of the Romans and duke of Cornwall, and the heirs of his body. ${ }^{10}$ It was unsuccessfully claimed in 13 I 5 by Corbet and Pomeray as representing co-heiresses. ${ }^{11}$ Another honour which grew out of the Mortain fief was the Cornish honour of Cardinan and Botardel. It consisted of all the Devonshire and Cornish estates held in I086 by Richard son of Turold or Torolf, whether held of the king in chief, such as

I Oliver, Hist. Exeter, I 84 ; Trans. Devon. Assoc. xxxiv, $576 . \quad$ Inq. p.m. 28 Edw. I, No. 44.

${ }^{3}$ Lib. Niger, 85, 98, 99.

' For proof of this see Trans. Devon. Assoc. xxxiv, 570. In 1346, when an ordinary fee paid 40 shillings, a Mortain fee paid 25 shillings (Feud. Aids, 385).

${ }^{5}$ Exchequer Domesday, I I 3, compared with Testa de Nevill (Rec. Com.), $184 a$ and $b$.

'John as count of Mortain confirmed the grant of Alison in South Tawton to Canonsleigh Priory, c. 1 I 90 (Oliver, Mon. p. 228).

${ }_{7}^{7}$ Lib. Niger, 131.

${ }^{8} \mathrm{Mr}$. Whale, in Trans. Devon. Assoc. xxxiii, 376, suggests that Valletort's one fee in Lib. Niger (p. I 28) was Clyst St. Laurence; but apart from the fact that Clyst St. Laurence was only half a fee it was held of the honour of Odcombe (Testa de Nevill (Rec. Com.), 200 ; Inq. p.m. 8 Edw. II, No. 56). Feud. Aids, 340, shews Sutton, Makerton, and Kingstamerton held by John de Vautard of the earl of Cornwall (successor to the earl of Mortain) for I fee. Inq. p.m. 2 Ric. II, No. 57, names Makre, Sutton, and Kingstamerton I fee among 'fees belonging to Trematon Castle.'

${ }^{9}$ Geldroll, fol. $69 b, \mathrm{~B} 2$, shows that the Hugh of the survey is Hugh de Valletort. See Testa de Nevill (Rec. Com.), 192a.

${ }^{10}$ Cal. Anc. D. A ro,842-4. Abbrev. Plac. (Rec. Com.), 324.

11 Peter Corbet had married Beatrice, one of the sisters of Roger de Valletort. See his Inq. p.m. I I Edw. II, No. 52 and 15 Edw. II, No. 34, and Trans. Devon. Assoc. xviii, 204. Henry de la Pomeray was grandson of Hawise de Valletort. They were found to be next heirs in Inq. p.m. 27 Edw. I, No. 32, and claimed it as such in 1300 (Abbrev. Plac. 33 Edw. I, Easter, Rot. 5), but in I 315 judgment was given against them (Abbrev. Plac. 9 Edw. II, Easter, Rot. 1 20). 


\section{FEUDAL BARONAGE}

Woodhuish, Notsworthy, and East Allington, ${ }^{1}$ or held of the count, such as St. Mary Church, Cotleigh, Little Bolbury, Shilston, and Little Modbury, ${ }^{2}$ together with Earl Hugh's estate of East Anstey. ${ }^{3}$

A third honour which sprung out of the Mortain fief was the Cornish honour of Mineli and Lantian in St. Sampson; to it belonged Shobrook in Devon. ${ }^{4}$ Yet another honour derived from the same source was that of Midellaund or Launceston Castle. It included not only Erchenbold's Domesday estates held under the count-Hele Satchvil, Culleigh, and Stockleigh Francis, ${ }^{5}$ Alverdiscot, Bratton Fleming, Croyde, Highbray and Bray, constituting the seven fees held by the Flemyngs - but also West Raddon, which in 124I was Geoffrey de Mandevil's ; ${ }^{7}$ Hamelin's two manors of Alwington, ${ }^{8}$ and Broad Hempston, ${ }^{8}$ and one estate, Webworthy, ${ }^{10}$ held by the king's crossbowman Nicolas.

From the same source sprang the Somersetshire HoNour of ODcombe, to which belonged all the Devonshire estates held of the count by Ansger the Breton (Brito)-Buckland Brewer, East Putford, Bulkworthy, ${ }^{11}$ and Smytham ; ${ }^{12}$ all those held by Alward the Englishman-probably neither Alward Tochisons mentioned fols. 337, 404, nor Alward Merta mentioned fols. 390,3906 , but the Alward Tabe of fol. 2 I 2-viz. Clyst St. Laurence, ${ }^{13}$ Northleigh, ${ }^{14}$ Hawkerland ${ }^{15}$ (Rocbebera, fol. 2 I6), Bickleigh, ${ }^{16}$ Stockleigh, and one estate held by Alvred the count's butler, viz. Sutton Satchvil and Upcot. ${ }^{17}$ Two other Somersetshire honours had their origin in the great Mortain fief ; that held in I 66 by the younger Drogo of Montacute and that of Walter de Ashul ${ }^{18}$ or Ashleigh. ${ }^{19}$ With Drogo's barony went three of the four Devonshire estates held at the time of the survey by Drogo or Drew, viz. Feniton, Corscombe, and Wombernford ${ }^{20}$ (the fourth, Honiton, had gone to the earl of Devon), and, excepting Sutton Satchvil and Upcot, all the estates held by Alvred the count's butler in Devon, viz. Monkleigh, Friseham, Wedfield, and Woodland (Liteltrorilanda, fol. 2 I I $b$ ), Matford Butter, ${ }^{21}$ Thornbury, ${ }^{22}$

1 Testa de Nevill (Rec. Com.), $192 a$ and $b$.

'Ibid. $184 a ;$ Feud. Aids, 325.

${ }^{4}$ Lib. Niger, p. 235, in the return of Richard de Luci has: "Oger the sewer (dapifer) one fee in the township of Scotebroc'; Inq. p.m. 18 Ric. II, No. 3 I: 'Shokebrok one fee pertaining to the manor of Lantyan.'

${ }^{5}$ Testa de Nevill (Rec. Com.), 184a. The complete list in Inq. p.m. 28 Edw. I, No. 44.

${ }^{6}$ By Archebold le Flemying in 1227 (Trans. Devon. Assoc. xxxiv, 567 ; by Richard le Flemyng in 1301 (Inq. p.m. $29 \mathrm{Edw}$. I, No. 46), by Baldwin le Fleming in 1346 (Feud. Aids, 437).

7 Testa de Nevill (Rec. Com.), $183 a$. ${ }^{8}$ Ibid. $184 a$.

${ }^{9}$ Inq. p. m. 28 Edw. I, No. 44.

${ }^{10}$ Testa de Nevill (Rec. Com.), $184 a$.

11 Testa de Nevill (Rec. Com.), $184 b, 200$; Inq. p.m. 36 Edw. III, No. 37.

12 Trans. Devon. Assoc. xxxiii, 376.

${ }^{13}$ Testa de Nevill (Rec. Com.), 200. Inq. p.m. 8 Edw. II, No. 56, says ' of the honour of Odcombe.' In 1286 it was held by Valletort of Briwer (Feud. Aids, p. 333).

${ }^{14}$ Testa de Nerill (Rec. Com.), I $83 b$; Inq. p.m. 8 Edw. II, No. 56.

${ }^{15}$ Ibid.; Trans. Devon. Assoc. xxxiv, 294. Part of it, as Testa de Nevill (Rec. Com.), igra and Inq. p.m. $8 \mathrm{Edw}$. II, state, was a Mortain fee held of the honour of Odcombe, whilst another part called Hoked in Oliver, Mon. 396, was given by Avice de Dun, successor of Richard Coc and Semer his brother, to Dunkswell Abbey (Oliver, Mon. p. 398). Alward.

${ }^{16}$ According to Lysons, ii, 46, Bickleigh belonged in Hen. II's time to Walter Croc, successor in title to

${ }_{17}$ Testa de Nevill (Rec. Com.), $1846,200$.

${ }^{18}$ Ibid. 1846 . Feud. Aids, 427, calls it 'Ystlegh.'

${ }^{19}$ Testa de Nevill (Rec. Com.), 179a.

${ }^{20}$ Ibid. p. 179a; Lib. Niger, 94: "William Malherbe in Devon 4 fees, and Robert son of William (Malherbe) has entered upon and wrongfully holds the land of Winburneford, which owes $\frac{1}{2}$ knight's service.' ${ }^{21}$ Tesia de Nevill (Rec. Com.), i $79 a$. ${ }^{23}$ Ibid. $184 b$. 


\section{A HISTORY OF DEVONSHIRE}

Chitterleigh, Stockleigh Luccombe, ${ }^{1}$ and Poughill, ${ }^{2}$ besides one held by Hugh de Valletort, viz. Much Bolbury alias Bolbury Beauchamp. ${ }^{3}$ To Ashleigh's barony belonged $5^{\frac{1}{2}}$ small fees in Devon," consisting of the three estates held by Bretel ${ }^{5}$ under the count at the time of the survey, viz. Charlton in Plymtree, ${ }^{6}$ Little Farringdon, and Holbrook Grindham alias Denbow ${ }^{7}$ (Colebroca, fol. 2I6), together with the two held by Mauger de Cartrey under the count, viz. Denson ${ }^{8}$ (Donicestona) in Clayhanger and Yalwinston alias Petton in Bampton ${ }^{9}$ (Alwinestona, fol. 2 I 3).

${ }^{1}$ Testa de Nevill (Rec. Com.), 191a, in 1243, says: "Held of Catharine de Montacute of the honour of Cheselbergh.' But Exon. Domesday, fol. 518, shows 'Ceselberia' in Somerset held by Alvred the count's butler.

Ibid. 191a.

${ }^{3}$ Ibid. I 92a. Feud. Aids, 324, says, 'held by Beauchamp of the king,' because Valletort's barony was then in abeyance.

${ }^{4}$ Trans. Devon. Assoc. xxxiii, 37 I.

${ }^{5}$ Bretel of St. Clare in La Manche, as appears from the geld-roll and the cartulary of Montacute Priory.

- Testa de Nevill (Rec. Com.), $179 a$.

${ }^{7}$ Ibid. I9Ia; Trans. Devon. Assoc. xxxv, 290 ; xxix, 506.

${ }^{8}$ Denson was given by Briwere to Tor Abbey (Oliver, Mon. 176, where it is written Dunnyngston). In Feud. Aids, 369, it appears as '[D]ammygescote I fee.'

${ }^{9}$ Testa de Nevill (Rec. Com.), 184b. Feud. Aids, 369: John de Radyngton holds in Pyaton 1 fee. Trans. Devol:. Assoc. xxx, 452. 



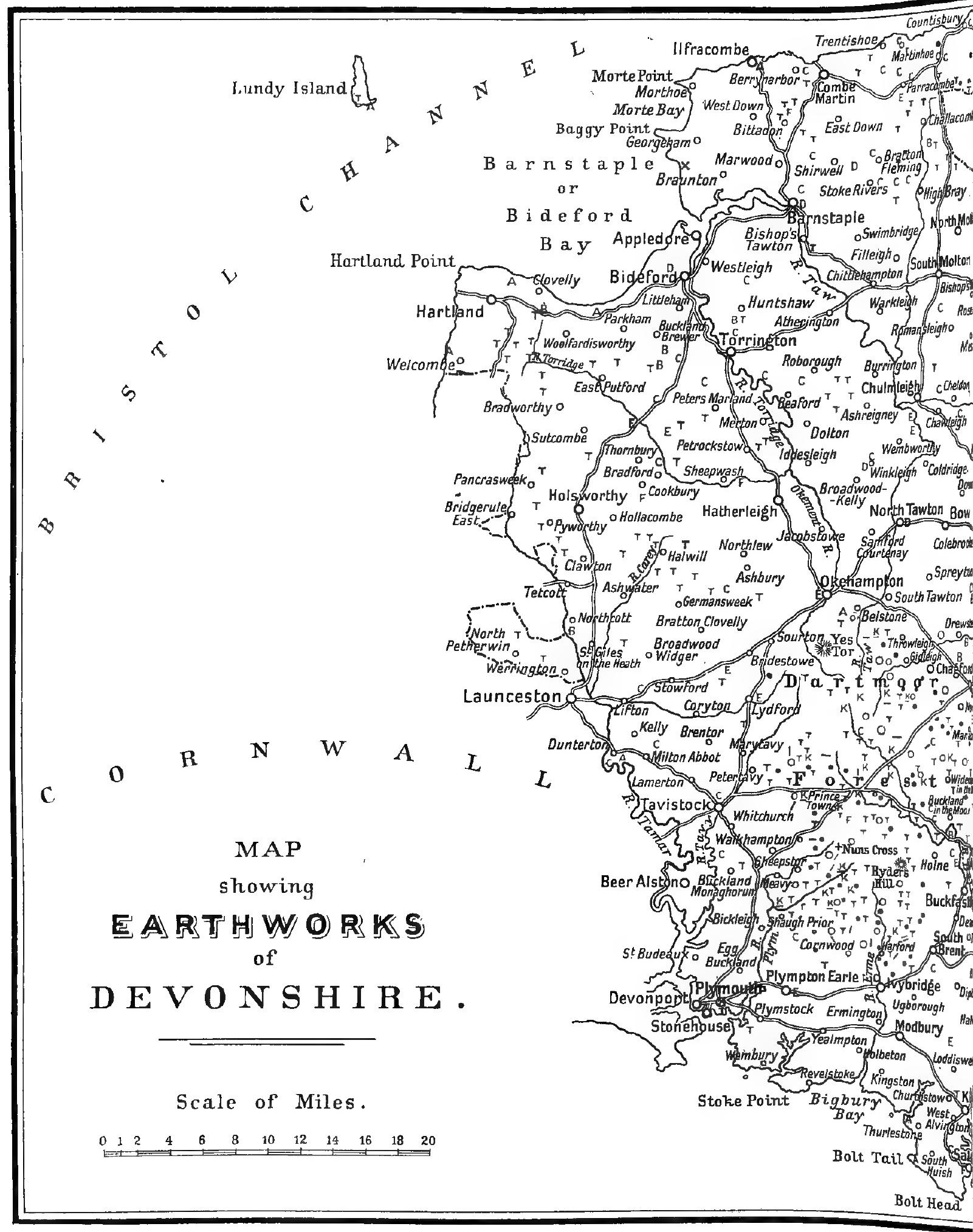




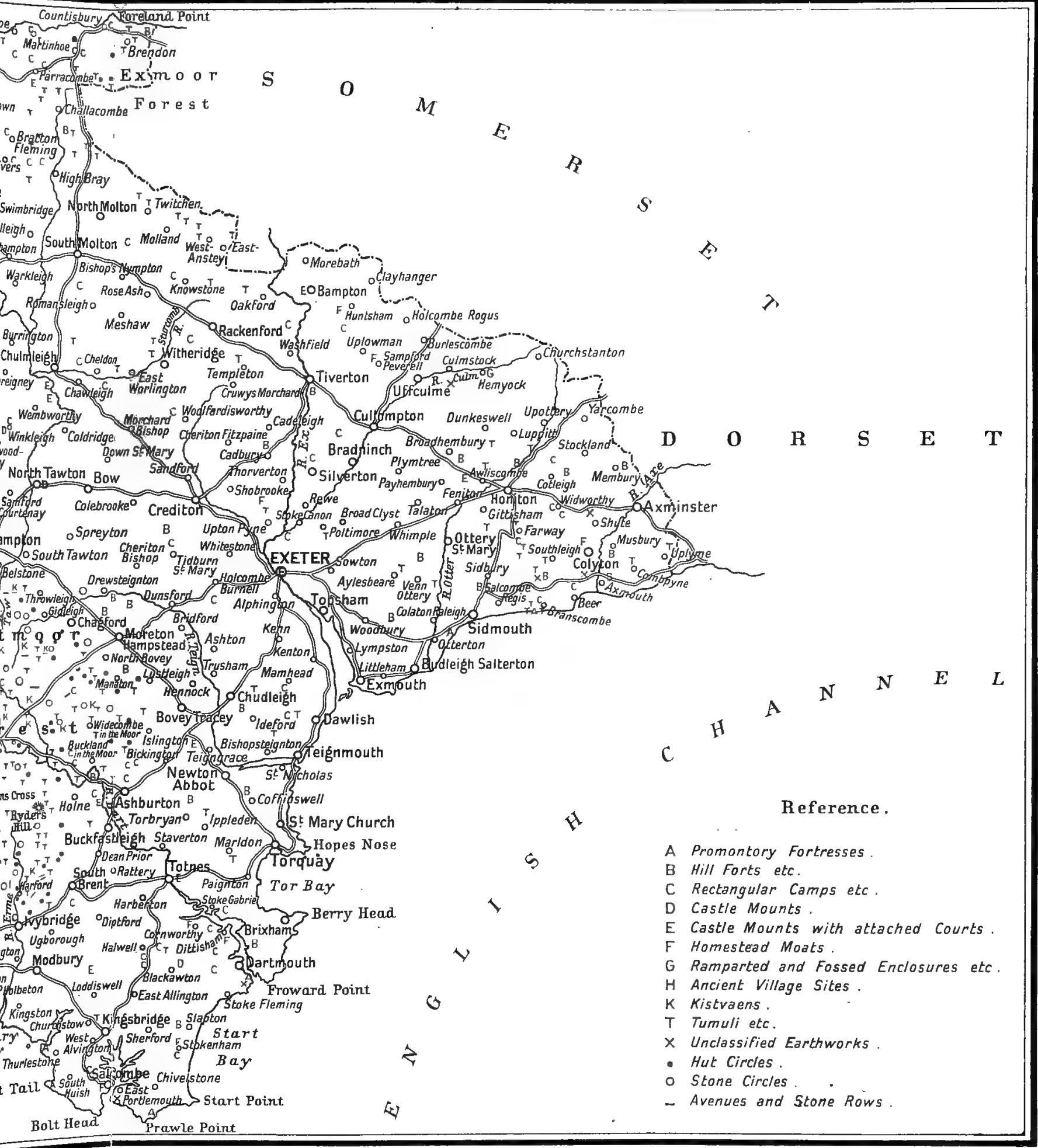





\section{ANCIENT EARTHWORKS}

\section{INTRODUCTION}

The study of the earthworks of Great Britain has been a much neglected branch of archaeology, and with few exceptions antiquaries have failed to use the mass of material still left to us to increase our knowledge of the defensive works thrown up by the early inhabitants of Britain to withstand the raids of neighbours or the invasions of foreign foes.

Thanks to the labours of a commission appointed by the Congress of Archaeological Societies we are now enabled to pursue this investigation on more systematic lines, and in considering the earthworks of Devonshire we follow the classification adopted by the Congress and published in 1903.

Class A.-Fortresses partly inaccessible, by reason of precipices, cliffs, or water, additionally defended by artificial works, usually known as promontory fortresses.

Class B.-Fortresses on hill-tops with artificial defences, following the natural line of the bill; or, though usually on high ground, less dependent on natural slopes for protection.

Class C.-Rectangular or other simple enclosures, including forts and towns of the RomanoBritish period.

Class D.-Forts consisting only of a mount with encircling ditch or fosse.

Class E.-Fortified mounts, either artificial or partly natural, with traces of an attached court or bailey, or of two or more such courts.

Class F.- Homestead moats, such as abound in some lowland districts, consisting of simple enclosures formed into artificial islands by water moats.

Class G.-Enclosures, mostly rectangular, partaking of the form of F, but protected by stronger defensive works, ramparted and fossed, and in some instances provided with outworks.

Class H.-Ancient village sites protected by walls, ramparts, or fosses.

Class $\mathbf{X}$.-Defensive works which fall under none of these headings.

Of Class A Devonshire has examples of a purely promontory type as on Bolt Tail, and also of others constructed on the edges of cliffs which are not exactly promontories, as may be seen at Berry Cliff, in Branscombe parish.

Class B is represented by many excellent examples, and the words of Tacitus describing a British position on a lofty hill the easier slopes of which were fortified with ramparts of stone, ${ }^{1}$ coupled with those of Caesar, who tells us that high ground was chosen by the Britons admirably fortified by nature and art, ${ }^{2}$ come forcibly home as we gaze on the stupendous ramparts of Hembury, near Honiton, or nature's defences at Hawkesdown; or note the strategical skill displayed by the engineers of Dumpdon. When the entrances of such strongholds were closed by felled trees, ${ }^{3}$ these fortresses may have been well-nigh impregnable.

Those falling under the second division of this class are very numerous; in some are curiously complicated entrances, including pit-falls, as at Holne Chase Castle ; in others we find successive defences enclosing large tracts of land divided into various courts providing ample accommodation for cattle, \&cc.

Class $\mathbf{C}$ includes camps which are square, such as Berry Castle in Witheridge parish, and the almost obliterated Broadbury Castle at Beaworthy;

$$
\text { 'Annals, xii, 33. } \quad{ }^{2} \text { De Bello Gallico, v, ix. }
$$




\section{A HISTORY OF DEVONSHIRE}

a square with rounded angles, as the camp in Charles parish; rectangular, of which a perfect but small example is in Chittlehampton parish, and one of beautiful construction in Shaugh Prior parish; circular, such as Castle Dyke near. Dawlish; elliptical, as Stanborough in Halwell parish ; or of irregular form, such as 'The Castle,' near Chulmleigh.

The defences of the foregoing classes were probably augmented by palisades; we know timber was used by both Britons and Romans, and one instance remains in Devonshire of such use by the former-in Bantham Camp, where the ends of the stakes, sharpened by fire, have been found.

Classes $\mathrm{D}$ and $\mathrm{E}$ are abundant in this county, where the mounts are at times found supporting mediaeval castles of later date than the earthworks. As freshly piled-up earth could not support the weight of stone buildings for a long period, timber structures alone were possible. ${ }^{1}$

Of the simple mounts of Class $D$ six examples remain, one of them, Kenwith Castle, is on the site of a battle, a description of which has been handed down to us.

Of the mount and bailey type (Class E) Devonshire has fifteen examples, the most noteworthy being Loddiswell Rings, and Hembury Castle, Buckfastleigh.

In most instances we find artificial mounts; but some are of nature's building, among them that at Bampton.

The mount in this type of fortification has in some cases a flat top, and in others a saucer-shaped summit.

Of Class F Devonshire has no complete examples and very few fragments.

Class $\mathrm{G}$ is represented by a solitary example in the parish of Hemyock.

Class $\mathrm{H}$ is not prolific in this county; but one great example at Grimspound is ample compensation for lack in numbers. It is situated on Dartmoor, which has justly been described as a place of surprises, for there the domestic, pastoral, industrial, or religious life is mapped out with marvellous distinctness.

In Class $\mathrm{X}$ are those series of segmental lines of parallel entrenchments found on headlands and elsewhere. The examples in Devonshire are not extensive; they are to be found at Braunton in the north, opposite Salcombe Harbour and elsewhere. In this class may also be included the curious work at Dartmouth known as Gallants' Bower.

The space at our disposal is insufficient to permit us to dwell on the story told or suggested by the series of earthworks of Devonshire, but we cannot close this introduction without a brief reference to the scheme of defence adopted by the Britons.

Devonshire was the main part of the possessions of the Dumnonii ; they had for neighbours the Carnabii of Western Cornwall on one side, and the Durotriges of Dorset on the east. The River Tamar formed a natural defence for a considerable part of the western boundary; here and there, however, are remains of strong fortresses; one at a bend of the river, placed to command the reaches of water, and others well situated for guarding against the incursions of the Carnabii.

On the east the ancient line of demarcation between the Dumnonii and the Durotriges, or Morini, of Dorsetshire was the river Axe-Stockland

1 Note illustrations of the fort at Dinan and other places, in the Bayeux tapestry. 


\section{ANCIENT EARTHWORKS}

and Dalwood were not transferred from Dorset to Devon until I 842-and here we find a series of immense strongholds in their respective territories on either side of that river. The Severn sea was an effective bulwark against the Silures of the opposite Welsh coast, and certain observation stations were all that was required until the incursions of the northmen necessitated greater strongholds.

The southern coast has an extensive seaboard, and ample opportunities were presented to piratical raiders by the numerous river mouths. Here we find that ancient fortifications were formerly constructed on the eastern side of most of the outlets and on many headlands, although comparatively few are left.

The fortresses of the interior largely followed the ancient British trackways, but isolated examples occur as the result of intertribal contests, or as remains of the refuge camps constructed in times of general invasion.

\section{PROMONTORY FORTRESSES}

\section{[Crass A]}

Branscombe (O.S. xcv, I and lxxxiii, 13 ).- To the west of Beer, on Littlecombe Hill-also called Berry Cliff-on ground locally known as Langham Field, is a rectangular camp.

The features of the locality have been so altered by the refuse from lime-burning that it is hard to distinguish nature's contours from the ancient entrenchments.

To the west of the mounds formed by the lime-burners the edge of the cliff bounds one side of the camp, while the other three sides are protected by ramparts and ditches, enclosing an area $952 \mathrm{ft}$.

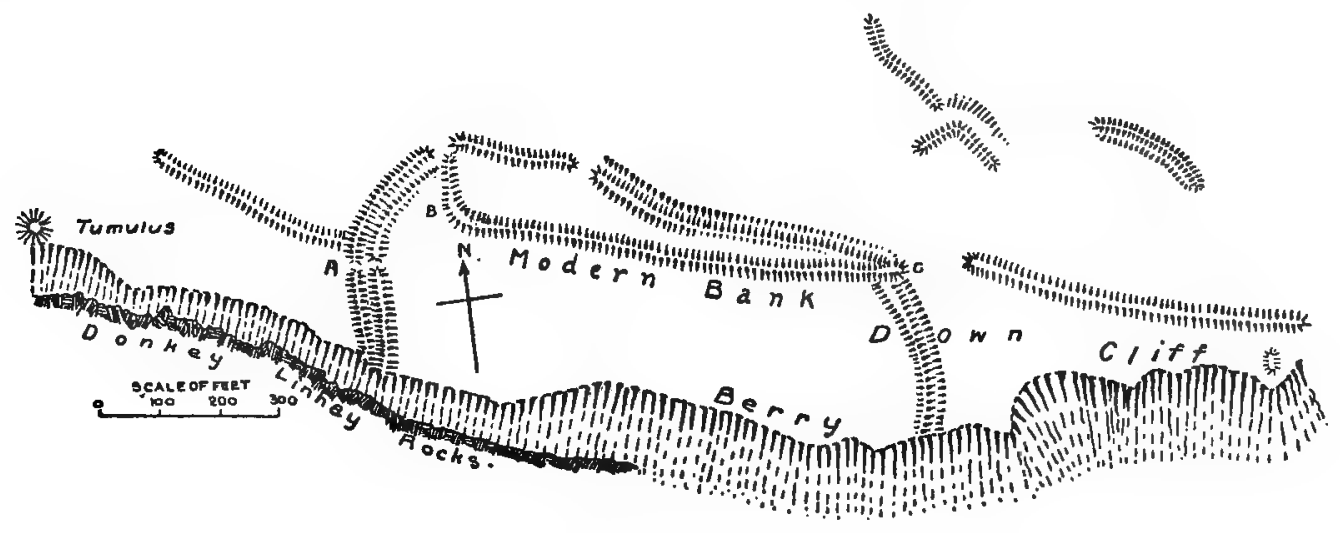

Earthwork on Littlecombe Hill, Branscombe.

in length, and $380 \mathrm{ft}$. at its widest part. The defences are strongest and most perfect at the west end, where a double vallum rises $\mathrm{I} g \mathrm{ft}$. from the fosse. The entrance $A$, on this side, is protected from a land assault by an agger extending over $350 \mathrm{ft}$. westwards.

In the long northern rampart are two gaps, and a track leads from the north-west angle; but these have every appearance of modern origin, and were probably made when the bank BC, which runs the length of the camp within the north vallum, was thrown up about twenty years since, to enclose a piece of land for attempted cultivation.

At $C$ was the principal entrance, and from this point an extension of the rampart follows an easterly course near the edge of the cliff; while north of the gateway, where the ground rapidly slopes down to a stream, are fragments of outworks to defend the approach. Two rectangular ramparts are on the ridge of a declivity, and another small entrenchment over $200 \mathrm{ft}$. in length, with an agger $5 \mathrm{ft} .6 \mathrm{in}$. high, and an internal ditch $\mathrm{Ift} .3$ in. deep, is supported at either end by natural ravines.

At each extremity of this stronghold is a tumulus on the edge of the cliff. 


\section{A HISTORY OF DEVONSHIRE}

Brixham.-Lysons ${ }^{1}$ speaks of a promontory fort on Berry Head, projecting into Torbay, where the vallum I $8 \mathrm{ft}$. in height stretched across the whole width of the promontory in a direct line. Here British pottery and Roman coins were found; but all is now destroyed, a fate which has

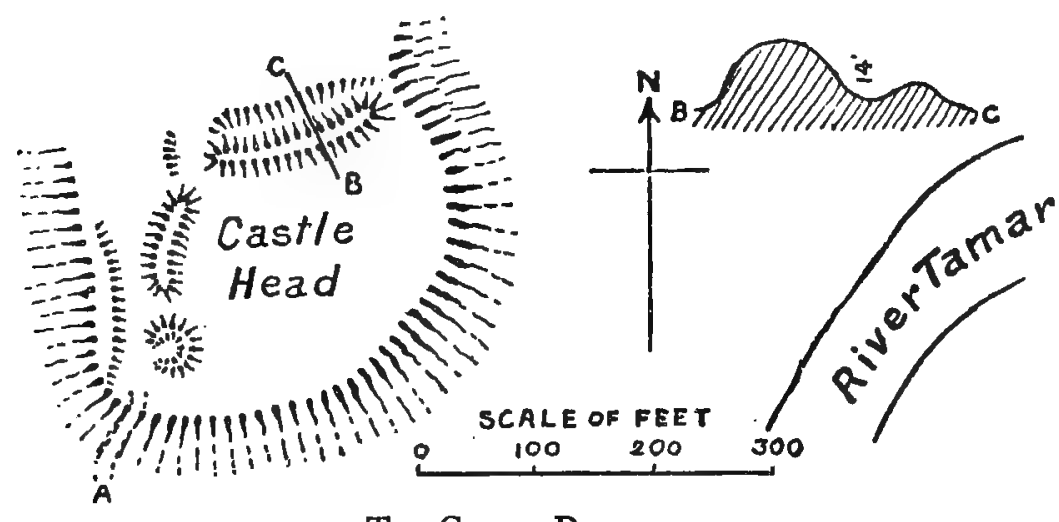

The Castre, Dunterton.

also overtaken another small entrenchment near Shorestone Point.

Chivelstone (O.S. cxxxix, 1).-Slight remains of entrenchments lie inland from Prawl Point.

Dartmouth, ST. Petrox (O.S. cxxviii, I3).-Dartmouth Castle. The successive engagements with the French in 1377 and 1404 ; the Wars of the Roses, when Dartmouth was held by the Lancastrians; and in the Great Rebellion, when it was besieged for a month in 1643 , and retaken in 1646 , caused such alterations to be made in the ancient defences that no earthworks of any definite period remain.

Above the cliff of Castle Point the remains of an entrenchment run southwards, following the outline of the cliff.

Upon Castle Head are a series of defences which have been more complete than at present appears. The remains consist of an agger $14 \mathrm{ft}$. in height, rising to a greater elevation at the eastern extremity, and a ditch $3 \mathrm{ft}$. deep extends across a full half of the neck of the headland. At the west of this vallum was the entrance; but the defences on the other side of the gateway are almost obliterated.

Within the entrance another agger, roo ft. long, extends at an obtuse angle to the first, and beyond it a rampart is on the margin of the height.

At the south-west extremity a circular guard-room or habitation is at the head of a narrow path which descends to the river bank.

Dunterton.- 'The Castle,' situated on Castle Head, is a strong frontier fort overlooking the Cornish territory. The position is a bold headland rising high from the surrounding country, and precipitately descending to the River Tamar, which makes a convex curve at its base ; it then commands two reaches of the river, and would have been a powerful deterrent to the raids of the Carnabii of the opposite bank.

HaRTLAND (O.S. xxvi, 8).Embury Beacon. On a projecting cliff on the west coast, between Nabor Point on the north and Knap on the south, is a stronghold of double defences; the inner workgreatly reduced by the fall of the cliff-is of arc form, with a rampart $5 \mathrm{ft}$. high, and $12 \mathrm{ft}$. wide, with an escarpment $10 \mathrm{ft}$. high descending to a ditch I $2 \mathrm{ft}$. wide. A circular mound $3 \mathrm{ft}$. in height with a table top has been called a tumulus, but

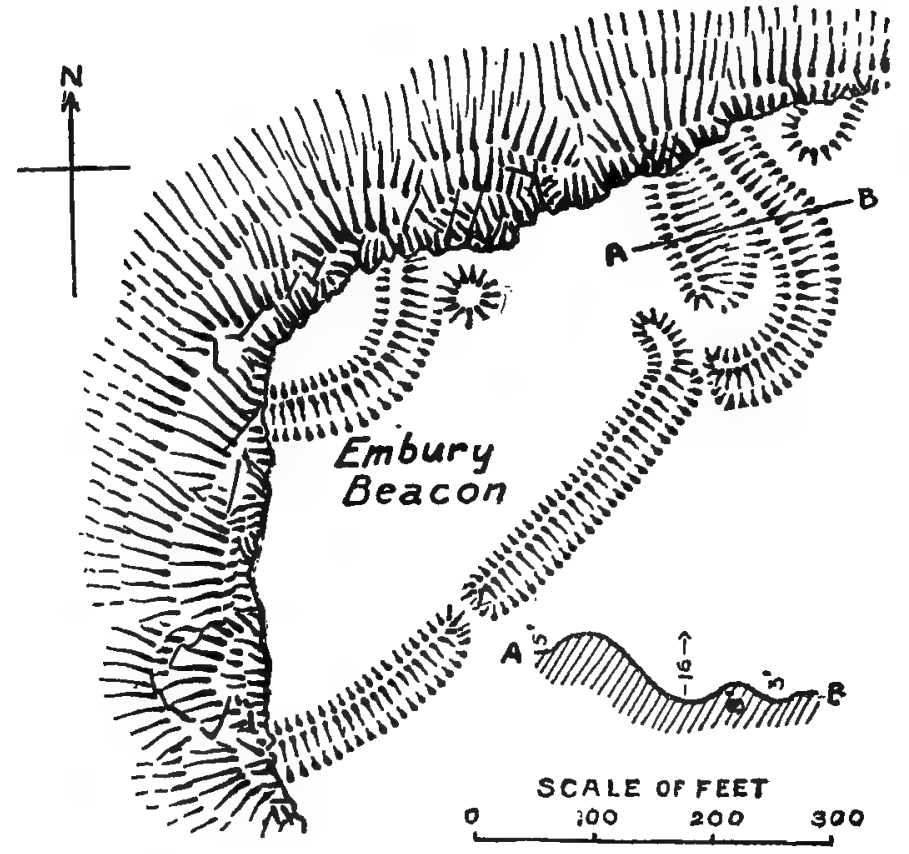

Embury Beacon, Hartland. unless future excavations prove otherwise, it may be assumed to be a defence to an entrance at this point. Beyond a court varying from 200 to $300 \mathrm{ft}$. in width is an outer vallum and fosse crossing the point in a north-easterly direction. The vallum $3 \mathrm{ft}$. high and $15 \mathrm{ft}$. wide has an escarpment

${ }^{1}$ Lysons, Mag. Brit. vi, $35 \mathrm{r}$. 


\section{ANCIENT EARTHWORKS}

Io ft. in depth, a fosse $8 \mathrm{ft}$. in width, and a counterscarp of $3 \mathrm{ft}$. About $200 \mathrm{ft}$. from its southwestern extremity is an entrance, and at a further distance of $300 \mathrm{ft}$. the vallum takes an inward curve and widens for the protection of an entrance at this point. From the angle of the curve, at the other side of the entrance, another vallum and fosse take a semicircular curve until they abut on the edge of the cliff. Within this vallum are an agger and ditch $130 \mathrm{ft}$. long, and of great strength, the agger being $16 \mathrm{ft}$. high from the bottom of the ditch. A portion of another mound outside this extremity remains on the margin of the cliff.

Hartland (O.S. xvii, 7).-Windbury Head. A second camp in Hartland parish is upon a bold promontory, also known as Windbury Point, projecting northwards into the Bristol Channel. The remains of the camp-which was certainly of larger proportions before the subsidence of the land into the sea-consist of half an ellipse, the edge of the cliff now forming its longer axis. At the west end is a fosse $40 \mathrm{ft}$. long and $\mathrm{r} 0 \mathrm{ft}$. wide, which evidently extended in former times to a greater distance than at present. The remaining defence is an artificial table-land which rises to $9 \mathrm{ft}$. at its highest point ; but even that is almost levelled at its eastern extremity.

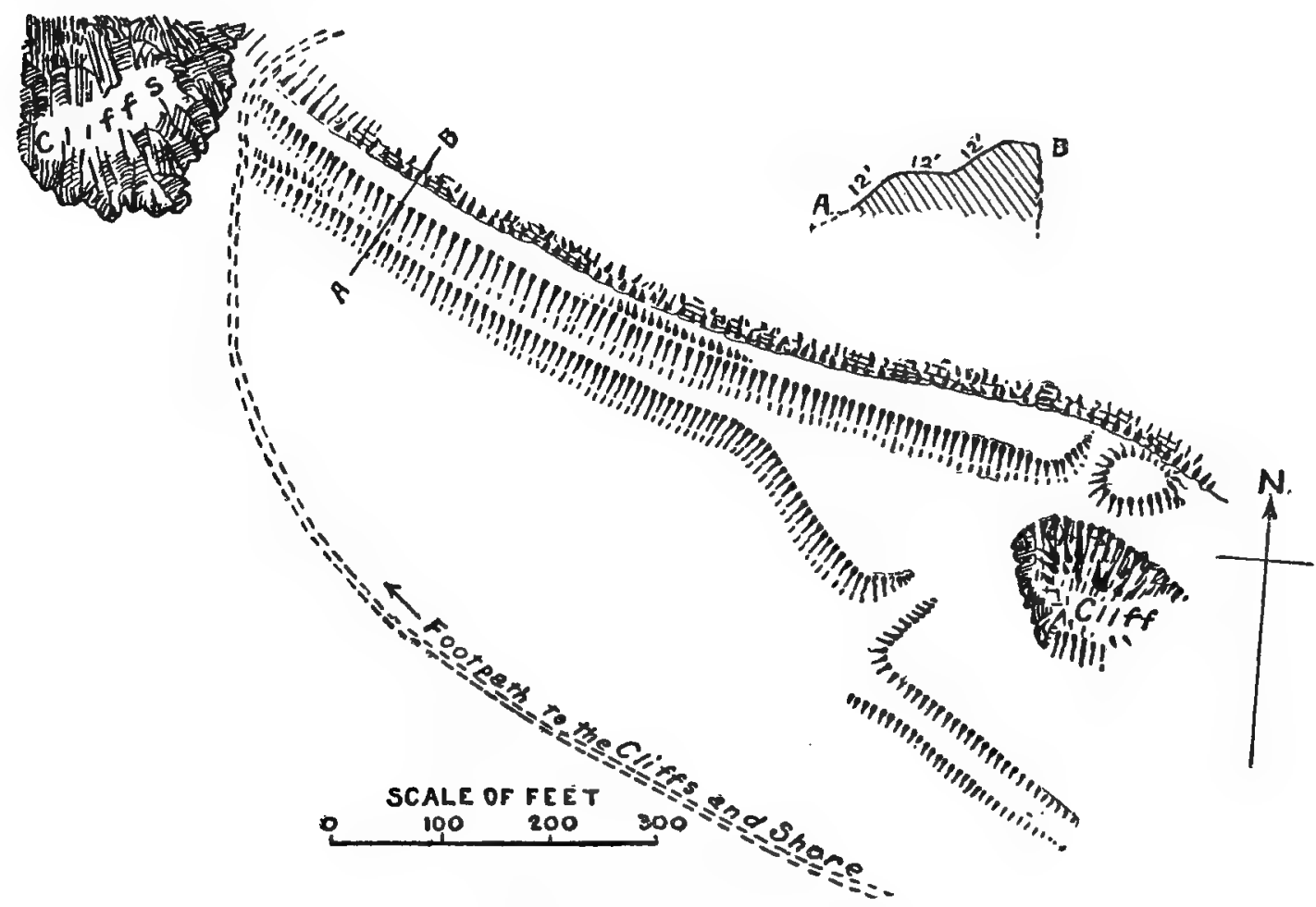

Earthwork at ILfRacombe.

ILFRACOMBE (O.S. v, I).- To the east of Ilfracombe, on the verge of the cliff, are two terraces formed on the inland slope; each of them has an escarpment of $12 \mathrm{ft}$., with an intermediate berme or platform $12 \mathrm{ft}$. wide, extending side by side for $550 \mathrm{ft}$., when the lower work, at its eastern extremity, inclines to the south-east for a distance of nearly $200 \mathrm{ft}$., with a final curve inwards at the entrance. A corresponding bank continuing at the other side of the entrance has a lower escarpment with a yet lower berme, which is lost in the slope of the hill. The highest work, $800 \mathrm{ft}$. long, returns to the edge of the cliff, and probably enclosed a wider area when the cliff was in a more perfect state. At the eastern end of this is a large mound, and a pile of natural rock supplied another mound defending the entrance.

The berme at the western extremity, which abuts on a precipitous cliff, has a bank on its outer edge for about $80 \mathrm{ft}$, and a similar bank in the middle of the uppermost work extends $150 \mathrm{ft}$.

Lundy IsLand.-Marisco Castle. Lundy Island is geographically included in the county of Devon, although 12 miles distant from Hartland Point, the nearest mainland, and 15 miles from Clovelly.

At the southern end of the island are the ruins of Marisco Castle, naturally defended by lofty and precipitous rocks, inaccessible except at a well-defended landing place on the eastern side. The castle was strengthened by considerable earthworks; on the north side a moat and portion of a rampart remain, which cannot be ascribed to a date earlier than that of the thirteenth-century outlaw by whose name it is known. 


\section{A HISTORY OF DEVONSHIRE}

Malborough (O.S. cxxxv, I2 and I6).-Bolt Tail, a headland jutting out on the west side of the most southern extremity of the county, is fortified in two places-the westerly pro-

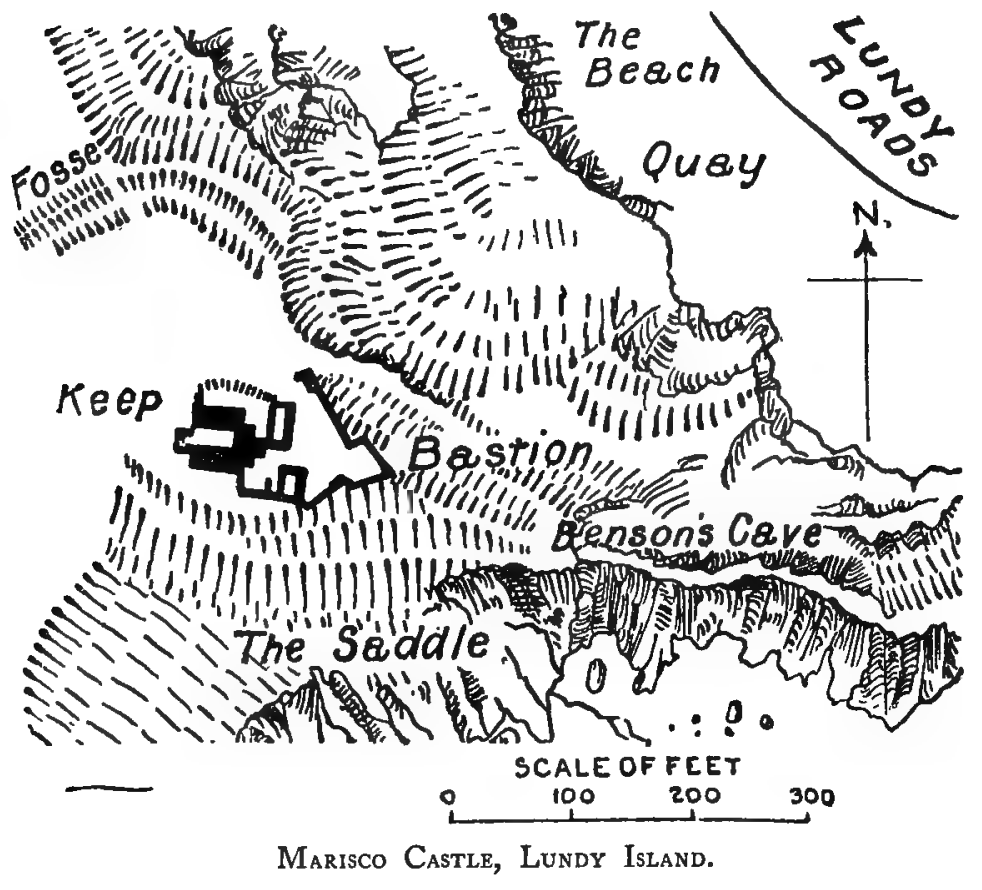
montory and the cliff facing northwards into Bigbury Bay. The first is an entrenchment $900 \mathrm{ft}$. long across the narrowest part of the promontory, which cuts off some 12 acres of land. The rampart-20 ft. thick at the base, and in places I $6 \mathrm{ft}$. high-is walled $4 \mathrm{ft}$. in height on its outer face. It is approached from a sweeping dip in the ground, well covered by the missiles of the defenders. The entrance is formed obliquely by the incurved ends of the rampart, which widens on the right-hand side. An advanced outwork of stone in the form of an arc is upon a mound $13 \mathrm{ft}$. high and $53 \mathrm{ft}$. long to the right of the entrance, and commands a hollow which has the appearance of having been a sunk road to a subsidiary entrenchment on a lower level.

This secondary stronghold is on a minor promontory facing Bigbury Bay and the entrance to a stream which empties itself at this point and provided fresh water to the garrison. A rampart $9 \mathrm{ft}$. high commences on the low ground at the southeast and continues a straight course north-west, where another small rampart on the edge of the cliff forms the exit which emerges on the sunk path leading to the stronger camp mentioned above. This smaller area is overlooked by the rising ground inland, but it provided a protected connexion with the drinking supply, and also with the boats in the bay until the defenders might possibly have to fall back on the great fort above.

Okehampton (O.S. lxxvii, 5).-On a high promontory formed by the curving of the East Okement river, which surrounds the north, east, and south of the termination of a spur of hills on the north-east side of Dartmoor, is a simple camp.

The promontory is crossed by a rampart and ditch of serpentine plan; the agger rises $8 \mathrm{ft}$. from the inside, and has an escarpment of $14 \mathrm{ft}$. into an outer fosse $6 \mathrm{ft}$. in depth.

The entrance is in the middle of the vallum, which slightly curves at each side in opposite directions. On the eastern extremity of the promontory, near the verge of the cleave, is the fragment of a breastwork following the line of the land, and a tabletopped mound $3 \mathrm{ft}$. high is in the south-east angle.

Outside the camp to the west traces of two hutcircles remain, and on the north side of the ridge flows the Moor Brook.

Otтterton (O.S. xciv, 5).-High Peak. One mile to the west of Sidmouth the destructive waves wash the base of a cliff which rises perpendicularly from the sea to a height of $5 \mathrm{II} \mathrm{ft}$, and has a summit of conical form. All that now remains of the stronghold that once crowned the cone of High Peak Hill is but a fragment of an earthwork. 


\section{ANCIENT EARTHWORKS}

A curved line of rampart about $250 \mathrm{ft}$. in length has an escarpment of $20 \mathrm{ft}$. perpendicular measurement, at the most perfect part and averages $35 \mathrm{ft}$. in width at the base. The slope of this

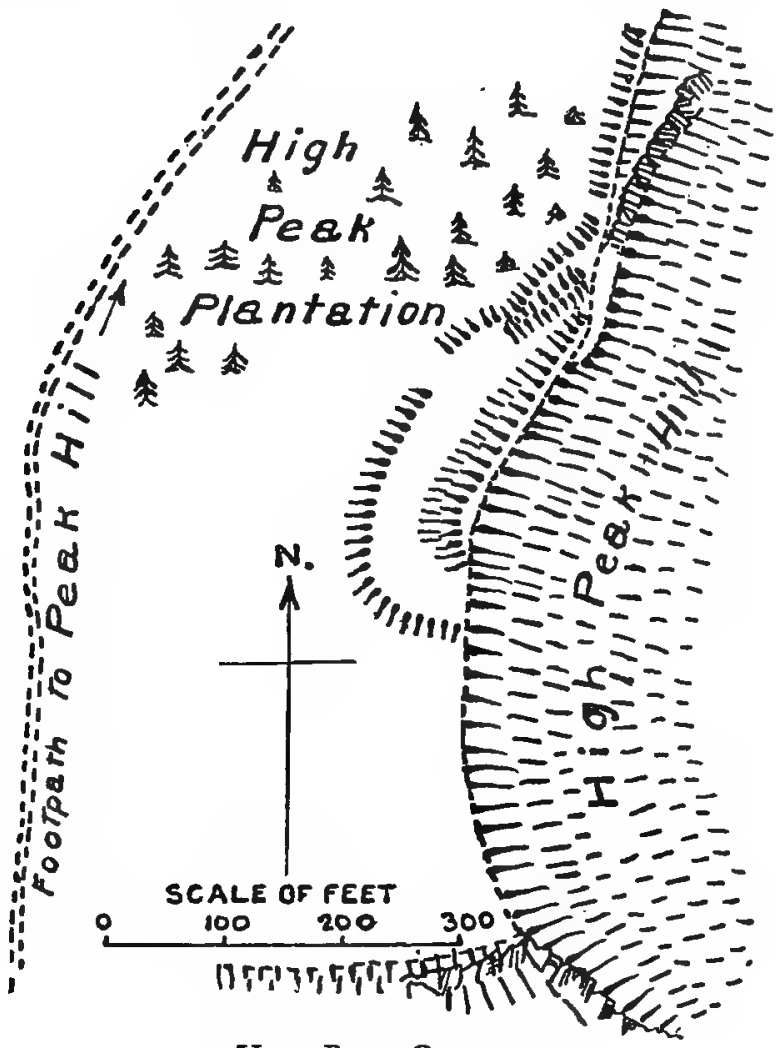

High Peak, Otterton. agger almost coincides with the angle of the hill as it rises from the valley. The fosse outside is filled nearly to the level; and beyond it is a plateau about $40 \mathrm{ft}$. wide. A succession of aggers at the northern extremity indicates a strongly defended entrance near this point. Mr. Hutchinson suggests ${ }^{1}$ that an entrance was gained from a road which has now perished. He draws attention to traces of an ancient road from Sidbury to High Peak, passing by Beacon Hill, Bulverton Hill, and Peak Hill, and lost on the edge of the cliff; but on the other side of the gap, at the foot of the cone of High Peak, it is again in evidence, following a westerly course to Otterton and Woodbury Hill Camp.

The irresistible sapping of the waves has destroyed much of this camp, but when it was constructed the cliff probably extended to the now detached rocks at sea.

This destruction has revealed certain details of the dwellers here, for in 1862 , at the north end of the great agger, about $3 \mathrm{ft}$. down the face of the cliff, a deposit of charcoal was exposed to view; the charcoal was of oak and fir, and the deposit extended for about $50 \mathrm{ft}$. Sling stones and pounders were found; fragments of pottery, coloured buff, dull red, and brown,

unglazed and decorated with incised lines; and pieces of haematite, the dust of which, mixed with grease, is supposed to have provided the natives with war-paint for their bodies.

The disintegration of the cliff carried various relics of those early times down to the beach, where many coins have been found at various times, among them a Constantine, a Claudius, and a Bactrian coin; while in 1840 , a bronze Roman centaur was picked up near the mouth of the Sid. ${ }^{2}$

Parkham (O.S. xviii, I 4).-On the edge of the cliff washed by the waters of Bideford Bay, facing due north, some 4 miles east of Clovelly, is an irregularly formed camp approaching an oblong.

An agger rising $3 \mathrm{ft}$. from the interior with an escarpment $8 \mathrm{ft}$., perpendicular measurement, defends the short eastern side and a portion of the south; the entrance on the east is a modern piercing. About the middle of the south side the breastwork is lost, but the ground declining towards the west, the camp assumes a commanding height of $23 \mathrm{ft}$. at the south-west.

The entrance is of a complicated character : a sunken path commences at $C$, between an outwork on the left and a circular chamber on the right, and winding round the south-western height enters the camp on the west, where the path gradually rises between two banks for a distance of $60 \mathrm{ft}$. to the interior camp level. The outwork on the left of the path beginning at $\mathrm{C}$ broadens into a

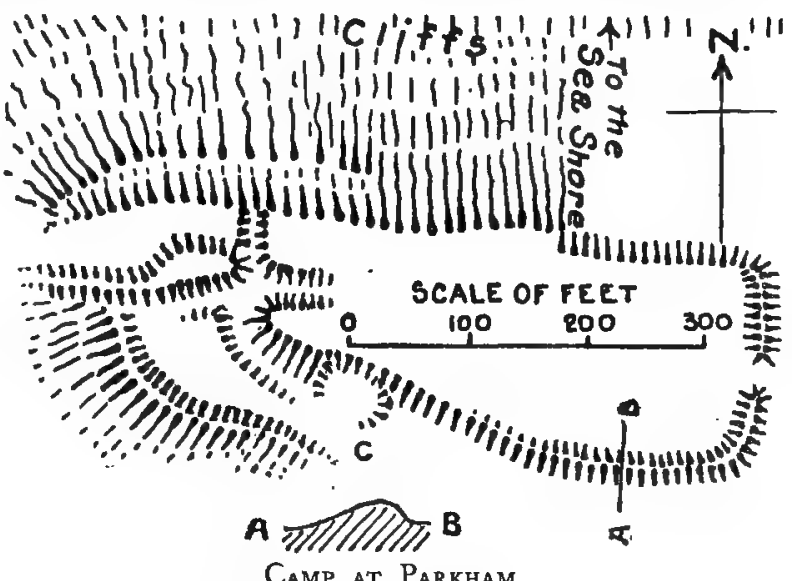
platform, with an outer vallum on the edge of the natural declivity, and another agger at right angles widens into a strong defence outside the entrance.

${ }^{1}$ Gent. Mag. Feb. 1849.

${ }^{2}$ Engraved in the Gentleman's Magazine, June, I 843 . 


\section{A HISTORY OF DEVONSHIRE}

Thurlestone.-Bantham Camp is situated on a sharp promontory at the mouth of the River Avon, which flows into Bigbury Bay, and is within easy signalling distance of the stronghold on Bolt Tail.

The entrenchments have been extensive, but the drifting sands have almost obliterated the lines of the camp, and it is difficult to estimate its original importance.

It depended for security not only on its sea-girt rocky coast, but also on the River Avon on the one side, and on the other a vast morass which is covered by the sea at each flow of the tide, and into which flows the Buckland Stream.

Bantham has been described as rectangular in plan, but the remains take no very definite form, the principal defence more nearly approaching an oval.

On the south side of the stream a low mound rises from the marsh, and a short length of rampart is seen near the sea line. Piles, pointed by burning, found in the marsh are evidence of very early occupation.

Fragments of other entrenchments are on a protruding rock at Long Stone, Butter Cove, south of Bantham; and at various points on Borough Island-a mile west of Bantham, across a stretch of sand off the mouth of the Avon-are defensive works retaining no special plan.
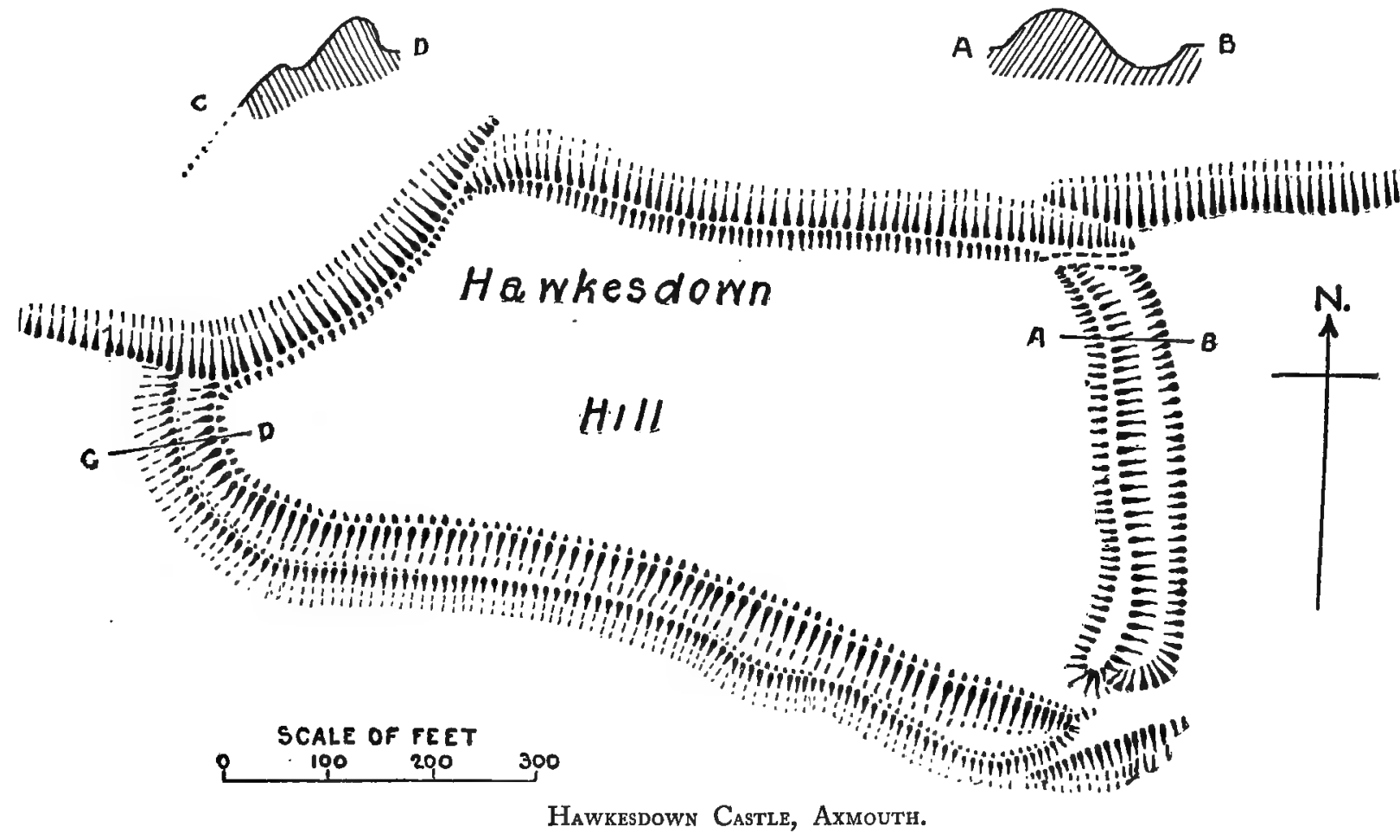

\section{HILL FORTS}

\section{[CLASS B (i)]}

Axmouth (O.S. 1xxxiii, 8).--Hawkesdown, or Hocksdon, Hill Castle ; also called Oxenden by Lysons, and Oxendown by Polwhele. The camp is formed on the extremity of a lofty ridge to the east of the River Axe, overlooking the village of Axmouth. It is an irregular quadrangle three sides of which take the form of the hill-top, and on those sides it depended for defence on the very precipitous descent of the hill ; artificial aid is, however, introduced and on the north side a strong agger crowns the breast of the steep; on the north-west nature needed but scant help to the chasm-like segment which is the chief cause of the irregularity of the camp; but from this point around the south a double vallum with a shallow intermediate ditch emphasizes the escarpment of the hill. At its eastern end the fosse widens under the controlling influence of nature, and thus provides a stronger defence to a narrow foot-entry. The work is strongest and most perfect on the eastern side, where an agger and fosse cross from side to side of the hill; the former rises Io ft. high from the interior of the camp and descends $15 \mathrm{ft}$., perpendicular measurement; into a fosse $5 \mathrm{ft}$. in depth. This side, which has an outwork $300 \mathrm{ft}$. beyond, is most easy of assault, as the top of the ridge is a level plateau. At each end of the eastern agger is an entrance; that on the south is now choked by vegetable growth, and another postern is found at the north-western angle. 


\section{ANCIENT EARTHWORKS}

The length of interior area is $852 \mathrm{ft}$. and the width $466 \mathrm{ft}$. at the east, and $420 \mathrm{ft}$. at the tarrowest part.

Sling stones have been found within its enclosure. It commands a view of the whole estuary If the Axe, and is in touch with the fortress at Musbury.

This camp was probเbly one of the border fortifi:ations of the territory of the Durotriges.

BuckLand B RE WER O.S. xxix, 9).-Hembury Zastle, also spelled 'Henbury' oo distinguish it from two ther earthworks bearing the same name in Devonshire.

This is one of a trio Jf camps, within sight of each ther, along the banks of the River Duntz, a tributary of the Yeo, the other two being respectively in Buckland Wood and Frithelstock parish.

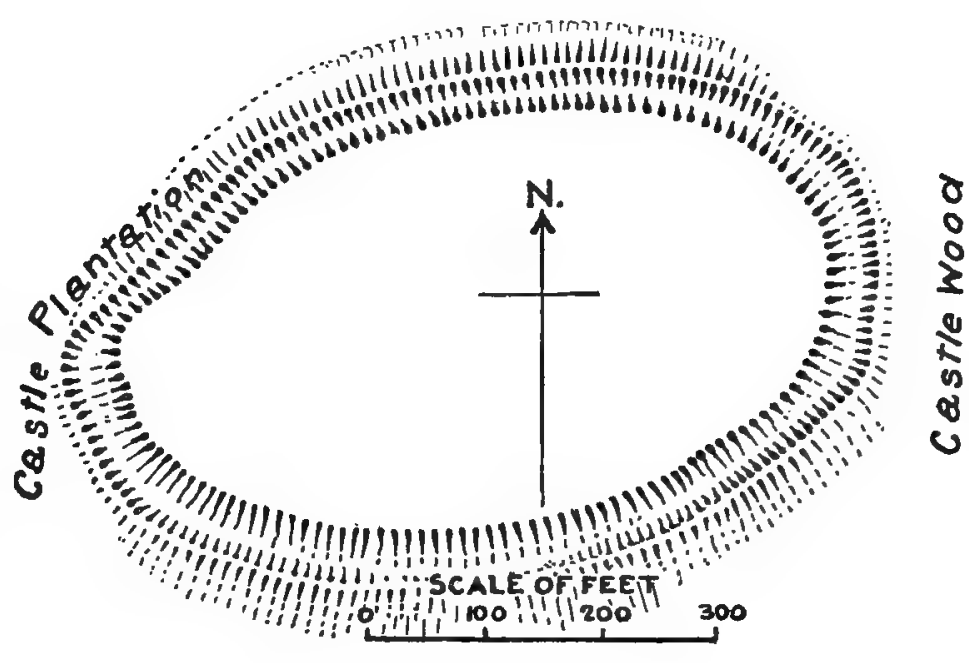

Situated on the summit of a hill 5 miles south-west of Torrington, Hembury is a small tableland of oblong form containing about 5 acres, now under cultivation. This is protected by an escarpment - the lower part of which is the natural slope- $\mathrm{IO} \mathrm{ft}$. perpendicular measurement, and a fosse from $16 \mathrm{ft}$. to $18 \mathrm{ft}$. wide; the earth removed for the formation of the fosse being thrown outwards formed a bank on the verge of the hill-side. The slope of the hill is very precipitous on the southern and eastern sides as it descends to two small streams which flow into the
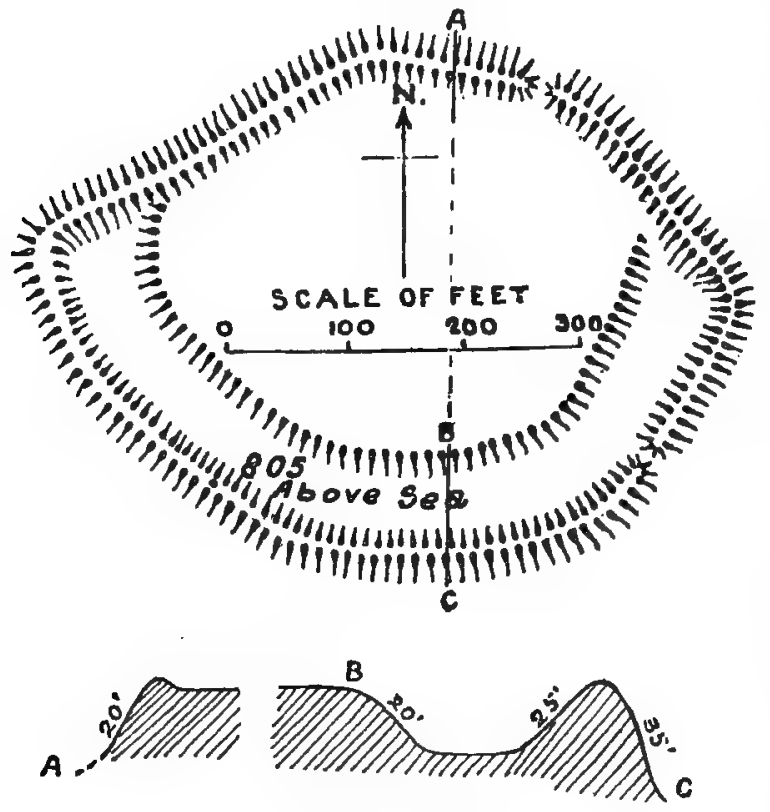

Cadbury Castre.

Duntz. The northern side, though difficult of approach, is the most accessible, and here are the remains of a crescent-formed outwork of rampart and fosse which join the main work at its two extremities on the west and the north-east.

In the ditches quantities of charred wood have been discovered; and a mound, formerly at the south-west of the interior area, was found to contain the bones of numerous skeletons, supposed to be the bodies of those who fell in the Civil War when the Royalist troops were fain to take refuge in these ancient entrenchments.

Cadbury (O.S. lv, 5).-Cadbury Castle. Crowning the summit of the most lofty of a group of isolated hills, nearly 2 miles from the River Exe, on its western bank, and the same distance south of Cadleigh, a British camp lies between Castle Plantation on the east and Northcastle Plantation on the north. Oval in form, $640 \mathrm{ft}$. in diameter from east to west, and $470 \mathrm{ft}$. from north to south, it consists of an elevated area $400 \mathrm{ft}$. by $300 \mathrm{ft}$. with a deep fosse on its west, south, and east sides, a bold agger surrounding the whole. The levelled camp area has a rampart only $2 \mathrm{ft}$. high on the north, but with an escarpment of $20 \mathrm{ft}$. on the exterior, otherwise depending on the steep declivity of the hill for its protection. From the east, round the 


\section{A HISTORY OF DEVONSHIRE}

south to the west, is no cresting bank, but an escarpment $25 \mathrm{ft}$. deep into the fosse of such width that it answers the purpose of a base-court. This is defended by a counterscarp of $20 \mathrm{ft}$., and a scarp of nearly $35 \mathrm{ft}$. descending on the precipitous hill-side. The agger is wider and heavier at the eastern extremity, which is approached by an easy acclivity. There are two entrances on the north-east and south-east respectively, and a postern or narrow way passes from the fosse to the inner camp. A heap of loose stones and earth was formerly near the south-eastern entrance within the fosse, which was thought by Polwhele to be the wreck of some work for the guarding of this gateway.

Near the centre of the inner area is a pit $12 \mathrm{ft}$. in diameter and now about $3 \mathrm{ft}$. deep. This has been cleared, and found to be a shaft $58 \mathrm{ft}$. in depth narrowing to $3 \mathrm{ft}$. wide at the base, which was formed like an inverted cone and puddled round with clay. As no spring is on the hill it has been suggested that the pit was for water storage. Diodorus Siculus, however, speaks of the construction of such shafts by the Britons in which the ears of wheat were stored, ${ }^{1}$ and it was probably for this purpose it was sunk at Cadbury. ${ }^{2}$

While removing the rubble with which the shaft was choked there were found a large ring set with an intaglio of light green antique paste, and other finger rings; beautiful armlets of bronze with gold ornamentation; a jet button, and glass and enamel beads. A fragment of an iron weapon, horses' teeth, pieces of cinerary urns of coarse pottery, charcoal, and charred human bones were also found ; fragments eloquent of various eras in which this camp was inhabited. ${ }^{3}$

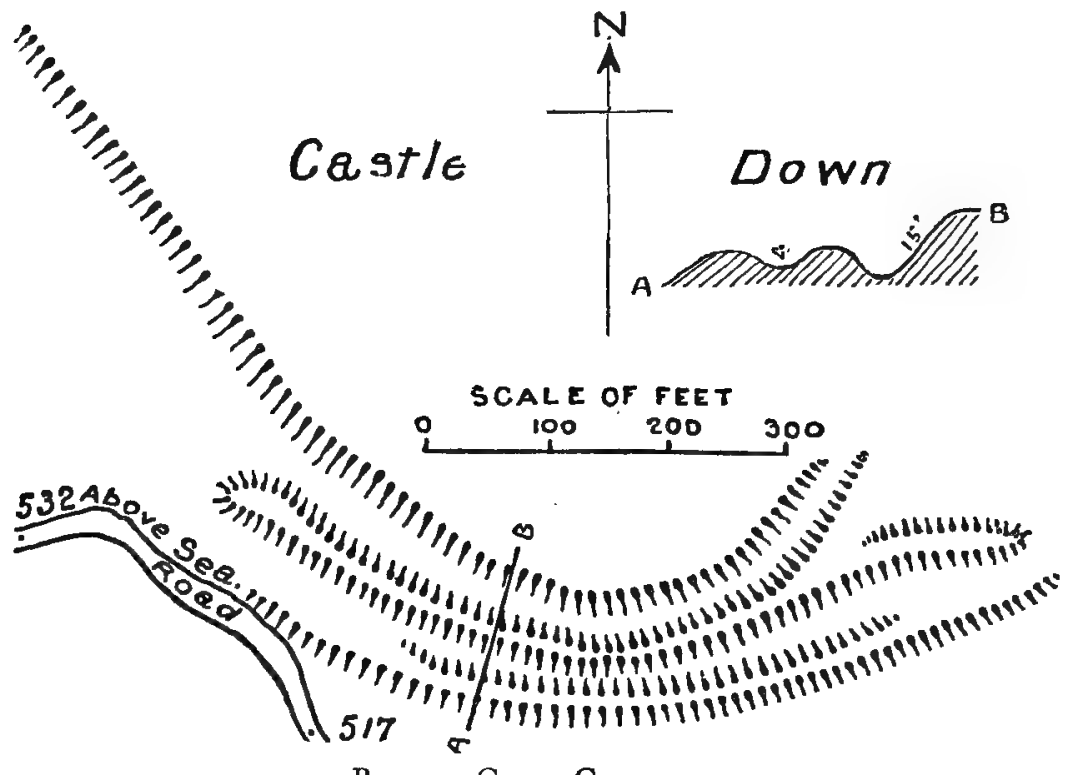

In the word ' $\mathrm{Cad}$ bury' the first syllable cot or cad is the Celtic equivalent to war and warrior, and is common to many names of persons and places, and, in this instance has led to a confusion with a place and camp of the same name in Somerset-a confusion intensified by the association of the place in both counties with other camps of 'Dolbury.'

The position of Cadbury Castle commands the valley and estuary of the Exe to the sea, and the heights

Posbury Camp, Crediton. of Dartmoor, with the strongholds of friend or

foe at Dane's Castle and Exeter Castle, Dolbury, Woodbury, Sidbury, Hembury, Dumpton, and Membury of the Dorsetshire tribes. By the ancient road they could also communicate with Ugbrooke, Musbury, Denbury and Milber, Mamhead and Castle Dyke, by which its strategic importance was considerable.

Cadbury Castle was occupied by the army of Fairfax in December, 1645. In the years I827 and 1848 , Roman and other articles were found on the site.

Crediton (O.S. lxvii, 9 and I3).--Posbury Camp, crowning Posbury Hill, on Castle Down, is above the west bank of the Culvery Brook, a tributary of the Yeo, $2 \frac{1}{2}$ miles south-west of the town of Crediton. This camp, with another formerly on Blackadown, opposite, commanded the ancient road from Crediton. Part only of these entrenchments remain, consisting of the southern defences. A scarp on the natural descent here falls $\mathrm{I} 5 \mathrm{ft}$. into a fosse $8 \mathrm{ft}$. deep. A curved agger, over $700 \mathrm{ft}$. in length, widens at either end, both of which turn outwards, the eastern extremity attaining its height above the base of the fosse in two grades. Outside the greater part of the length of this agger is a shallow fosse $4 \mathrm{ft}$. in depth, and an outer rampart, which, at its western extremity, has been mutilated by the cutting of a road.

LUPPITT (O.S. Iviii, 12).-Dumpton or Dumpdon Great Camp, over 2 miles north of Honiton, is on the summit of a round-backed eminence $879 \mathrm{ft}$. above the sea.

${ }^{1}$ Lib. v, 209 ; Munimenta Antiqua, i, 48.

Archaeologia, xlvi, 446.

${ }^{3}$ Some of these articles are illustrated in the Arch. Journ. v, 193-8. 


\section{ANCIENT EARTHWORKS}

The camp is kite-shaped with the point towards the south, and broad top running north-west, somewhat similar to Hembury Fort. The two sides and the point follow the formation of the hill, which declines precipitously, but the broad end cuts across the ridge where the ground slopes at a more moderate angle, and the lack of natural protection is compensated by artificial works. The area, $800 \mathrm{ft}$. long and $370 \mathrm{ft}$. wide at the broad end, is surrounded by a double vallum and single fosse, except on the northwest, where there is a double fosse.

The inner vallum, $6 \mathrm{ft}$. at its highest point from the interior, has on its north-west side an escarpment of $14 \mathrm{ft}$. perpendicular measurement, a fosse $2 \mathrm{ft}$. deep, and a platform defended by an outer rampart $4 \mathrm{ft}$. high, an escarpment $\mathrm{I}_{4} \mathrm{ft}$., and a fosse $5 \mathrm{ft} .6$ in. deep.

Around the other portions of the inner vallum is a narrow platform divided into two terraces; the lower one only has the imperfect remains of an outer rampart on the edge of the natural descent, which rises to $6 \mathrm{ft}$. in height at the southern point.

At the entrance on the east, about $120 \mathrm{ft}$. from the northern

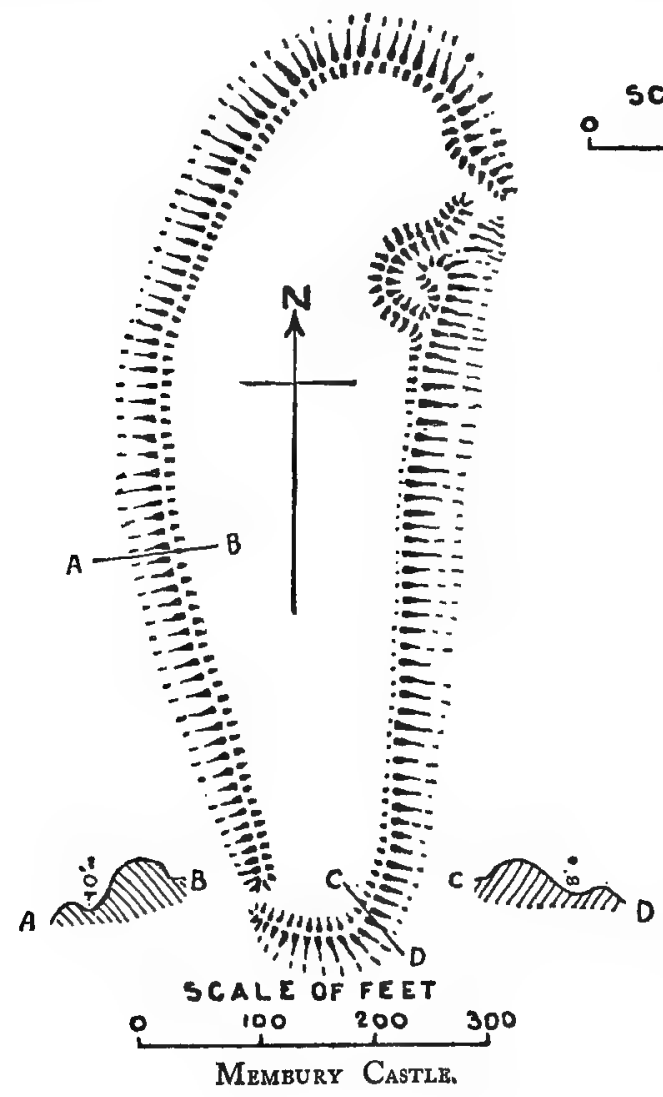

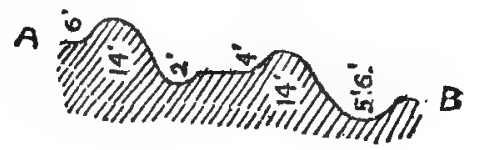

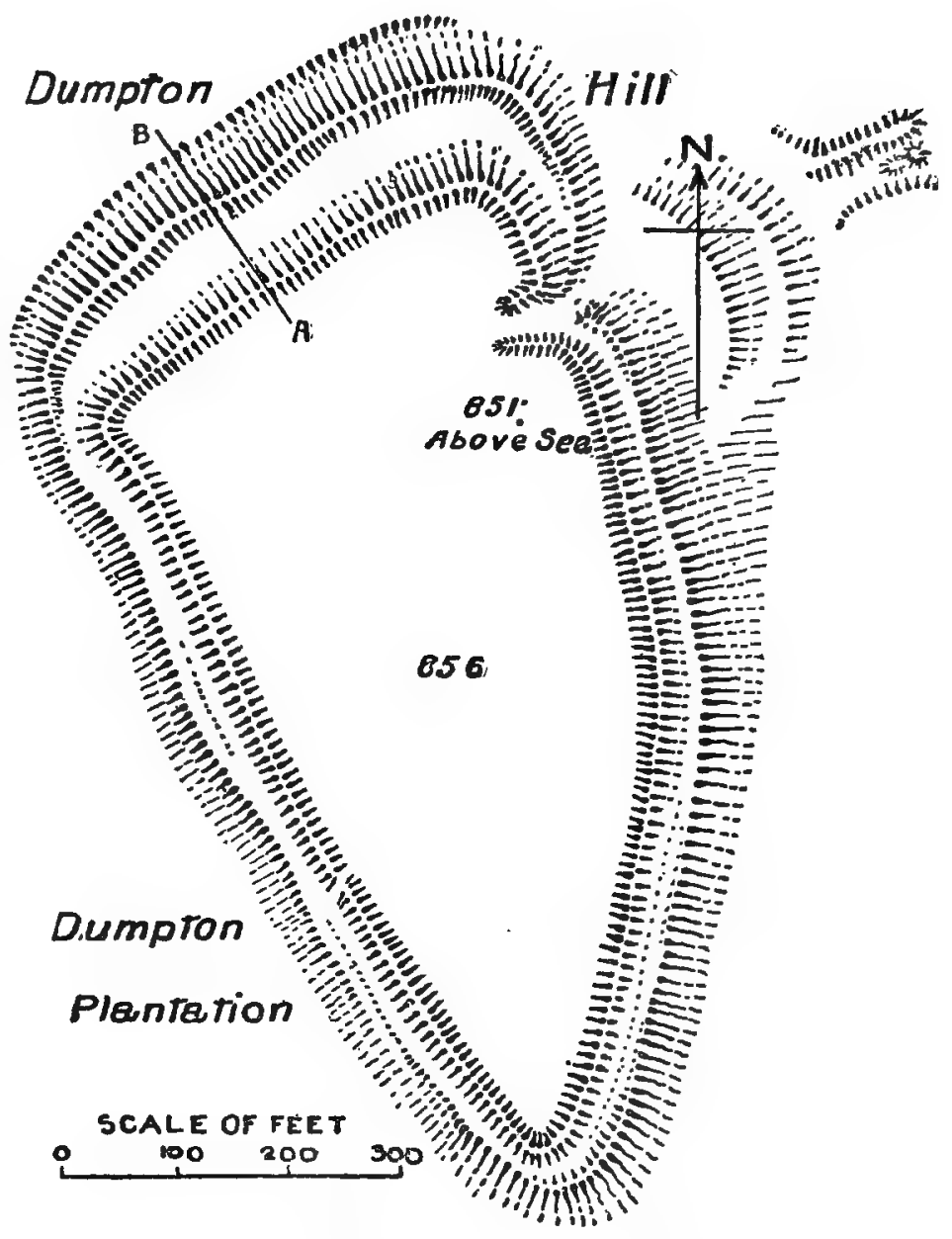

Dumpton Great Camp, Luppitt.

angle, the inner and outer ramparts on the north merge into one. Here the inner ramparts inflect, and are carried nearly $100 \mathrm{ft}$. into the body of the camp, forming a passage in which an intruding foe would be exposed to a crossfire. Beneath the entrance is a crescent-shaped defence, and yet lower another, before which are the remains of a well-defended entrance.

A mound, very like a tumulus, almost in the centre of the camp where the contour is marked, is said to have been thrown up by the Ordnance surveyors to obtain a level for their instruments, though no irregularities are around to show where they obtained the earth.

Membury (O.S. 1x, 9).-Membury Castle, 3 miles north of Axminster, one of the border fortresses of the Dumnonii, is of irregular oval form, surrounded by a single vallum enclosing between 2 and 3 acres. The rampart surrounding the crown of the hill varies in height, averaging $4 \mathrm{ft}$. from the inside except on the east side, where it rises but $\mathbf{I} \mathrm{ft}_{\text {., }}$ and that height is partially gained by the earth 583 


\section{A HISTORY OF DEVONSHIRE}

having been thrown up from the interior, thereby leaving a shallow depression. Around the other sides the vallum has been formed from the outside by which a shallow ditch has been left on the exterior $8 \mathrm{ft}$. below the agger at the south end, and $10 \mathrm{ft}$. on the west side. One entrance is at the south-west, but the feature of Membury is the intricately defended entrance on the east side towards the north. The main gateway is above a very steep incline, and is entered by an oblique path formed by the northern agger taking an outward curve; on the southern side the agger rises to a great height, curving in sickle-form into the main vallum, making a chamber from which missiles could be poured upon a foe at the gate. In the spot where the point of the sickle blade would be the agger widens, so that warriors upon it could not only rake the enemy, but, in case of necessity, cover the retreat of their fellows through the narrow path to the chamber, and hold the pursuers at bay whilst they climbed the agger into the main camp.

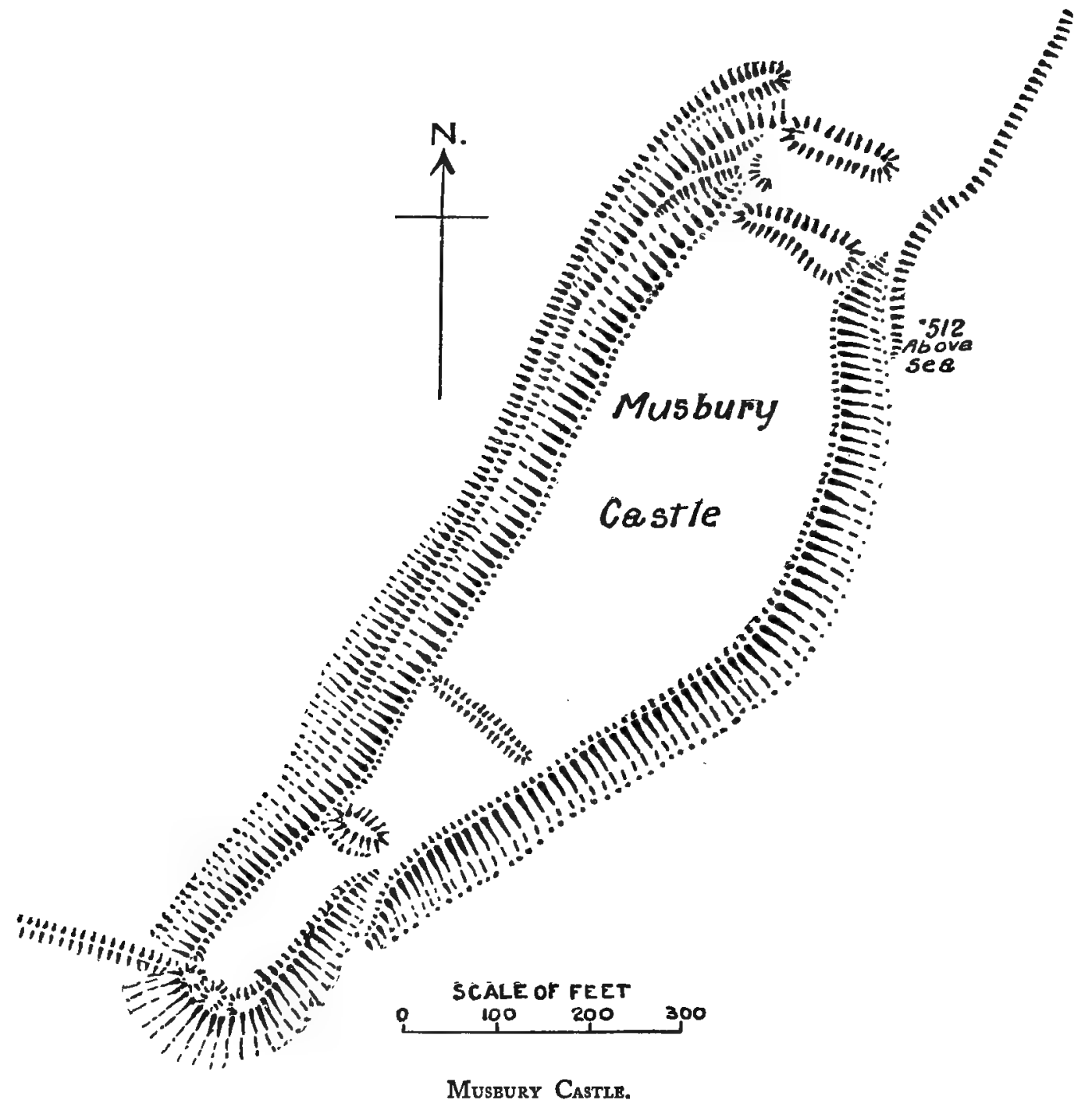

Camden calls Membury a Roman camp, afterwards occupied by the Danes; but without denying either of those people the hospitality of this stronghold, the origin must be attributed to earlier days. If the conjecture that the battle of Brunanburgh was fought in this locality be correct, Membury most probably played its part in weaving the laurel crown of victory for the brow of King Athelstan.

In 1809 some British pottery of a rude description was found here, and a cinerary urn with charcoal and bones; while in 18 I 4 a jar of Roman coins was unearthed.

Musbury (O.S. lxxxiv, I).-Musbury Castle, 3 miles south of Axminster, and the same distance from Hawksdown, is an irregular elliptical entrenchment following the formation of the ground on the extreme ridge of a lofty hill, surrounded by a single agger and fosse, except on the north-east, the most vulnerable spot, where the defences gradually increase in strength until they form a triple vallum at the northern termination. This portion of the fortifications may have misled Lysons, who 


\section{ANCIENT EARTHWORKS}

describes the entrenchments as double, whereas the greater part of the camp is cinctured only by a low bank on the crest of the deep escarpment of the natural hill-side. Adjoining the triple vallum two transverses cross the neck of the ridge; the outer one is $8 \mathrm{ft}$. in height, and the inner $4 \mathrm{ft}$., with an intermediate space of $120 \mathrm{ft}$. The inner transverse widens into a broad platform at its ' eastern end, where it commands an entrance into the camp and a path which descends the hill at this point.

The interior area, which comprises about 6 acres, assumes an elongated tongue-like form at its south-west extremity, where the vallum is broken by three apertures somewhat like embrasures for artillery, through which the defenders could approach the edge of the steep declivity for the discharge of missiles upon an enemy beneath. From the westernmost of these openings a long bank descends the hill-side to the valley to impede the massing of the foe. An entrance on the south enters the camp obliquely through the vallum, and is defended by an agger at the top, which also serves as a transverse, rising $4 \mathrm{ft}$. on the north-east, and descending $12 \mathrm{ft}$. into a ditch of $4 \mathrm{ft}$. on the south-west. Two hundred feet towards the centre of the camp is another transverse $4 \mathrm{ft}$. in height. Thus was the construction planned that should one end of the camp be forced a succession of defences would have to be carried before the besieged were overwhelmed.

In Lysons' time he tells us the vallum was much injured by the plough, and although the area is not now under cultivation, we cannot know how far this ingeniously conceived camp has been injured. The scene, doubtless, of feuds between the Dumnonii and the Durotriges, Musbury is supposed to have played a part in the famous battle of Brunanburgh in A.D. 937.

Ottery St. Mary (O.S. Ixxxi, 4).-Belbury, or Belsbury, Castle. Crowning an eminence on the West Hills-by which name the range is distinguished from the East Hills, on which Sidbury Castle is situatedI $\frac{1}{2}$ miles south-west of Ottery St. Mary, are the fragmentary remains of that which was once a formidable stronghold. The proportions of Belbury Castle are reduced, being partially destroyed about the year I 792, when the entrenchments were levelled, the earth from the ramparts being thrown into the central area to level the ground. It is described as having formerly had a great ditch all round the outside, and the present road on the southern and eastern sides occupies the site of the ancient fosse.

The site is now known as 'Castle Field,' and there an irregular rectangle $500 \mathrm{ft}$. long and $200 \mathrm{ft}$. at its widest part, more or less marks the position of the fort. From a careful survey of the road and the site, it is apparent that the entrance was on the south-east of the camp, but it is now impossible to ascertain the nature of its defence.

On the western side a sunk road runs due north and

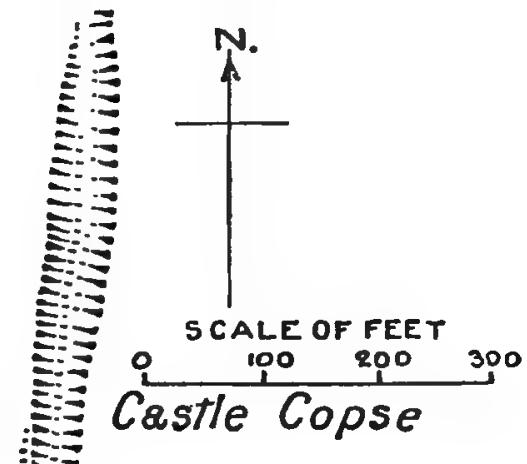

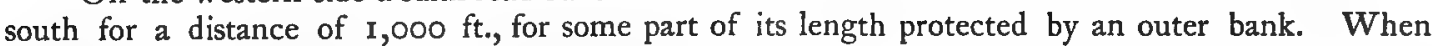
Mr. Orlando Hutchinson was making inquiries on the spot in $186 \mathrm{r}$, an old resident told him that he could remember when this road was perfect all the way northward to Streetway Head, and even at that time he could trace it in many places.

Paynembury (O.S. lviii, IO).- - Hembury Fort, 4 miles from Honiton, is the grandest monument of military skill and strategy of the Britons in the county of Devon. Crowning a bold spur of great height it is a marvellous exhibition of indomitable energy in those who raised such huge circumvallations of earth.

Of elongated egg-shape the area of Hembury- $1,085 \mathrm{ft}$. long and $330 \mathrm{ft}$. wide at the north end, tapering gradually towards the south-is surrounded by a double vallum, tripled on the north, west, and south, and quadrupled at the north-west corner. The inner vallum, varying from 4 ft. to $6 \mathrm{ft}$. in height, and rising to $10 \mathrm{ft}$. at the north, is incurved to protect an entrance from the fosse at the north-east; at the fosse level of this gateway is a raised semi-circular mound $\mathrm{C}$ for the defence of the same entry. Lower down on the same side two footpaths, D D, curving into one at the base, give access to a berme I I $\mathrm{ft}$. beneath the inner rampart and I $8 \mathrm{ft}$. above the fosse, which berme, 


\section{A HISTORY OF DEVONSHIRE}

or platform, extends only along a portion of this eastern side. A small mound, E, is built on the slope of the agger, from which the garrison could control the use of the path.

The fosse, $40 \mathrm{ft}$. below the inner vallum on the north side, rises at the north-west corner some $14 \mathrm{ft}$., and the second rampart rises with it until it attains the same height as the inner rampart, while the first rampart increases to $\mathrm{IO} \mathrm{ft}$. above the interior area at the north. The third great vallum encloses an intricate entry on the south, where a subsidiary earthwork occupies the broadened valley of the fosse: here a sunk path passes through the middle vallum from the inner fosse on to an ogee descent protected by a smaller fosse within the greater. It has been seen how the ramparts assume greater height on the north, this is because it is the position most easily assailed by reason of the land being less precipitous; the third vallum also rises to great height here, and at the north-west curve it widens into a broad platform edged by a low bank. Outside this angle is yet another defence in a fourth agger.

The entrance is near the middle of the west side, running the gauntlet of three ramparts and two ditches, with an agger parallel to the path protecting the entrance of the fosse on the right. This passes obliquely between the earthworks, and as it reaches the interior it is divided into two ways by a triangular mound; from the mound rise two defences which are the curved and widened ends of two lateral ramparts which cross the area from west to east.

The two transverses, somewhat south of the centre of the camp, have paths through them arranged obliquely. The southern bank is $3^{\circ} \mathrm{ft}$. high, rising to $5 \mathrm{ft}$. at a 


\section{ANCIENT EARTHWORKS}

circular mound in the middle, with a shallow ditch I $\mathrm{ft}$. deep on its south side. The northern bank, $5 \mathrm{ft}$. high, has no ditch.

Within the area, Polwhele says, are two praetoria in each division, but such a statement would not hold good even if it were a Roman camp; mounds such as he evidently referred to are nowhere visible in the northern part; but in the southern division, about the centre, is a circular platform $3 \mathrm{ft}$. high with a shallow ditch on the east side, which may possibly have been the site of the chieftain's hut. At the extreme south a low rampart encloses an almost circular area, about $100 \mathrm{ft}$. in diameter, which may have been for a beacon fire as suggested by Mr. Hutchinson, but was more probably for the watch.

Polwhele makes a plausible statement when he says that one division was for the accommodation of the foot and the other for the horse; certainly that branch of the entrance road into

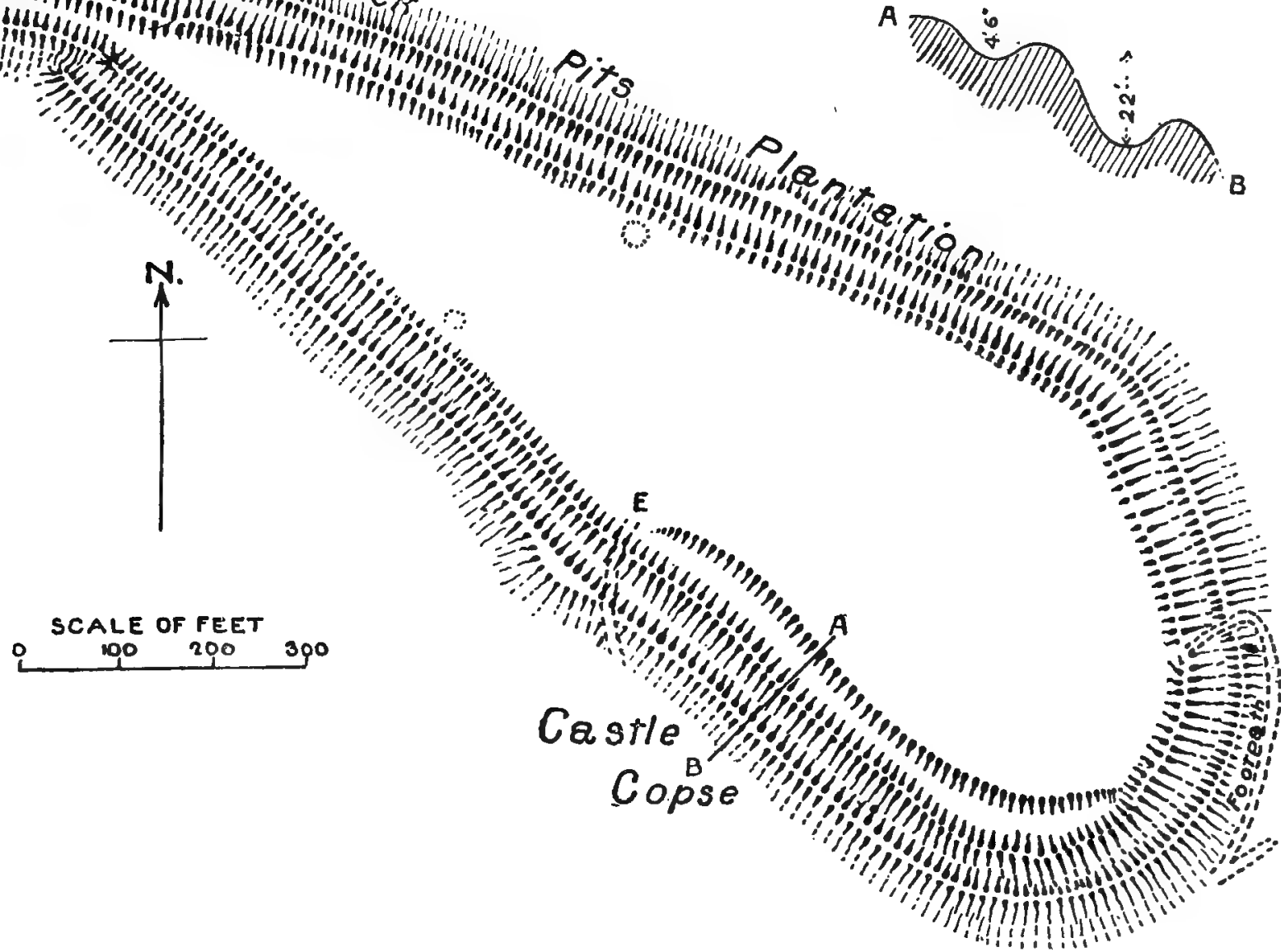

Sidbury Castie.

the northern partition is both straight and broad, while that into the southern is narrow and curved.

The discovery of Roman coins, and in I80I of a lar or household god, of iron, ${ }^{1}$ within this stronghold has been thought sufficient evidence by certain learned antiquaries to call Hembury a Roman camp; but while admitting a Roman occupation we plead for an ante-Roman origin for this most remarkable work. About I850 a well-preserved looped palstave was found within two miles of this fort, and in 1867 some long tapering blades of fine bronze were found in the vicinity.

SIDBURY (O.S. lxxxii, ro, I I).-Sidbury Castle, a large kite-shaped camp, with the point towards the north-west, is situated on the summit of one of the many lofty hills on the west bank of the Sid, $2 \frac{1}{2}$ miles north of Sidmouth.

' Figured in Archeologia, xiv, 279. 


\section{A HISTORY OF DEVONSHIRE}

The camp is encircled by a double vallum and fosse. The inner rampart, $4 \mathrm{ft}$. high, has an escarpment of $22 \mathrm{ft}$. perpendicular measurement, the fosse, $8 \mathrm{ft}$. greatest depth, divides the two

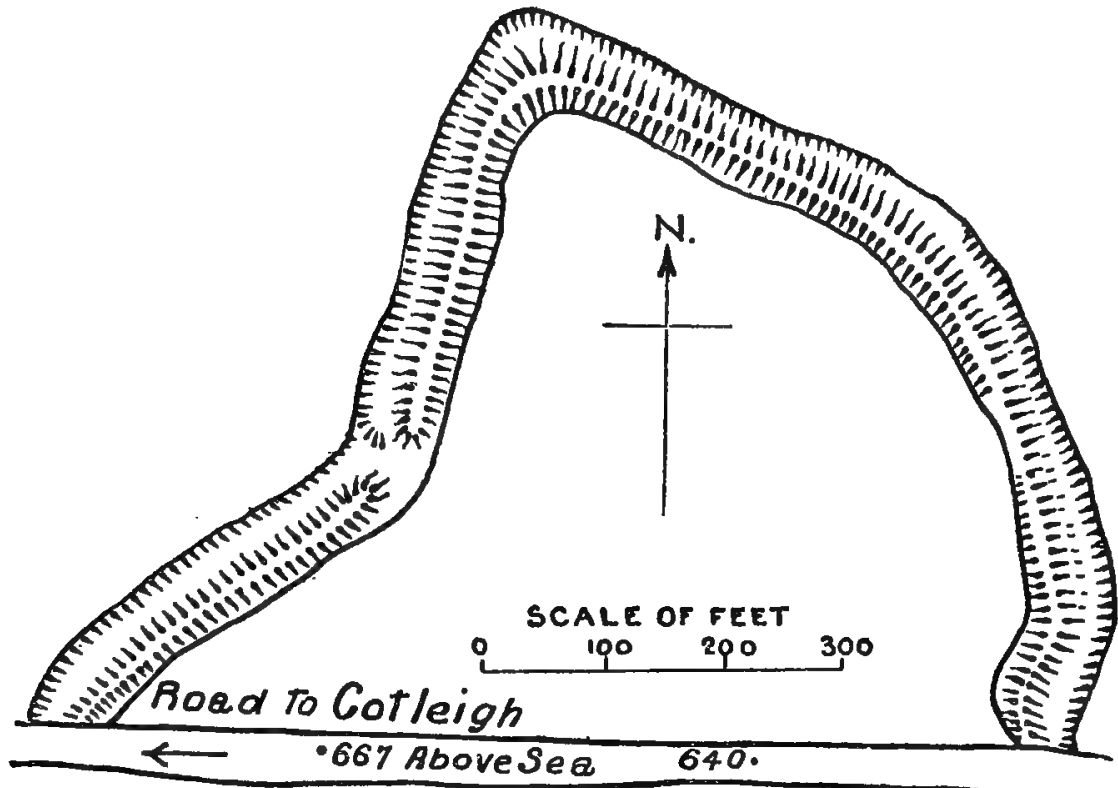

Stockland Great Castle. aggers, while the outer one falls off on a natural escarpment.

Almost in the middle of each of the long sides is a small semi-circular platform projecting from the outer vallum; that on the south-west was evidently constructed to defend a path which enters the entrenchments at this point; probably the platform on the north-east was for the same purpose, but the path is not apparent. Another path at the east is modern, but evidently on an ancient track.

The entrance is at the narrow north-west point, where a sunk road 200 feet long passes between an extension of the two ramparts, on a steep decline; at the outer extremity a triangular area formed by the northern agger creates an opportunity for a cross-fire against an invading force ; at the same time the defenders would be supported from the terminals of the outer aggers.

The interior area is larger than that of any other camp in this neighbourhood; it measures $\mathrm{I}, 300 \mathrm{ft}$. long, exclusive of the sunk road, and $400 \mathrm{ft}$. at its greatest width.

In the southern corner the higher ground of the hill-top has caused the formation of another fosse over 600 feet in length. Within the ramparts are two ponds or water-basins, one at each side, fed by perennial springs.

A field at the eastern base of the hill on which this camp stands contains signs of entrenching.

Near the eastern footpath are the remains of a cairn of flints, to which a tradition is attached that some great treasure is there buried, and it is locally known as the 'Treasure Heap' and the 'Money Heap.'

STOCKLAND (O.S. lix, Io and I 4).- -Stockland Great Castle is situated on the height ofStockland Hill, $667 \mathrm{ft}$.
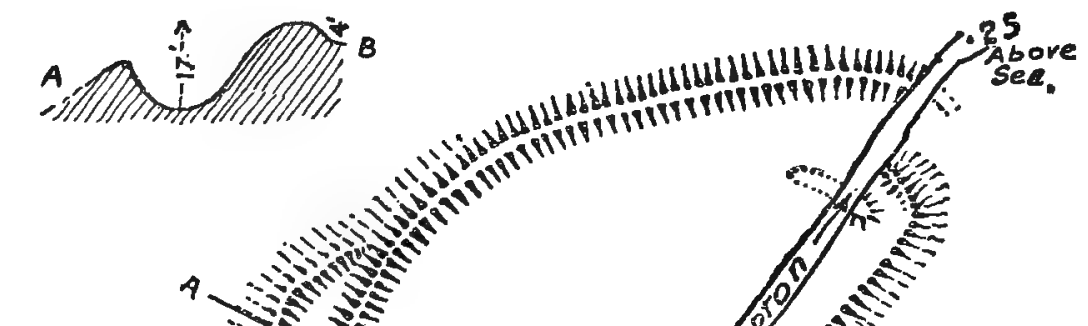
above the sea, to the north of Widworthy. The high road to Cotleigh, at this spot running east and west, has cut this camp in halves. The diameter at this destructive axis is $810 \mathrm{ft}$., and the longer axis from the 


\section{ANCIENT EARTHWORKS}

north to the south was originally $895 \mathrm{ft}$. ; from the northernmost point to the road it measures $5 \mathrm{r} 3 \mathrm{ft}$; the road is $42 \mathrm{ft}$. wide, and the southern half $340 \mathrm{ft}$. That part of the camp south of the road is entirely destroyed; but its site may be traced by the modern hedges.

The remaining northern half is of irregular form, defended by a single vallum and fosse, with an interior area of 12 acres. It is of great strength; the vallum, despite the levelling process of tillage, is still over $40 \mathrm{ft}$. high in certain places. One entrance is on the west side of this portion of the camp; but whether another one of greater importance ever existed it is now impossible to ascertain.

Many years ago large quantities of charcoal were found on the site, also a thumb scraper of flint and a great number of sling stones.

An outpost to this 'castle,' a circular camp, lies over a quarter of a mile to the north, and is known as 'Stockland Little Castle.'

Stokenham (O.S. cxxxiii, I4).-Slapton Castle, a little more than a mile inland from the famous Slapton Sands, is an irregular elliptical camp, enclosing about six acres, adjusting itself to the formation of the ground on the side of a hill, facing Start Bay in an eastern direction.

This camp has been of great strength; but the plough has obliterated all save the faintest traces of the south-eastern entrenchments. Apparently there was no attempt at ditching on this side,

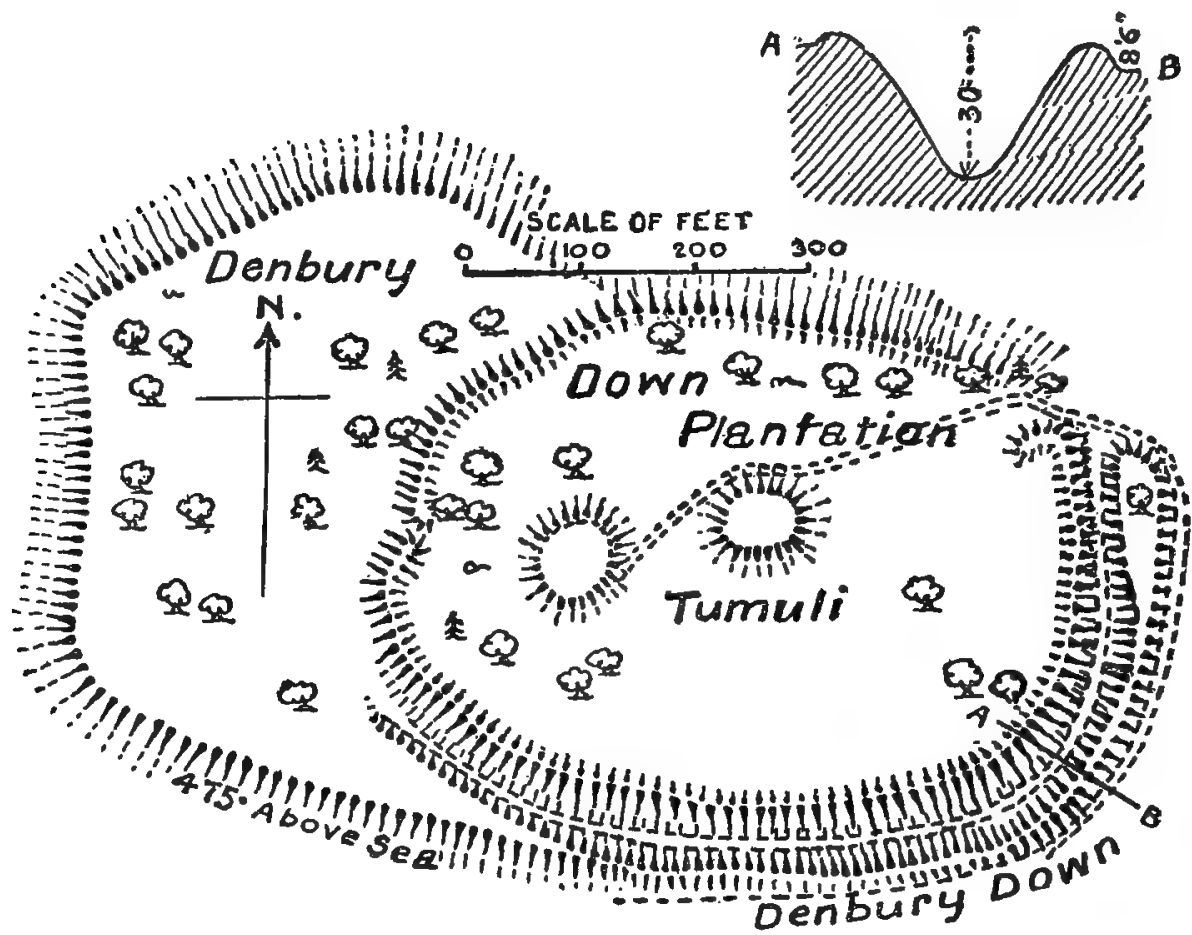

Denbury Camp, Torbryan.

and there remains but the border of an artificial tableland on the edge of the shoulder of the hill, which descends to a small stream. The northern part is defended by a vallum nearly $18 \mathrm{ft}$. high at parts and $48 \mathrm{ft}$. wide at the base; as the ground rises towards the west a fosse commences about the middle of this side, and gradually assumes vast strength on the due west, where the ditch, cut on the top of a natural ridge, is over eleven feet deep and $30 \mathrm{ft}$. wide, while the vallum rises over seventeen feet from the depth of the fosse.

The road down Darnacombe Hill cuts right through the camp, and at the north-eastern point it has greatly mutilated the original entrance. Here access was gained between the overlapping ends of the rampart, and the entry defended by an agger on the interior, of which a small portion only remains on the east of the road.

Numerous sling-stones have been found within the area.

Torbryan (O.S. cxv, 2).-Denbury Camp is $\frac{1}{2}$ a mile south-west of Denbury village, which lies 4 miles from Ashburton and 3 from Newton Abbot.

The entrenchments encircling the crown of this lofty igneous rock enclose 9 acres I rood and I perch, and consist of an elliptical camp with an outer court on the west side.

On the north a steep natural escarpment dispenses with the need for artificial works, and a low rampart only is found. On the south and east, however, the defences are very strong; the 


\section{A HISTORY OF DEVONSHIRE}

south, the most easy for assault, is provided with a fosse nearly $45 \mathrm{ft}$. on the slope of the escarpment, and $30 \mathrm{ft}$. perpendicular measurement, which is gradually reduced in depth as it approaches the courtyard. The entrance at the north-east corner is on the verge of the steep decline, and the southern side of it alone demanded the skill of the engineers. Here the outer vallum widens into a platform I6o ft. in length and $14 \mathrm{ft}$. above the ground level, while the inner vallum returns at a right angle into the camp. Between the two ramparts the fosse forms a sunken road through which the defenders could pour their reserves from the court on to the enemy at the gate, or pass

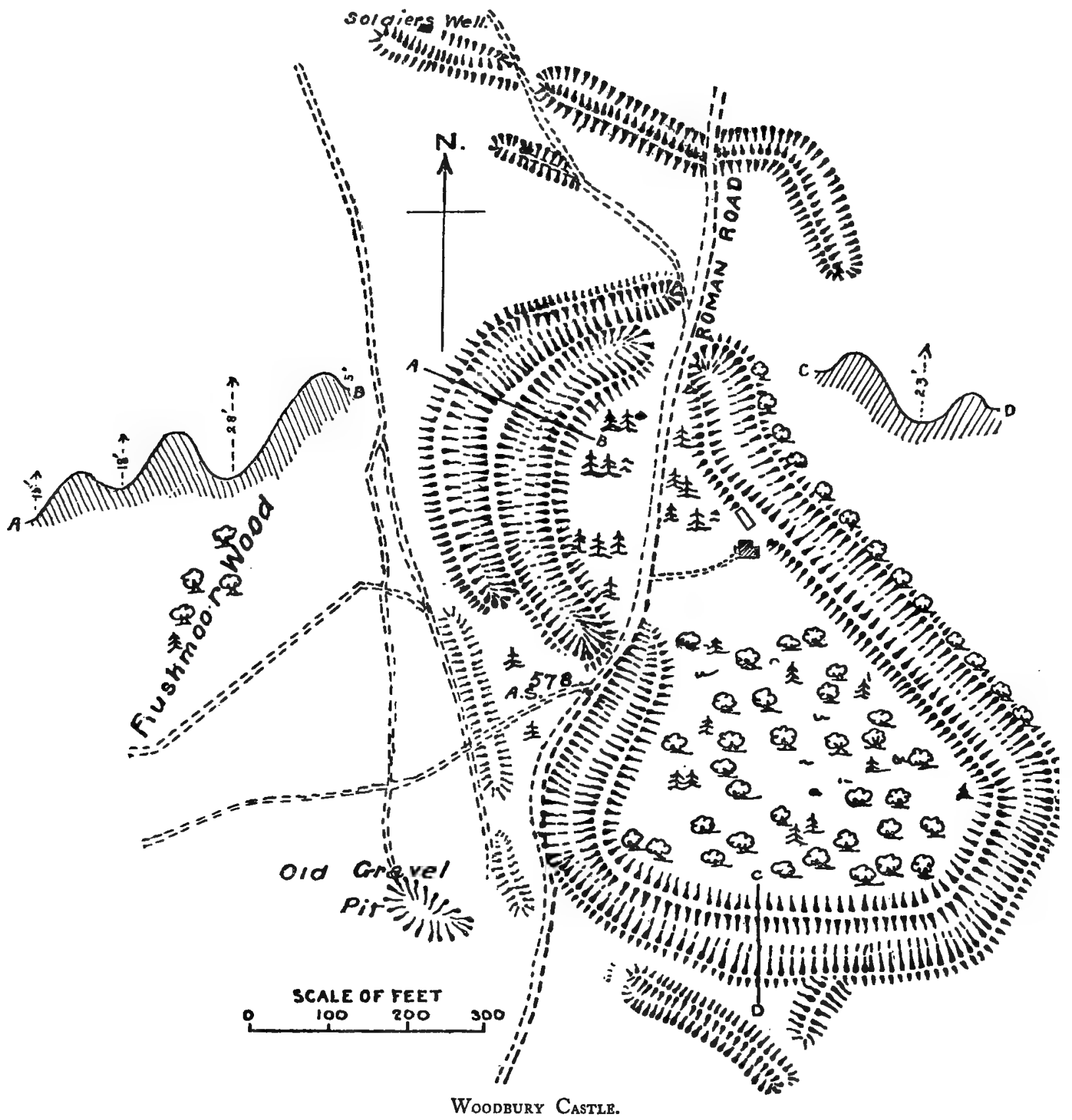

their cattle without taking them through the camp. At the middle of the west side is an entrance between the camp and the court where the agger on either side slightly incurves.

Within the central area are two large tumuli, or mounds.

Denbury was anciently known as Devenibyr, and Devenibyrie, names which gave rise to a questionable supposition that this camp was of Danish origin.

WoOdBury (O.S. xciii, 3)--Woodbury Castle has exceedingly strong entrenchments of eccentric form, being adapted to the formation of the ground. Situated on the north of Woodbury

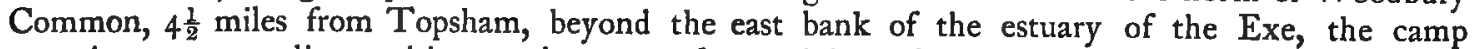
occupies a commanding position on the verge of a precipitous descent towards the river. 
A gradually sloping ancient road enters the trenches on the western side between defences which might turn a charge into a disaster; it passes through one side of the camp and emerges on the north. On the east of the road the interior area is almost surrounded by a double vallum and fosse, the latter $23 \mathrm{ft}$. deep perpendicular measurement, and on the west of the road two bold aggers are curved to the brow of the hill; the inner one, $45 \mathrm{ft}$. on the slope of the escarpment, descends into a fosse $16 \mathrm{ft}$. deep. These are succeeded by a vallum $18 \mathrm{ft}$. high, a fosse $7 \mathrm{ft}$. deep, and an outer vallum $15 \mathrm{ft}$. perpendicular measurement.

At the south-west entrance the crescentshaped agger meets the curve of the southern rampart at right-angles, while two aggers, I $20 \mathrm{ft}$. and $250 \mathrm{ft}$. respectively, flank the left side of the road, stretching in a line before the gateway. The north entrance has the end of the eastern vallum and broad fosse on its right, while on the left are the ends of the two crescent aggers commanding the gateway from independent points. Across this entrance, $140 \mathrm{ft}$. distant, a line of entrenchments is drawn, and other minor works are so arranged as to protect the path which leads to a spring of pure water.

Lysons' description of Woodbury as a

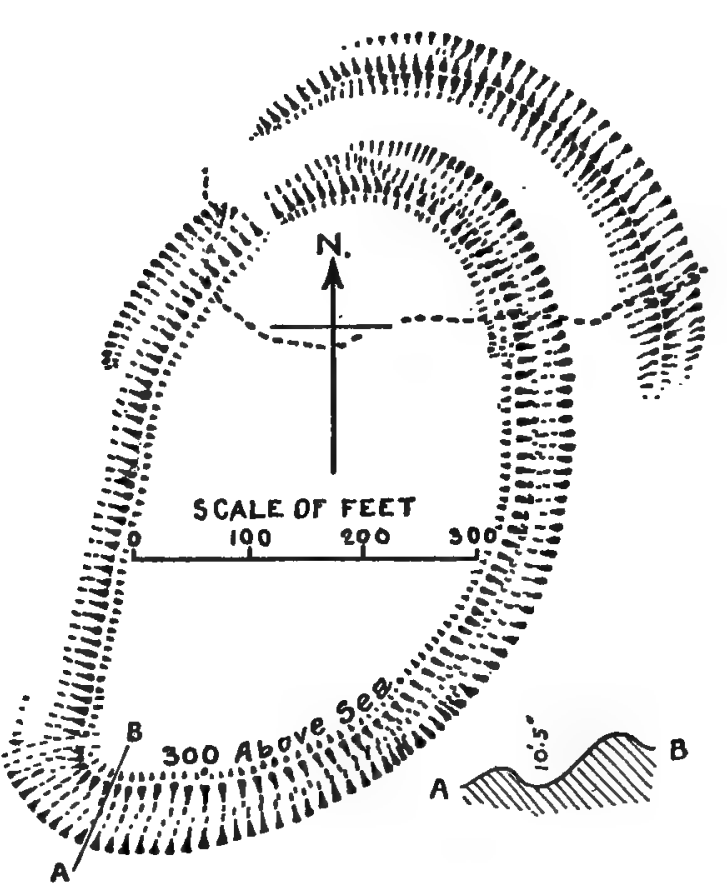

Greenway Camp, Brixham. small oval camp is misleading; but Polwhele, who gives the interior area as about five acres, tells us that the circumference round the outer edge of the ditch is $2,400 \mathrm{ft}$., and round the top of the rampart 2,100 ft.

Woodbury was occupied as a camp between 1798 and 1803 .

\section{[Class B (ii)]}

BRIXHAM (O.S. cxxviii, 5, 9).-Three miles south-west of the town of Brixham, on a steep slope descending to a tributary of the Dart, emptying itself on the east side of the estuary, is an

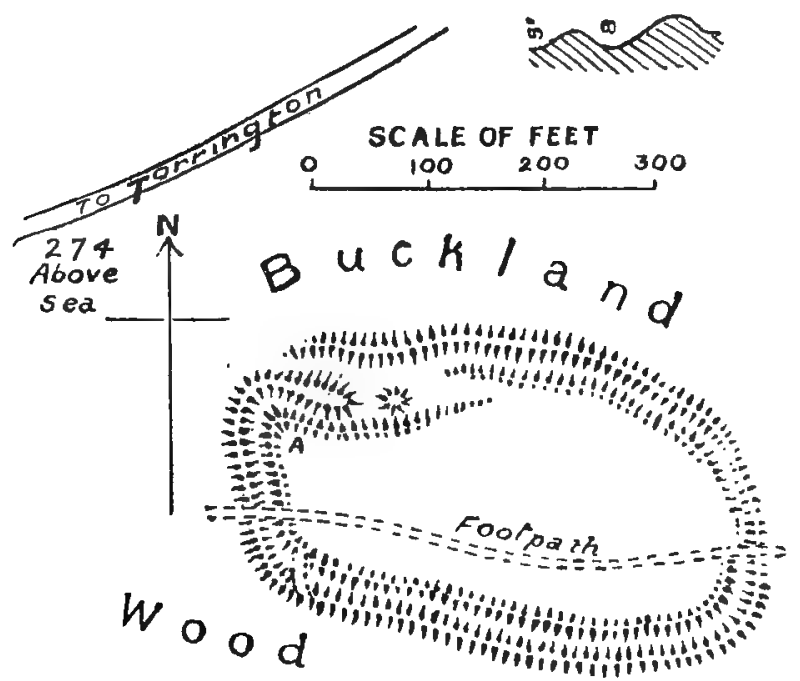

Camp at Buckiand Brewer. irregular oval camp.

The interior area of about three acres slopes towards the south, and is surrounded by a low vallum and broad fosse; the escarpment, assisted by the hill-side, is $10 \mathrm{ft}$. on the slope, but is lost on the western side. The main entrance is at the north-west, and from this point starts an outer vallum and fosse, which continue around the north to the east side, giving additional security towards the higher ground. A postern at the south-west opens on a path to the river.

This is supposed to be the work called 'Greenway Camp' in the Woolcombe manuscript. ${ }^{1}$

Flint flakes have been found here.

Buckland Brewer (O.S. xxix, 5). -One mile south-east of the village of that name and $3 \frac{1}{2}$ miles west of Great Torrington station, an irregular elliptical camp is situated in Buckland Wood on the steep western bank of the River Duntz, and between two small tributaries which flow on the north and south. The camp is protected by a double

' J. Davidson, Notes on the Antiquities of Devon before the Conquest. 


\section{A HISTORY OF DEVONSHIRE}

vallum and intermediate fosse. The inner vallum rises nearly four feet from the interior, and descends $8 \mathrm{ft}$. into the fosse, which has a counterscarp $5 \mathrm{ft}$. in height. At the east end, where the works are pierced by a modern path, both the ramparts and ditch are very shallow.

The entrance at the north-west corner is curiously defended. The outer agger incurves at right angles for a length of $100 \mathrm{ft}$., widening as it enters the camp, while the inner rampart taking the same direction rises to $12 \mathrm{ft}$. at the point marked $\mathrm{A}$ and extends more than twice that distance into the interior. The inner ramparts overlap the first for about eighty feet, and in the interval between the first-mentioned agger and the commencement of the inner northern rampart is a hollowed mound. An intruding enemy would therefore receive a double cross-fire for nearly $200 \mathrm{ft}$. before he could arrive within the camp.

A narrow path is cut obliquely through the entrenchments, by which water was apparently brought from the river.

Some distance to the west of the camp a tunnel $130 \mathrm{ft}$. in length running east and west, parallel with the river on the south of the camp, was discovered recently by the soil falling into it, leaving some twenty feet with the roof intact. This subterranean passage, $5 \mathrm{ft}$. wide and

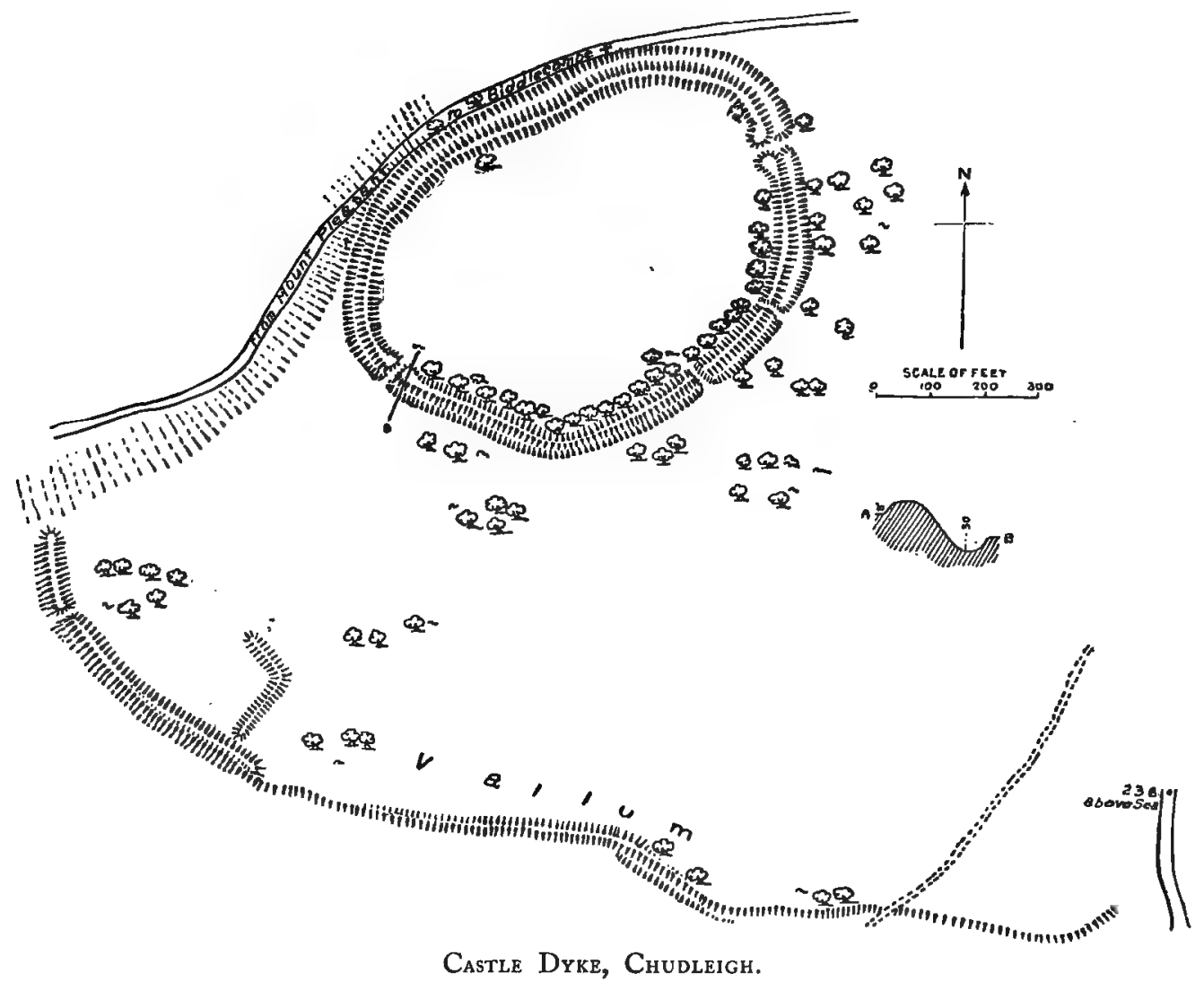

$3 \mathrm{ft}$. high, was about five feet beneath the surface; from it two branches turned to the south at the end nearest the camp, piercing the precipitous bank of the river, with the remains of a chamber between them. Evidently for the storage of grain, this chamber and passage may possibly have also served as a place of refuge.

One of a trio of earthworks, this camp was within 2 miles of Hembury Castle on the south, and the camp of Frithelstock on the other side of the River Duntz.

Chudleigh (O.S. ci, I2).-Castle Dyke, or Ugbrooke Park Camp, lies within Ugbrooke Park, $\frac{1}{2}$ a mile south-east from Chudleigh, and to the west of Smoothway Wood, on the east side of Katebrook, a tributary of the River Teign.

An elliptical camp of great strength, covering an area of 6 acres 2 roods II perches, being $780 \mathrm{ft}$ long by $580 \mathrm{ft}$. broad, occupies the crown of a hill, and is called by the natives the 'Round Field.' It is encompassed by a bold single vallum and fosse, the escarpment varying from forty-five to fifty feet on the slope, and the average perpendicular height being $30 \mathrm{ft}$.

Two entrances, one at each extremity of the longer axis-south-west to north-east-are both defended by the widening and incurving of the rampart, thereby creating a small platform at either side of the gateway. Polwhele may be right in assigning an opening through the middle of the southern rampart to another entrance, but there are two such posterns, the antiquity of which is doubtful. 


\section{ANCIENT EARTHWORKS}

The most easy approach is on the south, and on that side, about $900 \mathrm{ft}$. in advance of the camp, a long curved rampart and fosse runs from one steep natural escarpment on the south-east to another on the north-west. In front of the southern entrance it is a bold strong outwork, also pierced by a gateway; this extends for about $600 \mathrm{ft}$., and within this point is a right-angular defence. As it proceeds towards the east the declivity lessens and the ditch is lost.

Clovelly (O.S. xvii, I6).-Clovelly Dykes, Clovelly Dickens, or Dickenhills, was pronounced by Camden to be the largest Roman camp in the county. Whether it originated with that people or not, it certainly is of great size, embracing over 20 acres, although the area of the camp on Milber Down surpasses it.

This immense series of entrenchments is situated on high ground 2 miles south of Clovelly at the junction of the road from the fishing village with that between Bideford and Hartland.

The innermost entrenchment is an irregular oblong, tapering towards the south, with its axis $360 \mathrm{ft}$. and $300 \mathrm{ft}$. respectively; surrounded by a vallum $6 \mathrm{ft}$. in height from the interior, but $8 \mathrm{ft}$. on the exterior, except at the north-west corner $\mathrm{O}$ where it is $\mathrm{I} I \mathrm{ft}$. high. Around it is a flat court averaging I IO $\mathrm{ft}$. in width. The second entrenchment-losing its rectangular form-consists generally of a vallum and fosse, the former varying in height from fifteen to twenty-three feet, perpendicular measurement, from the base of the latter. At the north-west corner this vallum widens into a broad platform; but the fosse, very wide at this point, with a bank on the edge of the counterscarp, is gradually lost, and ceases altogether around the southern course. The area outside this second vallum is entered by a sunk road at the north-west and by a narrow neck on the east; but at the south-west it widens into a well-protected court, and in the extreme angle $\mathbf{N}$ is a recess in the outermost agger which rises $\mathrm{Io} \mathrm{ft}$. from the interior.

The third or middle rampart on the west side $(\mathrm{G}-\mathrm{H})$ has a dry wall of stone on its outer face, with a depth of $8 \mathrm{ft}$. into another court of a lower level ; this, however, extends only along the west side, and at the northern termination it has the protection of a ditch. The fourth line of defence is a rampart $4 \mathrm{ft}$. high from the interior, falling $7 \mathrm{ft}$. on the exterior; this has extended round the north and east, but a portion of the north side has largely perished, while the road to Clovelly has severed the eastern portion from the body of

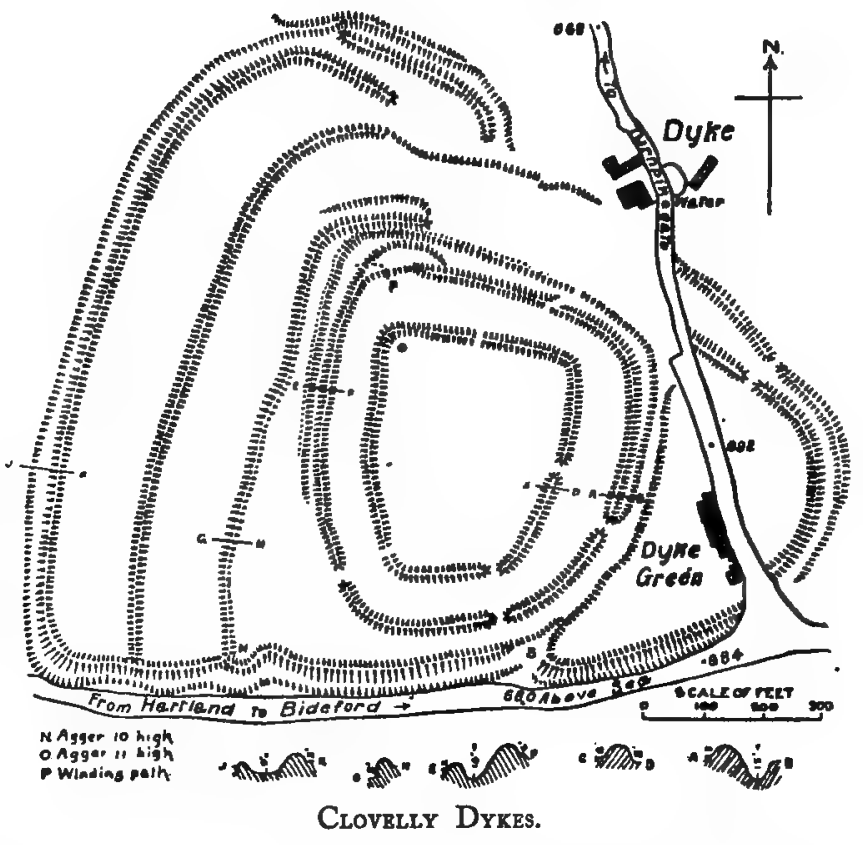
the fort. The fifth or outermost defence on the west consists of a vallum $9 \mathrm{ft}$. high, with an interior breastwork of $3 \mathrm{ft}$., and a wide fosse strengthened by a low bank on the height of its counterscarp; this continues round the south, and originally joined the severed portion on the east. On the north, where there was an entrance to the outer court, it is strengthened by two additional ramparts before merging in the single vallum of the fourth defence, an arrangement which transforms the interior oblong into an external round-angled triangle.

The extreme eastern defences of crescent form comprise a double vallum and fosse with the inner rampart from fifteen to twenty feet high and wide.

It will be seen that the north-west corners of the successive ramparts are of additional strength, and there lies the main entrance; another probable entrance is through the heavy agger on the south, at point $S$, where the works incurve. A study of the entries from one court to another is interesting and exhibits great strategical foresight. A comparison of the various levels also demonstrates the gradual rise to the central camp.

Various conjectures have assigned these works respectively to the Britons, Romans, and Saxons. Doubtless the expanse of the enclosures provided ample accommodation to those herds of cattle which both Caesar and Strabo tell us the Britons collected within their fortifications.

Coffinswell (O.S. cx, 13 and cxvi, I).--Milber Down Camp lies partly in the parish of Coffinswell and partly in Haccombe with Combe. This important camp is situated on the highest part of Milber Down, on the declivity of the hill sloping in a westerly direction, 2 miles south-east of Newton Abbot. 


\section{A HISTORY OF DEVONSHIRE}

It bears a strong resemblance to the plan of Clovelly Dykes, and has a triple vallum and fosse. The interior area is an irregular quadrangle $462 \mathrm{ft}$. long by $402 \mathrm{ft}$. wide, surrounded by a vallum $5 \mathrm{ft}$. above the interior, and a fosse varying from sixteen to twenty-three feet deep. The

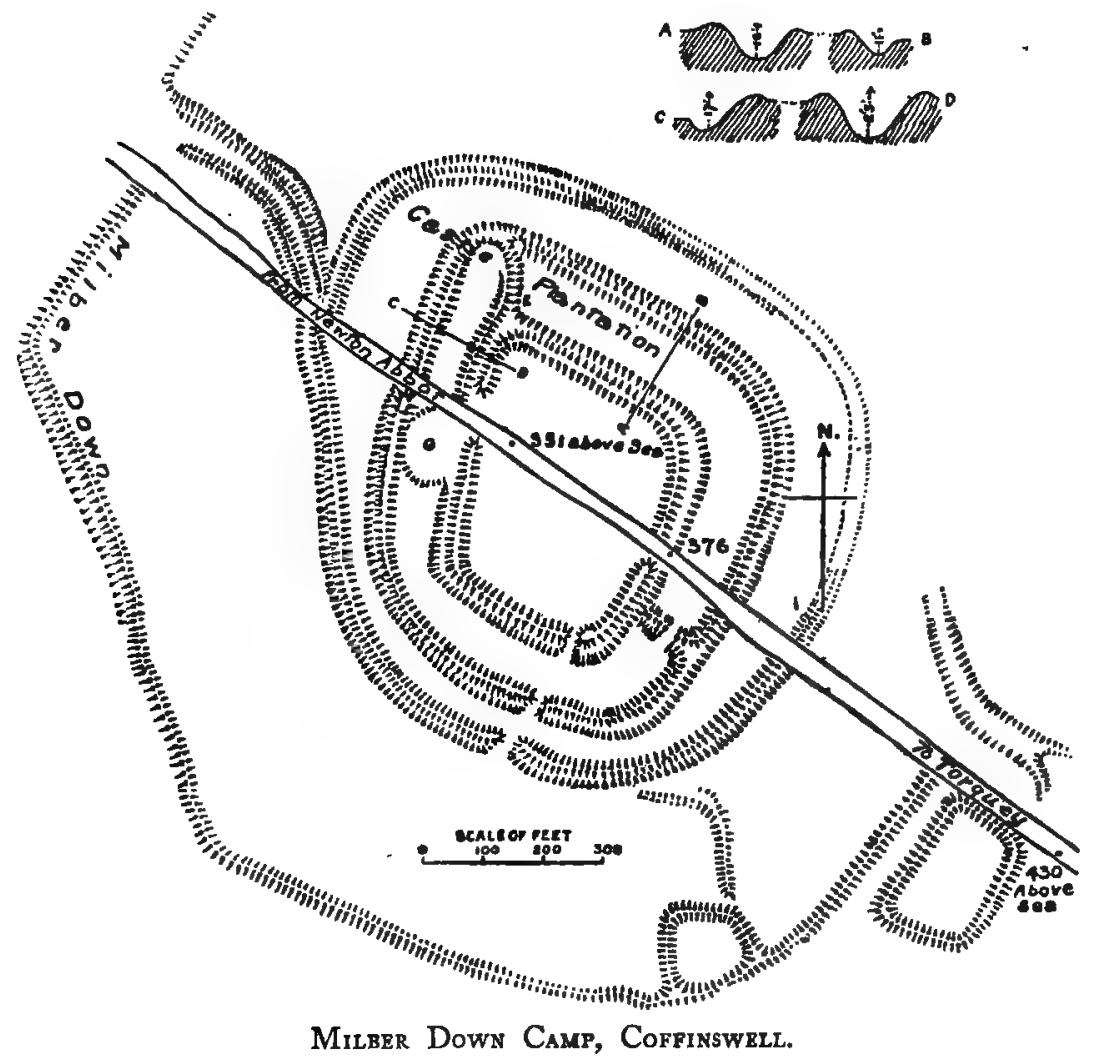

surrounding area, or courtyard, is from I $00 \mathrm{ft}$. to I $50 \mathrm{ft}$. wide. The second vallum, which departs from the square formation and has angles nearer a circular form, is surrounded by a fosse varying from eleven to seventeen feet deep; this is connected with the first fosse by a sunk road or ditch at $E$ in the north corner, and the remains of a transverse consisting of a ditch and double agger connect them at the south-east. On the western side of this intermediate area is a large dry pond, G. In the third vallum the square form is lost to a greater extent; this varies in distance from the second, the area being $50 \mathrm{ft}$. wide in some places and I $80 \mathrm{ft}$.

in others. The two entrances were probably on the north-west and south-east, where the road from Newton Abbot to Torquay cuts the camp in halves. That on the north-west was apparently through a sunk road, about five hundred feet in length, which curves on the north of the present road, and is flanked by the remains of two ditches. That on the south-east is not so easily traced, but it was evidently of the same nature though somewhat more involved, and on the south of it, outside the boundary wall, is an outpost in the form of a large flat mound.

The whole of this camp appears to have been surrounded by a vallum, or boundary wall, some four hundred and fifty feet beyond the entrenchments already described. On the west it is strengthened by $a$ fosse, and on the south by an almost square work and a curved transverse in addition to the outpost mentioned above. The north and west of this vallum has perished, but that portion at the north of the western entrance indicates the course it took. The entrances through the main entrenchments from the large court on the south to the interior of the camp take a slightly oblique direction.

The outermost vallum to the court is said to have been thrown up in 1688, when William III landed at Torbay and planted a park of artillery in this camp, but it appears to have been an integral part of the original scheme of defence.

About the year 1845 some coins, knives, and forks of the seventeenth century were found here, relics of William's occupation.
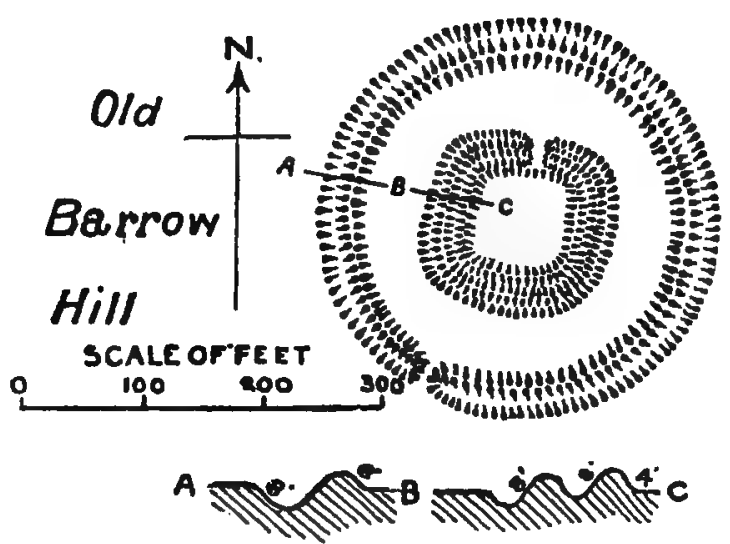

Old Barrow Camp, Countisbury.

Countisbury (O.S. iii, 12).-OOld Barrow

Camp, 4 miles east of Lynmouth, on Old Barrow Hill, is one of the most perfect camps in Devonshire.

Situated on the summit of a lofty hill rising out of the sea to a height of over $1,100 \mathrm{ft}$. though but little above the surrounding moors-the camp of Old Barrow is cinctured by a triple 


\section{ANCIENT EARTHWORKS}

vallum and fosse. The innermost vallum encloses an area, square with rounded angles, over $100 \mathrm{ft}$. in diameter; it varies from four to six feet in height, the highest part being towards the coast. This is surrounded by a fosse $8 \mathrm{ft}$. deep, and a second vallum $6 \mathrm{ft}$. high, with another fosse $8 \mathrm{ft}$. below. A surrounding area, about sixty feet wide, is defended by a circular vallum $9 \mathrm{ft}$. high on the south or land side, but only $6 \mathrm{ft}$. in height towards the sea, and a fosse $8 \mathrm{ft}$. deep. The entrance to the outer court is on the south-west, but that leading into the inner camp is at the north. Exactly in the centre of the camp is a small mound.

Drewsteignton (O.S. Ixxviii, 15).- Prestonbury Castle, three miles north-west of Moreton Hampstead, is one of a trio of camps above the River Teign. 'This 'castle,' high above the north bank of the river, on Prestonbury Common, has a triple vallum enclosing three separate areas.

The innermost camp, an irregular ellipse, measures $418 \mathrm{ft}$. on its long axis and $410 \mathrm{ft}$. on the short axis, and includes somewhat more ground than the apex of the hill. It is surrounded by a vallum $7 \mathrm{ft}$. $6 \mathrm{in}$. high, with two entrances at the ends of its long axis; at the eastern one the rampart widens on either side, but the other relies on the difficult nature of the ground to ensure its

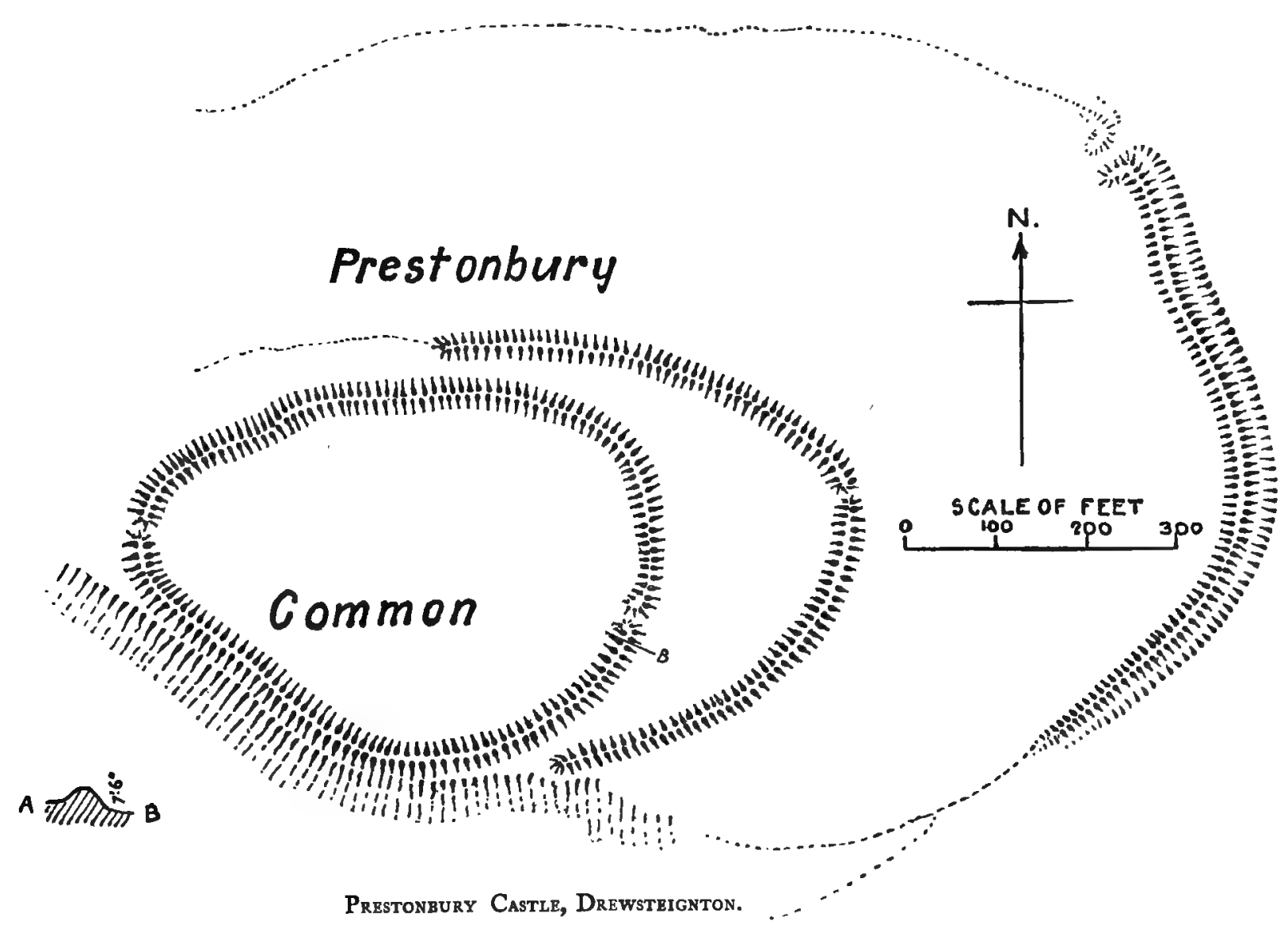

safety, while from that point along the southern side the vallum is on the verge of an almost perpendicular descent of $\mathrm{I}, 000 \mathrm{ft}$. to the river.

The second line of defence is also a vallum only, which forms a court on the east side, but closes in on the shorter axis of the ellipse to within fifty feet of the inner camp. A simple entrance is on the east, $267 \mathrm{ft}$. from the inner wall.

The third vallum, only partially extant, is placed $450 \mathrm{ft}$. from the second, giving ample accommodation to the cattle in time of need. The remaining portion of this rampart is very strong: it has a fosse $20 \mathrm{ft}$. broad cut into the solid rock, facing level ground, which makes this the most assailable side. At the entrance the agger returns inwards for the greater safety of the besieged; and it will be seen that the three successive entrances on this side are placed en échelon, or obliquely to each other, so that one direct charge could not carry them all by assault. This outer vallum divides into two lines on the southern slope as it approaches the precipitous part.

This camp is overlooked by Cranbury Castle on the opposite bank of the river.

High BRAY (O.S. x, 8).-Shoulsbury Castle, by Lysons called Shorsbury, is on the boundary of the parishes of High Bray and Challacombe, seven miles south of Lynton, on the border of Somersetshire. 


\section{A HISTORY OF DEVONSHIRE}

On high ground on Exmoor, with the treacherous bogs as nature's auxiliary, is a camp said by a late rector of the parish to be 'the only undoubted Roman camp in this neighbourhood,' but we see no sufficient evidence to attribute the work to the imperial rulers of Britain. Two swords,

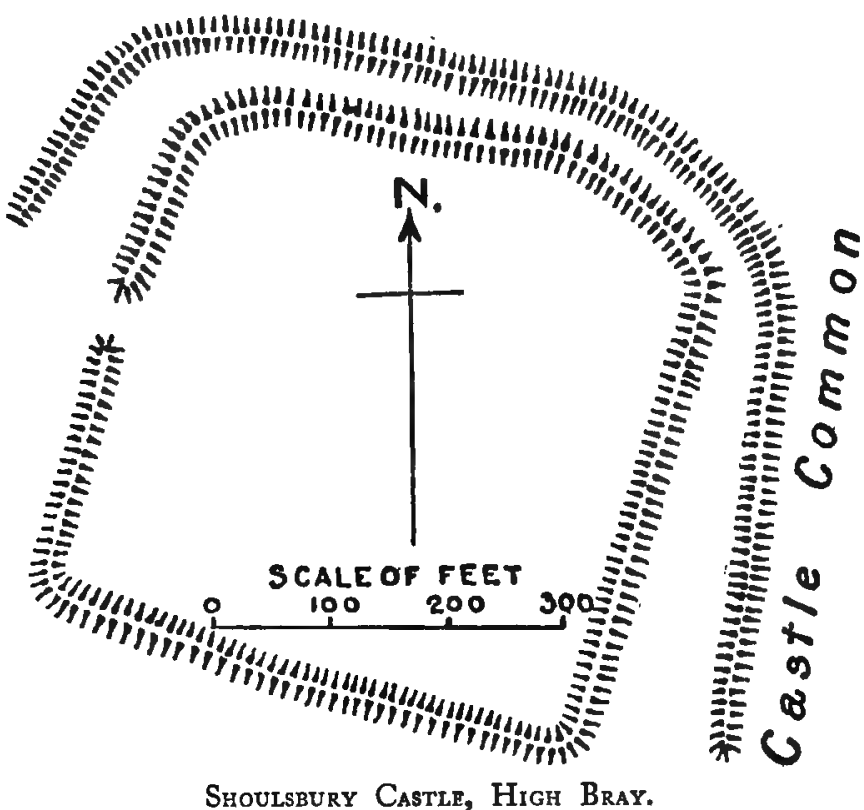

reported to be Roman, were found here, but they proved to be rapiers of the seventeenth century!

The camp is square-somewhat irregular on the north - with sides $480 \mathrm{ft}$. in length, and is surrounded by a vallum which varies from four to seven feet in height. From 50 to $\mathrm{I}$ oO ft. beyond, another vallum $3 \mathrm{ft}$. high protects the east and north sides, and half of the west, in the centre of which is the only entrance. The southern side is defended by a sudden fall in the ground.

In the north-east corner was a mound, about eighty feet in circumference, which was opened some years ago, but the results were barren.

The high position of this camp commands a view of the chain of forts from this point to Barnstaple and Braunton, and the group to the north, including Martinhoe.

A tradition is current that Alfred the Great held Shoulsbury Castle against the Danes.

Holne (O.S. cviii, 14).- Holne Chase Castle, $2 \frac{1}{2}$ miles north-west of Ashburton, is situated on ground $200 \mathrm{ft}$. above the River Dart, by which it is surrounded at some distance on the west, north, and east sides.

It is a very perfect irregular circular entrenchment of single vallum and fosse, enclosing an area of rather more than $2 \frac{3}{4}$ acres. The entrance at the south-west is defended on one side by the northern rampart curving eastwards and extending $60 \mathrm{ft}$. into the camp; while the end of the southern rampart, $12 \mathrm{ft}$. high at this point, is considerably wider, and within its inward curve nestles a circular excavation, $22 \mathrm{ft}$. in diameter, with an opening towards the entrance. This was originally lined with a dry wall which partially remains, and is said to be a guard-room or warder's hut, which savours too much of mediaevalism; it was probably the more primitive defence of a pit-fall, where an intruding enemy turning to the right-the only possible course considering the in-turned vallum on the left-would be at the mercy of the garrison upon the agger.

At the south-east is a minor entrance, a postern, protected by the broadened ends of the rampart.

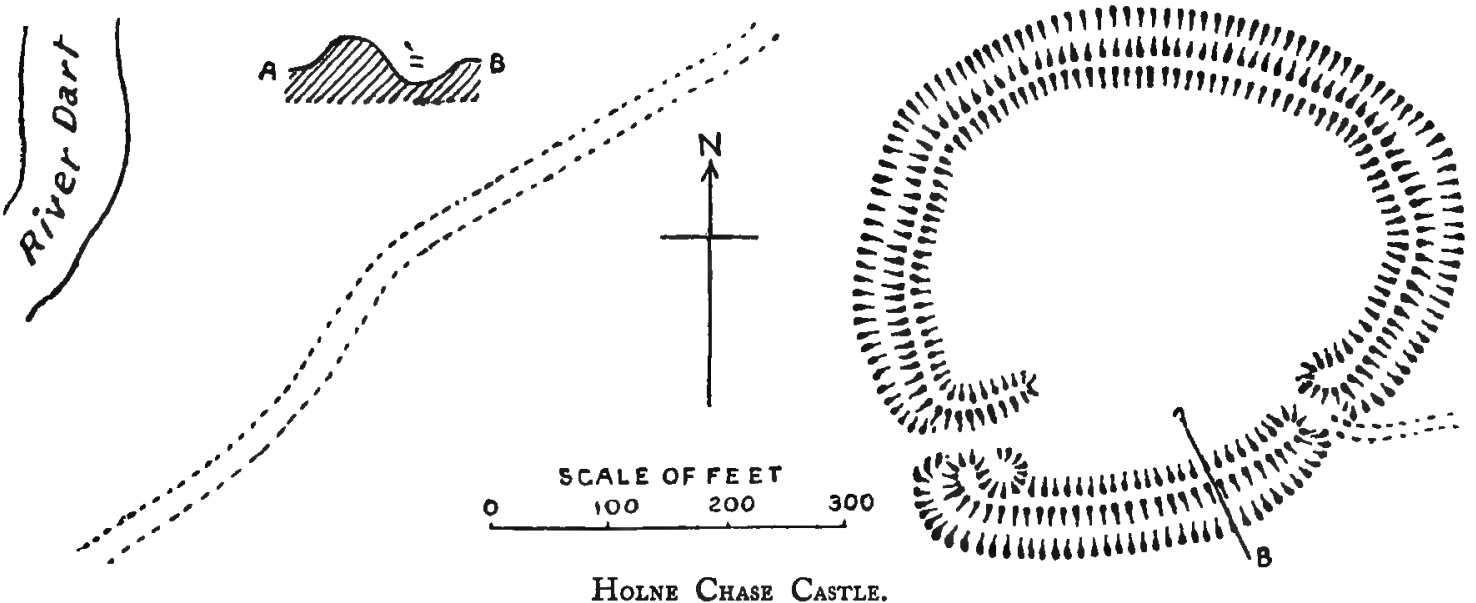

The easiest approach is on the south, and the defences towards that quarter are the strongest ; the agger rises eleven feet and over, from the bottom of the fosse, and the latter varies both in depth and width, from three to twelve feet deep and from thirty to forty feet wide. 


\section{ANCIENT EARTHWORKS}

Within the area large round pebbles have been found, some of them weighing from 7 to $12 \mathrm{lb}$., which were doubtless used as missiles, for, although too large for sling stones, they show no evidence of domestic use. In 1870 iron spear-heads were found between the camp and the river.

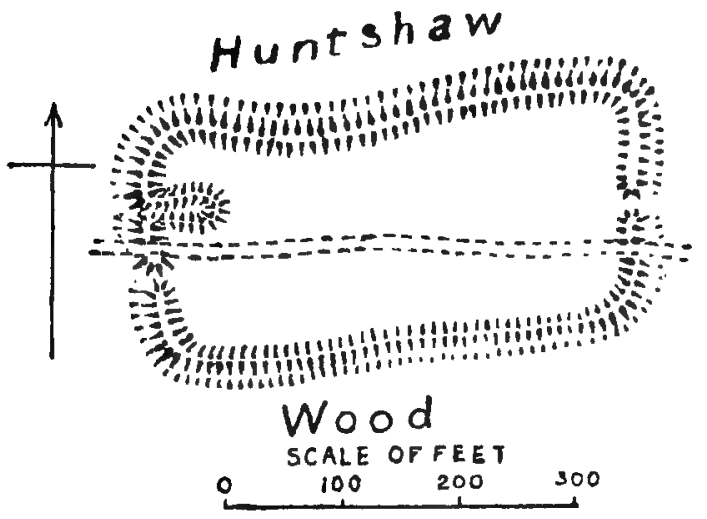

Berry Castle Camp, Huntshaw.

Slight indications of another small circular earthwork are visible considerably lower than the camp, on the edge of the river, nearer to Holne Bridge.

Within a short distance, on the southern spur of the same hill, are Hembury Castle and Gallantry Bower, but the ridge of the chase hides them from the view of Chase Castle.

Huntshaw (O.S. xix, I6).-Berry Castle Camp, one mile south-west of Huntshaw village, and $2 \frac{1}{2}$ miles north of Torrington, is situated on a promontory formed by a tributary of the Torridge and the Huntshaw Mill Lake.

It is an irregular quadrangle defended by a single vallum and fosse. The vallum averages six feet in height; but at the north, where the approach is unprotected by the rivers, it becomes very strong; while the fosse, $3 \mathrm{ft}$. deep, is very shallow on the south, or best naturally protected side. There are two entrances, on the east and west respectively; the eastern one is a simple opening, but at the northern side of the entrance on the west the agger rises to over eight feet high, which served not only to defend the entrance, but for another purpose which will shortly be apparent.

North of the western entrance is a curious loop of the rampart penetrating the camp a considerable distance. The incurve of the rampart as seen from the outside is a more conspicuous feature than the actual entrance; this would lead an enemy to conclude it was the entrance, especially as the rampart at each side was of greater height than that on the south of the real entrance; they would therefore concentrate their energies upon it, and if forcibly entered they would find themselves in a cul-de-sac surrounded by the defenders. It will thus be seen that this uncommon feature was constructed as a decoy to the invader.

Due south of Berry Castle, and in easy view of it, on the other side of the river, there was formerly another camp called 'Gaze Castle' which has been destroyed by the plough.

LUSTLEIGH (O.S. c, 3).-Situated on the wild rocky eminence called Lustleigh Cleave, 3 miles south of Moreton Hampstead, the remains of a camp are difficult to find amid the scattered boulders of granite; but within $100 \mathrm{ft}$. of the bold pile known as Hunter's Tor one of a very interesting nature may be located.

Three parts of an elliptical camp are extant, which apparently consisted of a broad rampart, and a ditch $6 \mathrm{ft}$. in depth; beyond this on the south-east is a second rampart which widens into a broad shallow platform, defended by a ditch now almost obliterated. At the end of this, on the east, is the entrance, which has on its northern side an enfeebled defence now consisting of little more than the indications of a rampart and ditch. The defences die out as they approach the north-west side, which is rendered almost impregnable by the abrupt declivity of a hill overlooking the River Bovey.

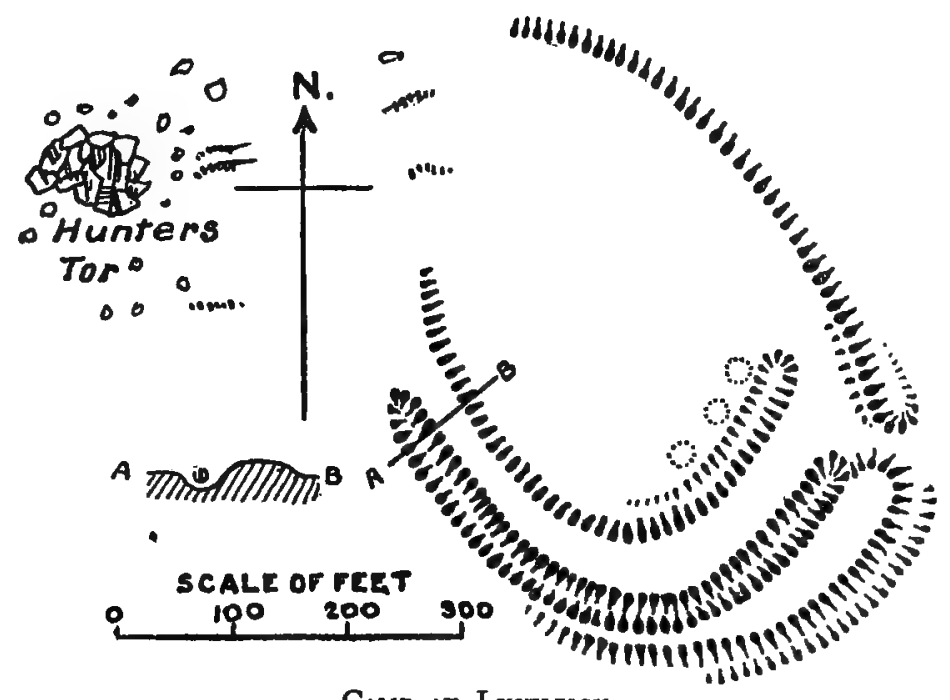

Camp at Lustueigh. Within the central area, at the south-east are the foundations of three hut circles, with a ditch at one side to drain them.

The clump of huge granite boulders known as Hunter's Tor, overlooking the ruins, provided an unequalled outlook, and also a vantage ground to destroy any raiders climbing up from the river. On the inner side, where the defences are strongest, the land gradually slopes. 


\section{A HISTORY OF DEVONSHIRE}

Moreton Hampstead (O.S. xc, 2).-Cranbrook Castle is in one of three great camps which dominate the River Teign by Fingle Bridge, being $1 \frac{2}{3}$ miles distant from Wooston and $\frac{3}{4}$ mile from Prestonbury, and about three miles north-west of Moreton Hampstead town. It is-broadly

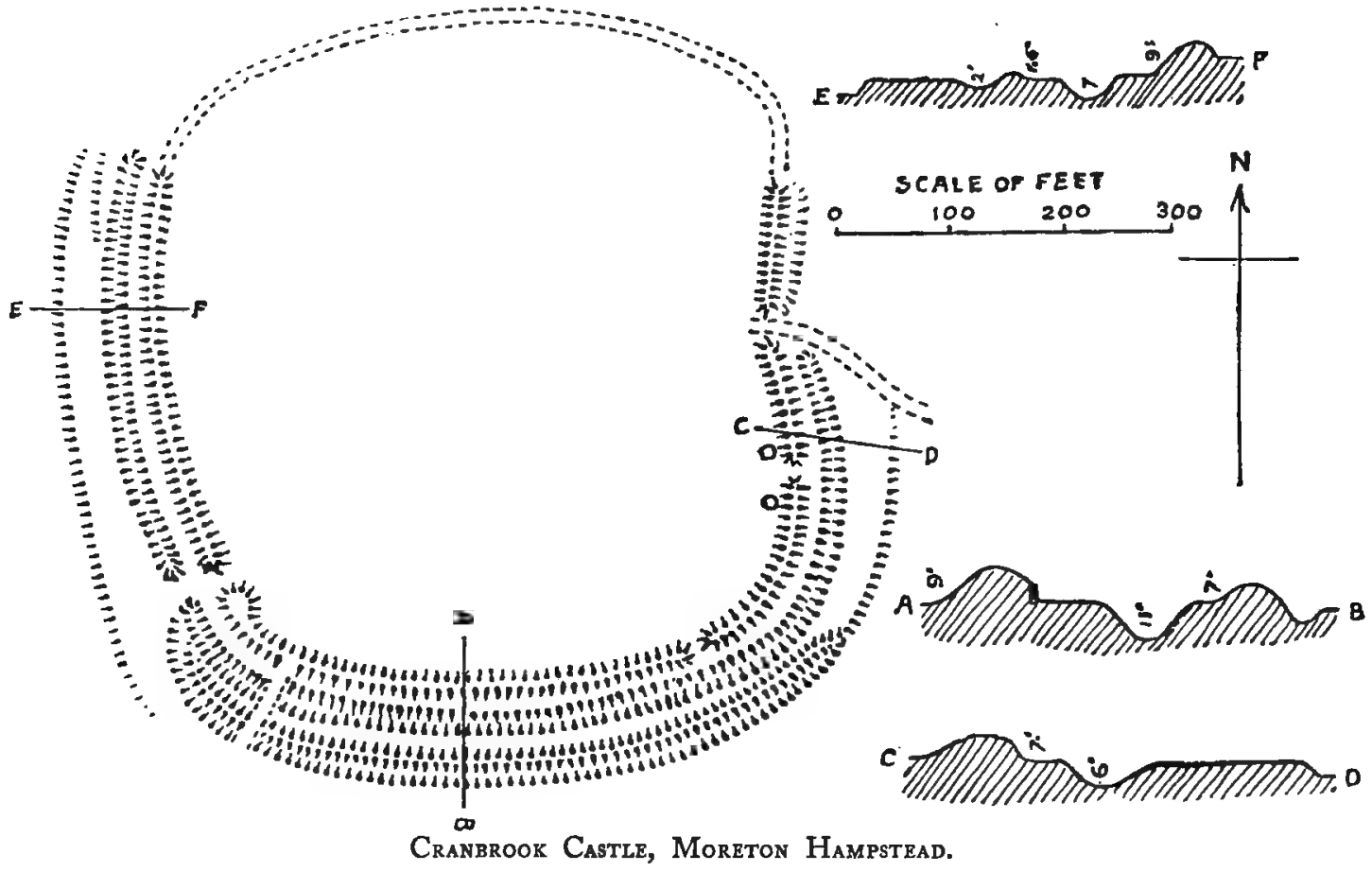

speaking-square in plan with rounded angles, $600 \mathrm{ft}$. by $550 \mathrm{ft}$., crowning an eminence $1,100 \mathrm{ft}$. above the level of the sea.

The approach from the south is not difficult, the eastern and western ascent is steeper, but the
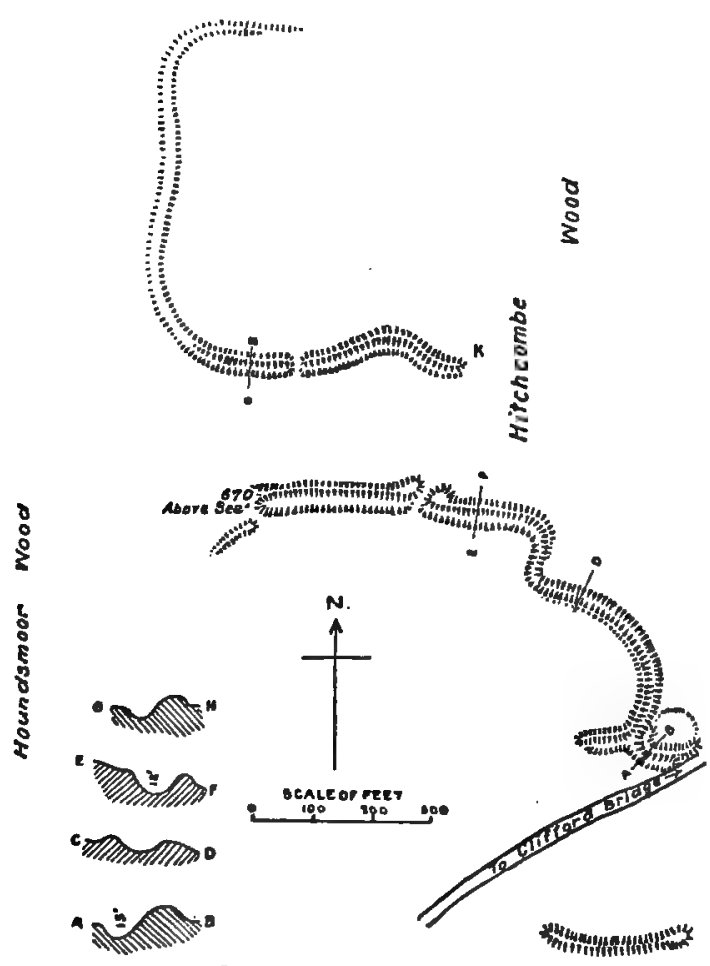

Wooston Castle, Moreton Hampstrad. northern side is above a precipitous descent to Fingle Bridge.

Easy of assault on the south, that side is more strongly defined than the others; here we find a double vallum and a double fosse with an inner and outer berm, or platform, one on either side of the fosse. The inner vallum, $9 \mathrm{ft}$. high and $2 \mathrm{I} \mathrm{ft}$. thick, was originally built with stone on the exterior to above half its height; outside this is a platform, then a fosse I I $\mathrm{ft}$. deep, an outer platform varying from six to sixteen feet wide, another vallum, and lastly a shallow fosse.

The main entrance is at the south-west, where the inner rampart slightly incurves and broadens. To the south of it a causeway across the entrenchments provides a narrow exit, and to the north of the entrance the outer fosse and vallum become shallow and almost give place to a broad platform of no great height. On the east side the same thing occurs; the outer vallum and fosse terminate at the bend and are replaced by a wide platform.

At either side of the south-east curve the inner vallum is pierced by a path giving access to the first platform.

In the middle of the east side is another entrance, at each side of which the entrench.

ments bend inwards. On the north of this gateway the agger and fosse only continue $120 \mathrm{ft}$.

The north side, we have seen, is the most difficult of access, and here there are no entrenchments, only a line of rubble, collected probably some $\mathrm{r}, 800$ years since, to form the core of a 


\section{ANCIENT EARTHWORKS}

projected vallum, but the earth which would have been thrown over the stones in the making of the fosse was never worked, and it has remained unfinished to the present day.

The contour of the ground of the interior area is so pronounced that the northern boundary cannot be seen from the southern ramparts.

No water is within the camp, the nearest spring being about $2,000 \mathrm{ft}$. distant.

Cranbrook Castle was explored in 1900 , when the fosse was found to be encumbered with about three feet of debris. Two hut-circles are within the east rampart with the remains of fireholes. Charcoal, fragments of pottery, the base of a quern, and sling stones were found.

This stronghold above the right bank of the Teign overlooks Prestonbury Castle, which is almost opposite on the left bank.

Moreton Hampstead (O.S. lxxviii, 15).-Wooston Castle: The castles of Wooston, Cranbrook, and Prestonbury together form a system of defence seldom equalled. The first two on

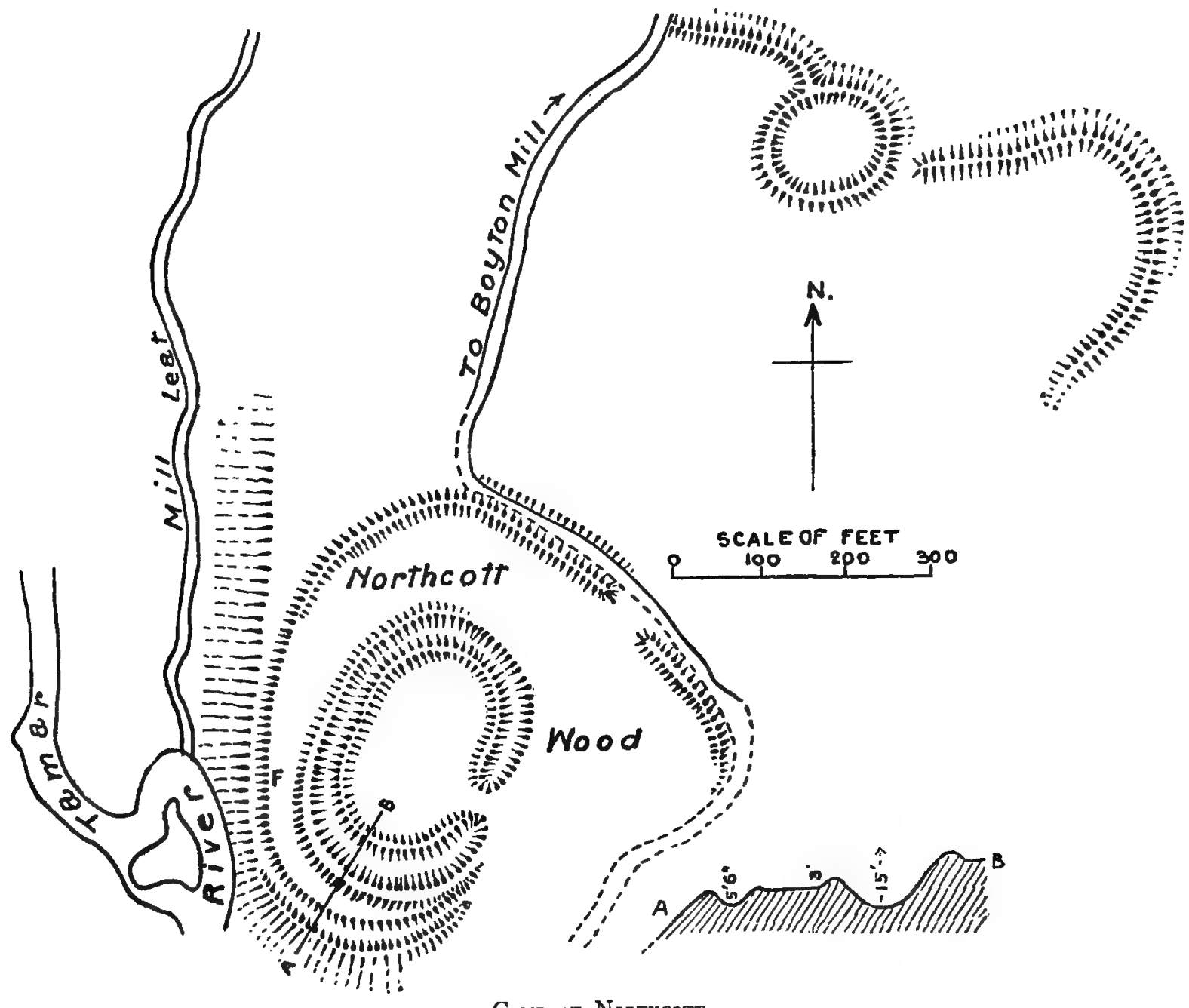

Camp at Northcott.

the south side of the Teign and the last on the north side form a triangle, and while the latter two are at the sides, Wooston is situated on a protecting curve commanding a view up the river.

Wooston Castle is an extensive camp on the slope of a hill descending to the brink of the deep river bank. The lower part, to the north, appears to have been a square area, but the outlines of the east side are lost in Hitchcombe Wood. The south side is an agger and fosse extending from the original entrance at the south-east to the south-west angle, where the fosse rises into a platform along the edge of the bank until it is lost on the north.

About $200 \mathrm{ft}$. to the south are another agger and fosse, the former $\mathrm{r} 2 \mathrm{ft}$. high, through which is a gateway with broadened ramparts, set obliquely with that which has been the entrance $K$ in the camp. In both of these defences the fosse is to the south of the agger, confronting an invader from the higher ground. The fosse now becomes a covertway partly lined with masonry as it curves serpent-like up the hill to a strong agger $15 \mathrm{ft}$. in height by the side of the present road. At the 


\section{A HISTORY OF DEVONSHIRE}

west side of the last curve a rampart and ditch extend $86 \mathrm{ft}$. to the left. Three hundred feet in advance an agger $240 \mathrm{ft}$. long provides an outwork on the top of the hill.

Wooston Castle is differently arranged from any other camp in Devonshire, and its features

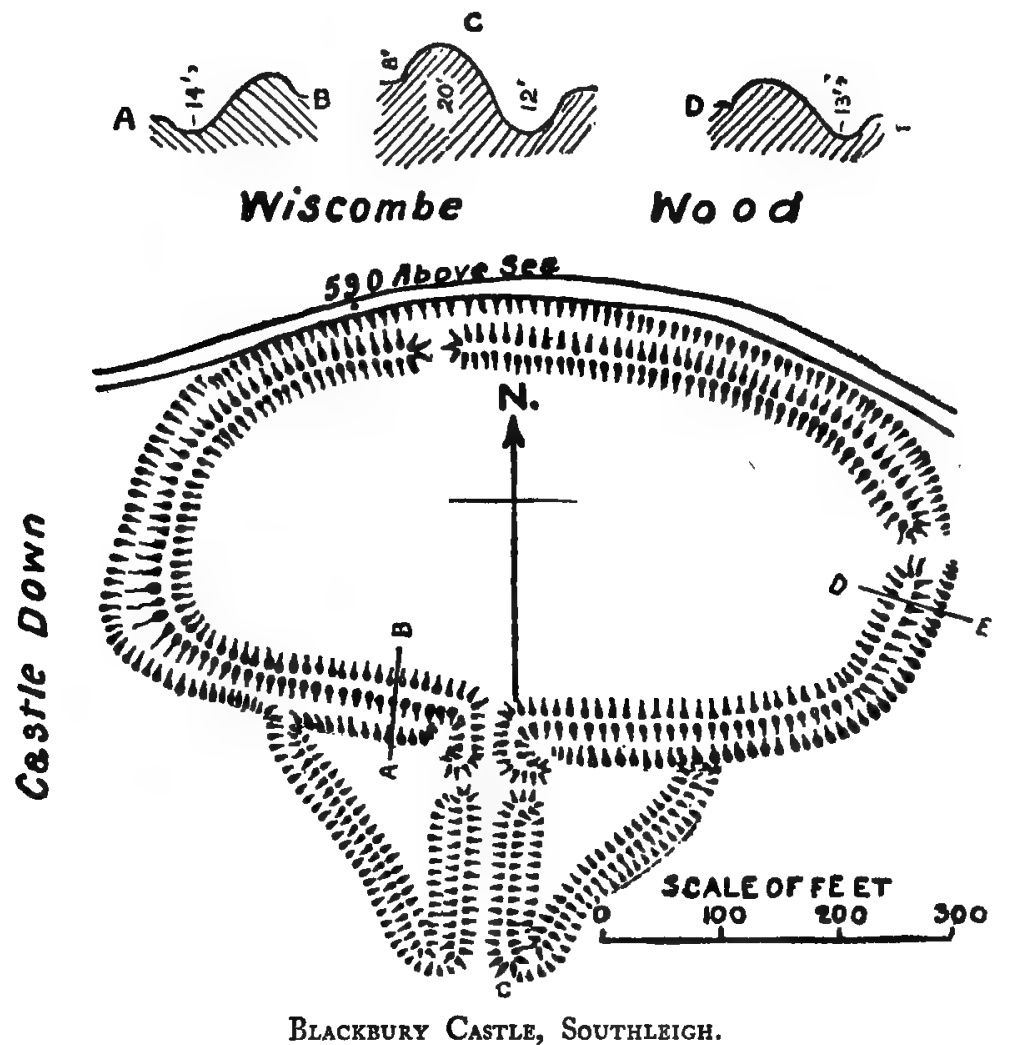
show a well-considered strategical plan.

NoRTHCOTT(O.S. Ixxiv, 9, 10, 13, 14).- This border camp of the Dumnonii appears to have been a very strong defence against the raids of the Carnabii. It is 6 miles north-west of Lifton Station; but it requires the help of the courteous farmer on whose land it lies to locate it.

Situated on the slope of a hill on the east side of the Tamar, where that river makes a course around a small island, the camp depended largely on the steepness of the bank to guard against surprise, additional strength being gained by a stream which flows at the foot of the hill.

The inner stronghold is oval, surrounded by a small bank above the escarpment, and a fosse $15 \mathrm{ft}$. deep and $28 \mathrm{ft}$. wide at the south. The counterscarp is surmounted by a bank $3 \mathrm{ft}$. high, beyond which is a level area with another bank, and fosse $5 \mathrm{ft} .6 \mathrm{in}$. deep before the hill descends to the river. The level area widens as it passes round the north to the east side, where it provides an ample court defended by a rampart which originally extended to the river-bank. The southern part, however, is destroyed. The north-east court wall is defended by a ditch as it faces the rising ground, and an entrance on this side led into another court.

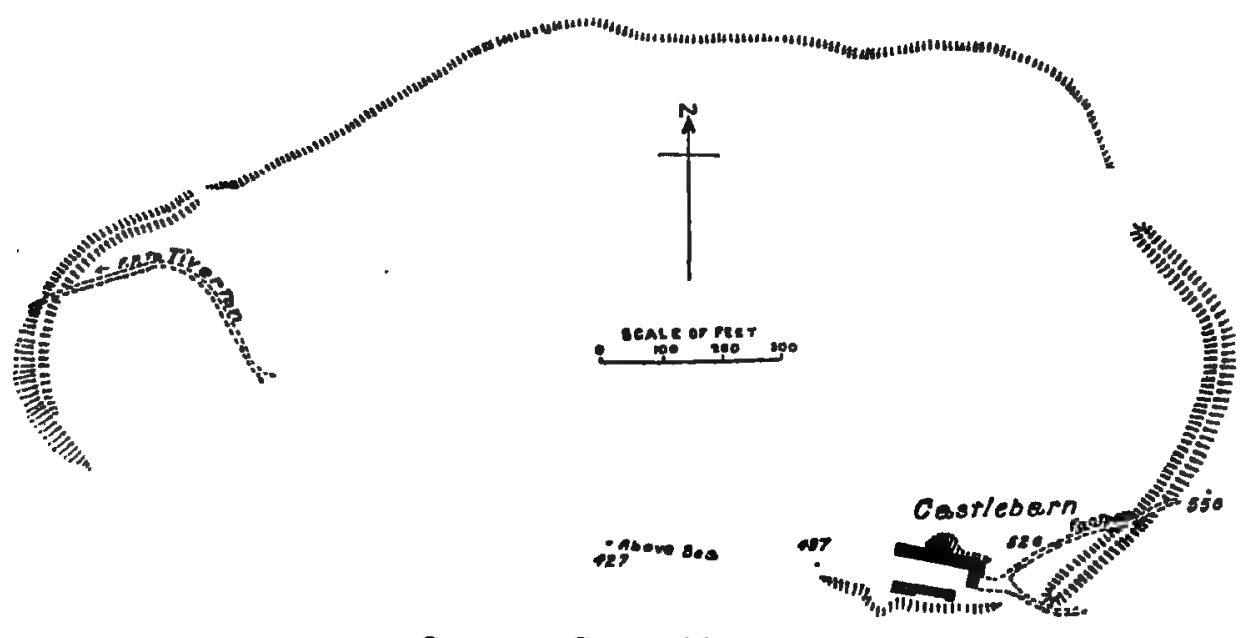

Cranmore Castle, Tiverton.

This outer area is only just traceable in a grass field known as 'Slimmond's Woods'; but not long since it was under the plough. On a higher level than the camp a circular mound surrounded by a ditch is clearly defined, but it is impossible to ascertain the former height of that mound. At 


\section{ANCIENT EARTHWORKS}

each side are the remains of a rampart which evidently extended to the river on the north and joined the inner defences on the south, enclosing an extensive area. The entrance was against one side of the mound.

At point $\mathrm{F}$ a path leads down to the river.

Silvertown (O.S. Ixviii, 4).-Dolbury: In the deer park of Killerton are the remains of a camp associated by legend with Cadbury Castle, distant 4 miles from it, with the valley of the Exe and Culm intervening.

It being but a poor fragment crowning the summit of a hill, we have not provided a plan. Roman coins were found here; and Polwhele considers it was a summer camp to Exeter.

SOUTHLEIGH. - Blackbury Castle, 3 miles north-west of Seaton, is separated from the camps in Branscombe parish by a deep valley. Towards the end of a ridge of lofty hills is

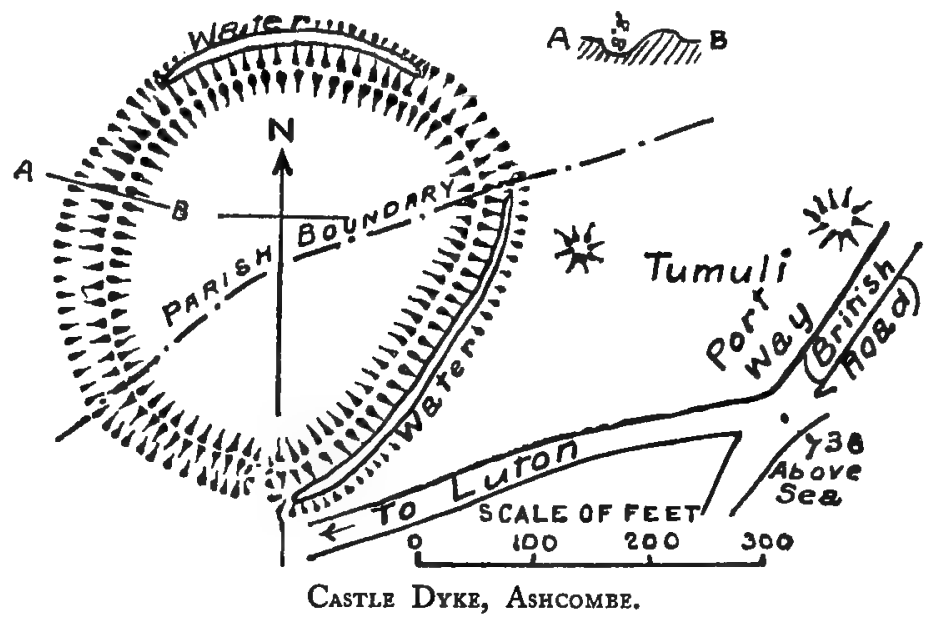
an oval camp $634 \mathrm{ft}$. long and $324 \mathrm{ft}$. wide, surrounded by a bold agger and fosse, the slope of the escarpment measuring $36 \mathrm{ft}$. at one point.

The feature of Blackbury is a remarkable entrance on the south. The vallum turns outwards and is extended on each side of a sunk road for $230 \mathrm{ft}$., with a fosse accompanying it the greater part of the distance. From the extreme end of this road the ramparts and ditches are deflected back at an angle of $40^{\circ}$ until they rejoin the main defences. Two large triangular areas are thus formed, one at either side of the entrance, each triangle being entered by a path through the rampart skirting the road, about seventy feet from the camp. The extreme south-west end of the road rampart rises to $8 \mathrm{ft}$. above the road and falls $20 \mathrm{ft}$. to the base of a fosse $12 \mathrm{ft}$. deep, making a very strong defence.

The entrances on the north and east are probably modern.

A large mound $900 \mathrm{ft}$. south-east of the camp is traditionally said to cover the bodies of those who fell in a great battle here; but an examination of the mound in 1861 yielded nothing to show that it was a tumulus, but rather a natural hill. A great mass of calcined flints and some charcoal found between the mound and the camp might, however, be taken

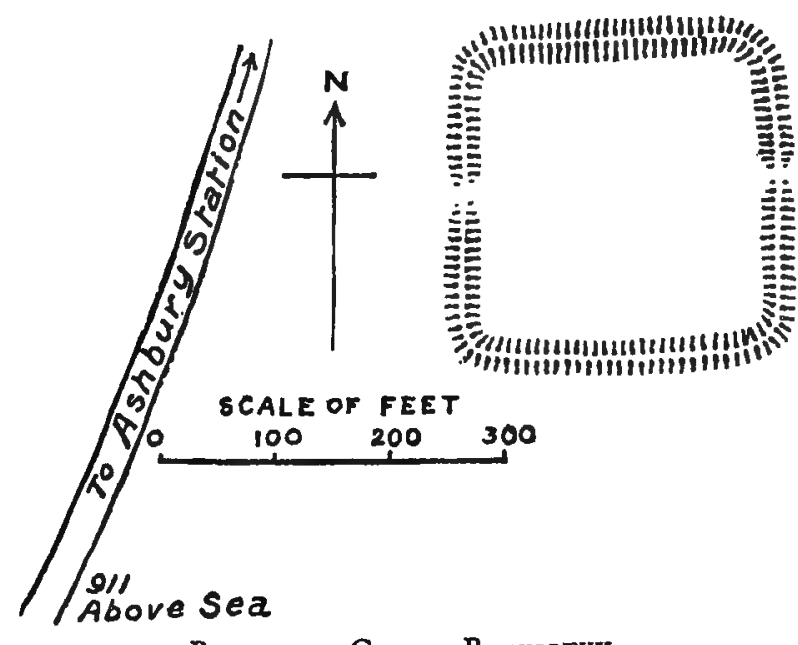

Broadbury Castle, Beaworthy. as evidence that the hill was used as a beacon.

A large hoard of bronze celts, palstaves, and various implements have been found in the vicinity.

Tiverton (O.S. xlv, 7).-Cranmore Castle lies half a mile south of the town, on the left bank of the River Exe.

The area enclosed is very extensive, and the defences of no great strength. The works remaining embrace three-quarters of an irregular ellipse, or egg-shape, the narrow end being towards the river at the west, where a rampart is on the crest of a natural escarpment. Along the north is an escarpment only, but at the east, on the top of the hill, is a curved agger Io ft. high.

Remains of buildings at the south-east, known as 'Cattle Barn,' have slight remains of banks both front and back.

During the disturbances in 1549 an engagement took place at Cranmore, when the insurgents were defeated by the king's troops. 


\section{A HISTORY OF DEVONSHIRE}

\section{SIMPLE DEFENSIVE ENCLOSURES}

[Class C]

AlvERDiscotT.-Four and a half miles east of Bideford the remains of an earthwork lie between Rookery Copse and Northclose Copse, east of Stoney Cross.

Ashburton (O.S. cviii, 15).--One mile north-east of Ashburton rises Tower Hill, a spur of Ashburton Down, upon which, between Woodencliff Wood and Place Wood, are very slight
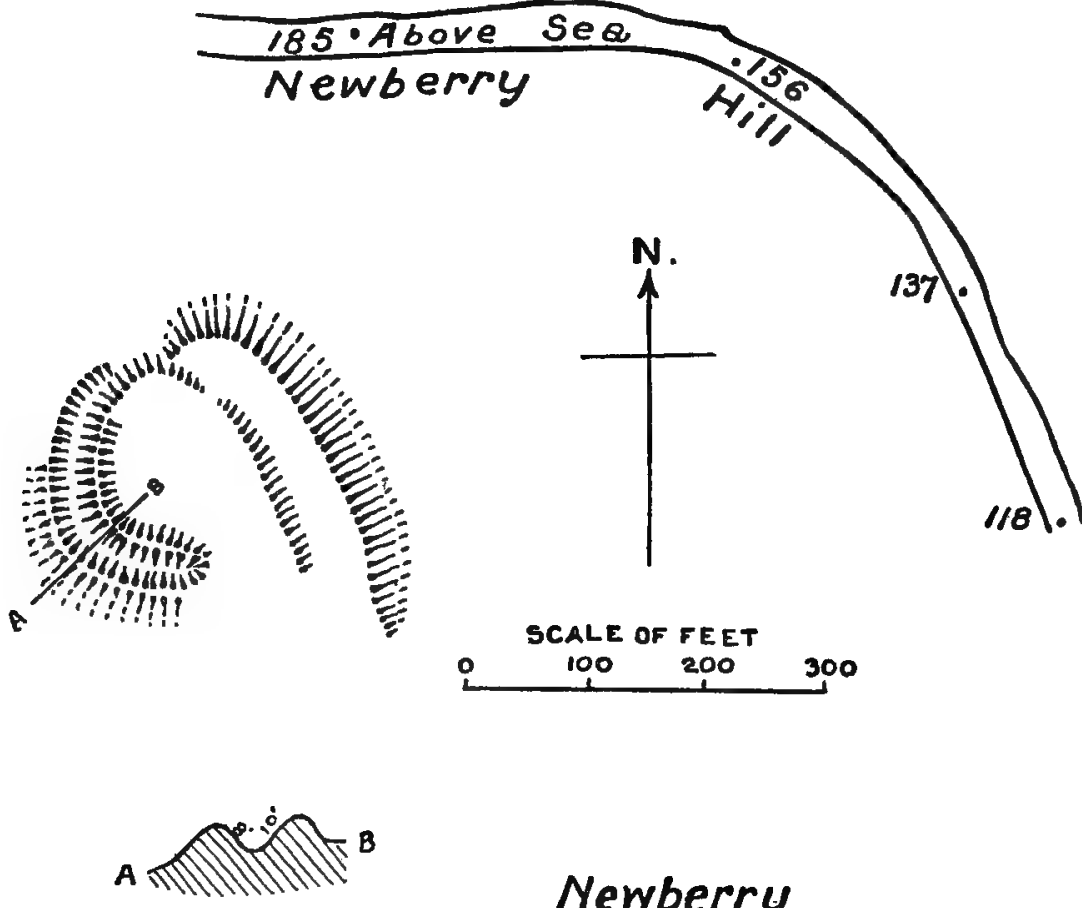
remains of a circular camp in two fields called Castle Parks. Traces of a fosse are perceptible from the north, by the east, to the south; but the escarpment a round that portion fails to give the impression of a strong defence such as the name implies, except for its natural position.

An ancient pathway is traceable down the hillside to the fords on the Dart.

Ashburton. -

Ashburton Down : Among the gorse are the scant remains of an agger and fosse behind Alston Plantation, which probably surrounded the

The Castle, Berrymarbor. western crest of the hill.

Ashburton.-On Storms Down, formerly known as 'Estdowne,' about half a mile distant from the last-mentioned camp, are the remains of another vallum and fosse, $80 \mathrm{ft}$. in length, defending the eastern crest of the hill. Both of these camps commanded an extensive view of the road from Ilsington to Ashburton, and would communicate with another enclosure in Borough Wood, on the other side of the Yeo.

Ashcombe (O.S. cii, I4).-Castle Dyke, or Castle Ditch, is situated on the boundary line of the parishes of Ashcombe and Dawlish, $2 \frac{1}{2}$ miles from the latter, to the west of the ancient Port Way, on Little Haldon Hill. It is a circular camp with single vallum and fosse, enclosing about $\mathrm{I} \frac{1}{2}$ acres. The rampart rises $4 \mathrm{ft}$. from the interior and descends from $7 \mathrm{ft} .6 \mathrm{in}$. to $8 \mathrm{ft} .8 \mathrm{in}$. into the ditch. The water in the ditch depends entirely on the rainfall. There is one entrance on the south. the camp.

Two tumuli lie on the eastern side of

Braford (O.S. xxx, 13).-On the east bank of the River Torridge, $1 \frac{1}{2}$ miles from Little Torrington, a camp is situated between Castle Hill and Castle Court.

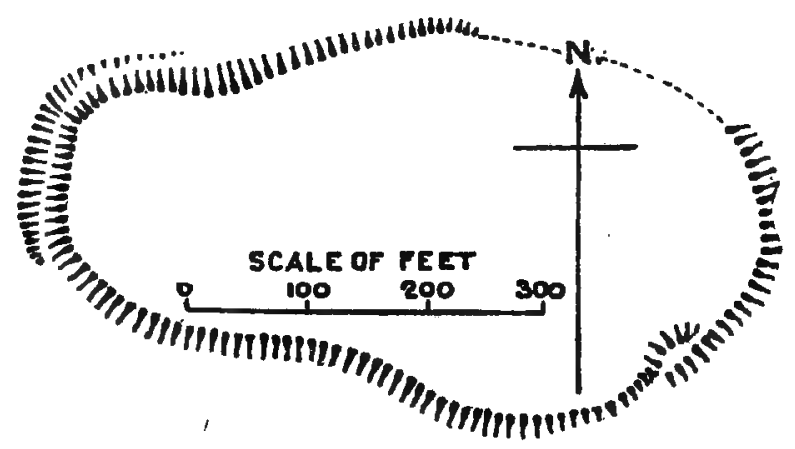

Camp at Bishops Nympton.

BeaWORTHY (O.S. lxxv, 4).-Broad-

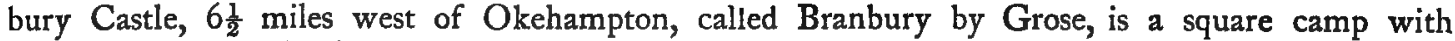
each side to a cardinal point of the compass. It was surrounded by a single vallum and fosse, the latter about twenty-five feet wide, and the vallum over fifteen feet high. There were four entrances, one in the middle of each side. Such was the camp until, about the year 1872 , a utilitarian farmer paid $\oint_{\mathrm{I}} 4$ to have it levelled; yet such is the stony nature of the ground that 


\section{ANCIENT EARTHWORKS}

wheat sown upon it fails to yield an adequate crop. At present, vallum and fosse may be traced by a slight rise and fall in the ground, and the sparseness of the growth; and the sites of two entrances are visible.

BERRYNARBor (O.S. v, 3).- ' The Castle' is an irregular camp on a height overlooking the harbour of Sandy Bay in Combe Martin Bay, on the north coast. The ground slopes down

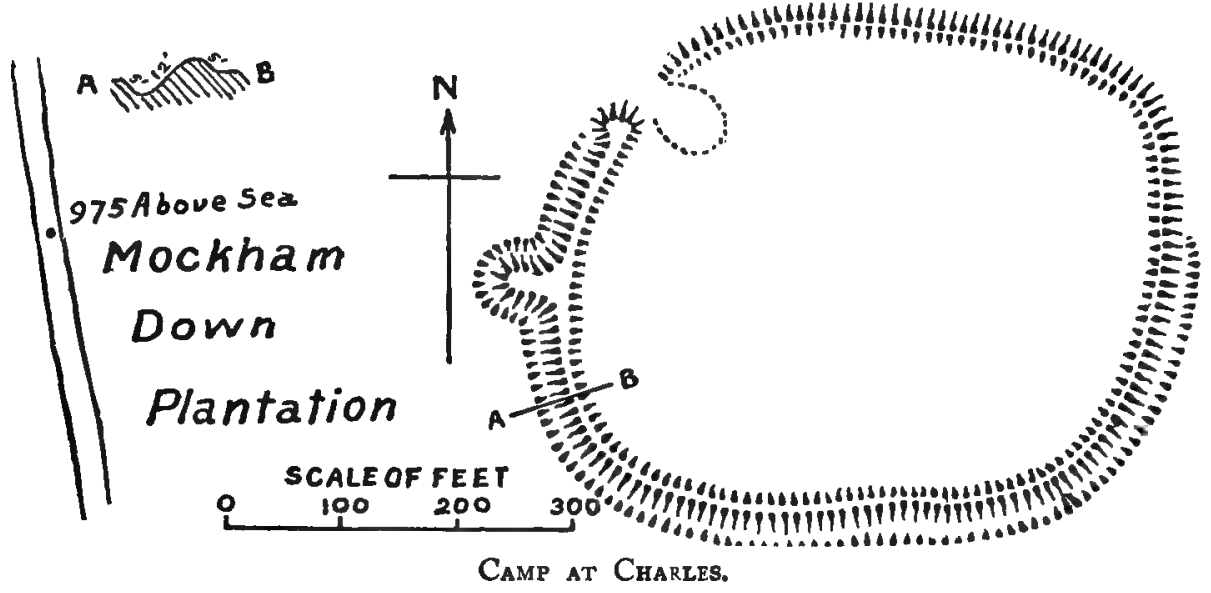

towards the south, on which side was the entrance, the west of which is defended by a strong curved agger with an escarpment of $10 \mathrm{ft}$. and a counterscarp $8 \mathrm{ft}$. in height. The highest part at the north is covered with a dense copse; but the natural hill was apparently considered impregnable; the east side also depends on nature for defence. A small stream fows at its eastern base.

Bishops Nympton (O.S. xxii, 3).- - Nearly two miles from Bishops Nympton Station, to the south of Exmoor; on the high ground half a mile north of the River Yeo is a camp of irregular elliptical form. It has consisted of a simple agger and fosse, though only a short piece of the latter is extant. At the south-east the entrance has been through the overlapping ends of the rampart.

Two small streams, one at either side, flow south into the Yeo, and assist in strengthening the position by isolation.

BRADFORD.- In Castle Wood, on the western bank of the River Torridge, a little over one mile north of Bradford village, the scant remains of a camp may be traced, of an irregular circular plan, called ' Hengiscot.'

Branscombe.-Castle Close : A camp of apparently quadrangular form, about one mile north of Berry Cliff, was all but destroyed about 1850 , and a portion of a trench then left has suffered the same fate. At the time of destruction numerous bones were dug out of the trench, with fragments of brown and yellow pottery. A barrow containing a cist was levelled at the same time. A Roman coin of Victorinus was also found.

\section{West Burridge}
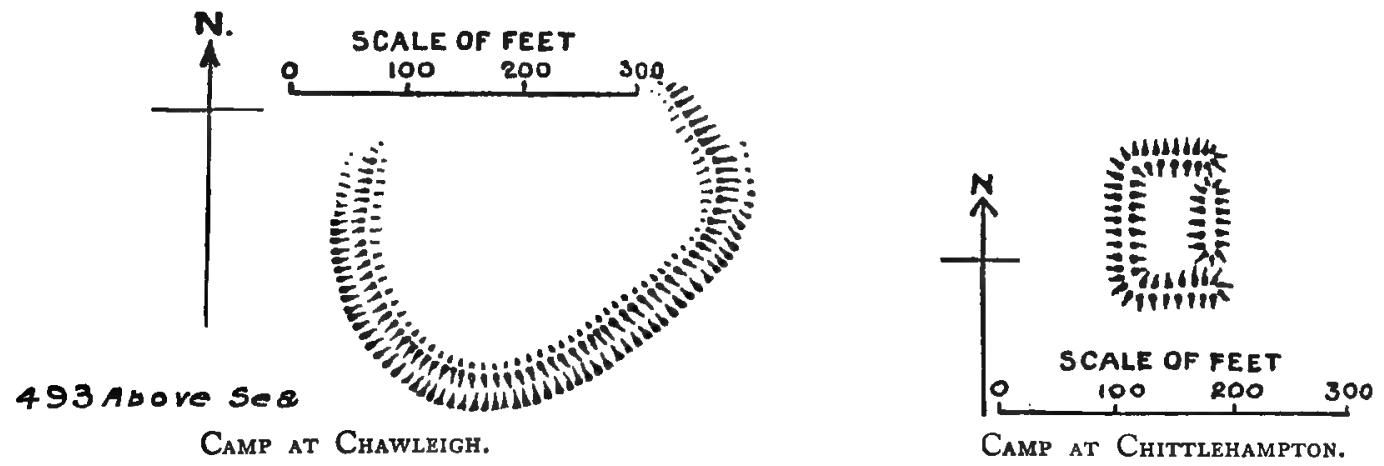

Buckland Monachorum (O.S. cxi, 4).- Three and half miles south of Tavistock the site of a small camp is situated on the east of the River Tavy; east of Balstone Wood and north-west of Higher Hele.

Charles (O.S. x, 15 ).-On very high ground in Mockham Down Plantation, fully two miles south-east of Bratton Fleming, is a camp we must describe as a rounded rectangle. It is surrounded 


\section{A HISTORY OF DEVONSHIRE}

by a vallum and by a fosse two-thirds of its circumvallation. The rampart is very considerably worn away, but in parts is still nearly five feet in height, the escarpment is $\mathbf{2} \mathbf{f t}$. and counterscarp $5 \mathrm{ft}$. On the west side is a protuberance from the vallum, with a somewhat broad top round

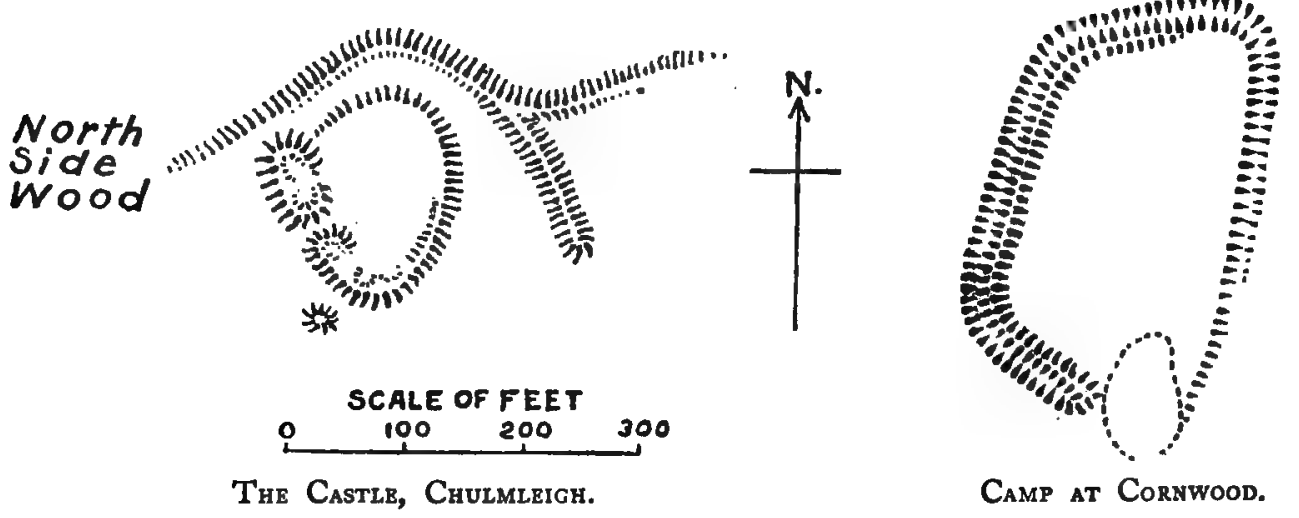

which the fosse is carried. At the north-west the rampart widens at the south of the entrance; but the place of entry is now excavated for a gravel-pit, of great size and $40 \mathrm{ft}$. in depth.

Two streams flow at the north of the camp.

Chawleigh (O.S. xliii, 6). - On the curve of a hill $493 \mathrm{ft}$. above the sea, but of no great height above the surrounding country, an earthwork near West Burridge Farm presents no special feature of a military character. The slightly raised mound with the ditch of an irregular crescentform, is only a portion of that which once existed. It is situated some distance from the left bank of the River Little Dart; but a spring which rises within $200 \mathrm{ft}$. would have supplied water to those occupying this simple enclosure. It is too mutilated to discern the place of entrance, which was possibly on the north side. Lysons speaks of a camp here with a double vallum.

Chittlemampton (O.S. xxi, 13 ).-A small rectangular camp is situated on the summit of a gentle slope above a stream to the north of Brightley Barton. It is only $120 \mathrm{ft}$. long by $32 \mathrm{ft}$. wide, and the interior accommodation is very limited, the surrounding vallum restricting the area to $102 \mathrm{ft}$. long by $14 \mathrm{ft}$. wide. The two entrances are on the east side, at the two extreme ends of the eastern rampart, which is $5 \mathrm{ft}$. in height. This cannot be regarded as an outpost, as there are no remains of a greater camp in the neighbourhood, but appears more likely to have been a shelter for a pastoral people in the midst of grazing grounds.

Chulmleigh (O.S. xliii, 1).- ' The Castle' is situated on the top of a ridge of hills 2 miles

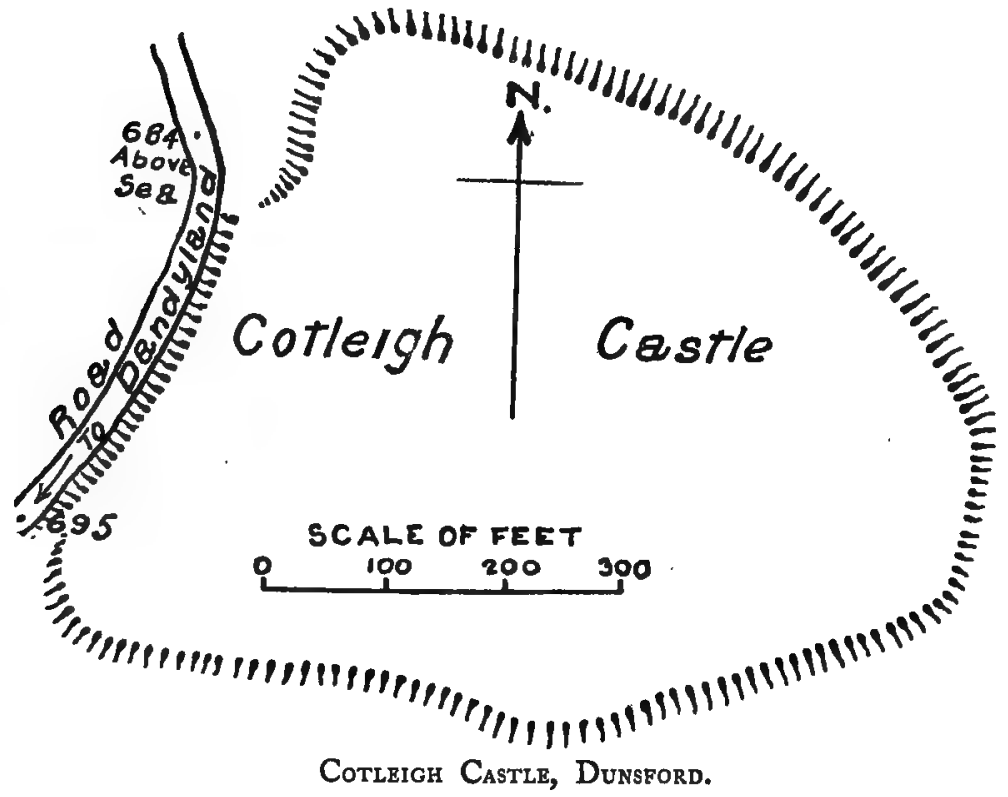
from Chulmleigh town. The position is naturally defended on the north-east and the south-west by the rapid declivity of the range. Here are the imperfect remains of a series of ramparts without ditches. The entrance on the west is flanked on each side by an agger ranging from three to five feet in height, returning inwards, and containing a circular chamber within its curve on each side. These were doubtless hut circles which, from the lack of stone, were of earth only. Another similar circle is within the northern curve of the agger, and two others in the southern curve. Beyond this habitable portion is a rampart nearly three feet in height from the outside, which has every appearance of having formed a perfect cincture, though now only two sides exist.

On the east, or the most vulnerable side, the rampart is heavier and an additional defence is projected. To the south of the entrance, on the exterior, is a mound or tumulus. 


\section{ANCIENT EARTHWORKS}

In the south valley is the Little Dart river, and a long stretch of marshy land prohibits the rapid approach of an enemy.

Cornwood (O.S. cxxv, 2).- -Six miles east of Plympton Earle, just above Ivybridge Station, a quadrilateral camp is upon Henlake Down. It has been defended by a single vallum and fosse,
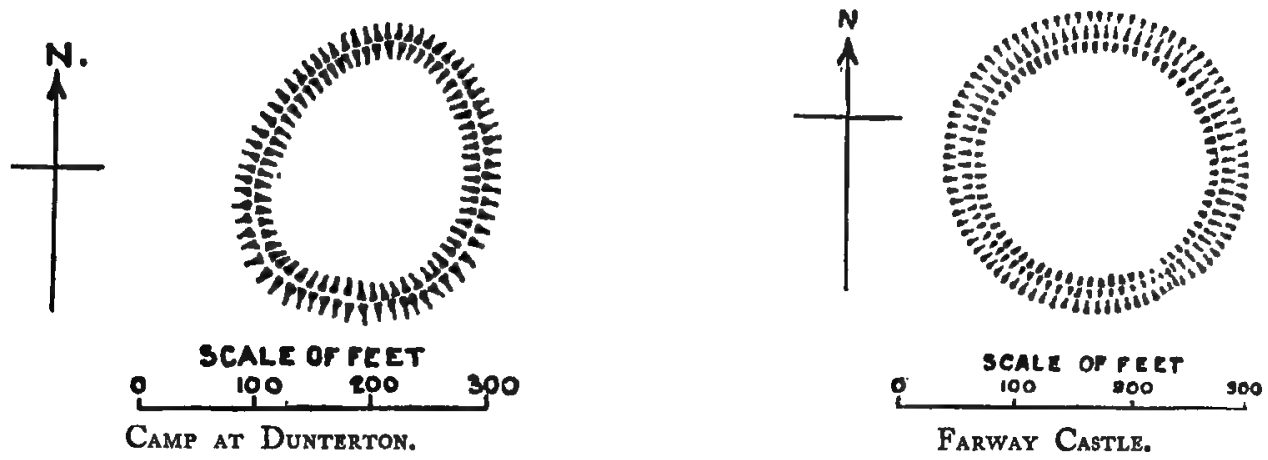

but a modern road has mutilated the south-eastern portion, and the entrance, formerly at the southern extremity, has been destroyed.

Its position upon the cliff-like heights was one of great security.

CoRnworthy.-Four-and-a-half miles south-east of Totnes are a few traces of a camp, said to be Roman.

CounTISBURY.-One-and-a-half miles north-east of Lynmouth the remains of an agger with

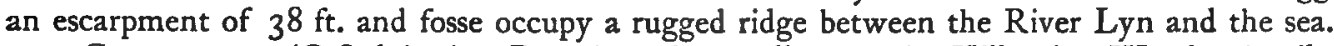

Cunlompton (O.S. lvi, 4).--Remains of a small camp in Hillersdon Wood, $3 \frac{1}{2}$ miles southeast of Tiverton.

Dittisham (O.S. cxxvii, I I).--North-east of Capton and near Bruckton Cross, is a plateau camp of simple structure, on a gentle swell of land overlooking the Dart from the north-east.

An irregular ellipse in plan, its only defence apparently consisted in its elevated position, and probably a palisade.

It is almost equidistant from the river, with another camp on the opposite bank in Brixham parish.

Dunsford (O.S. Ixxix, I5).-Cotleigh Castle, 4 miles south-west of Exeter, is a plateau camp of irregular plan. It is situated on a ridge of high grourd between the valleys of the Rivers Exe and Teign, to the east of Cotleigh Wood. The camp rises $12 \mathrm{ft}$. on the escarpment on the west side, and but half that height on the east, with the probable entrance by a sloping ascent at the south-west.

Duntrrton (O.S. xcvi, I 5). - On the high eastern bank of the River Tamar, about 800 feet from the stream, south-west of Woodtown Farm, and north-west of Palmerscleave Wood, is the
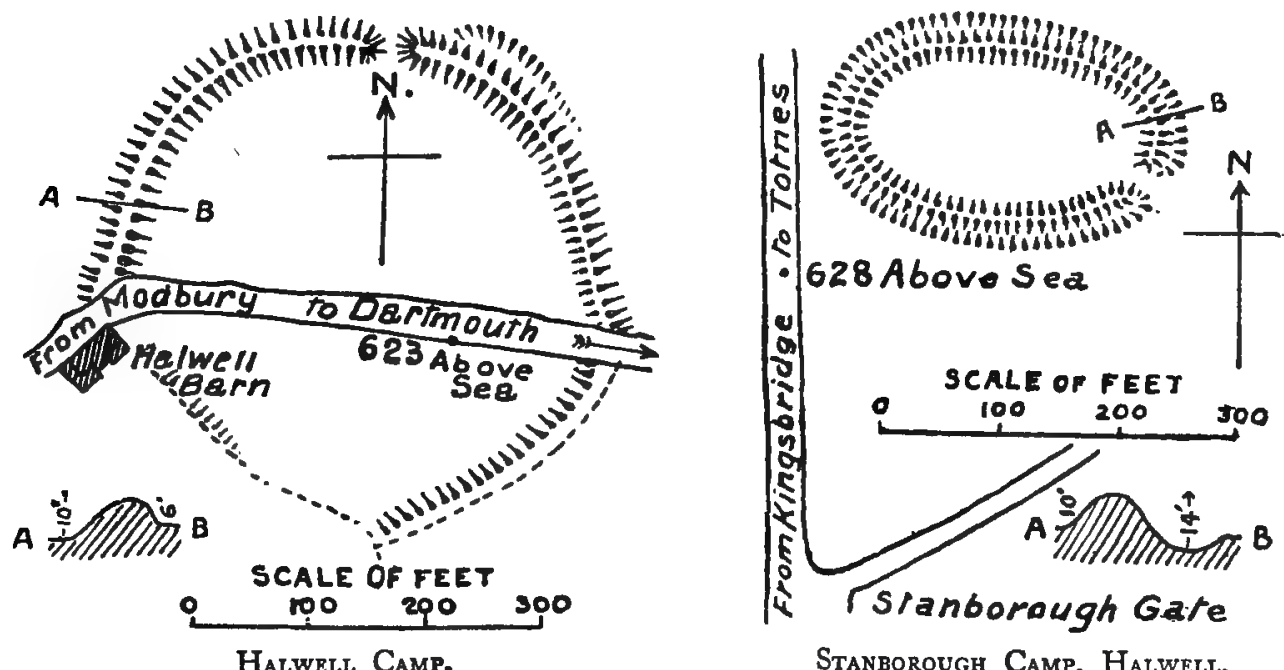

Stanborough Camp, Halwell.

site of a small circular camp, now almost levelled with the ground. At the time of inspection for this work the plough was being guided over the spot, where a gradually swelling mound reveals the position of the rampart, but all signs of an entrance are obliterated. 


\section{A HISTORY OF DEVONSHIRE}

This border camp is immediately opposite to another on the Cartha Martha, or Mount Hermitage, rocks on the Cornish side.

EXETER.-Though the chief remains appertain to the castle and its immediate surroundings

\section{Eqst}

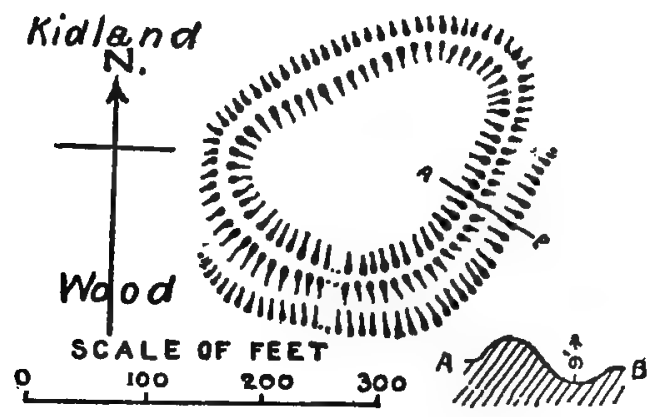

The Castle, Knowstone.

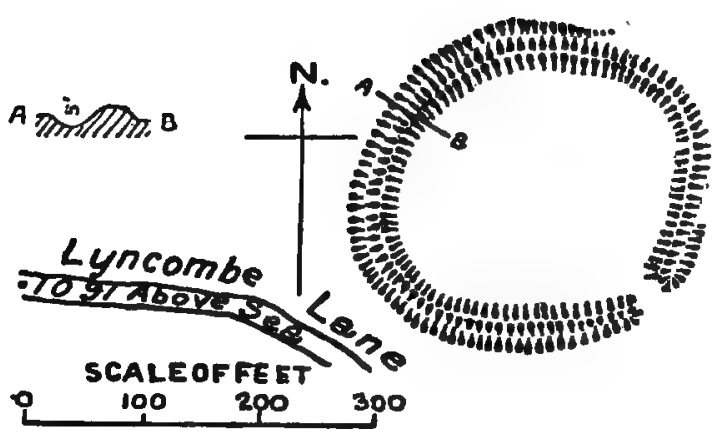

Roborough Castle, Lxnton.

and must presently be mentioned under Class $E$, it is well to note here that the line followed by the far earlier defences of the ancient town may be traced.

In this favoured position the Britons probably established a trading centre (Caer Isc), protecting themselves by constructing the usual rampart with outer fosse, excepting perhaps on the west, where the river or estuary afforded defence.

Numerous relics testify to the importance of the town in Roman times (Isca Damnorum) when

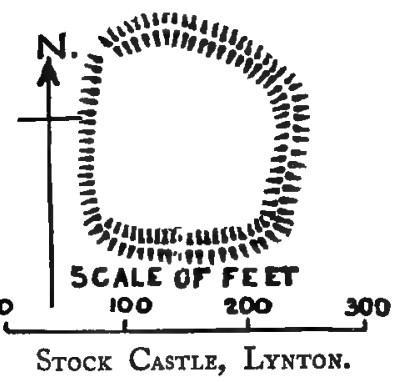
probably it was defended by a wall of masonry. It is said that Athelstan, about 926, fortified Exanceaster with a wall of stone and with towers, but probably he simply reinstated and strengthened the Roman wall.

Farway (O.S. lxx, 16).-Farway Castle, on the left of the ancient Icknield Street passing from Colyford, over Farway Hill towards Ottery St. Mary, stands on the flat of a hill on Broad Down, in the midst of numerous tumuli. It is a circular entrenchment $200 \mathrm{ft}$. in diameter, surrounded by an agger of low elevation and a shallow fosse. A bronze palstave was found near to it.

Frithrlstock (O.S. xxix, 6).-Due south of Buckland Wood and 4 miles west of Torrington, is the site of a camp called Ring Down. It was circular with a rampart and ditch, which have been ploughed over and are now scarcely traceable excipt by the sparseness of the crop on the stony ground. Situated on the slope of a high hill, east of the River Duntz, this camp was one of three neighbouring strongholds, Hembury Castle being I mile south-west, and the rectangular camp in Buckland Wood $\frac{3}{4}$ mile north-west.

Halwell (O.S. cxxvii, 9).-Halwell Camp, or Castle, is nearly 5 miles south of 'Totnes, on the road from Modbury to Dartmouth, which cuts through the centre of the circumvallation.

On the north of the road a strong vallum, rising $6 \mathrm{ft}$. from the interior, and $\mathrm{roft}$. perpendicular height on the exterior, de-

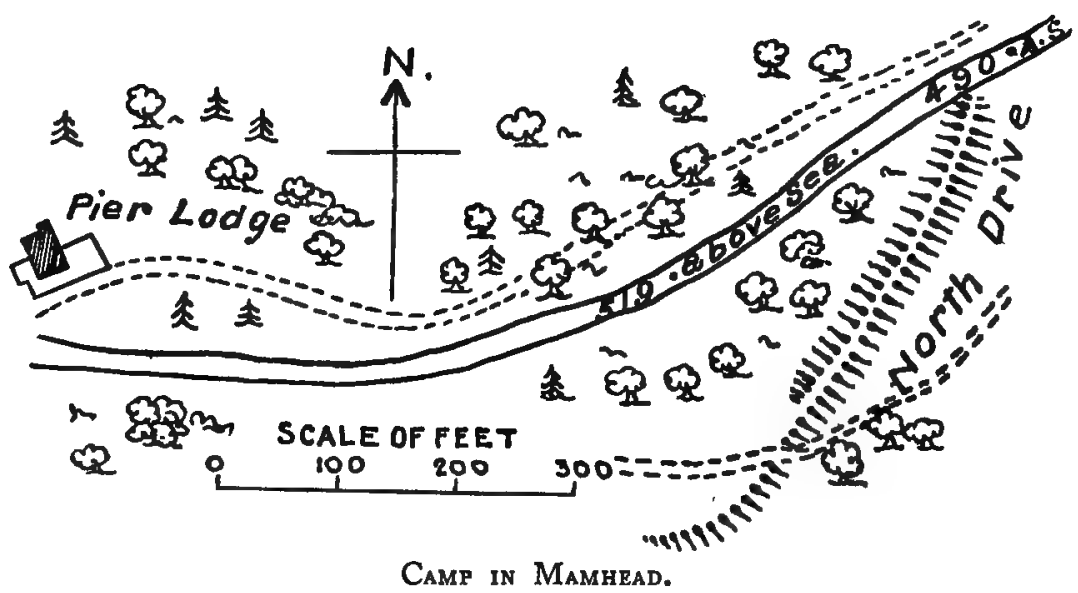
scribes a stilted semicircle. The entrance is due north, and eastward of it is the fragment of a fosse. South of the road portions of the rampart may be traced, but it is almost destroyed.

Halwell (O.S. cxxvi, 16).--Remains of small circular camp in Ritson Brake. 


\section{ANCIENT EARTHWORKS}

Halwell (O.S. cxxvi, I6).--Stanborough Camp, I $\frac{1}{2}$ miles south-west of the last, is a compact ellipse of single vallum and fosse, enclosing about $3 \frac{1}{2}$ acres. The height and depth of rampart and ditch vary considerably; the former rising $10 \mathrm{ft}$. from the interior, descends $\mathrm{I}_{4} \mathrm{ft}$. into the fosse,

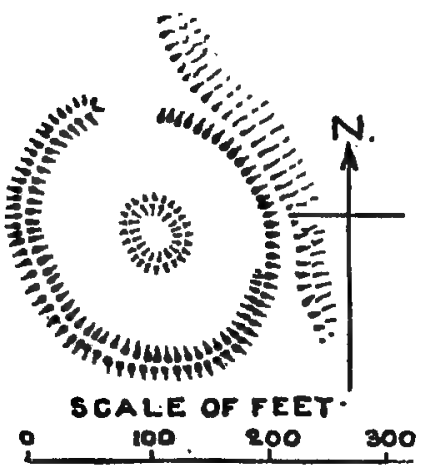

The Beacon, Martinhor. which is $3 \mathrm{ft}$. in some places and in others $5 \mathrm{ft}$. $6 \mathrm{in}$. Due east is the strongest defence, guarding the entrance on the south-east.

It is situated on the highest point of undulating ground commanding a view of the shipping in Start Bay, and was probably used as a signalling station between Slapton Castle, Woodbury Castle, Dartmouth and Dittisham, on one side, and the inland strongholds of Halwell and Blackdown. Coins and pottery are said to have been found within its area.

Several large tumuli are in an adjoining brake.

HoLNE.-Gallantry Bower, or Gallant-le-Bower, is situated between Holne Chase Castle and Hembury Castle. It is a circular camp surrounded by a rampart $4 \mathrm{ft}$. high and an outer fosse $2 \mathrm{ft}$. deep. There are two entrances to the north and east respectively; the former is approached by an easy slope, but the latter gives egress to a steep footpath descending to the River Dart, near Holne Bridge.

Knowstone (O.S. xxiii, 9).- 'The Castle,' 6 miles east of South Molton, lies in East Kidland Wood, on the south bank of the River Mole. It is an irregular elliptical camp forming one of a chain near the eastern boundary of the county. The surrounding vallum, $6 \mathrm{ft}$. in height, is in such bad condition that it is difficult to distinguish the original entrance, which was apparently on the south, where the escarpment of $9 \mathrm{ft}$. is strengthened by a fosse, which defends a little more than one-third of the camp.

LangTREe (O.S. xxix, 15).-Three miles south-west of Torrington, on Langtree Common, is the site of a small camp.

LOXHORE (O.S. x, 6).-A circular camp of about $200 \mathrm{ft}$. diameter, situated on the western bank of a tributary of the Yeo, south of Bratton Bridge, is one of a group of earthworks in this neighbourhood. It is a simple plateau, raised above the natural ground $8 \mathrm{ft}$. on the south-east ; but the plough has only left a swell of $3 \mathrm{ft}$. high on the north-west.

LyDFord (O.S. xcix).-East of Broad Down and north-west of Post Bridge, the site of a small camp lies on the bleak heights of Dartmoor.

Lynton (O.S. vii, I).- Roborough Castle is situated on a slope of Stock Common, descending on the east to Hoaroak Water, over two miles south of Lynmouth. Circular in form, it is surrounded by a rampart rising $4 \mathrm{ft}$. high, with an escarpment of $7 \mathrm{ft}$. to a fosse, except on the north-east, where the fosse is obliterated. It is within a mile of Stock Castle. Tumuli lie to the south of the camp.

LYNTON (O.S. vii, I).Stock Castle, $1 \frac{1}{2}$ miles south of Lynton, is on the east of the River Lyn, $960 \mathrm{ft}$. above the sea. It is a small irregular square of no great strength, defended on the north, east, and south by a rampart $6 \mathrm{ft}$. in height ; but on the west it has no such protection, cultivation has left it but a mound $4 \mathrm{ft}$. above the general level. The entrance was apparently on the south. Tumuli are situated to the south of the camp.

Mamhead (O.S. cii, 2).Three and a half miles northwest of Dawlish is a fragment of an earthwork on a wooded height; but it is only the remnant of a strong agger about

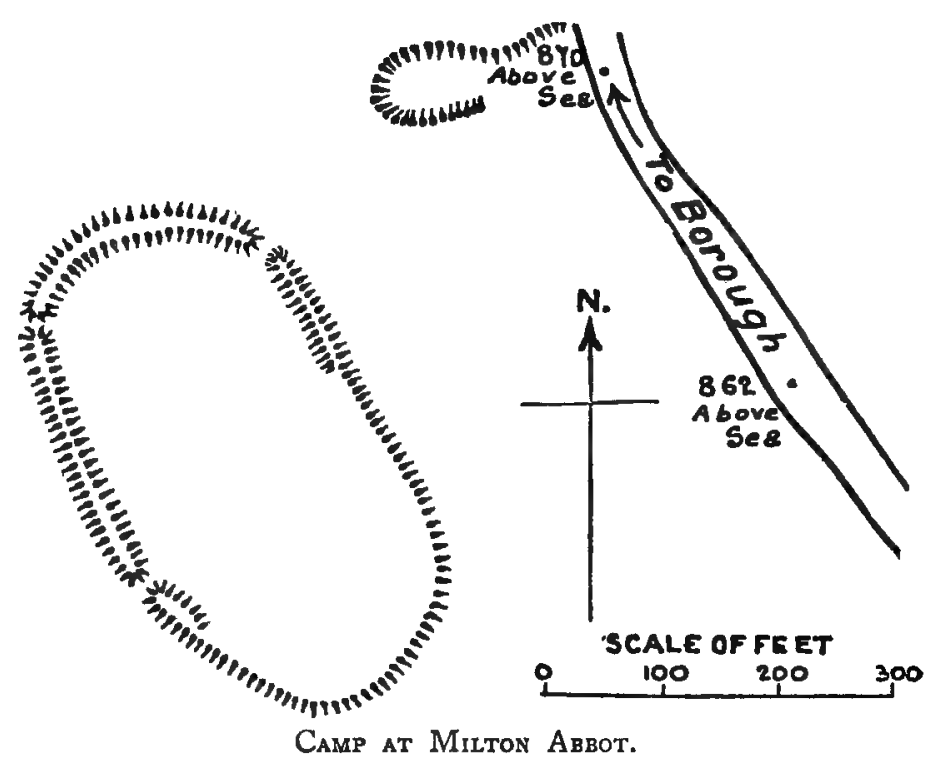
$450 \mathrm{ft}$. in length, curving at its southern extremity, which, it would seem, was the site of the original entrance.

Manaton.-The site of a small camp is in Hound Tor Wood, on the west side of the River Bovey and north of Becka Brook. 


\section{A HISTORY OF DEVONSHIRE}

Martinhoe (O.S. ii, II).- 'The Beacon' is an elliptical earthwork on the edge of the northern cliffs, to the east of the River Heddon. This was no doubt a signalling station.

A small enclosure in the centre of the camp has so frequently reverted to its ancient use of

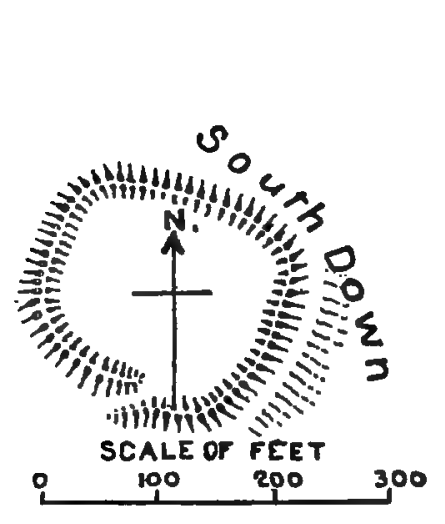

Camp at Parracombe.
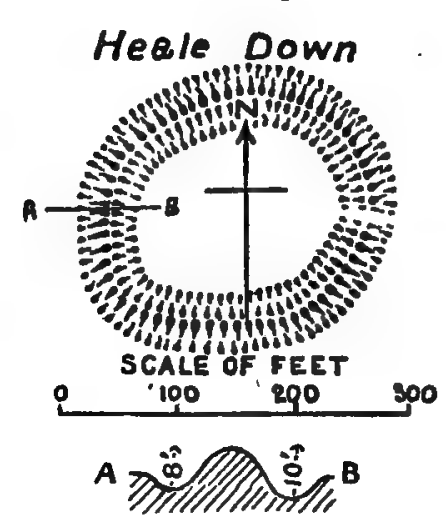

Volby Castle, Parracombe. encompassing a bonfire-though without its original purpose-and is subject to such constant incursions of modern vandals that its bank, $2 \mathrm{ft}$. 6 in. in height, is sadly mutilated. The outer vallum, $3 \mathrm{ft}$. high-also hardly used—surrounds the landward side, but its defence was unnecessary on the north-east where the cliff is its natural protection. The entrance was at the north.

Mreton Aвbot (O.S. xcvii, 9).- - On Ram Down, the highest spot on the undulating surface of this neighbourhood, is an elliptical camp of a pastoral people. The modest vallum is partly destroyed, but through it are three openings, all of which appear to be ancient. No running water is immediately at hand, but a depression in the ground was possibly a rainpond.

Parracombe (O.S. vi, 3).-On the boundary of the parishes of Parracombe and Martinhoe, upon a very high hill, is a small camp surrounded by a simple rampart $3 \mathrm{ft}$. high. On the east side the hill makes a rapid descent towards the River Heddon. The entrance on the south is protected by the overlapping ends of the rampart, making an oblique path from the south-west to the north-east.

From this lofty camp the stronghold of Voley Castle, $\frac{3}{4}$ mile distant, is overlooked.

Parracombe (O.S. vi, 8).- - Somewhat over one mile from Holwell Castle a small camp lies on Parracombe Common, to the south-west of Woolhanger Wood.

Parracombe (O.S. vi, 2).-Voley Castle, situate on Heale Down, on the west bank of the River Heddon, is in close proximity to two others in the parish, and the Beacon in the parish of Martinhoe. Though small it is very perfect and strong, being surrounded by a double fosse with an
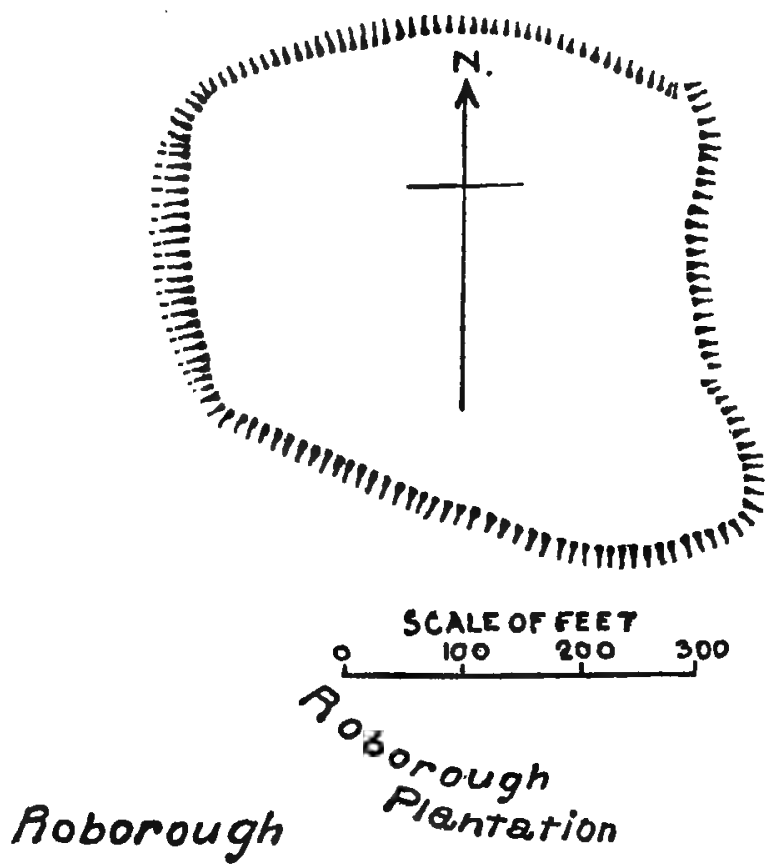

Roborough Camp, Pilton. intermediate vallum. The inner fosse being the deeper it is possible that this work is of sepulchral origin, and may be compared with Arbor Low in Derbyshire and others of like character. The entrance is on the east side towards the river.

Pilton (O.S. ix, 15).-Roborough Camp, I $\frac{1}{2}$ miles north of Barnstaple, is situated on high

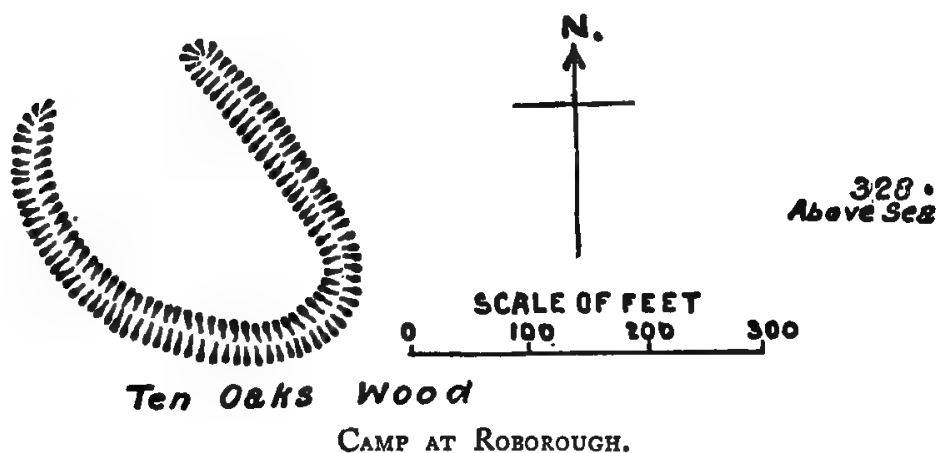

608 ground between the River Yeo and Bradiford Water, both of which enter the estuary of the Taw. It is a plateau camp of quadrangular form, rising $8 \mathrm{ft}$. above the ground. The south and eastern sides meet in a curved protuberance, where the entrance passes obliquely between the banks on the east.

A modern wall cuts across the whole of the northern banks from east to west. 


\section{ANCIENT EARTHWORKS}

A brass celt was found near this spot.

RoBorovgr (O.S. xxx, I5).-Eleven miles south of Barnstaple is the Ten Oaks camp in the midst of a wood, defended by a rampart and ditch, with an outside agger very perfect. It is $300 \mathrm{ft}$. by $200 \mathrm{ft}$., and an outer work embraces two-thirds of the camp on the north-west.

Sraton and Berr.-On Little Coochill, I mile west of Seaton, is the site of Hanna Ditches, popularly known as Honey Ditches, said to have received its name from Hanna, a Danish chief. This earthwork which was an oval, or nearly circular camp of vallum and fosse, containing about 3 acres, was destroyed in 1862 , and the name transferred to the site of a Roman villa with a hypocaust (excavated in 1859), lying in a field $\frac{1}{2}$ a mile distant. Hanna Ditches Camp is noticed by Stukeley, who also mentions a square camp with strong ditches $\frac{1}{2}$ a mile west of Seaton, of which Lysons said there were no traces in his time.

Shaugh PrIOR (O.S. cxviii, 7).-This interesting camp, north of Plympton Earle, is somewhat difficult to find. It is near Wotter, the Lee Moor Clay Works, and Cann Wood; between the River Plym and Lory Brook. Towards the south-west is an extensive view over the valley of the Plym, but to the north and the east the moor is bounded only by the skyline.

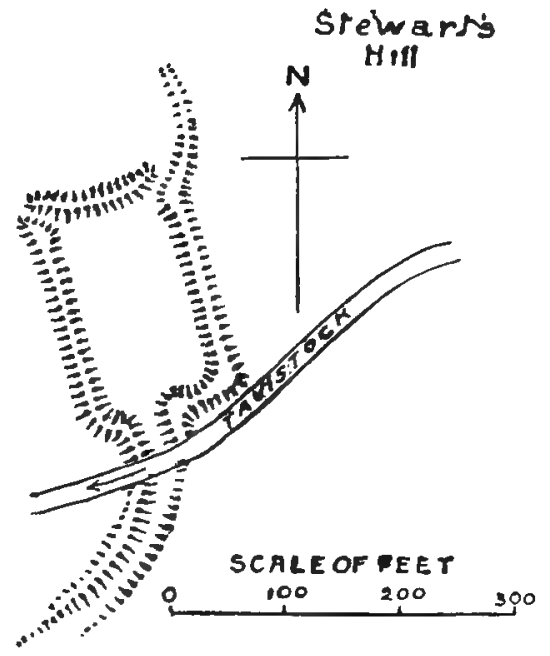

Camp at Shaugh Prior.

It is an oblong camp, locally known as the 'Bull

Ring,' surrounded by a vallum $20 \mathrm{ft}$. wide at the base and $8 \mathrm{ft}$. high, broadens at certain vulnerable points as may best be understood by the plan. The entrance on the south was by a sunk-road

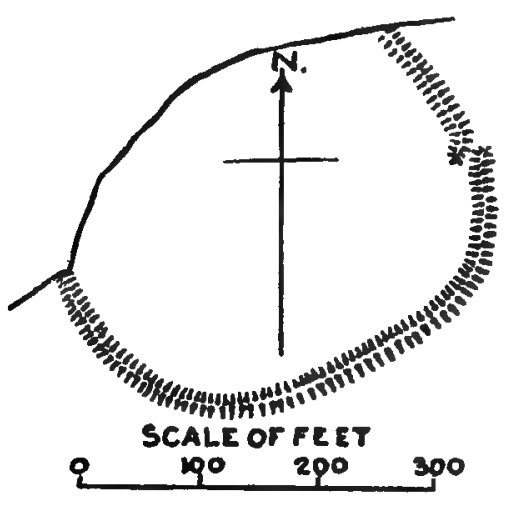

The Castle, Shirwell. $80 \mathrm{ft}$. wide, but the banks on either side have been largely destroyed, and a road severs it from the camp. At the northeast corner are the remains of a vallum which dies away in an arc; this was part of an enclosure which may be traced on the north and western sides; on the latter the court is $25 \mathrm{ft}$. wide, bordered by the fragment of a rampart I ft. in height and a fosse $4 \mathrm{ft} .6 \mathrm{in}$. in depth. At the two northern angles foot-paths lead into the external area, between the widened ends of the aggers. This was apparently a strongly defended habitation for a pastoral people, whose herds were folded within the outer enclosure.

ShebBrar (O.S. li, 1).-The site of a circular camp lies between Watergate and Waltersmoor.

SHIRweli (O.S. ix, 7).- 'The Castle' 4 miles northeast of Barnstaple, is a simply formed camp standing above Bradiford Water. The cutting of a modern lane has destroyed the north-western side, and that part remaining is surrounded by a vallum $6 \mathrm{ft}$. in height. The entrance on the east is between the incurving and the outcurving of the rampart, thus providing a slanting path up to the interior.

SILVERTON (O.S. lvi, I I ). - A small camp situated on a height above the east bank of the River Burn, lies 1 mile northwest of Silverton.

South Hursh (O.S. cxxxvi, Io).Burleigh Dolts, or Malborough Camp, close to the village of Malborough, in the south Hams, is on the slope of a hill declining towards Burleigh-Newhouse Farm. A strong vallum surrounds an area which we may term an irregular oval; that around the lower level towards the north is a rampart only, but from the east around the south, where it reaches its highest point, the illtreated vallum is still $14 \mathrm{ft}$. in height and $20 \mathrm{ft}$. wide at the base, and its strength is increased by a wide fosse. The entrance at the south-west has evidently been well

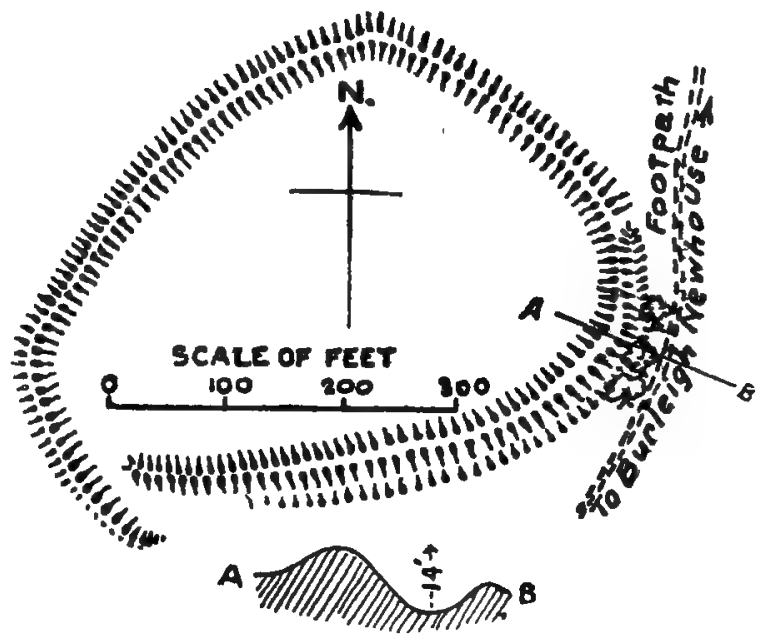

Burleigh Dòts, South Huish. 


\section{A HISTORY OF DEVONSHIRE}

guarded, for the western vallum may yet be seen extended in a curve, hugging the termination of the southern rampart and fosse.

Faint traces of an outer rampart appear on the side of the hill.

South Molton (O.S. xxii, I 3).- - Two miles south of the town of that name, to the south-east of East Trayne and north-west of South Woodhouse, on the north of the River Mole, is an irregular camp, called ' a post' by Lysons. It now consists of a broad rampart, broadened and lowered by the plough, which has obliterated the entrance.

Stockland, (O.S. lix, 9).-Stockland Little Castle, fully a quarter mile north of the 'Great Castle,' is circular, the axis northwest by south-east measuring $372 \mathrm{ft}$., and the other $33^{1} \mathrm{ft}$. It was encompassed by a single agger from 8 to Io $\mathrm{ft}$. high, composed of earth and stones, the interior being built with a dry wall of stone,

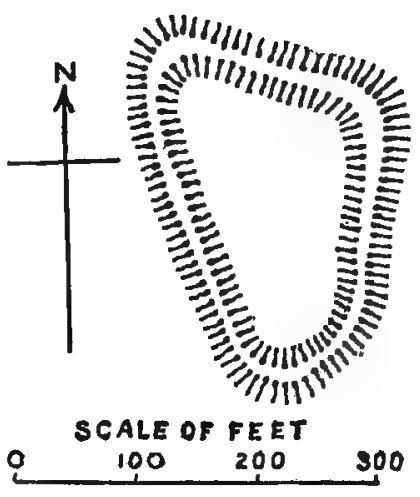

Camp at South Molton. but it is not known whether this was ancient, or whether the loose stones of the partially destroyed vallum were collected from the area and piled here in 1820 , when the site was first cultivated. The fosse has been filled in except a length of $\mathrm{I} 40 \mathrm{ft}$. on the

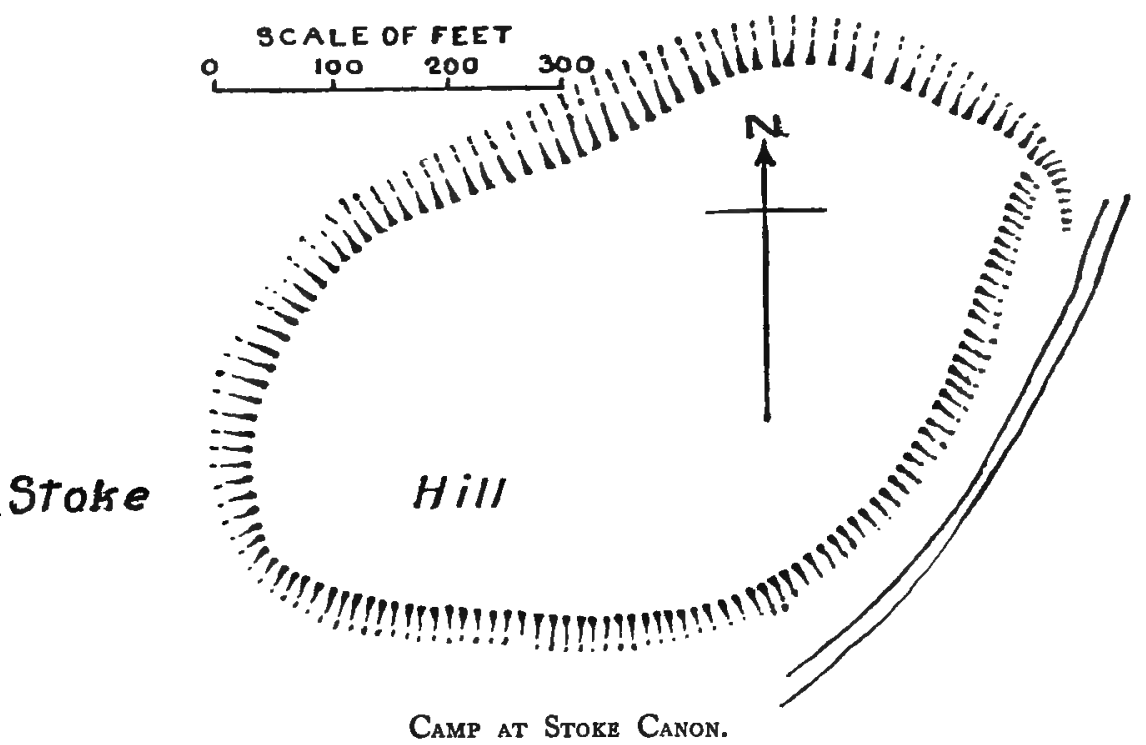
north side, where the escarpment was recently $35 \mathrm{ft}$. on the slope. The entrance was at the south-west.

STOKE CANON(O.S. lxviii, I4). -Cultivation has again made havoc with a camp situated nearly 2 miles north of Exeter, to the east of the Rivers Exe and Culme, on Stoke Hill. The escarpment partially remains, and the only indication of an entrance is on the eastern extremity.

Stoke Fleming (O.S. cxxvii, I 5).-Woodbury Camp, 2 miles west of Dartmouth, is an elliptical camp on a gradually sloping eminence. The north side, with its two angles, is defended by a rampart and fosse, the south-west by a shallow ditch only, and the south-east has been denuded of protection by the action of the plough. The strongest part lies some distance back, but facing the high road.

The northern vallum rises $9 \mathrm{ft}$. from the interior of the camp, and stands $14 \mathrm{ft}$. above the base of a fosse $5 \mathrm{ft}$. deep. At its eastern end it curves to the south, the rampart widens and the fosse terminates, an indication that an entrance was at this point.

At the north-west curve is a strong agger $200 \mathrm{ft}$. long, which rises $10 \mathrm{ft}$. from the interior and descends $28 \mathrm{ft}$. perpendicular measurement into a fosse $10 \mathrm{ft}$. deep ; at its northern end is another entrance, and at the southern the circumvallation consists of a trench only.

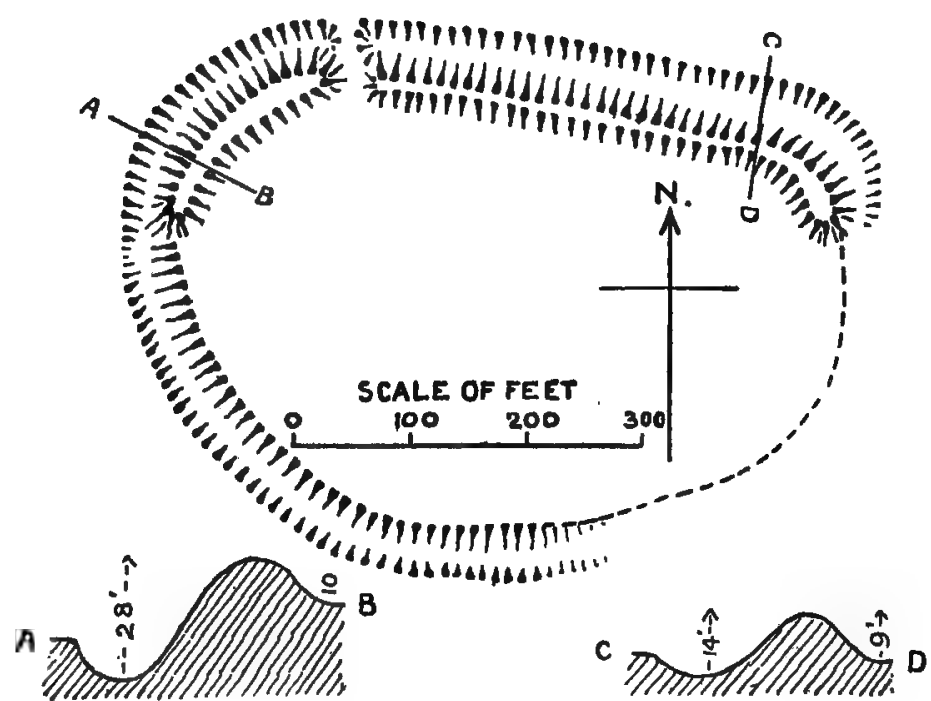

Woodbury Camp, Stoke Fleming. 


\section{ANCIENT EARTHWORKS}

The bold agger at the north-west is now in course of demolition, and will be levelled by the time these lines are published.

Stokenham.-In Widdecombe Wood, near Torcross, is a plateau camp of oval form; one long side-the western-only is left.

Stoke Gabrier (O.S. cxxi, I6).-On the eastern side of the estuary of the Dart the site of a simple camp is visible.

Stoke Rivers (O.S. $x, 14$ ).-Five miles north-east of Barnstaple, on high ground between Stoke Rivers and Beer. Nothing is left but a plateau, defended only by its height of $6 \mathrm{ft}$. above the surrounding soil. The road to Stoke Rivers has been cut to a depth of $8 \mathrm{ft}$. right through the camp. A tributary of the Yeo flows half a mile to the north.

Stokr RIVRrs (O.S. x, 15).-Within about one mile of the last another simple camp is to be seen by

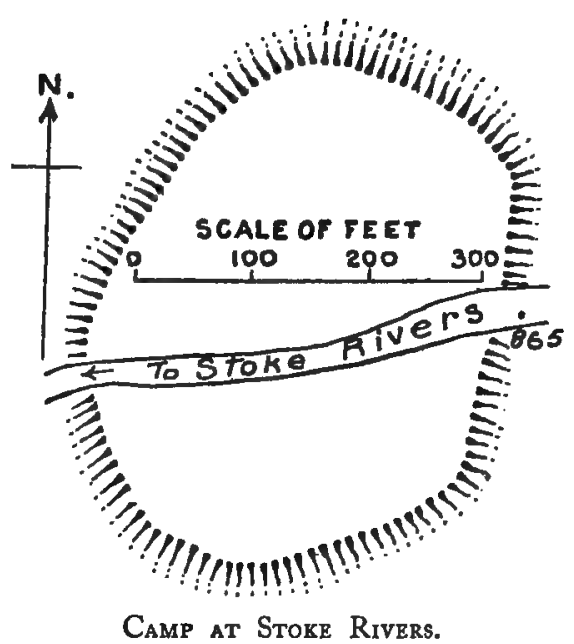
Smay's Wood. On a hill over I,000 ft. above the sea level is a small oval camp on the verge of a steep declivity, towards the tributary of the Yeo. Cultivation has levelled its defences, but on its east, south and western sides a fosse may be traced. The north descends precipitously some $60 \mathrm{ft}$., and forms the strongest defence. So far as it is possible to determine, the entrance was at the

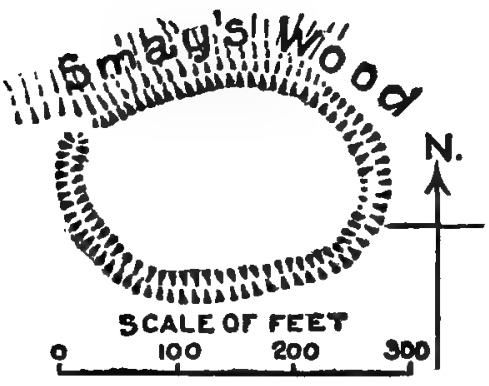

Camp at Stokg Rivers. north-west.

Stowford (O.S. lxxxvi, 12).-Near Castle Farm, to the east of Lifton, near the Cornish border, are the remains of a camp which has given the name to the farm. On the summit of a swell is all that is left of an ancient elliptical stronghold, divided into three portions by field fences, and as it is ploughed over year by year, it is a marvel that anything is now visible. The eastern side, under grass, is the most interesting. Here the ground rapidly descends to the edge of a precipitous bank of a small tributary of the River Thrushel, which flows into the Tamar. On this side, $12 \mathrm{ft}$. below the level of the camp, is a curved bank looking down a slightly hollowed channel towards the stream; and to the north of it, on the brink of the bank, is a circular excavation, apparently a sheltered position for obtaining water from below.

TAvistock (O.S. cv, 8).- To the north-east of Kelly College, in a valley outside the town, is an irregularly planned camp, divided in halves by the ancient high road from Tavistock. It is now scarcely distinguishable, and is unknown to the inhabitants of Tavistock. At the north of the western side, the most perfect, the rampart is nearly $6 \mathrm{ft}$. high, but is lost to the north; that at the south-east is $4 \mathrm{ft}$. high. A small stream, called the Waterbrook, flows north and east of the camp. (Plan on next page.)

TEDBURN ST. Mary (O.S. lxvii, 13).-Wigher Bury Camp, $3^{\frac{1}{2}}$ miles south-west of Crediton, and I mile south of Posbury Camp, is situated between the woods, respectively called Western Castle, Northern Castle, and Higher Longland. Here are the remains of a square camp with side $300 \mathrm{ft}$. in length, on ground sloping down to a river on the east. A rampart, $3 \mathrm{ft}$. in height, is extant on the north and west, and the simple escarpment on the east is lost towards the south. (Plan on next page.)

Tiverton (O.S. xxxiv, 8, and xxxv, 5).- - Huntsham Castle is on the boundary of the two parishes of Tiverton and Huntsham, 4 miles north-east of the

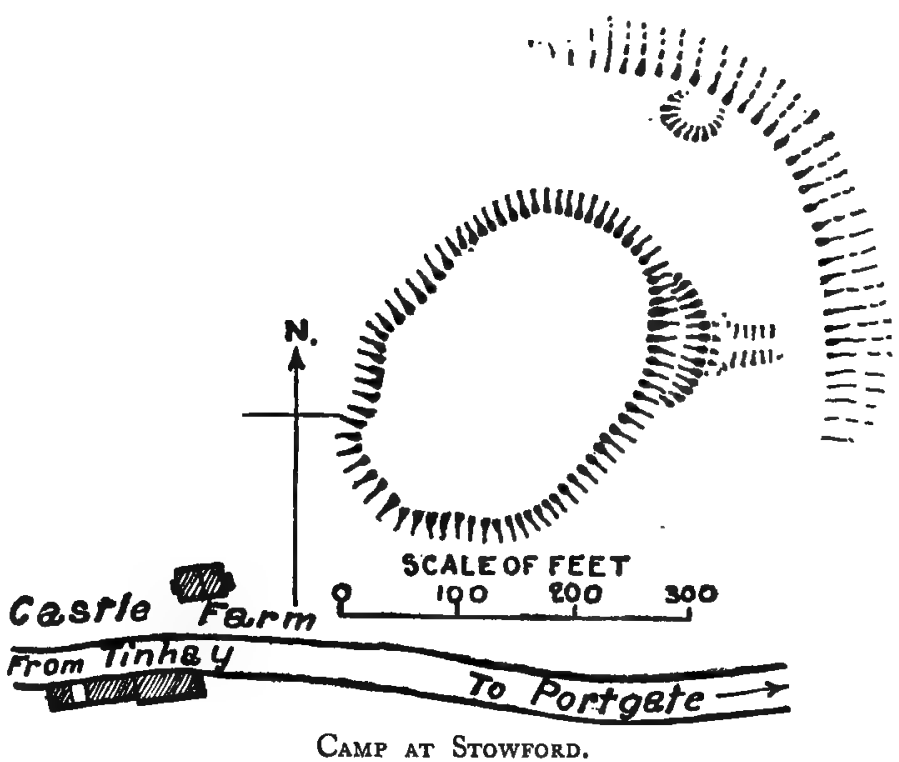

6 II 


\section{A HISTORY OF DEVONSHIRE}

town of Tiverton. Situated on high ground, very broken and wild, the camp of no nameable plan is surrounded by a vallum $8 \mathrm{ft}$. in height, except on the south-west, at which point a wooded steep descends in a valley through which a stream pursues its way to join the River Lowman.

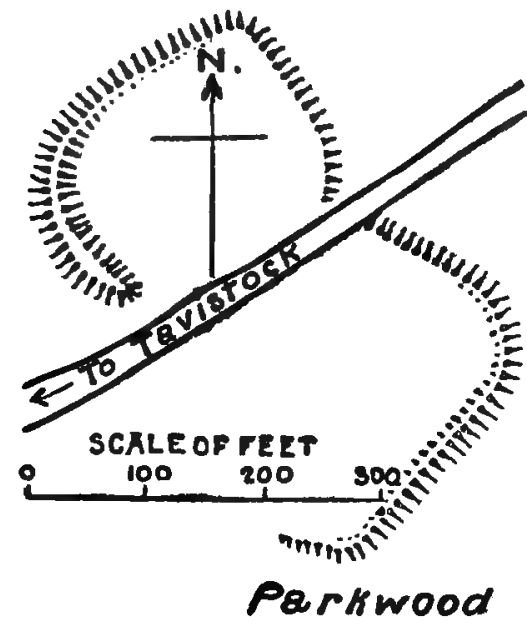

Camp at Tavistock.

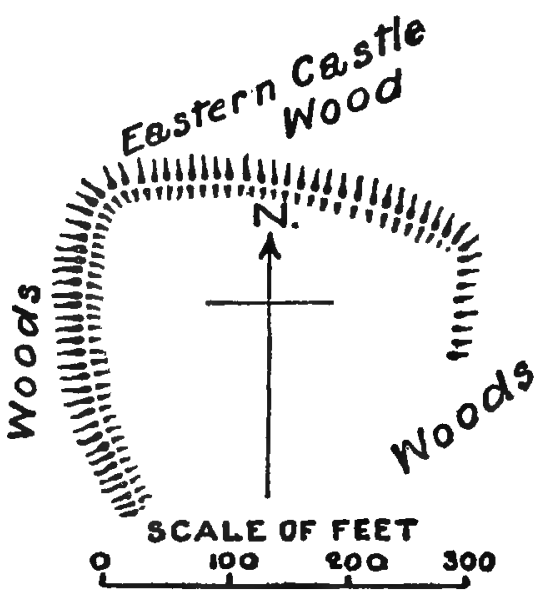

Higher Bury Camp, Tedrurn.

The entrance at the north-east is between the incurved ends of the vallum. The southern end, $60 \mathrm{ft}$. long, turns at an acute angle from the course of the rampart.

Torrington.-Gaze Castle, one mile south of Berry Castle camp in the neighbouring parish

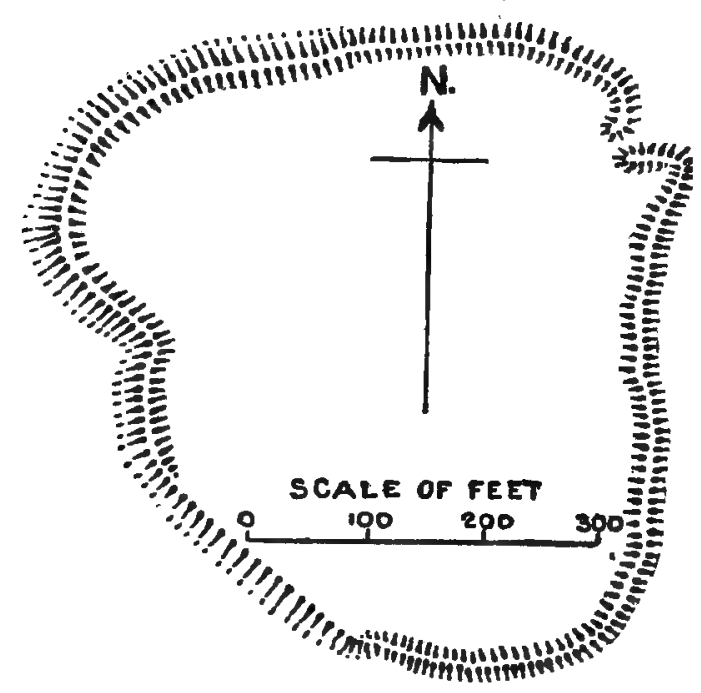

Huntsham Castle, Tiverton.

WASHFIELD (O.S. xxxiv, 6).- The site of a former camp may be traced in a field called 'Castle Close,' between Barehill Wood and Huntland Copse, nearly 2 miles north of Tiverton.

Two spear-heads of the 'leaf-shaped' type and a short sword were found in the entrenchment near the ford of the Exe at Worth.

WIDECOMBE IN THE Moor (O.S. cviii, 5).-Between 4 and 5 miles north-west of Ashburton are the remains of two entrenchments, both of Huntshaw, and in view of the same, is now destroyed and under cultivation; but $15 \mathrm{ft}$. of it may be traced through the crops. ${ }^{1}$

UGBOROUGH (O.S. cxx, I3).-Fully I mile south of South Brent and west of Turtley, a circular camp is upon a slight rising in a valley. It is strongest on its southern side, where a rampart rises $3 \mathrm{ft}$. from within, and has an encampment $6 \mathrm{ft}$. 6 in. with a fosse $3 \mathrm{ft} .6 \mathrm{in}$. deep. This agger guarded the entrance at the south-west, but in its easterly course it is gradually lost, leaving the remainder of the camp defended only by a ditch $8 \mathrm{ft}$. wide and $3 \mathrm{ft}$. deep; and even that is almost levelled at the north.

To the north of it flows the Glaze Brook, a tributary of the River Avon; while on the north and the west, rising from Brent Moor and Ugborough Moor, are the heights of Brent Hill, Ugborough Beacon, and Butterton Hill.

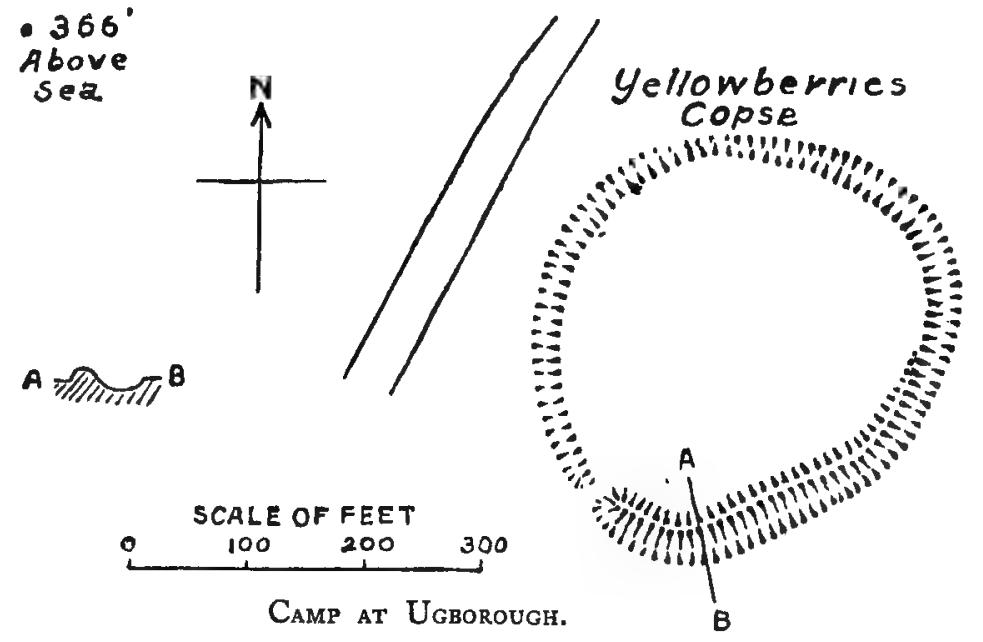

${ }^{1}$ Mrs. Armitage calls attention to an entry in the Cal. of Close, iii (1228), p. 67, for the razing to the ground of a castle at Torrington which had been erected without licence. 


\section{ANCIENT EARTHWORKS}

on Luesdon Common. One, near Lower Uppacott, is a slightly curved agger $300 \mathrm{ft}$. long, extending from the north-west to the south-east.

(O.S. cviii, 9).-The other, south-east of Lower Uppacott, is an agger forming the northern angle of an area, evidently part of a camp. These lie on high ground to the west of the River Webburn.

WIDworthy (O.S. lxxi, 6).-Castle Hill camp is about 3 miles east of Honiton, west of Widworthy church, and in Widworthy Park. It is now but a flat area terraced above the natural level; the north side of $103 \mathrm{ft}$. is nearly straight, as also is the west side, $90 \mathrm{ft}$. in length ; the south and east are portions of a circle which together measure $142 \mathrm{ft}$.

WIDworthy (O.S. lxxi, 6).- - On Widworthy Hill, $3 \frac{1}{2}$ miles east of Honiton, and nearly I mile south of the church, are the remains of a circular camp, $230 \mathrm{ft}$. on its axis east to west, and $225 \mathrm{ft}$. north to south. It is situated in the midst of a plantation.

WINKLEIGH. - Court Castle is one of the two earthworks at Winkleigh ; Croft Castle, with the plan of Court Castle, will be found in Class D. Four miles south-west of Eggesford station, Court Castle is situated north of Winkleigh church, but the plough has changed its configuration. The rampart has gone, leaving a plateau with an escarpment of $12 \mathrm{ft}$. on the south-west, broken by a terrace. The remnant of a fosse on the north makes a curve at its eastern extremity, and indicates an approach by a sunken road.

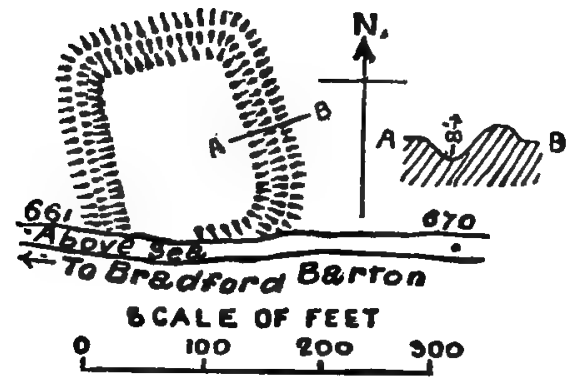

BerRy Castle, WithrRidge.

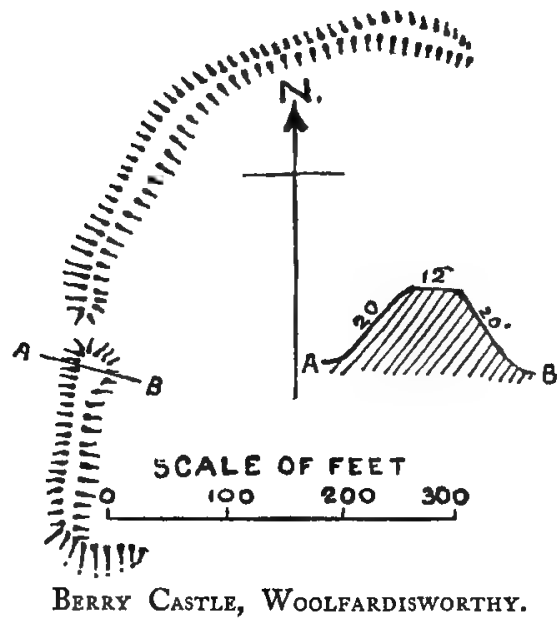

Witheridge (O.S. Xxxiii, IO).-Berry Castle, I I miles north of Crediton, on the east of Little Dart river. On wild undulating ground is a small quadrangular fortification, surrounded by a vallum and fosse enclosing $\frac{2}{3}$ of an acre. Contrary to the usual plan in the camps of Devonshire, Berry Castle has the fosse of $6 \mathrm{ft}$. deep inside the vallum of $8 \mathrm{ft}$. high.

The entrance must have been on the south side, and cut away in making the road to Bradford Borton, as there are no indications of an entrance through the remaining entrenchments.

Woolfardiswor'thy (O.S. xliv, I 3).-Berry Castle, 6 miles north-west of Crediton, and $5 \frac{1}{2}$ miles south-west of the last-mentioned fort of the same name, is situated on ground sloping down towards the west, and consists of a bold agger, $20 \mathrm{ft}$. on the scarp and $12 \mathrm{ft}$. broad at the top, embracing the north and west sides of a camp, and returning some $60 \mathrm{ft}$. towards the east at the southern extremity, where the agger gives place to a natural escarpment. The entrance is at the west, where the southern rampart considerably widens.

Near by rises a small stream which flows into the River Dalch.

When in a more perfect state this camp was described as circular, containing an area of 2 acres, divided into two parts by a bank.

\section{MOUNTS}

[CLAss D]

Barnstaple (O.S. xiii, 2).-Barnstaple Castle, also called Barum in Barnstaple, is said to have been a Saxon royal burgh, and for a time to have constituted the head quarters of King Athelstan whilst completing his subjugation of the Dumnonii. Long before his time, however, so advantageous a position was probably fortified.

Situated on the east bank of the River Taw, near its confluence with the Yeo, and easily accessible to the sea by the broad estuary of the Taw, a strong defence would be of vital necessity.

The very steep conical mount known as 'Castle Mound' was evidently the centre of a strongly fortified position some time before it was utilized for the keep of a feudal castle. The 


\section{A HISTORY OF DEVONSHIRE}

latter is wholly demolished, and the mound alone remains, cinctured by a winding ascent to the summit, some $60 \mathrm{ft}$. in height. The top is a flat area $40 \mathrm{ft}$. in diameter. Neither fosse nor other earth work remain, only this noble mound survives on the scene of many conflicts, ending in its surrender to General Fairfax in 1646.

Blackawton (O.S. cxxvii, I 4).- - The Mount,' also called 'The Beacon,' is a truncated cone $20 \mathrm{ft}$. in height and about $30 \mathrm{ft}$. in diameter at the base. It is situated $1 \frac{1}{2}$ miles west of Woodbury, at the junction of the roads to Blackawton and Strete.

EXETER.-Dane's Castle. A circular mount about $\mathrm{I} 50 \mathrm{ft}$. in diameter, with a cavity in the top and traces of a surrounding fosse, formerly stood in a field to the north of the city and opposite the castle, behind the county gaol ; but it was destroyed for the construction of a reservoir.

Loxhore (O.S. $x, 9)$.- Castle Roborough, to the east of Loxhore town, $5 \frac{1}{2}$ miles north-east of Barnstaple, and situated on a hill in the valley of the Yeo, is a simple mound, about $120 \mathrm{ft}$. in diameter at the base. It rises $15 \mathrm{ft}$. in height, and in its summit is a basin-shaped hollow over $7 \mathrm{ft}$. in depth.

This is one of a group of earthworks forming a chain across the narrow northern part of the county from Barnstaple to the morass defences of Exmoor, and is within signalling distance of camps in the adjacent parishes of Stoke Rivers, Bratton Fleming, and Shirwell.

Northam (O.S. xix, I).- Kenwith Castle, also called Cynwit, Cynuit and Kenwic, and locally known as Henni Castle and Henniborough, is situated at the upper end of a small valley on the north-west of Bideford, a little over a mile distant.

Kenwith was the scene of a stirring battle in the ninth century, a contest which proved one of the most decisive means of raising England from the humiliation of Danish domination, and we cannot but express our regret that the exigencies of space prohibit us from describing the event.

This historic fort is now a verdure-clad rock, an isolated mound rising precipitously on all sides, but the south-east having a rectangular base. Two small streams flow at the south-west, which apparently spread into a morass in former times. The entrance at the south-east slopes on to a platform $6 \mathrm{ft}$. wide which gradually rises as it passes round the southern and western sides, $12 \mathrm{ft}$. from the summit, and abruptly ends on the almost perpendicular northern side. From this platform the hill rapidly descends, although broken by a modern path at a lower level. From the entrance a steep incline on the eastern side curves at almost a right angle along the north until it attains the level top of the stronghold.

For evidence that this mound is the ancient Kenwith we may look to the south-west, where, on Silford Moor (Sel-fyrd=great camp), are slight remains of an earthwork nearly destroyed by tillage, which may possibly represent those entrenchments which Asser tells us were thrown up by the Danes for purposes of the blockade when the sortie of the Saxons stopped its completion. ${ }^{1}$

North Tawton (O.S. lxv, 3).-Castle Court, within the town of North Tawton, is a low mound about $100 \mathrm{ft}$. by $\mathrm{I} 30 \mathrm{ft}$. on its two axes. Its height is $9 \mathrm{ft}$., and a moat which formerly surrounded it is now scarcely perceptible in parts, but on the south, where it is the most prominent, the depth is $3 \mathrm{ft}$. Slight indications of an entrance may be traced on the south.

A little over $100 \mathrm{ft}$. to the north are the remains of another escarpment, but modern alterations and buildings, with the divisional fence of two gardens passing right over the centre of the mount, have largely destroyed the original features of this work.

WinkLEIGH (O.S. liii, 2). - Croft Castle. At either end of the town of Winkleigh is a defensive work, so placed as to recall the position of the two earthworks in Heywood Wood, by Eggersford.
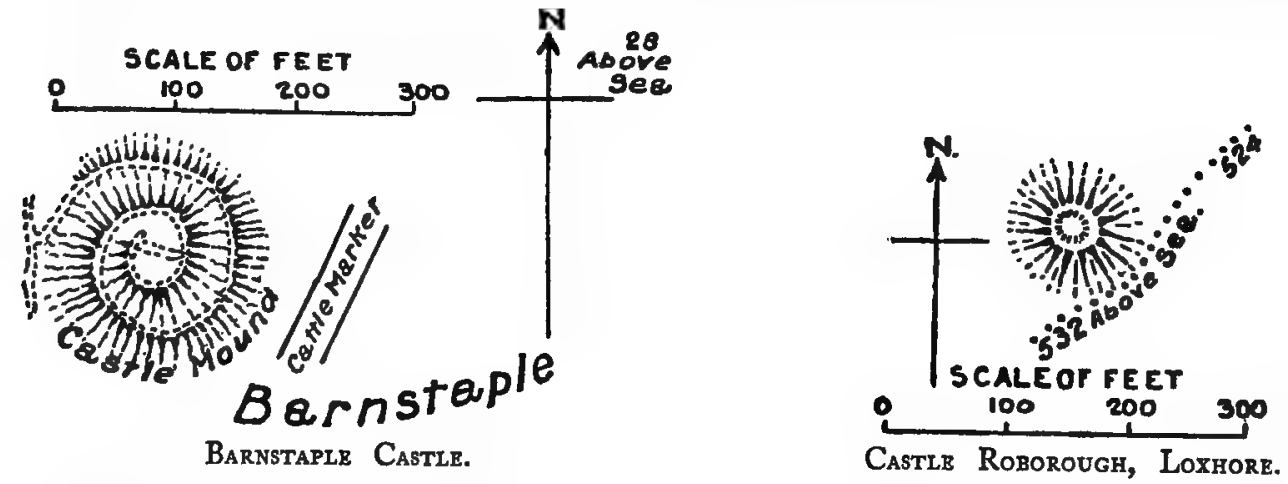

Croft Castle, on the south-west of the town, is a mount only with an escarpment $20 \mathrm{ft}$. in height. On the summit is a platform $6 \mathrm{ft}$. wide, surrounding a hollow which descends $12 \mathrm{ft}$. perpendicular measurement, into the heart of the mount.

${ }^{1}$ Asser, Life of Alfred, A.D. 878. 


\section{ANCIENT EARTHWORKS}

A very perfect specimen of a long tapering blade of fine bronze has been found near this spot. in Class C.

For the reference to the northern earthwork, shown on the plan, see under Winkleigh,

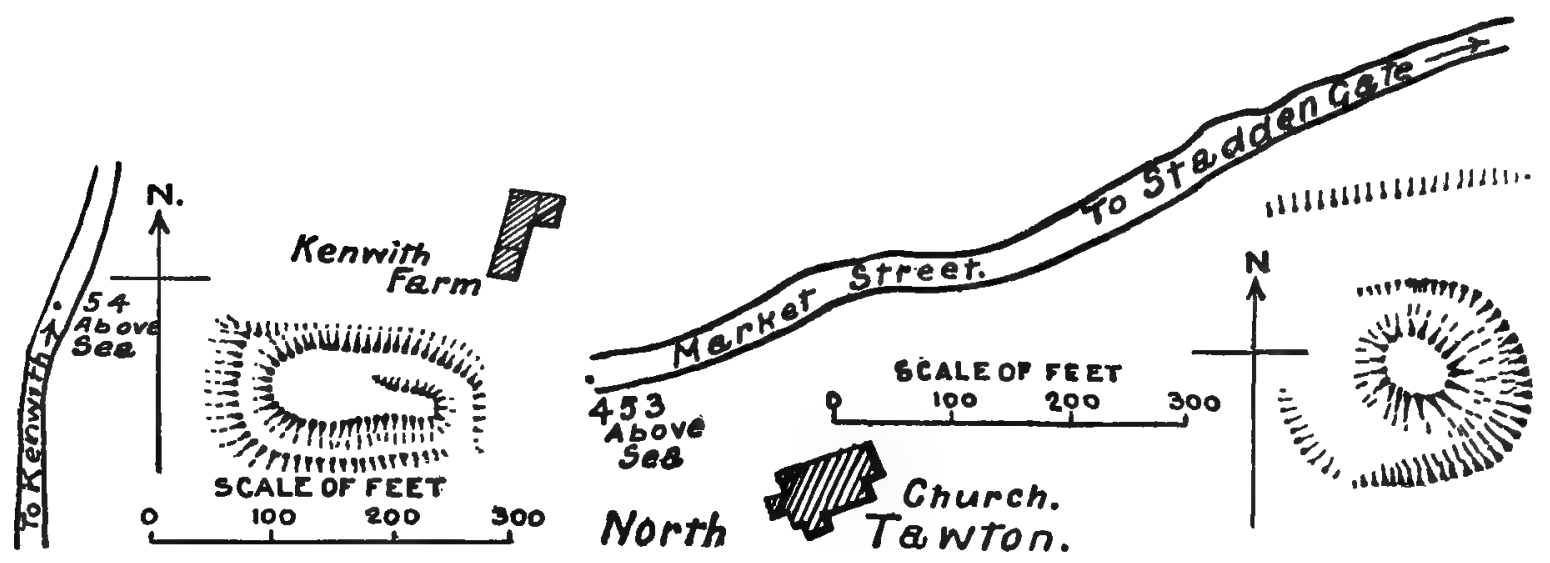

Kenwith Casteg, Northam.

Castle Court, North Tawton.

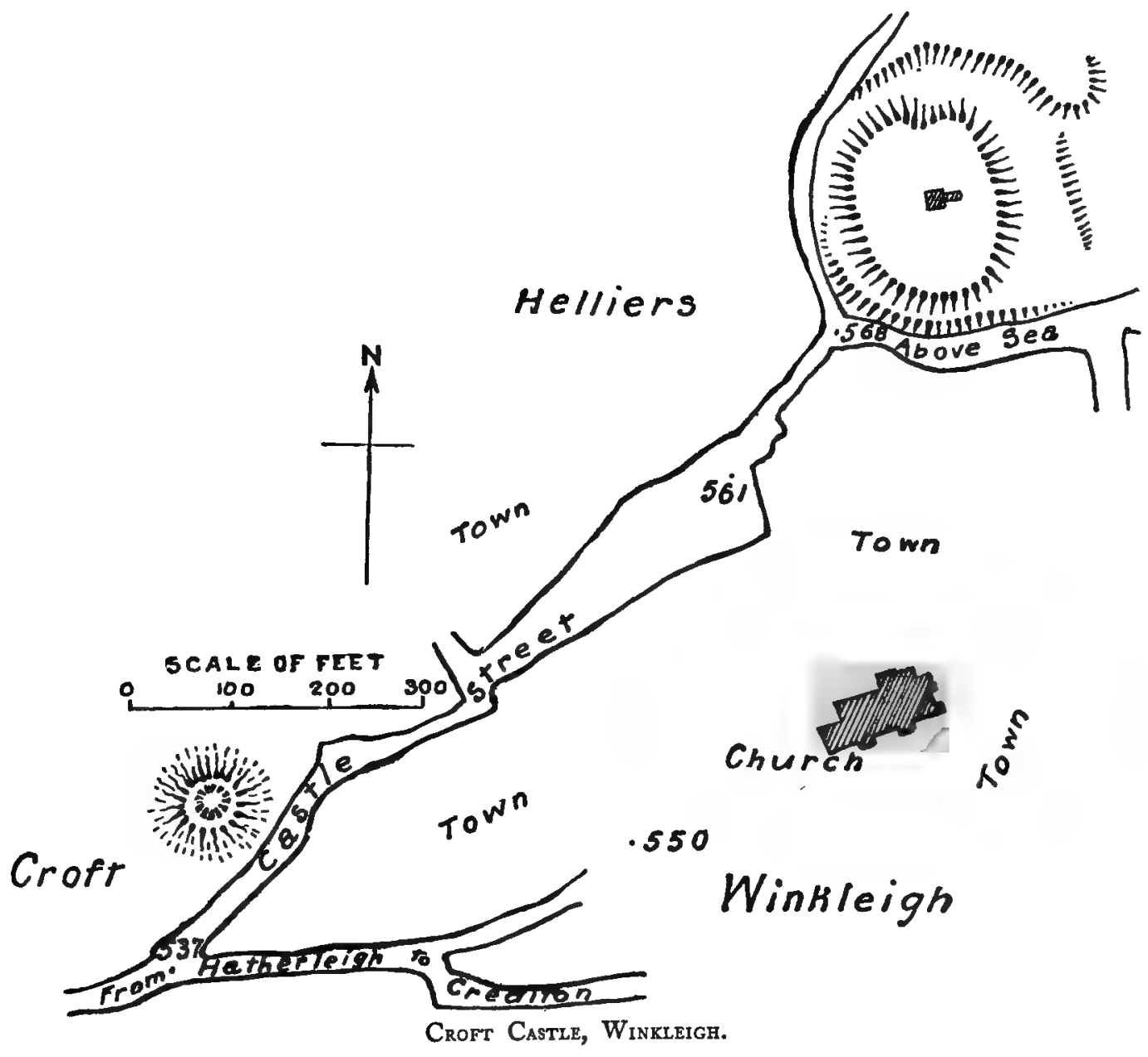

\section{MOUNTS WITH ONE OR MORE ATTACHED COURTS \\ [Class E]}

Bampton (O.S. xxiv, I 5).-Bampton Castle, locally known as 'The Mount,' is situated on the summit of a knoll at the junction of two roads, at the end of the present 'Castle Street.' It stands above the River Batham, or Bathern, a small tributary of the Exe, the confluence being about a quarter of a mile below the town. 


\section{A HISTORY OF DEVONSHIRE}

The mount, with an escarpment of $40 \mathrm{ft}$., is slightly hollowed at the top, having a breastwork $2 \mathrm{ft}$. in height. This occupies a position on the south-west of the site of the basecourt, which is of rectangular form $500 \mathrm{ft}$. by $400 \mathrm{ft}$., with its long axis north-west and south-east. The bailey largely depended for its defensive boundary on the natural height of the hill ; but the artificial protection on the east is of great strength, at which point the natural scarp is $50 \mathrm{ft}$, a broad fosse, and a counterscarp of $6 \mathrm{ft}$. is again strengthened by a natural escarpment of $30 \mathrm{ft}$.

Entrance was probably gained at the south, where the remains of an inner work above an incurved scarp immediately beneath the commanding height of the mount indicates strategical engineering. The incomplete state of the courtyard defences, however, forbids further surmise.

On this site a castle was erected

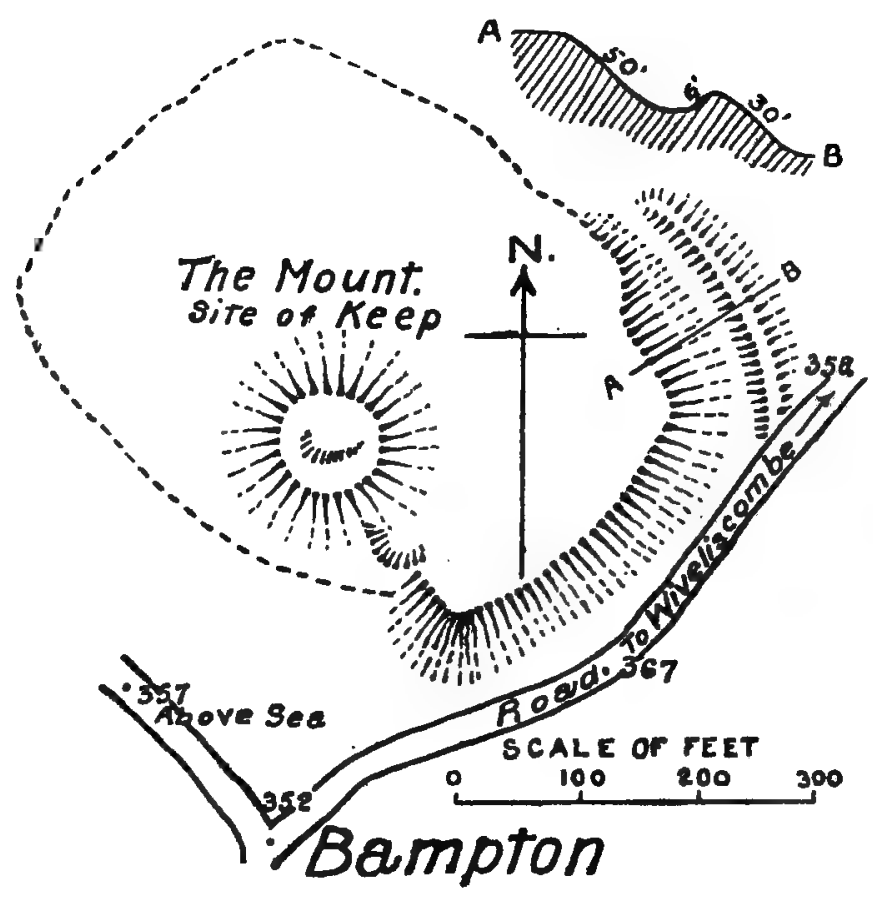

Bampton Castre. in 1336 by one of the Cogan family.

BRIDESTOWE (O.S. lxxxvii, 8).-U Upon the verge of a bold spur of hills of great height, at the foot of which flows the River Lew, is one of the strongest fortifications on the west side of Dartmoor. Within the dense growth of Burley Wood, north of Woodhead and east of Foxcombe Wood, we

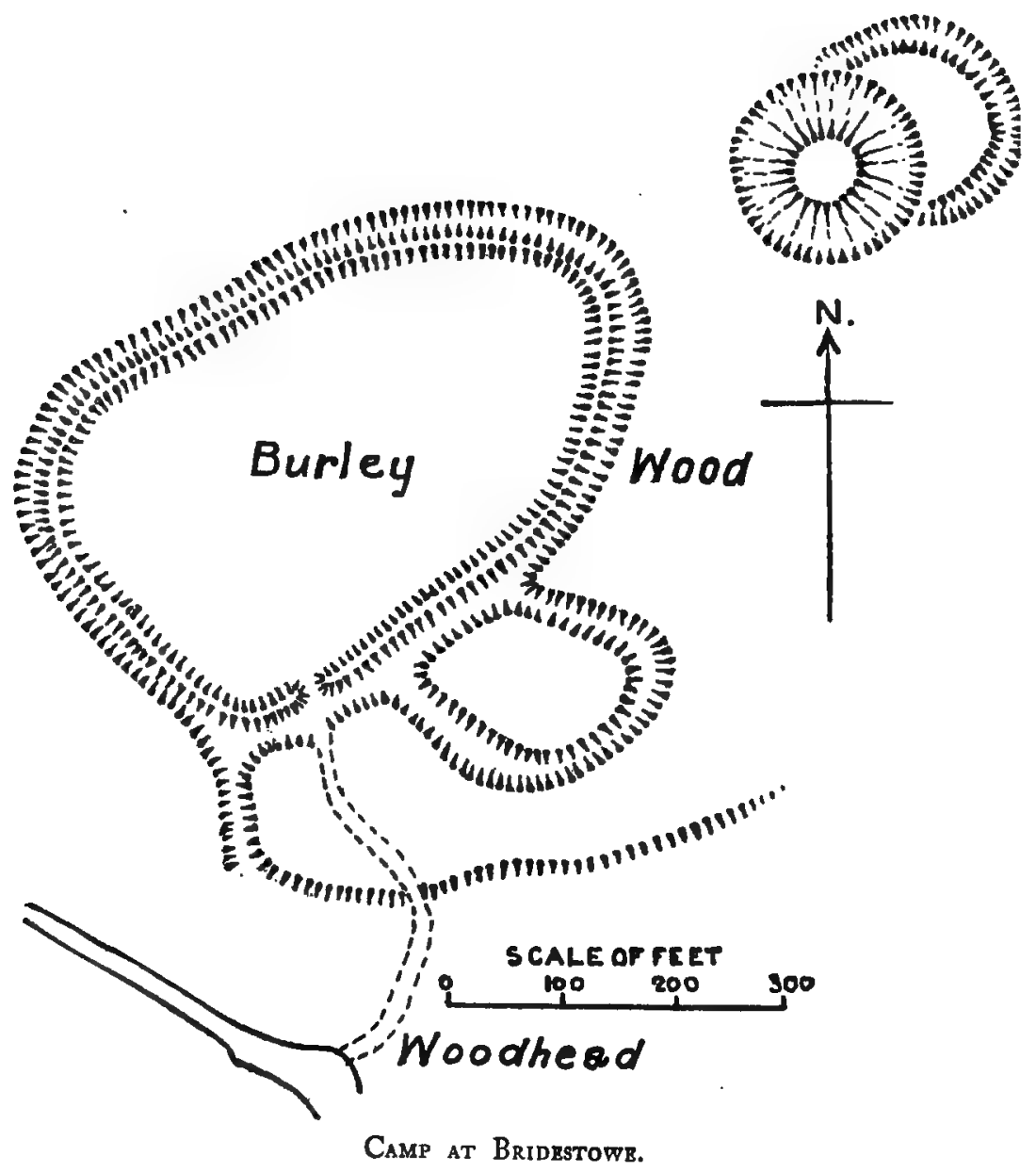

find not only a mount and bailey, but other extensive works which are marked as two separate camps on the Ordnance Survey. These formed but one camp originally, but it is now difficult to trace all the entrenchments. On the margin of the height stands a mount and ditch, with a bailey on the northeast, of crescent-shape, defended by a fosse which joins the circular fosse at its two extremities. On the opposite side - the south-west-is a large court of irregular oval form, surrounded by vallum and fosse; but the latter, on the south-east, extends around an almost quadrangular area, somewhat similar to that which will be seen on Milber Down. At the southernmost point the fosse again branches forth, pro- 

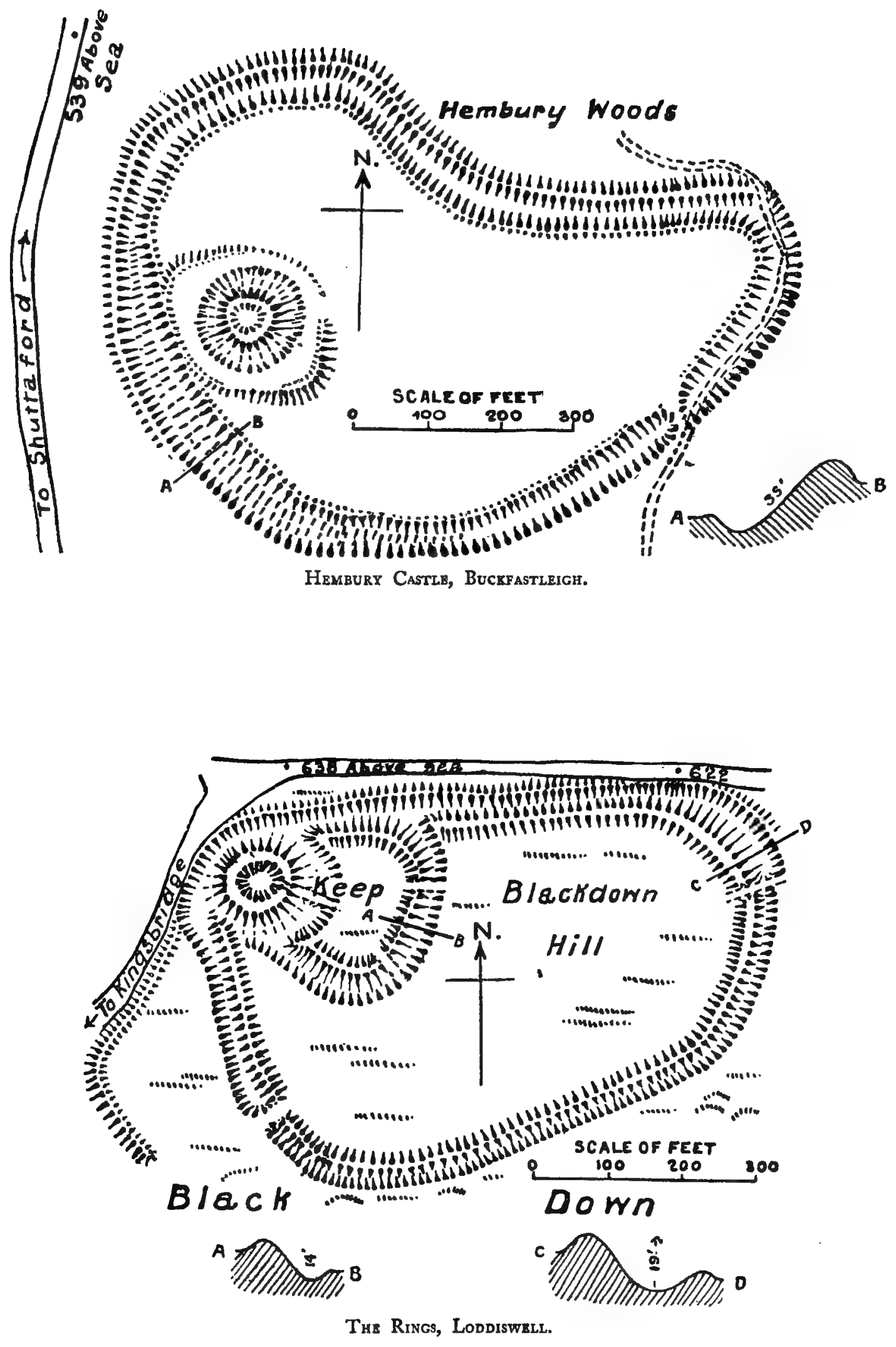


\section{A HISTORY OF DEVONSHIRE}

viding a sunken road to the edge of the hill, the natural scarp of which is assisted by man's handiwork for $500 \mathrm{ft}$. along the south of the camp. The entrance through the rampart lies between the two branches of the fosse, but the path descending from this point is evidently modern.

Buckerell.-Buckerell Knap, with Bushy Knap, is supposed by Mr. Orlando Hutchinson ${ }^{1}$ to have been an outpost to Hembury Fort, near Honiton. Occupying the north-west extremity of a lofty ridge is a circular mount about $200 \mathrm{ft}$. in diameter, rising with the natural scarp of the height. The ridge extends to the south-east, and on this side the mount is protected by three great ditches, cut in crescent form across the whole width of the ridge, the ends of them being supported by the steep declivity. These enclose two small base-courts, and the two inner ditches have each a bank on the edge of the counterscarp.

From the outer fosse the plateau on the top of the ridge gradually descends towards the southeastern extremity, known as 'Bushy Knap,' where another mound of smaller dimensions occupies the point.

Buckfastleigh (O.S. cxiv, 2).- - Hembury Castle, on the brow of Hembury Hill, which is the southern spur of the same range as that on which Chase Castle is situated, has been described as an irregular oblong, but its plan is that understood as kidney-shape. It lies 2 miles north-west of Buckfastleigh, on ground gradually sloping to the south, on the west side of the River Dart, where the natural escarpment greatly conduced to its strength.

'The mount locally called 'Dane's Castle,' at the western side of the camp, is some $44 \mathrm{ft}$. in diameter, and $28 \mathrm{ft}$. at its greatest height, with a hollow centre I $2 \mathrm{ft}$. in depth. An entrance to the centre on the south-east is probably worn by the feet of latter-day campers, who have left ample evidence of their picnics. A fosse encircles the base of the mount, and beyond it are the broken bounds of the first court. In Polwhele's time he tells us that five or six passages led to the praetorium -or mount-from different parts of the camp, but that the fosses had been destroyed and could not be traced; it can therefore be understood how far more difficult it is at the present time. The outer court, enclosing more than 7 acres, is protected on its most assailable side by a fosse $20 \mathrm{ft}$. deep and a heavy agger. Possibly the mount with its base-court is later than the great enclosure which encompasses it.

A bronze celt and sling stones have been found within this stronghold, and many fint implements were discovered in an adjoining field.

EXETER.-The earthworks of Rougemont, the celebrated castle of Exeter, though not strictly of the mount and court type, are kindred thereto, the essential feature being a high knoll, scarped and banked. Clark describes the work thus :-

The sides of the knoll have been scarped, and at the foot of its upper part a deep and broad ditch has been excavated, beyond which, to the north, a second scarp descends to the bottom of the valley. Towards the south, where the ground allowed of and required it, there was a second and outer ditch. The contents of the inner ditch were carried upwards and inwards to form a high bank round the original summit of the knoll. . . . The main ditch towards the north-east and north-west has been filled up and converted into a broad public walk and garden, but the outer or second scarp still remains, and descends to the valley side occupied by the L. and S.W. Railway. Towards the south-east and south the ditch remains unaltered. . . T Towards the east it seems to have been filled up. ${ }^{2}$

We need not here refer to the Norman gatehouse, the bastions, or the masonry erection against the upper bank.

HighweEK (cix, 2).-Castle Dyke, $1 \frac{1}{2}$ miles north-west of Newton Abbot, is the remnant of a mount and bailey fort, on the summit of a hill known as Castle Field. It is the smallest of this class in the county. The top of the mount is surrounded by a rampart, and the entrance to it is on the south-west. The entrance to the bailey was

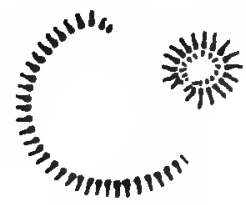

CAstre Dyke,

HiGHWEKK. originally on the north, but half the outer rampart has perished, as according to a plan in the Arcbaeologia, xix, $3^{1} 3$, the bailey surrounded the mount.

Loddiswell (O.S. cxxvi, I4).- ' The Rings,' Black Down Camp, is a strong and extensive fortification on Black Down, nearly $650 \mathrm{ft}$. above the sea at its highest part, $2 \frac{1}{2}$ miles north of Kingsbridge. This earthwork consists of a mount and two baileys.

The mount, with an interior hollow, is surrounded by a fosse crossed by a shallow earthen causeway, giving access to the inner bailey, enclosing about one acre. The latter is defended by a rampart with an escarpment $1_{4} \mathrm{ft}$. high perpendicular measurement, and a broad fosse which joins the ditch of the citadel; two outlets, equidistant from the centre, give egress to the outer court. The outer bailey, containing 10 acres, assumes an irregular elliptical form, and is surrounded by a very strong agger rising in places $16 \mathrm{ft}$. from the

${ }^{1}$ Arch. Assoc. Fourn. xviii, 62.

'Clark, Mediaeval Military Architecture (1884). 


\section{ANCIENT EARTHWORKS}

interior, and descending with an escarpment $30 \mathrm{ft}$. on the slope into a fosse $12 \mathrm{ft}$. wide, which, at the western extremity joins, and is incorporated into, the ditch around the mound.

The entrance on the south-west is protected by a strong outer rampart, supported at its northern end by the fosse of the mound, and curves to a parallel with the bailey defence some $150 \mathrm{ft}$. distant from it. The southern side depends largely upon its natural fastness, where it descends to the River Avon, while the northern-the most assailable side-has a double vallum.

LydFoRd (O.S. lxxxviii, I3).-Lydford Castle. The ancient importance of Lydford is far from demonstrated by the very small village which bears the name.

A portion of the mediaeval entrenchment and the site of one gate are still to be seen; but we may probably look to an earlier date for the most interesting remains extant in Lydford. The ruinous square keep of the mediaeval castle stands upon a mound $18 \mathrm{ft}$. high, which is possibly not long anterior, but the base-court is a small deeply entrenched defence lying to the north-west of the keep. Semi-elliptical in form, the short axis is the edge of a deep ravine, the bank of the Lyd. The keep shows no evidence of a moat, but the lower level of the bailey is protected on each side by a rampart $6 \mathrm{ft} .6 \mathrm{in}$. in height from the inner side, with an escarpment of $23 \mathrm{ft}$. in depth to the base of the fosse. On the south-west side an outer bank $3 \mathrm{ft}$. in height crests the counterscarp.

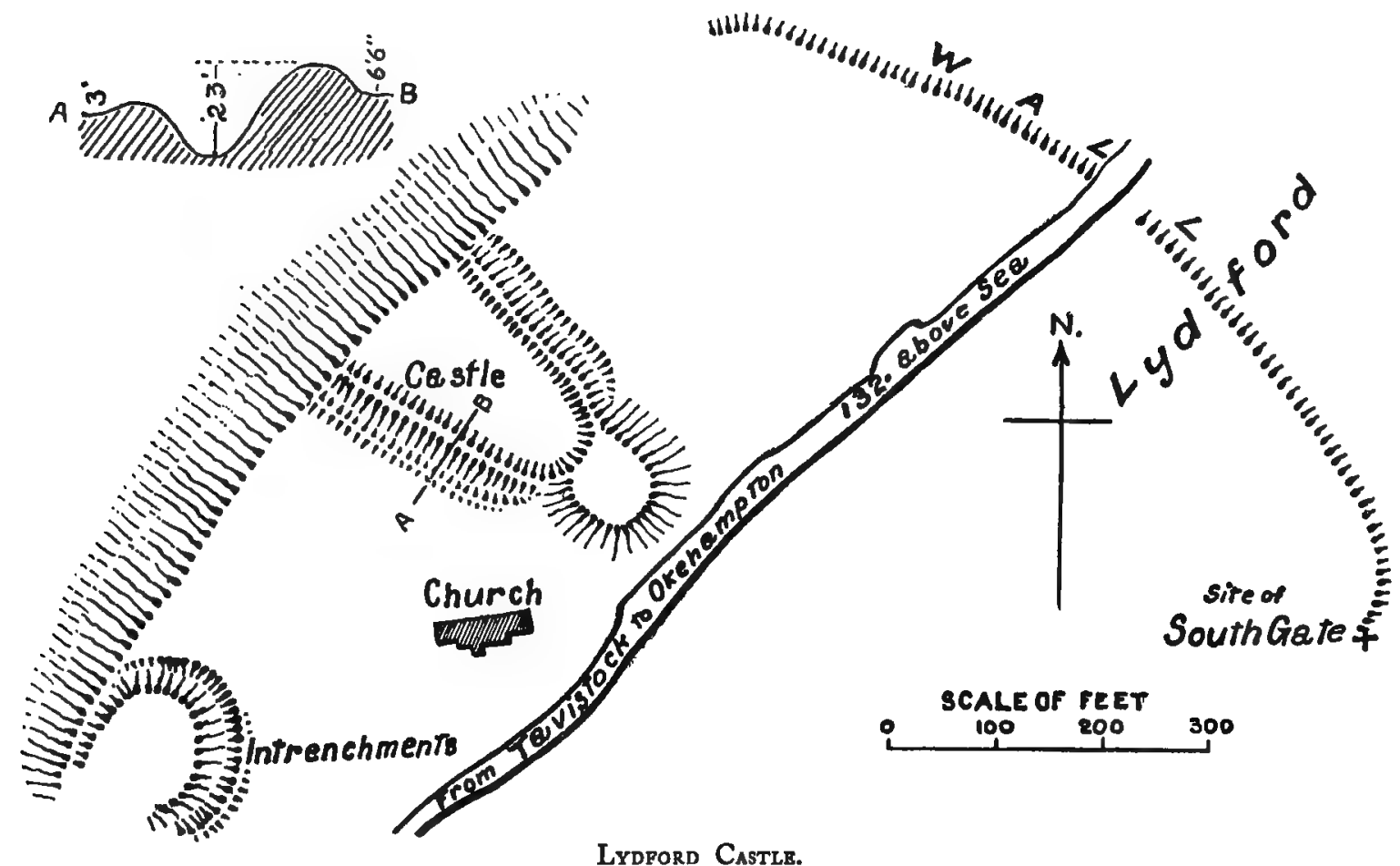

About $300 \mathrm{ft}$. south-west is another work, upon which no structure of the Middle Ages is seen. This consists of an earthern mount, also on the verge of the river bank. The mount is $22 \mathrm{ft}$. in height from the bottom of a fosse-which is $9 \mathrm{ft}$. deep-by which it was originally surrounded; the latter was also strengthened on the land side by a bank $3 \mathrm{ft}$. in height on the counterscarp, of which, part only remains on the east side. In both of these fortifications the ditches and ramparts abruptly terminate on the river height.

Milton Damerel (O.S. xxxix, 8 and 12).-Over 7 miles from Holsworthy, in the direction of Torrington, is a small camp which is included in this class of fortification. Situated on the west bank above the River Torridge to the north-east of Woodford Bridge, in the shade of Woodford Wood, is an entrenchment $200 \mathrm{ft}$. long by $\mathrm{r} 40 \mathrm{ft}$. wide. A bailey surrounded by a ditch is of almost right-angular form on the north-east, but at the opposite is semi-circular, within which is a shallow mount formed on one side by the curve of the ditch and on the side within the bailey by a crescent-shaped bank.

This forms one of a triangular group, the other two camps being respectively in the parishes of Shebbear and Bradford. (Plan on next page.)

Okenampton (O.S. Ixxvi, 8).-Okehampton Castle is situated on the summit of a tongue of rock about one mile south-west of the town, on the left bank of the West Okement. It occupies a very strong natural position which artificial works render well-nigh impregnable. These works 


\section{A HISTORY OF DEVONSHIRE}

consist of half the plan of a mound and base-court abutting on the natural precipitous bank on the south-eastern side, where no other defences are apparent. The lofty demi-mound is crowned by

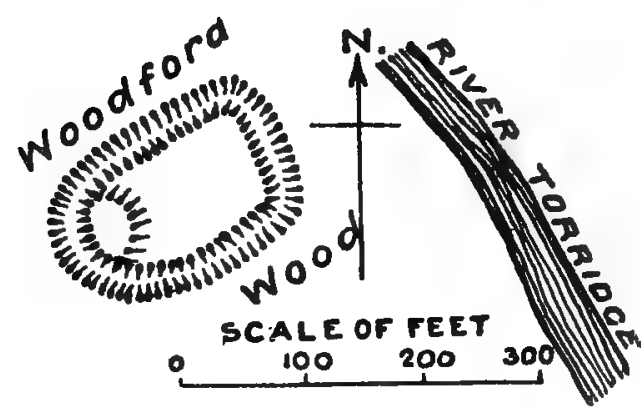

Camp at Militon Damerel. the mellowed ruins of the Norman keep of Baldwin de Brioniis, to whom the site was given by William $\mathrm{I}$, and within the bailey are the remains of the great hall and domestic buildings. The north side is protected by a wide fosse which has, however, lost much of its original appearance by subsequent alterations.

Parracombe.-Holwell Castle, to the south of Parracombe, is 5 miles south-west of Lynton. The mount, which rises to a height of $50 \mathrm{ft}$. on a steep escarpment and has a table top, was originally encompassed by a fosse $6 \mathrm{ft}$. in depth ; this, however, on those sides not touched by the bailey, has been destroyed by modern banking. The area of the base-court is protected by an agger $10 \mathrm{ft}$. in height from the interior and $20 \mathrm{ft}$. deep on the exterior ; this extends to the fosse of the mount at points almost at opposite ends of the axis of the latter.

At the entrance on the north-west the rampart incurves at either side.

Plympton Maurice (O.S. cxxiv, 7).-Plympton Castle on the south side of the Tory Brook, and $\mathbf{I} \frac{1}{2}$ miles from the River Plym, embodies the remains of an early camp of the keep and bailey class of fortifications, adapted by Richard de Redvers to his Norman stronghold.

The apparent lack of defences on the broad waters of Plymouth Sound may be accounted for by modern alterations on sites of great national importance; even the tumuli, which were probably numerous in this neighbourhood, have been obliterated except in two localities -one in the parish of Plymstock,

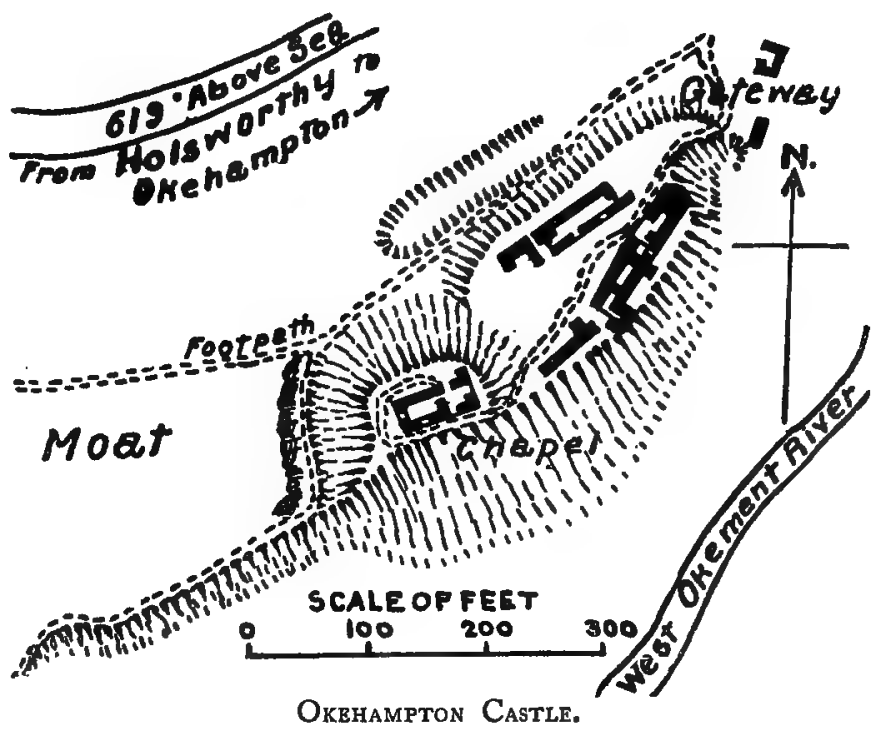
near the Sound, and others on Crownhill Down in the parish of Plympton St. Mary.

Plympton Earle, commemorating in its name the feudal period, has, however, preserved its early

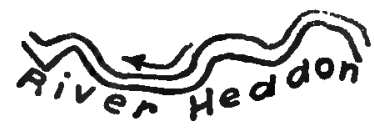

defences beneath its mediaeval robe. The conical mount, on which is a fragment of the Norman keep, retains a portion of its fosse on its western side, and on the same side extends the ancient base-court or bailey, forming a quadrangular area with its early rampart and fosse strengthened by subsequent

- Holy Well

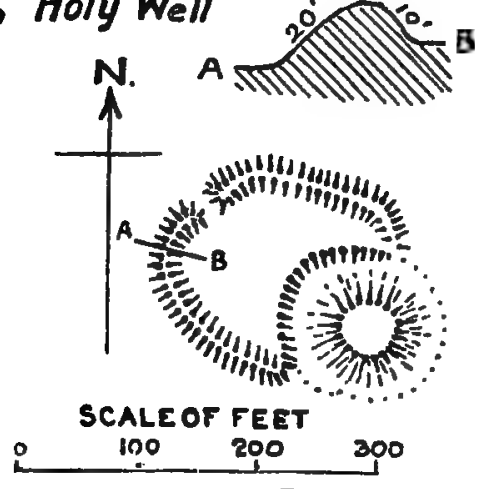

Holwell Castre, Parracombe. art and partially destroyed by succeeding improvements (!). The agger on the south side turns inward as it approaches the mount, but it cannot be fully traced because of latterday alterations.

ShebBear (O.S. $x l, 9)$.-Durpley Castle, almost due south of Hembury and Io miles from Bideford, is situated between Higher Combe and Moortown. 'The Castle' occupies the top of a very high conical hill, the apex of which forms the mount or keep, $18 \mathrm{ft}$. in height, perpendicular measurement. It has the crater-like hollow in its centre, nearly $30 \mathrm{ft}$. in diameter and $12 \mathrm{ft}$. deep. The mound is surrounded by a fosse, and a base-court on the western side, of an area a little over an acre, is defended by a double vallum. The bailey, of a crescent plan, has a breastwork rising between three and four feet, descending on the outer side $1 \circ \mathrm{ft}$., perpendicular measurement, into a fosse $6 \mathrm{ft}$. deep at its highest point ; this fosse runs into the fosse of the mount at the north and south junctions. From the outer vallum the ground 


\section{ANCIENT EARTHWORKS}

rapidly descends with a natural scarp, making the fort almost inaccessible by reason of its steepness and irregularities, except on the west side, from which the camp is easily approached by a gentle slope. On this side is the entrance into the fosse, but the gateway into the bailey is towards the south, thus allowing of no direct inlet.

The base of the hill is surrounded on three sides by two streams of the Coombe Lake which, joining on the southwest side, fow into the River Torridge. TOTNES (O.S. cxxi, 5).-Totnes Castle. Situated on the ancient road

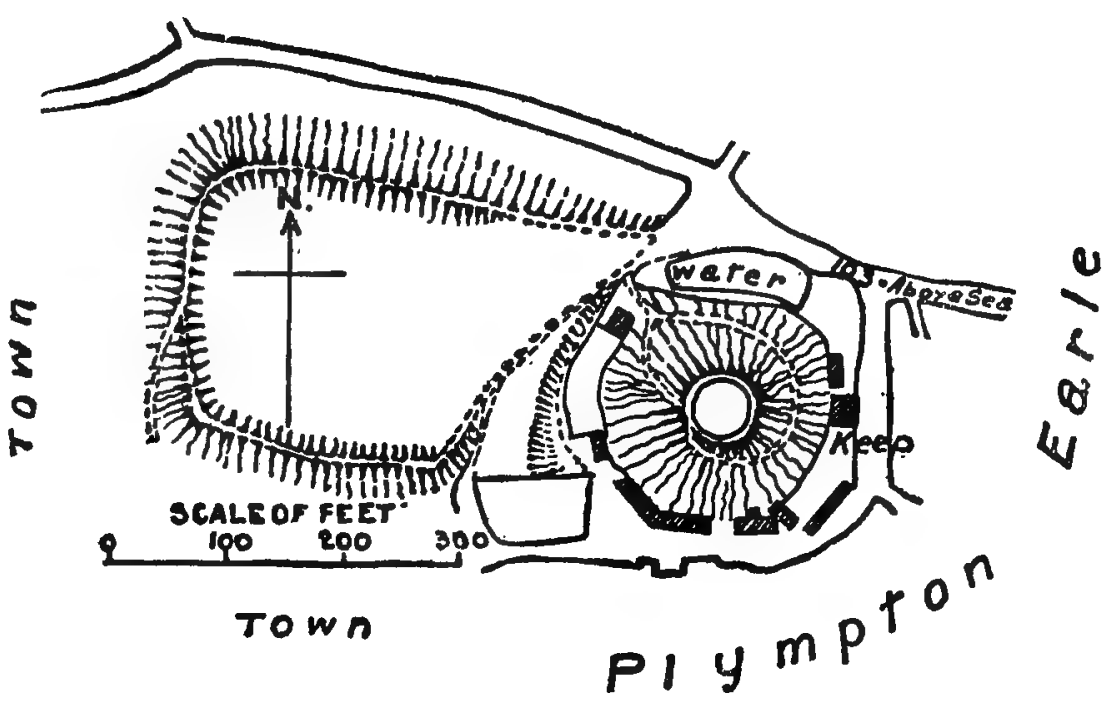
from Exeter to the

Tamar, the town of Totnes is built on the acclivity of the right bank of the River Dart.

At the north-west of the town and on the summit of the hill, stand the great mount with its bailey, and the castle built by the Norman baron Judhael, who assumed the surname of 'de Totnes.'

The fosse surrounding the mount is to a great extent destroyed, buildings being erected on part of its site, although we were able to trace a certain length of it in the gardens of the dwellinghouses. The fosse around the bailey to the north-west of the mount is exceedingly strong, varying in depth to $20 \mathrm{ft}$. A promenade beneath venerable trees has been made on the vallum since the last military occupation of the castle by General Goring during the Great Rebellion. From the rampart is a natural descent of very great depth to the town. The lines of a second and larger court at the base of the castle hill are nearly obliterated by buildings. (Plan on next page).

WembWorthy (O.S. xlii, 8). - In Heywood Wood, on the west bank of the River Taw, in the park of Eggesford House, 2 miles south-west of Chulmleigh, are two earthworks which come within this class, and also a small entrenchment which lies between them.

The northernmost of these is a circular mount and bailey, so covered in dense undergrowth that it was with difficulty the results were obtained. The mount, $160 \mathrm{ft}$. in diameter, is $26 \mathrm{ft}$. perpendicular height, and the summit, hollow, is surrounded by a rampart which, at its highest point on the west, rises $12 \mathrm{ft}$. from the interior. The fosse around the mount is generally $9 \mathrm{ft}$. in

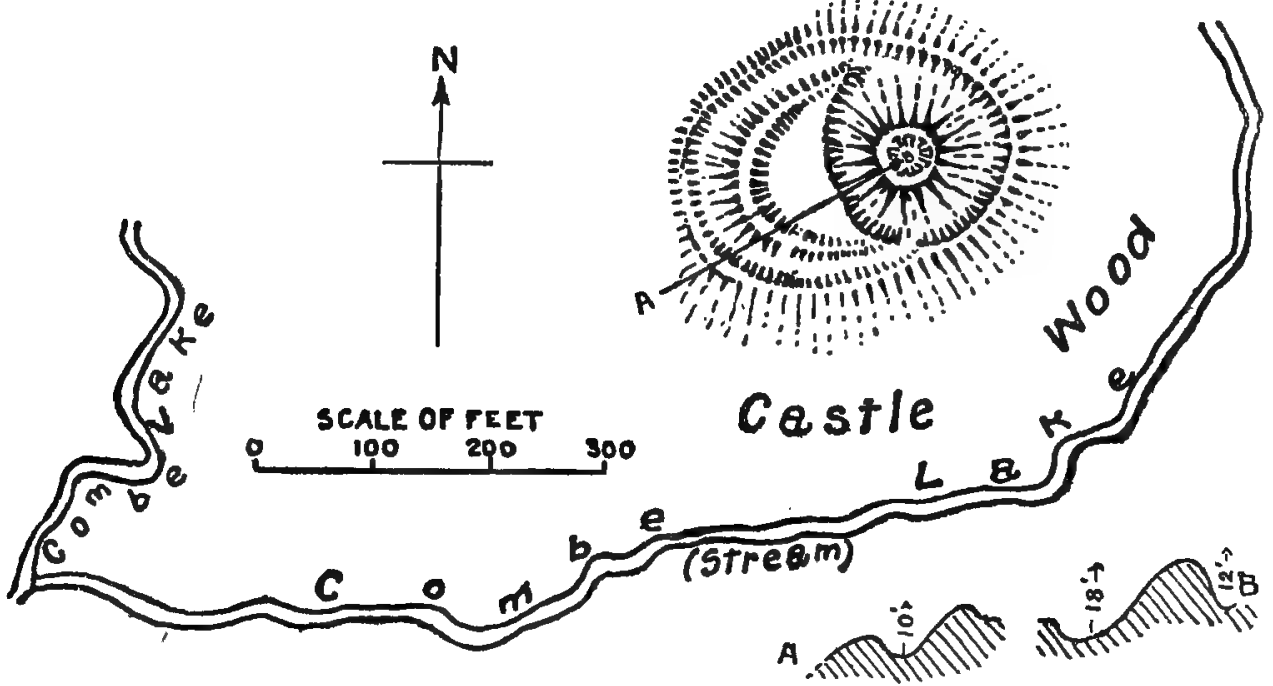

Durpley Castre, Shrbaear. 


\section{A HISTORY OF DEVONSHIRE}

depth, but on the north side it is $18 \mathrm{ft}$. deep and $30 \mathrm{ft}$. wide. The bailey, of horseshoe-form, lies on the north-eastby-east, and is entered from the keep at its north-east juncture with the mount-fosse. It is surrounded by a rampart from one to six feet in height, the lowest portion being on the south. The escarpment to the base of the fosse is $16 \mathrm{ft}$. perpendicular height, and the fosse is $5 \mathrm{ft}$. deep.

Two entrances pass through the defences of the court, at the north and at the south-east, both of them passing the ditch and rampart in an oblique direction as a better defence against assailants.

On the north side an additional defence was found to be necessary for this, the most exposed position. Here a rampart $2 \mathrm{ft}$. high and a ditch $3 \mathrm{ft}$. deep and the same width, makes an arc fully $200 \mathrm{ft}$. long, from the northern entrance to the fosse of the mount. ${ }^{1}$

(O.S. xlii, I 2).-Within the same wood the southern fort is also of the mount and court type, yet totally dissimilar to the last. In plan it is almost rectangular and the mount elliptical. On the edge of the steep bank of the Taw the natural features have been utilized and considered all the defence necessary; but on the west side, where the ground rises higher than the base-court of the camp, strong earthworks have been constructed.

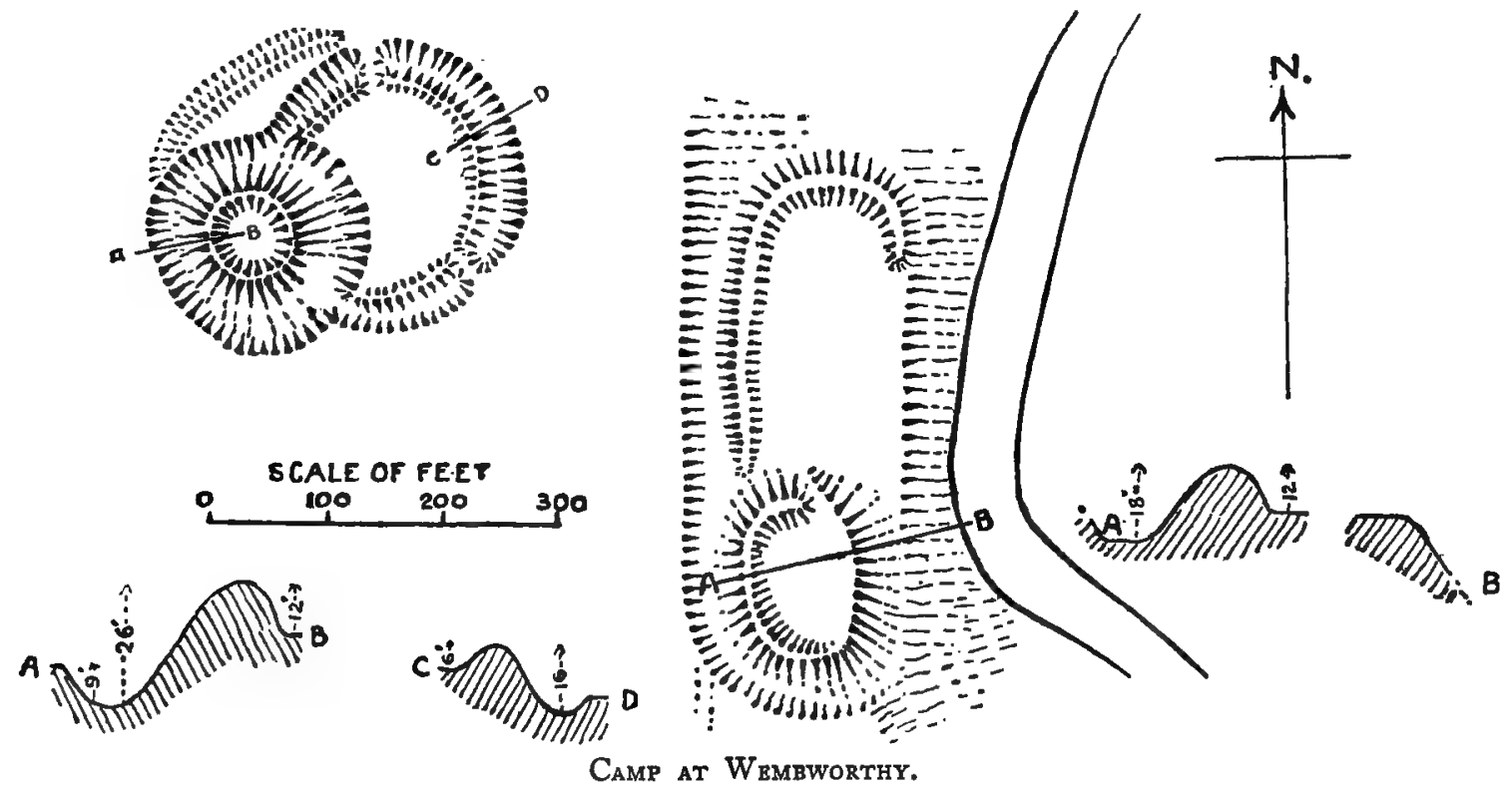

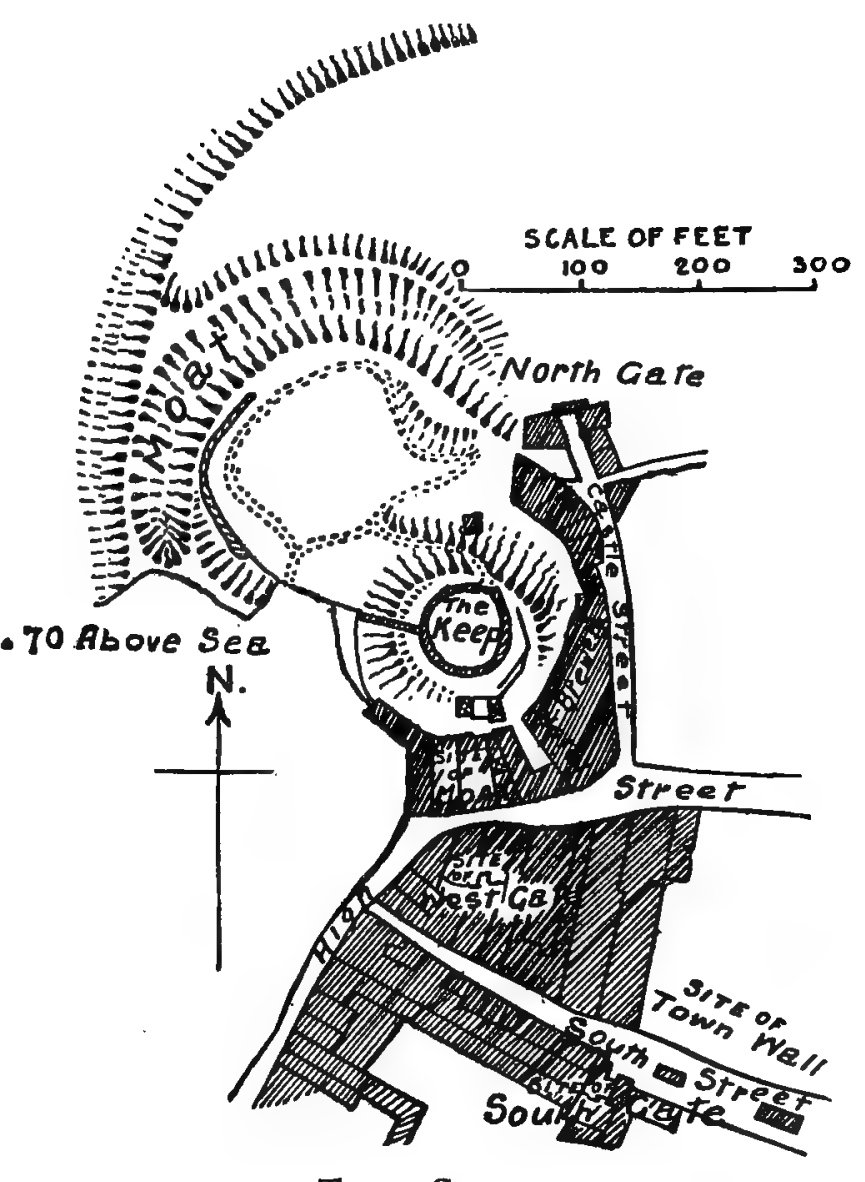

Totnes Castle.

A fosse or sunken road is cut into the sloping ground round the south end and west side of the fortress, each extremity of the fosse issuing upon the contour of the hill, and isolating the portion from the hill-side.

${ }^{1}$ It is possible that the two ways cut through the rampart of the bailey are modern and that the additional work on the north-west was a timber defended barbican covering the only access to the stronghold. Compare Mexborough Castle in Yorkshire. 


\section{ANCIENT EARTHWORKS}

At the south the mount and its western agger rise $18 \mathrm{ft}$. above the fosse, which height is attained by an inclined path rising from the base-court to the summit in a manner similar to that at Kenwith Castle; but the rampart of the mount, absent on the river side, rises from the south to west and north, where it is $12 \mathrm{ft}$. in height. The rectangular court is defended on its assailable sides by a strong vallum of varying height, but averaging $6 \mathrm{ft}$. The entrance is on the west, where a path curves round the base of the mount, passing from the sunk road into the court. Traces of an ancient path down to the river are still visible.

The fortress is skilfully planned, and although modern paths have been laid out around it, they have failed to efface the original scheme.

It is evident that these two fortifications were constructed to be used in conjunction, and were not hostile the one to the other, defending a long reach of the river and preserving communication with each other, for which purpose a slightly curved agger, $45 \mathrm{ft}$. long, was erected between them, facing the river.

\section{HOMESTEAD MOATS}

\section{[Class F]}

Insignificant fragments only remain of earthwork defences to residential mansions and bartons, and the lack of such may possibly be explained by the configuration of Devonshire, which spared the necessity of such surroundings as were required in the eastern and other lowland counties.

Remains of such works may be seen at :-

BRADFORD (O.S. 1, 12 and li, 9).- -South-west of Bramber Wood, near a large fish-pond.

Colyton (O.S. lxxi, I 5).-Colombe Castle.

CORnworthy (O.S. cxxvii, 2).-Cornworthy Court.

Ditrisham (O.S. cxxvii, 8).- Fragments of a moat of the Old Barton upon which site Dittisham Court now stands.

Highampton (O.S. li, 8 and lii, 5).-West Totleigh Mansion, south-west of Hele.

Huntsham (O.S. xxxiv, 4).-On Mason's Moor.

LYDFORD (O.S. cvii, 9).-Manor House, Bradninch.

Malborough.-Salcombe Castle.

Sampford Peverell (O.S. xxxv, 14).-Sampford Barton. The barton was destroyed in 1775 .

STokenham.-Near the Vicarage.

UPton Pyne (O.S. lxviii, I).-North-east of Shute.

\section{STRONGER ENCLOSURE OF 'HOMESTEAD' TYPE}

\section{[Class G]}

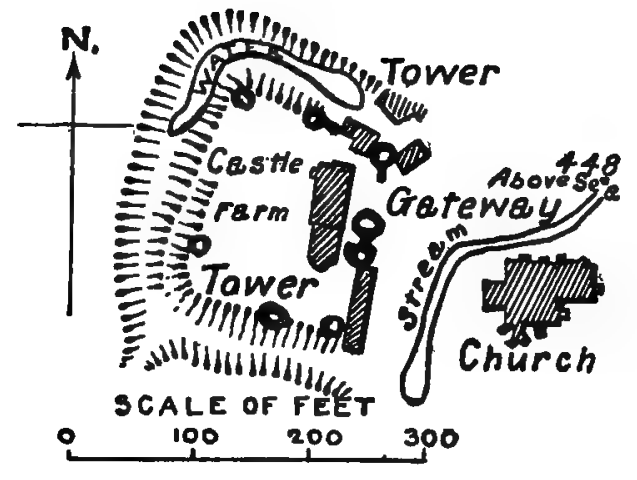

Hemyock Castle.

HeмYock (O.S. xlvii, 3).-Hemyock, or Hemmick Castle, in the vale of Hemyock at the foot of Black Down Hills, has considerable remains of its ancient earthwork extant.

Around its bastions on the north, west, and south, is a strong moat ro ft. deep, and on its western side the escarpment is continued $4 \mathrm{ft}$. higher above a path in its surface. This moat was fed by a stream which flows on the eastern side of the keep. There are indications that the moat was continued at the south-west corner, but its course cannot be followed.

Hemyock Castle is said to have been occupied by the Parliamentary forces.

At the north of the parish is a large cairn.

\section{VILLAGE SITES}

\section{[Class H]}

In the parishes of Ashburton, Bovey Tracey, Manaton, and Petertavy, are the sites of British villages, of which Grimspound, in the parish of Manaton, is the most marvellous example of a village of a pastoral people, probably of the Neolithic Age, but as the surrounding walls do not 


\section{A HISTORY OF DEVONSHIRE}

partake of the character of fortifications, only defending the inhabitants and their herds from the raids of the wolf and the boar, these will, in common with all those structures known as 'Pounds,' fall within the section devoted to Pre-historic Man.

\section{MISCELLANEOUS EARTHWORKS}

\section{[Class X]}

Under this category we include those ruins of segmental trenches found in places on the cliffs, and others which do not fall into the foregoing classes.

Braunton (O.S. viii, 10).-U Upon the shoulder of the cliff above Saunton Sands, on the southern side of a point projecting westwards into the Bristol Channelabout eight miles in a straight line from Barnstapleare five parallel banks extending I,400 ft., running north-west by west to south-east by east, with another bank obliquely crossing them. Their height varies from one to three feet, and the principal agger rises to $4 \mathrm{ft}$. at its south-

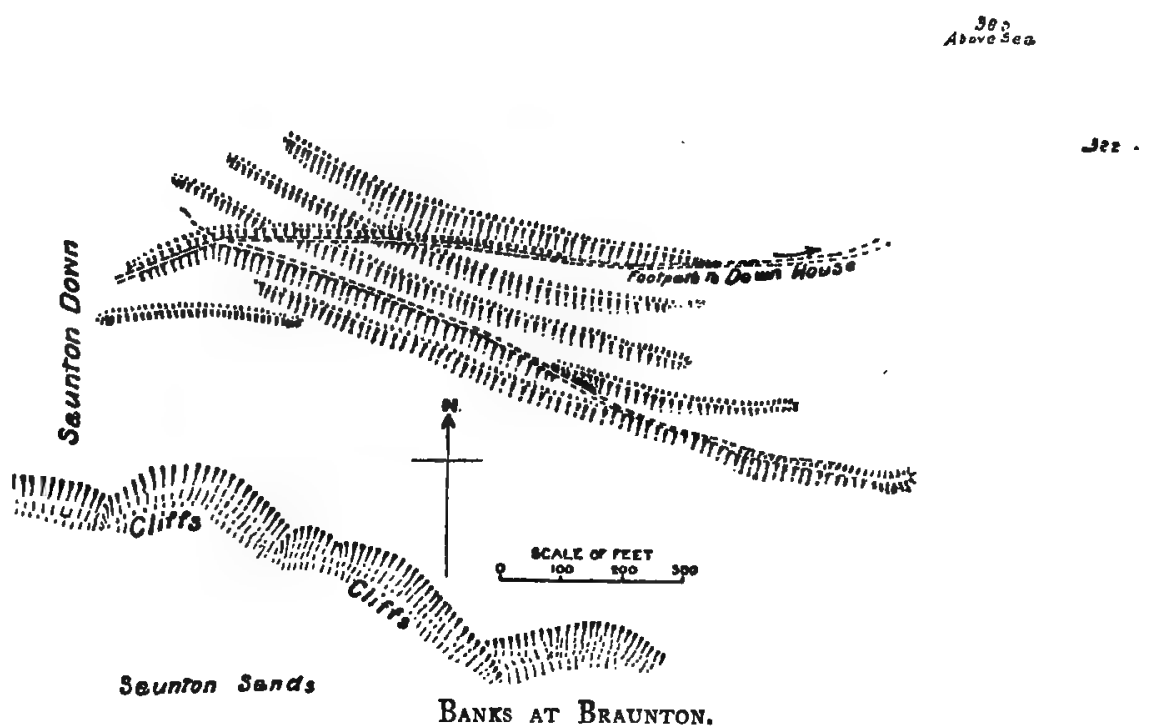
eastern extremity.

Bants at Braunton.

Above the most inland entrenchment the ground continues to rise, and below the outermost the rounded descent is so great that it is difficult to retain a foothold. The position overlooks the long stretch of sand dunes and burrows which provide an inviting beach for disembarkation and cover for an enemy.

That the defences have been reduced in strength in modern times is evident from the description left to us by Lysons, who says that in his day this camp, which was called the 'The Castle,' and covered an area of 4 acres, had a large vallum and fosse on the north, the only accessible side. ${ }^{1}$

Flint-flakes have been found in abundance at this camp.

Dartmouth (O.S. cxxxiv, r).-Gallants' Bower. Overlooking
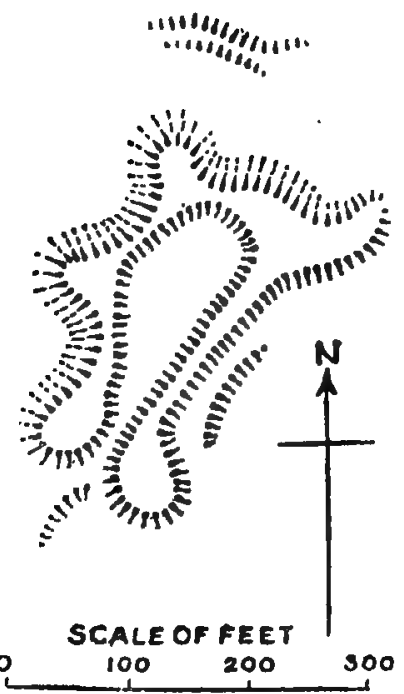

Gallayts Bowzr, Dartmouth. the entrance to Dartmouth Harbour on the east, some 300 feet above the town, and $600 \mathrm{ft}$. inland from Castle Cove, is an earthwork of somewhat fantastic shape. The camp is kite-shaped, with the entrance at the south-west, but the broad rampart widens into natural platforms at the four corners and in the middle of the west side. It is defended by a natural scarp on the north and west, and signs of additional works are at the entrance and two sides.

This camp is mentioned by Fairfax in one of his despatches to the Parliament.

East Portlemouth (O.S. cxxxviii, 3).-On Rickham Common, opposite Salcombe Harbour and Blackstone, are seventeen lines of entrenchments of the same character as those at Braunton. They run more or less parallel to the coast, from the south-west to the north-east. These banks range from $200 \mathrm{ft}$. to $600 \mathrm{ft}$. in length, and the one nearest to the line of the cliff has a semicircular terrace at the south-western end.

Southleigh (O.S. 1xxxiii, 5 and 9) and Branscombe.-Two miles inland from the south coast, and north of Berry Cliff Camp, stands the Three Horseshoes Inn, on the road from Ottery St. Mary to Colyford. Both north and south of this inn is an extensive

${ }^{1}$ Lysons, Mag. Brit. vi, 352. 


\section{ANCIENT EARTHWORKS}

earthwork originally consisting of a rampart and ditch. That to the south is now $900 \mathrm{ft}$. in length, and that to the north $960 \mathrm{ft}$. The end of the latter, on the declivity of a hill, turns slightly to the east. The inhabitants say that when the work was in a more perfect condition, the vallum was from twelve to fifteen feet high, and that the ditch was on the eastern side. The suggestion that this was the western side of a vast camp may be dismissed ; the wall was evidently a portion of a scheme of defence, forming part of a group of works, from Berry Cliff and Castle Close on the south to Blackbury Castle on the north. With the fosse on the east the probable enemy was looked for from the direction of the River Axe; while the Hanna ditches and Seaton Down trenches were possibly outposts. All these works are pressed into service for the battle of Brunanburgh by those who advocate this as the site of the engagement.

Seaton and BeER (O.S. lxxxiii, 7).-On the crown of the hill above Colyford, about I $\frac{1}{2}$ miles north-west of Seaton, and close to the great road from Colyford to Exeter, are the remains of two lines of entrenchments, drawn across Seaton Down. The first is an agger $770 \mathrm{ft}$. long, and $33 \mathrm{ft}$. on the slope of the escarpment, with a fosse on the south side. The second, $466 \mathrm{ft}$. to the north of the latter, is of the same character but only I $30 \mathrm{ft}$. in length. It has been suggested that the latter was never completed; but the cultivation surrounding it, and at the present time reducing its proportions, is probably accountable for its restricted limits.

ShUTE.-Upon Shute Hill, just above Icknield Street, traces of entrenchments are along its north-eastern side, opposite 'Dane's Hill.'

Uffculme.-Pixie Garden. Lysons mentions a small earthwork on Uffculme Down (about seven miles east of Tiverton) which was $60 \mathrm{ft}$. square, surrounded by banks $2 \mathrm{ft}$. high. It was divided into four compartments ; in the middle of each was a raised oval bank, and the square was open at the corners. ${ }^{1}$ This was destroyed early in the nineteenth century.

In $186 \mathrm{I}^{\prime} \mathrm{Mr}$. Hutchinson found an old man who remembered it, and described it as above with the addition of a mound in the centre. ${ }^{2}$

\section{TUMULI}

Asmburton.-Tumuli remains in Aweswell Wood on the E. side of the River Dart, and one on Horridge Common.

Ashbury.-A tumulus on Wadland Down.

Ashreigney.-Three large tumuli occupy a position on the SE. corner of Beaford Moor, and two others, one of which is of considerable size, are to the S. of Riddlecombe Moor, near the road from Torrington to Winkleigh.

Ashwater. - Two large barrows lie to the $W$. of Ash Cottage and Oak Cottage near the road leading to Hemscott, and another of a smaller size some distance to the S. Three large tumuli are on Langaford Moor, and one other to the N. of them.

Awliscombe. - A tumulus to the NW. of Rowbarrow Plantation.

Aylesbeare.-A tumulus near the Roman road from Exeter to Lyme Regis.

Beaworthy. - North of Halwill and Beaworthy Railway Station is the 'Great Halwill Barrow,' to the $\mathbf{N}$. of which other tumuli are situated.

Berrynarbor.-T Tumuli are on Berry Down, to the $W$. of the road from Barnstaple to Combe Martin ; and one to the SW. of Woolscott Barton.

Bishopsteignton.-Three tumuli N. of Humber Down.

Bishops Tawton.-A tumulus is at Codden Beacon, on Codden Hill.

Bittadon.-Eight large tumuli are situated to the N. of Narracote, near Centery Lane ; others to the S. of Clannaborough Plantation and N. of Frogmore; and one to the NE. of Gratton Plantation.

Bover $T_{\text {RACEY. }}$-To the $W$. of Owlacombe and NE. of Haytor Vale is the tumulus known as 'Owlacombe Barrow.'

Bradworthy.-A tumulus lies to the W. of West Down Village, and W. of the road from Bideford to Holsworthy, and another to the NE. of Dural.

Branscombe.-Tumuli are to the S. of Berry Barton, and one at the western extremity of Berry Down Camp.

Bratton Clovelly.-One barrow lies to the SE. of Northcombe Cottage, and another to the W. of Broadbury Castle.

Bratton Fleming.- - Tumuli are found in this parish on Bratton Down to the N. of Little Bray Cross and S. of 'Cape of Good Hope'; to the S. and SE. of Nightcott Cross. Three are situated to the E. and NE. of Stowford Cross, one lies between Kipscombe, and North Kipscombe, while others are to the N. of Ridge Gate.

$$
{ }^{1} \text { Lysons, Mag. Brit. vi, } 353 .
$$

arch. Assoc. Journ. xviii, 63. 


\section{A HISTORY OF DEVONSHIRE}

Brendon.-Two positions on Cheriton Ridge are each occupied by a tumulus, one is on Pig Hill to the N. of Halcomb Burrows, and another to the N. of Middle Hill, Farley Hill. Two tumuli are on Thornworthy Common, two are E. of Folly, and one on Brendon Common between Hoaroak Water and Farley Water.

BRIDGERULE EAST.-A barrow is situated to the SW. of Hollaton.

Broadwood WIDGer.-One tumulus is to the S. of Kennymoor Plantation and E. of the 'Old Quarry.'

BuckfastLEIGH.-Tumuli are over Buckfastleigh Moor.

Buckland Brewer.-A tumulus is on the W. of Ashbury and SW. of Hembury Castle.

Buckland Monachorum.-Two barrows are on Roborough Down.

Burrington.-At Moorland Gate on Burrington Moor two tumuli lie at the junction of the three parishes of Burrington, Roborough, and Ashreigney. There are other tumuli on the same moor to the SW. of Burrington Moor Cross.

Chagrord.-A tumulus is on Water Hill, W. of the road from Tavistock to Moreton Hampstead, on the bounds of the parishes of Chagford and Lydford.

Challacombe.- 'Chapman Barrows,' a dozen very large tumuli, some of them being $12 \mathrm{ft}$. in height, and $300 \mathrm{ft}$. in circumference, extending into Lynton Parish, to the N. of Radworthy and Challacombe Common. Others are on the same common, one known as 'Holwell Barrow' is near Holwell Rocks ; 'Longstone Barrow', consisting of three tumuli ; and 'Wood Barrow' to the S. of Woodbarrow Hangings. Others are on Challacombe Down, NW. of Higher Down.

Chudleigh.-A tumulus SE. of Harcombe.

Chulmleigh.-Two tumuli are on Horridge Moor.

Churchstow.-A tumulus is to the N. of Sorley.

CLAwton.-Tumuli are situated on Affaland Moor, and others farther N. to the W. and NW. of Leworthy.

Crovelty.-A tumulus is to the $\mathrm{W}$. of the Clovelly Dykes.

Combe Martin.- ' Holdstone Barrows' on Holdstone Hill, Holdstone Down.

CombPyne.-Tumuli are to the S. of Five Barrow Gate.

Cornwood.-On Penn Moor a single tumulus is S. of Broadall Head and W. of Dendle's Waste, and others are situated at 'Stalldown Barrow.' One is to the NE. of Yadsworthy, and another SE. of Shavercombe Head.

Countisbury.- Tumuli are on Cosgate Hill, between the Combe and Ashton Cleave; and one is on Countisbury Common to the NW. of Dogsworthy.

Dawlish.- Two tumuli are on the eastern side of the camp called 'Castle Dyke.'

Dunkeswell.- 'Row Barrow' Dunkeswell Turbary, and tumulus to the SW. corner of the same.

EAST ANstey.- 'Anstey Barrow' is situated on East Anstey Common on the Liscombe allotment. Another tumulus is to the W. of New Moor Cross, also one SE. of Bussell's Moor Cottage.

EAst Down.- - Two tumuli are on Mattock Down to the SW. of Long Lane Cottage, a spot which is supposed to have been the scene of a battle.

EAst PUTFord.-A tumulus is situated near Wrangworthy Cross to the N. of Middle Hills, and one by Common Moor Cottage. 'Bush Barrow' is on the Common Moor SW. of Wrangworthy, and another at Higher Narracott.

East WorLington.-One small and three large tumuli are to the $\mathrm{N}$. of Middle Burrow, and NE. of West Burrow Moor. There are also two others to the W. of Middle Burrow.

FARWaY.-Tumuli are found on Broad Down, some to the E. of Mincombe Posts Plantation, some NE. and W. of Runcombe Corner, and seven in a line-N. to S. - near the same spot. Others are on Farway Hill.

GermansweEk.-A barrow is on the NW. of Higher Eworthy.

Gitrisham.-Tumuli are on Gittisham Hill, near the road from Ottery St. Mary to Lyme Regis.

HaLweII.-Tumuli are in Stanborough Brake and Bickleigh Brake, near the two camps in this neighbourhood.

Halwill. - Foxhole Barrow' is to the SW. of Lord's Waste; 'Upcott Barrow' NW. of Rowdon and E. of Upcott Cross; 'Hender Barrow' is near the junction of Okehampton and Launceston Roads; and one tumulus is on. Halwill Moor.

HARFord.-Tumuli are on Piles Hill; and others to the N. of Sharp Tor on Butterton Hill and Wetherdon Hill.

HARTLAND.-Tumuli are scattered over this parish, one to the W. of East Yagland, one E. of West Yagland, one on the NW. of the road between those two villages, and one near Harbour Cross. Four lie to the W. of Welsford Moor, and three on Bursdon Moor to the $\mathrm{NE}$. of Lutsford. One is near the road from Stratton towards the E., one to the NW., and another to the SW. of Gorwin, and two S. of Welsford, and SW. of Higher Welsford. 


\section{ANCIENT EARTHWORKS}

High BraY.-Tumuli are on the NE. of Whitefield Down, and others, with 'Satta Barrow,' on Bray Common SE. of Muxworthy Ridge. Another is on Fullaford Down near the Five Cross Way.

Holne. - Tumuli are on Holne Moor to the S. and SW. of Venford Bridge, and at Holne Lee.

HoLswORTHY.- 'Ugworthy Barrow' is on Ugworthy Moor to the W. of Ugworthy; and another on the SW. of the village.

Hursh.-One tumulus is in this parish.

Huntshaw. - Tumuli are situated to the NW. of Darracott Moor, and to the S. of Haycroft.

Irsington.-Tumuli are situated near Rippon Tor. A brass celt was found in this parish.

IPPLEPEN.-One tumulus is to the S. of Darnafield Cross, and another NW. of Rowdon Plantation. A celt of copper was found in this parish in 1820 .

Knowstone.-Tumuli lie between New Moor Plantation and Owaldborough Moor, and on Whitefield Moor.

LeW Trenchard.-One barrow is to the SW. of Westdown Pool.

Lundy IsLAND.-A tumulus is to the W. of Three Quarter Wall, and one to the E. on Tibbet's Hill.

LuppitT.-A barrow is on Hartridge, E. of Shelf Farm.

LYDFORD.-Many tumuli remain in this extensive parish : 'Quintin's Man Tumulus' is to the W. of Varracombe, Dartmoor Forest ; one is on Hanging-stone Hill to the $\mathbf{N}$. of White Horse Hill ; and another NW. of Walern Oak. A tumulus is to the E. of Blacklake Brook; one on Dartmoor SW. of Moorlands ; and others to the S. of Lynch Tor, Dartmouth Forest. 'Huntingdon Barrow,' is at Huntingdon Warren; others on Lakebead Hill, and one north of Lydford at the junction of the Watergate and Hartwell Roads. Tumuli are also on Bridestow and Sourton Common, to the W. of Blackton Copse and W. of Dandgoot Tor.

A British coin of gold was found near Prince Town in the parish.

Lynton.-Tumuli to the S. of Shallowfield Common, others between Warcombe Water and Ruckham Combe; and one NE. of Dean.

Manaton.-Four tumuli are on Soussons Common and others near Black Hill.

MardDon.-A tumulus is on Beacon Hill.

MartinhoE. - Tumuli are situated to the SE. of the village.

MARWOoD.-One barrow is on Heuish Down, to the NW. of Honeywell, and another eastward of Heuish Down Plantation.

Marytavy.-Tumuli lie to the N. of Blackdown on the E. side of the high road from Tavistock to Okehampton.

Meavy.-One barrow is on Wifford Down.

Molland.-Two tumuli are on Molland Common, one between Black Ball on the N. and White Moor on the S., and one to the S. of Round Hill.

North Bovey.- 'King's Barrow' is situated on King Tor, and other tumuli are to the N. of Birch Tor, Headland Warren.

North Molton.- 'Five Barrows,' on Western Common, consist of seven tumuli, one of them has a diameter of $66 \mathrm{ft}$., and is nearly encircled by a trench. Other tumuli are on the W. of Twitchen Ball Corner, and one to the W. of Hangley Cleave.

North Petherwin.- - Two barrows, one of them a large size, are to the SE. of Brazacott, and SW. of Paynter's Moor Plantation.

OAKFORD.-A tumulus is on the SE. part of Blackenford Moor.

Okrhampton.-Tumuli are found on Black Down, Dartmoor; on Yes Tor; and one on Fordsland Ledge to the E. of Blackton Copse.

PANCRASWEEK. - Three tumuli are in position SE. of Highermoor.

Parracombe.- 'Roe Barrow' is on the S. of South Common, and another tumulus on Rowley Down.

Peters Marland.-Three tumuli are in Week Copse, to the SE. of Common Moor.

Petertayy. - Six tumuli are on Black Down, two to the $\mathrm{N}$. and four to the S. of White Hill. One on Standon Hill to the N. of Baggar Tor Brook; one on White Tor, Cudlipptown Down; and 'White Barrow' to the N. of Cocks Hill on the boundary of the parishes of Petertavy and Lydford.

Petrockstow.-A tumulus in Heathermoor Plantation.

Plymouth.-A Romano-British burial ground was found on Stamford Hill in 1864, when the new fort was constructed, with fragments of pottery and bronze implements.

Plympton St. Mary.-Tumuli are on Crownhill Down, near the Hemerdon China Clay Works.

Plymstock.-A tumulus on Barrow Hill, NW. of Higher Goosewell. Three daggers, sixteen bronze celts, weapons, etc., were found near Oreston, in this parish, 24 October, 1868 . 


\section{A HISTORY OF DEVONSHIRE}

Poltimore. - A barrow is in Mincimore Copse. This is near the supposed site of the battle between King Ethelred's troops and the Danes, A.D. Ioor. ${ }^{1}$

Rose Asr.-A tumulus lies to the SE. of West Catkill and E. of Catkill Cross; and others to the W. of Ditchett Moor and Great Ash Moor, to the NE. of Great Ash Cross.

Shaugh Prior.-One tumulus is on Lee Moor; one on Hentor Warren; one to the E. of Langcombe Head ; and another near Great Gnat's Head. One lies to the S. of Lee Moor ; and another called 'Emmet's Post,' is to the S. of Shaugh Moor, NE. of Blackaton Cross.

Shebrear. - A barrow lies on the borders of the parishes of Shebbear and Peters Marland.

SHEEPSTOR.-Several tumuli, one of them being known as 'Giant's Basin,' lie to the W. of Whitten Knowles Rocks. Others are on Ditsworthy Common, NW. of Giant's Hill, and on Ringmoor Down.

South Brent.- 'Eastern White Barrow' is situated on Zeal Plain; 'Western White Barrow' on Brent Moor, S. of the 'Abbots' Way'; and 'Knatta Barrow' to the S. of Petre's Pitt. Barbed spear-heads have been found in Bloody Pool, on the edge of Dartmoor.

SouthLerigh.-Tumuli lie to the W. and the NW. of Bullhall Wood; and 'Stone Barrow' on Lovehayne Farm, in a field called 'Stone Barrow Plot,' $\frac{3}{4}$ of a mile from Blackbury Castle, was opened in 1859 , when a cinerary urn and flint implements were found ${ }^{2}$ another, destroyed in 1763 , contained nearly a hundred Roman chisels for stone cutting.

South Tawton.-One barrow is on Cawsand Hill, and another near Taw Marsh.

Surcombe. - ' High Barrow Tumulus' is situated at Highbarrow Cross, NW. of Highbarrow Farm ; and another tumulus to the N. of Brendon.

Templeton.-Tumuli lie to the S. of Gibbet Moor Little Plantation.

TORBRYAN.-Two large tumuli are within the area of Denbury Camp.

TRentishoe.- 'Trentishoe Barrows' are on Trentishoe Down.

Twitchen.- 'Twitchen Barrows' are on Twitchen Ridge, to the N. of Blindwell.

UgBorough.-'Three Barrows,' 'Ugborough Beacon,' and one large tumulus NE. of Creber's Rock, are on Ugborough Moor. Tumuli lie to the N. of Cuckoo Ball, several others to the W. of Western Beacon, and one by Black Pool.

Uplyme.-_'Trinity Beacon' is on Trinity Hill.

UPTON PYNE.- 'Three Barrows' are situated to the SW. of Stevenstone, and one near 'No Man's Chapel,' to the S. of Shute.

Venn Ottery.-Tumuli are to be seen on Venn Ottery Hill.

Walkhampton.-A number of tumuli are on Walkhampton Common; and one barrow is close to Eylesbarrow Tin Mine.

WirRington.-One barrow to the SE. of Langdon and Beals.

West Anstey.- 'West Anstey Barrows' are on West Anstey Common, to the S. of Anstey Money Common; and other tumuli are on Woodland Common, near Ridges Road.

West BuckLand.-Tumuli are situated on Stoodley Down, SW. of Tossels Barton.

Widecombe in the Moor.- - Single Barrow,' 'Two Barrows,' and 'Hameldown Beacon' are on Hamel Down. Other tumuli are found on Blackslade Down; one on Corn Down, another on Cator Down, and 'Broad Barrow' is also in this parish.

Witheridge.-Two large tumuli are on West Yeo Moor to the N. of Dart Raffe Moor ; another on the same moor is to the E. of West Yeo Moor Cottages. Others occupy various positions on Witheridge Moor, one near Witheridge Moor Cross, one to the SW. of Ellworthy Cross, and two others to the SE. of the same cross.

\section{HUT CIRCLES}

Ashburton.-Horridge Common.

BREnDon.-Brendon Common, between Hoaroak Water and Farley Water; Cheriton Ridge, on two spots.

BuCKFASTLEIGH.-Near River Mardle; near Snowdon Brook.

Chagrord.-NW. of Metherall, near South Teign River; on Thornworthy Down, NE. of Fernworthy ; S. of Yardworthy; E. of Chagworthy Common; NE. of Chagford Common and others between Common and Shovel Down; on Bush Down, SE. and E. of North Walla Brook.

Cornwoon.-W. bank of River Erme ; on Penn Moor ; E. of Harford Moor and W. of River Erme ; E. of Dendle's Waste and Yealm River; E. of Penn Moor ; S. of High House Moor, and $\mathrm{N}$. of Ford Waste.

Countisbury.-On Cosgate Hill.

Dean PrIor.-Hickaton Hill ; others near Huntingdon Ford on River Avon.

'Angl.-Sax. Chron. and Florence of Worc., under the year roor. $\quad$ 'Fourn. Brit. Arch. Assoc. xviii, 58. 


\section{ANCIENT EARTHWORKS}

Gideligh.—N. of Gidleigh Common.

Harford.-On Erme Plain E. of River Erme ; Harford Moor; NE. of Hook Lake ; E. of Harford Moor and W. of River Erme; Highgate Piles (N. and S.) ; E. of River Erme; SW. of Harford Moor on either side of Butter Brook; SW. of Butterton Hill.

Holne.-Holne Moor, S. and SW. of Venford Bridge ; close to road from Hexworthy to Buckfastleigh ; SW. of Hexworthy ; S. of East Dart River ; one to W. of Combeston Tor.

Irsington.-Close to Grea Hill Tor; Haytor Down; near Haytor Quarries and Holwell Tor ; Mountsland Common; between Haytor and Bagtor Downs, and some- 'Seven Lords' Lands' - to N. of Hemsworthy Gate.

Lundy IsLand.-One on W. near Three Quarter Wall.

Lustreigh.-E. of Lustleigh Cleave; NE. Ermespound Circle.

LYDFORD.-W. of Wistman's Wood; on Lakebead Hill ; Bridestow and Sourton Common; Dartmoor, E. of Nodden Gate; others SE. of Great Nodden, and one to W. of Great Nodden; also NW. of Little Links Tor ; NW. of Walern Oak; SE. of Little Kneest; N. and NE. of Buckland Ford; E. of Beardown Hill ; near Saddle Bridge ; between West Dart River and Blackbrook River; Down Ridge; E. of Broad Down; Dartmoor Forest; S. of White Ridge; N. and NW. of Assycombe Hill ; E. of Sittaford Tor ; E. and W. of Lowton Brook; W. of Fernworth.

Lynton.-To S. of Shallowfield; between Warcombe Water and Ruckham Combe; Thorn Hill ; The Danes, or Valley of Rocks ; South Cleave; near Castle Rock on coast; Furzehill Common; one on Thornworthy Common ; one on Ilkerton Ridge.

Manaton.-Easdon Hill, near Whooping Rock; S. of Hayne Down; between Vogwell Down and Heytree Down; Cripdon Down; Houndtor Down ; near Becka Brook.

Meavy.-N. of Brisworthy Burrows ; Wifford Down, near clay works.

Moreton Hampstead.-Mardon Down, to SW. of Headless Cross.

North BoveY.-On Headland Warren, lie between Birch Tor Tin Mine and Golden Dagger Tin Mine; Shapley Common; Hookney Down; Easton Down; Dartmoor, N. of Headland Warren.

Petertavy.-On Cox Tor ; Shillpark; NE. and SE. of Wedlake, Dartmoor.

Plympton St. MarY.-Crownhill Down to N. and NW. of cottage of the same; also Tur.

Shavgr PrIor.-Shaugh Moor; N. and NW. of Saddlesborough; N. of Hawks Tor; on the Moor; W. of Hentnor Common; Trowlesworthy Warren; on Willings Walls Warren; one near Langcombe Brook.

SheEPSTOR.-S. of Blowing House ; one NW. and one S. of Gutter Tor ; Ringam Moor ; near Whitten Knowles Rocks; N. of Brisworthy (large) ; on Legiston Warren; on Ditsworthy Warren; 'Eylesbarrow' near tin mine of same name; near River Plym and NW. of Giant's Hill.

Soutr BRent.-Bishop's Meads; SW. of River Avon; 'The Rings,' Zeal Plain, N. of Brent Moor; W. of River Avon; Dockwell Ridge, E. of River Avon; near Petre's Pits Bottom; E. and W. sides of Balla Brook; N. of Hickley Plain; W. of River Avon; between Black Tur and Brentwood Clay Works on Brent Moor; Corringdon Ball, S. of Freeland Downs and E. of Glaze Brook.

South Tawton.-Between Taw Marsh, White Hill and Metheral Hill; on South Tawton Common.

Throwleigh.- Throwleigh Common, NW. of Shilstone.

Ugborough.-Between East Glaze Brook and West Glaze Brook; NW. Skitscombe ; Corringdon Wood; N. of Cuckoo Ball.

Walkhampton.-E. of Walkham River; Dartmoor, SE. of Merrivale Bridge; W. and NW. of Yellowmeade; Walkhampton Common, some near Rifle Range, and others near G.W.R. ; N. of Roundtrundle, S. of the common; N. and NE. of Stanlake; near Devonport Leat; E. of Black Tor ; Meavy River ; N. of Rounds Farm.

Widecombe In THe Moor.-Bone Hill Down, between Honeybag Tor and Clinkwell Tor ; E. of Widdecombe; W. of Blackaton Down; Brake Corner; Venton; one near road from Tavistock to Ashburton (large); N. of Shaugh Tor; NE. of Combestone Island, in East Dart River; one near Mell Tor to W. of Lower Torr; 'Foales Arrishes' on Blackslade Down; Yartor Down; Corn Down; E. of Dartmoor Cottage.

Hut circles are found within the pounds and enclosures at Grimspound, Legis Tor, Raddic Hill, Standon, Torr Town, and White Tor; also within the camps at Chulmleigh, Cranbrook, and Lustleigh.

\section{DOLMENS}

DRewstrignton.- 'The Spinsters' Rock,' 2 miles W. of the village. Morthoe.-One on Mort Point, and one on the cliffs to the S. 


\section{A HISTORY OF DEVONSHIRE}

\section{KISTVAENS}

Chagford.-Between Chagford Common and Shovel.Down.

HARFORD.-Erme Plain; E. of River Erme; Harford Moor.

LUNDY IsLAND.-On SW. point (large).

LYDFORD.-Stannon Tor; SE. of Archerton; and two near Fernworthy ; 'Childe's Tomb' by Fox Tor; I mile NE. of Princetown; near Two Bridges; NW. of Cholake Head; Blackbrook Down ; and Swincombe (3).

MeAvy.-Wigford Down.

Petertavy.-Langstone Moor.

Plymouth.-Within the town.

Shaugh Prior.-Lee Moor; W. of Langcombe Head ; and Little Gnat's Head.

SheEPSTOR.-Ditsworthy Warren.

South TaWton.-Cawsand Hill.

WALKHAMPTON.-SE. of Merrivale Bridge.

Widecombe in the Moor.-On Blackslade Down; Soussons Common.

\section{STONE CIRCLES}

Belstone.- 'Nine Stone Circle,' Belstone Common.

ChAGrord.-Batworthy, between Chagford Common and Shovel Down (triple).

Connwood.-Grimsgrove; Stall Moor; Yadsworthy; Cholwich Town.

DREwSteignton.- Near the Dolmen, W. of the village.

Gidleigh.—Gidleigh Common; and Buttern Hill.

HARFORD.- - Near Erme Head.

LyDFoRD.-'Grey Wethers' Sittaford Tor ; SW. of Sherberton; and Bellaford Tor.

Manaton.-Hound Tor.

Meavy.-N. of Brisworthy.

Petertavy.-Langstone Moor.

Shavgh Prior.-Shavercombe; and Trowlesworthy Tor.

Walkhampton.-Three circles at Drizzlecombe.

Widecombe in the Moor.—2 miles E. of Post Bridge; Soussons Common.

\section{MISCELLANEOUS}

Chagrord.-Stone rows at Batworthy, and Shovel Down; a 'Long Stone,' and Menhir.

ConNwood.-Stone rows at Stall Moor, and Yadsworthy; stone trackway on W. bank of River Erme; Avenue, Cholwich Town.

Drewsteignton.-Stone Avenue 2 miles W. of village.

HARFORD.-Stone row I mile E. of village ; enclosures on Erme Plain.

LydFord.-Stone rows, Longaford Tor; Assycombe Hill; a Menhir near Princetown ; rows and cairns, Conies Down.

Manaton.-Stone avenue with Menhir W. of Grimspound.

Petertavy.-Stone rows at Cudlipptown.

ShaUgh Prior.- - Stone avenue and enclosures.

ShEEPSTOR.-Stone avenue, Ditsworthy Warren.

South Tawron.-Stone rows, Cawsand Hill.

UGBOROUGH.-Stone rows, Ugborough Moor.

W ALKhampton.-Stone avenue, near Black Tor; and, with a Menhir, SE. of Merrivale Bridge ; Drizzlecombe. 







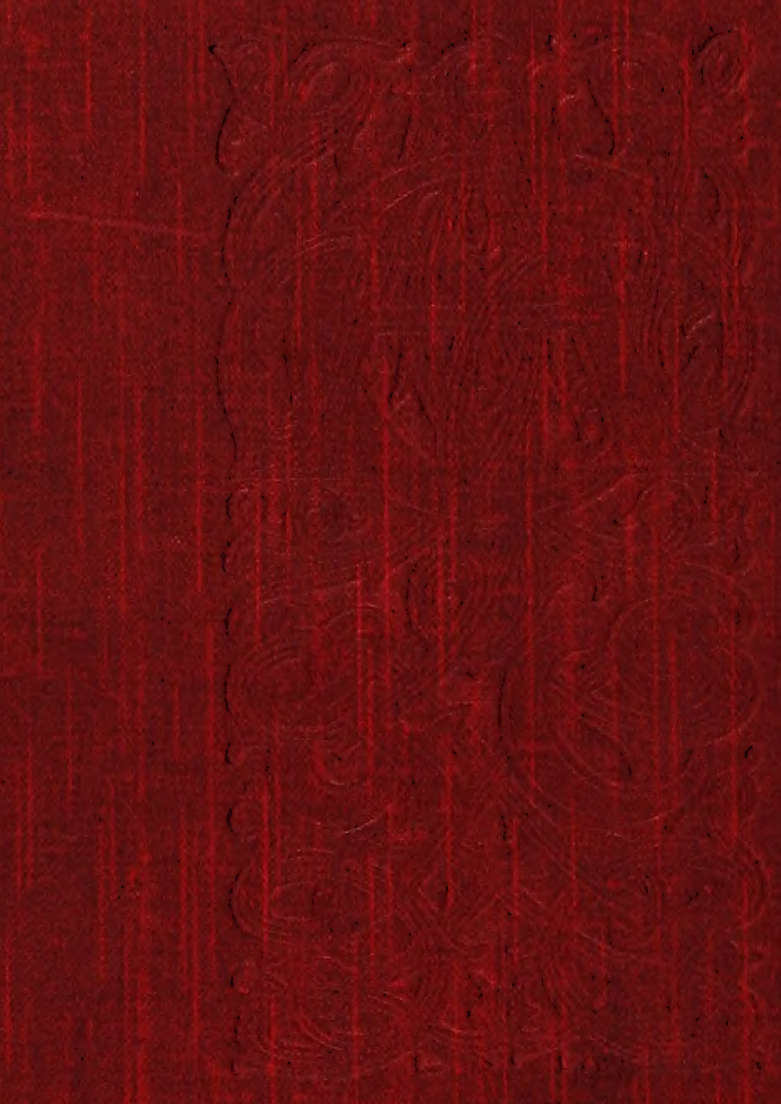

